\title{
Statistical Summaries of Streamflow Data and Characteristics of Drainage Basins for Selected Streamflow-Gaging Stations in Arizona Through Water Year 1996
}

Water-Resources Investigations Report 98-4225

Prepared in cooperation with the

Arizona Department of Water Resources,

Bureau of Reclamation,

Pima County Board of Sine ervisors:

Flood Control District of Maricopa County, and

Salt River Projects
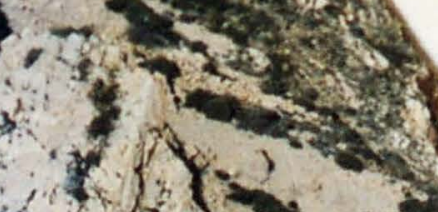

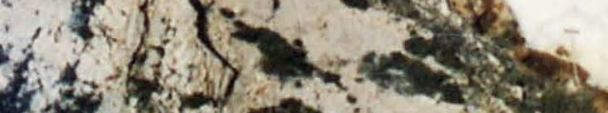
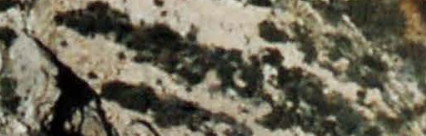

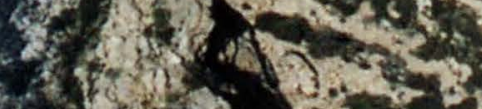

Xent:

S. 6

$7 x^{3}$

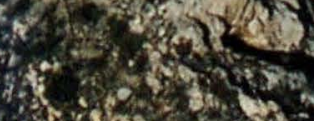

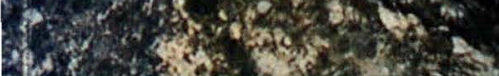


Cover photograph: Coolidge Dam (Photograph by J. Madrigal, Jr., Bureau of Reclamation, January 12,1993 ) 


\section{Statistical Summaries of Streamflow Data and Characteristics of Drainage Basins for Selected Streamflow-Gaging Stations in Arizona Through Water Year 1996}

By G.L. POPE, P.D. RIGAS, and C.F. SMITH

Water-Resources Investigations Report 98-4225

Prepared in cooperation with

Arizona Department of Water Resources,

Bureau of Reclamation,

Pima County Board of Supervisors,

U.S. GEOLOGICAL, SURVEY RESTON, WA.

MAR 081999

Flood Control District of Maricopa County, and

Salt River Project 


\title{
U.S. DEPARTMENT OF THE INTERIOR BRUCE BABBITT, Secretary
}

\author{
U.S. GEOLOGICAL SURVEY
}

Charles G. Groat, Director

The use of firm, trade, and brand names in this report is for identification purposes only and does not constitute endorsement by the U.S. Geological Survey

For additional information write to:

District Chief

U.S. Geological Survey

Water Resources Division

520 North Park Avenue, Suite 221

Tucson, AZ 85719-5035
Copies of this report can be purchased from:
U.S. Geological Survey
Information Services
Box 25286
Denver Federal Center
Denver, CO 80225-0046 


\section{CONTENTS}

Abstract

Introduction

History of the streamflow-gaging network in Arizona

Basin and climatic characteristics

Station records

Station identification numbers and downstream-order system

Location

Drainage area.

Period of record

Revised records

Gaging station

Remarks

Average discharge

Extremes for period of record

Extremes outside period of record

Rating tables

Explanation of statistical summaries.

Monthly and annual flow

Flow magnitude and probability.

Flood frequency

Flow duration

Discharge

Significant figures and rounding limits

Data use limitations.

References cited

Statistical summaries.

09371100 Teec Nos Pos Wash near Teec Nos Pos

09379030 Black Mountain Wash near Chinle

0937906

09379100

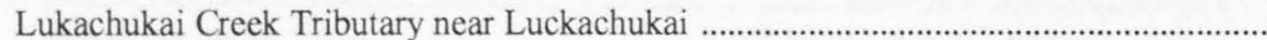

09379200 Long House Wash near Kayenta.

09379560 Chinle Creek near Mexican Water

El Capitan Wash near Kayenta.

09379980

09380000 Jack Bench Wash Tributary near Page

09382000 Paria River at Lees Ferry...

0938302

09383400

0938350

0938360

0938400

0938420

0938580 Colorado River at Lees Ferry

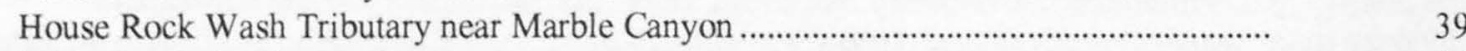

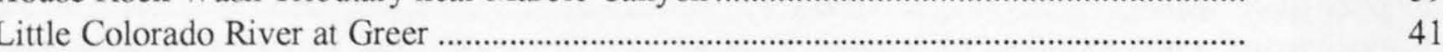

0938650

Nutrioso Creek above Nelson Reservoir, near Springerville.

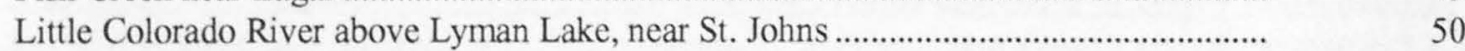

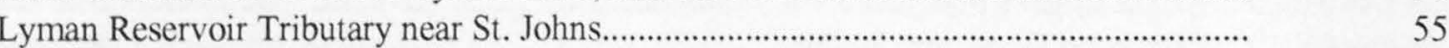

0938800

Little Colorado River Tributary near St. Johns

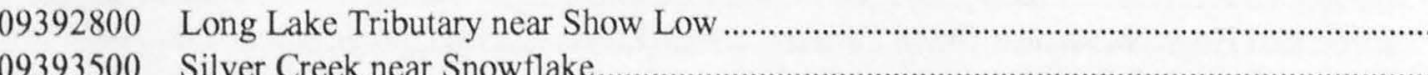


Statistical summaries - Continued

09394500 Little Colorado River at Woodruff...

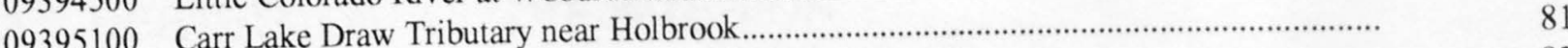

09395200 Decker Wash near Snowflake .......... 82

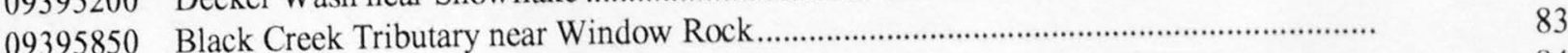

09395900 Black Creek near Lupton

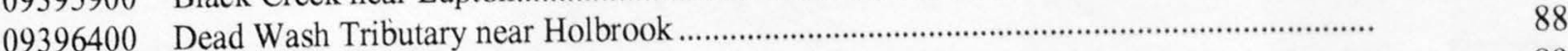

09396500 Puerco River near Adamana

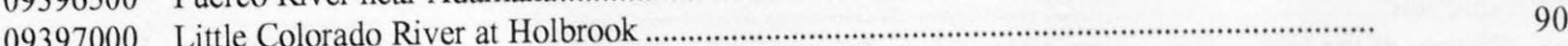

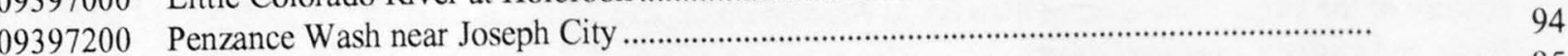

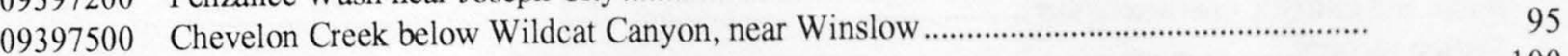

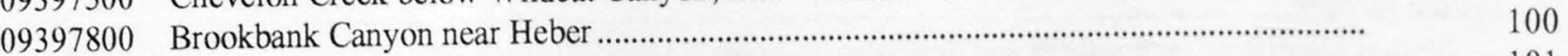

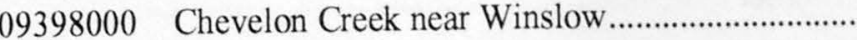

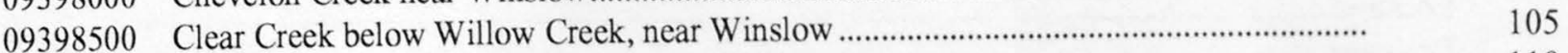

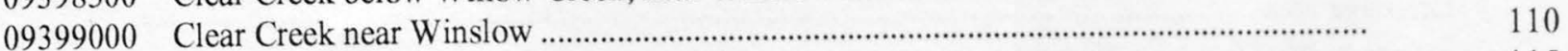

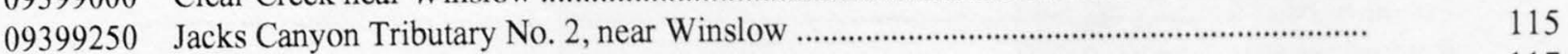

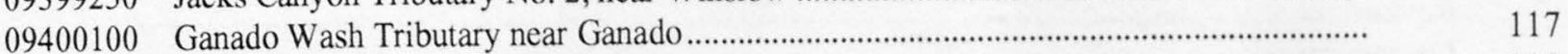

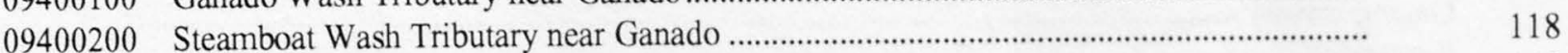

09400290 Teshbito Wash Tributary near Holbrook ....................................................................... 119

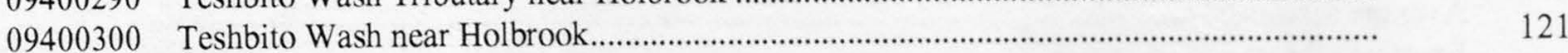

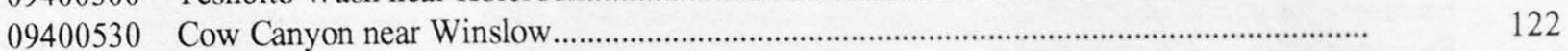

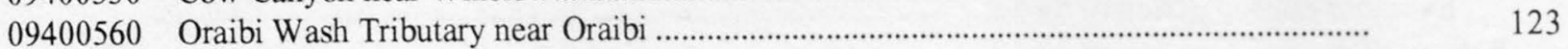

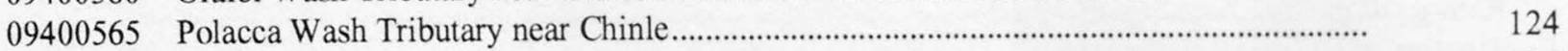

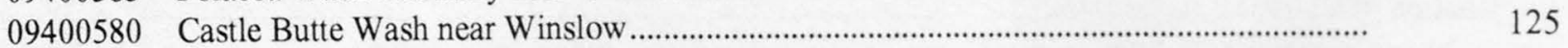

09400590 Rio De Flag at Hidden Hollow Road, at Flagstaff ....................................................... 126

09400595 Schultz Canyon at Flagstaff ........................................................................................... 127

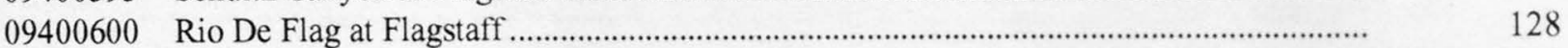

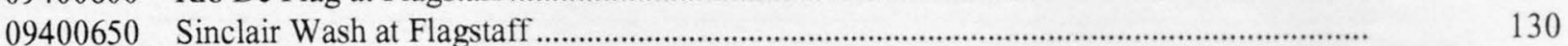

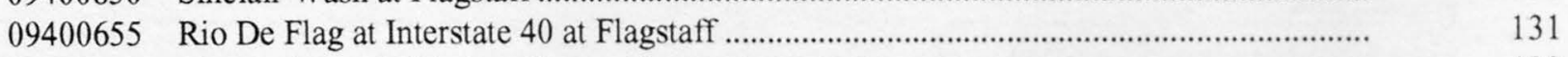

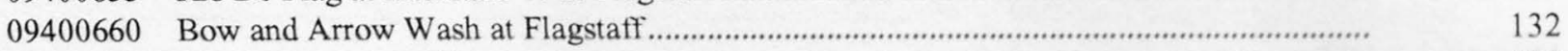

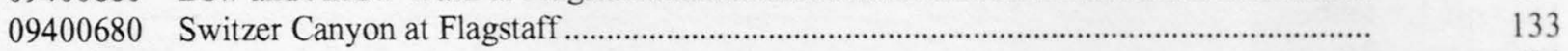

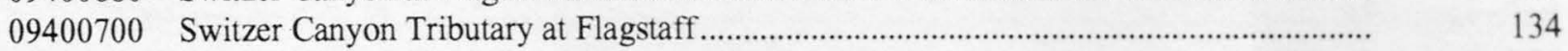

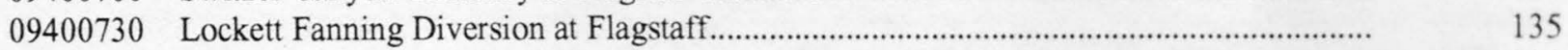

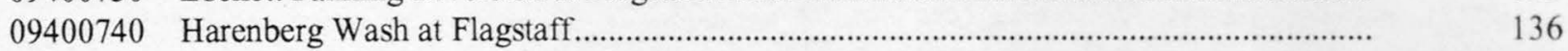

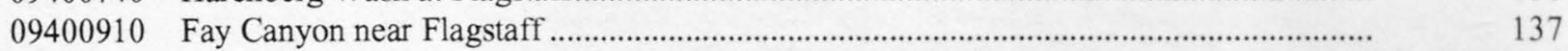

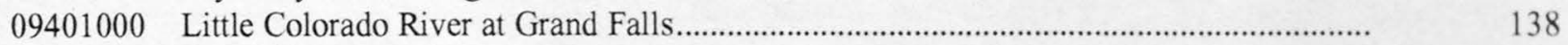

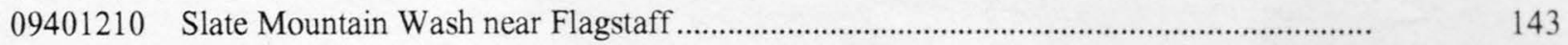

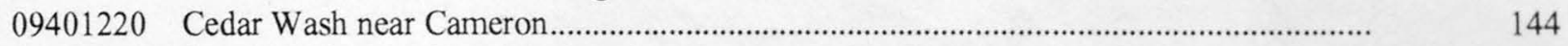

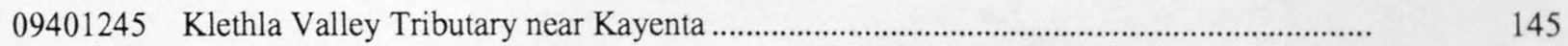

09401260 Moenkopi Wash at Moenkopi ........................................................................................ 146

09401300 Hamblin Wash Tributary near Cedar Ridge................................................................. 150

09401370 Hamblin Wash Tributary No. 2 near Tuba City .............................................................. 151

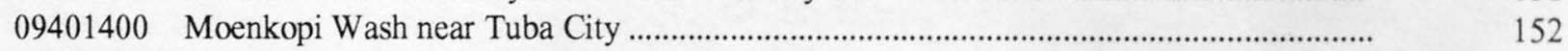

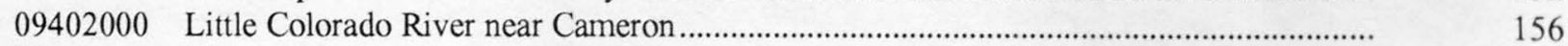

09402100 Forest Boundary Wash near Cameron …........................................................................ 161

09402500 Colorado River near Grand Canyon............................................................................. 162

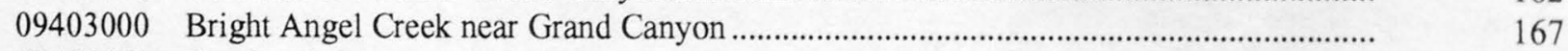

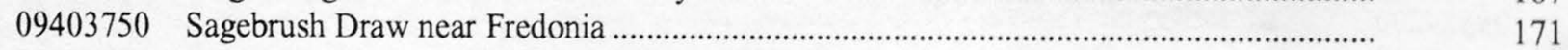

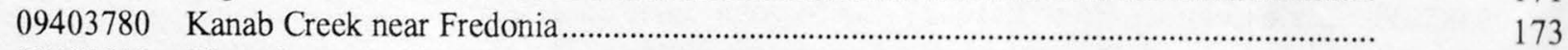

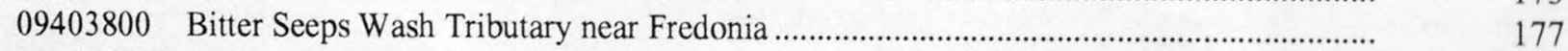

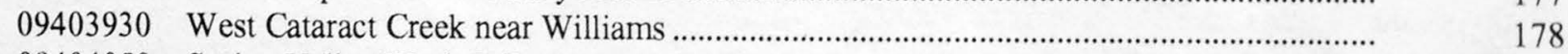

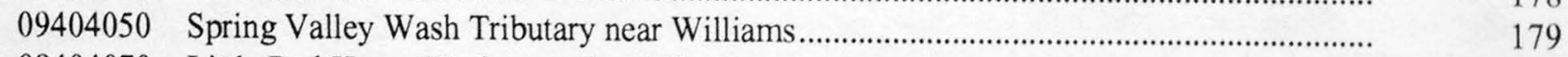

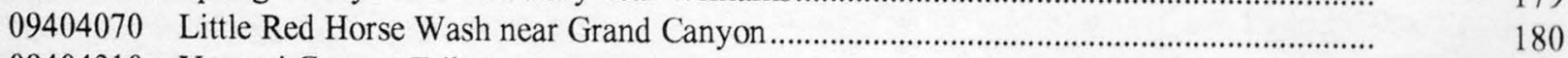

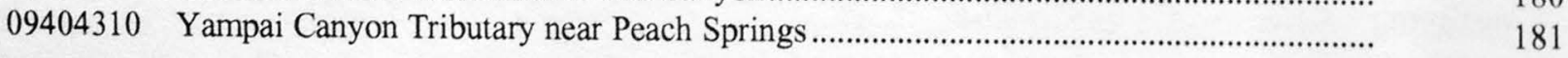


Statistical summaries - Continued

09404340 Truxton Wash at Valentine.

09404350 Valentine Wash at Valentine

09415000 Virgin River at Littlefield......

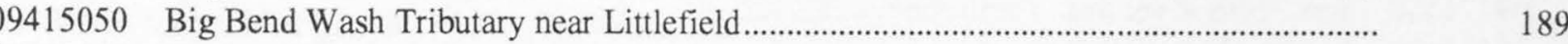

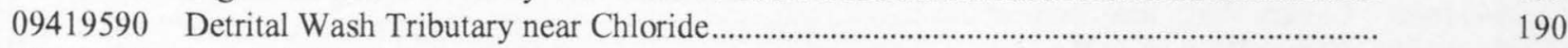

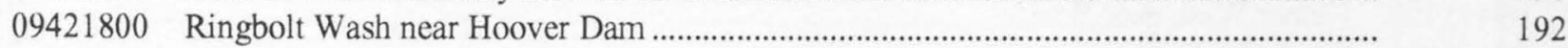

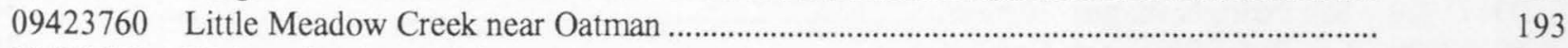

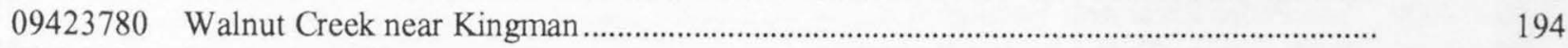

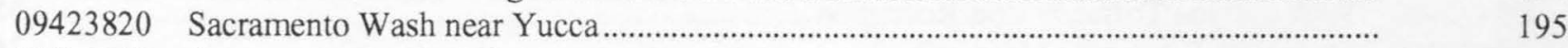

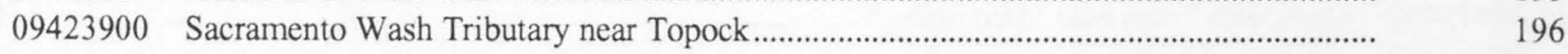

09424200 Willow Creek (Cottonwood Wash No. 1) near Kingman ................................................. 197

09424407 McGarrys Wash near Kingman ....................................................................................... 201

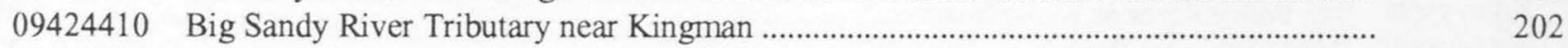

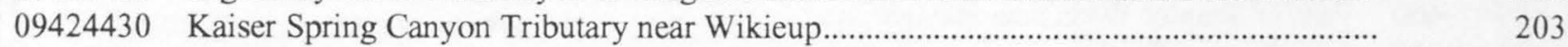

09424447 Burro Creek at Old U.S. 93 Bridge, near Bagdad .......................................................... 204

09424450 Big Sandy River near Wikieup................................................................................. 208

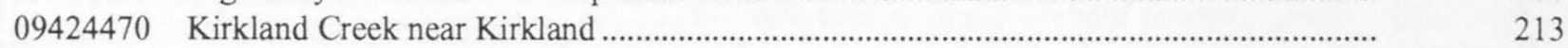

09424480 Ash Creek near Kirkland ...................................................................................... 215

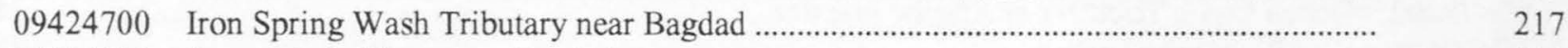

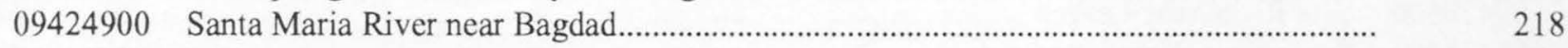

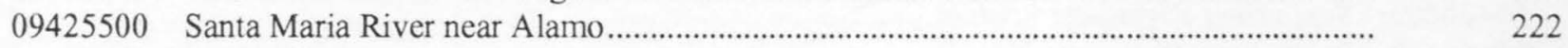

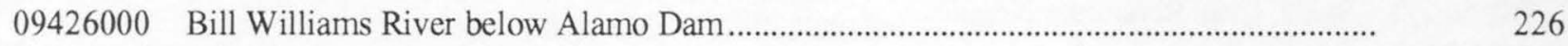

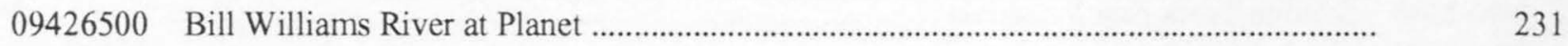

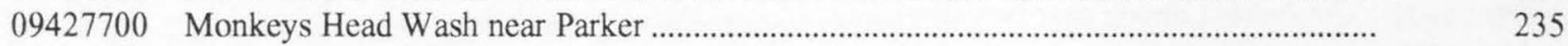

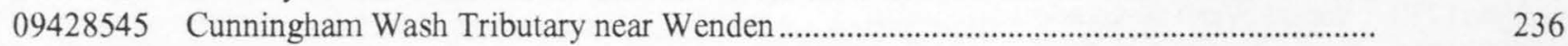

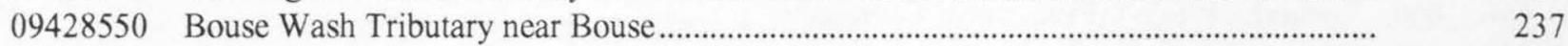

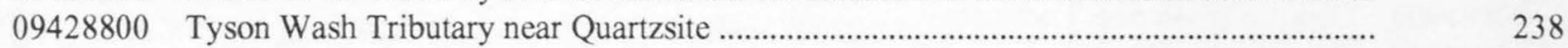

09429150 Creosote Wash near Ehrenberg .................................................................................... 239

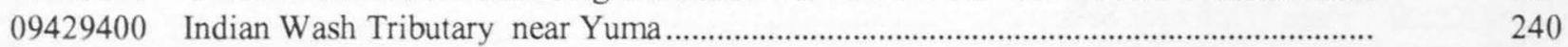

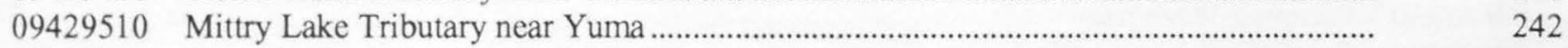

09432000 Gila River below Blue Creek near Virden, New Mexico................................................. 243

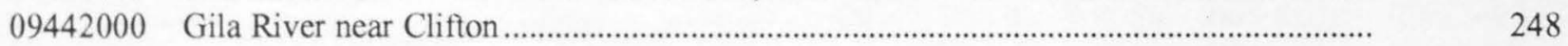

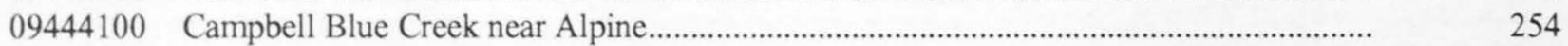

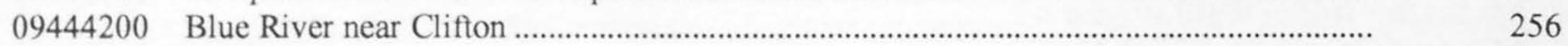

09444400 Chase Creek near Clifton ................................................................................................. 260

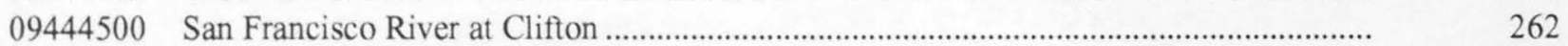

09445500 Willow Creek near Point of Pines, near Morenci.............................................................. 268

09446000 Willow Creek near Double Circle Ranch, near Morenci .................................................. 272

09446500 Eagle Creek near Double Circle Ranch, near Morenci .................................................... 276

09447000 Eagle Creek above pumping plant, near Morenci ............................................................ 280

09447800 Bonita Creek near Morenci ……........................................................................................ 285

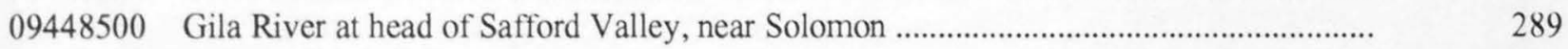

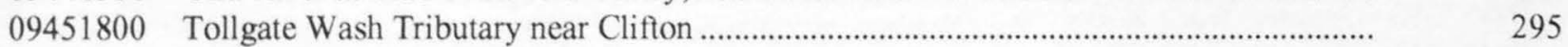

09456000 San Simon River near San Simon .................................................................................

09456400 Gold Gulch near Bowie ...................................................................................................

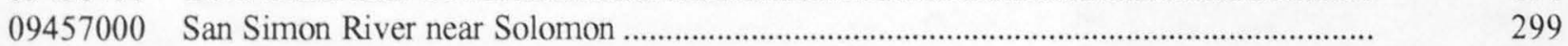

09458200 Deadman Creek near Safford .......................................................................................

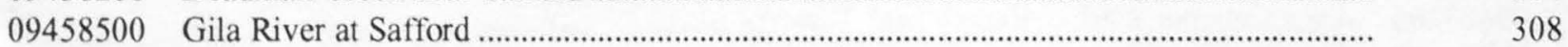

09460150 Frye Creek near Thatcher .............................................................................................. 312

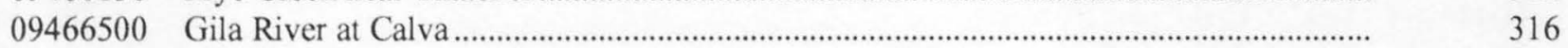

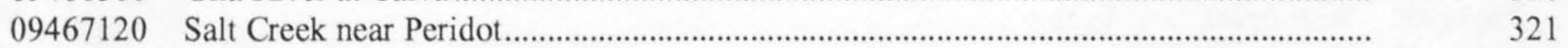

09468300 Sevenmile Wash Tributary near Globe ...................................................................... 322

09468500 San Carlos River near Peridot ...................................................................................... 
Statistical summaries-Continued

09470500 San Pedro River at Palominas

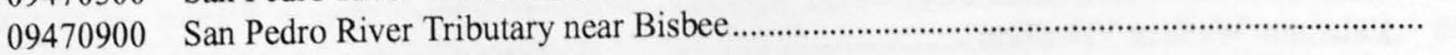

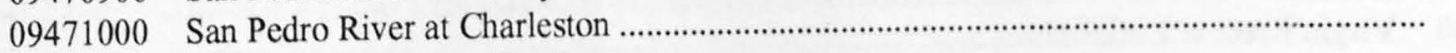

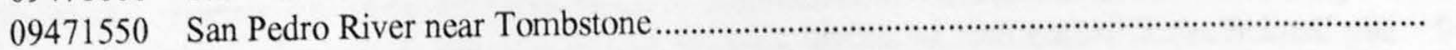

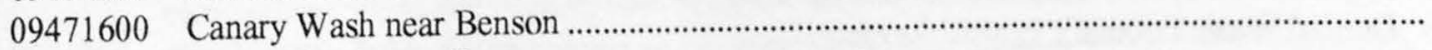

09471700

Fenner Wash near Benson.

09471800

San Pedro River near Benson.

09472000

San Pedro River near Redington.

09472100

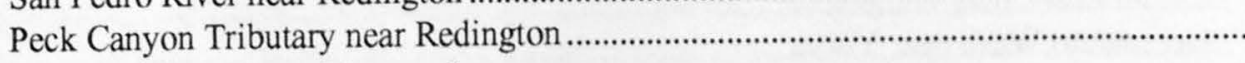

09472400

Mammoth Wash near Mammoth.

360

09473000

Aravaipa Creek near Mammoth.

362

09473200

Green Lantern Wash near Winkelman.

367

09473500

San Pedro River at Winkelman

368

09473600

Tam O'Shanter Wash near Hayden.

372

09474000

Gila River at Kelvin

373

09478200

Durham Wash near Florence.

379

09478500

Queen Creek at Whitlow Damsite, near Superior

381

09478600

Queen Creek Tributary No. 3 at Whitlow Dam....

09479200

Queen Creek Tributary at Apache Junction

09479500

Gila River near Laveen

09480000

Santa Cruz River near Lochiel.

385

386

388

393

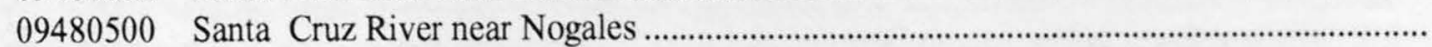

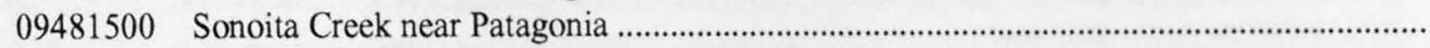

Calabasas Canyon near Nogales

407

09481800

Sopori Wash at Amado

408

09481900

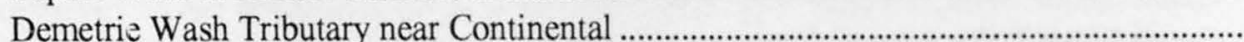

410

Ocotillo Wash near Continental ............................................................................................

09482000

Santa Cruz River at Continental

413

09482200

Flato Wash near Sahuarita

418

09482330

09482350

Pumping Wash near Vail....

420

09482370

South Fork Airport Wash ne

421

422

09482400

South Fork Airport Wash near Tucson

424

09482410

09482420

Airport Wash at Tucson

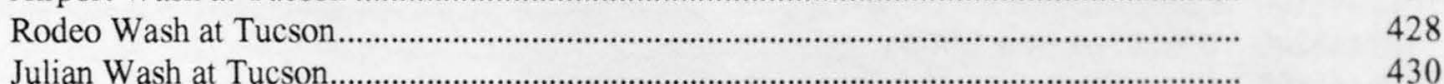

09482450

09482480

West Branch Santa Cruz

431

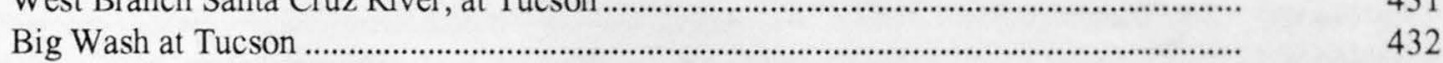

09482500

Santa Cruz River at Tucson

434

09482950

Railroad Wash at Tucson

440

09483000

09483010

Tucson Arroyo at Vine Avenue, at Tucson.

442

High School Wash at Tucson

446

450

09483030

Silvercroft Wash at Tucson

Anklam Wash at Tucson

09483042

09483045

09483100

09483200

09483250

09484000

09484200

West Speedway Wash near Tucson

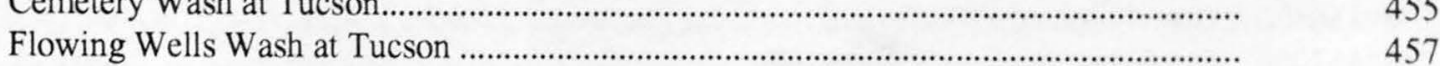

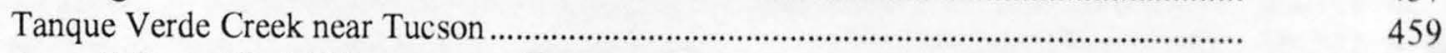

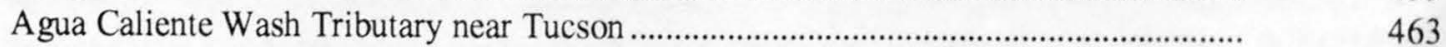

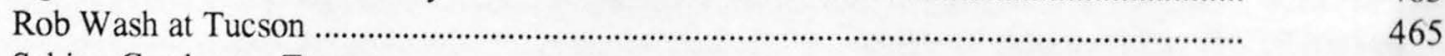

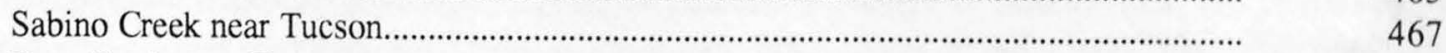

0948450

Bear Creek near Tucson

472

Tanque Verde Creek at Tucson.

09484510 Ventana Canyon Wash near Tucson 
Statistical summaries - Continued

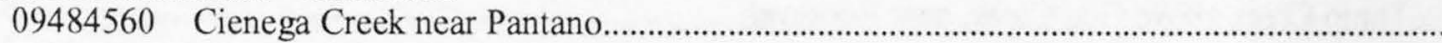

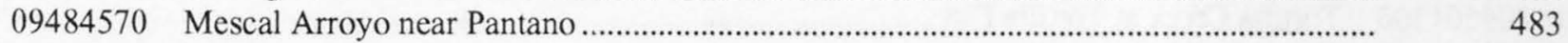

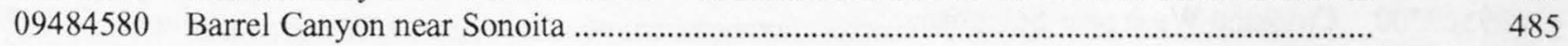

09484590 Davidson Canyon Wash near Vail ...................................................................................

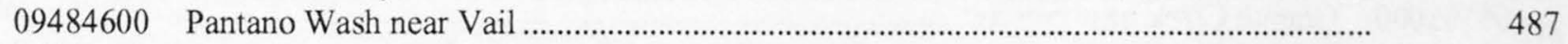

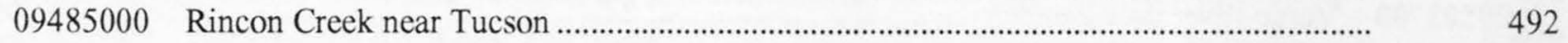

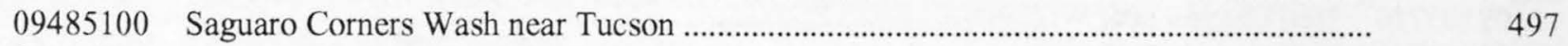

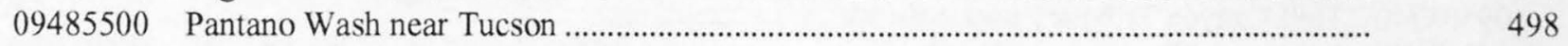

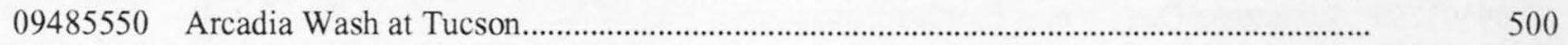

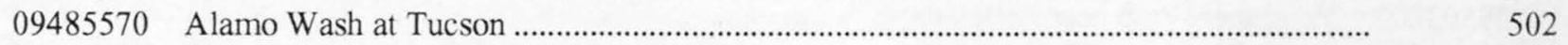

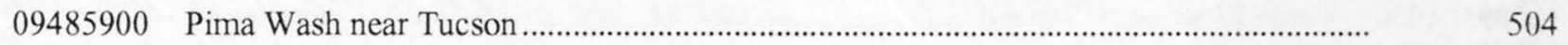

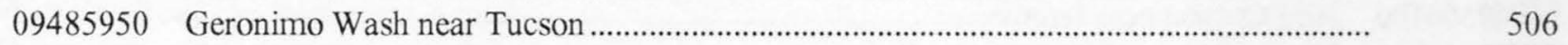

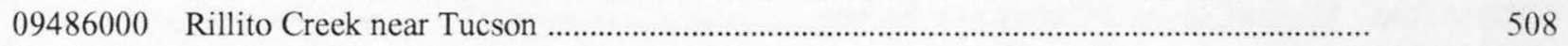

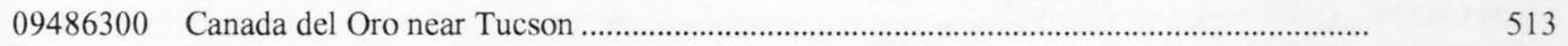

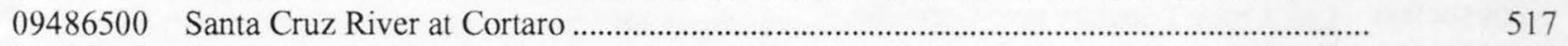

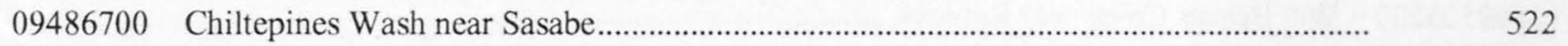

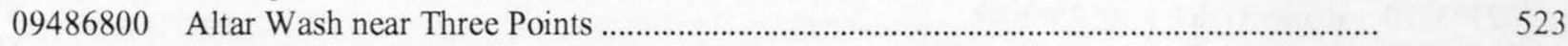

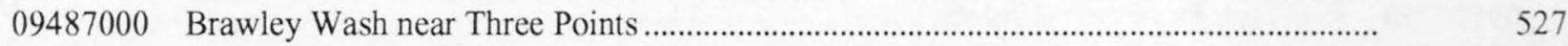

09487100 Little Brawley Wash near Three Points ........................................................................... 529

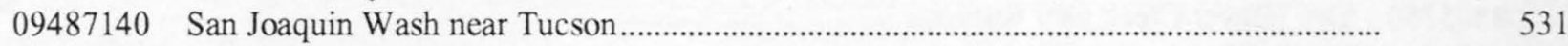

09487250 Los Robles Wash near Marana.....................................................................................

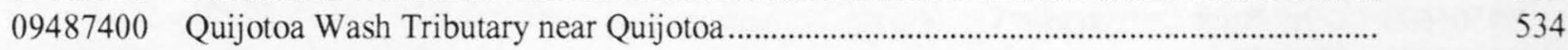

$09488500 \quad$ Santa Rosa Wash near Vaiva Vo, near Sells ............................................................

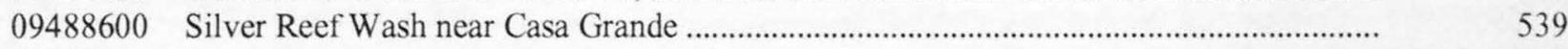

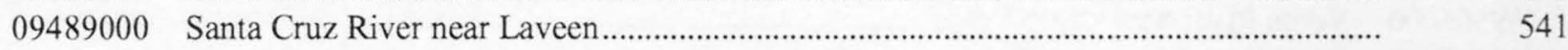

09489070 North Fork of East Fork Black River near Alpine ........................................................

09489080 Hannagan Creek near Hannagan Meadow …............................................................ 550

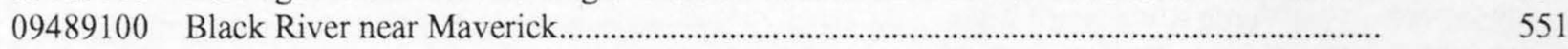

$09489200 \quad$ Pacheta Creek at Maverick ........................................................................................

09489500 Black River below Pumping Plant, near Point of Pines .................................................

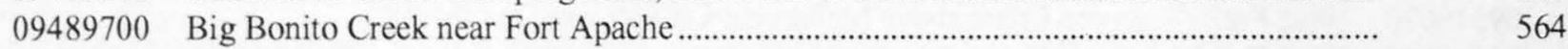

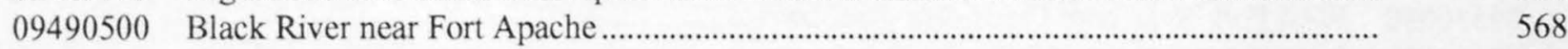

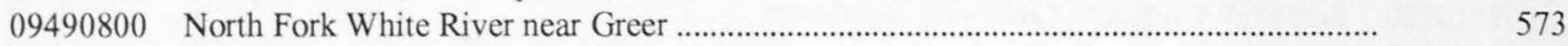

09491000 North Fork White River near McNary ........................................................................

09491800 North Fork White River Tributary near Whiteriver ........................................................ 579

$09492400 \quad$ East Fork White River near Fort Apache .................................................................... 580

09494000 White River near Fort Apache............................................................................................. 585

09494300 Carrizo Creek above Corduroy Creek, near Show Low.................................................

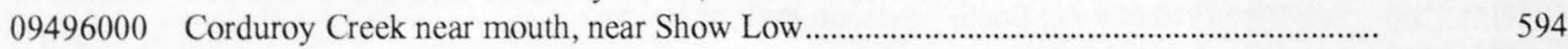

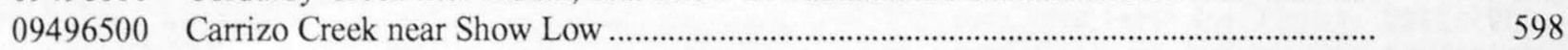

09496600 Cibecue No. 1, Tributary to Carrizo Creek, near Show Low ...........................................

09496700 Cibecue No. 2, Tributary to Carrizo Creek, near Show Low …….................................... $\quad 607$

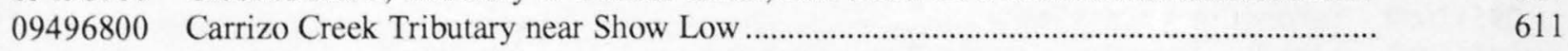

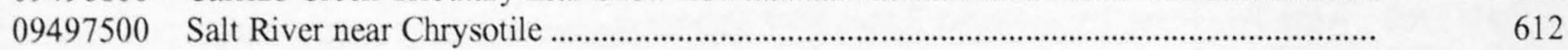

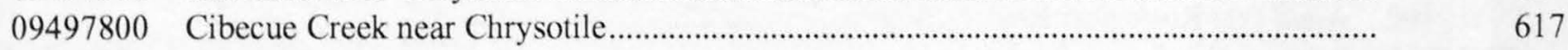

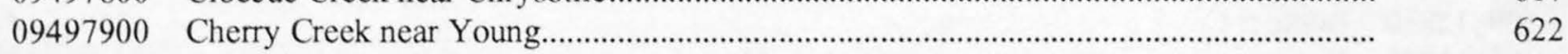

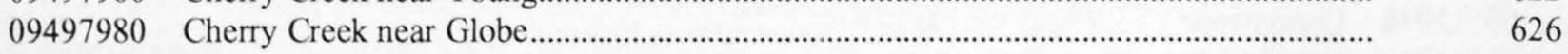

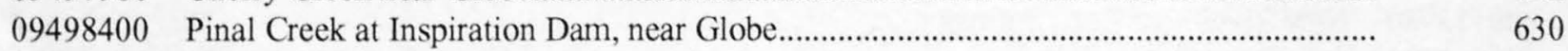

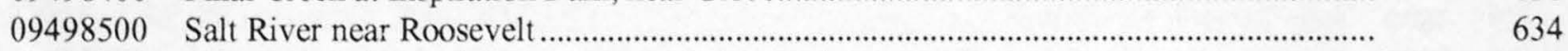

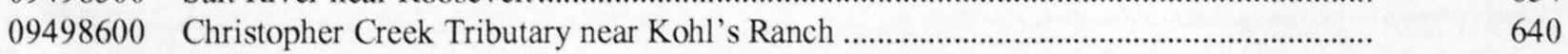

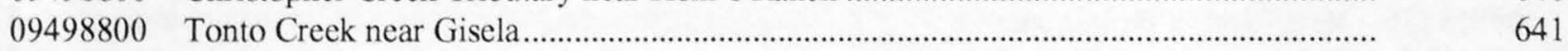

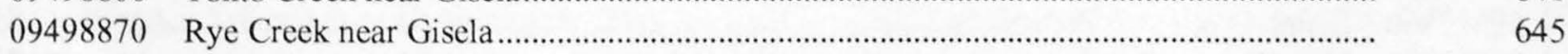

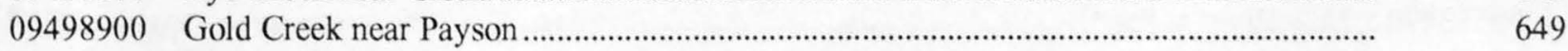


Statistical summaries-Continued

09499000 Tonto Creek above Gun Creek, near Roosevelt.

Page

0501300 Tortilla Creek at Tortilla Flat

09502700 Crookton Wash near Seligman.

09502800 Williamson Valley Wash near Paulden.

09503000 Granite Creek near Prescott.

09503700

09503720

Verde River near Paulden

09503740

Hell Canyon near Williams

09503750

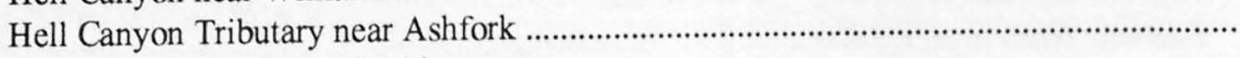

09503800

Limestone Canyon near Paulden

09504000

Volunteer Wash near Bellemont

09504100

Verde River near Clarkdale.

09504400

Hull Canyon near Jerome

09504500

Munds Canyon Tributary near Sedona.

09504800

Oak Creek near Cornville

09505200

Oak Creek Tributary near Cornville

09505220

Wet Beaver Creek near Rimrock

09505250

Rocky Gulch near Rimrock.

09505300

Red Tank Draw near Rimrock

09505350

Rattlesnake Canyon near Rimrock

09505550

Dry Beaver Creek near Rimrock

09505600

Verde River below Camp Verde

651

656

658

660

664

668

672

673

674

675

676

681

683

684

689

691

696

698

702

706

09505800

Dirty Neck Canyon near Clints Well

0950590

West Clear Creek near Camp Verde

Cottonwood Wash near Camp Verde

Verde River near Camp Verde

East Verde River near Pine

Webber Creek above West Fork Webber Creek, near Pine

East Verde River near Childs

Cacamore Creeknear Sunflower

09510180 Rock Creek near Sunflower

09512100 Indian Bend Wash at Scottsdale.

Salt River Tributary in South Mountain Park, at Phoenix

Cave Creek near Cave Creek

Agua Fria River near Mayer

Turkey Creek near Cleator... 
Statistical summaries - Continued

09513910 New River near Glendale

09513970 Agua Fria River at Avondale.

09514200 Waterman Wash near Buckeye

Hassayampa River at Box Damsite, near Wickenburg ...

832

0951580

Hartman Wash near Wickenburg.

834

09516500

Hassayampa River near Morristown

838

09516600 Ox Wash near Morristown

840

09516800 Jack Rabbit Wash near Tonopah

09517000 Hassayampa River near Arlington

0951720

Centennial Wash Tributary near Wenden

09517400 Winters Wash near Tonopah.

09517500 Centennial Wash near Arlington

0951960

Rainbow Wash Tributary near B

856

09519750

Bender Wash near Gila Bend

860

09519760 Sauceda Wash near Gila Bend.

862

09519780 Windmill Wash near Gila Bend

864

09520100

Military Wash near Sentinel.

866

09520110

09520130

Hot Shot Arroyo near Ajo.

868

09520160

Darby Arroyo near Ajo.

870

871

872

09520170 Rio Cornez near Ajo

874

09520200

Black Gap Wash near Ajo

878

09520230

Crater Range Wash near Ajo

880

09520300

Alamo Wash Tributary near Ajo

882

09520350 Mohawk Pass Wash at Mohawk

884

09520400 Ligurta Wash at Ligurta

09535100 San Simon Wash near Pisinimo .

09535200 Little Tucson Wash at Sells

09535300 Vamori Wash at Kom Vo

09536100 Pitchfork Canyon Tributary near Fort Grant ....................................................................

09536350 Surprise Canyon near Dos Cabezas.

898

09537200 Leslie Creek near McNeal

899

09537500 Whitewater Draw near Douglas

\section{FIGURES}

1. Map showing unregulated and partly regulated continuous-record streamflow-gaging stations in Arizona through water year 1996

2. Map showing unregulated and partly regulated peak-flow partial-record stations in Arizona through water year 1996.

3. Map showing basins and unregulated and partly regulated streamflow-gaging stations in southern Arizona through water year 1996

4. Map showing basins and unregulated and partly regulated streamflow-gaging stations in central Arizona through water year 1996

5. Map showing basins and unregulated and partly regulated streamflow-gaging stations in northern Arizona through water year 1996

6. Drawing showing explanation of boxplots 
TABLES

1. Streamflow-gaging stations in Arizona, 1996

2. Significant figures and rounding limits used for reporting monthly and annual mean discharge and probable discharge

\section{CONVERSION FACTORS}

\begin{tabular}{rll} 
Multiply & \multicolumn{1}{c}{ By } & To obtain \\
\hline inch (in.) & 25.4 & millimeter \\
foot $(\mathrm{ft})$ & 0.3048 & meter \\
mile $(\mathrm{mi})$ & 1.609 & kilometer \\
square $\mathrm{mile}\left(\mathrm{mi}^{2}\right)$ & 2.590 & square kilometer \\
acre & 0.4047 & hectare \\
acre-foot $(\mathrm{acre}-\mathrm{ft})$ & 0.001233 & cubic hectometer \\
cubic foot per second $\left(\mathrm{ft}^{3} / \mathrm{s}\right)$ & 0.02832 & cubic meter per second \\
gallon per minute $(\mathrm{gal} / \mathrm{min})$ & 0.06308 & liter per second \\
\hline
\end{tabular}

\section{VERTICAL DATUM}

Sea Level: In this report "sea level" refers to the National Geodetic Vertical Datum of 1929 (NGVD of 1929)-A geodetic datum derived from a general adjustment of the first-order level nets of both the United States and Canada, formerly called Sea Level Datum of 1929.

\section{DISCHARGE CODES}

C Urbanization has affected all or part of the record.

DF Discharge resulting from a dam failure.

ES Discharge is estimated.

HP Discharge associated with an isolated historic peak and is not part of the systematic record.

KR Discharge affected by diversion or regulation.

LT Discharge is less than reported value.

MD Average of the maximum daily discharge.

PF Discharge associated with a paleoflood peak and is not part of the systematic record.

UR Discharge is affected to an unknown degree by diversion and regulation.

\section{GLOSSARY OF TERMS}

Acre-foot (AC-FT, acre-ft)-The quantity of water required to cover 1 acre to a depth of 1 foot and is equivalent to 43,560 cubic feet or 325,851 gallons or $1,233.49$ cubic meters.

Annual high flow-The maximum mean daily discharge that occurred during the water year (used to calculate magnitude and probability statistics).

Annual low flow-The minimum mean daily discharge that occurred during the climatic year (used to calculate magnitude and probability statistics).

Annual peak discharge-The maximum instantaneous discharge during the water year.

Basin-boundary divide - The topographic divide separating one drainage basin from another. 
Basin characteristics - The physical characteristics that were selected for use in this report-main-channel slope, stream length, mean-basin elevation, forested area, and soil index.

Climatic year-Designated by the calendar year in which it begins and represents the 12-month period from April 1 through March 31.

Discharge - The volume of water (or more broadly, the volume of fluid plus suspended sediment) that passes a given point within a given period of time.

Daily mean discharge-Arithmetic mean of the individual increments of discharge in a day.

Instantaneous discharge - Discharge at a particular instant of time.

Mean discharge (mean)-Arithmetic mean of individual daily mean discharges during a specific period.

Annual 7-day minimum - Lowest mean discharge for 7 consecutive days for a calendar year or a water year.

Note that most low-flow frequency analyses of annual 7-day minimum flows use a climatic year

(April 1-March 31). The date shown in the summary statistics table is the initial date of the 7-day period

(The value should not be confused with the 7-day 10-year low-flow statistic.)

Discharge rating table-List discharge for specific gage heights over the middle to upper range of stage. The stage/discharge relation was based on channel conditions through the date indicated.

Drainage area-Area of a stream at a specific location is that area, measured in a horizontal plane, enclosed by a topographic divide from which direct surface runoff from precipitation normally drains by gravity into the river upstream from the specified point.

Drainage basin-A part of the surface of the earth that is occupied by a drainage system, which consists of a surface stream or a body of impounded surface water together with all tributary surface streams and bodies of impounded surface water.

Forested area (in percent)-A portion of the drainage area delineated on topographic maps as forested area and is computed as the ratio of forest coverage with respect to the total drainage area.

Gage datum - Elevation of the zero point of the reference gage from which gage height is determined as compared to sea level. This elevation is established by a system of levels from known bench marks or by approximation from topographic maps.

Gage height (G.H.)-The water-surface elevation referred to some arbitrary gage datum. Gage height is often used interchangeably with the more general term "stage," although gage height is more appropriate when used with a reading on a gage.

Gaging station-A particular site on a stream, canal, lake, or reservoir where systematic observations of hydrologic data are obtained.

Main-channel slope (feet per mile; $\mathrm{ft} / \mathrm{mi}$ )-An index of slope of the main channel and is computed with respect to location of the gaging station and the basin-boundary divide. Streambed elevation is computed from points that are 10 and 85 percent of this distance along the main channel between those two points.

Mean-Arithmetic average of a list of values. The arithmetic average is computed by summing the values and dividing the sum by the total number of values.

Mean annual precipitation (inches; in.)-The average annual precipitation that occurs within the drainage basin.

Mean-basin elevation (feet; $\mathrm{ft}$ )-Average elevation above sea level of representative points within the basin. Mean-basin elevation is the arithmetic average of the elevation and is computed at 50 to 100 points within the basin at intersections of equally spaced grid lines that are superimposed on a map.

Median - The middle value from a list of values that are listed in order from the minimum to the maximum. If the list contains an even number of values, the median is the average of the two middle values.

Partial-record station-A particular site where limited streamflow data are systematically collected over a period of years for use in hydrologic analyses.

Period of record-The period for which records have been published for the station or for an equivalent station. An equivalent station is a station that was in operation at a time that the present station was not in existence and whose location is such that records of flow at the station can reasonably be considered equivalent to flow at the present station.

Rainfall intensities (inches; in.)-Represents total rainfall in a 24-hour period with recurrence intervals of 2 and 50 years. 
Runoff in inches (inch, in.)-The depth to which the drainage area would be covered if all the runoff for a given period of time were uniformly distributed over the drainage area.

Soil index (inch; in.)-A numerical index that is proportional to the long-term infiltration rate.

Standard deviation-A measure of the variability of the values within a list of values. If all the values generally are equal, the standard deviation will be close to zero.

Streamflow-Discharge that occurs in a natural channel. Although the term "discharge" can be applied to the flow of a canal, the word "streamflow" uniquely describes the discharge in a surface stream course. The term "streamflow" is more general than "runoff," as streamflow may be applied to discharge whether or not it is affected by diversion or regulation.

Stream length (mile; mi)-Distance from the gaging station along the main channel to the basin-boundary divide.

Surface water-Water on the Earth surface.

Water year-In surface-water reports of the U.S. Geological Survey, the water year (October 1 through September 30) is designated by the calendar year in which the water year ends and that includes 9 of the 12 months. The year ending September 30, 1996, therefore, is called the "1996 water year." 


\title{
Statistical Summaries of Streamflow Data and Characteristics of Drainage Basins for Selected Streamflow-Gaging Stations in Arizona Through Water Year 1996
}

\author{
By G.L. Pope, P.D. Rigas, and C.F. Smith
}

\begin{abstract}
Statistical summaries of streamflow data are given for 142 unregulated or partly regulated continuous-record streamflow-gaging stations and 178 peak-flow partial-record stations in Arizona through water year 1996. Streamflow statistics were generated for stations with a minimum period of record of 10 years. Summaries for continuous-record stations include: (1) station description, (2) statistics of mean monthly and annual discharges, (3) magnitude and probability of annual peak discharge, (4) magnitude and probability of annual low and high flow, (5) mean daily flow duration, and (6) basin and climatic characteristics. Statistical summaries for peak-flow partial-record stations include: (1) station description, (2) magnitude and probability of annual peak discharge, and (3) basin and climatic characteristics.
\end{abstract}

\section{INTRODUCTION}

A significant part of the mission of the U.S. Geological Survey (USGS) is the systematic collection of data for determining the quantity as well as the quality and use of surface and ground water (Cardin and others, 1986). During the 1996 water year, the USGS determined surface-water discharge at 9,477 streamflow-gaging stations on major rivers and tributaries throughout the United States (Lew, 1998). The USGS operated 192 continuous streamflow-gaging stations and 18 peak-flow partial-record stations in Arizona during the 1996 water year. Statistical summaries of streamflow data in this report were computed for 142 continuous-record streamflow-gaging stations (active and discontinued) on unregulated and partly regulated streams and 178 peak-flow partial-record stations (active and discontinued) or unregulated streams in Arizona through water year 1996 (fig. 1,2) that have at least 10 years of systematic record.

Streamflow characteristics and statistical analysis of streamflow records are essential for the development and management of the available surface-water resources. Streamflow statistics and basin characteristics are used by hydrologists, engineers, and local planners for a variety of purposes to evaluate various land-use alternatives as well as hydrologic and hydraulic designs. This report was prepared in cooperation with the Arizona Department of Water Resources, Bureau of Reclamation, Pima County Board of Supervisors, Flood Control District of Maricopa County, and Salt River Project.

\section{History of the Streamflow-Gaging Network in Arizona}

The U.S. Geological Survey (USGS) was commissioned by an Act of Congress on March 3, 1879, to conduct the systematic and scientific "classification of the public lands, and examination of the geologic structure, mineral resources, and products of the national domain." Surface-water activities in the Arizona 


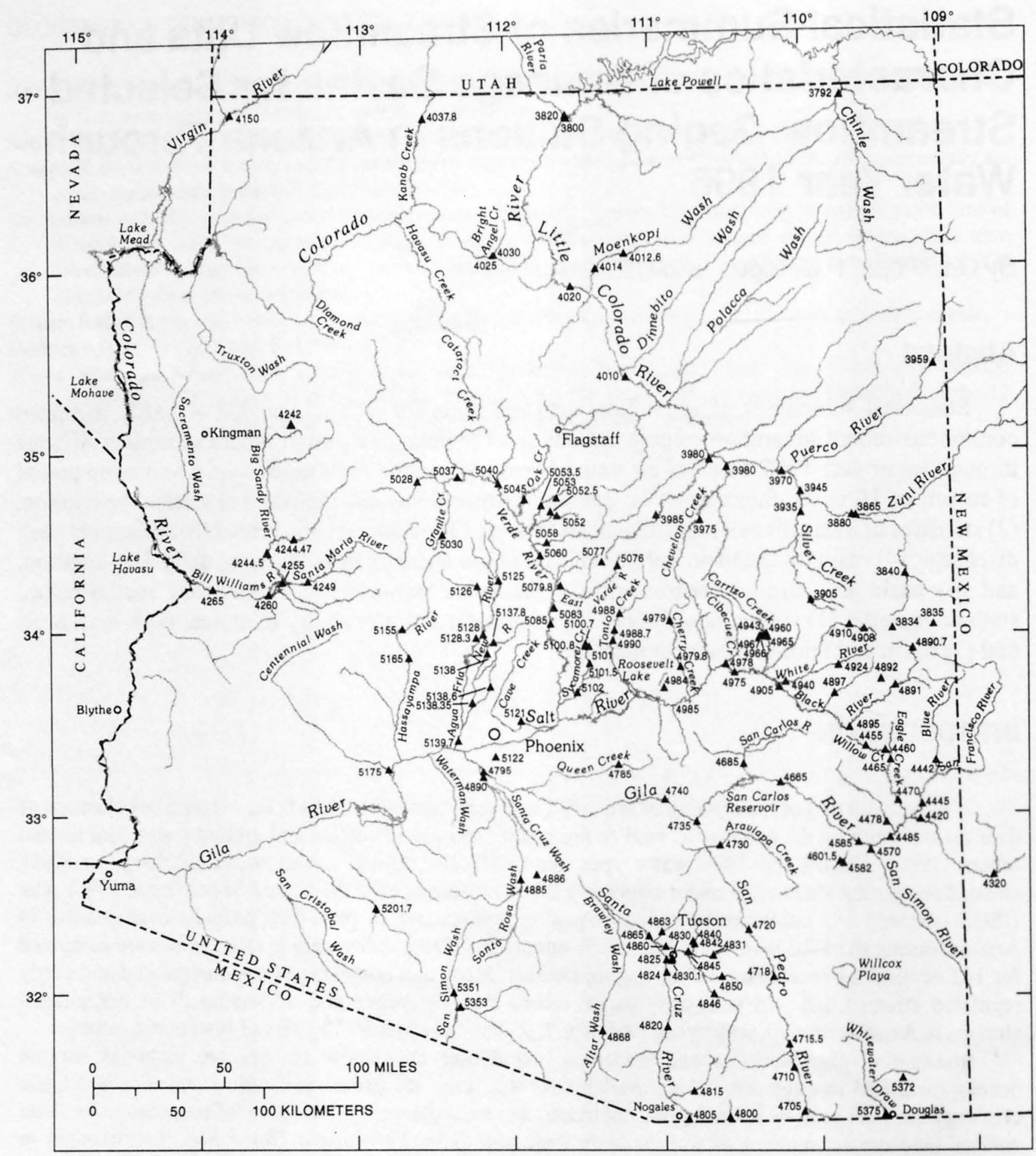

EXPLANATION

5155 STREAMFLOW-GAGING STATION AND

ABBREVIATED NUMBER-Complete

station number is 09515500

Figure 1. Unregulated and partly regulated continuous-record streamflow-gaging stations in Arizona through water year 1996 . 


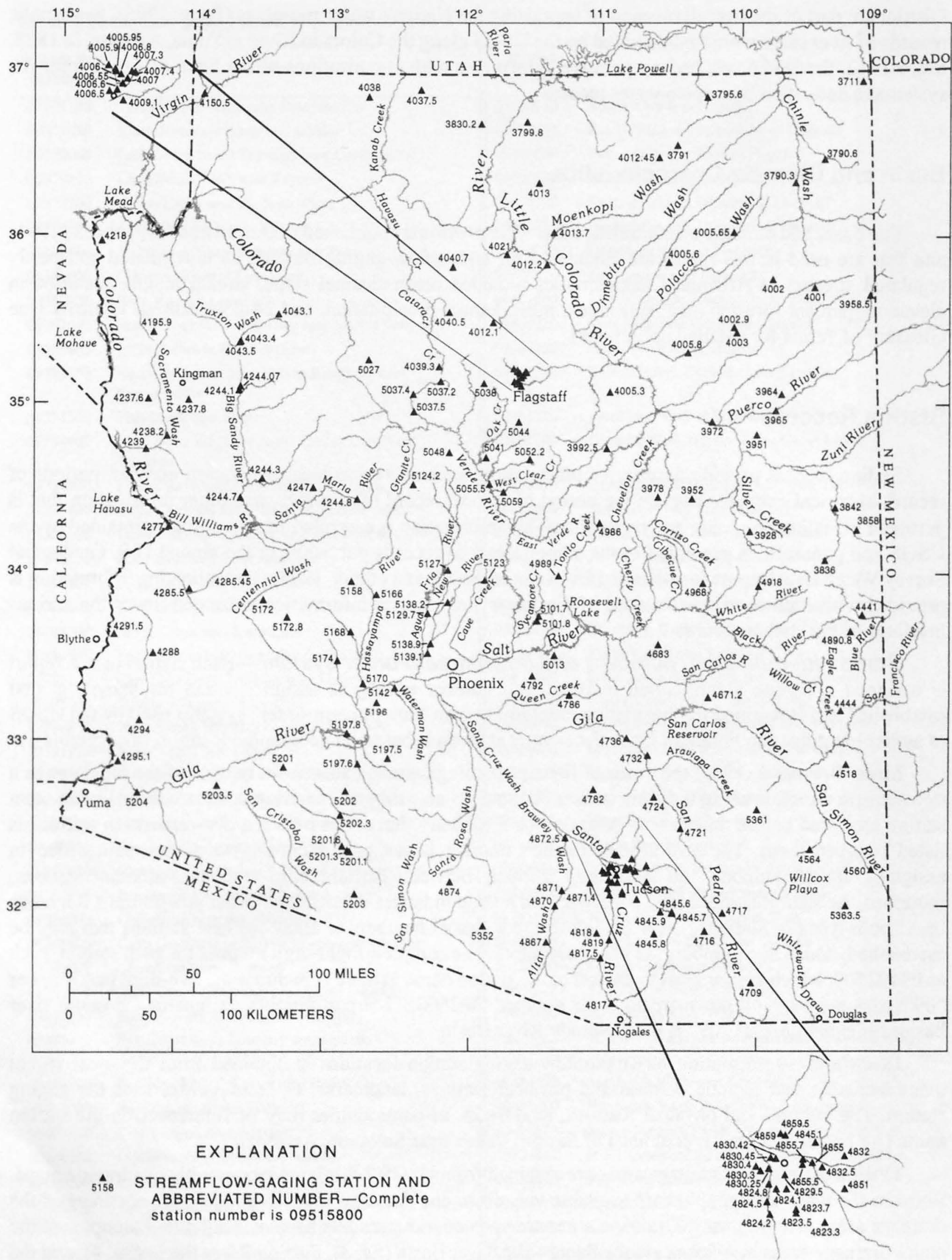

Figure 2. Unregulated and partly regulated peak-flow partial-record stations in Arizona through water year 1996. 
District are part of the overall mission of appraising the Nation's water resources (Pope, 1996). Systematic records of river stage were first obtained by the USGS along the Colorado River at Yuma, Arizona, in 1878. Since 1912, the USGS has had cooperative agreements with organizations of the State of Arizona for the systematic collection of surface-water records.

\section{Basin and Climatic Characteristics}

The basin and climatic characteristics that were previously published in Garrett and Gellenbeck (1991) and that are used in this report are calculated for streamflow-gaging stations on unregulated and partly regulated streams in Arizona. Characteristics included mean-channel slope, stream length, mean-basin elevation, percent forested area, soil index, mean annual precipitation, and 24-hour rainfall intensity (see Glossary of Terms for detailed definitions).

\section{Station Records}

Station records provide descriptive information, such as station location, station number, periods of record, historical extremes outside the period of record, record of accuracy, and other information that is pertinent to station operation and regulation. This information is compiled from records maintained by the USGS and generally is presented in the same format as records published in the annual U.S. Geological Survey Water-Data Report for Arizona (for example, Smith and others, 1997). The following information is provided with each continuous record of discharge and clarifies information presented under the various headings of the station records.

Station identification numbers and downstream-order system.-Each station in this report is assigned a unique identification number. The number usually is assigned when the station is first established and is retained for that station indefinitely. The "downstream-order" systems used by the USGS to assign identification numbers for surface-water stations is based on geographic location (fig. 1; table 1).

Since October 1, 1950, the order of listing hydrologic-station records in reports of the USGS is in a downstream direction along the main stream. All stations on a tributary entering upstream from a main-stem station are listed before that station. A station on a tributary that enters between two main-stem stations is listed between them. The station-identification number is assigned according to downstream order. In assigning station numbers, no distinction is made between partial-record stations and other stations; therefore, the station number for a partial-record station indicates downstream-order position in a list made up of both types of stations. Gaps are left in the series of numbers to allow for new stations that may be established; hence, the numbers are not consecutive. The complete eight-digit number for each station, such as 09402500, which appears just to the left of the station name in table 1, includes the two-digit part number "09": plus the six-digit downstream-order number "402500." The part number designates the major river basin; for example, part " 09 " is the Colorado River Basin.

Location.-Information on streamflow-gaging station locations is obtained from the most recent maps available that include cultural and physical features, landmarks, or communities near the gaging station. The cultural and physical features, landmarks, or communities may be referenced in the station name (for example, Gila River at head of Safford Valley near Solomon, Arizona).

Drainage area.-Drainage areas are obtained from USGS 7.5-minute topographic quadrangle maps. Because the types of maps that are available vary from one drainage basin to another, the accuracy of the drainage areas also may vary. Drainage areas are updated as better data become available. Examples of the major drainage areas in Arizona are the Santa Cruz River Basin (fig. 3), the Salt River Basin (fig. 4), and the Colorado River Basin (fig. 5). 
Table 1. Streamflow-gaging stations in Arizona, 1996

\begin{tabular}{|c|c|c|c|}
\hline $\begin{array}{l}\text { Station } \\
\text { number }\end{array}$ & Station name & $\begin{array}{l}\text { Station } \\
\text { number }\end{array}$ & Station name \\
\hline 09371100 & Teec Nos Pos Wash near Teec Nos Pos & 09400650 & Sinclair Wash at Flagstaff \\
\hline 09379030 & Black Mountain Wash near Chinle & 09400655 & Rio De Flag at Interstate 40 at Flagstaff \\
\hline 09379060 & Lukachukai Creek Tributary near Luckachukai & 09400660 & Bow and Arrow Wash at Flagstaff \\
\hline 09379100 & Long House Wash near Kayenta & 09400680 & Switzer Canyon at Flagstaff \\
\hline 09379200 & Chinle Creek near Mexican Water & 09400700 & Switzer Canyon Tributary at Flagstaff \\
\hline 09379560 & El Capitan Wash near Kayenta & 09400730 & Lockett Fanning Diversion at Flagstaff \\
\hline 09379980 & Jack Bench Wash Tributary near Page & 09400740 & Harenberg Wash at Flagstaff \\
\hline 09380000 & Colorado River at Lees Ferry & 09400910 & Fay Canyon near Flagstaff \\
\hline 09382000 & Paria River at Lees Ferry & 09401000 & Little Colorado River at Grand Falls \\
\hline 09383020 & House Rock Wash Tributary near Marble Canyon & 09401210 & Slate Mountain Wash near Flagstaff \\
\hline 09383400 & Little Colorado River at Greer & 09401220 & Cedar Wash near Cameron \\
\hline 09383500 & $\begin{array}{l}\text { Nutrioso Creek above Nelson Reservoir, near } \\
\text { Springerville }\end{array}$ & 09401245 & Klethla Valley Tributary near Kayenta \\
\hline 09383600 & Fish Creek near Eagar & 09401260 & Moenkopi Wash at Moenkopi \\
\hline 09384000 & $\begin{array}{l}\text { Little Colorado River above Lyman Lake, near } \\
\text { St. Johns }\end{array}$ & 09401300 & Hamblin Wash Tributary near Cedar Ridge \\
\hline 09384200 & Lyman Reservoir Tributary near St. Johns & 09401370 & Hamblin Wash Tributary No. 2 near Tuba City \\
\hline 09385800 & Little Colorado River Tributary near St. Johns & 09401400 & Moenkopi Wash near Tuba City \\
\hline 09386500 & Little Colorado River above Zuni River, near Hunt & 09402000 & Little Colorado River near Cameron \\
\hline 09388000 & Little Colorado River near Hunt & 09402100 & Forest Boundary Wash near Cameron \\
\hline 09390500 & Show Low Creek near Lakeside & 09402500 & Colorado River near Grand Canyon \\
\hline 09392800 & Long Lake Tributary near Show Low & 09403000 & Bright Angel Creek near Grand Canyon \\
\hline 09393500 & Silver Creek near Snowflake & 09403750 & Sagebrush Draw near Fredonia \\
\hline 09394500 & Little Colorado River at Woodruff & 09403780 & Kanab Creek near Fredonia \\
\hline 09395100 & Carr Lake Draw Tributary near Holbrook & 09403800 & Bitter Seeps Wash Tributary near Fredonia \\
\hline 09395200 & Decker Wash near Snowflake & 09403930 & West Cataract Creek near Williams \\
\hline 09395850 & Black Creek Tributary near Window Rock & 09404050 & Spring Valley Wash Tributary near Williams \\
\hline 09395900 & Black Creek near Lupton & 09404070 & Little Red Horse Wash near Grand Canyon \\
\hline 09396400 & Dead Wash Tributary near Holbrook & 09404310 & Yampai Canyon Tributary near Peach Springs \\
\hline 09396500 & Puerco River near Adamana & 09404340 & Truxton Wash at Valentine \\
\hline 09397000 & Little Colorado River at Holbrook & 09404350 & Valentine Wash at Valentine \\
\hline 09397200 & Penzance Wash near Joseph City & 09415000 & Virgin River at Littlefield \\
\hline 09397500 & Chevelon Creek below Wildcat Canyon, near Winslow & 09415050 & Big Bend Wash Tributary near Littlefield \\
\hline 09397800 & Brookbank Canyon near Heber & 09419590 & Detrital Wash Tributary near Chloride \\
\hline 09398000 & Chevelon Creek near Winslow & 09421800 & Ringbolt Wash near Hoover Dam \\
\hline 09398500 & Clear Creek below Willow Creek, near Winslow & 09423760 & Little Meadow Creek near Oatman \\
\hline 09399000 & Clear Creek near Winslow & 09423780 & Walnut Creek near Kingman \\
\hline 09399250 & Jacks Canyon Tributary No. 2, near Winslow & 09423820 & Sacramento Wash near Yucca \\
\hline 09400100 & Ganado Wash Tributary near Ganado & 09423900 & Sacramento Wash Tributary near Topock \\
\hline 09400200 & Steamboat Wash Tributary near Ganado & 09424200 & $\begin{array}{l}\text { Willow Creek (Cottonwood Wash No. 1) near King- } \\
\text { man }\end{array}$ \\
\hline 09400290 & Teshbito Wash Tributary near Holbrook & 09424407 & McGarrys Wash near Kingman \\
\hline 09400300 & Teshbito Wash near Holbrook & 09424410 & Big Sandy River Tributary near Kingman \\
\hline 09400530 & Cow Canyon near Winslow & 09424430 & Kaiser Spring Canyon Tributary near Wikieup \\
\hline 09400560 & Oraibi Wash Tributary near Oraibi & 09424447 & Burro Creek at Old U.S. 93 Bridge, near Bagdad \\
\hline 09400565 & Polacca Wash Tributary near Chinle & 09424450 & Big Sandy River near Wikieup \\
\hline 09400580 & Castle Butte Wash near Winslow & 09424470 & Kirkland Creek near Kirkland \\
\hline 09400590 & Rio De Flag at Hidden Hollow Road, at Flagstaff & 09424480 & Ash Creek near Kirkland \\
\hline 09400595 & Schultz Canyon at Flagstaff & 09424700 & Iron Spring Wash Tributary near Bagdad \\
\hline 09400600 & Rio De Flag at Flagstaff & 09424900 & Santa Maria River near Bagdad \\
\hline
\end{tabular}


Table 1. Streamflow-gaging stations in Arizona, 1996-Continued

\begin{tabular}{|c|c|c|c|}
\hline $\begin{array}{l}\text { Station } \\
\text { number }\end{array}$ & Station name & $\begin{array}{l}\text { Station } \\
\text { number }\end{array}$ & Station name \\
\hline 09425500 & Santa Maria River near Alamo & 09478500 & Queen Creek at Whitlow Damsite, near Superior \\
\hline 09426000 & Bill Williams River below Alamo Dam & 09478600 & Queen Creek Tributary No. 3 at Whitlow Dam \\
\hline 09426500 & Bill Williams River at Planet & 09479200 & Queen Creek Tributary at Apache Junction \\
\hline 09427700 & Monkeys Head Wash near Parker & 09479500 & Gila River near Laveen \\
\hline 09428545 & Cunningham Wash Tributary near Wenden & 09480000 & Santa Cruz River near Lochiel \\
\hline 09428550 & Bouse Wash Tributary near Bouse & 09480500 & Santa Cruz River near Nogales \\
\hline 09428800 & Tyson Wash Tributary near Quartzsite & 09481500 & Sonoita Creek near Patagonia \\
\hline 09429150 & Creosote Wash near Ehrenberg & 09481700 & Calabasas Canyon near Nogales \\
\hline 09429400 & Indian Wash Tributary near Yuma & 09481750 & Sopori Wash at Amado \\
\hline 09429510 & Mittry Lake Tributary near Yuma & 09481800 & Demetrie Wash Tributary near Continental \\
\hline 09432000 & Gila River below Blue Creek near Virden, New Mexico & 09481900 & Ocotillo Wash near Continental \\
\hline 09442000 & Gila River near Clifton & 09482000 & Santa Cruz River at Continental \\
\hline 09444100 & Campbell Blue Creek near Alpine & 09482200 & Flato Wash near Sahuarita \\
\hline 09444200 & Blue River near Clifton & 09482330 & Pumping Wash near Vail \\
\hline 09444400 & Chase Creek near Clifton & 09482350 & South Fork Airport Wash near Tucson \\
\hline 09444500 & San Francisco River at Clifton & 09482370 & North Fork Airport Wash near Tucson \\
\hline 09445500 & Willow Creek near Point of Pines, near Morenci & 09482400 & Airport Wash at Tucson \\
\hline 09446000 & Willow Creek near Double Circle Ranch, near Morenci & 09482410 & Rodeo Wash at Tucson \\
\hline 09446500 & Eagle Creek near Double Circle Ranch, near Morenci & 09482420 & Julian Wash at Tucson \\
\hline 09447000 & Eagle Creek above pumping plant, near Morenci & 09482450 & West Branch Santa Cruz River, at Tucson \\
\hline 09447800 & Bonita Creek near Morenci & 09482480 & Big Wash at Tucson \\
\hline 09448500 & Gila River at head of Safford Valley, near Solomon & 09482500 & Santa Cruz River at Tucson \\
\hline 09451800 & Tollgate Wash Tributary near Clifton & 09482950 & Railroad Wash at Tucson \\
\hline 09456000 & San Simon River near San Simon & 09483000 & Tucson Arroyo at Vine Avenue, at Tucson \\
\hline 09456400 & Gold Gulch near Bowie & 09483010 & High School Wash at Tucson \\
\hline 09457000 & San Simon River near Solomon & 09483025 & Silvercroft Wash at Tucson \\
\hline 09458200 & Deadman Creek near Safford & 09483030 & Anklam Wash at Tucson \\
\hline 09458500 & Gila River at Safford & 09483040 & West Speedway Wash near Tucson \\
\hline 09460150 & Frye Creek near Thatcher & 09483042 & Cemetery Wash at Tucson \\
\hline 09466500 & Gila River at Calva & 09483045 & Flowing Wells Wash at Tucson \\
\hline 09467120 & Salt Creek near Peridot & 09483100 & Tanque Verde Creek near Tucson \\
\hline 09468300 & Sevenmile Wash Tributary near Globe & 09483200 & Agua Caliente Wash Tributary near Tucson \\
\hline 09468500 & San Carlos River near Peridot & 09483250 & Rob Wash at Tucson \\
\hline 09470500 & San Pedro River at Palominas & 09484000 & Sabino Creek near Tucson \\
\hline 09470900 & San Pedro River Tributary near Bisbee & 09484200 & Bear Creek near Tucson \\
\hline 09471000 & San Pedro River at Charleston & 09484500 & Tanque Verde Creek at Tucson \\
\hline 09471550 & San Pedro River near Tombstone & 09484510 & Ventana Canyon Wash near Tucson \\
\hline 09471600 & Canary Wash near Benson & 09484560 & Cienega Creek near Pantano \\
\hline 09471700 & Fenner Wash near Benson & 09484570 & Mescal Arroyo near Pantano \\
\hline 09471800 & San Pedro River near Benson & 09484580 & Barrel Canyon near Sonoita \\
\hline 09472000 & San Pedro River near Redington & 09484590 & Davidson Canyon Wash near Vail \\
\hline 09472100 & Peck Canyon Tributary near Redington & 09484600 & Pantano Wash near Vail \\
\hline 09472400 & Mammoth Wash near Mammoth & 09485000 & Rincon Creek near Tucson \\
\hline 09473000 & Aravaipa Creek near Mammoth & 09485100 & Saguaro Corners Wash near Tucson \\
\hline 09473200 & Green Lantern Wash near Winkelman & 09485500 & Pantano Wash near Tucson \\
\hline 09473500 & San Pedro River at Winkelman & 09485550 & Arcadia Wash at Tucson \\
\hline 09473600 & Tam O'Shanter Wash near Hayden & 09485570 & Alamo Wash at Tucson \\
\hline 09474000 & Gila River at Kelvin & 09485900 & Pima Wash near Tucson \\
\hline 09478200 & Durham Wash near Florence & 09485950 & Geronimo Wash near Tucson \\
\hline
\end{tabular}


Table 1. Streamflow-gaging stations in Arizona, 1996-Continued

\begin{tabular}{|c|c|c|c|}
\hline $\begin{array}{l}\text { Station } \\
\text { number }\end{array}$ & Station name & $\begin{array}{l}\text { Station } \\
\text { number }\end{array}$ & Station name \\
\hline 09486000 & Rillito Creek near Tucson & 09503700 & Verde River near Paulden \\
\hline 09486300 & Canada del Oro near Tucson & 09503720 & Hell Canyon near Williams \\
\hline 09486500 & Santa Cruz River at Cortaro & 09503740 & Hell Canyon Tributary near Ashfork \\
\hline 09486700 & Chiltepines Wash near Sasabe & 09503750 & Limestone Canyon near Paulden \\
\hline 09486800 & Altar Wash near Three Points & 09503800 & Volunteer Wash near Bellemont \\
\hline 09487000 & Brawley Wash near Three Points & 09504000 & Verde River near Clarkdale \\
\hline 09487100 & Little Brawley Wash near Three Points & 09504100 & Hull Canyon near Jerome \\
\hline 09487140 & San Joaquin Wash near Tucson & 09504400 & Munds Canyon Tributary near Sedona \\
\hline 09487250 & Los Robles Wash near Marana & 09504500 & Oak Creek near Cornville \\
\hline 09487400 & Quijotoa Wash Tributary near Quijotoa & 09504800 & Oak Creek Tributary near Cornville \\
\hline 09488500 & Santa Rosa Wash near Vaiva Vo, near Sells & 09505200 & Wet Beaver Creek near Rimrock \\
\hline 09488600 & Silver Reef Wash near Casa Grande & 09505220 & Rocky Gulch near Rimrock \\
\hline 09489000 & Santa Cruz River near Laveen & 09505250 & Red Tank Draw near Rimrock \\
\hline 09489070 & North Fork of East Fork Black River near Alpine & 09505300 & Rattlesnake Canyon near Rimrock \\
\hline 09489080 & Hannagan Creek near Hannagan Meadow & 09505350 & Dry Beaver Creek near Rimrock \\
\hline 09489100 & Black River near Maverick & 09505550 & Verde River below Camp Verde \\
\hline 09489200 & Pacheta Creek at Maverick & 09505600 & Dirty Neck Canyon near Clints Well \\
\hline 09489500 & Black River below Pumping Plant, near Point of Pines & 09505800 & West Clear Creek near Camp Verde \\
\hline 09489700 & Big Bonito Creek near Fort Apache & 09505900 & Cottonwood Wash near Camp Verde \\
\hline 09490500 & Black River near Fort Apache & 09506000 & Verde River near Camp Verde \\
\hline 09490800 & North Fork White River near Greer & 09507600 & East Verde River near Pine \\
\hline 09491000 & North Fork White River near McNary & 09507700 & $\begin{array}{l}\text { Webber Creek above West Fork Webber Creek, near } \\
\text { Pine }\end{array}$ \\
\hline 09491800 & North Fork White River Tributary near Whiteriver & 09507980 & East Verde River near Childs \\
\hline 09492400 & East Fork White River near Fort Apache & 09508300 & Wet Bottom Creek near Childs \\
\hline 09494000 & White River near Fort Apache & 09508500 & $\begin{array}{l}\text { Verde River below Tangle Creek, above Horseshoe } \\
\text { Dam }\end{array}$ \\
\hline 09494300 & Carrizo Creek above Corduroy Creek, near Show Low & 09510070 & $\begin{array}{l}\text { West Fork Sycamore Creek above McFarland Canyon, } \\
\text { near Sunflower }\end{array}$ \\
\hline 09496000 & Corduroy Creek near mouth, near Show Low & 09510080 & West Fork Sycamore Creek near Sunflower \\
\hline 09496500 & Carrizo Creek near Show Low & 09510100 & East Fork Sycamore Creek near Sunflower \\
\hline 09496600 & $\begin{array}{l}\text { Cibecue No. 1, Tributary to Carrizo Creek, near Show } \\
\text { Low }\end{array}$ & 09510150 & Sycamore Creek near Sunflower \\
\hline 09496700 & $\begin{array}{l}\text { Cibecue No. 2, Tributary to Carrizo Creek, near Show } \\
\text { Low }\end{array}$ & 09510170 & Camp Creek near Sunflower \\
\hline 09496800 & Carrizo Creek Tributary near Show Low & 09510180 & Rock Creek near Sunflower \\
\hline 09497500 & Salt River near Chrysotile & 09510200 & Sycamore Creek near Fort McDowell \\
\hline 09497800 & Cibecue Creek near Chrysotile & 09512100 & Indian Bend Wash at Scottsdale \\
\hline 09497900 & Cherry Creek near Young & 09512200 & $\begin{array}{l}\text { Salt River Tributary in South Mountain Park, at } \\
\text { Phoenix }\end{array}$ \\
\hline 09497980 & Cherry Creek near Globe & 09512300 & Cave Creek near Cave Creek \\
\hline 09498400 & Pinal Creek at Inspiration Dam,near Globe & 09512420 & Lynx Creek Tributary near Prescott \\
\hline 09498500 & Salt River near Roosevelt & 09512500 & Agua Fria River near Mayer \\
\hline 09498600 & Christopher Creek Tributary near Kohl's Ranch & 09512600 & Turkey Creek near Cleator \\
\hline 09498800 & Tonto Creek near Gisela & 09512700 & Agua Fria River Tributary No. 2 near Rock Springs \\
\hline 09498870 & Rye Creek near Gisela & 09512800 & Agua Fria River near Rock Springs \\
\hline 09498900 & Gold Creek near Payson & 09512830 & Boulder Creek near Rock Springs \\
\hline 09499000 & Tonto Creek above Gun Creek, near Roosevelt & 09512970 & Cottonwood Creek near Waddell Dam \\
\hline 09501300 & Tortilla Creek at Tortilla Flat & 09513780 & New River near Rock Springs \\
\hline 09502700 & Crookton Wash near Seligman & 09513800 & New River at New River \\
\hline 09502800 & Williamson Valley Wash near Paulden & 09513820 & Deadman Wash near New River \\
\hline 09503000 & Granite Creek near Prescott & 09513835 & New River at Bell Road, near Peoria \\
\hline
\end{tabular}


Table 1. Streamflow-gaging stations in Arizona, 1996-Continued

\begin{tabular}{ll||ll}
\hline $\begin{array}{c}\text { Station } \\
\text { number }\end{array}$ & \multicolumn{1}{c||c}{ Station name } & $\begin{array}{c}\text { Station } \\
\text { number }\end{array}$ & \multicolumn{1}{c}{ Station name } \\
\hline 09513860 & Skunk Creek near Phoenix & 09519780 & Windmill Wash near Gila Bend \\
09513890 & New River at Peoria & 09520100 & Military Wash near Sentinel \\
09520110 & Hot Shot Arroyo near Ajo \\
09513910 & New River near Glendale & 09520130 & Darby Arroyo near Ajo \\
09513970 & Agua Fria River at Avondale & 09520160 & Gibson Arroyo at Ajo \\
09514200 & Waterman Wash near Buckeye & 09520170 & Rio Cornez near Ajo \\
09515500 & Hassayampa River at Box Damsite, near Wickenburg & 09520200 & Black Gap Wash near Ajo \\
09515800 & Hartman Wash near Wickenburg & 09520230 & Crater Range Wash near Ajo \\
09516500 & Hassayampa River near Morristown & 09520300 & Alamo Wash Tributary near Ajo \\
09516600 & Ox Wash near Morristown & 09520350 & Mohawk Pass Wash at Mohawk \\
09516800 & Jack Rabbit Wash near Tonopah & 09520400 & Ligurta Wash at Liguria \\
09517000 & Hassayampa River near Arlington & 09535100 & San Simon Wash near Pisinimo \\
09517200 & Centennial Wash Tributary near Wenden & 09535200 & Little Tucson Wash at Sells \\
09517280 & Tiger Wash near Aguila & 09535300 & Vamori Wash at Kom Vo \\
09517400 & Winters Wash near Tonopah & 09536100 & Pitchfork Canyon Tributary near Fort Grant \\
09517500 & Centennial Wash near Arlington & 09536350 & Surprise Canyon near Dos Cabezas \\
09519600 & Rainbow Wash Tributary near Buckeye & 09537200 & Leslie Creek near McNeal \\
09519750 & Bender Wash near Gila Bend & 09537500 & Whitewater Draw near Douglas \\
09519760 & Sauceda Wash near Gila Bend & & \\
\hline & &
\end{tabular}




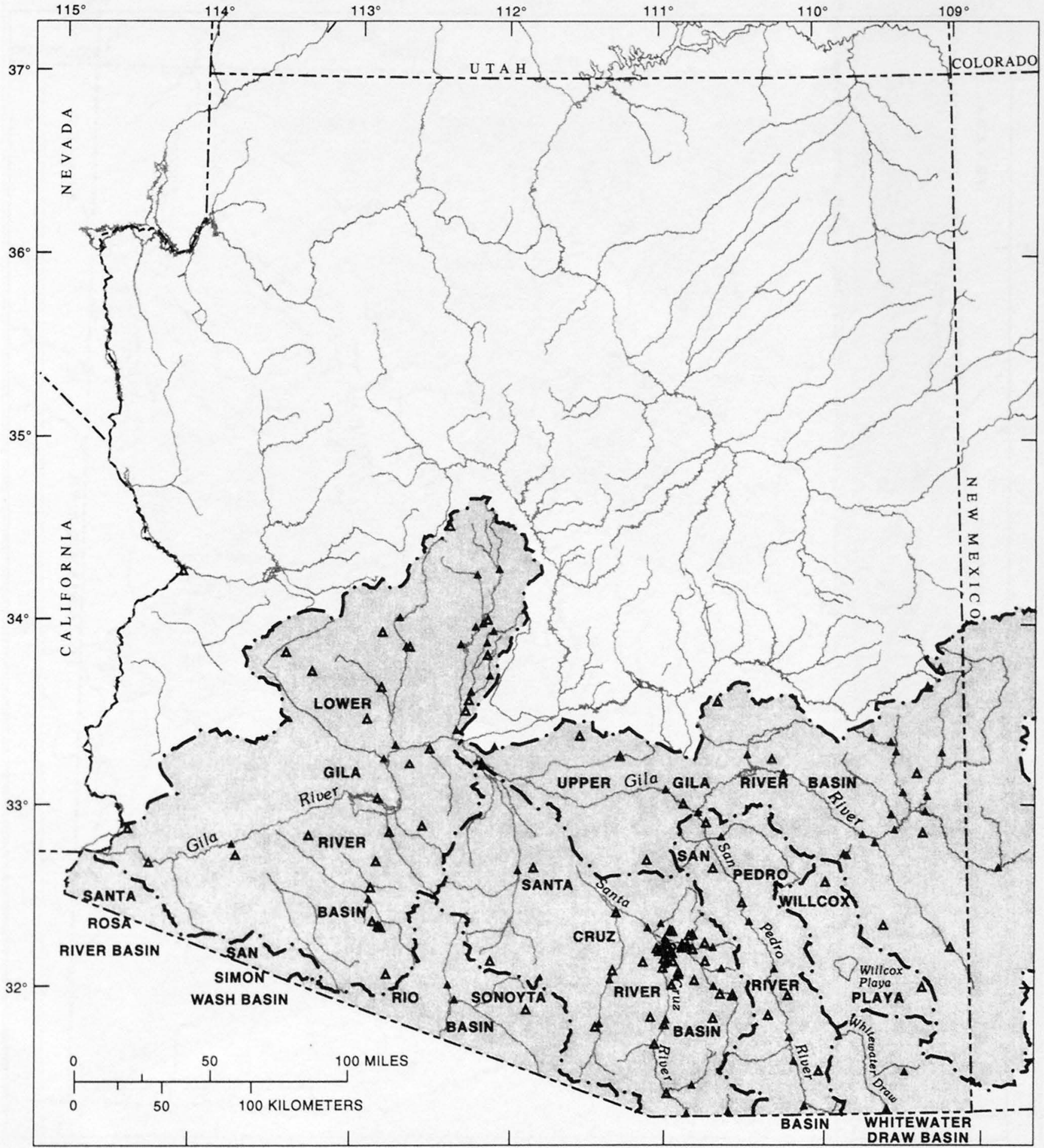

EXPLANATION

- CONTINUOUS-RECORD STREAMFLOW-GAGING STATION

$\triangle$ PEAK-FLOW PARTIAL-RECORD STREAMFLOWGAGING STATION

Figure 3. Basins and unregulated and partly regulated streamflow-gaging stations in southern Arizona through water year 1996. 


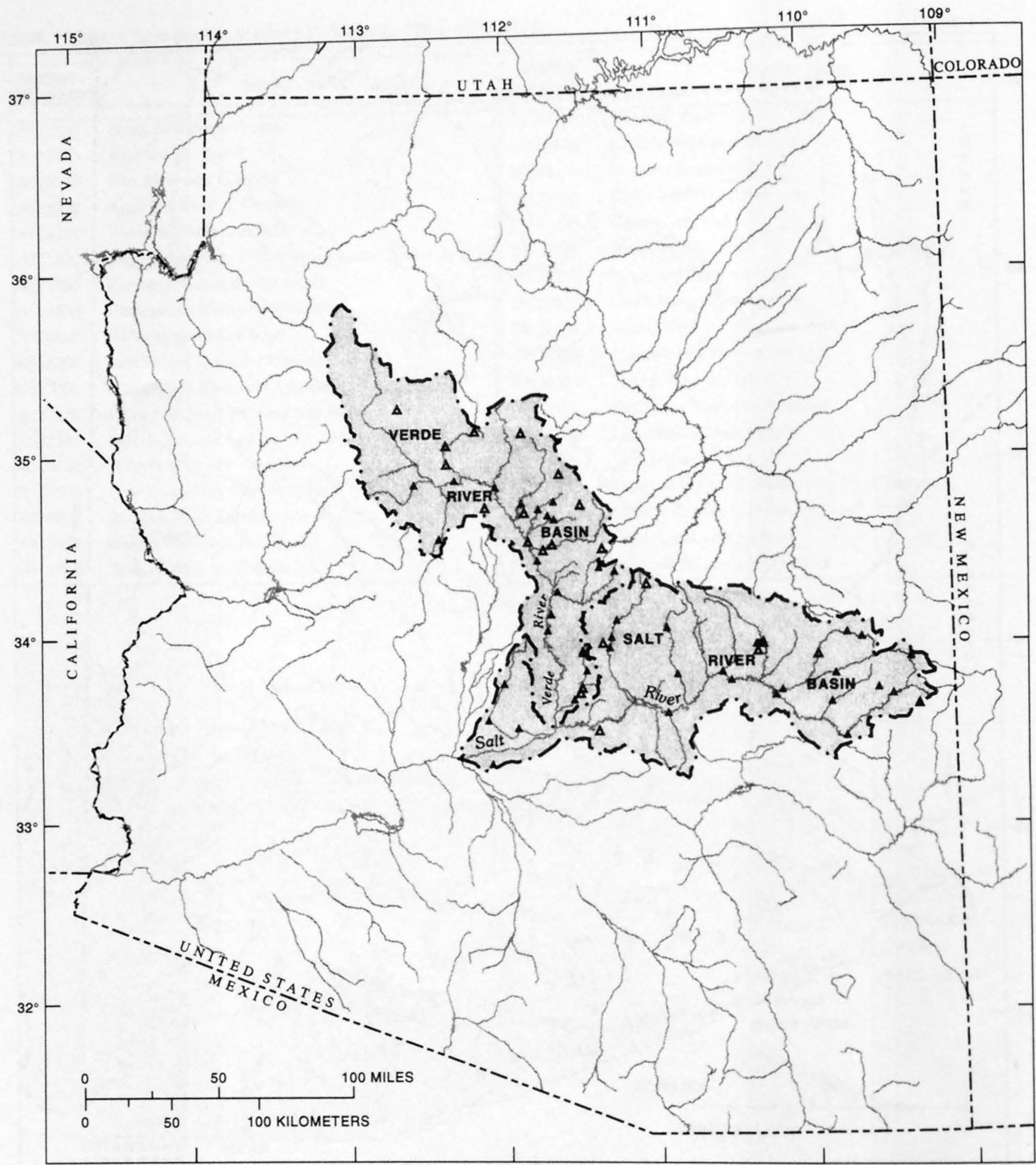

EXPLANATION

- CONTINUOUS-RECORD STREAMFLOW-GAGING STATION

$\triangle$ PEAK-FLOW PARTIAL-RECORD STREAMFLOW. GAGING STATION

Figure 4. Basins and unregulated and partly regulated streamflow-gaging stations in central Arizona through water year 1996. 


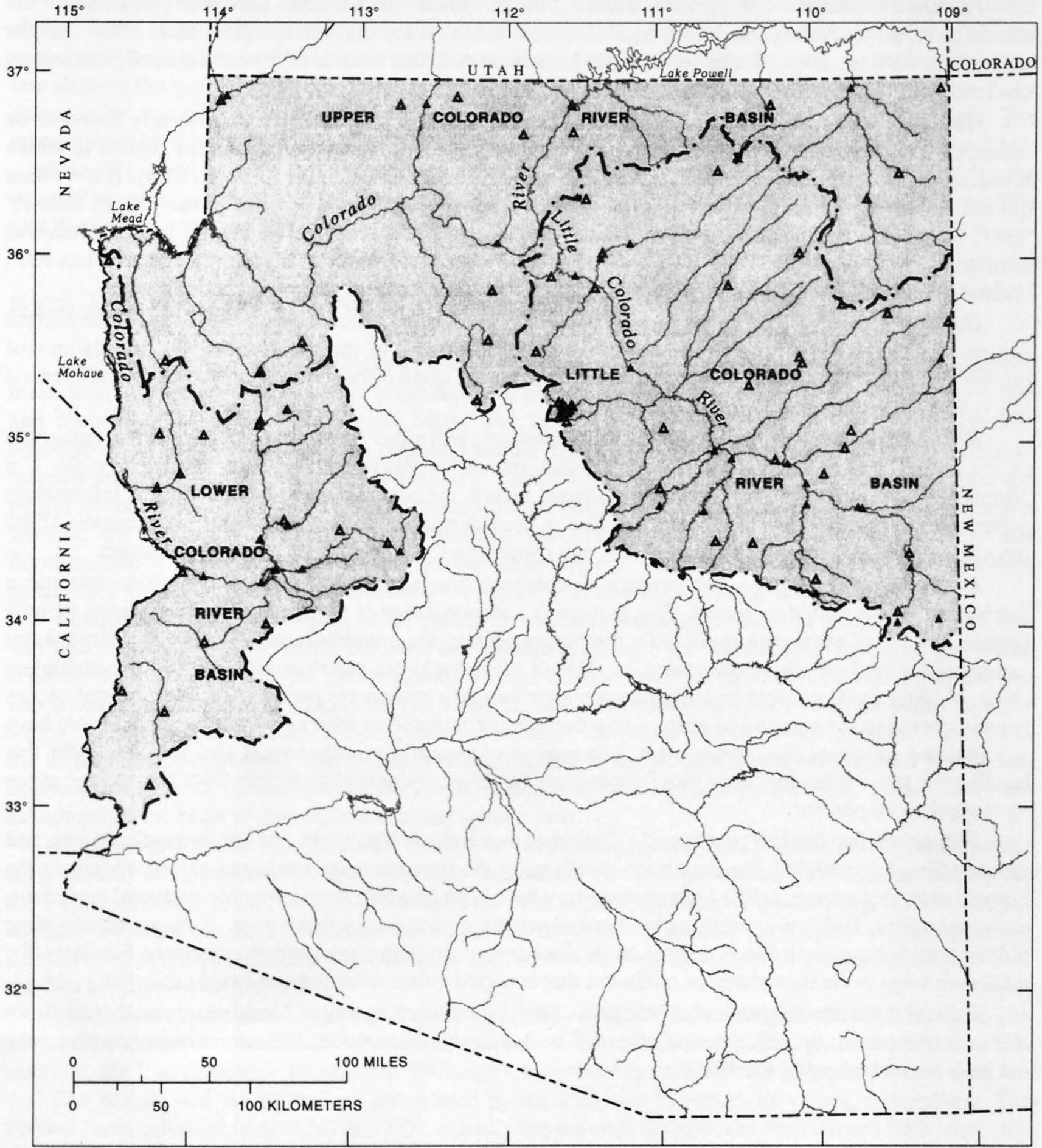

EXPLANATION

- CONTINUOUS-RECORD STREAMFLOW-GAGING STATION

$\triangle$ PEAK-FLOW PARTIAL-RECORD STREAMFLOWGAGING STATION

Figure 5. Basins and unregulated and partly regulated streamflow-gaging stations in northern Arizona through water year 1996. 
Period of record.-The period of record is the period for which records have been published for the station or for an equivalent station. An equivalent station is a station that was in operation at a time that the present station was not in existence and whose location is such that records of flow at the equivalent station can reasonably be considered equivalent to flow at the present station.

Revised records. - Because of new information, published records are occasionally found to be incorrect, and revisions are printed in later reports. Listed under this heading are all the reports in which revisions have been published for the station and the water years to which the revisions apply. If a revision did not include daily, monthly, or annual figures of discharge, that fact is noted after the year as follows: "(M)" means that only instantaneous maximum discharge was revised; " $(\mathrm{m})$ " that only the instantaneous minimum was revised; and " $(\mathrm{P})$ " that only peak discharges were revised. If the drainage area has been revised, the report in which the most recently revised drainage area was first published is given.

Gaging station.-The type of gaging station that is used to collect the data, the datum of the current gaging station referenced to sea level, and a condensed history of the types, locations, and datums of previous gaging stations used at that location. The datum of some discontinued stations may be referenced to a datum other than that of sea level.

Remarks.-All periods of estimated daily discharge will either be identified by date in this paragraph of the station description for the water-discharge stations or flagged in the daily discharge table. If a "Remarks" paragraph is used to identify estimated record, the paragraph will begin with this information presented as the first entry. The paragraph also is used to present information about the accuracy of the records, the special methods of computation, and conditions that affect natural flow at the station.

Average discharge.-The average discharge is the arithmetic mean of the water-year mean discharges for the period of record. This paragraph and calculation is only included if the station records contain periods of other than mean daily discharges such as the monthly mean. Average discharge is not computed for stations where diversions, storage, or other water-use practices would cause a meaningless value. If water developments that significantly alter flow at a station are put into use after the station has been in operation for a period of years, a new average is computed as soon as 5 water years of record have accumulated following the development. The median of annual mean discharges also is given under this heading for stations having 10 or more water years of record if the median differs from the average given by more than 10 percent.

Extremes for period of record.-Extremes may include maximum and minimum discharges, and unless otherwise qualified, the maximum discharge is the instantaneous maximum corresponding to the highest stage that occurred. The highest stage may have been obtained from a graphic or digital recorder, a crest-stage gage, high-water mark, or by direct observation of a nonrecording gage. If the maximum stage did not occur on the same day as the maximum discharge, maximum stage is given separately. Similarly, the minimum stage is the instantaneous minimum that occurred unless qualified and listed otherwise.

Extremes outside period of record.-Any information on major floods or unusually low flows that occurred outside the stated period of record are included in this section. The information, however, may not have been obtained by the USGS.

\section{Rating Tables}

The most recent discharge rating table is included with the data tables for each streamflow-gaging station. Some rating tables are not included because they were not available. The ratings can be used to relate discharges given in the various tables to their associated gage height at the streamflow-gaging station. These gage heights are referenced to the gage datum, which is an arbitrary datum selected to avoid negative gage heights. The gage datum and gage heights can be converted to elevation above mean sea level by adding the datum or elevation of the gaging station listed in the section titled "Gaging Stations" of the station description. 
Discharge ratings are updated periodically for active streamflow-gaging stations after a significant change in the stage-discharge relation has occurred. Because of the nature of the controls, the stability and duration of these ratings vary significantly from station to station. Generally the stage-discharge relation is less stable at the lower stages than it is at the higher stages. Sensitivity and unstable conditions at lower flows may require that discharge ratings be revised more frequently to account for these control changes. The rating tables in this report have taken this component into account and have been limited to the upper portions of the rating table that are more stable. Over time, many of the discharge rating tables for the streamflow-gaging stations will be superseded, and users of these data should contact the local office of the USGS to confirm the validity of the ratings.

\section{EXPLANATION OF STATISTICAL SUMMARIES}

Statistical summaries and associated plots for the 320 streamflow-gaging stations include statistics for mean monthly and annual discharge; magnitude and probability of annual high and low flows; magnitude and probability of instantaneous peak flows; and daily mean-flow duration and basin and climatic characteristics. Graphs and box plots represent annual peak discharge, annual mean discharge, and mean monthly and mean annual discharges respectively. Each station description and table heading identifies the period of record for which the statistical summaries are based. For continuous-record streamflow-gaging stations, the statistical summaries of streamflow are calculated from the daily mean values, and flood-frequency calculations are based on the instantaneous peak flows. Summaries for peak-flow partial-record stations only include the flood-frequency and recurrence-interval statistics.

Monthly and annual flow.-Mean monthly and annual discharge statistics include the minimum and maximum discharges, mean monthly and annual discharge, the standard deviation of the mean, coefficient of variation, and the percentage of average annual runoff for each month. The minimum and maximum mean monthly discharges are the minimum and maximum flow values of all the mean monthly values for that particular month. The coefficient of variation represents the ratio of the standard deviation to the mean. The percentage of the annual discharge runoff is the percent of the total annual runoff that occurred during each month. Except for low-flow magnitude and probability statistics, annual flows are calculated on the basis of data obtained during a water year.

Boxplots graphically summarize the characteristics of mean monthly and annual discharges and can be used to easily compare data sets. Boxplots were constructed for continuous-record stations and display (1) the median or center of the data, (2) variation of the data, and (3) skewness of the data (fig. 6). The boxplot in figure 6 summarizes the March mean discharges for the period of record at the streamflow-gaging station Blue River near Clifton (table 1). The column of data next to the boxplot in figure 6 is included to show how the data are distributed graphically.

Boxplots are constructed by computing the 10th, 25th, 50th, 75th, and 90th percentiles of the data. The percentile value represents the discharge which exceeds no more than the specified percent of the data. For example, the 10th percentile value is that discharge which exceeds no more than 10 percent of the data.

The bottom and top of the box are defined by the 25 th and 75 percentile values, respectively. The vertical lines, called whiskers (Tukey, 1977), extend from the ends of the box to the 10th and 90th percentile values. The 50th percentile value, referred to as the median, defines the line drawn within the box. In the example, the 10th, 25th, 50th, 75th, and 90th percentile values are 13.9, 35.9, 105.6, 314.3, and 429.3 cubic feet per second, respectively.

Flow magnitude and probability.-Computations for flow magnitudes and probability statistics are based on the log-Pearson Type III frequency distribution and provide the necessary data to plot standard flow-frequency curves. Magnitude and probability tables for annual low flow list the maximum and minimum average discharge for periods of $1,3,7,14,30,60,90,120$, and 183 consecutive days that correspond to selected recurrence intervals of $2,5,10,20,50$, and 100 years associated with annual nonexceedence probabilities of 50,20,10,5,2, and 1 percent. Recurrence intervals represent the average 
(28)

NUMBER OF OBSERVATIONS

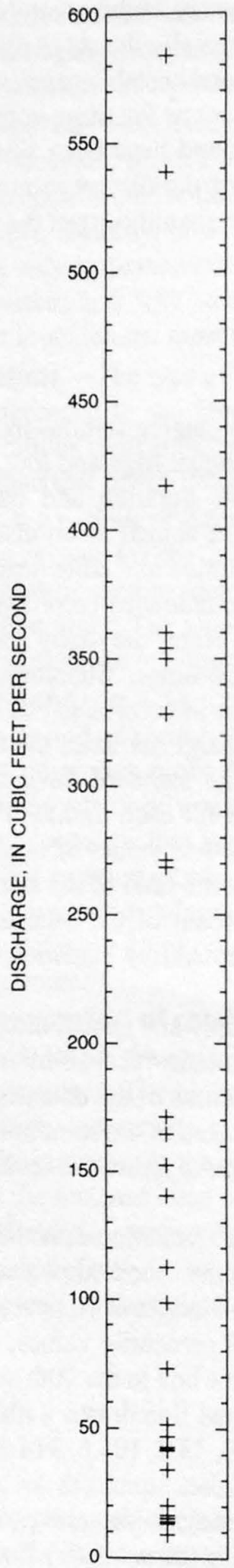

$90^{\text {th }}$ PERCENTILE VALUE

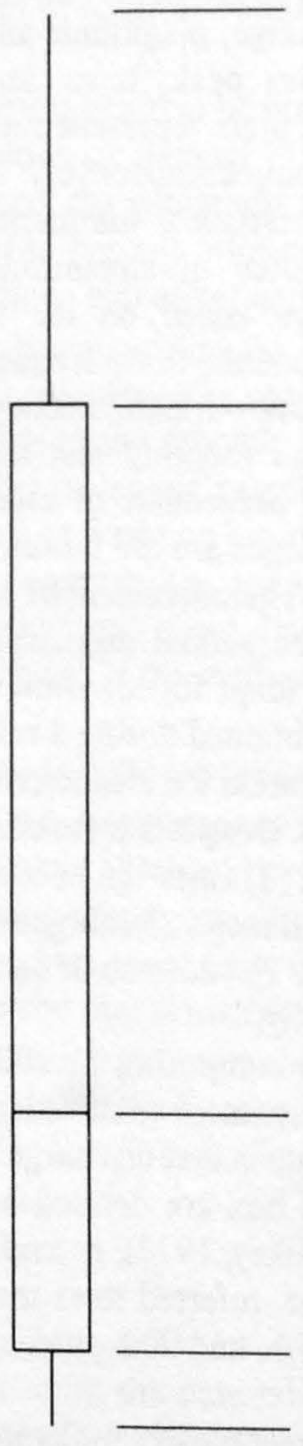

$75^{\text {th }}$ PERCENTILE VALUE

$50^{\text {th }}$ PERCENTILE VALUE

$25^{\text {th }}$ PERCENTILE VALUE

$10^{\text {th }}$ PERCENTILE VALUE

Figure 6. Explanation of boxplots. The values described by the boxplot in this explanation are the March mean discharges for the period of record at streamflow gaging station 09442000, Blue River near Clifton. 
length of time between annual minimum mean flows that are less than the stated magnitude of flow. Nonexceedence probability, expressed as a percentage, is the probability that the annual maximum and minimum mean flow will be less in any given year than the stated magnitude. Annual low-flow probabilities are computed on discharge values obtained during the climatic year.

Magnitude and probability tables for annual high flow list the maximum average discharge for periods of $1,3,7,15,30,60$, and 90 consecutive days that correspond to selective recurrence intervals of $2,5,10$, 25,50 , and 100 years associated with annual exceedance probabilities of 50,20,10,4,2, and 1 percent. Recurrence intervals represent the average length of time between annual maximum mean flows that are equal to or greater than the stated magnitude of flow. Exceedance probability, expressed as a percentage, is the probability that the annual maximum mean flow will be equal or greater in any given year than the stated magnitude. Annual high-flow probabilities are computed on discharge values obtained during the water year.

Flood frequency.-Flood-frequency relations and tabulations provide the necessary data to plot standard flood-frequency curves that are based on log-Pearson Type III frequency distribution, as recommended in the Water Resources Council Guidelines for determining floodflow frequency (Interagency Advisory Committee on Water Data, 1982). These data were calculated for streamflow-gaging stations on unregulated or partly regulated streams. Partly regulated streams are those in which flow is regulated or diverted to an unknown degree.

Flow duration.-Flow duration of daily mean discharge, expressed in percentage of time, are specified daily flows that were equaled or exceeded during the period of record. The tabulations show the discharges that were equaled or exceeded for a given percentage of time in $1,5,10,15,20,30,40,50,60$, $70,80,90,95,98,99,99.5$, and 99.9 percentiles. These data can be used to plot a standard flow-duration curve.

Discharge.-Annual peak discharge, mean monthly discharge and mean annual discharge are shown for all continuous-recording gaging stations. Annual peak discharges are included for peak-flow partial-record gaging stations.

Significant figures and rounding limits.-The number of significant figures used for reporting discharge in this report is based on the magnitude of the value and is not based on the accuracy of the data. Rounding criteria are used for reporting monthly and annual mean discharge, flow-duration, and probability values for individual streamflow-gaging stations (table 2).

Table 2. Significant figures and rounding limits used for reporting monthly and annual mean discharge and probable discharge

$[<$, less than; $\geq$, equal to or greater than $]$

\begin{tabular}{|c|c|c|c|c|c|}
\hline $\begin{array}{l}\text { Range in } \\
\text { discharge, } \\
\text { in cubic feet } \\
\text { per second }\end{array}$ & $\begin{array}{c}\text { Significant } \\
\text { figures }\end{array}$ & Rounding limits & $\begin{array}{c}\text { Range in } \\
\text { discharge, } \\
\text { in cubic feet } \\
\text { per second }\end{array}$ & $\begin{array}{l}\text { Significant } \\
\text { figures }\end{array}$ & Rounding limits \\
\hline \multicolumn{3}{|c|}{ Monthly and annual mean discharge } & \multicolumn{3}{|c|}{ Probable discharge } \\
\hline$<0.010$ & 1 & Thousandths & $<0.10$ & 1 & Hundredths \\
\hline $0.010-0.099$ & 2 & Thousandths & $0.10-0.99$ & 2 & Hundredths \\
\hline $0.10-0.99$ & 2 & Hundredths & $1.0-9.9$ & 2 & Tenths \\
\hline $1.00-9.99$ & 3 & Hundredths & $10-99$ & 2 & Units \\
\hline $10.0-99.9$ & 3 & Tenths & $\geq 100$ & 3 & Variable \\
\hline $100-999$ & 3 & Units & & & \\
\hline$\geq 1,000$ & 4 & Variable & & & \\
\hline
\end{tabular}




\section{DATA USE LIMITATIONS}

Statistical information presented in this report should be used for broad planning purposes and general basin investigations only. With the variability in the lengths of records for the streamflow-gaging stations and isolated factors that may affect streamflow other than regulation by reservoirs, site specific studies require a more detailed analysis of the streamflow data.

\section{REFERENCES CITED}

Cardin, C.W., and others, 1986, Water resources in the 1980's - A summary of activities and programs of the U.S. Geological Survey's Water Resources Division: U.S. Geological Survey Circular 1005, 80 p.

Ely, L.L., and Baker, V.R., 1985, Reconstructing paleoflood hydrology with slackwater deposits: Verde River, Arizona, Physical Geography, v. 6, no. 2, p. 103-126.

Garrett, J.M., and Gellenbeck, D.J., 1991, Basin characteristics and streamflow statistics in Arizona as of 1989: U.S. Geological Survey Water-Resources Investigations Report 91-4041, 612 p.

Interagency Advisory Committee on Water Data, 1982, Guidelines for determining floodflow frequency, Bulletin 17-B of the Hydrology Subcommittee: Reston, Virginia, U.S. Geological Survey, Office of Water Data Coordination, $183 \mathrm{p}$.

Lew, M., 1998, Operation of hydrologic data-collection stations by the United States Geological Survey in 1997: U.S. Geological Survey Open-File Report 97-832, 14 p.

O'Connor, J.E., Fuller, J.E., and Baker, V.R., 1986, Late Holocene flooding within the Salt River basin, central Arizona: Tucson, University of Arizona, Department of Geosciences, 84 p.

Partridge, J.B., and Baker, V.R., 1987, Paleoflood hydrology of the Salt River, Arizona: Earth Surface Processes and Landforms, v. 12, p. 109-125.

Pope, G.L., 1996, Surface-water quality-assurance plan for the Arizona District of the U.S. Geological Survey: U.S. Geological Survey Open-File Report 96-332, 25 p.

Roberts, L.K., 1987, Paleohydrologic reconstruction, hydraulics, and frequency-magnitude relationships of large flood events along Aravaipa Creek, Arizona: Tucson, University of Arizona, master's thesis, 63. p.

Smith, D.F., Duet, N.R., Fisk, G.G., McCormack, H.F., Partin, C.K., Pope, G.L., and Rigas, P.D., 1997, Water Resources Data, Arizona, water year 1996: U.S. Geological Survey Water-Data Report AZ-96-1, 328 p.

Tukey, J.W., 1977, Exploratory data analysis: Reading, Massachusetts, Addison-Wesley, 688 p. 


\section{STATISTICAL SUMMARIES}

NOTE: Disclaimer for text: Over time many of the discharge rating tables for the streamflow-gaging stations will be superceded, and users of these data should contact the local office of the USGS to confirm the validity of the ratings. For more information please contact:

U.S. Geological Survey, WRD

520 North Park Avenue, Suite 221

Tucson, Arizona 85719-5035,

Phone: (520) 670-6671,

FAX number, (520) 670-5592. 

09371100 TEEC NOS POS WASH NEAR TEEC NOS POS, AZ

LOCATION.--Lat $36^{\circ} 55^{\prime} 58^{\prime \prime}$, long $109^{\circ} 06^{\prime} 35$, in NE $\frac{1}{4} 4 \mathrm{sec} .27$, T.41 N., R.30 E., Apache County, Hydrologic Unit 14080201, at U.S. Highway 160 (renumbered), 1.5 mi northwest of Teec Nos Pos Trading Post.

DRAINAGE AREA.--16.0 $\mathrm{mi}^{2}$.

Annual peak discharges

\begin{tabular}{|c|c|c|c|c|c|c|c|}
\hline $\begin{array}{l}\text { Water } \\
\text { year }\end{array}$ & Date & $\begin{array}{c}\text { Annual peak } \\
\text { discharge } \\
\left(\mathrm{ft}^{3} / \mathrm{s}\right)\end{array}$ & $\begin{array}{l}\text { Discharge } \\
\text { codes }\end{array}$ & $\begin{array}{l}\text { Water } \\
\text { year }\end{array}$ & Date & $\begin{array}{l}\text { Annual peak } \\
\text { discharge } \\
\left(\mathrm{ft}^{3} / \mathrm{s}\right)\end{array}$ & $\begin{array}{c}\text { Discharge } \\
\text { codes }\end{array}$ \\
\hline 1967 & $00-00-67$ & 750 & & 1972 & $09-06-72$ & 810 & \\
\hline 1968 & $08-01-68$ & 400 & & 1973 & $10-19-72$ & 770 & \\
\hline 1969 & $07-18-69$ & 580 & & 1974 & $07-22-74$ & 100 & \\
\hline 1970 & $09-12-70$ & 1,350 & & 1975 & $09-00-75$ & 450 & \\
\hline 1971 & $08-00-71$ & 500 & & 1976 & $00-00-76$ & 300 & \\
\hline
\end{tabular}

Magnitude and probability of instantaneous peak flow based on period of record 1967-76

\begin{tabular}{|c|c|c|c|c|c|}
\hline \multicolumn{6}{|c|}{$\begin{array}{l}\text { Discharge, in } \mathrm{ft}^{3} / \mathrm{s} \text {, for indicated recurrence interval } \\
\text { in years, and exceedance probablility, in percent }\end{array}$} \\
\hline $\begin{array}{c}2 \\
50 \%\end{array}$ & $\begin{array}{c}5 \\
20 \%\end{array}$ & $\begin{array}{c}10 \\
10 \%\end{array}$ & $\begin{array}{l}25 \\
4 \%\end{array}$ & $\begin{array}{l}50 \dagger \\
2 \%\end{array}$ & $100 \dagger$ \\
\hline 557 & 841 & 1,050 & 1,320 & 1,530 & 1,760 \\
\hline $\begin{array}{l}\text { Weighted skew } \\
\text { Mean } \\
\text { Standard dev. }\end{array}$ & $\begin{array}{l}(\operatorname{logs})= \\
(\operatorname{logs})= \\
(\operatorname{logs})=\end{array}$ & $\begin{array}{l}0.03 \\
2.75 \\
0.21\end{array}$ & & & \\
\hline
\end{tabular}

f Reliability of values in column is uncertain, and potential errors are large.

\section{Basin characteristics}

Rainfall intensity, 24-hour

\begin{tabular}{cccccccc}
$\begin{array}{c}\text { Main } \\
\text { channel } \\
\text { slope } \\
(\mathrm{ft} / \mathrm{mi})\end{array}$ & $\begin{array}{c}\text { Stream } \\
\text { length } \\
\text { (mi) }\end{array}$ & $\begin{array}{c}\text { Mean } \\
\text { basin } \\
\text { elevation } \\
\text { (ft) }\end{array}$ & $\begin{array}{c}\text { Forested } \\
\text { area } \\
\text { (percent) }\end{array}$ & $\begin{array}{c}\text { Soil } \\
\text { index }\end{array}$ & $\begin{array}{c}\text { Mean } \\
\text { annual } \\
\text { precipitation } \\
\text { (in) }\end{array}$ & $\begin{array}{c}\text { 2-year } \\
\text { (in) }\end{array}$ & $\begin{array}{c}50 \text {-year } \\
\text { (in) }\end{array}$ \\
\hline 411 & 11.2 & 7,600 & 80.0 & 2.0 & 16.5 & 1.4 & 2.9 \\
\hline
\end{tabular}

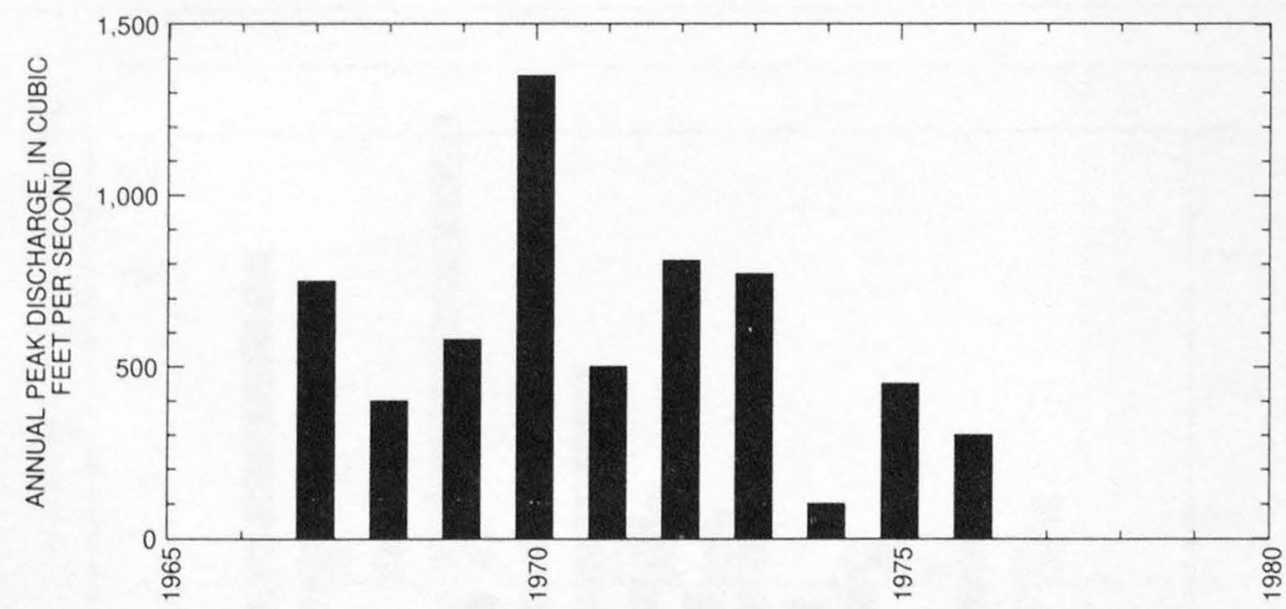


09379030 BLACK MOUNTAIN WASH NEAR CHINLE, AZ

LOCATION.--Lat 36 $20^{\prime} 00^{\prime \prime}$, long $109^{\circ} 37^{\prime} 25^{\prime \prime}$, Apache County, Hydrologic Unit 14080204, at State Highway 63, 1 mi south of Many Farms, and

13 mi north of Chinle.

DRAINAGE AREA.--80.7 $\mathrm{mi}^{2}$.

Annual peak discharges

\begin{tabular}{cccccrr}
\hline $\begin{array}{c}\text { Water } \\
\text { year }\end{array}$ & Date & $\begin{array}{c}\text { Annual peak } \\
\mathbf{d i s c h a r g e} \\
\left(\mathbf{f t}^{\mathbf{3}} / \mathbf{s}\right)\end{array}$ & $\begin{array}{c}\text { Discharge } \\
\text { codes }\end{array}$ & $\begin{array}{c}\text { Water } \\
\text { year }\end{array}$ & $\begin{array}{c}\text { Date } \\
\text { discharge } \\
\left(\mathbf{f t}^{\mathbf{3}} / \mathbf{s}\right)\end{array}$ \\
\hline 1963 & $08-06-63$ & 737 & & 1971 & $08-00-71$ & 2,180 \\
1964 & $08-13-64$ & 1,500 & & 1972 & $08-00-72$ & 900 \\
1965 & $08-02-65$ & 238 & & 1973 & $10-19-72$ & 1,040 \\
1966 & $07-31-66$ & 610 & & 1974 & $07-00-74$ & 140 \\
1967 & $08-00-67$ & 360 & 1975 & $08-20-75$ & 110 \\
1968 & $08-01-68$ & 1,560 & 1976 & $00-00-76$ & 1,900 \\
1969 & $08-03-69$ & 422 & & 1977 & $08-17-77$ & 3,100 \\
1970 & $08-20-70$ & 1,020 & & & & \\
\hline
\end{tabular}

Magnitude and probability of instantaneous peak flow based on period of record 1963-77

\begin{tabular}{cccccc}
\hline \multicolumn{5}{c}{ Discharge, in $\mathrm{ft}^{3}$ /s, for indicated recurrence interval } \\
in years, and exceedance probablility, in percent
\end{tabular}

Basin characteristics

\begin{tabular}{|c|c|c|c|c|c|c|c|}
\hline \multirow[b]{2}{*}{$\begin{array}{c}\text { Main } \\
\text { channel } \\
\text { slope } \\
(\mathrm{ft} / \mathrm{mi})\end{array}$} & \multirow[b]{2}{*}{$\begin{array}{l}\text { Stream } \\
\text { length } \\
\text { (mi) }\end{array}$} & \multirow[b]{2}{*}{$\begin{array}{c}\text { Mean } \\
\text { basin } \\
\text { elevation } \\
(\mathrm{ft})\end{array}$} & \multirow[b]{2}{*}{$\begin{array}{l}\text { Forested } \\
\text { area } \\
\text { (percent) }\end{array}$} & \multirow[b]{2}{*}{$\begin{array}{c}\text { Soil } \\
\text { index }\end{array}$} & \multirow[b]{2}{*}{$\begin{array}{c}\text { Mean } \\
\text { annual } \\
\text { precipitation } \\
\text { (in) }\end{array}$} & \multicolumn{2}{|c|}{ Rainfall intensity, 24-hour } \\
\hline & & & & & & $\begin{array}{l}\text { 2-year } \\
\text { (in) }\end{array}$ & $\begin{array}{l}\text { 50-year } \\
\text { (in) }\end{array}$ \\
\hline 46.2 & 14.4 & 5,920 & 2.1 & 3.0 & 10.9 & 1.3 & 2.7 \\
\hline
\end{tabular}

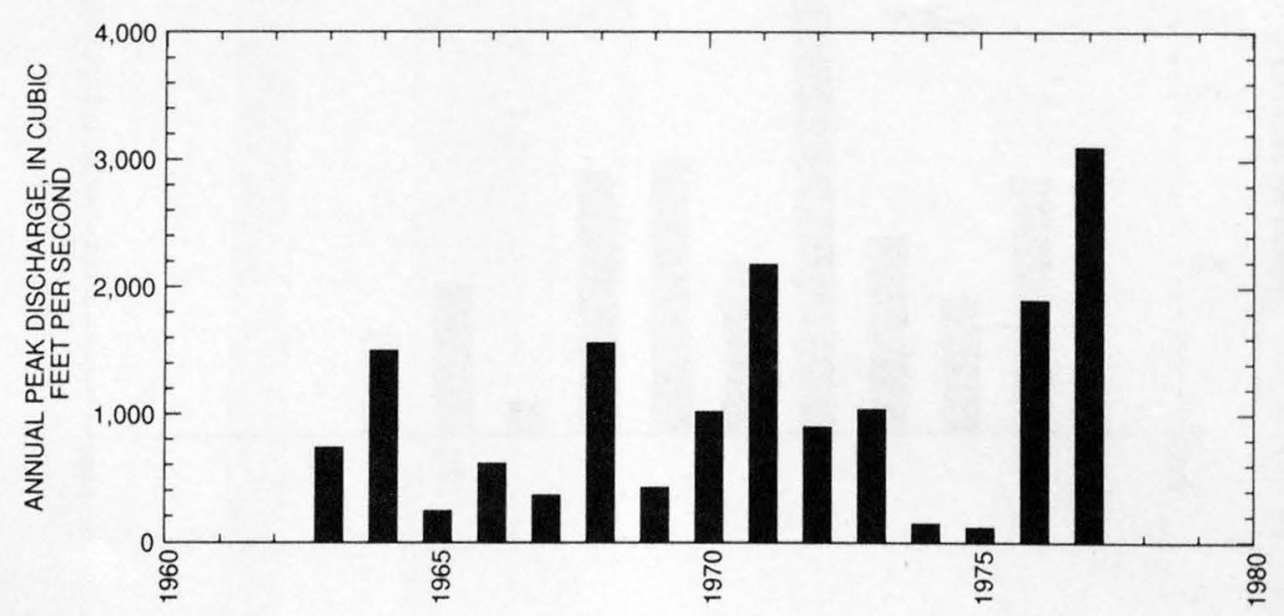


09379060 LUKACHUKAI CREEK TRIBUTARY NEAR LUKACHUKAI, AZ

LOCATION.--Lat $36^{\circ} 28^{\prime} 10^{\prime \prime}$, long $109^{\circ} 24^{\prime} 20^{\prime \prime}$, Apache County, Hydrologic Unit 14080204, at Navajo Highway 12, 6.8 mi southeast of Round Rock, and 10 mi northwest of Lukachukai.

DRAINAGE AREA.--1.37 $\mathrm{mi}^{2}$.

Annual peak discharges

\begin{tabular}{cccccccc}
\hline $\begin{array}{c}\text { Water } \\
\text { year }\end{array}$ & Date & $\begin{array}{c}\text { Annual peak } \\
\text { discharge } \\
\left(\mathrm{ft}^{3} / \mathbf{s}\right)\end{array}$ & $\begin{array}{c}\text { Discharge } \\
\text { codes }\end{array}$ & $\begin{array}{c}\text { Water } \\
\text { year }\end{array}$ & Date & $\begin{array}{c}\text { Annual peak } \\
\text { discharge } \\
\left(\mathrm{ft}^{3} / \mathbf{s}\right)\end{array}$ & $\begin{array}{c}\text { Discharge } \\
\text { codes }\end{array}$ \\
\hline 1963 & $08-31-63$ & 15 & & 1970 & $10-04-69$ & 36 & \\
1964 & $07-31-64$ & 33 & & 1971 & $08-00-71$ & 3.0 & ES \\
1965 & $01-06-65$ & 227 & ES & 1972 & $00-00-72$ & 0 & \\
1966 & $07-31-66$ & 10 & & 1973 & $00-00-73$ & 20 & \\
1967 & $00-00-67$ & 0 & & 1974 & $02-21-74$ & 10 & \\
1968 & $08-00-68$ & 30 & & 1975 & $00-00-75$ & 1.0 & ES \\
1969 & $07-18-69$ & 49 & & 1976 & $00-00-76$ & 43 & \\
\hline
\end{tabular}

Magnitude and probability of instantaneous peak flow based on period of record 1963-76

\begin{tabular}{|c|c|c|c|c|c|}
\hline \multicolumn{6}{|c|}{$\begin{array}{l}\text { Discharge, in } \mathrm{ft}^{3} / \mathrm{s} \text {, for indicated recurrence interval } \\
\text { in years, and exceedance probablility, in percent }\end{array}$} \\
\hline 2 & 5 & 10 & 25 & $50 t$ & $100 t$ \\
\hline $50 \%$ & $20 \%$ & $10 \%$ & $4 \%$ & $2 \%$ & $1 \%$ \\
\hline 17.9 & 46.8 & 79.5 & 143 & 210 & 301 \\
\hline Weighted skew & $(\log s)=$ & 0.26 & & & \\
\hline Mean & $(\log s)=$ & 1.27 & & & \\
\hline Standard dev. & $(\log s)=$ & 0.48 & & & \\
\hline
\end{tabular}

F Reliability of values in column is uncertain, and potential errors are large.

Basin characteristics

\begin{tabular}{cccccccc}
\hline $\begin{array}{c}\text { Main } \\
\text { channel } \\
\text { slope } \\
(\mathrm{ft} / \mathrm{mi})\end{array}$ & $\begin{array}{c}\text { Stream } \\
\text { length } \\
(\mathrm{mi})\end{array}$ & $\begin{array}{c}\text { Mean } \\
\text { basin } \\
\text { elevation } \\
(\mathrm{ft})\end{array}$ & $\begin{array}{c}\text { Forested } \\
\text { area } \\
\text { (percent) }\end{array}$ & $\begin{array}{c}\text { Roil } \\
\text { index }\end{array}$ & $\begin{array}{c}\text { Mean } \\
\text { annual } \\
\text { precipitation } \\
\text { (in) }\end{array}$ & $\begin{array}{c}\text { 2-year } \\
\text { (in) }\end{array}$ & $\begin{array}{c}50 \text {-year } \\
\text { (in) }\end{array}$ \\
\hline 72.2 & 2.4 & 5,820 & 0.0 & 3.0 & 9.8 & 1.2 \\
\hline
\end{tabular}

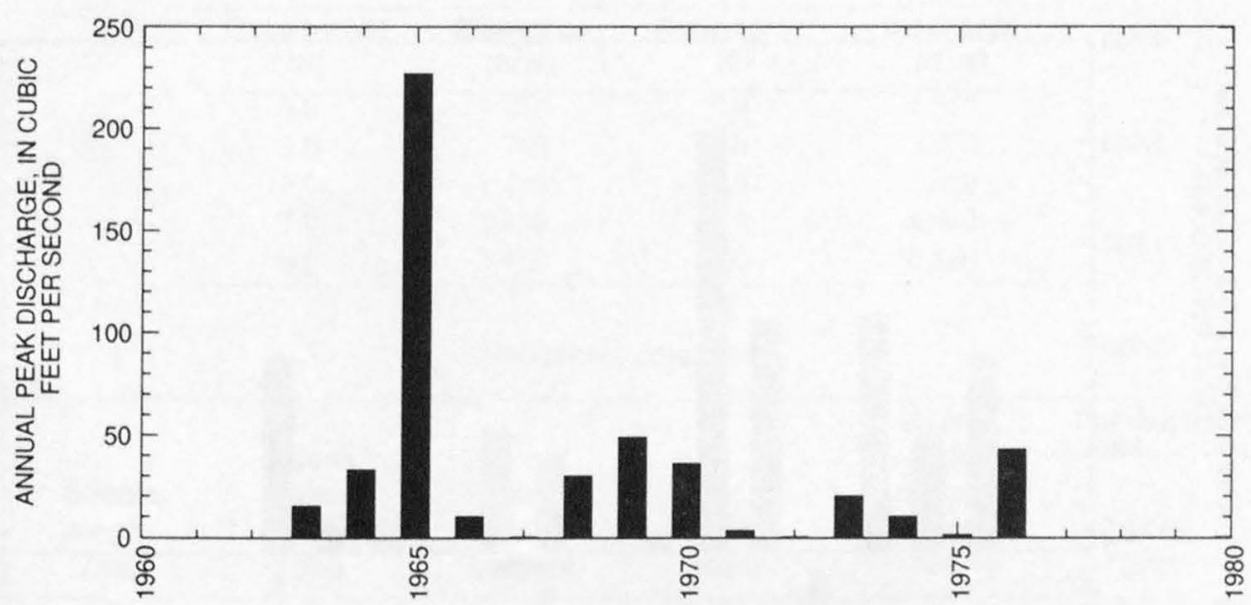




\section{LONG HOUSE WASH NEAR KAYENTA, AZ}

LOCATION.--Lat 36 34'02", long 110'29'17", Navajo County, Hydrologic Unit 15020018, at U.S. Highway 160,17 mi southwest of Kayenta. DRAINAGE AREA.--1.38 $\mathrm{mi}^{2}$.

Annual peak discharges

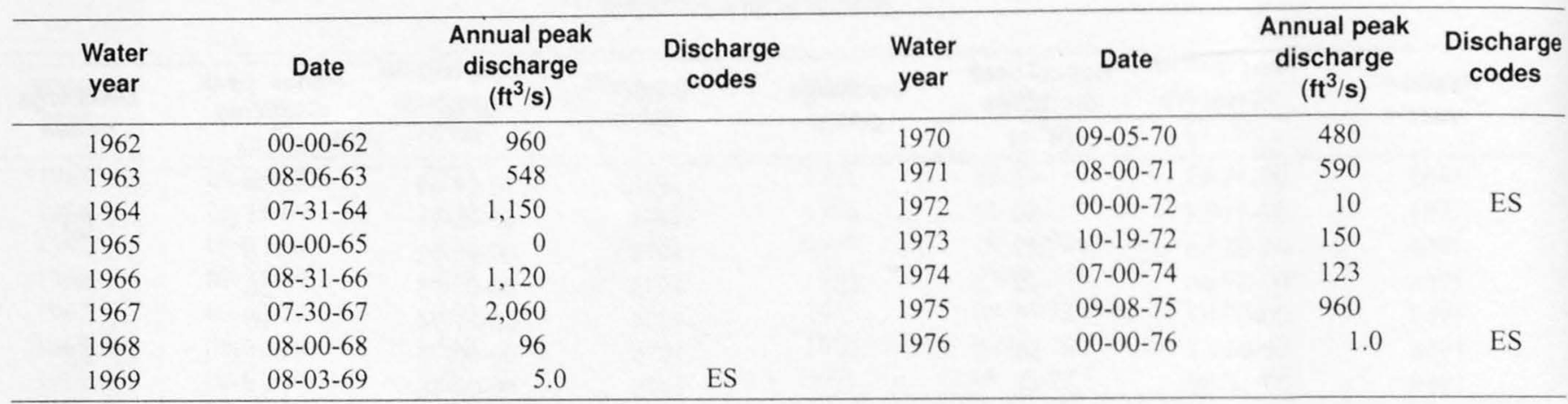

Magnitude and probability of instantaneous peak flow based on period of record 1962-76

\begin{tabular}{|c|c|c|c|c|c|}
\hline \multicolumn{6}{|c|}{$\begin{array}{l}\text { Discharge, in } \mathrm{ft}^{3} / \mathrm{s} \text {, for indicated recurrence interval } \\
\text { in years, and exceedance probablility, in percent }\end{array}$} \\
\hline $2 \dagger$ & $5 t$ & $10 \dagger$ & $25 \dagger$ & $50 \dagger$ & $100 t$ \\
\hline $50 \%$ & $20 \%$ & $10 \%$ & $4 \%$ & $2 \%$ & $1 \%$ \\
\hline 304 & 1,000 & 1,760 & 3,100 & 4,380 & 5,880 \\
\hline Weighted skew & $(\log s)=$ & -0.40 & & & \\
\hline Mean & $(\log s)=$ & 2.44 & & & \\
\hline Standard dev. & $(\log s)=$ & 0.66 & & & \\
\hline
\end{tabular}

f Reliability of values in column is uncertain, and potential errors are large.

Basin characteristics

\begin{tabular}{|c|c|c|c|c|c|c|c|}
\hline \multirow[b]{2}{*}{$\begin{array}{l}\text { Main } \\
\text { channel } \\
\text { slope } \\
(\mathrm{ft} / \mathrm{mi})\end{array}$} & \multirow[b]{2}{*}{$\begin{array}{l}\text { Stream } \\
\text { length } \\
\text { (mi) }\end{array}$} & \multirow[b]{2}{*}{$\begin{array}{c}\text { Mean } \\
\text { basin } \\
\text { elevation } \\
\text { (ft) }\end{array}$} & \multirow[b]{2}{*}{$\begin{array}{l}\text { Forested } \\
\text { area } \\
\text { (percent) }\end{array}$} & \multirow[b]{2}{*}{$\begin{array}{c}\text { Soil } \\
\text { index }\end{array}$} & \multirow[b]{2}{*}{$\begin{array}{c}\text { Mean } \\
\text { annual } \\
\text { precipitation } \\
\text { (in) }\end{array}$} & \multicolumn{2}{|c|}{ Rainfall intensity, 24-hour } \\
\hline & & & & & & $\begin{array}{l}\text { 2-year } \\
\text { (in) }\end{array}$ & $\begin{array}{c}\text { 50-year } \\
\text { (in) }\end{array}$ \\
\hline 236 & 1.1 & 6,920 & 57.0 & 3.0 & 12.0 & 1.4 & 2.9 \\
\hline
\end{tabular}

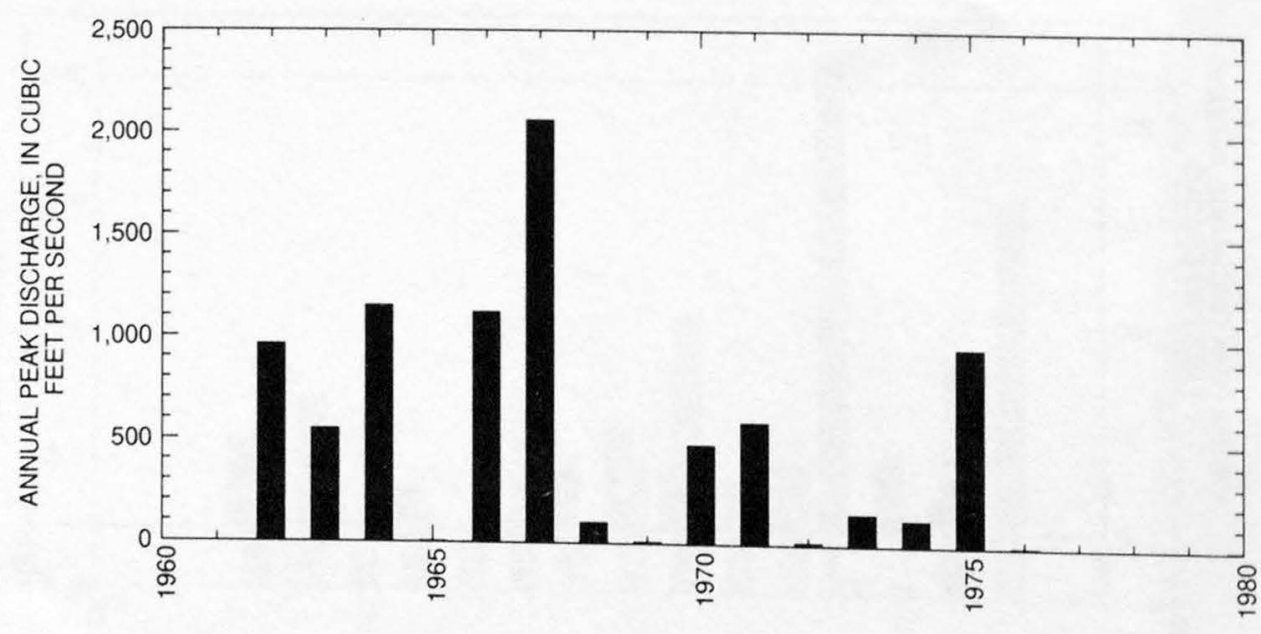


09379200 CHINLE CREEK NEAR MEXICAN WATER, AZ

LOCATION.--Lat 36 56'38", long 109'42'36", in sec.19, T.41 N., R.25 E. (unsurveyed), Apache County, Hydrologic Unit 14080204, in Navajo Indian Reservation, on right bank $150 \mathrm{ft}$ upstream from bridge on U.S. Highway 160, 3 mi upstream from Walker Creek, 4 mi southwest of Mexican Water, 5 mi downstream from confluence of Chinle Creek and Laguna Creek, and 6 mi upstream from Arizona-Utah State line.

DRAINAGE AREA.--3,650 $\mathrm{mi}^{2}$.

PERIOD OF RECORD.--October 1964 to current year (monthly discharge only for 1979). Prior to October 1970 published as Chinle Wash near Mexican Water.

REVISED RECORDS.--WDR AZ-88-1: Drainage area.

GAGE.--Water-stage recorder, crest-stage gage, and concrete control. Datum of gage is 4,720 ft above sea level.

REMARKS.--No estimated daily discharges. Records fair. Some diversions upstream for irrigation, livestock tanks, and domestic use. Many Farms Reservoir, about $25 \mathrm{mi}$ upstream, was built in 1939 with an original capacity of 25,000 acre-ft. The reservoir provides off-channel storage for irrigation of about 1,600 acres.

EXTREMES FOR PERIOD OF RECORD.--Maximum discharge, 12,000 ft $3 / \mathrm{s}$ Aug. 24, 1982, gage height, $13.87 \mathrm{ft}$, from rating curve extended above $3,100 \mathrm{ft}^{3} / \mathrm{s}$ on basis of slope-area measurement at gage height $12.50 \mathrm{ft}$; no flow at times in each year.

Annual peak discharges

\begin{tabular}{|c|c|c|c|c|c|c|c|}
\hline Water year & Date & $\begin{array}{c}\text { Annual peak } \\
\text { discharge } \\
\left(\mathrm{ft}^{3} / \mathrm{s}\right)\end{array}$ & $\begin{array}{c}\text { Discharge } \\
\text { codes }\end{array}$ & Water year & Date & $\begin{array}{c}\text { Annual peak } \\
\text { discharge } \\
\left(\mathrm{ft}^{3} / \mathrm{s}\right)\end{array}$ & $\begin{array}{l}\text { Discharge } \\
\text { codes }\end{array}$ \\
\hline 1963 & $00-00-63$ & 2,070 & ES & 1980 & $09-10-80$ & 1,630 & \\
\hline 1964 & $08-01-64$ & 3,280 & ES & 1981 & $07-14-81$ & 3,270 & \\
\hline 1965 & $05-15-65$ & 732 & & 1982 & $08-24-82$ & 12,000 & \\
\hline 1966 & $12-24-65$ & 650 & & 1983 & $07-28-83$ & 3,650 & \\
\hline 1967 & $08-10-67$ & 1,230 & & 1984 & $07-24-84$ & 7,500 & \\
\hline 1968 & $08-08-68$ & 1,040 & & 1985 & $04-30-85$ & 4,914 & \\
\hline 1969 & $01-15-69$ & 590 & & 1986 & $09-09-86$ & 1,720 & \\
\hline 1970 & $09-07-70$ & ${ }^{1} 9,880$ & & 1987 & $11-19-86$ & 5,800 & \\
\hline 1971 & $08-23-71$ & 1,050 & & 1988 & $11-06-87$ & 2,900 & \\
\hline 1972 & $08-28-72$ & 850 & & 1989 & $08-19-89$ & 2,940 & \\
\hline 1973 & $10-20-72$ & 984 & & 1990 & $07-18-90$ & 2,530 & \\
\hline 1974 & $03-02-74$ & 646 & & 1991 & $12-28-90$ & 3,590 & \\
\hline 1975 & $07-13-75$ & 3,680 & & 1992 & $08-0-792$ & 4,090 & \\
\hline 1976 & $09-25-76$ & 1,620 & & 1993 & $02-20-93$ & 6,870 & \\
\hline 1977 & $08-19-77$ & 7,120 & & 1994 & $10-06-93$ & 2,640 & \\
\hline 1978 & $07-18-78$ & 751 & & 1995 & $09-07-95$ & 2,720 & \\
\hline 1979 & $11-12-78$ & 1,390 & & 1996 & $08-26-96$ & 3,440 & \\
\hline
\end{tabular}

${ }^{1}$ Highest since 1950.

Discharge rating table developed January 1993

\begin{tabular}{cccc}
\hline $\begin{array}{c}\text { Gage height } \\
(\mathbf{f t})\end{array}$ & $\begin{array}{c}\text { Discharge } \\
\left(\mathbf{f t}^{\mathbf{3}} \mathbf{s}\right)\end{array}$ & $\begin{array}{c}\text { Gage height } \\
(\mathbf{f t})\end{array}$ & $\begin{array}{c}\text { Discharge } \\
\left(\mathrm{ft}^{\mathbf{3}} \mathbf{s}\right)\end{array}$ \\
\hline 4.0 & 330 & 9.0 & 4,550 \\
5.0 & 780 & 10.0 & 5,850 \\
6.0 & 1,440 & 11.0 & 7,310 \\
7.0 & 2,330 & 12.0 & 8,920 \\
8.0 & 3,410 & 13.0 & 10,860 \\
\hline
\end{tabular}

Basin characteristics

\begin{tabular}{cccccccc}
\hline $\begin{array}{c}\text { Main- } \\
\text { channel } \\
\text { slope } \\
(\mathrm{ft} / \mathrm{mi})\end{array}$ & $\begin{array}{c}\text { Stream } \\
\text { length } \\
(\mathrm{mi})\end{array}$ & $\begin{array}{c}\text { Mean } \\
\text { basin } \\
\text { elevation } \\
(\mathrm{mi})\end{array}$ & $\begin{array}{c}\text { Forested } \\
\text { area } \\
\text { (percent) }\end{array}$ & $\begin{array}{c}\text { Soil } \\
\text { index }\end{array}$ & $\begin{array}{c}\text { Mean } \\
\text { annual } \\
\text { precipit- } \\
\text { ation } \\
\text { (in) }\end{array}$ & $\begin{array}{c}\text { Rainfall intensity, 24-hour } \\
\text { (in) }\end{array}$ & $\begin{array}{c}50-\text { year } \\
\text { (in) }\end{array}$ \\
\hline 12.3 & 86.8 & 6,260 & 24.0 & 3.0 & 10.9 & 1.3 & 2.7 \\
\hline
\end{tabular}


09379200 CHINLE CREEK NEAR MEXICAN WATER, AZ - Continued

MEAN MONTHLY AND ANNUAL DISCHARGES $1965 \cdot 78,1980 \cdot 96$

\begin{tabular}{|c|c|c|c|c|c|c|}
\hline MONTH & $\begin{array}{l}\text { MAXIMUM } \\
\text { (FT3/S) }\end{array}$ & $\begin{array}{l}\text { MINIMUM } \\
\text { (FT3/S) }\end{array}$ & $\begin{array}{c}\text { MEAN } \\
(\mathrm{FT} 3 / \mathrm{S})\end{array}$ & $\begin{array}{l}\text { STAN - } \\
\text { DARD } \\
\text { DEVIA- } \\
\text { TION } \\
\text { (FT3/S) }\end{array}$ & $\begin{array}{l}\text { COEFFI- } \\
\text { CIENT OF } \\
\text { VARI- } \\
\text { ATION }\end{array}$ & $\begin{array}{c}\text { PERCENT } \\
\text { OF } \\
\text { ANNUAL } \\
\text { RUNOFF }\end{array}$ \\
\hline OCTOBER & 142 & 0.90 & 22 & 30 & 1.3 & 6.2 \\
\hline NOVEMBER & 144 & 0.41 & 16 & 28 & 1.8 & 4.3 \\
\hline DECEMBER & 41 & 1.1 & 9.4 & 9.9 & 1.1 & 2.6 \\
\hline JANUARY & 151 & 1.6 & 20 & 32 & 1.6 & 5.4 \\
\hline FEBRUARY & 169 & 2.3 & 31 & 47 & 1.5 & 8.4 \\
\hline MARCH & 215 & 0.67 & 27 & 45 & 1.7 & 7.4 \\
\hline APRIL & 402 & 0.53 & 60 & 95 & 1.6 & 16.5 \\
\hline MAY & 294 & 0.26 & 51 & 81 & 1.6 & 14.1 \\
\hline JUNE & 73 & 0.00 & 5.9 & 17 & 2.8 & 1.6 \\
\hline JULY & 129 & 0.00 & 26 & 32 & 1.3 & 7.1 \\
\hline AUGUST & 501 & 0.00 & 56 & 98 & 1.8 & 15.3 \\
\hline SEPTEMBER & 342 & 0.00 & 41 & 70 & 1.7 & 11.2 \\
\hline ANNUAL & 94 & 4.5 & 30 & 26 & 0.85 & 100 \\
\hline
\end{tabular}

MAGNITUDE AND PROBABILITY OF INSTANTANEOUS PEAK FLOW BASED ON PERIOD OF RECORD 1963.96

DISCHARGE, IN FT3/S, FOR INDICATED RECURRENCE INTERVAL IN YEARS, AND EXCEEDANCE PROBABILITY, IN PERCENT

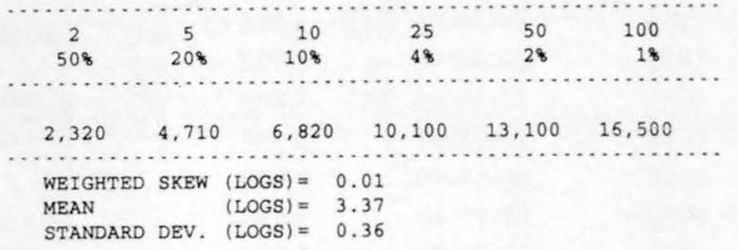

MAGNITUDE AND PROBABILITY OF ANNUAL LOW FLOW BASED ON PERIOD OF RECORD $1966 \cdot 78,1981.96$

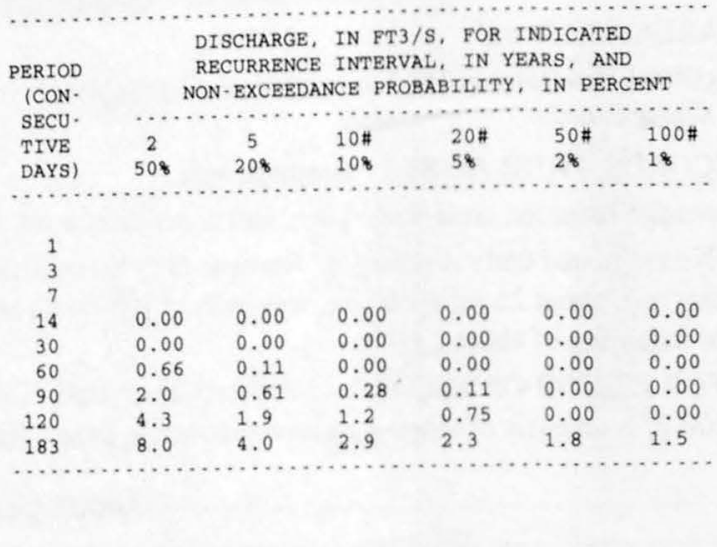

MAGNITUDE AND PROBABILITY OF ANNUAL HIGH FLOW BASED ON PERIOD OF RECORD $1965-78,1980.96$

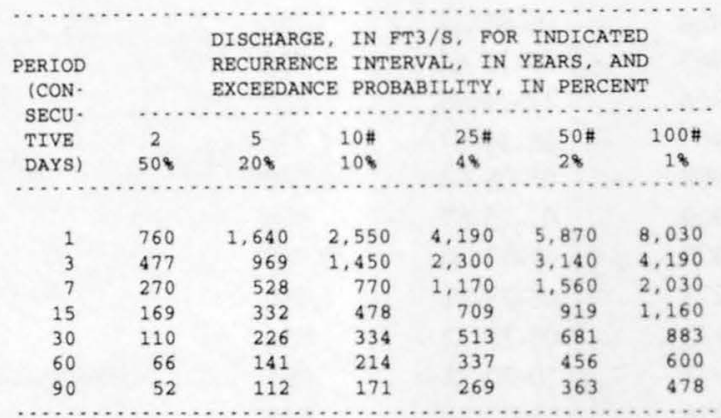

DURATION TABLE OF DAILY MEAN FLOW FOR PERIOD OF RECORD 1965-78, 1980-96

DISCHARGE, IN FT3/S, WHICH WAS EQUALED OR EXCEEDED FOR INDICATED PERCENT OF TTME

\begin{tabular}{|c|c|c|c|c|c|c|c|c|c|c|c|c|c|c|c|c|}
\hline 18 & 58 & 108 & 158 & 208 & 308 & 408 & 508 & 608 & 708 & 808 & 908 & 958 & 988 & 998 & 99.58 & 99.98 \\
\hline 00 & 158 & 63 & 29 & 17 & 7.9 & 4.8 & 3.3 & 2.1 & 1.1 & 0.46 & 0.00 & 0.00 & 0.00 & 0.00 & 0.00 & 0.00 \\
\hline
\end{tabular}

\# Reliability of values in column is uncertain, and potential errors are large. 
ANNUAL MEAN DISCHARGE, IN CUBIC FEET PER SECOND

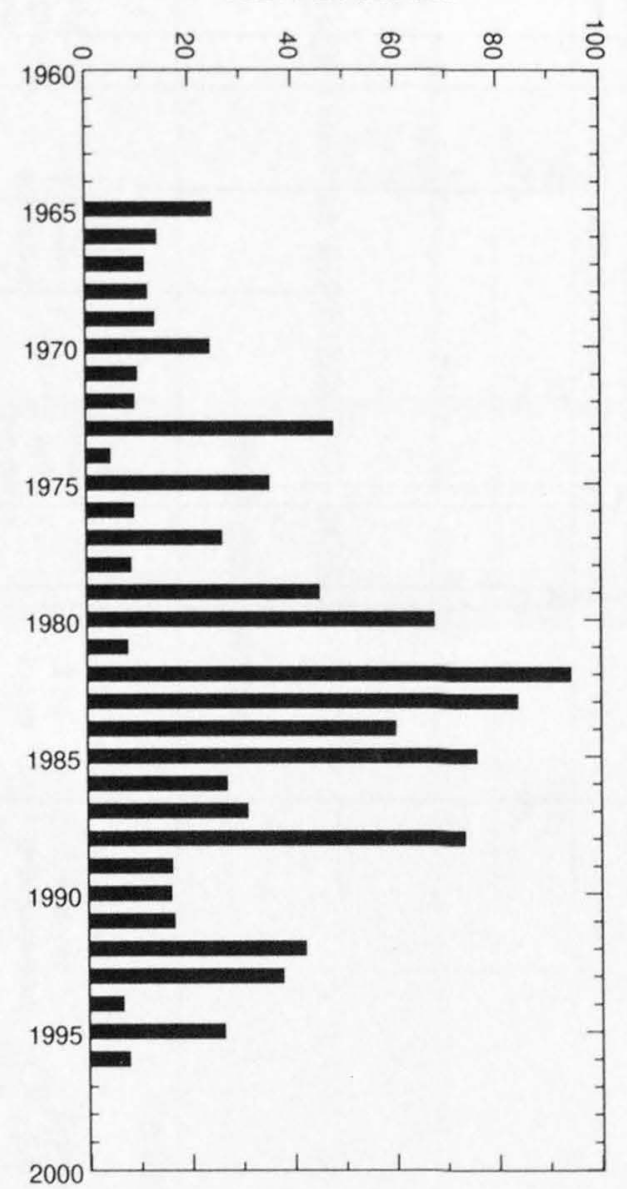

ANNUAL PEAK DISCHARGE, IN CUBIC

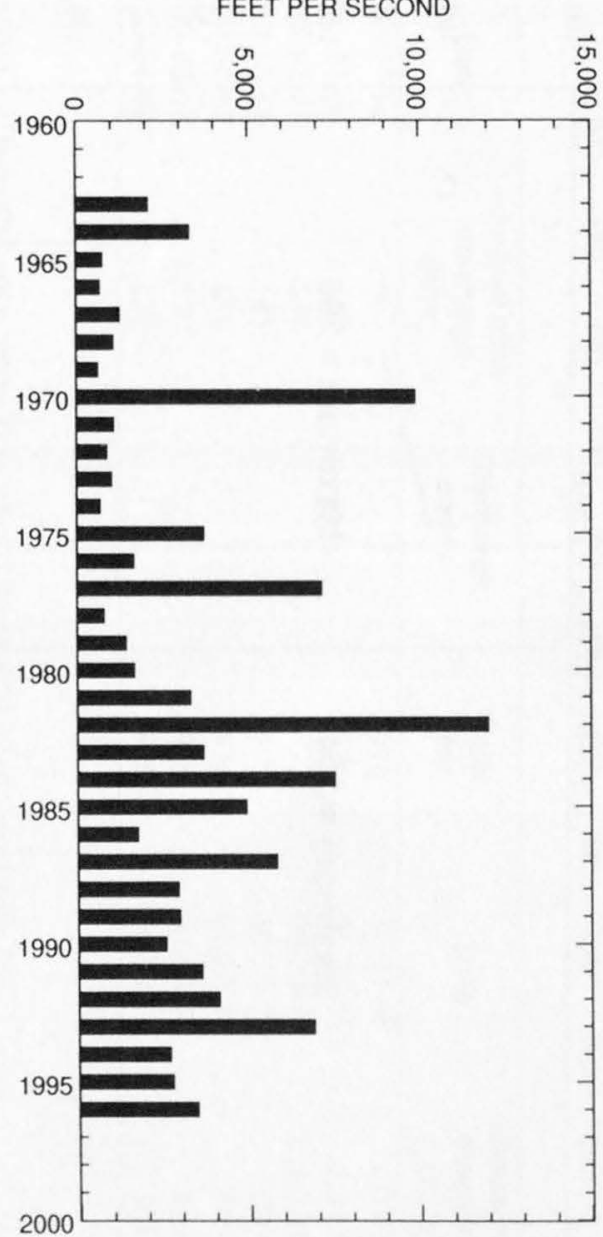


26

SAN JUAN RIVER BASIN

09379200 CHINLE CREEK NEAR MEXICAN WATER, AZ - Continued
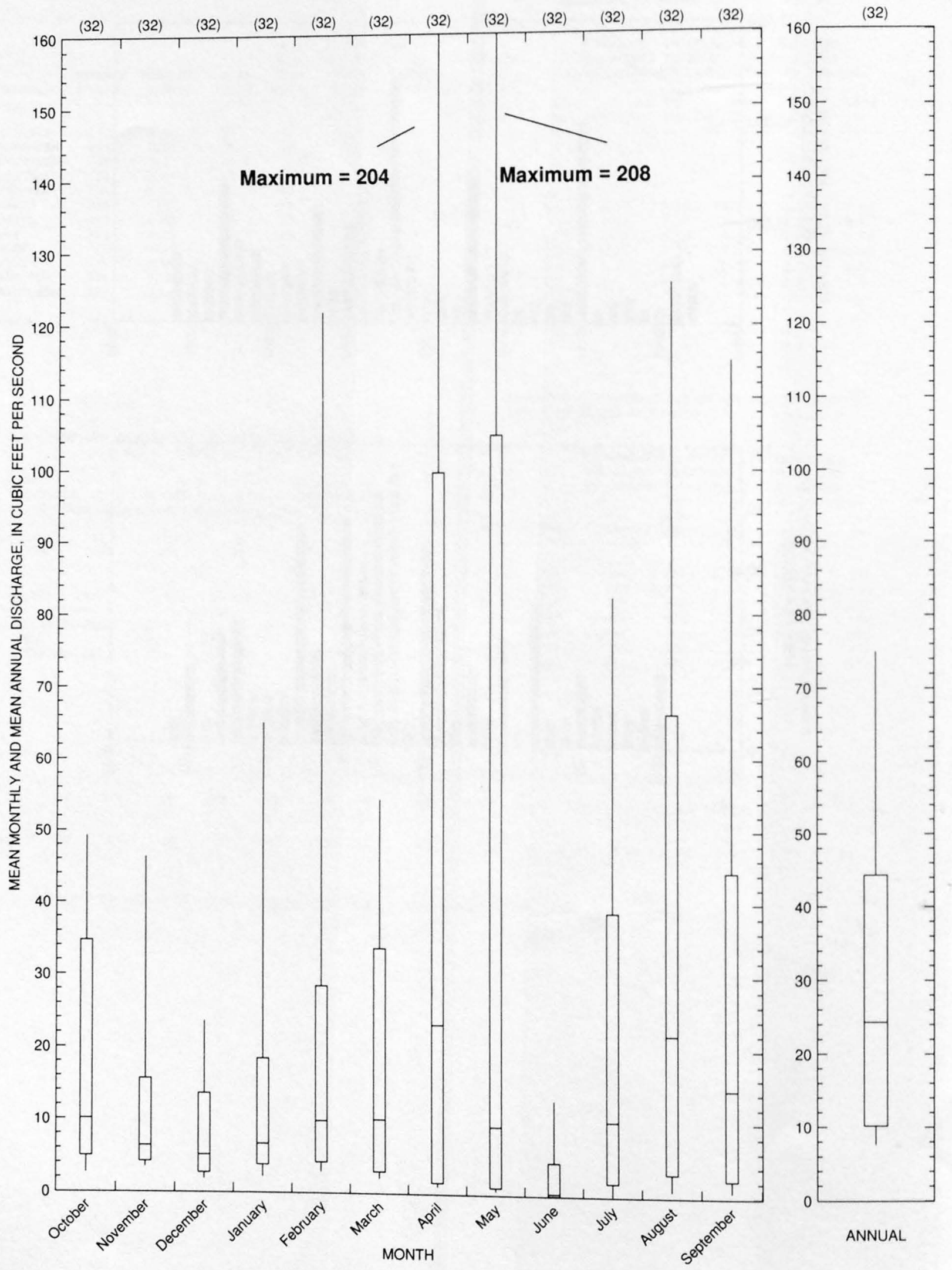
09379560 EL CAPITAN WASH NEAR KAYENTA, AZ

LOCATION.--Lat 36 51'32", long 110 15'55", Navajo County, Hydrologic Unit 14080205, at U.S. Highway 163, 12 mi north of Kayenta. DRAINAGE AREA.--5.88 $\mathrm{mi}^{2}$.

Annual peak discharges

\begin{tabular}{|c|c|c|c|c|c|c|c|}
\hline $\begin{array}{l}\text { Water } \\
\text { year }\end{array}$ & Date & $\begin{array}{c}\text { Annual peak } \\
\text { discharge } \\
\left(\mathrm{ft}^{3} / \mathrm{s}\right)\end{array}$ & $\begin{array}{l}\text { Discharge } \\
\text { codes }\end{array}$ & $\begin{array}{l}\text { Water } \\
\text { year }\end{array}$ & Date & $\begin{array}{c}\text { Annual peak } \\
\text { discharge } \\
\left(\mathrm{ft}^{3} / \mathrm{s}\right)\end{array}$ & $\begin{array}{c}\text { Discharge } \\
\text { codes }\end{array}$ \\
\hline 1963 & $08-30-63$ & 663 & & 1970 & $10-11-69$ & 390 & \\
\hline 1964 & $07-31-64$ & 181 & & 1971 & $08-26-71$ & 2,340 & \\
\hline 1965 & $08-02-65$ & 278 & & 1972 & $00-00-72$ & 1,320 & \\
\hline 1966 & $10-16-65$ & 185 & & 1973 & $10-19-72$ & 750 & \\
\hline 1967 & $09-00-67$ & 638 & & 1974 & $07-20-74$ & 145 & \\
\hline 1968 & $00-00-68$ & 1,220 & & 1975 & $07-07-75$ & 530 & \\
\hline 1969 & $10-04-68$ & 335 & & 1976 & $00-00-76$ & 301 & \\
\hline
\end{tabular}

Magnitude and probability of instantaneous peak flow based on period of record 1963-76

\begin{tabular}{|c|c|c|c|c|c|}
\hline \multicolumn{6}{|c|}{$\begin{array}{l}\text { Discharge, in } \mathrm{ft}^{3} / \mathbf{s} \text {, for indicated recurrence interval } \\
\text { in years, and exceedance probablility, in percent }\end{array}$} \\
\hline 2 & $\begin{array}{c}5 \\
20 \%\end{array}$ & $\begin{array}{c}10 \\
10 \%\end{array}$ & $\begin{array}{l}25 \\
4 \%\end{array}$ & $\begin{array}{l}50 \dagger \\
2 \%\end{array}$ & $\begin{array}{c}100 \dagger \\
1 \%\end{array}$ \\
\hline $00 \%$ & $20 \%$ & $10 \%$ & $4 \%$ & $2 \%$ & \\
\hline 470 & 952 & 1,390 & 2,100 & 2,760 & 3,530 \\
\hline Weighted skew & $(\log s)=$ & 0.14 & & & \\
\hline Mean & $(\log s)=$ & 2.68 & & & \\
\hline Standard dev. & $(\log s)=$ & 0.36 & & & \\
\hline
\end{tabular}

f Reliability of values in column is uncertain, and potential errors are large.

Basin characteristics

Rainfall intensity, 24-hour

\begin{tabular}{cccccccc}
$\begin{array}{c}\text { Main } \\
\text { channel } \\
\text { slope } \\
(\mathrm{ft} / \mathrm{mi})\end{array}$ & $\begin{array}{c}\text { Stream } \\
\text { length } \\
\text { (mi) }\end{array}$ & $\begin{array}{c}\text { Mean } \\
\text { basin } \\
\text { elevation } \\
\text { (ft) }\end{array}$ & $\begin{array}{c}\text { Forested } \\
\text { area } \\
\text { (percent) }\end{array}$ & $\begin{array}{c}\text { Soil } \\
\text { index }\end{array}$ & $\begin{array}{c}\text { Mean } \\
\text { annual } \\
\text { precipitation } \\
\text { (in) }\end{array}$ & $\begin{array}{c}\text { 2-year } \\
\text { (in) }\end{array}$ & $\begin{array}{c}\text { 50-year } \\
\text { (in) }\end{array}$ \\
\hline 78.4 & 3.4 & 5,690 & 8.0 & 3.0 & 9.0 & 1.2 & 2.4 \\
\hline
\end{tabular}

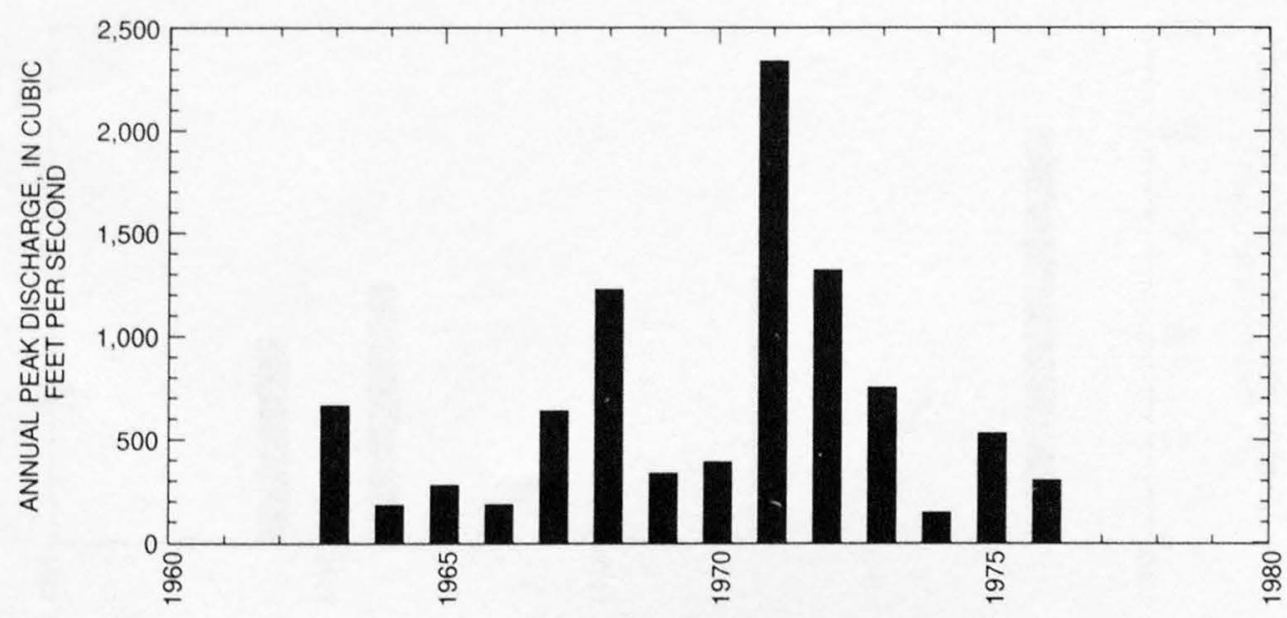


09379980 JACK BENCH WASH TRIBUTARY NEAR PAGE, AZ

LOCATION.--Lat 36 42'50", long 111 35'30", Coconino County, Hydrologic Unit 14070006, at U.S. Highway 89, 17 mi south of Page. DRAINAGE AREA.--0.98 $\mathrm{mi}^{2}$.

Annual peak discharges

\begin{tabular}{|c|c|c|c|c|c|c|c|}
\hline $\begin{array}{l}\text { Water } \\
\text { year }\end{array}$ & Date & $\begin{array}{c}\text { Annual peak } \\
\text { discharge } \\
\left(\mathrm{ft}^{3} / \mathrm{s}\right)\end{array}$ & $\begin{array}{l}\text { Discharge } \\
\text { codes }\end{array}$ & $\begin{array}{c}\text { Water } \\
\text { year }\end{array}$ & Date & $\begin{array}{c}\text { Annual peak } \\
\text { discharge } \\
\left(\mathrm{ft}^{3} / \mathrm{s}\right)\end{array}$ & $\begin{array}{c}\text { Discharge } \\
\text { codes }\end{array}$ \\
\hline 1962 & $00-00-62$ & 200 & & 1970 & $00-00-70$ & 0 & \\
\hline 1963 & $00-00-63$ & 2.0 & LT & 1971 & $00-00-71$ & 2.0 & LT \\
\hline 1964 & $08-29-64$ & 1.0 & ES & 1972 & $06-22-72$ & 10 & LT \\
\hline 1965 & $00-00-65$ & 0 & & 1973 & $00-00-73$ & .10 & LT \\
\hline 1966 & $00-00-66$ & 0 & & 1974 & $11-00-73$ & 125 & \\
\hline 1967 & $07-16-67$ & 128 & & 1975 & $00-00-75$ & 1.0 & LT \\
\hline 1968 & $00-00-68$ & 0 & & 1976 & $00-00-76$ & 99 & \\
\hline 1969 & $00-00-69$ & 0 & & & & & \\
\hline
\end{tabular}

Magnitude and probability of instantaneous peak flow based on period of record 1962-76

\begin{tabular}{|c|c|c|c|c|c|}
\hline \multicolumn{6}{|c|}{$\begin{array}{l}\text { Discharge, in } \mathrm{ft}^{3} / \mathrm{s} \text {, for indicated recurrence interval } \\
\text { in years, and exceedance probablility, in percent }\end{array}$} \\
\hline 2 & 5 & 10 & 25 & $50 t$ & $100 t$ \\
\hline $50 \%$ & $20 \%$ & $10 \%$ & $4 \%$ & $2 \%$ & $1 \%$ \\
\hline 2.6 & 37.1 & 141 & 560 & 1,330 & 2,850 \\
\hline Weighted skew & $(\log s)=$ & -0.21 & & & \\
\hline Mean & $(\log s)=$ & 0.36 & & & \\
\hline Standard dev. & $(\log s)=$ & 1.42 & & & \\
\hline
\end{tabular}

f Reliability of values in column is uncertain, and potential errors are large.

Basin characteristics

\begin{tabular}{cccccccc}
\hline $\begin{array}{c}\text { Main } \\
\text { channel } \\
\text { slope } \\
(\mathrm{ft} / \mathrm{mi})\end{array}$ & $\begin{array}{c}\text { Stream } \\
\text { length } \\
(\mathrm{mi})\end{array}$ & $\begin{array}{c}\text { Mean } \\
\text { basin } \\
\text { elevation } \\
(\mathrm{ft})\end{array}$ & $\begin{array}{c}\text { Forested } \\
\text { area } \\
\text { (percent) }\end{array}$ & $\begin{array}{c}\text { Soil } \\
\text { index }\end{array}$ & $\begin{array}{c}\text { Mean } \\
\text { annual } \\
\text { precipitation } \\
\text { (in) }\end{array}$ & $\begin{array}{c}\text { Rainfall intensity, 24-hour } \\
\text { (in) }\end{array}$ & $\begin{array}{c}50 \text {-year } \\
\text { (in) }\end{array}$ \\
\hline 133 & 2.6 & 6,180 & 22.0 & 3.0 & 8.8 & 1.3 \\
\hline
\end{tabular}

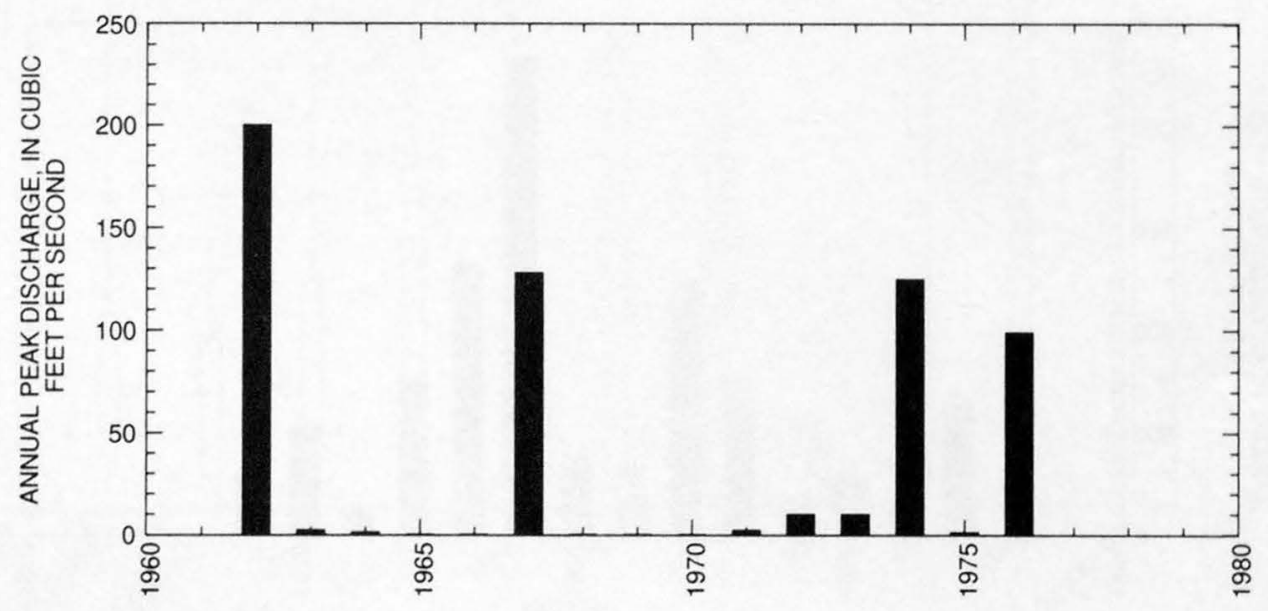




\section{COLORADO RIVER AT LEES FERRY, AZ}

LOCATION.--Lat 36.51'53", long $111^{\circ} 5^{\prime} 15^{\prime \prime}$, in NE $\frac{1}{4} \mathrm{SE}^{1} / 4 \mathrm{sec} .13$, T.40 N., R.7 E., Coconino County, Hydrologic Unit 14070006, in Navajo Indian Reservation, on left bank at head of Marble Gorge at Lees Ferry, just upstream from Paria River, 16 mi downstream from Glen Canyon Dam, $28 \mathrm{mi}$ downstream from Utah-Arizona State line, and $61.5 \mathrm{mi}$ upstream from Little Colorado River.

DRAINAGE AREA.--111,800 $\mathrm{mi}^{2}$, approximately, including 3,959 $\mathrm{mi}^{2}$ in Great Divide basin in southern Wyoming which is noncontributing. PERIOD OF RECORD.--January 1895 to current year. Estimates of monthly and annual discharge only for some periods, published in WSP 1313. REVISED RECORDS.--WSP 859: 1921-23. WSP 1313: 1914-21.

GAGE.--Water-stage recorder. Datum of gage is 3,106.16 ft above sea level. Prior to Jan. 19, 1923, nonrecording gages or reference points within $400 \mathrm{ft}$ of present gage, at different datums.

REMARKS.--No estimated daily discharge. Records good. Flow regulated since Mar. 13, 1963, by Lake Powell, 16 mi upstream. Many diversions above Lake Powell for irrigation, municipal, and industrial use. No diversions or inflow between Lake Powell and the gage.

AVERAGE DISCHARGE.--51 years (water years 1912-62), 17,850 ft3/s, 12,930,000 acre-ft/yr.

EXTREMES FOR PERIOD OF RECORD.--1895-1962: Maximum discharge, 220,000 ft3/s June 18, 1921, gage height, 26.5 ft, from floodmarks, from rating curve extended above $120,000 \mathrm{ft} 3 / \mathrm{s}$ on basis of discharge computed for station near Grand Canyon; minimum, $750 \mathrm{ft} 3 / \mathrm{s}$ Dec. $27,1924$.

1963-96: Maximum discharge, $97,300 \mathrm{ft}^{3} / \mathrm{s}$ June 29, 1983, gage height, $18.14 \mathrm{ft}$; minimum daily, $700 \mathrm{ft}^{3} / \mathrm{s} \mathrm{Jan} .23,24,1963$, result of closing coffer dam at Glen Canyon Dam.

EXTREMES OUTSIDE PERIOD OF RECORD.--Maximum discharge since at least 1868, about $300,000 \mathrm{ft}^{3} / \mathrm{s} \mathrm{July} 7,1884$, gage height, $31.5 \mathrm{ft}$, present site and datum, from floodmark at mouth of Paria River, from rating curve extended above $120,000 \mathrm{ft}^{3} / \mathrm{s}$ on basis of discharge computed for flood of June 18, 1921, for station near Grand Canyon. 
PARIA RIVER BASIN

09380000 COLORADO RIVER AT LEES FERRY, AZ--Continued

Annual peak discharges

\begin{tabular}{|c|c|c|c|c|c|c|c|}
\hline $\begin{array}{l}\text { Water } \\
\text { year }\end{array}$ & Date & $\begin{array}{l}\text { Annual peak } \\
\text { discharge } \\
\left(\mathrm{ft}^{3} / \mathrm{s}\right)\end{array}$ & $\begin{array}{l}\text { Discharge } \\
\text { codes }\end{array}$ & $\begin{array}{l}\text { Water } \\
\text { year }\end{array}$ & Date & $\begin{array}{c}\text { Annual peak } \\
\text { discharge } \\
\left(\mathrm{ft}^{3} / \mathrm{s}\right)\end{array}$ & $\begin{array}{l}\text { Discharge } \\
\text { codes }\end{array}$ \\
\hline 1884 & $07-07-84$ & 300,000 & ES,HP & 1959 & $06-19-59$ & 38,900 & \\
\hline 1921 & $06-18-21$ & 220,000 & & 1960 & $06-08-60$ & 46,700 & \\
\hline 1922 & $05-31-22$ & 116,000 & & 1961 & $06-05-61$ & 40,200 & \\
\hline 1923 & $05-31-23$ & 98,300 & & 1962 & $05-16-62$ & 85,000 & \\
\hline 1924 & $06-17-24$ & 76,200 & & 1963 & $10-21-62$ & 19,200 & $\mathrm{KR}$ \\
\hline 1925 & $06-03-25$ & 54,900 & & 1964 & $04-28-64$ & 20,200 & $\mathrm{KR}$ \\
\hline 1926 & $05-29-26$ & 86,500 & & 1965 & $06-15-65$ & 60,200 & $\mathrm{KR}$ \\
\hline 1927 & $07-01-27$ & 127,000 & & 1966 & $05-03-66$ & 21,100 & $\mathrm{KR}$ \\
\hline 1928 & $06-03-28$ & 115,000 & & 1967 & $04-19-67$ & 22,500 & KR \\
\hline 1929 & $05-29-29$ & 114,000 & & 1968 & $07-19-68$ & 26,800 & $\mathrm{KR}$ \\
\hline 1930 & $06-03-30$ & 73,300 & & 1969 & $08-28-69$ & 26,100 & KR \\
\hline 1931 & $05-21-31$ & 34,600 & & 1970 & $08-26-70$ & 27,300 & KR \\
\hline 1932 & $05-26-32$ & 102,000 & & 1971 & $04-05-71$ & 28,700 & $\mathrm{KR}$ \\
\hline 1933 & $06-05-33$ & 82,700 & & 1972 & $07-12-72$ & 30,600 & KR \\
\hline 1934 & $05-16-34$ & 25,300 & & 1973 & $03-28-73$ & 31,000 & $\mathrm{KR}$ \\
\hline 1935 & $06-19-35$ & 105,000 & & 1974 & $01-02-74$ & 27,700 & $\mathrm{KR}$ \\
\hline 1936 & $05-23-36$ & 76,300 & & 1975 & $05-07-75$ & 28,400 & $\mathrm{KR}$ \\
\hline 1937 & $05-20-37$ & 84,800 & & 1976 & $05-19-76$ & 27,100 & $\mathrm{KR}$ \\
\hline 1938 & $06-08-38$ & 101,000 & & 1977 & $09-07-77$ & 29,000 & KR \\
\hline 1939 & $05-26-39$ & 49,700 & & 1978 & $01-23-78$ & 28,400 & KR \\
\hline 1940 & $05-18-40$ & 47,200 & & 1979 & $01-29-79$ & 28,600 & $\mathrm{KR}$ \\
\hline 1941 & $05-17-41$ & 120,000 & & 1980 & $06-24-80$ & 44,800 & KR \\
\hline 1942 & $05-30-42$ & 92,800 & & 1981 & $07-20-81$ & 25,900 & $\mathrm{KR}$ \\
\hline 1943 & $06-05-43$ & 68,600 & & 1982 & $05-25-82$ & 29,700 & $\mathrm{KR}$ \\
\hline 1944 & $05-19-44$ & 94,400 & & 1983 & $06-29-83$ & 97,300 & $\mathrm{KR}$ \\
\hline 1945 & $05-17-45$ & 64,400 & & 1984 & $08-12-84$ & 58,200 & KR \\
\hline 1946 & $06-14-46$ & 50,400 & & 1985 & $06-02-85$ & 47,900 & KR \\
\hline 1947 & $05-13-47$ & 80,400 & & 1986 & $05-13-86$ & 53,200 & $\mathrm{KR}$ \\
\hline 1948 & $05-25-48$ & 92,400 & & 1987 & $12-03-86$ & 31,500 & KR \\
\hline 1949 & $06-22-49$ & 119,000 & & 1988 & $07-28-88$ & 27,100 & KR \\
\hline 1950 & $06-06-50$ & 60,600 & & 1989 & $08-28-89$ & 28,400 & $\mathrm{KR}$ \\
\hline 1951 & $06-01-51$ & 67,300 & & 1990 & $07-31-90$ & 29,500 & $\mathrm{KR}$ \\
\hline 1952 & $06-12-52$ & 123,000 & & 1991 & $06-17-91$ & 29,500 & $\mathrm{KR}$ \\
\hline 1953 & $06-17-53$ & 69,600 & & 1992 & $08-14-92$ & 18,700 & $\mathrm{KR}$ \\
\hline 1954 & $05-26-54$ & 34,300 & & 1993 & $08-16-93$ & 19,900 & $\mathrm{KR}$ \\
\hline 1955 & $06-13-55$ & 35,600 & & 1994 & $08-17-94$ & 22,200 & $\mathrm{KR}$ \\
\hline 1956 & $06-06-56$ & 69,600 & & 1995 & $06-01-95$ & 20,100 & $\mathrm{KR}$ \\
\hline 1957 & $06-12-57$ & 126,000 & & 1996 & $03-29-96$ & 45,900 & $\mathrm{KR}$ \\
\hline 1958 & $06-01-58$ & 105,600 & & & & & \\
\hline
\end{tabular}

Discharge rating table developed October 1990

\begin{tabular}{cccc}
\hline $\begin{array}{c}\text { Gage-Height } \\
(\mathbf{f t})\end{array}$ & $\begin{array}{c}\text { Discharge } \\
\left(\mathrm{ft}^{\mathbf{3}} \mathbf{/}\right)\end{array}$ & $\begin{array}{c}\text { Gage-Height } \\
(\mathbf{f t})\end{array}$ & $\begin{array}{c}\text { Discharge } \\
\left(\mathrm{ft}^{\mathbf{3}} / \mathbf{s}\right)\end{array}$ \\
\hline 5.0 & 1,270 & 10.0 & 16,750 \\
6.0 & 2,720 & 11.0 & 22,740 \\
7.0 & 4,900 & 12.0 & 29,910 \\
8.0 & 7,900 & 13.0 & 38,180 \\
9.0 & 11,820 & 14.0 & 46,150 \\
\hline
\end{tabular}


09380000 COLORADO RIVER AT LEES FERRY, AZ--Continued

MEAN MONTHLY AND ANNUAL DISCHARGES 1922.96

\begin{tabular}{|c|c|c|c|c|c|c|}
\hline MONTH & $\begin{array}{l}\text { MAXIMUM } \\
(\text { FT3/S) }\end{array}$ & $\begin{array}{l}\text { MINIMUM } \\
\text { (FT3/S) }\end{array}$ & $\begin{array}{c}\text { MEAN } \\
(\mathrm{FT} 3 / \mathrm{S})\end{array}$ & $\begin{array}{l}\text { STAN- } \\
\text { DARD } \\
\text { DEVIA- } \\
\text { TION } \\
\text { (FT3/S) }\end{array}$ & $\begin{array}{l}\text { COEFFI- } \\
\text { CIENT OF } \\
\text { VARI- } \\
\text { ATION }\end{array}$ & $\begin{array}{c}\text { PERCENT } \\
\text { OF } \\
\text { ANNUAL } \\
\text { RUNOFF }\end{array}$ \\
\hline OCTOBER & 29,200 & 1,000 & 9.200 & 4,720 & 0.51 & 5.1 \\
\hline NOVEMBER & 24,800 & 1,000 & 9,200 & 4,190 & 0.46 & 5.1 \\
\hline DECEMBER & 25,000 & 1.020 & 8,660 & 4,660 & 0.54 & 4.8 \\
\hline JANUARY & 26,300 & 1,150 & 8,800 & 5,290 & 0.60 & 4.9 \\
\hline FEBRUARY & 26,700 & 4,010 & 9.040 & 4,370 & 0.48 & 5.0 \\
\hline MARCH & 24.800 & 3.050 & 10,000 & 3.870 & 0.39 & 5.5 \\
\hline APRIL & 47.800 & 1.020 & 16,800 & 8,820 & 0.52 & 9.3 \\
\hline MAY & 82.600 & 1,010 & 30,900 & 21,300 & 0.69 & 17.0 \\
\hline JUNE & 94.900 & 1.000 & 36,300 & 24,700 & 0.68 & 20.0 \\
\hline JULY & 65,300 & 977 & 18,900 & 11,000 & 0.58 & 10.4 \\
\hline AUGUST & 30,800 & 1,010 & 12,800 & 6.720 & 0.53 & 7.1 \\
\hline SEPTEMBER & 34,200 & 1,010 & 10,800 & 6,540 & 0.61 & 5.9 \\
\hline ANNUAL & 28,200 & 3,330 & 15,100 & 5,460 & 0.36 & 100 \\
\hline
\end{tabular}

MAGNITUDE AND PROBABILITY OF INSTANTANEOUS PEAK FLOW BASED ON PERIOD OF RECORD

DISCHARGE, IN FT3/S, FOR INDICATED RECURRENCE INTERVAL

IN YEARS, AND EXCEEDANCE PROBABILITY, IN PERCENT

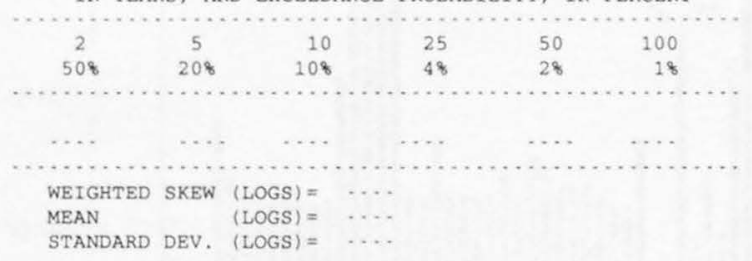

MAGNITUDE AND PROBABILITY OF ANNUAL LOW FLOW BASED ON PERIOD OF RECORD 1923-96

\begin{tabular}{|c|c|c|c|c|c|c|}
\hline \multirow{3}{*}{$\begin{array}{l}\text { PERIOD } \\
\text { ICON. } \\
\text { SECU. }\end{array}$} & \multicolumn{6}{|c|}{ DISCHARGE, IN FT3/S, FOR INDICATED } \\
\hline & \multicolumn{4}{|c|}{$\begin{array}{l}\text { RECURRENCE INTERVAL, IN } \\
\text { NON-EXCEEDANCE PROBABILI? }\end{array}$} & IN & CENT \\
\hline & & & & & & \\
\hline TIVE & 2 & 5 & 10 & 20 & 50 & 100 \\
\hline DAYS) & 508 & 208 & 108 & 58 & 28 & 18 \\
\hline 1 & 2,850 & 1,760 & 1,330 & 1,050 & 786 & 643 \\
\hline 3 & 3,380 & 2,030 & 1,510 & 1,160 & 852 & 685 \\
\hline 7 & 3,950 & 2,370 & 1,780 & 1.390 & 1,030 & 844 \\
\hline 14 & 4,560 & 2.730 & 2,030 & 1.570 & 1.160 & 937 \\
\hline 30 & 5,350 & 3,330 & 2,530 & 1,990 & 1,490 & 1,220 \\
\hline 60 & 6,320 & 3.940 & 2.950 & 2,280 & 1,660 & 1.330 \\
\hline 90 & 7,120 & 4,410 & 3,280 & 2,500 & 1,800 & 1,420 \\
\hline 120 & 7,580 & 4.790 & 3.610 & 2,800 & 2,060 & 1,650 \\
\hline 183 & 8,290 & 5,270 & 3,990 & 3,100 & 2,270 & 1,820 \\
\hline
\end{tabular}

MAGNITUDE AND PROBABILITY OF ANNUAL HIGH FLOW BASED ON PERIOD OF RECORD 1922.96

\begin{tabular}{|c|c|c|c|c|c|c|}
\hline \multirow{4}{*}{$\begin{array}{l}\text { PERIOD } \\
\text { (CON- } \\
\text { SECU- } \\
\text { TIVE } \\
\text { DAYS) }\end{array}$} & DIS & $\begin{array}{l}\text { CHARGE, } \\
\text { RECURRE } \\
\text { EXCEEDA }\end{array}$ & $\begin{array}{l}\text { IN FT3/S, } \\
\text { NCE INTER } \\
\text { NCE PROBA }\end{array}$ & $\begin{array}{l}\text { FOR IN } \\
\text { RVAL, IN } \\
\text { BILITY, }\end{array}$ & $\begin{array}{l}\text { ICATED } \\
\text { YEARS, A } \\
\text { IN PERCE }\end{array}$ & $\begin{array}{l}\text { AND } \\
\text { ENT }\end{array}$ \\
\hline & & & & & & \\
\hline & 2 & 5 & 10 & 25 & 50 & 100 \\
\hline & 508 & 208 & 108 & 48 & 28 & 18 \\
\hline 1 & 44,600 & 78,300 & 105,000 & 144,000 & 176,000 & 211,000 \\
\hline 3 & 43,300 & 76,600 & 103,000 & 141,000 & 173.000 & 207.000 \\
\hline 7 & 41,200 & 73,000 & 98,000 & 134,000 & 163,000 & 194,000 \\
\hline 15 & 38,200 & 67,200 & 89,800 & 122,000 & 148,000 & 176,000 \\
\hline 30 & 35,100 & 61,000 & 80,900 & 109,000 & 132,000 & 156,000 \\
\hline 60 & 30,600 & 51,700 & 67,800 & 90,300 & 109,000 & 128,000 \\
\hline 90 & 27,000 & 43,900 & 56,500 & 73,600 & 87,200 & 102,000 \\
\hline
\end{tabular}

DURATION TABLE OF DAILY MEAN FLOW FOR PERIOD OF RECORD 1922.96

DISCHARGE, IN FT3/S, WHICH WAS EQUALED OR EXCEEDED FOR INDICATED PERCENT OF TIME

\begin{tabular}{|c|c|c|c|c|c|c|c|c|c|c|c|c|c|c|c|c|}
\hline 18 & 58 & 108 & 158 & 208 & 308 & 408 & 508 & 608 & 708 & 808 & 908 & 958 & 988 & 998 & 99.58 & 99.98 \\
\hline .100 & 48,600 & 30,500 & 23,600 & 19,000 & 15,100 & 12,500 & 10,400 & 8,610 & 7,110 & 5,880 & 4,650 & 3,530 & 2,110 & 1.060 & 1.000 & 963 \\
\hline
\end{tabular}


ANNUAL MEAN DISCHARGE, IN CUBIC

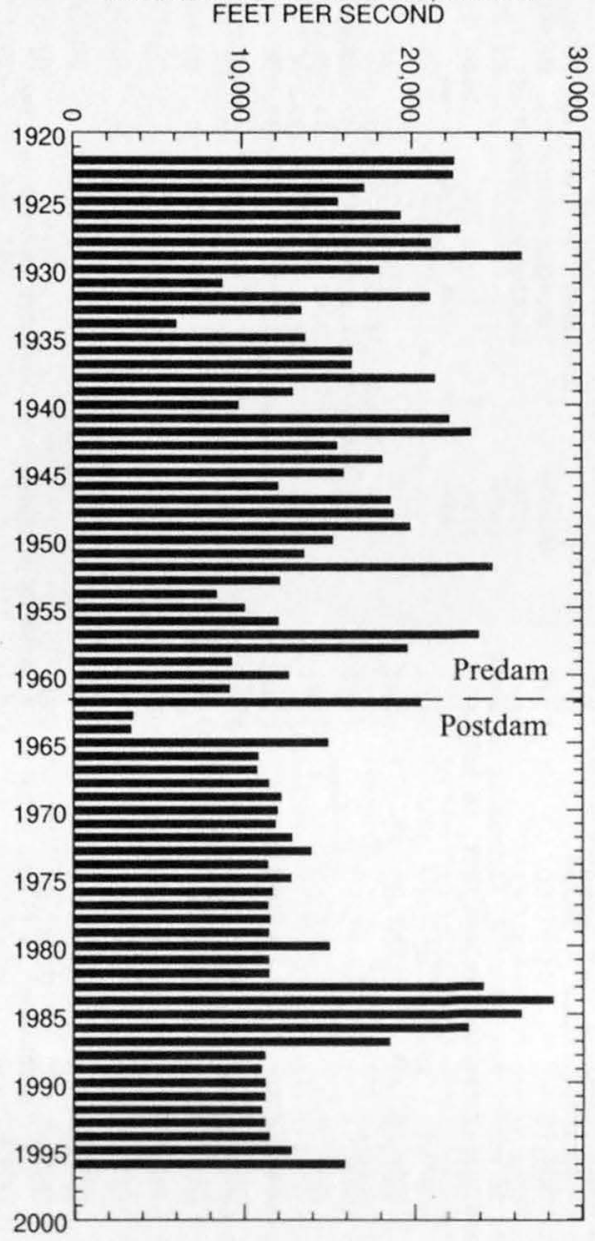

ANNUAL PEAK DISCHARGE, IN CUBIC

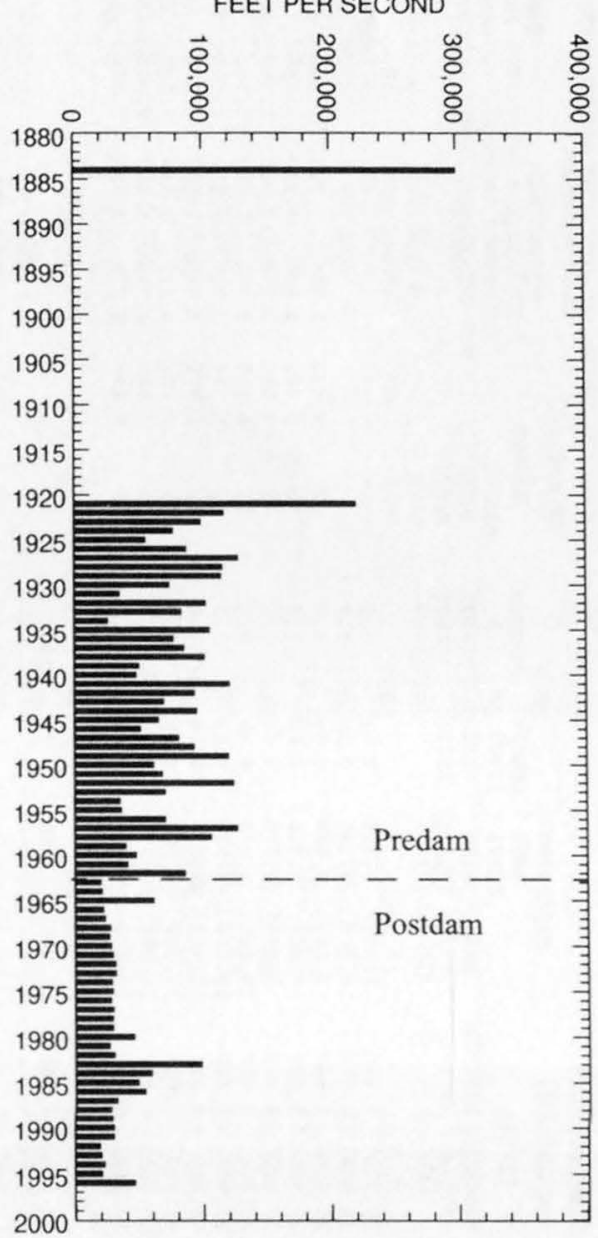


PARIA RIVER BASIN

09380000 COLORADO RIVER AT LEES FERRY, AZ--Continued

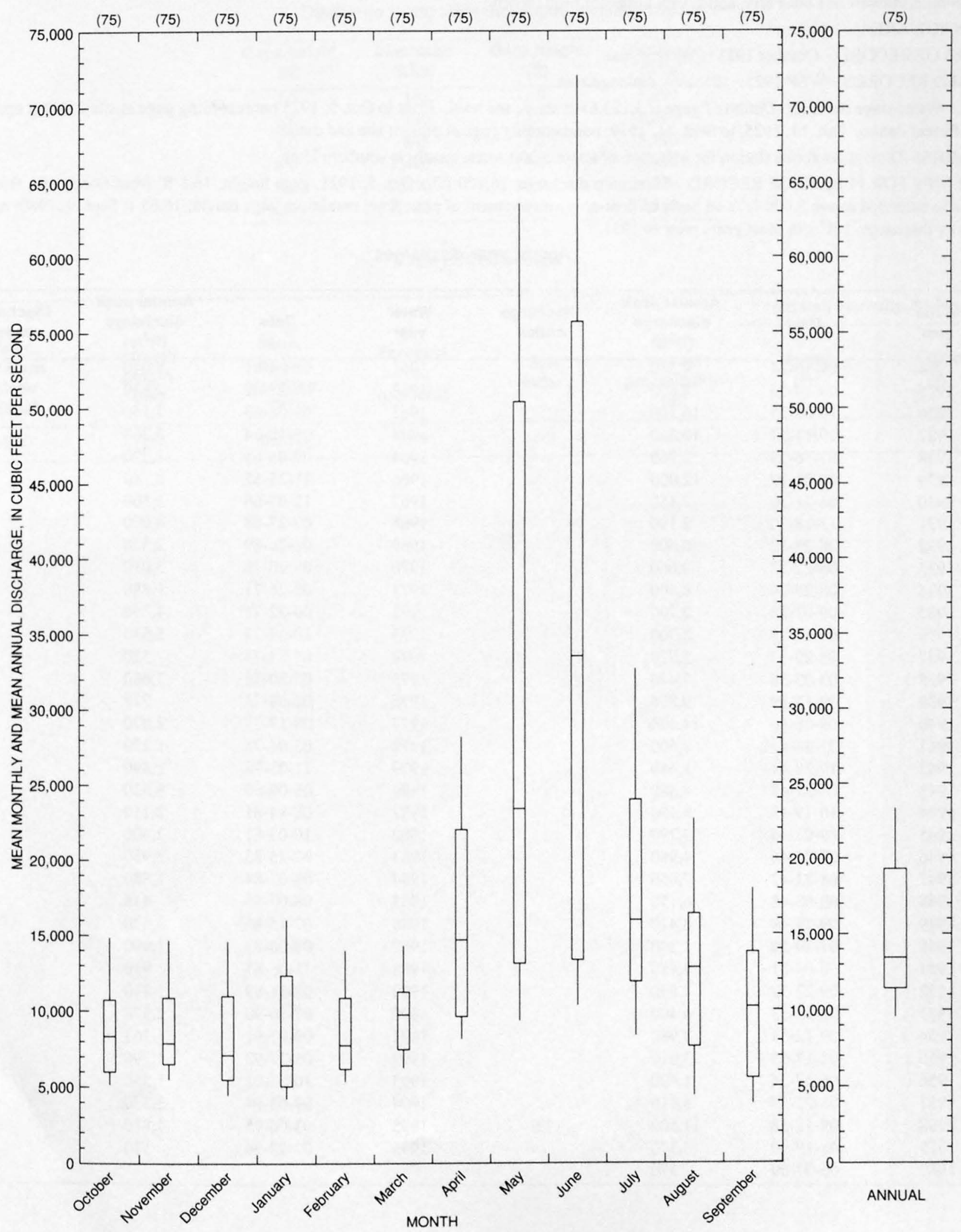




\section{PARIA RIVER AT LEES FERRY, AZ}

LOCATION.--Lat 36 52'20", long 111 $35^{\prime} 38^{\prime \prime}$, in NW1/4 NE $1 / 4$ sec.13, T.40 N., R.7 E., Coconino County, Hydrologic Unit 14070007, on left bank 0.6 mi northwest of Lees Ferry, and 1.1 mi upstream from mouth.

DRAINAGE AREA.--1,410 $\mathrm{mi}^{2}$.

PERIOD OF RECORD.--October 1923 to current year.

REVISED RECORDS.--WSP 1925: 1958(M), drainage area.

GAGE.--Water-stage recorder. Datum of gage is 3,123.68 ft above sea level. Prior to Oct, 5, 1925 nonrecording gage at site $2,000 \mathrm{ft}$ upstream at different datum. Oct. 13, 1925, to Sept. 11, 1929, nonrecording gage at present site and datum.

REMARKS.--Diversions above station for irrigation of about 3,300 acres, mostly in southern Utah.

EXTREMES FOR PERIOD OF RECORD.--Maximum discharge, 16,100 ft3/s Oct. 5, 1925, gage height, $16.3 \mathrm{ft}$, from floodmark, from rating curve extended above $2,000 \mathrm{ft}^{3} / \mathrm{s}$ on basis of float-area measurement of peak flow; maximum gage height, 16.65 $\mathrm{ft}$ Sept. 9 , 1980 ; minimum daily discharge, $1 \mathrm{ft}^{3} / \mathrm{s}$ in most years prior to 1931 .

Annual peak discharges

\begin{tabular}{|c|c|c|c|c|c|c|c|}
\hline $\begin{array}{l}\text { Water } \\
\text { year }\end{array}$ & Date & $\begin{array}{c}\text { Annual peak } \\
\text { discharge } \\
\left(\mathrm{ft}^{3} / \mathrm{s}\right)\end{array}$ & $\begin{array}{l}\text { Discharge } \\
\text { codes }\end{array}$ & $\begin{array}{l}\text { Water } \\
\text { year }\end{array}$ & Date & $\begin{array}{c}\text { Annual peak } \\
\text { discharge } \\
\left(\mathrm{ft}^{3} / \mathrm{s}\right)\end{array}$ & $\begin{array}{l}\text { Discharge } \\
\text { codes }\end{array}$ \\
\hline 1924 & $09-10-24$ & 4,330 & & 1961 & $08-04-61$ & 8,040 & \\
\hline 1925 & $09-19-25$ & 4,800 & & 1962 & $09-21-62$ & 2,830 & \\
\hline 1926 & $10-05-25$ & 16,100 & & 1963 & $09-01-63$ & 7,150 & \\
\hline 1927 & $09-13-27$ & 14,300 & & 1964 & $08-12-64$ & 2,360 & \\
\hline 1928 & $07-16-28$ & 2,960 & & 1964 & $09-06-65$ & 1,220 & \\
\hline 1929 & $09-02-29$ & 12,000 & & 1966 & $11-23-65$ & 2,140 & \\
\hline 1930 & $08-11-30$ & 7,150 & & 1967 & $12-07-66$ & 3,500 & \\
\hline 1931 & $11-18-30$ & 2,190 & & 1968 & $07-27-68$ & 4,090 & \\
\hline 1932 & $08-28-32$ & 10,500 & & 1969 & $01-26-69$ & 2,570 & \\
\hline 1933 & $08-22-33$ & 3,660 & & 1970 & $08-20-70$ & 3,010 & \\
\hline 1934 & $08-29-34$ & 8,400 & & 1971 & $08-26-71$ & 1,880 & \\
\hline 1935 & $09-01-35$ & 2,700 & & 1972 & $06-22-72$ & 4,750 & \\
\hline 1936 & $07-11-36$ & 8,700 & & 1973 & $10-19-72$ & 5,530 & \\
\hline 1937 & $08-29-37$ & 3,720 & & 1974 & $07-23-74$ & 520 & \\
\hline 1938 & $03-03-38$ & 7,440 & & 1975 & $07-30-75$ & 3,680 & \\
\hline 1939 & $09-13-39$ & 9,800 & & 1976 & $02-09-76$ & 718 & \\
\hline 1940 & $09-06-40$ & 14,000 & & 1977 & $08-17-77$ & 2,070 & \\
\hline 1941 & $07-24-41$ & 7,500 & & 1978 & $03-05-78$ & 1,270 & \\
\hline 1942 & $10-28-41$ & 1,680 & & 1979 & $11-03-78$ & 2,890 & \\
\hline 1943 & $08-22-43$ & 4,680 & & 1980 & $09-09-80$ & 8,520 & \\
\hline 1944 & $10-19-43$ & 8,400 & & 1981 & $08-14-81$ & 2,110 & \\
\hline 1945 & $09-03-45$ & 3,290 & & 1982 & $10-03-81$ & 2,400 & \\
\hline 1946 & $07-25-46$ & 4,980 & & 1983 & $07-25-83$ & 2,950 & \\
\hline 1947 & $08-22-47$ & 7,650 & & 1984 & $08-07-84$ & 1,880 & \\
\hline 1948 & $08-05-48$ & 6,150 & & 1985 & $08-07-85$ & 416 & \\
\hline 1949 & $09-29-49$ & 3,410 & & 1986 & $07-15-86$ & 3,520 & \\
\hline 1950 & $07-19-50$ & 1,340 & & 1987 & $08-08-87$ & 1,990 & \\
\hline 1951 & $08-04-51$ & 4,480 & & 1988 & $11-06-87$ & 910 & \\
\hline 1952 & $09-22-52$ & 1,830 & & 1989 & $08-11-89$ & 1,240 & \\
\hline 1953 & $08-27-53$ & 6,400 & & 1990 & $07-16-90$ & 2,370 & \\
\hline 1954 & $09-12-54$ & 3,980 & & 1991 & $09-07-91$ & 763 & \\
\hline 1955 & $08-17-55$ & 3,010 & & 1992 & $05-27-92$ & 1,790 & \\
\hline 1956 & $08-17-56$ & 1,420 & & 1993 & $10-25-92$ & 1,530 & $\therefore$ \\
\hline 1957 & $08-22-57$ & 3,310 & & 1994 & $09-03-94$ & 3,330 & \\
\hline 1958 & $09-12-58$ & 11,500 & ES & 1995 & $03-06-95$ & 1,810 & \\
\hline 1959 & $08-19-59$ & 5,370 & & 1996 & $02-22-96$ & 510 & \\
\hline 1960 & $06-07-60$ & 370 & & & & & \\
\hline
\end{tabular}


09382000 PARIA RIVER AT LEES FERRY, AZ--Continued

Discharge rating table developed January 1993

\begin{tabular}{cccc}
\hline $\begin{array}{c}\text { Gage height } \\
(\mathbf{f t})\end{array}$ & $\begin{array}{c}\text { Discharge } \\
\left(\mathbf{f t}^{3} / \mathbf{s}\right)\end{array}$ & $\begin{array}{c}\text { Gage height } \\
(\mathbf{f t})\end{array}$ & $\begin{array}{c}\text { Discharge } \\
\left(\mathbf{f t}^{\mathbf{3}} / \mathbf{s}\right)\end{array}$ \\
\hline 5.0 & 143 & 11.0 & 3,490 \\
6.0 & 390 & 12.0 & 4,280 \\
7.0 & 848 & 13.0 & 5,110 \\
8.0 & 1,410 & 14.0 & 5,990 \\
9.0 & 2,050 & 15.0 & 6,910 \\
10.0 & 2,750 & 16.0 & 7,860 \\
\hline
\end{tabular}

Basin characteristics

\begin{tabular}{|c|c|c|c|c|c|c|c|}
\hline \multirow[b]{2}{*}{$\begin{array}{l}\text { Main } \\
\text { channel } \\
\text { slope } \\
(\mathrm{ft} / \mathrm{mi})\end{array}$} & \multirow[b]{2}{*}{$\begin{array}{l}\text { Stream } \\
\text { length } \\
(\mathrm{mi})\end{array}$} & \multirow[b]{2}{*}{$\begin{array}{c}\text { Mean } \\
\text { basin } \\
\text { elevation } \\
(\mathrm{ft})\end{array}$} & \multirow[b]{2}{*}{$\begin{array}{l}\text { Forested } \\
\text { area } \\
\text { (percent) }\end{array}$} & \multirow[b]{2}{*}{$\begin{array}{c}\text { Soil } \\
\text { index }\end{array}$} & \multirow[b]{2}{*}{$\begin{array}{c}\text { Mean } \\
\text { annual } \\
\text { precipitation } \\
\text { (in) }\end{array}$} & \multicolumn{2}{|c|}{ Rainfall intensity, 24-hour } \\
\hline & & & & & & $\begin{array}{l}\text { 2-year } \\
\text { (in) }\end{array}$ & $\begin{array}{l}\text { 50-year } \\
\text { (in) }\end{array}$ \\
\hline 43.0 & 78.0 & 6,150 & 73.0 & 3.0 & 12.0 & 1.4 & 3.0 \\
\hline
\end{tabular}


PARIA RIVER BASIN

09382000 PARIA RIVER AT LEES FERRY, AZ--Continued

MEAN MONTHLY AND ANNUAL DISCHARGES $1924 \cdot 95$

\begin{tabular}{|c|c|c|c|c|c|c|}
\hline MONTH & $\begin{array}{l}\text { MAXIMUM } \\
\text { (FT3/S) }\end{array}$ & $\begin{array}{l}\text { MINIMUM } \\
(\mathrm{FT} 3 / \mathrm{S})\end{array}$ & $\begin{array}{c}\text { MEAN } \\
(\mathrm{FT} 3 / \mathrm{S})\end{array}$ & $\begin{array}{l}\text { STAN- } \\
\text { DARD } \\
\text { DEVIA- } \\
\text { TION } \\
\text { (FT3/S) }\end{array}$ & $\begin{array}{l}\text { COEFFI- } \\
\text { CIENT OF } \\
\text { VARI. } \\
\text { ATION }\end{array}$ & $\begin{array}{c}\text { PERCENT } \\
\text { OF } \\
\text { ANNUAL } \\
\text { RUNOFF }\end{array}$ \\
\hline OCTOBER & 288 & 6.0 & 30 & 41 & 1.4 & 8.6 \\
\hline NOVEMBER & 123 & 10 & 24 & 18 & 0.76 & 6.8 \\
\hline DECEMBER & 69 & 8.8 & 21 & 9.7 & 0.46 & 6.1 \\
\hline JANUARY & 97 & 8.0 & 22 & 13 & 0.58 & 6.5 \\
\hline FEBRUARY & 242 & 16 & 39 & 33 & 0.86 & 11.2 \\
\hline MARCH & 216 & 8.9 & 40 & 39 & 0.96 & 11.5 \\
\hline APRIL & 93 & 4.9 & 21 & 19 & 0.88 & 6.1 \\
\hline MAY & 52 & 2.0 & 11 & 10 & 0.93 & 3.1 \\
\hline JUNE & 58 & 2.0 & 7.3 & 9.2 & 1.3 & 2.1 \\
\hline JULY & 172 & 2.3 & 25 & 28 & 1.1 & 7.2 \\
\hline AUGUST & 237 & 4.5 & 56 & 49 & 0.89 & 16.0 \\
\hline SEPTEMBER & 424 & 4.2 & 52 & 80 & 1.5 & 15.0 \\
\hline ANNUAL & 65 & 11 & 29 & 12 & 0.41 & 100 \\
\hline
\end{tabular}

MAGNITUDE AND PROBABILITY OF INSTANTANEOUS PEAK FLOW BASED ON PERIOD OF RECORD 1924-95

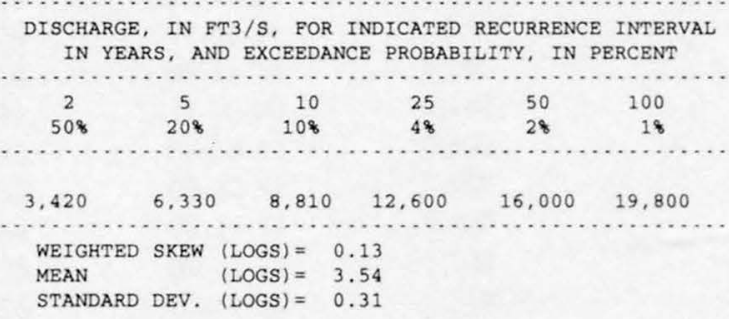

MAGNITUDE AND PROBABILITY OF ANNUAL LOW FLOW BASED ON PERIOD OF RECORD $1925 \cdot 95$

\begin{tabular}{|c|c|c|c|c|c|c|}
\hline \multirow{2}{*}{$\begin{array}{l}\text { PERIOD } \\
\text { (CON- } \\
\text { SECU. }\end{array}$} & \multicolumn{6}{|c|}{$\begin{array}{l}\text { DISCHARGE, IN FT3/S, FOR INDICATED } \\
\text { RECURRENCE INTERVAL, IN YEARS, AND } \\
\text { NON-EXCEEDANCE PROBABILITY, IN PERCENT }\end{array}$} \\
\hline & $\cdots$ & $\ldots \ldots$ & $\ldots \ldots$ & ...... & & \\
\hline TIVE & 2 & 5 & 10 & 20 & 50 & 100 \\
\hline DAYS) & 508 & 208 & 108 & 58 & 28 & 18 \\
\hline 1 & 2.4 & 1.8 & 1.5 & 1.3 & 1.0 & 0.91 \\
\hline 3 & 2.8 & 2.1 & 1.7 & 1.5 & 1.2 & 1.1 \\
\hline 7 & 3.0 & 2.3 & 2.0 & 1.7 & 1.4 & 1.3 \\
\hline 14 & 3.1 & 2.5 & 2.3 & 2.0 & 1.8 & 1.7 \\
\hline 30 & 3.5 & 2.8 & 2.5 & 2.4 & 2.2 & 2.1 \\
\hline 60 & 4.4 & 3.4 & 3.1 & 2.9 & 2.7 & 2.6 \\
\hline 90 & 6.4 & 4.4 & 3.8 & 3.3 & 3.9 & 2.7 \\
\hline 120 & 11 & 7.1 & 5.6 & 4.6 & 3.6 & 3.1 \\
\hline 183 & 18 & 13 & 10 & 8.5 & 5.8 & 5.9 \\
\hline
\end{tabular}

MAGNITUDE AND PROBABILITY OF ANNUAL HIGH FLOW BASED ON PERIOD OF RECORD $1924-95$

\begin{tabular}{|c|c|c|c|c|c|c|}
\hline PERIOD & & \multicolumn{5}{|c|}{$\begin{array}{l}\text { DISCHARGE, IN FT3/S, FOR INDICATED } \\
\text { RECURRENCE INTERVAL, IN YEARS, AND }\end{array}$} \\
\hline (CON. & & \multicolumn{3}{|c|}{ EXCEEDANCE PROBABILITY, } & \multicolumn{2}{|c|}{ IN PERCENT } \\
\hline SECU - & & …....... & ….... & & & \\
\hline TIVE & 2 & 5 & 10 & 25 & 50 & 100 \\
\hline DAYS) & 508 & 208 & 108 & 48 & 28 & 18 \\
\hline 1 & 776 & 1,680 & 2,590 & 4,220 & 5,870 & 7,980 \\
\hline 3 & 397 & 832 & 1,280 & 2,080 & 2,900 & 3,960 \\
\hline 7 & 216 & 431 & 643 & 1,010 & 1.380 & 1,850 \\
\hline 15 & 134 & 251 & 360 & 543 & 716 & 928 \\
\hline 30 & 91 & 163 & 226 & 324 & 413 & 518 \\
\hline 60 & 63 & 106 & 143 & 200 & 250 & 309 \\
\hline 90 & 49 & 79 & 105 & 145 & 179 & 219 \\
\hline
\end{tabular}

DURATION TABLE OF DAILY MEAN FLOW FOR PERIOD OF RECORD 1924.95

DISCHARGE, IN FT3/S, WHICH WAS EQUALED OR EXCEEDED FOR INDICATED PERCENT OF TIME

\begin{tabular}{|c|c|c|c|c|c|c|c|c|c|c|c|c|c|c|c|c|}
\hline 18 & 58 & 108 & 158 & 208 & 308 & 408 & 508 & 608 & 708 & 808 & 908 & 958 & 988 & 998 & 99.58 & 99.98 \\
\hline 22 & 79 & 44 & 33 & 28 & 22 & 18 & 14 & 10 & 6.8 & 4.9 & 3.8 & 3.2 & 2.5 & 2.0 & 1.8 & 1.4 \\
\hline
\end{tabular}


ANNUAL MEAN DISCHARGE, IN CUBIC EET PER SECOND

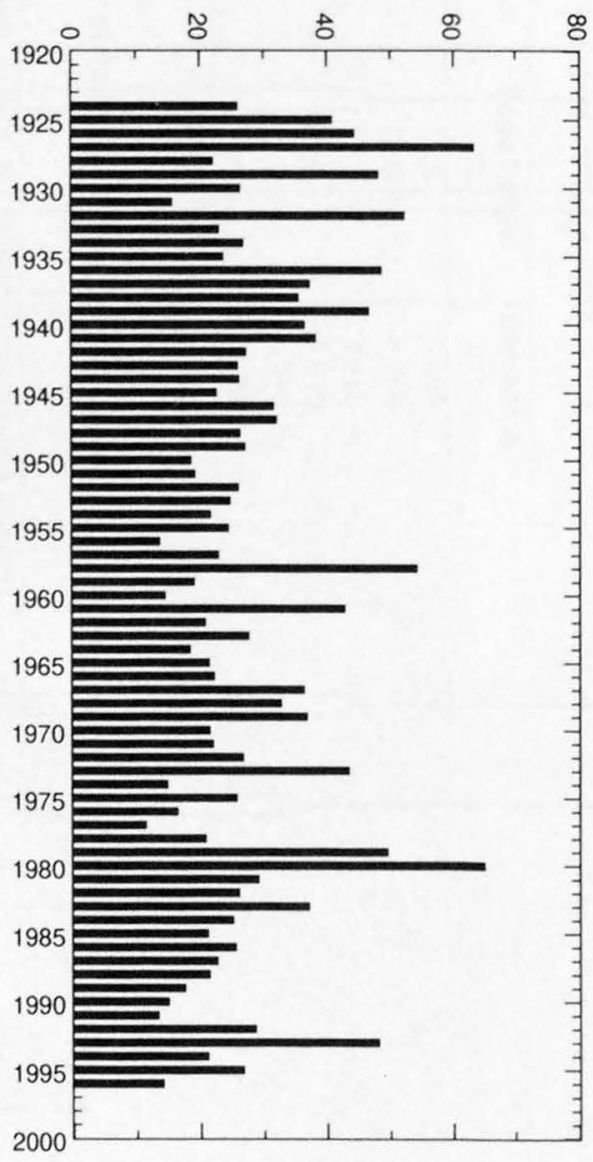

ANNUAL PEAK DISCHARGE, IN CUBIC FEET PER SECOND

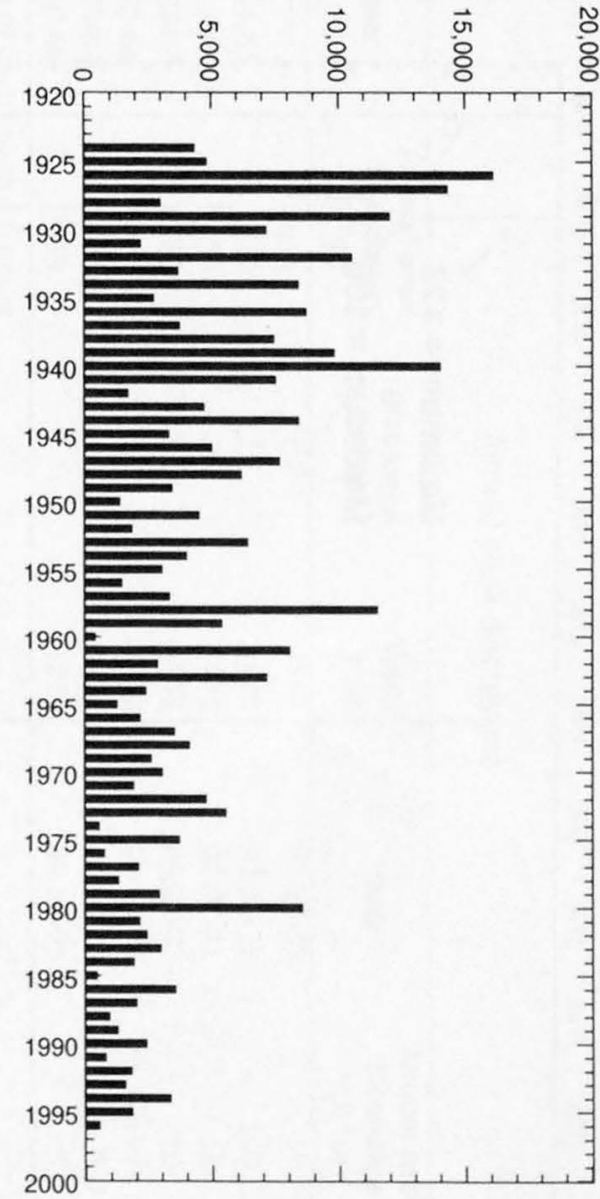


09382000 PARIA RIVER AT LEES FERRY, AZ--Continued
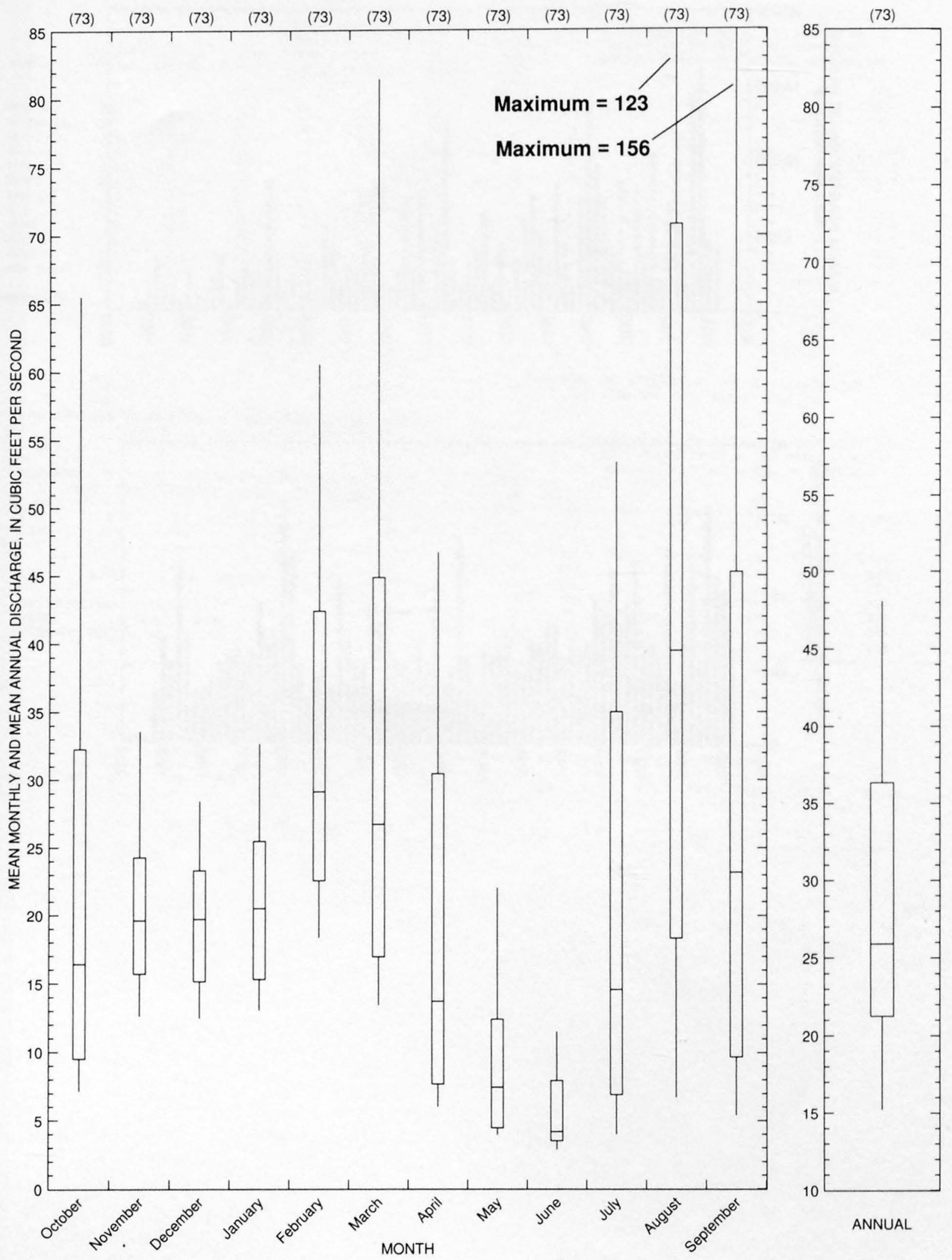


\section{HOUSE ROCK WASH TRIBUTARY NEAR MARBLE CANYON, AZ}

LOCATION.--Lat $36^{\circ} 42^{\prime} 05^{\prime \prime}$, long $111^{\circ} 55^{\prime} 45^{\prime \prime}$, in $\mathrm{SE}^{1} /{ }^{1} \mathrm{SE}^{1} / 4$, sec11, T.38 N., R.4 E., Coconino County, Hydrologic Unit 15010001 , at U.S. Highway 89 Alternate, 21 mi soutwest of Marble Canyon Post Office.

DRAINAGE AREA.--3.54 $\mathrm{mi}^{2}$.

Annual peak discharges

\begin{tabular}{cccccccc}
\hline $\begin{array}{c}\text { Water } \\
\text { year }\end{array}$ & Date & $\begin{array}{c}\text { Annual peak } \\
\text { discharge } \\
\left(\mathrm{ft}^{3} / \mathbf{s}\right)\end{array}$ & $\begin{array}{c}\text { Discharge } \\
\text { codes }\end{array}$ & $\begin{array}{c}\text { Water } \\
\text { year }\end{array}$ & Date & $\begin{array}{c}\text { Annual peak } \\
\text { discharge } \\
\left(\mathrm{ft}^{3} / \mathbf{s}\right)\end{array}$ & $\begin{array}{c}\text { Discharge } \\
\text { codes }\end{array}$ \\
\hline-- & $-1,610$ & HP & 1969 & $07-27-69$ & 19 & \\
1963 & $09-04-63$ & 10 & LT & 1970 & $07-23-70$ & 100 & ES \\
1964 & $09-13-64$ & 50 & ES & 1971 & $08-07-71$ & 58 & \\
1965 & $00-00-65$ & 10 & LT & 1972 & $06-22-72$ & 80 & \\
1966 & $00-00-66$ & 5.0 & LT & 1973 & $10-19-72$ & 197 & \\
1967 & $07-28-67$ & 24 & & 1974 & $11-04-73$ & 5.0 & LT \\
1968 & $00-00-68$ & 39 & & 1975 & $00-00-75$ & 5.0 & LT \\
\hline
\end{tabular}

${ }^{1}$ Highest since 1934, year of occurrence unknown.

Discharge rating table developed January 1993

\begin{tabular}{cccc}
\hline $\begin{array}{c}\text { Gage height } \\
(\mathbf{f t})\end{array}$ & $\begin{array}{c}\text { Discharge } \\
\left(\mathbf{f t}^{\mathbf{3}} \mathbf{s}\right)\end{array}$ & $\begin{array}{c}\text { Gage height } \\
(\mathbf{f t})\end{array}$ & $\begin{array}{c}\text { Discharge } \\
\left(\mathbf{f t}^{\mathbf{3}} \mathbf{s}\right)\end{array}$ \\
\hline 5.0 & 143 & 11.0 & 3,490 \\
6.0 & 390 & 12.0 & 4,280 \\
7.0 & 848 & 13.0 & 5,110 \\
8.0 & 1,410 & 14.0 & 5,990 \\
9.0 & 2,050 & 15.0 & 6,910 \\
10.0 & 2,750 & 16.0 & 7,860 \\
\hline
\end{tabular}

Magnitude and probability of instantaneous peak flow based on period of record 1934, 1963-75

\begin{tabular}{|c|c|c|c|c|c|}
\hline \multicolumn{6}{|c|}{$\begin{array}{l}\text { Discharge, in } \mathrm{ft}^{3} / \mathrm{s} \text {, for indicated recurrence interval } \\
\text { in years, and exceedance probablility, in percent }\end{array}$} \\
\hline 2 & 5 & 10 & 25 & $50 t$ & $100 \dagger$ \\
\hline $50 \%$ & $20 \%$ & $10 \%$ & $4 \%$ & $2 \%$ & $1 \%$ \\
\hline 24.7 & 81.8 & 161 & 342 & 569 & 913 \\
\hline Weighted skew & $(\log s)=$ & 0.37 & & & \\
\hline Mean & $(\log s)=$ & 1.43 & & & \\
\hline Standard dev. & $(\log s)=$ & 0.59 & & & \\
\hline
\end{tabular}

Basin characteristics

Rainfall intensity, 24-hour

\begin{tabular}{|c|c|c|c|c|c|c|c|}
\hline $\begin{array}{c}\begin{array}{c}\text { Main } \\
\text { channel } \\
\text { slope } \\
(\mathrm{ft} / \mathrm{mi})\end{array}\end{array}$ & $\begin{array}{c}\text { Stream } \\
\text { length } \\
(\mathrm{mi})\end{array}$ & $\begin{array}{c}\text { Mean } \\
\text { basin } \\
\text { elevation } \\
(\mathrm{ft})\end{array}$ & $\begin{array}{c}\text { Forested } \\
\text { area } \\
\text { (percent) }\end{array}$ & $\begin{array}{c}\text { Soil } \\
\text { index }\end{array}$ & $\begin{array}{c}\text { Mean } \\
\text { annual } \\
\text { precipitation } \\
\text { (in) }\end{array}$ & $\begin{array}{c}\text { 2-year } \\
\text { (in) }\end{array}$ & $\begin{array}{l}\text { 50-year } \\
\text { (in) }\end{array}$ \\
\hline 209 & 3.7 & 5,290 & 2.3 & 3.0 & 9.6 & 1.5 & 3.2 \\
\hline
\end{tabular}


40

HOUSE ROCK WASH BASIN

09383020 HOUSE ROCK WASH TRIBUTARY NEAR MARBLE CANYON, AZ

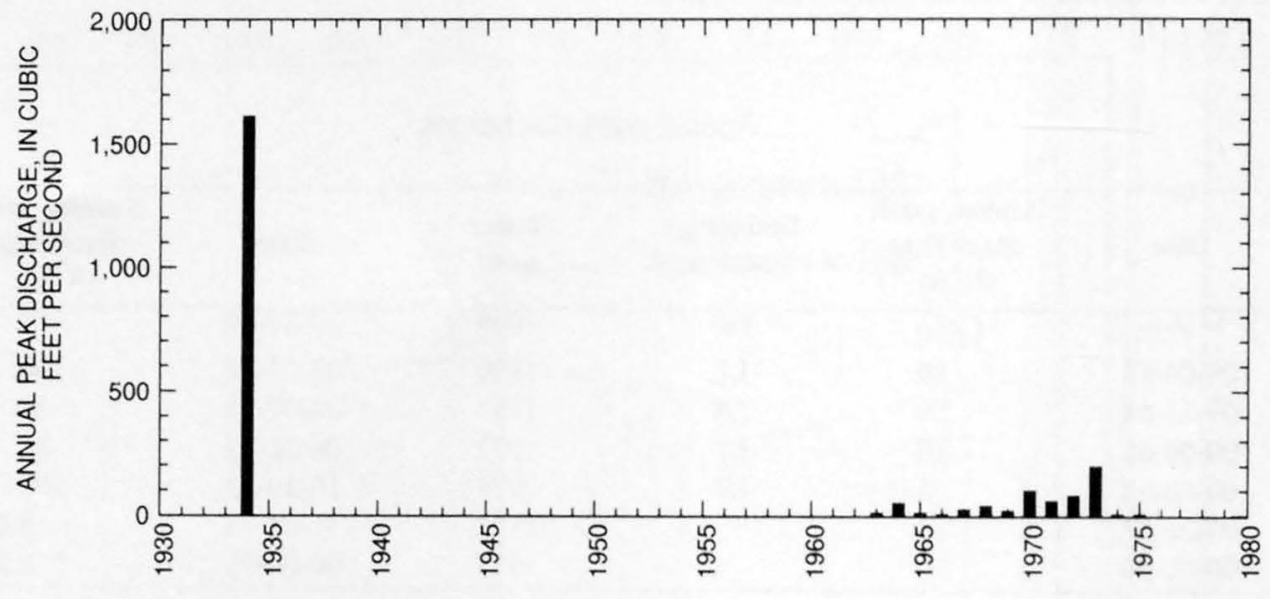




\section{LITTLE COLORADO RIVER AT GREER, AZ}

LOCATION.--Lat 34 $01^{\prime} 00^{\prime \prime}$, long 109.27'24", in NE $\frac{1}{4} \mathrm{SE}^{1} / 4$, sec.11, T.7 N., R.27 E., Apache County, Hydrologic Unit 15020001 , in ApacheSitgreaves National Forest, on upstream side of right abutment of culverts on State Highway 373, at Greer, 0.1 mi downstream from Filler ditch.

DRAINAGE AREA.--29.1 $\mathrm{mi}^{2}$.

REMARKS.--Filler ditch diverts about 1,700 acre- $\mathrm{ft} / \mathrm{yr}$ from river $0.1 \mathrm{mi}$ above station.

Annual peak discharges

\begin{tabular}{|c|c|c|c|c|c|c|c|}
\hline $\begin{array}{l}\text { Water } \\
\text { year }\end{array}$ & Date & $\begin{array}{c}\text { Annual peak } \\
\text { discharge } \\
\left(\mathrm{ft}^{3} / \mathrm{s}\right)\end{array}$ & $\begin{array}{l}\text { Discharge } \\
\text { codes }\end{array}$ & $\begin{array}{l}\text { Water } \\
\text { year }\end{array}$ & Date & $\begin{array}{c}\text { Annual peak } \\
\text { discharge } \\
\left(\mathrm{ft}^{3} / \mathrm{s}\right)\end{array}$ & $\begin{array}{c}\text { Discharge } \\
\text { codes }\end{array}$ \\
\hline 1961 & $04-05-61$ & 76 & & 1973 & $10-20-72$ & 615 & \\
\hline 1962 & $04-15-62$ & 277 & & 1974 & $03-30-74$ & 45 & \\
\hline 1963 & $03-28-63$ & 108 & & 1975 & $04-26-75$ & 231 & \\
\hline 1964 & $04-12-64$ & 135 & & 1976 & $04-10-76$ & 97 & \\
\hline 1965 & $04-22-65$ & 355 & & 1977 & $04-09-77$ & 174 & \\
\hline 1966 & $04-03-66$ & 326 & & 1978 & $04-07-78$ & 78 & \\
\hline 1967 & $08-11-67$ & 216 & & 1979 & $04-26-79$ & 247 & \\
\hline 1968 & $04-15-68$ & 316 & & 1980 & $04-22-80$ & 221 & \\
\hline 1969 & 09-08-69 & 414 & & 1981 & $04-10-81$ & 100 & \\
\hline 1970 & $09-06-70$ & 112 & & 1982 & $04-12-82$ & 212 & \\
\hline 1971 & $08-29-71$ & 42 & & 1984 & $10-02-83$ & 444 & HP \\
\hline 1972 & $10-24-71$ & 108 & & & & & \\
\hline
\end{tabular}

Basin characteristics

Rainfall intensity, 24-hour

\begin{tabular}{|c|c|c|c|c|c|c|c|}
\hline $\begin{array}{l}\text { Main } \\
\text { channel } \\
\text { slope } \\
\text { (ft/mi) }\end{array}$ & $\begin{array}{l}\text { Stream } \\
\text { length } \\
\text { (mi) }\end{array}$ & $\begin{array}{c}\text { Mean } \\
\text { basin } \\
\text { elevation } \\
\text { (ft) }\end{array}$ & $\begin{array}{c}\text { Forested } \\
\text { area } \\
\text { (percent) }\end{array}$ & $\begin{array}{c}\text { Soil } \\
\text { index }\end{array}$ & $\begin{array}{c}\text { Mean } \\
\text { annual } \\
\text { precipitation } \\
\text { (in) }\end{array}$ & $\begin{array}{l}\text { 2-year } \\
\text { (in) }\end{array}$ & $\begin{array}{c}\text { 50-year } \\
\text { (in) }\end{array}$ \\
\hline 180 & 10.4 & 9,400 & 69.0 & 2.9 & 31.2 & 2.8 & 5.1 \\
\hline
\end{tabular}


09383400 LITTLE COLORADO RIVER AT GREER, AZ--Continued

MEAN MONTHLY AND ANNUAL DISCHARGES 1961-82

\begin{tabular}{|c|c|c|c|c|c|c|}
\hline MONTH & $\begin{array}{l}\text { MAXIMUM } \\
(\mathrm{FT} 3 / \mathrm{S})\end{array}$ & $\begin{array}{l}\text { MINIMUM } \\
\text { (FT3/S) }\end{array}$ & $\begin{array}{c}\text { MEAN } \\
(\mathrm{FT} 3 / \mathrm{S})\end{array}$ & $\begin{array}{l}\text { STAN- } \\
\text { DARD } \\
\text { DEVIA- } \\
\text { TION } \\
\text { (FT3/S) }\end{array}$ & $\begin{array}{l}\text { COEFFI- } \\
\text { CIENT OF } \\
\text { VARI- } \\
\text { ATION }\end{array}$ & $\begin{array}{c}\text { PERCENT } \\
\text { OF } \\
\text { ANNUAL } \\
\text { RUNOFF }\end{array}$ \\
\hline OCTOBER & 35 & 0.64 & 5.9 & 7.3 & 1.2 & 3.2 \\
\hline NOVEMBER & 11 & 0.99 & 4.4 & 2.7 & 0.61 & 2.4 \\
\hline DECEMBER & 15 & 1.6 & 5.6 & 3.3 & 0.59 & 3.0 \\
\hline JANUARY & 18 & 2.3 & 6.2 & 3.2 & 0.51 & 3.3 \\
\hline FEBRUARY & 17 & 2.8 & 7.2 & 3.3 & 0.47 & 3.8 \\
\hline MARCH & 26 & 3.5 & 9.7 & 5.0 & 0.51 & 5.2 \\
\hline APRIL & 107 & 7.4 & 43 & 30 & 0.70 & 22.8 \\
\hline MAY & 163 & 7.3 & 41 & 37 & 0.89 & 22.0 \\
\hline JUNE & 96 & 6.4 & 26 & 24 & 0.91 & 13.9 \\
\hline JULY & 28 & 5.9 & 13 & 6.7 & 0.51 & 7.0 \\
\hline AUGUST & 54 & 5.7 & 14 & 9.9 & 0.69 & 7.7 \\
\hline SEPTEMBER & 37 & 5.0 & 11 & 7.4 & 0.67 & 5.9 \\
\hline ANNUAL & 38 & 6.0 & 16 & 8.3 & 0.53 & 100 \\
\hline
\end{tabular}

MAGNITUDE AND PROBABILITY OF ANNUAL LOW FLOW BASED ON PERIOD OF RECORD $1962-82$

\begin{tabular}{|c|c|c|c|c|c|c|}
\hline $\begin{array}{l}\text { PERIOD } \\
\text { (CON. }\end{array}$ & \multicolumn{6}{|c|}{$\begin{array}{l}\text { RECURRENCE INTERVAL, IN YEARS, AND } \\
\text { NON-EXCEEDANCE PROBABILITY, IN PERCENT }\end{array}$} \\
\hline SECU. & & & ..... & $\ldots \ldots$ & . & \\
\hline TIVE & 2 & 5 & 10 & 20 & $50 \#$ & $100 \#$ \\
\hline DAYS) & 508 & 208 & $10 \%$ & 58 & 28 & 18 \\
\hline 1 & 2.0 & 0.99 & 0.57 & 0.32 & 0.15 & 0.08 \\
\hline 3 & 2.2 & 1.1 & 0.61 & 0.34 & 0.15 & 0.08 \\
\hline 7 & 2.3 & 1.1 & 0.63 & 0.34 & 0.15 & 0.08 \\
\hline 14 & 2.5 & 1.2 & 0.66 & 0.36 & 0.16 & 0.08 \\
\hline 30 & 2.5 & 1.4 & 0.96 & 0.69 & 0.46 & 0.35 \\
\hline 60 & 3.0 & 1.8 & 1.3 & 1.0 & 0.77 & 0.63 \\
\hline 90 & 3.6 & 2.3 & 1.8 & 1.5 & 1.2 & 0.98 \\
\hline 120 & 4.4 & 3.1 & 2.6 & 2.2 & 1.8 & 1.5 \\
\hline 183 & 5.4 & 4.2 & 3.6 & 3.2 & 2.8 & 2.6 \\
\hline
\end{tabular}

MAGNITUDE AND PROBABILITY OF ANNUAL HIGH FLOW

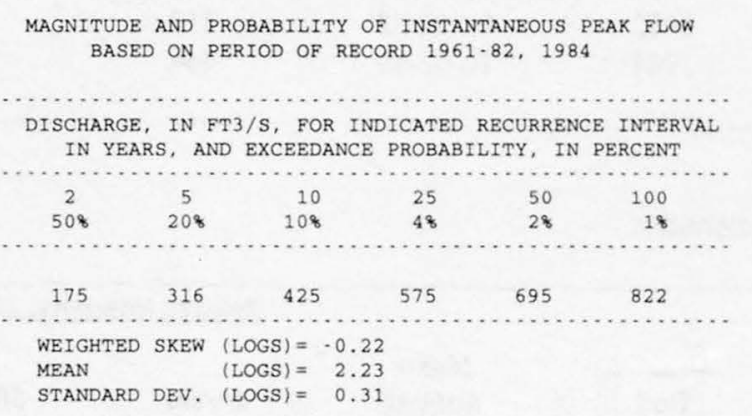

BASED ON PERIOD OF RECORD $1961-82$

\begin{tabular}{|c|c|c|c|c|c|c|}
\hline \multirow{4}{*}{$\begin{array}{l}\text { PERIOD } \\
\text { /CON- } \\
\text { SECU. }\end{array}$} & \multicolumn{6}{|c|}{ DISCHARGE, IN FT $3 / S$, FOR INDICATED } \\
\hline & & RECURRENCE & \multicolumn{4}{|c|}{ INTERVAL, IN YEARS, AND } \\
\hline & & EXCEEDANCE & \multicolumn{2}{|c|}{ PROBABILITY, } & IN PERC & \\
\hline & & & & & & ...... \\
\hline TIVE & 2 & 5 & 10 & 25 & $50 \#$ & $100 \#$ \\
\hline DAYS) & 508 & 208 & 108 & 48 & 28 & 18 \\
\hline 1 & 95 & 164 & 213 & 277 & 325 & 374 \\
\hline 3 & 82 & 146 & 193 & 255 & 303 & 352 \\
\hline 7 & 68 & 124 & 167 & 227 & 275 & 325 \\
\hline 15 & 55 & 105 & 147 & 208 & 259 & 315 \\
\hline 30 & 45 & 87 & 121 & 172 & 215 & 262 \\
\hline 60 & 37 & 70 & 97 & 137 & 169 & 205 \\
\hline 90 & 31 & 57 & 78 & 109 & 134 & 162 \\
\hline
\end{tabular}

DURATION TABLE OF DAILY MEAN FLOW FOR PERIOD OF RECORD 1961-82

DISCHARGE, IN FT3/S, WHICH WAS EQUALED OR EXCEEDED FOR INDICATED PERCENT OF TIME

18
18

\# Reliability of values in column is uncertain, and potential errors are large. 
09383400 LITTLE COLORADO RIVER AT GREER, AZ--Continued
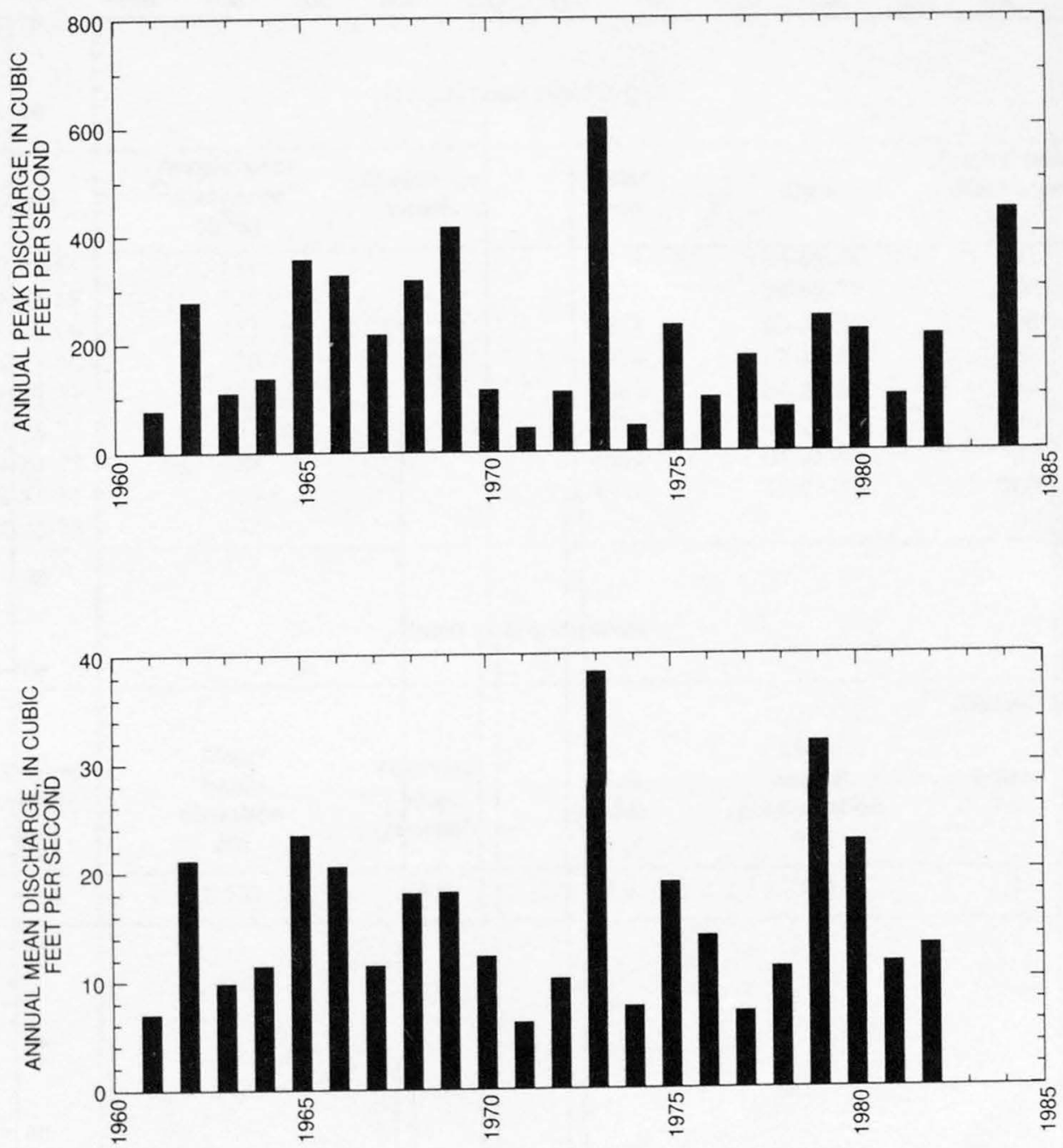
09383400 LITTLE COLORADO RIVER AT GREER, AZ--Continued
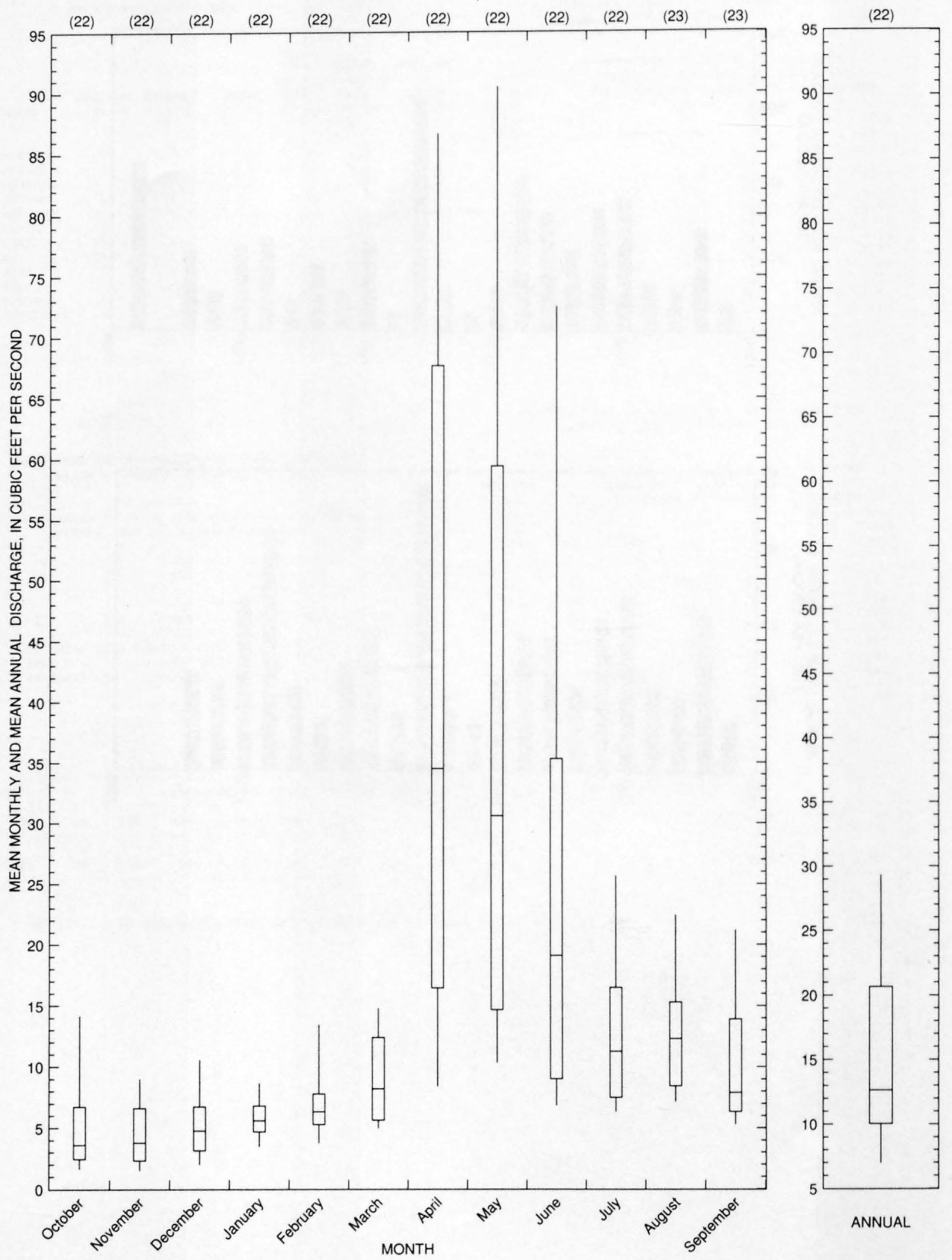
09383500 NUTRIOSO CREEK ABOVE NELSON RESERVOIR, NEAR SPRINGERVILLE, AZ

LOCATION.--Lat 34 01'49", long 109.11'09", in NE $1 / 4 \mathrm{SW}^{1} / 4$, sec.4, T.7 N., R.30 E., Apache County, Hydrologic Unit 15020001, in Apache-Sitgreaves National Forest, on right bank 2.4 mi upstream from dam on Nelson Reservoir and 9 mi southeast of Springerville.

DRAINAGE AREA.--83.3 $\mathrm{mi}^{2}$.

Annual peak discharges

\begin{tabular}{|c|c|c|c|c|c|c|c|}
\hline $\begin{array}{l}\text { Water } \\
\text { year }\end{array}$ & Date & $\begin{array}{c}\text { Annual peak } \\
\text { discharge } \\
\left(\mathrm{ft}^{3} / \mathrm{s}\right)\end{array}$ & $\begin{array}{c}\text { Discharge } \\
\text { codes }\end{array}$ & $\begin{array}{l}\text { Water } \\
\text { year }\end{array}$ & Date & $\begin{array}{c}\text { Annual peak } \\
\text { discharge } \\
\left(\mathrm{ft}^{3} / \mathrm{s}\right)\end{array}$ & $\begin{array}{c}\text { Discharge } \\
\text { codes }\end{array}$ \\
\hline 1967 & $09-05-67$ & 126 & & 1976 & $04-05-76$ & 41 & \\
\hline 1968 & $04-15-68$ & 121 & & 1977 & $09-03-77$ & 90 & \\
\hline 1969 & $09-08-69$ & 133 & & 1978 & $03-31-78$ & 88 & \\
\hline 1970 & $04-11-70$ & 30 & & 1979 & $12-18-78$ & 462 & \\
\hline 1971 & $09-01-71$ & 291 & & 1980 & $04-21-80$ & 174 & \\
\hline 1972 & $10-25-71$ & 67 & & 1981 & $04-10-81$ & 29 & \\
\hline 1973 & $04-28-73$ & 439 & & 1982 & $04-13-82$ & 37 & \\
\hline 1974 & $03-31-74$ & 7.1 & & 1984 & $10-02-83$ & 700 & HP \\
\hline 1975 & $04-25-75$ & 142 & & & & & \\
\hline
\end{tabular}

Basin characteristics

Rainfall intensity, 24-hour

\begin{tabular}{ccccccc}
$\begin{array}{c}\text { Main } \\
\text { channel } \\
\text { slope } \\
(\mathrm{ft} / \mathrm{mi})\end{array}$ & $\begin{array}{c}\text { Stream } \\
\text { length } \\
(\mathrm{mi})\end{array}$ & $\begin{array}{c}\text { Mean } \\
\text { basin } \\
\text { elevation } \\
(\mathrm{ft})\end{array}$ & $\begin{array}{c}\text { Forested } \\
\text { area } \\
\text { (percent) }\end{array}$ & $\begin{array}{c}\text { Soil } \\
\text { index }\end{array}$ & $\begin{array}{c}\text { Mean } \\
\text { annual } \\
\text { precipitation } \\
\text { (in) }\end{array}$ & $\begin{array}{c}\text { 2-year } \\
\text { (in) }\end{array}$ \\
\hline 78.0 & 12.6 & 8,550 & 75.0 & 3.0 & 20.0 & 1.7 \\
\hline
\end{tabular}


09383500 NUTRIOSO CREEK ABOVE NELSON RESERVOIR, NEAR SPRINGERVILLE, AZ--Continued

MEAN MONTHLY AND ANNUAL DISCHARGES 1968.82

\begin{tabular}{|c|c|c|c|c|c|c|}
\hline MONTH & $\begin{array}{l}\text { MAXIMUM } \\
\text { (FT } 3 / \mathrm{S})\end{array}$ & $\begin{array}{l}\text { MINIMUM } \\
(\text { FT } 3 / S)\end{array}$ & $\begin{array}{c}\text { MEAN } \\
(\mathrm{FT} 3 / \mathrm{S})\end{array}$ & $\begin{array}{l}\text { STAN- } \\
\text { DARD } \\
\text { DEVIA- } \\
\text { TION } \\
(\text { FT3/S) }\end{array}$ & $\begin{array}{l}\text { COEFFI- } \\
\text { CIBNT OF } \\
\text { VARI- } \\
\text { ATION }\end{array}$ & $\begin{array}{c}\text { PERCENT } \\
\text { OF } \\
\text { ANNUAL } \\
\text { RUNOFF }\end{array}$ \\
\hline OCTOBER & 16 & 0.00 & 2.4 & 4.3 & 1.8 & 3.3 \\
\hline NOVEMBER & 6.4 & 0.20 & 1.7 & 2.1 & 1.2 & 2.4 \\
\hline DECEMBER & 26 & 0.15 & 2.8 & 6.6 & 2.3 & 4.0 \\
\hline JANUARY & 9.5 & 0.17 & 2.2 & 2.7 & 1.2 & 3.1 \\
\hline FEBRUARY & 16 & 0.23 & 3.9 & 4.3 & 1.1 & 5.5 \\
\hline MARCH & 32 & 0.39 & 9.1 & 9.0 & 0.99 & 12.8 \\
\hline APRIL & 106 & 0.20 & 28 & 35 & 1.2 & 39.8 \\
\hline MAY & 117 & 0.12 & 15 & 30 & 2.0 & 20.8 \\
\hline JUNE & 12 & 0.01 & 2.0 & 3.7 & 1.8 & 2.9 \\
\hline JULY & 7.1 & 0.00 & 0.93 & 1.8 & 1.9 & 1.3 \\
\hline AUGUST & 5.0 & 0.00 & 1.2 & 1.3 & 1.1 & 1.6 \\
\hline SEPTEMBER & 11 & 0.00 & 1.8 & 2.8 & 1.6 & 2.5 \\
\hline ANNUAL & 25 & 0.56 & 5.9 & 7.1 & 1.2 & 100 \\
\hline
\end{tabular}

MAGNITUDE AND PROBABILITY OF INSTANTANEOUS PEAK FLOW BASED ON PERIOD OF RECORD $1967-82,1984$

DISCHARGE, IN FT3/S, FOR INDICATED RECURRENCE INTERVAL IN YEARS, AND EXCEEDANCE PROBABILITY, IN PERCENT

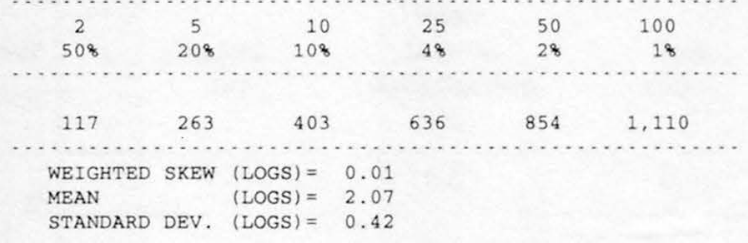

MAGNITUDE AND PROBABILITY OF ANNUAL LOW FLOW BASED ON PERIOD OF RECORD 1969-82

\begin{tabular}{|c|c|c|c|c|c|c|}
\hline \multirow{2}{*}{$\begin{array}{l}\text { PERIOD } \\
\text { (CON- } \\
\text { SECU. }\end{array}$} & \multicolumn{6}{|c|}{$\begin{array}{l}\text { RECURRENCE INTERVAL, IN YEARS, AND } \\
\text { NON-EXCEEDANCE PROBABILITY, IN PERCENT }\end{array}$} \\
\hline & $\cdots$ & $\cdots \cdots$ & $\ldots \ldots$ & …… & & $\cdots \cdots$ \\
\hline TIVE & 2 & 5 & $10 \#$ & $20 \#$ & $50 \#$ & $100 \#$ \\
\hline DAYS) & 508 & 208 & 108 & 58 & 28 & 18 \\
\hline 1 & & & & & & \\
\hline $\begin{array}{l}3 \\
7\end{array}$ & 0.00 & 0.00 & 0.00 & 0.00 & 0.00 & 0.00 \\
\hline 14 & 0.02 & 0.00 & 0.00 & 0.00 & 0.00 & 0.00 \\
\hline 30 & 0.09 & 0.00 & 0.00 & 0.00 & 0.00 & 0.00 \\
\hline 60 & 0.17 & 0,00 & 0.00 & 0.00 & 0.00 & 0.00 \\
\hline 90 & 0.19 & 0.04 & 0.01 & 0.00 & 0.00 & 0.00 \\
\hline 120 & 0.24 & 0.08 & 0.05 & 0.03 & 0.01 & 0.01 \\
\hline 183 & 0.48 & 0.22 & 0.15 & 0.11 & 0.07 & 0.05 \\
\hline
\end{tabular}

MAGNITUDE AND PROBABILITY OF ANNUAL HIGH FLOW BASED ON PERIOD OF RECORD 1968-82

\begin{tabular}{|c|c|c|c|c|c|c|}
\hline \multirow{3}{*}{$\begin{array}{l}\text { PERIOD } \\
\text { (CON- } \\
\text { SECU- }\end{array}$} & & \multicolumn{5}{|c|}{$\begin{array}{l}\text { DISCHARGE, IN FT } 3 / S \text {, FOR INDICATED } \\
\text { RECURRENCE INTERVAL, IN YEARS, AND }\end{array}$} \\
\hline & & & & & $\ldots \ldots$ & \\
\hline & 2 & 5 & $10 \#$ & $25 \#$ & $50 \#$ & $100 \#$ \\
\hline DAYS) & 508 & 208 & 108 & 48 & 28 & 18 \\
\hline 1 & 52 & 122 & 183 & 276 & 354 & 439 \\
\hline 3 & 40 & 99 & 160 & 264 & 364 & 486 \\
\hline 7 & 30 & 81 & 138 & 247 & 361 & 512 \\
\hline 15 & 23 & 67 & 118 & 223 & 339 & 497 \\
\hline 30 & 17 & 50 & 91 & 178 & 277 & 417 \\
\hline 60 & 11 & 34 & 63 & 122 & 189 & 283 \\
\hline 90 & 8.8 & 27 & 48 & 91 & 137 & 200 \\
\hline
\end{tabular}

DURATION TABLE OF DAILY MEAN FLOW FOR PERIOD OF RECORD 1968.82

DISCHARGE, IN FT3/S, WHICH WAS EQUALED OR EXCEEDED FOR INDICATED PERCENT OF TIME

\begin{tabular}{|c|c|c|c|c|c|c|c|c|c|c|c|c|c|c|c|c|}
\hline 18 & 58 & 108 & 158 & 208 & 308 & 408 & 508 & 608 & 708 & 808 & 908 & 958 & 988 & 998 & 99.58 & 99.98 \\
\hline 100 & 29 & 14 & 7.9 & 4.9 & 2.4 & 1.2 & 0.68 & 0.44 & 0.28 & 0.18 & 0.05 & 0.00 & 0.00 & 0.00 & 0.00 & 0.00 \\
\hline
\end{tabular}

\# Reliability of values in column is uncertain, and potential errors are large. 
09383500 NUTRIOSO CREEK ABOVE NELSON RESERVOIR, NEAR SPRINGERVILLE, AZ--Continued
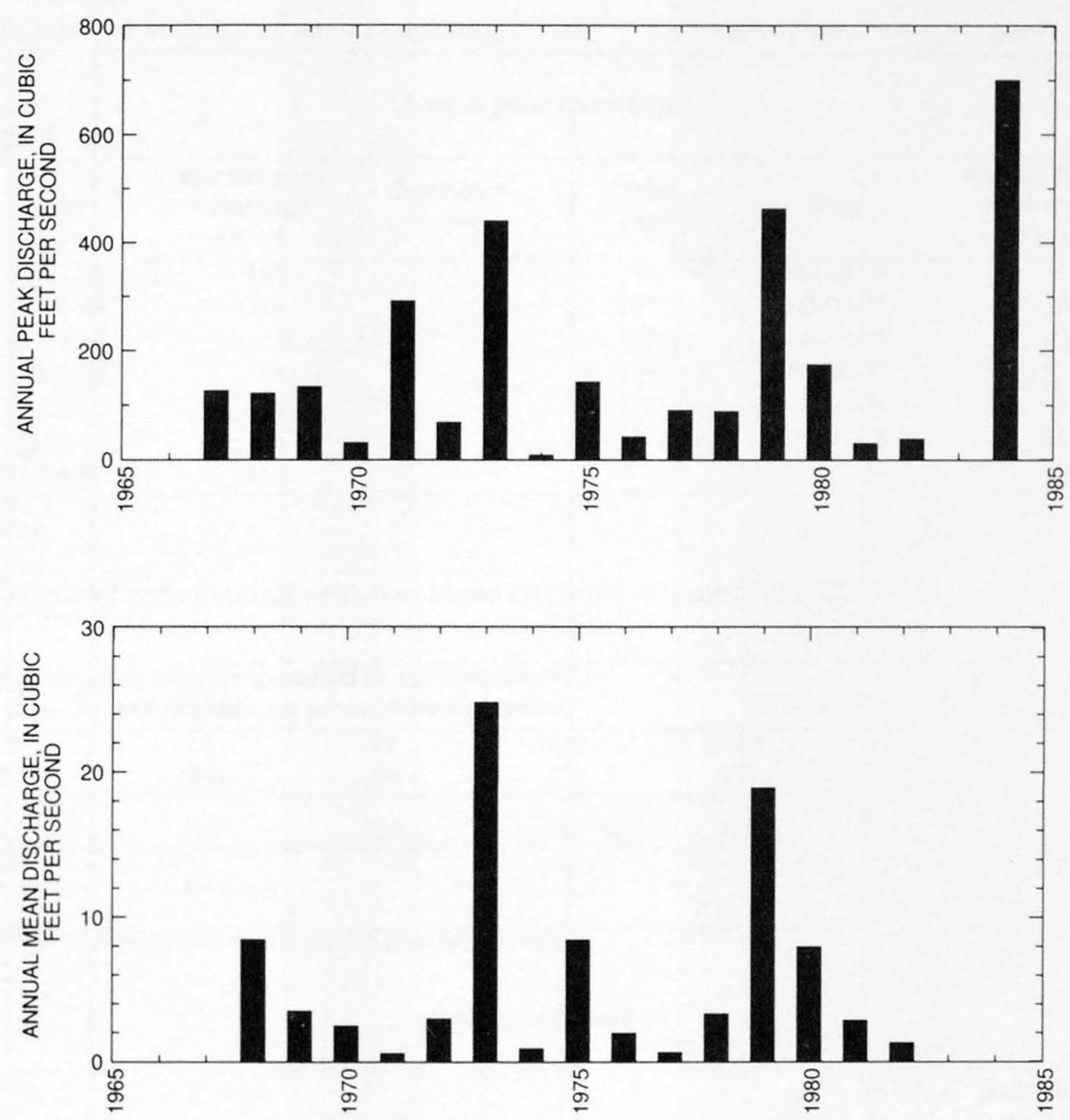
09383500 NUTRIOSO CREEK ABOVE NELSON RESERVOIR, NEAR SPRINGERVILLE, AZ--Continued
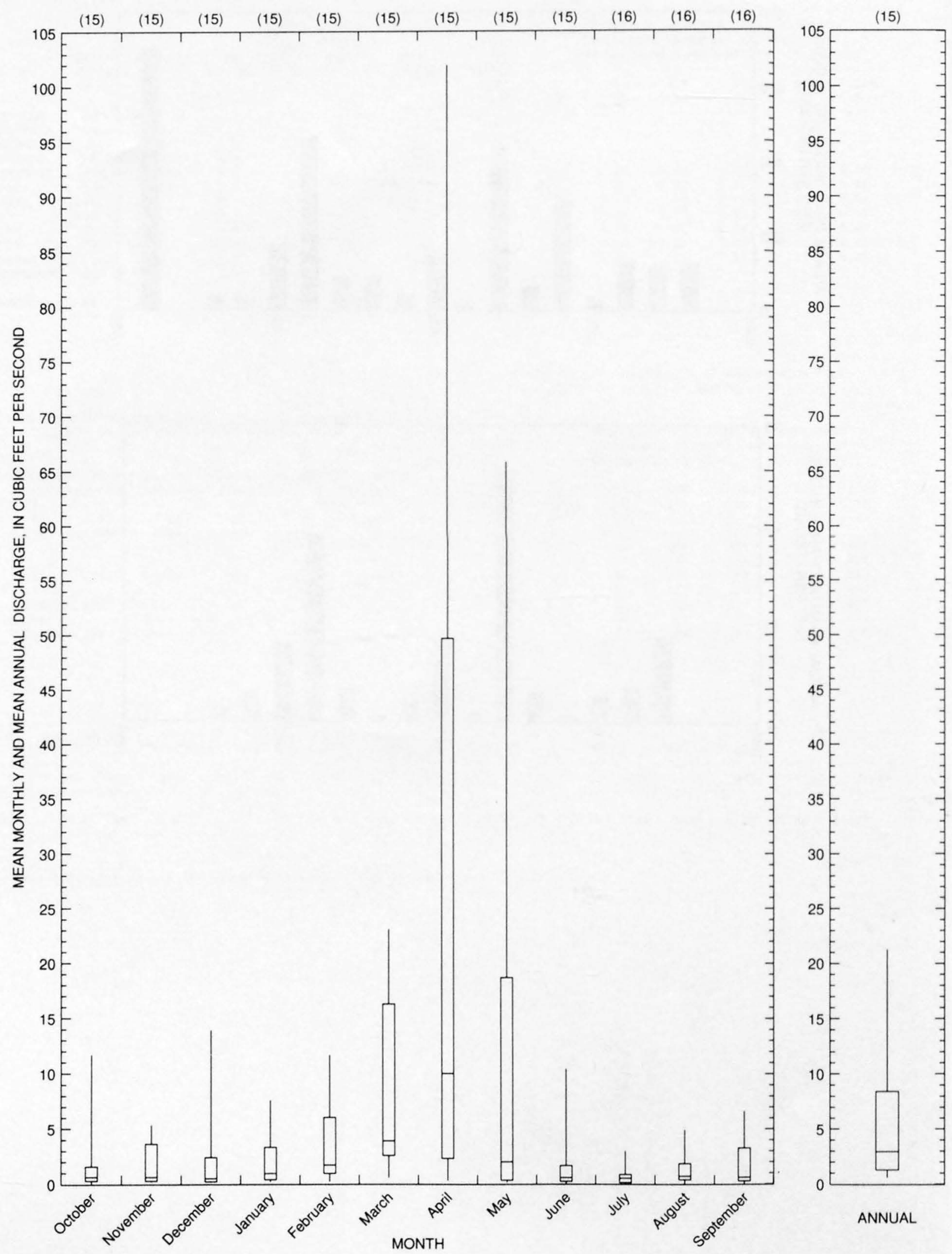
09383600 FISH CREEK NEAR EAGAR, AZ

LOCATION.--Lat 34 04'35", long 109'27'45", in $\mathrm{SW}^{1} / 4^{\circ} \mathrm{NE}^{1} / 4$, sec.23, T.8 N., R.27 E., Apache County, Hydrologic Unit 15020001, at State Highway 73, 10.5 mi west of Eagar.

DRAINAGE AREA.--16.9 $\mathrm{mi}^{2}$, of which $2.5 \mathrm{mi}^{2}$ is noncontributing.

Annual peak discharges

\begin{tabular}{|c|c|c|c|c|c|c|c|}
\hline $\begin{array}{l}\text { Water } \\
\text { year }\end{array}$ & Date & $\begin{array}{c}\text { Annual peak } \\
\text { discharge } \\
\left(\mathrm{ft}^{3} / \mathrm{s}\right)\end{array}$ & $\begin{array}{l}\text { Discharge } \\
\text { codes }\end{array}$ & $\begin{array}{l}\text { Water } \\
\text { year }\end{array}$ & Date & $\begin{array}{c}\text { Annual peak } \\
\text { discharge } \\
\left(\mathrm{ft}^{3} / \mathrm{s}\right)\end{array}$ & $\begin{array}{c}\text { Discharge } \\
\text { codes }\end{array}$ \\
\hline 1963 & $08-21-63$ & 168 & & 1970 & $00-00-70$ & 3.0 & ES \\
\hline 1964 & $08-01-64$ & 236 & & 1971 & $00-00-71$ & 3.0 & LT \\
\hline 1965 & $07-25-65$ & 81 & & 1972 & $00-00-72$ & 0 & \\
\hline 1966 & $04-03-66$ & 48 & & 1973 & $04-00-73$ & 153 & \\
\hline 1967 & $09-05-67$ & 139 & & 1974 & $00-00-74$ & 3.0 & ES \\
\hline 1968 & $00-00-68$ & 45 & & 1975 & $09-07-75$ & 81 & \\
\hline 1969 & $09-08-69$ & 215 & & & & & \\
\hline
\end{tabular}

Magnitude and probability of instantaneous peak flow based on period of record 1963-75

\begin{tabular}{|c|c|c|c|c|c|}
\hline \multicolumn{6}{|c|}{$\begin{array}{l}\text { Discharge, in } \mathrm{ft}^{3} / \mathrm{s} \text {, for indicated recurrence interval } \\
\text { in years, and exceedance probablility, in percent }\end{array}$} \\
\hline 2 & 5 & 10 & 25 & $50 t$ & $100 \dagger$ \\
\hline $50 \%$ & $20 \%$ & $10 \%$ & $4 \%$ & $2 \%$ & $1 \%$ \\
\hline 74.5 & 159 & 227 & 325 & 403 & 485 \\
\hline Weighted skew & $(\log s)=$ & -0.42 & & & \\
\hline Mean & $(\log s)=$ & 1.84 & & & \\
\hline Standard dev. & $(\log s)=$ & 0.42 & & & \\
\hline
\end{tabular}

† Reliability of values in column is uncertain, and potential errors are large.

Basin characteristics

Rainfall intensity, 24-hour

\begin{tabular}{cccccccc}
$\begin{array}{c}\text { Main } \\
\text { channel } \\
\text { slope } \\
\text { (ft/mi) }\end{array}$ & $\begin{array}{c}\text { Stream } \\
\text { length } \\
\text { (mi) }\end{array}$ & $\begin{array}{c}\text { Mean } \\
\text { basin } \\
\text { elevation } \\
\text { (ft) }\end{array}$ & $\begin{array}{c}\text { Forested } \\
\text { area } \\
\text { (percent) }\end{array}$ & $\begin{array}{c}\text { Soil } \\
\text { index }\end{array}$ & $\begin{array}{c}\text { Mean } \\
\text { annual } \\
\text { precipitation } \\
\text { (in) }\end{array}$ & $\begin{array}{c}\text { 2-year } \\
\text { (in) }\end{array}$ & $\begin{array}{c}50-\text { year } \\
\text { (in) }\end{array}$ \\
\hline 164 & 8.1 & 9,160 & 38.0 & 3.0 & 26.1 & 1.8 & 4.2 \\
\hline
\end{tabular}

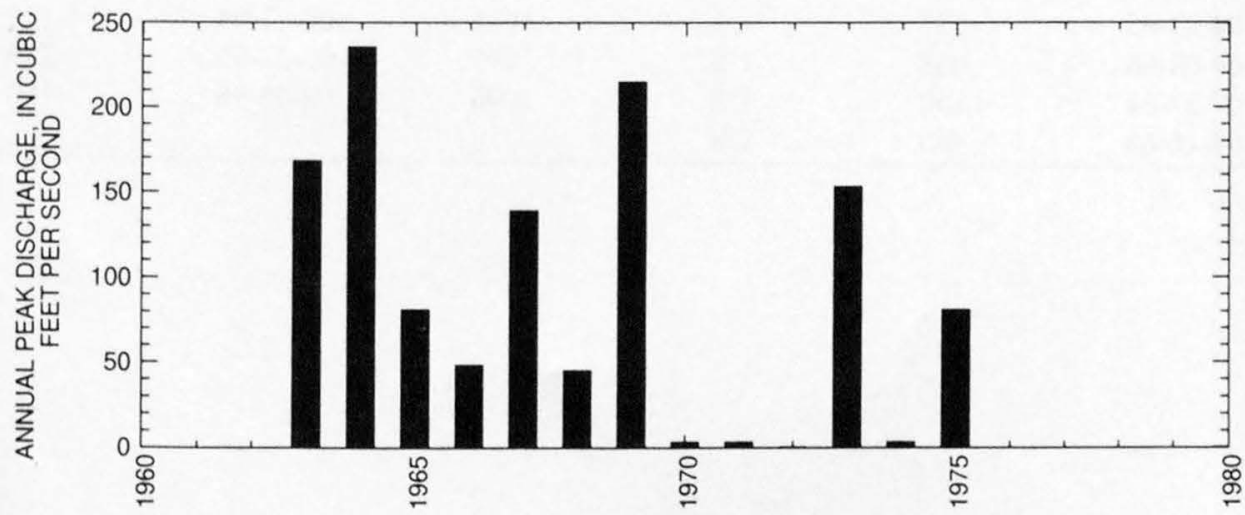


09384000 LITTLE COLORADO RIVER ABOVE LYMAN LAKE, NEAR ST. JOHNS, AZ

LOCATION.--Lat 34 $18^{\prime} 52^{\prime \prime}$, long 109 $21^{\prime} 42^{\prime \prime}$, in SW $1 / 4 \mathrm{SE}^{1} / 4$ sec.27, T.11 N., R.28 E., Apache County, Hydrologic Unit 15020001 , on left bani $0.75 \mathrm{mi}$ downstream from Coyote Creek, $6 \mathrm{mi}$ upstream from Lyman Dam, and $15 \mathrm{mi}$ south of St. Johns.

DRAINAGE AREA.--706 $\mathrm{mi}^{2}$, of which $2.5 \mathrm{mi} 2$ is noncontributing.

PERIOD OF RECORD.--April 1940 to current year. Prior to October 1975 published as "above Lyman Reservoir."

REVISED RECORDS.--WDR AZ-88-1: Drainage area.

GAGE.--Water-stage recorder and crest-stage gage. Elevation of gage is 6,010 ft above sea level, from topographic map. Prior to Dec. 7, 1976 water-stage recorder at site $0.4 \mathrm{mi}$ downstream at datum approximately $20 \mathrm{ft}$ lower, used as supplemental gage Mar. 21, 1980, to Apr. 2 1987. See WSP 1313 for history of changes prior to 1950.

REMARKS.--Flow regulated by many small reservoirs - combined capacity, about 15,500 acre-ft. Diversions for irrigation of about 6,700 acre above station.

EXTREMES FOR PERIOD OF RECORD.--Maximum discharge, 16,000 $\mathrm{ft}^{3} / \mathrm{s}$ July 25,1940 , gage height, $17.1 \mathrm{ft}$, datum then in use, from flood marks, by slope-area measurement of peak flow and reservoir inflow studies; maximum gage height, $18.6 \mathrm{ft}$, Sept. 12, 1975 , at previous sit (from graph recorded to $18.4 \mathrm{ft}$ ); no flow at times.

Annual peak discharges

\begin{tabular}{|c|c|c|c|c|c|c|c|}
\hline $\begin{array}{l}\text { Water } \\
\text { year }\end{array}$ & Date & $\begin{array}{c}\text { Annual peak } \\
\text { discharge } \\
\left(\mathrm{ft}^{3} / \mathrm{s}\right)\end{array}$ & $\begin{array}{l}\text { Discharge } \\
\text { codes }\end{array}$ & $\begin{array}{l}\text { Water } \\
\text { year }\end{array}$ & Date & $\begin{array}{c}\text { Annual peak } \\
\text { discharge } \\
\left(\mathrm{ft}^{3} / \mathrm{s}\right)\end{array}$ & $\begin{array}{l}\text { Discharge } \\
\text { codes }\end{array}$ \\
\hline 1940 & $07-25-40$ & ${ }^{1} 16,000$ & HP & 1969 & $07-26-69$ & 764 & UR \\
\hline 1942 & $08-10-42$ & 379 & UR & 1971 & $09-09-71$ & 229 & UR \\
\hline 1943 & $08-22-43$ & 2,360 & UR & 1972 & $08-29-72$ & 225 & UR \\
\hline 1946 & $08-04-46$ & 6,000 & UR & 1975 & $09-12-75$ & 1,600 & UR \\
\hline 1947 & $08-22-47$ & 1,620 & UR & 1976 & $04-09-76$ & 170 & UR \\
\hline 1948 & $04-17-48$ & 732 & UR & 1977 & $08-21-77$ & 389 & UR \\
\hline 1949 & $08-02-49$ & 1,000 & UR & 1978 & $08-01-78$ & 389 & UR \\
\hline 1950 & $07-18-50$ & 181 & UR & 1979 & $04-00-79$ & 1,500 & UR \\
\hline 1951 & $08-02-51$ & 3,200 & UR & 1980 & $04-23-80$ & 840 & UR \\
\hline 1956 & $08-18-56$ & 206 & UR & 1985 & $03-13-85$ & 695 & UR \\
\hline 1957 & $08-27-57$ & 2,850 & UR & 1986 & $08-26-86$ & 292 & UR \\
\hline 1958 & $04-23-58$ & 1,120 & UR & 1987 & $04-20-87$ & 1,090 & UR \\
\hline 1959 & $08-08-59$ & 1,340 & UR & 1988 & 09-01-88 & 414 & UR \\
\hline 1960 & $03-30-60$ & 323 & UR & 1989 & $07-28-89$ & 165 & UR \\
\hline 1961 & $08-11-61$ & 619 & UR & 1990 & $07-11-90$ & 1,020 & UR \\
\hline 1962 & $04-16-62$ & 736 & UR & 1991 & $04-11-91$ & 299 & UR \\
\hline 1963 & $08-26-63$ & 733 & UR & 1992 & $04-24-92$ & 665 & UR \\
\hline 1964 & $07-31-64$ & 1,160 & UR & 1993 & $08-29-93$ & 555 & UR \\
\hline 1965 & $04-23-65$ & 527 & UR & 1994 & $09-03-94$ & 1,120 & UR \\
\hline 1966 & $04-04-66$ & 658 & UR & 1995 & $03-22-95$ & 289 & UR \\
\hline
\end{tabular}

${ }^{\text {THighest since } 1900 .}$ 
09384000 LITTLE COLORADO RIVER ABOVE LYMAN LAKE, NEAR ST. JOHNS, AZ--Continued

Discharge rating table developed October 1993

\begin{tabular}{cccc}
\hline $\begin{array}{c}\text { Gage-Height } \\
(\mathrm{ft})\end{array}$ & $\begin{array}{c}\text { Discharge } \\
\left(\mathrm{ft}^{3} / \mathbf{s}\right)\end{array}$ & $\begin{array}{c}\text { Gage-Height } \\
(\mathrm{ft})\end{array}$ & $\begin{array}{c}\text { Discharge } \\
\left(\mathrm{ft}^{3} / \mathbf{s}\right)\end{array}$ \\
\hline 2.0 & 11.3 & 5.0 & 520 \\
3.0 & 83 & 6.0 & 873 \\
4.0 & 251 & 7.0 & 1,330 \\
\hline
\end{tabular}

Basin characteristics

Rainfall intensity, 24-hour

\begin{tabular}{|c|c|c|c|c|c|c|c|}
\hline $\begin{array}{l}\text { Main } \\
\text { channel } \\
\text { slope } \\
(\mathrm{ft} / \mathrm{mi})\end{array}$ & $\begin{array}{l}\text { Stream } \\
\text { length } \\
(\mathrm{mi})\end{array}$ & $\begin{array}{c}\text { Mean } \\
\text { basin } \\
\text { elevation } \\
(f t)\end{array}$ & $\begin{array}{l}\text { Forested } \\
\text { area } \\
\text { (percent) }\end{array}$ & $\begin{array}{c}\text { Soil } \\
\text { index }\end{array}$ & $\begin{array}{c}\text { Mean } \\
\text { annual } \\
\text { precipitation } \\
\text { (in) }\end{array}$ & $\begin{array}{l}\text { 2-year } \\
\text { (in) }\end{array}$ & $\begin{array}{c}50 \text {-year } \\
\text { (in) }\end{array}$ \\
\hline 89.5 & 41.7 & 7,760 & 39.0 & 2.9 & 20.0 & 1.6 & 3.3 \\
\hline
\end{tabular}


09384000 LITTLE COLORADO RIVER ABOVE LYMAN LAKE, NEAR ST. JOHNS, AZ--Continued

MEAN MONTHLY AND ANNUAL DISCHARGES 1941.96

\begin{tabular}{|c|c|c|c|c|c|c|}
\hline MONTH & $\begin{array}{l}\text { MAXIMUM } \\
(\text { FT3/S) }\end{array}$ & $\begin{array}{l}\text { MINIMUM } \\
(\text { FT } 3 / \mathrm{S})\end{array}$ & $\begin{array}{c}\text { MEAN } \\
(F T 3 / S)\end{array}$ & $\begin{array}{l}\text { STAN. } \\
\text { DARD } \\
\text { DEVIA. } \\
\text { TION } \\
\text { (FT3/S) }\end{array}$ & $\begin{array}{l}\text { COEFFI- } \\
\text { CIENT OF } \\
\text { VARI- } \\
\text { ATION }\end{array}$ & $\begin{array}{c}\text { PERCENT } \\
\text { OF } \\
\text { ANNUAL } \\
\text { RUNOFF }\end{array}$ \\
\hline OCTOBER & 213 & 0.07 & 10 & 28 & 2.8 & 3.6 \\
\hline NOVEMBER & 38 & 0.32 & 8.3 & 8.3 & 0.99 & 3.0 \\
\hline DECEMBER & 47 & 0.83 & 11 & 9.7 & 0.92 & 3.8 \\
\hline JANUARY & 39 & 2.1 & 11 & 8.0 & 0.72 & 4.0 \\
\hline FEBRUARY & 43 & 2.8 & 13 & 8.6 & 0.65 & 4.8 \\
\hline MARCH & 182 & 1.9 & 32 & 36 & 1.1 & 11.4 \\
\hline APRIL & 397 & 1.3 & 99 & 108 & 1.1 & 35.4 \\
\hline MAY & 374 & 0.73 & 38 & 70 & 1.8 & 13.7 \\
\hline JUNE & 95 & 0.01 & 10 & 17 & 1.7 & 3.6 \\
\hline JULY & 40 & 0.00 & 9.9 & 9.5 & 0.95 & 3.6 \\
\hline AUGUST & 143 & 0.78 & 23 & 27 & 1.2 & 8.3 \\
\hline SEPTEMBER & 105 & 0.02 & 13 & 19 & 1.4 & 4.8 \\
\hline ANNUAL & 72 & 2.9 & 23 & 18 & 0.77 & 100 \\
\hline
\end{tabular}

MAGNITUDE AND PROBABILITY OF INSTANTANEOUS PEAK FLOW BASED ON PERIOD OF RECORD 1940-96

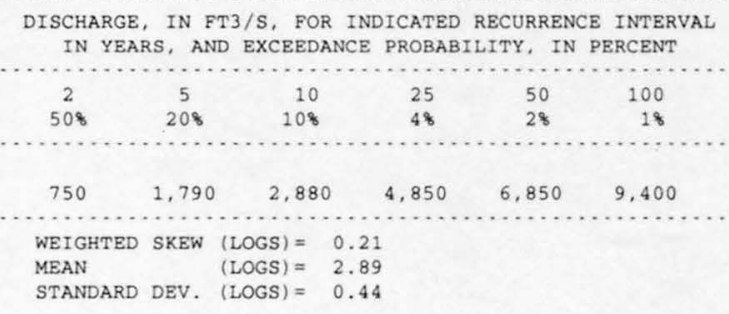

MAGNITUDE AND PROBABILITY OF ANNUAL LOW FLOW BASED ON PERIOD OF RECORD 1941.96

\begin{tabular}{|c|c|c|c|c|c|c|}
\hline \multirow{4}{*}{$\begin{array}{l}\text { PERIOD } \\
\text { (CON- } \\
\text { SECU- } \\
\text { TIVE } \\
\text { DAYS) }\end{array}$} & \multicolumn{6}{|c|}{$\begin{array}{l}\text { DISCHARGE, IN FT3/S, FOR INDICATED } \\
\text { RECURRENCE INTERVAL, IN YEARS, AND } \\
\text { NON-EXCEEDANCE PROBABILITY, IN PERCENT }\end{array}$} \\
\hline & $\cdots$ & & & ....... & & \\
\hline & 2 & 5 & 10 & 20 & 50 & $100 \#$ \\
\hline & 508 & 208 & 108 & 58 & 28 & 18 \\
\hline 1 & 0.56 & 0.00 & 0.00 & 0.00 & 0.00 & 0.00 \\
\hline 3 & 0.64 & 0.02 & 0.00 & 0.00 & 0.00 & 0.00 \\
\hline 7 & 0.83 & 0.04 & 0.00 & 0.00 & 0.00 & 0.00 \\
\hline 14 & 1.1 & 0.13 & 0.00 & 0.00 & 0.00 & 0.00 \\
\hline 30 & 1.6 & 0.32 & 0.06 & 0.00 & 0.00 & 0.00 \\
\hline 60 & 2.4 & 0.86 & 0.44 & 0.23 & 0.11 & 0.06 \\
\hline 90 & 3.2 & 1.4 & 0.84 & 0.54 & 0.32 & 0.22 \\
\hline 120 & 4.6 & 2.2 & 1.4 & 0.98 & 0.61 & 0.44 \\
\hline 183 & 6.4 & 3.3 & 2.3 & 1.7 & 1.2 & 0.90 \\
\hline
\end{tabular}

MAGNITUDE AND PROBABILITY OF ANNUAL HIGH FLOW BASED ON PERIOD OF RECORD 1941.96

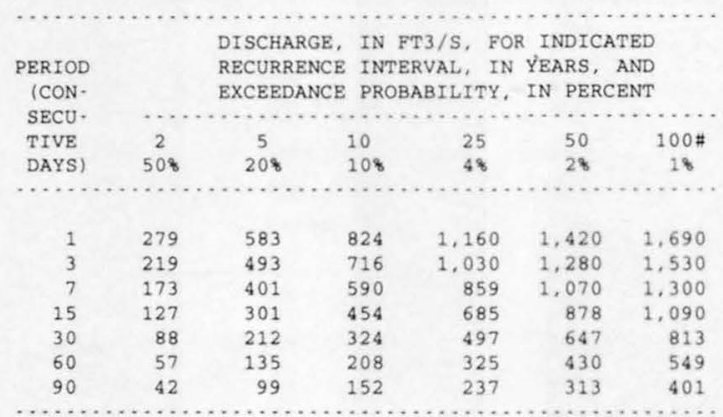

DURATION TABLE OF DAILY MEAN FLOW FOR PERIOD OF RECORD 1941.96

DISCHARGE, IN FT3/S, WHICH WAS EQUALED OR EXCEEDED FOR INDICATED PERCENT OF TIME

\begin{tabular}{|c|c|c|c|c|c|c|c|c|c|c|c|c|c|c|c|c|}
\hline 18 & 58 & 108 & 158 & 208 & 308 & 408 & 508 & 608 & 708 & 808 & 908 & 958 & 988 & 998 & 99.58 & 99.98 \\
\hline 335 & 97 & 47 & 30 & 21 & 14 & 9.5 & 7.4 & 5.6 & 4.1 & 2.6 & 1.3 & 0.57 & 0.10 & 0.00 & 0.00 & 0.00 \\
\hline
\end{tabular}

\# Reliability of values in column is uncertain, and potential errors are large. 
ANNUAL MEAN DISCHARGE, IN CUBIC

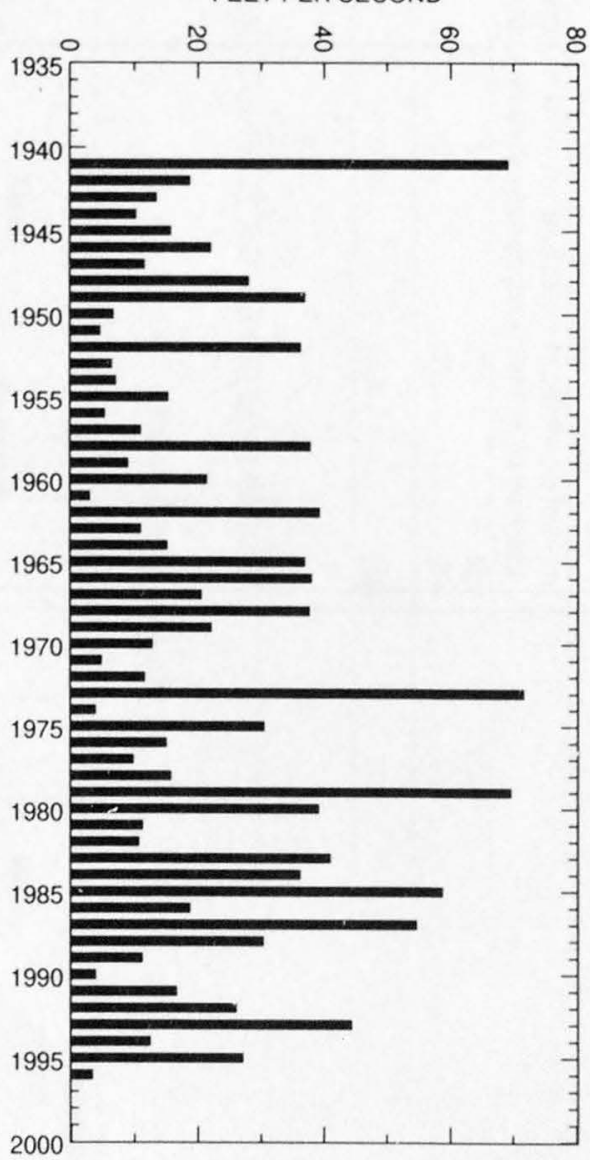

ANNUAL PEAK DISCHARGE, IN CUBIC FEET PER SECOND

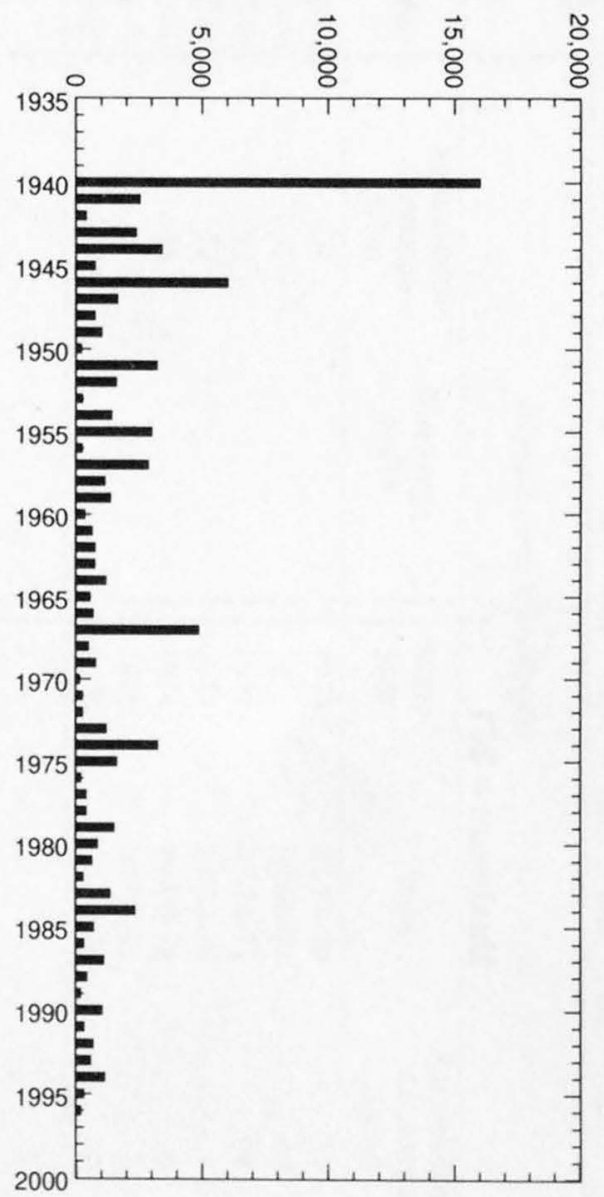


09384000 LITTLE COLORADO RIVER ABOVE LYMAN LAKE, NEAR ST. JOHNS, AZ--Continued
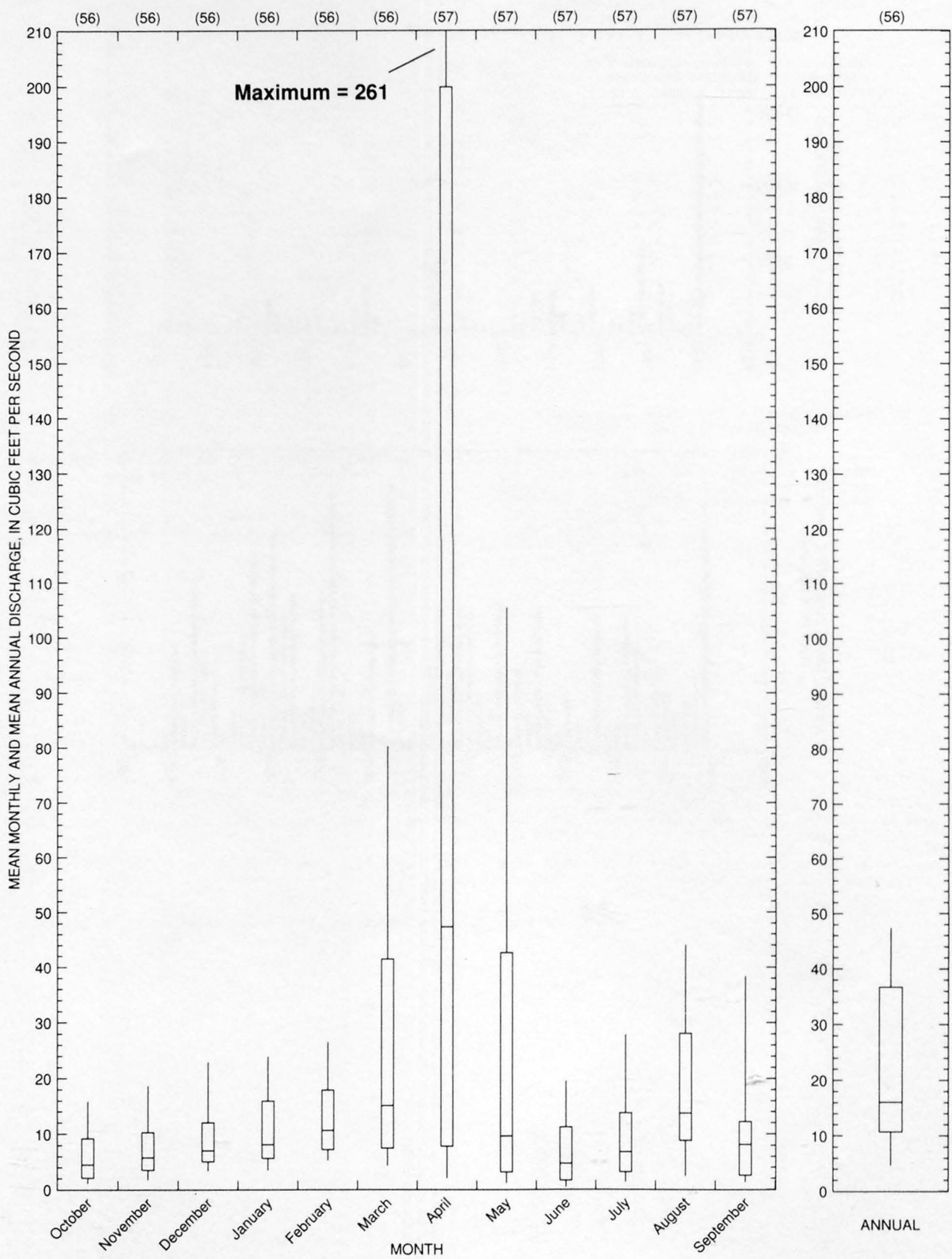
09384200 LYMAN RESERVOIR TRIBUTARY NEAR ST. JOHNS, AZ

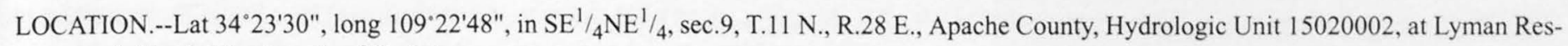
ervoir Road, 10 mi south of St. Johns.

DRAINAGE AREA.--0.24 $\mathrm{mi}^{2}$.

Annual peak discharges

\begin{tabular}{ccccccc}
\hline $\begin{array}{c}\text { Water } \\
\text { year }\end{array}$ & Date & $\begin{array}{c}\text { Annual peak } \\
\text { discharge } \\
\left(\mathrm{ft}^{3} / \mathbf{s}\right)\end{array}$ & $\begin{array}{c}\text { Discharge } \\
\text { codes }\end{array}$ & $\begin{array}{c}\text { Water } \\
\text { year }\end{array}$ & $\begin{array}{c}\text { Annual peak } \\
\text { discharge } \\
\left(\mathrm{ft}^{3} / \mathbf{s}\right)\end{array}$ & $\begin{array}{c}\text { Discharge } \\
\text { codes }\end{array}$ \\
\hline 1963 & $08-26-63$ & 101 & & 1970 & $07-20-70$ & 33 \\
1964 & $08-11-64$ & 53 & & 1971 & $08-00-71$ & 60 \\
1965 & $08-02-65$ & 42 & & 1972 & $07-24-72$ & 64 \\
1966 & $09-30-66$ & 52 & & 1973 & $07-00-73$ & 5.0 \\
1967 & $00-00-67$ & 48 & LT & 1974 & $00-00-74$ & 0 \\
1968 & $00-00-68$ & 1.0 & & 1975 & $09-06-75$ & 3.0 \\
1969 & $09-00-69$ & 7.0 & & & $00-00-76$ & 60 \\
\hline
\end{tabular}

Magnitude and probability of instantaneous peak flow based on period of record 1963-76

\begin{tabular}{|c|c|c|c|c|c|}
\hline \multicolumn{6}{|c|}{$\begin{array}{l}\text { Discharge, in } \mathrm{ft}^{3} / \mathrm{s} \text {, for indicated recurrence interval } \\
\text { in years, and exceedance probablility, in percent }\end{array}$} \\
\hline 2 & 5 & 10 & 25 & $50 \dagger$ & $100 \dagger$ \\
\hline $50 \%$ & $20 \%$ & $10 \%$ & $4 \%$ & $2 \%$ & $1 \%$ \\
\hline 42.8 & 62 & 74.9 & 91.4 & 104 & 116 \\
\hline Weighted skew & $(\log s)=$ & -0.11 & & & \\
\hline Mean & $(\log s)=$ & 1.63 & & & \\
\hline Standard dev. & $(\log s)=$ & 0.20 & & & \\
\hline
\end{tabular}

$\mp$ Reliability of values in column is uncertain, and potential errors are large.

Basin characteristics

\begin{tabular}{cccccccc}
\hline $\begin{array}{c}\text { Main } \\
\text { channel } \\
\text { slope } \\
(\mathrm{ft} / \mathrm{mi})\end{array}$ & $\begin{array}{c}\text { Stream } \\
\text { length } \\
(\mathrm{mi})\end{array}$ & $\begin{array}{c}\text { Mean } \\
\text { basin } \\
\text { elevation } \\
(\mathrm{ft})\end{array}$ & $\begin{array}{c}\text { Forested } \\
\text { area } \\
\text { (percent) }\end{array}$ & $\begin{array}{c}\text { Soil } \\
\text { index }\end{array}$ & $\begin{array}{c}\text { Mean } \\
\text { annual } \\
\text { precipitation } \\
\text { (in) }\end{array}$ & $\begin{array}{c}\text { Rainfall intensity, 24-hour } \\
\text { (in) }\end{array}$ & $\begin{array}{c}50 \text {-year } \\
\text { (in) }\end{array}$ \\
\hline 267 & 1.0 & 6,100 & 58.0 & 3.0 & 11.6 & 1.6 \\
\hline
\end{tabular}

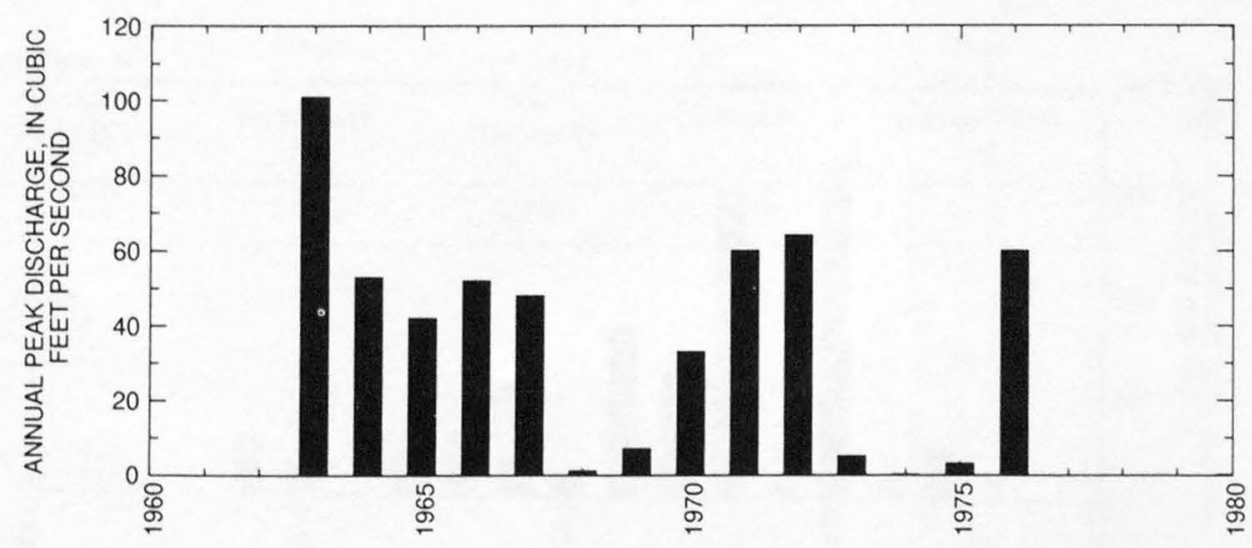




\section{LITTLE COLORADO RIVER TRIBUTARY NEAR ST. JOHNS, AZ}

LOCATION.--Lat $34^{\circ} 27^{\prime} 04^{\prime \prime}$, long $109^{\circ} 15^{\prime} 23^{\prime \prime}$, in NE $\frac{1}{4} \mathrm{SE}^{1} / 4$, sec.10, T.12 N., R.29 E., Apache County, Hydrologic Unit 15020002 , at county rodi

$7 \mathrm{mi}$ southeast of St. Johns.

DRAINAGE AREA.--0.35 $\mathrm{mi}^{2}$.

Annual peak discharges

\begin{tabular}{|c|c|c|c|c|c|c|c|}
\hline $\begin{array}{l}\text { Water } \\
\text { year }\end{array}$ & Date & $\begin{array}{c}\text { Annual peak } \\
\text { discharge } \\
\left(\mathrm{ft}^{3} / \mathrm{s}\right)\end{array}$ & $\begin{array}{l}\text { Discharge } \\
\text { codes }\end{array}$ & $\begin{array}{l}\text { Water } \\
\text { year }\end{array}$ & Date & $\begin{array}{l}\text { Annual peak } \\
\text { discharge } \\
\left(\mathrm{ft}^{3} / \mathrm{s}\right)\end{array}$ & $\begin{array}{c}\text { Discharge } \\
\text { codes }\end{array}$ \\
\hline 1963 & $08-00-63$ & 48 & & 1970 & $10-21-69$ & 24 & \\
\hline 1965 & $09-04-65$ & 326 & & 1972 & $12-26-71$ & 61 & \\
\hline 1966 & $08-30-66$ & 16 & & 1973 & $07-00-73$ & 42 & \\
\hline 1967 & $00-00-67$ & 300 & & 1974 & $00-00-74$ & 0 & \\
\hline 1969 & $07-00-69$ & 165 & & 1976 & $08-00-76$ & 57 & \\
\hline
\end{tabular}

Magnitude and probability of instantaneous peak flow based on period of record 1963-76

\begin{tabular}{|c|c|c|c|c|c|}
\hline \multicolumn{6}{|c|}{$\begin{array}{l}\text { Discharge, in } \mathrm{ft}^{3} / \mathrm{s} \text {, for indicated recurrence interval } \\
\text { in years, and exceedance probablility, in percent }\end{array}$} \\
\hline 2 & 5 & 10 & 25 & $50 \dagger$ & $100 t$ \\
\hline $50 \%$ & $20 \%$ & $10 \%$ & $4 \%$ & $2 \%$ & $1 \%$ \\
\hline 52 & 127 & 206 & 348 & 491 & 672 \\
\hline Weighted skew & $(\log s)=$ & 0.13 & & & \\
\hline Mean & $(\log s)=$ & 1.73 & & & \\
\hline Standard dev. & $(\log s)=$ & 0.46 & & & \\
\hline
\end{tabular}

f Reliability of values in column is uncertain, and potential errors are large.

Basin characteristics

\begin{tabular}{cccccccc}
\hline $\begin{array}{c}\text { Main } \\
\text { channel } \\
\text { slope } \\
(\mathrm{ft} / \mathrm{mi})\end{array}$ & $\begin{array}{c}\text { Stream } \\
\text { length } \\
\text { (mi) }\end{array}$ & $\begin{array}{c}\text { Mean } \\
\text { basin } \\
\text { elevation } \\
(\mathrm{ft})\end{array}$ & $\begin{array}{c}\text { Forested } \\
\text { area } \\
\text { (percent) }\end{array}$ & $\begin{array}{c}\text { Soil } \\
\text { index }\end{array}$ & $\begin{array}{c}\text { Mean } \\
\text { annual } \\
\text { precipitation } \\
\text { (in) }\end{array}$ & $\begin{array}{c}\text { Rainfall intensity, 24-hour } \\
\text { (in) }\end{array}$ & $\begin{array}{c}50 \text {-year } \\
\text { (in) }\end{array}$ \\
\hline 417 & 0.48 & 6,350 & 54.0 & 1.0 & 11.1 & 1.5 \\
\hline
\end{tabular}

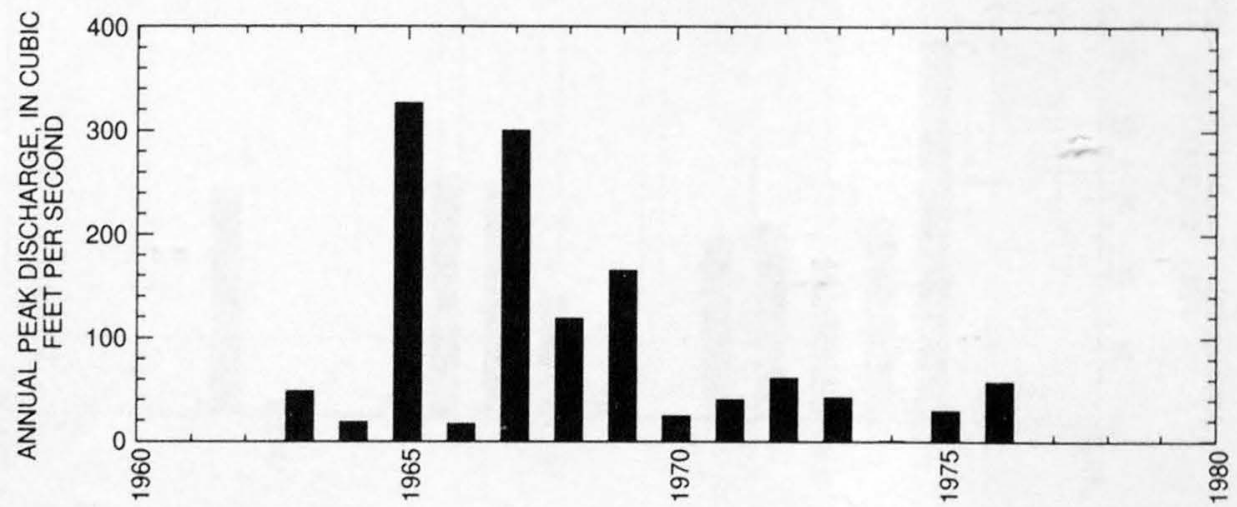


09386500 LITTLE COLORADO RIVER ABOVE ZUNI RIVER, NEAR HUNT, AZ

LOCATION.--Lat 34 38'30", long 109.40'35", in $\mathrm{SW}^{1} / 4^{\prime} \mathrm{NW}^{1} / 4$, sec.2, T.14 N., R.25 E. (unsurveyed), Apache County, Hydrologic Unit 15020002 , on right bank $500 \mathrm{ft}$ upstream from Zuni River and $3.6 \mathrm{mi}$ northwest of Hunt.

DRAINAGE AREA.--3,741 $\mathrm{mi}^{2}$, of which $184 \mathrm{mi}^{2}$ is noncontributing.

REMARKS.--Diversions for irrigation of about 11,000 acres above station. Considerable regulation by many reservoirs (combined capacity about 50,000 acre-ft), the largest of which is Lyman Lake, 40 mi upstream.

Annual peak discharges

\begin{tabular}{|c|c|c|c|c|c|c|c|}
\hline $\begin{array}{l}\text { Water } \\
\text { year }\end{array}$ & Date & $\begin{array}{c}\text { Annual peak } \\
\text { discharge } \\
\left(\mathrm{ft}^{3} / \mathrm{s}\right)\end{array}$ & $\begin{array}{l}\text { Discharge } \\
\text { codes }\end{array}$ & $\begin{array}{l}\text { Water } \\
\text { year }\end{array}$ & Date & $\begin{array}{c}\text { Annual peak } \\
\text { discharge } \\
\left(\mathrm{ft}^{3} / \mathrm{s}\right)\end{array}$ & $\begin{array}{l}\text { Discharge } \\
\text { codes }\end{array}$ \\
\hline 1940 & $07-26-40$ & 577 & UR & 1957 & $09-01-57$ & 476 & UR \\
\hline 1941 & $05-11-41$ & 516 & UR & 1958 & $08-21-58$ & 1,020 & UR \\
\hline 1942 & $10-07-41$ & 37 & UR & 1959 & $08-11-59$ & 130 & UR \\
\hline 1943 & $09-25-43$ & 11 & UR & 1960 & $10-30-59$ & 38 & UR \\
\hline 1944 & $09-26-44$ & 101 & UR & 1961 & $09-11-61$ & 2.6 & UR \\
\hline 1945 & $08-13-45$ & 1,100 & UR & 1962 & $01-31-62$ & 10 & UR,LT \\
\hline 1946 & $08-04-46$ & 745 & UR & 1963 & $09-04-63$ & 34 & UR \\
\hline 1947 & $08-04-47$ & 301 & UR & 1964 & $08-09-64$ & 233 & UR \\
\hline 1948 & $09-17-48$ & 75 & UR & 1965 & $09-08-65$ & 194 & UR \\
\hline 1949 & $08-10-49$ & 478 & UR & 1966 & $09-14-66$ & 56 & UR \\
\hline 1950 & $07-07-50$ & 140 & UR & 1967 & $08-01-67$ & 176 & UR \\
\hline 1951 & $08-28-51$ & 70 & UR & 1968 & $08-07-68$ & 179 & UR \\
\hline 1952 & $07-27-52$ & 67 & UR & 1969 & $07-23-69$ & 747 & UR \\
\hline 1953 & $07-27-53$ & 62 & UR & 1970 & $08-16-70$ & 52 & UR \\
\hline 1954 & $07-26-54$ & 136 & UR & 1971 & $09-03-71$ & 1,310 & UR,DF \\
\hline 1955 & $08-24-55$ & 831 & UR & 1972 & $10-01-71$ & 552 & UR \\
\hline 1956 & $02-19-56$ & 37 & UR & & & & \\
\hline
\end{tabular}

Discharge rating table developed October 1959

\begin{tabular}{cccc}
\hline $\begin{array}{c}\text { Gage height } \\
(\mathrm{ft})\end{array}$ & $\begin{array}{c}\text { Discharge } \\
\left(\mathrm{ft}^{3} / \mathbf{s}\right)\end{array}$ & $\begin{array}{c}\text { Gage height } \\
(\mathrm{ft})\end{array}$ & $\begin{array}{c}\text { Discharge } \\
\left(\mathrm{ft}^{3} / \mathbf{s}\right)\end{array}$ \\
\hline 2.0 & 85 & 3.0 & 420 \\
4.0 & 1,140 & & \\
\hline
\end{tabular}

Basin characteristics

\begin{tabular}{cccccccc}
\hline $\begin{array}{c}\text { Main } \\
\text { channel } \\
\text { slope } \\
\text { (ft/mi) }\end{array}$ & $\begin{array}{c}\text { Stream } \\
\text { length } \\
\text { (mi) }\end{array}$ & $\begin{array}{c}\text { Mean } \\
\text { basin } \\
\text { elevation } \\
\text { (ft) }\end{array}$ & $\begin{array}{c}\text { Forested } \\
\text { area } \\
\text { (percent) }\end{array}$ & $\begin{array}{c}\text { Soil } \\
\text { index }\end{array}$ & $\begin{array}{c}\text { Mean } \\
\text { annual } \\
\text { precipitation } \\
\text { (in) }\end{array}$ & $\begin{array}{c}\text { Rainfall intensity, 24-hour } \\
\text { (in) }\end{array}$ & $\begin{array}{c}\text { 50-year } \\
\text { (in) }\end{array}$ \\
\hline 40.2 & 87.9 & 7,160 & 25.0 & 2.9 & 14.6 & 1.5 \\
\hline
\end{tabular}


09386500 LITTLE COLORADO RIVER ABOVE ZUNI RIVER, NEAR HUNT, AZ--Continued

MEAN MONTHLY AND ANNUAL DISCHARGES 1941.72

\begin{tabular}{|c|c|c|c|c|c|c|}
\hline MONTH & $\begin{array}{l}\text { MAXIMUM } \\
\text { (FT3/S) }\end{array}$ & $\begin{array}{l}\text { MINIMUM } \\
\text { (FT3/S) }\end{array}$ & $\begin{array}{c}\text { MEAN } \\
(\text { FT3/S) }\end{array}$ & $\begin{array}{l}\text { STAN- } \\
\text { DARD } \\
\text { DEVIA- } \\
\text { TION } \\
\text { (FT3/S) }\end{array}$ & $\begin{array}{l}\text { COEFFI- } \\
\text { CIENT OF } \\
\text { VARI- } \\
\text { ATION }\end{array}$ & $\begin{array}{c}\text { PERCENT } \\
\text { OF } \\
\text { ANNUAL } \\
\text { RUNOFF }\end{array}$ \\
\hline OCTOBER & 41 & 0.00 & 3.3 & 7.9 & 2.4 & 5.4 \\
\hline NOVEMBER & 9.3 & 0.00 & 2.0 & 2.7 & 1.3 & 3.3 \\
\hline DECEMBER & 16 & 0.00 & 3.2 & 3.8 & 1.2 & 5.2 \\
\hline JANUARY & 15 & 0.00 & 3.8 & 4.1 & 1.1 & 6.1 \\
\hline FEBRUARY & 14 & 0.00 & 3.8 & 4.2 & 1.1 & 6.1 \\
\hline MARCH & 29 & 0.00 & 2.9 & 5.5 & 1.9 & 4.8 \\
\hline APRIL & 10 & 0.00 & 1.2 & 2.5 & 2.1 & 2.0 \\
\hline MAY & 148 & 0.00 & 5.0 & 26 & 5.2 & 8.2 \\
\hline JUNE & 7.6 & 0.00 & 0.44 & 1.3 & 3.0 & 0.7 \\
\hline JULY & 20 & 0.00 & 1.7 & 3.9 & 2.3 & 2.8 \\
\hline AUGUST & 338 & 0.00 & 25 & 64 & 2.6 & 40.6 \\
\hline SEPTEMBER & 71 & 0.00 & 9.2 & 17 & 1.9 & 14.9 \\
\hline ANNUAL & 30 & 0.01 & 5.2 & 6.3 & 1.2 & 100 \\
\hline
\end{tabular}

MAGNITUDE AND PROBABILITY OF INSTANTANEOUS PEAK FLOW BASED ON PERIOD OF RECORD 1940.72

DISCHARGE, IN FT3/S, FOR INDICATED RECURRENCE INTERVAL IN YEARS, AND EXCEEDANCE PROBABILITY, IN PERCENT

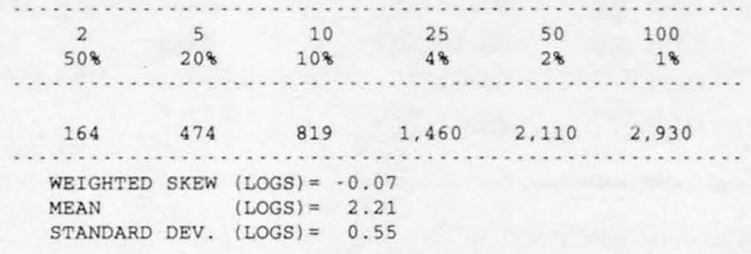

MAGNITUDE AND PROBABILITY OF ANNUAL LOW FLOW BASED ON PERIOD OF RECORD 1941.72

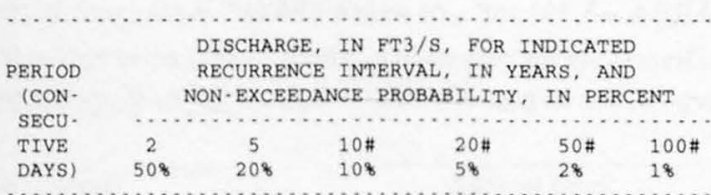

$\begin{array}{rllllll}1 & & & & & & \\ 3 & & & & & \\ 7 & 0.00 & 0.00 & 0.00 & 0.00 & 0.00 & 0.00 \\ 14 & 0.00 & 0.00 & 0.00 & 0.00 & 0.00 & 0.00 \\ 30 & 0.00 & 0.00 & 0.00 & 0.00 & 0.00 & 0.00 \\ 60 & 0.00 & 0.00 & 0.00 & 0.00 & 0.00 & 0.00 \\ 90 & 0.04 & 0.00 & 0.00 & 0.00 & 0.00 & 0.00 \\ 120 & 0.11 & 0.02 & 0.00 & 0.00 & 0.00 & 0.00 \\ 183 & 0.77 & 0.08 & 0.01 & 0.00 & 0.00 & 0.00\end{array}$

MAGNITUDE AND PROBABILITY OF ANNUAL HIGH FLOW BASED ON PERIOD OF RECORD 1941.72

\begin{tabular}{|c|c|c|c|c|c|c|}
\hline PERIOD & & \multicolumn{5}{|c|}{$\begin{array}{l}\text { DISCHARGE, IN FT3/S, FOR INDICATED } \\
\text { RECURRENCE INTERVAL, IN YEARS, AND }\end{array}$} \\
\hline (CON. & & EXCEEDANCB & PRC & BLLITY, & $\begin{array}{l}\text { YEARS, } \\
\text { IN PERC }\end{array}$ & \\
\hline SECU. & $\cdots$ & …… & . & ....... & $\ldots \ldots \ldots$ & $\ldots \ldots$ \\
\hline TIVE & 2 & 5 & $10 \#$ & $25 \#$ & $50 \#$ & $100 \#$ \\
\hline DAYS) & 508 & 208 & 108 & 48 & 28 & 18 \\
\hline 1 & 63 & 276 & 550 & 1,080 & 1,630 & 2,300 \\
\hline 3 & 46 & 213 & 439 & 903 & 1,400 & 2,030 \\
\hline 7 & 32 & 147 & 303 & 617 & 949 & 1,370 \\
\hline 15 & 22 & 98 & 194 & 373 & 546 & 751 \\
\hline 30 & 15 & 61 & 116 & 209 & 294 & 387 \\
\hline 60 & 10 & 39 & 68 & 111 & 145 & 177 \\
\hline 90 & 7.9 & 29 & 49 & 75 & 94 & 112 \\
\hline
\end{tabular}

DISCHARGE, IN FT3/S, WHICH WAS EQUALED OR EXCEEDED FOR INDICATED PERCENT OF TIME

\begin{tabular}{|c|c|c|c|c|c|c|c|c|c|c|c|c|c|c|c|c|}
\hline 18 & $5 \%$ & $10 \%$ & $15 \%$ & 208 & 308 & 408 & 508 & 608 & 708 & 808 & 908 & 958 & 988 & 998 & 99.58 & 99.98 \\
\hline 89 & 13 & 8.2 & 6.2 & 4.0 & 1.5 & 0.63 & 0.19 & 0.09 & 0.00 & 0.00 & 0.00 & 0.00 & 0.00 & 0.00 & 0.00 & 0.00 \\
\hline
\end{tabular}

\# Reliability of values in column is uncertain, and potential errors are large. 
09386500 LITTLE COLORADO RIVER ABOVE ZUNI RIVER, NEAR HUNT, AZ--Continued
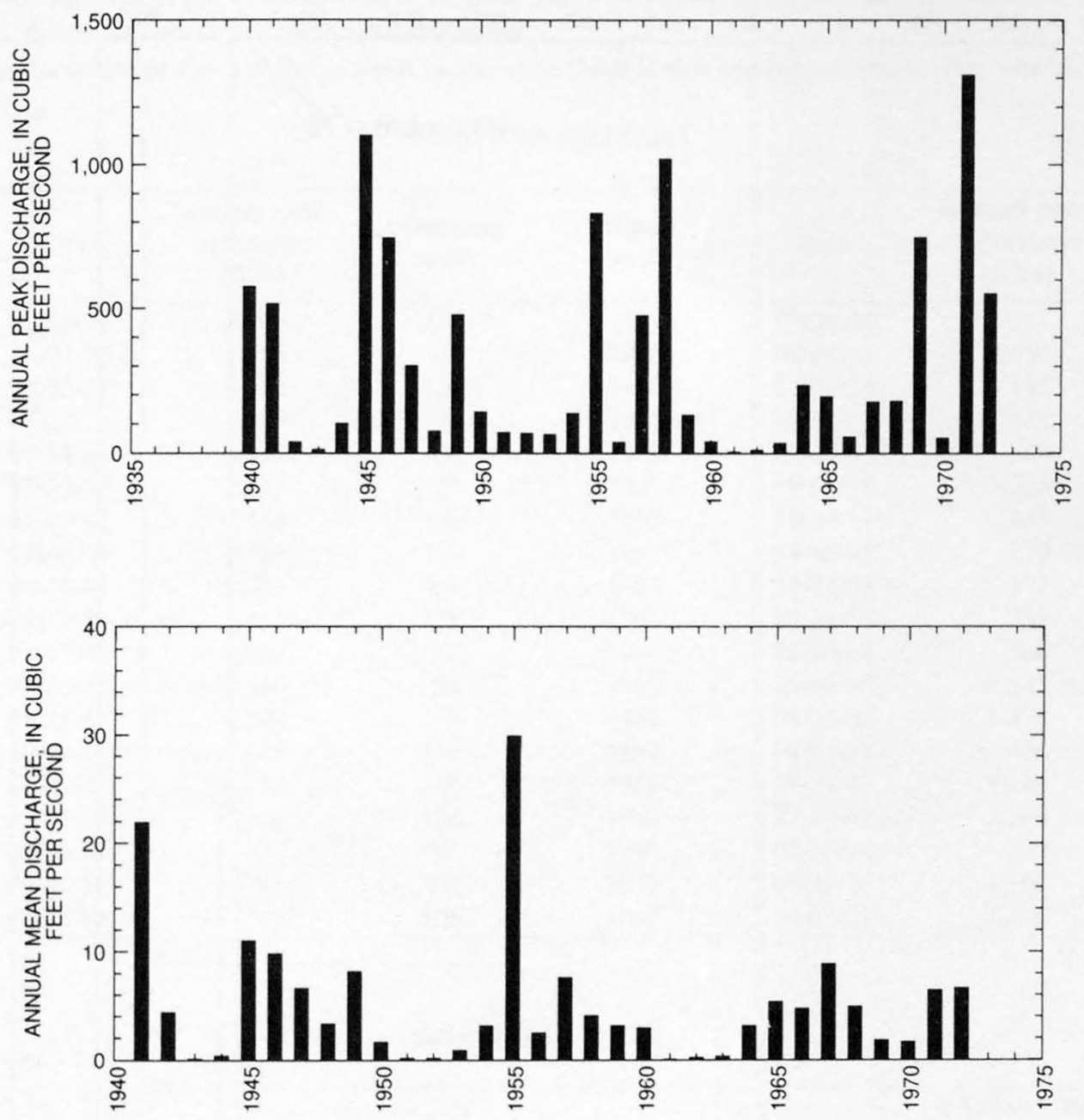
09386500 LITTLE COLORADO RIVER ABOVE ZUNI RIVER, NEAR HUNT, AZ--Continued
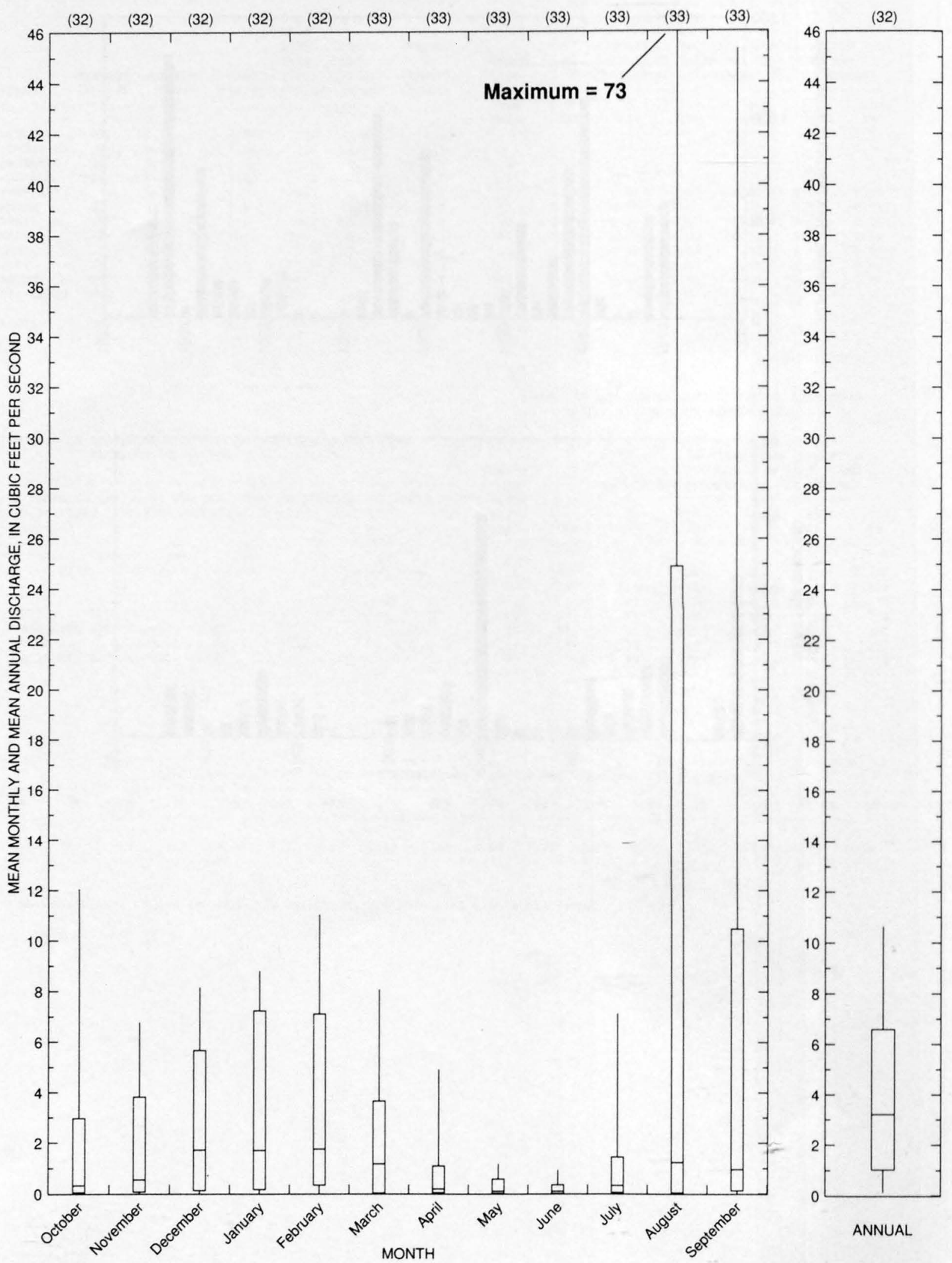


\section{LITTLE COLORADO RIVER NEAR HUNT, AZ}

LOCATION.--Lat $34^{\circ} 38^{\prime} 50^{\prime \prime}$, long $109^{\circ} 42^{\prime} 10^{\prime \prime}$, in NE $1 /{ }_{4} \mathrm{NW}^{1} / 4$, sec.4, T.14 N., R.25 E. (unsurveyed), Apache County, Hydrologic Unit 15020002 , near left bank on upstream side of pier of bridge on U.S. Highway 180, 2 mi downstream from Zuni River and 5 mi northwest of Hunt.

DRAINAGE AREA.--6,383 $\mathrm{mi}^{2}$, of which $210 \mathrm{mi}^{2}$ is noncontributing.

REMARKS.--Diversions for irrigation above station of about 14,000 acres. Considerable regulation by many reservoirs (combined capacity, about 59,000 acre-ft).

Annual peak discharges

\begin{tabular}{ccccccrc}
\hline $\begin{array}{c}\text { Water } \\
\text { year }\end{array}$ & Date & $\begin{array}{c}\text { Annual peak } \\
\text { discharge } \\
\left(\mathbf{f t}^{3} / \mathbf{s}\right)\end{array}$ & $\begin{array}{c}\text { Discharge } \\
\text { codes }\end{array}$ & $\begin{array}{c}\text { Water } \\
\text { year }\end{array}$ & Date & $\begin{array}{c}\text { Annual peak } \\
\text { discharge } \\
\left(\mathbf{f t}^{3} / \mathbf{s}\right)\end{array}$ & $\begin{array}{c}\text { Discharge } \\
\text { codes }\end{array}$ \\
\hline 1929 & $07-28-29$ & 8,000 & UR & 1954 & $07-26-54$ & 214 & UR \\
1930 & $08-07-30$ & 965 & UR & 1955 & $08-08-55$ & 2,550 & UR \\
1931 & $08-05-31$ & 3,600 & UR & 1956 & $07-22-56$ & 145 & UR \\
1932 & $08-29-32$ & 1,200 & UR & 1957 & $09-01-57$ & 882 & UR \\
1933 & $07-24-33$ & 3,600 & UR & 1958 & $08-21-58$ & 1,400 & UR \\
1940 & $07-26-40$ & 2,110 & UR & 1959 & $08-04-59$ & 235 & UR \\
1941 & $07-25-41$ & 1,560 & UR & 1960 & $10-30-59$ & 453 & UR \\
1942 & $10-04-41$ & 725 & UR & 1961 & $08-15-61$ & 272 & UR \\
1943 & $08-11-43$ & 508 & UR & 1962 & $10-31-61$ & 112 & UR \\
1944 & $09-30-44$ & 451 & UR & 1963 & $08-21-63$ & 232 & UR \\
1945 & $08-13-45$ & 1,590 & UR & 1964 & $08-04-64$ & 580 & UR \\
1946 & $08-05-46$ & 2,390 & UR & 1965 & $09-04-65$ & 545 & UR \\
1947 & $08-23-47$ & 1,290 & UR & 1966 & $08-15-66$ & 570 & UR \\
1948 & $10-14-47$ & 925 & UR & 1967 & $08-14-67$ & 748 & UR \\
1949 & $08-09-49$ & 4,050 & UR & 1968 & $08-04-68$ & 1,140 & UR \\
1950 & $07-24-50$ & 119 & UR & 1969 & $07-24-69$ & 2,360 & UR \\
1951 & $08-28-51$ & 531 & UR & 1970 & $08-17-70$ & 378 & UR \\
1952 & $09-22-52$ & 395 & UR & 1971 & $09-03-71$ & 1,900 & UR \\
1953 & $07-18-53$ & 42 & UR & 1972 & $10-01-71$ & 1,230 & UR \\
\hline
\end{tabular}

Basin characteristics

\begin{tabular}{ccccccccc}
\hline $\begin{array}{c}\text { Main } \\
\text { channel } \\
\text { slope } \\
(\mathrm{ft} / \mathrm{mi})\end{array}$ & $\begin{array}{c}\text { Stream } \\
\text { length } \\
(\mathrm{mi})\end{array}$ & $\begin{array}{c}\text { Mean } \\
\text { basin } \\
\text { elevation } \\
(\mathrm{ft})\end{array}$ & $\begin{array}{c}\text { Forested } \\
\text { area } \\
\text { (percent) }\end{array}$ & $\begin{array}{c}\text { Soil } \\
\text { index }\end{array}$ & $\begin{array}{c}\text { Mean } \\
\text { annual } \\
\text { precipitation } \\
\text { (in) }\end{array}$ & $\begin{array}{c}\text { 2-year } \\
\text { (in) }\end{array}$ & $\begin{array}{c}\text { 50-year } \\
\text { (in) }\end{array}$ \\
\hline 37.6 & 90.4 & 7,060 & 27.0 & 2.9 & 13.4 & 1.4 & 2.8 \\
\hline
\end{tabular}


MEAN MONTHLY AND ANNUAL DISCHARGES $1930-33,1941.72$

\begin{tabular}{|c|c|c|c|c|c|c|}
\hline MONTH & $\begin{array}{l}\text { MAXIMUM } \\
\text { (FT3/S) }\end{array}$ & $\begin{array}{l}\text { MINIMUM } \\
\text { (FT3/S) }\end{array}$ & $\begin{array}{c}\text { MEAN } \\
(\text { FT3/S) }\end{array}$ & $\begin{array}{l}\text { STAN - } \\
\text { DARD } \\
\text { DEVIA - } \\
\text { TION } \\
(\mathrm{FT} 3 / \mathrm{S})\end{array}$ & $\begin{array}{l}\text { COEFFI- } \\
\text { CIENT OF } \\
\text { VARI- } \\
\text { ATION }\end{array}$ & $\begin{array}{c}\text { PERCENT } \\
\text { OF } \\
\text { ANNUAL } \\
\text { RUNOFF }\end{array}$ \\
\hline OCTOBER & 105 & 0.00 & 11 & 24 & 2.1 & 6.6 \\
\hline NOVEMBER & 10 & 0.00 & 3.1 & 3.3 & 1.0 & 1.8 \\
\hline DECEMBER & 20 & 0.00 & 4.2 & 4.6 & 1.1 & 2.4 \\
\hline JANUARY & 33 & 0.00 & 5.4 & 6.7 & 1.2 & 3.2 \\
\hline FEBRUARY & 149 & 0.00 & 9.1 & 25 & 2.7 & 5.3 \\
\hline MARCH & 223 & 0.00 & 14 & 47 & 3.3 & 8.3 \\
\hline APRIL & 315 & 0.00 & 16 & 59 & 3.7 & 9.1 \\
\hline MAY & 239 & 0.00 & 7.6 & 40 & 5.3 & 4.4 \\
\hline JUNE & 9.1 & 0.00 & 0.93 & 1.8 & 1.9 & 0.5 \\
\hline JULY & 68 & 0.00 & 11 & 17 & 1.6 & 6.4 \\
\hline AUGUST & 524 & 0.00 & 63 & 105 & 1.7 & 36.4 \\
\hline SEPTEMBER & 170 & 0.00 & 27 & 42 & 1.6 & 15.5 \\
\hline ANNUAL & 79 & 0.44 & 14 & 18 & 1.2 & 100 \\
\hline
\end{tabular}

MAGNITUDE AND PROBABILITY OF INSTANTANEOUS PEAK FLOW BASED ON PERIOD OF RECORD $1929.33,1940.72$

DISCHARGE, IN FT3/S, FOR INDICATED RECURRENCE INTERVAL IN YEARS, AND EXCEEDANCE PROBABILITY, IN PERCENT

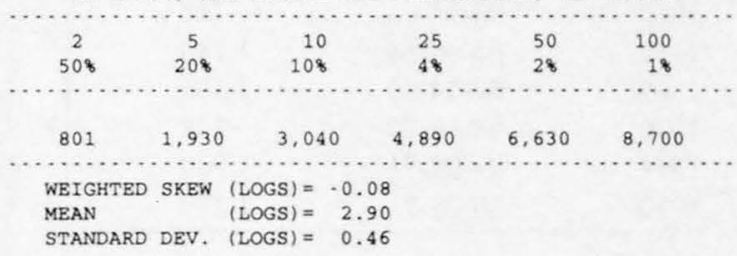

MAGNITUDE AND PROBABILITY OF ANNUAL LOW FLOW BASED ON PERIOD OF RECORD $1931.33,1941.72$

\begin{tabular}{|c|c|c|c|c|c|c|}
\hline \multirow{3}{*}{$\begin{array}{l}\text { PERIOD } \\
\text { (CON- } \\
\text { SECU- } \\
\text { TIVE } \\
\text { DAYS) }\end{array}$} & \multicolumn{6}{|c|}{$\begin{array}{l}\text { DISCHARGE, IN FT3/S, FOR INDICATED } \\
\text { RECURRENCE INTERVAL, IN YEARS, AND } \\
\text { NON-EXCEEDANCE PROBABILITY, IN PERCENT }\end{array}$} \\
\hline & 2 & 5 & 10 & $20 \#$ & $50 \#$ & $100 \#$ \\
\hline & 508 & 208 & 108 & 58 & 28 & 18 \\
\hline 1 & 0.00 & 0.00 & 0.00 & 0.00 & 0.00 & 0.00 \\
\hline 3 & 0.00 & 0.00 & 0.00 & 0.00 & 0.00 & 0.00 \\
\hline 7 & 0.00 & 0.00 & 0.00 & 0.00 & 0.00 & 0.00 \\
\hline 14 & 0.00 & 0.00 & 0.00 & 0.00 & 0.00 & 0.00 \\
\hline 30 & 0.00 & 0.00 & 0.00 & 0.00 & 0.00 & 0.00 \\
\hline 60 & 0.10 & 0.00 & 0.00 & 0.00 & 0.00 & 0.00 \\
\hline 90 & 0.19 & 0.00 & 0.00 & 0.00 & 0.00 & 0.00 \\
\hline 120 & 0.70 & 0.09 & 0.01 & 0.00 & 0.00 & 0.00 \\
\hline 183 & 2.4 & 0.47 & 0.15 & 0.00 & 0.00 & 0.00 \\
\hline
\end{tabular}

MAGNITUDE AND PROBABILITY OF ANNUAL HIGH FLOW BASED ON PERIOD OF RECORD $1930.33,1941.72$

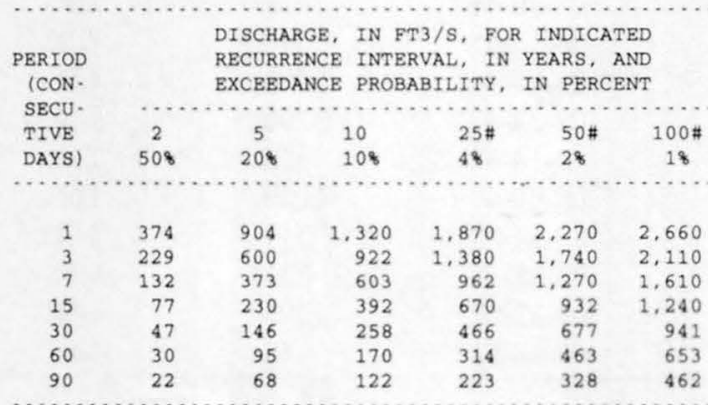

DURATION TABLE OF DAILY MEAN FLOW FOR PERIOD OF RECORD $1930 \cdot 33,1941-72$ DISCHARGE, IN FT3/S, WHICH WAS EQUALED OR EXCEEDED FOR INDICATED PERCBNT OF TIME

\begin{tabular}{|c|c|c|c|c|c|c|c|c|c|c|c|c|c|c|c|c|}
\hline 18 & 58 & 108 & 158 & 208 & 308 & 408 & 508 & 608 & 708 & 808 & 908 & 958 & 988 & 998 & 99.58 & 9 \\
\hline 347 & 49 & 16 & 10 & 7.7 & 3.3 & 1.5 & 0.67 & 0.23 & 0.11 & 0.00 & 0.00 & 0.00 & 0.00 & 0.00 & 0.00 & 0.00 \\
\hline
\end{tabular}

\# Reliability of values in column is uncertain, and potential errors are large. 
ANNUAL MEAN DISCHARGE, IN CUBIC

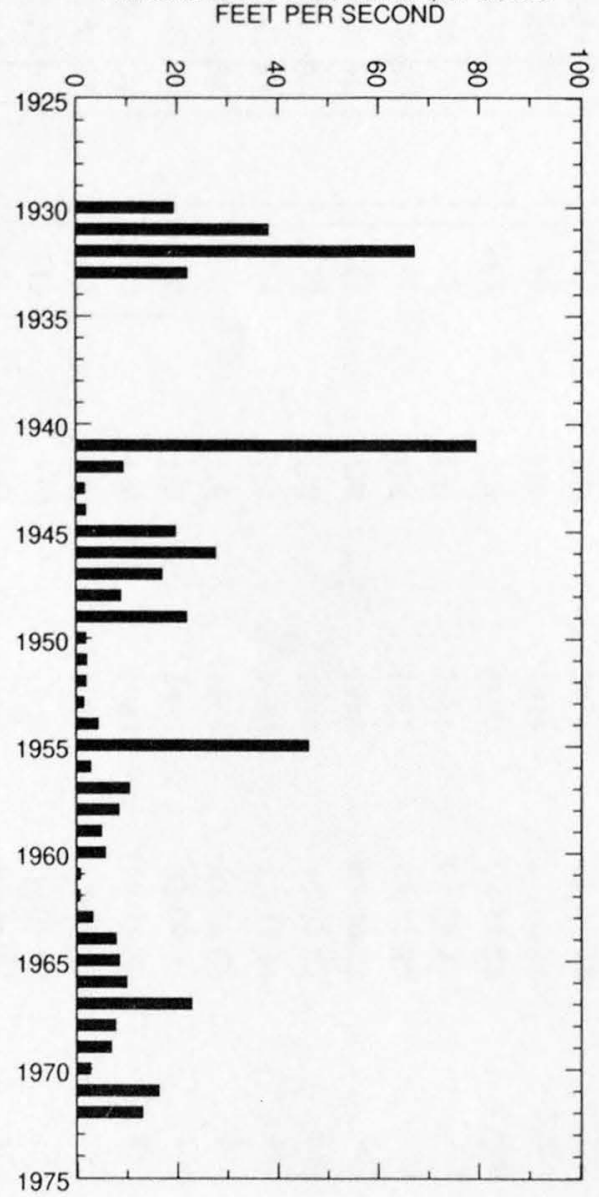

ANNUAL PEAK DISCHARGE, IN CUBIC

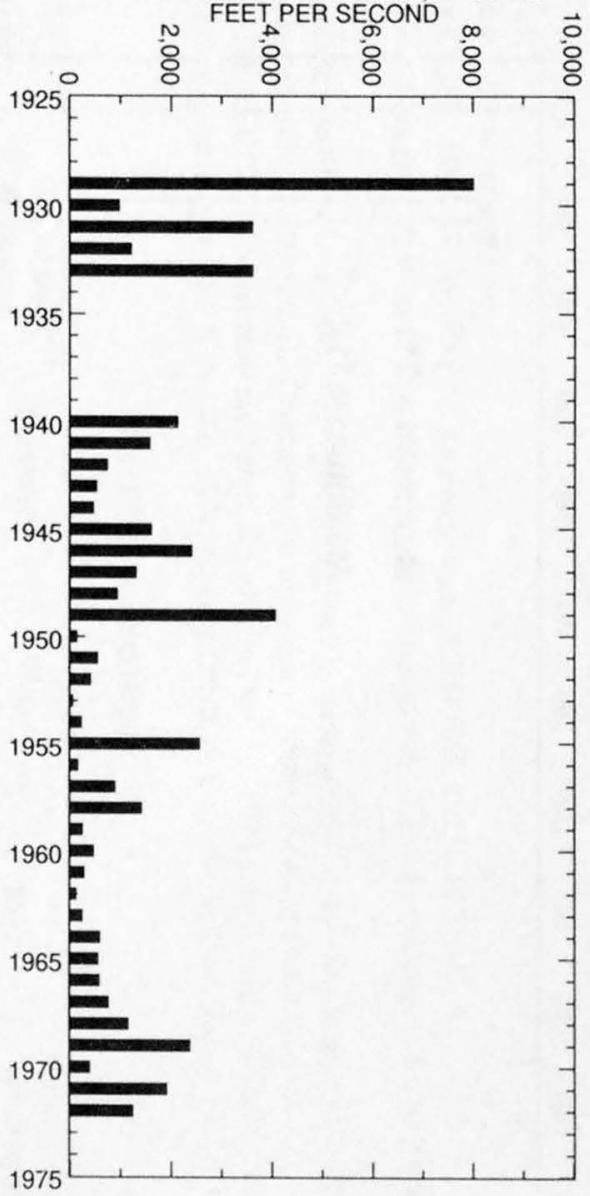


09388000 LITTLE COLORADO RIVER NEAR HUNT, AZ--Continued
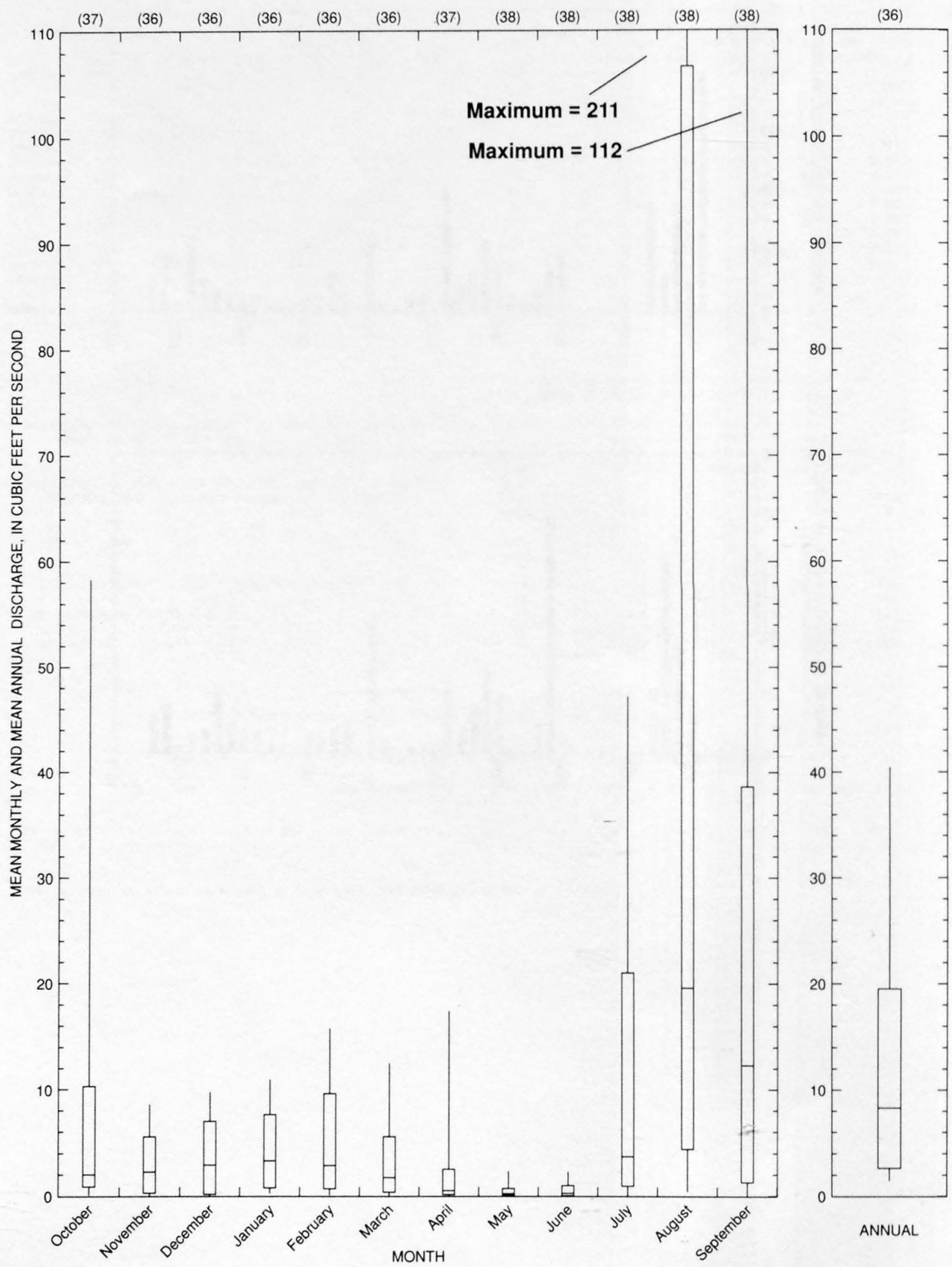
09390500 SHOW LOW CREEK NEAR LAKESIDE, AZ

LOCATION.--Lat 34'10'46", long 109.59'14", in SW1/4 NW $1 / 4$ sec.14, T.9 N., R.22 E., Navajo County, Hydrologic Unit 15020005 , on left bank 1 mi upstream from pumping plant on Show Low Lake, 1.9 mi northwest of Lakeside, 2.2 mi upstream from Jaques Dam, and 6 mi southeast of Show Low.

DRAINAGE AREA.--68.6 $\mathrm{mi}^{2}$.

PERIOD OF RECORD.--May 1953 to current year.

REVISED RECORDS.--WSP 1513: 1954-56. WSP 1926: Drainage area. WRD Ariz. 1971: 1970(M).

GAGE.--Water-stage recorder and concrete-dam control with V-notch sharp-crested weir. Elevation of gage is $6,610 \mathrm{ft}$ above sea level, from topographic map.

REMARKS.--Record shows inflow to Show Low Lake. Flow partly regulated by several small reservoirs, largest of which are Rainbow Lake and Scott Reservoir, combined capacity, 2,400 acre-ft. Diversions for irrigation of about 250 acres above station.

EXTREMES FOR PERIOD OF RECORD.--Maximum discharge, 5,550 $\mathrm{ft}^{3} / \mathrm{s} \mathrm{Dec.} \mathrm{18,} 1978$, gage height, $9.16 \mathrm{ft}$, from rating curve extended above $2,500 \mathrm{ft}^{3} / \mathrm{s}$; maximum gage height, $9.53 \mathrm{ft}$ Dec. 26, 1971; no flow Oct. 5, 6, Dec. 10-19, 1964, Jan. 4-15, 1970.

Annual peak discharges

\begin{tabular}{rcccccrr}
\hline $\begin{array}{l}\text { Water } \\
\text { year }\end{array}$ & Date & $\begin{array}{c}\text { Annual peak } \\
\text { discharge } \\
\left(\mathbf{f t}^{3} / \mathbf{s}\right)\end{array}$ & $\begin{array}{c}\text { Discharge } \\
\text { codes }\end{array}$ & $\begin{array}{c}\text { Water } \\
\text { year }\end{array}$ & Date & $\begin{array}{c}\text { Annual peak } \\
\text { discharge } \\
\left(\mathbf{f t}^{3} / \mathbf{s}\right)\end{array}$ & $\begin{array}{c}\text { Discharge } \\
\text { codes }\end{array}$ \\
\hline 1954 & $03-23-54$ & 2,040 & UR & 1976 & $04-20-76$ & 632 & UR \\
1955 & $08-27-55$ & 273 & UR & 1977 & $04-08-77$ & 35 & UR \\
1956 & $07-31-56$ & 103 & UR & 1978 & $03-01-78$ & 2,750 & UR \\
1957 & $02-09-57$ & 56 & UR & 1979 & $12-18-78$ & 5,550 & UR \\
1958 & $03-22-58$ & 867 & UR & 1980 & $02-15-80$ & 1,860 & UR \\
1959 & $08-28-59$ & 45 & UR & 1981 & $03-30-81$ & 31 & UR \\
1960 & $03-08-60$ & 487 & UR & 1982 & $03-13-82$ & 575 & UR \\
1961 & $04-01-61$ & 27 & UR & 1983 & $03-30-83$ & 442 & UR \\
1962 & $02-13-62$ & 930 & UR & 1984 & $10-02-83$ & 683 & UR \\
1963 & $02-22-63$ & 64 & UR & 1985 & $12-27-84$ & 5,430 & UR \\
1964 & $04-10-64$ & 30 & UR & 1986 & $02-16-86$ & 345 & UR \\
1965 & $01-07-65$ & 2,430 & UR & 1987 & $03-09-87$ & 500 & UR \\
1966 & $12-30-65$ & 3,880 & UR & 1988 & $08-31-88$ & 890 & UR \\
1967 & $08-02-67$ & 42 & UR & 1989 & $02-27-89$ & 92 & UR \\
1968 & $02-25-68$ & 345 & UR & 1990 & $09-01-90$ & 17 & UR \\
1969 & $03-19-69$ & 395 & UR & 1991 & $03-06-91$ & 582 & UR \\
1970 & $04-21-70$ & 30 & UR & 1992 & $05-28-92$ & 1,510 & UR \\
1971 & $08-10-71$ & 219 & UR & 1993 & $01-08-93$ & 4,370 & UR \\
1972 & $12-26-71$ & 5,450 & UR & 1994 & $03-20-94$ & 196 & UR \\
1973 & $05-05-73$ & 1,400 & UR & 1995 & $11-12-94$ & 1,490 & UR \\
1974 & $03-21-74$ & 60 & UR & 1996 & $01-17-96$ & 11 & UR \\
1975 & $03-09-75$ & 405 & & & & & \\
\hline
\end{tabular}

Discharge rating table developed October 1991

\begin{tabular}{cccc}
\hline $\begin{array}{c}\text { Gage height } \\
(\mathbf{t t})\end{array}$ & $\begin{array}{c}\text { Discharge } \\
\left(\mathbf{f t}^{\mathbf{3}} \mathbf{s}\right)\end{array}$ & $\begin{array}{c}\text { Gage height } \\
(\mathbf{f t})\end{array}$ & $\begin{array}{c}\text { Discharge } \\
\left(\mathbf{f t}^{\mathbf{3}} / \mathbf{s}\right)\end{array}$ \\
\hline 2.0 & 14.1 & 6.0 & 1,910 \\
3.0 & 89 & 7.0 & 2,940 \\
4.0 & 409 & 8.0 & 4,070 \\
5.0 & 1,040 & 9.5 & 6,000 \\
\hline
\end{tabular}


09390500 SHOW LOW CREEK NEAR LAKESIDE, AZ--Continued

Basin characteristics

\begin{tabular}{ccccccc}
\hline $\begin{array}{c}\text { Main } \\
\text { channel } \\
\text { slope } \\
(\mathrm{ft} / \mathrm{mi})\end{array}$ & $\begin{array}{c}\text { Stream } \\
\text { length } \\
(\mathrm{mi})\end{array}$ & $\begin{array}{c}\text { Mean } \\
\text { basin } \\
\text { elevation } \\
(\mathrm{ft})\end{array}$ & $\begin{array}{c}\text { Forested } \\
\text { area } \\
\text { (percent) }\end{array}$ & $\begin{array}{c}\text { Soil } \\
\text { index }\end{array}$ & $\begin{array}{c}\text { Mean } \\
\text { annual } \\
\text { precipitation } \\
\text { (in) }\end{array}$ & $\begin{array}{c}\text { Rainfall intensity, 24-hour } \\
\text { (in) }\end{array}$ \\
\hline 77.2 & 21.0 & 7,320 & 85.0 & 3.0 & 23.7 & $\begin{array}{c}50 \text {-year } \\
\text { (in) }\end{array}$ \\
\hline
\end{tabular}


09390500 SHOW LOW CREEK NEAR LAKESIDE, AZ--Continued

MEAN MONTHLY AND ANNUAL DISCHARGES 1954-96

\begin{tabular}{|c|c|c|c|c|c|c|}
\hline MONTH & $\begin{array}{l}\text { MAXIMUM } \\
\text { (FT3/S) }\end{array}$ & $\begin{array}{l}\text { MINIMUM } \\
\text { (FT3/S) }\end{array}$ & $\begin{array}{c}\text { MEAN } \\
(\text { FT3/S) }\end{array}$ & $\begin{array}{l}\text { STAN- } \\
\text { DARD } \\
\text { DEVIA- } \\
\text { TION } \\
(\text { FT3/S) }\end{array}$ & $\begin{array}{l}\text { COEFFI- } \\
\text { CIENT OF } \\
\text { VARI- } \\
\text { ATION }\end{array}$ & $\begin{array}{c}\text { PERCENT } \\
\text { OF } \\
\text { ANNUAL } \\
\text { RUNOFF }\end{array}$ \\
\hline OCTOBER & 57 & 0.73 & 6.2 & 11 & 1.8 & 3.4 \\
\hline NOVEMBER & 54 & 0.29 & 5.9 & 11 & 1.8 & 3.3 \\
\hline DECEMBER & 285 & 0.20 & 24 & 57 & 2.4 & 13.1 \\
\hline JANUARY & 200 & 0.10 & 15 & 32 & 2.2 & 8.2 \\
\hline FEBRUARY & 225 & 0.19 & 33 & 48 & 1.4 & 18.5 \\
\hline MARCH & 189 & 0.87 & 46 & 47 & 1.0 & 25.6 \\
\hline APRIL & 197 & 0.97 & 20 & 33 & 1.7 & 11.2 \\
\hline MAY & 72 & 1.7 & 7.7 & 11 & 1.5 & 4.3 \\
\hline JUNE & 13 & 1.8 & 6.2 & 2.5 & 0.40 & 3.4 \\
\hline JULY & 11 & 1.1 & 5.9 & 2.4 & 0.42 & 3.2 \\
\hline AUGUST & 20 & 1.4 & 5.6 & 3.8 & 0.68 & 3.1 \\
\hline SEPTEMBER & 19 & 1.3 & 4.9 & 3.0 & 0.62 & 2.7 \\
\hline ANNUAL & 57 & 1.6 & 15 & 14 & 0.90 & 100 \\
\hline
\end{tabular}

MAGNITUDE AND PROBABILITY OF ANNUAL LOW FLOW BASED ON PERIOD OF RECORD 1955-96

\begin{tabular}{|c|c|c|c|c|c|c|}
\hline $\begin{array}{l}\text { PERIOD } \\
(\mathrm{CON}-\end{array}$ & \multirow{2}{*}{\multicolumn{6}{|c|}{$\begin{array}{l}\text { DISCHARGE, IN FT3/S, FOR INDICATED } \\
\text { RECURRENCE INTERVAL, IN YEARS, AND } \\
\text { NON-EXCEEDANCE PROBABILITY, IN PERCENT }\end{array}$}} \\
\hline SECU. & & & & & & \\
\hline TIVE & 2 & 5 & 10 & 20 & 50 & $100 \#$ \\
\hline DAYS) & 508 & 208 & 108 & 58 & 28 & 18 \\
\hline 1 & 0.43 & 0.11 & 0.05 & 0.01 & 0.00 & 0.00 \\
\hline 3 & 0.46 & 0.13 & 0.05 & 0.01 & 0.00 & 0.00 \\
\hline 7 & 0.57 & 0.17 & 0.08 & 0.02 & 0.00 & 0.00 \\
\hline 14 & 0.84 & 0.24 & 0.11 & 0.05 & 0.02 & 0.01 \\
\hline 30 & 1.2 & 0.43 & 0.24 & 0.15 & 0.08 & 0.05 \\
\hline 60 & 1.6 & 0.64 & 0.38 & 0.24 & 0.14 & 0.09 \\
\hline 90 & 2.0 & 0.94 & 0.62 & 0.44 & 0.29 & 0.21 \\
\hline 120 & 2.4 & 1.3 & 0.87 & 0.63 & 0.44 & 0.34 \\
\hline 183 & 3.3 & 1.9 & 1.5 & 1.2 & 0.88 & 0.73 \\
\hline
\end{tabular}

MAGNITUDE AND PROBABILITY OF ANNUAL HIGH FLOW BASED ON PERIOD OF RECORD 1954.96

\begin{tabular}{|c|c|c|c|c|c|c|}
\hline PERIOD & & \multicolumn{5}{|c|}{$\begin{array}{l}\text { DISCHARGE, IN FT3/S, FOR INDICATED } \\
\text { RECURRENCE INTERVAL, IN YEARS, AND }\end{array}$} \\
\hline $\begin{array}{l}\text { PERIOD } \\
\text { (CON- }\end{array}$ & & \multicolumn{3}{|c|}{ EXCEEDANCE PROBABILITY, } & \multicolumn{2}{|c|}{ IN PERCENT } \\
\hline SECU. & & & & & . . . . & \\
\hline TIVE & 2 & 5 & 10 & 25 & 50 & $100 \#$ \\
\hline DAYS) & 508 & 208 & 108 & 48 & 28 & 18 \\
\hline 1 & 191 & 784 & 1,610 & 3,410 & 5,490 & 8,390 \\
\hline 3 & 135 & 497 & 954 & 1,870 & 2,850 & 4,140 \\
\hline 7 & 91 & 309 & 569 & 1,070 & 1.580 & 2,240 \\
\hline 15 & 61 & 188 & 330 & 587 & 843 & 1,160 \\
\hline 30 & 44 & 125 & 211 & 363 & 509 & 686 \\
\hline 60 & 31 & 83 & 136 & 227 & 314 & 419 \\
\hline 90 & 25 & 65 & 106 & 179 & 249 & 334 \\
\hline
\end{tabular}

DURATION TABLE OF DAILY MEAN FLOW FOR PERIOD OF RECORD 1954.96

DISCHARGE, IN FT3/S, WHICH WAS EQUALED OR EXCEEDED FOR INDICATED PERCENT OF TIME

\begin{tabular}{|c|c|c|c|c|c|c|c|c|c|c|c|c|c|c|c|c|}
\hline 18 & 58 & 108 & 158 & 208 & 308 & 408 & 508 & 608 & 708 & 808 & 908 & 958 & 988 & 998 & 99.58 & 99.98 \\
\hline 203 & 57 & 23 & 13 & 9.6 & 7.5 & 5.9 & 4.7 & 3.6 & 2.6 & 1.6 & 0.83 & 0.44 & 0.22 & 0.11 & 0.09 & 0.00 \\
\hline
\end{tabular}

\# Reliability of values in column is uncertain, and potential errors are large. 


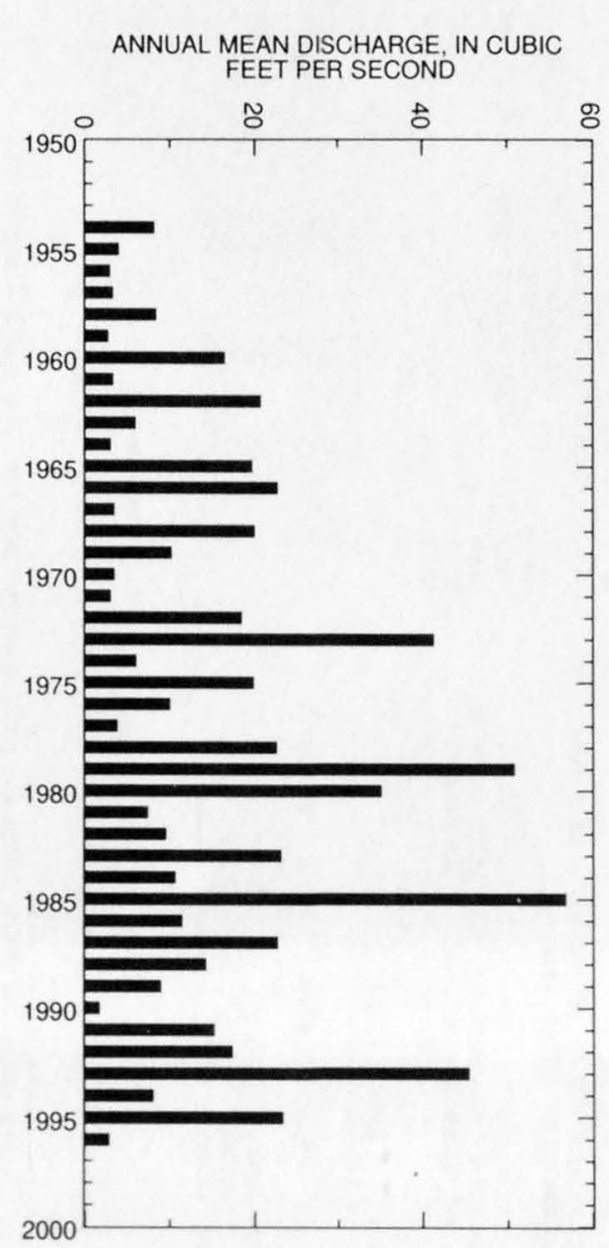

ANNUAL PEAK DISCHARGE, IN CUBIC

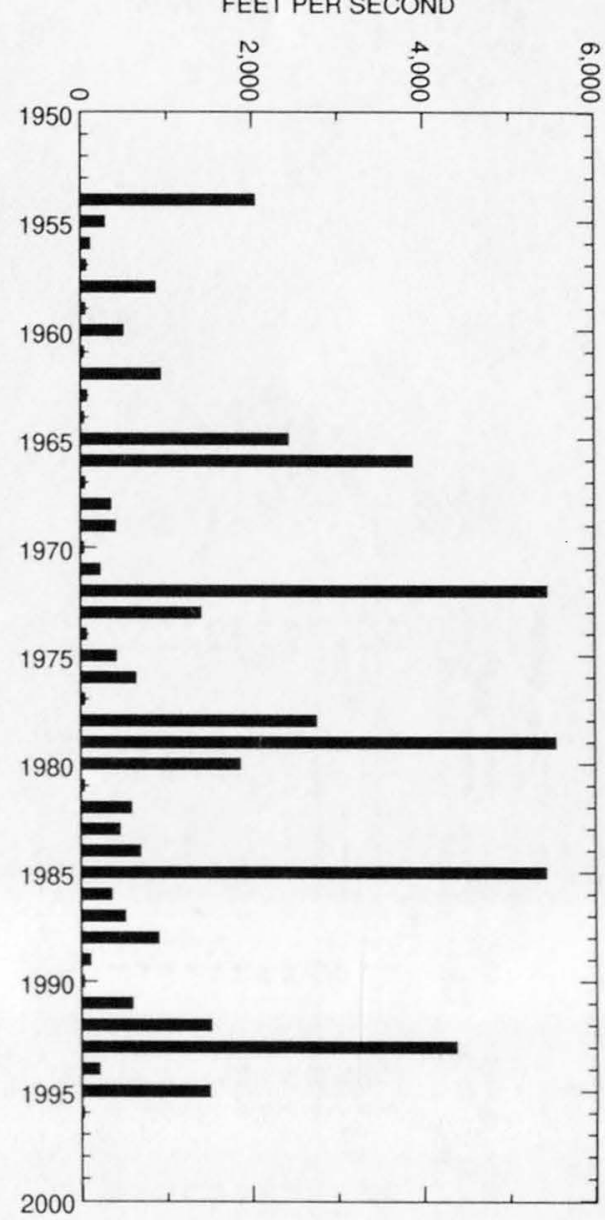


09390500 SHOW LOW CREEK NEAR LAKESIDE, AZ--Continued
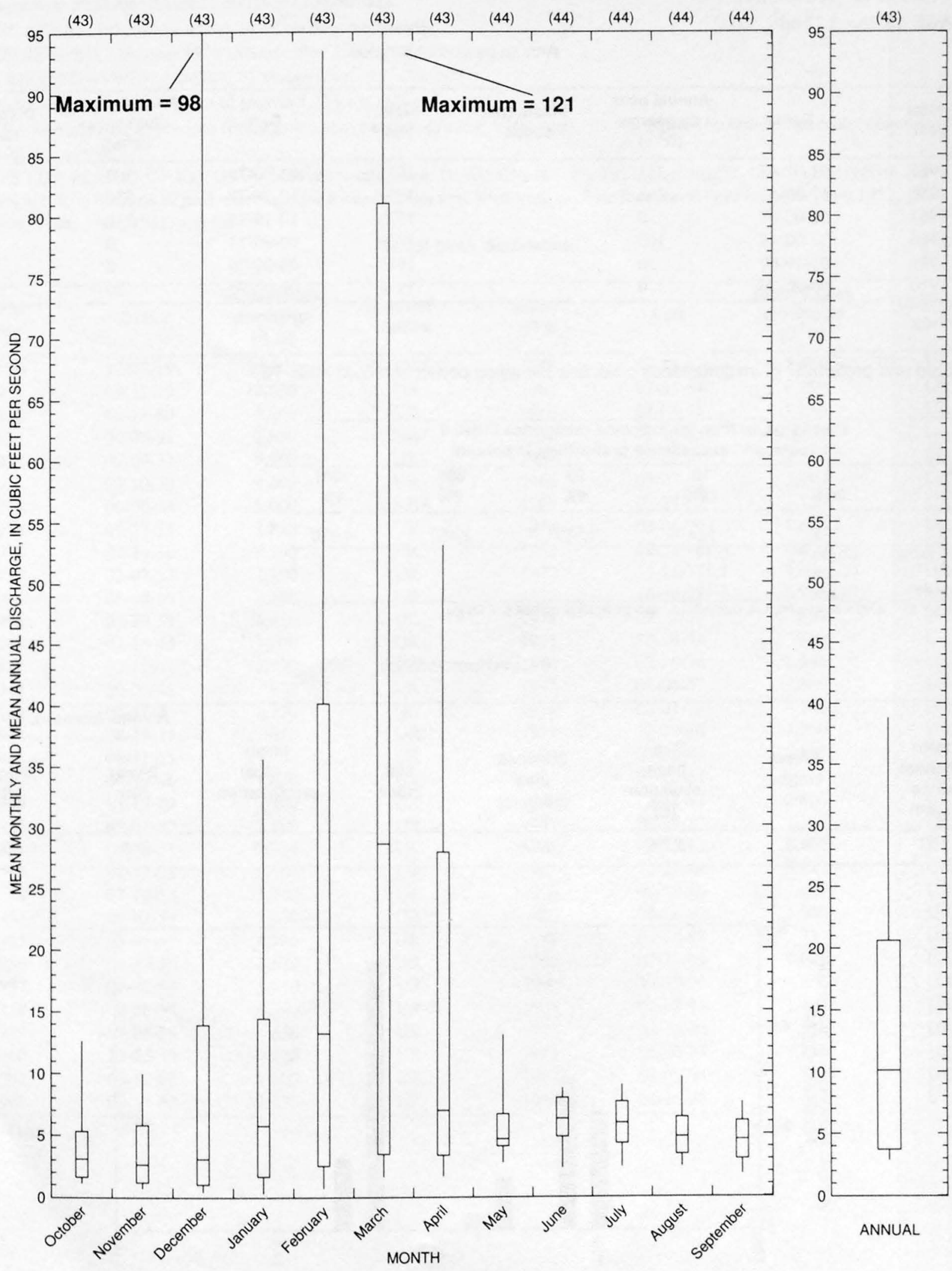
09392800 LONG LAKE TRIBUTARY NEAR SHOW LOW, AZ

LOCATION.--Lat $34^{\circ} 15^{\prime} 40^{\prime \prime}$, long 109.59'41", in NW $1 / 4 \mathrm{SE}^{1} / 4$, sec.15, T.10 N., R.22 E., Navajo County, Hydrologic Unit 15020005, at U.S. Hiy way 60,1 mi east of Show Low.

DRAINAGE AREA.--5.22 $\mathrm{mi}^{2}$.

Annual peak discharges

\begin{tabular}{|c|c|c|c|c|c|c|c|}
\hline $\begin{array}{l}\text { Water } \\
\text { year }\end{array}$ & Date & $\begin{array}{c}\text { Annual peak } \\
\text { discharge } \\
\left(\mathrm{ft}^{3} / \mathrm{s}\right)\end{array}$ & $\begin{array}{l}\text { Discharge } \\
\text { codes }\end{array}$ & $\begin{array}{l}\text { Water } \\
\text { year }\end{array}$ & Date & $\begin{array}{c}\text { Annual peak } \\
\text { discharge } \\
\left(\mathrm{ft}^{3} / \mathrm{s}\right)\end{array}$ & $\begin{array}{c}\text { Discharge } \\
\text { codes }\end{array}$ \\
\hline 1965 & $01-07-65$ & 231 & & 1971 & $00-00-71$ & 0 & \\
\hline 1966 & $12-30-65$ & 298 & & 1972 & $12-26-71$ & 530 & \\
\hline 1967 & $00-00-67$ & 0 & & 1973 & $10-19-72$ & 140 & \\
\hline 1968 & $02-00-68$ & 100 & & 1974 & $00-00-74$ & 0 & \\
\hline 1969 & $03-19-69$ & 30 & & 1975 & $00-00-75$ & 0 & \\
\hline 1970 & $00-00-70$ & 0 & & 1976 & $00-00-76$ & 20 & LT \\
\hline
\end{tabular}

Magnitude and probability of instantaneous peak flow based on period of record 1965-76

\begin{tabular}{|c|c|c|c|c|c|}
\hline \multicolumn{6}{|c|}{$\begin{array}{l}\text { Discharge, in } \mathrm{ft}^{3} / \mathrm{s} \text {, for indicated recurrence interval } \\
\text { in years, and exceedance probablility, in percent }\end{array}$} \\
\hline 2 & 5 & 10 & 25 & $50 t$ & $100 t$ \\
\hline $50 \%$ & $20 \%$ & $10 \%$ & $4 \%$ & $2 \%$ & $1 \%$ \\
\hline 23.4 & 195 & 524 & 1,380 & 2,460 & 4,030 \\
\hline Weighted skew & $(\log s)=$ & -0.49 & & & \\
\hline Mean & $(\log s)=$ & 1.27 & & & \\
\hline Standard dev. & $(\log s)=$ & 1.19 & & & \\
\hline
\end{tabular}

Basin characteristics

\begin{tabular}{cccccccc}
\hline $\begin{array}{c}\text { Main } \\
\text { channel } \\
\text { slope } \\
(\mathrm{ft} / \mathrm{mi})\end{array}$ & $\begin{array}{c}\text { Stream } \\
\text { length } \\
(\mathrm{mi})\end{array}$ & $\begin{array}{c}\text { Mean } \\
\text { basin } \\
\text { elevation } \\
\text { (ft) }\end{array}$ & $\begin{array}{c}\text { Forested } \\
\text { area } \\
\text { (percent) }\end{array}$ & $\begin{array}{c}\text { Soil } \\
\text { index }\end{array}$ & $\begin{array}{c}\text { Mean } \\
\text { annual } \\
\text { precipitation } \\
\text { (in) }\end{array}$ & $\begin{array}{c}\text { Rainfall intensity, 24-hour } \\
\text { (in) }\end{array}$ & $\begin{array}{c}50 \text {-year } \\
\text { (in) }\end{array}$ \\
\hline 133 & 4.0 & 6,700 & 66.0 & 3.0 & 13.5 & 1.8 \\
\hline
\end{tabular}

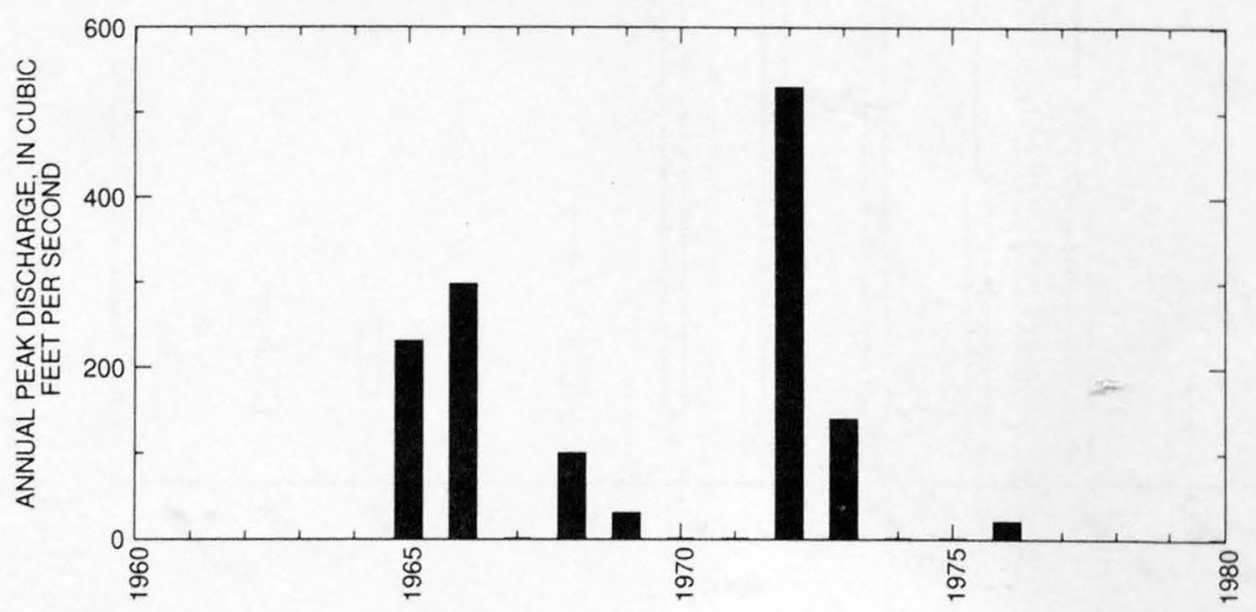




\section{SILVER CREEK NEAR SNOWFLAKE, AZ}

LOCATION.--Lat 34 40'00", long 110.02'30", in SW $1 / 4 \mathrm{NW}^{1 / 4} \mathrm{sec} .29$, T.15 N., R.22 E., Navajo County, Hydrologic Unit 15020005 , on left bank $6 \mathrm{mi}$ upstream from mouth and $11 \mathrm{mi}$ north of Snowflake.

DRAINAGE AREA.--925 $\mathrm{mi}^{2}$, of which $79 \mathrm{mi}^{2}$ is noncontributing.

PERIOD OF RECORD.--October 1950 to September 1995 (discontinued).

REVISED RECORDS.--WDR AZ-88-1: Drainage area.

GAGE.--Water-stage recorder. Datum of gage is $5,204.1 \mathrm{ft}$ above sea level.

REMARKS.--Records fair. Diversions for irrigation above station of about 6,600 acres. Flow regulated by several reservoirs - combined capacity, about 22,100 acre-ft.

EXTREMES FOR PERIOD OF RECORD.--Maximum discharge, 10,100 ft $3 / \mathrm{s} \mathrm{Jan.} \mathrm{19,} \mathrm{1952,} \mathrm{gage} \mathrm{height,} 18.0 \mathrm{ft}$, from rating curve extended above $4,400 \mathrm{ft}^{3} / \mathrm{s}$ on basis of peak discharge for former station near Woodruff; no flow for several days in water years 1971, 1974-78, 1980$82,1984,1986,1990,1991$, and 1994.

Annual peak discharges

\begin{tabular}{|c|c|c|c|c|c|c|c|}
\hline $\begin{array}{l}\text { Water } \\
\text { year }\end{array}$ & Date & $\begin{array}{c}\text { Annual peak } \\
\text { discharge } \\
\left(\mathrm{ft}^{3} / \mathrm{s}\right)\end{array}$ & $\begin{array}{l}\text { Discharge } \\
\text { codes }\end{array}$ & $\begin{array}{l}\text { Water } \\
\text { year }\end{array}$ & Date & $\begin{array}{c}\text { Annual peak } \\
\text { discharge } \\
\left(\mathrm{ft}^{3} / \mathrm{s}\right)\end{array}$ & $\begin{array}{c}\text { Discharge } \\
\text { codes }\end{array}$ \\
\hline 1920 & $12-05-19$ & 25,000 & ES,UR,HP & 1963 & $08-21-63$ & 5,860 & UR \\
\hline 1929 & $07-21-29$ & 10,500 & UR & 1964 & $07-31-64$ & 6,090 & UR \\
\hline 1930 & $08-11-30$ & 3,000 & UR & 1965 & $01-08-65$ & 1,260 & UR \\
\hline 1931 & $09-06-31$ & 2,850 & UR & 1966 & $12-30-65$ & 6,800 & UR \\
\hline 1932 & $02-09-32$ & 9,900 & UR & 1967 & $07-29-67$ & 4,070 & UR \\
\hline 1933 & $09-10-33$ & 4,600 & UR & 1968 & $08-05-68$ & 3,890 & UR \\
\hline 1934 & $00-00-34$ & 2,000 & ES,UR & 1969 & $07-24-69$ & 3,150 & UR \\
\hline 1935 & $09-27-35$ & 2,820 & UR & 1970 & $09-06-70$ & 1,690 & UR \\
\hline 1936 & $07-25-36$ & 4,300 & UR & 1971 & $09-30-71$ & 4,470 & UR \\
\hline 1937 & $02-07-37$ & 4,300 & UR & 1972 & $12-26-71$ & 4,780 & UR \\
\hline 1938 & $08-08-38$ & 3,100 & UR & 1973 & $10-20-72$ & 3,260 & UR \\
\hline 1939 & $08-29-39$ & 1,460 & UR & 1974 & $09-22-74$ & 558 & UR \\
\hline 1940 & $07-26-40$ & ${ }^{1} 11,000$ & UR & 1975 & $10-29-74$ & 990 & UR \\
\hline 1941 & $03-15-41$ & 3,700 & UR & 1976 & $02-10-76$ & 2,540 & UR \\
\hline 1942 & $10-03-41$ & 932 & UR & 1977 & $08-04-77$ & 1,840 & UR \\
\hline 1943 & $08-31-43$ & 3,120 & UR & 1978 & $03-01-78$ & 4,160 & UR \\
\hline 1944 & $08-25-44$ & 416 & UR & 1979 & $12-19-78$ & 7,700 & UR \\
\hline 1945 & $08-11-45$ & 3,230 & UR & 1980 & $02-20-80$ & 4460 & UR \\
\hline 1946 & $09-19-46$ & 1,680 & UR & 1981 & $07-15-81$ & 907 & UR \\
\hline 1949 & $01-13-49$ & 2,900 & UR & 1982 & $08-12-82$ & 5,090 & UR \\
\hline 1950 & $07-07-50$ & 1,160 & UR & 1983 & $09-29-83$ & 1,510 & UR \\
\hline 1951 & $08-28-51$ & 3,780 & UR & 1984 & $08-23-84$ & 3,160 & UR \\
\hline 1952 & $01-19-52$ & 10,100 & UR & 1985 & $12-27-84$ & 5,840 & UR \\
\hline 1953 & $07-16-53$ & 1,060 & UR & 1986 & $08-28-86$ & 409 & UR \\
\hline 1954 & $09-02-54$ & 7,670 & UR & 1987 & $08-06-87$ & 1,820 & UR \\
\hline 1955 & $08-06-55$ & 4,980 & UR & 1988 & $02-03-88$ & 737 & UR \\
\hline 1956 & $06-29-56$ & 3,620 & UR & 1989 & $07-23-89$ & 1,020 & UR \\
\hline 1957 & $08-05-57$ & 1,910 & UR & 1990 & $07-07-90$ & 635 & UR \\
\hline 1958 & $09-08-58$ & 4,340 & UR & 1991 & $03-02-91$ & 1,100 & UR \\
\hline 1959 & $07-05-59$ & 630 & UR & 1992 & $08-06-92$ & 1,800 & UR \\
\hline 1960 & $12-25-59$ & 2,120 & UR & 1993 & $02-20-93$ & 5,800 & UR \\
\hline 1961 & $08-17-61$ & 1,300 & UR & 1994 & $09-03-94$ & 628 & UR \\
\hline 1962 & $02-13-62$ & 1,030 & UR & 1995 & $02-15-95$ & 1,220 & UR \\
\hline
\end{tabular}

THighest since 1923. 
09393500 SILVER CREEK NEAR SNOWFLAKE, AZ--Continued

Discharge rating table developed January 1988

\begin{tabular}{cccc}
\hline $\begin{array}{c}\text { Gage height } \\
(\mathbf{f t})\end{array}$ & $\begin{array}{c}\text { Discharge } \\
\left(\mathbf{f t}^{\mathbf{3}} \mathbf{s}\right)\end{array}$ & $\begin{array}{c}\text { Gage height } \\
(\mathbf{f t})\end{array}$ & $\begin{array}{c}\text { Discharge } \\
\left(\mathbf{f t}^{\mathbf{3}} \mathbf{s}\right)\end{array}$ \\
\hline 3.0 & 135 & 14.0 & 6,510 \\
4.0 & 337 & 17.0 & 9,300 \\
5.0 & 642 & 20.0 & 12,490 \\
8.0 & 2,070 & 23.0 & 16,060 \\
11.0 & 4,030 & 26.0 & 20,000 \\
\hline
\end{tabular}

Basin characteristics

\begin{tabular}{cccccccc}
\hline $\begin{array}{c}\text { Main } \\
\text { channel } \\
\text { slope } \\
(\mathrm{ft} / \mathrm{mi})\end{array}$ & $\begin{array}{c}\text { Stream } \\
\text { length } \\
\text { (mi) }\end{array}$ & $\begin{array}{c}\text { Mean } \\
\text { basin } \\
\text { elevation } \\
\text { (ft) }\end{array}$ & $\begin{array}{c}\text { Forested } \\
\text { area } \\
\text { (percent) }\end{array}$ & $\begin{array}{c}\text { Soil } \\
\text { index }\end{array}$ & $\begin{array}{c}\text { Mean } \\
\text { annual } \\
\text { precipitation } \\
\text { (in) }\end{array}$ & $\begin{array}{c}\text { Rainfall intensity, 24-hour } \\
\text { (in) }\end{array}$ & $\begin{array}{c}50 \text {-year } \\
\text { (in) }\end{array}$ \\
\hline 35.0 & 53.3 & 6,400 & 53.0 & 3.0 & 16.7 & 1.8 \\
\hline
\end{tabular}


09393500 SILVER CREEK NEAR SNOWFLAKE, AZ--Continued

MEAN MONTHLY AND ANNUAL DISCHARGES 1951.95

\begin{tabular}{|c|c|c|c|c|c|c|}
\hline MONTH & $\begin{array}{l}\text { MAXIMUM } \\
\text { (FT3/S) }\end{array}$ & $\begin{array}{l}\text { MINIMUM } \\
(\text { FT3/S) }\end{array}$ & $\begin{array}{l}\text { MEAN } \\
(F T 3 / S)\end{array}$ & $\begin{array}{l}\text { STAN- } \\
\text { DARD } \\
\text { DEVIA- } \\
\text { TION } \\
(\text { FT3/S) }\end{array}$ & $\begin{array}{l}\text { COEFFI- } \\
\text { CIBNT OF } \\
\text { VARI- } \\
\text { ATION }\end{array}$ & $\begin{array}{c}\text { PERCENT } \\
\text { OF } \\
\text { ANNUAL } \\
\text { RUNOFF }\end{array}$ \\
\hline OCTOBER & 89 & 0.64 & 12 & 18 & 1.6 & 5.1 \\
\hline NOVEMBER & 109 & 0.82 & 7.9 & 18 & 2.3 & 3.5 \\
\hline DECEMBER & 276 & 0.88 & 23 & 52 & 2.2 & 10.2 \\
\hline JANUARY & 457 & 0.35 & 34 & 94 & 2.8 & 14.7 \\
\hline FEBRUARY & 468 & 0.01 & 37 & 97 & 2.6 & 16.3 \\
\hline MARCH & 195 & 0.00 & 32 & 50 & 1.5 & 14.1 \\
\hline APRIL & 212 & 0.00 & 10 & 32 & 3.1 & 4.5 \\
\hline MAY & 71 & 0.96 & 4.8 & 10 & 2.2 & 2.1 \\
\hline JUNE & 27 & 0.30 & 3.9 & 5.2 & 1.3 & 1.7 \\
\hline JULY & 67 & 0.86 & 15 & 16 & 1.1 & 6.4 \\
\hline AUGUST & 119 & 2.8 & 30 & 28 & 0.96 & 12.9 \\
\hline SEPTEMBER & 173 & 0.59 & 20 & 30 & 1.5 & 8.6 \\
\hline ANNUAL & 86 & 2.8 & 19 & 19 & 0.99 & 100 \\
\hline
\end{tabular}

MAGNITUDE AND PROBABILITY OF INSTANTANEOUS PEAK FLOW BASED ON PERIOD OF RECORD 1920, 1929-46, 1949.95

DISCHARGE, IN FT3/S, FOR INDICATED RECURRENCE INTERVAL IN YEARS, AND EXCEEDANCE PROBABILITY, IN PERCENT

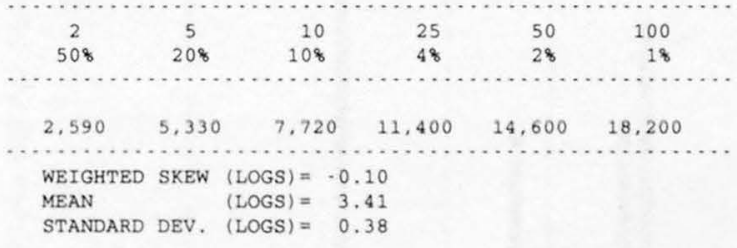

MAGNITUDE AND PROBABILITY OF ANNUAL LOW FLOW BASED ON PERIOD OF RECORD 1952-95

PERIOD
(CON-

MAGNITUDE AND PROBABILITY OF ANNUAL HIGH FLOW BASED ON PERIOD OF RECORD 1951.95

\begin{tabular}{|c|c|c|c|c|c|c|}
\hline PERIOD & & \multicolumn{5}{|c|}{$\begin{array}{l}\text { DISCHARGE, IN FT3/S, FOR INDICATED } \\
\text { RECURRENCE INTERVAL, IN YEARS, AND }\end{array}$} \\
\hline ICON. & & \multicolumn{3}{|c|}{ EXCEEDANCE PROBABILITY, } & \multicolumn{2}{|c|}{ IN PERCENT } \\
\hline SECU. & & $\ldots \ldots$ & $\cdots \cdots$ & $\ldots \ldots$ & $\ldots \ldots$ & . \\
\hline TIVE & 2 & 5 & 10 & 25 & 50 & $100 \#$ \\
\hline DAYS) & 508 & 208 & 108 & 48 & 28 & 18 \\
\hline 1 & 644 & 1,610 & 2,590 & 4,290 & 5,940 & 7,940 \\
\hline 3 & 336 & 859 & 1,430 & 2.500 & 3,610 & 5,050 \\
\hline 7 & 184 & 462 & 759 & 1.300 & 1.860 & 2,570 \\
\hline 15 & 107 & 263 & 428 & 727 & 1.030 & 1,410 \\
\hline 30 & 71 & 167 & 262 & 427 & 586 & 781 \\
\hline 60 & 44 & 105 & 167 & 279 & 392 & 534 \\
\hline 90 & 33 & 78 & 125 & 213 & 304 & 422 \\
\hline
\end{tabular}

DURATION TABLE OF DAILY MEAN FLOW FOR PERIOD OF RECORD 1951.95

DISCHARGE, IN FT3/S, WHICH WAS BQUALED OR EXCEEDED FOR INDICATED PERCENT OF TIME

\begin{tabular}{|c|c|c|c|c|c|c|c|c|c|c|c|c|c|c|c|c|}
\hline 18 & 58 & 108 & 158 & 208 & 308 & 408 & 508 & 608 & 708 & 808 & 908 & 958 & 988 & 998 & 99.58 & 99.98 \\
\hline 21 & 67 & 25 & 13 & 8.5 & 5.5 & 4.1 & 3.1 & 2.3 & 1.6 & 1.1 & 0.57 & 0.26 & 0.03 & 0.00 & 0.00 & 0.00 \\
\hline
\end{tabular}

\# Reliability of values in column is uncertain, and potential errors are large. 
ANNUAL MEAN DISCHARGE IN CUBIC

FEET PER SECOND

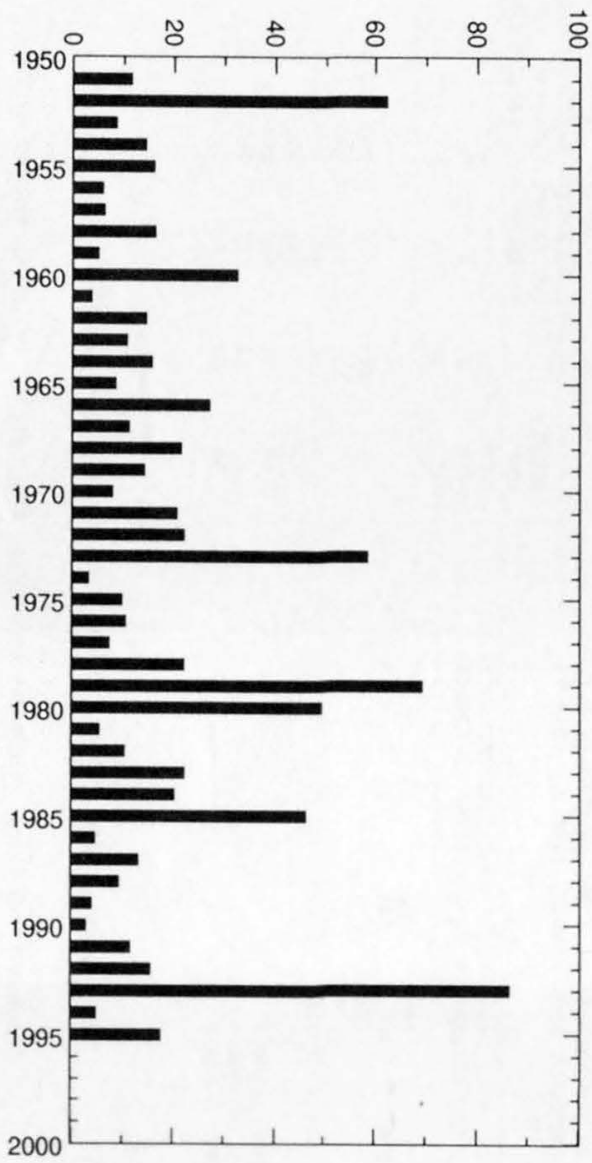

ANNUAL PEAK DISCHARGE, IN CUBIC

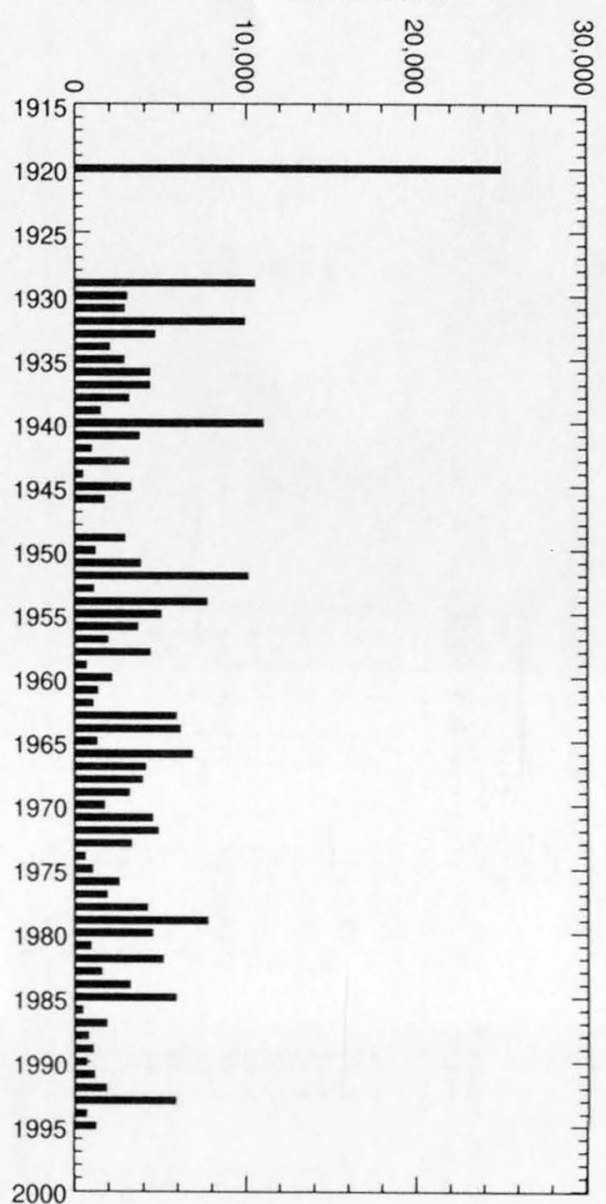


09393500 SILVER CREEK NEAR SNOWFLAKE, AZ--Continued
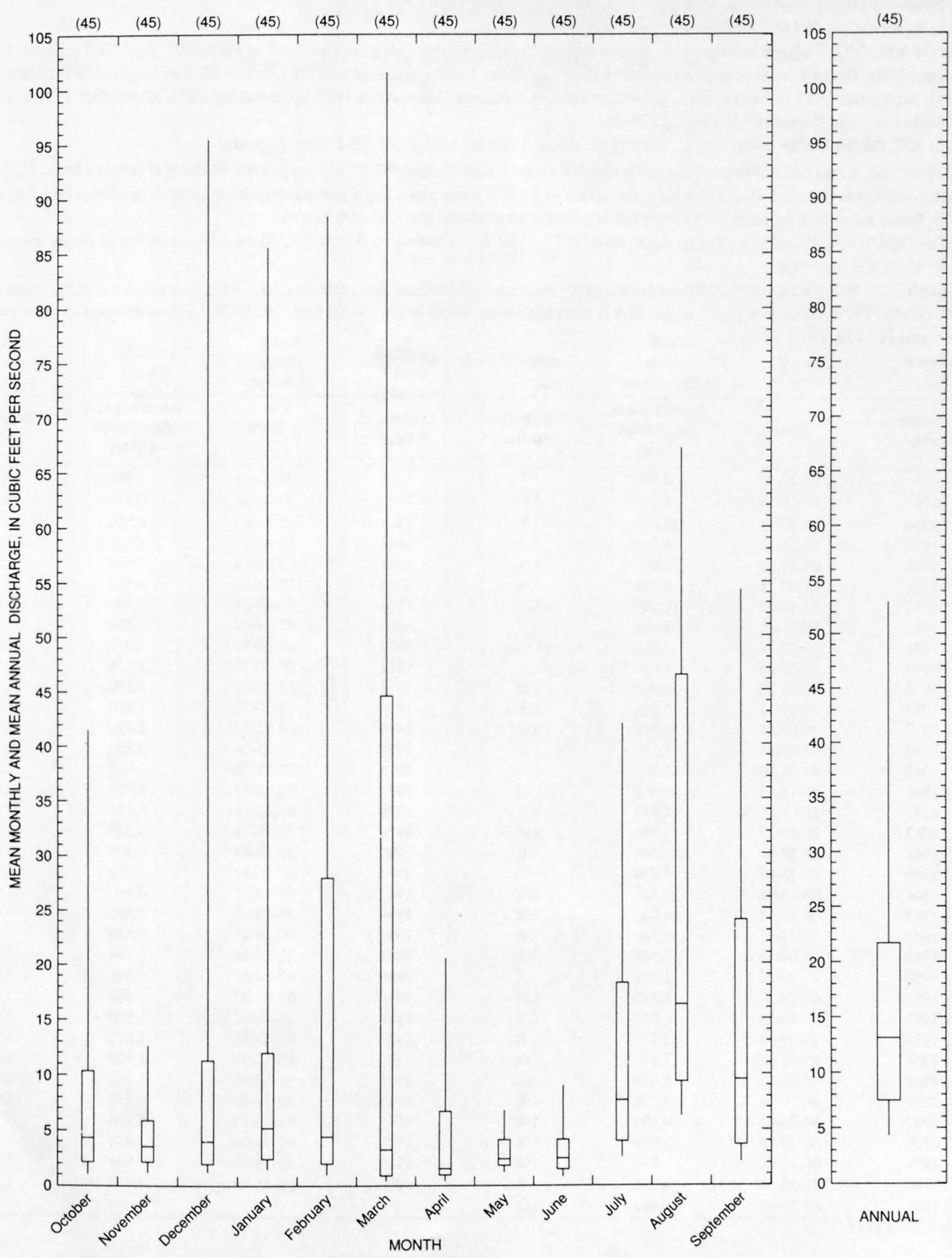


\section{LITTLE COLORADO RIVER AT WOODRUFF, AZ}

LOCATION.--Lat $34^{\circ} 46^{\prime} 58^{\prime \prime}$, long $110^{\circ} 02^{\prime} 37^{\prime \prime}$, in NE $\frac{1}{4} \mathrm{SW}^{1} / 4$ sec.17, T.16 N., R.22 E., Navajo County, Hydrologic Unit 15020002, on left at abandoned county road bridge in Woodruff, $3.7 \mathrm{mi}$ downstream from Silver Creek.

DRAINAGE AREA.--8,072 $\mathrm{mi}^{2}$, of which $297 \mathrm{mi}^{2}$ is noncontributing.

PERIOD OF RECORD.--March to May 1905; June to July 1905 (gage heights only); August 1905 to May 1907; July 1907 to April 1908, Jü October 1908, December 1908, and December 1915 to September 1916 (gage heights only); October 1916 to August 1917 (monthly disctir only); September 1917 to March 1918, December 1918 to December 1919, April 1929 to December 1933, September 1935 to currenty Published as "near Woodruff" 1916-19, 1929-48.

REVISED RECORDS.--WSP 1049: 1917. WSP 1213: 1906, 1919(M). WDR AZ-88-1: Drainage area.

GAGE.--Water-stage recorder. Datum of gage is $5,130.3 \mathrm{ft}$ above sea level. See WSP 1733 for history of changes prior to Sept. 22, 1949.

REMARKS.--Diversions above station for irrigation of about 22,000 acres, including a pump installation 1,000 ft upstream installed in sprin 1973. Some regulation by reservoirs above station; combined capacity, about 81,400 acre-ft.

AVERAGE DISCHARGE.--67 years (water years 1906, 1917, 1930-33, 1936-96), $51.3 \mathrm{ft}^{3} / \mathrm{s}, 37,200 \mathrm{acre}-\mathrm{ft} / \mathrm{yr}$; median of yearly mean dischar; $43 \mathrm{ft}^{3} / \mathrm{s}, 31,200$ acre-ft/yr.

EXTREMES FOR PERIOD OF RECORD.--Maximum discharge not determined, occurred Jan. 19, 1916; maximum discharge recorded, 25. $\mathrm{ft}^{3} / \mathrm{s}$ Dec. 5, 1919; maximum gage height, $22.9 \mathrm{ft}$ from high-water mark in gage well, Dec. 19, 1978; no flow at times in most years pric 1960 and in 1974, 1976, 1983.

Annual peak discharges

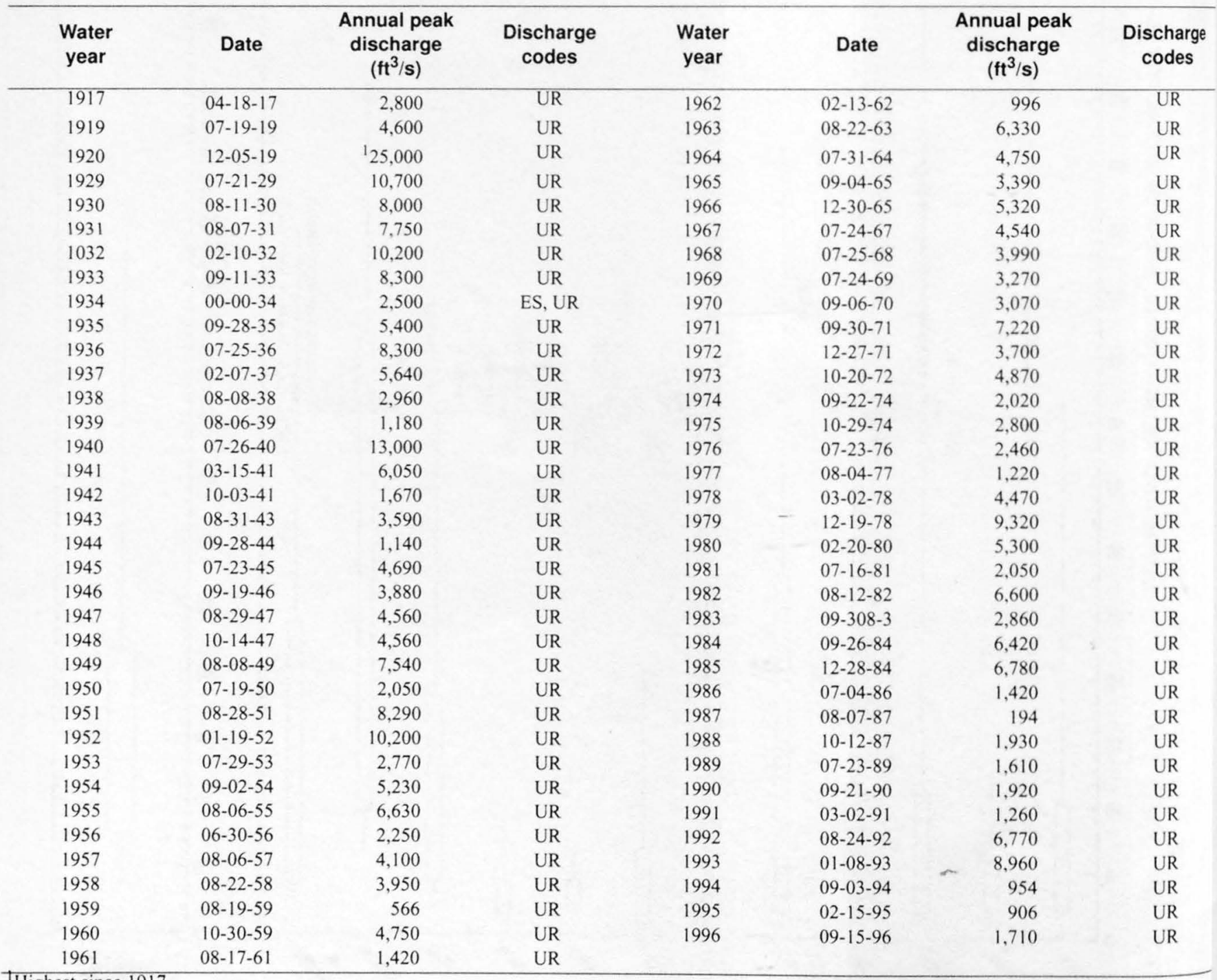

${ }^{\top}$ Highest since 1917. 
09394500 LITTLE COLORADO RIVER AT WOODRUFF, AZ--Continued

Discharge rating table developed January 1990

\begin{tabular}{cccc}
\hline $\begin{array}{c}\text { Gage height } \\
(\mathbf{f t})\end{array}$ & $\begin{array}{c}\text { Discharge } \\
\left(\mathrm{ft}^{\mathbf{3}} \mathbf{s}\right)\end{array}$ & $\begin{array}{c}\text { Gage height } \\
(\mathbf{f t})\end{array}$ & $\begin{array}{c}\text { Discharge } \\
\left(\mathbf{f t}^{\mathbf{3}} \mathbf{s}\right)\end{array}$ \\
\hline 6.0 & 103 & 14.0 & 3,680 \\
7.0 & 341 & 16.0 & 5,010 \\
8.0 & 660 & 18.0 & 6,460 \\
10.0 & 1,480 & 20.0 & 8,030 \\
12.0 & 2,500 & 23.0 & 10,600 \\
\hline
\end{tabular}

Basin characteristics

\begin{tabular}{ccccccccc}
\hline $\begin{array}{c}\text { Main } \\
\text { channel } \\
\text { slope } \\
(\mathrm{ft} / \mathrm{mi})\end{array}$ & $\begin{array}{c}\text { Stream } \\
\text { length }\end{array}$ & $\begin{array}{c}\text { Mean } \\
\text { (mi) }\end{array}$ & $\begin{array}{c}\text { basin } \\
\text { elevation } \\
\text { (ft) }\end{array}$ & $\begin{array}{c}\text { Forested } \\
\text { area } \\
\text { (percent) }\end{array}$ & $\begin{array}{c}\text { Soil } \\
\text { index }\end{array}$ & $\begin{array}{c}\text { Mean } \\
\text { annual } \\
\text { precipitation } \\
\text { (in) }\end{array}$ & $\begin{array}{c}\text { 2-year } \\
\text { (in) }\end{array}$ & $\begin{array}{c}\text { 50-year } \\
\text { (in) }\end{array}$ \\
\hline 25.2 & 122 & 6,810 & 28.0 & 2.9 & 13.4 & 1.5 & 3.0 \\
\hline
\end{tabular}


09394500 LITTLE COLORADO RIVER AT WOODRUFF, AZ--Continued

MEAN MONTHLY AND ANNUAL DISCHARGES $1906,1930.33,1936.96$

\begin{tabular}{|c|c|c|c|c|c|c|}
\hline MONTH & $\begin{array}{l}\text { MAXIMUM } \\
(\mathrm{FT} 3 / \mathrm{S})\end{array}$ & $\begin{array}{l}\text { MINIMUM } \\
\text { (FT3/S) }\end{array}$ & $\begin{array}{c}\text { MEAN } \\
(\mathrm{FT} 3 / \mathrm{S})\end{array}$ & $\begin{array}{l}\text { STAN- } \\
\text { DARD } \\
\text { DEVIA- } \\
\text { TION } \\
\text { (FT3/S) }\end{array}$ & $\begin{array}{l}\text { COEFFI- } \\
\text { CIENT OF } \\
\text { VARI- } \\
\text { ATION }\end{array}$ & $\begin{array}{c}\text { PERCENT } \\
\text { OF } \\
\text { ANNUAL } \\
\text { RUNOFF }\end{array}$ \\
\hline OCTOBER & 301 & 1.0 & 40 & 63 & 1.6 & 6.6 \\
\hline NOVEMBER & 543 & 3.0 & 25 & 71 & 2.8 & 4.1 \\
\hline DECEMBER & 349 & 2.3 & 28 & 54 & 1.9 & 4.7 \\
\hline JANUARY & 599 & 3.0 & 43 & 97 & 2.3 & 7.0 \\
\hline FEBRUARY & 827 & 2.5 & 61 & 134 & 2.2 & 9.9 \\
\hline MARCH & 610 & 1.3 & 74 & 121 & 1.6 & 12.0 \\
\hline APRIL & 414 & 0.00 & 42 & 87 & 2.1 & 6.8 \\
\hline MAY & 488 & 0.00 & 23 & 69 & 3.1 & 3.7 \\
\hline JUNE & 88 & 0.00 & 6.9 & 13 & 1.8 & 1.1 \\
\hline JULY & 238 & 0.53 & 57 & 59 & 1.0 & 9.3 \\
\hline AUGUST & 951 & 3.6 & 137 & 162 & 1.2 & 22.4 \\
\hline SEPTEMBER & 611 & 0.71 & 77 & 101 & 1.3 & 12.5 \\
\hline ANNUAL & 161 & 9.6 & 51 & 39 & 0.77 & 100 \\
\hline
\end{tabular}

MAGNITUDE AND PROBABILITY OF INSTANTANEOUS PEAK FLOW
BASED ON PERIOD OF RECORD $1917,1919 \cdot 20,1929.96$

DISCHARGE, IN FT $3 / S$, FOR INDICATED RECURRENCE INTERVAL IN YEARS, AND EXCEEDANCE PROBABILITY, IN PERCENT

\begin{tabular}{|c|c|c|c|c|c|}
\hline 2 & 5 & 10 & 25 & 50 & 100 \\
\hline 508 & 208 & 108 & 48 & 28 & 18 \\
\hline 3,790 & 6,920 & 9,340 & 12,700 & 15,400 & 18,300 \\
\hline WEIGHTED & SKEW & $($ LOGS $)=$ & & & \\
\hline MEAN & & $($ LOGS $)=$ & & & \\
\hline STANDARD & DEV. & $($ LOGS $)=$ & & & \\
\hline
\end{tabular}

MAGNITUDE AND PROBABILITY OF ANNUAE LOW FLOW BASED ON PERIOD OF RECORD 1907, 1931-33, 1937-96

\begin{tabular}{|c|c|c|c|c|c|c|}
\hline PERIOD & & $\begin{array}{l}\text { SCHAR } \\
\text { CURREI }\end{array}$ & $\begin{array}{l}\text { IN F } \\
\text { INTE }\end{array}$ & $\begin{array}{ll}\text { S, } & \text { FOI } \\
\text { L, IN }\end{array}$ & $\begin{array}{l}\text { NDICA } \\
\text { ARS, }\end{array}$ & \\
\hline (CON. & & EXCE & NCE P & ABILI & IN $P$ & ENT \\
\hline SECU. & & & & & & \\
\hline TIVE & 2 & 5 & 10 & 20 & 50 & $100 \#$ \\
\hline DAYS) & 508 & 208 & 108 & 58 & 28 & 18 \\
\hline 1 & 0.25 & 0.00 & 0.00 & 0.00 & 0.00 & 0.00 \\
\hline 3 & 0.49 & 0.00 & 0.00 & 0.00 & 0.00 & 0.00 \\
\hline 7 & 0.71 & 0,00 & 0.00 & 0.00 & 0.00 & 0.00 \\
\hline 14 & 0.85 & 0.00 & 0.00 & 0.00 & 0.00 & 0.00 \\
\hline 30 & 1.4 & 0.22 & 0.01 & 0.00 & 0.00 & 0.00 \\
\hline 60 & 2.6 & 0.82 & 0.38 & 0.18 & 0.03 & 0.00 \\
\hline 90 & 4.2 & 1.6 & 0.85 & 0.46 & 0.12 & 0.00 \\
\hline 120 & 7.7 & 4.6 & 3.6 & 3.1 & 2.6 & 2.3 \\
\hline 183 & 14 & 7.4 & 5.4 & 4.3 & 3.4 & 3.0 \\
\hline
\end{tabular}

MAGNITUDE AND PROBABILITY OF ANNUAL HIGH FLOW BASED ON PERIOD OF RECORD 1906, 1930-33, 1936.96

\begin{tabular}{|c|c|c|c|c|c|c|}
\hline \multirow{3}{*}{$\begin{array}{l}\text { PERIOD } \\
\text { /CON- } \\
\text { SECU. }\end{array}$} & \multicolumn{6}{|c|}{ DISCHARGE, IN FT3/S, FCR INDICATED } \\
\hline & & $\begin{array}{l}\text { RECURRE } \\
\text { EXCEEDA }\end{array}$ & CE INT: & VAL, II & YEARS, & $\begin{array}{l}\text { AND } \\
\text { ENT }\end{array}$ \\
\hline & - & ........ & …... & ….... & …... & $\ldots \ldots$ \\
\hline TIVE & 2 & 5 & 10 & 25 & 50 & $100 \#$ \\
\hline DAYS ) & 508 & 208 & 108 & 48 & 28 & 18 \\
\hline 1 & 1,770 & 3,520 & 4,930 & 6,910 & 8,510 & 10,200 \\
\hline 3 & 991 & 2,020 & 2,880 & 4,120 & 5,160 & 6,270 \\
\hline 7 & 553 & 1,090 & 1,520 & 2,130 & 2,620 & 3,130 \\
\hline 15 & 330 & 643 & 891 & 1,240 & 1.530 & 1.830 \\
\hline 30 & 217 & 421 & 588 & 830 & 1,030 & 1,250 \\
\hline 60 & 142 & 285 & 406 & 588 & 744 & 916 \\
\hline 90 & 106 & 214 & 307 & 447 & 569 & 705 \\
\hline
\end{tabular}

DURATION TABLE OF DAILY MEAN FLOW FOR PERIOD OF RECORD 1906, 1930.33, 1936.96

DISCHARGE, IN FT3/S, WHICH WAS EQUALED OR EXCEEDED FOR INDICATED PERCENT OF TIME

\begin{tabular}{|c|c|c|c|c|c|c|c|c|c|c|c|c|c|c|c|c|}
\hline 18 & 58 & 108 & 158 & 208 & 308 & 408 & 508 & 608 & 708 & 808 & 908 & 958 & 988 & 998 & 99.58 & 99.98 \\
\hline 87 & 229 & 99 & 51 & 32 & 16 & 10 & 7.3 & 5.5 & 4.3 & 3.0 & 1.2 & 0.30 & 0.00 & 0.00 & 0.00 & 0.00 \\
\hline
\end{tabular}

\# Reliability of values in column is uncertain, and potential errors are large. 
ANNUAL MEAN DISCHARGE, IN CUBIC FEET PER SECOND

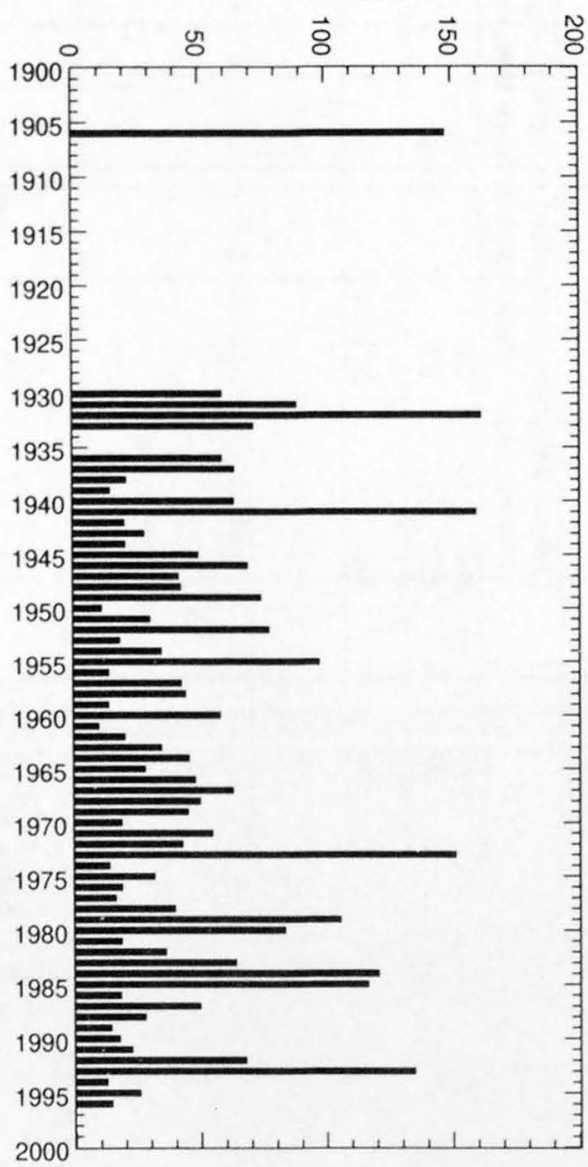

ANNUAL PEAK DISCHARGE, IN CUBIC FEET PER SECOND

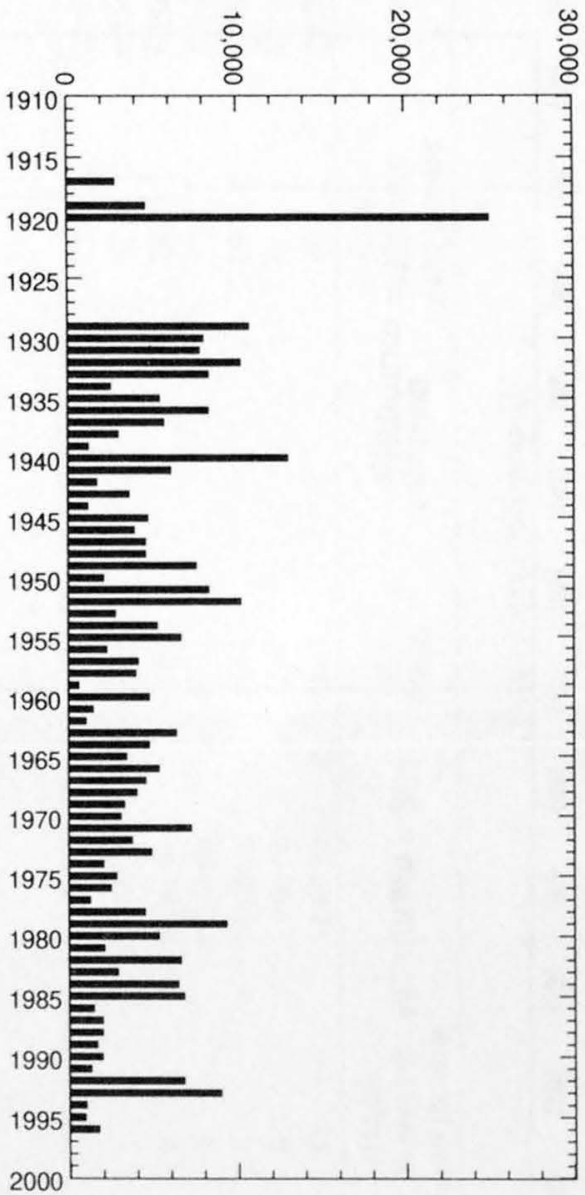


09394500 LITTLE COLORADO RIVER AT WOODRUFF, AZ--Continued

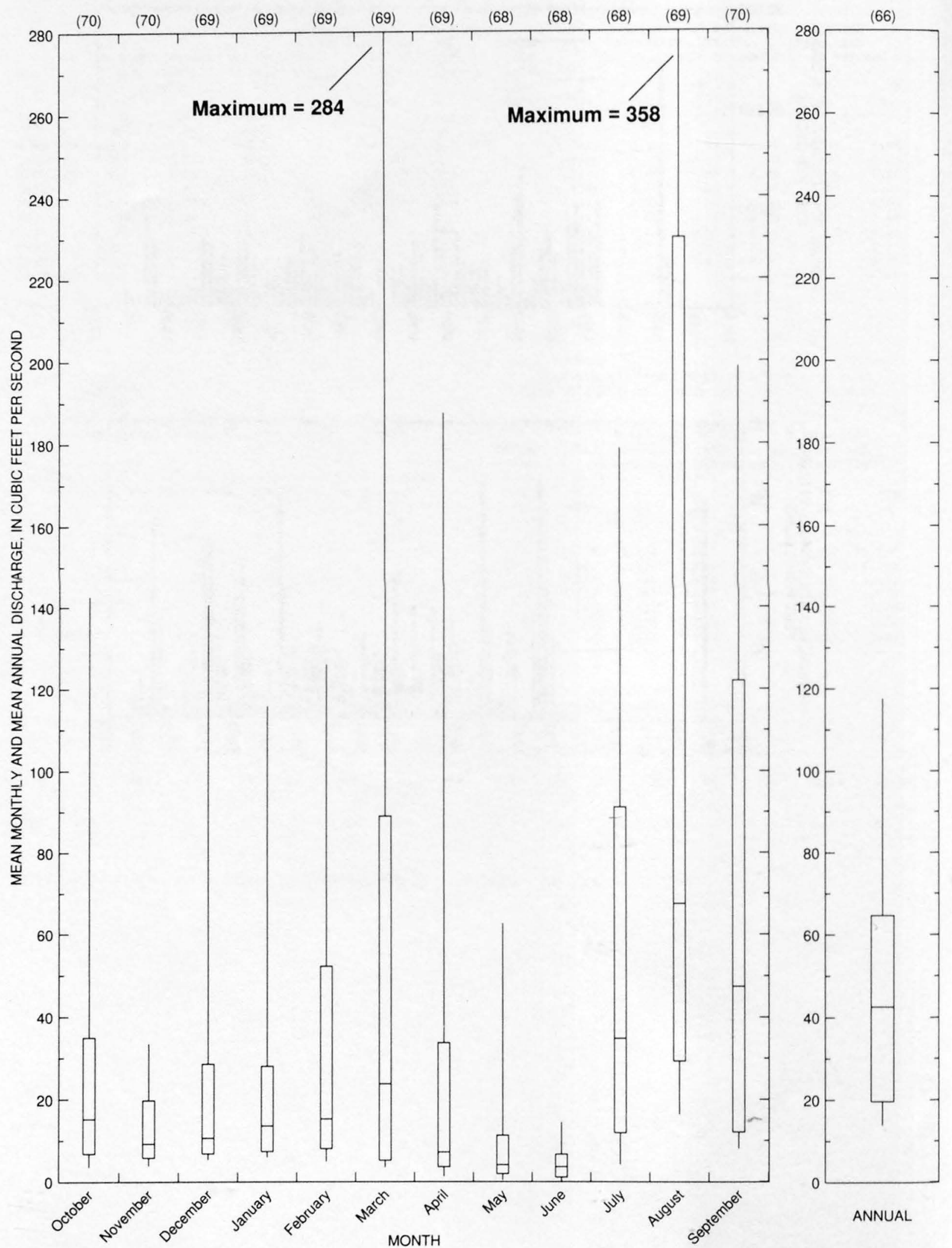


09395100 CARR LAKE DRAW TRIBUTARY NEAR HOLBROOK, AZ

LOCATION.--Lat 34 $50^{\prime} 05^{\prime \prime}$, long 109 $56^{\prime} 00^{\prime \prime}$, in $\mathrm{SE}^{1} / \mathrm{SE}^{1} / 4$, sec.30, T.17 N., R.23 E., Navajo County, Hydrologic Unit 15020005, at (former) U.S. Highway 180, 14 mi southeast of Holbrook.

DRAINAGE AREA.--1.28 $\mathrm{mi}^{2}$.

Annual peak discharges

\begin{tabular}{|c|c|c|c|c|c|c|c|}
\hline $\begin{array}{l}\text { Water } \\
\text { year }\end{array}$ & Date & $\begin{array}{c}\text { Annual peak } \\
\text { discharge } \\
\left(\mathrm{ft}^{3} / \mathbf{s}\right)\end{array}$ & $\begin{array}{c}\text { Discharge } \\
\text { codes }\end{array}$ & $\begin{array}{l}\text { Water } \\
\text { year }\end{array}$ & Date & $\begin{array}{c}\text { Annual peak } \\
\text { discharge } \\
\left(\mathrm{ft}^{3} / \mathrm{s}\right)\end{array}$ & $\begin{array}{c}\text { Discharge } \\
\text { codes }\end{array}$ \\
\hline 1964 & $07-28-64$ & 129 & & 1971 & $09-29-71$ & 45 & \\
\hline 1965 & $00-00-65$ & 0 & & 1972 & $00-00-72$ & 0 & \\
\hline 1966 & $08-12-66$ & 140 & & 1973 & $00-00-73$ & 5.0 & \\
\hline 1967 & $00-00-67$ & 35 & & 1974 & $00-00-74$ & 0 & \\
\hline 1968 & $08-00-68$ & 102 & & 1975 & $10-29-74$ & 4.0 & \\
\hline 1969 & $09-05-69$ & 39 & & 1976 & $09-00-76$ & 1.0 & \\
\hline 1970 & 09-06-70 & 52 & & & & & \\
\hline
\end{tabular}

Magnitude and probability of instantaneous peak flow based on period of record 1964-76

\begin{tabular}{|c|c|c|c|c|c|}
\hline \multicolumn{6}{|c|}{$\begin{array}{l}\text { Discharge, in } \mathrm{ft}^{3} / \mathrm{s} \text {, for indicated recurrence interval } \\
\text { in years, and exceedance probablility, in percent }\end{array}$} \\
\hline 2 & 5 & 10 & 25 & $50 \dagger$ & $100 \dagger$ \\
\hline $50 \%$ & $20 \%$ & $10 \%$ & $4 \%$ & $2 \%$ & $1 \%$ \\
\hline 27 & 73.8 & 120 & 196 & 266 & 345 \\
\hline Weighted skew & $(\log s)=$ & -0.34 & & & \\
\hline Mean & $(\log s)=$ & 1.40 & & & \\
\hline Standard dev. & $(\log s)=$ & 0.55 & & & \\
\hline
\end{tabular}

f Reliability of values in column is uncertain, and potential errors are large.

Basin characteristics

\begin{tabular}{cccccccc}
$\begin{array}{c}\text { Main } \\
\text { channel } \\
\text { slope } \\
(\mathrm{ft} / \mathrm{mi})\end{array}$ & $\begin{array}{c}\text { Stream } \\
\text { length } \\
(\mathrm{mi})\end{array}$ & $\begin{array}{c}\text { Mean } \\
\text { basin } \\
\text { elevation } \\
(\mathrm{ft})\end{array}$ & $\begin{array}{c}\text { Forested } \\
\text { area } \\
\text { (percent) }\end{array}$ & $\begin{array}{c}\text { Soil } \\
\text { index }\end{array}$ & $\begin{array}{c}\text { Mean } \\
\text { annual } \\
\text { precipitation } \\
\text { (in) }\end{array}$ & $\begin{array}{c}\text { Rainfall intensity, 24-hour } \\
\text { (in) }\end{array}$ & $\begin{array}{c}50 \text {-year } \\
\text { (in) }\end{array}$ \\
\hline 103 & 2.4 & 5,420 & 0.0 & 3.0 & 7.8 & 1.3 \\
\hline
\end{tabular}

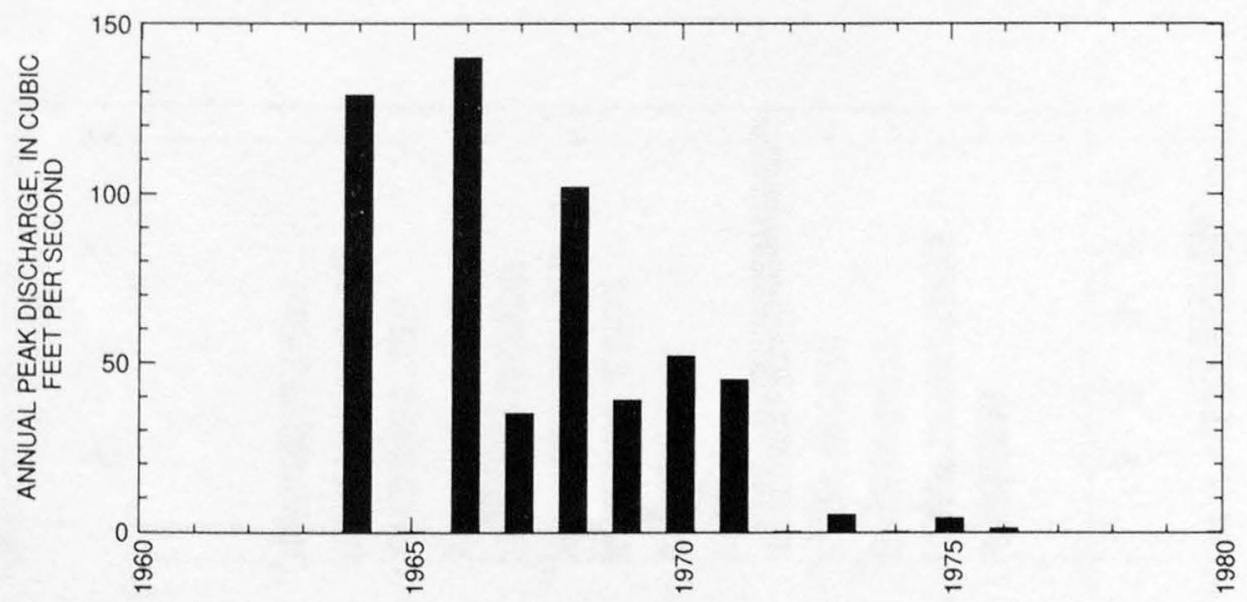


09395200 DECKER WASH NEAR SNOWFLAKE, AZ

LOCATION.--Lat 34 $27^{\prime} 40^{\prime \prime}$, long 110 $24^{\prime} 15^{\prime \prime}$, in SW $1 / 4$, sec.2, T.12 N., R.18 E., Navajo County, Hydrologic Unit 15020008, at State Highne $277,19 \mathrm{mi}$ west of Snowflake.

DRAINAGE AREA.--16.5 $\mathrm{mi}^{2}$.

Annual peak discharges

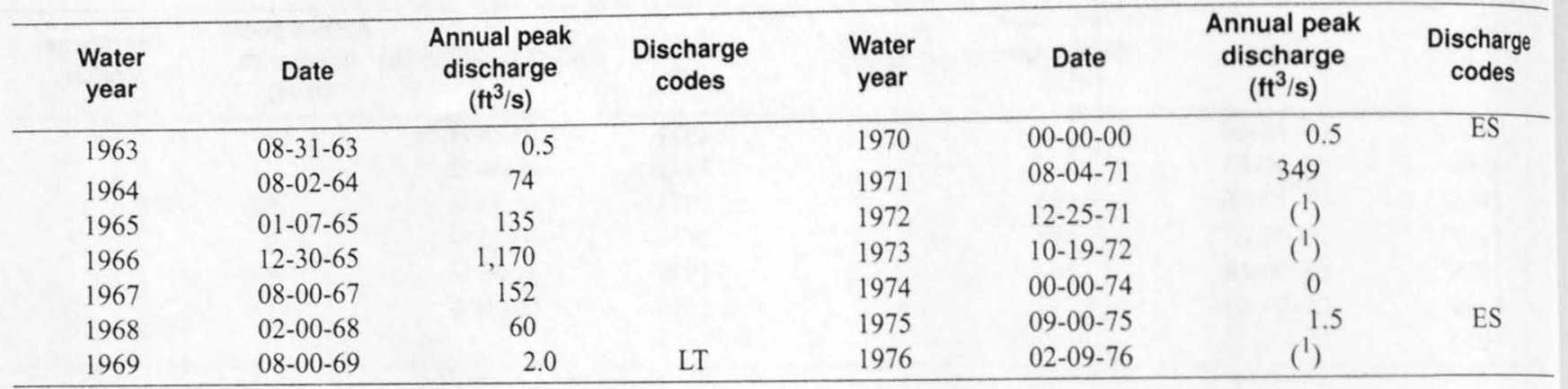

${ }^{1}$ Discharge unknown.

Magnitude and probability of instantaneous peak flow based on period of record

\begin{tabular}{|c|c|c|c|c|c|}
\hline \multicolumn{6}{|c|}{$\begin{array}{l}\text { Discharge, in } \mathrm{ft}^{3} / \mathrm{s} \text {, for indicated recurrence interval } \\
\text { in years, and exceedance probablility, in percent }\end{array}$} \\
\hline 2 & 5 & 10 & 25 & $50 t$ & $100 t$ \\
\hline $50 \%$ & $20 \%$ & $10 \%$ & $4 \%$ & $2 \%$ & $1 \%$ \\
\hline-- & -- & $-\cdot$ & $\cdots$ & -- & $\ldots$ \\
\hline Weighted skew & $(\log s)=$ & $\cdots$ & & & \\
\hline Mean & $(\log s)=$ & --- & & & \\
\hline Standard dev. & $(\log s)=$ & $\cdots$ & & & \\
\hline
\end{tabular}

Basin characteristics

\begin{tabular}{|c|c|c|c|c|c|c|c|c|}
\hline \multirow[b]{2}{*}{$\begin{array}{c}\text { Main } \\
\text { channel } \\
\text { slope } \\
(\mathrm{ft} / \mathrm{mi})\end{array}$} & \multirow{2}{*}{\multicolumn{2}{|c|}{$\begin{array}{l}\text { Stream } \\
\text { length } \\
\text { (mi) }\end{array}$}} & \multirow[b]{2}{*}{$\begin{array}{c}\text { Mean } \\
\text { basin } \\
\text { elevation } \\
\text { (ft) }\end{array}$} & \multirow[b]{2}{*}{$\begin{array}{l}\text { Forested } \\
\text { area } \\
\text { (percent) }\end{array}$} & \multirow[b]{2}{*}{$\begin{array}{c}\text { Soil } \\
\text { index }\end{array}$} & \multirow[b]{2}{*}{$\begin{array}{c}\text { Mean } \\
\text { annual } \\
\text { precipitation } \\
\text { (in) }\end{array}$} & \multicolumn{2}{|c|}{ Rainfall intensity, 24-hour } \\
\hline & & & & & & & $\begin{array}{l}\text { 2-year } \\
\text { (in) }\end{array}$ & $\begin{array}{l}\text { 50-year } \\
\text { (in) }\end{array}$ \\
\hline 31.1 & 14 & & 6,660 & 97.0 & 3.0 & 20.0 & 1.9 & 3.6 \\
\hline & 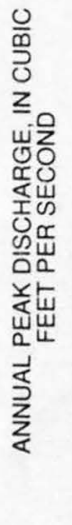 & 1,000- & & & $\begin{array}{l}\frac{1}{2} \\
\frac{0}{2}\end{array}$ & $\frac{\frac{1}{n}}{\frac{N}{2}}$ & & \\
\hline
\end{tabular}


09395850 BLACK CREEK TRIBUTARY NEAR WINDOW ROCK, AZ

LOCATION.--Lat 35 39'15", long 10905'20", in SE¹/4, sec.13, T.26 N., R.30 E., Apache County, Hydrologic Unit 15020006, at Navajo Highway 21, 0.2 mi south of State Highway 264, and 2.75 mi southwest of Window Rock.

DRAINAGE AREA.--0.33 $\mathrm{mi}^{2}$.

Annual peak discharges

\begin{tabular}{cccccrr}
\hline $\begin{array}{c}\text { Water } \\
\text { year }\end{array}$ & Date & $\begin{array}{c}\text { Annual peak } \\
\text { discharge } \\
\left(\mathrm{ft}^{3} / \mathbf{s}\right)\end{array}$ & $\begin{array}{c}\text { Discharge } \\
\text { codes }\end{array}$ & $\begin{array}{c}\text { Water } \\
\text { year }\end{array}$ & $\begin{array}{c}\text { Date } \\
\text { Annual peak } \\
\text { discharge } \\
\left(\mathrm{ft}^{3} / \mathbf{s}\right)\end{array}$ \\
\hline 1963 & $08-30-63$ & 134 & & 1970 & $08-07-70$ & 102 \\
1964 & $08-13-64$ & 145 & & 1971 & $08-27-71$ & 55 \\
1965 & $07-27-65$ & 125 & & 1972 & $09-00-72$ & 124 \\
1966 & $08-01-66$ & 12 & & 1973 & $08-00-73$ & 106 \\
1967 & $08-00-67$ & 141 & & 1974 & $08-05-74$ & 109 \\
1968 & $08-06-68$ & 171 & 1975 & $07-11-75$ & 156 \\
1969 & $08-00-69$ & 134 & & 1976 & $00-00-76$ & 81 \\
\hline
\end{tabular}

Magnitude and probability of instantaneous peak flow based on period of record

\begin{tabular}{|c|c|c|c|c|c|}
\hline \multicolumn{6}{|c|}{$\begin{array}{l}\text { Discharge, in } \mathrm{ft}^{3} / \mathrm{s} \text {, for indicated recurrence interval } \\
\text { in years, and exceedance probablility, in percent }\end{array}$} \\
\hline 2 & 5 & 10 & 25 & $50 t$ & $100+$ \\
\hline $50 \%$ & $20 \%$ & $10 \%$ & $4 \%$ & $2 \%$ & $1 \%$ \\
\hline -.. & -.. & $\ldots$ &.- & $\cdots$ & - \\
\hline Weighted skew & $(\log s)=$ & $\cdots$ & & & \\
\hline Mean & $(\log s)=$ & $\ldots$ & & & \\
\hline Standard dev. & $(\log s)=$ & $\ldots$ & & & \\
\hline
\end{tabular}

Basin characteristics

\begin{tabular}{cccccccc}
\hline $\begin{array}{c}\text { Main } \\
\text { channel } \\
\text { slope } \\
(\mathrm{ft} / \mathrm{mi})\end{array}$ & $\begin{array}{c}\text { Stream } \\
\text { length } \\
\text { (mi) }\end{array}$ & $\begin{array}{c}\text { Mean } \\
\text { basin } \\
\text { elevation } \\
\text { (ft) }\end{array}$ & $\begin{array}{c}\text { Forested } \\
\text { area } \\
\text { (percent) }\end{array}$ & $\begin{array}{c}\text { Soil } \\
\text { index }\end{array}$ & $\begin{array}{c}\text { Mean } \\
\text { annual } \\
\text { precipitation } \\
\text { (in) }\end{array}$ & $\begin{array}{c}\text { Rainfall intensity, 24-hour } \\
\text { (in) }\end{array}$ & $\begin{array}{c}50 \text {-year } \\
\text { (in) }\end{array}$ \\
\hline 53.3 & 0.75 & 6,830 & 0.0 & 1.0 & 14.1 & 1.2 \\
\hline
\end{tabular}

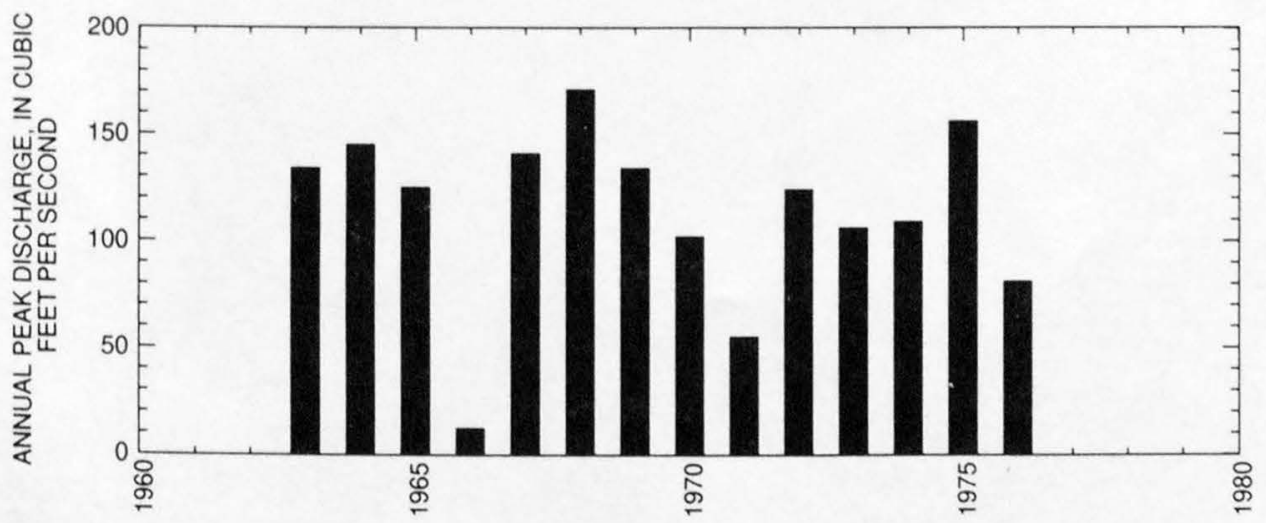




\section{BLACK CREEK NEAR LUPTON, AZ}

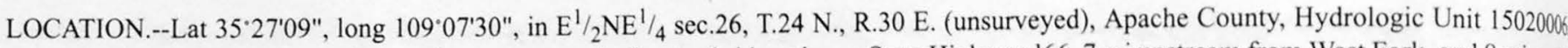
in Navajo Indian Reservation, on downstream end of center bridge pier on State Highway 166, 7 mi upstream from West Fork, and 8 mi north west of Lupton, and 16 mi south of Window Rock.

DRAINAGE AREA.--500 $\mathrm{mi}^{2}$, approximately.

PERIOD OF RECORD.--August 1964 to December 1972, May 1974 to September 1982 (discontinued). Monthly discharge only, October 197 to September 1979 .

GAGE.--Water-stage recorder. Altitude of gage is 6,550 ft, from topographic map. Prior to May 1974 at site $160 \mathrm{ft}$ downstream at same datum. REMARKS.--Records poor. Small diversions above station for irrigation and stock water. Red Lake, near headwaters 35 mi upstream, was bui in 1954, with capacity of 9,700 acre-ft, but silting may have reduced this amount.

AVERAGE DISCHARGE.-- 16 years, $8.26 \mathrm{ft}^{3} / \mathrm{s}, 5,980$ acre- $\mathrm{ft} / \mathrm{yr}$; median of yearly mean discharges, $7.2 \mathrm{ft}^{3} / \mathrm{s}, 5,200 \mathrm{acre}-\mathrm{ft} / \mathrm{yr}$.

EXTREMES FOR PERIOD OF RECORD.--Maximum discharge, 7,680 $\mathrm{ft}^{3} / \mathrm{s}$ Aug. 25, 1982, gage height, $9.58 \mathrm{ft}$, from rating curve extende above $410 \mathrm{ft}^{3} / \mathrm{s}$ on basis of slope-area measurement at gage height, $9.35 \mathrm{ft}$; no flow for many days each year.

Annual peak discharges

\begin{tabular}{ccccccc}
\hline $\begin{array}{c}\text { Water } \\
\text { year }\end{array}$ & Date & $\begin{array}{c}\text { Annual peak } \\
\text { discharge } \\
\left(\mathbf{f t}^{3} / \mathbf{s}\right)\end{array}$ & $\begin{array}{c}\text { Discharge } \\
\text { codes }\end{array}$ & $\begin{array}{c}\text { Water } \\
\text { year }\end{array}$ & $\begin{array}{c}\text { Annual peak } \\
\text { discharge } \\
\left(\mathrm{ft}^{3} / \mathbf{s}\right)\end{array}$ & $\begin{array}{c}\text { Discharge } \\
\text { codes }\end{array}$ \\
\hline 1964 & $08-01-64$ & 5,470 & & 1974 & $08-05-74$ & 1,600 \\
1965 & $07-29-65$ & 3,370 & & 1975 & $09-08-75$ & 865 \\
1966 & $09-15-66$ & 2,860 & & 1976 & $07-27-76$ & 830 \\
1967 & $07-16-67$ & 1,630 & 1977 & $08-17-77$ & 7,160 \\
1968 & $08-06-68$ & 3,750 & & 1978 & $03-01-78$ & 72 \\
1969 & $07-19-69$ & 4,280 & 1979 & $01-17-79$ & 1,340 \\
1970 & $09-06-70$ & 3,720 & 1980 & $02-20-80$ & 1,740 \\
1971 & $09-29-71$ & 3,090 & 1981 & $07-15-81$ & 2,220 \\
1972 & $08-26-72$ & 3,160 & & 1982 & $08-25-82$ & 7,680 \\
1973 & $10-07-72$ & 2,420 & & & \\
\hline
\end{tabular}

Basin characteristics

\begin{tabular}{cccccccc}
\hline $\begin{array}{c}\text { Main } \\
\text { channel } \\
\text { slope } \\
(\mathrm{ft} / \mathrm{mi})\end{array}$ & $\begin{array}{c}\text { Stream } \\
\text { length } \\
(\mathrm{mi})\end{array}$ & $\begin{array}{c}\text { Mean } \\
\text { basin } \\
\text { elevation } \\
\text { (ft) }\end{array}$ & $\begin{array}{c}\text { Forested } \\
\text { area } \\
\text { (percent) }\end{array}$ & $\begin{array}{c}\text { Soil } \\
\text { index }\end{array}$ & $\begin{array}{c}\text { Mean } \\
\text { annual } \\
\text { precipitation } \\
\text { (in) }\end{array}$ & $\begin{array}{c}\text { Rainfall intensity, 24-hour } \\
\text { (in) }\end{array}$ & $\begin{array}{c}50 \text {-year } \\
\text { (in) }\end{array}$ \\
\hline 11.2 & 47.6 & 7,500 & 50.0 & 2.0 & 15.8 & 1.4 \\
\hline
\end{tabular}


09395900 BLACK CREEK NEAR LUPTON, AZ--Continued

MEAN MONTHLY AND ANNUAL DISCHARGES $1965 \cdot 72,1975 \cdot 78,1980 \cdot 82$

\begin{tabular}{|c|c|c|c|c|c|c|}
\hline MONTH & $\begin{array}{l}\text { MAXIMUM } \\
(\text { FT } 3 / S)\end{array}$ & $\begin{array}{l}\text { MINIMUM } \\
(\text { FT3/S) }\end{array}$ & $\begin{array}{c}\text { MEAN } \\
(\mathrm{FT} 3 / \mathrm{S})\end{array}$ & $\begin{array}{l}\text { STAN- } \\
\text { DARD } \\
\text { DEVIA- } \\
\text { TION } \\
\text { (FT3/S) }\end{array}$ & $\begin{array}{l}\text { COEFFI- } \\
\text { CIENT OF } \\
\text { VARI- } \\
\text { ATION }\end{array}$ & $\begin{array}{c}\text { PERCENT } \\
\text { OF } \\
\text { ANNUAL } \\
\text { RUNOFF }\end{array}$ \\
\hline OCTOBER & 12 & 0.00 & 2.5 & 4.4 & 1.7 & 2.6 \\
\hline NOVEMBER & 4.8 & 0.00 & 0.86 & 1.2 & 1.4 & 0.9 \\
\hline DECEMBER & 11 & 0.02 & 1.8 & 2.8 & 1.6 & 1.9 \\
\hline JANUARY & 13 & 0.03 & 2.5 & 3.3 & 1.3 & 2.6 \\
\hline FEBRUARY & 75 & 0.18 & 12 & 22 & 1.8 & 12.6 \\
\hline MARCH & 27 & 0.00 & 8.3 & 9.2 & 1.1 & 8.6 \\
\hline APRIL & 57 & 0.00 & 8.0 & 16 & 2.0 & 8.3 \\
\hline MAY & 14 & 0.00 & 1.6 & 3.4 & 2.2 & 1.7 \\
\hline JUNE & 9.4 & 0.00 & 0.71 & 2.4 & 3.4 & 0.7 \\
\hline JULY & 81 & 0.03 & 15 & 21 & 1.4 & 15.8 \\
\hline AUGUST & 124 & 0.23 & 29 & 34 & 1.2 & 29.9 \\
\hline SEPTEMBER & 53 & 0.00 & 14 & 16 & 1.1 & 14.5 \\
\hline ANNUAL & 19 & 0.71 & 8.0 & 5.7 & 0.70 & 100 \\
\hline
\end{tabular}

MAGNITUDE AND PROBABILITY OF INSTANTANEOUS PEAK FLOW BASED ON PERIOD OF RECORD $1964 \cdot 82$

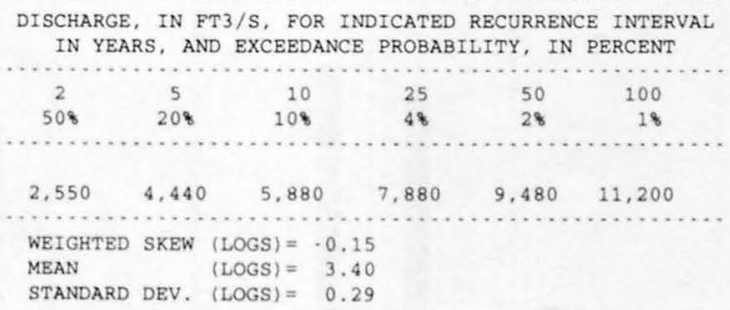

MAGNITUDE AND PROBABILITY OF ANNUAL LOW FLOW BASED ON PERIOD OF RECORD $1966-72,1976-78,1981.82$

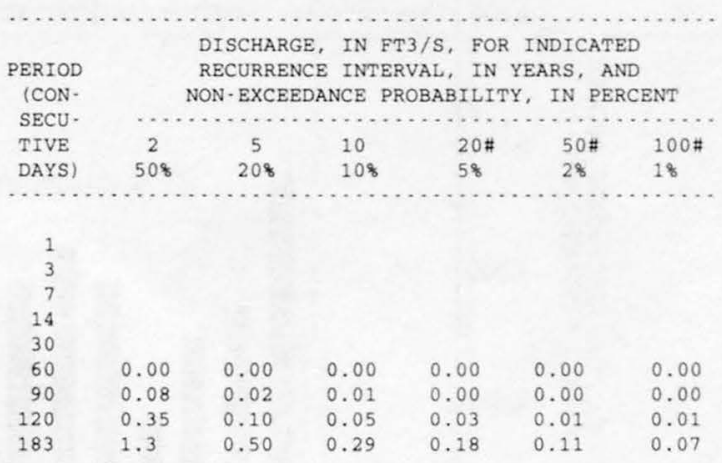

MAGNITUDE AND PROBABILITY OF ANNUAL HIGH FLOW BASED ON PERIOD OF RECORD $1965.72,1975.78,1980.82$

\begin{tabular}{|c|c|c|c|c|c|c|}
\hline $\begin{array}{l}\text { PERIOD } \\
\text { (CON. }\end{array}$ & & \multicolumn{5}{|c|}{$\begin{array}{l}\text { DISCHARGE, IN FT3/S, FOR INDICATED } \\
\text { RECURRENCE INTERVAL, IN YEARS, AND }\end{array}$} \\
\hline SECU. & & & \multicolumn{2}{|c|}{ EXCEEDANCE PROBABILITY, } & $\ldots \ldots$ & .... \\
\hline TIVE & 2 & 5 & 10 & $25 \#$ & $50 \#$ & $100 \#$ \\
\hline DAYS) & 508 & 208 & 108 & 48 & 28 & 18 \\
\hline 1 & 455 & 912 & 1,190 & 1,490 & 1,670 & 1,810 \\
\hline 3 & 224 & 477 & 668 & 917 & 1,100 & 1,280 \\
\hline 7 & 123 & 254 & 348 & 463 & 545 & 620 \\
\hline 15 & 69 & 140 & 189 & 249 & 291 & 330 \\
\hline 30 & 41 & 83 & 115 & 155 & 185 & 214 \\
\hline 60 & 26 & 54 & 74 & 100 & 119 & 136 \\
\hline 90 & 19 & 39 & 53 & 70 & 82 & 94 \\
\hline
\end{tabular}

DURATION TABLE OF DAILY MEAN FLOW FOR PERIOD OF RECORD $1965 \cdot 72,1975 \cdot 78,1980 \cdot 82$

DISCHARGE, IN FT3/S, WHICH WAS EQUALED OR EXCEEDED FOR INDICATED PERCENT OF TIME

\begin{tabular}{|c|c|c|c|c|c|c|c|c|c|c|c|c|c|c|c|c|}
\hline 18 & 58 & 108 & 158 & 208 & 308 & 408 & 508 & 608 & 708 & 808 & 908 & 958 & 988 & 998 & 99.58 & 99.98 \\
\hline 156 & 32 & 11 & 5.0 & 3.4 & 1.3 & 0.52 & 0.21 & 0.11 & 0.00 & 0.00 & 0.00 & 0.00 & 0.00 & 0.00 & 0.00 & 0.00 \\
\hline
\end{tabular}

\# Reliability of values in column is uncertain, and potential errors are large. 
ANNUAL MEAN DISCHARGE, IN CUBIC

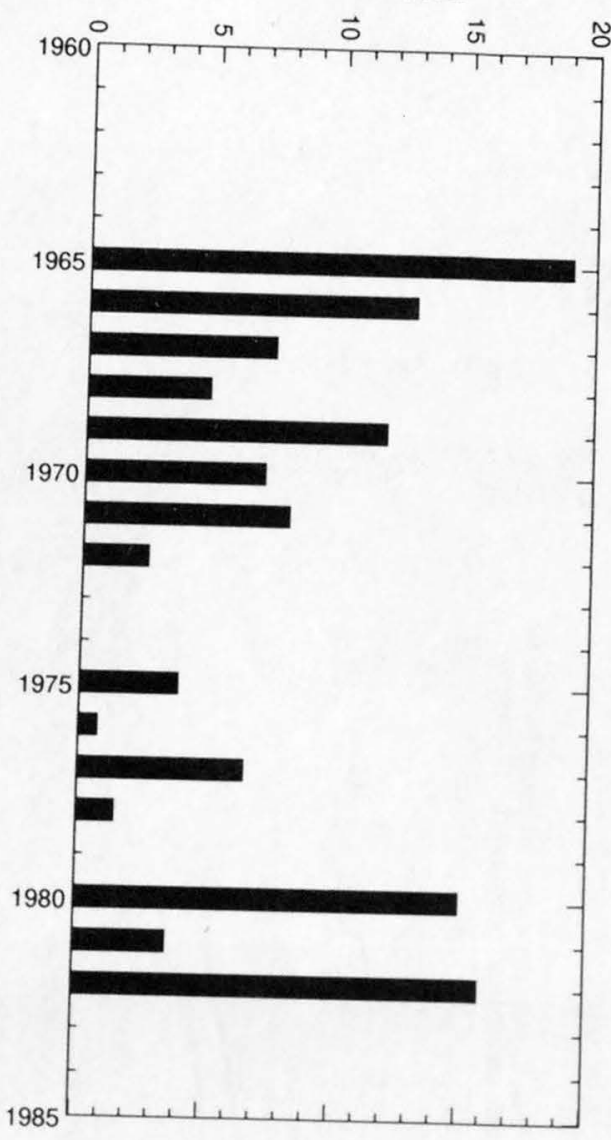

ANNUAL PEAK DISCHARGE, IN CUBIC

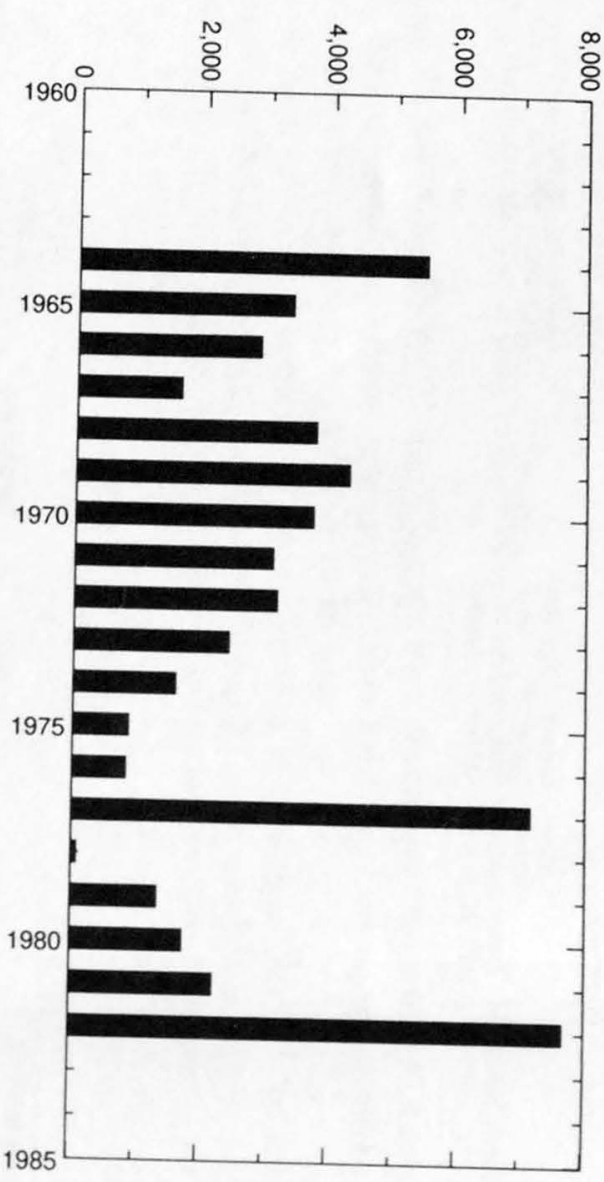


LITTLE COLORADO RIVER BASIN

87

09395900 BLACK CREEK NEAR LUPTON, AZ--Continued
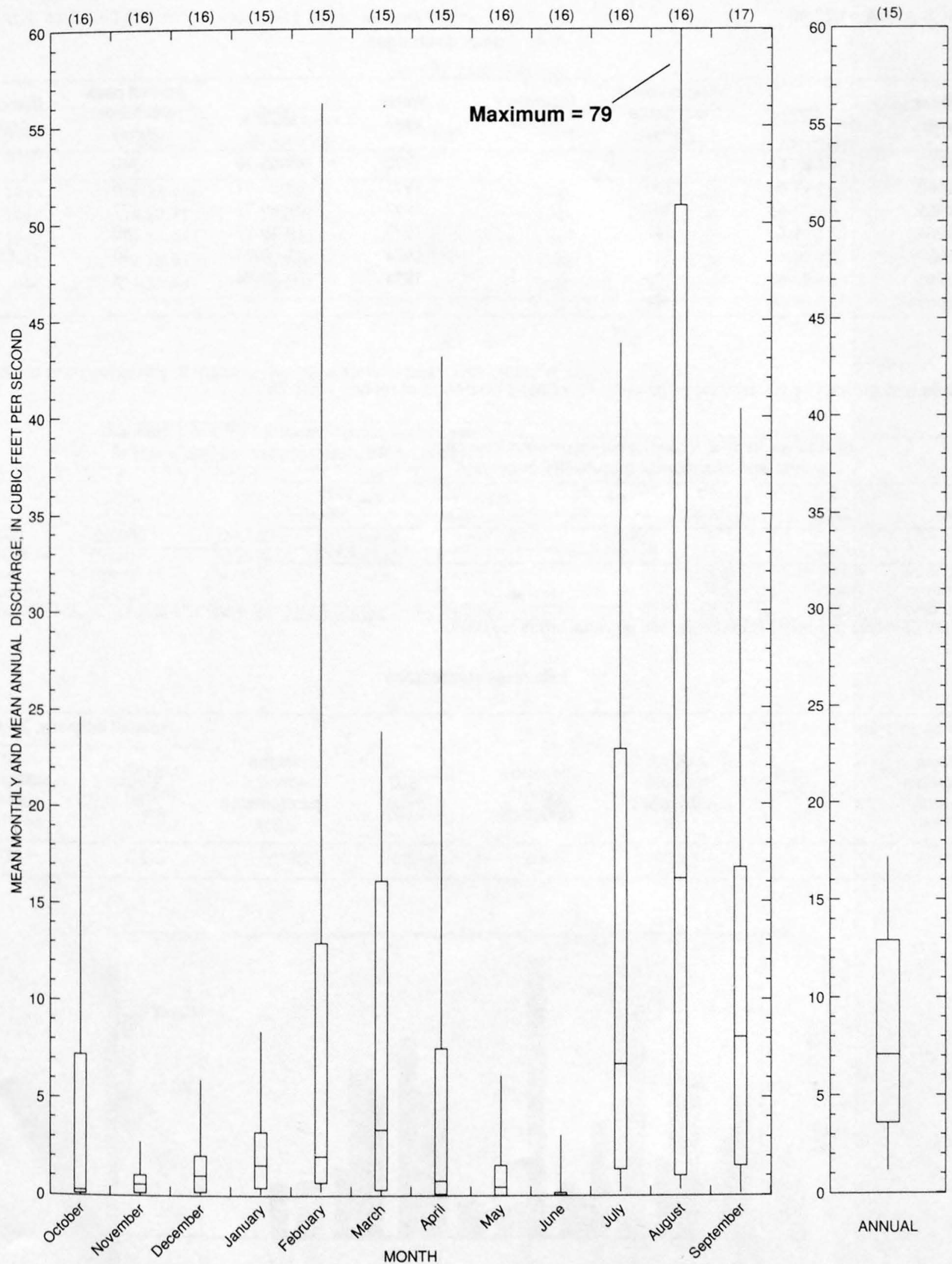

ANNUAL 


\section{DEAD WASH TRIBUTARY NEAR HOLBROOK, AZ}

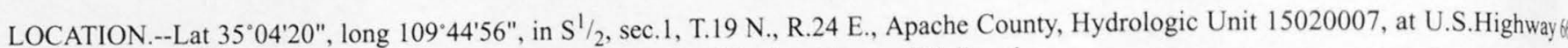
near east edge of Petrified Forest National Monument, and 26 mi northeast of Holbrook.

DRAINAGE AREA.--1.22 $\mathrm{mi}^{2}$.

Annual peak discharges

\begin{tabular}{ccccccc}
\hline $\begin{array}{c}\text { Water } \\
\text { year }\end{array}$ & Date & $\begin{array}{c}\text { Annual peak } \\
\text { discharge } \\
\left(\mathrm{ft}^{3} / \mathbf{s}\right)\end{array}$ & $\begin{array}{c}\text { Discharge } \\
\text { codes }\end{array}$ & $\begin{array}{c}\text { Water } \\
\text { year }\end{array}$ & $\begin{array}{c}\text { Date } \\
\text { Annual peak } \\
\text { discharge } \\
\left(\mathbf{f t}^{\mathbf{3}} / \mathbf{s}\right)\end{array}$ & $\begin{array}{c}\text { Discharge } \\
\text { codes }\end{array}$ \\
\hline 1963 & $09-00-63$ & 210 & & 1970 & $09-05-70$ & 340 \\
1964 & $08-12-64$ & 130 & & 1971 & $08-23-71$ & 526 \\
1965 & $09-04-65$ & 307 & & 1972 & $09-09-72$ & 377 \\
1966 & $12-21-65$ & 142 & & 1973 & $10-19-72$ & 180 \\
1967 & $08-00-67$ & 743 & & 1974 & $07-00-74$ & 30 \\
1968 & $03-00-68$ & 73 & & 1975 & $10-29-74$ & 138 \\
1969 & $10-03-68$ & 94 & & & & \\
\hline
\end{tabular}

Magnitude and probability of instantaneous peak flow based on period of record 1963-75

\begin{tabular}{|c|c|c|c|c|c|}
\hline \multicolumn{6}{|c|}{$\begin{array}{l}\text { Discharge, in } \mathrm{ft}^{3} / \mathrm{s} \text {, for indicated recurrence interval } \\
\text { in years, and exceedance probablility, in percent }\end{array}$} \\
\hline 2 & 5 & 10 & 25 & $50 \dagger$ & $100 t$ \\
\hline $50 \%$ & $20 \%$ & $10 \%$ & $4 \%$ & $2 \%$ & $1 \%$ \\
\hline 198 & 374 & 522 & 745 & 938 & 1,150 \\
\hline Weighted skew & $(\log s)=$ & 0.01 & & & \\
\hline Mean & $(\log s)=$ & 2.30 & & & \\
\hline Standard dev. & $(\log s)=$ & 0.33 & & & \\
\hline
\end{tabular}

7 Reliability of values in column is uncertain, and potential errors are large.

Basin characteristics

\begin{tabular}{cccccccc}
\hline $\begin{array}{c}\text { Main } \\
\text { channel } \\
\text { slope } \\
\text { (ft/mi) }\end{array}$ & $\begin{array}{c}\text { Stream } \\
\text { length } \\
(\mathrm{mi})\end{array}$ & $\begin{array}{c}\text { Mean } \\
\text { basin } \\
\text { elevation } \\
\text { (ft) }\end{array}$ & $\begin{array}{c}\text { Forested } \\
\text { area } \\
\text { (percent) }\end{array}$ & $\begin{array}{c}\text { Soil } \\
\text { index }\end{array}$ & $\begin{array}{c}\text { Mean } \\
\text { annual } \\
\text { precipitation } \\
\text { (in) }\end{array}$ & $\begin{array}{c}\text { Rainfall intensity, 24-hour } \\
\text { (in) }\end{array}$ & $\begin{array}{c}50 \text {-year } \\
\text { (in) }\end{array}$ \\
\hline 60.0 & 1.8 & 5,740 & 0.0 & 3.0 & 7.9 & 1.3 & 2.6 \\
\hline
\end{tabular}

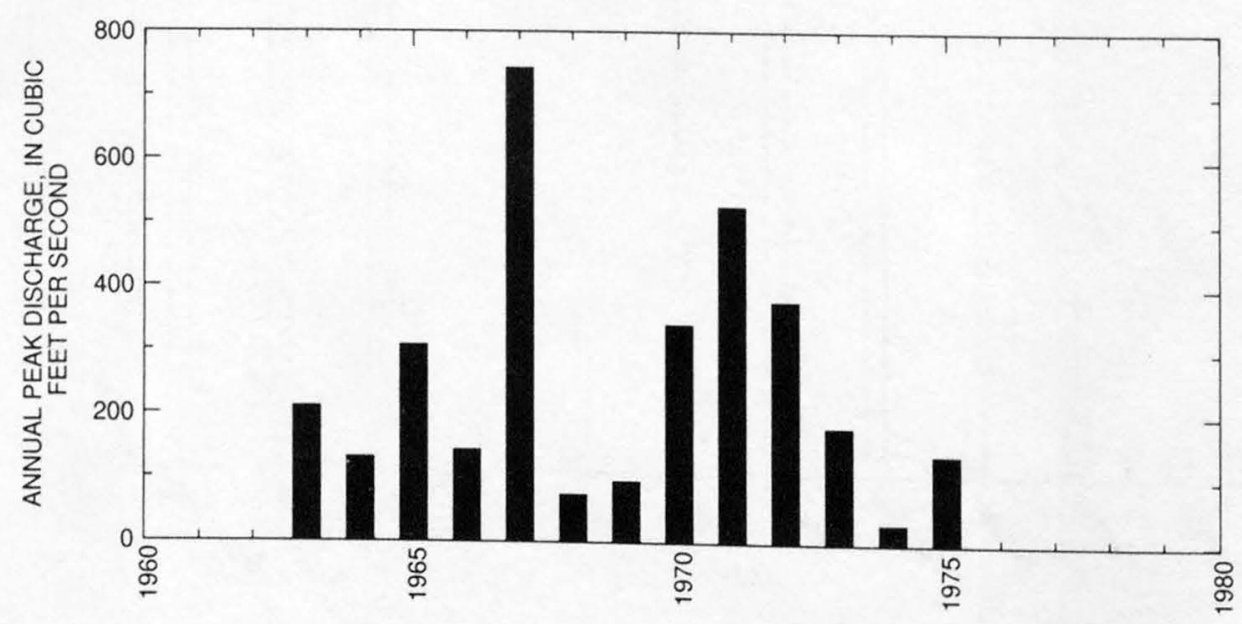


09396500 PUERCO RIVER NEAR ADAMANA, AZ

LOCATION.--Lat $34^{\circ} 58^{\prime} 45^{\prime \prime}$, long 109 $47^{\prime} 40^{\prime \prime}$, in NE $1 / 4$, sec.9, T.18 N., R.24 E., Apache County, Hydrologic Unit 15020007 , at highway bridge in Petrified Forest National Monument, $0.25 \mathrm{mi}$ downstream from Dead Wash and $1.5 \mathrm{mi}$ east of Adamana.

DRAINAGE AREA.--2,654 $\mathrm{mi}^{2}$, of which $50.5 \mathrm{mi}^{2}$ is noncontributing.

Annual peak discharges

\begin{tabular}{cccccrr}
\hline $\begin{array}{c}\text { Water } \\
\text { year }\end{array}$ & Date & $\begin{array}{c}\text { Annual peak } \\
\text { discharge } \\
\left(\mathbf{f t}^{3} / \mathbf{s}\right)\end{array}$ & $\begin{array}{c}\text { Discharge } \\
\text { codes }\end{array}$ & $\begin{array}{c}\text { Water } \\
\text { year }\end{array}$ & $\begin{array}{c}\text { Annual peak } \\
\text { Date } \\
\text { discharge } \\
\left(\mathrm{ft}^{3} / \mathbf{s}\right)\end{array}$ \\
\hline 1940 & $08-23-40$ & 7,500 & & 1945 & $02-03-45$ & 5,740 \\
1941 & $09-29-41$ & 22,600 & & 1946 & $08-12-46$ & 30,000 \\
1942 & $10-04-41$ & 19,400 & & 1947 & $08-10-47$ & 22,000 \\
1943 & $09-26-43$ & 4,800 & & 1948 & $10-14-47$ & 17,100 \\
1944 & $09-26-44$ & 4,700 & & 1949 & $08-08-49$ & 8,040 \\
\hline
\end{tabular}

Magnitude and probability of instantaneous peak flow based on period of record 1940-49

\begin{tabular}{|c|c|c|c|c|c|}
\hline \multicolumn{6}{|c|}{$\begin{array}{l}\text { Discharge, in } \mathrm{ft}^{3} / \mathrm{s} \text {, for indicated recurrence interval } \\
\text { in years, and exceedance probablility, in percent }\end{array}$} \\
\hline 2 & 5 & 10 & 25 & $50 t$ & $100+$ \\
\hline $50 \%$ & $20 \%$ & $10 \%$ & $4 \%$ & $2 \%$ & $1 \%$ \\
\hline 11,500 & 20,900 & 28,400 & 39,200 & 48,200 & 57,900 \\
\hline Weighted skew & $(\operatorname{logs})=$ & -0.08 & & & \\
\hline Mean & $(\log s)=$ & 4.06 & & & \\
\hline Standard dev. & $(\log s)=$ & 0.31 & & & \\
\hline
\end{tabular}

Basin characteristics

\begin{tabular}{ccccccc}
\hline $\begin{array}{c}\text { Main } \\
\text { channel } \\
\text { slope } \\
(\mathrm{ft} / \mathrm{mi})\end{array}$ & $\begin{array}{c}\text { Stream } \\
\text { length } \\
(\mathrm{mi})\end{array}$ & $\begin{array}{c}\text { Mean } \\
\text { basin } \\
\text { elevation } \\
(\mathrm{ft})\end{array}$ & $\begin{array}{c}\text { Forested } \\
\text { area } \\
\text { (percent) }\end{array}$ & $\begin{array}{c}\text { Soil } \\
\text { index }\end{array}$ & $\begin{array}{c}\text { Mean } \\
\text { annual } \\
\text { precipitation } \\
\text { (in) }\end{array}$ & $\begin{array}{c}\text { Rainfall intensity, 24-hour } \\
\text { (in) }\end{array}$ \\
\hline 15.7 & 115 & 6,730 & 29.0 & 2.8 & 12.6 & $\begin{array}{c}50 \text {-year } \\
\text { (in) }\end{array}$ \\
\hline
\end{tabular}

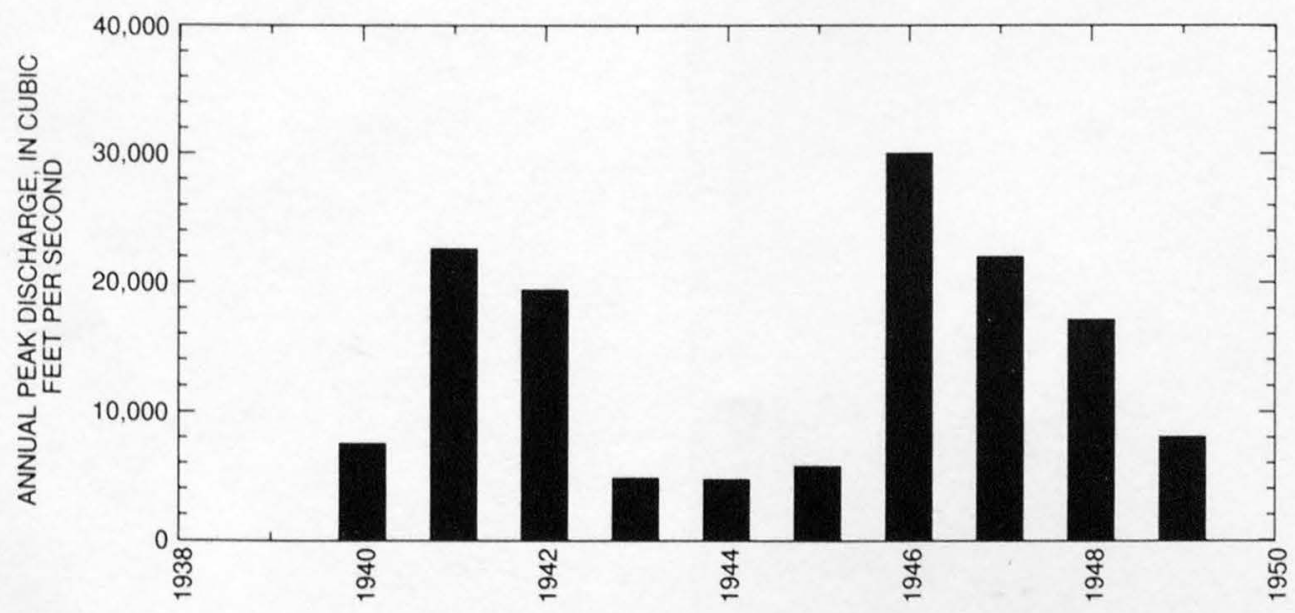




\section{LITTLE COLORADO RIVER AT HOLBROOK, AZ}

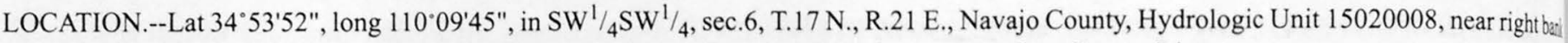
on downstream side of bridge on U.S. Highway 180 at Holbrook, 2.3 mi downstream from Puerco River.

DRAINAGE AREA.--11,462 $\mathrm{mi}^{2}$, of which $347 \mathrm{mi}^{2}$ is noncontributing.

Annual peak discharges

\begin{tabular}{|c|c|c|c|c|c|c|c|}
\hline $\begin{array}{l}\text { Water } \\
\text { year }\end{array}$ & Date & $\begin{array}{c}\text { Annual peak } \\
\text { discharge } \\
\left(\mathrm{ft}^{3} / \mathrm{s}\right)\end{array}$ & $\begin{array}{l}\text { Discharge } \\
\text { codes }\end{array}$ & $\begin{array}{l}\text { Water } \\
\text { year }\end{array}$ & Date & $\begin{array}{c}\text { Annual peak } \\
\text { discharge } \\
\left(\mathrm{ft}^{3} / \mathrm{s}\right)\end{array}$ & $\begin{array}{c}\text { Discharge } \\
\text { codes }\end{array}$ \\
\hline 1906 & $11-27-05$ & 20,200 & HP & 1961 & $08-16-61$ & 4,160 & \\
\hline 1923 & $09-19-23$ & ${ }^{1} 60,000$ & HP & 1962 & $10-31-61$ & 4,010 & \\
\hline 1950 & $07-19-50$ & 2,960 & & 1963 & $08-31-63$ & 9,370 & \\
\hline 1951 & $08-28-51$ & 8,700 & & 1964 & $09-09-64$ & 15,100 & \\
\hline 1952 & $01-19-52$ & 8,400 & & 1965 & $07-25-65$ & 14,800 & \\
\hline 1953 & $07-29-53$ & 6,030 & & 1966 & $08-13-66$ & 10,400 & \\
\hline 1954 & $07-22-54$ & 10,800 & & 1967 & $08-12-67$ & 14,100 & \\
\hline 1955 & $08-17-55$ & 10,500 & & 1968 & $08-12-68$ & 21,000 & \\
\hline 1956 & $06-30-56$ & 4,210 & & 1969 & $10-04-68$ & 24,200 & \\
\hline 1957 & $08-05-57$ & 21,800 & & 1970 & $09-06-70$ & 19,700 & \\
\hline 1958 & $09-14-58$ & 7,000 & & 1971 & $08-21-71$ & 13,200 & \\
\hline 1959 & 08-06-59 & 6,300 & & 1972 & $10-01-71$ & 20,300 & \\
\hline 1960 & $10-29-59$ & 11,400 & & 1973 & $10-20-72$ & 15,000 & \\
\hline
\end{tabular}

${ }^{\top}$ Highest since 1870 .

Basin characteristics

\begin{tabular}{cccccccc}
\hline $\begin{array}{c}\text { Main } \\
\text { channel } \\
\text { slope } \\
\text { (ft/mi) }\end{array}$ & $\begin{array}{c}\text { Stream } \\
\text { length } \\
\text { (mi) }\end{array}$ & $\begin{array}{c}\text { Mean } \\
\text { basin } \\
\text { elevation } \\
(\mathrm{ft})\end{array}$ & $\begin{array}{c}\text { Forested } \\
\text { area } \\
\text { (percent) }\end{array}$ & $\begin{array}{c}\text { Soil } \\
\text { index }\end{array}$ & $\begin{array}{c}\text { Mean } \\
\text { annual } \\
\text { precipitation } \\
\text { (in) }\end{array}$ & $\begin{array}{c}\text { Rainfall intensity, 24-hour } \\
\text { (in) }\end{array}$ & $\begin{array}{c}50 \text {-year } \\
\text { (in) }\end{array}$ \\
\hline 21.9 & 134 & 6,730 & 27.0 & 2.8 & 13.0 & 1.4 \\
\hline
\end{tabular}


09397000 LITTLE COLORADO RIVER AT HOLBROOK, AZ--Continued

MEAN MONTHLY AND ANNUAL DISCHARGES $1906,1950-73$

\begin{tabular}{|c|c|c|c|c|c|c|}
\hline MONTH & $\begin{array}{l}\text { MAXIMUM } \\
(\text { ET } 3 / S)\end{array}$ & $\begin{array}{l}\text { MINIMUM } \\
(\mathrm{FT} 3 / \mathrm{S})\end{array}$ & $\begin{array}{c}\text { MEAN } \\
(\text { FT3/S) }\end{array}$ & $\begin{array}{l}\text { STAN- } \\
\text { DARD } \\
\text { DEVIA- } \\
\text { TION } \\
\text { (FT3/S) }\end{array}$ & $\begin{array}{l}\text { COEFFI- } \\
\text { CIENT OF } \\
\text { VARI- } \\
\text { ATION }\end{array}$ & $\begin{array}{c}\text { PERCENI } \\
\text { OF } \\
\text { ANNUAI } \\
\text { RUNOF }\end{array}$ \\
\hline OCTOBER & 939 & 2.4 & 138 & 231 & 1.7 & 8.7 \\
\hline NOVEMBER & 1,160 & 4.4 & 75 & 229 & 3.1 & 4.7 \\
\hline DECEMBER & 529 & 3.9 & 51 & 110 & 2.1 & 3.2 \\
\hline JANUARY & 565 & 3.2 & 105 & 178 & 1.7 & 6.6 \\
\hline FEBRUARY & 312 & 2.8 & 58 & 81 & 1.4 & 3.6 \\
\hline MARCH & 1,270 & 3.4 & 112 & 272 & 2.4 & 7.1 \\
\hline APRIL & 1,020 & 2.3 & 64 & 206 & 3.2 & 4.1 \\
\hline MAY & 600 & 1.3 & 32 & 119 & 3.7 & 2.0 \\
\hline JUNE & 161 & 0.37 & 19 & 33 & 1.8 & 1.2 \\
\hline JULY & 638 & 2.4 & 157 & 190 & 1.2 & 9.9 \\
\hline AUGUST & 2.130 & 4.5 & 537 & 606 & 1.1 & 33.8 \\
\hline SEPTEMBER & 1.000 & 2.3 & 240 & 285 & 1.2 & 15.1 \\
\hline ANNUAL & 350 & 20 & 133 & 87 & 0.65 & 100 \\
\hline
\end{tabular}

MAGNITUDE AND PROBABILITY OF INSTANTANEOUS PEAK FLOW BASED ON PERIOD OF RECORD 1906, 1923, 1950-73

DISCHARGE, IN FT3/S, FOR INDICATED RECURRENCE INTERVAL IN YEARS, AND EXCEEDANCE PROBABILITY, IN PERCENT

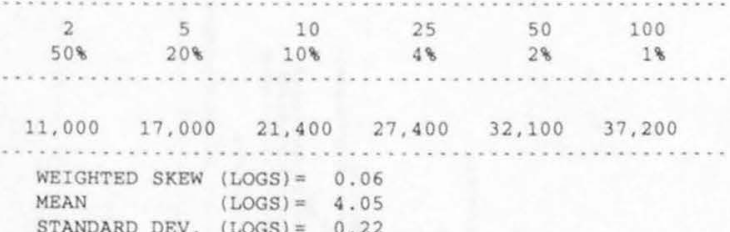

STANDARD DEV, (LOGS $)=0.22$
MAGNITUDE AND PROBABILITY OF ANNUAL LOW FLOW BASED ON PERIOD OF RECORD 1906-07, 1951.74

\begin{tabular}{|c|c|c|c|c|c|c|}
\hline $\begin{array}{l}\text { PERIOD } \\
\text { ICON. } \\
\text { SECU. }\end{array}$ & \multicolumn{6}{|c|}{ 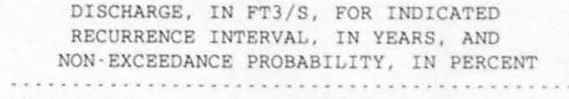 } \\
\hline TIVE & 2 & 5 & 10 & 20 & $50 \#$ & $100 \#$ \\
\hline DAYS) & 508 & 208 & 108 & 58 & 28 & 18 \\
\hline 1 & 0.38 & 0.07 & 0.00 & 0.00 & 0.00 & 0.00 \\
\hline 3 & 0.61 & 0.16 & 0.04 & 0.00 & 0.00 & 0.00 \\
\hline 7 & 1.1 & 0.35 & 0.15 & 0.04 & 0.00 & 0.00 \\
\hline 14 & 1.7 & 0.75 & 0.44 & 0.28 & 0.15 & 0.10 \\
\hline 30 & 2.4 & 1.0 & 0.68 & 0.47 & 0.31 & 0.23 \\
\hline 60 & 3.0 & 1.8 & 1.5 & 1.3 & 1.2 & 1.1 \\
\hline 90 & 5.1 & 2.6 & 1.9 & 1.5 & 1.2 & 1.1 \\
\hline 120 & 10 & 5.0 & 3.8 & 3.1 & 2.6 & 2.3 \\
\hline 183 & 28 & 12 & 7.9 & 5.7 & 4.1 & 3.2 \\
\hline
\end{tabular}

MAGNITUDE AND PROBABILITY OF ANNUAL HIGH FLOW BASED ON PERIOD OF RECORD 1906, 1950.73

\begin{tabular}{|c|c|c|c|c|c|c|}
\hline \multirow{3}{*}{$\begin{array}{l}\text { PERIOD } \\
\text { /CON- } \\
\text { SECU. }\end{array}$} & \multicolumn{6}{|c|}{ DISCHARGE, IN FT $3 / \mathrm{S}$, FOR INDICATED } \\
\hline & & EXCEEDA & NCE PROB & BILITY, & IN PERC & SNT \\
\hline & & $\cdots$ & 列 & .... & ......... & \\
\hline TIVE & 2 & 5 & 10 & 25 & $50 \#$ & $100 \#$ \\
\hline DAYS) & 508 & 208 & 108 & 48 & 28 & 18 \\
\hline 1 & 5,460 & 10,600 & 14,600 & 19,800 & 23,900 & 28,000 \\
\hline 3 & 3,290 & 6.030 & 7.820 & 9,940 & 11.400 & 12,700 \\
\hline 7 & 1,850 & 3,350 & 4,320 & 5,440 & 6,180 & 6,850 \\
\hline 15 & 1,110 & 2,030 & 2,670 & 3,480 & 4,070 & 4,640 \\
\hline 30 & 717 & 1,310 & 1,720 & 2,230 & 2,610 & 2,960 \\
\hline 60 & 457 & 875 & 1,180 & 1,580 & 1,890 & 2,190 \\
\hline 90 & 329 & 625 & 841 & 1,120 & 1,340 & 1,540 \\
\hline
\end{tabular}

DURATION TABLE OF DAILY MEAN FLOW FOR PERIOD OF RECORD 1906, 1950.73

DISCHARGE, IN FT3/S, WHICH WAS EQUALED OR EXCEEDED FOR INDICATED PERCENT OF TIME

\begin{tabular}{|c|c|c|c|c|c|c|c|c|c|c|c|c|c|c|c|}
\hline 58 & 108 & 158 & 208 & 308 & 408 & 508 & 608 & 708 & 808 & 908 & 958 & $98 \%$ & 998 & 99.58 & 99.98 \\
\hline 588 & 191 & 93 & 49 & 19 & 12 & 9.2 & 7.1 & 5.3 & 3.7 & 2.2 & 1.3 & 0.70 & 0.41 & 0.18 & 0.00 \\
\hline
\end{tabular}

\# Reliability of values in column is uncertain, and potential errors are large. 


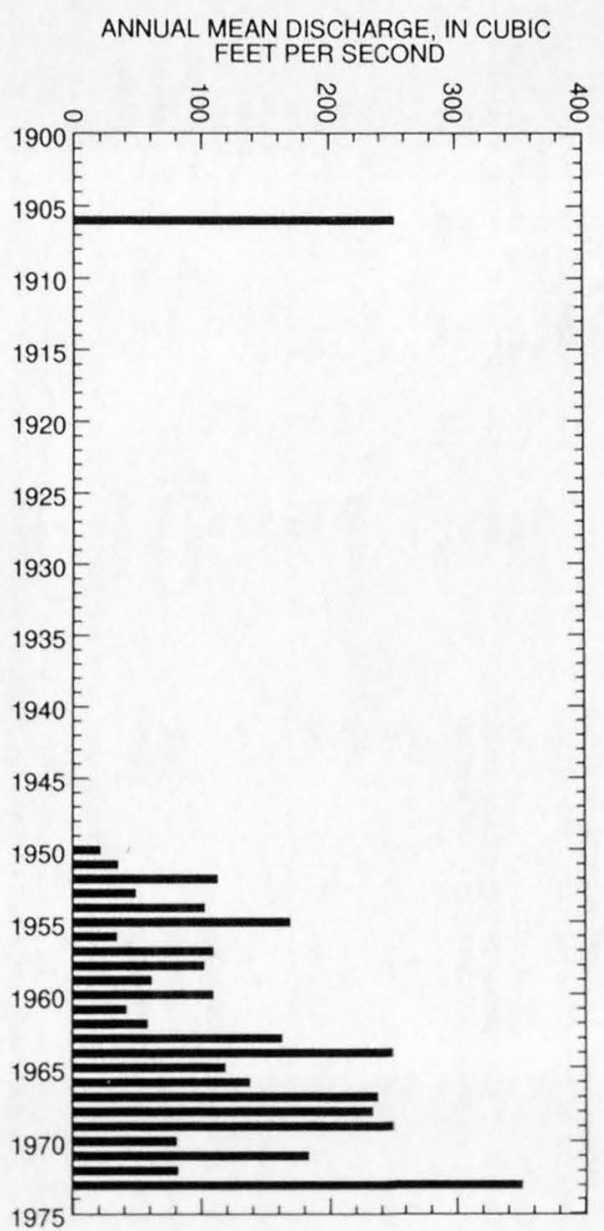

ANNUAL PEAK DISCHARGE, IN CUBIC FEET PER SECOND

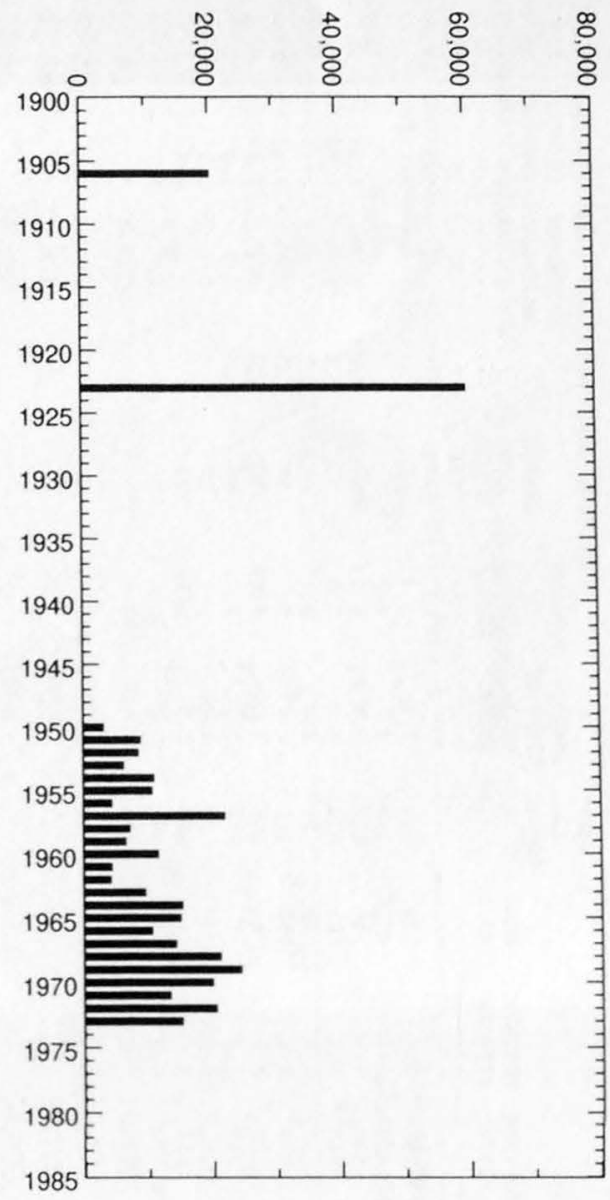


09397000 LITTLE COLORADO RIVER AT HOLBROOK, AZ--Continued
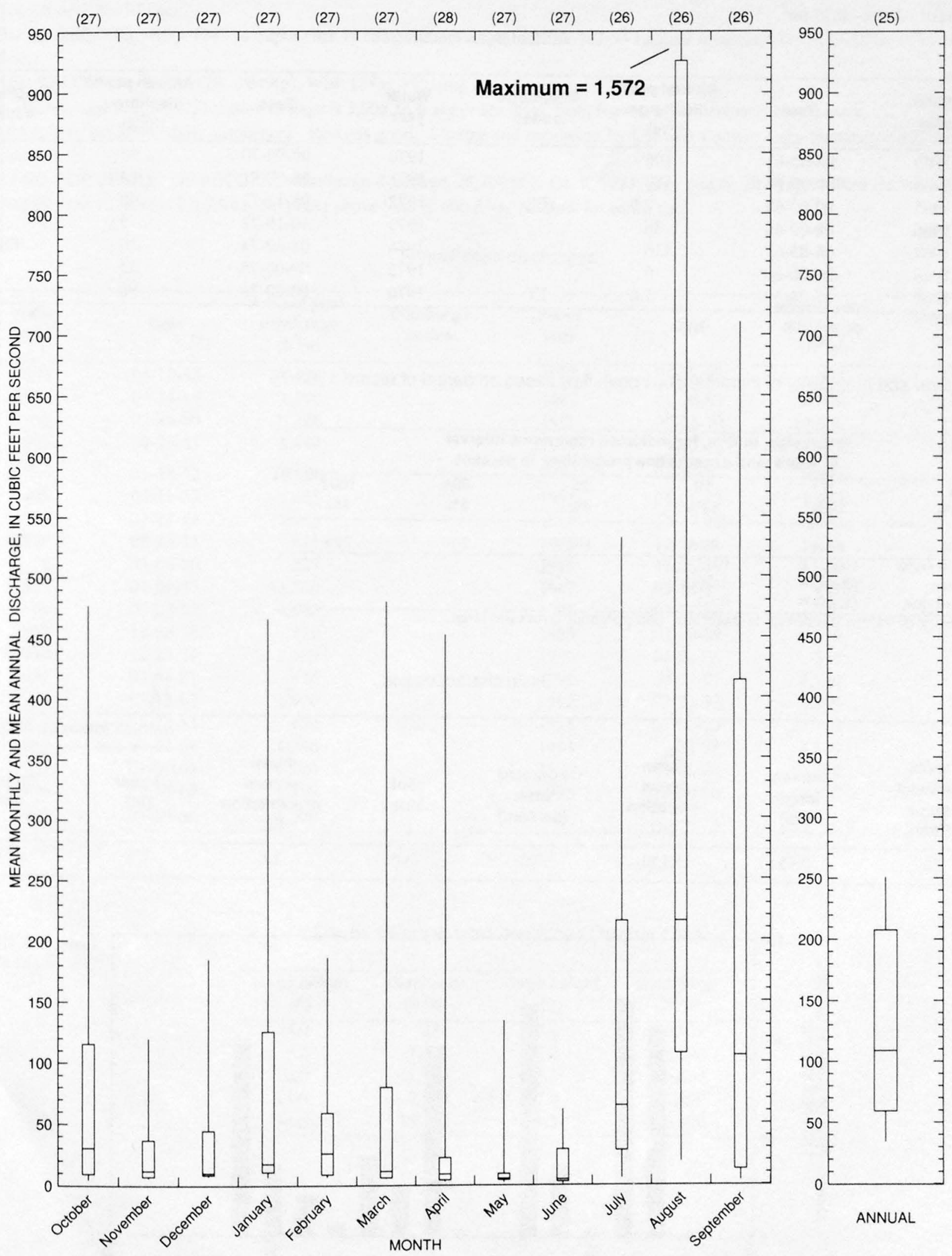
09397200 PENZANCE WASH NEAR JOSEPH CITY, AZ

LOCATION.--Lat $34^{\circ} 55^{\prime} 08^{\prime \prime}$, long $110^{\circ} 15^{\prime} 13^{\prime \prime}$, in NE $1 / 4$, sec.31, T.18 N., R.20 E., Navajo County, Hydrologic Unit 15020008, at U.S. Highwa 66, 5.6 mi southeast of Joseph City.

DRAINAGE AREA.--0.17 $\mathrm{mi}^{2}$.

Annual peak discharges

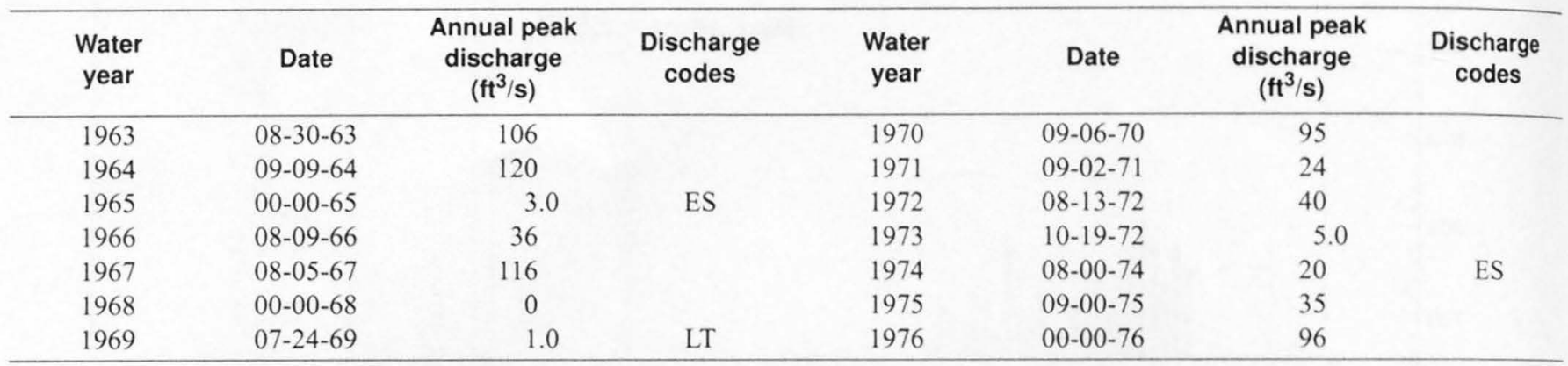

Magnitude and probability of instantaneous peak flow based on period of record 1963-76

\begin{tabular}{|c|c|c|c|c|c|}
\hline \multicolumn{6}{|c|}{$\begin{array}{l}\text { Discharge, in } \mathrm{ft}^{3} / \mathrm{s} \text {, for indicated recurrence interval } \\
\text { in years, and exceedance probablility, in percent }\end{array}$} \\
\hline 2 & 5 & 10 & 25 & $50 t$ & $100 t$ \\
\hline $50 \%$ & $20 \%$ & $10 \%$ & $4 \%$ & $2 \%$ & $1 \%$ \\
\hline 38 & 85.4 & 126 & 188 & 240 & 296 \\
\hline Weighted skew & $(\log s)=$ & -0.33 & & & \\
\hline Mean & $(\log s)=$ & 1.56 & & & \\
\hline Standard dev. & $(\log s)=$ & 0.44 & & & \\
\hline
\end{tabular}

Basin characteristics

\begin{tabular}{cccccccc}
\hline $\begin{array}{c}\text { Main } \\
\text { channel } \\
\text { slope } \\
(\mathrm{ft} / \mathrm{mi})\end{array}$ & $\begin{array}{c}\text { Stream } \\
\text { length } \\
(\mathrm{mi})\end{array}$ & $\begin{array}{c}\text { Mean } \\
\text { basin } \\
\text { elevation } \\
(\mathrm{ft})\end{array}$ & $\begin{array}{c}\text { Forested } \\
\text { area } \\
\text { (percent) }\end{array}$ & $\begin{array}{c}\text { Soil } \\
\text { index }\end{array}$ & $\begin{array}{c}\text { Mean } \\
\text { annual } \\
\text { precipitation } \\
\text { (in) }\end{array}$ & $\begin{array}{c}\text { Rainfall intensity, 24-hour } \\
\text { (in) }\end{array}$ & $\begin{array}{c}\text { 50-year } \\
\text { (in) }\end{array}$ \\
\hline 237 & 0.45 & 5,150 & 0.0 & 3.0 & 7.8 & 1.2 \\
\hline
\end{tabular}

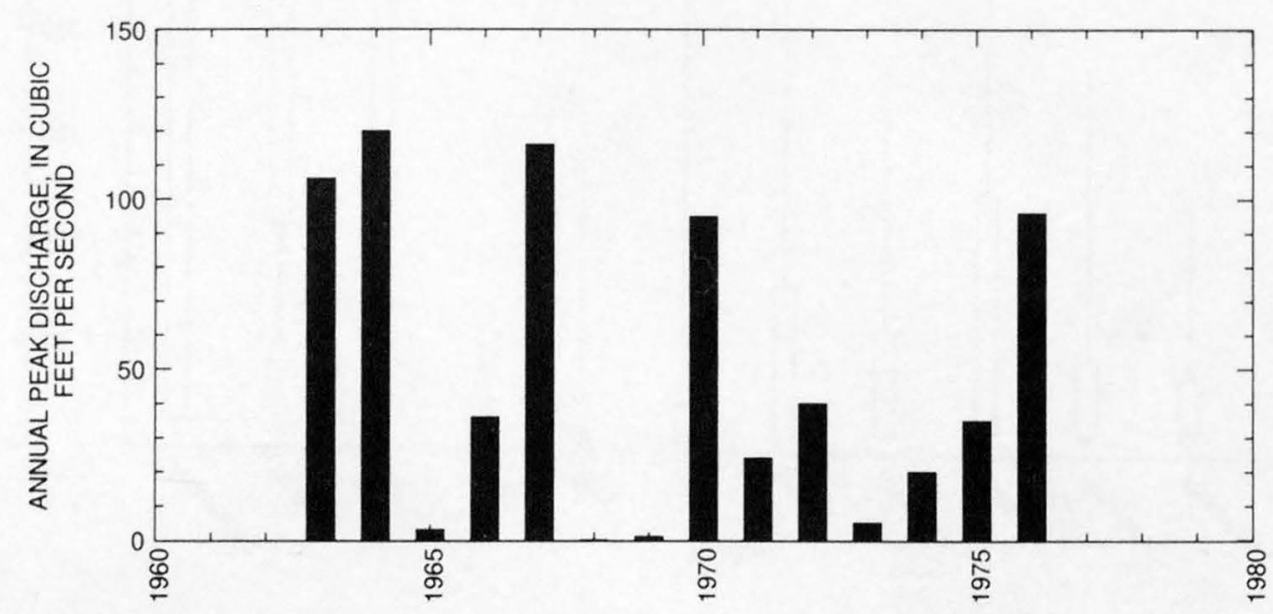


09397500 CHEVELON CREEK BELOW WILDCAT CANYON NEAR WINSLOW, AZ

LOCATION.--Lat $34^{\circ} 38^{\prime} 11^{\prime \prime}$, long $110^{\circ} 42^{\prime} 49^{\prime \prime}$, in SW $1 / 4 \sec .36$, T.15 N., R.15 E., Navajo County, Hydrologic Unit 15020010 , Sitgreaves National Forest, on right bank $0.4 \mathrm{mi}$ downstream from Wildcat Canyon and $25 \mathrm{mi}$ south of Winslow.

DRAINAGE AREA.--271 $\mathrm{mi}^{2}$.

PERIOD OF RECORD.--May 1947 to September 1970 (daily discharge), 1979, 1982-95 (annual maximum only), October 1995 to September 1996.

REVISED RECORDS.--WSP 1179: 1949(p), WSP 1283: 1951(m).

GAGE.--Water-stage recorder. Elevation of gage is 5,905.16 ft above sea level, from Bureau of Reclamation bench mark.

REMARKS.--No estimated daily discharges. Records good. Storage and regulation by Chevlon Canyon Lake (capacity 6,193 acre-ft) 17 mi upstream.

EXTREMES FOR PERIOD OF RECORD.--Maximum discharge, 24,700 $\mathrm{ft}^{3} / \mathrm{s} \mathrm{Jan} .8,1993$, gage height, $20.78 \mathrm{ft}$; no flow during most years.

EXTREMES FOR CURRENT YEAR.--No peaks above base of $400 \mathrm{ft}^{3} / \mathrm{s}$. No flow for entire year.

Annual peak discharges

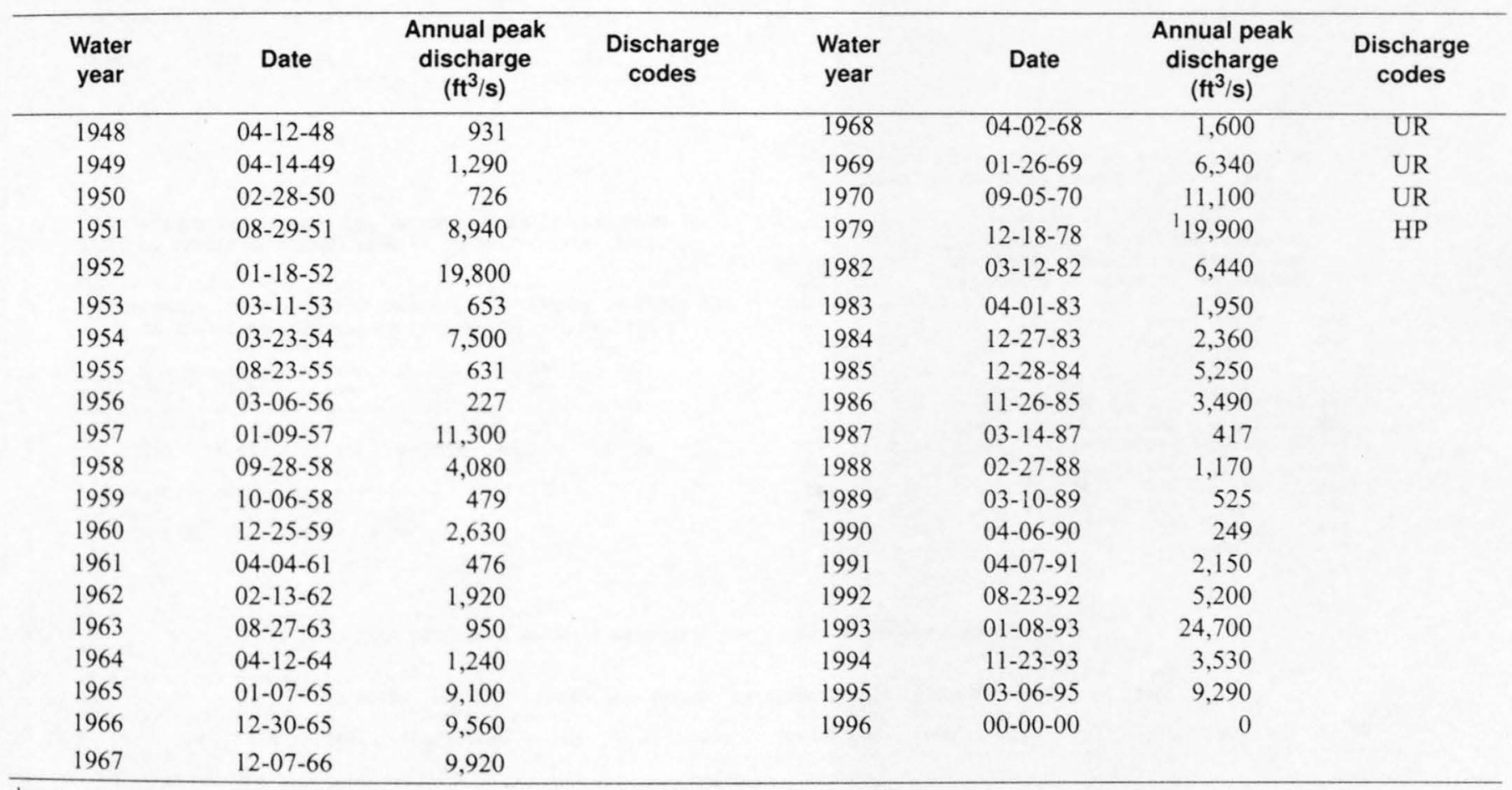

${ }^{1}$ Highest since 1929.

Discharge rating table developed October 1988

\begin{tabular}{crcc}
\hline $\begin{array}{c}\text { Gage height } \\
(\mathbf{f t})\end{array}$ & $\begin{array}{c}\text { Discharge } \\
\left(\mathbf{f t}^{\mathbf{3}} \mathbf{s}\right)\end{array}$ & $\begin{array}{c}\text { Gage height } \\
(\mathbf{f t})\end{array}$ & $\begin{array}{c}\text { Discharge } \\
\left(\mathbf{f t}^{\mathbf{3}} \mathbf{s}\right)\end{array}$ \\
\hline 4.0 & 231 & 19.0 & 19,920 \\
7.0 & 1,480 & 22.0 & 28,380 \\
10.0 & 3,980 & 25.0 & 36,060 \\
13.0 & 7,840 & 27.0 & 41,620 \\
16.0 & 13,130 & 28.5 & 46,000 \\
\hline
\end{tabular}


09397500 CHEVELON CREEK BELOW WILDCAT CANYON NEAR WINSLOW, AZ--Continued

Basin characteristics

\begin{tabular}{|c|c|c|c|c|c|c|c|}
\hline $\begin{array}{l}\text { Main } \\
\text { channel } \\
\text { slope } \\
\text { (ft/mi) }\end{array}$ & $\begin{array}{l}\text { Stream } \\
\text { length } \\
(\mathrm{mi})\end{array}$ & $\begin{array}{c}\text { Mean } \\
\text { basin } \\
\text { elevation } \\
(f t)\end{array}$ & $\begin{array}{l}\text { Forested } \\
\text { area } \\
\text { (percent) }\end{array}$ & $\begin{array}{c}\text { Soil } \\
\text { index }\end{array}$ & $\begin{array}{c}\text { Mean } \\
\text { annual } \\
\text { precipitation } \\
\text { (in) }\end{array}$ & $\begin{array}{l}\text { 2-year } \\
\text { (in) }\end{array}$ & $\begin{array}{l}\text { 50-year } \\
\text { (in) }\end{array}$ \\
\hline 54.4 & 39.2 & 7,030 & 99.0 & 3.0 & 24.0 & 2.5 & 4.6 \\
\hline
\end{tabular}


09397500 CHEVELON CREEK BELOW WILDCAT CANYON NEAR WINSLOW, AZ--Continued

MEAN MONTHLY AND ANNUAL DISCHARGES $1948-70,1996$

\begin{tabular}{|c|c|c|c|c|c|c|}
\hline MONTH & $\begin{array}{l}\text { MAXIMUM } \\
\text { (FT3/S) }\end{array}$ & $\begin{array}{l}\text { MINIMUM } \\
\text { (FT3/S) }\end{array}$ & $\begin{array}{c}\text { MEAN } \\
(\mathrm{FT} 3 / \mathrm{S})\end{array}$ & $\begin{array}{l}\text { STAN- } \\
\text { DARD } \\
\text { DEVIA- } \\
\text { TION } \\
\text { (FT3/S) }\end{array}$ & $\begin{array}{l}\text { COEFFI- } \\
\text { CIENT OF } \\
\text { VARI- } \\
\text { ATION }\end{array}$ & $\begin{array}{c}\text { PERCENT } \\
\text { OF } \\
\text { ANNUAL } \\
\text { RUNOFF }\end{array}$ \\
\hline OCTOBER & 46 & 0.00 & 2.6 & 9.5 & 3.6 & 0.5 \\
\hline NOVEMBER & 108 & 0.00 & 8.8 & 24 & 2.7 & 1.5 \\
\hline DECEMBER & 320 & 0.00 & 44 & 87 & 2.0 & 7.8 \\
\hline JANUARY & 523 & 0.00 & 92 & 170 & 1.8 & 16.1 \\
\hline FEBRUARY & 308 & 0.00 & 61 & 80 & 1.3 & 10,7 \\
\hline MARCH & 473 & 0.00 & 157 & 121 & 0.77 & 27.6 \\
\hline APRIL & 658 & 0.00 & 159 & 182 & 1.1 & 27.9 \\
\hline MAY & 47 & 0.00 & 11 & 16 & 1.5 & 1.9 \\
\hline JUNE & 1.7 & 0.00 & 0.09 & 0.35 & 4.0 & 0.0 \\
\hline JULY & 4.4 & 0.00 & 0.28 & 0.99 & 3.5 & 0.0 \\
\hline AUGUST & 205 & 0.00 & 17 & 46 & 2.8 & 2.9 \\
\hline SEPTEMBER & 210 & 0.00 & 18 & 50 & 2.8 & 3.2 \\
\hline ANNUAL & 132 & 0.00 & 47 & 34 & 0.71 & 100 \\
\hline
\end{tabular}

MAGNITUDE AND PROBABILITY OF INSTANTANEOUS PEAK FLOW BASED ON PERIOD OF RECORD $1948.67,1968 \cdot 70,1979,1982.96$

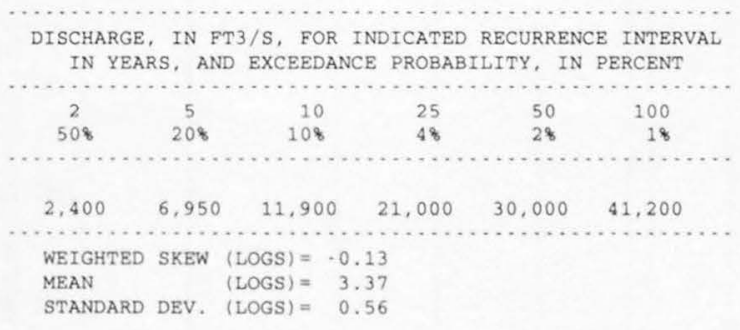

MAGNITUDE AND PROBABILITY OF ANNUAL LOW FLOW BASED ON PERIOD OF RECORD 1949.70

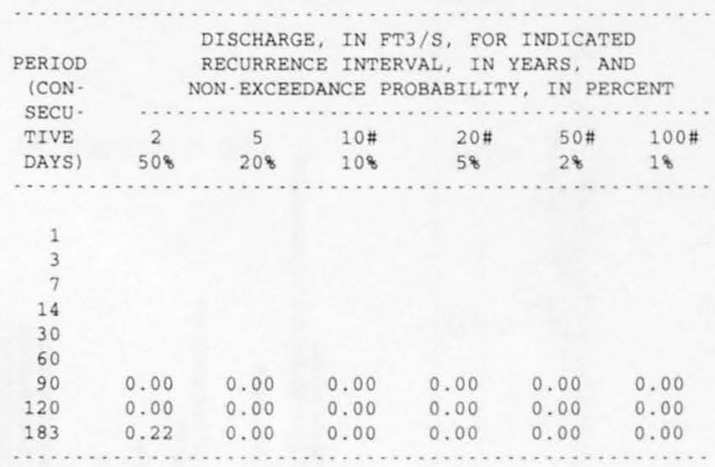

MAGNITUDE AND PROBABILITY OF ANNUAL HIGH FLOW BASED ON PERIOD OF RECORD $1948 \cdot 70,1996$

\begin{tabular}{|c|c|c|c|c|c|c|}
\hline \multirow{4}{*}{$\begin{array}{l}\text { PERIOD } \\
\text { (CON- } \\
\text { SECU- } \\
\text { TIVE } \\
\text { DAYS) }\end{array}$} & \multicolumn{6}{|c|}{$\begin{array}{l}\text { DISCHARGE, IN FT3/S, FOR INDICATED } \\
\text { RECURRENCE INTERVAL, IN YEARS, AND }\end{array}$} \\
\hline & $\cdots$ & $\ldots \ldots$ & …..... & $\ldots \ldots$. & ......... & ........ \\
\hline & 2 & 5 & $10 \#$ & $25 \#$ & $50 \#$ & $100 \#$ \\
\hline & 508 & 208 & 108 & 48 & 28 & 18 \\
\hline 1 & 1,310 & 3.530 & 5,850 & 9,920 & 14,000 & 19,000 \\
\hline 3 & 928 & 2,110 & 3,170 & 4,800 & 6.230 & 7,850 \\
\hline 7 & 640 & 1.220 & 1,620 & 2,100 & 2,440 & 2,750 \\
\hline 15 & 431 & 730 & 904 & 1,090 & 1.210 & 1,310 \\
\hline 30 & 286 & 473 & 586 & 711 & 793 & 867 \\
\hline 60 & 169 & 298 & 390 & 510 & 602 & 696 \\
\hline 90 & 126 & 234 & 316 & 425 & 512 & 602 \\
\hline
\end{tabular}

DURATION TABLE OF DAILY MEAN FLOW FOR PERIOD OF RECORD $1948 \cdot 70,1996$

DISCHARGE, IN FT3/S, WHICH WAS EQUALED OR EXCEEDED FOR INDICATED PERCENT OF TIME

\begin{tabular}{|c|c|c|c|c|c|c|c|c|c|c|c|c|c|c|c|c|}
\hline 18 & 58 & 108 & 158 & 208 & 308 & 408 & 508 & 608 & 708 & 808 & 908 & 958 & 988 & 998 & 99.58 & 99.98 \\
\hline 763 & 242 & 102 & 61 & 33 & 6.3 & 0.42 & 0.00 & 0.00 & 0.00 & 0.00 & 0.00 & 0.00 & 0.00 & 0.00 & 0.00 & 0.00 \\
\hline
\end{tabular}

\# Reliability of values in column is uncertain, and potential errors are large. 
ANNUAL MEAN DISCHARGE, IN CUBIC

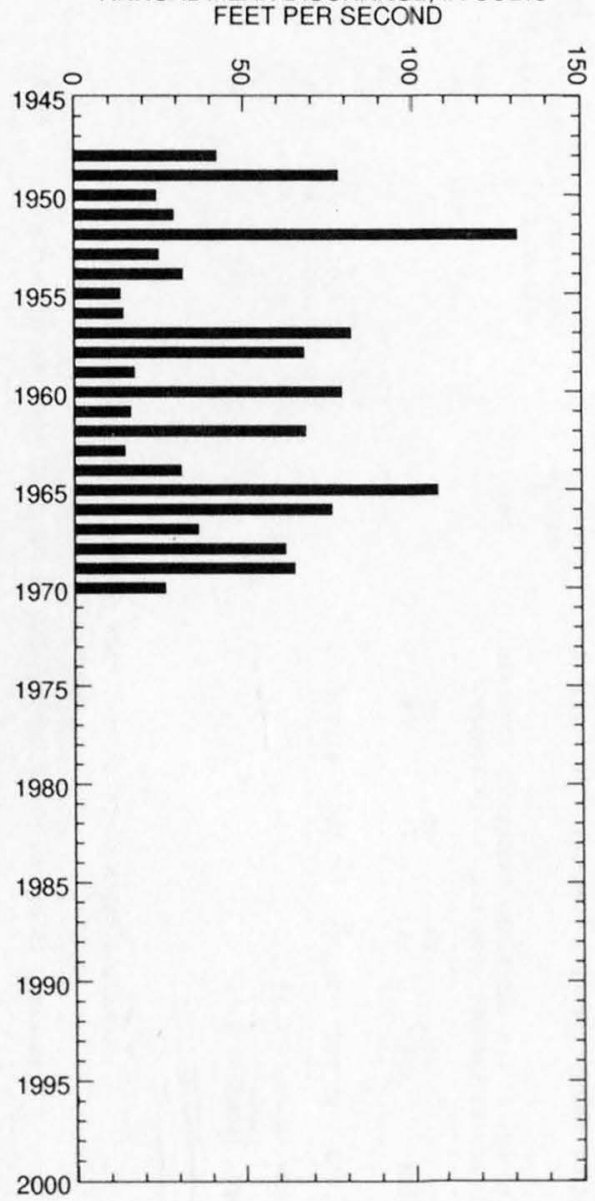

ANNUAL PEAK DISCHARGE IN CUBIC FEET PER SECOND

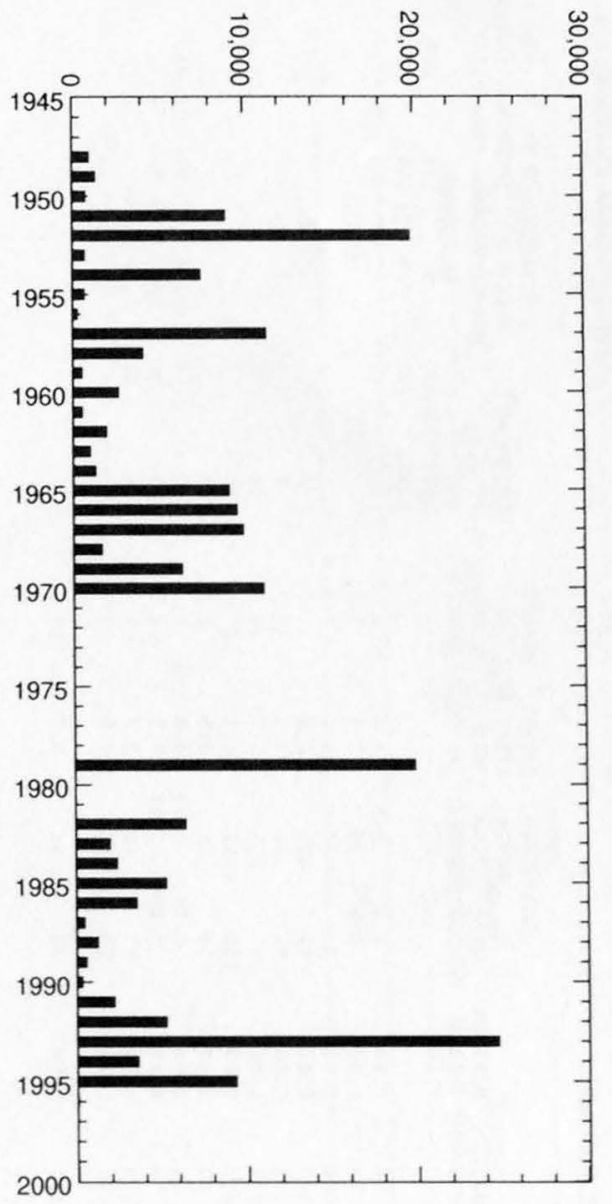


09397500 CHEVELON CREEK BELOW WILDCAT CANYON NEAR WINSLOW, AZ--Continued
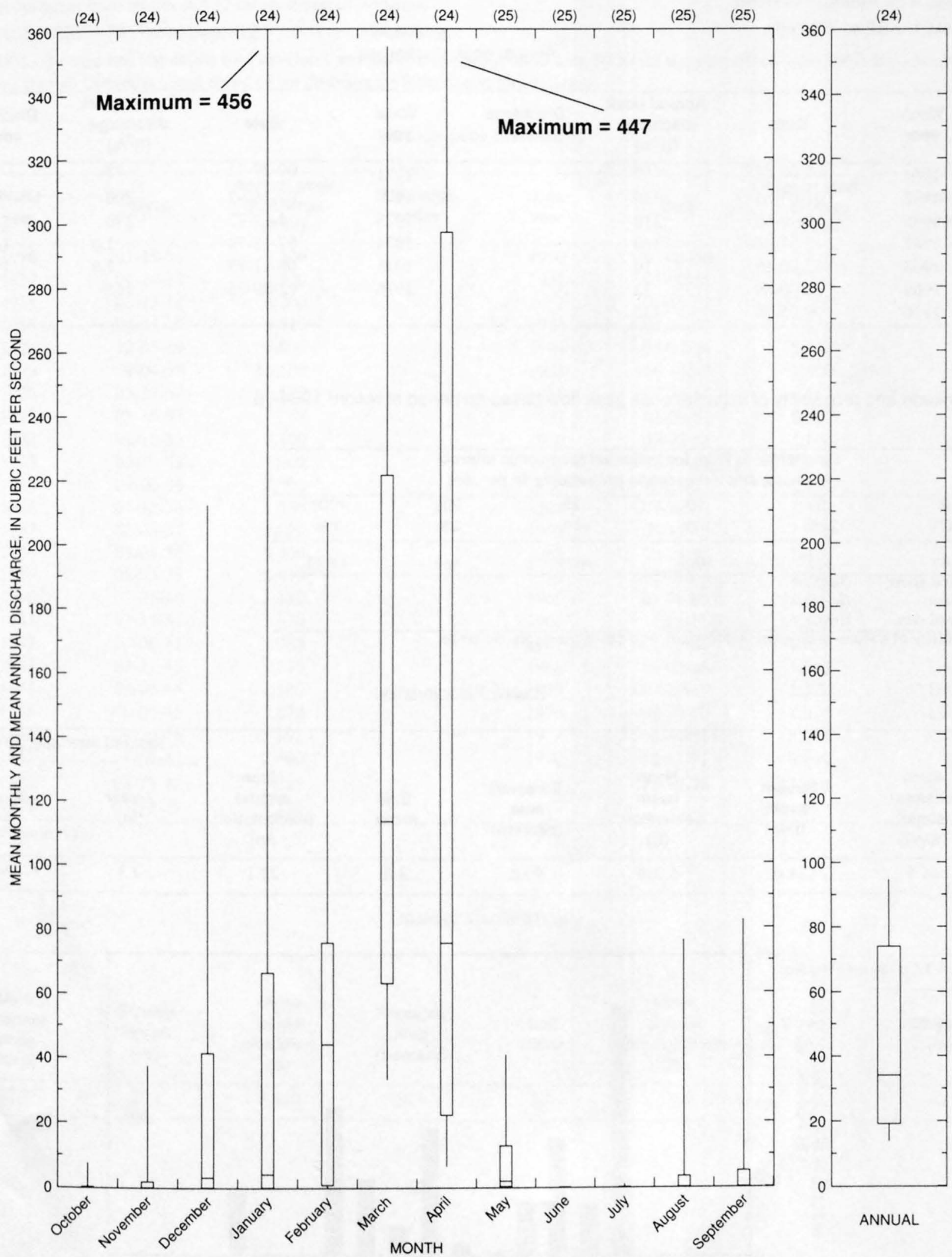
09397800 BROOKBANK CANYON NEAR HEBER, AZ

LOCATION.--Lat 34 28'20", long 110 38'50", in SE $1 / 4$, sec.33, T.13 N., R.16 E., Navajo County, Hydrologic Unit 15020010, at Heber-Winsin Road, 4 mi northwest of Heber.

DRAINAGE AREA.--27.9 $\mathrm{mi}^{2}$.

Annual peak discharges

\begin{tabular}{ccccccc}
\hline $\begin{array}{c}\text { Water } \\
\text { year }\end{array}$ & Date & $\begin{array}{c}\text { Annual peak } \\
\text { discharge } \\
\left(\mathrm{ft}^{3} / \mathbf{s}\right)\end{array}$ & $\begin{array}{c}\text { Discharge } \\
\text { codes }\end{array}$ & $\begin{array}{c}\text { Water } \\
\text { year }\end{array}$ & $\begin{array}{c}\text { Date } \\
\text { Annual peak } \\
\text { discharge } \\
\left(\mathrm{ft}^{3} / \mathbf{s}\right)\end{array}$ & $\begin{array}{c}\text { Discharge } \\
\text { codes }\end{array}$ \\
\hline 1964 & $08-00-64$ & 666 & & 1971 & $09-29-71$ & 78 \\
1965 & $01-07-65$ & 139 & & 1972 & $07-167-2$ & 200 \\
1966 & $12-30-65$ & 310 & & 1973 & $10-19-72$ & 390 \\
1967 & $12-07-66$ & 140 & & 1974 & $07-15-74$ & 1.0 \\
1968 & $02-00-68$ & 10 & & 1975 & $08-11-75$ & 2.0 \\
1969 & $03-00-69$ & 32 & & 1976 & $02-09-76$ & 165 \\
1970 & $09-05-70$ & 335 & & & & LT \\
\hline
\end{tabular}

Magnitude and probability of instantaneous peak flow based on period of record 1964-76

\begin{tabular}{cccccc}
\hline \multicolumn{5}{c}{$\begin{array}{l}\text { Discharge, in } \mathrm{ft}^{3} / \text { s, for indicated recurrence interval } \\
\text { in years, and exceedance probablility, in percent }\end{array}$} \\
\hline $\mathbf{2}$ & $\mathbf{5}$ & $\mathbf{1 0}$ & $\mathbf{2 5}$ & $\mathbf{5 0 \dagger}$ & $\mathbf{1 0 0 \dagger}$ \\
$\mathbf{5 0 \%}$ & $\mathbf{2 0 \%}$ & $\mathbf{1 0 \%}$ & $\mathbf{4} \%$ & $\mathbf{2 \%}$ & $\mathbf{1 \%}$ \\
\hline 147 & 315 & 460 & 679 & 866 & 1,070 \\
Weighted skew & $(\operatorname{logs})=$ & -0.23 & & \\
Mean & $(\operatorname{logs})=$ & 2.15 & & \\
Standard dev. & $(\operatorname{logs})=$ & 0.41 &
\end{tabular}

$\mp$ Reliability of values in column is uncertain, and potential errors are large.

Basin characteristics

\begin{tabular}{cccccccc}
\hline $\begin{array}{c}\text { Main } \\
\text { channel } \\
\text { slope } \\
(\mathrm{ft} / \mathrm{mi})\end{array}$ & $\begin{array}{c}\text { Stream } \\
\text { length } \\
\text { (mi) }\end{array}$ & $\begin{array}{c}\text { Mean } \\
\text { basin } \\
\text { elevation } \\
(\mathrm{ft})\end{array}$ & $\begin{array}{c}\text { Forested } \\
\text { area } \\
\text { (percent) }\end{array}$ & $\begin{array}{c}\text { Soil } \\
\text { index }\end{array}$ & $\begin{array}{c}\text { Mean } \\
\text { annual } \\
\text { precipitation } \\
\text { (in) }\end{array}$ & $\begin{array}{c}\text { Rainfall intensity, 24-hour } \\
\text { (in) }\end{array}$ & $\begin{array}{c}50 \text {-year } \\
\text { (in) }\end{array}$ \\
\hline 65.5 & 11.6 & 6,950 & 99.0 & 3.0 & 22.1 & 2.3 \\
\hline
\end{tabular}

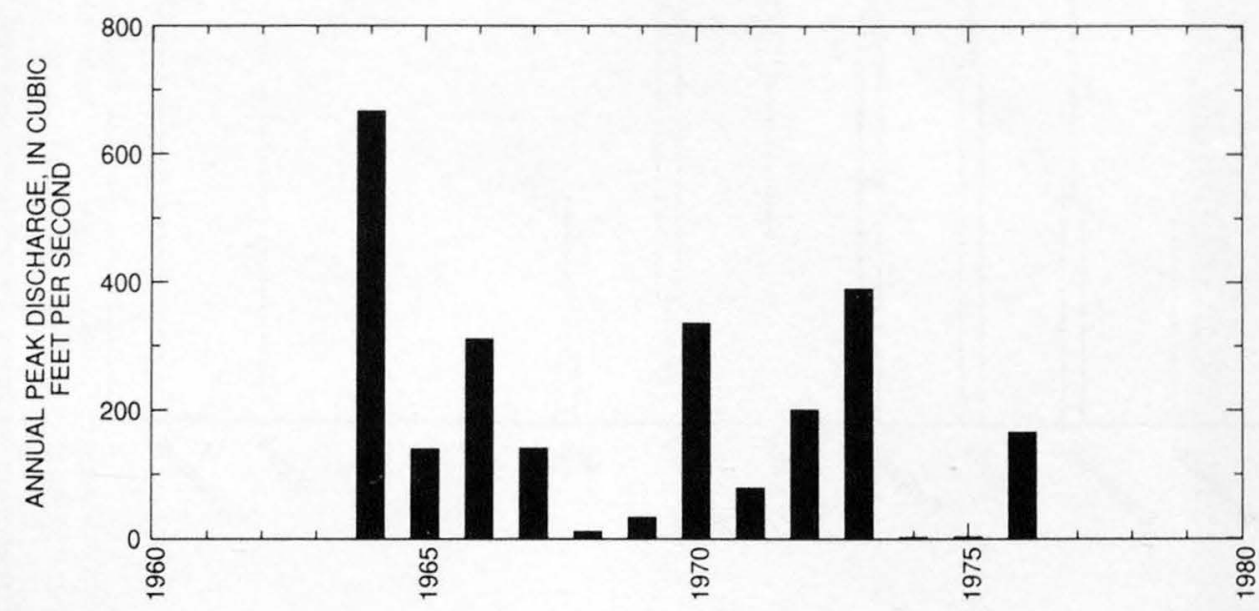


09398000 CHEVELON CREEK NEAR WINSLOW, AZ

LOCATION.--Lat $34^{\circ} 55^{\prime} 35^{\prime \prime}$, long $110^{\circ} 31^{\prime} 51^{\prime \prime}$, in $\mathrm{SE}^{1} / 4^{2} \mathrm{SW}^{1} / 4$, sec.27, T.18 N., R.17 E., Navajo County, Hydrologic Unit 15020010 , on right bank 3 mi upstream from mouth and 12 mi southeast of Winslow.

DRAINAGE AREA.--785 $\mathrm{mi}^{2}$, of which $3.9 \mathrm{mi}^{2}$ is noncontributing.

REMARKS.--Storage and regulation by Chevelon Canyon Lake (capacity, 6,193 acre-ft) $57 \mathrm{mi}$ upstream since June 1967. No known diversion above station. Chevelon Canal diverts 2 mi downstream from station for irrigation.

Annual peak discharges

\begin{tabular}{|c|c|c|c|c|c|c|c|}
\hline $\begin{array}{l}\text { Water } \\
\text { year }\end{array}$ & Date & $\begin{array}{c}\text { Annual peak } \\
\text { discharge } \\
\left(\mathrm{ft}^{3} / \mathrm{s}\right)\end{array}$ & $\begin{array}{l}\text { Discharge } \\
\text { codes }\end{array}$ & $\begin{array}{l}\text { Water } \\
\text { year }\end{array}$ & Date & $\begin{array}{c}\text { Annual peak } \\
\text { discharge } \\
\left(\mathrm{ft}^{3} / \mathrm{s}\right)\end{array}$ & $\begin{array}{c}\text { Discharge } \\
\text { codes }\end{array}$ \\
\hline 1916 & 01-19-16 & 9,500 & & 1950 & $03-01-50$ & 616 & \\
\hline 1917 & $04-24-17$ & 1,300 & & 1951 & $08-30-51$ & 7,200 & \\
\hline 1918 & $03-13-18$ & 6,200 & & 1952 & $01-19-52$ & 25,300 & \\
\hline 1919 & 04-01-19 & 1,110 & & 1953 & $03-12-53$ & 650 & \\
\hline 1920 & $12-05-19$ & 9,000 & & 1954 & $03-23-54$ & 5,730 & \\
\hline 1929 & $04-04-29$ & 16,100 & & 1955 & $06-135-5$ & 1,800 & \\
\hline 1930 & $03-27-30$ & 519 & & 1956 & $07-23-56$ & 562 & \\
\hline 1931 & $03-19-31$ & 548 & & 1957 & $01-09-57$ & 8,680 & \\
\hline 1932 & $02-10-32$ & 3,100 & & 1958 & $09-28-58$ & 2,140 & \\
\hline 1933 & $09-20-33$ & 1,060 & & 1959 & $08-14-59$ & 1,320 & \\
\hline 1934 & $00-00-34$ & 2,700 & & 1960 & $12-26-59$ & 2,640 & \\
\hline 1936 & $04-12-36$ & 1,350 & & 1961 & $07-30-61$ & 500 & \\
\hline 1937 & $02-08-37$ & 1,820 & & 1962 & $02-13-62$ & 1,540 & \\
\hline 1938 & $03-04-38$ & 9,400 & & 1963 & $08-21-63$ & 1,620 & \\
\hline 1939 & 08-03-39 & 2,410 & & 1964 & 08-01-64 & 1,680 & \\
\hline 1940 & $07-25-40$ & 1,180 & & 1965 & $01-08-65$ & 13,100 & \\
\hline 1941 & $03-15-41$ & 1,630 & & 1966 & $12-31-65$ & 13,300 & \\
\hline 1942 & $04-06-42$ & 985 & & 1967 & $12-07-66$ & 8,890 & \\
\hline 1943 & $03-11-43$ & 1,330 & & 1968 & $04-02-68$ & 1,640 & UR \\
\hline 1944 & $04-06-44$ & 1,180 & & 1969 & $01-2-669$ & 5,120 & UR \\
\hline 1945 & $08-04-45$ & 2,620 & & 1970 & 09-06-70 & 8,020 & UR \\
\hline 1946 & $09-20-46$ & 892 & & 1971 & $08-24-71$ & 4,150 & UR \\
\hline 1947 & $08-04-47$ & 2,460 & & 1972 & $12-27-71$ & 9,040 & UR \\
\hline 1948 & $04-13-48$ & 825 & & 1979 & $12-18-78$ & 133,600 & UR,HP \\
\hline 1949 & $04-14-49$ & 1,150 & & & & & \\
\hline
\end{tabular}

${ }^{1}$ Highest since 1923.

Basin characteristics

\begin{tabular}{|c|c|c|c|c|c|c|c|}
\hline \multirow[b]{2}{*}{$\begin{array}{c}\text { Main } \\
\text { channel } \\
\text { slope } \\
(\mathrm{ft} / \mathrm{mi})\end{array}$} & \multirow[b]{2}{*}{$\begin{array}{c}\text { Stream } \\
\text { length } \\
\text { (mi) }\end{array}$} & \multirow[b]{2}{*}{$\begin{array}{c}\text { Mean } \\
\text { basin } \\
\text { elevation } \\
(f t)\end{array}$} & \multirow[b]{2}{*}{$\begin{array}{l}\text { Forested } \\
\text { area } \\
\text { (percent) }\end{array}$} & \multirow[b]{2}{*}{$\begin{array}{c}\text { Soil } \\
\text { index }\end{array}$} & \multirow[b]{2}{*}{$\begin{array}{c}\text { Mean } \\
\text { annual } \\
\text { precipitation } \\
\text { (in) }\end{array}$} & \multicolumn{2}{|c|}{ Rainfall intensity, 24-hour } \\
\hline & & & & & & $\begin{array}{l}\text { 2-year } \\
\text { (in) }\end{array}$ & $\begin{array}{l}\text { 50-year } \\
\text { (in) }\end{array}$ \\
\hline 27.7 & 77.1 & 6,440 & 70.0 & 3.0 & 18.4 & 2.1 & 4.2 \\
\hline
\end{tabular}




\section{CHEVELON CREEK NEAR WINSLOW, AZ--Continued}

MEAN MONTHLY AND ANNUAL DISCHARGES 1917-19, 1930-33, 1936-72

\begin{tabular}{|c|c|c|c|c|c|c|}
\hline MONTH & $\begin{array}{l}\text { MAXIMUM } \\
\text { (FT3/S) }\end{array}$ & $\begin{array}{l}\text { MINIMUM } \\
\text { (FT3/S) }\end{array}$ & $\begin{array}{c}\text { MEAN } \\
(\text { FT3/S) }\end{array}$ & $\begin{array}{l}\text { STAN- } \\
\text { DARD } \\
\text { DEVIA- } \\
\text { TION } \\
(\mathrm{FT} 3 / \mathrm{S})\end{array}$ & $\begin{array}{l}\text { COEFFI- } \\
\text { CIENT OF } \\
\text { VARI- } \\
\text { ATION }\end{array}$ & $\begin{array}{c}\text { PERCENT } \\
\text { OF } \\
\text { ANNUAL } \\
\text { RUNOFF }\end{array}$ \\
\hline OCTOBER & 247 & 3.1 & 12 & 37 & 3.1 & 2.0 \\
\hline NOVEMBER & 137 & 2.7 & 14 & 26 & 1.9 & 2.3 \\
\hline DECEMBER & 347 & 2.8 & 37 & 76 & 2.1 & 6.1 \\
\hline JANUARY & 663 & 2.6 & 58 & 135 & 2.3 & 9.6 \\
\hline FEBRUARY & 338 & 2.7 & 64 & 89 & 1.4 & 10.6 \\
\hline MARCH & 527 & 3.5 & 171 & 138 & 0.80 & 28.2 \\
\hline APRIL & 733 & 3.9 & 181 & 188 & 1.0 & 29.9 \\
\hline MAY & 211 & 2.2 & 23 & 39 & 1.7 & 3.7 \\
\hline JUNE & 21 & 2.1 & 5.1 & 3.1 & 0.61 & 0.8 \\
\hline JULY & 25 & 3.0 & 7.1 & 4.1 & 0.57 & 1.2 \\
\hline AUGUST & 171 & 2.9 & 18 & 32 & 1.8 & 3.0 \\
\hline SEPTEMBER & 182 & 2.5 & 15 & 31 & 2.0 & 2.5 \\
\hline ANNUAL & 145 & 7.7 & 50 & 32 & 0.63 & 100 \\
\hline
\end{tabular}

MAGNITUDE AND PROBABILITY OF INSTANTANEOUS PEAK FLOW BASED ON PERIOD OF RECORD $1916 \cdot 20,1929 \cdot 34,1936-67,1968-72$

DISCHARGE, IN FT3/S, FOR INDICATED RECURRENCE INTERVAL IN YEARS, AND EXCEEDANCE PROBABILITY, IN PERCENT

\begin{tabular}{|c|c|c|c|c|c|}
\hline 2 & 5 & 10 & 25 & 50 & 100 \\
\hline $50 \%$ & 208 & 108 & 48 & 28 & 18 \\
\hline 2,360 & 5,860 & 9,670 & 16,800 & 24,200 & 33,800 \\
\hline
\end{tabular}

WEIGHTED SKEW (LOGS) $=0.24$

MEAN $\quad($ LOGS $)=3.39$

STANDARD DEV. $($ LOGS $)=0.46$
MAGNITUDE AND PROBABILITY OF ANNUAL LOW FLOW BASED ON PERIOD OF RECORD $1918-19,1930-33,1937-72$

\begin{tabular}{|c|c|c|c|c|c|c|}
\hline \multirow{4}{*}{$\begin{array}{l}\text { PERIOD } \\
\text { (CON- } \\
\text { SECU - } \\
\text { TIVE } \\
\text { DAYS) }\end{array}$} & \multirow{2}{*}{\multicolumn{3}{|c|}{$\begin{array}{r}\text { DISCHARGE, IN } \\
\text { RECURRENCE INI } \\
\text { NON-EXCEEDANCE } \\
\ldots \ldots \ldots \ldots \ldots \ldots\end{array}$}} & \multirow{2}{*}{\multicolumn{3}{|c|}{$\begin{array}{l}\text { N FT3/S, FOR INDICATED } \\
\text { NTERVAL, IN YEARS, AND } \\
\text { E PROBABILITY, IN PERCENT }\end{array}$}} \\
\hline & & & & & & \\
\hline & 2 & 5 & 10 & 20 & 50 & $100 \#$ \\
\hline & 508 & 208 & 108 & 58 & 28 & 18 \\
\hline 1 & 2.1 & 1.5 & 1.2 & 0.94 & 0.72 & 0.60 \\
\hline 3 & 2.3 & 1.7 & 1.4 & 1.2 & 0.95 & 0.82 \\
\hline 7 & 2.6 & 2.0 & 1.7 & 1.5 & 1.3 & 1.2 \\
\hline 14 & 2.8 & 2.3 & 2.0 & 1.9 & 1.7 & 1.5 \\
\hline 30 & 3.2 & 2.7 & 2.5 & 2.3 & 2.1 & 2.0 \\
\hline 60 & 3.7 & 3.2 & 2.9 & 2.7 & 2.5 & 2.3 \\
\hline 90 & 4.0 & 3.3 & 3.0 & 2.8 & 2.5 & 2.4 \\
\hline 120 & 4.1 & 3.5 & 3.4 & 3.3 & 3.3 & 3.3 \\
\hline 183 & 5.1 & 3.9 & 3.6 & 3.4 & 3.3 & 3.3 \\
\hline
\end{tabular}

MAGNITUDE AND PROBABILITY OF ANNUAL HIGH FLOW BASED ON PERIOD OF RECORD $1917 \cdot 19,1930-33,1936 \cdot 72$

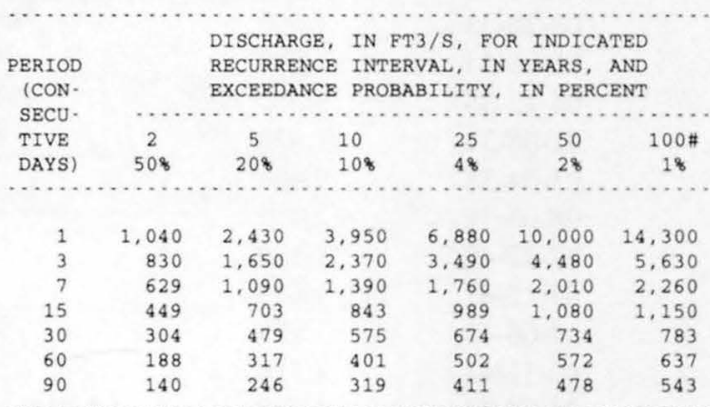

DURATION TABLE OF DAILY MEAN FLOW FOR PERIOD OF RECORD $1917 \cdot 19,1930 \cdot 33,1936 \cdot 72$ DISCHARGE, IN FT3/S, WHICH WAS EQUALED OR EXCEEDED FOR INDICATED PERCENT OF TIME

\begin{tabular}{|c|c|c|c|c|c|c|c|c|c|c|c|c|c|c|c|c|}
\hline 28 & 58 & 108 & 158 & 208 & 308 & 408 & 508 & 608 & 708 & 808 & 908 & 958 & 988 & 998 & 99.58 & 99.98 \\
\hline 768 & 267 & 113 & 60 & 33 & 9.0 & 5.6 & 5.1 & 4.7 & 4.3 & 3.8 & 3.3 & 3.0 & 2.1 & 1.8 & 1.7 & 1.1 \\
\hline
\end{tabular}

\# Reliability of values in column is uncertain, and potential errors are large. 
ANNUAL MEAN DISCHARGE, IN CUBIC
FEET PER SECOND

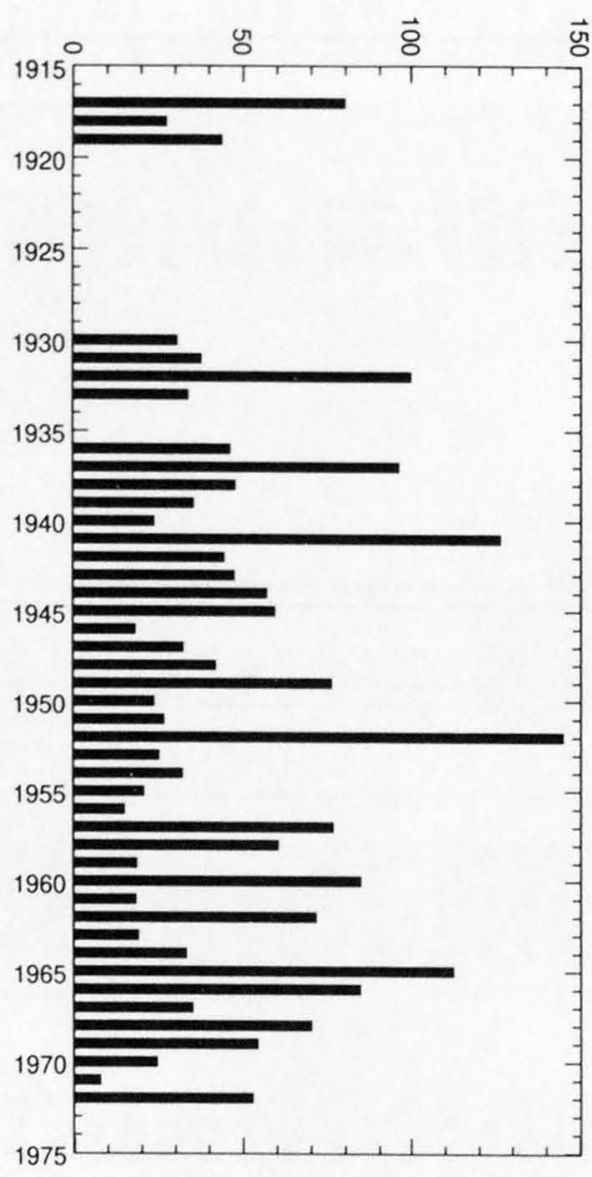

ANNUAL PEAK DISCHARGE, IN CUBIC

FEET PER SECOND

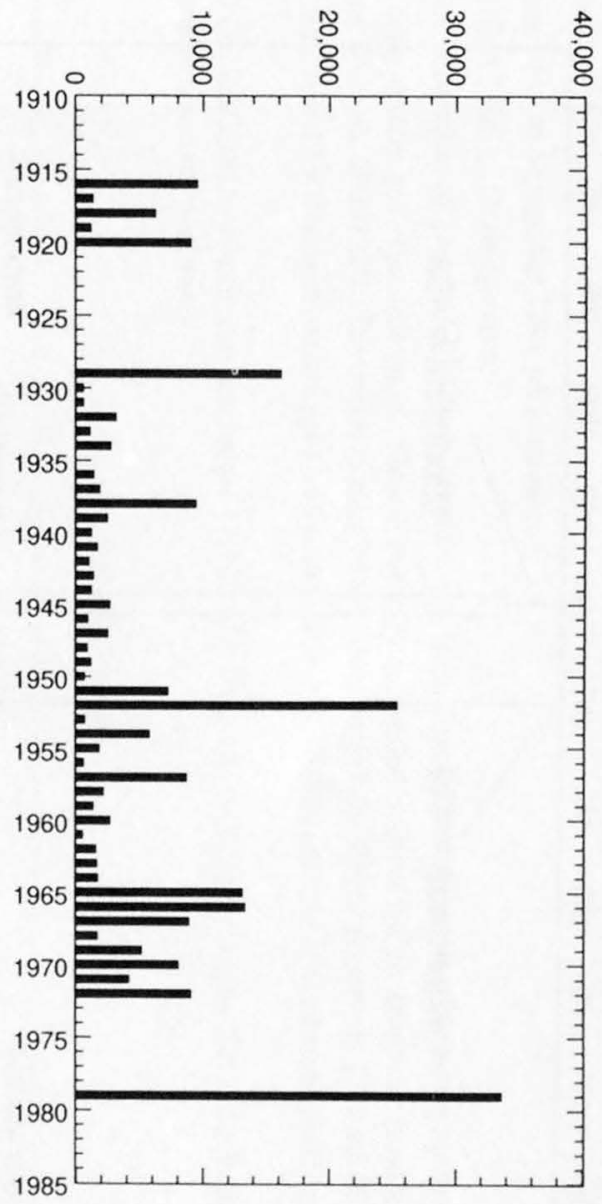


09398000 CHEVELON CREEK NEAR WINSLOW, AZ--Continued

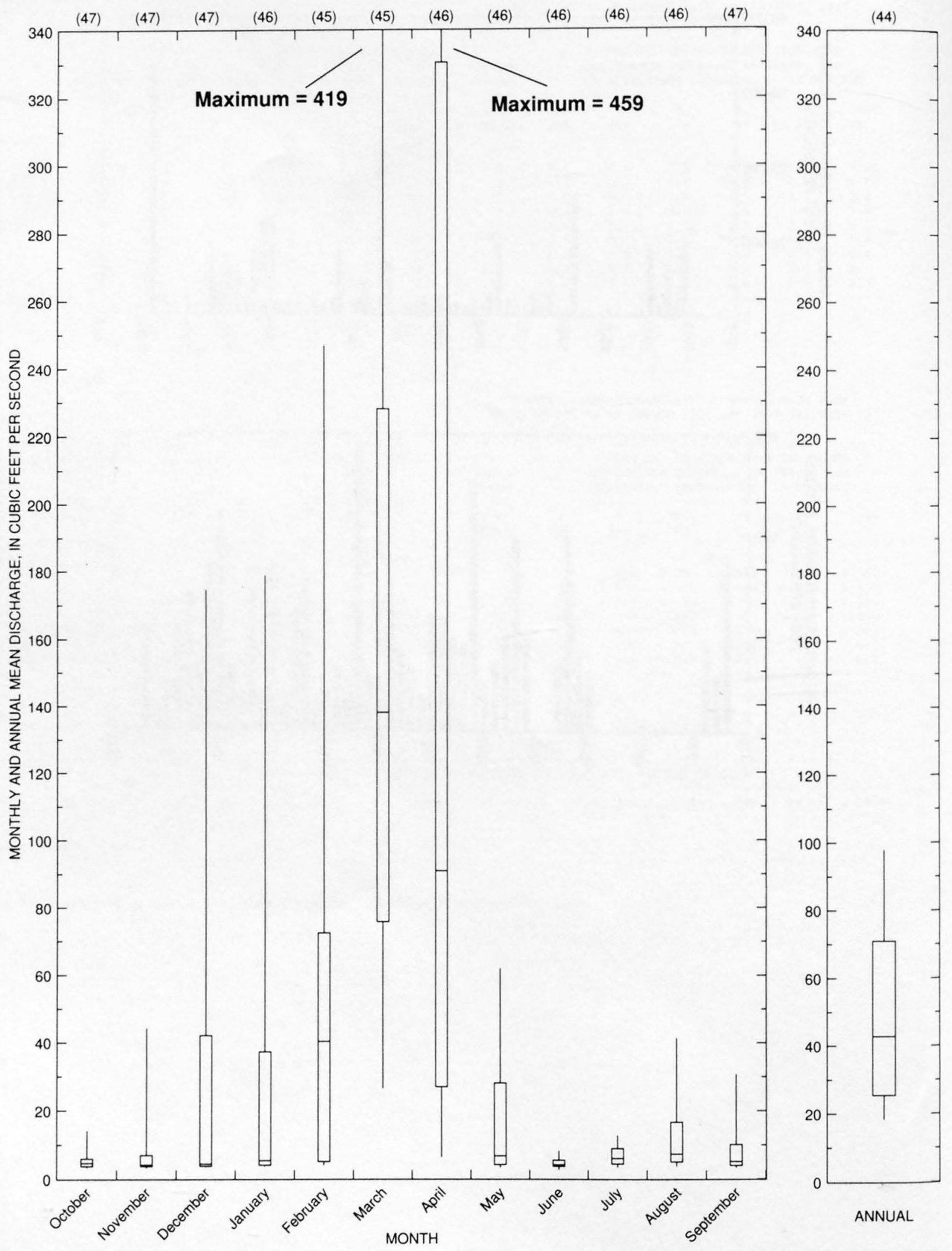




\section{CLEAR CREEK BELOW WILLOW CREEK, NEAR WINSLOW, AZ}

LOCATION.--Lat 34 40'03", long $111^{\circ} 00^{\prime} 25^{\prime \prime}$, in SW1/4 $\mathrm{SE}^{1} / 4$ sec.19, T.15 N., R.13 E., Coconino County, Hydrologic Unit 15020008 , in ApacheSitgreaves National Forest, on right bank 2 mi downstream from Willow Creek and 30 mi southwest of Winslow.

DRAINAGE AREA.--317 $\mathrm{mi}^{2}$.

PERIOD OF RECORD.--June 1947 to September 1991 (discontinued).

REVISED RECORDS.--WDR AZ-88-1: Drainage area.

GAGE.--Water-stage recorder. Elevation of gage is 5,957 ft above sea level, from Topographic Division by photogrammetry.

REMARKS.--No estimated daily discharges. Records good. Flow is partially controlled by Blue Ridge Reservoir (usable capacity, 15,000 acre-

$\mathrm{ft}$ ) about $20 \mathrm{mi}$ upstream. (See sta 09398300.) Diversion to East Verde River from Blue Ridge Reservoir. (See sta 09507580.)

AVERAGE DISCHARGE (unadjusted for diversion or storage).--44 years, $82.0 \mathrm{ft}^{3} / \mathrm{s}, 59,410$ acre- $\mathrm{ft} / \mathrm{yr}$; median of yearly mean discharges, $56 \mathrm{ft}^{3} /$ s, 40,600 acre-ft/yr.

EXTREMES FOR PERIOD OF RECORD.--Maximum discharge, 19,700 $\mathrm{ft}^{3} / \mathrm{s} \mathrm{Dec} .18,1978$, gage height, $22.32 \mathrm{ft}$, from rating curve extended above $6,000 \mathrm{ft}^{3} / \mathrm{s}$; no flow at times in most years.

Annual peak discharges

\begin{tabular}{|c|c|c|c|c|c|c|c|}
\hline $\begin{array}{l}\text { Water } \\
\text { year }\end{array}$ & Date & $\begin{array}{c}\text { Annual peak } \\
\text { discharge } \\
\left(\mathrm{ft}^{3} / \mathrm{s}\right)\end{array}$ & $\begin{array}{l}\text { Discharge } \\
\text { codes }\end{array}$ & $\begin{array}{l}\text { Water } \\
\text { year }\end{array}$ & Date & $\begin{array}{c}\text { Annual peak } \\
\text { discharge } \\
\left(\mathrm{ft}^{3} / \mathrm{s}\right)\end{array}$ & $\begin{array}{l}\text { Discharge } \\
\text { codes }\end{array}$ \\
\hline 1948 & $04-12-48$ & 1,810 & & 1971 & $08-27-71$ & 1,180 & UR \\
\hline 1949 & $04-15-49$ & 1,990 & & 1972 & $12-26-71$ & 5,840 & UR \\
\hline 1950 & $02-28-50$ & 1,090 & & 1973 & $10-20-72$ & 8,190 & UR \\
\hline 1951 & $08-29-51$ & 8,090 & & 1974 & $03-21-74$ & 589 & UR \\
\hline 1952 & $01-18-52$ & 16,400 & & 1975 & $04-26-75$ & 920 & UR \\
\hline 1953 & $03-11-53$ & 497 & & 1976 & $02-09-76$ & 4,170 & UR \\
\hline 1954 & $03-23-54$ & 5,730 & & 1977 & $04-09-77$ & 353 & UR \\
\hline 1955 & $06-14-55$ & 1,220 & & 1978 & 03-01-78 & 10,500 & UR \\
\hline 1956 & $03-26-56$ & 198 & & 1979 & $12-18-78$ & ${ }^{1} 19,700$ & UR \\
\hline 1957 & $01-10-57$ & 8,880 & & 1980 & $02-20-80$ & 8,140 & UR \\
\hline 1958 & $03-22-58$ & 2,920 & & 1981 & 04-08-81 & 250 & UR \\
\hline 1959 & $08-20-59$ & 296 & & 1982 & $03-12-82$ & 5,230 & UR \\
\hline 1960 & $12-25-59$ & 2,770 & & 1983 & $04-25-83$ & 2,020 & UR \\
\hline 1961 & $04-05-61$ & 1,080 & & 1984 & $12-27-83$ & 2,130 & UR \\
\hline 1962 & $02-13-62$ & 2,240 & & 1985 & $03-12-85$ & 4,750 & UR \\
\hline 1963 & $02-11-63$ & 403 & & 1986 & $11-26-85$ & 2,570 & UR \\
\hline 1964 & $04-16-64$ & 1,210 & & 1987 & $04-12-87$ & 1,070 & UR \\
\hline 1965 & $01-07-65$ & 5,600 & UR & 1988 & $11-01-87$ & 1,270 & UR \\
\hline 1966 & $12-30-65$ & 13,100 & UR & 1989 & $03-11-89$ & 602 & UR \\
\hline 1967 & $12-07-66$ & 9,970 & UR & 1990 & $04-06-90$ & 194 & UR \\
\hline 1968 & $04-02-68$ & 1,840 & UR & 1991 & $04-08-91$ & 2,770 & UR \\
\hline 1969 & $01-26-69$ & 5,550 & UR & 1993 & $01-08-93$ & 29,100 & UR \\
\hline 1970 & $09-06-70$ & 15,800 & UR & & & & \\
\hline
\end{tabular}

${ }^{1}$ Highest since 1939

Discharge rating table developed March 1990

\begin{tabular}{crcc}
\hline $\begin{array}{c}\text { Gage height } \\
(\mathbf{f t})\end{array}$ & $\begin{array}{c}\text { Discharge } \\
\left(\mathbf{f t}^{3} / \mathbf{s}\right)\end{array}$ & $\begin{array}{c}\text { Gage height } \\
(\mathbf{f t})\end{array}$ & $\begin{array}{c}\text { Discharge } \\
\left(\mathbf{f t}^{3} / \mathbf{s}\right)\end{array}$ \\
\hline 5.0 & 133 & 23.0 & 20,300 \\
8.0 & 890 & 26.0 & 27,260 \\
11.0 & 2,760 & 29.0 & 35,360 \\
14.0 & 5,600 & 32.0 & 44,630 \\
17.0 & 9,750 & 35.0 & 55,110 \\
20.0 & 14,600 & 39.0 & 71,000 \\
\hline
\end{tabular}




\section{LITTLE COLORADO RIVER BASIN}

09398500 CLEAR CREEK BELOW WILLOW CREEK, NEAR WINSLOW, AZ--Continued

Basin characteristics

\begin{tabular}{cccccccc}
\hline $\begin{array}{c}\text { Main } \\
\text { channel } \\
\text { slope } \\
(\mathrm{ft} / \mathrm{mi})\end{array}$ & $\begin{array}{c}\text { Stream } \\
\text { length } \\
\text { (mi) }\end{array}$ & $\begin{array}{c}\text { Mean } \\
\text { basin } \\
\text { elevation } \\
\text { (ft) }\end{array}$ & $\begin{array}{c}\text { Forested } \\
\text { area } \\
\text { (percent) }\end{array}$ & $\begin{array}{c}\text { Soil } \\
\text { index }\end{array}$ & $\begin{array}{c}\text { Mean } \\
\text { annual } \\
\text { precipitation } \\
\text { (in) }\end{array}$ & $\begin{array}{c}\text { Rainfall intensity, 24-hour } \\
\text { (in) }\end{array}$ & $\begin{array}{c}50 \text {-year } \\
\text { (in) }\end{array}$ \\
\hline 26.0 & 41.0 & 7,100 & 100 & 3.0 & 25.8 & 2.6 \\
\hline
\end{tabular}


09398500 CLEAR CREEK BELOW WILLOW CREEK, NEAR WINSLOW, AZ--Continued

MEAN MONTHLY AND ANNUAL DISCHARGES 1948-91

\begin{tabular}{|c|c|c|c|c|c|c|}
\hline MONTH & $\begin{array}{l}\text { MAXIMUM } \\
\text { (FT3/S) }\end{array}$ & $\begin{array}{l}\text { MINIMUM } \\
\text { (FT3/S) }\end{array}$ & $\begin{array}{c}\text { MBAN } \\
(\text { FT3/S) }\end{array}$ & $\begin{array}{l}\text { STAN- } \\
\text { DARD } \\
\text { DEVIA- } \\
\text { TION } \\
\text { (FT3/S) }\end{array}$ & $\begin{array}{l}\text { COEFFI- } \\
\text { CIENT OF } \\
\text { VARI- } \\
\text { ATION }\end{array}$ & $\begin{array}{c}\text { PERCENT } \\
\text { OF } \\
\text { ANNUAL } \\
\text { RUNOFF }\end{array}$ \\
\hline OCTOBER & 359 & 0.00 & 17 & 61 & 3.7 & 1.7 \\
\hline NOVEMBER & 202 & 0.00 & 24 & 50 & 2.1 & 2.4 \\
\hline DECEMBER & 720 & 0.00 & 71 & 148 & 2.1 & 7.2 \\
\hline JANUARY & 576 & 0.00 & 60 & 129 & 2.1 & 6.1 \\
\hline FEBRUARY & 721 & 0.00 & 89 & 138 & 1.6 & 9.0 \\
\hline MARCH & 1,250 & 4.0 & 253 & 252 & 1.0 & 25.6 \\
\hline APRIL & 1,330 & 0.00 & 370 & 372 & 1.0 & 37.5 \\
\hline MAY & 1,050 & 0.00 & 76 & 176 & 2.3 & 7.7 \\
\hline JUNE & 39 & 0.00 & 1.3 & 5.9 & 4.4 & 0.1 \\
\hline JULY & 4.3 & 0.00 & 0.24 & 0.88 & 3.6 & 0.0 \\
\hline AUGUST & 228 & 0.00 & 15 & 47 & 3.1 & 1.5 \\
\hline SEPTEMBER & 218 & 0.00 & 11 & 40 & 3.7 & 1.1 \\
\hline ANNUAL & 279 & 5.7 & 82 & 67 & 0.82 & 100 \\
\hline
\end{tabular}

MAGNITUDE AND PROBABILITY OF ANNUAL LOW FLOW BASED ON PERIOD OF RECORD 1949.91

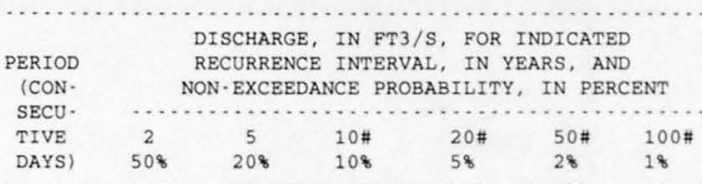

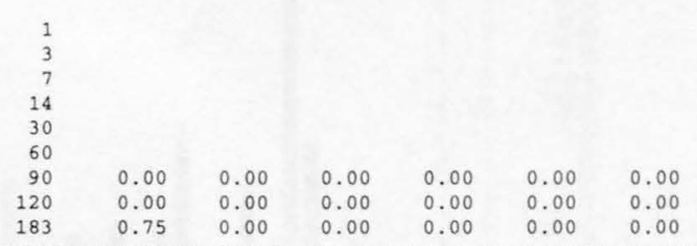

MAGNITUDE AND PROBABILITY OF ANNUAL HIGH FLOW BASED ON PERIOD OF RECORD 1948.91

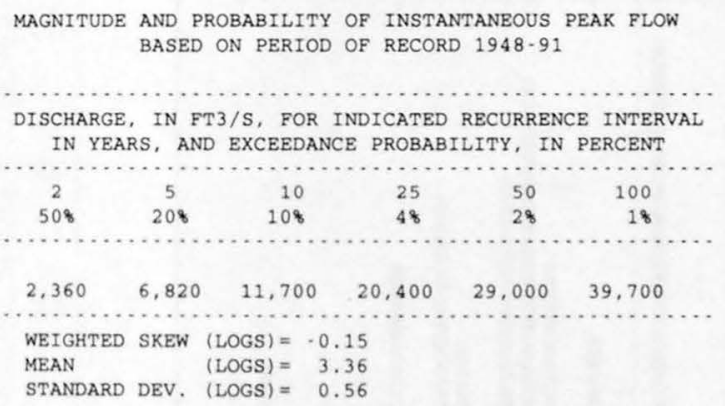

\begin{tabular}{|c|c|c|c|c|c|c|}
\hline \multirow{3}{*}{$\begin{array}{l}\text { PERIOD } \\
\text { ICON- } \\
\text { SECU. }\end{array}$} & \multicolumn{6}{|c|}{ DISCHARGE, IN FT $3 / S$, FOR INDICATED } \\
\hline & & EXCEEDA & CE PROB & BILITY, & IN PERC & ENT \\
\hline & & & & & & \\
\hline TIVE & 2 & 5 & $10 \#$ & $25 \#$ & $50 \#$ & $100 \#$ \\
\hline DAYS) & 508 & 208 & 108 & 48 & 28 & 18 \\
\hline 1 & 1,580 & 3,730 & 5,620 & 8,440 & 10,800 & 13,400 \\
\hline 3 & 1.220 & 2,590 & 3,650 & 5,080 & 6,160 & 7,250 \\
\hline 7 & 893 & 1,680 & 2,210 & 2,840 & 3,270 & 3,660 \\
\hline 15 & 605 & 1,120 & 1,470 & 1,910 & 2,210 & 2,510 \\
\hline 30 & 420 & 825 & 1,140 & 1,580 & 1,920 & 2,280 \\
\hline 60 & 267 & 548 & 777 & 1,100 & 1,370 & 1,650 \\
\hline 90 & 203 & 428 & 613 & 878 & 1,090 & 1,320 \\
\hline
\end{tabular}

DURATION TABLE OF DAILY MEAN FLOW FOR PERIOD OF RECORD 1948.91

DISCHARGE, IN FT3/S, WHICH WAS EQUALED OR EXCEEDED FOR INDICATED PERCENT OF TIME

\begin{tabular}{|c|c|c|c|c|c|c|c|c|c|c|c|c|c|c|c|c|}
\hline 18 & 58 & 108 & 158 & 208 & 308 & 408 & 508 & 608 & 708 & 808 & 908 & 958 & 988 & 998 & 99.58 & 99.98 \\
\hline 360 & 485 & 186 & 99 & 59 & 14 & 1.8 & 0.00 & 0.00 & 0.00 & 0.00 & 0.00 & 0.00 & 0.00 & 0.00 & 0.00 & 0.00 \\
\hline
\end{tabular}

\# Reliability of values in column is uncertain, and potential errors are large. 


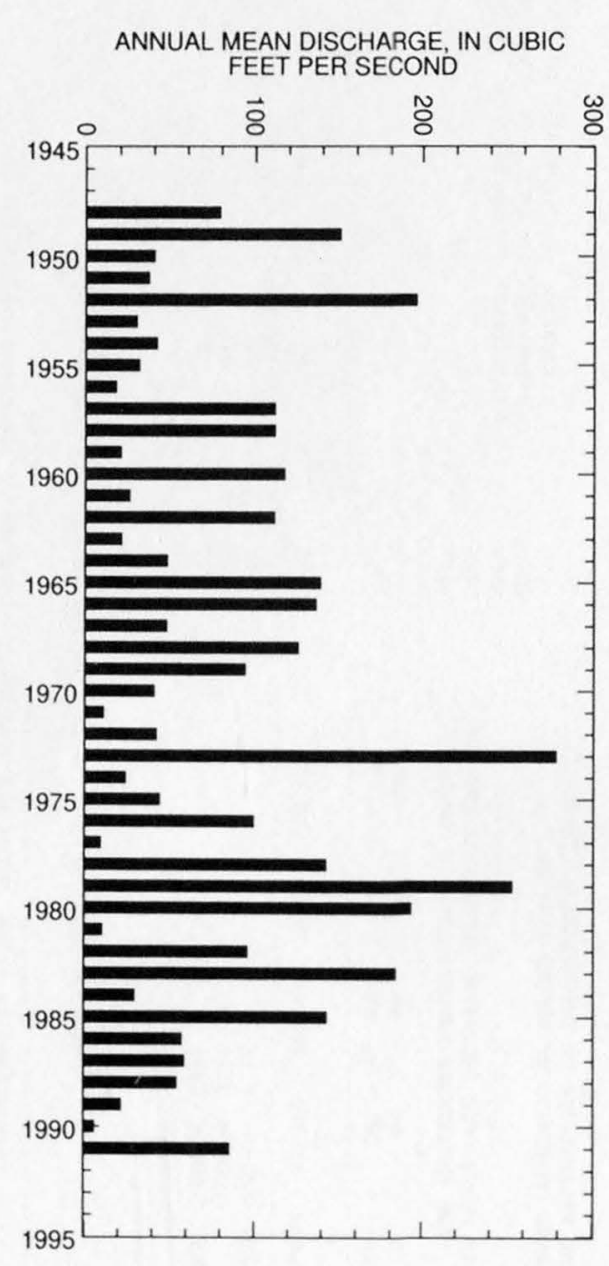

ANNUAL PEAK DISCHARGE, IN CUBIC

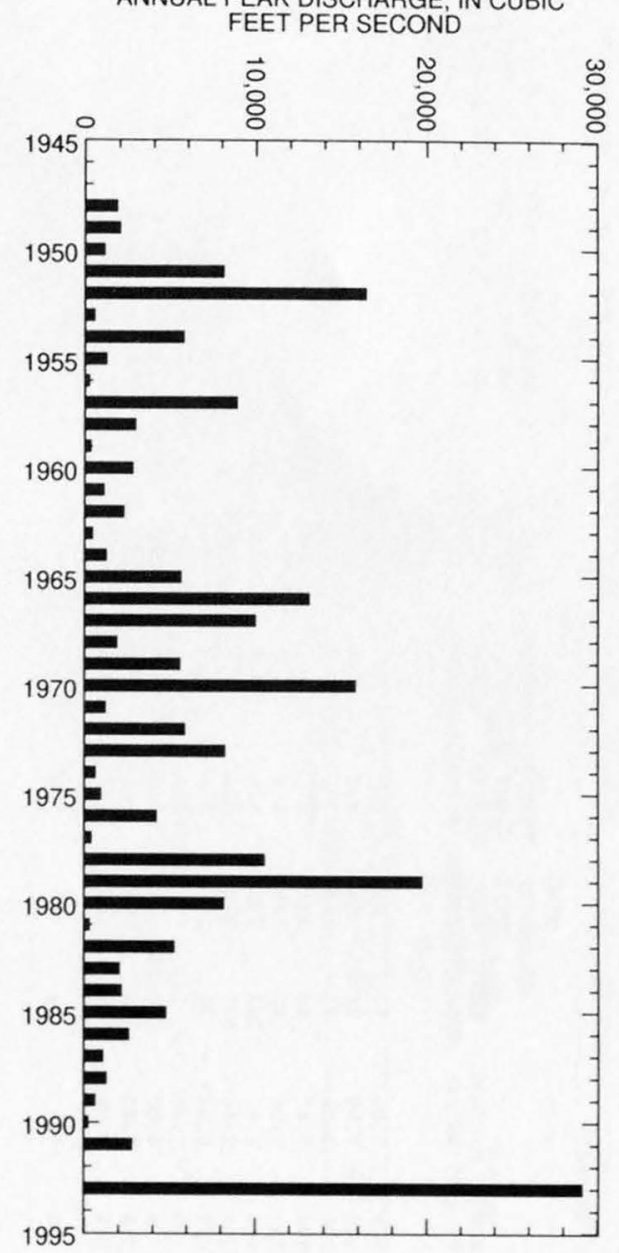


09398500 CLEAR CREEK BELOW WILLOW CREEK, NEAR WINSLOW, AZ--Continued
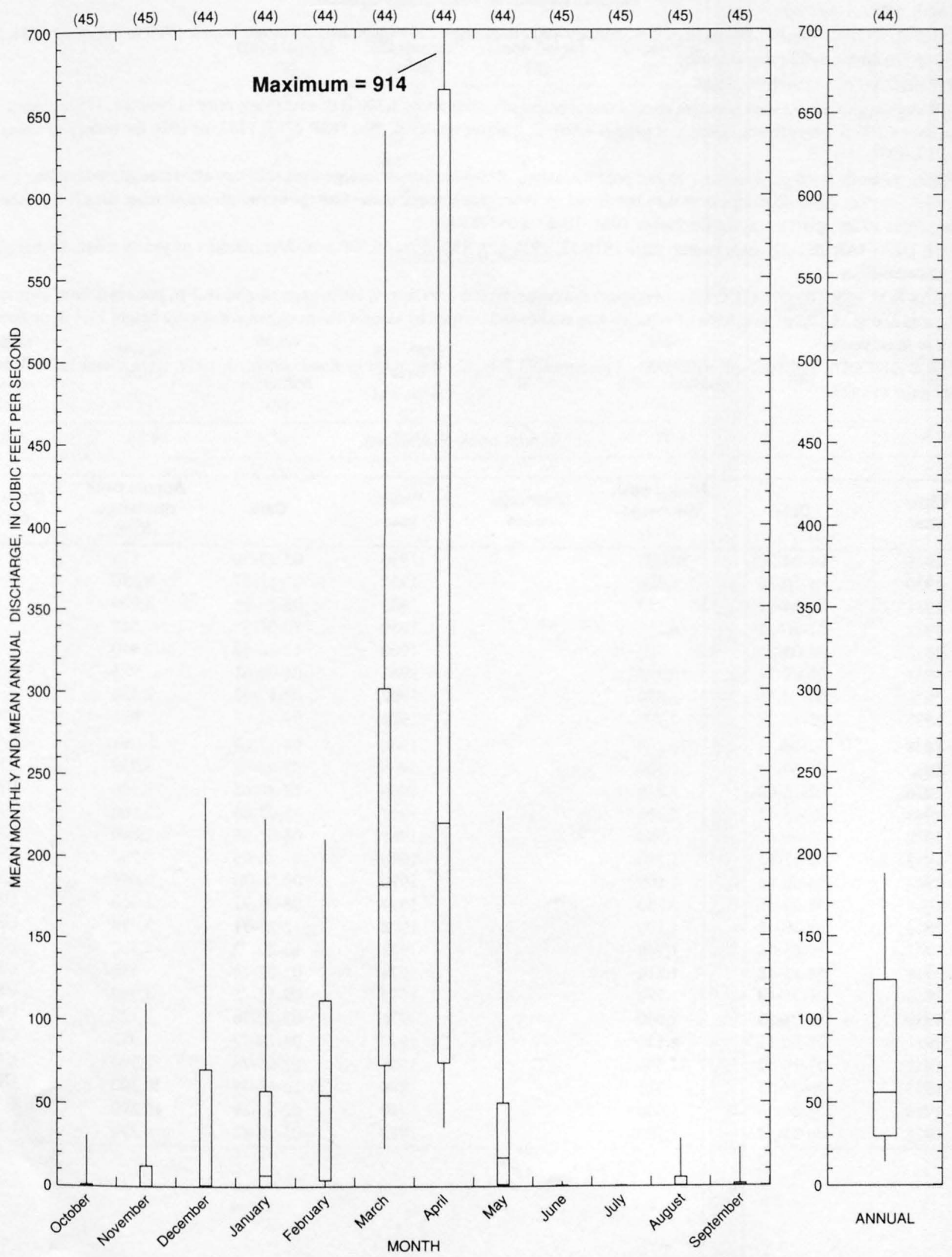


\section{CLEAR CREEK NEAR WINSLOW, AZ}

LOCATION.--Lat $34^{\circ} 58^{\prime} 10^{\prime \prime}$, long $110^{\circ} 38^{\prime} 40^{\prime \prime}$, in SE $1 / 4 \mathrm{SE}^{1} / 4$ sec.9, T.18 N., R.16 E., Navajo County, Hydrologic Unit 15020008 , on right bank 10 $\mathrm{ft}$ downstream from bridge on State Highway 99, $1.5 \mathrm{mi}$ upstream from mouth, and 5 mi southeast of Winslow.

DRAINAGE AREA.--607 $\mathrm{mi}^{2}$.

PERIOD OF RECORD.--June to December 1906, January 1907 to January 1909 (gage heights only), March 1929 to February 1934 , September 1935 to December 1982 (discontinued).

REVISED RECORDS.--WSP 859: 1929.

GAGE.--Water-stage recorder with concrete control and submerged orifice about 3,300 ft downstream; prior to Nov. 20, 1982, control was diversion dam 1,200 ft downstream. Datum of gage is 4,861.32 ft above sea level. See WSP 1713,1733 , or 1926 for history of changes prior to July 10, 1931.

REMARKS.--Records good prior to Nov. 20 and poor thereafter. Records show discharge over spillway and through outlet tube. Prior to Nov. 20, 1982, records show discharge over dam but do not show leakage through dam. Storage in and diversion from Blue Ridge Reservoir near Pine, about 50 mi upstream, since December 1964. (See sta 09398300.)

AVERAGE DISCHARGE.--51 years (water years 1930-33, 1936-82), $83.5 \mathrm{ft}^{3} / \mathrm{s}, 60,500 \mathrm{acre-ft/yr}$; median of yearly mean discharges, $60 \mathrm{ft}^{3} / \mathrm{s}$ 43,500 acre-ft/yr.

EXTREMES FOR PERIOD OF RECORD.--Maximum discharge, 50,000 $\mathrm{ft}^{3} / \mathrm{s} \mathrm{Apr.} \mathrm{4,} \mathrm{1929,} \mathrm{gage} \mathrm{height,} 18.1 \mathrm{ft}$, present datum, from rating curve extended above $13,500 \mathrm{ft}^{3} / \mathrm{s}$ on basis of velocity-area studies and verified by slope-area measurement at gage height $13.4 \mathrm{ft}$; no flow for many days in most years.

EXTREMES OUTSIDE PERIOD OF RECORD.--Floodmarks $3 \mathrm{ft}$ higher than stage of flood of Apr. 4, 1929, were found 1,850 $\mathrm{ft}$ downstream from gage in 1929.

Annual peak discharges

\begin{tabular}{|c|c|c|c|c|c|c|c|}
\hline $\begin{array}{l}\text { Water } \\
\text { year }\end{array}$ & Date & $\begin{array}{c}\text { Annual peak } \\
\text { discharge } \\
\left(\mathrm{ft}^{3} / \mathrm{s}\right)\end{array}$ & $\begin{array}{l}\text { Discharge } \\
\text { codes }\end{array}$ & $\begin{array}{l}\text { Water } \\
\text { year }\end{array}$ & Date & $\begin{array}{l}\text { Annual peak } \\
\text { discharge } \\
\left(\mathrm{ft}^{3} / \mathrm{s}\right)\end{array}$ & $\begin{array}{c}\text { Discharge } \\
\text { codes }\end{array}$ \\
\hline 1929 & 04-04-29 & 50,000 & & 1956 & $03-27-56$ & 173 & \\
\hline 1930 & $04-10-30$ & 1,080 & & 1957 & $01-11-57$ & 9,150 & \\
\hline 1931 & $03-24-31$ & 850 & & 1958 & $03-23-58$ & 2,920 & \\
\hline 1932 & $02-10-32$ & 6,100 & & 1959 & $10-01-58$ & 542 & \\
\hline 1933 & $04-05-33$ & 780 & & 1960 & $12-26-59$ & 2,440 & \\
\hline 1934 & $00-00-34$ & 6,300 & & 1961 & $04-06-61$ & 925 & \\
\hline 1936 & $04-14-36$ & 1,680 & & 1962 & $02-13-62$ & 2,330 & \\
\hline 1937 & $03-17-37$ & 2,790 & & 1963 & $09-02-63$ & 881 & \\
\hline 1938 & $03-04-38$ & 26,200 & & 1964 & $04-17-64$ & 1,060 & \\
\hline 1939 & 04-04-39 & 1,500 & & 1965 & $01-08-65$ & 5,930 & UR \\
\hline 1940 & $08-15-40$ & 1,840 & & 1966 & $12-30-65$ & 18,500 & UR \\
\hline 1941 & $03-15-41$ & 3,300 & & 1967 & $12-07-66$ & 12,500 & UR \\
\hline 1942 & $04-06-42$ & 1,940 & & 1968 & $04-02-68$ & 1,840 & UR \\
\hline 1943 & $03-11-43$ & 1,500 & & 1969 & $01-26-69$ & 5,700 & UR \\
\hline 1944 & $04-08-44$ & 1,500 & & 1970 & $09-06-70$ & 9,650 & UR \\
\hline 1945 & $04-22-45$ & 2,230 & & 1971 & $08-05-71$ & 1,460 & UR \\
\hline 1946 & $09-20-46$ & 1,100 & & 1972 & $12-27-71$ & 5,480 & UR \\
\hline 1947 & $11-25-46$ & 1,740 & & 1973 & $10-20-72$ & 9,350 & UR \\
\hline 1948 & $04-13-48$ & 1,810 & & 1974 & $03-22-74$ & 538 & UR \\
\hline 1949 & $04-16-49$ & 1,970 & & 1975 & $09-12-75$ & 3,940 & UR \\
\hline 1950 & $03-01-50$ & 1,000 & & 1976 & $02-10-76$ & 2,120 & UR \\
\hline 1951 & $08-30-51$ & 8,530 & & 1977 & $04-10-77$ & 372 & UR \\
\hline 1952 & $01-19-52$ & 22,500 & & 1978 & $03-01-78$ & 12,900 & UR \\
\hline 1953 & $08-27-53$ & 695 & & 1979 & $12-19-78$ & 36,300 & UR \\
\hline 1954 & $03-24-54$ & 5,800 & & 1980 & $02-20-80$ & 10,800 & UR \\
\hline 1955 & $08-25-55$ & 1,080 & & 1982 & $03-12-82$ & 6,330 & UR \\
\hline
\end{tabular}


09399000 CLEAR CREEK NEAR WINSLOW, AZ--Continued

Discharge rating table developed October 1983

\begin{tabular}{cccr}
\hline $\begin{array}{c}\text { Gage height } \\
(\mathbf{f t})\end{array}$ & $\begin{array}{c}\text { Discharge } \\
\left(\mathrm{ft}^{\mathbf{3}} \mathbf{s}\right)\end{array}$ & $\begin{array}{c}\text { Gage height } \\
(\mathbf{f t})\end{array}$ & $\begin{array}{c}\text { Discharge } \\
\left(\mathrm{ft}^{\mathbf{3}} \mathbf{s}\right)\end{array}$ \\
\hline 8.8 & 2.0 & 10.0 & 519 \\
9.2 & 12.5 & 10.3 & 933 \\
9.4 & 51.7 & 10.5 & 1,200 \\
9.7 & 208 & 10.7 & 1,500 \\
8.8 & 2.0 & 10.0 & 519 \\
\hline
\end{tabular}

Basin characteristics

\begin{tabular}{cccccccc}
\hline $\begin{array}{c}\text { Main } \\
\text { channel } \\
\text { slope } \\
(\mathrm{ft} / \mathrm{mi})\end{array}$ & $\begin{array}{c}\text { Stream } \\
\text { length } \\
\text { (mi) }\end{array}$ & $\begin{array}{c}\text { Mean } \\
\text { basin } \\
\text { elevation } \\
(\mathrm{ft})\end{array}$ & $\begin{array}{c}\text { Forested } \\
\text { area } \\
\text { (percent) }\end{array}$ & $\begin{array}{c}\text { Soil } \\
\text { index }\end{array}$ & $\begin{array}{c}\text { Mean } \\
\text { annual } \\
\text { precipitation } \\
\text { (in) }\end{array}$ & $\begin{array}{c}\text { Rainfall intensity, 24-hour } \\
\text { (in) }\end{array}$ & $\begin{array}{c}50-y e a r \\
\text { (in) }\end{array}$ \\
\hline 24.1 & 85.9 & 6,500 & 66.0 & 3.0 & 18.7 & 2.1 \\
\hline
\end{tabular}


09399000 CLEAR CREEK NEAR WINSLOW, AZ--Continued

MEAN MONTHLY AND ANNUAL DISCHARGES $1930 \cdot 33,1936 \cdot 78,1980 \cdot 82$

\begin{tabular}{|c|c|c|c|c|c|c|}
\hline MONTH & $\begin{array}{l}\text { MAXIMUM } \\
\text { (FT3/S) }\end{array}$ & $\begin{array}{l}\text { MINIMUM } \\
\text { (FT3/S) }\end{array}$ & $\begin{array}{c}\text { MEAN } \\
(\mathrm{FT} 3 / \mathrm{S})\end{array}$ & $\begin{array}{l}\text { STAN- } \\
\text { DARD } \\
\text { DEVIA- } \\
\text { TION } \\
\text { (FT3/S) }\end{array}$ & $\begin{array}{l}\text { COEFFI- } \\
\text { CIENT OF } \\
\text { VARI- } \\
\text { ATION }\end{array}$ & $\begin{array}{c}\text { PERCENT } \\
\text { OF } \\
\text { ANNUAL } \\
\text { RUNOFF }\end{array}$ \\
\hline OCTOBER & 348 & 0.00 & 13 & 52 & 4.2 & 1.3 \\
\hline NOVEMBER & 174 & 0.00 & 15 & 34 & 2.3 & 1.6 \\
\hline DECEMBER & 480 & 0.00 & 38 & 98 & 2.6 & 4.0 \\
\hline JANUARY & 784 & 0.00 & 54 & 142 & 2.6 & 5.7 \\
\hline FEBRUARY & 780 & 0.00 & 87 & 162 & 1.9 & 9.1 \\
\hline MARCH & 1,250 & 0.00 & 256 & 277 & 1.1 & 26.8 \\
\hline APRIL & 1,290 & 0.00 & 372 & 344 & 0.92 & 39.0 \\
\hline MAY & 1,090 & 0.00 & 93 & 190 & 2.1 & 9.7 \\
\hline JUNE & 25 & 0.00 & 1.5 & 4.0 & 2.7 & 0.2 \\
\hline JULY & 18 & 0.00 & 1.7 & 3.3 & 2.0 & 0.2 \\
\hline AUGUST & 207 & 0.00 & 11 & 37 & 3.3 & 1.2 \\
\hline SEPTEMBER & 192 & 0.00 & 12 & 34 & 2.9 & 1.2 \\
\hline ANNUAL & 271 & 6.5 & 79 & 65 & 0.82 & 100 \\
\hline
\end{tabular}

MAGNITUDE AND PROBABILITY OF INSTANTANEOUS PEAK FLOW BASED ON PERIOD OF RECORD $1929 \cdot 34,1936 \cdot 64,1965.82$

DISCHARGE, IN FT3/S, FOR INDICATED RECURRENCE INTERVAL IN YEARS, AND EXCEEDANCE PROBABILITY, IN PERCENT

\begin{tabular}{|c|c|c|c|c|c|}
\hline 2 & 5 & 10 & 25 & 50 & 100 \\
\hline 508 & 208 & 108 & 48 & 28 & 18 \\
\hline
\end{tabular}

$2,790 \quad 7,750 \quad 13,700 \quad 26,000 \quad 39,800 \quad 59,200$

WEIGHTED SKEW $($ LOGS $)=0.33$

MEAN $\quad$ (LOGS) $=3.47$

STANDARD DEV. $($ LOGS $)=0.51$
MAGNITUDE AND PROBABILITY OF ANNUAL LOW FLOW BASED ON PERIOD OF RECORD $1930 \cdot 33,1937 \cdot 78,1980 \cdot 82$

\begin{tabular}{|c|c|c|c|c|c|c|}
\hline \multirow{4}{*}{$\begin{array}{l}\text { PERIOD } \\
\text { (CON- } \\
\text { SECU- } \\
\text { TIVE } \\
\text { DAYS) }\end{array}$} & \multicolumn{6}{|c|}{$\begin{array}{l}\text { DISCHARGE, IN FT3/S, FOR INDICATED } \\
\text { RECURRENCE INTERVAL, IN YEARS, AND } \\
\text { NON-EXCEEDANCE PROBABILITY, IN PERCENT }\end{array}$} \\
\hline & $\ldots$ & …... & & & & $\ldots \ldots$ \\
\hline & 2 & 5 & $10 \#$ & $20 \#$ & $50 \#$ & $100=$ \\
\hline & 508 & $20 \%$ & 108 & 58 & 28 & 18 \\
\hline 1 & 0.00 & 0.00 & 0.00 & 0.00 & 0.00 & 0.00 \\
\hline 3 & 0.00 & 0.00 & 0.00 & 0.00 & 0.00 & 0.00 \\
\hline 7 & 0.00 & 0.00 & 0.00 & 0.00 & 0.00 & 0.00 \\
\hline 14 & 0.00 & 0.00 & 0.00 & 0.00 & 0.00 & 0.00 \\
\hline 30 & 0.00 & 0.00 & 0.00 & 0.00 & 0.00 & 0.00 \\
\hline 60 & 0.00 & 0.00 & 0.00 & 0.00 & 0.00 & 0.00 \\
\hline 90 & 0.04 & 0.00 & 0.00 & 0.00 & 0.00 & 0.00 \\
\hline 120 & 0.36 & 0.00 & 0.00 & 0.00 & 0.00 & 0.00 \\
\hline 183 & 0.84 & 0.05 & 0.00 & 0.00 & 0.00 & 0.00 \\
\hline
\end{tabular}

MAGNITUDE AND PROBABILITY OF ANNUAL HIGH FLOW BASED ON PERIOD OF RECORD $1930 \cdot 33,1936 \cdot 78,1980 \cdot 82$

\begin{tabular}{|c|c|c|c|c|c|c|}
\hline $\begin{array}{l}\text { PERIOD } \\
\text { (CON- }\end{array}$ & & $\begin{array}{l}\text { DISCHAR } \\
\text { RECURRE } \\
\text { EXCEEDA }\end{array}$ & $\begin{array}{ll}\text { 8, } & \text { IN F } \\
\text { CE } & \text { INTE } \\
\text { EE } & \text { PROB }\end{array}$ & $\begin{array}{l}\text { /S, FOP } \\
\text { AL, IN } \\
\text { ILITY, }\end{array}$ & $\begin{array}{l}\text { INDICA } \\
\text { YEARS, } \\
\text { IN PERC }\end{array}$ & $\begin{array}{l}\text { TED } \\
\text { AND } \\
\text { ENT }\end{array}$ \\
\hline SECU. & $\cdots$ & ........ & 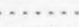 & $\ldots \ldots \ldots$ & $\ldots \ldots$ & …. \\
\hline TIVE & 2 & 5 & $10 \#$ & 25\# & $50 \#$ & $100 \#$ \\
\hline DAYS) & 508 & 208 & 108 & 48 & 28 & 18 \\
\hline 1 & 1,520 & 3,650 & 5,760 & 9.370 & 12,800 & 17,000 \\
\hline 3 & 1,200 & 2,440 & 3,420 & 4,780 & 5,850 & 6,960 \\
\hline 7 & 900 & 1,660 & 2,160 & 2,750 & 3,140 & 3,490 \\
\hline 15 & 637 & 1,160 & 1,490 & 1,880 & 2,140 & 2,380 \\
\hline 30 & 446 & 855 & 1,140 & 1,490 & 1,740 & 1,980 \\
\hline 60 & 282 & 577 & 796 & 1.080 & 1,300 & 1,510 \\
\hline 90 & 209 & 446 & 633 & 886 & 1,080 & 1,280 \\
\hline
\end{tabular}

DURATION TABLE OF DAILY MEAN FLOW FOR PERIOD OF RECORD $1930-33,1936-78,1980-82$

DISCHARGE, IN FT3/S, WHICH WAS EQUALED OR EXCEEDED FOR INDICATED PERCENT OF TIME

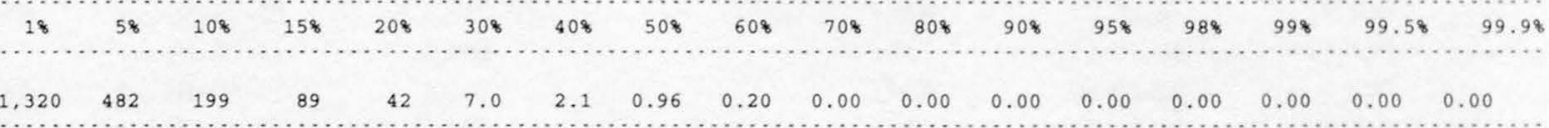

\# Reliability of values in column is uncertain, and potential errors are large. 


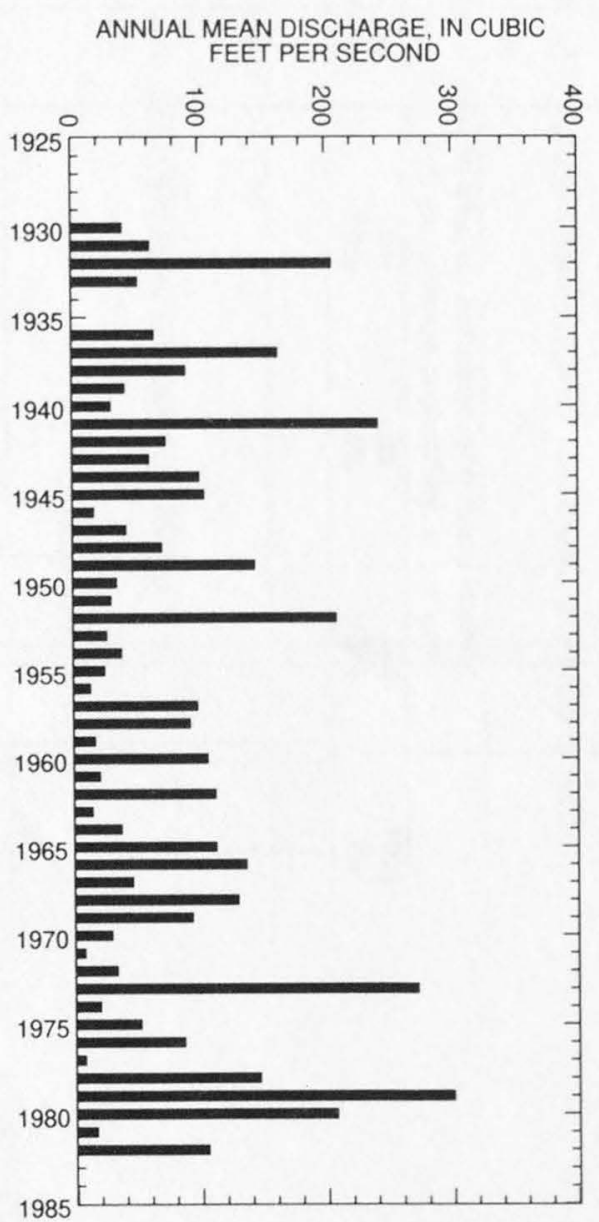

ANNUAL PEAK DISCHARGE, IN CUBIC FET PER SECOND

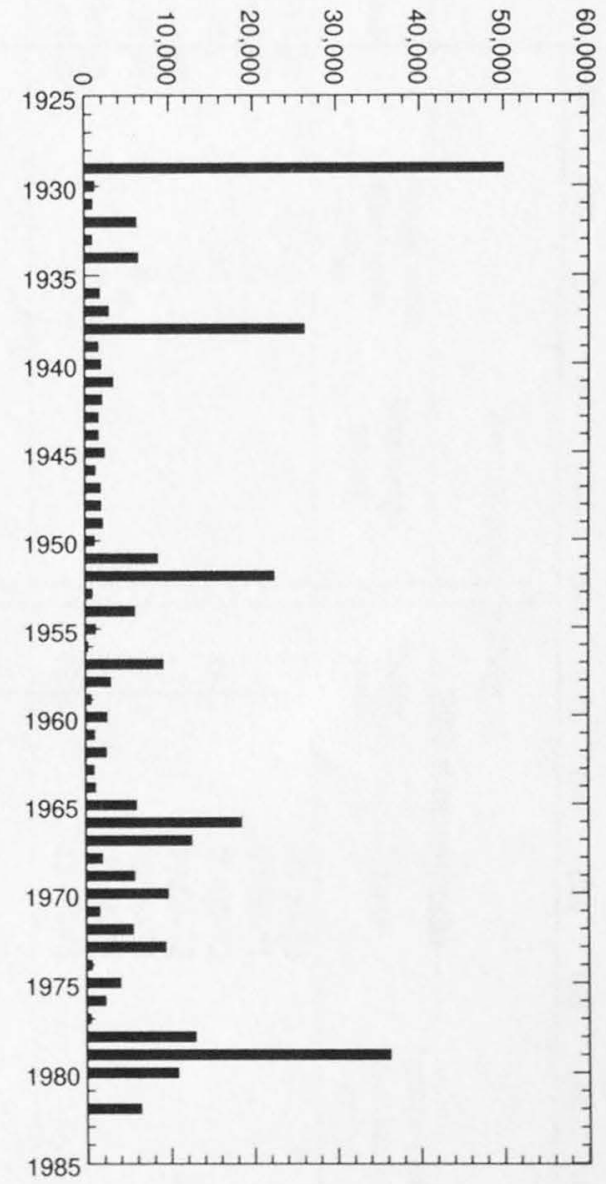


09399000 CLEAR CREEK NEAR WINSLOW, AZ--Continued
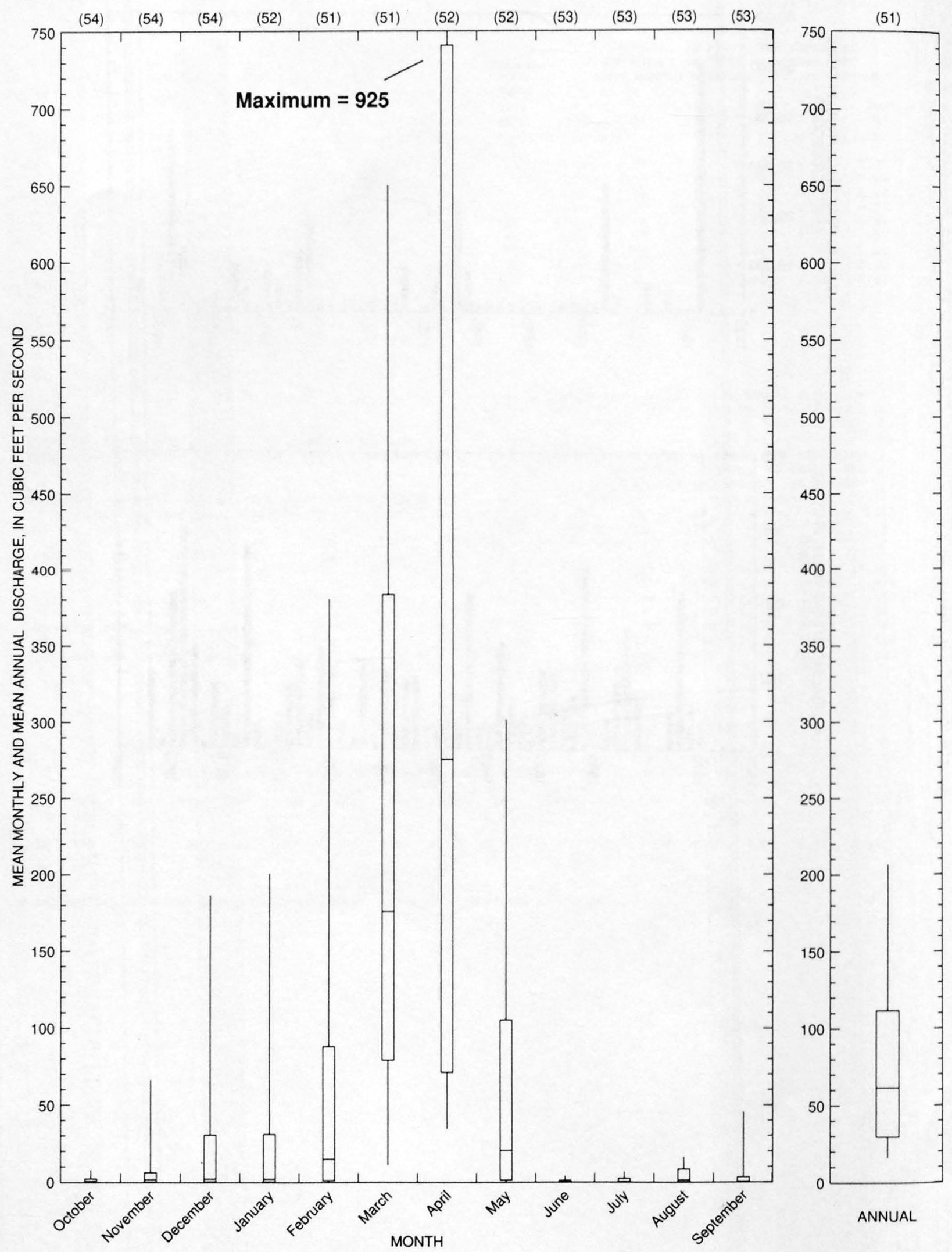
09399250 JACKS CANYON TRIBUTARY NO. 2 NEAR WINSLOW, AZ

LOCATION.--Lat 34 $45^{\prime} 56^{\prime \prime}$, long $111^{\circ} 00^{\prime} 44^{\prime \prime}$, in $\mathrm{NE}^{1} /{ }_{4} \mathrm{NW}^{1} / 4$, sec.19, T.16 N., R.13 E., Coconino County, Hydrologic Unit 15020008 , at State Highway 87, 27 mi soutwest of Winslow.

DRAINAGE AREA.--31.8 $\mathrm{mi}^{2}$.

Annual peak discharges

\begin{tabular}{|c|c|c|c|c|c|c|c|}
\hline $\begin{array}{l}\text { Water } \\
\text { year }\end{array}$ & Date & $\begin{array}{c}\text { Annual peak } \\
\text { discharge } \\
\left(\mathrm{ft}^{3} / \mathrm{s}\right)\end{array}$ & $\begin{array}{l}\text { Discharge } \\
\text { codes }\end{array}$ & $\begin{array}{l}\text { Water } \\
\text { year }\end{array}$ & Date & $\begin{array}{c}\text { Annual peak } \\
\text { discharge } \\
\left(\mathrm{ft}^{3} / \mathrm{s}\right)\end{array}$ & $\begin{array}{c}\text { Discharge } \\
\text { codes }\end{array}$ \\
\hline 1963 & $08-21-63$ & 9,330 & & 1970 & $00-00-70$ & 0 & \\
\hline 1964 & $00-00-64$ & 0 & & 1971 & $07-00-71$ & 67 & \\
\hline 1965 & $07-10-65$ & 544 & & 1972 & $00-00-72$ & 0 & \\
\hline 1966 & $00-00-66$ & 0 & & 1973 & $10-00-72$ & 2.0 & LT \\
\hline 1967 & $00-00-67$ & 0 & & 1974 & $00-00-74$ & 0 & \\
\hline 1968 & $00-00-68$ & 0 & & 1975 & $00-00-75$ & $\left({ }^{1}\right)$ & \\
\hline 1969 & $00-00-69$ & 0 & & 1979 & $12-18-78$ & ${ }^{2} 67$ & HP \\
\hline
\end{tabular}

${ }^{\top}$ Discharge unknown.

${ }^{2}$ Highest since 1976 .

Magnitude and probability of instantaneous peak flow based on period of record

\begin{tabular}{cccccc}
\hline \multicolumn{5}{c}{$\begin{array}{l}\text { Discharge, in } \mathrm{ft}^{3} / \mathrm{s} \text {, for indicated recurrence interval } \\
\text { in years, and exceedance probablility, in percent }\end{array}$} \\
\hline 2 & 5 & 10 & 25 & $50 \dagger$ & $100 \dagger$ \\
$50 \%$ & $20 \%$ & $10 \%$ & $4 \%$ & $2 \%$ & $1 \%$ \\
\hline & & & & & \\
Weighted skew & $(\operatorname{logs})=$ & --- & & \\
Mean & $(\operatorname{logs})=$ & --- & & & \\
Standard dev. & $(\operatorname{logs})=$ & -- & & & \\
\hline
\end{tabular}

FReliability of values in column is uncertain, and potential errors are large.

Basin characteristics

\begin{tabular}{cccccccc}
\hline $\begin{array}{c}\text { Main } \\
\text { channel } \\
\text { slope } \\
(\mathrm{ft} / \mathrm{mi})\end{array}$ & $\begin{array}{c}\text { Stream } \\
\text { length } \\
(\mathrm{mi})\end{array}$ & $\begin{array}{c}\text { Mean } \\
\text { basin } \\
\text { elevation } \\
(\mathrm{ft})\end{array}$ & $\begin{array}{c}\text { Forested } \\
\text { area } \\
\text { (percent) }\end{array}$ & $\begin{array}{c}\text { Soil } \\
\text { index }\end{array}$ & $\begin{array}{c}\text { Mean } \\
\text { annual } \\
\text { precipitation } \\
\text { (in) }\end{array}$ & $\begin{array}{c}\text { Rainfall intensity, 24-hour } \\
\text { (in) }\end{array}$ & $\begin{array}{c}50 \text {-year } \\
\text { (in) }\end{array}$ \\
\hline 71.6 & 10.8 & 6,530 & 71.0 & 3.0 & 19.2 & 2.1 \\
\hline
\end{tabular}


09399250 JACKS CANYON TRIBUTARY NO. 2 NEAR WINSLOW, AZ--Continued

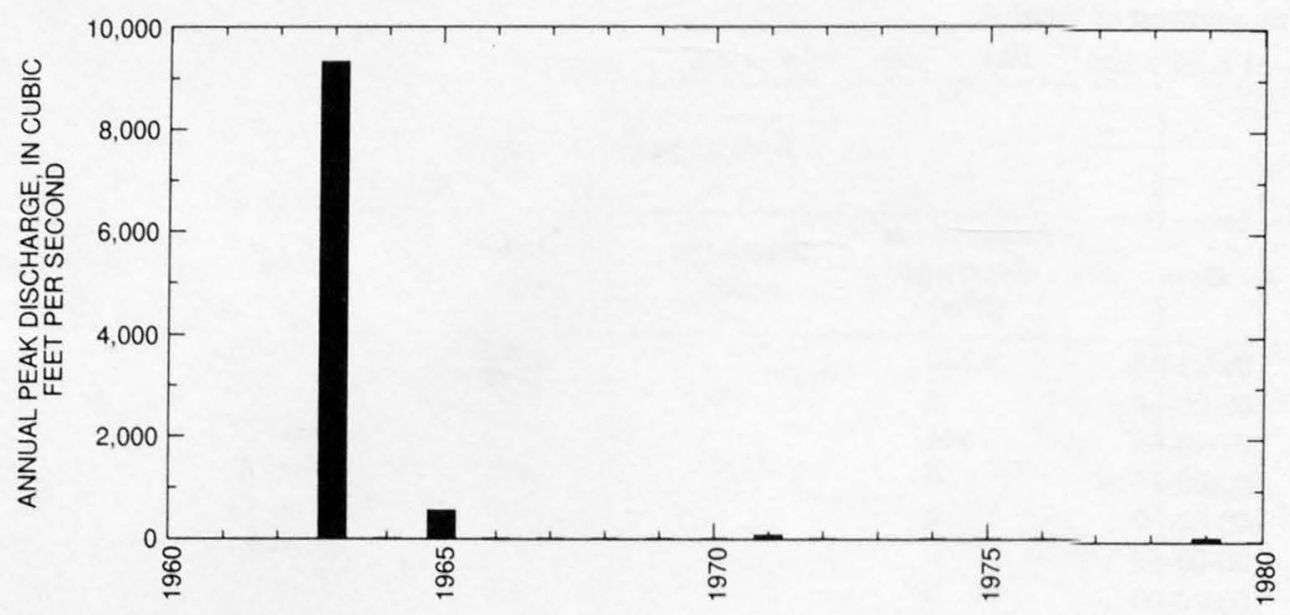


09400100 GANADO WASH TRIBUTARY NEAR GANADO, AZ

LOCATION.--Lat 35'42'40", long 109'29'50", Apache County, Hydrologic Unit 150200011, at State Highway 264, 2.4 mi east of Ganado. DRAINAGE AREA.--7.85 $\mathrm{mi}^{2}$.

Annual peak discharges

\begin{tabular}{cccccccc}
\hline $\begin{array}{c}\text { Water } \\
\text { year }\end{array}$ & Date & $\begin{array}{c}\text { Annual peak } \\
\text { discharge } \\
\left(\mathbf{f t}^{\mathbf{3}} \mathbf{s}\right)\end{array}$ & $\begin{array}{c}\text { Discharge } \\
\text { codes }\end{array}$ & $\begin{array}{c}\text { Water } \\
\text { year }\end{array}$ & Date & $\begin{array}{c}\text { Annual peak } \\
\text { discharge } \\
\left(\mathbf{f t}^{3} / \mathbf{s}\right)\end{array}$ & $\begin{array}{c}\text { Discharge } \\
\text { codes }^{-}\end{array}$ \\
\hline 1963 & $08-09-63$ & 231 & & 1970 & $09-06-70$ & 1.0 & ES \\
1964 & $08-26-64$ & 620 & & 1971 & $08-30-71$ & 35 & ES \\
1965 & $07-17-65$ & 1,680 & & 1972 & $06-00-72$ & 2.0 & \\
1966 & $00-00-66$ & 150 & & 1973 & $00-00-73$ & 287 & \\
1967 & $09-00-67$ & 456 & & 1974 & $07-16-74$ & 548 & \\
1968 & $00-00-68$ & 280 & & 1975 & $07-12-75$ & 418 & \\
1969 & $07-26-69$ & 10 & ES & 1976 & $00-00-76$ & 0 & \\
\hline
\end{tabular}

Magnitude and probability of instantaneous peak flow based on period of record 1963-76

\begin{tabular}{|c|c|c|c|c|c|}
\hline \multicolumn{6}{|c|}{$\begin{array}{l}\text { Discharge, in } \mathrm{ft}^{3} / \mathrm{s} \text {, for indicated recurrence interval } \\
\text { in years, and exceedance probablility, in percent }\end{array}$} \\
\hline 2 & 5 & 10 & 25 & $50 \dagger$ & $100 \dagger$ \\
\hline $50 \%$ & $20 \%$ & $10 \%$ & $4 \%$ & $2 \%$ & $1 \%$ \\
\hline 238 & 533 & 819 & 1,300 & 1,760 & 2,320 \\
\hline Weighted skew & $(\log s)=$ & 0.10 & & & \\
\hline Mean & $(\log s)=$ & 2.38 & & & \\
\hline Standard dev. & $(\log s)=$ & 0.41 & & & \\
\hline
\end{tabular}

Basin characteristics

\begin{tabular}{cccccccc}
\hline $\begin{array}{c}\text { Main } \\
\text { channel } \\
\text { slope } \\
(\mathrm{ft} / \mathrm{mi})\end{array}$ & $\begin{array}{c}\text { Stream } \\
\text { length } \\
(\mathrm{mi})\end{array}$ & $\begin{array}{c}\text { Mean } \\
\text { basin } \\
\text { elevation } \\
(\mathrm{ft})\end{array}$ & $\begin{array}{c}\text { Forested } \\
\text { area } \\
\text { (percent) }\end{array}$ & $\begin{array}{c}\text { Soil } \\
\text { index }\end{array}$ & $\begin{array}{c}\text { Mean } \\
\text { annual } \\
\text { precipitation } \\
\text { (in) }\end{array}$ & $\begin{array}{c}\text { Rainfall intensity, 24-hour } \\
\text { (in) }\end{array}$ & $\begin{array}{c}50 \text {-year } \\
\text { (in) }\end{array}$ \\
\hline 55.5 & 4.1 & 6,770 & 6.8 & 3.0 & 11.8 & 1.4 \\
\hline
\end{tabular}

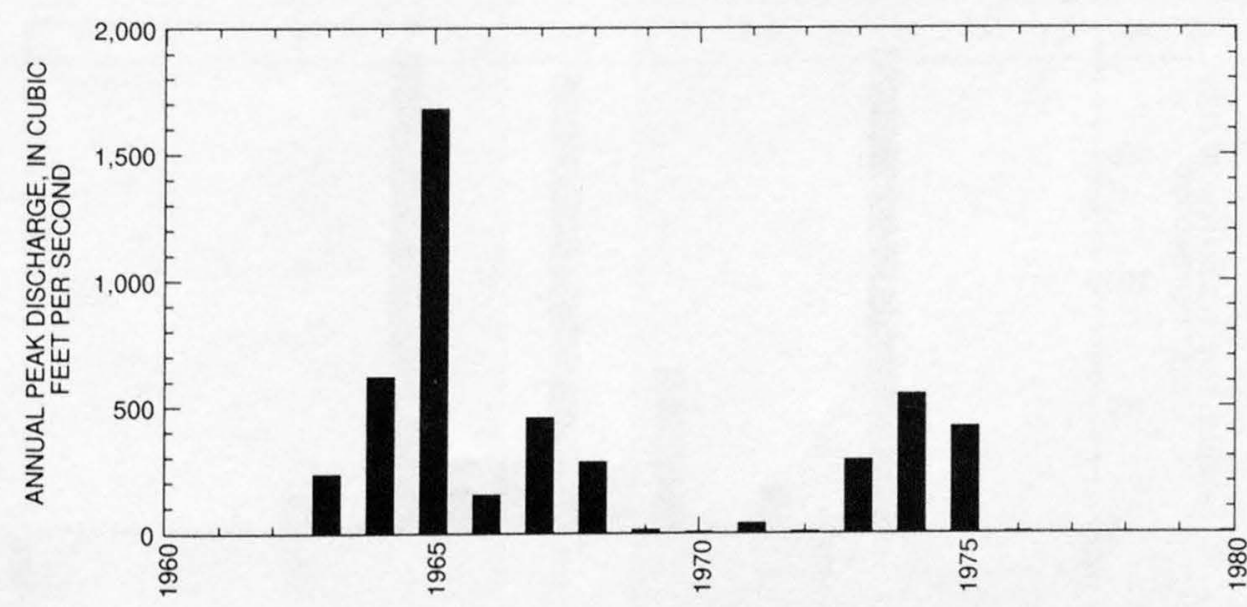




\section{STEAMBOAT WASH TRIBUTARY NEAR GANADO, AZ}

LOCATION.--Lat 35 45'50", long 109 48'00", Apache County, Hydrologic Unit 150200011, at State Highway 264, 15 mi west of Ganado DRAINAGE AREA.--0.32 $\mathrm{mi}^{2}$.

Annual peak discharges

\begin{tabular}{|c|c|c|c|c|c|c|c|}
\hline $\begin{array}{l}\text { Water } \\
\text { year }\end{array}$ & Date & $\begin{array}{c}\text { Annual peak } \\
\text { discharge } \\
\left(\mathrm{ft}^{3} / \mathrm{s}\right)\end{array}$ & $\begin{array}{l}\text { Discharge } \\
\text { codes }\end{array}$ & $\begin{array}{l}\text { Water } \\
\text { year }\end{array}$ & Date & $\begin{array}{c}\text { Annual peak } \\
\text { discharge } \\
\left(\mathrm{ft}^{3} / \mathrm{s}\right)\end{array}$ & $\begin{array}{l}\text { Discharg: } \\
\text { codes }\end{array}$ \\
\hline 1963 & $09-00-63$ & 1.0 & & 1970 & $09-05-70$ & 360 & \\
\hline 1964 & $08-13-64$ & 383 & & 1971 & $10-02-70$ & 55 & \\
\hline 1965 & $09-00-65$ & 1.0 & & 1972 & $06-00-72$ & 35 & \\
\hline 1966 & $08-00-66$ & 35 & & 1973 & $10-19-72$ & 380 & \\
\hline 1967 & $09-06-67$ & 5.0 & & 1974 & $08-00-74$ & 5.0 & \\
\hline 1968 & $08-00-68$ & 130 & & 1975 & $09-07-75$ & 28 & \\
\hline 1969 & $07-19-69$ & 49 & & & & & \\
\hline
\end{tabular}

Magnitude and probability of instantaneous peak flow based on period of record 1963-75

\begin{tabular}{|c|c|c|c|c|c|}
\hline \multicolumn{6}{|c|}{$\begin{array}{l}\text { Discharge, in } \mathrm{ft}^{3} / \mathrm{s} \text {, for indicated recurrence interval } \\
\text { in years, and exceedance probablility, in percent }\end{array}$} \\
\hline 2 & 5 & 10 & 25 & $50 \dagger$ & $100 t$ \\
\hline $50 \%$ & $20 \%$ & $10 \%$ & $4 \%$ & $2 \%$ & $1 \%$ \\
\hline 46.6 & 167 & 322 & 648 & 1,020 & 1,520 \\
\hline Weighted skew & $(\log s)=$ & -0.04 & & & \\
\hline Mean & $(\log s)=$ & 1.66 & & & \\
\hline Standard dev. & $(\log s)=$ & 0.66 & & & \\
\hline
\end{tabular}

Basin characteristics

\begin{tabular}{ccccccc}
\hline $\begin{array}{c}\text { Main } \\
\text { channel } \\
\text { slope } \\
(\mathrm{ft} / \mathrm{mi})\end{array}$ & $\begin{array}{c}\text { Stream } \\
\text { length } \\
\text { (mi) }\end{array}$ & $\begin{array}{c}\text { Mean } \\
\text { basin } \\
\text { elevation } \\
(\mathrm{ft})\end{array}$ & $\begin{array}{c}\text { Forested } \\
\text { area } \\
\text { (percent) }\end{array}$ & $\begin{array}{c}\text { Soil } \\
\text { index }\end{array}$ & $\begin{array}{c}\text { Mean } \\
\text { annual } \\
\text { precipitation } \\
\text { (in) }\end{array}$ & $\begin{array}{c}\text { Rainfall intensity, 24-hour } \\
\text { (in) }\end{array}$ \\
\hline 100 & 1.1 & 6,750 & 72.0 & 1.0 & 12.1 & $\begin{array}{c}50 \text {-year } \\
\text { (in) }\end{array}$ \\
\hline
\end{tabular}

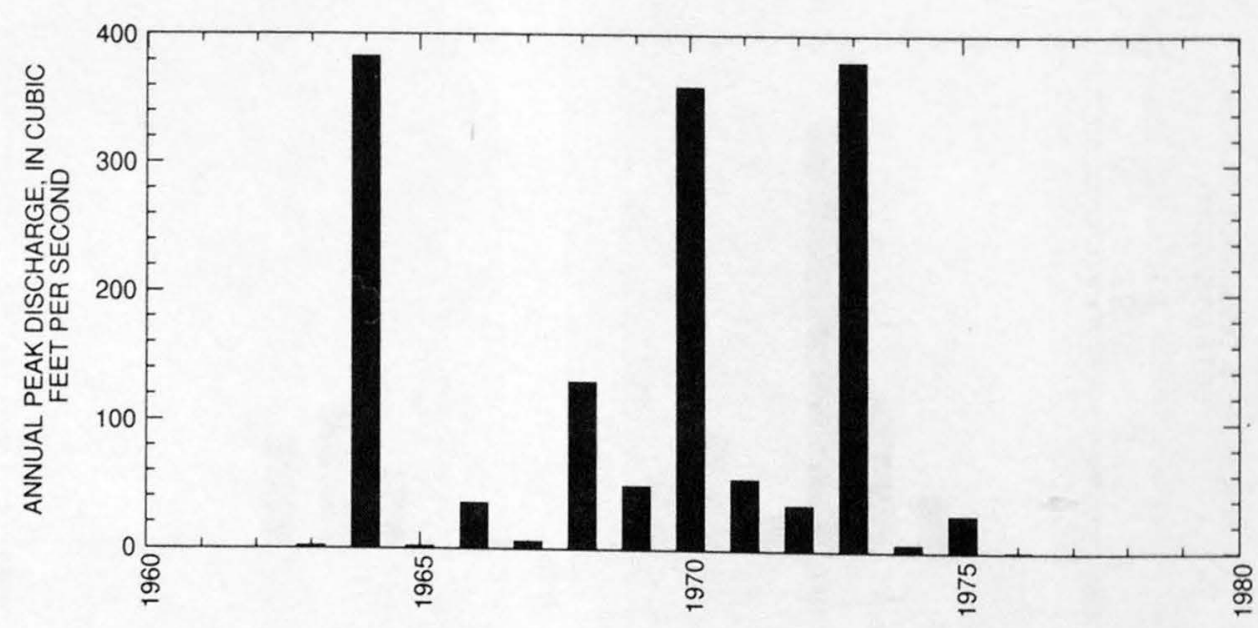


09400290 TESHBITO WASH TRIBUTARY NEAR HOLBROOK, AZ

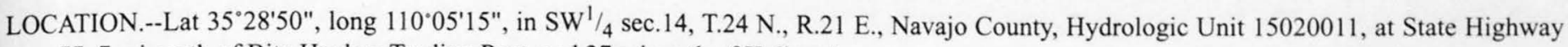

$77,7 \mathrm{mi}$ north of Bita Hochee Trading Post, and $37 \mathrm{mi}$ north of Holbrook.

DRAINAGE AREA.--20.0 $\mathrm{mi}^{2}$, of which $10.7 \mathrm{mi}^{2}$ is noncontributing.

Annual peak discharges

\begin{tabular}{|c|c|c|c|c|c|c|c|}
\hline $\begin{array}{l}\text { Water } \\
\text { year }\end{array}$ & Date & $\begin{array}{c}\text { Annual peak } \\
\text { discharge } \\
\left(\mathrm{ft}^{3} / \mathrm{s}\right)\end{array}$ & $\begin{array}{l}\text { Discharge } \\
\text { codes }\end{array}$ & $\begin{array}{l}\text { Water } \\
\text { year }\end{array}$ & Date & $\begin{array}{l}\text { Annual peak } \\
\text { discharge } \\
\left(\mathrm{ft}^{3} / \mathrm{s}\right)\end{array}$ & $\begin{array}{c}\text { Discharge } \\
\text { codes }\end{array}$ \\
\hline 1960 & $00-00-60$ & $(1)$ & HP & 1970 & $09-05-70$ & 890 & \\
\hline 1963 & $00-00-63$ & 700 & & 1971 & $08-31-71$ & 660 & \\
\hline 1964 & $08-00-64$ & 870 & & 1972 & $09-00-72$ & 30 & ES \\
\hline 1965 & $07-00-65$ & 787 & & 1973 & $10-19-72$ & 740 & \\
\hline 1966 & $08-01-66$ & 100 & & 1974 & $08-00-74$ & 370 & \\
\hline 1967 & $08-30-67$ & 600 & & 1975 & $07-15-75$ & 330 & \\
\hline 1968 & $08-11-68$ & 61 & & 1976 & $00-00-76$ & 160 & \\
\hline 1969 & $09-12-69$ & 640 & & & & & \\
\hline
\end{tabular}

Discharge unknown.

Magnitude and probability of instantaneous peak flow based on period of record 1963-76

\begin{tabular}{|c|c|c|c|c|c|}
\hline \multicolumn{6}{|c|}{$\begin{array}{l}\text { Discharge, in } \mathrm{ft}^{3} / \mathrm{s} \text {, for indicated recurrence interval } \\
\text { in years, and exceedance probablility, in percent }\end{array}$} \\
\hline 2 & 5 & 10 & 25 & $50 t$ & $100 \dagger$ \\
\hline $50 \%$ & $20 \%$ & $10 \%$ & $4 \%$ & $2 \%$ & $1 \%$ \\
\hline 371 & 874 & 1,310 & 1,950 & 2,470 & 3,030 \\
\hline Weighted skew & $(\log s)=$ & -0.46 & & & \\
\hline Mean & $(\log s)=$ & 2.53 & & & \\
\hline Standard dev. & $(\log s)=$ & 0.48 & & & \\
\hline
\end{tabular}

f Reliability of values in column is uncertain, and potential errors are large.

Basin characteristics

\begin{tabular}{cccccccc}
\hline $\begin{array}{c}\text { Main } \\
\text { channel } \\
\text { slope } \\
(\mathrm{ft} / \mathrm{mi})\end{array}$ & $\begin{array}{c}\text { Stream } \\
\text { length } \\
(\mathrm{mi})\end{array}$ & $\begin{array}{c}\text { Mean } \\
\text { basin } \\
\text { elevation } \\
(\mathrm{ft})\end{array}$ & $\begin{array}{c}\text { Forested } \\
\text { area } \\
\text { (percent) }\end{array}$ & $\begin{array}{c}\text { Soil } \\
\text { index }\end{array}$ & $\begin{array}{c}\text { Mean } \\
\text { annual } \\
\text { precipitation } \\
\text { (in) }\end{array}$ & $\begin{array}{c}\text { Rainfall intensity, 24-hour } \\
\text { 2-year } \\
\text { (in) }\end{array}$ & $\begin{array}{c}50 \text {-year } \\
\text { (in) }\end{array}$ \\
\hline 93.7 & 6.4 & 6,420 & 43.0 & 3.0 & 8.2 & 1.3 \\
\hline
\end{tabular}


09400290 TESHBITO WASH TRIBUTARY NEAR HOLBROOK, AZ--Continued

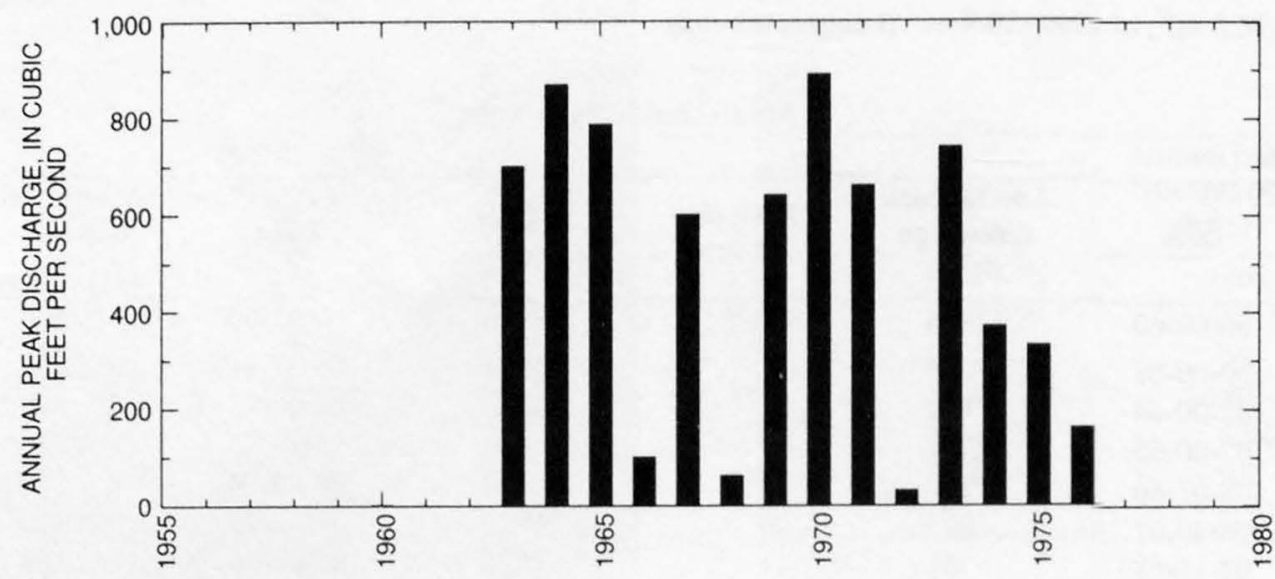


09400300 TESHBITO WASH NEAR HOLBROOK, AZ

LOCATION.--Lat 35²6'55", long $110^{\circ} 04^{\prime} 05^{\prime \prime}$, in NW1/4 sec.36, T.24 N., R.21 E., Navajo County, Hydrologic Unit 15020011 , at State Highway 77, 4.0 mi north of Bita Hochee Trading Post, 35 mi north of Holbrook.

DRAINAGE AREA.--60.3 $\mathrm{mi}^{2}$, of which $10.7 \mathrm{mi}^{2}$ is noncontributing.

Annual peak discharges

\begin{tabular}{cccccrr}
\hline $\begin{array}{c}\text { Water } \\
\text { year }\end{array}$ & Date & $\begin{array}{c}\text { Annual peak } \\
\text { discharge } \\
\left(\mathrm{ft}^{3} / \mathbf{s}\right)\end{array}$ & $\begin{array}{c}\text { Discharge } \\
\text { codes }\end{array}$ & $\begin{array}{c}\text { Water } \\
\text { year }\end{array}$ & $\begin{array}{c}\text { Annual peak } \\
\text { discharge } \\
\left(\mathrm{ft}^{3} / \mathbf{s}\right)\end{array}$ \\
\hline 1963 & $09-06-63$ & 1,220 & & 1970 & $09-05-70$ & $\begin{array}{c}\text { Discharge } \\
\text { codes }\end{array}$ \\
1964 & $08-13-64$ & 514 & & 1971 & $08-00-71$ & 1,280 \\
1965 & $07-20-65$ & 374 & & 1972 & $00-00-72$ & 68 \\
1966 & $08-01-66$ & 1,080 & & 1973 & $10-19-72$ & 628 \\
1967 & $00-00-67$ & 1,580 & & 1974 & $08-00-74$ & 256 \\
1968 & $08-11-68$ & 540 & & 1975 & $07-15-75$ & 700 \\
1969 & $09-12-69$ & 550 & & 1976 & $00-00-76$ & 100 \\
\hline
\end{tabular}

Magnitude and probability of instantaneous peak flow based on period of record 1963-76

\begin{tabular}{|c|c|c|c|c|c|}
\hline \multicolumn{6}{|c|}{$\begin{array}{l}\text { Discharge, in } \mathrm{ft}^{3} / \mathrm{s} \text {, for indicated recurrence interval } \\
\text { in years, and exceedance probablility, in percent }\end{array}$} \\
\hline 2 & 5 & 10 & 25 & $50 \dagger$ & $100 \dagger$ \\
\hline $50 \%$ & $20 \%$ & $10 \%$ & $4 \%$ & $2 \%$ & $1 \%$ \\
\hline 653 & 1,050 & 1,340 & 1,720 & 2,020 & 2,320 \\
\hline Weighted skew & $(\log s)=$ & -0.14 & & & \\
\hline Mean & $(\log s)=$ & 2.81 & & & \\
\hline Standard dev. & $(\log s)=$ & 0.25 & & & \\
\hline
\end{tabular}

f Reliability of values in column is uncertain, and potential errors are large.

\section{Basin characteristics}

\begin{tabular}{ccccccc}
\hline $\begin{array}{c}\text { Main } \\
\text { channel } \\
\text { slope } \\
\text { (ft/mi) }\end{array}$ & $\begin{array}{c}\text { Stream } \\
\text { length } \\
\text { (mi) }\end{array}$ & $\begin{array}{c}\text { Mean } \\
\text { basin } \\
\text { elevation } \\
\text { (ft) }\end{array}$ & $\begin{array}{c}\text { Forested } \\
\text { area } \\
\text { (percent) }\end{array}$ & $\begin{array}{c}\text { Soil } \\
\text { index }\end{array}$ & $\begin{array}{c}\text { Mean } \\
\text { annual } \\
\text { precipitation } \\
\text { (in) }\end{array}$ & $\begin{array}{c}\text { Rainfall intensity, 24-hour } \\
\text { (in) }\end{array}$ \\
\hline 57.7 & 10.4 & 6,280 & 16.0 & 3.0 & 9.2 & $\begin{array}{c}50 \text {-year } \\
\text { (in) }\end{array}$ \\
\hline
\end{tabular}

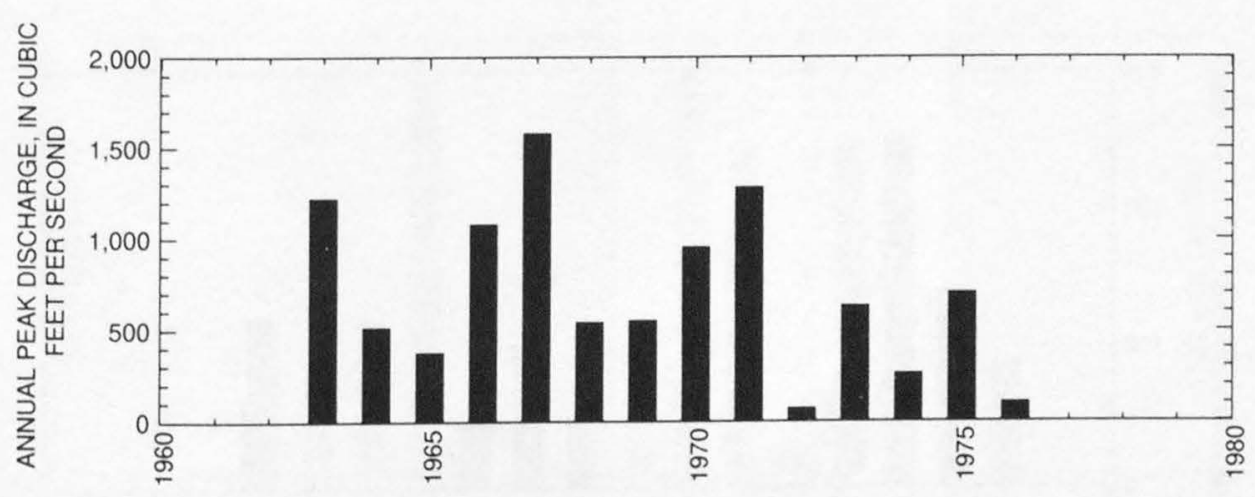


09400530 COW CANYON NEAR WINSLOW, AZ

LOCATION.--Lat 35 06'00", long 110 59'15", in SW $1 / 4$ sec.29, T.20 N., R.13 E., Coconino County, Hydrologic Unit 15020008, at U.S. Hig 66, $17 \mathrm{mi}$ west of Winslow.

DRAINAGE AREA.--7.53 $\mathrm{mi}^{2}$, of which $3.96 \mathrm{mi}^{2}$ is noncontributing.

Annual peak discharges

\begin{tabular}{|c|c|c|c|c|c|c|c|}
\hline $\begin{array}{l}\text { Water } \\
\text { year }\end{array}$ & Date & $\begin{array}{c}\text { Annual peak } \\
\text { discharge } \\
\left(\mathrm{ft}^{3} / \mathrm{s}\right)\end{array}$ & $\begin{array}{c}\text { Discharge } \\
\text { codes }\end{array}$ & $\begin{array}{l}\text { Water } \\
\text { year }\end{array}$ & Date & $\begin{array}{c}\text { Annual peak } \\
\text { discharge } \\
\left(\mathrm{ft}^{3} / \mathrm{s}\right)\end{array}$ & $\begin{array}{l}\text { Discharge } \\
\text { codes }\end{array}$ \\
\hline 1962 & $00-00-62$ & 90 & ES & 1970 & $00-00-70$ & 55 & \\
\hline 1963 & $08-30-63$ & 140 & & 1971 & $08-00-71$ & 102 & \\
\hline 1964 & $07-30-64$ & 253 & & 1972 & $07-16-72$ & 20 & \\
\hline 1965 & $00-00-65$ & 0 & & 1973 & $10-00-72$ & 61 & \\
\hline 1966 & $00-00-66$ & 0.1 & LT & 1974 & $00-00-74$ & 0 & \\
\hline 1967 & $00-00-67$ & 192 & & 1975 & $09-18-75$ & 8.0 & \\
\hline 1968 & $00-00-68$ & 82 & & 1976 & $00-00-76$ & 122 & \\
\hline 1969 & $12-02-68$ & 1.0 & LT & & & & \\
\hline
\end{tabular}

Magnitude and probability of instantaneous peak flow based on period of record 1962-76

\begin{tabular}{|c|c|c|c|c|c|}
\hline \multicolumn{6}{|c|}{$\begin{array}{l}\text { Discharge, in } \mathrm{ft}^{3} / \mathrm{s} \text {, for indicated recurrence interval } \\
\text { in years, and exceedance probablility, in percent }\end{array}$} \\
\hline 2 & 5 & 10 & 25 & $50 t$ & $100 \dagger$ \\
\hline $50 \%$ & $20 \%$ & $10 \%$ & $4 \%$ & $2 \%$ & $1 \%$ \\
\hline 64.8 & 129 & 181 & 257 & 319 & 387 \\
\hline Weighted skew & $(\log s)=$ & -0.23 & & & \\
\hline Mean & $(\log s)=$ & 1.80 & & & \\
\hline Standard dev. & $(\log s)=$ & 0.37 & & & \\
\hline
\end{tabular}

$\mp$ Reliability of values in column is uncertain, and potential errors are large.

Basin characteristics

\begin{tabular}{ccccccc}
\hline $\begin{array}{c}\text { Main } \\
\text { channel } \\
\text { slope } \\
(\mathrm{ft} / \mathrm{mi})\end{array}$ & $\begin{array}{c}\text { Stream } \\
\text { length } \\
(\mathrm{mi})\end{array}$ & $\begin{array}{c}\text { Mean } \\
\text { basin } \\
\text { elevation } \\
(\mathrm{ft})\end{array}$ & $\begin{array}{c}\text { Forested } \\
\text { area } \\
\text { (percent) }\end{array}$ & $\begin{array}{c}\text { Soil } \\
\text { index }\end{array}$ & $\begin{array}{c}\text { Mean } \\
\text { annual } \\
\text { precipitation } \\
\text { (in) }\end{array}$ & $\begin{array}{c}\text { Rainfall intensity, 24-hour } \\
\text { 2-year } \\
\text { (in) }\end{array}$ \\
\hline 61.8 & 4.1 & 5,380 & 0.0 & 3.0 & 10.0 & $\begin{array}{c}50 \text {-year } \\
\text { (in) }\end{array}$ \\
\hline
\end{tabular}

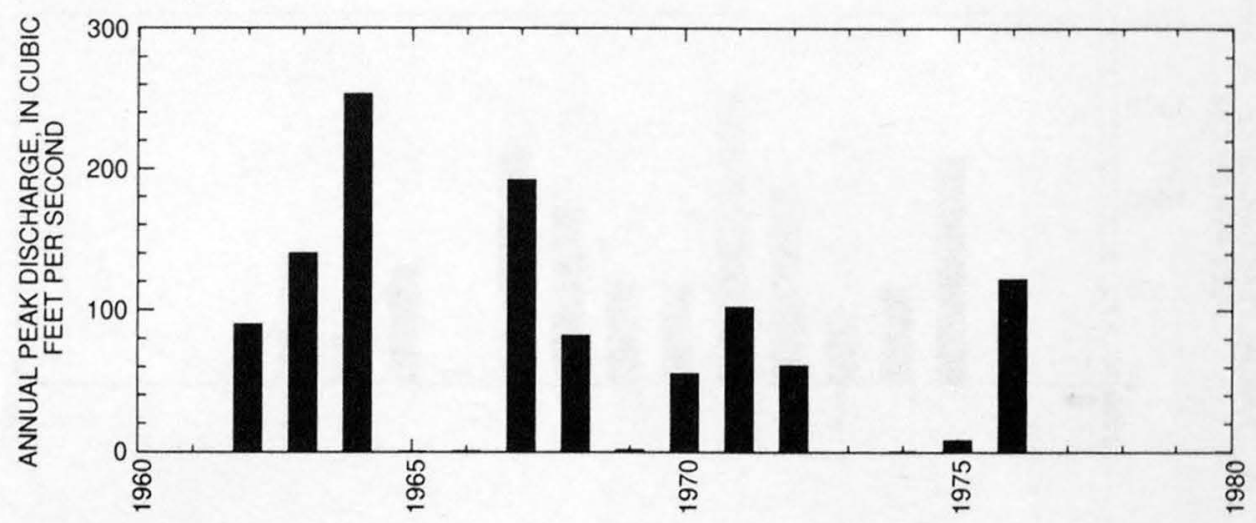


09400560 ORAIBI WASH TRIBUTARY NEAR ORAIBI, AZ

LOCATION.--Lat $35^{\circ} 52^{\prime 2} 20^{\prime \prime}$, long 110 33'20", in SW1/4 sec.31, T.29 N., R.17 E., Navajo County, Hydrologic Unit 15020012 , at State Highway $264,3.5 \mathrm{mi}$ east of Oraibi.

DRAINAGE AREA.--1.78 $\mathrm{mi}^{2}$.

Annual peak discharges

\begin{tabular}{cccccrr}
\hline $\begin{array}{c}\text { Water } \\
\text { year }\end{array}$ & Date & $\begin{array}{c}\text { Annual peak } \\
\text { discharge } \\
\left(\mathbf{f t}^{3} \mathbf{s}\right)\end{array}$ & $\begin{array}{c}\text { Discharge } \\
\text { codes }\end{array}$ & $\begin{array}{c}\text { Water } \\
\text { year }\end{array}$ & $\begin{array}{c}\text { Annual peak } \\
\text { discharge } \\
\left.\mathbf{f t}^{3} / \mathbf{s}\right)\end{array}$ & $\begin{array}{c}\text { Discharge } \\
\text { codes }\end{array}$ \\
\hline 1963 & $08-00-63$ & 96 & & 1970 & $09-05-70$ & 382 \\
1964 & $07-31-64$ & 142 & & 1971 & $00-00-71$ & 305 \\
1965 & $09-19-65$ & 383 & & 1972 & $07-00-72$ & 20 \\
1966 & $00-00-66$ & 112 & & 1973 & $10-19-72$ & 310 \\
1967 & $00-00-67$ & 65 & & 1974 & $07-19-74$ & 90 \\
1968 & $00-00-68$ & 7.0 & 135 & 1975 & $09-07-75$ & 100 \\
1969 & $07-19-69$ & & & 1976 & $00-00-76$ & 1.0 \\
\hline
\end{tabular}

Magnitude and probability of instantaneous peak flow based on period of record 1963-76

\begin{tabular}{|c|c|c|c|c|c|}
\hline \multicolumn{6}{|c|}{$\begin{array}{l}\text { Discharge, in } \mathrm{ft}^{3} / \mathrm{s} \text {, for indicated recurrence interval } \\
\text { in years, and exceedance probablility, in percent }\end{array}$} \\
\hline 2 & 5 & 10 & 25 & $50 t$ & $100 t$ \\
\hline $50 \%$ & $20 \%$ & $10 \%$ & $4 \%$ & $2 \%$ & $1 \%$ \\
\hline 124 & 240 & 339 & 490 & 622 & 770 \\
\hline Weighted skew & $(\log s)=$ & -0.00 & & & \\
\hline Mean & $(\log s)=$ & 2.09 & & & \\
\hline Standard dev. & $(\log s)=$ & 0.34 & & & \\
\hline
\end{tabular}

f Reliability of values in column is uncertain, and potential errors are large.

Basin characteristics

\begin{tabular}{|c|c|c|c|c|c|c|c|}
\hline \multirow[b]{2}{*}{$\begin{array}{l}\text { Main } \\
\text { channel } \\
\text { slope } \\
(\mathrm{ft} / \mathrm{mi})\end{array}$} & \multirow[b]{2}{*}{$\begin{array}{l}\text { Stream } \\
\text { length } \\
\text { (mi) }\end{array}$} & \multirow[b]{2}{*}{$\begin{array}{c}\text { Mean } \\
\text { basin } \\
\text { elevation } \\
(\mathrm{ft})\end{array}$} & \multirow[b]{2}{*}{$\begin{array}{l}\text { Forested } \\
\text { area } \\
\text { (percent) }\end{array}$} & \multirow[b]{2}{*}{$\begin{array}{c}\text { Soil } \\
\text { index }\end{array}$} & \multirow[b]{2}{*}{$\begin{array}{c}\text { Mean } \\
\text { annual } \\
\text { precipitation } \\
\text { (in) }\end{array}$} & \multicolumn{2}{|c|}{ Rainfall intensity, 24-hour } \\
\hline & & & & & & $\begin{array}{l}\text { 2-year } \\
\text { (in) }\end{array}$ & $\begin{array}{l}\text { 50-year } \\
\text { (in) }\end{array}$ \\
\hline 105 & 2.0 & 6,020 & 38.0 & 3.0 & 10.2 & 1.3 & 2.8 \\
\hline
\end{tabular}

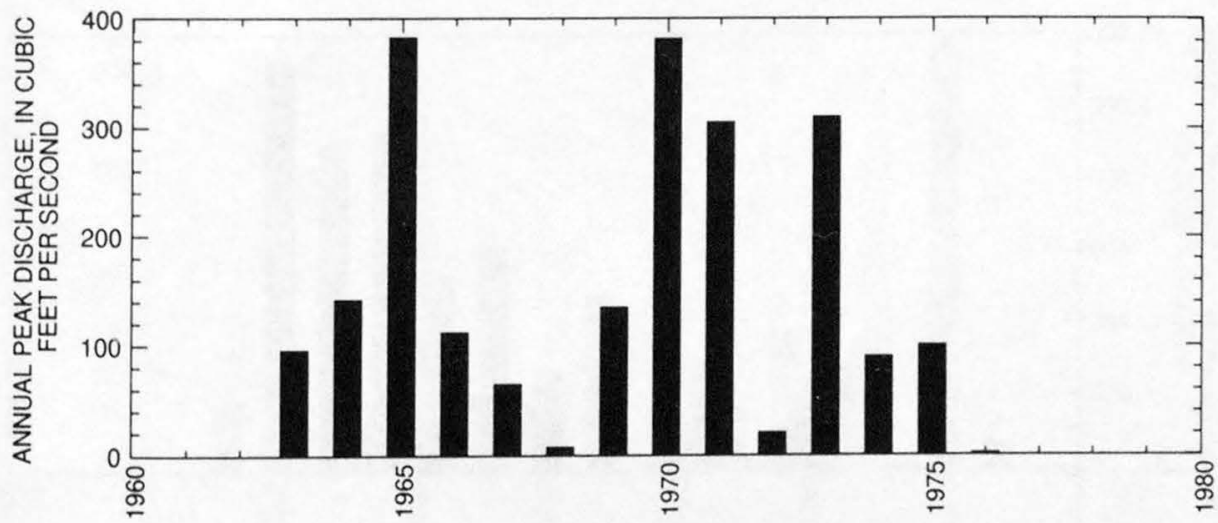


09400565 POLACCA WASH TRIBUTARY NEAR CHINLE, AZ

LOCATION.--Lat 36 02'50", long 110"04'50", Navajo County, Hydrologic Unit 15020013, at Navajo Highway No. 4 , 9 mi east of Pinon, and mi southwest of Chinle.

DRAINAGE AREA.--6.45 $\mathrm{mi}^{2}$.

Annual peak discharges

\begin{tabular}{|c|c|c|c|c|c|c|c|}
\hline $\begin{array}{l}\text { Water } \\
\text { year }\end{array}$ & Date & $\begin{array}{c}\text { Annual peak } \\
\text { discharge } \\
\left(\mathrm{ft}^{3} / \mathrm{s}\right)\end{array}$ & $\begin{array}{l}\text { Discharge } \\
\text { codes }\end{array}$ & $\begin{array}{l}\text { Water } \\
\text { year }\end{array}$ & Date & $\begin{array}{c}\text { Annual peak } \\
\text { discharge } \\
\left(\mathrm{ft}^{3} / \mathrm{s}\right)\end{array}$ & $\begin{array}{c}\text { Discharge } \\
\text { codes }\end{array}$ \\
\hline 1964 & $09-20-64$ & 7.0 & ES & 1971 & $08-26-71$ & 610 & \\
\hline 1965 & $09-19-65$ & 247 & & 1972 & $00-00-72$ & 540 & \\
\hline 1966 & $00-00-66$ & 43 & & 1973 & $10-19-72$ & 940 & \\
\hline 1967 & $09-00-67$ & 295 & & 1974 & $07-21-74$ & 900 & \\
\hline 1968 & $08-00-68$ & 20 & ES & 1975 & $09-07-75$ & 1,130 & \\
\hline 1969 & $09-11-69$ & 485 & & 1976 & $00-00-76$ & 180 & \\
\hline 1970 & $00-00-70$ & 275 & & & & & \\
\hline
\end{tabular}

Magnitude and probability of instantaneous peak flow based on period of record 1964-76

\begin{tabular}{|c|c|c|c|c|c|}
\hline \multicolumn{6}{|c|}{$\begin{array}{l}\text { Discharge, in } \mathrm{ft}^{3} / \mathrm{s} \text {, for indicated recurrence interval } \\
\text { in years, and exceedance probablility, in percent }\end{array}$} \\
\hline 2 & 5 & 10 & 25 & $50 t$ & $100 t$ \\
\hline $50 \%$ & $20 \%$ & $10 \%$ & $4 \%$ & $2 \%$ & $1 \%$ \\
\hline 361 & 708 & 995 & 1,420 & 1,770 & 2,150 \\
\hline Weighted skew & $(\log s)=$ & -0.17 & & & \\
\hline Mean & $(\log s)=$ & 2.55 & & & \\
\hline Standard dev. & $(\log s)=$ & 0.36 & & & \\
\hline
\end{tabular}

$\mp$ Reliability of values in column is uncertain, and potential errors are large.

Basin characteristics

\begin{tabular}{cccccccc}
\hline $\begin{array}{c}\text { Main } \\
\text { channel } \\
\text { slope } \\
(\mathrm{ft} / \mathrm{mi})\end{array}$ & $\begin{array}{c}\text { Stream } \\
\text { length } \\
\text { (mi) }\end{array}$ & $\begin{array}{c}\text { Mean } \\
\text { basin } \\
\text { elevation } \\
(\mathrm{ft})\end{array}$ & $\begin{array}{c}\text { Forested } \\
\text { area } \\
\text { (percent) }\end{array}$ & $\begin{array}{c}\text { Soil } \\
\text { index }\end{array}$ & $\begin{array}{c}\text { Mean } \\
\text { annual } \\
\text { precipitation } \\
\text { (in) }\end{array}$ & $\begin{array}{c}\text { R-year } \\
\text { (in) }\end{array}$ & $\begin{array}{c}\text { 24-hour } \\
\text { (in) }\end{array}$ \\
\hline 160 & 5.7 & 6,890 & 78.0 & 1.0 & 12.3 & 1.5 \\
\hline
\end{tabular}

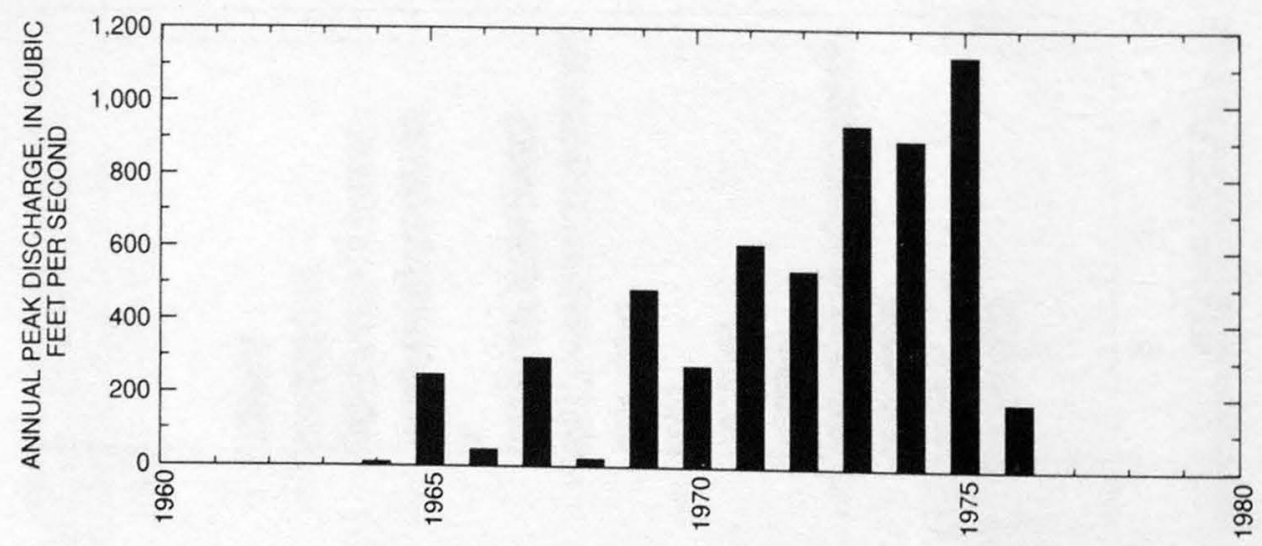


09400580 CASTLE BUTTE WASH NEAR WINSLOW, AZ

LOCATION.--Lat 35 $19^{\prime} 30^{\prime \prime}$, long $110^{\circ} 25^{\prime} 20^{\prime \prime}$, in SW $1 / 4$ sec.10, T.22 N., R.18 E., Navajo County, Hydrologic Unit 15020014, at State Highway

87,26 mi northwest of Winslow.

DRAINAGE AREA.--5.57 $\mathrm{mi}^{2}$.

Annual peak discharges

\begin{tabular}{cccccccc}
\hline $\begin{array}{c}\text { Water } \\
\text { year }\end{array}$ & Date & $\begin{array}{c}\text { Annual peak } \\
\text { discharge } \\
\left(\mathbf{f t}^{3} / \mathbf{s}\right)\end{array}$ & $\begin{array}{c}\text { Discharge } \\
\text { codes }\end{array}$ & $\begin{array}{c}\text { Water } \\
\text { year }\end{array}$ & Date & $\begin{array}{c}\text { Annual peak } \\
\text { discharge } \\
\left(\mathrm{ft}^{\mathbf{3}} / \mathbf{s}\right)\end{array}$ & $\begin{array}{c}\text { Discharge } \\
\text { codes }\end{array}$ \\
\hline 1964 & $09-09-64$ & 101 & & 1971 & $08-00-71$ & 127 \\
1965 & $07-00-65$ & 707 & & 1972 & $09-18-72$ & 280 & \\
1966 & $00-00-66$ & 5.0 & ES & 1973 & $10-19-72$ & 215 & \\
1967 & $00-00-67$ & 0 & & 1974 & $07-17-74$ & 2.0 & ES \\
1968 & $00-00-68$ & 0 & & 1975 & $07-15-75$ & 860 & \\
1969 & $10-04-68$ & 1.0 & LT & 1976 & $00-00-76$ & 58 & \\
1970 & $09-05-70$ & 117 & & & & & \\
\hline
\end{tabular}

Magnitude and probability of instantaneous peak flow based on period of record 1964-76

\begin{tabular}{|c|c|c|c|c|c|}
\hline \multicolumn{6}{|c|}{$\begin{array}{l}\text { Discharge, in } \mathrm{ft}^{3} / \mathrm{s} \text {, for indicated recurrence interval } \\
\text { in years, and exceedance probablility, in percent }\end{array}$} \\
\hline 2 & 5 & 10 & 25 & $50 \dagger$ & $100 t$ \\
\hline $50 \%$ & $20 \%$ & $10 \%$ & $4 \%$ & $2 \%$ & $1 \%$ \\
\hline 86 & 289 & 536 & 1,020 & 1,540 & 2,220 \\
\hline Weighted skew & $(\log s)=$ & -0.12 & & & \\
\hline Mean & $(\log s)=$ & 1.92 & & & \\
\hline Standard dev. & $(\log s)=$ & 0.64 & & & \\
\hline
\end{tabular}

† Reliability of values in column is uncertain, and potential errors are large.

Basin characteristics

\begin{tabular}{ccccccccc}
\hline $\begin{array}{c}\text { Main } \\
\text { channel } \\
\text { slope } \\
(\mathrm{ft} / \mathrm{mi})\end{array}$ & $\begin{array}{c}\text { Stream } \\
\text { length } \\
(\mathrm{mi})\end{array}$ & $\begin{array}{c}\text { Mean } \\
\text { basin } \\
\text { elevation } \\
(\mathrm{ft})\end{array}$ & $\begin{array}{c}\text { Forested } \\
\text { area } \\
\text { (percent) }\end{array}$ & $\begin{array}{c}\text { Soil } \\
\text { index }\end{array}$ & $\begin{array}{c}\text { Mean } \\
\text { annual } \\
\text { precipitation } \\
\text { (in) }\end{array}$ & $\begin{array}{c}\text { 2-year } \\
\text { (in) }\end{array}$ & $\begin{array}{c}\text { 50-year } \\
\text { (in) }\end{array}$ \\
\hline 83.8 & 3.2 & 5,820 & 0.0 & 1.0 & 8.6 & 1.3 & 2.7 \\
\hline
\end{tabular}

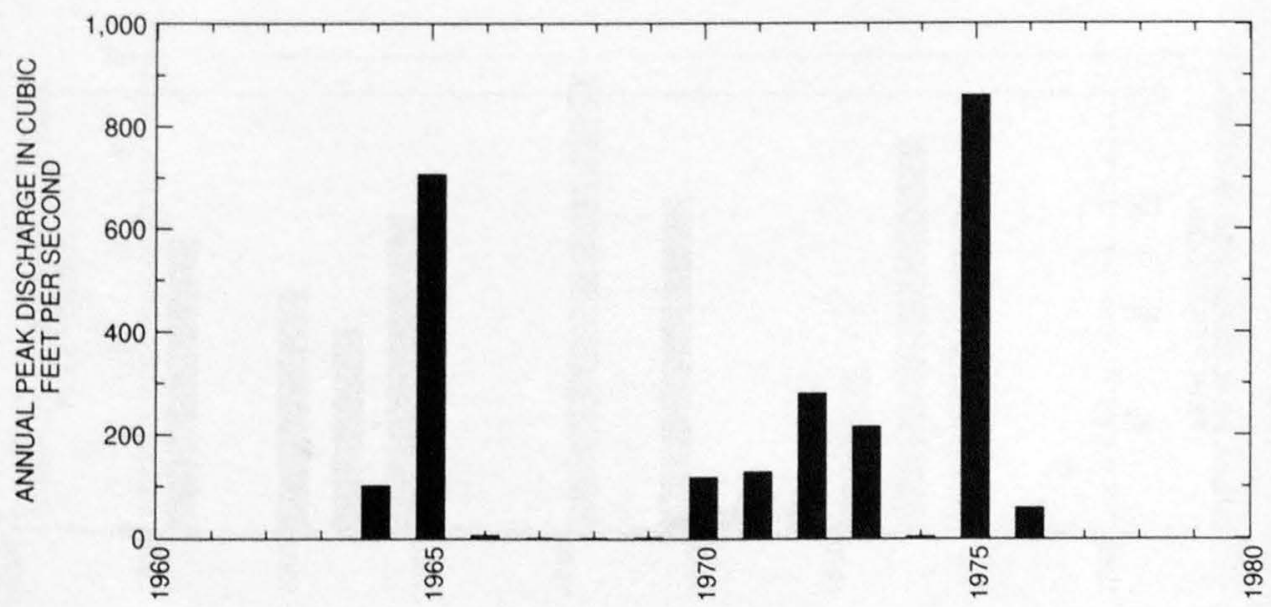




\section{RIO DE FLAG AT HIDDEN HOLLOW ROAD, AT FLAGSTAFF, AZ}

LOCATION.--Lat $35^{\circ} 14^{\prime} 31^{\prime \prime}$, long $111^{\circ} 41^{\prime} 02^{\prime \prime}$, in SW $1 / 4 \mathrm{SW}^{1} / 4 \mathrm{sec} .32$, T.22 N., R.7 E., Coconino County, Hydrologic Unit 15020015 , at Hide

Hollow Road, 1.4 mi northwest of the Museum of Northern Arizona, and 3.4 mi northwest of downtown Flagstaff.

DRAINAGE AREA.--31.5 $\mathrm{mi}^{2}$.

Annual peak discharges

\begin{tabular}{|c|c|c|c|c|c|c|c|}
\hline $\begin{array}{l}\text { Water } \\
\text { year }\end{array}$ & Date & $\begin{array}{c}\text { Annual peak } \\
\text { discharge } \\
\left(\mathrm{ft}^{3} / \mathrm{s}\right)\end{array}$ & $\begin{array}{l}\text { Discharge } \\
\text { codes }\end{array}$ & $\begin{array}{l}\text { Water } \\
\text { year }\end{array}$ & Date & $\begin{array}{c}\text { Annual peak } \\
\text { discharge } \\
\left(\mathrm{ft}^{3} / \mathrm{s}\right)\end{array}$ & $\begin{array}{c}\text { Discharge } \\
\text { codes }\end{array}$ \\
\hline 1970 & $08-03-70$ & 2.0 & ES & 1977 & $00-00-77$ & 1.0 & LT \\
\hline 1971 & $08-00-71$ & 0.1 & LT & 1978 & $04-00-78$ & 144 & \\
\hline 1972 & $12-26-71$ & 11 & & 1979 & $05-00-79$ & 93 & \\
\hline 1973 & $04-28-73$ & 153 & & 1980 & $02-20-80$ & 110 & \\
\hline 1974 & $00-00-74$ & 0 & & 1981 & $00-00-81$ & 1.0 & ES \\
\hline 1975 & $00-00-75$ & 0 & & 1982 & $03-12-82$ & 133 & \\
\hline 1976 & $00-00-76$ & 1.0 & LT & & & & \\
\hline
\end{tabular}

Magnitude and probability of instantaneous peak flow based on period of record

\begin{tabular}{|c|c|c|c|c|c|}
\hline \multicolumn{6}{|c|}{$\begin{array}{l}\text { Discharge, in } \mathrm{ft}^{3} / \mathrm{s} \text {, for indicated recurrence interval } \\
\text { in years, and exceedance probablility, in percent }\end{array}$} \\
\hline 2 & 5 & 10 & 25 & $50 t$ & $100 \dagger$ \\
\hline $50 \%$ & $20 \%$ & $10 \%$ & $4 \%$ & $2 \%$ & $1 \%$ \\
\hline --- & -.- & -- & -- & $\cdots$ & $\cdots$ \\
\hline Weighted skew & $(\log s)=$ & $-\cdots$ & & & \\
\hline Mean & $(\log s)=$ & --- & & & \\
\hline Standard dev. & $(\log s)=$ & -- & & & \\
\hline
\end{tabular}

Basin characteristics

\begin{tabular}{cccccccc}
\hline $\begin{array}{c}\text { Main } \\
\text { channel } \\
\text { slope } \\
(\mathrm{ft} / \mathrm{mi})\end{array}$ & $\begin{array}{c}\text { Stream } \\
\text { length } \\
(\mathrm{mi})\end{array}$ & $\begin{array}{c}\text { Mean } \\
\text { basin } \\
\text { elevation } \\
(\mathrm{ft})\end{array}$ & $\begin{array}{c}\text { Forested } \\
\text { area } \\
\text { (percent) }\end{array}$ & $\begin{array}{c}\text { Soil } \\
\text { index }\end{array}$ & $\begin{array}{c}\text { Mean } \\
\text { annual } \\
\text { precipitation } \\
\text { (in) }\end{array}$ & $\begin{array}{c}\text { R-year } \\
\text { (in) }\end{array}$ & $\begin{array}{c}50-\text { year } \\
\text { (in) }\end{array}$ \\
\hline 129 & 8.1 & 8,130 & 78.0 & 3.0 & 25.4 & 2.3 \\
\hline
\end{tabular}

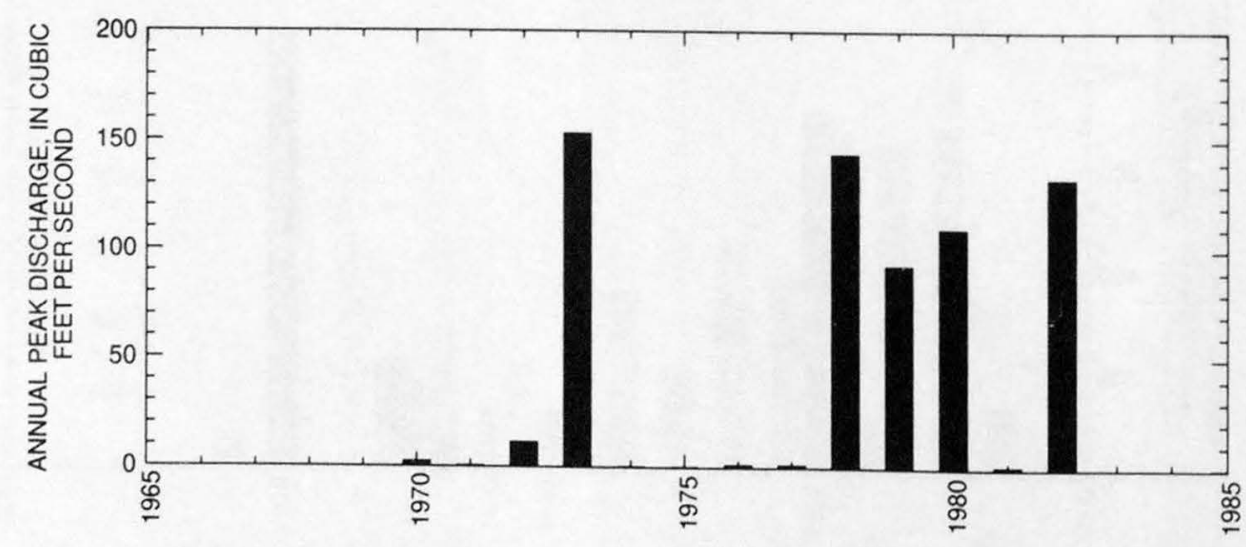


09400595 SCHULTZ CANYON AT FLAGSTAFF, AZ

LOCATION.--Lat 35 $13^{\prime} 37^{\prime \prime}$, long $111^{\circ} 39^{\prime} 29^{\prime \prime}$, in $\mathrm{SE}^{1} / \mathrm{SW}^{1} / 4$ sec.4, T.21 N., R.7 E., Coconino County, Hydrologic Unit 15020015 , at U.S. Highway $180,0.6 \mathrm{mi}$ south of the Museum of Northern Arizona in Flagstaff.

DRAINAGE AREA.--6.09 $\mathrm{mi}^{2}$.

Annual peak discharges

\begin{tabular}{cccccccc}
\hline $\begin{array}{c}\text { Water } \\
\text { year }\end{array}$ & Date & $\begin{array}{c}\text { Annual peak } \\
\text { discharge } \\
\left(\mathbf{f t}^{3} / \mathbf{s}\right)\end{array}$ & $\begin{array}{c}\text { Discharge } \\
\text { codes }\end{array}$ & $\begin{array}{c}\text { Water } \\
\text { year }\end{array}$ & Date & $\begin{array}{c}\text { Annual peak } \\
\text { discharge } \\
\left(\mathrm{ft}^{3} / \mathbf{s}\right)\end{array}$ & $\begin{array}{c}\text { Discharge } \\
\text { codes }\end{array}$ \\
\hline 1970 & $00-00-70$ & 0 & & 1976 & $00-00-76$ & 0 & \\
1971 & $00-00-71$ & 0 & 0 & 1977 & $00-00-77$ & 3.0 & LT \\
1972 & $00-00-72$ & 0 & & 1978 & $07-06-78$ & 17 & \\
1973 & $04-28-73$ & 48 & 1979 & $03-00-79$ & 41 & \\
1974 & $00-00-74$ & 0 & 1980 & $03-00-80$ & 35 & \\
1975 & $00-00-75$ & 0 & & & & & \\
\hline
\end{tabular}

Magnitude and probability of instantaneous peak flow based on period of record

\begin{tabular}{|c|c|c|c|c|c|}
\hline \multicolumn{6}{|c|}{$\begin{array}{l}\text { Discharge, in } \mathrm{ft}^{3} / \mathbf{s} \text {, for indicated recurrence interval } \\
\text { in years, and exceedance probablility, in percent }\end{array}$} \\
\hline 2 & 5 & 10 & 25 & $50 \dagger$ & $100 \dagger$ \\
\hline $50 \%$ & $20 \%$ & $10 \%$ & $4 \%$ & $2 \%$ & $1 \%$ \\
\hline --. & --. & --. & --. & -.- & --. \\
\hline Weighted skew & $(\log s)=$ & & & & \\
\hline Mean & $(\log s)=$ & ... & & & \\
\hline Standard dev. & $(\log s)=$ & --. & & & \\
\hline
\end{tabular}

Basin characteristics

\begin{tabular}{ccccccccc}
\hline $\begin{array}{c}\text { Main } \\
\text { channel }\end{array}$ & $\begin{array}{c}\text { Stream } \\
\text { slope } \\
\text { (tt/mi) }\end{array}$ & $\begin{array}{c}\text { length } \\
(\mathrm{mi})\end{array}$ & $\begin{array}{c}\text { Mean } \\
\text { basin } \\
\text { elevation } \\
\text { (ft) }\end{array}$ & $\begin{array}{c}\text { Forested } \\
\text { area } \\
\text { (percent) }\end{array}$ & $\begin{array}{c}\text { Soil } \\
\text { index }\end{array}$ & $\begin{array}{c}\text { Mean } \\
\text { annual } \\
\text { precipitation } \\
\text { (in) }\end{array}$ & $\begin{array}{c}\text { 2-year } \\
\text { (in) }\end{array}$ & $\begin{array}{c}\text { 50-year } \\
\text { (in) }\end{array}$ \\
\hline 296 & 5.8 & 8,060 & 89.0 & 3.0 & 21.9 & 2.1 & 4.0 \\
\hline
\end{tabular}

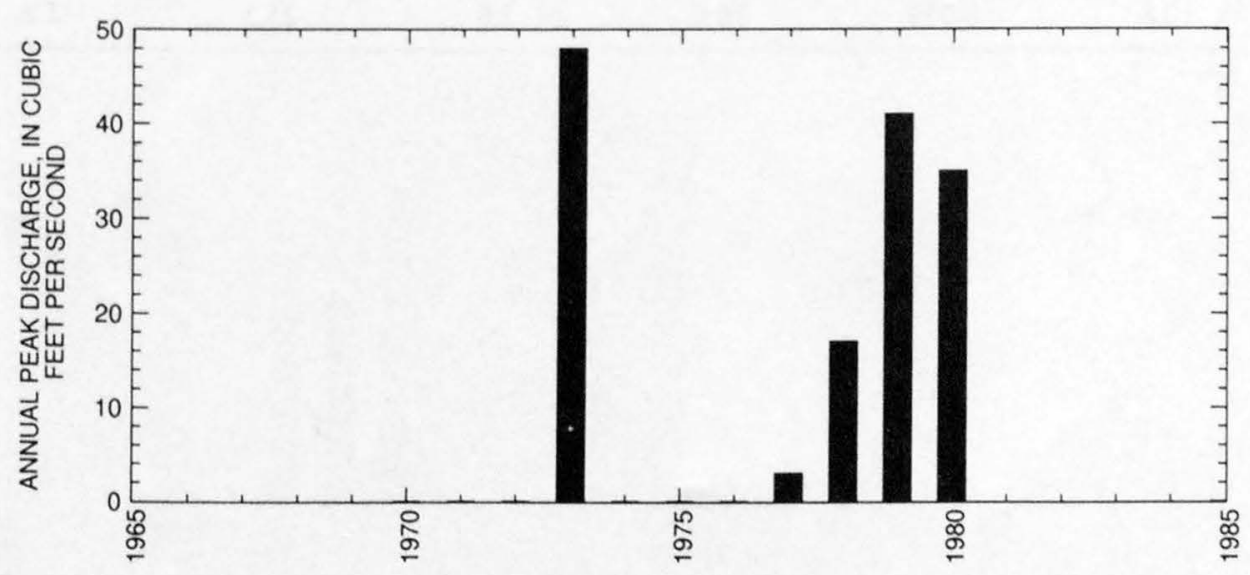




\section{LITTLE COLORADO RIVER BASIN}

09400600 RIO DE FLAG AT FLAGSTAFF, AZ

LOCATION.--Lat $35^{\circ} 13^{\prime} 18^{\prime \prime}$, long $111^{\circ} 39^{\prime} 24^{\prime \prime}$, in NW $\frac{1}{4} \mathrm{NE}^{1} / 4$ sec.9, T.21 N., R.7 E., Coconino County, Hydrologic Unit 15020015, at west sie of Crescent Drive in Flagstaff.

DRAINAGE AREA.--51.0 $\mathrm{mi}^{2}$.

Annual peak discharges

\begin{tabular}{cccccccc}
\hline $\begin{array}{c}\text { Water } \\
\text { year }\end{array}$ & Date & $\begin{array}{c}\text { Annual peak } \\
\text { discharge } \\
\left(\mathbf{f t}^{3} / \mathbf{s}\right)\end{array}$ & $\begin{array}{c}\text { Discharge } \\
\text { codes }\end{array}$ & $\begin{array}{c}\text { Water } \\
\text { year }\end{array}$ & Date & $\begin{array}{c}\text { Annual peak } \\
\text { discharge } \\
\left(\mathrm{ft}^{3} / \mathbf{s}\right)\end{array}$ & $\begin{array}{c}\text { Discharge } \\
\text { codes }\end{array}$ \\
\hline 1956 & $00-00-56$ & 0 & $\mathrm{C}$ & 1974 & $04-03-74$ & 3.0 & LT, C \\
1957 & $00-00-57$ & 0 & $\mathrm{C}$ & 1975 & $04-00-75$ & 10 & ES,C \\
1958 & $04-20-58$ & 56 & $\mathrm{C}$ & 1976 & $02-09-76$ & 35 & $\mathrm{C}$ \\
1959 & $00-00-59$ & 0 & $\mathrm{C}$ & 1977 & $05-15-77$ & 8.5 & $\mathrm{KR}, \mathrm{C}$ \\
1960 & $03-24-60$ & 11 & $\mathrm{C}$ & 1978 & $04-00-78$ & 128 & $\mathrm{C}$ \\
1970 & $08-03-70$ & 10 & $\mathrm{ES}, \mathrm{C}$ & 1979 & $05-00-79$ & 90 & $\mathrm{C}$ \\
1971 & $09-30-71$ & 10 & LT,C & 1980 & $07-00-80$ & 104 & $\mathrm{C}$ \\
1972 & $00-00-72$ & 0 & $\mathrm{C}$ & 1981 & $04-00-81$ & 14 & ES,C \\
1973 & $04-28-73$ & 235 & $\mathrm{C}$ & 1982 & $03-12-82$ & 240 & $\mathrm{C}$ \\
\hline
\end{tabular}

Magnitude and probability of instantaneous peak flow based on period of record 1956-60,

1970-82

\begin{tabular}{|c|c|c|c|c|c|}
\hline \multicolumn{6}{|c|}{$\begin{array}{l}\text { Discharge, in } \mathrm{ft}^{3} / \mathrm{s} \text {, for indicated recurrence interval } \\
\text { in years, and exceedance probablility, in percent }\end{array}$} \\
\hline 2 & 5 & 10 & 25 & $50 t$ & $100 \dagger$ \\
\hline $50 \%$ & $20 \%$ & $10 \%$ & $4 \%$ & $2 \%$ & $1 \%$ \\
\hline 17.2 & 74.5 & 167 & 340 & 557 & 861 \\
\hline Weighted skew & $(\log s)=$ & -0.13 & & & \\
\hline Mean & $(\log s)=$ & 1.22 & & & \\
\hline Standard dev. & $(\log s)=$ & 0.77 & & & \\
\hline
\end{tabular}

Basin characteristics

\begin{tabular}{cccccccc}
\hline $\begin{array}{c}\text { Main } \\
\text { channel } \\
\text { slope } \\
(\mathrm{ft} / \mathrm{mi})\end{array}$ & $\begin{array}{c}\text { Stream } \\
\text { length } \\
\text { (mi) }\end{array}$ & $\begin{array}{c}\text { Mean } \\
\text { basin } \\
\text { elevation } \\
(\mathrm{ft})\end{array}$ & $\begin{array}{c}\text { Forested } \\
\text { area } \\
\text { (percent) }\end{array}$ & $\begin{array}{c}\text { Soil } \\
\text { index }\end{array}$ & $\begin{array}{c}\text { Mean } \\
\text { annual } \\
\text { precipitation } \\
\text { (in) }\end{array}$ & $\begin{array}{c}\text { Rainfall intensity, 24-hour } \\
\text { (in) }\end{array}$ & $\begin{array}{c}50 \text {-year } \\
\text { (in) }\end{array}$ \\
\hline 106 & 10.8 & 8,050 & 76.0 & 3.0 & 25.3 & 2.2 \\
\hline
\end{tabular}


09400600 RIO DE FLAG AT FLAGSTAFF, AZ--Continued

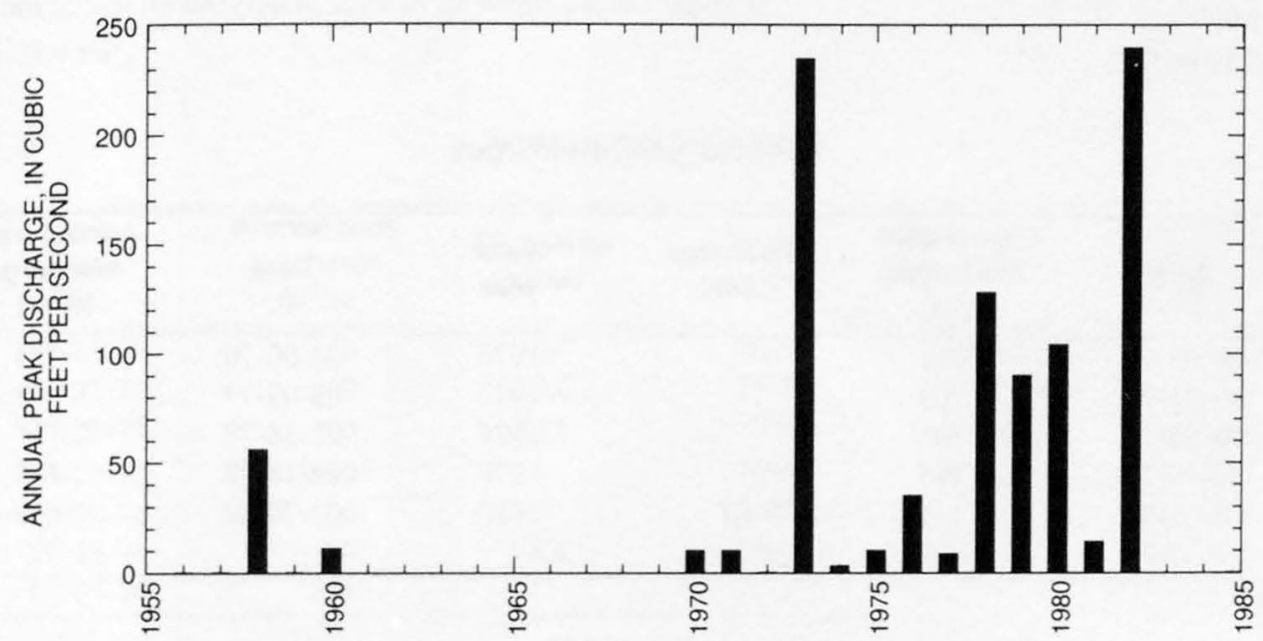


09400650 SINCLAIR WASH AT FLAGSTAFF, AZ

LOCATION.--Lat $35^{\circ} 09^{\prime} 50^{\prime \prime}$, long $111^{\circ} 40^{\prime} 48^{\prime \prime}$, in NW $1 /{ }_{4} \mathrm{NW}^{1} / 4 \mathrm{sec} .32, \mathrm{~T} .21 \mathrm{~N} ., \mathrm{R} .7$ E., Coconino County, at Holmes Avenue in the community Palmerville at Flagstaff.

DRAINAGE AREA.--8.11 $\mathrm{mi}^{2}$.

Annual peak discharges

\begin{tabular}{|c|c|c|c|c|c|c|c|}
\hline $\begin{array}{l}\text { Water } \\
\text { year }\end{array}$ & Date & $\begin{array}{c}\text { Annual peak } \\
\text { discharge } \\
\left(\mathrm{ft}^{3} / \mathrm{s}\right)\end{array}$ & $\begin{array}{c}\text { Discharge } \\
\text { codes }\end{array}$ & $\begin{array}{l}\text { Water } \\
\text { year }\end{array}$ & Date & $\begin{array}{c}\text { Annual peak } \\
\text { discharge } \\
\left(\mathrm{ft}^{3} / \mathrm{s}\right)\end{array}$ & $\begin{array}{c}\text { Discharge } \\
\text { codes }\end{array}$ \\
\hline 1970 & $09-05-70$ & $1_{401}$ & & 1976 & $04-00-76$ & 44 & \\
\hline 1971 & $07-21-71$ & 62 & & 1977 & $08-09-77$ & 23 & \\
\hline 1972 & $12-28-71$ & 105 & & 1978 & $02-28-78$ & 37 & \\
\hline 1973 & $10-19-72$ & 135 & & 1979 & $12-18-78$ & 295 & \\
\hline 1974 & $08-01-74$ & 1.0 & LT & 1980 & $02-20-80$ & 70 & \\
\hline 1975 & $10-30-74$ & 74 & & & & & \\
\hline
\end{tabular}

${ }^{1}$ Highest since 1944.

Magnitude and probability of instantaneous peak flow based on period of record 1970-80

\begin{tabular}{|c|c|c|c|c|c|}
\hline \multicolumn{6}{|c|}{$\begin{array}{l}\text { Discharge, in } \mathrm{ft}^{3} / \mathrm{s} \text {, for indicated recurrence interval } \\
\text { in years, and exceedance probablility, in percent }\end{array}$} \\
\hline 2 & 5 & 10 & 25 & $50 \dagger$ & $100 \dagger$ \\
\hline $50 \%$ & $20 \%$ & $10 \%$ & $4 \%$ & $2 \%$ & $1 \%$ \\
\hline 74.2 & 168 & 260 & 416 & 566 & 748 \\
\hline Weighted skew & $(\log s)=$ & 0.09 & & & \\
\hline Mean & $(\log s)=$ & 1.88 & & & \\
\hline Standard dev. & $(\log s)=$ & 0.42 & & & \\
\hline
\end{tabular}

\section{Basin characteristics}

\begin{tabular}{ccccccc}
\hline $\begin{array}{c}\text { Main } \\
\text { channel } \\
\text { slope } \\
(\mathrm{ft} / \mathrm{mi})\end{array}$ & $\begin{array}{c}\text { Stream } \\
\text { length } \\
\text { (mi) }\end{array}$ & $\begin{array}{c}\text { Mean } \\
\text { basin } \\
\text { elevation } \\
(\mathrm{ft})\end{array}$ & $\begin{array}{c}\text { Forested } \\
\text { area } \\
\text { (percent) }\end{array}$ & $\begin{array}{c}\text { Soil } \\
\text { index }\end{array}$ & $\begin{array}{c}\text { Mean } \\
\text { annual } \\
\text { precipitation } \\
\text { (in) }\end{array}$ & $\begin{array}{c}\text { Rainfall intensity, 24-hour } \\
\text { (in) }\end{array}$ \\
\hline 69.9 & 4.8 & 7,200 & 88.0 & 3.0 & 22.5 & $\begin{array}{c}50 \text {-year } \\
\text { (in) }\end{array}$ \\
\hline
\end{tabular}

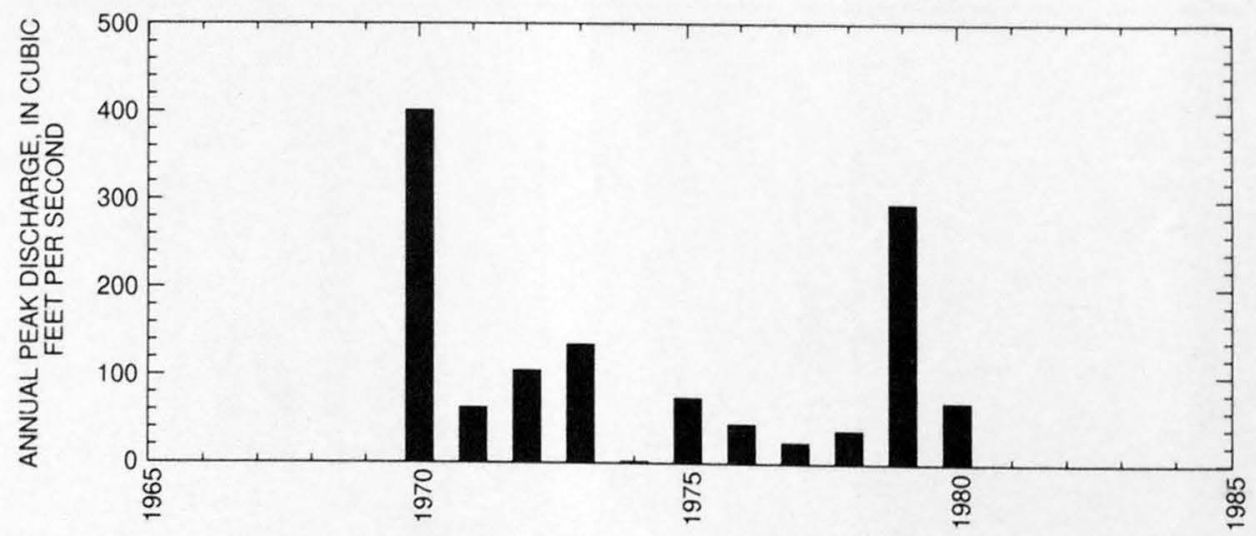


09400655 RIO DE FLAG AT INTERSTATE 40 AT FLAGSTAFF, AZ

LOCATION.--Lat $35^{\circ} 11^{\prime} 04^{\prime \prime}$, long $111^{\circ} 37^{\prime} 56^{\prime \prime}$, in SE $1 / 4 \mathrm{SE}^{1} / 4 \mathrm{sec} .22$, T.21 N., R.7 E., Coconino County, Hydrologic Unit 15020015 , on left bank

$80 \mathrm{ft}$ upstream from bridge for eastbound lanes of Interstate 40, in Flagstaff.

DRAINAGE AREA.--82.4 $\mathrm{mi}^{2}$.

Annual peak discharges

\begin{tabular}{|c|c|c|c|c|c|c|c|}
\hline $\begin{array}{l}\text { Water } \\
\text { year }\end{array}$ & Date & $\begin{array}{c}\text { Annual peak } \\
\text { discharge } \\
\left(\mathrm{ft}^{3} / \mathrm{s}\right)\end{array}$ & $\begin{array}{c}\text { Discharge } \\
\text { codes }\end{array}$ & $\begin{array}{l}\text { Water } \\
\text { year }\end{array}$ & Date & $\begin{array}{c}\text { Annual peak } \\
\text { discharge } \\
\left(\mathrm{ft}^{3} / \mathrm{s}\right)\end{array}$ & $\begin{array}{l}\text { Discharge } \\
\text { codes }\end{array}$ \\
\hline 1970 & $09-05-70$ & 350 & ES,C & 1977 & $00-00-77$ & 3.0 & LT,C \\
\hline 1971 & $07-21-71$ & 50 & ES,C & 1978 & $02-28-78$ & 153 & C \\
\hline 1972 & $12-28-71$ & 100 & ES,C & 1979 & $12-19-78$ & 421 & $\mathrm{C}$ \\
\hline 1973 & $04-25-73$ & $1_{300}$ & C & 1980 & $02-20-80$ & 165 & C \\
\hline 1974 & $00-00-74$ & 0 & & 1981 & $04-00-81$ & 150 & ES,C \\
\hline 1975 & $10-30-74$ & 70 & ES,C & 1982 & $03-12-82$ & 370 & C \\
\hline 1976 & $02-09-76$ & 134 & C & & & & \\
\hline
\end{tabular}

${ }^{1}$ Highest since 1938.

Magnitude and probability of instantaneous peak flow based on period of record

\begin{tabular}{|c|c|c|c|c|c|}
\hline \multicolumn{6}{|c|}{$\begin{array}{l}\text { Discharge, in } \mathrm{ft}^{3} / \mathrm{s} \text {, for indicated recurrence interval } \\
\text { in years, and exceedance probablility, in percent }\end{array}$} \\
\hline 2 & 5 & 10 & 25 & $50 t$ & $100 t$ \\
\hline $50 \%$ & $20 \%$ & $10 \%$ & $4 \%$ & $2 \%$ & $1 \%$ \\
\hline -.. & -.. & $\ldots$ & $\ldots$ & $\cdots$ & -.. \\
\hline Weighted skew & $(\log s)=$ & $\cdots$ & & & \\
\hline Mean & $(\log s)=$ & -.. & & & \\
\hline Standard dev. & $(\log s)=$ & -.- & & & \\
\hline
\end{tabular}

Basin characteristics

\begin{tabular}{|c|c|c|c|c|c|c|c|}
\hline \multirow[b]{2}{*}{$\begin{array}{l}\text { Main } \\
\text { channel } \\
\text { slope } \\
\text { (ft/mi) }\end{array}$} & \multirow[b]{2}{*}{$\begin{array}{l}\text { Stream } \\
\text { length } \\
(\mathrm{mi})\end{array}$} & \multirow[b]{2}{*}{$\begin{array}{c}\text { Mean } \\
\text { basin } \\
\text { elevation } \\
(f t)\end{array}$} & \multirow[b]{2}{*}{$\begin{array}{l}\text { Forested } \\
\text { area } \\
\text { (percent) }\end{array}$} & \multirow[b]{2}{*}{$\begin{array}{c}\text { Soil } \\
\text { index }\end{array}$} & \multirow[b]{2}{*}{$\begin{array}{c}\text { Mean } \\
\text { annual } \\
\text { precipitation } \\
\text { (in) }\end{array}$} & \multicolumn{2}{|c|}{ Rainfall intensity, 24-hour } \\
\hline & & & & & & $\begin{array}{c}\text { 2-year } \\
\text { (in) }\end{array}$ & $\begin{array}{l}\text { 50-year } \\
\text { (in) }\end{array}$ \\
\hline 55.5 & 5.9 & 7,840 & 97.0 & 3.0 & 20.0 & 1.9 & 3.6 \\
\hline
\end{tabular}

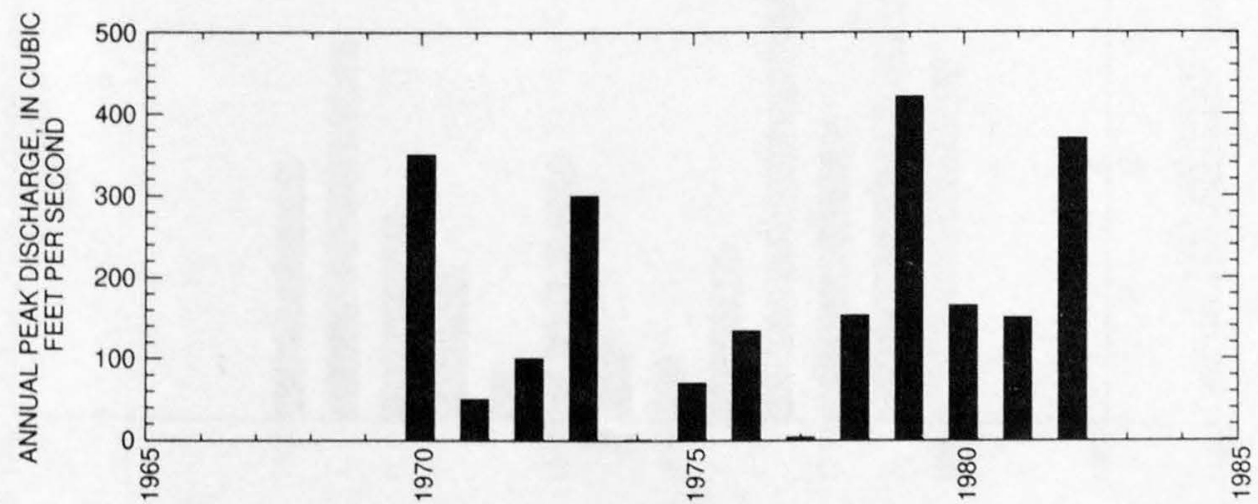


09400660 BOW AND ARROW WASH AT FLAGSTAFF, AZ

LOCATION.--Lat 35 09'58", long $111^{\circ} 39^{\prime} 10^{\prime \prime}$, in NW $1 / 4 \mathrm{NE}^{1} / 4 \mathrm{sec} .33$, T.21 N., R.7 E., Coconino County, Hydrologic Unit 15020015 , at Zuni Ros in Flagstaff.

DRAINAGE AREA.--2.06 $\mathrm{mi}^{2}$.

Annual peak discharges

\begin{tabular}{|c|c|c|c|c|c|c|c|}
\hline $\begin{array}{l}\text { Water } \\
\text { year }\end{array}$ & Date & $\begin{array}{l}\text { Annual peak } \\
\text { discharge } \\
\left(\mathrm{ft}^{3} / \mathrm{s}\right)\end{array}$ & $\begin{array}{l}\text { Discharge } \\
\text { codes }\end{array}$ & $\begin{array}{l}\text { Water } \\
\text { year }\end{array}$ & Date & $\begin{array}{c}\text { Annual peak } \\
\text { discharge } \\
\left(\mathrm{ft}^{3} / \mathrm{s}\right)\end{array}$ & $\begin{array}{c}\text { Discharge } \\
\text { codes }\end{array}$ \\
\hline 1969 & $00-00-69$ & 45 & $\mathrm{C}$ & 1975 & $00-00-75$ & 13 & $\mathrm{C}$ \\
\hline 1970 & $09-05-70$ & 42 & $\mathrm{C}$ & 1976 & $07-00-76$ & 7.0 & ES,C \\
\hline 1971 & $08-15-71$ & 73 & $\mathrm{C}$ & 1977 & $08-09-77$ & 24 & C \\
\hline 1972 & $12-28-71$ & 26 & $\mathrm{C}$ & 1978 & $10-06-77$ & 20 & $\mathrm{C}$ \\
\hline 1973 & $00-00-73$ & 10 & LT,C & 1979 & $11-11-78$ & 17 & $\mathrm{C}$ \\
\hline 1974 & $08-02-74$ & 12 & C & 1980 & $02-18-80$ & 40 & ES,C \\
\hline
\end{tabular}

Magnitude and probability of instantaneous peak flow based on period of record 1969-80

\begin{tabular}{|c|c|c|c|c|c|}
\hline \multicolumn{6}{|c|}{$\begin{array}{l}\text { Discharge, in } \mathrm{ft}^{3} / \mathrm{s} \text {, for indicated recurrence interval } \\
\text { in years, and exceedance probablility, in percent }\end{array}$} \\
\hline 2 & 5 & 10 & 25 & $50 t$ & $100 t$ \\
\hline $50 \%$ & $20 \%$ & $10 \%$ & $4 \%$ & $2 \%$ & $1 \%$ \\
\hline 22.1 & 39.7 & 53.9 & 74.7 & 92 & 111 \\
\hline Weighted skew & $(\log s)=$ & -0.02 & & & \\
\hline Mean & $(\log s)=$ & 1.34 & & & \\
\hline Standard dev. & $(\log s)=$ & 0.31 & & & \\
\hline
\end{tabular}

f Reliability of values in column is uncertain, and potential errors are large.

Basin characteristics

\begin{tabular}{cccccccc}
\hline $\begin{array}{c}\text { Main } \\
\text { channel } \\
\text { slope } \\
(\mathrm{ft} / \mathrm{mi})\end{array}$ & $\begin{array}{c}\text { Stream } \\
\text { length } \\
\text { (mi) }\end{array}$ & $\begin{array}{c}\text { Mean } \\
\text { basin } \\
\text { elevation } \\
(\mathrm{ft})\end{array}$ & $\begin{array}{c}\text { Forested } \\
\text { area } \\
\text { (percent) }\end{array}$ & $\begin{array}{c}\text { Soil } \\
\text { index }\end{array}$ & $\begin{array}{c}\text { Mean } \\
\text { annual } \\
\text { precipitation } \\
\text { (in) }\end{array}$ & $\begin{array}{c}\text { Rainfall intensity, 24-hour } \\
\text { (in) }\end{array}$ & $\begin{array}{c}50 \text {-year } \\
\text { (in) }\end{array}$ \\
\hline 46.5 & 2.9 & 6,990 & 75.0 & 3.0 & 19.4 & 2.0 \\
\hline
\end{tabular}

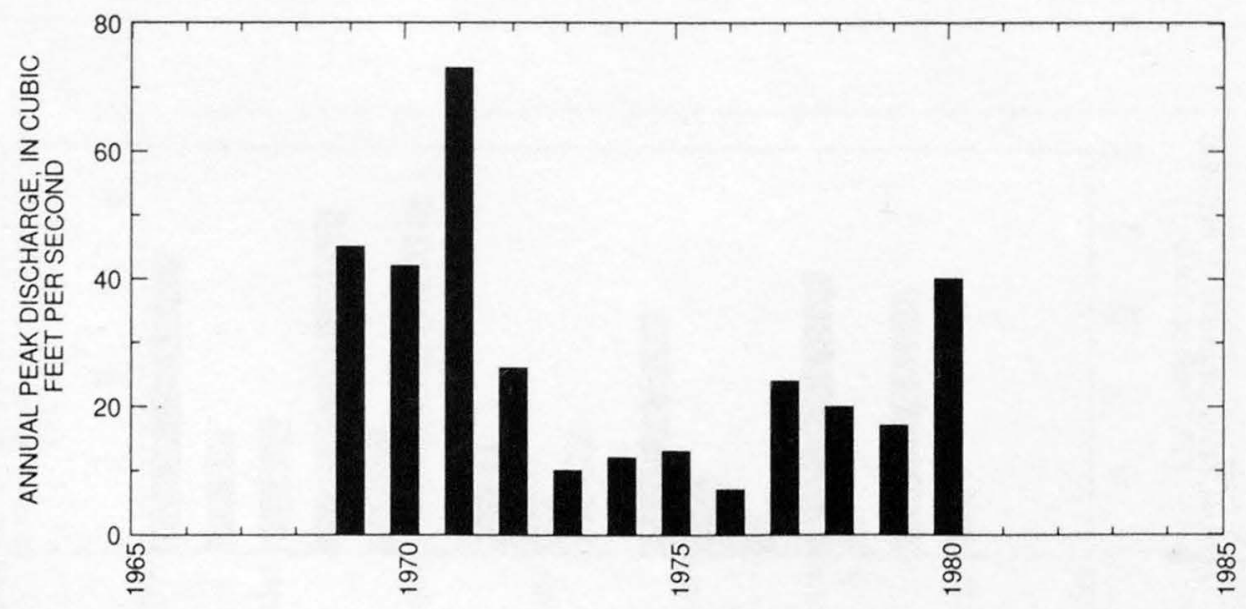


09400680 SWITZER CANYON AT FLAGSTAFF, AZ

LOCATION.--Lat $35^{\circ} 12^{\prime} 44^{\prime \prime}$, long $111^{\circ} 38^{\prime} 21^{\prime \prime}$, in SW $\mathrm{SW}_{4}^{1} \mathrm{SE}^{1} / 4$ sec.10, T.21 N., R.7 E., Coconino County, Hydrologic Unit 15020015 , at Turquoise and Oak Streets in Flagstaff.

DRAINAGE AREA.--1.87 $\mathrm{mi}^{2}$.

Annual peak discharges

\begin{tabular}{|c|c|c|c|c|c|c|c|}
\hline $\begin{array}{l}\text { Water } \\
\text { year }\end{array}$ & Date & $\begin{array}{c}\text { Annual peak } \\
\text { discharge } \\
\left(\mathrm{ft}^{3} / \mathrm{s}\right)\end{array}$ & $\begin{array}{l}\text { Discharge } \\
\text { codes }\end{array}$ & $\begin{array}{l}\text { Water } \\
\text { year }\end{array}$ & Date & $\begin{array}{c}\text { Annual peak } \\
\text { discharge } \\
\left(\mathrm{ft}^{3} / \mathrm{s}\right)\end{array}$ & $\begin{array}{c}\text { Discharge } \\
\text { codes }\end{array}$ \\
\hline 1969 & $09-12-69$ & 112 & & 1975 & $09-00-75$ & 10 & \\
\hline 1970 & $09-05-70$ & 61 & & 1976 & $02-09-76$ & 51 & \\
\hline 1971 & $08-03-71$ & 12 & & 1977 & $07-22-77$ & 5.0 & \\
\hline 1972 & $12-28-71$ & 15 & & 1978 & $02-28-78$ & 90 & \\
\hline 1973 & $04-13-73$ & 79 & & 1979 & $12-18-78$ & 135 & \\
\hline 1974 & $08-10-74$ & 18 & & 1980 & $02-19-80$ & 107 & \\
\hline
\end{tabular}

Magnitude and probability of instantaneous peak flow based on period of record 1969-80

\begin{tabular}{|c|c|c|c|c|c|}
\hline \multicolumn{6}{|c|}{$\begin{array}{l}\text { Discharge, in } \mathrm{ft}^{3} / \mathrm{s} \text {, for indicated recurrence interval } \\
\text { in years, and exceedance probablility, in percent }\end{array}$} \\
\hline 2 & 5 & 10 & 25 & $50 \dagger$ & $100 \dagger$ \\
\hline $50 \%$ & $20 \%$ & $10 \%$ & $4 \%$ & $2 \%$ & $1 \%$ \\
\hline 38.4 & 95.9 & 151 & 240 & 321 & 413 \\
\hline Weighted skew & $(\log s)=$ & -0.25 & & & \\
\hline Mean & $(\log s)=$ & 1.56 & & & \\
\hline Standard dev. & $(\log s)=$ & 0.49 & & & \\
\hline
\end{tabular}

Basin characteristics

\begin{tabular}{ccccccc}
\hline $\begin{array}{c}\text { Main } \\
\text { channel } \\
\text { slope } \\
(\mathrm{ft} / \mathrm{mi})\end{array}$ & $\begin{array}{c}\text { Stream } \\
\text { length } \\
(\mathrm{mi})\end{array}$ & $\begin{array}{c}\text { Mean } \\
\text { basin } \\
\text { elevation } \\
(\mathrm{ft})\end{array}$ & $\begin{array}{c}\text { Forested } \\
\text { area } \\
\text { (percent) }\end{array}$ & $\begin{array}{c}\text { Soil } \\
\text { index }\end{array}$ & $\begin{array}{c}\text { Mean } \\
\text { annual } \\
\text { precipitation } \\
\text { (in) }\end{array}$ & $\begin{array}{c}\text { Rainfall intensity, 24-hour } \\
\text { (in) }\end{array}$ \\
\hline 375 & 2.4 & 7,130 & 45.0 & 3.0 & 19.9 & $\begin{array}{c}50 \text {-year } \\
\text { (in) }\end{array}$ \\
\hline
\end{tabular}

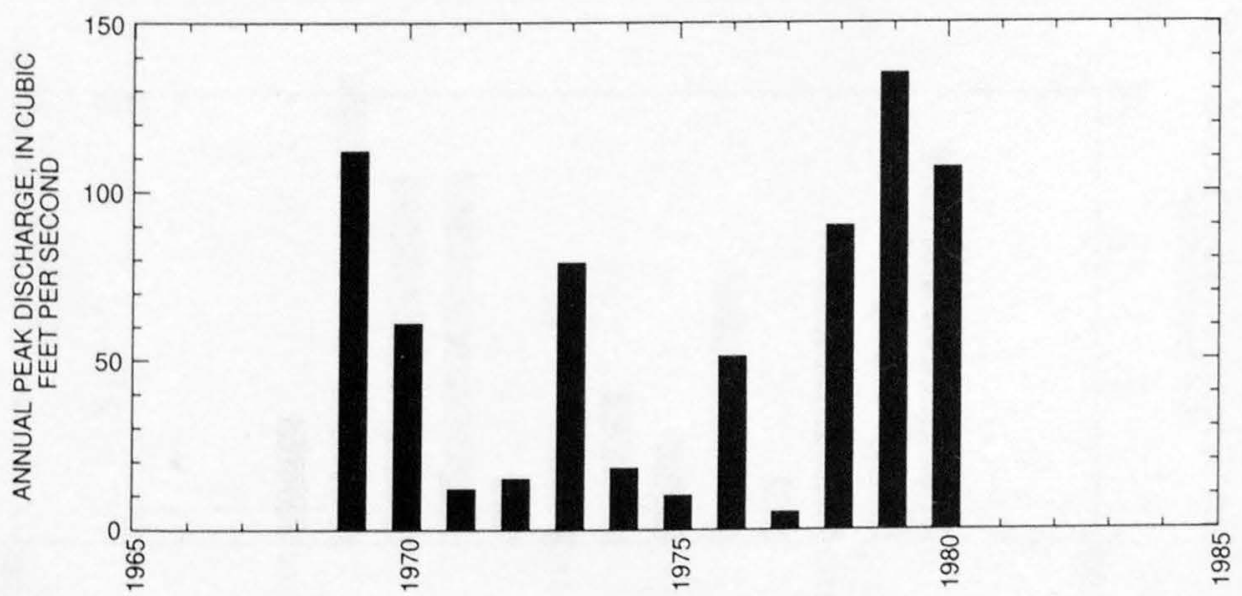




\section{LITTLE COLORADO RIVER BASIN}

\section{SWITZER CANYON TRIBUTARY AT FLAGSTAFF, AZ}

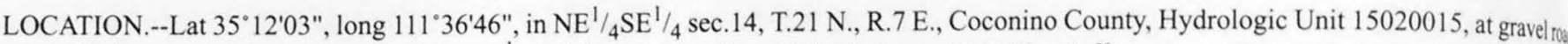

$500 \mathrm{ft}$ upstream from Interstate 40 , and $1 / 4$ mi downstream from U.S. Highway 66 in Flagstaff.

DRAINAGE AREA.--7.02 $\mathrm{mi}^{2}$, of which $2.50 \mathrm{mi}^{2}$ is noncontributing.

Annual peak discharges

\begin{tabular}{ccccccrc}
\hline $\begin{array}{c}\text { Water } \\
\text { year }\end{array}$ & Date & $\begin{array}{c}\text { Annual peak } \\
\text { discharge } \\
\left(\mathbf{f t}^{3} / \mathbf{s}\right)\end{array}$ & $\begin{array}{c}\text { Discharge } \\
\text { codes }\end{array}$ & $\begin{array}{c}\text { Water } \\
\text { year }\end{array}$ & Date & $\begin{array}{c}\text { Annual peak } \\
\text { discharge } \\
\left(\mathbf{f t}^{3} / \mathbf{s}\right)\end{array}$ & $\begin{array}{c}\text { Discharge } \\
\text { codes }\end{array}$ \\
\hline 1968 & $08-02-68$ & 262 & $\mathrm{C}$ & 1975 & $07-16-75$ & 65 & $\mathrm{C}$ \\
1969 & $09-12-69$ & 70 & $\mathrm{C}$ & 1976 & $02-09-76$ & 45 & $\mathrm{C}$ \\
1970 & $09-05-70$ & 178 & $\mathrm{C}$ & 1977 & $08-09-77$ & 47 & $\mathrm{C}$ \\
1971 & $08-03-71$ & 42 & $\mathrm{C}$ & 1978 & $07-15-78$ & 76 & $\mathrm{C}$ \\
1972 & $12-28-71$ & 15 & $\mathrm{C}$ & 1979 & $08-12-79$ & 103 & $\mathrm{C}$ \\
1973 & $07-16-73$ & 73 & $\mathrm{C}$ & 1980 & $02-19-80$ & 84 & $\mathrm{C}$ \\
1974 & $08-06-74$ & 100 & $\mathrm{C}$ & & & & \\
\hline
\end{tabular}

Magnitude and probability of instantaneous peak flow based on period of record 1968-80

\begin{tabular}{cccccc}
\hline \multicolumn{5}{c}{$\begin{array}{l}\text { Discharge, in } \mathrm{ft}^{3} / \mathrm{s} \text {, for indicated recurrence interval } \\
\text { in years, and exceedance probablility, in percent }\end{array}$} \\
\hline $\mathbf{2}$ & $\mathbf{5}$ & $\mathbf{1 0}$ & $\mathbf{2 5}$ & $\mathbf{5 0 \dagger}$ & $\mathbf{1 0 0 \dagger}$ \\
$\mathbf{5 0 \%}$ & $\mathbf{2 0 \%}$ & $\mathbf{1 0 \%}$ & $\mathbf{4} \%$ & $\mathbf{2 \%}$ & $\mathbf{1 \%}$ \\
\hline 75.2 & 124 & 163 & 221 & 271 & 326 \\
Weighted skew & $(\operatorname{logs})=$ & 0.28 & & \\
Mean & $(\operatorname{logs})=$ & 1.89 & \multicolumn{4}{l}{} \\
Standard dev. & $(\operatorname{logs})=$ & 0.25 & \\
F Reliability of values in column is uncertain, and potential errors are large.
\end{tabular}

Basin characteristics

\begin{tabular}{|c|c|c|c|c|c|c|c|}
\hline \multirow[b]{2}{*}{$\begin{array}{l}\text { Main } \\
\text { channel } \\
\text { slope } \\
(\mathrm{ft} / \mathrm{mi})\end{array}$} & \multirow[b]{2}{*}{$\begin{array}{l}\text { Stream } \\
\text { length } \\
\text { (mi) }\end{array}$} & \multirow[b]{2}{*}{$\begin{array}{c}\text { Mean } \\
\text { basin } \\
\text { elevation } \\
\text { (ft) }\end{array}$} & \multirow[b]{2}{*}{$\begin{array}{c}\text { Forested } \\
\text { area } \\
\text { (percent) }\end{array}$} & \multirow[b]{2}{*}{$\begin{array}{c}\text { Soil } \\
\text { index }\end{array}$} & \multirow[b]{2}{*}{$\begin{array}{c}\text { Mean } \\
\text { annual } \\
\text { precipitation } \\
\text { (in) }\end{array}$} & \multicolumn{2}{|c|}{ Rainfall intensity, 24-hour } \\
\hline & & & & & & $\begin{array}{l}\text { 2-year } \\
\text { (in) }\end{array}$ & $\begin{array}{l}\text { 50-year } \\
\text { (in) }\end{array}$ \\
\hline 267 & 4.1 & 6,950 & 78.0 & 3.0 & 20.0 & 2.0 & 4.0 \\
\hline
\end{tabular}

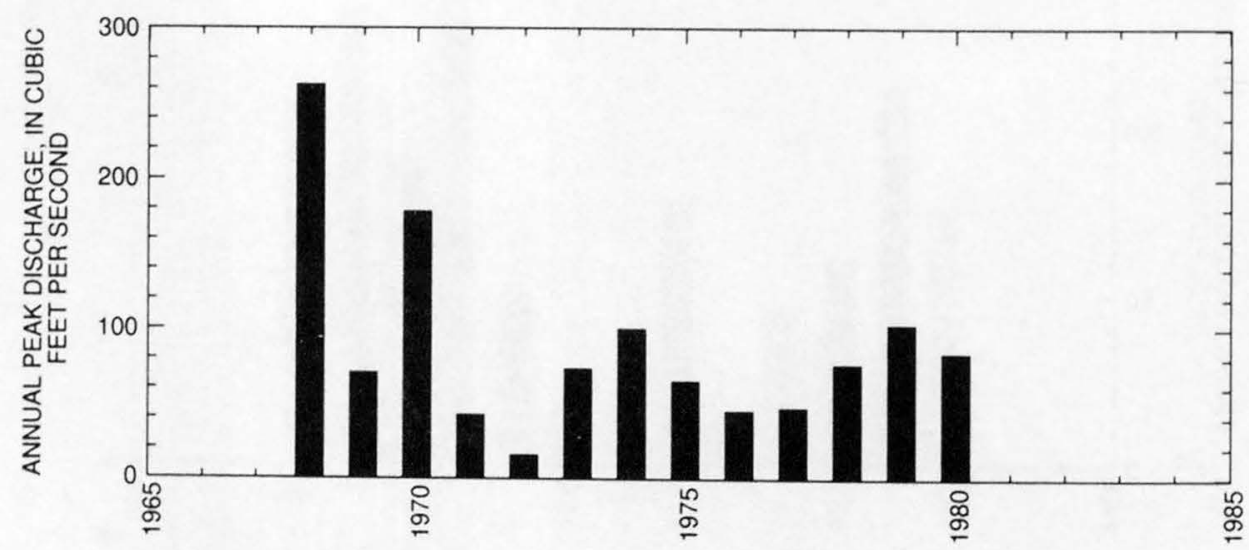


09400730 LOCKETT FANNING DIVERSION AT FLAGSTAFF, AZ

LOCATION.--Lat 35 13'19", long $111^{\circ} 35^{\prime} 58^{\prime \prime}$, in NW $1 / 4 \mathrm{NE}^{1} / 4$ sec.12, T.21 N., R.7 E., Coconino County, Hydrologic Unit 15020015, at Linda Vista Drive in Flagstaff.

DRAINAGE AREA.--1.05 $\mathrm{mi}^{2}$.

Annual peak discharges

\begin{tabular}{cccccccc}
\hline $\begin{array}{c}\text { Water } \\
\text { year }\end{array}$ & Date & $\begin{array}{c}\text { Annual peak } \\
\text { discharge } \\
\left(\mathrm{ft}^{3} \mathbf{s}\right)\end{array}$ & $\begin{array}{c}\text { Discharge } \\
\text { codes }\end{array}$ & $\begin{array}{c}\text { Water } \\
\text { year }\end{array}$ & Date & $\begin{array}{c}\text { Annual peak } \\
\text { discharge } \\
\left(\mathrm{ft}^{3} / \mathbf{s}\right)\end{array}$ & $\begin{array}{c}\text { Discharge } \\
\text { codes }\end{array}$ \\
\hline 1969 & $09-12-69$ & 85 & & 1975 & $00-00-75$ & 0 & \\
1970 & $09-05-70$ & 65 & & 1976 & $07-14-76$ & 46 & \\
1971 & $08-22-71$ & 66 & & 1977 & $08-09-77$ & 1.0 & ES \\
1972 & $00-00-72$ & 0 & & 1978 & $07-26-78$ & 54 & \\
1973 & $07-16-73$ & 17 & & 1979 & $12-18-78$ & 46 & \\
1974 & $08-06-74$ & 2.0 & ES & 1980 & $00-00-80$ & 0 & \\
\hline
\end{tabular}

Magnitude and probability of instantaneous peak flow based on period of record

\begin{tabular}{|c|c|c|c|c|c|}
\hline \multicolumn{6}{|c|}{$\begin{array}{l}\text { Discharge, in } \mathrm{ft}^{3} / \mathrm{s} \text {, for indicated recurrence interval } \\
\text { in years, and exceedance probablility, in percent }\end{array}$} \\
\hline 2 & 5 & 10 & 25 & 50 & 100 \\
\hline $50 \%$ & $20 \%$ & $10 \%$ & $4 \%$ & $2 \%$ & $1 \%$ \\
\hline$\ldots$ & -.- & -.. & $\cdots$ & $\ldots$ & $\cdots$ \\
\hline Weighted skew & $(\log s)=$ & $-\cdots$ & & & \\
\hline Mean & $(\log s)=$ & 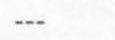 & & & \\
\hline Standard dev. & $(\log s)=$ & $\ldots$ & & & \\
\hline
\end{tabular}

Basin characteristics

\begin{tabular}{cccccccc}
\hline $\begin{array}{c}\text { Main } \\
\text { channel } \\
\begin{array}{c}\text { slope } \\
(\mathrm{ft} / \mathrm{mi})\end{array}\end{array}$ & $\begin{array}{c}\text { Stream } \\
\text { length } \\
(\mathrm{mi})\end{array}$ & $\begin{array}{c}\text { Mean } \\
\text { basin } \\
\text { elevation } \\
\text { (ft) }\end{array}$ & $\begin{array}{c}\text { Forested } \\
\text { area } \\
\text { (percent) }\end{array}$ & $\begin{array}{c}\text { Soil } \\
\text { index }\end{array}$ & $\begin{array}{c}\text { Mean } \\
\text { annual } \\
\text { precipitation } \\
\text { (in) }\end{array}$ & $\begin{array}{c}\text { Rainfall intensity, 24-hour } \\
\text { (in) }\end{array}$ & $\begin{array}{c}50 \text {-year } \\
\text { (in) }\end{array}$ \\
\hline 928 & 2.0 & 8,020 & 100 & 3.0 & 20.0 & 2.0 \\
\hline
\end{tabular}

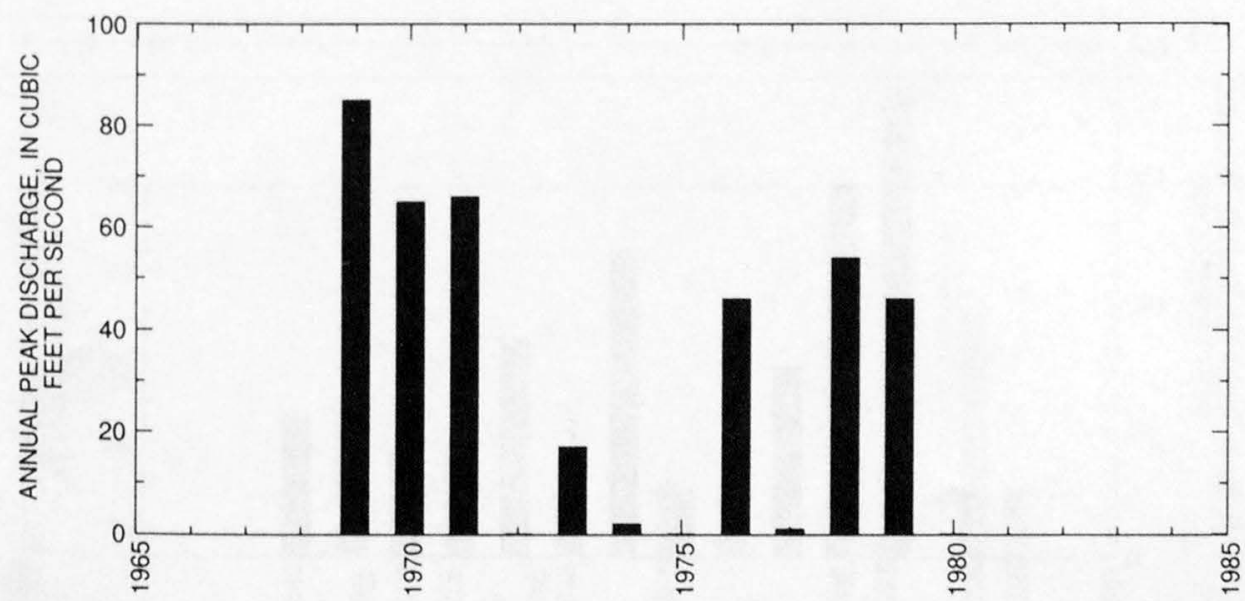


09400740 HARENBERG WASH AT FLAGSTAFF, AZ

LOCATION.--Lat 35 13'09", long $111^{\circ} 35^{\prime} 16^{\prime \prime}$, in SE $1 /{ }_{4} \mathrm{NW}^{1} / 4$ sec.7, T.21 N., R.8 E., Coconino County, Hydrologic Unit 15020015 , at Atchison Topeka, and Santa Fe railroad tracks at the east edge of Flagstaff.

DRAINAGE AREA.--2.41 $\mathrm{mi}^{2}$.

Annual peak discharges

\begin{tabular}{|c|c|c|c|c|c|c|c|}
\hline $\begin{array}{l}\text { Water } \\
\text { year }\end{array}$ & Date & $\begin{array}{c}\text { Annual peak } \\
\text { discharge } \\
\left(\mathrm{ft}^{3} / \mathrm{s}\right)\end{array}$ & $\begin{array}{l}\text { Discharge } \\
\text { codes }\end{array}$ & $\begin{array}{l}\text { Water } \\
\text { year }\end{array}$ & Date & $\begin{array}{c}\text { Annual peak } \\
\text { discharge } \\
\left(\mathrm{ft}^{3} / \mathrm{s}\right)\end{array}$ & $\begin{array}{c}\text { Discharge } \\
\text { codes }\end{array}$ \\
\hline 1969 & $09-12-69$ & 183 & $\mathrm{C}$ & 1975 & $09-00-75$ & 24 & $\mathrm{C}$ \\
\hline 1971 & $08-19-71$ & 74 & $\mathrm{C}$ & 1977 & $08-09-77$ & 44 & $\mathrm{C}$ \\
\hline 1972 & $07-24-72$ & 30 & C & 1978 & $02-28-78$ & 42 & $\mathrm{C}$ \\
\hline
\end{tabular}

Magnitude and probability of instantaneous peak flow based on period of record 1969-80

\begin{tabular}{|c|c|c|c|c|c|}
\hline \multicolumn{6}{|c|}{$\begin{array}{l}\text { Discharge, in } \mathrm{ft}^{3} / \mathrm{s} \text {, for indicated recurrence interval } \\
\text { in years, and exceedance probablility, in percent }\end{array}$} \\
\hline 2 & 5 & 10 & 25 & $50 t$ & $100 t$ \\
\hline $50 \%$ & $20 \%$ & $10 \%$ & $4 \%$ & $2 \%$ & $1 \%$ \\
\hline 60.2 & 107 & 144 & 198 & 243 & 293 \\
\hline Weighted skew & $(\log s)=$ & -0.02 & & & \\
\hline Mean & $(\log s)=$ & 1.78 & & & \\
\hline Standard dev. & $(\log s)=$ & 0.30 & & & \\
\hline
\end{tabular}

Basin characteristics

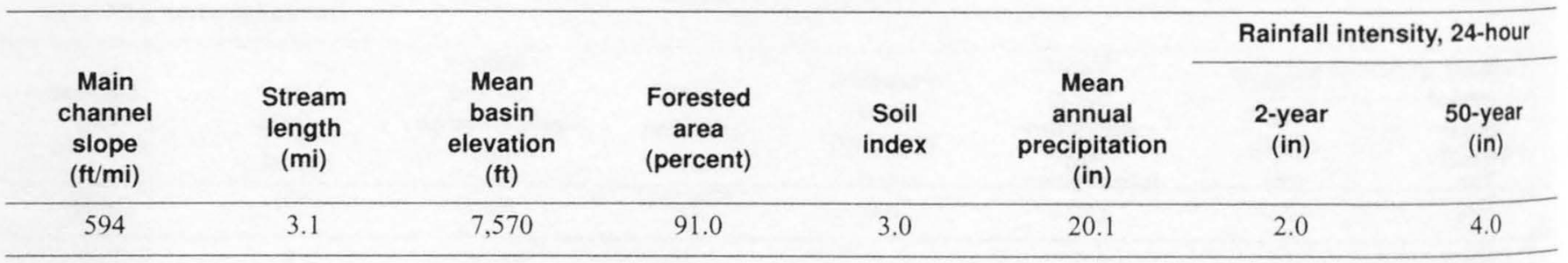

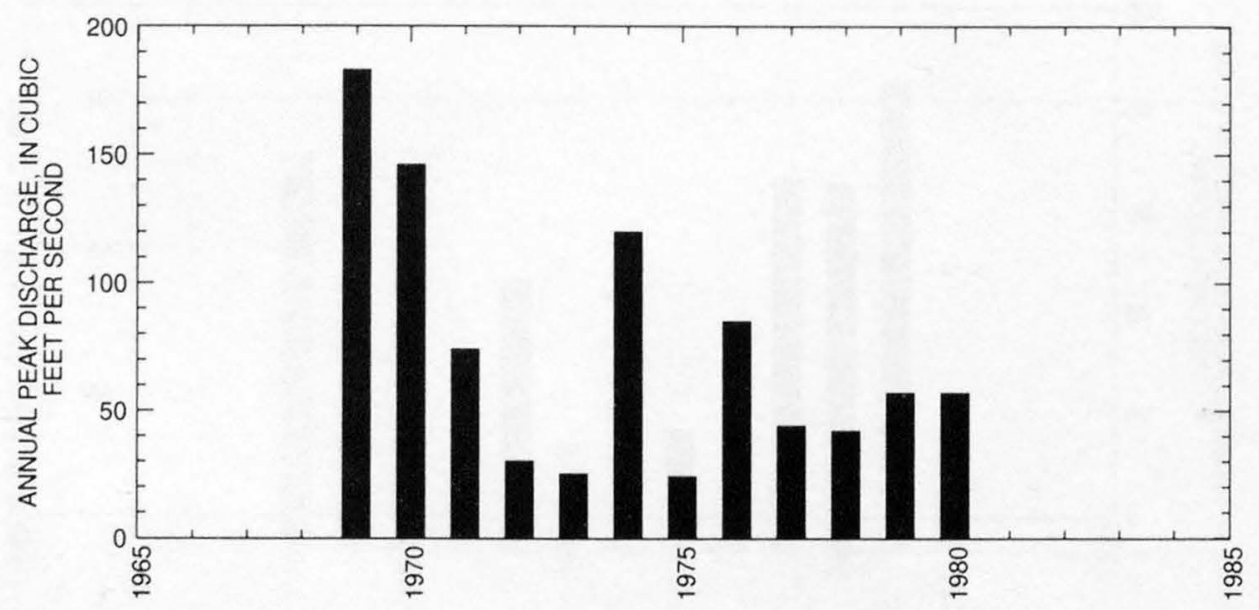


09400910 FAY CANYON NEAR FLAGSTAFF, AZ

LOCATION.--Lat $35^{\circ} 08^{\prime} 06^{\prime \prime}$, long $111^{\circ} 37^{\prime} 48^{\prime \prime}$, in NW $1 /{ }_{4} \mathrm{NW}^{1 / 4}$ sec.11, T.20 N., R.7 E., Coconino County, Hydrologic Unit 15020015 , at Lake Mary Road within corporate limits of Flagstaff.

DRAINAGE AREA.--3.28 $\mathrm{mi}^{2}$.

Annual peak discharges

\begin{tabular}{|c|c|c|c|c|c|c|c|}
\hline $\begin{array}{l}\text { Water } \\
\text { year }\end{array}$ & Date & $\begin{array}{c}\text { Annual peak } \\
\text { discharge } \\
\left(\mathrm{ft}^{3} / \mathrm{s}\right)\end{array}$ & $\begin{array}{l}\text { Discharge } \\
\text { codes }\end{array}$ & $\begin{array}{l}\text { Water } \\
\text { year }\end{array}$ & Date & $\begin{array}{c}\text { Annual peak } \\
\text { discharge } \\
\left(\mathrm{ft}^{3} / \mathrm{s}\right)\end{array}$ & $\begin{array}{c}\text { Discharge } \\
\text { codes }\end{array}$ \\
\hline 1964 & $00-00-64$ & 0.3 & LT & 1972 & $10-24-71$ & 31 & \\
\hline 1966 & $12-30-65$ & 87 & & 1974 & $08-06-74$ & 3.0 & LT \\
\hline 1967 & $00-00-67$ & 10 & LT & 1975 & $09-00-75$ & 1.0 & LT \\
\hline 1968 & $04-00-68$ & 4.0 & & 1976 & $00-00-76$ & 10 & ES \\
\hline 1970 & $09-05-70$ & 3.0 & ES & 1979 & $00-00-79$ & 70 & \\
\hline 1971 & $08-15-71$ & 2.0 & ES & 1980 & $02-19-80$ & 29 & \\
\hline
\end{tabular}

Magnitude and probability of instantaneous peak flow based on period of record 1964-77, $1979-80$

\begin{tabular}{|c|c|c|c|c|c|}
\hline \multicolumn{6}{|c|}{$\begin{array}{l}\text { Discharge, in } \mathrm{ft}^{3} / \mathrm{s} \text {, for indicated recurrence interval } \\
\text { in years, and exceedance probablility, in percent }\end{array}$} \\
\hline 2 & 5 & 10 & 25 & $50 \dagger$ & $100 \dagger$ \\
\hline $50 \%$ & $20 \%$ & $10 \%$ & $4 \%$ & $2 \%$ & $1 \%$ \\
\hline 8 & 36 & 66 & 98 & 128 & 182 \\
\hline Weighted skew & $(\log s)=$ & 0.01 & & & \\
\hline Mean & $(\log s)=$ & 0.96 & & & \\
\hline Standard dev. & $(\log s)=$ & 0.56 & & & \\
\hline
\end{tabular}

fReliability of values in column is uncertain, and potential errors are large.

Basin characteristics

\begin{tabular}{ccccccc}
\hline $\begin{array}{c}\text { Main } \\
\text { channel } \\
\text { slope } \\
(\mathrm{ft} / \mathrm{mi})\end{array}$ & $\begin{array}{c}\text { Stream } \\
\text { length } \\
(\mathrm{mi})\end{array}$ & $\begin{array}{c}\text { Mean } \\
\text { basin } \\
\text { elevation } \\
(\mathrm{ft})\end{array}$ & $\begin{array}{c}\text { Forested } \\
\text { area } \\
\text { (percent) }\end{array}$ & $\begin{array}{c}\text { Soil } \\
\text { index }\end{array}$ & $\begin{array}{c}\text { Mean } \\
\text { annual } \\
\text { precipitation } \\
\text { (in) }\end{array}$ & $\begin{array}{c}\text { Rainfall intensity, 24-hour } \\
\text { (in) }\end{array}$ \\
\hline 72.2 & 2.4 & 7,000 & 73.0 & 3.0 & 19.6 & 2.1 \\
\hline
\end{tabular}

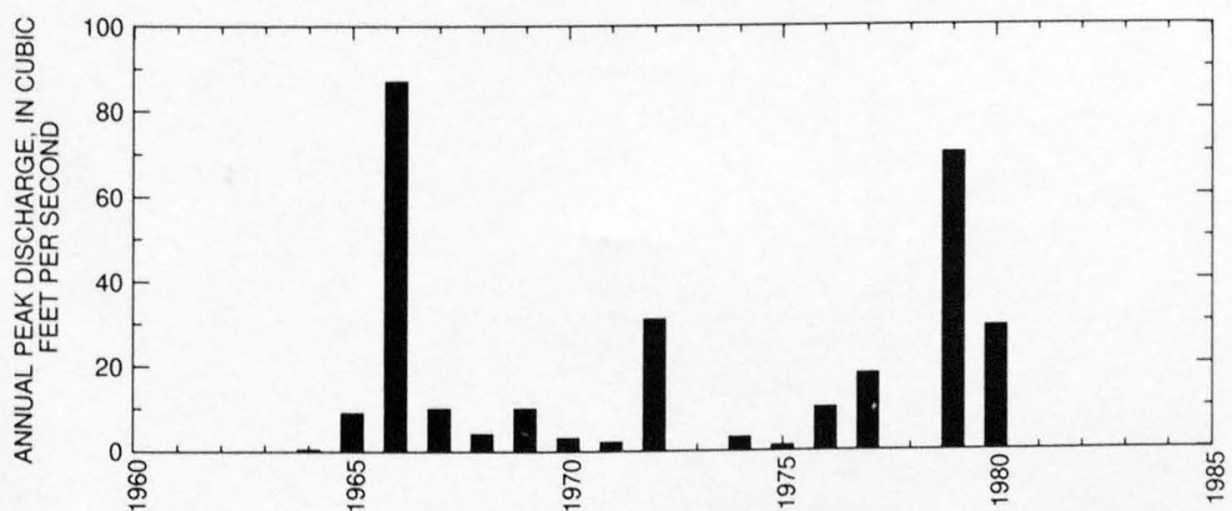




\section{LITTLE COLORADO RIVER AT GRAND FALLS, AZ}

LOCATION.--Lat 35'26'45", long 111 '12'12", in T.24 N., R.11 E. (unsurveyed), Coconino County, Hydrologic Unit 15020016, in Navajo Indian Reservation, on left bank 1,000 ft downstream from Grand Falls, 4.5 mi upstream from Dinnebito Wash, 30 mi northeast of Flagstaff, and 96 mi upstream from mouth.

DRAINAGE AREA.--21,068 $\mathrm{mi}^{2}$, of which $368 \mathrm{mi}^{2}$ are noncontributing.

PERIOD OF RECORD.--November 1925 to September 1951, October 1951 to September 1953 (peak discharges only), October 1953 to June 1960, October 1989 to September 1994 (discontinued). Monthly discharges only for January to September 1950, published in WSP 1313.

REVISED RECORDS.--WDR AZ-89-1: Drainage area.

GAGE.--Water-stage recorder. Datum of gage is $4,438.9 \mathrm{ft}$ above sea level.

REMARKS.-- Diversions above station for irrigation of about 29,000 acres. Some regulation by reservoirs above station (combined capacity, about 71,000 acre-feet in 1950, not including Long Pine Reservoir or Lake Mary).

AVERAGE DISCHARGE.--35 years (water years 1928-51, 1954-59, 1990-94), $247 \mathrm{ft}^{3} / \mathrm{s}, 181,800$ acre- $\mathrm{ft} / \mathrm{yr}$; median of yearly mean discharges, $210 \mathrm{ft}^{3} / \mathrm{s}, 152,000$ acre- $\mathrm{ft} / \mathrm{yr}$.

EXTREMES FOR PERIOD OF RECORD.--Maximum discharge, 50,500 $\mathrm{ft}^{3} / \mathrm{s} \mathrm{Apr}$. 5, 1929, gage height $30.0 \mathrm{ft}$, no flow at times each year.

EXTREMES OUTSIDE PERIOD OF RECORD.--A discharge of about $120,000 \mathrm{ft}^{3} / \mathrm{s}$, gage height $47.0 \mathrm{ft}$, from floodmarks, occurred on Sept. 19 , 1923, and is the highest since 1870 .

Annual peak discharges

\begin{tabular}{|c|c|c|c|c|c|c|c|}
\hline $\begin{array}{l}\text { Water } \\
\text { year }\end{array}$ & Date & $\begin{array}{c}\text { Annual peak } \\
\text { discharge } \\
\left(\mathrm{ft}^{3} / \mathrm{s}\right)\end{array}$ & $\begin{array}{l}\text { Discharge } \\
\text { codes }\end{array}$ & $\begin{array}{l}\text { Water } \\
\text { year }\end{array}$ & Date & $\begin{array}{c}\text { Annual peak } \\
\text { discharge } \\
\left(\mathrm{ft}^{3} / \mathrm{s}\right)\end{array}$ & $\begin{array}{l}\text { Discharge } \\
\text { codes }\end{array}$ \\
\hline 1923 & $09-19-23$ & ${ }^{1} 120,000$ & HP & 1947 & $08-24-47$ & 10,600 & \\
\hline 1926 & $09-27-26$ & 27,800 & & 1948 & $10-16-47$ & 12,400 & \\
\hline 1927 & $06-28-27$ & 28,800 & & 1949 & $08-09-49$ & 10,400 & \\
\hline 1928 & $02-07-28$ & 2,140 & & 1950 & $07-18-50$ & 3,500 & \\
\hline 1929 & $04-05-29$ & 50,500 & & 1951 & $08-30-51$ & 10,200 & \\
\hline 1930 & $07-19-30$ & 13,700 & & 1952 & $01-20-52$ & 26,100 & \\
\hline 1931 & $08-01-31$ & 6,530 & & 1953 & $07-31-53$ & 4,140 & \\
\hline 1932 & $02-10-32$ & 31,000 & - & 1954 & $03-25-54$ & 7,450 & \\
\hline 1933 & $09-12-33$ & 7,500 & & 1955 & $06-15-55$ & 9,020 & \\
\hline 1934 & $10-07-33$ & 4,920 & & 1956 & $08-17-56$ & 2,320 & \\
\hline 1935 & $04-10-35$ & 7,350 & & 1957 & $01-12-57$ & 8,390 & \\
\hline 1936 & $08-06-36$ & 5,430 & & 1958 & $08-23-58$ & 4,560 & \\
\hline 1937 & $02-09-37$ & 21,800 & & 1959 & 08-07-59 & 3,080 & \\
\hline 1938 & $03-05-38$ & 38,000 & & 1960 & $11-01-59$ & 7,960 & \\
\hline 1939 & $04-05-39$ & 6,680 & & 1970 & $09-06-70$ & 11,400 & $\mathrm{KR}, \mathrm{HP}$ \\
\hline 1940 & $07-27-40$ & 20,100 & & 1972 & $10-03-71$ & 13,200 & $\mathrm{KR}, \mathrm{HP}$ \\
\hline 1941 & $03-15-41$ & 17,000 & & 1990 & $08-17-90$ & 1,920 & KR \\
\hline 1942 & $10-04-41$ & 8,760 & & 1991 & $04-11-91$ & 3,320 & $\mathrm{KR}$ \\
\hline 1943 & $09-28-43$ & 3,900 & & 1992 & $08-29-92$ & 3,716 & KR \\
\hline 1944 & $09-29-44$ & 5,320 & & 1993 & $01-11-93$ & 16,600 & $\mathrm{KR}$ \\
\hline 1945 & $08-12-45$ & 4,650 & & 1994 & $03-23-94$ & 2,760 & KR \\
\hline 1946 & $09-19-46$ & 12,900 & & & & & \\
\hline
\end{tabular}

${ }^{1}$ Highest since 1870. 
09401000 LITTLE COLORADO RIVER AT GRAND FALLS, AZ--Continued

Discharge rating table developed October 1989

\begin{tabular}{cccc}
\hline $\begin{array}{c}\text { Gage height } \\
(\mathbf{f t})\end{array}$ & $\begin{array}{c}\text { Discharge } \\
\left(\mathbf{f t}^{\mathbf{3}} \mathbf{s}\right)\end{array}$ & $\begin{array}{c}\text { Gage height } \\
(\mathbf{f t})\end{array}$ & $\begin{array}{c}\text { Discharge } \\
\left(\mathbf{f t}^{3} / \mathbf{s}\right)\end{array}$ \\
\hline 7.0 & 390 & 25.0 & 34,790 \\
8.0 & 955 & 28.0 & 44,020 \\
10.0 & 2,680 & 31.0 & 54,080 \\
13.0 & 6,550 & 34.0 & 64,940 \\
16.0 & 11,820 & 38.0 & 80,600 \\
19.0 & 18,360 & 41.0 & 93,210 \\
22.0 & 26,100 & 43.0 & 102,000 \\
\hline
\end{tabular}

Basin characteristics

\begin{tabular}{cccccccc}
\hline $\begin{array}{c}\text { Main } \\
\text { channel } \\
\text { slope } \\
(\mathrm{ft} / \mathrm{mi})\end{array}$ & $\begin{array}{c}\text { Stream } \\
\text { length } \\
(\mathrm{mi})\end{array}$ & $\begin{array}{c}\text { Mean } \\
\text { basin } \\
\text { elevation } \\
(\mathrm{ft})\end{array}$ & $\begin{array}{c}\text { Forested } \\
\text { area } \\
\text { (percent) }\end{array}$ & $\begin{array}{c}\text { Soil } \\
\text { index }\end{array}$ & $\begin{array}{c}\text { Mean } \\
\text { annual } \\
\text { precipitation } \\
\text { (in) }\end{array}$ & $\begin{array}{c}\text { Rainfall intensity, 24-hour } \\
\text { (in) }\end{array}$ & $\begin{array}{c}50 \text {-year } \\
\text { (in) }\end{array}$ \\
\hline 10.5 & 234 & 6,440 & 33.0 & 2.7 & 12.9 & 1.5 & 2.9 \\
\hline
\end{tabular}


09401000 LITTLE COLORADO RIVER AT GRAND FALLS, AZ--Continued

MEAN MONTHLY AND ANNUAL DISCHARGES 1927-49, 1951, 1954-59, $1990-91,1993.94$

\begin{tabular}{|c|c|c|c|c|c|c|}
\hline MONTH & $\begin{array}{l}\text { MAXIMUM } \\
(\text { FT } 3 / S)\end{array}$ & $\begin{array}{l}\text { MINIMUM } \\
(\text { FT3/S) }\end{array}$ & $\begin{array}{c}\text { MEAN } \\
(\mathrm{FT} 3 / \mathrm{S})\end{array}$ & $\begin{array}{l}\text { STAN- } \\
\text { DARD } \\
\text { DEVIA- } \\
\text { TION } \\
\text { (FT3/S) }\end{array}$ & $\begin{array}{l}\text { COEFFI- } \\
\text { CIENT OF } \\
\text { VARI- } \\
\text { ATION }\end{array}$ & $\begin{array}{c}\text { PERCENT } \\
\text { OF } \\
\text { ANNUAL } \\
\text { RUNOFF }\end{array}$ \\
\hline OCTOBER & 927 & 0.00 & 116 & 229 & 2.0 & 3.7 \\
\hline NOVEMBER & 287 & 0.00 & 45 & 75 & 1.7 & 1.4 \\
\hline DECEMBER & 468 & 0.00 & 39 & 88 & 2.3 & 1.3 \\
\hline JANUARY & 4,100 & 0.00 & 211 & 716 & 3.4 & 6.7 \\
\hline FEBRUARY & 2,670 & 0.00 & 376 & 693 & 1.8 & 12.0 \\
\hline MARCH & 2,390 & 0.00 & 644 & 659 & 1.0 & 20.6 \\
\hline APRIL & 2,610 & 8.8 & 624 & 638 & 1.0 & 19.9 \\
\hline MAY & 1,410 & 0.00 & 97 & 249 & 2.6 & 3.1 \\
\hline JUNE & 622 & 0.00 & 30 & 117 & 3.9 & 1.0 \\
\hline JULY & 1,580 & 0.00 & 163 & 311 & 1.9 & 5.2 \\
\hline AUGUST & 1,990 & 0.00 & 475 & 482 & 1.0 & 15.2 \\
\hline SEPTEMBER & 1,940 & 0.00 & 309 & 427 & 1.4 & 9.9 \\
\hline ANNUAL & 811 & 26 & 260 & 204 & 0.78 & 100 \\
\hline
\end{tabular}

MAGNITUDE AND PROBABILITY OF ANNUAL LOW FLOW BASED ON PERIOD OF RECORD $1927-49,1955 \cdot 60,1991.92,1994$

PISCHARGE, IN FT3/S, FOR INDICATED
PERIOD
(CON

MAGNITUDE AND PROBABILITY OF ANNUAL HIGH FLOW BASED ON PERIOD OF RECORD 1927-49, 1951, 1954-59, 1990-91, 1993-94

MAGNITUDE AND PROBABILITY OF INSTANTANEOUS PEAK FLOW BASED ON PERIOD OF RECORD 1923, 1926-60,1990-91, 1993.94

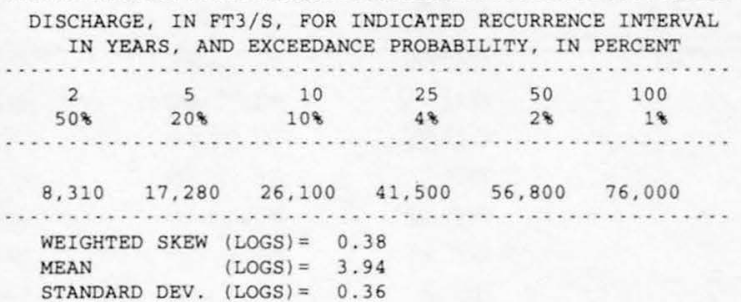

\begin{tabular}{|c|c|c|c|c|c|c|}
\hline PERIOD & & \multicolumn{5}{|c|}{ DISCHARGE, IN FT $3 / \mathrm{S}$, FOR INDICATED } \\
\hline (CON- & & \multicolumn{3}{|c|}{ EXCEEDANCE PROBABILITY, } & \multicolumn{2}{|c|}{ IN PERCENT } \\
\hline SECU - & & ...... & 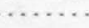 & ....... & ........ & .... \\
\hline TIVE & 2 & 5 & 10 & $25 \#$ & $50 \#$ & $100 \#$ \\
\hline DAYS) & 508 & 208 & 108 & 48 & 28 & 18 \\
\hline 1 & 5,170 & 10,100 & 14,400 & 21,100 & 27,000 & 33,700 \\
\hline 3 & 3,780 & 7,130 & 9,880 & 13,900 & 17.300 & 21,100 \\
\hline 7 & 2,520 & 4,400 & 5,690 & 7,320 & 8,500 & 9,640 \\
\hline 15 & 1,780 & 2,990 & 3,690 & 4,420 & 4,860 & 5,230 \\
\hline 30 & 1,210 & 2,040 & 2,540 & 3,080 & 3,430 & 3,720 \\
\hline 60 & 789 & 1,440 & 1,920 & 2,540 & 3,010 & 3,470 \\
\hline 90 & 566 & 1,080 & 1,490 & 2,070 & 2,550 & 3,060 \\
\hline
\end{tabular}

DURATION TABLE OF DAILY MEAN FLOW FOR PERIOD OF RECORD 1927.49, 1951, 1954.59, 1990.91, 1993.94

DISCHARGE, IN FT3/S, WHICH WAS EQUALED OR EXCEEDED FOR INDICATED PERCENT OF TIME

\begin{tabular}{|c|c|c|c|c|c|c|c|c|c|c|c|c|c|c|c|c|}
\hline 18 & 58 & 108 & 158 & 208 & 308 & 408 & 508 & 608 & 708 & 808 & 908 & 958 & 988 & 998 & 99.58 & 99.98 \\
\hline 390 & 1,440 & 745 & 402 & 226 & 79 & 25 & 7.7 & 0.17 & 0.00 & 0.00 & 0.00 & 0.00 & 0.00 & 0.00 & 0.00 & 0.00 \\
\hline
\end{tabular}

\# Reliability of values in column is uncertain, and potential errors are large. 
ANNUAL MEAN DISCHARGE, IN CUBIC FEET PER SECOND

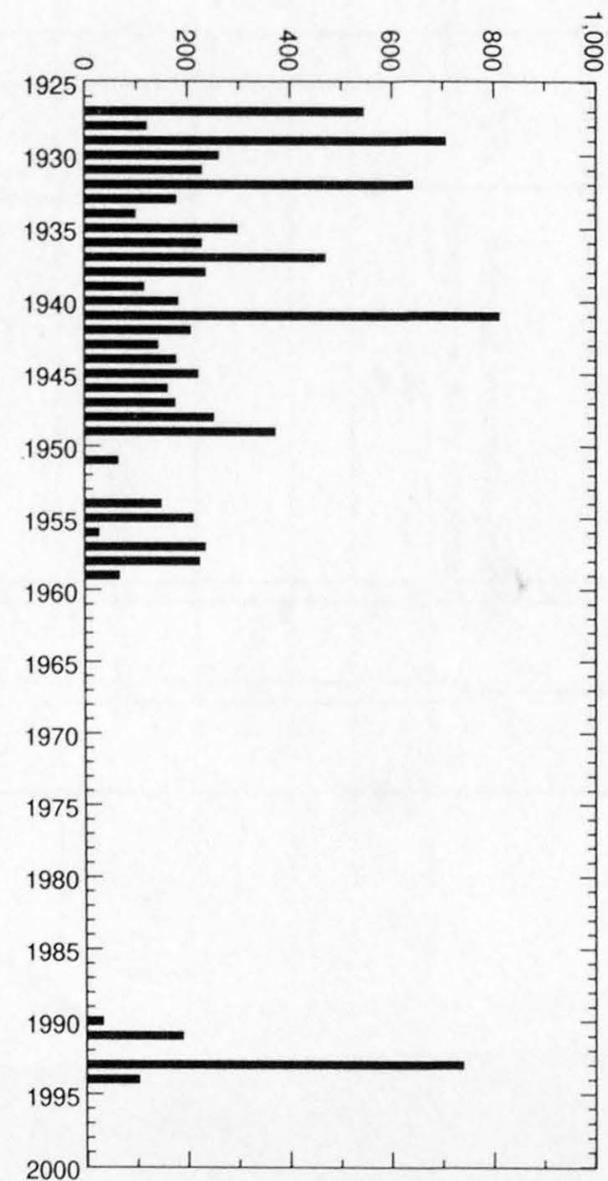

ANNUAL PEAK DISCHARGE, IN CUBIC FEET PER SECOND

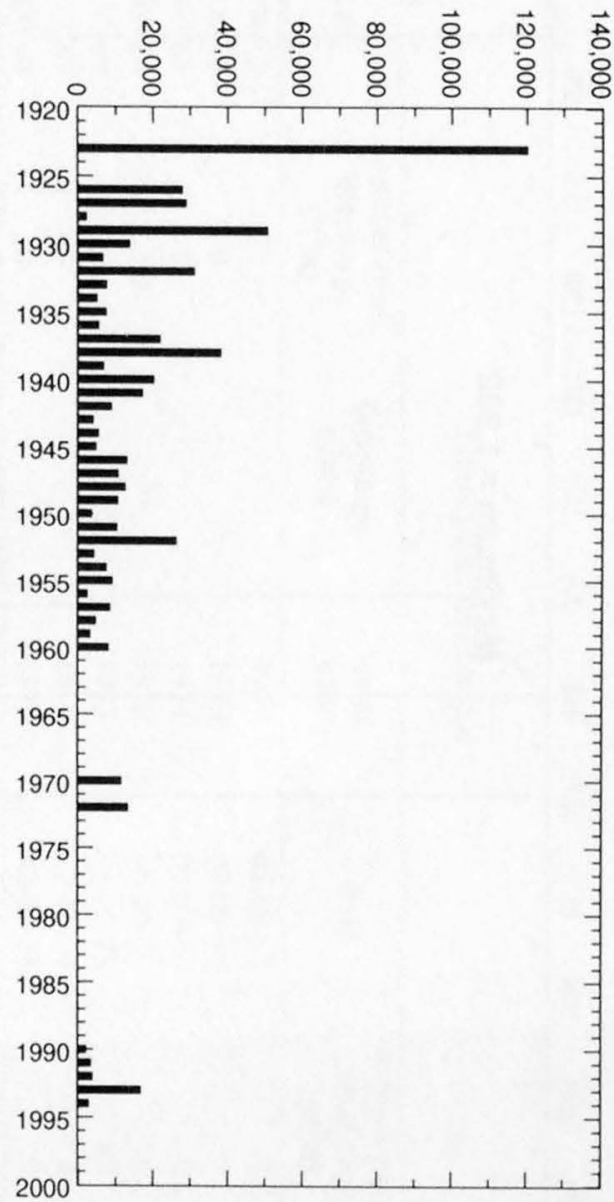


09401000 LITTLE COLORADO RIVER AT GRAND FALLS, AZ--Continued

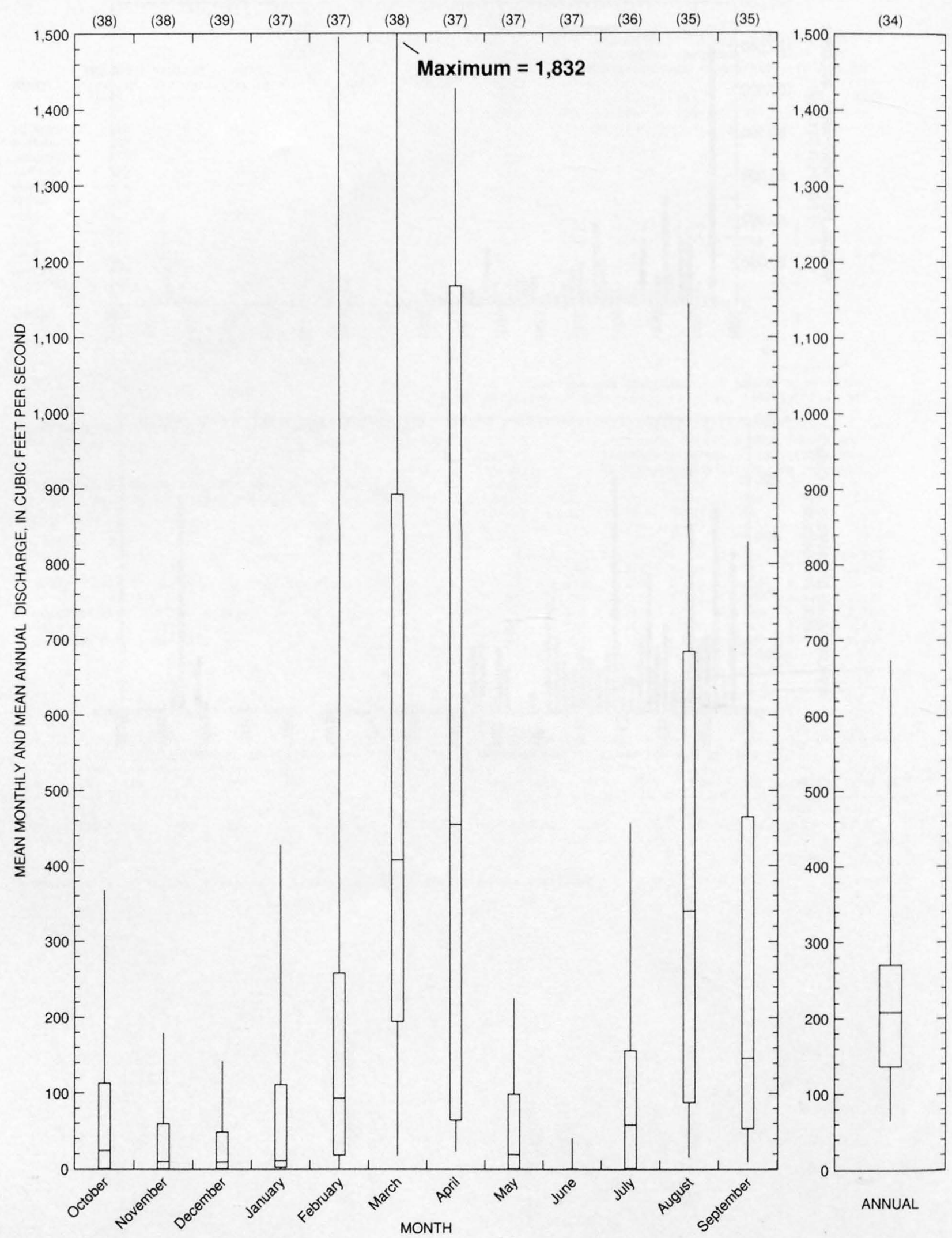


09401210 SLATE MOUNTAIN WASH NEAR FLAGSTAFF, AZ

LOCATION.--Lat 35 30'55", long 111 $50^{\prime} 55^{\prime \prime}$, in SW1/4 sec.26, T.25 N., R.5 E., Coconino County, at U.S. Highway 180, 24 mi northwest of Flagstaff.

DRAINAGE AREA.--5.43 $\mathrm{mi}^{2}$.

Annual peak discharges

\begin{tabular}{cccccccc}
\hline $\begin{array}{c}\text { Water } \\
\text { year }\end{array}$ & Date & $\begin{array}{c}\text { Annual peak } \\
\text { discharge } \\
\left(\mathrm{ft}^{3} / \mathbf{s}\right)\end{array}$ & $\begin{array}{c}\text { Discharge } \\
\text { codes }\end{array}$ & $\begin{array}{c}\text { Water } \\
\text { year }\end{array}$ & Date & $\begin{array}{c}\text { Annual peak } \\
\text { discharge } \\
\left(\mathbf{f t}^{3} / \mathbf{s}\right)\end{array}$ & $\begin{array}{c}\text { Discharge } \\
\text { codes }\end{array}$ \\
\hline 1962 & $00-00-62$ & 77 & & 1969 & $08-00-69$ & 1.0 & LT \\
1963 & $00-00-63$ & 0 & 1970 & $00-00-70$ & 13 & \\
1964 & $00-00-64$ & 0 & 1971 & $00-00-71$ & 0 & \\
1965 & $10-00-64$ & 5.0 & 1972 & $06-06-72$ & 40 & \\
1966 & $12-31-65$ & 37 & 1973 & $04-00-73$ & 88 & \\
1967 & $00-00-67$ & 66 & & 1974 & $00-00-74$ & 0 & \\
1968 & $00-00-68$ & 0 & & 1975 & $00-00-75$ & 0 & \\
\hline
\end{tabular}

Magnitude and probability of instantaneous peak flow based on period of record

\begin{tabular}{|c|c|c|c|c|c|}
\hline \multicolumn{6}{|c|}{$\begin{array}{l}\text { Discharge, in } \mathrm{ft}^{3} / \mathrm{s} \text {, for indicated recurrence interval } \\
\text { in years, and exceedance probablility, in percent }\end{array}$} \\
\hline 2 & 5 & 10 & 25 & 50 & 100 \\
\hline $50 \%$ & $20 \%$ & $10 \%$ & $4 \%$ & $2 \%$ & $1 \%$ \\
\hline -.- & --- & -.. & $\cdots$ & -- & -- \\
\hline Weighted skew & $(\log s)=$ & --- & & & \\
\hline Mean & $(\log s)=$ & ... & & & \\
\hline Standard dev. & $(\log s)=$ & $\ldots$ & & & \\
\hline
\end{tabular}

Basin characteristics

\begin{tabular}{cccccccc}
\hline $\begin{array}{c}\text { Main } \\
\text { channel } \\
\text { slope } \\
(\mathrm{ft} / \mathrm{mi})\end{array}$ & $\begin{array}{c}\text { Stream } \\
\text { length } \\
(\mathrm{mi})\end{array}$ & $\begin{array}{c}\text { Mean } \\
\text { basin } \\
\text { elevation } \\
(\mathrm{ft})\end{array}$ & $\begin{array}{c}\text { Forested } \\
\text { area } \\
\text { (percent) }\end{array}$ & $\begin{array}{c}\text { Soil } \\
\text { index }\end{array}$ & $\begin{array}{c}\text { Mean } \\
\text { annual } \\
\text { precipitation } \\
\text { (in) }\end{array}$ & $\begin{array}{c}\text { Rainfall intensity, 24-hour } \\
\text { (in) }\end{array}$ & $\begin{array}{c}50 \text {-year } \\
\text { (in) }\end{array}$ \\
\hline 46.4 & 9.8 & 7,350 & 60.0 & 3.0 & 19.7 & 2.1 \\
\hline
\end{tabular}

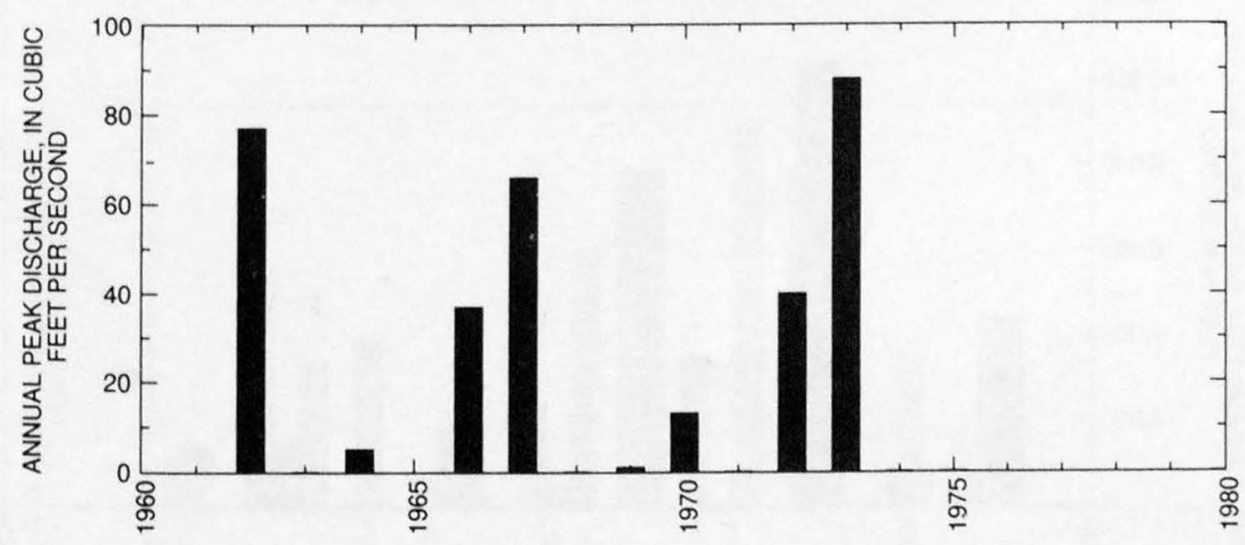




\section{CEDAR WASH NEAR CAMERON, AZ}

LOCATION.--Lat $35^{\circ} 51^{\prime} 31^{\prime \prime}$, long $111^{\circ} 26^{\prime} 32^{\prime \prime}$, in NW1/4 NW $1 / 4$ sec.33, T.29 N., R.9 E., Coconino County, Hydrologic Unit 15020016 downstream from State Highway 64 at the Tappan Spring, and 1 mi west of the intersection of State Highway 64 and U.S. Highway 89.

DRAINAGE AREA.--579 $\mathrm{mi}^{2}$.

Annual peak discharges

\begin{tabular}{|c|c|c|c|c|c|c|c|}
\hline $\begin{array}{l}\text { Water } \\
\text { year }\end{array}$ & Date & $\begin{array}{c}\text { Annual peak } \\
\text { discharge } \\
\left(\mathrm{ft}^{3} / \mathrm{s}\right)\end{array}$ & $\begin{array}{l}\text { Discharge } \\
\text { codes }\end{array}$ & $\begin{array}{l}\text { Water } \\
\text { year }\end{array}$ & Date & $\begin{array}{c}\text { Annual peak } \\
\text { discharge } \\
\left(\mathrm{ft}^{3} / \mathrm{s}\right)\end{array}$ & $\begin{array}{l}\text { Discharge } \\
\text { codes }\end{array}$ \\
\hline 1967 & $00-00-67$ & 4,400 & & 1972 & $07-18-72$ & 440 & \\
\hline 1968 & $07-00-68$ & 880 & & 1973 & $10-19-72$ & 1,950 & \\
\hline 1969 & $09-11-69$ & 10,400 & & 1974 & $09-05-74$ & 50 & \\
\hline 1970 & $09-05-70$ & 50 & ES & 1975 & $09-00-75$ & 1,490 & \\
\hline 1971 & $09-29-71$ & 7,900 & & 1976 & $00-00-76$ & 1,400 & \\
\hline
\end{tabular}

Magnitude and probability of instantaneous peak flow based on period of record 1967-76

\begin{tabular}{|c|c|c|c|c|c|}
\hline \multicolumn{6}{|c|}{$\begin{array}{l}\text { Discharge, in } \mathrm{ft}^{3} / \mathrm{s} \text {, for indicated recurrence interval } \\
\text { in years, and exceedance probablility, in percent }\end{array}$} \\
\hline 2 & 5 & 10 & 25 & $50 t$ & $100 \dagger$ \\
\hline $50 \%$ & $20 \%$ & $10 \%$ & $4 \%$ & $2 \%$ & $1 \%$ \\
\hline 1,510 & 4,550 & 8,040 & 14,600 & 21,400 & 30,200 \\
\hline Weighted skew & $(\log s)=$ & -0.08 & & & \\
\hline Mean & $(\log s)=$ & 3.17 & & & \\
\hline Standard dev. & $(\log s)=$ & 0.58 & & & \\
\hline
\end{tabular}

F Reliability of values in column is uncertain, and potential errors are large.

Basin characteristics

\begin{tabular}{cccccccc}
\hline $\begin{array}{c}\text { Main } \\
\text { channel } \\
\text { slope } \\
(\mathrm{ft} / \mathrm{mi})\end{array}$ & $\begin{array}{c}\text { Stream } \\
\text { length } \\
\text { (mi) }\end{array}$ & $\begin{array}{c}\text { Mean } \\
\text { basin } \\
\text { elevation } \\
\text { (ft) }\end{array}$ & $\begin{array}{c}\text { Forested } \\
\text { area } \\
\text { (percent) }\end{array}$ & $\begin{array}{c}\text { Soil } \\
\text { index }\end{array}$ & $\begin{array}{c}\text { Mean } \\
\text { annual } \\
\text { precipitation } \\
\text { (in) }\end{array}$ & $\begin{array}{c}\text { Rainfall intensity, 24-hour } \\
\text { (in) }\end{array}$ & $\begin{array}{c}50 \text {-year } \\
\text { (in) }\end{array}$ \\
\hline 123 & 32.4 & 6,430 & 37.0 & 3.0 & 13.7 & 1.7 \\
\hline
\end{tabular}

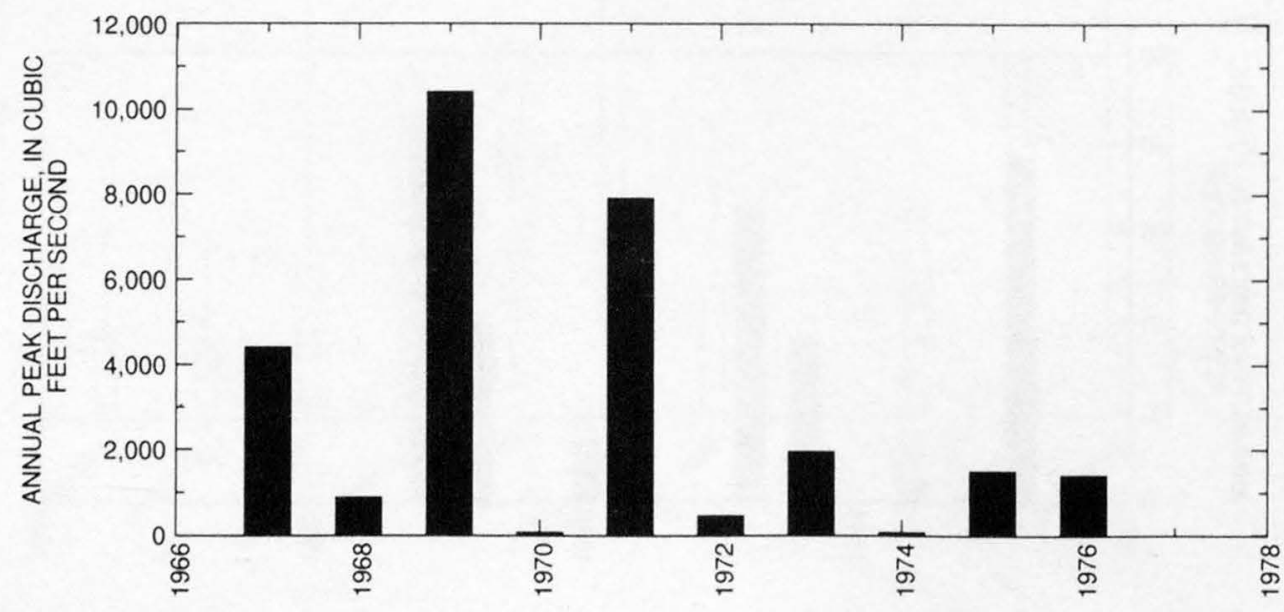


09401245 KETHLA VALLEY TRIBUTARY NEAR KAYENTA, AZ

LOCATION.--Lat 36'29'53", long 110³7'15", Navajo County, Hydrologic Unit 15020018, at State Highway 64, 15.5 mi southwest of Tsegi Trading Post, and $26 \mathrm{mi}$ southwest of Kayenta.

DRAINAGE AREA.--0.79 $\mathrm{mi}^{2}$.

Annual peak discharges

\begin{tabular}{|c|c|c|c|c|c|c|c|}
\hline $\begin{array}{l}\text { Water } \\
\text { year }\end{array}$ & Date & $\begin{array}{c}\text { Annual peak } \\
\text { discharge } \\
\left(\mathrm{ft}^{3} / \mathbf{s}\right)\end{array}$ & $\begin{array}{l}\text { Discharge } \\
\text { codes }\end{array}$ & $\begin{array}{l}\text { Water } \\
\text { year }\end{array}$ & Date & $\begin{array}{c}\text { Annual peak } \\
\text { discharge } \\
\left(\mathrm{ft}^{3} / \mathrm{s}\right)\end{array}$ & $\begin{array}{c}\text { Discharge } \\
\text { codes }\end{array}$ \\
\hline 1962 & $00-00-62$ & 175 & & 1970 & $09-05-70$ & 137 & \\
\hline 1963 & $08-06-63$ & 109 & & 1971 & $08-00-71$ & 290 & \\
\hline 1964 & $08-00-64$ & 128 & & 1972 & $09-02-72$ & 250 & \\
\hline 1965 & $00-00-65$ & 23 & & 1973 & $10-00-72$ & 225 & \\
\hline 1966 & $11-2-365$ & 12 & LT & 1974 & $07-22-74$ & 114 & \\
\hline 1967 & $07-30-67$ & 80 & & 1975 & $07-11-75$ & 57 & \\
\hline 1968 & $07-25-68$ & 195 & & 1976 & $00-00-76$ & 0.3 & ES \\
\hline 1969 & $09-00-69$ & 94 & & & & & \\
\hline
\end{tabular}

Magnitude and probability of instantaneous peak flow based on period of record 1962-76

\begin{tabular}{|c|c|c|c|c|c|}
\hline \multicolumn{6}{|c|}{$\begin{array}{l}\text { Discharge, in } \mathrm{ft}^{3} / \mathrm{s} \text {, for indicated recurrence interval } \\
\text { in years, and exceedance probablility, in percent }\end{array}$} \\
\hline 2 & 5 & 10 & 25 & $50 t$ & $100 \dagger$ \\
\hline $50 \%$ & $20 \%$ & $10 \%$ & $4 \%$ & $2 \%$ & $1 \%$ \\
\hline 121 & 193 & 244 & 312 & 365 & 419 \\
\hline Weighted skew & $(\log s)=$ & -0.14 & & & \\
\hline Mean & $(\log s)=$ & 2.08 & & & \\
\hline Standard dev. & $(\log s)=$ & 0.25 & & & \\
\hline
\end{tabular}

F Reliability of values in column is uncertain, and potential errors are large.

Basin characteristics

\begin{tabular}{cccccccc}
\hline $\begin{array}{c}\text { Main } \\
\text { channel } \\
\text { slope } \\
(\mathrm{ft} / \mathrm{mi})\end{array}$ & $\begin{array}{c}\text { Stream } \\
\text { length } \\
(\mathrm{mi})\end{array}$ & $\begin{array}{c}\text { Mean } \\
\text { basin } \\
\text { elevation } \\
(\mathrm{ft})\end{array}$ & $\begin{array}{c}\text { Forested } \\
\text { area } \\
\text { (percent) }\end{array}$ & $\begin{array}{c}\text { Soil } \\
\text { index }\end{array}$ & $\begin{array}{c}\text { Mean } \\
\text { annual } \\
\text { precipitation } \\
\text { (in) }\end{array}$ & $\begin{array}{c}\text { Rainfall intensity, 24-hour } \\
\text { (in) }\end{array}$ & $\begin{array}{c}50 \text {-year } \\
\text { (in) }\end{array}$ \\
\hline 87.7 & 2.3 & 6,730 & 21.0 & 3.0 & 10.2 & 1.3 \\
\hline
\end{tabular}

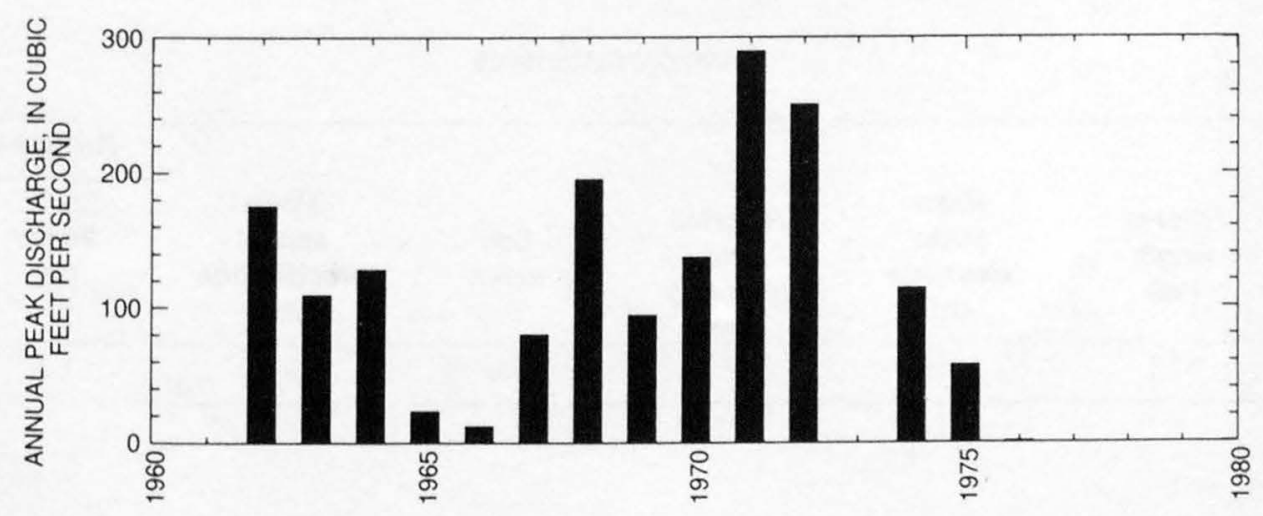




\section{MOENKOPI WASH AT MOENKOPI, AZ}

LOCATION.--Lat 36 $06^{\prime} 18^{\prime \prime}$, long $111^{\circ} 12^{\prime} 04^{\prime \prime}$, in NW $1 /{ }_{4} \mathrm{NE}^{1} / 4 \mathrm{sec} .3$, T.31 N., R.11 E. (unsurveyed), Coconino County, Hydrologic Unit 15020018 , in Hopi Indian Reservation on right bank, $100 \mathrm{ft}$ upstream from bridge on State Highway 264, 1.3 mi southeast of Moenkopi, 2.5 mi down. stream from former gaging station 09401250, and 12.5 mi downstream from Begashibito Wash.

DRAINAGE AREA.--1,629 $\mathrm{mi}^{2}$.

PERIOD OF RECORD.--July 1976 to current year. Records for October 1973 to July 1976, at site 2.5 mi upstream, not equivalent below 1.5 fi3 $\mathrm{s}$ due to channel losses.

REVISED RECORDS.--WDR AZ-88-1: Drainage area.

GAGE.--Water-stage recorder and crest stage gages. Elevation of gage is 4,610 ft above sea level, from topographic map.

REMARKS.--Records poor.

EXTREMES FOR PERIOD OF RECORD.--Maximum discharge, 10,100 ft $3 / \mathrm{s} \mathrm{Sept.} \mathrm{30,} \mathrm{1983,} \mathrm{gage} \mathrm{height,} 15.10 \mathrm{ft}$, from rating curve extended above $220 \mathrm{ft}^{3} / \mathrm{s}$ on basis of step-backwater computation at gage heights $12.2 \mathrm{ft}, 15.0 \mathrm{ft}$, and $17.8 \mathrm{ft}$; no flow at times each year.

EXTREMES OUTSIDE PERIOD OF RECORD.--A discharge of $15,100 \mathrm{ft}^{3} / \mathrm{s}$ occurred Aug. 4, 1929, at former gaging station site $3.5 \mathrm{mi}$ downstream.

Annual peak discharges

\begin{tabular}{|c|c|c|c|c|c|c|c|}
\hline $\begin{array}{l}\text { Water } \\
\text { year }\end{array}$ & Date & $\begin{array}{c}\text { Annual peak } \\
\text { discharge } \\
\left(\mathrm{ft}^{3} / \mathrm{s}\right)\end{array}$ & $\begin{array}{l}\text { Discharge } \\
\text { codes }\end{array}$ & $\begin{array}{l}\text { Water } \\
\text { year }\end{array}$ & Date & $\begin{array}{c}\text { Annual peak } \\
\text { discharge } \\
\left(\mathrm{ft}^{3} / \mathrm{s}\right)\end{array}$ & $\begin{array}{c}\text { Discharge } \\
\text { codes }\end{array}$ \\
\hline 1974 & $07-19-74$ & 2,340 & & 1986 & $09-09-86$ & 7,970 & \\
\hline 1975 & $09-13-75$ & 2,380 & & 1987 & $08-24-87$ & 3,990 & \\
\hline 1976 & $09-25-76$ & 5,420 & & 1988 & $08-27-88$ & 7,280 & \\
\hline 1977 & $07-21-77$ & 4,120 & & 1989 & 08-01-89 & 3,380 & \\
\hline 1978 & $09-25-78$ & 262 & & 1990 & $07-08-90$ & 2,090 & \\
\hline 1979 & $11-12-73$ & 330 & & 1991 & $10-02-90$ & 1,290 & \\
\hline 1980 & $09-10-80$ & 1,740 & & 1992 & $09-19-92$ & 3,110 & \\
\hline 1981 & $07-14-81$ & 4,640 & & 1993 & $02-20-93$ & 3,150 & \\
\hline 1982 & $10-02-81$ & 8,010 & & 1994 & $10-06-93$ & 2,970 & \\
\hline 1983 & $09-30-83$ & 10,100 & & 1995 & $07-14-95$ & 3,920 & \\
\hline 1984 & $08-18-84$ & 9,030 & & 1996 & $09-14-96$ & 737 & \\
\hline 1985 & $09-12-85$ & 520 & & & & & \\
\hline
\end{tabular}

Discharge rating table developed October 1991

\begin{tabular}{crcc}
\hline $\begin{array}{c}\text { Gage height } \\
\text { (ft) }\end{array}$ & $\begin{array}{c}\text { Discharge } \\
\left(\mathbf{f t}^{3} / \mathbf{s}\right)\end{array}$ & $\begin{array}{c}\text { Gage height } \\
(\mathbf{f t})\end{array}$ & $\begin{array}{c}\text { Discharge } \\
\left(\mathbf{f t}^{\mathbf{3}} \mathbf{s}\right)\end{array}$ \\
\hline 4.0 & 135 & 9.0 & 4,330 \\
5.0 & 560 & 10.0 & 5,520 \\
6.0 & 1,200 & 11.0 & 6,520 \\
7.0 & 2,070 & 12.0 & 7,520 \\
8.0 & 3,140 & 13.0 & 8,420 \\
\hline
\end{tabular}

Basin characteristics

\begin{tabular}{cccccccc}
$\begin{array}{c}\text { Main } \\
\text { channel } \\
\text { slope } \\
(\mathrm{ft} / \mathrm{mi})\end{array}$ & $\begin{array}{c}\text { Stream } \\
\text { length } \\
(\mathrm{mi})\end{array}$ & $\begin{array}{c}\text { Mean } \\
\text { basin } \\
\text { elevation } \\
(\mathrm{ft})\end{array}$ & $\begin{array}{c}\text { Forested } \\
\text { area } \\
\text { (percent) }\end{array}$ & $\begin{array}{c}\text { Soil } \\
\text { index }\end{array}$ & $\begin{array}{c}\text { Mean } \\
\text { annual } \\
\text { precipitation } \\
\text { (in) }\end{array}$ & $\begin{array}{c}\text { Rainfall intensity, 24-hour } \\
\text { (in) }\end{array}$ & $\begin{array}{c}50 \text {-year } \\
\text { (in) }\end{array}$ \\
\hline 24.8 & 87.1 & 5,850 & 47.2 & 3.0 & 9.5 & 1.3 \\
\hline
\end{tabular}


09401260 MOENKOPI WASH AT MOENKOPI, AZ--Continued

MEAN MONTHLY AND ANNUAL DISCHARGES 1977.96

\begin{tabular}{|c|c|c|c|c|c|c|}
\hline MONTH & $\begin{array}{l}\text { MAXIMUM } \\
\text { (FT3/S) }\end{array}$ & $\begin{array}{l}\text { MINIMUM } \\
\text { (FT3/S) }\end{array}$ & $\begin{array}{c}\text { MEAN } \\
(\text { FT } 3 / S)\end{array}$ & $\begin{array}{l}\text { STAN- } \\
\text { DARD } \\
\text { DEVIA- } \\
\text { TION } \\
(\text { PT } 3 / S)\end{array}$ & $\begin{array}{l}\text { COEFFI- } \\
\text { CIENT OF } \\
\text { VARI- } \\
\text { ATION }\end{array}$ & $\begin{array}{c}\text { PERCENT } \\
\text { OF } \\
\text { ANNUAL } \\
\text { RUNOFF }\end{array}$ \\
\hline OCTOBER & 82 & 0.24 & 9.2 & 19 & 2.0 & 8.7 \\
\hline NOVEMBER & 71 & 1.1 & 7.2 & 15 & 2.1 & 6.8 \\
\hline DECEMBER & 14 & 0.62 & 3.6 & 2.7 & 0.74 & 3.4 \\
\hline JANUARY & 28 & 2.0 & 5.3 & 5.6 & 1.1 & 5.0 \\
\hline FEBRUARY & 48 & 2.2 & 8.2 & 11 & 1.4 & 7.8 \\
\hline MARCH & 11 & 2.0 & 3.9 & 2.4 & 0.60 & 3.7 \\
\hline APRIL & 8.5 & 1.0 & 2.4 & 1.5 & 0.65 & 2.2 \\
\hline MAY & 16 & 0.31 & 2.3 & 3.8 & 1.6 & 2.2 \\
\hline JUNE & 11 & 0.00 & 0.75 & 2.3 & 3.2 & 0.7 \\
\hline JULY & 92 & 0.00 & 14 & 24 & 1.7 & 12.9 \\
\hline AUGUST & 129 & 0.00 & 24 & 33 & 1.3 & 22.9 \\
\hline SEPTEMBER & 134 & 0.00 & 25 & 33 & 1.3 & 23.6 \\
\hline ANNUAL & 19 & 2.1 & 8.8 & 5.6 & 0.64 & 100 \\
\hline
\end{tabular}

MAGNITUDE AND PROBABILITY OF INSTANTANEOUS PEAK FLOW BASED ON PERIOD OF RECORD 1974-96

DISCHARGE, IN FT3/S, FOR INDICATED RECURRENCE INTERVAL IN YEARS, AND EXCEEDANCE PROBABILITY, IN PERCENT

\begin{tabular}{|c|c|c|c|c|c|}
\hline 2 & 5 & 10 & 25 & 50 & 100 \\
\hline 508 & 208 & 108 & 48 & 28 & 18 \\
\hline 3,320 & 5.820 & 7,760 & 10,500 & 12,700 & 15,000 \\
\hline WEIGHTED & SKEW & $($ LOGS $)=-$ & & & \\
\hline MEAN & & $($ LOGS $)=$ & & & \\
\hline STANDARD & DEV. & $($ LOGS $)=$ & & & \\
\hline
\end{tabular}

MAGNITUDE AND PROBABILITY OF ANNUAL LOW FLOW BASED ON PERIOD OF RECORD 1978.96

\begin{tabular}{|c|c|c|c|c|c|c|}
\hline $\begin{array}{l}\text { PERIOD } \\
\text { (CON- }\end{array}$ & \multirow{2}{*}{\multicolumn{6}{|c|}{ 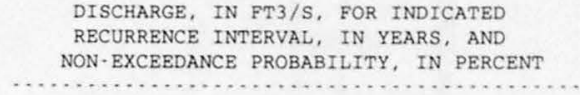 }} \\
\hline $\begin{array}{l}\text { (CON- } \\
\text { SECU. }\end{array}$ & & & & & & \\
\hline TIVE & 2 & 5 & 10 & $20 \#$ & $50 \#$ & $100 \#$ \\
\hline DAYS) & 508 & 208 & 108 & 58 & 28 & 18 \\
\hline \multicolumn{7}{|l|}{1} \\
\hline \multicolumn{7}{|l|}{3} \\
\hline \multicolumn{7}{|l|}{7} \\
\hline 14 & & & & & & \\
\hline 30 & 0.00 & 0.00 & 0.00 & 0.00 & 0.00 & 0.00 \\
\hline 60 & 0.17 & 0.03 & 0.00 & 0.00 & 0.00 & 0.00 \\
\hline 90 & 0.63 & 0.32 & 0.22 & 0.17 & 0.12 & 0.09 \\
\hline 120 & 1.6 & 0.70 & 0.44 & 0.30 & 0.18 & 0.13 \\
\hline 183 & 2.9 & 1.4 & 0.99 & 0.75 & 0.55 & 0.44 \\
\hline
\end{tabular}

MAGNITUDE AND PROBABILITY OF ANNUAL HIGH FLOW BASED ON PERIOD OF RECORD 1977.96

\begin{tabular}{|c|c|c|c|c|c|c|}
\hline \multirow{2}{*}{$\begin{array}{l}\text { PERIOD } \\
\text { /CON- } \\
\text { SECU- }\end{array}$} & \multicolumn{6}{|c|}{$\begin{array}{l}\text { DISCHARGE, IN FT3/S, FOR IND } \\
\text { RECURRENCE INTERVAL, IN YEARS, AND }\end{array}$} \\
\hline & & $\ldots \ldots$ & & & $\ldots \ldots$ & $\ldots \ldots$ \\
\hline TIVE & 2 & 5 & 10 & $25 \#$ & $50 \#$ & $100 \#$ \\
\hline DAYS) & 508 & 208 & 108 & 48 & 28 & 18 \\
\hline 1 & 539 & 1,270 & 2,000 & 3,260 & 4,480 & 5,960 \\
\hline 3 & 279 & 603 & 881 & 1.300 & 1,650 & 2,030 \\
\hline 7 & 136 & 290 & 415 & 591 & 733 & 881 \\
\hline 15 & 69 & 151 & 220 & 323 & 409 & 502 \\
\hline 30 & 40 & 85 & 121 & 173 & 216 & 261 \\
\hline 60 & 24 & 51 & 74 & 107 & 136 & 168 \\
\hline 90 & 18 & 38 & 54 & 78 & 98 & 120 \\
\hline
\end{tabular}

DURATION TABLE OF DAILY MEAN FLOW FOR PERIOD OF RECORD 1977.96

DISCHARGE, IN FT3/S, WHICH WAS EQUALED OR EXCEEDED FOR INDICATED PERCENT OF TIME

\begin{tabular}{|c|c|c|c|c|c|c|c|c|c|c|c|c|c|c|c|c|}
\hline 18 & 58 & 108 & 158 & 208 & 308 & 408 & 508 & 608 & 708 & 808 & 908 & 958 & 988 & 998 & 99.58 & 99.98 \\
\hline 146 & 18 & 7.4 & 5.0 & 3.9 & 3.1 & 2.4 & 2.0 & 1.4 & 0.67 & 0.00 & 0.00 & 0.00 & 0.00 & 0.00 & 0.00 & 0.00 \\
\hline
\end{tabular}

\# Reliability of values in column is uncertain, and potential errors are large. 
09401260 MOENKOPI WASH AT MOENKOPI, AZ--Continued
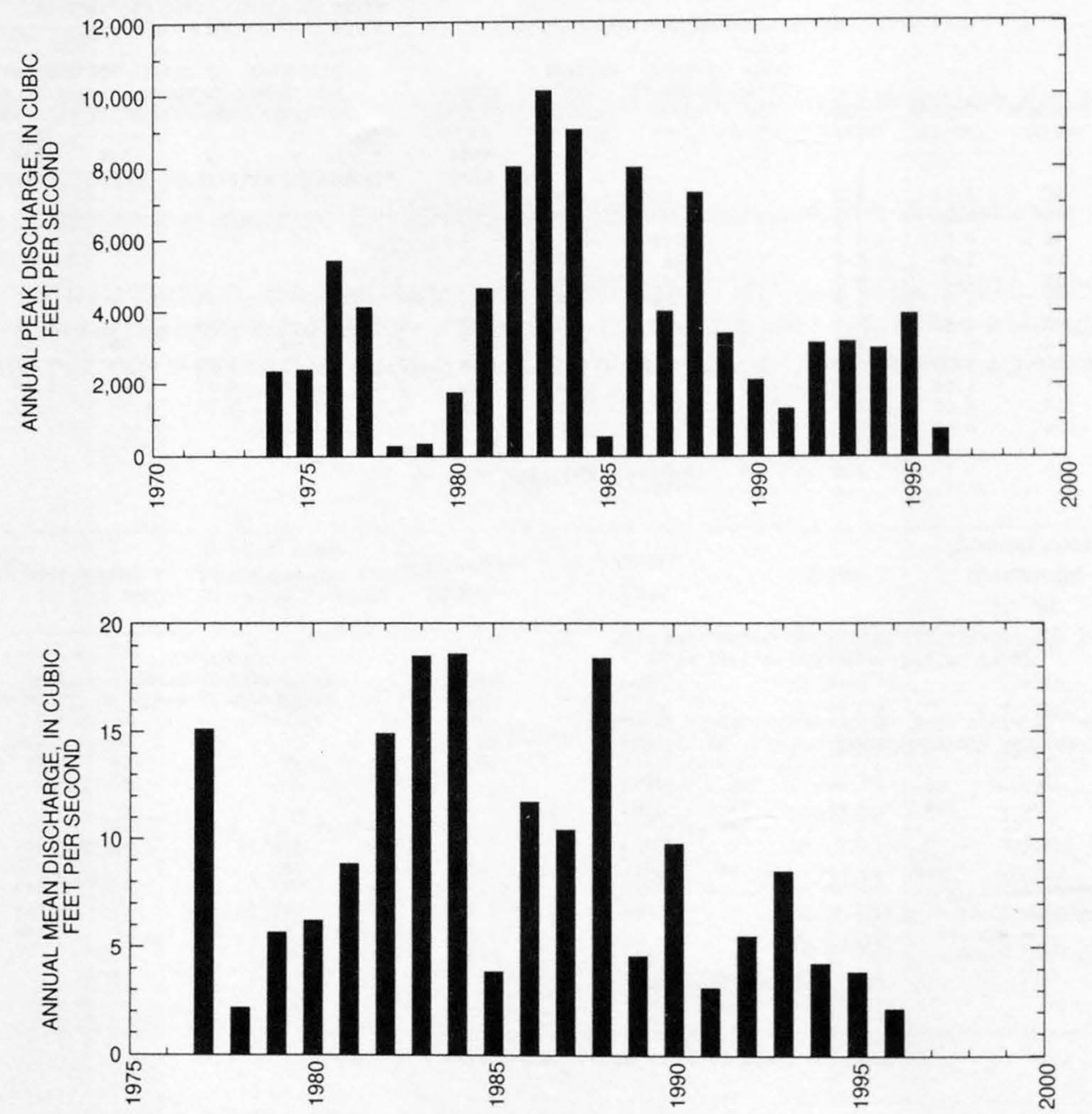
LITTLE COLORADO RIVER BASIN

149

09401260 MOENKOPI WASH AT MOENKOPI, AZ--Continued
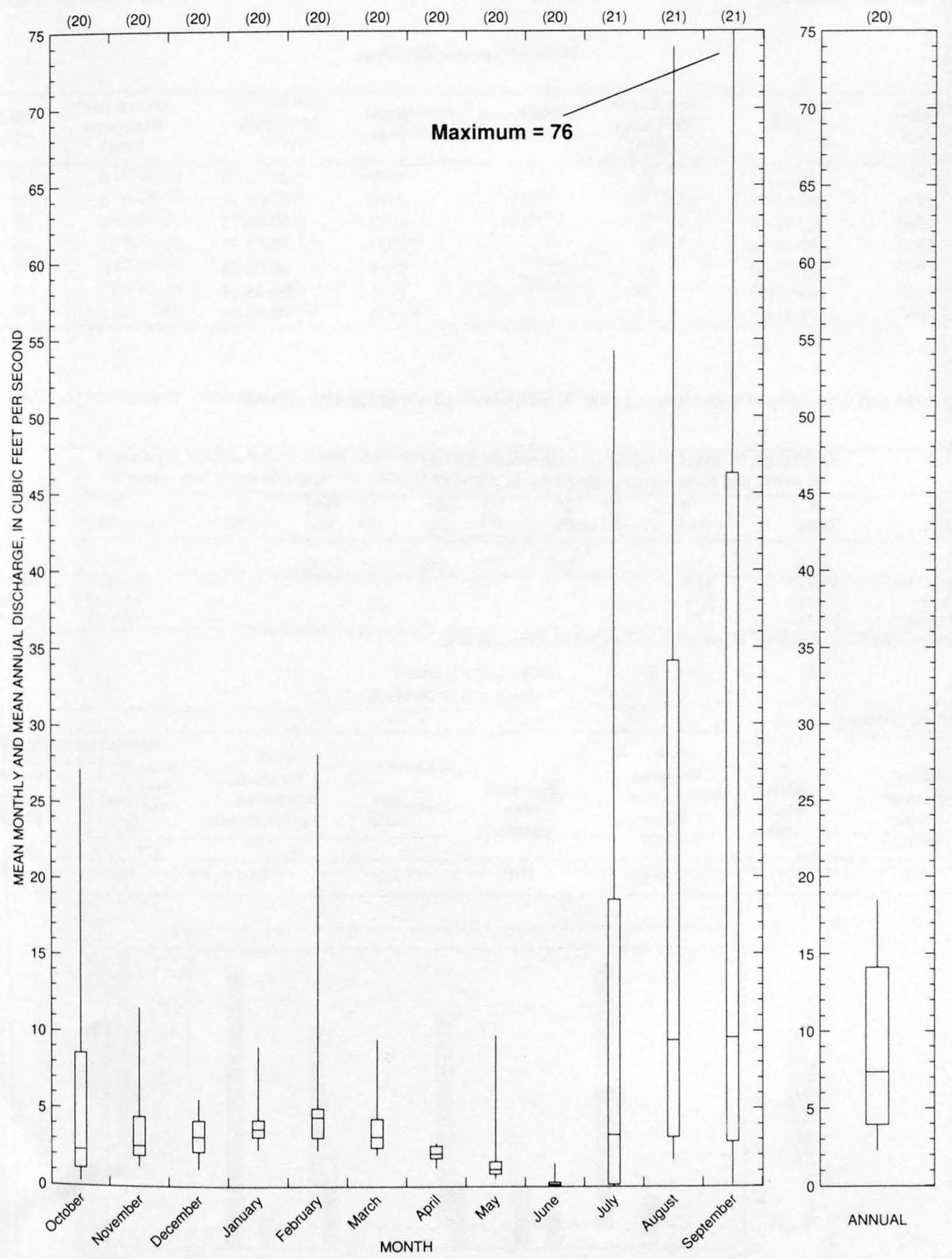


\section{HAMBLIN WASH TRIBUTARY NEAR CEDAR RIDGE, AZ}

LOCATION.--Lat 36²0'55", long 111 30'15", Coconino County, Hydrologic Unit 15020018, at U.S. Highway 89, 3.3 mi south of Cedar Ridge. DRAINAGE AREA.--0.10 $\mathrm{mi}^{2}$.

Annual peak discharges

\begin{tabular}{|c|c|c|c|c|c|c|c|}
\hline $\begin{array}{l}\text { Water } \\
\text { year }\end{array}$ & Date & $\begin{array}{c}\text { Annual peak } \\
\text { discharge } \\
\left(\mathrm{ft}^{3} / \mathrm{s}\right)\end{array}$ & $\begin{array}{c}\text { Discharge } \\
\text { codes }\end{array}$ & $\begin{array}{l}\text { Water } \\
\text { year }\end{array}$ & Date & $\begin{array}{c}\text { Annual peak } \\
\text { discharge } \\
\left(\mathrm{ft}^{3} / \mathrm{s}\right)\end{array}$ & $\begin{array}{c}\text { Discharge } \\
\text { codes }\end{array}$ \\
\hline 1963 & $09-07-63$ & 21 & & 1970 & $00-00-70$ & 0 & \\
\hline 1964 & $00-00-64$ & 0.3 & ES & 1971 & $00-00-71$ & 0 & \\
\hline 1965 & $00-00-65$ & 1.0 & LT & 1972 & $00-00-72$ & 0 & \\
\hline 1966 & $07-30-66$ & 15 & & 1973 & $10-19-72$ & 37 & \\
\hline 1967 & $09-25-67$ & 26 & & 1974 & $00-00-74$ & 0 & \\
\hline 1968 & $00-00-68$ & 0 & & 1975 & $09-08-75$ & 12 & \\
\hline 1969 & $09-11-69$ & 25 & & 1976 & $00-00-76$ & 110 & \\
\hline
\end{tabular}

Magnitude and probability of instantaneous peak flow based on period of record 1963-76

\begin{tabular}{|c|c|c|c|c|c|}
\hline \multicolumn{6}{|c|}{$\begin{array}{l}\text { Discharge, in } \mathrm{ft}^{3} / \mathrm{s} \text {, for indicated recurrence interval } \\
\text { in years, and exceedance probablility, in percent }\end{array}$} \\
\hline 2 & 5 & 10 & 25 & $50 t$ & $100 \dagger$ \\
\hline $50 \%$ & $20 \%$ & $10 \%$ & $4 \%$ & $2 \%$ & $1 \%$ \\
\hline 1.7 & 37.3 & 156 & 638 & 1,480 & 3,040 \\
\hline Weighted skew & $(\log s)=$ & -0.48 & & & \\
\hline Mean & $(\log s)=$ & 0.11 & & & \\
\hline Standard dev. & $(\log s)=$ & 1.71 & & & \\
\hline
\end{tabular}

Basin characteristics

\begin{tabular}{cccccccc}
\hline $\begin{array}{c}\text { Main } \\
\text { channel } \\
\text { slope } \\
(\mathrm{ft} / \mathrm{mi})\end{array}$ & $\begin{array}{c}\text { Stream } \\
\text { length } \\
(\mathrm{mi})\end{array}$ & $\begin{array}{c}\text { Mean } \\
\text { basin } \\
\text { elevation } \\
(\mathrm{ft})\end{array}$ & $\begin{array}{c}\text { Forested } \\
\text { area } \\
\text { (percent) }\end{array}$ & $\begin{array}{c}\text { Soil } \\
\text { index }\end{array}$ & $\begin{array}{c}\text { Mean } \\
\text { annual } \\
\text { precipitation } \\
\text { (in) }\end{array}$ & $\begin{array}{c}\text { Rainfall intensity, 24-hour } \\
\text { (in) }\end{array}$ & $\begin{array}{c}50 \text {-year } \\
\text { (in) }\end{array}$ \\
\hline 263 & 0.61 & 5,860 & 0.0 & 3.0 & 7.6 & 1.3 \\
\hline
\end{tabular}

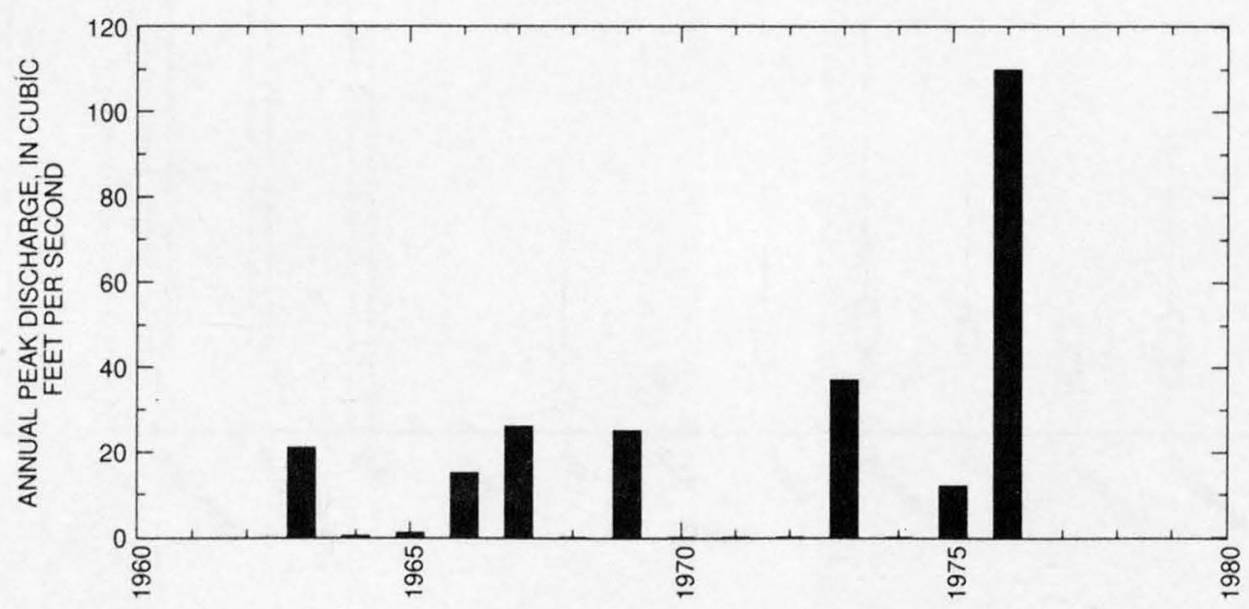


09401370 HAMBLIN WASH TRIBUTARY NO. 2 NEAR TUBA CITY, AZ

LOCATION.--Lat 36 $03^{\prime} 20^{\prime \prime}$, long 111'23'35", Coconino County, Hydrologic Unit 15020018, at U.S. Highway 89, 10 mi west of Tuba City. DRAINAGE AREA.--2.16 $\mathrm{mi}^{2}$.

Annual peak discharges

\begin{tabular}{|c|c|c|c|c|c|c|c|}
\hline $\begin{array}{l}\text { Water } \\
\text { year }\end{array}$ & Date & $\begin{array}{c}\text { Annual peak } \\
\text { discharge } \\
\left(\mathrm{ft}^{3} / \mathrm{s}\right)\end{array}$ & $\begin{array}{c}\text { Discharge } \\
\text { codes }\end{array}$ & $\begin{array}{l}\text { Water } \\
\text { year }\end{array}$ & Date & $\begin{array}{c}\text { Annual peak } \\
\text { discharge } \\
\left(\mathrm{ft}^{3} / \mathrm{s}\right)\end{array}$ & $\begin{array}{l}\text { Discharge } \\
\text { codes }\end{array}$ \\
\hline 1963 & $00-00-63$ & 10 & LT & 1970 & $10-21-69$ & 5.0 & LT \\
\hline 1964 & $00-00-64$ & 268 & & 1971 & $07-00-71$ & 14 & ES \\
\hline 1965 & $00-00-65$ & 0 & & 1972 & $07-18-72$ & 5.0 & LT \\
\hline 1966 & $10-16-65$ & 7.0 & ES & 1973. & $10-19-72$ & 350 & \\
\hline 1967 & $12-06-66$ & 0.5 & ES & 1974 & $00-00-74$ & 0 & \\
\hline 1968 & $08-07-68$ & 285 & & 1975 & $09-00-75$ & 7.0 & ES \\
\hline 1969 & $09-11-69$ & 5.0 & LT & & & & \\
\hline
\end{tabular}

Magnitude and probability of instantaneous peak flow based on period of record

\begin{tabular}{|c|c|c|c|c|c|}
\hline \multicolumn{6}{|c|}{$\begin{array}{l}\text { Discharge, in } \mathrm{ft}^{3} / \mathrm{s} \text {, for indicated recurrence interval } \\
\text { in years, and exceedance probablility, in percent }\end{array}$} \\
\hline 2 & 5 & 10 & 25 & 50 & 100 \\
\hline $50 \%$ & $20 \%$ & $10 \%$ & $4 \%$ & $2 \%$ & $1 \%$ \\
\hline --. & ... & $\cdots$ & $\ldots$ & $\cdots$ & -- \\
\hline Weighted skew & $(\log s)=$ & $\cdots$ & & & \\
\hline Mean & $(\log s)=$ & $\cdots$ & & & \\
\hline Standard dev. & $(\log s)=$ & -- & & & \\
\hline
\end{tabular}

Basin characteristics

\begin{tabular}{cccccccc}
\hline $\begin{array}{c}\text { Main } \\
\text { channel } \\
\text { slope } \\
(\mathrm{ft} / \mathrm{mi})\end{array}$ & $\begin{array}{c}\text { Stream } \\
\text { length } \\
(\mathrm{mi})\end{array}$ & $\begin{array}{c}\text { Mean } \\
\text { basin } \\
\text { elevation } \\
(\mathrm{ft})\end{array}$ & $\begin{array}{c}\text { Forested } \\
\text { area } \\
\text { (percent) }\end{array}$ & $\begin{array}{c}\text { Soil } \\
\text { index }\end{array}$ & $\begin{array}{c}\text { Mean } \\
\text { annual } \\
\text { precipitation } \\
\text { (in) }\end{array}$ & $\begin{array}{c}\text { Rainfall intensity, 24-hour } \\
\text { (in) }\end{array}$ & $\begin{array}{c}50-y=a r \\
\text { (in) }\end{array}$ \\
\hline 348 & 2.3 & 4,670 & 0.0 & 3.0 & 6.0 & 1.2 \\
\hline
\end{tabular}

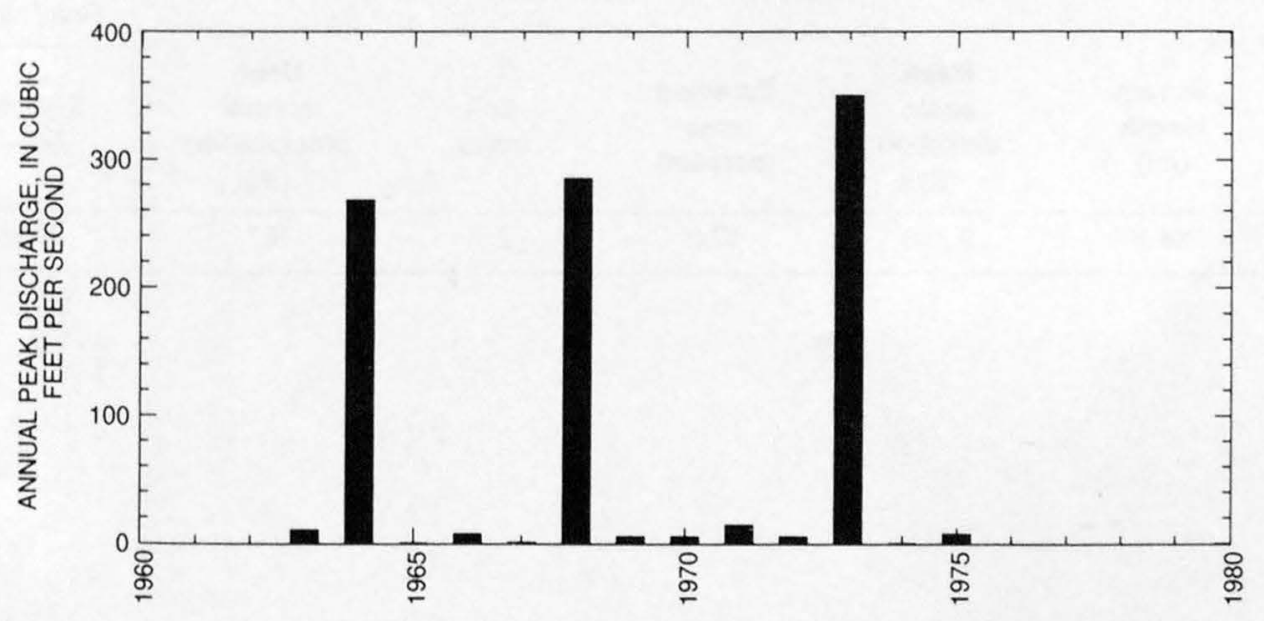


LOCATION.--Lat 36 01'25", long 111 '23'48", in sec.35, T.31 N., R.9 E. (unsurveyed), Coconino County, Hydrologic Unit 15020018, on Navajo Indian Reservation, on downstream side of bridge on U.S. Highway 89, 3,500 ft downstream from Hamblin Wash, 11 mi upstream from mouth, and 12 mi southwest of Tuba City.

DRAINAGE AREA.--2,500 $\mathrm{mi}^{2}$, approximately, of which about $1,200 \mathrm{mi}^{2}$ is partly or entirely noncontributing.

PERIOD OF RECORD.--June 1941 to December 1953 (published as "near Tuba"), February 1965 to September 1978 (discontinued). Records fo July 1926 to June 1941 at site 8 mi upstream not equivalent.

REVISED RECORDS.--WSP 1213: 1943(M). WSP 1926: Drainage area.

GAGE.--Water-stage recorder. Datum of gage is $4,309 \mathrm{ft}$ above sea level. June 23, 1941, to Dec. 10, 1953, at site 2,500 $\mathrm{ft}$ upstream at datum $4,310.96 \mathrm{ft}$ above sea level (State Highway Department bench mark).

REMARKS.--Records poor. Diversions above station for irrigation of about 500 acres.

AVERAGE DISCHARGE.--25 years (water years 1942-53, 1966-78), $14.7 \mathrm{ft}^{3} / \mathrm{s}, 10,650 \mathrm{acre}-\mathrm{ft} / \mathrm{yr}$.

EXTREMES FOR PERIOD OF RECORD.--Maximum discharge, 12,100 ft ${ }^{3} / \mathrm{s}$ Oct. 19, 1972, gage height, $16.98 \mathrm{ft}$, from floodmarks in recordef shelter; no flow for many days in each year.

EXTREMES OUTSIDE PERIOD OF RECORD.--A discharge of 15,100 $\mathrm{ft}^{3} / \mathrm{s}$ occurred $8 \mathrm{mi}$ upstream (published as "near Tuba City") Aug. 4 1929 , from rating curve extended above $200 \mathrm{ft}^{3} / \mathrm{s}$ on basis of slope-area measurement of peak flow; maximum discharge for period 1926-41.

Annual peak discharges

\begin{tabular}{|c|c|c|c|c|c|c|c|}
\hline $\begin{array}{l}\text { Water } \\
\text { year }\end{array}$ & Date & $\begin{array}{c}\text { Annual peak } \\
\text { discharge } \\
\left(\mathrm{ft}^{3} / \mathrm{s}\right)\end{array}$ & $\begin{array}{l}\text { Discharge } \\
\text { codes }\end{array}$ & $\begin{array}{l}\text { Water } \\
\text { year }\end{array}$ & Date & $\begin{array}{c}\text { Annual peak } \\
\text { discharge } \\
\left(\mathrm{ft}^{3} / \mathrm{s}\right)\end{array}$ & $\begin{array}{c}\text { Discharge } \\
\text { codes }\end{array}$ \\
\hline 1941 & $08-16-41$ & 5,240 & & 1966 & $07-29-66$ & 742 & \\
\hline 1942 & $10-13-41$ & 7,000 & & 1967 & $09-08-67$ & 3,790 & \\
\hline 1943 & $08-17-43$ & 7,150 & & 1968 & $08-12-68$ & 3,120 & \\
\hline 1944 & $09-27-44$ & 964 & & 1969 & $07-29-69$ & 1,900 & \\
\hline 1945 & $08-01-45$ & 6,290 & & 1970 & $09-06-70$ & 4,990 & \\
\hline 1946 & $08-12-46$ & 3,910 & & 1971 & $08-26-71$ & 5,410 & \\
\hline 1947 & $08-17-47$ & 2,860 & & 1972 & $08-27-72$ & 2,510 & \\
\hline 1948 & $10-13-47$ & 6,980 & & 1973 & $10-19-72$ & 12,100 & \\
\hline 1949 & $08-09-49$ & 2,210 & & 1974 & $07-19-74$ & 1,840 & \\
\hline 1950 & $07-25-50$ & 2,890 & & 1975 & $07-15-75$ & 1,200 & \\
\hline 1951 & $09-30-51$ & 5,000 & & 1976 & $09-25-76$ & 3,990 & \\
\hline 1952 & $09-21-52$ & 10,000 & & 1977 & $07-23-77$ & 3,360 & \\
\hline 1953 & $07-30-53$ & 5,700 & & 1978 & $06-28-78$ & 1,710 & \\
\hline 1965 & $07-30-65$ & 2,960 & & & & & \\
\hline
\end{tabular}

Basin characteristics

\begin{tabular}{cccccccc}
\hline $\begin{array}{c}\text { Main } \\
\text { channel } \\
\text { slope } \\
(\mathrm{ft} / \mathrm{mi})\end{array}$ & $\begin{array}{c}\text { Stream } \\
\text { length } \\
\text { (mi) }\end{array}$ & $\begin{array}{c}\text { Mean } \\
\text { basin } \\
\text { elevation } \\
\text { (ft) }\end{array}$ & $\begin{array}{c}\text { Forested } \\
\text { area } \\
\text { (percent) }\end{array}$ & $\begin{array}{c}\text { Soil } \\
\text { index }\end{array}$ & $\begin{array}{c}\text { Mean } \\
\text { annual } \\
\text { precipitation } \\
\text { (in) }\end{array}$ & $\begin{array}{c}\text { R-year } \\
\text { (in) }\end{array}$ & $\begin{array}{c}50-\text {-year } \\
\text { (in) }\end{array}$ \\
\hline 27.2 & 88.3 & 5,820 & 37.0 & 2.7 & 8.7 & 1.3 \\
\hline
\end{tabular}


09401400 MOENKOPI WASH NEAR TUBA CITY, AZ--Continued

MEAN MONTHLY AND ANNUAL DISCHARGES $1941.53,1966.78$

\begin{tabular}{|c|c|c|c|c|c|c|}
\hline MONTH & $\begin{array}{l}\text { MAXIMUM } \\
(\mathrm{FT} 3 / \mathrm{S})\end{array}$ & $\begin{array}{l}\text { MINIMUM } \\
\text { (FT3/S) }\end{array}$ & $\begin{array}{c}\text { MEAN } \\
(\mathrm{FT} 3 / \mathrm{S})\end{array}$ & $\begin{array}{l}\text { STAN- } \\
\text { DARD } \\
\text { DEVIA- } \\
\text { TION } \\
\text { (FT3/S) }\end{array}$ & $\begin{array}{l}\text { COEFFI- } \\
\text { CIENT OF } \\
\text { VARI- } \\
\text { ATION }\end{array}$ & $\begin{array}{c}\text { PERCENT } \\
\text { OF } \\
\text { ANNUAL } \\
\text { RUNOFF }\end{array}$ \\
\hline OCTOBER & 686 & 0.00 & 53 & 141 & 2.7 & 29.0 \\
\hline NOVEMBER & 7.9 & 0.33 & 3.2 & 1.6 & 0.50 & 1.8 \\
\hline DECEMBER & 20 & 0.70 & 5.0 & 4.1 & 0.83 & 2.8 \\
\hline JANUARY & 18 & 1.0 & 4.9 & 3.7 & 0.75 & 2.7 \\
\hline FEBRUARY & 25 & 0.80 & 5.1 & 5.6 & 1.1 & 2.8 \\
\hline MARCH & 17 & 0.43 & 4.1 & 4.0 & 0.96 & 2.3 \\
\hline APRIL & 14 & 0.00 & 1.4 & 3.2 & 2.2 & 0.8 \\
\hline MAY & 1.1 & 0.00 & 0.22 & 0.34 & 1.6 & 0.1 \\
\hline JUNE & 12 & 0.00 & 1.1 & 2.9 & 2.7 & 0.6 \\
\hline JULY & 174 & 0.00 & 26 & 39 & 1.5 & 14.3 \\
\hline AUGUST & 226 & 0.69 & 48 & 57 & 1.2 & 26.5 \\
\hline SEPTEMBER & 191 & 0.00 & 30 & 47 & 1.6 & 16.3 \\
\hline ANNUAL & 62 & 3.1 & 15 & 12 & 0.81 & 100 \\
\hline
\end{tabular}

MAGNITUDE AND PROBABILITY OF INSTANTANEOUS PEAK FLOW BASED ON PERIOD OF RECORD 1941-53, 1965-78

DISCHARGE, IN FT3/S, FOR INDICATED RECURRENCE INTERVAL IN YEARS, AND EXCEEDANCE PROBABILITY, IN PERCENT

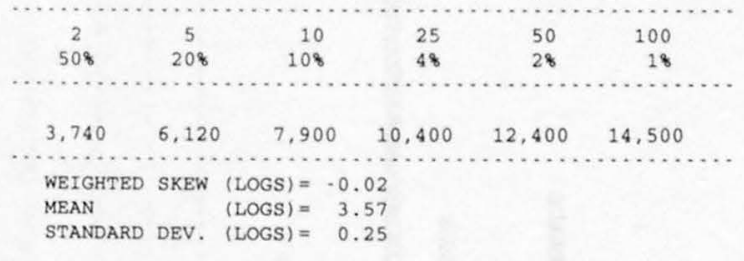

MAGNITUDE AND PROBABILITY OF ANNUAL LOW FLOW BASED ON PERIOD OF RECORD $1942 \cdot 53,1966 \cdot 78$

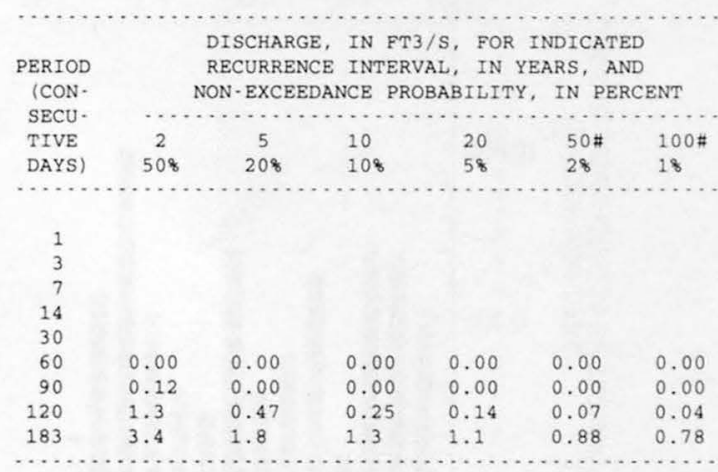

MAGNITUDE AND PROBABILITY OF ANNUAL HIGH FLOW BASED ON PERIOD OF RECORD 1941.53, 1966.78

\begin{tabular}{|c|c|c|c|c|c|c|}
\hline $\begin{array}{l}\text { PERIOD } \\
\text { (CON. }\end{array}$ & \multicolumn{6}{|c|}{$\begin{array}{l}\text { DISCHARGE, IN FT } 3 / S, \text { FOR INDICATED } \\
\text { RECURRENCE INTERVAL, IN YEARS, AND }\end{array}$} \\
\hline SECU - & & & & & & \\
\hline TIVE & 2 & 5 & 10 & $25 \#$ & $50 \#$ & $100 \#$ \\
\hline DAYS) & 508 & 208 & 108 & 48 & 28 & 18 \\
\hline 1 & 1,030 & 2,330 & 3,400 & 4,910 & 6,100 & 7,330 \\
\hline 3 & 513 & 1,170 & 1,760 & 2,670 & 3,450 & 4,330 \\
\hline 7 & 272 & 607 & 890 & 1,310 & 1,650 & 2,020 \\
\hline 15 & 146 & 341 & 514 & 777 & 1,000 & 1,250 \\
\hline 30 & 88 & 199 & 295 & 435 & 551 & 675 \\
\hline 60 & 51 & 112 & 165 & 242 & 306 & 375 \\
\hline 90 & 36 & 77 & 111 & 160 & 201 & 244 \\
\hline
\end{tabular}

DURATION TABLE OF DAILY MEAN FLOW FOR PERIOD OF RECORD $1941.53,1966.78$

DISCHARGE, IN FT3/S, WHICH WAS EQUALED OR EXCEEDED FOR INDICATED PERCENT OF TIME

\begin{tabular}{|c|c|c|c|c|c|c|c|c|c|c|c|c|c|c|c|c|}
\hline 18 & 58 & 108 & 158 & 208 & 308 & 408 & 508 & 608 & 708 & 808 & 908 & 958 & 988 & 998 & 99.58 & 99.98 \\
\hline 306 & 29 & 9.5 & 5.3 & 4.4 & 3.2 & 2.3 & 1.3 & 0.38 & 0.00 & 0.00 & 0.00 & 0.00 & 0.00 & 0.00 & 0.00 & 0.00 \\
\hline
\end{tabular}

\# Reliability of values in column is uncertain, and potential errors are large. 


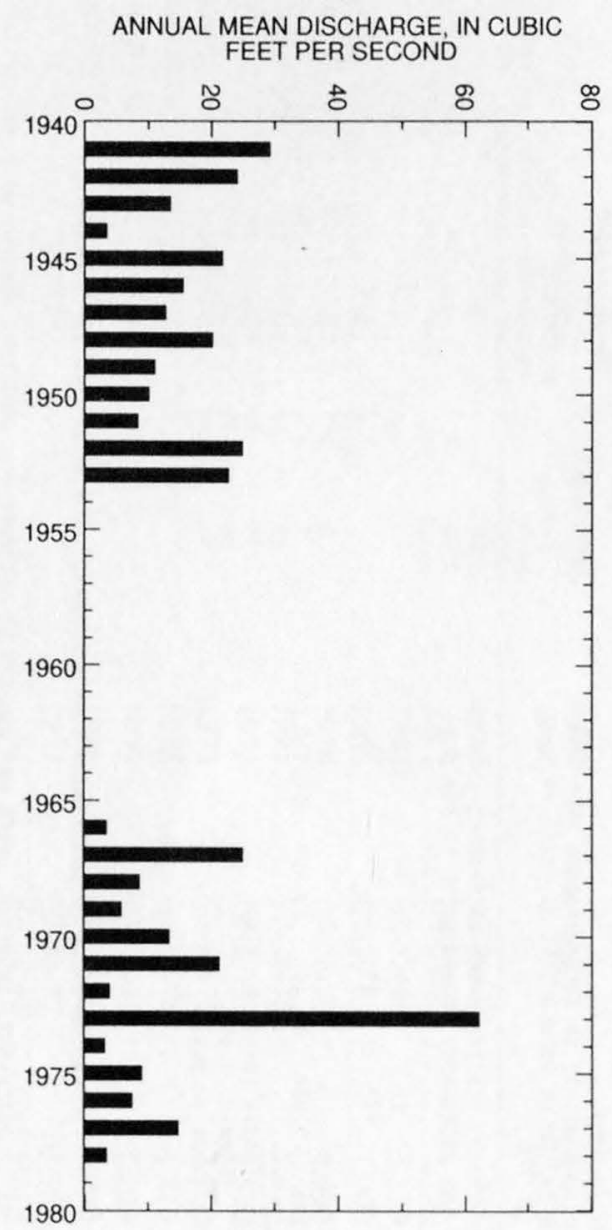

ANNUAL PEAK DISCHARGE, IN CUBIC FEET PER SECOND

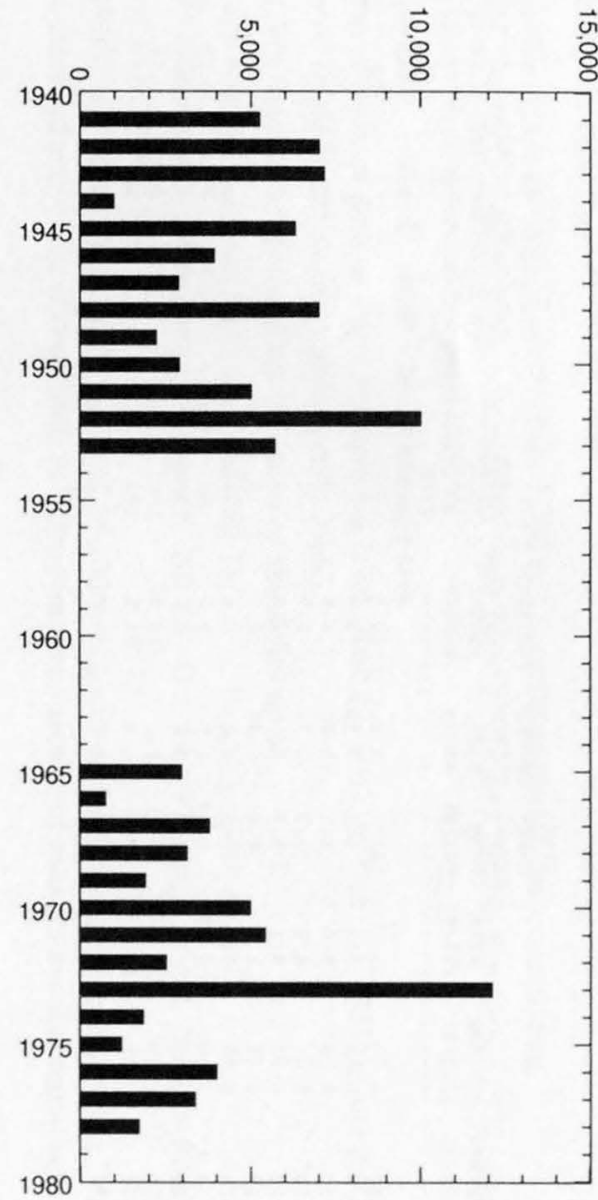


09401400 MOENKOPI WASH NEAR TUBA CITY, AZ--Continued
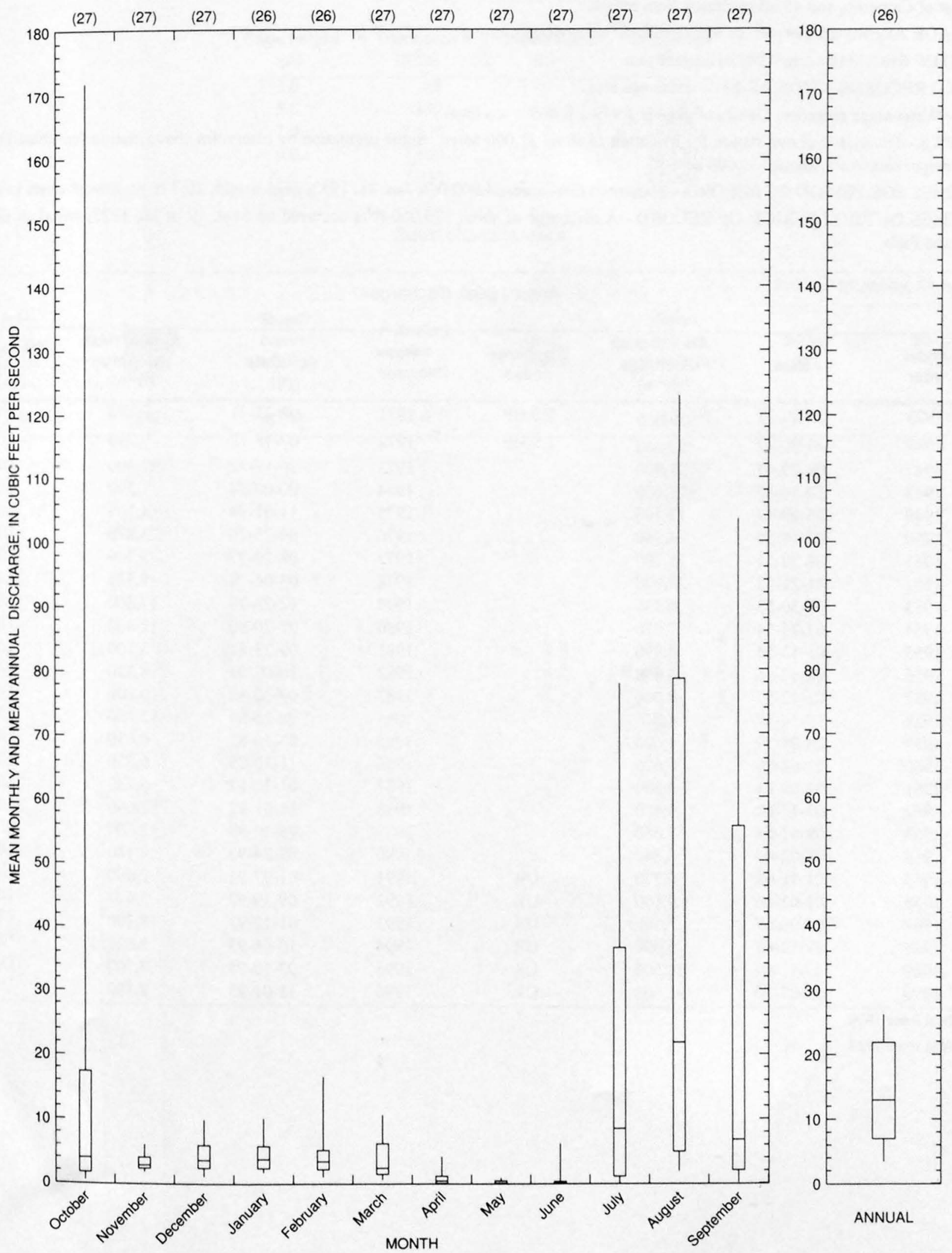


\section{LITTLE COLORADO RIVER NEAR CAMERON, AZ}

LOCATION.--Lat 35'55'35", long $111^{\prime} 34^{\prime} 00^{\prime \prime}$, in NW1/4 sec.5, T.29 N., R.8 E. (unsurveyed), Coconino County, Hydrologic Unit 15020016 , in Navajo Indian Reservation, on left bank 3 mi downstream from Coconino damsite, 9.5 mi downstream from Moenkopi Wash, 9.5 mi north. west of Cameron, and $45 \mathrm{mi}$ upstream from mouth.

DRAINAGE AREA.--26,459 $\mathrm{mi}^{2}$, of which $368 \mathrm{mi}^{2}$ are noncontributing.

PERIOD OF RECORD.--June 1947 to current year.

REVISED RECORDS.--WDR AZ-88-1: Drainage area.

GAGE.--Water-stage recorder. Datum of gage is 3,979.2 ft above sea level.

REMARKS.--Diversions above station for irrigation of about 32,000 acres. Some regulation by reservoirs above station (combined capacity of principal reservoirs, about 135,000 acre-ft).

EXTREMES FOR PERIOD OF RECORD.--Maximum discharge, 24,900 ft $3 / \mathrm{s} \mathrm{Jan.} \mathrm{21,} \mathrm{1952,} \mathrm{gage} \mathrm{height,} 20.7 \mathrm{ft}$; no flow at times in each year.

EXTREMES OUTSIDE PERIOD OF RECORD.--A discharge of about $120,000 \mathrm{ft}^{3}$ /s occurred on Sept. 19 or 20, 1923 , based on discharge at Grand Falls.

Annual peak discharges

\begin{tabular}{|c|c|c|c|c|c|c|c|}
\hline $\begin{array}{l}\text { Water } \\
\text { year }\end{array}$ & Date & $\begin{array}{c}\text { Annual peak } \\
\text { discharge } \\
\left(\mathrm{ft}^{3} / \mathrm{s}\right)\end{array}$ & $\begin{array}{l}\text { Discharge } \\
\text { codes }\end{array}$ & $\begin{array}{l}\text { Water } \\
\text { year }\end{array}$ & Date & $\begin{array}{c}\text { Annual peak } \\
\text { discharge } \\
\left(\mathrm{ft}^{3} / \mathrm{s}\right)\end{array}$ & $\begin{array}{c}\text { Discharge } \\
\text { codes }\end{array}$ \\
\hline 1923 & $09-20-23$ & ${ }^{\mathrm{l}} 120,000$ & ES,HP & 1971 & $08-27-71$ & 7,290 & UR \\
\hline 1929 & $04-06-29$ & ${ }^{2} 50,000$ & ES,HP & 1972 & $07-18-72$ & 9,250 & UR \\
\hline 1947 & $08-09-47$ & 21,900 & & 1973 & $10-19-72$ & 22,400 & UR \\
\hline 1948 & $10-14-47$ & 18,600 & & 1974 & $00-00-74$ & 1,590 & UR \\
\hline 1949 & $08-09-49$ & 12,400 & & 1975 & $11-01-74$ & 4,100 & UR \\
\hline 1950 & $07-18-50$ & 4,340 & & 1976 & $09-25-76$ & 3,870 & UR \\
\hline 1951 & $08-30-51$ & 11,700 & & 1977 & $08-20-77$ & 3,300 & UR \\
\hline 1952 & $01-21-52$ & 24,900 & & 1978 & $03-06-78$ & 9,540 & UR \\
\hline 1953 & $07-30-53$ & 6,230 & & 1979 & $12-23-78$ & 17,800 & UR \\
\hline 1954 & $03-25-54$ & 7,070 & & 1980 & $02-20-80$ & 12,400 & UR \\
\hline 1955 & $06-13-55$ & 8,990 & & 1981 & $09-23-81$ & 5,100 & UR \\
\hline 1956 & $08-17-56$ & 6,650 & & 1982 & $10-02-81$ & 8,320 & UR \\
\hline 1957 & $01-12-57$ & 8,060 & & 1983 & $09-30-83$ & 10,600 & UR \\
\hline 1958 & $10-14-57$ & 4,840 & & 1984 & $08-26-84$ & 12,400 & UR \\
\hline 1959 & $08-07-59$ & 4,600 & & 1985 & $03-16-85$ & 6,030 & UR \\
\hline 1960 & $11-02-59$ & 6,620 & & 1986 & $11-30-85$ & 6,530 & UR \\
\hline 1961 & 09-09-61 & 2,600 & & 1987 & $01-30-87$ & 6,730 & UR \\
\hline 1962 & $02-17-62$ & 3,470 & & 1988 & $11-01-87$ & 12,600 & UR \\
\hline 1963 & $09-01-63$ & 7,680 & & 1989 & $08-19-89$ & 12,800 & UR \\
\hline 1964 & $08-02-64$ & 8,540 & & 1990 & $09-24-90$ & 4,140 & UR \\
\hline 1965 & $01-11-65$ & 6,770 & UR & 1991 & 01-07-91 & 2,690 & UR \\
\hline 1966 & $01-03-66$ & 9,100 & UR & 1992 & $09-19-92$ & 5,620 & UR \\
\hline 1967 & $09-08-67$ & 7,580 & UR & 1993 & $01-12-93$ & 18,200 & UR \\
\hline 1968 & $08-12-68$ & 5,600 & UR & 1994 & $10-06-93$ & 8,820 & UR \\
\hline 1969 & $09-11-69$ & 11,600 & UR & 1995 & $03-10-95$ & 7,700 & UR \\
\hline 1970 & 09-07-70 & 12,600 & UR & 1996 & $11-01-95$ & 2,180 & UR \\
\hline
\end{tabular}

${ }^{1}$ Highest since 1870.

${ }^{2}$ Highest since 1923. 
09402000 LITTLE COLORADO RIVER NEAR CAMERON, AZ--Continued

Discharge rating table developed October ---

\begin{tabular}{crcc}
\hline $\begin{array}{c}\text { Gage height } \\
(\mathbf{f t})\end{array}$ & $\begin{array}{c}\text { Discharge } \\
\left(\mathrm{ft}^{\mathbf{3}} / \mathbf{s}\right)\end{array}$ & $\begin{array}{c}\text { Gage height } \\
(\mathbf{f t})\end{array}$ & $\begin{array}{c}\text { Discharge } \\
\left(\mathrm{ft}^{3} / \mathbf{s}\right)\end{array}$ \\
\hline 3.0 & 62 & 13.0 & 9,860 \\
5.0 & 880 & 15.0 & 13,060 \\
7.0 & 2,510 & 17.0 & 16,620 \\
9.0 & 4,600 & 19.0 & 20,530 \\
11.0 & 7,030 & 22.0 & 27,000 \\
\hline
\end{tabular}

Basin characteristics

\begin{tabular}{cccccccc}
\hline $\begin{array}{c}\text { Main } \\
\text { channel } \\
\text { slope } \\
(\mathrm{ft} / \mathrm{mi})\end{array}$ & $\begin{array}{c}\text { Stream } \\
\text { length } \\
\text { (mi) }\end{array}$ & $\begin{array}{c}\text { Mean } \\
\text { basin } \\
\text { elevation } \\
(\mathrm{ft})\end{array}$ & $\begin{array}{c}\text { Forested } \\
\text { area } \\
\text { (percent) }\end{array}$ & $\begin{array}{c}\text { Soil } \\
\text { index }\end{array}$ & $\begin{array}{c}\text { Mean } \\
\text { annual } \\
\text { precipitation } \\
\text { (in) }\end{array}$ & $\begin{array}{c}\text { Rainfall intensity, 24-hour } \\
\text { (in) }\end{array}$ & $\begin{array}{c}50 \text {-year } \\
\text { (in) }\end{array}$ \\
\hline 7.48 & 285 & 6,300 & 32.0 & 2.7 & 12.2 & 1.5 \\
\hline
\end{tabular}


09402000 LITTLE COLORADO RIVER NEAR CAMERON, AZ--Continued

MEAN MONTHLY AND ANNUAL DISCHARGES 1948.96

\begin{tabular}{|c|c|c|c|c|c|c|}
\hline MONTH & $\begin{array}{l}\text { MAXIMUM } \\
(\mathrm{FT} 3 / \mathrm{S})\end{array}$ & $\begin{array}{l}\text { MINIMUM } \\
\text { (FT3/S) }\end{array}$ & $\begin{array}{c}\text { MEAN } \\
(\text { FT3/S) }\end{array}$ & $\begin{array}{l}\text { STAN - } \\
\text { DARD } \\
\text { DEVIA. } \\
\text { TION } \\
\text { (FT3/S) }\end{array}$ & $\begin{array}{l}\text { COEFFI- } \\
\text { CIENT OF } \\
\text { VARI. } \\
\text { ATION }\end{array}$ & $\begin{array}{c}\text { PERCENT } \\
\text { OF } \\
\text { ANNUAL } \\
\text { RUNOFF }\end{array}$ \\
\hline OCTOBER & 4,190 & 0.00 & 218 & 636 & 2.9 & 7.5 \\
\hline NOVEMBER & 753 & 0.00 & 76 & 161 & 2.1 & 2.6 \\
\hline DECEMBER & 1,690 & 0.00 & 108 & 264 & 2.4 & 3.7 \\
\hline JANUARY & 4.690 & 0.00 & 250 & 730 & 2.9 & 8.7 \\
\hline FEBRUARY & 2,720 & 0.00 & 280 & 518 & 1.8 & 9.7 \\
\hline MARCH & 1,870 & 0.00 & 496 & 551 & 1.1 & 17.1 \\
\hline APRIL & 3,970 & 0.00 & 603 & 799 & 1.3 & 20.9 \\
\hline MAY & 2,880 & 0.00 & 140 & 427 & 3.1 & 4.8 \\
\hline JUNE & 595 & 0.00 & 17 & 85 & 4.9 & 0.6 \\
\hline JULY & 616 & 0.00 & 111 & 134 & 1.2 & 3.8 \\
\hline AUGUST & 2,260 & 0.00 & 373 & 469 & 1.3 & 12.9 \\
\hline SEPTEMBER & 832 & 0.00 & 219 & 219 & 1.0 & 7.6 \\
\hline ANNUAL & 1,130 & 24 & 241 & 206 & 0.86 & 100 \\
\hline
\end{tabular}

MAGNITUDE AND PROBABILITY OF INSTANTANEOUS PEAK FLOW BASED ON PERIOD OF RECORD 1947.64, 1965.96

DISCHARGE, IN FT3/S, FOR INDICATED RECURRENCE INTERVAL IN YEARS, AND EXCEEDANCE PROBABILITY, IN PERCENT

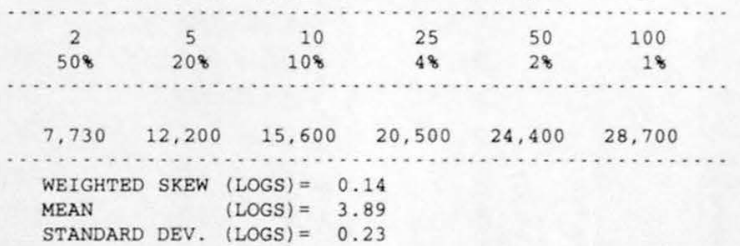

MAGNITUDE AND PROBABILITY OF ANNUAL LOW FLOW BASED ON PERIOD OF RECORD 1949.96

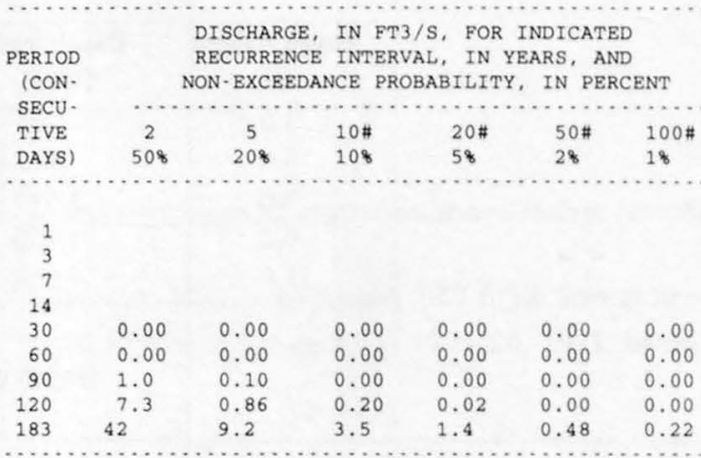

MAGNITUDE AND PROBABILITY OF ANNUAL HIGH FLOW BASED ON PERIOD OF RECORD 1948.96

\begin{tabular}{|c|c|c|c|c|c|c|}
\hline \multirow{2}{*}{$\begin{array}{l}\text { PERIOD } \\
\text { ICON- } \\
\text { SECU. }\end{array}$} & & $\begin{array}{l}\text { DISCHAF } \\
\text { RECURRE } \\
\text { EXCEEDA }\end{array}$ & $\begin{array}{ll}G E, & \text { IN } \\
\text { NCE } & \text { INT } \\
\text { NCE } & \text { PRO }\end{array}$ & $\begin{array}{l}\text { T3/S, FO } \\
\text { RVAL, IN } \\
\text { ABILITY, }\end{array}$ & $\begin{array}{l}\text { R INDICP } \\
\text { YEARS, } \\
\text { IN PERS }\end{array}$ & $\begin{array}{l}\text { TED } \\
\text { AND } \\
\text { ENT }\end{array}$ \\
\hline & & $\ldots \ldots$ & & & & \\
\hline TIVE & 2 & 5 & $10 \#$ & $25 \#$ & $50 \#$ & $100 \#$ \\
\hline DAYS) & $50 \%$ & 208 & 108 & 48 & 28 & 18 \\
\hline 1 & 4,210 & 7,660 & 10,500 & 14,600 & 18,200 & 22,100 \\
\hline 3 & 3,230 & 6,050 & 8,400 & 11,900 & 14.900 & 18,300 \\
\hline 7 & 2,190 & 3,990 & 5,400 & 7,410 & 9,050 & 10,800 \\
\hline 15 & 1,420 & 2.570 & 3.430 & 4,620 & 5.550 & 6.520 \\
\hline 30 & 991 & 1,860 & 2,540 & 3,490 & 4.250 & 5,060 \\
\hline 60 & 651 & 1,280 & 1,780 & 2,480 & 3,040 & 3,640 \\
\hline 90 & 502 & 1,010 & 1.410 & 1,980 & 2,430 & 2,890 \\
\hline
\end{tabular}

DURATION TABLE OF DAILY MEAN FLOW FOR PERIOD OF RECORD 1948.96

DISCHARGE, IN FT3/S, WHICH WAS EQUALED OR EXCEEDED FOR INDICATED PERCENT OF TIME

\begin{tabular}{|c|c|c|c|c|c|c|c|c|c|c|c|c|c|c|c|c|}
\hline 18 & 58 & 108 & 158 & 208 & 308 & 408 & 508 & 608 & 708 & 808 & 908 & 958 & 988 & 998 & 99.58 & 99.98 \\
\hline 020 & 1,360 & 687 & 361 & 195 & 66 & 19 & 3.1 & 0.23 & 0.00 & 0.00 & 0.00 & 0.00 & 0.00 & 0.00 & 0.00 & 0.00 \\
\hline
\end{tabular}

\# Reliability of values in column is uncertain, and potential errors are large. 

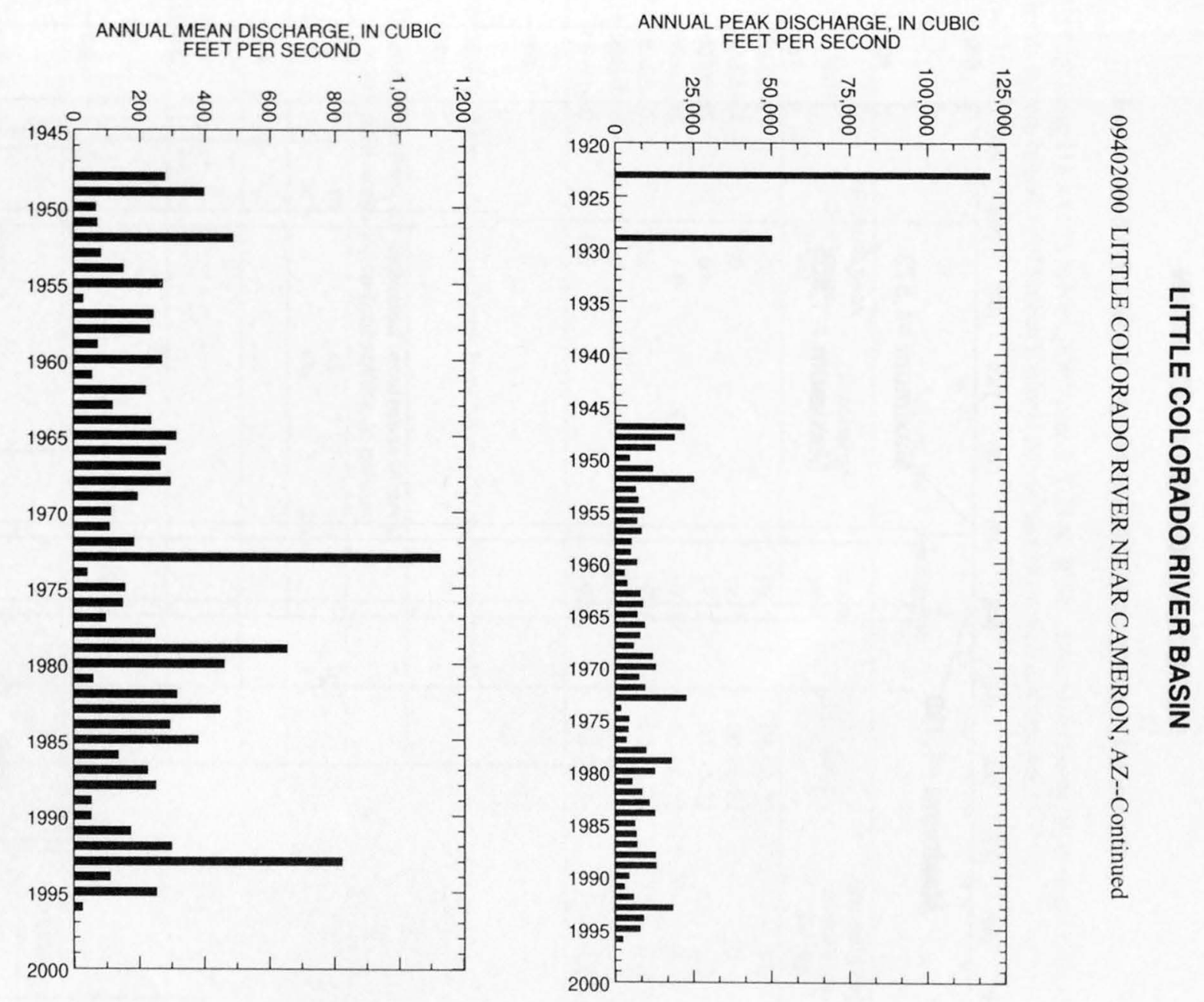
09402000 LITTLE COLORADO RIVER NEAR CAMERON, AZ--Continued
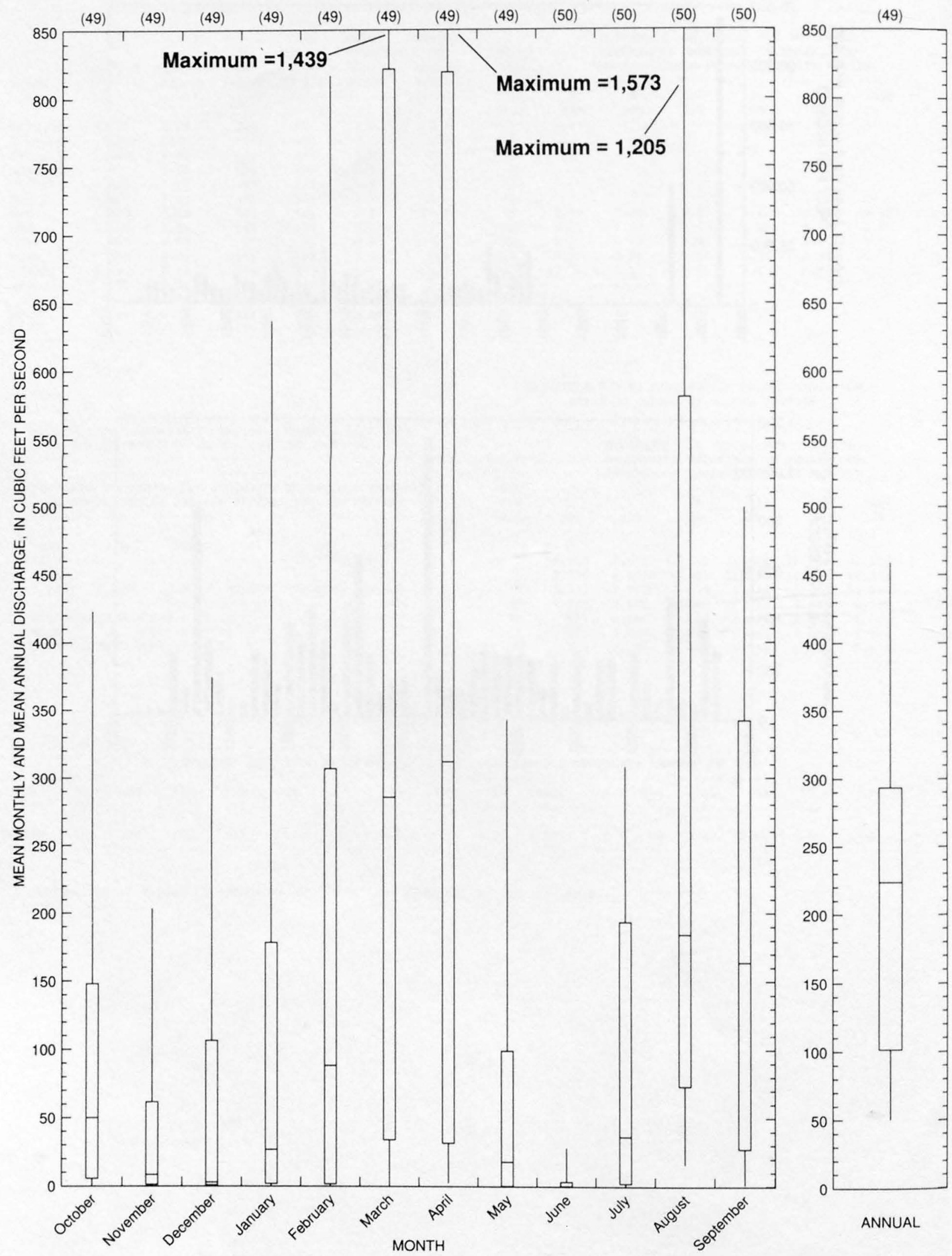
09402100 FOREST BOUNDARY WASH NEAR CAMERON, AZ

LOCATION.--Lat 35 55'25", long $111^{\circ} 44^{\prime} 15^{\prime \prime}$, in NE $1 / 4 \mathrm{SW}^{1} / 4$ sec.3, T.29 N., R.6 E., Coconino County, Hydrologic Unit 15020016, at State Highway $64,0.6 \mathrm{mi}$ inside eastern boundary of Kaibab National Forest, and $18 \mathrm{mi}$ west of Cameron.

DRAINAGE AREA.--0.72 $\mathrm{mi}^{2}$.

Annual peak discharges

\begin{tabular}{cccccccc}
\hline $\begin{array}{c}\text { Water } \\
\text { year }\end{array}$ & Date & $\begin{array}{c}\text { Annual peak } \\
\text { discharge } \\
\left(\mathbf{f t}^{3} / \mathbf{s}\right)\end{array}$ & $\begin{array}{c}\text { Discharge } \\
\text { codes }\end{array}$ & $\begin{array}{c}\text { Water } \\
\text { year }\end{array}$ & Date & $\begin{array}{c}\text { Annual peak } \\
\text { discharge } \\
\left(\mathbf{f t}^{3} \mathbf{s}\right)\end{array}$ & $\begin{array}{c}\text { Discharge } \\
\text { codes }\end{array}$ \\
\hline 1963 & $00-00-63$ & 0 & & 1970 & $08-00-70$ & 3.0 & \\
1964 & $00-00-64$ & 0 & & 1971 & $00-00-71$ & 0 & \\
1965 & $00-00-65$ & 0 & & 1972 & $07-18-72$ & 1.0 & ES \\
1966 & $00-00-66$ & 1.0 & LT & 1973 & $00-00-73$ & 0 & \\
1967 & $00-00-67$ & 12 & & 1974 & $00-00-74$ & 0 & \\
1968 & $00-00-68$ & 0 & & 1975 & $00-00-75$ & 0 & \\
1969 & $09-11-69$ & 115 & & 1976 & $00-00-76$ & 0.5 & ES \\
\hline
\end{tabular}

Magnitude and probability of instantaneous peak flow based on period of record

\begin{tabular}{|c|c|c|c|c|c|}
\hline \multicolumn{6}{|c|}{$\begin{array}{l}\text { Discharge, in } \mathrm{ft}^{3} / \mathrm{s} \text {, for indicated recurrence interval } \\
\text { in years, and exceedance probablility, in percent }\end{array}$} \\
\hline 2 & 5 & 10 & 25 & 50 & 100 \\
\hline $50 \%$ & $20 \%$ & $10 \%$ & $4 \%$ & $2 \%$ & $1 \%$ \\
\hline --. & --- & $\cdots$ & -.. & --- & -- \\
\hline Weighted skew & $(\log s)=$ & $-\cdots$ & & & \\
\hline Mean & $(\log s)=$ & --. & & & \\
\hline Standard dev. & $(\log s)=$ & -.- & & & \\
\hline
\end{tabular}

Basin characteristics

\begin{tabular}{cccccccc}
\hline $\begin{array}{c}\text { Main } \\
\text { channel } \\
\text { slope } \\
(\mathrm{ft} / \mathrm{mi})\end{array}$ & $\begin{array}{c}\text { Stream } \\
\text { length } \\
\text { (mi) }\end{array}$ & $\begin{array}{c}\text { Mean } \\
\text { basin } \\
\text { elevation } \\
\text { (ft) }\end{array}$ & $\begin{array}{c}\text { Forested } \\
\text { area } \\
\text { (percent) }\end{array}$ & $\begin{array}{c}\text { Soil } \\
\text { index }\end{array}$ & $\begin{array}{c}\text { Mean } \\
\text { annual } \\
\text { precipitation } \\
\text { (in) }\end{array}$ & $\begin{array}{c}\text { Rainfall intensity, 24-hour } \\
\text { (in) }\end{array}$ & $\begin{array}{c}50 \text {-year } \\
\text { (in) }\end{array}$ \\
\hline 167 & 1.6 & 6,810 & 94.0 & 3.0 & 11.9 & 1.4 \\
\hline
\end{tabular}

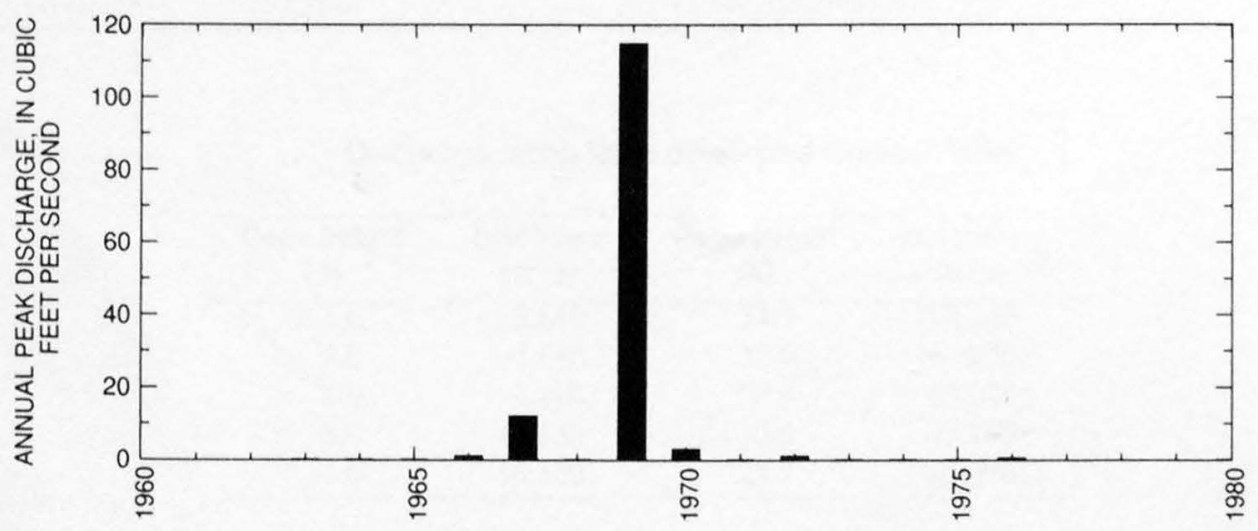


LOCATION.--Lat 36 06'05", long 112 $05^{\prime} 08^{\prime \prime}$, in sec.5, T.31 N., R.3 E. (unsurveyed), Coconino County, Hydrologic Unit 15010001 , in Grand Canyon National Park, on left bank 0.2 mi upstream from Kaibab Bridge, 0.4 mi upstream from Bright Angel Creek, 4.5 mi northeast of village of Grand Canyon, 26 mi downstream from Little Colorado River, and 267 mi upstream from Hoover Dam.

DRAINAGE AREA.--141,600 $\mathrm{mi}^{2}$ approximately, including 3,959 $\mathrm{mi}^{2}$ in Great Divide basin in southern Wyoming which is noncontributing.

PERIOD OF RECORD.--October 1922 to current year. Prior to 1944, published as "Colorado River at Bright Angel Creek, near Grand Canyon." Gage-height records collected 1.5 mi downstream 1908-13, published in reports of U.S. Weather Bureau.

GAGE.--Water-stage recorder. Datum of gage is $2,418.7 \mathrm{ft}$ above sea level.

REMARKS.--No estimated daily discharges. Records good. Flow completely regulated by Lake Powell, 104 mi upstream, since Mar. 13, 1963. (See elsewhere in this report.) Many diversions above station for irrigation, municipal, and industrial uses.

EXTREMES FOR PERIOD OF RECORD.--1922-62: Maximum discharge, 127,000 $\mathrm{ft}^{3} / \mathrm{s}$ July 2, 1927, gage height, $29.25 \mathrm{ft}$; minimum, $700 \mathrm{ft}^{3} /$ s Dec. 28, 1924, gage height, $-0.70 \mathrm{ft}$.

1963-96: Maximum discharge, $96,200 \mathrm{ft}^{3} / \mathrm{s}$ June 29, 1983, gage height, $26.26 \mathrm{ft}$; minimum, $850 \mathrm{ft}^{3} / \mathrm{s}$ Jan. 26, 1963, gage height, -0.55 ft, result of closing coffer dam at Glen Canyon Dam.

EXTREMES OUTSIDE PERIOD OF RECORD.--Maximum discharge since at least $1884,300,000 \mathrm{ft}^{3} / \mathrm{s}$ about July 8,1884 (computed on basis of flood studies at Lees Ferry). Crest discharge of flood of June 19, 1921, was 220,000 $\mathrm{ft}^{3} / \mathrm{s}$, gage height, $37.5 \mathrm{ft}$ from floodmarks, from rating curve extended above $120,000 \mathrm{ft}^{3} / \mathrm{s}$. 
09402500 COLORADO RIVER NEAR GRAND CANYON, AZ

Annual peak discharges

\begin{tabular}{|c|c|c|c|c|c|c|c|}
\hline $\begin{array}{l}\text { Water } \\
\text { year }\end{array}$ & Date & $\begin{array}{c}\text { Annual peak } \\
\text { discharge } \\
\left(\mathrm{ft}^{3} / \mathrm{s}\right)\end{array}$ & $\begin{array}{l}\text { Discharge } \\
\text { codes }\end{array}$ & $\begin{array}{l}\text { Water } \\
\text { year }\end{array}$ & Date & $\begin{array}{c}\text { Annual peak } \\
\text { discharge } \\
\left(\mathrm{ft}^{3} / \mathrm{s}\right)\end{array}$ & $\begin{array}{c}\text { Discharge } \\
\text { codes }\end{array}$ \\
\hline 1884 & $07-08-84$ & 300,000 & ES,HP & 1959 & $06-19-59$ & 38,300 & \\
\hline 1921 & $06-19-21$ & 220,000 & & 1960 & $06-10-60$ & 46,300 & \\
\hline 1922 & $06-01-22$ & 115,000 & ES & 1961 & $06-06-61$ & 39,800 & \\
\hline 1923 & $09-19-23$ & 112,000 & & 1962 & $05-17-62$ & 85,600 & \\
\hline 1924 & $06-18-24$ & 74,000 & & 1963 & $10-22-62$ & 20,700 & $\mathrm{KR}$ \\
\hline 1925 & $06-03-25$ & 53,700 & & 1964 & $04-29-64$ & 19,700 & $\mathrm{KR}$ \\
\hline 1926 & $05-29-26$ & 85,600 & & 1965 & $06-15-65$ & 58,400 & $\mathrm{KR}$ \\
\hline 1927 & $07-02-27$ & 127,000 & & 1966 & $05-04-66$ & 21,300 & $\mathrm{KR}$ \\
\hline 1928 & $06-03-28$ & 115,000 & & 1967 & $09-09-67$ & 23,900 & $\mathrm{KR}$ \\
\hline 1929 & $05-29-29$ & 111,000 & & 1968 & $07-20-68$ & 26,800 & $\mathrm{KR}$ \\
\hline 1930 & $06-04-30$ & 71,000 & & 1969 & $09-12-69$ & 30,800 & $\mathrm{KR}$ \\
\hline 1931 & $05-22-31$ & 34,600 & & 1970 & $08-27-70$ & 27,600 & $\mathrm{KR}$ \\
\hline 1932 & $05-26-32$ & 102,000 & & 1971 & $08-24-71$ & 33,400 & $\mathrm{KR}$ \\
\hline 1933 & $06-05-33$ & 81,500 & & 1972 & $05-26-72$ & 29,500 & $\mathrm{KR}$ \\
\hline 1934 & $05-17-34$ & 25,500 & & 1973 & $04-19-73$ & 38,300 & $\mathrm{KR}$ \\
\hline 1935 & $06-19-35$ & 105,000 & & 1974 & $08-21-74$ & 28,200 & $\mathrm{KR}$ \\
\hline 1936 & $05-24-36$ & 76,300 & & 1975 & $07-30-75$ & 28,900 & $\mathrm{KR}$ \\
\hline 1937 & $05-21-37$ & 85,300 & & 1976 & $05-20-76$ & 27,700 & $\mathrm{KR}$ \\
\hline 1938 & $06-08-38$ & 100,000 & & 1977 & $08-19-77$ & 32,100 & $\mathrm{KR}$ \\
\hline 1939 & $05-26-39$ & 49,000 & & 1978 & 09-09-78 & 29,400 & $\mathrm{KR}$ \\
\hline 1940 & $05-18-40$ & 46,800 & & 1979 & $12-23-78$ & 38,600 & $\mathrm{KR}$ \\
\hline 1941 & $05-17-41$ & 120,000 & & 1980 & $06-24-80$ & 45,000 & $\mathrm{KR}$ \\
\hline 1942 & $05-31-42$ & 91,800 & & 1981 & $07-30-81$ & 25,400 & $\mathrm{KR}$ \\
\hline 1943 & $06-06-43$ & 66,800 & & 1982 & $08-28-82$ & 27,900 & $\mathrm{KR}$ \\
\hline 1944 & $05-20-44$ & 93,400 & & 1983 & $06-29-83$ & 96,200 & $\mathrm{KR}$ \\
\hline 1945 & $05-17-45$ & 63,300 & & 1984 & $08-13-84$ & 47,600 & $\mathrm{KR}$ \\
\hline 1946 & $06-14-46$ & 50,100 & & 1985 & $06-02-85$ & 47,400 & $\mathrm{KR}$ \\
\hline 1947 & $05-14-47$ & 80,100 & & 1986 & $05-31-86$ & 53,500 & $\mathrm{KR}$ \\
\hline 1948 & $05-26-48$ & 89,800 & & 1987 & $01-31-87$ & 31,600 & $\mathrm{KR}$ \\
\hline 1949 & $06-22-49$ & 112,000 & & 1988 & $07-29-88$ & 25,500 & $\mathrm{KR}$ \\
\hline 1950 & $06-06-50$ & 58,400 & & 1989 & 08-19-89 & 31,300 & $\mathrm{KR}$ \\
\hline 1951 & $06-01-51$ & 63,700 & & 1990 & $08-01-90$ & 28,200 & $\mathrm{KR}$ \\
\hline 1952 & $06-12-52$ & 122,000 & & 1991 & $06-18-91$ & 29,000 & $\mathrm{KR}$ \\
\hline 1953 & $06-17-53$ & 68,500 & & 1992 & $07-24-92$ & 19,900 & $\mathrm{KR}$ \\
\hline 1954 & $05-27-54$ & 32,800 & & 1993 & $01-13-93$ & 34,100 & $\mathrm{KR}$ \\
\hline 1955 & $06-14-55$ & 40,400 & & 1994 & $08-18-94$ & 22,000 & $\mathrm{KR}$ \\
\hline 1956 & $06-06-56$ & 67,200 & & 1995 & $09-09-95$ & 25,400 & $\mathrm{KR}$ \\
\hline 1957 & $06-13-57$ & 125,000 & & 1996 & $03-31-96$ & 47,900 & $\mathrm{KR}$ \\
\hline 1958 & $06-02-58$ & 107,700 & & & & & \\
\hline
\end{tabular}

Discharge rating table developed October 1984

\begin{tabular}{cccc}
\hline $\begin{array}{c}\text { Gage height } \\
(\mathbf{f t})\end{array}$ & $\begin{array}{c}\text { Discharge } \\
\left(\mathbf{f t}^{3} / \mathbf{s}\right)\end{array}$ & $\begin{array}{c}\text { Gage height } \\
(\mathbf{f t})\end{array}$ & $\begin{array}{c}\text { Discharge } \\
\left(\mathbf{f t}^{3} \mathbf{s}\right)\end{array}$ \\
\hline 1.0 & 2,640 & 14.0 & 28,360 \\
2.0 & 3,640 & 17.0 & 40,220 \\
5.0 & 7,110 & 20.0 & 55,040 \\
8.0 & 12,130 & 23.0 & 73,130 \\
11.0 & 18,820 & 25.7 & 91,810 \\
\hline
\end{tabular}


09402500 COLORADO RIVER NEAR GRAND CANYON, AZ--Continued

MEAN MONTHLY AND ANNUAL DISCHARGES 1923.96

\begin{tabular}{|c|c|c|c|c|c|c|}
\hline MONTH & $\begin{array}{l}\text { MAXIMUM } \\
(\text { FT } 3 / S)\end{array}$ & $\begin{array}{l}\text { MINIMUM } \\
(\text { FT3/S) }\end{array}$ & $\begin{array}{c}\text { MEAN } \\
(\text { FT3/S) }\end{array}$ & $\begin{array}{l}\text { STAN- } \\
\text { DARD } \\
\text { DEVIA- } \\
\text { TION } \\
(\text { FT3/S) }\end{array}$ & $\begin{array}{l}\text { COEFFI- } \\
\text { CIENT OF } \\
\text { VARI- } \\
\text { ATION }\end{array}$ & $\begin{array}{c}\text { PERCENT } \\
\text { OF } \\
\text { ANNUAL } \\
\text { RUNOFF }\end{array}$ \\
\hline OCTOBER & 31,000 & 1,260 & 9,750 & 4,900 & 0.50 & 5.2 \\
\hline NOVEMBER & 25,000 & 1,280 & 9,650 & 4,220 & 0.44 & 5.2 \\
\hline DECEMBER & 25,200 & 1,260 & 9,170 & 4,760 & 0.52 & 4.9 \\
\hline JANUARY & 26,300 & 1,280 & 9,340 & 5,380 & 0.58 & 5.0 \\
\hline FEBRUARY & 27,200 & 4,260 & 9,680 & 4.540 & 0.47 & 5.2 \\
\hline MARCH & 25,800 & 3,300 & 10,700 & 4,030 & 0.38 & 5.7 \\
\hline APRIL & 46,400 & 1,200 & 17,300 & 8,870 & 0.51 & 9.3 \\
\hline MAY & 82,300 & 1.280 & 30,200 & 20,500 & 0.68 & 16.2 \\
\hline JUNE & 93,100 & 1,290 & 35,800 & 24,000 & 0.67 & 19.2 \\
\hline JULY & 65,600 & 1,370 & 19,400 & 11,100 & 0.57 & 10,4 \\
\hline AUGUST & 32,400 & 1,820 & 13,600 & 6,770 & 0.50 & 7.3 \\
\hline SEPTEMBER & 36,400 & 2,050 & 11,600 & 6,750 & 0.58 & 6.2 \\
\hline ANNUAL & 28,600 & 3,760 & 15,500 & 5,450 & 0.35 & 100 \\
\hline
\end{tabular}

MAGNITUDE AND PROBABILITY OF INSTANTANEOUS PEAK FLOW BASED ON PERIOD OF RECORD

DISCHARGE, IN FT3/S, FOR INDICATED RECURRENCE INTERVAL IN YEARS, AND EXCEEDANCE PROBABILITY, IN PERCENT

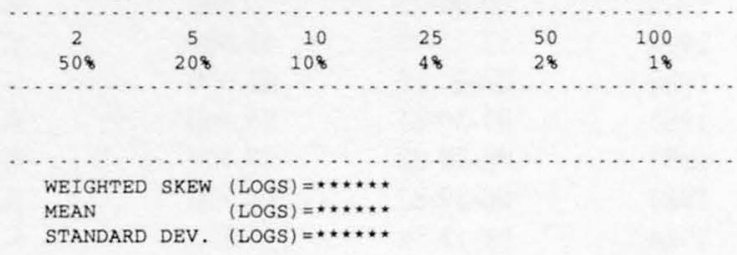

MAGNITUDE AND PROBABILITY OF ANNUAL LOW FLOW BASED ON PERIOD OF RECORD 1924-96

\begin{tabular}{|c|c|c|c|c|c|c|}
\hline \multirow{4}{*}{$\begin{array}{l}\text { PERIOD } \\
\text { (CON- } \\
\text { SECU- } \\
\text { TIVE }\end{array}$} & \multicolumn{6}{|c|}{ DISCHARGE, IN FT3/S, FOR INDICATED } \\
\hline & \multicolumn{6}{|c|}{$\begin{array}{l}\text { RECURRENCE INTERVAL, IN YEARS, AND } \\
\text { NON-EXCEEDANCE PROBABILITY, IN PERCENT }\end{array}$} \\
\hline & & . & & $\ldots \ldots \ldots$ & $\ldots \ldots \ldots$ & \\
\hline & 2 & 5 & 10 & 20 & 50 & 100 \\
\hline DAYS) & 508 & 208 & 108 & 58 & 28 & 18 \\
\hline 1 & 3,400 & 2,200 & 1,710 & 1,370 & 1,060 & 884 \\
\hline 3 & 3,850 & 2,460 & 1,910 & 1,530 & 1,180 & 985 \\
\hline 7 & 4,440 & 2,800 & 2,160 & 1,730 & 1.330 & 1,110 \\
\hline 14 & 5,040 & 3,160 & 2,420 & 1,930 & 1,470 & 1.220 \\
\hline 30 & 5,820 & 3,740 & 2.910 & 2,340 & 1,810 & 1.510 \\
\hline 60 & 6,780 & 4,350 & 3,340 & 2,640 & 2,000 & 1,640 \\
\hline 90 & 7,430 & 4.820 & 3,740 & 2,990 & 2,290 & 1,900 \\
\hline 120 & 7,940 & 5,210 & 4,070 & 3,270 & 2,520 & 2,100 \\
\hline 183 & 8,680 & 5,750 & 4,530 & 3,660 & 2,850 & 2,390 \\
\hline
\end{tabular}

MAGNITUDE AND PROBABILITY OF ANNUAL HIGH FLOW BASED ON PERIOD OF RECORD 1923.96

\begin{tabular}{|c|c|c|c|c|c|c|}
\hline \multirow{3}{*}{$\begin{array}{l}\text { PERIOD } \\
\text { (CON- }\end{array}$} & & \multicolumn{5}{|c|}{ DISCHARGE, IN FT3/S, FOR INDICATED } \\
\hline & & \multirow{2}{*}{\multicolumn{3}{|c|}{$\begin{array}{l}\text { RECURRENCE INTERVAL, IN } \\
\text { EXCEEDANCE PROBABILITY, }\end{array}$}} & $\begin{array}{l}\text { YEARS, } \\
\text { IN PERC }\end{array}$ & AND \\
\hline & & & & & IN PERC & ENT \\
\hline SECU - & & & & & & \\
\hline TIVE & 2 & 5 & 10 & 25 & 50 & 100 \\
\hline DAYS) & 508 & 208 & $10 \%$ & 48 & 28 & 18 \\
\hline 1 & 44,800 & 76,800 & 102,000 & 138,000 & 168,000 & 201,000 \\
\hline 3 & 43,400 & 75,100 & 100,000 & 136,000 & 165,000 & 197.000 \\
\hline 7 & 41,000 & 71,500 & 95,400 & 129,000 & 157,000 & 188,000 \\
\hline 15 & 37.900 & 65,800 & 87,500 & 118,000 & 144,000 & 171.000 \\
\hline 30 & 34,900 & 59,700 & 78,700 & 105,000 & 127,000 & 151,000 \\
\hline 60 & 30,500 & 50,700 & 66,000 & 87,400 & 105,000 & 123,000 \\
\hline 90 & 26,900 & 43,300 & 55,300 & 71,700 & 84,700 & 98,400 \\
\hline
\end{tabular}

DURATION TABLE OF DAILY MEAN FLOW FOR PERIOD OF RECORD 1923.96 DISCHARGE, IN FT3/S, WHICH WAS EQUALED OR EXCEEDED FOR INDICATED PERCENT OF TIME

\begin{tabular}{|c|c|c|c|c|c|c|c|c|c|c|c|c|c|c|c|c|}
\hline 18 & 58 & 108 & 158 & 208 & 308 & 408 & 508 & 608 & 708 & 808 & 908 & 958 & 988 & 998 & 99.58 & 99.98 \\
\hline .500 & 8,000 & 31,000 & 24,000 & 19,900 & 15,700 & 13,100 & 11,000 & 9,180 & 7,560 & 6,300 & 5,040 & 3,930 & 2,580 & 1,360 & 1,210 & 1,120 \\
\hline
\end{tabular}


ANNUAL MEAN DISCHARGE, IN CUBIC

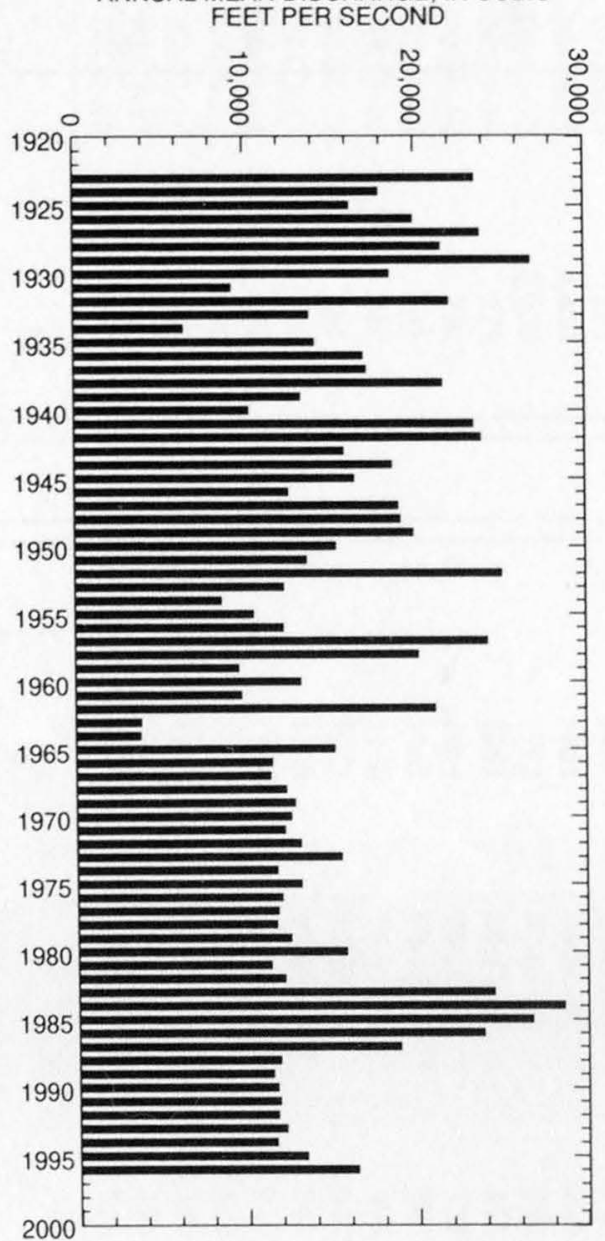

ANNUAL PEAK DISCHARGE, IN CUBIC FEET PER SECOND

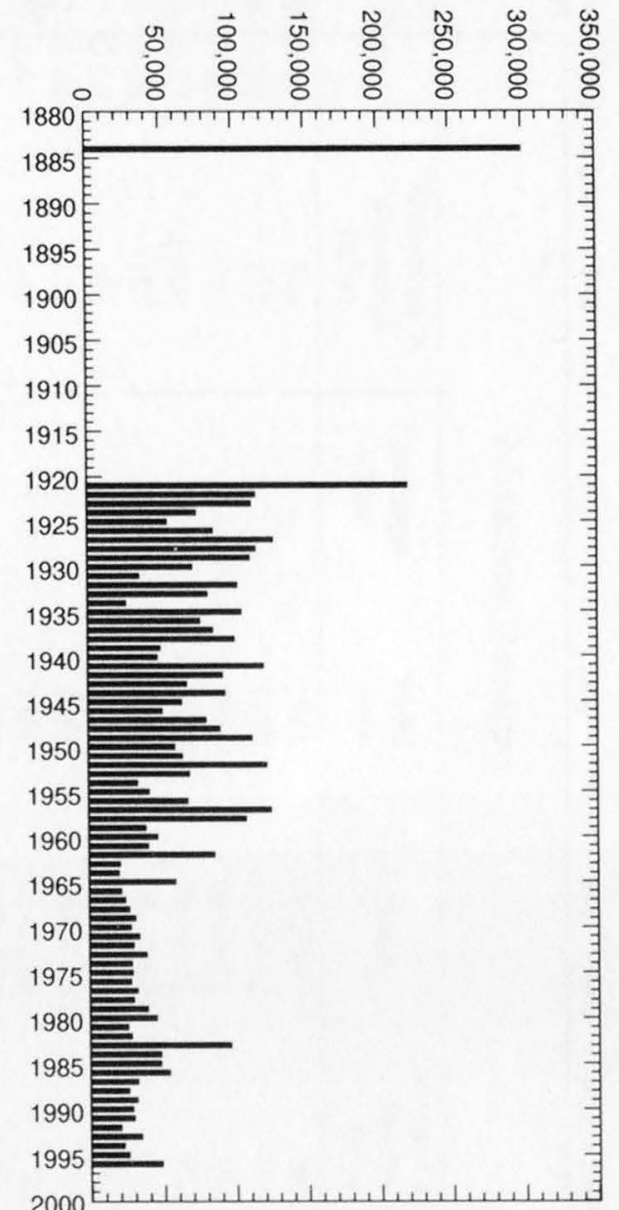


09402500 COLORADO RIVER NEAR GRAND CANYON, AZ--Continued

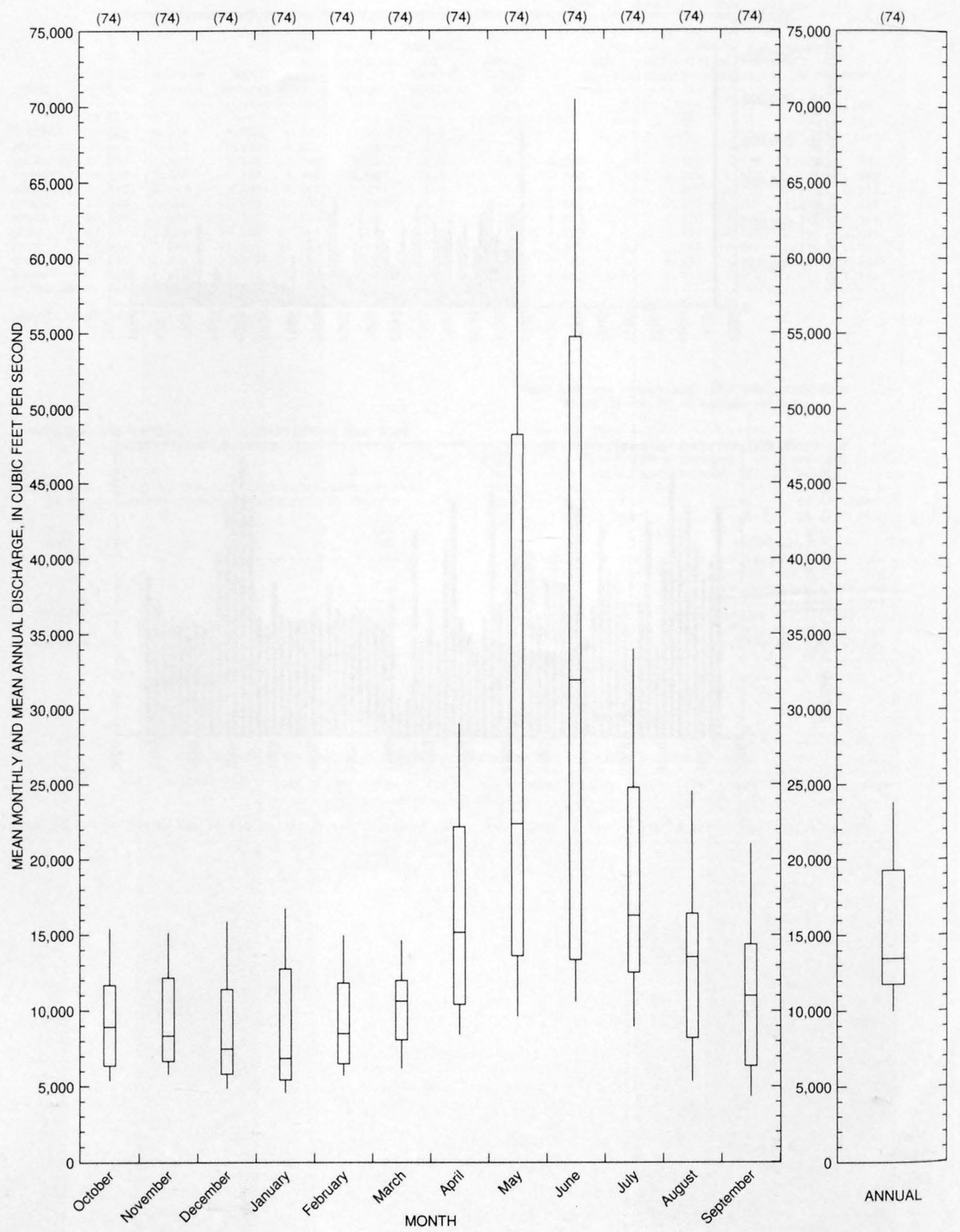


09403000 BRIGHT ANGEL CREEK NEAR GRAND CANYON, AZ

LOCATION.--Lat 36 06' $11^{\prime \prime}$, long $112^{\circ} 05^{\prime} 44^{\prime \prime}$, in sec.5, T.31 N., R.3 E. (unsurveyed), Coconino County, Hydrologic Unit 15010001 , in Grand Canyon National Park, on right bank 0.4 mi upstream from mouth and 4 mi northeast of Grand Canyon.

DRAINAGE AREA.--101 $\mathrm{mi}^{2}$.

Annual peak discharges

\begin{tabular}{|c|c|c|c|c|c|c|c|}
\hline $\begin{array}{l}\text { Water } \\
\text { year }\end{array}$ & Date & $\begin{array}{c}\text { Annual peak } \\
\text { discharge } \\
\left(\mathrm{ft}^{3} / \mathrm{s}\right)\end{array}$ & $\begin{array}{l}\text { Discharge } \\
\text { codes }\end{array}$ & $\begin{array}{l}\text { Water } \\
\text { year }\end{array}$ & Date & $\begin{array}{c}\text { Annual peak } \\
\text { discharge } \\
\left(\mathrm{ft}^{3} / \mathrm{s}\right)\end{array}$ & $\begin{array}{c}\text { Discharge } \\
\text { codes }\end{array}$ \\
\hline 1924 & $09-10-24$ & 530 & & 1949 & $04-28-49$ & 206 & \\
\hline 1925 & $09-17-25$ & 122 & & 1950 & $10-18-49$ & 197 & \\
\hline 1926 & $07-27-26$ & 1,000 & & 1951 & $08-29-51$ & 193 & \\
\hline 1927 & $09-16-27$ & 1,000 & & 1952 & $05-05-52$ & 672 & \\
\hline 1928 & $05-03-28$ & 187 & & 1953 & $08-27-53$ & 930 & \\
\hline 1929 & $07-10-29$ & 173 & & 1954 & $03-23-54$ & 446 & \\
\hline 1930 & $02-23-30$ & 113 & & 1955 & $06-13-55$ & 103 & \\
\hline 1931 & $06-25-31$ & 45 & & 1956 & $01-27-56$ & 77 & \\
\hline 1932 & $02-09-32$ & 500 & & 1957 & $08-05-57$ & 1,770 & \\
\hline 1933 & $08-07-33$ & 186 & & 1958 & $08-22-58$ & 900 & ES \\
\hline 1934 & $10-09-33$ & 250 & & 1959 & $08-11-59$ & 660 & \\
\hline 1935 & $07-20-35$ & 270 & & 1960 & $06-06-60$ & 240 & \\
\hline 1936 & $08-19-36$ & 4,400 & & 1961 & $08-30-61$ & 266 & \\
\hline 1937 & $07-29-37$ & 2,000 & & 1962 & $02-08-62$ & 240 & \\
\hline 1938 & $04-21-38$ & 575 & & 1963 & $08-17-63$ & 855 & \\
\hline 1939 & $09-06-39$ & 270 & & 1964 & $08-26-64$ & 353 & \\
\hline 1940 & $08-24-40$ & 602 & & 1965 & $08-15-65$ & 788 & \\
\hline 1941 & $05-13-41$ & 848 & & 1966 & $11-23-65$ & 484 & \\
\hline 1942 & $04-23-42$ & 264 & & 1967 & $12-06-66$ & 4,000 & \\
\hline 1943 & $04-23-43$ & 426 & & 1968 & $07-07-68$ & 240 & \\
\hline 1944 & $05-15-44$ & 199 & & 1969 & $01-25-69$ & 930 & \\
\hline 1945 & $07-30-45$ & 297 & & 1970 & $03-01-70$ & 1,180 & \\
\hline 1946 & $07-22-46$ & 840 & & 1971 & $07-19-71$ & 2,300 & \\
\hline 1947 & $08-27-47$ & 310 & & 1972 & $07-25-72$ & 126 & \\
\hline 1948 & $08-11-48$ & 1,900 & & 1973 & $05-12-73$ & 578 & \\
\hline
\end{tabular}

Discharge rating table developed October 1990

\begin{tabular}{cccc}
\hline $\begin{array}{c}\text { Gage height } \\
(\mathbf{f t})\end{array}$ & $\begin{array}{c}\text { Discharge } \\
\left(\mathbf{f t}^{3} / \mathbf{s}\right)\end{array}$ & $\begin{array}{c}\text { Gage height } \\
(\mathbf{f t})\end{array}$ & $\begin{array}{c}\text { Discharge } \\
\left(\mathbf{f t}^{3} / \mathbf{s}\right)\end{array}$ \\
\hline 3.8 & 10.0 & 5.2 & 150 \\
4.0 & 18.6 & 5.5 & 204 \\
4.3 & 38.4 & 5.8 & 268 \\
4.6 & 66.5 & 6.1 & 344 \\
4.9 & 104 & 6.4 & 430 \\
\hline
\end{tabular}

Basin characteristics

\begin{tabular}{cccccccc}
$\begin{array}{c}\text { Main } \\
\text { channel } \\
\begin{array}{c}\text { slope } \\
(\mathrm{ft} / \mathrm{mi})\end{array}\end{array}$ & $\begin{array}{c}\text { Stream } \\
\text { length } \\
(\mathrm{mi})\end{array}$ & $\begin{array}{c}\text { Mean } \\
\text { basin } \\
\text { elevation } \\
(\mathrm{ft})\end{array}$ & $\begin{array}{c}\text { Forested } \\
\text { area } \\
\text { (percent) }\end{array}$ & $\begin{array}{c}\text { Soil } \\
\text { index }\end{array}$ & $\begin{array}{c}\text { Mean } \\
\text { annual } \\
\text { precipitation } \\
\text { (in) }\end{array}$ & $\begin{array}{c}\text { Rainfall intensity, 24-hour } \\
\text { (in) }\end{array}$ & $\begin{array}{c}50 \text {-year } \\
\text { (in) }\end{array}$ \\
\hline 421 & 17.6 & 7,390 & 53 & 2.2 & 19.8 & 2.5 \\
\hline
\end{tabular}




\section{BRIGHT ANGEL CREEK NEAR GRAND CANYON, AZ--Continued}

MEAN MONTHLY AND ANNUAL DISCHARGES 1924.73

\begin{tabular}{|c|c|c|c|c|c|c|}
\hline MONTH & $\begin{array}{l}\text { MAXIMUM } \\
\text { (FT3/S) }\end{array}$ & $\begin{array}{l}\text { MINIMUM } \\
\text { (FT3/S) }\end{array}$ & $\begin{array}{c}\text { MEAN } \\
(\text { FT3/S) }\end{array}$ & $\begin{array}{l}\text { STAN- } \\
\text { DARD } \\
\text { DEVIA- } \\
\text { TION } \\
\text { (FT3/S) }\end{array}$ & $\begin{array}{l}\text { COEFFI- } \\
\text { CIENT OF } \\
\text { VARI- } \\
\text { ATION }\end{array}$ & $\begin{array}{c}\text { PERCENT } \\
\text { OF } \\
\text { ANNUAL } \\
\text { RUNOFF }\end{array}$ \\
\hline OCTOBER & 34 & 15 & 21 & 4.1 & 0.20 & 5.0 \\
\hline NOVEMBER & 34 & 15 & 21 & 4.2 & 0.20 & 5.1 \\
\hline DECEMBER & 181 & 16 & 25 & 23 & 0.90 & 6.0 \\
\hline JANUARY & 33 & 16 & 22 & 4.0 & 0.18 & 5.3 \\
\hline FEBRUARY & 51 & 16 & 24 & 7.6 & 0.31 & 5.8 \\
\hline MARCH & 64 & 16 & 27 & 9.8 & 0.36 & 6.5 \\
\hline APRIL & 217 & 15 & 77 & 51 & 0.66 & 18.3 \\
\hline MAY & 501 & 14 & 103 & 106 & 1.0 & 24.4 \\
\hline JUNE & 131 & 14 & 34 & 23 & 0.67 & 8.0 \\
\hline JULY & 44 & 13 & 23 & 5.8 & 0.25 & 5.5 \\
\hline AUGUST & 41 & 13 & 22 & 4.7 & 0.21 & 5.2 \\
\hline SEPTEMBER & 45 & 14 & 21 & 5.6 & 0.26 & 5.1 \\
\hline ANNUAL & 89 & 15 & 35 & 15 & 0.44 & 100 \\
\hline
\end{tabular}

MAGNITUDE AND PROBABILITY OF INSTANTANEOUS PEAK FLOW BASED ON PERIOD OF RECORD $1924-73$

DISCHARGE, IN FT3/S, FOR INDICATED RECURRENCE INTERVAI

IN YEARS, AND EXCEEDANCE PROBABILITY, IN PERCENT

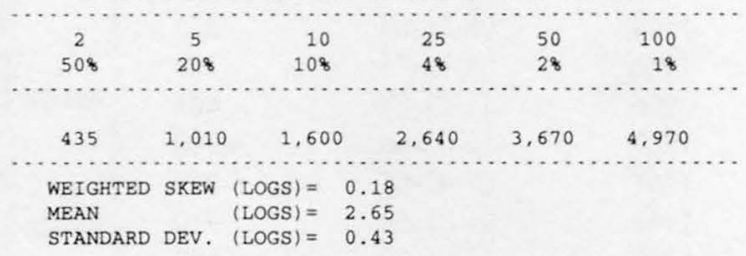

MAGNITUDE AND PROBABILITY OF ANNUAL LOW FLOW BASED ON PERIOD OF RECORD 1925.74

\begin{tabular}{|c|c|c|c|c|c|c|}
\hline \multirow{2}{*}{$\begin{array}{l}\text { PERIOD } \\
\text { (CON- } \\
\text { SECU. }\end{array}$} & \multicolumn{6}{|c|}{ DISCHARGE, IN FT3/S, FOR INDICATED } \\
\hline & \multicolumn{3}{|c|}{ NON-EXCEEDANCE } & \multicolumn{3}{|c|}{$\begin{array}{l}\text { IN YEARS, AND } \\
\text { BILITY, IN PERCENT }\end{array}$} \\
\hline SECU - & $\cdots$ & $\cdots$ & $\cdots$ & $\ldots$ & $\cdots$ & $\cdots$ \\
\hline TIVE & 2 & 5 & 10 & 20 & 50 & 100 \\
\hline DAYS) & 508 & 208 & 108 & 58 & 28 & 18 \\
\hline 1 & 17 & 14 & 13 & 12 & 11 & 11 \\
\hline 3 & 17 & 14 & 13 & 12 & 11 & 10 \\
\hline 7 & 17 & 15 & 13 & 12 & 12 & 11 \\
\hline 14 & 18 & 15 & 14 & 13 & 12 & 12 \\
\hline 30 & 18 & 16 & 15 & 14 & 13 & 12 \\
\hline 60 & 19 & 16 & 15 & 14 & 13 & 13 \\
\hline 90 & 19 & 17 & 16 & 15 & 14 & 13 \\
\hline 120 & 20 & 17 & 16 & 15 & 14 & 13 \\
\hline 183 & 20 & 18 & 16 & 15 & 14 & 14 \\
\hline
\end{tabular}

MAGNITUDE AND PROBABILITY OF ANNUAL HIGH FLOW BASED ON PERIOD OF RECORD $1924 \cdot 73$

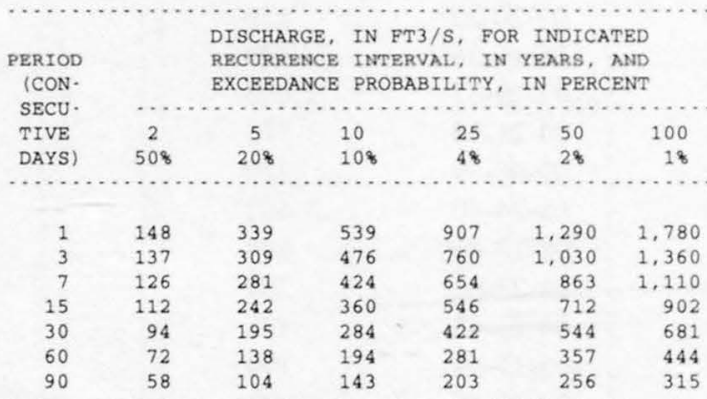

DURATION TABLE OF DAILY MEAN FLOW FOR PERIOD OF RECORD 1924-73

DISCHARGE, IN FT3/S, WHICH WAS EQUALED OR EXCEEDED FOR INDICATED PERCENT OF TIME

\begin{tabular}{|c|c|c|c|c|c|c|c|c|c|c|c|c|c|c|c|c|}
\hline 18 & 58 & 108 & 158 & 208 & 308 & 408 & 508 & 608 & 708 & 808 & 908 & 958 & 988 & 998 & 99.58 & 99.98 \\
\hline 294 & 100 & 54 & 37 & 32 & 27 & 25 & 23 & 22 & 20 & 19 & 17 & 16 & 15 & 14 & 13 & 12 \\
\hline
\end{tabular}


ANNUAL MEAN DISCHARGE, IN CUBIC

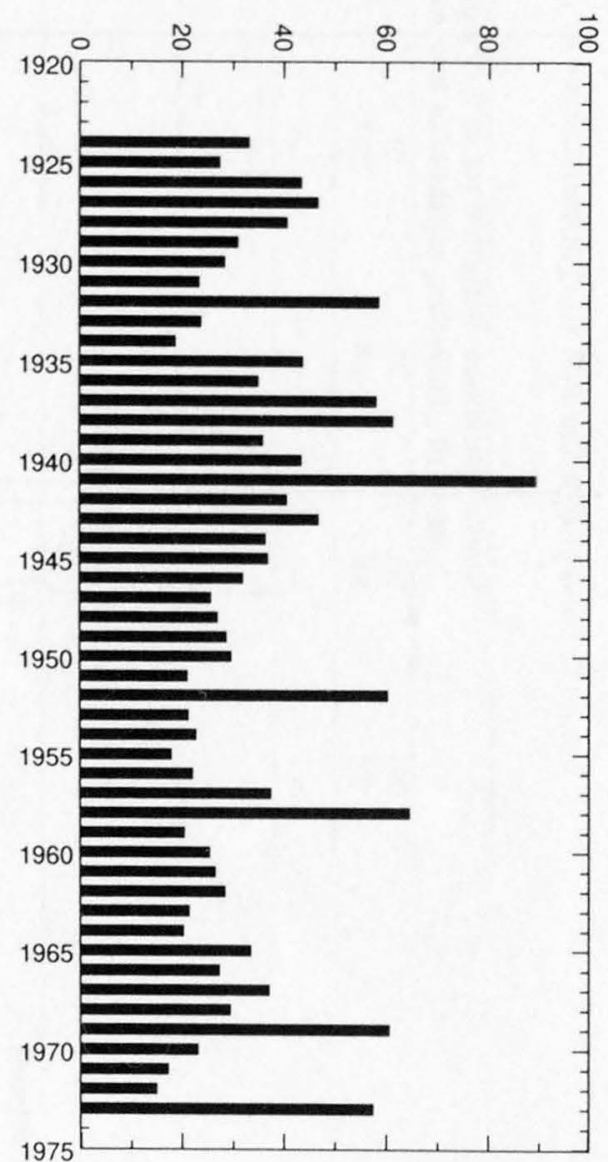

ANNUAL PEAK DISCHARGE, IN CUBIC

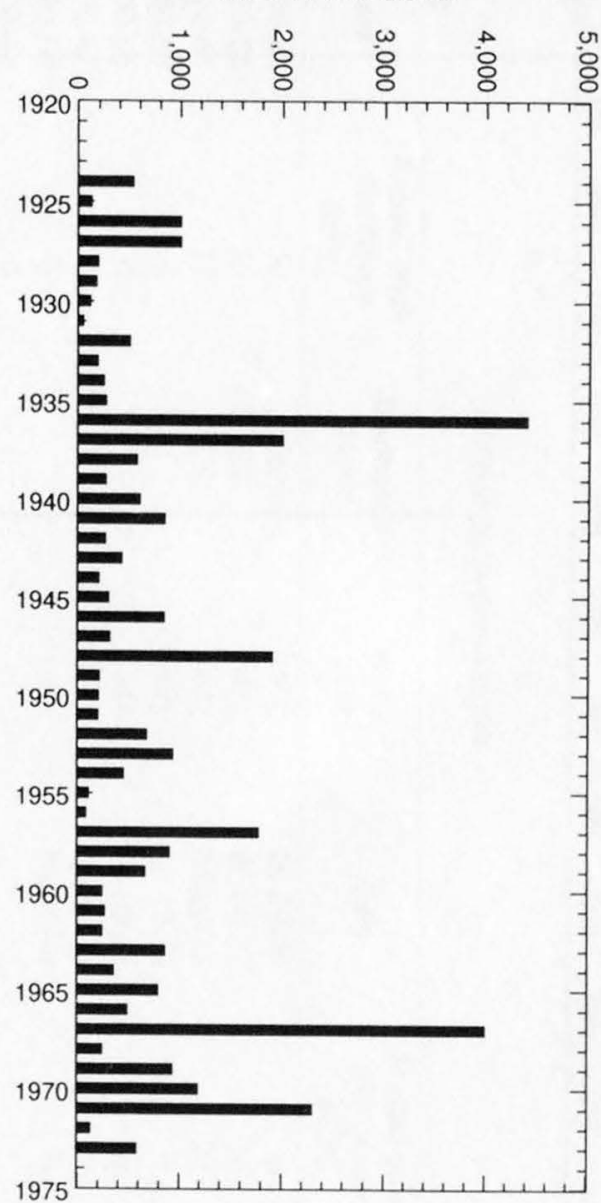


09403000 BRIGHT ANGEL CREEK NEAR GRAND CANYON, AZ--Continued
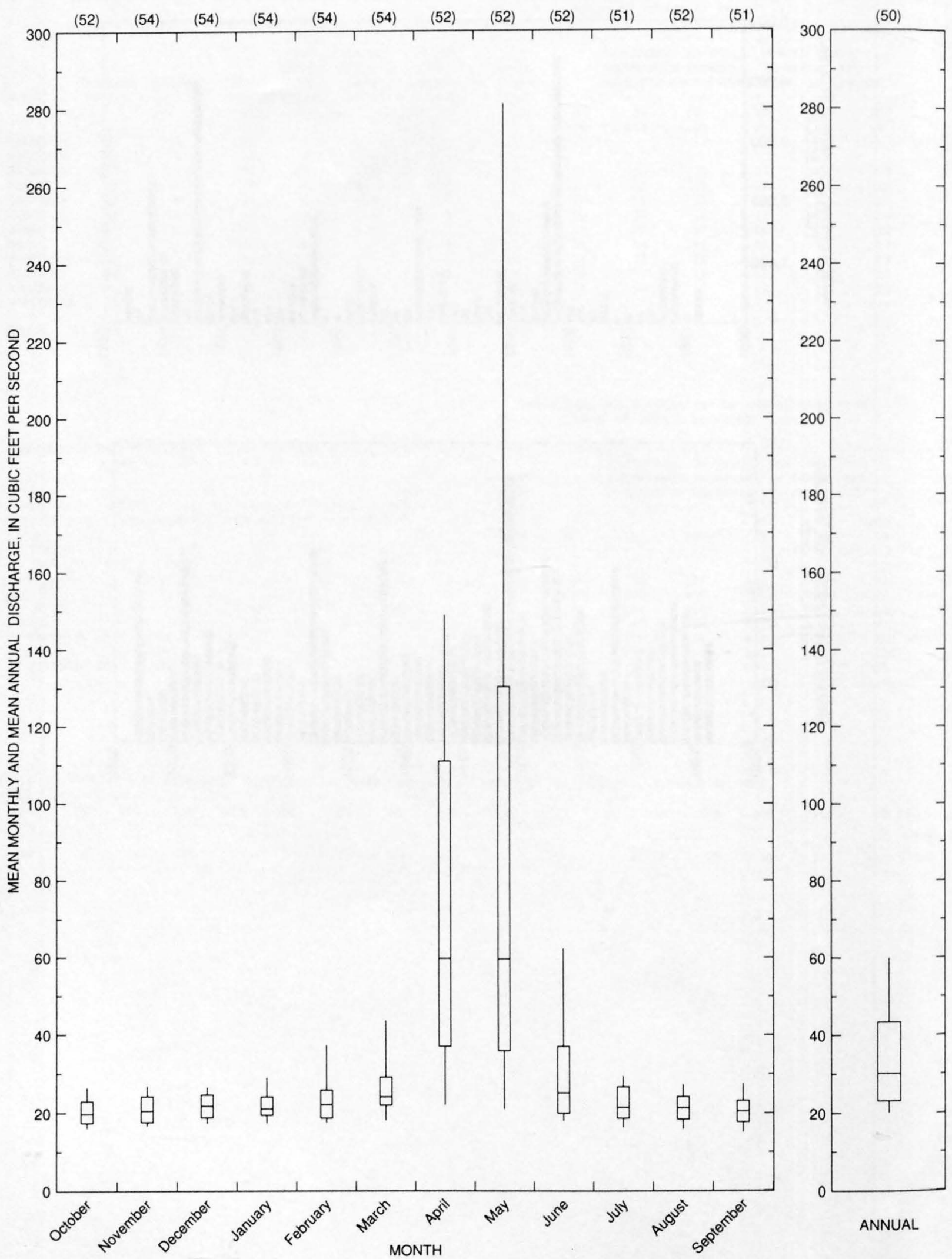


\section{SAGEBRUSH DRAW NEAR FREDONIA, AZ}

LOCATION.--Lat $36^{\circ} 54^{\prime} 05^{\prime \prime}$, long $112^{\circ} 22^{\prime} 35^{\prime \prime}$, in NE $1 /{ }_{4} \mathrm{NE}^{1} / 4$ sec.3, T.40 N., R.1 W. (unsurveyed), Coconino County, Hydrologic Unit 15010003 , at U.S. Highway 89 Alt., $9.5 \mathrm{mi}$ east of Fredonia.

DRAINAGE AREA.--0.68 $\mathrm{mi}^{2}$.

Annual peak discharges

\begin{tabular}{|c|c|c|c|c|c|c|c|}
\hline $\begin{array}{l}\text { Water } \\
\text { year }\end{array}$ & Date & $\begin{array}{l}\text { Annual peak } \\
\text { discharge } \\
\left(\mathrm{ft}^{3} / \mathrm{s}\right)\end{array}$ & $\begin{array}{l}\text { Discharge } \\
\text { codes }\end{array}$ & $\begin{array}{l}\text { Water } \\
\text { year }\end{array}$ & Date & $\begin{array}{c}\text { Annual peak } \\
\text { discharge } \\
\left(\mathrm{ft}^{3} / \mathrm{s}\right)\end{array}$ & $\begin{array}{c}\text { Discharge } \\
\text { codes }\end{array}$ \\
\hline 1960 & $00-00-60$ & 150 & HP & 1970 & $00-00-70$ & 0 & \\
\hline 1963 & $08-30-63$ & 50 & ES & 1971 & $02-00-71$ & 16 & ES \\
\hline 1964 & $07-14-64$ & 35 & ES & 1972 & $06-22-72$ & 20 & ES \\
\hline 1965 & $00-00-65$ & 0 & & 1973 & $10-19-72$ & 5.0 & ES \\
\hline 1966 & $00-00-66$ & 0 & & 1974 & $00-00-74$ & 0 & \\
\hline 1967 & $00-00-67$ & 0 & & 1975 & $08-21-75$ & 10 & LT \\
\hline 1968 & $00-00-68$ & 0 & & 1976 & $00-00-76$ & 0.1 & \\
\hline 1969 & $00-00-69$ & 0 & & & & & \\
\hline
\end{tabular}

Magnitude and probability of instantaneous peak flow based on period of record

\begin{tabular}{cccccc}
\hline \multicolumn{5}{c}{$\begin{array}{l}\text { Discharge, in } \mathrm{ft}^{3} / \mathbf{s} \text {, for indicated recurrence interval } \\
\text { in years, and exceedance probablility, in percent }\end{array}$} \\
\hline 2 & 5 & 10 & 25 & 50 & 100 \\
$50 \%$ & $20 \%$ & $10 \%$ & $4 \%$ & $2 \%$ & $1 \%$ \\
\hline & & & & & \\
Weighted skew & $(\operatorname{logs})=$ & --- & & \\
Mean & $(\operatorname{logs})=$ & -- & & & \\
Standard dev. & $(\operatorname{logs})=$ & -- &
\end{tabular}

Basin characteristics

\begin{tabular}{cccccccc}
\hline $\begin{array}{c}\text { Main } \\
\text { channel } \\
\text { slope } \\
(\mathrm{ft} / \mathrm{mi})\end{array}$ & $\begin{array}{c}\text { Stream } \\
\text { length } \\
(\mathbf{m i})\end{array}$ & $\begin{array}{c}\text { Mean } \\
\text { basin } \\
\text { elevation } \\
(\mathrm{ft})\end{array}$ & $\begin{array}{c}\text { Forested } \\
\text { area } \\
\text { (percent) }\end{array}$ & $\begin{array}{c}\text { Soil } \\
\text { index }\end{array}$ & $\begin{array}{c}\text { Mean } \\
\text { annual } \\
\text { precipitation } \\
\text { (in) }\end{array}$ & $\begin{array}{c}\text { Rainfall intensity, 24-hour } \\
\text { (in) }\end{array}$ & $\begin{array}{c}50 \text {-year } \\
\text { (in) }\end{array}$ \\
\hline 106 & 2.3 & 5,290 & 0.0 & 3.0 & 12.0 & 1.4 \\
\hline
\end{tabular}


09403750 SAGEBRUSH DRAW NEAR FREDONIA, AZ--Continued

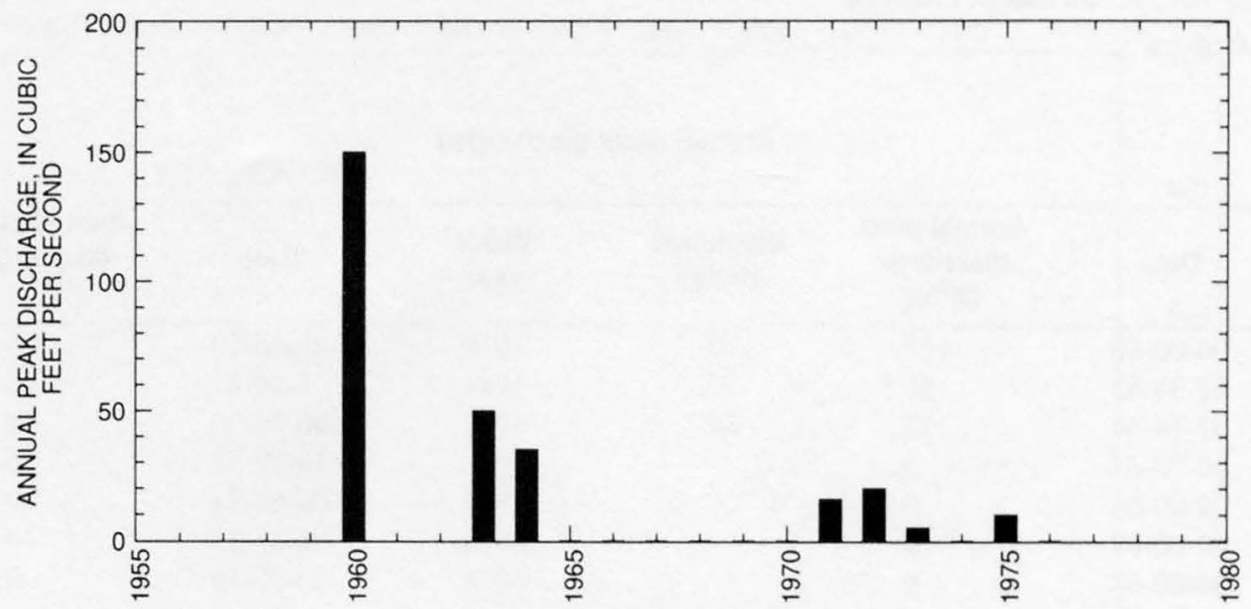


09403780 KANAB CREEK NEAR FREDONIA, AZ

LOCATION.--Lat 36 $51^{\prime} 50^{\prime \prime}$, long $112^{\circ} 34^{\prime} 45^{\prime \prime}$, in SE $\frac{1}{4}$ sec.14, T.40 N., R.3 W. (unsurveyed), Coconino County, Hydrologic Unit 15010003 , in

Kaibab Indian Reservation, at Nagles Crossing, on left bank 0.2 mi downstream from Johnson Wash and 6.5 mi southwest of Fredonia.

DRAINAGE AREA.--1,085 $\mathrm{mi}^{2}$.

PERIOD OF RECORD.--October 1963 to September 1980 (discontinued).

REVISED RECORDS.--WRD Ariz. 1974:

GAGE.--Water-stage recorder and concrete control. Altitude of gage is 4,500 ft, from topographic map.

REMARKS.--Records fair except those for periods of no gage-height record, which are poor. Diversions upstream for irrigation of about 1,400 acres in Utah and 800 acres in Arizona in 1967.

AVERAGE DISCHARGE.--17 years, $6.78 \mathrm{ft}^{3} / \mathrm{s}, 4,910$ acre- $\mathrm{ft} / \mathrm{yr}$.

EXTREMES FOR PERIOD OF RECORD.--Maximum discharge, 4,630 ft 3 /s Aug. 18, 1970, gage height, $9.11 \mathrm{ft}$, inside, $9.7 \mathrm{ft}$, from profile past gage, from rating curve extended above $850 \mathrm{ft}^{3} / \mathrm{s}$ on basis of slope-area measurement of peak flow; no flow for most of time.

Annual peak discharges

\begin{tabular}{ccccccc}
\hline $\begin{array}{l}\text { Water } \\
\text { year }\end{array}$ & Date & $\begin{array}{c}\text { Annual peak } \\
\text { discharge } \\
\left(\mathrm{ft}^{3} / \mathbf{s}\right)\end{array}$ & $\begin{array}{c}\text { Discharge } \\
\text { codes }\end{array}$ & $\begin{array}{c}\text { Water } \\
\text { year }\end{array}$ & $\begin{array}{c}\text { Annual peak } \\
\text { discharge } \\
\left(\mathrm{ft}^{3} / \mathbf{s}\right)\end{array}$ & $\begin{array}{c}\text { Discharge } \\
\text { codes }\end{array}$ \\
\hline 1550 & $00-00-50$ & 115,000 & ES,PF & 1972 & $09-19-72$ & 1,680 \\
1964 & $08-13-65$ & $\left({ }^{2}\right)$ & & 1973 & $04-14-73$ & 660 \\
1965 & $04-18-65$ & 250 & & 1974 & $07-23-74$ & 84 \\
1966 & $03-08-66$ & 668 & & 1975 & $07-13-75$ & 603 \\
1967 & $12-07-67$ & 2,960 & & 1976 & $09-25-76$ & 410 \\
1968 & $07-31-68$ & 1,130 & & 1977 & $07-23-77$ & 435 \\
1969 & $07-23-69$ & 1,330 & & 1978 & $04-11-78$ & 460 \\
1970 & $08-18-70$ & 4,630 & & 1979 & $02-14-79$ & 2,020 \\
1971 & $08-18-71$ & 1,340 & & 1980 & $02-20-80$ & 957 \\
\hline
\end{tabular}

${ }^{1}$ Highest since 1488 , Smith.

${ }^{2}$ Discharge not determined.

Basin characteristics

\begin{tabular}{|c|c|c|c|c|c|c|c|}
\hline $\begin{array}{l}\text { Main } \\
\text { channel } \\
\text { slope } \\
\text { (ft/mi) }\end{array}$ & $\begin{array}{l}\text { Stream } \\
\text { length } \\
\text { (mi) }\end{array}$ & $\begin{array}{c}\text { Mean } \\
\text { basin } \\
\text { elevation } \\
(f t)\end{array}$ & $\begin{array}{l}\text { Forested } \\
\text { area } \\
\text { (percent) }\end{array}$ & $\begin{array}{c}\text { Soil } \\
\text { index }\end{array}$ & $\begin{array}{c}\text { Mean } \\
\text { annual } \\
\text { precipitation } \\
\text { (in) }\end{array}$ & \multicolumn{2}{|c|}{ Rainfall intensity, 24-hour } \\
\hline 56.4 & 49.6 & 6,100 & 60.0 & 3.0 & 12.0 & 1.5 & 3.0 \\
\hline
\end{tabular}


09403780 KANAB CREEK NEAR FREDONIA, AZ--Continued

MEAN MONTHLY AND ANNUAL DISCHARGES $1964 \cdot 80$

\begin{tabular}{|c|c|c|c|c|c|c|}
\hline MONTH & $\begin{array}{l}\text { MAXIMUM } \\
(\text { FT } 3 / \mathrm{S})\end{array}$ & $\begin{array}{l}\text { MINIMUM } \\
\text { (FT3/S) }\end{array}$ & $\begin{array}{c}\text { MEAN } \\
(\mathrm{FT} 3 / \mathrm{S})\end{array}$ & $\begin{array}{l}\text { STAN:- } \\
\text { DARD } \\
\text { DEVIA- } \\
\text { TION } \\
\text { (FT3/S) }\end{array}$ & $\begin{array}{l}\text { COEFFI- } \\
\text { CIENT OF } \\
\text { VARI- } \\
\text { ATION }\end{array}$ & $\begin{array}{c}\text { PERCENT } \\
\text { OF } \\
\text { ANNUAL } \\
\text { RUNOFF }\end{array}$ \\
\hline OCTOBER & 19 & 0.00 & 1.8 & 4.6 & 2.6 & 2.2 \\
\hline NOVEMBER & 16 & 0.00 & 2.2 & 5.1 & 2.3 & 2.7 \\
\hline DECEMBER & 58 & 0.00 & 7.3 & 15 & 2.0 & 8.9 \\
\hline JT,UARY & 30 & 0.00 & 5.9 & 8.2 & 1.4 & 7.2 \\
\hline FEBRUARY & 49 & 0.00 & 9.9 & 14 & 1.5 & 12.2 \\
\hline MARCH & 90 & 0.00 & 17 & 24 & 1.5 & 20.5 \\
\hline APRIL & 80 & 0.00 & 21 & 30 & 1.4 & 25.8 \\
\hline MAY & 6.5 & 0.00 & 0.71 & 1.6 & 2.3 & 0.9 \\
\hline JUNE & 0.77 & 0.00 & 0.05 & 0.19 & 3.5 & 0.1 \\
\hline JULY & 20 & 0.00 & 3.5 & 6.4 & 1.8 & 4.3 \\
\hline AUGUST & 48 & 0.00 & 7.6 & 14 & 1.9 & 9.4 \\
\hline SEPTEMBER & 29 & 0.00 & 4.8 & 8.6 & 1.8 & 5.9 \\
\hline ANNUAL & 18 & 0.78 & 6.8 & 5.2 & 0.77 & 100 \\
\hline
\end{tabular}

MAGNITUDE AND PROBABILITY OF INSTANTANEOUS PEAK FLOW BASED ON PERIOD OF RECORD 1550, 1965.80

DISCHARGE, IN FT $3 / S$, FOR INDICATED RECURRENCE INTERVAI IN YEARS, AND EXCEEDANCE PROBABILITY, IN PERCENT

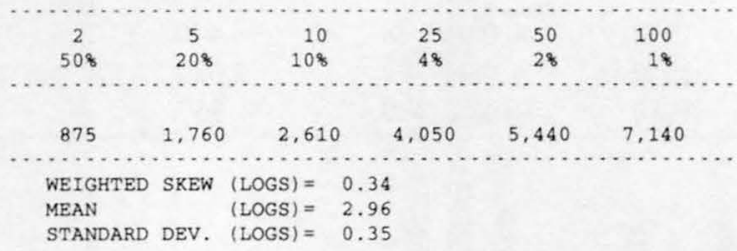

MAGNITUDE AND PROBABILITY OF ANNUAL LOW FLOW BASED ON PERIOD OF RECORD 1965.80

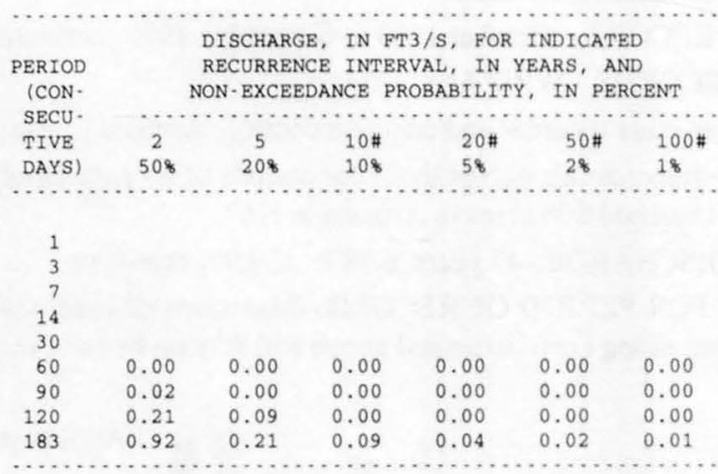

MAGNITUDE AND PROBABILITY OF ANNUAL HIGH FLOW BASED ON PERIOD OF RECORD $1964 \cdot 80$

\begin{tabular}{|c|c|c|c|c|c|c|}
\hline \multirow{3}{*}{$\begin{array}{l}\text { PERIOD } \\
\text { (CON- } \\
\text { SECU. }\end{array}$} & \multirow{2}{*}{\multicolumn{2}{|c|}{$\begin{array}{l}\text { DISCHARGE, } \\
\text { RECURRENCE } \\
\text { BXCEEDANCE }\end{array}$}} & \multicolumn{4}{|c|}{ IN $\mathrm{FT} 3 / \mathrm{S}$, FOR INDICATED } \\
\hline & & & Q PRO & ILITY, & IN PERC & \\
\hline & & ............ & $\ldots \ldots$ & ....... & .......... & \\
\hline TIVE & 2 & 5 & $10 \#$ & $25 \#$ & $50 \#$ & $100 \#$ \\
\hline DAYS) & 508 & 208 & 108 & 48 & 28 & 18 \\
\hline 1 & 223 & 482 & 679 & 936 & 1,130 & 1,310 \\
\hline 3 & 139 & 290 & 397 & 529 & 620 & 704 \\
\hline 7 & 85 & 179 & 244 & 319 & 369 & 414 \\
\hline 15 & 50 & 116 & 166 & 232 & 280 & 327 \\
\hline 30 & 32 & 74 & 108 & 154 & 188 & 221 \\
\hline 60 & 19 & 46 & 67 & 97 & 119 & 142 \\
\hline 90 & 14 & 35 & 53 & 78 & 99 & 121 \\
\hline
\end{tabular}

DURATION TABLE OF DAILY MEAN FLOW FOR PERIOD OF RECORD 1964-80

DISCHARGE, IN FT3/S, WHICH WAS BQUALED OR EXCEEDED FOR INDICATED PERCENT OF TIME

\begin{tabular}{|c|c|c|c|c|c|c|c|c|c|c|c|c|c|c|c|c|}
\hline 18 & 58 & 108 & 158 & 208 & 308 & 408 & 508 & 608 & 708 & 808 & 908 & 958 & 988 & 998 & 99.58 & 99.98 \\
\hline 146 & 28 & 11 & 5.6 & 2.9 & 0.69 & 0.10 & 0.00 & 0.00 & 0.00 & 0.00 & 0.00 & 0.00 & 0.00 & 0.00 & 0.00 & 0.00 \\
\hline
\end{tabular}

\# Reliability of values in column is uncertain, and potential errors are large. 
09403780 KANAB CREEK NEAR FREDONIA, AZ--Continued
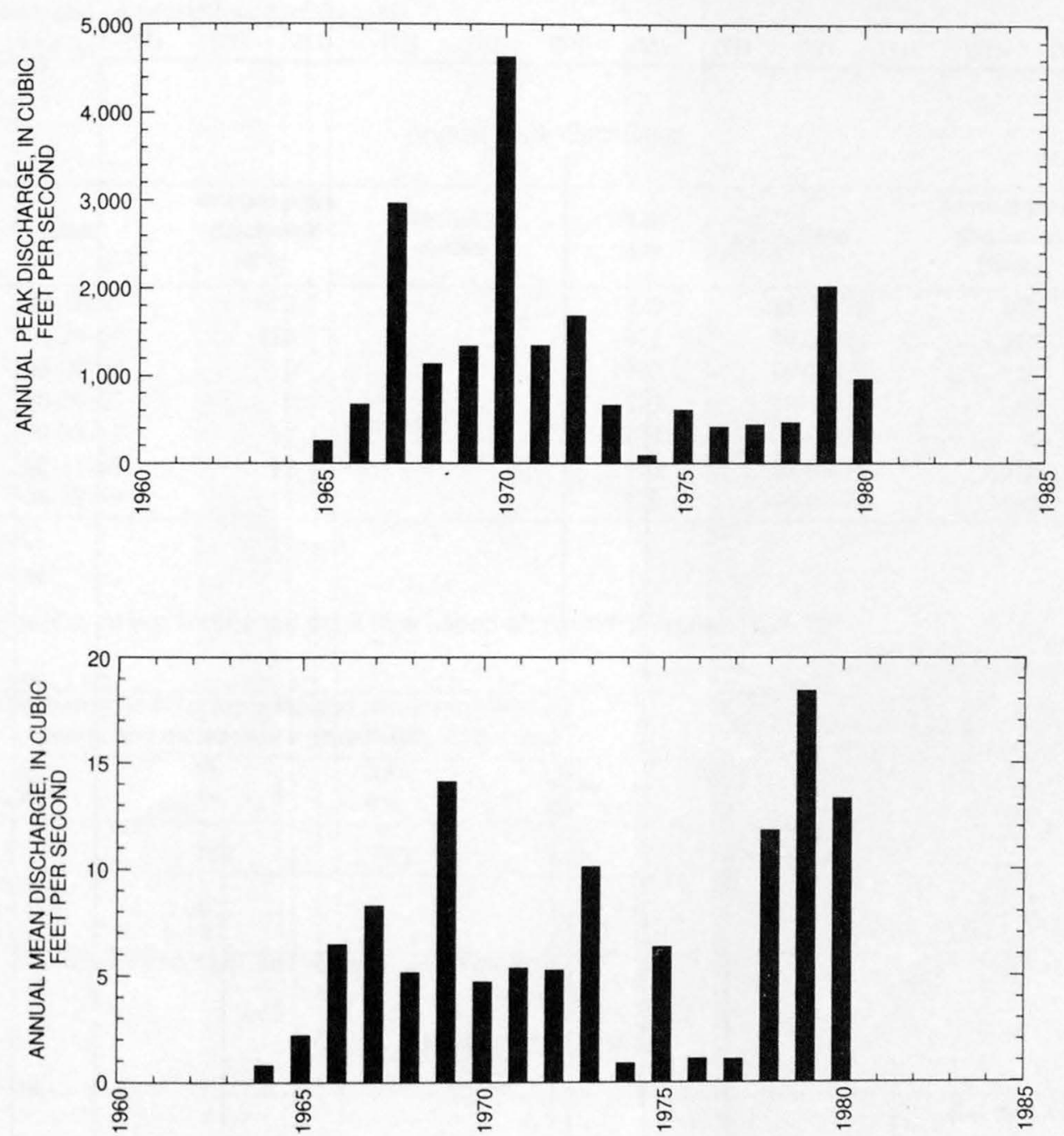
09403780 KANAB CREEK NEAR FREDONIA, AZ--Continued
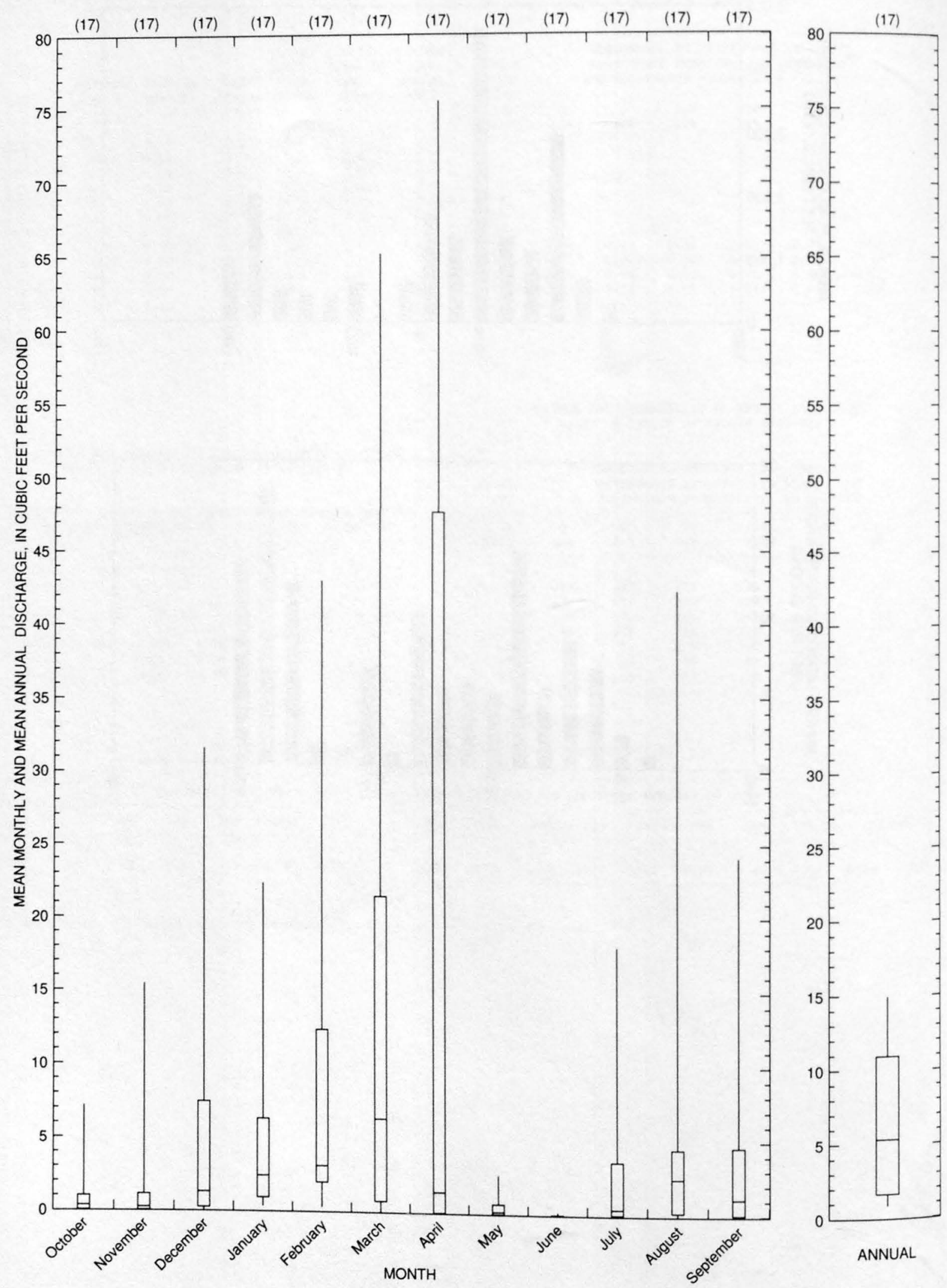
09403800 BITTER SEEPS WASH TRIBUTARY NEAR FREDONIA, AZ

LOCATION.--Lat 36 $51^{\prime} 25^{\prime \prime}$, long $112^{\circ} 45^{\prime} 30^{\prime \prime}$, in NE $\frac{1}{4}$ sec. 19 , T.40 N., R.4 W., Mohave County, at State Highway 389,1 mi west of Pipe Spring National Monument, and $14 \mathrm{mi}$ southwest of Fredonia.

DRAINAGE AREA.--2.85 $\mathrm{mi}^{2}$.

Annual peak discharges

\begin{tabular}{|c|c|c|c|c|c|c|c|}
\hline $\begin{array}{l}\text { Water } \\
\text { year }\end{array}$ & Date & $\begin{array}{c}\text { Annual peak } \\
\text { discharge } \\
\left(\mathrm{ft}^{3} / \mathrm{s}\right)\end{array}$ & $\begin{array}{c}\text { Discharge } \\
\text { codes }\end{array}$ & $\begin{array}{l}\text { Water } \\
\text { year }\end{array}$ & Date & $\begin{array}{c}\text { Annual peak } \\
\text { discharge } \\
\left(\mathrm{ft}^{3} / \mathrm{s}\right)\end{array}$ & $\begin{array}{c}\text { Discharge } \\
\text { codes }\end{array}$ \\
\hline 1963 & $08-30-63$ & 622 & & 1970 & $08-18-70$ & 670 & \\
\hline 1964 & $07-24-64$ & 736 & & 1971 & $08-23-71$ & 1,950 & \\
\hline 1965 & $00-00-65$ & 0 & & 1972 & $09-20-72$ & 50 & ES \\
\hline 1966 & $00-00-66$ & 0 & & 1973 & $10-19-72$ & 30 & \\
\hline 1967 & $09-23-67$ & 55 & & 1974 & $00-00-74$ & 0 & \\
\hline 1968 & $07-31-68$ & 57 & & 1975 & $07-08-75$ & 1,050 & \\
\hline 1969 & $08-29-69$ & 137 & & 1976 & $00-00-76$ & 165 & \\
\hline
\end{tabular}

Magnitude and probability of instantaneous peak flow based on period of record 1963-76

\begin{tabular}{|c|c|c|c|c|c|}
\hline \multicolumn{6}{|c|}{$\begin{array}{l}\text { Discharge, in } \mathrm{ft}^{3} / \mathrm{s} \text {, for indicated recurrence interval } \\
\text { in years, and exceedance probablility, in percent }\end{array}$} \\
\hline 2 & 5 & 10 & 25 & $50 t$ & $100 \dagger$ \\
\hline $50 \%$ & $20 \%$ & $10 \%$ & $4 \%$ & $2 \%$ & $1 \%$ \\
\hline 127 & 575 & 1,240 & 2,780 & 4,660 & 7,350 \\
\hline Weighted skew & $(\log s)=$ & -0.11 & & & \\
\hline Mean & $(\log s)=$ & 2.09 & & & \\
\hline Standard dev. & $(\log s)=$ & 0.79 & & & \\
\hline
\end{tabular}

\section{Basin characteristics}

\begin{tabular}{cccccccc}
\hline $\begin{array}{c}\text { Main } \\
\text { channel } \\
\begin{array}{c}\text { slope } \\
(\mathrm{ft} / \mathrm{mi})\end{array}\end{array}$ & $\begin{array}{c}\text { Stream } \\
\text { length } \\
(\mathrm{mi})\end{array}$ & $\begin{array}{c}\text { Mean } \\
\text { basin } \\
\text { elevation } \\
(\mathrm{ft})\end{array}$ & $\begin{array}{c}\text { Forested } \\
\text { area } \\
\text { (percent) }\end{array}$ & $\begin{array}{c}\text { Soil } \\
\text { index }\end{array}$ & $\begin{array}{c}\text { Mean } \\
\text { annual } \\
\text { precipitation } \\
\text { (in) }\end{array}$ & $\begin{array}{c}\text { Rainfall intensity, 24-hour } \\
\text { (in) }\end{array}$ & $\begin{array}{c}50 \text {-year } \\
\text { (in) }\end{array}$ \\
\hline 129 & 2.9 & 5,120 & 40.0 & 3.0 & 12.0 & 1.5 \\
\hline
\end{tabular}

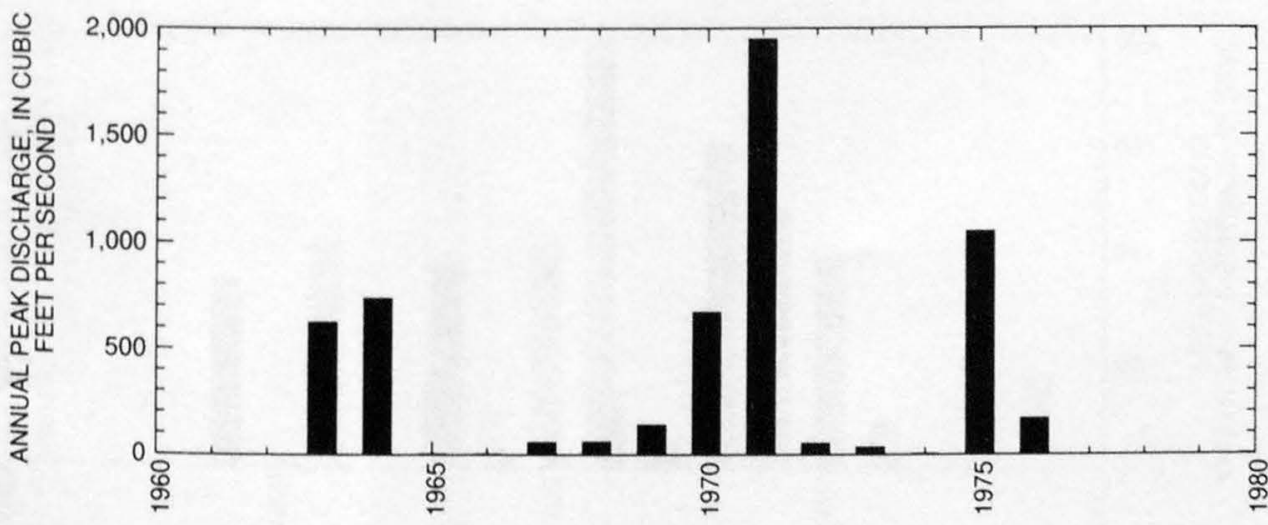




\section{WEST CATARACT CREEK NEAR WILLIAMS, AZ}

LOCATION.--Lat $35^{\circ} 14^{\prime} 52^{\prime \prime}$, long $112^{\circ} 13^{\prime} 28^{\prime \prime}$, in NW $1 / 4$ sec.31, T.22 N., R.2 E., Coconino County, Hydrologic Unit 15010004 , at Country Club Road, $0.25 \mathrm{mi}$ above Cataract Lake, and $1 \mathrm{mi}$ west of Williams city limits.

DRAINAGE AREA.--3.18 $\mathrm{mi}^{2}$.

Annual peak discharges

\begin{tabular}{|c|c|c|c|c|c|c|c|}
\hline $\begin{array}{l}\text { Water } \\
\text { year }\end{array}$ & Date & $\begin{array}{c}\text { Annual peak } \\
\text { discharge } \\
\left(\mathrm{ft}^{3} / \mathrm{s}\right)\end{array}$ & $\begin{array}{l}\text { Discharge } \\
\text { codes }\end{array}$ & $\begin{array}{l}\text { Water } \\
\text { year }\end{array}$ & Date & $\begin{array}{c}\text { Annual peak } \\
\text { discharge } \\
\left(\mathrm{ft}^{3} / \mathrm{s}\right)\end{array}$ & $\begin{array}{c}\text { Discharge } \\
\text { codes }\end{array}$ \\
\hline 1964 & $08-26-64$ & 21 & & 1971 & $00-00-71$ & 4.0 & \\
\hline 1965 & $04-00-65$ & 99 & & 1972 & $12-26-71$ & 60 & \\
\hline 1966 & $11-25-65$ & 122 & & 1973 & $10-00-72$ & 96 & \\
\hline 1967 & $12-06-66$ & 151 & & 1974 & $07-00-74$ & 1.0 & LT \\
\hline 1968 & $00-00-68$ & 12 & & 1975 & $04-00-75$ & 2.0 & \\
\hline 1969 & 03-00-69 & 36 & & 1976 & $00-00-76$ & 88 & \\
\hline 1970 & $00-00-70$ & 5.0 & & & & & \\
\hline
\end{tabular}

Magnitude and probability of instantaneous peak flow based on period of record 1964-76

\begin{tabular}{|c|c|c|c|c|c|}
\hline \multicolumn{6}{|c|}{$\begin{array}{l}\text { Discharge, in } \mathrm{ft}^{3} / \mathrm{s} \text {, for indicated recurrence interval } \\
\text { in years, and exceedance probablility, in percent }\end{array}$} \\
\hline 2 & 5 & 10 & 25 & $50 t$ & $100 \dagger$ \\
\hline $50 \%$ & $20 \%$ & $10 \%$ & $4 \%$ & $2 \%$ & $1 \%$ \\
\hline 26.2 & 96.4 & 181 & 342 & 505 & 708 \\
\hline Weighted skew & $(\log s)=$ & -0.34 & & & \\
\hline Mean & $(\log s)=$ & 1.38 & & & \\
\hline Standard dev. & $(\log s)=$ & 0.71 & & & \\
\hline
\end{tabular}

f Reliability of values in column is uncertain, and potential errors are large.

\section{Basin characteristics}

\begin{tabular}{|c|c|c|c|c|c|c|c|}
\hline \multirow[b]{2}{*}{$\begin{array}{c}\text { Main } \\
\text { channel } \\
\text { slope } \\
(\mathrm{ft} / \mathrm{mi})\end{array}$} & \multirow[b]{2}{*}{$\begin{array}{l}\text { Stream } \\
\text { length } \\
\text { (mi) }\end{array}$} & \multirow[b]{2}{*}{$\begin{array}{c}\text { Mean } \\
\text { basin } \\
\text { elevation } \\
\text { (ft) }\end{array}$} & \multirow[b]{2}{*}{$\begin{array}{l}\text { Forested } \\
\text { area } \\
\text { (percent) }\end{array}$} & \multirow[b]{2}{*}{$\begin{array}{c}\text { Soil } \\
\text { index }\end{array}$} & \multirow[b]{2}{*}{$\begin{array}{c}\text { Mean } \\
\text { annual } \\
\text { precipitation } \\
\text { (in) }\end{array}$} & \multicolumn{2}{|c|}{ Rainfall intensity, 24-hour } \\
\hline & & & & & & $\begin{array}{l}\text { 2-year } \\
\text { (in) }\end{array}$ & $\begin{array}{l}50 \text {-year } \\
\text { (in) }\end{array}$ \\
\hline 465 & 3.9 & 7,190 & 82.0 & 3.0 & 23.5 & 2.0 & 4.2 \\
\hline
\end{tabular}

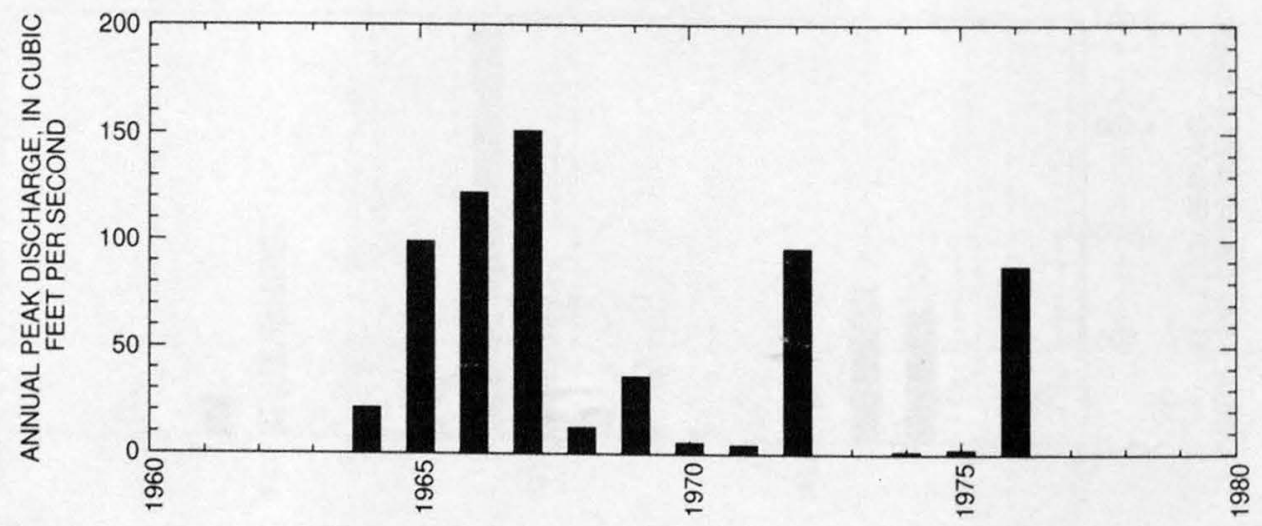


09404050 SPRING VALLEY WASH TRIBUTARY NEAR WILLIAMS, AZ

LOCATION.--Lat 35'34'28", long $112^{\circ} 09^{\prime} 12^{\prime \prime}$, in SW $1 / 4$ sec.2, T.25 N., R.2 E., Coconino County, Hydrologic Unit 15010004, at State Highway

64,6 mi southeast of Valle, and $22.5 \mathrm{mi}$ north of Williams.

DRAINAGE AREA.--5.00 $\mathrm{mi}^{2}$, of which $1.07 \mathrm{mi}^{2}$ is noncontributing.

Annual peak discharges

\begin{tabular}{|c|c|c|c|c|c|c|c|}
\hline $\begin{array}{l}\text { Water } \\
\text { year }\end{array}$ & Date & $\begin{array}{c}\text { Annual peak } \\
\text { discharge } \\
\left(\mathrm{ft}^{3} / \mathrm{s}\right)\end{array}$ & $\begin{array}{c}\text { Discharge } \\
\text { codes }\end{array}$ & $\begin{array}{l}\text { Water } \\
\text { year }\end{array}$ & Date & $\begin{array}{c}\text { Annual peak } \\
\text { discharge } \\
\left(\mathrm{ft}^{3} / \mathrm{s}\right)\end{array}$ & $\begin{array}{c}\text { Discharge } \\
\text { codes }\end{array}$ \\
\hline 1963 & $08-24-63$ & 100 & & 1970 & $07-22-70$ & 30 & ES \\
\hline 1964 & $07-00-64$ & 25 & ES & 197.1 & $00-00-71$ & 1.0 & LT \\
\hline 1965 & $09-06-65$ & 35 & ES & 1972 & $07-17-72$ & 8.0 & LT \\
\hline 1966 & $00-00-66$ & 20 & ES & 1973 & $00-00-73$ & 1.0 & LT \\
\hline 1967 & $00-00-67$ & 98 & & 1974 & $00-00-74$ & 0 & \\
\hline 1968 & $00-00-68$ & 190 & & 1975 & $00-00-75$ & 0 & \\
\hline 1969 & $00-00-69$ & 1.0 & ES & 1976 & $09-26-76$ & 32 & \\
\hline
\end{tabular}

Magnitude and probability of instantaneous peak flow based on period of record 1963-76

\begin{tabular}{|c|c|c|c|c|c|}
\hline \multicolumn{6}{|c|}{$\begin{array}{l}\text { Discharge, in } \mathrm{ft}^{3} / \mathrm{s} \text {, for indicated recurrence interval } \\
\text { in years, and exceedance probablility, in percent }\end{array}$} \\
\hline 2 & 5 & 10 & 25 & $50 \dagger$ & $100 \dagger$ \\
\hline $50 \%$ & $20 \%$ & $10 \%$ & $4 \%$ & $2 \%$ & $1 \%$ \\
\hline 17.7 & 61.2 & 113 & 213 & 317 & 449 \\
\hline Weighted skew & $(\log s)=$ & -0.23 & & & \\
\hline Mean & $(\log s)=$ & 1.22 & & & \\
\hline Standard dev. & $(\log s)=$ & 0.66 & & & \\
\hline
\end{tabular}

† Reliability of values in column is uncertain, and potential errors are large.

Basin characteristics

\begin{tabular}{ccccccc}
\hline $\begin{array}{c}\text { Main } \\
\text { channel } \\
\text { slope } \\
\text { (ft/mi) }\end{array}$ & $\begin{array}{c}\text { Stream } \\
\text { length } \\
\text { (mi) }\end{array}$ & $\begin{array}{c}\text { Mean } \\
\text { basin } \\
\text { elevation } \\
\text { (ft) }\end{array}$ & $\begin{array}{c}\text { Forested } \\
\text { area } \\
\text { (percent) }\end{array}$ & $\begin{array}{c}\text { Soil } \\
\text { index }\end{array}$ & $\begin{array}{c}\text { Mean } \\
\text { annual } \\
\text { precipitation } \\
\text { (in) }\end{array}$ & $\begin{array}{c}\text { Rainfall intensity, 24-hour } \\
\text { (in) }\end{array}$ \\
\hline 40.5 & 2.4 & 6,750 & 9.2 & 3.0 & 12.2 & $\begin{array}{c}50 \text {-year } \\
\text { (in) }\end{array}$ \\
\hline
\end{tabular}

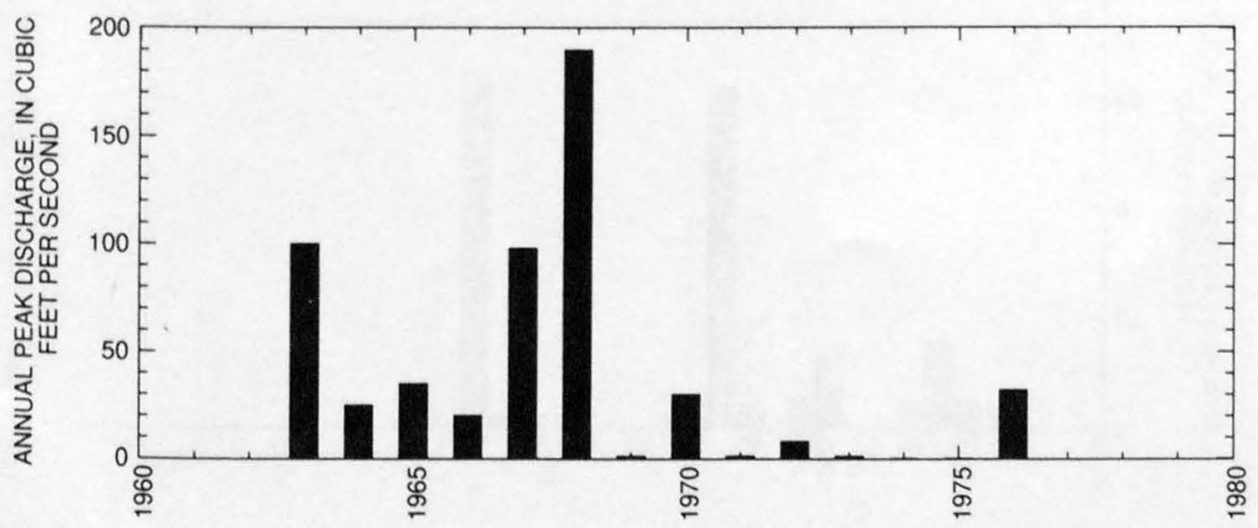


09404070 LITTLE RED HORSE WASH NEAR GRAND CANYON, AZ

LOCATION.--Lat $35^{\circ} 50^{\prime} 45^{\prime \prime}$, long $112^{\circ} 07^{\prime} 55^{\prime \prime}$, in NW $1 / 4$ sec.1, T.28 N., R.2 E., Coconino County, Hydrologic Unit 15010004 , at State Highway

64, 0.1 mi south of road to Old Grand Canyon airport, and $15 \mathrm{mi}$ south of Grand Canyon Village.

DRAINAGE AREA.--21.8 $\mathrm{mi}^{2}$.

Annual peak discharges

\begin{tabular}{ccccccc}
\hline $\begin{array}{c}\text { Water } \\
\text { year }\end{array}$ & Date & $\begin{array}{c}\text { Annual peak } \\
\text { discharge } \\
\left(\mathbf{f t}^{3} / \mathbf{s}\right)\end{array}$ & $\begin{array}{c}\text { Discharge } \\
\text { codes }\end{array}$ & $\begin{array}{c}\text { Water } \\
\text { year }\end{array}$ & $\begin{array}{c}\text { Annual peak } \\
\text { discharge } \\
\left(\mathbf{f t}^{3} / \mathbf{s}\right)\end{array}$ & $\begin{array}{c}\text { Discharge } \\
\text { codes }\end{array}$ \\
\hline 1963 & $08-24-63$ & 16 & & 1970 & $00-00-70$ & 0 \\
1964 & $00-00-64$ & 0.1 & 1971 & $00-00-71$ & 2.0 \\
1965 & $10-17-64$ & 15 & 0 & 1972 & $00-00-72$ & 0 \\
1966 & $00-00-66$ & 62 & 1973 & $00-00-73$ & 0 \\
1967 & $00-00-67$ & 0 & 1974 & $00-00-74$ & 0 \\
1968 & $00-00-68$ & 0 & & 1975 & $00-00-75$ & 0 \\
1969 & $00-00-69$ & 0 & 1976 & $00-00-76$ & 0 \\
\hline
\end{tabular}

Magnitude and probability of instantaneous peak flow based on period of record

\begin{tabular}{|c|c|c|c|c|c|}
\hline \multicolumn{6}{|c|}{$\begin{array}{l}\text { Discharge, in } \mathrm{ft}^{3} / \mathrm{s} \text {, for indicated recurrence interval } \\
\text { in years, and exceedance probablility, in percent }\end{array}$} \\
\hline 2 & 5 & 10 & 25 & 50 & 100 \\
\hline $50 \%$ & $20 \%$ & $10 \%$ & $4 \%$ & $2 \%$ & $1 \%$ \\
\hline -.. & -.. & $\ldots$ & ... & -.. & ... \\
\hline $\begin{array}{l}\text { Weighted skew } \\
\text { Mean } \\
\text { Standard dev. }\end{array}$ & $\begin{array}{l}(\operatorname{logs})= \\
(\operatorname{logs})= \\
(\operatorname{logs})=\end{array}$ & $\begin{array}{ll}-- \\
\cdots- \\
\cdots-\end{array}$ & & & \\
\hline
\end{tabular}

Basin characteristics

\begin{tabular}{cccccccc}
\hline $\begin{array}{c}\text { Main } \\
\text { channel } \\
\text { slope } \\
(\mathrm{ft} / \mathrm{mi})\end{array}$ & $\begin{array}{c}\text { Stream } \\
\text { length } \\
(\mathrm{mi})\end{array}$ & $\begin{array}{c}\text { Mean } \\
\text { basin } \\
\text { elevation } \\
(\mathrm{ft})\end{array}$ & $\begin{array}{c}\text { Forested } \\
\text { area } \\
\text { (percent) }\end{array}$ & $\begin{array}{c}\text { Soil } \\
\text { index }\end{array}$ & $\begin{array}{c}\text { Mean } \\
\text { annual } \\
\text { precipitation } \\
\text { (in) }\end{array}$ & $\begin{array}{c}\text { Rainfall intensity, 24-hour } \\
\text { (in) }\end{array}$ & $\begin{array}{c}50 \text {-year } \\
\text { (in) }\end{array}$ \\
\hline 56.9 & 9.6 & 6,780 & 87.0 & 3.0 & 13.2 & 1.7 \\
\hline
\end{tabular}

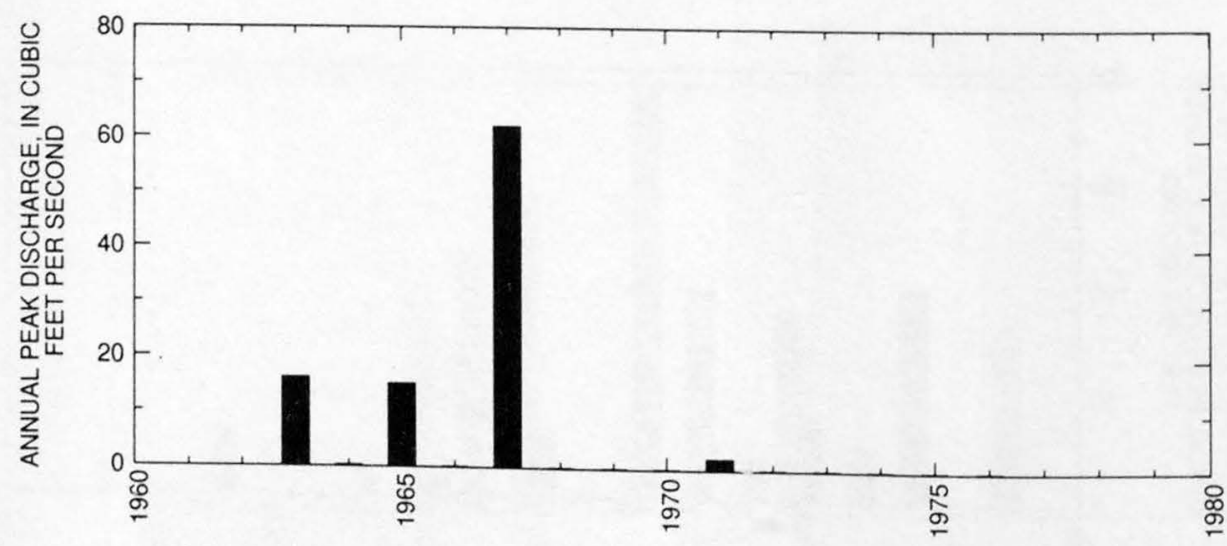


09404310 YAMPAI CANYON TRIBUTARY NEAR PEACH SPRINGS, AZ

LOCATION.--Lat 35 33'07", long 113 23'17", in SE $1 /{ }_{4} \mathrm{NW}^{1} / 4$ sec.18, T.25 N., R.10 W., Mohave County, Hydrologic Unit 15010002, at U.S. Highway $66,2.8$ mi northeast of the Peach Springs Post Office.

DRAINAGE AREA.--0.20 $\mathrm{mi}^{2}$.

Annual peak discharges

\begin{tabular}{|c|c|c|c|c|c|c|c|}
\hline $\begin{array}{l}\text { Water } \\
\text { year }\end{array}$ & Date & $\begin{array}{c}\text { Annual peak } \\
\text { discharge } \\
\left(\mathrm{ft}^{3} / \mathrm{s}\right)\end{array}$ & $\begin{array}{c}\text { Discharge } \\
\text { codes }\end{array}$ & $\begin{array}{l}\text { Water } \\
\text { year }\end{array}$ & Date & $\begin{array}{c}\text { Annual peak } \\
\text { discharge } \\
\left(\mathrm{ft}^{3} / \mathrm{s}\right)\end{array}$ & $\begin{array}{c}\text { Discharge } \\
\text { codes }\end{array}$ \\
\hline 1964 & $00-00-64$ & 0 & & 1971 & $08-10-71$ & 21 & \\
\hline 1965 & $00-00-65$ & 0 & & 1972 & $08-12-72$ & 27 & \\
\hline 1966 & $07-30-66$ & 177 & & 1973 & $00-00-73$ & 0 & \\
\hline 1967 & $09-03-67$ & 17 & & 1974 & $07-21-74$ & 25 & ES \\
\hline 1968 & $08-04-68$ & 19 & & 1975 & $07-06-75$ & 25 & \\
\hline 1969 & $07-29-69$ & 6.0 & ES & 1976 & $00-00-76$ & 5.0 & \\
\hline 1970 & $07-00-70$ & 27 & & & & & \\
\hline
\end{tabular}

Magnitude and probability of instantaneous peak flow based on period of record 1964-76

\begin{tabular}{|c|c|c|c|c|c|}
\hline \multicolumn{6}{|c|}{$\begin{array}{l}\text { Discharge, in } \mathrm{ft}^{3} / \mathrm{s} \text {, for indicated recurrence interval } \\
\text { in years, and exceedance probablility, in percent }\end{array}$} \\
\hline 2 & 5 & 10 & 25 & $50 \dagger$ & $100 t$ \\
\hline $50 \%$ & $20 \%$ & $10 \%$ & $4 \%$ & $2 \%$ & $1 \%$ \\
\hline 13.8 & 37.2 & 63.5 & 114 & 167 & 237 \\
\hline Weighted skew & $(\log s)=$ & 0.16 & & & \\
\hline Mean & $(\log s)=$ & 1.15 & & & \\
\hline Standard dev. & $(\log s)=$ & 0.50 & & & \\
\hline
\end{tabular}

Basin characteristics

\begin{tabular}{cccccccc}
\hline $\begin{array}{c}\text { Main } \\
\text { channel } \\
\text { slope } \\
(\mathrm{ft} / \mathrm{mi})\end{array}$ & $\begin{array}{c}\text { Stream } \\
\text { length } \\
(\mathrm{mi})\end{array}$ & $\begin{array}{c}\text { Mean } \\
\text { basin } \\
\text { elevation } \\
(\mathrm{ft})\end{array}$ & $\begin{array}{c}\text { Forested } \\
\text { area } \\
\text { (percent) }\end{array}$ & $\begin{array}{c}\text { Soil } \\
\text { index }\end{array}$ & $\begin{array}{c}\text { Mean } \\
\text { annual } \\
\text { precipitation } \\
\text { (in) }\end{array}$ & $\begin{array}{c}\text { R-year } \\
\text { (in) }\end{array}$ & $\begin{array}{c}50 \text {-year } \\
\text { (in) }\end{array}$ \\
\hline 444 & 0.30 & 5,360 & 0.0 & 3.0 & 12.2 & 1.5 \\
\hline
\end{tabular}

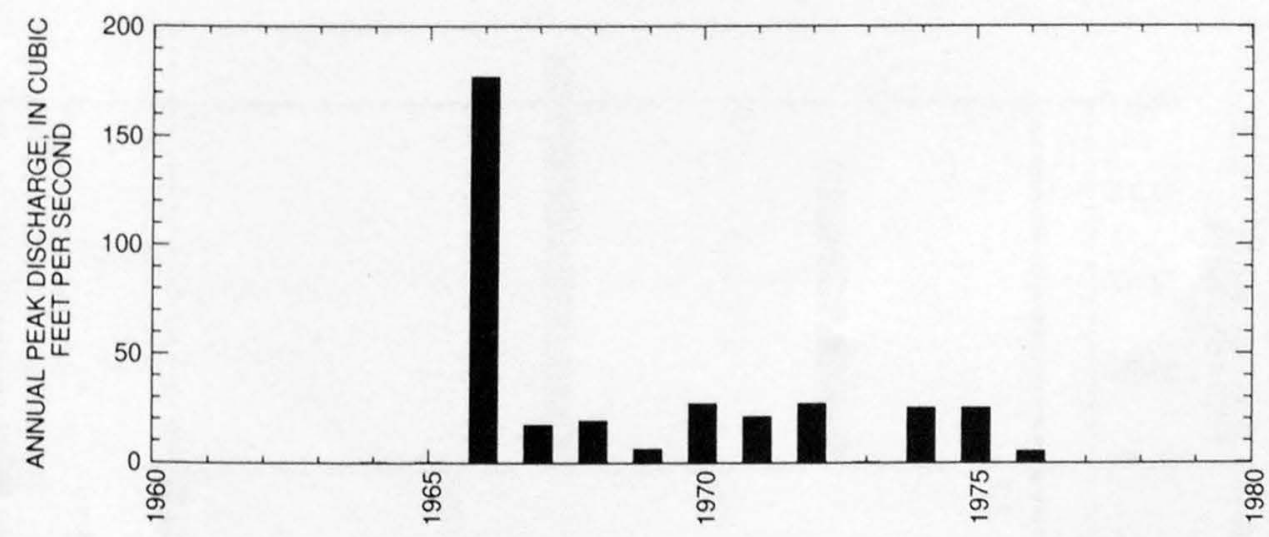


09404340 TRUXTON WASH AT VALENTINE, AZ

LOCATION.--Lat 35'23'10", long 113.39'20", in SE $\frac{1}{4}$ sec.10, T.23 N., R.13 W., Mohave County, Hydrologic Unit 15010007, in Hualapai India

Reservation, at Valentine.

DRAINAGE AREA.--370 $\mathrm{mi}^{2}$.

Annual peak discharges

\begin{tabular}{ccccccc}
\hline $\begin{array}{c}\text { Water } \\
\text { year }\end{array}$ & Date & $\begin{array}{c}\text { Annual peak } \\
\text { discharge } \\
\left(\mathrm{ft}^{3} / \mathbf{s}\right)\end{array}$ & $\begin{array}{c}\text { Discharge } \\
\text { codes }\end{array}$ & $\begin{array}{c}\text { Water } \\
\text { year }\end{array}$ & $\begin{array}{c}\text { Annual peak } \\
\text { discharge } \\
\left(\mathrm{ft}^{3} / \mathbf{s}\right)\end{array}$ & $\begin{array}{c}\text { Discharge } \\
\text { codes }\end{array}$ \\
\hline 1904 & $07-30-04$ & 49,000 & ES,HP & 1971 & $08-21-71$ & 1,130 \\
1965 & $07-29-65$ & 250 & & 1972 & $09-19-72$ & 40 \\
1966 & $08-18-66$ & 1,960 & & 1973 & $07-08-73$ & 380 \\
1967 & $09-15-67$ & 1,640 & & 1974 & $07-20-74$ & 6,500 \\
1968 & $08-04-68$ & 8,760 & & 1975 & $00-00-75$ & 1,400 \\
1969 & $07-19-69$ & 900 & & 1976 & $00-00-76$ & 5.0 \\
1970 & $07-22-70$ & 2,650 & & & & \\
\hline
\end{tabular}

${ }^{1}$ Highest since 1898.

Magnitude and probability of instantaneous peak flow based on period of record 1904 ,

1965-76

\begin{tabular}{|c|c|c|c|c|c|}
\hline \multicolumn{6}{|c|}{$\begin{array}{l}\text { Discharge, in } \mathrm{ft}^{3} / \mathrm{s} \text {, for indicated recurrence interval } \\
\text { in years, and exceedance probablility, in percent }\end{array}$} \\
\hline 2 & 5 & 10 & 25 & $50 \dagger$ & $100 \dagger$ \\
\hline $50 \%$ & $20 \%$ & $10 \%$ & $4 \%$ & $2 \%$ & $1 \%$ \\
\hline 1,150 & 3,510 & 6,330 & 12,000 & 18,100 & 26,300 \\
\hline Weighted skew & $(\log s)=$ & 0.06 & & & \\
\hline Mean & $(\log s)=$ & 3.07 & & & \\
\hline Standard dev. & $(\log s)=$ & 0.57 & & & \\
\hline
\end{tabular}

f Reliability of values in column is uncertain, and potential errors are large.

Basin characteristics

\begin{tabular}{cccccccc}
\hline $\begin{array}{c}\text { Main } \\
\text { channel } \\
\text { slope } \\
(\mathrm{ft} / \mathrm{mi})\end{array}$ & $\begin{array}{c}\text { Stream } \\
\text { length } \\
(\mathrm{mi})\end{array}$ & $\begin{array}{c}\text { Mean } \\
\text { basin } \\
\text { elevation } \\
(\mathrm{ft})\end{array}$ & $\begin{array}{c}\text { Forested } \\
\text { area } \\
\text { (percent) }\end{array}$ & $\begin{array}{c}\text { Soil } \\
\text { index }\end{array}$ & $\begin{array}{c}\text { Mean } \\
\text { annual } \\
\text { precipitation } \\
\text { (in) }\end{array}$ & $\begin{array}{c}\text { R-year } \\
\text { (in) }\end{array}$ & $\begin{array}{c}50 \text {-year } \\
\text { (in) }\end{array}$ \\
\hline 57.0 & 30.4 & 4,630 & 72.0 & 2.0 & 12.1 & 1.5 \\
\hline
\end{tabular}

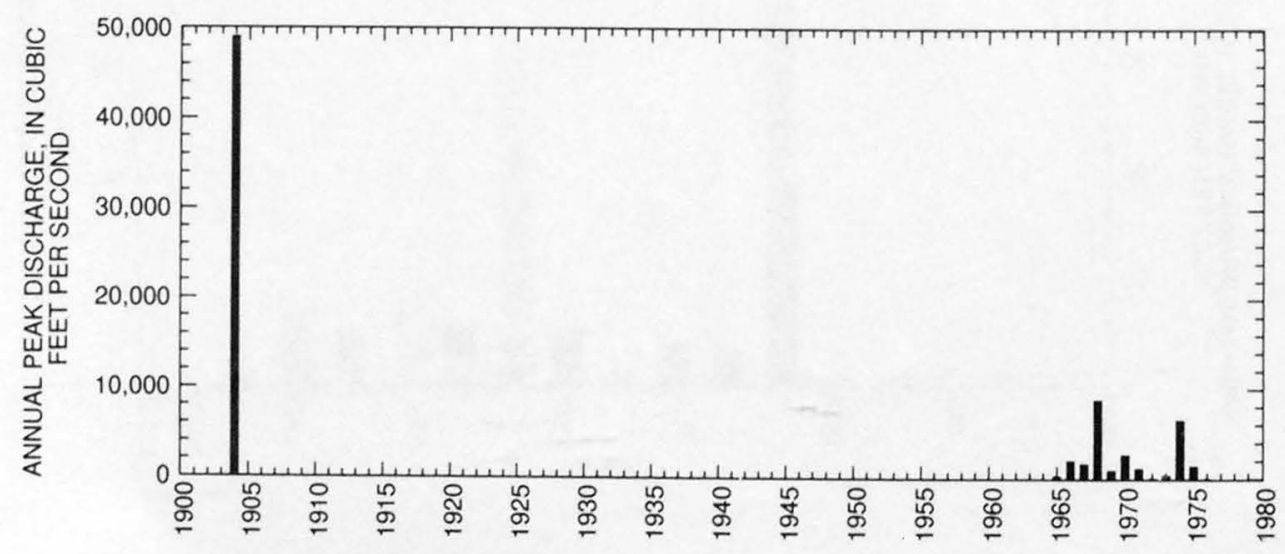


09404350 VALENTINE WASH AT VALENTINE, AZ

LOCATION.--Lat 35 23'00", long 113³9'45", in SW¹/4 sec.15, T.23 N., R.13 W., Mohave County, Hydrologic Unit 15010007, at U.S. Highway $66,0.3 \mathrm{mi}$ southwest of Valentine.

DRAINAGE AREA.--3.15 $\mathrm{mi}^{2}$.

Annual peak discharges

\begin{tabular}{|c|c|c|c|c|c|c|c|}
\hline $\begin{array}{l}\text { Water } \\
\text { year }\end{array}$ & Date & $\begin{array}{c}\text { Annual peak } \\
\text { discharge } \\
\left(\mathrm{ft}^{3} / \mathrm{s}\right)\end{array}$ & $\begin{array}{l}\text { Discharge } \\
\text { codes }\end{array}$ & $\begin{array}{l}\text { Water } \\
\text { year }\end{array}$ & Date & $\begin{array}{c}\text { Annual peak } \\
\text { discharge } \\
\left(\mathrm{ft}^{3} / \mathrm{s}\right)\end{array}$ & $\begin{array}{c}\text { Discharge } \\
\text { codes }\end{array}$ \\
\hline 1963 & $00-00-63$ & 0 & & 1970 & $07-00-70$ & 30 & ES \\
\hline 1964 & $08-00-64$ & 5.0 & ES & 1971 & $00-00-71$ & 15 & ES \\
\hline 1965 & $10-17-64$ & 20 & ES & 1972 & $08-12-72$ & 2,800 & \\
\hline 1966 & $12-09-65$ & 4.0 & ES & 1973 & $03-12-73$ & 25 & ES \\
\hline 1967 & $08-20-67$ & 3,800 & & 1974 & $00-00-74$ & 0 & \\
\hline 1968 & $08-04-68$ & 50 & ES & 1975 & $00-00-75$ & 1.0 & ES \\
\hline 1969 & $07-29-69$ & 2.0 & LT & 1976 & $00-00-76$ & 10 & LT \\
\hline
\end{tabular}

Magnitude and probability of instantaneous peak flow based on period of record 1963-76

\begin{tabular}{|c|c|c|c|c|c|}
\hline \multicolumn{6}{|c|}{$\begin{array}{l}\text { Discharge, in } \mathrm{ft}^{3} / \mathrm{s} \text {, for indicated recurrence interval } \\
\text { in years, and exceedance probablility, in percent }\end{array}$} \\
\hline 2 & 5 & 10 & 25 & $50 t$ & $100 \dagger$ \\
\hline $50 \%$ & $20 \%$ & $10 \%$ & $4 \%$ & $2 \%$ & $1 \%$ \\
\hline 12.7 & 142 & 552 & 2,540 & 7,090 & 18,400 \\
\hline Weighted skew & $(\log s)=$ & 0.37 & & & \\
\hline Mean & $(\log s)=$ & 1.18 & & & \\
\hline Standard dev. & $(\log s)=$ & 1.19 & & & \\
\hline
\end{tabular}

Basin characteristics

\begin{tabular}{|c|c|c|c|c|c|c|c|}
\hline \multirow[b]{2}{*}{$\begin{array}{l}\text { Main } \\
\text { channel } \\
\text { slope } \\
\text { (ft/mi) }\end{array}$} & \multirow[b]{2}{*}{$\begin{array}{l}\text { Stream } \\
\text { length } \\
(\mathrm{mi})\end{array}$} & \multirow[b]{2}{*}{$\begin{array}{c}\text { Mean } \\
\text { basin } \\
\text { elevation } \\
\text { (ft) }\end{array}$} & \multirow[b]{2}{*}{$\begin{array}{l}\text { Forested } \\
\text { area } \\
\text { (percent) }\end{array}$} & \multirow[b]{2}{*}{$\begin{array}{c}\text { Soil } \\
\text { index }\end{array}$} & \multirow[b]{2}{*}{$\begin{array}{c}\text { Mean } \\
\text { annual } \\
\text { precipitation } \\
\text { (in) }\end{array}$} & \multicolumn{2}{|c|}{ Rainfall intensity, 24-hour } \\
\hline & & & & & & $\begin{array}{l}\text { 2-year } \\
\text { (in) }\end{array}$ & $\begin{array}{l}50-\text { year } \\
\text { (in) }\end{array}$ \\
\hline 378 & 3.1 & 4,490 & 63.0 & 1.0 & 12.1 & 1.5 & 3.5 \\
\hline
\end{tabular}

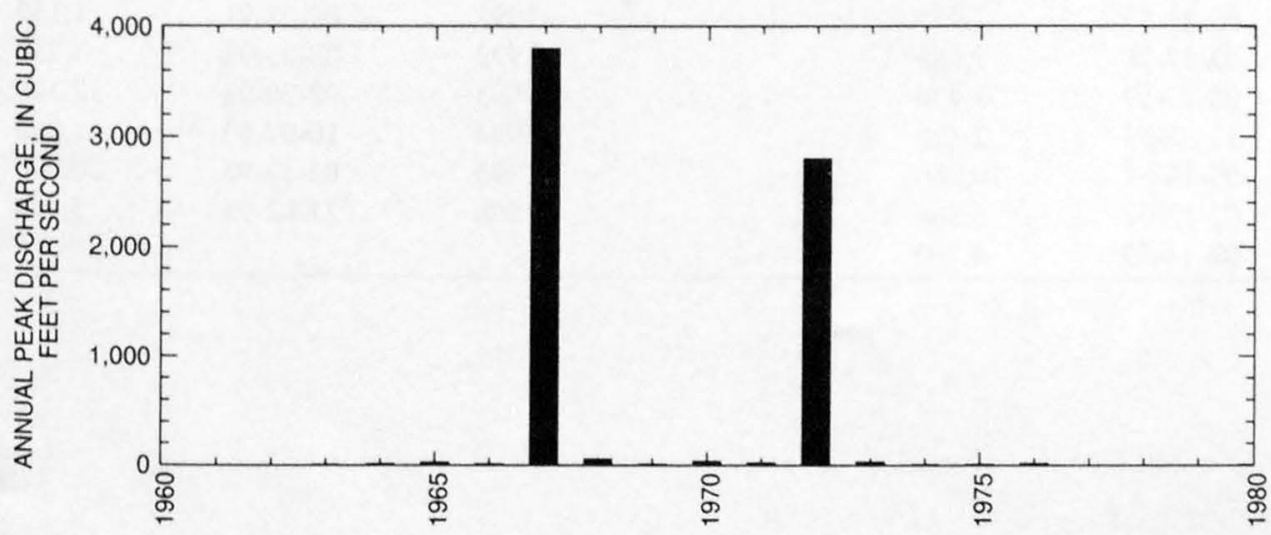




\section{VIRGIN RIVER AT LITTLEFIELD, AZ}

LOCATION.--Lat 36 53'30", long 113.55'25", in SW $1 / 4 \mathrm{SW}^{\prime} / 4$ sec.4, T.40 N., R.15 W., Mohave County, Hydrologic Unit 1115010010 , on right bank, $0.5 \mathrm{mi}$ downstream from Beaver Dam Wash, 0.4 mi upstream from Littlefield, and 36 mi upstream from Lake Mead.

DRAINAGE AREA.--5,090 $\mathrm{mi}^{2}$, approximately.

PERIOD OF RECORD.--October 1929 to current year.

REVISED RECORDS.--WSP 959: 1932. WSP 979: 1930-31, 1933-37. WSP 1313: 1940 (M).

GAGE.--Water-stage recorder. Datum of gage is $1,763.68 \mathrm{ft}$ above sea level. Prior to May 28, 1933, nonrecording gage at site $300 \mathrm{ft}$ upstrean and May 28, 1933, to November 7, 1939, at same site, both at datum 2.53 ft higher. November 8, 1939, to March 31, 1942, nonrecordin gage at same site at datum $2.00 \mathrm{ft}$ higher. April 1, 1942, to September 30, 1970, water-stage recorder at same site at same datum. October 1970 , to August 7, 1979, at site $300 \mathrm{ft}$ upstream at same datum.

REMARKS.--Records fair.

Annual peak discharges

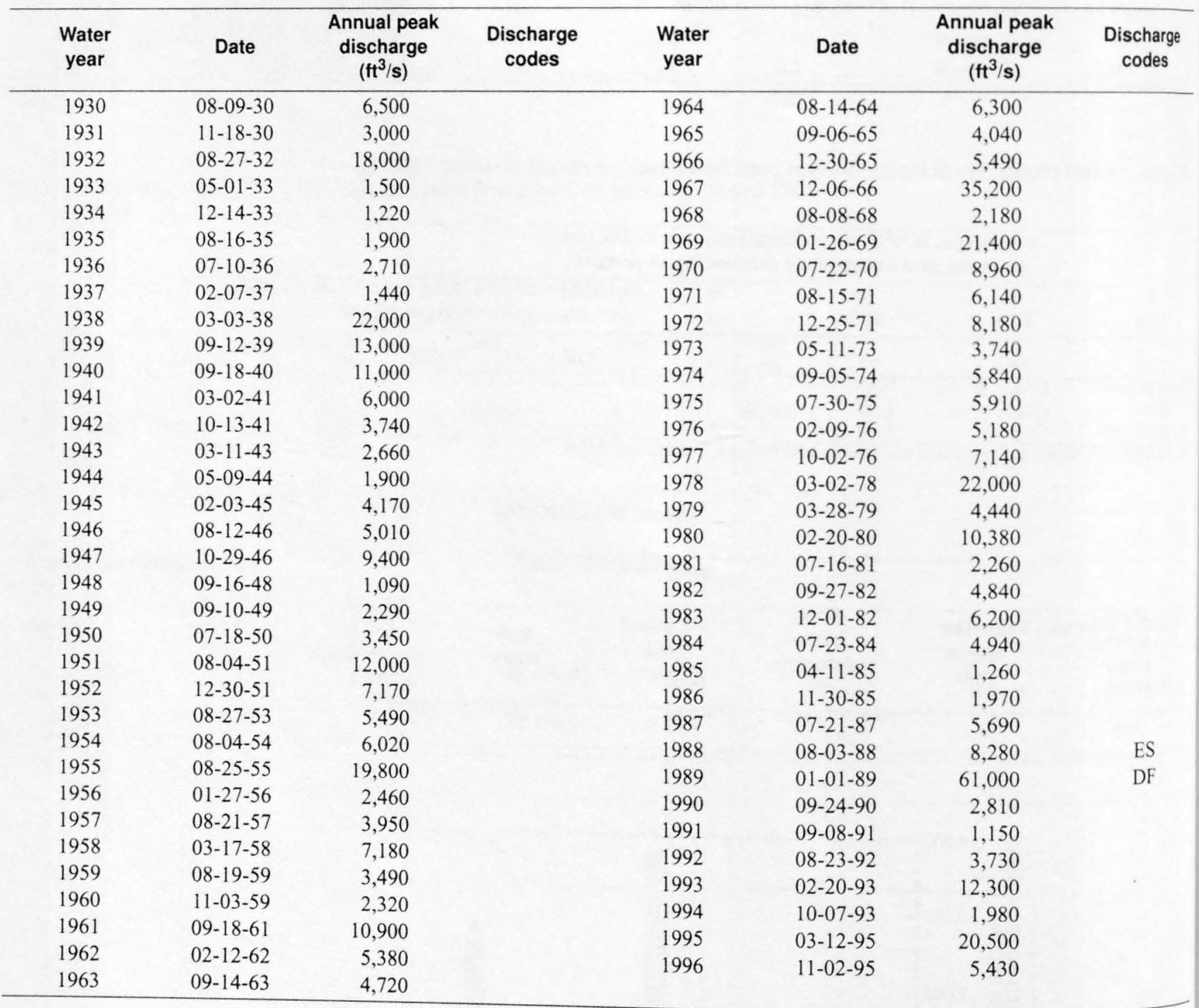


09415000 VIRGIN RIVER AT LITTLEFIELD, AZ--Continued

Discharge rating table developed October 1990

\begin{tabular}{crcc}
\hline $\begin{array}{c}\text { Gage height } \\
(\mathbf{f t})\end{array}$ & $\begin{array}{c}\text { Discharge } \\
\left(\mathbf{f t}^{\mathbf{3}} / \mathbf{s}\right)\end{array}$ & $\begin{array}{c}\text { Gage height } \\
(\mathbf{f t})\end{array}$ & $\begin{array}{c}\text { Discharge } \\
\left(\mathrm{ft}^{3} / \mathbf{s}\right)\end{array}$ \\
\hline 4.0 & 104 & 14.0 & 18,680 \\
6.0 & 1,000 & 16.0 & 26,170 \\
8.0 & 3,130 & 18.0 & 35,240 \\
10.0 & 7,180 & 20.0 & 45,980 \\
12.0 & 12,660 & 22.3 & 60,520 \\
\hline
\end{tabular}

Basin characteristics

\begin{tabular}{|c|c|c|c|c|c|c|c|}
\hline \multirow[b]{2}{*}{$\begin{array}{l}\text { Main } \\
\text { channel } \\
\text { slope } \\
\text { (ft/mi) }\end{array}$} & \multirow[b]{2}{*}{$\begin{array}{c}\text { Stream } \\
\text { length } \\
\text { (mi) }\end{array}$} & \multirow[b]{2}{*}{$\begin{array}{c}\text { Mean } \\
\text { basin } \\
\text { elevation } \\
(f t)\end{array}$} & \multirow[b]{2}{*}{$\begin{array}{c}\text { Forested } \\
\text { area } \\
\text { (percent) }\end{array}$} & \multirow[b]{2}{*}{$\begin{array}{c}\text { Soil } \\
\text { index }\end{array}$} & \multirow[b]{2}{*}{$\begin{array}{c}\text { Mean } \\
\text { annual } \\
\text { precipitation } \\
\text { (in) }\end{array}$} & \multicolumn{2}{|c|}{ Rainfall intensity, 24-hour } \\
\hline & & & & & & $\begin{array}{l}\text { 2-year } \\
\text { (in) }\end{array}$ & $\begin{array}{c}\text { 50-year } \\
\text { (in) }\end{array}$ \\
\hline 38.4 & 112 & 5,500 & 50.0 & 3.0 & 16.0 & 1.8 & 3.4 \\
\hline
\end{tabular}


09415000 VIRGIN RIVER AT LITTLEFIELD, AZ--Continued

MEAN MONTHLY AND ANNUAL DISCHARGES 1930-95

\begin{tabular}{|c|c|c|c|c|c|c|}
\hline MONTH & $\begin{array}{l}\text { MAXIMUM } \\
\text { (FT3/S) }\end{array}$ & $\begin{array}{l}\text { MINIMUM } \\
(\mathrm{FT} 3 / \mathrm{S})\end{array}$ & $\begin{array}{c}\text { MEAN } \\
(\text { FT3 } 3 / S \text { ) }\end{array}$ & $\begin{array}{l}\text { STAN- } \\
\text { DARD } \\
\text { DEVIA- } \\
\text { TION } \\
\text { (FT3/S) }\end{array}$ & $\begin{array}{l}\text { COEFFI- } \\
\text { CIENT OF } \\
\text { VARI- } \\
\text { ATION }\end{array}$ & $\begin{array}{c}\text { PERCENT } \\
\text { OF } \\
\text { ANNUAL } \\
\text { RUNOFF }\end{array}$ \\
\hline OCTOBER & 602 & 53 & 147 & 89 & 0.61 & 5.0 \\
\hline NOVEMBER & 552 & 101 & 190 & 78 & 0.41 & 6.5 \\
\hline DECEMBER & 1,250 & 111 & 226 & 148 & 0.65 & 7.7 \\
\hline JANUARY & 775 & 108 & 237 & 124 & 0.52 & 8.1 \\
\hline FEBRUARY & 2,330 & 110 & 324 & 331 & 1.0 & 11.1 \\
\hline MARCH & 1.810 & 85 & 377 & 390 & 1.0 & 12.9 \\
\hline APRIL & 1,390 & 62 & 418 & 378 & 0.90 & 14.3 \\
\hline MAY & 2,120 & 50 & 434 & 517 & 1.2 & 14.8 \\
\hline JUNE & 1,120 & 47 & 143 & 165 & 1.2 & 4.9 \\
\hline JULY & 381 & 52 & 107 & 70 & 0.65 & 3.7 \\
\hline AUGUST & 976 & 50 & 181 & 168 & 0.93 & 6.2 \\
\hline SEPTEMBER & 737 & 53 & 146 & 127 & 0.87 & 5.0 \\
\hline ANNUAL & 697 & 100 & 244 & 139 & 0.57 & 100 \\
\hline
\end{tabular}

MAGNITUDE AND PROBABILITY OF INSTANTANEOUS PEAK FLOW BASED ON PERIOD OF RECORD 1930.96

DISCHARGE, IN FT3/S, FOR INDICATED RECURRENCE INTERVAL IN YEARS, AND EXCEEDANCE PROBABILITY, IN PERCENT

\begin{tabular}{|c|c|c|c|c|c|}
\hline $\begin{array}{c}2 \\
508\end{array}$ & $\begin{array}{c}5 \\
208\end{array}$ & $\begin{array}{r}10 \\
108\end{array}$ & $\begin{array}{l}25 \\
48\end{array}$ & $\begin{array}{l}50 \\
28\end{array}$ & $\begin{array}{r}100 \\
18\end{array}$ \\
\hline 4,920 & 9,810 & 14,200 & 21,300 & 27,800 & 35,500 \\
\hline $\begin{array}{l}\text { WEIGHTED } \\
\text { MEAN } \\
\text { STANDARD }\end{array}$ & $\begin{array}{l}\text { SKEW } \\
\text { DEV. }\end{array}$ & $\begin{array}{l}\text { LOGS) }= \\
\text { LOGS) }= \\
\text { LOGS) }=\end{array}$ & $\begin{array}{l}14 \\
70 \\
35\end{array}$ & & \\
\hline
\end{tabular}

DURATION TABLE OF DAILY MEAN FLOW FOR PERIOD OF RECORD 1930-95
MAGNTTUDE AND PROBABILITY OF ANNUAL LOW FLOW BASED ON PERIOD OF RECORD 1931.95

\begin{tabular}{|c|c|c|c|c|c|c|}
\hline \multirow{3}{*}{$\begin{array}{l}\text { PERIOD } \\
\text { (CON- } \\
\text { SECU- } \\
\text { TIVE }\end{array}$} & \multicolumn{3}{|c|}{$\begin{array}{l}\text { DISCHARGE, IN } \\
\text { RECURRENCE IN } \\
\text { NON-EXCEEDANCE }\end{array}$} & CN FT3/S, & 3. FOR INDICATED & $\begin{array}{l}\text { IN YEARS, AND } \\
\text { ILITY, IN PERCENT }\end{array}$ \\
\hline & $\cdots$ & … & ..... & .... & ..... & $\cdots \cdots$ \\
\hline & 2 & 5 & 10 & 20 & 50 & 100 \\
\hline DAYS ) & 508 & 208 & 108 & 58 & 28 & 18 \\
\hline 1 & 56 & 50 & 47 & 44 & 42 & 40 \\
\hline 3 & 57 & 51 & 47 & 45 & 42 & 41 \\
\hline 7 & 58 & 52 & 49 & 46 & 44 & 42 \\
\hline 14 & 59 & 53 & 50 & 48 & 46 & 45 \\
\hline 30 & 61 & 55 & 53 & 52 & 51 & 51 \\
\hline 60 & 68 & 59 & 56 & 55 & 54 & 53 \\
\hline 90 & 83 & 64 & 58 & 54 & 51 & 49 \\
\hline 120 & 92 & 71 & 63 & 58 & 54 & 52 \\
\hline 183 & 120 & 92 & 81 & 74 & 66 & 62 \\
\hline
\end{tabular}

MAGNITUDE AND PROBABILITY OF ANNUAL HIGH FLOW BASED ON PERIOD OF RECORD 1930.95

\begin{tabular}{|c|c|c|c|c|c|c|}
\hline \multirow{2}{*}{$\begin{array}{l}\text { PERIOD } \\
\text { (CON- } \\
\text { SECU- }\end{array}$} & & \multicolumn{5}{|c|}{$\begin{array}{l}\text { DISCHARGE, IN FT3/S, FOR INDICATED } \\
\text { RECURRENCE INTERVAL, IN YEARS, AND }\end{array}$} \\
\hline & & $\ldots \ldots$ & & & & \\
\hline TIVE & 2 & 5 & 10 & 25 & 50 & 100 \\
\hline DAYS) & 508 & 208 & 108 & 48 & 28 & 18 \\
\hline 1 & 2,180 & 4.630 & 7,280 & 12,300 & 17,800 & 25,100 \\
\hline 3 & 1,370 & 2,880 & 4,510 & 7,650 & 11,100 & 15,700 \\
\hline 7 & 915 & 1,880 & 2,870 & 4,700 & 6,590 & 9,080 \\
\hline 15 & 670 & 1.320 & 1,960 & 3,090 & 4,210 & 5,620 \\
\hline 30 & 516 & 984 & 1.430 & 2,180 & 2,910 & 3,820 \\
\hline 60 & 406 & 759 & 1,100 & 1,670 & 2.230 & 2.920 \\
\hline 90 & 355 & 654 & 941 & 1,440 & 1,920 & 2,540 \\
\hline
\end{tabular}

DISCHARGE, IN FT3/S, WHICH WAS EQUALED OR EXCEEDED FOR INDICATED PERCENT OF TIME

18
$\ldots \ldots$


ANNUAL MEAN DISCHARGE, IN CUBIC

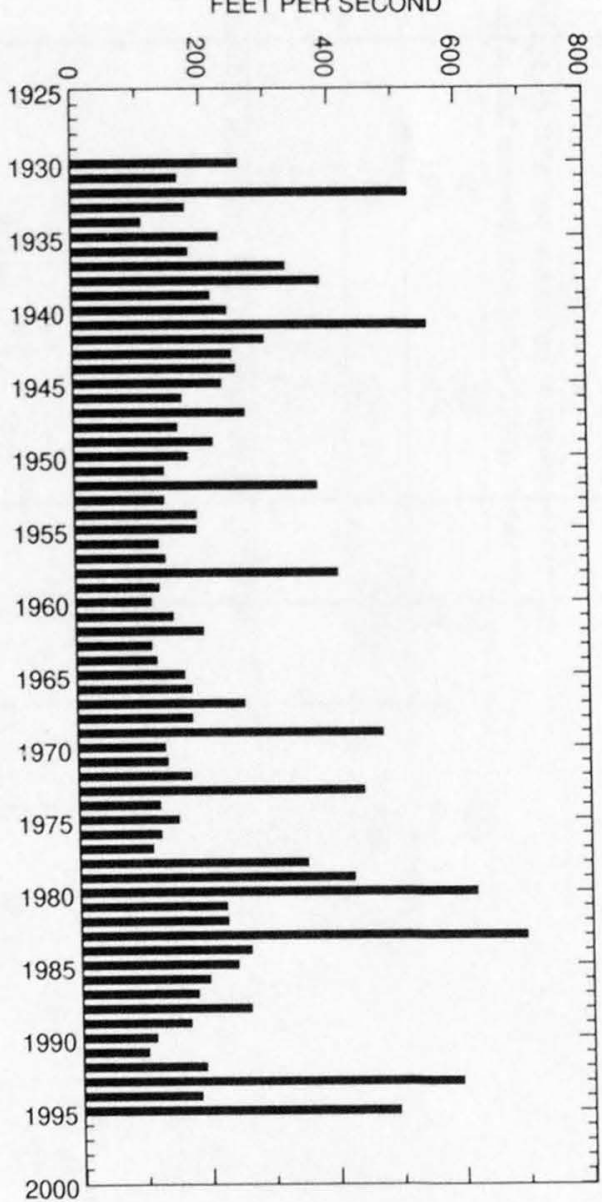

ANNUAL PEAK DISCHARGE, IN CUBIC FEET PER SECOND

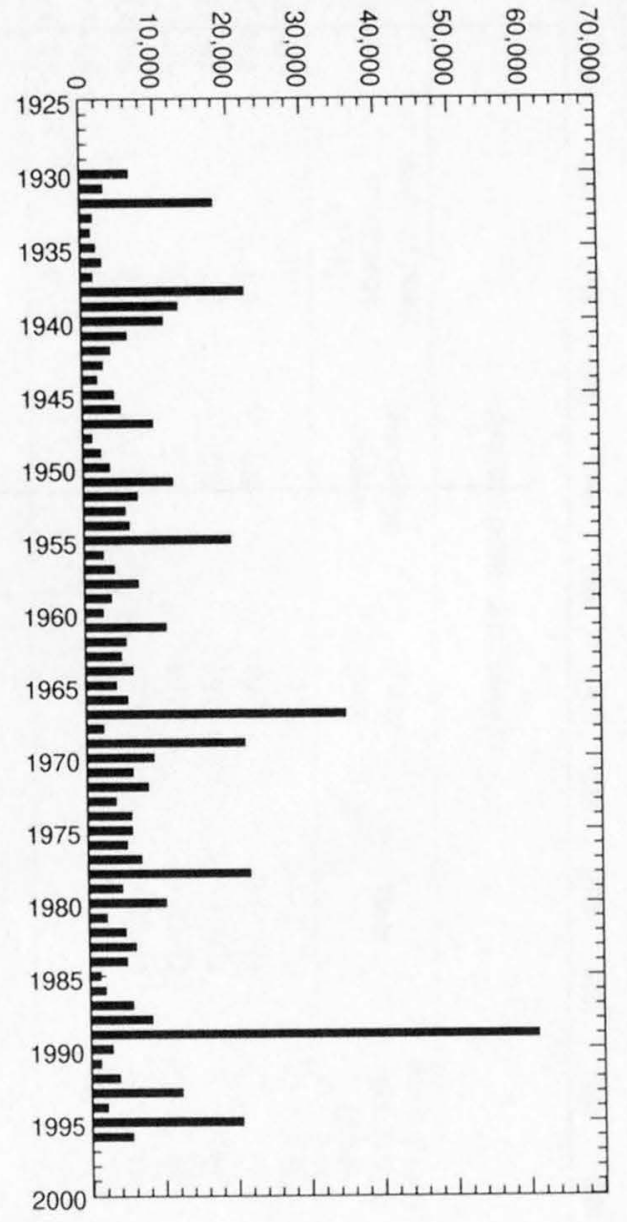


09415000 VIRGIN RIVER AT LITTLEFIELD, AZ--Continued

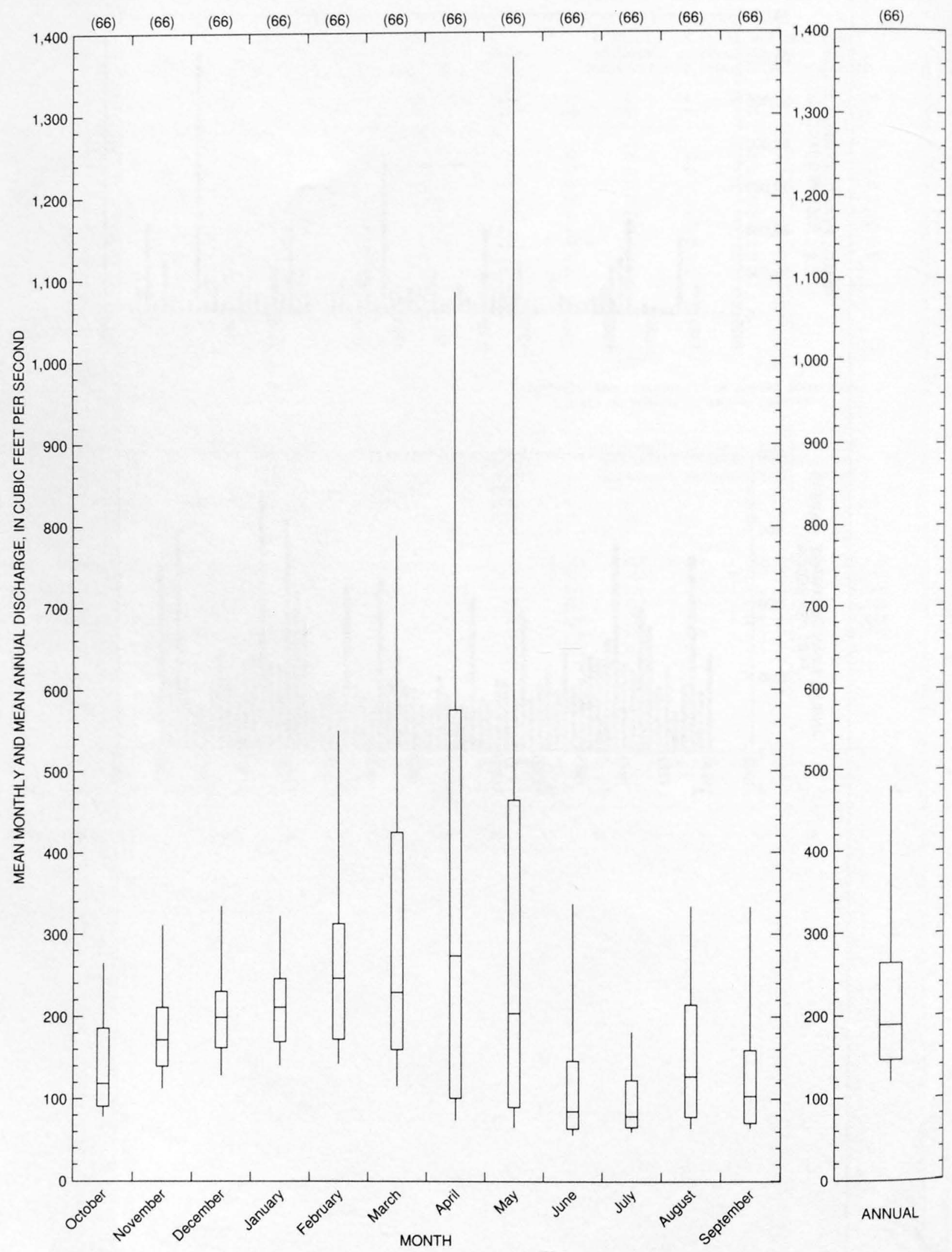


09415050 BIG BEND WASH TRIBUTARY NEAR LITTLEFIELD, AZ

LOCATION.--Lat $36^{\circ} 51^{\prime} 45^{\prime \prime}$, long $113^{\circ} 58^{\prime} 05^{\prime \prime}$, in $\mathrm{SE}^{1} / 4 \mathrm{sec} .13$, T.40 N., R.16 W., Mohave County, Hydrologic Unit 15010010 , at U.S. Highway 91, $2.7 \mathrm{mi}$ southest of Littlefield.

DRAINAGE AREA.--7.27 $\mathrm{mi}^{2}$.

Annual peak discharges

\begin{tabular}{|c|c|c|c|c|c|c|c|}
\hline $\begin{array}{l}\text { Water } \\
\text { year }\end{array}$ & Date & $\begin{array}{c}\text { Annual peak } \\
\text { discharge } \\
\left(\mathrm{ft}^{3} / \mathrm{s}\right)\end{array}$ & $\begin{array}{l}\text { Discharge } \\
\text { codes }\end{array}$ & $\begin{array}{c}\text { Water } \\
\text { year }\end{array}$ & Date & $\begin{array}{c}\text { Annual peak } \\
\text { discharge } \\
\left(\mathrm{ft}^{3} / \mathrm{s}\right)\end{array}$ & $\begin{array}{c}\text { Discharge } \\
\text { codes }\end{array}$ \\
\hline 1963 & $09-18-63$ & 248 & & 1970 & $00-00-70$ & 250 & \\
\hline 1964 & $07-00-64$ & 1.5 & ES & 1971 & $00-00-71$ & 0 & \\
\hline 1965 & $08-00-65$ & 3.0 & ES & 1972 & $09-19-72$ & 160 & \\
\hline 1966 & $11-23-65$ & 2.0 & ES & 1973 & $10-09-72$ & 10 & ES \\
\hline 1967 & $12-06-66$ & 2.0 & ES & 1974 & $00-00-74$ & 0 & \\
\hline 1968 & $00-00-68$ & 0.1 & LT & 1975 & $11-00-74$ & 1.0 & LT \\
\hline 1969 & $00-00-69$ & 0 & & & & & \\
\hline
\end{tabular}

Magnitude and probability of instantaneous peak flow based on period of record 1963-75

\begin{tabular}{|c|c|c|c|c|c|}
\hline \multicolumn{6}{|c|}{$\begin{array}{l}\text { Discharge, in } \mathrm{ft}^{3} / \mathrm{s} \text {, for indicated recurrence interval } \\
\text { in years, and exceedance probablility, in percent }\end{array}$} \\
\hline 2 & 5 & 10 & 25 & $50 \dagger$ & $100 t$ \\
\hline $50 \%$ & $20 \%$ & $10 \%$ & $4 \%$ & $2 \%$ & $1 \%$ \\
\hline 1.7 & 32.8 & 152 & 759 & 2,120 & 5,300 \\
\hline Weighted skew & $(\log s)=$ & -0.09 & & & \\
\hline Mean & $(\log s)=$ & 0.20 & & & \\
\hline Standard dev. & $(\log s)=$ & 1.56 & & & \\
\hline
\end{tabular}

fReliability of values in column is uncertain, and potential errors are large.

Basin characteristics

\begin{tabular}{cccccccc}
\hline $\begin{array}{c}\text { Main } \\
\text { channel } \\
\text { slope } \\
(\mathrm{ft} / \mathrm{mi})\end{array}$ & $\begin{array}{c}\text { Stream } \\
\text { length } \\
(\mathrm{mi})\end{array}$ & $\begin{array}{c}\text { Mean } \\
\text { basin } \\
\text { elevation } \\
\text { (ft) }\end{array}$ & $\begin{array}{c}\text { Forested } \\
\text { area } \\
\text { (percent) }\end{array}$ & $\begin{array}{c}\text { Soil } \\
\text { index }\end{array}$ & $\begin{array}{c}\text { Mean } \\
\text { annual } \\
\text { precipitation } \\
\text { (in) }\end{array}$ & $\begin{array}{c}\text { Rainfall intensity, 24-hour } \\
\text { (in) }\end{array}$ & $\begin{array}{c}50 \text {-year } \\
\text { (in) }\end{array}$ \\
\hline 55.8 & 7.4 & 2,240 & 0.0 & 3.0 & 7.6 & 1.5 \\
\hline
\end{tabular}

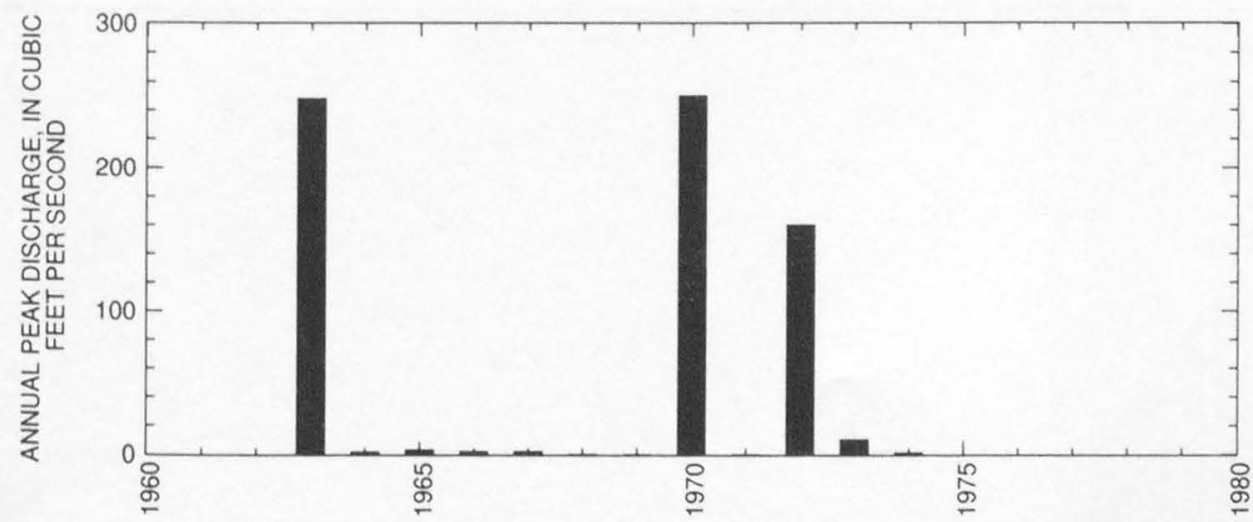


09419590 DETRITAL WASH TRIBUTARY NEAR CHLORIDE, AZ

LOCATION.--Lat $35^{\circ} 25^{\prime} 55^{\prime \prime}$, long $114^{\circ} 17^{\prime} 05^{\prime \prime}$, in NW1/4 sec.35, T.24 N., R.19 W., Mohave County, Hydrologic Unit 15010014, at U.S. Highwz 93, 5.5 mi northwest of Chloride.

DRAINAGE AREA.--1.23 $\mathrm{mi}^{2}$.

Annual peak discharges

\begin{tabular}{|c|c|c|c|c|c|c|c|}
\hline $\begin{array}{l}\text { Water } \\
\text { year }\end{array}$ & Date & $\begin{array}{c}\text { Annual peak } \\
\text { discharge } \\
\left(\mathrm{ft}^{3} / \mathrm{s}\right)\end{array}$ & $\begin{array}{l}\text { Discharge } \\
\text { codes }\end{array}$ & $\begin{array}{l}\text { Water } \\
\text { year }\end{array}$ & Date & $\begin{array}{c}\text { Annual peak } \\
\text { discharge } \\
\left(\mathrm{ft}^{3} / \mathrm{s}\right)\end{array}$ & $\begin{array}{c}\text { Discharge } \\
\text { codes }\end{array}$ \\
\hline 1963 & $09-17-63$ & 25 & ES & 1971 & $08-12-71$ & 470 & \\
\hline 1964 & $08-12-64$ & 50 & ES & 1972 & $09-19-72$ & 92 & \\
\hline 1965 & $04-03-65$ & 0.2 & ES & 1973 & $00-00-73$ & 0 & \\
\hline 1966 & $08-16-66$ & 73 & & 1974 & $07-21-74$ & 46 & \\
\hline 1967 & $09-00-67$ & 117 & & 1975 & $00-00-75$ & 0 & \\
\hline 1968 & $00-00-68$ & 20 & ES & 1976 & $09-10-76$ & 460 & \\
\hline 1969 & $07-27-69$ & 42 & & 1980 & $00-00-80$ & ${ }^{1} 30$ & ES,HP \\
\hline 1970 & $00-00-70$ & 0 & & & & & \\
\hline
\end{tabular}

'Highest since 1976.

Magnitude and probability of instantaneous peak flow based on period of record 1963-76,

1980

\begin{tabular}{|c|c|c|c|c|c|}
\hline \multicolumn{6}{|c|}{$\begin{array}{l}\text { Discharge, in } \mathrm{ft}^{3} / \mathrm{s} \text {, for indicated recurrence interval } \\
\text { in years, and exceedance probablility, in percent }\end{array}$} \\
\hline 2 & 5 & 10 & 25 & $50 \dagger$ & $100 \dagger$ \\
\hline $50 \%$ & $20 \%$ & $10 \%$ & $4 \%$ & $2 \%$ & $1 \%$ \\
\hline 41.4 & 126 & 233 & 457 & 715 & 1,080 \\
\hline Weighted skew & $(\log s)=$ & 0.23 & & & \\
\hline Mean & $(\log s)=$ & 1.64 & & & \\
\hline Standard dev. & $(\log s)=$ & 0.56 & & & \\
\hline
\end{tabular}

$\mp$ Reliability of values in column is uncertain, and potential errors are large.

Basin characteristics

\begin{tabular}{ccccccc}
\hline $\begin{array}{c}\text { Main } \\
\text { channel } \\
\text { slope } \\
(\mathrm{ft} / \mathrm{mi})\end{array}$ & $\begin{array}{c}\text { Stream } \\
\text { length } \\
\text { (mi) }\end{array}$ & $\begin{array}{c}\text { Mean } \\
\text { basin } \\
\text { elevation } \\
\text { (ft) }\end{array}$ & $\begin{array}{c}\text { Forested } \\
\text { area } \\
\text { (percent) }\end{array}$ & $\begin{array}{c}\text { Soil } \\
\text { index }\end{array}$ & $\begin{array}{c}\text { Mean } \\
\text { annual } \\
\text { precipitation } \\
\text { (in) }\end{array}$ & $\begin{array}{c}\text { Rainfall intensity, 24-hour } \\
\text { (in) }\end{array}$ \\
\hline 200 & 2.4 & 3,710 & 0.0 & 3.0 & 10.1 & 1.4 \\
\hline
\end{tabular}


09419590 DETRITAL WASH TRIBUTARY NEAR CHLORIDE, AZ--Continued

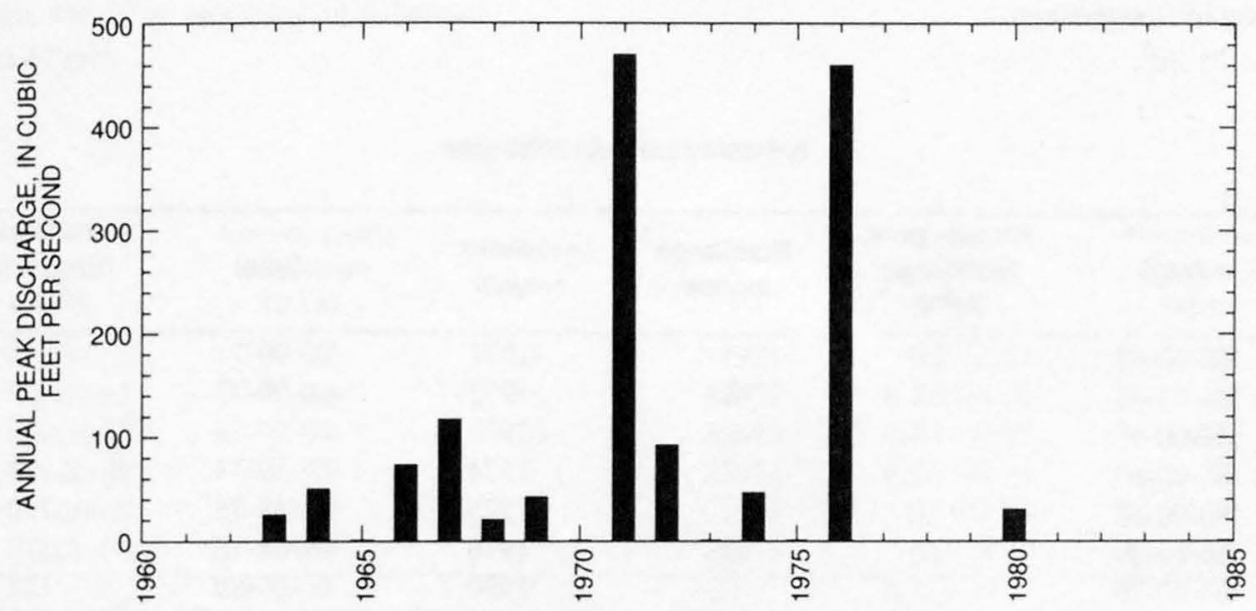


09421800 RINGBOLT WASH NEAR HOOVER DAM, AZ

LOCATION.--Lat $35^{\circ} 58^{\prime} 05^{\prime \prime}$, long $114^{\circ} 41^{\prime} 00^{\prime \prime}$, in SW $1 / 4$ sec.19, T.30 N., R.22 W., Mohave County, Hydrologic Unit 15030101, at U.S. Highwa 93, 5.9 mi southeast of Hoover Dam.

DRAINAGE AREA.--1.21 $\mathrm{mi}^{2}$.

Annual peak discharges

\begin{tabular}{|c|c|c|c|c|c|c|c|}
\hline $\begin{array}{l}\text { Water } \\
\text { year }\end{array}$ & Date & $\begin{array}{c}\text { Annual peak } \\
\text { discharge } \\
\left(\mathrm{ft}^{3} / \mathrm{s}\right)\end{array}$ & $\begin{array}{c}\text { Discharge } \\
\text { codes }\end{array}$ & $\begin{array}{l}\text { Water } \\
\text { year }\end{array}$ & Date & $\begin{array}{c}\text { Annual peak } \\
\text { discharge } \\
\left(\mathrm{ft}^{3} / \mathrm{s}\right)\end{array}$ & $\begin{array}{c}\text { Discharge } \\
\text { codes }\end{array}$ \\
\hline 1964 & $00-00-64$ & 0 & & 1971 & $00-00-71$ & 16 & ES \\
\hline 1965 & $04-03-65$ & 1.0 & ES & 1972 & $00-00-72$ & 0 & \\
\hline 1966 & $12-00-65$ & 1.0 & ES & 1973 & $00-00-73$ & 0 & \\
\hline 1967 & $08-00-67$ & 2.0 & ES & 1974 & $07-19-74$ & 1.0 & LT \\
\hline 1968 & $00-00-68$ & 0 & & 1975 & $08-19-75$ & 250 & ES \\
\hline 1969 & $00-00-69$ & 10 & ES & 1976 & $09-08-76$ & 310 & \\
\hline 1970 & $08-00-70$ & 1.0 & ES & 1980 & $00-00-80$ & ${ }^{1} 125$ & HP \\
\hline
\end{tabular}

${ }^{1}$ Highest since 1976.

Magnitude and probability of instantaneous peak flow based on period of record

\begin{tabular}{cccccc}
\hline \multicolumn{5}{c}{$\begin{array}{l}\text { Discharge, in } \mathrm{ft}^{3} / \mathrm{s} \text {, for indicated recurrence interval } \\
\text { in years, and exceedance probablility, in percent }\end{array}$} \\
\hline 2 & 5 & 10 & 25 & 50 & 100 \\
$50 \%$ & $20 \%$ & $10 \%$ & $4 \%$ & $2 \%$ & $1 \%$ \\
\hline & & & & & \\
Weighted skew & $(\operatorname{logs})=$ & -- & & \\
Mean & $(\operatorname{logs})=$ & -- & & \\
Standard dev. & $(\operatorname{logs})=$ & -- &
\end{tabular}

Basin characteristics

\begin{tabular}{ccccccc}
\hline $\begin{array}{c}\text { Main } \\
\text { channel } \\
\text { slope } \\
(\mathrm{ft} / \mathrm{mi})\end{array}$ & $\begin{array}{c}\text { Stream } \\
\text { length } \\
\text { (mi) }\end{array}$ & $\begin{array}{c}\text { Mean } \\
\text { basin } \\
\text { elevation } \\
\text { (ft) }\end{array}$ & $\begin{array}{c}\text { Forested } \\
\text { area } \\
\text { (percent) }\end{array}$ & $\begin{array}{c}\text { Soil } \\
\text { index }\end{array}$ & $\begin{array}{c}\text { Mean } \\
\text { annual } \\
\text { precipitation } \\
\text { (in) }\end{array}$ & $\begin{array}{c}\text { Rainfall intensity, 24-hour } \\
\text { (in) }\end{array}$ \\
\hline 428 & 3.0 & 2,590 & 0.0 & 1.0 & 5.8 & $\begin{array}{c}50 \text {-year } \\
\text { (in) }\end{array}$ \\
\hline
\end{tabular}

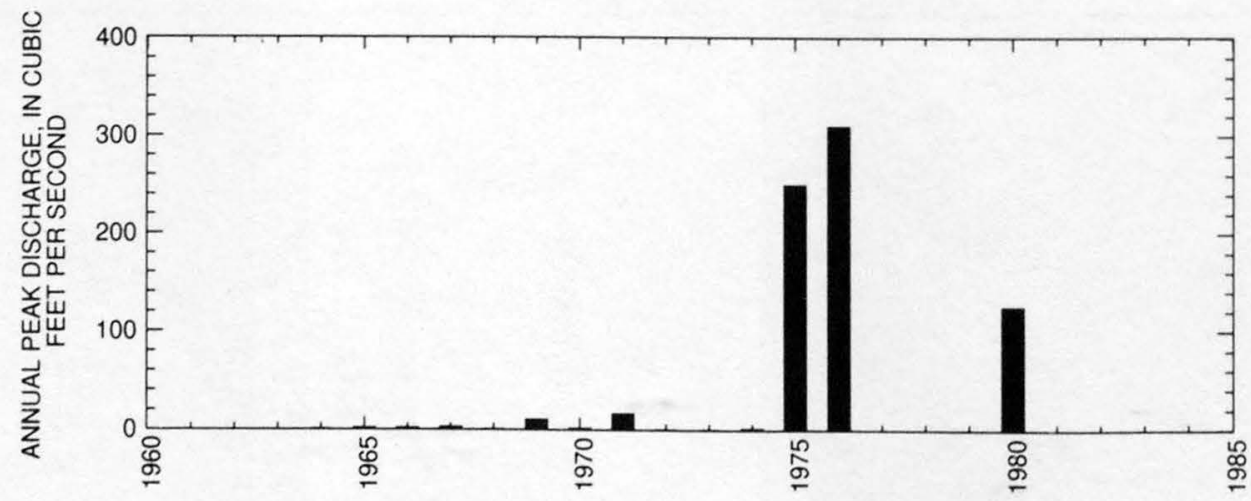


09423760 LITTLE MEADOW CREEK NEAR OATMAN, AZ

LOCATION.--Lat 35 $01^{\prime} 50^{\prime \prime}$, long 114 $18^{\prime} 30^{\prime \prime}$, in NE $\frac{1}{4} \mathrm{SW}^{1} / 4$ sec.16, T.19 N., R. 19 W., Mohave County, Hydrologic Unit 15030103, at Eds Camp,

4 mi east of Oatman, and $19 \mathrm{mi}$ southwest of Kingman.

DRAINAGE AREA.--8.47 $\mathrm{mi}^{2}$.

Annual peak discharges

\begin{tabular}{ccccccrr}
\hline $\begin{array}{c}\text { Water } \\
\text { year }\end{array}$ & Date & $\begin{array}{c}\text { Annual peak } \\
\text { discharge } \\
\left(\mathrm{ft}^{3} / \mathbf{s}\right)\end{array}$ & $\begin{array}{c}\text { Discharge } \\
\text { codes }\end{array}$ & $\begin{array}{c}\text { Water } \\
\text { year }\end{array}$ & Date & $\begin{array}{c}\text { Annual peak } \\
\text { discharge } \\
\left(\mathrm{ft}^{3} / \mathbf{s}\right)\end{array}$ & $\begin{array}{c}\text { Discharge } \\
\text { codes }\end{array}$ \\
\hline 1965 & $04-00-65$ & 10 & LT & 1971 & $08-12-71$ & 50 & ES \\
1966 & $12-09-65$ & 20 & ES & 1972 & $09-00-72$ & 50 & ES \\
1967 & $08-06-67$ & 0.5 & ES & 1973 & $11-00-72$ & 600 & ES \\
1968 & $00-00-68$ & 0 & & 1974 & $07-19-74$ & 182 & \\
1969 & $00-00-69$ & 0 & & 1975 & $00-00-75$ & 0 & \\
1970 & $08-00-70$ & 869 & & 1976 & $02-08-76$ & 105 & \\
\hline
\end{tabular}

Magnitude and probability of instantaneous peak flow based on period of record 1965-76

\begin{tabular}{|c|c|c|c|c|c|}
\hline \multicolumn{6}{|c|}{$\begin{array}{l}\text { Discharge, in } \mathrm{ft}^{3} / \mathrm{s} \text {, for indicated recurrence interval } \\
\text { in years, and exceedance probablility, in percent }\end{array}$} \\
\hline 2 & 5 & 10 & 25 & $50 t$ & $100 t$ \\
\hline $50 \%$ & $20 \%$ & $10 \%$ & $4 \%$ & $2 \%$ & $1 \%$ \\
\hline 28.7 & 194 & 508 & 1,390 & 2,610 & 4,590 \\
\hline Weighted skew & $(\log s)=$ & -0.16 & & & \\
\hline Mean & $(\log s)=$ & 1.43 & & & \\
\hline Standard dev. & $(\log s)=$ & 1.01 & & & \\
\hline
\end{tabular}

Basin characteristics

\begin{tabular}{ccccccccc}
\hline $\begin{array}{c}\text { Main } \\
\text { channel } \\
\text { slope }\end{array}$ & $\begin{array}{c}\text { Stream } \\
\text { (ft/mi) }\end{array}$ & $\begin{array}{c}\text { length } \\
\text { (mi) }\end{array}$ & $\begin{array}{c}\text { Mean } \\
\text { basin } \\
\text { elevation } \\
\text { (ft) }\end{array}$ & $\begin{array}{c}\text { Forested } \\
\text { area } \\
\text { (percent) }\end{array}$ & $\begin{array}{c}\text { Soil } \\
\text { index }\end{array}$ & $\begin{array}{c}\text { Mean } \\
\text { annual } \\
\text { precipitation } \\
\text { (in) }\end{array}$ & $\begin{array}{c}\text { 2-year } \\
\text { (in) }\end{array}$ & $\begin{array}{c}\text { 50-year } \\
\text { (in) }\end{array}$ \\
\hline 241 & 3.8 & 3,400 & 0.0 & 1.0 & 12.0 & 1.6 & 3.9 \\
\hline
\end{tabular}

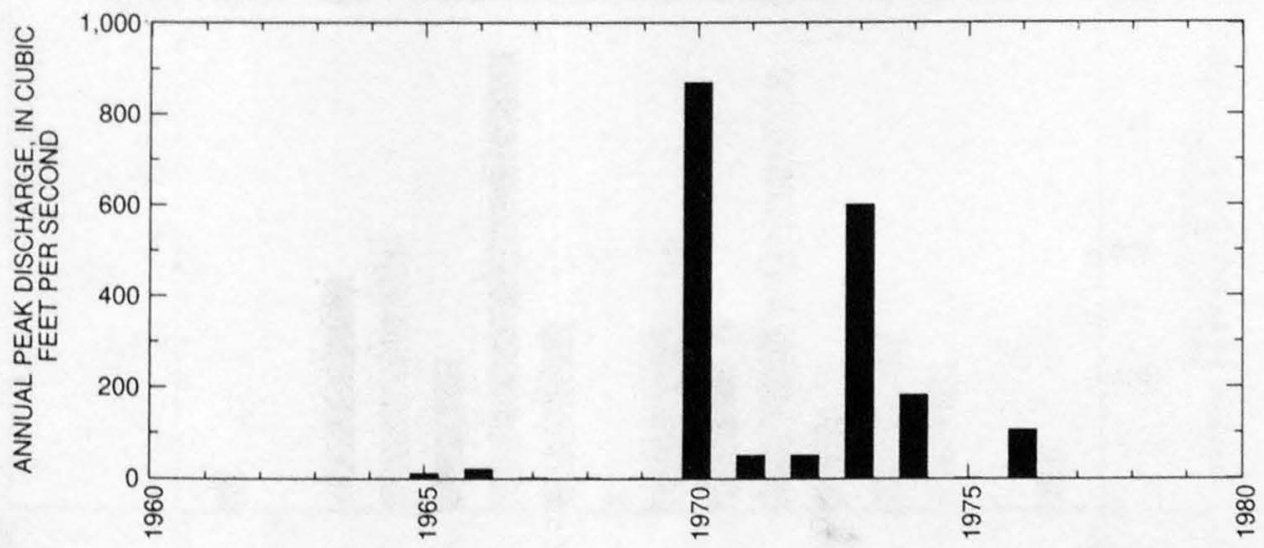


09423780 WALNUT CREEK NEAR KINGMAN, AZ

LOCATION.--Lat $35^{\circ} 02^{\prime} 00^{\prime \prime}$, long $114^{\circ} 01^{\prime} 05^{\prime \prime}$, in SE $1 /{ }_{4} \mathrm{NW}^{1} / 4$ sec.18, T.19 N., R.16 W., Mohave County, Hydrologic Unit 15030103 , 11 mi sou of Kingman.

DRAINAGE AREA.--31.3 $\mathrm{mi}^{2}$.

Annual peak discharges

\begin{tabular}{ccccccc}
\hline $\begin{array}{c}\text { Water } \\
\text { year }\end{array}$ & Date & $\begin{array}{c}\text { Annual peak } \\
\text { discharge } \\
\left(\mathrm{ft}^{3} / \mathbf{s}\right)\end{array}$ & $\begin{array}{c}\text { Discharge } \\
\text { codes }\end{array}$ & $\begin{array}{c}\text { Water } \\
\text { year }\end{array}$ & $\begin{array}{c}\text { Annual peak } \\
\text { discharge } \\
\left(\mathrm{ft}^{3} / \mathbf{s}\right)\end{array}$ & $\begin{array}{c}\text { Discharge } \\
\text { codes }\end{array}$ \\
\hline 1965 & $08-00-65$ & 152 & & 1971 & $08-12-71$ & 715 \\
1966 & $07-20-66$ & 288 & & 1972 & $06-06-72$ & 235 \\
1967 & $00-00-67$ & 228 & & 1973 & $07-00-73$ & 425 \\
1968 & $00-00-68$ & 289 & LT & 1974 & $09-00-74$ & 360 \\
1969 & $09-13-69$ & 2.0 & & 1975 & $00-00-75$ & 0 \\
1970 & $08-15-70$ & 290 & & & $00-00-76$ & 60 \\
\hline
\end{tabular}

Magnitude and probability of instantaneous peak flow based on period of record 1965-76

\begin{tabular}{|c|c|c|c|c|c|}
\hline \multicolumn{6}{|c|}{$\begin{array}{l}\text { Discharge, in } \mathrm{ft}^{3} / \mathrm{s} \text {, for indicated recurrence interval } \\
\text { in years, and exceedance probablility, in percent }\end{array}$} \\
\hline $\begin{array}{c}2 \\
50 \%\end{array}$ & $\begin{array}{c}5 \\
20 \%\end{array}$ & $\begin{array}{c}10 \\
10 \%\end{array}$ & $\begin{array}{l}25 \\
4 \%\end{array}$ & $\begin{array}{l}50 \dagger \\
2 \%\end{array}$ & $\begin{array}{c}100 \dagger \\
1 \%\end{array}$ \\
\hline 247 & 389 & 496 & 644 & 764 & 892 \\
\hline $\begin{array}{l}\text { Weighted skew } \\
\text { Mean } \\
\text { Standard dev. }\end{array}$ & $\begin{array}{l}(\operatorname{logs})= \\
(\log s)= \\
(\log s)=\end{array}$ & $\begin{array}{l}0.08 \\
2.40 \\
0.23\end{array}$ & & & \\
\hline
\end{tabular}

$\mp$ Reliability of values in column is uncertain, and potential errors are large.

Basin characteristics

\begin{tabular}{cccccccc}
\hline $\begin{array}{c}\text { Main } \\
\text { channel } \\
\text { slope } \\
(\mathrm{ft} / \mathrm{mi})\end{array}$ & $\begin{array}{c}\text { Stream } \\
\text { length } \\
\text { (mi) }\end{array}$ & $\begin{array}{c}\text { Mean } \\
\text { basin } \\
\text { elevation } \\
\text { (ft) }\end{array}$ & $\begin{array}{c}\text { Forested } \\
\text { area } \\
\text { (percent) }\end{array}$ & $\begin{array}{c}\text { Soil } \\
\text { index }\end{array}$ & $\begin{array}{c}\text { Mean } \\
\text { annual } \\
\text { precipitation } \\
\text { (in) }\end{array}$ & $\begin{array}{c}\text { Rainfall intensity, 24-hour } \\
\text { (in) }\end{array}$ & $\begin{array}{c}50 \text {-year } \\
\text { (in) }\end{array}$ \\
\hline 255 & 9.3 & 5,040 & 50.0 & 1.0 & 13.9 & 1.8 \\
\hline
\end{tabular}

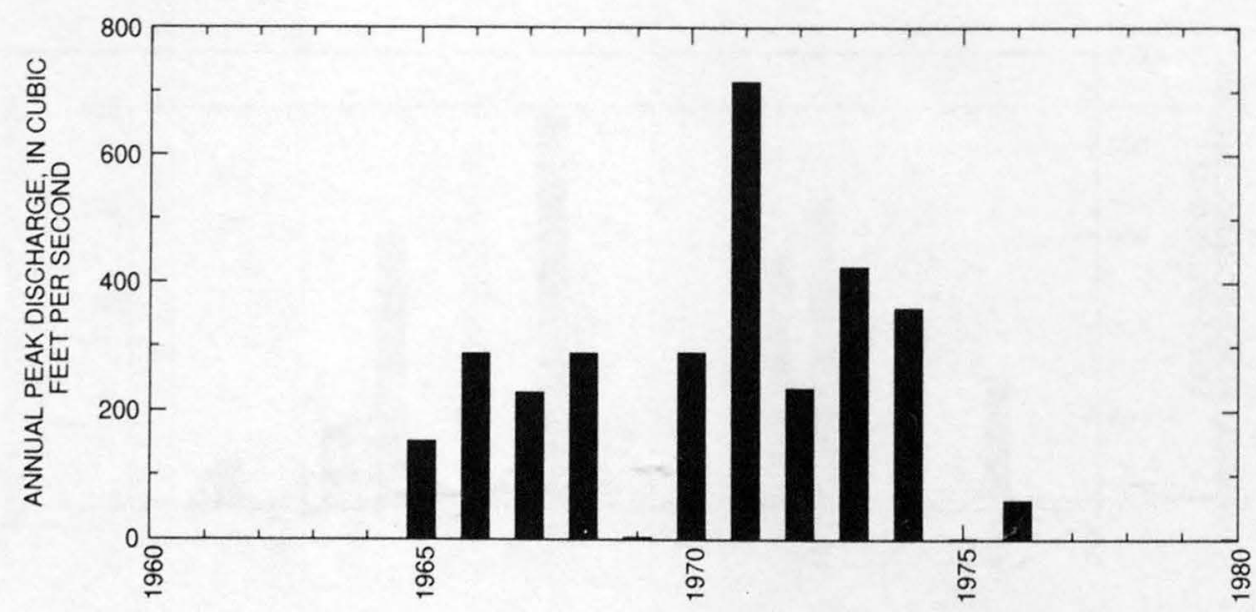


09423820 SACRAMENTO WASH NEAR YUCCA, AZ

LOCATION.--Lat 34 48'40", long $114^{\circ} 09^{\prime} 40^{\prime \prime}$, in SE $1 /{ }_{4} \mathrm{SE}^{1} / 4 \mathrm{sec} .35$, T.17 N., R.18 W., Mohave County, Hydrologic Unit 15030103 , at Sante Fe Railroad bridge, $5 \mathrm{mi}$ south of Yucca.

DRAINAGE AREA.--787 $\mathrm{mi}^{2}$.

Annual peak discharges

\begin{tabular}{ccccccc}
\hline $\begin{array}{l}\text { Water } \\
\text { year }\end{array}$ & Date & $\begin{array}{c}\text { Annual peak } \\
\text { discharge } \\
\left(\mathbf{f t}^{\mathbf{3}} / \mathbf{s}\right)\end{array}$ & $\begin{array}{c}\text { Discharge } \\
\text { codes }\end{array}$ & $\begin{array}{c}\text { Water } \\
\text { year }\end{array}$ & $\begin{array}{c}\text { Annual peak } \\
\text { discharge } \\
\left(\mathbf{f t}^{3} / \mathbf{s}\right)\end{array}$ & $\begin{array}{c}\text { Discharge } \\
\text { codes }\end{array}$ \\
\hline 1965 & $00-00-65$ & 0 & & 1971 & $08-12-71$ & 13,000 \\
1966 & $12-09-65$ & 2,060 & & 1972 & $06-00-72$ & 3,010 \\
1967 & $09-00-67$ & 800 & & 1973 & $11-16-72$ & 5,200 \\
1968 & $07-00-68$ & 520 & & 1974 & $07-19-74$ & 4,260 \\
1969 & $09-16-69$ & 8,030 & & 1975 & $00-00-75$ & 0 \\
1970 & $00-00-70$ & 3,000 & & 1976 & $09-25-76$ & 2,100 \\
\hline
\end{tabular}

Magnitude and probability of instantaneous peak flow based on period of record 1965-76

\begin{tabular}{|c|c|c|c|c|c|}
\hline \multicolumn{6}{|c|}{$\begin{array}{l}\text { Discharge, in } \mathrm{ft}^{3} / \mathrm{s} \text {, for indicated recurrence interval } \\
\text { in years, and exceedance probablility, in percent }\end{array}$} \\
\hline 2 & 5 & 10 & 25 & $50 \dagger$ & $100 t$ \\
\hline $50 \%$ & $20 \%$ & $10 \%$ & $4 \%$ & $2 \%$ & $1 \%$ \\
\hline 2,640 & 5,540 & 8,200 & 12,500 & 16,500 & 21,200 \\
\hline Weighted skew & $(\log s)=$ & 0.07 & & & \\
\hline Mean & $(\log s)=$ & 3.43 & & & \\
\hline Standard dev. & $(\log s)=$ & 0.38 & & & \\
\hline
\end{tabular}

fReliability of values in column is uncertain, and potential errors are large.

Basin characteristics

\begin{tabular}{cccccccc}
\hline $\begin{array}{c}\text { Main } \\
\text { channel } \\
\text { slope } \\
(\mathrm{ft} / \mathrm{mi})\end{array}$ & $\begin{array}{c}\text { Stream } \\
\text { length } \\
(\mathrm{mi})\end{array}$ & $\begin{array}{c}\text { Mean } \\
\text { basin } \\
\text { elevation } \\
(\mathrm{ft})\end{array}$ & $\begin{array}{c}\text { Forested } \\
\text { area } \\
\text { (percent) }\end{array}$ & $\begin{array}{c}\text { Soil } \\
\text { index }\end{array}$ & $\begin{array}{c}\text { Mean } \\
\text { annual } \\
\text { precipitation } \\
\text { (in) }\end{array}$ & $\begin{array}{c}\text { Rainfall intensity, 24-hour } \\
\text { (in) }\end{array}$ & $\begin{array}{c}50-\text { year } \\
\text { (in) }\end{array}$ \\
\hline 45.2 & 48.4 & 3,400 & 6.4 & 2.5 & 10.1 & 1.6 \\
\hline
\end{tabular}

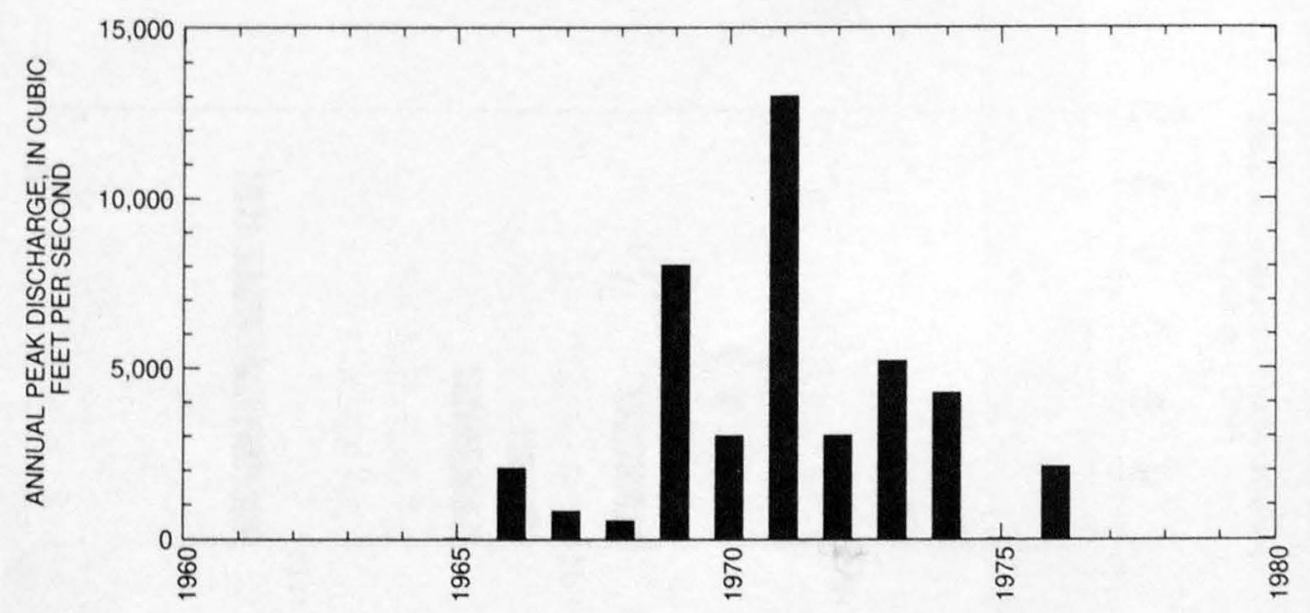


09423900 SACRAMENTO WASH TRIBUTARY NEAR TOPOCK, AZ

LOCATION.--Lat $34^{\circ} 43^{\prime} 47^{\prime \prime}$, long $114^{\circ} 18^{\prime} 45^{\prime \prime}$, in $\mathrm{SW}^{1} / 4^{\mathrm{NW}}{ }^{1} / 4 \mathrm{sec} .13$, T.16 N., R.20 W., Mohave County, Hydrologic Unit 15030103 , at U.S. Highway $66,9.7$ mi east of Topock.

DRAINAGE AREA.--14.7 $\mathrm{mi}^{2}$.

Arınual peak discharges

\begin{tabular}{|c|c|c|c|c|c|c|c|}
\hline $\begin{array}{l}\text { Water } \\
\text { year }\end{array}$ & Date & $\begin{array}{c}\text { Annual peak } \\
\text { discharge } \\
\left(\mathrm{ft}^{3} / \mathrm{s}\right)\end{array}$ & $\begin{array}{c}\text { Discharge } \\
\text { codes }\end{array}$ & $\begin{array}{l}\text { Water } \\
\text { year }\end{array}$ & Date & $\begin{array}{c}\text { Annual peak } \\
\text { discharge } \\
\left(\mathrm{ft}^{3} / \mathrm{s}\right)\end{array}$ & $\begin{array}{c}\text { Discharge } \\
\text { codes }\end{array}$ \\
\hline 1963 & $08-00-63$ & 70 & & 1970 & $08-00-70$ & 15 & ES \\
\hline 1964 & $07-26-64$ & 128 & & 1971 & $08-00-71$ & 320 & \\
\hline 1965 & $08-10-65$ & 10 & ES & 1972 & $00-00-72$ & 500 & ES \\
\hline 1966 & $12-09-65$ & 20 & ES & 1973 & $10-00-72$ & 240 & ES \\
\hline 1967 & $00-00-67$ & 0 & & 1974 & $01-00-74$ & 1.0 & ES \\
\hline 1968 & $11-21-67$ & 10 & LT & 1975 & $00-00-75$ & 0 & \\
\hline 1969 & $07-17-69$ & 430 & & 1976 & $09-10-76$ & 1,030 & \\
\hline
\end{tabular}

Magnitude and probability of instantaneous peak flow based on period of record 1963-76

\begin{tabular}{|c|c|c|c|c|c|}
\hline \multicolumn{6}{|c|}{$\begin{array}{l}\text { Discharge, in } \mathrm{ft}^{3} / \mathrm{s} \text {, for indicated recurrence interval } \\
\text { in years, and exceedance probablility, in percent }\end{array}$} \\
\hline 2 & 5 & 10 & 25 & $50 t$ & $100 t$ \\
\hline $50 \%$ & $20 \%$ & $10 \%$ & $4 \%$ & $2 \%$ & $1 \%$ \\
\hline 48.7 & 291 & 710 & 1,780 & 3,180 & 5,290 \\
\hline Weighted skew & $(\log s)=$ & -0.20 & & & \\
\hline Mean & $(\log s)=$ & 1.66 & & & \\
\hline Standard dev. & $(\log s)=$ & 0.95 & & & \\
\hline
\end{tabular}

f Reliability of values in column is uncertain, and potential errors are large.

Basin characteristics

\begin{tabular}{ccccccc}
\hline $\begin{array}{c}\text { Main } \\
\text { channel } \\
\text { slope } \\
(\mathrm{ft} / \mathrm{mi})\end{array}$ & $\begin{array}{c}\text { Stream } \\
\text { length } \\
(\mathrm{mi})\end{array}$ & $\begin{array}{c}\text { Mean } \\
\text { basin } \\
\text { elevation } \\
(\mathrm{ft})\end{array}$ & $\begin{array}{c}\text { Forested } \\
\text { area } \\
\text { (percent) }\end{array}$ & $\begin{array}{c}\text { Soil } \\
\text { index }\end{array}$ & $\begin{array}{c}\text { Mean } \\
\text { annual } \\
\text { precipitation } \\
\text { (in) }\end{array}$ & $\begin{array}{c}\text { R-year } \\
\text { (in) }\end{array}$ \\
\hline 98.2 & 7.6 & 1,450 & 0.0 & 3.0 & 6.2 & $\begin{array}{c}50 \text {-year } \\
\text { (in) }\end{array}$ \\
\hline
\end{tabular}

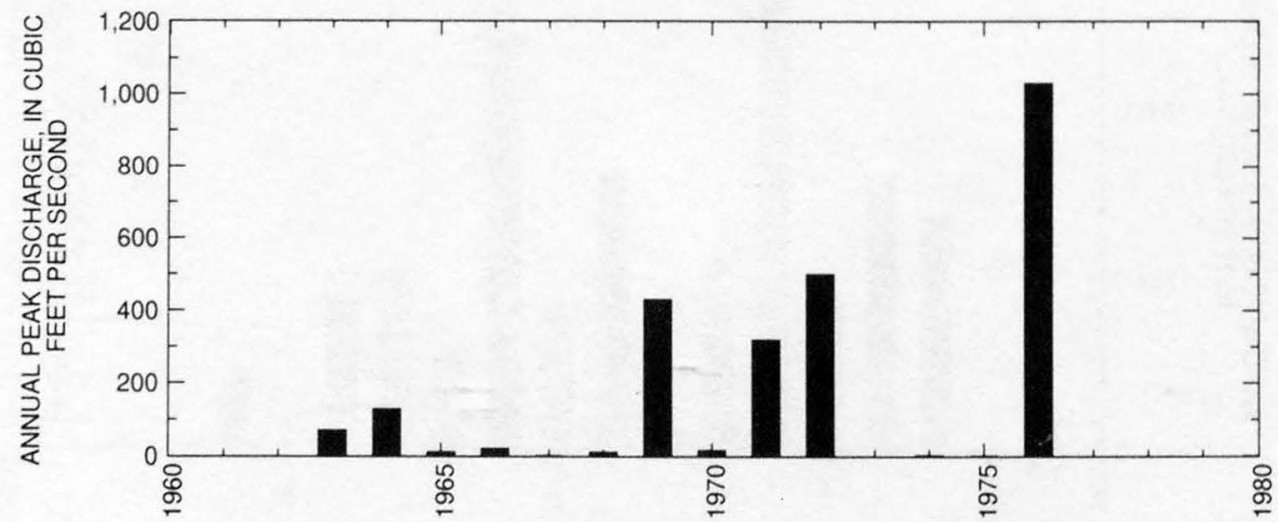




\section{WILLOW CREEK (COTTONWOOD WASH NO. 1) NEAR KINGMAN, AZ}

LOCATION.--Lat 35'10'52", long 113'28'08", in NW $1 / 4$ sec.29, T.21 N., R.11 W., Mohave County, Hydrologic Unit 15030201 , on right bank, 13 mi upstream from mouth, and 34 mi east of Kingman.

DRAINAGE AREA.--143 $\mathrm{mi}^{2}$.

PERIOD OF RECORD.--January 1959 to August 1963 (fragmentary low flow only published in WSP 1858), February 1964 to September 1978 (discontinued).

GAGE.--Water-stage recorder and concrete control. Datum of gage is 4,545 ft, from special U.S. Geological Survey project map. Prior to June 20,1969 , at site $10 \mathrm{ft}$ downstream at datum $2.72 \mathrm{ft}$ lower.

REMARKS.--Records poor.

AVERAGE DISCHARGE.--14 years, $4.69 \mathrm{ft}^{3} / \mathrm{s}, 3,400$ acre- $\mathrm{ft} / \mathrm{yr}$.

EXTREMES FOR PERIOD OF RECORD.--Maximum discharge, 7,000 $\mathrm{ft}^{3} / \mathrm{s}$ July 31 , 1964, gage height, $7.36 \mathrm{ft}$ at former site and datum of supplementary gage, from rating curve extended above $400 \mathrm{ft}^{3} / \mathrm{s}$ on basis of slope-area measurements at gage heights 3.82 and $7.36 \mathrm{ft}$; minimum daily, $0.10 \mathrm{ft}^{3} / \mathrm{s}$ many days in $1972,1974-78$.

Annual peak discharges

\begin{tabular}{|c|c|c|c|c|c|c|c|}
\hline $\begin{array}{l}\text { Water } \\
\text { year }\end{array}$ & Date & $\begin{array}{c}\text { Annual peak } \\
\text { discharge } \\
\left(\mathrm{ft}^{3} / \mathrm{s}\right)\end{array}$ & $\begin{array}{l}\text { Discharge } \\
\text { codes }\end{array}$ & $\begin{array}{l}\text { Water } \\
\text { year }\end{array}$ & Date & $\begin{array}{c}\text { Annual peak } \\
\text { discharge } \\
\left(\mathrm{ft}^{3} / \mathrm{s}\right)\end{array}$ & $\begin{array}{l}\text { Discharge } \\
\text { codes }\end{array}$ \\
\hline 1964 & $07-31-64$ & 7,000 & & 1972 & $06-07-72$ & 436 & \\
\hline 1965 & $08-15-65$ & 820 & & 1973 & $06-13-73$ & 1,720 & \\
\hline 1966 & $08-18-66$ & 6,700 & & 1974 & $07-21-74$ & 6,450 & \\
\hline 1967 & $08-19-67$ & 6,300 & & 1975 & $07-27-75$ & 870 & \\
\hline 1968 & $10-05-67$ & 3,640 & & 1976 & $07-24-76$ & 5,250 & \\
\hline 1969 & $09-13-69$ & 5,580 & & 1977 & $09-10-77$ & 2,620 & \\
\hline 1970 & $08-19-70$ & 3,120 & & 1978 & $03-01-78$ & 3,080 & \\
\hline 1971 & $08-21-71$ & 4,020 & & & & & \\
\hline
\end{tabular}

Basin characteristics

\begin{tabular}{cccccccc}
\hline $\begin{array}{c}\text { Main } \\
\text { channel } \\
\text { slope } \\
\text { (ft/mi) }\end{array}$ & $\begin{array}{c}\text { Stream } \\
\text { length } \\
(\mathrm{mi})\end{array}$ & $\begin{array}{c}\text { Mean } \\
\text { basin } \\
\text { elevation } \\
(\mathrm{ft})\end{array}$ & $\begin{array}{c}\text { Forested } \\
\text { area } \\
\text { (percent) }\end{array}$ & $\begin{array}{c}\text { Soil } \\
\text { index }\end{array}$ & $\begin{array}{c}\text { Mean } \\
\text { annual } \\
\text { precipitation } \\
\text { (in) }\end{array}$ & $\begin{array}{c}\text { R-year } \\
\text { (in) }\end{array}$ & $\begin{array}{c}50 \text {-year } \\
\text { (in) }\end{array}$ \\
\hline 67.0 & 17.6 & 5,350 & 90.0 & 1.0 & 14.0 & 1.7 \\
\hline
\end{tabular}


09424200 WILLOW CREEK (COTTONWOOD WASH NO. 1) NEAR KINGMAN, AZ--Continued

MEAN MONTHLY AND ANNUAL DISCHARGES $1965 \cdot 78$

\begin{tabular}{|c|c|c|c|c|c|c|}
\hline MONTH & $\begin{array}{l}\text { MAXIMUM } \\
\text { (FT3/S) }\end{array}$ & $\begin{array}{l}\text { MINIMUM } \\
\text { (FT3/S) }\end{array}$ & $\begin{array}{c}\text { MEAN } \\
(\mathrm{FT} 3 / \mathrm{S})\end{array}$ & $\begin{array}{l}\text { STAN- } \\
\text { DARD } \\
\text { DEVIA- } \\
\text { TION } \\
(\text { FT3/S) }\end{array}$ & $\begin{array}{l}\text { COEFFI- } \\
\text { CIENT OF } \\
\text { VARI- } \\
\text { ATION }\end{array}$ & $\begin{array}{c}\text { PERCENT } \\
\text { OF } \\
\text { ANNUAL } \\
\text { RUNOFF }\end{array}$ \\
\hline OCTOBER & 7.8 & 0.27 & 1.4 & 1.9 & 1.3 & 2.5 \\
\hline NOVEMBER & 2.0 & 0.65 & 1.1 & 0.38 & 0.35 & 1.9 \\
\hline DECEMBER & 38 & 0.68 & 4.3 & 10 & 2.3 & 7.7 \\
\hline JANUARY & 6.6 & 0.61 & 1.7 & 1.5 & 0.86 & 3.1 \\
\hline FEBRUARY & 82 & 0.91 & 8.8 & 22 & 2.4 & 15.7 \\
\hline MARCH & 88 & 0.51 & 10 & 24 & 2.3 & 18.7 \\
\hline APRIL & 18 & 0.15 & 2.4 & 4.7 & 1.9 & 4.3 \\
\hline MAY & 1.3 & 0.18 & 0.74 & 0.33 & 0.45 & 1.3 \\
\hline JUNE & 4.2 & 0.10 & 0.94 & 1.1 & 1.2 & 1.7 \\
\hline JULY & 21 & 0.13 & 5.1 & 6.3 & 1.3 & 9.0 \\
\hline AUGUST & 32 & 0.21 & 12 & 11 & 0.99 & 20.7 \\
\hline SEPTEMBER & 31 & 0.20 & 7.6 & 11 & 1.5 & 13.4 \\
\hline NNUAL & 11 & 0.86 & 4.7 & 3.4 & 0.72 & 100 \\
\hline
\end{tabular}

MAGNITUDE AND PROBABILITY OF INSTANTANEOUS PEAK FLOW BASED ON PERIOD OF RECORD 1964-78

DISCHARGE, IN FT3/S, FOR INDICATED RECURRENCE INTERVAL IN YEARS, AND EXCEBDANCE PROBABILITY, IN PERCENT

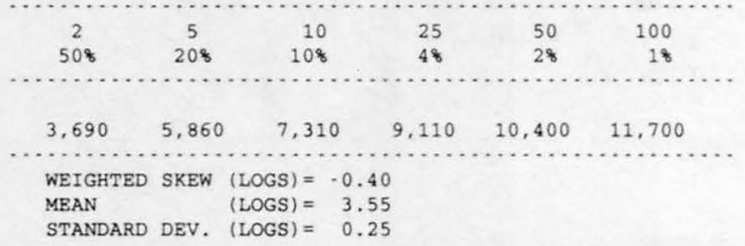

MAGNITUDE AND PROBABILITY OF ANNUAL LOW FLOW BASED ON PERIOD OF RECORD 1965.78

\begin{tabular}{|c|c|c|c|c|c|c|}
\hline \multirow{4}{*}{$\begin{array}{l}\text { PERIOD } \\
\text { (CON- } \\
\text { SECU- } \\
\text { TIVE } \\
\text { DAYS) }\end{array}$} & \multicolumn{6}{|c|}{$\begin{array}{l}\text { DISCHARGE, IN FT3/S, FOR INDICATED } \\
\text { RECURRENCE INTERVAL, IN YEARS, AND } \\
\text { NON-EXCEEDANCE PROBABILITY, IN PERCENT }\end{array}$} \\
\hline & $\cdots$ & $\ldots \ldots$ & & & $\ldots \ldots$ & \\
\hline & 2 & 5 & 10 & 20 & $50 \#$ & $100 \#$ \\
\hline & 508 & 208 & 108 & 58 & 28 & 18 \\
\hline 1 & 0.27 & 0.13 & 0.09 & 0.06 & 0.04 & 0.03 \\
\hline 3 & 0.29 & 0.14 & 0.09 & 0.07 & 0.05 & 0.04 \\
\hline 7 & 0.30 & 0.14 & 0.10 & 0.07 & 0.05 & 0.03 \\
\hline 14 & 0.31 & 0.14 & 0.09 & 0.07 & 0.05 & 0.03 \\
\hline 30 & 0.34 & 0.16 & 0.10 & 0.07 & 0.05 & 0.03 \\
\hline 60 & 0.49 & 0.25 & 0.16 & 0.11 & 0.07 & 0.05 \\
\hline 90 & 0.54 & 0.29 & 0.20 & 0.14 & 0.09 & 0.07 \\
\hline 120 & 0.81 & 0.50 & 0.37 & 0.28 & 0.20 & 0.15 \\
\hline 183 & 1.2 & 0.82 & 0.69 & 0.61 & 0.54 & 0.50 \\
\hline
\end{tabular}

MAGNITUDE AND PROBABILITY OF ANNUAL HIGH FLOW BASED ON PBRIOD OF RECORD 1965.78

\begin{tabular}{|c|c|c|c|c|c|c|}
\hline $\begin{array}{l}\text { PERIOD } \\
\text { (CON- }\end{array}$ & & \multicolumn{5}{|c|}{$\begin{array}{l}\text { DISCHARGE, IN FT3/S, FOR INDICATED } \\
\text { RECURRENCE INTERVAL, IN YEARS, AND }\end{array}$} \\
\hline SECU - & $\ldots \ldots$ & ....... & ........ & $\ldots . .$. & $\ldots \ldots$ & $\ldots \ldots$ \\
\hline TIVE & 2 & 5 & 10 & 25 & $50 \#$ & $100 \#$ \\
\hline DAYS) & 508 & 208 & 108 & 48 & 28 & 18 \\
\hline 1 & 298 & 697 & 1,060 & 1,640 & 2,160 & 2,740 \\
\hline 3 & 143 & 322 & 469 & 679 & 848 & 1.020 \\
\hline 7 & 76 & 175 & 254 & 363 & 447 & 532 \\
\hline 15 & 42 & 93 & 131 & 179 & 213 & 246 \\
\hline 30 & 24 & 52 & 73 & 99 & 118 & 135 \\
\hline 60 & 14 & 29 & 41 & 55 & 65 & 75 \\
\hline 90 & 9.3 & 20 & 28 & 38 & 46 & 53 \\
\hline
\end{tabular}

DURATION TABLE OF DAILY MEAN FLOW FOR PERIOD OF RECORD 1965-78 DISCHARGE, IN FT3/S, WHICH WAS EQUALED OR EXCEEDED FOR INDICATED PERCENT OF TIME

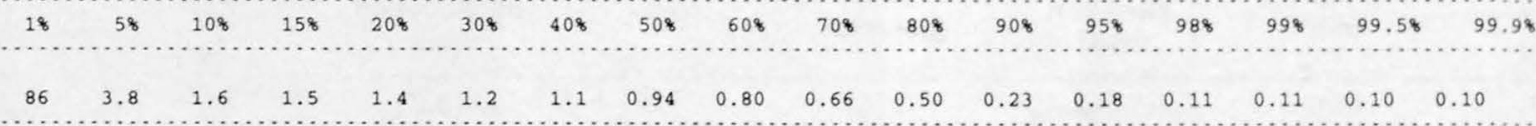

\# Reliability of values in column is uncertain, and potential errors are large. 
09424200 WILLOW CREEK (COTTONWOOD WASH NO. 1) NEAR KINGMAN, AZ--Continued
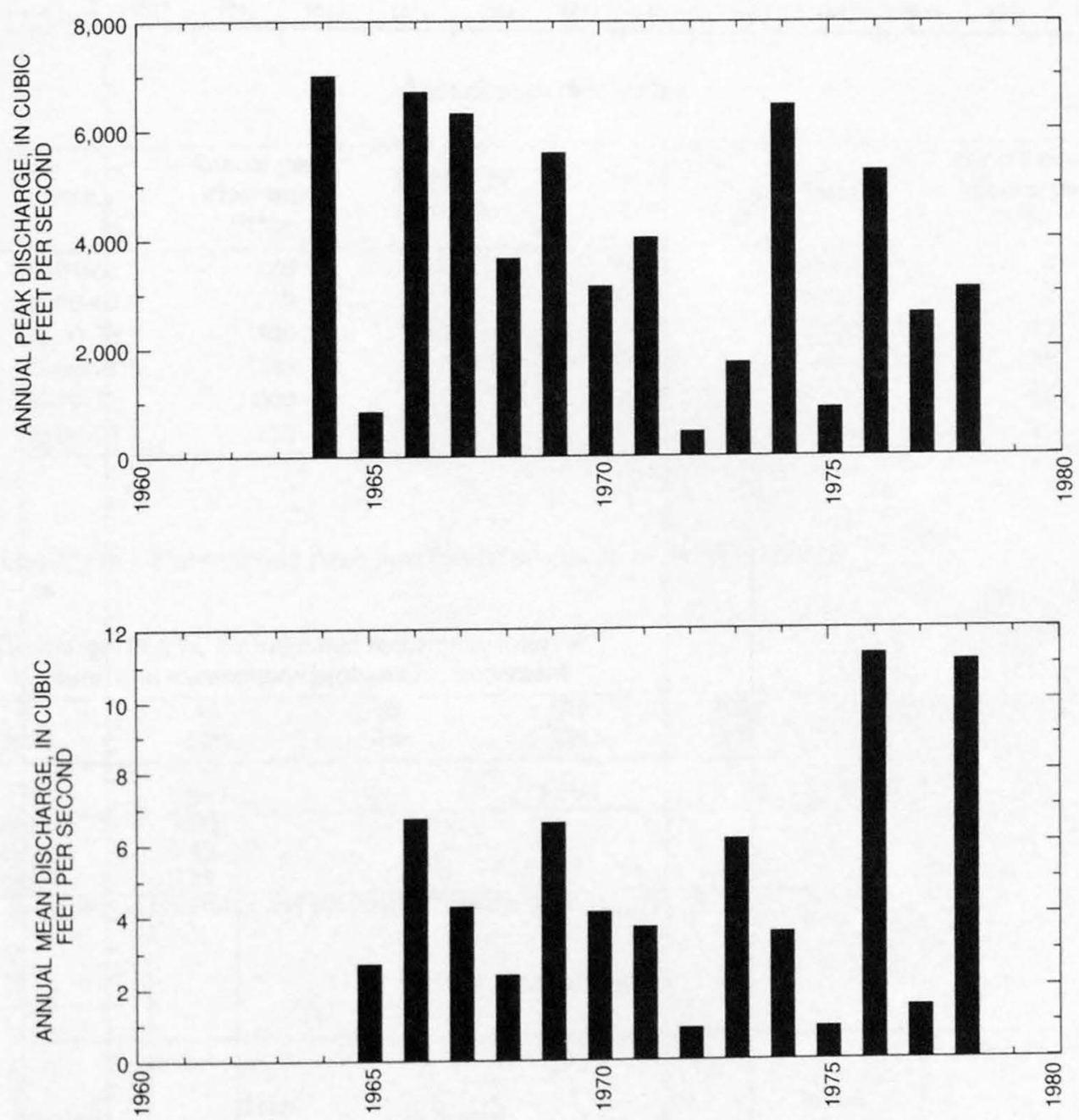
200

BILL WILLIAMS RIVER BASIN

09424200 WILLOW CREEK (COTTONWOOD WASH NO. 1) NEAR KINGMAN, AZ--Continued
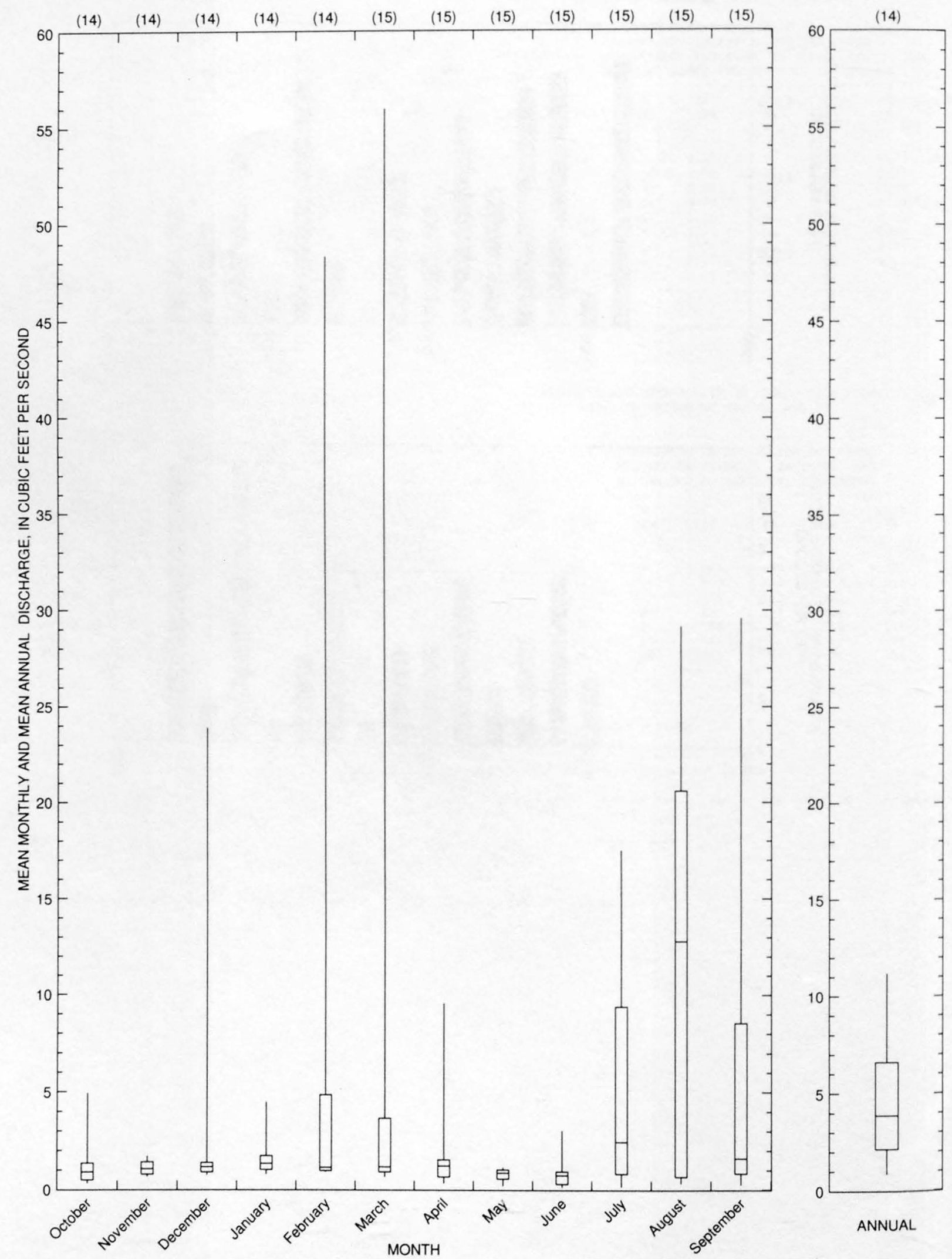
09424407 MCGARRYS WASH NEAR KINGMAN, AZ

LOCATION.--Lat 35 07'00", long $113^{\circ} 39^{\prime} 00^{\prime \prime}$, in sec.16, T.20 N., R.13 W., Mohave County, Hydrologic Unit 15030201, at U.S. Highway 93, 1.2 mi north of junction of U.S. Highway 93 and Hackberry Road, and 23 mi southeast of Kingman.

DRAINAGE AREA.--13.5 $\mathrm{mi}^{2}$.

Annual peak discharges

\begin{tabular}{|c|c|c|c|c|c|c|c|}
\hline $\begin{array}{l}\text { Water } \\
\text { year }\end{array}$ & Date & $\begin{array}{c}\text { Annual peak } \\
\text { discharge } \\
\left(\mathrm{ft}^{3} / \mathrm{s}\right)\end{array}$ & $\begin{array}{l}\text { Discharge } \\
\text { codes }\end{array}$ & $\begin{array}{l}\text { Water } \\
\text { year }\end{array}$ & Date & $\begin{array}{c}\text { Annual peak } \\
\text { discharge } \\
\left(\mathrm{ft}^{3} / \mathrm{s}\right)\end{array}$ & $\begin{array}{c}\text { Discharge } \\
\text { codes }\end{array}$ \\
\hline 1968 & 08-04-68 & 20 & ES & 1974 & $00-00-74$ & 0 & \\
\hline 1969 & $00-00-69$ & 0 & & 1975 & $10-29-74$ & 2 & ES \\
\hline 1970 & $08-00-70$ & 400 & ES & 1976 & $00-00-76$ & 20 & LT \\
\hline 1971 & $07-00-71$ & 40 & ES & 1977 & $00-00-77$ & 25 & LT \\
\hline 1972 & $09-19-72$ & 1,000 & & 1978 & $00-00-78$ & 25 & LT \\
\hline 1973 & $00-00-73$ & 150 & & 1979 & $00-00-79$ & 25 & LT \\
\hline
\end{tabular}

Magnitude and probability of instantaneous peak flow based on period of record 1968-79

Discharge, in $\mathrm{ft}^{3} / \mathrm{s}$, for indicated recurrence interval

in years, and exceedance probablility, in percent

\begin{tabular}{cccccc}
\hline 2 & $\mathbf{5}$ & $\mathbf{1 0}$ & $\mathbf{2 5}$ & $\mathbf{5 0 \dagger}$ & $\mathbf{1 0 0 \dagger}$ \\
$\mathbf{5 0 \%}$ & $\mathbf{2 0 \%}$ & $\mathbf{1 0 \%}$ & $\mathbf{4 \%}$ & $\mathbf{2 \%}$ & $\mathbf{1 \%}$ \\
\hline 26.9 & 150 & 369 & 963 & 1,790 & 3,130 \\
\hline $\begin{array}{c}\text { Weighted skew } \\
\text { Mean }\end{array}$ & $(\operatorname{logs})=$ & 0.00 & & & \\
Standard dev. & $(\log )=$ & 1.43 & & & \\
\hline
\end{tabular}

f Reliability of values in column is uncertain, and potential errors are large.

Basin characteristics

\begin{tabular}{cccccccc}
\hline $\begin{array}{c}\text { Main } \\
\text { channel } \\
\text { slope } \\
\text { (ft/mi) }\end{array}$ & $\begin{array}{c}\text { Stream } \\
\text { length } \\
\text { (mi) }\end{array}$ & $\begin{array}{c}\text { Mean } \\
\text { basin } \\
\text { elevation } \\
\text { (ft) }\end{array}$ & $\begin{array}{c}\text { Forested } \\
\text { area } \\
\text { (percent) }\end{array}$ & $\begin{array}{c}\text { Soil } \\
\text { index }\end{array}$ & $\begin{array}{c}\text { Mean } \\
\text { annual } \\
\text { precipitation } \\
\text { (in) }\end{array}$ & $\begin{array}{c}\text { Rainfall intensity, 24-hour } \\
\text { (in) }\end{array}$ & $\begin{array}{c}50 \text {-year } \\
\text { (in) }\end{array}$ \\
\hline 203 & 12.1 & 4,610 & 40.0 & 3.0 & 12.0 & 1.5 \\
\hline
\end{tabular}

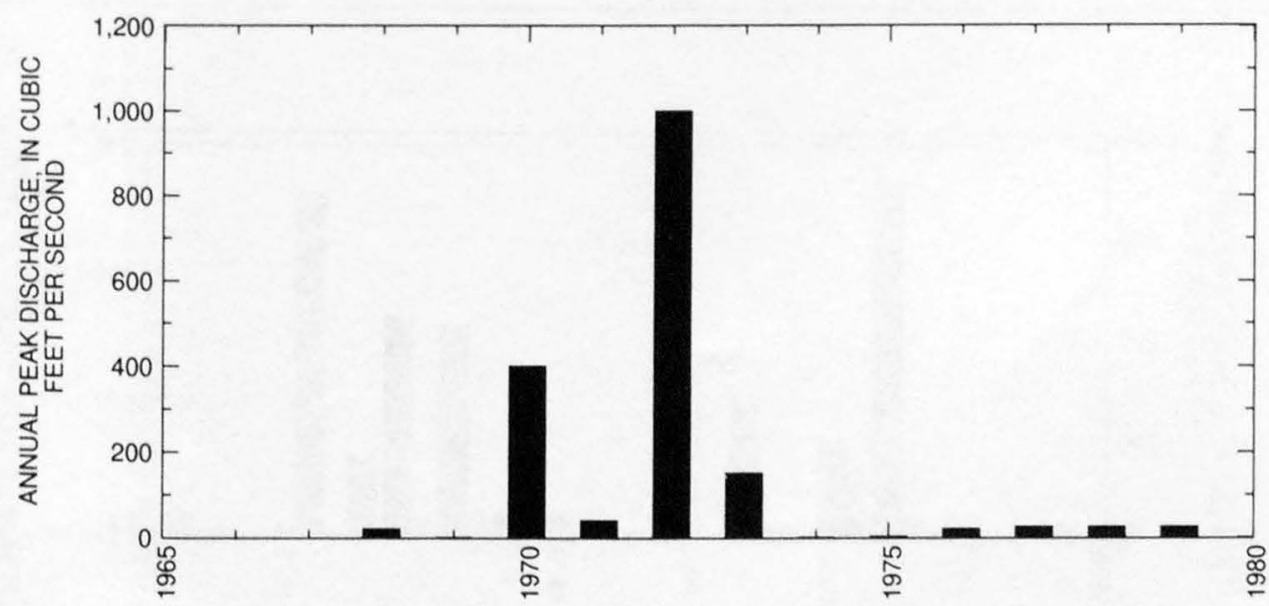




\section{BIG SANDY RIVER TRIBUTARY NEAR KINGMAN, AZ}

LOCATION.--Lat $35^{\circ} 05^{\prime} 30^{\prime \prime}$, long $113^{\circ} 39^{\prime} 30^{\prime \prime}$, in NE $1 / 4$ sec.28, T.20 N., R.13 W., Mohave County, Hydrologic Unit 15030201, at U.S. Highway 93, 21 mi southeast of Kingman.

DRAINAGE AREA.--1.99 $\mathrm{mi}^{2}$.

Annual peak discharges

\begin{tabular}{|c|c|c|c|c|c|c|c|}
\hline $\begin{array}{l}\text { Water } \\
\text { year }\end{array}$ & Date & $\begin{array}{c}\text { Annual peak } \\
\text { discharge } \\
\left(\mathrm{ft}^{3} / \mathrm{s}\right)\end{array}$ & $\begin{array}{c}\text { Discharge } \\
\text { codes }\end{array}$ & $\begin{array}{l}\text { Water } \\
\text { year }\end{array}$ & Date & $\begin{array}{c}\text { Annual peak } \\
\text { discharge } \\
\left(\mathrm{ft}^{3} / \mathrm{s}\right)\end{array}$ & $\begin{array}{c}\text { Discharge } \\
\text { codes }\end{array}$ \\
\hline 1963 & $08-00-63$ & 10 & ES & 1971 & $08-00-71$ & 25 & ES \\
\hline 1964 & $08-02-64$ & 353 & & 1972 & $09-19-72$ & 210 & \\
\hline 1965 & $08-29-65$ & 100 & ES & 1973 & $08-00-73$ & 220 & \\
\hline 1966 & $07-30-66$ & 10 & ES & 1974 & $00-00-74$ & 0 & \\
\hline 1967 & $12-06-66$ & 5.0 & LT & 1975 & $11-02-74$ & 10 & ES \\
\hline 1968 & $08-04-68$ & 1.0 & ES & 1976 & $02-09-76$ & 7.0 & ES \\
\hline 1969 & $07-18-69$ & 5.0 & ES & 1977 & $00-00-77$ & 25 & LT \\
\hline 1970 & $08-00-70$ & 30 & ES & 1978 & $03-01-78$ & 25 & ES \\
\hline
\end{tabular}

Magnitude and probability of instantaneous peak flow based on period of record 1963-78

\begin{tabular}{|c|c|c|c|c|c|}
\hline \multicolumn{6}{|c|}{$\begin{array}{l}\text { Discharge, in } \mathrm{ft}^{3} / \mathrm{s} \text {, for indicated recurrence interval } \\
\text { in years, and exceedance probablility, in percent }\end{array}$} \\
\hline 2 & 5 & 10 & 25 & $50 t$ & $100 t$ \\
\hline $50 \%$ & $20 \%$ & $10 \%$ & $4 \%$ & $2 \%$ & $1 \%$ \\
\hline 18.6 & 80.2 & 173 & 394 & 672 & 1,090 \\
\hline Weighted skew & $(\log s)=$ & 0.03 & & & \\
\hline Mean & $(\log s)=$ & 1.27 & & & \\
\hline Standard dev. & $(\log s)=$ & 0.75 & & & \\
\hline
\end{tabular}

Basin characteristics

\begin{tabular}{cccccccc}
\hline $\begin{array}{c}\text { Main } \\
\text { channel } \\
\text { slope } \\
(\mathrm{ft} / \mathrm{mi})\end{array}$ & $\begin{array}{c}\text { Stream } \\
\text { length } \\
(\mathrm{mi})\end{array}$ & $\begin{array}{c}\text { Mean } \\
\text { basin } \\
\text { elevation } \\
(\mathrm{ft})\end{array}$ & $\begin{array}{c}\text { Forested } \\
\text { area } \\
\text { (percent) }\end{array}$ & $\begin{array}{c}\text { Soil } \\
\text { index }\end{array}$ & $\begin{array}{c}\text { Mean } \\
\text { annual } \\
\text { precipitation } \\
\text { (in) }\end{array}$ & $\begin{array}{c}\text { Rainfall intensity, 24-hour } \\
\text { (in) }\end{array}$ & $\begin{array}{c}50 \text {-year } \\
\text { (in) }\end{array}$ \\
\hline 214 & 4.3 & 3,700 & 60.0 & 3.0 & 12.0 & 1.5 \\
\hline
\end{tabular}

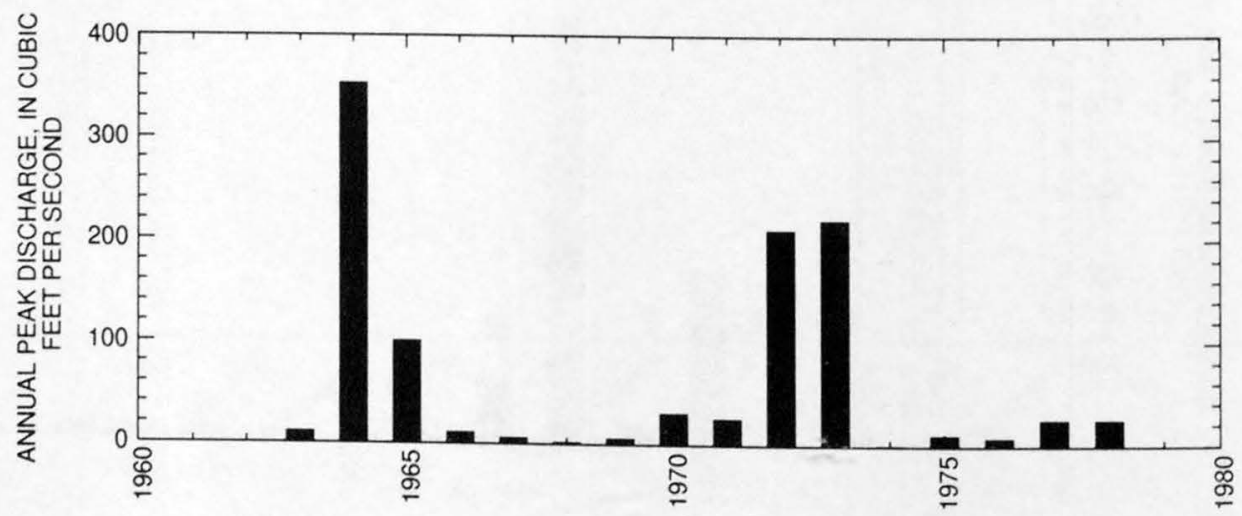


09424430 KAISER SPRING CANYON TRIBUTARY NEAR WIKIEUP, AZ

LOCATION.--Lat $34^{\circ} 34^{\prime} 20^{\prime \prime}$, long $113^{\circ} 28^{\prime} 40^{\prime \prime}$, in NW $1 / 4$ sec.12, T.14 N., R.12 W., Mohave County, Hydrologic Unit 15030202 , at U.S. Highway 93, 13 mi southeast of Wikieup.

DRAINAGE AREA.--1.7 $\mathrm{mi}^{2}$.

Annual peak discharges

\begin{tabular}{|c|c|c|c|c|c|c|c|}
\hline $\begin{array}{l}\text { Water } \\
\text { year }\end{array}$ & Date & $\begin{array}{c}\text { Annual peak } \\
\text { discharge } \\
\left(\mathrm{ft}^{3} / \mathrm{s}\right)\end{array}$ & $\begin{array}{c}\text { Discharge } \\
\text { codes }\end{array}$ & $\begin{array}{l}\text { Water } \\
\text { year }\end{array}$ & Date & $\begin{array}{c}\text { Annual peak } \\
\text { discharge } \\
\left(\mathrm{ft}^{3} / \mathrm{s}\right)\end{array}$ & $\begin{array}{l}\text { Discharge } \\
\text { codes }\end{array}$ \\
\hline 1963 & $08-22-63$ & 1,310 & & 1971 & $08-19-71$ & 510 & \\
\hline 1964 & $08-02-64$ & 290 & & 1972 & $00-00-72$ & 0 & \\
\hline 1965 & $00-00-65$ & 50 & LT & 1973 & $05-31-73$ & 250 & ES \\
\hline 1966 & $12-10-65$ & 90 & & 1974 & $09-00-74$ & 50 & ES \\
\hline 1967 & $00-00-67$ & 50 & LT & 1975 & $00-00-75$ & 0 & \\
\hline 1968 & $08-04-68$ & 2.0 & ES & 1976 & $00-00-76$ & 0 & \\
\hline 1969 & $00-00-69$ & 0 & & 1977 & $00-00-77$ & 30 & LT \\
\hline 1970 & $08-00-70$ & 50 & ES & 1978 & $00-00-78$ & 30 & ES \\
\hline
\end{tabular}

Magnitude and probability of instantaneous peak flow based on period of record 1963-78

Discharge, in $\mathrm{ft}^{3} / \mathrm{s}$, for indicated recurrence interval

in years, and exceedance probablility, in percent

\begin{tabular}{|c|c|c|c|c|c|}
\hline $\begin{array}{c}2 \\
50 \%\end{array}$ & $\begin{array}{c}5 \\
20 \%\end{array}$ & $\begin{array}{c}10 \\
10 \%\end{array}$ & $\begin{array}{l}25 \\
4 \%\end{array}$ & $\begin{array}{l}50 \dagger \\
2 \%\end{array}$ & $\begin{array}{c}100 \dagger \\
1 \%\end{array}$ \\
\hline 49.2 & 196 & 413 & 933 & 1,600 & 2,610 \\
\hline $\begin{array}{l}\text { Weighted skew } \\
\text { Mean } \\
\text { Standard dey }\end{array}$ & $\begin{array}{l}(\log s)= \\
(\log s)= \\
(\log s)=\end{array}$ & $\begin{array}{l}0.17 \\
1.71 \\
0.70\end{array}$ & & & \\
\hline
\end{tabular}

fReliability of values in column is uncertain, and potential errors are large.

Basin characteristics

\begin{tabular}{|c|c|c|c|c|c|c|c|}
\hline \multirow[b]{2}{*}{$\begin{array}{l}\text { Main } \\
\text { channel } \\
\text { slope } \\
\text { (ft/mi) }\end{array}$} & \multirow[b]{2}{*}{$\begin{array}{c}\text { Stream } \\
\text { length } \\
\text { (mi) }\end{array}$} & \multirow[b]{2}{*}{$\begin{array}{c}\text { Mean } \\
\text { basin } \\
\text { elevation } \\
(\mathrm{ft})\end{array}$} & \multirow[b]{2}{*}{$\begin{array}{l}\text { Forested } \\
\text { area } \\
\text { (percent) }\end{array}$} & \multirow[b]{2}{*}{$\begin{array}{c}\text { Soil } \\
\text { index }\end{array}$} & \multirow[b]{2}{*}{$\begin{array}{c}\text { Mean } \\
\text { annual } \\
\text { precipitation } \\
\text { (in) }\end{array}$} & \multicolumn{2}{|c|}{ Rainfall intensity, 24-hour } \\
\hline & & & & & & $\begin{array}{l}\text { 2-year } \\
\text { (in) }\end{array}$ & $\begin{array}{l}\text { 50-year } \\
\text { (in) }\end{array}$ \\
\hline 615 & 1.3 & 3,520 & 0.0 & 1.0 & 11.2 & 1.7 & 3.9 \\
\hline
\end{tabular}

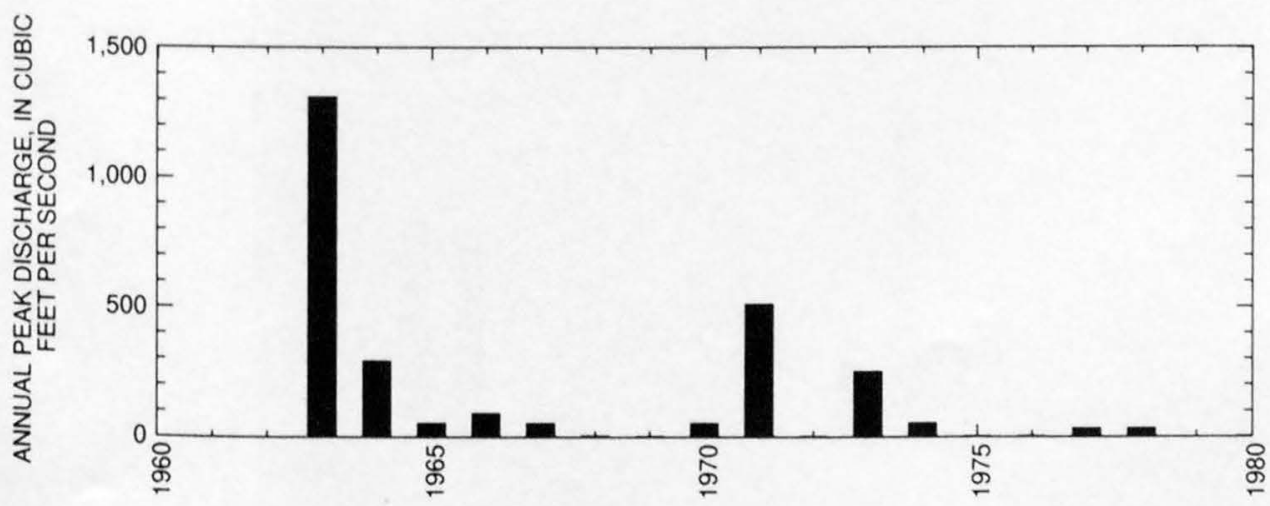




\section{BURRO CREEK AT OLD U.S. 93 BRIDGE, NEAR BAGDAD, AZ}

LOCATION.--Lat 34'32'30", long 113'26'40", in SW'1/4 sec.19, T.14 N., R.11 W., Mohave County, Hydrologic Unit 15030202, on left bank $15 \mathrm{~m}$ upstream from confluence with Big Sandy River, and 15 mi southwest of Bagdad.

DRAINAGE AREA.--611 mi ${ }^{2}$, of which $10 \mathrm{mi}^{2}$ is noncontributing.

PERIOD OF RECORD.--August 1980 to current year.

REVISED RECORDS.--WDR AZ-88-1: Drainage area.

GAGE.--Water-stage recorder. Elevation of gage is 1,880 ft above sea level, from topographic map.

EXTREMES FOR PERIOD OF RECORD.--Maximum discharge, 55,300 $\mathrm{ft}^{3} / \mathrm{s}$ Feb. 8, 1993, gage height, $16.30 \mathrm{ft}$; no flow at times in some years EXTREMES OUTSIDE PERIOD OF RECORD.--Maximum discharge, 44,700 $\mathrm{ft}^{3} / \mathrm{s} \mathrm{Feb.} \mathrm{14,} \mathrm{1980,} \mathrm{from} \mathrm{slope-area} \mathrm{measurement} \mathrm{of} \mathrm{peak} \mathrm{flow} \mathrm{at}$ present site and datum, gage height, $15.6 \mathrm{ft}$, from high-water mark in vicinity of gage.

Annual peak discharges

\begin{tabular}{|c|c|c|c|c|c|c|c|}
\hline $\begin{array}{l}\text { Water } \\
\text { year }\end{array}$ & Date & $\begin{array}{c}\text { Annual peak } \\
\text { discharge } \\
\left(\mathrm{ft}^{3} / \mathrm{s}\right)\end{array}$ & $\begin{array}{l}\text { Discharge } \\
\text { codes }\end{array}$ & $\begin{array}{l}\text { Water } \\
\text { year }\end{array}$ & Date & $\begin{array}{c}\text { Annual peak } \\
\text { discharge } \\
\left(\mathrm{ft}^{3} / \mathrm{s}\right)\end{array}$ & $\begin{array}{c}\text { Discharge } \\
\text { codes }\end{array}$ \\
\hline 1980 & $02-14-80$ & 47,400 & & 1987 & $03-05-87$ & 565 & 1987 \\
\hline 1981 & $09-05-81$ & 728 & & 1988 & $08-27-88$ & 6,410 & 1988 \\
\hline 1982 & $02-11-82$ & 5,400 & & 1989 & $01-05-89$ & 798 & 1989 \\
\hline 1983 & $03-03-83$ & 30,600 & & 1990 & $09-18-90$ & 1,410 & 1990 \\
\hline 1984 & $08-24-84$ & 3,950 & & 1991 & $03-01-91$ & 29,900 & 1991 \\
\hline 1985 & $12-27-84$ & 12,400 & & 1992 & $02-13-92$ & 12,300 & 1992 \\
\hline 1986 & $11-30-85$ & 6,210 & & 1993 & $02-08-93$ & 55,300 & 1993 \\
\hline
\end{tabular}

Discharge rating table developed September 1990

\begin{tabular}{cccc}
\hline $\begin{array}{c}\text { Gage height } \\
(\mathbf{f t})\end{array}$ & $\begin{array}{c}\text { Discharge } \\
\left(\mathbf{f t}^{\mathbf{3}} / \mathbf{s}\right)\end{array}$ & $\begin{array}{c}\text { Gage height } \\
(\mathbf{f t})\end{array}$ & $\begin{array}{c}\text { Discharge } \\
\left(\mathbf{f t}^{3} / \mathbf{s}\right)\end{array}$ \\
\hline 4.0 & 7.0 & 12.0 & 18,140 \\
5.0 & 176 & 13.0 & 24,540 \\
7.0 & 1,660 & 14.0 & 32,220 \\
9.0 & 5,580 & 15.0 & 41,270 \\
10.0 & 8,770 & 16.0 & 51,820 \\
11.0 & 12,920 & 16.4 & 56,500 \\
\hline
\end{tabular}


09424447 BURRO CREEK AT OLD U.S. 93 BRIDGE, NEAR BAGDAD, AZ--Continued

MEAN MONTHLY AND ANNUAL DISCHARGES 1981.93

\begin{tabular}{|c|c|c|c|c|c|c|}
\hline MONTH & $\begin{array}{l}\text { MAXIMUM } \\
(\text { FT3/S) }\end{array}$ & $\begin{array}{l}\text { MINIMUM } \\
\text { (FT3/S) }\end{array}$ & $\begin{array}{c}\text { MEAN } \\
(\text { FT3/S) }\end{array}$ & $\begin{array}{l}\text { STAN- } \\
\text { DARD } \\
\text { DEVIA- } \\
\text { TION } \\
\text { (FT3/S) }\end{array}$ & $\begin{array}{l}\text { COEFFI- } \\
\text { CIENT OF } \\
\text { VARI- } \\
\text { ATION }\end{array}$ & $\begin{array}{c}\text { PERCENT } \\
\text { OF } \\
\text { ANNUAL } \\
\text { RUNOFF }\end{array}$ \\
\hline OCTOBER & 69 & 0.00 & 6.2 & 19 & 3.1 & 0.5 \\
\hline NOVEMBER & 112 & 0.00 & 19 & 38 & 2.0 & 1.7 \\
\hline DECEMBER & 710 & 0.00 & 102 & 205 & 2.0 & 8.9 \\
\hline JANUARY & 2,610 & 3.3 & 256 & 715 & 2.8 & 22.4 \\
\hline FEBRUARY & 2,090 & 5.8 & 325 & 568 & 1.8 & 28.4 \\
\hline MARCH & 1,370 & 4.9 & 336 & 458 & 1.4 & 29.3 \\
\hline APRIL & 93 & 1.6 & 30 & 34 & 1.1 & 2.6 \\
\hline MAY & 15 & 0.56 & 3.8 & 3.9 & 1.0 & 0.3 \\
\hline JUNE & 4.4 & 0.04 & 0.93 & 1.2 & 1.2 & 0.1 \\
\hline JULY & 6.2 & 0.00 & 1.5 & 2.2 & 1.4 & 0.1 \\
\hline AUGUST & 188 & 0.06 & 28 & 58 & 2.1 & 2.4 \\
\hline SEPTEMBER & 403 & 0.00 & 38 & 110 & 2.9 & 3.3 \\
\hline ANNUAL & 414 & 5.6 & 95 & 116 & 1.2 & 100 \\
\hline
\end{tabular}

MAGNITUDE AND PROBABILITY OF ANNUAL LOW FLOW BASED ON PERIOD OF RECORD 1982.93

\begin{tabular}{|c|c|c|c|c|c|c|}
\hline $\begin{array}{l}\text { PERIOD } \\
\text { (CON. }\end{array}$ & \multirow{2}{*}{\multicolumn{6}{|c|}{$\begin{array}{c}\text { DISCHARGE, IN FT3/S, FOR INDICATED } \\
\text { RECURRENCE INTERVAL, IN YEARS, AND } \\
\text { NON-EXCEEDANCE PROBABILITY, IN PERCENT } \\
\ldots \ldots \ldots \ldots \ldots \ldots \ldots\end{array}$}} \\
\hline SECU - & & & & & & \\
\hline TIVE & 2 & 5 & 10 & $20 \#$ & $50 \#$ & $100 \#$ \\
\hline DAYS) & 508 & 208 & 108 & 58 & 28 & 18 \\
\hline 1 & 0.00 & 0.00 & 0.00 & 0.00 & 0.00 & 0.00 \\
\hline 3 & 0.03 & 0.00 & 0.00 & 0.00 & 0.00 & 0.00 \\
\hline 7 & 0.05 & 0.00 & 0.00 & 0.00 & 0.00 & 0.00 \\
\hline 14 & 0.10 & 0.00 & 0.00 & 0.00 & 0.00 & 0.00 \\
\hline 30 & 0.13 & 0.00 & 0.00 & 0.00 & 0.00 & 0.00 \\
\hline 60 & 0.24 & 0.00 & 0.00 & 0.00 & 0.00 & 0.00 \\
\hline 90 & 0.34 & 0.09 & 0.02 & 0.00 & 0.00 & 0.00 \\
\hline 120 & 1.0 & 0.23 & 0.04 & 0.00 & 0.00 & 0.00 \\
\hline 183 & 2.5 & 0.45 & 0.17 & 0.07 & 0.02 & 0.01 \\
\hline
\end{tabular}

MAGNITUDE AND PROBABILITY OF ANNUAL HIGH FLOW BASED ON PERIOD OF RECORD 1981.93

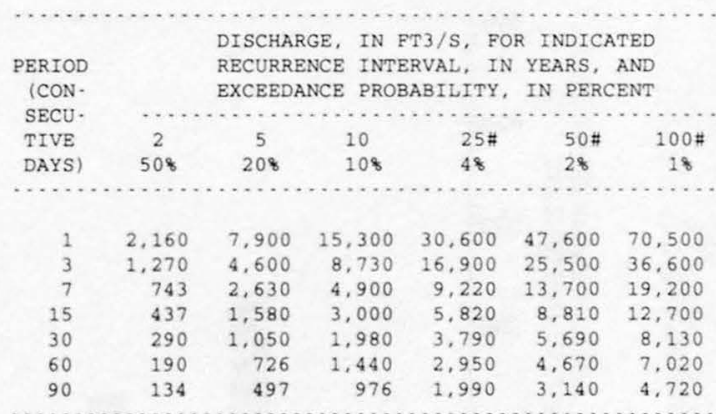

MAGNITUDE AND PROBABILITY OF INSTANTANEOUS PEAK FLOW BASED ON PERIOD OF RECORD

DISCHARGE, IN FT3/S, FOR INDICATED RECURRENCE INTERVAL IN YEARS, AND EXCEEDANCE PROBABILITY, IN PERCENT

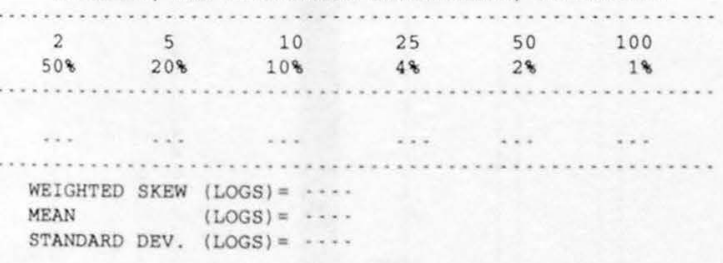

DURATION TABLE OF DAILY MEAN FLOW FOR PBRIOD OF RECORD 1981.93

DISCHARGE, IN FT3/S, WHICH WAS EQUALED OR EXCEEDED FOR INDICATED PERCENT OF TIME

\begin{tabular}{|c|c|c|c|c|c|c|c|c|c|c|c|c|c|c|c|c|}
\hline 18 & 58 & 108 & 158 & 208 & 308 & 408 & 508 & 608 & 708 & 808 & 908 & $95 \%$ & 988 & 998 & 99.58 & 99.98 \\
\hline 80 & 236 & 62 & 28 & 18 & 10 & 6.0 & 2.8 & 1.4 & 0.69 & 0.30 & 0.08 & 0.00 & 0.00 & 0.00 & 0.00 & 0.00 \\
\hline
\end{tabular}

\# Reliability of values in column is uncertain, and potential errors are large. 
09424447 BURRO CREEK AT OLD U.S. 93 BRIDGE, NEAR BAGDAD, AZ--Continued
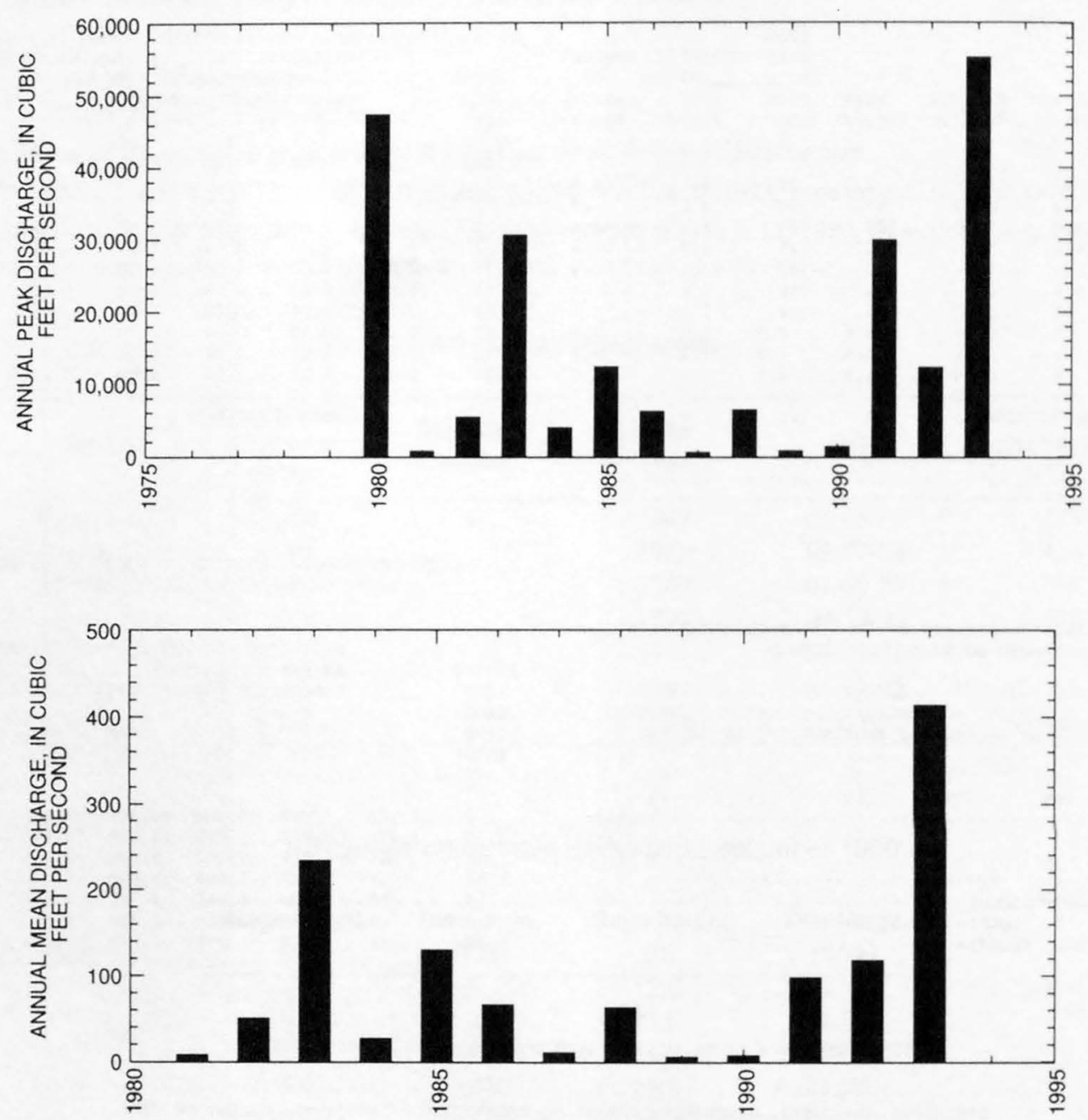
09424447 BURRO CREEK AT OLD U.S. 93 BRIDGE, NEAR BAGDAD, AZ--Continued
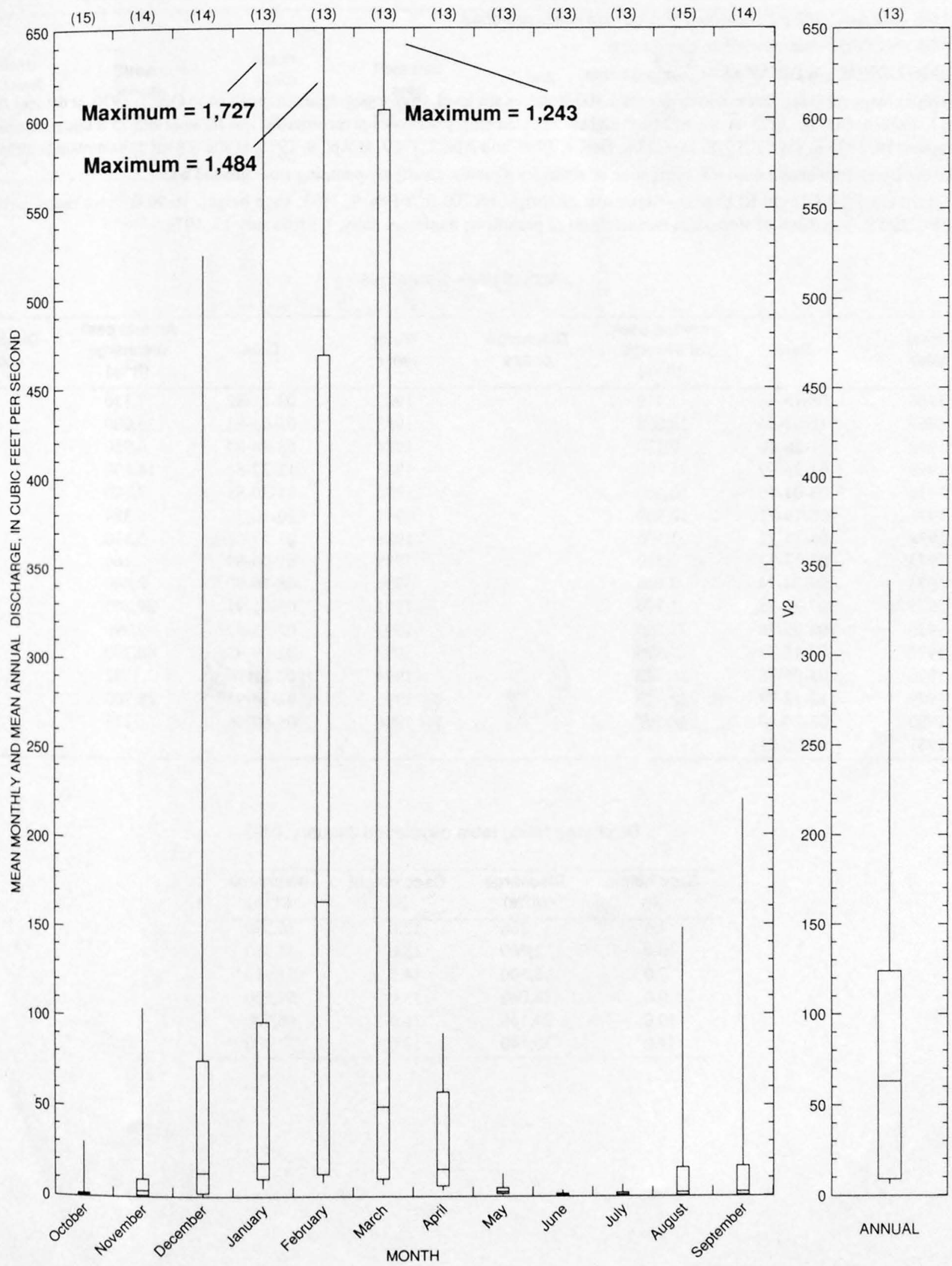


\section{BIG SANDY RIVER NEAR WIKIEUP, AZ}

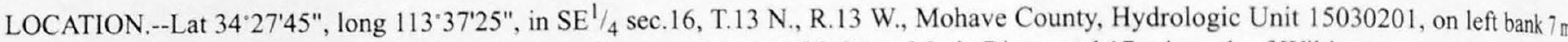
downstream from Burro Creek, 15 mi upstream from confluence with Santa Maria River, and 17 mi south of Wikieup.

DRAINAGE AREA.--2,742 $\mathrm{mi}^{2}$, of which $10.1 \mathrm{mi}^{2}$ are noncontributing.

PERIOD OF RECORD.--March 1966 to current year.

REVISED RECORDS.--WDR AZ-88-1: Drainage area.

GAGE.--Water-stage recorder. Elevation of gage is $1,400 \mathrm{ft}$, above sea level, from topographic map. Prior to Oct. 1, 1970, at datum $3.06 \mathrm{fthighe}$ Oct. 1, 1970, to Oct. 10, 1973, at datum $2.06 \mathrm{ft}$ higher. Supplementary water-stage recorder for low flows at site $75 \mathrm{ft}$ upstream at same datur from Apr. 10, 1975 to Mar. 1, 1978; Mar. 28 to Dec. 7, 1966, and Apr. 2, 1969, to Apr. 9, 1975, at site 0.8 mi downstream at different datum REMARKS.--Diversions above station for irrigation of about 3,800 acres, mostly by pumping from ground water.

EXTREMES FOR PERIOD OF RECORD.--Maximum discharge, $68,700 \mathrm{ft}^{3} / \mathrm{s}$ Feb. 9, 1993, gage height, $16.00 \mathrm{ft}$, from rating curve extende above $2,200 \mathrm{ft}^{3} / \mathrm{s}$ on basis of slope-area measurement of peak flow; minimum daily, $1.3 \mathrm{ft}^{3} / \mathrm{s} \mathrm{July} 13,1974$.

Annual peak discharges

\begin{tabular}{|c|c|c|c|c|c|c|c|}
\hline $\begin{array}{l}\text { Water } \\
\text { year }\end{array}$ & Date & $\begin{array}{c}\text { Annual peak } \\
\text { discharge } \\
\left(\mathrm{ft}^{3} / \mathrm{s}\right)\end{array}$ & $\begin{array}{c}\text { Discharge } \\
\text { codes }\end{array}$ & $\begin{array}{l}\text { Water } \\
\text { year }\end{array}$ & Date & $\begin{array}{c}\text { Annual peak } \\
\text { discharge } \\
\left(\mathrm{ft}^{3} / \mathrm{s}\right)\end{array}$ & $\begin{array}{c}\text { Discharge } \\
\text { codes }\end{array}$ \\
\hline 1966 & $08-15-66$ & 1,410 & & 1982 & $02-11-82$ & 7,330 & \\
\hline 1967 & $12-07-66$ & 28,000 & & 1983 & $03-03-83$ & 25,000 & \\
\hline 1968 & $01-28-68$ & 9,520 & & 1984 & $08-18-84$ & 4,950 & \\
\hline 1969 & $01-26-69$ & 11,100 & & 1985 & $12-27-84$ & 14,600 & \\
\hline 1970 & $03-01-70$ & 10,000 & & 1986 & $11-30-85$ & 7,680 & \\
\hline 1971 & $08-19-71$ & 10,300 & & 1987 & $10-10-86$ & 384 & \\
\hline 1972 & $08-13-72$ & 1,300 & & 1988 & $08-27-88$ & 5,310 & \\
\hline 1973 & $03-13-73$ & 5,310 & & 1989 & $01-06-89$ & 166 & \\
\hline 1974 & $09-24-74$ & 3,000 & & 1990 & $08-16-90$ & 2,040 & \\
\hline 1975 & $07-29-75$ & 1,720 & & 1991 & $03-01-91$ & 29,800 & \\
\hline 1976 & $02-09-76$ & 23,700 & & 1992 & $02-13-92$ & 9,990 & \\
\hline 1977 & $08-15-77$ & 3,370 & & 1993 & $02-09-93$ & 68,700 & \\
\hline 1978 & $03-01-78$ & 36,500 & & 1994 & $02-11-94$ & 52 & \\
\hline 1979 & $12-18-78$ & 28,400 & & 1995 & $02-14-95$ & 28,700 & \\
\hline 1980 & $02-20-80$ & 38,500 & & 1996 & $09-09-96$ & 275 & \\
\hline 1981 & $09-06-81$ & 437 & & & & & \\
\hline
\end{tabular}

Discharge rating table developed January 1993

\begin{tabular}{cccc}
\hline $\begin{array}{c}\text { Gage height } \\
(\mathbf{f t})\end{array}$ & $\begin{array}{c}\text { Discharge } \\
\left(\mathbf{f t}^{3} / \mathbf{s}\right)\end{array}$ & $\begin{array}{c}\text { Gage height } \\
(\mathbf{f t})\end{array}$ & $\begin{array}{c}\text { Discharge } \\
\left(\mathrm{ft}^{3} / \mathbf{s}\right)\end{array}$ \\
\hline 3.0 & 256 & 12.0 & 36,350 \\
5.0 & 2,700 & 13.0 & 43,330 \\
7.0 & 8,500 & 14.0 & 51,910 \\
9.0 & 18,060 & 15.0 & 59,550 \\
10.0 & 24,160 & 16.0 & 68,700 \\
11.0 & 30,140 & 17.0 & 77,000 \\
\hline
\end{tabular}


09424450 BIG SANDY RIVER NEAR WIKIEUP, AZ--Continued

Basin characteristics

\begin{tabular}{ccccccc}
\hline $\begin{array}{c}\text { Main } \\
\text { channel } \\
\text { slope } \\
(\mathrm{ft} / \mathrm{mi})\end{array}$ & $\begin{array}{c}\text { Stream } \\
\text { length } \\
\text { (mi) }\end{array}$ & $\begin{array}{c}\text { Mean } \\
\text { basin } \\
\text { elevation } \\
(\mathrm{ft})\end{array}$ & $\begin{array}{c}\text { Forested } \\
\text { area } \\
\text { (percent) }\end{array}$ & $\begin{array}{c}\text { Soil } \\
\text { index }\end{array}$ & $\begin{array}{c}\text { Mean } \\
\text { annual } \\
\text { precipitation } \\
\text { (in) }\end{array}$ & $\begin{array}{c}\text { R-year } \\
\text { (in) }\end{array}$ \\
\hline 41.9 & 58.8 & 4,490 & 46.0 & 1.7 & 14.2 & $\begin{array}{c}50 \text {-year } \\
\text { (in) }\end{array}$ \\
\hline
\end{tabular}




\section{BIG SANDY RIVER NEAR WIKIEUP, AZ--Continued}

MEAN MONTHLY AND ANNUAL DISCHARGES 1967-96

\begin{tabular}{|c|c|c|c|c|c|c|}
\hline MONTH & $\begin{array}{l}\text { MAXIMUM } \\
\text { (ET3/S) }\end{array}$ & $\begin{array}{l}\text { MINIMUM } \\
\text { (FT3/S) }\end{array}$ & $\begin{array}{c}\text { MEAN } \\
(\text { FT } 3 / \mathrm{S})\end{array}$ & $\begin{array}{l}\text { STAN- } \\
\text { DARD } \\
\text { DEVIA- } \\
\text { TION } \\
(\text { FT } 3 / S)\end{array}$ & $\begin{array}{l}\text { COEFFI- } \\
\text { CIENT OF } \\
\text { VARI- } \\
\text { ATION }\end{array}$ & $\begin{array}{c}\text { PERCENT } \\
\text { OF } \\
\text { ANNUAL } \\
\text { RUNOFF }\end{array}$ \\
\hline OCTOBER & 100 & 2.7 & 10 & 21 & 2.1 & 0.9 \\
\hline NOVEMBER & 252 & 2.4 & 22 & 53 & 2.4 & 2.0 \\
\hline DECEMBER & 737 & 2.8 & 84 & 186 & 2.2 & 7.5 \\
\hline JANUARY & 2,670 & 2.4 & 195 & 508 & 2.6 & 17.3 \\
\hline FEBRUARY & 3,890 & 3.5 & 396 & 862 & 2.2 & 35.1 \\
\hline MARCH & 1,940 & 4.1 & 323 & 479 & 1.5 & 28.6 \\
\hline APRIL & 153 & 3.6 & 36 & 42 & 1.2 & 3.2 \\
\hline MAY & 36 & 2.0 & 9.2 & 8.2 & 0.89 & 0.8 \\
\hline JUNE & 14 & 2.1 & 5.2 & 2.6 & 0.50 & 0.5 \\
\hline JULY & 21 & 1.9 & 5.6 & 4.1 & 0.73 & 0.5 \\
\hline AUGUST & 178 & 2.7 & 24 & 43 & 1.8 & 2.1 \\
\hline SEPTEMBER & 226 & 2.8 & 18 & 40 & 2.3 & 1.6 \\
\hline ANNUAL & 586 & 3.9 & 93 & 125 & 1.3 & 100 \\
\hline
\end{tabular}

MAGNITUDE AND PROBABILITY OF INSTANTANEOUS PEAK FLOW BASED ON PERIOD OF RECORD 1966.96

DISCHARGE, IN FT3/S, FOR INDICATED RECURRENCE INTERVAL IN YEARS, AND EXCEEDANCE PROBABILITY, IN PERCENT

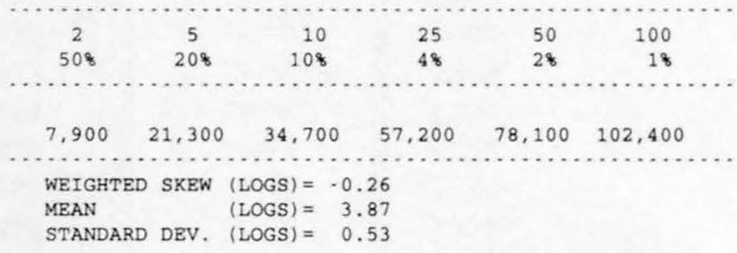

MAGNITUDE AND PROBABILITY OF ANNUAL LOW FLOW BASED ON PERIOD OF RECORD 1967.96

\begin{tabular}{|c|c|c|c|c|c|c|}
\hline \multirow{3}{*}{$\begin{array}{l}\text { PERIOD } \\
\text { (CON. } \\
\text { SECU. }\end{array}$} & \multicolumn{6}{|c|}{ DISCHARGE, IN FT $3 / S$, FOR INDICATED } \\
\hline & & $\begin{array}{l}\text { RECURRENCE INTERVAL, I } \\
\text { NON-EXCEEDANCE PROBABII }\end{array}$ & NCE & ABILI & \multicolumn{2}{|c|}{$\begin{array}{l}\text { ARS, AND } \\
\text { IN PERCENT }\end{array}$} \\
\hline & & $\ldots \ldots$ & $\cdots \cdots$ & …. & .... & \\
\hline TIVE & 2 & 5 & 10 & 20 & 50 & $100 \#$ \\
\hline DAYS) & 508 & 208 & 108 & 58 & 28 & 18 \\
\hline 1 & 2.5 & 1.9 & 1.6 & 1.4 & 1.2 & 1.1 \\
\hline 3 & 2.7 & 2.0 & 1.7 & 1.5 & 1.3 & 1.1 \\
\hline 7 & 2.8 & 2.2 & 1.8 & 1.6 & 1.4 & 1.2 \\
\hline 14 & 3.0 & 2.3 & 2.0 & 1.7 & 1.5 & 1.3 \\
\hline 30 & 3.3 & 2.5 & 2.2 & 1.9 & 1.7 & 1.5 \\
\hline 60 & 3.7 & 3.0 & 2.6 & 2.3 & 2.0 & 1.8 \\
\hline 90 & 4.1 & 3.3 & 2.9 & 2.6 & 2.3 & 2.1 \\
\hline 120 & 4.3 & 3.4 & 3.1 & 2.8 & 2.6 & 2.4 \\
\hline 183 & 5.3 & 3.9 & 3.6 & 3.4 & 3.3 & 3.3 \\
\hline
\end{tabular}

MAGNITUDE AND PROBABILITY OF ANNUAL HIGH FLOW BASED ON PERIOD OF RECORD 1967.96

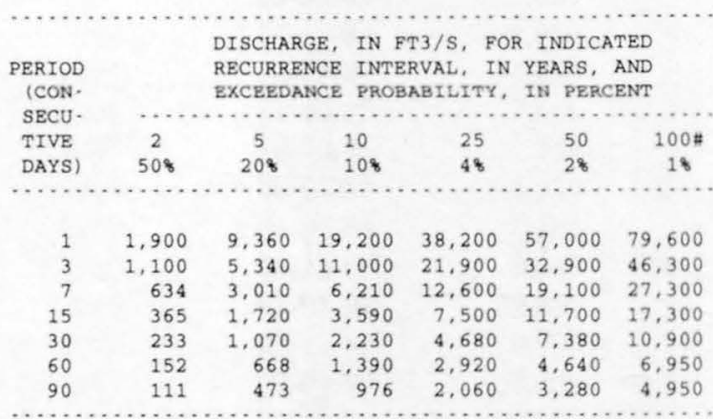

DURATION TABLE OF DAILY MEAN FLOW FOR PERIOD OF RECORD 1967.96

DISCHARGE, IN FT3/S, WHICH WAS EQUALED OR EXCEEDED FOR INDICATED PERCENT OF TIME

\begin{tabular}{|c|c|c|c|c|c|c|c|c|c|c|c|c|c|c|c|c|}
\hline 18 & 58 & 108 & 158 & 208 & 308 & 408 & 508 & 608 & 708 & 808 & 908 & 958 & 988 & 998 & 99.58 & 99.98 \\
\hline 880 & 218 & 64 & 29 & 18 & 8.9 & 6.3 & 5.3 & 4.8 & 4.3 & 3.7 & 3.1 & 2.6 & 2.2 & 1.9 & 1.8 & 1.5 \\
\hline
\end{tabular}

\# Reliability of values in column is uncertain, and potential errors are large. 
BILL WILLIAMS RIVER BASIN

09424450 BIG SANDY RIVER NEAR WIKIEUP, AZ--Continued
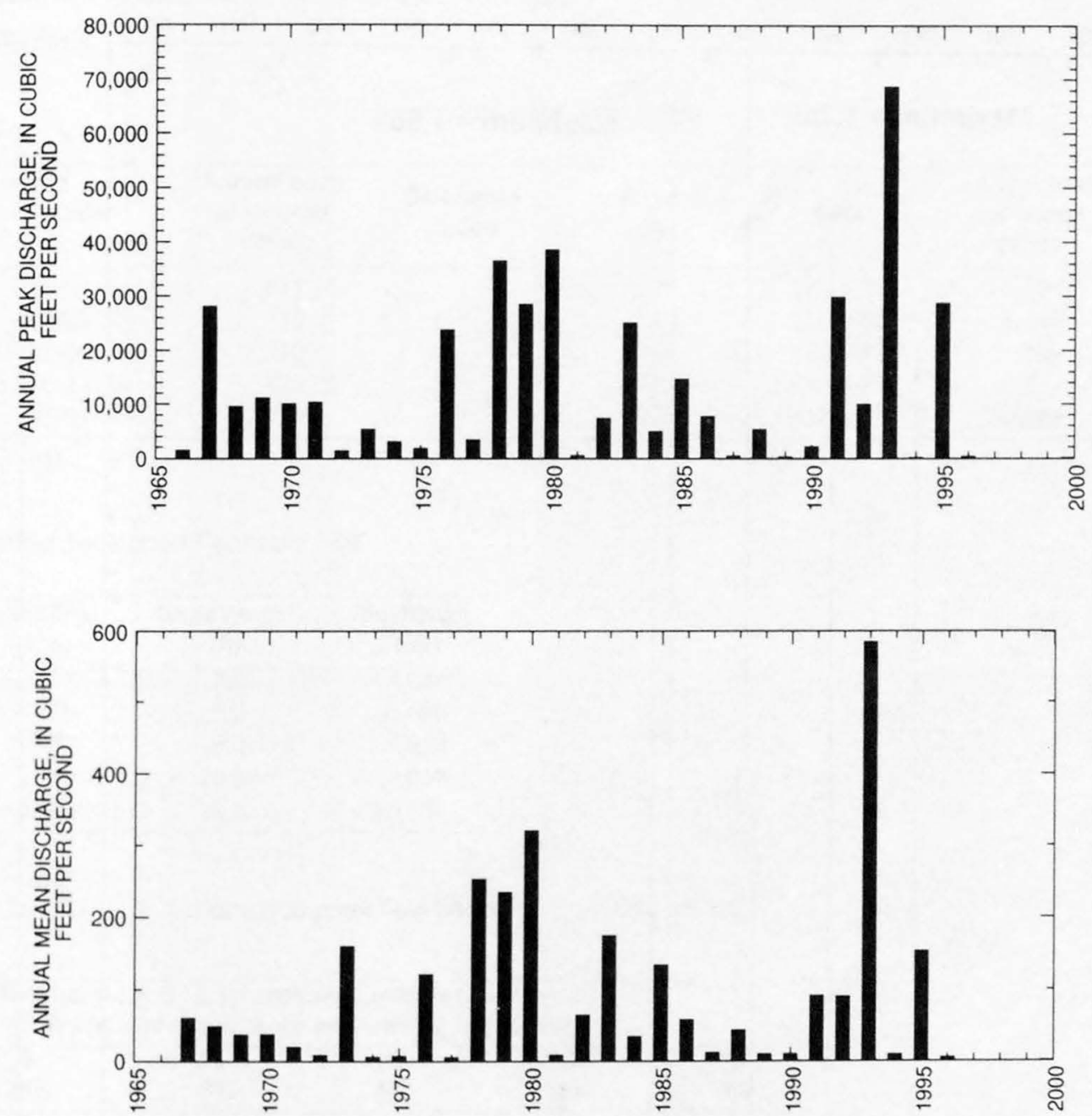
09424450 BIG SANDY RIVER NEAR WIKIEUP, AZ--Continued

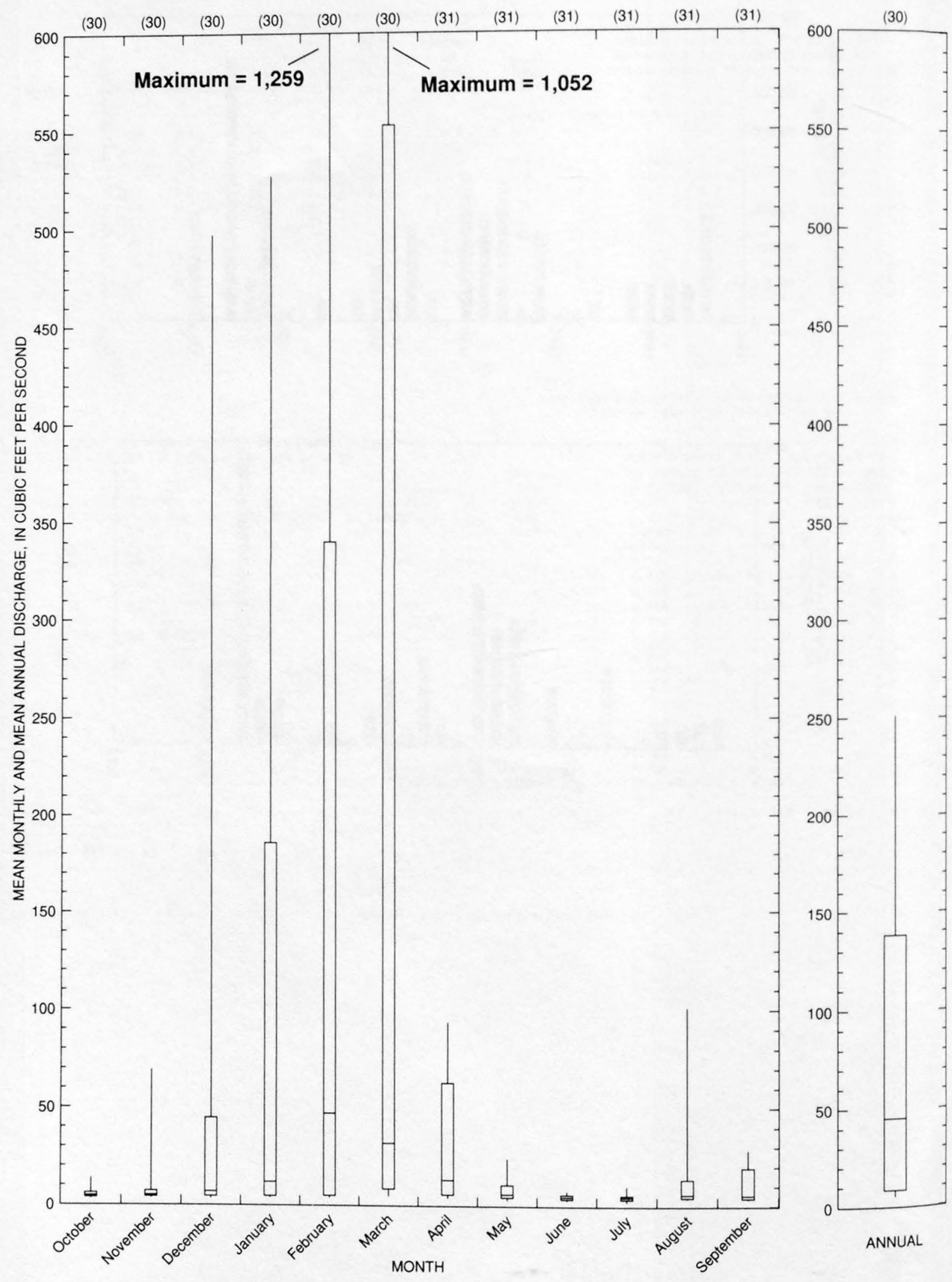




\section{KIRKLAND CREEK NEAR KIRKLAND, AZ}

LOCATION.--Lat 34'23'38", long 112 43'19", in SW'/4 sec.7, T.12 N., R.4 W., Yavapai County, Hydrologic Unit 15030203 , on right bank 1.3 mi upstream from Skull Valley Wash and $1.7 \mathrm{mi}$ southwest of Kirkland.

DRAINAGE AREA.-- $109 \mathrm{mi}^{2}$.

Annual peak discharges

\begin{tabular}{ccccccc}
\hline $\begin{array}{c}\text { Water } \\
\text { year }\end{array}$ & Date & $\begin{array}{c}\text { Annual peak } \\
\text { discharge } \\
\left(\mathbf{f t}^{3} / \mathbf{s}\right)\end{array}$ & $\begin{array}{c}\text { Discharge } \\
\text { codes }\end{array}$ & $\begin{array}{c}\text { Water } \\
\text { year }\end{array}$ & $\begin{array}{c}\text { Annual peak } \\
\text { discharge } \\
\left(\mathrm{ft}^{3} / \mathbf{s}\right)\end{array}$ & $\begin{array}{c}\text { Discharge } \\
\mathbf{c o d e s}^{-2}\end{array}$ \\
\hline 1974 & $08-02-74$ & 615 & & 1979 & $11-11-78$ & 10,300 \\
1975 & $07-28-75$ & 776 & & 1980 & $02-19-80$ & 8,440 \\
1976 & $02-09-76$ & 2,710 & & 1981 & $07-11-81$ & 706 \\
1977 & $10-23-76$ & 328 & & 1982 & $03-15-82$ & 520 \\
1978 & $03-01-78$ & 7,890 & & 1983 & $03-03-83$ & 2,980 \\
\hline
\end{tabular}

Discharge rating table developed February 1980

\begin{tabular}{cccc}
\hline $\begin{array}{c}\text { Gage height } \\
(\mathrm{ft})\end{array}$ & $\begin{array}{c}\text { Discharge } \\
\left(\mathrm{ft}^{3} / \mathbf{s}\right)\end{array}$ & $\begin{array}{c}\text { Gage height } \\
(\mathrm{ft})\end{array}$ & $\begin{array}{c}\text { Discharge } \\
\left(\mathrm{ft}^{3} / \mathbf{s}\right)\end{array}$ \\
\hline 2.0 & 152 & 7.0 & 4,040 \\
3.0 & 450 & 8.0 & 5,680 \\
4.0 & 957 & 9.0 & 7,650 \\
5.0 & 1,710 & 10.0 & 10,000 \\
6.0 & 2,730 & 10.2 & 10,500 \\
\hline
\end{tabular}

Magnitude and probability of instantaneous peak flow based on period of record

\begin{tabular}{|c|c|c|c|c|c|}
\hline \multicolumn{6}{|c|}{$\begin{array}{l}\text { Discharge, in } \mathrm{ft}^{3} / \mathrm{s} \text {, for indicated recurrence interval } \\
\text { in years, and exceedance probablility, in percent }\end{array}$} \\
\hline 2 & 5 & 10 & 25 & $50 t$ & $100 t$ \\
\hline $50 \%$ & $20 \%$ & $10 \%$ & $4 \%$ & $2 \%$ & $1 \%$ \\
\hline … & -.. & $\ldots$ & $\ldots$ & $\ldots$ &.-- \\
\hline $\begin{array}{l}\text { Weighted skew } \\
\text { Mean } \\
\text { Standard dev. }\end{array}$ & $\begin{array}{l}(\log s)= \\
(\log s)= \\
(\log s)=\end{array}$ & $-\cdots$ & & & \\
\hline
\end{tabular}

Basin characteristics

\begin{tabular}{cccccccc}
\hline $\begin{array}{c}\text { Main } \\
\text { channel } \\
\text { slope } \\
(\mathrm{ft} / \mathrm{mi})\end{array}$ & $\begin{array}{c}\text { Stream } \\
\text { length } \\
(\mathrm{mi})\end{array}$ & $\begin{array}{c}\text { Mean } \\
\text { basin } \\
\text { elevation } \\
(\mathrm{ft})\end{array}$ & $\begin{array}{c}\text { Forested } \\
\text { area } \\
\text { (percent) }\end{array}$ & $\begin{array}{c}\text { Soil } \\
\text { index }\end{array}$ & $\begin{array}{c}\text { Mean } \\
\text { annual } \\
\text { precipitation } \\
\text { (in) }\end{array}$ & $\begin{array}{c}\text { R-year } \\
\text { (in) }\end{array}$ & $\begin{array}{c}50 \text {-year } \\
\text { (in) }\end{array}$ \\
\hline 40.5 & 23 & 4,665 & 72.0 & 1.0 & 18.4 & 1.4 \\
\hline
\end{tabular}


09424470 KIRKLAND CREEK NEAR KIRKLAND, AZ--Continued

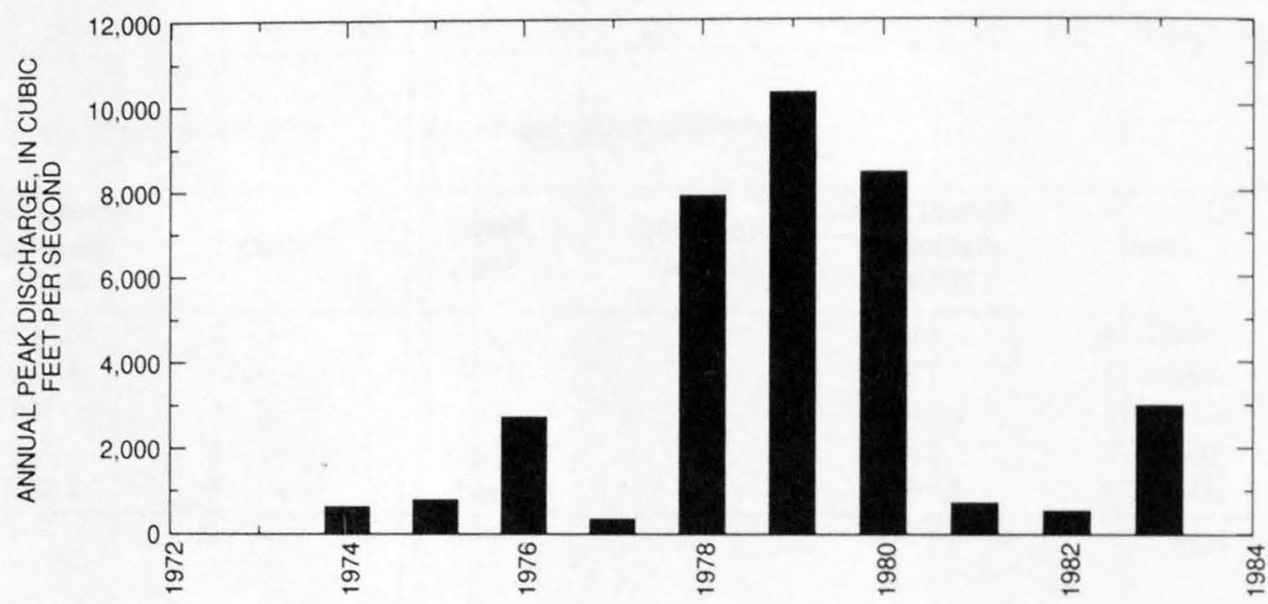


09424480 ASH CREEK NEAR KIRKLAND, AZ

LOCATION.--Lat $34^{\circ} 27^{\prime} 12^{\prime \prime}$, long $112^{\circ} 47^{\prime} 45^{\prime \prime}$, in NW $\frac{1}{4}$ sec.21, T.13 N., R.5 W., Yavapai County, Hydrologic Unit 15030203 , at State Highway 96, 5.5 mi west of Kirkland.

DRAINAGE AREA.--6.95 $\mathrm{mi}^{2}$.

Annual peak discharges

\begin{tabular}{|c|c|c|c|c|c|c|c|}
\hline $\begin{array}{l}\text { Water } \\
\text { year }\end{array}$ & Date & $\begin{array}{c}\text { Annual peak } \\
\text { discharge } \\
\left(\mathrm{ft}^{3} / \mathrm{s}\right)\end{array}$ & $\begin{array}{c}\text { Discharge } \\
\text { codes }\end{array}$ & $\begin{array}{l}\text { Water } \\
\text { year }\end{array}$ & Date & $\begin{array}{c}\text { Annual peak } \\
\text { discharge } \\
\left(\mathrm{ft}^{3} / \mathrm{s}\right)\end{array}$ & $\begin{array}{l}\text { Discharge } \\
\text { codes }\end{array}$ \\
\hline 1960 & $00-00-60$ & 4,000 & HP & 1971 & $08-19-71$ & 300 & ES \\
\hline 1963 & $08-20-63$ & 109 & & 1972 & $08-13-72$ & 20 & \\
\hline 1964 & $09-13-64$ & 1,020 & & 1973 & $10-07-72$ & 60 & ES \\
\hline 1965 & $09-03-65$ & 140 & & 1974 & $00-00-74$ & 15 & ES \\
\hline 1966 & $12-10-65$ & 230 & & 1975 & $11-02-74$ & 20 & ES \\
\hline 1967 & $07-15-67$ & 225 & & 1976 & $09-25-76$ & 240 & \\
\hline 1968 & $08-12-68$ & 140 & & 1978 & $03-01-78$ & 300 & \\
\hline 1969 & $08-19-69$ & 250 & & 1979 & $12-18-78$ & 205 & \\
\hline 1970 & $08-00-70$ & 15 & ES & & & & \\
\hline
\end{tabular}

Magnitude and probability of instantaneous peak flow based on period of record 1960 , 1963-76, 1978-79

\begin{tabular}{|c|c|c|c|c|c|}
\hline \multicolumn{6}{|c|}{$\begin{array}{l}\text { Discharge, in } \mathrm{ft}^{3} / \mathrm{s} \text {, for indicated recurrence interval } \\
\text { in years, and exceedance probablility, in percent }\end{array}$} \\
\hline 2 & 5 & 10 & 25 & $50 \dagger$ & $100 t$ \\
\hline $50 \%$ & $20 \%$ & $10 \%$ & $4 \%$ & $2 \%$ & $1 \%$ \\
\hline 133 & 459 & 882 & 1,780 & 2,810 & 4,250 \\
\hline Weighted skew & $(\log s)=$ & 0.05 & & & \\
\hline Mean & $(\log s)=$ & 2.13 & & & \\
\hline Standard dev. & $(\log s)=$ & 0.63 & & & \\
\hline
\end{tabular}

Basin characteristics

\begin{tabular}{cccccccc}
\hline $\begin{array}{c}\text { Main } \\
\text { channel } \\
\text { slope } \\
(\mathrm{ft} / \mathrm{mi})\end{array}$ & $\begin{array}{c}\text { Stream } \\
\text { length } \\
(\mathrm{mi})\end{array}$ & $\begin{array}{c}\text { Mean } \\
\text { basin } \\
\text { elevation } \\
(\mathrm{ft})\end{array}$ & $\begin{array}{c}\text { Forested } \\
\text { area } \\
\text { (percent) }\end{array}$ & $\begin{array}{c}\text { Soil } \\
\text { index }\end{array}$ & $\begin{array}{c}\text { Mean } \\
\text { annual } \\
\text { precipitation } \\
\text { (in) }\end{array}$ & $\begin{array}{c}\text { Rainfall intensity, 24-hour } \\
\text { (in) }\end{array}$ & $\begin{array}{c}\text { 50-year } \\
\text { (in) }\end{array}$ \\
\hline 278 & 5.3 & 4,680 & 1.4 & 1.0 & 10.4 & 1.9 \\
\hline
\end{tabular}


09424480 ASH CREEK NEAR KIRKLAND, AZ--Continued

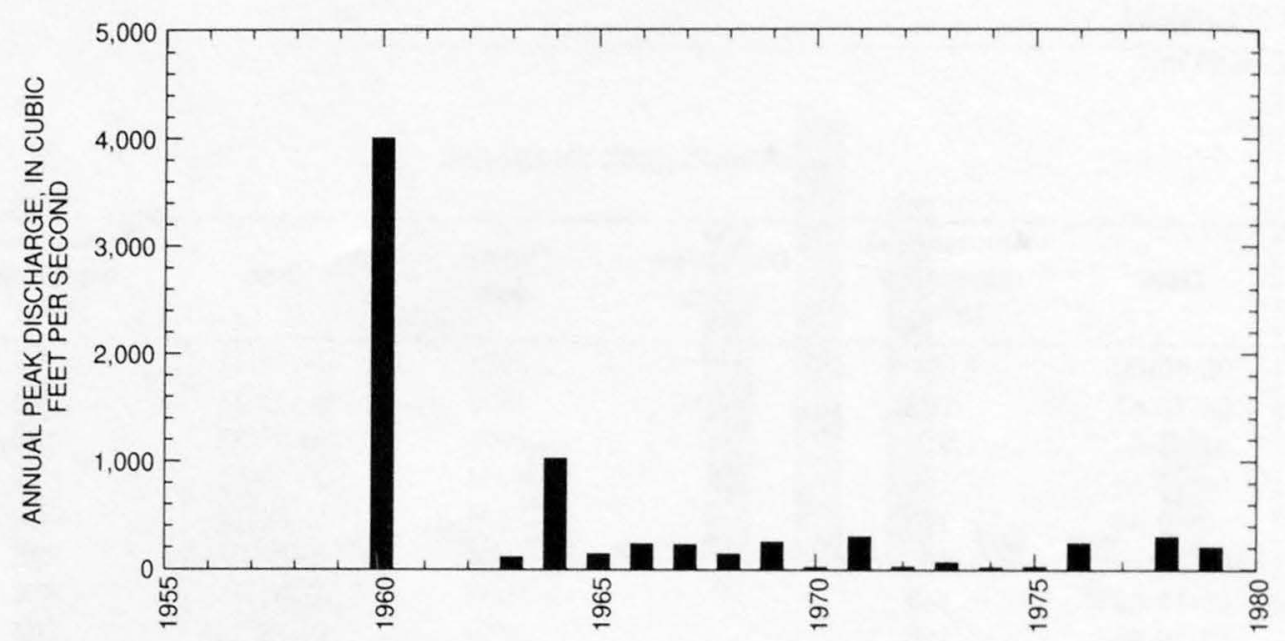


09424700 IRON SPRING WASH TRIBUTARY NEAR BAGDAD, AZ

LOCATION.--Lat $34^{\circ} 31^{\prime} 20^{\prime \prime}$, long $113^{\circ} 06^{\prime} 43^{\prime \prime}$, in NE $1 / 4 \mathrm{SE}^{1} / 4 \mathrm{sec} .29$, T.14 N., R.8 W., Yavapai County, Hydrologic Unit 15030203 , at State Highway $96,6.6$ mi southeast of Bagdad.

DRAINAGE AREA.--0.64 $\mathrm{mi}^{2}$.

Annual peak discharges

\begin{tabular}{|c|c|c|c|c|c|c|c|}
\hline $\begin{array}{l}\text { Water } \\
\text { year }\end{array}$ & Date & $\begin{array}{c}\text { Annual peak } \\
\text { discharge } \\
\left(\mathrm{ft}^{3} / \mathrm{s}\right)\end{array}$ & $\begin{array}{c}\text { Discharge } \\
\text { codes }\end{array}$ & $\begin{array}{l}\text { Water } \\
\text { year }\end{array}$ & Date & $\begin{array}{c}\text { Annual peak } \\
\text { discharge } \\
\left(\mathrm{ft}^{3} / \mathrm{s}\right)\end{array}$ & $\begin{array}{c}\text { Discharge } \\
\text { codes }\end{array}$ \\
\hline 1964 & $09-25-64$ & 150 & & 1972 & $08-00-72$ & 0.5 & \\
\hline 1965 & $04-04-65$ & 10 & ES & 1973 & $10-00-72$ & 25 & ES \\
\hline 1966 & $11-23-65$ & 65 & & 1974 & $08-05-74$ & 4.0 & ES \\
\hline 1967 & $12-06-66$ & 10 & & 1975 & $11-02-74$ & 1.0 & ES \\
\hline 1968 & $08-12-68$ & 85 & & 1976 & $02-06-76$ & 9.0 & \\
\hline 1969 & $01-14-69$ & 5.0 & ES & 1978 & $03-01-78$ & 180 & \\
\hline 1970 & $08-12-70$ & 6.0 & ES & 1979 & $00-00-79$ & 125 & \\
\hline 1971 & $08-00-71$ & 0.6 & ES & & & & \\
\hline
\end{tabular}

Magnitude and probability of instantaneous peak flow based on period of record 1964-76, 1978-79

\begin{tabular}{|c|c|c|c|c|c|}
\hline \multicolumn{6}{|c|}{$\begin{array}{l}\text { Discharge, in } \mathrm{ft}^{3} / \mathrm{s} \text {, for indicated recurrence interval } \\
\text { in years, and exceedance probablility, in percent }\end{array}$} \\
\hline 2 & 5 & 10 & 25 & $50 t$ & $100 t$ \\
\hline $50 \%$ & $20 \%$ & $10 \%$ & $4 \%$ & $2 \%$ & $1 \%$ \\
\hline 12.4 & 63.5 & 146 & 348 & 607 & 993 \\
\hline Weighted skew & $(\log s)=$ & -0.11 & & & \\
\hline Mean & $(\log s)=$ & 1.08 & & & \\
\hline Standard dev. & $(\log s)=$ & 0.86 & & & \\
\hline
\end{tabular}

fReliability of values in column is uncertain, and potential errors are large.

Basin characteristics

\begin{tabular}{|c|c|c|c|c|c|c|c|}
\hline \multirow[b]{2}{*}{$\begin{array}{l}\text { Main } \\
\text { channel } \\
\text { slope } \\
\text { (ft/mi) }\end{array}$} & \multirow[b]{2}{*}{$\begin{array}{l}\text { Stream } \\
\text { length } \\
\text { (mi) }\end{array}$} & \multirow[b]{2}{*}{$\begin{array}{c}\text { Mean } \\
\text { basin } \\
\text { elevation } \\
\text { (ft) }\end{array}$} & \multirow[b]{2}{*}{$\begin{array}{l}\text { Forested } \\
\text { area } \\
\text { (percent) }\end{array}$} & \multirow[b]{2}{*}{$\begin{array}{c}\text { Soil } \\
\text { index }\end{array}$} & \multirow[b]{2}{*}{$\begin{array}{c}\text { Mean } \\
\text { annual } \\
\text { precipitation } \\
\text { (in) }\end{array}$} & \multicolumn{2}{|c|}{ Rainfall intensity, 24-hour } \\
\hline & & & & & & $\begin{array}{l}\text { 2-year } \\
\text { (in) }\end{array}$ & $\begin{array}{l}\text { 50-year } \\
\text { (in) }\end{array}$ \\
\hline 431 & 2.6 & 3,470 & 0.0 & 1.0 & 12.1 & 1.9 & 4.0 \\
\hline
\end{tabular}

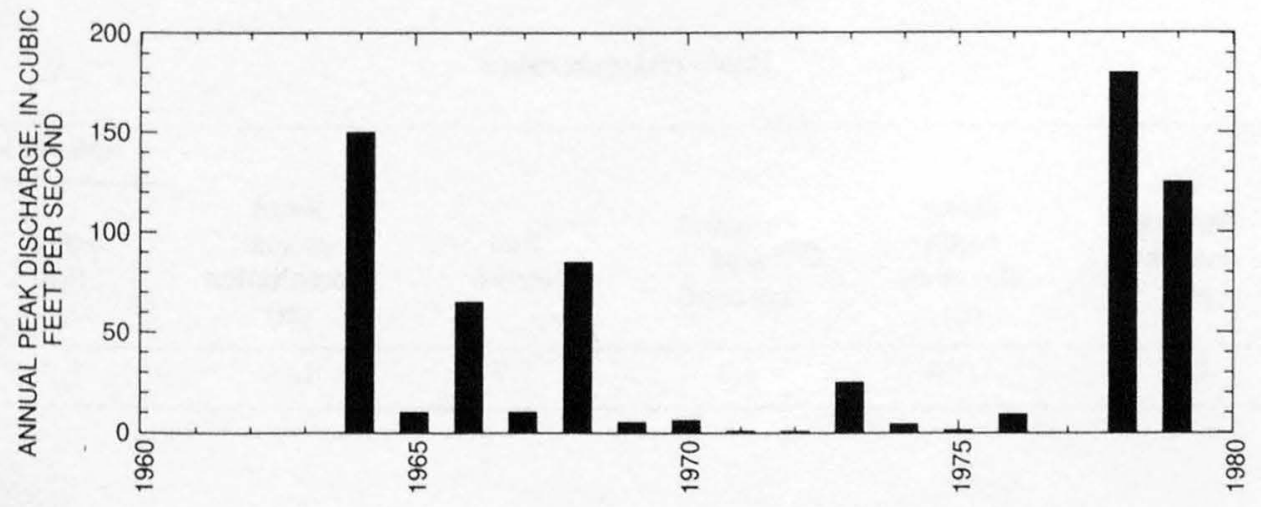




\section{SANTA MARIA RIVER NEAR BAGDAD, AZ}

LOCATION.--Lat 34'18'21", long 113'20'47", in SE $\frac{1}{4}$ sec.12, T.11 N., R.11 W., Mohave County, Hydrologic Unit 15030203 , on right bank 4.0 mi east of Palmerita Ranch, 12 mi upstream from confluence with Big Sandy River, and 21 mi southwest of Bagdad.

DRAINAGE AREA.--1,129 $\mathrm{mi}^{2}$.

PERIOD OF RECORD.--April 1966 to September 1985, October 1988 to current year.

REVISED RECORDS.--WDR AZ-89-1: Drainage area.

GAGE.--Water-stage recorder. Elevation of gage is $1,360 \mathrm{ft}$ above sea level, from topographic map.

REMARKS.--Records fair. Diversions above station for irrigation of about 5,300 acres, most of which is by pumping from ground water.

EXTREMES FOR PERIOD OF RECORD.--Maximum discharge, 23,100 ft $3 / \mathrm{s} \mathrm{Mar.} \mathrm{1,} \mathrm{1978,} \mathrm{gage} \mathrm{height,} 7.82 \mathrm{ft}$, from rating curve extended above $5,000 \mathrm{ft}^{3} / \mathrm{s}$ on basis of step-backwater computations and slope-area measurements at gage heights 5.50 and $7.82 \mathrm{ft}$; no flow for many days in most years.

Annual peak discharges

\begin{tabular}{|c|c|c|c|c|c|c|c|}
\hline $\begin{array}{l}\text { Water } \\
\text { year }\end{array}$ & Date & $\begin{array}{c}\text { Annual peak } \\
\text { discharge } \\
\left(\mathrm{ft}^{3} / \mathrm{s}\right)\end{array}$ & $\begin{array}{l}\text { Discharge } \\
\text { codes }\end{array}$ & $\begin{array}{l}\text { Water } \\
\text { year }\end{array}$ & Date & $\begin{array}{c}\text { Annual peak } \\
\text { discharge } \\
\left(\mathrm{ft}^{3} / \mathrm{s}\right)\end{array}$ & $\begin{array}{c}\text { Discharge } \\
\text { codes }\end{array}$ \\
\hline 1967 & $12-07-66$ & 13,500 & \multirow{14}{*}{ ES } & 1981 & $03-06-81$ & 191 & \multirow{14}{*}{ HP } \\
\hline 1968 & $01-28-68$ & 6,500 & & 1982 & $02-11-82$ & 6,750 & \\
\hline 1969 & $01-26-69$ & 7,200 & & 1983 & $09-24-83$ & 15,200 & \\
\hline 1970 & $03-03-70$ & 1,420 & & 1984 & $08-17-84$ & 5,450 & \\
\hline 1971 & $08-25-71$ & 2,940 & & 1985 & $02-10-85$ & 10,400 & \\
\hline 1972 & $08-13-72$ & 1,350 & & 1989 & $02-06-89$ & 394 & \\
\hline 1973 & $10-19-72$ & 11,000 & & 1990 & $10-04-89$ & 6,650 & \\
\hline 1974 & $08-05-74$ & 600 & & 1991 & $03-01-91$ & 18,900 & \\
\hline 1975 & $07-29-75$ & 1,890 & & 1992 & $08-23-92$ & 9,310 & \\
\hline 1976 & $02-09-76$ & 11,800 & & 1993 & $02-09-93$ & 15,700 & \\
\hline 1977 & $09-11-77$ & 1,500 & & 1994 & 09-03-94 & 2,170 & \\
\hline 1978 & 03-01-78 & 23,100 & & 1995 & $02-14-95$ & 8,790 & \\
\hline 1979 & $12-18-78$ & 17,200 & & 1996 & $00-00-96$ & 0 & \\
\hline 1980 & $01-30-80$ & 19,800 & & & & & \\
\hline
\end{tabular}

Discharge rating table developed January 1993

\begin{tabular}{crcc}
\hline $\begin{array}{c}\text { Gage height } \\
(\mathbf{f t})\end{array}$ & $\begin{array}{c}\text { Discharge } \\
\left(\mathbf{f t}^{\mathbf{3}} \mathbf{s}\right)\end{array}$ & $\begin{array}{c}\text { Gage height } \\
(\mathbf{f t})\end{array}$ & $\begin{array}{c}\text { Discharge } \\
\left(\mathbf{f t}^{\mathbf{3}} \mathbf{s}\right)\end{array}$ \\
\hline 1.8 & 233 & 8.0 & 16,360 \\
2.0 & 350 & 9.0 & 21,000 \\
3.0 & 1,340 & 10.0 & 26,330 \\
4.0 & 3,040 & 11.0 & 31,950 \\
5.0 & 5,420 & 12.0 & 37,670 \\
6.0 & 8,450 & 13.0 & 43,810 \\
7.0 & 12,130 & 13.8 & 49,000 \\
\hline
\end{tabular}

Basin characteristics

\begin{tabular}{cccccccc}
\hline $\begin{array}{c}\text { Main } \\
\text { channel } \\
\text { slope } \\
\text { (ft/mi) }\end{array}$ & $\begin{array}{c}\text { Stream } \\
\text { length } \\
\text { (mi) }\end{array}$ & $\begin{array}{c}\text { Mean } \\
\text { basin } \\
\text { elevation } \\
\text { (ft) }\end{array}$ & $\begin{array}{c}\text { Forested } \\
\text { area } \\
\text { (percent) }\end{array}$ & $\begin{array}{c}\text { Soil } \\
\text { index }\end{array}$ & $\begin{array}{c}\text { Mean } \\
\text { annual } \\
\text { precipitation } \\
\text { (in) }\end{array}$ & $\begin{array}{c}\text { Rainfall intensity, 24-hour } \\
\text { (in) }\end{array}$ & $\begin{array}{c}50 \text {-year } \\
\text { (in) }\end{array}$ \\
\hline 91.9 & 46.4 & 4,010 & 1.0 & 1.7 & 14.0 & 1.9 \\
\hline
\end{tabular}


09424900 SANTA MARIA RIVER NEAR BAGDAD, AZ--Continued

MEAN MONTHLY AND ANNUAL DISCHARGES 1967-85, 1989, 1991-96

\begin{tabular}{|c|c|c|c|c|c|c|}
\hline MONTH & $\begin{array}{l}\text { MAXIMUM } \\
\text { (FT3/S) }\end{array}$ & $\begin{array}{l}\text { MINIMUM } \\
\text { (FT3/S) }\end{array}$ & $\begin{array}{c}\text { MEAN } \\
(\mathrm{FT} 3 / \mathrm{S})\end{array}$ & $\begin{array}{l}\text { STAN- } \\
\text { DARD } \\
\text { DEVIA- } \\
\text { TION } \\
\text { (FT3/S) }\end{array}$ & $\begin{array}{l}\text { COEFFI- } \\
\text { CIENT OF } \\
\text { VARI- } \\
\text { ATION }\end{array}$ & $\begin{array}{c}\text { PERCENT } \\
\text { OF } \\
\text { ANNUAL } \\
\text { RUNOFF }\end{array}$ \\
\hline OCTOBER & 505 & 0.00 & 25 & 102 & 4.0 & 3.0 \\
\hline NOVEMBER & 392 & 0.00 & 23 & 82 & 3.5 & 2.8 \\
\hline DECEMBER & 461 & 0.00 & 86 & 151 & 1.8 & 10.4 \\
\hline JANUARY & 936 & 0.00 & 137 & 244 & 1.8 & 16.5 \\
\hline FEBRUARY & 1,520 & 0.00 & 234 & 352 & 1.5 & 28.1 \\
\hline MARCH & 1,040 & 0.00 & 243 & 346 & 1.4 & 29.2 \\
\hline APRIL & 204 & 0.00 & 37 & 51 & 1.4 & 4.5 \\
\hline MAY & 37 & 0.00 & 8.2 & 12 & 1.5 & 1.0 \\
\hline JUNE & 17 & 0.00 & 2.1 & 5.0 & 2.4 & 0.3 \\
\hline JULY & 6.7 & 0.00 & 0.52 & 1.5 & 2.9 & 0.1 \\
\hline AUGUST & 198 & 0.00 & 18 & 48 & 2.7 & 2.1 \\
\hline SEPTEMBER & 355 & 0.00 & 18 & 70 & 3.9 & 2.1 \\
\hline ANNUAL & 232 & 0.00 & 69 & 76 & 1.1 & 100 \\
\hline
\end{tabular}

MAGNITUDE AND PROBABILITY OF INSTANTANEOUS PEAK FLOW BASED ON PERIOD OF RECORD 1967.96

DISCHARGE, IN FT3/S, FOR INDICATED RECURRENCE INTERVAL IN YEARS, AND EXCEEDANCE PROBABILITY, IN PERCENT

\begin{tabular}{|c|c|c|c|c|c|}
\hline 2 & 5 & 10 & 25 & 50 & 100 \\
\hline 508 & 208 & 108 & 48 & 28 & 18 \\
\hline 7.510 & 13,400 & 17,700 & 23,500 & 28,000 & 32,600 \\
\hline
\end{tabular}

WEIGHTED SKEW $($ LOGS $)=-0.33$

MEAN (LOGS) $=3.86$

STANDARD DEV. $($ LOGS $)=0.31$
MAGNITUDE AND PROBABILITY OF ANNUAL LOW FLOW BASED ON PERIOD OF RECORD $1968.85,1992.96$

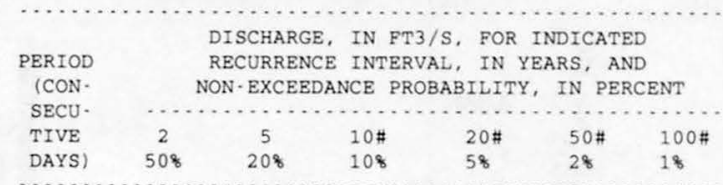

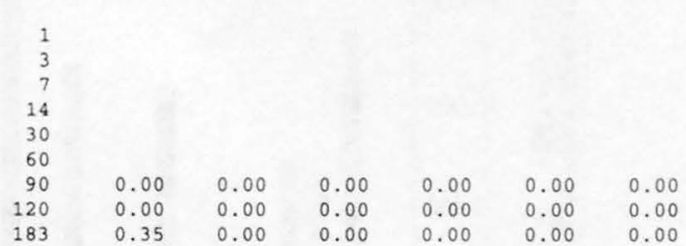

MAGNTTUDE AND PROBABILITY OF ANNUAL HIGH FLOW BASED ON PERIOD OF RECORD 1967-85, 1989, 1991-96

\begin{tabular}{|c|c|c|c|c|c|c|}
\hline & & \multicolumn{5}{|c|}{ DISCHARGE, IN FT3/S, FOR INDICATED } \\
\hline PERIOD & & RECURRE & CE INTE & RVAL, IN & YEARS, & AND \\
\hline (CON. & & EXCEEDA & CE PROE & ABILITY, & IN PERC & ENT \\
\hline SECU- & & …..... & …… & .......... & $\cdots \cdots$ & …… \\
\hline TIVE & 2 & 5 & $10 \#$ & $25 \#$ & $50 \#$ & $100 \#$ \\
\hline DAYS) & 508 & 208 & 108 & 48 & 28 & 18 \\
\hline 1 & 1,630 & 5,210 & 8,760 & 14,300 & 19,100 & 24,400 \\
\hline 3 & 911 & 3,140 & 5,480 & 9,300 & 12,700 & 16,500 \\
\hline 7 & 518 & 1,890 & 3,390 & 5,900 & 8,190 & 10,800 \\
\hline 15 & 306 & 1,210 & 2,250 & 4,010 & 5,650 & 7,530 \\
\hline 30 & 189 & 785 & 1.480 & 2,660 & 3,760 & 5,020 \\
\hline 60 & 125 & 555 & 1,060 & 1.930 & 2.730 & 3,640 \\
\hline 90 & 93 & 417 & 800 & 1,460 & 2,060 & 2,730 \\
\hline
\end{tabular}

DURATION TABLE OF DAILY MEAN FLOW FOR PERIOD OF RECORD 1967.85, $1989,1991.96$

DISCHARGE, IN FT3/S, WHICH WAS EQUALED OR EXCEEDED FOR INDICATED PERCENT OF TIME

\begin{tabular}{|c|c|c|c|c|c|c|c|c|c|c|c|c|c|c|c|c|}
\hline 18 & 58 & 108 & 158 & 208 & 308 & 408 & 508 & 608 & 708 & 808 & 908 & 958 & 988 & 998 & 99.58 & 99.98 \\
\hline 650 & 265 & 87 & 36 & 19 & 2.0 & 0.00 & 0.00 & 0.00 & 0.00 & 0.00 & 0.00 & 0.00 & 0.00 & 0.00 & 0.00 & 0.00 \\
\hline
\end{tabular}

\# Reliability of values in column is uncertain, and potential errors are large. 
09424900 SANTA MARIA RIVER NEAR BAGDAD, AZ--Continued
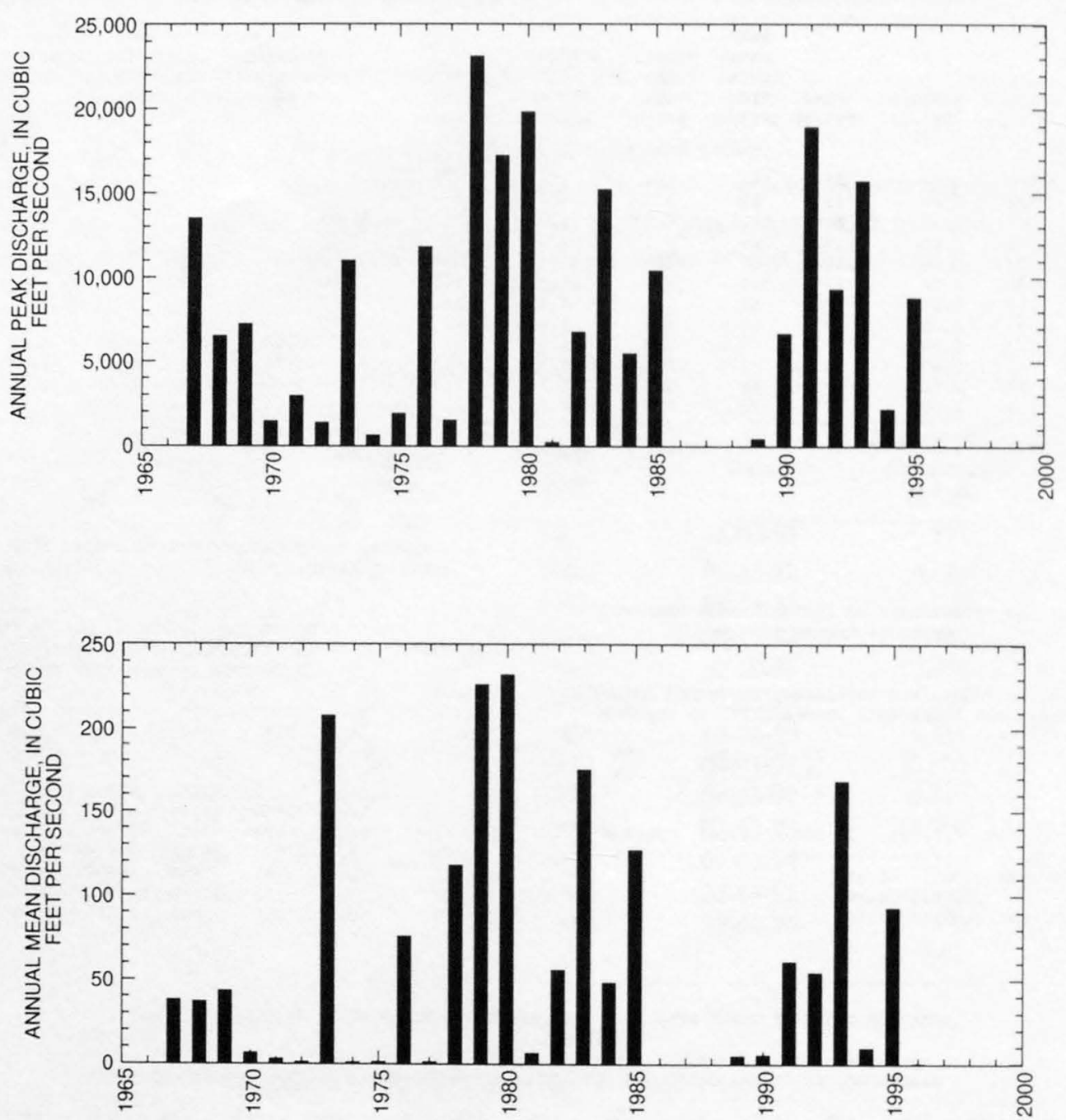
BILL WILLIAMS RIVER BASIN

221

09424900 SANTA MARIA RIVER NEAR BAGDAD, AZ--Continued
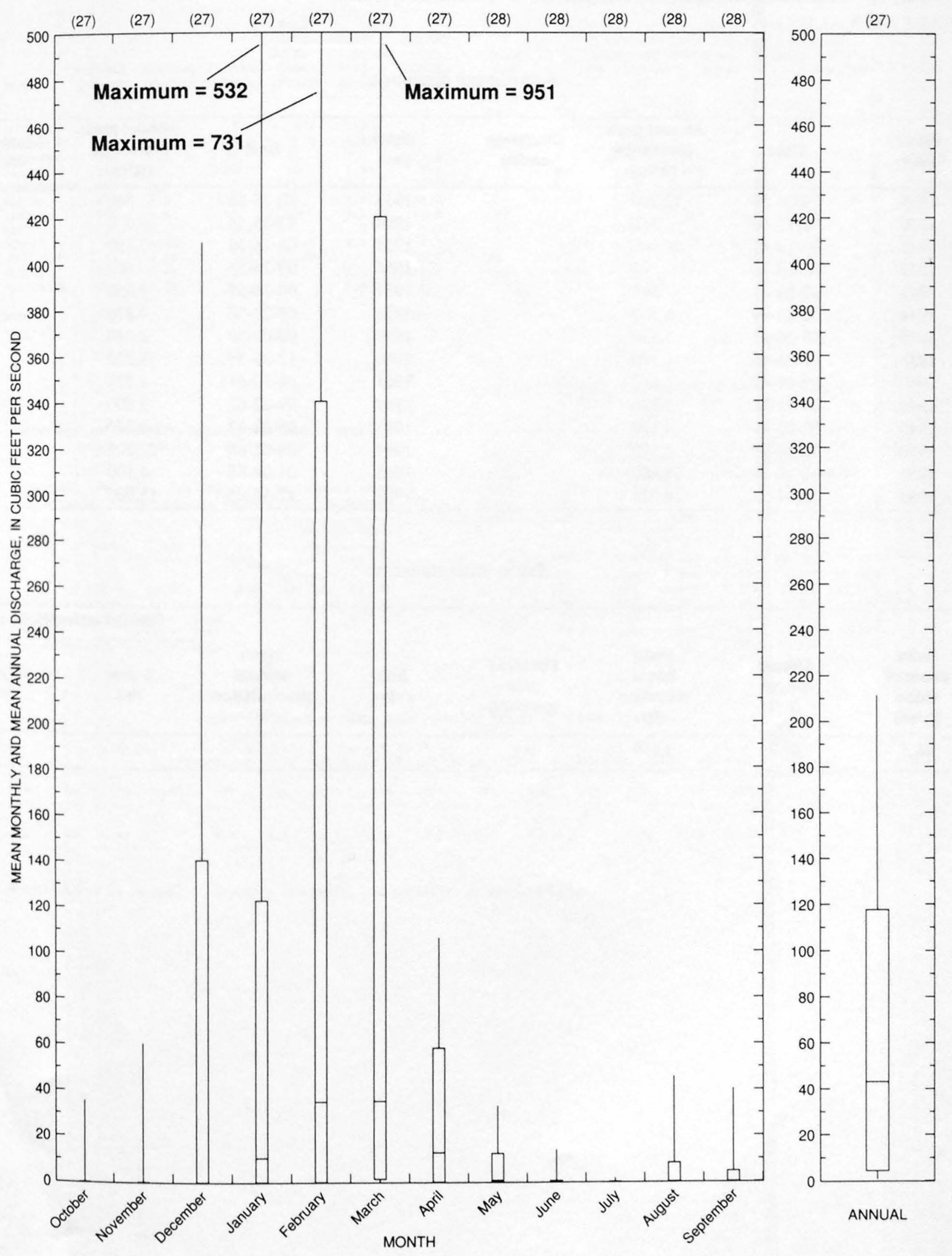


\section{SANTA MARIA RIVER NEAR ALAMO, AZ}

LOCATION.--Lat $34^{\circ} 18^{\prime}$, long $113^{\circ} 31^{\prime}$, in NE $1 / 4 \mathrm{SW}^{1} / 4$ sec.9, T.11 N., R.12 W., Mohave County, Hydrologic Unit 15030204 , on right bank 0.5 mi upstream from confluence with Big Sandy River and 5.25 mi upstream from Alamo.

DRAINAGE AREA.--1,439 $\mathrm{mi}^{2}$.

Annual peak discharges

\begin{tabular}{|c|c|c|c|c|c|c|c|}
\hline $\begin{array}{l}\text { Water } \\
\text { year }\end{array}$ & Date & $\begin{array}{c}\text { Annual peak } \\
\text { discharge } \\
\left(\mathrm{ft}^{3} / \mathrm{s}\right)\end{array}$ & $\begin{array}{l}\text { Discharge } \\
\text { codes }\end{array}$ & $\begin{array}{l}\text { Water } \\
\text { year }\end{array}$ & Date & $\begin{array}{c}\text { Annual peak } \\
\text { discharge } \\
\left(\mathrm{ft}^{3} / \mathrm{s}\right)\end{array}$ & $\begin{array}{c}\text { Discharge } \\
\text { codes }\end{array}$ \\
\hline 1939 & 09-06-39 & 22,300 & & 1953 & $08-28-53$ & 560 & \\
\hline 1941 & $03-14-41$ & 20,600 & & 1955 & $08-18-55$ & 7,180 & \\
\hline 1942 & $01-14-42$ & 91 & & 1956 & $07-24-56$ & 107 & \\
\hline 1943 & $08-04-43$ & 544 & & 1957 & $08-20-57$ & 2,050 & \\
\hline 1945 & $03-26-45$ & 1,530 & & 1959 & 08-03-59 & 2,940 & \\
\hline 1946 & $07-24-46$ & 1,170 & & 1960 & $12-26-59$ & 3,220 & \\
\hline 1947 & $09-19-47$ & 1,610 & & 1961 & $08-30-61$ & 1,720 & \\
\hline 1948 & $08-05-48$ & 1,520 & & 1962 & $09-27-62$ & 3,800 & \\
\hline 1949 & $02-25-49$ & 1,100 & & 1963 & $08-23-63$ & 4,240 & \\
\hline 1950 & $10-18-49$ & 1,570 & & 1964 & $08-02-64$ & 22,500 & \\
\hline
\end{tabular}

Basin characteristics

\begin{tabular}{cccccccc}
\hline $\begin{array}{c}\text { Main } \\
\text { channel } \\
\text { slope } \\
(\mathrm{ft} / \mathrm{mi})\end{array}$ & $\begin{array}{c}\text { Stream } \\
\text { length } \\
(\mathrm{mi})\end{array}$ & $\begin{array}{c}\text { Mean } \\
\text { basin } \\
\text { elevation } \\
\text { (ft) }\end{array}$ & $\begin{array}{c}\text { Forested } \\
\text { area } \\
\text { (percent) }\end{array}$ & $\begin{array}{c}\text { Soil } \\
\text { index }\end{array}$ & $\begin{array}{c}\text { Mean } \\
\text { annual } \\
\text { precipitation } \\
\text { (in) }\end{array}$ & $\begin{array}{c}\text { R-year } \\
\text { (in) }\end{array}$ & $\begin{array}{c}50-y e a r \\
\text { (in) }\end{array}$ \\
\hline 46.5 & 81.7 & 3,650 & 0.9 & 1.7 & 14.4 & 1.9 \\
\hline
\end{tabular}


09425500 SANTA MARIA RIVER NEAR ALAMO, AZ--Continued

MEAN MONTHLY AND ANNUAL DISCHARGES 1941.65

\begin{tabular}{|c|c|c|c|c|c|c|}
\hline MONTH & $\begin{array}{l}\text { MAXIMUM } \\
(\text { FT3/S) }\end{array}$ & $\begin{array}{l}\text { MINIMUM } \\
\text { (FT3/S) }\end{array}$ & $\begin{array}{c}\text { MEAN } \\
(\mathrm{FT} 3 / \mathrm{S})\end{array}$ & $\begin{array}{l}\text { STAN - } \\
\text { DARD } \\
\text { DEVIA- } \\
\text { TION } \\
(\mathrm{PT} 3 / \mathrm{S})\end{array}$ & $\begin{array}{l}\text { COEFFI- } \\
\text { CIENT OF } \\
\text { VARI- } \\
\text { ATION }\end{array}$ & $\begin{array}{c}\text { PERCENT } \\
\text { OF } \\
\text { ANNUAL } \\
\text { RUNOFF }\end{array}$ \\
\hline OCTOBER & 34 & 0.55 & 6.4 & 9.2 & 1.4 & 1.7 \\
\hline NOVEMBER & 13 & 0.92 & 3.9 & 3.3 & 0.85 & 1.0 \\
\hline DECEMBER & 228 & 2.1 & 20 & 50 & 2.5 & 5.5 \\
\hline JANUARY & 160 & 2.7 & 20 & 39 & 1.9 & 5.4 \\
\hline FEBRUARY & 397 & 1.9 & 47 & 101 & 2.2 & 12.6 \\
\hline MARCH & 1,070 & 3.3 & 129 & 259 & 2.0 & 34.6 \\
\hline APRIL & 880 & 2.6 & 65 & 199 & 3.1 & 17.6 \\
\hline MAY & 34 & 1.4 & 4.8 & 7.1 & 1.5 & 1.3 \\
\hline JUNE & 38 & 0.79 & 3.4 & 7.3 & 2.2 & 0.9 \\
\hline JULY & 38 & 0.60 & 3.4 & 7.2 & 2.1 & 0.9 \\
\hline AUGUST & 624 & 0.74 & 50 & 131 & 2.7 & 13.4 \\
\hline SEPTEMBER & 257 & 0.53 & 19 & 52 & 2.8 & 5.1 \\
\hline ANNUAL & 255 & 2.4 & 31 & 52 & 1.7 & 100 \\
\hline
\end{tabular}

MAGNITUDE AND PROBABILITY OF ANNUAL LOW FLOW BASED ON PERIOD OF RECORD 1941.66

\begin{tabular}{|c|c|c|c|c|c|c|}
\hline \multirow{3}{*}{$\begin{array}{l}\text { PERIOD } \\
\text { (CON- } \\
\text { SECU. }\end{array}$} & \multicolumn{2}{|c|}{$\begin{array}{l}\text { DISCHARGE } \\
\text { RECURRENC: }\end{array}$} & \multicolumn{4}{|c|}{ IN $\mathrm{FT} 3 / \mathrm{S}$, FOR INDICATED } \\
\hline & \multirow{2}{*}{\multicolumn{6}{|c|}{$\begin{array}{c}\text { NON-EXCEEDANCE PROBABILITY, IN PERCENT } \\
\ldots\end{array}$}} \\
\hline & & & & & & \\
\hline TIVE & 2 & 5 & 10 & 20 & 50 & $100 \#$ \\
\hline DAYS) & 508 & 208 & 108 & 58 & 28 & 18 \\
\hline 1 & 0.71 & 0.34 & 0.22 & 0.15 & 0.09 & 0.07 \\
\hline 3 & 0.77 & 0.38 & 0.25 & 0.17 & 0.11 & 0.08 \\
\hline 7 & 0.88 & 0.45 & 0.30 & 0.20 & 0.13 & 0.09 \\
\hline 14 & 0.95 & 0.52 & 0.37 & 0.26 & 0.18 & 0.13 \\
\hline 30 & 1.0 & 0.68 & 0.54 & 0.44 & 0.35 & 0.30 \\
\hline 60 & 1.3 & 0.92 & 0.76 & 0.65 & 0.55 & 0.48 \\
\hline 90 & 1.5 & 1.0 & 0.89 & 0.78 & 0.68 & 0.62 \\
\hline 120 & 1.7 & 1.2 & 1.1 & 0.94 & 0.83 & 0.77 \\
\hline 183 & 2.7 & 1.7 & 1.5 & 1.4 & 1.3 & 1.3 \\
\hline
\end{tabular}

MAGNITUDE AND PROBABILITY OF ANNUAL HIGH FLOW BASED ON PERIOD OF RECORD 1941.65

\begin{tabular}{|c|c|c|c|c|c|c|}
\hline \multirow{4}{*}{$\begin{array}{l}\text { PERIOD } \\
\text { /CON- } \\
\text { SECU. }\end{array}$} & \multicolumn{6}{|c|}{ DISCHARGE, IN FT3/S, FOR INDICATED } \\
\hline & & RECURRE & CE INT: & RVAL, IN & YEARS, & AND \\
\hline & & EXCEEDA & CE PRO: & ABILITY, & IN PERC & ENT \\
\hline & & 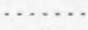 & & & ........... & \\
\hline TIVE & 2 & 5 & 10 & 25 & 50 & $100 \#$ \\
\hline DAYS) & 508 & 208 & 108 & 48 & 28 & 18 \\
\hline 1 & 633 & 2,810 & 5,930 & 12,800 & 20,700 & 31,600 \\
\hline 3 & 359 & 1.550 & 3,260 & 7,040 & 11.500 & 17.600 \\
\hline 7 & 200 & 871 & 1.860 & 4,170 & 6,980 & 11,100 \\
\hline 15 & 126 & 537 & 1,140 & 2,560 & 4,300 & 6,850 \\
\hline 30 & 78 & 318 & 672 & 1,500 & 2.530 & 4,060 \\
\hline 60 & 48 & 186 & 386 & 855 & 1,440 & 2,320 \\
\hline 90 & 36 & 133 & 271 & 591 & 991 & 1.590 \\
\hline
\end{tabular}

DURATION TABLE OF DAILY MEAN FLOW FOR PERIOD OF RECORD 1941.65

DISCHARGE, IN FT3/S, WHICH WAS EQUALED OR EXCEEDED FOR INDICATED PERCENT OF TIME

\begin{tabular}{|c|c|c|c|c|c|c|c|c|c|c|c|c|c|c|c|c|}
\hline 18 & 58 & 108 & 158 & 208 & 308 & 408 & 508 & 608 & 708 & 808 & 908 & 958 & 988 & 998 & 99.58 & 99.98 \\
\hline 662 & 49 & 13 & 7.9 & 6.0 & 4. 6 & 3.7 & 2.9 & 2.3 & 2.0 & 1.6 & 1.1 & 0.84 & 0.56 & 0.46 & 0.41 & 0.21 \\
\hline
\end{tabular}

\# Reliability of values in column is uncertain, and potential errors are large. 
09425500 SANTA MARIA RIVER NEAR ALAMO, AZ--Continued
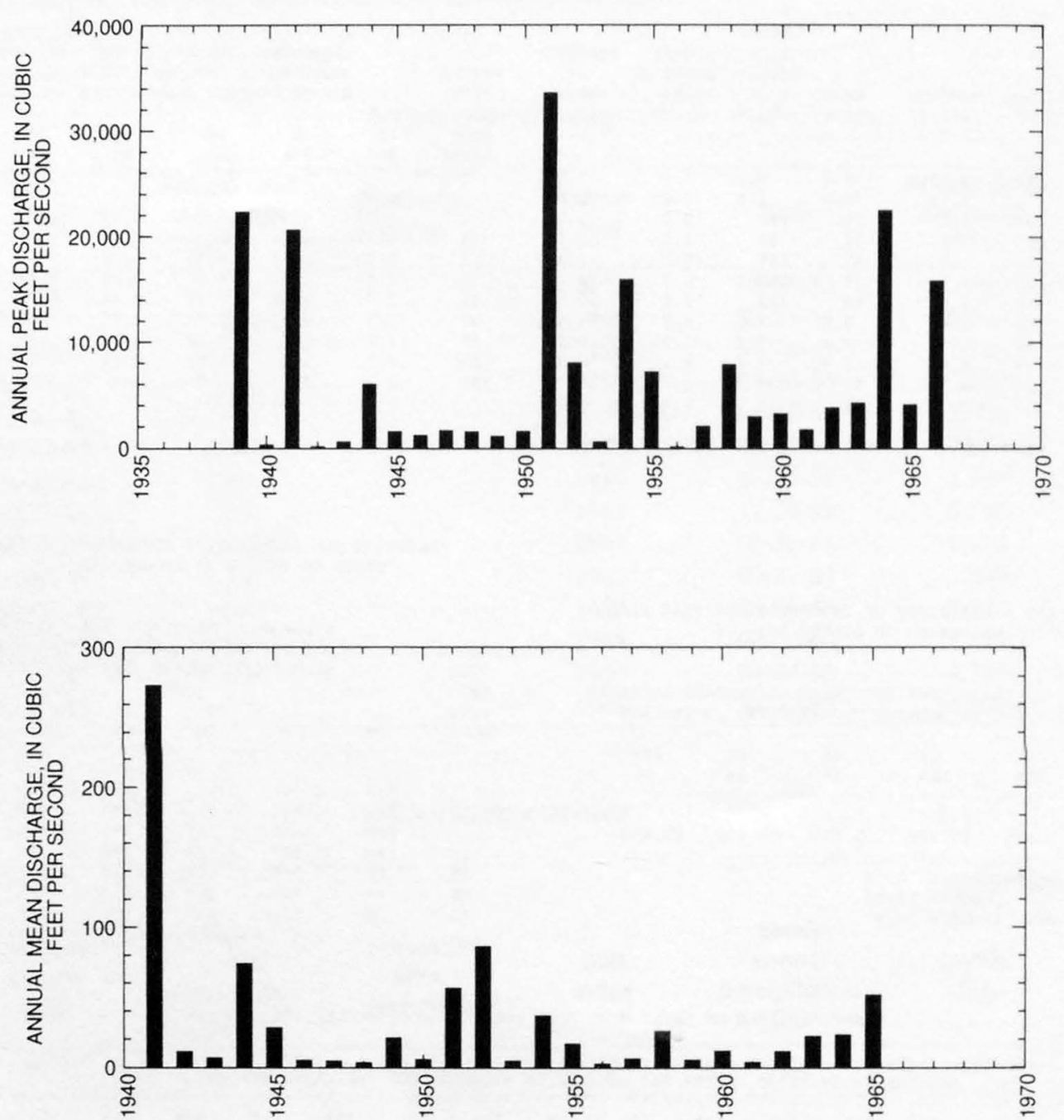
09425500 SANTA MARIA RIVER NEAR ALAMO, AZ--Continued
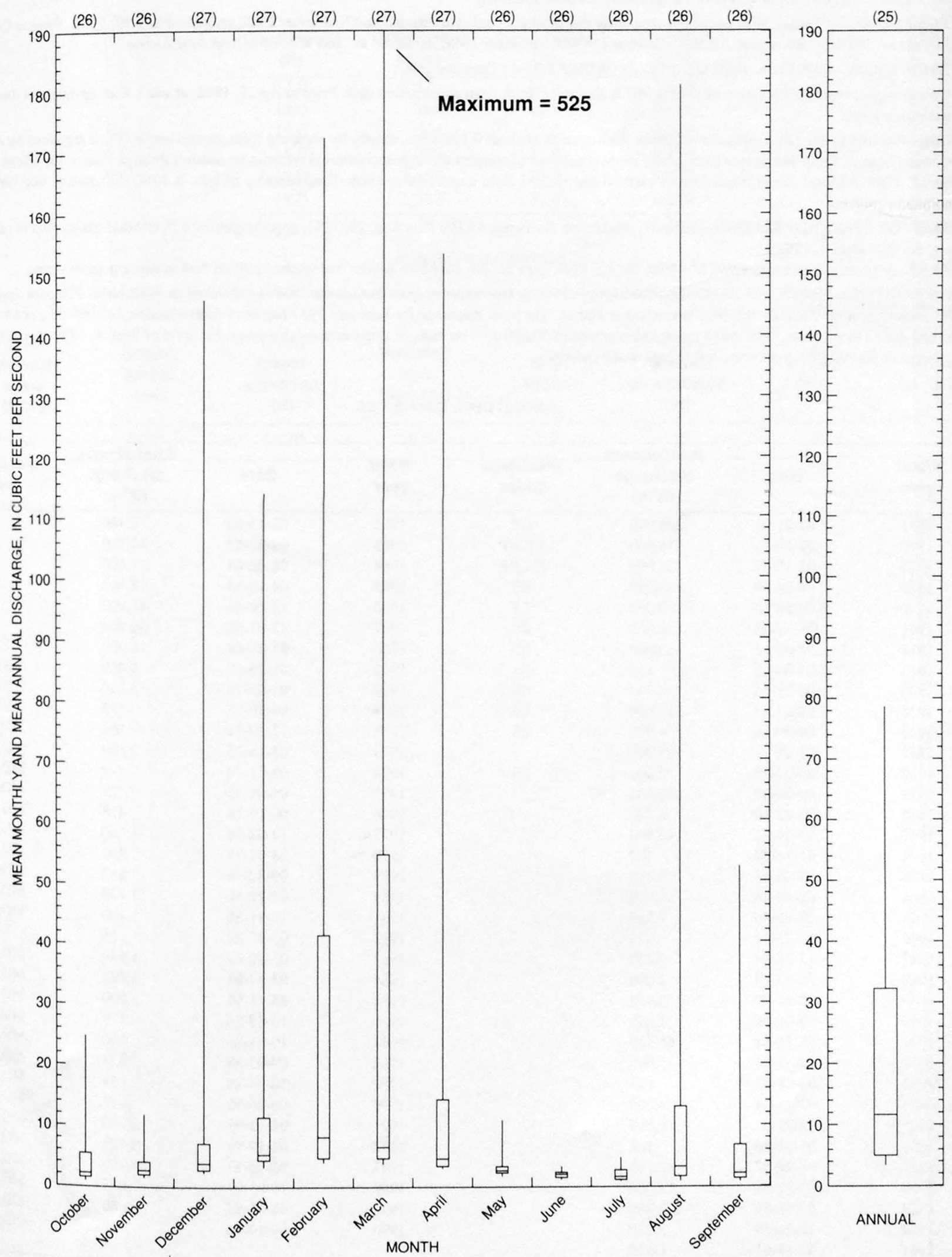


\section{BILL WILLIAMS RIVER BELOW ALAMO DAM, AZ}

LOCATION.--Lat 34'13'51", long 113'36'29", in SE $\frac{1}{4}$ SE $^{1} / 4$ sec.4, T.10 N., R.13 W., La Paz County, Hydrologic Unit 15030204, on left bank 0.6 mi downstream from Alamo Dam, 3.7 mi downstream from Bullard Wash, and $8 \mathrm{mi}$ downstream from confluence of Santa Maria and Big Sandy Rivers.

DRAINAGE AREA.--4,633 $\mathrm{mi}^{2}$, of which $10 \mathrm{mi}^{2}$ probably is noncontributing.

PERIOD OF RECORD.--October 1939 to currẹt year. Monthly discharge only for October and November 1939, published in WSP 1313. Prior to October 1943, published as "Williams River near Alamo." October 1943 to September 1967, published as "Bill Williams River near Alamo."

REVISED RECORDS.--WSP 1213: 1939(M). 1941(P). WDR AZ-89-1: Drainage area.

GAGE.--Water-stage recorder. Elevation of gage is $967 \mathrm{ft}$ above sea level, from construction data. Prior to Apr. 9, 1968, at site $1.7 \mathrm{mi}$ upstream at datum 1,002.95 $\mathrm{ft}$ above sea level.

REMARKS.--Records good. Diversions above station for irrigation of about 9,100 acres, mostly by pumping from ground water. Flow regulated by Alamo Lake, beginning Mar. 2, 1969. Temporary storage and slight regulation of releases through uncontrolled rectangular conduit through Alamo Dam June 23, 1968, to Mar. 27, 1969. Alamo Lake is formed by an earthfill and rockfill dam, completed in 1968. Total capacity of lake is 1,043,000 acre-ft. See table below for monthend contents.

EXTREMES FOR PERIOD OF RECORD.--1940-68: Maximum discharge, $65,100 \mathrm{ft}^{3} / \mathrm{s}$ Aug. 29, 1951, gage height, $30.8 \mathrm{ft}$, site and datum then in use; minimum daily, $1.1 \mathrm{ft}^{3} / \mathrm{s}$ Sept. $4,1958$.

1969-95: Maximum discharge, 6,980 $\mathrm{ft}^{3} / \mathrm{s}$ Mar. 16, 22, 1993, gage height, unknown as weir had washed out; no flow at times in most years.

EXTREMES OUTSIDE PERIOD OF RECORD.--The history of floods that occurred prior to October 1939 is published in WSP 1683 . The peak discharges have been correlated with those for Bill Williams River at Planet. The peak discharge for February 1937 has been determined as $105,000 \mathrm{ft}^{3} / \mathrm{s}$ at a stage of $46 \mathrm{ft}$, site and datum then in use, from rating curve extended above $50,000 \mathrm{ft}^{3} / \mathrm{s}$ on basis of slope-area measurement for flood of Sept. 6,1939 , at a stage of $39.6 \mathrm{ft}$, discharge of $86,000 \mathrm{ft}^{3} / \mathrm{s}$ and known stable high-water control.

Annual peak discharges

\begin{tabular}{|c|c|c|c|c|c|c|c|}
\hline $\begin{array}{l}\text { Water } \\
\text { year }\end{array}$ & Date & $\begin{array}{c}\text { Annual peak } \\
\text { discharge } \\
\left(\mathrm{ft}^{3} / \mathrm{s}\right)\end{array}$ & $\begin{array}{c}\text { Discharge } \\
\text { codes }\end{array}$ & $\begin{array}{l}\text { Water } \\
\text { year }\end{array}$ & Date & $\begin{array}{c}\text { Annual peak } \\
\text { discharge } \\
\left(\mathrm{ft}^{3} / \mathrm{s}\right)\end{array}$ & $\begin{array}{c}\text { Discharge } \\
\text { codes }\end{array}$ \\
\hline 1891 & $02-21-91$ & ${ }^{1} 200,000$ & HP & 1962 & $02-13-62$ & 8,400 & \\
\hline 1916 & $01-19-16$ & 175,000 & $\mathrm{ES}, \mathrm{HP}$ & 1963 & $08-22-63$ & 10,300 & \\
\hline 1927 & $02-16-27$ & 125,000 & ES,HP & 1964 & $08-02-64$ & 25,600 & \\
\hline 1929 & $09-04-29$ & 35,000 & ES & 1965 & $04-10-65$ & 12,300 & \\
\hline 1930 & $09-08-30$ & 90,000 & ES & 1966 & $12-10-65$ & 41,900 & \\
\hline 1931 & $08-05-31$ & 100,000 & ES & 1967 & $12-07-66$ & 38,900 & \\
\hline 1932 & $02-09-32$ & 60,000 & ES & 1968 & $01-28-68$ & 16,000 & ES \\
\hline 1933 & $03-04-33$ & 150 & ES & 1969 & $01-26-69$ & 4,950 & KR \\
\hline 1934 & $08-29-34$ & 2,000 & ES & 1970 & $03-03-70$ & 2,240 & KR \\
\hline 1935 & $02-07-35$ & 20,000 & ES & 1971 & $08-30-71$ & 732 & KR \\
\hline 1936 & $08-09-36$ & 4,000 & ES & 1972 & $12-29-71$ & 108 & $\mathrm{MD}, \mathrm{KR}$ \\
\hline 1937 & $02-07-37$ & 105,000 & & 1973 & $03-16-73$ & 2,150 & $\mathrm{MD}, \mathrm{KR}$ \\
\hline 1938 & $03-04-38$ & 70,000 & ES & 1974 & $03-11-74$ & 13 & $\mathrm{MD}, \mathrm{KR}$ \\
\hline 1939 & 09-06-39 & 86,000 & & 1975 & $04-04-75$ & 27 & $\mathrm{MD}, \mathrm{KR}$ \\
\hline 1940 & $02-03-40$ & 2,700 & & 1976 & $02-19-76$ & 418 & $\mathrm{MD}, \mathrm{KR}$ \\
\hline 1941 & $03-14-41$ & 46,000 & & 1977 & $12-08-76$ & 60 & $\mathrm{MD}, \mathrm{KR}$ \\
\hline 1942 & $01-14-42$ & 407 & & 1978 & $03-04-78$ & 286 & $\mathrm{MD}, \mathrm{KR}$ \\
\hline 1943 & $03-05-43$ & 2,480 & & 1979 & $09-30-79$ & 652 & $\mathrm{MD}, \mathrm{KR}$ \\
\hline 1944 & $02-24-44$ & 11,000 & & 1980 & $03-22-80$ & 3,400 & $M D, K R$ \\
\hline 1945 & $03-16-45$ & 7,380 & & 1981 & $10-11-80$ & 1,100 & $\mathrm{MD}, \mathrm{KR}$ \\
\hline 1946 & $07-25-46$ & 972 & & 1982 & $03-12-82$ & 25 & KR \\
\hline 1947 & $12-28-46$ & 7,230 & & 1983 & $03-30-83$ & 1,930 & $\mathrm{MD}, \mathrm{KR}$ \\
\hline 1948 & $08-05-48$ & 2,070 & & 1984 & $03-14-84$ & 2,000 & $\mathrm{MD}, \mathrm{KR}$ \\
\hline 1949 & $02-25-49$ & 2,900 & & 1985 & $03-21-85$ & 2,000 & $M D, K R$ \\
\hline 1950 & $09-06-50$ & 1,850 & & 1986 & $10-15-85$ & 379 & $\mathrm{MD}, \mathrm{KR}$ \\
\hline 1951 & $08-29-51$ & 65,100 & & 1987 & $10-01-86$ & 300 & $M D, K R$ \\
\hline 1952 & $12-31-51$ & 37,600 & & 1988 & $09-03-88$ & 328 & $M D, K R$ \\
\hline 1953 & $08-28-53$ & 193 & & 1989 & $10-04-89$ & 54 & $\mathrm{MD}, \mathrm{KR}$ \\
\hline 1954 & $03-23-54$ & 34,700 & & 1990 & $06-06-90$ & 13 & $\mathrm{MD}, \mathrm{KR}$ \\
\hline 1955 & $08-23-55$ & 4,610 & & 1991 & $04-13-91$ & 2,280 & $M D, K R$ \\
\hline 1956 & $07-24-56$ & 162 & & 1992 & $02-19-92$ & 2,120 & $\mathrm{MD}, \mathrm{KR}$ \\
\hline 1957 & $08-20-57$ & 12,100 & & 1993 & $03-16-93$ & 6,980 & $\mathrm{MD}, \mathrm{KR}$ \\
\hline 1958 & $03-22-58$ & 13,000 & & 1994 & $10-01-93$ & 255 & $\mathrm{MD}, \mathrm{KR}$ \\
\hline 1959 & $08-18-59$ & 2,900 & & 1995 & $02-16-95$ & 6,680 & $\mathrm{MD}, \mathrm{KR}$ \\
\hline 1960 & $12-26-59$ & 3,420 & & 1996 & $09-10-96$ & 48 & $\mathrm{MD}, \mathrm{KR}$ \\
\hline 1961 & $09-13-61$ & 1,630 & & & & & \\
\hline
\end{tabular}

${ }^{1}$ Highest since 1861 . 
09426000 BILL WILLIAMS RIVER BELOW ALAMO DAM, AZ--Continued

Discharge rating table developed February 1995

\begin{tabular}{cccc}
\hline $\begin{array}{c}\text { Gage height } \\
(\mathbf{f t})\end{array}$ & $\begin{array}{c}\text { Discharge } \\
\left(\mathbf{f t}^{3} / \mathbf{s}\right)\end{array}$ & $\begin{array}{c}\text { Gage height } \\
(\mathbf{f t})\end{array}$ & $\begin{array}{c}\text { Discharge } \\
\left(\mathrm{ft}^{3} / \mathbf{s}\right)\end{array}$ \\
\hline 13.0 & 170 & 19.0 & 3,120 \\
14.0 & 368 & 20.0 & 4,010 \\
15.0 & 664 & 21.0 & 5,020 \\
16.0 & 1,090 & 22.0 & 6,160 \\
17.0 & 1,660 & 23.0 & 7,440 \\
18.0 & 2,330 & 23.4 & 8,000 \\
\hline
\end{tabular}

Basin characteristics

\begin{tabular}{cccccccc}
\hline $\begin{array}{c}\text { Main } \\
\text { channel } \\
\text { slope } \\
(\mathrm{ft} / \mathrm{mi})\end{array}$ & $\begin{array}{c}\text { Stream } \\
\text { length } \\
\text { (mi) }\end{array}$ & $\begin{array}{c}\text { Mean } \\
\text { basin } \\
\text { elevation } \\
\text { (ft) }\end{array}$ & $\begin{array}{c}\text { Forested } \\
\text { area } \\
\text { (percent) }\end{array}$ & $\begin{array}{c}\text { Soil } \\
\text { index }\end{array}$ & $\begin{array}{c}\text { Mean } \\
\text { annual } \\
\text { precipitation } \\
\text { (in) }\end{array}$ & $\begin{array}{c}\text { R-year } \\
\text { (in) }\end{array}$ & $\begin{array}{c}50 \text {-year } \\
\text { (in) }\end{array}$ \\
\hline 42.6 & 125 & 4,120 & 28.0 & 1.7 & 13.7 & 1.8 \\
\hline
\end{tabular}


09426000 BILL WILLIAMS RIVER BELOW ALAMO DAM, AZ--Continued

MEAN MONTHLY AND ANNUAL DISCHARGES $1941.89,1991.96$

\begin{tabular}{|c|c|c|c|c|c|c|}
\hline MONTH & $\begin{array}{l}\text { MAXIMUM } \\
(\mathrm{FT} 3 / \mathrm{S})\end{array}$ & $\begin{array}{l}\text { MINIMUM } \\
(\text { FT } 3 / S)\end{array}$ & $\begin{array}{c}\text { MEAN } \\
(\text { FT } 3 / S)\end{array}$ & $\begin{array}{l}\text { STAN- } \\
\text { DARD } \\
\text { DEVIA- } \\
\text { TION } \\
\text { (FT3/S) }\end{array}$ & $\begin{array}{l}\text { COEFFI- } \\
\text { CIENT OF } \\
\text { VARI- } \\
\text { ATION }\end{array}$ & $\begin{array}{c}\text { PERCENT } \\
\text { OF } \\
\text { ANNUAL } \\
\text { RUNOFF }\end{array}$ \\
\hline OCTOBER & 456 & 0.00 & 47 & 101 & 2.2 & 3.1 \\
\hline NOVEMBER & 576 & 0.00 & 46 & 111 & 2.4 & 3.0 \\
\hline DECEMBER & 1,980 & 1.3 & 119 & 317 & 2.7 & 7.9 \\
\hline JANUARY & 1,470 & 1.5 & 100 & 239 & 2.4 & 6.6 \\
\hline FEBRUARY & 3,390 & 2.7 & 223 & 529 & 2.4 & 14.7 \\
\hline MARCH & 4,790 & 5.0 & 504 & 936 & 1.9 & 33.2 \\
\hline APRIL & 2,160 & 2.1 & 160 & 385 & 2.4 & 10.6 \\
\hline MAY & 719 & 0.00 & 46 & 123 & 2.7 & 3.0 \\
\hline JUNE & 494 & 0.00 & 34 & 86 & 2.5 & 2.2 \\
\hline JULY & 1,420 & 0.00 & 61 & 204 & 3.4 & 4.0 \\
\hline AUGUST & 964 & 0.00 & 101 & 200 & 2.0 & 6.6 \\
\hline SEPTEMBER & 1,110 & 0.05 & 78 & 171 & 2.2 & 5.1 \\
\hline ANNUAL & 955 & 2.1 & 126 & 197 & 1.6 & 100 \\
\hline
\end{tabular}

MAGNITUDE AND PROBABILITY OF INSTANTANEOUS PEAK FLOW BASED ON PERIOD OF RECORD

DISCHARGE, IN FT3/S, FOR INDICATED RECURRENCE INTERVAL IN YEARS, AND EXCEEDANCE PROBABILITY, IN PERCENT

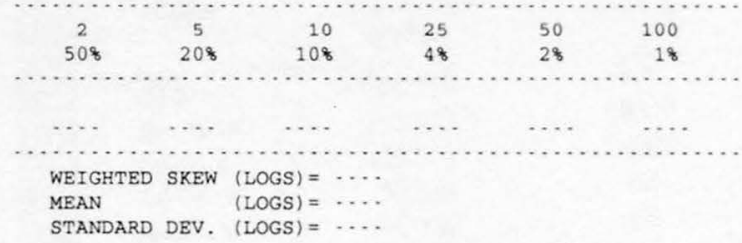

DURATION TABLE OF DAILY MEAN FLOW FOR PERIOD OF RECORD 1941-89, 1991.96
MAGNITUDE AND PROBABILITY OF ANNUAL LOW FLOW BASED ON PERIOD OF RECORD 1941-89, 1992.96

\begin{tabular}{|c|c|c|c|c|c|c|}
\hline \multirow{3}{*}{$\begin{array}{l}\text { PERIOD } \\
\text { (CON- } \\
\text { SECU- } \\
\text { TIVE } \\
\text { DAYS) }\end{array}$} & \multicolumn{6}{|c|}{ 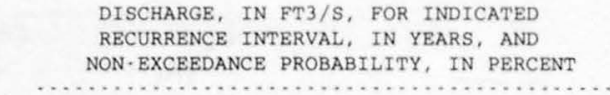 } \\
\hline & 2 & 5 & 10 & 20 & 50 & $100 \#$ \\
\hline & 508 & 208 & 108 & 58 & 28 & 18 \\
\hline 1 & 3.5 & 0.24 & 0.00 & 0.00 & 0.00 & 0. \\
\hline 3 & 3.7 & 0.25 & 0.00 & 0.00 & 0.00 & 0.0 \\
\hline 7 & 3.8 & 0.43 & 0.00 & 0.00 & 0.00 & 0.0 \\
\hline 14 & 4.9 & 0.81 & 0.00 & 0.00 & 0.00 & 0. \\
\hline 30 & 5.1 & 1.4 & 0.18 & 0.00 & 0.00 & \\
\hline 60 & 5.1 & 2.3 & 1.1 & 0.00 & 0.00 & 0.0 \\
\hline 90 & 7.6 & 1.6 & 0.47 & 0.07 & 0.00 & 0. \\
\hline 120 & 8.2 & 2.3 & 1.0 & 0.49 & 0.20 & \\
\hline 183 & 13 & 4.4 & 2.5 & 1.6 & 0.91 & \\
\hline
\end{tabular}

MAGNITUDE AND PROBABILITY OF ANNUAL HIGH FLOW BASED ON PERIOD OF RECORD 1941.89,1991.96

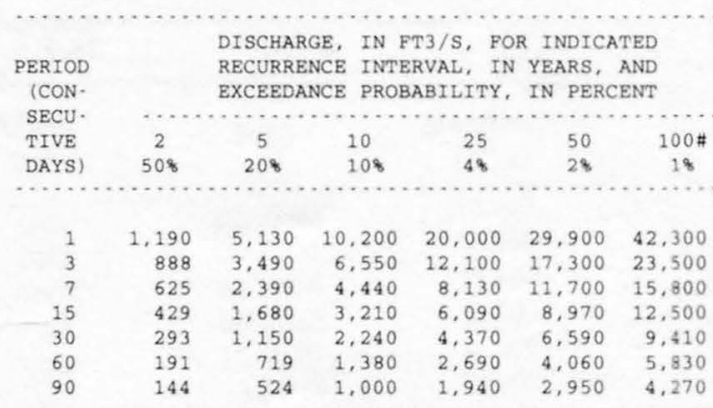

DISCHARGE, IN FT3/S, WHICH WAS EQUALED OR EXCEEDED FOR INDICATED PERCENT OF TIME

\begin{tabular}{|c|c|c|c|c|c|c|c|c|c|c|c|c|c|c|c|c|}
\hline 18 & 58 & 108 & 158 & 208 & 308 & 408 & 508 & 608 & 708 & 808 & 908 & 958 & 988 & 998 & 99.58 & 99.98 \\
\hline 360 & 511 & 248 & 81 & 43 & 21 & 15 & 11 & 9.6 & 7.8 & 6.0 & 3.8 & 1.0 & 0.00 & 0.00 & 0.00 & 0.00 \\
\hline
\end{tabular}

\# Reliability of values in column is uncertain, and potential errors are large. 
ANNUAL MEAN DISCHARGE, IN CUBIC FEET PER SECOND

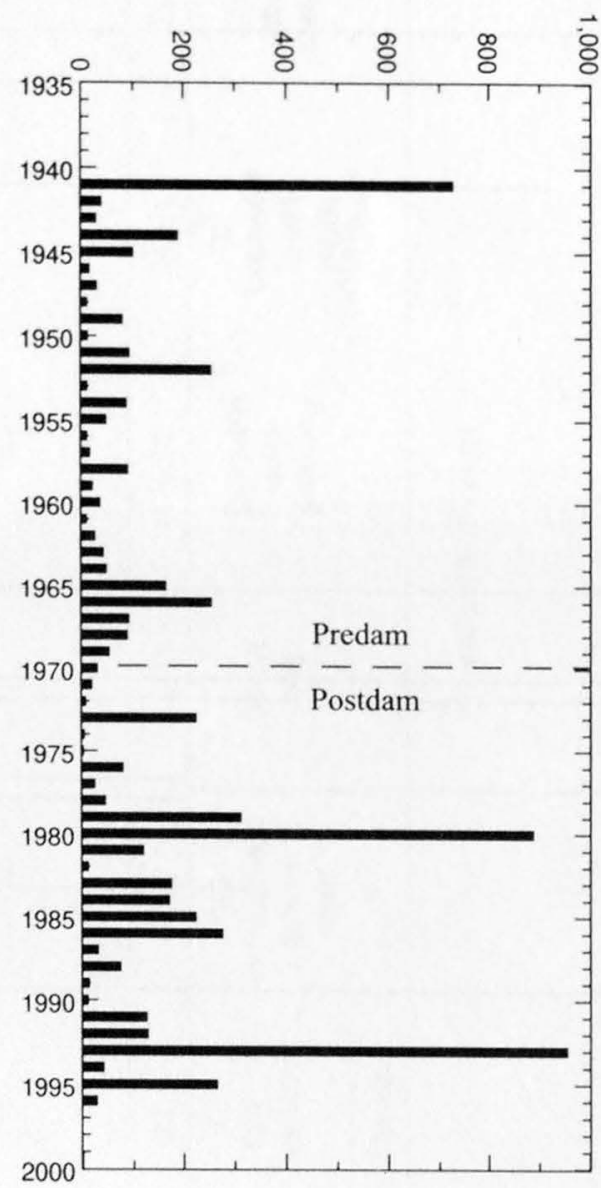

ANNUAL PEAK DISCHARGE IN CUBIC FEET PER SECOND

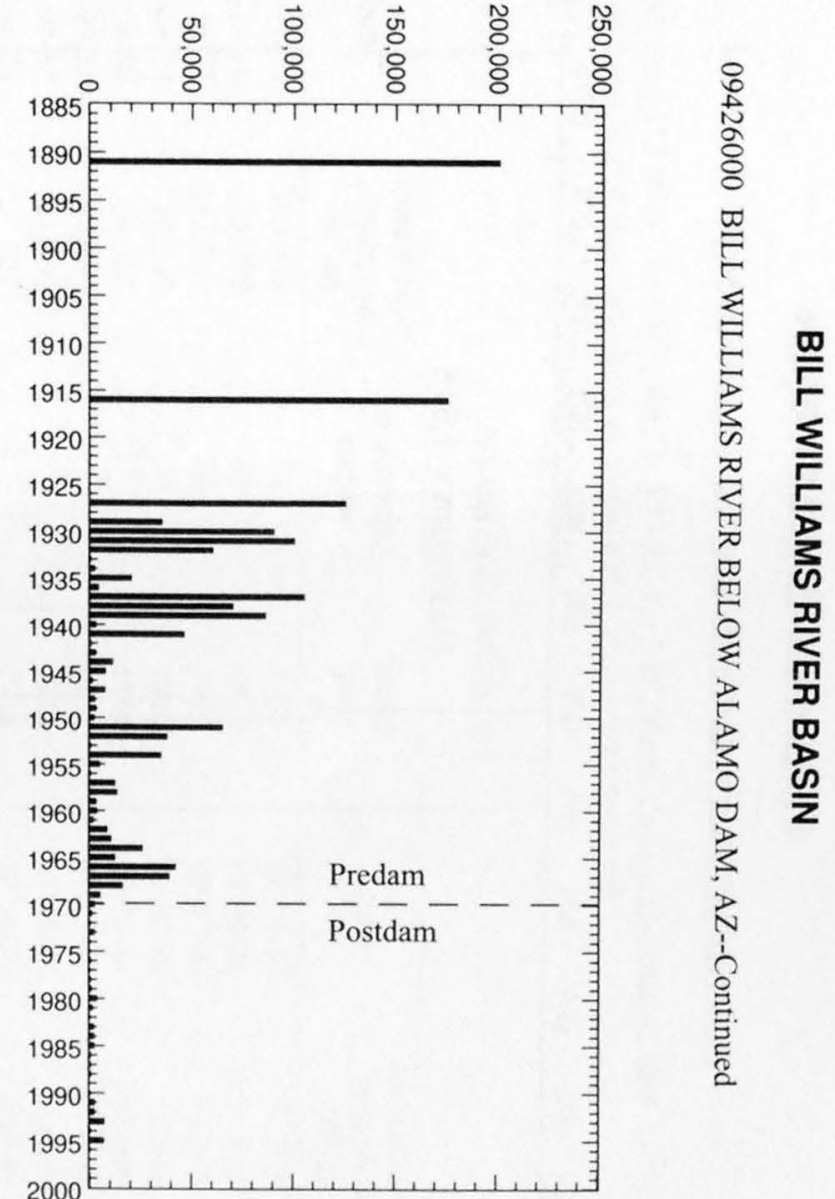


09426000 BILL WILLIAMS RIVER BELOW ALAMO DAM, AZ--Continued

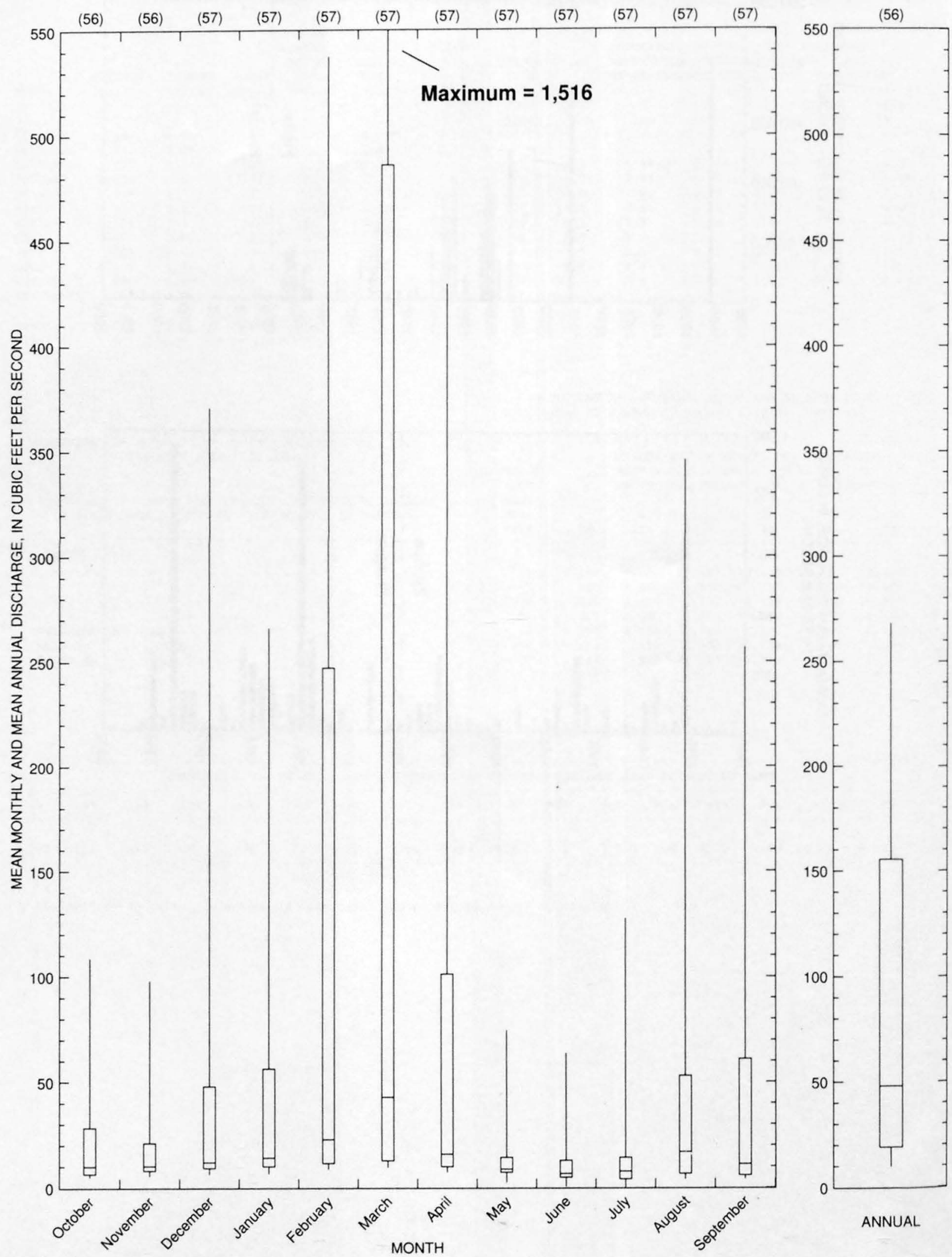




\section{BILL WILLIAMS RIVER AT PLANET, AZ}

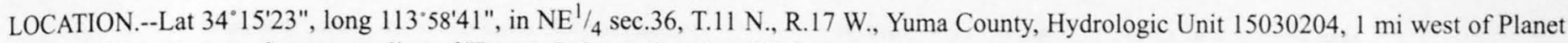
and $6 \mathrm{mi}$ upstream from water line of Havasu Lake at elevation $450 \mathrm{ft}$ above mean sea level.

DRAINAGE AREA.--5,054 $\mathrm{mi}^{2}$, of which $10.1 \mathrm{mi}^{2}$ is noncontributing.

Annual peak discharges

\begin{tabular}{|c|c|c|c|c|c|c|c|}
\hline $\begin{array}{l}\text { Water } \\
\text { year }\end{array}$ & Date & $\begin{array}{c}\text { Annual peak } \\
\text { discharge } \\
\left(\mathrm{ft}^{3} / \mathrm{s}\right)\end{array}$ & $\begin{array}{l}\text { Discharge } \\
\text { codes }\end{array}$ & $\begin{array}{l}\text { Water } \\
\text { year }\end{array}$ & Date & $\begin{array}{c}\text { Annual peak } \\
\text { discharge } \\
\left(\mathrm{ft}^{3} / \mathrm{s}\right)\end{array}$ & $\begin{array}{l}\text { Discharge } \\
\text { codes }\end{array}$ \\
\hline 1891 & $02-21-91$ & 200,000 & HP & 1937 & $02-07-37$ & 92,500 & \\
\hline 1916 & $01-19-16$ & 175,000 & HP & 1938 & $03-04-38$ & 61,000 & \\
\hline 1927 & $02-16-27$ & 125,000 & $\mathrm{HP}$ & 1939 & $09-07-39$ & 73,000 & \\
\hline 1929 & $09-04-29$ & 25,000 & & 1940 & $02-03-40$ & 2,600 & \\
\hline 1930 & $09-08-30$ & 64,000 & & 1941 & $03-02-41$ & 42,600 & \\
\hline 1931 & $08-05-31$ & 80,000 & & 1942 & $01-15-42$ & 300 & \\
\hline 1932 & $02-09-32$ & 51,000 & & 1943 & $03-05-43$ & 1,580 & \\
\hline 1933 & $03-04-33$ & 107 & & 1944 & $02-24-44$ & 10,800 & \\
\hline 1934 & $08-29-34$ & 1,470 & & 1945 & $03-16-45$ & 4,520 & \\
\hline 1935 & $02-07-35$ & 15,900 & & 1946 & $07-22-46$ & 328 & \\
\hline 1936 & $08-09-36$ & 2,900 & & & & & \\
\hline
\end{tabular}

\section{Basin characteristics}

\begin{tabular}{ccccccc}
\hline $\begin{array}{c}\text { Main } \\
\text { channel } \\
\text { slope } \\
\text { (ft/mi) }\end{array}$ & $\begin{array}{c}\text { Stream } \\
\text { length } \\
\text { (mi) }\end{array}$ & $\begin{array}{c}\text { Mean } \\
\text { basin } \\
\text { elevation } \\
\text { (ft) }\end{array}$ & $\begin{array}{c}\text { Forested } \\
\text { area } \\
\text { (percent) }\end{array}$ & $\begin{array}{c}\text { Soil } \\
\text { index }\end{array}$ & $\begin{array}{c}\text { Mean } \\
\text { annual } \\
\text { precipitation } \\
\text { (in) }\end{array}$ & $\begin{array}{c}\text { R-year } \\
\text { (in) }\end{array}$ \\
\hline 38.6 & 152 & 3,900 & 26.0 & 1.7 & 13.2 & $\begin{array}{c}50-\text { year } \\
\text { (in) }\end{array}$ \\
\hline
\end{tabular}




\section{BILL WILLIAMS RIVER AT PLANET, AZ--Continued}

MEAN MONTHLY AND ANNUAL DISCHARGES 1915, 1929.46

\begin{tabular}{|c|c|c|c|c|c|c|}
\hline MONTH & $\begin{array}{l}\text { MAXIMUM } \\
\text { (FT3/S) }\end{array}$ & $\begin{array}{l}\text { MINIMUM } \\
\text { (FT3/S) }\end{array}$ & $\begin{array}{c}\text { MEAN } \\
(\text { FT3/S) }\end{array}$ & $\begin{array}{l}\text { STAN- } \\
\text { DARD } \\
\text { DEVIA- } \\
\text { TION } \\
\text { (FT3/S) }\end{array}$ & $\begin{array}{l}\text { COEFFI- } \\
\text { CIENT OF } \\
\text { VARI. } \\
\text { ATION }\end{array}$ & $\begin{array}{c}\text { PERCEN } \\
\text { OF } \\
\text { ANNUAI } \\
\text { RUNOF: }\end{array}$ \\
\hline OCTOBER & 46 & 9.2 & 21 & 8.5 & 0.40 & 1.1 \\
\hline NOVEMBER & 26 & 10 & 19 & 3.8 & 0.20 & 1.0 \\
\hline DECEMBER & 659 & 13 & 68 & 150 & 2.2 & 3.7 \\
\hline JANUARY & 445 & 13 & 78 & 125 & 1.6 & 4.2 \\
\hline FEBRUARY & 4,680 & 13 & 687 & 1.320 & 1.9 & 36.9 \\
\hline MARCH & 2,780 & 13 & 425 & 730 & 1.7 & 22.9 \\
\hline APRIL & 1,830 & 12 & 121 & 414 & 3.4 & 6.5 \\
\hline MAY & 122 & 11 & 28 & 29 & 1.0 & 1.5 \\
\hline JUNE & 95 & 11 & 21 & 18 & 0.86 & 1.1 \\
\hline JULY & 80 & 9.5 & 22 & 15 & 0.70 & 1.2 \\
\hline AUGUST & 1,180 & 14 & 93 & 264 & 2.8 & 5.0 \\
\hline SEPTEMBER & 3,660 & 13 & 276 & 832 & 3.0 & 14.8 \\
\hline ANNUAL & 603 & 16 & 152 & 164 & 1.1 & 100 \\
\hline
\end{tabular}

MAGNITUDE AND PROBABILITY OF INSTANTANEOUS PEAK FLOW BASED ON PERTOD OF RECORD

DISCHARGE, IN FT3/S, FOR INDICATED RECURRENCE INTERVAL IN YEARS, AND EXCEEDANCE PROBABILITY, IN PERCENT

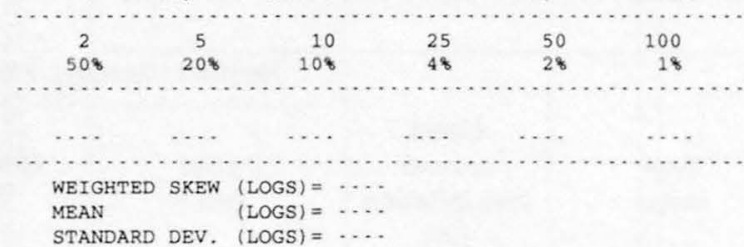

MAGNITUDE AND PROBABILITY OF ANNUAL LOW FLOW BASED ON PERIOD OF RECORD 1930.46

\begin{tabular}{|c|c|c|c|c|c|c|}
\hline PERIOD & & $\begin{array}{l}\text { SCHAR } \\
\text { CURRE }\end{array}$ & $\begin{array}{l}\text { IN F } \\
\text { INTE }\end{array}$ & $\begin{array}{ll}\text { S, } & \text { FOP } \\
\text { L, IN }\end{array}$ & $\begin{array}{l}\text { DICAT } \\
\text { RS, A }\end{array}$ & \\
\hline $1 \mathrm{CON}-$ & & $-\mathrm{EXCE}$ & NCE P & ABILI? & IN PE & ENT \\
\hline SECU- & $\cdots$ & $\ldots \ldots$ & $\ldots \ldots$ & $\cdots \cdots$ & $\cdots \cdots$ & $\ldots \ldots$ \\
\hline TIVE & 2 & 5 & 10 & 20 & $50 \#$ & $100 \#$ \\
\hline DAYS) & 508 & 208 & 108 & 58 & 28 & 18 \\
\hline 1 & 12 & 10 & 9.3 & 8.6 & 7.8 & 7.3 \\
\hline 3 & 13 & 10 & 9.4 & 8.5 & 7.6 & 7.1 \\
\hline 7 & 14 & 11 & 10 & 9.3 & 8.2 & 7.5 \\
\hline 14 & 14 & 12 & 11 & 9.6 & 8.6 & 7.9 \\
\hline 30 & 15 & 13 & 11 & 10 & 8.9 & 8.1 \\
\hline 60 & 16 & 13 & 12 & 11 & 9.3 & 8.4 \\
\hline 90 & 17 & 14 & 12 & 11 & 9.7 & 8.9 \\
\hline 120 & 17 & 14 & 13 & 12 & 12 & 11 \\
\hline 183 & 23 & 15 & 13 & 12 & 12 & 11 \\
\hline
\end{tabular}

MAGNITUDE AND PROBABILITY OF ANNUAL HIGH FLOW BASED ON PERIOD OF RECORD 1915, 1929-46

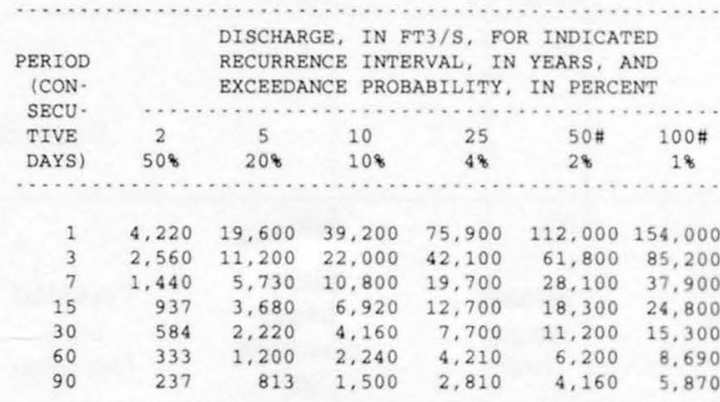

DURATION TABLE OF DAILY MEAN FLOW FOR PERIOD OF RECORD 1915, $1929-46$ DISCHARGE, IN FT3/S, WHICH WAS EQUALED OR EXCEEDED FOR INDICATED PERCENT OF TIME

\begin{tabular}{|c|c|c|c|c|c|c|c|c|c|c|c|c|c|c|c|c|}
\hline 18 & 58 & 108 & 158 & 208 & 308 & 408 & 508 & 608 & 708 & 808 & 908 & 958 & $98 \%$ & 998 & 99.58 & 99.98 \\
\hline & 275 & 82 & 39 & 28 & 23 & 21 & 20 & 18 & 17 & 15 & 13 & 12 & 10 & 10 & 9.0 & 8.2 \\
\hline
\end{tabular}

\# Reliability of values in column is uncertain, and potential errors are large. 
09426500 BILL WILLIAMS RIVER AT PLANET, AZ--Continued
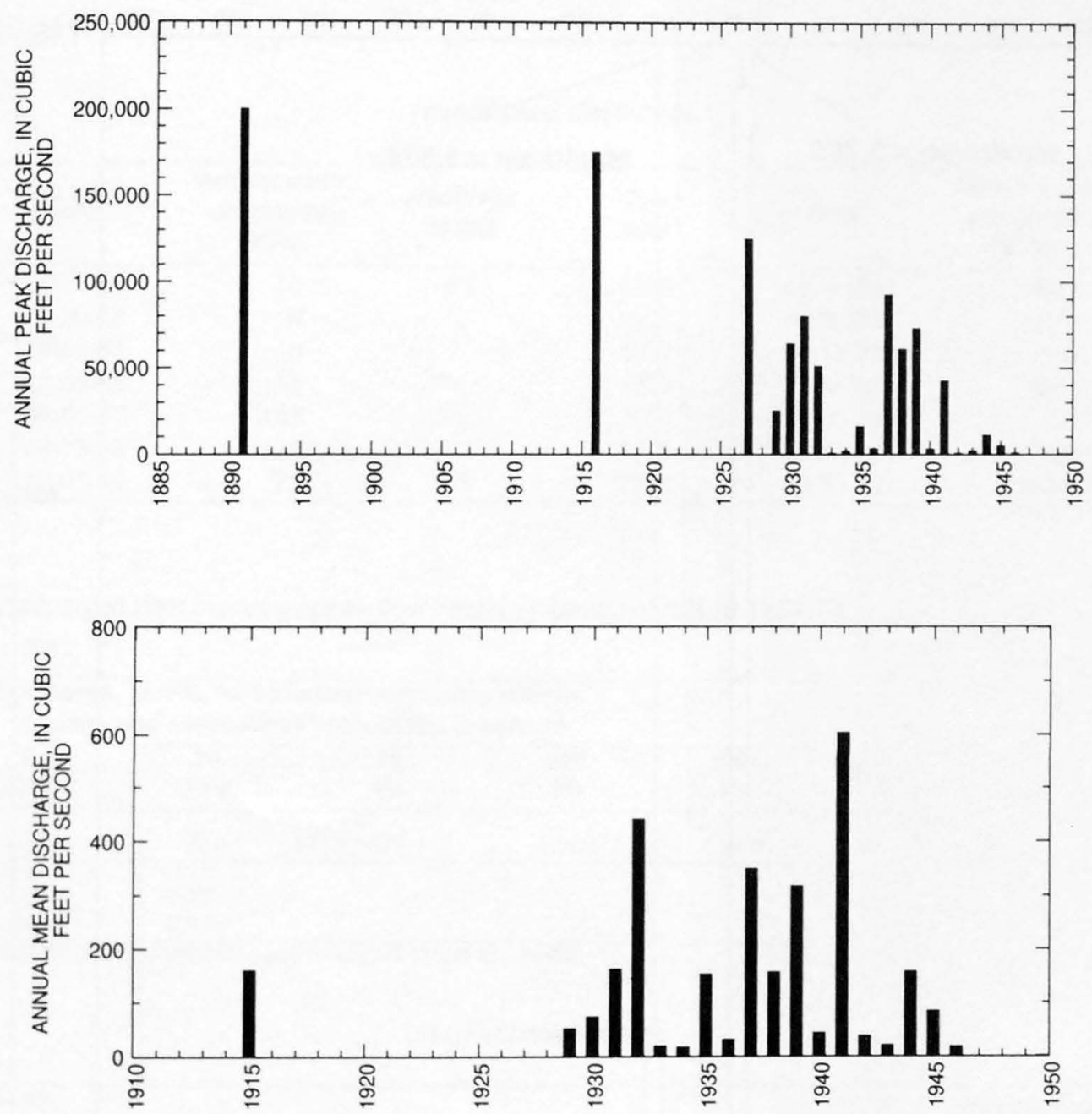
09426500 BILL WILLIAMS RIVER AT PLANET, AZ--Continued
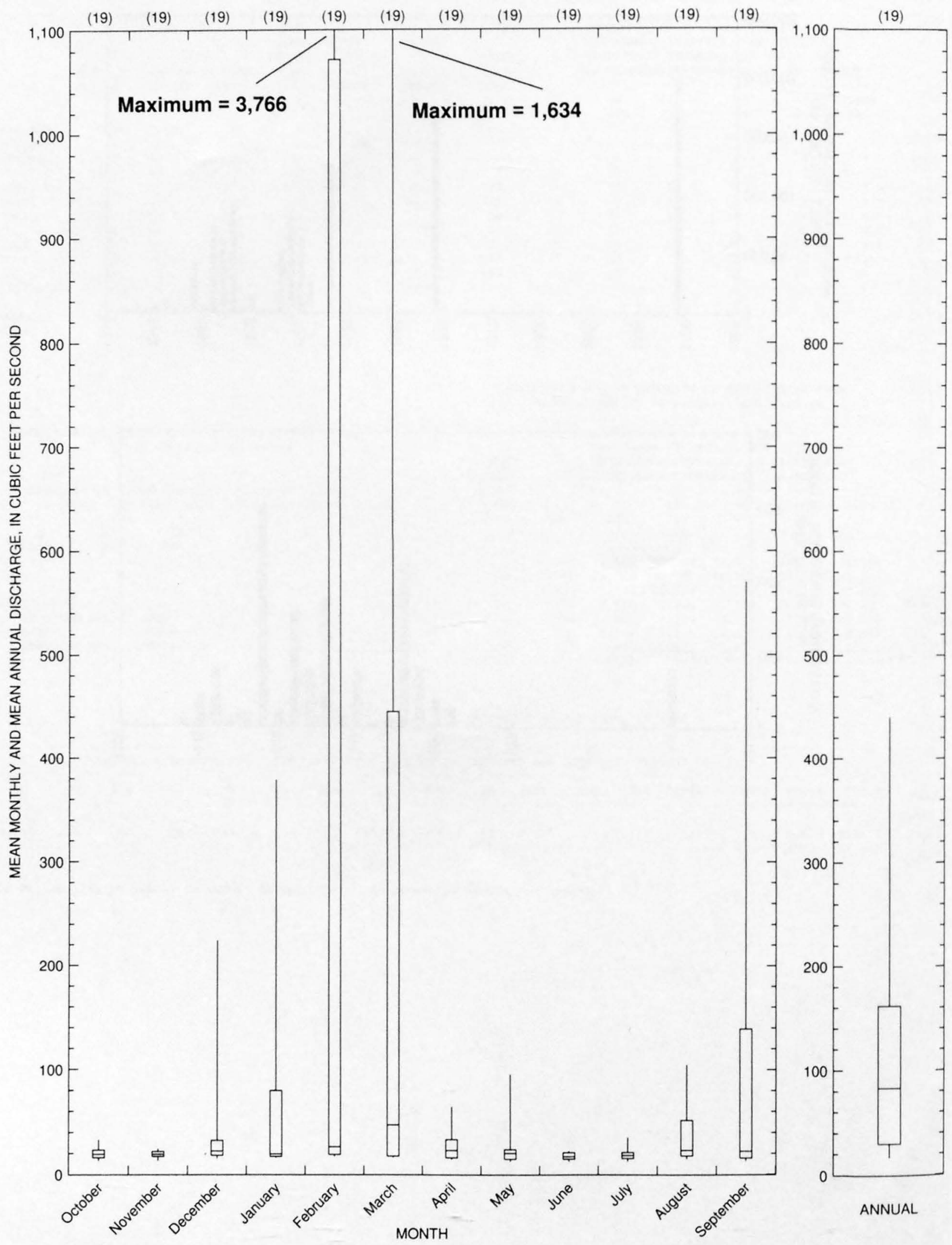
09427700 MONKEYS HEAD WASH NEAR PARKER, AZ

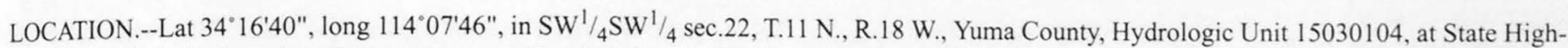
way $172,1.5$ mi south of Parker Dam, and 13 mi northeast of Parker.

DRAINAGE AREA.--1.84 $\mathrm{mi}^{2}$.

Annual peak discharges

\begin{tabular}{|c|c|c|c|c|c|c|c|}
\hline $\begin{array}{l}\text { Water } \\
\text { year }\end{array}$ & Date & $\begin{array}{c}\text { Annual peak } \\
\text { discharge } \\
\left(\mathrm{ft}^{3} / \mathrm{s}\right)\end{array}$ & $\begin{array}{l}\text { Discharge } \\
\text { codes }\end{array}$ & $\begin{array}{c}\text { Water } \\
\text { year }\end{array}$ & Date & $\begin{array}{c}\text { Annual peak } \\
\text { discharge } \\
\left(\mathrm{ft}^{3} / \mathrm{s}\right)\end{array}$ & $\begin{array}{c}\text { Discharge } \\
\text { codes }\end{array}$ \\
\hline 1963 & $08-16-63$ & 10 & ES & 1970 & $03-0-170$ & 35 & \\
\hline 1964 & $00-00-64$ & 0 & & 1971 & $00-00-71$ & 265 & \\
\hline 1965 & $00-00-65$ & 0 & & 1972 & $09-18-72$ & 5.0 & ES \\
\hline 1966 & $12-09-65$ & 12 & & 1973 & $11-16-72$ & 60 & \\
\hline 1967 & $09-00-67$ & 103 & & 1974 & $00-00-74$ & 0 & \\
\hline 1968 & $08-13-68$ & 320 & & 1975 & $08-00-75$ & 0.5 & LT \\
\hline 1969 & $10-03-68$ & 12 & ES & 1976 & $09-25-76$ & 160 & \\
\hline
\end{tabular}

Magnitude and probability of instantaneous peak flow based on period of record 1963-76

\begin{tabular}{|c|c|c|c|c|c|}
\hline \multicolumn{6}{|c|}{$\begin{array}{l}\text { Discharge, in } \mathrm{ft}^{3} / \mathrm{s} \text {, for indicated recurrence interval } \\
\text { in years, and exceedance probablility, in percent }\end{array}$} \\
\hline 2 & 5 & 10 & 25 & $50 t$ & $100 \dagger$ \\
\hline $50 \%$ & $20 \%$ & $10 \%$ & $4 \%$ & $2 \%$ & $1 \%$ \\
\hline 18.2 & 100 & 236 & 572 & 1,000 & 1,640 \\
\hline Weighted skew & $(\log s)=$ & -0.18 & & & \\
\hline Mean & $(\log s)=$ & 1.23 & & & \\
\hline Standard dev. & $(\log s)=$ & 0.91 & & & \\
\hline
\end{tabular}

Basin characteristics

\begin{tabular}{cccccccc}
\hline $\begin{array}{c}\text { Main } \\
\text { channel } \\
\begin{array}{c}\text { slope } \\
(\mathrm{ft} / \mathrm{mi})\end{array}\end{array}$ & $\begin{array}{c}\text { Stream } \\
\text { length } \\
(\mathrm{mi})\end{array}$ & $\begin{array}{c}\text { Mean } \\
\text { basin } \\
\text { elevation } \\
(\mathrm{ft})\end{array}$ & $\begin{array}{c}\text { Forested } \\
\text { area } \\
\text { (percent) }\end{array}$ & $\begin{array}{c}\text { Soil } \\
\text { index }\end{array}$ & $\begin{array}{c}\text { Mean } \\
\text { annual } \\
\text { precipitation } \\
\text { (in) }\end{array}$ & $\begin{array}{c}\text { R-year } \\
\text { (in) }\end{array}$ & $\begin{array}{c}50 \text {-year } \\
\text { (in) }\end{array}$ \\
\hline 415 & 2.7 & 1,130 & 0.0 & 3.0 & 5.5 & 1.4 \\
\hline
\end{tabular}

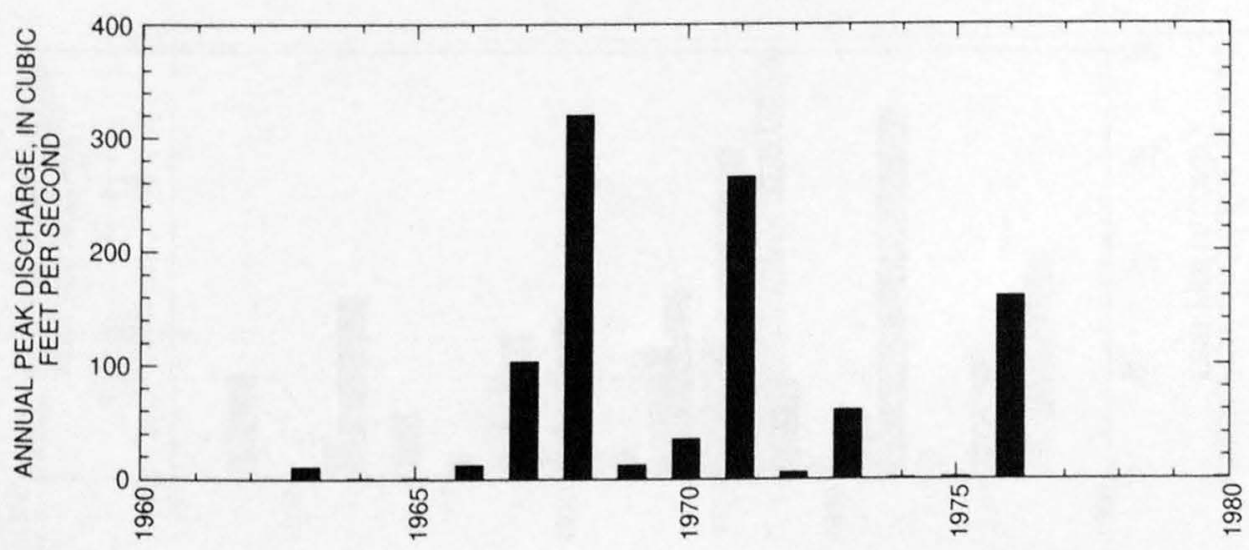


09428545 CUNNINGHAM WASH TRIBUTARY NEAR WENDEN, AZ

LOCATION.--Lat 34 00'35", long 113 34'40", in SE $1 / 4^{\prime} \mathrm{NE}^{1} / 4$ sec.26, T.8 N., R.13 W., Yuma County, Hydrologic Unit 15030105 , Alamo Dan access road, $13 \mathrm{mi}$ north of Wenden.

DRAINAGE AREA.--0.77 $\mathrm{mi}^{2}$.

Annual peak discharges

\begin{tabular}{|c|c|c|c|c|c|c|c|}
\hline $\begin{array}{l}\text { Water } \\
\text { year }\end{array}$ & Date & $\begin{array}{c}\text { Annual peak } \\
\text { discharge } \\
\left(\mathrm{ft}^{3} / \mathrm{s}\right)\end{array}$ & $\begin{array}{c}\text { Discharge } \\
\text { codes }\end{array}$ & $\begin{array}{l}\text { Water } \\
\text { year }\end{array}$ & Date & $\begin{array}{c}\text { Annual peak } \\
\text { discharge } \\
\left(\mathrm{ft}^{3} / \mathrm{s}\right)\end{array}$ & $\begin{array}{c}\text { Discharge } \\
\text { codes }\end{array}$ \\
\hline 1964 & $08-02-64$ & 173 & & 1971 & $00-00-71$ & 68 & \\
\hline 1965 & $04-04-65$ & 5.0 & ES & 1972 & $09-17-72$ & 12 & \\
\hline 1966 & $08-18-66$ & 46 & & 1973 & $10-06-72$ & 31 & \\
\hline 1967 & $08-20-67$ & 155 & & 1974 & $07-20-74$ & 85 & \\
\hline 1968 & $08-06-68$ & 88 & & 1975 & $07-25-75$ & 0.4 & ES \\
\hline 1969 & $08-00-69$ & 15 & & 1976 & $09-25-76$ & 48 & \\
\hline 1970 & $08-10-70$ & 80 & ES & & & & \\
\hline
\end{tabular}

Magnitude and probability of instantaneous peak flow based on period of record 1964-76

\begin{tabular}{|c|c|c|c|c|c|}
\hline \multicolumn{6}{|c|}{$\begin{array}{l}\text { Discharge, in } \mathrm{ft}^{3} / \mathrm{s} \text {, for indicated recurrence interval } \\
\text { in years, and exceedance probablility, in percent }\end{array}$} \\
\hline 2 & 5 & 10 & 25 & $50 t$ & $100 \dagger$ \\
\hline $50 \%$ & $20 \%$ & $10 \%$ & $4 \%$ & $2 \%$ & $1 \%$ \\
\hline 52.1 & 101 & 140 & 198 & 245 & 296 \\
\hline Weighted skew & $(\log s)=$ & -0.19 & & & \\
\hline Mean & $(\log s)=$ & 1.71 & & & \\
\hline Standard dev. & $(\log s)=$ & 0.35 & & & \\
\hline
\end{tabular}

Basin characteristics

\begin{tabular}{ccccccc}
\hline $\begin{array}{c}\text { Main } \\
\text { channel } \\
\text { slope } \\
(\mathrm{ft} / \mathrm{mi})\end{array}$ & $\begin{array}{c}\text { Stream } \\
\text { length } \\
(\mathrm{mi})\end{array}$ & $\begin{array}{c}\text { Mean } \\
\text { basin } \\
\text { elevation } \\
(\mathrm{ft})\end{array}$ & $\begin{array}{c}\text { Forested } \\
\text { area } \\
\text { (percent) }\end{array}$ & $\begin{array}{c}\text { Soil } \\
\text { index }\end{array}$ & $\begin{array}{c}\text { Mean } \\
\text { annual } \\
\text { precipitation } \\
\text { (in) }\end{array}$ & $\begin{array}{c}\text { Rainfall intensity, 24-hour } \\
\text { (in) }\end{array}$ \\
\hline 365 & 1.8 & 2,330 & 0.0 & 3.0 & 8.1 & $\begin{array}{c}50 \text {-year } \\
\text { (in) }\end{array}$ \\
\hline
\end{tabular}

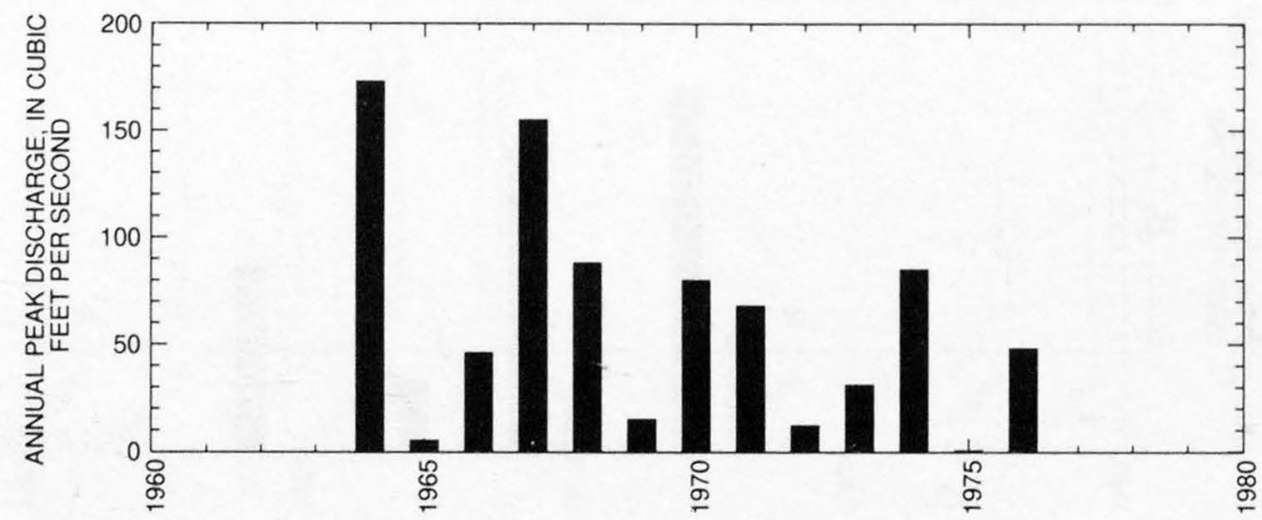


09428550 BOUSE WASH TRIBUTARY NEAR BOUSE, AZ

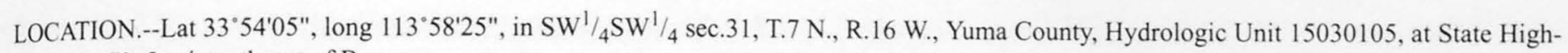
way 72,3 mi southeast of Bouse.

DRAINAGE AREA.-- $14.6 \mathrm{mi}^{2}$.

Annual peak discharges

\begin{tabular}{cccccccc}
\hline $\begin{array}{c}\text { Water } \\
\text { year }\end{array}$ & Date & $\begin{array}{c}\text { Annual peak } \\
\text { discharge } \\
\left(\mathbf{f t}^{3} / \mathbf{s}\right)\end{array}$ & $\begin{array}{c}\text { Discharge } \\
\text { codes }\end{array}$ & $\begin{array}{c}\text { Water } \\
\text { year }\end{array}$ & Date & $\begin{array}{c}\text { Annual peak } \\
\text { discharge } \\
\left(\mathbf{f t}^{3} / \mathbf{s}\right)\end{array}$ & $\begin{array}{c}\text { Discharge } \\
\text { codes }\end{array}$ \\
\hline 1963 & $09-17-63$ & 248 & & 1970 & $08-02-70$ & 1,000 & ES \\
1964 & $10-18-63$ & 75 & & 1971 & $08-10-71$ & 2,920 & \\
1965 & $04-03-65$ & 20 & ES & 1972 & $09-17-72$ & 76 & \\
1966 & $09-20-66$ & 678 & & 1973 & $08-16-73$ & 390 & \\
1967 & $10-04-66$ & 170 & & 1974 & $08-03-74$ & 120 & \\
1968 & $08-03-68$ & 180 & & 1975 & $07-29-75$ & 828 & \\
1969 & $08-08-69$ & 890 & & 1976 & $09-25-76$ & 1,500 & \\
\hline
\end{tabular}

Magnitude and probability of instantaneous peak flow based on period of record 1963-76

\begin{tabular}{|c|c|c|c|c|c|}
\hline \multicolumn{6}{|c|}{$\begin{array}{l}\text { Discharge, in } \mathrm{ft}^{3} / \mathrm{s} \text {, for indicated recurrence interval } \\
\text { in years, and exceedance probablility, in percent }\end{array}$} \\
\hline $\begin{array}{c}2 \\
50 \%\end{array}$ & $\begin{array}{c}5 \\
20 \%\end{array}$ & $\begin{array}{c}10 \\
10 \%\end{array}$ & $\begin{array}{l}25 \\
4 \%\end{array}$ & $\begin{array}{l}50 \dagger \\
2 \%\end{array}$ & $\begin{array}{c}100 \dagger \\
1 \%\end{array}$ \\
\hline 344 & 998 & 1,730 & 3,070 & 4,440 & 6,170 \\
\hline $\begin{array}{l}\text { Weighted skew } \\
\text { Mean } \\
\text { Standard dev. }\end{array}$ & $\begin{array}{l}(\log s)= \\
(\operatorname{logs})= \\
(\operatorname{logs})=\end{array}$ & $\begin{array}{r}-0.08 \\
2.53 \\
0.56\end{array}$ & & & \\
\hline
\end{tabular}

Basin characteristics

\begin{tabular}{|c|c|c|c|c|c|c|c|}
\hline \multirow[b]{2}{*}{$\begin{array}{l}\text { Main } \\
\text { channel } \\
\text { slope } \\
(\mathrm{ft} / \mathrm{mi})\end{array}$} & \multirow[b]{2}{*}{$\begin{array}{l}\text { Stream } \\
\text { length } \\
(\mathrm{mi})\end{array}$} & \multirow[b]{2}{*}{$\begin{array}{c}\text { Mean } \\
\text { basin } \\
\text { elevation } \\
\text { (ft) }\end{array}$} & \multirow[b]{2}{*}{$\begin{array}{c}\text { Forested } \\
\text { area } \\
\text { (percent) }\end{array}$} & \multirow[b]{2}{*}{$\begin{array}{c}\text { Soil } \\
\text { index }\end{array}$} & \multirow[b]{2}{*}{$\begin{array}{c}\text { Mean } \\
\text { annual } \\
\text { precipitation } \\
\text { (in) }\end{array}$} & \multicolumn{2}{|c|}{ Rainfall intensity, 24-hour } \\
\hline & & & & & & $\begin{array}{l}\text { 2-year } \\
\text { (in) }\end{array}$ & $\begin{array}{l}50 \text {-year } \\
\text { (in) }\end{array}$ \\
\hline 50.0 & 8.3 & 1,230 & 0.0 & 3.0 & 6.5 & 1.5 & 3.7 \\
\hline
\end{tabular}

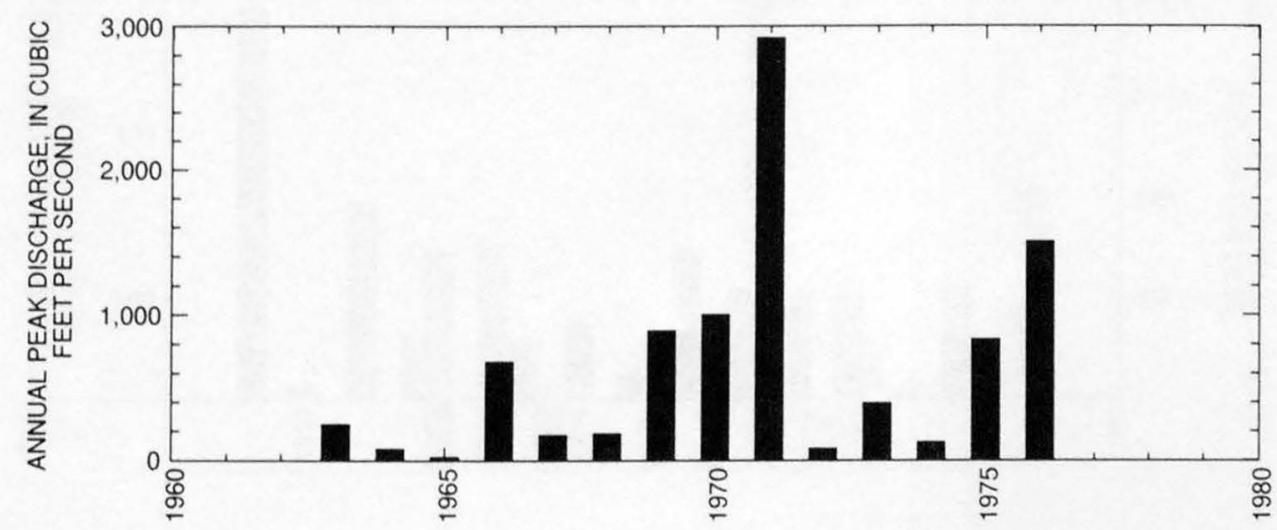


09428800 TYSON WASH TRIBUTARY NEAR QUARTZSITE, AZ

LOCATION.--Lat 33 $30^{\prime} 45^{\prime \prime}$, long $114^{\circ} 13^{\prime} 00^{\prime \prime}$, in SW $\mathrm{SW}_{4}^{1}$ sec.15, T.2 N., R.19 W., Yuma County, Hydrologic Unit 15030106, at U.S. Highway 95. $10.7 \mathrm{mi}$ south of Quartzsite.

DRAINAGE AREA.--13.7 $\mathrm{mi}^{2}$, contributing drainage area not determined.

Annual peak discharges

\begin{tabular}{|c|c|c|c|c|c|c|c|}
\hline $\begin{array}{l}\text { Water } \\
\text { year }\end{array}$ & Date & $\begin{array}{c}\text { Annual peak } \\
\text { discharge } \\
\left(\mathrm{ft}^{3} / \mathrm{s}\right)\end{array}$ & $\begin{array}{l}\text { Discharge } \\
\text { codes }\end{array}$ & $\begin{array}{l}\text { Water } \\
\text { year }\end{array}$ & Date & $\begin{array}{c}\text { Annual peak } \\
\text { discharge } \\
\left(\mathrm{ft}^{3} / \mathrm{s}\right)\end{array}$ & $\begin{array}{l}\text { Discharge } \\
\text { codes }\end{array}$ \\
\hline 1963 & $09-17-63$ & 554 & & 1970 & $08-00-70$ & 365 & \\
\hline 1964 & $08-01-64$ & 55 & & 1971 & $08-19-71$ & 300 & \\
\hline 1965 & $08-19-65$ & 503 & & 1972 & $08-08-72$ & 5.0 & ES \\
\hline 1966 & $09-13-66$ & 510 & & 1973 & $08-04-73$ & 300 & \\
\hline 1967 & $10-04-66$ & 535 & & 1974 & $08-03-74$ & 980 & \\
\hline 1968 & $07-05-68$ & 740 & & 1975 & $09-07-75$ & 80 & \\
\hline 1969 & $09-13-69$ & 120 & & 1976 & $09-25-76$ & 1,950 & \\
\hline
\end{tabular}

Magnitude and probability of instantaneous peak flow based on period of record

\begin{tabular}{cccccc}
\hline \multicolumn{5}{c}{$\begin{array}{l}\text { Discharge, in } \mathrm{ft}^{3} / \mathrm{s} \text {, for indicated recurrence interval } \\
\text { in years, and exceedance probablility, in percent }\end{array}$} \\
\hline 2 & 5 & 10 & 25 & 50 & 100 \\
$50 \%$ & $20 \%$ & $10 \%$ & $4 \%$ & $2 \%$ & $1 \%$ \\
\hline & & & & & \\
Weighted skew & $(\operatorname{logs})=$ & $-\cdots$ & & & \\
Mean & $(\operatorname{logs})=$ & --- & & \\
Standard dev. & $(\log )=$ & $-\cdots$ & & & \\
\hline
\end{tabular}

Basin characteristics

\begin{tabular}{ccccccc}
\hline $\begin{array}{c}\text { Main } \\
\text { channel } \\
\text { slope } \\
(\mathrm{ft} / \mathrm{mi})\end{array}$ & $\begin{array}{c}\text { Stream } \\
\text { length } \\
(\mathrm{mi})\end{array}$ & $\begin{array}{c}\text { Mean } \\
\text { basin } \\
\text { elevation } \\
(\mathrm{ft})\end{array}$ & $\begin{array}{c}\text { Forested } \\
\text { area } \\
\text { (percent) }\end{array}$ & $\begin{array}{c}\text { Soil } \\
\text { index }\end{array}$ & $\begin{array}{c}\text { Mean } \\
\text { annual } \\
\text { precipitation } \\
\text { (in) }\end{array}$ & $\begin{array}{c}\text { R-year } \\
\text { (in) }\end{array}$ \\
\hline 75 & 8.0 & 1,520 & 0.0 & 3.0 & 6.0 & $\begin{array}{c}50-y e a r \\
\text { (in) }\end{array}$ \\
\hline
\end{tabular}

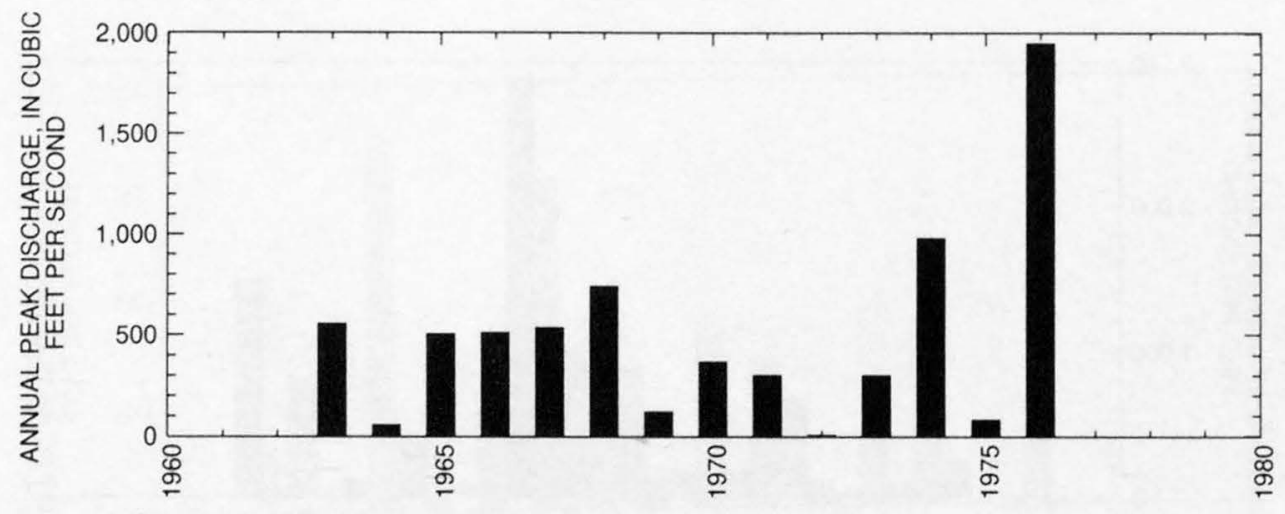


09429150 CREOSOTE WASH NEAR EHRENBERG, AZ

LOCATION.--Lat $33^{\circ} 37^{\prime} 15^{\prime \prime}$, long $114^{\circ} 29^{\prime} 41^{\prime \prime}$, in NE $\frac{1}{4}$ sec.2, T.3 N., R.22 W., Yuma County, Hydrologic Unit 15030104, at Parker Valley Road,

2.5 mi northeast of Ehrenberg, and 6 mi northeast of Blythe, California.

DRAINAGE AREA.--1.98 $\mathrm{mi}^{2}$.

Annual peak discharges

\begin{tabular}{|c|c|c|c|c|c|c|c|}
\hline $\begin{array}{l}\text { Water } \\
\text { year }\end{array}$ & Date & $\begin{array}{c}\text { Annual peak } \\
\text { discharge } \\
\left(\mathrm{ft}^{3} / \mathrm{s}\right)\end{array}$ & $\begin{array}{c}\text { Discharge } \\
\text { codes }\end{array}$ & $\begin{array}{l}\text { Water } \\
\text { year }\end{array}$ & Date & $\begin{array}{c}\text { Annual peak } \\
\text { discharge } \\
\left(\mathrm{ft}^{3} / \mathrm{s}\right)\end{array}$ & $\begin{array}{l}\text { Discharge } \\
\text { codes }\end{array}$ \\
\hline 1965 & $08-16-65$ & 259 & & 1971 & $08-10-71$ & 580 & \\
\hline 1966 & $12-09-65$ & 265 & & 1972 & $08-08-72$ & 72 & \\
\hline 1967 & $08-14-67$ & 98 & & 1973 & $08-16-73$ & 125 & \\
\hline 1968 & $07-06-68$ & 43 & & 1974 & $08-00-74$ & 5.0 & LT \\
\hline 1969 & $07-17-69$ & 65 & & 1975 & $09-16-75$ & 28 & \\
\hline 1970 & $02-28-70$ & 34 & & 1976 & $09-25-76$ & 325 & \\
\hline
\end{tabular}

Magnitude and probability of instantaneous peak flow based on period of record 1965-76

\begin{tabular}{|c|c|c|c|c|c|}
\hline \multicolumn{6}{|c|}{$\begin{array}{l}\text { Discharge, in } \mathrm{ft}^{3} / \mathrm{s} \text {, for indicated recurrence interval } \\
\text { in years, and exceedance probablility, in percent }\end{array}$} \\
\hline 2 & 5 & 10 & $25 \dagger$ & $50 t$ & $100 t$ \\
\hline $50 \%$ & $20 \%$ & $10 \%$ & $4 \%$ & $2 \%$ & $1 \%$ \\
\hline 95.6 & 239 & 390 & 660 & 932 & 1,270 \\
\hline Weighted skew & $(\log s)=$ & 0.09 & & & \\
\hline Mean & $(\operatorname{logs})=$ & 1.99 & & & \\
\hline Standard dev. & $(\log s)=$ & 0.47 & & & \\
\hline
\end{tabular}

fReliability of values in column is uncertain, and potential errors are large.

Basin characteristics

\begin{tabular}{cccccccc}
\hline $\begin{array}{c}\text { Main } \\
\text { channel } \\
\text { slope } \\
(\mathrm{ft} / \mathrm{mi})\end{array}$ & $\begin{array}{c}\text { Stream } \\
\text { length } \\
\text { (mi) }\end{array}$ & $\begin{array}{c}\text { Mean } \\
\text { basin } \\
\text { elevation } \\
(\mathrm{ft})\end{array}$ & $\begin{array}{c}\text { Forested } \\
\text { area } \\
\text { (percent) }\end{array}$ & $\begin{array}{c}\text { Soil } \\
\text { index }\end{array}$ & $\begin{array}{c}\text { Mean } \\
\text { annual } \\
\text { precipitation } \\
\text { (in) }\end{array}$ & $\begin{array}{c}\text { Rainfall intensity, 24-hour } \\
\text { (in) }\end{array}$ & $\begin{array}{c}50 \text {-year } \\
\text { (in) }\end{array}$ \\
\hline 95.2 & 4.2 & 509 & 0.0 & 3.0 & 5.5 & 1.2 \\
\hline
\end{tabular}

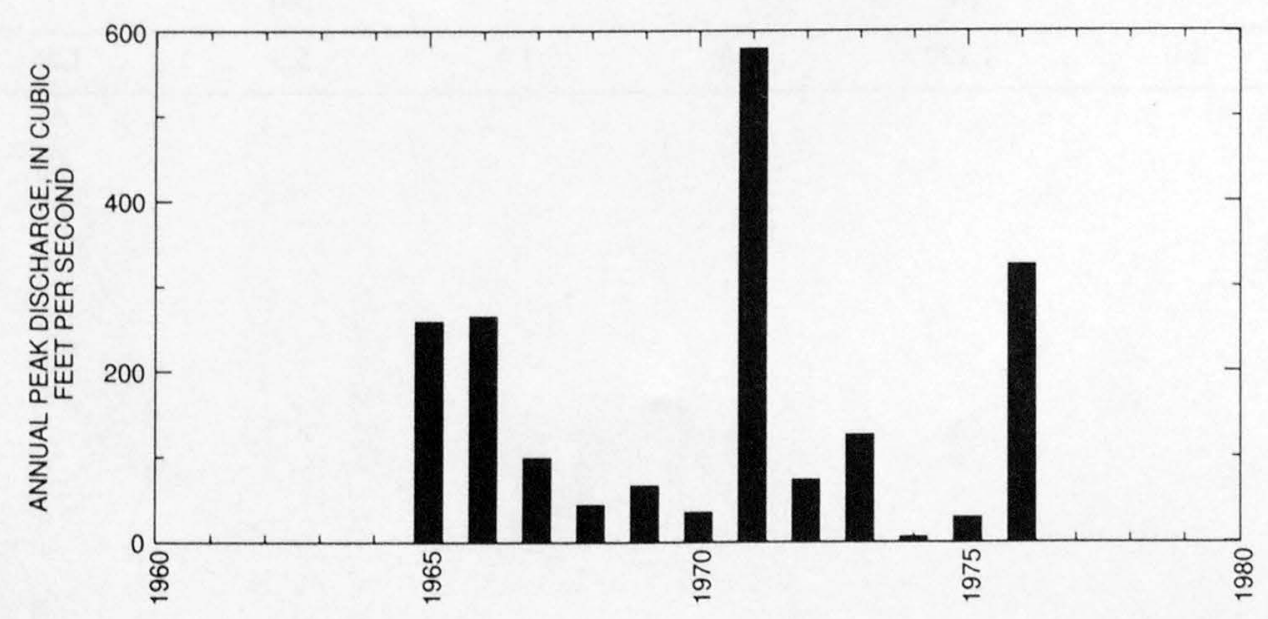


09429400 INDIAN WASH TRIBUTARY NEAR YUMA, AZ

LOCATION.--Lat $33^{\circ} 06^{\prime} 33^{\prime \prime}$, long $114^{\circ} 17^{\prime} 41^{\prime \prime}$, in NW $\frac{1}{4}$ sec.2, T.4 N., R.20 W. (unsurveyed), Yuma County, Hydrologic Unit 15030104 , at U.S. Highway 95,33 mi northeast of Dome.

DRAINAGE AREA.--2.56 $\mathrm{mi}^{2}$.

Annual peak discharges

\begin{tabular}{|c|c|c|c|c|c|c|c|}
\hline $\begin{array}{l}\text { Water } \\
\text { year }\end{array}$ & Date & $\begin{array}{c}\text { Annual peak } \\
\text { discharge } \\
\left(\mathrm{ft}^{3} / \mathrm{s}\right)\end{array}$ & $\begin{array}{c}\text { Discharge } \\
\text { codes }\end{array}$ & $\begin{array}{l}\text { Water } \\
\text { year }\end{array}$ & Date & $\begin{array}{c}\text { Annual peak } \\
\text { discharge } \\
\left(\mathrm{ft}^{3} / \mathrm{s}\right)\end{array}$ & $\begin{array}{l}\text { Discharge } \\
\text { codes }\end{array}$ \\
\hline 1963 & $09-17-63$ & 72 & & 1971 & $09-29-71$ & 45 & \\
\hline 1964 & $10-18-63$ & 24 & & 1972 & $08-00-72$ & 29 & \\
\hline 1965 & $04-03-65$ & 37 & & 1973 & $10-06-72$ & 37 & \\
\hline 1966 & $09-13-66$ & 90 & & 1974 & $07-21-74$ & 57 & \\
\hline 1967 & $10-03-66$ & 24 & & 1975 & $00-00-75$ & 40 & \\
\hline 1968 & $11-00-67$ & 50 & & 1976 & $09-25-76$ & 61 & \\
\hline 1969 & $09-06-69$ & 43 & & 1980 & $00-00-80$ & ${ }^{1} 98$ & HP \\
\hline 1970 & $03-01-70$ & 36 & & & & & \\
\hline
\end{tabular}

${ }^{1}$ Highest since 1976.

Magnitude and probability of instantaneous peak flow based on period of record 1963-76, 1980

\begin{tabular}{|c|c|c|c|c|c|}
\hline \multicolumn{6}{|c|}{$\begin{array}{l}\text { Discharge, in } \mathrm{ft}^{3} / \mathrm{s} \text {, for indicated recurrence interval } \\
\text { in years, and exceedance probablility, in percent }\end{array}$} \\
\hline $2+$ & $5 \dagger$ & $10 t$ & $25 \dagger$ & $50 t$ & $100 \dagger$ \\
\hline $50 \%$ & $20 \%$ & $10 \%$ & $4 \%$ & $2 \%$ & $1 \%$ \\
\hline 44.7 & 64.8 & 79.3 & 99 & 115 & 131 \\
\hline Weighted skew & $(\log s)=$ & 0.19 & & & \\
\hline Mean & $(\log s)=$ & 1.66 & & & \\
\hline Standard dev. & $(\log s)=$ & 0.19 & & & \\
\hline
\end{tabular}

$\mp$ Reliability of values in column is uncertain, and potential errors are large.

Basin characteristics

\begin{tabular}{ccccccc}
\hline $\begin{array}{c}\text { Main } \\
\text { channel } \\
\text { slope } \\
(\mathrm{ft} / \mathrm{mi})\end{array}$ & $\begin{array}{c}\text { Stream } \\
\text { length } \\
\text { (mi) }\end{array}$ & $\begin{array}{c}\text { Mean } \\
\text { basin } \\
\text { elevation } \\
(\mathrm{ft})\end{array}$ & $\begin{array}{c}\text { Forested } \\
\text { area } \\
\text { (percent) }\end{array}$ & $\begin{array}{c}\text { Soil } \\
\text { index }\end{array}$ & $\begin{array}{c}\text { Mean } \\
\text { annual } \\
\text { precipitation } \\
\text { (in) }\end{array}$ & $\begin{array}{c}\text { Rainfall intensity, 24-hour } \\
\text { (in) }\end{array}$ \\
\hline 66.7 & 5.0 & 1,190 & 0.0 & 3.0 & 5.5 & $\begin{array}{c}50 \text {-year } \\
\text { (in) }\end{array}$ \\
\hline
\end{tabular}


09429400 INDIAN WASH TRIBUTARY NEAR YUMA, AZ--Continued

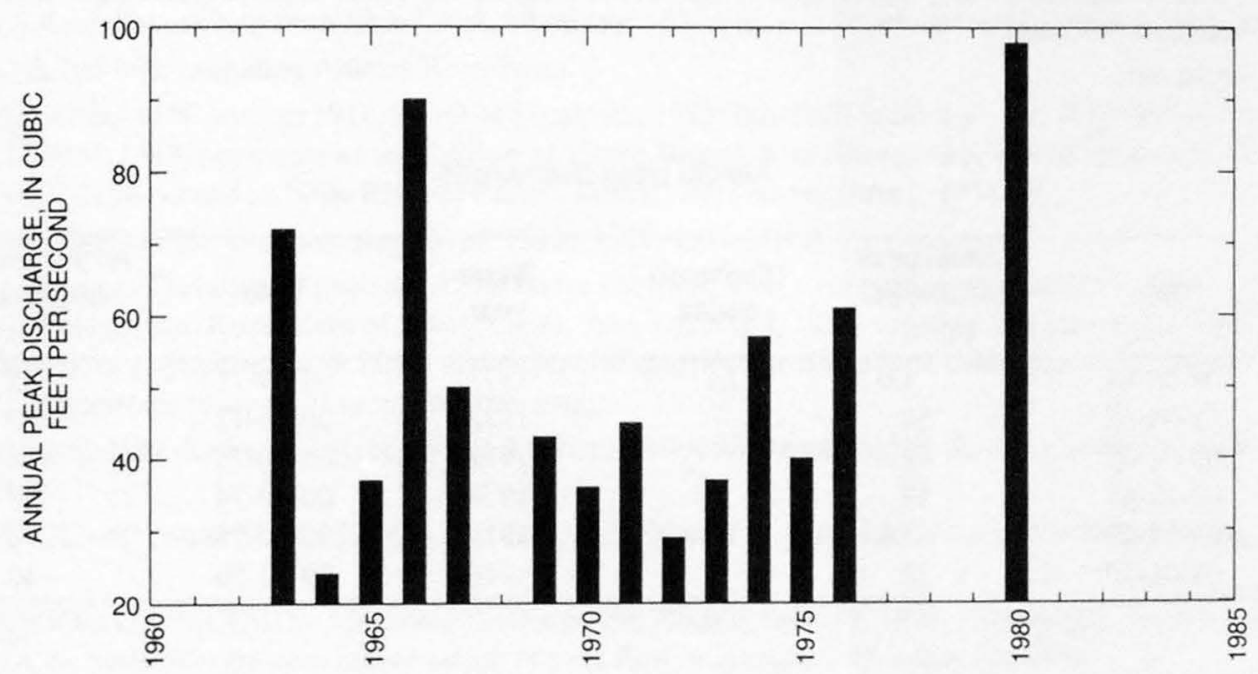


09429510 MITTRY LAKE TRIBUTARY NEAR YUMA, AZ

LOCATION.--Lat $32^{\circ} 51^{\prime} 35^{\prime \prime}$, long $114^{\circ} 26^{\prime} 05^{\prime \prime}$, in SWl/4 $\mathrm{SE}^{1} / 4$ sec.32, T.6 S., R.21 W., Yuma County, Hydrologic Unit 15030107 , at Yuma Test Station, $14.5 \mathrm{mi}$ northeast of Yuma.

DRAINAGE AREA.--0.30 $\mathrm{mi}^{2}$.

Annual peak discharges

\begin{tabular}{cccccrr}
\hline $\begin{array}{c}\text { Water } \\
\text { year }\end{array}$ & Date & $\begin{array}{c}\text { Annual peak } \\
\text { discharge } \\
\left(\mathbf{f t}^{3} / \mathbf{s}\right)\end{array}$ & $\begin{array}{c}\text { Discharge } \\
\text { codes }\end{array}$ & $\begin{array}{c}\text { Water } \\
\text { year }\end{array}$ & $\begin{array}{c}\text { Date } \\
\text { Annual peak } \\
\text { discharge } \\
\left(\mathbf{f t}^{3} / \mathbf{s}\right)\end{array}$ \\
\hline 1965 & $04-03-65$ & 1.0 & LT & 1971 & $09-29-71$ & 45 \\
1966 & $12-09-65$ & 50 & & 1972 & $06-06-72$ & 55 \\
1967 & $09-02-67$ & 42 & & 1973 & $08-16-73$ & 165 \\
1968 & $02-12-68$ & 48 & ES & 1974 & $00-00-74$ & 0 \\
1969 & $01-14-69$ & 3.0 & & 1975 & $00-00-75$ & 0 \\
1970 & $03-01-70$ & 22 & & 1976 & $09-24-76$ & 10 \\
\hline
\end{tabular}

Magnitude and probability of instantaneous peak flow based on period of record 1965-76

\begin{tabular}{|c|c|c|c|c|c|}
\hline \multicolumn{6}{|c|}{$\begin{array}{l}\text { Discharge, in } \mathrm{ft}^{3} / \mathrm{s} \text {, for indicated recurrence interval } \\
\text { in years, and exceedance probablility, in percent }\end{array}$} \\
\hline $\begin{array}{c}2 \dagger \\
50 \%\end{array}$ & $\begin{array}{c}5 \dagger \\
20 \%\end{array}$ & $\begin{array}{r}10 \dagger \\
10 \%\end{array}$ & $\begin{array}{l}25 \dagger \\
4 \%\end{array}$ & $\begin{array}{l}50 \dagger \\
2 \%\end{array}$ & $\begin{array}{c}100 \dagger \\
1 \%\end{array}$ \\
\hline 22 & 62.8 & 106 & 182 & 256 & 346 \\
\hline $\begin{array}{l}\text { Weighted skew } \\
\text { Mean }\end{array}$ & $\begin{array}{l}(\operatorname{logs})= \\
(\log s)= \\
(\operatorname{logs})=\end{array}$ & $\begin{array}{r}-0.19 \\
1.33 \\
0.56\end{array}$ & & & \\
\hline
\end{tabular}

千 Reliability of values in column is uncertain, and potential errors are large.

Basin characteristics

\begin{tabular}{ccccccc}
\hline $\begin{array}{c}\text { Main } \\
\text { channel } \\
\text { slope } \\
\text { (ft/mi) }\end{array}$ & $\begin{array}{c}\text { Stream } \\
\text { length } \\
\text { (mi) }\end{array}$ & $\begin{array}{c}\text { Mean } \\
\text { basin } \\
\text { elevation } \\
\text { (ft) }\end{array}$ & $\begin{array}{c}\text { Forested } \\
\text { area } \\
\text { (percent) }\end{array}$ & $\begin{array}{c}\text { Soil } \\
\text { index }\end{array}$ & $\begin{array}{c}\text { Mean } \\
\text { annual } \\
\text { precipitation } \\
\text { (in) }\end{array}$ & $\begin{array}{c}\text { Rainfall intensity, 24-hour } \\
\text { (in) }\end{array}$ \\
\hline 122 & 0.98 & 346 & 0.0 & 3.0 & 4.2 & $\begin{array}{c}50 \text {-year } \\
\text { (in) }\end{array}$ \\
\hline
\end{tabular}

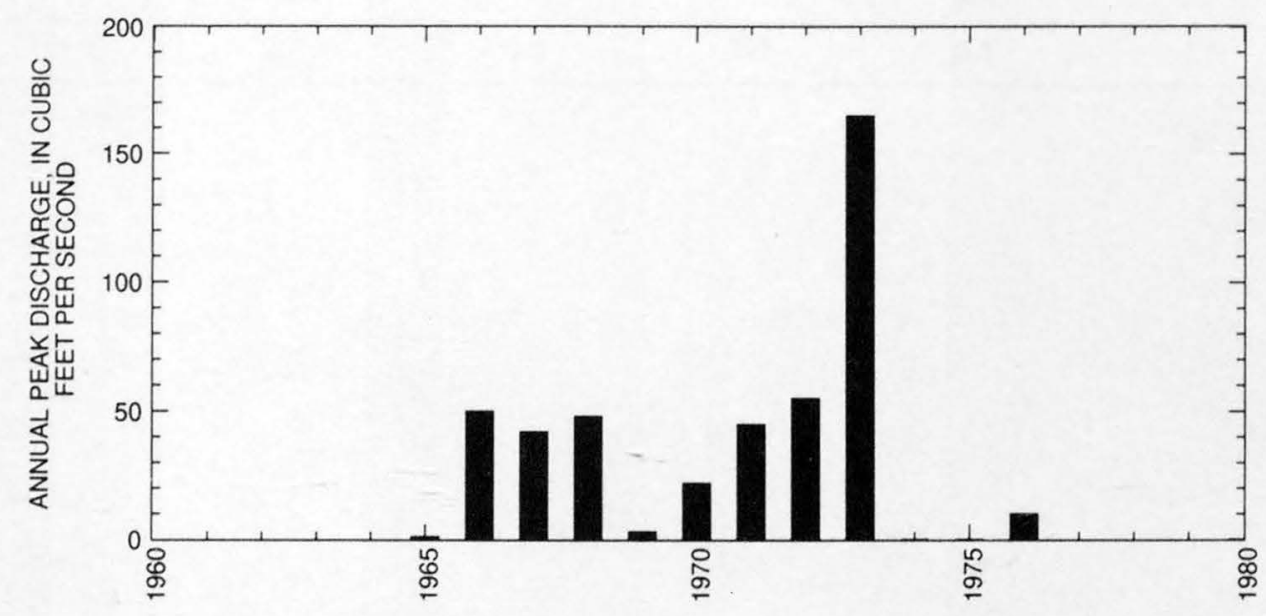




\section{GILA RIVER BELOW BLUE CREEK, NEAR VIRDEN, NM}

LOCATION.--Lat 32'38'53", long 108 50'43", in SE $1 /{ }_{4} \mathrm{SW}^{1} / 4 \mathrm{sec} .18$, T.19 S., R. 19 W., Grant County, Hydrologic Unit 15040002 , on left bank at head of canyon, $1.4 \mathrm{mi}$ downstream from Blue Creek, $10 \mathrm{mi}$ east of Virden, and $16 \mathrm{mi}$ upstream from New Mexico-Arizona State line.

DRAINAGE AREA.--3,203 $\mathrm{mi}^{2}$, excluding Animas River basin.

PERIOD OF RECORD.--May to November 1914, March to September 1915, July 1927 to current year. July 1927 to May 1931 monthly discharge only, published in WSP 1313, computed as sum of flow at Virden Bridge, 9 mi downstream, and in Sunset Canal. Published as "Gila River near Duncan, Ariz.," 1914-15 and as "Gila River at Fuller's Ranch, near Duncan, Ariz.," 1931-38.

REVISED RECORDS.--WSP 1283: Drainage area. WSP 1313: 1929, 1931-32(M).

GAGE.--Water-stage recorder. Elevation of gage is 3,875 ft above sea level, from river-profile map. May 11, 1914, to Sept. 30, 1915, at site 6 mi downstream, 1,000 ft upstream from intake of Sunset Canal. June 1 to July 7, 1931, nonrecording gage at present site and datum. Since April 18,1980 , supplementary gage on left bank $800 \mathrm{ft}$ downstream at same datum. Since June 1980, crest-stage gages at supplementary gage site. Since Nov. 1990, water-stage recorder at supplementary gage.

REMARKS.--No estimated daily discharges. Records fair. Station is above all Duncan Valley diversions. Diversions for irrigation of about 6,200 acres above station.

AVERAGE DISCHARGE.--69 (water years 1928-96), $215 \mathrm{ft}^{3} / \mathrm{s}, 155,800 \mathrm{acre}-\mathrm{ft} / \mathrm{yr}$; median of yearly mean discharges, $150 \mathrm{ft}^{3} / \mathrm{s}, 109,000 \mathrm{acre}^{\mathrm{ft}} /$ yr.

EXTREMES FOR PERIOD OF RECORD.--Maximum discharge, 58,700 $\mathrm{ft}^{3} / \mathrm{s}$ Dec. 19, 1978, gage height, $29.00 \mathrm{ft}$, from rating curve extended above $38,000 \mathrm{ft}^{3} / \mathrm{s}$ on basis of slope-area measurement of peak flow; minimum, $1 \mathrm{ft}^{3} / \mathrm{s} \mathrm{July} 14,1934$.

Annual peak discharges

\begin{tabular}{|c|c|c|c|c|c|c|c|}
\hline $\begin{array}{l}\text { Water } \\
\text { year }\end{array}$ & Date & $\begin{array}{c}\text { Annual peak } \\
\text { discharge } \\
\left(\mathrm{ft}^{3} / \mathrm{s}\right)\end{array}$ & $\begin{array}{l}\text { Discharge } \\
\text { codes }\end{array}$ & $\begin{array}{l}\text { Water } \\
\text { year }\end{array}$ & Date & $\begin{array}{c}\text { Annual peak } \\
\text { discharge } \\
\left(\mathrm{ft}^{3} / \mathrm{s}\right)\end{array}$ & $\begin{array}{l}\text { Discharge } \\
\text { codes }\end{array}$ \\
\hline 1927 & $07-04-27$ & 1,800 & & 1962 & $09-26-62$ & 3,920 & \\
\hline 1928 & $07-26-28$ & 1,630 & & 1963 & $08-31-63$ & 7,320 & \\
\hline 1929 & $07-30-29$ & 5,700 & & 1964 & $07-25-64$ & 4,480 & \\
\hline 1930 & $08-11-30$ & 7,400 & & 1965 & $07-24-65$ & 2,540 & \\
\hline 1931 & $08-03-31$ & 8,000 & & 1966 & $12-23-65$ & 10,900 & \\
\hline 1932 & $07-30-32$ & 6,800 & & 1967 & $08-12-67$ & 11,500 & \\
\hline 1933 & $09-08-33$ & 5,650 & & 1968 & $02-15-68$ & 2,920 & \\
\hline 1934 & $08-2634$ & 8,920 & & 1969 & $09-02-69$ & 1,790 & \\
\hline 1935 & $09-27-35$ & 8,600 & & 1970 & $09-19-70$ & 1,130 & \\
\hline 1936 & $06-11-36$ & 3,600 & & 1971 & $09-18-71$ & 3,730 & \\
\hline 1937 & $02-17-37$ & 9,070 & & 1972 & $10-26-71$ & 5,700 & \\
\hline 1938 & $08-31-38$ & 6,400 & & 1973 & $10-20-72$ & 27,200 & \\
\hline 1939 & $09-16-39$ & 1,630 & & 1974 & 08-04-74 & 7,560 & \\
\hline 1940 & $09-06-40$ & 11,000 & & 1975 & $09-08-75$ & 7,720 & \\
\hline 1941 & $09-29-41$ & 141,700 & & 1976 & $09-15-76$ & 3,700 & \\
\hline 1942 & $09-13-42$ & 3,140 & & 1977 & $08-13-77$ & 4,450 & \\
\hline 1943 & $09-27-43$ & 1,600 & & 1978 & 03-03-78 & 7,800 & \\
\hline 1944 & $08-19-44$ & 4,010 & & 1979 & $12-19-78$ & 58,700 & \\
\hline 1945 & $08-11-45$ & 5,370 & & 1980 & $09-10-80$ & 4,300 & \\
\hline 1946 & $10-08-45$ & 10,600 & & 1981 & $08-18-81$ & 1,890 & \\
\hline 1947 & $08-22-47$ & 3,400 & & 1982 & $10-02-81$ & 3,680 & \\
\hline 1948 & $08-12-48$ & 2,240 & & 1983 & $02-04-83$ & 5,870 & \\
\hline 1949 & $01-14-49$ & 15,600 & & 1984 & $10-02-83$ & 15,500 & \\
\hline 1950 & $09-24-50$ & 2,190 & & 1985 & $12-28-84$ & 37,000 & \\
\hline 1951 & $08-28-51$ & 440 & & 1986 & $10-11-85$ & 6,670 & \\
\hline 1952 & $01-19-52$ & 6,100 & & 1987 & $08-11-87$ & 2,680 & \\
\hline 1953 & $08-21-53$ & 3,330 & & 1988 & $09-22-88$ & 9,000 & \\
\hline 1954 & $08-21-54$ & 6,670 & & 1989 & $07-30-89$ & 696 & \\
\hline 1955 & $07-28-55$ & 5,280 & & 1990 & $08-23-90$ & 710 & \\
\hline 1956 & $08-13-56$ & 2,660 & & 1991 & $03-02-91$ & 10,200 & \\
\hline 1957 & $08-05-57$ & 6,710 & & 1992 & $02-14-92$ & 4,430 & \\
\hline 1958 & $03-23-58$ & 4,550 & & 1993 & $01-11-93$ & 30,000 & \\
\hline 1959 & $08-13-59$ & 16,400 & & 1994 & $09-06-94$ & 783 & \\
\hline 1960 & $01-12-60$ & 5,220 & & 1995 & $12-06-94$ & 22,700 & \\
\hline 1961 & $08-15-61$ & 1,920 & & 1996 & $08-21-96$ & 11,200 & \\
\hline
\end{tabular}

${ }^{1}$ Highest since 1891 . 


\section{GILA RIVER BASIN}

09432000 GILA RIVER BELOW BLUE CREEK, NEAR VIRDEN, NM--Continued

Discharge rating table developed November 1994

\begin{tabular}{crcc}
\hline $\begin{array}{c}\text { Gage height } \\
(\mathbf{f t})\end{array}$ & $\begin{array}{c}\text { Discharge } \\
\left(\mathbf{f t}^{3} / \mathbf{s}\right)\end{array}$ & $\begin{array}{c}\text { Gage height } \\
(\mathbf{f t})\end{array}$ & $\begin{array}{c}\text { Discharge } \\
\left(\mathrm{ft}^{\mathbf{3}} \mathbf{s} \mathbf{s}\right)\end{array}$ \\
\hline 4.0 & 207 & 18.0 & 26,480 \\
6.0 & 1,200 & 20.0 & 31,570 \\
8.0 & 4,200 & 22.0 & 37,010 \\
10.0 & 9,850 & 24.0 & 42,800 \\
12.0 & 13,450 & 26.0 & 48,920 \\
14.0 & 17,400 & 28.0 & 55,360 \\
16.0 & 21,750 & 29.0 & 58,700 \\
\hline
\end{tabular}

Basin characteristics

\begin{tabular}{ccccccccc}
\hline $\begin{array}{c}\text { Main } \\
\text { channel } \\
\text { slope } \\
\text { (ft/mi) }\end{array}$ & $\begin{array}{c}\text { Stream } \\
\text { length } \\
\text { (mi) }\end{array}$ & $\begin{array}{c}\text { Mean } \\
\text { basin } \\
\text { elevation } \\
\text { (ft) }\end{array}$ & $\begin{array}{c}\text { Forested } \\
\text { area } \\
\text { (percent) }\end{array}$ & $\begin{array}{c}\text { Soil } \\
\text { index }\end{array}$ & $\begin{array}{c}\text { Mean } \\
\text { annual } \\
\text { precipitation } \\
\text { (in) }\end{array}$ & $\begin{array}{c}\text { 2-year } \\
\text { (in) }\end{array}$ & $\begin{array}{c}\text { Ro-year } \\
\text { (in) }\end{array}$ \\
\hline 35.8 & 115 & 6,690 & 54.0 & 3.0 & 16.2 & 1.6 & 3.3 \\
\hline
\end{tabular}


09432000 GILA RIVER BELOW BLUE CREEK, NEAR VIRDEN, NM--Continued

MEAN MONTHLY AND ANNUAL DISCHARGES 1991, 1995.96

\begin{tabular}{|c|c|c|c|c|c|c|}
\hline MONTH & $\begin{array}{l}\text { MAXIMUM } \\
\text { (FT3/S) }\end{array}$ & $\begin{array}{l}\text { MINIMUM } \\
(\text { FT3/S) }\end{array}$ & $\begin{array}{c}\text { MEAN } \\
(\mathrm{FT} 3 / \mathrm{S})\end{array}$ & $\begin{array}{l}\text { STAN - } \\
\text { DARD } \\
\text { DEVIA- } \\
\text { TION } \\
(\text { FT } 3 / S)\end{array}$ & $\begin{array}{l}\text { COEFFI- } \\
\text { CIENT OF } \\
\text { VARI- } \\
\text { ATION }\end{array}$ & $\begin{array}{c}\text { PERCENT } \\
\text { OF } \\
\text { ANNUAL } \\
\text { RUNOFF }\end{array}$ \\
\hline OCTOBER & 120 & 41 & 70 & 43 & 0.62 & 1.6 \\
\hline NOVEMBER & 1,040 & 67 & 409 & 547 & 1.3 & 9.3 \\
\hline DECEMBER & 1,550 & 85 & 673 & 773 & 1.2 & 15.2 \\
\hline JANUARY & 1,640 & 103 & 745 & 798 & 1.1 & 16.9 \\
\hline FEBRUARY & 1,350 & 116 & 584 & 670 & 1.2 & 13.2 \\
\hline MARCH & 1,390 & 91 & 699 & 656 & 0.94 & 15.8 \\
\hline APRIL & 722 & 58 & 311 & 359 & 1.2 & 7.0 \\
\hline MAY & 246 & 34 & 126 & 109 & 0.86 & 2.9 \\
\hline JUNE & 99 & 11 & 56 & 44 & 0.78 & 1.3 \\
\hline JULY & 122 & 52 & 89 & 35 & 0.39 & 2.0 \\
\hline AUGUST & 537 & 125 & 320 & 207 & 0.65 & 7.2 \\
\hline SEPTEMBER & 538 & 86 & 335 & 230 & 0.69 & 7.6 \\
\hline ANNUAL & 564 & 130 & 367 & 220 & 0.60 & 100 \\
\hline
\end{tabular}

MAGNITUDE AND PROBABILITY OF INSTANTANEOUS PEAK FLOW BASED ON PERIOD OF RECORD 1927.96

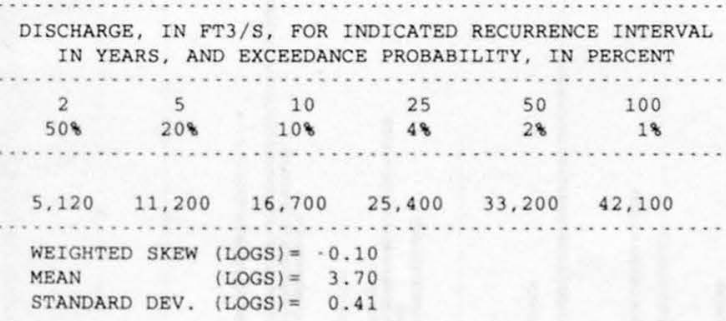

MAGNITUDE AND PROBABILITY OF ANNUAL LOW FLOW BASED ON PERIOD OF RECORD 1996

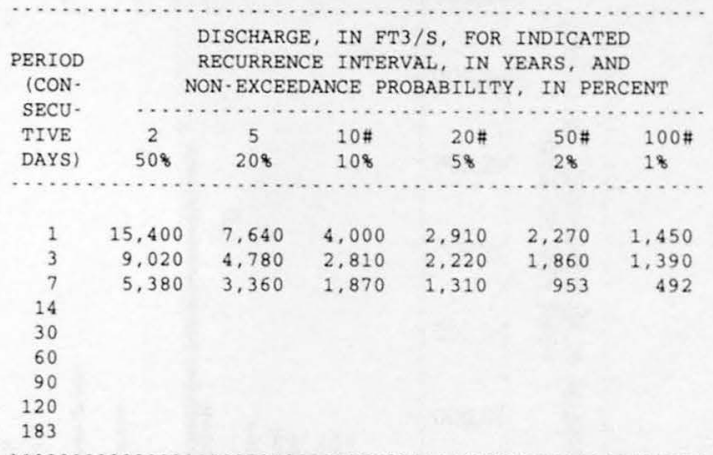

MAGNITUDE AND PROBABILITY OF ANNUAL HIGH FLOW BASED ON PERIOD OF RECORD 1991, 1995.96

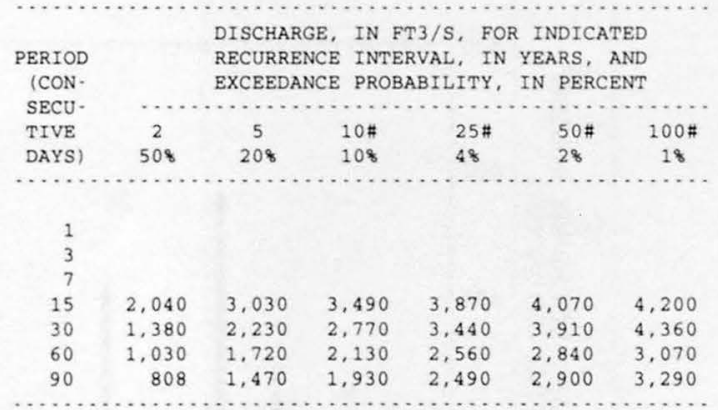

DURATION TABLE OF DAILY MEAN FLOW FOR PERIOD OF RECORD 1991, 1995.96

DISCHARGE, IN FT3/S, WHICH WAS EQUALED OR EXCEEDED FOR INDICATED PERCENT OF TIME

\begin{tabular}{|c|c|c|c|c|c|c|c|c|c|c|c|c|c|c|c|c|}
\hline 18 & 58 & 108 & 158 & 208 & 308 & 408 & 508 & 608 & 708 & 808 & 908 & 958 & 988 & 998 & 99.58 & 99.98 \\
\hline 070 & 1,260 & 747 & 577 & 451 & 243 & 156 & 120 & 99 & 77 & 59 & 42 & 30 & 13 & 9.4 & 8.3 & 7.5 \\
\hline
\end{tabular}

\# Reliability of values in column is uncertain, and potential errors are large. 
ANNUAL MEAN DISCHARGE, IN CUBIC

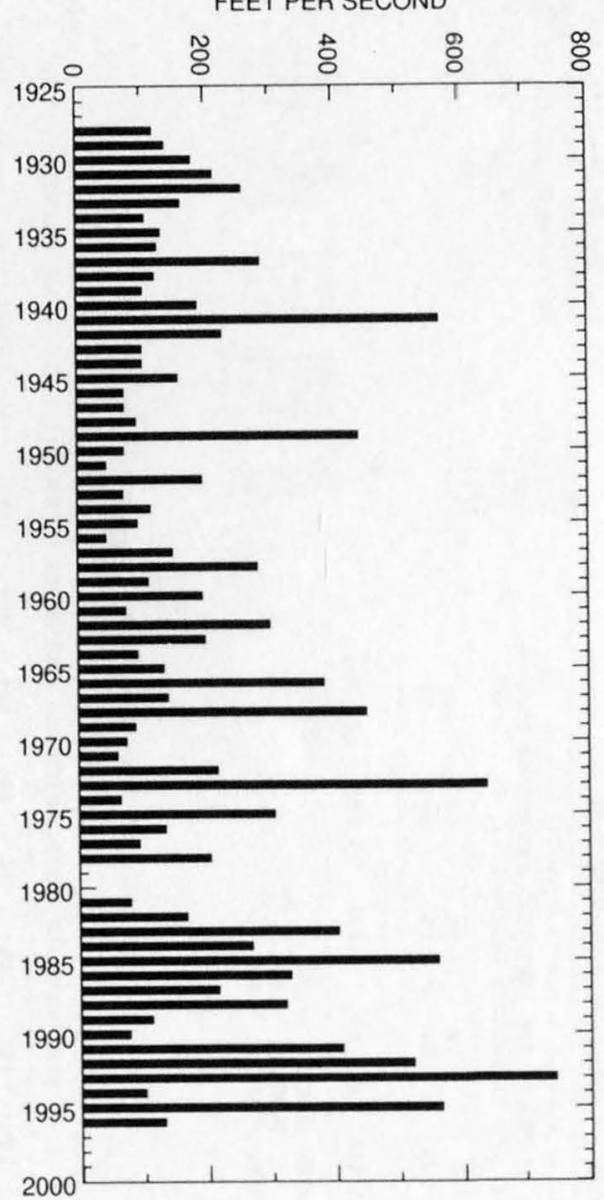

ANNUAL PEAK DISCHARGE, IN CUBIC FEET PER SECOND

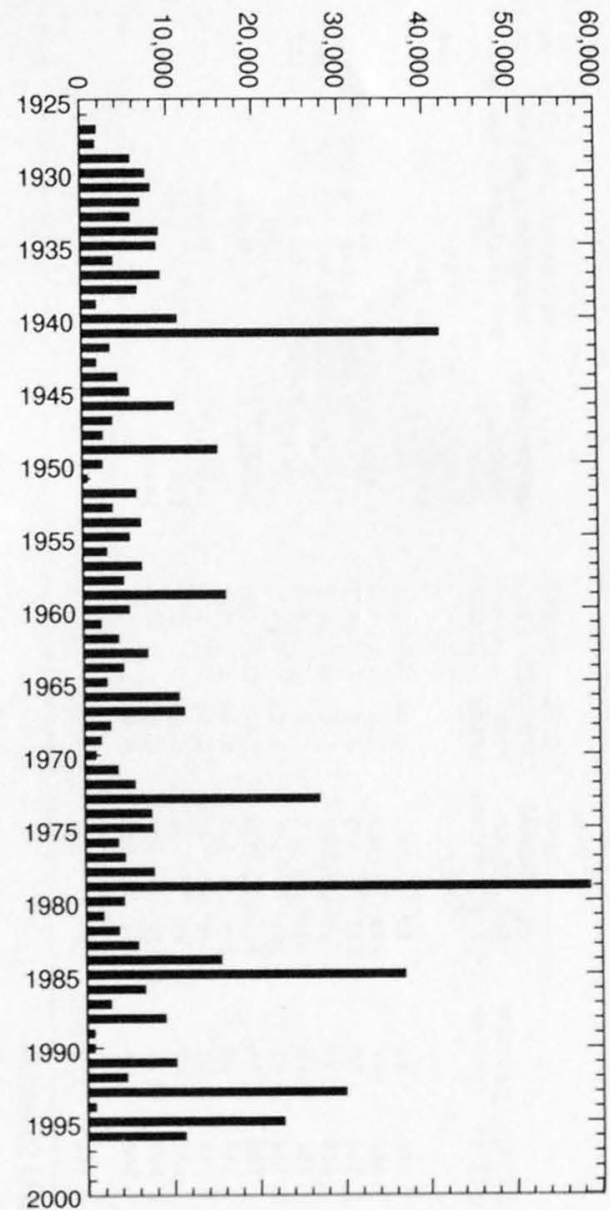


09432000 GILA RIVER BELOW BLUE CREEK, NEAR VIRDEN, NM--Continued

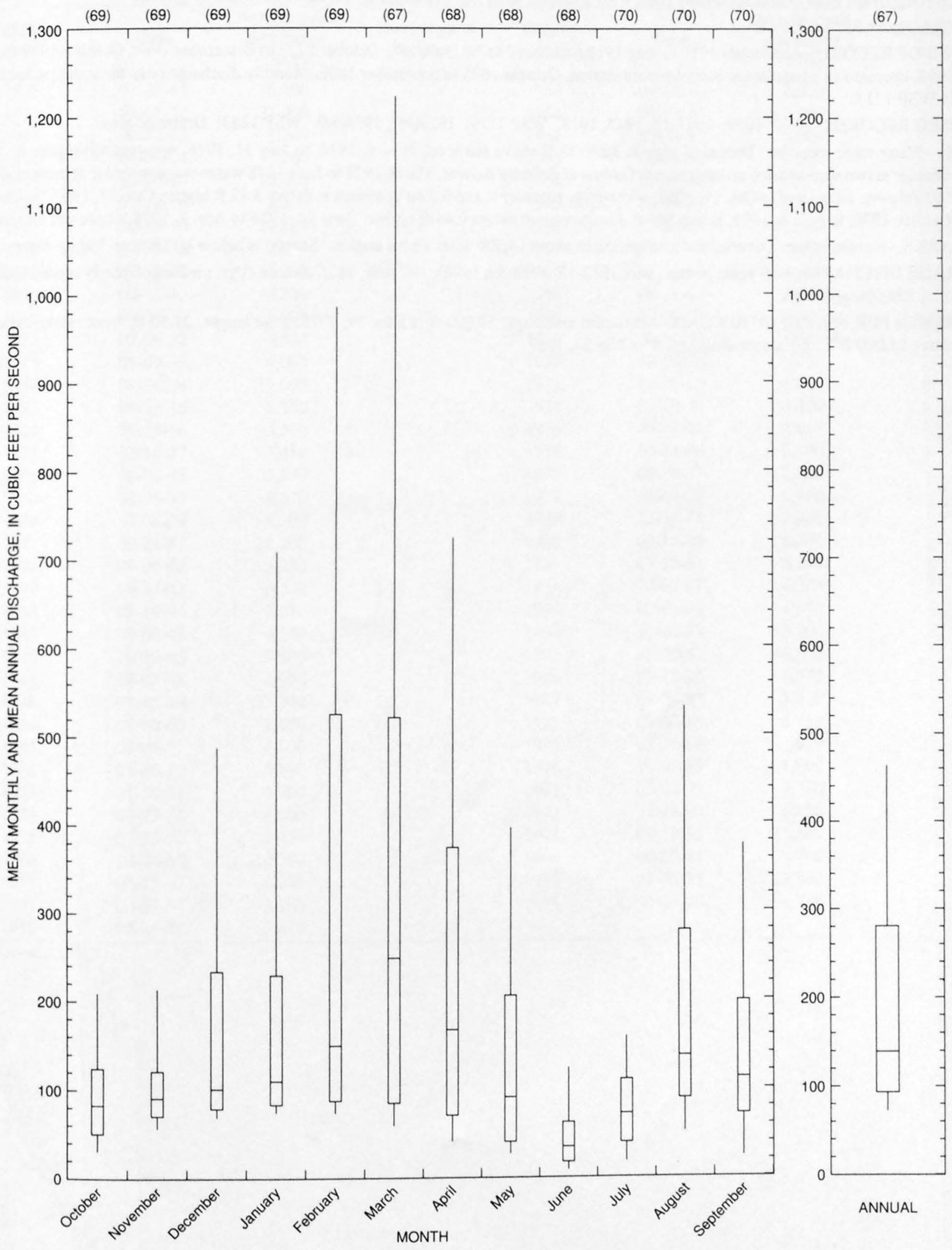


LOCATION.--Lat 32.57'57", long 109'18'35", in NE $\frac{1}{4} \mathrm{SE}^{1} / 4 \mathrm{sec} .25$, T.5 S., R.29 E., Greenlee County, Hydrologic Unit 15040002 , on right bank $60 \mathrm{ft}$ upstream from bridge on county road, 6 mi upstream from San Francisco River, and 6 mi south of Clifton.

DRAINAGE AREA.--4,010 $\mathrm{mi}^{2}$.

PERIOD OF RECORD.--November 1910 to July 1918 (published as "at Guthrie"), October 1927 to September 1989 , October 1989 to September 1995, operated as a crest-stage partial-record station, October 1995 to September 1996. Monthly discharge only for some periods, published in WSP 1313.

REVISED RECORDS.--WSP 1059: 1911-12, 1915, 1917. WSP 1179: 1929(M), 1934(M). WSP 1283: Drainage area.

GAGE.--Water-stage recorder. Datum of gage is $3,336.38 \mathrm{ft}$ above sea level. Nov. 6, 1910, to July 11 , 1918, non-recording gage or water-stage recorder at two sites about $6 \mathrm{mi}$ upstream at Guthrie at different datums. March 1928 to June 1948 water-stage recorder at present site at datum $0.91 \mathrm{ft}$ lower. June 1948 to Oct. 17, 1967, water-stage recorder at site 0.2 mi upstream at datum $3.12 \mathrm{ft}$ higher. Oct. 18, 1967, to June 23 , 1974, Apr. 10, 1978, to Feb. 6, 1979, at site $500 \mathrm{ft}$ downstream at datum $0.44 \mathrm{ft}$ higher. June 24, 1974 to Apr. 9, 1978, at present site and datum.

REMARKS.--Records poor. Diversions for irrigation of about 14,300 acres above station. Station is below all Duncan Valley diversions.

AVERAGE DISCHARGE.--69 years (water years 1912-17, 1928-89, 1996), $197 \mathrm{ft}^{3} / \mathrm{s}, 142,700$ acre-ft/yr; median of yearly mean discharges, 140 $\mathrm{ft}^{3} / \mathrm{s}, 101,000$ acre- $\mathrm{ft} / \mathrm{yr}$.

EXTREMES FOR PERIOD OF RECORD.--Maximum discharge, 57,000 $\mathrm{ft}^{3} / \mathrm{s}$ Dec. 19, 1978, gage height, $23.80 \mathrm{ft}$, from rating curve extended above $28,000 \mathrm{ft}^{3} / \mathrm{s}$; minimum daily, $3.7 \mathrm{ft}^{3} / \mathrm{s}$ July $27,1987$. 
GILA RIVER BASIN

09442000 GILA RIVER NEAR CLIFTON, AZ--Continued

Annual peak discharges

\begin{tabular}{|c|c|c|c|c|c|c|c|}
\hline $\begin{array}{l}\text { Water } \\
\text { year }\end{array}$ & Date & $\begin{array}{c}\text { Annual peak } \\
\text { discharge } \\
\left(\mathrm{ft}^{3} / \mathrm{s}\right)\end{array}$ & $\begin{array}{c}\text { Discharge } \\
\text { codes }\end{array}$ & $\begin{array}{l}\text { Water } \\
\text { year }\end{array}$ & Date & $\begin{array}{c}\text { Annual peak } \\
\text { discharge } \\
\left(\mathrm{ft}^{3} / \mathrm{s}\right)\end{array}$ & $\begin{array}{l}\text { Discharge } \\
\text { codes }\end{array}$ \\
\hline 1911 & $07-25-11$ & 16,000 & & 1960 & $01-13-60$ & 4,000 & \\
\hline 1912 & $03-11-12$ & 21,000 & & 1961 & $08-13-61$ & 2,400 & \\
\hline 1913 & $09-22-13$ & 1,200 & & 1962 & $09-26-62$ & 8,980 & \\
\hline 1914 & $08-06-14$ & 5,700 & & 1963 & $08-31-63$ & 3,580 & \\
\hline 1915 & $12-20-14$ & 12,000 & & 1964 & $07-15-64$ & 5,070 & \\
\hline 1916 & $01-18-16$ & 7,600 & & 1965 & $09-03-65$ & 3,310 & \\
\hline 1917 & $10-15-16$ & 19,500 & & 1966 & $12-24-65$ & 10,700 & \\
\hline 1928 & $07-31-28$ & 2,870 & & 1967 & $08-12-67$ & 11,100 & \\
\hline 1929 & $07-30-29$ & 13,200 & & 1968 & $03-11-68$ & 4,380 & \\
\hline 1930 & $08-11-30$ & 6,300 & & 1969 & $09-11-69$ & 3,610 & \\
\hline 1931 & $09-04-31$ & 6,900 & & 1970 & $08-05-70$ & 4,220 & \\
\hline 1932 & $07-09-32$ & 4,500 & & 1971 & $10-02-70$ & 5,010 & \\
\hline 1933 & 09-09-33 & 4,000 & & 1972 & $09-03-72$ & 6,160 & \\
\hline 1934 & $08-26-34$ & 17,000 & & 1973 & $10-21-72$ & ${ }^{1} 33,000$ & \\
\hline 1935 & $08-31-35$ & 3,100 & & 1974 & $07-19-74$ & 3,460 & \\
\hline 1936 & $08-28-36$ & 4,300 & & 1975 & $09-08-75$ & 4,660 & \\
\hline 1937 & $02-18-37$ & 7,450 & & 1976 & $02-11-76$ & 2,390 & \\
\hline 1938 & 08-06-38 & 5,930 & & 1977 & $08-13-77$ & 2,820 & \\
\hline 1939 & $08-05-39$ & 8,670 & & 1978 & $03-04-78$ & 8,420 & \\
\hline 1940 & $10-08-39$ & 6,300 & & 1979 & $12-19-78$ & 57,000 & \\
\hline 1941 & $09-29-41$ & 28,200 & & 1980 & $09-10-80$ & 8,500 & \\
\hline 1942 & $08-06-42$ & 3,280 & & 1981 & $07-12-81$ & 8,190 & \\
\hline 1943 & $09-27-43$ & 6,770 & & 1982 & $10-02-81$ & 4,520 & \\
\hline 1944 & $08-19-44$ & 2,610 & & 1983 & $02-04-83$ & 4,980 & \\
\hline 1945 & $08-08-45$ & 4,540 & & 1984 & $10-02-83$ & 15,300 & \\
\hline 1946 & $10-09-45$ & 5,800 & & 1985 & $12-29-84$ & 48,800 & \\
\hline 1947 & $08-03-48$ & 1,090 & & 1986 & $10-18-85$ & 6,270 & \\
\hline 1948 & $01-15-49$ & 13,900 & & 1987 & $07-21-87$ & 3,020 & \\
\hline 1950 & $07-30-50$ & 1,680 & & 1988 & $09-22-88$ & 6,710 & \\
\hline 1951 & $08-03-51$ & 4,600 & & 1989 & 07-31-89 & 620 & \\
\hline 1952 & $01-20-52$ & 4,280 & & 1990 & $10-05-89$ & 1,690 & \\
\hline 1953 & $07-30-53$ & 3,700 & & 1991 & $03-02-91$ & 9,730 & \\
\hline 1954 & $08-23-54$ & 6,000 & & 1992 & $12-21-91$ & 6,460 & \\
\hline 1955 & $07-23-55$ & 9,450 & & 1993 & 01-19-93 & 35,500 & \\
\hline 1956 & $10-04-55$ & 12,700 & & 1994 & $09-07-94$ & 700 & \\
\hline 1957 & $08-29-57$ & 8,070 & & 1995 & $01-05-95$ & 24,800 & \\
\hline 1958 & $03-24-58$ & 3,980 & & 1996 & $09-14-96$ & 4,780 & \\
\hline 1959 & $08-26-59$ & 5,610 & & & & & \\
\hline
\end{tabular}

${ }^{1}$ Highest since 1891. 
09442000 GILA RIVER NEAR CLIFTON, AZ--Continued

Discharge rating table developed October 1996

\begin{tabular}{cccc}
\hline $\begin{array}{c}\text { Gage height } \\
(\mathbf{f t})\end{array}$ & $\begin{array}{c}\text { Discharge } \\
\left(\mathrm{ft}^{\mathbf{3}} \mathbf{/ s}\right)\end{array}$ & $\begin{array}{c}\text { Gage height } \\
(\mathbf{f t})\end{array}$ & $\begin{array}{c}\text { Discharge } \\
\left(\mathrm{ft}^{\mathbf{3}} \mathbf{/ s}\right)\end{array}$ \\
\hline 2.0 & 146 & 12.0 & 13,090 \\
4.0 & 1,150 & 13.0 & 15,410 \\
6.0 & 2,990 & 14.0 & 17,910 \\
8.0 & 5,610 & 15.0 & 20,580 \\
9.0 & 7,210 & 16.0 & 23,420 \\
10.0 & 8,990 & 17.0 & 26,430 \\
11.0 & 10,950 & 17.5 & 28,000 \\
\hline
\end{tabular}

Basin characteristics

\begin{tabular}{ccccccc}
\hline $\begin{array}{c}\text { Main } \\
\text { channel } \\
\text { slope } \\
(\mathrm{ft} / \mathrm{mi})\end{array}$ & $\begin{array}{c}\text { Stream } \\
\text { length } \\
(\mathrm{mi})\end{array}$ & $\begin{array}{c}\text { Mean } \\
\text { basin } \\
\text { elevation } \\
\text { (ft) }\end{array}$ & $\begin{array}{c}\text { Forested } \\
\text { area } \\
\text { (percent) }\end{array}$ & $\begin{array}{c}\text { Soil } \\
\text { index }\end{array}$ & $\begin{array}{c}\text { Mean } \\
\text { annual } \\
\text { precipitation } \\
\text { (in) }\end{array}$ & $\begin{array}{c}\text { Rainfall intensity, 24-hour } \\
\text { (in) }\end{array}$ \\
\hline 29.0 & 158 & 6,250 & 44.0 & 2.9 & 15.4 & $\begin{array}{c}50-y e a r \\
\text { (in) }\end{array}$ \\
\hline
\end{tabular}


09442000 GILA RIVER NEAR CLIFTON, AZ--Continued

MEAN MONTHLY AND ANNUAL DISCHARGES 1912-17, $1929 \cdot 33$, $1936-46,1949-89,1996$

\begin{tabular}{|c|c|c|c|c|c|c|}
\hline MONTH & $\begin{array}{l}\text { MAXIMUM } \\
(\text { FT3/S) }\end{array}$ & $\begin{array}{l}\text { MINIMUM } \\
\text { (FT3/S) }\end{array}$ & $\begin{array}{c}\text { MEAN } \\
(\mathrm{FT} 3 / \mathrm{S})\end{array}$ & $\begin{array}{l}\text { STAN- } \\
\text { DARD } \\
\text { DEVIA- } \\
\text { TION } \\
\text { (FT3/S) }\end{array}$ & $\begin{array}{l}\text { COEFFI- } \\
\text { CIENT OF } \\
\text { VARI- } \\
\text { ATION }\end{array}$ & $\begin{array}{c}\text { PERCENT } \\
\text { OF } \\
\text { ANNUAL } \\
\text { RUNOFF }\end{array}$ \\
\hline OCTOBER & 1,750 & 8.7 & 192 & 334 & 1.7 & 7.8 \\
\hline NOVEMBER & 564 & 11 & 110 & 105 & 0.95 & 4.5 \\
\hline DECEMBER & 2,390 & 17 & 235 & 455 & 1.9 & 9.6 \\
\hline JANUARY & 1,360 & 43 & 256 & 327 & 1.3 & 10.4 \\
\hline FBBRUARY & 1,670 & 24 & 321 & 365 & 1.1 & 13.0 \\
\hline MARCH & 1.770 & 21 & 390 & 435 & 1.1 & 15.8 \\
\hline APRIL & 1.690 & 12 & 246 & 302 & 1.2 & 10.0 \\
\hline MAY & 874 & 12 & 119 & 157 & 1.3 & 4.8 \\
\hline JUNE & 171 & 9.4 & 41 & 38 & 0.93 & 1.7 \\
\hline JULY & 934 & 13 & 119 & 166 & 1.4 & 4.8 \\
\hline AUGUST & 898 & 17 & 235 & 216 & 0.92 & 9.6 \\
\hline SEPTEMBER & 1.210 & 8.2 & 198 & 235 & 1.2 & 8.0 \\
\hline ANNUAL & 930 & 43 & 205 & 166 & 0.81 & 100 \\
\hline
\end{tabular}

MAGNITUDE AND PROBABILITY OF ANNUAL LOW FLOW BASED ON PERIOD OF RECORD 1912-18, 1929-33, 1937-47, 1950-89

\begin{tabular}{|c|c|c|c|c|c|c|}
\hline \multirow{4}{*}{$\begin{array}{l}\text { PERIOD } \\
\text { (CON- } \\
\text { SECU- } \\
\text { TIVE }\end{array}$} & \multirow{3}{*}{\multicolumn{3}{|c|}{$\begin{array}{c}\text { DISCHARGE, IN } \\
\text { RECURRENCE IN } \\
\text { NON-EXCEEDANCE } \\
\ldots \ldots \ldots \ldots \ldots \ldots\end{array}$}} & \multicolumn{3}{|c|}{, FOR INDICATED } \\
\hline & & & & \multicolumn{3}{|c|}{$\begin{array}{l}\text { IN YEARS, AND } \\
\text { ILITY, IN PERCENT }\end{array}$} \\
\hline & & & & $\ldots \ldots$ & $\ldots \ldots$ & $\ldots \ldots$ \\
\hline & 2 & 5 & 10 & 20 & 50 & 100 \\
\hline DAYS) & 508 & 208 & 108 & 58 & 28 & 18 \\
\hline 1 & 14 & 8.9 & 6.9 & 5.5 & 4.3 & 3.6 \\
\hline 3 & 15 & 9.4 & 7.4 & 6.1 & 4.8 & 4.1 \\
\hline 7 & 15 & 10 & 8.3 & 7.0 & 5.8 & 5.1 \\
\hline 14 & 17 & 11 & 9.2 & 7.8 & 6.4 & 5.7 \\
\hline 30 & 20 & 13 & 10 & 8.8 & 7.3 & 6.4 \\
\hline 60 & 26 & 16 & 12 & 10 & 8.2 & 7.1 \\
\hline 90 & 37 & 21 & 16 & 12 & 9.1 & 7.5 \\
\hline 120 & 53 & 31 & 23 & 18 & 14 & 11 \\
\hline 183 & 85 & 50 & 38 & 30 & 23 & 20 \\
\hline
\end{tabular}

MAGNITUDE AND PROBABILITY OF ANNUAL HIGH FLOW BASED ON PERIOD OF RECORD $1912 \cdot 17,1929 \cdot 33,1936-46,1949 \cdot 89,199$

MAGNITUDE AND PROBABILITY OF INSTANTANEOUS PEAK FLOW BASED ON PERIOD OF RECORD 1911-17, 1928-46, 1948-89

DISCHARGE, IN FT $3 / S$, FOR INDICATED RECURRENCE INTERVAL IN YEARS, AND EXCEEDANCE PROBABILITY, IN PERCENT

\begin{tabular}{|c|c|c|c|c|c|}
\hline 2 & 5 & 10 & 25 & 50 & 100 \\
\hline 508 & 208 & 108 & 48 & 28 & 18 \\
\hline
\end{tabular}

$6,000 \quad 12,600 \quad 18,600 \quad 28,300 \quad 37,300 \quad 47,700$

WEIGHTED SKEW (LOGS) $=0.06$

MEAN (LOGS) $=3.78$

STANDARD DEV. $($ LOGS $)=0.38$

\begin{tabular}{|c|c|c|c|c|c|c|}
\hline \multirow{4}{*}{$\begin{array}{l}\text { PERIOD } \\
\text { (CON- } \\
\text { SECU- }\end{array}$} & \multicolumn{6}{|c|}{ DISCHARGE, IN FT3/S, FOR INDICATED } \\
\hline & & RECURRE & CE INTE & RVAL, IN & YEARS, & AND \\
\hline & & EXCEEDA & CE PROE & ABILITY, & IN PER & ENT \\
\hline & & $\cdots \ldots$ & & & & \\
\hline TIVE & 2 & 5 & 10 & 25 & 50 & 100 \\
\hline DAYS) & $50 \%$ & 208 & 108 & 48 & 28 & 18 \\
\hline 1 & 2,690 & 6,150 & 9.750 & 16,300 & 23,100 & 31,70 \\
\hline 3 & 1,800 & 4,060 & 6,320 & 10,300 & 14,200 & 19.00 \\
\hline 7 & 1,210 & 2,600 & 3,920 & 6,120 & 8,210 & 10,700 \\
\hline 15 & 827 & 1,700 & 2,480 & 3,700 & 4,780 & 6,020 \\
\hline 30 & 589 & 1,160 & 1,630 & 2,310 & 2,870 & 3,470 \\
\hline 60 & 412 & 818 & 1,160 & 1,680 & 2,120 & 2.610 \\
\hline 90 & 326 & 660 & 961 & 1,440 & 1,880 & 2,380 \\
\hline
\end{tabular}

DURATION TABLE OF DAILY MEAN FLOW FOR PERIOD OF RECORD $1912 \cdot 17,1929 \cdot 33,1936 \cdot 46,1949 \cdot 89,1996$

\begin{tabular}{|c|c|c|c|c|c|c|c|c|c|c|c|c|c|c|c|c|}
\hline 18 & 58 & 108 & 158 & 208 & 308 & 408 & 508 & 608 & 708 & 808 & 908 & 958 & 988 & 998 & 99.58 & 99.98 \\
\hline 2,010 & 813 & 452 & 310 & 227 & 144 & 102 & 79 & 61 & 43 & 28 & 18 & 13 & 10 & 9.0 & 8.3 & 6.5 \\
\hline
\end{tabular}


ANNUAL MEAN DISCHARGE IN CUBIC FEET PER SECOND

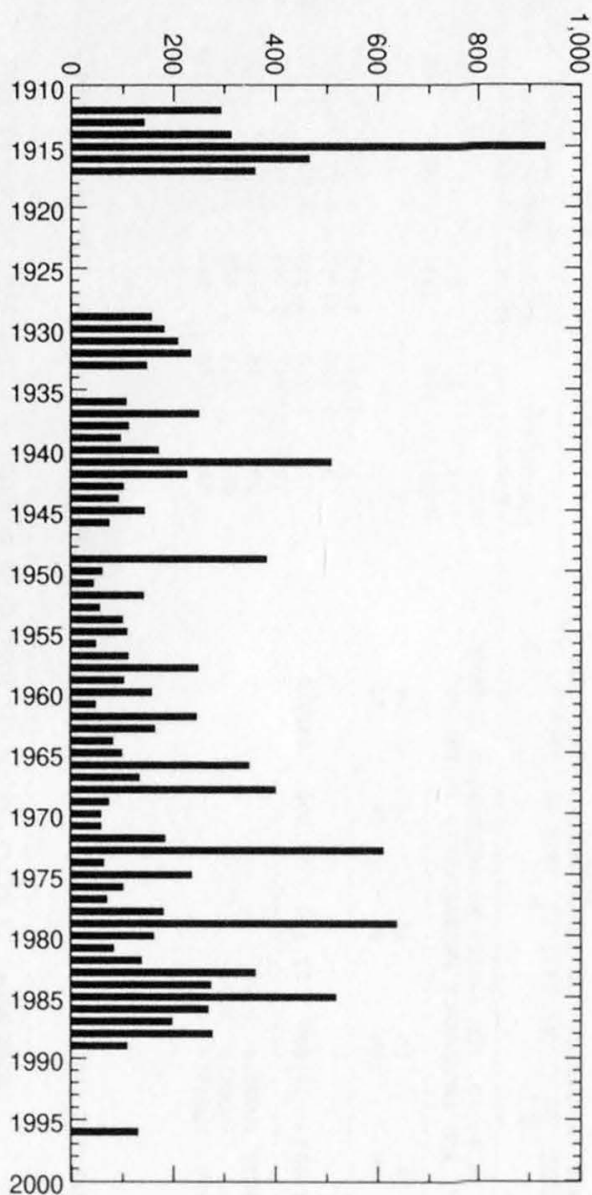

ANNUAL PEAK DISCHARGE, IN CUBIC FEET PER SECOND

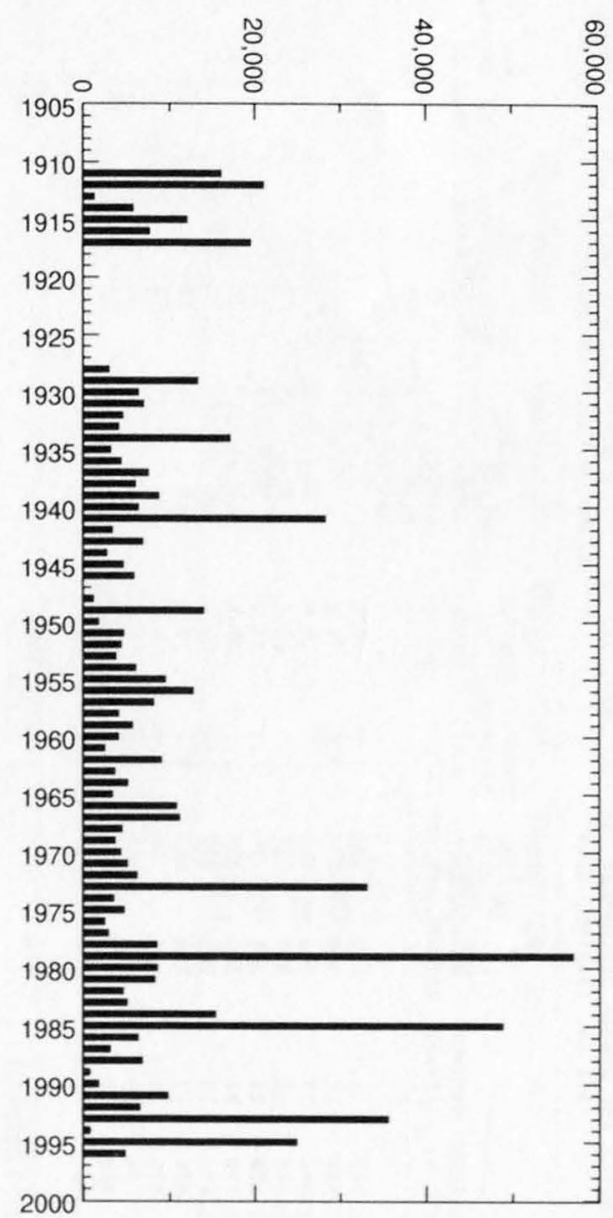


09442000 GILA RIVER NEAR CLIFTON, AZ--Continued

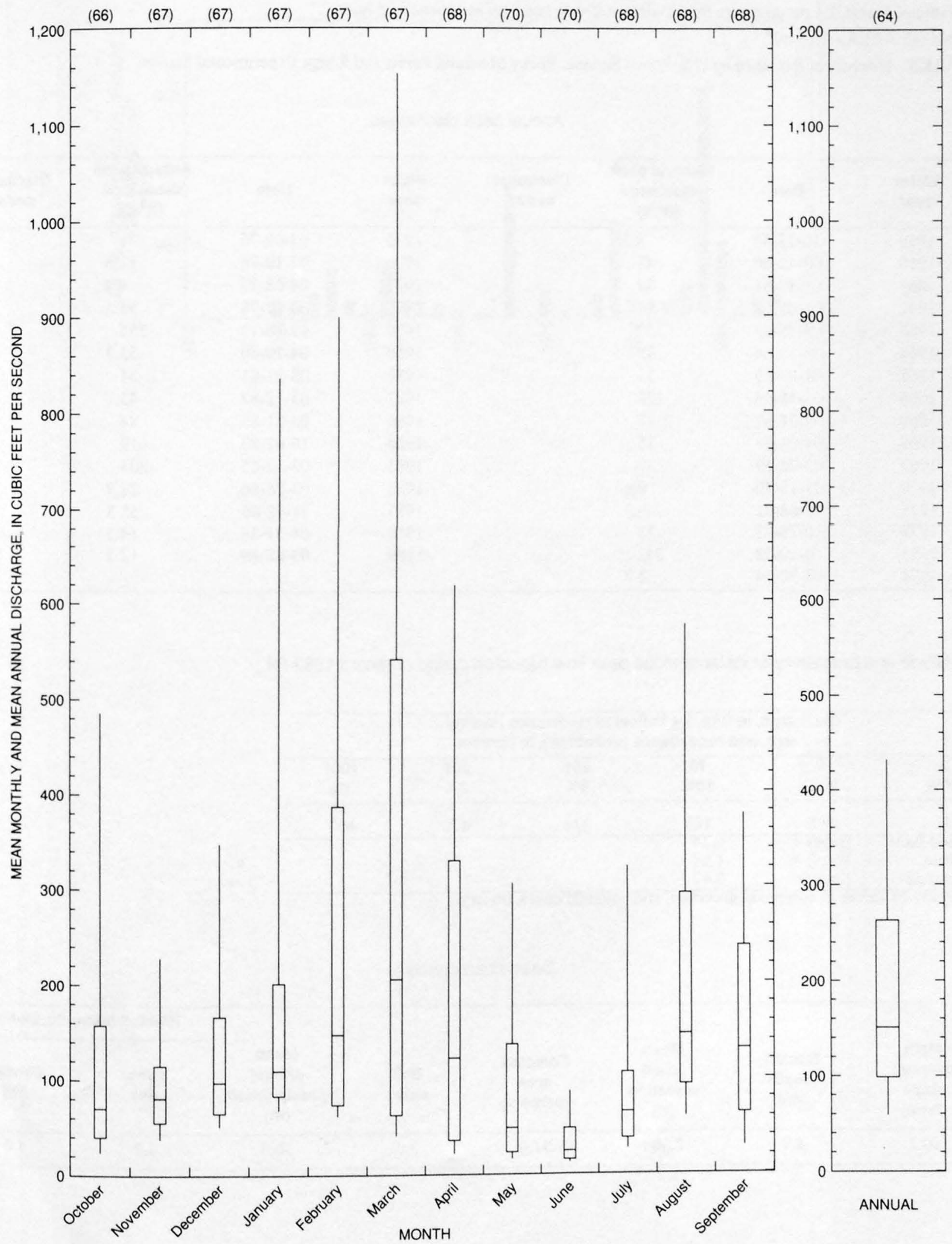


09444100 CAMPBELL BLUE CREEK NEAR ALPINE, AZ

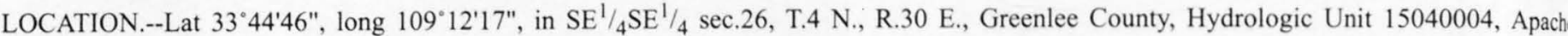

National Forest, 2.5 mi upstream from Coleman Creek, and 8 mi southwest of Alpine.

DRAINAGE AREA.--11.6 $\mathrm{mi}^{2}$.

REMARKS.--Discharges furnished by U.S. Forest Service, Rocky Mountain Forest and Range Experimental Station.

Annual peak discharges

\begin{tabular}{|c|c|c|c|c|c|c|c|}
\hline $\begin{array}{l}\text { Water } \\
\text { year }\end{array}$ & Date & $\begin{array}{c}\text { Annual peak } \\
\text { discharge } \\
\left(\mathrm{ft}^{3} / \mathrm{s}\right)\end{array}$ & $\begin{array}{l}\text { Discharge } \\
\text { codes }\end{array}$ & $\begin{array}{l}\text { Water } \\
\text { year }\end{array}$ & Date & $\begin{array}{c}\text { Annual peak } \\
\text { discharge } \\
\left(\mathrm{ft}^{3} / \mathrm{s}\right)\end{array}$ & $\begin{array}{c}\text { Discharge } \\
\text { codes }\end{array}$ \\
\hline 1959 & $10-13-58$ & 8.2 & & 1975 & $03-08-75$ & 71 & \\
\hline 1960 & $03-12-60$ & 41 & & 1976 & $02-10-76$ & 13.6 & \\
\hline 1961 & $08-23-61$ & 32 & & 1977 & $04-08-77$ & 4.2 & \\
\hline 1962 & $01-05-62$ & 61 & & 1978 & $03-22-78$ & 58.5 & \\
\hline 1963 & $08-25-63$ & 132 & & 1979 & $12-18-78$ & 255 & \\
\hline 1964 & $08-10-64$ & 48 & & 1980 & $04-10-80$ & 53.3 & \\
\hline 1965 & $01-07-65$ & 33 & & 1981 & $08-01-81$ & 34 & \\
\hline 1966 & $03-18-66$ & 123 & & 1982 & $03-12-82$ & 43 & \\
\hline 1967 & $07-31-67$ & 17 & & 1983 & $03-31-83$ & 84 & \\
\hline 1968 & 04-01-68 & 35 & & 1984 & $10-02-83$ & 619 & \\
\hline 1969 & $03-28-69$ & 37 & & 1985 & $03-12-85$ & 204 & \\
\hline 1970 & $03-15-70$ & 9.6 & & 1986 & $03-26-86$ & 29.9 & \\
\hline 1971 & $09-08-71$ & 3.3 & & 1987 & $11-18-86$ & 53.3 & \\
\hline 1972 & $10-26-71$ & 23 & & 1988 & $08-31-88$ & 54.3 & \\
\hline 1973 & $10-20-72$ & 342 & & 1989 & $03-08-89$ & 12.3 & \\
\hline 1974 & $03-20-74$ & 3.7 & & & & & \\
\hline
\end{tabular}

Magnitude and probability of instantaneous peak flow based on period of record 1959-89

\begin{tabular}{|c|c|c|c|c|c|}
\hline \multicolumn{6}{|c|}{$\begin{array}{l}\text { Discharge, in } \mathrm{ft}^{3} / \mathrm{s} \text {, for indicated recurrence interval } \\
\text { in years, and exceedance probablility, in percent }\end{array}$} \\
\hline 2 & 5 & 10 & $25 \dagger$ & $50 \dagger$ & $100 \dagger$ \\
\hline $50 \%$ & $20 \%$ & $10 \%$ & $4 \%$ & $2 \%$ & $1 \%$ \\
\hline 42 & 108 & 181 & 321 & 470 & 667 \\
\hline Weighted skew & $(\log s)=$ & 0.24 & & & \\
\hline Mean & $(\log s)=$ & 1.64 & & & \\
\hline Standard dev. & $(\log s)=$ & 0.47 & & & \\
\hline
\end{tabular}

Basin characteristics

\begin{tabular}{cccccccc}
\hline $\begin{array}{c}\text { Main } \\
\text { channel } \\
\text { slope } \\
(\mathrm{ft} / \mathrm{mi})\end{array}$ & $\begin{array}{c}\text { Stream } \\
\text { length } \\
\text { (mi) }\end{array}$ & $\begin{array}{c}\text { Mean } \\
\text { basin } \\
\text { elevation } \\
(\mathrm{ft})\end{array}$ & $\begin{array}{c}\text { Forested } \\
\text { area } \\
\text { (percent) }\end{array}$ & $\begin{array}{c}\text { Soil } \\
\text { index }\end{array}$ & $\begin{array}{c}\text { Mean } \\
\text { annual } \\
\text { precipitation } \\
\text { (in) }\end{array}$ & $\begin{array}{c}\text { Rainfall intensity, 24-hour } \\
\text { (in) }\end{array}$ & $\begin{array}{c}50 \text {-year } \\
\text { (in) }\end{array}$ \\
\hline 90.2 & 8.9 & 8,300 & 91.0 & 3.0 & 20.0 & 2.2 \\
\hline
\end{tabular}


09444100 CAMPBELL BLUE CREEK NEAR ALPINE, AZ--Continued

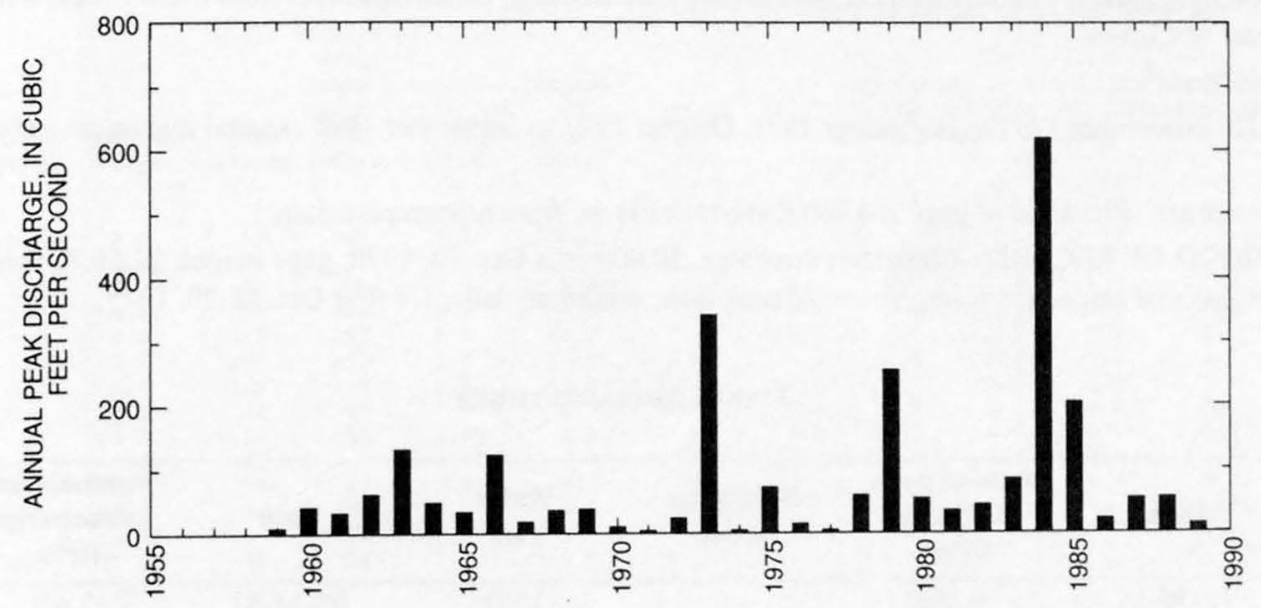


09444200 BLUE RIVER NEAR CLIFTON, AZ

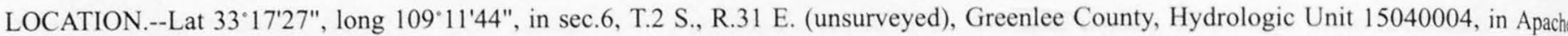
National Forest, on right bank $0.1 \mathrm{mi}$ downstream from county road crossing, 0.9 mi upstream from Clear Creek, 8 mi upstream from mouth and $17 \mathrm{mi}$ northeast of Clifton.

DRAINAGE AREA.--506 $\mathrm{mi}^{2}$.

PERIOD OF RECORD.--November 1967 to September 1991, October 1992 to September 1995 (annual maximum only), October 1995 to September 1996.

GAGE.--Water-stage recorder. Elevation of gage is 4,160 ft above sea level, from topographic map.

EXTREMES FOR PERIOD OF RECORD.--Maximum discharge, 30,000 $\mathrm{ft}^{3} / \mathrm{s}$ Oct. 20, 1972, gage height, $22.56 \mathrm{ft}$, from rating curve extended above $960 \mathrm{ft}^{3} / \mathrm{s}$ on basis of slope-area measurement of peak flow; minimum daily, $1.4 \mathrm{ft}^{3} / \mathrm{s}$ Oct. 18-20, 1978.

Annual peak discharges

\begin{tabular}{cccccrr}
\hline $\begin{array}{c}\text { Water } \\
\text { year }\end{array}$ & Date & $\begin{array}{c}\text { Annual peak } \\
\text { discharge } \\
\left(\mathbf{f t}^{3} / \mathbf{s}\right)\end{array}$ & $\begin{array}{c}\text { Discharge } \\
\text { codes }\end{array}$ & $\begin{array}{c}\text { Water } \\
\text { year }\end{array}$ & $\begin{array}{c}\text { Date } \\
\text { Annual peak } \\
\text { discharge } \\
\left(\mathrm{ft}^{3} / \mathbf{s}\right)\end{array}$ & $\begin{array}{c}\text { Discharge } \\
\text { codes }\end{array}$ \\
\hline 1966 & $12-30-65$ & 9,380 & & 1982 & $08-14-82$ & 2,620 \\
1968 & $08-09-68$ & 6,290 & 1983 & $07-27-83$ & 2,040 \\
1969 & $08-07-69$ & 1,360 & 1984 & $10-01-83$ & 24,300 \\
1970 & $07-28-70$ & 1,180 & 1985 & $12-28-84$ & 7,630 \\
1971 & $10-03-70$ & 4,240 & 1986 & $07-16-86$ & 2,040 \\
1972 & $10-25-71$ & 2,520 & 1987 & $07-30-87$ & 2,910 \\
1973 & $10-20-72$ & 30,000 & 1988 & $08-31-88$ & 6,410 \\
1974 & $08-23-74$ & 2,380 & 1989 & $09-22-89$ & 1,380 \\
1975 & $09-08-75$ & 25,500 & 1990 & $07-23-90$ & 2,220 \\
1976 & $02-10-76$ & 2,550 & 1991 & $03-02-91$ & 3,820 \\
1977 & $08-19-77$ & 1,570 & 1992 & $02-14-92$ & 1,700 \\
1978 & $03-02-78$ & 3,660 & 1993 & $01-19-93$ & 17,000 \\
1979 & $11-24-78$ & 14,700 & 1994 & $09-03-94$ & 835 \\
1980 & $02-15-80$ & 5,570 & 1995 & $11-24-94$ & 7,290 \\
1981 & $08-07-81$ & 1,910 & 1996 & $08-29-96$ & 2,430 \\
\hline
\end{tabular}

${ }^{1}$ Highest since 1885 .

Discharge rating table developed October 1996

\begin{tabular}{crcc}
\hline $\begin{array}{c}\text { Gage height } \\
(\mathbf{f t})\end{array}$ & $\begin{array}{c}\text { Discharge } \\
\left(\mathrm{ft}^{\mathbf{3}} \mathbf{s}\right)\end{array}$ & $\begin{array}{c}\text { Gage height } \\
(\mathbf{f t})\end{array}$ & $\begin{array}{c}\text { Discharge } \\
\left(\mathrm{ft}^{\mathbf{3}} \mathbf{s}\right)\end{array}$ \\
\hline 6.0 & 370 & 18.0 & 15,900 \\
8.0 & 1,240 & 20.0 & 21,200 \\
10.0 & 2,730 & 22.0 & 27,600 \\
12.0 & 4,900 & 24.0 & 35,000 \\
14.0 & 7,810 & 26.0 & 43,200 \\
16.0 & 11,400 & 26.4 & 45,000 \\
\hline
\end{tabular}

Basin characteristics

\begin{tabular}{|c|c|c|c|c|c|c|c|}
\hline \multirow[b]{2}{*}{$\begin{array}{c}\text { Main } \\
\text { channel } \\
\text { slope } \\
(\mathrm{ft} / \mathrm{mi})\end{array}$} & \multirow[b]{2}{*}{$\begin{array}{l}\text { Stream } \\
\text { length } \\
\text { (mi) }\end{array}$} & \multirow[b]{2}{*}{$\begin{array}{c}\text { Mean } \\
\text { basin } \\
\text { elevation } \\
(\mathrm{ft})\end{array}$} & \multirow[b]{2}{*}{$\begin{array}{l}\text { Forested } \\
\text { area } \\
\text { (percent) }\end{array}$} & \multirow[b]{2}{*}{$\begin{array}{c}\text { Soil } \\
\text { index }\end{array}$} & \multirow[b]{2}{*}{$\begin{array}{c}\text { Mean } \\
\text { annual } \\
\text { precipitation } \\
\text { (in) }\end{array}$} & \multicolumn{2}{|c|}{ Rainfall intensity, 24-hour } \\
\hline & & & & & & $\begin{array}{l}\text { 2-year } \\
\text { (in) }\end{array}$ & $\begin{array}{l}\text { 50-year } \\
\text { (in) }\end{array}$ \\
\hline 65.3 & 40.8 & 6,910 & 85.0 & 3.0 & 20.7 & 1.8 & 3.6 \\
\hline
\end{tabular}


09444200 BLUE RIVER NEAR CLIFTON, AZ--Continued

MEAN MONTHLY AND ANNUAL DISCHARGES 1969.90, 1996

\begin{tabular}{|c|c|c|c|c|c|c|}
\hline MONTH & $\begin{array}{l}\text { MAXIMUM } \\
\text { (FT3/S) }\end{array}$ & $\begin{array}{l}\text { MINIMUM } \\
\text { (FT3/S) }\end{array}$ & $\begin{array}{c}\text { MEAN } \\
(\mathrm{FT} 3 / \mathrm{S})\end{array}$ & $\begin{array}{l}\text { STAN- } \\
\text { DARD } \\
\text { DEVIA- } \\
\text { TION } \\
\text { (FT3/S) }\end{array}$ & $\begin{array}{l}\text { COEFFI- } \\
\text { CIENT OF } \\
\text { VARI- } \\
\text { ATION }\end{array}$ & $\begin{array}{c}\text { PERCENT } \\
\text { OF } \\
\text { ANNUAL } \\
\text { RUNOFF }\end{array}$ \\
\hline OCTOBER & 1,030 & 2.6 & 99 & 249 & 2.5 & 11.4 \\
\hline NOVEMBER & 443 & 3.9 & 44 & 91 & 2.1 & 5.1 \\
\hline DECEMBER & 616 & 3.7 & 82 & 155 & 1.9 & 9.4 \\
\hline JANUARY & 569 & 5.4 & 68 & 123 & 1.8 & 7.9 \\
\hline FEBRUARY & 707 & 8.0 & 115 & 167 & 1.5 & 13.2 \\
\hline MARCH & 584 & 8.9 & 157 & 179 & 1.1 & 18.1 \\
\hline APRIL & 488 & 6.7 & 122 & 147 & 1.2 & 14.0 \\
\hline MAY & 338 & 4.1 & 58 & 80 & 1.4 & 6.7 \\
\hline JUNE & 46 & 2.9 & 12 & 12 & 0.95 & 1.4 \\
\hline JULY & 71 & 7.6 & 25 & 17 & 0.67 & 2.9 \\
\hline AUGUST & 108 & 8.7 & 39 & 23 & 0.60 & 4.5 \\
\hline SEPTEMBER & 366 & 7.4 & 47 & 78 & 1.7 & 5.4 \\
\hline ANNUAL & 243 & 10 & 72 & 72 & 1.0 & 100 \\
\hline
\end{tabular}

MAGNITUDE AND PROBABILITY OF INSTANTANEOUS PEAK FLOW BASED ON PERIOD OF RECORD 1966, 1968.96

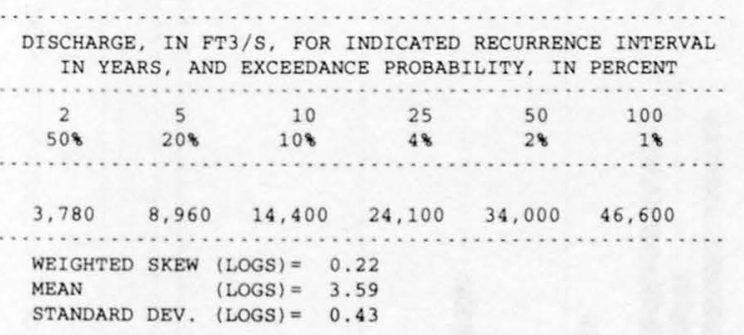

MAGNITUDE AND PROBABILITY OF ANNUAL LOW FLOW BASED ON PERIOD OF RECORD 1969.90

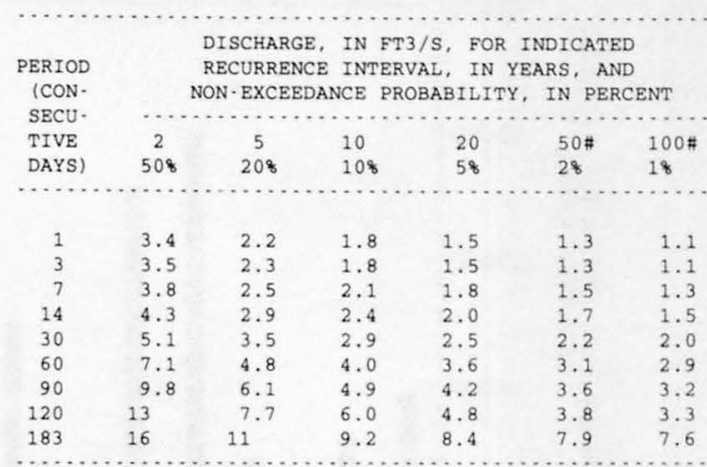

MAGNITUDE AND PROBABILITY OF ANNUAL HIGH FLOW BASED ON PERIOD OF RECORD 1969.90, 1996

\begin{tabular}{|c|c|c|c|c|c|c|}
\hline \multirow{4}{*}{$\begin{array}{l}\text { PERIOD } \\
\text { (CON. } \\
\text { SECU. }\end{array}$} & \multirow{2}{*}{\multicolumn{6}{|c|}{ DISCHARGE, IN FT3/S, FOR INDICATED }} \\
\hline & & RECURR: & $\mathrm{CE}$ INTE & RVAL，IN & & \\
\hline & & EXCEED & CE PROE & ABILITY, & IN PER & $\mathrm{ENT}$ \\
\hline & & & & & & \\
\hline TIVE & 2 & 5 & 10 & 25 & $50 \#$ & $100 \#$ \\
\hline DAYS) & 508 & 208 & 108 & 48 & 28 & 18 \\
\hline 1 & 790 & 2,990 & 6.240 & 14,200 & 24,600 & 40,700 \\
\hline 3 & 494 & 1,820 & 3.750 & 8,370 & 14,300 & 23,500 \\
\hline 7 & 324 & 1,080 & 2,080 & 4,280 & 6,880 & 10,600 \\
\hline 15 & 223 & 666 & 1,200 & 2.250 & 3,420 & 4,980 \\
\hline 30 & 159 & 433 & 745 & 1,340 & 1,980 & 2,810 \\
\hline 60 & 113 & 296 & 500 & 885 & 1,290 & 1,820 \\
\hline 90 & 94 & 249 & 419 & 740 & 1,080 & 1,510 \\
\hline
\end{tabular}

DURATION TABLE OF DAILY MEAN FLOW FOR PERIOD OF RECORD 1969-90, 1996

DISCHARGE, IN FT3/S, WHICH WAS EQUALED OR EXCEEDED FOR INDICATED PERCENT OF TIME

\begin{tabular}{|c|c|c|c|c|c|c|c|c|c|c|c|c|c|c|c|c|}
\hline 18 & 58 & 108 & 158 & 208 & 308 & 408 & 508 & 608 & 708 & 808 & 908 & 958 & 988 & 998 & 99.58 & 99.98 \\
\hline 682 & 316 & 164 & 97 & 67 & 41 & 28 & 20 & 14 & 11 & 7.9 & 5.1 & 3.8 & 3.0 & 2.6 & 2.4 & 1.8 \\
\hline
\end{tabular}

\# Reliability of values in column is uncertain, and potential errors are large. 
09444200 BLUE RIVER NEAR CLIFTON, AZ--Continued
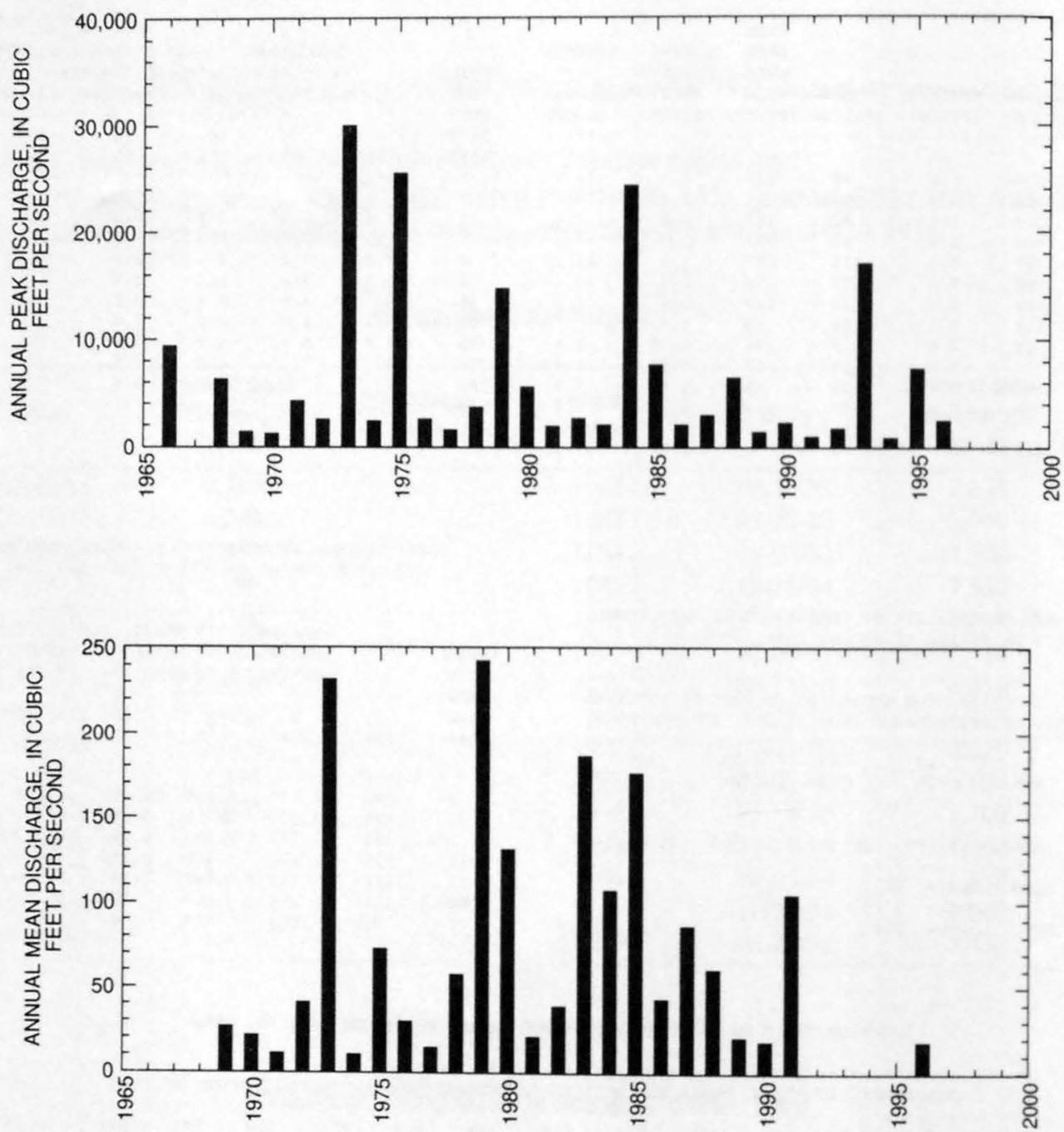
GILA RIVER BASIN

259

09444200 BLUE RIVER NEAR CLIFTON, AZ--Continued
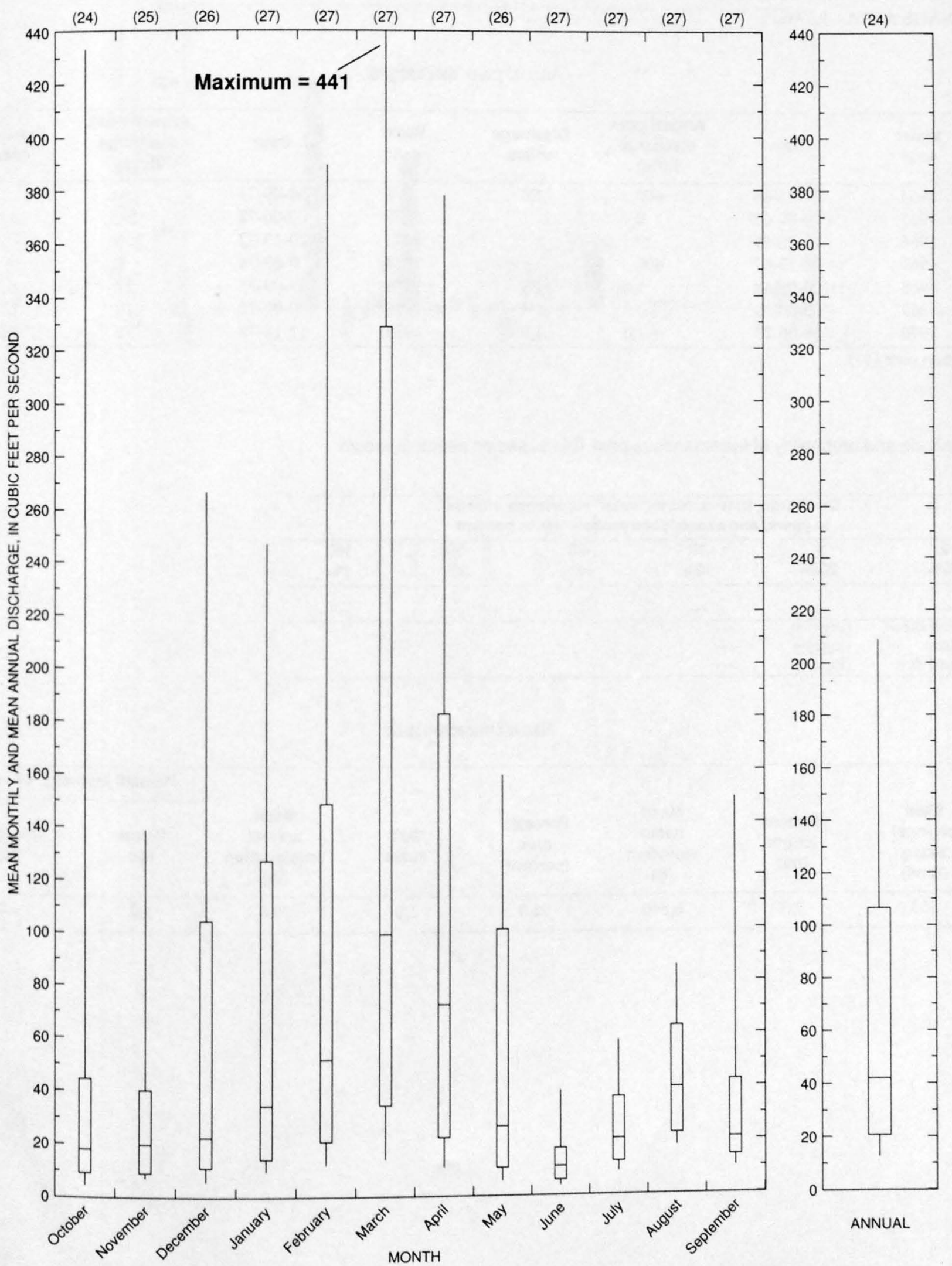


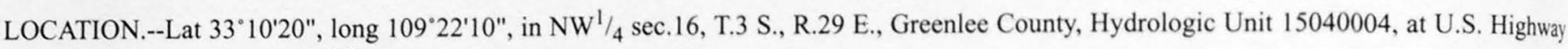
666,9 mi northwest of Clifton.

DRAINAGE AREA.--1.37 $\mathrm{mi}^{2}$.

Annual peak discharges

\begin{tabular}{|c|c|c|c|c|c|c|c|}
\hline $\begin{array}{l}\text { Water } \\
\text { year }\end{array}$ & Date & $\begin{array}{c}\text { Annual peak } \\
\text { discharge } \\
\left(\mathrm{ft}^{3} / \mathrm{s}\right)\end{array}$ & $\begin{array}{c}\text { Discharge } \\
\text { codes }\end{array}$ & $\begin{array}{l}\text { Water } \\
\text { year }\end{array}$ & Date & $\begin{array}{c}\text { Annual peak } \\
\text { discharge } \\
\left(\mathrm{ft}^{3} / \mathrm{s}\right)\end{array}$ & $\begin{array}{c}\text { Discharge } \\
\text { codes }\end{array}$ \\
\hline 1964 & $07-25-64$ & 600 & ES & 1971 & $00-00-71$ & 0 & \\
\hline 1965 & $00-00-65$ & 0 & & 1972 & $00-00-72$ & 0 & \\
\hline 1966 & $12-22-65$ & 29 & & 1973 & $10-19-72$ & 150 & \\
\hline 1967 & $08-12-67$ & 400 & & 1974 & $00-00-74$ & 0 & \\
\hline 1968 & $00-00-68$ & 1.0 & ES & 1975 & $09-09-75$ & 22 & \\
\hline 1969 & $00-00-69$ & 0 & & 1976 & $00-00-76$ & 10 & LT \\
\hline 1970 & $08-06-70$ & 1.0 & LT & 1979 & $12-18-78$ & ${ }^{I} 72$ & HP \\
\hline
\end{tabular}

${ }^{1}$ Highest since 1973.

Magnitude and probability of instantaneous peak flow based on period of record

\begin{tabular}{cccccc}
\hline \multicolumn{5}{c}{$\begin{array}{c}\text { Discharge, in } \mathrm{ft}^{3} / \mathrm{s} \text {, for indicated recurrence interval } \\
\text { in years, and exceedance probablility, in percent }\end{array}$} \\
\hline 2 & 5 & 10 & 25 & 50 & 100 \\
$50 \%$ & $20 \%$ & $10 \%$ & $4 \%$ & $2 \%$ & $1 \%$ \\
\hline & & & & & \\
Weighted skew & $(\operatorname{logs})=$ & $\cdots$ & & \\
Mean & $(\operatorname{logs})=$ & $\ldots-$ & & \\
Standard dev. & $(\operatorname{logs})=$ & $\cdots$ &
\end{tabular}

Basin characteristics

\begin{tabular}{cccccccc}
\hline $\begin{array}{c}\text { Main } \\
\text { channel } \\
\text { slope } \\
(\mathrm{ft} / \mathrm{mi})\end{array}$ & $\begin{array}{c}\text { Stream } \\
\text { length } \\
\text { (mi) }\end{array}$ & $\begin{array}{c}\text { Mean } \\
\text { basin } \\
\text { elevation } \\
(\mathrm{ft})\end{array}$ & $\begin{array}{c}\text { Forested } \\
\text { area } \\
\text { (percent) }\end{array}$ & $\begin{array}{c}\text { Soil } \\
\text { index }\end{array}$ & $\begin{array}{c}\text { Mean } \\
\text { annual } \\
\text { precipitation } \\
\text { (in) }\end{array}$ & $\begin{array}{c}\text { Rainfall intensity, 24-hour } \\
\text { (in) }\end{array}$ & $\begin{array}{c}50-y e a r \\
\text { (in) }\end{array}$ \\
\hline 363 & 2.5 & 6,840 & 98.0 & 1.0 & 20.0 & 2.2 \\
\hline
\end{tabular}


09444400 CHASE CREEK NEAR CLIFTON, AZ

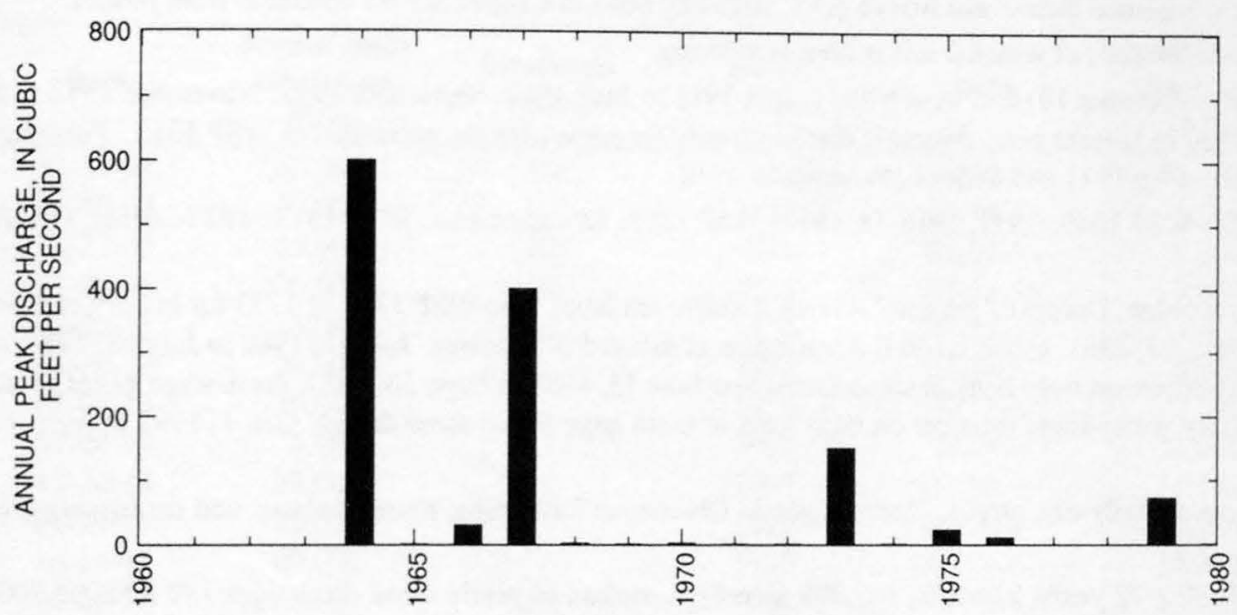




\section{SAN FRANCISCO RIVER AT CLIFTON, AZ}

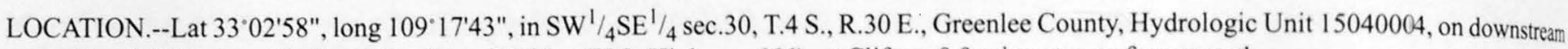
side of right pier at Railroad Boulevard Bridge (U.S. Highway 666), at Clifton, 9.9 mi upstream from mouth.

DRAINAGE AREA.--2,766 $\mathrm{mi}^{2}$, of which $2 \mathrm{mi}^{2}$ is noncontributing.

PERIOD OF RECORD.--October 1910 to March 1911, July 1911 to June 1912, September 1912, November 1912 to March 1913 , May 1913 to July 1918, July 1927 to current year. Monthly discharge only for some periods, published in WSP 1313. Published as "San Francisco River at dam above Clifton" in 1911 and under both names in 1912.

REVISED RECORDS.--WSP 1049: 1911, 1913-15, 1917. WSP 1283: Drainage area. WSP 1313: 1927-30(M), 1932(M), 1934(M). WRD Ariz 1972: $1917(\mathrm{M})$.

GAGE.--Water-stage recorder. Datum of gage is 3,436.16 ft above sea level. See WSP 1713 or 1733 for history of changes prior to Apr. 7, 1959 . Apr. 7, 1959, to Mar. 23, 1961, at site 1,140 ft downstream at datum $5.37 \mathrm{ft}$ lower. July 18, 1980 to July 28, 1983, supplementary water-stage recorder $0.4 \mathrm{mi}$ upstream on right bank at same datum and June 15, 1981 to Sept. 30, 1983, crest-stage gages at site. Aug. 4, 1983 to Mar. I, 1985, supplementary water-stage recorder on right bank at main gage site at same datum, Oct. 1, 1992 at main gage site, at datum $10.00 \mathrm{ft}$ higher.

REMARKS.--No estimated daily discharges. Records good. Diversions for mining, municipal use, and for irrigation of about 2,700 acres above station.

AVERAGE DISCHARGE.--72 years, $224 \mathrm{ft}^{3} / \mathrm{s}, 162,300$ acre- $\mathrm{ft} / \mathrm{yr}$; median of yearly mean discharges $130 \mathrm{ft}^{3} / \mathrm{s}, 94,200 \mathrm{acre}-\mathrm{ft} / \mathrm{yr}$.

EXTREMES FOR PERIOD OF RECORD.--Maximum discharge, 90,900 ft $3 / \mathrm{s}$ Oct. 2, 1983, gage height, $19.72 \mathrm{ft}$, from high-water mark, from rating curve extended above $30,000 \mathrm{ft}^{3} / \mathrm{s}$ on basis of slope-area measurement at gage height $17.0 \mathrm{ft}$; minimum daily, $6.1 \mathrm{ft} / \mathrm{s} J u n e ~ 21,1971$. 
09444500 SAN FRANCISCO RIVER AT CLIFTON, AZ--Continued

Annual peak discharges

\begin{tabular}{|c|c|c|c|c|c|c|c|}
\hline $\begin{array}{l}\text { Water } \\
\text { year }\end{array}$ & Date & $\begin{array}{c}\text { Annual peak } \\
\text { discharge } \\
\left(\mathrm{ft}^{3} / \mathrm{s}\right)\end{array}$ & $\begin{array}{c}\text { Discharge } \\
\text { codes }\end{array}$ & $\begin{array}{l}\text { Water } \\
\text { year }\end{array}$ & Date & $\begin{array}{c}\text { Annual peak } \\
\text { discharge } \\
\left(\mathrm{ft}^{3} / \mathrm{s}\right)\end{array}$ & $\begin{array}{l}\text { Discharge } \\
\text { codes }\end{array}$ \\
\hline 1891 & $02-21-91$ & 65,000 & HP & 1952 & $01-19-52$ & 15,800 & \\
\hline 1905 & $01-10-05$ & 60,000 & HP & 1953 & $08-18-53$ & 6,090 & \\
\hline 1906 & $11-27-05$ & 65,000 & HP & 1954 & $08-07-54$ & 7,280 & \\
\hline 1907 & $12-03-06$ & ${ }^{1} 70,000$ & HP & 1955 & $07-23-55$ & 8,450 & \\
\hline 1911 & $03-07-11$ & 15,000 & & 1956 & $10-04-55$ & 5,820 & \\
\hline 1912 & $03-10-12$ & 20,000 & & 1957 & $07-26-57$ & 5,230 & \\
\hline 1913 & $07-00-13$ & 10,000 & & 1958 & $09-12-58$ & 7,000 & \\
\hline 1914 & $07-04-14$ & 5,000 & & 1959 & $08-2859$ & 11,600 & \\
\hline 1915 & $12-20-14$ & 23,000 & & 1960 & $01-12-60$ & 11,800 & \\
\hline 1916 & $01-19-16$ & 59,000 & & 1961 & $09-10-61$ & 7,100 & \\
\hline 1917 & $10-14-16$ & 60,000 & & 1962 & $09-26-62$ & 14,300 & \\
\hline 1918 & $00-00-18$ & 3,000 & ES & 1963 & $10-18-62$ & 12,200 & \\
\hline 1919 & $00-00-19$ & 15,000 & ES & 1964 & $07-31-64$ & 8,670 & \\
\hline 1920 & $00-00-20$ & 5,500 & ES & 1965 & $08-02-65$ & 5,640 & \\
\hline 1921 & $00-00-21$ & 16,000 & ES & 1966 & $12-23-65$ & 30,500 & \\
\hline 1922 & $00-00-22$ & 3,500 & ES & 1967 & $08-12-67$ & 34,700 & \\
\hline 1923 & $00-00-23$ & 10,000 & ES & 1968 & $12-20-67$ & 9,480 & \\
\hline 1924 & $00-00-24$ & 10,000 & ES & 1969 & 09-01-69 & 1,270 & \\
\hline 1925 & $00-00-25$ & 16,000 & ES & 1970 & $10-21-69$ & 902 & \\
\hline 1926 & $00-00-26$ & 5,000 & ES & 1971 & $10-04-70$ & 5,420 & \\
\hline 1927 & $09-12-27$ & 4,060 & & 1972 & $10-25-71$ & 9,200 & \\
\hline 1928 & $07-15-28$ & 3,380 & & 1973 & $10-20-72$ & ${ }^{2} 64,000$ & \\
\hline 1929 & $09-23-29$ & 5,200 & & 1974 & $07-21-74$ & 964 & \\
\hline 1930 & $08-11-30$ & 3,420 & & 1975 & $09-09-75$ & 30,000 & \\
\hline 1931 & $09-29-31$ & 3,330 & & 1976 & $02-10-76$ & 3,100 & \\
\hline 1932 & $02-10-32$ & 10,000 & & 1977 & $09-05-77$ & 2,520 & \\
\hline 1933 & $07-23-33$ & 3,800 & & 1978 & $03-03-78$ & 9,500 & \\
\hline 1934 & $08-26-34$ & 11,700 & & 1979 & $12-19-78$ & 56,000 & \\
\hline 1935 & $09-01-35$ & 2,450 & & 1980 & $02-16-80$ & 9,900 & \\
\hline 1936 & $02-17-36$ & 3,700 & & 1981 & $07-09-81$ & 1,570 & \\
\hline 1937 & $02-08-37$ & 12,400 & & 1982 & $03-13-82$ & 2,020 & \\
\hline 1938 & $03-04-38$ & 4,540 & & 1983 & $03-25-83$ & 6,060 & \\
\hline 1939 & 04-06-39 & 1,230 & & 1984 & $10-02-83$ & 90,900 & \\
\hline 1940 & $09-06-40$ & 8,700 & & 1985 & $12-28-84$ & 27,400 & \\
\hline 1941 & $12-31-40$ & 8,700 & & 1986 & $10-17-85$ & 3,590 & \\
\hline 1942 & $12-11-41$ & 7,930 & & 1987 & $11-03-86$ & 1,940 & \\
\hline 1943 & $03-05-43$ & 1,580 & & 1988 & $08-31-88$ & 3,630 & \\
\hline 1944 & $09-26-44$ & 3,800 & & 1989 & $10-15-88$ & 882 & \\
\hline 1945 & $08-22-45$ & 2,820 & & 1990 & $08-14-90$ & 952 & \\
\hline 1946 & $09-05-46$ & 1,380 & & 1991 & $03-02-91$ & 13,800 & \\
\hline 1947 & $08-23-47$ & 5,860 & & 1992 & $02-14-92$ & 6,420 & \\
\hline 1948 & $06-01-48$ & 5,850 & & 1993 & $01-18-93$ & 42,900 & \\
\hline 1949 & $01-13-49$ & 24,100 & & 1994 & 09-04-94 & 972 & \\
\hline 1950 & $07-27-50$ & 825 & & 1995 & $01-05-95$ & 22,200 & \\
\hline 1951 & $08-29-51$ & 735 & & 1996 & $08-30-96$ & 1,750 & \\
\hline
\end{tabular}

Highest since 1870 .

'Highest since 1907. 
09444500 SAN FRANCISCO RIVER AT CLIFTON, AZ--Continued

Discharge rating table developed October 1995

\begin{tabular}{crrr}
\hline $\begin{array}{c}\text { Gage height } \\
(\mathbf{f t})\end{array}$ & $\begin{array}{c}\text { Discharge } \\
\left(\mathbf{f t}^{3} \mathbf{s}\right)\end{array}$ & $\begin{array}{c}\text { Gage height } \\
(\mathbf{f t})\end{array}$ & $\begin{array}{c}\text { Discharge } \\
\left(\mathbf{f t}^{3} / \mathbf{s}\right)\end{array}$ \\
\hline 10.0 & 91 & 24.0 & 32,200 \\
12.0 & 983 & 26.0 & 42,250 \\
14.0 & 2,970 & 28.0 & 53,790 \\
16.0 & 6,160 & 30.0 & 66,840 \\
18.0 & 10,630 & 33.0 & 89,320 \\
20.0 & 16,420 & 36.0 & 115,400 \\
22.0 & 23,600 & 39.0 & 145,000 \\
\hline
\end{tabular}

Basin characteristics

\begin{tabular}{ccccccc}
\hline $\begin{array}{c}\text { Main } \\
\text { channel } \\
\text { slope } \\
(\mathrm{ft} / \mathrm{mi})\end{array}$ & $\begin{array}{c}\text { Stream } \\
\text { length } \\
(\mathrm{mi})\end{array}$ & $\begin{array}{c}\text { Mean } \\
\text { basin } \\
\text { elevation } \\
(\mathrm{ft})\end{array}$ & $\begin{array}{c}\text { Forested } \\
\text { area } \\
\text { (percent) }\end{array}$ & $\begin{array}{c}\text { Soil } \\
\text { index }\end{array}$ & $\begin{array}{c}\text { Mean } \\
\text { annual } \\
\text { precipitation } \\
\text { (in) }\end{array}$ & $\begin{array}{c}\text { Rainfall intensity, 24-hour } \\
\text { (in) }\end{array}$ \\
\hline 48.9 & 93.2 & 6,880 & 87.0 & 2.9 & 18.1 & $\begin{array}{c}50 \text {-year } \\
\text { (in) }\end{array}$ \\
\hline
\end{tabular}


09444500 SAN FRANCISCO RIVER AT CLIFTON, AZ--Continued

MEAN MONTHLY AND ANNUAL DISCHARGES $1914 \cdot 15,1917,1928 \cdot 33,1936-96$

MAGNITUDE AND PROBABILITY OF ANNUAL LOW FLOW BASED ON PERIOD OF RECORD 1915, 1917-18, 1929-33, 1937.96

\begin{tabular}{|c|c|c|c|c|c|c|}
\hline MONTH & $\begin{array}{l}\text { MAXIMUM } \\
(\mathrm{FT} 3 / \mathrm{S})\end{array}$ & $\begin{array}{l}\text { MINIMUM } \\
\text { (FT3/S) }\end{array}$ & $\begin{array}{c}\text { MEAN } \\
(\text { FT3/S) }\end{array}$ & $\begin{array}{l}\text { STAN- } \\
\text { DARD } \\
\text { DEVIA- } \\
\text { TION } \\
\text { (FT3/S) }\end{array}$ & $\begin{array}{l}\text { COEFFI- } \\
\text { CIENT OF } \\
\text { VARI- } \\
\text { ATION }\end{array}$ & $\begin{array}{c}\text { PERCEN } \\
\text { OF } \\
\text { ANNUAI } \\
\text { RUNOF }\end{array}$ \\
\hline OCTOBER & 4.290 & 23 & 228 & 624 & 2.7 & 8.4 \\
\hline NOVEMBER & 1,450 & 28 & 126 & 201 & 1.6 & 4.6 \\
\hline DECEMBER & 2,450 & 34 & 267 & 485 & 1.8 & 9.8 \\
\hline JANUARY & 4,200 & 37 & 314 & 598 & 1.9 & 11.5 \\
\hline FEBRUARY & 2,430 & 39 & 349 & 468 & 1.3 & 12.8 \\
\hline MARCH & 2,140 & 44 & 443 & 496 & 1.1 & 16.2 \\
\hline APRIL & 2,250 & 36 & 332 & 407 & 1.2 & 12.2 \\
\hline MAY & 1,240 & 24 & 165 & 215 & 1.3 & 6.0 \\
\hline JUNE & 310 & 11 & 57 & 47 & 0.81 & 2.1 \\
\hline JULY & 657 & 29 & 103 & 96 & 0.93 & 3.8 \\
\hline AUGUST & 1,360 & 41 & 198 & 201 & 1.0 & 7.2 \\
\hline SEPTEMBER & 816 & 22 & 148 & 141 & 0.95 & 5.4 \\
\hline ANNUAL & 937 & 42 & 227 & 202 & 0.89 & 100 \\
\hline
\end{tabular}

\begin{tabular}{|c|c|c|c|c|c|c|}
\hline \multirow{3}{*}{$\begin{array}{l}\text { PERIOD } \\
\text { (CON- } \\
\text { SECU- }\end{array}$} & & \multicolumn{2}{|c|}{ DISCHARGE, IN FT3/S, FOR INDICATED } & $\begin{array}{l}\text { FOR } \\
\text { IN }\end{array}$ & & \\
\hline & \multicolumn{6}{|c|}{ NON-EXCEEDANCE PROBABILITY, IN PERCENT } \\
\hline & 2 & 5 & 10 & 20 & 50 & 100 \\
\hline DAYS) & 508 & 208 & 108 & 58 & 28 & 18 \\
\hline 1 & 21 & 14 & 11 & 9.5 & 7.7 & 6.7 \\
\hline 3 & 23 & 16 & 13 & 11 & 9.0 & 8.0 \\
\hline 7 & 25 & 17 & 14 & 12 & 10 & 9,2 \\
\hline 14 & 28 & 20 & 16 & 14 & 12 & 11 \\
\hline 30 & 33 & 23 & 19 & 17 & 14 & 12 \\
\hline 60 & 41 & 29 & 24 & 21 & 18 & 16 \\
\hline 90 & 50 & 35 & 30 & 26 & 22 & 20 \\
\hline 120 & 58 & 42 & 36 & 32 & 29 & 27 \\
\hline 183 & 75 & 52 & 45 & 40 & 36 & 34 \\
\hline
\end{tabular}

MAGNTTUDE AND PROBABILITY OF ANNUAL HIGH FLON BASED ON PERIOD OF RECORD $1914 \cdot 15,1917,1928-33,1936-96$

MAGNITUDE AND PROBABILITY OF INSTANTANEOUS PEAK FLOW BASED ON PERIOD OF RECORD 1891, 1905.07, 1911.96

DISCHARGE, IN FT3/S, FOR INDICATED RECURRENCE INTERVAL IN YEARS, AND EXCEEDANCE PROBABILITY, IN PERCENT

\begin{tabular}{|c|c|c|c|c|c|}
\hline 2 & 5 & 10 & 25 & 50 & 100 \\
\hline 508 & 208 & 108 & 48 & 28 & 18 \\
\hline
\end{tabular}

$6,780 \quad 18,300 \quad 31,100 \quad 55,400 \quad 80,800 \quad 113,900$

WEIGHTED SKEW (LOGS) $=0.12$ MEAN (LOGS) $=3.84$ STANDARD DEV. $($ LOGS $)=0.50$

\begin{tabular}{|c|c|c|c|c|c|c|}
\hline & & \multicolumn{5}{|c|}{ DISCHARGE, IN FT3/S, FOR INDICATED } \\
\hline PERIOD & & \multicolumn{5}{|c|}{ RECURRENCE INTERVAL, IN YEARS, AND } \\
\hline $1 \mathrm{CON}-$ & & \multicolumn{3}{|c|}{ EXCEEDANCE PROBABILITY, } & \multicolumn{2}{|c|}{ IN PERCENT } \\
\hline SECU - & & $\cdots \cdot$ & & $\cdots$ & & (2) \\
\hline TIVE & 2 & 5 & 10 & 25 & 50 & 100 \\
\hline DAYS) & 508 & 208 & 108 & 48 & 28 & 18 \\
\hline 1 & 2,550 & 7,870 & 14,600 & 28,800 & 45,200 & 68,400 \\
\hline 3 & 1,660 & 4,870 & 8,790 & 16,800 & 26,000 & 38,600 \\
\hline 7 & 1,100 & 2,950 & 5,090 & 9,310 & 13,900 & 20,200 \\
\hline 15 & 745 & 1,920 & 3,230 & 5,740 & 8,420 & 12,000 \\
\hline 30 & 537 & 1,300 & 2,100 & 3,550 & 5,030 & 6,910 \\
\hline 60 & 389 & 914 & 1,450 & 2,410 & 3,360 & 4,560 \\
\hline 90 & 316 & 747 & 1,200 & 2,010 & 2,850 & 3,910 \\
\hline
\end{tabular}

DURATION TABLE OF DAILY MEAN FLOW FOR PERIOD OF RECORD $1914 \cdot 15,1917,1928 \cdot 33,1936 \cdot 96$

DISCHARGE, IN FT3/S, WHICH WAS EOUALED OR EXCEEDED FOR INDICATED PERCENT OF TIME

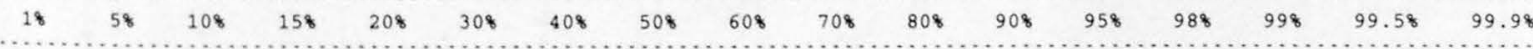

$\begin{array}{llllllllllllllll}2,220 & 829 & 449 & 299 & 218 & 139 & 100 & 78 & 65 & 55 & 46 & 35 & 27 & 21 & 18 & 15\end{array}$




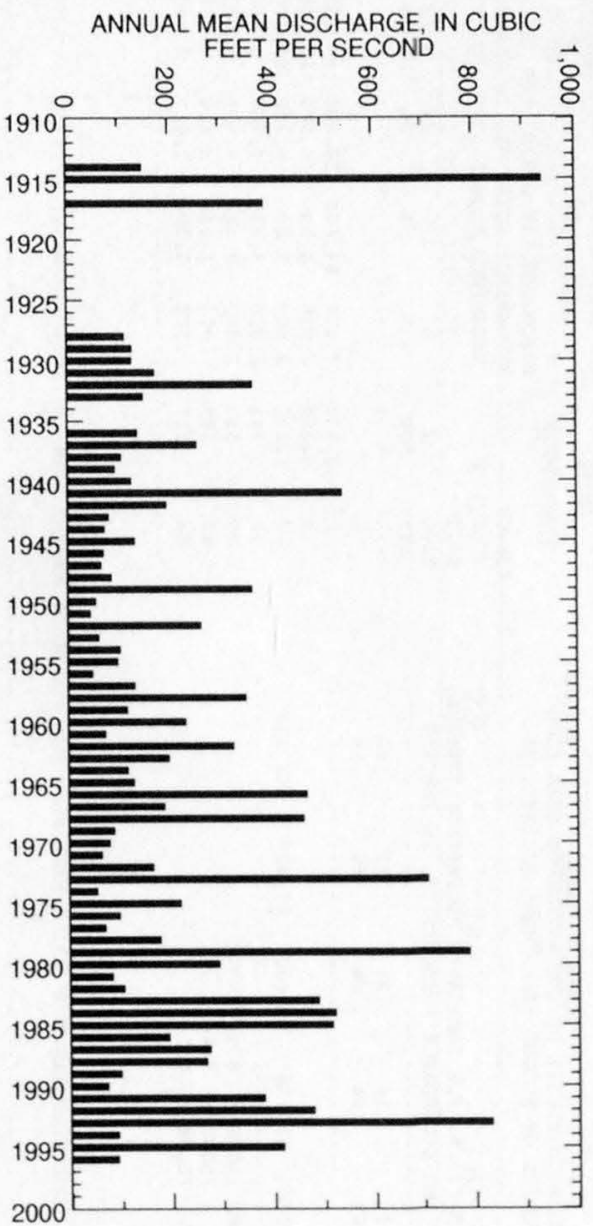

ANNUAL PEAK DISCHARGE, IN CUBIC FEET PER SECOND

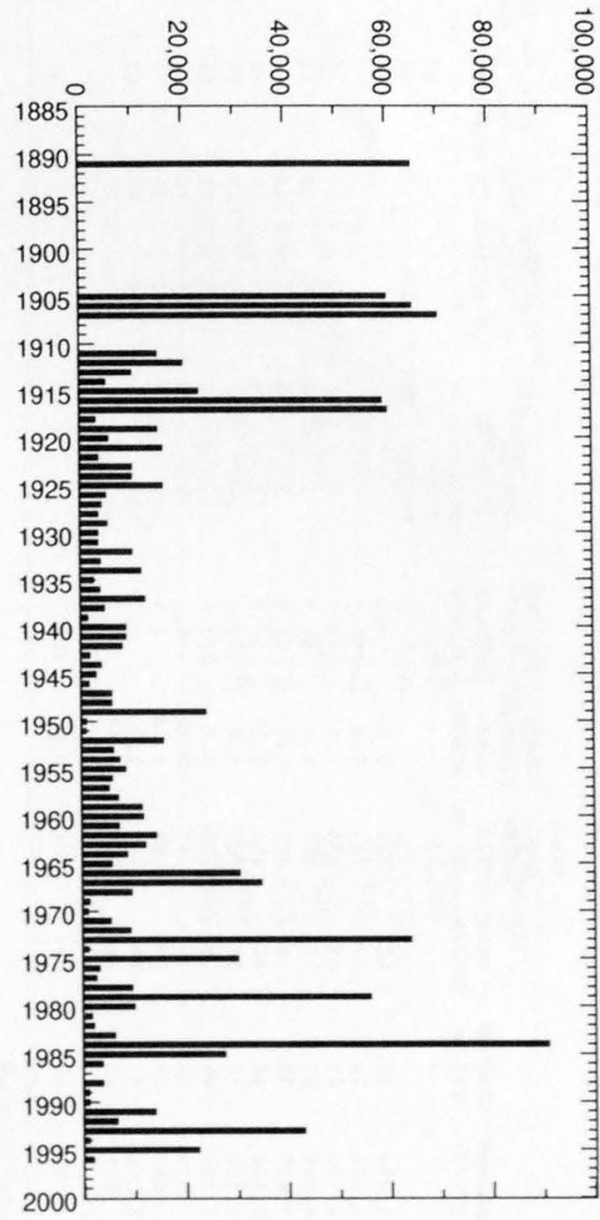


09444500 SAN FRANCISCO RIVER AT CLIFTON, AZ--Continued

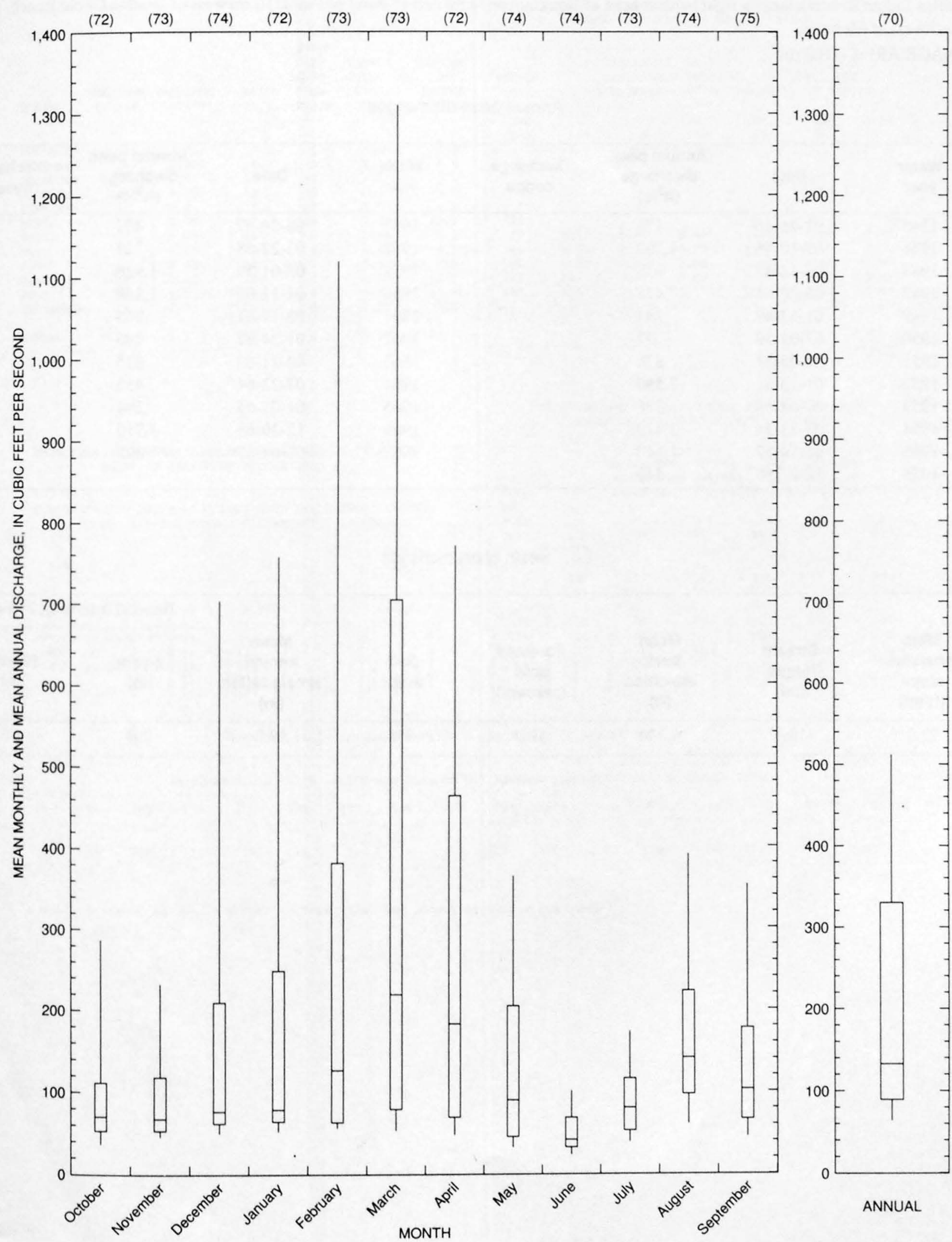


09445500 WILLOW CREEK NEAR POINT OF PINES, NEAR MORENCI, AZ

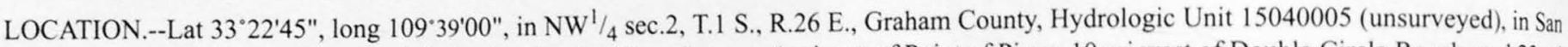
Carlos Indian Reservation, on right bank at head of Box Canyon, 4 mi east of Point of Pines, 10 mi west of Double Circle Ranch, and 23 mi northwest of Morenci.

DRAINAGE AREA.--102 mi ${ }^{2}$.

Annual peak discharges

\begin{tabular}{|c|c|c|c|c|c|c|c|}
\hline $\begin{array}{l}\text { Water } \\
\text { year }\end{array}$ & Date & $\begin{array}{c}\text { Annual peak } \\
\text { discharge } \\
\left(\mathrm{ft}^{3} / \mathrm{s}\right)\end{array}$ & $\begin{array}{l}\text { Discharge } \\
\text { codes }\end{array}$ & $\begin{array}{l}\text { Water } \\
\text { year }\end{array}$ & Date & $\begin{array}{c}\text { Annual peak } \\
\text { discharge } \\
\left(\mathrm{ft}^{3} / \mathrm{s}\right)\end{array}$ & $\begin{array}{c}\text { Discharge } \\
\text { codes }\end{array}$ \\
\hline 1945 & $03-26-45$ & 178 & & 1957 & $08-24-57$ & 459 & \\
\hline 1946 & $07-10-46$ & 1,390 & & 1958 & $03-22-58$ & 727 & \\
\hline 1947 & $08-12-47$ & 935 & & 1959 & 08-01-59 & 1,920 & \\
\hline 1948 & $08-20-48$ & 428 & & 1960 & $01-11-60$ & 1,140 & \\
\hline 1949 & $01-13-49$ & 744 & & 1961 & $08-17-61$ & 245 & \\
\hline 1950 & $07-01-50$ & 37 & & 1962 & $01-24-62$ & 195 & \\
\hline 1951 & $08-05-51$ & 878 & & 1963 & $08-21-63$ & 825 & \\
\hline 1952 & $01-13-52$ & 2,590 & & 1964 & $07-22-64$ & 435 & \\
\hline 1953 & $07-08-53$ & 378 & & 1965 & $01-07-65$ & 294 & \\
\hline 1954 & $03-23-54$ & 1,410 & & 1966 & $12-30-65$ & 3,710 & \\
\hline 1955 & $08-10-55$ & 1,140 & & 1967 & $09-04-67$ & 895 & \\
\hline 1956 & $10-02-55$ & 440 & & & & & \\
\hline
\end{tabular}

Basin characteristics

\begin{tabular}{cccccccc}
\hline $\begin{array}{c}\text { Main } \\
\text { channel } \\
\text { slope } \\
(\mathrm{ft} / \mathrm{mi})\end{array}$ & $\begin{array}{c}\text { Stream } \\
\text { length } \\
(\mathrm{mi})\end{array}$ & $\begin{array}{c}\text { Mean } \\
\text { basin } \\
\text { elevation } \\
(\mathrm{ft})\end{array}$ & $\begin{array}{c}\text { Forested } \\
\text { area } \\
\text { (percent) }\end{array}$ & $\begin{array}{c}\text { Soil } \\
\text { index }\end{array}$ & $\begin{array}{c}\text { Mean } \\
\text { annual } \\
\text { precipitation } \\
\text { (in) }\end{array}$ & $\begin{array}{c}\text { Rainfall intensity, 24-hour } \\
\text { (in) }\end{array}$ & $\begin{array}{c}\text { 50-year } \\
\text { (in) }\end{array}$ \\
\hline 73.0 & 18.3 & 6,340 & 59.0 & 3.0 & 19.8 & 2.0 \\
\hline
\end{tabular}


09445500 WILLOW CREEK NEAR POINT OF PINES, NEAR MORENCI, AZ--Continued

MEAN MONTHLY AND ANNUAL DISCHARGES $1945-67$

\begin{tabular}{|c|c|c|c|c|c|c|}
\hline MONTH & $\begin{array}{l}\text { MAXIMUM } \\
(\text { FT } 3 / \mathrm{S})\end{array}$ & $\begin{array}{l}\text { MINIMUM } \\
\text { (FT3/S) }\end{array}$ & $\begin{array}{c}\text { MEAN } \\
(\mathrm{FT} 3 / \mathrm{S})\end{array}$ & $\begin{array}{l}\text { STAN- } \\
\text { DARD } \\
\text { DEVIA- } \\
\text { TION } \\
\text { (FT3/S) }\end{array}$ & $\begin{array}{l}\text { COEFFI- } \\
\text { CIENT OF } \\
\text { VARI- } \\
\text { ATION }\end{array}$ & $\begin{array}{c}\text { PERCENT } \\
\text { OF } \\
\text { ANNUAL } \\
\text { RUNOFF }\end{array}$ \\
\hline OCTOBER & 21 & 0.00 & 9.6 & 6.6 & 0.69 & 7.0 \\
\hline NOVEMBER & 17 & 0.00 & 6.8 & 5.7 & 0.84 & 5.0 \\
\hline DECEMBER & 157 & 0.00 & 11 & 32 & 2.9 & 8.1 \\
\hline JANUARY & 103 & 0.00 & 12 & 25 & 2.1 & 8.6 \\
\hline FEBRUARY & 33 & 0.00 & 6.1 & 10 & 1.6 & 4.5 \\
\hline MARCH & 76 & 0.00 & 17 & 18 & 1.1 & 12.4 \\
\hline APRIL & 21 & 0.00 & 13 & 5.6 & 0.44 & 9.3 \\
\hline MAY & 19 & 0.00 & 12 & 4.8 & 0.41 & 8.7 \\
\hline JUNE & 22 & 0.00 & 12 & 5.9 & 0.49 & 8.8 \\
\hline JULY & 25 & 0.06 & 12 & 6.0 & 0.50 & 8.7 \\
\hline AUGUST & 35 & 4.1 & 15 & 6.2 & 0.42 & 10.9 \\
\hline SEPTEMBER & 19 & 0.00 & 11 & 6.0 & 0.55 & 7.9 \\
\hline ANNUAL & 32 & 1.0 & 11 & 6.3 & 0.55 & 100 \\
\hline
\end{tabular}

MAGNITUDE AND PROBABILITY OF INSTANTANEOUS PEAK FLOW BASED ON PERIOD OF RECORD 1945-67

DISCHARGE, IN FT3/S, FOR INDICATED RECURRENCE INTERVAL IN YEARS, AND EXCEEDANCE PROBABILITY, IN PERCENT

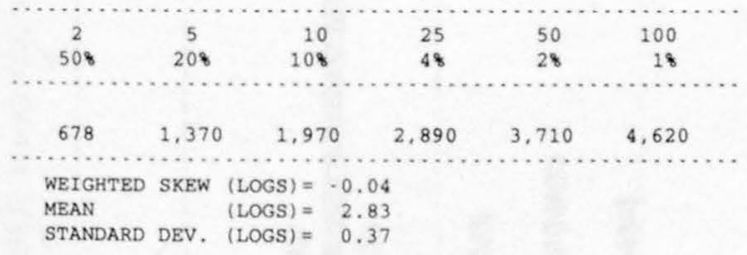

MAGNITUDE AND PROBABILITY OF ANNUAL LOW FLOW BASED ON PERIOD OF RECORD 1946-67

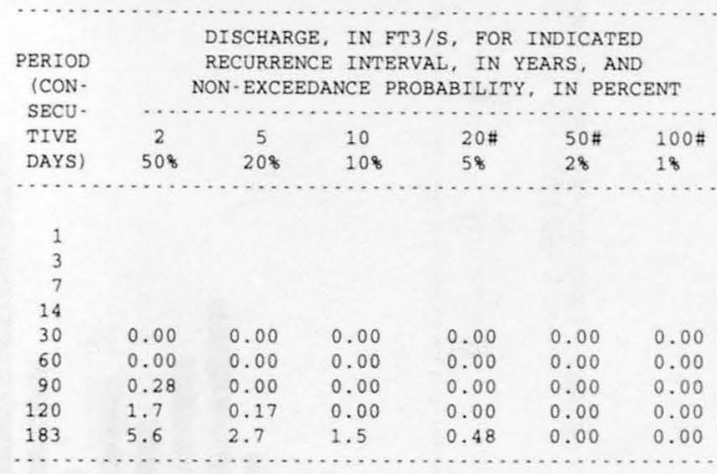

MAGNITUDE AND PROBABILITY OF ANNUAL HIGH FLOW BASED ON PERIOD OF RECORD 1945.67

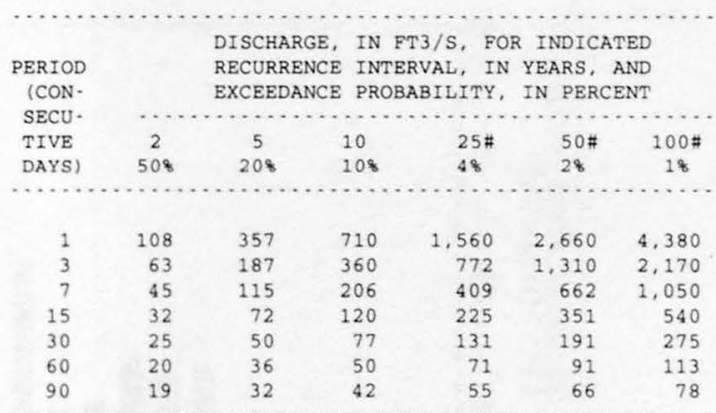

DURATION TABLE OF DAILY MEAN FLOW FOR PERIOD OF RECORD 1945.67

DISCHARGE, IN FT3/S, WHICH WAS EQUALED OR EXCEEDED FOR INDICATED PERCENT OF TIME

\begin{tabular}{|c|c|c|c|c|c|c|c|c|c|c|c|c|c|c|c|c|}
\hline 18 & 58 & 108 & 158 & 208 & 308 & 408 & 508 & 608 & 708 & 808 & 908 & 958 & 988 & 998 & 99.58 & 99.98 \\
\hline 66 & 24 & 20 & 18 & 17 & 15 & 13 & 10 & 6.3 & 0.49 & 0.00 & 0.00 & 0.00 & 0.00 & 0.00 & 0.00 & 0.00 \\
\hline
\end{tabular}

\# Reliability of values in column is uncertain, and potential errors are large. 
09445500 WILLOW CREEK NEAR POINT OF PINES, NEAR MORENCI, AZ--Continued
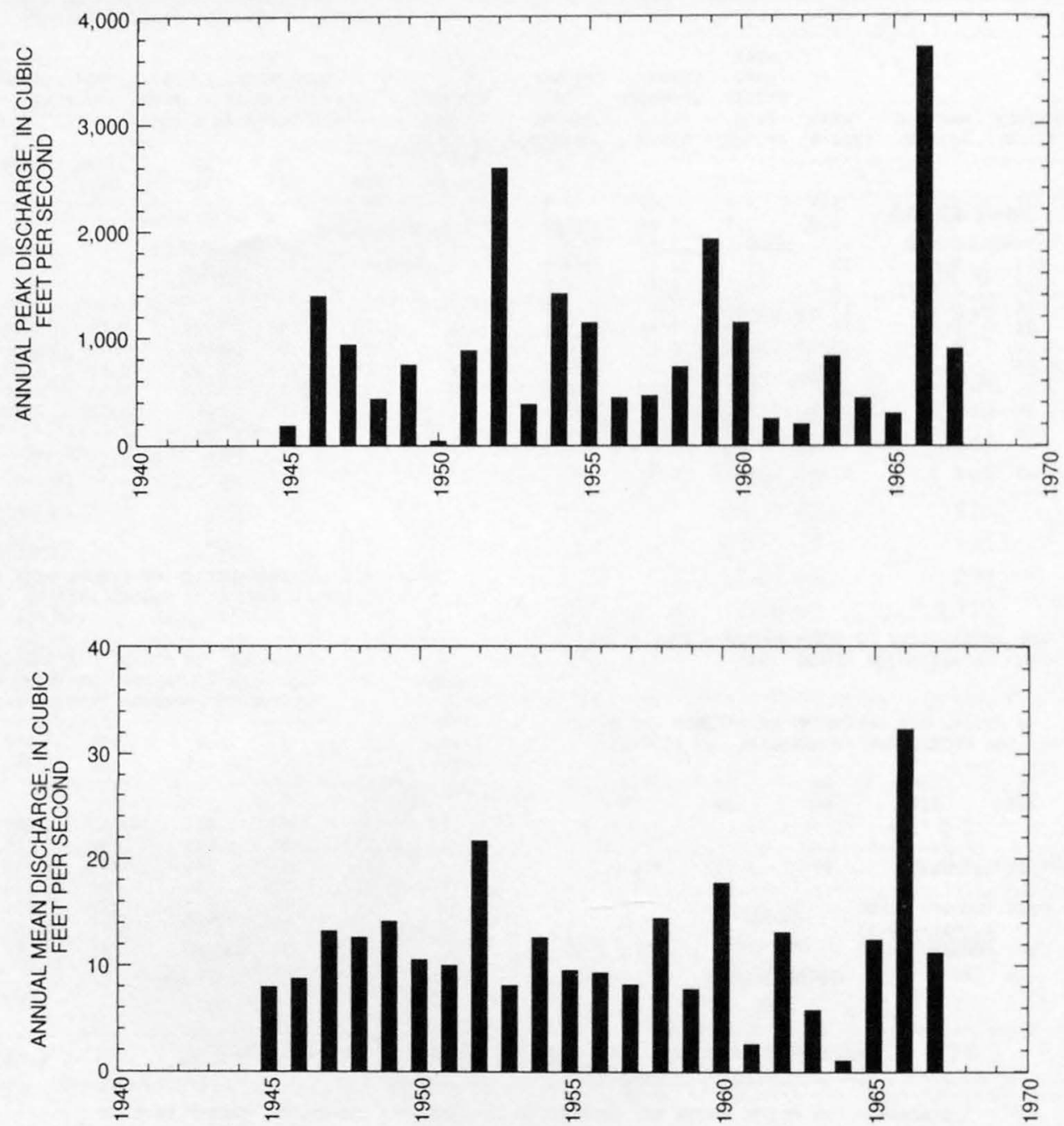
09445500 WILLOW CREEK NEAR POINT OF PINES, NEAR MORENCI, AZ--Continued
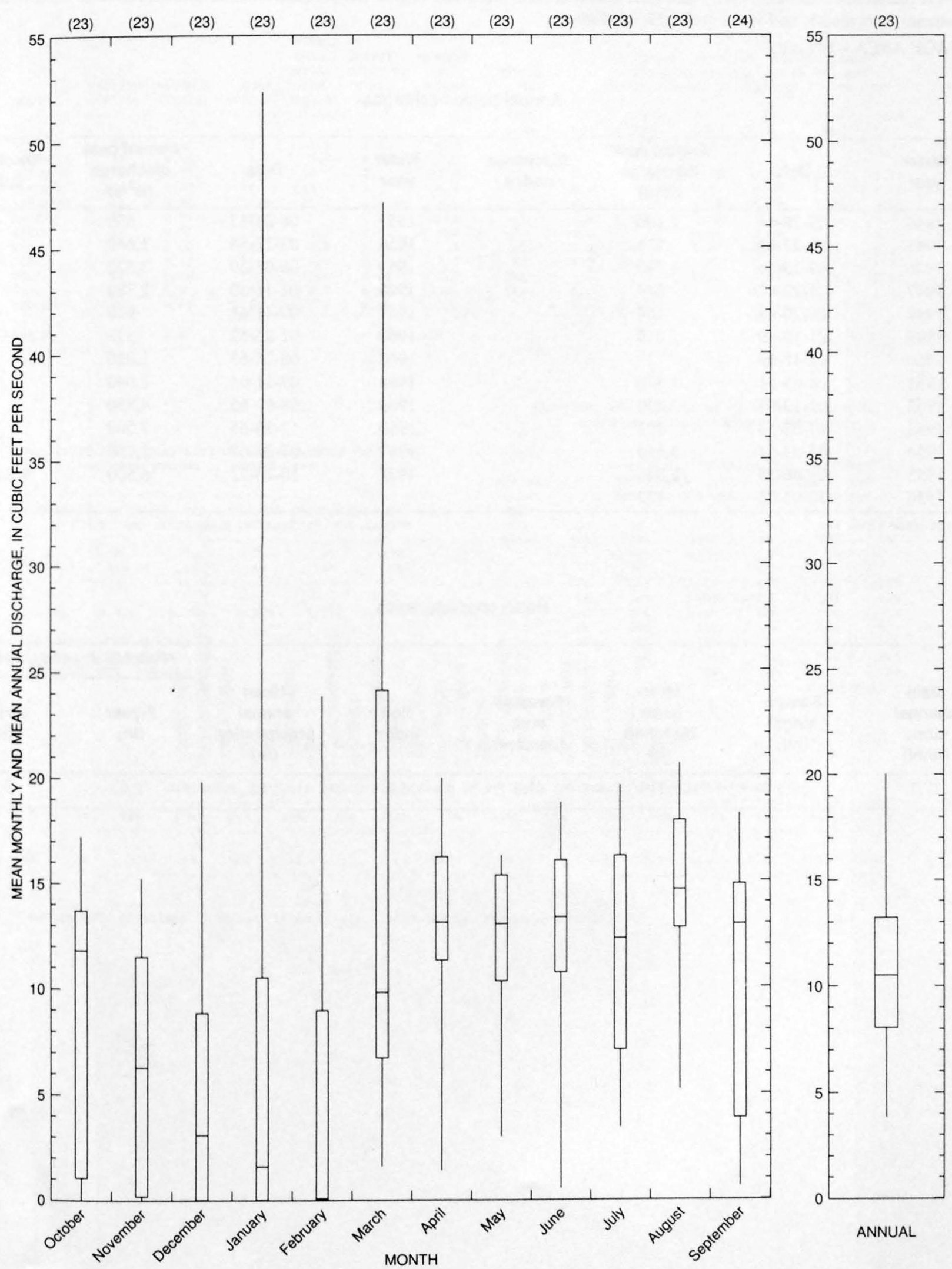


\section{WILLOW CREEK NEAR DOUBLE CIRCLE RANCH, NEAR MORENCI, AZ}

LOCATION.--Lat 33²1'15", long 109'31'30", in NE/4 sec.13, T.1 S., R.27 E., Graham County, Hydrologic Unit 15040005, (unsurveyed), in San Carlos Indian Reservation, on left bank $1 \mathrm{mi}$ upstream from lower end of Box Canyon, 2.25 mi northwest of Double Circle Ranch, $2.5 \mathrm{mi}$ upstream from mouth, and $19 \mathrm{mi}$ northwest of Morenci.

DRAINAGE AREA.--149 $\mathrm{mi}^{2}$.

Annual peak discharges

\begin{tabular}{|c|c|c|c|c|c|c|c|}
\hline $\begin{array}{l}\text { Water } \\
\text { year }\end{array}$ & Date & $\begin{array}{c}\text { Annual peak } \\
\text { discharge } \\
\left(\mathrm{ft}^{3} / \mathrm{s}\right)\end{array}$ & $\begin{array}{l}\text { Discharge } \\
\text { codes }\end{array}$ & $\begin{array}{l}\text { Water } \\
\text { year }\end{array}$ & Date & $\begin{array}{c}\text { Annual peak } \\
\text { discharge } \\
\left(\mathrm{ft}^{3} / \mathrm{s}\right)\end{array}$ & $\begin{array}{c}\text { Discharge } \\
\text { codes }\end{array}$ \\
\hline 1944 & $09-28-44$ & 1,100 & & 1957 & $08-24-57$ & 630 & \\
\hline 1945 & $03-27-45$ & 134 & & 1958 & $03-22-58$ & 1,640 & \\
\hline 1946 & $07-10-46$ & 1,240 & & 1959 & $08-01-59$ & 3,520 & \\
\hline 1947 & $07-22-47$ & 629 & & 1960 & $01-11-60$ & 2,380 & \\
\hline 1948 & $08-20-48$ & 164 & & 1961 & $09-11-61$ & 950 & \\
\hline 1949 & $01-13-49$ & 2,010 & & 1962 & $01-25-62$ & 378 & \\
\hline 1950 & $12-11-49$ & 28 & & 1963 & $08-22-63$ & 1,020 & \\
\hline 1951 & $08-05-51$ & 1,370 & & 1964 & $07-31-64$ & 2,040 & \\
\hline 1952 & $01-13-52$ & 4,230 & & 1965 & $08-01-65$ & 4,880 & \\
\hline 1953 & $07-08-53$ & 202 & & 1966 & $12-30-65$ & 7,500 & \\
\hline 1954 & $03-23-54$ & 1,610 & & 1967 & $07-24-67$ & 1,750 & \\
\hline 1955 & $08-06-55$ & 2,840 & & 1973 & $10-20-72$ & ${ }^{1} 6,500$ & KR,HP \\
\hline 1956 & $10-03-55$ & 122 & & & & & \\
\hline
\end{tabular}

${ }^{1}$ Highest since 1944.

Basin characteristics

\begin{tabular}{ccccccc}
\hline $\begin{array}{c}\text { Main } \\
\text { channel } \\
\text { slope } \\
(\mathrm{ft} / \mathrm{mi})\end{array}$ & $\begin{array}{c}\text { Stream } \\
\text { length } \\
(\mathrm{mi})\end{array}$ & $\begin{array}{c}\text { Mean } \\
\text { basin } \\
\text { elevation } \\
(\mathrm{ft})\end{array}$ & $\begin{array}{c}\text { Forested } \\
\text { area } \\
\text { (percent) }\end{array}$ & $\begin{array}{c}\text { Soil } \\
\text { index }\end{array}$ & $\begin{array}{c}\text { Mean } \\
\text { annual } \\
\text { precipitation } \\
\text { (in) }\end{array}$ & $\begin{array}{c}\text { R-year } \\
\text { (in) }\end{array}$ \\
\hline 77.7 & 27.5 & 6,310 & 63.0 & 3.0 & 19.2 & $\begin{array}{c}50 \text {-year } \\
\text { (in) }\end{array}$ \\
\hline
\end{tabular}


09446000 WILLOW CREEK NEAR DOUBLE CIRCLE RANCH, NEAR MORENCI, AZ--Continued

MEAN MONTHLY AND ANNUAL DISCHARGES 1945-67

\begin{tabular}{|c|c|c|c|c|c|c|}
\hline MONTH & $\begin{array}{l}\text { MAXIMUM } \\
\text { (FT3/S) }\end{array}$ & $\begin{array}{l}\text { MINIMUM } \\
\text { (FT3/S) }\end{array}$ & $\begin{array}{c}\text { MEAN } \\
(\mathrm{FT} 3 / \mathrm{S})\end{array}$ & $\begin{array}{l}\text { STAN- } \\
\text { DARD } \\
\text { DEVIA- } \\
\text { TION } \\
\text { (FT3/S) }\end{array}$ & $\begin{array}{l}\text { COEFFI- } \\
\text { CIENT OF } \\
\text { VARI- } \\
\text { ATION }\end{array}$ & $\begin{array}{c}\text { PERCENT } \\
\text { OF } \\
\text { ANNUAL } \\
\text { RUNOFF }\end{array}$ \\
\hline OCTOBER & 22 & 0.84 & 10 & 6.1 & 0.61 & 6.3 \\
\hline NOVEMBER & 19 & 0.86 & 8.1 & 5.6 & 0.69 & 5.0 \\
\hline DECEMBER & 273 & 0.93 & 18 & 56 & 3.1 & 11.2 \\
\hline JANUARY & 163 & 0.90 & 19 & 40 & 2.1 & 11.8 \\
\hline FEBRUARY & 36 & 0.86 & 7.9 & 11 & 1.4 & 4.9 \\
\hline MARCH & 103 & 0.79 & 19 & 23 & 1.2 & 11.8 \\
\hline APRIL & 23 & 0.76 & 13 & 5.4 & 0.41 & 8.3 \\
\hline MAY & 19 & 0.59 & 12 & 4.8 & 0.42 & 7.2 \\
\hline JUNE & 20 & 0.03 & 11 & 5.7 & 0.51 & 7.0 \\
\hline JULY & 24 & 0.40 & 13 & 5.6 & 0.44 & 7.9 \\
\hline AUGUST & 55 & 6.3 & 19 & 9.8 & 0.52 & 11.6 \\
\hline SEPTEMBER & 20 & 0.43 & 11 & 5.4 & 0.48 & 7.0 \\
\hline ANNUAL & 42 & 2.8 & 13 & 8.3 & 0.62 & 100 \\
\hline
\end{tabular}

MAGNITUDE AND PROBABILITY OF INSTANTANEOUS PEAK FLOW BASED ON PERIOD OF RECORD $1944-67,1973$

DISCHARGE, IN FT3/S, FOR INDICATED RECURRENCE INTERVAL IN YEARS, AND EXCEEDANCE PROBABILITY, IN PERCENT

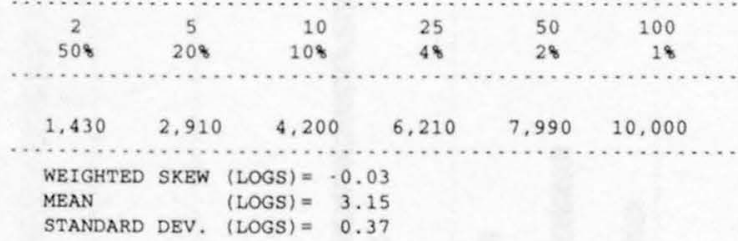

MAGNITUDE AND PROBABILITY OF ANNUAL LOW FLOW BASED ON PERIOD OF RECORD $1946 \cdot 67$

\begin{tabular}{|c|c|c|c|c|c|c|}
\hline \multirow{4}{*}{$\begin{array}{l}\text { PERIOD } \\
\text { (CON- } \\
\text { SECU- } \\
\text { TIVE }\end{array}$} & \multicolumn{2}{|c|}{$\begin{array}{l}\text { DISCHARGE } \\
\text { RECURRENC: }\end{array}$} & \multicolumn{4}{|c|}{ IN FT3/S, FOR INDICATED } \\
\hline & & - EXCEB & \multicolumn{4}{|c|}{$\begin{array}{l}\text { INTERVAL, IN YEARS, AND } \\
\text { NCE PROBABILITY, IN PERCENT }\end{array}$} \\
\hline & & $\cdots \cdots$ & $\cdots \cdots$ & $\cdots \ldots$ & $\cdots \cdots$ & \\
\hline & 2 & 5 & 10 & 20 & $50 \#$ & $100 \#$ \\
\hline DAYS) & 508 & 208 & 108 & 58 & 28 & 18 \\
\hline 1 & 0.71 & 0.19 & 0.02 & 0.00 & 0.00 & 0.00 \\
\hline 3 & 0.77 & 0.22 & 0.02 & 0.00 & 0.00 & 0.00 \\
\hline 7 & 0.87 & 0.28 & 0.05 & 0.00 & 0.00 & 0.00 \\
\hline 14 & 0.87 & 0.32 & 0.10 & 0.00 & 0.00 & 0.00 \\
\hline 30 & 1.0 & 0.46 & 0.24 & 0.00 & 0.00 & 0.00 \\
\hline 60 & 1.4 & 0.59 & 0.37 & 0.25 & 0.16 & 0.12 \\
\hline 90 & 1.9 & 0.94 & 0.69 & 0.54 & 0.43 & 0.37 \\
\hline 120 & 3.0 & 1.7 & 1.2 & 0.97 & 0.76 & 0.65 \\
\hline 183 & 6.8 & 3.8 & 2.7 & 2.0 & 1.3 & 1.0 \\
\hline
\end{tabular}

MAGNITUDE AND PROBABILITY OF ANNUAL HIGH FLOW BASED ON PERIOD OF RECORD $1945-67$

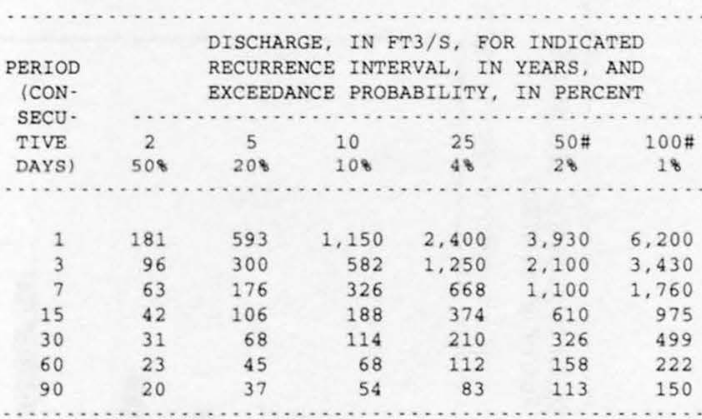

DURATION TABLE OF DAILY MEAN FLOW FOR PERIOD OF RECORD 1945-67

DISCHARGE, IN FT3/S, WHICH WAS EQUALED OR EXCEEDED FOR INDICATED PERCENT OF TIME

\begin{tabular}{|c|c|c|c|c|c|c|c|c|c|c|c|c|c|c|c|c|}
\hline 18 & 58 & 108 & 158 & 208 & 308 & 408 & 508 & 608 & 708 & 808 & 908 & 958 & 988 & 998 & 99.58 & 99.98 \\
\hline 83 & 26 & 21 & 18 & 16 & 14 & 12 & 10 & 7.3 & 2.9 & 1.4 & 0.97 & 0.67 & 0.27 & 0.00 & 0.00 & .00 \\
\hline
\end{tabular}

\# Reliability of values in column is uncertain, and potential errors are large. 

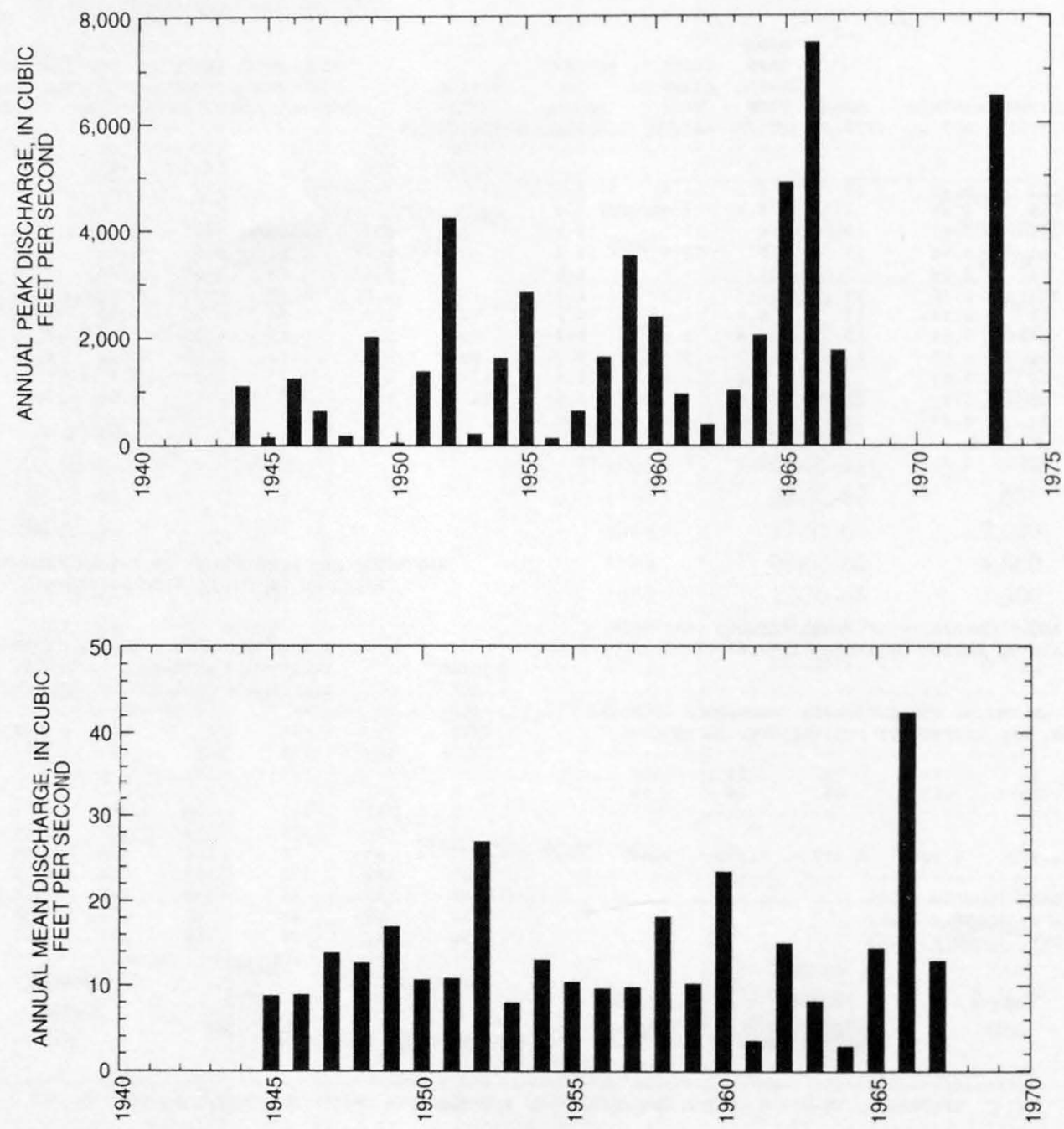
09446000 WILLOW CREEK NEAR DOUBLE CIRCLE RANCH, NEAR MORENCI, AZ--Continued
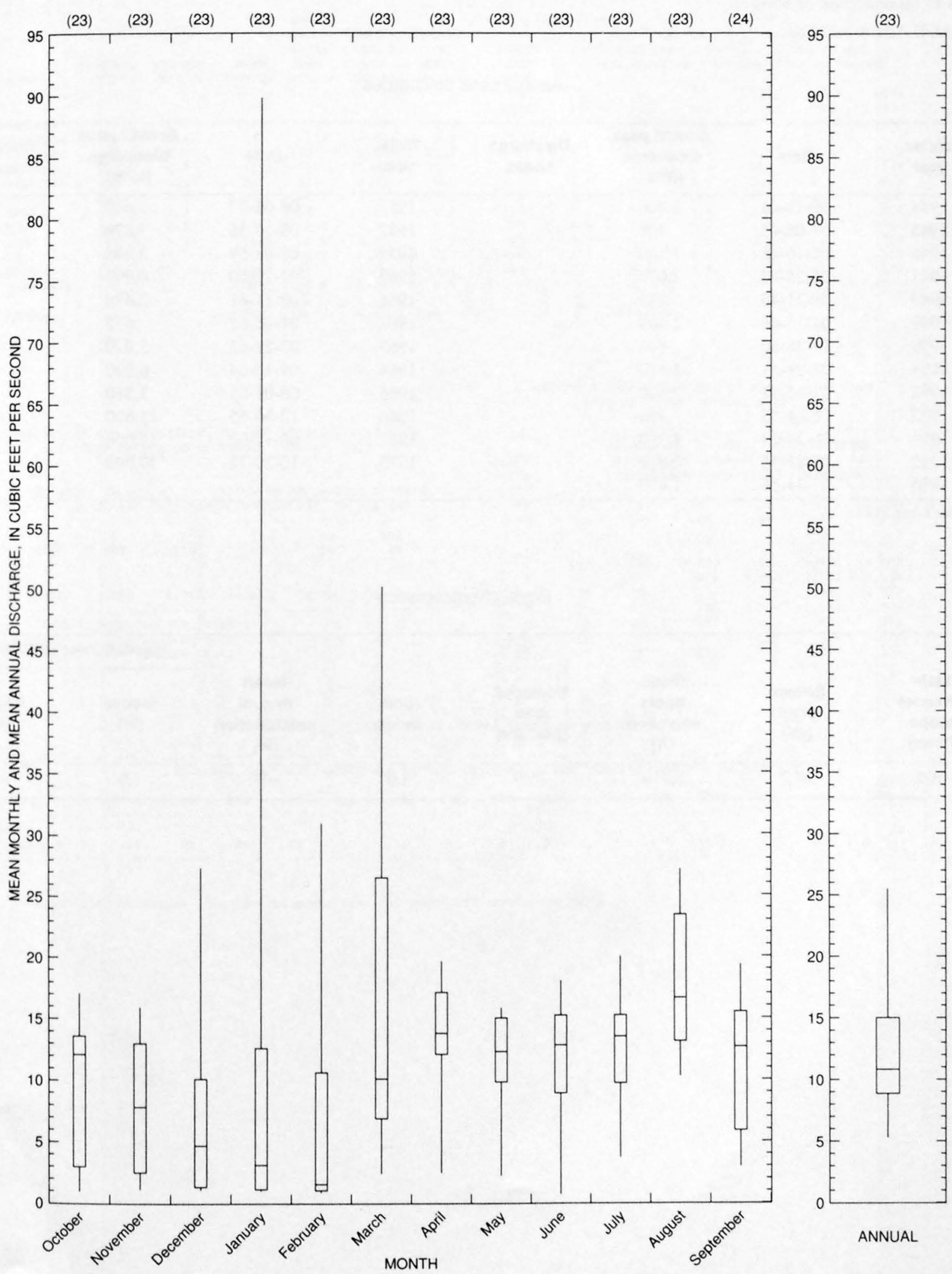


\section{EAGLE CREEK NEAR DOUBLE CIRCLE RANCH, NEAR MORENCI, AZ}

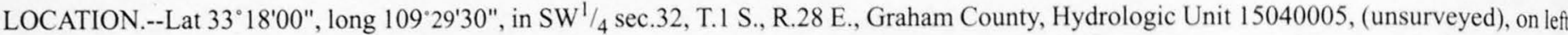
bank 0.5 mi upstream from head of Box Canyon, 2.75 mi downstream from Willow Creek, 3.25 mi downstream from Double Circle Ranch, and $17 \mathrm{mi}$ northwest of Morenci.

DRAINAGE AREA.--377 mi².

Annual peak discharges

\begin{tabular}{|c|c|c|c|c|c|c|c|}
\hline $\begin{array}{l}\text { Water } \\
\text { year }\end{array}$ & Date & $\begin{array}{c}\text { Annual peak } \\
\text { discharge } \\
\left(\mathrm{ft}^{3} / \mathrm{s}\right)\end{array}$ & $\begin{array}{c}\text { Discharge } \\
\text { codes }\end{array}$ & $\begin{array}{c}\text { Water } \\
\text { year }\end{array}$ & Date & $\begin{array}{c}\text { Annual peak } \\
\text { discharge } \\
\left(\mathrm{ft}^{3} / \mathrm{s}\right)\end{array}$ & $\begin{array}{l}\text { Discharge } \\
\text { codes }\end{array}$ \\
\hline 1944 & $09-11-44$ & 2,400 & & 1957 & $08-01-57$ & 1,610 & \\
\hline 1945 & $08-06-45$ & 798 & & 1958 & $09-10-58$ & 7,270 & \\
\hline 1946 & $07-10-46$ & 1,160 & & 1959 & $08-01-59$ & 3,200 & \\
\hline 1947 & $08-25-47$ & 2,070 & & 1960 & $01-12-60$ & 4,990 & \\
\hline 1948 & $08-21-48$ & 135 & & 1961 & $09-11-61$ & 2,470 & \\
\hline 1949 & $01-13-49$ & 2,400 & & 1962 & $01-25-62$ & 612 & \\
\hline 1950 & $07-28-50$ & 874 & & 1963 & $08-21-63$ & 3,920 & \\
\hline 1951 & $08-28-51$ & 1,470 & & 1964 & $09-10-64$ & 6,390 & \\
\hline 1952 & $01-13-52$ & 7,000 & & 1965 & $08-01-65$ & 3,510 & \\
\hline 1953 & $08-01-53$ & 456 & & 1966 & $12-30-65$ & 13,600 & \\
\hline 1954 & $08-24-54$ & 4,380 & & 1967 & $08-11-67$ & 6,000 & \\
\hline 1955 & $08-21-55$ & 2,680 & & 1973 & $10-20-72$ & ${ }^{1} 30,000$ & HP \\
\hline 1956 & $07-31-56$ & 1,410 & & & & & \\
\hline
\end{tabular}

THighest since 1944.

Basin characteristics

\begin{tabular}{cccccccc}
\hline $\begin{array}{c}\text { Main } \\
\text { channel } \\
\text { slope } \\
(\mathrm{ft} / \mathrm{mi})\end{array}$ & $\begin{array}{c}\text { Stream } \\
\text { length } \\
(\mathrm{mi})\end{array}$ & $\begin{array}{c}\text { Mean } \\
\text { basin } \\
\text { elevation } \\
(\mathrm{ft})\end{array}$ & $\begin{array}{c}\text { Forested } \\
\text { area } \\
\text { (percent) }\end{array}$ & $\begin{array}{c}\text { Soil } \\
\text { index }\end{array}$ & $\begin{array}{c}\text { Mean } \\
\text { annual } \\
\text { precipitation } \\
\text { (in) }\end{array}$ & $\begin{array}{c}\text { Rainfall intensity, 24-hour } \\
\text { (in) }\end{array}$ & $\begin{array}{c}50-y e a r \\
\text { (in) }\end{array}$ \\
\hline 100 & 29.2 & 6,410 & 75.0 & 3.0 & 20.0 & 2.0 \\
\hline
\end{tabular}


09446500 EAGLE CREEK NEAR DOUBLE CIRCLE RANCH, NEAR MORENCI, AZ--Continued

MEAN MONTHLY AND ANNUAL DISCHARGES $1945 \cdot 67$

\begin{tabular}{|c|c|c|c|c|c|c|}
\hline MONTH & $\begin{array}{l}\text { MAXIMUM } \\
\text { (FT3/S) }\end{array}$ & $\begin{array}{l}\text { MINIMUM } \\
\text { (FT3/S) }\end{array}$ & $\begin{array}{c}\text { MEAN } \\
(\mathrm{FT} 3 / \mathrm{S})\end{array}$ & $\begin{array}{l}\text { STAN- } \\
\text { DARD } \\
\text { DEVIA- } \\
\text { TION } \\
(\mathrm{FT} 3 / \mathrm{S})\end{array}$ & $\begin{array}{l}\text { COEFFI- } \\
\text { CIENT OF } \\
\text { VARI- } \\
\text { ATION }\end{array}$ & $\begin{array}{c}\text { PERCENT } \\
\text { OF } \\
\text { ANNUAL } \\
\text { RUNOFF }\end{array}$ \\
\hline OCTOBER & 33 & 5.7 & 18 & 5.9 & 0.33 & 5.7 \\
\hline NOVEMBER & 22 & 5.2 & 14 & 5.3 & 0.38 & 4.5 \\
\hline DECEMBER & 502 & 4.7 & 36 & 103 & 2.9 & 11.6 \\
\hline JANUARY & 310 & 4.7 & 41 & 76 & 1.8 & 13.3 \\
\hline FEBRUARY & 101 & 4.1 & 22 & 27 & 1.2 & 7.1 \\
\hline MARCH & 213 & 5.9 & 41 & 48 & 1.2 & 13.1 \\
\hline APRIL & 89 & 4.3 & 27 & 18 & 0.64 & 8.9 \\
\hline MAY & 25 & 5.3 & 18 & 4.3 & 0.24 & 5.7 \\
\hline JUNE & 25 & 3.7 & 16 & 4.6 & 0.30 & 5.0 \\
\hline JULY & 36 & 13 & 21 & 6.3 & 0.30 & 6.7 \\
\hline AUGUST & 93 & 13 & 38 & 25 & 0.66 & 12.1 \\
\hline SEPTEMBER & 42 & 11 & 20 & 8.8 & 0.45 & 6.4 \\
\hline ANNUAL & 81 & 11 & 26 & 16 & 0.62 & 100 \\
\hline
\end{tabular}

MAGNITUDE AND PROBABILITY OF INSTANTANEOUS PEAK FLOW BASED ON PERIOD OF RECORD $1944-67,1973$

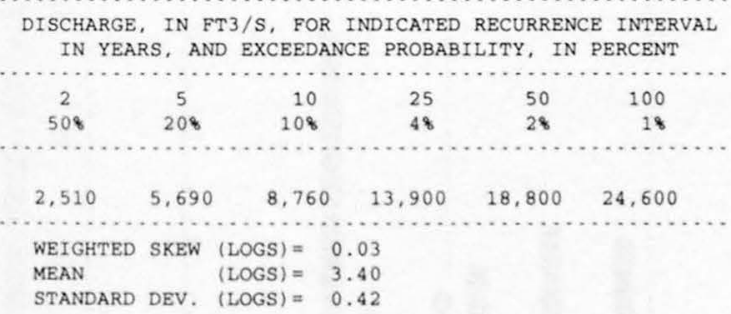

MAGNITUDE AND PROBABILITY OF ANNUAL LOW FLOW BASED ON PERIOD OF RECORD 1946.67

\begin{tabular}{|c|c|c|c|c|c|c|}
\hline \multirow{4}{*}{$\begin{array}{l}\text { PERIOD } \\
\text { (CON- } \\
\text { SECU. }\end{array}$} & \multicolumn{6}{|c|}{ DISCHARGE, IN FT3/S, FOR INDICATED } \\
\hline & \multirow{2}{*}{\multicolumn{6}{|c|}{$\begin{array}{l}\text { RECURRENCE INTERVAL, IN YEARS, AND } \\
\text { NON-EXCEEDANCE PROBABILITY, IN PERCENT }\end{array}$}} \\
\hline & & & & & & \\
\hline & 2 & 5 & 10 & 20 & $50 \#$ & $100 \#$ \\
\hline DAYS) & $50 \%$ & 208 & 108 & 58 & 28 & 18 \\
\hline 1 & 4.1 & 3.1 & 2.7 & 2.3 & 2.0 & 1.9 \\
\hline 3 & 4.2 & 3.1 & 2.7 & 2.4 & 2.1 & 1.9 \\
\hline 7 & 4.4 & 3.3 & 2.9 & 2.6 & 2.3 & 2.2 \\
\hline 14 & 5.1 & 3.9 & 3.4 & 3.1 & 2.8 & 2.7 \\
\hline 30 & 5.8 & 4.5 & 4.0 & 3.8 & 3.5 & 3.4 \\
\hline 60 & 6.8 & 5.0 & 4.4 & 3.9 & 3.6 & 3.3 \\
\hline 90 & 7.5 & 5.4 & 4.6 & 4.1 & 3.6 & 3.4 \\
\hline 120 & 9.1 & 6.6 & 5.6 & 5.0 & 4.3 & 3.9 \\
\hline 183 & 12 & 9.4 & 8.2 & 7.3 & 6.5 & 6.0 \\
\hline
\end{tabular}

MAGNITUDE AND PROBABILITY OF ANNUAL HIGH FLOW BASED ON PERIOD OF RECORD 1945-67

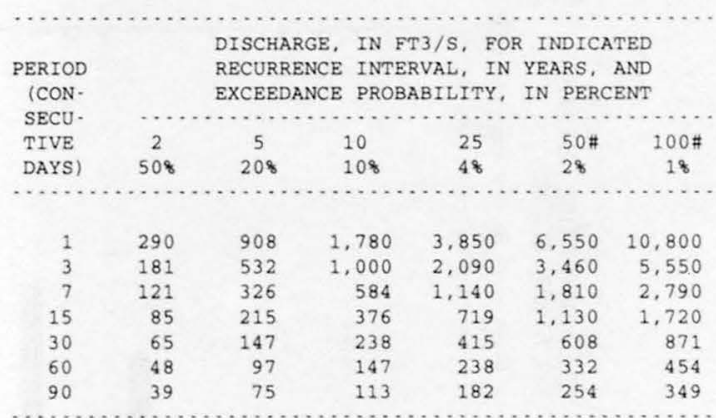

DURATION TABLE OF DAILY MEAN FLOW FOR PERIOD OF RECORD 1945.67

DISCHARGE, IN FT3/S, WHICH WAS EQUALED OR EXCEEDED FOR INDICATED PERCENT OF TIME

18
18

\# Reliability of values in column is uncertain, and potential errors are large. 
09446500 EAGLE CREEK NEAR DOUBLE CIRCLE RANCH, NEAR MORENCI, AZ--Continued
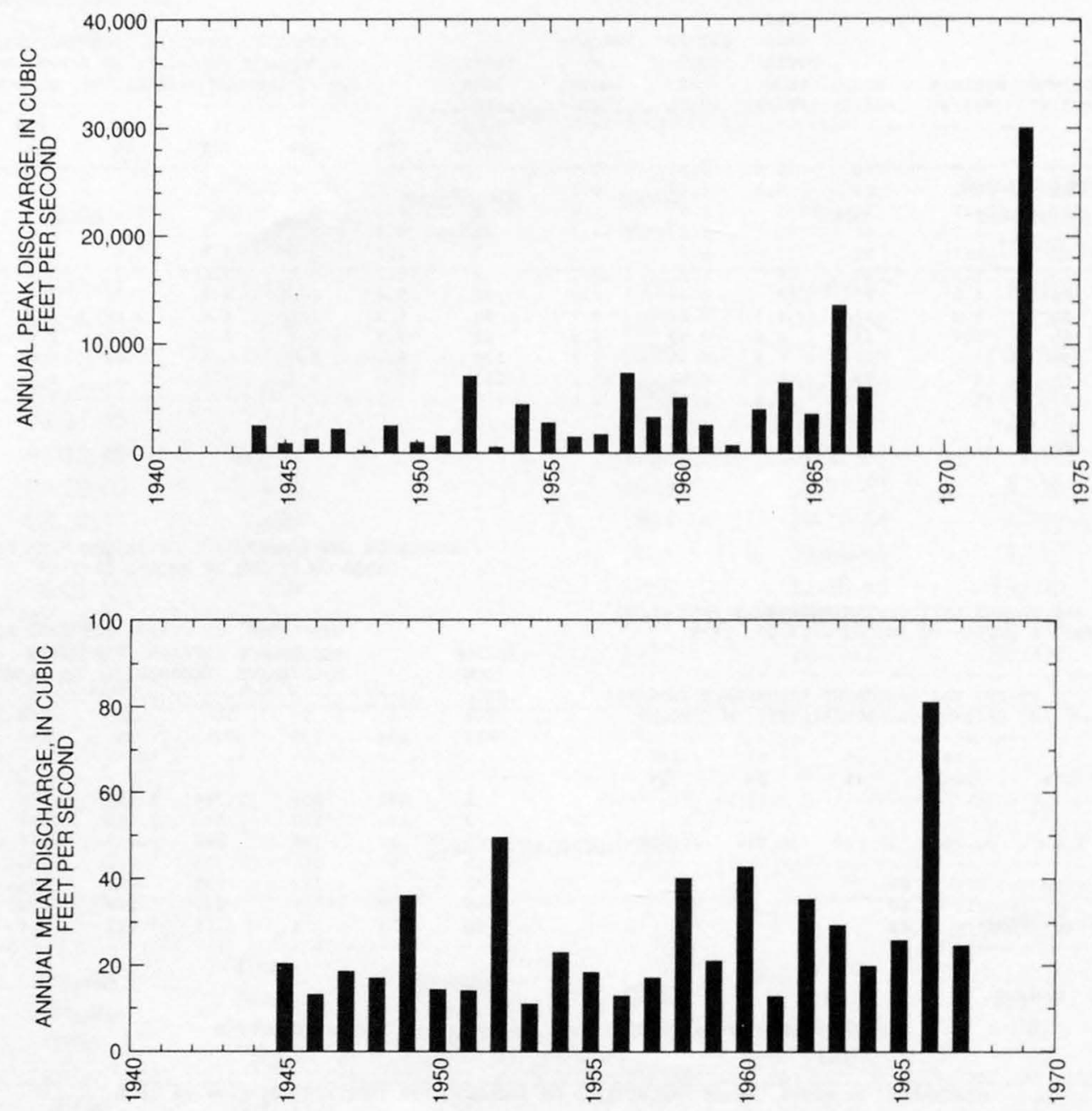
09446500 EAGLE CREEK NEAR DOUBLE CIRCLE RANCH, NEAR MORENCI, AZ--Continued
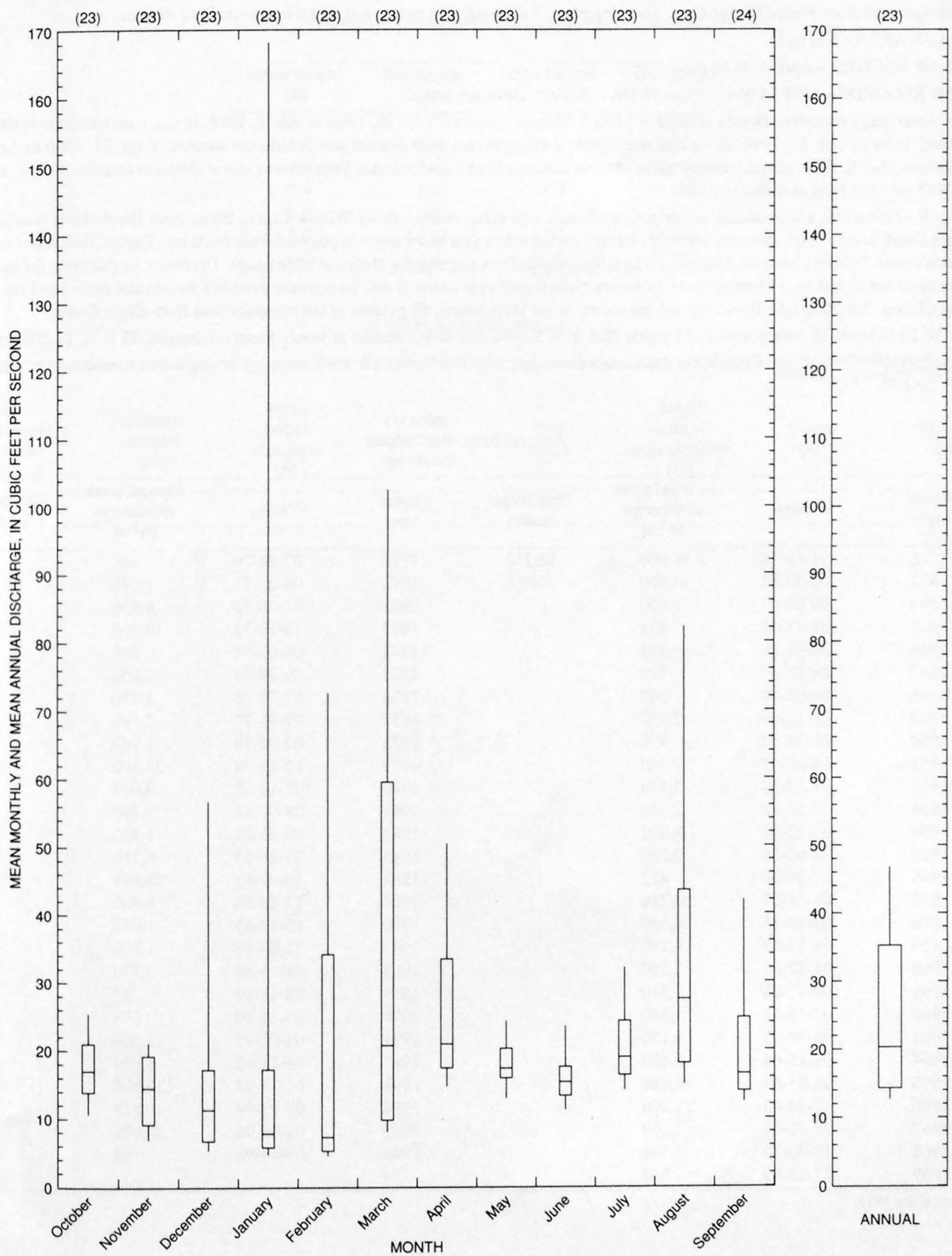
LOCATION.--Lat 33 $03^{\prime} 52^{\prime \prime}$, long 109'26'30", in $\mathrm{SW}^{1} / 4^{1} \mathrm{SE}^{1} / 4 \mathrm{sec} .23$, T.4 S., R.28 E., Greenlee County, Hydrologic Unit 15040005 , on right bank 2 mi upstream from Phelps Dodge Corp. pumping plant, 5 mi west of Morenci, and 12 mi upstream from mouth.

DRAINAGE AREA.--622 $\mathrm{mi}^{2}$.

PERIOD OF RECORD.--April 1944 to current year.

REVISED RECORDS.--WSP 1850-C: 1966. WDR AZ-88-1: Drainage area.

GAGE.--Water-stage recorder. Datum of gage is 3,673.5 ft above sea level. Oct. 25, 1984 to Mar. 6, 1986, at site 1 mi upstream at datum $24.1 \mathrm{ft}$ higher. Prior to Oct. 25, 1984, at various sites within 1 mi upstream from present site at different datums. Aug. 23, 1950 to Aug. 1, 1981 , and since Mar. 6, 1984, supplementary gages at various sites within 1 mi upstream from present site at different datums. Feb. 7, 1993 to July 2, 1993 on right bank at different datum.

REMARKS.--Diversions above station for irrigation of about 500 acres, mostly above Willow Creek. Water from Black River was pumped into Eagle Creek basin, $52 \mathrm{mi}$ upstream from this station, for the entire year and water was pumped from wells into Eagle Creek near Double Circle Ranch below Willow Creek for 7 months. The monthly quantities pumped are shown in table below. Diversion by pumping for industrial and municipal use in and near Morenci and Clifton are made from Eagle Creek, 3 mi downstream from this station and from San Francisco River near Clifton. Monthly quantities diverted are shown in the table below; 98 percent of the pumpage was from Eagle Creek.

AVERAGE DISCHARGE (unadjusted).--52 years, $70.5 \mathrm{ft}^{3} / \mathrm{s}, 51,080$ acre- $\mathrm{ft} / \mathrm{yr}$; median of yearly mean discharges, $38 \mathrm{ft}^{3} / \mathrm{s}, 27,500 \mathrm{acre}-\mathrm{ft} / \mathrm{yr}$.

EXTREMES FOR PERIOD OF RECORD.--Maximum discharge, $36,800 \mathrm{ft}^{3} / \mathrm{s} \mathrm{Jan} .18,1993$, on basis of slope-area measurement; minimum, 2.9 $\mathrm{ft}^{3} / \mathrm{s}$ June $25,1982$.

Annual peak discharges

\begin{tabular}{|c|c|c|c|c|c|c|c|}
\hline $\begin{array}{l}\text { Water } \\
\text { year }\end{array}$ & Date & $\begin{array}{c}\text { Annual peak } \\
\text { discharge } \\
\left(\mathrm{ft}^{3} / \mathrm{s}\right)\end{array}$ & $\begin{array}{l}\text { Discharge } \\
\text { codes }\end{array}$ & $\begin{array}{l}\text { Water } \\
\text { year }\end{array}$ & Date & $\begin{array}{c}\text { Annual peak } \\
\text { discharge } \\
\left(\mathrm{ft}^{3} / \mathrm{s}\right)\end{array}$ & $\begin{array}{c}\text { Discharge } \\
\text { codes }\end{array}$ \\
\hline 1916 & $01-18-16$ & 36,000 & ES,HP & 1970 & $07-23-70$ & 560 & \\
\hline 1932 & $02-10-32$ & 13,000 & HP & 1971 & $08-22-71$ & 1,680 & \\
\hline 1944 & $00-00-44$ & 7,500 & & 1972 & $07-16-72$ & 6,650 & \\
\hline 1945 & $08-11-45$ & 433 & & 1973 & $10-19-72$ & 14,000 & \\
\hline 1946 & $08-07-46$ & 384 & & 1974 & $08-03-74$ & 630 & \\
\hline 1947 & $08-08-47$ & 710 & & 1975 & $09-09-75$ & 1,550 & \\
\hline 1948 & $08-05-48$ & 300 & & 1976 & $07-29-76$ & 2,250 & \\
\hline 1949 & $01-13-49$ & 2,500 & & 1977 & $07-31-77$ & 2,190 & \\
\hline 1950 & $07-28-50$ & 470 & & 1978 & $03-02-78$ & 3,900 & \\
\hline 1951 & $08-28-51$ & 1,260 & & 1979 & $12-18-78$ & 24,500 & \\
\hline 1952 & $01-14-52$ & 5,340 & & 1980 & $02-15-80$ & 9,000 & \\
\hline 1953 & $07-25-53$ & 2,780 & & 1981 & $08-07-81$ & 3,380 & \\
\hline 1954 & $07-22-54$ & 4,930 & & 1982 & $08-23-82$ & 1,720 & \\
\hline 1955 & $08-06-55$ & 3,260 & & 1983 & $03-25-83$ & 6,210 & \\
\hline 1956 & $07-30-56$ & 452 & & 1984 & $10-02-83$ & 36,400 & \\
\hline 1957 & $07-26-57$ & 4,210 & & 1985 & $12-28-84$ & 8,400 & \\
\hline 1958 & $09-10-58$ & 6,150 & & 1986 & $10-17-85$ & 1,030 & \\
\hline 1959 & $08-17-59$ & 4,780 & & 1987 & $11-03-86$ & 1,990 & \\
\hline 1960 & $01-12-60$ & 5,350 & & 1988 & $08-15-88$ & 3,770 & \\
\hline 1961 & $09-12-61$ & 1,210 & & 1989 & $08-18-89$ & 97 & \\
\hline 1962 & $07-18-62$ & 1,850 & & 1990 & $07-16-90$ & 698 & \\
\hline 1963 & $08-30-63$ & 6,150 & & 1991 & $03-02-91$ & 13,500 & \\
\hline 1964 & $07-15-64$ & 8,620 & & 1992 & $02-14-92$ & 6,920 & \\
\hline 1965 & $08-01-65$ & 3,080 & & 1993 & $01-18-93$ & $\mathrm{I}_{36,800}$ & \\
\hline 1966 & $12-30-65$ & 21,000 & & 1994 & $09-03-94$ & 513 & \\
\hline 1967 & $08-12-67$ & 7,650 & & 1995 & $01-05-95$ & 20,000 & \\
\hline 1968 & $12-06-70$ & 3,300 & & 1996 & $09-14-96$ & 95 & \\
\hline 1969 & $07-25-69$ & 250 & & & & & \\
\hline
\end{tabular}

${ }^{1}$ Highest since 1916. 
09447000 EAGLE CREEK ABOVE PUMPING PLANT, NEAR MORENCI, AZ--Continued

Discharge rating table developed October 1994

\begin{tabular}{crcr}
\hline $\begin{array}{c}\text { Gage height } \\
(\mathbf{f t})\end{array}$ & $\begin{array}{c}\text { Discharge } \\
\left(\mathbf{f t}^{3} / \mathbf{s}\right)\end{array}$ & $\begin{array}{c}\text { Gage height } \\
(\mathrm{ft})\end{array}$ & $\begin{array}{c}\text { Discharge } \\
\left(\mathrm{ft}^{3} / \mathbf{s}\right)\end{array}$ \\
\hline 2.0 & 42 & 9.0 & 7,220 \\
3.0 & 64 & 10.0 & 9,550 \\
4.0 & 164 & 11.0 & 12,100 \\
5.0 & 655 & 12.0 & 14,870 \\
6.0 & 1,800 & 13.0 & 17,830 \\
7.0 & 3,320 & 14.0 & 20,990 \\
8.0 & 5,130 & 14.4 & 22,300 \\
\hline
\end{tabular}

Basin characteristics

\begin{tabular}{cccccccc}
\hline $\begin{array}{c}\text { Main } \\
\text { channel } \\
\text { slope } \\
(\mathrm{ft} / \mathrm{mi})\end{array}$ & $\begin{array}{c}\text { Stream } \\
\text { length } \\
(\mathrm{mi})\end{array}$ & $\begin{array}{c}\text { Mean } \\
\text { basin } \\
\text { elevation } \\
(\mathrm{ft})\end{array}$ & $\begin{array}{c}\text { Forested } \\
\text { area } \\
\text { (percent) }\end{array}$ & $\begin{array}{c}\text { Soil } \\
\text { index }\end{array}$ & $\begin{array}{c}\text { Mean } \\
\text { annual } \\
\text { precipitation } \\
\text { (in) }\end{array}$ & $\begin{array}{c}\text { Rainfall intensity, 24-hour } \\
\text { (in) }\end{array}$ & $\begin{array}{c}50 \text {-year } \\
\text { (in) }\end{array}$ \\
\hline 60.9 & 52.5 & 6,060 & 64.0 & 2.8 & 19.2 & 2.0 \\
\hline
\end{tabular}


09447000 EAGLE CREEK ABOVE PUMPING PLANT, NEAR MORENCI, AZ--Continued

MEAN MONTHLY AND ANNUAL DISCHARGES 1945.96

\begin{tabular}{|c|c|c|c|c|c|c|}
\hline MONTH & $\begin{array}{l}\text { MAXIMUM } \\
\text { (FT3/S) }\end{array}$ & $\begin{array}{c}\text { MINIMUM } \\
\text { (FT3/S) }\end{array}$ & $\begin{array}{c}\text { MEAN } \\
\text { (FT3/S) }\end{array}$ & $\begin{array}{l}\text { STAN- } \\
\text { DARD } \\
\text { DEVIA- } \\
\text { TION } \\
(\text { FT3/S) }\end{array}$ & $\begin{array}{l}\text { COEFFI- } \\
\text { CIENT OF } \\
\text { VARI- } \\
\text { ATION }\end{array}$ & $\begin{array}{c}\text { PERCENT } \\
\text { OF } \\
\text { ANNUAL } \\
\text { RUNOFF }\end{array}$ \\
\hline OCTOBER & 1.170 & 13 & 60 & 164 & 2.8 & 7.1 \\
\hline NOVEMBER & 228 & 10 & 35 & 35 & 1.0 & 4.2 \\
\hline DECEMBER & 884 & 11 & 87 & 160 & 1.8 & 10.2 \\
\hline JANUARY & 4,440 & 11 & 184 & 630 & 3.4 & 21.7 \\
\hline FEBRUARY & 1,760 & 11 & 135 & 290 & 2.1 & 16.0 \\
\hline MARCH & 709 & 14 & 109 & 151 & 1.4 & 12.9 \\
\hline APRIL & 214 & 11 & 53 & 41 & 0.78 & 6.3 \\
\hline MAY & 84 & 9.2 & 33 & 16 & 0.50 & 3.9 \\
\hline JUNE & 49 & 5.3 & 25 & 9.4 & 0.38 & 2.9 \\
\hline JULY & 98 & 16 & 37 & 17 & 0.45 & 4.4 \\
\hline AUGUST & 203 & 19 & 55 & 38 & 0.70 & 6.5 \\
\hline SEPTEMBER & 114 & 13 & 35 & 19 & 0.54 & 4.1 \\
\hline ANNUAL & 568 & 17 & 71 & 86 & 1.2 & 100 \\
\hline
\end{tabular}

MAGNITUDE AND PROBABILITY OF INSTANTANEOUS PEAK FLOW BASED ON PERIOD OF RECORD 1944.96

DISCHARGE, IN FT3/S, FOR INDICATED RECURRENCE INTERVAL IN YEARS, AND EXCEEDANCE PROBABILITY, IN PERCENT

$\begin{array}{cccccc}2 & 5 & 10 & 25 & 50 & 100 \\ 508 & 208 & 108 & 48 & 28 & 18 \\ 2.730 & 8,300 & 14,400 & 24,900 & 35,000 & 47,000\end{array}$

WEIGHTED SKEW (LOGS) $=-0.32$

MEAN (LOGS) $=3.40$

STANDARD DEV. (LOGS) $=0.61$
MAGNITUDE AND PROBABILITY OF ANNUAL LOW FLOW BASED ON PERIOD OF RECORD $1945 \cdot 96$

\begin{tabular}{|c|c|c|c|c|c|c|}
\hline \multirow{3}{*}{$\begin{array}{l}\text { PERIOD } \\
\text { (CON- } \\
\text { SECU- } \\
\text { TIVE }\end{array}$} & \multicolumn{6}{|c|}{$\begin{array}{l}\text { DISCHARGE, IN FT3/S, FOR INDICATED } \\
\text { RECURRENCE INTERVAL, IN YEARS, AND } \\
\text { NON-EXCEEDANCE PROBABILITY, IN PERCENT }\end{array}$} \\
\hline & $\cdots$ & 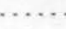 & $\cdots \cdots$ & $\ldots \ldots$ & $\cdots \cdots$ & $\cdots \cdots$ \\
\hline & 2 & 5 & 10 & 20 & 50 & 100 \\
\hline DAYS) & 508 & 208 & 108 & $5 \%$ & 28 & 18 \\
\hline 1 & 12 & 7.2 & 5.4 & 4.2 & 3.2 & 2.6 \\
\hline 3 & 12 & 7.6 & 5.7 & 4.5 & 3.4 & 2.7 \\
\hline 7 & 14 & 8.4 & 6.3 & 4.9 & 3.7 & 3.0 \\
\hline 14 & 15 & 9.8 & 7.5 & 5.9 & 4.5 & 3.7 \\
\hline 30 & 17 & 12 & 9.0 & 7.2 & 5.5 & 4.6 \\
\hline 60 & 19 & 13 & 11 & 8.9 & 7.2 & 6.2 \\
\hline 90 & 20 & 14 & 12 & 9.9 & 8.1 & 7.1 \\
\hline 120 & 23 & 16 & 13 & 11 & 9.6 & 8.5 \\
\hline 183 & 26 & 20 & 17 & 15 & 13 & 12 \\
\hline
\end{tabular}

MAGNITUDE AND PROBABILITY OF ANNUAL HIGH FLOW BASED ON PERIOD OF RECORD 1945.96

\begin{tabular}{|c|c|c|c|c|c|c|}
\hline $\begin{array}{l}\text { PERIOD } \\
\text { (CON- }\end{array}$ & & \multicolumn{5}{|c|}{$\begin{array}{l}\text { DISCHARGE, IN FT } 3 / \mathrm{S} \text {, FOR INDICATED } \\
\text { RECURRENCE INTERVAL, IN YEARS, AND }\end{array}$} \\
\hline SECU- & $\cdots$ & 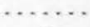 & . & $\ldots \ldots \ldots$ & … & $\ldots \ldots \ldots$ \\
\hline TIVE & 2 & 5 & 10 & 25 & 50 & 100 \\
\hline DAYS) & 508 & 208 & 108 & 48 & 28 & 18 \\
\hline 1 & 708 & 2,900 & 6,320 & 14,900 & 26,400 & 44,700 \\
\hline 3 & 428 & 1,700 & 3,690 & 8,860 & 16,000 & 27,700 \\
\hline 7 & 271 & 987 & 2,080 & 4,860 & 8,670 & 14,900 \\
\hline 15 & 180 & 605 & 1,240 & 2.870 & 5.120 & 8,840 \\
\hline 30 & 132 & 394 & 756 & 1,610 & 2,730 & 4,480 \\
\hline 60 & 96 & 267 & 500 & 1,050 & 1,750 & 2,870 \\
\hline 90 & 79 & 208 & 379 & 769 & 1,260 & 2,030 \\
\hline
\end{tabular}

DURATION TABLE OF DAILY MEAN FLOW FOR PERIOD OF RECORD 1945.96

DISCHARGE, IN FT3/S, WHICH WAS EQUALED OR EXCEEDED FOR INDICATED PERCENT OF TIME

\begin{tabular}{|c|c|c|c|c|c|c|c|c|c|c|c|c|c|c|c|c|}
\hline 18 & 58 & 108 & 158 & 208 & $30 \%$ & 408 & 508 & $60 \%$ & 708 & 808 & 908 & 958 & 988 & 998 & 99.58 & 99.98 \\
\hline 686 & 168 & 88 & 58 & 46 & 38 & 33 & 30 & 26 & 23 & 19 & 15 & 12 & 10 & 9.0 & 7.3 & 4. 2 \\
\hline
\end{tabular}




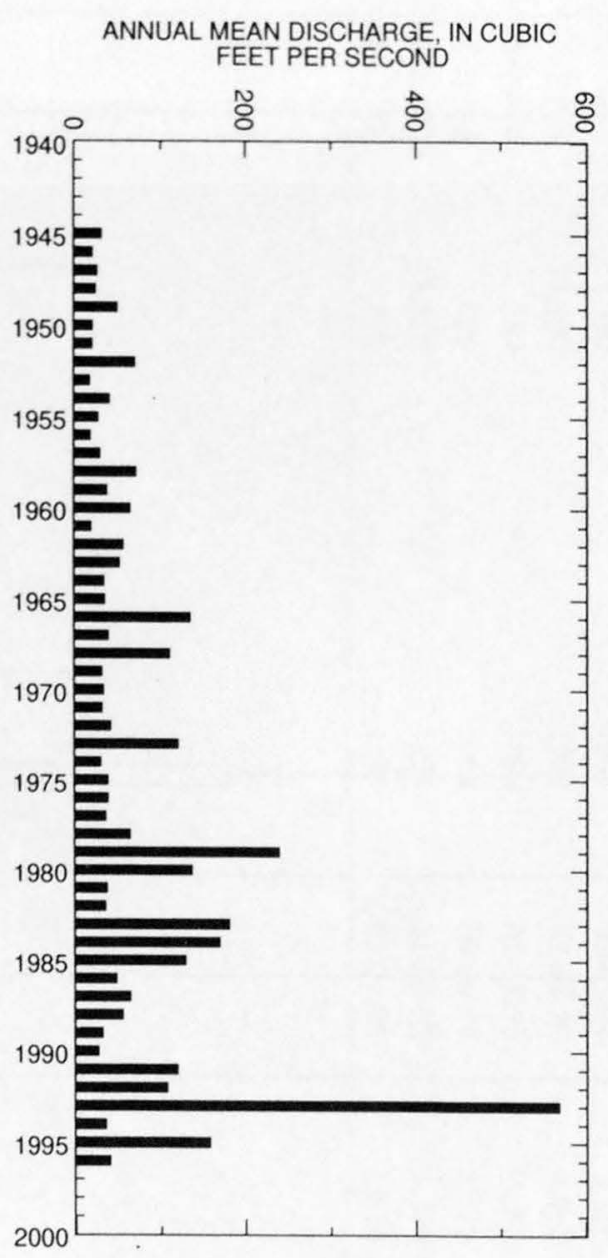

ANNUAL PEAK DISCHARGE, IN CUBIC

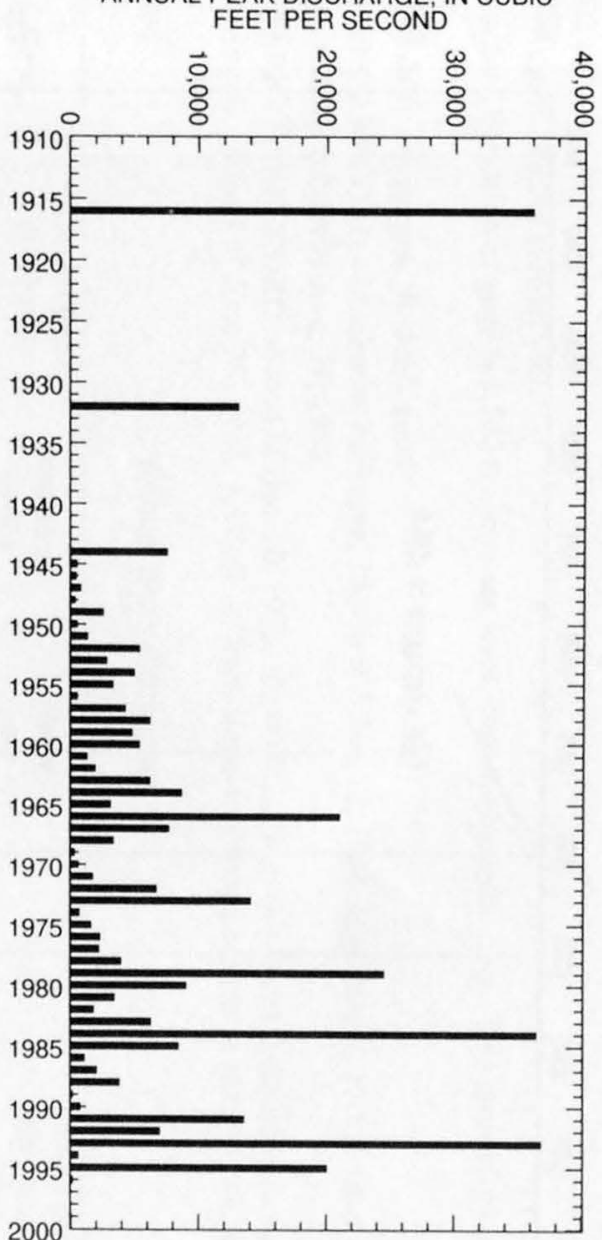


09447000 EAGLE CREEK ABOVE PUMPING PLANT, NEAR MORENCI, AZ--Continued

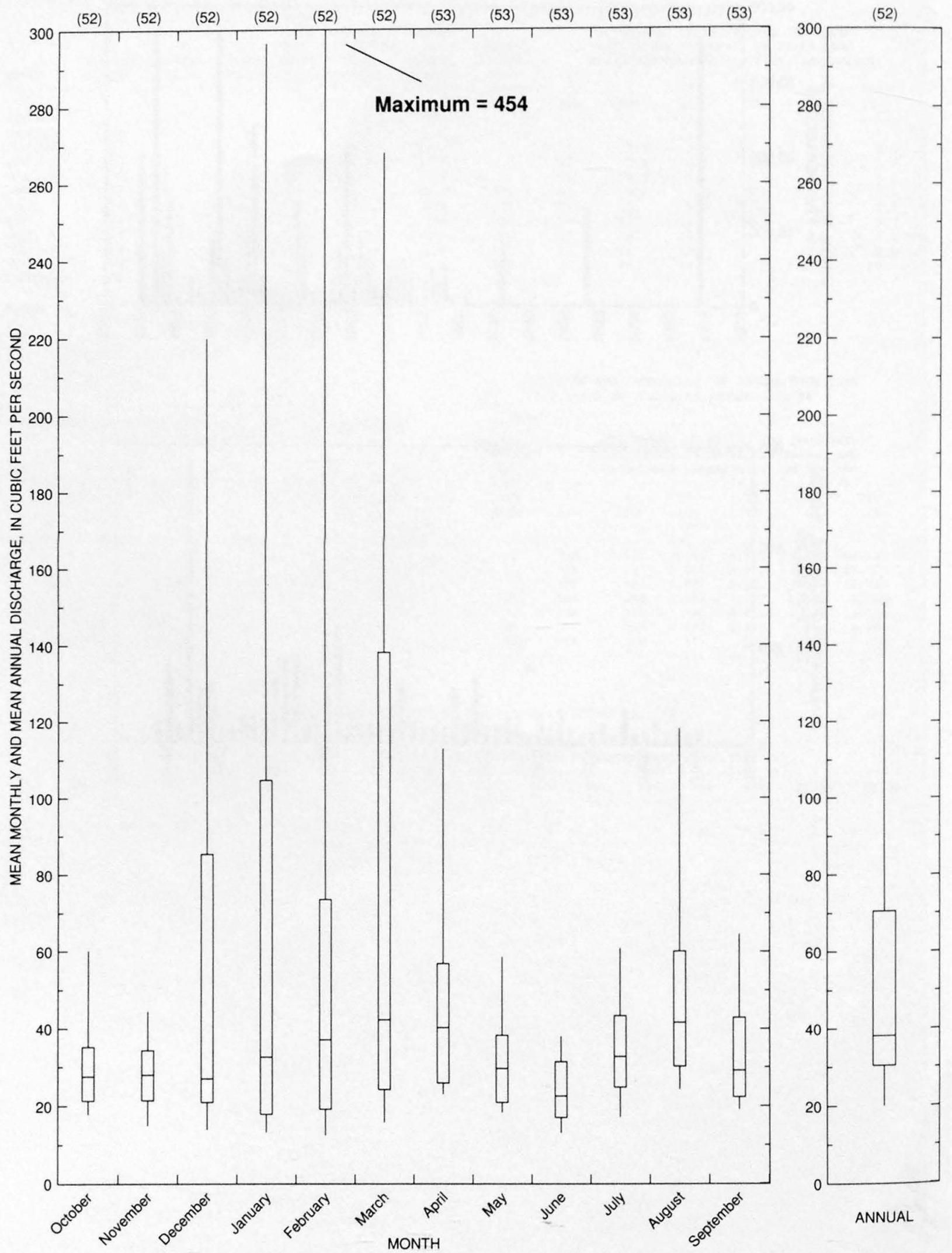




\section{BONITA CREEK NEAR MORENCI, AZ}

LOCATION.--Lat 3257'20", long 109 31'50", in SE $1 / 4 \mathrm{NW}^{1 / 4} \mathrm{sec} .36$, T.5 S., R.27 E., Graham County, Hydrologic Unit 15040005, on left bank $2.0 \mathrm{mi}$ upstream from intake of City of Safford water supply, $6.3 \mathrm{mi}$ upstream from mouth, and 12.8 mi southwest of Morenci.

DRAINAGE AREA.--302 $\mathrm{mi}^{2}$.

PERIOD OF RECORD.--August 1981 to current year.

GAGE.--Water-stage recorder. Elevation of gage is 3,500 ft above sea level, from topographic map. Two crest-stage gages $440 \mathrm{ft}$ upstream on right and left banks.

REMARKS.--No estimated daily discharges. Records good.

EXTREMES FOR PERIOD OF RECORD.--Maximum discharge, 19,500 $\mathrm{ft}^{3} / \mathrm{s}$ Jan. 18, 1993, gage height, 16.5 $\mathrm{ft}$, from slope-area measurement of peak flow; minimum daily, $0.66 \mathrm{ft}^{3} / \mathrm{s}$ Aug. 31, 1988.

EXTREMES OUTSIDE PERIOD OF RECORD.--Flood of Dec. 20, 1972, 10,000 $\mathrm{ft}^{3} / \mathrm{s}$, from slope-area measurement made by City of Safford at site about $2 \mathrm{mi}$ downstream. Flood of June 27, 1981, 1,340 ft $\mathrm{t}^{3} / \mathrm{s}$, from slope-area measurement at present site, gage height, $5.6 \mathrm{ft}$, from floodmark.

Annual peak discharges

\begin{tabular}{|c|c|c|c|c|c|c|c|}
\hline $\begin{array}{l}\text { Water } \\
\text { year }\end{array}$ & Date & $\begin{array}{l}\text { Annual peak } \\
\text { discharge } \\
\left(\mathrm{ft}^{3} / \mathrm{s}\right)\end{array}$ & $\begin{array}{l}\text { Discharge } \\
\text { codes }\end{array}$ & $\begin{array}{l}\text { Water } \\
\text { year }\end{array}$ & Date & $\begin{array}{l}\text { Annual peak } \\
\text { discharge } \\
\left(\mathrm{ft}^{3} / \mathrm{s}\right)\end{array}$ & $\begin{array}{c}\text { Discharge } \\
\text { codes }\end{array}$ \\
\hline 1981 & $08-10-81$ & 1,340 & & 1989 & $08-07-89$ & 367 & \\
\hline 1982 & $07-29-82$ & 595 & & 1990 & $08-21-90$ & 1,680 & \\
\hline 1983 & $02-04-83$ & 950 & & 1991 & $09-05-91$ & 3,220 & \\
\hline 1984 & $10-02-83$ & 19,400 & & 1992 & $02-14-92$ & 2,000 & \\
\hline 1985 & $12-28-84$ & 269 & & 1993 & 01-18-93 & 19,500 & \\
\hline 1986 & $08-20-86$ & 456 & & 1994 & $09-04-94$ & 1,910 & \\
\hline 1987 & $07-21-87$ & 3,510 & & 1995 & 01-05-95 & 5,300 & \\
\hline 1988 & $09-12-88$ & 798 & & 1996 & $09-11-96$ & 2,020 & \\
\hline
\end{tabular}




\section{BONITA CREEK NEAR MORENCI, AZ--Continued}

MEAN MONTHLY AND ANNUAL DISCHARGES 1981.96

\begin{tabular}{|c|c|c|c|c|c|c|}
\hline MONTH & $\begin{array}{l}\text { MAXIMUM } \\
\text { (FT3/S) }\end{array}$ & $\begin{array}{l}\text { MINIMUM } \\
\text { (FT3/S) }\end{array}$ & $\begin{array}{c}\text { MEAN } \\
\text { (FT3/S) }\end{array}$ & $\begin{array}{l}\text { STAN- } \\
\text { DARD } \\
\text { DEVIA- } \\
\text { TION } \\
\text { (FT3/S) }\end{array}$ & $\begin{array}{l}\text { COEFFI- } \\
\text { CIENT OF } \\
\text { VARI- } \\
\text { ATION }\end{array}$ & $\begin{array}{c}\text { PERCENT } \\
\text { OF } \\
\text { ANNUAL } \\
\text { RUNOFF }\end{array}$ \\
\hline OCTOBER & 176 & 1.5 & 16 & 44 & 2.7 & 8.5 \\
\hline NOVEMBER & 22 & 1.9 & 6.3 & 4.9 & 0.78 & 3.3 \\
\hline DECEMBER & 30 & 5.0 & 11 & 7.4 & 0.68 & 5.7 \\
\hline JANUARY & 769 & 5.7 & 71 & 197 & 2.8 & 37 \\
\hline FEBRUARY & 165 & 4.2 & 34 & 47 & 1.4 & 18 \\
\hline MARCH & 54 & 3.5 & 16 & 17 & 1.0 & 8.6 \\
\hline APRIL & 8.3 & 2.0 & 5.1 & 1.9 & 0.36 & 2.7 \\
\hline MAY & 6.3 & 2.1 & 4.1 & 1.5 & 0.36 & 2.2 \\
\hline JUNE & 5.9 & 1.3 & 3.2 & 1,2 & 0.38 & 1.7 \\
\hline JULY & 14 & 2.3 & 5.5 & 3.5 & 0.63 & 2.9 \\
\hline AUGUST & 14 & 4.3 & 8.3 & 3.4 & 0.41 & 4.4 \\
\hline SEPTEMBER & 29 & 2.5 & 8.7 & 7.3 & 0.84 & 4.6 \\
\hline ANNUAL & 84 & 4.2 & 16 & 20 & 1.3 & 100 \\
\hline
\end{tabular}

MAGNITUDE AND PROBABILITY OF INSTANTANEOUS PEAK FLOW BASED ON PERIOD OF RECORD 1981.96

DISCHARGE, IN FT3/S, FOR INDICATED RECURRENCE INTERVAL IN YEARS, AND EXCEEDANCE PROBABILITY, IN PERCENT

\begin{tabular}{|c|c|c|c|c|c|}
\hline 2 & 5 & 10 & 25 & 50 & 100 \\
\hline 508 & 208 & 108 & 48 & 28 & 18 \\
\hline 2,320 & 5,680 & 9,070 & 15,000 & 20,600 & 27,600 \\
\hline
\end{tabular}

WEIGHTED SKEW (LOGS) $=0.00$

MEAN $\quad$ LOGS) $=3.36$

STANDARD DEV. $($ LOGS $)=0.46$
MAGNITUDE AND PROBABILITY OF ANNUAL LOW FLOW BASED ON PERIOD OF RECORD 1981.96

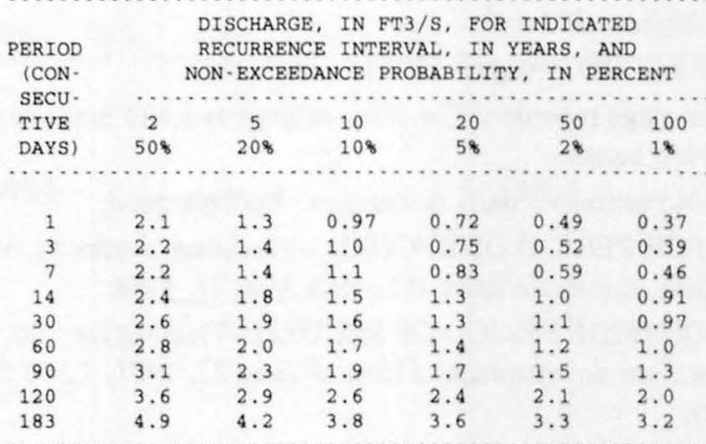

MAGNITUDE AND PROBABILITY OF ANNUAL HIGH FLOW BASED ON PERIOD OF RECORD 1981.96

\begin{tabular}{|c|c|c|c|c|c|c|}
\hline PERIOD & & \multicolumn{5}{|c|}{ DISCHARGE，IN FT3/S，FOR INDICATED } \\
\hline$(\mathrm{CON}-$ & & EXCEED & CE PROB & BILITY, & IN PER & ENT \\
\hline SECU - & & ........ & & & & \\
\hline TIVE & 2 & 5 & 10 & 25 & 50 & 100 \\
\hline DAYS) & 508 & 208 & 108 & 48 & 28 & 18 \\
\hline 1 & 271 & 1,140 & 2,720 & 7,640 & 15,700 & 31,200 \\
\hline 3 & 157 & 644 & 1.490 & 3,920 & 7,670 & 14.500 \\
\hline 7 & 96 & 359 & 771 & 1,850 & 3,350 & 5,840 \\
\hline 15 & 55 & 202 & 438 & 1,080 & 2,020 & 3,660 \\
\hline 30 & 33 & 111 & 230 & 545 & 996 & 1.770 \\
\hline 60 & 23 & 71 & 142 & 324 & 581 & 1,020 \\
\hline 90 & 18 & 53 & 103 & 228 & 402 & 691 \\
\hline
\end{tabular}

DURATION TABLE OF DAILY MEAN FLOW FOR PERIOD OF RECORD 1981.96

DISCHARGE, IN FT3/S, WHICH WAS EQUALED OR EXCEEDED FOR INDICATED PERCENT OF TIME

\begin{tabular}{|c|c|c|c|c|c|c|c|c|c|c|c|c|c|c|c|c|}
\hline 18 & $5 \%$ & 108 & 158 & 208 & 308 & 408 & 508 & 608 & 708 & 808 & 908 & 958 & 988 & 998 & 99.58 & 99.98 \\
\hline 189 & 23 & 9.9 & 7.2 & 6.1 & 5.7 & 5.3 & 4.6 & 4.2 & 3.1 & 3.1 & 2.4 & 1.9 & 0.00 & 0.00 & 0.00 & 0.00 \\
\hline
\end{tabular}


09447800 BONITA CREEK NEAR MORENCI, AZ--Continued
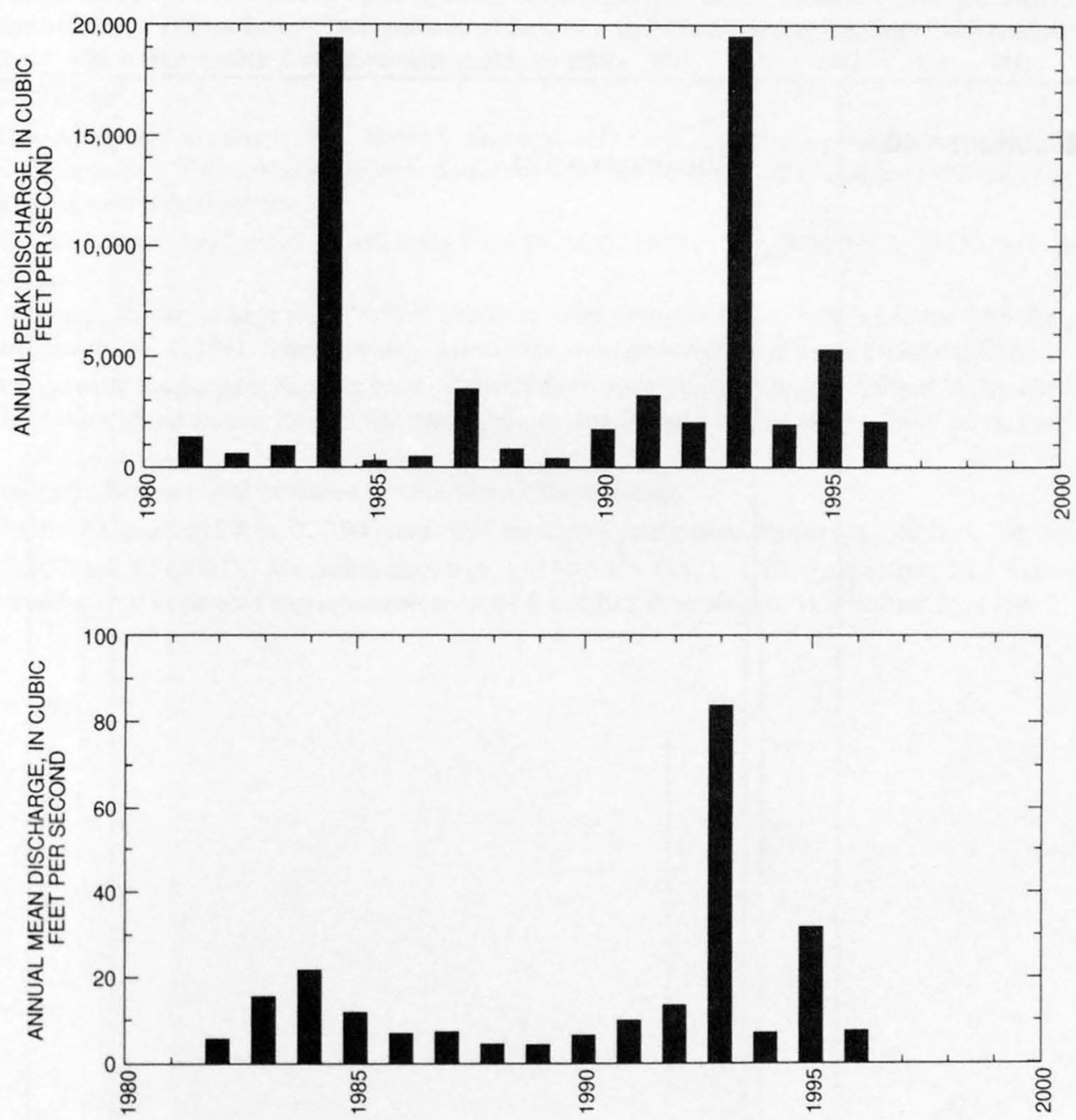
09447800 BONITA CREEK NEAR MORENCI, AZ--Continued
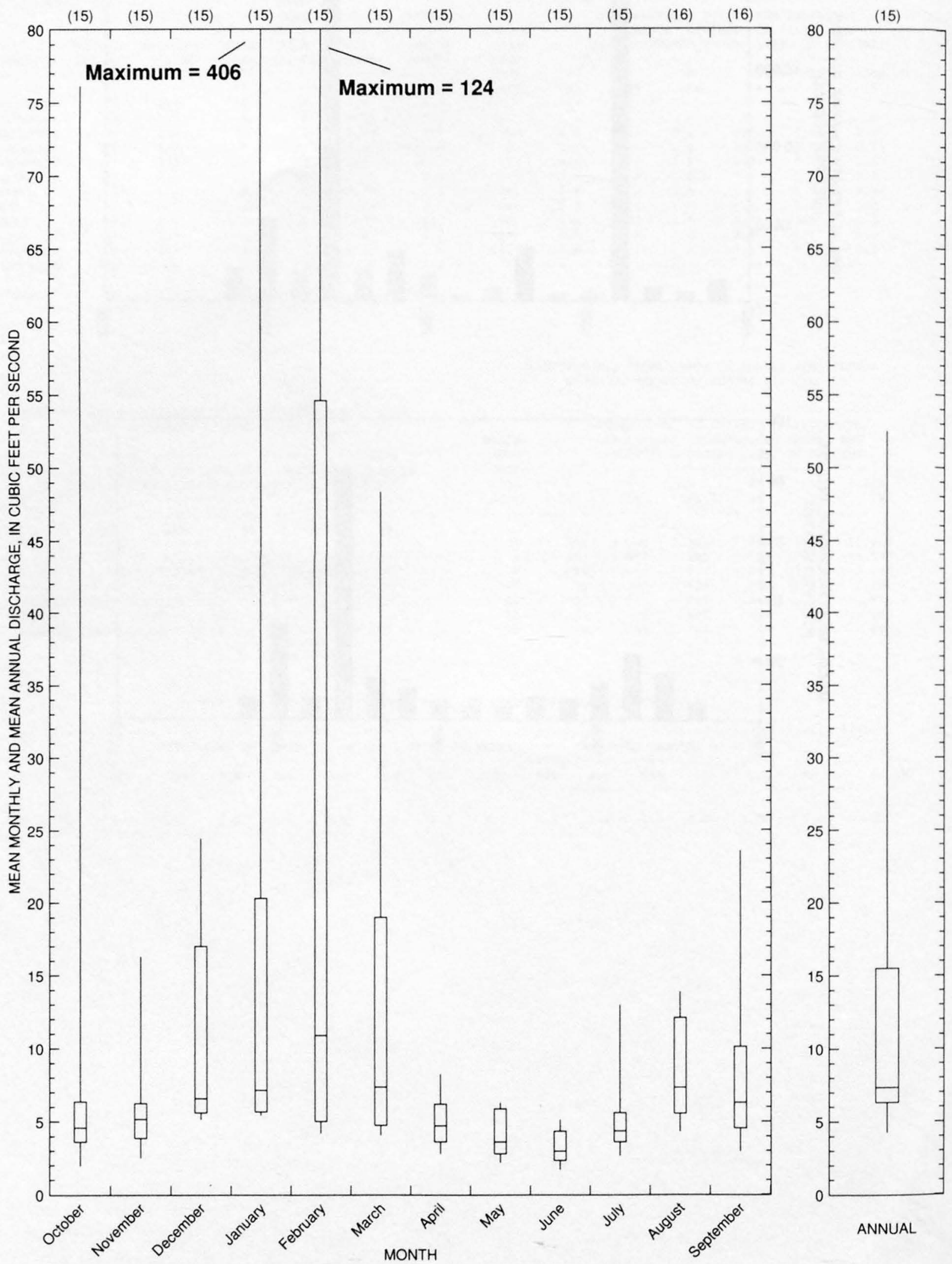


\section{GILA RIVER AT HEAD OF SAFFORD VALLEY, NEAR SOLOMON, AZ}

LOCATION.--Lat 32'52'06", long 109'30'38", in SE $\frac{1}{4} \mathrm{NE}^{1} / 4$ sec.31, T.6 S., R.28 E., Graham County, Hydrologic Unit 15040005 , on left bank 0.6 mi downstream from intake of Brown Canal, 8 mi northeast of Solomon, and $17 \mathrm{mi}$ downstream from San Francisco River. Records include flow of Brown Canal, which is measured 2,000 ft downstream from intake.

DRAINAGE AREA.--7,896 $\mathrm{mi}^{2}$.

PERIOD OF RECORD.--April 1914 to current year. Monthly discharge only for some periods, published in WSP 1313 . Prior to October 1932 and October 1940 to September 1949 published as "near Solomonsville" and October 1932 to October 1933 and May 1935 to September 1940 as "below Bonita Creek near Solomonsville."

REVISED RECORDS.--WSP 1059: 1914, 1916-17, 1923(M), 1924-25, 1927, 1929-31(M). WSP 1179: 1915, 1918-19(M). WSP 1313 : 1934. WSP 1733: 1923.

GAGE.--Water-stage recorder. Datum of gage is 3,059.92 ft above sea level. Prior to July 8, 1980, at datum $4.96 \mathrm{ft}$ higher. See WSP 1733 for history of changes prior to Jan. 1, 1941. Supplementary water-stage recorder and Parshall flume on Brown Canal.

REMARKS.--No estimated daily discharges. Records good. Records show water reaching head of Safford Valley and include water diverted to Brown Canal. Diversions above station for mining, municipal use, and for irrigation of about 17,500 acres, much of it by pumping from ground water.

COOPERATION.--Record for Brown Canal furnished by Gila Water Commissioner.

AVERAGE DISCHARGE.--82 years, $512 \mathrm{ft}^{3} / \mathrm{s}, 370,900$ acre- $\mathrm{ft} / \mathrm{yr}$; median of yearly mean discharges, $340 \mathrm{ft}^{3} / \mathrm{s}, 246,000 \mathrm{acre}-\mathrm{ft} / \mathrm{yr}$.

EXTREMES FOR PERIOD OF RECORD.--Maximum discharge, 132,000 ft $3 / \mathrm{s}$ Oct. 2, 1983, gage height, $20.8 \mathrm{ft}$, from rating curve extended above $52,000 \mathrm{ft}^{3} / \mathrm{s}$ on basis of slope-area measurements at $14.40 \mathrm{ft}$ and $20.8 \mathrm{ft}$; minimum, $11 \mathrm{ft}^{3} / \mathrm{s} \mathrm{June} 25,1956$. 
GILA RIVER BASIN

09448500 GILA RIVER AT HEAD OF SAFFORD VALLEY, NEAR SOLOMON, AZ--Continued

Annual peak discharges

\begin{tabular}{|c|c|c|c|c|c|c|c|}
\hline $\begin{array}{l}\text { Water } \\
\text { year }\end{array}$ & Date & $\begin{array}{l}\text { Annual peak } \\
\text { discharge } \\
\left(\mathrm{ft}^{3} / \mathbf{s}\right)\end{array}$ & $\begin{array}{l}\text { Discharge } \\
\text { codes }\end{array}$ & $\begin{array}{l}\text { Water } \\
\text { year }\end{array}$ & Date & $\begin{array}{c}\text { Annual peak } \\
\text { discharge } \\
\left(\mathrm{ft}^{3} / \mathrm{s}\right)\end{array}$ & $\begin{array}{l}\text { Discharge } \\
\text { codes }\end{array}$ \\
\hline 1914 & $08-21-14$ & 9,000 & & 1956 & $10-04-55$ & 13,300 & \\
\hline 1915 & $12-20-14$ & 50,000 & & 1957 & $07-26-57$ & 5,980 & \\
\hline 1916 & $01-19-16$ & ${ }^{1} 100,000$ & & 1958 & $03-23-58$ & 9,060 & \\
\hline 1917 & $10-14-16$ & 67,900 & & 1959 & $08-28-59$ & 7,860 & \\
\hline 1918 & $07-01-18$ & 2,700 & & 1960 & $01-12-60$ & 16,700 & \\
\hline 1919 & 08-03-19 & 15,000 & & 1961 & $09-10-61$ & 4,800 & \\
\hline 1920 & $12-05-19$ & 7,620 & & 1962 & $09-26-62$ & 16,100 & \\
\hline 1921 & $08-21-21$ & 15,700 & & 1963 & $10-19-62$ & 9,350 & \\
\hline 1922 & $08-15-22$ & 3,780 & & 1964 & $07-15-64$ & 9,880 & \\
\hline 1923 & $08-12-23$ & 12,600 & & 1965 & $08-02-65$ & 4,800 & \\
\hline 1924 & $12-28-23$ & 10,600 & & 1966 & $12-22-65$ & 43,000 & \\
\hline 1925 & $09-03-25$ & 15,900 & & 1967 & $08-12-67$ & 34,800 & \\
\hline 1926 & $04-07-26$ & 5,660 & & 1968 & $12-20-67$ & 9,280 & \\
\hline 1927 & $09-13-27$ & 9,320 & & 1969 & $09-11-69$ & 2,460 & \\
\hline 1928 & $08-01-28$ & 3,230 & & 1970 & $08-06-70$ & 2,250 & \\
\hline 1929 & $07-30-29$ & 12,700 & & 1971 & $10-03-70$ & 4,510 & \\
\hline 1930 & $08-11-30$ & 10,100 & & 1972 & $10-25-71$ & 10,200 & \\
\hline 1931 & $02-15-31$ & 10,500 & & 1973 & $10-20-72$ & 82,400 & \\
\hline 1932 & $02-10-32$ & 24,000 & & 1974 & $08-16-74$ & 3,280 & \\
\hline 1933 & $09-09-33$ & 9,600 & & 1975 & $09-09-75$ & 35,000 & \\
\hline 1934 & $08-27-34$ & 23,000 & & 1976 & $02-11-76$ & 3,400 & \\
\hline 1935 & $09-01-35$ & 5,550 & & 1977 & $08-13-77$ & 2,540 & \\
\hline 1936 & $02-17-36$ & 8,000 & & 1978 & $03-02-78$ & 21,600 & \\
\hline 1937 & $02-08-37$ & 23,700 & & 1979 & $12-19-78$ & 100,000 & \\
\hline 1938 & $03-04-38$ & 4,690 & & 1980 & $02-16-80$ & 25,300 & \\
\hline 1939 & 08-06-39 & 7,370 & & 1981 & $07-12-81$ & 7,000 & \\
\hline 1940 & $09-06-40$ & 9,840 & & 1982 & $10-03-81$ & 5,240 & \\
\hline 1941 & $09-30-41$ & 31,900 & & 1983 & $03-25-83$ & 11,300 & \\
\hline 1942 & $12-12-41$ & 7,730 & & 1984 & $10-02-83$ & ${ }^{2} 132,000$ & \\
\hline 1943 & $09-27-43$ & 6,680 & & 1985 & $12-29-84$ & 60,200 & \\
\hline 1944 & $09-25-44$ & 15,800 & & 1986 & $10-17-85$ & 7,690 & \\
\hline 1945 & $08-11-45$ & 4,820 & & 1987 & $11-0-386$ & 3,020 & \\
\hline 1946 & $10-09-45$ & 5,100 & & 1988 & $09-23-88$ & 7,820 & \\
\hline 1947 & $08-30-47$ & 9,250 & & 1989 & $10-15-88$ & 891 & \\
\hline 1948 & $06-01-48$ & 2,540 & & 1990 & $08-16-90$ & 2,240 & \\
\hline 1949 & $01-14-49$ & 25,200 & & 1991 & $03-02-91$ & 26,200 & \\
\hline 1950 & $07-30-50$ & 1,240 & & 1992 & $02-14-92$ & 17900 & \\
\hline 1951 & $08-03-51$ & 4,240 & & 1993 & $01-19-93$ & 86,200 & \\
\hline 1952 & $01-19-52$ & 19,700 & & 1994 & $09-04-94$ & 1,760 & \\
\hline 1953 & $07-30-53$ & 3,040 & & 1995 & 01-05-95 & 62,400 & \\
\hline 1954 & $03-24-54$ & 9,850 & & 1996 & $08-10-96$ & 7,470 & \\
\hline 1955 & $07-24-55$ & 11,700 & & & & & \\
\hline
\end{tabular}

${ }^{1}$ Highest since 1906.

${ }^{2}$ Highest since 1916. 
09448500 GILA RIVER AT HEAD OF SAFFORD VALLEY, NEAR SOLOMON, AZ--Continued

Discharge rating table developed October 1994

\begin{tabular}{cccc}
\hline $\begin{array}{c}\text { Gage height } \\
(\mathbf{f t})\end{array}$ & $\begin{array}{c}\text { Discharge } \\
\left(\mathbf{f t}^{\mathbf{3}} / \mathbf{s}\right)\end{array}$ & $\begin{array}{c}\text { Gage height } \\
(\mathbf{f t})\end{array}$ & $\begin{array}{c}\text { Discharge } \\
\left(\mathbf{f t}^{\mathbf{3}} / \mathbf{s}\right)\end{array}$ \\
\hline 9.0 & 2,570 & 15.0 & 32,700 \\
9.5 & 3,320 & 16.0 & 42,000 \\
10.0 & 4,200 & 17.0 & 54,900 \\
11.0 & 6,500 & 18.0 & 70,500 \\
12.0 & 9,800 & 19.0 & 89,200 \\
13.0 & 14,900 & 20.0 & 111,400 \\
14.0 & 22,600 & 20.8 & 132,000 \\
\hline
\end{tabular}

Basin characteristics

\begin{tabular}{cccccccc}
\hline $\begin{array}{c}\text { Main } \\
\text { channel } \\
\text { slope } \\
\text { (ft/mi) }\end{array}$ & $\begin{array}{c}\text { Stream } \\
\text { length } \\
(\mathrm{mi})\end{array}$ & $\begin{array}{c}\text { Mean } \\
\text { basin } \\
\text { elevation } \\
(\mathrm{ft})\end{array}$ & $\begin{array}{c}\text { Forested } \\
\text { area } \\
\text { (percent) }\end{array}$ & $\begin{array}{c}\text { Soil } \\
\text { index }\end{array}$ & $\begin{array}{c}\text { Mean } \\
\text { annual } \\
\text { precipitation } \\
\text { (in) }\end{array}$ & $\begin{array}{c}\text { Rainfall intensity, 24-hour } \\
\text { (in) }\end{array}$ & $\begin{array}{c}50 \text {-year } \\
\text { (in) }\end{array}$ \\
\hline 26.4 & 177 & 6,360 & 58.0 & 2.8 & 16.7 & 1.7 \\
\hline
\end{tabular}


09448500 GILA RIVER AT HEAD OF SAFFORD VALLEY, NEAR SOLOMON, AZ--Continued

MEAN MONTHLY AND ANNUAL DISCHARGES 1921-33, 1936-96

\begin{tabular}{|c|c|c|c|c|c|c|}
\hline MONTH & $\begin{array}{l}\text { MAXIMUM } \\
(\text { FT } 3 / S)\end{array}$ & $\begin{array}{l}\text { MINIMUM } \\
\text { (FT3/S) }\end{array}$ & $\begin{array}{c}\text { MEAN } \\
(\text { FT3/S) }\end{array}$ & $\begin{array}{l}\text { STAN- } \\
\text { DARD } \\
\text { DEVIA- } \\
\text { TION } \\
(\mathrm{FT} 3 / \mathrm{S})\end{array}$ & $\begin{array}{l}\text { COEFFI- } \\
\text { CIENT OF } \\
\text { VARI- } \\
\text { ATION }\end{array}$ & $\begin{array}{c}\text { PERCENT } \\
\text { OF } \\
\text { ANNUAL } \\
\text { RUNOFF }\end{array}$ \\
\hline OCTOBER & 7,450 & 40 & 380 & 1,010 & 2.7 & 6.7 \\
\hline NOVEMBER & 2,230 & 49 & 258 & 326 & 1.3 & 4.5 \\
\hline DECEMBER & 5,800 & 60 & 538 & 932 & 1.7 & 9.5 \\
\hline JANUARY & 14,000 & 93 & 737 & 1,760 & 2.4 & 12.9 \\
\hline FEBRUARY & 5,510 & 103 & 779 & 1,050 & 1.3 & 13.7 \\
\hline MARCH & 3,630 & 82 & 873 & 980 & 1.1 & 15.3 \\
\hline APRIL & 2,780 & 64 & 592 & 629 & 1.1 & 10.4 \\
\hline MAY & 2.040 & 38 & 313 & 404 & 1.3 & 5.5 \\
\hline JUNE & 716 & 20 & 110 & 104 & 0.95 & 1.9 \\
\hline JULY & 735 & 44 & 210 & 148 & 0.70 & 3.7 \\
\hline AUGUST & 2,500 & 66 & 511 & 505 & 0.99 & 9.0 \\
\hline SEPTEMBER & 2,080 & 36 & 392 & 389 & 0.99 & 6.9 \\
\hline ANNUAL & 2,230 & 101 & 474 & 404 & 0.85 & 100 \\
\hline
\end{tabular}

MAGNITUDE AND PROBABILITY OF INSTANTANEOUS PEAK FLOW BASED ON PERIOD OF RECORD 1914-96

DISCHARGE, IN FT3/S, FOR INDICATED RECURRENCE INTERVAL

IN YEARS, AND EXCEEDANCE PROBABITITY, IN PERCENT

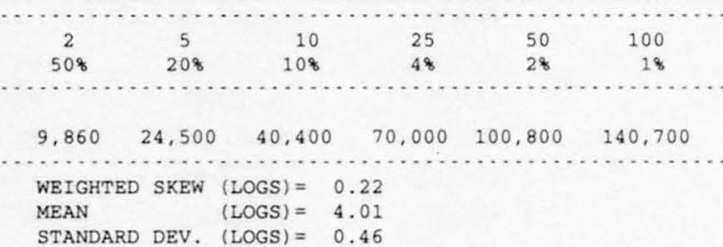

DURATION TABLE OF DAILY MEAN FLOW FOR PERIOD OF RECORD $1921-33,1936-96$
MAGNITUDE AND PROBABILITY OF ANNUAL LOW FLOW BASED ON PERIOD OF RECORD $1922 \cdot 33,1937 \cdot 96$

\begin{tabular}{|c|c|c|c|c|c|c|}
\hline $\begin{array}{l}\text { PERIOD } \\
\text { (CON- }\end{array}$ & \multirow{2}{*}{\multicolumn{6}{|c|}{$\begin{array}{l}\text { DISCHARGE, IN FT } 3 / \mathrm{S}, \text { FOR INDICATED } \\
\text { RECURRENCE INTERVAL, IN YEARS, AND } \\
\text { NON-EXCEEDANCE PROBABILITY, IN PERCENT }\end{array}$}} \\
\hline SECU. & ... & & & & & \\
\hline TIVE & 2 & 5 & 10 & 20 & 50 & 100 \\
\hline DAYS) & 508 & 208 & 108 & 58 & 28 & 18 \\
\hline 1 & 42 & 27 & 21 & 18 & 14 & 12 \\
\hline 3 & 44 & 28 & 23 & 19 & 15 & 13 \\
\hline 7 & 46 & 31 & 25 & 21 & 17 & 15 \\
\hline 14 & 50 & 34 & 27 & 23 & 19 & 17 \\
\hline 30 & 60 & 40 & 32 & 27 & 22 & 19 \\
\hline 60 & 78 & 51 & 42 & 35 & 29 & 26 \\
\hline 90 & 101 & 66 & 54 & 45 & 37 & 32 \\
\hline 120 & 130 & 89 & 72 & 61 & 50 & 44 \\
\hline 183 & 176 & 120 & 100 & 87 & 76 & 70 \\
\hline
\end{tabular}

MAGNITUDE AND PROBABILITY OF ANNUAL HIGH FLOW BASED ON PERIOD OF RECORD $1921-33,1936.96$

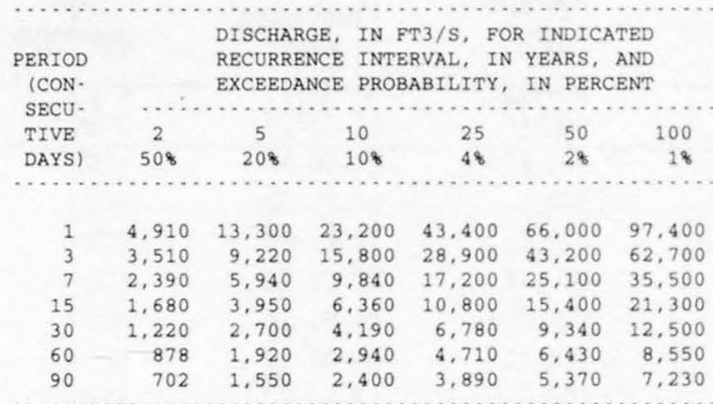

DISCHARGE, IN FT3/S, WHICH WAS EQUALED OR EXCEEDED FOR INDICATED PERCENT OF TIME

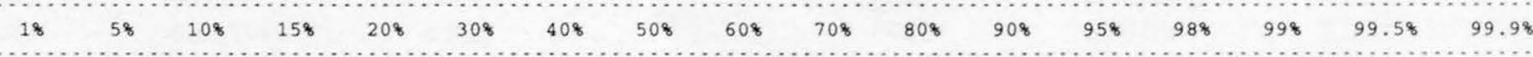

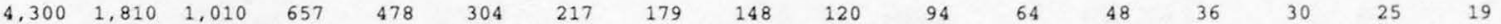



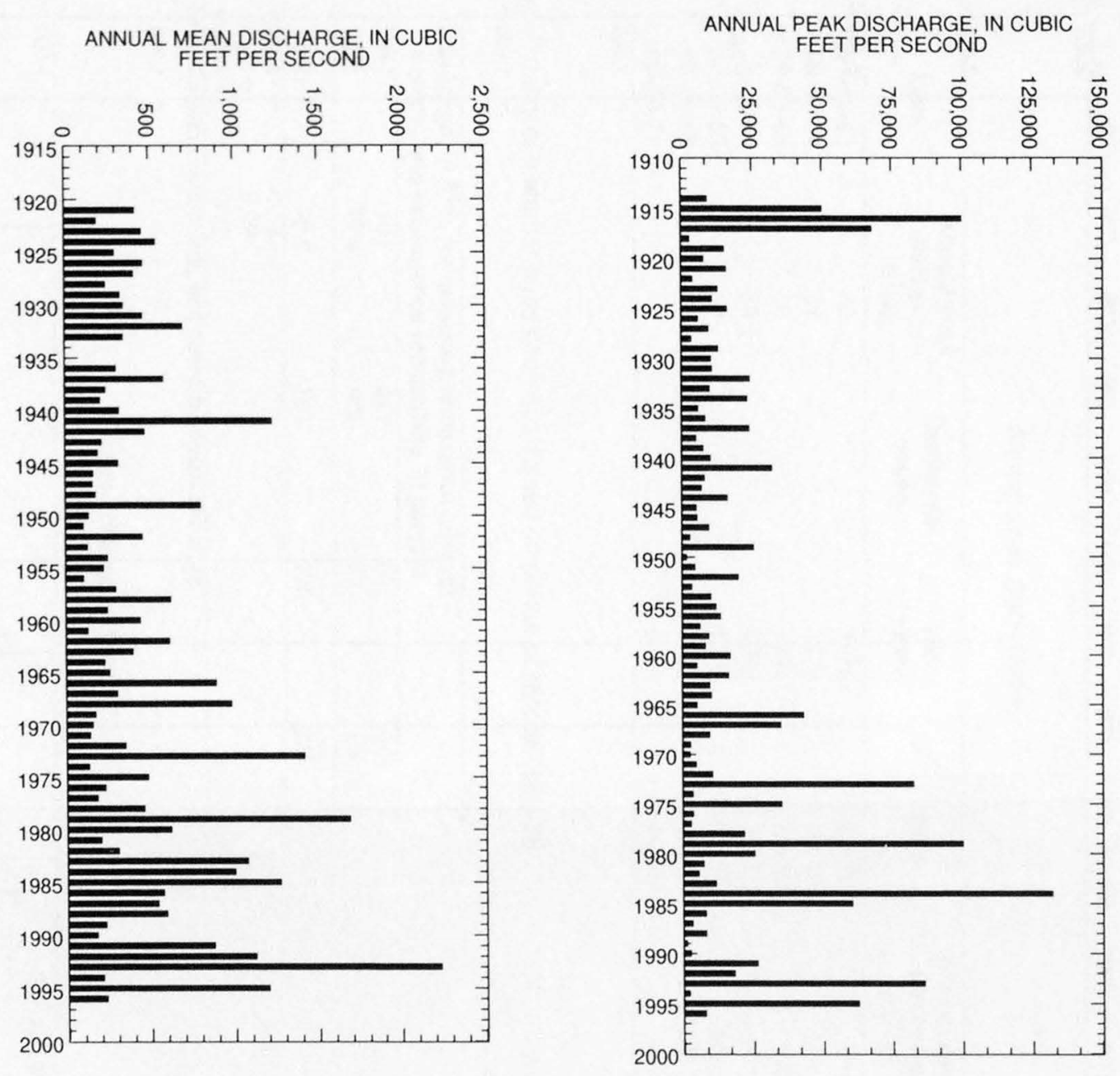
09448500 GILA RIVER AT HEAD OF SAFFORD VALLEY, NEAR SOLOMON, AZ--Continued

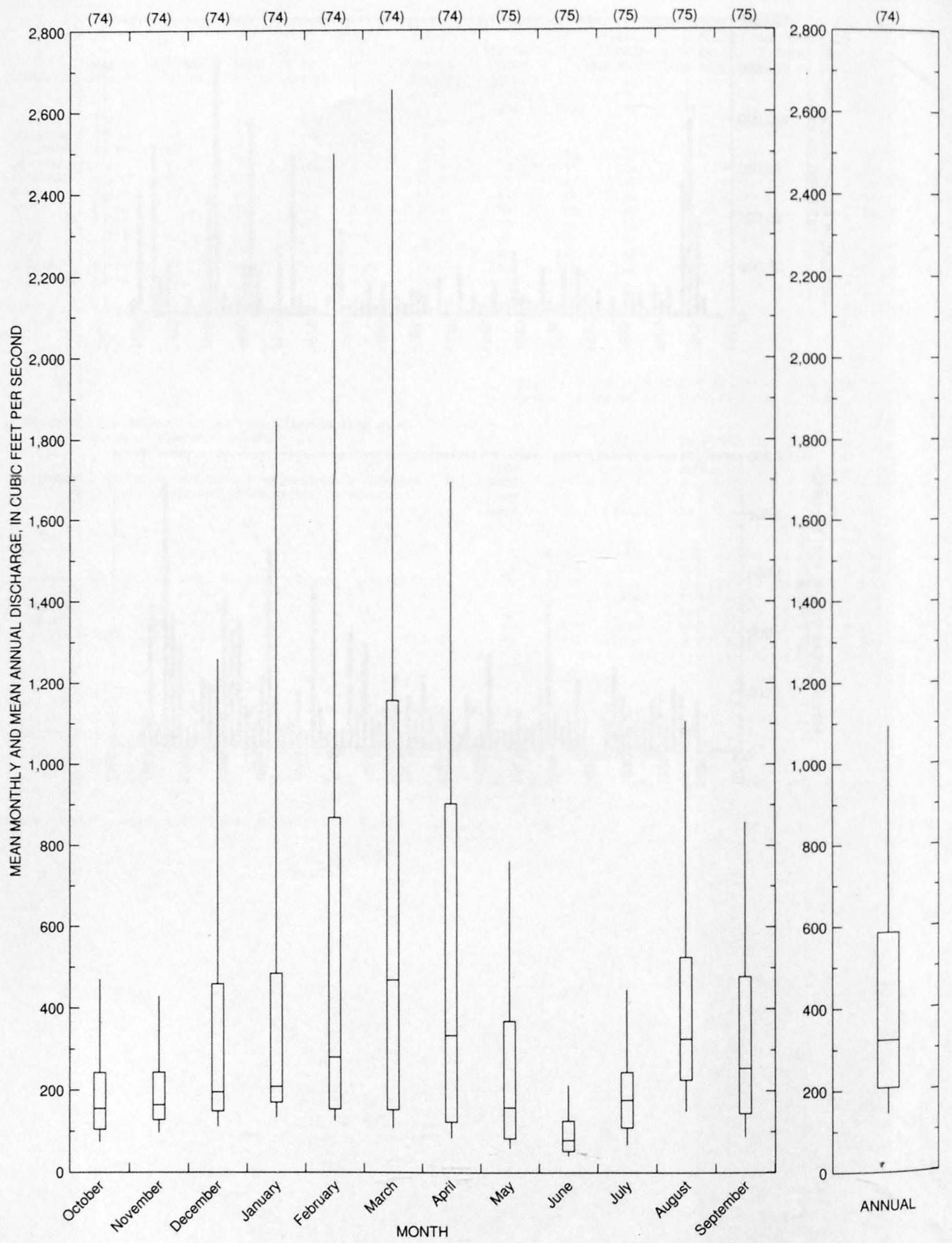


09451800 TOLLGATE WASH TRIBUTARY NEAR CLIFTON, AZ

LOCATION.--Lat $32^{\circ} 51^{\prime} 00^{\prime \prime}$, long $109^{\circ} 20^{\prime} 15^{\prime \prime}$, in SW $1 / 4$ sec.1, T.7 S., R.29 E., Graham County, Hydrologic Unit 15040005, at U.S. Highway 666, at Graham-Greenlee County line $14 \mathrm{mi}$ south of Clifton.

DRAINAGE AREA.--0.12 $\mathrm{mi}^{2}$.

Annual peak discharges

\begin{tabular}{|c|c|c|c|c|c|c|c|}
\hline $\begin{array}{l}\text { Water } \\
\text { year }\end{array}$ & Date & $\begin{array}{c}\text { Annual peak } \\
\text { discharge } \\
\left(\mathrm{ft}^{3} / \mathrm{s}\right)\end{array}$ & $\begin{array}{c}\text { Discharge } \\
\text { codes }\end{array}$ & $\begin{array}{l}\text { Water } \\
\text { year }\end{array}$ & Date & $\begin{array}{c}\text { Annual peak } \\
\text { discharge } \\
\left(\mathrm{ft}^{3} / \mathrm{s}\right)\end{array}$ & $\begin{array}{l}\text { Discharge } \\
\text { codes }\end{array}$ \\
\hline 1963 & $07-19-63$ & 62 & & 1970 & $00-00-70$ & 0 & \\
\hline 1964 & $00-00-64$ & 29 & & 1971 & $10-02-70$ & 15 & \\
\hline 1965 & $09-03-65$ & 10 & & 1972 & $00-00-72$ & 8.0 & LT \\
\hline 1966 & $09-15-66$ & 34 & & 1973 & $10-19-72$ & 63 & \\
\hline 1967 & $00-00-67$ & 0 & & 1974 & $08-23-74$ & 56 & \\
\hline 1968 & $00-00-68$ & 0 & & 1975 & $07-24-75$ & 22 & \\
\hline 1969 & $00-00-69$ & 0 & & 1976 & $00-00-76$ & 0 & \\
\hline
\end{tabular}

Magnitude and probability of instantaneous peak flow based on period of record 1963-76

\begin{tabular}{|c|c|c|c|c|c|}
\hline \multicolumn{6}{|c|}{$\begin{array}{l}\text { Discharge, in } \mathrm{ft}^{3} / \mathrm{s} \text {, for indicated recurrence interval } \\
\text { in years, and exceedance probablility, in percent }\end{array}$} \\
\hline $\begin{array}{c}2 \dagger \\
50 \%\end{array}$ & $\begin{array}{c}5 \dagger \\
20 \%\end{array}$ & $\begin{array}{c}10 \dagger \\
10 \%\end{array}$ & $\begin{array}{l}25 \dagger \\
4 \%\end{array}$ & $\begin{array}{l}50 \dagger \\
2 \%\end{array}$ & $\begin{array}{c}100 t \\
1 \%\end{array}$ \\
\hline 11.4 & 39.7 & 70.5 & 123 & 170 & 225 \\
\hline $\begin{array}{l}\text { Weighted skew } \\
\text { Mean } \\
\text { Standard dev. }\end{array}$ & $\begin{array}{l}(\log s)= \\
(\log s)= \\
(\log s)=\end{array}$ & $\begin{array}{c}-0.54 \\
0.99 \\
0.71\end{array}$ & & & \\
\hline
\end{tabular}

Basin characteristics

\begin{tabular}{cccccccc}
\hline $\begin{array}{c}\text { Main } \\
\text { channel } \\
\text { slope } \\
(\mathrm{ft} / \mathrm{mi})\end{array}$ & $\begin{array}{c}\text { Stream } \\
\text { length } \\
(\mathrm{mi})\end{array}$ & $\begin{array}{c}\text { Mean } \\
\text { basin } \\
\text { elevation } \\
(\mathrm{ft})\end{array}$ & $\begin{array}{c}\text { Forested } \\
\text { area } \\
\text { (percent) }\end{array}$ & $\begin{array}{c}\text { Soil } \\
\text { index }\end{array}$ & $\begin{array}{c}\text { Mean } \\
\text { annual } \\
\text { precipitation } \\
\text { (in) }\end{array}$ & $\begin{array}{c}\text { Rainfall intensity, 24-hour } \\
\text { (in) }\end{array}$ & $\begin{array}{c}50 \text {-year } \\
\text { (in) }\end{array}$ \\
\hline 878 & 0.60 & 4,800 & 0.0 & 1.0 & 13.5 & 1.8 \\
\hline
\end{tabular}


09451800 TOLLGATE WASH TRIBUTARY NEAR CLIFTON, AZ--Continued

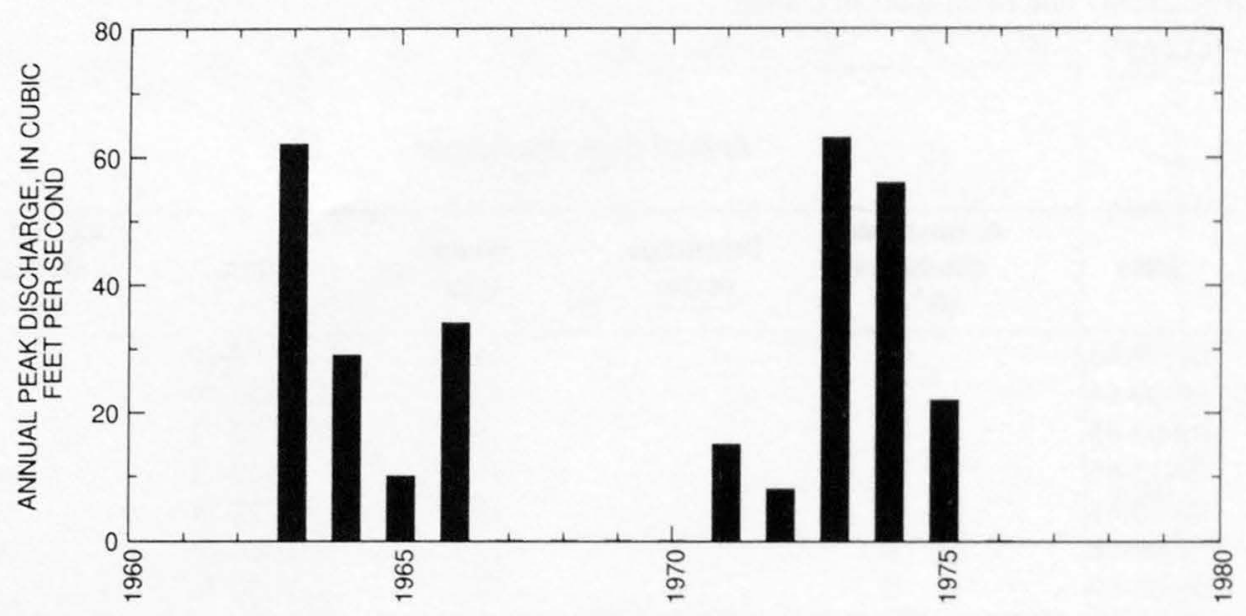


09456000 SAN SIMON RIVER NEAR SAN SIMON, AZ

LOCATION.--Lat $32^{\circ} 13^{\prime} 30^{\prime \prime}$, long 109 10'30", in SW $1 / 4$ sec.10, T.14 S., R.31 E., Cochise County, Hydrologic Unit $15040006,4.5$ mi southeast of San Simon.

DRAINAGE AREA.--814 $\mathrm{mi}^{2}$.

Annual peak discharges

\begin{tabular}{cccccrr}
\hline $\begin{array}{c}\text { Water } \\
\text { year }\end{array}$ & Date & $\begin{array}{c}\text { Annual peak } \\
\text { discharge } \\
\left(\mathrm{ft}^{3} / \mathbf{s}\right)\end{array}$ & $\begin{array}{c}\text { Discharge } \\
\text { codes }\end{array}$ & $\begin{array}{c}\text { Water } \\
\text { year }\end{array}$ & $\begin{array}{c}\text { Date } \\
\text { Annual peak } \\
\text { discharge } \\
\left(\mathrm{ft}^{3} / \mathbf{s}\right)\end{array}$ \\
\hline 1923 & $07-21-23$ & 5,350 & HP & 1936 & $08-18-36$ & 4,190 \\
1931 & $08-10-31$ & 4,500 & & 1937 & $08-09-37$ & 548 \\
1932 & $07-25-32$ & 1,250 & & 1938 & $06-29-38$ & 2,280 \\
1933 & $08-04-33$ & 1,550 & & 1939 & $08-13-39$ & 2,840 \\
1934 & $08-00-34$ & 4,550 & & 1940 & $06-29-40$ & 4,280 \\
1935 & $08-28-35$ & 5,020 & & 1941 & $08-15-41$ & 1,870 \\
\hline
\end{tabular}

Magnitude and probability of instantaneous peak flow based on period of record 1923 , $1931-41$

\begin{tabular}{|c|c|c|c|c|c|}
\hline \multicolumn{6}{|c|}{$\begin{array}{l}\text { Discharge, in } \mathrm{ft}^{3} / \mathrm{s} \text {, for indicated recurrence interval } \\
\text { in years, and exceedance probablility, in percent }\end{array}$} \\
\hline 2 & 5 & 10 & $25 t$ & $50 t$ & $100 t$ \\
\hline $50 \%$ & $20 \%$ & $10 \%$ & $4 \%$ & $2 \%$ & $1 \%$ \\
\hline 2,920 & 4,520 & 5,570 & 6,860 & 7,770 & 8,660 \\
\hline Weighted skew & $(\log s)=$ & -0.41 & & & \\
\hline Mean & $(\log s)=$ & 3.45 & & & \\
\hline Standard dev. & $(\log s)=$ & 0.24 & & & \\
\hline
\end{tabular}

fReliability of values in column is uncertain, and potential errors are large.

Basin characteristics

\begin{tabular}{ccccccc}
\hline $\begin{array}{c}\text { Main } \\
\text { channel } \\
\text { slope } \\
(\mathrm{ft} / \mathrm{mi})\end{array}$ & $\begin{array}{c}\text { Stream } \\
\text { length } \\
(\mathrm{mi})\end{array}$ & $\begin{array}{c}\text { Mean } \\
\text { basin } \\
\text { elevation } \\
(\mathrm{ft})\end{array}$ & $\begin{array}{c}\text { Forested } \\
\text { area } \\
\text { (percent) }\end{array}$ & $\begin{array}{c}\text { Soil } \\
\text { index }\end{array}$ & $\begin{array}{c}\text { Mean } \\
\text { annual } \\
\text { precipitation } \\
\text { (in) }\end{array}$ & $\begin{array}{c}\text { R-year } \\
\text { (in) }\end{array}$ \\
\hline 23.8 & 64.6 & 4,830 & 23.0 & 2.3 & 14.9 & 1.9 \\
\hline
\end{tabular}

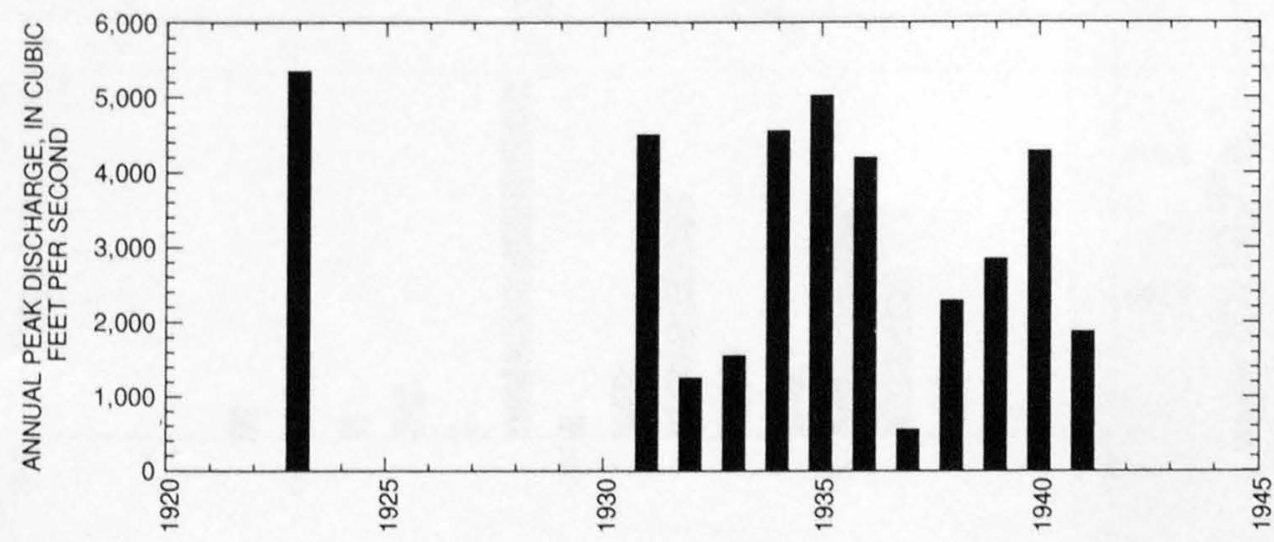


09456400 GOLD GULCH NEAR BOWIE, AZ

LOCATION.--Lat 32 $20^{\prime} 52^{\prime \prime}$, long 109 $36^{\prime} 10^{\prime \prime}$, in SW $1 /{ }_{4} \mathrm{NW}^{1 / 4}$ sec.33, T.12 S., R.27 E., Cochise County, Hydrologic Unit 15040006 , 100 ft upstream from State Highway $86,7 \mathrm{mi}$ west of Bowie.

DRAINAGE AREA.--15.0 $\mathrm{mi}^{2}$.

Annual peak discharges

\begin{tabular}{|c|c|c|c|c|c|c|c|}
\hline $\begin{array}{l}\text { Water } \\
\text { year }\end{array}$ & Date & $\begin{array}{c}\text { Annual peak } \\
\text { discharge } \\
\left(\mathrm{ft}^{3} / \mathrm{s}\right)\end{array}$ & $\begin{array}{c}\text { Discharge } \\
\text { codes }\end{array}$ & $\begin{array}{l}\text { Water } \\
\text { year }\end{array}$ & Date & $\begin{array}{c}\text { Annual peak } \\
\text { discharge } \\
\left(\mathrm{ft}^{3} / \mathrm{s}\right)\end{array}$ & $\begin{array}{c}\text { Discharge } \\
\text { codes }\end{array}$ \\
\hline 1963 & $08-23-63$ & 420 & & 1970 & $07-20-70$ & 150 & ES \\
\hline 1964 & $08-18-64$ & 1,180 & & 1971 & $08-18-71$ & 2,550 & \\
\hline 1965 & $08-29-65$ & 1,770 & & 1972 & $00-00-72$ & 90 & LT \\
\hline 1966 & $08-07-66$ & 450 & ES & 1973 & $10-00-72$ & 400 & \\
\hline 1967 & $07-07-67$ & 640 & & 1974 & $08-24-74$ & 147 & \\
\hline 1968 & $12-20-67$ & 1,740 & & 1975 & $07-22-75$ & 630 & \\
\hline 1969 & $09-11-69$ & 520 & & 1976 & $00-00-76$ & 225 & \\
\hline
\end{tabular}

Magnitude and probability of instantaneous peak flow based on period of record 1963-76

\begin{tabular}{|c|c|c|c|c|c|}
\hline \multicolumn{6}{|c|}{$\begin{array}{l}\text { Discharge, in } \mathrm{ft}^{3} / \mathrm{s} \text {, for indicated recurrence interval } \\
\text { in years, and exceedance probablility, in percent }\end{array}$} \\
\hline 2 & 5 & 10 & 25 & $50 t$ & $100 t$ \\
\hline $50 \%$ & $20 \%$ & $10 \%$ & $4 \%$ & $2 \%$ & $1 \%$ \\
\hline 527 & 1,180 & 1,790 & 2,760 & 3,630 & 4,640 \\
\hline Weighted skew & $(\log s)=$ & -0.11 & & & \\
\hline Mean & $(\log s)=$ & 2.71 & & & \\
\hline Standard dev. & $(\log s)=$ & 0.42 & & & \\
\hline
\end{tabular}

$\mp$ Reliability of values in column is uncertain, and potential errors are large.

Basin characteristics

\begin{tabular}{ccccccc}
\hline $\begin{array}{c}\text { Main } \\
\text { channel } \\
\text { slope } \\
(\mathrm{ft} / \mathrm{mi})\end{array}$ & $\begin{array}{c}\text { Stream } \\
\text { length } \\
(\mathrm{mi})\end{array}$ & $\begin{array}{c}\text { Mean } \\
\text { basin } \\
\text { elevation } \\
(\mathrm{ft})\end{array}$ & $\begin{array}{c}\text { Forested } \\
\text { area } \\
\text { (percent) }\end{array}$ & $\begin{array}{c}\text { Soil } \\
\text { index }\end{array}$ & $\begin{array}{c}\text { Mean } \\
\text { annual } \\
\text { precipitation } \\
\text { (in) }\end{array}$ & $\begin{array}{c}\text { R-year } \\
\text { (in) }\end{array}$ \\
\hline 201 & 9.6 & 5,170 & 0.0 & 2.0 & 10.9 & $\begin{array}{c}50 \text {-year } \\
\text { (in) }\end{array}$ \\
\hline
\end{tabular}

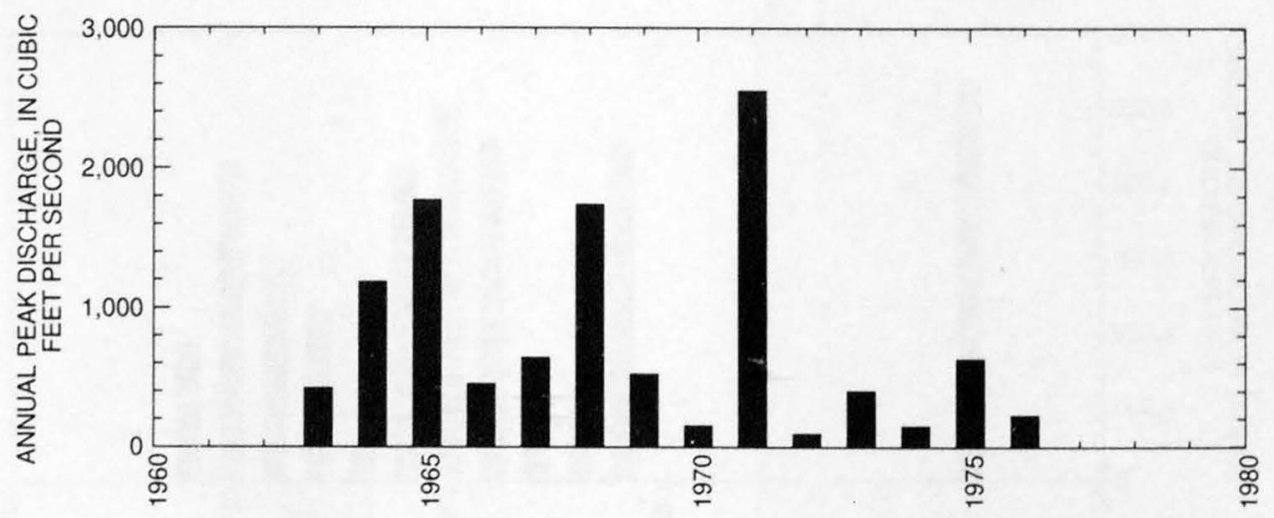




\section{SAN SIMON RIVER NEAR SOLOMON, AZ}

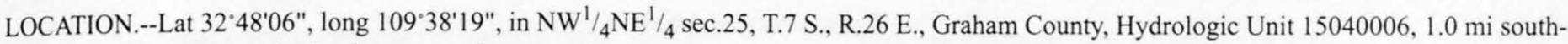
west of Solomon and 2.2 mi upstream from mouth.

DRAINAGE AREA.--2,192 $\mathrm{mi}^{2}$.

PERIOD OF RECORD.--June 1931 to September 1932, May 1935 to September 1982 (discontinued). Published as "San Simon Creek near Solomonsville" prior to October 1949 and as "San Simon Creek near Solomon" October 1949 to September 1961.

GAGE.--Water-stage recorder. Datum of gage is 2,960.15 ft above sea level.

REMARKS.--Records poor. Records do not include waste water passing station from San Jose Canal, which diverts from Gila River. Irrigation of about 34,000 acres above station, mostly by pumping from ground water. Floodflows are partly regulated by flood-control detention structures on main stream and tributaries; combined maximum capacity in excess of 10,500 acre-ft.

AVERAGE DISCHARGE.--48 years, $11.7 \mathrm{ft}^{3} / \mathrm{s}, 8,480 \mathrm{acre}-\mathrm{ft} / \mathrm{yr}$; median of yearly mean discharges, $8.4 \mathrm{ft}^{3} / \mathrm{s} 6,100 \mathrm{acre}-\mathrm{ft} / \mathrm{yr}$.

EXTREMES FOR PERIOD OF RECORD.--Maximum discharge, 27,500 $\mathrm{ft}^{3} / \mathrm{s}$ Aug. 9, 1931, gage height, $19.0 \mathrm{ft}$, by slope-area measurement of peak flow; no flow for most of time.

Annual peak discharges

\begin{tabular}{|c|c|c|c|c|c|c|c|}
\hline $\begin{array}{l}\text { Water } \\
\text { year }\end{array}$ & Date & $\begin{array}{c}\text { Annual peak } \\
\text { discharge } \\
\left(\mathrm{ft}^{3} / \mathrm{s}\right)\end{array}$ & $\begin{array}{c}\text { Discharge } \\
\text { codes }\end{array}$ & $\begin{array}{l}\text { Water } \\
\text { year }\end{array}$ & Date & $\begin{array}{c}\text { Annual peak } \\
\text { discharge } \\
\left(\mathrm{ft}^{3} / \mathrm{s}\right)\end{array}$ & $\begin{array}{l}\text { Discharge } \\
\text { codes }\end{array}$ \\
\hline 1931 & $08-09-31$ & ${ }^{1} 27,500$ & & 1958 & $08-16-58$ & 4,250 & UR \\
\hline 1932 & $07-30-32$ & 8,800 & & 1959 & $08-23-59$ & 4,610 & UR \\
\hline 1933 & $07-16-33$ & 4,000 & & 1960 & $09-09-60$ & 3,260 & UR \\
\hline 1934 & $08-00-34$ & 11,500 & & 1961 & $08-22-61$ & 7,750 & UR \\
\hline 1935 & $08-01-35$ & 12,000 & & 1962 & $09-26-62$ & 3,970 & UR \\
\hline 1936 & $09-10-36$ & 10,600 & & 1963 & $08-26-63$ & 5,170 & UR \\
\hline 1937 & $08-21-37$ & 2,370 & & 1964 & $07-14-64$ & 5,800 & UR \\
\hline 1938 & $07-12-38$ & 4,500 & & 1965 & $08-29-65$ & 4,880 & UR \\
\hline 1939 & $08-14-39$ & 2,140 & & 1966 & $09-15-66$ & 2,250 & UR \\
\hline 1940 & $09-05-40$ & 6,080 & & 1967 & $08-12-67$ & 5,570 & UR \\
\hline 1941 & $08-17-41$ & 13,000 & & 1968 & $07-02-68$ & 3,870 & UR \\
\hline 1942 & $09-11-42$ & 5,000 & & 1969 & $07-10-69$ & 2,310 & UR \\
\hline 1943 & $08-15-43$ & 6,430 & & 1970 & $08-17-70$ & 1,010 & UR \\
\hline 1944 & $09-25-44$ & 5,900 & & 1971 & $09-23-71$ & 3,310 & UR \\
\hline 1945 & $08-10-45$ & 7,350 & & 1972 & $08-26-72$ & 4,570 & UR \\
\hline 1946 & $08-30-46$ & 4,820 & & 1973 & $10-19-72$ & 1,820 & UR \\
\hline 1947 & $06-18-47$ & 2,700 & & 1974 & $08-15-74$ & 4,840 & UR \\
\hline 1948 & $08-06-48$ & 5,880 & & 1975 & $09-08-75$ & 3,690 & UR \\
\hline 1949 & $08-08-49$ & 8,100 & & 1976 & $09-24-76$ & 3,070 & UR \\
\hline 1950 & $09-18-50$ & 2,060 & & 1977 & $08-22-77$ & 3,010 & UR \\
\hline 1951 & $08-02-51$ & 7,390 & & 1978 & $07-22-78$ & 1,630 & UR \\
\hline 1952 & $08-17-52$ & 5,100 & & 1979 & $08-14-79$ & 4,370 & UR \\
\hline 1953 & $07-07-53$ & 3,970 & UR & 1980 & $08-13-80$ & 484 & UR \\
\hline 1954 & $09-12-54$ & 6,980 & UR & 1981 & $09-17-81$ & 3,560 & UR \\
\hline 1955 & $07-30-55$ & 6,400 & UR & 1982 & $07-26-82$ & 4,430 & UR \\
\hline 1956 & $10-04-55$ & 1,520 & UR & 1984 & $10-02-83$ & 5,500 & UR,HP \\
\hline 1957 & $08-30-57$ & 8,950 & UR & & & & \\
\hline
\end{tabular}

'Highest since 1880. 
09457000 SAN SIMON RIVER NEAR SOLOMON, AZ--Continued

Basin characteristics

\begin{tabular}{cccccccc}
\hline $\begin{array}{c}\text { Main } \\
\text { channel } \\
\text { slope } \\
(\mathrm{ft} / \mathrm{mi})\end{array}$ & $\begin{array}{c}\text { Stream } \\
\text { length } \\
\text { (mi) }\end{array}$ & $\begin{array}{c}\text { Mean } \\
\text { basin } \\
\text { elevation } \\
(\mathrm{ft})\end{array}$ & $\begin{array}{c}\text { Forested } \\
\text { area } \\
\text { (percent) }\end{array}$ & $\begin{array}{c}\text { Soil } \\
\text { index }\end{array}$ & $\begin{array}{c}\text { Mean } \\
\text { annual } \\
\text { precipitation } \\
\text { (in) }\end{array}$ & $\begin{array}{c}\text { Rainfall intensity, 24-hour } \\
\text { (in) }\end{array}$ & $\begin{array}{c}5-\text {-year } \\
\text { (in) }\end{array}$ \\
\hline 14.6 & 119 & 4,270 & 11.0 & 2.3 & 12.2 & 1.8 \\
\hline
\end{tabular}


09457000 SAN SIMON RIVER NEAR SOLOMON, AZ--Continued

MEAN MONTHLY AND ANNUAL DISCHARGES $1932,1936.82$

\begin{tabular}{|c|c|c|c|c|c|c|}
\hline MONTH & $\begin{array}{l}\text { MAXIMUM } \\
\text { (FT3/S) }\end{array}$ & $\begin{array}{l}\text { MINIMUM } \\
\text { (FT3/S) }\end{array}$ & $\begin{array}{c}\text { MEAN } \\
(\text { FT3/S) }\end{array}$ & $\begin{array}{l}\text { STAN- } \\
\text { DARD } \\
\text { DEVIA- } \\
\text { TION } \\
(\text { FT3/S) }\end{array}$ & $\begin{array}{l}\text { COEFFI- } \\
\text { CIENT OF } \\
\text { VARI- } \\
\text { ATION }\end{array}$ & $\begin{array}{c}\text { PERCENT } \\
\text { OF } \\
\text { ANNUAL } \\
\text { RUNOFF }\end{array}$ \\
\hline OCTOBER & 71 & 0.00 & 8.8 & 17 & 1.9 & 6.3 \\
\hline NOVEMBER & 8.0 & 0.00 & 0.65 & 1.9 & 2.9 & 0.5 \\
\hline DECEMBER & 25 & 0.00 & 1.1 & 4.1 & 3.6 & 0.8 \\
\hline JANUARY & 8.0 & 0.00 & 0.45 & 1.6 & 3.5 & 0.3 \\
\hline PEBRUARY & 14 & 0.00 & 0.86 & 3.0 & 3.4 & 0.6 \\
\hline MARCH & 5.5 & 0.00 & 0.20 & 0.85 & 4.4 & 0.1 \\
\hline APRIL & 15 & 0.00 & 0.52 & 2.5 & 4.8 & 0.4 \\
\hline MAY & 11 & 0.00 & 0.44 & 1.7 & 3.9 & 0.3 \\
\hline JUNE & 20 & 0.00 & 1.6 & 4.1 & 2.6 & 1.2 \\
\hline JULY & 274 & 0.00 & 32 & 44 & 1.4 & 23.0 \\
\hline AUGUST & 328 & 0.00 & 68 & 74 & 1.1 & 49.1 \\
\hline SEPTEMBER & 176 & 0.00 & 24 & 32 & 1.4 & 17.3 \\
\hline ANNUAL & 38 & 1.4 & 12 & 8.8 & 0.75 & 100 \\
\hline
\end{tabular}

MAGNITUDE AND PROBABILITY OF INSTANTANEOUS PEAK FLOW BASED ON PERIOD OF RECORD $1931 \cdot 82,1984$

DISCHARGE, IN FT3/S, FOR INDICATED RECURRENCE INTERVAL IN YEARS, AND EXCEEDANCE PROBABILITY, IN PERCENT

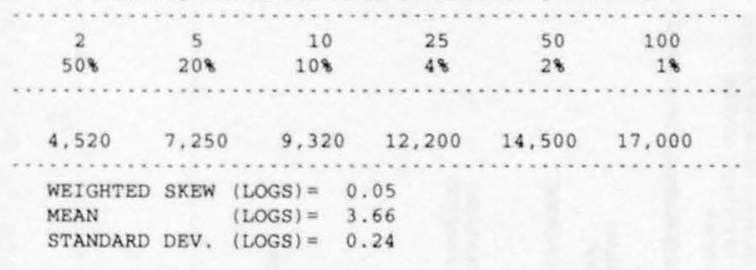

MAGNITUDE AND PROBABILITY OF ANNUAL LOW FLOW BASED ON PERIOD OF RECORD $1937-82$

\begin{tabular}{|c|c|c|c|c|c|c|}
\hline $\begin{array}{l}\text { PERIOD } \\
\text { /CON- } \\
\text { SECU. }\end{array}$ & & $\begin{array}{l}\text { ISCHA: } \\
\text { RECURR: } \\
\text { ON-EXCI } \\
\ldots \ldots\end{array}$ & $\begin{array}{l}\text { IN F } \\
\text { INTE } \\
\text { ANCE } \\
\ldots . . .\end{array}$ & $\begin{array}{l}\text { S, FOR } \\
\text { AL, IN } \\
\text { BABILIT } \\
\cdots\end{array}$ & $\begin{array}{l}\text { INDICAT } \\
\text { EARS, } \\
\text { IN PE }\end{array}$ & $\begin{array}{l}\text { D } \\
\text { CENT } \\
\cdots \ldots .\end{array}$ \\
\hline TIVE & 2 & 5 & $10 \#$ & $20 \#$ & $50 \#$ & $100 \#$ \\
\hline DAYS) & 508 & 208 & 108 & 58 & 28 & 18 \\
\hline $\begin{array}{r}1 \\
3 \\
7 \\
14 \\
30 \\
60\end{array}$ & & & & & & \\
\hline 90 & 0.00 & 0.00 & 0.00 & 0.00 & 0.00 & 0.00 \\
\hline 120 & 0.00 & 0.00 & 0.00 & 0.00 & 0.00 & 0.00 \\
\hline 183 & 0.58 & 0.00 & 0.00 & 0.00 & 0.00 & 0.00 \\
\hline
\end{tabular}

MAGNITUDE AND PROBABILITY OF ANNUAL HIGH FLOW BASED ON PERIOD OF RECORD 1932, 1936.82

\begin{tabular}{|c|c|c|c|c|c|c|}
\hline $\begin{array}{l}\text { PERIOD } \\
\text { (CON- }\end{array}$ & & \multicolumn{5}{|c|}{$\begin{array}{l}\text { DISCHARGE, IN FT3/S, FOR INDICATED } \\
\text { RECURRENCE INTERVAL, IN YEARS, AND }\end{array}$} \\
\hline SECU- & & & & & & \\
\hline TIVE & 2 & 5 & $10 \#$ & $25 \#$ & $50 \#$ & $100 \#$ \\
\hline DAYS) & 508 & 208 & 108 & 48 & 28 & 18 \\
\hline 1 & 721 & 1,260 & 1,670 & 2,230 & 2,670 & 3,130 \\
\hline 3 & 381 & 725 & 1.010 & 1,420 & 1,770 & 2,160 \\
\hline 7 & 197 & 389 & 556 & 817 & 1,050 & 1,310 \\
\hline 15 & 117 & 237 & 340 & 498 & 635 & 790 \\
\hline 30 & 72 & 147 & 212 & 312 & 401 & 501 \\
\hline 60 & 45 & 91 & 130 & 188 & 239 & 295 \\
\hline 90 & 32 & 63 & 89 & 129 & 163 & 201 \\
\hline
\end{tabular}

DURATION TABLE OF DAILY MEAN FLOW FOR PERIOD OF RECORD 1932, 1936-82

DISCHARGE, IN FT3/S, WHICH WAS EQUALED OR EXCEEDED FOR INDICATED PERCENT OF TIME

18
$\ldots$

\# Reliability of values in column is uncertain, and potential errors are large. 
ANNUAL MEAN DISCHARGE, IN CUBIC

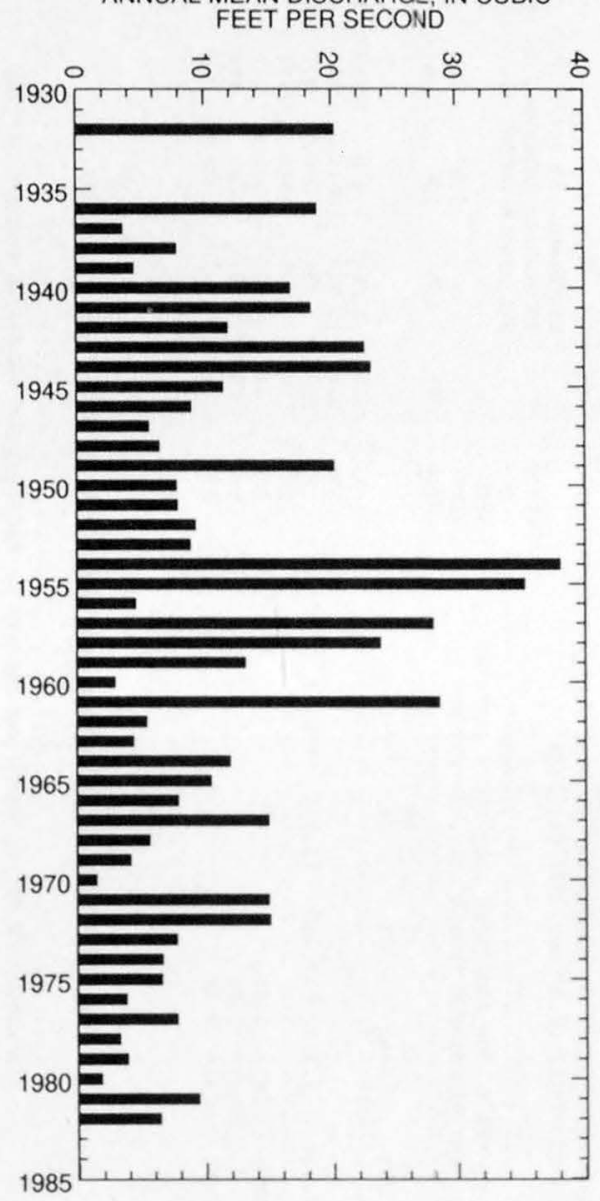

ANNUAL PEAK DISCHARGE, IN CUBIC

FEET PER SECOND

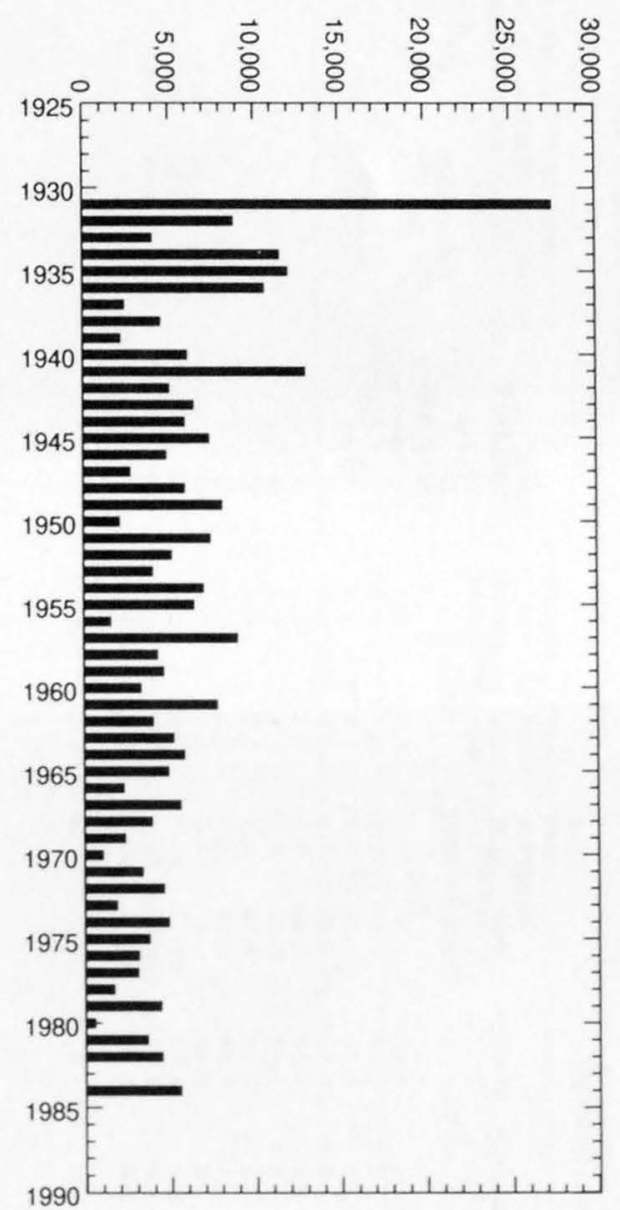


09457000 SAN SIMON RIVER NEAR SOLOMON, AZ--Continued
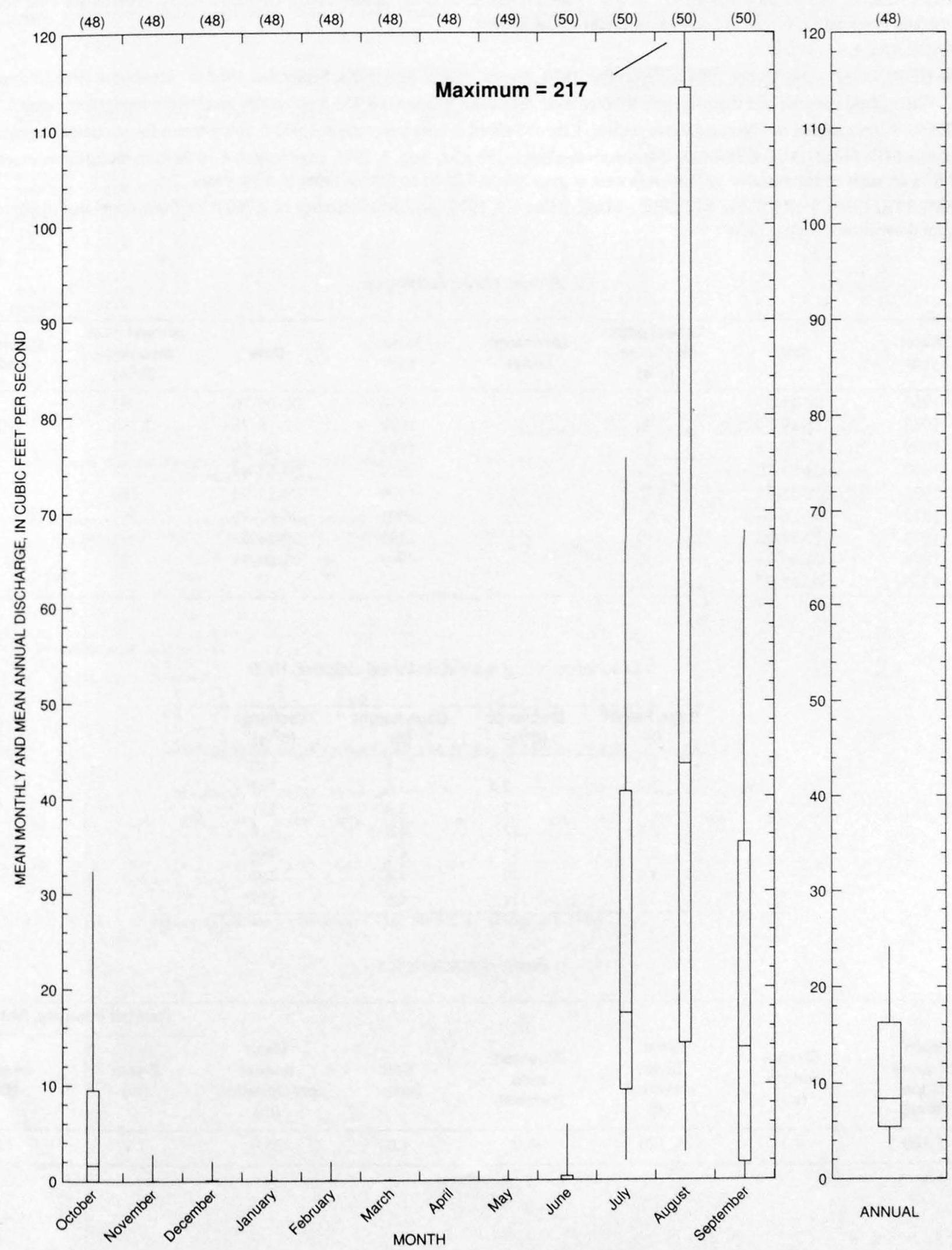


\section{DEADMAN CREEK NEAR SAFFORD, AZ}

LOCATION.--Lat 32 43'59", long 109 48'57", in SW1/4 sec.17, T.8 S., R.25 E., (unsurveyed), Graham County, Hydrologic Unit 15040005, in Coronado National Forest, on left bank $9 \mathrm{mi}$ southwest of Safford.

DRAINAGE AREA.--4.78 $\mathrm{mi}^{2}$.

PERIOD OF RECORD.--November 1966 to September 1976, August 1986 to May 1988, September 1988 to September 1993 (discontinued). GAGE.--Water-stage recorder and sharp-crested V-notch weir. Elevation of gage is 4,950 ft above sea level, from topographic map. REMARKS.--No regulation or diversion above station. City of Safford diverts water about 1,000 ft downstream for municipal supply.

EXTREMES FOR PERIOD OF RECORD.--Maximum discharge, $393 \mathrm{ft}^{3} / \mathrm{s}$, Aug. 7, 1991, gage height, $4.30 \mathrm{ft}$, from rating curve extended above $90 \mathrm{ft}^{3} / \mathrm{s}$ on basis of computation of flow over weir, at gage height $3.25 \mathrm{ft}$; no flow at times in most years.

EXTREMES OUTSIDE PERIOD OF RECORD.--Flood of Dec. 19, 1978, reached a discharge of $2,760 \mathrm{ft}^{3} / \mathrm{s}$, from slope-area measurement $1 / 4$ mi downstream from station.

Annual peak discharges

\begin{tabular}{|c|c|c|c|c|c|c|c|}
\hline $\begin{array}{l}\text { Water } \\
\text { year }\end{array}$ & Date & $\begin{array}{c}\text { Annual peak } \\
\text { discharge } \\
\left(\mathrm{ft}^{3} / \mathrm{s}\right)\end{array}$ & $\begin{array}{c}\text { Discharge } \\
\text { codes }\end{array}$ & $\begin{array}{l}\text { Water } \\
\text { year }\end{array}$ & Date & $\begin{array}{c}\text { Annual peak } \\
\text { discharge } \\
\left(\mathrm{ft}^{3} / \mathrm{s}\right)\end{array}$ & $\begin{array}{l}\text { Discharge } \\
\text { codes }\end{array}$ \\
\hline 1967 & $08-11-67$ & 89 & & 1976 & $02-09-76$ & 43 & \\
\hline 1968 & $12-19-67$ & 96 & & 1979 & $12-18-78$ & 2,760 & HP \\
\hline 1969 & $12-26-68$ & 12 & & 1987 & $11-03-86$ & 33 & \\
\hline 1970 & $08-07-70$ & 21 & & 1989 & 09-04-89 & 74 & \\
\hline 1971 & $09-08-71$ & 12 & & 1990 & $09-23-90$ & 186 & \\
\hline 1972 & $08-26-72$ & 82 & & 1991 & $08-07-91$ & 393 & \\
\hline 1973 & $10-19-72$ & 119 & & 1992 & $08-24-92$ & 92 & \\
\hline 1974 & $08-06-74$ & 57 & & 1993 & $01-08-93$ & 97 & \\
\hline 1975 & $09-06-75$ & 82 & & & & & \\
\hline
\end{tabular}

Discharge rating table developed October 1990

\begin{tabular}{cccc}
\hline $\begin{array}{c}\text { Gage height } \\
(\mathrm{ft})\end{array}$ & $\begin{array}{c}\text { Discharge } \\
\left(\mathrm{ft}^{3} / \mathbf{s}\right)\end{array}$ & $\begin{array}{c}\text { Gage height } \\
(\mathrm{ft})\end{array}$ & $\begin{array}{c}\text { Discharge } \\
\left(\mathrm{ft}^{3} / \mathbf{s}\right)\end{array}$ \\
\hline 2.0 & 2.3 & 3.4 & 150 \\
2.2 & 7.4 & 3.6 & 197 \\
2.4 & 17 & 3.8 & 251 \\
2.6 & 32 & 4.0 & 314 \\
2.8 & 52 & 4.2 & 386 \\
3.0 & 79 & 4.4 & 467 \\
3.2 & 111 & 4.6 & 557 \\
\hline
\end{tabular}

Basin characteristics

\begin{tabular}{ccccccc}
\hline $\begin{array}{c}\text { Main } \\
\text { channel } \\
\text { slope } \\
(\mathrm{ft} / \mathrm{mi})\end{array}$ & $\begin{array}{c}\text { Stream } \\
\text { length } \\
(\mathrm{mi})\end{array}$ & $\begin{array}{c}\text { Mean } \\
\text { basin } \\
\text { elevation } \\
(\mathrm{ft})\end{array}$ & $\begin{array}{c}\text { Forested } \\
\text { area } \\
\text { (percent) }\end{array}$ & $\begin{array}{c}\text { Soil } \\
\text { index }\end{array}$ & $\begin{array}{c}\text { Mean } \\
\text { annual } \\
\text { precipitation } \\
\text { (in) }\end{array}$ & $\begin{array}{c}\text { Rainfall intensity, 24-hour } \\
\text { (in) }\end{array}$ \\
\hline 1,100 & 4.1 & 7,520 & 48.0 & 1.0 & 25.0 & $\begin{array}{c}50 \text {-year } \\
\text { (in) }\end{array}$ \\
\hline
\end{tabular}


09458200 DEADMAN CREEK NEAR SAFFORD, AZ--Continued

MEAN MONTHLY AND ANNUAL DISCHARGES $1968 \cdot 76,1987,1989 \cdot 93$

\begin{tabular}{|c|c|c|c|c|c|c|}
\hline MONTH & $\begin{array}{l}\text { MAXIMUM } \\
(\text { FT3/S) }\end{array}$ & $\begin{array}{l}\text { MINIMUM } \\
(\text { FT3/S) }\end{array}$ & $\begin{array}{c}\text { MEAN } \\
\text { (FT3/S) }\end{array}$ & $\begin{array}{l}\text { STAN- } \\
\text { DARD } \\
\text { DEVIA- } \\
\text { TION } \\
(\text { FT3/S) }\end{array}$ & $\begin{array}{l}\text { COEFFI- } \\
\text { CIENT OF } \\
\text { VARI- } \\
\text { ATION }\end{array}$ & $\begin{array}{c}\text { PERCENT } \\
\text { OF } \\
\text { ANNUAL } \\
\text { RUNOFF }\end{array}$ \\
\hline OCTOBER & 4.1 & 0.00 & 0.98 & 1.3 & 1.4 & 4.4 \\
\hline NOVEMBER & 3.3 & 0.08 & 0.66 & 0.89 & 1.3 & 3.0 \\
\hline DECEMBER & 4.5 & 0.09 & 1.7 & 1.7 & 1.0 & 7.8 \\
\hline JANUARY & 18 & 0.13 & 2.4 & 4.5 & 1.8 & 11.1 \\
\hline FEBRUARY & 9.9 & 0.14 & 2.5 & 3.1 & 1.2 & 11.5 \\
\hline MARCH & 9.5 & 0.12 & 3.0 & 3.0 & 1.0 & 13.5 \\
\hline APRIL & 8.5 & 0.13 & 3.1 & 2.9 & 0.96 & 14.0 \\
\hline MAY & 13 & 0.03 & 4.1 & 4.0 & 0.97 & 18.9 \\
\hline JUNE & 4.1 & 0.00 & 1.5 & 1.6 & 1.1 & 6.6 \\
\hline JULY & 0.73 & 0.00 & 0.27 & 0.23 & 0.86 & 1.2 \\
\hline AUGUST & 2.4 & 0.00 & 0.57 & 0.70 & 1.2 & 2.6 \\
\hline SEPTEMBER & 6.7 & 0.03 & 1.2 & 2.1 & 1.8 & 5.3 \\
\hline ANNUAL & 4.7 & 0.11 & 1.8 & 1.6 & 0.86 & 100 \\
\hline
\end{tabular}

MAGNITUDE AND PROBABILITY OF INSTANTANEOUS PEAK FLOW BASED ON PERIOD OF RECORD

DISCHARGE, IN FT3/S, FOR INDICATED RECURRENCE INTERVAL IN YEARS, AND EXCEEDANCE PROBABILITY, IN PERCENT

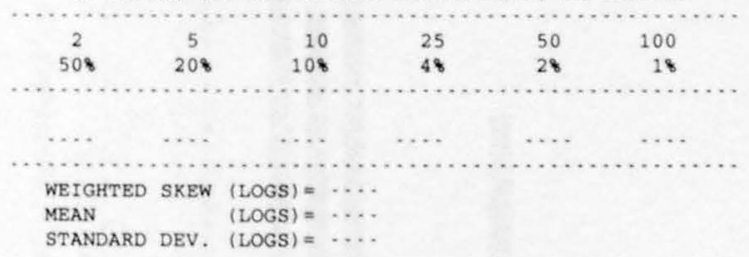

MAGNITUDE AND PROBABILITY OF ANNUAL LOW FLOW BASED ON PERIOD OF RECORD $1968.76,1988,1990.93$

\begin{tabular}{|c|c|c|c|c|c|c|}
\hline \multirow{3}{*}{$\begin{array}{l}\text { PERIOD } \\
\text { (CON- } \\
\text { SECU- } \\
\text { TIVE }\end{array}$} & \multicolumn{6}{|c|}{$\begin{array}{l}\text { DISCHARGE, IN FT3/S, FOR INDICATED } \\
\text { RECURRENCE INTERVAL, IN YEARS, AND }\end{array}$} \\
\hline & $\cdots$ & $\ldots \ldots$ & $\ldots \ldots$ & - & $\ldots \ldots$ & $\cdots \ldots$ \\
\hline & 2 & 5 & $10 \#$ & $20 \#$ & $50 \#$ & $100 \#$ \\
\hline DAYS) & 508 & 208 & 108 & 58 & 28 & 18 \\
\hline 1 & 0.00 & 0.00 & 0.00 & 0.00 & 0.00 & 0.00 \\
\hline 3 & 0.00 & 0.00 & 0.00 & 0.00 & 0.00 & 0.00 \\
\hline 7 & 0.00 & 0.00 & 0.00 & 0.00 & 0.00 & 0.00 \\
\hline 14 & 0.00 & 0.00 & 0.00 & 0.00 & 0.00 & 0.00 \\
\hline 30 & 0.00 & 0.00 & 0.00 & 0.00 & 0.00 & 0.00 \\
\hline 60 & 0.06 & 0.00 & 0.00 & 0.00 & 0.00 & 0.00 \\
\hline 90 & 0.10 & 0.03 & 0.00 & 0.00 & 0.00 & 0.00 \\
\hline 120 & 0.17 & 0.06 & 0.02 & 0.00 & 0.00 & 0.00 \\
\hline 183 & 0.29 & 0.12 & 0.08 & 0.05 & 0.03 & 0.02 \\
\hline
\end{tabular}

MAGNITUDE AND PROBABILITY OF ANNUAL HIGH FLOW BASED ON PERIOD OF RECORD $1968 \cdot 76,1987,1989.93$

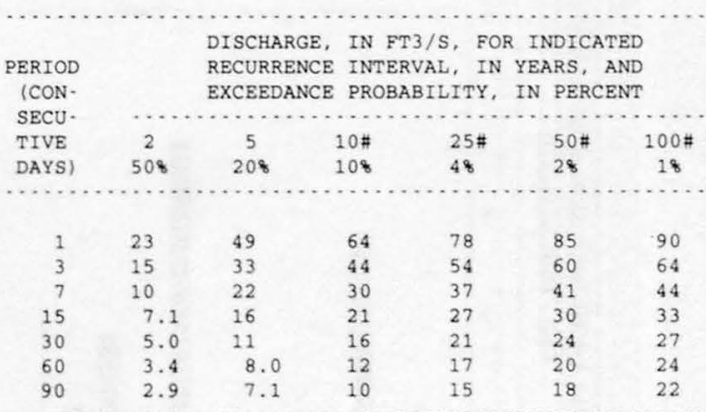

DURATION TABLE OF DAILY MEAN FLOW FOR PERIOD OF RECORD $1968 \cdot 76,1987,1989.93$

DISCHARGE, IN FT3/S, WHICH WAS EQUALED OR EXCEEDED FOR INDICATED PERCENT OF TIME

\begin{tabular}{|c|c|c|c|c|c|c|c|c|c|c|c|c|c|c|c|c|}
\hline 18 & 58 & 108 & 158 & 208 & 308 & 408 & 508 & 608 & 708 & 808 & 908 & 958 & 988 & 998 & 99.58 & 99.98 \\
\hline 18 & 8.3 & 5.7 & 4.1 & 2.7 & 1.2 & 0.63 & 0.42 & 0.28 & 0.19 & 0.12 & 0.02 & 0.00 & 0.00 & 0.00 & 0.00 & 0.00 \\
\hline
\end{tabular}


ANNUAL MEAN DISCHARGE, IN CUBIC FEET PER SECOND

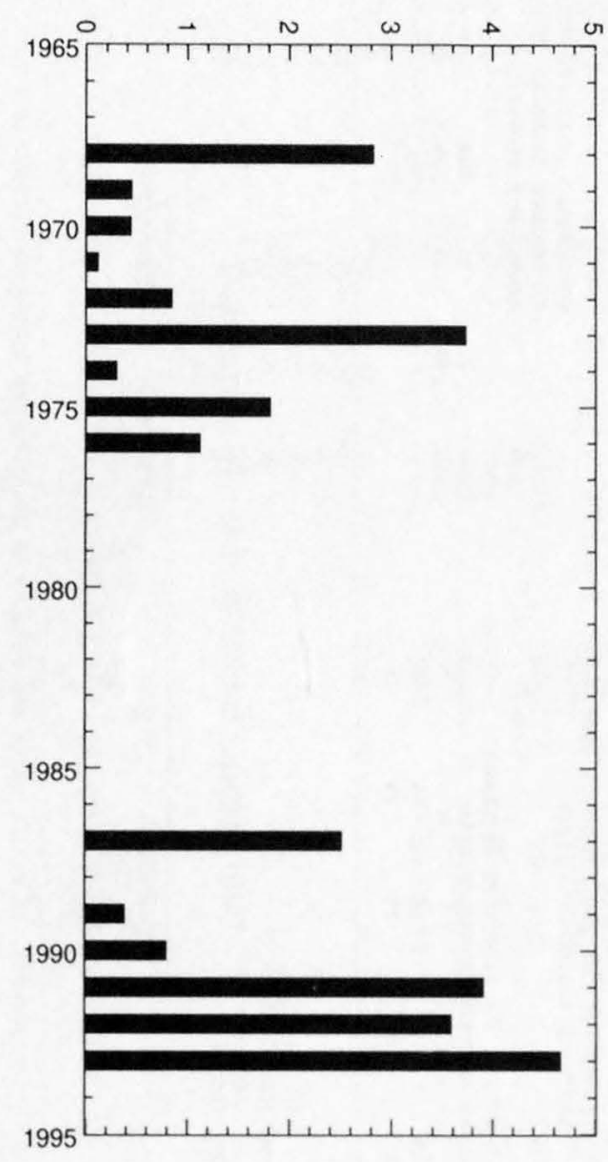

ANNUAL PEAK DISCHARGE, IN CUBIC FEET PER SECOND

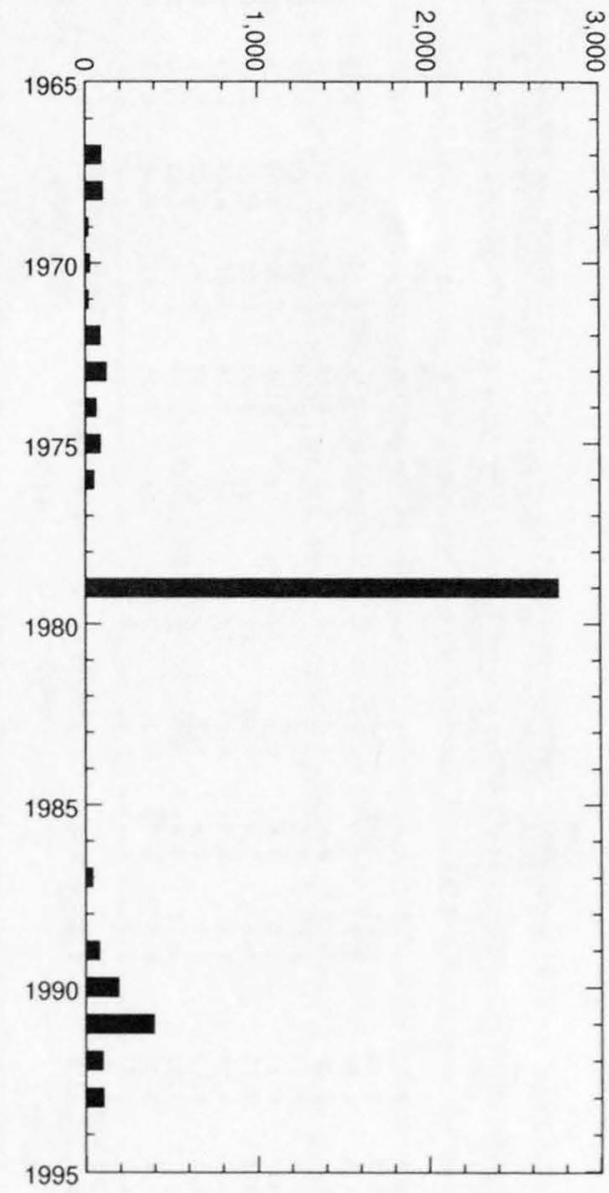


09458200 DEADMAN CREEK NEAR SAFFORD, AZ--Continued
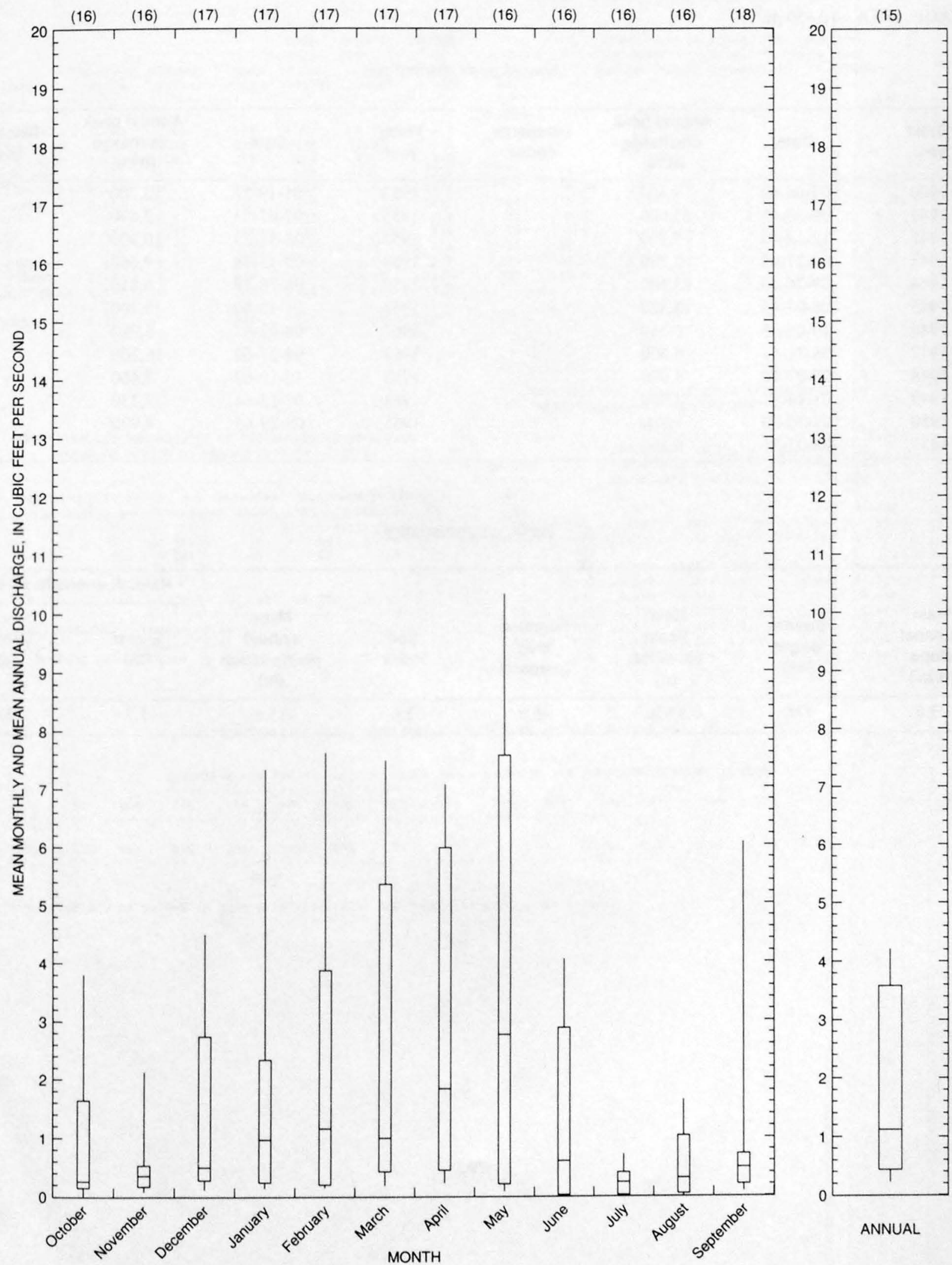
LOCATION.--Lat $32^{\circ} 50^{\prime} 50^{\prime \prime}$, long $109^{\circ} 42^{\prime} 55^{\prime \prime}$, in SW $1 / 4 \mathrm{SW}^{1} / 4$ sec.5, T.7 S., R.26 E., Graham County, Hydrologic Unit 15040005 , on downstream side of highway bridge $1 \mathrm{mi}$ north of Safford and $4.5 \mathrm{mi}$ downstream from San Simon River.

DRAINAGE AREA.--10,459 $\mathrm{mi}^{2}$.

Annual peak discharges

\begin{tabular}{|c|c|c|c|c|c|c|c|}
\hline $\begin{array}{l}\text { Water } \\
\text { year }\end{array}$ & Date & $\begin{array}{c}\text { Annual peak } \\
\text { discharge } \\
\left(\mathrm{ft}^{3} / \mathrm{s}\right)\end{array}$ & $\begin{array}{l}\text { Discharge } \\
\text { codes }\end{array}$ & $\begin{array}{l}\text { Water } \\
\text { year }\end{array}$ & Date & $\begin{array}{c}\text { Annual peak } \\
\text { discharge } \\
\left(\mathrm{ft}^{3} / \mathrm{s}\right)\end{array}$ & $\begin{array}{l}\text { Discharge } \\
\text { codes }\end{array}$ \\
\hline 1940 & $09-06-40$ & 8,600 & & 1952 & $01-19-52$ & 15,700 & \\
\hline 1941 & $09-30-41$ & 33,000 & & 1953 & $07-07-53$ & 2,670 & \\
\hline 1942 & $12-12-41$ & 7,800 & & 1957 & $08-31-57$ & 10,300 & \\
\hline 1943 & $09-27-43$ & 5,780 & & 1958 & $09-13-58$ & 9,660 & \\
\hline 1944 & $09-26-44$ & 13,600 & & 1959 & $08-28-59$ & 6,810 & \\
\hline 1945 & $08-03-45$ & 5,320 & & 1960 & $01-12-60$ & 15,400 & \\
\hline 1946 & $10-09-45$ & 6,340 & & 1961 & $08-22-61$ & 6,990 & \\
\hline 1947 & $08-31-47$ & 4,600 & & 1962 & $09-27-62$ & 16,200 & \\
\hline 1948 & $08-07-48$ & 6,090 & & 1963 & $10-19-62$ & 7,460 & \\
\hline 1949 & $01-14-49$ & 23,900 & & 1964 & $07-15-64$ & 7,330 & \\
\hline 1950 & $07-30-50$ & 1,860 & & 1965 & $08-29-65$ & 4,900 & \\
\hline 1951 & $08-03-51$ & 6,390 & & & & & \\
\hline
\end{tabular}

Basin characteristics

\begin{tabular}{cccccccc}
\hline $\begin{array}{c}\text { Main } \\
\text { channel } \\
\text { slope } \\
\text { (ft/mi) }\end{array}$ & $\begin{array}{c}\text { Stream } \\
\text { length } \\
\text { (mi) }\end{array}$ & $\begin{array}{c}\text { Mean } \\
\text { basin } \\
\text { elevation } \\
\text { (ft) }\end{array}$ & $\begin{array}{c}\text { Forested } \\
\text { area } \\
\text { (percent) }\end{array}$ & $\begin{array}{c}\text { Soil } \\
\text { index }\end{array}$ & $\begin{array}{c}\text { Mean } \\
\text { annual } \\
\text { precipitation } \\
\text { (in) }\end{array}$ & $\begin{array}{c}\text { Rainfall intensity, 24-hour } \\
\text { (in) }\end{array}$ & $\begin{array}{c}50 \text {-year } \\
\text { (in) }\end{array}$ \\
\hline 23.6 & 186 & 5,830 & 46.0 & 2.6 & 15.6 & 1.7 \\
\hline
\end{tabular}


09458500 GILA RIVER AT SAFFORD, AZ--Continued

MEAN MONTHLY AND ANNUAL DISCHARGES $1941-46,1957-65$

\begin{tabular}{|c|c|c|c|c|c|c|}
\hline MONTH & $\begin{array}{l}\text { MAXIMUM } \\
\text { (FT3/S) }\end{array}$ & $\begin{array}{l}\text { MINIMUM } \\
\text { (FT3/S) }\end{array}$ & $\begin{array}{c}\text { MEAN } \\
(\text { FT3 } / S)\end{array}$ & $\begin{array}{l}\text { STAN- } \\
\text { DARD } \\
\text { DEVIA- } \\
\text { TION } \\
\text { (FT3/S) }\end{array}$ & $\begin{array}{l}\text { COEFFI- } \\
\text { CIENT OF } \\
\text { VARI- } \\
\text { ATION }\end{array}$ & $\begin{array}{c}\text { PERCENT } \\
\text { OF } \\
\text { ANNUAL } \\
\text { RUNOFF }\end{array}$ \\
\hline OCTOBER & 1,180 & 0.16 & 194 & 292 & 1.5 & 5.7 \\
\hline NOVEMBER & 403 & 0.28 & 139 & 128 & 0.92 & 4.1 \\
\hline DECEMBER & 1,400 & 1.3 & 288 & 423 & 1.5 & 8.4 \\
\hline JANUARY & 2.040 & 74 & 445 & 574 & 1.3 & 13.0 \\
\hline FEBRUARY & 2,170 & 27 & 458 & 640 & 1.4 & 13.4 \\
\hline MARCH & 2,660 & 13 & 491 & 796 & 1.6 & 14.3 \\
\hline APRIL & 1,680 & 4.7 & 337 & 533 & 1.6 & 9.8 \\
\hline MAY & 1.560 & 0.13 & 154 & 393 & 2.6 & 4.5 \\
\hline JUNE & 130 & 0.00 & 15 & 33 & 2.2 & 0.4 \\
\hline JULY & 442 & 5.6 & 94 & 115 & 1.2 & 2.7 \\
\hline AUGUST & 1,660 & 11 & 428 & 417 & 0.98 & 12.5 \\
\hline SEPTEMBER & 1,180 & 16 & 380 & 332 & 0.87 & 11.1 \\
\hline ANNUAL & 1,120 & 87 & 284 & 263 & 0.92 & 100 \\
\hline
\end{tabular}

MAGNITUDE AND PROBABILITY OF INSTANTANEOUS PEAK FLOW BASED ON PERIOD OF RECORD $1940.53,1957.65$

DISCHARGE, IN FT3/S, FOR INDICATED RECURRENCE INTERVAL IN YEARS, AND EXCEEDANCE PROBABILITY, IN PERCENT

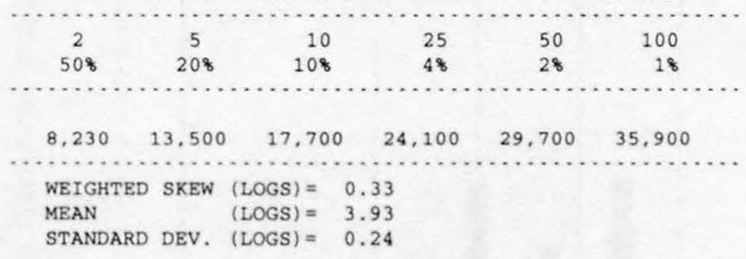

MAGNITUDE AND PROBABILITY OF ANNUAL LOW FLOW BASED ON PERIOD OF RECORD $1942 \cdot 47,1958 \cdot 65$

\begin{tabular}{|c|c|c|c|c|c|c|}
\hline \multirow{4}{*}{$\begin{array}{l}\text { PERIOD } \\
\text { (CON- } \\
\text { SECU- }\end{array}$} & \multicolumn{6}{|c|}{ DISCHARGE, IN FT3/S, FOR INDICATED } \\
\hline & \multicolumn{6}{|c|}{$\begin{array}{l}\text { RECURRENCE INTERVAL, IN YEARS, AND } \\
\text { NON-EXCEEDANCE PROBABILITY, IN PERCENT }\end{array}$} \\
\hline & \multicolumn{6}{|c|}{ 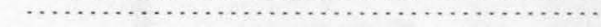 } \\
\hline & 2 & 5 & $10 \#$ & $20 \#$ & $50 \#$ & $100 \#$ \\
\hline DAYS) & 508 & 208 & 108 & 58 & 28 & 18 \\
\hline \multicolumn{7}{|l|}{1} \\
\hline 3 & 0.00 & 0.00 & 0.00 & 0.00 & 0.00 & 0.00 \\
\hline 7 & 0.00 & 0.00 & 0.00 & 0.00 & 0.00 & 0.00 \\
\hline 14 & 0.01 & 0.00 & 0.00 & 0.00 & 0.00 & 0.00 \\
\hline 30 & 0.51 & 0.00 & 0.00 & 0.00 & 0.00 & 0.00 \\
\hline 60 & 4.3 & 0.75 & 0.27 & 0.11 & 0.04 & 0.02 \\
\hline 90 & 14 & 5.2 & 3.4 & 2.4 & 1.7 & 1.3 \\
\hline 120 & 36 & 19 & 14 & 11 & 8.3 & 7.0 \\
\hline 183 & 116 & 57 & 38 & 26 & 17 & 13 \\
\hline
\end{tabular}

MAGNITUDE AND PROBABILTTY OF ANNUAL HIGH FLOW BASED ON PERIOD OF RECORD $1941-46,1957.65$

\begin{tabular}{|c|c|c|c|c|c|c|}
\hline $\begin{array}{l}\text { PERIOD } \\
\text { (CON. }\end{array}$ & & \multicolumn{5}{|c|}{$\begin{array}{l}\text { DISCHARGE, IN FT3/S, FOR INDICATED } \\
\text { RECURRENCE INTERVAL, IN YEARS, AND }\end{array}$} \\
\hline SECU. & & $\cdots \cdots$ & & . & & $\ldots \ldots \ldots$ \\
\hline TIVE & 2 & 5 & $10 \#$ & $25 \#$ & $50 \#$ & $100 \#$ \\
\hline DAYS) & 508 & 208 & 108 & 48 & 28 & 18 \\
\hline 1 & 4,090 & 7,800 & 11,500 & 17,900 & 24,400 & 32,600 \\
\hline 3 & 2,900 & 5,340 & 7,490 & 10,900 & 14,000 & 17,700 \\
\hline 7 & 1,940 & 3,490 & 4,700 & 6,440 & 7,870 & 9,400 \\
\hline 15 & 1,310 & 2,390 & 3,250 & 4,500 & 5,540 & 6,660 \\
\hline 30 & 873 & 1,640 & 2,290 & 3,300 & 4,190 & 5,210 \\
\hline 60 & 610 & 1,180 & 1,700 & 2,560 & 3,380 & 4,360 \\
\hline 90 & 479 & 967 & 1,440 & 2,250 & 3,050 & 4,030 \\
\hline
\end{tabular}

DURATION TABLE OF DAILY MEAN FLOW FOR PERIOD OF RECORD 1941.46, 1957.65

DISCHARGE, IN FT3/S, WHICH WAS EQUALED OR EXCEEDED FOR INDICATED PERCENT OF TIME

\begin{tabular}{|c|c|c|c|c|c|c|c|c|c|c|c|c|c|c|c|c|}
\hline 18 & 58 & 108 & 158 & 208 & 308 & 408 & 508 & 608 & 708 & 808 & 908 & 958 & 988 & 998 & 99.58 & 99.98 \\
\hline 480 & 1,360 & 694 & 442 & 322 & 176 & 103 & 66 & 40 & 21 & 7.9 & 0.52 & 0.00 & 0.00 & 0.00 & 0.00 & 0.00 \\
\hline
\end{tabular}

\# Reliability of values in column is uncertain, and potential errors are large. 
ANNUAL MEAN DISCHARGE, IN CUBIC FEET PER SECOND

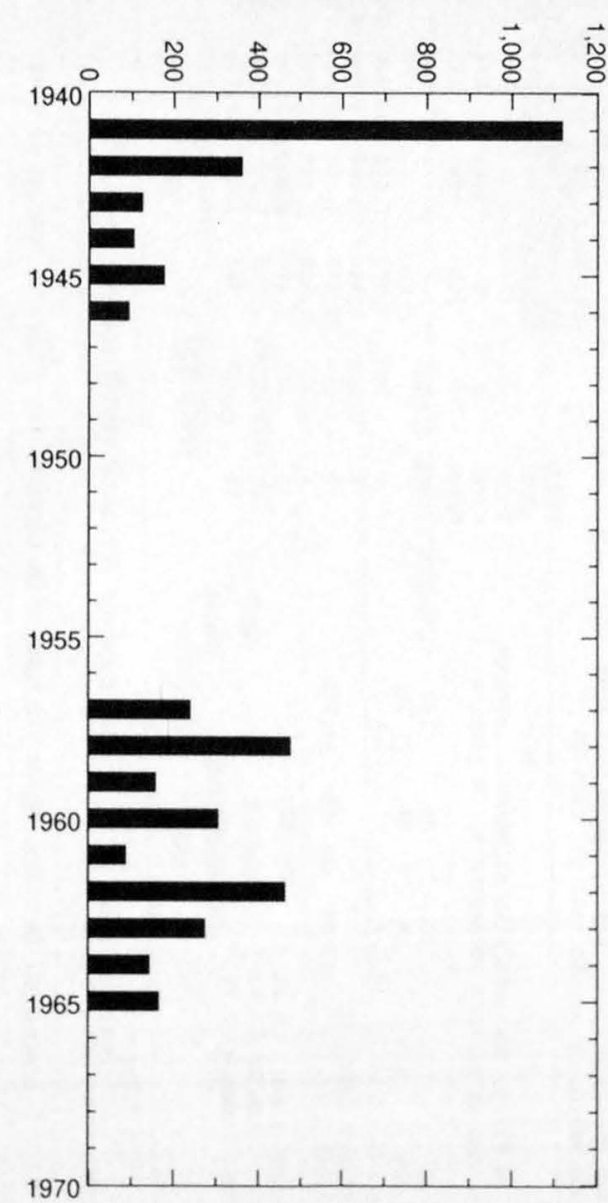

ANNUAL PEAK DISCHARGE, IN CUBIC

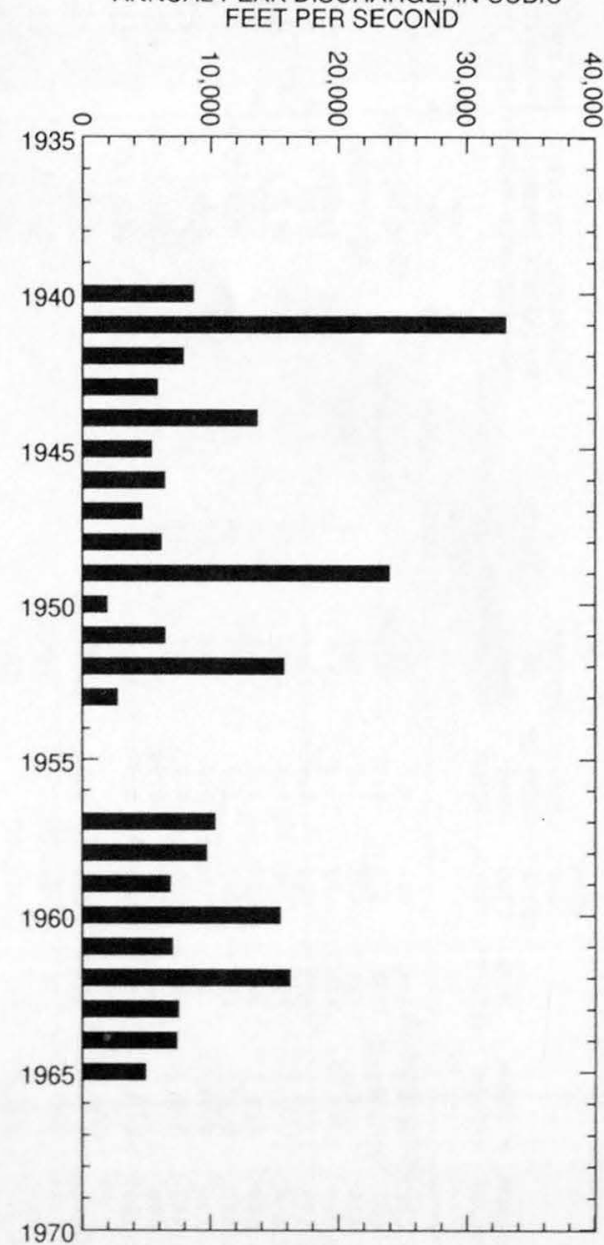


09458500 GILA RIVER AT SAFFORD, AZ--Continued
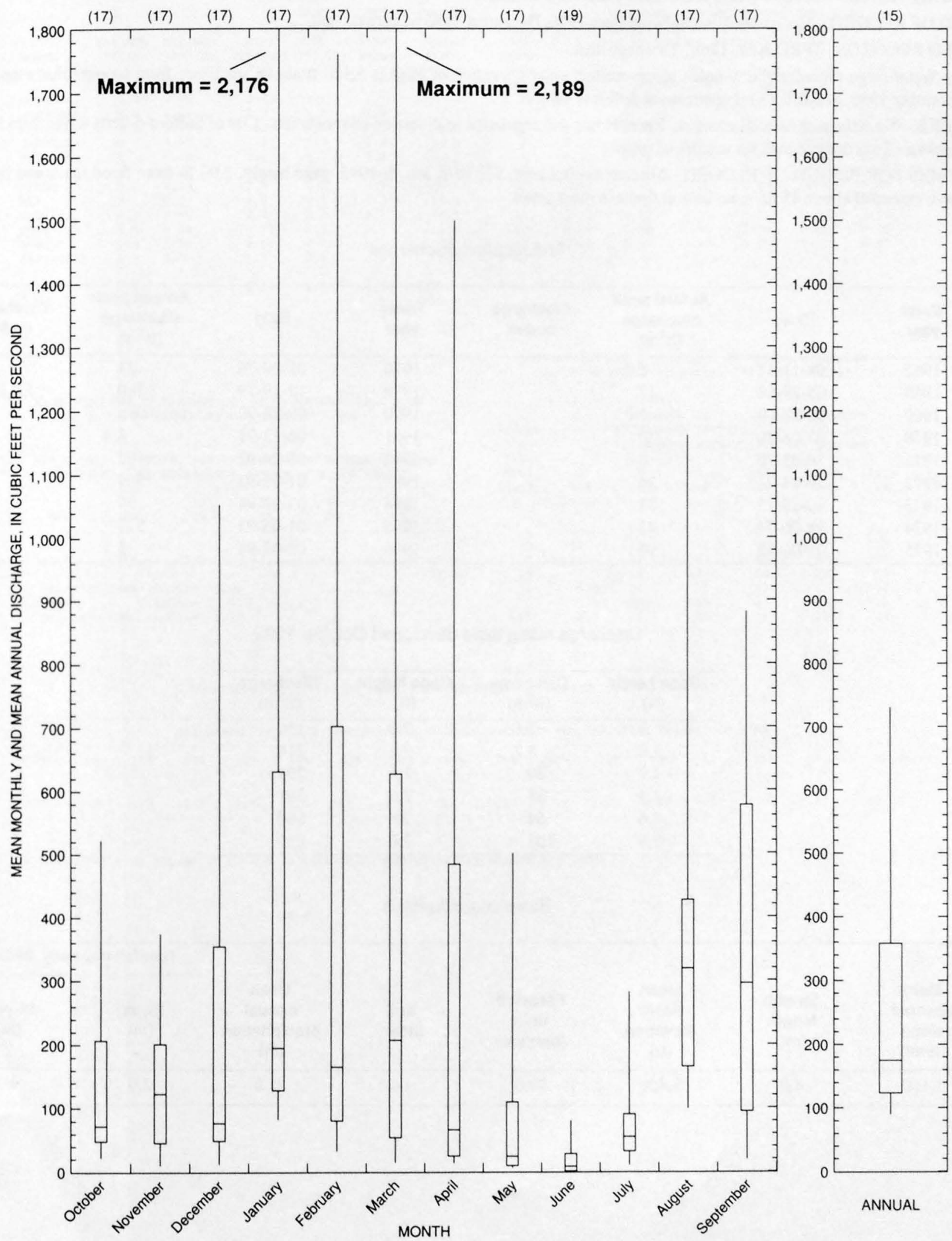


\section{FRYE CREEK NEAR THATCHER, AZ}

LOCATION.--Lat 32 44'38", long 109 $50^{\prime} 15^{\prime \prime}$, in NE $1 / 4$ sec.13, T.8 S., R.24 E. (unsurveyed), Graham County, Hydrologic Unit 15040005 , in Coronado National Forest, on left bank $8.5 \mathrm{mi}$ southwest of Thatcher.

DRAINAGE AREA.--4.02 $\mathrm{mi}^{2}$. (Area at site used 1966-76, 3.91 mi2.)

PERIOD OF RECORD.--December 1966 to September 1976, December 1988 to current year.

REVISED RECORDS.--WRD Ariz. 1968: Drainage area.

GAGE.--Water-stage recorder and V-notch sharp-crested weir. Elevation of gage is 5,580 $\mathrm{ft}$ above sea level, from topographic map. Prior to December 1988 , at site 0.25 mi upstream at different datum.

REMARKS.--No estimated daily discharges. Records fair. No regulation or diversion above station. City of Safford diverts water from Frye Mesa Reservoir 1 mi downstream for municipal supply.

EXTREMES FOR PERIOD OF RECORD.--Maximum discharge, $577 \mathrm{ft}^{3} / \mathrm{s}$, Jan. 5, 1995, gage height, $2.90 \mathrm{ft}$, from flood mark and from rating curve extended above $45 \mathrm{ft}^{3} / \mathrm{s}$; no flow at times in most years.

Annual peak discharges

\begin{tabular}{|c|c|c|c|c|c|c|c|}
\hline $\begin{array}{l}\text { Water } \\
\text { year }\end{array}$ & Date & $\begin{array}{c}\text { Annual peak } \\
\text { discharge } \\
\left(\mathrm{ft}^{3} / \mathrm{s}\right)\end{array}$ & $\begin{array}{l}\text { Discharge } \\
\text { codes }\end{array}$ & $\begin{array}{l}\text { Water } \\
\text { year }\end{array}$ & Date & $\begin{array}{c}\text { Annual peak } \\
\text { discharge } \\
\left(\mathrm{ft}^{3} / \mathrm{s}\right)\end{array}$ & $\begin{array}{c}\text { Discharge } \\
\text { codes }\end{array}$ \\
\hline 1967 & $08-11-67$ & 2.2 & & 1976 & $02-09-76$ & 24 & \\
\hline 1968 & $05-29-68$ & 17 & & 1979 & $12-19-79$ & 2,300 & ES,HP \\
\hline 1969 & $05-23-69$ & 3.2 & & 1990 & $09-23-90$ & unknown & \\
\hline 1970 & $09-06-70$ & 30 & & 1991 & $06-13-91$ & 8.4 & \\
\hline 1971 & $10-03-70$ & 0.8 & & 1992 & $08-24-92$ & 102 & \\
\hline 1972 & $10-24-71$ & 36 & & 1993 & $01-08-93$ & 509 & \\
\hline 1973 & $10-19-72$ & 51 & & 1994 & $03-20-94$ & 37 & \\
\hline 1974 & $08-06-74$ & 42 & & 1995 & $01-05-95$ & 577 & \\
\hline 1975 & $09-06-75$ & 96 & & 1996 & 08-09-96 & 5.2 & \\
\hline
\end{tabular}

Discharge rating table developed October 1992

\begin{tabular}{cccc}
\hline $\begin{array}{c}\text { Gage height } \\
(\mathbf{f t})\end{array}$ & $\begin{array}{c}\text { Discharge } \\
\left(\mathrm{ft}^{3} / \mathbf{s}\right)\end{array}$ & $\begin{array}{c}\text { Gage height } \\
(\mathbf{f t})\end{array}$ & $\begin{array}{c}\text { Discharge } \\
\left(\mathrm{ft}^{3} / \mathbf{s}\right)\end{array}$ \\
\hline .8 & 3.1 & 2.0 & 150 \\
1.0 & 8.2 & 2.2 & 214 \\
1.2 & 20 & 2.4 & 293 \\
1.4 & 38 & 2.6 & 391 \\
1.6 & 64 & 2.8 & 509 \\
1.8 & 101 & 3.0 & 650 \\
\hline
\end{tabular}

Basin characteristics

\begin{tabular}{ccccccc}
\hline $\begin{array}{c}\text { Main } \\
\text { channel } \\
\text { slope } \\
\text { (ft/mi) }\end{array}$ & $\begin{array}{c}\text { Stream } \\
\text { length } \\
\text { (mi) }\end{array}$ & $\begin{array}{c}\text { Mean } \\
\text { basin } \\
\text { elevation } \\
\text { (ft) }\end{array}$ & $\begin{array}{c}\text { Forested } \\
\text { area } \\
\text { (percent) }\end{array}$ & $\begin{array}{c}\text { Soil } \\
\text { index }\end{array}$ & $\begin{array}{c}\text { Mean } \\
\text { annual } \\
\text { precipitation } \\
\text { (in) }\end{array}$ & $\begin{array}{c}\text { Rainfall intensity, 24-hour } \\
\text { (in) }\end{array}$ \\
\hline 1,160 & 4.0 & 8,400 & 79.0 & 1.0 & 25.0 & $\begin{array}{c}50 \text {-year } \\
\text { (in) }\end{array}$ \\
\hline
\end{tabular}


09460150 FRYE CREEK NEAR THATCHER, AZ--Continued

MEAN MONTHLY AND ANNUAL DISCHARGES 1968-76, 1990.95

\begin{tabular}{|c|c|c|c|c|c|c|}
\hline MONTH & $\begin{array}{l}\text { MAXIMUM } \\
\text { (FT3/S) }\end{array}$ & $\begin{array}{l}\text { MINIMUM } \\
\text { (FT3/S) }\end{array}$ & $\begin{array}{c}\text { MEAN } \\
\text { (FT3/S) }\end{array}$ & $\begin{array}{l}\text { STAN- } \\
\text { DARD } \\
\text { DEVIA- } \\
\text { TION } \\
(\mathrm{FT} 3 / \mathrm{S})\end{array}$ & $\begin{array}{l}\text { COEFFI- } \\
\text { CIENT OF } \\
\text { VARI- } \\
\text { ATION }\end{array}$ & $\begin{array}{c}\text { PERCENT } \\
\text { OF } \\
\text { ANNUAL } \\
\text { RUNOFF }\end{array}$ \\
\hline OCTOBER & 5.9 & 0.00 & 0.88 & 1.5 & 1.7 & 3.6 \\
\hline NOVEMBER & 9.3 & 0.00 & 1.2 & 2.3 & 1.9 & 4.9 \\
\hline DECEMBER & 5.1 & 0.09 & 1.2 & 1.5 & 1.3 & 5.1 \\
\hline JANUARY & 29 & 0.17 & 3.5 & 7.7 & 2.2 & 14.4 \\
\hline FEBRUARY & 11 & 0.18 & 2.4 & 3.3 & 1.4 & 10.0 \\
\hline MARCH & 11 & 0.19 & 2.4 & 2.7 & 1.2 & 9.8 \\
\hline APRIL & 9.4 & 0.15 & 2.5 & 2.5 & 0.99 & 10.2 \\
\hline MAY & 17 & 0.07 & 4.9 & 5.1 & 1.0 & 19.9 \\
\hline JUNE & 7.4 & 0.00 & 2.7 & 2.8 & 1.1 & 10.9 \\
\hline JULY & 4.3 & 0.00 & 0.72 & 1.1 & 1.5 & 3.0 \\
\hline AUGUST & 2.0 & 0.00 & 0.66 & 0.72 & 1.1 & 2.7 \\
\hline SEPTEMBER & 6.9 & 0.00 & 1.3 & 2.1 & 1.6 & 5.5 \\
\hline ANNUAL & 5.4 & 0.13 & 2.0 & 1.8 & 0.86 & 100 \\
\hline
\end{tabular}

MAGNITUDE AND PROBABILITY OF INSTANTANEOUS PEAK FLOW BASED ON PERIOD OF RECORD 1968.76, 1990.96

DISCHARGE, IN FT3/S, FOR INDICATED RECURRENCE INTERVAL IN YEARS, AND EXCEEDANCE PROBABILITY, IN PERCENT

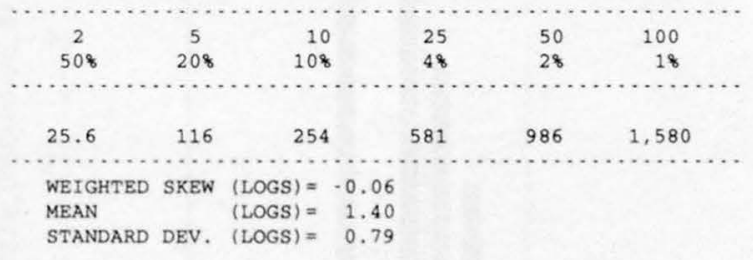

MAGNITUDE AND PROBABILITY OF ANNUAL LOW FLOW BASED ON PERIOD OF RECORD 1968-76, 1991-95

\begin{tabular}{|c|c|c|c|c|c|c|}
\hline \multirow{4}{*}{$\begin{array}{l}\text { PERIOD } \\
\text { (CON- } \\
\text { SECU- } \\
\text { TIVE } \\
\text { DAYS) }\end{array}$} & \multicolumn{6}{|c|}{$\begin{array}{l}\text { DISCHARGE, IN FT3/S, FOR INDICATED } \\
\text { RECURRENCE INTERVAL, IN YEARS, AND } \\
\text { NON-EXCEEDANCE PROBABILITY, IN PERCENT }\end{array}$} \\
\hline & $\cdots$ & ........ & $\ldots \ldots$ & $\ldots \ldots$ & $\ldots \ldots$ & $\ldots \ldots$ \\
\hline & 2 & 5 & 10 & $20 \#$ & $50 \#$ & $100 \#$ \\
\hline & 508 & 208 & 108 & 58 & 28 & 18 \\
\hline 1 & 0.00 & 0.00 & 0.00 & 0.00 & 0.00 & 0.00 \\
\hline 3 & 0.00 & 0.00 & 0.00 & 0.00 & 0.00 & 0.00 \\
\hline 7 & 0.00 & 0.00 & 0.00 & 0.00 & 0.00 & 0.00 \\
\hline 14 & 0.00 & 0.00 & 0.00 & 0.00 & 0.00 & 0,00 \\
\hline 30 & 0.00 & 0.00 & 0.00 & 0.00 & 0.00 & 0.00 \\
\hline 60 & 0.07 & 0.00 & 0.00 & 0.00 & 0.00 & 0.00 \\
\hline 90 & 0.18 & 0.04 & 0.01 & 0.00 & 0.00 & 0.00 \\
\hline 120 & 0.26 & 0.09 & 0.03 & 0.00 & 0.00 & 0.00 \\
\hline 183 & 0.44 & 0.15 & 0.07 & 0.04 & 0.02 & 0.01 \\
\hline
\end{tabular}

MAGNITUDE AND PROBABILITY OF ANNUAL HIGH FLOW BASED ON PERIOD OF RECORD 1968.76, 1990.95

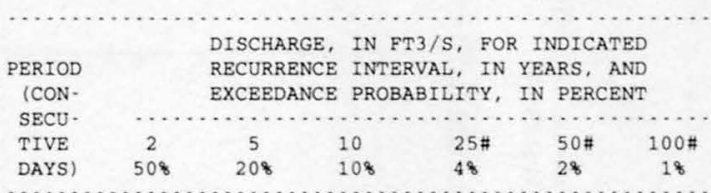

\begin{tabular}{|c|c|c|c|c|c|c|}
\hline 1 & 21 & 58 & 91 & 137 & 174 & 211 \\
\hline 3 & 15 & 40 & 61 & 92 & 117 & 143 \\
\hline 7 & 11 & 27 & 42 & 64 & 83 & 104 \\
\hline 15 & 7.8 & 19 & 29 & 44 & 56 & 69 \\
\hline 30 & 5.6 & 14 & 21 & 33 & 42 & 52 \\
\hline 60 & 4.1 & 10 & 15 & 23 & 29 & 36 \\
\hline 90 & 3.4 & 8.1 & 12 & 18 & 23 & 28 \\
\hline
\end{tabular}

DURATION TABLE OF DAILY MEAN FLOW FOR PERIOD OF RECORD 1968.76, 1990.95

DISCHARGE, IN FT3/S, WHICH WAS EQUALED OR EXCEEDED FOR INDICATED PERCENT OF TIME

\begin{tabular}{|c|c|c|c|c|c|c|c|c|c|c|c|c|c|c|c|c|}
\hline 18 & 58 & 108 & 158 & 208 & 308 & 408 & 508 & 608 & 708 & 808 & 908 & 958 & 988 & 998 & 99.58 & 99.98 \\
\hline 21 & 7.7 & 4.7 & 3.2 & 2.6 & 1.4 & 0.87 & 0.60 & 0.41 & 0.29 & 0.18 & 0.04 & 0.00 & 0.00 & 0.00 & 0.00 & 0.00 \\
\hline
\end{tabular}

\# Reliability of values in column is uncertain, and potential errors are large. 
09460150 FRYE CREEK NEAR THATCHER, AZ--Continued
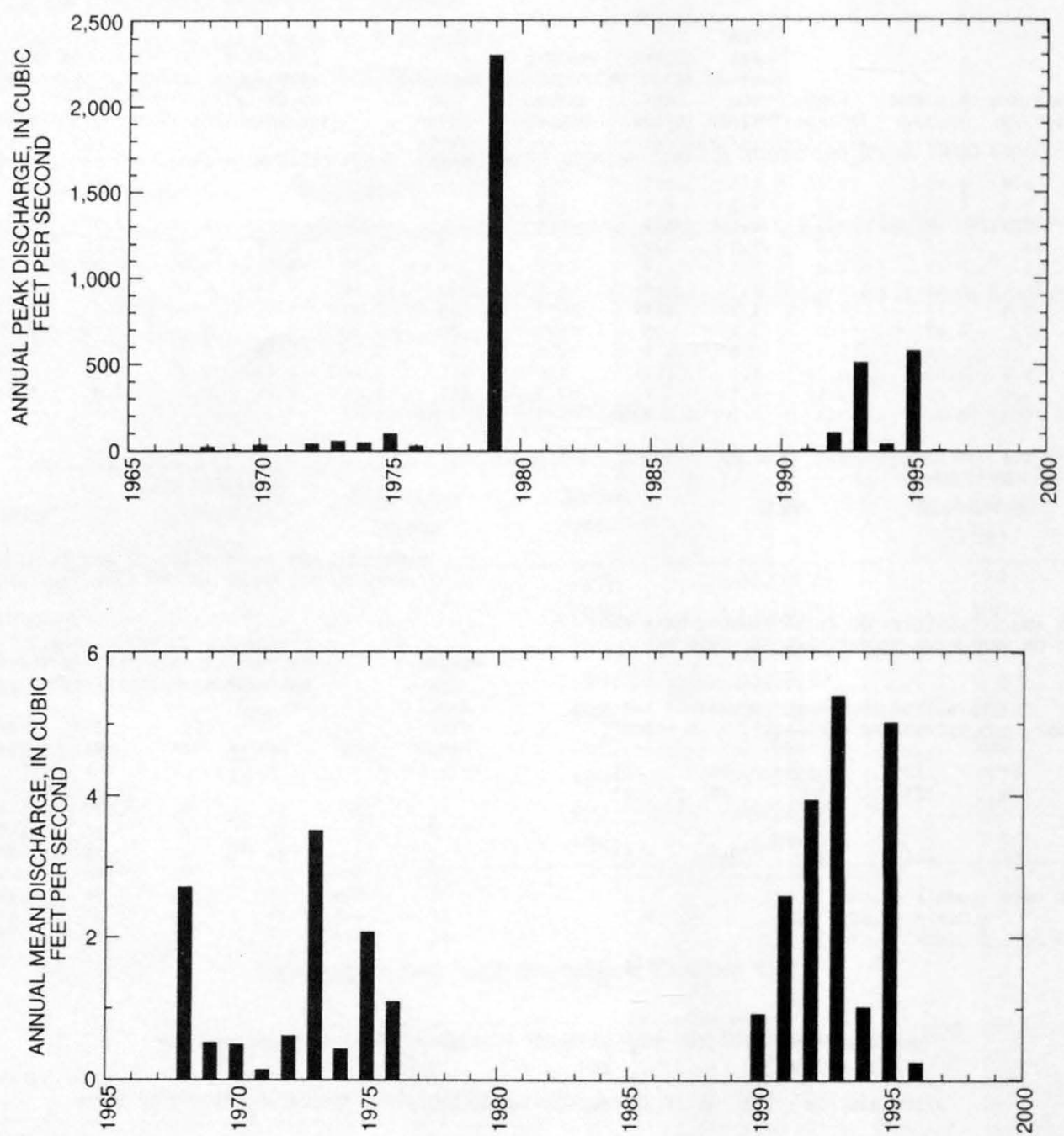
GILA RIVER BASIN

315

09460150 FRYE CREEK NEAR THATCHER, AZ--Continued
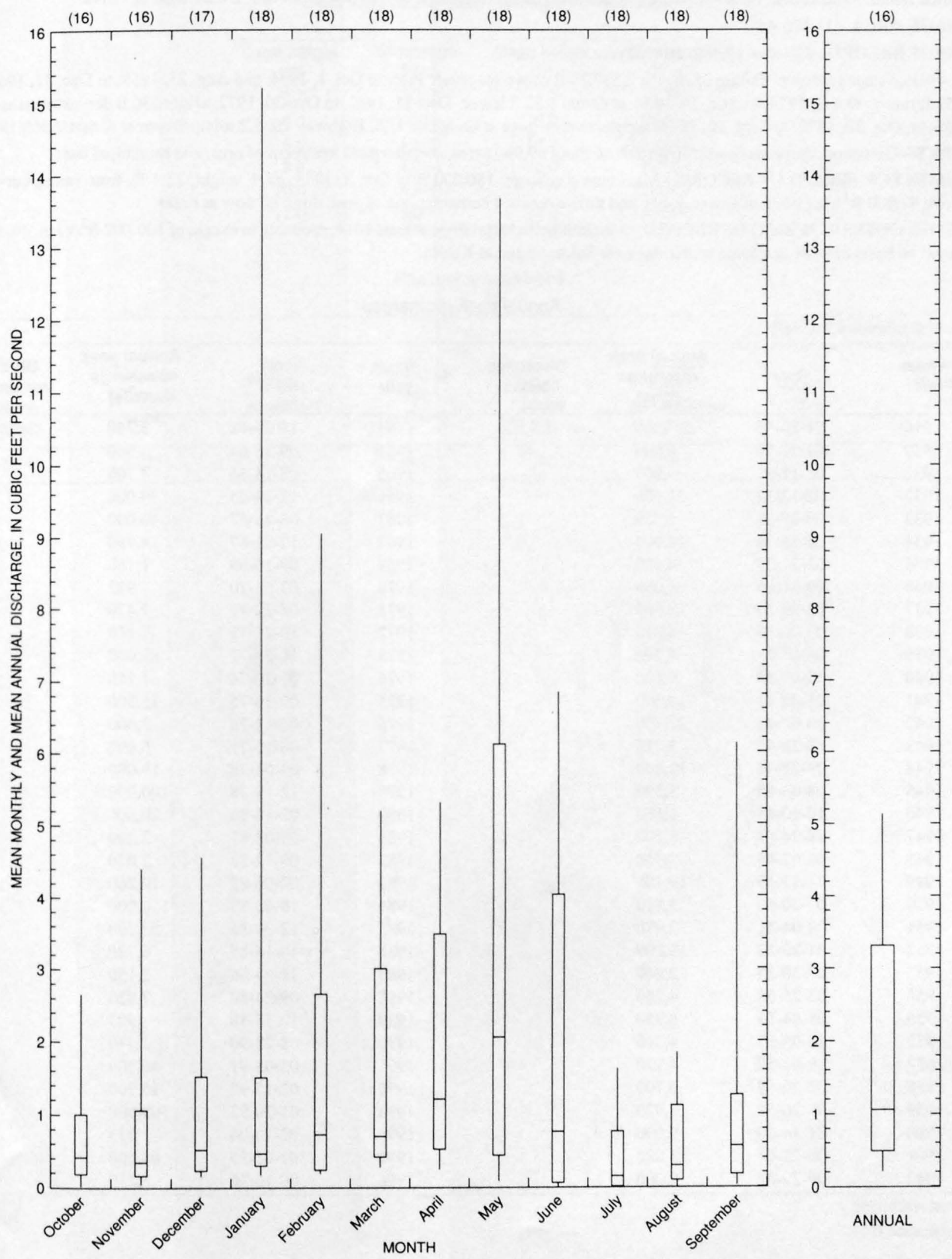


\section{GILA RIVER AT CALVA, AZ}

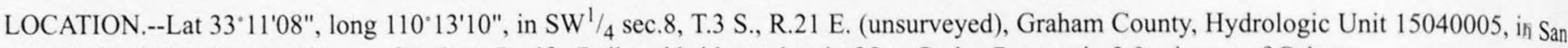
Carlos Indian Reservation, on Southern Pacific Railroad bridge at head of San Carlos Reservoir, 2.0 mi west of Calva.

DRAINAGE AREA.--11,470 $\mathrm{mi}^{2}$.

PERIOD OF RECORD,--October 1929 to current year.

GAGE.--Water-stage recorder. Datum of gage is 2,517.29 ft above sea level. Prior to Oct. 1, 1954, and Aug. 25, 1958, to Dec. 31, 1962, at datum $2.52 \mathrm{ft}$ lower. Oct. 1, 1954, to Aug. 24, 1958, at datum $5.52 \mathrm{ft}$ lower. Dec. 31, 1962, to Oct. 20, 1972, at site $530 \mathrm{ft}$ downstream at datum 3.65 ft lower. Oct. 20, 1972, to Sept. 30, 1974, supplementary gage at bridge on U.S. Highway 70, 6.2 mi upstream at datum 2,560.19 ft, NGVD. REMARKS.--Diversion above station for irrigation of about 69,000 acres, metallurgical treatment of ores, and municipal uses.

EXTREMES FOR PERIOD OF RECORD.--Maximum discharge, 150,000 ft $3 / \mathrm{s}$ Oct. 3, 1983, gage height, $23.1 \mathrm{ft}$, from rating curve extended above $87,000 \mathrm{ft}^{3} / \mathrm{s}$ on basis of area-velocity and flow-over-road computations of peak flow; no flow at times.

EXTREMES OUTSIDE PERIOD OF RECORD.--Maximum discharge since at least 1914, probably in excess of $100,000 \mathrm{ft}^{3} / \mathrm{s}^{\mathrm{J}} \mathrm{jan} .20,1916$, determined on basis of peak discharge at stations near Solomon and at Kelvin.

Annual peak discharges

\begin{tabular}{|c|c|c|c|c|c|c|c|}
\hline $\begin{array}{l}\text { Water } \\
\text { year }\end{array}$ & Date & $\begin{array}{c}\text { Annual peak } \\
\text { discharge } \\
\left(\mathrm{ft}^{3} / \mathrm{s}\right)\end{array}$ & $\begin{array}{l}\text { Discharge } \\
\text { codes }\end{array}$ & $\begin{array}{l}\text { Water } \\
\text { year }\end{array}$ & Date & $\begin{array}{l}\text { Annual peak } \\
\text { discharge } \\
\left(\mathrm{ft}^{3} / \mathrm{s}\right)\end{array}$ & $\begin{array}{l}\text { Discharge } \\
\text { codes }\end{array}$ \\
\hline 1916 & $01-20-16$ & ${ }^{\mathrm{I}} 100,000$ & ES,HP & 1963 & $10-20-62$ & 3,240 & \\
\hline 1930 & $07-29-30$ & 9,600 & & 1964 & $09-26-64$ & 3,060 & \\
\hline 1931 & $08-11-31$ & 9,900 & & 1965 & $08-14-65$ & 4,700 & \\
\hline 1932 & $02-12-32$ & 21,500 & & 1966 & $12-24-65$ & 39,000 & \\
\hline 1933 & $09-09-33$ & 6,560 & & 1967 & $08-13-67$ & 40,000 & \\
\hline 1934 & $08-28-34$ & 18,000 & & 1968 & $12-21-67$ & 8,960 & \\
\hline 1935 & $07-31-35$ & 4,470 & & 1969 & $09-14-69$ & 1,160 & \\
\hline 1936 & $09-11-36$ & 6,000 & & 1970 & $03-03-70$ & 982 & \\
\hline 1937 & $02-09-37$ & 12,800 & & 1971 & $08-22-71$ & 7,470 & \\
\hline 1938 & $03-05-38$ & 4,310 & & 1972 & $10-28-71$ & 7,160 & \\
\hline 1939 & $08-07-39$ & 4,260 & & 1973 & $10-20-72$ & 80,000 & \\
\hline 1940 & $10-09-39$ & 5,620 & & 1974 & $07-20-74$ & 1,160 & \\
\hline 1941 & $01-02-41$ & 14,300 & & 1975 & $09-10-75$ & 15,800 & \\
\hline 1942 & $10-01-41$ & 27,900 & & 1976 & $02-12-76$ & 2,600 & \\
\hline 1943 & $09-28-43$ & 3,710 & & 1977 & $08-15-77$ & 6,090 & \\
\hline 1944 & $09-27-44$ & 12,800 & & 1978 & 03-04-78 & 19,000 & \\
\hline 1945 & $08-03-45$ & 3,390 & & 1979 & $12-19-78$ & 100,000 & \\
\hline 1946 & $10-10-45$ & 4,680 & & 1980 & $02-16-80$ & 20,600 & \\
\hline 1947 & $08-24-47$ & 3,200 & & 1981 & $08-02-81$ & 2,200 & \\
\hline 1948 & $08-07-48$ & 2,570 & & 1982 & $09-12-82$ & 2,020 & \\
\hline 1949 & $01-15-49$ & 19,400 & & 1983 & $02-06-83$ & 10,260 & \\
\hline 1950 & $07-30-50$ & 3,210 & & 1984 & $10-03-83$ & ${ }^{2} 150,000$ & \\
\hline 1951 & $08-04-51$ & 2,970 & & 1985 & $12-29-84$ & 53,700 & \\
\hline 1952 & $01-20-52$ & 13,200 & & 1986 & $10-18-85$ & 6,720 & \\
\hline 1953 & $07-30-53$ & 2,040 & & 1987 & $11-04-86$ & 2,150 & \\
\hline 1954 & $03-25-54$ & 4,260 & & 1988 & $09-24-88$ & 7,820 & \\
\hline 1955 & $08-04-55$ & 4,950 & & 1989 & $10-16-88$ & 903 & \\
\hline 1956 & $10-05-55$ & 4,240 & & 1990 & $08-23-90$ & 1,110 & \\
\hline 1957 & $09-01-57$ & 4,220 & & 1991 & 03-03-91 & 46,400 & \\
\hline 1958 & $03-26-58$ & 6,700 & & 1992 & $02-15-92$ & 15,700 & \\
\hline 1959 & $08-26-59$ & 3,920 & & 1993 & $01-20-93$ & 109,000 & \\
\hline 1960 & $01-14-60$ & 9,090 & & 1994 & $02-10-94$ & 553 & \\
\hline 1961 & $08-23-61$ & 3,080 & & 1995 & 01-06-95 & 64,500 & \\
\hline 1962 & $09-29-62$ & 9,000 & & 1996 & $09-16-96$ & 3,250 & \\
\hline
\end{tabular}

${ }^{1}$ Highest since 1906.

${ }^{2}$ Highest since 1907. 
09466500 GILA RIVER AT CALVA, AZ--Continued

Discharge rating table developed October 1996

\begin{tabular}{crcr}
\hline $\begin{array}{c}\text { Gage height } \\
(\mathbf{f t})\end{array}$ & $\begin{array}{c}\text { Discharge } \\
\left(\mathbf{f t}^{\mathbf{3}} / \mathbf{s}\right)\end{array}$ & $\begin{array}{c}\text { Gage height } \\
(\mathbf{f t})\end{array}$ & $\begin{array}{c}\text { Discharge } \\
\left(\mathbf{f t}^{\mathbf{3}} / \mathbf{s}\right)\end{array}$ \\
\hline 2.0 & 109 & 14.0 & 23,300 \\
4.0 & 618 & 16.0 & 38,000 \\
6.0 & 1,800 & 18.0 & 60,000 \\
8.0 & 4,250 & 20.0 & 91,000 \\
10.0 & 8,400 & 22.0 & 130,000 \\
12.0 & 15,000 & 24.0 & 180,000 \\
\hline
\end{tabular}

Basin characteristics

\begin{tabular}{cccccccc}
\hline $\begin{array}{c}\text { Main } \\
\text { channel } \\
\text { slope } \\
(\mathrm{ft} / \mathrm{mi})\end{array}$ & $\begin{array}{c}\text { Stream } \\
\text { length } \\
(\mathrm{mi})\end{array}$ & $\begin{array}{c}\text { Mean } \\
\text { basin } \\
\text { elevation } \\
(\mathrm{ft})\end{array}$ & $\begin{array}{c}\text { Forested } \\
\text { area } \\
\text { (percent) }\end{array}$ & $\begin{array}{c}\text { Soil } \\
\text { index }\end{array}$ & $\begin{array}{c}\text { Mean } \\
\text { annual } \\
\text { precipitation } \\
\text { (in) }\end{array}$ & $\begin{array}{c}\text { 2-year } \\
\text { (in) }\end{array}$ & $\begin{array}{c}50 \text {-year } \\
\text { (in) }\end{array}$ \\
\hline 20.4 & 225 & 5,650 & 44.0 & 2.6 & 15.5 & 1.7 \\
\hline
\end{tabular}


09466500 GILA RIVER AT CALVA, AZ--Continued

MEAN MONTHLY AND ANNUAL DISCHARGES 1930.96

\begin{tabular}{|c|c|c|c|c|c|c|}
\hline MONTH & $\begin{array}{l}\text { MAXIMUM } \\
(\text { FT3/S) }\end{array}$ & $\begin{array}{l}\text { MINIMUM } \\
\text { (FT3/S) }\end{array}$ & $\begin{array}{c}\text { MEAN } \\
(\text { FT3/S) }\end{array}$ & $\begin{array}{l}\text { STAN- } \\
\text { DARD } \\
\text { DEVIA- } \\
\text { TION } \\
\text { (FT3/S) }\end{array}$ & $\begin{array}{l}\text { COEFFI- } \\
\text { CIENT OF } \\
\text { VARI- } \\
\text { ATION }\end{array}$ & $\begin{array}{c}\text { PERCENT } \\
\text { OF } \\
\text { ANNUAL } \\
\text { RUNOFF }\end{array}$ \\
\hline OCTOBER & 8,490 & 0.00 & 348 & 1,180 & 3.4 & 7.5 \\
\hline NOVEMBER & 2,270 & 0.00 & 203 & 352 & 1.7 & 4.4 \\
\hline DECEMBER & 5,650 & 0.00 & 469 & 941 & 2.0 & 10.1 \\
\hline JANUARY & 16,300 & 22 & 796 & 2,110 & 2.7 & 17,1 \\
\hline FEBRUARY & 6,230 & 29 & 762 & 1.150 & 1.5 & 16.4 \\
\hline MARCH & 3,760 & 10 & 749 & 997 & 1.3 & 16.1 \\
\hline APRIL & 2,620 & 1.3 & 410 & 612 & 1.5 & 8.8 \\
\hline MAY & 3,080 & 1.3 & 229 & 503 & 2.2 & 4.9 \\
\hline JUNE & 1,270 & 0.00 & 54 & 163 & 3.0 & 1.2 \\
\hline JULY & 838 & 0.00 & 82 & 140 & 1.7 & 1.8 \\
\hline AUGUST & 1,660 & 0.00 & 308 & 392 & 1.3 & 6.6 \\
\hline SEPTEMBER & 1,680 & 0.00 & 247 & 334 & 1.3 & 5.3 \\
\hline ANNUAL & 2,450 & 29 & 387 & 453 & 1.2 & 100 \\
\hline
\end{tabular}

MAGNITUDE AND PROBABILITY OF INSTANTANEOUS PEAK FLOW BASED ON PERIOD OF RECORD 1916, 1930.96

DISCHARGE, IN FT3/S, FOR INDICATED RECURRENCE INTERVAL IN YEARS, AND EXCEEDANCE PROBABILITY, IN PERCENT

\begin{tabular}{|c|c|c|c|c|c|}
\hline 2 & 5 & 10 & 25 & 50 & 100 \\
\hline . & & & & & \\
\hline 6,760 & 19.600 & 35,500 & 68,700 & 106,900 & 161,000 \\
\hline WEIGHTED & D SKEW & $($ LOGS $)=$ & & & \\
\hline MEAN & & (LOGS $)=$ & & & \\
\hline STANDARD & D DEV. & $($ LOGS $)=$ & 53 & & \\
\hline
\end{tabular}

MAGNITUDE AND PROBABILITY OF ANNUAL LOW FLOW BASED ON PERIOD OF RECORD 1931.96

\begin{tabular}{|c|c|c|c|c|c|c|}
\hline $\begin{array}{l}\text { PERIOD } \\
\text { (CON- }\end{array}$ & \multirow{2}{*}{\multicolumn{6}{|c|}{$\begin{array}{r}\text { DISCHARGE, IN FT3/S, FOR INDICATED } \\
\text { RECURRENCE INTERVAL, IN YEARS, AND } \\
\text { NON-EXCEEDANCE PROBABILITY, IN PERCENT }\end{array}$}} \\
\hline SECU. & & & & & & \\
\hline TIVE & 2 & 5 & 10 & 20 & 50 & $100 \#$ \\
\hline DAYS) & 508 & 208 & 108 & 58 & 28 & 18 \\
\hline 1 & 0.00 & 0.00 & 0.00 & 0.00 & 0.00 & 0.00 \\
\hline 3 & 0.00 & 0.00 & 0.00 & 0.00 & 0.00 & 0.00 \\
\hline 7 & 0.15 & 0.00 & 0.00 & 0.00 & 0.00 & 0.00 \\
\hline 14 & 0.78 & 0.00 & 0.00 & 0.00 & 0.00 & 0.00 \\
\hline 30 & 2.2 & 0.02 & 0.00 & 0.00 & 0.00 & 0.00 \\
\hline 60 & 8.0 & 1.7 & 0.56 & 0.06 & 0.00 & 0.00 \\
\hline 90 & 18 & 5.7 & 2.8 & 0.79 & 0.00 & 0.00 \\
\hline 120 & 39 & 12 & 4.7 & 1.5 & 0.00 & 0.00 \\
\hline 183 & 82 & 35 & 22 & 14 & 9.0 & 6.5 \\
\hline
\end{tabular}

MAGNITUDE AND PROBABILITY OF ANNUAL HIGH FLOW BASED ON PERIOD OF RECORD 1930.96

\begin{tabular}{|c|c|c|c|c|c|c|}
\hline \multirow{4}{*}{$\begin{array}{l}\text { PERIOD } \\
\text { ICON- } \\
\text { SECU. }\end{array}$} & & \multicolumn{5}{|c|}{ DISCHARGE, IN FT3/S, FOR INDICATED } \\
\hline & & \multirow{2}{*}{\multicolumn{3}{|c|}{$\begin{array}{l}\text { RECURRENCE INTERVAL, I } \\
\text { EXCEEDANCE PROBABILITY }\end{array}$}} & \multicolumn{2}{|c|}{ YEARS, AND } \\
\hline & & & NCE PRO & ABILITY, & IN PER & ENT \\
\hline & & . & . & . & ....... & $\ldots \ldots \ldots$ \\
\hline TIVE & 2 & 5 & 10 & 25 & 50 & $100 \#$ \\
\hline DAYS) & 508 & 208 & 108 & 48 & 28 & 18 \\
\hline 1 & 4,300 & 13,300 & 25.100 & 51,100 & 82,400 & 128,000 \\
\hline 3 & 3,140 & 9,400 & 17,200 & 33,600 & 52,500 & 79,200 \\
\hline 7 & 2,130 & 6,080 & 10,700 & 19,800 & 29,700 & 43,000 \\
\hline 15 & 1,440 & 3,980 & 6,860 & 12,400 & 18,300 & 26.000 \\
\hline 30 & 983 & 2,590 & 4,350 & 7,640 & 11,000 & 15,400 \\
\hline 60 & 675 & 1,780 & 3,000 & 5,320 & 7,750 & 10,900 \\
\hline 90 & 529 & 1,410 & 2,410 & 4,330 & 6,380 & 9,100 \\
\hline
\end{tabular}

DURATION TABLE OF DAILY MEAN FLOW FOR PERIOD OF RECORD 1930.96

DISCHARGE, IN FT3/S, WHICH WAS EQUALED OR EXCEEDED FOR INDICATED PERCENT OF TIME

\begin{tabular}{|c|c|c|c|c|c|c|c|c|c|c|c|c|c|c|c|c|}
\hline 18 & 58 & 108 & 158 & 208 & 308 & 408 & 508 & 608 & 708 & 808 & 908 & 958 & $98 \%$ & 998 & 99.58 & 99.98 \\
\hline 300 & 1,770 & 826 & 513 & 360 & 198 & 115 & 69 & 43 & 26 & 12 & 2.3 & 0.00 & 0.00 & 0.00 & 0.00 & 0.00 \\
\hline
\end{tabular}

\# Reliability of values in column is uncertain, and potential errors are large. 
ANNUAL MEAN DISCHARGE, IN CUBIC FEET PER SECOND

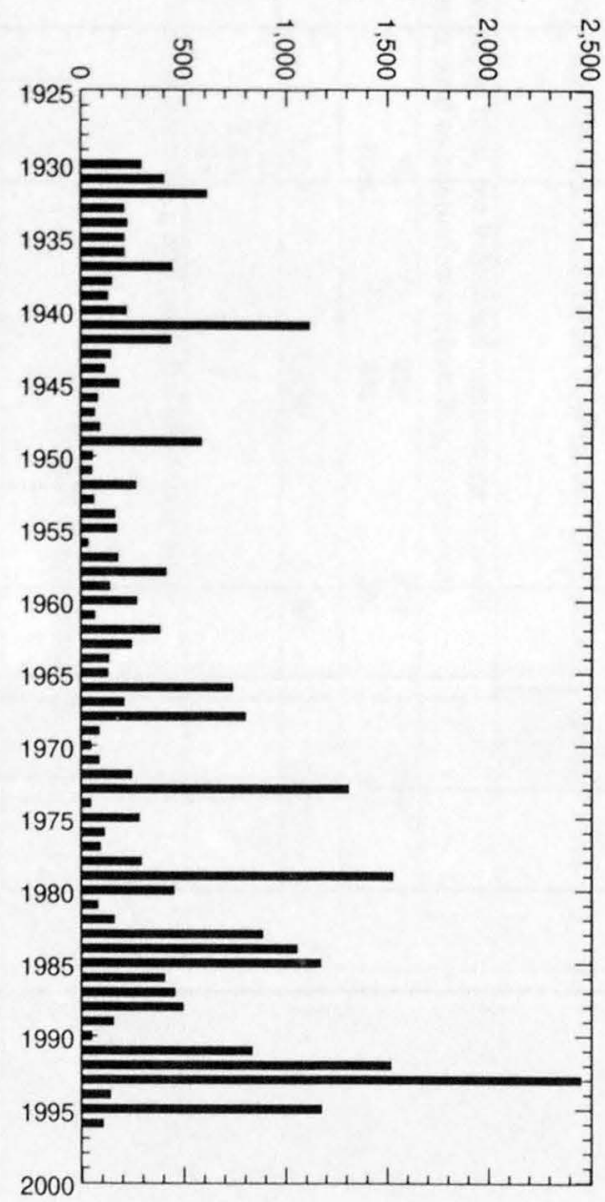

ANNUAL PEAK DISCHARGE, IN CUBIC FEET PER SECOND

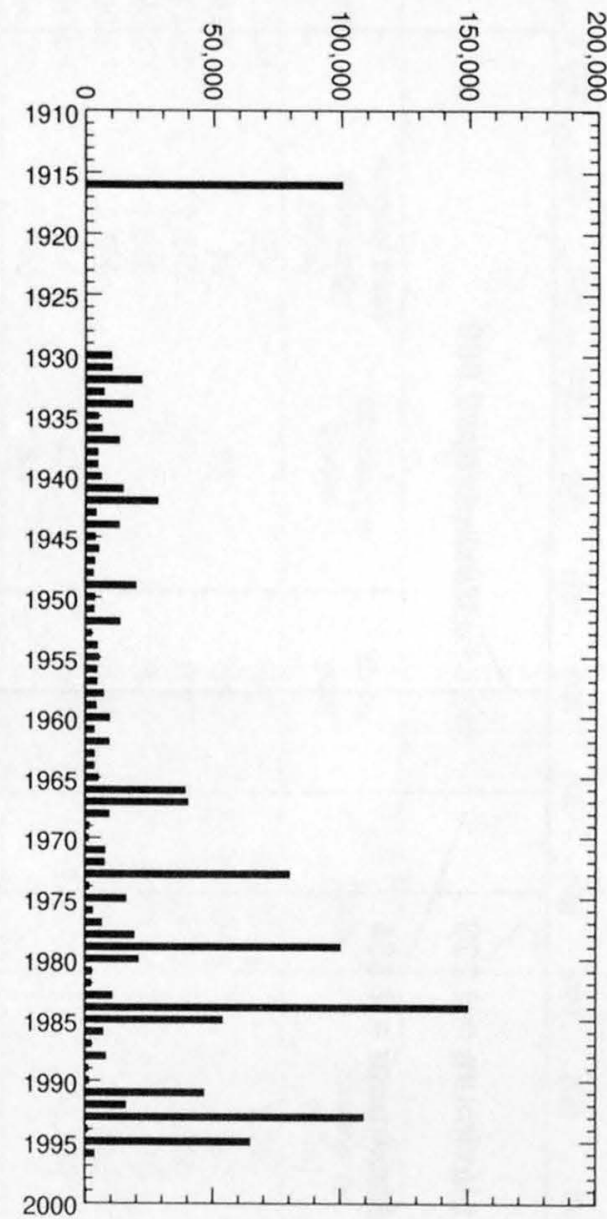


09466500 GILA RIVER AT CALVA, AZ--Continued

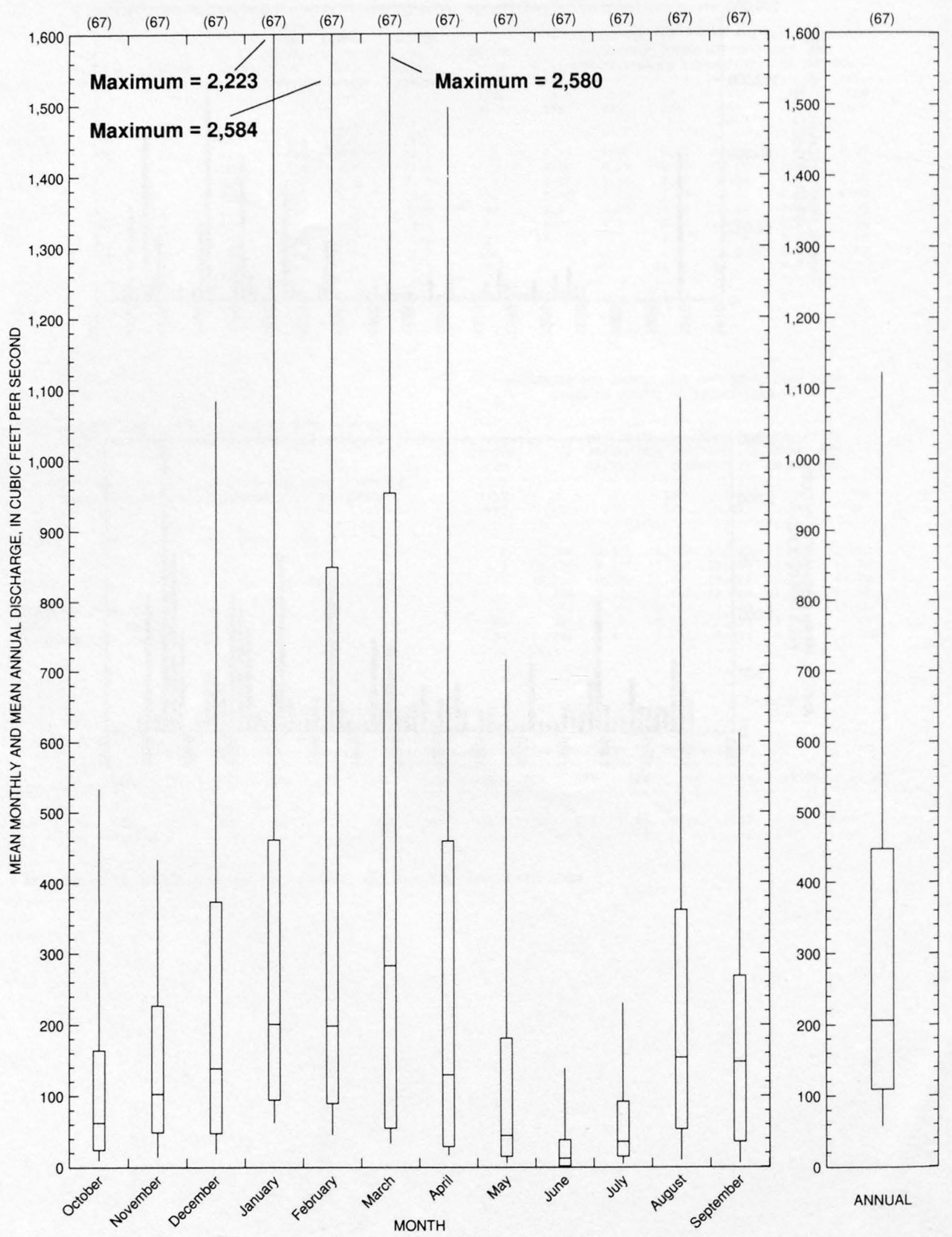


09467120 SALT CREEK NEAR PERIDOT, AZ

LOCATION.--Lat $33^{\circ} 16^{\prime} 15^{\prime \prime}$, long $110^{\circ} 18^{\prime} 15^{\prime \prime}$, Graham County, Hydrologic Unit 15040005 , at U.S. Highway $70,4 \mathrm{mi}$ above mouth, and $9.5 \mathrm{mi}$ southeast of Peridot.

DRAINAGE AREA.--35.1 $\mathrm{mi}^{2}$, of which $4.9 \mathrm{mi}^{2}$ is noncontributing.

Annual peak discharges

\begin{tabular}{|c|c|c|c|c|c|c|c|}
\hline $\begin{array}{l}\text { Water } \\
\text { year }\end{array}$ & Date & $\begin{array}{c}\text { Annual peak } \\
\text { discharge } \\
\left(\mathrm{ft}^{3} / \mathrm{s}\right)\end{array}$ & $\begin{array}{l}\text { Discharge } \\
\text { codes }\end{array}$ & $\begin{array}{l}\text { Water } \\
\text { year }\end{array}$ & Date & $\begin{array}{c}\text { Annual peak } \\
\text { discharge } \\
\left(\mathrm{ft}^{3} / \mathbf{s}\right)\end{array}$ & $\begin{array}{c}\text { Discharge } \\
\text { codes }\end{array}$ \\
\hline 1964 & $08-13-64$ & 604 & & 1971 & $09-30-71$ & 2,300 & \\
\hline 1965 & $01-03-65$ & 17 & ES & 1972 & $10-00-71$ & 517 & \\
\hline 1966 & $12-22-65$ & 1,270 & & 1973 & $10-19-72$ & 3,200 & \\
\hline 1967 & $08-06-67$ & 1,650 & & 1974 & $00-00-74$ & 0 & \\
\hline 1968 & $12-20-67$ & 2,500 & & 1975 & $07-20-75$ & 880 & \\
\hline 1969 & $00-00-69$ & 100 & LT & 1979 & $12-18-78$ & ${ }^{1} 2,200$ & HP \\
\hline 1970 & $09-06-70$ & 40 & ES & & & & \\
\hline
\end{tabular}

${ }^{1}$ Highest since 1975.

Magnitude and probability of instantaneous peak flow based on period of record 1964-75, 1979

\begin{tabular}{|c|c|c|c|c|c|}
\hline \multicolumn{6}{|c|}{$\begin{array}{l}\text { Discharge, in } \mathrm{ft}^{3} / \mathrm{s} \text {, for indicated recurrence interval } \\
\text { in years, and exceedance probablility, in percent }\end{array}$} \\
\hline 2 & 5 & 10 & $25 \dagger$ & $50 t$ & $100 \dagger$ \\
\hline $50 \%$ & $20 \%$ & $10 \%$ & $4 \%$ & $2 \%$ & $1 \%$ \\
\hline 810 & 1,920 & 2,890 & 4,350 & 5,580 & 6,910 \\
\hline Weighted skew & $(\log s)=$ & -0.40 & & & \\
\hline Mean & $(\log s)=$ & 2.88 & & & \\
\hline Standard dev. & $(\log s)=$ & 0.48 & & & \\
\hline
\end{tabular}

fReliability of values in column is uncertain, and potential errors are large.

Basin characteristics

\begin{tabular}{cccccccc}
\hline $\begin{array}{c}\text { Main } \\
\text { channel } \\
\text { slope } \\
(\mathrm{ft} / \mathrm{mi})\end{array}$ & $\begin{array}{c}\text { Stream } \\
\text { length } \\
(\mathrm{mi})\end{array}$ & $\begin{array}{c}\text { Mean } \\
\text { basin } \\
\text { elevation } \\
(\mathrm{ft})\end{array}$ & $\begin{array}{c}\text { Forested } \\
\text { area } \\
\text { (percent) }\end{array}$ & $\begin{array}{c}\text { Soil } \\
\text { index }\end{array}$ & $\begin{array}{c}\text { Mean } \\
\text { annual } \\
\text { precipitation } \\
\text { (in) }\end{array}$ & $\begin{array}{c}\text { Rainfall intensity, 24-hour } \\
\text { (in) }\end{array}$ & $\begin{array}{c}50 \text {-year } \\
\text { (in) }\end{array}$ \\
\hline 169 & 14.4 & 3,490 & 2.3 & 1.0 & 16.0 & 1.9 \\
\hline
\end{tabular}

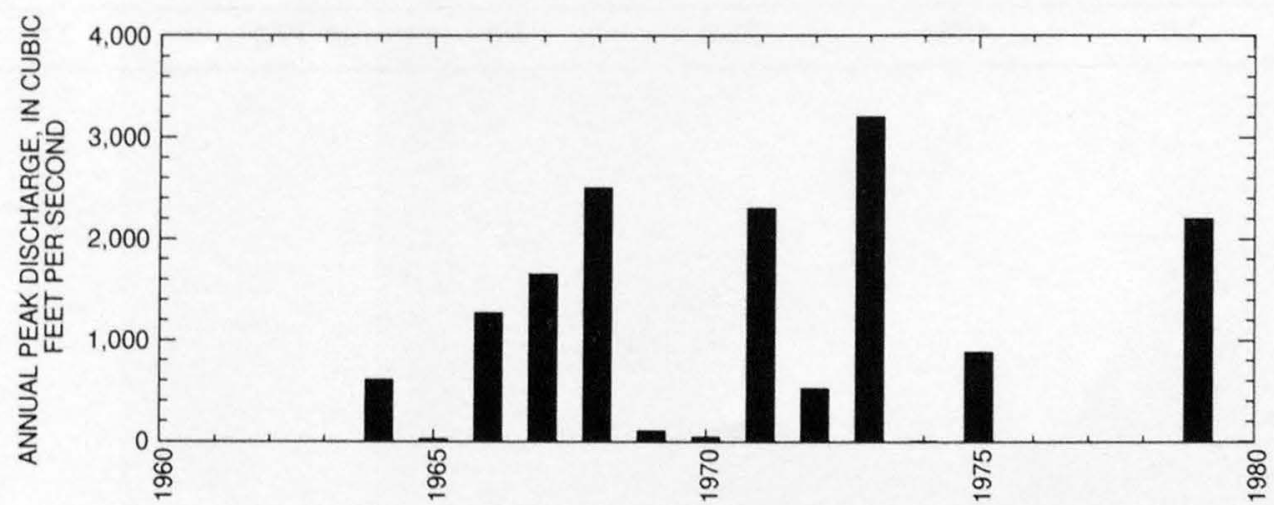


09468300 SEVENMILE WASH TRIBUTARY NEAR GLOBE, AZ

LOCATION.--Lat 33 35'10", long 110 39'00", Gila County, Hydrologic Unit 15040007, at U.S. Highway 60, 0.2 mi south of Sevenmile Wash, and

$15 \mathrm{mi}$ northwest of Globe.

DRAINAGE AREA.--0.83 $\mathrm{mi}^{2}$.

Annual peak discharges

\begin{tabular}{|c|c|c|c|c|c|c|c|}
\hline $\begin{array}{l}\text { Water } \\
\text { year }\end{array}$ & Date & $\begin{array}{c}\text { Annual peak } \\
\text { discharge } \\
\left(\mathrm{ft}^{3} / \mathrm{s}\right)\end{array}$ & $\begin{array}{l}\text { Discharge } \\
\text { codes }\end{array}$ & $\begin{array}{l}\text { Water } \\
\text { year }\end{array}$ & Date & $\begin{array}{c}\text { Annual peak } \\
\text { discharge } \\
\left(\mathrm{ft}^{3} / \mathrm{s}\right)\end{array}$ & $\begin{array}{l}\text { Discharge } \\
\text { codes }\end{array}$ \\
\hline$\ldots$ & -....... & $1_{526}$ & HP & 1970 & $08-00-70$ & 150 & \\
\hline 1962 & $00-00-62$ & 38 & & 1971 & $00-00-71$ & 50 & LT \\
\hline 1963 & $08-17-63$ & 22 & & 1972 & $10-17-71$ & 5.0 & ES \\
\hline 1964 & $07-31-64$ & 486 & & 1973 & $10-19-72$ & 245 & \\
\hline 1965 & $00-00-65$ & 0 & & 1974 & $07-19-74$ & 4.0 & \\
\hline 1966 & $12-10-65$ & 30 & & 1975 & $09-08-75$ & 0.1 & ES \\
\hline 1967 & $00-00-67$ & 0 & & 1976 & $00-00-76$ & 5.0 & ES \\
\hline 1968 & $08-04-68$ & 135 & & 1979 & $12-18-78$ & ${ }^{2} 640$ & HP \\
\hline 1969 & $08-27-69$ & 35 & & & & & \\
\hline
\end{tabular}

${ }^{1}$ Highest since 1933, year of occurrence unknown.

${ }^{2}$ Highest since 1933.

Magnitude and probability of instantaneous peak flow based on period of record 1933 ,

1962-76, 1979

\begin{tabular}{|c|c|c|c|c|c|}
\hline \multicolumn{6}{|c|}{$\begin{array}{l}\text { Discharge, in } \mathrm{ft}^{3} / \mathrm{s} \text {, for indicated recurrence interval } \\
\text { in years, and exceedance probablility, in percent }\end{array}$} \\
\hline 2 & 5 & 10 & 25 & $50 t$ & $100 \dagger$ \\
\hline $50 \%$ & $20 \%$ & $10 \%$ & $4 \%$ & $2 \%$ & $1 \%$ \\
\hline 27.3 & 134 & 295 & 660 & 1,090 & 1,690 \\
\hline Weighted skew & $(\log s)=$ & -0.26 & & & \\
\hline Mean & $(\log s)=$ & 1.40 & & & \\
\hline Standard dev. & $(\log s)=$ & 0.86 & & & \\
\hline
\end{tabular}

$\mp$ Reliability of values in column is uncertain, and potential errors are large.

Basin characteristics

\begin{tabular}{cccccccc}
\hline $\begin{array}{c}\text { Main } \\
\text { channel } \\
\text { slope } \\
(\mathrm{ft} / \mathrm{mi})\end{array}$ & $\begin{array}{c}\text { Stream } \\
\text { length } \\
(\mathrm{mi})\end{array}$ & $\begin{array}{c}\text { Mean } \\
\text { basin } \\
\text { elevation } \\
(\mathrm{ft})\end{array}$ & $\begin{array}{c}\text { Forested } \\
\text { area } \\
\text { (percent) }\end{array}$ & $\begin{array}{c}\text { Soil } \\
\text { index }\end{array}$ & $\begin{array}{c}\text { Mean } \\
\text { annual } \\
\text { precipitation } \\
\text { (in) }\end{array}$ & $\begin{array}{c}\text { R-year } \\
\text { (in) }\end{array}$ & $\begin{array}{c}\text { 50-year } \\
\text { (in) }\end{array}$ \\
\hline 280 & 2.0 & 4,410 & 36.0 & 3.0 & 19.0 & 2.0 \\
\hline
\end{tabular}


09468300 SEVENMILE WASH TRIBUTARY NEAR GLOBE, AZ--Continued

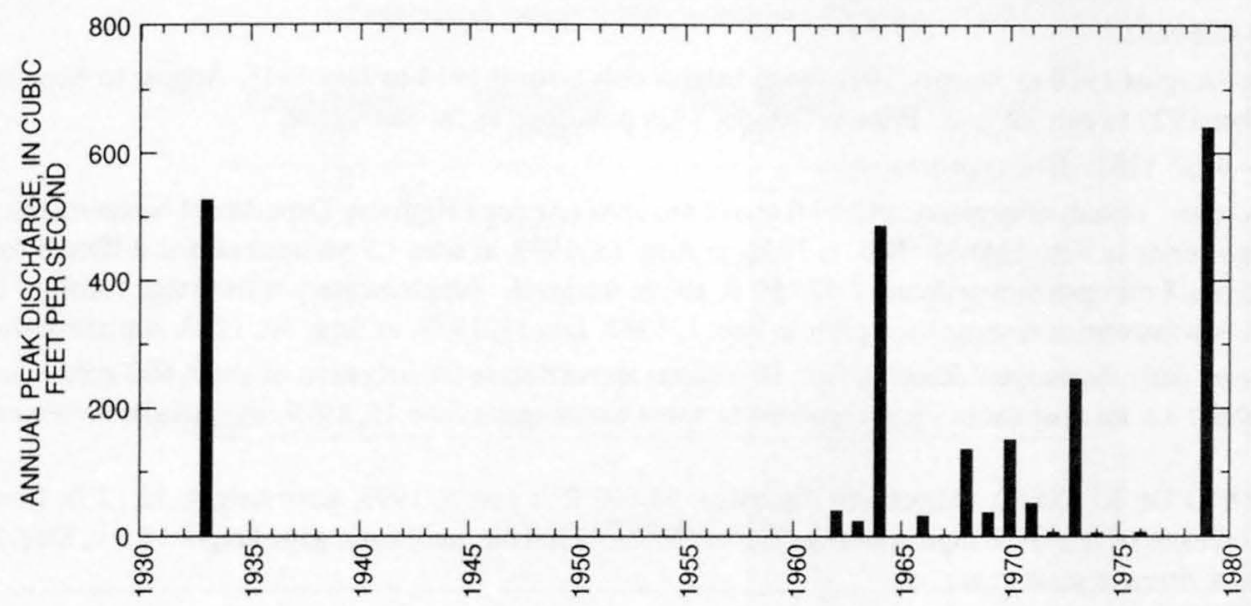




\section{SAN CARLOS RIVER NEAR PERIDOT, AZ}

LOCATION.--Lat 33'17'47", long 110 $27^{\prime} 03^{\prime \prime}$, in SE $1 / 4$ sec.36, T.1 S., R.18 E. (unsurveyed), Gila County, Hydrologic Unit 15040007 , in San Carlos Indian Reservation, on U.S. Highway 70 bridge, 0.9 mi south of Peridot.

DRAINAGE AREA.--1,026 $\mathrm{mi}^{2}$.

PERIOD OF RECORD.--August 1910 to January 1911 (gage heights only), April 1914 to July 1915, August to September 1915 (monthly discharge only), October 1929 to current year. Prior to October 1929 published as "at San Carlos."

REVISED RECORDS.--WSP 1283: Drainage area.

GAGE.--Water-stage recorder. Datum of gage is 2,542.29 ft above sea level (Arizona Highway Department bench mark). See WSP 1713 or 1733 for history of changes prior to Feb. 1, 1942. Feb. 1, 1942, to Aug. 13, 1970, at sites 1.9 mi upstream at different datums. Aug 14, 1970, to Sept. 30, 1980, at site $1.8 \mathrm{mi}$ upstream at datum 2,578.90 ft, above sea level. Supplementary water-stage recorder Dec. 21, 1967 , to July 2, 1968, at site $2.2 \mathrm{mi}$ downstream at datum in use prior to Feb. 1, 1942; Jan. 31, 1979, to Sept. 30, 1980, at present site and datum.

REMARKS.--No estimated daily discharges. Records fair. Diversions above station for irrigation of about 600 acres. Small inflow from sewage treatment system about 3.6 mi upstream. Flow regulated to some extent since June 15, 1979, by Talkalai Reservoir; capacity, about 6,000 acre-ft.

EXTREMES FOR PERIOD OF RECORD.--Maximum discharge, 54,800 $\mathrm{ft}^{3} / \mathrm{s}$ Jan. 8, 1993, gage height, $12.12 \mathrm{ft}$, from rating curve extended above $23,000 \mathrm{ft}^{3} / \mathrm{s}$ on basis of rate of change in storage in San Carlos Reservoir; maximum gage height 14.8 ft, Dec. 22, 1965, site and datum then in use; no flow at times in most years.

Annual peak discharges

\begin{tabular}{|c|c|c|c|c|c|c|c|}
\hline $\begin{array}{l}\text { Water } \\
\text { year }\end{array}$ & Date & $\begin{array}{c}\text { Annual peak } \\
\text { discharge } \\
\left(\mathrm{ft}^{3} / \mathrm{s}\right)\end{array}$ & $\begin{array}{c}\text { Discharge } \\
\text { codes }\end{array}$ & $\begin{array}{l}\text { Water } \\
\text { year }\end{array}$ & Date & $\begin{array}{c}\text { Annual peak } \\
\text { discharge } \\
\left(\mathrm{ft}^{3} / \mathrm{s}\right)\end{array}$ & $\begin{array}{c}\text { Discharge } \\
\text { codes }\end{array}$ \\
\hline 1916 & $01-18-16$ & 25,000 & ES,HP & 1963 & $02-11-63$ & 9,740 & \\
\hline 1930 & $03-17-30$ & 5,700 & & 1964 & $07-25-64$ & 6,610 & \\
\hline 1931 & $08-07-31$ & 7,000 & & 1965 & $01-08-65$ & 1,710 & \\
\hline 1932 & $02-10-32$ & 12,000 & & 1966 & $12-22-65$ & 36,300 & \\
\hline 1933 & 09-08-33 & 11,000 & & 1967 & $07-29-67$ & 16,100 & \\
\hline 1934 & $08-18-34$ & 8,200 & & 1968 & $12-20-67$ & 32,000 & \\
\hline 1935 & $02-07-35$ & 13,800 & & 1969 & $01-22-69$ & 4,580 & \\
\hline 1936 & $02-17-36$ & 14,400 & & 1970 & $09-06-70$ & 5,080 & \\
\hline 1937 & $02-07-37$ & 29,400 & & 1971 & $08-14-71$ & 7,930 & \\
\hline 1938 & $03-04-38$ & 8,640 & & 1972 & $10-17-71$ & 4,970 & \\
\hline 1939 & 08-03-39 & 10,200 & & 1973 & $10-19-72$ & 25,000 & \\
\hline 1940 & $08-03-40$ & 6,000 & & 1974 & $07-20-74$ & 7,800 & \\
\hline 1941 & $03-14-41$ & 40,600 & & 1975 & $04-11-75$ & 1,960 & \\
\hline 1942 & $12-12-41$ & 2,520 & & 1976 & $09-25-76$ & 12,000 & \\
\hline 1943 & $09-26-43$ & 5,060 & & 1977 & $09-11-77$ & 5,400 & \\
\hline 1944 & $09-27-44$ & 795 & & 1978 & $03-02-78$ & 18,600 & \\
\hline 1945 & $08-09-45$ & 3,200 & & 1979 & $12-18-78$ & 22,500 & \\
\hline 1946 & $07-27-46$ & 4,530 & & 1980 & $02-15-80$ & 12,300 & \\
\hline 1947 & $08-08-47$ & 15,000 & & 1981 & $09-22-81$ & 565 & \\
\hline 1948 & $08-02-48$ & 2,850 & & 1982 & $02-11-82$ & 6,260 & \\
\hline 1949 & $01-09-49$ & 3,260 & & 1983 & $03-25-83$ & 6,260 & \\
\hline 1950 & $07-21-50$ & 2,150 & & 1984 & $10-01-83$ & 10,300 & \\
\hline 1951 & $08-29-51$ & 2,940 & & 1985 & $12-27-84$ & 4,470 & \\
\hline 1952 & $01-13-52$ & 39,200 & & 1986 & $07-16-86$ & 3,240 & \\
\hline 1953 & $08-27-53$ & 860 & & 1987 & $12-07-86$ & 4,130 & \\
\hline 1954 & $03-23-54$ & 23,500 & & 1988 & $08-27-88$ & 3,400 & \\
\hline 1955 & $08-06-55$ & 14,600 & & 1989 & $08-03-89$ & 3,160 & \\
\hline 1956 & $01-29-56$ & 9,300 & & 1990 & $08-14-90$ & 12,900 & \\
\hline 1957 & $07-26-57$ & 7,310 & & 1991 & $03-01-91$ & 13,900 & \\
\hline 1958 & $03-22-58$ & 7,670 & & 1992 & $02-13-92$ & 13,200 & \\
\hline 1959 & $08-18-59$ & 2,280 & & 1993 & $01-08-93$ & 54,800 & \\
\hline 1960 & $12-26-59$ & 14,300 & & 1994 & $03-20-94$ & 2,940 & \\
\hline 1961 & $07-22-61$ & 5,510 & & 1995 & $01-05-95$ & 27,900 & \\
\hline 1962 & $09-26-62$ & 4,400 & & 1996 & $09-11-96$ & 1,990 & \\
\hline
\end{tabular}


09468500 SAN CARLOS RIVER NEAR PERIDOT, AZ--Continued

Discharge rating table developed October 1996

\begin{tabular}{crcr}
\hline $\begin{array}{c}\text { Gage height } \\
\text { (ft) }\end{array}$ & $\begin{array}{c}\text { Discharge } \\
\left(\mathbf{f t}^{\mathbf{3}} \mathbf{/ s}\right)\end{array}$ & $\begin{array}{c}\text { Gage height } \\
(\mathbf{f t})\end{array}$ & $\begin{array}{c}\text { Discharge } \\
\left(\mathbf{f t}^{\mathbf{3}} / \mathbf{s}\right)\end{array}$ \\
\hline 6.0 & 276 & 10.0 & 33,500 \\
7.0 & 2,150 & 10.5 & 45,150 \\
7.5 & 4,210 & 11.0 & 59,300 \\
8.0 & 7,300 & 11.5 & 71,930 \\
8.5 & 11,550 & 12.0 & 86,000 \\
9.0 & 17,200 & 12.5 & 102,200 \\
9.5 & 24,450 & 13.0 & 120,000 \\
\hline
\end{tabular}

Basin characteristics

\begin{tabular}{cccccccc}
\hline $\begin{array}{c}\text { Main } \\
\text { channel } \\
\text { slope } \\
\text { (ft/mi) }\end{array}$ & $\begin{array}{c}\text { Stream } \\
\text { length } \\
\text { (mi) }\end{array}$ & $\begin{array}{c}\text { Mean } \\
\text { basin } \\
\text { elevation } \\
\text { (ft) }\end{array}$ & $\begin{array}{c}\text { Forested } \\
\text { area } \\
\text { (percent) }\end{array}$ & $\begin{array}{c}\text { Soil } \\
\text { index }\end{array}$ & $\begin{array}{c}\text { Mean } \\
\text { annual } \\
\text { precipitation } \\
\text { (in) }\end{array}$ & $\begin{array}{c}\text { Rainfall intensity, 24-hour } \\
\text { (in) }\end{array}$ & $\begin{array}{c}50 \text {-year } \\
\text { (in) }\end{array}$ \\
\hline 29.4 & 56.7 & 4,480 & 10 & 2.0 & 17.2 & 2.1 \\
\hline
\end{tabular}


09468500 SAN CARLOS RIVER NEAR PERIDOT, AZ--Continued

MEAN MONTHLY AND ANNUAL DISCHARGES 1930.96

\begin{tabular}{|c|c|c|c|c|c|c|}
\hline MONTH & $\begin{array}{l}\text { MAXIMUM } \\
\text { (FT3/S) }\end{array}$ & $\begin{array}{l}\text { MINIMUM } \\
\text { (FT3/S) }\end{array}$ & $\begin{array}{c}\text { MEAN } \\
(\mathrm{FT} 3 / \mathrm{S})\end{array}$ & $\begin{array}{l}\text { STAN- } \\
\text { DARD } \\
\text { DEVIA- } \\
\text { TION } \\
\text { (FT3/S) }\end{array}$ & $\begin{array}{l}\text { COEFFI- } \\
\text { CIENT OF } \\
\text { VARI- } \\
\text { ATION }\end{array}$ & $\begin{array}{c}\text { PBRCENT } \\
\text { OF } \\
\text { ANNUAL } \\
\text { RUNOFF }\end{array}$ \\
\hline OCTOBER & 519 & 0.20 & 30 & 83 & 2.8 & 3.9 \\
\hline NOVEMBER & 178 & 2.7 & 19 & 25 & 1.3 & 2.5 \\
\hline DECEMBER & 1,580 & 5.1 & 111 & 245 & 2.2 & 14.5 \\
\hline JANUARY & 3,210 & 5.8 & 151 & 421 & 2.8 & 19.8 \\
\hline FEBRUARY & 1,500 & 7.0 & 170 & 280 & 1.6 & 22.3 \\
\hline MARCH & 1,260 & 4.8 & 149 & 254 & 1.7 & 19.6 \\
\hline APRIL & 170 & 2.2 & 24 & 31 & 1.3 & 3.1 \\
\hline MAY & 42 & 0.03 & 7.8 & 7.6 & 0.97 & 1.0 \\
\hline JUNE & 20 & 0.00 & 3.6 & 4.4 & 1.2 & 0.5 \\
\hline JULY & 85 & 0.00 & 19 & 20 & 1.1 & 2.4 \\
\hline AUGUST & 320 & 1.6 & 55 & 66 & 1.2 & 7.2 \\
\hline SEPTEMBER & 166 & 0.00 & 25 & 29 & 1.2 & 3.2 \\
\hline ANNUAL & 426 & 8.2 & 63 & 71 & 1.1 & 100 \\
\hline
\end{tabular}

MAGNITUDE AND PROBABILITY OF ANNUAL LOW FLOW BASED ON PERIOD OF RECORD 1931.96

PERIOD
(CON -

MAGNITUDE AND PROBABILITY OF ANNUAL HIGH FLOW BASED ON PERIOD OF RECORD 1930-96

\begin{tabular}{|c|c|c|c|c|c|c|}
\hline \multirow{4}{*}{$\begin{array}{l}\text { PERIOD } \\
\text { (CON- } \\
\text { SECU- }\end{array}$} & \multicolumn{6}{|c|}{ DISCHARGE, IN FT3/S, FOR INDICATED } \\
\hline & & RECURR & CE INTI & RVAL， IN & YEARS, & AND \\
\hline & & EXCEED & CE PROF & ABILITY, & IN PER & ENT \\
\hline & & 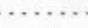 & & & (ence & encose \\
\hline TIVE & 2 & 5 & 10 & 25 & 50 & $100 \#$ \\
\hline DAYS) & 508 & 208 & 108 & 48 & 28 & 18 \\
\hline 1 & 1.910 & 5,390 & 9,400 & 17,200 & 25,500 & 36,500 \\
\hline 3 & 1,070 & 3,110 & 5,520 & 10,300 & 15,500 & 22,500 \\
\hline 7 & 592 & 1,680 & 2,950 & 5,430 & 8,120 & 11.700 \\
\hline 15 & 341 & 938 & 1,630 & 2,990 & 4,470 & 6,470 \\
\hline 30 & 210 & 562 & 963 & 1,750 & 2,590 & 3,720 \\
\hline 60 & 135 & 357 & 609 & 1,100 & 1,630 & 2,330 \\
\hline 90 & 101 & 270 & 463 & 842 & 1,250 & 1,810 \\
\hline
\end{tabular}

MAGNITUDE AND PROBABILITY OF INSTANTANEOUS PEAK FLOW BASED ON PERIOD OF RECORD 1930.96

DISCHARGE, IN FT3/S, FOR INDICATED RECURRENCE INTERVAI IN YEARS, AND EXCEEDANCE PROBABILITY, IN PERCENT

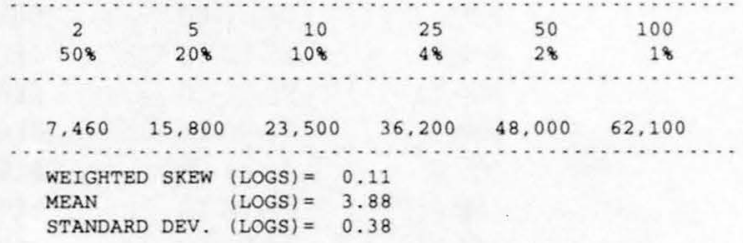

DURATION TABLE OF DAILY MEAN FLOW FOR PERIOD OF RECORD 1930.96

DISCHARGE, IN FT3/S, WHICH WAS EQUALED OR EXCEEDED FOR INDICATED PERCENT OF TIME

\begin{tabular}{|c|c|c|c|c|c|c|c|c|c|c|c|c|c|c|c|c|}
\hline 18 & 58 & 108 & 158 & 208 & 308 & 408 & 508 & 608 & 708 & 808 & 908 & 958 & 988 & 998 & 99.58 & 99.98 \\
\hline 90 & 162 & 69 & 41 & 28 & 18 & 13 & 10 & 7.6 & 5.4 & 3.4 & 1.1 & 0.00 & 0.00 & 0.00 & 0.00 & 0.00 \\
\hline
\end{tabular}

\# Reliability of values in column is uncertain, and potential errors are large. 
ANNUAL MEAN DISCHARGE, IN CUBIC
FEET PER SECOND

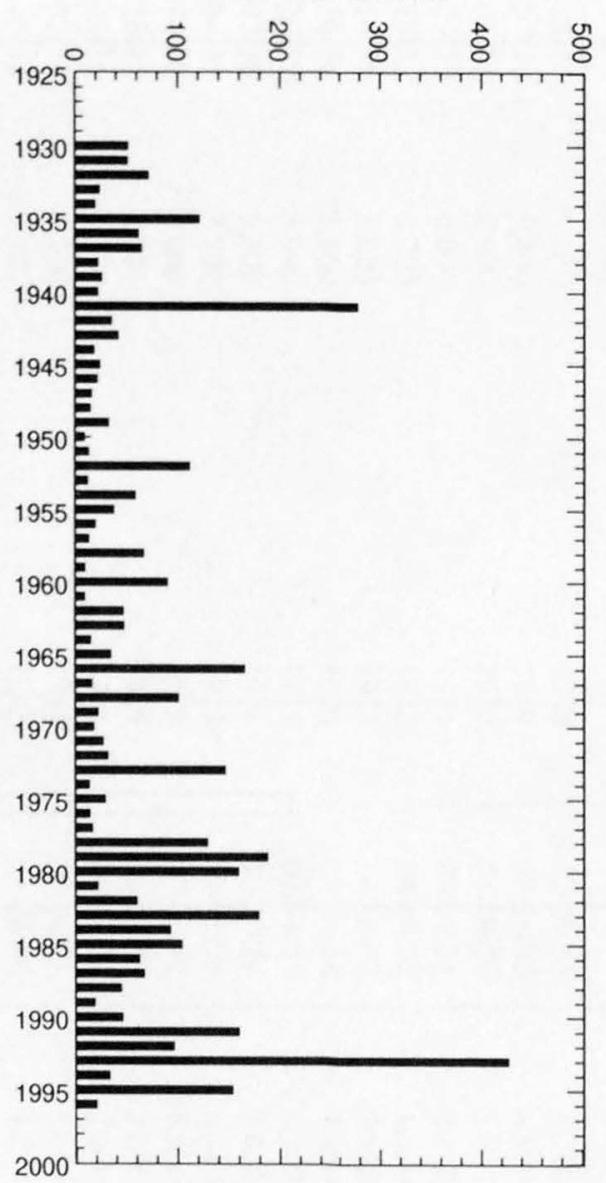

ANNUAL PEAK DISCHARGE, IN CUBIC FEET PER SECOND

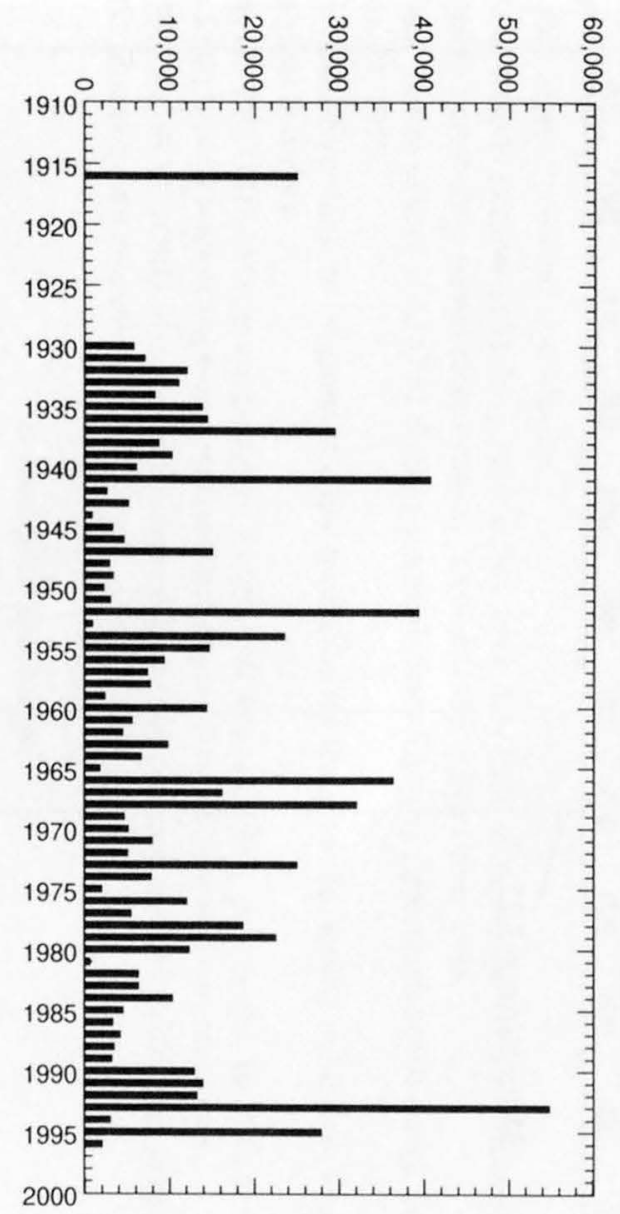


09468500 SAN CARLOS RIVER NEAR PERIDOT, AZ--Continued
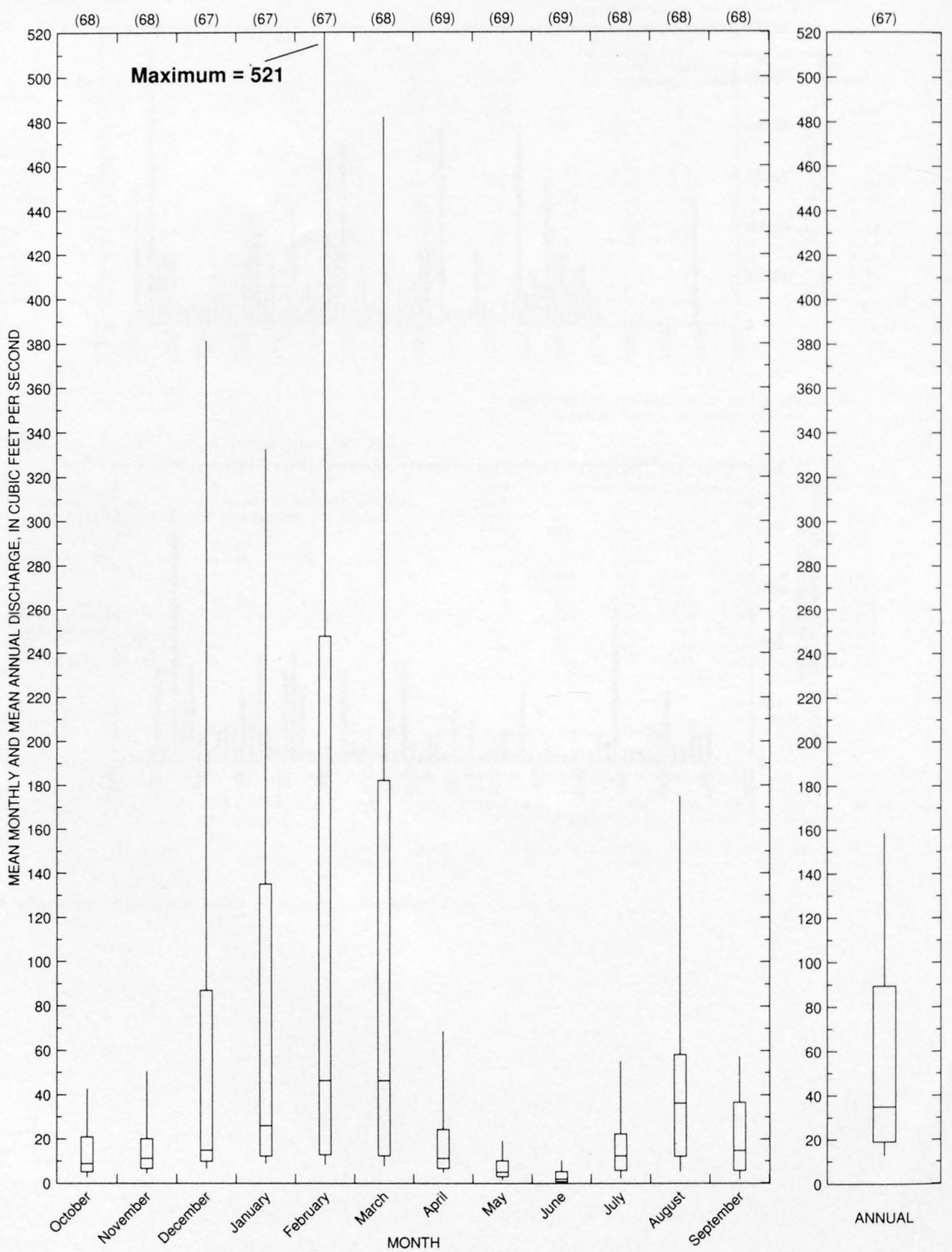


\section{SAN PEDRO RIVER AT PALOMINAS, AZ}

LOCATION.--Lat 31 '22'48", long 110'06'38", in SW $1 / 4 \mathrm{SE}^{1} / 4$ sec.33, T.23 S., R.22 E., Cochise County, Hydrologic Unit 15050202, near left bank on downstream side of pier of bridge on State Highway 92, 0.7 mi east of Palominas, 2.5 mi upstream from Green Brush Draw, 4.5 mi downstream from international boundary, and 12 mi southwest of Bisbee.

DRAINAGE AREA.--741 $\mathrm{mi}^{2}$, of which $649 \mathrm{mi}^{2}$ is in Mexico.

PERIOD OF RECORD.--May 1930 to October 1933, May 1935 to July 1941, July 1950 to September 30, 1981 (discontinued as a continuousrecord station; converted to a crest-stage partial-record station). October 1995 to September 1996.

GAGE.--Water-stage recorder. Datum of gage is $4,187.62 \mathrm{ft}$ above sea level (State Highway Department bench mark). See WSP 1733 for history of changes prior to Nov. 24, 1955.

REMARKS.--Records fair. Small diversions for irrigation of a few hundred acres above station, mostly in Mexico. Records show approximate flow of river at international boundary.

EXTREMES FOR PERIOD OF RECORD.--Maximum discharge, 22,000 ft ${ }^{3} / \mathrm{s}$ Aug. 14, 1940, gage height, $16.16 \mathrm{ft}$, present datum, from rating curve extended above $5,600 \mathrm{ft}^{3} / \mathrm{s}$ on basis of slope-area measurement of peak flow; no flow at times most years.

EXTREMES OUTSIDE PERIOD OF RECORD.--Greatest flood since at least 1906 occurred Sept. 28, 1926, gage height, about $23.9 \mathrm{ft}$, present datum, from floodmarks; discharge not determined.

Annual peak discharges

\begin{tabular}{|c|c|c|c|c|c|c|c|}
\hline $\begin{array}{l}\text { Water } \\
\text { year }\end{array}$ & Date & $\begin{array}{c}\text { Annual peak } \\
\text { discharge } \\
\left(\mathrm{ft}^{3} / \mathrm{s}\right)\end{array}$ & $\begin{array}{l}\text { Discharge } \\
\text { codes }\end{array}$ & $\begin{array}{l}\text { Water } \\
\text { year }\end{array}$ & Date & $\begin{array}{c}\text { Annual peak } \\
\text { discharge } \\
\left(\mathrm{ft}^{3} / \mathbf{s}\right)\end{array}$ & $\begin{array}{l}\text { Discharge } \\
\text { codes }\end{array}$ \\
\hline 1926 & $09-28-26$ & $(1)$ & HP & 1968 & $12-20-67$ & 6,500 & \\
\hline 1930 & $08-07-30$ & 9,400 & & 1969 & $07-28-69$ & 4,000 & ES \\
\hline 1931 & $08-08-31$ & 8,900 & & 1970 & $08-09-70$ & 5,870 & \\
\hline 1932 & $08-09-32$ & 6,000 & & 1971 & $08-11-71$ & 6,380 & \\
\hline 1933 & $09-19-33$ & 4,700 & & 1972 & $08-26-72$ & 1,830 & \\
\hline 1935 & $08-14-35$ & 3,000 & & 1973 & $10-18-72$ & 2,900 & \\
\hline 1936 & $09-10-36$ & 13,500 & & 1974 & $07-30-74$ & 7,360 & \\
\hline 1937 & $08-20-37$ & 8,090 & & 1975 & $09-14-75$ & 6,840 & \\
\hline 1938 & $08-07-38$ & 6,300 & & 1976 & $07-27-76$ & 5,000 & \\
\hline 1939 & 08-06-39 & 7,500 & & 1977 & $07-31-77$ & 3,310 & \\
\hline 1940 & $08-14-40$ & ${ }^{2} 22,000$ & & 1978 & $10-09-77$ & 14,500 & \\
\hline 1941 & $01-28-41$ & 5,900 & & 1979 & $01-18-79$ & 12,000 & \\
\hline 1950 & $07-05-50$ & 6,270 & & 1980 & $08-04-80$ & 2,000 & \\
\hline 1951 & $07-02-51$ & 5,710 & & 1981 & $07-10-81$ & 3,360 & \\
\hline 1952 & $08-16-52$ & 7,400 & & 1982 & $09-10-82$ & 4,260 & \\
\hline 1953 & $07-07-53$ & 11,900 & & 1983 & $02-04-83$ & 1,940 & \\
\hline 1954 & $08-14-54$ & 6,510 & & 1984 & $10-02-83$ & 8,180 & \\
\hline 1955 & $07-31-55$ & 6,250 & & 1985 & $12-28-84$ & 10,600 & \\
\hline 1956 & $07-17-56$ & 4,640 & & 1986 & $08-18-86$ & 4,000 & \\
\hline 1957 & $08-20-57$ & 2,540 & & 1987 & $08-10-87$ & 978 & \\
\hline 1958 & $08-05-58$ & 16,500 & & 1988 & $09-11-88$ & 7,020 & \\
\hline 1959 & $07-27-59$ & 13,000 & & 1989 & $08-04-89$ & 1,550 & \\
\hline 1960 & $08-16-60$ & 3,410 & & 1990 & $07-16-90$ & 2,260 & \\
\hline 1961 & $07-29-61$ & 3,820 & & 1991 & $03-02-91$ & 1,680 & \\
\hline 1962 & $07-26-62$ & 4,130 & & 1992 & $08-24-92$ & 2,320 & \\
\hline 1963 & $07-27-63$ & 6,340 & & 1993 & $01-19-93$ & 11,200 & \\
\hline 1964 & $08-14-64$ & 11,000 & & 1994 & $08-21-94$ & 2,050 & \\
\hline 1965 & $07-28-65$ & 4,530 & & 1995 & $11-12-94$ & 10,700 & \\
\hline 1966 & $07-28-66$ & 3,610 & & 1996 & $08-03-96$ & 6,310 & \\
\hline 1967 & $07-26-67$ & 5,560 & & & & & \\
\hline
\end{tabular}

${ }^{1}$ Highest since 1906; discharge unknown.

${ }^{2}$ Highest since 1927. 
09470500 SAN PEDRO RIVER AT PALOMINAS, AZ--Continued

Discharge rating table developed October 1995

\begin{tabular}{crcr}
\hline $\begin{array}{c}\text { Gage height } \\
(\mathbf{f t})\end{array}$ & $\begin{array}{c}\text { Discharge } \\
\left(\mathbf{f t}^{\mathbf{3}} / \mathbf{s}\right)\end{array}$ & $\begin{array}{c}\text { Gage height } \\
(\mathbf{f t})\end{array}$ & $\begin{array}{c}\text { Discharge } \\
\left(\mathbf{f t}^{3} / \mathbf{s}\right)\end{array}$ \\
\hline 4.0 & 125 & 10.0 & 3,600 \\
5.0 & 350 & 11.0 & 4,710 \\
6.0 & 700 & 12.0 & 6,000 \\
7.0 & 1,200 & 13.0 & 7,460 \\
8.0 & 1,830 & 14.0 & 9,110 \\
9.0 & 2,640 & 14.5 & 10,000 \\
\hline
\end{tabular}

Basin characteristics

\begin{tabular}{cccccccc}
\hline $\begin{array}{c}\text { Main } \\
\text { channel } \\
\text { slope } \\
\text { (ft/mi) }\end{array}$ & $\begin{array}{c}\text { Stream } \\
\text { length } \\
\text { (mi) }\end{array}$ & $\begin{array}{c}\text { Mean } \\
\text { basin } \\
\text { elevation } \\
\text { (ft) }\end{array}$ & $\begin{array}{c}\text { Forested } \\
\text { area } \\
\text { (percent) }\end{array}$ & $\begin{array}{c}\text { Soil } \\
\text { index }\end{array}$ & $\begin{array}{c}\text { Mean } \\
\text { annual } \\
\text { precipitation } \\
\text { (in) }\end{array}$ & $\begin{array}{c}\text { Rainfall intensity, 24-hour } \\
\text { (in) }\end{array}$ & $\begin{array}{c}50 \text {-year } \\
\text { (in) }\end{array}$ \\
\hline 23.5 & 35.4 & 4,950 & 12.0 & 2.3 & 17.9 & 1.9 \\
\hline
\end{tabular}


09470500 SAN PEDRO RIVER AT PALOMINAS, AZ--Continued

MEAN MONTHLY AND ANNUAL DISCHARGES $1931-33,1936-40,1951-81,1996$

\begin{tabular}{|c|c|c|c|c|c|c|}
\hline MONTH & $\begin{array}{l}\text { MAXIMUM } \\
(\text { FT } 3 / S)\end{array}$ & $\begin{array}{l}\text { MINIMUM } \\
\text { (FT3/S) }\end{array}$ & $\begin{array}{c}\text { MEAN } \\
\text { (FT3/S) }\end{array}$ & $\begin{array}{l}\text { STAN - } \\
\text { DARD } \\
\text { DEVIA - } \\
\text { TION } \\
\text { (FT3/S) }\end{array}$ & $\begin{array}{l}\text { COEFFI- } \\
\text { CIENT OF } \\
\text { VARI- } \\
\text { ATION }\end{array}$ & $\begin{array}{c}\text { PERCENT } \\
\text { OF } \\
\text { ANNUAI } \\
\text { RUNOFF }\end{array}$ \\
\hline OCTOBER & 770 & 0.00 & 25 & 121 & 4.8 & 6.7 \\
\hline NOVEMBER & 43 & 0.00 & 4.9 & 7.8 & 1.6 & 1.3 \\
\hline DECEMBER & 414 & 0.10 & 22 & 70 & 3.2 & 5.8 \\
\hline JANUARY & 452 & 0.04 & 21 & 73 & 3.4 & 5.6 \\
\hline FEBRUARY & 74 & 0.07 & 11 & 16 & 1.5 & 2.9 \\
\hline MARCH & 76 & 0.22 & 8.1 & 14 & 1.8 & 2.2 \\
\hline APRIL & 15 & 0.00 & 2.6 & 3.1 & 1.2 & 0.7 \\
\hline MAY & 7.0 & 0.00 & 1.2 & 1.5 & 1.3 & 0.3 \\
\hline JUNE & 23 & 0.00 & 3.9 & 6.4 & 1.6 & 1.0 \\
\hline JULY & 280 & 3.0 & 89 & 70 & 0.80 & 23.6 \\
\hline AUGUST & 591 & 2.7 & 151 & 165 & 1.1 & 40.4 \\
\hline SEPTEMBER & 275 & 0.19 & 35 & 52 & 1.5 & 9.4 \\
\hline ANNUAL & 93 & 7.1 & 32 & 22 & 0.69 & 100 \\
\hline
\end{tabular}

MAGNITUDE AND PROBABILITY OF INSTANTANEOUS PEAK FLOW BASED ON PERIOD OF RECORD 1926, 1930-33, 1935-41, 1950-96

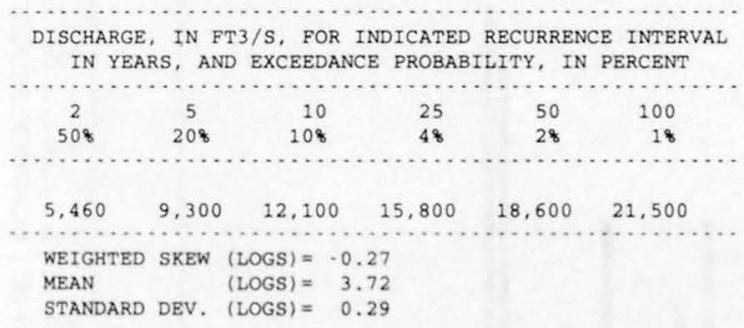

MAGNITUDE AND PROBABILITY OF ANNUAL LOW FLOW BASED ON PERIOD OF RECORD 1932-33, 1937-41, 1952-81
MAGNITUDE AND PROBABILITY OF ANNUAL HIGH FLOW BASED ON PERIOD OF RECORD 1931-33, 1936-40, 1951-81, 1996

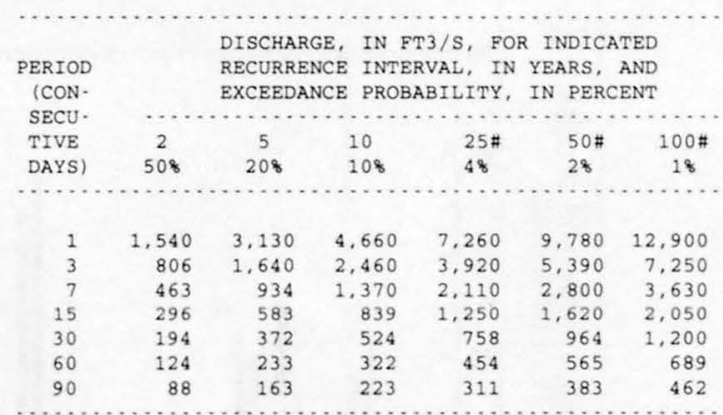

DURATION TABLE OF DAILY MEAN FLOW FOR PERIOD OF RECORD $1931 \cdot 33,1936 \cdot 40,1951 \cdot 81,1996$ DISCHARGE, IN FT3/S, WHICH WAS EQUALED OR EXCEEDED FOR INDICATED PERCENT OF TIME

\begin{tabular}{|c|c|c|c|c|c|c|c|c|c|c|c|c|c|c|c|c|}
\hline 18 & 58 & 108 & $15 \%$ & 208 & 308 & 408 & 508 & 608 & 708 & 808 & 908 & 958 & 988 & 998 & 99.58 & 99.98 \\
\hline 630 & 108 & 35 & 17 & 11 & 6.5 & 4.2 & 2.6 & 1.3 & 0.50 & 0.20 & 0.00 & 0.00 & 0.00 & 0.00 & 0.00 & 0.00 \\
\hline
\end{tabular}

\# Reliability of values in column is uncertain, and potential errors are large. 
ANNUAL MEAN DISCHARGE, IN CUBIC

FEET PER SECOND

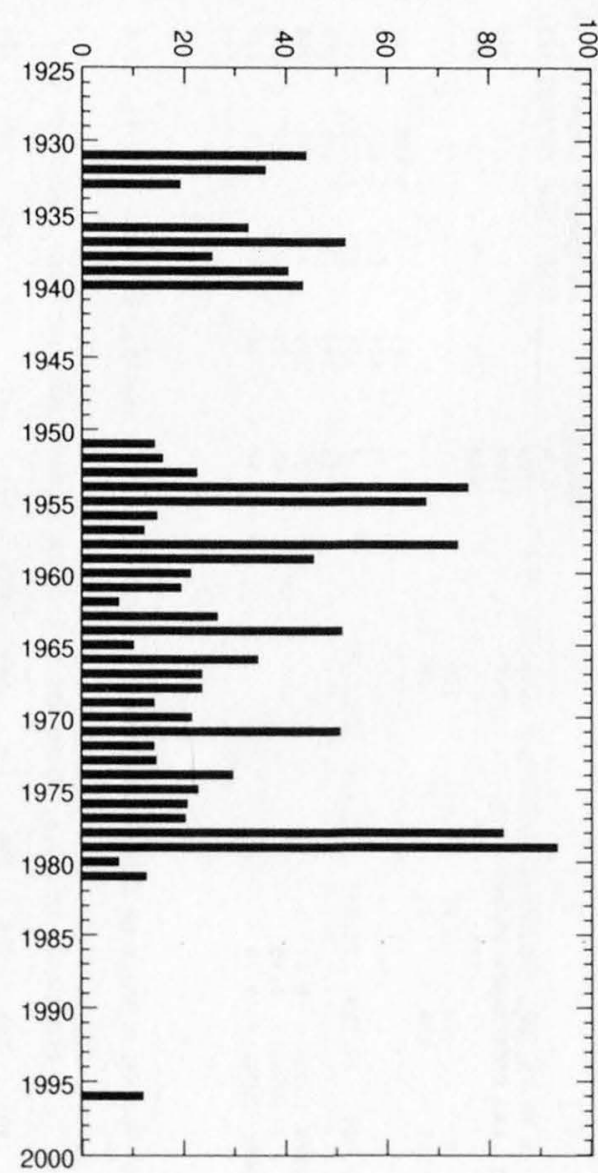

ANNUAL PEAK DISCHARGE IN CUBIC FEET PER SECOND

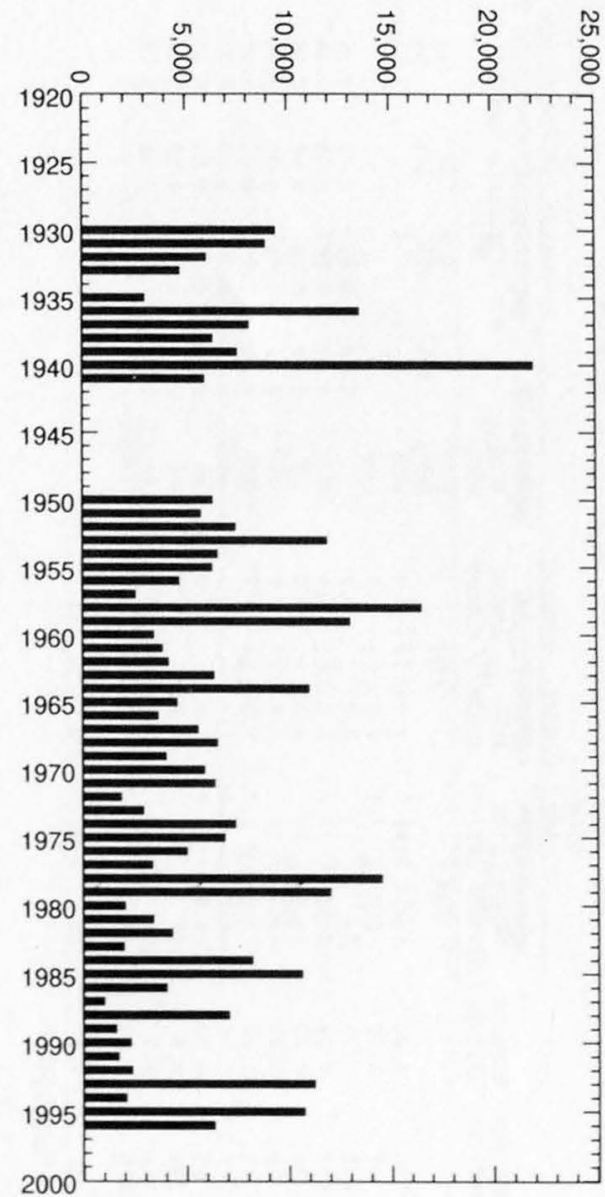


09470500 SAN PEDRO RIVER AT PALOMINAS, AZ--Continued
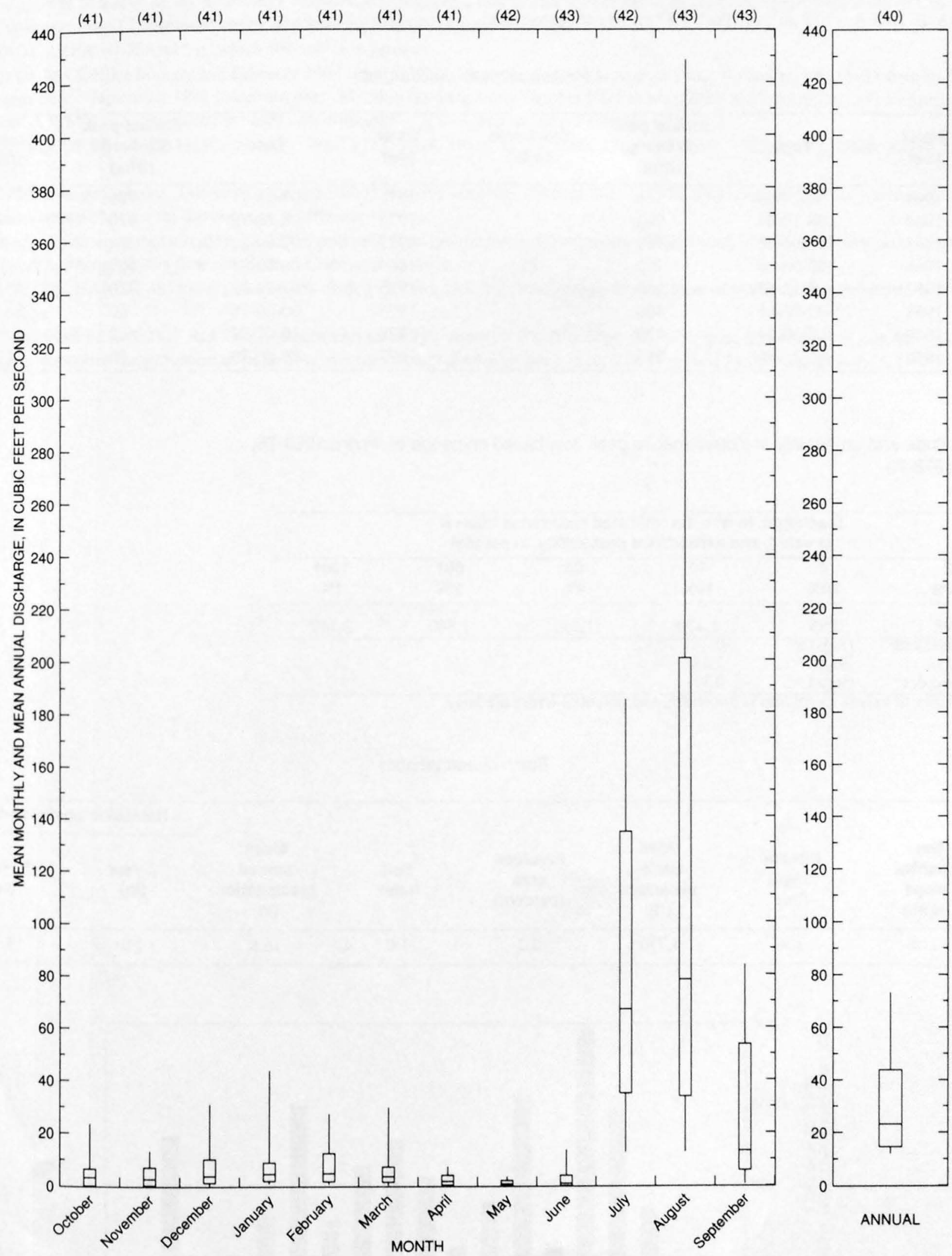
09470900 SAN PEDRO RIVER TRIBUTARY NEAR BISBEE, AZ

LOCATION.--Lat $31^{\circ} 34^{\prime} 12^{\prime \prime}$, long $110^{\circ} 01^{\prime} 36^{\prime \prime}, \mathrm{SW}^{1} / 4 \mathrm{SE}^{1} / 4$ sec.29. T.21 S., R.23 E., Cochise County, Hydrologic Unit 15050202, at U.S. Highwa

80,11 mi northwest of Bisbee.

DRAINAGE AREA.--7.12 $\mathrm{mi}^{2}$, of which $1.87 \mathrm{mi}^{2}$ is noncontributing.

Annual peak discharges

\begin{tabular}{|c|c|c|c|c|c|c|c|}
\hline $\begin{array}{l}\text { Water } \\
\text { year }\end{array}$ & Date & $\begin{array}{c}\text { Annual peak } \\
\text { discharge } \\
\left(\mathrm{ft}^{3} / \mathrm{s}\right)\end{array}$ & $\begin{array}{c}\text { Discharge } \\
\text { codes }\end{array}$ & $\begin{array}{l}\text { Water } \\
\text { year }\end{array}$ & Date & $\begin{array}{c}\text { Annual peak } \\
\text { discharge } \\
\left(\mathrm{ft}^{3} / \mathrm{s}\right)\end{array}$ & $\begin{array}{c}\text { Discharge } \\
\text { codes }\end{array}$ \\
\hline 1963 & $09-20-63$ & 360 & & 1971 & $07-23-71$ & 780 & \\
\hline 1964 & $08-10-64$ & 962 & & 1972 & $08-26-72$ & 620 & \\
\hline 1965 & $09-04-65$ & 1,460 & & 1973 & $07-00-73$ & 280 & \\
\hline 1966 & $00-00-66$ & 150 & LT & 1974 & $00-00-74$ & 1,000 & \\
\hline 1967 & $08-12-67$ & 1,060 & & 1975 & $07-24-75$ & 320 & \\
\hline 1968 & $07-00-68$ & 400 & & 1976 & $00-00-76$ & 60 & \\
\hline 1969 & $08-30-69$ & 120 & & 1978 & $10-07-77$ & 800 & \\
\hline 1970 & $07-28-70$ & 556 & & 1979 & $00-00-79$ & 20 & LT \\
\hline
\end{tabular}

Magnitude and probability of instantaneous peak flow based on period of record 1963-76, 1978-79

\begin{tabular}{|c|c|c|c|c|c|}
\hline \multicolumn{6}{|c|}{$\begin{array}{l}\text { Discharge, in } \mathrm{ft}^{3} / \mathrm{s} \text {, for indicated recurrence interval } \\
\text { in years, and exceedance probablility, in percent }\end{array}$} \\
\hline 2 & 5 & 10 & 25 & $50 t$ & $100 t$ \\
\hline $50 \%$ & $20 \%$ & $10 \%$ & $4 \%$ & $2 \%$ & $1 \%$ \\
\hline 498 & 885 & 1,170 & 1,550 & 1,840 & 2,140 \\
\hline Weighted skew & $(\log s)=$ & -0.34 & & & \\
\hline Mean & $(\log s)=$ & 2.68 & & & \\
\hline Standard dev. & $(\log s)=$ & 0.31 & & & \\
\hline
\end{tabular}

F Reliability of values in column is uncertain, and potential errors are large.

Basin characteristics

\begin{tabular}{|c|c|c|c|c|c|c|c|}
\hline \multirow[b]{2}{*}{$\begin{array}{c}\text { Main } \\
\text { channel } \\
\text { slope } \\
(\mathrm{ft} / \mathrm{mi})\end{array}$} & \multirow[b]{2}{*}{$\begin{array}{l}\text { Stream } \\
\text { length } \\
\text { (mi) }\end{array}$} & \multirow[b]{2}{*}{$\begin{array}{c}\text { Mean } \\
\text { basin } \\
\text { elevation } \\
(\mathrm{ft})\end{array}$} & \multirow[b]{2}{*}{$\begin{array}{l}\text { Forested } \\
\text { area } \\
\text { (percent) }\end{array}$} & \multirow[b]{2}{*}{$\begin{array}{c}\text { Soil } \\
\text { index }\end{array}$} & \multirow[b]{2}{*}{$\begin{array}{c}\text { Mean } \\
\text { annual } \\
\text { precipitation } \\
\text { (in) }\end{array}$} & \multicolumn{2}{|c|}{ Rainfall intensity, 24-hour } \\
\hline & & & & & & $\begin{array}{l}\text { 2-year } \\
\text { (in) }\end{array}$ & $\begin{array}{l}\text { 50-year } \\
\text { (in) }\end{array}$ \\
\hline 129 & 3.9 & 4,770 & 2.2 & 1.0 & 16.0 & 1.9 & 4.1 \\
\hline
\end{tabular}

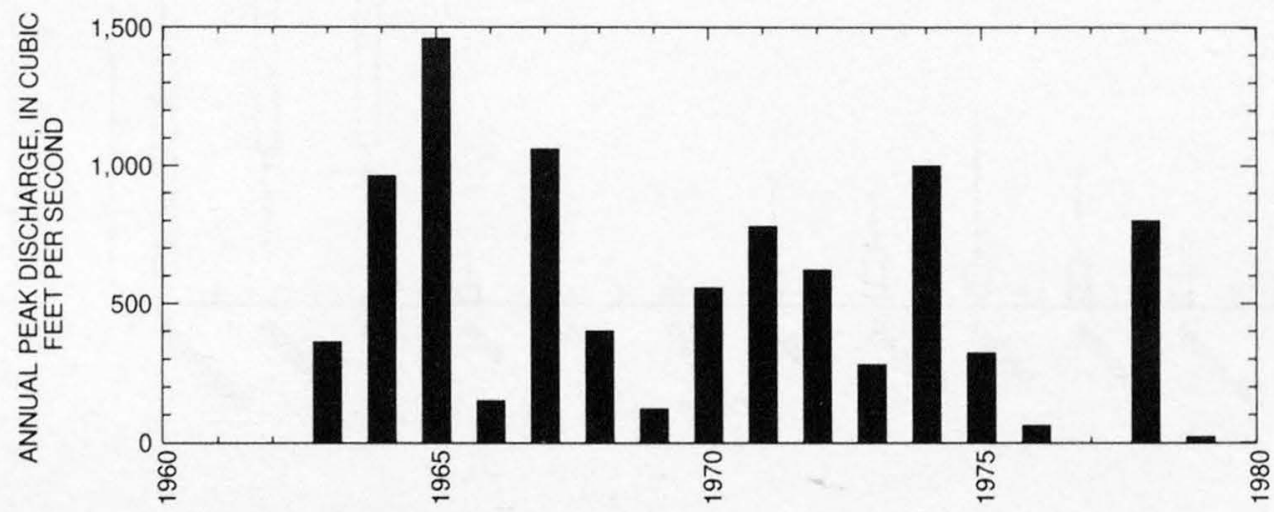


09471000 SAN PEDRO RIVER AT CHARLESTON, AZ

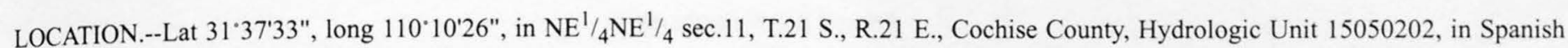
land grant of San Juan de las Boquillas y Nogales, at downstream side of pier near center of highway bridge, 0.3 mi south of Charleston, 1.5 mi upstream from Charleston damsite, and 9 mi upstream from Babocomari River.

DRAINAGE AREA.--1,234 $\mathrm{mi}^{2}$, of which $696 \mathrm{mi}^{2}$ is in Mexico.

PERIOD OF RECORD.--January and February 1904 (gage heights only); March 1904 to August 1906; November 1910 to December 1911 (gage heights only); September 1912 to current year. Monthly discharge only October 1926 to May 1928 and December 1933 to April 1935, published in WSP 1313. Published as "near Lewis Springs" 1910-11, and as "near Fairbank" 1911-26.

REVISED RECORDS.--WSP 1119: 1939(M). WSP 1213: 1914, 1916(M), 1918(M), 1919, 1920(M), 1922-23(M), WDR AZ-90-1: Drainage area.

GAGE.--Water-stage recorder. Datum of gage is 3,954.01 ft above sea level. Prior to Dec. 1, 1942, nonrecording gage or water-stage recorder at various sites within $6.5 \mathrm{mi}$ downstream at different datums.

REMARKS.--Diversions above station, mostly by pumping from ground water, for irrigation of 3,200 acres in 1978, excluding an unknown amount in Mexico. Record shows flow available at Charleston damsite.

AVERAGE DISCHARGE.--85 years (water years 1905, 1913-96), $56.0 \mathrm{ft}^{3} / \mathrm{s}, 40,570 \mathrm{acre-ft} / \mathrm{yr}$; median of yearly mean discharges, $46 \mathrm{ft}^{3} / \mathrm{s}, 33,300$ acre-ft/yr.

EXTREMES FOR PERIOD OF RECORD.--Maximum discharge, about $98,000 \mathrm{ft}^{3} / \mathrm{s} \mathrm{Sept.} \mathrm{28,} \mathrm{1926,} \mathrm{gage} \mathrm{height,} 21.9 \mathrm{ft}$, site and datum then in use, by slope-area measurement of peak flow; minimum daily discharge since 1928, $0.06 \mathrm{ft}^{3} / \mathrm{s} \mathrm{June} \mathrm{11,} \mathrm{1996,} \mathrm{gage} \mathrm{height,} 1.75 \mathrm{ft}$. 
GILA RIVER BASIN

09471000 SAN PEDRO RIVER AT CHARLESTON, AZ--Continued

Annual peak discharges

\begin{tabular}{|c|c|c|c|c|c|c|c|}
\hline $\begin{array}{l}\text { Water } \\
\text { year }\end{array}$ & Date & $\begin{array}{c}\text { Annual peak } \\
\text { discharge } \\
\left(\mathrm{ft}^{3} / \mathrm{s}\right)\end{array}$ & $\begin{array}{c}\text { Discharge } \\
\text { codes }\end{array}$ & $\begin{array}{l}\text { Water } \\
\text { year }\end{array}$ & Date & $\begin{array}{c}\text { Annual peak } \\
\text { discharge } \\
\left(\mathrm{ft}^{3} / \mathrm{s}\right)\end{array}$ & $\begin{array}{c}\text { Discharge } \\
\text { codes }\end{array}$ \\
\hline 1916 & $08-16-16$ & 7,700 & \multirow{11}{*}{ ES } & 1957 & $07-25-57$ & 6,000 & \\
\hline 1917 & $08-12-17$ & 13,000 & & 1958 & $08-05-58$ & 8,400 & \\
\hline 1918 & $07-01-18$ & 4,000 & & 1959 & $07-27-59$ & 7,480 & \\
\hline 1919 & $08-16-19$ & 25,100 & & 1960 & $08-11-60$ & 3,900 & \\
\hline 1920 & $09-05-20$ & 4,500 & & 1961 & $07-30-61$ & 3,620 & \\
\hline 1921 & $07-19-21$ & 19,000 & & 1962 & $07-28-62$ & 3,580 & \\
\hline 1922 & $09-09-22$ & 3,720 & & 1963 & $07-27-63$ & 6,460 & \\
\hline 1923 & $08-12-23$ & 5,200 & & 1964 & $08-14-64$ & 7,690 & \\
\hline 1924 & $07-24-24$ & 1,900 & & 1965 & $09-04-65$ & 4,180 & \\
\hline 1925 & $08-06-25$ & 11,900 & & 1966 & $08-03-66$ & 4,400 & \\
\hline 1926 & $09-28-26$ & $\mathrm{l}_{98,000}$ & & 1967 & $07-26-67$ & 6,010 & \\
\hline 1927 & $10-09-26$ & 5,100 & \multirow[t]{7}{*}{ ES } & 1968 & $12-20-67$ & 5,050 & \\
\hline 1928 & $07-15-28$ & 3,800 & & 1969 & $07-28-69$ & 3,920 & \\
\hline 1929 & $07-29-29$ & 10,400 & & 1970 & $08-09-70$ & 4,600 & \\
\hline 1930 & $08-07-30$ & 9,740 & & 1971 & $08-10-71$ & 5,920 & \\
\hline 1931 & $08-09-31$ & 24,500 & & 1972 & $08-26-72$ & 5,950 & \\
\hline 1932 & $08-09-32$ & 7,000 & & 1973 & $07-15-73$ & 3,340 & \\
\hline 1933 & $07-22-33$ & 9,600 & & 1974 & $07-20-74$ & 13,100 & \\
\hline 1934 & $00-00-34$ & 5,000 & \multirow[t]{23}{*}{ ES } & 1975 & $09-14-75$ & 4,020 & \\
\hline 1935 & $08-28-35$ & 8,600 & & 1976 & $09-05-76$ & 3,620 & \\
\hline 1936 & $09-11-36$ & 13,000 & & 1977 & $08-23-77$ & 5,200 & \\
\hline 1937 & $08-20-37$ & 9,430 & & 1978 & $10-09-77$ & 23,700 & \\
\hline 1938 & $08-07-38$ & 7,450 & & 1979 & $01-18-79$ & 11,800 & \\
\hline 1939 & $08-07-39$ & 9,370 & & 1980 & $08-15-80$ & 990 & \\
\hline 1940 & $08-13-40$ & 31,000 & & 1981 & $09-03-81$ & 3,210 & \\
\hline 1941 & $08-16-41$ & 10,800 & & 1982 & $09-10-82$ & 8,800 & \\
\hline 1942 & $07-24-42$ & 2,870 & & 1983 & $09-12-83$ & 3,100 & \\
\hline 1943 & $08-09-43$ & 8,650 & & 1984 & $10-03-83$ & 8,560 & \\
\hline 1944 & $08-18-44$ & 3,430 & & 1985 & $12-28-84$ & 13,000 & \\
\hline 1945 & $08-09-45$ & 7,670 & & 1986 & $08-18-86$ & 5,020 & \\
\hline 1946 & $08-04-46$ & 12,000 & & 1987 & $08-04-87$ & 3,290 & \\
\hline 1947 & $08-09-47$ & 10,100 & & 1988 & $09-12-88$ & 3,640 & \\
\hline 1948 & $08-03-48$ & 7,850 & & 1989 & $08-04-89$ & 1,680 & \\
\hline 1949 & $07-24-49$ & 6,720 & & 1990 & $08-12-90$ & 2,820 & \\
\hline 1950 & $07-06-50$ & 6,070 & & 1991 & $03-02-91$ & 1,950 & \\
\hline 1951 & $07-02-51$ & 5,730 & & 1992 & $08-24-92$ & 1,680 & \\
\hline 1952 & $08-17-52$ & 7,850 & & 1993 & $01-19-93$ & 11,500 & \\
\hline 1953 & $07-07-53$ & 8,590 & & 1994 & $09-10-94$ & 6,260 & \\
\hline 1954 & $08-15-54$ & 23,600 & & 1995 & $11-12-94$ & 9,760 & \\
\hline 1955 & $08-06-55$ & 14,400 & & 1996 & $07-15-96$ & 2,070 & \\
\hline 1956 & $07-18-56$ & 6,550 & & & & & \\
\hline
\end{tabular}

${ }^{1}$ Highest since 1906. 
09471000 SAN PEDRO RIVER AT CHARLESTON, AZ--Continued

Discharge rating table developed October 1995

\begin{tabular}{crcr}
\hline $\begin{array}{c}\text { Gage height } \\
(\mathbf{f t})\end{array}$ & $\begin{array}{c}\text { Discharge } \\
\left(\mathbf{f t}^{\mathbf{3}} \mathbf{s}\right)\end{array}$ & $\begin{array}{c}\text { Gage height } \\
(\mathbf{f t})\end{array}$ & $\begin{array}{c}\text { Discharge } \\
\left(\mathbf{f t}^{\mathbf{3}} / \mathbf{s}\right)\end{array}$ \\
\hline 3.0 & 45 & 8.0 & 6,040 \\
4.0 & 297 & 9.0 & 9,190 \\
5.0 & 938 & 10.0 & 13,290 \\
6.0 & 2,070 & 11.0 & 18,470 \\
7.0 & 3,700 & 11.3 & 20,250 \\
\hline
\end{tabular}

Basin characteristics

\begin{tabular}{ccccccc}
\hline $\begin{array}{c}\text { Main } \\
\text { channel } \\
\text { slope } \\
\text { (ft/mi) }\end{array}$ & $\begin{array}{c}\text { Stream } \\
\text { length } \\
\text { (mi) }\end{array}$ & $\begin{array}{c}\text { Mean } \\
\text { basin } \\
\text { elevation } \\
\text { (ft) }\end{array}$ & $\begin{array}{c}\text { Forested } \\
\text { area } \\
\text { (percent) }\end{array}$ & $\begin{array}{c}\text { Soil } \\
\text { index }\end{array}$ & $\begin{array}{c}\text { Mean } \\
\text { annual } \\
\text { precipitation } \\
\text { (in) }\end{array}$ & $\begin{array}{c}\text { Rainfall intensity, 24-hour } \\
\text { (in) }\end{array}$ \\
\hline 18.4 & 56.2 & 4,840 & 12.0 & 2.0 & 16.5 & $\begin{array}{c}50 \text {-year } \\
\text { (in) }\end{array}$ \\
\hline
\end{tabular}


09471000 SAN PEDRO RIVER AT CHARLESTON, AZ--Continued

MEAN MONTHLY AND ANNUAL DISCHARGES 1905, 1913-26, 1929-33, 1936.96

\begin{tabular}{|c|c|c|c|c|c|c|}
\hline MONTH & $\begin{array}{l}\text { MAXIMUM } \\
(\mathrm{FT} 3 / \mathrm{S})\end{array}$ & $\begin{array}{c}\text { MINIMUM } \\
(\mathrm{FT} 3 / \mathrm{S})\end{array}$ & $\begin{array}{c}\text { MEAN } \\
(\mathrm{FT} 3 / \mathrm{S})\end{array}$ & $\begin{array}{l}\text { STAN - } \\
\text { DARD } \\
\text { DEVIA- } \\
\text { TION } \\
\text { (FT3/S) }\end{array}$ & $\begin{array}{l}\text { COEFFI- } \\
\text { CIENT OF } \\
\text { VARI- } \\
\text { ATION }\end{array}$ & $\begin{array}{c}\text { PERCENT } \\
\text { OF } \\
\text { ANNUAL } \\
\text { RUNOFF }\end{array}$ \\
\hline OCTOBER & 1,090 & 2.9 & 37 & 127 & 3.5 & 5.5 \\
\hline NOVEMBER & 128 & 5.0 & 18 & 17 & 0.97 & 2.6 \\
\hline DECEMBER & 1.230 & 6.0 & 49 & 147 & 3.0 & 7.2 \\
\hline JANUARY & 507 & 9.5 & 42 & 80 & 1.9 & 6.2 \\
\hline FEBRUARY & 217 & 7.2 & 29 & 33 & 1.2 & 4.3 \\
\hline MARCH & 160 & 8.1 & 25 & 28 & 1.1 & 3.7 \\
\hline APRIL & 67 & 3.0 & 13 & 9.1 & 0.68 & 2.0 \\
\hline MAY & 37 & 2.4 & 8.3 & 5.4 & 0.65 & 1.2 \\
\hline JUNE & 167 & 1.2 & 12 & 22 & 1.8 & 1.8 \\
\hline JULY & 876 & 3.1 & 138 & 150 & 1.1 & 20.6 \\
\hline AUGUST & 968 & 10 & 216 & 225 & 1.0 & 32.2 \\
\hline SEPTEMBER & 1,890 & 4.1 & 85 & 212 & 2.5 & 12.6 \\
\hline ANNUAL & 206 & 13 & 56 & 37 & 0.66 & 100 \\
\hline
\end{tabular}

MAGNITUDE AND PROBABILITY OF INSTANTANEOUS PEAK FLOW BASED ON PERIOD OF RECORD 1916.96

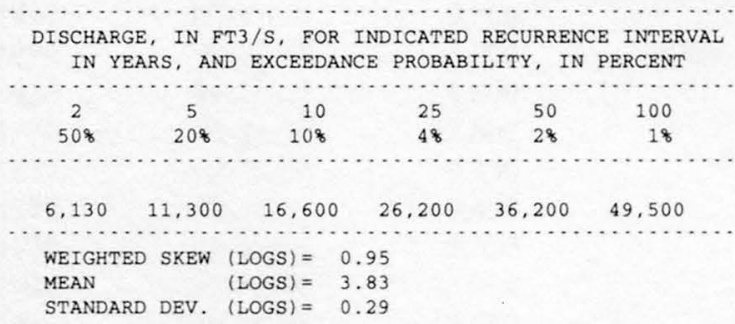

MAGNITUDE AND PROBABILITY OF ANNUAL LOW FLOW BASED ON PERIOD OF RECORD $1914 \cdot 26,1930 \cdot 33,1937 \cdot 96$

\begin{tabular}{|c|c|c|c|c|c|c|}
\hline PERIOD & \multicolumn{6}{|c|}{ DISCHARGE, IN FT3/S, FOR INDICATED } \\
\hline $1 \mathrm{CON}-$ & \multicolumn{6}{|c|}{$\begin{array}{l}\text { RECURRENCE INTERVAL, IN YEARS, AND } \\
\text { NON-EXCEEDANCE PROBABILITY, IN PERCENT }\end{array}$} \\
\hline SECU - & & & & & & \\
\hline TIVE & 2 & 5 & 10 & 20 & 50 & 100 \\
\hline DAYS) & 508 & 208 & 108 & 58 & 28 & 18 \\
\hline 1 & 2.1 & 1.0 & 0.63 & 0.40 & 0.21 & 0.14 \\
\hline 3 & 2.3 & 1.1 & 0.70 & 0.43 & 0.23 & 0.15 \\
\hline 7 & 2.5 & 1.3 & 0.82 & 0.51 & 0.28 & 0.18 \\
\hline 14 & 2.6 & 1.5 & 1.0 & 0.74 & 0.48 & 0.36 \\
\hline 30 & 3.2 & 2.1 & 1.6 & 1.3 & 1.0 & 0.89 \\
\hline 60 & 4.6 & 3.2 & 2.7 & 2.3 & 1.9 & 1.7 \\
\hline 90 & 6.6 & 4.7 & 4.0 & 3.4 & 3.0 & 2.7 \\
\hline 120 & 12 & 8.2 & 6.7 & 5.7 & 4.8 & 4.4 \\
\hline 183 & 16 & 10 & 8.8 & 7.9 & 7.2 & 6.9 \\
\hline
\end{tabular}

MAGNITUDE AND PROBABILITY OF ANNUAL HIGH FLOW BASED ON PERIOD OF RECORD $1905,1913.26,1929 \cdot 33,1936.96$

\begin{tabular}{|c|c|c|c|c|c|c|}
\hline $\begin{array}{l}\text { PERIOD } \\
(\mathrm{CON}-\end{array}$ & & \multicolumn{5}{|c|}{$\begin{array}{l}\text { DISCHARGE, IN FT } 3 / S \text {, FOR INDICATED } \\
\text { RECURRENCE INTERVAL, IN YEARS, AND }\end{array}$} \\
\hline SECU- & & . . . . . & . . . . & ....... & $\ldots \ldots \ldots$ & $\ldots \ldots$ \\
\hline TIVE & 2 & 5 & 10 & 25 & 50 & 100 \\
\hline DAYS) & 508 & 208 & 108 & 48 & 28 & 18 \\
\hline 1 & 1,860 & 3,950 & 6,070 & 9,850 & 13,700 & 18,600 \\
\hline 3 & 1,030 & 2,190 & 3,390 & 5,620 & 7,960 & 11,000 \\
\hline 7 & 606 & 1,280 & 1,960 & 3.180 & 4,400 & 5,960 \\
\hline 15 & 394 & 818 & 1,230 & 1,960 & 2,670 & 3,550 \\
\hline 30 & 269 & 536 & 783 & 1,190 & 1,560 & 2,020 \\
\hline 60 & 182 & 347 & 487 & 700 & 887 & 1,100 \\
\hline 90 & 135 & 252 & 348 & 491 & 613 & 747 \\
\hline
\end{tabular}

DURATION TABLE OF DAILY MEAN FLOW FOR PERIOD OF RECORD 1905, 1913-26, $1929 \cdot 33,1936 \cdot 96$

DISCHARGE, IN FT3/S, WHICH WAS EQUALED OR EXCEEDED FOR INDICATED PERCENT OF TIME

\begin{tabular}{|c|c|c|c|c|c|c|c|c|c|c|c|c|c|c|c|c|}
\hline 18 & 58 & 108 & 158 & 208 & 308 & 408 & 508 & 608 & 708 & 808 & 908 & 958 & 988 & 998 & 99.58 & 99.98 \\
\hline 02 & 184 & 74 & 42 & 31 & 22 & 17 & 14 & 12 & 9.0 & 6.3 & 3.8 & 2.6 & 1.8 & 1.4 & 1.0 & 0.42 \\
\hline
\end{tabular}


ANNUAL MEAN DISCHARGE, IN CUBIC FEET PER SECOND

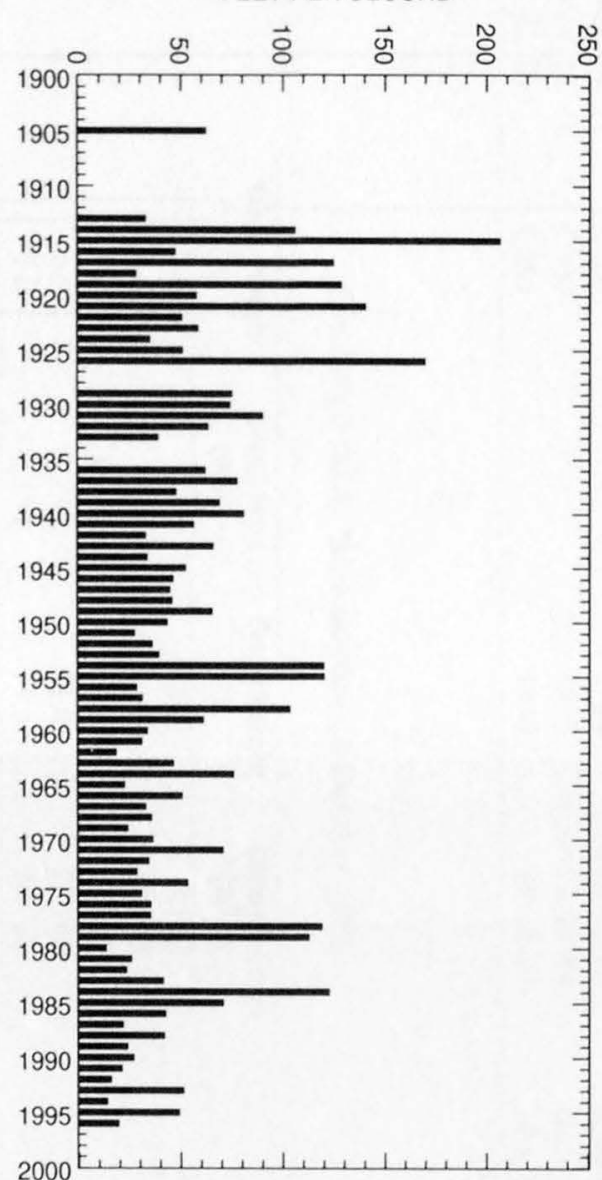

ANNUAL PEAK DISCHARGE, IN CUBIC FEET PER SECOND

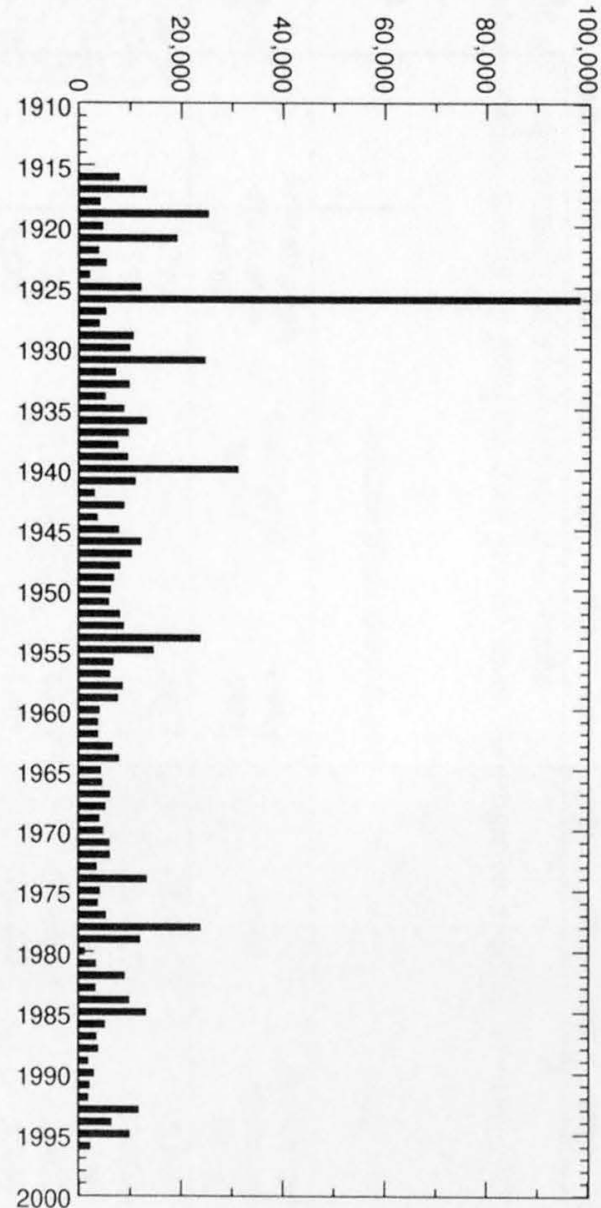


09471000 SAN PEDRO RIVER AT CHARLESTON, AZ--Continued

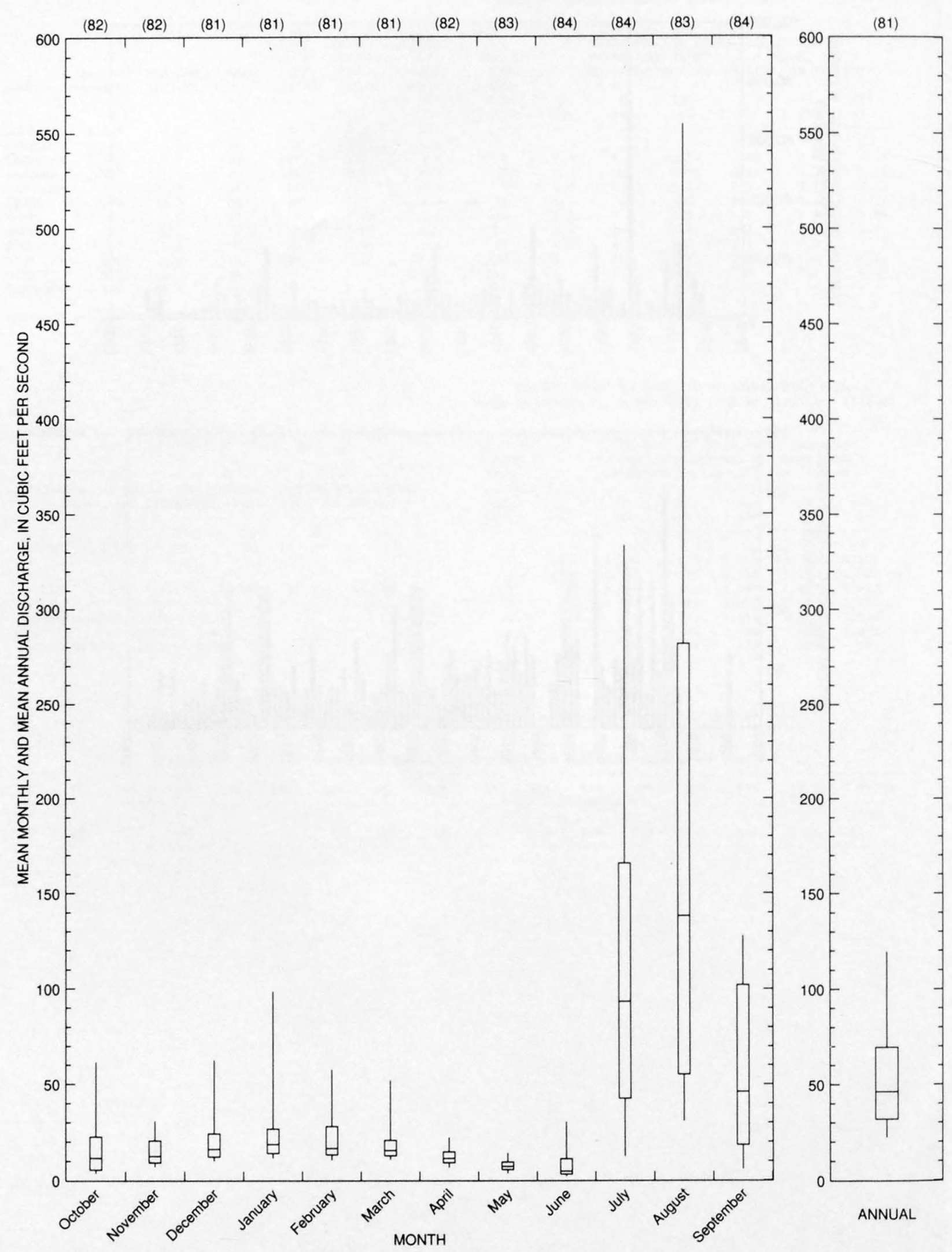


09471550 SAN PEDRO RIVER NEAR TOMBSTONE, AZ

LOCATION.--Lat 31 '45'03", long 110 12'02", in SE $\frac{1}{4} 4$ sec.28, T.19 S., R.21 E., Cochise County, Hydrologic Unit 15050202 , in Spanish land grant of San Juan de las Boquillas y Nogales, at right bank 0.5 mi downstream from Willow Wash, 2.6 mi north of Fairbank, and 8 mi northwest of Tombstone.

DRAINAGE AREA.--1,730 $\mathrm{mi}^{2}$, approximately, of which $696 \mathrm{mi}^{2}$ is in Mexico.

REMARKS.--Diversions above station, mostly by pumping from ground water, for irrigation of 3,200 acres in 1978 , excluding an unknown amount in Mexico.

Annual peak discharges

\begin{tabular}{|c|c|c|c|c|c|c|c|}
\hline $\begin{array}{l}\text { Water } \\
\text { year }\end{array}$ & Date & $\begin{array}{c}\text { Annual peak } \\
\text { discharge } \\
\left(\mathrm{ft}^{3} / \mathrm{s}\right)\end{array}$ & $\begin{array}{l}\text { Discharge } \\
\text { codes }\end{array}$ & $\begin{array}{l}\text { Water } \\
\text { year }\end{array}$ & Date & $\begin{array}{c}\text { Annual peak } \\
\text { discharge } \\
\left(\mathrm{ft}^{3} / \mathrm{s}\right)\end{array}$ & $\begin{array}{l}\text { Discharge } \\
\text { codes }\end{array}$ \\
\hline 1967 & $07-26-67$ & 7,790 & & 1977 & $08-23-77$ & 8,900 & \\
\hline 1968 & $12-20-67$ & 7,340 & & 1978 & $10-09-77$ & 24,200 & \\
\hline 1969 & $07-28-69$ & 2,950 & & 1979 & $01-18-79$ & 10,200 & \\
\hline 1970 & $08-03-70$ & 5,400 & & 1980 & $08-15-80$ & 1,400 & \\
\hline 1971 & $08-10-71$ & 9,220 & & 1981 & $08-01-81$ & 5,640 & \\
\hline 1972 & $08-12-72$ & 12,900 & & 1982 & $09-10-82$ & 6,500 & \\
\hline 1973 & $08-21-73$ & 1,880 & & 1983 & $09-10-83$ & 4,720 & \\
\hline 1974 & $07-20-74$ & 18,500 & & 1984 & $10-02-83$ & 13,600 & \\
\hline 1975 & $09-14-75$ & 4,500 & & 1985 & $12-28-84$ & 10,500 & \\
\hline 1976 & $07-27-76$ & 8,580 & & 1986 & $08-18-86$ & 4,410 & \\
\hline
\end{tabular}

Discharge rating table developed October 1986

\begin{tabular}{crcc}
\hline $\begin{array}{c}\text { Gage height } \\
(\mathbf{f t})\end{array}$ & $\begin{array}{c}\text { Discharge } \\
\left(\mathbf{f t}^{\mathbf{3}} \mathbf{s}\right)\end{array}$ & $\begin{array}{c}\text { Gage height } \\
(\mathbf{f t})\end{array}$ & $\begin{array}{c}\text { Discharge } \\
\left(\mathbf{f t}^{\mathbf{3}} \mathbf{s}\right)\end{array}$ \\
\hline 3.0 & 32 & 8.0 & 9,190 \\
4.0 & 656 & 9.0 & 12,780 \\
5.0 & 1,940 & 10.0 & 17,210 \\
6.0 & 3,790 & 11.0 & 22,100 \\
7.0 & 6,250 & 11.5 & 24,700 \\
\hline
\end{tabular}

Basin characteristics

\begin{tabular}{cccccccc}
\hline $\begin{array}{c}\text { Main } \\
\text { channel } \\
\text { slope } \\
(\mathrm{ft} / \mathrm{mi})\end{array}$ & $\begin{array}{c}\text { Stream } \\
\text { length } \\
(\mathrm{mi})\end{array}$ & $\begin{array}{c}\text { Mean } \\
\text { basin } \\
\text { elevation } \\
(\mathrm{ft})\end{array}$ & $\begin{array}{c}\text { Forested } \\
\text { area } \\
\text { (percent) }\end{array}$ & $\begin{array}{c}\text { Soil } \\
\text { index }\end{array}$ & $\begin{array}{c}\text { Mean } \\
\text { annual } \\
\text { precipitation } \\
\text { (in) }\end{array}$ & $\begin{array}{c}\text { Rainfall intensity, 24-hour } \\
\text { (in) }\end{array}$ & $\begin{array}{c}50 \text {-year } \\
\text { (in) }\end{array}$ \\
\hline 18.0 & 67.4 & 4,820 & 13.0 & 2.0 & 16.2 & 1.9 \\
\hline
\end{tabular}


09471550 SAN PEDRO RIVER NEAR TOMBSTONE, AZ--Continued

MEAN MONTHLY AND ANNUAL DISCHARGES 1968.86

\begin{tabular}{|c|c|c|c|c|c|c|}
\hline MONTH & $\begin{array}{l}\text { MAXIMUM } \\
(\mathrm{FT} 3 / \mathrm{S})\end{array}$ & $\begin{array}{l}\text { MINIMUM } \\
\text { (FT3/S) }\end{array}$ & $\begin{array}{c}\text { MEAN } \\
(\mathrm{FT} 3 / \mathrm{S})\end{array}$ & $\begin{array}{l}\text { STAN- } \\
\text { DARD } \\
\text { DEVIA- } \\
\text { TION } \\
(\mathrm{FT} 3 / \mathrm{S})\end{array}$ & $\begin{array}{l}\text { COEFFI- } \\
\text { CIENT OF } \\
\text { VARI- } \\
\text { ATION }\end{array}$ & $\begin{array}{c}\text { PERCENT } \\
\text { OF } \\
\text { ANNUAL } \\
\text { RUNOFF }\end{array}$ \\
\hline OCTOBER & 998 & 0.00 & 95 & 247 & 2.6 & 14.4 \\
\hline NOVEMBER & 61 & 2.8 & 16 & 14 & 0.88 & 2.4 \\
\hline DECEMBER & 375 & 6.4 & 63 & 112 & 1.8 & 9.6 \\
\hline JANUARY & 450 & 9.7 & 56 & 105 & 1.9 & 8.5 \\
\hline FEBRUARY & 214 & 9.1 & 45 & 53 & 1.2 & 6.7 \\
\hline MARCH & 179 & 9.1 & 37 & 42 & 1.1 & 5.7 \\
\hline APRIL & 44 & 4.2 & 15 & 9.4 & 0.63 & 2.3 \\
\hline MAY & 21 & 1.7 & 7.9 & 4.9 & 0.62 & 1.2 \\
\hline JUNE & 22 & 0.00 & 3.8 & 5.8 & 1.5 & 0.6 \\
\hline JULY & 369 & 1.8 & 104 & 102 & 0.98 & 15.8 \\
\hline AUGUST & 820 & 15 & 160 & 188 & 1.2 & 24.1 \\
\hline SEPTEMBER & 177 & 0.09 & 58 & 56 & 0.96 & 8.8 \\
\hline ANNUAL & 157 & 13 & 56 & 36 & 0.64 & 100 \\
\hline
\end{tabular}

MAGNITUDE AND PROBABILITY OF INSTANTANEOUS PEAK FLOW BASED ON PERIOD OF RECORD 1967.86

DISCHARGE, IN $F T 3 / S$, FOR INDICATED RECURRENCE INTERVAL IN YEARS, AND EXCEEDANCE PROBABILITY, IN PERCENT

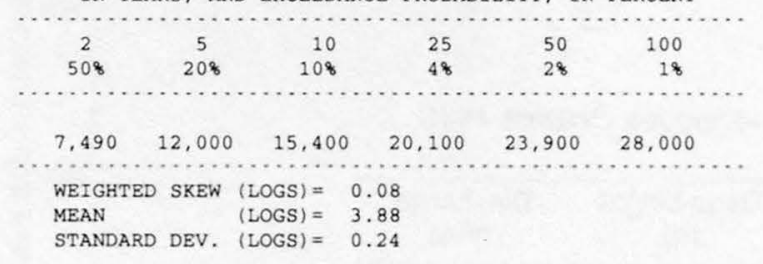

MAGNITUDE AND PROBABILITY OF ANNUAL LOW FLOW BASED ON PERIOD OF RECORD $1969 \cdot 86$

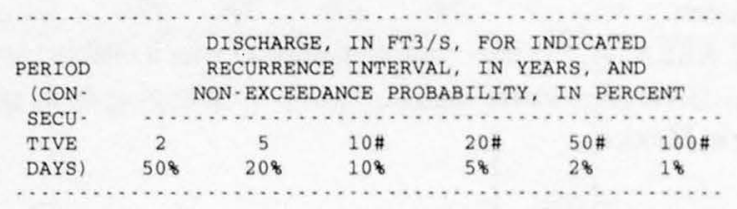

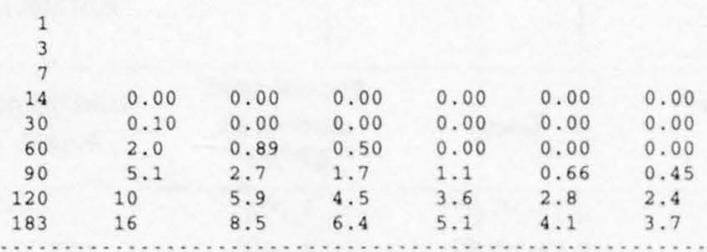

MAGNITUDE AND PROBABILITY OF ANNUAL HIGH FLOW BASED ON PERIOD OF RECORD 1968.86

\begin{tabular}{|c|c|c|c|c|c|c|}
\hline & & \multicolumn{5}{|c|}{ DISCHARGE, IN FT3/S, FOR INDICATED } \\
\hline PERIOD & & \multirow{2}{*}{\multicolumn{3}{|c|}{$\begin{array}{l}\text { RECURRENCE } \\
\text { EXCEEDANCE PROBABILITY, }\end{array}$}} & YEARS, & AND \\
\hline $1 \mathrm{CON}-$ & & & & & \multicolumn{2}{|c|}{ IN PERCENT } \\
\hline SECU - & & .... & & & (1) & ......... \\
\hline TIVE & 2 & 5 & $10 \#$ & $25 \#$ & $50 \#$ & $100 \#$ \\
\hline DAYS) & 508 & 208 & 108 & 48 & 28 & 18 \\
\hline 1 & 1,840 & 4,060 & 6,440 & 10,900 & 15,600 & 21.900 \\
\hline 3 & 1,120 & 2,370 & 3,670 & 6,030 & 8,470 & 11,600 \\
\hline 7 & 654 & 1,320 & 1,940 & 2,950 & 3,890 & 5,020 \\
\hline 15 & 431 & 816 & 1,130 & 1,590 & 1,980 & 2,410 \\
\hline 30 & 285 & 501 & 666 & 896 & 1.080 & 1,280 \\
\hline 60 & 198 & 332 & 420 & 527 & 602 & 674 \\
\hline 90 & 145 & 237 & 294 & 358 & 402 & 441 \\
\hline
\end{tabular}

DURATION TABLE OF DAILY MEAN FLOW FOR PERIOD OF RECORD 1968-86

DISCHARGE, IN FT3/S, WHICH WAS EQUALED OR EXCEEDED FOR INDICATED PERCENT OF TIME

\begin{tabular}{|c|c|c|c|c|c|c|c|c|c|c|c|c|c|c|c|c|}
\hline $1 \%$ & 58 & $10 \%$ & 158 & 208 & 308 & 408 & 508 & 608 & 708 & 808 & 908 & 958 & 988 & 998 & 99.58 & 99.98 \\
\hline 898 & 182 & 82 & 49 & 36 & 23 & 17 & 13 & 10 & 6.9 & 3.7 & 0.03 & 0.00 & 0.00 & 0.00 & 0.00 & 0.00 \\
\hline
\end{tabular}

\# Reliability of values in column is uncertain, and potential errors are large. 
09471550 SAN PEDRO RIVER NEAR TOMBSTONE, AZ--Continued
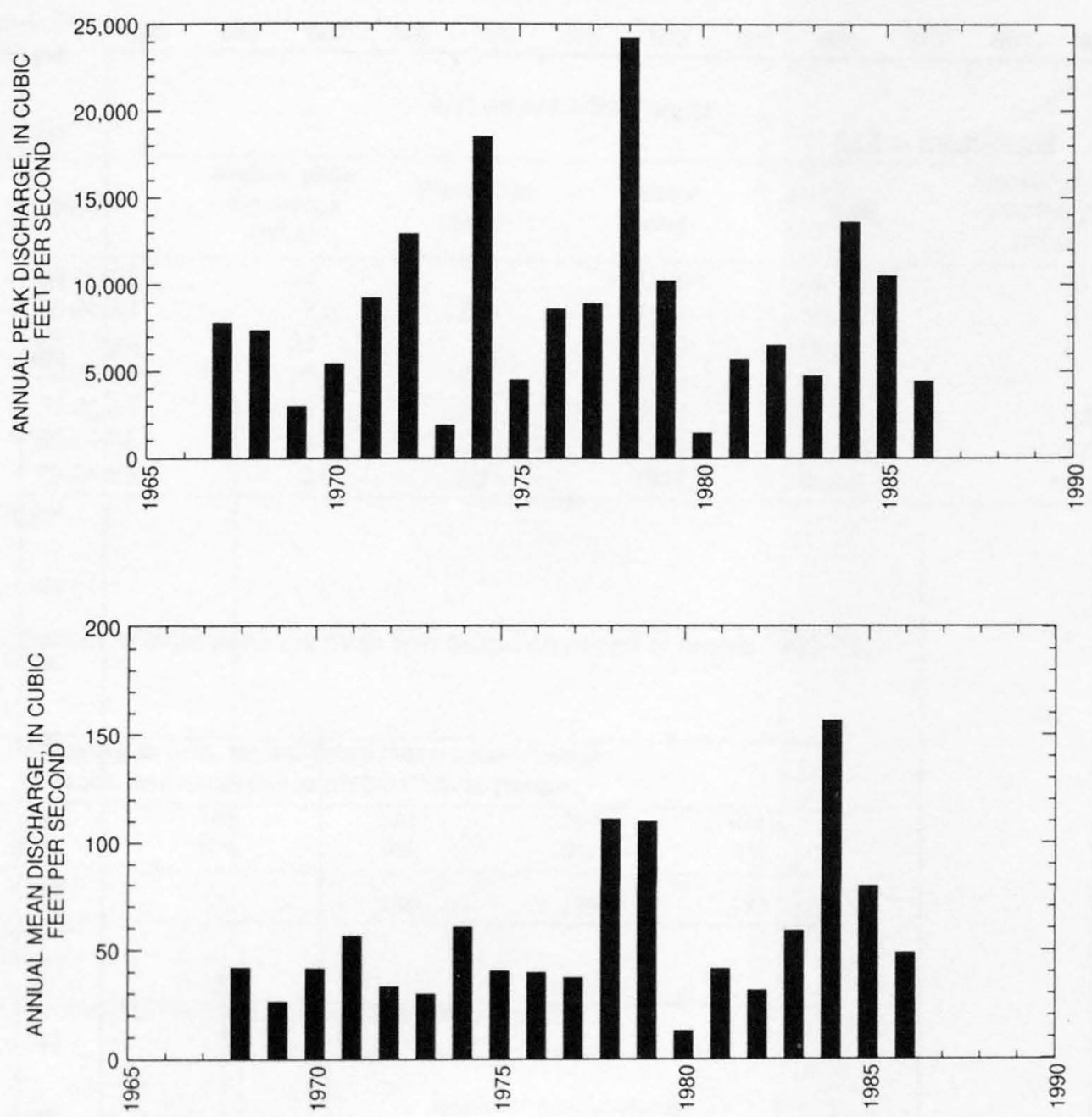
09471550 SAN PEDRO RIVER NEAR TOMBSTONE, AZ--Continued
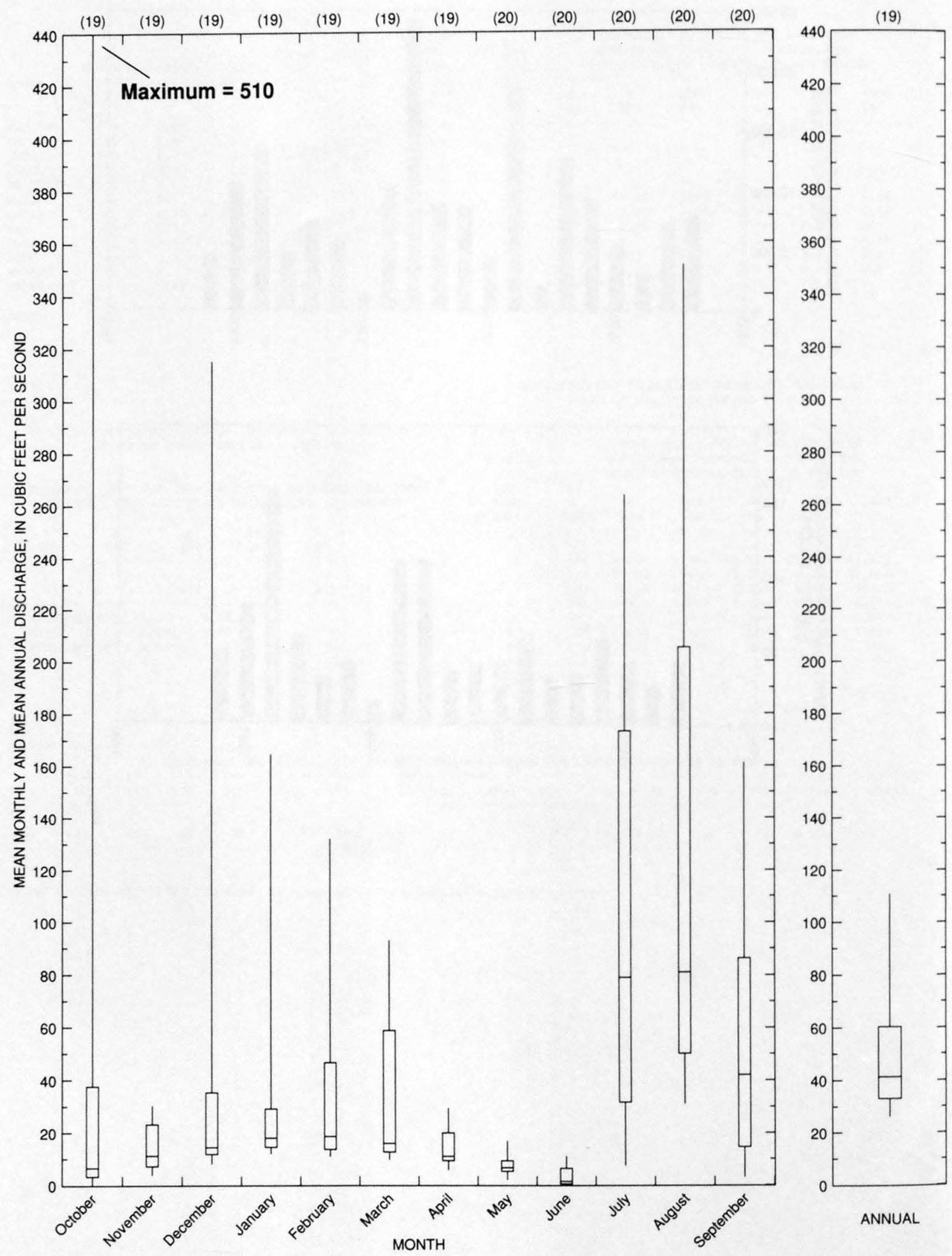
09471600 CANARY WASH NEAR BENSON, AZ

LOCATION.--Lat $31^{\circ} 52^{\prime} 35^{\prime \prime}$, long $110^{\circ} 20^{\prime} 30^{\prime \prime}, \mathrm{NW}^{1} \frac{1}{4}$ sec.18, T.18 S., R.20 E., Cochise County, Hydrologic Unit 15050202, at State Highway 90 , $6.5 \mathrm{mi}$ southwest of Benson.

DRAINAGE AREA.--0.79 $\mathrm{mi}^{2}$.

Annual peak discharges

\begin{tabular}{cccccccc}
\hline $\begin{array}{c}\text { Water } \\
\text { year }\end{array}$ & Date & $\begin{array}{c}\text { Annual peak } \\
\text { discharge } \\
\left(\mathbf{f t}^{\mathbf{3}} \mathbf{/ s}\right)\end{array}$ & $\begin{array}{c}\text { Discharge } \\
\text { codes }\end{array}$ & $\begin{array}{c}\text { Water } \\
\text { year }\end{array}$ & Date & $\begin{array}{c}\text { Annual peak } \\
\text { discharge } \\
\left(\mathrm{ft}^{3} / \mathbf{s}\right)\end{array}$ & $\begin{array}{c}\text { Discharge } \\
\text { codes }\end{array}$ \\
\hline 1963 & $00-00-63$ & 84 & & 1970 & $00-00-70$ & 0 & \\
1964 & $00-00-64$ & 2.0 & ES & 1971 & $00-00-71$ & 0 & \\
1965 & $08-13-65$ & 59 & & 1972 & $00-00-72$ & 0 & \\
1966 & $08-00-66$ & 20 & ES & 1973 & $00-00-73$ & 0 & ES \\
1967 & $07-00-67$ & 15 & ES & 1974 & $07-19-74$ & 2.0 & ES \\
1968 & $00-00-68$ & 10 & LT & 1975 & $10-00-74$ & 2.0 & HP \\
1969 & $09-06-69$ & 2.0 & ES & 1978 & $00-00-78$ & ${ }^{5} 55$ & \\
\hline
\end{tabular}

${ }^{1}$ Highest since 1976.

Magnitude and probability of instantaneous peak flow based on period of record 1963-75, 1978

\begin{tabular}{|c|c|c|c|c|c|}
\hline \multicolumn{6}{|c|}{$\begin{array}{l}\text { Discharge, in } \mathrm{ft}^{3} / \mathrm{s} \text {, for indicated recurrence interval } \\
\text { in years, and exceedance probablility, in percent }\end{array}$} \\
\hline $\begin{array}{c}2 \dagger \\
50 \%\end{array}$ & $\begin{array}{c}5 \dagger \\
20 \%\end{array}$ & $\begin{array}{c}10 \dagger \\
10 \%\end{array}$ & $\begin{array}{l}25 \dagger \\
4 \%\end{array}$ & $\begin{array}{l}50 \dagger \\
2 \%\end{array}$ & $\begin{array}{c}100+ \\
1 \%\end{array}$ \\
\hline 4.4 & 24.7 & 58 & 139 & 239 & 385 \\
\hline Weighted skew & $(\log s)=$ & -0.26 & & & \\
\hline Mean & $(\log s)=$ & 0.60 & & & \\
\hline Standard dev. & $(\log s)=$ & 0.93 & & & \\
\hline
\end{tabular}

Basin characteristics

\begin{tabular}{cccccccc}
\hline $\begin{array}{c}\text { Main } \\
\text { channel } \\
\text { slope } \\
(\mathrm{ft} / \mathrm{mi})\end{array}$ & $\begin{array}{c}\text { Stream } \\
\text { length } \\
(\mathrm{mi})\end{array}$ & $\begin{array}{c}\text { Mean } \\
\text { basin } \\
\text { elevation } \\
\text { (ft) }\end{array}$ & $\begin{array}{c}\text { Forested } \\
\text { area } \\
\text { (percent) }\end{array}$ & $\begin{array}{c}\text { Soil } \\
\text { index }\end{array}$ & $\begin{array}{c}\text { Mean } \\
\text { annual } \\
\text { precipitation } \\
\text { (in) }\end{array}$ & $\begin{array}{c}\text { Rainfall intensity, 24-hour } \\
\text { (in) }\end{array}$ & $\begin{array}{c}50 \text {-year } \\
\text { (in) }\end{array}$ \\
\hline 472 & 2.6 & 5,240 & 66.0 & 3.0 & 15.0 & 1.7 \\
\hline
\end{tabular}


09471600 CANARY WASH NEAR BENSON, AZ--Continued

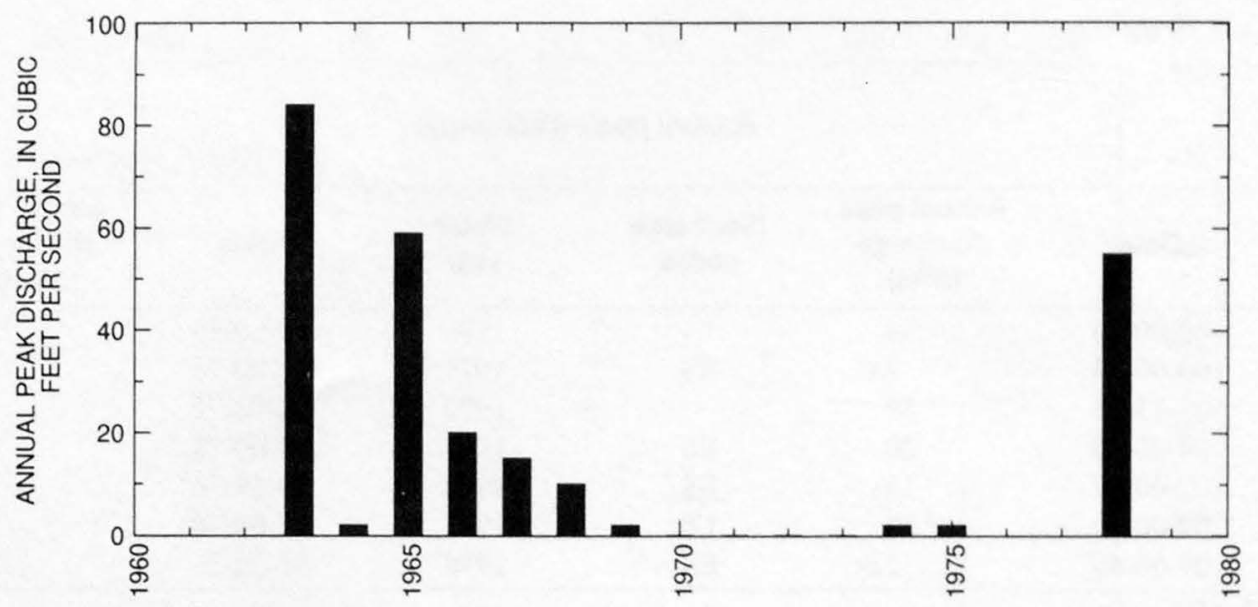


09471700 FENNER WASH NEAR BENSON, AZ

LOCATION.--Lat $31^{\circ} 58^{\prime} 49^{\prime \prime}$, long $110^{\circ} 12^{\prime} 57^{\prime \prime}, \mathrm{SE}^{1} / \mathrm{SE}^{1} / 4 \mathrm{sec} .5$, T.17 S., R.21 E., Cochise County, Hydrologic Unit 15050202 , at Interstate 10 ,

4.3 mi east of Benson.

DRAINAGE AREA.--2.71 $\mathrm{mi}^{2}$.

Annual peak discharges

\begin{tabular}{|c|c|c|c|c|c|c|c|}
\hline $\begin{array}{l}\text { Water } \\
\text { year }\end{array}$ & Date & $\begin{array}{c}\text { Annual peak } \\
\text { discharge } \\
\left(\mathrm{ft}^{3} / \mathrm{s}\right)\end{array}$ & $\begin{array}{c}\text { Discharge } \\
\text { codes }\end{array}$ & $\begin{array}{c}\text { Water } \\
\text { year }\end{array}$ & Date & $\begin{array}{c}\text { Annual peak } \\
\text { discharge } \\
\left(\mathrm{ft}^{3} / \mathrm{s}\right)\end{array}$ & $\begin{array}{c}\text { Discharge } \\
\text { codes }\end{array}$ \\
\hline 1962 & $00-00-62$ & 1950 & & 1970 & $07-00-70$ & 615 & \\
\hline 1963 & $07-30-63$ & 896 & & 1971 & $08-03-71$ & 250 & \\
\hline 1964 & $00-00-64$ & 110 & ES & 1972 & $09-07-72$ & 649 & \\
\hline 1965 & $10-17-64$ & 70 & ES & 1973 & $00-00-73$ & 48 & \\
\hline 1966 & $08-00-66$ & 300 & ES & 1974 & $07-19-74$ & 225 & \\
\hline 1967 & $09-24-67$ & 330 & & 1975 & $07-22-75$ & 50 & \\
\hline 1968 & $10-03-67$ & 27 & & 1976 & $00-00-76$ & 220 & \\
\hline 1969 & $08-30-69$ & 40 & & 1978 & $00-00-78$ & ${ }^{2} 290$ & HP \\
\hline
\end{tabular}

THighest since 1958.

${ }^{2}$ Highest since 1972 .

Magnitude and probability of instantaneous peak flow based on period of record 1962-76, 1978

\begin{tabular}{|c|c|c|c|c|c|}
\hline \multicolumn{6}{|c|}{$\begin{array}{l}\text { Discharge, in } \mathrm{ft}^{3} / \mathrm{s} \text {, for indicated recurrence interval } \\
\text { in years, and exceedance probablility, in percent }\end{array}$} \\
\hline 2 & 5 & 10 & 25 & $50 t$ & $100 t$ \\
\hline $50 \%$ & $20 \%$ & $10 \%$ & $4 \%$ & $2 \%$ & $1 \%$ \\
\hline 196 & 501 & 798 & 1,290 & 1,740 & 2,270 \\
\hline Weighted skew & $(\log s)=$ & -0.22 & & & \\
\hline Mean & $(\log s)=$ & 2.27 & & & \\
\hline Standard dev. & $(\log s)=$ & 0.50 & & & \\
\hline
\end{tabular}

† Reliability of values in column is uncertain, and potential errors are large.

Basin characteristics

\begin{tabular}{cccccccr}
\hline $\begin{array}{c}\text { Main } \\
\text { channel } \\
\text { slope } \\
\text { (ft/mi) }\end{array}$ & $\begin{array}{c}\text { Stream } \\
\text { length } \\
\text { (mi) }\end{array}$ & $\begin{array}{c}\text { Mean } \\
\text { basin } \\
\text { elevation } \\
\text { (ft) }\end{array}$ & $\begin{array}{c}\text { Forested } \\
\text { area } \\
\text { (percent) }\end{array}$ & $\begin{array}{c}\text { Soil } \\
\text { index }\end{array}$ & $\begin{array}{c}\text { Mean } \\
\text { annual } \\
\text { precipitation } \\
\text { (in) }\end{array}$ & $\begin{array}{c}\text { Rainfall intensity, 24-hour } \\
\text { 2-year } \\
\text { (in) }\end{array}$ & $\begin{array}{c}50 \text {-year } \\
\text { (in) }\end{array}$ \\
\hline 93.8 & 5.4 & 4,180 & 0.0 & 1.0 & 12.3 & 1.7 \\
\hline
\end{tabular}


09471700 FENNER WASH NEAR BENSON, AZ--Continued

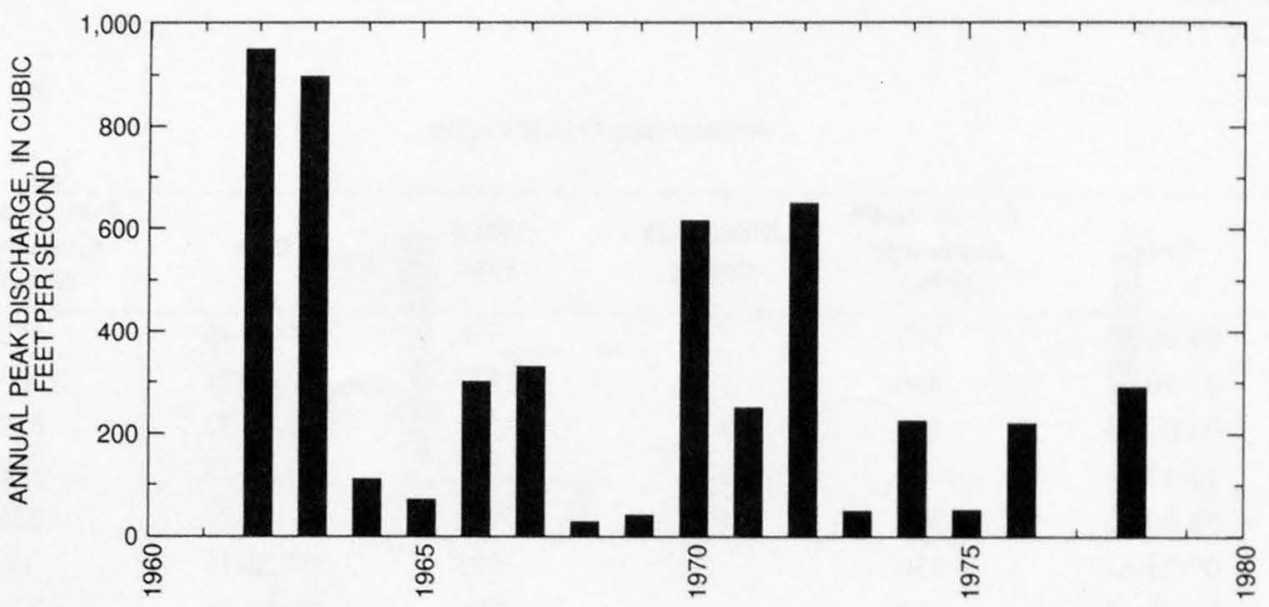


09471800 SAN PEDRO RIVER NEAR BENSON, AZ

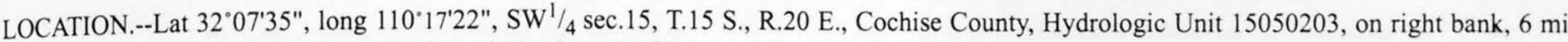
downstream from Tres Alamo Wash, and 11 mi north of Benson.

DRAINAGE AREA.--2,490 $\mathrm{mi}^{2}$, of which $696 \mathrm{mi}^{2}$ is in Mexico.

Annual peak discharges

\begin{tabular}{ccccccc}
\hline $\begin{array}{c}\text { Water } \\
\text { year }\end{array}$ & Date & $\begin{array}{c}\text { Annual peak } \\
\text { discharge } \\
\left(\mathbf{f t}^{\mathbf{3}} \mathbf{s}\right)\end{array}$ & $\begin{array}{c}\text { Discharge } \\
\text { codes }\end{array}$ & $\begin{array}{c}\text { Water } \\
\text { year }\end{array}$ & $\begin{array}{c}\text { Annual peak } \\
\text { discharge } \\
\left(\mathbf{f t}^{3} / \mathbf{s}\right)\end{array}$ \\
\hline 1966 & $08-06-66$ & 5,470 & & 1972 & $08-26-72$ & 9,800 \\
1967 & $07-26-67$ & 4,560 & & 1973 & $07-16-73$ & 2,140 \\
1968 & $08-10-68$ & 5,900 & & 1974 & $07-20-74$ & 9,520 \\
1969 & $07-28-69$ & 2,640 & & 1975 & $07-23-85$ & 8,920 \\
1970 & $07-20-70$ & 8,200 & & 1976 & $07-28-76$ & 5,110 \\
1971 & $08-11-71$ & 7,390 & & & & \\
\hline
\end{tabular}

Basin characteristics

\begin{tabular}{cccccccc}
\hline $\begin{array}{c}\text { Main } \\
\text { channel } \\
\text { slope } \\
\text { (ft/mi) }\end{array}$ & $\begin{array}{c}\text { Stream } \\
\text { length } \\
\text { (mi) }\end{array}$ & $\begin{array}{c}\text { Mean } \\
\text { basin } \\
\text { elevation } \\
\text { (ft) }\end{array}$ & $\begin{array}{c}\text { Forested } \\
\text { area } \\
\text { (percent) }\end{array}$ & $\begin{array}{c}\text { Soil } \\
\text { index }\end{array}$ & $\begin{array}{c}\text { Mean } \\
\text { annual } \\
\text { precipitation } \\
\text { (in) }\end{array}$ & $\begin{array}{c}\text { Rainfall intensity, 24-hour } \\
\text { (in) }\end{array}$ & $\begin{array}{c}50 \text {-year } \\
\text { (in) }\end{array}$ \\
\hline 17.3 & 84.0 & 4,800 & 12.0 & 1.9 & 15.6 & 1.9 \\
\hline
\end{tabular}




\section{GILA RIVER BASIN}

\section{SAN PEDRO RIVER NEAR BENSON, AZ--Continued}

MEAN MONTHLY AND ANNUAL DISCHARGES 1967.76

\begin{tabular}{|c|c|c|c|c|c|c|}
\hline MONTH & $\begin{array}{l}\text { MAXIMUM } \\
\text { (FT3/S) }\end{array}$ & $\begin{array}{l}\text { MINIMUM } \\
\text { (FT3/S) }\end{array}$ & $\begin{array}{c}\text { MEAN } \\
(\mathrm{FT} 3 / \mathrm{S})\end{array}$ & $\begin{array}{l}\text { STAN- } \\
\text { DARD } \\
\text { DEVIA- } \\
\text { TION } \\
(\mathrm{FT} 3 / \mathrm{S})\end{array}$ & $\begin{array}{l}\text { COEFFI- } \\
\text { CIENT OF } \\
\text { VARI- } \\
\text { ATION }\end{array}$ & $\begin{array}{c}\text { PERCENT } \\
\text { OF } \\
\text { ANNUAL } \\
\text { RUNOFF }\end{array}$ \\
\hline OCTOBER & 71 & 0.00 & 8.6 & 22 & 2.6 & 2.3 \\
\hline NOVEMBER & 23 & 0.00 & 2.3 & 7.2 & 3.2 & 0.6 \\
\hline DECEMBER & 196 & 0.00 & 20 & 62 & 3.1 & 5.4 \\
\hline JANUARY & 28 & 0.00 & 2.8 & 8.9 & 3.1 & 0.8 \\
\hline FEBRUARY & 47 & 0.00 & 8.2 & 18 & 2.1 & 2.2 \\
\hline MARCH & 46 & 0.00 & 8.1 & 17 & 2.1 & 2.1 \\
\hline APRIL & 0.01 & 0.00 & 0.00 & 0.00 & 3.2 & 0.0 \\
\hline MAY & 0.08 & 0.00 & 0.01 & 0.03 & 2.3 & 0.0 \\
\hline JUNE & 34 & 0.00 & 4.6 & 11 & 2.3 & 1.2 \\
\hline JULY & 260 & 11 & 124 & 85 & 0.68 & 32.8 \\
\hline AUGUST & 555 & 2.0 & 165 & 177 & 1.1 & 43.6 \\
\hline SEPTEMBER & 81 & 0.00 & 34 & 25 & 0.74 & 9.0 \\
\hline ANNUAL & 61 & 15 & 32 & 14 & 0.43 & 100 \\
\hline
\end{tabular}

MAGNITUDE AND PROBABILITY OF ANNUAL LOW FLOW BASED ON PERIOD OF RECORD 1967.76

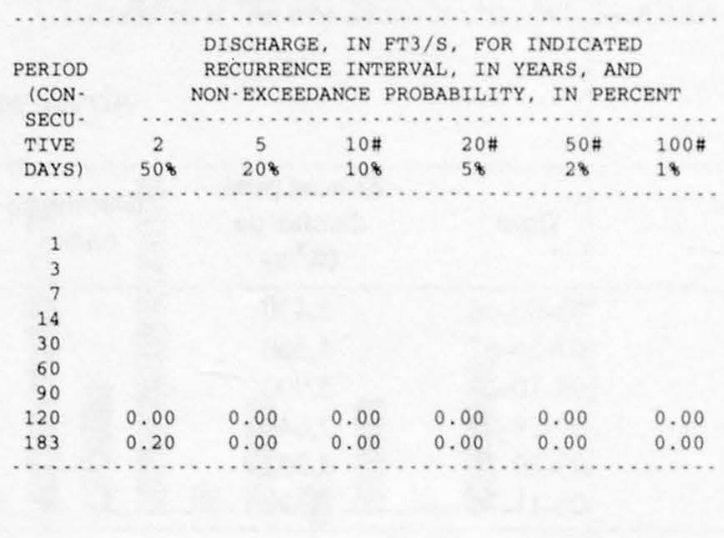

MAGNITUDE AND PROBABILITY OF ANNUAL HIGH FLOW BASED ON PERIOD OF RECORD 1967.76

\begin{tabular}{|c|c|c|c|c|c|c|}
\hline $\begin{array}{l}\text { PERIOD } \\
\text { /CON. }\end{array}$ & & \multicolumn{5}{|c|}{$\begin{array}{l}\text { DISCHARGE, IN FT3/S, FOR INDICATED } \\
\text { RECURRENCE INTERVAL, IN YEARS, AND }\end{array}$} \\
\hline SECU. & $\cdots$ & $\ldots \ldots \ldots$ & $\ldots \ldots$ & $\ldots \ldots \ldots$ & $\ldots \ldots \ldots$ & \\
\hline TIVE & 2 & 5 & $10 \#$ & $25 \#$ & $50 \#$ & $100 \#$ \\
\hline DAYS) & 508 & 208 & 108 & 48 & 28 & 18 \\
\hline 1 & 1.970 & 2.910 & 3,440 & 4.030 & 4,400 & 4.730 \\
\hline 3 & 1,100 & 1,620 & 1,930 & 2,260 & 2,470 & 2,660 \\
\hline 7 & 608 & 915 & 1,100 & 1.330 & 1,480 & 1.620 \\
\hline 15 & 392 & 619 & 774 & 974 & 1,120 & 1,270 \\
\hline 30 & 251 & 414 & 532 & 688 & 810 & 934 \\
\hline 60 & 153 & 243 & 302 & 374 & 427 & 477 \\
\hline 90 & 110 & 170 & 207 & 250 & 279 & 306 \\
\hline
\end{tabular}

DURATION TABLE OF DAILY MEAN FLOW FOR PERIOD OF RECORD 1967.76

DISCHARGE, IN FT3/S, WHICH WAS EQUALED OR EXCEEDED FOR INDICATED PERCENT OF TIME

\begin{tabular}{|c|c|c|c|c|c|c|c|c|c|c|c|c|c|c|c|c|}
\hline 18 & 58 & 108 & 158 & 208 & 308 & 408 & 508 & 608 & 708 & 808 & 908 & 958 & 988 & 998 & 99.58 & 99.98 \\
\hline & 126 & 41 & 16 & 2.3 & 0.00 & 0.00 & 0.00 & 0.00 & 0.00 & 0.00 & 0.00 & 0.00 & 0.00 & 0.00 & 0.00 & 0.00 \\
\hline
\end{tabular}

\# Reliability of values in column is uncertain, and potential errors are large. 

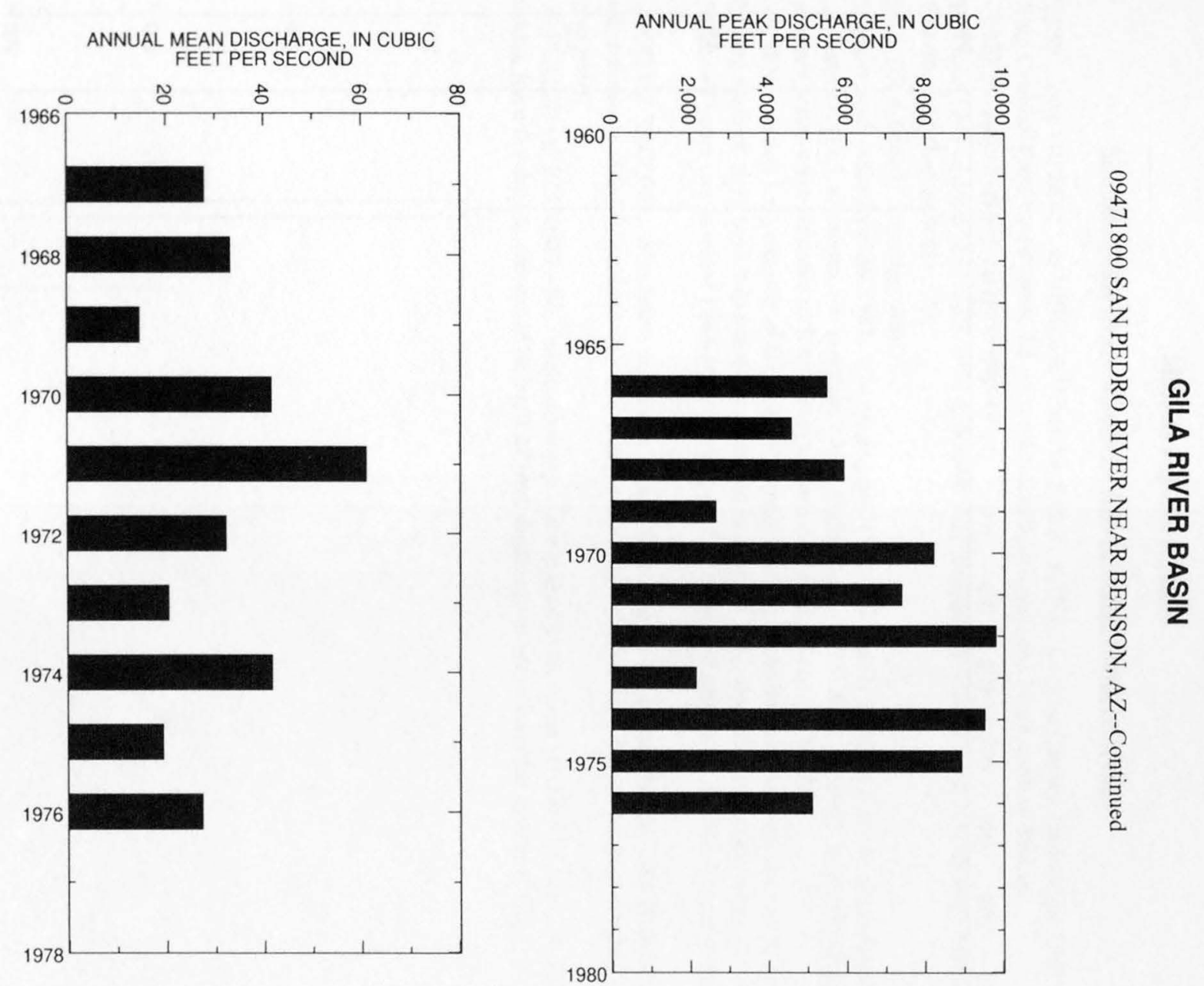
09471800 SAN PEDRO RIVER NEAR BENSON, AZ--Continued
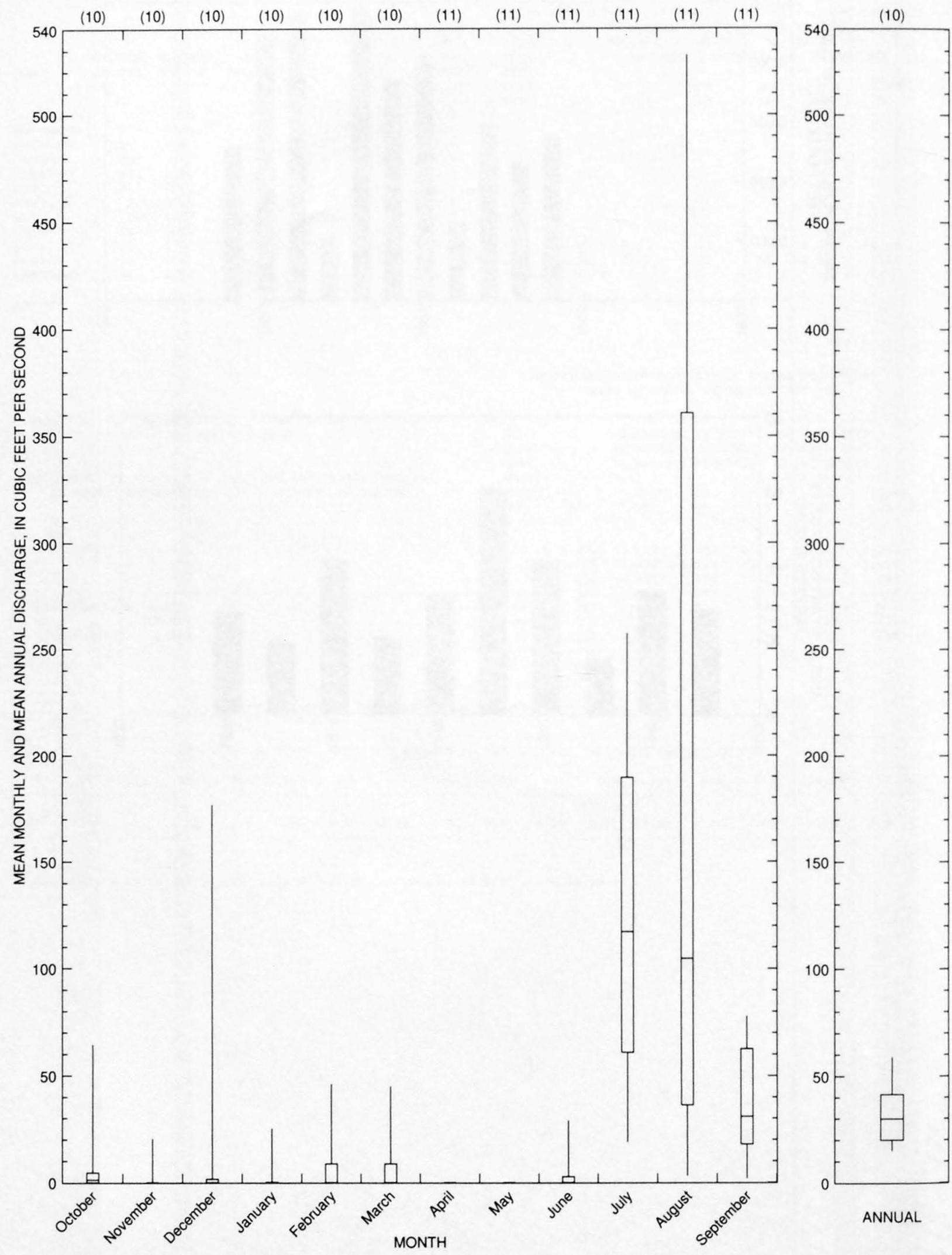


\section{SAN PEDRO RIVER NEAR REDINGTON, AZ}

LOCATION.--Lat 32'22'50", long 110'26'45", in NE $1 / 4^{2} \mathrm{NW}^{1} / 4$ sec.19, T.12 S., R.19 E., Cochise County, Hydrologic Unit 15050203, on left bank, $0.3 \mathrm{mi}$ upstream from Cochise-Pima County line, $4.3 \mathrm{mi}$ southeast of Redington, and $30 \mathrm{mi}$ north of Benson.

DRAINAGE AREA.--2,927 $\mathrm{mi}^{2}$, of which $696 \mathrm{mi}^{2}$ is in Mexico.

PERIOD OF RECORD.--June 1943 to June 1947, July 1950 to current year (monthly discharge only, July 1954 to March 1955). Also extremes for water years 1948-50, published in WSP 1179.

REVISED RECORDS.--WDR AZ-90-1: Drainage area.

GAGE.--Water-stage recorder, and, since Aug. 26, 1981, crest-stage gage. Datum of gage is 2,930.04 ft above sea level. June 1943 to June 1947 , and Oct. 1, 1962, to June 27, 1963, at present site at datum $7.49 \mathrm{ft}$ higher. July 1950 to Sept. 30, 1962, at site $400 \mathrm{ft}$ upstream at datum 10.47 $\mathrm{ft}$ higher. Supplementary water-stage recorder $50 \mathrm{ft}$ upstream at same datum since June 29, 1977.

REMARKS.--Diversions above station for irrigation of about 10,800 acres in 1978, excluding an unknown amount in Mexico. Diversion above gage into formerly used ditch on right bank was placed in operation in January 1972; diversion was measured Oct. 20, Mar. 2 , and May 5.

AVERAGE DISCHARGE.--49 years (water years 1944-46, 1951-96), $43.7 \mathrm{ft}^{3} / \mathrm{s}, 31,660 \mathrm{acre}-\mathrm{ft} / \mathrm{yr}$; median of yearly mean discharges, $27 \mathrm{ft}^{3} / \mathrm{s}$, $19,600 \mathrm{acre}-\mathrm{ft} / \mathrm{yr}$.

EXTREMES FOR PERIOD OF RECORD.--Maximum discharge, 28,600 ft3 $3 / \mathrm{s}$ Aug. 2, 1951, gage height, $20.2 \mathrm{ft}$, present site and datum, from rating curve extended above $16,000 \mathrm{ft}^{3} / \mathrm{s}$ on basis of slope-area measurement of peak flow, maximum gage height $21.60 \mathrm{ft}$, Oct. 2, 1983; no flow at times in most years.

EXTREMES OUTSIDE PERIOD OF RECORD.--Maximum discharge since at least 1906, about 90,000 ft $3 / \mathrm{s}$ Sept. 28, 1926, gage height, $29.0 \mathrm{ft}$, present site and datum, from floodmark, computed on basis of peak discharge of same flood for station at Charleston and for Gila River at Kelvin. 
GILA RIVER BASIN

09472000 SAN PEDRO RIVER NEAR REDINGTON, AZ--Continued

Annual peak discharges

\begin{tabular}{|c|c|c|c|c|c|c|c|}
\hline $\begin{array}{l}\text { Water } \\
\text { year }\end{array}$ & Date & $\begin{array}{l}\text { Annual peak } \\
\text { discharge } \\
\left(\mathrm{ft}^{3} / \mathrm{s}\right)\end{array}$ & $\begin{array}{l}\text { Discharge } \\
\text { codes }\end{array}$ & $\begin{array}{l}\text { Water } \\
\text { year }\end{array}$ & Date & $\begin{array}{c}\text { Annual peak } \\
\text { discharge } \\
\left(\mathrm{ft}^{3} / \mathrm{s}\right)\end{array}$ & $\begin{array}{l}\text { Discharge } \\
\text { codes }\end{array}$ \\
\hline 1926 & $09-28-26$ & ${ }^{1} 90,000$ & ES,HP & 1964 & $08-15-64$ & 6,070 & \\
\hline 1931 & $08-10-31$ & 18,000 & ES & 1965 & $08-14-65$ & 2,140 & \\
\hline 1932 & $10-02-31$ & 19,400 & ES & 1966 & $07-29-66$ & 5,890 & \\
\hline 1933 & $07-23-33$ & 13,500 & ES & 1967 & $09-25-67$ & 7,800 & \\
\hline 1934 & $08-04-34$ & 7,400 & ES & 1968 & $12-20-67$ & 5,000 & \\
\hline 1935 & $08-24-35$ & 16,300 & ES & 1969 & $08-07-69$ & 2,480 & \\
\hline 1936 & $09-11-36$ & 10,400 & ES & 1970 & $07-21-70$ & 8,490 & \\
\hline 1937 & $08-30-37$ & 14,100 & ES & 1971 & $08-11-71$ & 8,600 & \\
\hline 1938 & $08-05-38$ & 7,800 & ES & 1972 & $08-27-72$ & 11,400 & \\
\hline 1939 & $08-02-39$ & 9,920 & ES & 1973 & $10-19-72$ & 1,680 & \\
\hline 1940 & $08-14-40$ & 50,000 & ES & 1974 & $07-20-74$ & 12,100 & \\
\hline 1941 & $01-29-41$ & 10,100 & ES & 1975 & $07-23-75$ & 8,030 & \\
\hline 1943 & $08-09-43$ & 7,090 & & 1976 & $07-28-76$ & 6,550 & \\
\hline 1944 & $09-24-44$ & 19,000 & & 1977 & $08-24-77$ & 1,980 & \\
\hline 1945 & $08-10-45$ & 14,600 & & 1978 & $10-10-77$ & 23,000 & \\
\hline 1946 & $08-04-46$ & 9,000 & & 1979 & $01-18-79$ & 10,800 & \\
\hline 1947 & $08-08-47$ & 23,000 & & 1980 & $08-14-80$ & 392 & \\
\hline 1948 & $09-26-48$ & 11,500 & ES & 1981 & $07-29-81$ & 3,460 & \\
\hline 1949 & $00-00-49$ & 10,000 & ES & 1982 & $09-11-82$ & 7,190 & \\
\hline 1950 & $07-30-50$ & 8,800 & & 1983 & $09-28-83$ & 6,880 & \\
\hline 1951 & $08-02-51$ & 28,600 & & 1984 & $10-02-83$ & 25,400 & \\
\hline 1952 & $08-16-52$ & 4,470 & & 1985 & $12-29-84$ & 7,000 & \\
\hline 1953 & $07-07-53$ & 7,290 & & 1986 & $08-18-86$ & 2,140 & \\
\hline 1954 & $08-01-54$ & 18,500 & & 1987 & $08-22-87$ & 410 & \\
\hline 1955 & $08-07-55$ & 18,800 & & 1988 & $09-12-88$ & 1,020 & \\
\hline 1956 & $07-30-56$ & 3,160 & & 1989 & $10-20-88$ & 2,210 & \\
\hline 1957 & $08-18-57$ & 9,300 & & 1990 & $07-24-90$ & 2,960 & \\
\hline 1958 & $08-17-58$ & 10,800 & & 1991 & $03-02-91$ & 5,060 & \\
\hline 1959 & $07-27-59$ & 8,580 & & 1992 & $08-24-92$ & 3,980 & \\
\hline 1960 & $09-05-60$ & 1,980 & & 1993 & $01-19-93$ & 19,100 & \\
\hline 1961 & $07-30-61$ & 3,800 & & 1994 & $08-27-94$ & 1,090 & \\
\hline 1962 & $07-28-62$ & 2,050 & & 1995 & $01-05-95$ & 5,970 & \\
\hline 1963 & $08-26-63$ & 5,530 & & 1996 & $09-02-96$ & 1,680 & \\
\hline
\end{tabular}

${ }^{1}$ Highest since 1906. 
09472000 SAN PEDRO RIVER NEAR REDINGTON, AZ--Continued

Discharge rating table developed January 1993

\begin{tabular}{cccc}
\hline $\begin{array}{c}\text { Gage height } \\
(\mathrm{ft})\end{array}$ & $\begin{array}{c}\text { Discharge } \\
\left(\mathrm{ft}^{\mathbf{3}} / \mathbf{s}\right)\end{array}$ & $\begin{array}{c}\text { Gage height } \\
(\mathrm{ft})\end{array}$ & $\begin{array}{c}\text { Discharge } \\
\left(\mathrm{ft}^{\mathbf{3}} / \mathbf{s}\right)\end{array}$ \\
\hline 8.0 & 171 & 14.0 & 6,330 \\
9.0 & 491 & 15.0 & 8,610 \\
10.0 & 1,040 & 16.0 & 11,330 \\
11.0 & 1,860 & 17.0 & 14,520 \\
12.0 & 2,990 & 18.0 & 18,220 \\
13.0 & 4,470 & 19.0 & 22,450 \\
\hline
\end{tabular}

Basin characteristics

\begin{tabular}{cccccccc}
\hline $\begin{array}{c}\text { Main } \\
\text { channel } \\
\text { slope } \\
(\mathrm{ft} / \mathrm{mi})\end{array}$ & $\begin{array}{c}\text { Stream } \\
\text { length } \\
(\mathrm{mi})\end{array}$ & $\begin{array}{c}\text { Mean } \\
\text { basin } \\
\text { elevation } \\
(\mathrm{ft})\end{array}$ & $\begin{array}{c}\text { Forested } \\
\text { area } \\
\text { (percent) }\end{array}$ & $\begin{array}{c}\text { Soil } \\
\text { index }\end{array}$ & $\begin{array}{c}\text { Mean } \\
\text { annual } \\
\text { precipitation } \\
\text { (in) }\end{array}$ & $\begin{array}{c}\text { Rainfall intensity, 24-hour } \\
\text { (in) }\end{array}$ & $\begin{array}{c}50 \text {-year } \\
\text { (in) }\end{array}$ \\
\hline 15.3 & 124 & 4,660 & 13.0 & 1.8 & 15.5 & 1.9 \\
\hline
\end{tabular}


09472000 SAN PEDRO RIVER NEAR REDINGTON, AZ--Continued

MEAN MONTHLY AND ANNUAL DISCHARGES $1944-46,1951.96$

\begin{tabular}{|c|c|c|c|c|c|c|}
\hline MONTH & $\begin{array}{l}\text { MAXIMUM } \\
\text { (FT3/S) }\end{array}$ & $\begin{array}{l}\text { MINIMUM } \\
\text { (FT3/S) }\end{array}$ & $\begin{array}{c}\text { MEAN } \\
(\mathrm{FT} 3 / \mathrm{S})\end{array}$ & $\begin{array}{l}\text { STAN - } \\
\text { DARD } \\
\text { DEVIA - } \\
\text { TION } \\
(\mathrm{FT} 3 / \mathrm{S})\end{array}$ & $\begin{array}{l}\text { COEFFI- } \\
\text { CIENT OF } \\
\text { VARI- } \\
\text { ATION }\end{array}$ & $\begin{array}{c}\text { PERCENT } \\
\text { OF } \\
\text { ANNUAL } \\
\text { RUNOFF }\end{array}$ \\
\hline OCTOBER & 939 & 0.00 & 44 & 173 & 4.0 & 8.4 \\
\hline NOVEMBER & 108 & 0.00 & 5.4 & 17 & 3.2 & 1.0 \\
\hline DECEMBER & 532 & 0.00 & 38 & 111 & 2.9 & 7.4 \\
\hline JANUARY & 1,160 & 0.00 & 58 & 198 & 3.4 & 11.2 \\
\hline FEBRUARY & 223 & 0.00 & 25 & 52 & 2.1 & 4.7 \\
\hline MARCH & 193 & 0.00 & 19 & 40 & 2.1 & 3.6 \\
\hline APRIL & 59 & 0.00 & 3.7 & 9.5 & 2.6 & 0.7 \\
\hline MAY & 19 & 0.00 & 1.3 & 3.7 & 3.0 & 0.2 \\
\hline JUNE & 26 & 0.00 & 1.6 & 4.4 & 2.8 & 0.3 \\
\hline JULY & 621 & 0.00 & 90 & 115 & 1.3 & 17.3 \\
\hline AUGUST & 1,480 & 0.08 & 188 & 278 & 1.5 & 36.3 \\
\hline SEPTEMBER & 367 & 0.07 & 45 & 70 & 1.5 & 8.7 \\
\hline ANNUAL & 179 & 2.9 & 44 & 42 & 0.96 & 100 \\
\hline
\end{tabular}
MAGNITUDE AND PROBABILITY OF INSTANTANEOUS PEAK FLOW
BASED ON PERIOD OF RECORD 1926, 1931-41, 1943.96

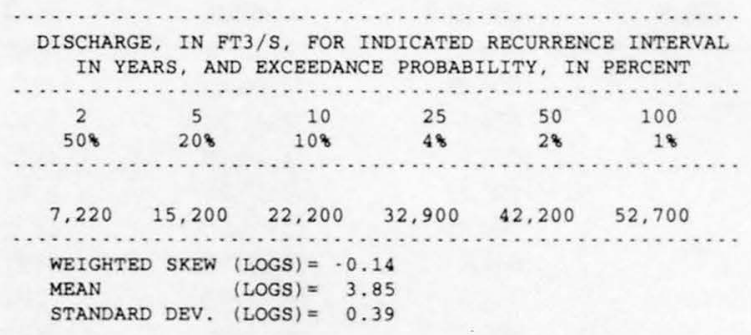

MAGNITUDE AND PROBABILITY OF ANNUAL LOW FLOW BASED ON PERIOD OF RECORD $1945 \cdot 47,1952 \cdot 96$

\begin{tabular}{|c|c|c|c|c|c|c|}
\hline \multirow{2}{*}{$\begin{array}{l}\text { PERIOD } \\
\text { (CON- } \\
\text { SECU- } \\
\text { TIVB }\end{array}$} & \multicolumn{6}{|c|}{$\begin{array}{l}\text { DISCHARGE, IN FT3/S, FOR INDICATED } \\
\text { RECURRENCE INTERVAL, IN YEARS, AND }\end{array}$} \\
\hline & 2 & 5 & $10 \#$ & $20 \#$ & $50 \#$ & $100 \#$ \\
\hline DAYS) & 508 & 208 & 108 & 58 & 28 & 18 \\
\hline $\begin{array}{l}1 \\
3\end{array}$ & & & & & & \\
\hline 7 & 0.00 & 0.00 & 0.00 & 0.00 & 0.00 & 0.00 \\
\hline 14 & 0.00 & 0.00 & 0.00 & 0.00 & 0.00 & 0.00 \\
\hline 30 & 0.00 & 0.00 & 0.00 & 0.00 & 0.00 & 0.00 \\
\hline 60 & 0.00 & 0.00 & 0.00 & 0.00 & 0.00 & 0.00 \\
\hline 90 & 0.08 & 0.00 & 0.00 & 0.00 & 0.00 & 0.00 \\
\hline 120 & 1.0 & 0.09 & 0.00 & 0.00 & 0.00 & 0.00 \\
\hline 183 & 3.2 & 0.52 & 0.16 & 0.05 & 0.00 & 0.00 \\
\hline
\end{tabular}

DURATION TABLE OF DAILY MEAN FLOW FOR PERIOD OF RECORD 1944-46, 1951.96

DISCHARGE, IN FT3/S, WHICH WAS EQUALED OR EXCEEDED FOR INDICATED PERCENT OF TIME

\begin{tabular}{|c|c|c|c|c|c|c|c|c|c|c|c|c|c|c|c|c|}
\hline 18 & 58 & 108 & 158 & 208 & 308 & 408 & 508 & 608 & 708 & 808 & 908 & 958 & 988 & 998 & 99.58 & 99.98 \\
\hline 41 & 168 & 52 & 24 & 11 & 3.0 & 1.2 & 0.46 & 0.10 & 0.00 & 0.00 & 0.00 & 0.00 & 0.00 & 0.00 & 0.00 & 0.00 \\
\hline
\end{tabular}

\# Reliability of values in column is uncertain, and potential errors are large. 


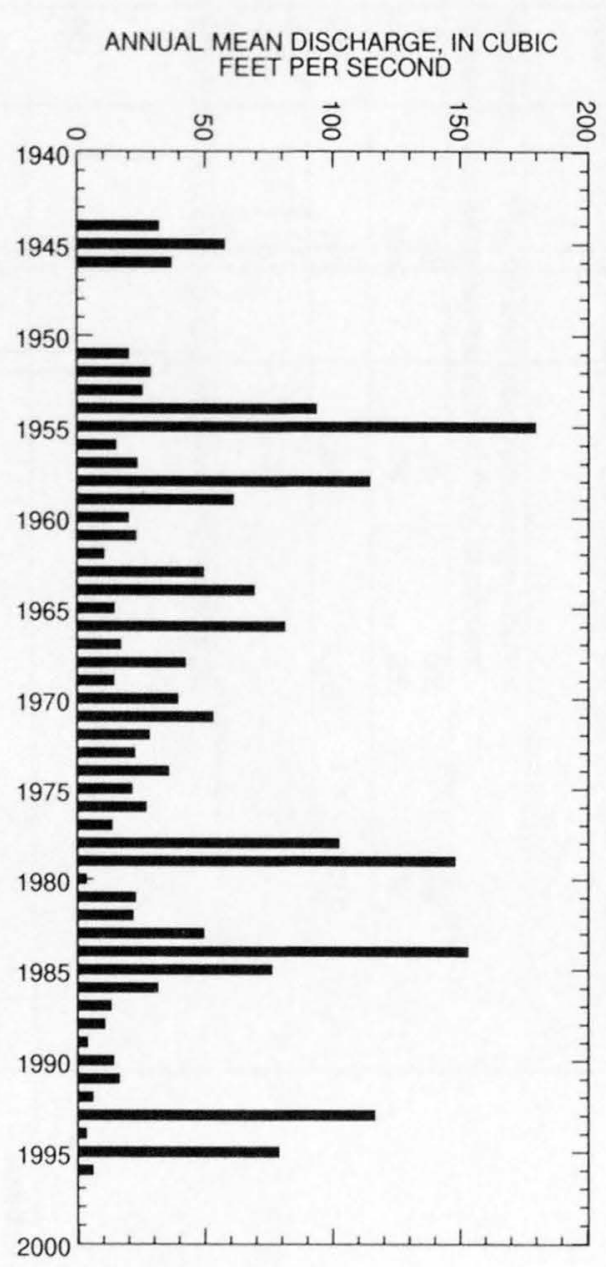

ANNUAL PEAK DISCHARGE, IN CUBIC

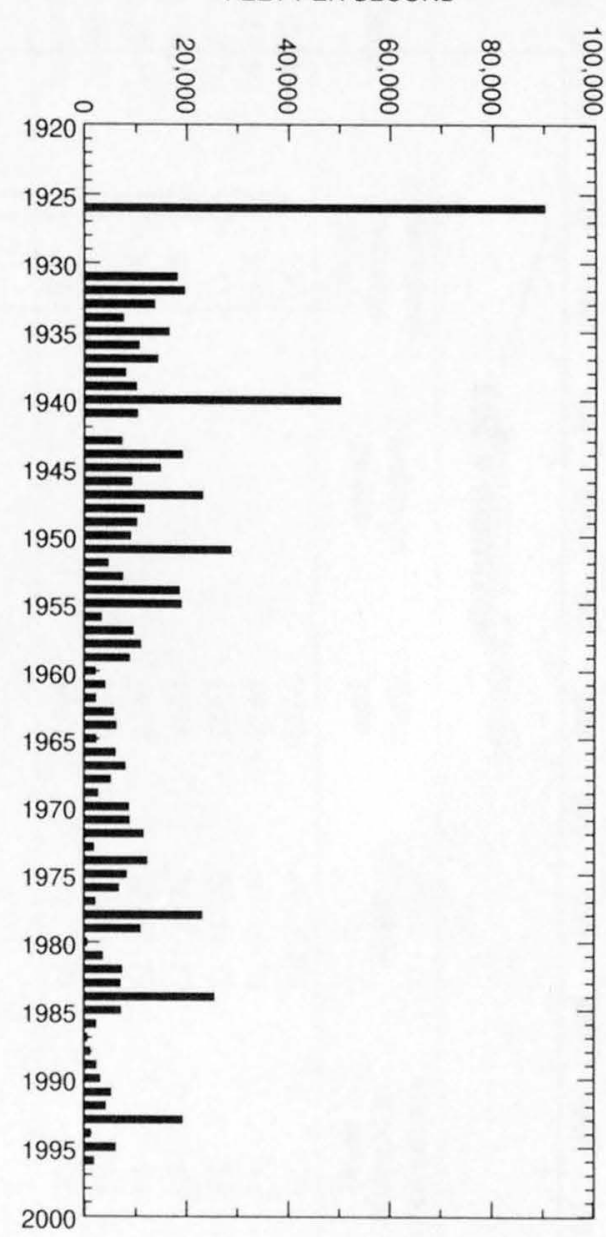


09472000 SAN PEDRO RIVER NEAR REDINGTON, AZ--Continued
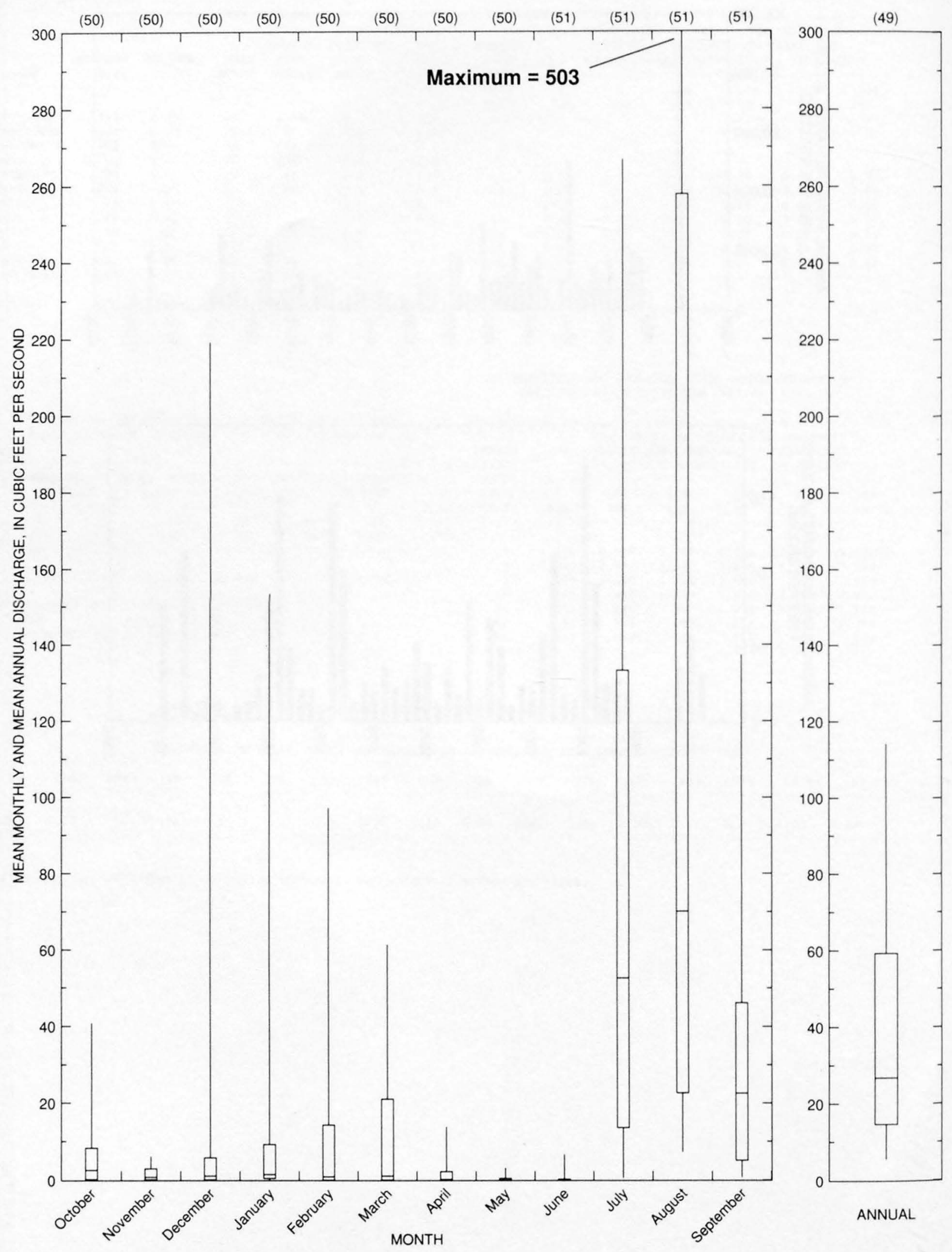
09472100 PECK CANYON TRIBUTARY NEAR REDINGTON, AZ

LOCATION.--Lat $32^{\circ} 29^{\prime} 12^{\prime \prime}$, long $110^{\circ} 30^{\prime} 00^{\prime \prime}, \mathrm{SW}^{1} / \mathrm{SW}^{1} / 4 \mathrm{sec} .10$, T.11 S., R.18 E., Pima County, Hydrologic Unit 15050203 , on left bank 0.2 mi upstream from mouth and $4 \mathrm{mi}$ north of Redington.

DRAINAGE AREA.--8.02 $\mathrm{mi}^{2}$.

Annual peak discharges

\begin{tabular}{|c|c|c|c|c|c|c|c|}
\hline $\begin{array}{l}\text { Water } \\
\text { year }\end{array}$ & Date & $\begin{array}{c}\text { Annual peak } \\
\text { discharge } \\
\left(\mathrm{ft}^{3} / \mathrm{s}\right)\end{array}$ & $\begin{array}{c}\text { Discharge } \\
\text { codes }\end{array}$ & $\begin{array}{l}\text { Water } \\
\text { year }\end{array}$ & Date & $\begin{array}{c}\text { Annual peak } \\
\text { discharge } \\
\left(\mathrm{ft}^{3} / \mathrm{s}\right)\end{array}$ & $\begin{array}{l}\text { Discharge } \\
\text { codes }\end{array}$ \\
\hline 1968 & $08-09-68$ & 650 & & 1975 & $10-00-74$ & 70 & \\
\hline 1969 & $08-28-69$ & 150 & & 1976 & $07-19-76$ & 110 & \\
\hline 1970 & $08-14-70$ & 73 & & 1977 & $08-14-77$ & 58 & \\
\hline 1971 & $07-29-71$ & 442 & & 1978 & $10-07-77$ & 69 & \\
\hline 1972 & $08-12-72$ & 4,340 & & 1979 & 08-07-79 & 94 & \\
\hline 1973 & $10-00-72$ & 10 & ES & 1980 & $08-11-80$ & 51 & \\
\hline 1974 & $00-00-74$ & 120 & & 1981 & $07-20-81$ & 100 & \\
\hline
\end{tabular}

Magnitude and probability of instantaneous peak flow based on period of record 1968-81

\begin{tabular}{|c|c|c|c|c|c|}
\hline \multicolumn{6}{|c|}{$\begin{array}{l}\text { Discharge, in } \mathrm{ft}^{3} / \mathrm{s} \text {, for indicated recurrence interval } \\
\text { in years, and exceedance probablility, in percent }\end{array}$} \\
\hline 2 & 5 & 10 & 25 & $50 t$ & $100 t$ \\
\hline $50 \%$ & $20 \%$ & $10 \%$ & $4 \%$ & $2 \%$ & $1 \%$ \\
\hline 118 & 402 & 793 & 1,680 & 2,780 & 4,410 \\
\hline Weighted skew & $(\log s)=$ & 0.27 & & & \\
\hline Mean & $(\log s)=$ & 2.10 & & & \\
\hline Standard dev. & $(\log s)=$ & 0.61 & & & \\
\hline
\end{tabular}

fReliability of values in column is uncertain, and potential errors are large.

\section{Basin characteristics}

\begin{tabular}{cccccccc}
\hline $\begin{array}{c}\text { Main } \\
\text { channel } \\
\text { slope } \\
(\mathrm{ft} / \mathrm{mi})\end{array}$ & $\begin{array}{c}\text { Stream } \\
\text { length } \\
(\mathrm{mi})\end{array}$ & $\begin{array}{c}\text { Mean } \\
\text { basin } \\
\text { elevation } \\
(\mathrm{ft})\end{array}$ & $\begin{array}{c}\text { Forested } \\
\text { area } \\
\text { (percent) }\end{array}$ & $\begin{array}{c}\text { Soil } \\
\text { index }\end{array}$ & $\begin{array}{c}\text { Mean } \\
\text { annual } \\
\text { precipitation } \\
\text { (in) }\end{array}$ & $\begin{array}{c}\text { R-year } \\
\text { (in) }\end{array}$ & $\begin{array}{c}50 \text {-year } \\
\text { (in) }\end{array}$ \\
\hline 146 & 9.3 & 3,680 & 0.0 & 1.0 & 11.7 & 1.7 \\
\hline
\end{tabular}

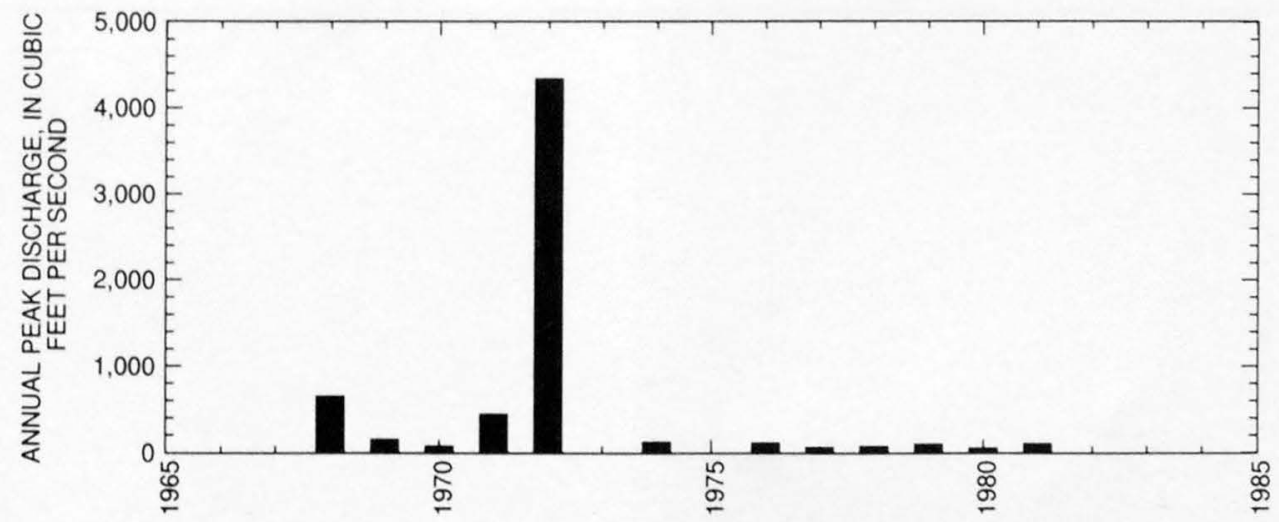


09472400 MAMMOTH WASH NEAR MAMMOTH, AZ

LOCATION.--Lat 3240'35", long $110^{\circ} 41^{\prime} 05^{\prime \prime}$, SW $1 / 4$ sec.2, T.9 S., R.16 E., Pinal County, Hydrologic Unit 15050203, at State Highway 76,3 mi southwest of Mammoth..

DRAINAGE AREA.--2.40 $\mathrm{mi}^{2}$.

Annual peak discharges

\begin{tabular}{|c|c|c|c|c|c|c|c|}
\hline $\begin{array}{l}\text { Water } \\
\text { year }\end{array}$ & Date & $\begin{array}{c}\text { Annual peak } \\
\text { discharge } \\
\left(\mathrm{ft}^{3} / \mathrm{s}\right)\end{array}$ & $\begin{array}{l}\text { Discharge } \\
\text { codes }\end{array}$ & $\begin{array}{l}\text { Water } \\
\text { year }\end{array}$ & Date & $\begin{array}{c}\text { Annual peak } \\
\text { discharge } \\
\left(\mathrm{ft}^{3} / \mathrm{s}\right)\end{array}$ & $\begin{array}{c}\text { Discharge } \\
\text { codes }\end{array}$ \\
\hline$\ldots$ & -....- & $1_{3,200}$ & HP & 1970 & $03-03-70$ & 1.0 & LT \\
\hline 1963 & $00-00-63$ & 1,290 & & 1971 & $09-29-71$ & 240 & \\
\hline 1964 & $00-00-64$ & 736 & & 1972 & $12-00-71$ & 15 & \\
\hline 1965 & $07-16-65$ & 300 & ES & 1973 & $08-05-73$ & 1,060 & \\
\hline 1966 & $12-10-65$ & 10 & LT & 1974 & $09-00-74$ & 236 & \\
\hline 1967 & $07-17-67$ & 510 & & 1975 & $08-13-75$ & 40 & ES \\
\hline 1968 & $08-26-68$ & 100 & & 1976 & $00-00-76$ & 260 & \\
\hline 1969 & $09-05-69$ & 12 & ES & & & & \\
\hline
\end{tabular}

${ }^{1}$ Highest since 1956, year of occurrence unknown.

Magnitude and probability of instantaneous peak flow based on period of record 1956, 1963-76

\begin{tabular}{|c|c|c|c|c|c|}
\hline \multicolumn{6}{|c|}{$\begin{array}{l}\text { Discharge, in } \mathrm{ft}^{3} / \mathrm{s} \text {, for indicated recurrence interval } \\
\text { in years, and exceedance probablility, in percent }\end{array}$} \\
\hline 2 & 5 & 10 & 25 & $50 t$ & $100 t$ \\
\hline $50 \%$ & $20 \%$ & $10 \%$ & $4 \%$ & $2 \%$ & $1 \%$ \\
\hline 167 & 752 & 1,540 & 3,130 & 4,810 & 6,960 \\
\hline Weighted skew & $(\log s)=$ & -0.42 & & & \\
\hline Mean & $(\log s)=$ & 2.16 & & & \\
\hline Standard dev. & $(\log s)=$ & 0.83 & & & \\
\hline
\end{tabular}

7 Reliability of values in column is uncertain, and potential errors are large.

Basin characteristics

\begin{tabular}{cccccccc}
\hline $\begin{array}{c}\text { Main } \\
\text { channel } \\
\text { slope } \\
(\mathrm{ft} / \mathrm{mi})\end{array}$ & $\begin{array}{c}\text { Stream } \\
\text { length } \\
\text { (mi) }\end{array}$ & $\begin{array}{c}\text { Mean } \\
\text { basin } \\
\text { elevation } \\
(\mathrm{ft})\end{array}$ & $\begin{array}{c}\text { Forested } \\
\text { area } \\
\text { (percent) }\end{array}$ & $\begin{array}{c}\text { Soil } \\
\text { index }\end{array}$ & $\begin{array}{c}\text { Mean } \\
\text { annual } \\
\text { precipitation } \\
\text { (in) }\end{array}$ & $\begin{array}{c}\text { R-year } \\
\text { (in) }\end{array}$ & $\begin{array}{c}50 \text {-year } \\
\text { (in) }\end{array}$ \\
\hline 181 & 4.2 & 3,700 & 0.0 & 1.0 & 13.8 & 1.8 \\
\hline
\end{tabular}


09472400 MAMMOTH WASH NEAR MAMMOTH, AZ--Continued

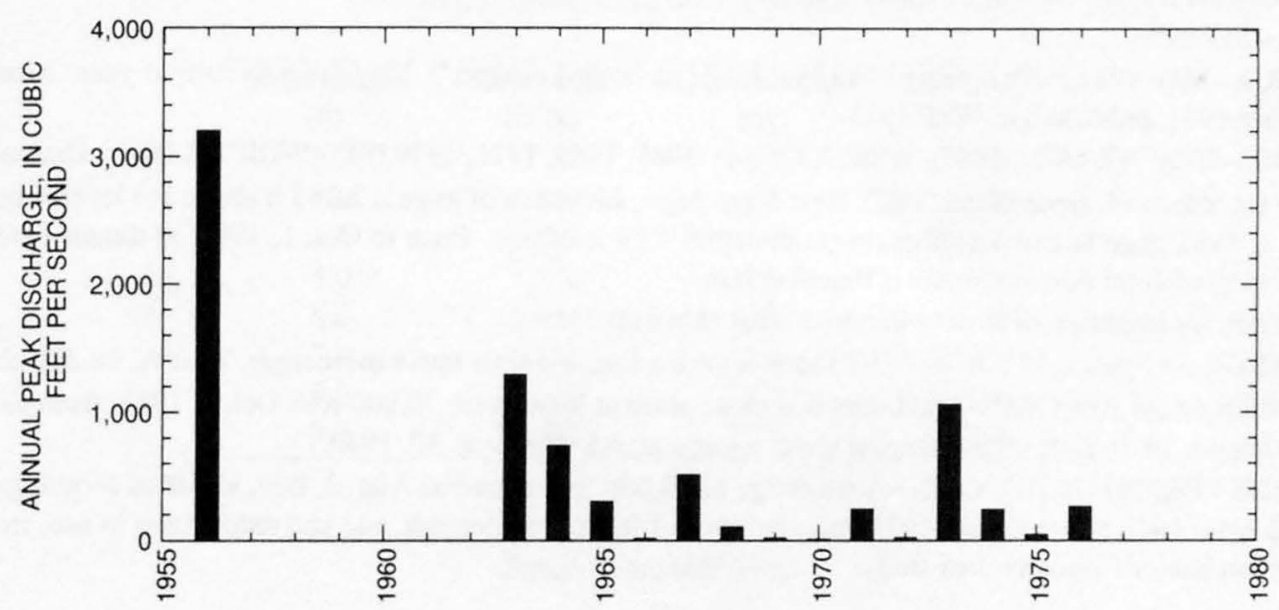




\section{ARAVAIPA CREEK NEAR MAMMOTH, AZ}

LOCATION.--Lat $32^{\circ} 50^{\prime} 37^{\prime \prime}$, long $110^{\circ} 37^{\prime} 09^{\prime \prime}$, in NW $1 /{ }_{4} \mathrm{NW}^{1} / 4$ sec.9, T.7 S., R.17 E., Pinal County, Hydrologic Unit 15050203 , on right bank 6 mi upstream from mouth and 9 mi north of Mammoth.

DRAINAGE AREA.--537 $\mathrm{mi}^{2}$.

PERIOD OF RECORD.--May 1931 to December 1942 (published as "near Feldman"), May 1966 to current year. Monthly discharge only July 1941 to September 1941, published in WSP 1313.

REVISED RECORDS.--WDR AZ-68-1: 1967. WDR AZ-82-1: 1968, 1969, 1973, 1979 (M). WDR AZ-90-1: Drainage area.

GAGE.--Water-stage recorder and, since March 1980, crest-stage gage. Elevation of gage is 2,345 ft above sea level, from topographic map. Oct. 1, 1981 to Oct. 1, 1983 gage at site $300 \mathrm{ft}$ upstream at datum $4.19 \mathrm{ft}$ higher. Prior to Oct. 1, 1981, at datum $1.00 \mathrm{ft}$ higher. May 1931 to December 1942 at site $0.3 \mathrm{mi}$ downstream at different datum.

REMARKS.--Diversions for irrigation of several hundred acres above station.

AVERAGE DISCHARGE.--41 years, $37.2 \mathrm{ft}^{3} / \mathrm{s}, 26,950$ acre- $\mathrm{ft} / \mathrm{yr}$; median of yearly mean discharges, $26 \mathrm{ft}^{3} / \mathrm{s}, 18,800 \mathrm{acre}-\mathrm{ft} / \mathrm{yr}$.

EXTREMES FOR PERIOD OF RECORD.--Maximum discharge since at least 1919, 70,800 $\mathrm{ft}^{3} / \mathrm{s}$ Oct. 1, 1983, from slope-area measurement of peak flow, gage height, $16.76 \mathrm{ft}$, from profile past gage; minimum, $0.3 \mathrm{ft}^{3} / \mathrm{s} \mathrm{Aug.} 30,1940$.

EXTREMES OUTSIDE PERIOD OF RECORD.--A discharge of 20,000 ft $3 / \mathrm{s}$ occurred Aug. 2, 1919, at site of former gaging station $6 \mathrm{mi}$ downstream, operated April 1919 to September 1921, gage height, $6.3 \mathrm{ft}$, from floodmark, site and datum then in use, from rating curve extended above $5,100 \mathrm{ft}^{3} / \mathrm{s}$ on basis of velocity-area study.

Annual peak discharges

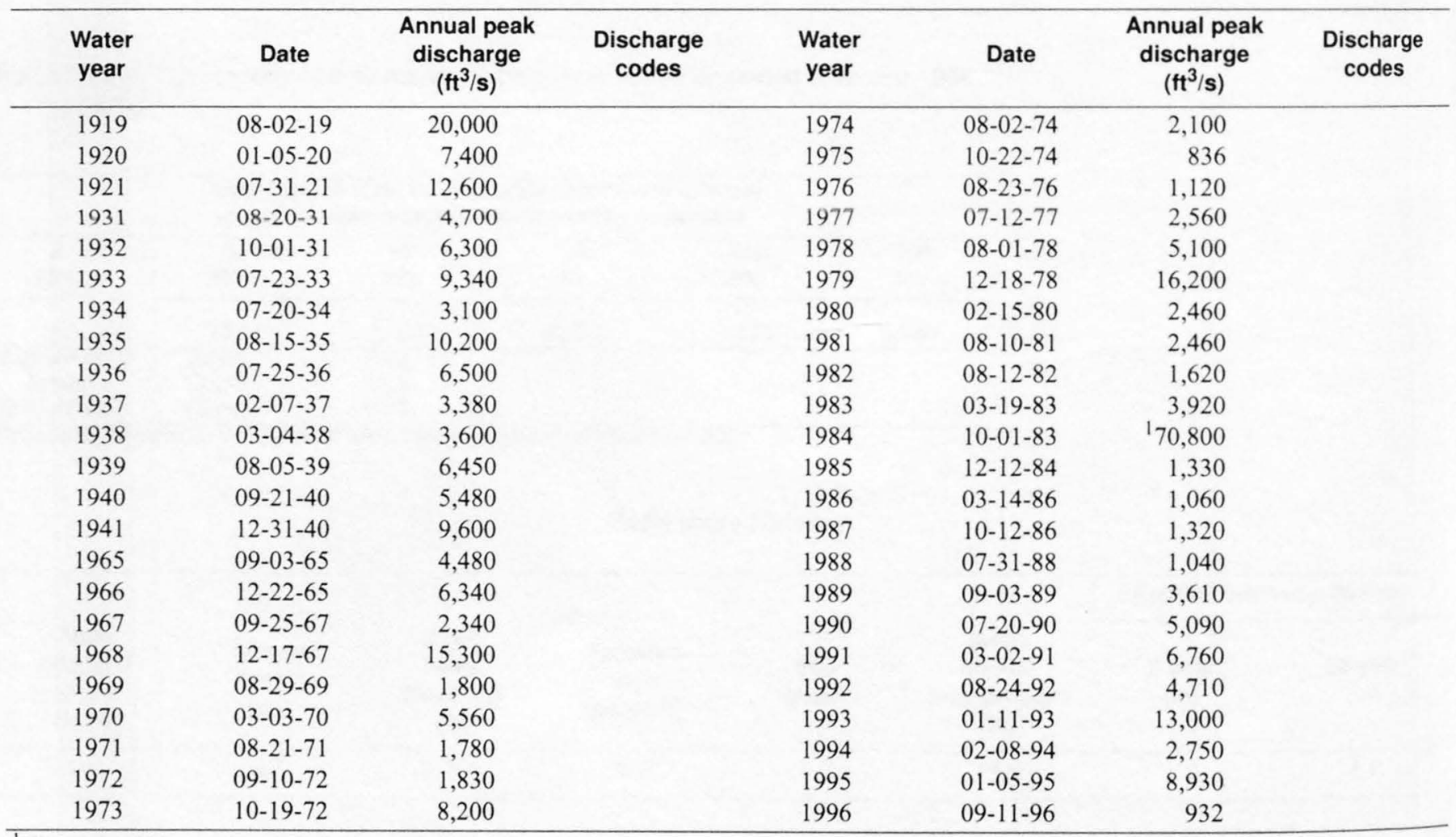

${ }^{1}$ Highest in 1100 years based on Roberts (1987). 
09473000 ARAVAIPA CREEK NEAR MAMMOTH, AZ--Continued

Discharge rating table developed October 1995

\begin{tabular}{crcc}
\hline $\begin{array}{c}\text { Gage height } \\
(\mathbf{f t})\end{array}$ & $\begin{array}{c}\text { Discharge } \\
\left(\mathrm{ft}^{\mathbf{3}} \mathbf{/}\right)\end{array}$ & $\begin{array}{c}\text { Gage height } \\
(\mathbf{f t})\end{array}$ & $\begin{array}{c}\text { Discharge } \\
\left(\mathbf{f t}^{\mathbf{3}} \mathbf{s}\right)\end{array}$ \\
\hline 2.7 & 58 & 10.0 & 17,000 \\
3.0 & 136 & 11.0 & 22,300 \\
4.0 & 686 & 12.0 & 28,400 \\
5.0 & 1,770 & 13.0 & 35,400 \\
6.0 & 3,460 & 14.0 & 43,400 \\
7.0 & 5,830 & 15.0 & 52,400 \\
8.0 & 8,930 & 16.0 & 62,400 \\
9.0 & 12,600 & 17.0 & 73,000 \\
\hline
\end{tabular}

Basin characteristics

\begin{tabular}{cccccccr}
\hline $\begin{array}{c}\text { Main } \\
\text { channel } \\
\text { slope } \\
\text { (ft/mi) }\end{array}$ & $\begin{array}{c}\text { Stream } \\
\text { length } \\
\text { (mi) }\end{array}$ & $\begin{array}{c}\text { Mean } \\
\text { basin } \\
\text { elevation } \\
\text { (ft) }\end{array}$ & $\begin{array}{c}\text { Forested } \\
\text { area } \\
\text { (percent) }\end{array}$ & $\begin{array}{c}\text { Soil } \\
\text { index }\end{array}$ & $\begin{array}{c}\text { Mean } \\
\text { annual } \\
\text { precipitation } \\
\text { (in) }\end{array}$ & $\begin{array}{c}\text { Rainfall intensity, 24-hour } \\
\text { (in) }\end{array}$ & $\begin{array}{c}50 \text {-year } \\
\text { (in) }\end{array}$ \\
\hline 45.7 & 55.4 & 4,530 & 24.0 & 2.0 & 16.2 & 2.0 \\
\hline
\end{tabular}


09473000 ARAVAIPA CREEK NEAR MAMMOTH, AZ--Continued

MEAN MONTHLY AND ANNUAL DISCHARGES $1932.40,1942,1967.96$

\begin{tabular}{|c|c|c|c|c|c|c|}
\hline MONTH & $\begin{array}{l}\text { MAXIMUM } \\
\text { (FT3/S) }\end{array}$ & $\begin{array}{l}\text { MINIMUM } \\
\text { (FT3/S) }\end{array}$ & $\begin{array}{c}\text { MEAN } \\
(\mathrm{FT} 3 / \mathrm{S})\end{array}$ & $\begin{array}{l}\text { STAN- } \\
\text { DARD } \\
\text { DEVIA- } \\
\text { TION } \\
(\mathrm{FT} 3 / \mathrm{S})\end{array}$ & $\begin{array}{l}\text { COEFFI- } \\
\text { CIENT OF } \\
\text { VARI- } \\
\text { ATION }\end{array}$ & $\begin{array}{c}\text { PERCENT } \\
\text { OF } \\
\text { ANNUAL } \\
\text { RUNOFF }\end{array}$ \\
\hline OCTOBER & 1,100 & 6.2 & 52 & 173 & 3.3 & 12.0 \\
\hline NOVEMBER & 91 & 8.7 & 24 & 16 & 0.69 & 5.4 \\
\hline DECEMBER & 474 & 9.7 & 47 & 78 & 1.7 & 10.8 \\
\hline JANUARY & 682 & 10 & 57 & 111 & 2.0 & 13.1 \\
\hline FEBRUARY & 215 & 11 & 64 & 60 & 0.93 & 14.8 \\
\hline MARCH & 349 & 9.5 & 60 & 76 & 1.3 & 13.8 \\
\hline APRIL & 53 & 7.2 & 21 & 12 & 0.58 & 4.8 \\
\hline MAY & 45 & 4.3 & 15 & 10 & 0.67 & 3.5 \\
\hline JUNE & 40 & 1.9 & 13 & 9.7 & 0.77 & 2.9 \\
\hline JULY & 115 & 7.1 & 25 & 22 & 0.89 & 5.7 \\
\hline AUGUST & 133 & 7.8 & 32 & 24 & 0.75 & 7.5 \\
\hline SEPTEMBER & 56 & 5.4 & 25 & 13 & 0.51 & 5.7 \\
\hline ANNUAL & 140 & 9.6 & 36 & 28 & 0.77 & 100 \\
\hline
\end{tabular}

MAGNITUDE AND PROBABILITY OF INSTANTANEOUS PEAK FLOW BASED ON PERIOD OF RECORD 1888, 1919-21, 1931-41, 1965-96

DISCHARGE, IN FT3/S, FOR INDICATED RECURRENCE INTERVAL IN YEARS, AND EXCEEDANCE PROBABILITY, IN PERCENT

\begin{tabular}{|c|c|c|c|c|c|}
\hline 2 & 5 & 10 & 25 & 50 & 100 \\
\hline 508 & 208 & 108 & 48 & 28 & 18 \\
\hline 3,980 & 8,000 & 11,500 & 16,800 & 21,600 & 26,900 \\
\hline WEIGHTED & SKEW & LOGS $)=-$ & & & \\
\hline MEAN & & LOGS $)=$ & & & \\
\hline STANDARD & DEV. & LOGS) $=$ & & & \\
\hline
\end{tabular}

MAGNITUDE AND PROBABILITY OF ANNUAL LOW FLOW BASED ON PERIOD OF RECORD 1933.41, 1968.96

\begin{tabular}{|c|c|c|c|c|c|c|}
\hline \multirow{2}{*}{$\begin{array}{l}\text { PERIOD } \\
\text { (CON- } \\
\text { SECU- } \\
\text { TIVE }\end{array}$} & \multicolumn{3}{|c|}{$\begin{array}{l}\text { DISCHARGE, IN } \\
\text { RECURRENCE IN } \\
\text { NON-EXCEEDANCE }\end{array}$} & $\begin{array}{l}\text { FOR } \\
\text { IN } \\
\text { BILIT } \\
\ldots . . .\end{array}$ & $\begin{array}{l}\text { IDICAT } \\
\text { RS, A } \\
\text { IN PE } \\
\ldots . .\end{array}$ & $\begin{array}{l}\text { ENT } \\
\cdots\end{array}$ \\
\hline & 2 & 5 & 10 & 20 & 50 & $100 \#$ \\
\hline DAYS) & 508 & 208 & 108 & 58 & 28 & 18 \\
\hline 1 & 4.1 & 1.6 & 1.0 & 0.68 & 0.43 & 0.32 \\
\hline 3 & 4.4 & 1.8 & 1.1 & 0.76 & 0.49 & 0.36 \\
\hline 7 & 4.9 & 2.1 & 1.3 & 0.90 & 0.59 & 0.44 \\
\hline 14 & 5.6 & 2.6 & 1.8 & 1.3 & 0.90 & 0.71 \\
\hline 30 & 6.9 & 3.5 & 2.5 & 1.9 & 1.4 & 1.1 \\
\hline 60 & 8.6 & 4.9 & 3.7 & 2.9 & 2.2 & 1.9 \\
\hline 90 & 11 & 6.6 & 5.3 & 4.4 & 3.6 & 3.1 \\
\hline 120 & 13 & 9.2 & 7.6 & 6.6 & 5.7 & 5.2 \\
\hline 183 & 18 & 13 & 11 & 9.8 & 8.6 & 7.9 \\
\hline
\end{tabular}

MAGNITUDE AND PROBABILITY OF ANNUAL HIGH FLOW BASED ON PERIOD OF RECORD $1932.40,1942,1967.96$

\begin{tabular}{|c|c|c|c|c|c|c|}
\hline \multirow{3}{*}{$\begin{array}{l}\text { PERIOD } \\
\text { (CON- } \\
\text { SECU. }\end{array}$} & & \multicolumn{5}{|c|}{ DISCHARGE, IN FT $3 / S$, FOR INDICATED } \\
\hline & & EXCEEDA & CE PRO & BILITY, & IN PERC & ENT \\
\hline & & 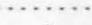 & & & ....... & \\
\hline TIVE & 2 & 5 & 10 & 25 & 50 & $100 \#$ \\
\hline DAYS) & 508 & 208 & 108 & 48 & 28 & 18 \\
\hline 1 & 726 & 2.020 & 3,640 & 7,130 & 11,300 & 17,300 \\
\hline 3 & 400 & 1,130 & 2,080 & 4,140 & 6,640 & 10,300 \\
\hline 7 & 233 & 624 & 1,090 & 2,070 & 3,180 & 4,750 \\
\hline 15 & 138 & 343 & 583 & 1,070 & 1,620 & 2,390 \\
\hline 30 & 90 & 212 & 350 & 620 & 919 & 1,330 \\
\hline 60 & 63 & 141 & 227 & 395 & 577 & 825 \\
\hline 90 & 51 & 110 & 173 & 289 & 411 & 572 \\
\hline
\end{tabular}

DURATION TABLE OF DAILY MEAN FLOW FOR PERIOD OF RECORD 1932-40, 1942, 1967.96

DISCHARGE, IN FT3/S, WHICH WAS EQUALED OR EXCEEDED FOR INDICATED PERCENT OF TIME

\begin{tabular}{|c|c|c|c|c|c|c|c|c|c|c|c|c|c|c|c|c|}
\hline 18 & 58 & 108 & 158 & 208 & 308 & 408 & 508 & 608 & 708 & 808 & 908 & 958 & 988 & 998 & 99.58 & 99.98 \\
\hline 379 & 83 & 48 & 37 & 32 & 25 & 21 & 17 & 14 & 12 & 9.2 & 6.2 & 4.2 & 2.8 & 2.2 & 1.5 & 0.93 \\
\hline
\end{tabular}

\# Reliability of values in column is uncertain, and potential errors are large. 
ANNUAL MEAN DISCHARGE, IN CUBIC

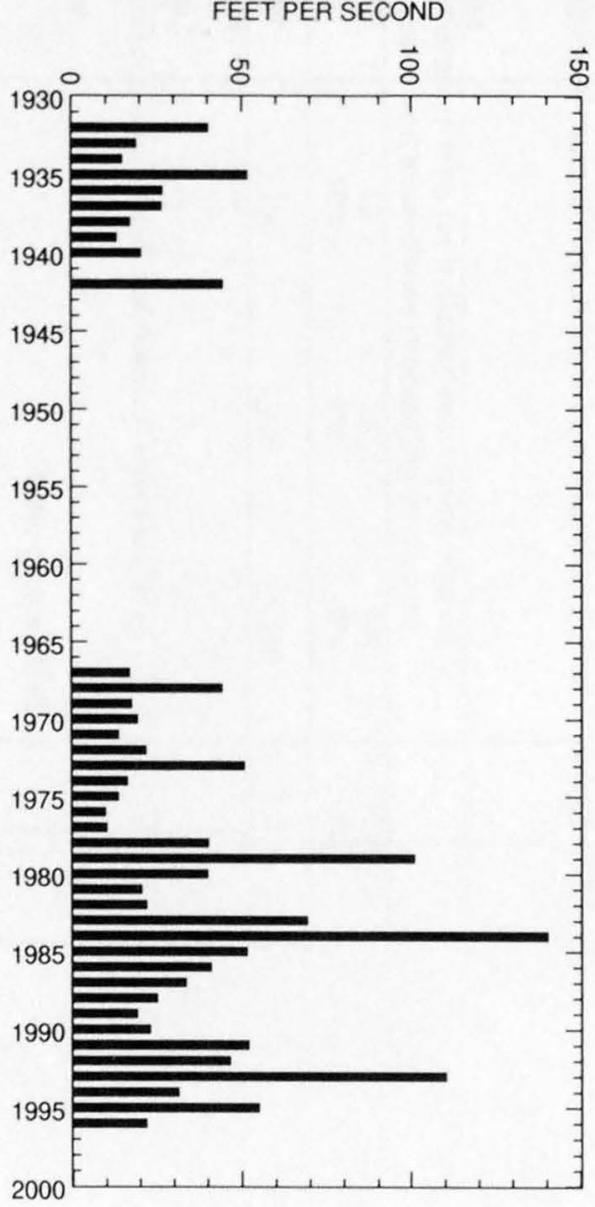

ANNUAL PEAK DISCHARGE, IN CUBIC FEET PER SECOND

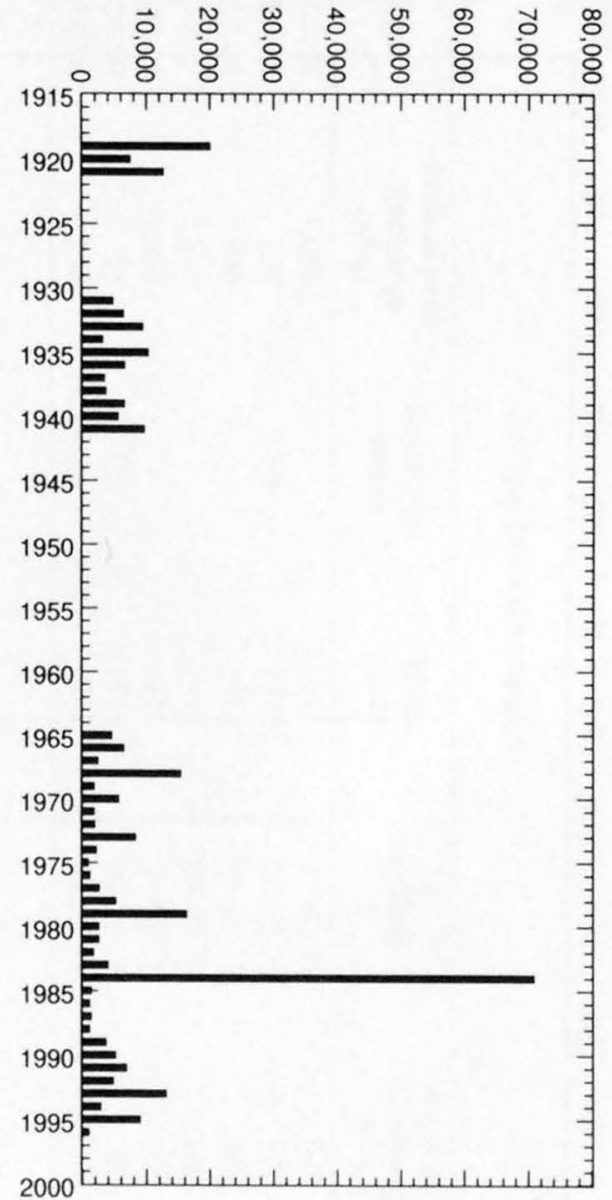


09473000 ARAVAIPA CREEK NEAR MAMMOTH, AZ--Continued
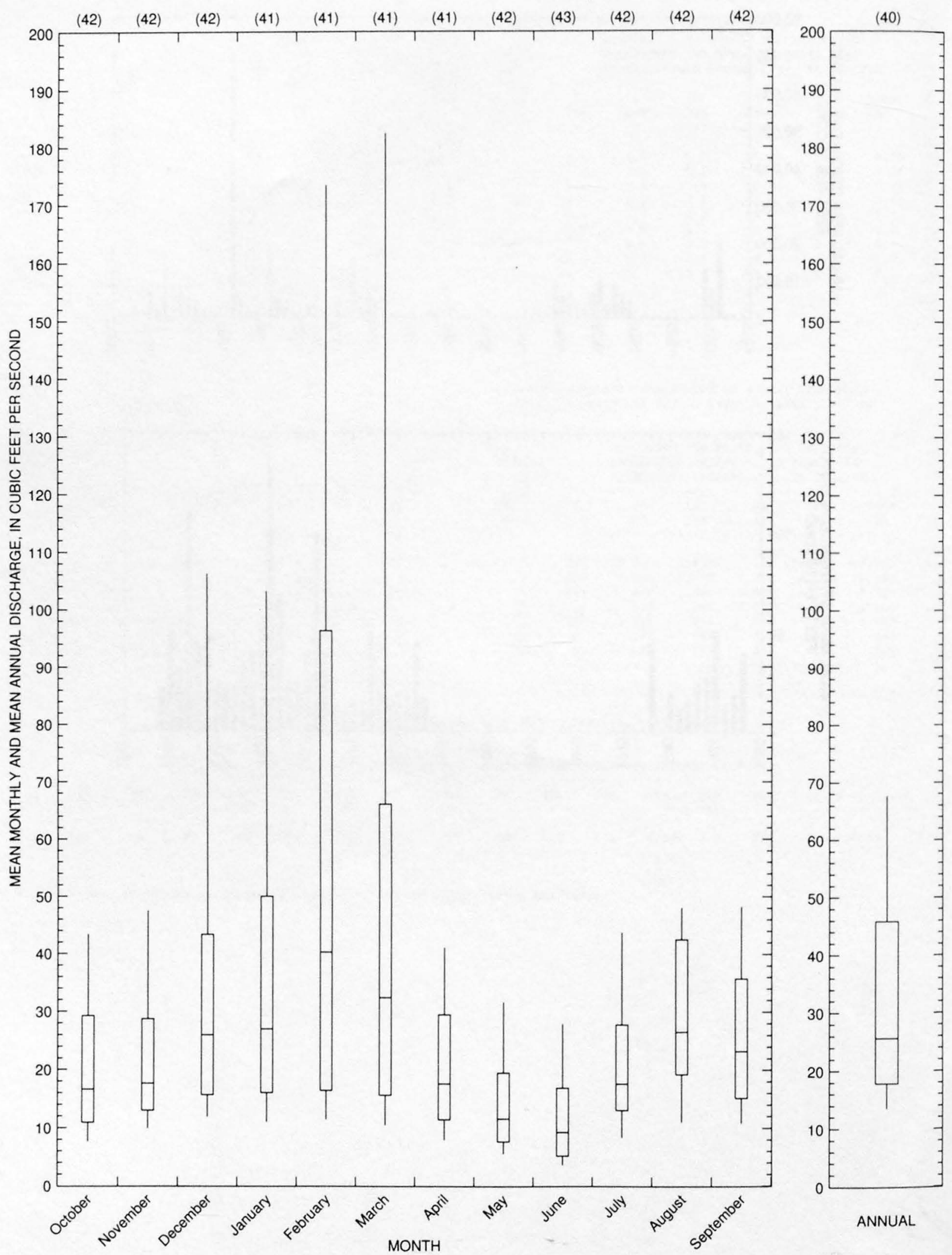
09473200 GREEN LANTERN WASH NEAR WINKELMAN, AZ

LOCATION.--Lat 32 55'30", long $110^{\circ} 43^{\prime} 35^{\prime \prime}, \mathrm{NE}^{1} /{ }_{4} \mathrm{SE}^{1} / 4 \mathrm{sec} .8$, T.6 S., R.16 E., Pinal County, Hydrologic Unit 15050203 , at State Highway 77 , 5 mi southeast of Winkelman.

DRAINAGE AREA.--3.63 $\mathrm{mi}^{2}$.

Annual peak discharges

\begin{tabular}{|c|c|c|c|c|c|c|c|}
\hline $\begin{array}{l}\text { Water } \\
\text { year }\end{array}$ & Date & $\begin{array}{c}\text { Annual peak } \\
\text { discharge } \\
\left(\mathrm{ft}^{3} / \mathrm{s}\right)\end{array}$ & $\begin{array}{c}\text { Discharge } \\
\text { codes }\end{array}$ & $\begin{array}{l}\text { Water } \\
\text { year }\end{array}$ & Date & $\begin{array}{c}\text { Annual peak } \\
\text { discharge } \\
\left(\mathrm{ft}^{3} / \mathrm{s}\right)\end{array}$ & $\begin{array}{l}\text { Discharge } \\
\text { codes }\end{array}$ \\
\hline 1964 & $08-00-64$ & 1,060 & & 1971 & $09-30-71$ & 600 & \\
\hline 1965 & $07-23-65$ & 200 & ES & 1972 & $08-12-72$ & 170 & \\
\hline 1966 & $09-13-66$ & 900 & & 1973 & $10-00-72$ & 440 & \\
\hline 1967 & $07-17-67$ & 2,650 & & 1974 & $07-00-74$ & 100 & \\
\hline 1968 & $08-03-68$ & 1,960 & & 1975 & $09-00-75$ & 1,200 & \\
\hline 1969 & $08-29-69$ & 15 & ES & 1976 & $09-25-76$ & 200 & ES \\
\hline 1970 & $08-16-70$ & 250 & ES & 1981 & $05-01-81$ & $1_{3,700}$ & HP \\
\hline
\end{tabular}

${ }^{1}$ Highest since 1964.

Magnitude and probability of instantaneous peak flow based on period of record 1964-76, 1981

\begin{tabular}{cccccc}
\hline \multicolumn{7}{c}{$\begin{array}{l}\text { Discharge, in } \mathrm{ft}^{3} / \mathrm{s} \text {, for indicated recurrence interval } \\
\text { in years, and exceedance probablility, in percent }\end{array}$} \\
\hline 2 & $\mathbf{5}$ & $\mathbf{1 0}$ & $\mathbf{2 5}$ & $\mathbf{5 0 \dagger}$ & $\mathbf{1 0 0 \dagger}$ \\
$\mathbf{5 0 \%}$ & $\mathbf{2 0 \%}$ & $\mathbf{1 0 \%}$ & $\mathbf{4} \%$ & $\mathbf{2 \%}$ & $\mathbf{1 \%}$ \\
\hline 509 & 1,380 & 2,290 & 3,920 & 5,520 & 7,490 \\
Weighted skew & $(\operatorname{logs})=$ & -0.09 & & & \\
Mean & $(\operatorname{logs})=$ & 2.70 & & & \\
Standard dev. & $(\operatorname{logs})=$ & 0.52 &
\end{tabular}

$\mp$ Reliability of values in column is uncertain, and potential errors are large.

Basin characteristics

\begin{tabular}{|c|c|c|c|c|c|c|c|}
\hline \multirow[b]{2}{*}{$\begin{array}{l}\text { Main } \\
\text { channel } \\
\text { slope } \\
(\mathrm{ft} / \mathrm{mi})\end{array}$} & \multirow[b]{2}{*}{$\begin{array}{l}\text { Stream } \\
\text { length } \\
\text { (mi) }\end{array}$} & \multirow[b]{2}{*}{$\begin{array}{c}\text { Mean } \\
\text { basin } \\
\text { elevation } \\
\text { (ft) }\end{array}$} & \multirow[b]{2}{*}{$\begin{array}{l}\text { Forested } \\
\text { area } \\
\text { (percent) }\end{array}$} & \multirow[b]{2}{*}{$\begin{array}{c}\text { Soil } \\
\text { index }\end{array}$} & \multirow[b]{2}{*}{$\begin{array}{c}\text { Mean } \\
\text { annual } \\
\text { precipitation } \\
\text { (in) }\end{array}$} & \multicolumn{2}{|c|}{ Rainfall intensity, 24-hour } \\
\hline & & & & & & $\begin{array}{l}\text { 2-year } \\
\text { (in) }\end{array}$ & $\begin{array}{l}\text { 50-year } \\
\text { (in) }\end{array}$ \\
\hline 189 & 4.8 & 2,590 & 0.0 & 1.0 & 14.0 & 1.9 & 4.1 \\
\hline
\end{tabular}

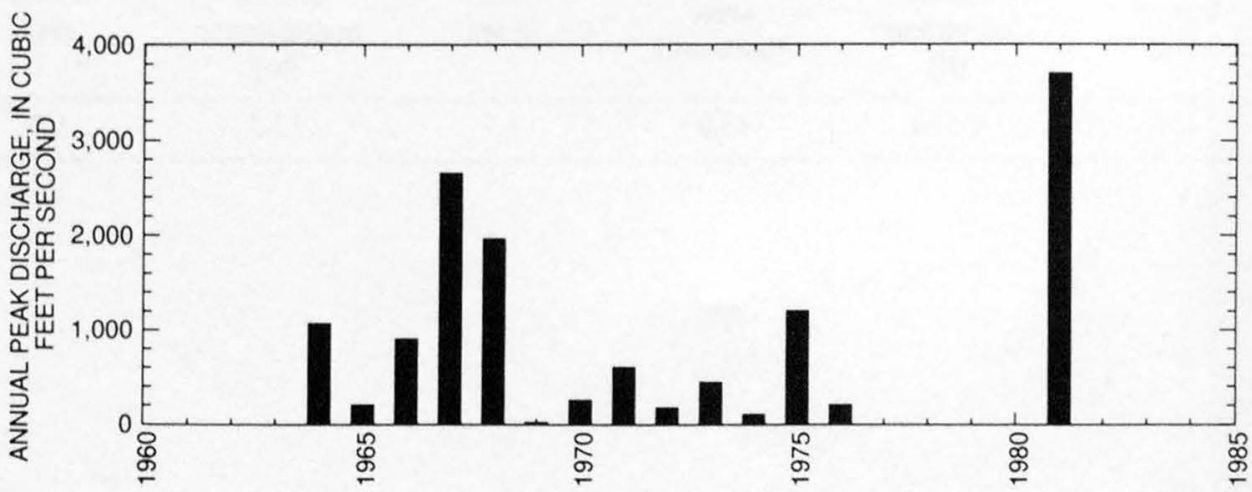


09473500 SAN PEDRO RIVER AT WINKELMAN, AZ

LOCATION.--Lat 32 58'38", long $110^{\circ} 46^{\prime} 11^{\prime \prime}, \mathrm{SE}^{1} / \mathrm{SW}^{1} / 4 \mathrm{sec} .24$, T.5 S., R.15 E., Pinal County, Hydrologic Unit 15050203 , on right bank $0.7 \mathrm{mi}$ south of Winkelman, and $1.0 \mathrm{mi}$ upstream from mouth.

DRAINAGE AREA.--4,453 $\mathrm{mi}^{2}$.

Annual peak discharges

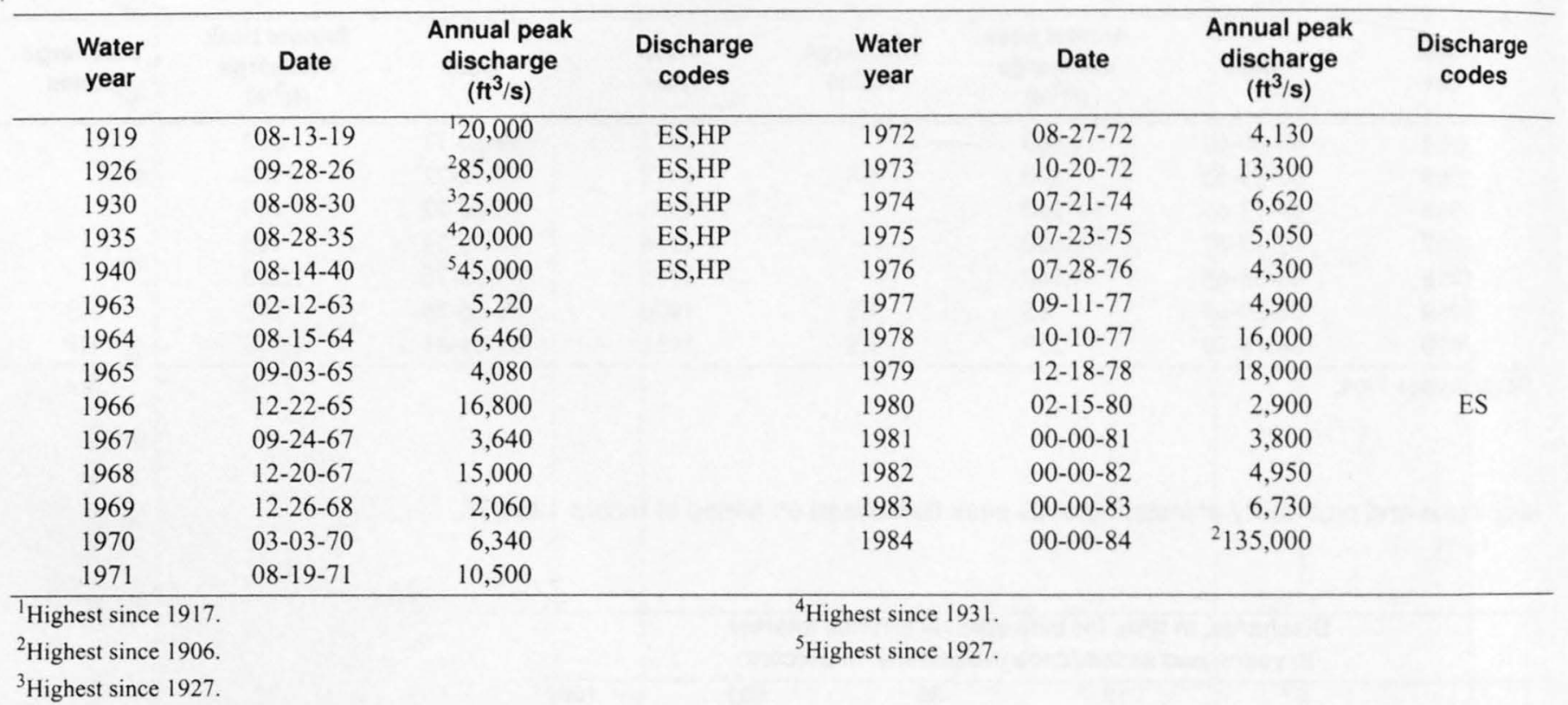

Discharge rating table developed January ---

\begin{tabular}{crcc}
\hline $\begin{array}{c}\text { Gage height } \\
(\mathbf{f t})\end{array}$ & $\begin{array}{c}\text { Discharge } \\
\left(\mathbf{f t}^{3} / \mathbf{s}\right)\end{array}$ & $\begin{array}{c}\text { Gage height } \\
(\mathbf{f t})\end{array}$ & $\begin{array}{c}\text { Discharge } \\
\left(\mathrm{ft}^{3} / \mathbf{s}\right)\end{array}$ \\
\hline 6.0 & 36 & 11.0 & 5,780 \\
7.0 & 371 & 12.0 & 8,400 \\
8.0 & 1,070 & 13.0 & 11,830 \\
9.0 & 2,180 & 14.0 & 16,080 \\
10.0 & 3,730 & 14.5 & 18,500 \\
\hline
\end{tabular}

Basin characteristics

\begin{tabular}{cccccccc}
\hline $\begin{array}{c}\text { Main } \\
\text { channel } \\
\text { slope } \\
(\mathrm{ft} / \mathrm{mi})\end{array}$ & $\begin{array}{c}\text { Stream } \\
\text { length } \\
(\mathrm{mi})\end{array}$ & $\begin{array}{c}\text { Mean } \\
\text { basin } \\
\text { elevation } \\
(\mathrm{ft})\end{array}$ & $\begin{array}{c}\text { Forested } \\
\text { area } \\
\text { (percent) }\end{array}$ & $\begin{array}{c}\text { Soil } \\
\text { index }\end{array}$ & $\begin{array}{c}\text { Mean } \\
\text { annual } \\
\text { precipitation } \\
\text { (in) }\end{array}$ & $\begin{array}{c}\text { R-year } \\
\text { (in) }\end{array}$ & $\begin{array}{c}\text { Ro-year } \\
\text { (in) }\end{array}$ \\
\hline 18.0 & 176 & 4,520 & 15.0 & 1.7 & 15.3 & 1.9 \\
\hline
\end{tabular}


09473500 SAN PEDRO RIVER AT WINKELMAN, AZ--Continued

MEAN MONTHLY AND ANNUAL DISCHARGES 1967.78

\begin{tabular}{|c|c|c|c|c|c|c|}
\hline MONTH & $\begin{array}{l}\text { MAXIMUM } \\
(\text { FT3/S) }\end{array}$ & $\begin{array}{l}\text { MINIMUM } \\
\text { (FT3/S) }\end{array}$ & $\begin{array}{c}\text { MEAN } \\
(\text { FT3 } / \mathrm{S})\end{array}$ & $\begin{array}{l}\text { STAN - } \\
\text { DARD } \\
\text { DEVIA- } \\
\text { TION } \\
(\text { FT3/S) }\end{array}$ & $\begin{array}{l}\text { COEFFI- } \\
\text { CIENT OF } \\
\text { VARI- } \\
\text { ATION }\end{array}$ & $\begin{array}{c}\text { PERCENT } \\
\text { OF } \\
\text { ANNUAI } \\
\text { RUNOFF }\end{array}$ \\
\hline OCTOBER & 565 & 0.00 & 80 & 174 & 2.2 & 15.2 \\
\hline NOVBMBER & 25 & 0.01 & 6.9 & 7.6 & 1.1 & 1.3 \\
\hline DECEMBER & 538 & 0.34 & 55 & 153 & 2.8 & 10.5 \\
\hline JANUARY & 48 & 2.3 & 15 & 17 & 1.1 & 2.9 \\
\hline FEBRUARY & 206 & 0.76 & 43 & 73 & 1.7 & 8.1 \\
\hline MARCH & 459 & 0.00 & 74 & 133 & 1.8 & 14.1 \\
\hline APRIL & 35 & 0.00 & 8.2 & 11 & 1.4 & 1.6 \\
\hline MAY & 7.2 & 0.00 & 1.6 & 2.2 & 1.4 & 0.3 \\
\hline JUNE & 16 & 0.00 & 2.3 & 5.4 & 2.3 & 0.4 \\
\hline JULY & 184 & 10 & 66 & 59 & 0.89 & 12.5 \\
\hline AUGUST & 586 & 3.4 & 134 & 156 & 1.2 & 25.6 \\
\hline SEPTEMBER & 81 & 0.13 & 39 & 27 & 0.68 & 7.5 \\
\hline ANNUAL & 104 & 13 & 44 & 30 & 0.67 & 100 \\
\hline
\end{tabular}

MAGNITUDE AND PROBABILITY OF ANNUAL LOW FLOW BASED ON PERIOD OF RECORD 1967.78

\begin{tabular}{|c|c|c|c|c|c|c|}
\hline $\begin{array}{l}\text { PERIOD } \\
\text { (CON- }\end{array}$ & \multirow{2}{*}{\multicolumn{6}{|c|}{ 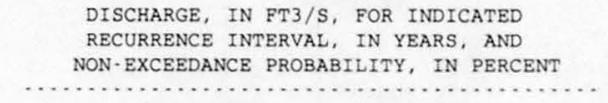 }} \\
\hline SECU - & & & & & & \\
\hline TIVE & 2 & 5 & 10 & 20 & $50 \#$ & $100 \#$ \\
\hline DAYS) & 508 & 208 & 108 & 58 & 28 & 18 \\
\hline $\begin{array}{r}1 \\
3 \\
7 \\
14 \\
30\end{array}$ & & & & & & \\
\hline 60 & 0.02 & 0.00 & 0.00 & 0.00 & 0.00 & 0.00 \\
\hline 90 & 0.49 & 0.05 & 0.00 & 0.00 & 0.00 & 0.00 \\
\hline 120 & 4.9 & 1.6 & 0.82 & 0.46 & 0.23 & 0.14 \\
\hline 183 & 9.4 & 2.6 & 1.2 & 0.63 & 0.29 & 0.17 \\
\hline
\end{tabular}

MAGNITUDE AND PROBABILITY OF ANNUAL HIGH FLOW BASED ON PERIOD OF RECORD $1967 \cdot 78$

\begin{tabular}{|c|c|c|c|c|c|c|}
\hline \multirow{3}{*}{$\begin{array}{l}\text { PERIOD } \\
\text { (CON- } \\
\text { SECU. }\end{array}$} & \multicolumn{6}{|c|}{ DISCHARGE, IN FT3/S, FOR INDICATED } \\
\hline & & EXCEED? & CE PRO & ABILITY & IN PER & ENT \\
\hline & $\cdots$ & $\ldots \ldots \ldots$ & $\ldots \ldots \ldots$ & $\cdots \ldots \ldots$ & $\cdots \ldots$ & $\ldots \ldots$ \\
\hline TIVE & 2 & 5 & 10 & $25 \#$ & $50 \#$ & $100 \#$ \\
\hline DAYS) & 508 & 208 & 108 & 48 & 28 & 18 \\
\hline 1 & 2,000 & 4,460 & 7,020 & 11,700 & 16,600 & 22,900 \\
\hline 3 & 1,130 & 2,380 & 3,650 & 5,950 & 8,290 & 11,300 \\
\hline 7 & 587 & 1,230 & 1,880 & 3,050 & 4,230 & 5,750 \\
\hline 15 & 362 & 715 & 1,040 & 1,590 & 2,100 & 2,720 \\
\hline 30 & 236 & 425 & 578 & 802 & 993 & 1,200 \\
\hline 60 & 147 & 244 & 323 & 439 & 539 & 650 \\
\hline 90 & 105 & 173 & 228 & 309 & 379 & 456 \\
\hline
\end{tabular}

DURATION TABLE OF DAILY MEAN FLOW FOR PERIOD OF RECORD 1967.78

DISCHARGE, IN FT3/S, WHICH WAS EQUALED OR EXCEEDED FOR INDICATED PERCENT OF TIME

\begin{tabular}{|c|c|c|c|c|c|c|c|c|c|c|c|c|c|c|c|c|}
\hline 18 & 58 & 108 & 158 & 208 & 308 & 408 & 508 & 608 & 708 & 808 & 908 & 958 & 988 & 998 & 99.58 & 99.98 \\
\hline 810 & 145 & 52 & 28 & 20 & 10 & 5.5 & 3.0 & 1.1 & 0.18 & 0.00 & 0.00 & 0.00 & 0.00 & 0.00 & 0.00 & 0.00 \\
\hline
\end{tabular}

\# Reliability of values in column is uncertain, and potential errors are large. 
ANNUAL MEAN DISCHARGE, IN CUBIC

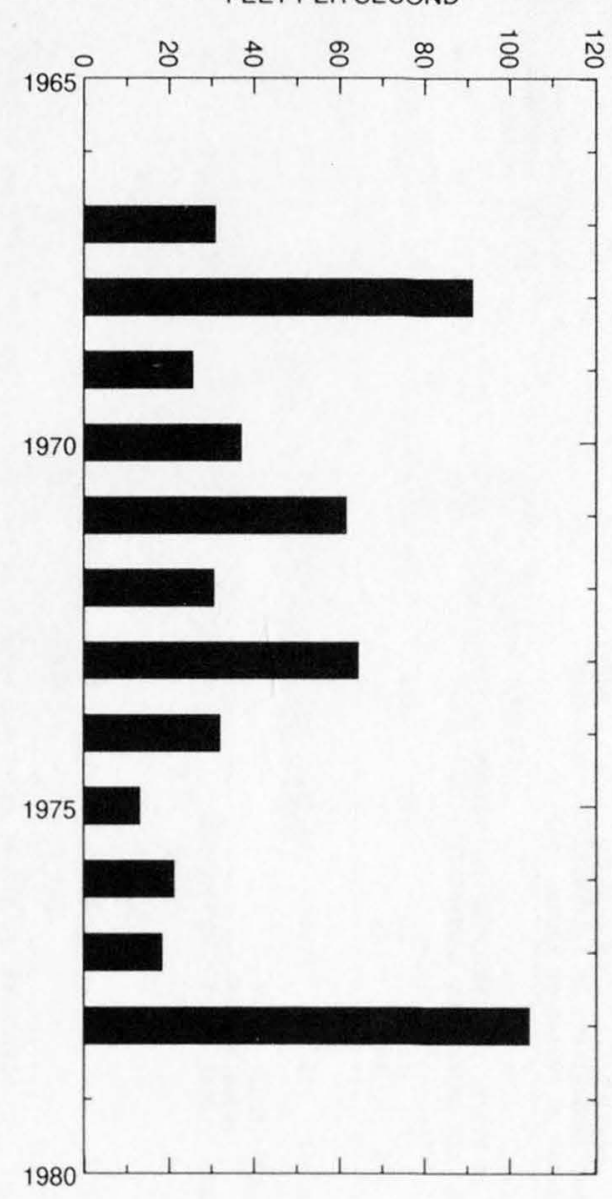

ANNUAL PEAK DISCHARGE, IN CUBIC

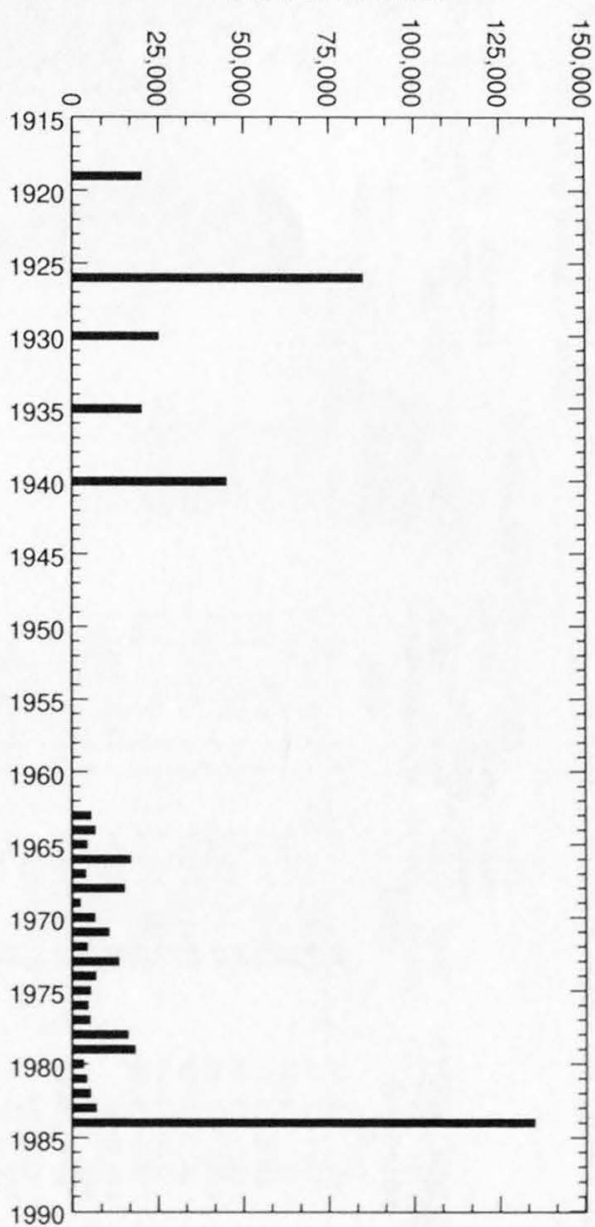


09473500 SAN PEDRO RIVER AT WINKELMAN, AZ--Continued
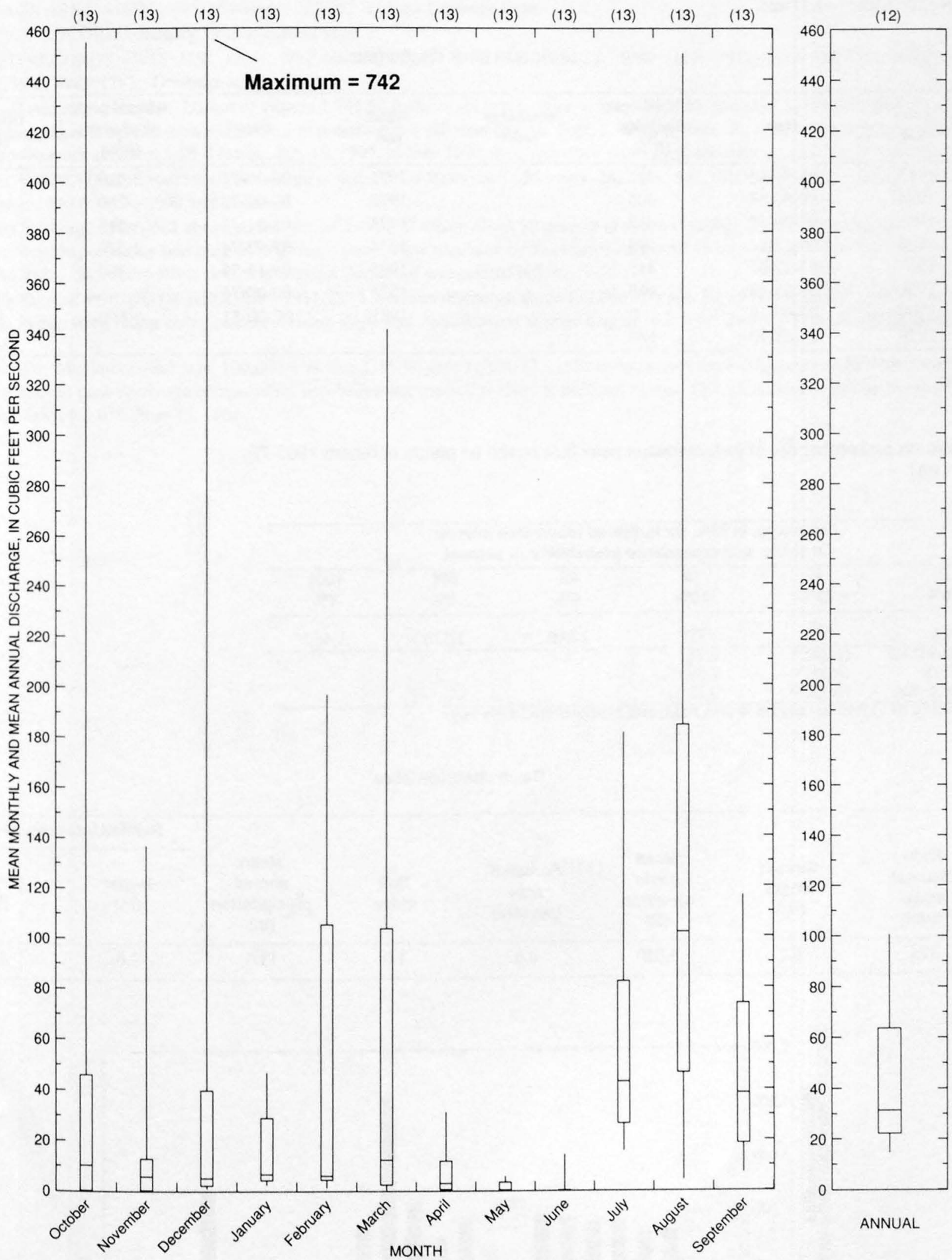
09473600 TAM O'SHANTER WASH NEAR HAYDEN, AZ

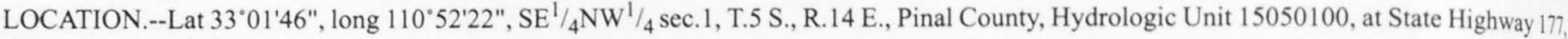
$6 \mathrm{mi}$ west of Hayden.

DRAINAGE AREA.--4.37 $\mathrm{mi}^{2}$.

Annual peak discharges

\begin{tabular}{|c|c|c|c|c|c|c|c|}
\hline $\begin{array}{l}\text { Water } \\
\text { year }\end{array}$ & Date & $\begin{array}{c}\text { Annual peak } \\
\text { discharge } \\
\left(\mathrm{ft}^{3} / \mathrm{s}\right)\end{array}$ & $\begin{array}{c}\text { Discharge } \\
\text { codes }\end{array}$ & $\begin{array}{l}\text { Water } \\
\text { year }\end{array}$ & Date & $\begin{array}{c}\text { Annual peak } \\
\text { discharge } \\
\left(\mathrm{ft}^{3} / \mathrm{s}\right)\end{array}$ & $\begin{array}{c}\text { Discharge } \\
\text { codes }\end{array}$ \\
\hline 1963 & $08-00-63$ & 322 & & 1971 & $09-30-71$ & 410 & \\
\hline 1965 & $07-00-65$ & 412 & & 1973 & $05-31-73$ & 615 & \\
\hline 1966 & $09-13-66$ & 399 & & 1974 & $08-02-74$ & 1,570 & \\
\hline 1969 & $00-00-69$ & 0 & & 1981 & $00-00-81$ & ' 690 & HP \\
\hline 1970 & $03-03-70$ & 180 & & & & & \\
\hline
\end{tabular}

${ }^{1}$ Highest since 1974.

Magnitude and probability of instantaneous peak flow based on period of record 1963-76, 1981

\begin{tabular}{|c|c|c|c|c|c|}
\hline \multicolumn{6}{|c|}{$\begin{array}{l}\text { Discharge, in } \mathrm{ft}^{3} / \mathrm{s} \text {, for indicated recurrence interval } \\
\text { in years, and exceedance probablility, in percent }\end{array}$} \\
\hline 2 & 5 & 10 & 25 & $50 \dagger$ & $100 \dagger$ \\
\hline $50 \%$ & $20 \%$ & $10 \%$ & $4 \%$ & $2 \%$ & $1 \%$ \\
\hline 368 & 586 & 759 & 1,010 & 1,220 & 1,460 \\
\hline Weighted skew & $(\log s)=$ & 0.28 & & & \\
\hline Mean & $(\log s)=$ & 2.58 & & & \\
\hline Standard dev. & $(\log s)=$ & 0.23 & & & \\
\hline
\end{tabular}

f Reliability of values in column is uncertain, and potential errors are large.

Basin characteristics

\begin{tabular}{cccccccc}
\hline $\begin{array}{c}\text { Main } \\
\text { channel } \\
\text { slope } \\
(\mathrm{ft} / \mathrm{mi})\end{array}$ & $\begin{array}{c}\text { Stream } \\
\text { length } \\
(\mathrm{mi})\end{array}$ & $\begin{array}{c}\text { Mean } \\
\text { basin } \\
\text { elevation } \\
(\mathrm{ft})\end{array}$ & $\begin{array}{c}\text { Forested } \\
\text { area } \\
\text { (percent) }\end{array}$ & $\begin{array}{c}\text { Soil } \\
\text { index }\end{array}$ & $\begin{array}{c}\text { Mean } \\
\text { annual } \\
\text { precipitation } \\
\text { (in) }\end{array}$ & $\begin{array}{c}\text { Rainfall intensity, 24-hour } \\
\text { (in) }\end{array}$ & $\begin{array}{c}50 \text {-year } \\
\text { (in) }\end{array}$ \\
\hline 324 & 6.1 & 3,050 & 0.0 & 1.0 & 15.6 & 2.0 \\
\hline
\end{tabular}

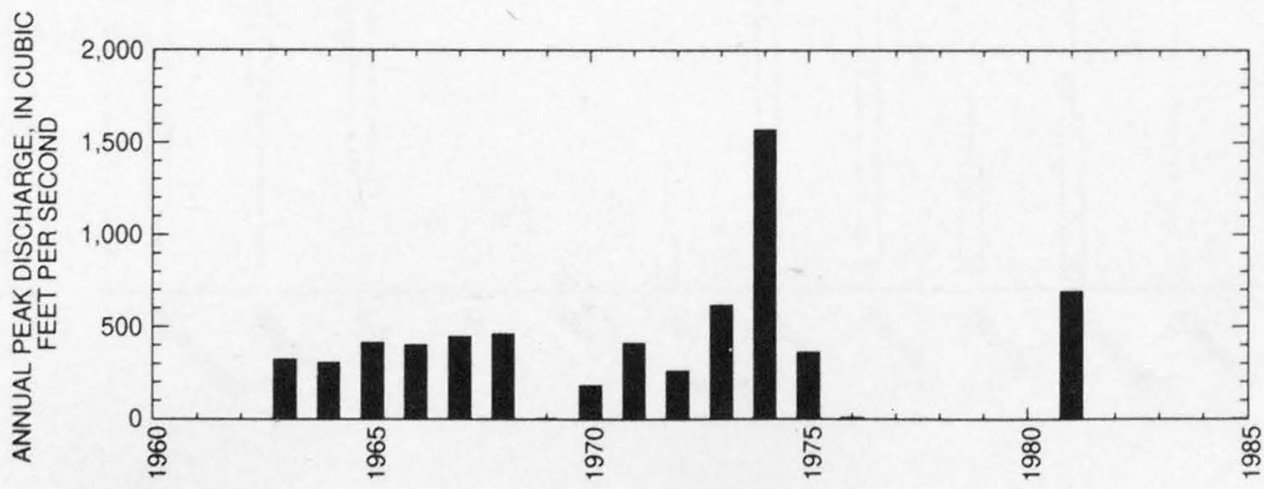




\section{GILA RIVER AT KELVIN, AZ}

LOCATION.--Lat 33'06'10", long $110^{\circ} 58^{\prime} 33^{\prime \prime}$, in NE $\frac{1}{4}{ }_{4} \mathrm{NW}^{1} / 4$ sec.12, T.4 S., R.13 E., Pinal County, Hydrologic Unit 15050100 , on left bank at Kelvin, $500 \mathrm{ft}$ downstream from Mineral Creek, $18 \mathrm{mi}$ downstream from San Pedro River, and $19 \mathrm{mi}$ upstream from Ashurst-Hayden Dam. DRAINAGE AREA.--18,011 $\mathrm{mi}^{2}$, of which $5,125 \mathrm{mi}^{2}$ is below Coolidge Dam.

PERIOD OF RECORD.--January 1911 to current year.

REVISED RECORDS.--WSP 329: 1911. WSP 609: 1916(M). WSP 629: 1914-17. WSP 1119: 1913, 1915, 1917(M), 1921(M), 1922-23, 1927(M). WSP 1283: Drainage area.

GAGE.--Water-stage recorder. Datum of gage is $1,745.02 \mathrm{ft}$ above sea level. Prior to June 15, 1914, and Dec. 1, 1914, to Aug. 31, 1915, nonrecording gages at several sites within $2 \mathrm{mi}$ of present site at different datums. Sept. 1, 1915, to Sept. 30, 1963, water-stage recorder at site 900 $\mathrm{ft}$ downstream at datum $1.80 \mathrm{ft}$ lower. Jan. 16, 1985, to June 1990, supplementary water-stage recorder at same site and datum.

AVERAGE DISCHARGE (adjusted for storage in San Carlos Reservoir).--85 years, $542 \mathrm{ft}^{3} / \mathrm{s}, 392,700 \mathrm{acre-ft} / \mathrm{yr}$; median of yearly mean discharges, $340 \mathrm{ft}^{3} / \mathrm{s}, 246,000$ acre- $\mathrm{ft} / \mathrm{yr}$.

REMARKS.--Large diversions above station for irrigation, of which about 90 percent is above Coolidge Dam. About 82,000 acres irrigated, a considerable portion by pumping from ground water. Flow regulated by San Carlos Reservoir 49 mi upstream since Nov. 15, 1928. (See sta 09469000.) San Pedro River contributes major portion of unregulated inflow.

EXTREMES FOR PERIOD OF RECORD.--1911-28: Maximum discharge, about 132,000 ft $3 / \mathrm{s} \mathrm{Jan.} \mathrm{20,1916,} \mathrm{gage} \mathrm{height,} 19.5 \mathrm{ft}$, site and datum then in use, from rating curve extended above slope-area measurement at gage height, $16.2 \mathrm{ft}$ for flood of Sept. 28, 1926; no flow Feb. 25, 1913.

1929-96: Maximum discharge, 100,000 ft $3 / \mathrm{s}$ Oct. 2, 1983, gage height, $33.0 \mathrm{ft}$ from floodmark, from rating curve extended above $12,000 \mathrm{ft}^{3} /$ $\mathrm{s}$ on basis of peak discharge computed by step-backwater method at Hayden Railroad Bridge, 17.8 mi upstream, and by flood-routing; minimum daily, $0.1 \mathrm{ft}^{3} / \mathrm{s}$ June 25,1961 . 
GILA RIVER BASIN

09474000 GILA RIVER AT KELVIN, AZ--Continued

Annual peak discharges

\begin{tabular}{|c|c|c|c|c|c|c|c|}
\hline $\begin{array}{l}\text { Water } \\
\text { year }\end{array}$ & Date & $\begin{array}{c}\text { Annual peak } \\
\text { discharge } \\
\left(\mathrm{ft}^{3} / \mathrm{s}\right)\end{array}$ & $\begin{array}{l}\text { Discharge } \\
\text { codes }\end{array}$ & $\begin{array}{l}\text { Water } \\
\text { year }\end{array}$ & Date & $\begin{array}{c}\text { Annual peak } \\
\text { discharge } \\
\left(\mathrm{ft}^{3} / \mathrm{s}\right)\end{array}$ & $\begin{array}{l}\text { Discharge } \\
\text { codes }\end{array}$ \\
\hline 1913 & $02-25-13$ & 4,400 & & 1955 & $08-08-55$ & 9,860 & \\
\hline 1914 & $09-21-14$ & 18,000 & & 1956 & $08-17-56$ & 1,800 & \\
\hline 1915 & $12-24-14$ & 67,300 & ES & 1957 & $08-19-57$ & 4,540 & \\
\hline 1916 & $01-20-16$ & 132,000 & & 1958 & $08-06-58$ & 5,310 & \\
\hline 1917 & $10-15-16$ & 55,000 & ES & 1959 & $08-17-59$ & 5,930 & \\
\hline 1918 & 08-06-18 & 15,100 & ES & 1960 & $12-26-59$ & 11,200 & \\
\hline 1919 & 08-03-19 & 20,800 & ES & 1961 & $07-22-61$ & 9,600 & \\
\hline 1920 & $12-05-19$ & 25,800 & ES & 1962 & $12-16-61$ & 4,910 & \\
\hline 1921 & $07-31-21$ & 24,000 & ES & 1963 & $02-12-63$ & 5,880 & \\
\hline 1922 & $07-26-22$ & 7,520 & & 1964 & $08-15-64$ & 4,150 & \\
\hline 1923 & $07-14-23$ & 11,700 & & 1965 & $08-17-65$ & 4,980 & \\
\hline 1924 & $12-29-23$ & 11,700 & & 1966 & $12-23-65$ & 26,300 & \\
\hline 1925 & $09-04-25$ & 11,200 & & 1967 & $09-24-67$ & 4,220 & \\
\hline 1926 & $09-28-26$ & 82,000 & & 1968 & $12-20-67$ & 27,700 & \\
\hline 1927 & $02-18-27$ & 8,570 & ES & 1969 & $01-22-69$ & 5,800 & \\
\hline 1928 & $08-02-28$ & 12,000 & & 1970 & $03-03-70$ & 6,600 & \\
\hline 1929 & $09-24-29$ & 11,600 & & 1971 & $08-20-71$ & 3,120 & \\
\hline 1930 & $08-08-30$ & 42,800 & & 1972 & $10-01-71$ & 3,930 & \\
\hline 1931 & $08-30-31$ & 28,600 & & 1973 & $10-20-72$ & 10,300 & \\
\hline 1932 & $10-02-31$ & 12,800 & & 1974 & $08-02-74$ & 3,880 & \\
\hline 1933 & $07-24-33$ & 8,800 & & 1975 & $07-23-75$ & 2,650 & \\
\hline 1934 & $08-23-34$ & 6,750 & & 1976 & $07-28-76$ & 2,510 & \\
\hline 1935 & $08-29-35$ & 21,000 & & 1977 & $09-11-77$ & 2,540 & ES \\
\hline 1936 & $09-11-36$ & 12,600 & & 1978 & $10-11-77$ & 16,100 & \\
\hline 1937 & $08-21-37$ & 10,200 & & 1979 & $12-19-78$ & 27,000 & \\
\hline 1938 & $08-05-38$ & 5,660 & & 1980 & $02-15-80$ & 6,950 & \\
\hline 1939 & 08-07-39 & 9,320 & & 1981 & $08-01-81$ & 3,320 & \\
\hline 1940 & $08-14-40$ & 38,200 & & 1982 & $09-12-82$ & 3,530 & \\
\hline 1941 & $12-31-40$ & 23,300 & & 1983 & $03-19-83$ & 4,490 & \\
\hline 1942 & $08-09-42$ & 3,300 & & 1984 & $10-02-83$ & 100,000 & \\
\hline 1943 & $09-26-43$ & 6,290 & & 1985 & $12-30-84$ & 6,150 & \\
\hline 1944 & $08-09-44$ & 28,000 & & 1986 & $03-18-86$ & 4,270 & \\
\hline 1945 & $08-10-45$ & 9,200 & & 1987 & $08-04-87$ & 1,670 & \\
\hline 1946 & $08-05-46$ & 6,440 & & 1988 & $07-25-88$ & 4,080 & \\
\hline 1947 & $08-08-47$ & 10,000 & & 1989 & $07-24-89$ & 1,720 & \\
\hline 1948 & $08-03-48$ & 5,850 & & 1990 & $08-14-90$ & 5,120 & \\
\hline 1949 & $09-15-49$ & 5,610 & & 1991 & 03-03-91 & 11,100 & \\
\hline 1950 & $07-30-50$ & 6,920 & & 1992 & $02-14-92$ & 5,150 & \\
\hline 1951 & $08-03-51$ & 13,200 & & 1993 & $01-19-93$ & 74,900 & \\
\hline 1952 & $01-14-52$ & 5,30 & & 1994 & 02-08-94 & 2,010 & \\
\hline 1953 & $07-07-53$ & 4,210 & & 1995 & $01-06-95$ & 19,700 & \\
\hline 1954 & $08-05-54$ & 17,800 & & 1996 & $07-26-96$ & 2,370 & \\
\hline
\end{tabular}


09474000 GILA RIVER AT KELVIN, AZ--Continued

Discharge rating table developed October 1995

\begin{tabular}{rrrr}
\hline $\begin{array}{c}\text { Gage height } \\
\text { (ft) }\end{array}$ & $\begin{array}{c}\text { Discharge } \\
\left(\mathbf{f t}^{\mathbf{3}} \mathbf{s}\right)\end{array}$ & $\begin{array}{c}\text { Gage height } \\
(\mathbf{f t})\end{array}$ & $\begin{array}{c}\text { Discharge } \\
\left(\mathbf{f t}^{\mathbf{3}} \mathbf{s}\right)\end{array}$ \\
\hline 3.0 & 218 & 25.0 & 30,000 \\
4.0 & 468 & 28.0 & 43,000 \\
7.0 & 1,950 & 31.0 & 67,000 \\
10.0 & 4,600 & 34.0 & 122,300 \\
13.0 & 7,800 & 37.0 & 148,000 \\
16.0 & 11,200 & 40.0 & 178,700 \\
19.0 & 15,200 & 43.0 & 213,000 \\
22.0 & 21,700 & 46.0 & 250,000 \\
\hline
\end{tabular}

Basin characteristics

\begin{tabular}{cccccccc}
\hline $\begin{array}{c}\text { Main } \\
\text { channel } \\
\text { slope } \\
(\mathrm{ft} / \mathrm{mi})\end{array}$ & $\begin{array}{c}\text { Stream } \\
\text { length } \\
(\mathrm{mi})\end{array}$ & $\begin{array}{c}\text { Mean } \\
\text { basin } \\
\text { elevation } \\
(\mathrm{ft})\end{array}$ & $\begin{array}{c}\text { Forested } \\
\text { area } \\
\text { (percent) }\end{array}$ & $\begin{array}{c}\text { Soil } \\
\text { index }\end{array}$ & $\begin{array}{c}\text { Mean } \\
\text { annual } \\
\text { precipitation } \\
\text { (in) }\end{array}$ & $\begin{array}{c}\text { Rainfall intensity, 24-hour } \\
\text { (in) }\end{array}$ & $\begin{array}{c}50 \text {-year } \\
\text { (in) }\end{array}$ \\
\hline 17.8 & 284 & 5,150 & 13.0 & 2.2 & 15.7 & 1.9 \\
\hline
\end{tabular}


GILA RIVER BASIN

09474000 GILA RIVER AT KELVIN, AZ--Continued

MEAN MONTHLY AND ANNUAL DISCHARGES 1912.96

\begin{tabular}{|c|c|c|c|c|c|c|}
\hline MONTH & $\begin{array}{l}\text { MAXIMUM } \\
(\text { FT3/S) }\end{array}$ & $\begin{array}{l}\text { MINIMUM } \\
\text { (FT3/S) }\end{array}$ & $\begin{array}{c}\text { MEAN } \\
(\mathrm{FT} 3 / \mathrm{S})\end{array}$ & $\begin{array}{l}\text { STAN- } \\
\text { DARD } \\
\text { DEVIA- } \\
\text { TION } \\
\text { (FT3/S) }\end{array}$ & $\begin{array}{l}\text { COEFFI- } \\
\text { CIENT OF } \\
\text { VARI- } \\
\text { ATION }\end{array}$ & $\begin{array}{c}\text { PERCENT } \\
\text { OF } \\
\text { ANNUAL } \\
\text { RUNOFF }\end{array}$ \\
\hline OCTOBER & 4,370 & 1.0 & 277 & 603 & 2.2 & 4.3 \\
\hline NOVEMBER & 810 & 3.1 & 152 & 165 & 1.1 & 2.4 \\
\hline DECEMBER & 12,400 & 7.1 & 469 & 1.370 & 2.9 & 7.3 \\
\hline JANUARY & 17,100 & 9.3 & 702 & 2,370 & 3.4 & 10.9 \\
\hline FEBRUARY & 7,210 & 22 & 577 & 1,130 & 2.0 & 9.0 \\
\hline MARCH & 5,820 & 38 & 749 & 914 & 1.2 & 11.6 \\
\hline APRIL & 3.930 & 9.2 & 569 & 642 & 1.1 & 8.8 \\
\hline MAY & 1.270 & 2.5 & 411 & 315 & 0.77 & 6.4 \\
\hline JUNE & 1,610 & 0.46 & 456 & 405 & 0.89 & 7.1 \\
\hline JULY & 3,460 & 2.7 & 710 & 557 & 0.78 & 11.0 \\
\hline AUGUST & 4,590 & 43 & 895 & 655 & 0.73 & 13.9 \\
\hline SEPTEMBER & 1.970 & 2.1 & 476 & 334 & 0.70 & 7.4 \\
\hline ANNUAL & 3,280 & 78 & 538 & 513 & 0.95 & 100 \\
\hline
\end{tabular}

MAGNITUDE AND PROBABILITY OF INSTANTANEOUS PEAK FLOW BASED ON PERIOD OF RECORD

DISCHARGE, IN FT3/S, POR INDICATED RECURRENCE INTERYAL IN YEARS, AND EXCEEDANCE PROBABILITY, IN PERCENT

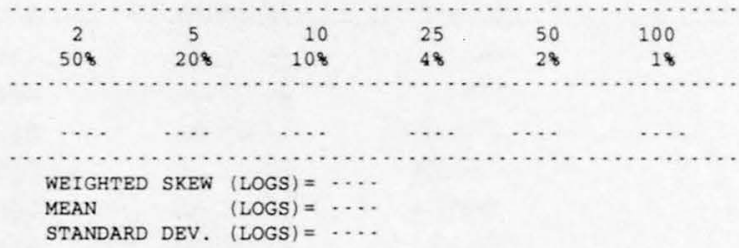

MAGNITUDE AND PROBABILITY OF ANNUAL LOW FLOW BASED ON PERIOD OF RECORD 1913.96

\begin{tabular}{|c|c|c|c|c|c|c|}
\hline \multirow{5}{*}{$\begin{array}{l}\text { PERIOD } \\
\text { (CON- } \\
\text { SECU- } \\
\text { TIVE } \\
\text { DAYS) }\end{array}$} & \multirow{2}{*}{\multicolumn{3}{|c|}{$\begin{array}{l}\text { DISCHARGE, IN } \\
\text { RECURRENCE IN } \\
\text { NON-EXCEEDANCE }\end{array}$}} & \multicolumn{3}{|c|}{ IN FT3/S, FOR INDICATED } \\
\hline & & & & \multicolumn{3}{|c|}{$\begin{array}{l}\text { INTERVAL, IN YEARS, AND } \\
\text { CE PROBABILITY, IN PERCENT }\end{array}$} \\
\hline & & ........ & ….... & ….... & & …... \\
\hline & 2 & 5 & 10 & 20 & 50 & 100 \\
\hline & 508 & 208 & 108 & 58 & 28 & 18 \\
\hline 1 & 13 & 2.3 & 0.72 & 0.25 & 0.04 & 0.00 \\
\hline 3 & 14 & 2.3 & 0.76 & 0.26 & 0.04 & 0.00 \\
\hline 7 & 15 & 2.6 & 0.86 & 0.30 & 0.05 & 0.00 \\
\hline 14 & 18 & 3.2 & 1.2 & 0.45 & 0.15 & 0.06 \\
\hline 30 & 27 & 5.4 & 2.1 & 0.84 & 0.29 & 0.13 \\
\hline 60 & 53 & 13 & 5.4 & 2.4 & 0.90 & 0.44 \\
\hline 90 & 90 & 28 & 13 & 6.5 & 2.7 & 1.4 \\
\hline 120 & 132 & 53 & 29 & 16 & 7.8 & 4.6 \\
\hline 183 & 218 & 104 & 66 & 43 & 25 & 17 \\
\hline
\end{tabular}

MAGNITUDE AND PROBABILITY OF ANNUAL HIGH FLOW BASED ON PERIOD OF RECORD 1912.96

\begin{tabular}{|c|c|c|c|c|c|c|}
\hline & & \multicolumn{5}{|c|}{ DISCHARGE, IN FT3/S, FOR INDICATED } \\
\hline $\begin{array}{l}\text { PERIOD } \\
\text { (CON- }\end{array}$ & & $\begin{array}{l}\text { RECURRE } \\
\text { EXCEEDA }\end{array}$ & $\begin{array}{l}\text { NCE INTE } \\
\text { NCE PROE }\end{array}$ & $\begin{array}{l}\text { RVAL, IN } \\
\text { BILITY, }\end{array}$ & $\begin{array}{l}\text { YEARS, } \\
\text { IN PER }\end{array}$ & $\begin{array}{l}\text { AND } \\
\text { ENT }\end{array}$ \\
\hline TIVE & 2 & 5 & 10 & 25 & 50 & 100 \\
\hline DAYS) & 508 & 208 & 108 & 48 & 28 & 18 \\
\hline 1 & 4,070 & 10,500 & 18,800 & 37,400 & 60,600 & 95,800 \\
\hline 3 & 2,690 & 6.760 & 12,000 & 23,800 & 38,600 & 61,500 \\
\hline 7 & 1,770 & 4,350 & 7,720 & 15,500 & 25,400 & 40,900 \\
\hline 15 & 1.260 & 2,970 & 5,150 & 10,000 & 16,200 & 25,700 \\
\hline 30 & 1,020 & 2,220 & 3,630 & 6.570 & 10,000 & 15,100 \\
\hline 60 & 832 & 1,720 & 2,690 & 4,540 & 6,570 & 9,340 \\
\hline 90 & 726 & 1,490 & 2,270 & 3.660 & 5,070 & 6,900 \\
\hline
\end{tabular}

DURATION TABLE OF DAILY MEAN FLOW FOR PERIOD OF RECORD 1912.96

DISCHARGE, IN FT3/S, WHICH WAS EQUALED OR EXCEEDED FOR INDICATED PERCENT OF TIME

\begin{tabular}{|c|c|c|c|c|c|c|c|c|c|c|c|c|c|c|c|c|}
\hline 18 & 58 & 108 & 158 & 208 & 308 & 408 & 508 & 608 & 708 & 808 & 908 & 958 & 988 & 998 & 99.58 & 99.98 \\
\hline 570 & 1,470 & 1,090 & 889 & 740 & 527 & 379 & 270 & 189 & 128 & 71 & 26 & 8.3 & 1.9 & 0.90 & 0.64 & 0.17 \\
\hline
\end{tabular}


ANNUAL MEAN DISCHARGE, IN CUBIC

FEET PER SECOND

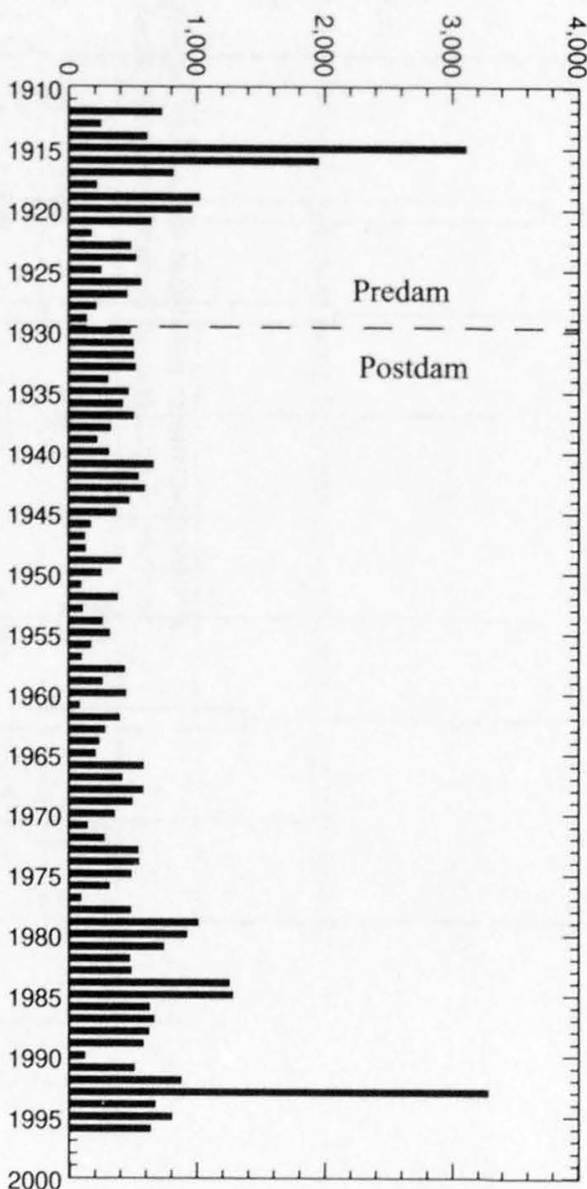

ANNUAL PEAK DISCHARGE, IN CUBIC FEET PER SECOND

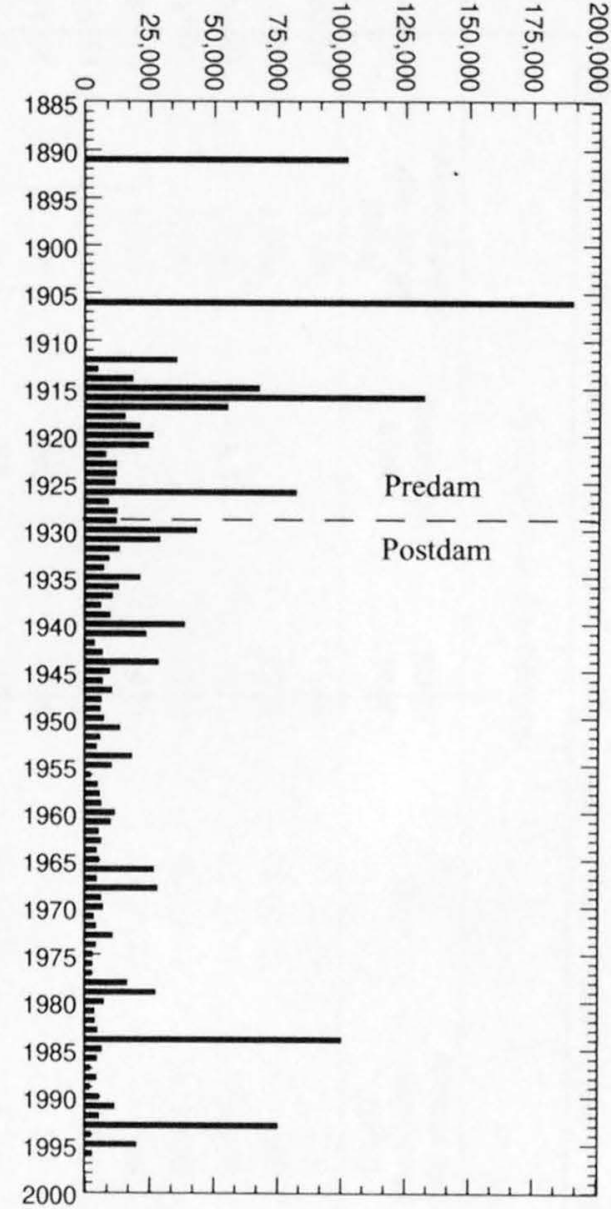


09474000 GILA RIVER AT KELVIN, AZ--Continued

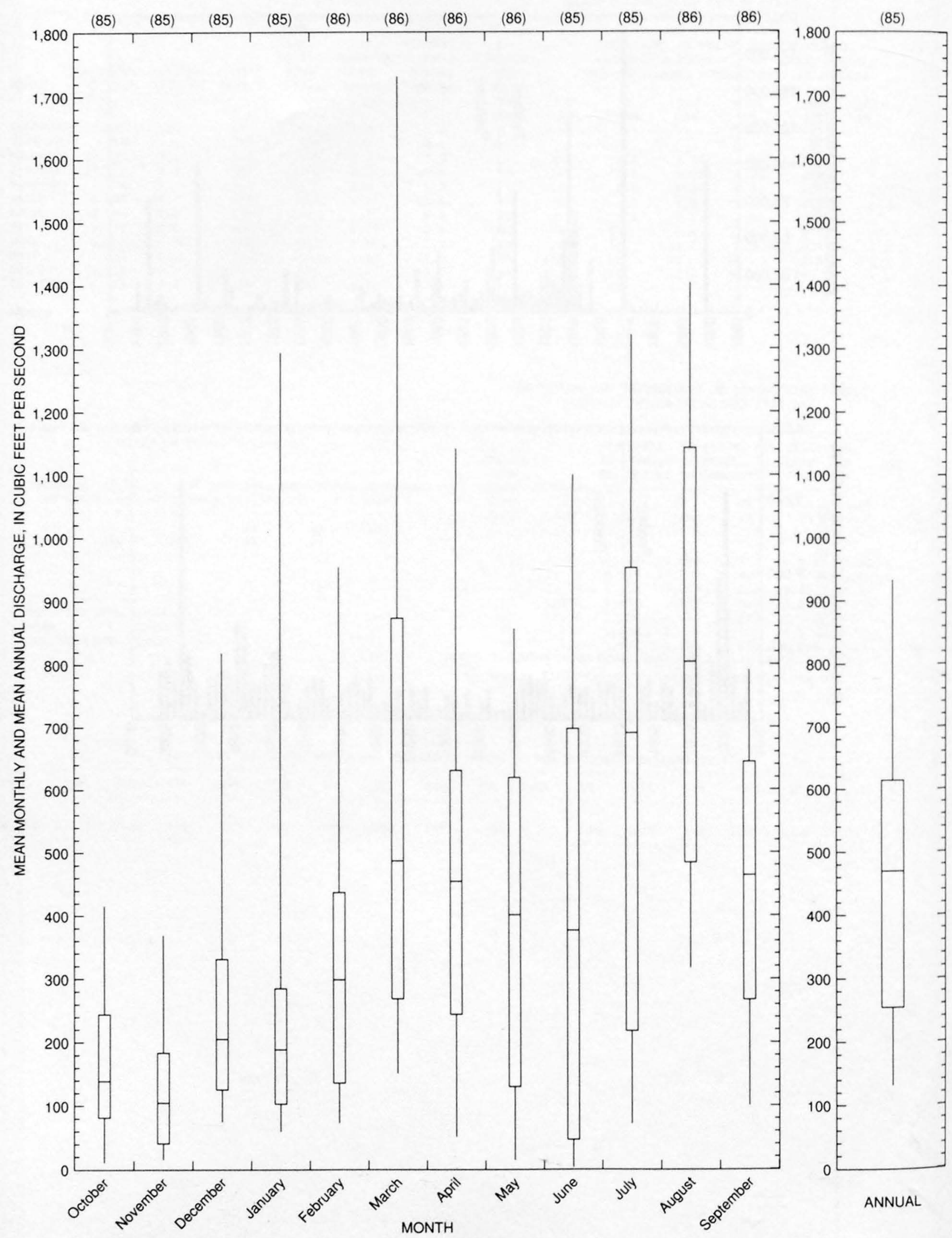


09478200 DURHAM WASH NEAR FLORENCE, AZ

LOCATION.--Lat 3243'20", long $111^{\circ} 06^{\prime} 30^{\prime \prime}, \mathrm{NE}^{1} / 4$ sec.21, T.8 S., R.12 E., Pinal County, Hydrologic Unit 15050100, at U.S. Highway 80, 27 mi southeast of Florence.

DRAINAGE AREA.--15.6 $\mathrm{mi}^{2}$.

Annual peak discharges

\begin{tabular}{|c|c|c|c|c|c|c|c|}
\hline $\begin{array}{l}\text { Water } \\
\text { year }\end{array}$ & Date & $\begin{array}{c}\text { Annual peak } \\
\text { discharge } \\
\left(\mathrm{ft}^{3} / \mathrm{s}\right)\end{array}$ & $\begin{array}{l}\text { Discharge } \\
\text { codes }\end{array}$ & $\begin{array}{l}\text { Water } \\
\text { year }\end{array}$ & Date & $\begin{array}{c}\text { Annual peak } \\
\text { discharge } \\
\left(\mathrm{ft}^{3} / \mathrm{s}\right)\end{array}$ & $\begin{array}{c}\text { Discharge } \\
\text { codes }\end{array}$ \\
\hline 1954 & $07-20-54$ & 3,340 & & 1969 & $00-00-69$ & 310 & \\
\hline 1955 & $08-00-55$ & 1,000 & & 1970 & $08-00-70$ & 290 & \\
\hline 1956 & $07-00-56$ & 100 & ES & 1971 & $08-20-71$ & $2_{3,500}$ & \\
\hline 1957 & $00-00-57$ & 2,700 & & 1972 & $06-00-72$ & 1,450 & \\
\hline 1963 & $09-01-63$ & 1,080 & & 1973 & $10-19-72$ & 100 & ES \\
\hline 1964 & $09-14-64$ & ${ }^{1} 1,790$ & & 1974 & $08-02-74$ & 550 & \\
\hline 1965 & $09-08-65$ & 40 & ES & 1975 & $09-13-75$ & 570 & \\
\hline 1966 & $12-00-65$ & 50 & ES & 1976 & $09-25-76$ & 1,100 & \\
\hline 1967 & $09-25-67$ & 230 & & 1980 & $07-00-80$ & ${ }^{3} 500$ & HP \\
\hline 1968 & $00-00-68$ & 500 & & & & & \\
\hline
\end{tabular}

${ }^{1}$ Highest since 1957.

${ }^{2}$ Highest since 1954 .

${ }^{3}$ Highest since 1976.

Magnitude and probability of instantaneous peak flow based on period of record 1954-57,

1963-76, 1980

\begin{tabular}{|c|c|c|c|c|c|}
\hline \multicolumn{6}{|c|}{$\begin{array}{l}\text { Discharge, in } \mathrm{ft}^{3} / \mathrm{s} \text {, for indicated recurrence interval } \\
\text { in years, and exceedance probablility, in percent }\end{array}$} \\
\hline 2 & 5 & 10 & 25 & $50 t$ & $100 t$ \\
\hline $50 \%$ & $20 \%$ & $10 \%$ & $4 \%$ & $2 \%$ & $1 \%$ \\
\hline 561 & 1,320 & 2,050 & 3,260 & 4,390 & 5,710 \\
\hline Weighted skew & $(\log s)=$ & -0.08 & & & \\
\hline Mean & $(\log s)=$ & 2.74 & & & \\
\hline Standard dev. & $(\log s)=$ & 0.45 & & & \\
\hline
\end{tabular}

†Reliability of values in column is uncertain, and potential errors are large.

Basin characteristics

\begin{tabular}{|c|c|c|c|c|c|c|c|}
\hline \multirow[b]{2}{*}{$\begin{array}{l}\text { Main } \\
\text { channel } \\
\text { slope } \\
\text { (ft/mi) }\end{array}$} & \multirow[b]{2}{*}{$\begin{array}{l}\text { Stream } \\
\text { length } \\
\text { (mi) }\end{array}$} & \multirow[b]{2}{*}{$\begin{array}{c}\text { Mean } \\
\text { basin } \\
\text { elevation } \\
(f t)\end{array}$} & \multirow[b]{2}{*}{$\begin{array}{l}\text { Forested } \\
\text { area } \\
\text { (percent) }\end{array}$} & \multirow[b]{2}{*}{$\begin{array}{c}\text { Soil } \\
\text { index }\end{array}$} & \multirow[b]{2}{*}{$\begin{array}{c}\text { Mean } \\
\text { annual } \\
\text { precipitation } \\
\text { (in) }\end{array}$} & \multicolumn{2}{|c|}{ Rainfall intensity, 24-hour } \\
\hline & & & & & & $\begin{array}{l}\text { 2-year } \\
\text { (in) }\end{array}$ & $\begin{array}{l}\text { 50-year } \\
\text { (in) }\end{array}$ \\
\hline 143 & 11.0 & 3,670 & 0.0 & 3.0 & 12.1 & 1.9 & 4.3 \\
\hline
\end{tabular}


09478200 DURHAM WASH NEAR FLORENCE, AZ--Continued

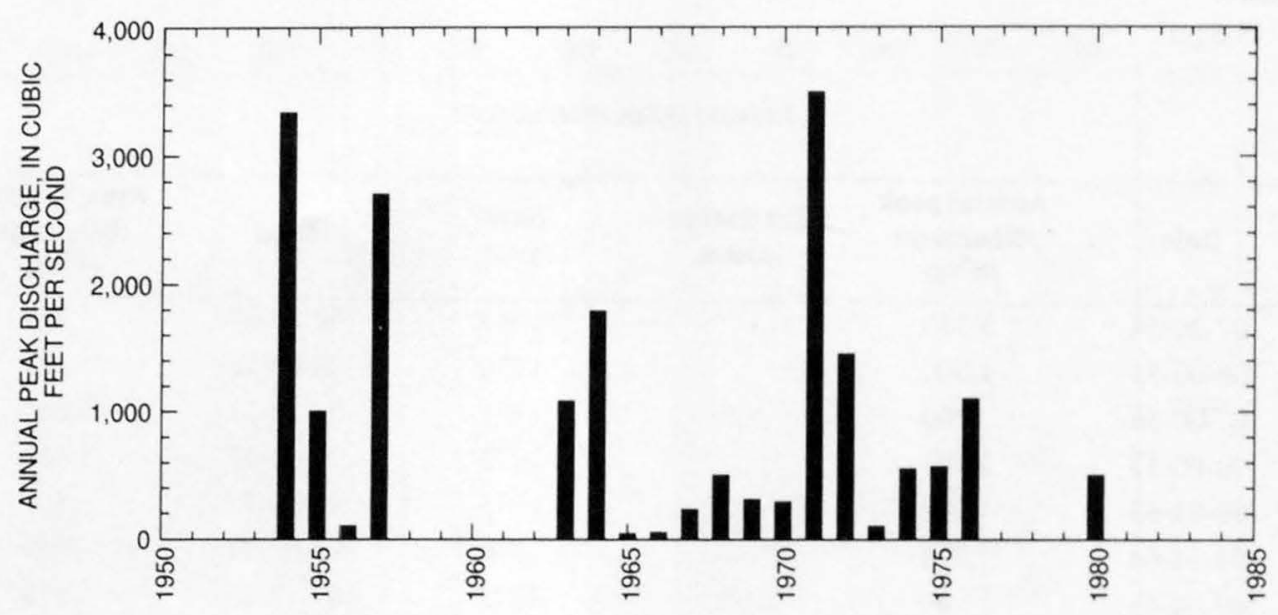


09478500 QUEEN CREEK AT WHITLOW DAMSITE, NEAR SUPERIOR, AZ

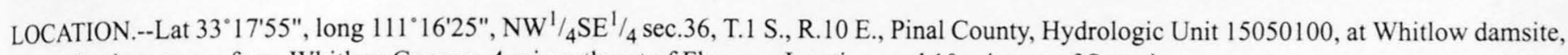
2.5 mi upstream from Whitlow Canyon, 4 mi northeast of Florence Junction, and 10 mi west of Superior.

DRAINAGE AREA.--144 $\mathrm{mi}^{2}$.

Annual peak discharges

\begin{tabular}{|c|c|c|c|c|c|c|c|}
\hline $\begin{array}{l}\text { Water } \\
\text { year }\end{array}$ & Date & $\begin{array}{c}\text { Annual peak } \\
\text { discharge } \\
\left(\mathrm{ft}^{3} / \mathrm{s}\right)\end{array}$ & $\begin{array}{l}\text { Discharge } \\
\text { codes }\end{array}$ & $\begin{array}{l}\text { Water } \\
\text { year }\end{array}$ & Date & $\begin{array}{c}\text { Annual peak } \\
\text { discharge } \\
\left(\mathrm{ft}^{3} / \mathrm{s}\right)\end{array}$ & $\begin{array}{c}\text { Discharge } \\
\text { codes }\end{array}$ \\
\hline 1917 & $05-20-17$ & 2,800 & & 1952 & $01-18-52$ & 1,170 & \\
\hline 1918 & $08-05-18$ & 5,000 & & 1953 & $07-29-53$ & 1,780 & \\
\hline 1919 & $08-01-19$ & 10,000 & & 1954 & $08-19-54$ & 42,900 & \\
\hline 1920 & $02-20-20$ & 750 & & 1955 & $08-03-55$ & 5,430 & \\
\hline 1939 & $08-06-39$ & 13,200 & & 1956 & $08-17-56$ & 4,100 & \\
\hline 1948 & $07-21-48$ & 676 & & 1957 & $08-19-57$ & 8,260 & \\
\hline 1949 & $07-22-49$ & 2,630 & & 1958 & $03-22-58$ & 3,970 & \\
\hline 1950 & $07-18-50$ & 5,100 & & 1959 & $08-17-59$ & 30,000 & ES \\
\hline 1951 & $08-03-51$ & 1,510 & & & & & \\
\hline
\end{tabular}

Basin characteristics

\begin{tabular}{cccccccc}
\hline $\begin{array}{c}\text { Main } \\
\text { channel } \\
\text { slope } \\
(\mathrm{ft} / \mathrm{mi})\end{array}$ & $\begin{array}{c}\text { Stream } \\
\text { length } \\
(\mathrm{mi})\end{array}$ & $\begin{array}{c}\text { Mean } \\
\text { basin } \\
\text { elevation } \\
(\mathrm{ft})\end{array}$ & $\begin{array}{c}\text { Forested } \\
\text { area } \\
\text { (percent) }\end{array}$ & $\begin{array}{c}\text { Soil } \\
\text { index }\end{array}$ & $\begin{array}{c}\text { Mean } \\
\text { annual } \\
\text { precipitation } \\
\text { (in) }\end{array}$ & $\begin{array}{c}\text { R-year } \\
\text { (in) }\end{array}$ & $\begin{array}{c}\text { 24-hour } \\
\text { (in) }\end{array}$ \\
\hline 137 & 19.4 & 3,180 & 5.6 & 1.0 & 17.9 & 2.4 \\
\hline
\end{tabular}


09478500 QUEEN CREEK AT WHITLOW DAMSITE, NEAR SUPERIOR, AZ--Continued

MEAN MONTHLY AND ANNUAL DISCHARGES 1949.58

\begin{tabular}{|c|c|c|c|c|c|c|}
\hline MONTH & $\begin{array}{l}\text { MAXIMUM } \\
(\text { FT3/S) }\end{array}$ & $\begin{array}{l}\text { MINIMUM } \\
\text { (FT3/S) }\end{array}$ & $\begin{array}{c}\text { MEAN } \\
(\mathrm{FT} 3 / \mathrm{S})\end{array}$ & $\begin{array}{l}\text { STAN- } \\
\text { DARD } \\
\text { DEVIA. } \\
\text { TION } \\
(\mathrm{FT} 3 / \mathrm{S})\end{array}$ & $\begin{array}{l}\text { COEFFI- } \\
\text { CIENT OF } \\
\text { VARI- } \\
\text { ATION }\end{array}$ & $\begin{array}{l}\text { PERCENT } \\
\text { OF } \\
\text { ANNUAL } \\
\text { RUNOFF }\end{array}$ \\
\hline OCTOBER & 8.5 & 0.00 & 1.1 & 2.7 & 2.4 & 2.3 \\
\hline NOVEMBER & 3.8 & 0.00 & 0.61 & 1.2 & 2.0 & 1.2 \\
\hline DECEMBER & 13 & 0.00 & 2.0 & 4.0 & 2.0 & 4.1 \\
\hline JANUARY & 17 & 0.00 & 3.8 & 5.1 & 1.4 & 7.7 \\
\hline FEBRUARY & 4.4 & 0.00 & 0.65 & 1.4 & 2.1 & 1.3 \\
\hline MARCH & 50 & 0.00 & 13 & 18 & 1.4 & 25.6 \\
\hline APRIL & 8.9 & 0.00 & 1.7 & 3.3 & 2.0 & 3.5 \\
\hline MAY & 2.4 & 0.00 & 0.55 & 0.87 & 1.6 & 1.1 \\
\hline JUNE & 10 & 0.00 & 1.4 & 3.2 & 2.2 & 2.9 \\
\hline JULY & 19 & 0.30 & 5.6 & 6.8 & 1.2 & 11.4 \\
\hline AUGUST & 106 & 0.03 & 18 & 32 & 1.8 & 37.4 \\
\hline SEPTEMBER & 2.8 & 0.00 & 0.75 & 1.2 & 1.6 & 1.5 \\
\hline ANNUAL & 14 & 1.3 & 4.1 & 3.7 & 0.89 & 100 \\
\hline
\end{tabular}

MAGNITUDE AND PROBABILITY OF INSTANTANEOUS PEAK FLOW BASED ON PERIOD OF RECORD 1917-20, 1939, 1948-59

DISCHARGE, IN FT3/S, FOR INDICATED RECURRENCE INTERVAL IN YEARS, AND EXCEEDANCE PROBABILITY, IN PERCENT

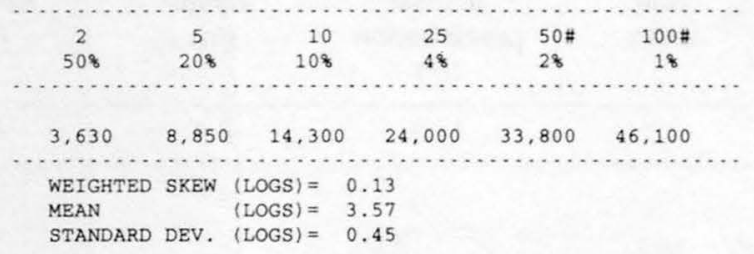

MAGNITUDE AND PROBABILITY OF ANNUAL LOW FLOW BASED ON PERIOD OF RECORD 1950.58

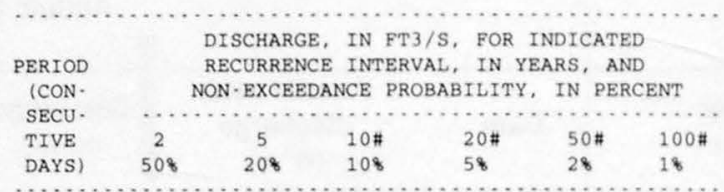

$\begin{array}{rrrrrrr}1 & & & & & & \\ 3 & & & & & & \\ 7 & & & & & & \\ 14 & & & & & & \\ 30 & & & & & & \\ 60 & & & & & \\ 90 & 0.00 & 0.00 & 0.00 & 0.00 & 0.00 & 0.00 \\ 120 & 0.14 & 0.00 & 0.00 & 0.00 & 0.00 & 0.00 \\ 183 & 0.84 & 0.23 & 0.10 & 0.04 & 0.02 & 0.01\end{array}$

MAGNITUDE AND PROBABILITY OF ANNUAL HIGH FLOW BASED ON PERIOD OF RECORD 1949.58

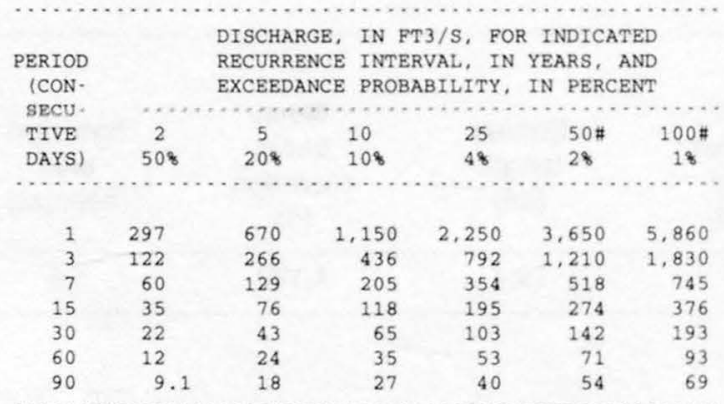

DURATION TABLE OF DAILY MEAN FLOW FOR PERIOD OF RECORD 1949.58

DISCHARGE, IN FT3/S, WHICH WAS EQUALED OR EXCEEDED FOR INDICATED PERCENT OF TIME

\begin{tabular}{|c|c|c|c|c|c|c|c|c|c|c|c|c|c|c|c|c|}
\hline 18 & 58 & 108 & 158 & 208 & 308 & 408 & 508 & 608 & 708 & 808 & 908 & 958 & 988 & 998 & 99.58 & 99.98 \\
\hline 88 & 3.3 & 1.7 & 0.96 & 0.53 & 0.19 & 0.00 & 0.00 & 0.00 & 0.00 & 0.00 & 0.00 & 0.00 & 0.00 & 0.00 & 0.00 & 0.00 \\
\hline
\end{tabular}

\# Reliability of values in column is uncertain, and potential errors are large. 
09478500 QUEEN CREEK AT WHITLOW DAMSITE, NEAR SUPERIOR, AZ--Continued
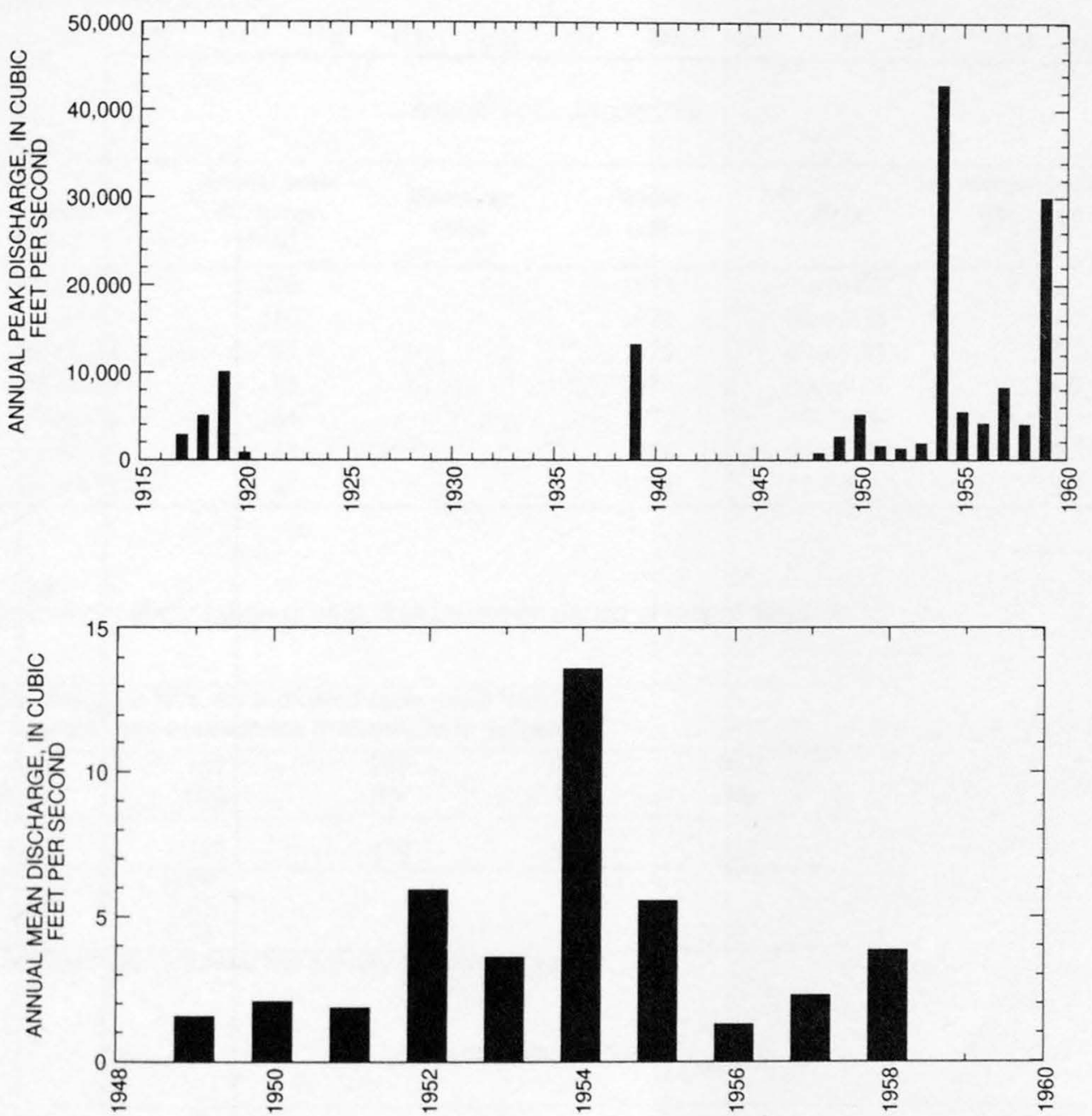
09478500 QUEEN CREEK AT WHITLOW DAMSITE, NEAR SUPERIOR, AZ--Continued
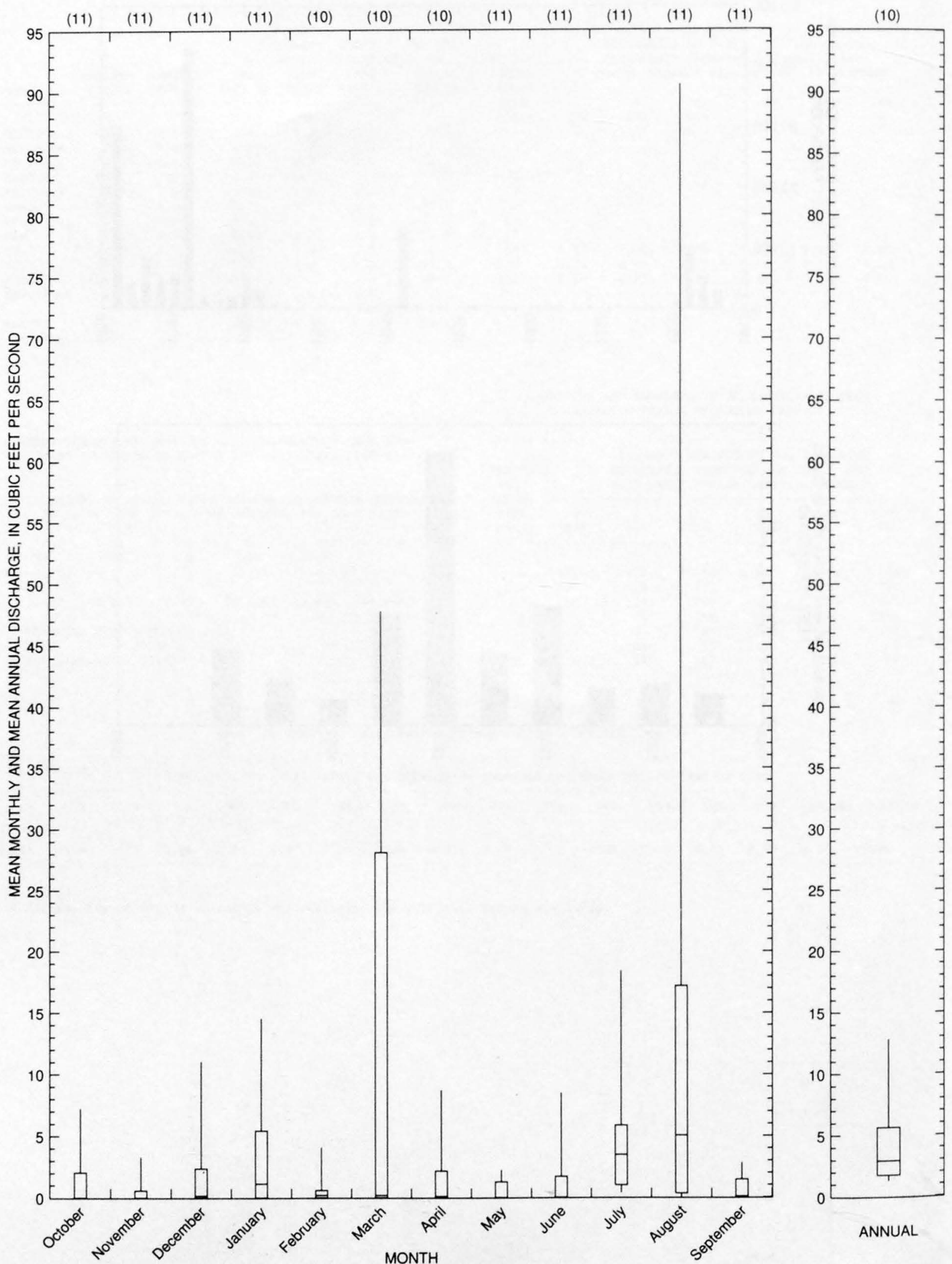
09478600 QUEEN CREEK TRIBUTARY NO. 3 AT WHITLOW DAM, AZ

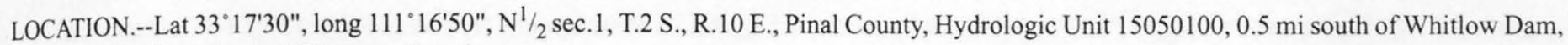
and $4.5 \mathrm{mi}$ northeast of Florence Junction.

DRAINAGE AREA.--0.37 $\mathrm{mi}^{2}$.

Annual peak discharges

\begin{tabular}{|c|c|c|c|c|c|c|c|}
\hline $\begin{array}{l}\text { Water } \\
\text { year }\end{array}$ & Date & $\begin{array}{c}\text { Annual peak } \\
\text { discharge } \\
\left(\mathrm{ft}^{3} / \mathrm{s}\right)\end{array}$ & $\begin{array}{c}\text { Discharge } \\
\text { codes }\end{array}$ & $\begin{array}{l}\text { Water } \\
\text { year }\end{array}$ & Date & $\begin{array}{c}\text { Annual peak } \\
\text { discharge } \\
\left(\mathrm{ft}^{3} / \mathrm{s}\right)\end{array}$ & $\begin{array}{c}\text { Discharge } \\
\text { codes }\end{array}$ \\
\hline 1966 & $09-13-66$ & 280 & & 1973 & $10-19-72$ & 80 & \\
\hline 1967 & $08-00-67$ & 110 & & 1974 & $00-00-74$ & 0 & \\
\hline 1968 & $08-00-68$ & 95 & & 1975 & $09-05-75$ & 35 & \\
\hline 1969 & $09-16-69$ & 85 & & 1976 & $04-16-76$ & 4.0 & \\
\hline 1970 & $08-09-70$ & 64 & & 1977 & $10-23-76$ & 115 & \\
\hline 1971 & $08-00-71$ & 63 & & 1978 & $03-02-78$ & 240 & \\
\hline 1972 & $08-00-72$ & 44 & & 1979 & $12-18-78$ & 5.0 & ES \\
\hline
\end{tabular}

Magnitude and probability of instantaneous peak flow based on period of record 1966-79

\begin{tabular}{|c|c|c|c|c|c|}
\hline \multicolumn{6}{|c|}{$\begin{array}{l}\text { Discharge, in } \mathrm{ft}^{3} / \mathrm{s} \text {, for indicated recurrence interval } \\
\text { in years, and exceedance probablility, in percent }\end{array}$} \\
\hline $2 \dagger$ & $5 t$ & $10 t$ & $25 \dagger$ & $50+$ & $100+$ \\
\hline $50 \%$ & $20 \%$ & $10 \%$ & $4 \%$ & $2 \%$ & $1 \%$ \\
\hline 71.3 & 135 & 189 & 270 & 339 & 417 \\
\hline Weighted skew & $(\log s)=$ & -0.00 & & & \\
\hline Mean & $(\log s)=$ & 1.85 & & & \\
\hline Standard dev. & $(\log s)=$ & 0.33 & & & \\
\hline
\end{tabular}

Basin characteristics

\begin{tabular}{cccccccc}
\hline $\begin{array}{c}\text { Main } \\
\text { channel } \\
\begin{array}{c}\text { slope } \\
(\mathrm{ft} / \mathrm{mi})\end{array}\end{array}$ & $\begin{array}{c}\text { Stream } \\
\text { length } \\
(\mathrm{mi})\end{array}$ & $\begin{array}{c}\text { Mean } \\
\text { basin } \\
\text { elevation } \\
(\mathrm{ft})\end{array}$ & $\begin{array}{c}\text { Forested } \\
\text { area } \\
\text { (percent) }\end{array}$ & $\begin{array}{c}\text { Soil } \\
\text { index }\end{array}$ & $\begin{array}{c}\text { Mean } \\
\text { annual } \\
\text { precipitation } \\
\text { (in) }\end{array}$ & $\begin{array}{c}\text { Rainfall intensity, 24-hour } \\
\text { (in) }\end{array}$ & $\begin{array}{c}50 \text {-year } \\
\text { (in) }\end{array}$ \\
\hline 187 & 1.1 & 2,320 & 0.0 & 1.0 & 12.0 & 1.8 \\
\hline
\end{tabular}

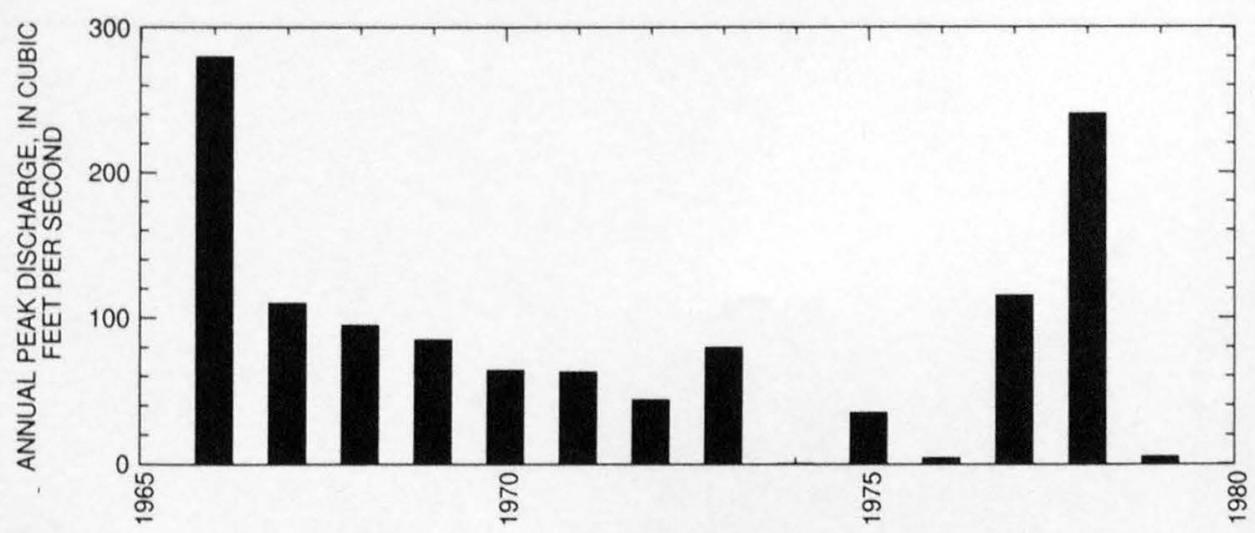


LOCATION.--Lat 33 $24^{\prime} 13^{\prime \prime}$, long $111^{\circ} 32^{\prime} 27^{\prime \prime}, \mathrm{NE}^{1} / \mathrm{SW}^{1} / 4 \mathrm{sec} .21$, T.1 S., R.8 E., Pinal County, Hydrologic Unit 15050100, at eastbound lane of

U.S. Highway 60, $0.6 \mathrm{mi}$ southeast of Apache Junction.

DRAINAGE AREA.--0.51 $\mathrm{mi}^{2}$.

Annual peak discharges

\begin{tabular}{|c|c|c|c|c|c|c|c|}
\hline $\begin{array}{l}\text { Water } \\
\text { year }\end{array}$ & Date & $\begin{array}{c}\text { Annual peak } \\
\text { discharge } \\
\left(\mathrm{ft}^{3} / \mathrm{s}\right)\end{array}$ & $\begin{array}{l}\text { Discharge } \\
\text { codes }\end{array}$ & $\begin{array}{l}\text { Water } \\
\text { year }\end{array}$ & Date & $\begin{array}{c}\text { Annual peak } \\
\text { discharge } \\
\left(\mathrm{ft}^{3} / \mathrm{s}\right)\end{array}$ & $\begin{array}{c}\text { Discharge } \\
\text { codes }\end{array}$ \\
\hline 1961 & $08-18-61$ & 179 & & 1971 & $09-30-71$ & 262 & \\
\hline 1962 & $11-21-61$ & 39 & & 1972 & $00-00-72$ & 0 & \\
\hline 1963 & $08-17-63$ & 46 & & 1973 & $10-18-72$ & 109 & \\
\hline 1964 & $09-14-64$ & 74 & & 1974 & $07-07-74$ & 22 & \\
\hline 1965 & $08-17-65$ & 1.7 & & 1975 & $09-05-75$ & 54 & \\
\hline 1966 & $08-30-66$ & 126 & & 1976 & $07-24-76$ & 176 & \\
\hline 1967 & $07-11-67$ & 42 & & 1977 & $00-00-77$ & 0 & \\
\hline 1968 & $08-03-68$ & 70 & & 1978 & $00-00-78$ & 0 & \\
\hline 1969 & $09-16-69$ & 19 & & 1979 & $00-00-79$ & 0 & \\
\hline 1970 & $09-05-70$ & 137 & & & & & \\
\hline
\end{tabular}

Magnitude and probability of instantaneous peak flow based on period of record 1961-79

\begin{tabular}{|c|c|c|c|c|c|}
\hline \multicolumn{6}{|c|}{$\begin{array}{l}\text { Discharge, in } \mathrm{ft}^{3} / \mathrm{s} \text {, for indicated recurrence interval } \\
\text { in years, and exceedance probablility, in percent }\end{array}$} \\
\hline $2 \dagger$ & $5 \dagger$ & $10 t$ & $25 t$ & $50 \dagger$ & $100 \dagger$ \\
\hline $50 \%$ & $20 \%$ & $10 \%$ & $4 \%$ & $2 \%$ & $1 \%$ \\
\hline 52.5 & 120 & 179 & 269 & 346 & 431 \\
\hline Weighted skew & $(\log s)=$ & -0.31 & & & \\
\hline Mean & $(\log s)=$ & 1.70 & & & \\
\hline Standard dev. & $(\log s)=$ & 0.45 & & & \\
\hline
\end{tabular}

Basin characteristics

\begin{tabular}{cccccccc}
\hline $\begin{array}{c}\text { Main } \\
\text { channel } \\
\text { slope } \\
(\mathrm{ft} / \mathrm{mi})\end{array}$ & $\begin{array}{c}\text { Stream } \\
\text { length } \\
(\mathrm{mi})\end{array}$ & $\begin{array}{c}\text { Mean } \\
\text { basin } \\
\text { elevation } \\
(\mathrm{ft})\end{array}$ & $\begin{array}{c}\text { Forested } \\
\text { area } \\
\text { (percent) }\end{array}$ & $\begin{array}{c}\text { Soil } \\
\text { index }\end{array}$ & $\begin{array}{c}\text { Mean } \\
\text { annual } \\
\text { precipitation } \\
\text { (in) }\end{array}$ & $\begin{array}{c}\text { R-year } \\
\text { (in) }\end{array}$ & $\begin{array}{c}50 \text {-year } \\
\text { (in) }\end{array}$ \\
\hline 76.2 & 1.4 & 1,760 & 0.0 & 1.0 & 10.5 & 1.6 \\
\hline
\end{tabular}


09479200 QUEEN CREEK TRIBUTARY AT APACHE JUNCTION, AZ--Continued

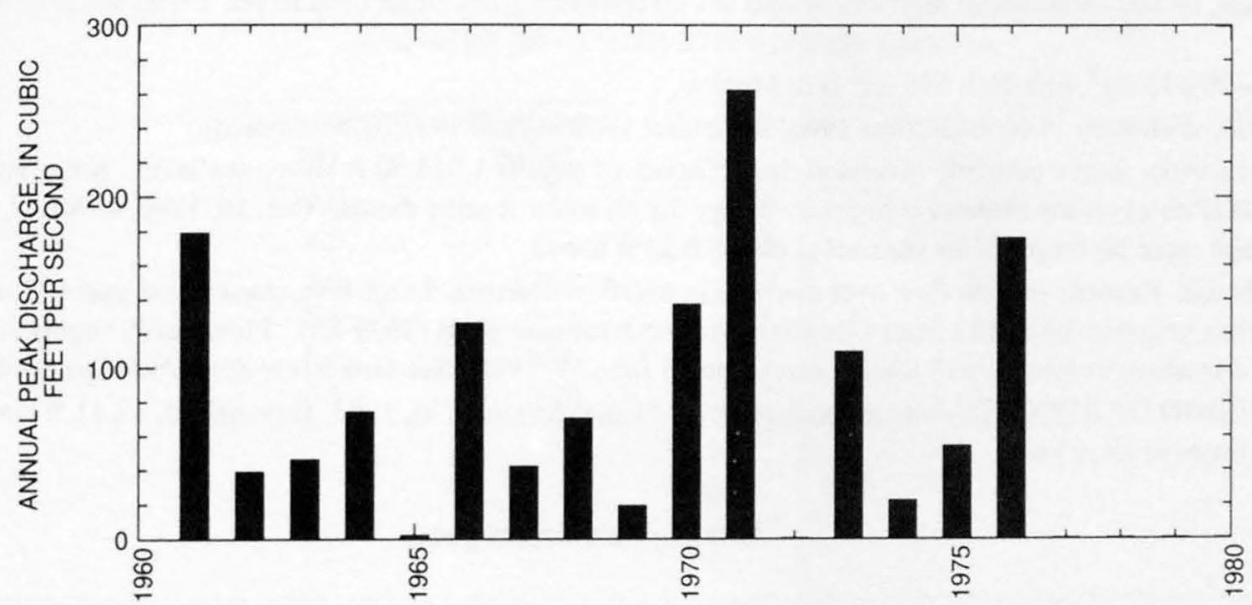




\section{GILA RIVER NEAR LAVEEN, AZ}

LOCATION.--Lat 33.15'25", long $112^{\circ} 09^{\prime} 59^{\prime \prime}$, in SW $1 /{ }_{4} \mathrm{NW}^{1 / 4}$ sec.16, T.2 S., R.2 E., Pinal County, Hydrologic Unit 15050100 , on Gila River Indian Reservation, on left abutment of highway bridge, 2.1 mi upstream from Santa Cruz River, 2.6 mi south of Komatke, and 7.3 mi south of Laveen.

DRAINAGE AREA.--20,615 $\mathrm{mi}^{2}$, of which $696 \mathrm{mi}^{2}$ is in Mexico.

PERIOD OF RECORD.--February 1940 to October 1946, December 1947 to June 1995 (discontinued).

GAGE.--Water-stage recorder above concrete diversion dam. Datum of gage is $1,018.90 \mathrm{ft}$ above sea level. Since July 9 , 1969, supplementary water-stage recorder on overflow channel at highway bridge $0.2 \mathrm{mi}$ south at same datum. Oct. 16, 1940, to July 8, 1969, supplementary staff gage or water-stage recorder on overflow channel at datum $0.23 \mathrm{ft}$ lower.

REMARKS.--Records fair. Records include flow over dam and in overflow channel. Large diversions above station for irrigation. Most low flow is waste water from irrigated lands and from Chandler, Arizona treatment plant (1979-83). Flow partly regulated by storage in San Carlos Reservoir. (See elsewhere in this report.) Station discontinued June 19, 1995. See Gila River near Maricopa (09479350).

EXTREMES FOR PERIOD OF RECORD.--Maximum discharge, 41,600 $\mathrm{ft}^{3} / \mathrm{s}$ Jan. 20, 1993, gage height, $12.41 \mathrm{ft}$ main gage, $12.12 \mathrm{ft}$ overflow gage; no flow at times in most years.

Annual peak discharges

\begin{tabular}{|c|c|c|c|c|c|c|c|}
\hline $\begin{array}{l}\text { Water } \\
\text { year }\end{array}$ & Date & $\begin{array}{c}\text { Annual peak } \\
\text { discharge } \\
\left(\mathrm{ft}^{3} / \mathrm{s}\right)\end{array}$ & $\begin{array}{l}\text { Discharge } \\
\text { codes }\end{array}$ & $\begin{array}{l}\text { Water } \\
\text { year }\end{array}$ & Date & $\begin{array}{c}\text { Annual peak } \\
\text { discharge } \\
\left(\mathrm{ft}^{3} / \mathrm{s}\right)\end{array}$ & $\begin{array}{c}\text { Discharge } \\
\text { codes }\end{array}$ \\
\hline 1916 & $01-20-16$ & ${ }^{\prime} 65,000$ & ES,HP & 1968 & $12-23-68$ & 5,890 & UR \\
\hline 1926 & $09-28-26$ & ${ }^{2} 40,000$ & ES,HP & 1969 & $00-00-69$ & 0 & UR \\
\hline 1940 & $08-17-40$ & 8,740 & UR & 1970 & $03-05-70$ & 178 & UR \\
\hline 1941 & $01-02-41$ & 11,900 & UR & 1971 & $08-23-71$ & 1,130 & UR \\
\hline 1942 & $12-12-42$ & 1,170 & UR & 1972 & $10-03-72$ & 544 & UR \\
\hline 1943 & $09-27-43$ & 1,570 & UR & 1973 & $10-23-73$ & 1,500 & UR \\
\hline 1944 & $08-11-44$ & 1,330 & UR & 1974 & $08-07-74$ & 1,220 & UR \\
\hline 1945 & $08-13-45$ & 2,800 & UR & 1975 & $11-02-75$ & 19 & UR \\
\hline 1946 & $09-20-46$ & 1,260 & UR & 1976 & $09-25-76$ & 397 & UR \\
\hline 1948 & $08-05-48$ & 1,430 & UR & 1977 & $10-22-77$ & 430 & UR \\
\hline 1949 & $08-10-49$ & 1,250 & UR & 1978 & $10-13-78$ & 6,360 & UR \\
\hline 1950 & $08-02-50$ & 1,500 & UR & 1979 & $12-21-79$ & 9,720 & UR \\
\hline 1951 & $08-29-51$ & 1,210 & UR & 1980 & $02-23-80$ & 545 & UR \\
\hline 1952 & $01-20-52$ & 1,070 & UR & 1981 & $03-03-81$ & 20 & $\mathrm{MD}, \mathrm{U}$ \\
\hline 1953 & $07-31-53$ & 565 & UR & 1982 & $09-15-82$ & 194 & $\mathrm{MD}, \mathrm{U}$ \\
\hline 1954 & $08-08-54$ & 4,510 & UR & 1983 & $02-09-83$ & 385 & UR \\
\hline 1955 & $08-24-55$ & 3,230 & UR & 1984 & $10-04-84$ & 35,000 & UR \\
\hline 1956 & $01-31-56$ & 46 & UR & 1985 & $01-11-85$ & 2,080 & $\mathrm{MD}, \mathrm{U}$ \\
\hline 1957 & $08-20-57$ & 446 & UR & 1986 & $12-10-86$ & 10 & UR \\
\hline 1958 & $08-19-58$ & 995 & UR & 1987 & $02-25-87$ & 7.1 & UR \\
\hline 1959 & $08-19-59$ & 934 & UR & 1988 & $12-18-87$ & 16 & UR \\
\hline 1960 & $01-14-60$ & 1,760 & UR & 1989 & $01-05-89$ & 12 & UR \\
\hline 1961 & $08-25-61$ & 655 & UR & 1990 & $08-17-90$ & 456 & UR \\
\hline 1962 & $12-18-62$ & 1,020 & UR & 1991 & $03-06-91$ & 203 & UR \\
\hline 1963 & $02-14-63$ & 798 & UR & 1992 & $07-24-92$ & 245 & UR \\
\hline 1964 & $08-17-64$ & 996 & UR & 1993 & $01-20-93$ & ${ }^{3} 41,600$ & UR \\
\hline 1965 & $02-09-65$ & 85 & UR & 1994 & $11-17-93$ & 1,230 & UR \\
\hline 1966 & $12-26-66$ & 10,900 & UR & 1995 & $01-07-95$ & 13,090 & UR \\
\hline 1967 & $09-28-67$ & 350 & UR & & & & \\
\hline
\end{tabular}

\footnotetext{
${ }^{1}$ Highest since 1907.

${ }^{2}$ Highest since 1916.

${ }^{3}$ Highest since 1926.
} 
09479500 GILA RIVER NEAR LAVEEN, AZ--Continued

Discharge rating table developed January -.--

\begin{tabular}{crcc}
\hline $\begin{array}{c}\text { Gage height } \\
(\mathbf{f t})\end{array}$ & $\begin{array}{c}\text { Discharge } \\
\left(\mathbf{f t}^{\mathbf{3}} \mathbf{s}\right)\end{array}$ & $\begin{array}{c}\text { Gage height } \\
(\mathbf{f t})\end{array}$ & $\begin{array}{c}\text { Discharge } \\
\left(\mathbf{f t}^{\mathbf{3}} / \mathbf{s}\right)\end{array}$ \\
\hline 4.0 & 26 & 7.0 & 414 \\
4.5 & 212 & 7.5 & 512 \\
5.0 & 264 & 8.0 & 788 \\
5.5 & 299 & 8.5 & 1,180 \\
6.0 & 327 & 9.0 & 1,680 \\
6.5 & 357 & 9.7 & 2,630 \\
\hline
\end{tabular}


09479500 GILA RIVER NEAR LAVEEN, AZ--Continued

MEAN MONTHLY AND ANNUAL DISCHARGES $1941.46,1949.91,1993.94$

\begin{tabular}{|c|c|c|c|c|c|c|}
\hline MONTH & $\begin{array}{l}\text { MAXIMUM } \\
\text { (FT3/S) }\end{array}$ & $\begin{array}{l}\text { MINIMUM } \\
\text { (FT3/S) }\end{array}$ & $\begin{array}{c}\text { MEAN } \\
\text { (FT3/S) }\end{array}$ & $\begin{array}{l}\text { STAN- } \\
\text { DARD } \\
\text { DEVIA- } \\
\text { TION } \\
\text { (FT3/S) }\end{array}$ & $\begin{array}{l}\text { COEFFI- } \\
\text { CIENT OF } \\
\text { VARI- } \\
\text { ATION }\end{array}$ & $\begin{array}{c}\text { PERCENT } \\
\text { OF } \\
\text { ANNUAL } \\
\text { RUNOFF }\end{array}$ \\
\hline OCTOBER & 2,660 & 0.00 & 61 & 372 & 6.1 & 8.0 \\
\hline NOVEMBER & 182 & 0.00 & 8.6 & 27 & 3.2 & 1.1 \\
\hline DECEMBER & 518 & 0.00 & 39 & 111 & 2.8 & 5.2 \\
\hline JANUARY & 9,730 & 0.00 & 255 & 1,370 & 5.4 & 33.5 \\
\hline FEBRUARY & 5,020 & 0.00 & 152 & 722 & 4.8 & 20.0 \\
\hline MARCH & 3,210 & 0.00 & 111 & 474 & 4.3 & 14.6 \\
\hline APRIL & 1,760 & 0.00 & 41 & 246 & 6.1 & 5.3 \\
\hline MAY & 26 & 0.00 & 1.8 & 4.4 & 2.4 & 0.2 \\
\hline JUNE & 12 & 0.00 & 1.0 & 2.3 & 2.2 & 0.1 \\
\hline JULY & 249 & 0.00 & 14 & 40 & 2.9 & 1.8 \\
\hline AUGUST & 895 & 0.00 & 63 & 154 & 2.5 & 8.2 \\
\hline SEPTEMBER & 106 & 0.00 & 13 & 26 & 1.9 & 1.8 \\
\hline ANNUAL & 1,630 & 0.00 & 63 & 232 & 3.7 & 100 \\
\hline
\end{tabular}

MAGNITUDE AND PROBABILITY OF INSTANTANEOUS PEAK FLOW BASED ON PERIOD OF RECORD 1941.46, 1949.91, 1993.94

DISCHARGE, IN $F T 3 / S$, FOR INDICATED RECURRENCE INTERVAL IN YEARS, AND EXCEEDANCE PROBABILITY, IN PERCENT

\begin{tabular}{|c|c|c|c|c|c|}
\hline 2 & 5 & 10 & 25 & 50 & 100 \\
\hline 508 & 208 & 108 & 48 & 28 & 18 \\
\hline 1,020 & 3,760 & 8,040 & 19,200 & 34,800 & 60,900 \\
\hline WEIGHTED & SKEW & $($ LOGS $)=$ & & & \\
\hline MEAN & & $($ LOGS) = & & & \\
\hline
\end{tabular}

MAGNITUDE AND PROBABILITY OF ANNUAL LOW FLOW BASED ON PERIOD OF RECORD 1941-46, 1949-91, 1994.95

\begin{tabular}{|c|c|c|c|c|c|c|}
\hline \multirow{3}{*}{$\begin{array}{l}\text { PERIOD } \\
\text { (CON- } \\
\text { SECU- } \\
\text { TIVE }\end{array}$} & $\begin{array}{l}\text { RECURRENCE INTERVAL, IN YEARS, AND } \\
\text { NON-EXCEEDANCE PROBABILITY, IN PERCENT }\end{array}$ & \multicolumn{5}{|c|}{ DISCHARGE, IN FT3/S, FOR INDICATED } \\
\hline & & $\ldots \ldots$ & & 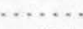 & & \\
\hline & 2 & 5 & 10 & $20 \#$ & $50 \#$ & $100 \#$ \\
\hline DAYS) & 508 & 208 & 108 & 58 & 28 & 18 \\
\hline 1 & 0.00 & 0.00 & 0.00 & 0.00 & 0.00 & 0.00 \\
\hline 3 & 0.00 & 0.00 & 0.00 & 0.00 & 0.00 & 0.00 \\
\hline 7 & 0.00 & 0.00 & 0.00 & 0.00 & 0.00 & 0.00 \\
\hline 14 & 0.00 & 0.00 & 0.00 & 0.00 & 0.00 & 0.00 \\
\hline 30 & 0.00 & 0.00 & 0.00 & 0.00 & 0.00 & 0.00 \\
\hline 60 & 0.00 & 0.00 & 0.00 & 0.00 & 0.00 & 0.00 \\
\hline 90 & 0.00 & 0.00 & 0.00 & 0.00 & 0.00 & 0.00 \\
\hline 120 & 0.00 & 0.00 & 0.00 & 0.00 & 0.00 & 0.00 \\
\hline 183 & 0.75 & 0.00 & 0.00 & 0.00 & 0.00 & 0.00 \\
\hline
\end{tabular}

MAGNITUDE AND PROBABILITY OF ANNUAL HIGH FLOW BASED ON PERIOD OF RECORD $1941.46,1949 \cdot 91,1993.94$

\begin{tabular}{|c|c|c|c|c|c|c|}
\hline $\begin{array}{l}\text { PERIOD } \\
\text { (CON. }\end{array}$ & & \multicolumn{5}{|c|}{$\begin{array}{l}\text { DISCHARGE, IN FT3/S, FOR INDICATED } \\
\text { RECURRENCE INTERVAL, IN YEARS, AND }\end{array}$} \\
\hline SECU - & & & & & & \\
\hline TIVE & 2 & 5 & 10 & $25 \#$ & $50 \#$ & $100 \#$ \\
\hline DAYS) & 508 & 208 & 108 & 48 & 28 & 18 \\
\hline 1 & 499 & 2,620 & 5,710 & 12,300 & 19,600 & 29,200 \\
\hline 3 & 311 & 1.770 & 4,000 & 8,930 & 14,500 & 22,100 \\
\hline 7 & 165 & 1,010 & 2,400 & 5,620 & 9,440 & 14,800 \\
\hline 15 & 88 & 587 & 1,460 & 3,640 & 6.390 & 10,400 \\
\hline 30 & 55 & 376 & 947 & 2,380 & 4,200 & 6.880 \\
\hline 60 & 32 & 228 & 590 & 1,530 & 2,780 & 4,660 \\
\hline 90 & 23 & 164 & 433 & 1,160 & 2,160 & 3,710 \\
\hline
\end{tabular}

DURATION TABLE OF DAILY MEAN FLOW FOR PERIOD OF RECORD $1941.46,1949.91,1993.94$

DISCHARGE, IN FT3/S, WHICH WAS EQUALED OR EXCEEDED FOR INDICATED PERCENT OF TIME

\begin{tabular}{|c|c|c|c|c|c|c|c|c|c|c|c|c|c|c|c|c|}
\hline 18 & 58 & 108 & 158 & 208 & 308 & 408 & 508 & 608 & 708 & 808 & 908 & 958 & 988 & 998 & 99.58 & 99.98 \\
\hline 390 & 55 & 16 & 8.6 & 5.8 & 1.8 & 0.00 & 0.00 & 0.00 & 0.00 & 0.00 & 0.00 & 0.00 & 0.00 & 0.00 & 0.00 & 0.00 \\
\hline
\end{tabular}

\# Reliability of values in column is uncertain, and potential errors are large. 
ANNUAL MEAN DISCHARGE, IN CUBIC FEET PER SECOND

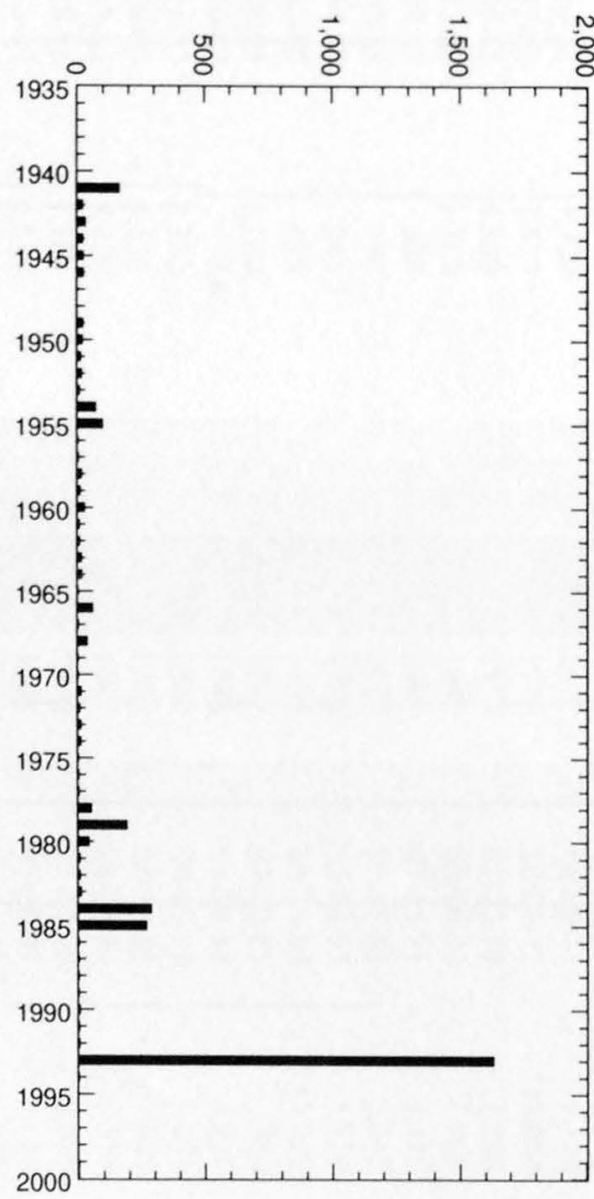
ANNUAL PEAK DISCHARGE, IN CUBIC
FEET PER SECOND

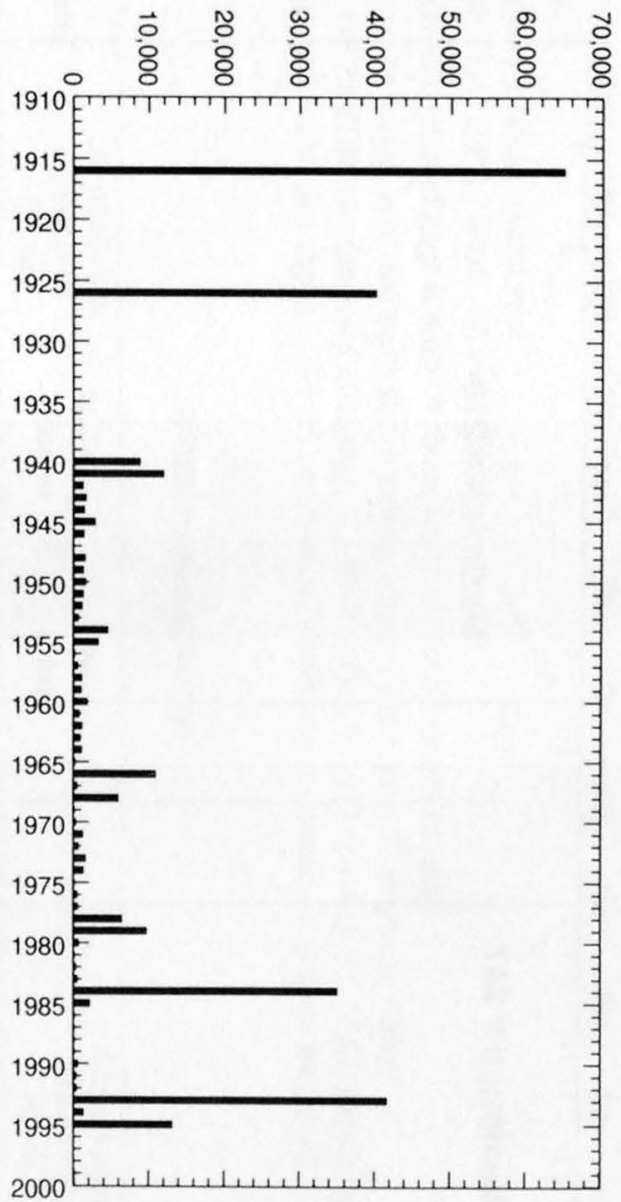


09479500 GILA RIVER NEAR LAVEEN, AZ--Continued
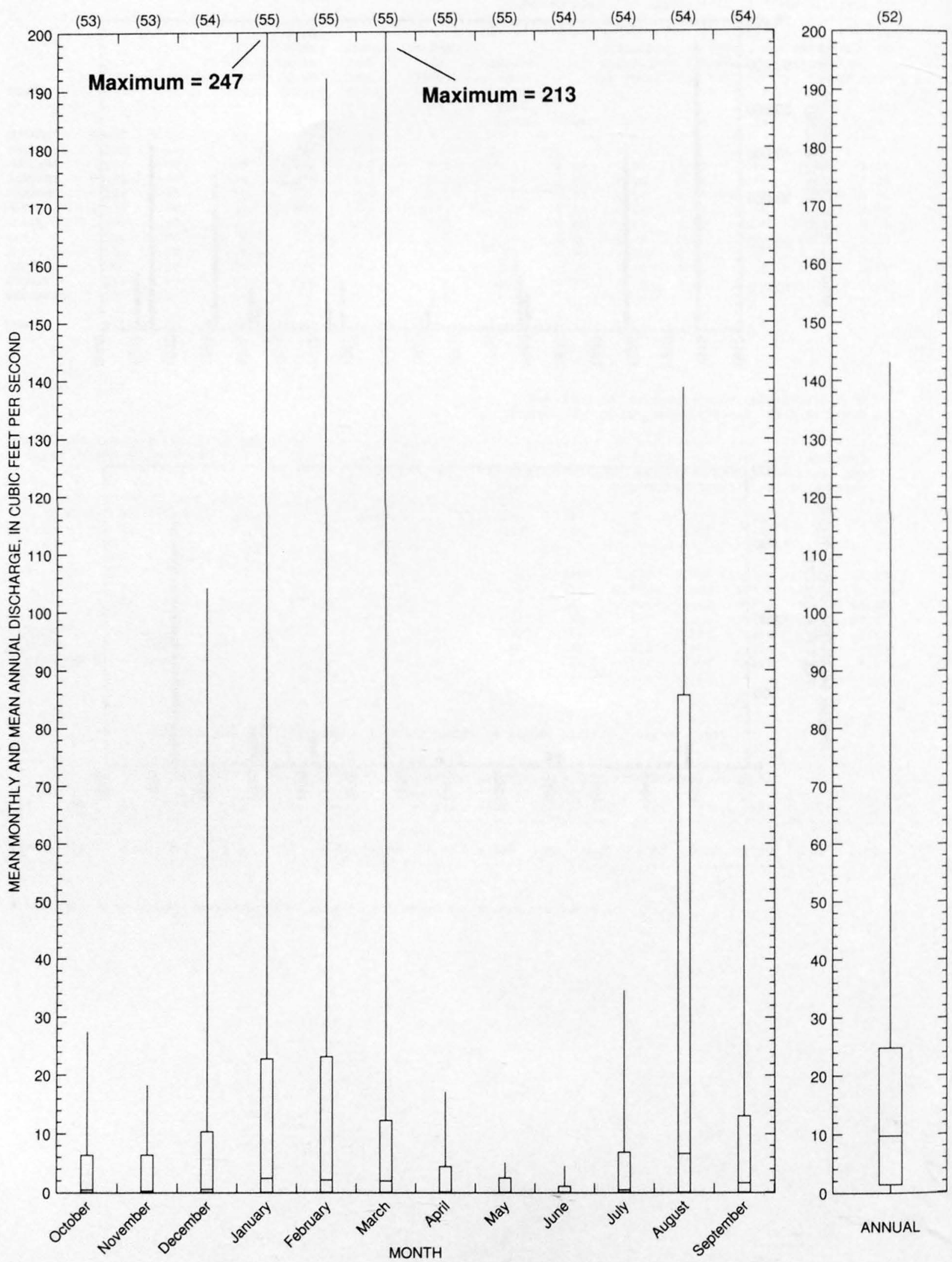
09480000 SANTA CRUZ RIVER NEAR LOCHIEL, AZ

LOCATION.--Lat 31'21'19", long 110'35'20", in SW1/4 sec.11, T.24 S., R.17 E. ( unsurveyed), Santa Cruz County, Hydrologic Unit 15050301, on southern border of Spanish land grant of San Rafael, near left bank on downstream side of pier of bridge on county road, $1.7 \mathrm{mi}$ upstream from international boundary, and 2.5 mi northeast of Lochiel.

DRAINAGE AREA.--82.2 $\mathrm{mi}^{2}$.

PERIOD OF RECORD.--January 1949 to current year.

REVISED RECORDS.--WSP 1733: 1951. WDR AZ 94-1: 1993.

GAGE.--Water-stage recorder. Elevation of gage is $4,620 \mathrm{ft}$ above sea level, from topographic map.

REMARKS.--Small diversions for irrigation of 200 acres above station, mostly by pumping from ground water.

EXTREMES FOR PERIOD OF RECORD.--Maximum discharge, 12,000 f $\mathrm{ft}^{3} / \mathrm{s}$ Oct. 9, 1977 and Aug. 15, 1984, gage height, $10.21 \mathrm{ft}$ and $10.2 \mathrm{ft}$ respectively, from rating curve extended above $1,600 \mathrm{ft}^{3} / \mathrm{s}$ on basis of slope-area measurement at gage height $10.21 \mathrm{ft}$; no flow at times in most years.

Annual peak discharges

\begin{tabular}{|c|c|c|c|c|c|c|c|}
\hline $\begin{array}{l}\text { Water } \\
\text { year }\end{array}$ & Date & $\begin{array}{c}\text { Annual peak } \\
\text { discharge } \\
\left(\mathrm{ft}^{3} / \mathrm{s}\right)\end{array}$ & $\begin{array}{l}\text { Discharge } \\
\text { codes }\end{array}$ & $\begin{array}{l}\text { Water } \\
\text { year }\end{array}$ & Date & $\begin{array}{c}\text { Annual peak } \\
\text { discharge } \\
\left(\mathrm{ft}^{3} / \mathrm{s}\right)\end{array}$ & $\begin{array}{c}\text { Discharge } \\
\text { codes }\end{array}$ \\
\hline 1949 & $09-13-49$ & 1,650 & & 1973 & $06-30-73$ & 1,490 & \\
\hline 1950 & $07-30-50$ & 4,520 & & 1974 & $08-04-74$ & 1,730 & \\
\hline 1951 & $08-02-51$ & 2,560 & & 1975 & $07-22-75$ & 3,330 & \\
\hline 1952 & $08-16-52$ & 550 & & 1976 & $07-22-76$ & 3,540 & \\
\hline 1953 & $07-14-53$ & 3,320 & & 1977 & $09-05-77$ & 1,130 & \\
\hline 1954 & $07-22-54$ & 1,570 & & 1978 & $10-09-77$ & ${ }^{1} 12,000$ & \\
\hline 1955 & $08-06-55$ & 4,300 & & 1979 & $01-25-79$ & 1,060 & \\
\hline 1956 & $07-17-56$ & 1,360 & & 1980 & $06-30-80$ & 406 & \\
\hline 1957 & $08-09-57$ & 688 & & 1981 & $07-15-81$ & 1,110 & \\
\hline 1958 & $08-07-58$ & 380 & & 1982 & $08-11-82$ & 2,640 & \\
\hline 1959 & $08-14-59$ & 243 & & 1983 & $03-04-83$ & 1,120 & \\
\hline 1960 & $07-30-60$ & 625 & & 1984 & $08-15-84$ & 12,000 & \\
\hline 1961 & $08-08-61$ & 1,120 & & 1985 & $07-19-85$ & 850 & \\
\hline 1962 & $07-29-62$ & 7.6 & & 1986 & $08-29-86$ & 4,210 & \\
\hline 1963 & $08-25-63$ & 2,390 & & 1987 & $08-10-87$ & 291 & \\
\hline 1964 & $09-09-64$ & 2,330 & & 1988 & $08-23-88$ & 804 & \\
\hline 1965 & $09-12-65$ & 4,810 & & 1989 & $08-04-89$ & 871 & \\
\hline 1966 & $08-18-66$ & 1,780 & & 1990 & $07-17-90$ & 3,510 & \\
\hline 1967 & $08-03-67$ & 1,870 & & 1991 & $07-26-91$ & 17 & \\
\hline 1968 & $12-20-67$ & 986 & & 1992 & $08-01-92$ & 483 & \\
\hline 1969 & $08-05-69$ & 484 & & 1993 & $01-18-93$ & 7,320 & \\
\hline 1970 & $08-03-70$ & 880 & & 1994 & $08-30-94$ & 478 & \\
\hline 1971 & $08-10-71$ & 2,830 & & 1995 & $07-12-95$ & 2,020 & \\
\hline 1972 & $07-16-72$ & 2,070 & & 1996 & $07-10-96$ & 1,860 & \\
\hline
\end{tabular}

'Highest since 1926.

Discharge rating table developed January 1993

\begin{tabular}{crcc}
\hline $\begin{array}{c}\text { Gage height } \\
(\mathbf{f t})\end{array}$ & $\begin{array}{c}\text { Discharge } \\
\left(\mathbf{f t}^{\mathbf{3}} / \mathbf{s}\right)\end{array}$ & $\begin{array}{c}\text { Gage height } \\
(\mathbf{f t})\end{array}$ & $\begin{array}{c}\text { Discharge } \\
\left(\mathbf{f t}^{\mathbf{3}} / \mathbf{s}\right)\end{array}$ \\
\hline 2.0 & 265 & 5.0 & 2,100 \\
2.5 & 465 & 5.5 & 2,540 \\
3.0 & 710 & 6.0 & 3,020 \\
3.5 & 999 & 6.5 & 3,520 \\
4.0 & 1,330 & 7.0 & 4,070 \\
4.5 & 1,700 & 7.7 & 4,880 \\
\hline
\end{tabular}




\section{GILA RIVER BASIN}

09480000 SANTA CRUZ RIVER NEAR LOCHIEL, AZ--Continued

Basin characteristics

\begin{tabular}{|c|c|c|c|c|c|c|c|}
\hline \multirow[b]{2}{*}{$\begin{array}{l}\text { Main } \\
\text { channel } \\
\text { slope } \\
(\mathrm{ft} / \mathrm{mi})\end{array}$} & \multirow[b]{2}{*}{$\begin{array}{l}\text { Stream } \\
\text { length } \\
\text { (mi) }\end{array}$} & \multirow[b]{2}{*}{$\begin{array}{c}\text { Mean } \\
\text { basin } \\
\text { elevation } \\
(f t)\end{array}$} & \multirow[b]{2}{*}{$\begin{array}{l}\text { Forested } \\
\text { area } \\
\text { (percent) }\end{array}$} & \multirow[b]{2}{*}{$\begin{array}{c}\text { Soil } \\
\text { index }\end{array}$} & \multirow[b]{2}{*}{$\begin{array}{c}\text { Mean } \\
\text { annual } \\
\text { precipitation } \\
\text { (in) }\end{array}$} & \multicolumn{2}{|c|}{ Rainfall intensity, 24-hour } \\
\hline & & & & & & $\begin{array}{l}\text { 2-year } \\
\text { (in) }\end{array}$ & $\begin{array}{l}\text { 50-year } \\
\text { (in) }\end{array}$ \\
\hline 42.2 & 12.0 & 5,150 & 31.0 & 2.3 & 18.2 & 1.9 & 4.3 \\
\hline
\end{tabular}


09480000 SANTA CRUZ RIVER NEAR LOCHIEL, AZ--Continued

MEAN MONTHLY AND ANNUAL DISCHARGES 1950.96

\begin{tabular}{|c|c|c|c|c|c|c|}
\hline MONTH & $\begin{array}{l}\text { MAXIMUM } \\
\text { (FT3/S) }\end{array}$ & $\begin{array}{l}\text { MINIMUM } \\
\text { (FT3/S) }\end{array}$ & $\begin{array}{c}\text { MEAN } \\
(\text { FT3/S) }\end{array}$ & $\begin{array}{l}\text { STAN- } \\
\text { DARD } \\
\text { DEVIA- } \\
\text { TION } \\
(\text { FT3/S) }\end{array}$ & $\begin{array}{l}\text { COEFFI- } \\
\text { CIENT OF } \\
\text { VARI- } \\
\text { ATION }\end{array}$ & $\begin{array}{c}\text { PERCENT } \\
\text { OF } \\
\text { ANNUAL } \\
\text { RUNOFF }\end{array}$ \\
\hline OCTOBER & 77 & 0.00 & 4.6 & 15 & 3.4 & 10.0 \\
\hline NOVEMBER & 6.8 & 0.00 & 1.1 & 1.4 & 1.3 & 2.3 \\
\hline DECEMBER & 18 & 0.00 & 1.8 & 3.5 & 1.9 & 3.9 \\
\hline JANUARY & 116 & 0.02 & 5.0 & 18 & 3.6 & 10.9 \\
\hline FEBRUARY & 18 & 0.03 & 1.9 & 3.5 & 1.8 & 4.2 \\
\hline MARCH & 34 & 0.01 & 2.0 & 5.2 & 2.7 & 4.3 \\
\hline APRIL & 8.7 & 0.00 & 0.93 & 1.6 & 1.7 & 2.0 \\
\hline MAY & 2.8 & 0.00 & 0.46 & 0.68 & 1.5 & 1.0 \\
\hline JUNE & 2.8 & 0.00 & 0.33 & 0.62 & 1.9 & 0.7 \\
\hline JULY & 69 & 0.03 & 7.7 & 14 & 1.9 & 16.7 \\
\hline AUGUST & 187 & 0.00 & 15 & 36 & 2.3 & 33.5 \\
\hline SEPTEMBER & 44 & 0.00 & 4.8 & 8.9 & 1.9 & 10.3 \\
\hline ANNUAL & 29 & 0.31 & 3.9 & 5.1 & 1.3 & 100 \\
\hline
\end{tabular}
BASED ON PERIOD OF RECORD 1949.96

DISCHARGE, IN FT $3 / S$, FOR INDICATED RECURRENCE INTERVAL IN YEARS, AND EXCEEDANCE PROBABILITY, IN PERCENT

\begin{tabular}{|c|c|c|c|c|c|}
\hline 2 & 5 & 10 & 25 & 50 & 100 \\
\hline 508 & 208 & 108 & 48 & 28 & 18 \\
\hline 1,450 & 3,020 & 4,460 & 6,820 & 9,010 & 11,600 \\
\hline
\end{tabular}

WEIGHTED SKEW $($ LOGS $)=0.12$

MEAN $\quad($ LOGS $)=3.17$

STANDARD DEV. $($ LOGS $)=0.37$
MAGNITUDE AND PROBABILITY OF ANNUAL LOW FLOW BASED ON PERIOD OF RECORD 1950-96

\begin{tabular}{|c|c|c|c|c|c|c|}
\hline \multirow{3}{*}{$\begin{array}{l}\text { PERIOD } \\
\text { (CON- } \\
\text { SECU - } \\
\text { TIVE }\end{array}$} & \multicolumn{6}{|c|}{$\begin{array}{l}\text { DISCHARGE, IN FT3/S, FOR INDICATED } \\
\text { RECURRENCE INTERVAL, IN YEARS, AND } \\
\text { NON-EXCEEDANCE PROBABILITY, IN PERCENT }\end{array}$} \\
\hline & & & & & & \\
\hline & 2 & 5 & 10 & 20 & $50 \#$ & $100 \#$ \\
\hline DAYS) & 508 & 208 & 108 & 58 & 28 & 18 \\
\hline 1 & 0.00 & 0.00 & 0.00 & 0.00 & 0.00 & 0.00 \\
\hline 3 & 0.00 & 0.00 & 0.00 & 0.00 & 0.00 & 0.00 \\
\hline 7 & 0.00 & 0.00 & 0.00 & 0.00 & 0.00 & 0.00 \\
\hline 14 & 0.00 & 0.00 & 0.00 & 0.00 & 0.00 & 0.00 \\
\hline 30 & 0.03 & 0.00 & 0.00 & 0.00 & 0.00 & 0.00 \\
\hline 60 & 0.09 & 0.00 & 0.00 & 0.00 & 0.00 & 0.00 \\
\hline 90 & 0.14 & 0.02 & 0.00 & 0.00 & 0.00 & 0.00 \\
\hline 120 & 0.46 & 0.15 & 0.07 & 0.02 & 0.00 & 0.00 \\
\hline 183 & 0.77 & 0.24 & 0.12 & 0.06 & 0.03 & 0.02 \\
\hline
\end{tabular}

MAGNITUDE AND PROBABILITY OF ANNUAL HIGH FLOW BASED ON PERIOD OF RECORD 1950-96

\begin{tabular}{|c|c|c|c|c|c|c|}
\hline \multirow{3}{*}{$\begin{array}{l}\text { PERIOD } \\
\text { /CON- } \\
\text { SECU- }\end{array}$} & \multicolumn{2}{|r|}{$\begin{array}{l}\text { DISCHARGE, } \\
\text { RECURRENCE }\end{array}$} & \multicolumn{4}{|c|}{ IN FT3/S, FOR INDICATED } \\
\hline & & EXCEEDANCE & $\mathrm{PRO}$ & BILITY, & IN PERC & NT \\
\hline & & $\ldots \ldots \ldots \ldots$ & & & ........ & \\
\hline TIVE & 2 & 5 & 10 & 25 & $50 \#$ & $100 \#$ \\
\hline DAYS) & 508 & 208 & 108 & 48 & 28 & 18 \\
\hline 1 & 150 & 441 & 721 & 1,160 & 1.530 & 1,930 \\
\hline 3 & 66 & 206 & 361 & 640 & 912 & 1,240 \\
\hline 7 & 34 & 109 & 198 & 373 & 558 & 801 \\
\hline 15 & 20 & 62 & 113 & 214 & 324 & 470 \\
\hline 30 & 12 & 38 & 69 & 132 & 202 & 296 \\
\hline 60 & 8.0 & 23 & 42 & 78 & 117 & 169 \\
\hline 90 & 5.8 & 16 & 29 & 54 & 81 & 118 \\
\hline
\end{tabular}

DURATION TABLE OF DAILY MEAN FLOW FOR PERIOD OF RECORD 1950.96 DISCHARGE, IN FT3/S, WHICH WAS EQUALED OR EXCEEDED FOR INDICATED PERCENT OF TIME

\begin{tabular}{|c|c|c|c|c|c|c|c|c|c|c|c|c|c|c|c|c|}
\hline 18 & 58 & 108 & 158 & 208 & 308 & 408 & 508 & 608 & 708 & 808 & 908 & 958 & 988 & 998 & 99.58 & 99.98 \\
\hline 55 & 8.8 & 4.4 & 2.4 & 1.6 & 1.0 & 0.72 & 0.54 & 0.37 & 0.24 & 0.11 & 0.00 & 0.00 & 0.00 & 0.00 & 0.00 & 0.00 \\
\hline
\end{tabular}

\# Reliability of values in column is uncertain, and potential errors are large. 


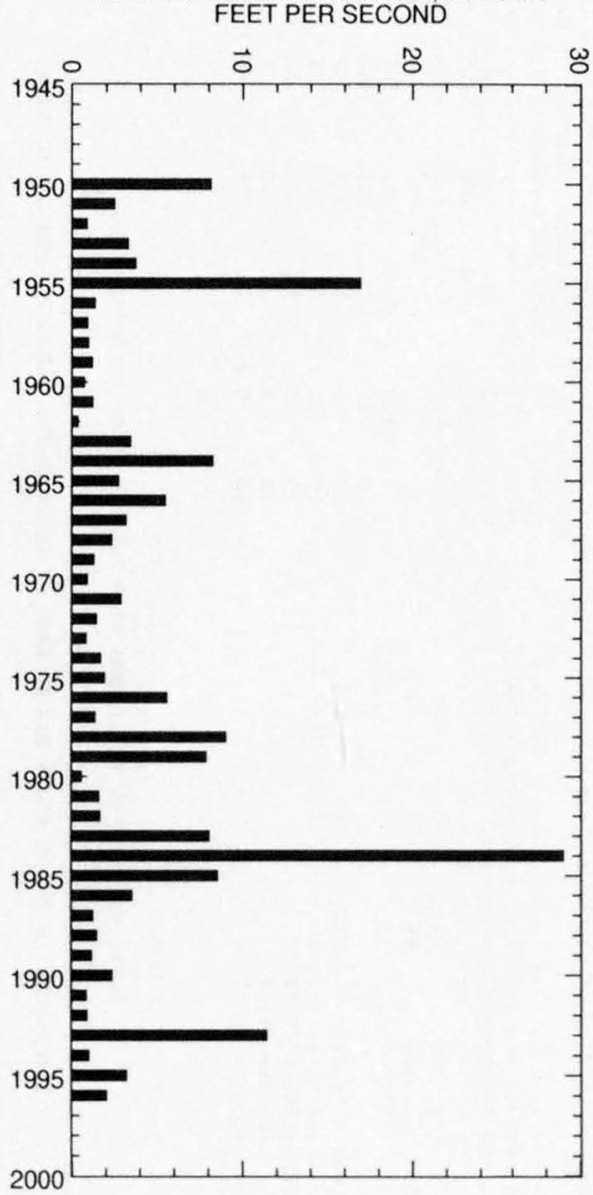

ANNUAL PEAK DISCHARGE IN CUBIC FEET PER SECOND

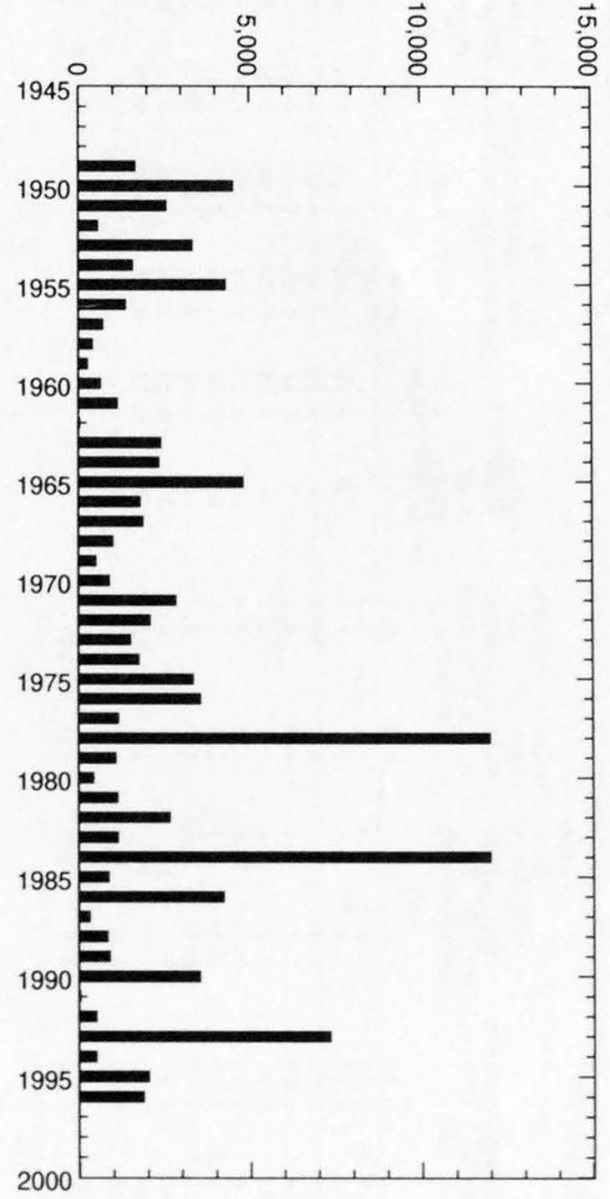


GILA RIVER BASIN

397

09480000 SANTA CRUZ RIVER NEAR LOCHIEL, AZ--Continued
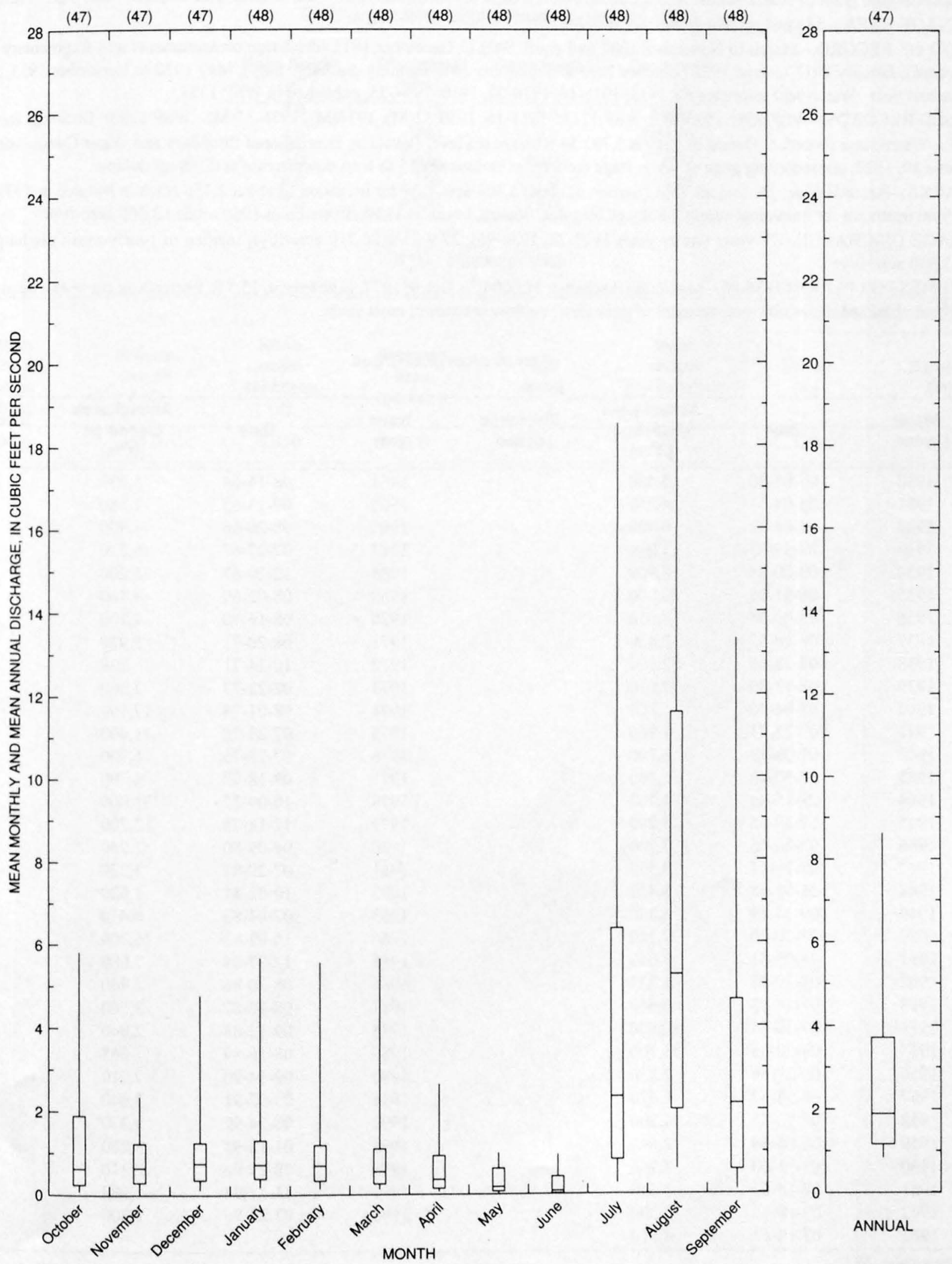

ANNUAL 
LOCATION.--Lat 31'20'40", long 110 51'03", in NW1/4 sec.18, T.24 S., R.15 E. (unsurveyed), Santa Cruz County, Hydrologic Unit 15050301, in Spanish land grant of Maria Santisima del Carmen, on left bank 0.8 mi downstream from international boundary and 5.5 mi east of Nogales.

DRAINAGE AREA.--533 $\mathrm{mi}^{2}$, of which $348 \mathrm{mi}^{2}$ is in Mexico.

PERIOD OF RECORD.--March to November 1907 and April 1909 to December 1912 (discharge measurements and fragmentary gage-height record), January 1913 to June 1922 (October 1915 to September 1916 monthly discharge only), May 1930 to December 1933, July 1935 to current year. Water-year estimates for 1913, 1915-16, 1920-22, 1930, 1934-35, published in WSP 1733.

REVISED RECORDS.--WSP 959: 1935(M). WSP 1213: 1915-16, 1930-32(M), 1934(M), 1936-37(M). WSP 1283: Drainage area.

GAGE.--Water-stage recorder. Datum of gage is $3,702.54 \mathrm{ft}$ above sea level (levels by International Boundary and Water Commission). Prior to June 30, 1922, nonrecording gage or water-stage recorder at various sites 5 to $6 \mathrm{mi}$ downstream at different datums.

REMARKS.--Records poor. Diversions above station of about 4,300 acre- $\mathrm{ft} / \mathrm{yr}$ for irrigation of about 2,150 acres in Mexico in 1977. Diversion 19 mi upstream for municipal supply of city of Nogales, Sonora, began in 1949; diversion in 1968 totaled 3,500 acre-ft/yr.

AVERAGE DISCHARGE.--77 years (water years 1913-22, 1930-96), $27.9 \mathrm{ft}^{3} / \mathrm{s}, 20,210$ acre- $\mathrm{ft} / \mathrm{yr}$; median of yearly mean discharges, $19 \mathrm{ft}^{3} / \mathrm{s}$, $13,800 \mathrm{acre}-\mathrm{ft} / \mathrm{yr}$.

EXTREMES FOR PERIOD 1930-96.--Maximum discharge, 31,000 ft $3 / \mathrm{s}$ Oct. 9, 1977, gage height, $15.5 \mathrm{ft}$, from rating curve extended above 1,660 $\mathrm{ft}^{3} / \mathrm{s}$ on basis of slope-area measurement of peak flow; no flow at times in most years.

Annual peak discharges

\begin{tabular}{|c|c|c|c|c|c|c|c|}
\hline $\begin{array}{l}\text { Water } \\
\text { year }\end{array}$ & Date & $\begin{array}{c}\text { Annual peak } \\
\text { discharge } \\
\left(\mathrm{ft}^{3} / \mathrm{s}\right)\end{array}$ & $\begin{array}{l}\text { Discharge } \\
\text { codes }\end{array}$ & $\begin{array}{l}\text { Water } \\
\text { year }\end{array}$ & Date & $\begin{array}{c}\text { Annual peak } \\
\text { discharge } \\
\left(\mathrm{ft}^{3} / \mathrm{s}\right)\end{array}$ & $\begin{array}{c}\text { Discharge } \\
\text { codes }\end{array}$ \\
\hline 1930 & $08-07-30$ & 5,400 & & 1964 & $08-14-64$ & 5,630 & \\
\hline 1931 & $08-04-31$ & 4,150 & & 1965 & $09-13-65$ & 1,580 & \\
\hline 1932 & $07-08-32$ & 6,400 & & 1966 & $08-20-66$ & 4,400 & \\
\hline 1933 & $09-19-33$ & 1,900 & & 1967 & $07-27-67$ & 6,310 & \\
\hline 1934 & $08-00-34$ & 5,900 & & 1968 & $12-20-67$ & 15,200 & \\
\hline 1935 & $08-31-35$ & 12,000 & & 1969 & $08-02-69$ & 4,460 & \\
\hline 1936 & $08-09-36$ & 4,050 & & 1970 & $08-16-70$ & 4,100 & \\
\hline 1937 & $08-16-37$ & 2,400 & & 1971 & $08-20-71$ & 2,930 & \\
\hline 1938 & $07-28-38$ & 2,200 & & 1972 & $10-24-71$ & 738 & \\
\hline 1939 & $08-13-39$ & 7,010 & & 1973 & $02-22-73$ & 2,300 & \\
\hline 1940 & $08-04-40$ & 1,800 & & 1974 & $08-01-74$ & 17,100 & \\
\hline 1941 & $07-21-41$ & 1,980 & & 1975 & $07-22-75$ & 11,400 & \\
\hline 1942 & $07-08-42$ & 8,200 & & 1976 & $07-22-76$ & 6,700 & \\
\hline 1943 & $07-30-43$ & 5,300 & & 1977 & $08-18-77$ & 6,700 & \\
\hline 1944 & $08-15-44$ & 4,700 & & 1978 & $10-09-77$ & ${ }^{1} 31,000$ & \\
\hline 1945 & $07-30-45$ & 3,290 & & 1979 & $12-18-78$ & 12,700 & \\
\hline 1946 & $07-26-46$ & 7,200 & & 1980 & 08-09-80 & 1,950 & \\
\hline 1947 & $08-29-47$ & 2,550 & & 1981 & $07-29-81$ & 3,220 & \\
\hline 1948 & $08-01-48$ & 3,410 & & 1982 & $10-02-81$ & 1,620 & \\
\hline 1949 & $09-14-49$ & 6,350 & & 1983 & $02-04-83$ & 6,410 & \\
\hline 1950 & $07-20-50$ & 7,210 & & 1984 & $10-02-83$ & 16,200 & \\
\hline 1951 & $08-03-51$ & 3,040 & & 1985 & $12-27-84$ & 7,080 & \\
\hline 1952 & $07-29-52$ & 2,330 & & 1986 & $08-30-86$ & 2,440 & \\
\hline 1953 & $07-14-53$ & 3,500 & & 1987 & $08-10-87$ & 3,560 & \\
\hline 1954 & $07-10-54$ & 10,600 & & 1988 & $09-12-88$ & 2,940 & \\
\hline 1955 & $08-20-55$ & 11,100 & & 1989 & $08-16-89$ & 663 & \\
\hline 1956 & $06-28-56$ & 2,530 & & 1990 & $09-14-90$ & 7,010 & \\
\hline 1957 & $08-18-57$ & 1,620 & & 1991 & $03-02-91$ & 3,640 & \\
\hline 1958 & $08-13-58$ & 4,000 & & 1992 & $08-24-92$ & 9,370 & \\
\hline 1959 & $08-06-59$ & 2,640 & & 1993 & $01-18-93$ & 8,800 & \\
\hline 1960 & $01-11-60$ & 2,760 & & 1994 & $08-21-94$ & 2,310 & \\
\hline 1961 & $08-15-61$ & 1,640 & & 1995 & $11-12-94$ & 1,980 & \\
\hline 1962 & $08-19-62$ & 2,390 & & 1996 & $07-25-96$ & 1,800 & \\
\hline 1963 & $07-10-63$ & 4,510 & & & & & \\
\hline
\end{tabular}

${ }^{1}$ Highest since 1892. 
09480500 SANTA CRUZ RIVER NEAR NOGALES, AZ--Continued

Discharge rating table developed January 1993

\begin{tabular}{crcr}
\hline $\begin{array}{c}\text { Gage height } \\
(\mathbf{f t})\end{array}$ & $\begin{array}{c}\text { Discharge } \\
\left(\mathbf{f t}^{\mathbf{3}} / \mathbf{s}\right)\end{array}$ & $\begin{array}{c}\text { Gage height } \\
(\mathbf{f t})\end{array}$ & $\begin{array}{c}\text { Discharge } \\
\left(\mathrm{ft}^{3} / \mathbf{s}\right)\end{array}$ \\
\hline 3.0 & 177 & 8.0 & 6,800 \\
4.0 & 800 & 9.0 & 9,130 \\
5.0 & 1,800 & 10.0 & 11,600 \\
6.0 & 3,150 & 11.0 & 14,400 \\
7.0 & 4,820 & 12.0 & 17,150 \\
\hline
\end{tabular}

Basin characteristics

\begin{tabular}{|c|c|c|c|c|c|c|c|}
\hline \multirow[b]{2}{*}{$\begin{array}{l}\text { Main } \\
\text { channel } \\
\text { slope } \\
(\mathrm{ft} / \mathrm{mi})\end{array}$} & \multirow[b]{2}{*}{$\begin{array}{l}\text { Stream } \\
\text { length } \\
\text { (mi) }\end{array}$} & \multirow[b]{2}{*}{$\begin{array}{c}\text { Mean } \\
\text { basin } \\
\text { elevation } \\
(\mathrm{ft})\end{array}$} & \multirow[b]{2}{*}{$\begin{array}{l}\text { Forested } \\
\text { area } \\
\text { (percent) }\end{array}$} & \multirow[b]{2}{*}{$\begin{array}{c}\text { Soil } \\
\text { index }\end{array}$} & \multirow[b]{2}{*}{$\begin{array}{c}\text { Mean } \\
\text { annual } \\
\text { precipitation } \\
\text { (in) }\end{array}$} & \multicolumn{2}{|c|}{ Rainfall intensity, 24-hour } \\
\hline & & & & & & $\begin{array}{l}\text { 2-year } \\
\text { (in) }\end{array}$ & $\begin{array}{l}\text { 50-year } \\
\text { (in) }\end{array}$ \\
\hline 26.0 & 51.2 & 4,850 & 28.0 & 2.1 & 18.7 & 2.0 & 4.3 \\
\hline
\end{tabular}


MEAN MONTHLY AND ANNUAL DISCHARGES $1914,1917 \cdot 19,1931 \cdot 33,1936.96$ MAGNITUDE AND PROBABILITY OF ANNUAL LOW FLOW BASED ON PERIOD OF RECORD 1914, 1917-19, 1931.33, 1936.96

\begin{tabular}{|c|c|c|c|c|c|c|}
\hline MONTH & $\begin{array}{l}\text { MAXIMUM } \\
(\text { FT3/S) }\end{array}$ & $\begin{array}{l}\text { MINIMUM } \\
\text { (FT3/S) }\end{array}$ & $\begin{array}{c}\text { MEAN } \\
(\text { FT3/S) }\end{array}$ & $\begin{array}{l}\text { STAN- } \\
\text { DARD } \\
\text { DEVIA- } \\
\text { TION } \\
\text { (FT3/S) }\end{array}$ & $\begin{array}{l}\text { COEFFI- } \\
\text { CIENT OF } \\
\text { VARI- } \\
\text { ATION }\end{array}$ & $\begin{array}{c}\text { PERCENT } \\
\text { OF } \\
\text { ANNUAL } \\
\text { RUNOFF }\end{array}$ \\
\hline OCTOBER & 905 & 0.00 & 25 & 113 & 4.6 & 7.2 \\
\hline NOVEMBER & 120 & 0.00 & 8.9 & 17 & 1.9 & 2.6 \\
\hline DECEMBER & 542 & 0.00 & 36 & 99 & 2.8 & 10.6 \\
\hline JANUARY & 493 & 0.00 & 43 & 101 & 2.3 & 12.6 \\
\hline FEBRUARY & 370 & 0.00 & 34 & 67 & 2.0 & 10.0 \\
\hline MARCH & 318 & 0.00 & 25 & 52 & 2.1 & 7.4 \\
\hline APRIL & 58 & 0.00 & 7.6 & 12 & 1.6 & 2.2 \\
\hline MAY & 17 & 0.00 & 1.9 & 3.5 & 1.8 & 0.56 \\
\hline JUNE & 24 & 0.00 & 1.4 & 3.7 & 2.6 & 0.41 \\
\hline JULY & 254 & 0.00 & 42 & 53 & 1.3 & 12.4 \\
\hline AUGUST & 745 & 1.5 & 89 & 120 & 1.3 & 26.2 \\
\hline SEPTEMBER & 159 & 0.00 & 27 & 35 & 1.3 & 7.9 \\
\hline ANNUAL & 123 & 2.1 & 27.9 & 29.3 & 1.1 & 100 \\
\hline
\end{tabular}

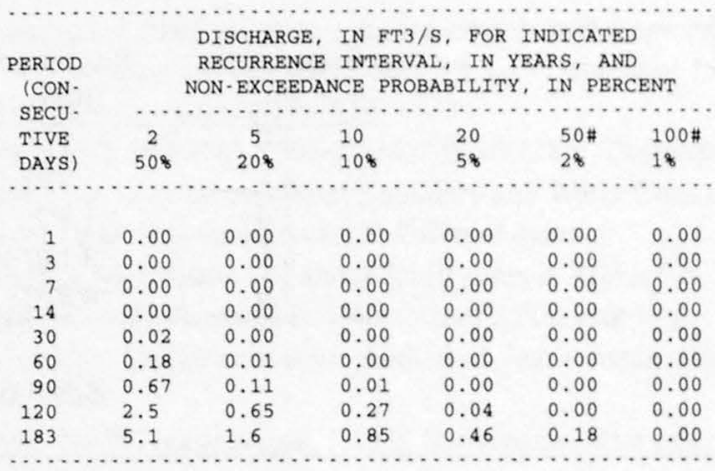

MAGNITUDE AND PROBABILITY OF INSTANTANEOUS PEAK FLOW BASED ON PERIOD OF RECORD 1930.96

DISCHARGE, IN FT3/S, FOR INDICATED RECURRENCE INTERVAL IN YEARS, AND EXCEEDANCE PROBABILITY, IN PERCENT

\begin{tabular}{|c|c|c|c|c|c|}
\hline 2 & 5 & 10 & 25 & 50 & 100 \\
\hline 508 & 208 & 108 & 48 & 28 & 18 \\
\hline 4,090 & 7,610 & 10,600 & 15,000 & 18,900 & 23,200 \\
\hline WEIGHTED & SKEW & $($ LOGS $)=$ & & & \\
\hline MEAN & & $($ LOGS $)=$ & 61 & & \\
\hline STANDARD & DEV. & $($ LOGS $)=$ & 32 & & \\
\hline
\end{tabular}

MAGNITUDE AND PROBABILITY OF ANNUAL HIGH FLOW BASED ON PERIOD OF RECORD 1914, 1917-19,1931-33, 1936-96

\begin{tabular}{|c|c|c|c|c|c|c|}
\hline \multirow{2}{*}{$\begin{array}{l}\text { PERIOD } \\
\text { (CON- } \\
\text { SECU- }\end{array}$} & & $\begin{array}{l}\text { DISCHA } \\
\text { RECURR } \\
\text { EXCEED }\end{array}$ & $\begin{array}{ll}\text { GE, IN } \\
\text { NCE } & \text { IN? } \\
\text { NCE } & \text { PRC }\end{array}$ & $\begin{array}{l}3 / \mathrm{S}, \text { FO } \\
\text { VAL, IN } \\
\text { BILITY, }\end{array}$ & $\begin{array}{l}\text { INDIC, } \\
\text { YEARS, } \\
\text { IN PER }\end{array}$ & $\begin{array}{l}\text { TED } \\
\text { AND } \\
\text { ENT }\end{array}$ \\
\hline & & $\ldots \ldots$ & $\ldots$. & . . . . . & $\ldots \ldots$ & \\
\hline TIVE & 2 & 5 & 10 & 25 & $50 \#$ & $100 \#$ \\
\hline DAYS) & 508 & 208 & 108 & 48 & 28 & 18 \\
\hline 1 & 735 & 1,950 & 3,320 & 5,910 & 8,660 & 12,270 \\
\hline 3 & 419 & 1,110 & 1,890 & 3,370 & 4,940 & 7,010 \\
\hline 7 & 255 & 649 & 1,070 & 1,830 & 2,610 & 3,600 \\
\hline 15 & 166 & 406 & 649 & 1,080 & 1,490 & 2,000 \\
\hline 30 & 112 & 264 & 414 & 667 & 908 & 1,200 \\
\hline 60 & 73 & 174 & 272 & 439 & 597 & 787 \\
\hline 90 & 53 & 126 & 198 & 320 & 436 & 576 \\
\hline
\end{tabular}

DURATION TABLE OF DAILY MEAN FLOW FOR PERIOD OF RECORD 1914, 1917.19, 1931.33, 1936.96

DISCHARGE, IN FT3/S, WHICH WAS EQUALED OR EXCERDED FOR INDICATED PERCENT OF TIME

\begin{tabular}{|c|c|c|c|c|c|c|c|c|c|c|c|c|c|c|c|c|}
\hline 18 & 58 & 108 & $15 \%$ & 208 & 308 & 408 & 508 & 608 & 708 & 808 & 908 & 958 & 988 & 998 & 99.58 & 99.98 \\
\hline 545 & 102 & 43 & 26 & 17 & 7.2 & 4.2 & 2.7 & 1.0 & 0.66 & 0.19 & 0.00 & 0.00 & 0.00 & 0.00 & 0.00 & 0.00 \\
\hline
\end{tabular}

\# Reliability of values in column is uncertain, and potential errors are large. 
ANNUAL MEAN DISCHARGE, IN CUBIC FEET PER SECOND

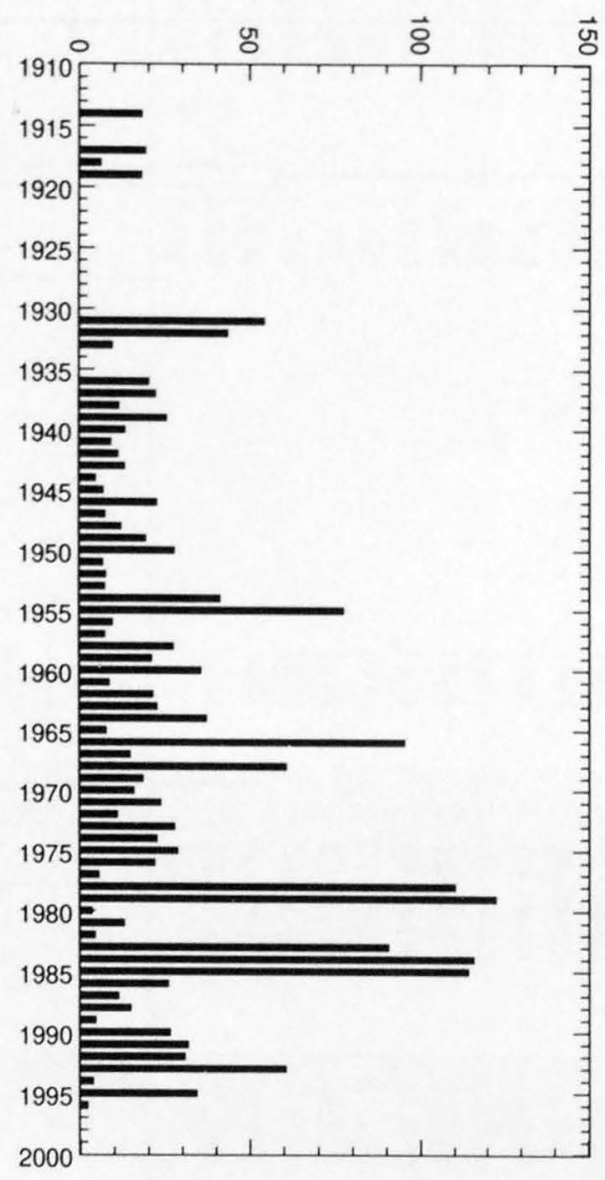

ANNUAL PEAK DISCHARGE, IN CUBIC

FEET PER SECOND

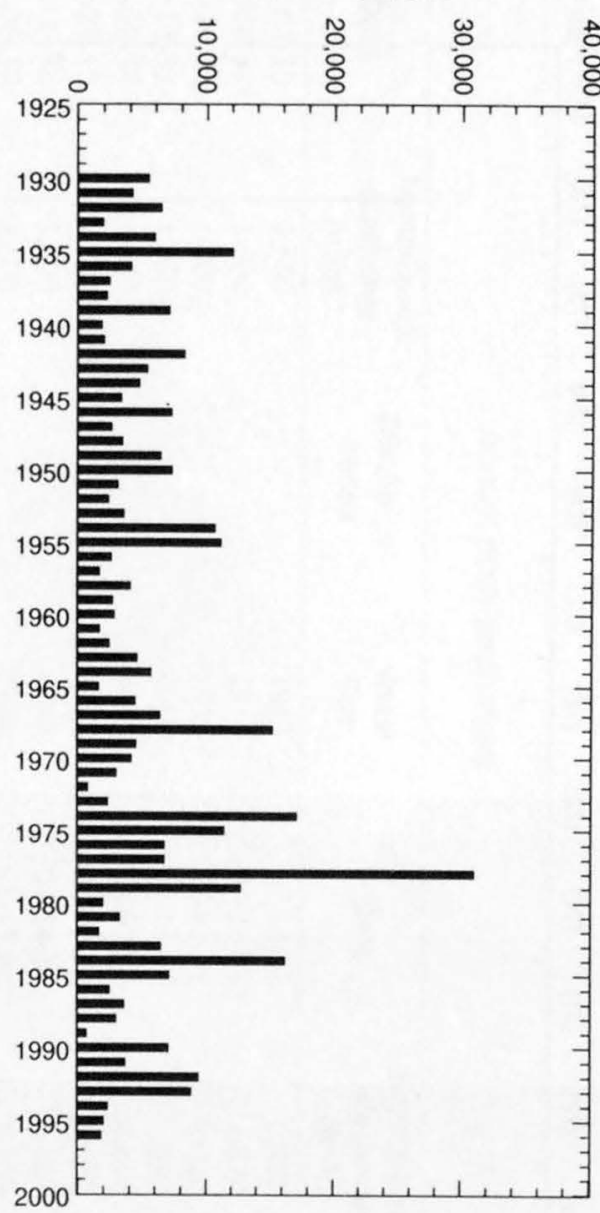


09480500 SANTA CRUZ RIVER NEAR NOGALES, AZ--Continued

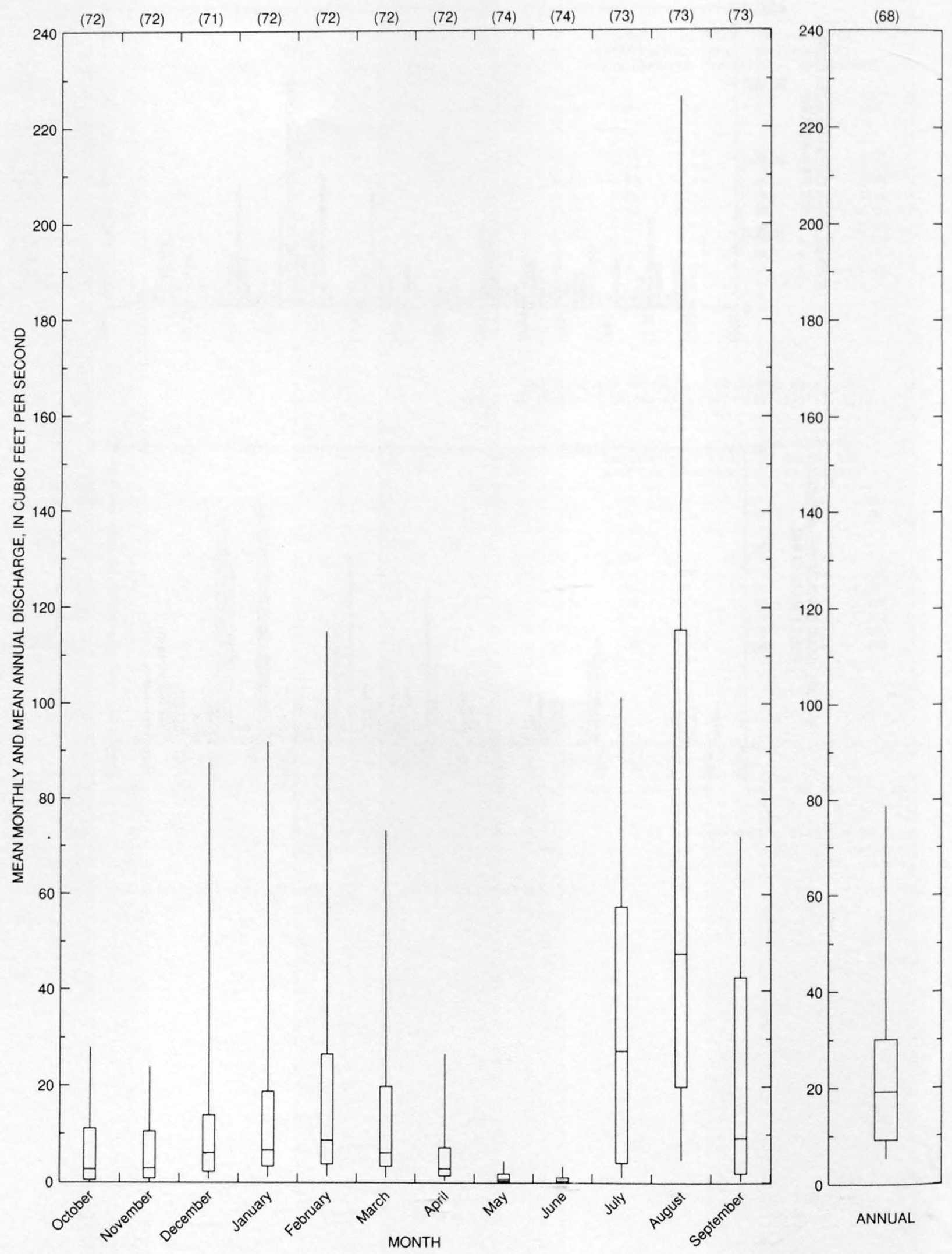


09481500 SONOITA CREEK NEAR PATAGONIA, AZ

LOCATION.--Lat $31^{\circ} 30^{\prime} 00^{\prime \prime}$, long $110^{\circ} 49^{\prime} 00^{\prime \prime}, \mathrm{SE}^{1} / \mathrm{SW}^{1} / 4 \mathrm{sec} .21$, T.22 S., R.15 E., Santa Cruz County, Hydrologic Unit 15050301 , on left abutment of former railroad bridge, $5 \mathrm{mi}$ downstream from Patagonia.

DRAINAGE AREA.--209 $\mathrm{mi}^{2}$.

Annual peak discharges

\begin{tabular}{|c|c|c|c|c|c|c|c|}
\hline $\begin{array}{l}\text { Water } \\
\text { year }\end{array}$ & Date & $\begin{array}{c}\text { Annual peak } \\
\text { discharge } \\
\left(\mathrm{ft}^{3} / \mathrm{s}\right)\end{array}$ & $\begin{array}{l}\text { Discharge } \\
\text { codes }\end{array}$ & $\begin{array}{l}\text { Water } \\
\text { year }\end{array}$ & Date & $\begin{array}{c}\text { Annual peak } \\
\text { discharge } \\
\left(\mathrm{ft}^{3} / \mathrm{s}\right)\end{array}$ & $\begin{array}{c}\text { Discharge } \\
\text { codes }\end{array}$ \\
\hline 1930 & $08-07-30$ & 2,600 & & 1953 & $07-14-53$ & 2,870 & \\
\hline 1931 & $07-28-31$ & 1,900 & & 1954 & $07-20-54$ & 4,670 & \\
\hline 1932 & $07-26-32$ & 1,700 & & 1955 & $08-12-55$ & 6,920 & \\
\hline 1933 & $07-15-33$ & 1,050 & & 1956 & $07-19-56$ & 780 & \\
\hline 1934 & $08-00-34$ & 11,000 & & 1957 & $08-02-57$ & 4,860 & \\
\hline 1935 & $08-23-35$ & 4,700 & & 1958 & $07-05-58$ & 5,590 & \\
\hline 1936 & $08-09-36$ & 3,600 & & 1959 & $08-24-59$ & 2,310 & \\
\hline 1937 & $09-06-37$ & 3,600 & & 1960 & $08-13-60$ & 1,550 & \\
\hline 1938 & $09-09-38$ & 3,400 & & 1961 & $10-09-60$ & 2,760 & \\
\hline 1939 & 08-08-39 & 3,300 & & 1962 & $12-15-61$ & 680 & \\
\hline 1940 & $08-13-40$ & 2,580 & & 1963 & $08-26-63$ & 4,320 & \\
\hline 1941 & $08-09-41$ & 2,150 & & 1964 & $09-10-64$ & 2,640 & \\
\hline 1942 & $09-12-42$ & 1,000 & & 1965 & $09-08-65$ & 806 & \\
\hline 1943 & $08-28-43$ & 4,530 & & 1966 & $08-18-66$ & 4,120 & \\
\hline 1944 & $08-09-44$ & 669 & & 1967 & $07-03-67$ & 2,060 & \\
\hline 1945 & $08-06-45$ & 3,140 & & 1968 & $12-20-67$ & 5,410 & \\
\hline 1946 & $09-30-46$ & 14,000 & & 1969 & $08-24-69$ & 450 & \\
\hline 1947 & $08-12-47$ & 2,360 & & 1970 & $08-03-70$ & 622 & \\
\hline 1948 & $08-15-48$ & 4,750 & & 1971 & $08-11-71$ & 2,860 & \\
\hline 1949 & $08-08-49$ & 5,790 & & 1972 & $09-09-72$ & 368 & \\
\hline 1950 & $07-30-50$ & 7,300 & & 1978 & $10-09-77$ & ${ }^{17,380}$ & HP \\
\hline 1951 & $08-02-51$ & 5,030 & & 1984 & $10-02-83$ & ${ }^{2} 16,000$ & HP \\
\hline 1952 & $08-14-52$ & 3,630 & & & & & \\
\hline
\end{tabular}

${ }^{1}$ Highest since 1946.

${ }^{2}$ Highest since 1930.

Basin characteristics

\begin{tabular}{cccccccc}
\hline $\begin{array}{c}\text { Main } \\
\text { channel } \\
\text { slope } \\
(\mathrm{ft} / \mathrm{mi})\end{array}$ & $\begin{array}{c}\text { Stream } \\
\text { length } \\
(\mathrm{mi})\end{array}$ & $\begin{array}{c}\text { Mean } \\
\text { basin } \\
\text { elevation } \\
(\mathrm{ft})\end{array}$ & $\begin{array}{c}\text { Forested } \\
\text { area } \\
\text { (percent) }\end{array}$ & $\begin{array}{c}\text { Soil } \\
\text { index }\end{array}$ & $\begin{array}{c}\text { Mean } \\
\text { annual } \\
\text { precipitation } \\
\text { (in) }\end{array}$ & $\begin{array}{c}\text { Rainfall intensity, 24-hour } \\
\text { (in) }\end{array}$ & $\begin{array}{c}50 \text {-year } \\
\text { (in) }\end{array}$ \\
\hline 76.7 & 21.7 & 4,800 & 52.0 & 2.0 & 19.3 & 2.0 \\
\hline
\end{tabular}


09481500 SONOITA CREEK NEAR PATAGONIA, AZ--Continued

MEAN MONTHLY AND ANNUAL DISCHARGES $1931 \cdot 33,1936.72$

\begin{tabular}{|c|c|c|c|c|c|c|}
\hline MONTH & $\begin{array}{l}\text { MAXIMUM } \\
(\text { FT } 3 / S)\end{array}$ & $\begin{array}{l}\text { MINIMUM } \\
(\mathrm{FT} 3 / \mathrm{S})\end{array}$ & $\begin{array}{c}\text { MBAN } \\
(\mathrm{FT} 3 / \mathrm{S})\end{array}$ & $\begin{array}{l}\text { STAN- } \\
\text { DARD } \\
\text { DEVIA- } \\
\text { TION } \\
(\text { FT3/S) }\end{array}$ & $\begin{array}{l}\text { COEFFI- } \\
\text { CIENT OF } \\
\text { VARI- } \\
\text { ATION }\end{array}$ & $\begin{array}{c}\text { PERCENT } \\
\text { OF } \\
\text { ANNUAL } \\
\text { RUNOFF }\end{array}$ \\
\hline OCTOBER & 20 & 0.03 & 3.9 & 3.9 & 0.99 & 4.0 \\
\hline NOVEMBER & 18 & 0.32 & 4.0 & 3.3 & 0.83 & 4.1 \\
\hline DECEMBER & 107 & 0.99 & 10 & 21 & 2.1 & 10.5 \\
\hline JANUARY & 52 & 1.1 & 7.5 & 8.9 & 1.2 & 7.8 \\
\hline FEBRUARY & 96 & 0.99 & 9.9 & 18 & 1.8 & 10.2 \\
\hline MARCH & 16 & 0.87 & 5.5 & 3.3 & 0.61 & 5.7 \\
\hline APRIL & 12 & 0.49 & 4.1 & 2.9 & 0.70 & 4.3 \\
\hline MAY & 10 & 0.06 & 2.5 & 2.4 & 0.95 & 2.6 \\
\hline JUNE & 8.6 & 0.00 & 1.6 & 2.1 & 1.3 & 1.7 \\
\hline JULY & 112 & 0.06 & 13 & 19 & 1.4 & 13.5 \\
\hline AUGUST & 151 & 1.5 & 25 & 27 & 1.1 & 26.1 \\
\hline SEPTEMBER & 71 & 0.05 & 9.2 & 13 & 1.4 & 9.5 \\
\hline ANNUAL & 33 & 1.9 & 8.1 & 5.6 & 0.69 & 100 \\
\hline
\end{tabular}

MAGNITUDE AND PROBABILITY OF INSTANTANEOUS PEAK FLOW BASED ON PERIOD OF RECORD 1930.72, 1978, 1984

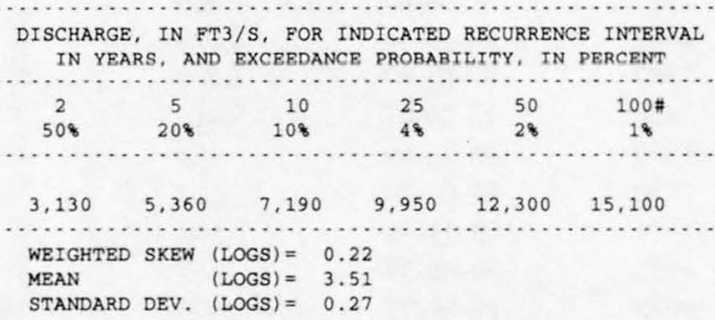

MAGNITUDE AND PROBABILITY OF ANNUAL LOW FLOW BASED ON PERIOD OF RECORD $1932 \cdot 33,1937.72$

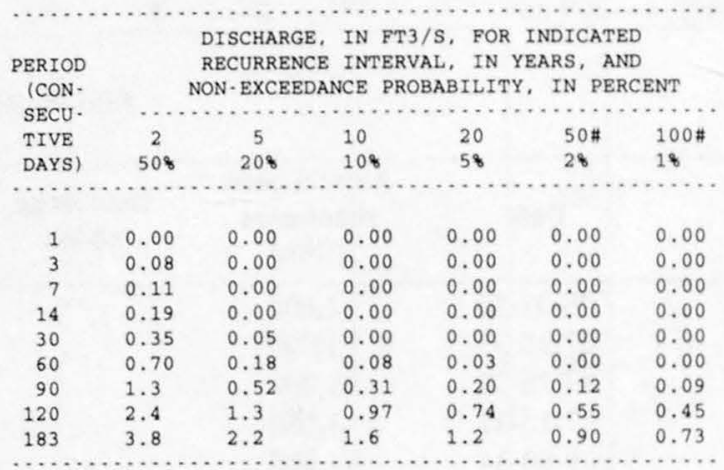

MAGNITUDE AND PROBABILITY OF ANNUAL HIGH FLOW BASED ON PERIOD OF RECORD $1931.33,1936.72$

\begin{tabular}{|c|c|c|c|c|c|c|}
\hline \multirow{3}{*}{$\begin{array}{l}\text { PERIOD } \\
\text { (CON- } \\
\text { SECU. } \\
\text { TIVE }\end{array}$} & & $\begin{array}{l}\text { DISCH: } \\
\text { RECUR: } \\
\text { EXCEE: }\end{array}$ & $\begin{array}{l}\text { IN } \\
\text { INI } \\
\text { PRO }\end{array}$ & $\begin{array}{l}\text { 3/S, FO } \\
\text { VAL, IN } \\
\text { BILITY, }\end{array}$ & $\begin{array}{l}\text { INDIC } \\
\text { YEARS, } \\
\text { IN PERC }\end{array}$ & \\
\hline & $\cdots$ & $\cdots \cdots$ & . & 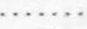 & $\ldots \ldots$ & $\ldots \ldots$ \\
\hline & 2 & 5 & 10 & 25 & $50 \mathrm{H}$ & $100 \mathrm{n}$ \\
\hline DAYS) & 508 & 208 & 108 & 48 & 28 & 18 \\
\hline 1 & 277 & 599 & 881 & 1,310 & 1,680 & 2,090 \\
\hline 3 & 132 & 285 & 415 & 608 & 771 & 948 \\
\hline 7 & 74 & 156 & 225 & 329 & 418 & 516 \\
\hline 15 & 44 & 92 & 138 & 212 & 282 & 364 \\
\hline 30 & 29 & 59 & 87 & 131 & 171 & 218 \\
\hline 60 & 20 & 39 & 54 & 79 & 100 & 124 \\
\hline 90 & 15 & 28 & 39 & 56 & 71 & 87 \\
\hline
\end{tabular}

DURATION TABLE OF DAILY MEAN FLOW FOR PERIOD OF RECORD $1931 \cdot 33,1936 \cdot 72$

DISCHARGE, IN FT3/S, WHICH WAS EQUALED OR EXCEEDED FOR INDICATED PERCENT OF TIME

\begin{tabular}{|c|c|c|c|c|c|c|c|c|c|c|c|c|c|c|c|c|}
\hline 18 & 58 & 108 & 158 & 208 & 308 & 408 & 508 & 608 & 708 & 808 & 908 & 958 & 988 & 998 & 99.58 & 99.98 \\
\hline & 19 & 11 & 8.0 & 7.1 & 5.3 & 4.0 & 3.2 & 2.6 & 2.1 & 1.2 & 0.45 & 0.10 & 0.00 & 0.00 & 0.00 & 0.00 \\
\hline
\end{tabular}

\# Reliability of values in column is uncertain, and potential errors are large. 


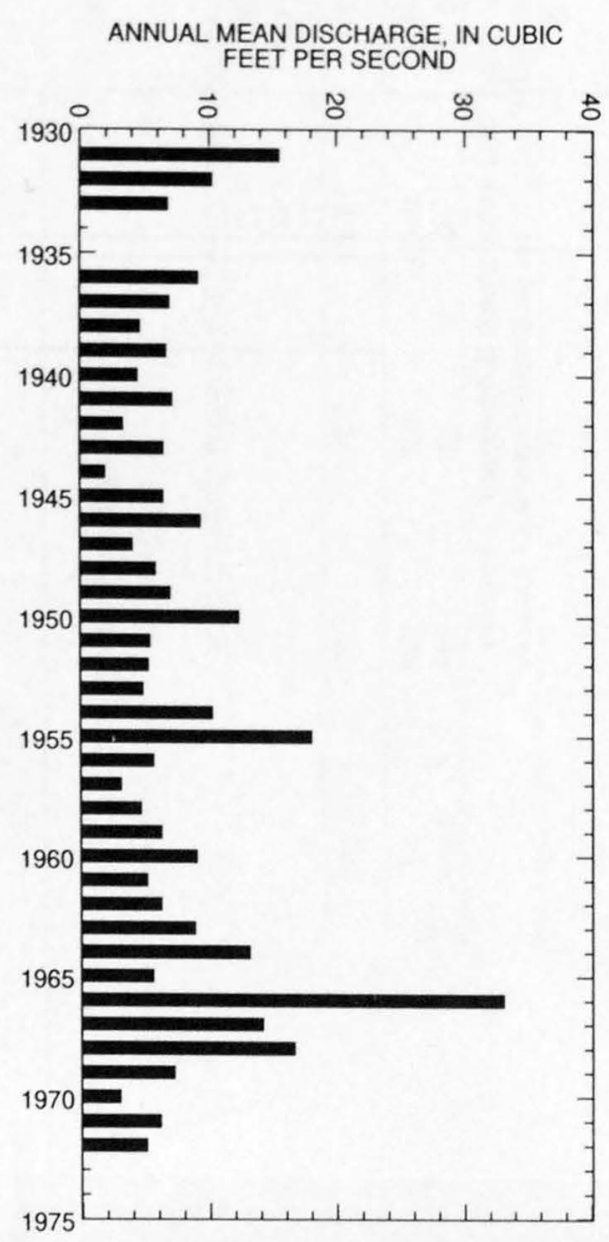

ANNUAL PEAK DISCHARGE, IN CUBIC

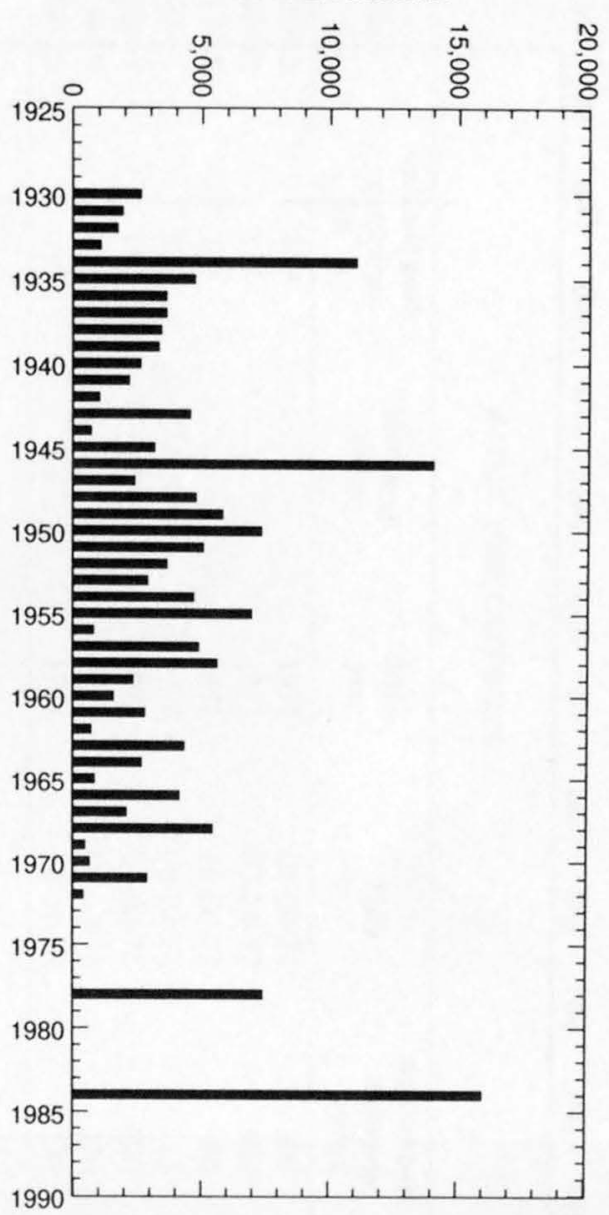


09481500 SONOITA CREEK NEAR PATAGONIA, AZ--Continued
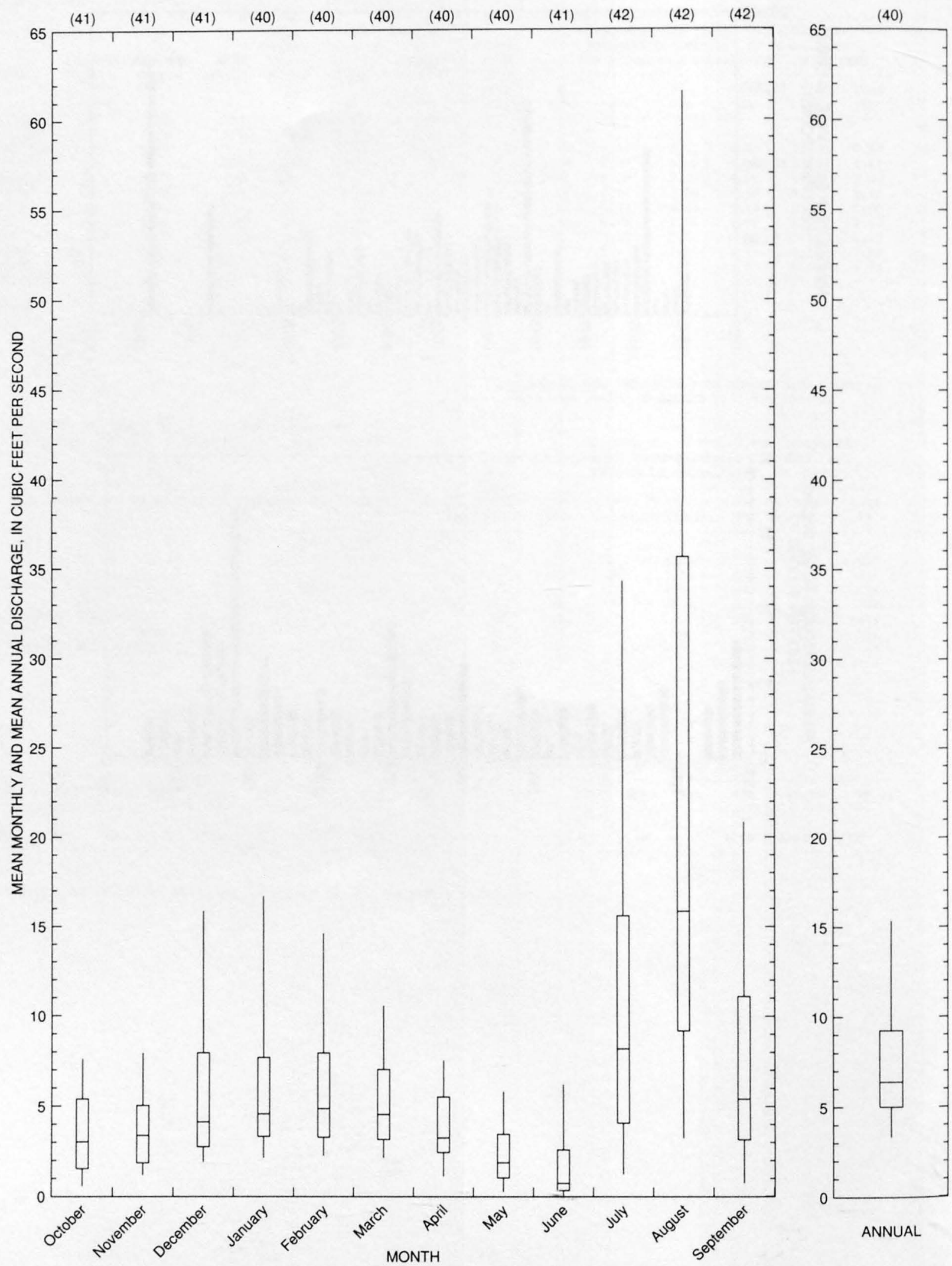
09481700 CALABASAS CANYON NEAR NOGALES, AZ

LOCATION.--Lat $31^{\circ} 27^{\prime} 25^{\prime \prime}$, long $110^{\circ} 59^{\prime} 09^{\prime \prime}, \mathrm{SE}^{1}{ }_{4} \mathrm{NW}^{1} / 4$ sec.2, T.23 S., R. 13 E., Santa Cruz County, Hydrologic Unit 15050301 , at U.S. Highway $89,8.5 \mathrm{mi}$ north of Nogales.

DRAINAGE AREA.--10.3 $\mathrm{mi}^{2}$.

Annual peak discharges

\begin{tabular}{|c|c|c|c|c|c|c|c|}
\hline $\begin{array}{l}\text { Water } \\
\text { year }\end{array}$ & Date & $\begin{array}{c}\text { Annual peak } \\
\text { discharge } \\
\left(\mathrm{ft}^{3} / \mathrm{s}\right)\end{array}$ & $\begin{array}{l}\text { Discharge } \\
\text { codes }\end{array}$ & $\begin{array}{l}\text { Water } \\
\text { year }\end{array}$ & Date & $\begin{array}{c}\text { Annual peak } \\
\text { discharge } \\
\left(\mathrm{ft}^{3} / \mathrm{s}\right)\end{array}$ & $\begin{array}{c}\text { Discharge } \\
\text { codes }\end{array}$ \\
\hline 1963 & $08-05-63$ & 179 & & 1971 & $08-00-71$ & 700 & \\
\hline 1964 & $07-00-64$ & 813 & & 1972 & $07-24-72$ & 300 & \\
\hline 1965 & $07-17-65$ & 263 & & 1973 & $10-00-72$ & 80 & \\
\hline 1967 & $07-00-67$ & 520 & & 1974 & $08-02-74$ & 115 & \\
\hline 1968 & $12-20-67$ & 150 & ES & 1975 & $07-00-75$ & 600 & \\
\hline 1969 & $09-00-69$ & 1,000 & & 1976 & $00-00-76$ & 220 & \\
\hline 1970 & $08-08-70$ & 50 & LT & 1978 & $10-09-77$ & 1,200 & HP \\
\hline
\end{tabular}

Magnitude and probability of instantaneous peak flow based on period of record 1963-76,

1978

\begin{tabular}{cccccc}
\hline \multicolumn{5}{c}{ Discharge, in $\mathrm{ft}^{3} / \mathbf{s}$, for indicated recurrence interval } \\
in years, and exceedance probablility, in percent \\
\hline 2 & 5 & 10 & 25 & $50 \dagger$ & $100 \dagger$ \\
$50 \%$ & $20 \%$ & $10 \%$ & $4 \%$ & $2 \%$ & $1 \%$ \\
\hline 309 & 691 & 1,030 & 1,560 & 2,020 & 2,540 \\
Weighted skew & $(\operatorname{logs}=$ & -0.21 & & & \\
Mean & $(\operatorname{logs})=$ & 2.48 & & \\
Standard dev. & $(\operatorname{logs})=$ & 0.43 &
\end{tabular}

fReliability of values in column is uncertain, and potential errors are large.

Basin characteristics

\begin{tabular}{|c|c|c|c|c|c|c|c|}
\hline \multirow[b]{2}{*}{$\begin{array}{l}\text { Main } \\
\text { channel } \\
\text { slope } \\
\text { (ft/mi) }\end{array}$} & \multirow[b]{2}{*}{$\begin{array}{l}\text { Stream } \\
\text { length } \\
\text { (mi) }\end{array}$} & \multirow[b]{2}{*}{$\begin{array}{c}\text { Mean } \\
\text { basin } \\
\text { elevation } \\
(\mathrm{ft})\end{array}$} & \multirow[b]{2}{*}{$\begin{array}{l}\text { Forested } \\
\text { area } \\
\text { (percent) }\end{array}$} & \multirow[b]{2}{*}{$\begin{array}{c}\text { Soil } \\
\text { index }\end{array}$} & \multirow[b]{2}{*}{$\begin{array}{c}\text { Mean } \\
\text { annual } \\
\text { precipitation } \\
\text { (in) }\end{array}$} & \multicolumn{2}{|c|}{ Rainfall intensity, 24-hour } \\
\hline & & & & & & $\begin{array}{l}\text { 2-year } \\
\text { (in) }\end{array}$ & $\begin{array}{c}\text { 50-year } \\
\text { (in) }\end{array}$ \\
\hline 75.4 & 11.5 & 4,360 & 17.0 & 3.0 & 15.8 & 2.0 & 4.3 \\
\hline
\end{tabular}

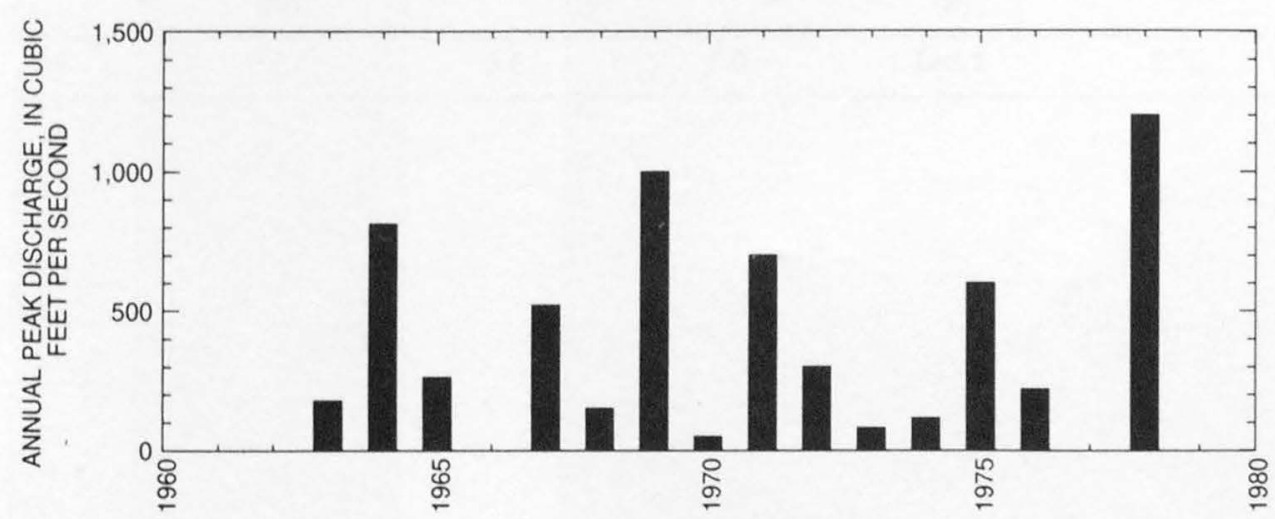


09481750 SOPORI WASH AT AMADO, AZ

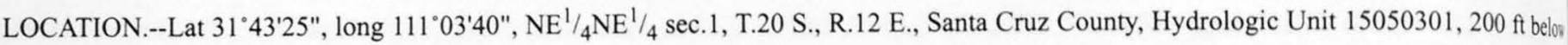
bridge on State Highway $89,1.1$ mi north of Amado.

DRAINAGE AREA.--176 $\mathrm{mi}^{2}$.

Annual peak discharges

\begin{tabular}{|c|c|c|c|c|c|c|c|}
\hline $\begin{array}{l}\text { Water } \\
\text { year }\end{array}$ & Date & $\begin{array}{c}\text { Annual peak } \\
\text { discharge } \\
\left(\mathrm{ft}^{3} / \mathrm{s}\right)\end{array}$ & $\begin{array}{l}\text { Discharge } \\
\text { codes }\end{array}$ & $\begin{array}{l}\text { Water } \\
\text { year }\end{array}$ & Date & $\begin{array}{c}\text { Annual peak } \\
\text { discharge } \\
\left(\mathrm{ft}^{3} / \mathrm{s}\right)\end{array}$ & $\begin{array}{c}\text { Discharge } \\
\text { codes }\end{array}$ \\
\hline 1948 & $08-15-48$ & 16,000 & HP & 1968 & $00-00-68$ & 500 & LT \\
\hline 1954 & $00-00-54$ & 6,500 & & 1969 & $00-00-69$ & 500 & LT \\
\hline 1955 & $08-00-55$ & 2,700 & & 1970 & $09-04-70$ & 1,800 & \\
\hline 1956 & $00-00-56$ & 500 & ES & 1971 & 09-01-71 & 2,900 & \\
\hline 1957 & $00-00-57$ & 100 & ES & 1972 & $07-14-72$ & 27,300 & \\
\hline 1958 & $00-00-58$ & ${ }^{1} 8,000$ & & 1973 & $07-00-73$ & 400 & \\
\hline 1964 & $09-10-64$ & 3,800 & & 1974 & $07-23-74$ & 3,900 & \\
\hline 1965 & $00-00-65$ & 500 & LT & 1975 & $08-23-75$ & 2,700 & \\
\hline 1966 & $12-22-65$ & 1500 & & 1976 & $09-25-76$ & 2,500 & \\
\hline 1967 & $00-00-67$ & 500 & LT & 1978 & $10-00-77$ & 2,300 & HP \\
\hline
\end{tabular}

${ }^{1}$ Highest since 1949.

${ }^{2}$ Highest since 1958.

Magnitude and probability of instantaneous peak flow based on period of record 1948 , $1954-58,1964-76,1978$

\begin{tabular}{|c|c|c|c|c|c|}
\hline \multicolumn{6}{|c|}{$\begin{array}{l}\text { Discharge, in } \mathrm{ft}^{3} / \mathrm{s} \text {, for indicated recurrence interval } \\
\text { in years, and exceedance probablility, in percent }\end{array}$} \\
\hline 2 & 5 & 10 & 25 & $50 t$ & $100 t$ \\
\hline $50 \%$ & $20 \%$ & $10 \%$ & $4 \%$ & $2 \%$ & $1 \%$ \\
\hline 2,230 & 4,610 & 6,780 & 10,200 & 13,400 & 17,100 \\
\hline Weighted skew & $(\log s)=$ & 0.05 & & & \\
\hline Mean & $(\log s)=$ & 3.35 & & & \\
\hline Standard dev. & $(\log s)=$ & 0.37 & & & \\
\hline
\end{tabular}

f Reliability of values in column is uncertain, and potential errors are large.

Basin characteristics

\begin{tabular}{|c|c|c|c|c|c|c|c|}
\hline \multirow[b]{2}{*}{$\begin{array}{l}\text { Main } \\
\text { channel } \\
\text { slope } \\
\text { (ft/mi) }\end{array}$} & \multirow[b]{2}{*}{$\begin{array}{l}\text { Stream } \\
\text { length } \\
(\mathrm{mi})\end{array}$} & \multirow[b]{2}{*}{$\begin{array}{c}\text { Mean } \\
\text { basin } \\
\text { elevation } \\
(f t)\end{array}$} & \multirow[b]{2}{*}{$\begin{array}{l}\text { Forested } \\
\text { area } \\
\text { (percent) }\end{array}$} & \multirow[b]{2}{*}{$\begin{array}{c}\text { Soil } \\
\text { index }\end{array}$} & \multirow[b]{2}{*}{$\begin{array}{c}\text { Mean } \\
\text { annual } \\
\text { precipitation } \\
\text { (in) }\end{array}$} & \multicolumn{2}{|c|}{ Rainfall intensity, 24-hour } \\
\hline & & & & & & $\begin{array}{c}\text { 2-year } \\
\text { (in) }\end{array}$ & $\begin{array}{l}\text { 50-year } \\
\text { (in) }\end{array}$ \\
\hline 35.9 & 27.3 & 3,840 & 0.3 & 3.0 & 15.5 & 2.2 & 4.3 \\
\hline
\end{tabular}


09481750 SOPORI WASH AT AMADO, AZ--Continued

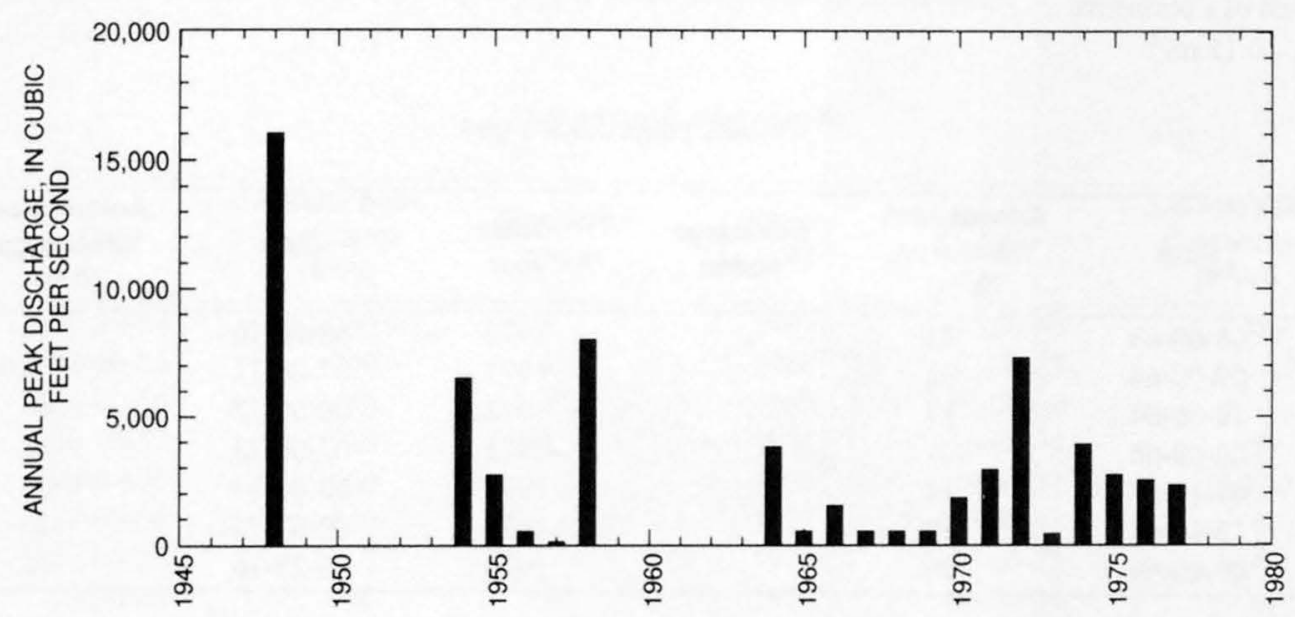


09481800 DEMETRIE WASH TRIBUTARY NEAR CONTINENTAL, AZ

LOCATION.--Lat $31^{\circ} 52^{\prime} 15^{\prime \prime}$, long $111^{\circ} 05^{\prime} 15^{\prime \prime}, \mathrm{SW}^{1} / \mathrm{SE}^{1} / 4 \mathrm{sec} .11$, T.18 S., R.12 E., Pima County, Hydrologic Unit 15050301, at Duval Mine

Road, 6.5 mi west of Continental.

DRAINAGE AREA.--0.15 $\mathrm{mi}^{2}$.

Annual peak discharges

\begin{tabular}{cccccccc}
\hline $\begin{array}{c}\text { Water } \\
\text { year }\end{array}$ & Date & $\begin{array}{c}\text { Annual peak } \\
\text { discharge } \\
\left(\mathrm{ft}^{3} / \mathbf{s}\right)\end{array}$ & $\begin{array}{c}\text { Discharge } \\
\text { codes }\end{array}$ & $\begin{array}{c}\text { Water } \\
\text { year }\end{array}$ & Date & $\begin{array}{c}\text { Annual peak } \\
\text { discharge } \\
\left(\mathrm{ft}^{3} / \mathbf{s}\right)\end{array}$ & $\begin{array}{c}\text { Discharge } \\
\text { codes }\end{array}$ \\
\hline 1963 & $08-08-63$ & 71 & & 1970 & $08-00-70$ & 10 & \\
1964 & $00-00-64$ & 71 & 1971 & $07-00-71$ & 4.0 & ES \\
1965 & $10-00-64$ & 17 & 1972 & $00-00-72$ & 60 & \\
1966 & $00-00-66$ & 11 & 1973 & $07-00-73$ & 17 & \\
1967 & $07-17-67$ & 19 & 1974 & $00-00-74$ & 63 & \\
1968 & $12-15-67$ & 10 & 1975 & $09-07-75$ & 110 & \\
1969 & $09-00-69$ & 20 & & 1976 & $09-25-76$ & 36 & \\
\hline
\end{tabular}

Magnitude and probability of instantaneous peak flow based on period of record 1963-76

\begin{tabular}{|c|c|c|c|c|c|}
\hline \multicolumn{6}{|c|}{$\begin{array}{l}\text { Discharge, in } \mathrm{ft}^{3} / \mathrm{s} \text {, for indicated recurrence interval } \\
\text { in years, and exceedance probablility, in percent }\end{array}$} \\
\hline 2 & 5 & 10 & 25 & $50 \dagger$ & $100 t$ \\
\hline $50 \%$ & $20 \%$ & $10 \%$ & $4 \%$ & $2 \%$ & $1 \%$ \\
\hline 25.6 & 56.9 & 85.2 & 130 & 169 & 214 \\
\hline Weighted skew & $(\log s)=$ & -0.15 & & & \\
\hline Mean & $(\log s)=$ & 1.40 & & & \\
\hline Standard dev. & $(\log s)=$ & 0.42 & & & \\
\hline
\end{tabular}

f Reliability of values in column is uncertain, and potential errors are large.

Basin characteristics

\begin{tabular}{|c|c|c|c|c|c|c|c|}
\hline \multirow[b]{2}{*}{$\begin{array}{l}\text { Main } \\
\text { channel } \\
\text { slope } \\
(\mathrm{ft} / \mathrm{mi})\end{array}$} & \multirow[b]{2}{*}{$\begin{array}{l}\text { Stream } \\
\text { length } \\
\text { (mi) }\end{array}$} & \multirow[b]{2}{*}{$\begin{array}{c}\text { Mean } \\
\text { basin } \\
\text { elevation } \\
(\mathrm{ft})\end{array}$} & \multirow[b]{2}{*}{$\begin{array}{l}\text { Forested } \\
\text { area } \\
\text { (percent) }\end{array}$} & \multirow[b]{2}{*}{$\begin{array}{c}\text { Soil } \\
\text { index }\end{array}$} & \multirow[b]{2}{*}{$\begin{array}{c}\text { Mean } \\
\text { annual } \\
\text { precipitation } \\
\text { (in) }\end{array}$} & \multicolumn{2}{|c|}{ Rainfall intensity, 24-hour } \\
\hline & & & & & & $\begin{array}{l}\text { 2-year } \\
\text { (in) }\end{array}$ & $\begin{array}{l}\text { 50-year } \\
\text { (in) }\end{array}$ \\
\hline 100 & 0.80 & 3,620 & 0.0 & 1.0 & 14.5 & 2.3 & 4.7 \\
\hline
\end{tabular}

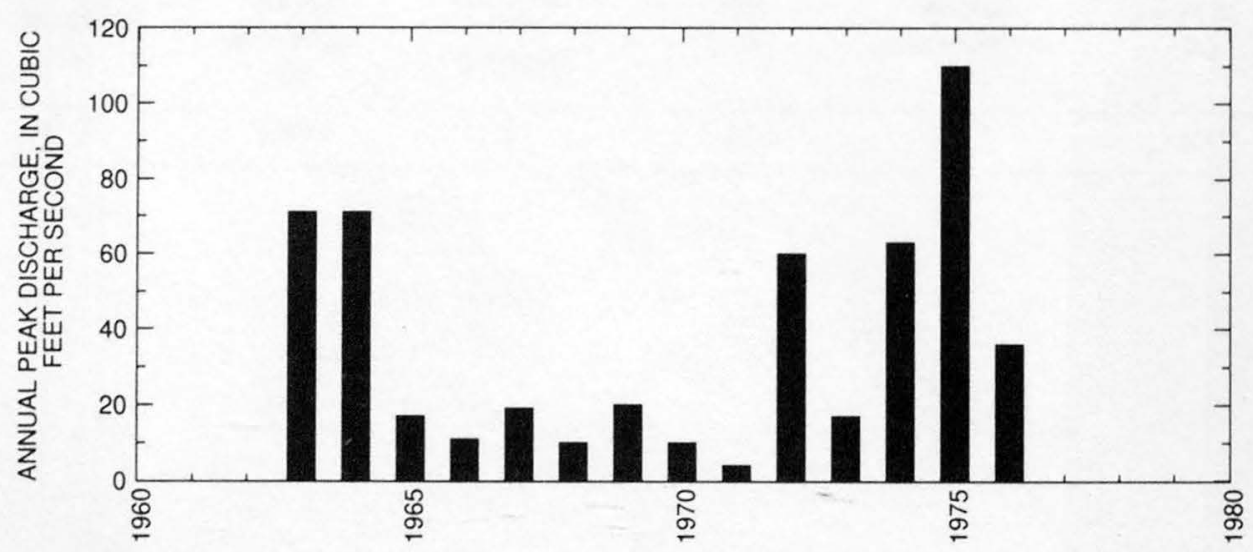


09481900 OCOTILLO WASH NEAR CONTINENTAL, AZ

LOCATION.--Lat $31^{\circ} 50^{\prime} 00^{\prime \prime}$, long $111^{\circ} 00^{\prime} 00^{\prime \prime}, \mathrm{SE}^{1} \frac{1}{4} \mathrm{sec} .27$, T.18 S., R.12 E. (unsurveyed), Pima County, Hydrologic Unit 15050301, in Spanish Land Grant of San Ignacio del la Canoa at U.S. Highway 89, 1.5 mi southwest of Continental.

DRAINAGE AREA.--3.60 $\mathrm{mi}^{2}$.

Annual peak discharges

\begin{tabular}{|c|c|c|c|c|c|c|c|}
\hline $\begin{array}{l}\text { Water } \\
\text { year }\end{array}$ & Date & $\begin{array}{c}\text { Annual peak } \\
\text { discharge } \\
\left(\mathrm{ft}^{3} / \mathbf{s}\right)\end{array}$ & $\begin{array}{l}\text { Discharge } \\
\text { codes }\end{array}$ & $\begin{array}{l}\text { Water } \\
\text { year }\end{array}$ & Date & $\begin{array}{l}\text { Annual peak } \\
\text { discharge } \\
\left(\mathrm{ft}^{3} / \mathrm{s}\right)\end{array}$ & $\begin{array}{c}\text { Discharge } \\
\text { codes }\end{array}$ \\
\hline 1954 & $07-20-54$ & 1,250 & & 1967 & $00-00-67$ & 20 & ES \\
\hline 1955 & $00-00-55$ & 200 & ES & 1968 & $12-00-67$ & 1.0 & LT \\
\hline 1956 & $00-00-56$ & 90 & ES & 1969 & $00-00-69$ & 0 & \\
\hline 1957 & $00-00-57$ & 35 & ES & 1970 & $00-00-70$ & 0 & $\mathrm{KR}$ \\
\hline 1963 & $08-0-563$ & ${ }^{1} 1,660$ & & 1971 & $08-00-71$ & 12 & $\mathrm{KR}$ \\
\hline 1964 & $07-00-64$ & ${ }^{2} 1,840$ & & 1972 & $00-00-72$ & 0 & $\mathrm{KR}$ \\
\hline 1965 & $00-00-65$ & 0 & & 1973 & $00-00-73$ & 10 & $\mathrm{ES}, \mathrm{KR}$ \\
\hline 1966 & $00-00-66$ & 40 & ES & 1974 & $00-00-74$ & 0 & KR \\
\hline
\end{tabular}

THighest since 1954.

${ }^{2}$ Highest since 1954 .

Magnitude and probability of instantaneous peak flow based on period of record

\begin{tabular}{|c|c|c|c|c|c|}
\hline \multicolumn{6}{|c|}{$\begin{array}{l}\text { Discharge, in } \mathrm{ft}^{3} / \mathrm{s} \text {, for indicated recurrence interval } \\
\text { in years, and exceedance probablility, in percent }\end{array}$} \\
\hline 2 & 5 & 10 & 25 & 50 & 100 \\
\hline $50 \%$ & $20 \%$ & $10 \%$ & $4 \%$ & $2 \%$ & $1 \%$ \\
\hline -.- & $\ldots$ & $\ldots$ & $\ldots$ & $\ldots$ & -..- \\
\hline Weighted skew & $(\log s)=$ & $\cdots$ & & & \\
\hline Mean & $(\operatorname{logs})=$ & $\cdots$ & & & \\
\hline Standard dev. & $(\log s)=$ & $\cdots$ & & & \\
\hline
\end{tabular}

Basin characteristics

\begin{tabular}{cccccccc}
\hline $\begin{array}{c}\text { Main } \\
\text { channel } \\
\text { slope } \\
(\mathrm{ft} / \mathrm{mi})\end{array}$ & $\begin{array}{c}\text { Stream } \\
\text { length } \\
(\mathrm{mi})\end{array}$ & $\begin{array}{c}\text { Mean } \\
\text { basin } \\
\text { elevation } \\
(\mathrm{ft})\end{array}$ & $\begin{array}{c}\text { Forested } \\
\text { area } \\
\text { (percent) }\end{array}$ & $\begin{array}{c}\text { Soil } \\
\text { index }\end{array}$ & $\begin{array}{c}\text { Mean } \\
\text { annual } \\
\text { precipitation } \\
\text { (in) }\end{array}$ & $\begin{array}{c}\text { Rainfall intensity, 24-hour } \\
\text { (in) }\end{array}$ & $\begin{array}{c}50 \text {-year } \\
\text { (in) }\end{array}$ \\
\hline 106 & 5.99 & 3,280 & 0.0 & 3.0 & 14.1 & 2.1 \\
\hline
\end{tabular}


09481900 OCOTILLO WASH NEAR CONTINENTAL, AZ--Continued

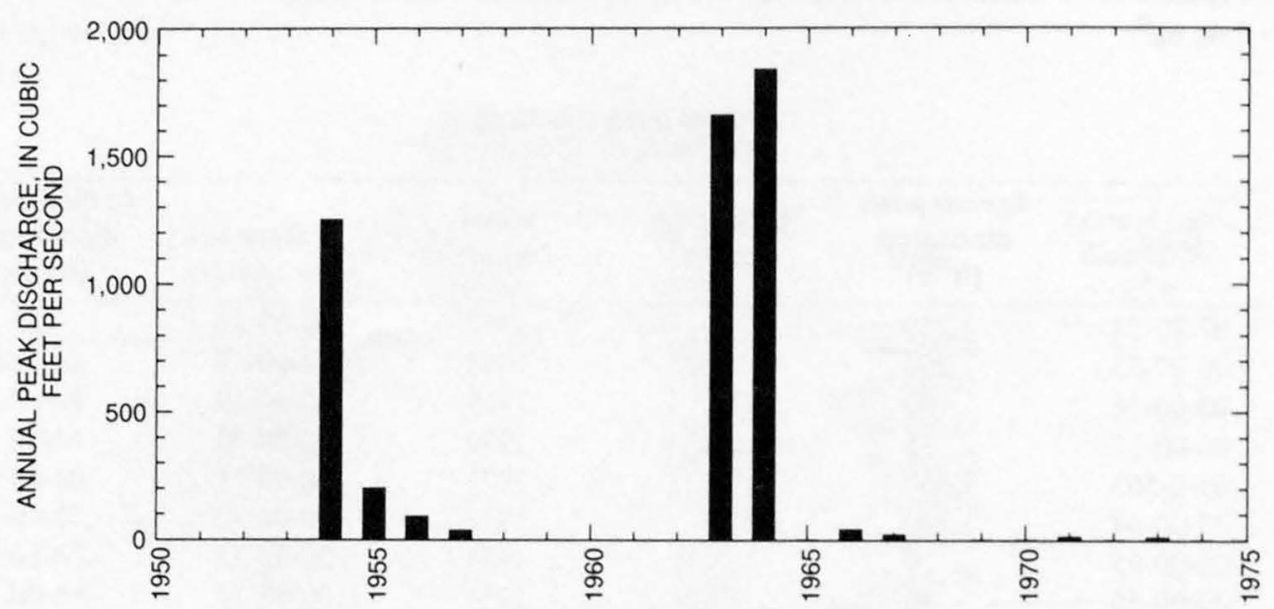




\section{SANTA CRUZ RIVER AT CONTINENTAL, AZ}

LOCATION.--Lat 31 '52'17", long 110 58'46", in $\mathrm{SE}^{1} / \mathrm{SEE}^{1} / 4 \mathrm{sec} .11$, T.18 S., R.13 E. (unsurveyed), Pima County, Hydrologic Unit 15050301 , in Spanish land grant of San Ignacio de la Canoa, on right bank 0.8 mi northeast of Green Valley Post Office, and 1.5 mi north of Continental. Prior to Feb. 13, 1981, at site 1.5 mi upstream.

DRAINAGE AREA.--1,682 $\mathrm{mi}^{2}$, revised, of which $395 \mathrm{mi}^{2}$ is in Mexico.

PERIOD OF RECORD.--May 1940 to December 1946, October 1951 to September 1985, October 1991 to current year (monthly discharge only for 1985-86), (crest-stage partial record station for 1987-1990). Low-flow records not equivalent prior to Feb. 13, 1981, due to undetermined amount of underflow between sites.

REVISED RECORDS.--WSP 1283: Drainage area.

GAGE.--Water-stage recorder and crest-stage gages. Datum of gage is 2,806.61 ft above sea level. Prior to Feb. 13, 1981 , at site 1.5 mi upstream. July 21, 1940 to Sept. 8, 1965 at datum $17.28 \mathrm{ft}$ higher; Sept. 8, 1965 to present at datum $13.21 \mathrm{ft} \mathrm{higher}$. Old site used as supplementary gage until Oct. 29, 1985.

REMARKS.--Records poor. Irrigation above station of about 12,500 acres including about 2,300 acres in Mexico, mostly by pumping from ground water.

AVERAGE DISCHARGE.--46 years, $27.1 \mathrm{ft}^{3} / \mathrm{s}, 19,630$ acre-ft/yr; median of yearly mean discharges, $9.5 \mathrm{ft}^{3} / \mathrm{s}, 6,900 \mathrm{acre}-\mathrm{ft} / \mathrm{yr}$.

EXTREMES FOR PERIOD OF RECORD.--Maximum discharge, $45,000 \mathrm{ft}^{3} / \mathrm{s}$ Oct. 2, 1983, gage height, $16.34 \mathrm{ft}$ from rating curve extended above $530 \mathrm{ft}^{3} / \mathrm{s}$ on basis of float-area measurement at gage height $7.75 \mathrm{ft}$ and slope-area measurement of peak flow, maximum gage height $16.70 \mathrm{ft}$ Oct. 9, 1977, site and datum then in use; no flow for most of each year.

Annual peak discharges

\begin{tabular}{|c|c|c|c|c|c|c|c|}
\hline $\begin{array}{l}\text { Water } \\
\text { year }\end{array}$ & Date & $\begin{array}{c}\text { Annual peak } \\
\text { discharge } \\
\left(\mathrm{ft}^{3} / \mathrm{s}\right)\end{array}$ & $\begin{array}{l}\text { Discharge } \\
\text { codes }\end{array}$ & $\begin{array}{l}\text { Water } \\
\text { year }\end{array}$ & Date & $\begin{array}{c}\text { Annual peak } \\
\text { discharge } \\
\left(\mathrm{ft}^{3} / \mathrm{s}\right)\end{array}$ & $\begin{array}{l}\text { Discharge } \\
\text { codes }\end{array}$ \\
\hline 1940 & $08-14-40$ & 12,100 & & 1971 & $08-20-71$ & 3,270 & \\
\hline 1941 & $08-09-41$ & 3,670 & & 1972 & $07-14-72$ & 3,290 & \\
\hline 1942 & $07-28-42$ & 2,700 & & 1973 & $03-14-73$ & 2,130 & \\
\hline 1943 & $08-01-43$ & 4,000 & & 1974 & $09-03-74$ & 3,450 & \\
\hline 1944 & $08-12-44$ & 4,440 & & 1975 & $09-01-75$ & 3,350 & \\
\hline 1945 & $08-09-45$ & 7,820 & & 1976 & $07-12-76$ & 3,800 & \\
\hline 1946 & $09-09-46$ & 4,120 & & 1977 & $07-18-77$ & 3,290 & \\
\hline 1947 & $10-01-46$ & 5,330 & & 1978 & $10-09-77$ & 26,500 & \\
\hline 1952 & $08-15-52$ & 1,820 & & 1979 & $12-18-78$ & 16,000 & \\
\hline 1953 & $07-14-53$ & 4,910 & & 1980 & $08-25-80$ & 2,360 & \\
\hline 1954 & $08-05-54$ & 14,600 & & 1981 & $09-05-81$ & 3,350 & \\
\hline 1955 & $08-19-55$ & 17,500 & & 1982 & $08-15-82$ & 2,160 & \\
\hline 1956 & $07-29-56$ & 3,090 & & 1983 & $02-04-83$ & 4,800 & \\
\hline 1957 & $08-21-57$ & 1,690 & & 1984 & $10-02-83$ & $\mathrm{I}_{45,000}$ & \\
\hline 1958 & $08-05-58$ & 5,620 & & 1985 & $12-28-84$ & 11,600 & \\
\hline 1959 & $08-17-59$ & 3,900 & & 1986 & $07-16-86$ & 840 & \\
\hline 1960 & $01-12-60$ & 3,740 & & 1987 & $08-05-87$ & 340 & \\
\hline 1961 & $08-23-61$ & 4,820 & & 1988 & $07-28-88$ & 930 & \\
\hline 1962 & $01-25-62$ & 2,480 & & 1989 & 09-03-89 & 1,200 & \\
\hline 1963 & $08-06-63$ & 4,220 & & 1990 & $10-05-89$ & 1,790 & \\
\hline 1964 & $09-10-64$ & 14,000 & & 1991 & 09-01-91 & 1,270 & \\
\hline 1965 & $09-12-65$ & 370 & & 1992 & $08-24-92$ & 4,120 & \\
\hline 1966 & $12-23-65$ & 5,990 & & 1993 & $01-19-93$ & 32,400 & \\
\hline 1967 & $07-27-67$ & 3,730 & & 1994 & $08-22-94$ & 707 & \\
\hline 1968 & $12-20-67$ & 18,000 & & 1995 & 01-06-95 & 2,350 & \\
\hline 1969 & $08-05-69$ & 1,680 & & 1996 & $09-03-96$ & 1,520 & \\
\hline 1970 & $07-20-70$ & 3,720 & & & & & \\
\hline
\end{tabular}

${ }^{1}$ Highest since 1892 . 
09482000 SANTA CRUZ RIVER AT CONTINENTAL, AZ--Continued

Discharge rating table developed January 1993

\begin{tabular}{crcc}
\hline $\begin{array}{c}\text { Gage height } \\
(\mathbf{f t})\end{array}$ & $\begin{array}{c}\text { Discharge } \\
\left(\mathbf{f t}^{\mathbf{3}} \mathbf{s}\right)\end{array}$ & $\begin{array}{c}\text { Gage height } \\
(\mathbf{f t})\end{array}$ & $\begin{array}{c}\text { Discharge } \\
\left(\mathbf{f t}^{\mathbf{3}} \mathbf{s}\right)\end{array}$ \\
\hline 2.5 & 57 & 9.0 & 7,130 \\
3.0 & 147 & 10.0 & 9,910 \\
4.0 & 460 & 11.0 & 13,300 \\
5.0 & 1,030 & 12.0 & 17,360 \\
6.0 & 1,920 & 13.0 & 22,140 \\
7.0 & 3,190 & 14.0 & 27,700 \\
8.0 & 4,910 & 14.7 & 32,070 \\
\hline
\end{tabular}

Basin characteristics

\begin{tabular}{ccccccc}
\hline $\begin{array}{c}\text { Main } \\
\text { channel } \\
\text { slope } \\
(\mathrm{ft} / \mathrm{mi})\end{array}$ & $\begin{array}{c}\text { Stream } \\
\text { length } \\
(\mathrm{mi})\end{array}$ & $\begin{array}{c}\text { Mean } \\
\text { basin } \\
\text { elevation } \\
(\mathrm{ft})\end{array}$ & $\begin{array}{c}\text { Forested } \\
\text { area } \\
\text { (percent) }\end{array}$ & $\begin{array}{c}\text { Soil } \\
\text { index }\end{array}$ & $\begin{array}{c}\text { Mean } \\
\text { annual } \\
\text { precipitation } \\
\text { (in) }\end{array}$ & $\begin{array}{c}\text { Rainfall intensity, 24-hour } \\
\text { (in) }\end{array}$ \\
\hline 21.0 & 99.2 & 4,350 & 22.0 & 2.0 & 18.1 & $\begin{array}{c}50 \text {-year } \\
\text { (in) }\end{array}$ \\
\hline
\end{tabular}


09482000 SANTA CRUZ RIVER AT CONTINENTAL, AZ--Continued

MEAN MONTHLY AND ANNUAL DISCHARGES $1941.46,1952 \cdot 85,1992.96$

\begin{tabular}{|c|c|c|c|c|c|c|}
\hline MONTH & $\begin{array}{l}\text { MAXIMUM } \\
\text { (FT3/S) }\end{array}$ & $\begin{array}{l}\text { MINIMUM } \\
\text { (FT3/S) }\end{array}$ & $\begin{array}{c}\text { MEAN } \\
(\text { FT3 } / \mathrm{S})\end{array}$ & $\begin{array}{l}\text { STAN- } \\
\text { DARD } \\
\text { DEVIA- } \\
\text { TION } \\
(\mathrm{FT} 3 / \mathrm{S})\end{array}$ & $\begin{array}{l}\text { COEFFI- } \\
\text { CIENT OF } \\
\text { VARI- } \\
\text { ATION }\end{array}$ & $\begin{array}{c}\text { PERCENT } \\
\text { OF } \\
\text { ANNUAL } \\
\text { RUNOFF }\end{array}$ \\
\hline OCTOBER & 1,530 & 0.00 & 53 & 251 & 4.8 & 16.1 \\
\hline NOVEMBER & 133 & 0.00 & 3.3 & 20 & 6.1 & 1.0 \\
\hline DECEMBER & 658 & 0.00 & 47 & 146 & 3.1 & 14.3 \\
\hline JANUARY & 1,390 & 0.00 & 56 & 223 & 4.0 & 17.1 \\
\hline FEBRUARY & 207 & 0.00 & 18 & 48 & 2.7 & 5.4 \\
\hline MARCH & 181 & 0.00 & 12 & 37 & 3.2 & 3.6 \\
\hline APRIL & 32 & 0.00 & 0.72 & 4.7 & 6.5 & 0.2 \\
\hline MAY & 1.3 & 0.00 & 0.03 & 0.20 & 6.3 & 0.0 \\
\hline JUNE & 6.2 & 0.00 & 0.34 & 1.2 & 3.4 & 0.1 \\
\hline JULY & 227 & 0.00 & 32 & 45 & 1.4 & 9.9 \\
\hline AUGUST & 753 & 0.00 & 86 & 160 & 1.8 & 26.3 \\
\hline SEPTEMBER & 285 & 0.00 & 20 & 45 & 2.3 & 6.1 \\
\hline ANNUAL & 206 & 0.26 & 28 & 41 & 1.5 & 100 \\
\hline
\end{tabular}

MAGNITUDE AND PROBABILITY OF ANNUAL LOW FLOW BASED ON PERIOD OF RECORD $1942-46,1953.85,1993-96$

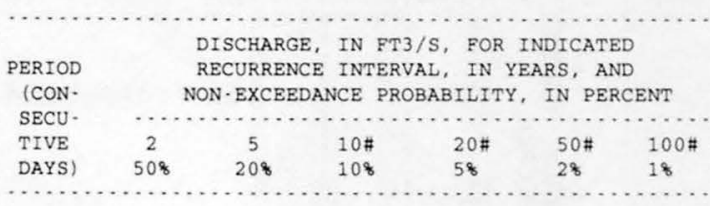

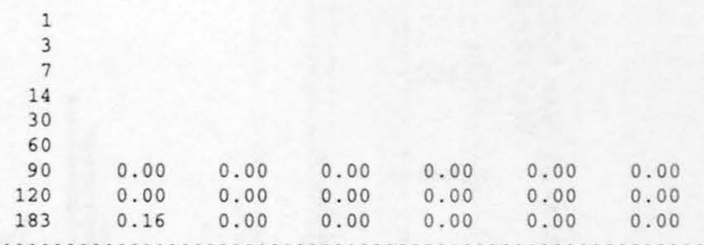

MAGNITUDE AND PROBABILITY OF ANNUAL HIGH FLOW BASED ON PERIOD OF RECORD $1941 \cdot 46,1952 \cdot 85,1992 \cdot 96$

\begin{tabular}{|c|c|c|c|c|c|c|}
\hline \multirow{3}{*}{$\begin{array}{l}\text { PERIOD } \\
\text { (CON- } \\
\text { SECU- } \\
\text { TIVE }\end{array}$} & & $\begin{array}{l}\text { DISCHAF } \\
\text { RECURRE } \\
\text { EXCEEDF }\end{array}$ & $\begin{array}{ll}\text { E, } & \text { IN } \\
C E & \text { INT: } \\
C E & \text { PRO }\end{array}$ & $\begin{array}{l}\text { T3/S, FO } \\
\text { RVAL, IN } \\
\text { ABILITY, }\end{array}$ & $\begin{array}{l}\text { R INDIC } \\
\text { YEARS, } \\
\text { IN PER }\end{array}$ & $\begin{array}{l}\text { TED } \\
\text { AND } \\
\text { ENT }\end{array}$ \\
\hline & & & & & & \\
\hline & 2 & 5 & $10 \#$ & $25 \#$ & $50 \#$ & $100=$ \\
\hline DAYS) & 508 & 208 & 108 & 48 & 28 & 18 \\
\hline 1 & 1,000 & 3,260 & 6,180 & 12,400 & 19,700 & 30,000 \\
\hline 3 & 518 & 1,750 & 3,390 & 6,920 & 11,100 & 17,000 \\
\hline 7 & 270 & 920 & 1,780 & 3,640 & 5,830 & 8,950 \\
\hline 15 & 159 & 533 & 1,010 & 2,010 & 3,150 & 4,730 \\
\hline 30 & 105 & 332 & 598 & 1,110 & 1,640 & 2,320 \\
\hline 60 & 63 & 196 & 348 & 634 & 928 & 1,300 \\
\hline 90 & 44 & 136 & 241 & 439 & 643 & 902 \\
\hline
\end{tabular}

DURATION TABLE OF DAILY MEAN FLOW FOR PERIOD OF RECORD $1941 \cdot 46,1952 \cdot 85,1992 \cdot 96$

DISCHARGE, IN FT3/S, WHICH WAS EQUALED OR EXCEEDED FOR INDICATED PERCENT OF TIME

\begin{tabular}{|c|c|c|c|c|c|c|c|c|c|c|c|c|c|c|c|c|}
\hline 18 & 58 & 108 & 158 & 208 & 308 & 408 & 508 & 608 & 708 & 808 & 908 & 958 & 988 & 998 & 99.58 & 99.98 \\
\hline 600 & 52 & 2.9 & 0.00 & 0.00 & 0.00 & 0.00 & 0.00 & 0.00 & 0.00 & 0.00 & 0.00 & 0.00 & 0.00 & 0.00 & 0.00 & 0.00 \\
\hline
\end{tabular}

\# Reliability of values in column is uncertain, and potential errors are large. 
ANNUAL MEAN DISCHARGE, IN CUBIC PER SECOND

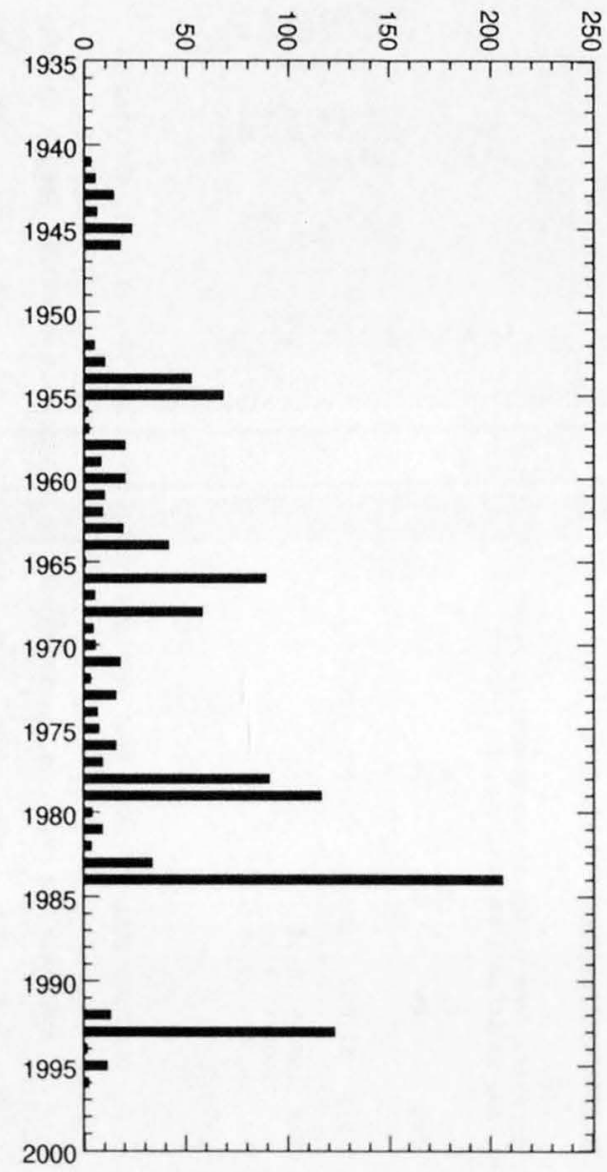

ANNUAL PEAK DISCHARGE, IN CUBIC

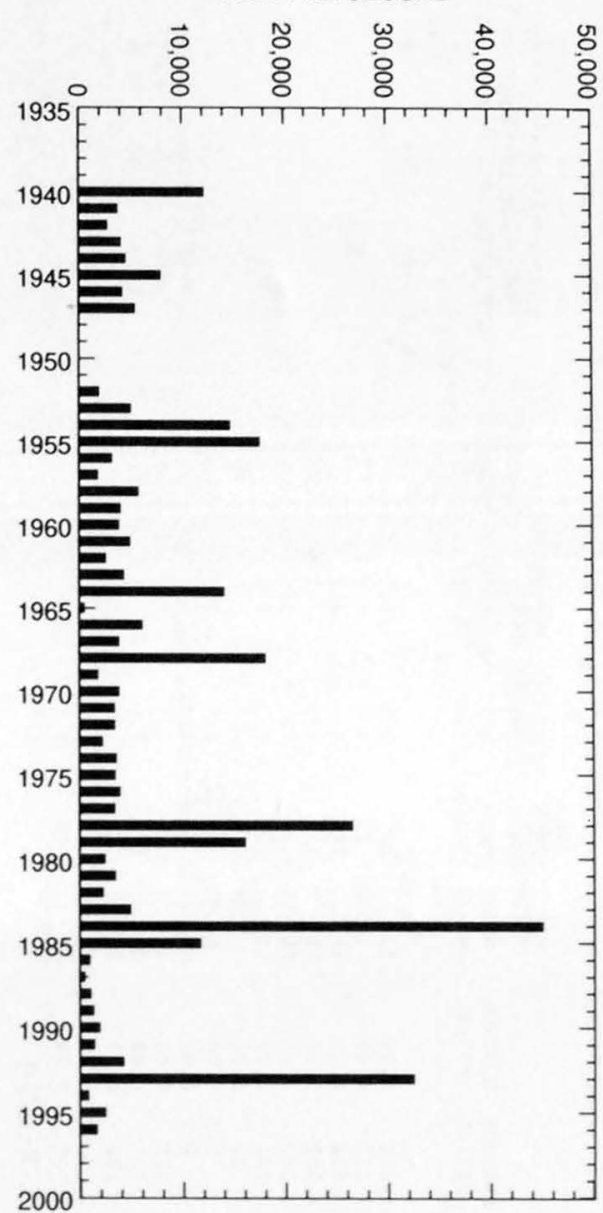


09482000 SANTA CRUZ RIVER AT CONTINENTAL, AZ--Continued
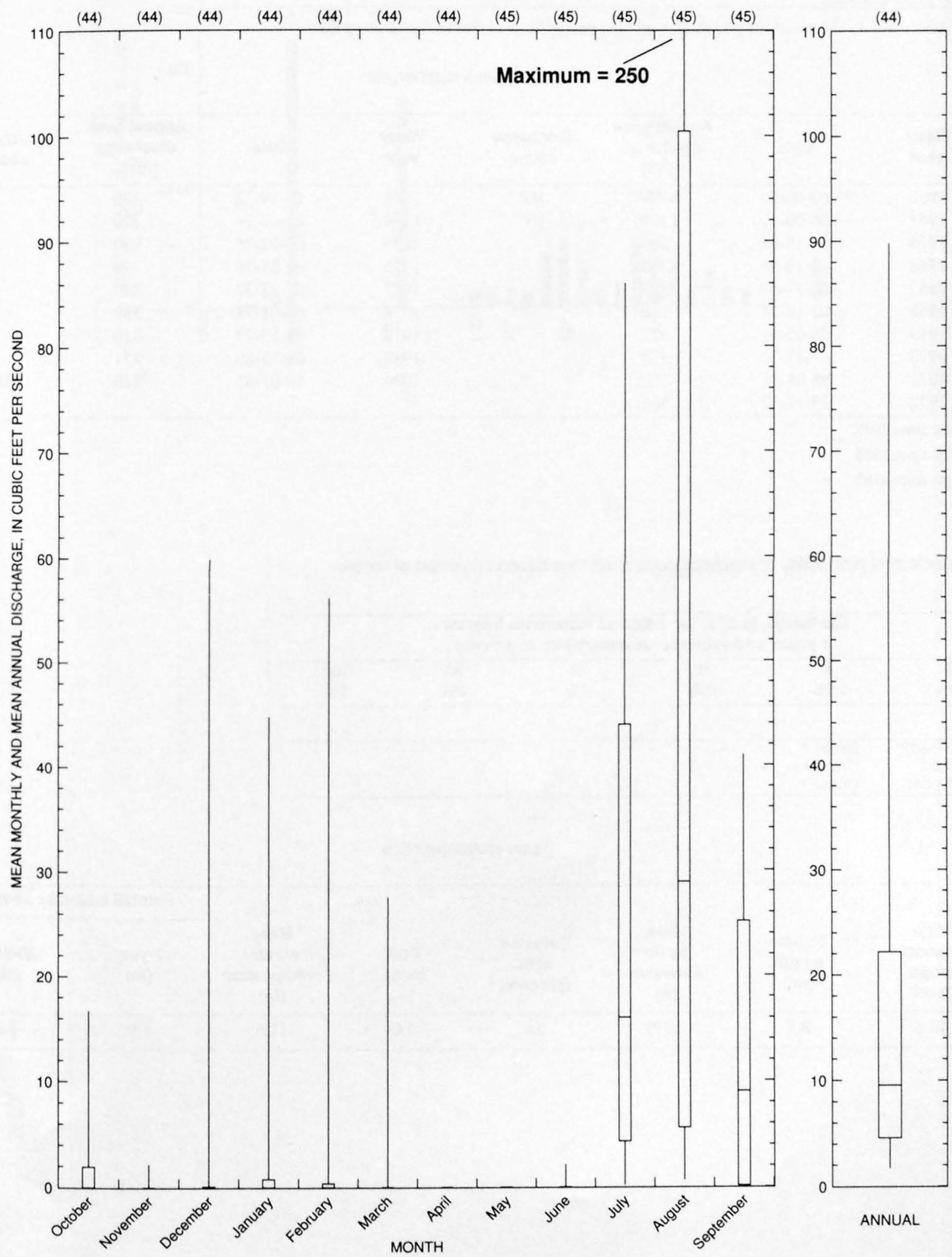


\section{FLATO WASH NEAR SAHUARITA, AZ}

LOCATION.--Lat $32^{\circ} 02^{\prime} 43^{\prime \prime}$, long $110^{\circ} 57^{\prime} 00^{\prime \prime}, \mathrm{SW}^{1} / \mathrm{SE}^{1} / 4$ sec.7, T.16 S., R. 14 E., Pima County, Hydrologic Unit 15050301 , at U.S. Highway 89 6 mi north Sahuarita.

DRAINAGE AREA.--30.1 $\mathrm{mi}^{2}$, of which $23.4 \mathrm{mi}^{2}$ is noncontributing and/or distributary flow.

Annual peak discharges

\begin{tabular}{|c|c|c|c|c|c|c|c|}
\hline $\begin{array}{l}\text { Water } \\
\text { year }\end{array}$ & Date & $\begin{array}{c}\text { Annual peak } \\
\text { discharge } \\
\left(\mathrm{ft}^{3} / \mathrm{s}\right)\end{array}$ & $\begin{array}{c}\text { Discharge } \\
\text { codes }\end{array}$ & $\begin{array}{l}\text { Water } \\
\text { year }\end{array}$ & Date & $\begin{array}{c}\text { Annual peak } \\
\text { discharge } \\
\left(\mathrm{ft}^{3} / \mathrm{s}\right)\end{array}$ & $\begin{array}{c}\text { Discharge } \\
\text { codes }\end{array}$ \\
\hline 1955 & $00-00-55$ & 14,500 & HP & 1973 & $10-19-72$ & 370 & \\
\hline 1961 & $00-00-61$ & $2_{3,500}$ & HP & 1974 & $07-07-74$ & 1,170 & \\
\hline 1965 & $08-30-65$ & 250 & & 1975 & $09-07-75$ & 960 & \\
\hline 1966 & $08-19-66$ & 340 & & 1976 & $09-25-76$ & 790 & \\
\hline 1967 & $07-17-67$ & 307 & & 1977 & $07-22-77$ & 390 & \\
\hline 1968 & $02-12-68$ & 230 & & 1978 & $08-01-78$ & 580 & \\
\hline 1969 & $08-07-69$ & 913 & & 1979 & $08-15-79$ & 310 & \\
\hline 1970 & $07-21-70$ & 1,150 & & 1980 & $08-13-80$ & 271 & \\
\hline 1971 & $08-08-71$ & 760 & & 1984 & $10-01-83$ & ${ }^{3} 820$ & HP \\
\hline 1972 & $09-12-72$ & 365 & & & & & \\
\hline
\end{tabular}

${ }^{1}$ Highest since 1928.

${ }^{2}$ Highest since 1955.

${ }^{3}$ Highest since 1980 .

Magnitude and probability of instantaneous peak flow based on period of record

\begin{tabular}{|c|c|c|c|c|c|}
\hline \multicolumn{6}{|c|}{$\begin{array}{l}\text { Discharge, in } \mathrm{ft}^{3} / \mathrm{s} \text {, for indicated recurrence interval } \\
\text { in years, and exceedance probablility, in percent }\end{array}$} \\
\hline 2 & 5 & 10 & 25 & 50 & 100 \\
\hline $50 \%$ & $20 \%$ & $10 \%$ & $4 \%$ & $2 \%$ & $1 \%$ \\
\hline --. & -.- & --. & -- &.-- & -.. \\
\hline Weighted skew & $(\log s)=$ & $\cdots$ & & & \\
\hline Mean & $(\log s)=$ & -.. & & & \\
\hline Standard dev. & $(\log s)=$ & -- & & & \\
\hline
\end{tabular}

Basin characteristics

\begin{tabular}{|c|c|c|c|c|c|c|c|}
\hline \multirow[b]{2}{*}{$\begin{array}{c}\text { Main } \\
\text { channel } \\
\text { slope } \\
(\mathrm{ft} / \mathrm{mi})\end{array}$} & \multirow[b]{2}{*}{$\begin{array}{l}\text { Stream } \\
\text { length } \\
\text { (mi) }\end{array}$} & \multirow[b]{2}{*}{$\begin{array}{c}\text { Mean } \\
\text { basin } \\
\text { elevation } \\
\text { (ft) }\end{array}$} & \multirow[b]{2}{*}{$\begin{array}{l}\text { Forested } \\
\text { area } \\
\text { (percent) }\end{array}$} & \multirow[b]{2}{*}{$\begin{array}{c}\text { Soil } \\
\text { index }\end{array}$} & \multirow[b]{2}{*}{$\begin{array}{c}\text { Mean } \\
\text { annual } \\
\text { precipitation } \\
\text { (in) }\end{array}$} & \multicolumn{2}{|c|}{ Rainfall intensity, 24-hour } \\
\hline & & & & & & $\begin{array}{l}\text { 2-year } \\
\text { (in) }\end{array}$ & $\begin{array}{l}\text { 50-year } \\
\text { (in) }\end{array}$ \\
\hline 40.4 & 8.8 & 2,770 & 0.0 & 3.0 & 11.6 & 1.9 & 4.3 \\
\hline
\end{tabular}


09482200 FLATO WASH NEAR SAHUARITA, AZ--Continued

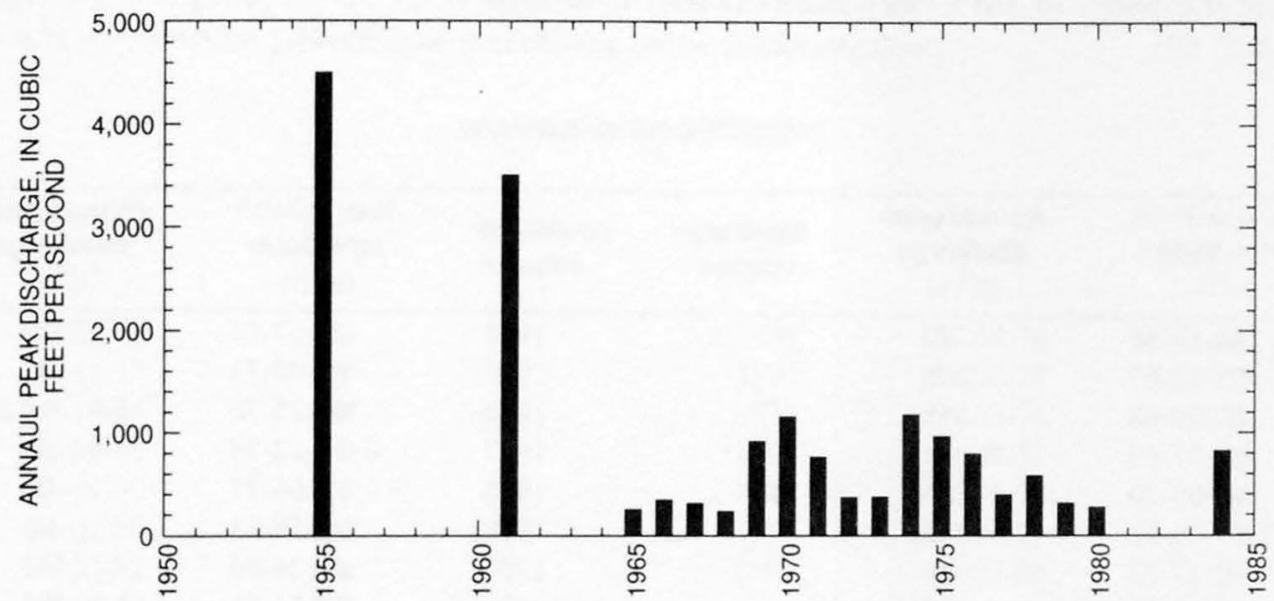


09482330 PUMPING WASH NEAR VAIL, AZ

LOCATION.--Lat $32^{\circ} 04^{\prime} 10^{\prime \prime}$, long $110^{\circ} 48^{\prime} 23^{\prime \prime}, \mathrm{SW}^{1} /{ }_{4} \mathrm{NW}^{1} / 4 \mathrm{sec} .3$, T.16 S., R.15 E., Pima County, Hydrologic Unit 15050301 , at road to pumping station $1.1 \mathrm{mi}$ south of Interstate 10 , and $5.7 \mathrm{mi}$ west of Vail.

DRAINAGE AREA.--0.81 $\mathrm{mi}^{2}$.

Annual peak discharges

\begin{tabular}{ccccccc}
\hline $\begin{array}{c}\text { Water } \\
\text { year }\end{array}$ & Date & $\begin{array}{c}\text { Annual peak } \\
\text { discharge } \\
\left(\mathrm{ft}^{3} / \mathbf{s}\right)\end{array}$ & $\begin{array}{c}\text { Discharge } \\
\text { codes }\end{array}$ & $\begin{array}{c}\text { Water } \\
\text { year }\end{array}$ & $\begin{array}{c}\text { Annual peak } \\
\text { discharge } \\
\left(\mathrm{ft}^{3} / \mathbf{s}\right)\end{array}$ & $\begin{array}{c}\text { Discharge } \\
\text { codes }\end{array}$ \\
\hline 1966 & $08-16-66$ & 90 & & 1974 & $07-07-74$ & 210 \\
1967 & $07-17-67$ & 205 & & 1975 & $07-00-75$ & 43 \\
1968 & $00-00-68$ & 135 & 33 & 1976 & $09-25-76$ & 9.0 \\
1969 & $08-07-69$ & 220 & & 1977 & $10-22-76$ & 67 \\
1970 & $08-00-70$ & 337 & 1978 & $10-06-77$ & 90 \\
1971 & $07-00-71$ & 30 & & 1979 & $12-18-78$ & 41 \\
1972 & $07-17-72$ & 142 & & 1980 & $08-24-80$ & 190 \\
1973 & $10-05-72$ & & & 1981 & $07-25-81$ & 195 \\
\hline
\end{tabular}

Magnitude and probability of instantaneous peak flow based on period of record 1966-81

\begin{tabular}{|c|c|c|c|c|c|}
\hline \multicolumn{6}{|c|}{$\begin{array}{l}\text { Discharge, in } \mathrm{ft}^{3} / \mathrm{s} \text {, for indicated recurrence interval } \\
\text { in years, and exceedance probablility, in percent }\end{array}$} \\
\hline 2 & 5 & 10 & 25 & $50 t$ & $100 \dagger$ \\
\hline $50 \%$ & $20 \%$ & $10 \%$ & $4 \%$ & $2 \%$ & $1 \%$ \\
\hline 102 & 198 & 274 & 382 & 469 & 560 \\
\hline Weighted skew & $(\log s)=$ & -0.31 & & & \\
\hline Mean & $(\log s)=$ & 1.99 & & & \\
\hline Standard dev. & $(\log s)=$ & 0.36 & & & \\
\hline
\end{tabular}

7 Reliability of values in column is uncertain, and potential errors are large.

Basin characteristics

\begin{tabular}{cccccccc}
\hline $\begin{array}{c}\text { Main } \\
\text { channel } \\
\text { slope } \\
(\mathrm{ft} / \mathrm{mi})\end{array}$ & $\begin{array}{c}\text { Stream } \\
\text { length } \\
\text { (mi) }\end{array}$ & $\begin{array}{c}\text { Mean } \\
\text { basin } \\
\text { elevation } \\
(\mathrm{ft})\end{array}$ & $\begin{array}{c}\text { Forested } \\
\text { area } \\
\text { (percent) }\end{array}$ & $\begin{array}{c}\text { Soil } \\
\text { index }\end{array}$ & $\begin{array}{c}\text { Mean } \\
\text { annual } \\
\text { precipitation } \\
\text { (in) }\end{array}$ & $\begin{array}{c}\text { Rainfall intensity, 24-hour } \\
\text { (in) }\end{array}$ & $\begin{array}{c}50 \text {-year } \\
\text { (in) }\end{array}$ \\
\hline 57.7 & 2.3 & 3,010 & 0.0 & 1.0 & 11.2 & 1.8 \\
\hline
\end{tabular}

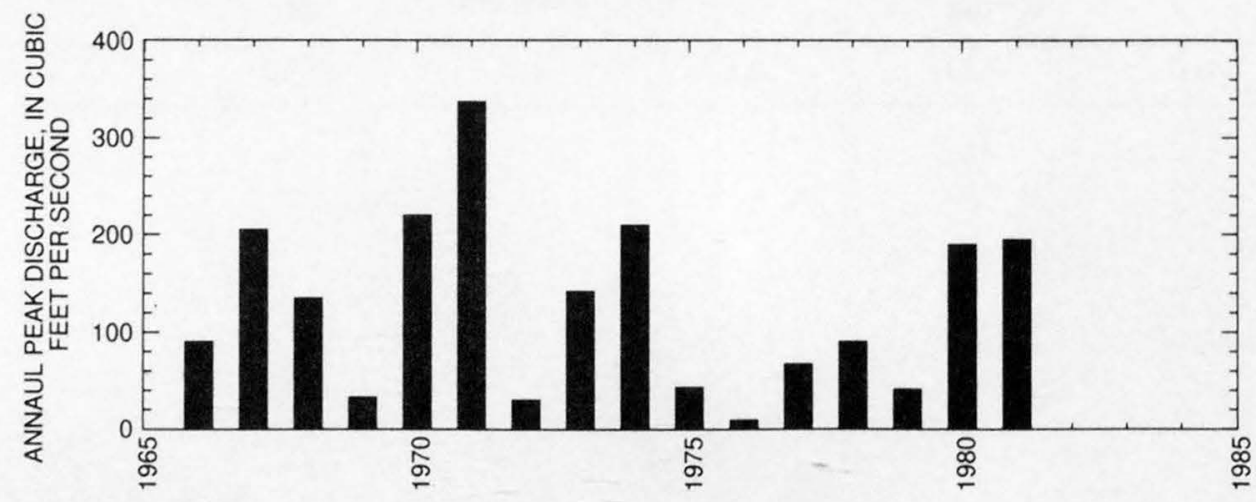


09482350 SOUTH FORK AIRPORT WASH NEAR TUCSON, AZ

LOCATION.--Lat 32 06'00", long 110 54'30", SE $\frac{1}{4} \mathrm{NE}^{1 / 4}$ sec.28, T.15 S., R.14 E., Pima County, Hydrologic Unit 15050301 , at Hughes Access

Road, $3.25 \mathrm{mi}$ south of U.S. Highway 80 , and 1.5 mi southeast of Tucson city limits.

DRAINAGE AREA.--9.78 $\mathrm{mi}^{2}$, of which $5.40 \mathrm{mi}^{2}$ is noncontributing and/or distributary flow.

Annual peak discharges

\begin{tabular}{|c|c|c|c|c|c|c|c|}
\hline $\begin{array}{l}\text { Water } \\
\text { year }\end{array}$ & Date & $\begin{array}{c}\text { Annual peak } \\
\text { discharge } \\
\left(\mathrm{ft}^{3} / \mathrm{s}\right)\end{array}$ & $\begin{array}{c}\text { Discharge } \\
\text { codes }\end{array}$ & $\begin{array}{l}\text { Water } \\
\text { year }\end{array}$ & Date & $\begin{array}{c}\text { Annual peak } \\
\text { discharge } \\
\left(\mathrm{ft}^{3} / \mathrm{s}\right)\end{array}$ & $\begin{array}{c}\text { Discharge } \\
\text { codes }\end{array}$ \\
\hline 1966 & $07-28-66$ & 225 & & 1974 & $07-08-74$ & ${ }^{1} 1,890$ & \\
\hline 1967 & $07-17-67$ & 450 & & 1975 & $07-00-75$ & 10 & LT \\
\hline 1968 & $08-19-68$ & 180 & & 1976 & $09-25-76$ & 980 & \\
\hline 1969 & $07-28-69$ & 8.0 & & 1977 & $01-29-77$ & 69 & \\
\hline 1970 & $07-19-70$ & 216 & & 1978 & $01-15-78$ & 59 & \\
\hline 1971 & $10-02-70$ & 480 & & 1979 & $00-00-79$ & 0.0 & \\
\hline 1972 & $08-12-72$ & 180 & & 1980 & $09-07-80$ & 620 & \\
\hline 1973 & $10-19-72$ & 250 & & & & & \\
\hline
\end{tabular}

THighest since 1956.

Magnitude and probability of instantaneous peak flow based on period of record

\begin{tabular}{|c|c|c|c|c|c|}
\hline \multicolumn{6}{|c|}{$\begin{array}{l}\text { Discharge, in } \mathrm{ft}^{3} / \mathrm{s} \text {, for indicated recurrence interval } \\
\text { in years, and exceedance probablility, in percent }\end{array}$} \\
\hline 2 & 5 & 10 & 25 & 50 & 100 \\
\hline $50 \%$ & $20 \%$ & $10 \%$ & $4 \%$ & $2 \%$ & $1 \%$ \\
\hline$\ldots$ & -.. & -.. & $\cdots$ & -- & -.. \\
\hline Weighted skew & $(\log s)=$ & $\cdots$ & & & \\
\hline Mean & $(\log s)=$ & -- & & & \\
\hline Standard dev. & $(\log s)=$ & $\cdots$ & & & \\
\hline
\end{tabular}

Basin characteristics

\begin{tabular}{|c|c|c|c|c|c|c|c|}
\hline \multirow[b]{2}{*}{$\begin{array}{l}\text { Main } \\
\text { channel } \\
\text { slope } \\
(\mathrm{ft} / \mathrm{mi})\end{array}$} & \multirow[b]{2}{*}{$\begin{array}{c}\text { Stream } \\
\text { length } \\
(\mathrm{mi})\end{array}$} & \multirow[b]{2}{*}{$\begin{array}{c}\text { Mean } \\
\text { basin } \\
\text { elevation } \\
(\mathrm{ft})\end{array}$} & \multirow[b]{2}{*}{$\begin{array}{l}\text { Forested } \\
\text { area } \\
\text { (percent) }\end{array}$} & \multirow[b]{2}{*}{$\begin{array}{c}\text { Soil } \\
\text { index }\end{array}$} & \multirow[b]{2}{*}{$\begin{array}{c}\text { Mean } \\
\text { annual } \\
\text { precipitation } \\
\text { (in) }\end{array}$} & \multicolumn{2}{|c|}{ Rainfall intensity, 24-hour } \\
\hline & & & & & & $\begin{array}{l}\text { 2-year } \\
\text { (in) }\end{array}$ & $\begin{array}{l}\text { 50-year } \\
\text { (in) }\end{array}$ \\
\hline 36.5 & 3.1 & 2,740 & 0.0 & 1.0 & 11.0 & 1.8 & 3.9 \\
\hline
\end{tabular}

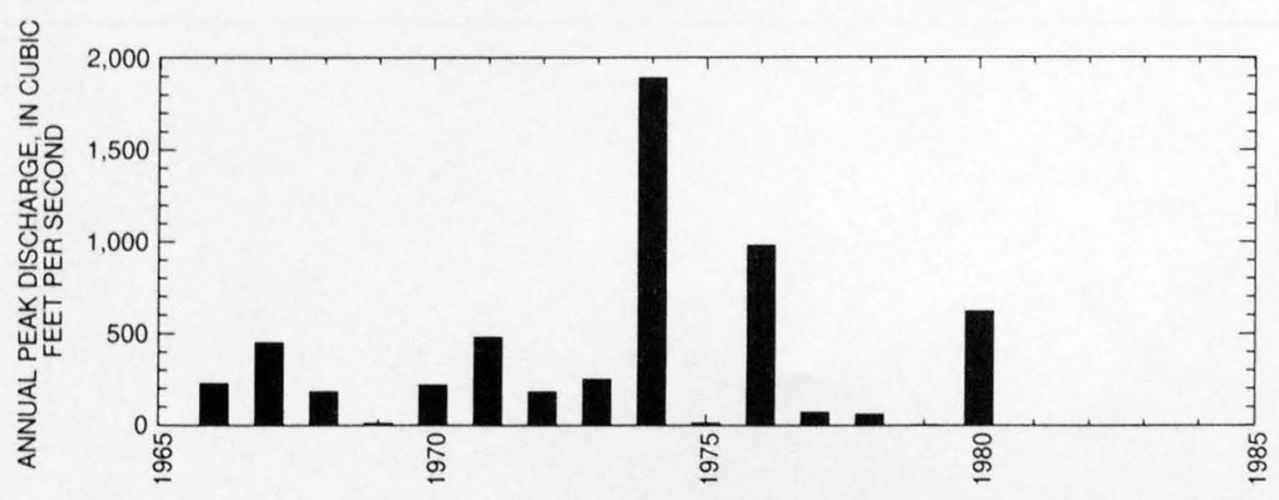




\section{NORTH FORK AIRPORT WASH NEAR TUCSON, AZ}

LOCATION.--Lat 32 06'40", long 110 54'30", NE $1 / 4 \mathrm{SE}^{1} / 4$ sec.21, T.15 S., R.14 E., Pima County, Hydrologic Unit 15050301 , at Hughes Access Road, 2.5 mi south of U.S. Highway 80 , and 1 mi east of Tucson city limits.

DRAINAGE AREA.--6.65 $\mathrm{mi}^{2}$, of which $1.37 \mathrm{mi}^{2}$ is noncontributing and/or distributary flow.

Annual peak discharges

\begin{tabular}{|c|c|c|c|c|c|c|c|}
\hline $\begin{array}{l}\text { Water } \\
\text { year }\end{array}$ & Date & $\begin{array}{c}\text { Annual peak } \\
\text { discharge } \\
\left(\mathrm{ft}^{3} / \mathrm{s}\right)\end{array}$ & $\begin{array}{c}\text { Discharge } \\
\text { codes }\end{array}$ & $\begin{array}{l}\text { Water } \\
\text { year }\end{array}$ & Date & $\begin{array}{c}\text { Annual peak } \\
\text { discharge } \\
\left(\mathrm{ft}^{3} / \mathrm{s}\right)\end{array}$ & $\begin{array}{c}\text { Discharge } \\
\text { codes }\end{array}$ \\
\hline 1961 & $08-22-61$ & ${ }^{1} 1,350$ & HP & 1973 & $10-19-72$ & 230 & \\
\hline 1965 & $00-00-65$ & ${ }^{2} 650$ & & 1974 & $07-08-74$ & ${ }^{1} 1,030$ & \\
\hline 1966 & $07-28-66$ & 100 & ES & 1975 & $07-12-75$ & 10 & LT \\
\hline 1967 & $07-17-67$ & 30 & ES & 1976 & $09-25-76$ & 700 & \\
\hline 1968 & $08-19-68$ & 370 & & 1977 & $11-11-76$ & 70 & \\
\hline 1969 & $09-15-69$ & 40 & & 1978 & $01-15-78$ & 115 & \\
\hline 1970 & $07-19-70$ & 190 & & 1979 & $00-00-79$ & 0 & \\
\hline 1971 & $10-02-70$ & 450 & & 1980 & $09-07-80$ & 460 & \\
\hline 1972 & $08-12-72$ & 130 & & & & & \\
\hline
\end{tabular}

${ }^{1}$ Highest since 1956.

${ }^{2}$ Highest since 1961.

Magnitude and probability of instantaneous peak flow based on period of record

\begin{tabular}{|c|c|c|c|c|c|}
\hline \multicolumn{6}{|c|}{$\begin{array}{l}\text { Discharge, in } \mathrm{ft}^{3} / \mathrm{s} \text {, for indicated recurrence interval } \\
\text { in years, and exceedance probablility, in percent }\end{array}$} \\
\hline 2 & 5 & 10 & 25 & 50 & 100 \\
\hline $50 \%$ & $20 \%$ & $10 \%$ & $4 \%$ & $2 \%$ & $1 \%$ \\
\hline -.- & -- & -- &.-- & $-\cdots$ & -.. \\
\hline Weighted skew & $(\log s)=$ & -- & & & \\
\hline Mean & $(\log s)=$ & -- & & & \\
\hline Standard dev. & $(\log s)=$ & -- & & & \\
\hline
\end{tabular}

Basin characteristics

\begin{tabular}{cccccccc}
\hline $\begin{array}{c}\text { Main } \\
\text { channel } \\
\text { slope } \\
\text { (ft/mi) }\end{array}$ & $\begin{array}{c}\text { Stream } \\
\text { length } \\
\text { (mi) }\end{array}$ & $\begin{array}{c}\text { Mean } \\
\text { basin } \\
\text { elevation } \\
\text { (ft) }\end{array}$ & $\begin{array}{c}\text { Forested } \\
\text { area } \\
\text { (percent) }\end{array}$ & $\begin{array}{c}\text { Soil } \\
\text { index }\end{array}$ & $\begin{array}{c}\text { Mean } \\
\text { annual } \\
\text { precipitation } \\
\text { (in) }\end{array}$ & $\begin{array}{c}\text { Rainfall intensity, 24-hour } \\
\text { (in) }\end{array}$ & $\begin{array}{c}50 \text {-year } \\
\text { (in) }\end{array}$ \\
\hline 40.6 & 4.6 & 2,780 & 3.0 & 1.0 & 10.8 & 1.8 \\
\hline
\end{tabular}


09482370 NORTH FORK AIRPORT WASH NEAR TUCSON, AZ--Continued

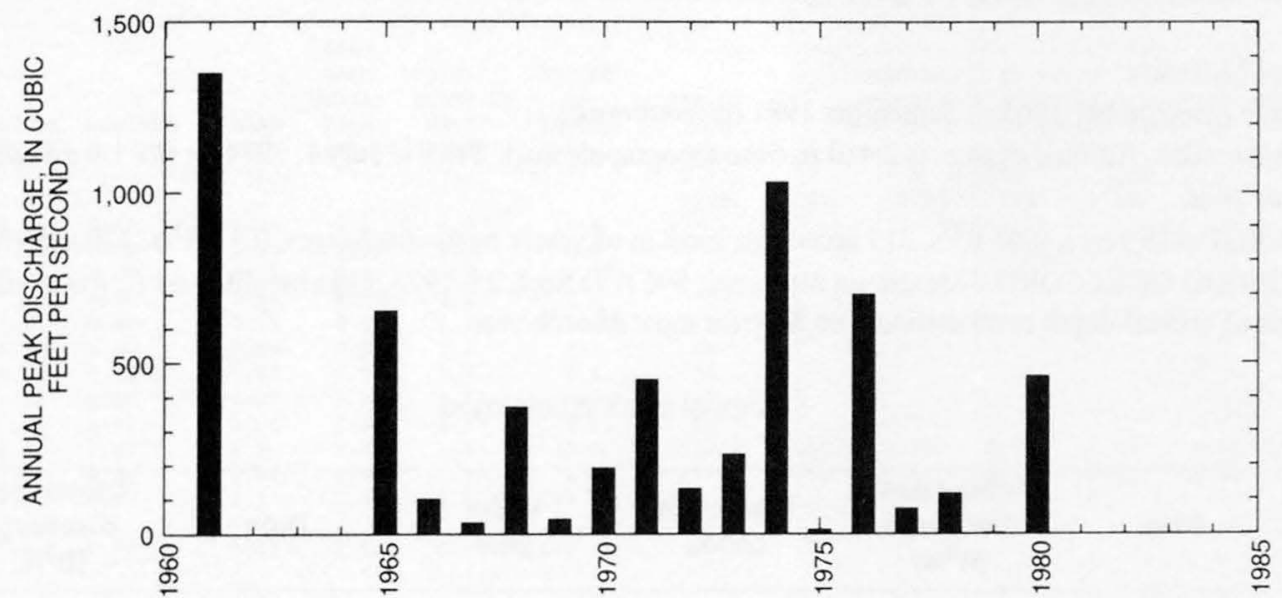


LOCATION.--Lat 32.09'09", long 110 58'52", in NE $1 / 4 \mathrm{SE}^{1} / 4$ sec.2, T.15 S., R.13 E., Pima County, Hydrologic Unit 15050301 , $25 \mathrm{ft}$ upstream from Santa Clara Avenue, $0.7 \mathrm{mi}$ upstream from mouth, $4.3 \mathrm{mi}$ downstream from confluence of North and South Forks, and 4.9 mi south of city hall in Tucson.

DRAINAGE AREA.--23.0 $\mathrm{mi}^{2}$.

PERIOD OF RECORD.--September 1965 to September 1981 (discontinued).

GAGE.--Water-stage recorder. Altitude of gage is 2,460 ft, from topographic map. Prior to July 1, 1974, at site $1.8 \mathrm{mi}$ upstream at different datum REMARKS.--Records poor.

AVERAGE DISCHARGE.--16 years, $0.43 \mathrm{ft}^{3} / \mathrm{s}, 312$ acre-ft/yr; median of yearly mean discharges, $0.37 \mathrm{ft}^{3} / \mathrm{s}, 270 \mathrm{acre}-\mathrm{ft} / \mathrm{yr}$.

EXTREMES FOR PERIOD OF RECORD.--Maximum discharge, $896 \mathrm{ft}^{3} / \mathrm{s}$ Sept. 25,1976 , gage height, $2.64 \mathrm{ft}$, from rating curve extended above

$150 \mathrm{ft}^{3} / \mathrm{s}$ on basis of critical-depth computations; no flow for most of each year.

Annual peak discharges

\begin{tabular}{|c|c|c|c|c|c|c|c|}
\hline $\begin{array}{l}\text { Water } \\
\text { year }\end{array}$ & Date & $\begin{array}{c}\text { Annual peak } \\
\text { discharge } \\
\left(\mathrm{ft}^{3} / \mathrm{s}\right)\end{array}$ & $\begin{array}{l}\text { Discharge } \\
\text { codes }\end{array}$ & $\begin{array}{l}\text { Water } \\
\text { year }\end{array}$ & Date & $\begin{array}{c}\text { Annual peak } \\
\text { discharge } \\
\left(\mathrm{ft}^{3} / \mathrm{s}\right)\end{array}$ & $\begin{array}{c}\text { Discharge } \\
\text { codes }\end{array}$ \\
\hline 1966 & $09-11-66$ & 322 & $\mathrm{C}$ & 1975 & $07-12-75$ & 377 & $\mathrm{C}$ \\
\hline 1967 & $07-17-67$ & 106 & $\mathrm{C}$ & 1976 & $09-25-76$ & 896 & $\mathrm{C}$ \\
\hline 1968 & $08-20-68$ & 385 & $\mathrm{C}$ & 1977 & $09-09-77$ & 304 & $\mathrm{C}$ \\
\hline 1969 & $08-28-69$ & 118 & $\mathrm{C}$ & 1978 & $09-21-78$ & 405 & $\mathrm{C}$ \\
\hline 1970 & $07-20-70$ & 823 & $\mathrm{C}$ & 1979 & $10-21-78$ & 279 & $\mathrm{C}$ \\
\hline 1971 & $10-02-70$ & 549 & $\mathrm{C}$ & 1980 & $09-07-80$ & 378 & $\mathrm{C}$ \\
\hline 1972 & $07-16-72$ & 310 & $\mathrm{C}$ & 1981 & $07-27-81$ & 385 & $\mathrm{C}$ \\
\hline 1973 & $10-19-72$ & 159 & $\mathrm{C}$ & 1984 & $10-01-83$ & 2,900 & $\mathrm{HP}, \mathrm{C}$ \\
\hline 1974 & $07-07-74$ & 689 & $\mathrm{C}$ & 1988 & $08-23-88$ & 1,200 & C \\
\hline
\end{tabular}

Discharge rating table developed April 1987

\begin{tabular}{cccc}
\hline $\begin{array}{c}\text { Gage height } \\
(\mathrm{ft})\end{array}$ & $\begin{array}{c}\text { Discharge } \\
\left(\mathrm{ft}^{3} / \mathbf{s}\right)\end{array}$ & $\begin{array}{c}\text { Gage height } \\
(\mathrm{ft})\end{array}$ & $\begin{array}{c}\text { Discharge } \\
\left(\mathrm{ft}^{3} / \mathbf{s}\right)\end{array}$ \\
\hline 1.0 & 2.4 & 2.0 & 372 \\
1.2 & 16 & 2.2 & 528 \\
1.4 & 69 & 2.4 & 680 \\
1.6 & 146 & 2.6 & 860 \\
1.8 & 250 & & \\
\hline
\end{tabular}

\section{Basin characteristics}

\begin{tabular}{ccccccc}
\hline $\begin{array}{c}\text { Main } \\
\text { channel } \\
\text { slope } \\
(\mathrm{ft} / \mathrm{mi})\end{array}$ & $\begin{array}{c}\text { Stream } \\
\text { length } \\
\text { (mi) }\end{array}$ & $\begin{array}{c}\text { Mean } \\
\text { basin } \\
\text { elevation } \\
(\mathrm{ft})\end{array}$ & $\begin{array}{c}\text { Forested } \\
\text { area } \\
\text { (percent) }\end{array}$ & $\begin{array}{c}\text { Soil } \\
\text { index }\end{array}$ & $\begin{array}{c}\text { Mean } \\
\text { annual } \\
\text { precipitation } \\
\text { (in) }\end{array}$ & $\begin{array}{c}\text { R-year } \\
\text { (in) }\end{array}$ \\
\hline 37.0 & 10.8 & 2,700 & 1.1 & 1.0 & 10.8 & $\begin{array}{c}50 \text {-year } \\
\text { (in) }\end{array}$ \\
\hline
\end{tabular}


09482400 AIRPORT WASH AT TUCSON, AZ--Continued

MEAN MONTHLY AND ANNUAL DISCHARGES $1966 \cdot 81$

\begin{tabular}{|c|c|c|c|c|c|c|}
\hline MONTH & $\begin{array}{l}\text { MAXIMUM } \\
\text { (FT3/S) }\end{array}$ & $\begin{array}{l}\text { MINIMUM } \\
\text { (FT3/S) }\end{array}$ & $\begin{array}{c}\text { MEAN } \\
(\mathrm{FT} 3 / \mathrm{S})\end{array}$ & $\begin{array}{l}\text { STAN- } \\
\text { DARD } \\
\text { DEVIA- } \\
\text { TION } \\
\text { (FT3/S) }\end{array}$ & $\begin{array}{l}\text { COEFFI- } \\
\text { CIENT OF } \\
\text { VARI- } \\
\text { ATION }\end{array}$ & $\begin{array}{c}\text { PERCENT } \\
\text { OF } \\
\text { ANNUAL } \\
\text { RUNOFF }\end{array}$ \\
\hline OCTOBER & 3.4 & 0.00 & 0.54 & 1.1 & 2.0 & 10.6 \\
\hline NOVEMBER & 0.67 & 0.00 & 0.10 & 0.20 & 2.0 & 2.0 \\
\hline DECEMBER & 0.97 & 0.00 & 0.19 & 0.32 & 1.7 & 3.7 \\
\hline JANUARY & 0.53 & 0.00 & 0.09 & 0.18 & 2.0 & 1.7 \\
\hline FEBRUARY & 1.4 & 0.00 & 0.13 & 0.35 & 2.7 & 2.6 \\
\hline MARCH & 0.41 & 0.00 & 0.06 & 0.12 & 1.8 & 1.2 \\
\hline APRIL & 0.24 & 0.00 & 0.02 & 0.06 & 3.0 & 0.4 \\
\hline MAY & 0.05 & 0.00 & 0.00 & 0.01 & 3.4 & 0.1 \\
\hline JUNE & 0.19 & 0.00 & 0.01 & 0.05 & 3.8 & 0.2 \\
\hline JULY & 9.4 & 0.00 & 1.9 & 2.7 & 1.4 & 38.0 \\
\hline AUGUST & 3.0 & 0.00 & 0.81 & 0.80 & 0.98 & 16.0 \\
\hline SEPTEMBER & 6.0 & 0.00 & 1.2 & 1.8 & 1.5 & 23.5 \\
\hline ANNUAL & 1.1 & 0.08 & 0.43 & 0.31 & 0.72 & 100 \\
\hline
\end{tabular}

MAGNITUDE AND PROBABILITY OF INSTANTANEOUS PEAK FLOW BASED ON PERIOD OF RECORD $1966 \cdot 81,1984$

DISCHARGE, IN FT3/S, FOR INDICATED RECURRENCE INTERVAL IN YEARS, AND EXCEEDANCE PROBABILITY, IN PERCENT

\begin{tabular}{|c|c|c|c|c|c|}
\hline 2 & 5 & 10 & 25 & $50 \#$ & $100 \#$ \\
\hline 508 & 208 & 108 & 48 & 28 & 18 \\
\hline 403 & 666 & 889 & 1,230 & 1,540 & 1,900 \\
\hline WEIGHTED & SKEW & $($ LOGS $)=$ & 0.47 & & \\
\hline MEAN & & (LOGS) $=$ & 2.63 & & \\
\hline STANDARD & DEV. & $($ LOGS) $=$ & 0.25 & & \\
\hline
\end{tabular}

MAGNITUDE AND PROBABILITY OF ANNUAL LOW FLOW BASED ON PERIOD OF RECORD 1967-81

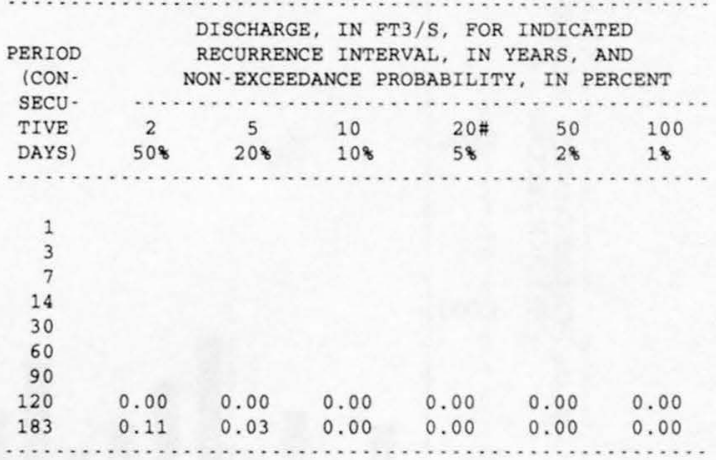

MAGNITUDE AND PROBABILITY OF ANNUAL HIGH FLOW BASED ON PERIOD OF RECORD $1966 \cdot 81$

\begin{tabular}{|c|c|c|c|c|c|c|}
\hline $\begin{array}{l}\text { PERIOD } \\
\text { (CON- }\end{array}$ & & \multicolumn{5}{|c|}{$\begin{array}{l}\text { DISCHARGE, IN FT3/S, FOR INDICATED } \\
\text { RECURRENCE INTERVAL, IN YEARS, AND }\end{array}$} \\
\hline SECU- & & $\ldots \ldots \ldots$ & & & $\ldots \ldots \ldots$ & \\
\hline TIVE & 2 & 5 & 10 & $25 \#$ & $50 \#$ & $100 \#$ \\
\hline DAYS) & 508 & 208 & 108 & 48 & 28 & 18 \\
\hline 1 & 41 & 81 & 118 & 176 & 229 & 291 \\
\hline 3 & 17 & 38 & 58 & 92 & 124 & 162 \\
\hline 7 & 7.8 & 17 & 27 & 42 & 57 & 74 \\
\hline 15 & 3.9 & 8.7 & 13 & 20 & 27 & 35 \\
\hline 30 & 2.5 & 5.3 & 7.8 & 12 & 15 & 19 \\
\hline 60 & 1.5 & 3.1 & 4.6 & 7.0 & 9.1 & 11 \\
\hline 90 & 1,0 & 2.1 & 3.1 & 4.6 & 6.0 & 7.6 \\
\hline
\end{tabular}

DURATION TABLE OF DAILY MEAN FLOW FOR PERIOD OF RECORD 1966.81

DISCHARGE, IN FT3/S, WHICH WAS EQUALED OR EXCEEDED FOR INDICATED PERCENT OF TIME

\begin{tabular}{|c|c|c|c|c|c|c|c|c|c|c|c|c|c|c|c|c|}
\hline 18 & 58 & 108 & 158 & 208 & 308 & 408 & 508 & 608 & 708 & 808 & 908 & 958 & 988 & 998 & 99.58 & 99.98 \\
\hline 9.3 & 0.18 & 0.00 & 0.00 & 0.00 & 0.00 & 0.00 & 0.00 & 0.00 & 0.00 & 0.00 & 0.00 & 0.00 & 0.00 & 0.00 & 0.00 & 0.00 \\
\hline
\end{tabular}

\# Reliability of values in column is uncertain, and potential errors are large. 
09482400 AIRPORT WASH AT TUCSON, AZ--Continued
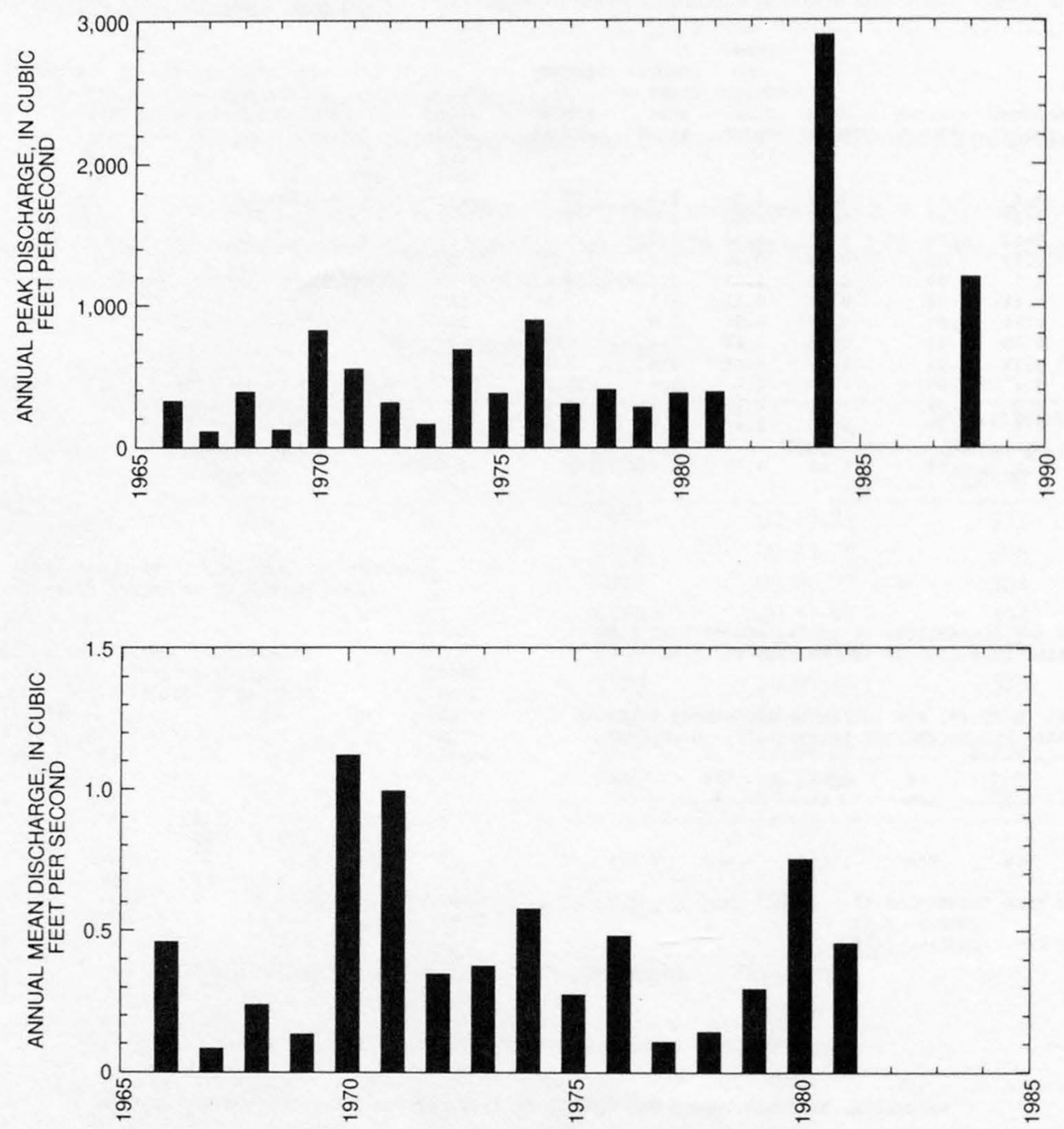
GILA RIVER BASIN

427

09482400 AIRPORT WASH AT TUCSON, AZ--Continued
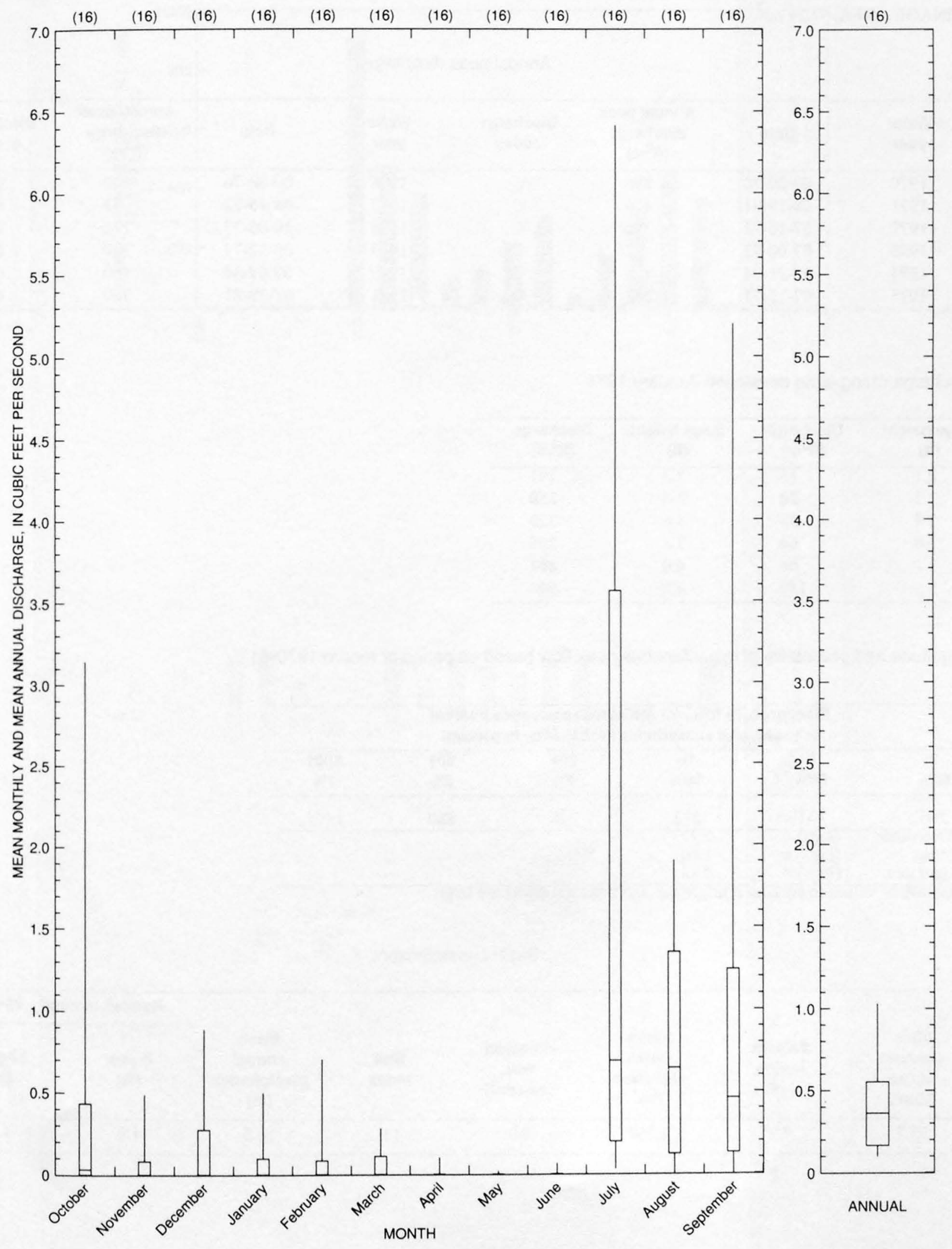


\section{RODEO WASH AT TUCSON, AZ}

LOCATION.--Lat $32^{\circ} 10^{\prime} 20^{\prime \prime}$, long $110^{\circ} 58^{\prime} 35^{\prime \prime}, \mathrm{SW}^{1} /{ }_{4} \mathrm{NW}^{1} / 4$ sec.36, T.14 S., R.13 E., Pima County, Hydrologic Unit 15050301, at South 12 th Ave. nue, $0.8 \mathrm{mi}$ above mouth in Tucson city limits.

DRAINAGE AREA.--7.24 $\mathrm{mi}^{2}$.

Annual peak discharges

\begin{tabular}{|c|c|c|c|c|c|c|c|}
\hline $\begin{array}{l}\text { Water } \\
\text { year }\end{array}$ & Date & $\begin{array}{c}\text { Annual peak } \\
\text { discharge } \\
\left(\mathrm{ft}^{3} / \mathrm{s}\right)\end{array}$ & $\begin{array}{c}\text { Discharge } \\
\text { codes }\end{array}$ & $\begin{array}{l}\text { Water } \\
\text { year }\end{array}$ & Date & $\begin{array}{c}\text { Annual peak } \\
\text { discharge } \\
\left(\mathrm{ft}^{3} / \mathrm{s}\right)\end{array}$ & $\begin{array}{l}\text { Discharge } \\
\text { codes }\end{array}$ \\
\hline 1970 & $07-20-70$ & 898 & $\mathrm{C}$ & 1976 & $00-00-76$ & 300 & $\mathrm{C}$ \\
\hline 1971 & $08-19-71$ & 476 & $\mathrm{C}$ & 1977 & $08-15-77$ & 32 & $\mathrm{C}$ \\
\hline 1972 & $07-16-72$ & 380 & $\mathrm{C}$ & 1978 & $10-06-77$ & 170 & $\mathrm{C}$ \\
\hline 1973 & $07-00-73$ & 97 & $\mathrm{C}$ & 1979 & $08-12-79$ & 320 & $\mathrm{C}$ \\
\hline 1974 & $07-21-74$ & 125 & $\mathrm{C}$ & 1980 & $09-07-80$ & 460 & $\mathrm{C}$ \\
\hline 1975 & $07-12-75$ & 212 & $\mathrm{C}$ & 1981 & $07-29-81$ & 360 & $\mathrm{C}$ \\
\hline
\end{tabular}

Discharge rating table developed January 1971

\begin{tabular}{crcc}
\hline $\begin{array}{c}\text { Gage height } \\
(\mathbf{f t})\end{array}$ & $\begin{array}{c}\text { Discharge } \\
\left(\mathbf{f t}^{\mathbf{3}} \mathbf{s}\right)\end{array}$ & $\begin{array}{c}\text { Gage height } \\
(\mathbf{f t})\end{array}$ & $\begin{array}{c}\text { Discharge } \\
\left(\mathrm{ft}^{\mathbf{3}} \mathbf{s}\right)\end{array}$ \\
\hline 2.1 & 13 & 3.2 & 191 \\
2.2 & 20 & 3.4 & 250 \\
2.4 & 38 & 3.6 & 320 \\
2.6 & 64 & 3.8 & 398 \\
2.8 & 96 & 4.0 & 488 \\
3.0 & 139 & 4.2 & 590 \\
\hline
\end{tabular}

Magnitude and probability of instantaneous peak flow based on period of record 1970-81

\begin{tabular}{|c|c|c|c|c|c|}
\hline \multicolumn{6}{|c|}{$\begin{array}{l}\text { Discharge, in } \mathrm{ft}^{3} / \mathrm{s} \text {, for indicated recurrence interval } \\
\text { in years, and exceedance probablility, in percent }\end{array}$} \\
\hline 2 & 5 & 10 & $25 t$ & $50 t$ & $100 t$ \\
\hline $50 \%$ & $20 \%$ & $10 \%$ & $4 \%$ & $2 \%$ & $1 \%$ \\
\hline 260 & 441 & 572 & 746 & 880 & 1,020 \\
\hline Weighted skew & $(\log s)=$ & -0.27 & & & \\
\hline Mean & $(\log s)=$ & 2.40 & & & \\
\hline Standard dev. & $(\log s)=$ & 0.28 & & & \\
\hline
\end{tabular}

$千$ Reliability of values in column is uncertain, and potential errors are large.

Basin characteristics

\begin{tabular}{ccccccc}
\hline $\begin{array}{c}\text { Main } \\
\text { channel } \\
\text { slope } \\
(\mathrm{ft} / \mathrm{mi})\end{array}$ & $\begin{array}{c}\text { Stream } \\
\text { length } \\
\text { (mi) }\end{array}$ & $\begin{array}{c}\text { Mean } \\
\text { basin } \\
\text { elevation } \\
(\mathrm{ft})\end{array}$ & $\begin{array}{c}\text { Forested } \\
\text { area } \\
\text { (percent) }\end{array}$ & $\begin{array}{c}\text { Soil } \\
\text { index }\end{array}$ & $\begin{array}{c}\text { Mean } \\
\text { annual } \\
\text { precipitation } \\
\text { (in) }\end{array}$ & $\begin{array}{c}\text { Rainfall intensity, 24-hour } \\
\text { (in) }\end{array}$ \\
\hline 33.7 & 7.9 & 2,560 & 0.0 & 1.0 & 10.6 & $\begin{array}{c}50 \text {-year } \\
\text { (in) }\end{array}$ \\
\hline
\end{tabular}


09482410 RODEO WASH AT TUCSON, AZ--Continued

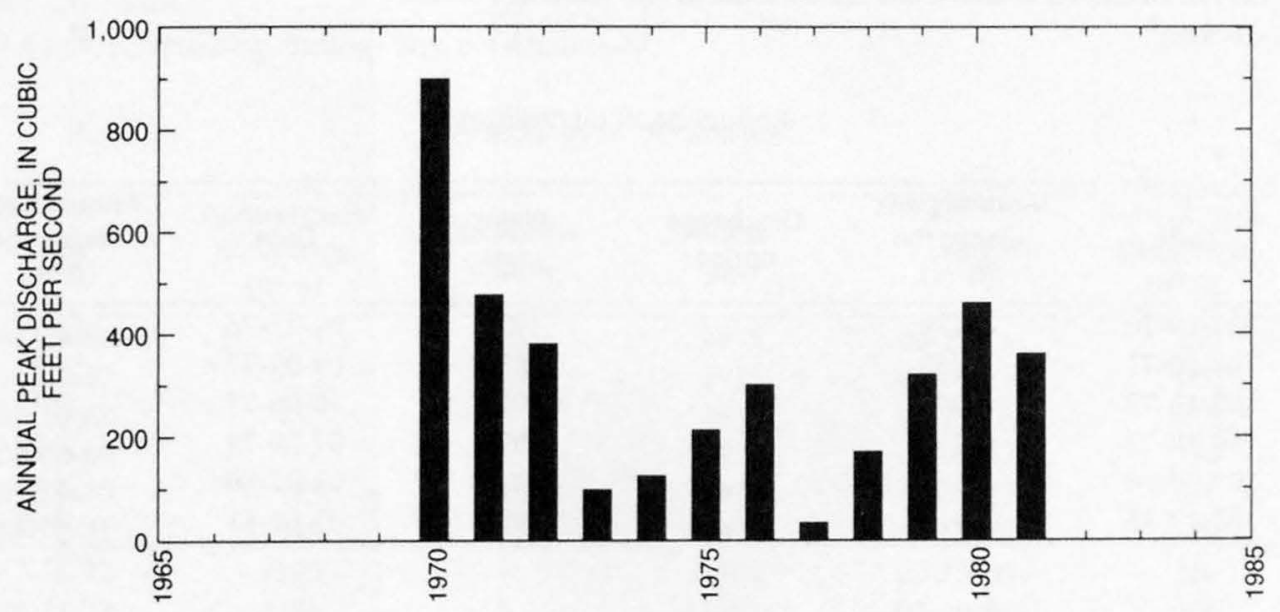




\section{JULIAN WASH AT TUCSON, AZ}

LOCATION.--Lat $32^{\circ} 10^{\prime} 15^{\prime \prime}$, long $110^{\circ} 56^{\prime} 25^{\prime \prime}, \mathrm{SW}^{1} / \mathrm{NW}^{1} / 4 \mathrm{sec} .32$, T.14 S., R.14 E., Pima County, Hydrologic Unit 15050302, 1,600 ft above confluence with Tucson Diversion channel, and $0.5 \mathrm{mi}$ south of Ajo Road in Tucson.

DRAINAGE AREA.--26.5 $\mathrm{mi}^{2}$.

Annual peak discharges

\begin{tabular}{ccccccc}
\hline $\begin{array}{l}\text { Water } \\
\text { year }\end{array}$ & Date & $\begin{array}{c}\text { Annual peak } \\
\text { discharge } \\
\left(\mathbf{f t}^{3} / \mathbf{s}\right)\end{array}$ & $\begin{array}{c}\text { Discharge } \\
\text { codes }\end{array}$ & $\begin{array}{c}\text { Water } \\
\text { year }\end{array}$ & $\begin{array}{c}\text { Annual peak } \\
\text { discharge } \\
\left(\mathrm{ft}^{3} / \mathbf{s}\right)\end{array}$ & $\begin{array}{c}\text { Discharge } \\
\text { codes }\end{array}$ \\
\hline 1970 & $07-19-70$ & 1,270 & & 1976 & $09-25-76$ & 1,050 \\
1971 & $08-20-71$ & 1,050 & & 1977 & $09-09-77$ & 680 \\
1972 & $07-16-72$ & 440 & & 1978 & $10-06-77$ & 395 \\
1973 & $10-19-72$ & 330 & & 1979 & $07-29-79$ & 190 \\
1974 & $07-07-74$ & 130 & & 1980 & $09-07-80$ & 435 \\
1975 & $07-12-75$ & 112 & & 1981 & $03-02-81$ & 400 \\
\hline
\end{tabular}

Magnitude and probability of instantaneous peak flow based on period of record 1970-81

\begin{tabular}{|c|c|c|c|c|c|}
\hline \multicolumn{6}{|c|}{$\begin{array}{l}\text { Discharge, in } \mathrm{ft}^{3} / \mathrm{s} \text {, for indicated recurrence interval } \\
\text { in years, and exceedance probablility, in percent }\end{array}$} \\
\hline 2 & 5 & 10 & $25 \dagger$ & $50 \dagger$ & $100 t$ \\
\hline $50 \%$ & $20 \%$ & $10 \%$ & $4 \%$ & $2 \%$ & $1 \%$ \\
\hline 428 & 817 & 1,130 & 1,570 & 1,930 & 2,310 \\
\hline Weighted skew & $(\log s)=$ & -0.22 & & & \\
\hline Mean & $(\log s)=$ & 2.62 & & & \\
\hline Standard dev. & $(\log s)=$ & 0.34 & & & \\
\hline
\end{tabular}

Basin characteristics

\begin{tabular}{cccccccc}
\hline $\begin{array}{c}\text { Main } \\
\text { channel } \\
\text { slope } \\
(\mathrm{ft} / \mathrm{mi})\end{array}$ & $\begin{array}{c}\text { Stream } \\
\text { length } \\
(\mathrm{mi})\end{array}$ & $\begin{array}{c}\text { Mean } \\
\text { basin } \\
\text { elevation } \\
(\mathrm{ft})\end{array}$ & $\begin{array}{c}\text { Forested } \\
\text { area } \\
\text { (percent) }\end{array}$ & $\begin{array}{c}\text { Soil } \\
\text { index }\end{array}$ & $\begin{array}{c}\text { Mean } \\
\text { annual } \\
\text { precipitation } \\
\text { (in) }\end{array}$ & $\begin{array}{c}\text { Rainfall intensity, 24-hour } \\
\text { (in) }\end{array}$ & $\begin{array}{c}50 \text {-year } \\
\text { (in) }\end{array}$ \\
\hline 38.9 & 17.3 & 2,900 & 1.9 & 1.0 & 11.0 & 1.7 \\
\hline
\end{tabular}

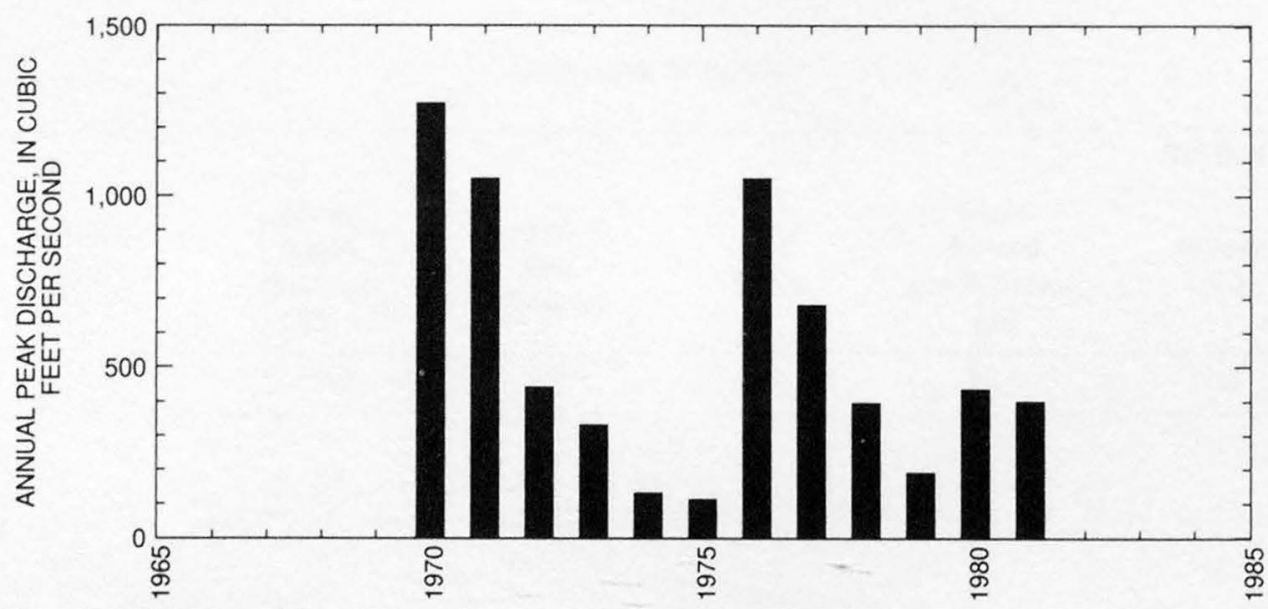


09482450 WEST BRANCH SANTA CRUZ RIVER AT TUCSON, AZ

LOCATION.--Lat $32^{\circ} 08^{\prime} 00^{\prime \prime}$, long $111^{\circ} 00^{\prime} 30^{\prime \prime}, \mathrm{NE}^{1} / 4^{\mathrm{NE}}{ }^{1} / 4$ sec.16, T.15 S., R.13 E., Pima County, Hydrologic Unit 15050301 , at Valencia Road,

$0.4 \mathrm{mi}$ west of Tucson city limits.

DRAINAGE AREA.--23.6 $\mathrm{mi}^{2}$, contributing drainage area not determined.

Annual peak discharges

\begin{tabular}{|c|c|c|c|c|c|c|c|}
\hline $\begin{array}{l}\text { Water } \\
\text { year }\end{array}$ & Date & $\begin{array}{c}\text { Annual peak } \\
\text { discharge } \\
\left(\mathrm{ft}^{3} / \mathbf{s}\right)\end{array}$ & $\begin{array}{c}\text { Discharge } \\
\text { codes }\end{array}$ & $\begin{array}{l}\text { Water } \\
\text { year }\end{array}$ & Date & $\begin{array}{c}\text { Annual peak } \\
\text { discharge } \\
\left(\mathrm{ft}^{3} / \mathrm{s}\right)\end{array}$ & $\begin{array}{c}\text { Discharge } \\
\text { codes }\end{array}$ \\
\hline 1966 & $08-19-66$ & 75 & ES & 1974 & $06-25-74$ & 110 & \\
\hline 1967 & $07-17-67$ & 130 & ES & 1975 & $09-09-75$ & 190 & \\
\hline 1968 & $08-10-68$ & 480 & & 1976 & $09-25-76$ & 910 & \\
\hline 1969 & 08-08-69 & 318 & & 1977 & $09-09-77$ & 350 & \\
\hline 1970 & $07-19-70$ & 75 & ES & 1978 & $10-06-77$ & 910 & \\
\hline 1971 & $08-17-71$ & 540 & & 1979 & $12-18-78$ & 50 & \\
\hline 1972 & $07-16-72$ & 190 & & 1980 & $09-25-80$ & 150 & \\
\hline 1973 & $08-23-73$ & 80 & & 1981 & $07-29-81$ & 760 & \\
\hline
\end{tabular}

Magnitude and probability of instantaneous peak flow based on period of record

\begin{tabular}{cccccc}
\hline \multicolumn{5}{c}{$\begin{array}{l}\text { Discharge, in } \mathrm{ft}^{3} / \mathrm{s} \text {, for indicated recurrence interval } \\
\text { in years, and exceedance probablility, in percent }\end{array}$} \\
\hline 2 & 5 & 10 & 25 & 50 & 100 \\
$50 \%$ & $20 \%$ & $10 \%$ & $4 \%$ & $2 \%$ & $1 \%$ \\
\hline & & & & & \\
Weighted skew & $(\operatorname{logs})=$ & $\ldots$ & & \\
Mean & $(\operatorname{logs})=$ & $\ldots-$ & & \\
Standard dev. & $(\operatorname{logs})=$ & $\ldots-$ &
\end{tabular}

Basin characteristics

\begin{tabular}{ccccccc}
\hline $\begin{array}{c}\text { Main } \\
\text { channel } \\
\text { slope } \\
(\mathrm{ft} / \mathrm{mi})\end{array}$ & $\begin{array}{c}\text { Stream } \\
\text { length } \\
(\mathrm{mi})\end{array}$ & $\begin{array}{c}\text { Mean } \\
\text { basin } \\
\text { elevation } \\
(\mathrm{ft})\end{array}$ & $\begin{array}{c}\text { Forested } \\
\text { area } \\
\text { (percent) }\end{array}$ & $\begin{array}{c}\text { Soil } \\
\text { index }\end{array}$ & $\begin{array}{c}\text { Mean } \\
\text { annual } \\
\text { precipitation } \\
\text { (in) }\end{array}$ & $\begin{array}{c}\text { Rainfall intensity, 24-hour } \\
\text { (in) }\end{array}$ \\
\hline 80.9 & 15.3 & 2,800 & 0.3 & 3.0 & 11.2 & $\begin{array}{c}50 \text {-year } \\
\text { (in) }\end{array}$ \\
\hline
\end{tabular}

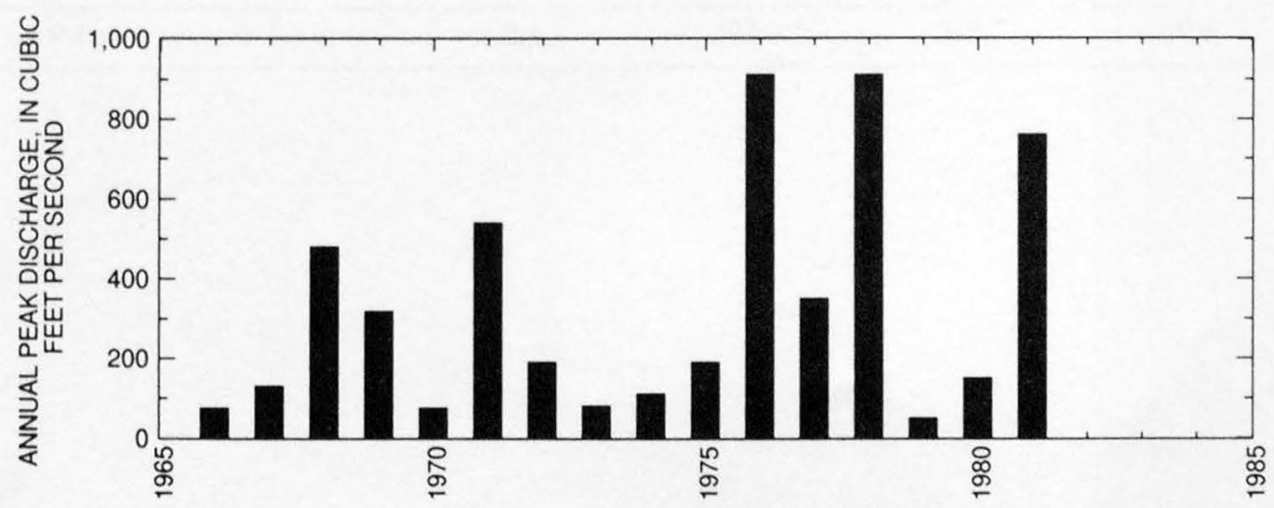




\section{BIG WASH AT TUCSON, AZ}

LOCATION.--Lat $32^{\circ} 11^{\prime} 10^{\prime \prime}$, long $111^{\circ} 00^{\prime} 07^{\prime \prime}, \mathrm{SW}^{1 /} / \mathrm{NE}^{1 / 4} \mathrm{sec} .27$, T.14 S., R. 13 E., Pima County, at Mission Road, $0.6 \mathrm{mi}$ north of State Highway 86 , in Tucson.

DRAINAGE AREA.--2.94 $\mathrm{mi}^{2}$.

Annual peak discharge

\begin{tabular}{|c|c|c|c|c|c|c|c|}
\hline $\begin{array}{l}\text { Water } \\
\text { year }\end{array}$ & Date & $\begin{array}{c}\text { Annual peak } \\
\text { discharge } \\
\left(\mathrm{ft}^{3} / \mathrm{s}\right)\end{array}$ & $\begin{array}{c}\text { Discharge } \\
\text { codes }\end{array}$ & $\begin{array}{l}\text { Water } \\
\text { year }\end{array}$ & Date & $\begin{array}{c}\text { Annual peak } \\
\text { discharge } \\
\left(\mathrm{ft}^{3} / \mathrm{s}\right)\end{array}$ & $\begin{array}{c}\text { Discharge } \\
\text { codes }\end{array}$ \\
\hline 1965 & $07-10-65$ & 1,000 & ES & 1974 & $00-00-74$ & 0 & \\
\hline 1966 & $08-12-66$ & 240 & & 1975 & $00-00-75$ & 0 & \\
\hline 1967 & $07-17-67$ & 50 & & 1976 & $09-25-76$ & 120 & \\
\hline 1968 & $08-10-68$ & 65 & & 1977 & $00-00-77$ & 0 & \\
\hline 1969 & $00-00-69$ & 0 & & 1978 & $10-06-77$ & 380 & \\
\hline 1970 & $07-19-70$ & ${ }^{1} 1,070$ & & 1979 & $08-12-79$ & 20 & \\
\hline 1971 & $08-17-71$ & 23,000 & & 1980 & $09-07-80$ & 3.0 & \\
\hline 1972 & $07-16-72$ & 285 & & 1981 & $09-18-81$ & 29 & \\
\hline 1973 & $07-00-73$ & 300 & ES & & & & \\
\hline
\end{tabular}

${ }^{1}$ Highest since 1963.

${ }^{2}$ Highest since 1920 .

Magnitude and probability of instantaneous peak flow based on period of record 1965-81

\begin{tabular}{|c|c|c|c|c|c|}
\hline \multicolumn{6}{|c|}{$\begin{array}{l}\text { Discharge, in } \mathrm{ft}^{3} / \mathrm{s} \text {, for indicated recurrence interval } \\
\text { in years, and exceedance probablility, in percent }\end{array}$} \\
\hline 2 & 5 & 10 & 25 & $50 t$ & $100 t$ \\
\hline $50 \%$ & $20 \%$ & $10 \%$ & $4 \%$ & $2 \%$ & $1 \%$ \\
\hline 63.0 & 390 & 887 & 1,940 & 3,060 & 4,460 \\
\hline Weighted skew & $(\log s)=$ & -0.62 & & & \\
\hline Mean & $(\log s)=$ & 1.69 & & & \\
\hline Standard dev. & $(\log s)=$ & 1.05 & & & \\
\hline
\end{tabular}

$\mp$ Reliability of values in column is uncertain, and potential errors are large.

Basin characteristics

\begin{tabular}{cccccccr}
\hline $\begin{array}{c}\text { Main } \\
\text { channel } \\
\text { slope } \\
(\mathrm{ft} / \mathrm{mi})\end{array}$ & $\begin{array}{c}\text { Stream } \\
\text { length } \\
\text { (mi) }\end{array}$ & $\begin{array}{c}\text { Mean } \\
\text { basin } \\
\text { elevation } \\
\text { (ft) }\end{array}$ & $\begin{array}{c}\text { Forested } \\
\text { area } \\
\text { (percent) }\end{array}$ & $\begin{array}{c}\text { Soil } \\
\text { index }\end{array}$ & $\begin{array}{c}\text { Mean } \\
\text { annual } \\
\text { precipitation } \\
\text { (in) }\end{array}$ & $\begin{array}{c}\text { Rainfall intensity, 24-hour } \\
\text { (in) }\end{array}$ & $\begin{array}{c}50 \text {-year } \\
\text { (in) }\end{array}$ \\
\hline 95.2 & 4.9 & 2,850 & 0.0 & 1.0 & 11.0 & 1.9 \\
\hline
\end{tabular}


09482480 BIG WASH AT TUCSON, AZ--Continued

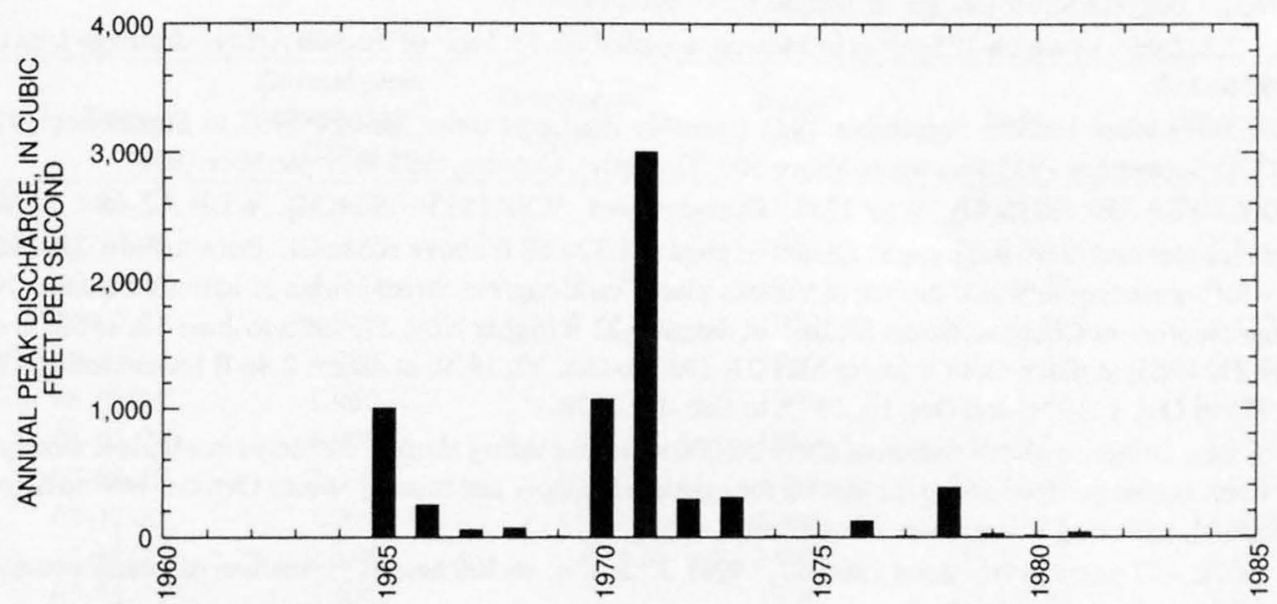




\section{SANTA CRUZ RIVER AT TUCSON, AZ}

LOCATION.--Lat $32^{\circ} 13^{\prime} 19^{\prime \prime}$, long $110^{\circ} 58^{\prime} 52^{\prime \prime}$, in SE $\frac{1}{4} \mathrm{SE}^{1} / 4$ sec.11, T.14 S., R.13 E., Pima County, Hydrologic Unit 15050301 , on right bank, 300 $\mathrm{ft}$ downstream from Congress Street Bridge, in Tucson.

DRAINAGE AREA.--2,222 $\mathrm{mi}^{2}$, of which $395 \mathrm{mi}^{2}$ is in Mexico, adjusted for $15.2 \mathrm{mi}^{2}$ of Tucson Arroyo drainage area contributing to this station effective July 1956.

PERIOD OF RECORD.--October 1905 to September 1981 (monthly discharge only, January 1907 to September 1912, January to September 1914), June 1986 to September 1995 (discharge above $500 \mathrm{ft} 3 / \mathrm{s}$ only), October 1995 to September 1996.

REVISED RECORDS.--WSP 859: 1915(M). WSP 1283: Drainage area. WSP 1313: 1939(M). WDR AZ-88-1: 1986-87(M).

GAGE.--Water-stage recorder and crest-stage gage. Datum of gage is 2,320.68 ft above sea level. Prior to Nov. 27, 1929, nonrecording gages or reference points for measuring to water surface at various places on Congress Street bridge at various datums. Nov. 27,1929 to Sept. 30 , 1981, water-stage recorder at Congress Street bridge: at datum $6.22 \mathrm{ft}$ higher Nov. 27, 1929 to June 18, 1958; at datum $2.22 \mathrm{ft}$ higher June 18, 1958 to May 21, 1963; at datum $3.48 \mathrm{ft}$ lower May 21, 1963 to Oct. 27, 1970; at datum $2.86 \mathrm{ft}$ lower Oct. 1, 1971 to Sept. 30,1981 . No gage Oct. 27, 1970 to Oct. 1, 1971, and Oct. 10, 1977, to Feb. 14, 1978.

REMARKS.--Records fair. Irrigation above station of about 26,000 acres, including about 2,300 acres in Mexico, mostly by pumping from ground water. Ground water is also pumped above the station for municipal supply and mining. From October 1969 to September 1981 , all flow past station was published, including waste water when known.

AVERAGE DISCHARGE.--77 years (water years 1906-81, 1996), $22.5 \mathrm{ft}^{3} / \mathrm{s}, 16,300 \mathrm{acre}-\mathrm{ft} / \mathrm{yr}$; median of yearly mean discharges, $13 \mathrm{ft}^{3} / \mathrm{s}, 9,400$ acre-ft/yr.

EXTREMES FOR PERIOD OF RECORD.--Maximum discharge, 37,400 $\mathrm{ft}^{3} / \mathrm{s} \mathrm{Jan} .19,1993$, gage height, $11.67 \mathrm{ft}$; no flow for most of each year.

EXTREMES OUTSIDE PERIOD OF RECORD.--Maximum discharge since at least $1892,52,700 \mathrm{ft}^{3} / \mathrm{s}$, from slope-area measurement of peak flow, Oct. 2, 1983; gage height, $22.2 \mathrm{ft}$, from floodmark, at site and datum used in 1981 .

Maximum discharge during the 1985 water year was $10,000 \mathrm{ft}^{3} / \mathrm{s}$ Dec. 28, 1984; gage height, $12.5 \mathrm{ft}$, at site and datum used in 1981 . 
GILA RIVER BASIN

09482500 SANTA CRUZ RIVER AT TUCSON, AZ--Continued

Annual peak discharges

\begin{tabular}{|c|c|c|c|c|c|c|c|}
\hline $\begin{array}{l}\text { Water } \\
\text { year }\end{array}$ & Date & $\begin{array}{c}\text { Annual peak } \\
\text { discharge } \\
\left(\mathrm{ft}^{3} / \mathrm{s}\right)\end{array}$ & $\begin{array}{l}\text { Discharge } \\
\text { codes }\end{array}$ & $\begin{array}{l}\text { Water } \\
\text { year }\end{array}$ & Date & $\begin{array}{l}\text { Annual peak } \\
\text { discharge } \\
\left(\mathrm{ft}^{3} / \mathrm{s}\right)\end{array}$ & $\begin{array}{l}\text { Discharge } \\
\text { codes }\end{array}$ \\
\hline 1915 & $12-23-14$ & ${ }^{1} 15,000$ & & 1956 & $07-29-56$ & 2,610 & \\
\hline 1916 & $01-20-16$ & 5,000 & & 1957 & $08-31-57$ & 3,050 & \\
\hline 1917 & $09-08-17$ & 7,500 & & 1958 & $07-29-58$ & 6,350 & \\
\hline 1918 & $08-07-18$ & 4,900 & & 1959 & $08-20-59$ & 4,420 & \\
\hline 1919 & $08-02-19$ & 4,700 & & 1960 & $08-10-60$ & 6,140 & \\
\hline 1920 & $08-09-20$ & 1,950 & & 1961 & $08-23-61$ & 16,600 & \\
\hline 1921 & $08-01-21$ & 4,000 & & 1962 & $09-26-62$ & 4,980 & \\
\hline 1922 & $07-20-22$ & 2,000 & & 1963 & $08-26-63$ & 4,670 & \\
\hline 1923 & $08-17-23$ & 1,900 & & 1964 & $09-10-64$ & 13,000 & \\
\hline 1924 & $11-17-23$ & 2,050 & & 1965 & $07-16-65$ & 1,190 & \\
\hline 1925 & $09-18-25$ & 3,400 & & 1966 & $08-19-66$ & 5,500 & \\
\hline 1926 & $09-28-26$ & 11,400 & & 1967 & $07-17-67$ & 5,860 & \\
\hline 1927 & $09-07-27$ & 1,950 & & 1968 & $12-20-67$ & 16,100 & \\
\hline 1928 & $08-01-28$ & 1,600 & & 1969 & $08-06-69$ & 8,710 & \\
\hline 1929 & $09-24-29$ & 10,400 & & 1970 & $07-20-70$ & 8,530 & \\
\hline 1930 & $08-07-30$ & 1,770 & & 1971 & $08-17-71$ & 8,000 & \\
\hline 1931 & $08-10-31$ & 9,200 & & 1972 & $07-15-72$ & 3,470 & \\
\hline 1932 & $07-30-32$ & 4,200 & & 1973 & $10-19-72$ & 4,710 & \\
\hline 1933 & $08-21-33$ & 6,100 & & 1974 & $07-08-74$ & 7,930 & \\
\hline 1934 & $08-23-34$ & 6,000 & & 1975 & $07-12-75$ & 2,480 & \\
\hline 1935 & $09-01-35$ & 10,300 & & 1976 & $09-25-76$ & 7,100 & \\
\hline 1936 & $07-26-36$ & 5,400 & & 1977 & $08-15-77$ & 2,660 & \\
\hline 1937 & $07-10-37$ & 3,280 & & 1978 & $10-10-77$ & 23,700 & \\
\hline 1938 & $08-05-38$ & 9,000 & & 1979 & $12-19-78$ & 13,500 & \\
\hline 1939 & 08-03-39 & 8,000 & & 1980 & $08-13-80$ & 2,760 & \\
\hline 1940 & $08-14-40$ & 11,300 & & 1981 & $07-27-81$ & 2,700 & \\
\hline 1941 & $08-14-41$ & 2,490 & & 1982 & $00-00-82$ & 10,000 & \\
\hline 1942 & $08-09-42$ & 1,670 & & 1983 & $00-00-83$ & 7,000 & \\
\hline 1943 & $08-02-43$ & 4,510 & & 1984 & $10-02-83$ & ${ }^{2} 52,700$ & \\
\hline 1944 & $08-16-44$ & 6,530 & & 1985 & $12-28-84$ & 10,000 & \\
\hline 1945 & $08-10-45$ & 10,800 & & 1986 & $07-21-86$ & 1,920 & HP \\
\hline 1946 & $08-04-46$ & 4,260 & & 1987 & $08-02-87$ & 1,500 & \\
\hline 1947 & $10-01-46$ & 2,960 & & 1988 & $08-23-88$ & 10,700 & \\
\hline 1948 & $08-16-48$ & 3,860 & & 1989 & $10-20-88$ & 2,960 & \\
\hline 1949 & 08-08-49 & 3,800 & & 1990 & $07-24-90$ & 9,430 & \\
\hline 1950 & $07-30-50$ & 9,490 & & 1991 & 08-09-91 & 2,130 & \\
\hline 1951 & $08-02-51$ & 5,020 & & 1992 & $08-06-92$ & 5,970 & \\
\hline 1952 & $08-16-52$ & 3,820 & & 1993 & $01-19-93$ & 37,400 & \\
\hline 1953 & $07-15-53$ & 5,900 & & 1994 & $07-28-94$ & 4,890 & \\
\hline 1954 & $07-24-54$ & 9,570 & & 1995 & $02-16-95$ & 576 & \\
\hline 1955 & $08-03-55$ & 10,900 & & 1996 & $09-03-97$ & 9,370 & \\
\hline
\end{tabular}

'Highest since 1905.

${ }^{2}$ Highest since 1892. 
09482500 SANTA CRUZ RIVER AT TUCSON, AZ--Continued

Discharge rating table developed October 1992

\begin{tabular}{crcc}
\hline $\begin{array}{c}\text { Gage height } \\
(\mathrm{ft})\end{array}$ & $\begin{array}{c}\text { Discharge } \\
\left(\mathrm{ft}^{3} / \mathbf{s}\right)\end{array}$ & $\begin{array}{c}\text { Gage height } \\
(\mathbf{f t})\end{array}$ & $\begin{array}{c}\text { Discharge } \\
\left(\mathrm{ft}^{3} / \mathbf{s}\right)\end{array}$ \\
\hline 2.0 & 73 & 7.0 & 9,600 \\
2.5 & 263 & 8.0 & 14,000 \\
3.0 & 570 & 9.0 & 19,400 \\
4.0 & 1,700 & 10.0 & 25,000 \\
5.0 & 3,550 & 11.0 & 32,500 \\
6.0 & 6,100 & 12.0 & 40,000 \\
\hline
\end{tabular}

Basin characteristics

\begin{tabular}{cccccccc}
\hline $\begin{array}{c}\text { Main } \\
\text { channel } \\
\text { slope } \\
(\mathrm{ft} / \mathrm{mi})\end{array}$ & $\begin{array}{c}\text { Stream } \\
\text { length } \\
\text { (mi) }\end{array}$ & $\begin{array}{c}\text { Mean } \\
\text { basin } \\
\text { elevation } \\
\text { (ft) }\end{array}$ & $\begin{array}{c}\text { Forested } \\
\text { area } \\
\text { (percent) }\end{array}$ & $\begin{array}{c}\text { Soil } \\
\text { index }\end{array}$ & $\begin{array}{c}\text { Mean } \\
\text { annual } \\
\text { precipitation } \\
\text { (in) }\end{array}$ & $\begin{array}{c}\text { Rainfall intensity, 24-hour } \\
\text { (in) }\end{array}$ & $\begin{array}{c}50 \text {-year } \\
\text { (in) }\end{array}$ \\
\hline 20.1 & 128 & 4,050 & 17.0 & 2.0 & 16.9 & 2.1 \\
\hline
\end{tabular}


09482500 SANTA CRUZ RIVER AT TUCSON, AZ--Continued

MEAN MONTHLY AND ANNUAL DISCHARGES 1906, 1913, 1915-81, 1996

\begin{tabular}{|c|c|c|c|c|c|c|}
\hline MONTH & $\begin{array}{l}\text { MAXIMUM } \\
(\mathrm{PT} 3 / \mathrm{S})\end{array}$ & $\begin{array}{l}\text { MINIMUM } \\
\text { (FT3/S) }\end{array}$ & $\begin{array}{c}\text { MEAN } \\
(\mathrm{FT} 3 / \mathrm{S})\end{array}$ & $\begin{array}{l}\text { STAN- } \\
\text { DARD } \\
\text { DEVIA- } \\
\text { TION } \\
\text { (FT3/S) }\end{array}$ & $\begin{array}{l}\text { COEFFI- } \\
\text { CIENT OF } \\
\text { VARI- } \\
\text { ATION }\end{array}$ & $\begin{array}{c}\text { PERCENT } \\
\text { OF } \\
\text { ANNUAL } \\
\text { RUNOFF }\end{array}$ \\
\hline OCTOBER & 656 & 0.00 & 13 & 79 & 6.0 & 4.9 \\
\hline NOVEMBER & 215 & 0.00 & 6.5 & 28 & 4.3 & 2.4 \\
\hline DECEMBER & 895 & 0.00 & 35 & 145 & 4.1 & 13.0 \\
\hline JANUARY & 518 & 0.00 & 21 & 81 & 3.8 & 7.8 \\
\hline FEBRUARY & 202 & 0.00 & 11 & 37 & 3.3 & 4.1 \\
\hline MARCH & 102 & 0.00 & 4.8 & 17 & 3.6 & 1. 8 \\
\hline APRIL & 1.7 & 0.00 & 0.10 & 0.28 & 2.7 & 0.0 \\
\hline MAY & 2.3 & 0.00 & 0.09 & 0.34 & 3.8 & 0.0 \\
\hline JUNE & 25 & 0.00 & 1.4 & 4.3 & 3.2 & 0.5 \\
\hline JULY & 430 & 0.00 & 51 & 71 & 1.4 & 18.8 \\
\hline AUGUST & 682 & 0.00 & 93 & 114 & 1.2 & 34.4 \\
\hline SEPTEMBER & 312 & 0.00 & 33 & 60 & 1.8 & 12.3 \\
\hline ANNUAL & 112 & 1.3 & 23 & 23 & 1.0 & 100 \\
\hline
\end{tabular}

MAGNITUDE AND PROBABILITY OF INSTANTANEOUS PEAK FLOW BASED ON PERIOD OF RECORD $1915 \cdot 81,1984.96$

DISCHARGE, IN FT3/S, FOR INDICATED RECURRENCE INTERVA IN YEARS, AND EXCEEDANCE PROBABILITY, IN PERCENT

\begin{tabular}{|c|c|c|c|c|c|}
\hline 2 & 5 & 10 & 25 & 50 & 100 \\
\hline 508 & 208 & 108 & 48 & 28 & 18 \\
\hline 5,110 & 9,720 & 13,800 & 20,300 & 26,300 & 33,300 \\
\hline
\end{tabular}

WEIGHTED SKEW $($ LOGS $)=0.22$

MEAN $\quad($ LOGS $)=3.72$

STANDARD DEV, $($ LOGS $)=0.32$
MAGNITUDE AND PROBABILITY OF ANNUAL LOW FLOW BASED ON PERIOD OF RECORD $1916 \cdot 81$

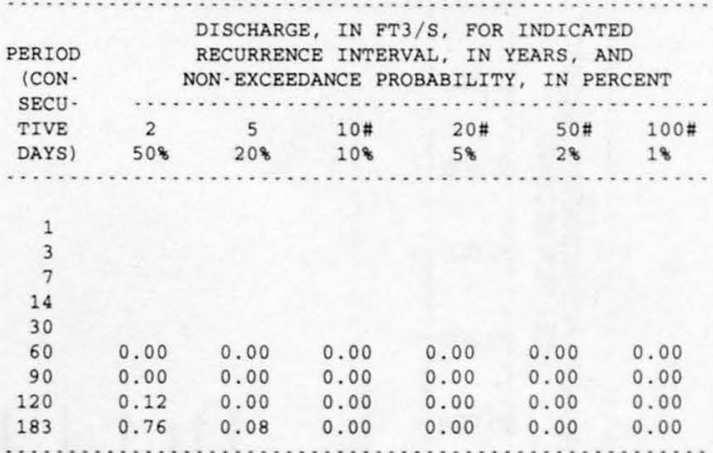

MAGNITUDE AND PROBABILITY OF ANNUAL HIGH FLOW BASED ON PERIOD OF RECORD 1906, 1913,1915.81, 1996

\begin{tabular}{|c|c|c|c|c|c|c|}
\hline \multirow{4}{*}{$\begin{array}{l}\text { PERIOD } \\
\text { ICON- } \\
\text { SECU. }\end{array}$} & \multicolumn{6}{|c|}{ DISCHARGE, IN FT3/S, FOR INDICATED } \\
\hline & & RECURR: & CE INTI & VAL, IN & YEARS, & AND \\
\hline & . & EXCEED & CE PROI & BILITY, & IN PERC & ENT \\
\hline & & & & & & $\ldots \ldots$ \\
\hline TIVE & 2 & 5 & $10 \#$ & $25 \#$ & $50 \#$ & $100 \#$ \\
\hline DAYS) & 508 & 208 & 108 & 48 & 28 & 18 \\
\hline 1 & 1,410 & 3,230 & 4,840 & 7,330 & 9,480 & 11,900 \\
\hline 3 & 703 & 1,640 & 2,480 & 3,770 & 4,890 & 6,130 \\
\hline 7 & 364 & 852 & 1,300 & 2,000 & 2,610 & 3,310 \\
\hline 15 & 211 & 484 & 730 & 1,110 & 1,450 & 1,820 \\
\hline 30 & 131 & 291 & 434 & 654 & 845 & 1,060 \\
\hline 60 & 79 & 174 & 262 & 403 & 533 & 684 \\
\hline 90 & 56 & 122 & 183 & 282 & 371 & 475 \\
\hline
\end{tabular}

DURATION TABLE OF DAILY MEAN FLOW FOR PERIOD OF RECORD 1906, 1913, 1915-81, 1996

DISCHARGE, IN FT3/S, WHICH WAS EQUALED OR EXCEEDED FOR INDICATED PERCENT OF TIME

\begin{tabular}{|c|c|c|c|c|c|c|c|c|c|c|c|c|c|c|c|c|}
\hline 18 & 58 & 108 & 158 & 208 & 308 & 408 & 508 & 608 & 708 & 808 & 908 & 958 & 988 & 998 & 99.58 & 99.98 \\
\hline 535 & 50 & 5.9 & 0.00 & 0.00 & 0.00 & 0.00 & 0.00 & 0.00 & 0.00 & 0.00 & 0.00 & 0.00 & 0.00 & 0.00 & 0.00 & 0.00 \\
\hline
\end{tabular}

\# Reliability of values in column is uncertain, and potential errors are large. 
ANNUAL MEAN DISCHARGE, IN CUBIC

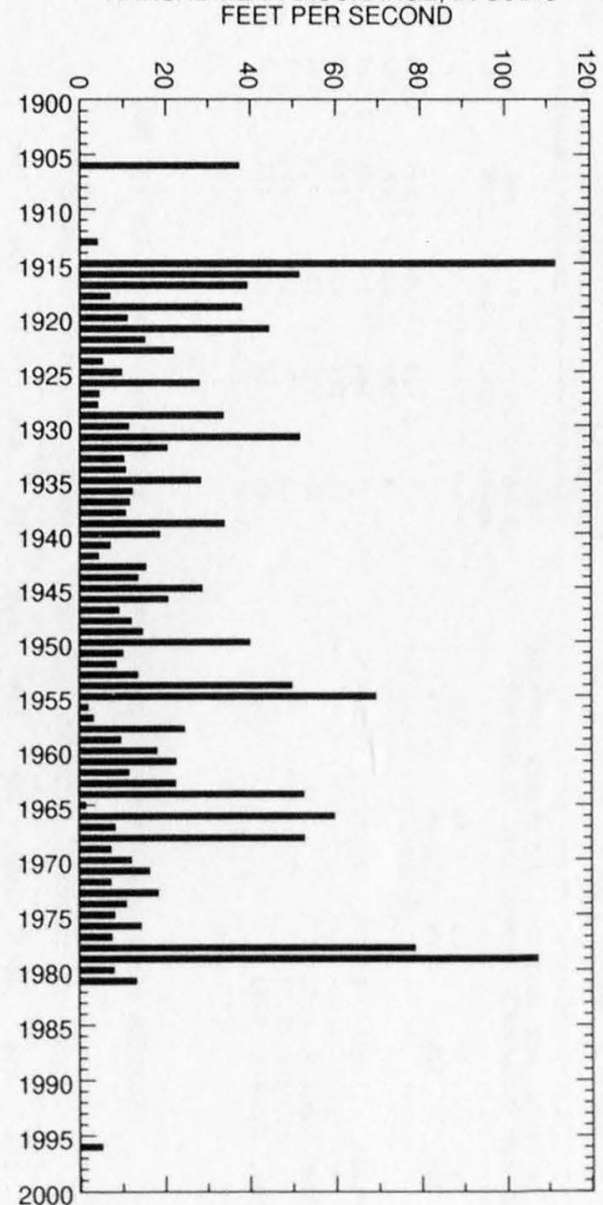

ANNUALPEAK DISCHARGE IN CUBIC

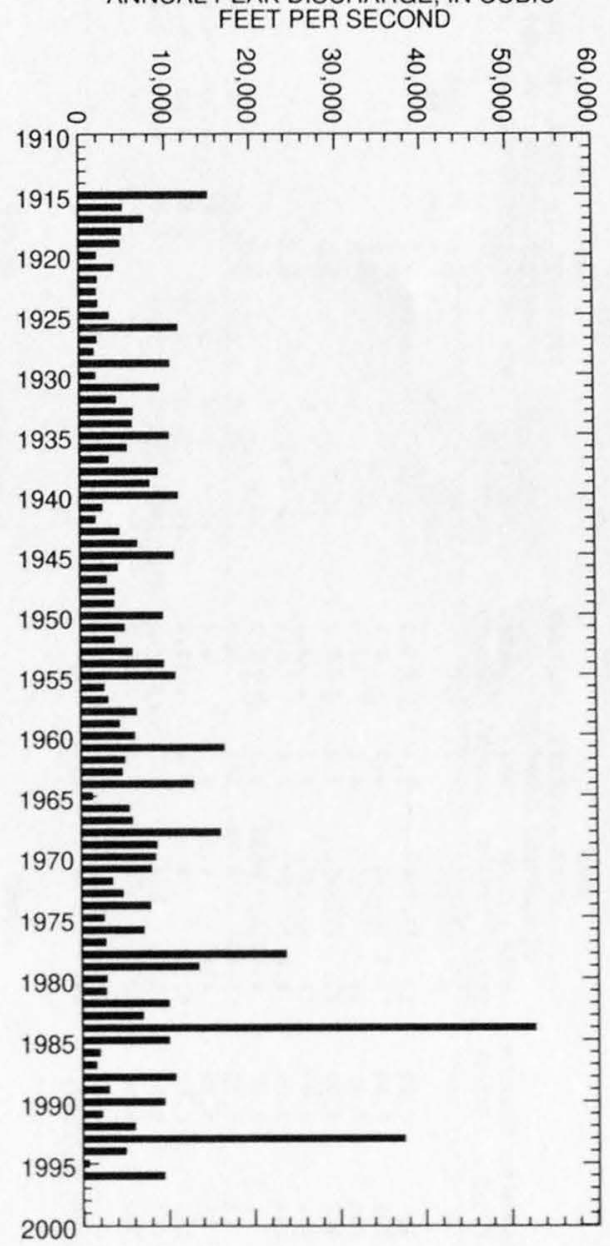


09482500 SANTA CRUZ RIVER AT TUCSON, AZ--Continued
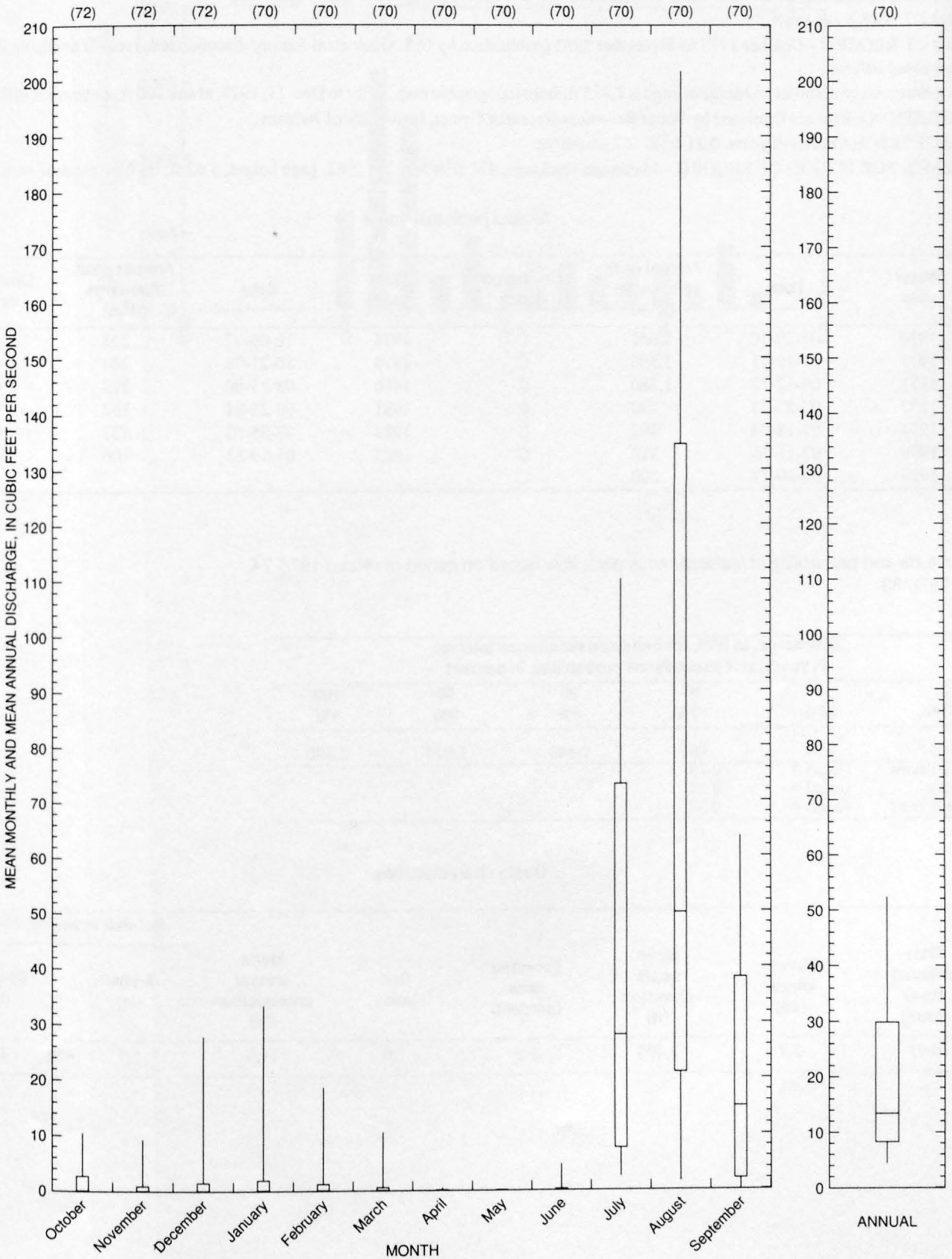


\section{RAILROAD WASH AT TUCSON, AZ}

LOCATION.--Lat $32^{\prime} 12^{\prime} 50^{\prime \prime}$, long $110^{\circ} 56^{\prime} 45^{\prime \prime}$, in NE $1 / 4 \mathrm{SE}^{1} / 4$ sec.18, T.14 S., R.14 E., Pima County, Hydrologic Unit 15050301 , on center of cul. vert on upstream side of Winsett Street in Tucson. Prior to Dec. 11, 1978, at site $200 \mathrm{ft}$ upstream.

DRAINAGE AREA.--2.3 $\mathrm{mi}^{2}$.

PERIOD OF RECORD.--October 1975 to September 1983 (publication by U.S. Geological Survey discontinued; records available from cooperator listed below).

GAGE.--Water-stage recorder. Altitude of gage is 2,425 ft, from topographic map. Prior to Dec. 11, 1978, at site $200 \mathrm{ft}$ upstream at different datum. COOPERATION.--Records furnished by Water Resources Research Center, University of Arizona.

AVERAGE DISCHARGE.--8 years, $0.21 \mathrm{ft}^{3} / \mathrm{s}, 152$ acre- $\mathrm{ft} / \mathrm{yr}$.

EXTREMES FOR PERIOD OF RECORD.--Maximum discharge, $421 \mathrm{ft}^{3} / \mathrm{s}$ July 25,1982 , gage height, $5.62 \mathrm{ft}$; no flow most of each year.

Annual peak discharge

\begin{tabular}{|c|c|c|c|c|c|c|c|}
\hline $\begin{array}{l}\text { Water } \\
\text { year }\end{array}$ & Date & $\begin{array}{c}\text { Annual peak } \\
\text { discharge } \\
\left(\mathrm{ft}^{3} / \mathrm{s}\right)\end{array}$ & $\begin{array}{l}\text { Discharge } \\
\text { codes }\end{array}$ & $\begin{array}{l}\text { Water } \\
\text { year }\end{array}$ & Date & $\begin{array}{c}\text { Annual peak } \\
\text { discharge } \\
\left(\mathrm{ft}^{3} / \mathrm{s}\right)\end{array}$ & $\begin{array}{c}\text { Discharge } \\
\text { codes }\end{array}$ \\
\hline 1970 & $07-20-70$ & 642 & $\mathrm{C}$ & 1978 & $10-06-77$ & 223 & $\mathrm{C}$ \\
\hline 1971 & $07-19-71$ & 1,590 & $\mathrm{C}$ & 1979 & $10-21-78$ & 243 & $\mathrm{C}$ \\
\hline 1972 & $08-12-72$ & 1,280 & $\mathrm{C}$ & 1980 & $08-23-80$ & 222 & $\mathrm{C}$ \\
\hline 1973 & $07-27-73$ & 220 & $\mathrm{C}$ & 1981 & $06-25-81$ & 162 & $\mathrm{C}$ \\
\hline 1974 & $07-18-74$ & 482 & $\mathrm{C}$ & 1982 & $07-25-82$ & 421 & $\mathrm{C}$ \\
\hline 1976 & $07-11-76$ & 312 & $\mathrm{C}$ & 1983 & $01-29-83$ & 106 & $\mathrm{C}$ \\
\hline 1977 & $09-10-77$ & 300 & $\mathrm{C}$ & & & & \\
\hline
\end{tabular}

Magnitude and probability of instantaneous peak flow based on period of record 1970-74, 1976-83

\begin{tabular}{|c|c|c|c|c|c|}
\hline \multicolumn{6}{|c|}{$\begin{array}{l}\text { Discharge, in } \mathrm{ft}^{3} / \mathrm{s} \text {, for indicated recurrence interval } \\
\text { in years, and exceedance probablility, in percent }\end{array}$} \\
\hline 2 & 5 & 10 & 25 & 50 & 100 \\
\hline $50 \%$ & $20 \%$ & $10 \%$ & $4 \%$ & $2 \%$ & $1 \%$ \\
\hline 340 & 666 & 961 & 1,440 & 1,870 & 2,390 \\
\hline Weighted skew & $(\log s)=$ & 0.19 & & & \\
\hline Mean & $(\log s)=$ & 2.54 & & & \\
\hline Standard dev. & $(\log s)=$ & 0.34 & & & \\
\hline
\end{tabular}

Basin characteristics

\begin{tabular}{cccccccc}
\hline $\begin{array}{c}\text { Main } \\
\text { channel } \\
\text { slope } \\
(\mathrm{ft} / \mathrm{mi})\end{array}$ & $\begin{array}{c}\text { Stream } \\
\text { length } \\
\text { (mi) }\end{array}$ & $\begin{array}{c}\text { Mean } \\
\text { basin } \\
\text { elevation } \\
(\mathrm{ft})\end{array}$ & $\begin{array}{c}\text { Forested } \\
\text { area } \\
\text { (percent) }\end{array}$ & $\begin{array}{c}\text { Soil } \\
\text { index }\end{array}$ & $\begin{array}{c}\text { Mean } \\
\text { annual } \\
\text { precipitation } \\
\text { (in) }\end{array}$ & $\begin{array}{c}\text { Rainfall intensity, 24-hour } \\
\text { (in) }\end{array}$ & $\begin{array}{c}50 \text {-year } \\
\text { (in) }\end{array}$ \\
\hline 0.73 & 2.7 & 2,490 & 0.0 & 1.0 & 11.0 & 1.7 \\
\hline
\end{tabular}


09482950 RAILROAD WASH AT TUCSON, AZ--Continued

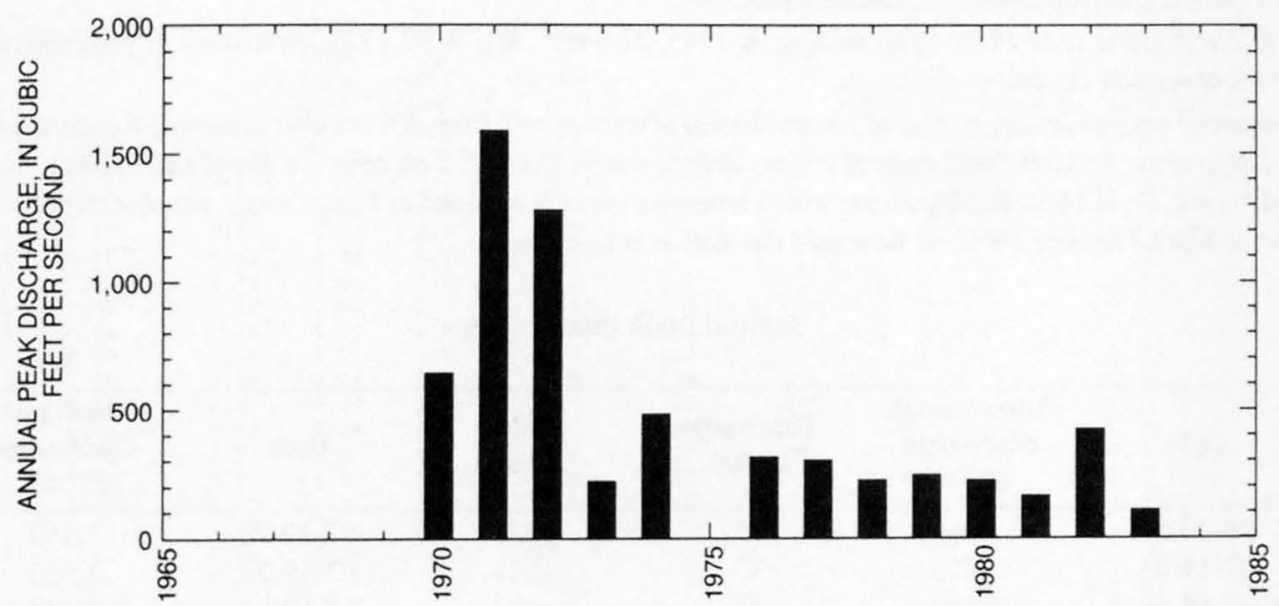


09483000 TUCSON ARROYO AT VINE AVENUE, TUCSON, AZ

LOCATION.--Lat $32^{\circ} 13^{\prime} 00^{\prime \prime}$, long $110^{\circ} 56^{\prime} 54^{\prime \prime}, \mathrm{SW}^{1} / 4^{\mathrm{NE}} \mathrm{N}^{1 / 4} \mathrm{sec} .18$, T.14 S., R. 14 E., Pima County, Hydrologic Unit 15050301 , on right bank at Vine Avenue in Tucson, 0.2 mi downstream from Arroyo Chico.

DRAINAGE AREA.--8.2 $\mathrm{mi}^{2}$ since June 1956. Prior to August 1945, $27.0 \mathrm{mi}^{2}$. See WSP 1733 for history of progressive reduction of drainage area by flood-control diversion structures.

REMARKS.--A flood-control project, at upper end of natural basin, diverts runoff from $3.6 \mathrm{mi}$ into Lakeside Reservoir (Atterbury), which is in Pantano Wash drainage area. Another flood-control project diverts runoff from $15.2 \mathrm{mi}$ near the upper end of basin into a flood-control detention reservoir in $\mathrm{SE}^{1} / 4 \mathrm{sec} .29$, T.14 S., R.14 E., from which reservoir water is released to Julian Wash, which enters Santa Cruz River upstream from Tucson Arroyo. Since October 1969, all flow past the station is published.

Annual peak discharges

\begin{tabular}{cccccccc}
\hline $\begin{array}{c}\text { Water } \\
\text { year }\end{array}$ & Date & $\begin{array}{c}\text { Annual peak } \\
\text { discharge } \\
\left(\mathbf{f t}^{3} / \mathbf{s}\right)\end{array}$ & $\begin{array}{c}\text { Discharge } \\
\text { codes }\end{array}$ & $\begin{array}{c}\text { Water } \\
\text { year }\end{array}$ & Date & $\begin{array}{c}\text { Annual peak } \\
\text { discharge } \\
\left(\mathbf{f t}^{3} / \mathbf{s}\right)\end{array}$ & $\begin{array}{c}\text { Discharge } \\
\text { codes }\end{array}$ \\
\hline 1956 & $08-26-56$ & 364 & $\mathrm{C}$ & 1970 & $07-20-70$ & 1,550 & $\mathrm{C}$ \\
1957 & $07-17-57$ & 716 & $\mathrm{C}$ & 1971 & $07-19-71$ & 2,930 & $\mathrm{C}$ \\
1959 & $08-20-59$ & 2,540 & $\mathrm{C}$ & 1972 & $08-12-72$ & 2,950 & $\mathrm{C}$ \\
1960 & $08-20-60$ & 609 & $\mathrm{C}$ & 1973 & $10-18-72$ & 720 & $\mathrm{C}$ \\
1961 & $08-22-61$ & 15,000 & UR,C & 1974 & $07-18-74$ & 332 & $\mathrm{C}$ \\
1962 & $09-26-62$ & 1,060 & $\mathrm{C}$ & 1975 & $07-25-75$ & 760 & $\mathrm{C}$ \\
1963 & $09-03-63$ & 208 & $\mathrm{C}$ & 1976 & $09-04-76$ & 446 & $\mathrm{C}$ \\
1964 & $08-12-64$ & 1,060 & $\mathrm{C}$ & 1977 & $09-10-77$ & 1,480 & $\mathrm{C}$ \\
1965 & $07-16-65$ & 1,220 & $\mathrm{C}$ & 1978 & $10-06-77$ & 764 & $\mathrm{C}$ \\
1966 & $09-13-66$ & 593 & $\mathrm{C}$ & 1979 & $10-21-78$ & 1,040 & $\mathrm{C}$ \\
1967 & $05-24-67$ & 350 & $\mathrm{C}$ & 1980 & $08-13-80$ & 816 & $\mathrm{C}$ \\
1968 & $08-19-68$ & 644 & $\mathrm{C}$ & 1981 & $06-25-81$ & 746 & $\mathrm{C}$ \\
1969 & $08-01-69$ & 800 & $\mathrm{C}$ & & & & 76 \\
\hline
\end{tabular}

${ }^{1}$ Highest since 1940.

Discharge rating table developed October 1992

\begin{tabular}{crcc}
\hline $\begin{array}{c}\text { Gage height } \\
(\mathbf{f t})\end{array}$ & $\begin{array}{c}\text { Discharge } \\
\left(\mathbf{f t}^{\mathbf{3}} \mathbf{s}\right)\end{array}$ & $\begin{array}{c}\text { Gage height } \\
(\mathbf{f t})\end{array}$ & $\begin{array}{c}\text { Discharge } \\
\left(\mathbf{f t}^{\mathbf{3}} / \mathbf{s}\right)\end{array}$ \\
\hline 2.0 & 73 & 7.0 & 9,600 \\
2.5 & 263 & 8.0 & 14,000 \\
3.0 & 570 & 9.0 & 19,400 \\
4.0 & 1,700 & 10.0 & 25,000 \\
5.0 & 3,550 & 11.0 & 32,500 \\
6.0 & 6,100 & 12.0 & 40,000 \\
\hline
\end{tabular}

Basin characteristics

\begin{tabular}{ccccccc}
\hline $\begin{array}{c}\text { Main } \\
\text { channel } \\
\text { slope } \\
(\mathrm{ft} / \mathrm{mi})\end{array}$ & $\begin{array}{c}\text { Stream } \\
\text { length } \\
(\mathrm{mi})\end{array}$ & $\begin{array}{c}\text { Mean } \\
\text { basin } \\
\text { elevation } \\
(\mathrm{ft})\end{array}$ & $\begin{array}{c}\text { Forested } \\
\text { area } \\
\text { (percent) }\end{array}$ & $\begin{array}{c}\text { Soil } \\
\text { index }\end{array}$ & $\begin{array}{c}\text { Mean } \\
\text { annual } \\
\text { precipitation } \\
\text { (in) }\end{array}$ & $\begin{array}{c}\text { Rainfall intensity, 24-hour } \\
\text { (in) }\end{array}$ \\
\hline 37.0 & 5.5 & 2,510 & 0.0 & 1.0 & 11.0 & $\begin{array}{c}50 \text {-year } \\
\text { (in) }\end{array}$ \\
\hline
\end{tabular}


09483000 TUCSON ARROYO AT VINE AVENUE, TUCSON, AZ--Continued

MEAN MONTHLY AND ANNUAL DISCHARGES $1957 \cdot 81$

\begin{tabular}{|c|c|c|c|c|c|c|}
\hline MONTH & $\begin{array}{l}\text { MAXIMUM } \\
(\mathrm{FT} 3 / \mathrm{S})\end{array}$ & $\begin{array}{l}\text { MINIMUM } \\
(\text { FT } 3 / S)\end{array}$ & $\begin{array}{c}\text { MEAN } \\
(\mathrm{FT} 3 / \mathrm{S})\end{array}$ & $\begin{array}{l}\text { STAN- } \\
\text { DARD } \\
\text { DEVIA- } \\
\text { TION } \\
(\mathrm{FT} 3 / \mathrm{S})\end{array}$ & $\begin{array}{l}\text { COEPFI- } \\
\text { CIENT OF } \\
\text { VARI- } \\
\text { ATION }\end{array}$ & $\begin{array}{c}\text { PERCENT } \\
\text { OF } \\
\text { ANNUAL } \\
\text { RUNOFF }\end{array}$ \\
\hline OCTOBER & 8.7 & 0.00 & 0.81 & 1.7 & 2.1 & 7.8 \\
\hline NOVEMBER & 4.4 & 0.00 & 0.43 & 0.82 & 1.9 & 4.2 \\
\hline DECEMBER & 8.5 & 0.00 & 0.79 & 1.8 & 2.2 & 7.5 \\
\hline JANUARY & 3.8 & 0.00 & 0.56 & 0.94 & 1.7 & 5.4 \\
\hline FEBRUARY & 3.8 & 0.00 & 0.50 & 0.94 & 1.9 & 4.8 \\
\hline MARCH & 2.0 & 0.00 & 0.36 & 0.57 & 1.6 & 3.5 \\
\hline APRIL & 0.69 & 0.00 & 0.09 & 0.15 & 1.8 & 0.8 \\
\hline MAY & 1.0 & 0.00 & 0.07 & 0.19 & 2.8 & 0.7 \\
\hline JUNE & 1.3 & 0.00 & 0.10 & 0.24 & 2.5 & 1.0 \\
\hline JULY & 12 & 0.00 & 2.8 & 2.9 & 1.0 & 26.4 \\
\hline AUGUST & 15 & 0.00 & 2.9 & 3.7 & 1.3 & 27.8 \\
\hline SEPTEMBER & 5.7 & 0.00 & 1.1 & 1.5 & 1.4 & 10.2 \\
\hline ANNUAL & 1.8 & 0.12 & 0.88 & 0.45 & 0.51 & 100 \\
\hline
\end{tabular}

MAGNITUDE AND PROBABILITY OF INSTANTANEOUS PEAK FLOW BASED ON PERIOD OF RECORD $1956 \cdot 81$

DISCHARGE, IN FT3/S, FOR INDICATED RECURRENCE INTERVAL IN YEARS, AND EXCEEDANCE PROBABILITY, IN PERCENT

\begin{tabular}{|c|c|c|c|c|c|}
\hline 2 & 5 & 10 & 25 & 50 & $100 \#$ \\
\hline 508 & 208 & 108 & 48 & 28 & 18 \\
\hline 842 & 1,540 & 2,150 & 3.090 & 3,920 & 4,890 \\
\hline NEIGHTED & SKEW & (LOGS) $=$ & & & \\
\hline MEAN & & (LOGS) $=$ & & & \\
\hline STANDARD & DEV. & $($ LOGS $)=$ & & & \\
\hline
\end{tabular}

MAGNITUDE AND PROBABILITY OF ANNUAL LOW FLOW BASED ON PERIOD OF RECORD $1958 \cdot 81$

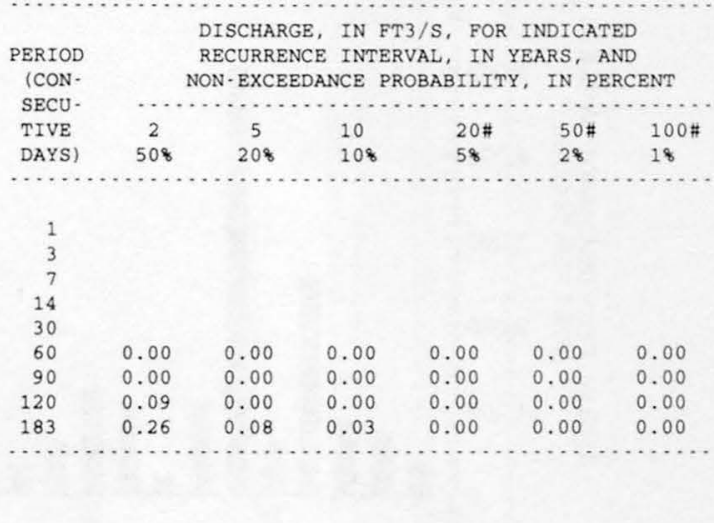

MAGNITUDE AND PROBABILITY OF ANNUAL HIGH FLOW BASED ON PERIOD OF RECORD 1957-81

\begin{tabular}{|c|c|c|c|c|c|c|}
\hline \multirow{2}{*}{$\begin{array}{l}\text { PERIOD } \\
\text { ICON- } \\
\text { SECU. }\end{array}$} & & $\begin{array}{r}\text { D } \\
\text { RECURRE } \\
\text { EXCEEDA }\end{array}$ & $\begin{array}{l}\text { SCHARGE } \\
\text { CE INTE } \\
\text { EE PROB }\end{array}$ & $\begin{array}{l}\text { IN FT3, } \\
\text { VAL, IN } \\
\text { BLITY, }\end{array}$ & $\begin{array}{l}\text { S, FOR } \\
\text { YEARS, } \\
\text { IN PERC }\end{array}$ & $\begin{array}{l}\text { NDICATED } \\
\text { ND } \\
\text { NT }\end{array}$ \\
\hline & $\ldots$. & ........ & . . . . . & ........... & ........ & ........ \\
\hline TIVE & 2 & 5 & 10 & $25 \#$ & $50 \#$ & $100 \#$ \\
\hline DAYS) & 508 & 208 & 108 & 48 & 28 & 18 \\
\hline 1 & 79 & 153 & 216 & 316 & 404 & 506 \\
\hline 3 & 31 & 58 & 81 & 116 & 147 & 181 \\
\hline 7 & 14 & 27 & 38 & 54 & 68 & 83 \\
\hline 15 & 8.1 & 15 & 21 & 30 & 37 & 45 \\
\hline 30 & 5.1 & 9.2 & 12 & 16 & 20 & 23 \\
\hline 60 & 3.1 & 5.4 & 7.0 & 9.1 & 11 & 12 \\
\hline 90 & 2.3 & 3.9 & 5.1 & 6.5 & 7.5 & 8.5 \\
\hline
\end{tabular}

DURATION TABLE OF DAILY MEAN FLOW FOR PERIOD OF RECORD 1957-81

DISCHARGE, IN FT3/S, WHICH WAS EQUALED OR EXCEEDED FOR INDICATED PERCENT OF TIME

\begin{tabular}{|c|c|c|c|c|c|c|c|c|c|c|c|c|c|c|c|c|}
\hline 18 & 58 & 108 & 158 & 208 & 308 & 408 & 508 & 608 & 708 & 808 & 908 & 958 & 988 & 998 & 99.58 & 99.98 \\
\hline & 1.2 & 0.02 & 0.00 & 0.00 & 0.00 & 0.00 & 0.00 & 0.00 & 0.00 & 0.00 & 0.00 & 0.00 & 0.00 & 0.00 & 0.00 & 0.00 \\
\hline
\end{tabular}

\# Reliability of values in column is uncertain, and potential errors are large. 


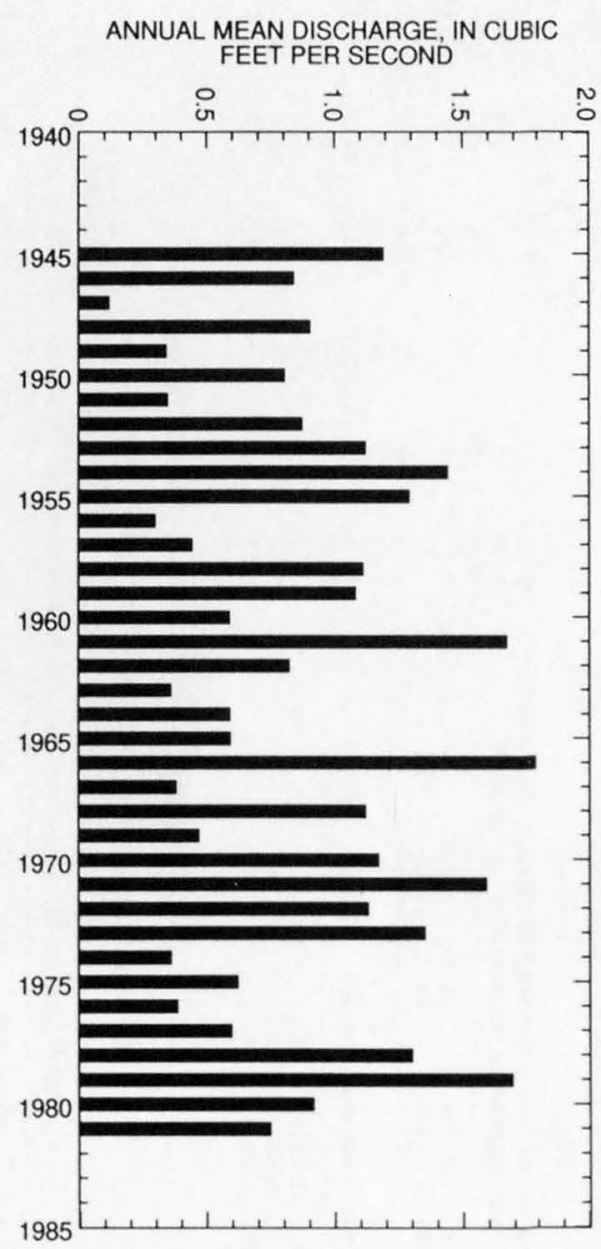

ANNUAL PEAK DISCHARGE, IN CUBIC

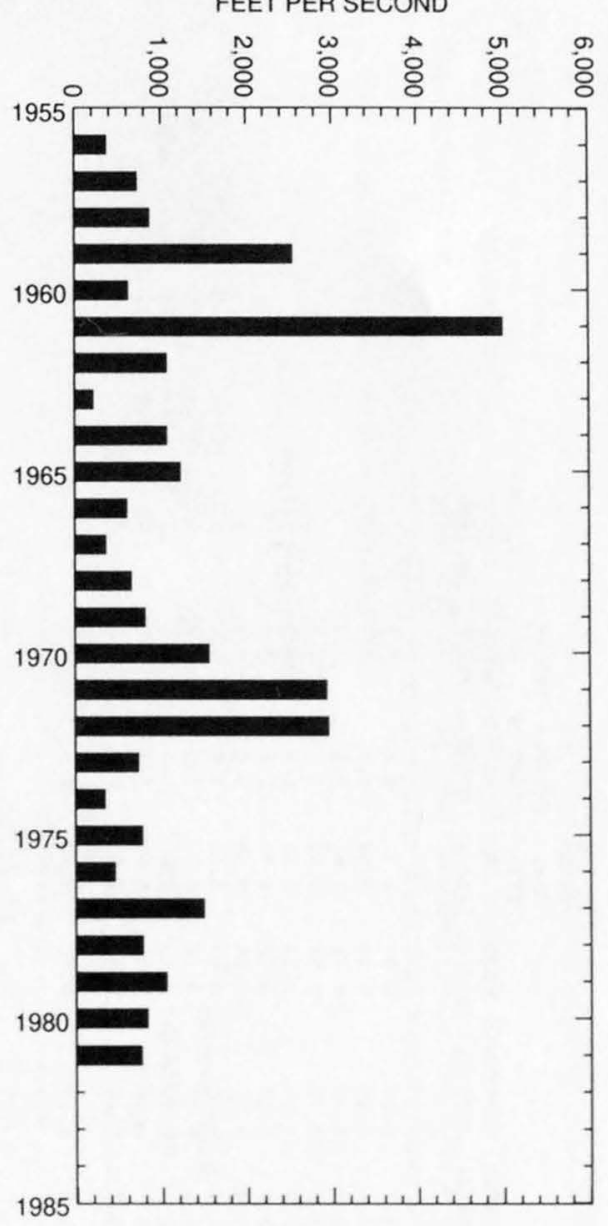


09483000 TUCSON ARROYO AT VINE AVENUE, TUCSON, AZ--Continued
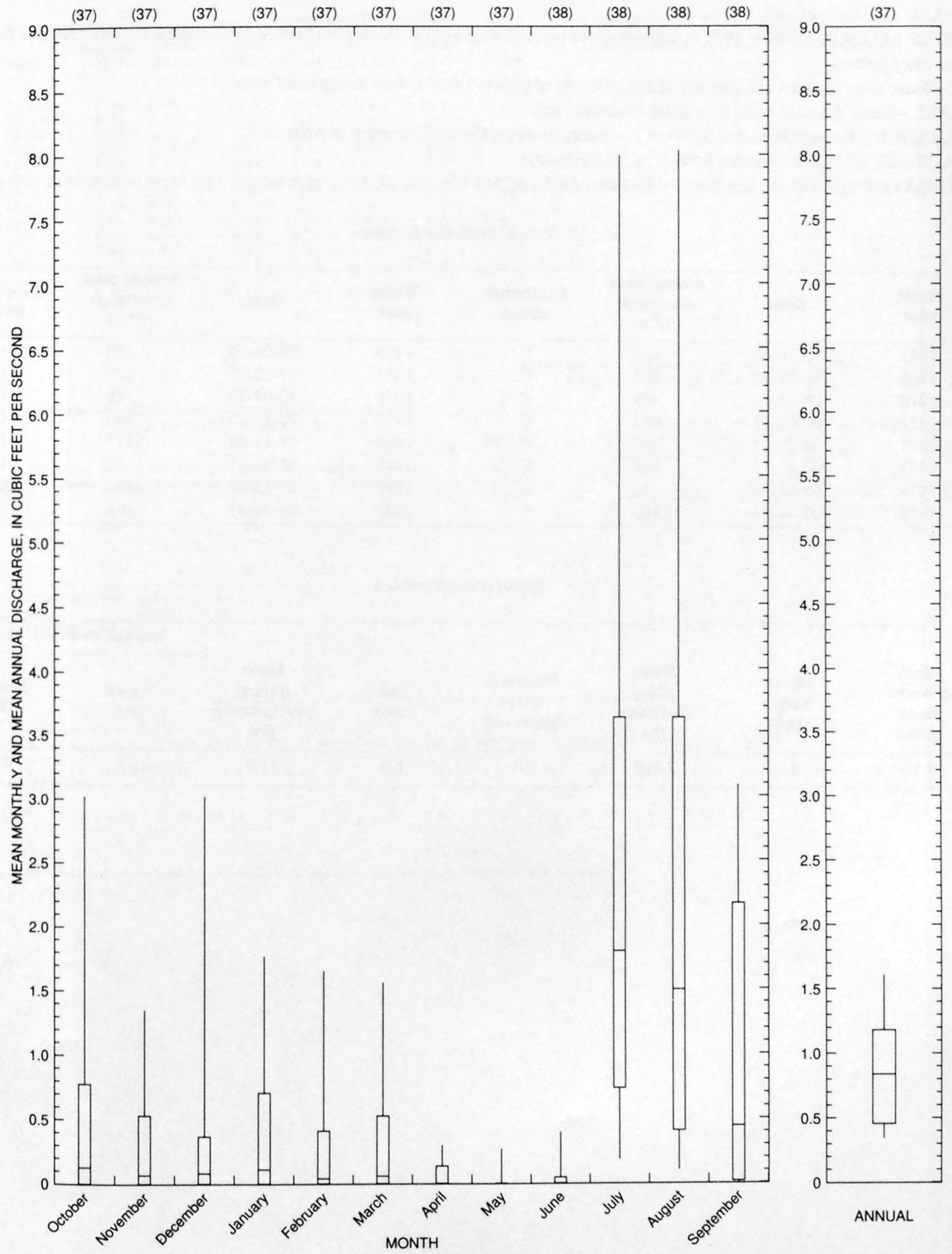


\section{HIGH SCHOOL WASH AT TUCSON, AZ}

LOCATION.--Lat 32 13'28", long $110^{\circ} 56^{\prime} 48^{\prime \prime}$, in SE $1 / 4 \mathrm{SE}^{1} / 4$ sec.7, T.14 S., R.14 E., Pima County, Hydrologic Unit 15050301 , on right bank 200 ft upstream from Cherry Avenue in Tucson.

DRAINAGE AREA.--0.95 $\mathrm{mi}^{2}$.

PERIOD OF RECORD.--October 1973 to September 1983 (publication by U.S. Geological Survey discontinued; records available from cooperator listed below).

GAGE.--Water-stage recorder and concrete flume. Altitude of gage is 2,415 ft, from topographic map.

REMARKS.--Entire drainage basin is an urban residential area.

COOPERATION.--Records furnished by Water Resources Research Center, University of Arizona.

AVERAGE DISCHARGE.--10 years, $0.109 \mathrm{ft}^{3} / \mathrm{s}, 79.0$ acre- $\mathrm{ft} / \mathrm{yr}$.

EXTREMES FOR PERIOD OF RECORD.--Maximum discharge, $464 \mathrm{ft}^{3} / \mathrm{s} \mathrm{July} 25,1982$, gage height, $5.00 \mathrm{ft}$; no flow most of each year.

Annual peak discharges

\begin{tabular}{|c|c|c|c|c|c|c|c|}
\hline $\begin{array}{l}\text { Water } \\
\text { year }\end{array}$ & Date & $\begin{array}{c}\text { Annual peak } \\
\text { discharge } \\
\left(\mathrm{ft}^{3} / \mathrm{s}\right)\end{array}$ & $\begin{array}{c}\text { Discharge } \\
\text { codes }\end{array}$ & $\begin{array}{l}\text { Water } \\
\text { year }\end{array}$ & Date & $\begin{array}{c}\text { Annual peak } \\
\text { discharge } \\
\left(\mathrm{ft}^{3} / \mathrm{s}\right)\end{array}$ & $\begin{array}{c}\text { Discharge } \\
\text { codes }\end{array}$ \\
\hline 1968 & $08-10-68$ & 486 & $\mathrm{C}$ & 1976 & $09-04-76$ & 156 & C \\
\hline 1969 & 08-01-69 & 341 & $\mathrm{C}$ & 1977 & $07-22-77$ & 129 & $\mathrm{C}$ \\
\hline 1970 & $08-11-70$ & 409 & $\mathrm{C}$ & 1978 & $10-07-77$ & 178 & $\mathrm{C}$ \\
\hline 1971 & $08-08-71$ & 664 & $\mathrm{C}$ & 1979 & $08-12-79$ & 346 & $\mathrm{C}$ \\
\hline 1972 & $08-12-72$ & 800 & $\mathrm{C}$ & 1980 & $08-13-80$ & 357 & $\mathrm{C}$ \\
\hline 1973 & $07-07-73$ & 204 & $\mathrm{C}$ & 1981 & $05-01-81$ & 85 & $\mathrm{C}$ \\
\hline 1974 & $08-02-74$ & 126 & $\mathrm{C}$ & 1982 & $07-25-82$ & 464 & $\mathrm{C}$ \\
\hline 1975 & $07-16-75$ & 195 & $\mathrm{C}$ & 1983 & $08-16-83$ & 163 & $\mathrm{C}$ \\
\hline
\end{tabular}

Basin characteristics

\begin{tabular}{cccccccc}
\hline $\begin{array}{c}\text { Main } \\
\text { channel } \\
\text { slope } \\
(\mathrm{ft} / \mathrm{mi})\end{array}$ & $\begin{array}{c}\text { Stream } \\
\text { length } \\
(\mathrm{mi})\end{array}$ & $\begin{array}{c}\text { Mean } \\
\text { basin } \\
\text { elevation } \\
(\mathrm{ft})\end{array}$ & $\begin{array}{c}\text { Forested } \\
\text { area } \\
\text { (percent) }\end{array}$ & $\begin{array}{c}\text { Soil } \\
\text { index }\end{array}$ & $\begin{array}{c}\text { Mean } \\
\text { annual } \\
\text { precipitation } \\
\text { (in) }\end{array}$ & $\begin{array}{c}\text { Rainfall intensity, 24-hour } \\
\text { (in) }\end{array}$ & $\begin{array}{c}50 \text {-year } \\
\text { (in) }\end{array}$ \\
\hline 1.11 & 1.6 & 2,460 & 0.0 & 1.0 & 11.0 & 1.7 \\
\hline
\end{tabular}


09483010 HIGH SCHOOL WASH AT TUCSON, AZ--Continued

MEAN MONTHLY AND ANNUAL DISCHARGES $1974 \cdot 83$

\begin{tabular}{|c|c|c|c|c|c|c|}
\hline MONTH & $\begin{array}{l}\text { MAXIMUM } \\
(\text { FT3/S) }\end{array}$ & $\begin{array}{l}\text { MINIMUM } \\
\text { (FT3/S) }\end{array}$ & $\begin{array}{c}\text { MEAN } \\
(\text { FT3/S) }\end{array}$ & $\begin{array}{l}\text { STAN- } \\
\text { DARD } \\
\text { DEVIA- } \\
\text { TION } \\
(\text { FT3/S) }\end{array}$ & $\begin{array}{l}\text { COEFFI- } \\
\text { CIENT OF } \\
\text { VARI- } \\
\text { ATION }\end{array}$ & $\begin{array}{c}\text { PERCENT } \\
\text { OF } \\
\text { ANNUAL } \\
\text { RUNOFF }\end{array}$ \\
\hline OCTOBER & 0.38 & 0.00 & 0.10 & 0.16 & 1.5 & 8.0 \\
\hline NOVEMBER & 0.20 & 0.00 & 0.05 & 0.07 & 1.4 & 4.0 \\
\hline DECEMBER & 0.35 & 0.00 & 0.07 & 0.11 & 1.5 & 5.7 \\
\hline JANUARY & 0.28 & 0.00 & 0.12 & 0.11 & 0.92 & 9.5 \\
\hline FEBRUARY & 0.31 & 0.00 & 0.07 & 0.12 & 1.6 & 5.6 \\
\hline MARCH & 0.26 & 0.00 & 0.09 & 0.07 & 0.78 & 7.1 \\
\hline APRIL & 0.10 & 0.00 & 0.02 & 0.03 & 2.0 & 1.2 \\
\hline MAY & 0.10 & 0.00 & 0.02 & 0.03 & 1.9 & 1.4 \\
\hline JUNE & 0.09 & 0.00 & 0.02 & 0.03 & 1.5 & 1.5 \\
\hline JULY & 0.57 & 0.04 & 0.23 & 0.19 & 0.81 & 17.8 \\
\hline AUGUST & 0.82 & 0.01 & 0.27 & 0.30 & 1.1 & 20.9 \\
\hline SEPTEMBER & 0.78 & 0.00 & 0.22 & 0.24 & 1.1 & 17.4 \\
\hline ANNUAL & 0.20 & 0.05 & 0.11 & 0.05 & 0.43 & 100 \\
\hline
\end{tabular}

MAGNITUDE AND PROBABILITY OF INSTANTANEOUS PEAK FLOW BASED ON PERIOD OF RECORD 1968.83

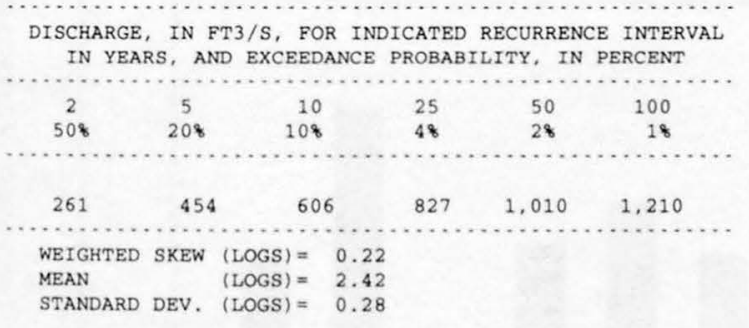

MAGNITUDE AND PROBABILITY OF ANNUAL LOW FLOW BASED ON PERIOD OF RECORD $1975 \cdot 83$

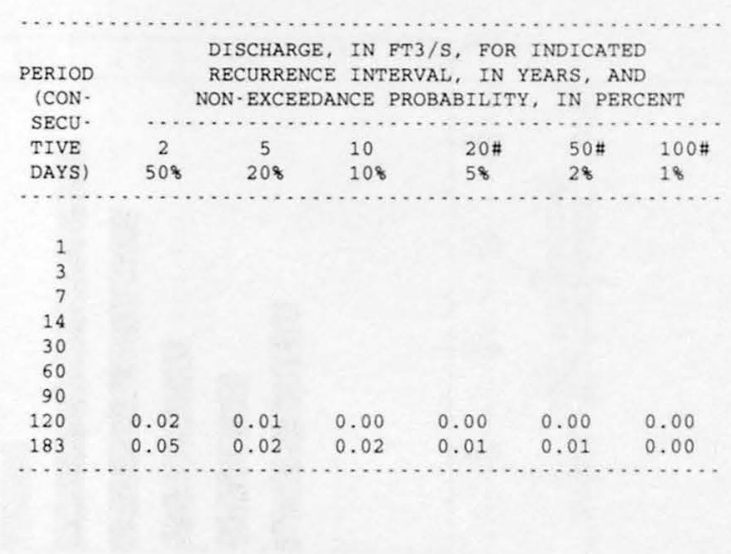

MAGNITUDE AND PROBABILITY OF ANNUAL HIGH FLOW BASED ON PERIOD OF RECORD 1974-83

\begin{tabular}{|c|c|c|c|c|c|c|}
\hline \multirow{2}{*}{$\begin{array}{l}\text { PERIOD } \\
\text { (CON- } \\
\text { SECU. }\end{array}$} & & $\begin{array}{r}\text { DI } \\
\text { RECURREI } \\
\text { EXCEEDAI }\end{array}$ & $\begin{array}{l}\text { CHARGE } \\
\text { INTE } \\
\text { PROB }\end{array}$ & $\begin{array}{l}\text { IN FT3, } \\
\text { AL, IN } \\
\text { ILITY, }\end{array}$ & $\begin{array}{l}\text { S, FOR } \\
\text { YEARS, } \\
\text { IN PERC }\end{array}$ & $\begin{array}{l}\text { DICATED } \\
\text { D } \\
\text { T }\end{array}$ \\
\hline & & & & & ....... & \\
\hline TIVE & 2 & 5 & 10 & $25 \#$ & $50=$ & $100 \#$ \\
\hline DAYS) & 508 & 208 & 108 & 48 & 28 & 18 \\
\hline 1 & 6.0 & 10 & 14 & 20 & 25 & 31 \\
\hline 3 & 2.6 & 4.6 & 6.3 & 9.0 & 11 & 14 \\
\hline 7 & 1.3 & 2.2 & 2.9 & 4.0 & 4.9 & 5.8 \\
\hline 15 & 0.72 & 1.2 & 1.5 & 2.0 & 2.3 & 2.8 \\
\hline 30 & 0.43 & 0.72 & 0.98 & 1.4 & 1.8 & 2.3 \\
\hline 60 & 0.30 & 0.52 & 0.72 & 1.0 & 1.4 & 1.7 \\
\hline 90 & 0.25 & 0.41 & 0.53 & 0.71 & 0.86 & 1.0 \\
\hline
\end{tabular}

DURATION TABLE OF DAILY MEAN FLOW FOR PERIOD OF RECORD $1974 \cdot 83$ DISCHARGE, IN FT3/S, WHICH WAS EQUALED OR EXCEEDED FOR INDICATED PERCENT OF TIME

\begin{tabular}{|c|c|c|c|c|c|c|c|c|c|c|c|c|c|c|c|c|}
\hline 18 & 58 & 108 & 158 & 208 & 308 & 408 & 508 & 608 & 708 & 808 & 908 & 958 & 988 & 998 & 99.58 & 99.98 \\
\hline 3.0 & 0.34 & 0.00 & 0.00 & 0.00 & 0.00 & 0.00 & 0.00 & 0.00 & 0.00 & 0.00 & 0.00 & 0.00 & 0.00 & 0.00 & 0.00 & 0.00 \\
\hline
\end{tabular}

\# Reliability of values in column is uncertain, and potential errors are large. 
09483010 HIGH SCHOOL WASH AT TUCSON, AZ--Continued
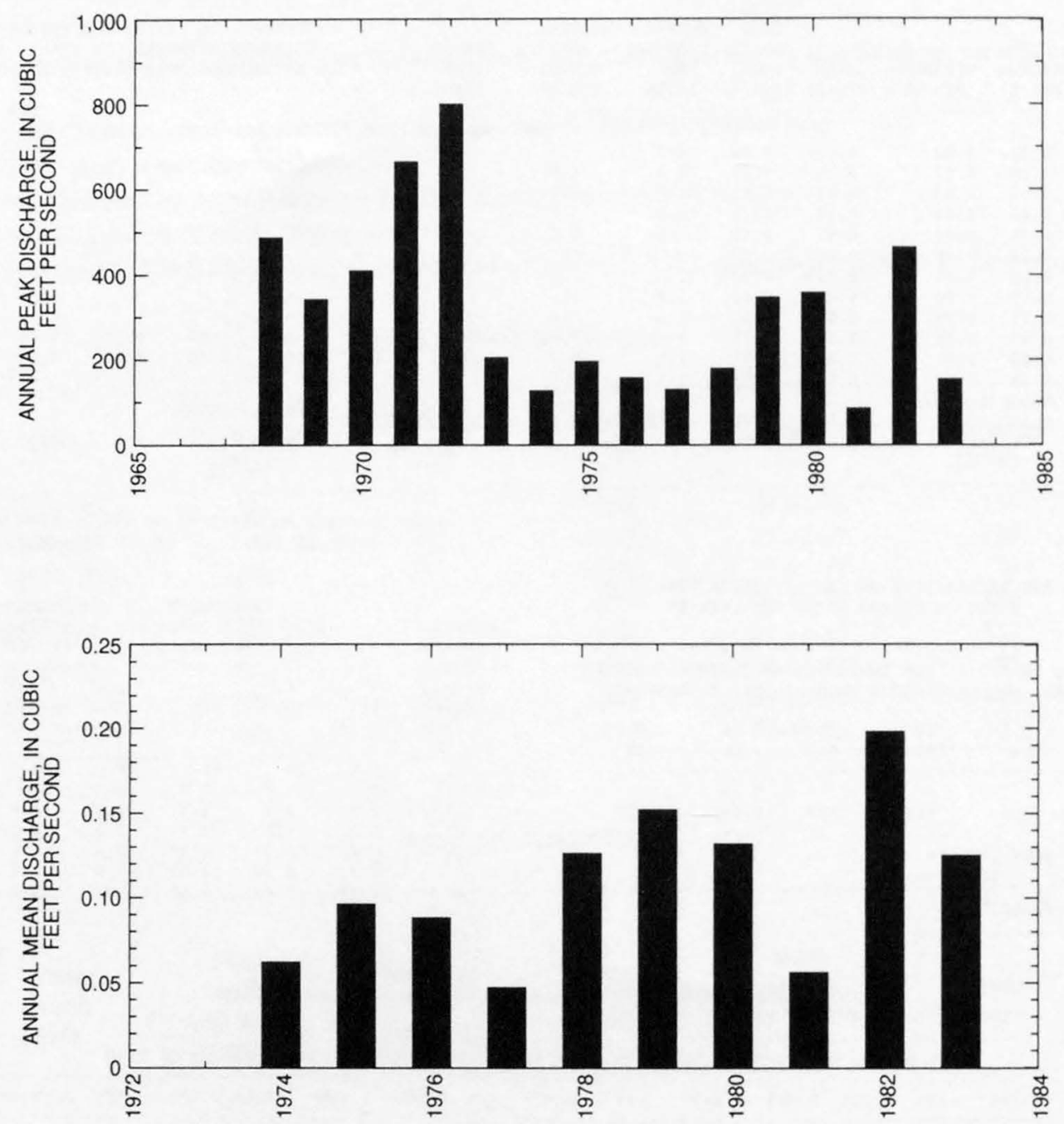
09483010 HIGH SCHOOL WASH AT TUCSON, AZ--Continued
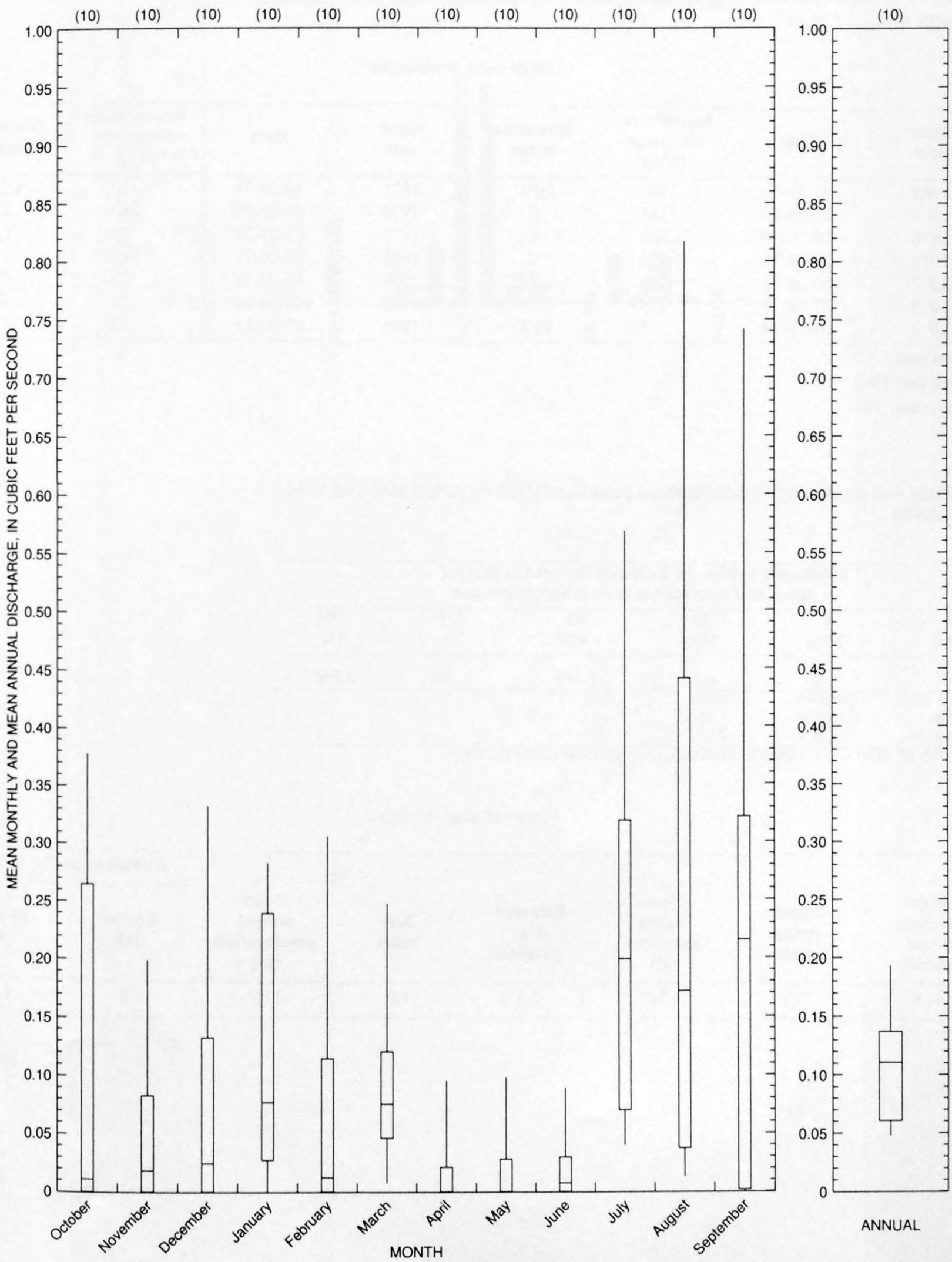


\section{SILVERCROFT WASH AT TUCSON, AZ}

LOCATION.--Lat $32^{\circ} 13^{\prime} 53^{\prime \prime}$, long $111^{\circ} 00^{\prime} 10^{\prime \prime}$, NW $1 / 4$ sec.10, T.14 S., R.13 E., Pima County, Hydrologic Unit $15050301,0.1$ mi west of Silverbell.

Road, 0.3 mi northwest of St. Mary's Hospital, and 0.4 mi north of Anklam Road at Tucson.

DRAINAGE AREA.--2.74 $\mathrm{mi}^{2}$.

Annual peak discharges

\begin{tabular}{|c|c|c|c|c|c|c|c|}
\hline $\begin{array}{l}\text { Water } \\
\text { year }\end{array}$ & Date & $\begin{array}{c}\text { Annual peak } \\
\text { discharge } \\
\left(\mathrm{ft}^{3} / \mathrm{s}\right)\end{array}$ & $\begin{array}{c}\text { Discharge } \\
\text { codes }\end{array}$ & $\begin{array}{l}\text { Water } \\
\text { year }\end{array}$ & Date & $\begin{array}{c}\text { Annual peak } \\
\text { discharge } \\
\left(\mathrm{ft}^{3} / \mathrm{s}\right)\end{array}$ & $\begin{array}{c}\text { Discharge } \\
\text { codes }\end{array}$ \\
\hline 1965 & $07-10-65$ & ${ }^{1} 560$ & HP,C & 1975 & $10-30-74$ & 10 & LT,C \\
\hline 1969 & $08-00-69$ & 2365 & C & 1976 & $09-25-76$ & 310 & C \\
\hline 1970 & $07-20-70$ & ${ }^{3} 1,500$ & $\mathrm{C}$ & 1977 & $01-01-77$ & 63 & $\mathrm{C}$ \\
\hline 1971 & $08-00-71$ & 1,450 & C & 1978 & $10-06-77$ & 290 & C \\
\hline 1972 & $00-00-72$ & 100 & ES,C & 1979 & $07-20-79$ & 19 & $\mathrm{C}$ \\
\hline 1973 & $07-00-73$ & 115 & C & 1980 & $08-24-80$ & 72 & $\mathrm{C}$ \\
\hline 1974 & $07-20-74$ & 5.0 & LT,C & 1981 & $07-21-81$ & 22 & $\mathrm{C}$ \\
\hline
\end{tabular}

${ }^{1}$ Highest since 1961.

${ }^{2}$ Highest since 1965 .

${ }^{3}$ Highest since 1961 .

Magnitude and probability of instantaneous peak flow based on period of record 1965 1969-81

\begin{tabular}{|c|c|c|c|c|c|}
\hline \multicolumn{6}{|c|}{$\begin{array}{l}\text { Discharge, in } \mathrm{ft}^{3} / \mathrm{s} \text {, for indicated recurrence interval } \\
\text { in years, and exceedance probablility, in percent }\end{array}$} \\
\hline 2 & 5 & 10 & 25 & $50 t$ & $100 t$ \\
\hline $50 \%$ & $20 \%$ & $10 \%$ & $4 \%$ & $2 \%$ & $1 \%$ \\
\hline 90 & 332 & 659 & 1,370 & 2,200 & 3,360 \\
\hline Weighted skew & $(\log s)=$ & 0.01 & & & \\
\hline Mean & $(\log s)=$ & 1.96 & & & \\
\hline Standard dev. & $(\log s)=$ & 0.67 & & & \\
\hline
\end{tabular}

Basin characteristics

\begin{tabular}{|c|c|c|c|c|c|c|c|}
\hline \multirow[b]{2}{*}{$\begin{array}{l}\text { Main } \\
\text { channel } \\
\text { slope } \\
\text { (ft/mi) }\end{array}$} & \multirow[b]{2}{*}{$\begin{array}{l}\text { Stream } \\
\text { length } \\
\text { (mi) }\end{array}$} & \multirow[b]{2}{*}{$\begin{array}{c}\text { Mean } \\
\text { basin } \\
\text { elevation } \\
(\mathrm{ft})\end{array}$} & \multirow[b]{2}{*}{$\begin{array}{l}\text { Forested } \\
\text { area } \\
\text { (percent) }\end{array}$} & \multirow[b]{2}{*}{$\begin{array}{c}\text { Soil } \\
\text { index }\end{array}$} & \multirow[b]{2}{*}{$\begin{array}{c}\text { Mean } \\
\text { annual } \\
\text { precipitation } \\
\text { (in) }\end{array}$} & \multicolumn{2}{|c|}{ Rainfall intensity, 24-hour } \\
\hline & & & & & & $\begin{array}{l}\text { 2-year } \\
\text { (in) }\end{array}$ & $\begin{array}{l}\text { 50-year } \\
\text { (in) }\end{array}$ \\
\hline 44.6 & 3.3 & 2,540 & 0.0 & 1.0 & 11.8 & 1.9 & 4.4 \\
\hline
\end{tabular}


09483025 SILVERCROFT WASH AT TUCSON, AZ--Continued

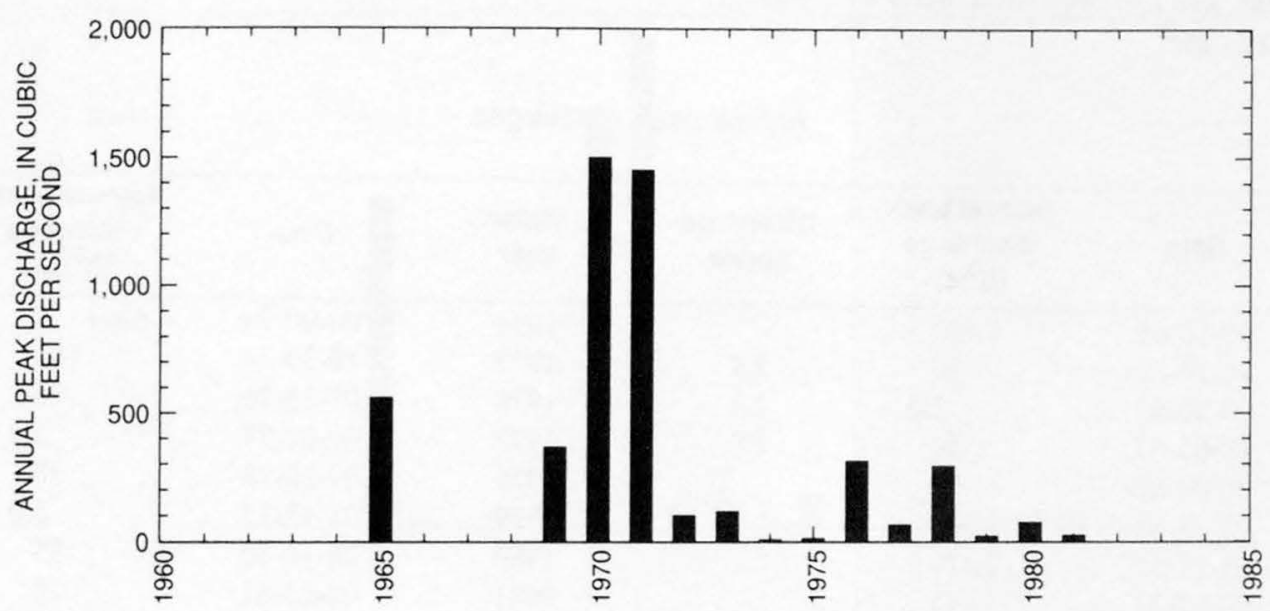




\section{ANKLAM WASH AT TUCSON, AZ}

LOCATION.--Lat $32^{\circ} 13^{\prime} 30^{\prime \prime}$, long $111^{\circ} 01^{\prime} 50^{\prime \prime}, \mathrm{SE}^{1} / 4^{\mathrm{sec}} \mathrm{s}, \mathrm{T} .14 \mathrm{~S} ., \mathrm{R} .13$ E., Pima County, Hydrologic Unit 15050301, at Anklam Road, 1 mi west of Tucson city limits, and 2 mi west of St. Mary's Hospital.

DRAINAGE AREA.--2.11 $\mathrm{mi}^{2}$.

Annual peak discharges

\begin{tabular}{cccccccc}
\hline $\begin{array}{c}\text { Water } \\
\text { year }\end{array}$ & Date & $\begin{array}{c}\text { Annual peak } \\
\text { discharge } \\
\left(\mathbf{f t}^{3} / \mathbf{s}\right)\end{array}$ & $\begin{array}{c}\text { Discharge } \\
\text { codes }\end{array}$ & $\begin{array}{c}\text { Water } \\
\text { year }\end{array}$ & Date & $\begin{array}{c}\text { Annual peak } \\
\text { discharge } \\
\left(\mathrm{ft}^{3} / \mathbf{s}\right)\end{array}$ & $\begin{array}{c}\text { Discharge } \\
\text { codes }\end{array}$ \\
\hline 1965 & $07-10-65$ & 1,600 & & 1974 & $00-00-74$ & 0 & \\
1966 & $12-10-65$ & 30 & ES & 1975 & $10-30-74$ & 160 & \\
1967 & $00-00-67$ & 2.0 & ES & 1976 & $09-25-76$ & 75 & \\
1968 & $10-03-67$ & 50 & ES & 1977 & $01-01-77$ & 5.0 & ES \\
1969 & $08-00-69$ & 98 & & 1978 & $07-30-78$ & 38 & \\
1970 & $07-19-70$ & 2,000 & & 1979 & $01-18-79$ & 5.0 & \\
1971 & $08-17-71$ & 2,420 & & 1980 & $08-14-80$ & 35 & \\
1972 & $09-07-72$ & 75 & ES & 1981 & $08-13-81$ & & 76 \\
1973 & $07-15-73$ & 140 & ES & & & & \\
\hline
\end{tabular}

Magnitude and probability of instantaneous peak flow based on period of record 1965-81

\begin{tabular}{|c|c|c|c|c|c|}
\hline \multicolumn{6}{|c|}{$\begin{array}{l}\text { Discharge, in } \mathrm{ft}^{3} / \mathrm{s} \text {, for indicated recurrence interval } \\
\text { in years, and exceedance probablility, in percent }\end{array}$} \\
\hline $\begin{array}{c}2 \dagger \\
50 \%\end{array}$ & $\begin{array}{c}5 t \\
20 \%\end{array}$ & $\begin{array}{r}10 \dagger \\
10 \%\end{array}$ & $\begin{array}{l}25 t \\
4 \%\end{array}$ & $\begin{array}{l}50 \dagger \\
2 \%\end{array}$ & $\begin{array}{c}100 \dagger \\
1 \%\end{array}$ \\
\hline 75.6 & 362 & 855 & 2,210 & 4,140 & 7,390 \\
\hline Weighted skew & $(\log s)=$ & 0.24 & & & \\
\hline Mean & $(\log s)=$ & 1.91 & & & \\
\hline Standard dev. & $(\log s)=$ & 0.78 & & & \\
\hline
\end{tabular}

Basin characteristics

\begin{tabular}{ccccccccc}
\hline $\begin{array}{c}\text { Main } \\
\text { channel } \\
\text { slope } \\
(\mathrm{ft} / \mathrm{mi})\end{array}$ & $\begin{array}{c}\text { Stream } \\
\text { length } \\
\text { (mi) }\end{array}$ & $\begin{array}{c}\text { Mean } \\
\text { basin } \\
\text { elevation } \\
\text { (ft) }\end{array}$ & $\begin{array}{c}\text { Forested } \\
\text { area } \\
\text { (percent) }\end{array}$ & $\begin{array}{c}\text { Soil } \\
\text { index }\end{array}$ & $\begin{array}{c}\text { Mean } \\
\text { annual } \\
\text { precipitation } \\
\text { (in) }\end{array}$ & $\begin{array}{c}\text { 2-year } \\
\text { (in) }\end{array}$ & $\begin{array}{c}\text { 50-year } \\
\text { (in) }\end{array}$ \\
\hline 298 & 2.1 & 2,700 & 0.0 & 1.0 & 11.8 & 1.9 & 4.4 \\
\hline
\end{tabular}


09483030 ANKLAM WASH AT TUCSON, AZ--Continued

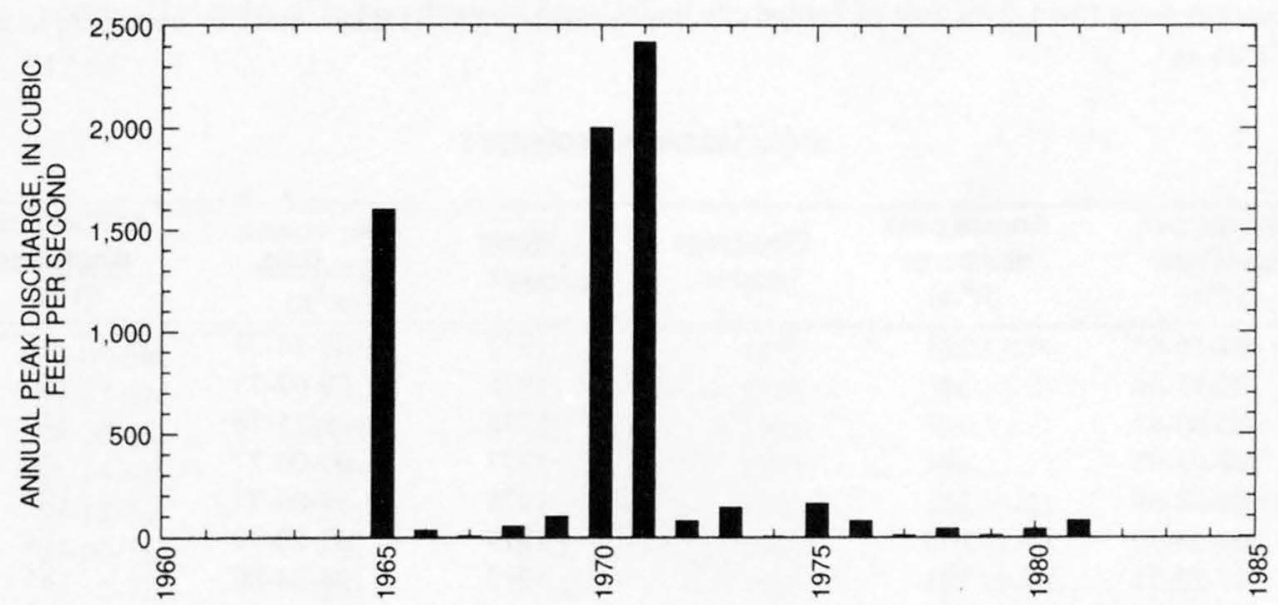




\section{WEST SPEEDWAY WASH NEAR TUCSON, AZ}

LOCATION.--Lat $32^{\circ} 14^{\prime} 20^{\prime \prime}$, long $111^{\circ} 02^{\prime} 43^{\prime \prime}, \mathrm{SE}^{1} / \mathrm{SE}^{1} / 4 \mathrm{sec} .6$, T.14 S., R. 13 E., Pima County, Hydrologic Unit 15050301, at driveway to Power substation off West Speedway Road, 2 mi west of Tucson city limits, and 3 mi northwest of St. Mary's Hospital.

DRAINAGE AREA.--0.46 $\mathrm{mi}^{2}$.

Annual peak discharges

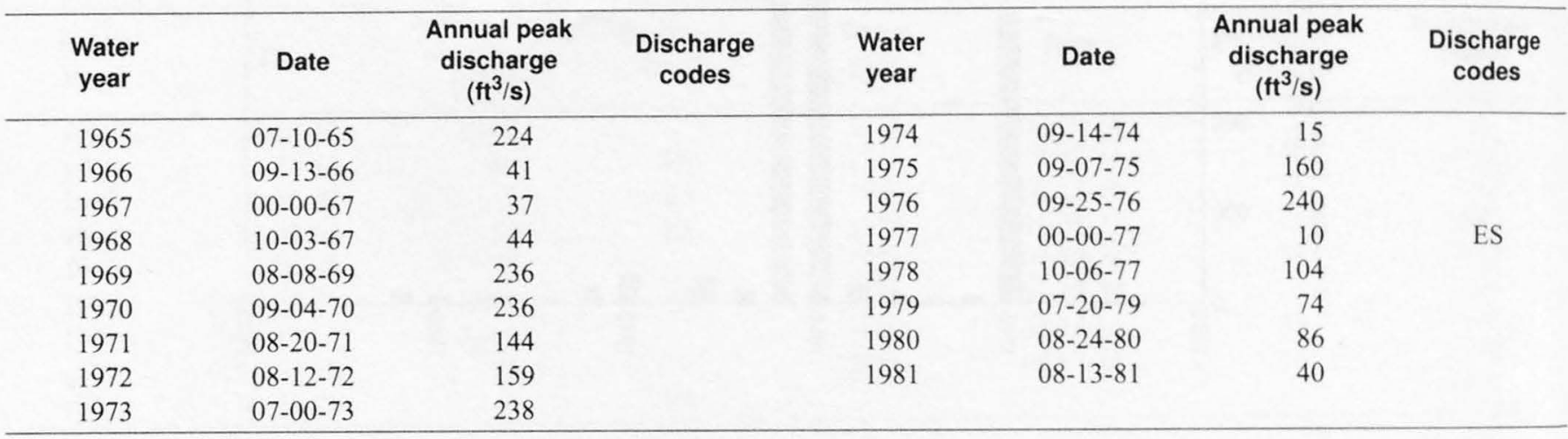

Magnitude and probability of instantaneous peak flow based on period of record 1965-81

\begin{tabular}{|c|c|c|c|c|c|}
\hline \multicolumn{6}{|c|}{$\begin{array}{l}\text { Discharge, in } \mathrm{ft}^{3} / \mathrm{s} \text {, for indicated recurrence interval } \\
\text { in years, and exceedance probablility, in percent }\end{array}$} \\
\hline 2 & 5 & 10 & 25 & $50 t$ & $100 t$ \\
\hline $50 \%$ & $20 \%$ & $10 \%$ & $4 \%$ & $2 \%$ & $1 \%$ \\
\hline 92.2 & 202 & 291 & 419 & 521 & 627 \\
\hline Weighted skew & $(\log s)=$ & -0.05 & & & \\
\hline Mean & $(\log s)=$ & 1.93 & & & \\
\hline Standard dev. & $(\log s)=$ & 0.43 & & & \\
\hline
\end{tabular}

Basin characteristics

\begin{tabular}{cccccccc}
\hline $\begin{array}{c}\text { Main } \\
\text { channel } \\
\text { slope } \\
(\mathrm{ft} / \mathrm{mi})\end{array}$ & $\begin{array}{c}\text { Stream } \\
\text { length } \\
(\mathrm{mi})\end{array}$ & $\begin{array}{c}\text { Mean } \\
\text { basin } \\
\text { elevation } \\
(\mathrm{ft})\end{array}$ & $\begin{array}{c}\text { Forested } \\
\text { area } \\
\text { (percent) }\end{array}$ & $\begin{array}{c}\text { Soil } \\
\text { index }\end{array}$ & $\begin{array}{c}\text { Mean } \\
\text { annual } \\
\text { precipitation } \\
\text { (in) }\end{array}$ & $\begin{array}{c}\text { R-year } \\
\text { (in) }\end{array}$ & $\begin{array}{c}50 \text {-year } \\
\text { (in) }\end{array}$ \\
\hline 364 & 1.1 & 2,750 & 0.0 & 1.0 & 11.8 & 1.9
\end{tabular}

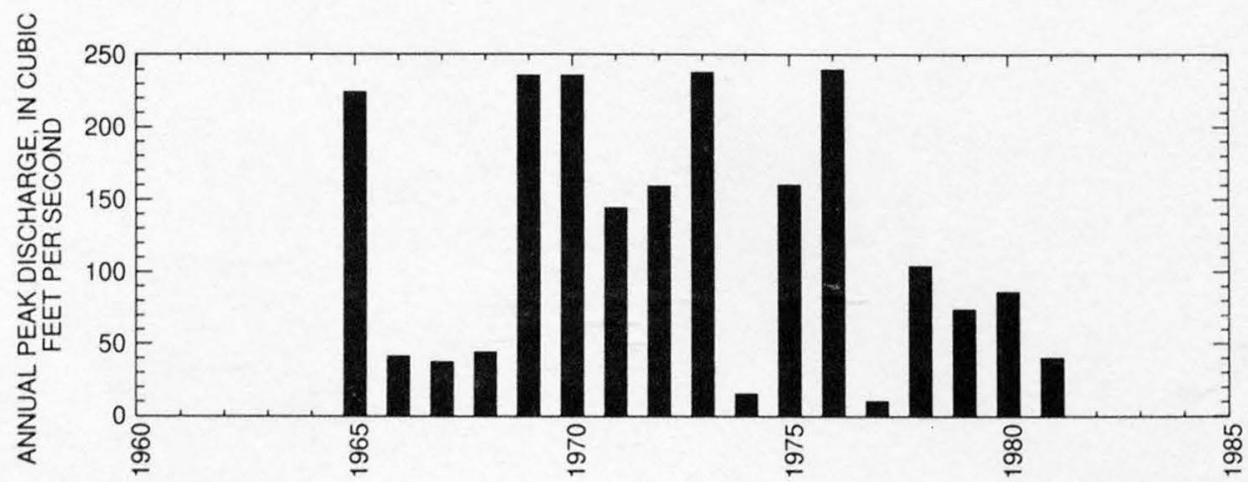


09483042 CEMETERY WASH AT TUCSON, AZ

LOCATION.--Lat $32^{\circ} 15^{\prime} 50^{\prime \prime}$, long $110^{\circ} 58^{\prime} 38^{\prime \prime}$, NW $1 / 4 \mathrm{NW}^{1} / 4$ sec.36, T.13 S., R. 13 E., Pima County, Hydrologic Unit 15050301 , at U.S. Highway $89,0.25$ mi north of junction with State Highway 84 in Tucson.

DRAINAGE AREA.--1.17 $\mathrm{mi}^{2}$.

Annual peak discharges

\begin{tabular}{|c|c|c|c|c|c|c|c|}
\hline $\begin{array}{l}\text { Water } \\
\text { year }\end{array}$ & Date & $\begin{array}{c}\text { Annual peak } \\
\text { discharge } \\
\left(\mathrm{ft}^{3} / \mathrm{s}\right)\end{array}$ & $\begin{array}{l}\text { Discharge } \\
\text { codes }\end{array}$ & $\begin{array}{l}\text { Water } \\
\text { year }\end{array}$ & Date & $\begin{array}{c}\text { Annual peak } \\
\text { discharge } \\
\left(\mathrm{ft}^{3} / \mathrm{s}\right)\end{array}$ & $\begin{array}{c}\text { Discharge } \\
\text { codes }\end{array}$ \\
\hline 1966 & $08-00-66$ & 229 & $\mathrm{C}$ & 1979 & $08-12-79$ & 375 & $\mathrm{C}$ \\
\hline 1967 & $07-17-67$ & 396 & $\mathrm{C}$ & 1980 & $08-14-80$ & 225 & $\mathrm{C}$ \\
\hline 1968 & $08-20-68$ & 600 & $\mathrm{C}$ & 1981 & $04-30-81$ & 156 & $\mathrm{C}$ \\
\hline 1969 & $11-14-68$ & 570 & $\mathrm{C}$ & 1982 & $08-23-82$ & 540 & $\mathrm{C}$ \\
\hline 1970 & $08-18-70$ & 285 & C & 1983 & $08-15-83$ & 209 & $\mathrm{C}$ \\
\hline 1971 & $08-00-71$ & 100 & $\mathrm{ES}, \mathrm{C}$ & 1984 & $10-02-83$ & 456 & $\mathrm{C}$ \\
\hline 1972 & $08-12-72$ & 400 & $\mathrm{ES}, \mathrm{C}$ & 1985 & $00-00-85$ & 172 & $\mathrm{C}$ \\
\hline 1973 & $07-00-73$ & 150 & ES,C & 1986 & $07-15-86$ & 264 & $\mathrm{C}$ \\
\hline 1974 & $09-06-74$ & 580 & C & 1987 & $02-25-87$ & 52 & $\mathrm{C}$ \\
\hline 1975 & $07-17-75$ & 290 & $\mathrm{C}$ & 1988 & $08-20-88$ & 216 & $\mathrm{C}$ \\
\hline 1976 & $09-25-76$ & 360 & $\mathrm{C}$ & 1989 & $07-26-89$ & 262 & $\mathrm{C}$ \\
\hline 1977 & $09-10-77$ & 205 & $\mathrm{C}$ & 1990 & $07-24-90$ & 302 & $\mathrm{C}$ \\
\hline 1978 & $07-30-78$ & 160 & $\mathrm{C}$ & & & & \\
\hline
\end{tabular}

Discharge rating table developed October 1981

\begin{tabular}{crcc}
\hline $\begin{array}{c}\text { Gage height } \\
(\mathbf{f t})\end{array}$ & $\begin{array}{c}\text { Discharge } \\
\left(\mathrm{ft}^{3} / \mathbf{s}\right)\end{array}$ & $\begin{array}{c}\text { Gage height } \\
(\mathbf{f t})\end{array}$ & $\begin{array}{c}\text { Discharge } \\
\left(\mathbf{f t}^{3} / \mathbf{s}\right)\end{array}$ \\
\hline .8 & 47 & 3.0 & 278 \\
1.0 & 63 & 3.5 & 346 \\
1.5 & 108 & 4.0 & 418 \\
2.0 & 159 & 4.5 & 493 \\
2.5 & 215 & 4.8 & 540 \\
\hline
\end{tabular}

Magnitude and probability of instantaneous peak flow based on period of record 1966-90

\begin{tabular}{|c|c|c|c|c|c|}
\hline \multicolumn{6}{|c|}{$\begin{array}{l}\text { Discharge, in } \mathrm{ft}^{3} / \mathrm{s} \text {, for indicated recurrence interval } \\
\text { in years, and exceedance probablility, in percent }\end{array}$} \\
\hline 2 & 5 & 10 & 25 & $50 t$ & $100 \dagger$ \\
\hline $50 \%$ & $20 \%$ & $10 \%$ & $4 \%$ & $2 \%$ & $1 \%$ \\
\hline 274 & 418 & 517 & 646 & 743 & 842 \\
\hline Weighted skew & $(\log s)=$ & -0.15 & & & \\
\hline Mean & $(\log s)=$ & 2.43 & & & \\
\hline Standard dev. & $(\log s)=$ & 0.22 & & & \\
\hline
\end{tabular}


09483042 CEMETERY WASH AT TUCSON, AZ--Continued

Basin characteristics

\begin{tabular}{cccccccc}
\hline $\begin{array}{c}\text { Main } \\
\text { channel } \\
\text { slope } \\
(\mathrm{ft} / \mathrm{mi})\end{array}$ & $\begin{array}{c}\text { Stream } \\
\text { length } \\
(\mathrm{mi})\end{array}$ & $\begin{array}{c}\text { Mean } \\
\text { basin } \\
\text { elevation } \\
(\mathrm{ft})\end{array}$ & $\begin{array}{c}\text { Forested } \\
\text { area } \\
\text { (percent) }\end{array}$ & $\begin{array}{c}\text { Soil } \\
\text { index }\end{array}$ & $\begin{array}{c}\text { Mean } \\
\text { annual } \\
\text { precipitation } \\
\text { (in) }\end{array}$ & $\begin{array}{c}\text { Rainfall intensity, 24-hour } \\
\text { 2-year } \\
\text { (in) }\end{array}$ & $\begin{array}{c}50-\text { year } \\
\text { (in) }\end{array}$ \\
\hline 40.0 & 2.2 & 2,370 & 0.0 & 1.0 & 11.0 & 1.9 \\
\hline
\end{tabular}

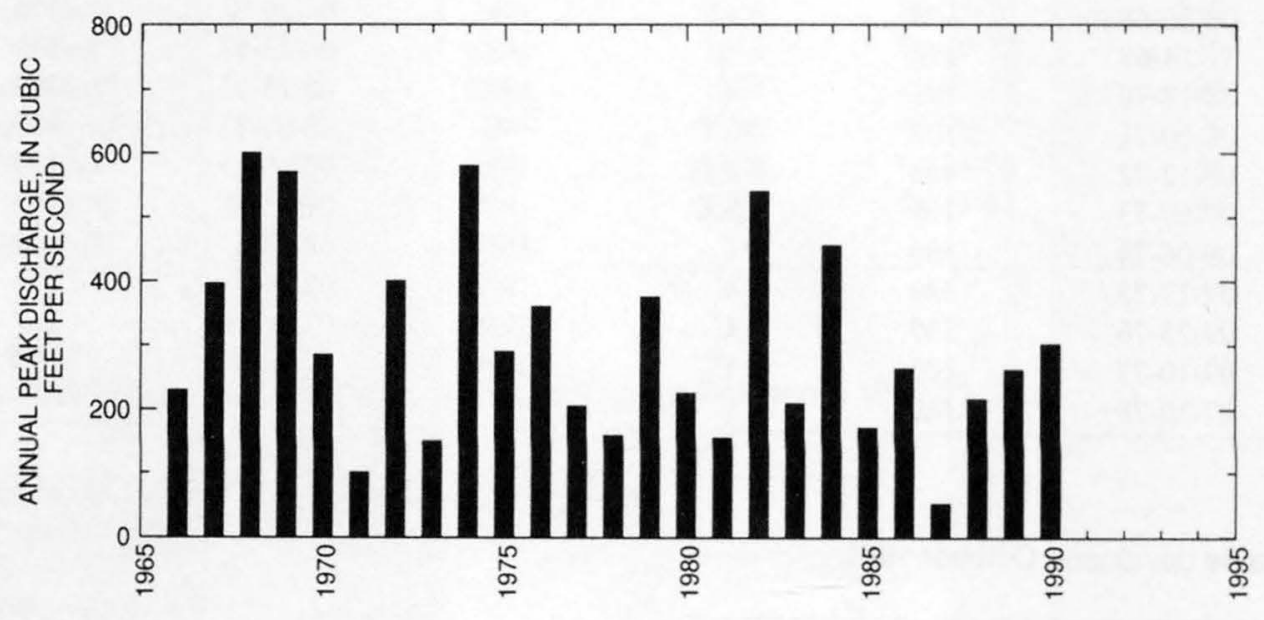


09483045 FLOWING WELLS WASH AT TUCSON, AZ

LOCATION.--Lat $32^{\circ} 15^{\prime} 55^{\prime \prime}$, long $110^{\circ} 59^{\prime} 40^{\prime \prime}, \mathrm{SW}^{1} / \mathrm{SW}^{1} / 4 \mathrm{sec} .26$, T.13 S., R.13 E., Pima County, Hydrologic Unit 15050301 , at intersection of

Ft. Lowell Road and Flowing Wells Road in Tucson city limits.

DRAINAGE AREA.-- $3.53 \mathrm{mi}^{2}$.

Annual peak discharges

\begin{tabular}{ccccccrr}
\hline $\begin{array}{c}\text { Water } \\
\text { year }\end{array}$ & Date & $\begin{array}{c}\text { Annual peak } \\
\text { discharge } \\
\left(\mathbf{f t}^{\mathbf{3}} \mathbf{s}\right)\end{array}$ & $\begin{array}{c}\text { Discharge } \\
\text { codes }\end{array}$ & $\begin{array}{c}\text { Water } \\
\text { year }\end{array}$ & Date & $\begin{array}{c}\text { Annual peak } \\
\text { discharge } \\
\left(\mathbf{f t}^{\mathbf{3}} / \mathbf{s}\right)\end{array}$ & $\begin{array}{c}\text { Discharge } \\
\text { codes }\end{array}$ \\
\hline 1971 & $08-00-71$ & 1,250 & $\mathrm{C}$ & 1981 & $04-30-81$ & 220 & $\mathrm{C}$ \\
1972 & $08-12-72$ & 870 & $\mathrm{C}$ & 1982 & $08-23-82$ & 1,470 & $\mathrm{C}$ \\
1973 & $00-00-73$ & 430 & $\mathrm{C}$ & 1983 & $08-09-83$ & 943 & $\mathrm{C}$ \\
1974 & $09-06-74$ & 1,150 & $\mathrm{C}$ & 1984 & $07-22-84$ & 995 & $\mathrm{C}$ \\
1975 & $07-12-75$ & 620 & $\mathrm{C}$ & 1985 & $00-00-85$ & 356 & $\mathrm{C}$ \\
1976 & $07-11-76$ & 920 & $\mathrm{C}$ & 1986 & $07-15-86$ & 310 & $\mathrm{C}$ \\
1977 & $09-10-77$ & 225 & $\mathrm{C}$ & 1987 & $02-25-87$ & 81 & $\mathrm{C}$ \\
1978 & $07-30-78$ & 185 & $\mathrm{C}$ & 1988 & $08-19-88$ & 438 & $\mathrm{C}$ \\
1979 & $08-12-79$ & 490 & $\mathrm{C}$ & 1989 & $07-26-89$ & 532 & $\mathrm{C}$ \\
1980 & $02-13-80$ & 330 & $\mathrm{C}$ & 1990 & $07-24-90$ & 674 & $\mathrm{C}$ \\
\hline
\end{tabular}

Discharge rating table developed January 1979

\begin{tabular}{crcc}
\hline $\begin{array}{c}\text { Gage height } \\
(\mathbf{f t})\end{array}$ & $\begin{array}{c}\text { Discharge } \\
\left(\mathbf{f t}^{3} / \mathbf{s}\right)\end{array}$ & $\begin{array}{c}\text { Gage height } \\
(\mathbf{f t})\end{array}$ & $\begin{array}{c}\text { Discharge } \\
\left(\mathbf{f t}^{3} / \mathbf{s}\right)\end{array}$ \\
\hline 2.0 & 80 & 6.0 & 800 \\
3.0 & 199 & 7.0 & 1,060 \\
4.0 & 364 & 8.0 & 1,370 \\
5.0 & 565 & 9.0 & 1,720 \\
\hline
\end{tabular}

Magnitude and probability of instantaneous peak flow based on period of record 1971-90

\begin{tabular}{|c|c|c|c|c|c|}
\hline \multicolumn{6}{|c|}{$\begin{array}{l}\text { Discharge, in } \mathrm{ft}^{3} / \mathrm{s} \text {, for indicated recurrence interval } \\
\text { in years, and exceedance probablility, in percent }\end{array}$} \\
\hline 2 & 5 & 10 & 25 & $50 \dagger$ & $100 t$ \\
\hline $50 \%$ & $20 \%$ & $10 \%$ & $4 \%$ & $2 \%$ & $1 \%$ \\
\hline 524 & 911 & 1,200 & 1,610 & 1,920 & 2,260 \\
\hline Weighted skew & $(\log s)=$ & -0.17 & & & \\
\hline Mean & $(\log s)=$ & 2.71 & & & \\
\hline Standard dev. & $(\log s)=$ & 0.29 & & & \\
\hline
\end{tabular}

TReliability of values in column is uncertain, and potential errors are large.

Basin characteristics

\begin{tabular}{|c|c|c|c|c|c|c|c|}
\hline \multirow[b]{2}{*}{$\begin{array}{l}\text { Main } \\
\text { channel } \\
\text { slope } \\
(\mathrm{ft} / \mathrm{mi})\end{array}$} & \multirow[b]{2}{*}{$\begin{array}{c}\text { Stream } \\
\text { length } \\
\text { (mi) }\end{array}$} & \multirow[b]{2}{*}{$\begin{array}{c}\text { Mean } \\
\text { basin } \\
\text { elevation } \\
\text { (ft) }\end{array}$} & \multirow[b]{2}{*}{$\begin{array}{c}\text { Forested } \\
\text { area } \\
\text { (percent) }\end{array}$} & \multirow[b]{2}{*}{$\begin{array}{c}\text { Soil } \\
\text { index }\end{array}$} & \multirow[b]{2}{*}{$\begin{array}{c}\text { Mean } \\
\text { annual } \\
\text { precipitation } \\
\text { (in) }\end{array}$} & \multicolumn{2}{|c|}{ Rainfall intensity, 24-hour } \\
\hline & & & & & & $\begin{array}{c}\text { 2-year } \\
\text { (in) }\end{array}$ & $\begin{array}{c}\text { 50-year } \\
\text { (in) }\end{array}$ \\
\hline 32.1 & 4.2 & 2,380 & 0.0 & 1.0 & 11.0 & 1.9 & 4.2 \\
\hline
\end{tabular}


09483045 FLOWING WELLS WASH AT TUCSON, AZ--Continued

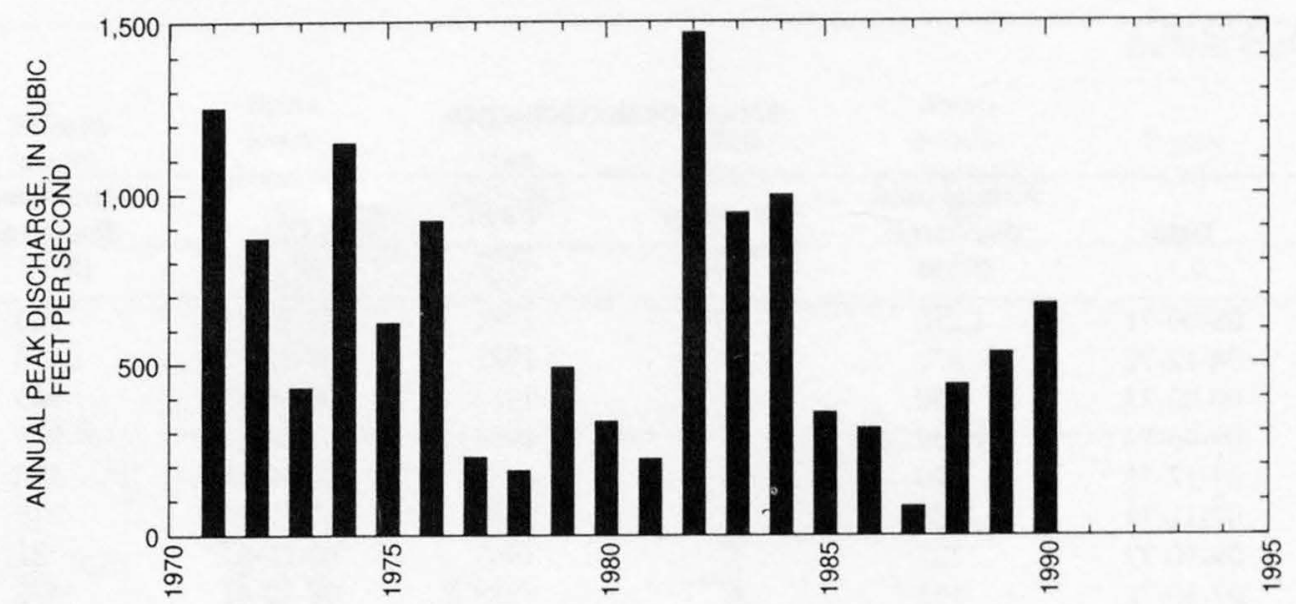


09483100 TANQUE VERDE CREEK NEAR TUCSON, AZ

LOCATION.--Lat $32^{\circ} 14^{\prime} 48^{\prime \prime}$, long $110^{\circ} 40^{\prime} 46^{\prime \prime}, \mathrm{NE}^{1} / \mathrm{N}^{\mathrm{NW}} \frac{1}{4} \mathrm{sec} .2$, T.14 S., R.16 E., Pima County, Hydrologic Unit 15050302 , 4.4 mi east of Tanque Verde School, $7.4 \mathrm{mi}$ upstream from Agua Caliente Wash, and $17.5 \mathrm{mi}$ east of city hall in Tucson.

DRAINAGE AREA.-- $43.0 \mathrm{mi}^{2}$.

Annual peak discharges

\begin{tabular}{ccccccc}
\hline $\begin{array}{c}\text { Water } \\
\text { year }\end{array}$ & Date & $\begin{array}{c}\text { Annual peak } \\
\text { discharge } \\
\left(\mathbf{f t}^{3} / \mathbf{s}\right)\end{array}$ & $\begin{array}{c}\text { Discharge } \\
\text { codes }\end{array}$ & $\begin{array}{c}\text { Water } \\
\text { year }\end{array}$ & $\begin{array}{c}\text { Date } \\
\text { discharge } \\
\left(\mathbf{f t}^{\mathbf{3}} / \mathbf{s}\right)\end{array}$ \\
\hline 1960 & $01-11-60$ & 789 & & 1973 & $10-19-72$ & 2,120 \\
1961 & $09-08-61$ & 1,260 & & 1974 & $07-08-74$ & 804 \\
1962 & $12-16-61$ & 925 & & 1975 & $00-00-75$ & 210 \\
1963 & $02-11-63$ & 1,520 & 1976 & $09-05-76$ & 300 \\
1964 & $09-10-64$ & 2,630 & 1977 & $01-01-77$ & 420 \\
1965 & $09-04-65$ & 828 & 1978 & $07-25-78$ & 1,280 \\
1966 & $12-22-65$ & 2,760 & 1979 & $12-18-78$ & 4,100 \\
1967 & $07-16-67$ & 1,260 & 1980 & $02-14-80$ & 1,150 \\
1968 & $12-20-67$ & 3,080 & 1981 & $07-30-81$ & 6,700 \\
1969 & $01-15-69$ & 278 & 1982 & $08-23-82$ & 2,460 \\
1970 & $03-02-70$ & 1,060 & 1983 & $02-03-83$ & 1,100 \\
1971 & $08-21-71$ & 2,350 & 1984 & $10-02-83$ & 8,600 \\
1972 & $07-16-72$ & 1,190 & & 1985 & $12-28-84$ & 7,880 \\
\hline
\end{tabular}

Discharge rating table developed October 1980

\begin{tabular}{crcr}
\hline $\begin{array}{c}\text { Gage height } \\
(\mathrm{ft})\end{array}$ & $\begin{array}{c}\text { Discharge } \\
\left(\mathrm{ft}^{\mathbf{3}} / \mathbf{s}\right)\end{array}$ & $\begin{array}{c}\text { Gage height } \\
(\mathrm{ft})\end{array}$ & $\begin{array}{c}\text { Discharge } \\
\left(\mathrm{ft}^{\mathbf{3}} / \mathbf{s}\right)\end{array}$ \\
\hline 3.0 & 668 & 6.0 & 4,900 \\
3.5 & 1,060 & 6.5 & 6,100 \\
4.0 & 1,570 & 7.0 & 7,470 \\
4.5 & 2,190 & 7.5 & 9,000 \\
5.0 & 2,960 & 8.0 & 10,710 \\
5.5 & 3,860 & 8.5 & 12,600 \\
\hline
\end{tabular}

Basin characteristics

\begin{tabular}{|c|c|c|c|c|c|c|c|}
\hline \multirow[b]{2}{*}{$\begin{array}{l}\text { Main } \\
\text { channel } \\
\text { slope } \\
(\mathrm{ft} / \mathrm{mi})\end{array}$} & \multirow[b]{2}{*}{$\begin{array}{l}\text { Stream } \\
\text { length } \\
\text { (mi) }\end{array}$} & \multirow[b]{2}{*}{$\begin{array}{c}\text { Mean } \\
\text { basin } \\
\text { elevation } \\
(f t)\end{array}$} & \multirow[b]{2}{*}{$\begin{array}{l}\text { Forested } \\
\text { area } \\
\text { (percent) }\end{array}$} & \multirow[b]{2}{*}{$\begin{array}{c}\text { Soil } \\
\text { index }\end{array}$} & \multirow[b]{2}{*}{$\begin{array}{c}\text { Mean } \\
\text { annual } \\
\text { precipitation } \\
\text { (in) }\end{array}$} & \multicolumn{2}{|c|}{ Rainfall intensity, 24-hour } \\
\hline & & & & & & $\begin{array}{l}\text { 2-year } \\
\text { (in) }\end{array}$ & $\begin{array}{l}\text { 50-year } \\
\text { (in) }\end{array}$ \\
\hline 156 & 11.0 & 4,780 & 21.0 & 1.0 & 17.0 & 2.0 & 4.0 \\
\hline
\end{tabular}




\section{TANQUE VERDE CREEK NEAR TUCSON, AZ--Continued}

MEAN MONTHLY AND ANNUAL DISCHARGES 1960.74

\begin{tabular}{|c|c|c|c|c|c|c|}
\hline MONTH & $\begin{array}{l}\text { MAXIMUM } \\
\text { (FT3/S) }\end{array}$ & $\begin{array}{l}\text { MINIMUM } \\
\text { (FT3/S) }\end{array}$ & $\begin{array}{c}\text { MEAN } \\
(\text { FT3/S) }\end{array}$ & $\begin{array}{l}\text { STAN- } \\
\text { DARD } \\
\text { DEVIA- } \\
\text { TION } \\
\text { (FT3/S) }\end{array}$ & $\begin{array}{l}\text { COEFFI- } \\
\text { CIENT OF } \\
\text { VARI- } \\
\text { ATION }\end{array}$ & $\begin{array}{c}\text { PERCENT } \\
\text { OF } \\
\text { ANNUAL } \\
\text { RUNOFF }\end{array}$ \\
\hline OCTOBER & 44 & 0.00 & 4.6 & 12 & 2.6 & 4.3 \\
\hline NOVEMBER & 12 & 0.00 & 2.1 & 3.4 & 1.7 & 1.9 \\
\hline DECEMBER & 198 & 0.00 & 25 & 51 & 2.1 & 23.1 \\
\hline JANUARY & 94 & 0.00 & 15 & 24 & 1.6 & 13.8 \\
\hline FEBRUARY & 83 & 0.00 & 21 & 31 & 1.5 & 19.8 \\
\hline MARCH & 100 & 0.00 & 15 & 26 & 1.7 & 14.0 \\
\hline APRIL & 19 & 0.00 & 3.4 & 5.2 & 1.5 & 3.2 \\
\hline MAY & 3.5 & 0.00 & 0.31 & 0.90 & 2.9 & 0.3 \\
\hline JUNE & 0.82 & 0.00 & 0.05 & 0.21 & 3.9 & 0.1 \\
\hline JULY & 16 & 0.00 & 3.2 & 4.8 & 1.5 & 3.0 \\
\hline AUGUST & 46 & 0.00 & 8.2 & 12 & 1.5 & 7.7 \\
\hline SEPTEMBER & 69 & 0.00 & 9.6 & 19 & 2.0 & 9.0 \\
\hline ANNUAL & 32 & 1.1 & 8.9 & 8.5 & 0.96 & 100 \\
\hline
\end{tabular}

MAGNITUDE AND PROBABILITY OF INSTANTANEOUS PEAK FLOW BASED ON PERIOD OF RECORD 1960-85

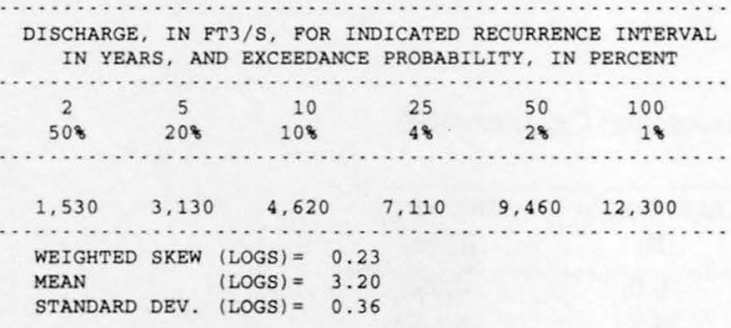

MAGNITUDE AND PROBABILITY OF ANNUAL LOW FLOW BASED ON PERIOD OF RECORD 1961.74

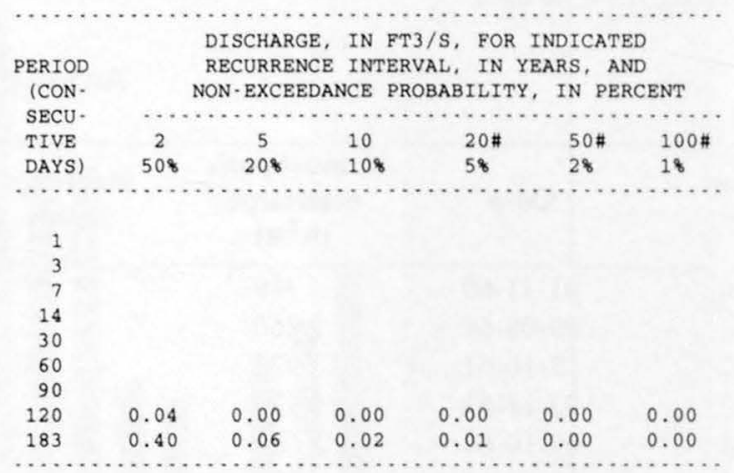

MAGNITUDE AND PROBABILITY OF ANNUAL HIGH FLOW BASED ON PERIOD OF RECORD 1960.74

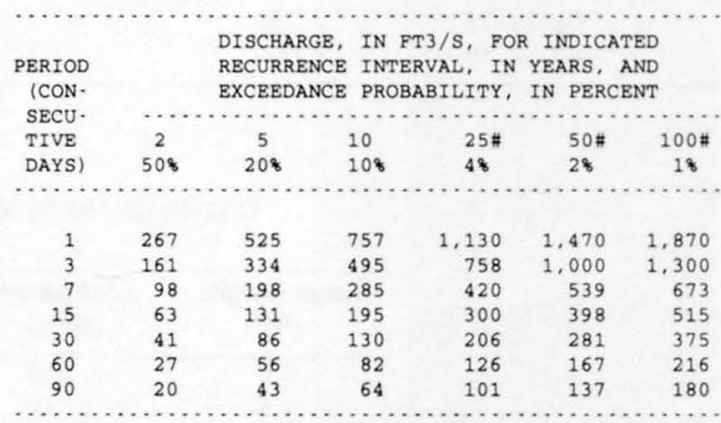

DURATION TABLE OF DAILY MEAN FLOW FOR PERIOD OF RECORD 1960.74

DISCHARGE, IN FT3/S, WHICH WAS EQUALED OR EXCEEDED FOR INDICATED PERCENT OF TIM

\begin{tabular}{|c|c|c|c|c|c|c|c|c|c|c|c|c|c|c|c|c|}
\hline 18 & 58 & 108 & 158 & 208 & 308 & 408 & 508 & 608 & 708 & 808 & 908 & 958 & 988 & 998 & 99.58 & 99.98 \\
\hline 181 & 38 & 16 & 8.9 & 5.4 & 1.2 & 0.18 & 0.07 & 0.00 & 0.00 & 0.00 & 0.00 & 0.00 & 0.00 & 0.00 & 0.00 & 0.00 \\
\hline
\end{tabular}

\# Reliability of values in column is uncertain, and potential errors are large. 
09483100 TANQUE VERDE CREEK NEAR TUCSON, AZ--Continued
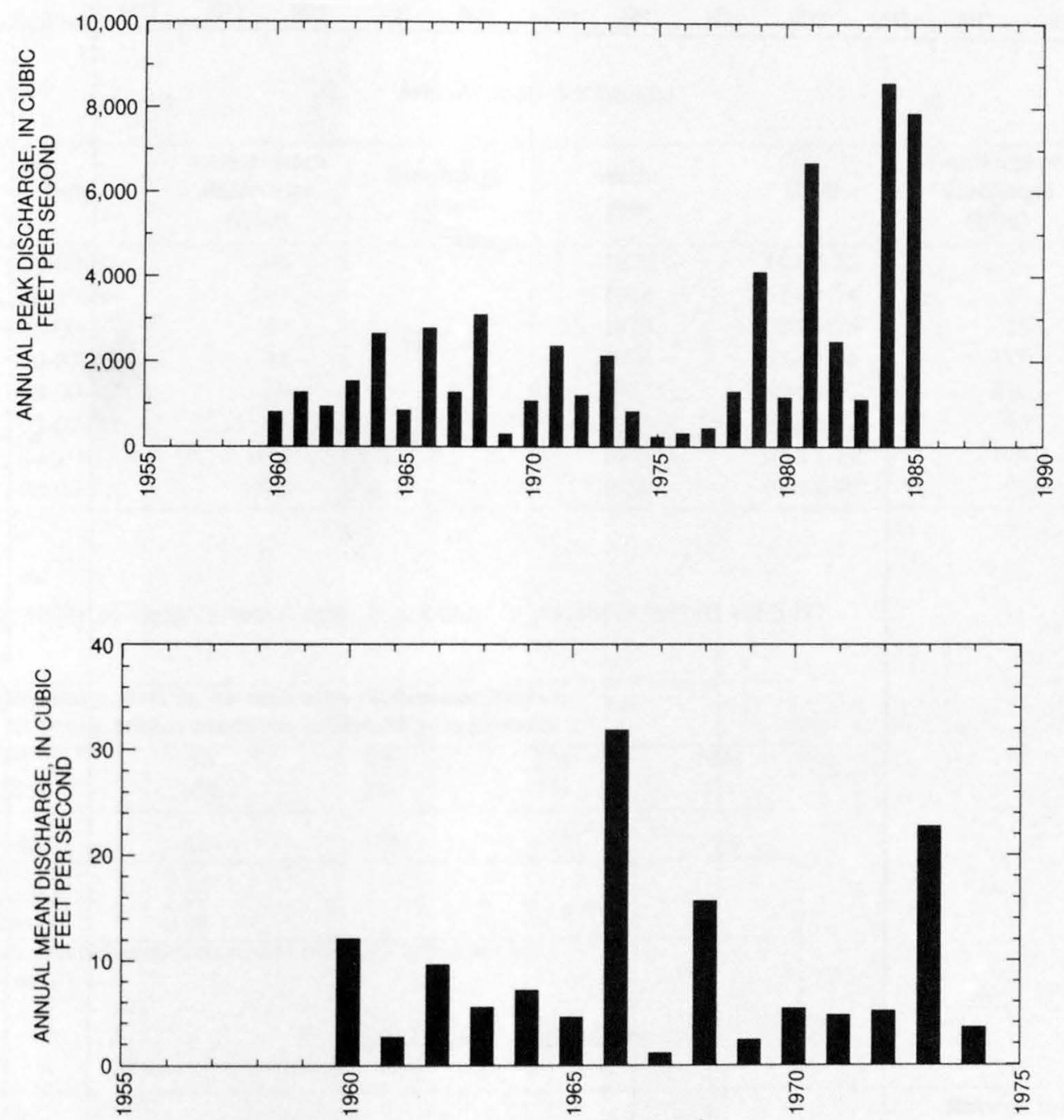
09483100 TANQUE VERDE CREEK NEAR TUCSON, AZ--Continued
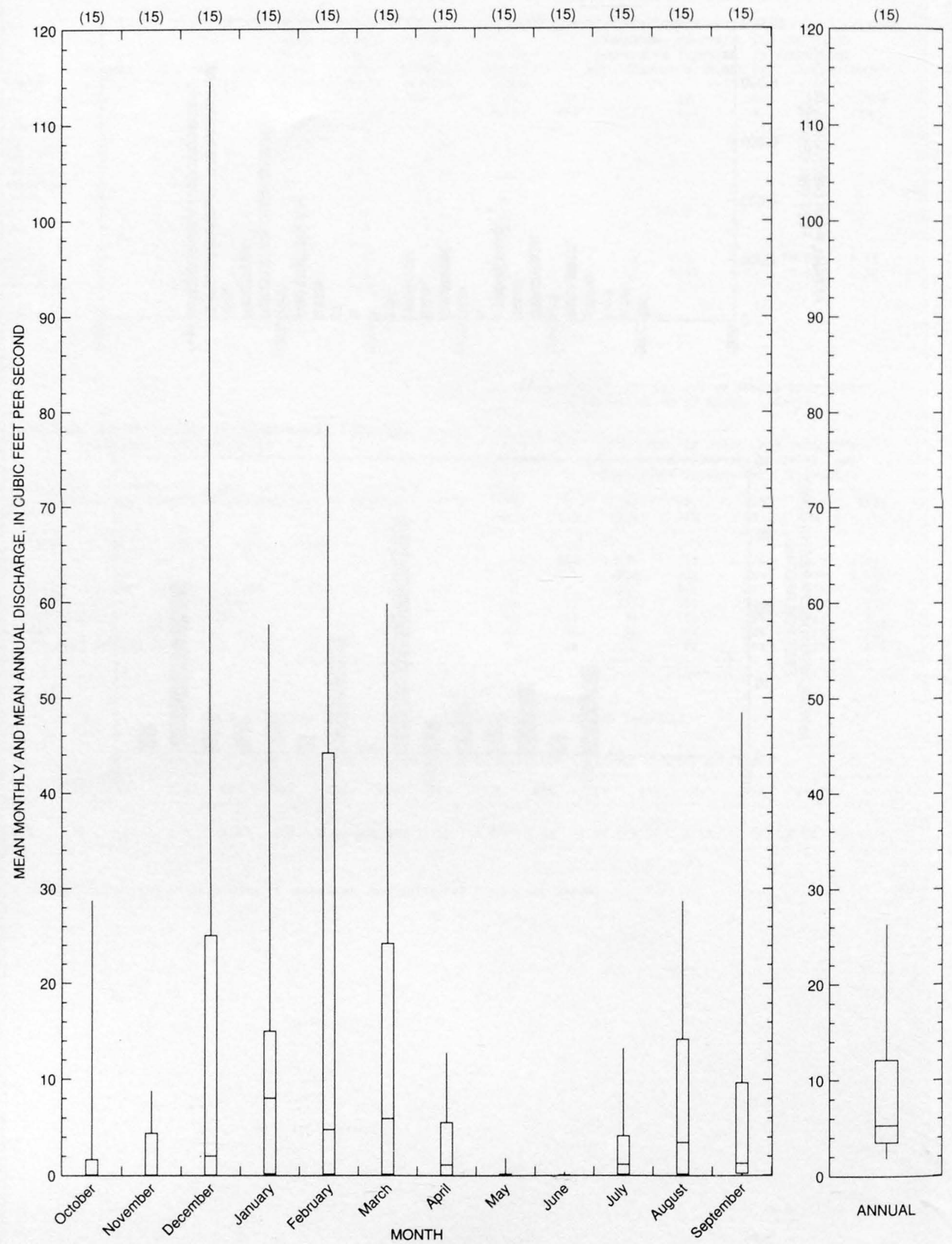
09483200 AGUA CALIENTE WASH TRIBUTARY NEAR TUCSON, AZ

LOCATION.--Lat $32^{\circ} 16^{\prime} 07^{\prime \prime}$, long $110^{\circ} 44^{\prime} 15^{\prime \prime}, \mathrm{SW}^{1} / \mathrm{SW}^{1} / 4 \mathrm{sec} .29$, T.13 S., R.16 E., Pima County, Hydrologic Unit 15050302 , at Soldier Trail,

1.4 mi north of Tanque Verde Road, and 5 mi northeast of Tucson City Limits.

DRAINAGE AREA.--2.04 $\mathrm{mi}^{2}$.

Annual peak discharges

\begin{tabular}{|c|c|c|c|c|c|c|c|}
\hline $\begin{array}{l}\text { Water } \\
\text { year }\end{array}$ & Date & $\begin{array}{c}\text { Annual peak } \\
\text { discharge } \\
\left(\mathrm{ft}^{3} / \mathrm{s}\right)\end{array}$ & $\begin{array}{l}\text { Discharge } \\
\text { codes }\end{array}$ & $\begin{array}{l}\text { Water } \\
\text { year }\end{array}$ & Date & $\begin{array}{c}\text { Annual peak } \\
\text { discharge } \\
\left(\mathrm{ft}^{3} / \mathrm{s}\right)\end{array}$ & $\begin{array}{c}\text { Discharge } \\
\text { codes }\end{array}$ \\
\hline 1965 & $08-00-65$ & 86 & & 1973 & $10-00-72$ & 210 & \\
\hline 1967 & $08-00-67$ & 57 & & 1975 & $07-25-75$ & 25 & \\
\hline 1968 & $10-03-67$ & 44 & & 1976 & $07-17-76$ & 175 & \\
\hline 1971 & $08-19-71$ & 430 & & 1979 & $08-12-79$ & 48 & \\
\hline 1972 & $08-00-72$ & 430 & & 1980 & $08-13-80$ & 76 & \\
\hline
\end{tabular}

Magnitude and probability of instantaneous peak flow based on period of record 1965-80

\begin{tabular}{|c|c|c|c|c|c|}
\hline \multicolumn{6}{|c|}{$\begin{array}{l}\text { Discharge, in } \mathrm{ft}^{3} / \mathrm{s} \text {, for indicated recurrence interval } \\
\text { in years, and exceedance probablility, in percent }\end{array}$} \\
\hline 2 & 5 & 10 & 25 & $50 t$ & $100 t$ \\
\hline $50 \%$ & $20 \%$ & $10 \%$ & $4 \%$ & $2 \%$ & $1 \%$ \\
\hline 98.9 & 208 & 309 & 470 & 619 & 793 \\
\hline Weighted skew & $(\operatorname{logs})=$ & 0.05 & & & \\
\hline Mean & $(\log s)=$ & 2.00 & & & \\
\hline Standard dev. & $(\log s)=$ & 0.38 & & & \\
\hline
\end{tabular}

Basin characteristics

\begin{tabular}{cccccccc}
\hline $\begin{array}{c}\text { Main } \\
\text { channel } \\
\text { slope } \\
(\mathrm{ft} / \mathrm{mi})\end{array}$ & $\begin{array}{c}\text { Stream } \\
\text { length } \\
(\mathrm{mi})\end{array}$ & $\begin{array}{c}\text { Mean } \\
\text { basin } \\
\text { elevation } \\
(\mathrm{ft})\end{array}$ & $\begin{array}{c}\text { Forested } \\
\text { area } \\
\text { (percent) }\end{array}$ & $\begin{array}{c}\text { Soil } \\
\text { index }\end{array}$ & $\begin{array}{c}\text { Mean } \\
\text { annual } \\
\text { precipitation } \\
\text { (in) }\end{array}$ & $\begin{array}{c}\text { R-year } \\
\text { (in) }\end{array}$ & $\begin{array}{c}50 \text {-year } \\
\text { (in) }\end{array}$ \\
\hline 368 & 4.6 & 3,300 & 7.8 & 1.0 & 14.0 & 1.9 \\
\hline
\end{tabular}


09483200 AGUA CALIENTE WASH TRIBUTARY NEAR TUCSON, AZ--Continued

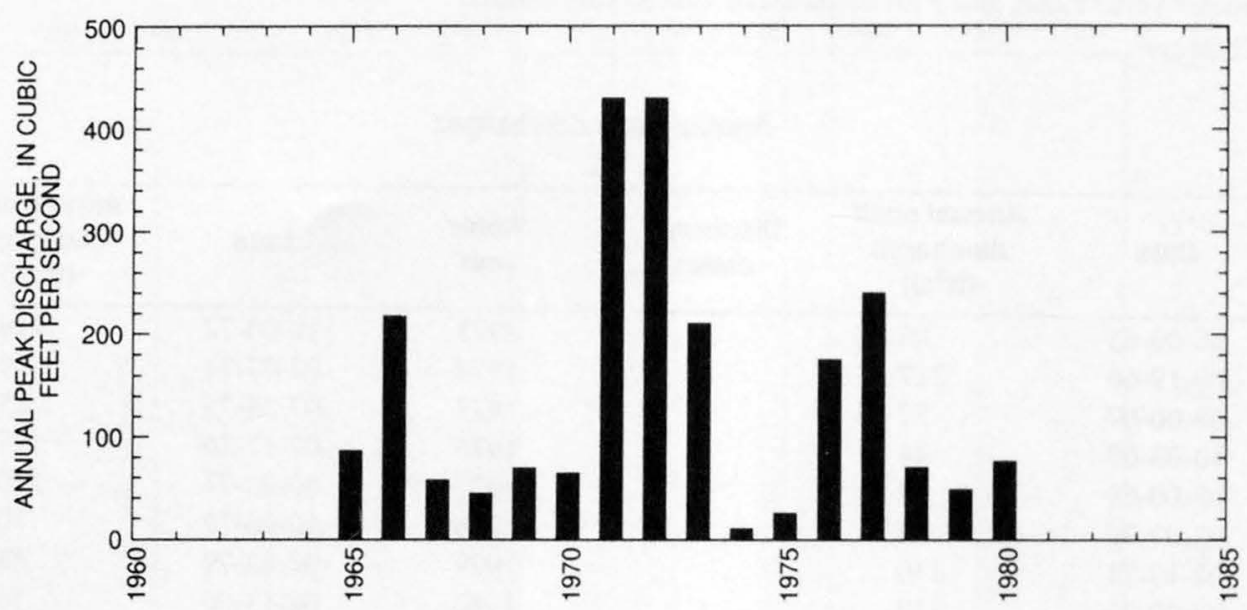


09483250 ROB WASH AT TUCSON, AZ

LOCATION.--Lat $32^{\circ} 14^{\prime} 08^{\prime \prime}$, long $110^{\circ} 48^{\prime} 58^{\prime \prime}$, NE $1 /{ }^{1} \mathrm{NW}^{1} / 4$ sec.9, T.14 S., R. 15 E., Pima County, Hydrologic Unit 15050302 , at Speedway Blvd., $0.4 \mathrm{mi}$ west of Pantano Road, and $1 \mathrm{mi}$ north of East Broadway in Tucson city limits.

DRAINAGE AREA.--2.08 $\mathrm{mi}^{2}$.

Annual peak discharges

\begin{tabular}{|c|c|c|c|c|c|c|c|}
\hline $\begin{array}{l}\text { Water } \\
\text { year }\end{array}$ & Date & $\begin{array}{c}\text { Annual peak } \\
\text { discharge } \\
\left(\mathrm{ft}^{3} / \mathrm{s}\right)\end{array}$ & $\begin{array}{l}\text { Discharge } \\
\text { codes }\end{array}$ & $\begin{array}{l}\text { Water } \\
\text { year }\end{array}$ & Date & $\begin{array}{c}\text { Annual peak } \\
\text { discharge } \\
\left(\mathrm{ft}^{3} / \mathrm{s}\right)\end{array}$ & $\begin{array}{l}\text { Discharge } \\
\text { codes }\end{array}$ \\
\hline 1971 & $00-00-71$ & 1,400 & ES,C & 1981 & $07-19-81$ & 305 & $\mathrm{C}$ \\
\hline 1972 & $00-00-72$ & 600 & ES,C & 1982 & $08-23-82$ & 1,900 & $\mathrm{C}$ \\
\hline 1973 & $00-00-73$ & 180 & C & 1983 & $09-23-83$ & 156 & C \\
\hline 1974 & $01-00-74$ & 200 & $\mathrm{C}$ & 1984 & $07-16-84$ & 405 & $\mathrm{C}$ \\
\hline 1975 & $07-12-75$ & 400 & $\mathrm{C}$ & 1985 & $00-00-85$ & 55 & $\mathrm{C}$ \\
\hline 1976 & $09-25-76$ & 330 & $\mathrm{C}$ & 1986 & $10-10-86$ & 65 & $\mathrm{C}$ \\
\hline 1977 & $08-01-77$ & 100 & C & 1987 & $01-31-86$ & 277 & $\mathrm{C}$ \\
\hline 1978 & $12-28-77$ & 50 & $\mathrm{ES}, \mathrm{C}$ & 1988 & $08-20-88$ & 1,300 & $\mathrm{C}$ \\
\hline 1979 & $08-12-79$ & 450 & C & 1989 & $07-21-89$ & 207 & $\mathrm{C}$ \\
\hline 1980 & $07-26-80$ & 190 & $\mathrm{C}$ & 1990 & $07-24-90$ & 896 & $\mathrm{C}$ \\
\hline
\end{tabular}

Discharge rating table developed January 1979

\begin{tabular}{cccc}
\hline $\begin{array}{c}\text { Gage height } \\
(\mathrm{ft})\end{array}$ & $\begin{array}{c}\text { Discharge } \\
\left(\mathrm{ft}^{3} \mathbf{s}\right)\end{array}$ & $\begin{array}{c}\text { Gage height } \\
(\mathbf{f t})\end{array}$ & $\begin{array}{c}\text { Discharge } \\
\left(\mathrm{ft}^{\mathbf{3}} / \mathbf{s}\right)\end{array}$ \\
\hline 1.8 & 138 & 4.0 & 1,310 \\
2.0 & 200 & 4.5 & 1,720 \\
2.5 & 400 & 5.0 & 2,170 \\
3.0 & 660 & 5.5 & 2,640 \\
3.5 & 970 & 6.0 & 3,140 \\
\hline
\end{tabular}

Magnitude and probability of instantaneous peak flow based on period of record 1971-86

\begin{tabular}{|c|c|c|c|c|c|}
\hline \multicolumn{6}{|c|}{$\begin{array}{l}\text { Discharge, in } \mathrm{ft}^{3} / \mathrm{s} \text {, for indicated recurrence interval } \\
\text { in years, and exceedance probablility, in percent }\end{array}$} \\
\hline 2 & 5 & 10 & 25 & $50 t$ & $100 \dagger$ \\
\hline $50 \%$ & $20 \%$ & $10 \%$ & $4 \%$ & $2 \%$ & $1 \%$ \\
\hline 267 & 622 & 973 & 1,580 & 2,160 & 2,870 \\
\hline Weighted skew & $(\log s)=$ & 0.06 & & & \\
\hline Mean & $(\log s)=$ & 2.43 & & & \\
\hline Standard dev. & $(\log s)=$ & 0.43 & & & \\
\hline
\end{tabular}

fReliability of values in column is uncertain, and potential errors are large.

Basin characteristics

\begin{tabular}{cccccccc}
\hline $\begin{array}{c}\text { Main } \\
\text { channel } \\
\text { slope } \\
\text { (ft/mi) }\end{array}$ & $\begin{array}{c}\text { Stream } \\
\text { length } \\
\text { (mi) }\end{array}$ & $\begin{array}{c}\text { Mean } \\
\text { basin } \\
\text { elevation } \\
\text { (ft) }\end{array}$ & $\begin{array}{c}\text { Forested } \\
\text { area } \\
\text { (percent) }\end{array}$ & $\begin{array}{c}\text { Soil } \\
\text { index }\end{array}$ & $\begin{array}{c}\text { Mean } \\
\text { annual } \\
\text { precipitation } \\
\text { (in) }\end{array}$ & $\begin{array}{c}\text { Rainfall intensity, 24-hour } \\
\text { (in) }\end{array}$ & $\begin{array}{c}\text { 50-year } \\
\text { (in) }\end{array}$ \\
\hline 55.6 & 4.7 & 2,710 & 0.0 & 1.0 & 11.0 & 1.9 \\
\hline
\end{tabular}


09483250 ROB WASH AT TUCSON, AZ--Continued

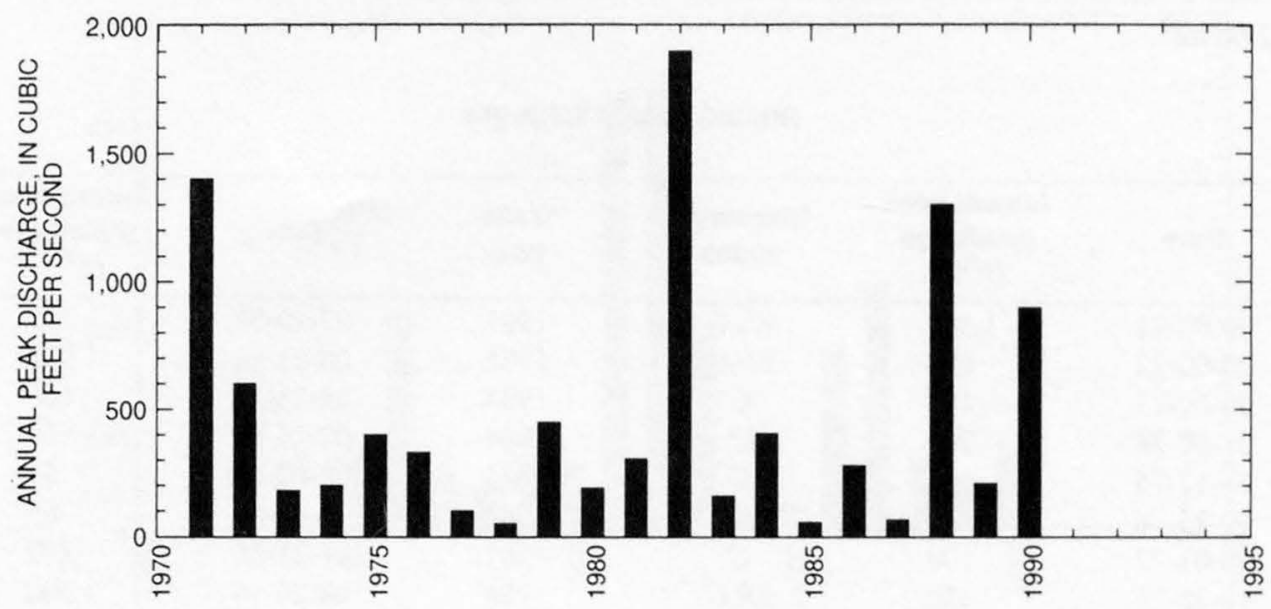




\section{SABINO CREEK NEAR TUCSON, AZ}

LOCATION.--Lat 32'19'00", long 110 48'35", in SE $\frac{1}{4} \mathrm{NE}^{1} / 4$ sec.9, T.13 S., R.15 E., Pima County, Hydrologic Unit 15050302 , on left bank, $30 \mathrm{ft}$ upstream from Lower Sabino Dam, 0.5 mi north of Coronado National Forest boundary and 12 mi northeast of Tucson City Hall.

DRAINAGE AREA.--35.5 $\mathrm{mi}^{2}$.

PERIOD OF RECORD.--July 1904 to June 1912 (monthly discharge only); June 1932 to September 1974 (continuous record station); October 1974 to September 1989 (crest-stage partial-record station); October 1989 to current year.

REVISED RECORDS.--WSP 1213: 1938, 1946. WSP 1283: Drainage area.

GAGE.--Water-stage recorder and concrete control. Elevation of gage is 2,720 ft above sea level, from topographic map. July 1904 to June 1912 , water-stage recorder and sharp-crested weir at site $0.7 \mathrm{mi}$ upstream at different datum. June 1932 to September 1974 (water-stage recorder) and October 1974 to August 1981 (crest-stage gage) at site 1,000 ft upstream at different datum.

REMARKS.--Records poor. No diversion above station except for domestic supply.

AVERAGE DISCHARGE.--56 years (water years 1905-11, 1933-74, 1990-96), $14.7 \mathrm{ft}^{3} / \mathrm{s}, 10,650$ acre- $\mathrm{ft} / \mathrm{yr}$; median of yearly mean discharges 8.9 $\mathrm{ft}^{3} / \mathrm{s}, 6,400$ acre- $\mathrm{ft} / \mathrm{yr}$.

EXTREMES FOR PERIOD OF RECORD.--Maximum discharge, 12,900 $\mathrm{ft}^{3} / \mathrm{s} \mathrm{Jan.} \mathrm{8,} \mathrm{1993,} \mathrm{gage} \mathrm{height} 7.60 \mathrm{ft}$ from high water marks, from rating curve extended above $3,000 \mathrm{ft}^{3} / \mathrm{s}$ on basis of slope-area measurement at gage height $9.65 \mathrm{ft}$; no flow at times in most years.

Annual peak discharges

\begin{tabular}{|c|c|c|c|c|c|c|c|}
\hline $\begin{array}{l}\text { Water } \\
\text { year }\end{array}$ & Date & $\begin{array}{c}\text { Annual peak } \\
\text { discharge } \\
\left(\mathrm{ft}^{3} / \mathrm{s}\right)\end{array}$ & $\begin{array}{l}\text { Discharge } \\
\text { codes }\end{array}$ & $\begin{array}{l}\text { Water } \\
\text { year }\end{array}$ & Date & $\begin{array}{c}\text { Annual peak } \\
\text { discharge } \\
\left(\mathrm{ft}^{3} / \mathrm{s}\right)\end{array}$ & $\begin{array}{c}\text { Discharge } \\
\text { codes }\end{array}$ \\
\hline 1932 & $07-15-32$ & 706 & & 1965 & $02-07-65$ & 244 & \\
\hline 1933 & $09-10-33$ & 510 & & 1966 & $08-10-66$ & 6,400 & \\
\hline 1934 & $09-22-34$ & 472 & & 1967 & $07-17-67$ & 788 & \\
\hline 1935 & $02-06-35$ & 540 & & 1968 & $12-19-67$ & 2,340 & \\
\hline 1936 & $01-29-36$ & 500 & & 1969 & $01-14-69$ & 310 & \\
\hline 1937 & $02-07-37$ & 2,020 & & 1970 & $09-06-70$ & 7,730 & \\
\hline 1938 & $03-03-38$ & 3,200 & & 1971 & $08-10-71$ & 660 & \\
\hline 1939 & $08-06-39$ & 385 & & 1972 & $10-01-71$ & 1,710 & \\
\hline 1940 & $02-23-40$ & 904 & & 1973 & $10-19-72$ & 2,750 & \\
\hline 1941 & $12-30-40$ & 3,180 & & 1974 & $07-20-74$ & 117 & \\
\hline 1942 & $09-10-42$ & 449 & & 1975 & $00-00-75$ & 70 & LT \\
\hline 1943 & $03-05-43$ & 567 & & 1976 & $09-26-76$ & 580 & \\
\hline 1944 & $07-08-44$ & 175 & & 1977 & $07-10-77$ & 480 & \\
\hline 1945 & $07-30-45$ & 916 & & 1978 & $03-02-78$ & 3,160 & \\
\hline 1946 & $08-23-46$ & 2,000 & & 1979 & $12-18-78$ & 7,400 & \\
\hline 1947 & $12-26-46$ & 227 & & 1980 & $02-14-80$ & 2,290 & \\
\hline 1948 & $08-06-48$ & 380 & & 1981 & $07-31-81$ & 1,420 & \\
\hline 1949 & $08-08-49$ & 1,430 & & 1982 & $08-13-82$ & 2,000 & \\
\hline 1950 & $07-07-50$ & 2,260 & & 1983 & $02-03-83$ & 1,340 & \\
\hline 1951 & $08-02-51$ & 750 & & 1984 & $10-01-83$ & 6,500 & \\
\hline 1952 & $01-13-52$ & 1,640 & & 1985 & $12-28-84$ & 2,350 & \\
\hline 1953 & $07-16-53$ & 861 & & 1986 & $02-16-86$ & 910 & \\
\hline 1954 & $03-23-54$ & 5,110 & & 1987 & $08-13-87$ & 866 & \\
\hline 1955 & $08-03-55$ & 2,000 & & 1988 & $08-20-88$ & 350 & \\
\hline 1956 & $08-11-56$ & 55 & & 1989 & $08-07-89$ & 350 & \\
\hline 1957 & $01-09-57$ & 2,030 & & 1990 & $07-20-90$ & 1,290 & \\
\hline 1958 & $03-22-58$ & 1,500 & & 1991 & $03-01-91$ & 2,600 & \\
\hline 1959 & $07-26-59$ & 4,240 & & 1992 & $02-13-92$ & 2,000 & \\
\hline 1960 & $12-24-59$ & 1,600 & & 1993 & 01-08-93 & 12,900 & \\
\hline 1961 & $08-30-61$ & 910 & & 1994 & $09-03-94$ & 564 & \\
\hline 1962 & $09-26-62$ & 1,010 & & 1995 & $02-15-95$ & 3,530 & \\
\hline 1963 & $08-15-63$ & 2,070 & & 1996 & $07-27-96$ & 2,900 & \\
\hline 1964 & $09-13-64$ & 1,310 & & & & & \\
\hline
\end{tabular}


09484000 SABINO CREEK NEAR TUCSON, AZ--Continued

Discharge rating table developed October 1989

\begin{tabular}{crrr}
\hline $\begin{array}{c}\text { Gage height } \\
(\mathbf{f t})\end{array}$ & $\begin{array}{c}\text { Discharge } \\
\left(\mathbf{f t}^{\mathbf{3}} \mathbf{s}\right)\end{array}$ & $\begin{array}{c}\text { Gage height } \\
(\mathbf{f t})\end{array}$ & $\begin{array}{c}\text { Discharge } \\
\left(\mathbf{f t}^{\mathbf{3}} \mathbf{s}\right)\end{array}$ \\
\hline 2.0 & 413 & 7.0 & 10,820 \\
3.0 & 1,250 & 8.0 & 14,420 \\
4.0 & 2,470 & 9.0 & 18,490 \\
5.0 & 4,320 & 10.0 & 23,020 \\
6.0 & 7,680 & 11.0 & 28,000 \\
\hline
\end{tabular}

Basin characteristics

\begin{tabular}{cccccccc}
\hline $\begin{array}{c}\text { Main } \\
\text { channel } \\
\text { slope } \\
(\mathrm{ft} / \mathrm{mi})\end{array}$ & $\begin{array}{c}\text { Stream } \\
\text { length } \\
(\mathrm{mi})\end{array}$ & $\begin{array}{c}\text { Mean } \\
\text { basin } \\
\text { elevation } \\
(\mathrm{ft})\end{array}$ & $\begin{array}{c}\text { Forested } \\
\text { area } \\
\text { (percent) }\end{array}$ & $\begin{array}{c}\text { Soil } \\
\text { index }\end{array}$ & $\begin{array}{c}\text { Mean } \\
\text { annual } \\
\text { precipitation } \\
\text { (in) }\end{array}$ & $\begin{array}{c}\text { Rainfall intensity, 24-hour } \\
\text { (in) }\end{array}$ & $\begin{array}{c}50 \text {-year } \\
\text { (in) }\end{array}$ \\
\hline 482 & 13.0 & 6,300 & 85.0 & 1.0 & 22.6 & 2.1 \\
\hline
\end{tabular}


09484000 SABINO CREEK NEAR TUCSON, AZ--Continued

MEAN MONTHLY AND ANNUAL DISCHARGES 1933-74, 1990-96

\begin{tabular}{|c|c|c|c|c|c|c|}
\hline MONTH & $\begin{array}{l}\text { MAXIMUM } \\
(\mathrm{FT} 3 / \mathrm{S})\end{array}$ & $\begin{array}{l}\text { MINIMUM } \\
(\mathrm{FT} 3 / \mathrm{S})\end{array}$ & $\begin{array}{c}\text { MEAN } \\
(\text { FT } 3 / S)\end{array}$ & $\begin{array}{l}\text { STAN- } \\
\text { DARD } \\
\text { DEVIA- } \\
\text { TION } \\
(\text { FT3/S) }\end{array}$ & $\begin{array}{l}\text { COEFFI- } \\
\text { CIENT OF } \\
\text { VARI- } \\
\text { ATION }\end{array}$ & $\begin{array}{c}\text { PERCENT } \\
\text { OF } \\
\text { ANNUAL } \\
\text { RUNOFF }\end{array}$ \\
\hline OCTOBER & 84 & 0.00 & 4.4 & 14 & 3.1 & 2.7 \\
\hline NOVEMBER & 40 & 0.00 & $4 \cdot 3$ & 7.9 & 1.8 & 2.6 \\
\hline DECEMBER & 217 & 0.00 & 20 & 39 & 2.0 & 11.9 \\
\hline JANUARY & 441 & 0.03 & 27 & 66 & 2.4 & 16.5 \\
\hline FEBRUARY & 211 & 0.02 & 29 & 41 & 1.5 & 17.4 \\
\hline MARCH & 311 & 0.30 & 35 & 52 & 1.5 & 21.6 \\
\hline APRIL & 97 & 0.03 & 13 & 18 & 1.4 & 7.7 \\
\hline MAY & 17 & 0.00 & 2.2 & 3.7 & 1.7 & 1.3 \\
\hline JUNE & 6.4 & 0.00 & 0.43 & 1.2 & 2.9 & 0.3 \\
\hline JULY & 60 & 0.00 & 5.9 & 11 & 1.8 & 3.6 \\
\hline AUGUST & 84 & 0.09 & 14 & 18 & 1.3 & 8.4 \\
\hline SEPTEMBER & 105 & 0.01 & 9.8 & 22 & 2.2 & 6.0 \\
\hline ANNUAL & 65 & 0.52 & 14 & 14 & 1.1 & 100 \\
\hline
\end{tabular}

MAGNITUDE AND PROBABILITY OF INSTANTANEOUS PEAK FLOW BASED ON PERIOD OF RECORD 1932.96

DISCHARGE, IN FT3/S, FOR INDICATED RECURRENCE INTERVAL IN YEARS, AND EXCEEDANCE PROBABILITY, IN PERCENT

$\begin{array}{cccccc}2 & 5 & 10 & 25 & 50 & 100 \\ 508 & 208 & 108 & 48 & 28 & 18 \\ 1,190 & 2,780 & 4,310 & 6,850 & 9,220 & 12,000\end{array}$

WEIGHTED SKEW (LOGS) $=.0 .06$

MEAN $\quad($ LOGS $)=3.07$

STANDARD DEV. $($ LOGS $)=0.44$
MAGNITUDE AND PROBABILITY OF ANNUAL LOW FLOW BASED ON PERIOD OF RECORD 1934-74, 1991.96

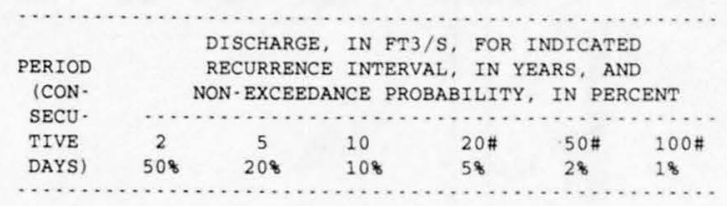

$\begin{array}{rllllll}1 & & & & & & \\ 3 & & & & & & \\ 7 & & & & & & \\ 14 & & & & & \\ 30 & 0.00 & 0.00 & 0.00 & 0.00 & 0.00 & 0.00 \\ 60 & 0.00 & 0.00 & 0.00 & 0.00 & 0.00 & 0.00 \\ 90 & 0.11 & 0.00 & 0.00 & 0.00 & 0.00 & 0.00 \\ 120 & 0.63 & 0.07 & 0.00 & 0.00 & 0.00 & 0.00 \\ 183 & 2.0 & 0.64 & 0.31 & 0.16 & 0.07 & 0.04\end{array}$

MAGNITUDE AND PROBABILITY OF ANNUAL HIGH FLOW BASED ON PERIOD OF RECORD 1933-74, 1990.96

\begin{tabular}{|c|c|c|c|c|c|c|}
\hline \multirow{3}{*}{$\begin{array}{l}\text { PERIOD } \\
\text { ICON- } \\
\text { SECU- }\end{array}$} & \multicolumn{6}{|c|}{ DISCHARGE, IN FT $3 / S$, FOR INDICATED } \\
\hline & & \multicolumn{5}{|c|}{ RECURRENCE INTERVAL, IN YEARS, AND } \\
\hline & $\cdots$ & & & $\ldots . .$. & ........ & $\begin{array}{ll}\cdots \\
\cdots\end{array}$ \\
\hline TIVE & 2 & 5 & 10 & $25 \#$ & $50 \#$ & $100 \#$ \\
\hline DAYS) & 508 & $20 \%$ & 108 & 48 & 28 & 18 \\
\hline 1 & 308 & 783 & 1,250 & 2,040 & 2,780 & 3,650 \\
\hline 3 & 184 & 459 & 728 & 1,180 & 1,590 & 2,090 \\
\hline 7 & 108 & 269 & 429 & 699 & 954 & 1.260 \\
\hline 15 & 67 & 161 & 254 & 413 & 565 & 749 \\
\hline 30 & 47 & 110 & 170 & 269 & 360 & 466 \\
\hline 60 & 31 & 74 & 114 & 179 & 238 & 307 \\
\hline 90 & 24 & 59 & 90 & . 142 & 187 & 240 \\
\hline
\end{tabular}

DURATION TABLE OF DAILY MEAN FLOW FOR PERIOD OF RECORD 1933-74, 1990.96

DISCHARGE, IN FT3/S, WHICH WAS EQUALED OR EXCEEDED FOR INDICATED PERCENT OF TIME

\begin{tabular}{|c|c|c|c|c|c|c|c|c|c|c|c|c|c|c|c|c|}
\hline 18 & 58 & 108 & 158 & 208 & 308 & 408 & 508 & 608 & 708 & 808 & 908 & 958 & 988 & 998 & 99.58 & 99.98 \\
\hline 196 & 56 & 32 & 18 & 11 & 4.8 & 2.0 & 0.75 & 0.24 & 0.04 & 0.01 & 0.00 & 0.00 & 0.00 & 0.00 & 0.00 & 0.00 \\
\hline
\end{tabular}

\# Reliability of values in column is uncertain, and potential errors are large. 
ANNUAL MEAN DISCHARGE, IN CUBIC

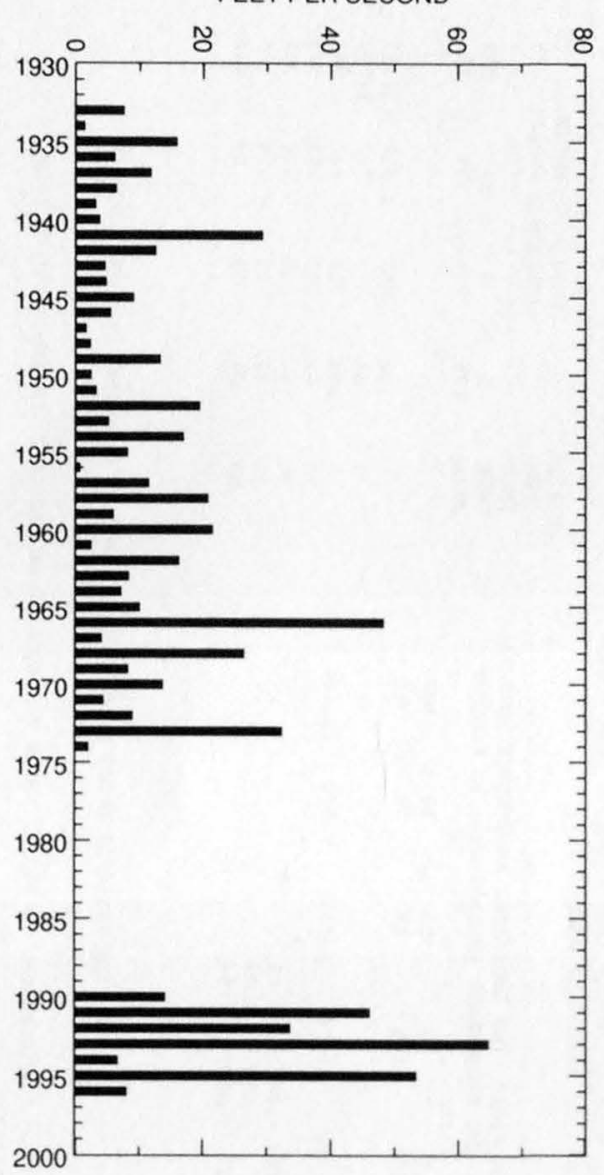

ANNUAL PEAK DISCHARGE, IN CUBIC

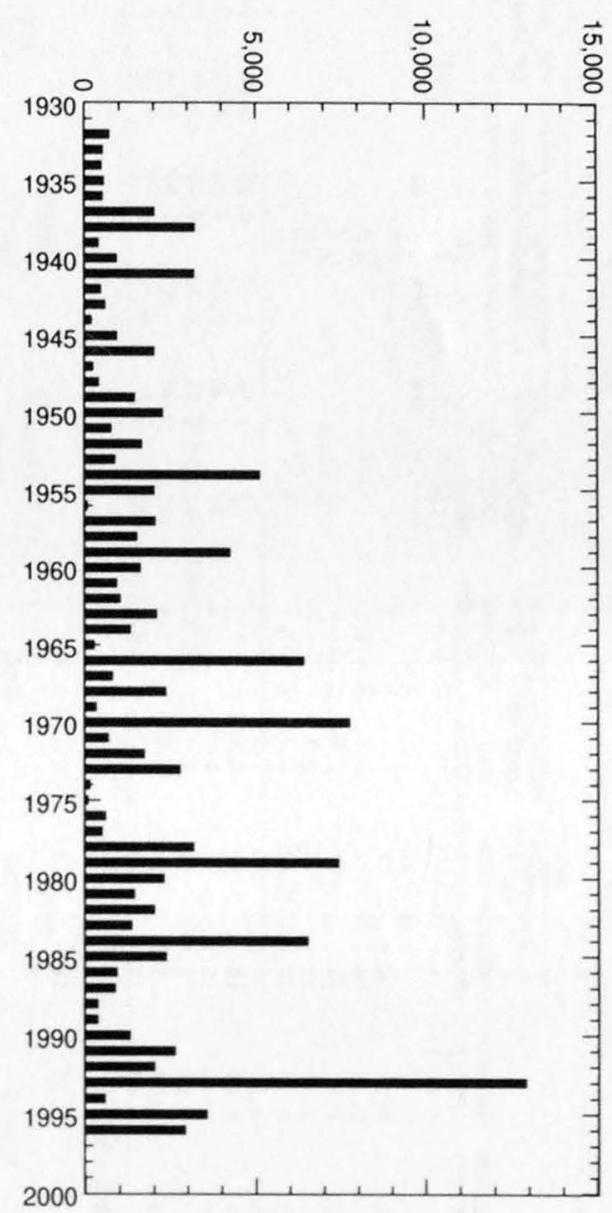


GILA RIVER BASIN

471

09484000 SABINO CREEK NEAR TUCSON, AZ--Continued
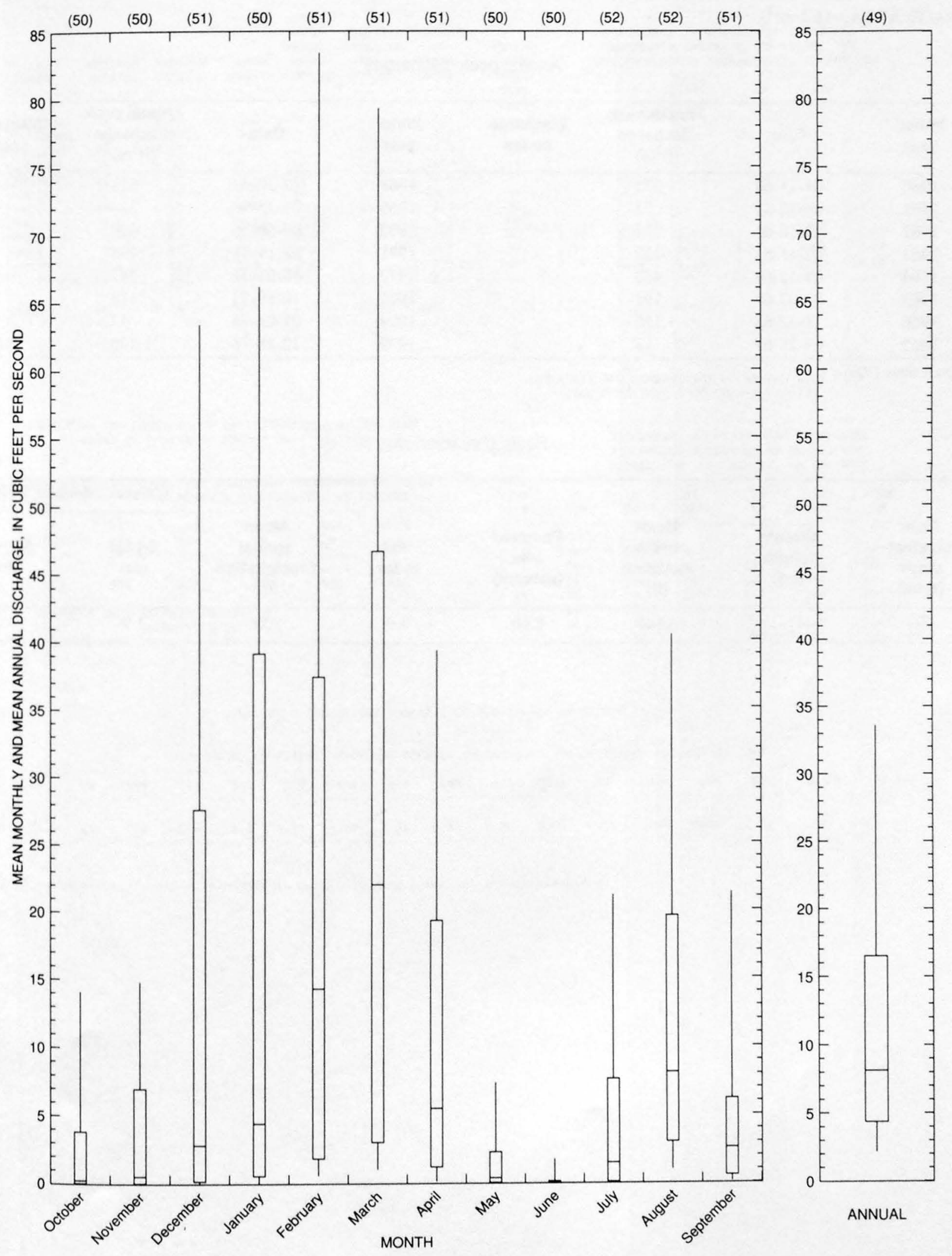
09484200 BEAR CREEK NEAR TUCSON, AZ

LOCATION.--Lat $32^{\circ} 18^{\prime} 22^{\prime \prime}$, long $110^{\circ} 48^{\prime} 03^{\prime \prime}$, NW $\frac{1}{4}$ sec.15, T.13 S., R.15 E., Pima County, Hydrologic Unit 15050302 , on left bank $0.8 \mathrm{mi}$ upstream from mouth and $15 \mathrm{mi}$ northeast of city hall in Tucson.

DRAINAGE AREA.-- $16.3 \mathrm{mi}^{2}$.

Annual peak discharges

\begin{tabular}{|c|c|c|c|c|c|c|c|}
\hline $\begin{array}{l}\text { Water } \\
\text { year }\end{array}$ & Date & $\begin{array}{c}\text { Annual peak } \\
\text { discharge } \\
\left(\mathrm{ft}^{3} / \mathrm{s}\right)\end{array}$ & $\begin{array}{c}\text { Discharge } \\
\text { codes }\end{array}$ & $\begin{array}{c}\text { Water } \\
\text { year }\end{array}$ & Date & $\begin{array}{c}\text { Annual peak } \\
\text { discharge } \\
\left(\mathrm{ft}^{3} / \mathrm{s}\right)\end{array}$ & $\begin{array}{c}\text { Discharge } \\
\text { codes }\end{array}$ \\
\hline 1960 & $01-11-60$ & 575 & & 1968 & $12-20-67$ & 621 & \\
\hline 1961 & $09-12-61$ & 53 & & 1969 & $01-15-69$ & 214 & \\
\hline 1962 & $12-16-61$ & 225 & & 1970 & $09-06-70$ & 670 & \\
\hline 1963 & $02-11-63$ & 357 & & 1971 & $08-19-71$ & 495 & \\
\hline 1964 & $09-13-64$ & 433 & & 1972 & $10-01-71$ & 247 & \\
\hline 1965 & $02-07-65$ & 192 & & 1973 & $10-19-72$ & 618 & \\
\hline 1966 & $12-22-65$ & 1,150 & & 1974 & $01-09-74$ & 57 & \\
\hline 1967 & $09-25-67$ & 13 & & 1979 & $12-18-78$ & ${ }^{1} 1,400$ & HP \\
\hline
\end{tabular}

${ }^{1}$ Highest since 1959.

Basin characteristics

\begin{tabular}{|c|c|c|c|c|c|c|c|}
\hline \multirow[b]{2}{*}{$\begin{array}{c}\text { Main } \\
\text { channel } \\
\text { slope } \\
(\mathrm{ft} / \mathrm{mi})\end{array}$} & \multirow[b]{2}{*}{$\begin{array}{c}\text { Stream } \\
\text { length } \\
\text { (mi) }\end{array}$} & \multirow[b]{2}{*}{$\begin{array}{c}\text { Mean } \\
\text { basin } \\
\text { elevation } \\
(\mathrm{ft})\end{array}$} & \multirow[b]{2}{*}{$\begin{array}{c}\text { Forested } \\
\text { area } \\
\text { (percent) }\end{array}$} & \multirow[b]{2}{*}{$\begin{array}{c}\text { Soil } \\
\text { index }\end{array}$} & \multirow[b]{2}{*}{$\begin{array}{c}\text { Mean } \\
\text { annual } \\
\text { precipitation } \\
\text { (in) }\end{array}$} & \multicolumn{2}{|c|}{ Rainfall intensity, 24-hour } \\
\hline & & & & & & $\begin{array}{c}\text { 2-year } \\
\text { (in) }\end{array}$ & $\begin{array}{l}\text { 50-year } \\
\text { (in) }\end{array}$ \\
\hline 502 & 11.0 & 5,860 & 82.0 & 1.0 & 20.6 & 1.9 & 4.0 \\
\hline
\end{tabular}


09484200 BEAR CREEK NEAR TUCSON, AZ--Continued

MEAN MONTHLY AND ANNUAL DISCHARGES $1960-74$

\begin{tabular}{|c|c|c|c|c|c|c|}
\hline MONTH & $\begin{array}{l}\text { MAXIMUM } \\
\text { (FT3/S) }\end{array}$ & $\begin{array}{l}\text { MINIMUM } \\
(\mathrm{FT} 3 / \mathrm{S})\end{array}$ & $\begin{array}{c}\text { MEAN } \\
(\mathrm{FT} 3 / \mathrm{S})\end{array}$ & $\begin{array}{l}\text { STAN- } \\
\text { DARD } \\
\text { DEVIA- } \\
\text { TION } \\
\text { (FT3/S) }\end{array}$ & $\begin{array}{l}\text { COEFFI- } \\
\text { CIENT OF } \\
\text { VARI- } \\
\text { ATION }\end{array}$ & $\begin{array}{c}\text { PERCENT } \\
\text { OF } \\
\text { ANNUAL } \\
\text { RUNOFF }\end{array}$ \\
\hline OCTOBER & 27 & 0.00 & 3.2 & 7.4 & 2.3 & 5.6 \\
\hline NOVEMBER & 4.0 & 0.00 & 1.0 & 1.4 & 1.4 & 1.8 \\
\hline DECEMBER & 89 & 0.00 & 13 & 23 & 1.8 & 22.4 \\
\hline JANUARY & 57 & 0.00 & 10 & 15 & 1.5 & 18.0 \\
\hline FEBRUARY & 42 & 0.00 & 12 & 15 & 1.2 & 21.8 \\
\hline MARCH & 41 & 0.00 & 8.8 & 12 & 1.3 & 15.6 \\
\hline APRIL & 13 & 0.00 & 2.1 & 3.5 & 1.7 & 3.7 \\
\hline MAY & 2.6 & 0.00 & 0.22 & 0.67 & 3.1 & 0.4 \\
\hline JUNE & 0.02 & 0.00 & 0.00 & 0.01 & 3.9 & 0.0 \\
\hline JULY & 7.9 & 0.00 & 0.66 & 2.0 & 3.1 & 1.2 \\
\hline AUGUST & 7.4 & 0.00 & 1.8 & 2.4 & 1.3 & 3.2 \\
\hline SEPTEMBER & 20 & 0.00 & 3.6 & 6.7 & 1.8 & 6.4 \\
\hline ANNUAL & 16 & 0.14 & 4.7 & 4.6 & 0.99 & 100 \\
\hline
\end{tabular}

MAGNITUDE AND PROBABILITY OF INSTANTANEOUS PEAK FLOW BASED ON PERIOD OF RECORD $1960.74,1979$

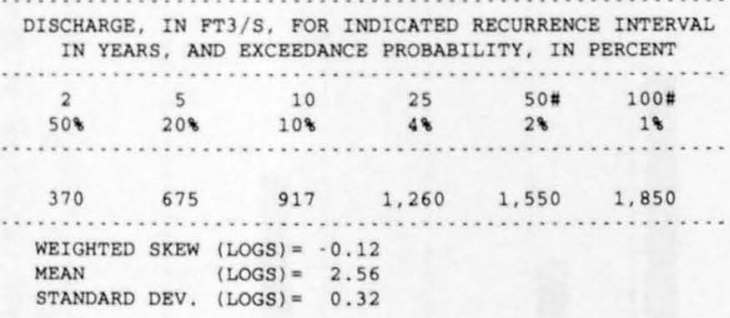

MAGNITUDE AND PROBABILITY OF ANNUAL LOW FLOW BASED ON PERIOD OF RECORD 1961-74

\begin{tabular}{|c|c|c|c|c|c|c|}
\hline \multirow{3}{*}{$\begin{array}{l}\text { PERIOD } \\
\text { (CON- } \\
\text { SECU- } \\
\text { TIVE } \\
\text { DAYS) }\end{array}$} & \multicolumn{6}{|c|}{ 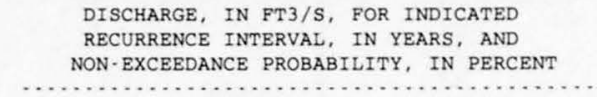 } \\
\hline & 2 & 5 & $10 \#$ & 20 & $50 \#$ & $100 \#$ \\
\hline & 508 & 208 & 108 & 58 & 28 & 18 \\
\hline $\begin{array}{r}1 \\
3 \\
7 \\
14 \\
30 \\
60\end{array}$ & & & & & & \\
\hline 90 & 0.00 & 0.00 & 0.00 & 0.00 & 0.00 & 0.00 \\
\hline 120 & 0.00 & 0.00 & 0.00 & 0.00 & 0.00 & $\begin{array}{l}0.00 \\
0.00\end{array}$ \\
\hline 183 & 0.19 & 0.00 & 0.00 & 0.00 & 0.00 & 0.00 \\
\hline
\end{tabular}

MAGNITUDE AND PROBABILITY OF ANNUAL HIGH FLOW BASED ON PERIOD OF RECORD 1960-74

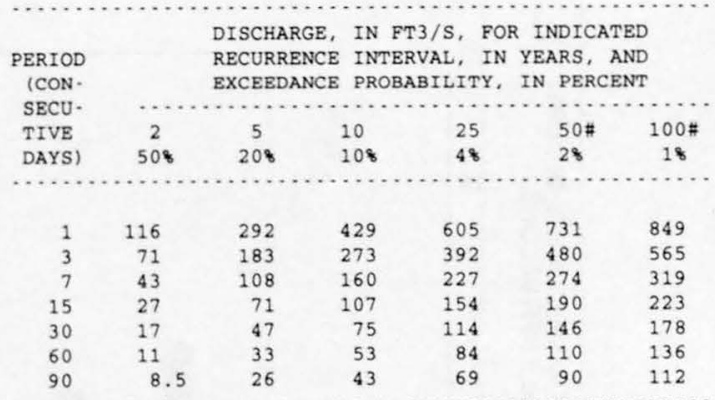

DURATION TABLE OF DAILY MEAN FLOW FOR PERIOD OF RECORD 1960-74

DISCHARGE, IN FT3/S, WHICH WAS EQUALED OR EXCEEDED FOR INDICATED PERCENT OF TIME

\begin{tabular}{|c|c|c|c|c|c|c|c|c|c|c|c|c|c|c|c|c|}
\hline 18 & 58 & 108 & 158 & 208 & 308 & 408 & 508 & 608 & 708 & 808 & 908 & $95 \%$ & 988 & 998 & 99.58 & 99.98 \\
\hline 71 & 25 & 12 & 5.4 & 2.8 & 0.83 & 0.23 & 0.05 & 0.00 & 0.00 & 0.00 & 0.00 & 0.00 & 0.00 & 0.00 & 0.00 & 0.00 \\
\hline
\end{tabular}

\# Reliability of values in column is uncertain, and potential errors are large. 
ANNUAL MEAN DISCHARGE, IN CUBIC FEET PER SECOND

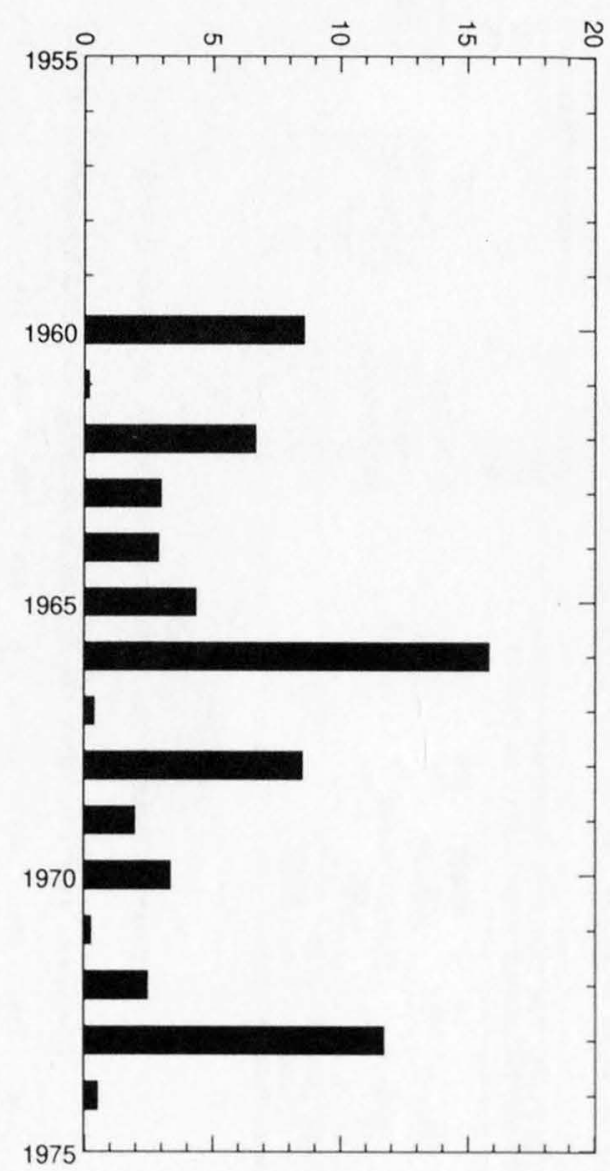

ANNUAL PEAK DISCHARGE, IN CUBIC FEET PER SECOND

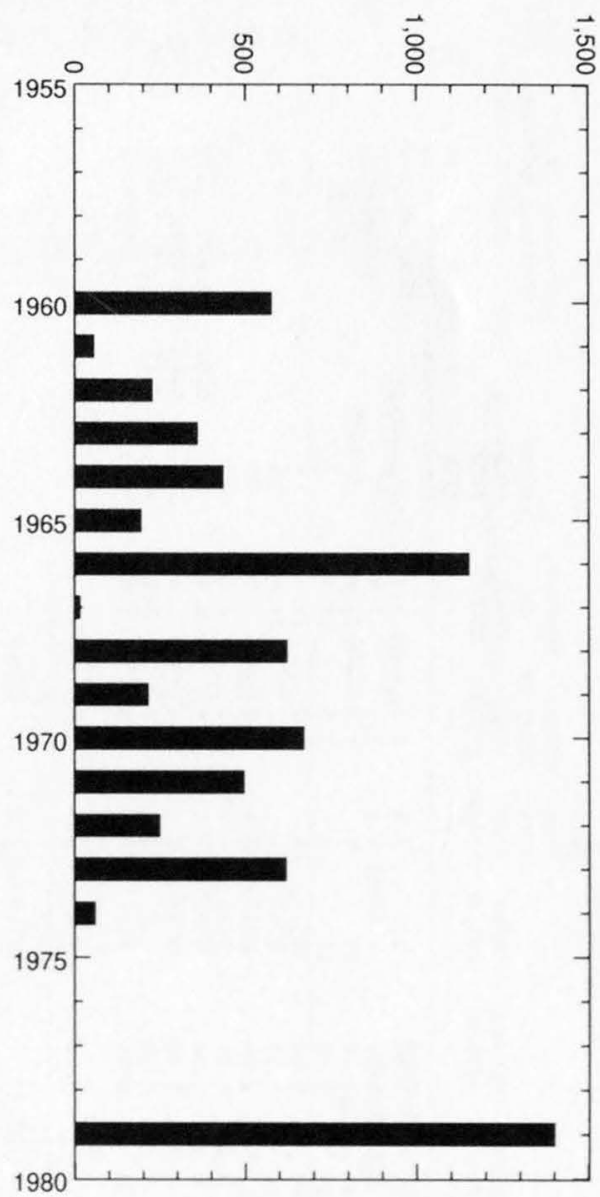


GILA RIVER BASIN

475

09484200 BEAR CREEK NEAR TUCSON, AZ--Continued

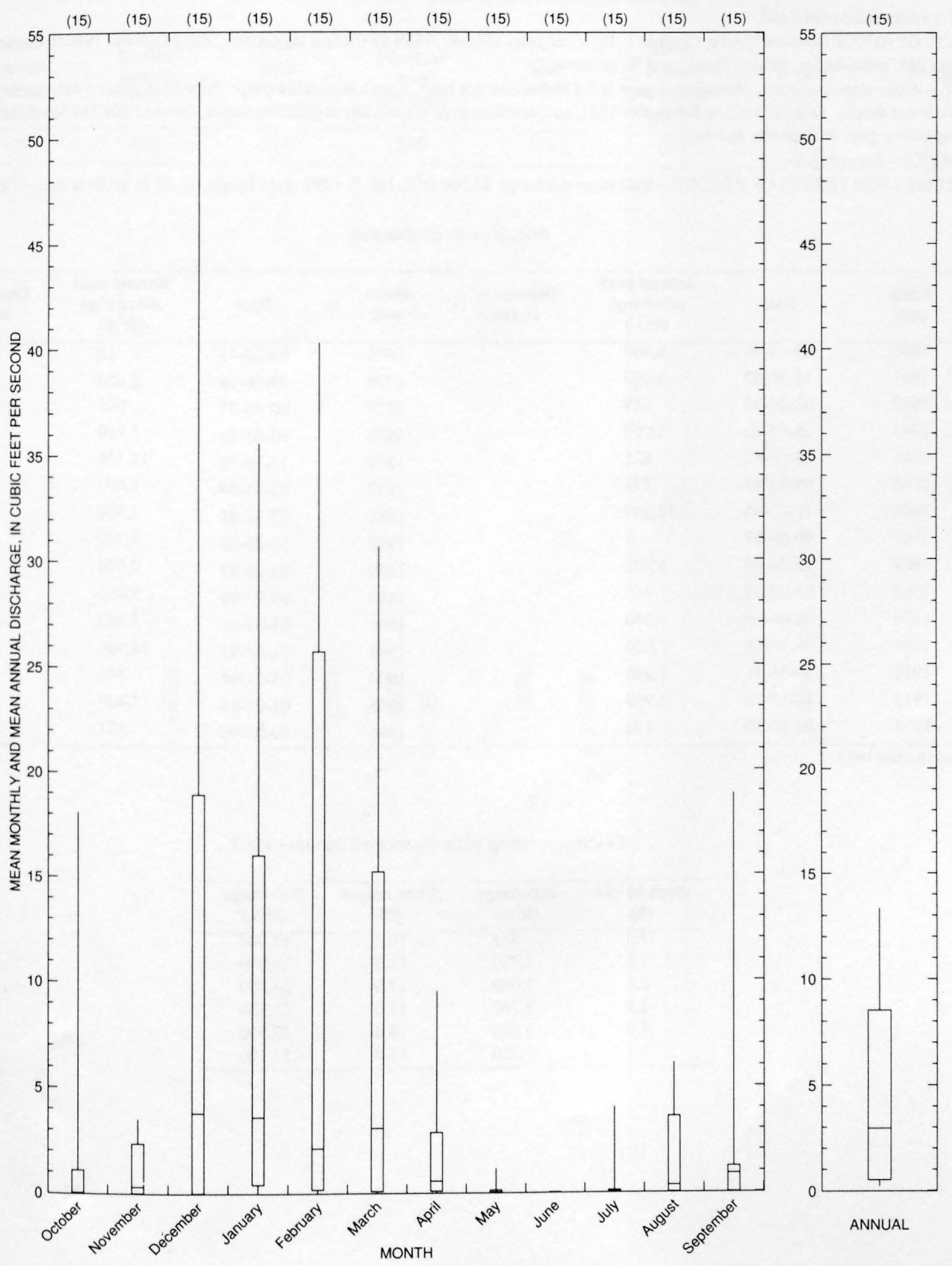


09484500 TANQUE VERDE CREEK AT TUCSON, AZ

LOCATION.--Lat 32'15'57", long 110 50'27", in SE $1 / 4 \mathrm{SE}^{1} / 4$ sec.31, T.13 S., R.15 E., Pima County, Hydrologic Unit 15050302, at Sabino Canyo Road, 1 mi downstream from Sabino Creek, and 0.8 mi north of Tucson city limits.

DRAINAGE AREA.--219 $\mathrm{mi}^{2}$.

PERIOD OF RECORD.--June 1940 to October 1945; water years 1966-81, 1988-90 (annual maximums only); October 1990 to current year. Pri to 1945 , published as "Rillito Creek near Wrightstown."

GAGE.--Water-stage recorder. Elevation of gage is 2,470 ft above sea level, from topographic map. Prior to October 1945, at same location different datum. October 1965 to September 1981, nonrecording gage at same site at different datum. October 1987 to September 1990, non recording gage at same site and datum.

REMARKS.--Records poor.

EXTREMES FOR PERIOD OF RECORD.--Maximum discharge, $24,500 \mathrm{ft}^{3} / \mathrm{s}$, Jan. 8,1993 , gage height, $11.85 \mathrm{ft}$; no flow most of each year.

Annual peak discharges

\begin{tabular}{|c|c|c|c|c|c|c|c|}
\hline $\begin{array}{l}\text { Water } \\
\text { year }\end{array}$ & Date & $\begin{array}{l}\text { Annual peak } \\
\text { discharge } \\
\left(\mathrm{ft}^{3} / \mathrm{s}\right)\end{array}$ & $\begin{array}{l}\text { Discharge } \\
\text { codes }\end{array}$ & $\begin{array}{l}\text { Water } \\
\text { year }\end{array}$ & Date & $\begin{array}{l}\text { Annual peak } \\
\text { discharge } \\
\left(\mathrm{ft}^{3} / \mathrm{s}\right)\end{array}$ & $\begin{array}{l}\text { Discharge } \\
\text { codes }\end{array}$ \\
\hline 1940 & $08-13-40$ & 6,400 & & 1975 & $04-24-75$ & 10 & ES \\
\hline 1941 & $12-30-40$ & 9,000 & & 1976 & $09-26-76$ & 2,020 & \\
\hline 1942 & $02-28-42$ & 639 & & 1977 & $00-00-77$ & 600 & \\
\hline 1943 & $03-05-43$ & 1,090 & & 1978 & $03-02-78$ & 3,880 & \\
\hline 1944 & $08-09-44$ & 825 & & 1979 & $12-18-78$ & ${ }^{1} 12,700$ & \\
\hline 1945 & $08-09-45$ & 573 & & 1980 & $02-14-80$ & 1,600 & \\
\hline 1966 & $12-22-65$ & 12,200 & & 1981 & $07-31-81$ & 3,500 & \\
\hline 1967 & $00-00-67$ & 0 & & 1988 & $08-20-88$ & 3,700 & \\
\hline 1968 & $12-20-67$ & 6,300 & & 1989 & $08-18-89$ & 2,070 & \\
\hline 1969 & $03-22-69$ & 460 & & 1990 & $07-24-90$ & 5,030 & \\
\hline 1970 & $09-06-70$ & 7,340 & & 1991 & $03-01-91$ & 5,980 & \\
\hline 1971 & $08-19-71$ & 7,000 & & 1993 & $01-08-93$ & 24,500 & \\
\hline 1972 & $10-01-71$ & 1,240 & & 1994 & $03-21-94$ & 841 & \\
\hline 1973 & $10-19-72$ & 4,930 & & 1995 & 01-05-95 & 7,400 & \\
\hline 1974 & $09-21-74$ & 420 & & 1996 & $09-03-96$ & 387 & \\
\hline
\end{tabular}

${ }^{1}$ Highest since 1940.

Discharge rating table developed January 1993

\begin{tabular}{crcc}
\hline $\begin{array}{c}\text { Gage height } \\
(\mathbf{f t})\end{array}$ & $\begin{array}{c}\text { Discharge } \\
\left(\mathbf{f t}^{\mathbf{3}} / \mathbf{s}\right)\end{array}$ & $\begin{array}{c}\text { Gage height } \\
(\mathbf{f t})\end{array}$ & $\begin{array}{c}\text { Discharge } \\
\left(\mathbf{f t}^{3} / \mathbf{s}\right)\end{array}$ \\
\hline 7.0 & 855 & 10.0 & 12,000 \\
7.5 & 1,780 & 11.0 & 18,040 \\
8.0 & 3,080 & 12.0 & 24,990 \\
8.5 & 4,760 & 13.0 & 33,130 \\
9.0 & 6,820 & 14.0 & 42,500 \\
9.5 & 9,220 & 15.0 & 53,100 \\
\hline
\end{tabular}


09484500 TANQUE VERDE CREEK AT TUCSON, AZ--Continued

Basin characteristics

Rainfall intensity, 24-hour

\begin{tabular}{cccccccc}
$\begin{array}{c}\text { Main } \\
\text { channel } \\
\text { slope } \\
\text { (ft/mi) }\end{array}$ & $\begin{array}{c}\text { Stream } \\
\text { length } \\
\text { (mi) }\end{array}$ & $\begin{array}{c}\text { Mean } \\
\text { basin } \\
\text { elevation } \\
\text { (ft) }\end{array}$ & $\begin{array}{c}\text { Forested } \\
\text { area } \\
\text { (percent) }\end{array}$ & $\begin{array}{c}\text { Soil } \\
\text { index }\end{array}$ & $\begin{array}{c}\text { Mean } \\
\text { annual } \\
\text { precipitation } \\
\text { (in) }\end{array}$ & $\begin{array}{c}\text { 2-year } \\
\text { (in) }\end{array}$ & $\begin{array}{c}50 \text {-year } \\
\text { (in) }\end{array}$ \\
\hline 109 & 19.0 & 4,340 & 36.0 & 1.0 & 16.7 & 2.0 & 4.2 \\
\hline
\end{tabular}


09484500 TANQUE VERDE CREEK AT TUCSON, AZ--Continued

MEAN MONTHLY AND ANNUAL DISCHARGES $1940-45,1966-81,1988-96$

\begin{tabular}{|c|c|c|c|c|c|c|}
\hline MONTH & $\begin{array}{l}\text { MAXIMUM } \\
\text { (FT3/S) }\end{array}$ & $\begin{array}{l}\text { MINIMUM } \\
\text { (FT3/S) }\end{array}$ & $\begin{array}{c}\text { MEAN } \\
(\mathrm{FT} 3 / \mathrm{S})\end{array}$ & $\begin{array}{l}\text { STAN- } \\
\text { DARD } \\
\text { DEVIA- } \\
\text { TION } \\
(\text { FT } 3 / S)\end{array}$ & $\begin{array}{l}\text { COEFFI- } \\
\text { CIENT OF } \\
\text { VARI- } \\
\text { ATION }\end{array}$ & $\begin{array}{c}\text { PERCENT } \\
\text { OF } \\
\text { ANNUAL } \\
\text { RUNOFF }\end{array}$ \\
\hline OCTOBER & 1.1 & 0.00 & 0.10 & 0.31 & 3.0 & 0.03 \\
\hline NOVEMBER & 36 & 0.00 & 3.8 & 10 & 2.7 & 1.0 \\
\hline DECEMBER & 248 & 0.00 & 48 & 77 & 1.6 & 13 \\
\hline JANUARY & 1,295 & 0.00 & 154 & 366 & 2.4 & 42 \\
\hline FEBRUARY & 212 & 0.00 & 62 & 84 & 1.4 & 17 \\
\hline MARCH & 277 & 0.00 & 78 & 89 & 1.1 & 21 \\
\hline APRIL & 54 & 0.00 & 15 & 19 & 1.3 & 4.0 \\
\hline MAY & 3.9 & 0.00 & 0.99 & 1.6 & 1.7 & 0.27 \\
\hline JUNE & 0.00 & 0.00 & 0.00 & 0.00 & $\cdots$ & 0.0 \\
\hline JULY & 11 & 0.00 & 1.4 & 3.2 & 2.4 & 0.37 \\
\hline AUGUST & 13 & 0.00 & 2.9 & 4.0 & 1.4 & 0.78 \\
\hline SEPTEMBER & 3.8 & 0.00 & 0.43 & 1.1 & 2.7 & 0.12 \\
\hline ANNUAL & 147 & 0.48 & 33 & 44 & 1.3 & 100 \\
\hline
\end{tabular}

MAGNITUDE AND PROBABILITY OF ANNUAL LOW FLOW BASED ON PERIOD OF RECORD $1940 \cdot 45,1966-81,1988 \cdot 96$

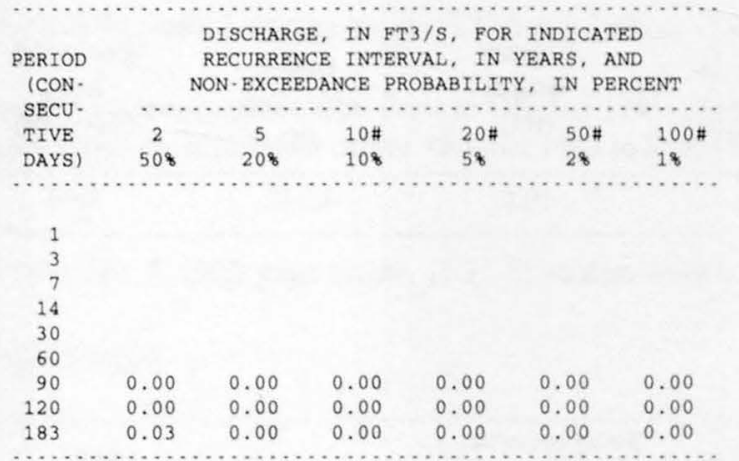

MAGNITUDE AND PROBABILITY OF ANNUAL HIGH FLOW BASED ON PERIOD OF RECORD 1940-45, 1966-81, 1988.96

\begin{tabular}{|c|c|c|c|c|c|c|}
\hline \multirow{4}{*}{$\begin{array}{l}\text { PERIOD } \\
\text { (CON- } \\
\text { SECU. }\end{array}$} & & \multicolumn{5}{|c|}{ DISCHARGE, IN FT3/S, FOR INDICATED } \\
\hline & & \multirow{2}{*}{\multicolumn{3}{|c|}{$\begin{array}{l}\text { RECURRENCE INTERVAL, IN } \\
\text { BXCEEDANCE PROBABILITY, }\end{array}$}} & \multicolumn{2}{|c|}{ YEARS, AND } \\
\hline & & & & ABILITY, & IN PERC & ENT \\
\hline & $\cdots$ & $\cdots \cdots \cdot$ & ....... & $\ldots \ldots \ldots$ & $\ldots \ldots \ldots$ & $\cdots \ldots$ \\
\hline TIVE & 2 & 5 & $10 \#$ & $25 \#$ & $50 \#$ & $100 \#$ \\
\hline DAYS ) & 508 & 208 & 108 & 48 & 28 & 18 \\
\hline 1 & 653 & 3,060 & 6,730 & 15,400 & 26,200 & 42,100 \\
\hline 3 & 365 & 1,740 & 3,830 & 8,720 & 14.700 & 23,300 \\
\hline 7 & 208 & 955 & 2,080 & 4,710 & 7,910 & 12,600 \\
\hline 15 & 117 & 541 & 1,190 & 2.770 & 4,750 & 7,700 \\
\hline 30 & 77 & 333 & 711 & 1.580 & 2,640 & 4,180 \\
\hline 60 & 52 & 229 & 477 & 1,010 & 1,620 & 2,450 \\
\hline 90 & 43 & 190 & 397 & 839 & 1,330 & 2,000 \\
\hline
\end{tabular}

DURATION TABLE OF DAILY MEAN FLOW FOR PERIOD OF RECORD $1940 \cdot 45,1966 \cdot 81,1988 \cdot 96$

DISCHARGE, IN FT3/S, WHICH WAS EQUALED OR EXCEEDED FOR INDICATED PERCENT OF TIME

\begin{tabular}{|c|c|c|c|c|c|c|c|c|c|c|c|c|c|c|c|c|}
\hline 18 & 58 & 108 & 158 & 208 & 308 & 408 & 508 & 608 & 708 & 808 & 908 & 958 & 988 & 998 & 99.58 & 99.98 \\
\hline 77 & 94 & 36 & 16 & 6.1 & 0.00 & 0.00 & 0.00 & 0.00 & 0.00 & 0.00 & 0.00 & 0.00 & 0.00 & 0.00 & 0.00 & 0.00 \\
\hline
\end{tabular}

\# Reliability of values in column is uncertain, and potential errors are large. 
ANNUAL MEAN DISCHARGE, IN CUBIC EET PER SECOND

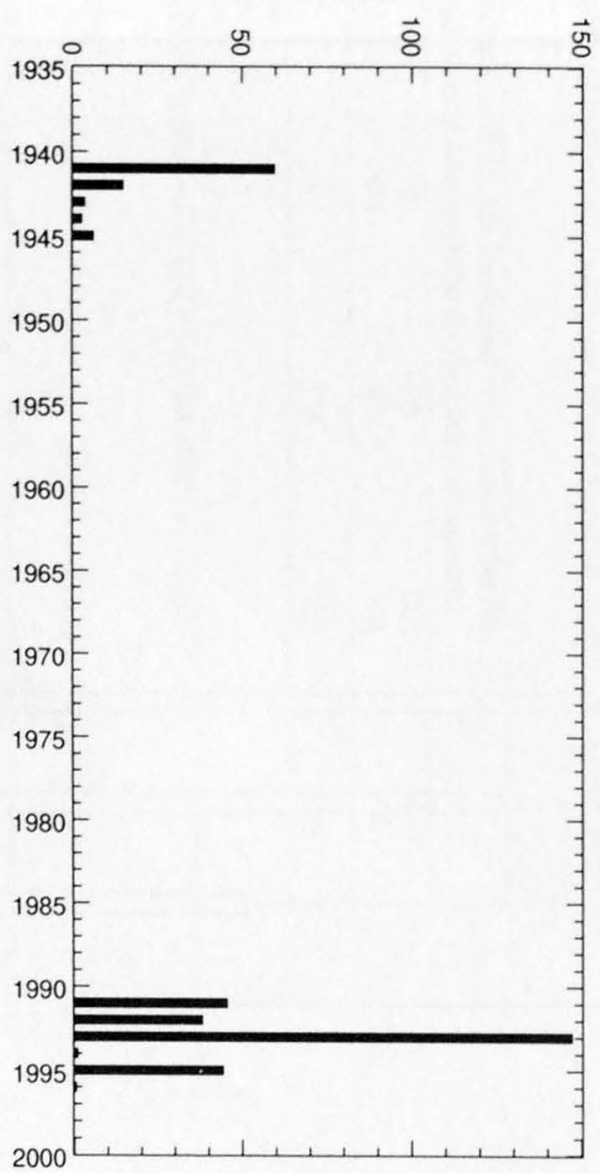

ANNUAL PEAK DISCHARGE IN CUBIC FEET PER SECOND

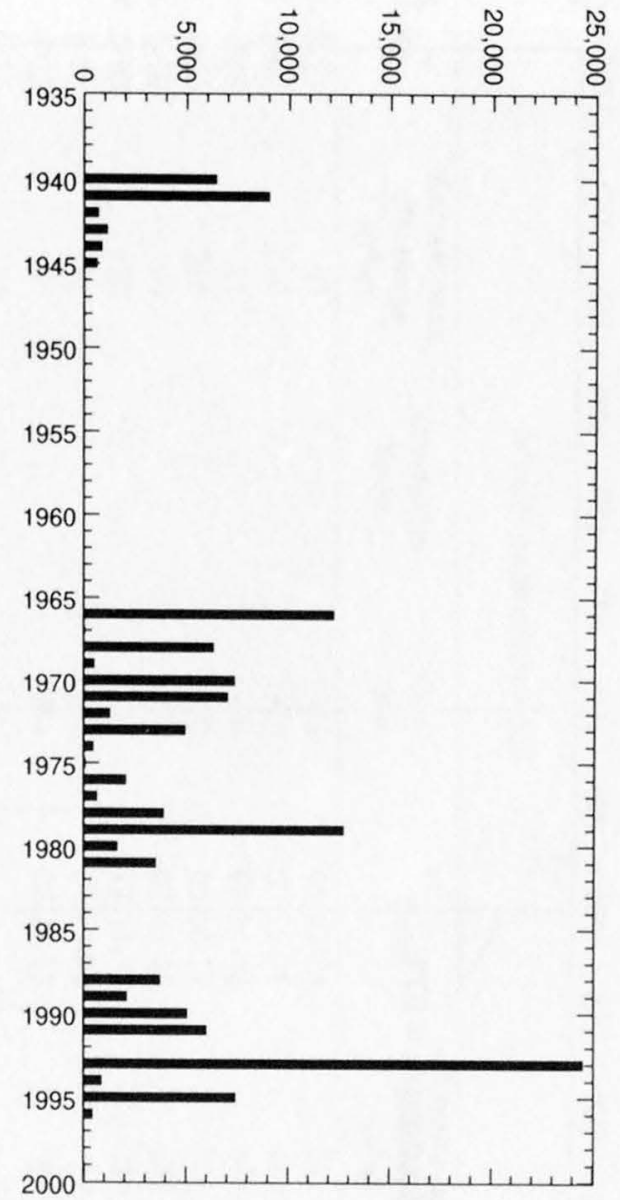


09484500 TANQUE VERDE CREEK AT TUCSON, AZ--Continued

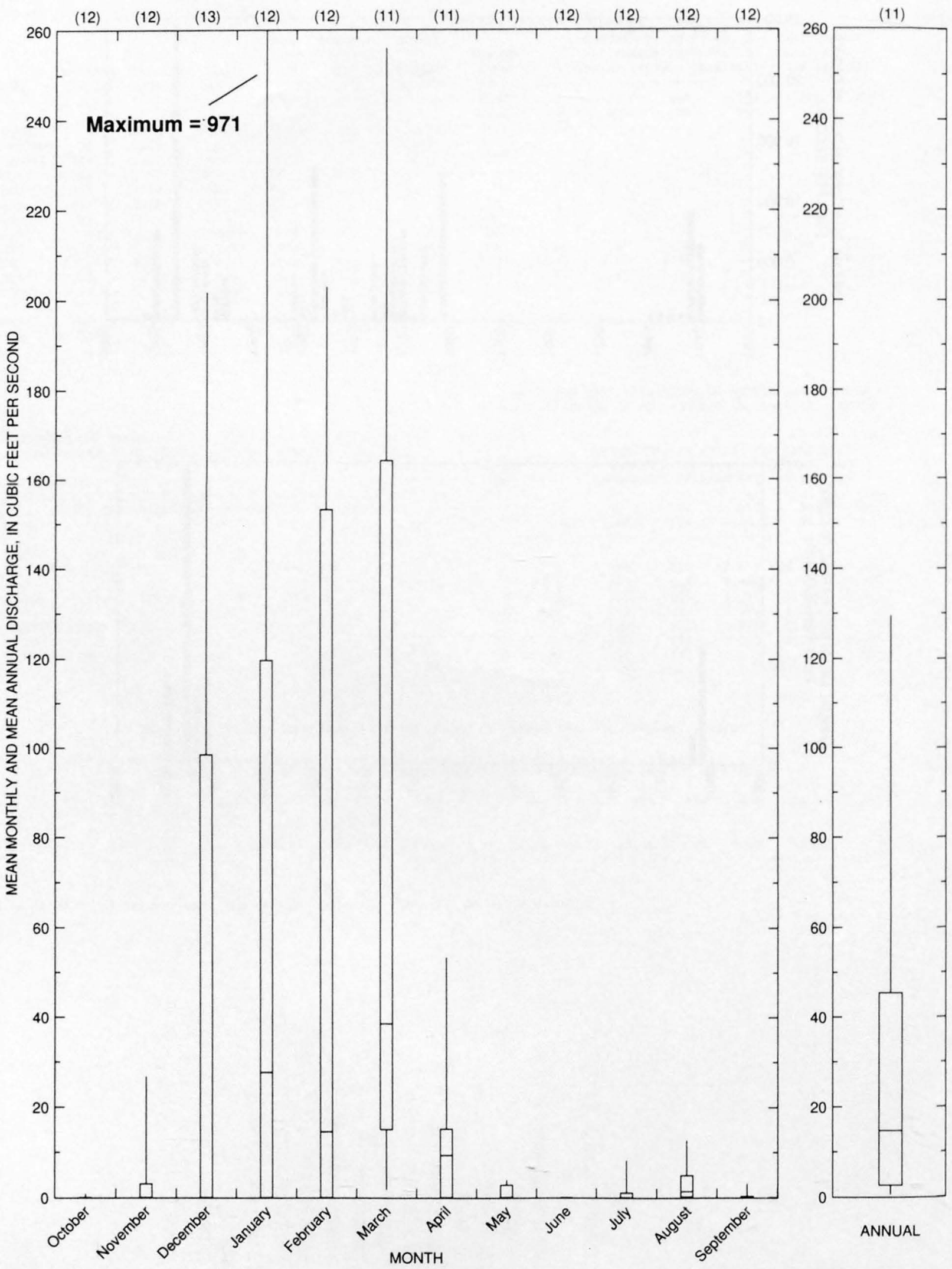


09484510 VENTANA CANYON WASH NEAR TUCSON, AZ

LOCATION.--Lat $32^{\circ} 18^{\prime} 35^{\prime \prime}$, long $110^{\circ} 50^{\prime} 20^{\prime \prime}, \mathrm{SW}^{1} /{ }_{4} \mathrm{SW}^{1} / 4$ sec.8, T.13 S., R. 15 E., Pima County, Hydrologic Unit 15050302 , at Sunrise Drive

(Sabino Canyon West Road), $0.5 \mathrm{mi}$ above Esperero Wash, and 4 mi northeast of Tucson city limits.

DRAINAGE AREA.--6.46 $\mathrm{mi}^{2}$.

Annual peak discharges

\begin{tabular}{|c|c|c|c|c|c|c|c|}
\hline $\begin{array}{l}\text { Water } \\
\text { year }\end{array}$ & Date & $\begin{array}{c}\text { Annual peak } \\
\text { discharge } \\
\left(\mathrm{ft}^{3} / \mathrm{s}\right)\end{array}$ & $\begin{array}{c}\text { Discharge } \\
\text { codes }\end{array}$ & $\begin{array}{c}\text { Water } \\
\text { year }\end{array}$ & Date & $\begin{array}{c}\text { Annual peak } \\
\text { discharge } \\
\left(\mathrm{ft}^{3} / \mathrm{s}\right)\end{array}$ & $\begin{array}{l}\text { Discharge } \\
\text { codes }\end{array}$ \\
\hline 1965 & 09-08-65 & 93 & & 1974 & $08-02-74$ & 131 & \\
\hline 1966 & $12-22-65$ & 260 & & 1975 & $07-25-75$ & 120 & \\
\hline 1967 & $10-04-66$ & 21 & & 1976 & $09-25-76$ & 195 & \\
\hline 1968 & $12-20-67$ & 216 & & 1977 & $00-00-77$ & 145 & \\
\hline 1969 & $08-01-69$ & 95 & & 1978 & $07-25-78$ & 185 & \\
\hline 1970 & $09-06-70$ & 180 & & 1979 & $12-18-78$ & 234 & \\
\hline 1971 & $08-19-71$ & 195 & & 1980 & $07-13-80$ & 32 & \\
\hline 1972 & $07-16-72$ & 180 & & 1981 & $07-31-81$ & 86 & \\
\hline 1973 & $10-19-72$ & 125 & & & & & \\
\hline
\end{tabular}

Magnitude and probability of instantaneous peak flow based on period of record 1965-81

\begin{tabular}{|c|c|c|c|c|c|}
\hline \multicolumn{6}{|c|}{$\begin{array}{l}\text { Discharge, in } \mathrm{ft}^{3} / \mathrm{s} \text {, for indicated recurrence interval } \\
\text { in years, and exceedance probablility, in percent }\end{array}$} \\
\hline 2 & 5 & 10 & 25 & $50 t$ & $100 t$ \\
\hline $50 \%$ & $20 \%$ & $10 \%$ & $4 \%$ & $2 \%$ & $1 \%$ \\
\hline 147 & 202 & 235 & 275 & 303 & 329 \\
\hline Weighted skew & $(\log s)=$ & -0.32 & & & \\
\hline Mean & $(\log s)=$ & 2.16 & & & \\
\hline Standard dev. & $(\log s)=$ & 0.17 & & & \\
\hline
\end{tabular}

Basin characteristics

\begin{tabular}{|c|c|c|c|c|c|c|c|}
\hline \multirow[b]{2}{*}{$\begin{array}{l}\text { Main } \\
\text { channel } \\
\text { slope } \\
(\mathrm{ft} / \mathrm{mi})\end{array}$} & \multirow[b]{2}{*}{$\begin{array}{l}\text { Stream } \\
\text { length } \\
(\mathrm{mi})\end{array}$} & \multirow[b]{2}{*}{$\begin{array}{c}\text { Mean } \\
\text { basin } \\
\text { elevation } \\
\text { (ft) }\end{array}$} & \multirow[b]{2}{*}{$\begin{array}{l}\text { Forested } \\
\text { area } \\
\text { (percent) }\end{array}$} & \multirow[b]{2}{*}{$\begin{array}{c}\text { Soil } \\
\text { index }\end{array}$} & \multirow[b]{2}{*}{$\begin{array}{c}\text { Mean } \\
\text { annual } \\
\text { precipitation } \\
\text { (in) }\end{array}$} & \multicolumn{2}{|c|}{ Rainfall intensity, 24-hour } \\
\hline & & & & & & $\begin{array}{c}\text { 2-year } \\
\text { (in) }\end{array}$ & $\begin{array}{l}\text { 50-year } \\
\text { (in) }\end{array}$ \\
\hline 553 & 5.4 & 4,600 & 51.0 & 1.0 & 13.0 & 1.8 & 4.2 \\
\hline
\end{tabular}

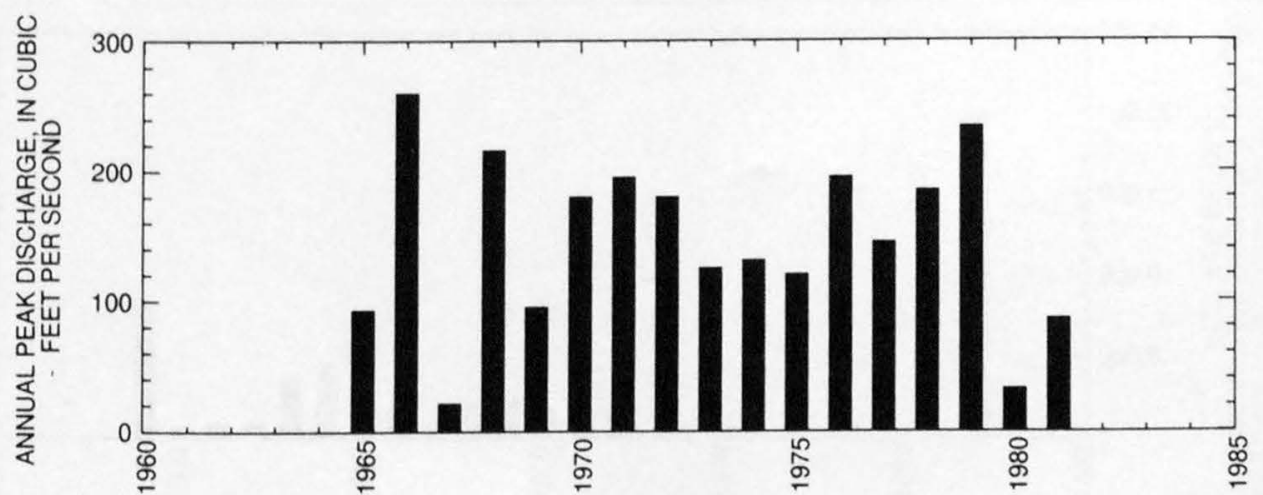


09484560 CIENEGA CREEK NEAR PANTANO, AZ

LOCATION.--Lat $31^{\circ} 59^{\prime} 08^{\prime \prime}$, long $110^{\circ} 33^{\prime} 57^{\prime \prime}, \mathrm{NW}^{1} / 4$ sec.1, T.17 S., R.17 E., Pima County, Hydrologic Unit 15050302 , on downstream end of first pier from right bank of bridge on Interstate Highway 10, and 1.2 mi southeast of Pantano.

DRAINAGE AREA.--289 $\mathrm{mi}^{2}$.

Annual peak discharges

\begin{tabular}{ccccccc}
\hline $\begin{array}{c}\text { Water } \\
\text { year }\end{array}$ & Date & $\begin{array}{c}\text { Annual peak } \\
\text { discharge } \\
\left(\mathbf{f t}^{3} / \mathbf{s}\right)\end{array}$ & $\begin{array}{c}\text { Discharge } \\
\text { codes }\end{array}$ & $\begin{array}{c}\text { Water } \\
\text { year }\end{array}$ & $\begin{array}{c}\text { Date } \\
\text { Dnual peak } \\
\text { discharge } \\
\left(\mathbf{f t}^{3} / \mathbf{s}\right)\end{array}$ \\
\hline 1958 & $08-11-58$ & 20,000 & ES,HP & 1975 & $09-02-75$ & 1,550 \\
1968 & $07-26-68$ & 1,870 & & 1976 & $08-10-76$ & 4,650 \\
1969 & $07-22-69$ & 9990 & & 1977 & $09-11-77$ & 3,800 \\
1970 & $07-20-70$ & 1,770 & & 1978 & $10-06-77$ & 900 \\
1971 & $08-03-71$ & 2,240 & & 1979 & $08-12-79$ & 860 \\
1972 & $09-13-72$ & 1,930 & & 1980 & $09-07-80$ & 630 \\
1973 & $02-22-73$ & 878 & & 1981 & $07-06-81$ & 8,310 \\
1974 & $07-19-74$ & 2,570 & & & & \\
\hline
\end{tabular}

Magnitude and probability of instantaneous peak flow based on period of record 1958, 1968-81

\begin{tabular}{|c|c|c|c|c|c|}
\hline \multicolumn{6}{|c|}{$\begin{array}{l}\text { Discharge, in } \mathrm{ft}^{3} / \mathrm{s} \text {, for indicated recurrence interval } \\
\text { in years, and exceedance probablility, in percent }\end{array}$} \\
\hline 2 & 5 & 10 & 25 & $50 \dagger$ & $100 \dagger$ \\
\hline $50 \%$ & $20 \%$ & $10 \%$ & $4 \%$ & $2 \%$ & $1 \%$ \\
\hline 1,880 & 4,020 & 6,150 & 9,930 & 13,700 & 18,500 \\
\hline Weighted skew & $(\log s)=$ & 0.36 & & & \\
\hline Mean & $(\log s)=$ & 2.30 & & & \\
\hline Standard dev. & $(\log s)=$ & 0.37 & & & \\
\hline
\end{tabular}

fReliability of values in column is uncertain, and potential errors are large.

\section{Basin characteristics}

\begin{tabular}{ccccccc}
\hline $\begin{array}{c}\text { Main } \\
\text { channel } \\
\text { slope } \\
(\mathrm{ft} / \mathrm{mi})\end{array}$ & $\begin{array}{c}\text { Stream } \\
\text { length } \\
(\mathbf{m i})\end{array}$ & $\begin{array}{c}\text { Mean } \\
\text { basin } \\
\text { elevation } \\
(\mathrm{ft})\end{array}$ & $\begin{array}{c}\text { Forested } \\
\text { area } \\
\text { (percent) }\end{array}$ & $\begin{array}{c}\text { Soil } \\
\text { index }\end{array}$ & $\begin{array}{c}\text { Mean } \\
\text { annual } \\
\text { precipitation } \\
\text { (in) }\end{array}$ & $\begin{array}{c}\text { Rainfall intensity, 24-hour } \\
\text { (in) }\end{array}$ \\
\hline 59.8 & 31.2 & 4,890 & 13.0 & 2.5 & 16.6 & $\begin{array}{c}50 \text {-year } \\
\text { (in) }\end{array}$ \\
\hline
\end{tabular}

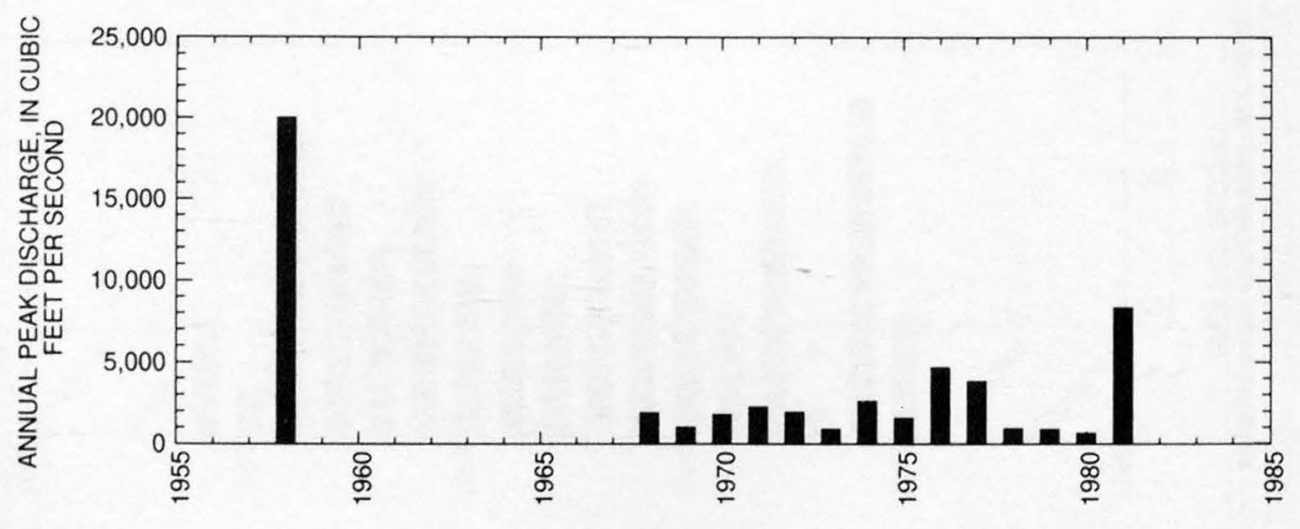


09484570 MESCAL ARROYO NEAR PANTANO, AZ

LOCATION.--Lat $31^{\circ} 59^{\prime} 23^{\prime \prime}$, long $110^{\circ} 33^{\prime} 52^{\prime \prime}, \mathrm{NE}^{1} /{ }_{4} \mathrm{NW}^{1} / 4$ sec.1, T.17 S., R.17 E., Pima County, Hydrologic Unit 15050302 , at county road, 0.25 mi above mouth, and $1.1 \mathrm{mi}$ southeast of Pantano.

DRAINAGE AREA.--38.4 $\mathrm{mi}^{2}$.

Annual peak discharges

\begin{tabular}{|c|c|c|c|c|c|c|c|}
\hline $\begin{array}{l}\text { Water } \\
\text { year }\end{array}$ & Date & $\begin{array}{c}\text { Annual peak } \\
\text { discharge } \\
\left(\mathrm{ft}^{3} / \mathrm{s}\right)\end{array}$ & $\begin{array}{l}\text { Discharge } \\
\text { codes }\end{array}$ & $\begin{array}{l}\text { Water } \\
\text { year }\end{array}$ & Date & $\begin{array}{c}\text { Annual peak } \\
\text { discharge } \\
\left(\mathrm{ft}^{3} / \mathrm{s}\right)\end{array}$ & $\begin{array}{l}\text { Discharge } \\
\text { codes }\end{array}$ \\
\hline 1958 & $08-11-58$ & 127,000 & HP & 1973 & $07-00-73$ & 320 & \\
\hline 1965 & $09-12-65$ & 1,600 & & 1974 & $08-19-74$ & 620 & \\
\hline 1966 & $08-13-66$ & 1,610 & & 1975 & $09-06-75$ & 510 & \\
\hline 1967 & $07-12-67$ & 290 & & 1976 & $07-29-76$ & 385 & \\
\hline 1968 & $08-03-68$ & 430 & & 1977 & $09-10-77$ & 1,700 & \\
\hline 1969 & $08-05-69$ & 950 & & 1978 & $10-06-77$ & 2,100 & \\
\hline 1970 & $07-21-70$ & 680 & & 1979 & $08-12-79$ & 130 & \\
\hline 1971 & $08-19-71$ & 6,140 & & 1980 & $09-07-80$ & 15 & \\
\hline 1972 & $09-00-72$ & 520 & & 1981 & $07-06-81$ & 3,500 & \\
\hline
\end{tabular}

${ }^{\mathrm{I}}$ Highest since 1930.

Magnitude and probability of instantaneous peak flow based on period of record 1958 , 1965-81

\begin{tabular}{|c|c|c|c|c|c|}
\hline \multicolumn{6}{|c|}{$\begin{array}{l}\text { Discharge, in } \mathrm{ft}^{3} / \mathrm{s} \text {, for indicated recurrence interval } \\
\text { in years, and exceedance probablility, in percent }\end{array}$} \\
\hline 2 & 5 & 10 & 25 & $50 t$ & $100 t$ \\
\hline $50 \%$ & $20 \%$ & $10 \%$ & $4 \%$ & $2 \%$ & $1 \%$ \\
\hline 753 & 2,060 & 3,620 & 6,800 & 10,400 & 15,400 \\
\hline Weighted skew & $(\log s)=$ & 0.35 & & & \\
\hline Mean & $(\log s)=$ & 2.91 & & & \\
\hline Standard dev. & $(\log s)=$ & 0.50 & & & \\
\hline
\end{tabular}

fReliability of values in column is uncertain, and potential errors are large.

Basin characteristics

\begin{tabular}{ccccccc}
\hline $\begin{array}{c}\text { Main } \\
\text { channel } \\
\text { slope } \\
(\mathrm{ft} / \mathrm{mi})\end{array}$ & $\begin{array}{c}\text { Stream } \\
\text { length } \\
(\mathrm{mi})\end{array}$ & $\begin{array}{c}\text { Mean } \\
\text { basin } \\
\text { elevation } \\
(\mathrm{ft})\end{array}$ & $\begin{array}{c}\text { Forested } \\
\text { area } \\
\text { (percent) }\end{array}$ & $\begin{array}{c}\text { Soil } \\
\text { index }\end{array}$ & $\begin{array}{c}\text { Mean } \\
\text { annual } \\
\text { precipitation } \\
\text { (in) }\end{array}$ & $\begin{array}{c}\text { R-year } \\
\text { (in) }\end{array}$ \\
\hline 82.9 & 11.9 & 4,260 & 0.0 & 1.0 & 15.0 & $\begin{array}{c}50 \text {-year } \\
\text { (in) }\end{array}$ \\
\hline
\end{tabular}


09484570 MESCAL ARROYO NEAR PANTANO, AZ--Continued

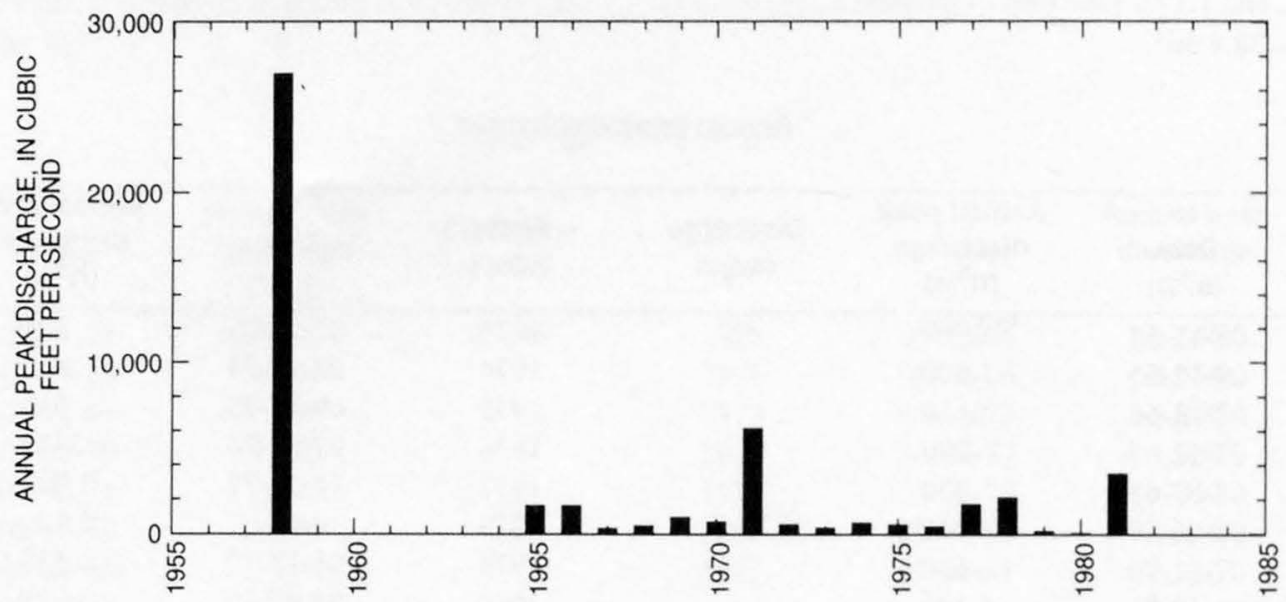




\section{BARREL CANYON NEAR SONOITA, AZ}

LOCATION.--Lat $31^{\circ} 51^{\prime} 42^{\prime \prime}$, long $110^{\circ} 41^{\prime} 25^{\prime \prime}, \mathrm{SE}^{1} / 4 \mathrm{SE}^{1} / 4 \mathrm{sec} .15$, T.18 S., R.16 E., Pima County, Hydrologic Unit 15050302 , at State Highway

$83,13 \mathrm{mi}$ north of Sonoita.

DRAINAGE AREA.--14.1 $\mathrm{mi}^{2}$.

Annual peak discharges

\begin{tabular}{|c|c|c|c|c|c|c|c|}
\hline $\begin{array}{l}\text { Water } \\
\text { year }\end{array}$ & Date & $\begin{array}{c}\text { Annual peak } \\
\text { discharge } \\
\left(\mathrm{ft}^{3} / \mathrm{s}\right)\end{array}$ & $\begin{array}{l}\text { Discharge } \\
\text { codes }\end{array}$ & $\begin{array}{l}\text { Water } \\
\text { year }\end{array}$ & Date & $\begin{array}{c}\text { Annual peak } \\
\text { discharge } \\
\left(\mathrm{ft}^{3} / \mathrm{s}\right)\end{array}$ & $\begin{array}{l}\text { Discharge } \\
\text { codes }\end{array}$ \\
\hline 1962 & $00-00-62$ & 140 & & 1970 & $07-20-70$ & 1,350 & \\
\hline 1963 & $00-00-63$ & 145 & & 1971 & $08-00-71$ & 1,900 & \\
\hline 1964 & $09-10-64$ & 879 & & 1972 & $07-00-72$ & 240 & \\
\hline 1965 & $09-08-65$ & 480 & & 1973 & $00-00-73$ & 10 & LT \\
\hline 1966 & $00-00-66$ & 260 & & 1974 & $09-21-74$ & 1,350 & \\
\hline 1967 & $09-00-67$ & 323 & & 1975 & $09-13-75$ & 980 & \\
\hline 1968 & $07-26-68$ & 1,600 & & 1976 & $08-00-76$ & 1,100 & \\
\hline 1969 & $07-23-69$ & 15 & LT & & & & \\
\hline
\end{tabular}

Magnitude and probability of instantaneous peak flow based on period of record 1962-76

Discharge, in $\mathrm{ft}^{3} / \mathrm{s}$, for indicated recurrence interval

in years, and exceedance probablility, in percent

\begin{tabular}{cccccc}
\hline 2 & $\mathbf{5}$ & $\mathbf{1 0}$ & $\mathbf{2 5}$ & $\mathbf{5 0 \dagger}$ & $\mathbf{1 0 0 \dagger}$ \\
$\mathbf{5 0 \%}$ & $\mathbf{2 0 \%}$ & $\mathbf{1 0 \%}$ & $\mathbf{4 \%}$ & $\mathbf{2 \%}$ & $\mathbf{1 \%}$ \\
\hline 504 & 1,190 & 1,810 & 2,760 & 3,580 & 4,480 \\
Weighted skew & $(\operatorname{logs})=$ & -0.34 & & & \\
Mean & $(\log )=$ & 2.68 & & & \\
Standard dev. & $(\operatorname{logs})=$ & 0.47 & &
\end{tabular}

fReliability of values in column is uncertain, and potential errors are large.

Basin characteristics

\begin{tabular}{cccccccc}
\hline $\begin{array}{c}\text { Main } \\
\text { channel } \\
\text { slope } \\
(\mathrm{ft} / \mathrm{mi})\end{array}$ & $\begin{array}{c}\text { Stream } \\
\text { length } \\
(\mathrm{mi})\end{array}$ & $\begin{array}{c}\text { Mean } \\
\text { basin } \\
\text { elevation } \\
(\mathrm{ft})\end{array}$ & $\begin{array}{c}\text { Forested } \\
\text { area } \\
\text { (percent) }\end{array}$ & $\begin{array}{c}\text { Soil } \\
\text { index }\end{array}$ & $\begin{array}{c}\text { Mean } \\
\text { annual } \\
\text { precipitation } \\
\text { (in) }\end{array}$ & $\begin{array}{c}\text { Rainfall intensity, 24-hour } \\
\text { (in) }\end{array}$ & $\begin{array}{c}50 \text {-year } \\
\text { (in) }\end{array}$ \\
\hline 190 & 5.9 & 5,000 & 0.0 & 3.0 & 16.0 & 1.9 \\
\hline
\end{tabular}

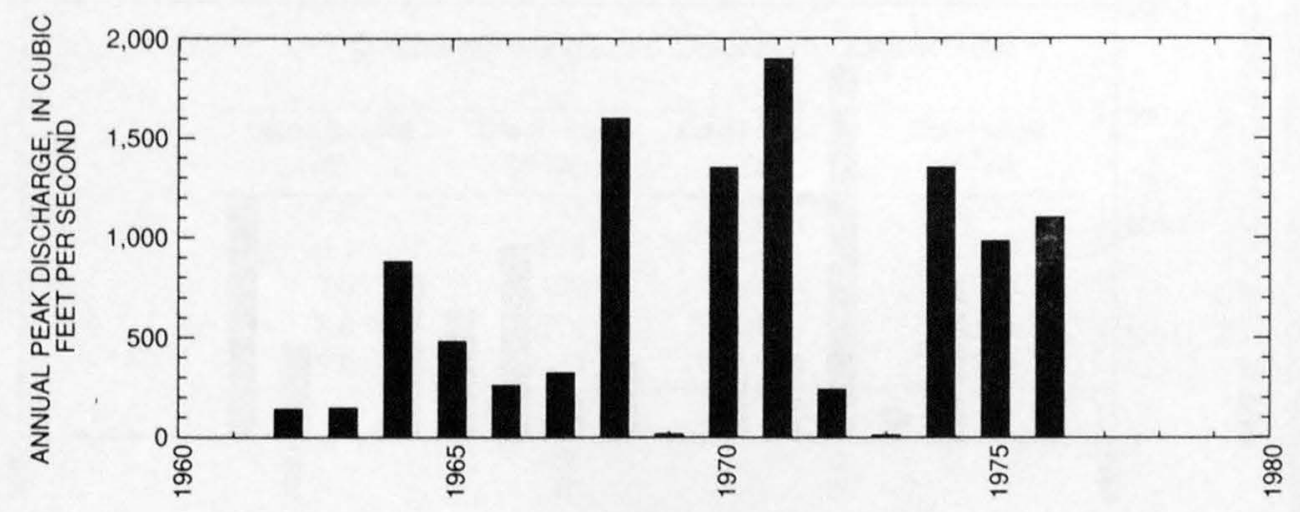


09484590 DAVIDSON CANYON WASH NEAR VAIL, AZ

LOCATION.--Lat $31^{\circ} 59^{\prime} 37^{\prime \prime}$, long $110^{\circ} 38^{\prime} 40^{\prime \prime}, \mathrm{SW}^{1} / \mathrm{SE}^{1} / 4$ sec.31, T.16 S., R.17 E., Hydrologic Unit 15050302 , on right bank 0.3 mi upstrean from Interstate Highway 10, $2.0 \mathrm{mi}$ upstream from mouth, and $5.5 \mathrm{mi}$ southeast of Vail.

DRAINAGE AREA.--50.5 $\mathrm{mi}^{2}$.

Annual peak discharges

\begin{tabular}{cccccrr}
\hline $\begin{array}{c}\text { Water } \\
\text { year }\end{array}$ & Date & $\begin{array}{c}\text { Annual peak } \\
\text { discharge } \\
\left(\mathrm{ft}^{3} / \mathbf{s}\right)\end{array}$ & $\begin{array}{c}\text { Discharge } \\
\text { codes }\end{array}$ & $\begin{array}{c}\text { Water } \\
\text { year }\end{array}$ & $\begin{array}{c}\text { Annual peak } \\
\text { discharge } \\
\left(\mathrm{ft}^{3} / \mathbf{s}\right)\end{array}$ & $\begin{array}{c}\text { Discharge } \\
\text { codes }\end{array}$ \\
\hline 1968 & $07-26-68$ & 3,040 & & 1975 & $07-08-75$ & 708 \\
1969 & $08-05-69$ & 587 & & 1976 & $07-12-76$ & 3,550 \\
1970 & $07-20-70$ & 6,860 & & 1977 & $09-10-77$ & 2,400 \\
1971 & $08-10-71$ & 1,490 & 1978 & $10-06-77$ & 3,040 \\
1972 & $09-07-72$ & 1,320 & & 1979 & $08-15-79$ & 105 \\
1973 & $10-19-72$ & 28 & & 1980 & $09-07-80$ & 1,740 \\
1974 & $09-21-74$ & 1,460 & & 1981 & $07-27-81$ & 4,480 \\
\hline
\end{tabular}

Magnitude and probability of instantaneous peak flow based on period of record 1968-81

\begin{tabular}{|c|c|c|c|c|c|}
\hline \multicolumn{6}{|c|}{$\begin{array}{l}\text { Discharge, in } \mathrm{ft}^{3} / \mathrm{s} \text {, for indicated recurrence interval } \\
\text { in years, and exceedance probablility, in percent }\end{array}$} \\
\hline 2 & 5 & 10 & 25 & $50 t$ & $100 t$ \\
\hline $50 \%$ & $20 \%$ & $10 \%$ & $4 \%$ & $2 \%$ & $1 \%$ \\
\hline 1,900 & 3,430 & 4,640 & 6,380 & 7,800 & 9,340 \\
\hline Weighted skew & $(\log s)=$ & -0.11 & & & \\
\hline Mean & $(\log s)=$ & 3.27 & & & \\
\hline Standard dev. & $(\log s)=$ & 0.31 & & & \\
\hline
\end{tabular}

$\mathrm{f}$ Reliability of values in column is uncertain, and potential errors are large.

\section{Basin characteristics}

\begin{tabular}{cccccccc}
\hline $\begin{array}{c}\text { Main } \\
\text { channel } \\
\text { slope } \\
(\mathrm{ft} / \mathrm{mi})\end{array}$ & $\begin{array}{c}\text { Stream } \\
\text { length } \\
(\mathrm{mi})\end{array}$ & $\begin{array}{c}\text { Mean } \\
\text { basin } \\
\text { elevation } \\
(\mathrm{ft})\end{array}$ & $\begin{array}{c}\text { Forested } \\
\text { area } \\
\text { (percent) }\end{array}$ & $\begin{array}{c}\text { Soil } \\
\text { index }\end{array}$ & $\begin{array}{c}\text { Mean } \\
\text { annual } \\
\text { precipitation } \\
\text { (in) }\end{array}$ & $\begin{array}{c}\text { Rainfall intensity, 24-hour } \\
\text { (in) }\end{array}$ & $\begin{array}{c}50 \text {-year } \\
\text { (in) }\end{array}$ \\
\hline 86.2 & 13.6 & 4,340 & 0.0 & 1.0 & 14.9 & 1.9 \\
\hline
\end{tabular}

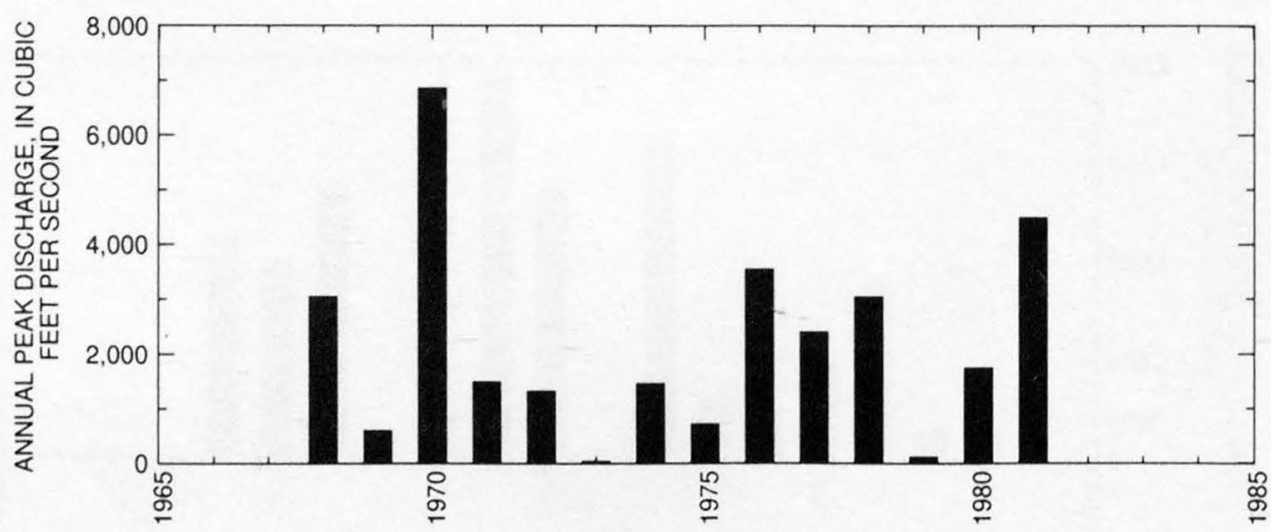


09484600 PANTANO WASH NEAR VAIL, AZ

LOCATION.--Lat 32'02'09", long 110 40'37", in SW $1 / 4 \mathrm{SE}^{1} / 4$ sec.14, T.16 S., R. 16 E., Pima County, Hydrologic Unit 15050302 , on right bank 60 $\mathrm{ft}$ upstream from dam, $2.2 \mathrm{mi}$ southeast of Vail, and 20 mi southeast of Tucson City Hall.

DRAINAGE AREA.--457 $\mathrm{mi}^{2}$.

PERIOD OF RECORD.--January 1959 to September 1974, water years 1975-89 (annual maximums only), October 1989 to current year.

GAGE.--Water-stage recorder and concrete weir. Elevation of gage is 3,205 ft above sea level, from topographic map. January 1959 to September 1974 (water-stage recorder) and October 1974 to September 1989 (crest-stage gage) at same site and datum.

REMARKS.--Records poor. No known diversion above station. Records published herein represent flow by gage. Infiltration flow is not included. Base runoff past gage station consists of downvalley underflow that is brought to the surface by the concrete dam $60 \mathrm{ft}$ downstream which extends to bedrock.

EXTREMES FOR PERIOD OF RECORD.--Maximum discharge, $12,000 \mathrm{ft}^{3} / \mathrm{s}$ Oct. 1 or 2, 1983, gage height, $15.25 \mathrm{ft}$, from inside high-water mark, from rating curve extended above $2,000 \mathrm{ft}^{3} / \mathrm{s}$ on basis of slope-area measurements at gage heights 10.9 and $24 \mathrm{ft}$; no flow June 26 to July 13, Aug. 7, 1971, result of work on infiltration gallery, June 27 to July 13, 1973, result of ponding during construction work on dam, and May 28 to June 12, July 12, 13, 17, 18, 1974.

EXTREMES OUTSIDE PERIOD OF RECORD.--Maximum discharge since at least 1930, about $38,000 \mathrm{ft}^{3} / \mathrm{s}$, Aug. 11, 1958, gage height, about $24 \mathrm{ft}$, from floodmark, from slope-area measurement.

Annual peak discharges

\begin{tabular}{|c|c|c|c|c|c|c|c|}
\hline $\begin{array}{l}\text { Water } \\
\text { year }\end{array}$ & Date & $\begin{array}{l}\text { Annual peak } \\
\text { discharge } \\
\left(\mathrm{ft}^{3} / \mathrm{s}\right)\end{array}$ & $\begin{array}{l}\text { Discharge } \\
\text { codes }\end{array}$ & $\begin{array}{l}\text { Water } \\
\text { year }\end{array}$ & Date & $\begin{array}{c}\text { Annual peak } \\
\text { discharge } \\
\left(\mathrm{ft}^{3} / \mathbf{s}\right)\end{array}$ & $\begin{array}{l}\text { Discharge } \\
\text { codes }\end{array}$ \\
\hline 1958 & $08-11-58$ & ${ }^{1} 38,000$ & $\mathrm{ES}, \mathrm{HP}$ & 1978 & $10-06-77$ & 1,300 & \\
\hline 1959 & $08-17-59$ & 9,310 & & 1979 & $12-18-78$ & 790 & \\
\hline 1960 & $08-09-60$ & 7,300 & & 1980 & $09-07-80$ & 1,300 & \\
\hline 1961 & $08-28-61$ & 5,280 & & 1981 & $09-22-81$ & 13,000 & \\
\hline 1962 & $09-26-62$ & 1,500 & & 1982 & $08-23-82$ & 3,400 & \\
\hline 1963 & $08-25-63$ & 9,700 & & 1983 & $08-03-83$ & 1,840 & \\
\hline 1964 & $09-10-64$ & 9,960 & & 1984 & $10-02-83$ & 12,000 & \\
\hline 1965 & $09-12-65$ & 5,880 & & 1985 & $08-20-85$ & 363 & \\
\hline 1966 & $08-13-66$ & 7,410 & & 1986 & $08-17-86$ & 1,020 & \\
\hline 1967 & $08-18-67$ & 7,680 & & 1987 & $09-24-87$ & 1,370 & \\
\hline 1968 & $12-20-67$ & 2,640 & & 1988 & $07-29-88$ & 7,420 & \\
\hline 1969 & $08-05-69$ & 857 & & 1989 & $07-21-89$ & 803 & \\
\hline 1970 & $07-20-70$ & 6,850 & & 1990 & $07-24-90$ & 3,960 & \\
\hline 1971 & $08-19-71$ & 8,700 & & 1991 & $03-02-91$ & 129 & \\
\hline 1972 & $09-07-72$ & 1,460 & & 1992 & $07-10-92$ & 834 & \\
\hline 1973 & $10-04-72$ & 371 & & 1993 & $07-11-93$ & 1,840 & \\
\hline 1974 & $07-20-74$ & 1,780 & & 1994 & $09-11-94$ & 2,370 & \\
\hline 1975 & $09-02-75$ & 1,200 & & 1995 & $01-05-95$ & 650 & \\
\hline 1976 & $07-25-76$ & 5,200 & & 1996 & 09-01-96 & 2,250 & \\
\hline 1977 & $09-10-77$ & 1,600 & & & & & \\
\hline
\end{tabular}

${ }^{1}$ Highest since 1930.

Discharge rating table developed October 1992

\begin{tabular}{crcc}
\hline $\begin{array}{c}\text { Gage height } \\
(\mathbf{f t})\end{array}$ & $\begin{array}{c}\text { Discharge } \\
\left(\mathbf{f t}^{\mathbf{3}} / \mathbf{s}\right)\end{array}$ & $\begin{array}{c}\text { Gage height } \\
(\mathbf{f t})\end{array}$ & $\begin{array}{c}\text { Discharge } \\
\left(\mathbf{f t}^{\mathbf{3}} \mathbf{/ s}\right)\end{array}$ \\
\hline 6.0 & 558 & 10.0 & 3,520 \\
6.5 & 818 & 11.0 & 4,600 \\
7.0 & 1,100 & 12.0 & 5,790 \\
8.0 & 1,770 & 13.0 & 7,110 \\
9.0 & 2,580 & 13.7 & 8,100 \\
\hline
\end{tabular}




\section{GILA RIVER BASIN}

09484600 PANTANO WASH NEAR VAIL, AZ--Continued

Basin characteristics

\begin{tabular}{cccccccc}
\hline $\begin{array}{c}\text { Main } \\
\text { channel } \\
\text { slope } \\
(\mathrm{ft} / \mathrm{mi})\end{array}$ & $\begin{array}{c}\text { Stream } \\
\text { length } \\
(\mathrm{mi})\end{array}$ & $\begin{array}{c}\text { Mean } \\
\text { basin } \\
\text { elevation } \\
(\mathrm{ft})\end{array}$ & $\begin{array}{c}\text { Forested } \\
\text { area } \\
\text { (percent) }\end{array}$ & $\begin{array}{c}\text { Soil } \\
\text { index }\end{array}$ & $\begin{array}{c}\text { Mean } \\
\text { annual } \\
\text { precipitation } \\
\text { (in) }\end{array}$ & $\begin{array}{c}\text { R-year } \\
\text { (in) }\end{array}$ & $\begin{array}{c}50 \text {-year } \\
\text { (in) }\end{array}$ \\
\hline 46.3 & 43.5 & 4,500 & 15.0 & 1.75 & 15.4 & 1.9 \\
\hline
\end{tabular}


09484600 PANTANO WASH NEAR VAIL, AZ--Continued

MEAN MONTHLY AND ANNUAL DISCHARGES $1960.74,1990.96$

\begin{tabular}{|c|c|c|c|c|c|c|}
\hline MONTH & $\begin{array}{l}\text { MAXIMUM } \\
\text { (FT3/S) }\end{array}$ & $\begin{array}{l}\text { MINIMUM } \\
(\text { FT3/S) }\end{array}$ & $\begin{array}{c}\text { MEAN } \\
(\mathrm{FT} 3 / \mathrm{S})\end{array}$ & $\begin{array}{l}\text { STAN- } \\
\text { DARD } \\
\text { DEVIA- } \\
\text { TION } \\
(\text { FT3/S) }\end{array}$ & $\begin{array}{l}\text { COBFFI- } \\
\text { CIENT OF } \\
\text { VARI- } \\
\text { ATION }\end{array}$ & $\begin{array}{c}\text { PERCENT } \\
\text { OF } \\
\text { ANNUAL } \\
\text { RUNOFF }\end{array}$ \\
\hline OCTOBER & 6.7 & 0.10 & 1.9 & 1.9 & 1.0 & 2.5 \\
\hline NOVBMBER & 3.0 & 0.10 & 1.2 & 0.73 & 0.59 & 1.7 \\
\hline DECEMBER & 50 & 0.10 & 6.4 & 14 & 2.1 & 8.7 \\
\hline JANUARY & 111 & 0.10 & 8.4 & 24 & 2.8 & 11.3 \\
\hline FEBRUARY & 36 & 0.10 & 4.7 & 7.9 & 1.7 & 6.4 \\
\hline MARCH & 18 & 0.12 & 3.4 & 3.9 & 1.2 & 4.6 \\
\hline APRIL & 5.2 & 0.32 & 2.1 & 1.3 & 0.62 & 2.8 \\
\hline MAY & 2.0 & 0.19 & 1.3 & 0.48 & 0.37 & 1.7 \\
\hline JUNE & 3.6 & 0.07 & 1.2 & 0.83 & 0.68 & 1.6 \\
\hline JULY & 50 & 0.66 & 12 & 14 & 1.2 & 16.0 \\
\hline AUGUST & 93 & 0.52 & 20 & 26 & 1.3 & 27.0 \\
\hline SEPTEMBER & 105 & 0.16 & 12 & 21 & 1.8 & 15.7 \\
\hline ANNUAL & 13 & 1.8 & 6.2 & 3.8 & 0.61 & 100 \\
\hline
\end{tabular}

MAGNITUDE AND PROBABILITY OF INSTANTANEOUS PEAK FLOW BASED ON PERIOD OF RECORD 1958.96

DISCHARGE, IN FT3/S, FOR INDICATED RECURRENCE INTERVAL IN YEARS, AND EXCEEDANCB PROBABILITY, IN PERCENT

\begin{tabular}{|c|c|c|c|c|c|}
\hline 2 & 5 & 10 & 25 & 50 & 100 \\
\hline 508 & 208 & 108 & 48 & 28 & 18 \\
\hline 2,600 & 6,450 & 10,400 & 17,200 & 23,900 & 32,100 \\
\hline WEIGHTED & SKEW & (LOGS) $=$ & 00 & & \\
\hline MEAN & & $($ LOGS $)=$ & 41 & & \\
\hline STANDARD & DEV. & (LOGS) $=$ & 47 & & \\
\hline
\end{tabular}

DURATION TABLE OF DAILY MEAN FLOW FOR PERIOD OF RECORD 1960-74, 1990-96
MAGNITUDE AND PROBABILITY OF ANNUAL LOW FLOW BASED ON PERIOD OF RECORD 1960.74, 1991.96

\begin{tabular}{|c|c|c|c|c|c|c|}
\hline $\begin{array}{l}\text { PERIOD } \\
\text { ICON- } \\
\text { SECU - }\end{array}$ & & $\begin{array}{l}\text { DISCHARGE, IN FT3/S, FOR INDICATED } \\
\text { RECURRENCE INTERVAL, IN YEARS, AND }\end{array}$ & $\begin{array}{l}\text { IN } \\
\text { E INTE } \\
\text { ANCE } \\
\ldots \ldots\end{array}$ & $\begin{array}{l}\text { /S, FO } \\
\text { AL, IN } \\
\text { BABILI } \\
\ldots \ldots\end{array}$ & $\begin{array}{l}\text { INDICAT } \\
\text { EARS, A } \\
, \text { IN PE } \\
\ldots . .\end{array}$ & ENT \\
\hline TIVE & 2 & 5 & 10 & 20 & $50 \#$ & $100 \#$ \\
\hline DAYS) & 508 & 208 & 108 & 58 & 28 & 18 \\
\hline 1 & 0.43 & 0.19 & 0.06 & 0.00 & 0.00 & 0.00 \\
\hline 3 & 0.46 & 0.20 & 0.05 & 0.00 & 0.00 & 0.00 \\
\hline 7 & 0.54 & 0.23 & 0.05 & 0.00 & 0.00 & 0.00 \\
\hline 14 & 0.61 & 0.31 & 0.11 & 0.00 & 0.00 & 0.00 \\
\hline 30 & 0.69 & 0.36 & 0.23 & 0.16 & 0.09 & 0.06 \\
\hline 60 & 0.82 & 0.44 & 0.29 & 0.19 & 0.11 & 0.08 \\
\hline 90 & 1.0 & 0.58 & 0.37 & 0.24 & 0.13 & 0.08 \\
\hline 120 & 1.4 & 0.65 & 0.40 & 0.26 & 0.15 & 0.10 \\
\hline 183 & 2.1 & 0.86 & 0.49 & 0.29 & 0.15 & 0.09 \\
\hline
\end{tabular}

MAGNITUDE AND PROBABILITY OF ANNUAL HIGH FLOW BASED ON PERIOD OF RECORD $1960.74,1990.96$

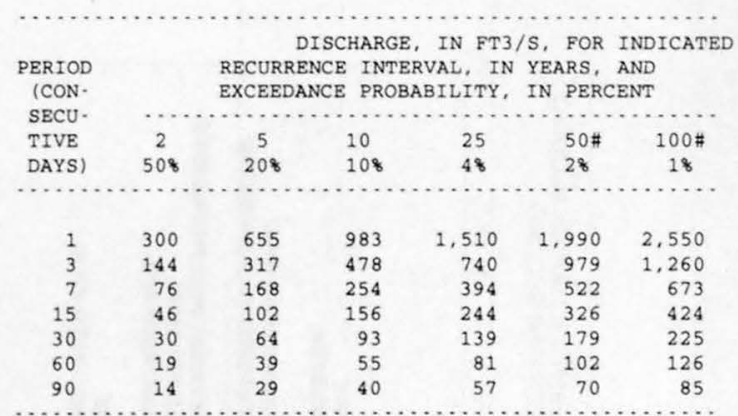

ISCHARGE, IN FT3/S, WHICH WAS EQUALED OR EXCEEDED FOR INDICATED PERCENT OF TIME

\begin{tabular}{|c|c|c|c|c|c|c|c|c|c|c|c|c|c|c|c|c|}
\hline 18 & 58 & 108 & 158 & 208 & 308 & 408 & 508 & 608 & 708 & 808 & 908 & 958 & 988 & 998 & 99.58 & 99.98 \\
\hline 30 & 15 & 4.7 & 3.3 & 2.7 & 2.0 & 1.6 & 1.4 & 1.2 & 0.93 & 0.71 & 0.43 & 0.22 & 0.09 & 0.08 & 0.00 & 0.00 \\
\hline
\end{tabular}

\# Reliability of values in column is uncertain, and potential errors are large. 
ANNUAL MEAN DISCHARGE, IN CUBIC

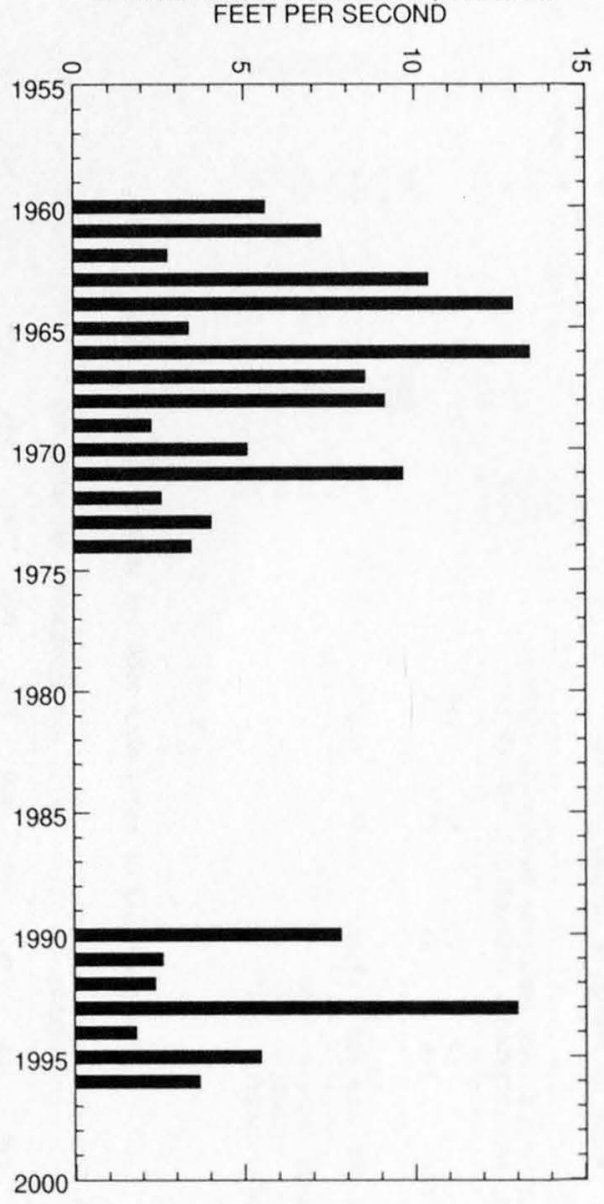

ANNUAL PEAK DISCHARGE, IN CUBIC

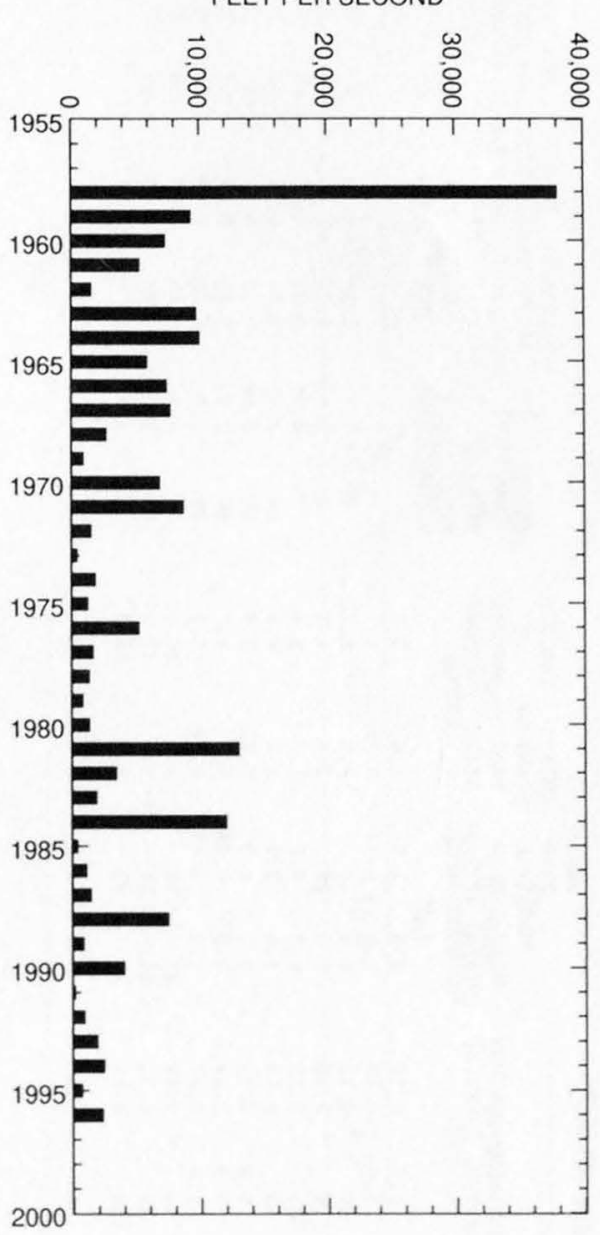


GILA RIVER BASIN

491

09484600 PANTANO WASH NEAR VAIL, AZ--Continued
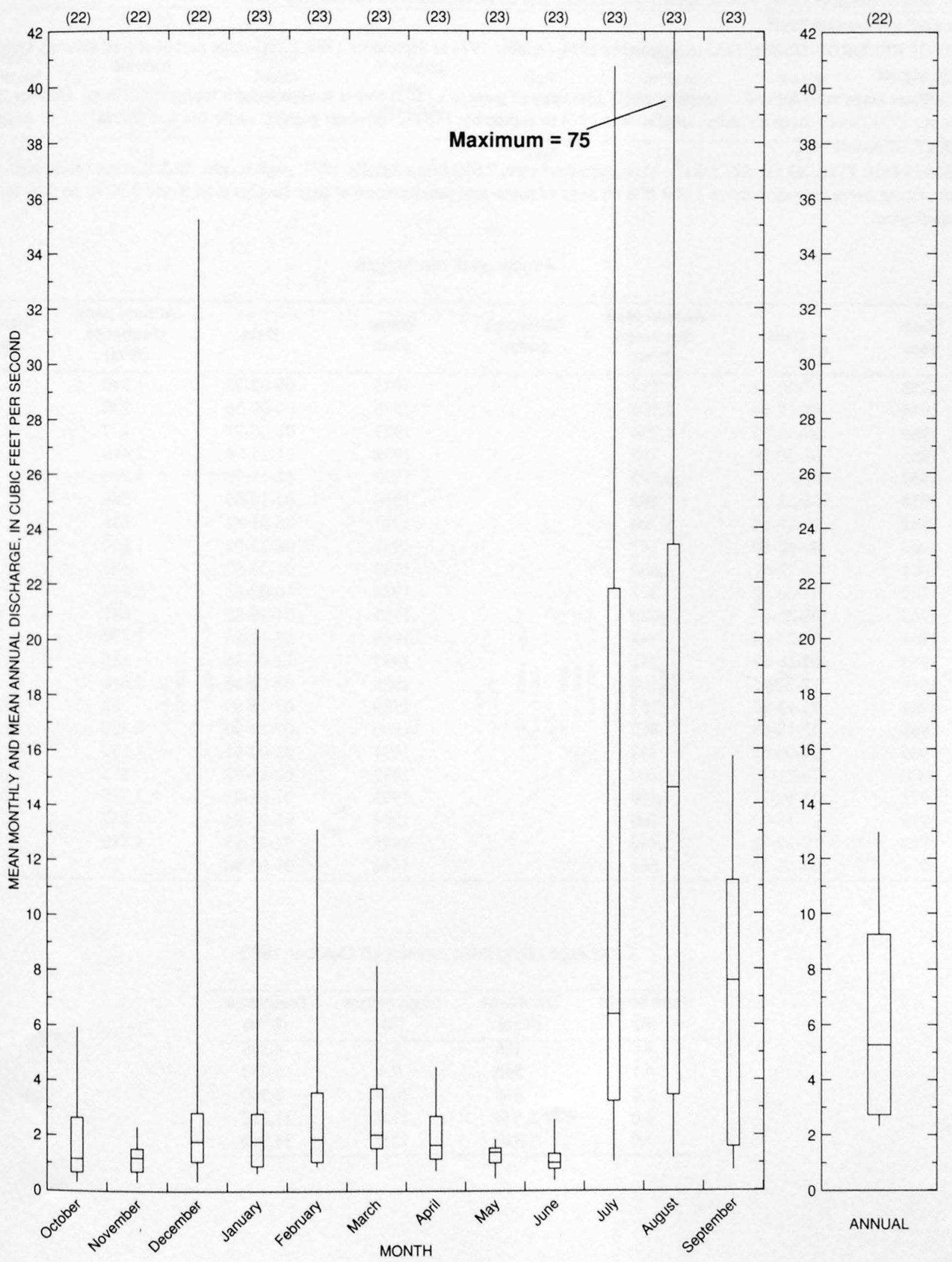
09485000 RINCON CREEK NEAR TUCSON, AZ

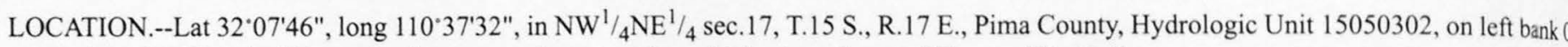
mi north of Sentinel Butte, 9 mi upstream from mouth, and 22 mi southeast of Tucson City Hall.

DRAINAGE AREA.--44.8 $\mathrm{mi}^{2}$.

PERIOD OF RECORD.--October 1952 to September 1974, October 1974 to September 1989, (crest-stage partial-record station); October 1989 current year.

GAGE.--Water-stage recorder and concrete control. Elevation of gage is 3,120 ft above sea level, from topographic map. October 1952 to Se tember 1974 (water-stage recorder) and October 1974 to September 1989 (crest-stage gage) at same site and datum.

REMARKS.--Records fair.

EXTREMES FOR PERIOD OF RECORD.--Maximum discharge, 9,660 $\mathrm{ft}^{3} / \mathrm{s} \mathrm{Aug.} \mathrm{19,} \mathrm{1971,} \mathrm{gage} \mathrm{height,} 10.5 \mathrm{ft}$, from inside high-water mar from rating curve extended above $1,800 \mathrm{ft}^{3} / \mathrm{s}$ on basis of slope-area measurement at gage heights $6.50 \mathrm{ft}$ and $9.90 \mathrm{ft}$; no flow for many dar in each year.

Annual peak discharges

\begin{tabular}{|c|c|c|c|c|c|c|c|}
\hline $\begin{array}{l}\text { Water } \\
\text { year }\end{array}$ & Date & $\begin{array}{c}\text { Annual peak } \\
\text { discharge } \\
\left(\mathrm{ft}^{3} / \mathrm{s}\right)\end{array}$ & $\begin{array}{l}\text { Discharge } \\
\text { codes }\end{array}$ & $\begin{array}{l}\text { Water } \\
\text { year }\end{array}$ & Date & $\begin{array}{c}\text { Annual peak } \\
\text { discharge } \\
\left(\mathrm{ft}^{3} / \mathrm{s}\right)\end{array}$ & $\begin{array}{c}\text { Discharge } \\
\text { codes }\end{array}$ \\
\hline 1953 & $07-30-53$ & 194 & & 1975 & $09-02-75$ & 340 & \\
\hline 1954 & $08-19-54$ & 2,160 & & 1976 & $00-00-76$ & 230 & \\
\hline 1955 & $08-03-55$ & 8,250 & & 1977 & $01-00-77$ & 127 & \\
\hline 1956 & $07-20-56$ & 150 & & 1978 & $01-15-78$ & 2,440 & \\
\hline 1957 & $01-09-57$ & 3,570 & & 1979 & $12-18-78$ & 4,890 & \\
\hline 1958 & $03-22-58$ & 492 & & 1980 & $02-14-80$ & 586 & \\
\hline 1959 & $10-21-58$ & 5,220 & & 1981 & $08-01-81$ & 236 & \\
\hline 1960 & $01-12-60$ & 747 & & 1982 & $08-23-82$ & 1,800 & \\
\hline 1961 & $08-22-61$ & 2,600 & & 1983 & $01-30-83$ & 688 & \\
\hline 1962 & $01-24-62$ & 227 & & 1984 & $10-02-83$ & 5,640 & \\
\hline 1963 & $08-25-63$ & 3,420 & & 1985 & $01-26-85$ & 647 & \\
\hline 1964 & $09-23-64$ & 948 & & 1986 & $07-15-86$ & 1,170 & \\
\hline 1965 & $08-18-65$ & 311 & & 1987 & $12-07-86$ & 535 & \\
\hline 1966 & $12-22-65$ & 3,100 & & 1988 & $08-02-88$ & 2,640 & \\
\hline 1967 & $08-13-67$ & 157 & & 1989 & $07-25-89$ & 88 & \\
\hline 1968 & $02-12-68$ & 1,860 & & 1990 & $07-24-90$ & 6,320 & \\
\hline 1969 & $09-06-69$ & 548 & & 1991 & $03-02-91$ & 1,180 & \\
\hline 1970 & $08-01-70$ & 1,200 & & 1992 & $02-13-92$ & 871 & \\
\hline 1971 & $08-19-71$ & 9,660 & & 1993 & $01-08-93$ & 3,720 & \\
\hline 1972 & $07-16-72$ & 360 & & 1994 & $03-20-94$ & 237 & \\
\hline 1973 & $10-19-72$ & 1,440 & & 1995 & $02-15-95$ & 1,880 & \\
\hline 1974 & $08-01-74$ & 664 & & 1996 & $09-04-96$ & 27 & \\
\hline
\end{tabular}

Discharge rating table developed October 1993

\begin{tabular}{crcc}
\hline $\begin{array}{c}\text { Gage height } \\
(\mathbf{f t})\end{array}$ & $\begin{array}{c}\text { Discharge } \\
\left(\mathbf{f t}^{3} \mathbf{s}\right)\end{array}$ & $\begin{array}{c}\text { Gage height } \\
(\mathbf{f t})\end{array}$ & $\begin{array}{c}\text { Discharge } \\
\left(\mathbf{f t}^{3} / \mathbf{s}\right)\end{array}$ \\
\hline 4.0 & 266 & 8.0 & 4,340 \\
4.5 & 540 & 9.0 & 6,250 \\
5.0 & 846 & 10.0 & 8,560 \\
6.0 & 1,670 & 11.0 & 11,310 \\
7.0 & 2,820 & 12.0 & 14,500 \\
\hline
\end{tabular}


GILA RIVER BASIN

493

09485000 RINCON CREEK NEAR TUCSON, AZ--Continued

Basin characteristics

\begin{tabular}{ccccccc}
\hline $\begin{array}{c}\text { Main } \\
\text { channel } \\
\text { slope } \\
(\mathrm{ft} / \mathrm{mi})\end{array}$ & $\begin{array}{c}\text { Stream } \\
\text { length } \\
(\mathrm{mi})\end{array}$ & $\begin{array}{c}\text { Mean } \\
\text { basin } \\
\text { elevation } \\
(\mathrm{ft})\end{array}$ & $\begin{array}{c}\text { Forested } \\
\text { area } \\
\text { (percent) }\end{array}$ & $\begin{array}{c}\text { Soil } \\
\text { index }\end{array}$ & $\begin{array}{c}\text { Mean } \\
\text { annual } \\
\text { precipitation } \\
\text { (in) }\end{array}$ & $\begin{array}{c}\text { Rainfall intensity, 24-hour } \\
\text { (in) }\end{array}$ \\
\hline 612 & 9.0 & 4,850 & 57.0 & 1.09 & 19.2 & $\begin{array}{c}50 \text {-year } \\
\text { (in) }\end{array}$ \\
\hline
\end{tabular}


09485000 RINCON CREEK NEAR TUCSON, AZ--Continued

MEAN MONTHLY AND ANNUAL DISCHARGES 1953-96

\begin{tabular}{|c|c|c|c|c|c|c|}
\hline MONTH & $\begin{array}{l}\text { MAXIMUM } \\
(\text { FT } 3 / \mathrm{S})\end{array}$ & $\begin{array}{l}\text { MINIMUM } \\
\text { (FT3/S) }\end{array}$ & $\begin{array}{c}\text { MEAN } \\
(\mathrm{FT} 3 / \mathrm{S})\end{array}$ & $\begin{array}{l}\text { STAN- } \\
\text { DARD } \\
\text { DEVIA- } \\
\text { TION } \\
(\text { FT3/S) }\end{array}$ & $\begin{array}{l}\text { COEFFI- } \\
\text { CIENT OF } \\
\text { VARI- } \\
\text { ATION }\end{array}$ & $\begin{array}{l}\text { PERCENT } \\
\text { OF } \\
\text { ANNUAL } \\
\text { RUNOFF }\end{array}$ \\
\hline OCTOBER & 23 & 0.00 & 1.6 & 5.0 & 3.2 & 1.9 \\
\hline NOVEMBER & 18 & 0.00 & 1.1 & 3.4 & 3.1 & 1.3 \\
\hline DECEMBER & 130 & 0.00 & 11 & 26 & 2.4 & 12.7 \\
\hline JANUARY & 247 & 0.00 & 20 & 49 & 2.4 & 23.9 \\
\hline FEBRUARY & 75 & 0.00 & 15 & 25 & 1.6 & 18.2 \\
\hline MARCH & 74 & 0.00 & 14 & 20 & 1.5 & 16.1 \\
\hline APRIL & 18 & 0.00 & 3.1 & 5.3 & 1.7 & 3.7 \\
\hline MAY & 2.0 & 0.00 & 0.17 & 0.38 & 2.2 & 0.2 \\
\hline JUNE & 1.5 & 0.00 & 0.07 & 0.27 & 4.2 & 0.1 \\
\hline JULY & 82 & 0.00 & 3.6 & 15 & 4.2 & 4.3 \\
\hline AUGUST & 64 & 0.00 & 11 & 16 & 1.4 & 13.6 \\
\hline SEPTEMBER & 19 & 0.00 & 3.4 & 5.6 & 1.7 & 4.0 \\
\hline ANNUAL & 33 & 0.07 & 7.0 & 8.0 & 1.2 & 100 \\
\hline
\end{tabular}

MAGNITUDE AND PROBABILITY OF INSTANTANEOUS PEAK FLOW BASED ON PERIOD OF RECORD 1953.96

DISCHARGE, IN FT3/S, FOR INDICATED RECURRENCE INTERVAL IN YEARS, AND EXCEEDANCE PROBABILITY, IN PERCENT

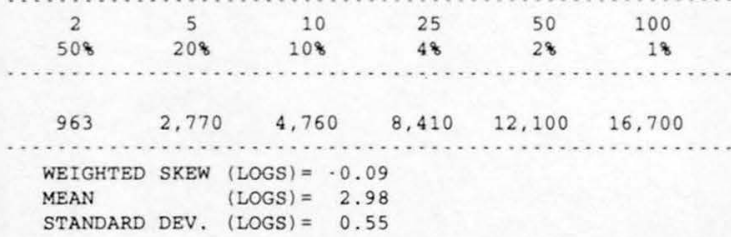

MAGNITUDE AND PROBABILITY OF ANNUAL LOW FLOW BASED ON PERIOD OF RECORD 1954.96

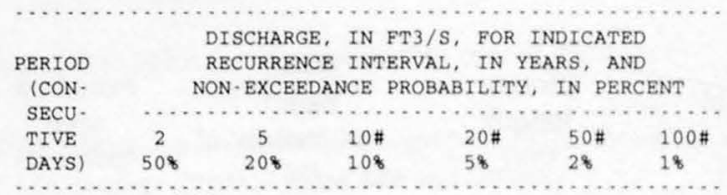

MAGNITUDE AND PROBABILITY OF ANNUAL HIGH FLOW BASED ON PERIOD OF RECORD 1953.96

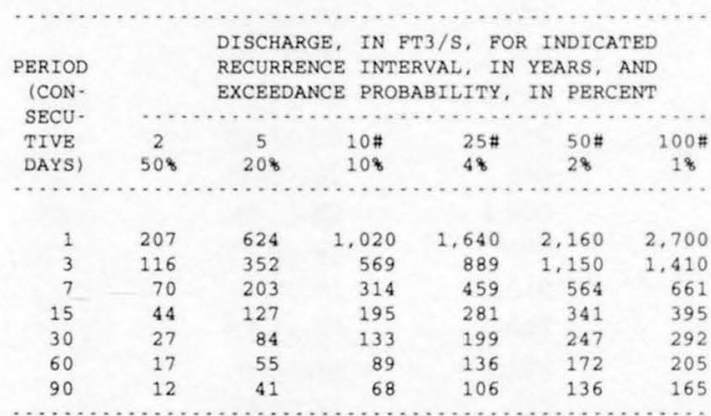

DURATION TABLE OF DAILY MEAN FLOW FOR PERIOD OF RECORD 1953.96

DISCHARGE, IN FT3/S, WHICH WAS EQUALED OR EXCEEDED FOR INDICATED PERCENT OF TIME

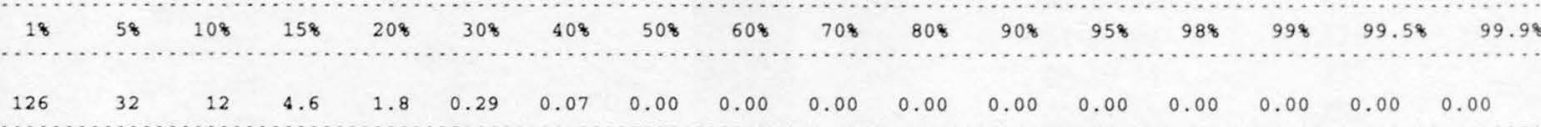

\# Reliability of values in column is uncertain, and potential errors are large. 
ANNUAL MEAN DISCHARGE, IN CUBIC

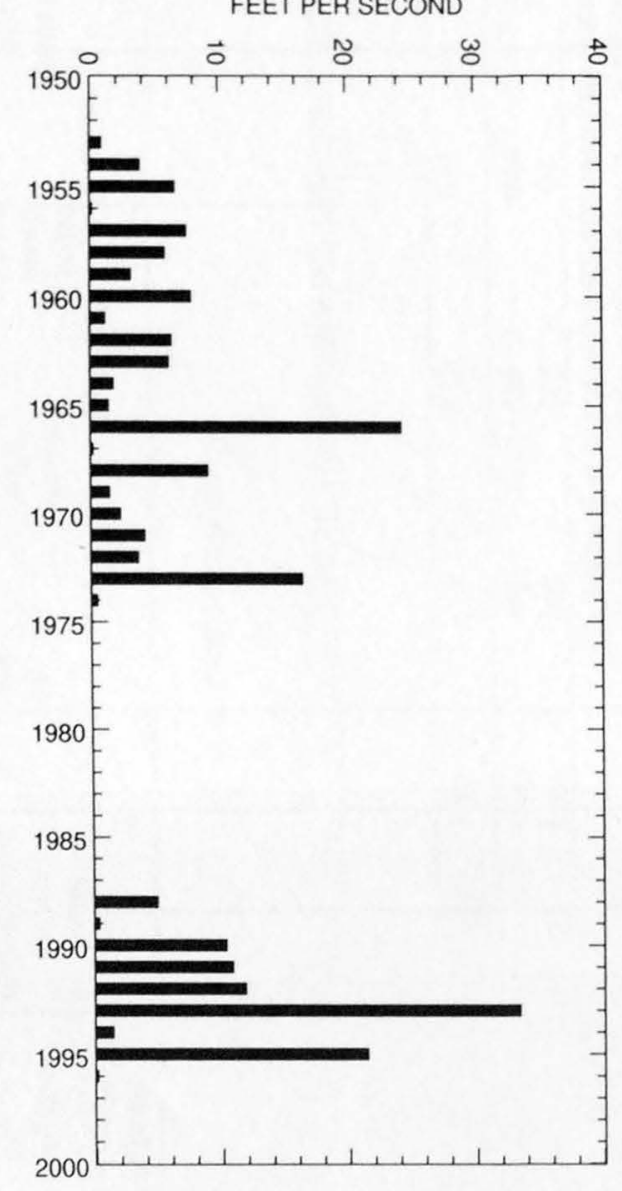

ANNUAL PEAK DISCHARGE, IN CUBIC FEET PER SECOND

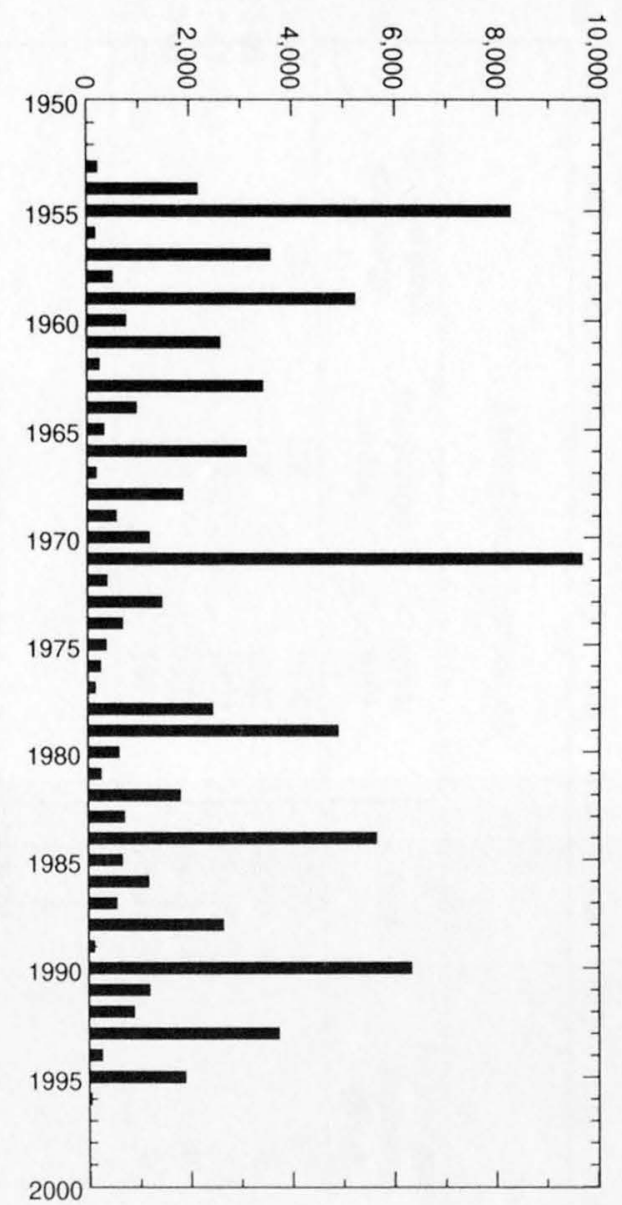


496

GILA RIVER BASIN

09485000 RINCON CREEK NEAR TUCSON, AZ--Continued
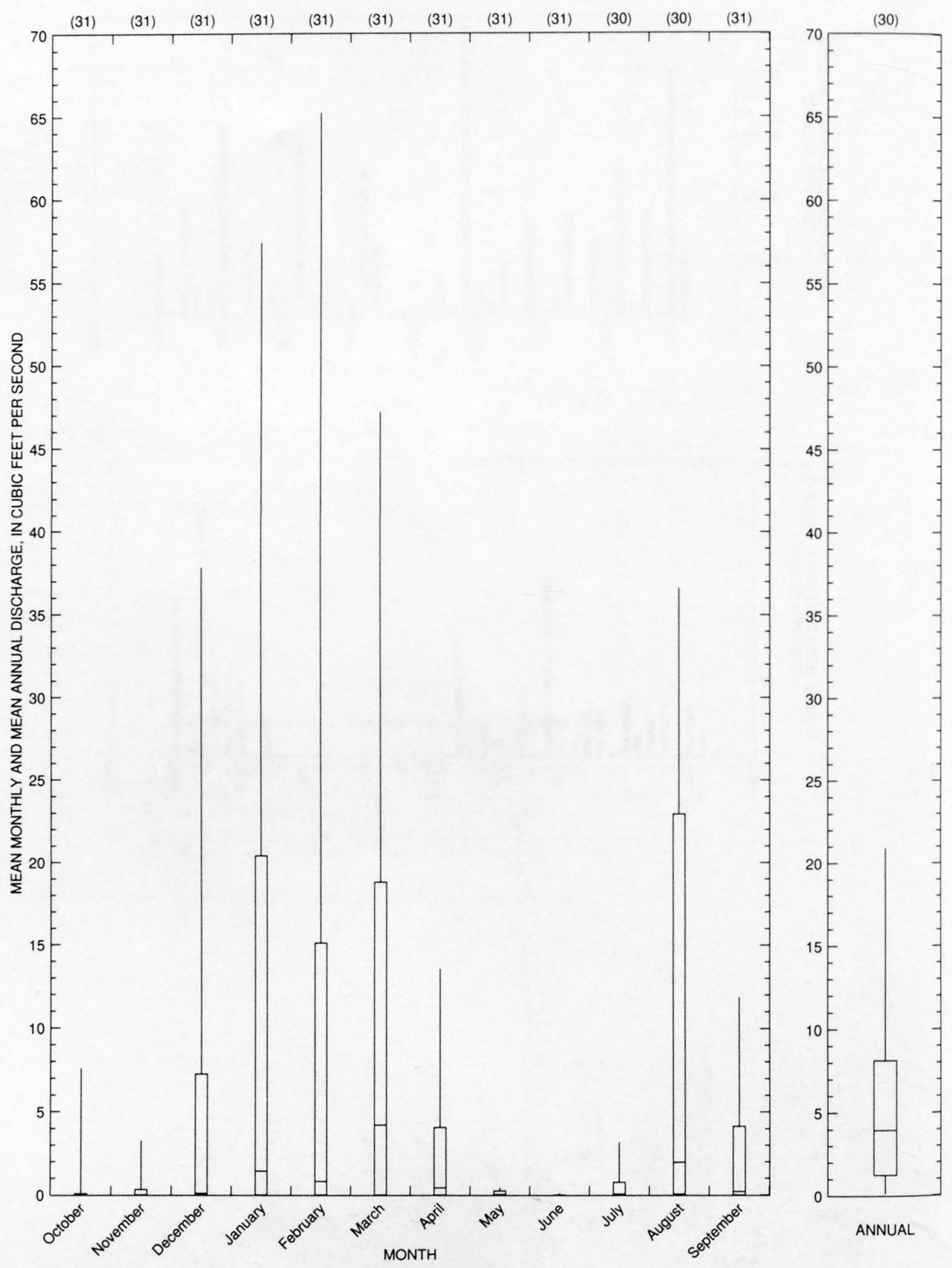
09485100 SAGUARO CORNERS WASH NEAR TUCSON, AZ

LOCATION.--Lat $32^{\circ} 10^{\prime} 11^{\prime \prime}$, long 110 44'15", SW $1 / 4 \mathrm{NW}^{1} / 4 \mathrm{sec} .32$, T.14 S., R.16 E., Pima County, Hydrologic Unit 15050302, at Freeman Road, 0.9 mi south of Old Spanish Trail, and $4 \mathrm{mi}$ southeast of Tucson city limits.

DRAINAGE AREA.--0.17 $\mathrm{mi}^{2}$.

Annual peak discharges

\begin{tabular}{|c|c|c|c|c|c|c|c|}
\hline $\begin{array}{l}\text { Water } \\
\text { year }\end{array}$ & Date & $\begin{array}{c}\text { Annual peak } \\
\text { discharge } \\
\left(\mathrm{ft}^{3} / \mathrm{s}\right)\end{array}$ & $\begin{array}{l}\text { Discharge } \\
\text { codes }\end{array}$ & $\begin{array}{l}\text { Water } \\
\text { year }\end{array}$ & Date & $\begin{array}{c}\text { Annual peak } \\
\text { discharge } \\
\left(\mathrm{ft}^{3} / \mathrm{s}\right)\end{array}$ & $\begin{array}{c}\text { Discharge } \\
\text { codes }\end{array}$ \\
\hline 1965 & $09-12-65$ & 29 & UR & 1970 & $07-20-70$ & 29 & UR \\
\hline 1966 & $09-12-66$ & 23 & UR & 1971 & $08-00-71$ & 30 & UR \\
\hline 1967 & $07-17-67$ & 33 & UR & 1972 & $08-12-72$ & 4.4 & UR \\
\hline 1968 & $08-00-68$ & 49 & UR & 1973 & $08-00-73$ & 10 & UR \\
\hline 1969 & 09-09-69 & 28 & UR & 1974 & $00-00-74$ & 0 & UR \\
\hline
\end{tabular}

Magnitude and probability of instantaneous peak flow based on period of record 1965-74

\begin{tabular}{|c|c|c|c|c|c|}
\hline \multicolumn{6}{|c|}{$\begin{array}{l}\text { Discharge, in } \mathrm{ft}^{3} / \mathrm{s} \text {, for indicated recurrence interval } \\
\text { in years, and exceedance probablility, in percent }\end{array}$} \\
\hline 2 & 5 & 10 & $25 \dagger$ & $50 \dagger$ & $100 \dagger$ \\
\hline $50 \%$ & $20 \%$ & $10 \%$ & $4 \%$ & $2 \%$ & $1 \%$ \\
\hline 27.4 & 34.4 & 39.1 & 44.8 & 49.1 & 53.4 \\
\hline Weighted skew & $(\log s)=$ & 0.21 & & & \\
\hline Mean & $(\log s)=$ & 1.44 & & & \\
\hline Standard dev. & $(\log s)=$ & 0.11 & & & \\
\hline
\end{tabular}

Basin characteristics

\begin{tabular}{cccccccc}
\hline $\begin{array}{c}\text { Main } \\
\text { channel } \\
\text { slope } \\
(\mathrm{ft} / \mathrm{mi})\end{array}$ & $\begin{array}{c}\text { Stream } \\
\text { length } \\
(\mathrm{mi})\end{array}$ & $\begin{array}{c}\text { Mean } \\
\text { basin } \\
\text { elevation } \\
(\mathrm{ft})\end{array}$ & $\begin{array}{c}\text { Forested } \\
\text { area } \\
\text { (percent) }\end{array}$ & $\begin{array}{c}\text { Soil } \\
\text { index }\end{array}$ & $\begin{array}{c}\text { Mean } \\
\text { annual } \\
\text { precipitation } \\
\text { (in) }\end{array}$ & $\begin{array}{c}\text { R-year } \\
\text { (in) }\end{array}$ & $\begin{array}{c}\text { 50-year } \\
\text { (in) }\end{array}$ \\
\hline 117 & 0.4 & 3,040 & 0.0 & 1.0 & 12.0 & 1.8 \\
\hline
\end{tabular}

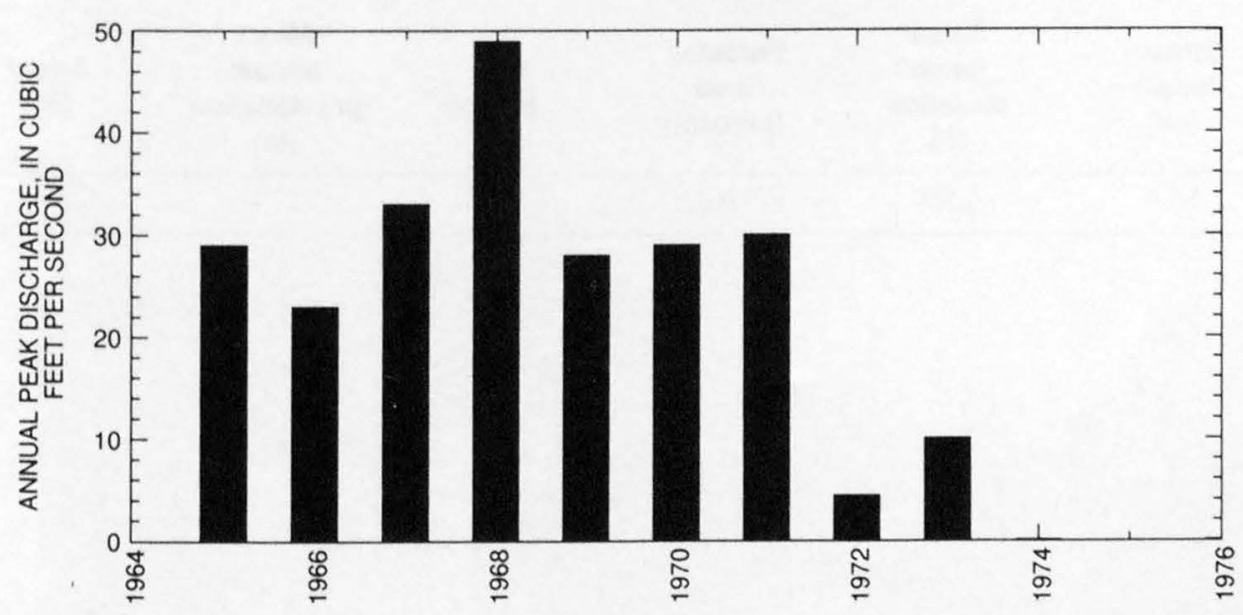




\section{PANTANO WASH NEAR TUCSON, AZ}

LOCATION.--Lat $32^{\circ} 14^{\prime} 57^{\prime \prime}$, long $110^{\circ} 50^{\prime} 53^{\prime \prime}, \mathrm{NW}^{1} / \mathrm{NE}^{1} / 4$ sec.6, T.14 S., R.15 E., Pima County, Hydrologic Unit 15050302 , at Tanque Verde Road 0.7 mi northeast of Tucson city limits, and $1.75 \mathrm{mi}$ above mouth.

DRAINAGE AREA.--602 $\mathrm{mi}^{2}$.

Annual peak discharge

\begin{tabular}{|c|c|c|c|c|c|c|c|}
\hline $\begin{array}{l}\text { Water } \\
\text { year }\end{array}$ & Date & $\begin{array}{c}\text { Annual peak } \\
\text { discharge } \\
\left(\mathrm{ft}^{3} / \mathrm{s}\right)\end{array}$ & $\begin{array}{l}\text { Discharge } \\
\text { codes }\end{array}$ & $\begin{array}{l}\text { Water } \\
\text { year }\end{array}$ & Date & $\begin{array}{c}\text { Annual peak } \\
\text { discharge } \\
\left(\mathrm{ft}^{3} / \mathrm{s}\right)\end{array}$ & $\begin{array}{c}\text { Discharge } \\
\text { codes }\end{array}$ \\
\hline 1940 & $08-13-40$ & 9,200 & HP & 1972 & $09-07-72$ & 180 & \\
\hline 1958 & $08-12-58$ & ${ }^{1} 20,000$ & HP & 1973 & $10-19-72$ & 500 & ES \\
\hline 1965 & $08-18-65$ & 250 & & 1974 & $07-20-74$ & 600 & ES \\
\hline 1966 & $08-13-66$ & 2,820 & & 1975 & $07-16-75$ & 3,490 & \\
\hline 1967 & $07-12-67$ & 4,120 & & 1976 & $08-10-76$ & 3,080 & \\
\hline 1968 & $12-20-67$ & 2,420 & & 1979 & $00-00-79$ & 1,530 & \\
\hline 1969 & $08-05-69$ & 1,250 & & 1980 & $00-00-80$ & 1,000 & \\
\hline 1970 & $07-20-70$ & 6,480 & & 1981 & $00-00-81$ & 9,700 & \\
\hline 1971 & $08-20-71$ & ${ }^{2} 12,800$ & & 1984 & $10-01-83$ & 11,000 & HP \\
\hline
\end{tabular}

${ }^{1}$ Highest since 1930.

${ }^{2}$ Highest since 1958.

Magnitude and probability of instantaneous peak flow based on period of record 1940 ,

$1958,1965-76,1979-81,1984$

\begin{tabular}{|c|c|c|c|c|c|}
\hline \multicolumn{6}{|c|}{$\begin{array}{l}\text { Discharge, in } \mathrm{ft}^{3} / \mathrm{s} \text {, for indicated recurrence interval } \\
\text { in years, and exceedance probablility, in percent }\end{array}$} \\
\hline 2 & 5 & 10 & 25 & $50 \dagger$ & $100 t$ \\
\hline $50 \%$ & $20 \%$ & $10 \%$ & $4 \%$ & $2 \%$ & $1 \%$ \\
\hline 1,720 & 4,620 & 7,540 & 12,500 & 17,100 & 22,600 \\
\hline Weighted skew & $(\log s)=$ & -0.22 & & & \\
\hline Mean & $(\log s)=$ & 3.22 & & & \\
\hline Standard dev. & $(\log s)=$ & 0.53 & & & \\
\hline
\end{tabular}

Basin characteristics

\begin{tabular}{ccccccc}
\hline $\begin{array}{c}\text { Main } \\
\text { channel } \\
\text { slope } \\
(\mathrm{ft} / \mathrm{mi})\end{array}$ & $\begin{array}{c}\text { Stream } \\
\text { length } \\
\text { (mi) }\end{array}$ & $\begin{array}{c}\text { Mean } \\
\text { basin } \\
\text { elevation } \\
(\mathrm{ft})\end{array}$ & $\begin{array}{c}\text { Forested } \\
\text { area } \\
\text { (percent) }\end{array}$ & $\begin{array}{c}\text { Soil } \\
\text { index }\end{array}$ & $\begin{array}{c}\text { Mean } \\
\text { annual } \\
\text { precipitation } \\
\text { (in) }\end{array}$ & $\begin{array}{c}\text { Rainfall intensity, 24-hour } \\
\text { (in) }\end{array}$ \\
\hline 56.4 & 54.4 & 4,560 & 16.0 & 1.1 & 17.1 & $\begin{array}{c}50 \text {-year } \\
\text { (in) }\end{array}$ \\
\hline
\end{tabular}


09485500 PANTANO WASH NEAR TUCSON, AZ--Continued

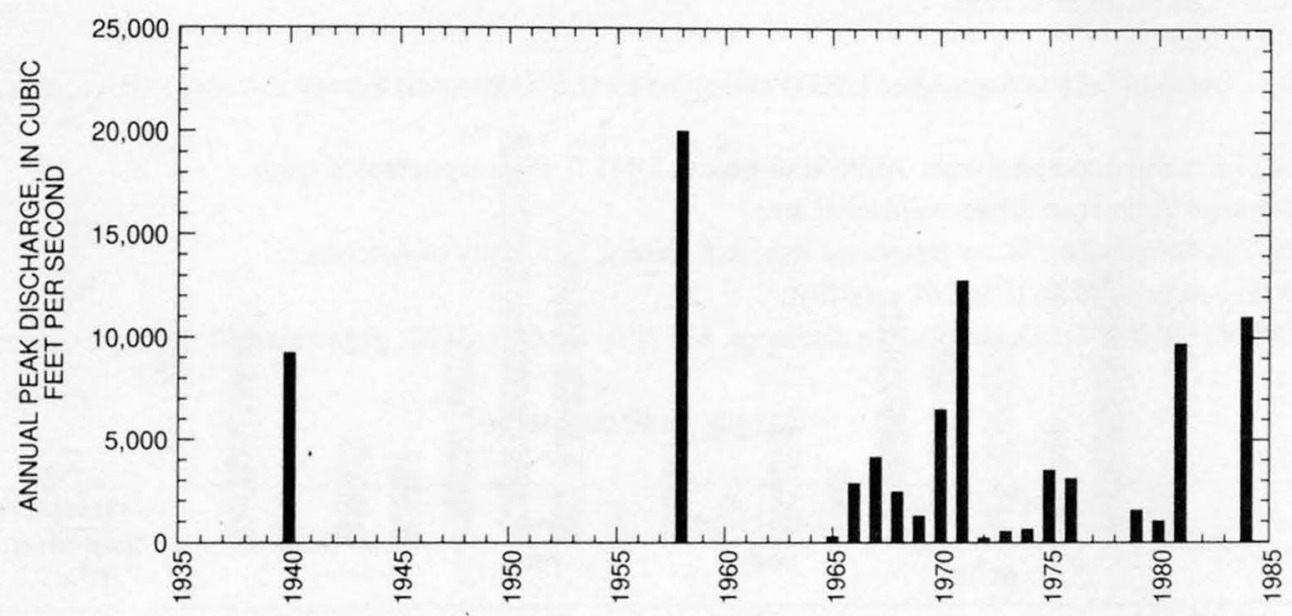


09485550 ARCADIA WASH AT TUCSON, AZ

LOCATION.--Lat 32 $14^{\prime} 37^{\prime \prime}$, long $110^{\circ} 53^{\prime} 05^{\prime \prime}$, in $\mathrm{SE}^{1} /{ }_{4} \mathrm{NW}^{1} / 4 \mathrm{sec} .2$, T.14 S., R.14 E., Pima County, Hydrologic Unit 15050302 , on right bank 150 ft downstream from Pima Street in Tucson.

DRAINAGE AREA.--2.72 $\mathrm{mi}^{2}$.

PERIOD OF RECORD.--October 1975 to September 1983 (Publication by U.S. Geological Survey discontinued; records available from cooperator listed below).

GAGE.--Water-stage recorder and concrete flume. Altitude of gage is $2,485 \mathrm{ft}$, from topographic map.

REMARKS.--Entire drainage basin is an urban, residential area.

COOPERATION.--Records furnished by Water Resources Research Center, University of Arizona.

AVERAGE DISCHARGE.--8 years, $0.36 \mathrm{ft}^{3} / \mathrm{s}, 261$ acre-ft/yr.

EXTREMES FOR PERIOD OF RECORD.--Maximum discharge, $836 \mathrm{ft}^{3} / \mathrm{s}$, Sept. 11, 1982, gage height, $5.36 \mathrm{ft}$; no flow most of each year.

Annual peak discharge

\begin{tabular}{cccccccc}
\hline $\begin{array}{c}\text { Water } \\
\text { year }\end{array}$ & Date & $\begin{array}{c}\text { Annual peak } \\
\text { discharge } \\
\left(\mathbf{f t}^{\mathbf{3}} \mathbf{s}\right)\end{array}$ & $\begin{array}{c}\text { Discharge } \\
\text { codes }\end{array}$ & $\begin{array}{c}\text { Water } \\
\text { year }\end{array}$ & Date & $\begin{array}{c}\text { Annual peak } \\
\text { discharge } \\
\left(\mathbf{f t}^{\mathbf{3}} / \mathbf{s}\right)\end{array}$ & $\begin{array}{c}\text { Discharge } \\
\text { codes }\end{array}$ \\
\hline 1966 & $07-19-66$ & 658 & $\mathrm{HP}, \mathrm{C}$ & 1976 & $09-25-76$ & 342 & $\mathrm{C}$ \\
1968 & $08-19-68$ & 310 & $\mathrm{C}$ & 1977 & $09-10-77$ & 220 & $\mathrm{C}$ \\
1969 & $08-05-69$ & 265 & $\mathrm{C}$ & 1978 & $09-21-78$ & 661 & $\mathrm{C}$ \\
1970 & $08-11-70$ & 594 & $\mathrm{C}$ & 1979 & $08-12-79$ & 647 & $\mathrm{C}$ \\
1971 & $08-17-71$ & 11,210 & $\mathrm{C}$ & 1980 & $08-13-80$ & 595 & $\mathrm{C}$ \\
1972 & $08-12-72$ & 940 & $\mathrm{C}$ & 1981 & $06-25-81$ & 330 & $\mathrm{C}$ \\
1973 & $07-07-73$ & 121 & $\mathrm{C}$ & 1982 & $09-11-82$ & 836 & $\mathrm{C}$ \\
1974 & $07-18-74$ & 264 & $\mathrm{C}$ & 1983 & $11-30-83$ & 157 & $\mathrm{C}$ \\
1975 & $07-16-75$ & 134 & $\mathrm{C}$ & & & & \\
\hline
\end{tabular}

${ }^{1}$ Highest since 1963.

Magnitude and probability of instantaneous peak flow based on period of record 1966, 1968-83

\begin{tabular}{|c|c|c|c|c|c|}
\hline \multicolumn{6}{|c|}{$\begin{array}{l}\text { Discharge, in } \mathrm{ft}^{3} / \mathrm{s} \text {, for indicated recurrence interval } \\
\text { in years, and exceedance probablility, in percent }\end{array}$} \\
\hline 2 & 5 & 10 & 25 & $50 t$ & $100 t$ \\
\hline $50 \%$ & $20 \%$ & $10 \%$ & $4 \%$ & $2 \%$ & $1 \%$ \\
\hline 395 & 707 & 953 & 1,310 & 1,600 & 1,920 \\
\hline Weighted skew & $(\log s)=$ & -0.07 & & & \\
\hline Mean & $(\log s)=$ & 2.59 & & & \\
\hline Standard dev. & $(\log s)=$ & 0.30 & & & \\
\hline
\end{tabular}

$\mp$ Reliability of values in column is uncertain, and potential errors are large.

Basin characteristics

\begin{tabular}{cccccccc}
\hline $\begin{array}{c}\text { Main } \\
\text { channel } \\
\text { slope } \\
(\mathrm{ft} / \mathrm{mi})\end{array}$ & $\begin{array}{c}\text { Stream } \\
\text { length } \\
(\mathrm{mi})\end{array}$ & $\begin{array}{c}\text { Mean } \\
\text { basin } \\
\text { elevation } \\
(\mathrm{ft})\end{array}$ & $\begin{array}{c}\text { Forested } \\
\text { area } \\
\text { (percent) }\end{array}$ & $\begin{array}{c}\text { Soil } \\
\text { index }\end{array}$ & $\begin{array}{c}\text { Mean } \\
\text { annual } \\
\text { precipitation } \\
\text { (in) }\end{array}$ & $\begin{array}{c}\text { R-year } \\
\text { (in) }\end{array}$ & $\begin{array}{c}50 \text {-year } \\
\text { (in) }\end{array}$ \\
\hline 0.77 & 5.54 & 2,560 & 0.0 & 1.0 & 11.0 & 1.7 \\
\hline
\end{tabular}


09485550 ARCADIA WASH AT TUCSON, AZ--Continued

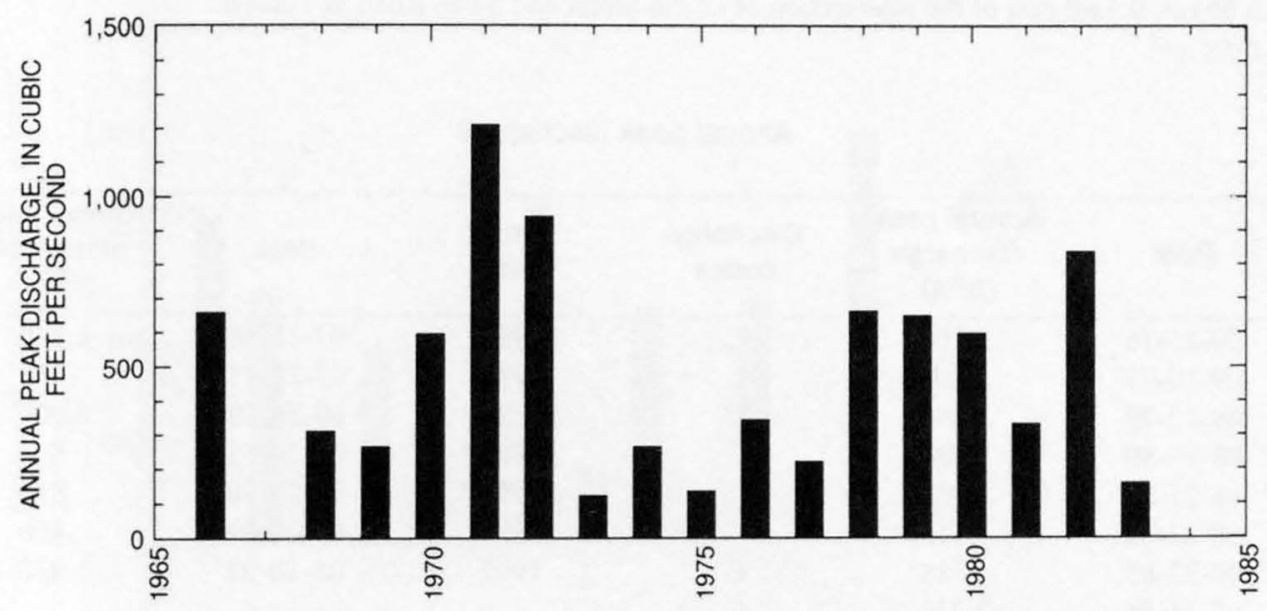


LOCATION.--Lat $32^{\circ} 15^{\prime} 31^{\prime \prime}$, long $110^{\circ} 53^{\prime} 01^{\prime \prime}, \mathrm{SE}^{1} /{ }_{4} \mathrm{SE}^{1} /{ }_{4} \mathrm{NW}^{1} / 4$ sec.35, T.13 S., R.14 E., Pima County, Hydrologic Unit 15050302, $270 \mathrm{ft}$ down. stream from Glenn Street, 0.5 mi east of the intersection of Glenn Street and Swan Road in Tucson.

DRAINAGE AREA.--9.58 $\mathrm{mi}^{2}$.

Annual peak discharges

\begin{tabular}{|c|c|c|c|c|c|c|c|}
\hline $\begin{array}{l}\text { Water } \\
\text { year }\end{array}$ & Date & $\begin{array}{c}\text { Annual peak } \\
\text { discharge } \\
\left(\mathrm{ft}^{3} / \mathrm{s}\right)\end{array}$ & $\begin{array}{l}\text { Discharge } \\
\text { codes }\end{array}$ & $\begin{array}{l}\text { Water } \\
\text { year }\end{array}$ & Date & $\begin{array}{c}\text { Annual peak } \\
\text { discharge } \\
\left(\mathrm{ft}^{3} / \mathrm{s}\right)\end{array}$ & $\begin{array}{c}\text { Discharge } \\
\text { codes }\end{array}$ \\
\hline 1976 & $09-25-76$ & 3,200 & $\mathrm{C}$ & 1986 & $07-21-86$ & 2,340 & $\mathrm{C}$ \\
\hline 1977 & $09-10-77$ & 2,500 & $\mathrm{C}$ & 1987 & $07-26-87$ & 432 & $\mathrm{C}$ \\
\hline 1979 & $08-12-79$ & 1,890 & $\mathrm{C}$ & 1988 & $08-20-88$ & 4,000 & $\mathrm{C}$ \\
\hline 1980 & $08-13-80$ & 820 & $\mathrm{C}$ & 1989 & $07-26-89$ & 853 & $\mathrm{C}$ \\
\hline 1981 & $06-25-81$ & 800 & $\mathrm{C}$ & 1990 & $07-24-90$ & 870 & $\mathrm{C}$ \\
\hline 1982 & $08-23-82$ & 3,310 & $\mathrm{C}$ & 1991 & $01-05-91$ & 466 & $\mathrm{C}$ \\
\hline 1983 & $09-23-83$ & 714 & $\mathrm{C}$ & 1992 & $05-10-92$ & 420 & $\mathrm{C}$ \\
\hline 1984 & $07-16-84$ & 2,280 & $\mathrm{C}$ & & & & \\
\hline
\end{tabular}

Discharge rating table developed October 1986

\begin{tabular}{crcc}
\hline $\begin{array}{c}\text { Gage height } \\
(\mathbf{f t})\end{array}$ & $\begin{array}{c}\text { Discharge } \\
\left(\mathrm{ft}^{\mathbf{3}} / \mathbf{s}\right)\end{array}$ & $\begin{array}{c}\text { Gage height } \\
(\mathbf{f t})\end{array}$ & $\begin{array}{c}\text { Discharge } \\
\left(\mathrm{ft}^{\mathbf{3}} / \mathbf{s}\right)\end{array}$ \\
\hline 2.0 & 249 & 5.0 & 2,530 \\
2.5 & 534 & 5.5 & 3,020 \\
3.0 & 911 & 6.0 & 3,540 \\
3.5 & 1,280 & 6.5 & 4,100 \\
4.0 & 1,660 & 7.0 & 4,680 \\
4.5 & 2,080 & 7.1 & 4,800 \\
\hline
\end{tabular}

Magnitude and probability of instantaneous peak flow based on period of record 1976-84, 1986-92

\begin{tabular}{|c|c|c|c|c|c|}
\hline \multicolumn{6}{|c|}{$\begin{array}{l}\text { Discharge, in } \mathrm{ft}^{3} / \mathrm{s} \text {, for indicated recurrence interval } \\
\text { in years, and exceedance probablility, in percent }\end{array}$} \\
\hline 2 & 5 & 10 & 25 & $50 t$ & $100 t$ \\
\hline $50 \%$ & $20 \%$ & $10 \%$ & $4 \%$ & $2 \%$ & $1 \%$ \\
\hline 1,600 & 2,900 & 3,890 & 5,260 & 6,340 & 7,460 \\
\hline Weighted skew & $(\operatorname{logs})=$ & -0.27 & & & \\
\hline Mean & $(\log s)=$ & 3.18 & & & \\
\hline Standard dev. & $(\log s)=$ & 0.32 & & & \\
\hline
\end{tabular}

f Reliability of values in column is uncertain, and potential errors are large.

Basin characteristics

\begin{tabular}{cccccccc}
\hline $\begin{array}{c}\text { Main } \\
\text { channel } \\
\text { slope } \\
(\mathrm{ft} / \mathrm{mi})\end{array}$ & $\begin{array}{c}\text { Stream } \\
\text { length } \\
(\mathrm{mi})\end{array}$ & $\begin{array}{c}\text { Mean } \\
\text { basin } \\
\text { elevation } \\
(\mathrm{ft})\end{array}$ & $\begin{array}{c}\text { Forested } \\
\text { area } \\
\text { (percent) }\end{array}$ & $\begin{array}{c}\text { Soil } \\
\text { index }\end{array}$ & $\begin{array}{c}\text { Mean } \\
\text { annual } \\
\text { precipitation } \\
\text { (in) }\end{array}$ & $\begin{array}{c}\text { Rainfall intensity, 24-hour } \\
\text { (in) }\end{array}$ & $\begin{array}{c}50 \text {-year } \\
\text { (in) }\end{array}$ \\
\hline 46.2 & 6.4 & 2,590 & 0.0 & 1.0 & 11.0 & 3.8 \\
\hline
\end{tabular}


09485570 ALAMO WASH AT TUCSON, AZ--Continued

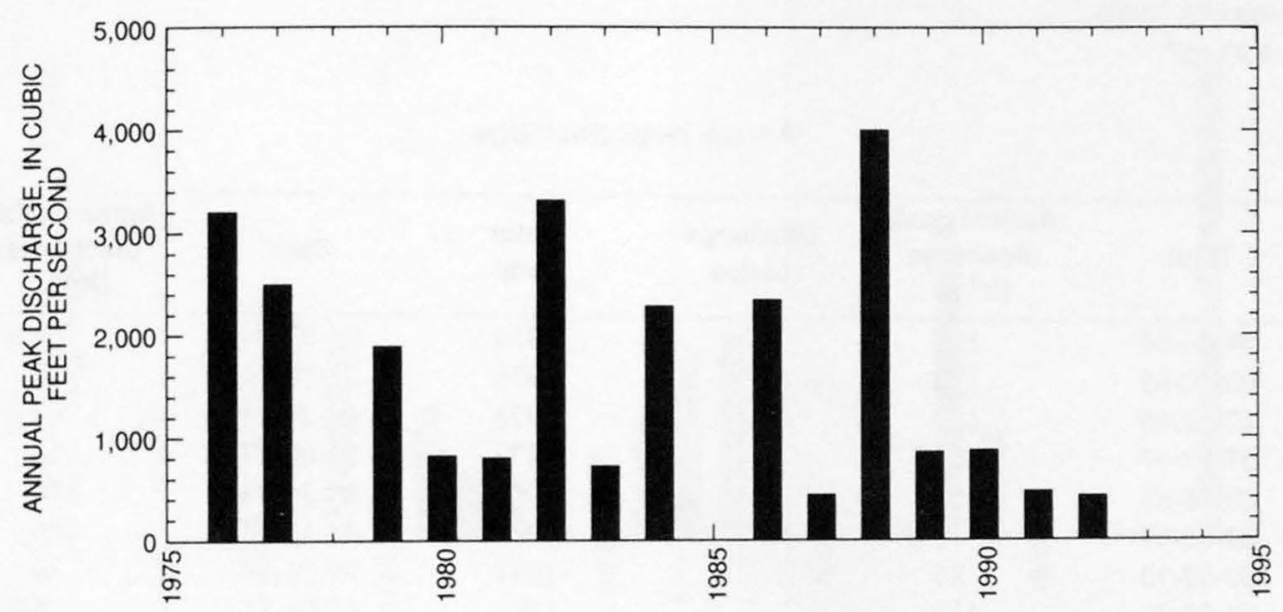


09485900 PIMA WASH NEAR TUCSON, AZ

LOCATION.--Lat $32^{\circ} 20^{\prime} 15^{\prime \prime}$, long $110^{\circ} 57^{\prime} 35^{\prime \prime}, \mathrm{SW}^{1} / \mathrm{SW}^{1} / 4$ sec.31, T.12 S., R.14 E., Pima County, Hydrologic Unit 15050302, at Ina Road, and 4 mi north of Tucson city limits.

DRAINAGE AREA.--4.93 $\mathrm{mi}^{2}$.

Annual peak discharge

\begin{tabular}{|c|c|c|c|c|c|c|c|}
\hline $\begin{array}{l}\text { Water } \\
\text { year }\end{array}$ & Date & $\begin{array}{c}\text { Annual peak } \\
\text { discharge } \\
\left(\mathrm{ft}^{3} / \mathrm{s}\right)\end{array}$ & $\begin{array}{l}\text { Discharge } \\
\text { codes }\end{array}$ & $\begin{array}{c}\text { Water } \\
\text { year }\end{array}$ & Date & $\begin{array}{c}\text { Annual peak } \\
\text { discharge } \\
\left(\mathrm{ft}^{3} / \mathrm{s}\right)\end{array}$ & $\begin{array}{c}\text { Discharge } \\
\text { codes }\end{array}$ \\
\hline 1964 & $09-06-64$ & 195 & & 1974 & $09-07-74$ & 5.0 & LT \\
\hline 1965 & $00-00-65$ & 120 & LT & 1975 & $10-30-74$ & 10 & LT \\
\hline 1966 & $12-22-65$ & 125 & & 1976 & $09-26-76$ & 2.0 & ES \\
\hline 1967 & $07-17-67$ & 65 & & 1977 & $01-00-77$ & 10 & ES \\
\hline 1968 & $08-06-68$ & 50 & & 1978 & $07-26-78$ & 300 & \\
\hline 1969 & $09-06-69$ & 2.0 & ES & 1979 & $12-18-78$ & 100 & \\
\hline 1970 & $00-00-70$ & 80 & & 1980 & $09-26-80$ & 30 & \\
\hline 1971 & $08-12-71$ & 117 & & 1981 & $05-01-81$ & 5.0 & \\
\hline 1972 & $09-01-72$ & 170 & & 1984 & $10-01-83$ & ${ }^{1} 460$ & HP \\
\hline 1973 & $10-19-72$ & 195 & & & & & \\
\hline
\end{tabular}

${ }^{1}$ Highest since 1963.

Magnitude and probability of instantaneous peak flow based on period of record 1964-81, 1984

\begin{tabular}{|c|c|c|c|c|c|}
\hline \multicolumn{6}{|c|}{$\begin{array}{l}\text { Discharge, in } \mathrm{ft}^{3} / \mathrm{s} \text {, for indicated recurrence interval } \\
\text { in years, and exceedance probablility, in percent }\end{array}$} \\
\hline 2 & 5 & 10 & 25 & $50 \dagger$ & $100 t$ \\
\hline $50 \%$ & $20 \%$ & $10 \%$ & $4 \%$ & $2 \%$ & $1 \%$ \\
\hline 74.2 & 177 & 269 & 407 & 524 & 652 \\
\hline Weighted skew & $(\log s)=$ & -0.39 & & & \\
\hline Mean & $(\log s)=$ & 1.84 & & & \\
\hline Standard dev. & $(\log s)=$ & 0.48 & & & \\
\hline
\end{tabular}

\section{Basin characteristics}

\begin{tabular}{ccccccc}
\hline $\begin{array}{c}\text { Main } \\
\text { channel } \\
\text { slope } \\
(\mathrm{ft} / \mathrm{mi})\end{array}$ & $\begin{array}{c}\text { Stream } \\
\text { length } \\
\text { (mi) }\end{array}$ & $\begin{array}{c}\text { Mean } \\
\text { basin } \\
\text { elevation } \\
\text { (ft) }\end{array}$ & $\begin{array}{c}\text { Forested } \\
\text { area } \\
\text { (percent) }\end{array}$ & $\begin{array}{c}\text { Soil } \\
\text { index }\end{array}$ & $\begin{array}{c}\text { Mean } \\
\text { annual } \\
\text { precipitation } \\
\text { (in) }\end{array}$ & $\begin{array}{c}\text { R-year } \\
\text { (in) }\end{array}$ \\
\hline 533 & 5.5 & 4,430 & 18.0 & 1.0 & 16.0 & $\begin{array}{c}50 \text {-year } \\
\text { (in) }\end{array}$ \\
\hline
\end{tabular}


09485900 PIMA WASH NEAR TUCSON, AZ--Continued

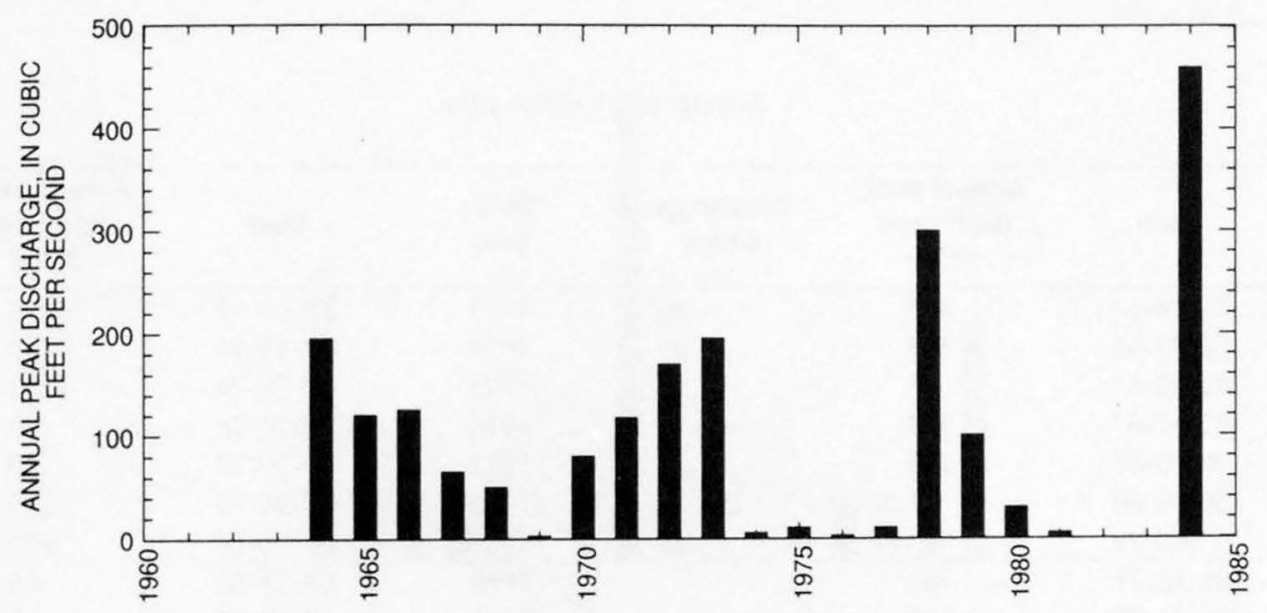




\section{GERONIMO WASH NEAR TUCSON, AZ}

LOCATION.--Lat 32 $199^{\prime} 56^{\prime \prime}$, long $110^{\circ} 56^{\prime} 37^{\prime \prime}, \mathrm{SE}^{1} /{ }_{4} \mathrm{NE}^{1} / 4 \mathrm{sec} .6, \mathrm{~T} .13 \mathrm{~S} ., \mathrm{R} .14$ E., Pima County, Hydrologic Unit 15050302 , at Skyline Drive, 0. mi southeast of Ina Road, and 3.5 mi north of Tucson city limits.

DRAINAGE AREA.--2.15 $\mathrm{mi}^{2}$.

Annual peak discharge

\begin{tabular}{|c|c|c|c|c|c|c|c|}
\hline $\begin{array}{l}\text { Water } \\
\text { year }\end{array}$ & Date & $\begin{array}{c}\text { Annual peak } \\
\text { discharge } \\
\left(\mathrm{ft}^{3} / \mathrm{s}\right)\end{array}$ & $\begin{array}{l}\text { Discharge } \\
\text { codes }\end{array}$ & $\begin{array}{l}\text { Water } \\
\text { year }\end{array}$ & Date & $\begin{array}{c}\text { Annual peak } \\
\text { discharge } \\
\left(\mathrm{ft}^{3} / \mathrm{s}\right)\end{array}$ & $\begin{array}{c}\text { Discharge } \\
\text { codes }\end{array}$ \\
\hline 1964 & $09-06-64$ & 445 & & 1973 & $10-00-72$ & 195 & \\
\hline 1965 & $08-02-65$ & 135 & & 1974 & $07-19-74$ & 10 & LT \\
\hline 1966 & $12-22-65$ & 65 & & 1975 & $10-29-74$ & 35 & \\
\hline 1967 & $07-17-67$ & 139 & & 1976 & $09-26-76$ & 90 & \\
\hline 1968 & $08-10-68$ & 120 & & 1977 & $09-10-77$ & 80 & \\
\hline 1969 & $09-06-69$ & 2.0 & ES & 1978 & $07-26-78$ & 225 & \\
\hline 1970 & $09-00-70$ & 95 & & 1979 & $08-12-79$ & 370 & \\
\hline 1971 & $08-12-71$ & 705 & & 1980 & $08-13-80$ & 65 & \\
\hline 1972 & $09-01-72$ & 385 & & 1981 & $07-10-81$ & 4.0 & \\
\hline
\end{tabular}

Magnitude and probability of instantaneous peak flow based on period of record 1964-81

\begin{tabular}{|c|c|c|c|c|c|}
\hline \multicolumn{6}{|c|}{$\begin{array}{l}\text { Discharge, in } \mathrm{ft}^{3} / \mathrm{s} \text {, for indicated recurrence interval } \\
\text { in years, and exceedance probablility, in percent }\end{array}$} \\
\hline 2 & 5 & 10 & 25 & $50 t$ & $100 \dagger$ \\
\hline $50 \%$ & $20 \%$ & $10 \%$ & $4 \%$ & $2 \%$ & $1 \%$ \\
\hline 118 & 271 & 414 & 643 & 850 & 1,090 \\
\hline Weighted skew & $(\log s)=$ & -0.14 & & & \\
\hline Mean & $(\log s)=$ & 2.06 & & & \\
\hline Standard dev. & $(\log s)=$ & 0.44 & & & \\
\hline
\end{tabular}

Basin characteristics

\begin{tabular}{cccccccc}
\hline $\begin{array}{c}\text { Main } \\
\text { channel } \\
\text { slope } \\
(\mathrm{ft} / \mathrm{mi})\end{array}$ & $\begin{array}{c}\text { Stream } \\
\text { length } \\
\text { (mi) }\end{array}$ & $\begin{array}{c}\text { Mean } \\
\text { basin } \\
\text { elevation } \\
\text { (ft) }\end{array}$ & $\begin{array}{c}\text { Forested } \\
\text { area } \\
\text { (percent) }\end{array}$ & $\begin{array}{c}\text { Soil } \\
\text { index }\end{array}$ & $\begin{array}{c}\text { Mean } \\
\text { annual } \\
\text { precipitation } \\
\text { (in) }\end{array}$ & $\begin{array}{c}\text { Rainfall intensity, 24-hour } \\
\text { (in) }\end{array}$ & $\begin{array}{c}50 \text {-year } \\
\text { (in) }\end{array}$ \\
\hline 699 & 3.6 & 3,600 & 0.0 & 1.0 & 15.0 & 1.9 \\
\hline
\end{tabular}


09485950 GERONIMO WASH NEAR TUCSON, AZ--Continued

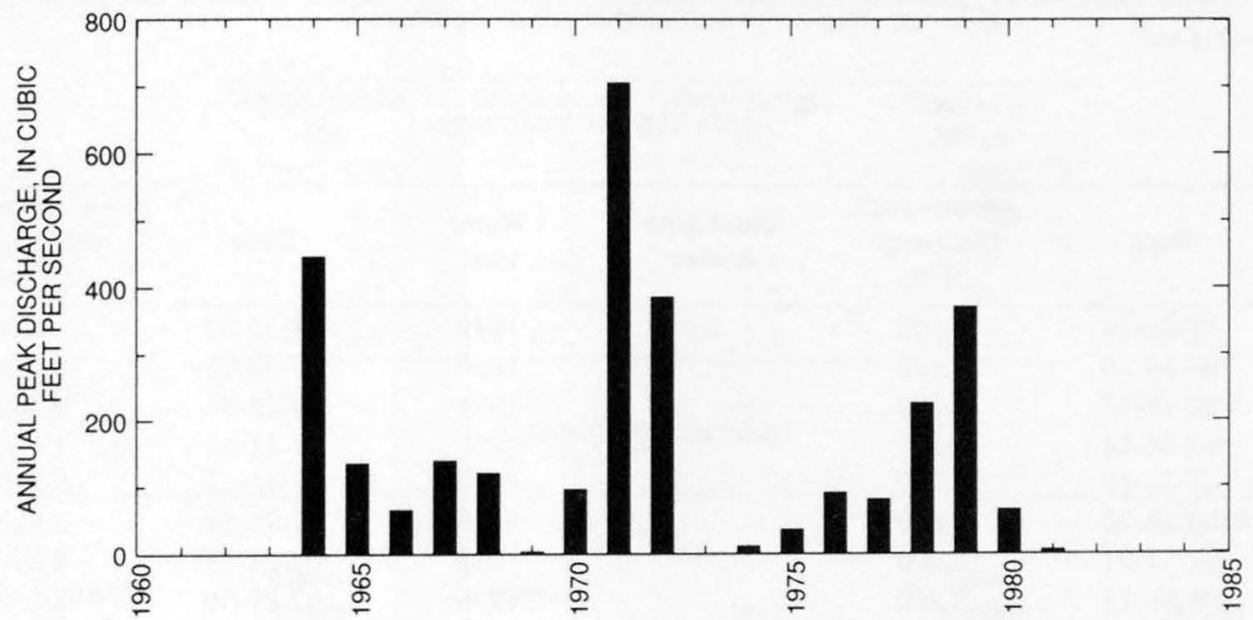




\section{RILLITO CREEK NEAR TUCSON, AZ}

LOCATION.--Lat 32 17'41", long $110^{\circ} 59^{\prime} 00^{\prime \prime}$, in SW $1 / 4 \mathrm{SE}^{1} / 4$ sec.14, T.13 S., R.13 E., Pima County, Hydrologic Unit 15050302, on right bank $600 \mathrm{ft}$ downstream from Pima Wash, 1,800 ft downstream from U.S. Highway $89,5 \mathrm{mi}$ above mouth, and $5.4 \mathrm{mi}$ north of city hall in Tucson DRAINAGE AREA.--918 $\mathrm{mi}^{2}$.

Annual peak discharge

\begin{tabular}{|c|c|c|c|c|c|c|c|}
\hline $\begin{array}{l}\text { Water } \\
\text { year }\end{array}$ & Date & $\begin{array}{c}\text { Annual peak } \\
\text { discharge } \\
\left(\mathrm{ft}^{3} / \mathrm{s}\right)\end{array}$ & $\begin{array}{l}\text { Discharge } \\
\text { codes }\end{array}$ & $\begin{array}{l}\text { Water } \\
\text { year }\end{array}$ & Date & $\begin{array}{c}\text { Annual peak } \\
\text { discharge } \\
\left(\mathrm{ft}^{3} / \mathrm{s}\right)\end{array}$ & $\begin{array}{c}\text { Discharge } \\
\text { codes }\end{array}$ \\
\hline 1915 & $12-23-14$ & 17,000 & & 1949 & $09-15-49$ & 1,640 & \\
\hline 1916 & $01-19-16$ & 7,620 & & 1950 & $07-30-50$ & 9,490 & \\
\hline 1917 & $08-11-17$ & 10,000 & & 1951 & $07-25-51$ & 9,500 & \\
\hline 1918 & 03-01-18 & 5,300 & & 1952 & $11-11-51$ & 1,630 & \\
\hline 1919 & $07-27-19$ & 9,250 & & 1953 & $07-16-53$ & 5,470 & \\
\hline 1920 & $02-21-20$ & 7,800 & & 1954 & $07-24-54$ & 7,680 & \\
\hline 1921 & $07-31-21$ & 16,000 & & 1955 & $07-21-55$ & 8,070 & \\
\hline 1922 & $08-09-22$ & 3,250 & & 1956 & $07-29-56$ & 2,050 & \\
\hline 1923 & $08-26-23$ & 4,000 & & 1957 & $01-09-57$ & 4,500 & \\
\hline 1924 & $12-26-23$ & 1,980 & & 1958 & $08-12-58$ & 8,930 & \\
\hline 1925 & $09-17-25$ & 3,500 & & 1959 & $08-17-59$ & 7,710 & \\
\hline 1926 & $09-27-26$ & 1,750 & & 1960 & $01-12-60$ & 3,610 & \\
\hline 1927 & $09-12-27$ & 2,200 & & 1961 & $07-22-61$ & 4,140 & \\
\hline 1928 & $08-01-28$ & 4,500 & & 1962 & $09-26-62$ & 2,690 & \\
\hline 1929 & $09-23-29$ & 24,000 & & 1963 & $08-26-63$ & 7,640 & \\
\hline 1930 & $08-08-30$ & 4,600 & & 1964 & $09-10-64$ & 9,420 & \\
\hline 1931 & $08-10-31$ & 7,200 & & 1965 & $09-12-65$ & 754 & \\
\hline 1932 & $07-29-32$ & 7,200 & & 1966 & $12-22-65$ & 12,400 & \\
\hline 1933 & $09-10-33$ & 4,400 & & 1967 & $08-19-67$ & 3,100 & ES \\
\hline 1934 & $07-17-34$ & 3,000 & & 1968 & $02-12-68$ & 7,740 & \\
\hline 1935 & $08-31-35$ & 13,400 & & 1969 & $08-05-69$ & 2,220 & \\
\hline 1936 & $08-17-36$ & 4,500 & & 1970 & $09-06-70$ & 7,000 & \\
\hline 1937 & $08-17-37$ & 2,980 & & 1971 & $08-20-71$ & 9,290 & \\
\hline 1938 & $03-04-38$ & 3,000 & & 1972 & $08-12-72$ & 1,820 & \\
\hline 1939 & 08-03-39 & 9,710 & & 1973 & $10-20-72$ & 5,160 & \\
\hline 1940 & $08-13-40$ & 13,200 & & 1974 & $08-02-74$ & 1,440 & \\
\hline 1941 & $12-31-40$ & 9,900 & & 1975 & $07-16-75$ & 2,270 & \\
\hline 1942 & $09-14-42$ & 1,600 & & 1976 & $09-25-76$ & 9,400 & \\
\hline 1943 & $08-15-43$ & 3,850 & & 1977 & $09-05-77$ & 1,200 & \\
\hline 1944 & $08-09-44$ & 4,100 & & 1978 & $03-02-78$ & 7,500 & \\
\hline 1945 & $08-10-45$ & 7,000 & & 1979 & $00-00-79$ & 16,400 & \\
\hline 1946 & $08-31-46$ & 4,160 & & 1980 & $00-00-80$ & 2,300 & \\
\hline 1947 & $08-15-47$ & 7,660 & & 1981 & $00-00-81$ & 4,600 & \\
\hline 1948 & $09-26-48$ & 779 & & 1984 & $10-02-83$ & ${ }^{1} 29,700$ & HP \\
\hline
\end{tabular}

${ }^{1}$ Highest since 1915. 
09486000 RILLITO CREEK NEAR TUCSON, AZ--Continued

Discharge rating table developed October 1950

\begin{tabular}{rrrr}
\hline $\begin{array}{c}\text { Gage height } \\
\text { (ft) }\end{array}$ & $\begin{array}{c}\text { Discharge } \\
\left(\mathbf{f t}^{3} / \mathbf{s}\right)\end{array}$ & $\begin{array}{c}\text { Gage height } \\
(\mathbf{f t})\end{array}$ & $\begin{array}{c}\text { Discharge } \\
\left(\mathbf{f t}^{\mathbf{3}} / \mathbf{s}\right)\end{array}$ \\
\hline 3.0 & 32 & 5.5 & 976 \\
3.5 & 110 & 6.0 & 1,300 \\
4.0 & 245 & 6.5 & 1,640 \\
4.5 & 456 & 7.0 & 2,000 \\
5.0 & 680 & 7.4 & 2,320 \\
\hline
\end{tabular}

Basin characteristics

\begin{tabular}{ccccccccc}
\hline $\begin{array}{c}\text { Main } \\
\text { channel } \\
\text { slope } \\
(\mathrm{ft} / \mathrm{mi})\end{array}$ & $\begin{array}{c}\text { Stream } \\
\text { length } \\
(\mathrm{mi})\end{array}$ & $\begin{array}{c}\text { Mean } \\
\text { basin } \\
\text { elevation } \\
(\mathrm{ft})\end{array}$ & $\begin{array}{c}\text { Forested } \\
\text { area } \\
\text { (percent) }\end{array}$ & $\begin{array}{c}\text { Soil } \\
\text { index }\end{array}$ & $\begin{array}{c}\text { Mean } \\
\text { annual } \\
\text { precipitation } \\
\text { (in) }\end{array}$ & $\begin{array}{c}\text { 2-year } \\
\text { (in) }\end{array}$ & $\begin{array}{c}\text { 50-year } \\
\text { (in) }\end{array}$ \\
\hline 45.5 & 69.7 & 4,400 & 22.0 & 1.5 & 15.5 & 1.9 & 4.0 \\
\hline
\end{tabular}


09486000 RILLITO CREEK NEAR TUCSON, AZ--Continued

MEAN MONTHLY AND ANNUAL DISCHARGES $1914,1916-75$

\begin{tabular}{|c|c|c|c|c|c|c|}
\hline MONTH & $\begin{array}{l}\text { MAXIMUM } \\
\text { (FT3/S) }\end{array}$ & $\begin{array}{l}\text { MINIMUM } \\
\text { (FT3/S) }\end{array}$ & $\begin{array}{c}\text { MEAN } \\
(\mathrm{FT} 3 / \mathrm{S})\end{array}$ & $\begin{array}{l}\text { STAN- } \\
\text { DARD } \\
\text { DEVIA- } \\
\text { TION } \\
\text { (FT } 3 / S \text { ) }\end{array}$ & $\begin{array}{l}\text { COBFFI- } \\
\text { CIENT OF } \\
\text { VARI- } \\
\text { ATION }\end{array}$ & $\begin{array}{c}\text { PERCENT } \\
\text { OF } \\
\text { ANNUAL } \\
\text { RUNOFF }\end{array}$ \\
\hline OCTOBER & 116 & 0.00 & 2.8 & 15 & 5.3 & 1.7 \\
\hline NOVEMBER & 40 & 0.00 & 2.0 & 7.5 & 3.8 & 1.2 \\
\hline DECEMBER & 556 & 0.00 & 18 & 77 & 4.2 & 11.2 \\
\hline JANUARY & 603 & 0.00 & 18 & 80 & 4. 5 & 10.9 \\
\hline FEBRUARY & 203 & 0.00 & 21 & 46 & 2.2 & 12.6 \\
\hline MARCH & 183 & 0.00 & 17 & 37 & 2.2 & 10.3 \\
\hline APRIL & 13 & 0.00 & 0.89 & 2.8 & 3.1 & 0.5 \\
\hline MAY & 69 & 0.00 & 1.1 & 8.8 & 7.8 & 0.7 \\
\hline JUNE & 9.7 & 0.00 & 0.54 & 1.9 & 3.5 & 0.3 \\
\hline JULY & 500 & 0.00 & 29 & 84 & 2.9 & 17.7 \\
\hline AUGUST & 263 & 0.00 & 37 & 49 & 1.3 & 22.7 \\
\hline SEPTEMBER & 302 & 0.00 & 17 & 44 & 2.7 & 10.1 \\
\hline ANNUAL & 74 & 0.43 & 14 & 17 & 1.2 & 100 \\
\hline
\end{tabular}

MAGNITUDE AND PROBABILITY OF INSTANTANEOUS PEAK FLOW BASED ON PERIOD OF RECORD $1915.81,1984$

DISCHARGE, IN FT3/S, FOR INDICATED RECURRENCE INTERVAL IN YEARS, AND EXCEEDANCE PROBABILITY, IN PERCENT

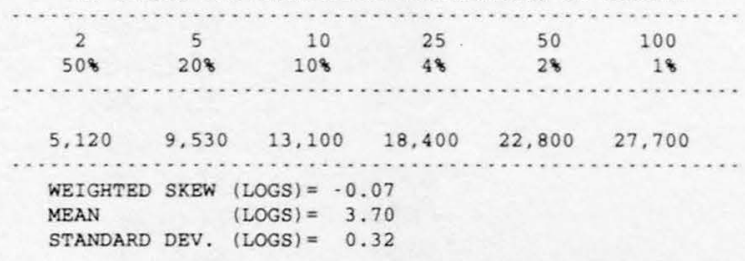

MAGNITUDE AND PROBABILITY OF ANNUAL LOW FLOW BASED ON PERIOD OF RECORD 1915, 1917.75

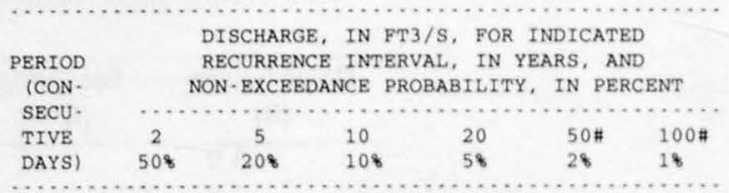

MAGNITUDE AND PROBABILITY OF ANNUAL HIGH FLOW BASED ON PERIOD OF RECORD 1914, 1916.75

\begin{tabular}{|c|c|c|c|c|c|c|}
\hline $\begin{array}{l}\text { PERIOD } \\
(C O N-\end{array}$ & & \multicolumn{5}{|c|}{$\begin{array}{l}\text { DISCHARGE, IN FT3/S, FOR INDICATED } \\
\text { RECURRENCE INTERVAL, IN YEARS, AND }\end{array}$} \\
\hline SECU. & & & test & $\ldots \ldots$ & $\ldots . .$. & $\cdots$ \\
\hline TIVE & 2 & 5 & 10 & 25 & $50 \#$ & $100=$ \\
\hline DAYSI & 508 & 208 & 108 & 48 & 28 & 18 \\
\hline 1 & 863 & 2,020 & 3,040 & 4,560 & 5,840 & 7,220 \\
\hline 3 & 380 & 999 & 1,630 & 2.700 & 3.730 & 4,960 \\
\hline 7 & 186 & 493 & 811 & 1.370 & 1,910 & 2.570 \\
\hline 15 & 104 & 280 & 464 & 786 & 1.100 & 1,480 \\
\hline 30 & 62 & 166 & 275 & 463 & 645 & 866 \\
\hline 60 & 36 & 94 & 154 & 258 & 357 & 476 \\
\hline 90 & 25 & 68 & 112 & 190 & 266 & 360 \\
\hline
\end{tabular}

DURATION TABLE OF DAILY MEAN FLOW FOR PERIOD OF RECORD 1960.74, 1990.96

DISCHARGE, IN FT3/S, WHICH WAS EQUALED OR EXCEEDED FOR INDICATED PERCENT OF TIME

\begin{tabular}{|c|c|c|c|c|c|c|c|c|c|c|c|c|c|c|c|c|}
\hline 18 & 58 & 108 & 158 & 208 & 308 & 408 & 508 & 608 & 708 & 808 & 908 & 958 & 988 & 998 & 99.58 & $99.9^{\circ}$ \\
\hline 30 & 23 & 0.01 & 0.01 & 0.01 & 0.01 & 0.01 & 0.01 & 0.00 & 0.00 & 0.00 & 0.00 & 0.00 & 0.00 & 0.00 & 0.00 & 0.00 \\
\hline
\end{tabular}

\# Reliability of values in column is uncertain, and potential errors are large. 


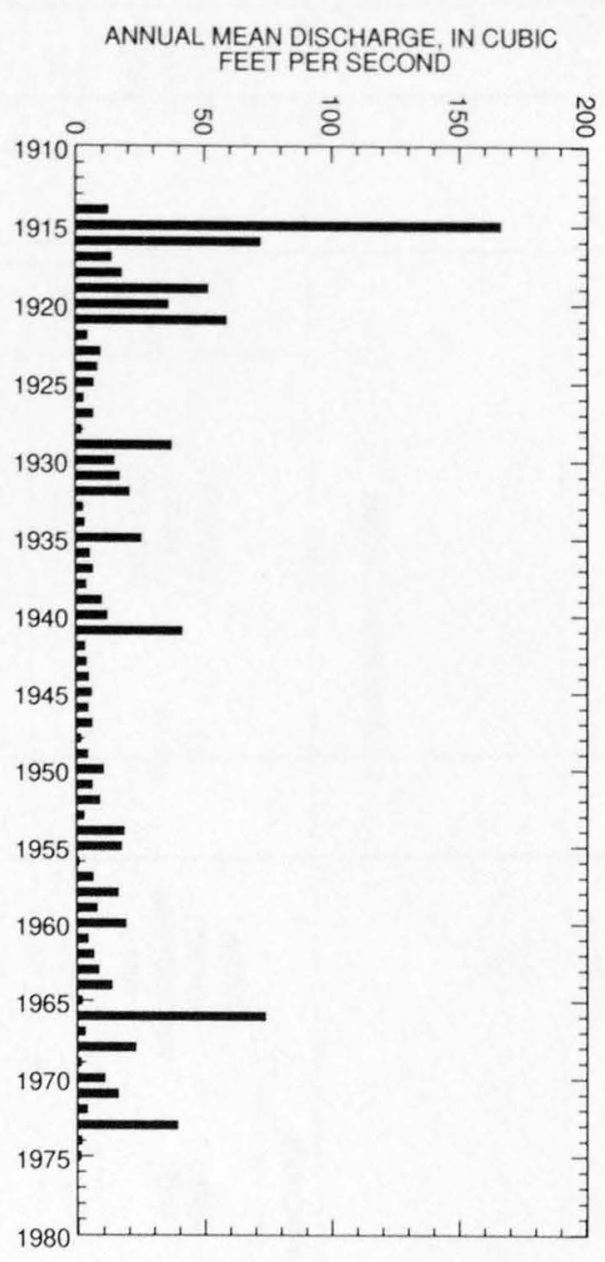

ANNUAL PEAK DISCHARGE, IN CUBIC

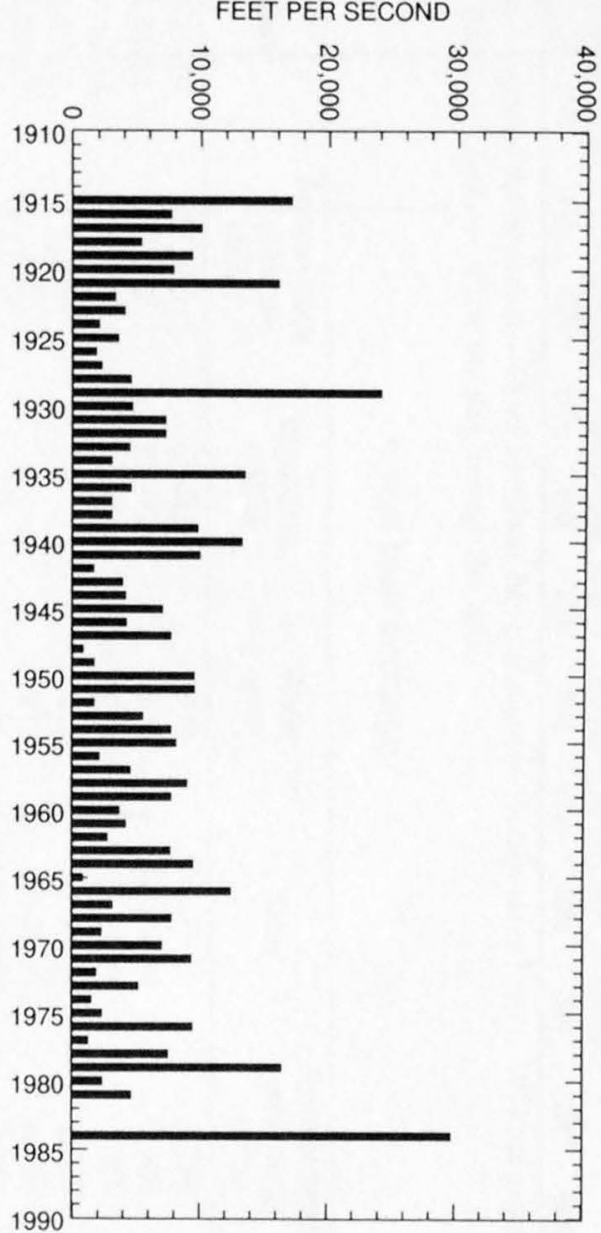


09486000 RILLITO CREEK NEAR TUCSON, AZ--Continued

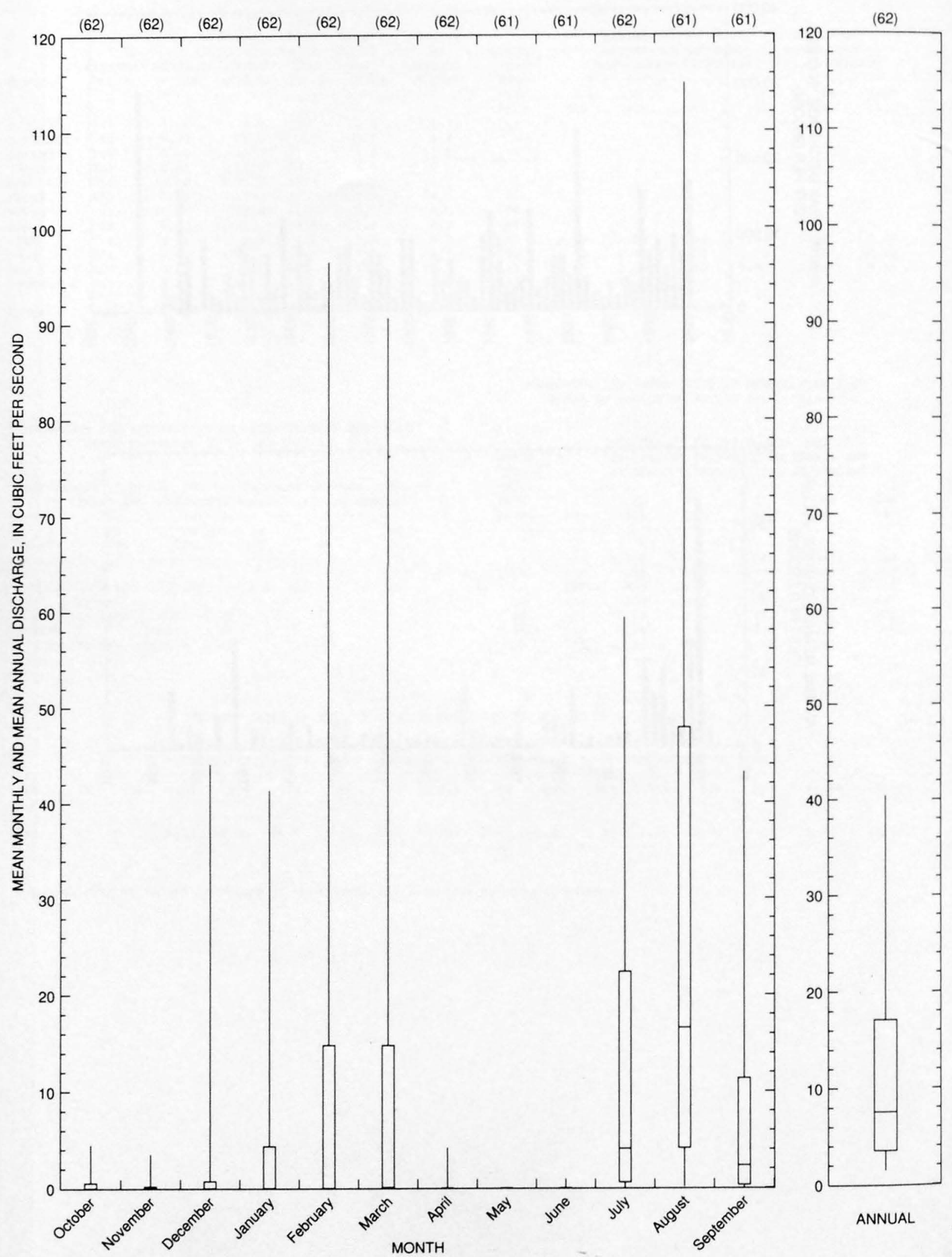




\section{CANADA DEL ORO NEAR TUCSON, AZ}

LOCATION.--Lat 32'22'27", long $11^{\circ} 00^{\prime} 31^{\prime \prime}$, in SW $1 / 2 \mathrm{NW}^{1} / 2$ sec.22, T.12 S., R.13 E., Pima County, Hydrologic Unit 15050301 , on right bank at upstream side of Overton Road, 4.7 mi upstream from mouth, and 10.5 mi north of city hall in Tucson.

DRAINAGE AREA.--250 $\mathrm{mi}^{2}$.

REMARKS.--Lago del Oro-capacity 9,400 acre-ft-16 mi upstream, has contained no storage since May 4, 1971, as gates were opened by court order; however, peak flows are regulated while passing through the lake.

Annual peak discharge

\begin{tabular}{|c|c|c|c|c|c|c|c|}
\hline $\begin{array}{l}\text { Water } \\
\text { year }\end{array}$ & Date & $\begin{array}{c}\text { Annual peak } \\
\text { discharge } \\
\left(\mathrm{ft}^{3} / \mathrm{s}\right)\end{array}$ & $\begin{array}{c}\text { Discharge } \\
\text { codes }\end{array}$ & $\begin{array}{l}\text { Water } \\
\text { year }\end{array}$ & Date & $\begin{array}{c}\text { Annual peak } \\
\text { discharge } \\
\left(\mathrm{ft}^{3} / \mathrm{s}\right)\end{array}$ & $\begin{array}{l}\text { Discharge } \\
\text { codes }\end{array}$ \\
\hline 1959 & $07-21-59$ & ${ }^{1} 17,000$ & ES,HP & 1973 & $10-19-72$ & 3,750 & \\
\hline 1961 & $09-01-61$ & 12,000 & ES,HP & 1974 & $07-20-74$ & 7,700 & \\
\hline 1964 & $09-10-64$ & 8,000 & ES,HP & 1975 & $09-04-75$ & 454 & \\
\hline 1966 & $12-22-65$ & 2,290 & & 1976 & $09-05-76$ & 2,220 & \\
\hline 1967 & $08-05-67$ & 652 & & 1977 & $08-09-77$ & 4,500 & \\
\hline 1968 & $12-20-67$ & ${ }^{2} 13,900$ & & 1978 & $01-16-78$ & 2,070 & \\
\hline 1969 & $07-22-69$ & 454 & & 1979 & $11-25-78$ & 1,380 & \\
\hline 1970 & $08-18-70$ & 1,930 & & 1980 & $07-19-80$ & 4,240 & \\
\hline 1971 & $08-17-71$ & 4,200 & & 1981 & $07-25-81$ & 5,900 & \\
\hline 1972 & $08-12-72$ & 728 & & 1984 & $10-01-83$ & 6,600 & \\
\hline
\end{tabular}

${ }^{1}$ Highest since 1950.

${ }^{2}$ Highest since 1959.

Basin characteristics

\begin{tabular}{cccccccc}
\hline $\begin{array}{c}\text { Main } \\
\text { channel } \\
\text { slope } \\
(\mathrm{ft} / \mathrm{mi})\end{array}$ & $\begin{array}{c}\text { Stream } \\
\text { length } \\
(\mathrm{mi})\end{array}$ & $\begin{array}{c}\text { Mean } \\
\text { basin } \\
\text { elevation } \\
(\mathrm{ft})\end{array}$ & $\begin{array}{c}\text { Forested } \\
\text { area } \\
\text { (percent) }\end{array}$ & $\begin{array}{c}\text { Soil } \\
\text { index }\end{array}$ & $\begin{array}{c}\text { Mean } \\
\text { annual } \\
\text { precipitation } \\
\text { (in) }\end{array}$ & $\begin{array}{c}\text { Rainfall intensity, 24-hour } \\
\text { (in) }\end{array}$ & $\begin{array}{c}50 \text {-year } \\
\text { (in) }\end{array}$ \\
\hline 108 & 28.4 & 4,000 & 21.0 & 2.0 & 16.4 & 2.0 \\
\hline
\end{tabular}


09486300 CANADA DEL ORO NEAR TUCSON, AZ--Continued

MEAN MONTHLY AND ANNUAL DISCHARGES $1966-78$

\begin{tabular}{|c|c|c|c|c|c|c|}
\hline MONTH & $\begin{array}{l}\text { MAXIMUM } \\
(\text { FT3/S) }\end{array}$ & $\begin{array}{l}\text { MINIMUM } \\
(\text { ET3/S) }\end{array}$ & $\underset{(\mathrm{FT} 3 / \mathrm{S})}{\operatorname{MEAN}}$ & $\begin{array}{l}\text { STAN- } \\
\text { DARD } \\
\text { DEVIA- } \\
\text { TION } \\
(\mathrm{FT} 3 / \mathrm{S})\end{array}$ & $\begin{array}{l}\text { COEFFI- } \\
\text { CIENT OF } \\
\text { VARI. } \\
\text { ATION }\end{array}$ & $\begin{array}{c}\text { PERCENT } \\
\text { OF } \\
\text { ANNUAL } \\
\text { RUNOFF }\end{array}$ \\
\hline OCTOBER & 19 & 0.00 & 2.0 & 5.5 & 2.7 & 10.0 \\
\hline NOVEMBER & 0.00 & 0.00 & 0.00 & 0.00 & & 0.0 \\
\hline DECEMBER & 88 & 0.00 & 9.8 & 26 & 2.6 & 49.1 \\
\hline JANUARY & 6.2 & 0.00 & 0.48 & 1.7 & 3.6 & 2.4 \\
\hline FEBRUARY & 2.5 & 0.00 & 0.34 & 0.79 & 2.3 & 1.7 \\
\hline MARCH & 5.9 & 0.00 & 0.47 & 1.7 & 3.5 & 2.4 \\
\hline APRIL & 0.00 & 0.00 & 0.00 & 0.00 & & 0.0 \\
\hline MAY & 0.00 & 0.00 & 0.00 & 0.00 & & 0.0 \\
\hline JUNE & 0.00 & 0.00 & 0.00 & 0.00 & & 0.0 \\
\hline JULY & 13 & 0.00 & 1.2 & 3.6 & 2.9 & 6.2 \\
\hline AUGUST & 30 & 0.00 & 3.5 & 8.3 & 2.4 & 17.4 \\
\hline SEPTEMBER & 14 & 0.00 & 2.2 & 3.9 & 1.8 & 10.9 \\
\hline ANNUAL & 7.5 & 0.06 & 1.7 & 2.0 & 1.2 & 100 \\
\hline
\end{tabular}

MAGNITUDE AND PROBABILITY OF INSTANTANEOUS PEAK FLOW BASED ON PERIOD OF RECORD 1959, 1961, 1966-81, 1984

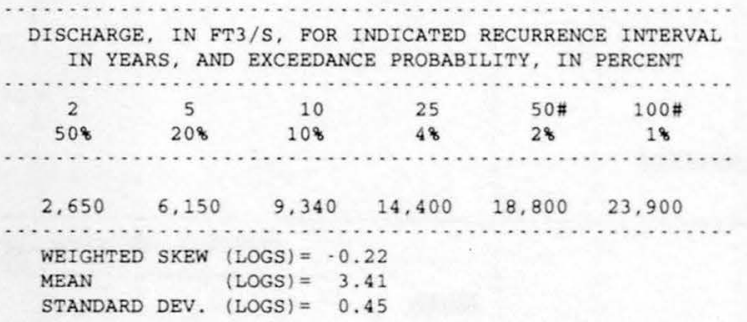

MAGNITUDE AND PROBABILITY OF ANNUAL LOW FLOW BASED ON PERIOD OF RECORD 1967.78

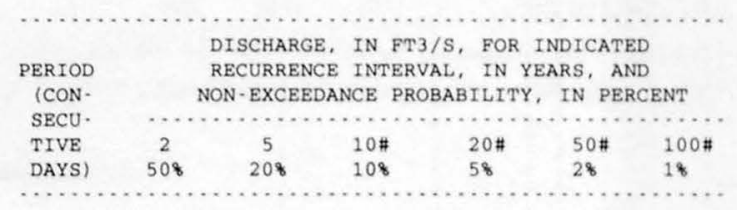

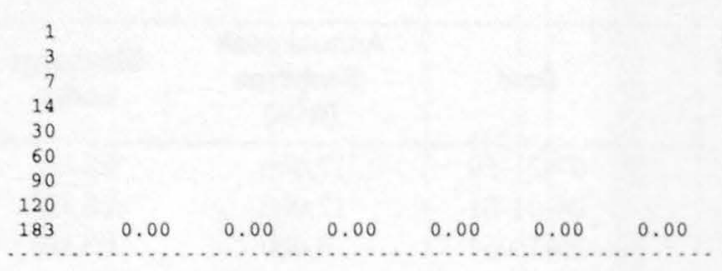

MAGNITUDE AND PROBABILITY OF ANNUAL HIGH FLOW BASED ON PERIOD OF RECORD $1966 \cdot 78$

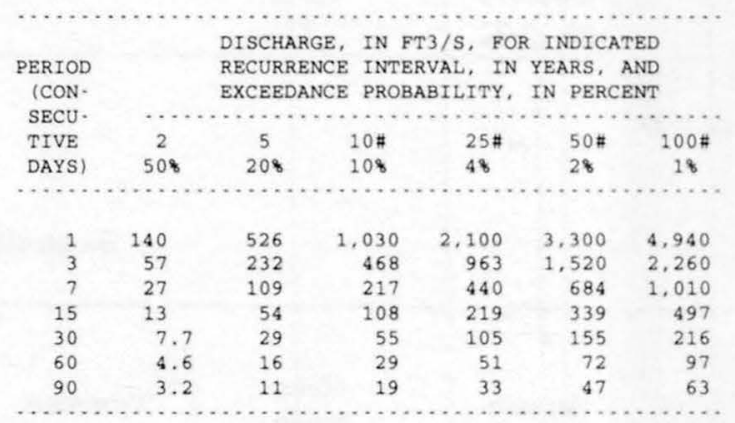

DURATION TABLE OF DAILY MEAN FLOW FOR PERIOD OF RECORD 1966.78

DISCHARGE, IN FT3/S, WHICH WAS EQUALED OR EXCEEDED FOR INDICATED PERCENT OF TIME

\begin{tabular}{|c|c|c|c|c|c|c|c|c|c|c|c|c|c|c|c|c|}
\hline 18 & 58 & 108 & 158 & 208 & 308 & 408 & 508 & 608 & 708 & 808 & 908 & 958 & 988 & 998 & 99.58 & 99.98 \\
\hline 15 & 0.00 & 0.00 & 0.00 & 0.00 & 0.00 & 0.00 & 0.00 & 0.00 & 0.00 & 0.00 & 0.00 & 0.00 & 0.00 & 0.00 & 0.00 & 0.00 \\
\hline
\end{tabular}

\# Reliability of values in column is uncertain, and potential errors are large. 
09486300 CANADA DEL ORO NEAR TUCSON, AZ--Continued
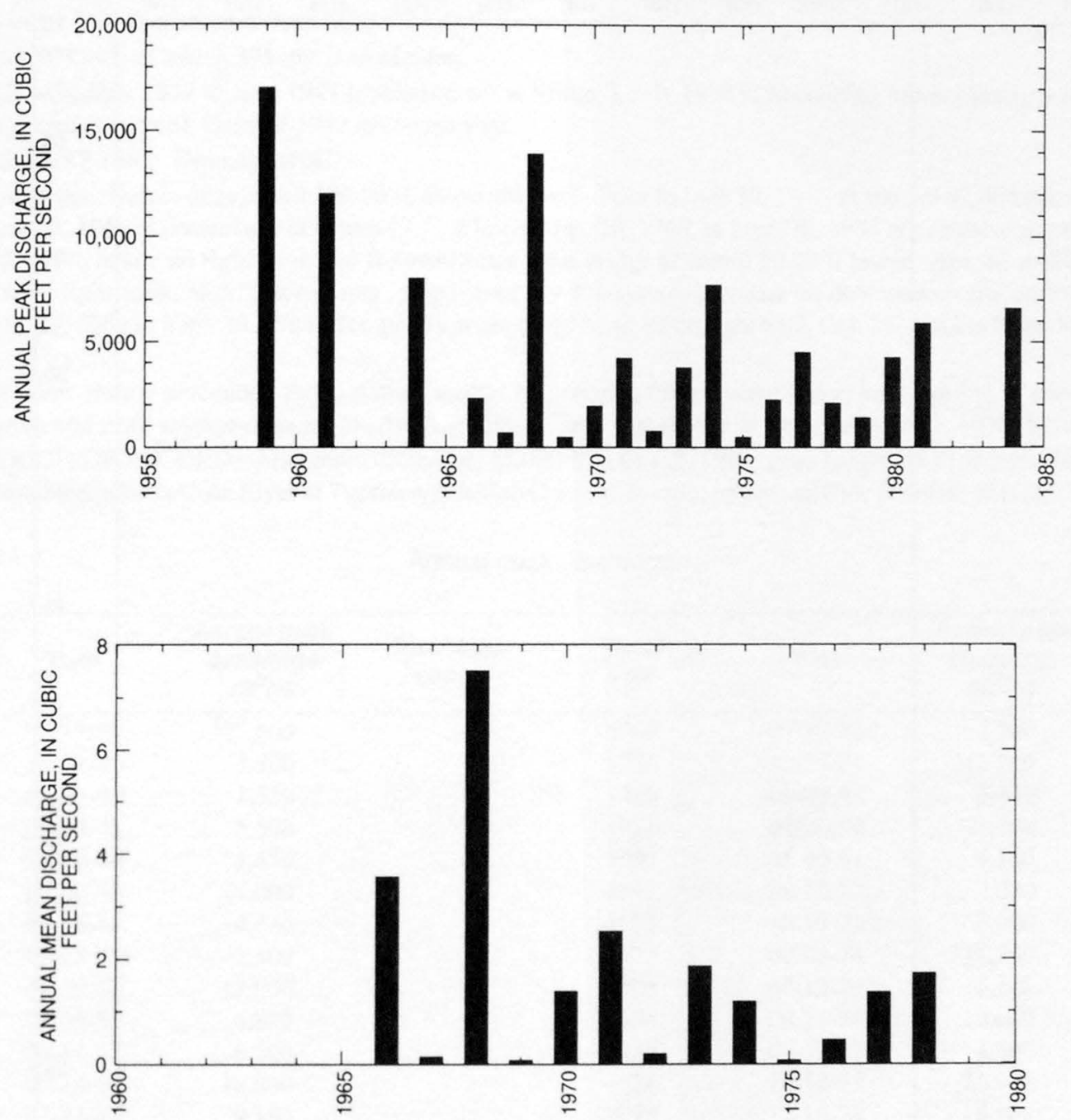
09486300 CANADA DEL ORO NEAR TUCSON, AZ--Continued
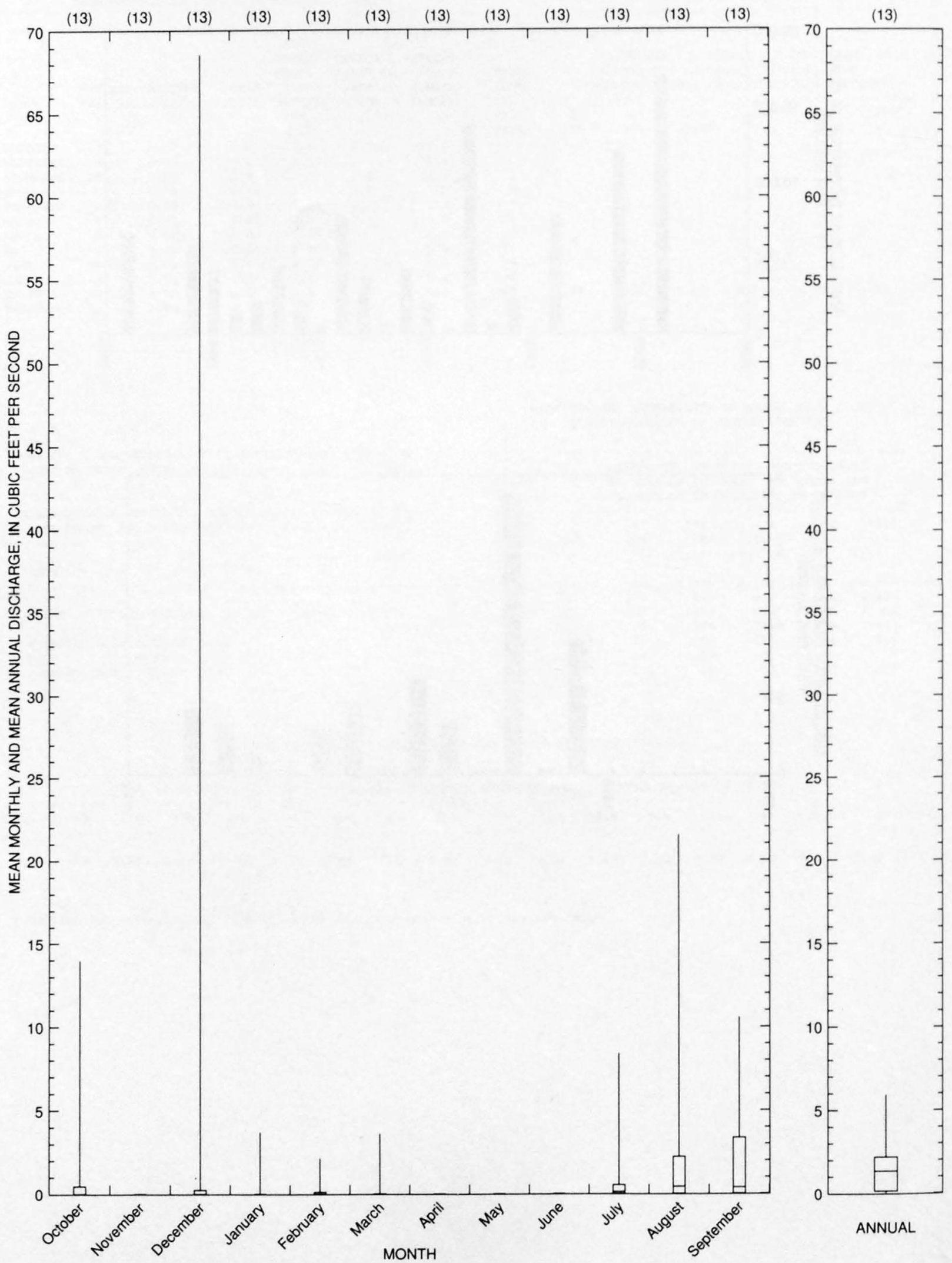
09486500 SANTA CRUZ RIVER AT CORTARO, AZ

LOCATION.--Lat 32 $21^{\prime} 04^{\prime \prime}$, long $111^{\circ} 05^{\prime} 38^{\prime \prime}$, in NW"1/4 NW $1 / 4 \mathrm{NW}^{1} / 4 \mathrm{sec} .35$, T.12 S., R.12 E., Pima County, Hydrologic Unit 15050302 , at center column of bridge pier on left bank, $0.5 \mathrm{mi}$ southwest of Cortaro, $1.0 \mathrm{mi}$ downstream from Ina Road treatment plant, $2.6 \mathrm{mi}$ downstream from Canada del Oro, and $3.7 \mathrm{mi}$ downstream from Rillito Creek.

DRAINAGE AREA.--3,503 $\mathrm{mi}^{2}$, of which $395 \mathrm{mi}^{2}$ is in Mexico.

PERIOD OF RECORD.--October 1939 to June 1947 (published as "at Rillito"), July 1950 to September 1984, March to June 1990, July to September 1990 (fragmentary record), October 1990 to current year.

REVISED RECORDS.--WSP 1283: Drainage area.

GAGE.--Water-stage recorder. Datum of gage is 2,100.00 ft, above sea level. Prior to June 30, 1947, at site $5.5 \mathrm{mi}$ downstream at different datum. July 8, 1950 to Jan. 20, 1966 at present site at datum $19.11 \mathrm{ft}$ lower. Jan. 20, 1966, to Sept. 30,1984 at present site and datum $23.11 \mathrm{ft}$ lower. Aug. 1 to Oct. 19,1990 , at site on right bank $600 \mathrm{ft}$ downstream from bridge at datum $30.20 \mathrm{ft}$ lower. Apr. 10 to May 17,1991 , at site on bridge, $200 \mathrm{ft}$ toward right bank, at different datum. Supplementary water-stage recorder on downstream site on left bridge pier at datum $19.11 \mathrm{ft}$ lower Aug. 29, 1969 to Sept. 30, 1984. Temporary water-stage recorder on right bank Oct. 27,1983 to Sept. 30,1984 at datum 20.80 ft lower.

REMARKS.--Records poor. Many diversions above station, mostly by pumping from ground water, for irrigation of about 34,000 acres. Waste water from irrigation and from sewage-disposal plants is included in flow past station in water years 1951, 1952, 1970-82, 1990-95.

EXTREMES FOR PERIOD OF RECORD.--Maximum discharge, $65,000 \mathrm{ft}^{3} / \mathrm{s}$ Oct. 2, 1983, gage height $16.57 \mathrm{ft}$ from floodmark, computed by flood-routing method from Santa Cruz River at Tucson and Rillito Creek at Tucson; no natural flow for most of each year. (See REMARKS)

Annual peak discharge

\begin{tabular}{|c|c|c|c|c|c|c|c|}
\hline $\begin{array}{l}\text { Water } \\
\text { year }\end{array}$ & Date & $\begin{array}{c}\text { Annual peak } \\
\text { discharge } \\
\left(\mathrm{ft}^{3} / \mathrm{s}\right)\end{array}$ & $\begin{array}{l}\text { Discharge } \\
\text { codes }\end{array}$ & $\begin{array}{l}\text { Water } \\
\text { year }\end{array}$ & Date & $\begin{array}{c}\text { Annual peak } \\
\text { discharge } \\
\left(\mathrm{ft}^{3} / \mathrm{s}\right)\end{array}$ & $\begin{array}{l}\text { Discharge } \\
\text { codes }\end{array}$ \\
\hline 1940 & $08-14-40$ & ${ }^{1} 17,000$ & & 1967 & $07-17-67$ & 5,740 & \\
\hline 1941 & $12-31-40$ & 7,800 & & 1968 & $12-21-67$ & 15,800 & \\
\hline 1942 & $08-09-42$ & 1,550 & & 1969 & $08-06-69$ & 8,400 & \\
\hline 1943 & $09-24-43$ & 5,500 & & 1970 & $07-20-70$ & 11,200 & \\
\hline 1944 & $08-16-44$ & 5,650 & & 1971 & $08-20-71$ & 9,100 & \\
\hline 1945 & $08-10-45$ & 14,000 & & 1972 & $08-12-72$ & 7,050 & \\
\hline 1946 & $08-04-46$ & 4,440 & & 1973 & $10-19-72$ & 9,000 & \\
\hline 1947 & $08-15-47$ & 7,500 & & 1974 & $07-08-74$ & 11,700 & \\
\hline 1950 & $07-30-50$ & 12,900 & & 1975 & $07-12-75$ & 5,200 & \\
\hline 1951 & $07-25-51$ & 6,820 & & 1976 & $09-25-76$ & 10,600 & \\
\hline 1952 & $08-14-52$ & 6,100 & & 1977 & $09-10-77$ & 4,700 & \\
\hline 1953 & $07-14-53$ & 10,800 & & 1978 & $10-10-77$ & 23,000 & \\
\hline 1954 & $07-24-54$ & 9,150 & & 1979 & $12-18-78$ & 18,800 & \\
\hline 1955 & $08-03-55$ & 16,600 & & 1980 & $07-19-80$ & 2,650 & \\
\hline 1956 & $07-29-56$ & 3,150 & & 1981 & $09-22-81$ & 4,310 & \\
\hline 1957 & $09-01-57$ & 4,400 & & 1982 & $08-23-82$ & 13,300 & \\
\hline 1958 & $08-12-58$ & 7,890 & & 1983 & $02-04-83$ & 7,620 & \\
\hline 1959 & $08-20-59$ & 8,000 & & 1984 & $10-02-83$ & ${ }^{2} 65,000$ & HP \\
\hline 1960 & $08-11-60$ & 6,420 & & 1985 & $12-28-84$ & 13,000 & \\
\hline 1961 & $08-23-61$ & 14,700 & & 1990 & $07-24-90$ & 27,500 & \\
\hline 1962 & $09-26-62$ & 11,200 & & 1991 & $03-01-91$ & 11,600 & \\
\hline 1963 & $08-26-63$ & 7,240 & & 1992 & $08-24-92$ & 4,670 & \\
\hline 1964 & $09-10-64$ & 15,900 & & 1995 & $02-15-95$ & 6,170 & \\
\hline 1965 & $07-16-65$ & 2,710 & & 1996 & $09-03-96$ & 16,400 & \\
\hline 1966 & $12-22-65$ & 16,800 & & & & & \\
\hline
\end{tabular}

\footnotetext{
${ }^{1}$ Highest since 1935
}

${ }^{2}$ Highest since 1959. 


\section{GILA RIVER BASIN}

09486500 SANTA CRUZ RIVER AT CORTARO, AZ--Continued

Discharge rating table developed October 1993

\begin{tabular}{cccc}
\hline $\begin{array}{c}\text { Gage height } \\
(\mathbf{f t})\end{array}$ & $\begin{array}{c}\text { Discharge } \\
\left(\mathbf{f t}^{\mathbf{3}} \mathbf{/} \mathbf{s}\right)\end{array}$ & $\begin{array}{c}\text { Gage height } \\
(\mathbf{f t})\end{array}$ & $\begin{array}{c}\text { Discharge } \\
\left(\mathbf{f t}^{\mathbf{3}} \mathbf{s} \mathbf{s}\right)\end{array}$ \\
\hline 6.0 & 1,700 & 9.5 & 9,410 \\
6.5 & 2,290 & 10.0 & 11,400 \\
7.0 & 3,020 & 10.5 & 13,700 \\
7.5 & 3,900 & 11.0 & 16,200 \\
8.0 & 4,960 & 11.5 & 19,200 \\
8.5 & 6,220 & 12.0 & 22,500 \\
9.0 & 7,690 & 12.5 & 26,100 \\
\hline
\end{tabular}

Basin characteristics

\begin{tabular}{|c|c|c|c|c|c|c|c|}
\hline \multirow[b]{2}{*}{$\begin{array}{l}\text { Main } \\
\text { channel } \\
\text { slope } \\
(\mathrm{ft} / \mathrm{mi})\end{array}$} & \multirow[b]{2}{*}{$\begin{array}{l}\text { Stream } \\
\text { length } \\
\text { (mi) }\end{array}$} & \multirow[b]{2}{*}{$\begin{array}{c}\text { Mean } \\
\text { basin } \\
\text { elevation } \\
\text { (ft) }\end{array}$} & \multirow[b]{2}{*}{$\begin{array}{l}\text { Forested } \\
\text { area } \\
\text { (percent) }\end{array}$} & \multirow[b]{2}{*}{$\begin{array}{c}\text { Soil } \\
\text { index }\end{array}$} & \multirow[b]{2}{*}{$\begin{array}{c}\text { Mean } \\
\text { annual } \\
\text { precipitation } \\
\text { (in) }\end{array}$} & \multicolumn{2}{|c|}{ Rainfall intensity, 24-hour } \\
\hline & & & & & & $\begin{array}{l}\text { 2-year } \\
\text { (in) }\end{array}$ & $\begin{array}{l}\text { 50-year } \\
\text { (in) }\end{array}$ \\
\hline 20.3 & 140 & 4,000 & 18.0 & 1.8 & 16.3 & 2.0 & 4.2 \\
\hline
\end{tabular}


09486500 SANTA CRUZ RIVER AT CORTARO, AZ--Continued

MEAN MONTHLY AND ANNUAL DISCHARGES $1940.46,1951.82,1991.96$

\begin{tabular}{|c|c|c|c|c|c|c|}
\hline MONTH & $\begin{array}{l}\text { MAXIMUM } \\
(\text { FT3/S) }\end{array}$ & $\begin{array}{l}\text { MINIMUM } \\
\text { (FT3/S) }\end{array}$ & $\begin{array}{c}\text { MEAN } \\
\text { (FT3/S) }\end{array}$ & $\begin{array}{l}\text { STAN- } \\
\text { DARD } \\
\text { DEVIA- } \\
\text { TION } \\
\text { (FT3/S) }\end{array}$ & $\begin{array}{l}\text { COEFFI- } \\
\text { CIENT OF } \\
\text { VARI- } \\
\text { ATION }\end{array}$ & $\begin{array}{c}\text { PERCENT } \\
\text { OF } \\
\text { ANNUAL } \\
\text { RUNOFF }\end{array}$ \\
\hline OCTOBER & 744 & 0.00 & 40 & 114 & 2.8 & 6.4 \\
\hline NOVEMBER & 168 & 0.00 & 22 & 32 & 1.5 & 3.5 \\
\hline DECEMBER & 1.040 & 0.00 & 86 & 217 & 2.5 & 13.5 \\
\hline JANUARY & 2,490 & 0.00 & 105 & 383 & 3.6 & 16.7 \\
\hline FEBRUARY & 252 & 0.00 & 43 & 60 & 1.4 & 6.8 \\
\hline MARCH & 496 & 0.00 & 38 & 79 & 2.1 & 6.1 \\
\hline APRIL & 60 & 0.00 & 15 & 20 & 1.3 & 2.4 \\
\hline MAY & 53 & 0.00 & 14 & 19 & 1.3 & 2.2 \\
\hline JUNE & 61 & 0.00 & 15 & 18 & 1.2 & 2.4 \\
\hline JULY & 393 & 1.7 & 72 & 79 & 1.1 & 11.4 \\
\hline AUGUST & 868 & 2.0 & 122 & 142 & 1.2 & 19.4 \\
\hline SEPTEMBER & 358 & 0.00 & 59 & 70 & 1.2 & 9.3 \\
\hline ANNUAL & 262 & 2.6 & 53 & 50 & 0.95 & 100 \\
\hline
\end{tabular}

MAGNITUDE AND PROBABILITY OF ANNUAL LOW FLOW BASED ON PERIOD OF RECORD $1941 \cdot 47,1952 \cdot 82,1992 \cdot 96$

\begin{tabular}{|c|c|c|c|c|c|c|}
\hline \multirow{4}{*}{$\begin{array}{l}\text { PERIOD } \\
\text { (CON- } \\
\text { SECU- } \\
\text { TIVE }\end{array}$} & \multicolumn{6}{|c|}{ DISCHARGE, IN FT3/S, FOR INDICATED } \\
\hline & \multicolumn{6}{|c|}{$\begin{array}{l}\text { RECURRENCE INTERVAL, IN YEARS, AND } \\
\text { NON-EXCEEDANCE PROBABILITY, IN PERCENT }\end{array}$} \\
\hline & $\cdots$ & & & & $\cdots \cdots$ & $\cdots \cdots$ \\
\hline & 2 & 5 & 10 & 20 & $50 \#$ & $100 \#$ \\
\hline DAYS) & 508 & 208 & 108 & $5 \%$ & 28 & 18 \\
\hline 1 & 0.00 & 0.00 & 0.00 & 0.00 & 0.00 & 0.00 \\
\hline 3 & 0.00 & 0.00 & 0.00 & 0.00 & 0.00 & 0.00 \\
\hline 7 & 0.00 & 0.00 & 0.00 & 0.00 & 0.00 & 0.00 \\
\hline 14 & 0.00 & 0.00 & 0.00 & 0.00 & 0.00 & 0.00 \\
\hline 30 & 0.00 & 0.00 & 0.00 & 0.00 & 0.00 & 0.00 \\
\hline 60 & 0.00 & 0.00 & 0.00 & 0.00 & 0.00 & 0.00 \\
\hline 90 & 0.43 & 0.00 & 0.00 & 0.00 & 0.00 & 0.00 \\
\hline 120 & 3.8 & 0.00 & 0.00 & 0.00 & 0.00 & 0.00 \\
\hline 183 & 11 & 1.5 & 0.35 & 0.07 & 0.00 & 0.00 \\
\hline
\end{tabular}

MAGNITUDE AND PROBABILITY OF ANNUAL HIGH FLOW BASED ON PERIOD OF RECORD $1940.46,1951.82,1991.96$

\begin{tabular}{|c|c|c|c|c|c|c|}
\hline \multirow{3}{*}{$\begin{array}{l}\text { PERIOD } \\
\text { /CON- } \\
\text { SECU- }\end{array}$} & \multicolumn{6}{|c|}{ DISCHARGE, IN FT3/S, FOR INDICATED } \\
\hline & & EXCEEDA & CE PROE & ABILITY, & IN PERC & ENT \\
\hline & & $\cdots \ldots$ & $\cdots \cdots$ & $\ldots \ldots$ & $\ldots \ldots$ & …... \\
\hline TIVE & 2 & 5 & 10 & $25 \#$ & $50 \#$ & $100 \#$ \\
\hline DAYS) & 508 & 208 & 108 & 48 & 28 & 18 \\
\hline 1 & 2,040 & 5,050 & 8,080 & 13,300 & 18,300 & 24,400 \\
\hline 3 & 1,020 & 2,560 & 4,180 & 7,090 & 10,000 & 13,700 \\
\hline 7 & 541 & 1,330 & 2,130 & 3,530 & 4,900 & 6,580 \\
\hline 15 & 314 & 760 & 1.210 & 2,010 & 2,790 & 3,750 \\
\hline 30 & 202 & 464 & 714 & 1,130 & 1,520 & 1,970 \\
\hline 60 & 132 & 290 & 432 & 652 & 846 & 1,060 \\
\hline 90 & 101 & 218 & 316 & 460 & 579 & 707 \\
\hline
\end{tabular}

DURATION TABLE OF DAILY MEAN FLOW FOR PERIOD OF RECORD 1940-46, 1951-82, 1991-96

DISCHARGE, IN FT3/S, WHICH WAS EQUALED OR EXCEEDED FOR INDICATED PERCENT OF TIME

\begin{tabular}{|c|c|c|c|c|c|c|c|c|c|c|c|c|c|c|c|c|}
\hline 18 & 58 & 108 & 158 & 208 & 308 & 408 & 508 & 608 & 708 & $80 \%$ & 908 & 958 & 988 & 998 & 99.58 & 99.98 \\
\hline 833 & 105 & 65 & 54 & 47 & 35 & 21 & 0.16 & 0.00 & 0.00 & 0.00 & 0.00 & 0.00 & 0.00 & 0.00 & 0.00 & 0.00 \\
\hline
\end{tabular}

\# Reliability of values in column is uncertain, and potential errors are large. 

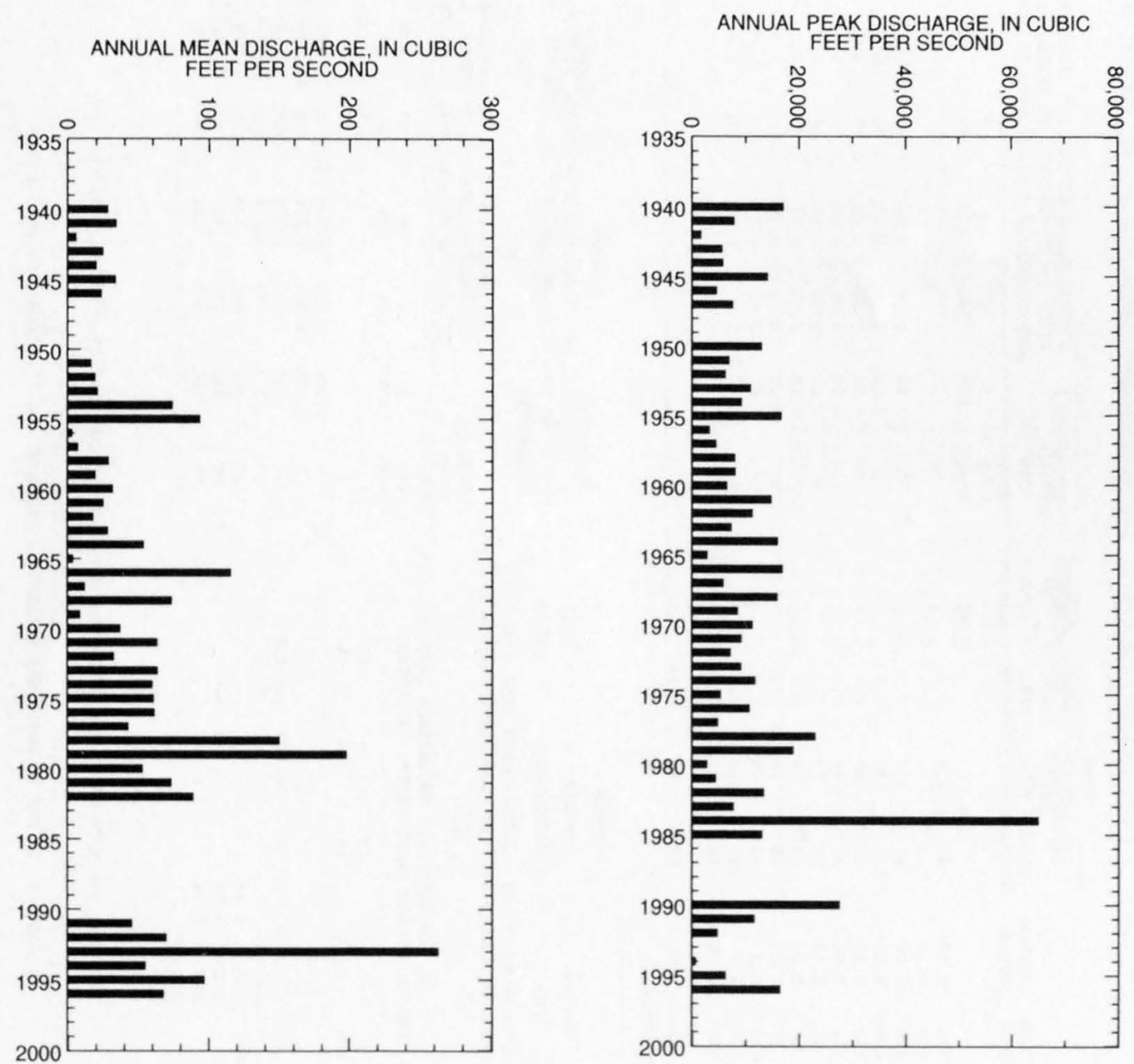
09486500 SANTA CRUZ RIVER AT CORTARO, AZ--Continued

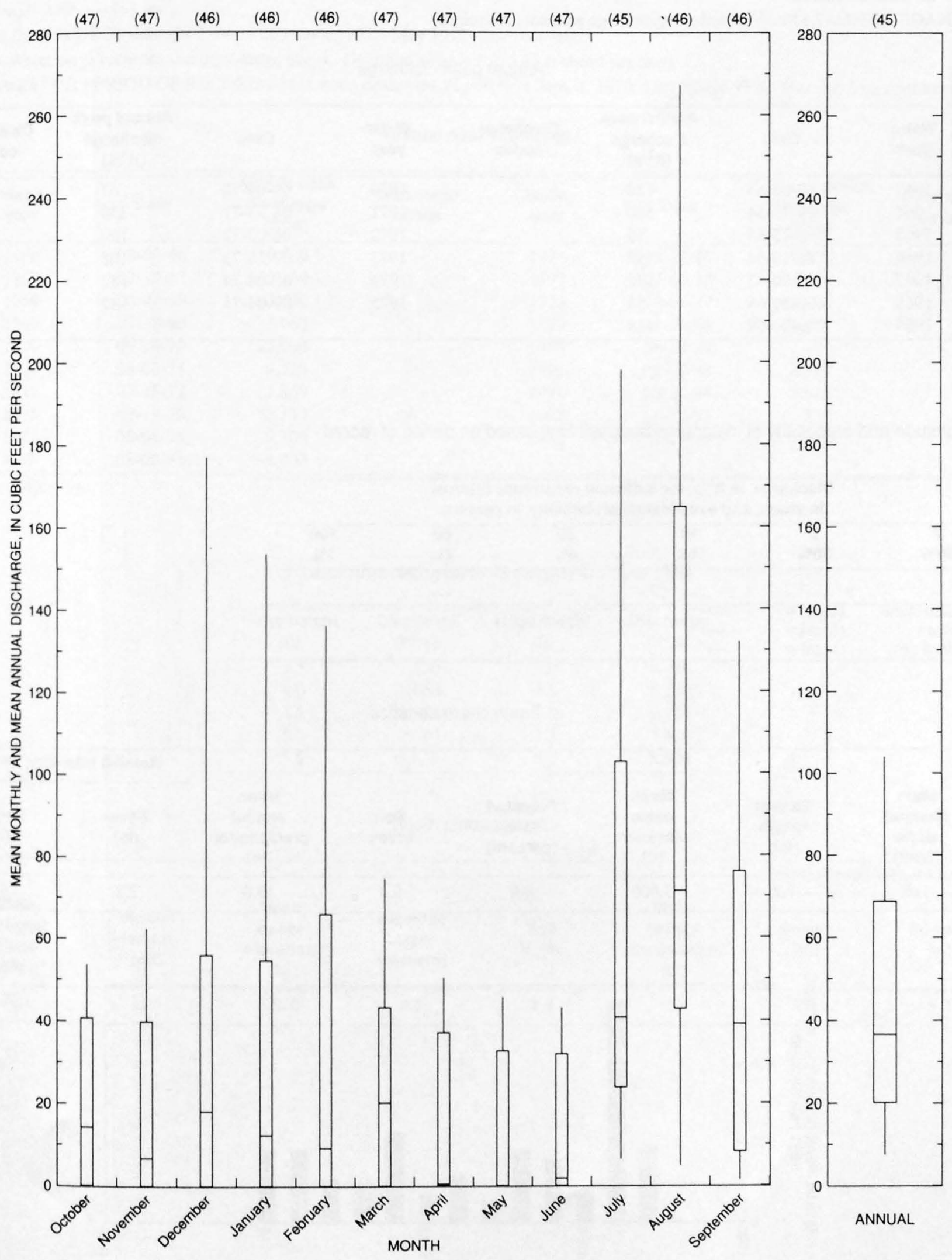


09486700 CHILTEPINES WASH NEAR SASABE, AZ

LOCATION.--Lat $31^{\circ} 49^{\prime} 08^{\prime \prime}$, long $11^{\circ} 26^{\prime} 16^{\prime \prime}, \mathrm{NE}^{1} /{ }_{4} \mathrm{SE}^{1} / 4 \mathrm{sec} .32$, T.18 S., R.9 E., Pima County, Hydrologic Unit 15050304 , at State Highway 286 24 mi north of Sasabe.

DRAINAGE AREA.--7.13 $\mathrm{mi}^{2}$, contributing drainage area not determined.

Annual peak discharge

\begin{tabular}{ccccccc}
\hline $\begin{array}{c}\text { Water } \\
\text { year }\end{array}$ & Date & $\begin{array}{c}\text { Annual peak } \\
\text { discharge } \\
\left(\mathrm{ft}^{3} / \mathbf{s}\right)\end{array}$ & $\begin{array}{c}\text { Discharge } \\
\text { codes }\end{array}$ & $\begin{array}{c}\text { Water } \\
\text { year }\end{array}$ & $\begin{array}{c}\text { Date } \\
\text { Annual peak } \\
\text { discharge } \\
\left(\mathrm{ft}^{3} / \mathbf{s}\right)\end{array}$ \\
\hline 1963 & $08-05-63$ & 189 & & 1970 & $09-04-70$ & 67 \\
1964 & $09-10-64$ & 560 & & 1971 & $08-03-71$ & 230 \\
1965 & $09-15-65$ & 85 & & 1972 & $08-00-72$ & 100 \\
1966 & $08-19-66$ & 158 & & 1973 & $07-15-73$ & 108 \\
1967 & $00-00-67$ & 182 & & 1974 & $08-04-74$ & 182 \\
1968 & $00-00-68$ & 53 & & 1975 & $09-04-75$ & 182 \\
1969 & $00-00-69$ & 118 & & & \\
\hline
\end{tabular}

Magnitude and probability of instantaneous peak flow based on period of record

\begin{tabular}{|c|c|c|c|c|c|}
\hline \multicolumn{6}{|c|}{$\begin{array}{l}\text { Discharge, in } \mathrm{ft}^{3} / \mathrm{s} \text {, for indicated recurrence interval } \\
\text { in years, and exceedance probablility, in percent }\end{array}$} \\
\hline 2 & 5 & 10 & 25 & 50 & 100 \\
\hline $50 \%$ & $20 \%$ & $10 \%$ & $4 \%$ & $2 \%$ & $1 \%$ \\
\hline -- & --. & -- & -- & $\cdots$ & $\ldots$ \\
\hline Weighted skew & $(\log s)=$ & -- & & & \\
\hline Mean & $(\log s)=$ &.-- & & & \\
\hline Standard dev. & $(\log s)=$ & $\cdots$ & & & \\
\hline
\end{tabular}

Basin characteristics

\begin{tabular}{|c|c|c|c|c|c|c|c|}
\hline \multirow[b]{2}{*}{$\begin{array}{l}\text { Main } \\
\text { channel } \\
\text { slope } \\
(\mathrm{ft} / \mathrm{mi})\end{array}$} & \multirow[b]{2}{*}{$\begin{array}{l}\text { Stream } \\
\text { length } \\
\text { (mi) }\end{array}$} & \multirow[b]{2}{*}{$\begin{array}{c}\text { Mean } \\
\text { basin } \\
\text { elevation } \\
(f t)\end{array}$} & \multirow[b]{2}{*}{$\begin{array}{l}\text { Forested } \\
\text { area } \\
\text { (percent) }\end{array}$} & \multirow[b]{2}{*}{$\begin{array}{c}\text { Soil } \\
\text { index }\end{array}$} & \multirow[b]{2}{*}{$\begin{array}{c}\text { Mean } \\
\text { annual } \\
\text { precipitation } \\
\text { (in) }\end{array}$} & \multicolumn{2}{|c|}{ Rainfall intensity, 24-hour } \\
\hline & & & & & & $\begin{array}{l}\text { 2-year } \\
\text { (in) }\end{array}$ & $\begin{array}{l}\text { 50-year } \\
\text { (in) }\end{array}$ \\
\hline 116 & 7.2 & 3,660 & 0.8 & 3.0 & 13.0 & 2.2 & 4.0 \\
\hline
\end{tabular}

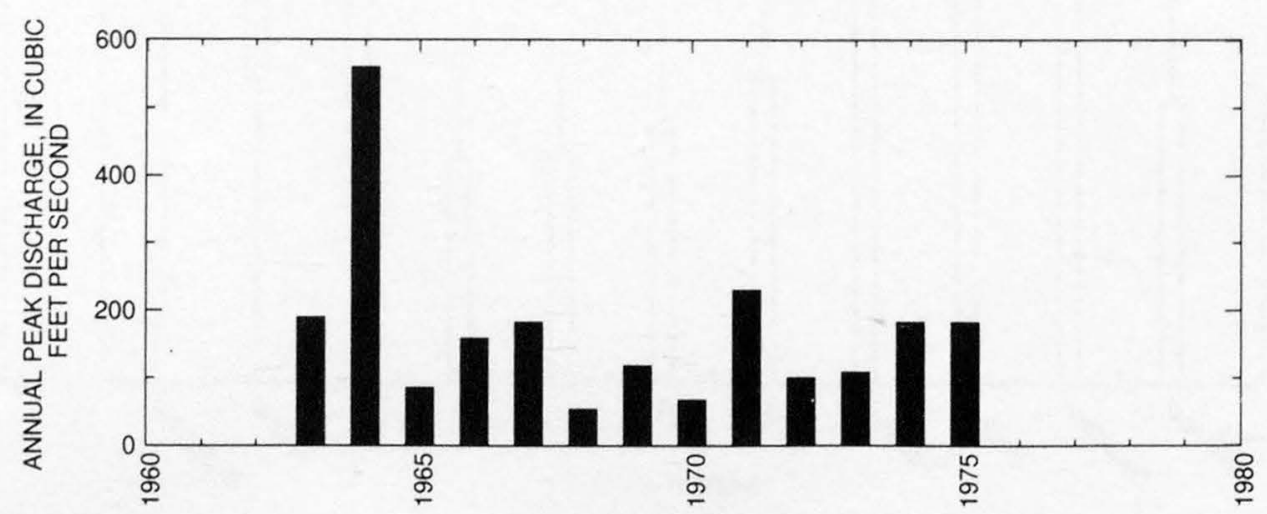


09486800 ALTAR WASH NEAR THREE POINTS, AZ

LOCATION.--Lat 31 '50'20", long $111^{\circ} 24^{\prime} 13^{\prime \prime}$, in SE $\frac{1}{4} \mathrm{NE}^{1} / 4^{\mathrm{NE}} / 4$ sec.27, T.18 S., R.9 E., Pima County, Hydrologic Unit 15050304 , on right bank attached to downstream side of bridge on State Highway 286, 0.3 mi below mouth of Chiltipines Wash and 18 mi south of Three Points. DRAINAGE AREA.--463 $\mathrm{mi}^{2}$.

PERIOD OF RECORD.--January 1966 to September 1975, May 1992 to current year.

GAGE.--Water stage recorder and crest-stage gages. Datum of gage is 2,975.15 ft above sea level.

EXTREMES FOR PERIOD OF RECORD.--Maximum discharge, 22,000 ft $3 / \mathrm{s} \mathrm{Sept.} \mathrm{4,} \mathrm{1970,} \mathrm{gage} \mathrm{height} 13.85 \mathrm{ft}$ at site $2 \mathrm{mi}$ upstream.

Annual peak discharges

\begin{tabular}{|c|c|c|c|c|c|c|c|}
\hline $\begin{array}{l}\text { Water } \\
\text { year }\end{array}$ & Date & $\begin{array}{c}\text { Annual peak } \\
\text { discharge } \\
\left(\mathrm{ft}^{3} / \mathrm{s}\right)\end{array}$ & $\begin{array}{l}\text { Discharge } \\
\text { codes }\end{array}$ & $\begin{array}{l}\text { Water } \\
\text { year }\end{array}$ & Date & $\begin{array}{c}\text { Annual peak } \\
\text { discharge } \\
\left(\mathrm{ft}^{3} / \mathrm{s}\right)\end{array}$ & $\begin{array}{c}\text { Discharge } \\
\text { codes }\end{array}$ \\
\hline 1966 & $08-10-66$ & 10,700 & & 1976 & $08-22-76$ & 7,000 & \\
\hline 1967 & $07-15-67$ & 2,360 & & 1977 & $07-00-77$ & 4,500 & \\
\hline 1968 & $08-02-68$ & 3,430 & & 1978 & $10-06-77$ & 10,100 & \\
\hline 1969 & $07-18-69$ & 3,060 & & 1979 & $11-25-78$ & 1,480 & \\
\hline 1970 & $09-04-70$ & ${ }^{1} 22,000$ & & 1980 & $08-13-80$ & 1,400 & \\
\hline 1971 & $08-03-71$ & 4,220 & & 1993 & $08-27-93$ & 6,390 & \\
\hline 1972 & $07-15-72$ & 3,360 & & 1994 & $08-21-94$ & 7,660 & \\
\hline 1973 & $07-14-73$ & 2,130 & & 1995 & $08-17-95$ & 835 & \\
\hline 1974 & $08-04-74$ & 9,200 & & 1996 & $07-07-96$ & 4,600 & \\
\hline 1975 & $08-08-75$ & 9,700 & & & & & \\
\hline
\end{tabular}

${ }^{1}$ Highest since 1940.

Discharge rating table developed October 1994

\begin{tabular}{cccc}
\hline $\begin{array}{c}\text { Gage height } \\
(\mathbf{f t})\end{array}$ & $\begin{array}{c}\text { Discharge } \\
\left(\mathrm{ft}^{\mathbf{3}} / \mathbf{s}\right)\end{array}$ & $\begin{array}{c}\text { Gage height } \\
(\mathbf{f t})\end{array}$ & $\begin{array}{c}\text { Discharge } \\
\left(\mathrm{ft}^{\mathbf{3}} / \mathbf{s}\right)\end{array}$ \\
\hline 3.5 & 1,170 & 6.0 & 4,440 \\
4.0 & 1,660 & 6.5 & 5,340 \\
4.5 & 2,230 & 7.0 & 6,310 \\
5.0 & 2,890 & 7.5 & 7,410 \\
5.5 & 3,630 & 8.0 & 8,600 \\
\hline
\end{tabular}

Basin characteristics

\begin{tabular}{cccccccc}
$\begin{array}{c}\text { Main } \\
\text { channel } \\
\text { slope } \\
(\mathrm{ft} / \mathrm{mi})\end{array}$ & $\begin{array}{c}\text { Stream } \\
\text { length } \\
\text { (mi) }\end{array}$ & $\begin{array}{c}\text { Mean } \\
\text { basin } \\
\text { elevation } \\
\text { (ft) }\end{array}$ & $\begin{array}{c}\text { Forested } \\
\text { area } \\
\text { (percent) }\end{array}$ & $\begin{array}{c}\text { Soil } \\
\text { index }\end{array}$ & $\begin{array}{c}\text { Mean } \\
\text { annual } \\
\text { precipitation } \\
\text { (in) }\end{array}$ & $\begin{array}{c}\text { Rainfall intensity, 24-hour } \\
\text { (in) }\end{array}$ & $\begin{array}{c}50 \text {-year } \\
\text { (in) }\end{array}$ \\
\hline 74.1 & 32.4 & 3,920 & 6.5 & 2.0 & 15.6 & 2.2 \\
\hline
\end{tabular}




\section{ALTAR WASH NEAR THREE POINTS, AZ--Continued}

MEAN MONTHLY AND ANNUAL DISCHARGES $1967-75,1993.96$

\begin{tabular}{|c|c|c|c|c|c|c|}
\hline MONTH & $\begin{array}{l}\text { MAXIMUM } \\
\text { (FT3/S) }\end{array}$ & $\begin{array}{l}\text { MINIMUM } \\
\text { (FT3/S) }\end{array}$ & $\begin{array}{c}\text { MEAN } \\
\text { (FT3/S) }\end{array}$ & $\begin{array}{l}\text { STAN- } \\
\text { DARD } \\
\text { DEVIA- } \\
\text { TION } \\
(\mathrm{FT} 3 / \mathrm{S})\end{array}$ & $\begin{array}{l}\text { COEFFI- } \\
\text { CIENT OF } \\
\text { VARI- } \\
\text { ATION }\end{array}$ & $\begin{array}{c}\text { PERCENT } \\
\text { OF } \\
\text { ANNUAL } \\
\text { RUNOFF }\end{array}$ \\
\hline OCTOBER & 6.7 & 0.00 & 0.87 & 2.0 & 2.3 & 1.2 \\
\hline NOVEMBER & 0.62 & 0.00 & 0.05 & 0.17 & 3.3 & 0.1 \\
\hline DECEMBER & 39 & 0.00 & 3.0 & 11 & 3.5 & 4.1 \\
\hline JANUARY & 0.00 & 0.00 & 0.00 & 0.00 & & 0.0 \\
\hline FEBRUARY & 0.13 & 0.00 & 0.01 & 0.04 & 3.6 & 0.0 \\
\hline MARCH & 15 & 0.00 & 1.3 & 4.2 & 3.2 & 1.8 \\
\hline APRIL & 0.00 & 0.00 & 0.00 & 0.00 & & 0.0 \\
\hline MAY & 0.05 & 0.00 & 0.00 & 0.01 & 3.6 & 0.0 \\
\hline JUNE & 3.3 & 0.00 & 0.29 & 0.92 & 3.2 & 0.4 \\
\hline JULY & 56 & 0.00 & 14 & 15 & 1.0 & 19.5 \\
\hline AUGUST & 74 & 3.8 & 27 & 21 & 0.77 & 36.6 \\
\hline SEPTEMBER & 210 & 0.00 & 27 & 57 & 2.2 & 36.3 \\
\hline ANNUAL & 20 & 0.53 & 6.2 & 5.5 & 0.90 & 100 \\
\hline
\end{tabular}

MAGNITUDE AND PROBABILITY OF INSTANTANEOUS PEAK FLOW BASED ON PERIOD OF RECORD $1966 \cdot 80,1993.96$

DISCHARGE, IN FT3/S, FOR INDICATED RECURRENCE INTERVAL IN YEARS, AND EXCEEDANCE PROBABILITY, IN PERCENT

\begin{tabular}{|c|c|c|c|c|c|}
\hline $\begin{array}{c}2 \\
508\end{array}$ & $\begin{array}{c}5 \\
208\end{array}$ & $\begin{array}{r}10 \\
108\end{array}$ & $\begin{array}{l}25 \\
48\end{array}$ & $\begin{array}{l}50 \\
28\end{array}$ & $\begin{array}{r}100 \\
\end{array}$ \\
\hline 4,360 & 8,180 & 11,200 & 15,400 & 18,800 & 22,400 \\
\hline $\begin{array}{l}\text { WEIGHTED } \\
\text { MEAN } \\
\text { STANDARD }\end{array}$ & $\begin{array}{l}\text { SKEW } \\
\text { DEV. }\end{array}$ & $\begin{array}{l}(\text { LOGS })=\cdot \\
(\text { LOGS })= \\
(\text { LOGS })=\end{array}$ & & & \\
\hline
\end{tabular}

MAGNITUDE AND PROBABILITY OF ANNUAL LOW FLOW BASED ON PERIOD OF RECORD $1967.75,1994.96$

\begin{tabular}{|c|c|c|c|c|c|c|}
\hline \multirow{2}{*}{$\begin{array}{l}\text { PERIOD } \\
\text { (CON- }\end{array}$} & \multicolumn{3}{|c|}{$\begin{array}{ll}\text { DISCHARGE, IN } \\
\text { RECURRENCE IN? }\end{array}$} & \multicolumn{3}{|c|}{ - FOR INDICATED } \\
\hline & \multirow{2}{*}{\multicolumn{3}{|c|}{ NON-EXCEEDANCE }} & BILIT & IN PI & ENT \\
\hline SECU- & & & & .......... & .... & \\
\hline TIVE & 2 & 5 & $10 \#$ & $20 \#$ & $50 \#$ & $100 \#$ \\
\hline DAYS) & 508 & 208 & 108 & 58 & 28 & 18 \\
\hline
\end{tabular}

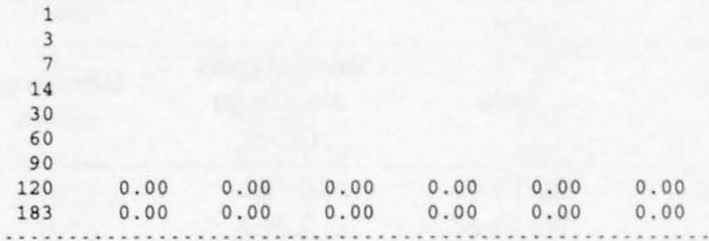

DURATION TABLE OF DAILY MEAN FLOW FOR PERIOD OF RECORD 1967-75, 1993-96 DISCHARGE, IN FT3/S, WHICH WAS EQUALED OR EXCEEDED FOR INDICATED PERCENT OF TIME

\begin{tabular}{|c|c|c|c|c|c|c|c|c|c|c|c|c|c|c|c|c|}
\hline 18 & 58 & 108 & 158 & 208 & 308 & 408 & 508 & 608 & 708 & 808 & 908 & 958 & 988 & 998 & 99.58 & 99.98 \\
\hline 132 & 1.6 & 0.00 & 0.00 & 0.00 & 0.00 & 0.00 & 0.00 & 0.00 & 0.00 & 0.00 & 0.00 & 0.00 & 0.00 & 0.00 & 0.00 & 0.00 \\
\hline
\end{tabular}

\# Reliability of values in column is uncertain, and potential errors are large. 
09486800 ALTAR WASH NEAR THREE POINTS, AZ--Continued
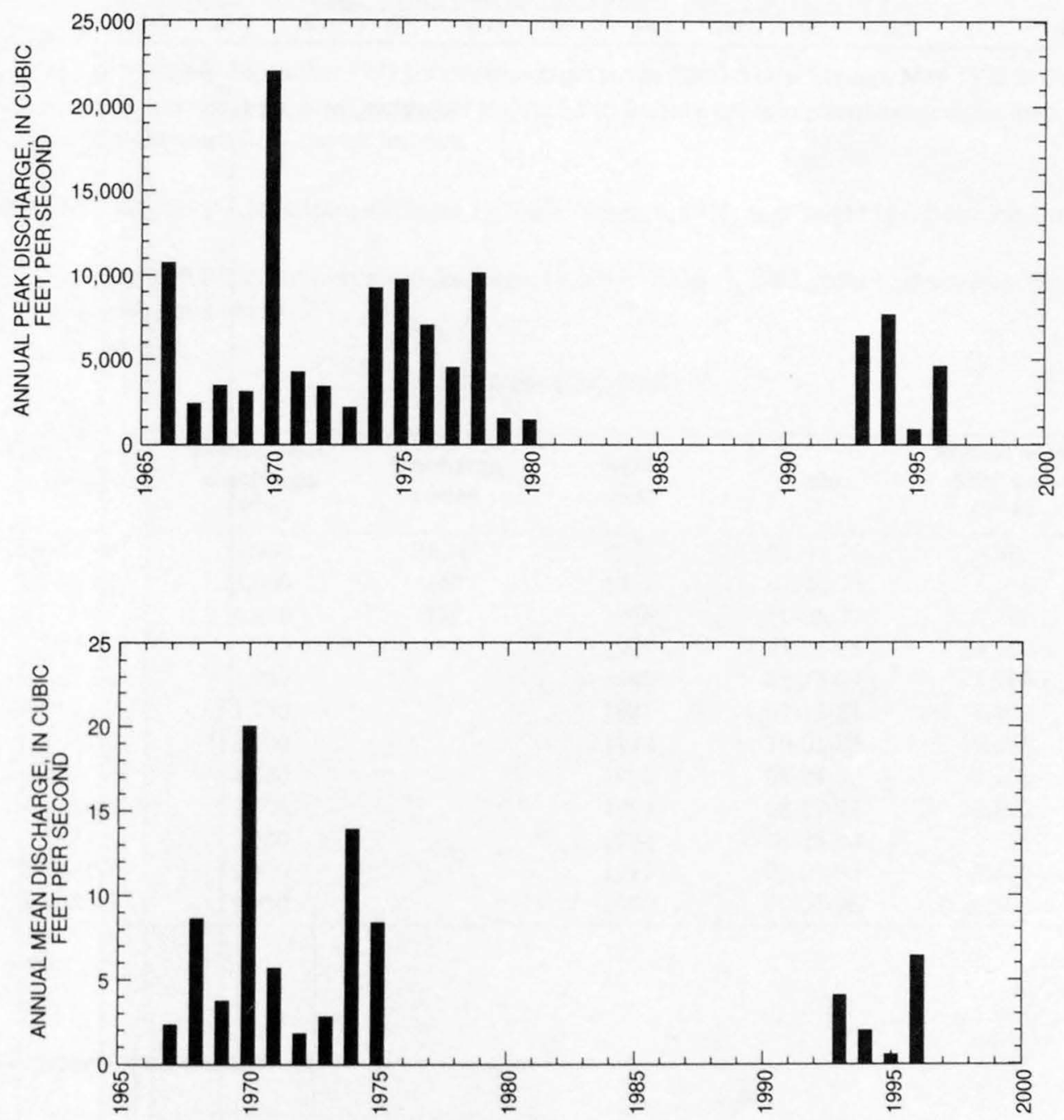
09486800 ALTAR WASH NEAR THREE POINTS, AZ--Continued
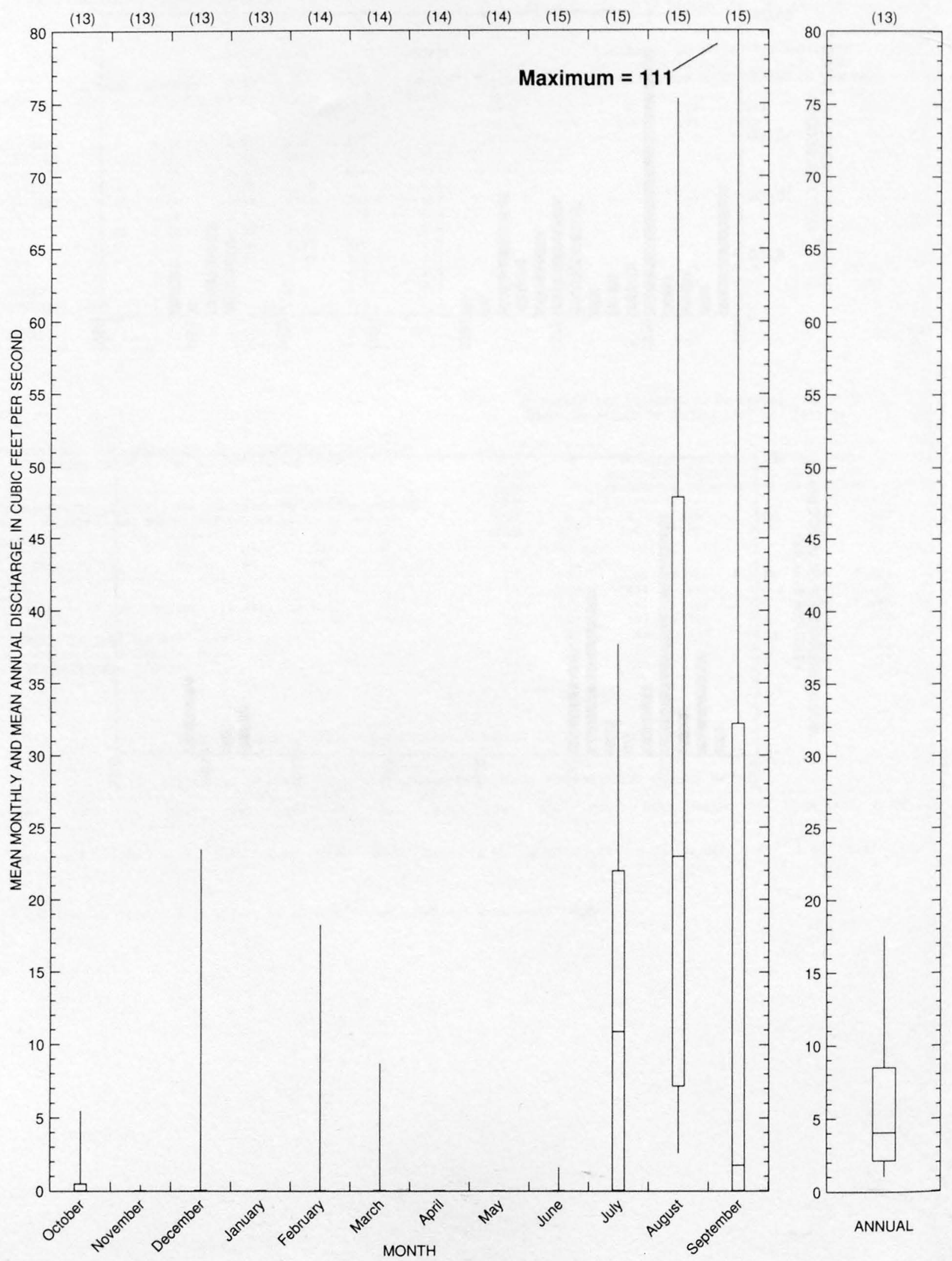


\section{BRAWLEY WASH NEAR THREE POINTS, AZ}

LOCATION.--Lat 32.04'32", long 111'20'17", in $\mathrm{SE}^{1} /{ }_{4} \mathrm{NE}^{1} /{ }_{4} \mathrm{SW}^{1} / 4 \mathrm{sec} .32$, T.15 S., R.10 E., Pima County, Hydrologic Unit 15050302, on right bank downstream side of State Highway 86 bridge, 1.6 mi west of Three Points, and 23 mi west of Tucson.

DRAINAGE AREA.-- $776 \mathrm{mi}^{2}$.

PERIOD OF RECORD.--October 1966 to September 1981 (crest-stage gage) at site 1,000 ft downstream, May 1992 to current year.

GAGE.--Water-stage recorder and crest-stage gages. Elevation of gage is 2,540 ft above sea level, from topographic map. Prior to May 19, 1992 gage was located $1,000 \mathrm{ft}$ downstream from current location.

REMARKS.--Records poor.

EXTREMES FOR PERIOD OF RECORD.--Maximum discharge 13,700 ft $3 / \mathrm{s}$ Sept. 4, 1970, gage height $15.8 \mathrm{ft}$ site and datum then in use; no flow most of each year.

EXTREMES OUTSIDE PERIOD OF RECORD.--Maximum discharge, $19,100 \mathrm{ft}^{3} / \mathrm{s}$ Oct. 1, 1983, from contracted opening measurement of peak flow, gage height $12.07 \mathrm{ft}$ from flood marks.

Annual peak discharges

\begin{tabular}{cccccccc}
\hline $\begin{array}{c}\text { Water } \\
\text { year }\end{array}$ & Date & $\begin{array}{c}\text { Annual peak } \\
\text { discharge } \\
\left(\mathbf{f t}^{3} / \mathbf{s}\right)\end{array}$ & $\begin{array}{c}\text { Discharge } \\
\text { codes }\end{array}$ & $\begin{array}{c}\text { Water } \\
\text { year }\end{array}$ & Date & $\begin{array}{c}\text { Annual peak } \\
\text { discharge } \\
\left(\mathbf{f t}^{3} / \mathbf{s}\right)\end{array}$ & $\begin{array}{c}\text { Discharge } \\
\text { codes }\end{array}$ \\
\hline 1940 & $08-14-40$ & 13,000 & ES,HP & 1976 & $09-05-76$ & 3,600 & \\
1962 & $09-26-62$ & 13,000 & HP & 1977 & $07-26-77$ & 1,400 & ES \\
1966 & $12-24-65$ & 6,600 & DF & 1978 & $10-06-77$ & 7,300 & \\
1967 & $07-17-67$ & 2,250 & & 1979 & $11-25-78$ & 3,600 & \\
1968 & $12-21-67$ & 1,950 & & 1980 & $08-13-80$ & 1,900 & \\
1969 & $07-18-69$ & 2,250 & & 1981 & $07-13-81$ & 4,400 & \\
1970 & $09-04-70$ & 13,700 & & 1984 & $10-01-83$ & 19,100 & \\
1971 & $08-03-71$ & 4,830 & & 1992 & $08-24-92$ & 1,150 & \\
1972 & $07-15-72$ & 3,300 & & 1993 & $08-27-93$ & 10,800 & \\
1973 & $07-14-73$ & 3,060 & & 1994 & $06-28-94$ & 58 & \\
1974 & $08-04-74$ & 6,910 & & 1995 & $01-01-95$ & 2,560 & \\
1975 & $08-00-75$ & 5,750 & & 1996 & $09-03-96$ & 5,500 & \\
\hline
\end{tabular}

${ }^{1}$ Highest since 1955.

Discharge rating table developed October 1996

\begin{tabular}{cccc}
\hline $\begin{array}{c}\text { Gage height } \\
\text { (ft) }\end{array}$ & $\begin{array}{c}\text { Discharge } \\
\left(\mathrm{ft}^{\mathbf{3}} / \mathbf{s}\right)\end{array}$ & $\begin{array}{c}\text { Gage height } \\
(\mathbf{f t})\end{array}$ & $\begin{array}{c}\text { Discharge } \\
\left(\mathrm{ft}^{\mathbf{3}} / \mathbf{s}\right)\end{array}$ \\
\hline 8.0 & 1,160 & 12.0 & 8,970 \\
8.5 & 1,760 & 13.0 & 11,950 \\
9.0 & 2,470 & 14.0 & 15,320 \\
10.0 & 4,220 & 15.0 & 19,070 \\
11.0 & 6,390 & 16.0 & 23,200 \\
\hline
\end{tabular}


Magnitude and probability of instantaneous peak flow based on period of record 1940 ,

$1962,1966-81,1984,1992-96$

\begin{tabular}{|c|c|c|c|c|c|}
\hline \multicolumn{6}{|c|}{$\begin{array}{l}\text { Discharge, in } \mathrm{ft}^{3} / \mathrm{s} \text {, for indicated recurrence interval } \\
\text { in years, and exceedance probablility, in percent }\end{array}$} \\
\hline $\begin{array}{c}2 \\
50 \%\end{array}$ & $\begin{array}{c}5 \\
20 \%\end{array}$ & $\begin{array}{c}10 \\
10 \%\end{array}$ & $\begin{array}{l}25 \\
4 \%\end{array}$ & $\begin{array}{l}50 \dagger \\
2 \%\end{array}$ & $\begin{array}{c}100 \dagger \\
1 \%\end{array}$ \\
\hline 3,570 & 6,630 & 9,260 & 13,300 & 17,000 & 21,100 \\
\hline $\begin{array}{l}\text { Weighted skew } \\
\text { Mean } \\
\text { Standard dev. }\end{array}$ & $\begin{array}{l}(\log s)= \\
(\log s)= \\
(\log s)=\end{array}$ & $\begin{array}{l}0.16 \\
3.56 \\
0.31\end{array}$ & & & \\
\hline
\end{tabular}

Basin characteristics

\begin{tabular}{cccccccc}
\hline $\begin{array}{c}\text { Main } \\
\text { channel } \\
\text { slope } \\
\text { (ft/mi) }\end{array}$ & $\begin{array}{c}\text { Stream } \\
\text { length } \\
\text { (mi) }\end{array}$ & $\begin{array}{c}\text { Mean } \\
\text { basin } \\
\text { elevation } \\
\text { (ft) }\end{array}$ & $\begin{array}{c}\text { Forested } \\
\text { area } \\
\text { (percent) }\end{array}$ & $\begin{array}{c}\text { Soil } \\
\text { index }\end{array}$ & $\begin{array}{c}\text { Mean } \\
\text { annual } \\
\text { precipitation } \\
\text { (in) }\end{array}$ & $\begin{array}{c}\text { R-year } \\
\text { (in) }\end{array}$ & $\begin{array}{c}\text { 24-year } \\
\text { (in) }\end{array}$ \\
\hline 30.8 & 53.8 & 3,710 & 6.0 & 2.0 & 14.6 & 2.2 \\
\hline
\end{tabular}

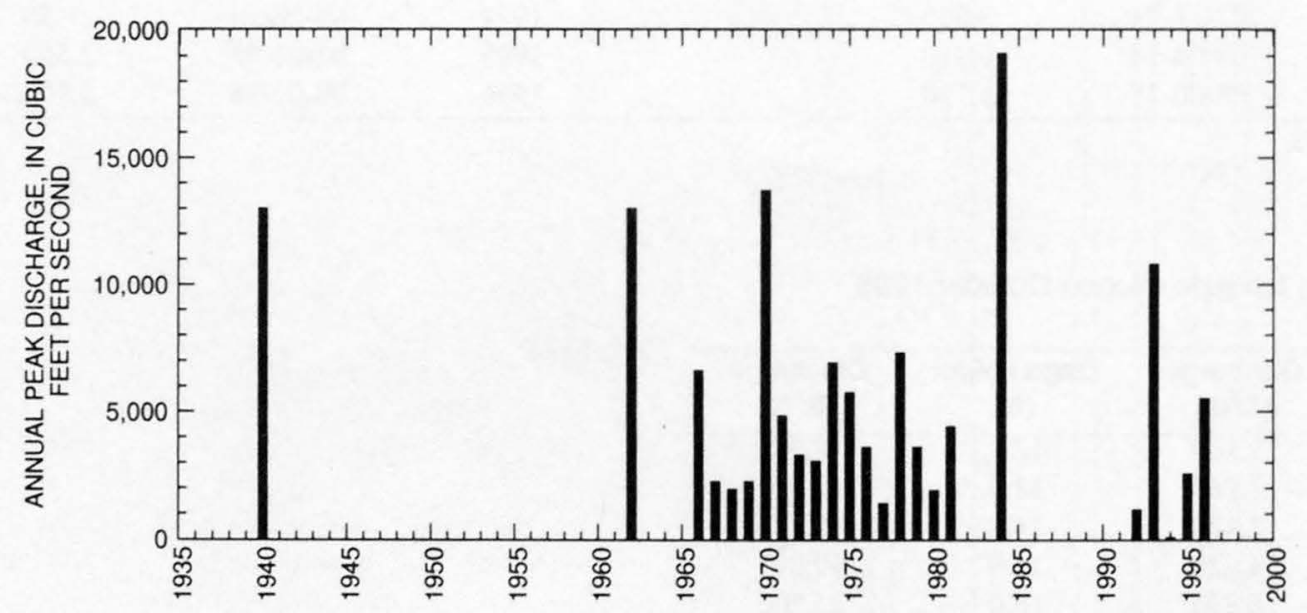


09487100 LITTLE BRAWLEY WASH NEAR THREE POINTS, AZ

LOCATION.--Lat $32^{\circ} 07^{\prime} 25^{\prime \prime}$, long $111^{\circ} 19^{\prime} 45^{\prime \prime}, \mathrm{SE}^{1} /{ }_{4} \mathrm{NW}^{1} / 4$ sec.16, T.15 S., R.10 E., Pima County, Hydrologic Unit $15050304,3.4$ mi north of Three Points.

DRAINAGE AREA.--11.9 $\mathrm{mi}^{2}$.

Annual peak discharges

\begin{tabular}{|c|c|c|c|c|c|c|c|}
\hline $\begin{array}{l}\text { Water } \\
\text { year }\end{array}$ & Date & $\begin{array}{c}\text { Annual peak } \\
\text { discharge } \\
\left(\mathrm{ft}^{3} / \mathrm{s}\right)\end{array}$ & $\begin{array}{l}\text { Discharge } \\
\text { codes }\end{array}$ & $\begin{array}{l}\text { Water } \\
\text { year }\end{array}$ & Date & $\begin{array}{c}\text { Annual peak } \\
\text { discharge } \\
\left(\mathrm{ft}^{3} / \mathbf{s}\right)\end{array}$ & $\begin{array}{l}\text { Discharge } \\
\text { codes }\end{array}$ \\
\hline 1962 & $09-26-62$ & ${ }^{1} 13,800$ & HP & 1975 & $08-00-75$ & 1,150 & \\
\hline 1968 & $08-00-68$ & 450 & & 1976 & $09-25-76$ & 2,230 & \\
\hline 1969 & $08-16-69$ & 388 & & 1977 & $00-00-77$ & 0 & \\
\hline 1970 & $00-00-70$ & 700 & & 1978 & $10-06-77$ & 2,500 & \\
\hline 1971 & $00-00-71$ & 1,440 & & 1979 & $08-15-79$ & 566 & \\
\hline 1972 & $00-00-72$ & 310 & & 1980 & $06-30-80$ & 893 & \\
\hline 1973 & $00-00-73$ & 1,500 & & 1981 & $09-05-81$ & 1,150 & \\
\hline 1974 & $00-00-74$ & 335 & & & & & \\
\hline
\end{tabular}

${ }^{1}$ Highest since 1462.

Magnitude and probability of instantaneous peak flow based on period of record 1962, 1968-81

\begin{tabular}{|c|c|c|c|c|c|}
\hline \multicolumn{6}{|c|}{$\begin{array}{l}\text { Discharge, in } \mathrm{ft}^{3} / \mathrm{s} \text {, for indicated recurrence interval } \\
\text { in years, and exceedance probablility, in percent }\end{array}$} \\
\hline 2 & 5 & 10 & 25 & $50 t$ & $100 t$ \\
\hline $50 \%$ & $20 \%$ & $10 \%$ & $4 \%$ & $2 \%$ & $1 \%$ \\
\hline 777 & 1,450 & 2,010 & 2,860 & 3,600 & 4,440 \\
\hline Weighted skew & $(\log s)=$ & 0.05 & & & \\
\hline Mean & $(\log s)=$ & 2.89 & & & \\
\hline Standard dev. & $(\log s)=$ & 0.32 & & & \\
\hline
\end{tabular}

fReliability of values in column is uncertain, and potential errors are large.

Basin characteristics

\begin{tabular}{cccccccc}
\hline $\begin{array}{c}\text { Main } \\
\text { channel } \\
\text { slope } \\
(\mathrm{ft} / \mathrm{mi})\end{array}$ & $\begin{array}{c}\text { Stream } \\
\text { length } \\
(\mathrm{mi})\end{array}$ & $\begin{array}{c}\text { Mean } \\
\text { basin } \\
\text { elevation } \\
(\mathrm{ft})\end{array}$ & $\begin{array}{c}\text { Forested } \\
\text { area } \\
\text { (percent) }\end{array}$ & $\begin{array}{c}\text { Soil } \\
\text { index }\end{array}$ & $\begin{array}{c}\text { Mean } \\
\text { annual } \\
\text { precipitation } \\
\text { (in) }\end{array}$ & $\begin{array}{c}\text { R-year } \\
\text { (in) }\end{array}$ & $\begin{array}{c}50 \text {-year } \\
\text { (in) }\end{array}$ \\
\hline 112 & 5.9 & 2,800 & 0.0 & 3.0 & 13.0 & 2.1 \\
\hline
\end{tabular}


09487100 LITTLE BRAWLEY WASH NEAR THREE POINTS, AZ--Continued

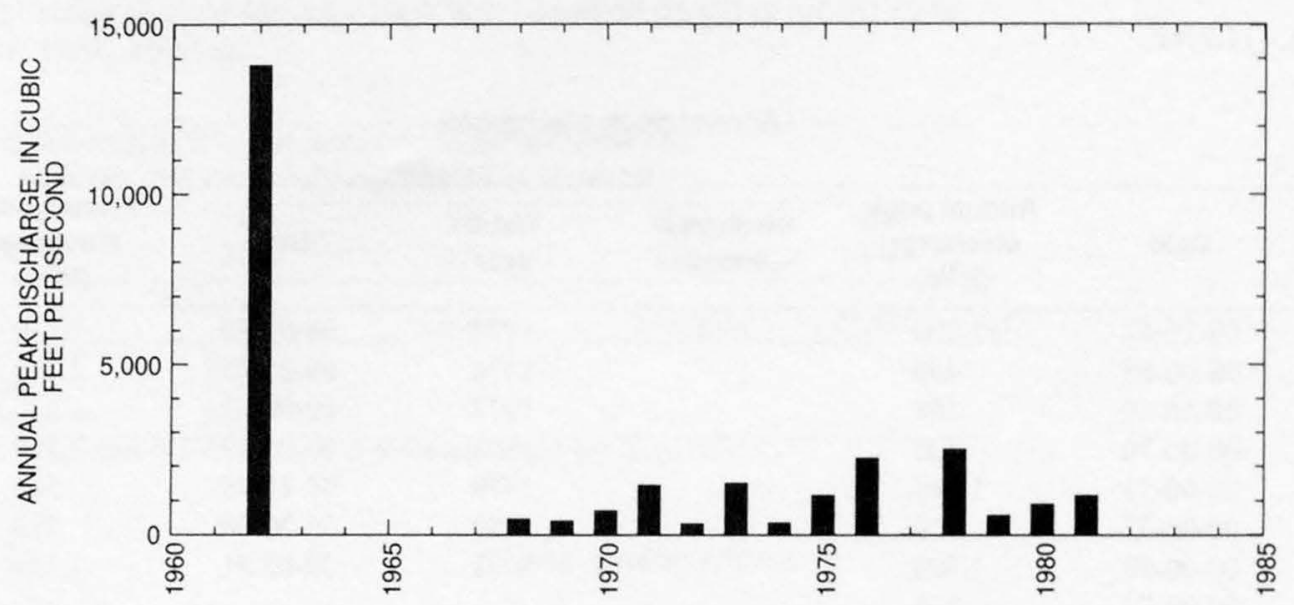


09487140 SAN JOAQUIN WASH NEAR TUCSON, AZ

LOCATION.--Lat $32^{\circ} 10^{\prime} 07^{\prime \prime}$, long $111^{\circ} 07^{\prime} 58^{\prime \prime}, \mathrm{NE}^{1} / \mathrm{S}^{\mathrm{S}} \mathrm{S}^{1} / 4 \mathrm{sec} .32$, T.14 S., R.12 E., Pima County, Hydrologic Unit 15050301 , 1.1 mi northwest of the intersection of San Joaquin Road and the Tucson-Ajo Highway.

DRAINAGE AREA.--0.45 $\mathrm{mi}^{2}$.

Annual peak discharges

\begin{tabular}{cccccrr}
\hline $\begin{array}{l}\text { Water } \\
\text { year }\end{array}$ & Date & $\begin{array}{c}\text { Annual peak } \\
\text { discharge } \\
\left(\mathbf{f t}^{3} / \mathbf{s}\right)\end{array}$ & $\begin{array}{c}\text { Discharge } \\
\text { codes }\end{array}$ & $\begin{array}{c}\text { Water } \\
\text { year }\end{array}$ & $\begin{array}{c}\text { Annual peak } \\
\text { discharge } \\
\left(\mathbf{f t}^{3} / \mathbf{s}\right)\end{array}$ \\
\hline 1969 & $08-02-69$ & 102 & & 1976 & $09-25-76$ & 480 \\
1970 & $07-19-70$ & 487 & & 1977 & $07-22-77$ & 220 \\
1971 & $08-17-71$ & 370 & & 1978 & $01-15-78$ & 127 \\
1972 & $10-17-71$ & 190 & & 1979 & $11-24-78$ & 230 \\
1973 & $07-00-73$ & 115 & & 1980 & $00-00-80$ & 0 \\
1974 & $07-00-74$ & 135 & & 1981 & $07-28-81$ & 520 \\
1975 & $08-20-75$ & 60 & & & & \\
\hline
\end{tabular}

Magnitude and probability of instantaneous peak flow based on period of record 1969-81

\begin{tabular}{|c|c|c|c|c|c|}
\hline \multicolumn{6}{|c|}{$\begin{array}{l}\text { Discharge, in } \mathrm{ft}^{3} / \mathrm{s} \text {, for indicated recurrence interval } \\
\text { in years, and exceedance probablility, in percent }\end{array}$} \\
\hline 2 & 5 & 10 & 25 & $50 t$ & $100 \dagger$ \\
\hline $50 \%$ & $20 \%$ & $10 \%$ & $4 \%$ & $2 \%$ & $1 \%$ \\
\hline 191 & 356 & 486 & 672 & 824 & 987 \\
\hline Weighted skew & $(\log s)=$ & -0.17 & & & \\
\hline Mean & $(\log s)=$ & 2.27 & & & \\
\hline Standard dev. & $(\log s)=$ & 0.33 & & & \\
\hline
\end{tabular}

†Reliability of values in column is uncertain, and potential errors are large.

Basin characteristics

\begin{tabular}{cccccccc}
\hline $\begin{array}{c}\text { Main } \\
\text { channel } \\
\text { slope } \\
(\mathrm{ft} / \mathrm{mi})\end{array}$ & $\begin{array}{c}\text { Stream } \\
\text { length } \\
(\mathrm{mi})\end{array}$ & $\begin{array}{c}\text { Mean } \\
\text { basin } \\
\text { elevation } \\
(\mathrm{ft})\end{array}$ & $\begin{array}{c}\text { Forested } \\
\text { area } \\
\text { (percent) }\end{array}$ & $\begin{array}{c}\text { Soil } \\
\text { index }\end{array}$ & $\begin{array}{c}\text { Mean } \\
\text { annual } \\
\text { precipitation } \\
\text { (in) }\end{array}$ & $\begin{array}{c}\text { Rainfall intensity, 24-hour } \\
\text { (in) }\end{array}$ & $\begin{array}{c}50 \text {-year } \\
\text { (in) }\end{array}$ \\
\hline 69.3 & 2.5 & 2,530 & 0.0 & 3.0 & 11.0 & 2.1 \\
\hline
\end{tabular}

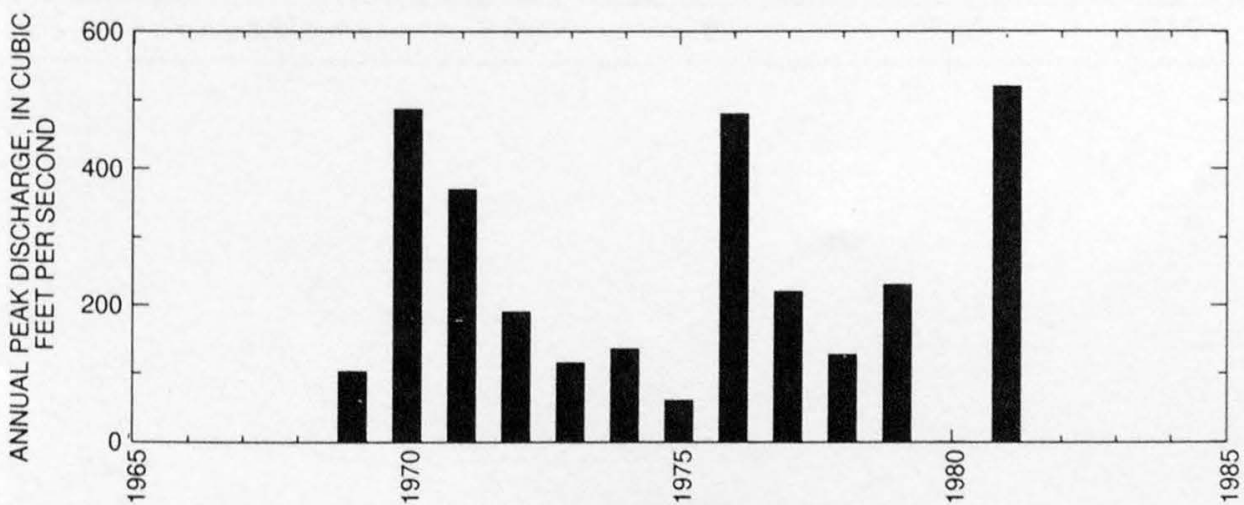




\section{LOS ROBLES WASH NEAR MARANA, AZ}

LOCATION.--Lat $32^{\circ} 26^{\prime} 16^{\prime \prime}$, long $111^{\circ} 18^{\prime} 13^{\prime \prime}, \mathrm{SE}^{1} / \mathrm{SE}^{1} / 4 \mathrm{sec} .27, \mathrm{~T} .11$ S., R.10 E., Pima County, Hydrologic Unit 15050304 , at Trico Road, 0.75 mi downstream from confluence of Brawley Wash and China Draw, 3 mi upstream Blanco Wash, and 5 mi southwest of Marana.

DRAINAGE AREA.--1,170 $\mathrm{mi}^{2}$.

Annual peak discharges

\begin{tabular}{|c|c|c|c|c|c|c|c|}
\hline $\begin{array}{l}\text { Water } \\
\text { year }\end{array}$ & Date & $\begin{array}{c}\text { Annual peak } \\
\text { discharge } \\
\left(\mathrm{ft}^{3} / \mathrm{s}\right)\end{array}$ & $\begin{array}{l}\text { Discharge } \\
\text { codes }\end{array}$ & $\begin{array}{l}\text { Water } \\
\text { year }\end{array}$ & Date & $\begin{array}{c}\text { Annual peak } \\
\text { discharge } \\
\left(\mathrm{ft}^{3} / \mathrm{s}\right)\end{array}$ & $\begin{array}{l}\text { Discharge } \\
\text { codes }\end{array}$ \\
\hline 1962 & $09-26-62$ & $1_{32,000}$ & HP & 1973 & $10-19-72$ & 1,910 & \\
\hline 1966 & $00-00-66$ & 2,500 & & 1974 & $07-08-74$ & 630 & \\
\hline 1967 & $07-1-767$ & 80 & & 1975 & $08-09-75$ & 260 & \\
\hline 1968 & $12-20-67$ & 1,000 & & 1976 & $09-25-76$ & 1,950 & \\
\hline 1969 & 09-09-69 & 105 & & 1977 & $00-00-77$ & 900 & \\
\hline 1970 & $09-05-70$ & 4,490 & & 1978 & $10-06-77$ & 2,400 & \\
\hline 1971 & $08-18-71$ & 1,770 & & 1984 & $10-02-83$ & ${ }^{2} 12,500$ & HP \\
\hline 1972 & $08-00-72$ & 2,750 & & & & & \\
\hline
\end{tabular}

\footnotetext{
${ }^{1}$ Highest since 1885.
}

${ }^{2}$ Highest since 1962.

Magnitude and probability of instantaneous peak flow based on period of record 1962 , 1966-78, 1984

\begin{tabular}{|c|c|c|c|c|c|}
\hline \multicolumn{6}{|c|}{$\begin{array}{l}\text { Discharge, in } \mathrm{ft}^{3} / \mathrm{s} \text {, for indicated recurrence interval } \\
\text { in years, and exceedance probablility, in percent }\end{array}$} \\
\hline 2 & 5 & 10 & 25 & $50 t$ & $100 t$ \\
\hline $50 \%$ & $20 \%$ & $10 \%$ & $4 \%$ & $2 \%$ & $1 \%$ \\
\hline 1,140 & 3,090 & 4,900 & 7,670 & 10,000 & 12,500 \\
\hline Weighted skew & $(\log s)=$ & -0.52 & & & \\
\hline Mean & $(\log s)=$ & 3.01 & & & \\
\hline Standard dev. & $(\log s)=$ & 0.56 & & & \\
\hline
\end{tabular}

Basin characteristics

\begin{tabular}{cccccccc}
\hline $\begin{array}{c}\text { Main } \\
\text { channel } \\
\text { slope } \\
\text { (ft/mi) }\end{array}$ & $\begin{array}{c}\text { Stream } \\
\text { length } \\
\text { (mi) }\end{array}$ & $\begin{array}{c}\text { Mean } \\
\text { basin } \\
\text { elevation } \\
\text { (ft) }\end{array}$ & $\begin{array}{c}\text { Forested } \\
\text { area } \\
\text { (percent) }\end{array}$ & $\begin{array}{c}\text { Soil } \\
\text { index }\end{array}$ & $\begin{array}{c}\text { Mean } \\
\text { annual } \\
\text { precipitation } \\
\text { (in) }\end{array}$ & $\begin{array}{c}\text { R-year } \\
\text { (in) }\end{array}$ & $\begin{array}{c}50 \text {-year } \\
\text { (in) }\end{array}$ \\
\hline 25.9 & 77.2 & 3,350 & 4.0 & 2.0 & 11.8 & 2.2
\end{tabular}


09487250 LOS ROBLES WASH NEAR MARANA, AZ--Continued

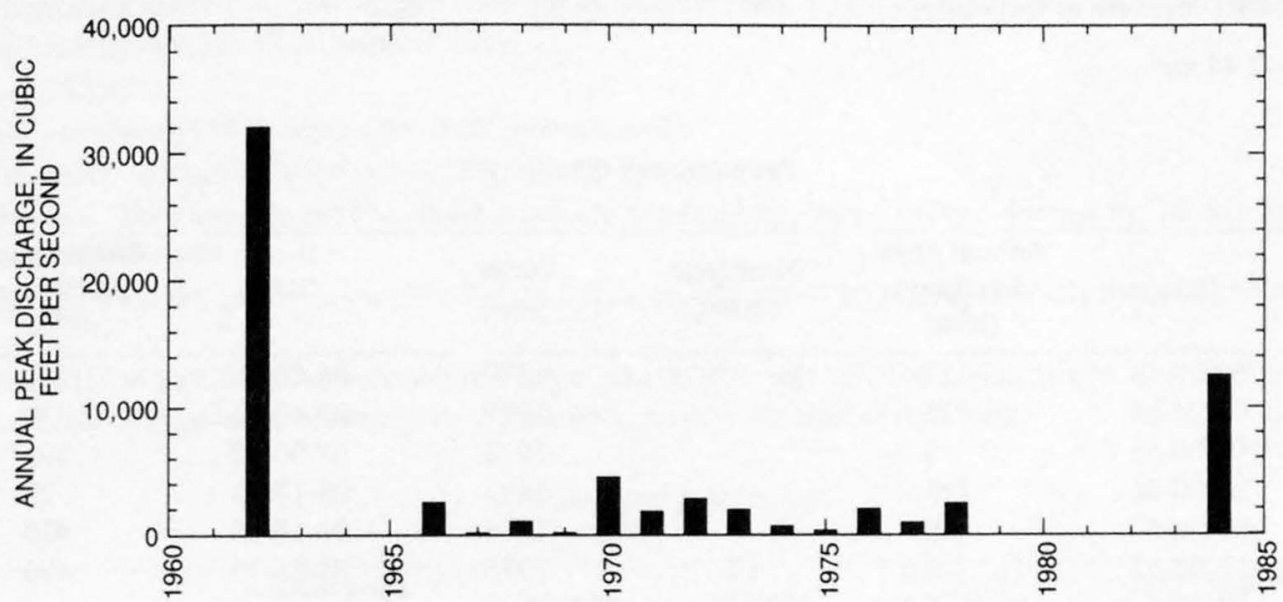


09487400 QUIJOTOA WASH TRIBUTARY NEAR QUIJOTOA, AZ

LOCATION.--Lat $32^{\circ} 10^{\prime} 25^{\prime \prime}$, long $112^{\circ} 06^{\prime} 30^{\prime \prime}$, Pima County, Hydrologic Unit 15050306, on the Papago Indian Reservation, at the Quijotoa-Cas

Grande Road, and 1.1 mi north of Quijotoa.

DRAINAGE AREA.--2.44 $\mathrm{mi}^{2}$.

Annual peak discharges

\begin{tabular}{|c|c|c|c|c|c|c|c|}
\hline $\begin{array}{l}\text { Water } \\
\text { year }\end{array}$ & Date & $\begin{array}{c}\text { Annual peak } \\
\text { discharge } \\
\left(\mathrm{ft}^{3} / \mathrm{s}\right)\end{array}$ & $\begin{array}{l}\text { Discharge } \\
\text { codes }\end{array}$ & $\begin{array}{l}\text { Water } \\
\text { year }\end{array}$ & Date & $\begin{array}{c}\text { Annual peak } \\
\text { discharge } \\
\left(\mathrm{ft}^{3} / \mathrm{s}\right)\end{array}$ & $\begin{array}{l}\text { Discharge } \\
\text { codes }\end{array}$ \\
\hline 1963 & $00-00-63$ & 127 & & 1970 & $08-00-70$ & 200 & \\
\hline 1964 & $07-24-64$ & 715 & & 1971 & $00-00-71$ & 370 & \\
\hline 1965 & $00-00-65$ & 0 & & 1972 & $07-00-72$ & 190 & \\
\hline 1966 & $08-00-66$ & 225 & & 1973 & $08-17-73$ & 20 & ES \\
\hline 1967 & $00-00-67$ & 0 & & 1974 & $09-26-74$ & 420 & \\
\hline 1968 & $12-00-67$ & 5.0 & LT & 1975 & $08-26-75$ & 470 & \\
\hline 1969 & $08-05-69$ & 5.0 & LT & & & & \\
\hline
\end{tabular}

Magnitude and probability of instantaneous peak flow based on period of record 1963-75

\begin{tabular}{cccccc}
\hline \multicolumn{5}{c}{$\begin{array}{l}\text { Discharge, in } \mathrm{ft}^{3} / \mathbf{s} \text {, for indicated recurrence interval } \\
\text { in years, and exceedance probablility, in percent }\end{array}$} \\
\hline $\mathbf{2}$ & $\mathbf{5}$ & $\mathbf{1 0}$ & $\mathbf{2 5}$ & $\mathbf{5 0 \dagger}$ & $\mathbf{1 0 0 \dagger}$ \\
$\mathbf{5 0 \%}$ & $\mathbf{2 0 \%}$ & $\mathbf{1 0 \%}$ & $\mathbf{4} \%$ & $\mathbf{2 \%}$ & $\mathbf{1 \%}$ \\
\hline 168 & 370 & 546 & 813 & 1,040 & 1,300 \\
Weighted skew & $\begin{array}{l}\text { (logs) } \\
\text { Mean }\end{array}$ & $\begin{array}{c}\text { (logs) } \\
\text { (logs) }\end{array}$ & 2.21 & & \\
Standard dev. & 0.42 & & \\
F Reliability of values in column is uncertain, and potential errors are large.
\end{tabular}

f Reliability of values in column is uncertain, and potential errors are large.

Basin characteristics

\begin{tabular}{cccccccc}
\hline $\begin{array}{c}\text { Main } \\
\text { channel } \\
\text { slope } \\
(\mathrm{ft} / \mathrm{mi})\end{array}$ & $\begin{array}{c}\text { Stream } \\
\text { length } \\
\text { (mi) }\end{array}$ & $\begin{array}{c}\text { Mean } \\
\text { basin } \\
\text { elevation } \\
\text { (ft) }\end{array}$ & $\begin{array}{c}\text { Forested } \\
\text { area } \\
\text { (percent) }\end{array}$ & $\begin{array}{c}\text { Soil } \\
\text { index }\end{array}$ & $\begin{array}{c}\text { Mean } \\
\text { annual } \\
\text { precipitation } \\
\text { (in) }\end{array}$ & $\begin{array}{c}\text { R-year } \\
\text { (in) }\end{array}$ & $\begin{array}{c}50 \text {-year } \\
\text { (in) }\end{array}$ \\
\hline 88.9 & 3.6 & 2,800 & 0.0 & 1.0 & 10.1 & 2.1 \\
\hline
\end{tabular}

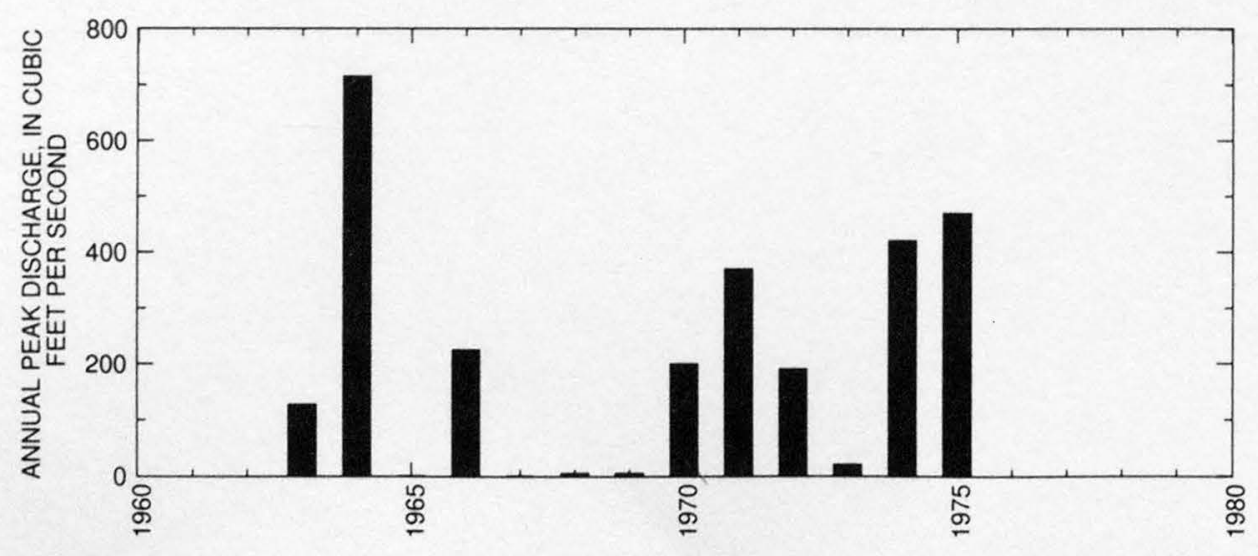




\section{SANTA ROSA WASH NEAR VAIVA VO, NEAR SELLS, AZ}

LOCATION.--Lat 32 40'03", long $111^{\circ} 55^{\prime} 39^{\prime \prime}$, in SW $1 / 4 \mathrm{SW}^{1} / 4$ sec.2, T.9 S., R.4 E., Pinal County, Hydrologic Unit 15050306 , in Papago Indian Reservation, on right bank about $1 \mathrm{mi}$ downstream from Tat Momolikot Dam, $3.3 \mathrm{mi}$ south of Vaiva Vo, 10 mi southwest of Chuichu, $12 \mathrm{mi}$ downstream from Gu Komelik and 52 mi north of Sells.

DRAINAGE AREA.--1,782 $\mathrm{mi}^{2}$.

PERIOD OF RECORD.--October 1954 to September 1980 (discontinued).

GAGE.--Water-stage recorder. Altitude of gage is $1,470 \mathrm{ft}$, from topographic map.

REMARKS.--Records poor. Beginning July 1974, flood flows are regulated by Lake St. Clair, formed by Tat Momolikot Dam, about 1 mi upstream--total capacity, 384,000 acre-ft.

AVERAGE DISCHARGE.--20 years, (water years 1955-74), $14.0 \mathrm{ft}^{3} / \mathrm{s}, 10,140 \mathrm{acre}-\mathrm{ft} / \mathrm{yr}$; median of yearly mean discharges, $8.1 \mathrm{ft} 3 / \mathrm{s}, 5,900$ acre$\mathrm{ft} / \mathrm{yr}$.

EXTREMES FOR PERIOD OF RECORD.--Maximum discharge, $53,100 \mathrm{ft}^{3} / \mathrm{s}$ Sept. 27, 1962, gage height, $16.9 \mathrm{ft}$, from rating curve extended above $840 \mathrm{ft}^{3} / \mathrm{s}$ on basis of slope-area measurement of peak flow; no flow for most of each year.

\section{Annual peak discharges}

\begin{tabular}{|c|c|c|c|c|c|c|c|}
\hline $\begin{array}{l}\text { Water } \\
\text { year }\end{array}$ & Date & $\begin{array}{c}\text { Annual peak } \\
\text { discharge } \\
\left(\mathrm{ft}^{3} / \mathrm{s}\right)\end{array}$ & $\begin{array}{l}\text { Discharge } \\
\text { codes }\end{array}$ & $\begin{array}{l}\text { Water } \\
\text { year }\end{array}$ & Date & $\begin{array}{c}\text { Annual peak } \\
\text { discharge } \\
\left(\mathrm{ft}^{3} / \mathrm{s}\right)\end{array}$ & $\begin{array}{c}\text { Discharge } \\
\text { codes }\end{array}$ \\
\hline 1955 & $08-08-55$ & 1,150 & & 1969 & $08-08-69$ & 514 & \\
\hline 1956 & $07-24-56$ & 740 & & 1970 & $08-10-70$ & 865 & \\
\hline 1957 & $08-12-57$ & 492 & & 1971 & $08-04-71$ & 6,110 & \\
\hline 1958 & $11-01-57$ & 10,000 & & 1972 & $08-14-72$ & 410 & \\
\hline 1959 & $07-13-59$ & 4,120 & & 1973 & $10-21-72$ & 762 & \\
\hline 1960 & $07-30-60$ & 805 & & 1974 & $08-02-74$ & 364 & KR \\
\hline 1961 & $07-27-61$ & 892 & & 1975 & $07-17-75$ & 580 & KR \\
\hline 1962 & $09-27-62$ & ${ }^{1} 53,100$ & & 1976 & $09-25-76$ & 390 & $\mathrm{KR}$ \\
\hline 1963 & $09-14-63$ & 4,180 & & 1977 & $07-17-77$ & 291 & KR \\
\hline 1964 & $07-25-64$ & 6,760 & & 1978 & $02-13-78$ & 66 & KR \\
\hline 1965 & $09-04-65$ & 433 & & 1979 & $01-17-79$ & 51 & KR \\
\hline 1966 & $09-14-66$ & 1,820 & & 1980 & $08-24-80$ & 105 & KR \\
\hline 1967 & $06-26-67$ & 302 & & 1984 & $10-04-83$ & 1,890 & HP,KR \\
\hline 1968 & $07-28-68$ & 840 & & & & & \\
\hline
\end{tabular}

'Highest since 1885

Basin characteristics

\begin{tabular}{cccccccc}
\hline $\begin{array}{c}\text { Main } \\
\text { channel } \\
\text { slope } \\
(\mathrm{ft} / \mathrm{mi})\end{array}$ & $\begin{array}{c}\text { Stream } \\
\text { length } \\
\text { (mi) }\end{array}$ & $\begin{array}{c}\text { Mean } \\
\text { basin } \\
\text { elevation } \\
\text { (ft) }\end{array}$ & $\begin{array}{c}\text { Forested } \\
\text { area } \\
\text { (percent) }\end{array}$ & $\begin{array}{c}\text { Soil } \\
\text { index }\end{array}$ & $\begin{array}{c}\text { Mean } \\
\text { annual } \\
\text { precipitation } \\
\text { (in) }\end{array}$ & $\begin{array}{c}\text { Rainfall intensity, 24-hour } \\
\text { (in) }\end{array}$ & $\begin{array}{c}50 \text {-year } \\
\text { (in) }\end{array}$ \\
\hline 25.5 & 55.0 & 2,340 & 0.3 & 2.0 & 10.2 & 1.9 \\
\hline
\end{tabular}


09488500 SANTA ROSA WASH NEAR VAIVA VO, NEAR SELLS, AZ--Continued

MEAN MONTHLY AND ANNUAL DISCHARGES $1955 \cdot 80$

\begin{tabular}{|c|c|c|c|c|c|c|}
\hline MONTH & $\begin{array}{l}\text { MAXIMUM } \\
(\text { FT3/S) }\end{array}$ & $\begin{array}{l}\text { MINIMUM } \\
\text { (FT3/S) }\end{array}$ & $\underset{\text { MEAN }}{\operatorname{MT} 3 / \mathrm{S})}$ & $\begin{array}{l}\text { STAN- } \\
\text { DARD } \\
\text { DEVIA. } \\
\text { TION } \\
\text { (FT3/S) }\end{array}$ & $\begin{array}{l}\text { COEFFI- } \\
\text { CIENT OF } \\
\text { VARI- } \\
\text { ATION }\end{array}$ & $\begin{array}{c}\text { PERCENT } \\
\text { OF } \\
\text { ANNUAL } \\
\text { RUNOFF }\end{array}$ \\
\hline OCTOBER & 84 & 0.00 & 7.1 & 18 & 2.6 & 5.5 \\
\hline NOVBMBER & 150 & 0.00 & 6.6 & 29 & 4.4 & 5.1 \\
\hline DECEMBER & 89 & 0.00 & 7.5 & 22 & 3.0 & 5.8 \\
\hline JANUARY & 8.5 & 0.00 & 0.74 & 2.3 & 3.1 & 0.6 \\
\hline FEBRUARY & 92 & 0.00 & 5.9 & 21 & 3.5 & 4.6 \\
\hline MARCH & 40 & 0.00 & 1.8 & 7.8 & 4.3 & 1.4 \\
\hline APRIL & 1.9 & 0.00 & 0.07 & 0.37 & 4.9 & 0.1 \\
\hline MAY & 0.81 & 0.00 & 0.03 & 0.16 & 4.8 & 0.0 \\
\hline JUNE & 4.8 & 0.00 & 0.60 & 1.5 & 2.6 & 0.5 \\
\hline JULY & 129 & 0.00 & 19 & 34 & 1.8 & 14.5 \\
\hline AUGUST & 236 & 0.00 & 41 & 68 & 1.7 & 31.5 \\
\hline SEPTEMBER & 838 & 0.00 & 39 & 163 & 4.1 & 30.5 \\
\hline ANNUAL & 69 & 0.01 & 11 & 16 & 1.5 & 100 \\
\hline
\end{tabular}

MAGNITUDE AND PROBABILITY OF INSTANTANEOUS PEAK FLOW BASED ON PERIOD OF RECORD $1955 \cdot 80,1984$

DISCHARGE, IN FT3/S, FOR INDICATED RECURRENCE INTERVAL IN YEARS, AND EXCEEDANCE PROBABILITY, IN PERCENT

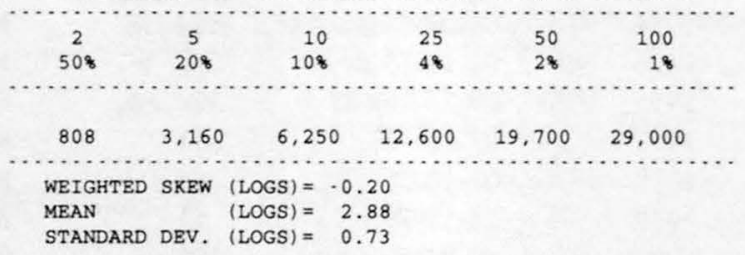

MAGNITUDE AND PROBABILITY OE ANNUAL LOW FLOW BASED ON PERIOD OF RECORD $1956-80$

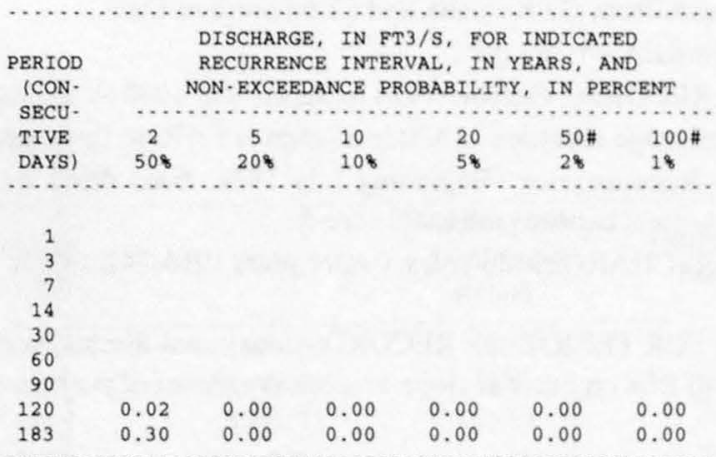

MAGNITUDE AND PROBABILITY OF ANNUAL HIGH FLOW BASED ON PERIOD OF RECORD 1955.80

\begin{tabular}{|c|c|c|c|c|c|c|}
\hline \multirow{3}{*}{$\begin{array}{l}\text { PERIOD } \\
\text { ICON- } \\
\text { SECU- }\end{array}$} & & \multicolumn{5}{|c|}{ DISCHARGE, IN FT $3 / \mathrm{S}$, FOR INDICATED } \\
\hline & & EXCEEDA & $C E$ PROI & BILITY, & IN PER & ENT \\
\hline & $\cdots$ & $\ldots \ldots$ & $\ldots \ldots$ & $\ldots \ldots \ldots$ & ....... & $\ldots \ldots$ \\
\hline TIVE & 2 & 5 & 10 & 25 & $50 \#$ & $100 \#$ \\
\hline DAYS) & 508 & 208 & 108 & 48 & 28 & 18 \\
\hline 1 & 268 & 1,560 & 3,620 & 8,370 & 13,900 & 21,600 \\
\hline 3 & 149 & 892 & 2,000 & 4,330 & 6,810 & 9,910 \\
\hline 7 & 75 & 444 & 982 & 2,080 & 3,200 & 4,580 \\
\hline 15 & 43 & 263 & 587 & 1,240 & 1,900 & 2,700 \\
\hline 30 & 26 & 159 & 350 & 728 & 1,100 & 1,550 \\
\hline 60 & 15 & 91 & 198 & 403 & 603 & 835 \\
\hline 90 & 11 & 64 & 135 & 268 & 394 & 535 \\
\hline
\end{tabular}

DURATION TABLE OF DAILY MEAN FLOW FOR PERIOD OF RECORD $1955-80$

DISCHARGE, IN FT3/S, WHICH WAS EQUALED OR EXCEEDED FOR INDICATED PERCENT OF TIME

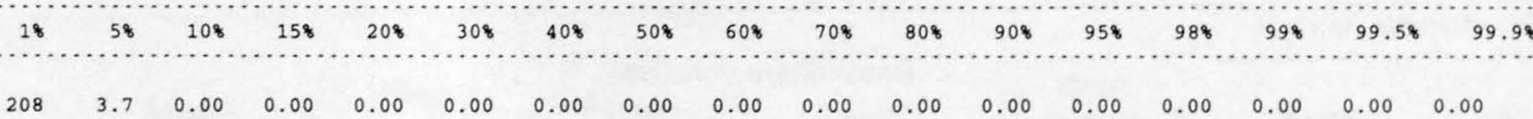

\# Reliability of values in column is uncertain, and potential errors are large. 
09488500 SANTA ROSA WASH NEAR VAIVA VO, NEAR SELLS, AZ--Continued
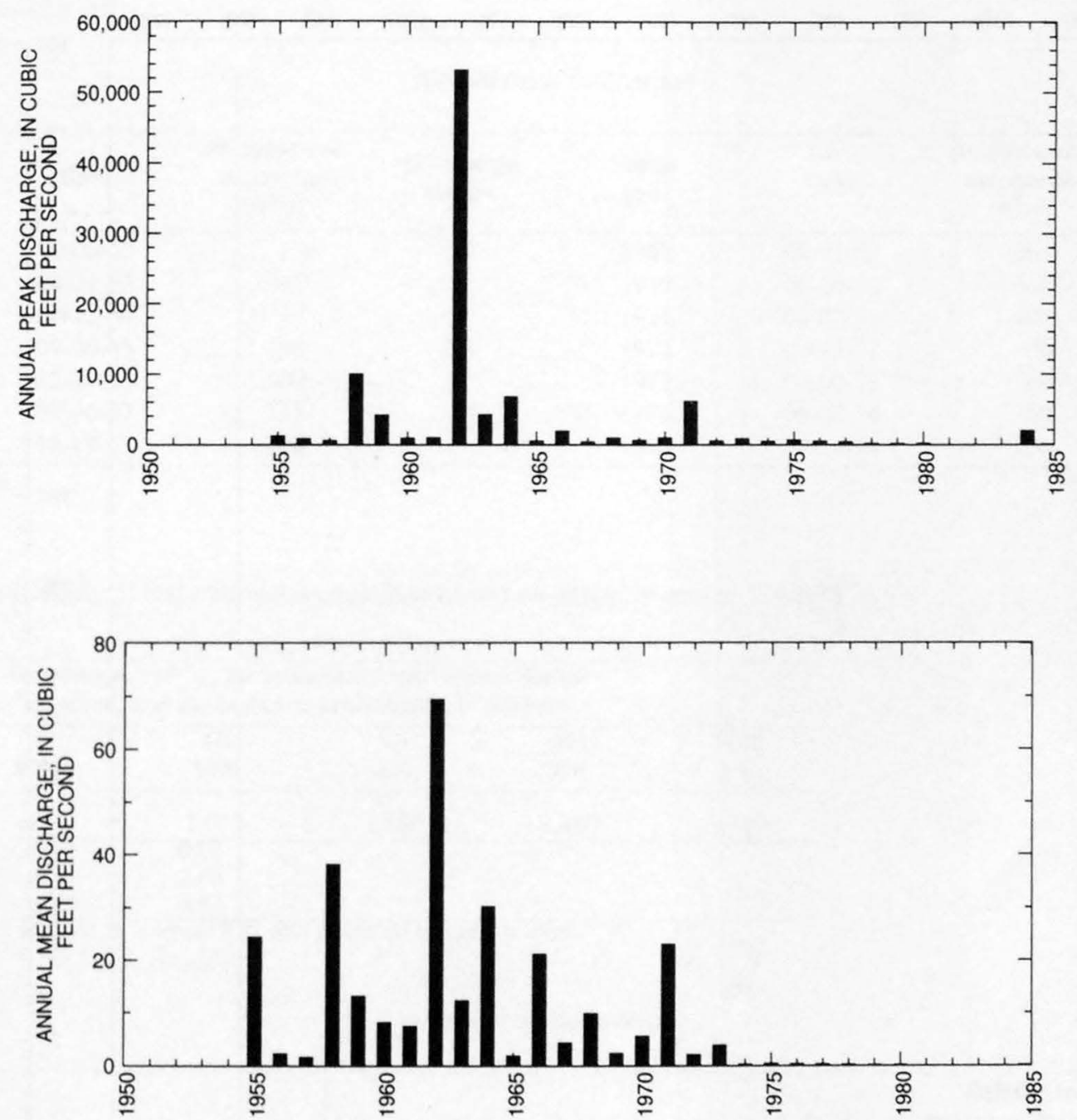
538

GILA RIVER BASIN

09488500 SANTA ROSA WASH NEAR VAIVA VO, NEAR SELLS, AZ--Continued
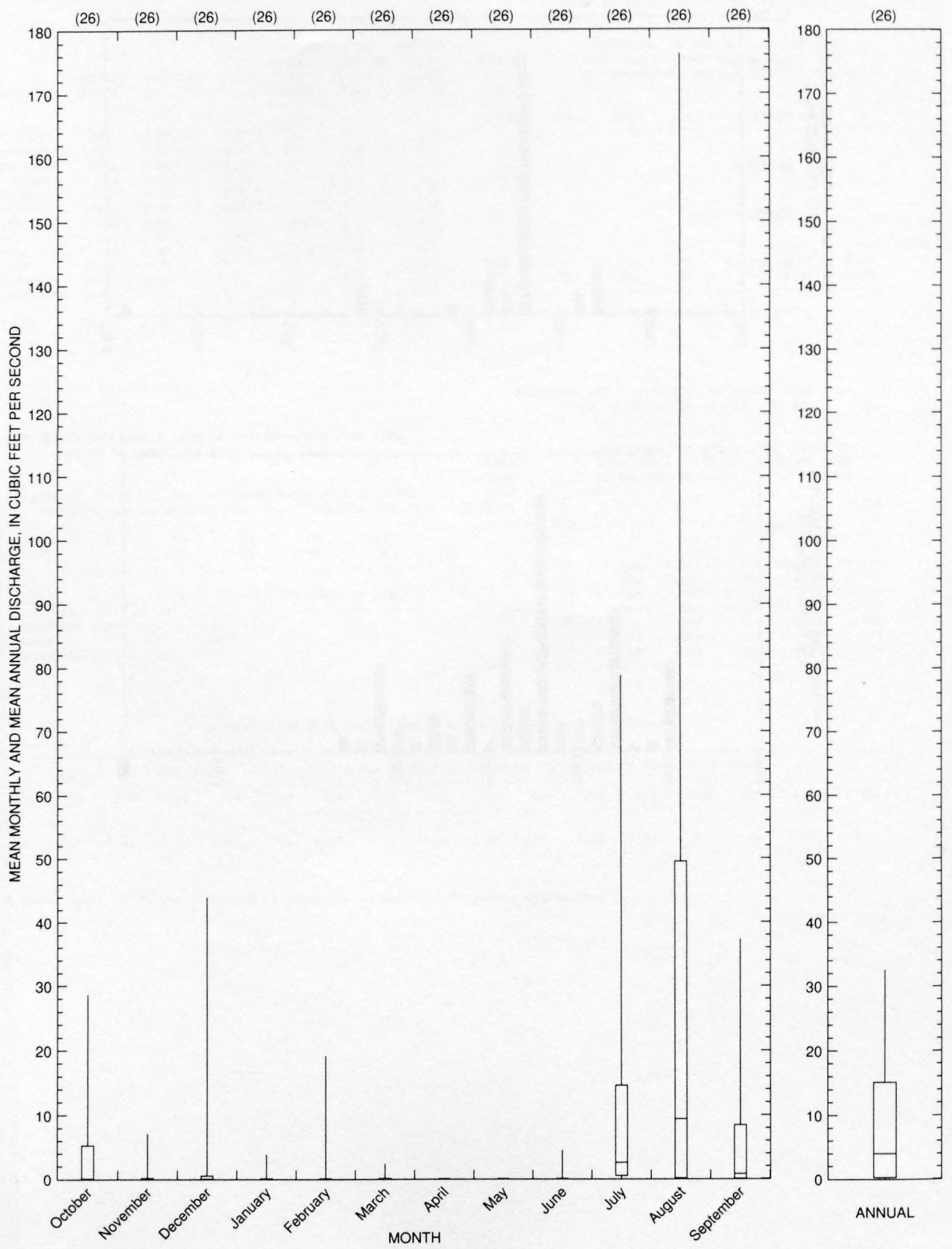
09488600 SILVER REEF WASH NEAR CASA GRANDE, AZ

LOCATION.--Lat $32^{\circ} 40^{\prime} 56^{\prime \prime}$, long $111^{\circ} 50^{\prime} 03^{\prime \prime}, \mathrm{SW}^{\frac{1}{1}} / \mathrm{SE}^{1} / 4$ sec.34, T.8 S., R.5 E., Pinal County, Hydrologic Unit 15050306 , at Quijotoa-Casa Grande Road, 14 mi southwest of Casa Grande.

DRAINAGE AREA.-- $12.8 \mathrm{mi}^{2}$.

Annual peak discharges

\begin{tabular}{rrrrrrrr}
\hline $\begin{array}{c}\text { Water } \\
\text { year }\end{array}$ & Date & $\begin{array}{c}\text { Annual peak } \\
\text { discharge } \\
\left(\mathbf{f t}^{3} \mathbf{s}\right)\end{array}$ & $\begin{array}{c}\text { Discharge } \\
\text { codes }\end{array}$ & $\begin{array}{c}\text { Water } \\
\text { year }\end{array}$ & Date & $\begin{array}{c}\text { Annual peak } \\
\text { discharge } \\
\left(\mathbf{f t}^{3} / \mathbf{s}\right)\end{array}$ & $\begin{array}{c}\text { Discharge } \\
\text { codes }\end{array}$ \\
\hline 1950 & $00-00-50$ & $\left({ }^{1}\right)$ & HP & 1969 & $08-11-69$ & 165 & \\
1963 & $08-21-63$ & 662 & & 1970 & $00-00-70$ & 630 & \\
1964 & $08-12-64$ & 1,170 & & 1971 & $08-03-71$ & 1,400 & \\
1965 & $09-00-65$ & 100 & ES & 1972 & $08-12-72$ & 90 & \\
1966 & $12-00-65$ & 600 & & 1973 & $00-00-73$ & 100 & ES \\
1967 & $00-00-67$ & 135 & & 1974 & $09-00-74$ & 60 & \\
1968 & $12-19-67$ & 490 & & 1975 & $10-28-74$ & 160 & \\
\hline
\end{tabular}

${ }^{1}$ Discharge unknown.

Magnitude and probability of instantaneous peak flow based on period of record 1963-75

\begin{tabular}{|c|c|c|c|c|c|}
\hline \multicolumn{6}{|c|}{$\begin{array}{l}\text { Discharge, in } \mathrm{ft}^{3} / \mathrm{s} \text {, for indicated recurrence interval } \\
\text { in years, and exceedance probablility, in percent }\end{array}$} \\
\hline 2 & 5 & 10 & 25 & $50 t$ & $100 \dagger$ \\
\hline $50 \%$ & $20 \%$ & $10 \%$ & $4 \%$ & $2 \%$ & $1 \%$ \\
\hline 268 & 663 & 1,070 & 1,780 & 2,480 & 3,340 \\
\hline Weighted skew & $(\log s)=$ & 0.03 & & & \\
\hline Mean & $(\log s)=$ & 2.43 & & & \\
\hline Standard dev. & $(\log s)=$ & 0.47 & & & \\
\hline
\end{tabular}

$\mp$ Reliability of values in column is uncertain, and potential errors are large.

\section{Basin characteristics}

\begin{tabular}{cccccccc}
\hline $\begin{array}{c}\text { Main } \\
\text { channel } \\
\text { slope } \\
\text { (ft/mi) }\end{array}$ & $\begin{array}{c}\text { Stream } \\
\text { length } \\
(\mathrm{mi})\end{array}$ & $\begin{array}{c}\text { Mean } \\
\text { basin } \\
\text { elevation } \\
(\mathrm{ft})\end{array}$ & $\begin{array}{c}\text { Forested } \\
\text { area } \\
\text { (percent) }\end{array}$ & $\begin{array}{c}\text { Soil } \\
\text { index }\end{array}$ & $\begin{array}{c}\text { Mean } \\
\text { annual } \\
\text { precipitation } \\
\text { (in) }\end{array}$ & $\begin{array}{c}\text { Rainfall intensity, 24-hour } \\
\text { (in) }\end{array}$ & $\begin{array}{c}50 \text {-year } \\
\text { (in) }\end{array}$ \\
\hline 67.4 & 8.6 & 1,620 & 0.0 & 3.0 & 8.5 & 1.6 \\
\hline
\end{tabular}


09488600 SILVER REEF WASH NEAR CASA GRANDE, AZ--Continued

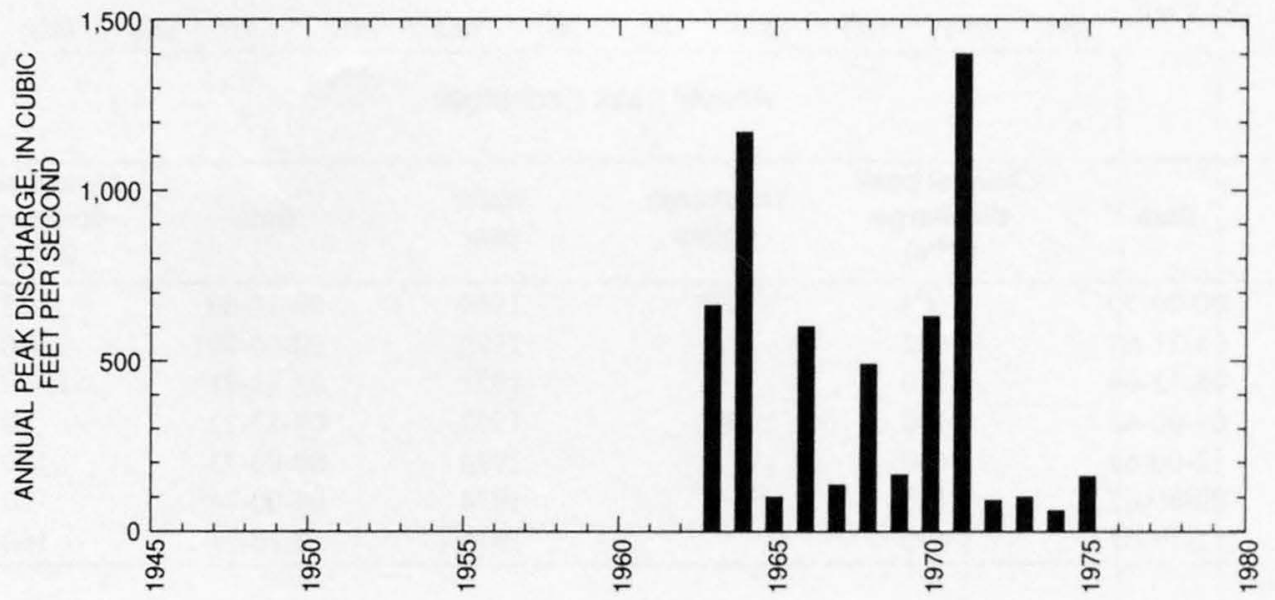




\section{SANTA CRUZ RIVER NEAR LAVEEN, AZ}

LOCATION.--Lat 33'13'56", long $112^{\circ} 10^{\prime} 08^{\prime \prime}$, in NE $1 /{ }_{4} \mathrm{NE}^{1 / 4} \mathrm{sec} .29$, T.2 S., R.2 E., Pinal County, Hydrologic Unit 15050303 , in Gila River Indian Reservation, on downstream side of highway bridge, 3.4 mi upstream from mouth, 4.3 mi south of Komatke, and 9 mi south of Laveen. DRAINAGE AREA.--8,581 $\mathrm{mi}^{2}$.

PERIOD OF RECORD.--January 1940 to September 1946, December 1947 to current year.

REVISED RECORDS.--WSP 1283: Drainage area.

GAGE.--Water-stage recorder. Datum of gage is $1,020.86 \mathrm{ft}$ above sea level.

REMARKS.--Many diversions above station, mostly by pumping from ground water, for municipal uses and for irrigation of about 240,000 acres, not including San Carlos Project. Much of the low flow passing this station is drainage and wasteway return from irrigated lands upstream and pumpage from ground water.

EXTREMES FOR PERIOD OF RECORD.--Maximum discharge, 33,000 $\mathrm{ft}^{3} / \mathrm{s}$ Oct. 4, 1983, gage height, $19.74 \mathrm{ft}$, from flow-routing computation; no flow for most of time in recent years.

\section{Annual peak discharges}

\begin{tabular}{|c|c|c|c|c|c|c|c|}
\hline $\begin{array}{l}\text { Water } \\
\text { year }\end{array}$ & Date & $\begin{array}{c}\text { Annual peak } \\
\text { discharge } \\
\left(\mathrm{ft}^{3} / \mathrm{s}\right)\end{array}$ & $\begin{array}{l}\text { Discharge } \\
\text { codes }\end{array}$ & $\begin{array}{l}\text { Water } \\
\text { year }\end{array}$ & Date & $\begin{array}{c}\text { Annual peak } \\
\text { discharge } \\
\left(\mathrm{ft}^{3} / \mathrm{s}\right)\end{array}$ & $\begin{array}{c}\text { Discharge } \\
\text { codes }\end{array}$ \\
\hline 1940 & $09-18-40$ & 1,200 & UR & 1969 & $11-14-68$ & 152 & UR \\
\hline 1941 & $03-15-41$ & 1,580 & UR & 1970 & $09-09-70$ & 1,010 & UR \\
\hline 1942 & $07-15-42$ & 1,890 & UR & 1971 & $08-22-71$ & 2,440 & $\mathrm{R}$ \\
\hline 1943 & $09-28-43$ & 1,200 & UR & 1972 & $08-07-72$ & 112 & UR \\
\hline 1944 & $02-25-44$ & 217 & UR & 1973 & $10-22-72$ & 1,650 & UR \\
\hline 1945 & $08-11-45$ & 1,200 & UR & 1974 & $07-20-74$ & 144 & KR \\
\hline 1946 & $09-21-46$ & 5,020 & UR & 1975 & $07-14-75$ & 203 & KR \\
\hline 1948 & $08-07-48$ & 1,200 & UR & 1976 & $09-25-76$ & 583 & $\mathrm{KR}$ \\
\hline 1949 & $09-17-49$ & 1,780 & UR & 1977 & $10-23-76$ & 472 & $\mathrm{KR}$ \\
\hline 1950 & $08-11-50$ & 685 & UR & 1978 & $10-13-77$ & 2,010 & KR \\
\hline 1951 & $08-28-51$ & 5,060 & UR & 1979 & $12-22-78$ & 4,120 & KR \\
\hline 1952 & $08-15-52$ & 1,860 & UR & 1980 & $02-20-80$ & 115 & KR \\
\hline 1953 & $07-17-53$ & 555 & UR & 1981 & $07-16-81$ & 368 & $\mathrm{KR}$ \\
\hline 1954 & $08-09-54$ & 726 & UR & 1982 & $09-15-82$ & 751 & KR \\
\hline 1955 & $08-10-55$ & 2,180 & UR & 1983 & $03-08-83$ & 1,910 & KR \\
\hline 1956 & $01-30-56$ & 90 & UR & 1984 & $10-04-83$ & 33,000 & KR \\
\hline 1957 & $08-20-57$ & 1,040 & UR & 1985 & $12-31-84$ & 2,030 & $\mathrm{KR}$ \\
\hline 1958 & $11-03-57$ & 3,360 & UR & 1986 & $07-21-86$ & 456 & KR \\
\hline 1959 & $08-12-59$ & 3,010 & UR & 1987 & $02-25-87$ & 110 & KR \\
\hline 1960 & $01-15-60$ & 707 & UR & 1988 & $08-27-88$ & 310 & KR \\
\hline 1961 & $08-15-61$ & 547 & UR & 1989 & $10-15-88$ & 688 & KR \\
\hline 1962 & $09-29-62$ & 9,200 & UR & 1990 & $07-16-90$ & 1,910 & KR \\
\hline 1963 & $08-17-63$ & 608 & UR & 1991 & $03-05-91$ & 257 & KR \\
\hline 1964 & $08-14-64$ & 2,520 & UR & 1992 & $07-24-92$ & 360 & $\mathrm{KR}$ \\
\hline 1965 & $06-23-65$ & 309 & UR & 1993 & $01-21-93$ & 11,000 & KR \\
\hline 1966 & $12-26-65$ & 2,940 & UR & 1994 & $11-15-93$ & 850 & KR \\
\hline 1967 & $09-06-67$ & 448 & UR & 1995 & $02-18-95$ & 726 & KR \\
\hline 1968 & $12-23-67$ & 3,820 & UR & 1996 & $07-27-96$ & 176 & KR \\
\hline
\end{tabular}


09489000 SANTA CRUZ RIVER NEAR LAVEEN, AZ--Continued

Discharge rating table developed October 1992

\begin{tabular}{crcr}
\hline $\begin{array}{c}\text { Gage height } \\
(\mathbf{f t})\end{array}$ & $\begin{array}{c}\text { Discharge } \\
\left(\mathbf{f t}^{\mathbf{3}} \mathbf{s}\right)\end{array}$ & $\begin{array}{c}\text { Gage height } \\
(\mathbf{f t})\end{array}$ & $\begin{array}{c}\text { Discharge } \\
\left(\mathbf{f t}^{\mathbf{3}} / \mathbf{s}\right)\end{array}$ \\
\hline 12.5 & 995 & 15.5 & 3,440 \\
13.0 & 1,280 & 16.0 & 4,300 \\
13.5 & 1,590 & 16.5 & 5,400 \\
14.0 & 1,940 & 17.0 & 6,700 \\
14.5 & 2,350 & 17.5 & 8,500 \\
15.0 & 2,810 & 18.1 & 11,500 \\
\hline
\end{tabular}

Basin characteristics

\begin{tabular}{cccccccc}
\hline $\begin{array}{c}\text { Main } \\
\text { channel } \\
\text { slope } \\
(\mathrm{ft} / \mathrm{mi})\end{array}$ & $\begin{array}{c}\text { Stream } \\
\text { length } \\
(\mathrm{mi})\end{array}$ & $\begin{array}{c}\text { Mean } \\
\text { basin } \\
\text { elevation } \\
(\mathrm{ft})\end{array}$ & $\begin{array}{c}\text { Forested } \\
\text { area } \\
\text { (percent) }\end{array}$ & $\begin{array}{c}\text { Soil } \\
\text { index }\end{array}$ & $\begin{array}{c}\text { Mean } \\
\text { annual } \\
\text { precipitation } \\
\text { (in) }\end{array}$ & $\begin{array}{c}\text { Rainfall intensity, 24-hour } \\
\text { (in) }\end{array}$ & $\begin{array}{c}50 \text {-year } \\
\text { (in) }\end{array}$ \\
\hline 15.2 & 241 & 3,060 & 7.8 & 2.0 & 13.0 & 1.9 \\
\hline
\end{tabular}


09489000 SANTA CRUZ RIVER NEAR LAVEEN, AZ--Continued

MEAN MONTHLY AND ANNUAL DISCHARGES 1975.96

\begin{tabular}{|c|c|c|c|c|c|c|}
\hline MONTH & $\begin{array}{l}\text { MAXIMUM } \\
\text { (FT3/S) }\end{array}$ & $\begin{array}{l}\text { MINIMUM } \\
\text { (FT3/S) }\end{array}$ & $\begin{array}{c}\text { MEAN } \\
(\mathrm{FT} 3 / \mathrm{S})\end{array}$ & $\begin{array}{l}\text { STAN- } \\
\text { DARD } \\
\text { DEVIA- } \\
\text { TION } \\
\text { (FT3/S) }\end{array}$ & $\begin{array}{l}\text { COEFFI- } \\
\text { CIENT OF } \\
\text { VARI- } \\
\text { ATION }\end{array}$ & $\begin{array}{c}\text { PERCENT } \\
\text { OF } \\
\text { ANNUAL } \\
\text { RUNOFF }\end{array}$ \\
\hline OCTOBER & 1,810 & 0.00 & 90 & 385 & 4.3 & 33 \\
\hline NOVEMBER & 28.9 & 0.00 & 2.8 & 8.0 & 2.7 & 1.1 \\
\hline DECEMBER & 248 & 0.00 & 20 & 56 & 2.8 & 7.2 \\
\hline JANUARY & 1,180 & 0.00 & 85 & 264 & 3.1 & 31 \\
\hline FEBRUARY & 187 & 0.00 & 19 & 42 & 2.2 & 6.8 \\
\hline MARCH & 160 & 0.00 & 14 & 38 & 2.7 & 5.2 \\
\hline APRIL & 13 & 0.00 & 1.8 & 3.1 & 1.7 & 0.65 \\
\hline MAY & 12 & 0.00 & 1.7 & 3.1 & 1.8 & 0.62 \\
\hline JUNE & 3.9 & 0.00 & 0.48 & 1. 0 & 2.1 & 0.17 \\
\hline JULY & 193 & 0.00 & 14 & 41 & 3.0 & 4.9 \\
\hline AUGUST & 129 & 0.00 & 17 & 36 & 2.1 & 6.3 \\
\hline SEPTEMBER & 53 & 0.00 & 9.7 & 15 & 1.6 & 3.5 \\
\hline ANNUAL & 170 & 0.47 & 23 & 41 & 1.8 & 100 \\
\hline
\end{tabular}

MAGNITUDE AND PROBABILITY OF INSTANTANEOUS PEAK FLOW BASED ON PERIOD OF RECORD

DISCHARGE, IN FT3/S, FOR INDICATED RECURRENCE INTERVAL IN YEARS, AND EXCEEDANCE PROBABILITY, IN PERCENT

\begin{tabular}{|c|c|c|c|c|c|}
\hline 2 & 5 & 10 & 25 & 50 & 100 \\
\hline 508 & 208 & 108 & 48 & 28 & 18 \\
\hline 986 & 2,600 & 4,270 & 7,160 & 9,940 & 13,300 \\
\hline WEIGHTED & SKEW & $($ LOGS $)=$ & .0 .13 & & \\
\hline MEAN & & (LOGS) $=$ & 2.98 & & \\
\hline STANDARD & DEV. & (LOGS) $=$ & 0.51 & & \\
\hline
\end{tabular}

MAGNITUDE AND PROBABILITY OF ANNUAL LOW FLOW BASED ON PERIOD OF RECORD 1975-96

\begin{tabular}{|c|c|c|c|c|c|c|}
\hline \multirow{2}{*}{$\begin{array}{l}\text { PERIOD } \\
\text { ICON- } \\
\text { SECU. }\end{array}$} & \multicolumn{6}{|c|}{ DISCHARGE， IN FT $3 / S$, FOR INDICATED } \\
\hline & $\cdots$ & & & & & …... \\
\hline TIVE & 2 & 5 & 10 & 20 & $50 \#$ & $100 \#$ \\
\hline DAYS) & 508 & 208 & 108 & 58 & 28 & 18 \\
\hline 1 & & & & & & \\
\hline 3 & & & & & & \\
\hline 7 & & & & & & \\
\hline 14 & & & & & & \\
\hline 30 & & & & & & \\
\hline 60 & & & & & & \\
\hline 90 & 0.04 & 0.00 & 0.00 & 0.00 & 0.00 & 0.00 \\
\hline 120 & 0.36 & 0.00 & 0.00 & 0.00 & 0.00 & 0.00 \\
\hline 183 & 1.6 & 0.27 & 0.08 & 0.01 & 0.00 & 0.00 \\
\hline
\end{tabular}

MAGNITUDE AND PROBABIUITY OF ANNUAL HIGH FLOW BASED ON PERIOD OF RECORD 1975.89

\begin{tabular}{|c|c|c|c|c|c|c|}
\hline $\begin{array}{c}\text { PERIOD } \\
\text { (CON- }\end{array}$ & DISC & $\begin{array}{l}\text { GE, IN } \\
\text { RECURR } \\
\text { EXCEED }\end{array}$ & $\begin{array}{l}\text { ET3/S, } \\
\text { NCE INI } \\
\text { NCE PRO }\end{array}$ & $\begin{array}{l}\text { R INDIC } \\
\text { VAL, IN } \\
\text { BILITY, }\end{array}$ & $\begin{array}{l}\text { ATED } \\
\text { YEARS, } \\
\text { IN PERC }\end{array}$ & $\begin{array}{l}\text { AND } \\
\text { ENT }\end{array}$ \\
\hline SECU- & & & & & (2) & ….... \\
\hline TIVE & 2 & 5 & 10 & $25 \#$ & $50 \#$ & $100 \#$ \\
\hline DAYS) & 508 & 208 & 108 & 48 & 28 & 18 \\
\hline 1 & 510 & 1,760 & 3,450 & 7,250 & 11.800 & 18,600 \\
\hline 3 & 320 & 1,240 & 2,590 & 5,810 & 9.890 & 16,100 \\
\hline 7 & 175 & 690 & 1,440 & 3,210 & 5,430 & 8,770 \\
\hline 15 & 98 & 399 & 846 & 1,910 & 3.250 & 5,270 \\
\hline 30 & 58 & 235 & 489 & 1,070 & 1.760 & 2.780 \\
\hline 60 & 35 & 137 & 279 & 592 & 958 & 1,480 \\
\hline 90 & 25 & 96 & 193 & 402 & 646 & 987 \\
\hline
\end{tabular}

DURATION TABLE OF DAILY MEAN FLOW FOR PERIOD OF RECORD 1975-89

DISCHARGE, IN FT3/S, WHICH WAS EQUALED OR EXCEEDED FOR INDICATED PERCENT OF TIME

\begin{tabular}{|c|c|c|c|c|c|c|c|c|c|c|c|c|c|c|c|c|}
\hline 18 & 58 & 108 & 158 & 208 & 308 & 408 & 508 & 608 & 708 & 808 & 908 & 958 & 988 & 998 & 99.58 & 99.98 \\
\hline 03 & 32 & 8.9 & 4.7 & 300 & 1.2 & 0.49 & 0.04 & 0.00 & 0.00 & 0.00 & 0.00 & 0.00 & 0.00 & 0.00 & 0.00 & 0.00 \\
\hline
\end{tabular}

\# Reliability of values in column is uncertain, and potential errors are large. 
ANNUAL MEAN DISCHARGE, IN CUBIC

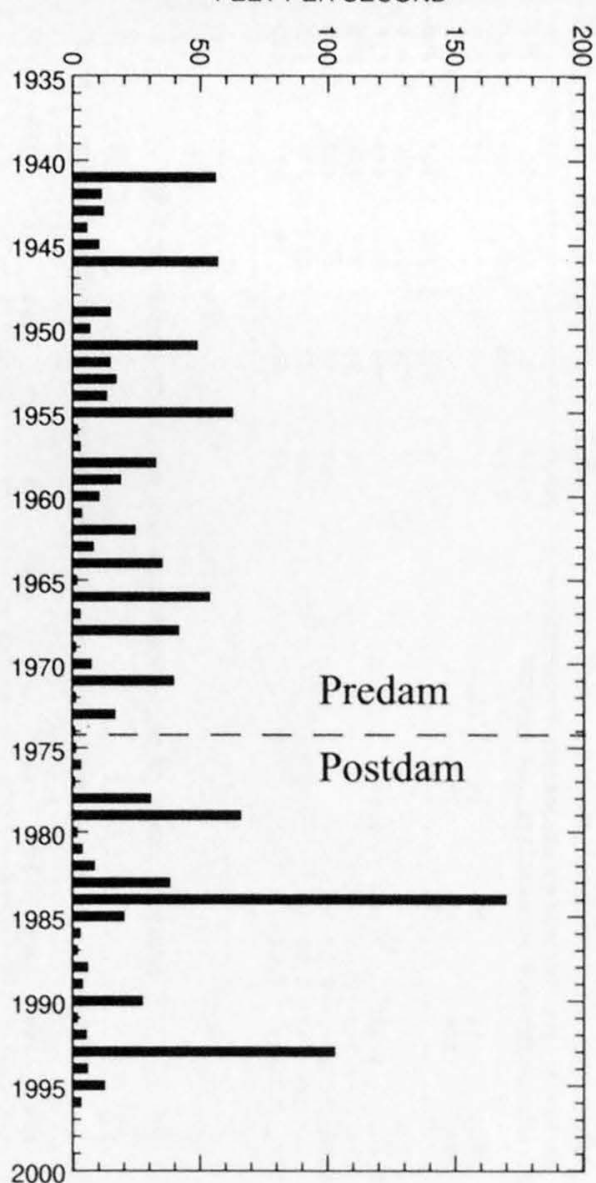

ANNUAL PEAK DISCHARGE IN CUBIC FEET PER SECOND

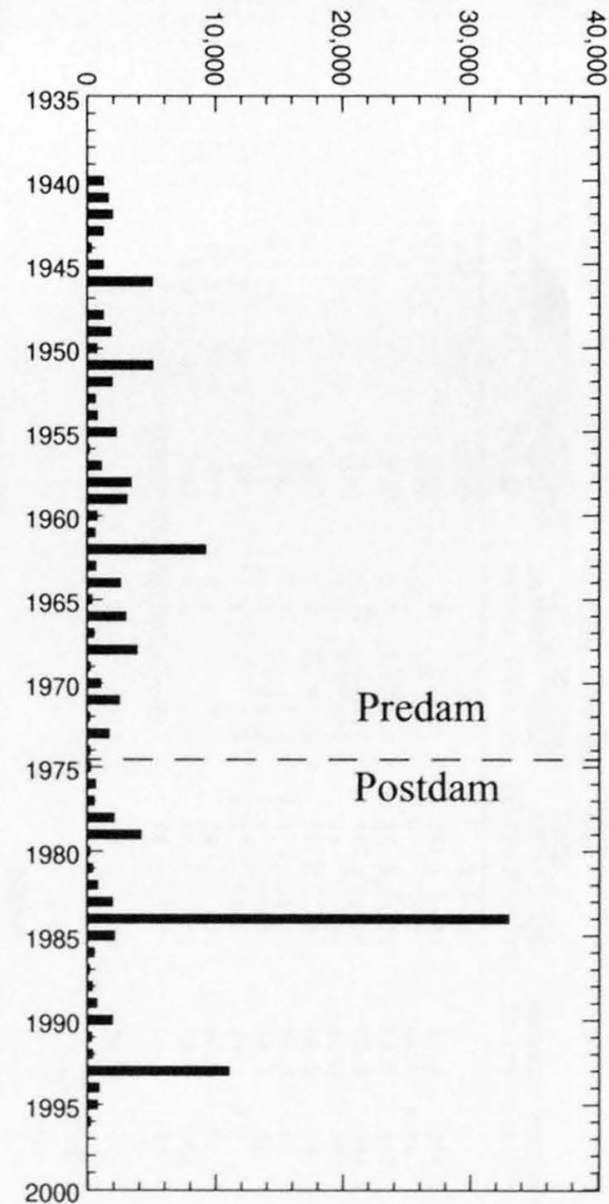


09489000 SANTA CRUZ RIVER NEAR LAVEEN, AZ--Continued
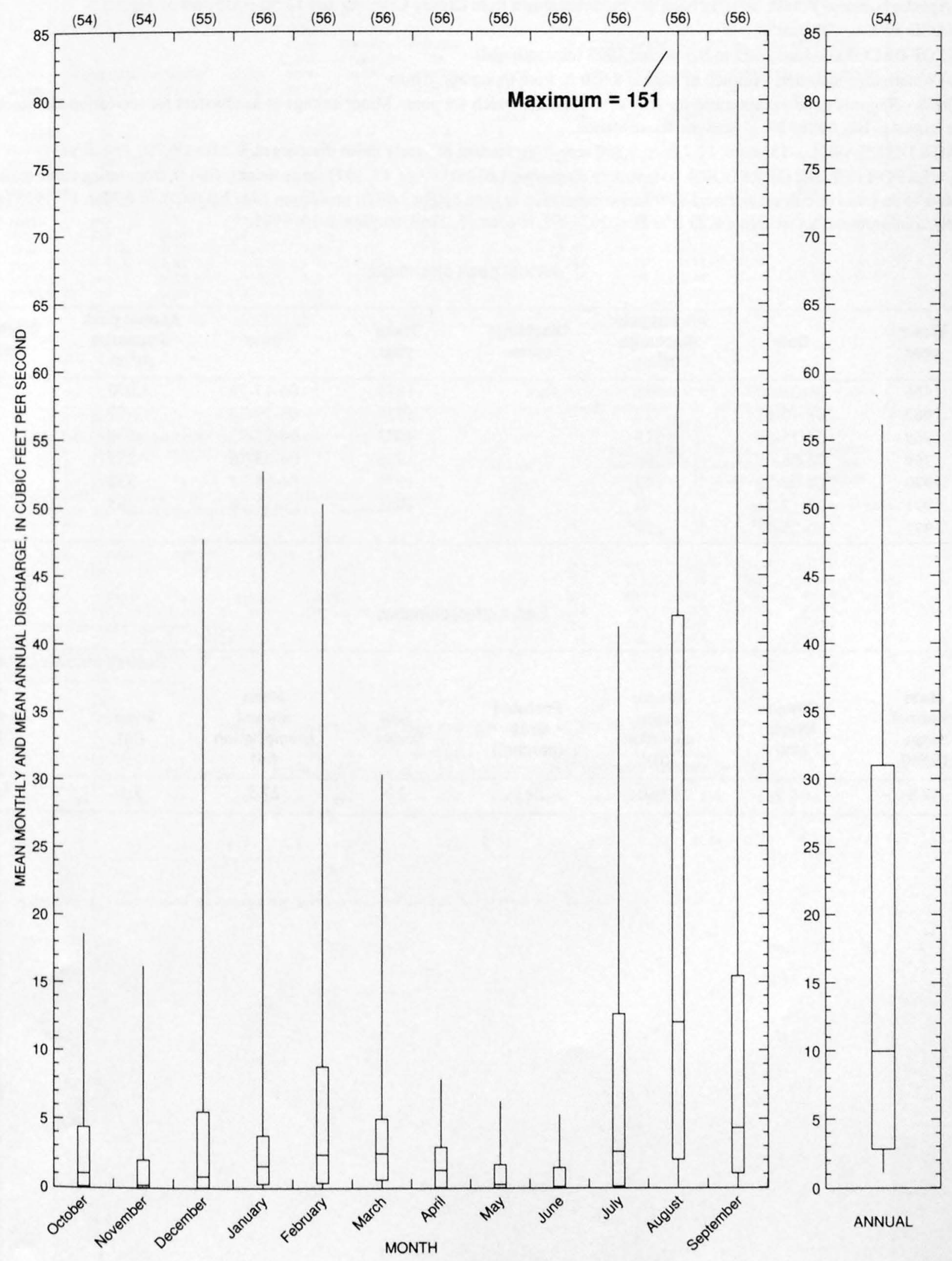


\section{NORTH FORK OF EAST FORK BLACK RIVER NEAR ALPINE, AZ}

LOCATION.--Lat 33.54'11", long 109.19'20", in SW $1 / 4 \mathrm{NE}^{1} / 4$ sec.19, T.6 N., R.29 E. (unsurveyed), Apache County, Hydrologic Unit 15060101, in Apache National Forest, on right bank $1.4 \mathrm{mi}$ downstream from Crosby Crossing and 12 mi northwest of Alpine.

DRAINAGE AREA.--38.1 $\mathrm{mi}^{2}$.

PERIOD OF RECORD.--June 1965 to September 1978 (discontinued).

GAGE.--Water-stage recorder. Altitude of gage is $8,650 \mathrm{ft}$, from topographic map.

REMARKS.--Records good except those for Jan. 16 to Mar. 23, which are poor. Minor storage at headwaters for recreation and stock purposes; the largest is Big Lake. No diversions above station.

AVERAGE DISCHARGE.--13 years, $12.7 \mathrm{ft}^{3} / \mathrm{s}, 9,200 \mathrm{acre}-\mathrm{ft} / \mathrm{yr}$; median of yearly mean discharges, $9.2 \mathrm{ft} 3 / \mathrm{s} 6,700 \mathrm{acre}-\mathrm{ft} / \mathrm{yr}$.

EXTREMES FOR PERIOD OF RECORD.--Maximum discharge, 1,070 $\mathrm{ft}^{3} / \mathrm{s} \mathrm{Apr.} \mathrm{17,} \mathrm{1973,} \mathrm{gage} \mathrm{height,} 4.64 \mathrm{ft}$, from rating curve extended above $260 \mathrm{ft}^{3} / \mathrm{s}$ on basis of culvert and road overflow computation at gage height $3.80 \mathrm{ft}$; maximum gage height, $4.75 \mathrm{ft}$ Mar. 11,1975 (result of ice jam); minimum daily discharge, $0.20 \mathrm{ft}^{3} / \mathrm{s}$ Dec. 20, 1968, to Mar. 15, 1969, and Jan. 6-10, 1971.

Annual peak discharges

\begin{tabular}{|c|c|c|c|c|c|c|c|}
\hline $\begin{array}{l}\text { Water } \\
\text { year }\end{array}$ & Date & $\begin{array}{c}\text { Annual peak } \\
\text { discharge } \\
\left(\mathrm{ft}^{3} / \mathrm{s}\right)\end{array}$ & $\begin{array}{c}\text { Discharge } \\
\text { codes }\end{array}$ & $\begin{array}{l}\text { Water } \\
\text { year }\end{array}$ & Date & $\begin{array}{c}\text { Annual peak } \\
\text { discharge } \\
\left(\mathrm{ft}^{3} / \mathrm{s}\right)\end{array}$ & $\begin{array}{l}\text { Discharge } \\
\text { codes }\end{array}$ \\
\hline 1966 & $04-03-66$ & 556 & & 1973 & $04-17-73$ & 1,070 & \\
\hline 1967 & $08-10-67$ & 27 & & 1974 & $03-29-74$ & 77 & \\
\hline 1968 & $04-15-68$ & 515 & & 1975 & $04-25-75$ & 577 & \\
\hline 1969 & 04-06-69 & 366 & & 1976 & $04-05-76$ & 283 & \\
\hline 1970 & 04-06-70 & 142 & & 1977 & $04-08-77$ & 332 & \\
\hline 1971 & $03-12-71$ & 39 & & 1978 & $03-31-78$ & 383 & \\
\hline 1972 & $10-25-71$ & 218 & & & & & \\
\hline
\end{tabular}

Basin characteristics

\begin{tabular}{ccccccc}
\hline $\begin{array}{c}\text { Main } \\
\text { channel } \\
\text { slope } \\
(\mathrm{ft} / \mathrm{mi})\end{array}$ & $\begin{array}{c}\text { Stream } \\
\text { length } \\
\text { (mi) }\end{array}$ & $\begin{array}{c}\text { Mean } \\
\text { basin } \\
\text { elevation } \\
(\mathrm{ft})\end{array}$ & $\begin{array}{c}\text { Forested } \\
\text { area } \\
\text { (percent) }\end{array}$ & $\begin{array}{c}\text { Soil } \\
\text { index }\end{array}$ & $\begin{array}{c}\text { Mean } \\
\text { annual } \\
\text { precipitation } \\
\text { (in) }\end{array}$ & $\begin{array}{c}\text { Rainfall intensity, 24-hour } \\
\text { (in) }\end{array}$ \\
\hline 48.8 & 10.1 & 9,060 & 24.0 & 3.0 & 27.5 & $\begin{array}{c}50 \text {-year } \\
\text { (in) }\end{array}$ \\
\hline
\end{tabular}


09489070 NORTH FORK OF EAST FORK BLACK RIVER NEAR ALPINE, AZ--Continued

MEAN MONTHLY AND ANNUAL DISCHARGES $1966 \cdot 78$

\begin{tabular}{|c|c|c|c|c|c|c|}
\hline MONTH & $\begin{array}{l}\text { MAXIMUM } \\
\text { (FT3/S) }\end{array}$ & $\begin{array}{l}\text { MINIMUM } \\
(\mathrm{FT} 3 / \mathrm{S})\end{array}$ & $\begin{array}{c}\text { MEAN } \\
(\mathrm{FT} 3 / \mathrm{S})\end{array}$ & $\begin{array}{l}\text { STAN- } \\
\text { DARD } \\
\text { DEVIA- } \\
\text { TION } \\
(\text { FT3/S) }\end{array}$ & $\begin{array}{l}\text { COEFFI- } \\
\text { CIENT OF } \\
\text { VARI- } \\
\text { ATION }\end{array}$ & $\begin{array}{c}\text { PERCENT } \\
\text { OF } \\
\text { ANNUAL } \\
\text { RUNOFF }\end{array}$ \\
\hline OCTOBER & 33 & 0.83 & 5.8 & 9.9 & 1.7 & 3.8 \\
\hline NOVEMBER & 8.7 & 0.67 & 3.0 & 2.9 & 0.97 & 1.9 \\
\hline DECEMBER & 16 & 0.33 & 2.5 & 4.2 & 1.7 & 1.6 \\
\hline JANUARY & 13 & 0.20 & 1.9 & 3.3 & 1.7 & 1.3 \\
\hline FEBRUARY & 15 & 0.20 & 2.7 & 4.1 & 1.5 & 1.8 \\
\hline MARCH & 103 & 2.4 & 23 & 29 & 1.2 & 15.1 \\
\hline APRIL & 362 & 1.2 & 83 & 102 & 1.2 & 54.0 \\
\hline MAY & 134 & 0.49 & 21 & 37 & 1.8 & 13.5 \\
\hline JUNE & 9.8 & 0.29 & 2.0 & 2.5 & 1.2 & 1.3 \\
\hline JULY & 4.7 & 0.41 & 1.8 & 1.2 & 0.69 & 1.1 \\
\hline AUGUST & 13 & 0.56 & 3.9 & 3.9 & 1.0 & 2.5 \\
\hline SEPTEMBER & 13 & 0.38 & 3.0 & 3.5 & 1.2 & 2.0 \\
\hline ANNUAL & 50 & 1.2 & 13 & 13 & 1.0 & 100 \\
\hline
\end{tabular}

MAGNITUDE AND PROBABILITY OF ANNUAL LOW FLOW BASED ON PERIOD OF RECORD $1967-78$

\begin{tabular}{|c|c|c|c|c|c|c|}
\hline $\begin{array}{l}\text { PERIOD } \\
\text { (CON. }\end{array}$ & \multirow{2}{*}{\multicolumn{6}{|c|}{$\begin{array}{l}\text { DISCHARGE, IN FT } 3 / S \text {, FOR INDICATED } \\
\text { RECURRENCE INTERVAL, IN YEARS, AND } \\
\text { NON-EXCEEDANCE PROBABILITY, IN PERCENT }\end{array}$}} \\
\hline SECU. & & & & & & \\
\hline TIVE & 2 & 5 & 10 & 20 & $50 \#$ & $100 \#$ \\
\hline DAYS) & 508 & 208 & 108 & 58 & 28 & 18 \\
\hline 1 & 0.37 & 0.24 & 0.20 & 0.17 & 0.14 & 0.13 \\
\hline 3 & 0.39 & 0.25 & 0.21 & 0.17 & 0.14 & 0.13 \\
\hline 7 & 0.40 & 0.27 & 0.22 & 0.19 & 0.16 & 0.15 \\
\hline 14 & 0.42 & 0.29 & 0.24 & 0.21 & 0.18 & 0.16 \\
\hline 30 & 0.47 & 0.31 & 0.25 & 0.22 & 0.18 & 0.16 \\
\hline 60 & 0.55 & 0.36 & 0.28 & 0.23 & 0.19 & 0.16 \\
\hline 90 & 0.63 & 0.40 & 0.31 & 0.25 & 0.20 & 0.18 \\
\hline 120 & 0.66 & 0.46 & 0.41 & 0.38 & 0.36 & 0.35 \\
\hline 183 & 0.96 & 0.75 & 0.70 & 0.68 & 0.66 & 0.66 \\
\hline
\end{tabular}

MAGNITUDE AND PROBABILITY OF ANNUAL HIGH FLOW BASED ON PERIOD OF RECORD 1966.78

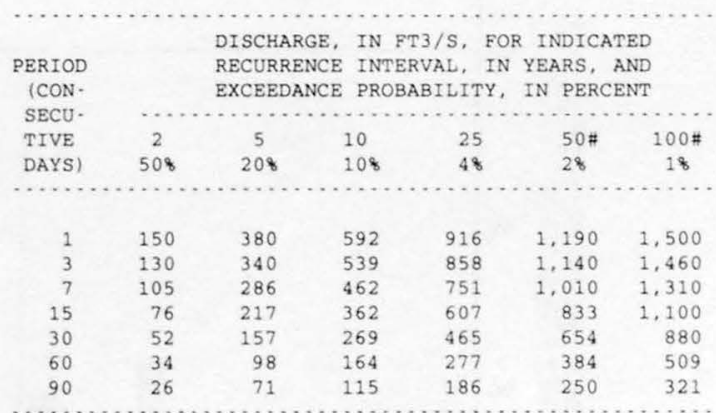

MAGNITUDE AND PROBABILITY OF INSTANTANEOUS PEAK FLOW BASED ON PERIOD OF RECORD 1966.78

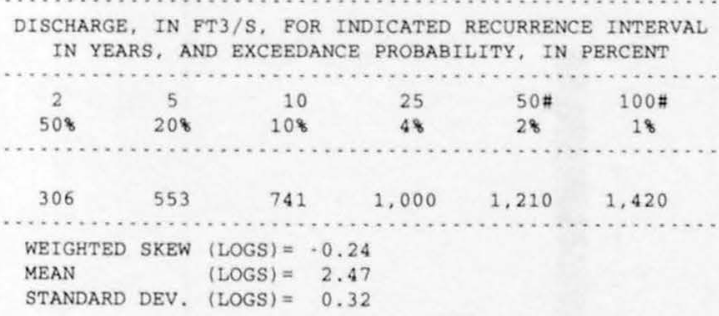

DURATION TABLE OF DAILY MEAN FLOW FOR PERIOD OF RECORD 1966-78

\begin{tabular}{|c|c|c|c|c|c|c|c|c|c|c|c|c|c|c|c|c|}
\hline 18 & 58 & 108 & 158 & 208 & 308 & 408 & 508 & 608 & 708 & 808 & 908 & 958 & 988 & 998 & 99.58 & 99.98 \\
\hline 253 & 53 & 22 & 12 & 6.7 & 3.4 & 2.2 & 1.5 & 1.2 & 0.91 & 0.66 & 0.46 & 0.33 & 0.26 & 0.23 & 0.21 & 0.20 \\
\hline
\end{tabular}

\# Reliability of values in column is uncertain, and potential errors are large. 
09489070 NORTH FORK OF EAST FORK BLACK RIVER NEAR ALPINE, AZ--Continued
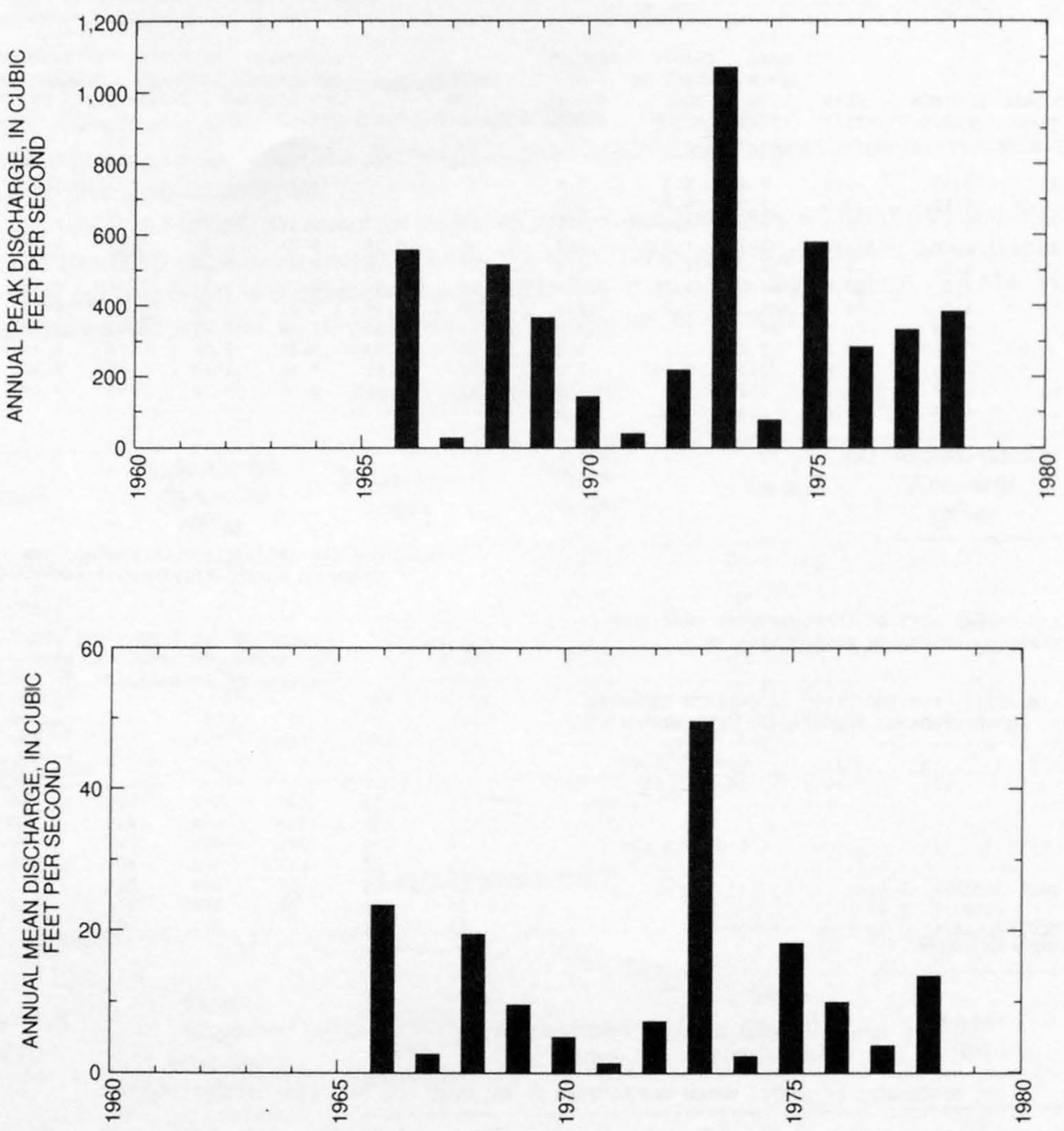
09489070 NORTH FORK OF EAST FORK BLACK RIVER NEAR ALPINE, AZ--Continued
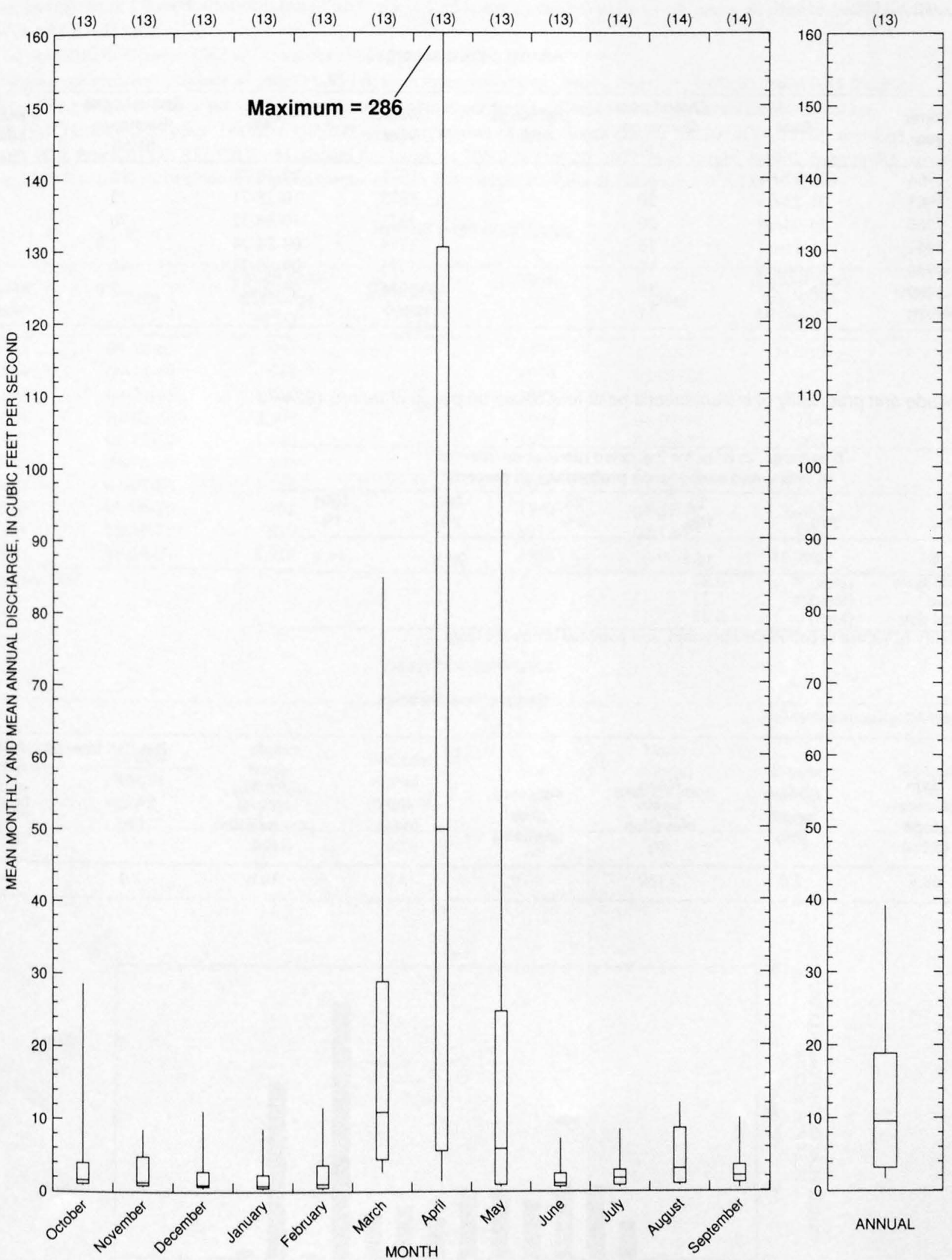
09489080 HANNAGAN CREEK NEAR HANNAGAN MEADOW, AZ

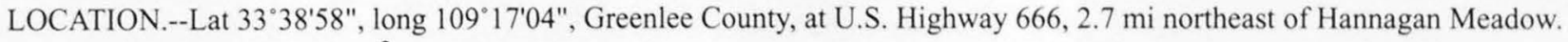
DRAINAGE AREA.--1.61 $\mathrm{mi}^{2}$.

Annual peak discharges

\begin{tabular}{|c|c|c|c|c|c|c|c|}
\hline $\begin{array}{l}\text { Water } \\
\text { year }\end{array}$ & Date & $\begin{array}{c}\text { Annual peak } \\
\text { discharge } \\
\left(\mathrm{ft}^{3} / \mathrm{s}\right)\end{array}$ & $\begin{array}{c}\text { Discharge } \\
\text { codes }\end{array}$ & $\begin{array}{l}\text { Water } \\
\text { year }\end{array}$ & Date & $\begin{array}{c}\text { Annual peak } \\
\text { discharge } \\
\left(\mathrm{ft}^{3} / \mathrm{s}\right)\end{array}$ & $\begin{array}{c}\text { Discharge } \\
\text { codes }\end{array}$ \\
\hline 1964 & $09-21-64$ & 10 & & 1971 & $09-30-71$ & 14 & \\
\hline 1965 & $04-22-65$ & 20 & & 1972 & $10-25-71$ & 12 & \\
\hline 1966 & 04-04-66 & 20 & & 1973 & $10-19-72$ & 70 & \\
\hline 1967 & $08-11-67$ & 16 & & 1974 & $04-24-74$ & 1.0 & \\
\hline 1968 & $04-00-68$ & 19 & & 1975 & $09-00-75$ & 48 & \\
\hline 1969 & 09-03-69 & 19 & & 1976 & $08-00-76$ & 2.0 & \\
\hline 1970 & $04-00-70$ & 11 & & & & & \\
\hline
\end{tabular}

Magnitude and probability of instantaneous peak flow based on period of record 1964-76

\begin{tabular}{|c|c|c|c|c|c|}
\hline \multicolumn{6}{|c|}{$\begin{array}{l}\text { Discharge, in } \mathrm{ft}^{3} / \mathrm{s} \text {, for indicated recurrence interval } \\
\text { in years, and exceedance probablility, in percent }\end{array}$} \\
\hline 2 & 5 & 10 & 25 & $50 \dagger$ & $100 \dagger$ \\
\hline $50 \%$ & $20 \%$ & $10 \%$ & $4 \%$ & $2 \%$ & $1 \%$ \\
\hline 16.5 & 29 & 39.7 & 56.1 & 70.6 & 87.3 \\
\hline Weighted skew & $(\log s)=$ & 0.27 & & & \\
\hline Mean & $(\log s)=$ & 1.23 & & & \\
\hline Standard dev. & $(\log s)=$ & 0.28 & & & \\
\hline
\end{tabular}

Basin characteristics

\begin{tabular}{|c|c|c|c|c|c|c|c|}
\hline \multirow[b]{2}{*}{$\begin{array}{l}\text { Main } \\
\text { channel } \\
\text { slope } \\
(\mathrm{ft} / \mathrm{mi})\end{array}$} & \multirow[b]{2}{*}{$\begin{array}{l}\text { Stream } \\
\text { length } \\
\text { (mi) }\end{array}$} & \multirow[b]{2}{*}{$\begin{array}{c}\text { Mean } \\
\text { basin } \\
\text { elevation } \\
(f t)\end{array}$} & \multirow[b]{2}{*}{$\begin{array}{l}\text { Forested } \\
\text { area } \\
\text { (percent) }\end{array}$} & \multirow[b]{2}{*}{$\begin{array}{c}\text { Soil } \\
\text { index }\end{array}$} & \multirow[b]{2}{*}{$\begin{array}{c}\text { Mean } \\
\text { annual } \\
\text { precipitation } \\
\text { (in) }\end{array}$} & \multicolumn{2}{|c|}{ Rainfall intensity, 24-hou } \\
\hline & & & & & & $\begin{array}{l}\text { 2-year } \\
\text { (in) }\end{array}$ & $\begin{array}{l}\text { 50-year } \\
\text { (in) }\end{array}$ \\
\hline 68.6 & 2.0 & 9,160 & 90.0 & 3.0 & 30.0 & 2.4 & 4.0 \\
\hline
\end{tabular}

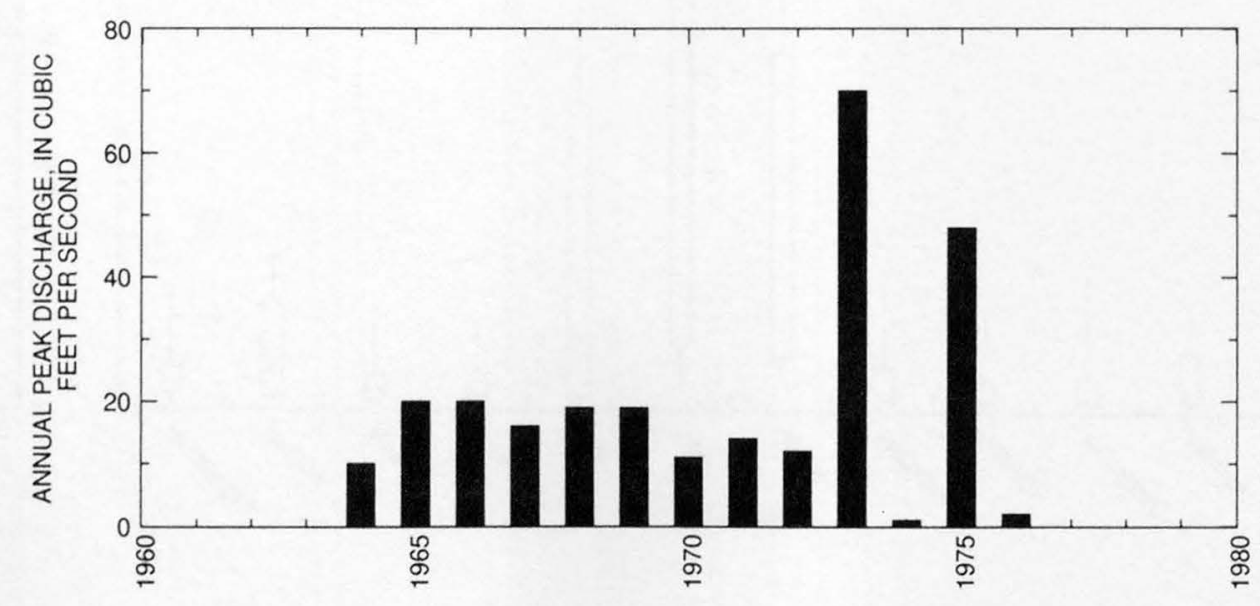




\section{BLACK RIVER NEAR MAVERICK, AZ}

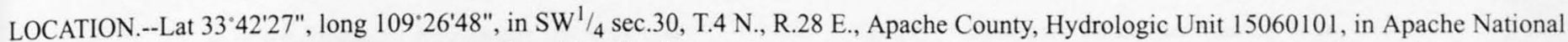
Forest, on right bank $1.0 \mathrm{mi}$ downstream from Fish Creek, 1.1 mi upstream from Conklin Creek, and 6 mi southeast of Maverick.

DRAINAGE AREA.--315 $\mathrm{mi}^{2}$.

PERIOD OF RECORD.--October 1962 to September 1982 (discontinued).

GAGE.--Water-stage recorder. Altitude of gage is 6,850 ft, from topographic map. Prior to Sept. 19, 1973, at datum $0.85 \mathrm{ft}$ higher.

REMARKS.--Records good. Minor storage for recreational and stock purposes near headwaters. No diversion above station.

AVERAGE DISCHARGE.--20 years, $141 \mathrm{ft}^{3} / \mathrm{s}, 102,200 \mathrm{acre}-\mathrm{ft} / \mathrm{yr}$; median of yearly mean discharges, $107 \mathrm{ft} 3 / \mathrm{s}, 77,500 \mathrm{acre}-\mathrm{ft} / \mathrm{yr}$.

EXTREMES FOR PERIOD OF RECORD.--Maximum discharge, 11,100 ft ${ }^{3} / \mathrm{s}$ Oct. 20, 1972, gage height, $8.99 \mathrm{ft}$, from rating curve extended above $2,100 \mathrm{ft}^{3} / \mathrm{s}$ on basis of slope-area measurement of peak flow; minimum daily discharge, $9.6 \mathrm{ft}^{3} / \mathrm{s} \mathrm{Dec.} 7,1976$.

Annual peak discharges

\begin{tabular}{|c|c|c|c|c|c|c|c|}
\hline $\begin{array}{l}\text { Water } \\
\text { year }\end{array}$ & Date & $\begin{array}{c}\text { Annual peak } \\
\text { discharge } \\
\left(\mathrm{ft}^{3} / \mathrm{s}\right)\end{array}$ & $\begin{array}{l}\text { Discharge } \\
\text { codes }\end{array}$ & $\begin{array}{l}\text { Water } \\
\text { year }\end{array}$ & Date & $\begin{array}{c}\text { Annual peak } \\
\text { discharge } \\
\left(\mathrm{ft}^{3} / \mathrm{s}\right)\end{array}$ & $\begin{array}{c}\text { Discharge } \\
\text { codes }\end{array}$ \\
\hline 1963 & $09-10-63$ & 911 & & 1973 & $10-20-72$ & 11,100 & \\
\hline 1964 & $09-15-64$ & 946 & & 1974 & $03-31-74$ & 342 & \\
\hline 1965 & $04-21-65$ & 2,010 & & 1975 & $03-08-75$ & 2,360 & \\
\hline 1966 & $04-02-66$ & 2,300 & & 1976 & $04-05-76$ & 714 & \\
\hline 1967 & $08-12-67$ & 1,040 & & 1977 & $04-10-77$ & 700 & \\
\hline 1968 & $04-16-68$ & 1,890 & & 1978 & $03-01-78$ & 2,390 & \\
\hline 1969 & 04-07-69 & 1,740 & & 1979 & $12-18-78$ & 10,300 & \\
\hline 1970 & 09-06-70 & 402 & & 1980 & $04-21-80$ & 3,400 & \\
\hline 1971 & $08-29-71$ & 580 & & 1982 & $04-13-82$ & 1,550 & \\
\hline 1972 & $10-24-71$ & 2,910 & & 1984 & $10-02-83$ & ${ }^{1} 14,000$ & HP \\
\hline
\end{tabular}

${ }^{1}$ Highest since 1954.

Basin characteristics

\begin{tabular}{cccccccc}
\hline $\begin{array}{c}\text { Main } \\
\text { channel } \\
\text { slope } \\
(\mathrm{ft} / \mathrm{mi})\end{array}$ & $\begin{array}{c}\text { Stream } \\
\text { length } \\
\text { (mi) }\end{array}$ & $\begin{array}{c}\text { Mean } \\
\text { basin } \\
\text { elevation } \\
(\mathrm{ft})\end{array}$ & $\begin{array}{c}\text { Forested } \\
\text { area } \\
\text { (percent) }\end{array}$ & $\begin{array}{c}\text { Soil } \\
\text { index }\end{array}$ & $\begin{array}{c}\text { Mean } \\
\text { annual } \\
\text { precipitation } \\
\text { (in) }\end{array}$ & $\begin{array}{c}\text { R-year } \\
\text { (in) }\end{array}$ & $\begin{array}{c}50 \text {-year } \\
\text { (in) }\end{array}$ \\
\hline 69.4 & 38.4 & 8,700 & 82.0 & 3.0 & 27.2 & 2.4 \\
\hline
\end{tabular}


09489100 BLACK RIVER NEAR MAVERICK, AZ--Continued

MEAN MONTHLY AND ANNUAL DISCHARGES 1963.82

\begin{tabular}{|c|c|c|c|c|c|c|}
\hline MONTH & $\begin{array}{l}\text { MAXIMUM } \\
(\text { FT3/S) }\end{array}$ & $\begin{array}{l}\text { MINIMUM } \\
\text { (FT3/S) }\end{array}$ & $\begin{array}{c}\text { MEAN } \\
\text { (FT3/S) }\end{array}$ & $\begin{array}{l}\text { STAN- } \\
\text { DARD } \\
\text { DEVIA- } \\
\text { TION } \\
\text { (FT3/S) }\end{array}$ & $\begin{array}{l}\text { COEFFI- } \\
\text { CIENT OF } \\
\text { VARI- } \\
\text { ATION }\end{array}$ & $\begin{array}{c}\text { PERCENT } \\
\text { OF } \\
\text { ANNUAL } \\
\text { RUNOFF }\end{array}$ \\
\hline OCTOBER & 726 & 20 & 91 & 173 & 1.9 & 5.4 \\
\hline NOVEMBER & 219 & 18 & 60 & 62 & 1.0 & 3.6 \\
\hline DECEMBER & 609 & 15 & 72 & 134 & 1.9 & 4.2 \\
\hline JANUARY & 205 & 18 & 61 & 60 & 0.99 & 3.6 \\
\hline FEBRUARY & 290 & 21 & 99 & 86 & 0.87 & 5.8 \\
\hline MARCH & 934 & 44 & 307 & 262 & 0.85 & 18.1 \\
\hline APRIL & 1,480 & 32 & 559 & 462 & 0.83 & 33.0 \\
\hline MAY & 1,330 & 22 & 224 & 309 & 1.4 & 13.2 \\
\hline JUNE & 147 & 17 & 45 & 37 & 0.82 & 2.6 \\
\hline JULY & 107 & 21 & 38 & 22 & 0.58 & 2.2 \\
\hline AUGUST & 224 & 24 & 66 & 44 & 0.68 & 3.9 \\
\hline SEPTEMBER & 274 & 21 & 72 & 70 & 0.97 & 4.3 \\
\hline ANNUAL & 392 & 37 & 141 & 98 & 0.70 & 100 \\
\hline
\end{tabular}

MAGNITUDE AND PROBABILITY OF INSTANTANEOUS PEAK FLOW BASED ON PERIOD OF RECORD $1963 \cdot 80,1982,1984$

DISCHARGE, IN FT3/S, FOR INDICATED RECURRENCE INTERVAI IN YEARS, AND EXCBEDANCE PROBABILITY, IN PERCENT

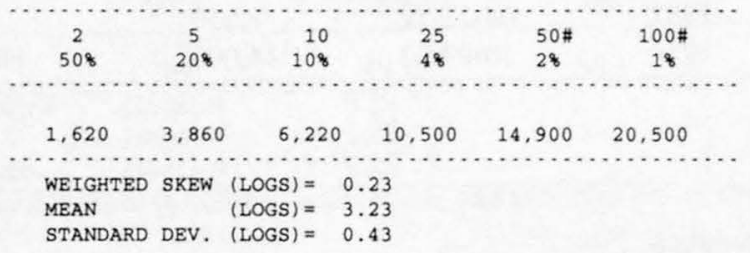

MAGNITUDE AND PROBABILITY OF ANNUAL LOW FLOW BASED ON PERIOD OF RECORD $1964 \cdot 82$

\begin{tabular}{|c|c|c|c|c|c|c|}
\hline \multirow{2}{*}{$\begin{array}{l}\text { PERIOD } \\
\text { (CON- }\end{array}$} & \multicolumn{6}{|c|}{ DISCHARGE， IN FT3/S，FOR INDICATED } \\
\hline & \multicolumn{6}{|c|}{$\begin{array}{l}\text { RECURRENCE INTERVAL, IN YEARS, AND } \\
\text { NON-EXCEEDANCE PROBABILITY, IN PERCENT }\end{array}$} \\
\hline SECU - & ... & $\cdots \cdots$ & $\cdots \cdots$ & & & $\cdots \cdots$ \\
\hline TIVE & 2 & 5 & 10 & 20 & $50 \#$ & $100 \#$ \\
\hline DAYS) & 508 & 208 & 108 & 58 & 28 & 18 \\
\hline 1 & 14 & 12 & 11 & 10 & 9.3 & 8.8 \\
\hline 3 & 15 & 13 & 12 & 12 & 11 & 11 \\
\hline 7 & 16 & 14 & 13 & 12 & 11 & 11 \\
\hline 14 & 17 & 15 & 14 & 14 & 13 & 13 \\
\hline 30 & 19 & 17 & 16 & 15 & 14 & 14 \\
\hline 60 & 21 & 19 & 18 & 17 & 16 & 16 \\
\hline 90 & 23 & 20 & 19 & 18 & 17 & 16 \\
\hline 120 & 25 & 21 & 20 & 19 & 18 & 18 \\
\hline 183 & 35 & 27 & 24 & 21 & 20 & 18 \\
\hline
\end{tabular}

MAGNITUDE AND PROBABILITY OF ANNUAL HIGH FLOW BASED ON PERIOD OF RECORD 1963.82

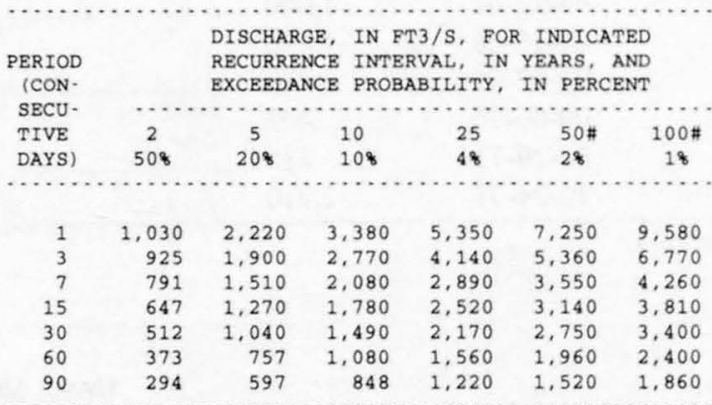

DURATION TABLE OF DAILY MEAN FLOW FOR PERIOD OF RECORD 1963.82

DISCHARGE, IN FT3/S, WHICH WAS EQUALED OR EXCEEDED FOR INDICATED PERCENT OF TIME

\begin{tabular}{|c|c|c|c|c|c|c|c|c|c|c|c|c|c|c|c|c|}
\hline 18 & 58 & 108 & 158 & 208 & 308 & 408 & 508 & 608 & 708 & 808 & 908 & 958 & 988 & 998 & 99.58 & 99.98 \\
\hline 500 & 633 & 354 & 224 & 154 & 84 & 54 & 40 & 31 & 27 & 24 & 20 & 18 & 16 & 15 & 13 & 11 \\
\hline
\end{tabular}

\# Reliability of values in column is uncertain, and potential errors are large. 


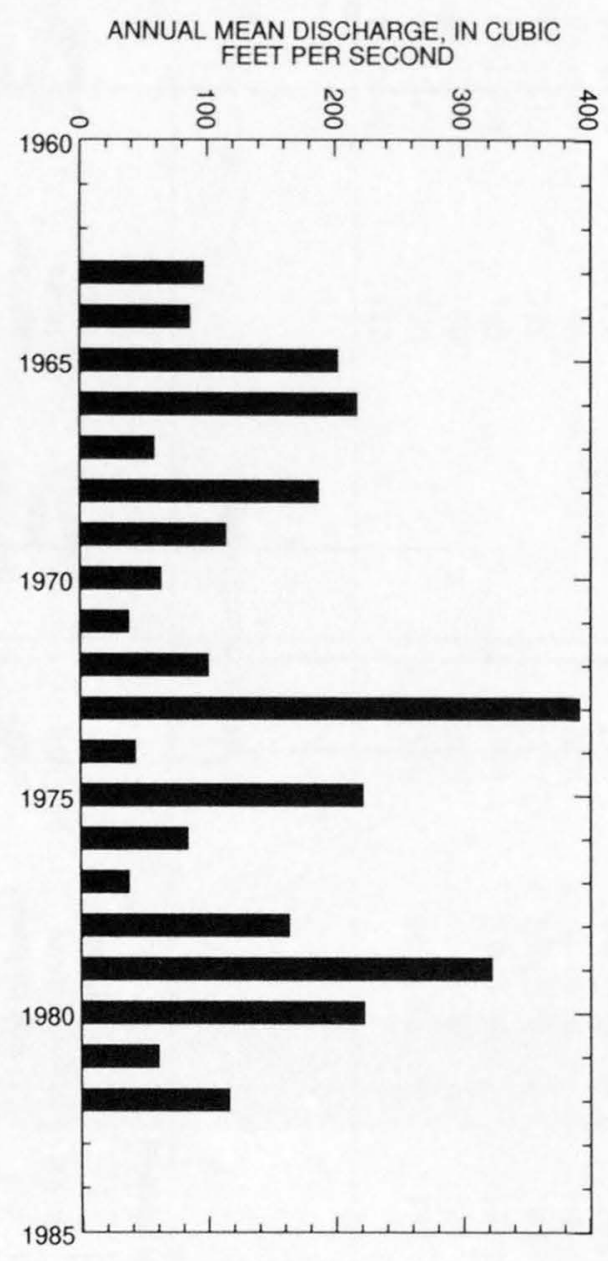

ANNUAL PEAK DISCHARGE, IN CUBIC FEET PER SECOND

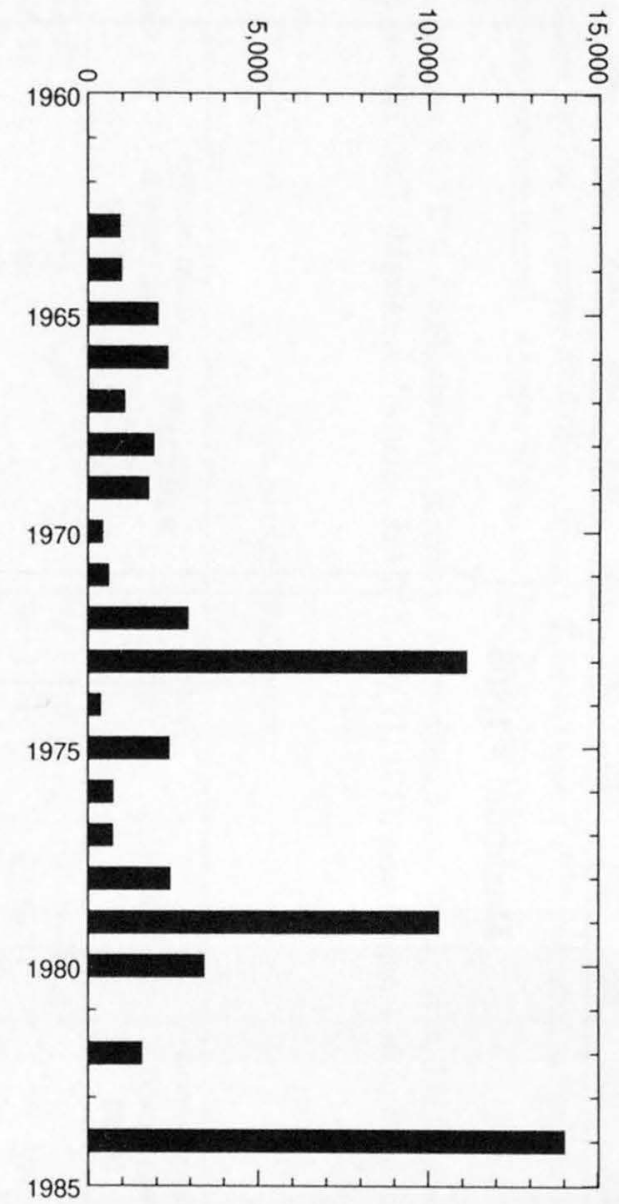


09489100 BLACK RIVER NEAR MAVERICK, AZ--Continued
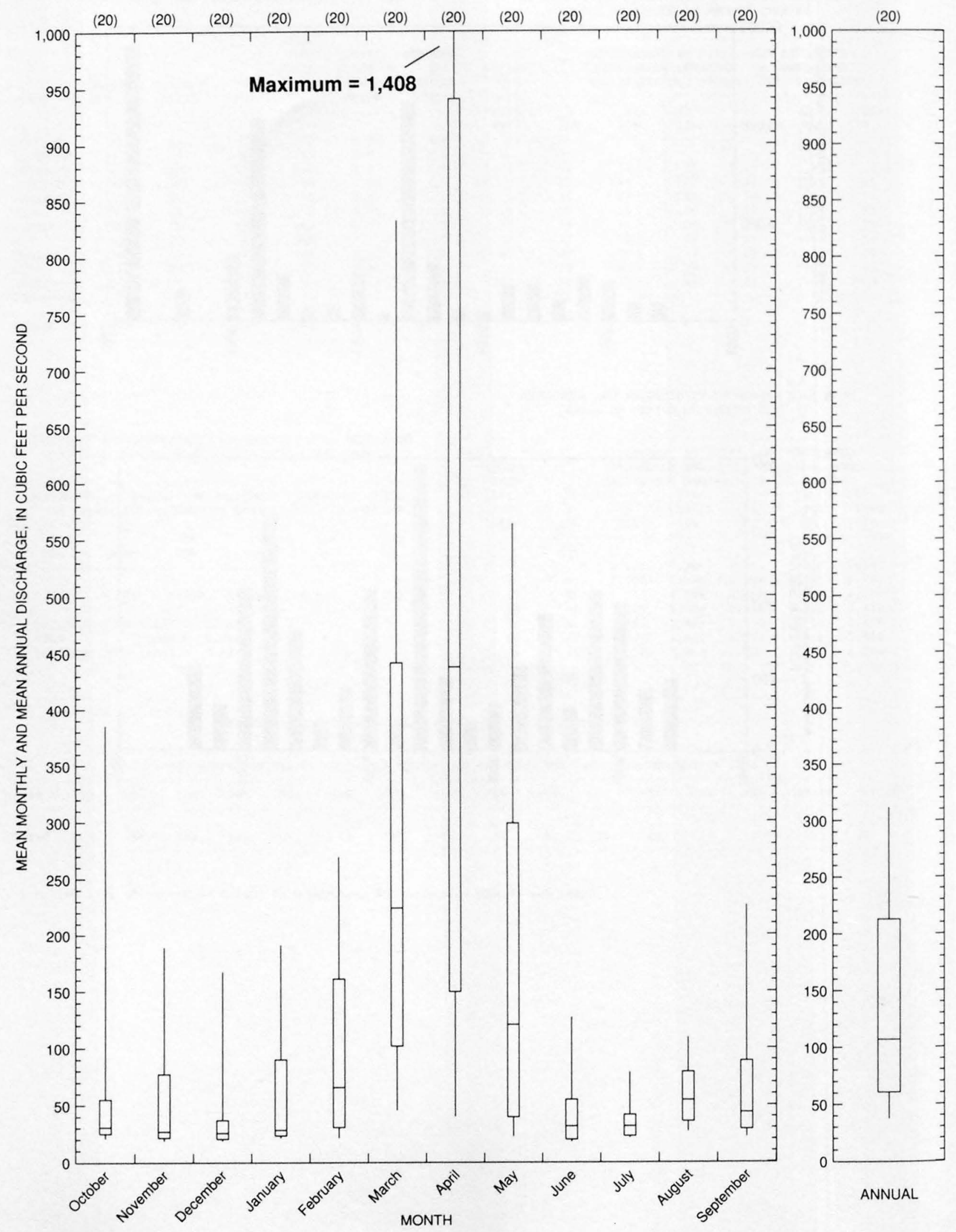
09489200 PACHETA CREEK AT MAVERICK, AZ

LOCATION.--Lat 33'44'23", long 109'32'24", at corner of secs.28, 29, 32, 33, T.4 N., R.27 E. (unsurveyed), Apache County, Hydrologic Unit 15060101 , in Fort Apache Indian Reservation, on left bank 0.5 mi southeast of Maverick.

DRAINAGE AREA.--14.8 $\mathrm{mi}^{2}$.

PERIOD OF RECORD.--October 1957 to September 1980 (discontinued). Prior to October 1970 published as Pachete Creek at Maverick.

GAGE.--Water-stage recorder and concrete control. Altitude of gage is $7,850 \mathrm{ft}$, by barometer.

REMARKS.--Records good.

AVERAGE DISCHARGE.--23 years, $9.15 \mathrm{ft}^{3} / \mathrm{s}, 6,630$ acre- $\mathrm{ft} / \mathrm{yr}$; median of yearly mean discharges, $8.0 \mathrm{ft}^{3} / \mathrm{s}, 5,800 \mathrm{acre}-\mathrm{ft} / \mathrm{yr}$.

EXTREMES FOR PERIOD OF RECORD.--Maximum discharge, $323 \mathrm{ft}^{3} / \mathrm{s}$ May 13,1973 , gage height, $4.36 \mathrm{ft}$; minimum daily, $0.2 \mathrm{ft}^{3} / \mathrm{s}$ many days in 1959-60.

Annual peak discharges

\begin{tabular}{|c|c|c|c|c|c|c|c|}
\hline $\begin{array}{l}\text { Water } \\
\text { year }\end{array}$ & Date & $\begin{array}{c}\text { Annual peak } \\
\text { discharge } \\
\left(\mathrm{ft}^{3} / \mathrm{s}\right)\end{array}$ & $\begin{array}{l}\text { Discharge } \\
\text { codes }\end{array}$ & $\begin{array}{l}\text { Water } \\
\text { year }\end{array}$ & Date & $\begin{array}{c}\text { Annual peak } \\
\text { discharge } \\
\left(\mathrm{ft}^{3} / \mathrm{s}\right)\end{array}$ & $\begin{array}{c}\text { Discharge } \\
\text { codes }\end{array}$ \\
\hline 1958 & $04-22-58$ & 312 & & 1970 & $04-11-70$ & 71 & \\
\hline 1959 & $10-06-58$ & 140 & & 1971 & $09-30-71$ & 39 & \\
\hline 1960 & $03-21-60$ & 102 & & 1972 & $10-24-71$ & 69 & \\
\hline 1961 & $08-08-61$ & 18 & & 1973 & $05-13-73$ & 323 & \\
\hline 1962 & $04-09-62$ & 179 & & 1974 & $08-05-74$ & 39 & \\
\hline 1963 & $08-19-63$ & 118 & & 1975 & $04-25-75$ & 132 & \\
\hline 1964 & $08-14-64$ & 95 & & 1976 & $04-10-76$ & 47 & \\
\hline 1965 & $04-23-65$ & 128 & & 1977 & $08-11-77$ & 45 & \\
\hline 1966 & $03-22-66$ & 145 & & 1978 & $03-30-78$ & 173 & \\
\hline 1967 & $08-27-67$ & 60 & & 1979 & $12-18-78$ & 224 & \\
\hline 1968 & $04-15-68$ & 120 & & 1980 & $04-21-80$ & 158 & \\
\hline 1969 & $04-06-69$ & 122 & & & & & \\
\hline
\end{tabular}

Basin characteristics

\begin{tabular}{|c|c|c|c|c|c|c|c|}
\hline \multirow[b]{2}{*}{$\begin{array}{c}\text { Main } \\
\text { channel } \\
\text { slope } \\
(\mathrm{ft} / \mathrm{mi})\end{array}$} & \multirow[b]{2}{*}{$\begin{array}{l}\text { Stream } \\
\text { length } \\
(\mathrm{mi})\end{array}$} & \multirow[b]{2}{*}{$\begin{array}{c}\text { Mean } \\
\text { basin } \\
\text { elevation } \\
(\mathrm{ft})\end{array}$} & \multirow[b]{2}{*}{$\begin{array}{c}\text { Forested } \\
\text { area } \\
\text { (percent) }\end{array}$} & \multirow[b]{2}{*}{$\begin{array}{c}\text { Soil } \\
\text { index }\end{array}$} & \multirow[b]{2}{*}{$\begin{array}{c}\text { Mean } \\
\text { annual } \\
\text { precipitation } \\
\text { (in) }\end{array}$} & \multicolumn{2}{|c|}{ Rainfall intensity, 24-hour } \\
\hline & & & & & & $\begin{array}{l}\text { 2-year } \\
\text { (in) }\end{array}$ & $\begin{array}{l}\text { 50-year } \\
\text { (in) }\end{array}$ \\
\hline 160 & 14.2 & 8,810 & 88.0 & 3.0 & 30.3 & 2.2 & 5.2 \\
\hline
\end{tabular}


MEAN MONTHLY AND ANNUAL DISCHARGES $1958 \cdot 80$

\begin{tabular}{|c|c|c|c|c|c|c|}
\hline MONTH & $\begin{array}{l}\text { MAXIMUM } \\
\text { (FT3/S) }\end{array}$ & $\begin{array}{l}\text { MINIMUM } \\
\text { (FT3/S) }\end{array}$ & $\begin{array}{c}\text { MEAN } \\
(\text { FT3/S) }\end{array}$ & $\begin{array}{l}\text { STAN- } \\
\text { DARD } \\
\text { DEVIA- } \\
\text { TION } \\
\text { (FT3/S) }\end{array}$ & $\begin{array}{l}\text { COEFFI- } \\
\text { CIENT OF } \\
\text { VARI- } \\
\text { ATION }\end{array}$ & $\begin{array}{c}\text { PERCENT } \\
\text { OF } \\
\text { ANNUAL } \\
\text { RUNOFF }\end{array}$ \\
\hline OCTOBER & 24 & 0.53 & 3.3 & 5.2 & 1.6 & 3.0 \\
\hline NOVEMBER & 12 & 0.35 & 2.5 & 2.5 & 1.0 & 2.2 \\
\hline DECEMBER & 27 & 0.68 & 3.3 & 5.7 & 1.7 & 3.0 \\
\hline JANUARY & 15 & 0.59 & 3.3 & 4.3 & 1.3 & 3.0 \\
\hline FEBRUARY & 14 & 0.59 & 4.2 & 4.0 & 0.94 & 3.8 \\
\hline MARCH & 61 & 1.6 & 17 & 17 & 1.0 & 15.3 \\
\hline APRIL & 102 & 1.5 & 44 & 36 & 0.82 & 39.8 \\
\hline MAY & 131 & 0.50 & 21 & 30 & 1.4 & 19.5 \\
\hline JUNE & 19 & 0.27 & 3.4 & 4.6 & 1.3 & 3.1 \\
\hline JULY & 6.4 & 0.25 & 2.0 & 1.5 & 0.76 & 1.8 \\
\hline AUGUST & 12 & 0.37 & 2.6 & 2.2 & 0.87 & 2.3 \\
\hline SEPTEMBER & 15 & 0.33 & 3.3 & 3.7 & 1.1 & 3.0 \\
\hline ANNUAL & 28 & 0.96 & 9.1 & 7.0 & 0.76 & 100 \\
\hline
\end{tabular}

MAGNITUDE AND PROBABILITY OF INSTANTANEOUS PEAK FLOW BASED ON PERIOD OF RECORD $1958 \cdot 80$

DISCHARGE, IN FT3/S, FOR INDICATED RECURRENCE INTERVAL IN YEARS, AND EXCEEDANCE PROBABILITY, IN PERCENT

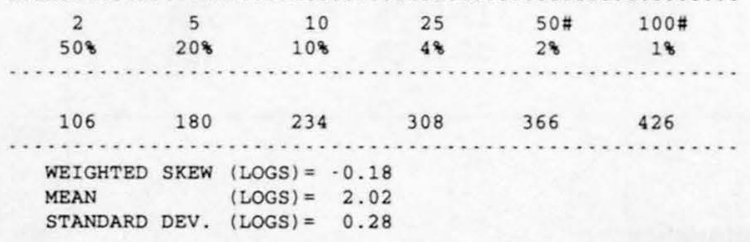

MAGNITUDE AND PROBABILITY OF ANNUAL LOW FLOW BASED ON PERIOD OF RECORD $1959 \cdot 80$

\begin{tabular}{|c|c|c|c|c|c|c|}
\hline \multirow{3}{*}{$\begin{array}{l}\text { PERIOD } \\
\text { ICON- } \\
\text { SECU- } \\
\text { TIVE }\end{array}$} & \multicolumn{6}{|c|}{$\begin{array}{l}\text { DISCHARGE, IN FT3/S, FOR INDICATED } \\
\text { RECURRENCE INTERVAL, IN YEARS, AND }\end{array}$} \\
\hline & & $N-\mathrm{BXC}$ & NON-EXCEEDANCE PROBABILITY, IN PERCENT & BABILI & $\therefore \mathrm{IN} P \mathrm{~PB}$ & \\
\hline & 2 & 5 & 10 & 20 & $50 \#$ & $100 \#$ \\
\hline DAYS) & 508 & 208 & 108 & 58 & 28 & 18 \\
\hline 1 & 0.89 & 0,50 & 0.34 & 0.23 & 0.14 & 0.10 \\
\hline 3 & 0.89 & 0.50 & 0.34 & 0.23 & 0.14 & 0.10 \\
\hline 7 & 0.92 & 0.52 & 0.35 & 0.24 & 0.14 & 0.10 \\
\hline 14 & 0.95 & 0.55 & 0.38 & 0.26 & 0.16 & 0.11 \\
\hline 30 & 0.99 & 0.61 & 0.43 & 0.31 & 0.20 & 0.14 \\
\hline 60 & 1.1 & 0.69 & 0.50 & 0.37 & 0.25 & 0.18 \\
\hline 90 & 1.2 & 0.77 & 0.57 & 0.42 & 0.28 & 0.21 \\
\hline 120 & 1.4 & 0.86 & 0.63 & 0.47 & 0.32 & 0.24 \\
\hline 183 & 1.6 & 0.95 & 0.70 & 0.54 & 0.39 & 0.32 \\
\hline
\end{tabular}

MAGNITUDE AND PROBABILITY OF ANNUAL HIGH FLOW BASED ON PERIOD OF RECORD $1958-80$

\begin{tabular}{|c|c|c|c|c|c|c|}
\hline $\begin{array}{l}\text { PERIOD } \\
\text { (CON- }\end{array}$ & & \multicolumn{4}{|c|}{$\begin{array}{l}\text { DISCHARGE, IN FT3/S, FOR INDICATED } \\
\text { RECURRENCE INTERVAL, IN YEARS, AND }\end{array}$} & \\
\hline SECU - & & 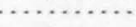 & & & 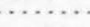 & \\
\hline TIVE & 2 & 5 & 10 & 25 & $50 \#$ & $100 \#$ \\
\hline DAYS) & 508 & 208 & 108 & 48 & 28 & 18 \\
\hline 1 & 73 & 146 & 199 & 265 & 312 & 356 \\
\hline 3 & 66 & 139 & 191 & 254 & 298 & 339 \\
\hline 7 & 58 & 127 & 176 & 235 & 275 & 312 \\
\hline 15 & 48 & 109 & 155 & 213 & 255 & 294 \\
\hline 30 & 38 & 89 & 131 & 187 & 230 & 273 \\
\hline 60 & 26 & 63 & 94 & 139 & 175 & 212 \\
\hline 90 & 20 & 47 & 70 & 103 & 129 & 157 \\
\hline
\end{tabular}

DURATION TABLE OF DAILY MEAN FLOW FOR PERIOD OF RECORD $1958 \cdot 80$

DISCHARGE, IN FT3/S, WHICH WAS EQUALED OR EXCEEDED FOR INDICATED PERCENT OF TIME

\begin{tabular}{|c|c|c|c|c|c|c|c|c|c|c|c|c|c|c|c|c|}
\hline 18 & 58 & 108 & 158 & 208 & 308 & 408 & 508 & 608 & 708 & 808 & 908 & 958 & 988 & 998 & 99.58 & 99.98 \\
\hline 116 & 49 & 23 & 14 & 8.4 & 3.9 & 2.4 & 1.9 & 1.6 & 1.4 & 1.2 & 0.83 & 0.56 & 0.31 & 0.26 & 0.23 & 0.21 \\
\hline
\end{tabular}

\# Reliability of values in column is uncertain, and potential errors are large. 
09489200 PACHETA CREEK AT MAVERICK, AZ--Continued
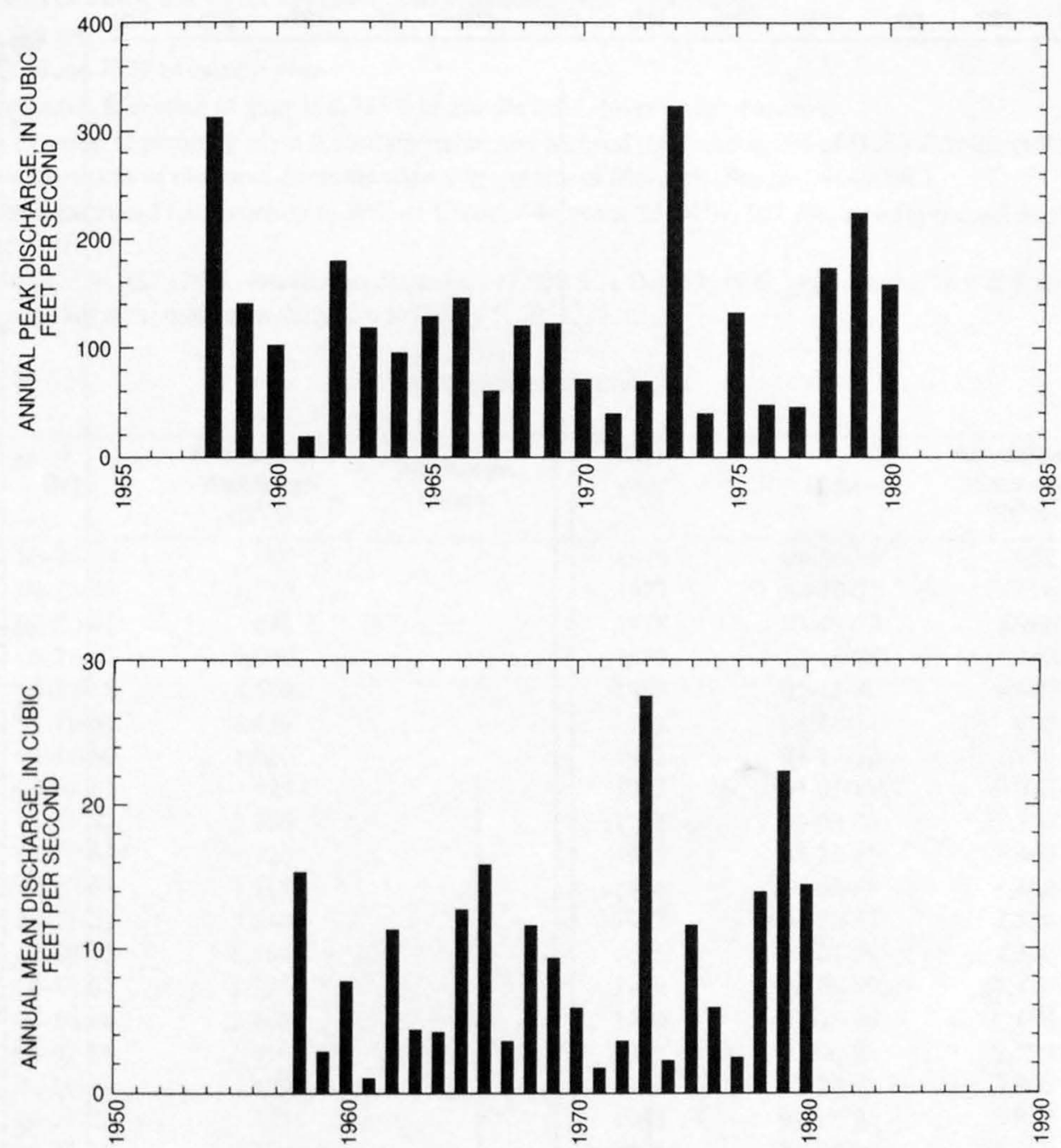
09489200 PACHETA CREEK AT MAVERICK, AZ--Continued
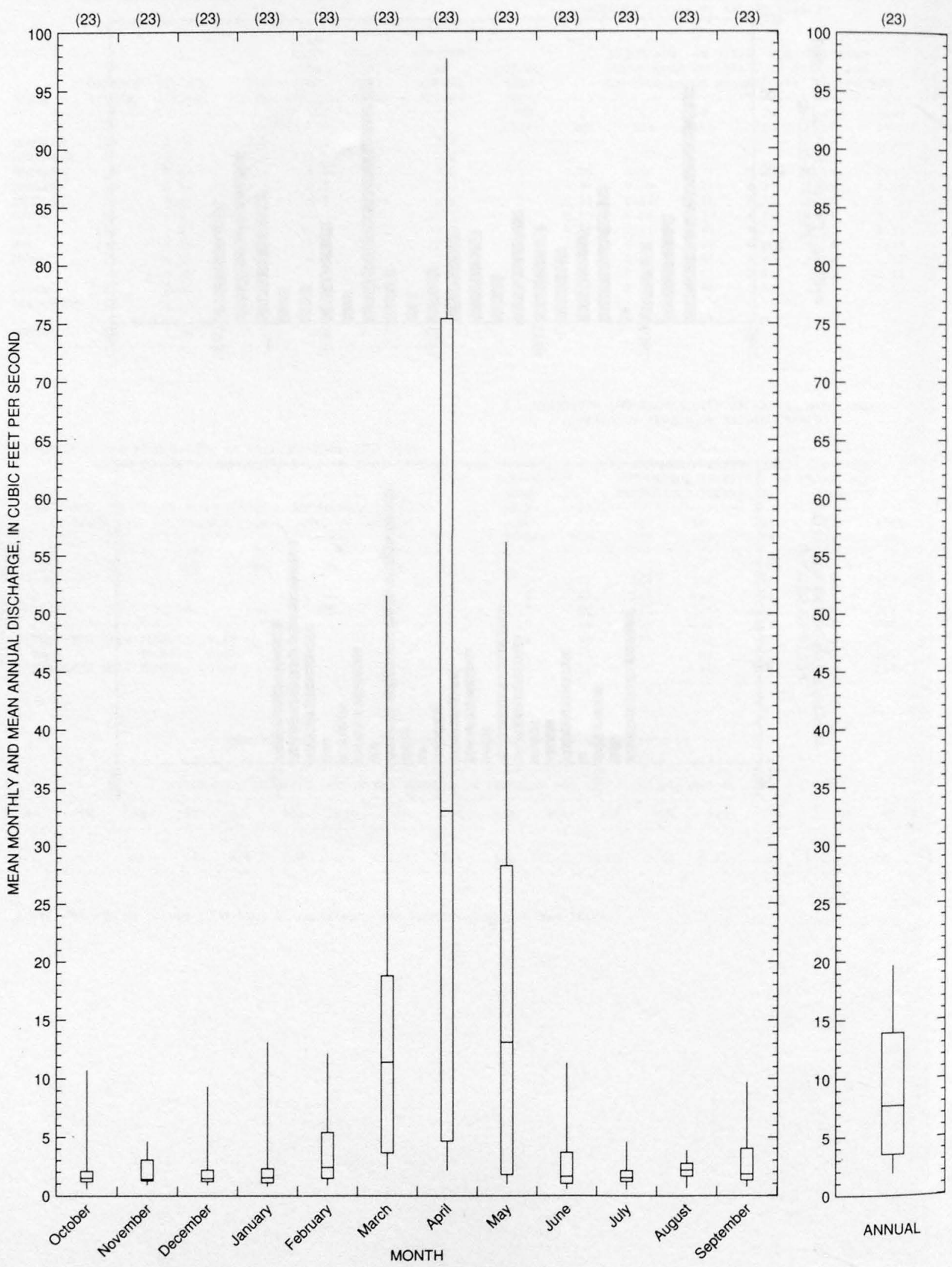
09489500 BLACK RIVER BELOW PUMPING PLANT, NEAR POINT OF PINES, AZ

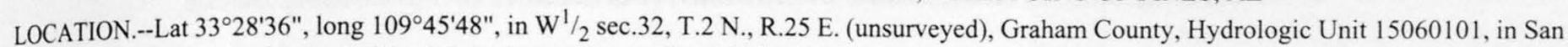
Carlos Indian Reservation, on left bank 0.9 mi downstream from Phelps Dodge Corp. pumping plant, 1.3 mi downstream from Freezeout Creek, 8 mi northwest of Point of Pines, and $63 \mathrm{mi}$ upstream from confluence with White River.

DRAINAGE AREA.--560 $\mathrm{mi}^{2}$.

PERIOD OF RECORD.--June 1953 to current year.

GAGE.--Water-stage recorder. Elevation of gage is $5,725 \mathrm{ft}$ above sea level, from topographic map.

REMARKS.--Water is diverted at pumping plant 0.9 mi upstream and pumped into headwaters of Willow Creek (tributary of Eagle Creek) for mining, metallurgical treatment of ores, and domestic supply in vicinity of Morenci. (See sta 09445000.)

AVERAGE DISCHARGE (adjusted for diversion to Willow Creek).--44 years, $231 \mathrm{ft}^{3} / \mathrm{s}, 167,400$ acre-ft/yr; median of yearly mean discharges, $190 \mathrm{ft}^{3} / \mathrm{s}, 138,000$ acre-ft/yr.

EXTREMES FOR PERIOD OF RECORD.--Maximum discharge, 17,900 $\mathrm{ft}^{3} / \mathrm{s}$ Oct. 19, 1972, gage height, $18.0 \mathrm{ft}$, from floodmarks, from rating curve extended above $5,000 \mathrm{ft}^{3} / \mathrm{s}$; minimum daily, $2.6 \mathrm{ft}^{3} / \mathrm{s}$ July $5,1974$.

Annual peak discharges

\begin{tabular}{|c|c|c|c|c|c|c|c|}
\hline $\begin{array}{l}\text { Water } \\
\text { year }\end{array}$ & Date & $\begin{array}{c}\text { Annual peak } \\
\text { discharge } \\
\left(\mathrm{ft}^{3} / \mathrm{s}\right)\end{array}$ & $\begin{array}{c}\text { Discharge } \\
\text { codes }\end{array}$ & $\begin{array}{l}\text { Water } \\
\text { year }\end{array}$ & Date & $\begin{array}{c}\text { Annual peak } \\
\text { discharge } \\
\left(\mathrm{ft}^{3} / \mathrm{s}\right)\end{array}$ & $\begin{array}{c}\text { Discharge } \\
\text { codes }\end{array}$ \\
\hline 1954 & $03-23-54$ & 5,000 & & 1976 & $04-10-76$ & 782 & \\
\hline 1955 & $08-21-55$ & 1,310 & & 1977 & $04-10-77$ & 610 & \\
\hline 1956 & $03-20-56$ & 642 & & 1978 & $03-01-78$ & 5,980 & \\
\hline 1957 & $08-26-57$ & 2,060 & & 1979 & $12-19-78$ & 12,400 & \\
\hline 1958 & $04-23-58$ & 4,590 & & 1980 & $02-15-80$ & 6,640 & \\
\hline 1959 & $08-19-59$ & 4,820 & & 1981 & $04-14-81$ & 820 & \\
\hline 1960 & $03-14-60$ & 1,820 & & 1982 & $04-13-82$ & 1,750 & \\
\hline 1961 & $04-04-61$ & 495 & & 1983 & $04-01-83$ & 3,020 & \\
\hline 1962 & $04-11-62$ & 2,950 & & 1984 & $10-02-83$ & 17,300 & \\
\hline 1963 & $08-29-63$ & 1,720 & & 1985 & $03-12-85$ & 7,440 & \\
\hline 1964 & $10-20-63$ & 1,110 & & 1986 & $04-03-86$ & 1,460 & \\
\hline 1965 & $04-22-65$ & 2,640 & & 1987 & $04-18-87$ & 2,530 & \\
\hline 1966 & $12-30-65$ & 6,380 & & 1988 & $09-01-88$ & 2,360 & \\
\hline 1967 & $08-13-67$ & 1,330 & & 1989 & $08-05-89$ & 1,140 & \\
\hline 1968 & $04-16-68$ & 2,440 & & 1990 & $07-18-90$ & 808 & \\
\hline 1969 & $04-07-69$ & 2,010 & & 1991 & $03-06-91$ & 2,790 & \\
\hline 1970 & $04-12-70$ & 479 & & 1992 & $08-24-92$ & 2,960 & \\
\hline 1971 & $08-21-71$ & 542 & & 1993 & $01-08-93$ & 8,940 & \\
\hline 1972 & $10-25-71$ & 3,210 & & 1994 & $03-20-94$ & 2,550 & \\
\hline 1973 & $10-19-72$ & 17,900 & & 1995 & $02-15-95$ & 5,450 & \\
\hline 1974 & $08-02-74$ & 404 & & 1996 & $08-26-96$ & 391 & \\
\hline 1975 & $03-09-75$ & 2,840 & & & & & \\
\hline
\end{tabular}


09489500 BLACK RIVER BELOW PUMPING PLANT, NEAR POINT OF PINES, AZ--Continued

Discharge rating table developed October 1993

\begin{tabular}{cccc}
\hline $\begin{array}{c}\text { Gage height } \\
(\mathbf{f t})\end{array}$ & $\begin{array}{c}\text { Discharge } \\
\left(\mathbf{f t}^{3} / \mathbf{s}\right)\end{array}$ & $\begin{array}{c}\text { Gage height } \\
(\mathbf{f t})\end{array}$ & $\begin{array}{c}\text { Discharge } \\
\left(\mathbf{f t}^{3} / \mathbf{s}\right)\end{array}$ \\
\hline 5.4 & 1,590 & 11.0 & 8,200 \\
5.8 & 1,920 & 13.0 & 12,800 \\
7.0 & 3,080 & 15.0 & 20,300 \\
9.0 & 5,270 & 17.0 & 32,000 \\
\hline
\end{tabular}

Basin characteristics

\begin{tabular}{cccccccc}
\hline $\begin{array}{c}\text { Main } \\
\text { channel } \\
\text { slope } \\
\text { (ft/mi) }\end{array}$ & $\begin{array}{c}\text { Stream } \\
\text { length } \\
(\mathrm{mi})\end{array}$ & $\begin{array}{c}\text { Mean } \\
\text { basin } \\
\text { elevation } \\
\text { (ft) }\end{array}$ & $\begin{array}{c}\text { Forested } \\
\text { area } \\
\text { (percent) }\end{array}$ & $\begin{array}{c}\text { Soil } \\
\text { index }\end{array}$ & $\begin{array}{c}\text { Mean } \\
\text { annual } \\
\text { precipitation } \\
\text { (in) }\end{array}$ & $\begin{array}{c}\text { R-year } \\
\text { (in) }\end{array}$ & $\begin{array}{c}\text { 50-year } \\
\text { (in) }\end{array}$ \\
\hline 51.1 & 69.2 & 8,000 & 86.0 & 2.9 & 25.3 & 2.3 \\
\hline
\end{tabular}


09489500 BLACK RIVER BELOW PUMPING PLANT, NEAR POINT OF PINES, AZ--Continued

MEAN MONTHLY AND ANNUAL DISCHARGES 1954.96

\begin{tabular}{|c|c|c|c|c|c|c|}
\hline MONTH & $\begin{array}{l}\text { MAXIMUM } \\
\text { (FT3/S) }\end{array}$ & $\begin{array}{l}\text { MINIMUM } \\
\text { (FT3/S) }\end{array}$ & $\begin{array}{c}\text { MEAN } \\
(\text { FT3/S) }\end{array}$ & $\begin{array}{l}\text { STAN- } \\
\text { DARD } \\
\text { DEVIA- } \\
\text { TION } \\
\text { (FT3/S) }\end{array}$ & $\begin{array}{l}\text { COEFFI- } \\
\text { CIENT OF } \\
\text { VARI- } \\
\text { ATION }\end{array}$ & $\begin{array}{c}\text { PERCENT } \\
\text { OF } \\
\text { ANNUAL } \\
\text { RUNOFF }\end{array}$ \\
\hline OCTOBER & 1,210 & 14 & 114 & 228 & 2.0 & 4.3 \\
\hline NOVEMBER & 505 & 23 & 92 & 101 & 1.1 & 3.5 \\
\hline DECEMBER & 915 & 20 & 126 & 174 & 1.4 & 4.8 \\
\hline JANUARY & 1,570 & 23 & 154 & 251 & 1.6 & 5.8 \\
\hline FEBRUARY & 1,040 & 35 & 250 & 266 & 1.1 & 9.4 \\
\hline MARCH & 1,860 & 30 & 568 & 475 & 0.84 & 21.4 \\
\hline APRIL & 2,250 & 32 & 751 & 667 & 0.89 & 28.3 \\
\hline MAY & 1,930 & 23 & 230 & 375 & 1.2 & 11.3 \\
\hline JUNE & 244 & 14 & 63 & 63 & 1.0 & 2.4 \\
\hline JULY & 122 & 14 & 43 & 21 & 0.50 & 1.6 \\
\hline AUGUST & 378 & 18 & 101 & 93 & 0.92 & 3.8 \\
\hline SEPTEMBER & 385 & 9.4 & 89 & 85 & 0.96 & 3.4 \\
\hline ANNUAL & 617 & 38 & 221 & 161 & 0.73 & 100 \\
\hline
\end{tabular}

MAGNITUDE AND PROBABILITY OF INSTANTANEOUS PEAK FLOW BASED ON PERIOD OF RECORD 1954-96

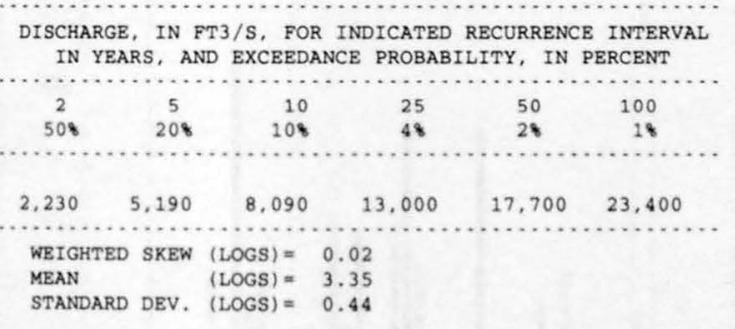

MAGNITUDE AND PROBABILITY OF ANNUAL LOW FLOW BASED ON PERIOD OF RECORD 1955.96

\begin{tabular}{|c|c|c|c|c|c|c|}
\hline \multirow[t]{2}{*}{$\begin{array}{l}\text { PERIOD } \\
\text { (CON- } \\
\text { SECU- }\end{array}$} & \multicolumn{6}{|c|}{$\begin{array}{l}\text { DISCHARGE, IN FT3/S, FOR INDICATED } \\
\text { RECURRENCE INTERVAL, IN YEARS, AND }\end{array}$} \\
\hline & \multicolumn{6}{|c|}{$\begin{array}{c}\text { NON-EXCEEDANCE PROBABILITY, IN PERCENT } \\
\cdots\end{array}$} \\
\hline $\begin{array}{l}\text { TIVE } \\
\text { DAYS) }\end{array}$ & $\begin{array}{c}2 \\
508\end{array}$ & $\begin{array}{c}5 \\
208\end{array}$ & $\begin{array}{l}10 \\
108\end{array}$ & $\begin{array}{l}20 \\
58\end{array}$ & $\begin{array}{l}50 \# \\
28\end{array}$ & $\begin{array}{l}100 \# \\
18\end{array}$ \\
\hline 1 & 12 & 7.0 & 5.2 & 4.0 & 3.0 & 2.5 \\
\hline 3 & 13 & 7.9 & 5.9 & 4.7 & 3.6 & 3.0 \\
\hline 7 & 15 & 9.9 & 7.9 & 6.7 & 5.5 & 4.8 \\
\hline 14 & 18 & 12 & 9.7 & 8.1 & 6.6 & 5.8 \\
\hline 30 & 22 & 15 & 13 & 11 & 9.1 & 8.1 \\
\hline 60 & 27 & 20 & 17 & 15 & 13 & 12 \\
\hline 90 & 33 & 25 & 21 & 19 & 17 & 16 \\
\hline 120 & 38 & 28 & 24 & 22 & 19 & 18 \\
\hline 183 & 50 & 35 & 30 & 26 & 23 & 22 \\
\hline
\end{tabular}

MAGNITUDE AND PROBABILITY OF ANNUAL HIGH FLOW BASED ON PERIOD OF RECORD $1954-96$

\begin{tabular}{|c|c|c|c|c|c|c|}
\hline & & \multicolumn{5}{|c|}{ DISCHARGE，IN FT3/S，FOR INDICATED } \\
\hline $\begin{array}{l}\text { PERIOD } \\
\text { (CON- }\end{array}$ & & \multicolumn{5}{|c|}{ RECURRENCE INTERVAL, IN YEARS, AND } \\
\hline SECU. & & $\cdots \cdots$ & & & ........ & ...... \\
\hline TIVE & 2 & 5 & 10 & 25 & $50 \#$ & $100 \#$ \\
\hline DAYS) & 508 & 208 & 108 & 48 & 28 & 18 \\
\hline 1 & 1,610 & 3,660 & 5,500 & 8,340 & 10,800 & 13,600 \\
\hline 3 & 1,400 & 2,950 & 4,200 & 5,970 & 7,390 & 8,870 \\
\hline 7 & 1,170 & 2,300 & 3,130 & 4,210 & 5,020 & 5,810 \\
\hline 15 & 945 & 1.850 & 2,520 & 3,390 & 4.040 & 4,670 \\
\hline 30 & 743 & 1,500 & 2,090 & 2,910 & 3.550 & 4,210 \\
\hline 60 & 546 & 1,140 & 1,630 & 2.330 & 2,900 & 3,500 \\
\hline 90 & 432 & 919 & 1,330 & 1,930 & 2,420 & 2,960 \\
\hline
\end{tabular}

DURATION TABLE OF DAILY MEAN FLOW FOR PERIOD OF RECORD 1954-96 DISCHARGE, IN FT3/S, WHICH WAS EQUALED OR EXCEEDED FOR INDICATED PERCENT OF TIME

\begin{tabular}{|c|c|c|c|c|c|c|c|c|c|c|c|c|c|c|c|c|}
\hline 18 & 58 & 108 & 158 & 208 & 308 & 408 & 508 & 608 & 708 & 808 & 908 & 958 & 988 & 998 & 99.58 & 99.98 \\
\hline 2,240 & 1,060 & 596 & 368 & 255 & 142 & 87 & 61 & 49 & 40 & 32 & 23 & 19 & 13 & 10 & 8.8 & 5.4 \\
\hline
\end{tabular}

\# Reliablifty of values in column is uncertain, and potential errors are large. 

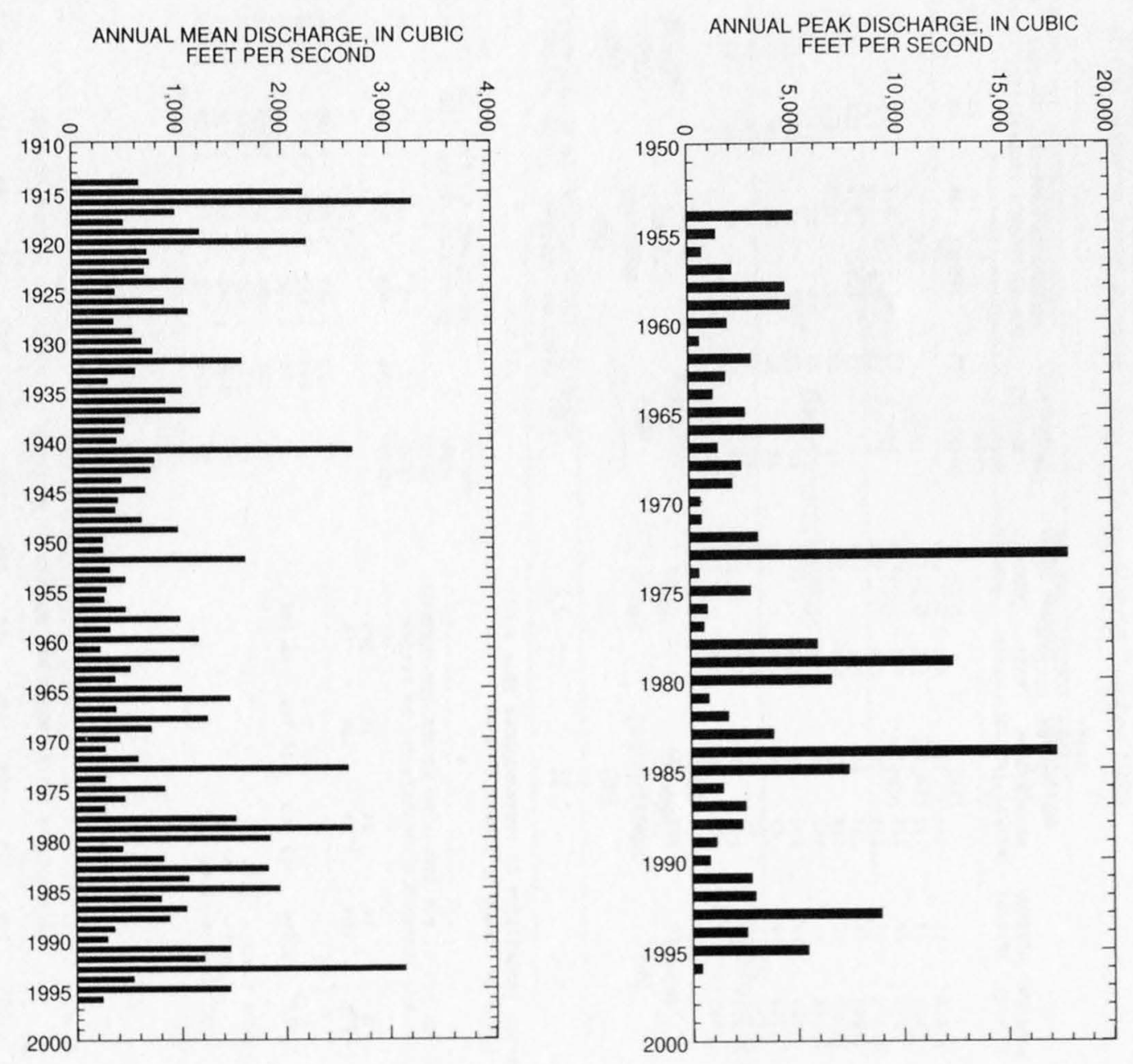
09489500 BLACK RIVER BELOW PUMPING PLANT, NEAR POINT OF PINES, AZ--Continued

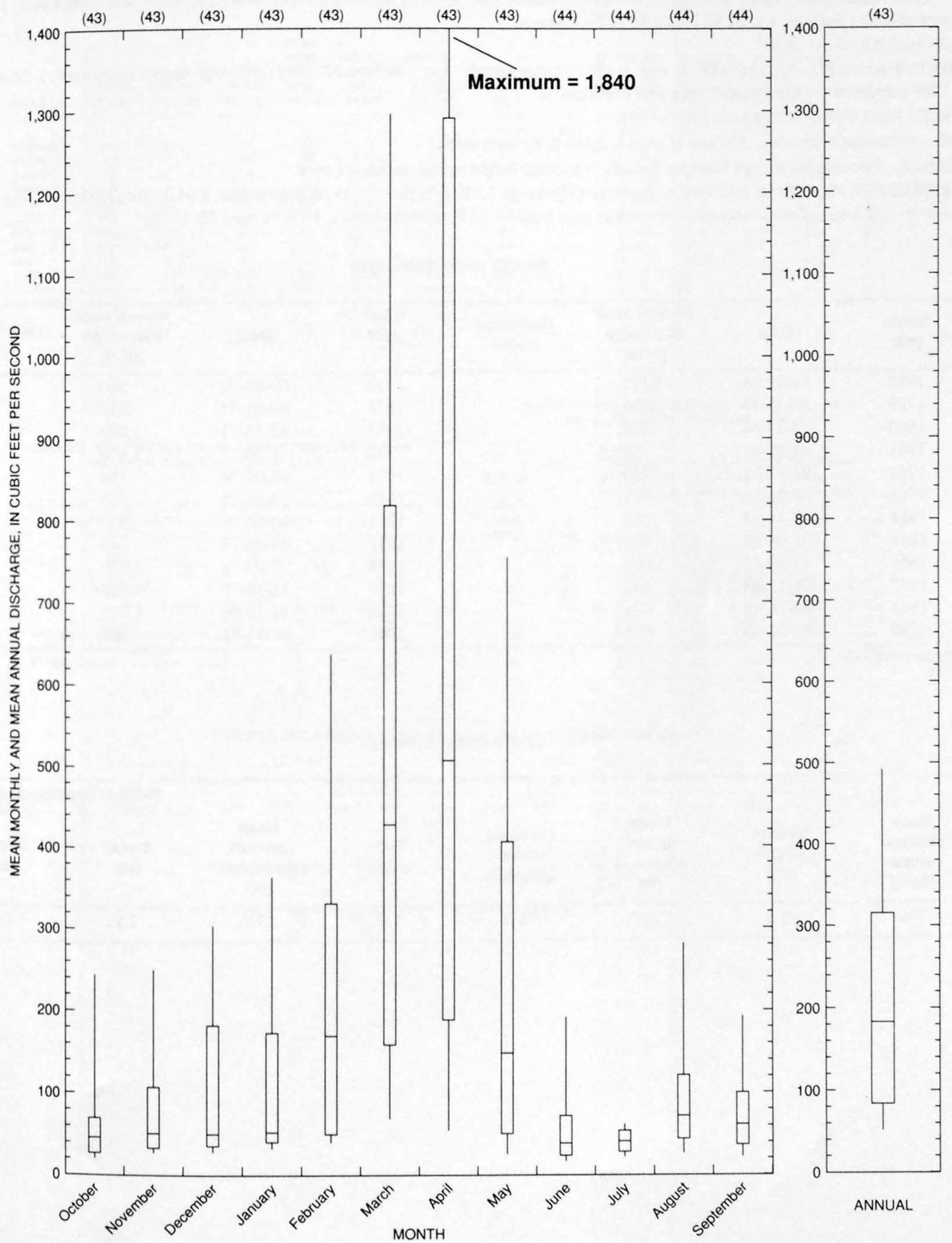




\section{GILA RIVER BASIN}

\section{BIG BONITO CREEK NEAR FORT APACHE, AZ}

LOCATION.--Lat 33 40'02", long 109 50'46", in NE $1 / 4$ sec.28, T.4 N., R.24 E. (unsurveyed), Apache County, Hydrologic Unit 15060101 , in Fort Apache Indian Reservation, near right bank on downstream side of pier of highway bridge, 1.9 mi upstream from Tonto Creek, 3.7 mi south. east of Chino Springs, and $12 \mathrm{mi}$ southeast of Fort Apache.

DRAINAGE AREA.--119 $\mathrm{mi}^{2}$.

PERIOD OF RECORD.--October 1957 to Apr. 1, 1981 (discontinued). Apr. 2 to Sept. 30, 1981 (discharge measurements only). Prior to October 1969 published as "Big Bonita Creek near Fort Apache."

REVISED RECORDS.--WRD Ariz. 1969: 1967.

GAGE.--Water-stage recorder. Altitude of gage is $5,910 \mathrm{ft}$, by barometer.

REMARKS.--Records fair except those for periods of no gage-height record, which are poor.

EXTREMES FOR PERIOD OF RECORD.--Maximum discharge, $4,510 \mathrm{ft}^{3} / \mathrm{s}$ Dec. 18, 1978, gage height, $9.04 \mathrm{ft}$; from rating curve extended above $640 \mathrm{ft}^{3} / \mathrm{s}$ on basis of slope-area measurement at gage height $7.77 \mathrm{ft}$; minimum daily, $4.2 \mathrm{ft}^{3} / \mathrm{s}$ Nov. 30,1975 .

Annual peak discharges

\begin{tabular}{|c|c|c|c|c|c|c|c|}
\hline $\begin{array}{l}\text { Water } \\
\text { year }\end{array}$ & Date & $\begin{array}{c}\text { Annual peak } \\
\text { discharge } \\
\left(\mathrm{ft}^{3} / \mathrm{s}\right)\end{array}$ & $\begin{array}{c}\text { Discharge } \\
\text { codes }\end{array}$ & $\begin{array}{l}\text { Water } \\
\text { year }\end{array}$ & Date & $\begin{array}{c}\text { Annual peak } \\
\text { discharge } \\
\left(\mathrm{ft}^{3} / \mathrm{s}\right)\end{array}$ & $\begin{array}{c}\text { Discharge } \\
\text { codes }\end{array}$ \\
\hline 1958 & $03-22-58$ & 1,120 & & 1970 & $09-06-70$ & 560 & \\
\hline 1959 & $08-15-59$ & 1,200 & & 1971 & 09-01-71 & 312 & \\
\hline 1960 & $03-27-60$ & 356 & & 1972 & $12-26-71$ & 1,090 & \\
\hline 1961 & $04-06-61$ & 83 & & 1973 & $10-20-72$ & 1,870 & \\
\hline 1962 & $04-17-62$ & 710 & & 1974 & $08-02-74$ & 194 & \\
\hline 1963 & $08-26-63$ & 557 & & 1975 & $04-26-75$ & 453 & \\
\hline 1964 & $08-14-64$ & 885 & & 1976 & $02-10-76$ & 194 & \\
\hline 1965 & $01-08-65$ & 973 & & 1977 & $08-19-77$ & 243 & \\
\hline 1966 & $12-30-65$ & 1,640 & & 1978 & $03-03-78$ & ${ }^{\prime} 2,870$ & \\
\hline 1967 & $08-12-67$ & 448 & & 1979 & $12-18-78$ & ${ }^{1} 4,510$ & \\
\hline 1968 & $04-16-68$ & 426 & & 1980 & $02-15-80$ & 3,440 & \\
\hline 1969 & 04-01-69 & 678 & & 1981 & $04-01-81$ & 153 & \\
\hline
\end{tabular}

'Highest since 1952.

Basin characteristics

\begin{tabular}{ccccccc}
\hline $\begin{array}{c}\text { Main } \\
\text { channel } \\
\text { slope } \\
(\mathrm{ft} / \mathrm{mi})\end{array}$ & $\begin{array}{c}\text { Stream } \\
\text { length } \\
(\mathrm{mi})\end{array}$ & $\begin{array}{c}\text { Mean } \\
\text { basin } \\
\text { elevation } \\
(\mathrm{ft})\end{array}$ & $\begin{array}{c}\text { Forested } \\
\text { area } \\
\text { (percent) }\end{array}$ & $\begin{array}{c}\text { Soil } \\
\text { index }\end{array}$ & $\begin{array}{c}\text { Mean } \\
\text { annual } \\
\text { precipitation } \\
\text { (in) }\end{array}$ & $\begin{array}{c}\text { R-year } \\
\text { (in) }\end{array}$ \\
\hline 106 & 33.5 & 7,920 & 95.0 & 3.0 & 27.9 & $\begin{array}{c}50 \text {-year } \\
\text { (in) }\end{array}$ \\
\hline
\end{tabular}


09489700 BIG BONITO CREEK NEAR FORT APACHE, AZ--Continued

MEAN MONTHLY AND ANNUAL DISCHARGES 1958-80

\begin{tabular}{|c|c|c|c|c|c|c|}
\hline MONTH & $\begin{array}{l}\text { MAXIMUM } \\
\text { (FT3/S) }\end{array}$ & $\begin{array}{l}\text { MINIMUM } \\
\text { (FT3/S) }\end{array}$ & $\begin{array}{c}\text { MEAN } \\
(\text { FT3/S })\end{array}$ & $\begin{array}{l}\text { STAN- } \\
\text { DARD } \\
\text { DEVIA- } \\
\text { TION } \\
\text { (FT3/S) }\end{array}$ & $\begin{array}{l}\text { COEFFI- } \\
\text { CIENT OF } \\
\text { VARI- } \\
\text { ATION }\end{array}$ & $\begin{array}{c}\text { PERCENT } \\
\text { OF } \\
\text { ANNUAL } \\
\text { RUNOFF }\end{array}$ \\
\hline OCTOBER & 157 & 9.2 & 30 & 40 & 1.3 & 3.7 \\
\hline NOVEMBER & 69 & 10 & 28 & 19 & 0.71 & 3.3 \\
\hline DECEMBER & 251 & 11 & 39 & 57 & 1.5 & 4.8 \\
\hline JANUARY & 115 & 11 & 40 & 35 & 0.86 & 4.9 \\
\hline FEBRUARY & 316 & 10 & 65 & 68 & 1.0 & 7.9 \\
\hline MARCH & 521 & 21 & 133 & 119 & 0.90 & 16.2 \\
\hline APRIL & 540 & 23 & 224 & 168 & 0.75 & 27.2 \\
\hline MAY & 610 & 11 & 138 & 149 & 1.1 & 16.8 \\
\hline JUNE & 148 & 6.3 & 34 & 39 & 1.1 & 4.1 \\
\hline JULY & 50 & 6.5 & 20 & 11 & 0.56 & 2.4 \\
\hline AUGUST & 122 & 7.8 & 40 & 29 & 0.73 & 4.9 \\
\hline SEPTEMBER & 112 & 7.0 & 31 & 26 & 0.83 & 3.8 \\
\hline ANNUAL & 167 & 17 & 69 & 43 & 0.62 & 100 \\
\hline
\end{tabular}

MAGNITUDE AND PROBABILITY OF INSTANTANEOUS PEAK FLOW BASED ON PERIOD OF RECORD 1958-81

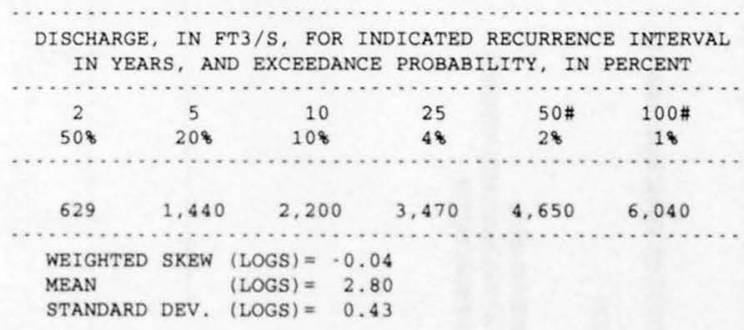

MAGNITUDE AND PROBABILITY OF ANNUAL LOW FLOW BASED ON PERIOD OF RECORD $1959 \cdot 81$

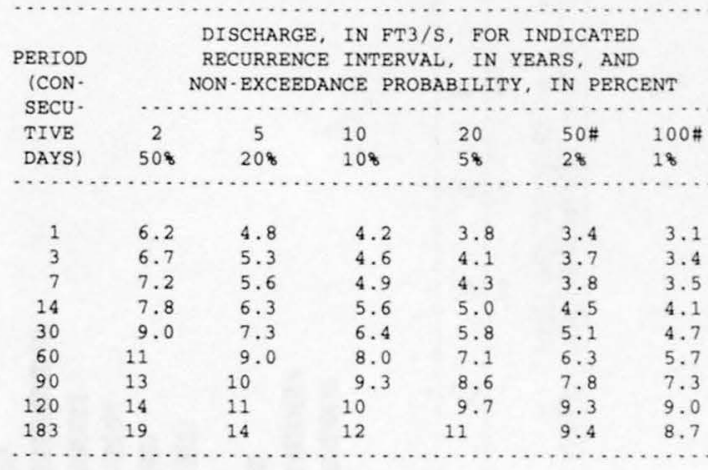

MAGNITUDE AND PROBABILITY OF ANNUAL HIGH FLOW BASED ON PERIOD OF RECORD 1958-80

\begin{tabular}{|c|c|c|c|c|c|c|}
\hline $\begin{array}{l}\text { PERIOD } \\
\text { ICON- }\end{array}$ & & \multicolumn{5}{|c|}{$\begin{array}{l}\text { DISCHARGE, IN FT3/S, FOR INDICATED } \\
\text { RECURRENCE INTERVAL, IN YEARS, AND }\end{array}$} \\
\hline SECU. & & & & & $\ldots \ldots \ldots$ & \\
\hline TIVE & 2 & 5 & 10 & 25 & $50 \#$ & $100 \#$ \\
\hline DAYS) & 508 & 208 & 108 & 48 & 28 & 18 \\
\hline 1 & 453 & 969 & 1,440 & 2,190 & 2,860 & 3,640 \\
\hline 3 & 391 & 791 & 1,140 & 1,670 & 2,130 & 2,650 \\
\hline 7 & 328 & 623 & 859 & 1,190 & 1,470 & 1,760 \\
\hline 15 & 269 & 503 & 684 & 937 & 1,140 & 1,350 \\
\hline 30 & 219 & 416 & 576 & 806 & 997 & 1,200 \\
\hline 60 & 172 & 330 & 456 & 638 & 786 & 945 \\
\hline 90 & 140 & 271 & 375 & 520 & 638 & 761 \\
\hline
\end{tabular}

DURATION TABLE OF DAILY MEAN FLOW FOR PERIOD OF RECORD 1958-80

DISCHARGE, IN FT3/S, WHICH WAS EQUALED OR EXCEEDED FOR INDICATED PERCENT OF TIME

18
$\ldots$

\# Reliability of values in column is uncertain, and potential errors are large. 
ANNUAL MEAN DISCHARGE, IN CUBIC

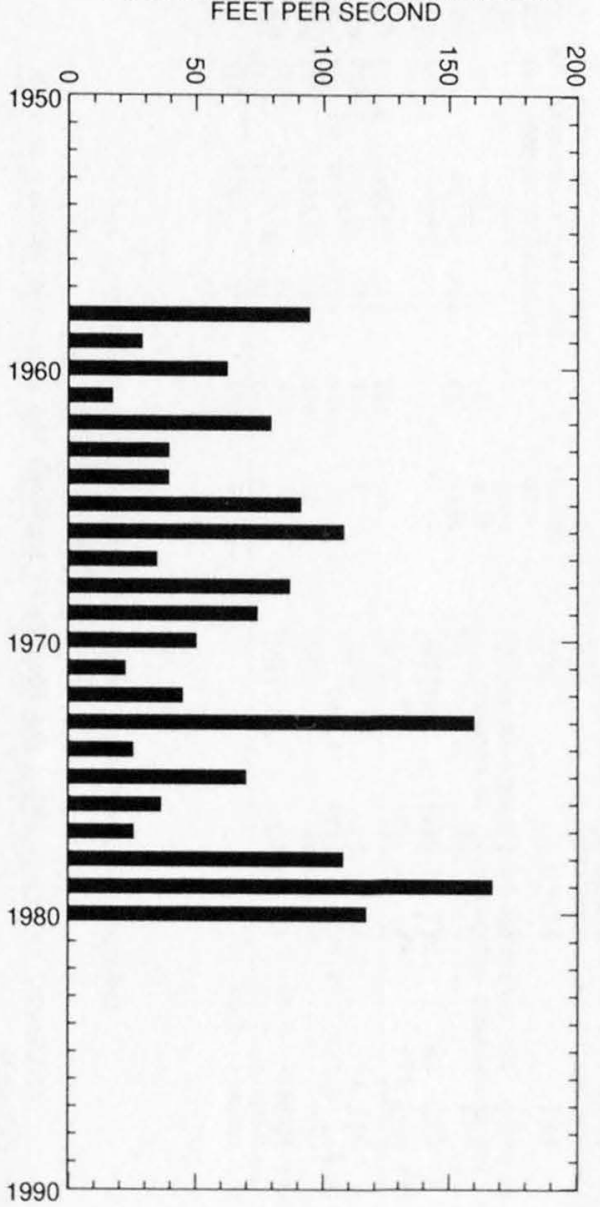

ANNUAL PEAK DISCHARGE, IN CUBIC FEET PER SECOND

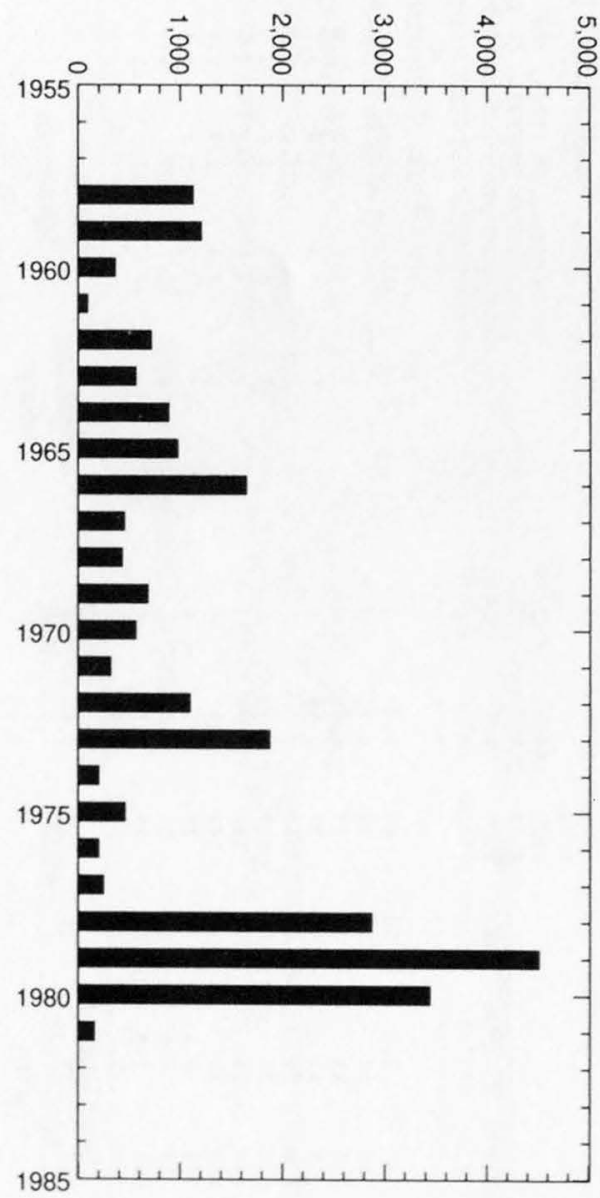


09489700 BIG BONITO CREEK NEAR FORT APACHE, AZ--Continued
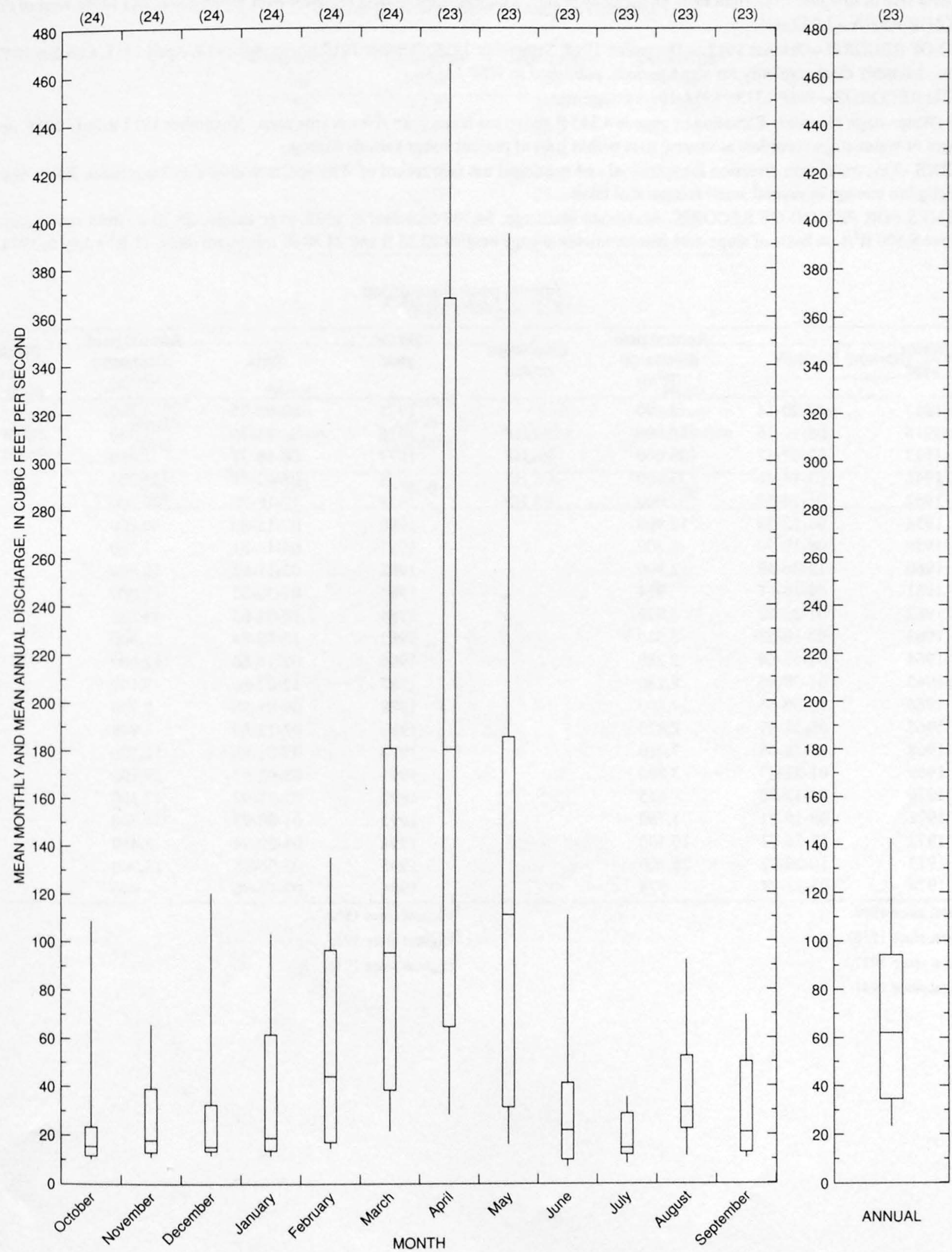

ANNUAL 


\section{BLACK RIVER NEAR FORT APACHE, AZ}

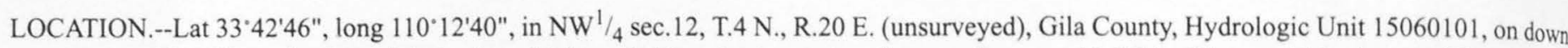
stream side of first pier from right bank on highway bridge, 5 mi upstream from confluence with White River and 14 mi west of Fort Apache.

DRAINAGE AREA.--1,232 $\mathrm{mi}^{2}$.

PERIOD OF RECORD.--October 1912 to December 1915, September 1916, October 1917 to January 1918, April 1918, October 1957 to curren year. Monthly discharge only for somè periods, published in WSP 1313.

REVISED RECORDS.--WSP 1313: 1914-15, drainage area.

GAGE.--Water-stage recorder. Elevation of gage is 4,345 ft above sea level, from river-profile map. November 1912 to July 1918 , nonrecording gages or water-stage recorders at several sites within $1 \mathrm{mi}$ of present site at various datums.

REMARKS.--One transbasin diversion for industrial and municipal use (see record of Willow Creek diversion from Black River, near Morenci) Negligible storage in several small recreational lakes.

EXTREMES FOR PERIOD OF RECORD.--Maximum discharge, 54,700 $\mathrm{ft}^{3} / \mathrm{s} \mathrm{Jan.} \mathrm{8,} \mathrm{1993,} \mathrm{gage} \mathrm{height,} 28.10 \mathrm{ft}$, from rating curve extended above $8,500 \mathrm{ft}^{3} / \mathrm{s}$ on basis of slope-area measurements at gage heights $22.33 \mathrm{ft}$ and $24.80 \mathrm{ft}$; minimum daily, $11 \mathrm{ft}^{3} / \mathrm{s} \mathrm{July} 6,1974$.

Annual peak discharges

\begin{tabular}{|c|c|c|c|c|c|c|c|}
\hline $\begin{array}{l}\text { Water } \\
\text { year }\end{array}$ & Date & $\begin{array}{c}\text { Annual peak } \\
\text { discharge } \\
\left(\mathrm{ft}^{3} / \mathrm{s}\right)\end{array}$ & $\begin{array}{c}\text { Discharge } \\
\text { codes }\end{array}$ & $\begin{array}{l}\text { Water } \\
\text { year }\end{array}$ & Date & $\begin{array}{c}\text { Annual peak } \\
\text { discharge } \\
\left(\mathrm{ft}^{3} / \mathrm{s}\right)\end{array}$ & $\begin{array}{c}\text { Discharge } \\
\text { codes }\end{array}$ \\
\hline 1915 & $12-20-14$ & 18,000 & & 1975 & $03-09-75$ & 4,360 & \\
\hline 1916 & $01-19-16$ & ${ }^{1} 50,000$ & ES,HP & 1976 & $02-11-76$ & 1,560 & \\
\hline 1937 & $02-07-37$ & 235,000 & ES,HP & 1977 & $08-18-77$ & 1,030 & \\
\hline 1941 & $03-14-41$ & ${ }^{3} 35,000$ & $\mathrm{ES}, \mathrm{HP}$ & 1978 & $03-02-78$ & 533,200 & \\
\hline 1952 & $01-14-52$ & $4_{35,000}$ & ES,HP & 1979 & $12-18-78$ & ${ }^{6} 40,200$ & \\
\hline 1958 & $03-22-58$ & 12,900 & & 1980 & $02-15-80$ & 40,000 & \\
\hline 1959 & $08-18-59$ & 8,300 & & 1981 & $04-14-81$ & 1,260 & \\
\hline 1960 & $12-26-59$ & 12,900 & & 1982 & $02-11-82$ & 10,800 & \\
\hline 1961 & $09-14-61$ & 914 & & 1983 & $01-30-83$ & 12,000 & \\
\hline 1962 & $01-25-62$ & 4,920 & & 1984 & $10-02-83$ & 44,200 & \\
\hline 1963 & $02-10-63$ & 5,580 & & 1985 & $12-28-84$ & 21,400 & \\
\hline 1964 & $08-15-64$ & 2,280 & & 1986 & $02-16-86$ & 12,600 & \\
\hline 1965 & $01-08-65$ & 8,180 & & 1987 & $12-07-86$ & 7,140 & \\
\hline 1966 & $12-30-65$ & 24,800 & & 1988 & $09-01-88$ & 8,790 & \\
\hline 1967 & $08-11-67$ & 2,870 & & 1989 & $03-12-89$ & 948 & \\
\hline 1968 & $01-28-68$ & 7,010 & & 1990 & $07-21-90$ & 12,500 & \\
\hline 1969 & $01-22-69$ & 3,860 & & 1991 & $03-02-91$ & 29,100 & \\
\hline 1970 & $04-13-70$ & 675 & & 1992 & $02-13-92$ & 17,300 & \\
\hline 1971 & $08-19-71$ & 1,780 & & 1993 & $01-08-93$ & ${ }^{7} 54,700$ & \\
\hline 1972 & $12-26-71$ & 10,500 & & 1994 & $03-20-94$ & 5,460 & \\
\hline 1973 & $10-20-72$ & 28,400 & & 1995 & $03-06-95$ & 15,000 & \\
\hline 1974 & $03-22-74$ & 574 & & 1996 & $07-12-96$ & 946 & \\
\hline
\end{tabular}

${ }^{1}$ Highest since 1906.

${ }^{2}$ Highest since 1916.

${ }^{3}$ Highest since 1937.

${ }^{4}$ Highest since 1941.

\footnotetext{
${ }^{5}$ Highest since 1952.

${ }^{6}$ Highest since 1916.

${ }^{7}$ Highest since 1916.
} 
09490500 BLACK RIVER NEAR FORT APACHE, AZ--Continued

Discharge rating table developed January 1993

\begin{tabular}{cccc}
\hline $\begin{array}{c}\text { Gage height } \\
(\mathbf{f t})\end{array}$ & $\begin{array}{c}\text { Discharge } \\
\left(\mathbf{f t}^{\mathbf{3}} / \mathbf{s}\right)\end{array}$ & $\begin{array}{c}\text { Gage height } \\
(\mathbf{f t})\end{array}$ & $\begin{array}{c}\text { Discharge } \\
\left(\mathbf{f t}^{\mathbf{3}} / \mathbf{s}\right)\end{array}$ \\
\hline 5.0 & 1,490 & 18.0 & 21,780 \\
6.0 & 2,240 & 20.0 & 27,170 \\
8.0 & 4,040 & 22.0 & 33,200 \\
10.0 & 6,370 & 24.0 & 39,650 \\
12.0 & 9,290 & 26.0 & 46,670 \\
14.0 & 12,840 & 28.0 & 54,290 \\
16.0 & 17,000 & 28.4 & 55,880 \\
\hline
\end{tabular}

Basin characteristics

\begin{tabular}{cccccccc}
\hline $\begin{array}{c}\text { Main } \\
\text { channel } \\
\text { slope } \\
(\mathrm{ft} / \mathrm{mi})\end{array}$ & $\begin{array}{c}\text { Stream } \\
\text { length } \\
(\mathrm{mi})\end{array}$ & $\begin{array}{c}\text { Mean } \\
\text { basin } \\
\text { elevation } \\
(\mathrm{ft})\end{array}$ & $\begin{array}{c}\text { Forested } \\
\text { area } \\
\text { (percent) }\end{array}$ & $\begin{array}{c}\text { Soil } \\
\text { index }\end{array}$ & $\begin{array}{c}\text { Mean } \\
\text { annual } \\
\text { precipitation } \\
\text { (in) }\end{array}$ & $\begin{array}{c}\text { Rainfall intensity, 24-hour } \\
\text { 2-year } \\
\text { (in) }\end{array}$ & $\begin{array}{c}50 \text {-year } \\
\text { (in) }\end{array}$ \\
\hline 36.2 & 120 & 7,200 & 81.0 & 3.0 & 23.4 & 2.2 \\
\hline
\end{tabular}


09490500 BLACK RIVER NEAR FORT APACHE, AZ--Continued

MEAN MONTHLY AND ANNUAL DISCHARGES 1915, 1958.96

\begin{tabular}{|c|c|c|c|c|c|c|}
\hline MONTH & $\begin{array}{l}\text { MAXIMUM } \\
(\text { FT3/S) }\end{array}$ & $\begin{array}{l}\text { MINIMUM } \\
(\text { FT3/S) }\end{array}$ & $\begin{array}{c}\text { MEAN } \\
(\text { FT3/S) }\end{array}$ & $\begin{array}{l}\text { STAN - } \\
\text { DARD } \\
\text { DEVIA- } \\
\text { TION } \\
(\mathrm{FT} 3 / \mathrm{S})\end{array}$ & $\begin{array}{l}\text { COEFFI- } \\
\text { CIENT OF } \\
\text { VARI- } \\
\text { ATION }\end{array}$ & $\begin{array}{c}\text { PERCENT } \\
\text { OF } \\
\text { ANNUAL } \\
\text { RUNOFF }\end{array}$ \\
\hline OCTOBER & 2.730 & 31 & 226 & 507 & 2.2 & 4.3 \\
\hline NOVEMBER & 769 & 37 & 158 & 167 & 1.1 & 3.0 \\
\hline DECEMBER & 2,450 & 38 & 400 & 594 & 1.5 & 7.6 \\
\hline JANUARY & 4,900 & 37 & 462 & 796 & 1.7 & 8.8 \\
\hline FEBRUARY & 3,150 & 57 & 640 & 684 & 1.1 & 12.2 \\
\hline MARCH & 3,860 & 57 & 1,100 & 954 & 0.87 & 20.9 \\
\hline APRIL & 4,420 & 48 & 1,220 & 1.050 & 0.86 & 23.2 \\
\hline MAY & 3,110 & 29 & 517 & 608 & 1.2 & 9.8 \\
\hline JUNE & 448 & 17 & 114 & 114 & 1.0 & 2.2 \\
\hline JULY & 763 & 23 & 89 & 115 & 1.3 & 1.7 \\
\hline AUGUST & 659 & 35 & 187 & 172 & 0.92 & 3.5 \\
\hline SEPTEMBER & 650 & 31 & 150 & 138 & 0.92 & 2.9 \\
\hline JNUAL & 1,200 & 62 & 438 & 323 & 0.74 & 100 \\
\hline
\end{tabular}

MAGNITUDE AND PROBABILITY OF INSTANTANEOUS PEAK FLOW BASED ON PERIOD OF RECORD 1958.95

DISCHARGE, IN FT3/S, FOR INDICATED RECURRENCE INTERVAL IN YEARS, AND EXCEEDANCE PROBABILITY, IN PERCENT

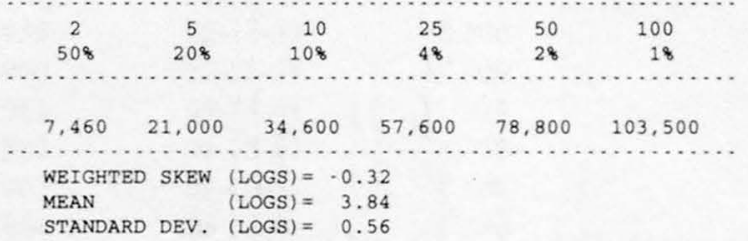

DURATION TABLE OF DAILY MEAN FLOW FOR PERIOD OF RECORD 1915, 1958.96
MAGNITUDE AND PROBABILITY OF ANNUAL LOW FLOW BASED ON PERIOD OF RECORD 1915, 1959.96

\begin{tabular}{|c|c|c|c|c|c|c|}
\hline \multirow{3}{*}{$\begin{array}{l}\text { PERIOD } \\
\text { (CON- } \\
\text { SECU- } \\
\text { TIVE }\end{array}$} & \multirow{2}{*}{\multicolumn{6}{|c|}{$\begin{array}{l}\text { DISCHARGE, IN FT3/S, FOR INDICATED } \\
\text { RECURRENCE INTERVAL, IN YEARS, AND } \\
\text { NON-EXCEBDANCE PROBABILITY, IN PERCENT }\end{array}$}} \\
\hline & & & & & & ......... \\
\hline & 2 & 5 & 10 & 20 & 50 & $100 \#$ \\
\hline DAYS) & 508 & 208 & 108 & 58 & 28 & 18 \\
\hline 1 & 25 & 18 & 15 & 13 & 11 & 9.7 \\
\hline 3 & 26 & 19 & 16 & 14 & 12 & 11 \\
\hline 7 & 28 & 20 & 17 & 15 & 13 & 12 \\
\hline 14 & 31 & 22 & 19 & 16 & 14 & 13 \\
\hline 30 & 36 & 26 & 22 & 19 & 17 & 15 \\
\hline 60 & 44 & 33 & 29 & 26 & 24 & 23 \\
\hline 90 & 54 & 40 & 35 & 32 & 29 & 27 \\
\hline 120 & 65 & 46 & 40 & 36 & 32 & 30 \\
\hline 183 & 83 & 58 & 50 & 46 & 43 & 41 \\
\hline
\end{tabular}

MAGNITUDE AND PROBABILITY OF ANNUAL HIGH FLOW BASED ON PERIOD OF RECORD 1915, 1958.96

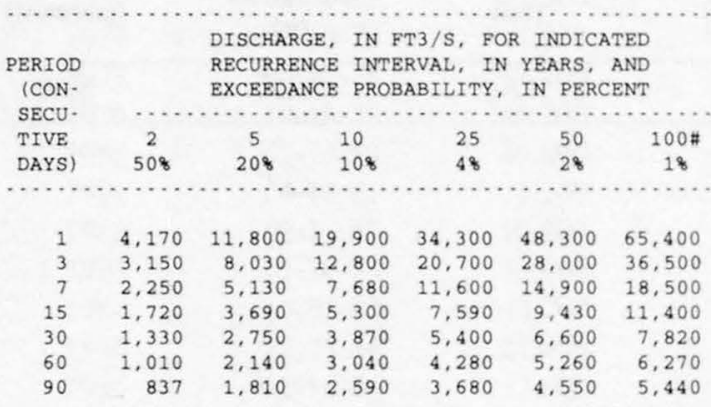

\begin{tabular}{|c|c|c|c|c|c|c|c|c|c|c|c|c|c|c|c|c|}
\hline 18 & 58 & 108 & 158 & 208 & 308 & 408 & 508 & 608 & 708 & 808 & 908 & 958 & 988 & 998 & 99.58 & 99.98 \\
\hline 4,160 & 1,970 & 1,230 & 775 & 505 & 266 & 161 & 109 & 82 & 65 & 52 & 39 & 31 & 24 & 21 & 18 & 13 \\
\hline
\end{tabular}

\# Reliability of values in column is uncertain, and potential errors are large. 
ANNUAL MEAN DISCHARGE, IN CUBIC FEET PER SECOND

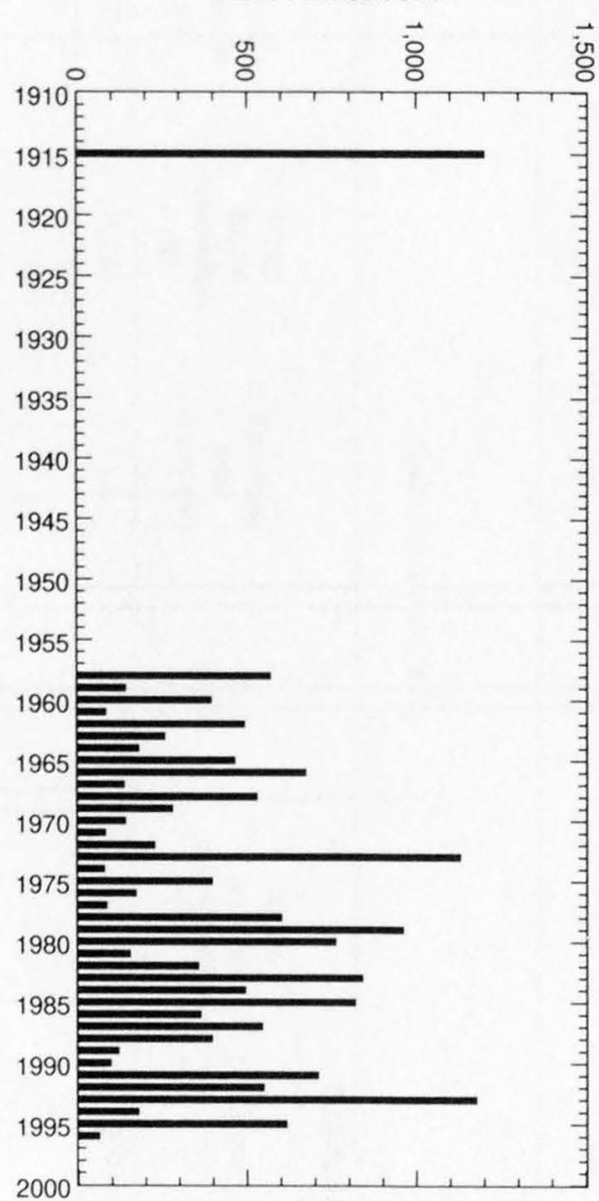

ANNUAL PEAK DISCHARGE, IN CUBIC FEET PER SECOND

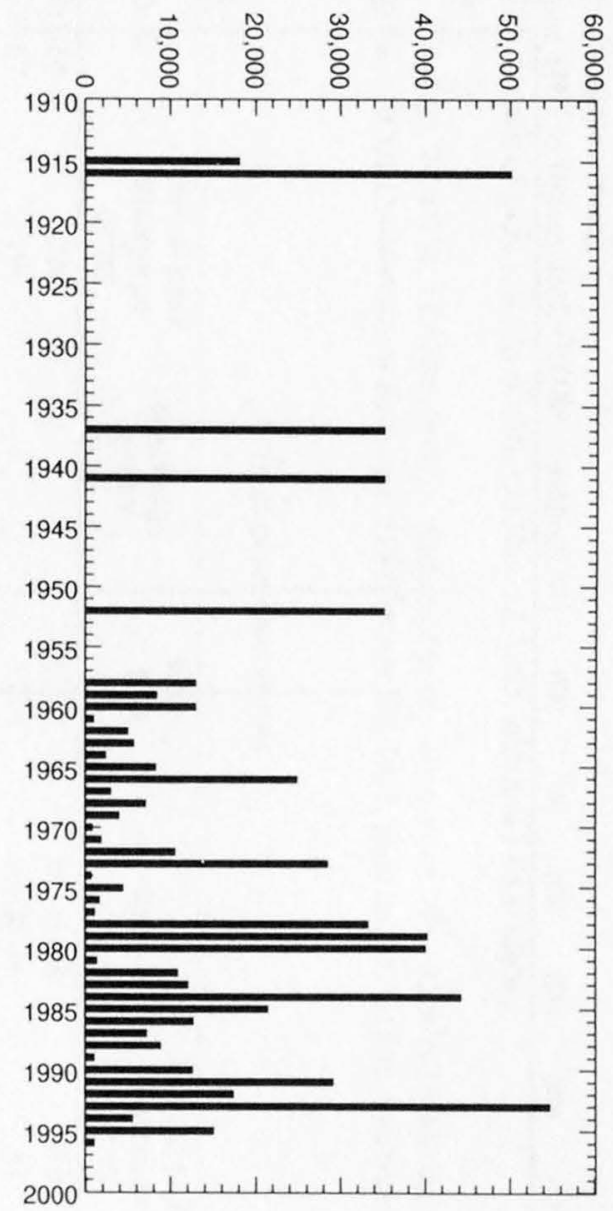


09490500 BLACK RIVER NEAR FORT APACHE, AZ--Continued

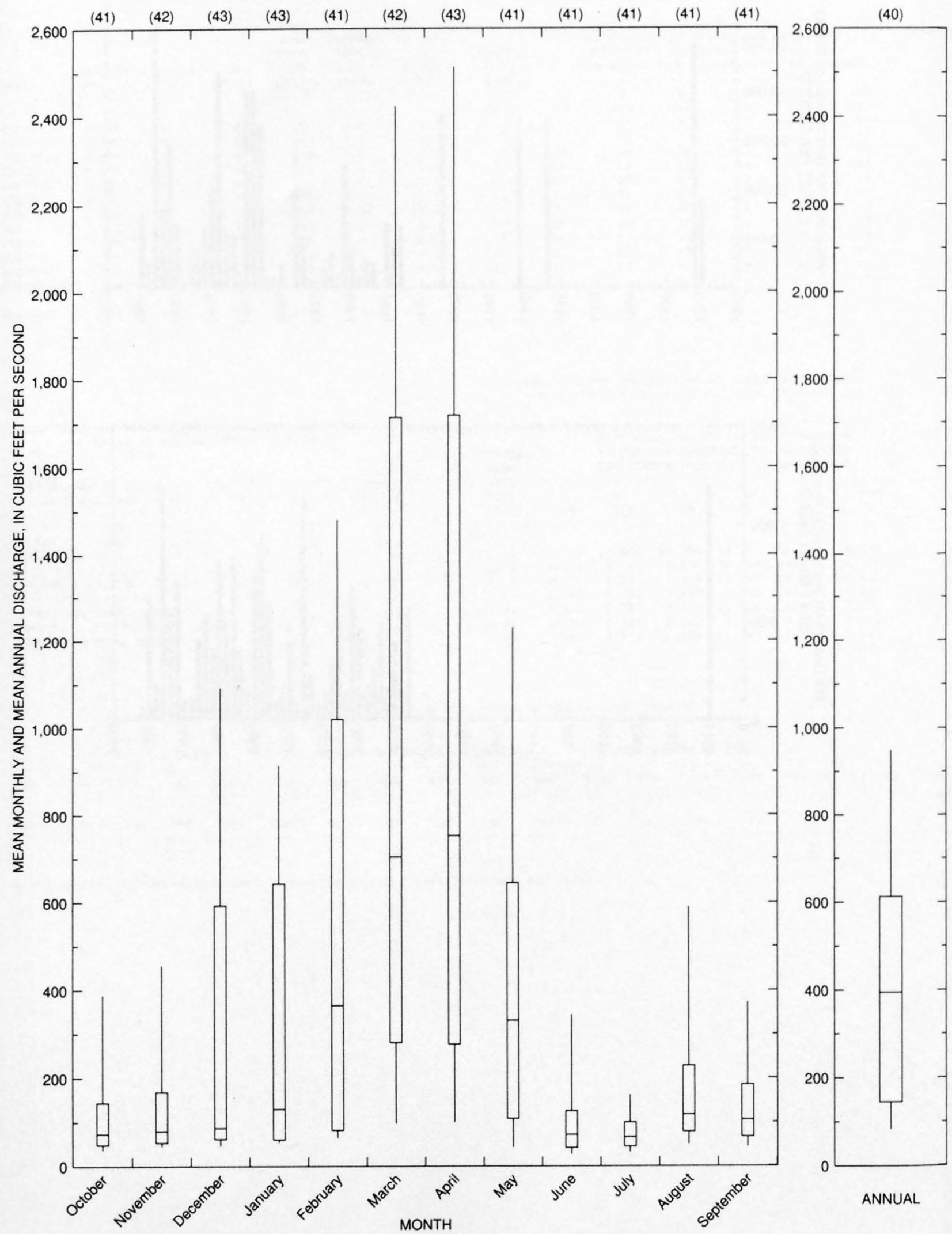




\section{NORTH FORK WHITE RIVER NEAR GREER, AZ}

LOCATION.--Lat 34'00'55", long 109'38'37", in SW¹/4 sec.7, T.7 N., R.26 E. (unsurveyed), Apache County, Hydrologic Unit 15060102, in Fort Apache Indian Reservation, on right bank $300 \mathrm{ft}$ upstream from Bear Cienega Creek and 11 mi west of Greer.

DRAINAGE AREA.--40.2 $\mathrm{mi}^{2}$, approximately.

PERIOD OF RECORD.--June 1965 to September 1978 (discontinued).

GAGE.--Water-stage recorder. Datum of gage is 8,372 ft. Prior to Nov. 13, 1965, at datum $1.08 \mathrm{ft}$ higher.

REMARKS.--Records poor.

AVERAGE DISCHARGE.--13 years, $24.6 \mathrm{ft}^{3} / \mathrm{s}, 17,820$ acre- $\mathrm{ft} / \mathrm{yr}$; median of yearly mean discharges, $22 \mathrm{ft}^{3} / \mathrm{s} 15,900$ acre- $\mathrm{ft} / \mathrm{yr}$.

EXTREMES FOR PERIOD OF RECORD.--Maximum discharge, $510 \mathrm{ft}^{3} / \mathrm{s} \mathrm{Apr.} \mathrm{28,} \mathrm{1973,} \mathrm{gage} \mathrm{height,} 4.15 \mathrm{ft}$; minimum daily, $4.6 \mathrm{ft}^{3} / \mathrm{s} \mathrm{July} 29$, 1978.

Annual peak discharges

\begin{tabular}{|c|c|c|c|c|c|c|c|}
\hline $\begin{array}{l}\text { Water } \\
\text { year }\end{array}$ & Date & $\begin{array}{l}\text { Annual peak } \\
\text { discharge } \\
\left(\mathrm{ft}^{3} / \mathrm{s}\right)\end{array}$ & $\begin{array}{l}\text { Discharge } \\
\text { codes }\end{array}$ & $\begin{array}{l}\text { Water } \\
\text { year }\end{array}$ & Date & $\begin{array}{l}\text { Annual peak } \\
\text { discharge } \\
\left(\mathrm{ft}^{3} / \mathrm{s}\right)\end{array}$ & $\begin{array}{c}\text { Discharge } \\
\text { codes }\end{array}$ \\
\hline 1966 & $04-02-66$ & 299 & & 1973 & $04-28-73$ & 510 & \\
\hline 1967 & $03-09-67$ & 194 & & 1974 & $03-30-74$ & 81 & \\
\hline 1968 & $04-15-68$ & 183 & & 1975 & $05-15-75$ & 157 & \\
\hline 1969 & 04-06-69 & 177 & & 1976 & $04-09-76$ & 139 & \\
\hline 1970 & $04-10-70$ & 242 & & 1977 & $04-09-77$ & 205 & \\
\hline 1971 & $09-30-71$ & 150 & & 1978 & $03-30-78$ & 153 & \\
\hline 1972 & $10-01-71$ & 162 & & & & & \\
\hline
\end{tabular}

Basin characteristics

\begin{tabular}{ccccccccc}
\hline $\begin{array}{c}\text { Main } \\
\text { channel } \\
\text { slope } \\
(\mathrm{ft} / \mathrm{mi})\end{array}$ & $\begin{array}{c}\text { Stream } \\
\text { length }\end{array}$ & $\begin{array}{c}\text { Mean } \\
\text { (mi) }\end{array}$ & $\begin{array}{c}\text { basin } \\
\text { elevation } \\
\text { (ft) }\end{array}$ & $\begin{array}{c}\text { Forested } \\
\text { area } \\
\text { (percent) }\end{array}$ & $\begin{array}{c}\text { Soil } \\
\text { index }\end{array}$ & $\begin{array}{c}\text { Mean } \\
\text { annual } \\
\text { precipitation } \\
\text { (in) }\end{array}$ & $\begin{array}{c}\text { 2-year } \\
\text { (in) }\end{array}$ & $\begin{array}{c}\text { 50-year } \\
\text { (in) }\end{array}$ \\
\hline 216 & 10.1 & 9,520 & 74.0 & 3.0 & 34.2 & 2.9 & 5.5 \\
\hline
\end{tabular}


09490800 NORTH FORK WHITE RIVER NEAR GREER, AZ--Continued

MEAN MONTHLY AND ANNUAL DISCHARGES $1966 \cdot 78$

\begin{tabular}{|c|c|c|c|c|c|c|}
\hline MONTH & $\begin{array}{l}\text { MAXIMUM } \\
\text { (FT3/S) }\end{array}$ & $\begin{array}{l}\text { MINIMUM } \\
\text { (FT3/S) }\end{array}$ & $\begin{array}{c}\text { MEAN } \\
(F T 3 / S)\end{array}$ & $\begin{array}{l}\text { STAN- } \\
\text { DARD } \\
\text { DEVIA- } \\
\text { TION } \\
\text { (FT3/S) }\end{array}$ & $\begin{array}{l}\text { COEFFI- } \\
\text { CIENT OF } \\
\text { VARI- } \\
\text { ATION }\end{array}$ & $\begin{array}{c}\text { PERCENT } \\
\text { OF } \\
\text { ANNUAL } \\
\text { RUNOFF }\end{array}$ \\
\hline OCTOBER & 54 & 8.7 & 20 & 15 & 0.76 & 6.7 \\
\hline NOVEMBER & 27 & 6.6 & 13 & 7.4 & 0.55 & 4.5 \\
\hline DECEMBER & 25 & 6.1 & 11 & 5.3 & 0.49 & 3.7 \\
\hline JANUARY & 19 & 6.0 & 9.6 & 3.3 & 0.35 & 3.3 \\
\hline FEBRUARY & 21 & 6.0 & 10 & 3.7 & 0.35 & 3.5 \\
\hline MARCH & 54 & 9.8 & 21 & 13 & 0.62 & 7.0 \\
\hline APRIL & 97 & 17 & 52 & 28 & 0.53 & 17.6 \\
\hline MAY & 233 & 14 & 63 & 57 & 0.90 & 21.4 \\
\hline JUNE & 152 & 7.9 & 39 & 39 & 0.98 & 13.3 \\
\hline JULY & 44 & 8.4 & 17 & 9.5 & 0.55 & 5.9 \\
\hline AUGUST & 47 & 9.9 & 20 & 9.9 & 0.49 & 6.9 \\
\hline SEPTEMBER & 43 & 9.0 & 18 & 10 & 0.57 & 6.2 \\
\hline ANNUAL & 57 & 13 & 25 & 12 & 0.47 & 100 \\
\hline
\end{tabular}

MAGNITUDE AND PROBABILITY OF INSTANTANEOUS PEAK FLOW BASED ON PERIOD OF RECORD 1966.78

DISCHARGE, IN FT3/S, FOR INDICATED RECURRENCE INTERVAL IN YEARS, AND EXCEEDANCE PROBABILITY, IN PERCENT

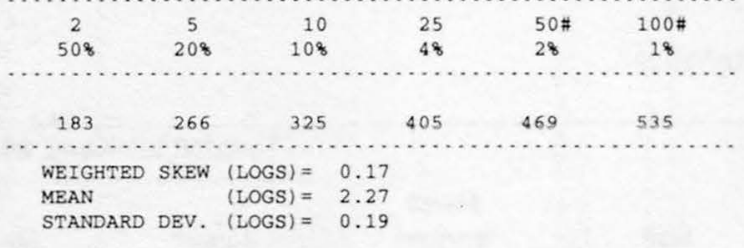

MAGNITUDE AND PROBABILITY OF ANNUAL LOW FLOW BASED ON PERIOD OF RECORD 1967.78

\begin{tabular}{|c|c|c|c|c|c|c|}
\hline $\begin{array}{l}\text { PERIOD } \\
\left(\mathrm{CON}^{-}\right.\end{array}$ & \multirow{2}{*}{\multicolumn{6}{|c|}{$\begin{array}{l}\text { DISCHARGE, IN FT3/S, FOR INDICATED } \\
\text { RECURRENCE INTERVAL, IN YEARS, AND } \\
\text { NON-EXCEEDANCE PROBABILITY, IN PERCENT }\end{array}$}} \\
\hline SECU- & & & & & & \\
\hline TIVE & 2 & 5 & 10 & 20 & $50 \#$ & $100 \#$ \\
\hline DAYS) & 508 & 208 & 108 & 58 & 28 & 18 \\
\hline 1 & 6.4 & 5.3 & 4.8 & 4.3 & 3.9 & 3.6 \\
\hline 3 & 6.5 & 5.5 & 5.0 & 4.6 & 4.2 & 4.0 \\
\hline 7 & 6.7 & 5.8 & 5.3 & 4.9 & 4.5 & 4.3 \\
\hline 14 & 6.8 & 6.1 & 5.7 & 5.4 & 5.1 & 4.9 \\
\hline 30 & 7.6 & 6.9 & 6.5 & 6.1 & 5.8 & 5.5 \\
\hline 60 & 8.1 & 7.3 & 6.8 & 6.4 & 5.9 & 5.5 \\
\hline 90 & 8.6 & 7.6 & 7.0 & 6.5 & 5.9 & 5.5 \\
\hline 120 & 8.9 & 8.0 & 7.6 & 7.4 & 7.0 & 6.8 \\
\hline 183 & 11 & 9.5 & 9.1 & 8.8 & 8.5 & 8.3 \\
\hline
\end{tabular}

MAGNITUDE AND PROBABILITY OF ANNUAL HIGH FLOW BASED ON PERIOD OF RECORD 1966.78

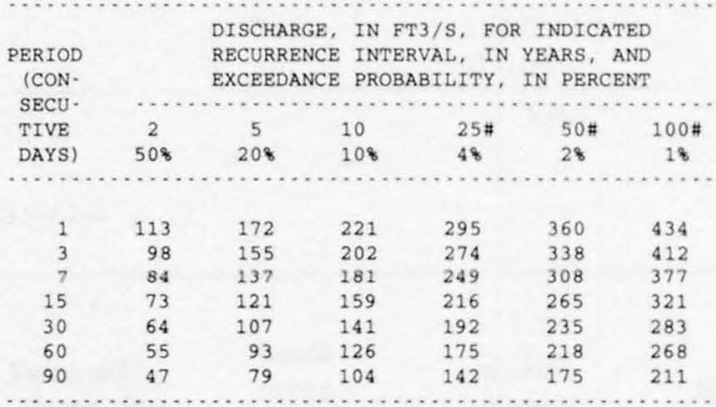

DURATION TABLE OF DAILY MEAN FLOW FOR PERIOD OF RECORD 1966.78

DISCHARGE, IN FT3/S, WHICH WAS EQUALED OR EXCEEDED FOR INDICATED PERCENT OF TIME

\begin{tabular}{|c|c|c|c|c|c|c|c|c|c|c|c|c|c|c|c|c|}
\hline 18 & 58 & 108 & 158 & 208 & 308 & 408 & 508 & 608 & 708 & 808 & 908 & 958 & 988 & 998 & 99.58 & 99.98 \\
\hline 93 & 79 & 59 & 42 & 32 & 22 & 17 & 14 & 12 & 10 & 8.9 & 7.9 & 7.2 & 6.4 & 6.0 & 5.3 & 4.7 \\
\hline
\end{tabular}

\# Reliability of values in column is uncertain, and potential errors are large. 
09490800 NORTH FORK WHITE RIVER NEAR GREER, AZ--Continued
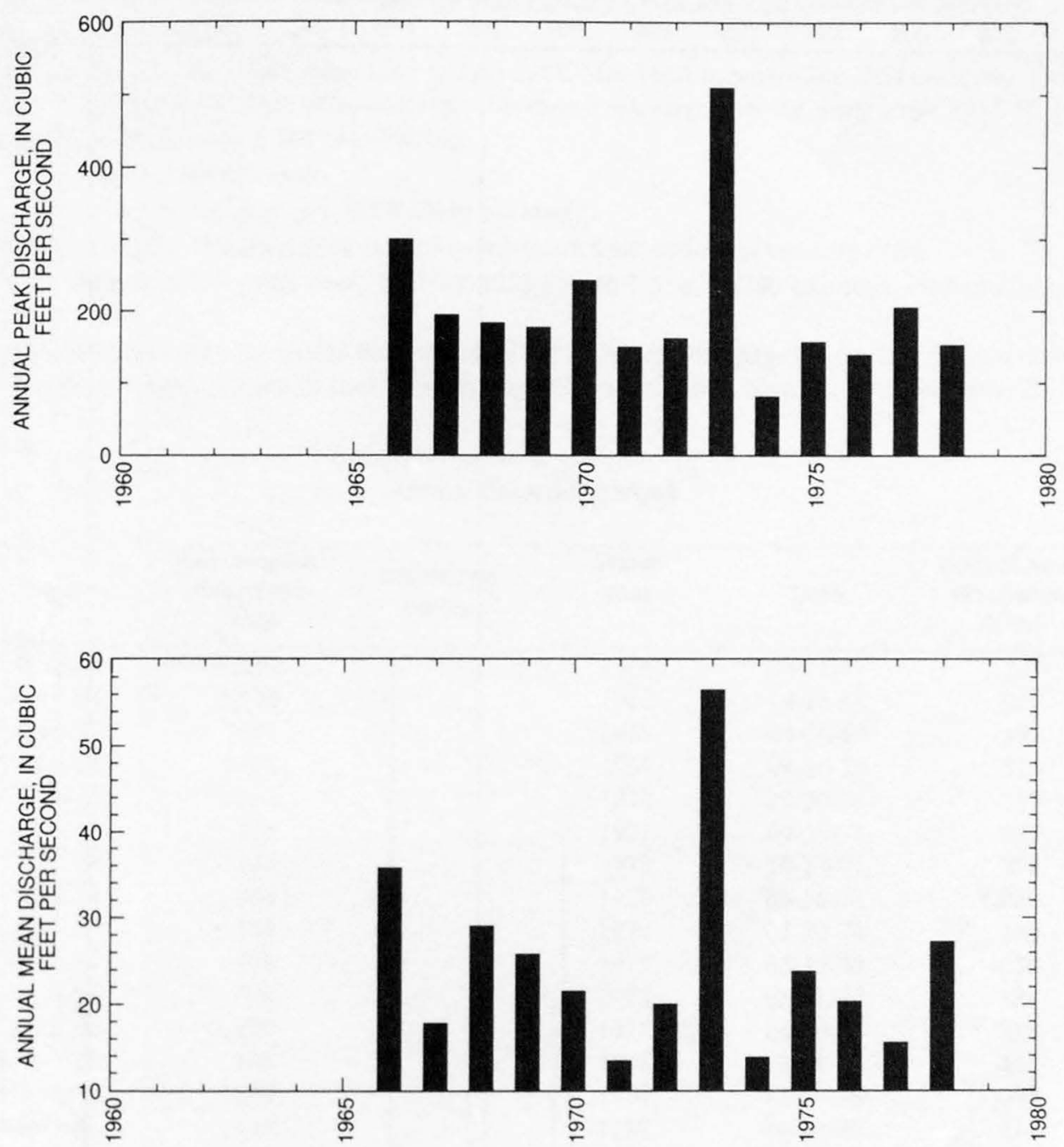
09490800 NORTH FORK WHITE RIVER NEAR GREER, AZ--Continued
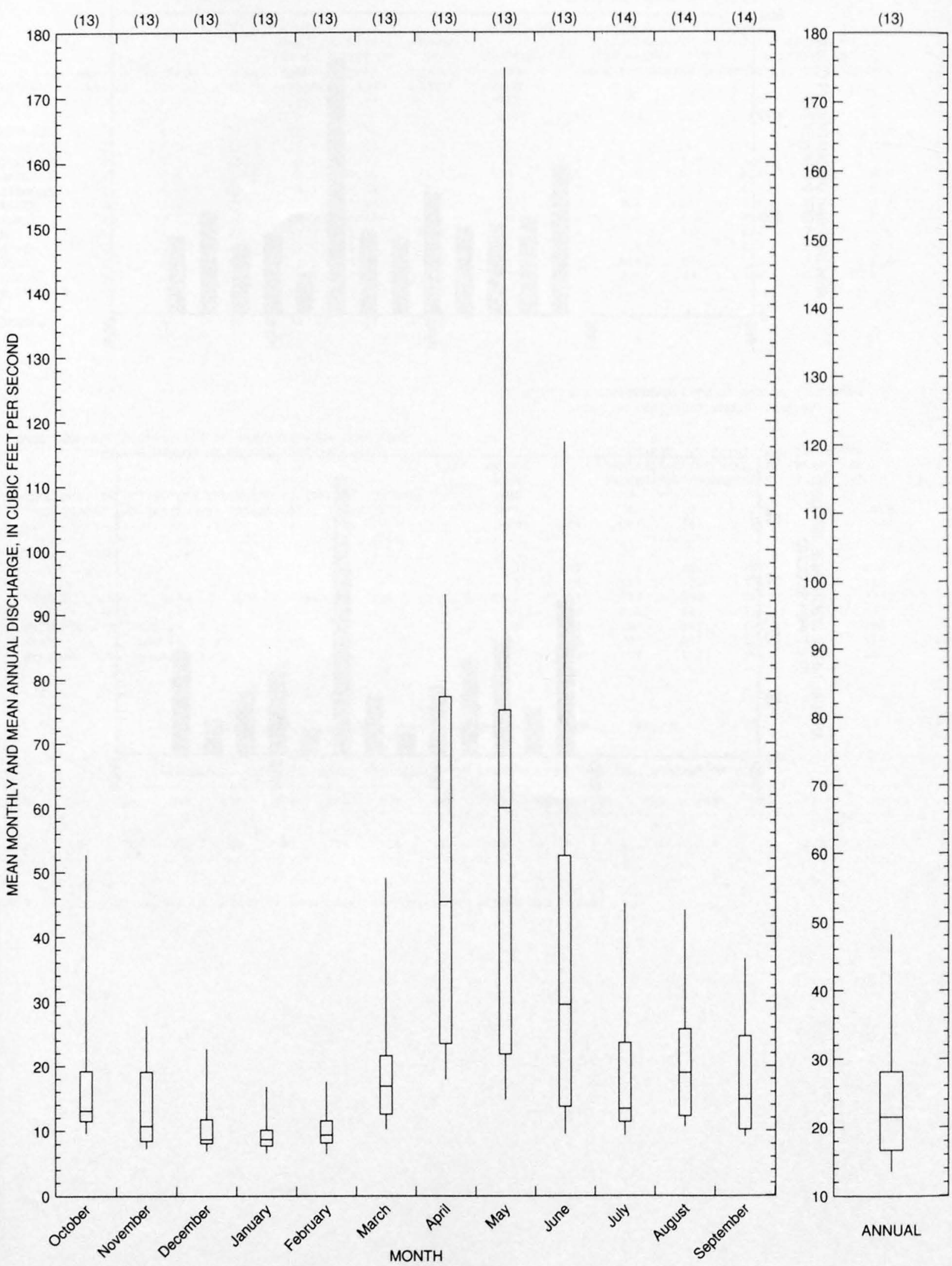


\section{NORTH FORK WHITE RIVER NEAR McNARY, AZ}

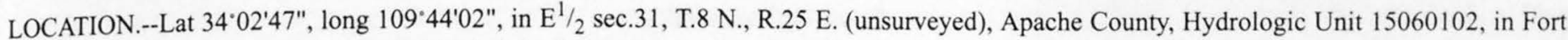
Apache Indian Reservation, on left bank 1.9 mi downstream from Paradise Creek and 7 mi southeast of McNary.

DRAINAGE AREA.--66 $\mathrm{mi}^{2}$, approximately.

PERIOD OF RECORD.--June 1945 to June 1947, May 1948 to June 1949, May 1950 to September 1954 (monthly discharge only, July to September 1954), June 1957 to September 1985 (discontinued). Maximum discharge only for water years 1955-56, published in WSP 1513. Prior to Oct. 1, 1963, published as White River near McNary.

REVISED RECORDS.--WSP 1243: Drainage area.

GAGE.--Water-stage recorder. Elevation of gage is 7,723 ft above sea level.

REMARKS.--No storage above station. Water diverted about $5 \mathrm{mi}$ upstream from station for use at McNary.

AVERAGE DISCHARGE.--33 years (water years 1946, 1951-53, 1958-85), $48.7 \mathrm{ft}^{3} / \mathrm{s}, 35,280$ acre-ft/yr; median of yearly mean discharges, 46 $\mathrm{ft}^{3} / \mathrm{s}, 33,300$ acre-ft/yr.

EXTREMES FOR PERIOD OF RECORD.--Maximum discharge, 2,310 $\mathrm{ft}^{3} / \mathrm{s}$ Oct. 2, 1983, gage height, $6.78 \mathrm{ft}$, from rating curve extended above $470 \mathrm{ft}^{3} / \mathrm{s}$ on basis of slope-area measurement of peak flow; minimum recorded, $4 \mathrm{ft}^{3} / \mathrm{s}$ Nov. 19, 1948, and Nov. 29, 1950, caused by ice jam upstream.

Annual peak discharges

\begin{tabular}{|c|c|c|c|c|c|c|c|}
\hline $\begin{array}{l}\text { Water } \\
\text { year }\end{array}$ & Date & $\begin{array}{c}\text { Annual peak } \\
\text { discharge } \\
\left(\mathrm{ft}^{3} / \mathrm{s}\right)\end{array}$ & $\begin{array}{l}\text { Discharge } \\
\text { codes }\end{array}$ & $\begin{array}{l}\text { Water } \\
\text { year }\end{array}$ & Date & $\begin{array}{c}\text { Annual peak } \\
\text { discharge } \\
\left(\mathrm{ft}^{3} / \mathrm{s}\right)\end{array}$ & $\begin{array}{l}\text { Discharge } \\
\text { codes }\end{array}$ \\
\hline 1946 & $09-19-46$ & 1,290 & & 1966 & $08-11-67$ & 271 & \\
\hline 1947 & $04-16-48$ & 1,120 & & 1967 & $04-15-68$ & 351 & \\
\hline 1948 & $04-15-49$ & 656 & & 1968 & $04-06-69$ & 393 & \\
\hline 1949 & $03-00-50$ & 188 & & 1969 & $04-10-70$ & 310 & \\
\hline 1950 & $08-28-51$ & 167 & & 1970 & $09-30-71$ & 257 & \\
\hline 1951 & 04-06-52 & 748 & & 1971 & $09-30-71$ & 257 & \\
\hline 1952 & $03-29-53$ & 152 & & 1972 & $10-24-71$ & 352 & \\
\hline 1953 & $03-23-54$ & 304 & & 1973 & $04-28-73$ & 1,000 & ES \\
\hline 1954 & $00-00-55$ & 145 & & 1974 & $03-30-74$ & 140 & \\
\hline 1955 & $00-00-56$ & 170 & & 1975 & $05-15-75$ & 350 & \\
\hline 1956 & $08-24-57$ & 729 & & 1976 & $05-21-76$ & 184 & \\
\hline 1957 & $04-22-58$ & 1,230 & & 1977 & $04-09-77$ & 316 & \\
\hline 1958 & $10-05-58$ & 148 & & 1978 & $03-31-78$ & 455 & \\
\hline 1959 & $03-26-60$ & 390 & & 1979 & $12-18-78$ & 1,060 & \\
\hline 1960 & $04-05-61$ & 248 & & 1980 & $06-09-80$ & 273 & \\
\hline 1961 & $04-16-62$ & 680 & & 1981 & $04-13-81$ & 397 & \\
\hline 1962 & $09-10-63$ & 385 & & 1982 & $04-12-82$ & 505 & \\
\hline 1963 & $04-12-64$ & 444 & & 1983 & $06-01-83$ & 552 & \\
\hline 1964 & $04-23-65$ & 791 & & 1984 & $10-02-83$ & 2,310 & \\
\hline 1965 & $04-03-66$ & 512 & & 1985 & $03-12-85$ & 754 & \\
\hline
\end{tabular}

Magnitude and probability of instantaneous peak flow based on period of record 1946, 1948-85

\begin{tabular}{|c|c|c|c|c|c|}
\hline \multicolumn{6}{|c|}{$\begin{array}{l}\text { Discharge, in } \mathrm{ft}^{3} / \mathrm{s} \text {, for indicated recurrence interval } \\
\text { in years, and exceedance probablility, in percent }\end{array}$} \\
\hline $\begin{array}{c}2 \\
50 \%\end{array}$ & $\begin{array}{c}5 \\
20 \%\end{array}$ & $\begin{array}{c}10 \\
10 \%\end{array}$ & $\begin{array}{l}25 \\
4 \%\end{array}$ & $\begin{array}{l}50 \\
2 \%\end{array}$ & $\begin{array}{c}100 \dagger \\
1 \%\end{array}$ \\
\hline $\begin{array}{l}407 \\
\text { Weighted skew } \\
\text { Mean } \\
\text { Standard dev. }\end{array}$ & $\begin{array}{c}742 \\
(\log s)= \\
(\log s)= \\
(\log s)=\end{array}$ & $\begin{array}{l}1,030 \\
0.16 \\
2.62 \\
0.30\end{array}$ & 1,460 & 1,850 & 2,280 \\
\hline
\end{tabular}

fReliability of values in column is uncertain, and potential errors are large. 
09491000 NORTH FORK WHITE RIVER NEAR McNARY, AZ--Continued

Basin characteristics

\begin{tabular}{|c|c|c|c|c|c|c|c|}
\hline \multirow[b]{2}{*}{$\begin{array}{l}\text { Main } \\
\text { channel } \\
\text { slope } \\
(\mathrm{ft} / \mathrm{mi})\end{array}$} & \multirow[b]{2}{*}{$\begin{array}{c}\text { Stream } \\
\text { length } \\
\text { (mi) }\end{array}$} & \multirow[b]{2}{*}{$\begin{array}{c}\text { Mean } \\
\text { basin } \\
\text { elevation } \\
(\mathrm{ft})\end{array}$} & \multirow[b]{2}{*}{$\begin{array}{c}\text { Forested } \\
\text { area } \\
\text { (percent) }\end{array}$} & \multirow[b]{2}{*}{$\begin{array}{c}\text { Soil } \\
\text { index }\end{array}$} & \multirow[b]{2}{*}{$\begin{array}{c}\text { Mean } \\
\text { annual } \\
\text { precipitation } \\
\text { (in) }\end{array}$} & \multicolumn{2}{|c|}{ Rainfall intensity, 24-hour } \\
\hline & & & & & & $\begin{array}{l}\text { 2-year } \\
\text { (in) }\end{array}$ & $\begin{array}{l}\text { 50-year } \\
\text { (in) }\end{array}$ \\
\hline 153 & 18.5 & 9,320 & 79.7 & 3.0 & 32.2 & 2.9 & 5.5 \\
\hline
\end{tabular}

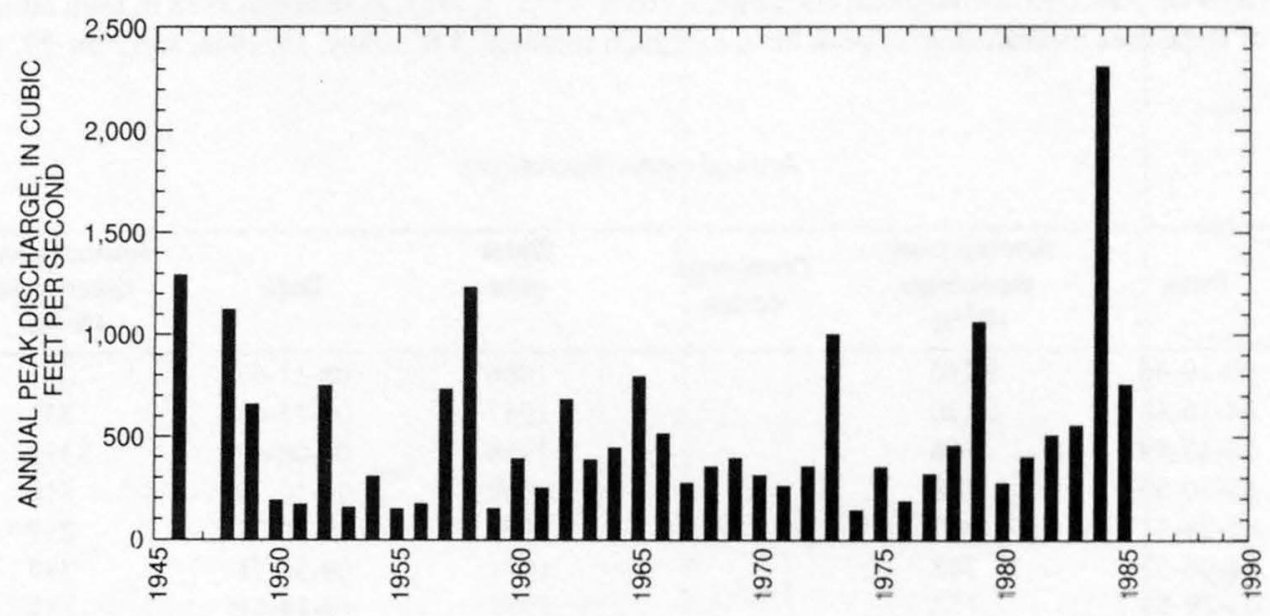


09491800 NORTH FORK WHITE RIVER TRIBUTARY NEAR WHITERIVER, AZ

LOCATION.--Lat 33 $55^{\prime} 44^{\prime \prime}$, long 109 56'19", in SE $1 / 4$ sec.8, T.6 N., R.23 E. (unsurveyed), Navajo County, Hydrologic Unit 15060102, at State Highway $73,6.5 \mathrm{mi}$ north of Whiteriver.

DRAINAGE AREA.--2.27 $\mathrm{mi}^{2}$.

Annual peak discharges

\begin{tabular}{cccccrr}
\hline $\begin{array}{l}\text { Water } \\
\text { year }\end{array}$ & Date & $\begin{array}{c}\text { Annual peak } \\
\mathbf{d i s c h a r g e} \\
\left(\mathbf{f t}^{3} / \mathbf{s}\right)\end{array}$ & $\begin{array}{c}\text { Discharge } \\
\text { codes }\end{array}$ & $\begin{array}{c}\text { Water } \\
\text { year }\end{array}$ & $\begin{array}{c}\text { Date } \\
\text { Annual peak } \\
\text { discharge } \\
\left(\mathbf{f t}^{3} / \mathbf{s}\right)\end{array}$ \\
\hline 1965 & $00-00-65$ & 120 & LT & 1971 & $08-00-71$ & 110 \\
1966 & $12-22-65$ & 24 & & 1972 & $00-00-72$ & 0 \\
1967 & $00-00-67$ & 0 & DT & 1973 & $10-19-72$ & 25 \\
1968 & $11-00-67$ & 0.1 & & 1974 & $00-00-74$ & 0 \\
1969 & $00-00-69$ & 0 & & & $07-00-75$ & 56 \\
1970 & $00-00-70$ & 0 & & & & \\
\hline
\end{tabular}

Magnitude and probability of instantaneous peak flow based on period of record

\begin{tabular}{|c|c|c|c|c|c|}
\hline \multicolumn{6}{|c|}{$\begin{array}{l}\text { Discharge, in } \mathrm{ft}^{3} / \mathrm{s} \text {, for indicated recurrence interval } \\
\text { in years, and exceedance probablility, in percent }\end{array}$} \\
\hline 2 & 5 & 10 & 25 & 50 & 100 \\
\hline $50 \%$ & $20 \%$ & $10 \%$ & $4 \%$ & $2 \%$ & $1 \%$ \\
\hline --. & -.- & -.. & -- & -- & -.. \\
\hline Weighted skew & $(\log s)=$ & -- & & & \\
\hline Mean & $(\log s)=$ & $-\cdots$ & & & \\
\hline Standard dev. & $(\log s)=$ & -. & & & \\
\hline
\end{tabular}

\section{Basin characteristics}

\begin{tabular}{cccccccc}
\hline $\begin{array}{c}\text { Main } \\
\text { channel } \\
\text { slope } \\
(\mathrm{ft} / \mathrm{mi})\end{array}$ & $\begin{array}{c}\text { Stream } \\
\text { length } \\
\text { (mi) }\end{array}$ & $\begin{array}{c}\text { Mean } \\
\text { basin } \\
\text { elevation } \\
\text { (ft) }\end{array}$ & $\begin{array}{c}\text { Forested } \\
\text { area } \\
\text { (percent) }\end{array}$ & $\begin{array}{c}\text { Soil } \\
\text { index }\end{array}$ & $\begin{array}{c}\text { Mean } \\
\text { annual } \\
\text { precipitation } \\
\text { (in) }\end{array}$ & $\begin{array}{c}\text { R-year } \\
\text { (in) }\end{array}$ & $\begin{array}{c}50 \text {-year } \\
\text { (in) }\end{array}$ \\
\hline 272 & 3.6 & 6,290 & 99.0 & 3.0 & 20.5 & 2.1 \\
\hline
\end{tabular}

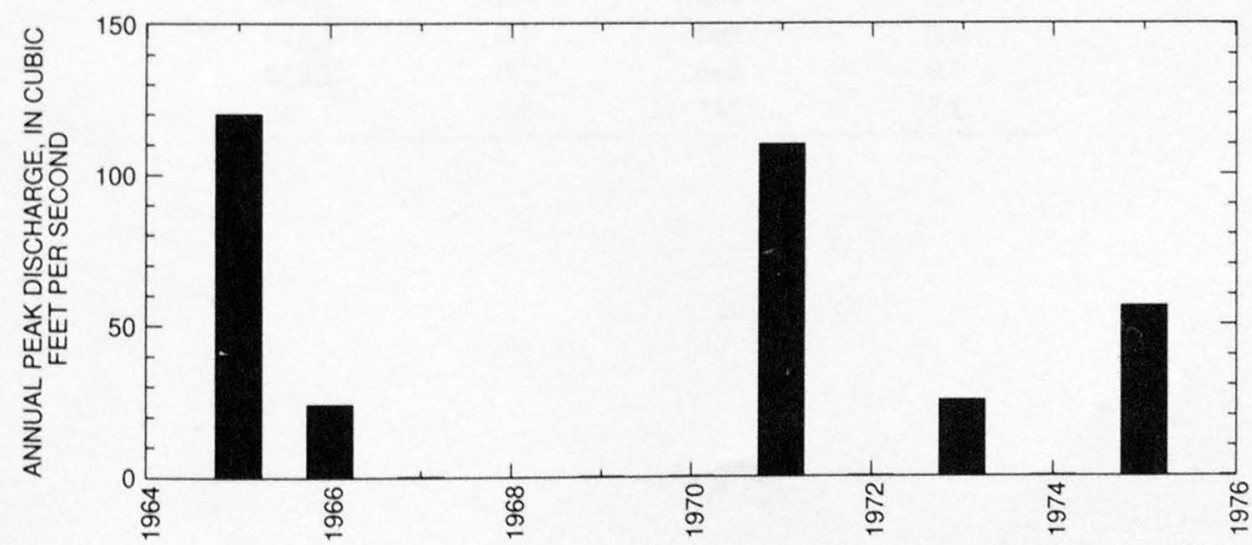


LOCATION.--Lat 33.49'20", long 109.48'50", in SE $1 / 4$ sec.16, T.5 N., R.24 E. (unsurveyed), Apache County, Hydrologic Unit 15060102, in Fon Apache Indian Reservation, on left bank $600 \mathrm{ft}$ downstream from highway bridge, 0.1 mi upstream from Rock Creek, and 10 mi east of Fon Apache.

DRAINAGE AREA.--38.8 $\mathrm{mi}^{2}$.

PERIOD OF RECORD,--August 1957 to current year.

GAGE.--Water-stage recorder. Elevation of gage is $6,050 \mathrm{ft}$ above sea level. Prior to Dec. 29, 1960, at site $600 \mathrm{ft}$ upstream at datum $12.78 \mathrm{ft}$ higher Dec. 29, 1960, to Sept. 28, 1962, at site $600 \mathrm{ft}$ upstream at datum $12.92 \mathrm{ft}$ higher.

EXTREMES FOR PERIOD OF RECORD.--Maximum discharge, 2,700 ft $3 / \mathrm{s}$ Oct. 1, 1983, gage height, $5.40 \mathrm{ft}$, from rating curve extended above $1,000 \mathrm{ft}^{3} / \mathrm{s} ;$ minimum daily, $4.0 \mathrm{ft}^{3} / \mathrm{s}$ Nov. $29,1975$.

Annual peak discharges

\begin{tabular}{|c|c|c|c|c|c|c|c|}
\hline $\begin{array}{l}\text { Water } \\
\text { year }\end{array}$ & Date & $\begin{array}{c}\text { Annual peak } \\
\text { discharge } \\
\left(\mathrm{ft}^{3} / \mathrm{s}\right)\end{array}$ & $\begin{array}{l}\text { Discharge } \\
\text { codes }\end{array}$ & $\begin{array}{l}\text { Water } \\
\text { year }\end{array}$ & Date & $\begin{array}{c}\text { Annual peak } \\
\text { discharge } \\
\left(\mathrm{ft}^{3} / \mathrm{s}\right)\end{array}$ & $\begin{array}{c}\text { Discharge } \\
\text { codes }\end{array}$ \\
\hline 1958 & $04-22-58$ & 411 & & 1978 & $03-01-78$ & 318 & \\
\hline 1959 & $10-06-58$ & 330 & & 1979 & $12-18-78$ & 751 & \\
\hline 1960 & $05-13-60$ & 207 & & 1980 & $05-22-80$ & 372 & \\
\hline 1961 & $08-17-61$ & 663 & & 1981 & $05-03-81$ & 167 & \\
\hline 1962 & $05-12-62$ & 300 & ES & 1982 & $05-02-82$ & 283 & \\
\hline 1963 & $08-30-63$ & 116 & & 1983 & $06-01-83$ & 388 & \\
\hline 1964 & $08-09-64$ & 83 & & 1984 & $10-01-83$ & 2,700 & \\
\hline 1965 & $05-02-65$ & 204 & & 1985 & $03-12-85$ & 481 & \\
\hline 1966 & $11-25-65$ & 270 & & 1986 & $07-16-86$ & 154 & \\
\hline 1967 & $08-03-67$ & 758 & & 1987 & $04-17-87$ & 235 & \\
\hline 1968 & $08-05-68$ & 352 & & 1988 & $04-28-88$ & 211 & \\
\hline 1969 & $05-21-69$ & 194 & & 1989 & $03-14-89$ & 122 & \\
\hline 1970 & $09-06-70$ & 396 & & 1990 & $04-29-90$ & 81 & \\
\hline 1971 & $09-01-71$ & 205 & & 1991 & $05-10-91$ & 212 & \\
\hline 1972 & $10-01-71$ & 266 & & 1992 & $08-24-92$ & 776 & \\
\hline 1973 & $10-20-72$ & 732 & & 1993 & $01-08-93$ & 563 & \\
\hline 1974 & $05-15-74$ & 89 & & 1994 & 09-03-94 & 326 & \\
\hline 1975 & $05-16-75$ & 270 & & 1995 & $03-06-95$ & 387 & \\
\hline 1976 & $07-30-76$ & 166 & & 1996 & $09-14-96$ & 155 & \\
\hline 1977 & $08-16-77$ & 122 & & & & & \\
\hline
\end{tabular}

Discharge rating table developed October 1988

\begin{tabular}{cccc}
\hline $\begin{array}{c}\text { Gage height } \\
(\mathbf{f t})\end{array}$ & $\begin{array}{c}\text { Discharge } \\
\left(\mathbf{f t}^{3} / \mathbf{s}\right)\end{array}$ & $\begin{array}{c}\text { Gage height } \\
(\mathbf{f t})\end{array}$ & $\begin{array}{c}\text { Discharge } \\
\left(\mathrm{ft}^{\mathbf{3}} / \mathbf{s}\right)\end{array}$ \\
\hline 2.0 & 166 & 4.0 & 1,050 \\
2.5 & 306 & 4.5 & 1,510 \\
3.0 & 496 & 5.0 & 2,120 \\
3.5 & 741 & 5.4 & 2,700 \\
\hline
\end{tabular}


09492400 EAST FORK WHITE RIVER NEAR FORT APACHE, AZ--Continued

Basin characteristics

\begin{tabular}{cccccccc}
\hline $\begin{array}{c}\text { Main } \\
\text { channel } \\
\text { slope } \\
(\mathrm{ft} / \mathrm{mi})\end{array}$ & $\begin{array}{c}\text { Stream } \\
\text { length } \\
\text { (mi) }\end{array}$ & $\begin{array}{c}\text { Mean } \\
\text { basin } \\
\text { elevation } \\
(\mathrm{ft})\end{array}$ & $\begin{array}{c}\text { Forested } \\
\text { area } \\
\text { (percent) }\end{array}$ & $\begin{array}{c}\text { Soil } \\
\text { index }\end{array}$ & $\begin{array}{c}\text { Mean } \\
\text { annual } \\
\text { precipitation } \\
\text { (in) }\end{array}$ & $\begin{array}{c}\text { R-year } \\
\text { (in) }\end{array}$ & $\begin{array}{c}\text { 50-year } \\
\text { (in) }\end{array}$ \\
\hline 239 & 17.9 & 8,580 & 96.0 & 3.0 & 31.2 & 2.8 \\
\hline
\end{tabular}


09492400 EAST FORK WHITE RIVER NEAR FORT APACHE, AZ--Continued

MEAN MONTHLY AND ANNUAL DISCHARGES 1958.96

\begin{tabular}{|c|c|c|c|c|c|c|}
\hline MONTH & $\begin{array}{l}\text { MAXIMUM } \\
\text { (FT3/S) }\end{array}$ & $\begin{array}{l}\text { MINIMUM } \\
\text { (FT3/S) }\end{array}$ & $\begin{array}{c}\text { MEAN } \\
(\text { FT3/S) }\end{array}$ & $\begin{array}{l}\text { STAN- } \\
\text { DARD } \\
\text { DEVIA- } \\
\text { TION } \\
\text { (FT3/S) }\end{array}$ & $\begin{array}{l}\text { COEFFI- } \\
\text { CIENT OF } \\
\text { VARI- } \\
\text { ATION }\end{array}$ & $\begin{array}{c}\text { PERCENT } \\
\text { OF } \\
\text { ANNUAL } \\
\text { RUNOFF }\end{array}$ \\
\hline OCTOBER & 127 & 8.7 & 23 & 24 & 1.1 & 5.2 \\
\hline NOVEMBER & 44 & 7.7 & 17 & 9.1 & 0.53 & 3.9 \\
\hline DECEMBER & 57 & 7.8 & 17 & 10 & 0.62 & 3.8 \\
\hline JANUARY & 34 & 7.1 & 17 & 13 & 0.79 & 3.8 \\
\hline FEBRUARY & 66 & 7.7 & 22 & 14 & 0.63 & 4.9 \\
\hline MARCH & 103 & 10 & 40 & 25 & 0.62 & 9.1 \\
\hline APRIL & 192 & 15 & 84 & 46 & 0.55 & 19 \\
\hline MAY & 284 & 8.4 & 104 & 69 & 0.67 & 23 \\
\hline JUNE & 172 & 5.0 & 48 & 46 & 0.96 & 11 \\
\hline JULY & 46 & 7.7 & 19 & 10 & 0.54 & 4. 3 \\
\hline AUGUST & 91 & 11 & 28 & 17 & 0.61 & 6.4 \\
\hline SEPTEMBER & 66 & 6.9 & 25 & 13 & 0.53 & 5.5 \\
\hline ANNUAL & 75 & 12 & 37 & 17 & 0.45 & 100 \\
\hline
\end{tabular}

MAGNITUDE AND PROBABILITY OF INSTANTANEOUS PEAK FLOW BASED ON PERIOD OF RECORD 1958.96

DISCHARGE, IN FT3/S, FOR INDICATED RECURRENCE INTERVAL IN YEARS, AND EXCEEDANCE PROBABILITY, IN PERCENT

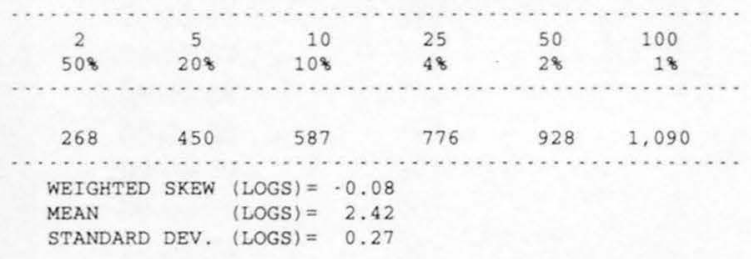

MAGNITUDE AND PROBABILITY OF ANNUAL LOW FLOW BASED ON PERIOD OF RECORD 1959.96

\begin{tabular}{|c|c|c|c|c|c|c|}
\hline \multirow{5}{*}{$\begin{array}{l}\text { PERIOD } \\
\text { (CON- } \\
\text { SECU- } \\
\text { TIVE } \\
\text { DAYS) }\end{array}$} & \multicolumn{6}{|c|}{ DISCHARGE, IN FT3/S, FOR INDICATED } \\
\hline & \multicolumn{6}{|c|}{$\begin{array}{l}\text { RECURRENCE INTERVAL, IN YEARS, AND } \\
\text { NON-EXCEEDANCE PROBABILITY, IN PERCENT }\end{array}$} \\
\hline & $\cdots$ & $\ldots \ldots \ldots$ & $\cdots \cdots$ & $\ldots \ldots$ & $\cdots \cdots$ & $\ldots \ldots$ \\
\hline & 2 & 5 & $10 \#$ & $20 \#$ & $50 \#$ & $100 \#$ \\
\hline & 508 & 208 & 108 & 58 & 28 & 18 \\
\hline 1 & 6.7 & 5.4 & 4.9 & 4.6 & 4.3 & 4.1 \\
\hline 3 & 7.2 & 6.0 & 5.5 & 5.3 & 5.0 & 4.9 \\
\hline 7 & 7.7 & 6.4 & 5.9 & 5.6 & 5.3 & 5.1 \\
\hline 14 & 8.2 & 6.9 & 6.4 & 6.1 & 5.8 & 5.6 \\
\hline 30 & 8.9 & 7.5 & 7.0 & 6.7 & 6.4 & 6.3 \\
\hline 60 & 9.8 & 8.3 & 7.7 & 7.4 & 7.1 & 6.9 \\
\hline 90 & 11 & 9.2 & 8.4 & 7.9 & 7.4 & 7.2 \\
\hline 120 & 13 & 10 & 9.0 & 8.3 & 7.7 & 7.3 \\
\hline 183 & 15 & 12 & 10 & 9.3 & 8.4 & 8,0 \\
\hline
\end{tabular}

MAGNITUDE AND PROBABILITY OF ANNUAL HIGH FLOW BASED ON PERIOD OF RECORD 1958.96

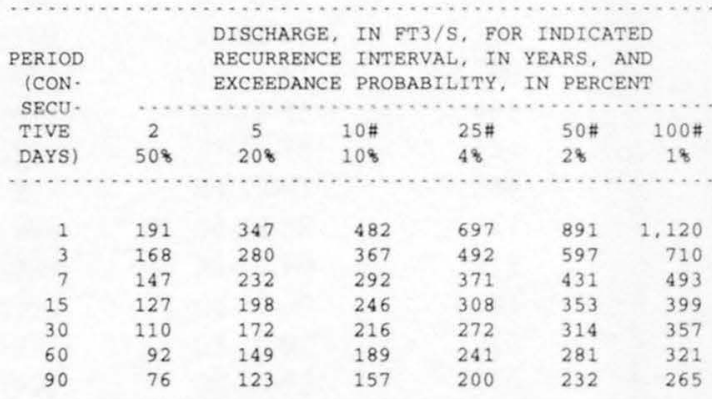

DURATION TABLE OF DAILY MEAN FLOW FOR PERIOD OF RECORD 1958.96

DISCHARGE, IN FT3/S, WHICH WAS EQUALED OR EXCEEDED FOR INDICATED PERCENT OF TIME

\begin{tabular}{|c|c|c|c|c|c|c|c|c|c|c|c|c|c|c|c|c|}
\hline 18 & 58 & 108 & 158 & 208 & 308 & 408 & 508 & 608 & 708 & 808 & 908 & 958 & 988 & 998 & 99.58 & 99.98 \\
\hline 237 & 135 & 91 & 64 & 50 & 33 & 25 & 20 & 16 & 13 & 11 & 9.0 & 7.8 & 0.00 & 0.00 & 0.00 & 0.00 \\
\hline
\end{tabular}

\# Reliability of values in column is uncertain, and potential errors are large. 
ANNUAL MEAN DISCHARGE, IN CUBIC

FEET PER SECOND

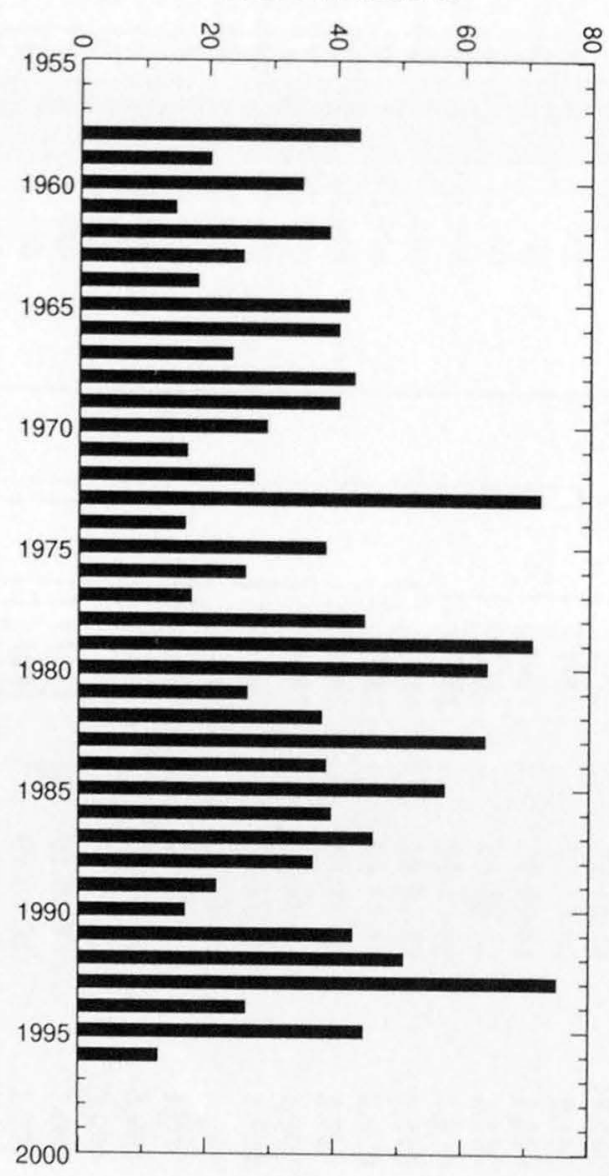

ANNUAL PEAK DISCHARGE, IN CUBIC

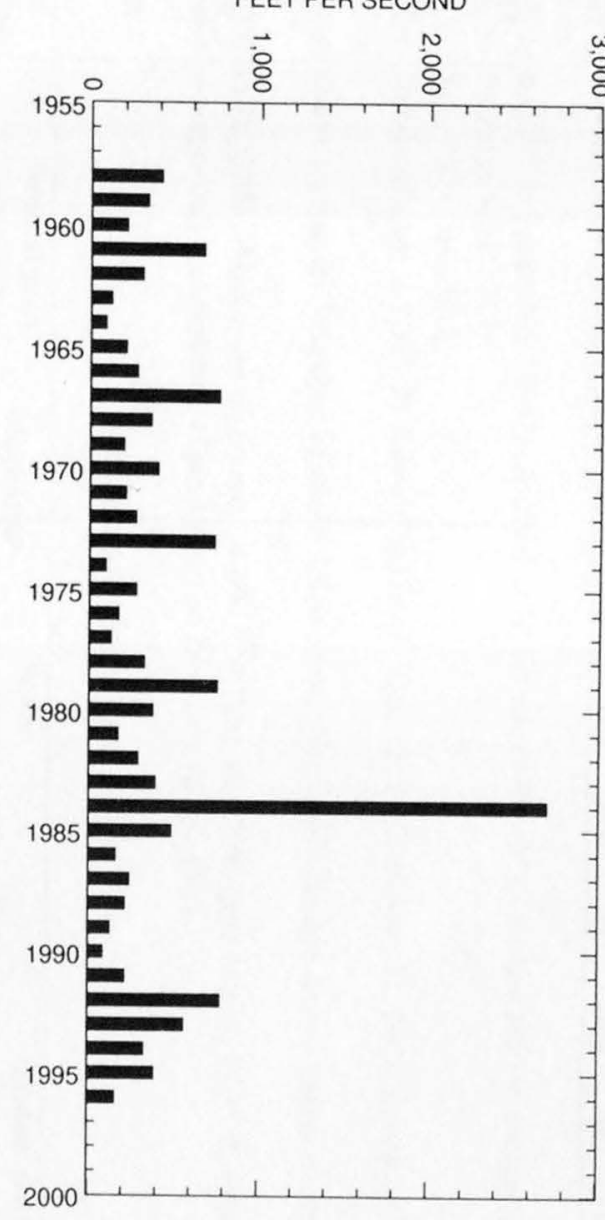


09492400 EAST FORK WHITE RIVER NEAR FORT APACHE, AZ--Continued
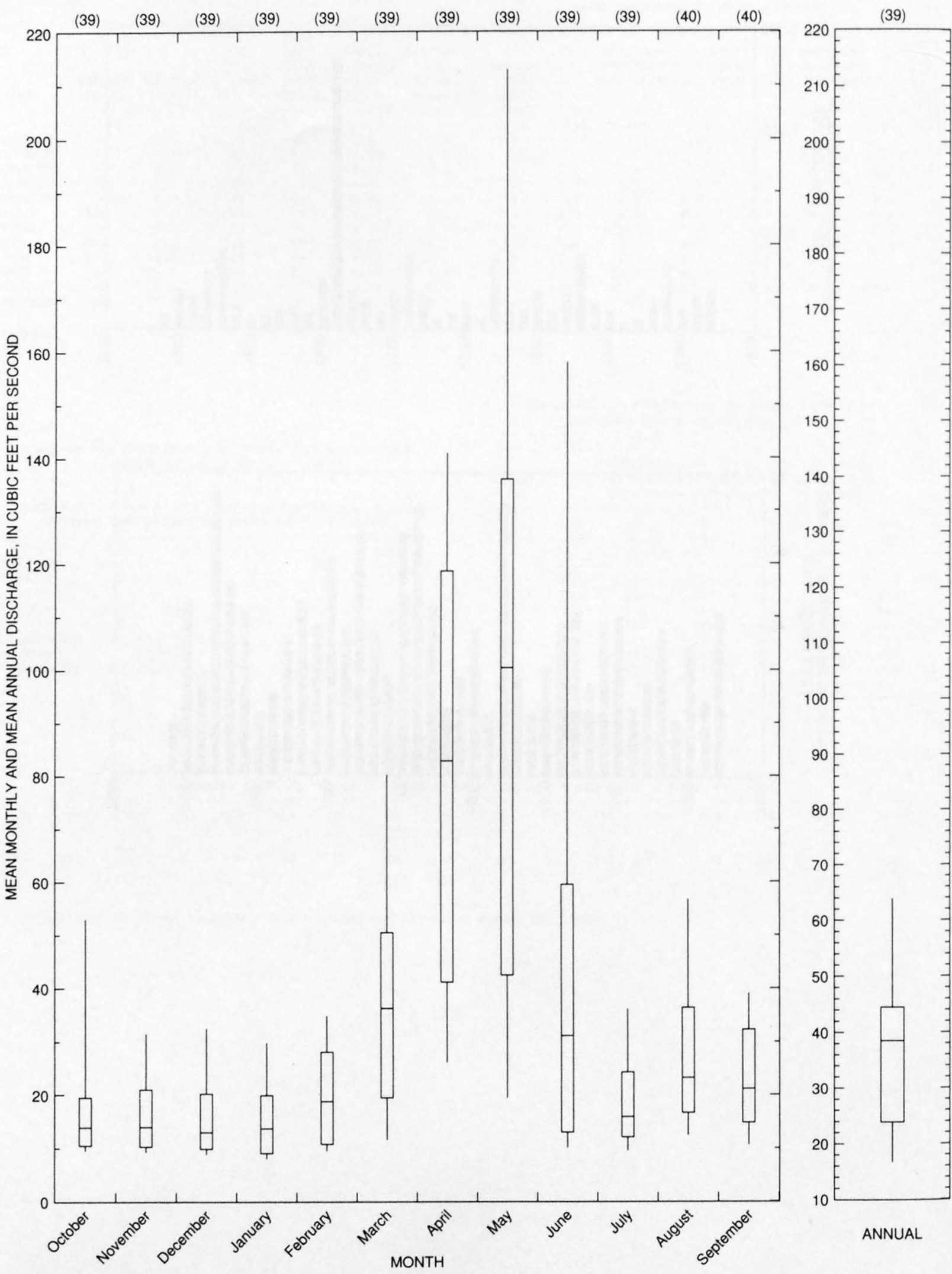
09494000 WHITE RIVER NEAR FORT APACHE, AZ

LOCATION.--Lat 33.44'11", long 110.09'58", in SE $1 / 4$ sec.32, T.4 N., R.21 E. (unsurveyed), Gila County, Hydrologic Unit 15060102, in Fort Apache Indian Reservation, on right bank 2,200 ft downstream from highway bridge, 4.5 mi upstream from confluence with Black River, and $11 \mathrm{mi}$ west of Fort Apache.

DRAINAGE AREA.--632 $\mathrm{mi}^{2}$.

PERIOD OF RECORD.--October 1917 to September 1918 (published as "at Wanslee's Ranch"), October 1957 to current year. Monthly discharge only for some periods, published in WSP 1313.

REVISED RECORDS.--WRD Ariz. 1971: 1967(M).

GAGE.--Water-stage recorder. Datum of gage is 4,365.99 ft above sea level. Oct. 12, 1917, to Aug. 31, 1918, nonrecording gage at site $2,100 \mathrm{ft}$ upstream at different datum.

REMARKS.--Small diversions above station for irrigation of about 1,460 acres. Negligible storage above station in several small recreational lakes.

EXTREMES FOR PERIOD OF RECORD.--Maximum discharge, 14,600 $\mathrm{ft}^{3} / \mathrm{s} \mathrm{Dec} .18,1978$, gage height, $15.71 \mathrm{ft}$, from rating curve extended above $7,800 \mathrm{ft}^{3} / \mathrm{s}$ on basis of slope-area measurement of peak flow; no flow July $18-21,1963$.

Annual peak discharges

\begin{tabular}{|c|c|c|c|c|c|c|c|}
\hline $\begin{array}{l}\text { Water } \\
\text { year }\end{array}$ & Date & $\begin{array}{c}\text { Annual peak } \\
\text { discharge } \\
\left(\mathrm{ft}^{3} / \mathrm{s}\right)\end{array}$ & $\begin{array}{l}\text { Discharge } \\
\text { codes }\end{array}$ & $\begin{array}{l}\text { Water } \\
\text { year }\end{array}$ & Date & $\begin{array}{c}\text { Annual peak } \\
\text { discharge } \\
\left(\mathrm{ft}^{3} / \mathrm{s}\right)\end{array}$ & $\begin{array}{c}\text { Discharge } \\
\text { codes }\end{array}$ \\
\hline 1958 & $03-22-58$ & 2,700 & & 1978 & $03-01-78$ & 6,590 & \\
\hline 1959 & $07-28-59$ & 4,900 & & 1979 & $12-18-78$ & 14,600 & \\
\hline 1960 & $12-26-59$ & 2,580 & & 1980 & $02-15-80$ & 8,160 & \\
\hline 1961 & $08-29-61$ & 3,590 & & 1981 & $08-31-81$ & 1,240 & \\
\hline 1962 & $04-16-62$ & 2,090 & & 1982 & $03-13-82$ & 2,130 & \\
\hline 1963 & $08-26-63$ & 1,970 & & 1983 & $04-26-83$ & 1,870 & \\
\hline 1964 & $07-25-64$ & 4,480 & & 1984 & $10-02-83$ & 9,410 & \\
\hline 1965 & $07-28-65$ & 2,870 & & 1985 & $03-12-85$ & 8,900 & \\
\hline 1966 & $12-30-65$ & 4,360 & & 1986 & $08-27-86$ & 3,780 & \\
\hline 1967 & $07-22-67$ & 8,180 & & 1987 & $04-18-87$ & 2,000 & \\
\hline 1968 & $04-16-68$ & 1,390 & & 1988 & $08-31-88$ & 1,590 & \\
\hline 1969 & 04-07-69 & 1,190 & & 1989 & $03-14-89$ & 688 & \\
\hline 1970 & $08-13-70$ & 1,850 & & 1990 & $07-07-90$ & 1,640 & \\
\hline 1971 & $08-12-71$ & 8,670 & & 1991 & $08-27-91$ & 2,980 & \\
\hline 1972 & $12-26-71$ & 5,170 & & 1992 & $08-24-92$ & 3,020 & \\
\hline 1973 & $04-29-73$ & 4,680 & & 1993 & $01-08-93$ & 12,600 & \\
\hline 1974 & $08-01-74$ & 3,110 & & 1994 & 09-03-94 & 1,770 & \\
\hline 1975 & $04-27-75$ & 1,930 & & 1995 & $03-06-95$ & 5,730 & \\
\hline 1976 & $07-14-76$ & 2,220 & & 1996 & 09-03-96 & 1,770 & \\
\hline 1977 & $07-24-77$ & 1,980 & & & & & \\
\hline
\end{tabular}

Discharge rating table developed January 1993

\begin{tabular}{cccc}
\hline $\begin{array}{c}\text { Gage height } \\
(\mathbf{f t})\end{array}$ & $\begin{array}{c}\text { Discharge } \\
\left(\mathrm{ft}^{\mathbf{3}} / \mathbf{s}\right)\end{array}$ & $\begin{array}{c}\text { Gage height } \\
(\mathrm{ft})\end{array}$ & $\begin{array}{c}\text { Discharge } \\
\left(\mathbf{f t}^{3} / \mathbf{s}\right)\end{array}$ \\
\hline 5.0 & 1,480 & 11.0 & 7,230 \\
6.0 & 2,140 & 12.0 & 8,590 \\
7.0 & 2,930 & 13.0 & 10,060 \\
8.0 & 3,830 & 14.0 & 11,650 \\
9.0 & 4,850 & 15.0 & 13,350 \\
10.0 & 5,980 & 15.7 & 14,600 \\
\hline
\end{tabular}


586

GILA RIVER BASIN

09494000 WHITE RIVER NEAR FORT APACHE, AZ--Continued

Basin characteristics

\begin{tabular}{cccccccc}
\hline $\begin{array}{c}\text { Main } \\
\text { channel } \\
\text { slope } \\
(\mathrm{ft} / \mathrm{mi})\end{array}$ & $\begin{array}{c}\text { Stream } \\
\text { length } \\
\text { (mi) }\end{array}$ & $\begin{array}{c}\text { Mean } \\
\text { basin } \\
\text { elevation } \\
(\mathrm{ft})\end{array}$ & $\begin{array}{c}\text { Forested } \\
\text { area } \\
\text { (percent) }\end{array}$ & $\begin{array}{c}\text { Soil } \\
\text { index }\end{array}$ & $\begin{array}{c}\text { Mean } \\
\text { annual } \\
\text { precipitation } \\
\text { (in) }\end{array}$ & $\begin{array}{c}\text { Rainfall intensity, 24-hour } \\
\text { (in) }\end{array}$ & $\begin{array}{c}50-\text {-year } \\
\text { (in) }\end{array}$ \\
\hline 76.8 & 62.5 & 7,400 & 83.0 & 3.0 & 25.4 & 2.3 & 4.6 \\
\hline
\end{tabular}


09494000 WHITE RIVER NEAR FORT APACHE, AZ--Continued

MEAN MONTHLY AND ANNUAL DISCHARGES 1958.96

\begin{tabular}{|c|c|c|c|c|c|c|}
\hline MONTH & $\begin{array}{l}\text { MAXIMUM } \\
(\text { FT } 3 / S)\end{array}$ & $\begin{array}{l}\text { MINIMUM } \\
\text { (FT3/S) }\end{array}$ & $\begin{array}{c}\text { MEAN } \\
(\mathrm{FT} 3 / \mathrm{S})\end{array}$ & $\begin{array}{l}\text { STAN- } \\
\text { DARD } \\
\text { DEVIA- } \\
\text { TION } \\
\text { (FT3/S) }\end{array}$ & $\begin{array}{l}\text { COEFFI- } \\
\text { CIENT OF } \\
\text { VARI- } \\
\text { ATION }\end{array}$ & $\begin{array}{c}\text { PERCENT } \\
\text { OF } \\
\text { ANNUAL } \\
\text { RUNOFF }\end{array}$ \\
\hline OCTOBER & 774 & 31 & 110 & 150 & 1.4 & 4.3 \\
\hline NOVEMBER & 218 & 35 & 87 & 55 & 0.63 & 3.4 \\
\hline DECEMBER & 715 & 35 & 124 & 142 & 1.2 & 4.9 \\
\hline JANUARY & 1,120 & 32 & 136 & 181 & 1.3 & 5.4 \\
\hline FEBRUARY & 787 & 33 & 186 & 180 & 0.97 & 7.3 \\
\hline MARCH & 1,160 & 49 & 377 & 290 & 0.77 & 15 \\
\hline APRIL & 1.450 & 55 & 607 & 409 & 0.67 & 24 \\
\hline MAY & 2.070 & 22 & 462 & 410 & 0.89 & 18 \\
\hline JUNE & 602 & 6.9 & 162 & 161 & 0.99 & 6.4 \\
\hline JULY & 187 & 3.9 & 72 & 44 & 0.62 & 2.8 \\
\hline AUGUST & 388 & 26 & 122 & 84 & 0.69 & 4.8 \\
\hline SEPTEMBER & 293 & 19 & 106 & 68 & 0.64 & 4.2 \\
\hline ANNUAL & 487 & 50 & 212 & 124 & 0.59 & 100 \\
\hline
\end{tabular}

MAGNITUDE AND PROBABILITY OF ANNUAL LOW FLOW BASED ON PERIOD OF RECORD 1959.96

\begin{tabular}{|c|c|c|c|c|c|c|}
\hline $\begin{array}{l}\text { PERIOD } \\
\text { (CON. }\end{array}$ & \multirow{2}{*}{\multicolumn{6}{|c|}{$\begin{array}{r}\text { DISCHARGE, IN FT3/S, FOR INDICATED } \\
\text { RECURRENCE INTERVAL, IN YEARS, AND } \\
\text { NON-EXCEEDANCE PROBABILITY, IN PERCENT }\end{array}$}} \\
\hline SECU. & & $\ldots \ldots$ & & & & \\
\hline TIVE & 2 & 5 & $10 \#$ & $20 \#$ & $50 \#$ & $100 \#$ \\
\hline DAYS) & 508 & 208 & 108 & 58 & 28 & 18 \\
\hline 1 & 22 & 9.9 & 5.9 & 3.4 & 0.00 & 0.00 \\
\hline 3 & 23 & 11 & 6.8 & 4.2 & 0.00 & 0.00 \\
\hline 7 & 29 & 12 & 6.2 & 3.2 & 1.4 & 0.73 \\
\hline 14 & 31 & 14 & 8.2 & 4.9 & 2.5 & 1.6 \\
\hline 30 & 34 & 18 & 12 & 8.2 & 5.1 & 3.7 \\
\hline 60 & 42 & 26 & 20 & 16 & 12 & 9.8 \\
\hline 90 & 47 & 34 & 29 & 26 & 23 & 21 \\
\hline 120 & 54 & 39 & 34 & 30 & 27 & 25 \\
\hline 183 & 68 & 48 & 41 & 37 & 33 & 31 \\
\hline
\end{tabular}

MAGNITUDE AND PROBABILITY OF ANNUAL HIGH FLOW BASED ON PERTOD OF RECORD 1958.96

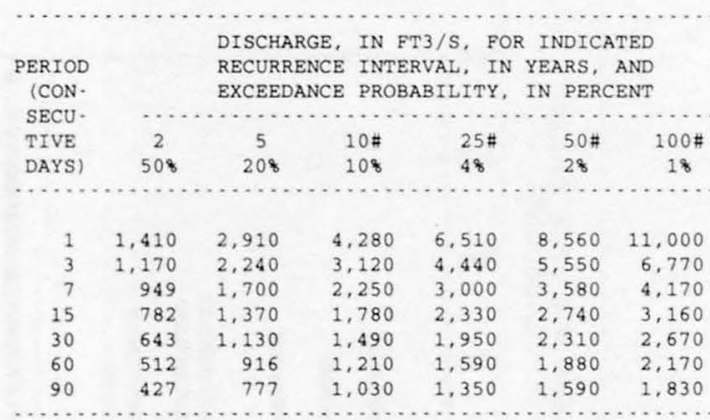

DURATION TABLE OF DAILY MEAN FLOW FOR PERIOD OF RECORD 19584.96

DISCHARGE, IN FT3/S, WHICH WAS EQUALED OR EXCEEDED FOR INDICATED PERCENT OF TIME

\begin{tabular}{|c|c|c|c|c|c|c|c|c|c|c|c|c|c|c|c|c|}
\hline 18 & 58 & 108 & 158 & 208 & 308 & 408 & 508 & 608 & 708 & 808 & 908 & 958 & 988 & 998 & 99.58 & 99.98 \\
\hline 880 & 856 & 567 & 407 & 297 & 172 & 122 & 90 & 70 & 56 & 46 & 35 & 25 & 0.00 & 0.00 & 0.00 & 0.00 \\
\hline
\end{tabular}


ANNUAL MEAN DISCHARGE, IN CUBIC

FEET PER SECOND

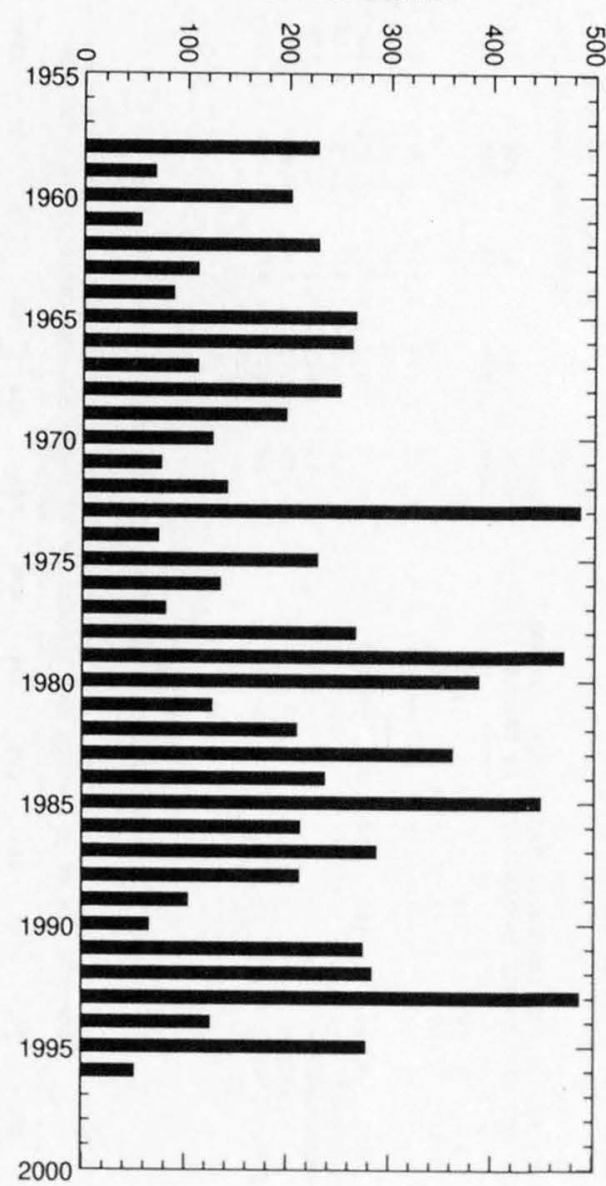

ANNUAL PEAK DISCHARGE, IN CUBIC FEET PER SECOND

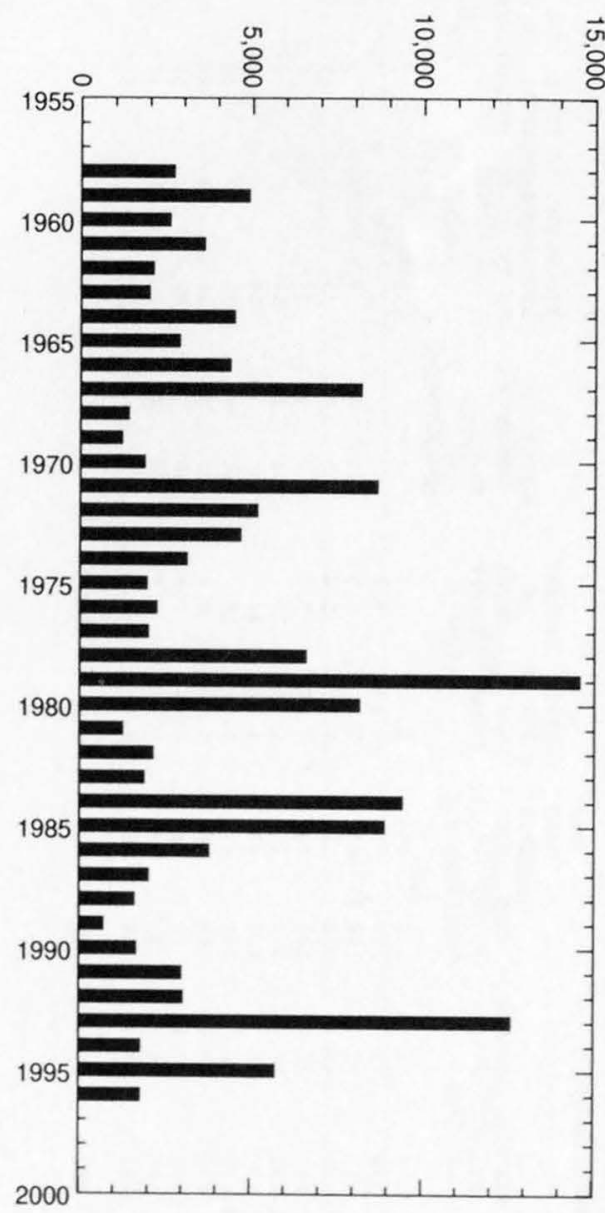


09494000 WHITE RIVER NEAR FORT APACHE, AZ--Continued

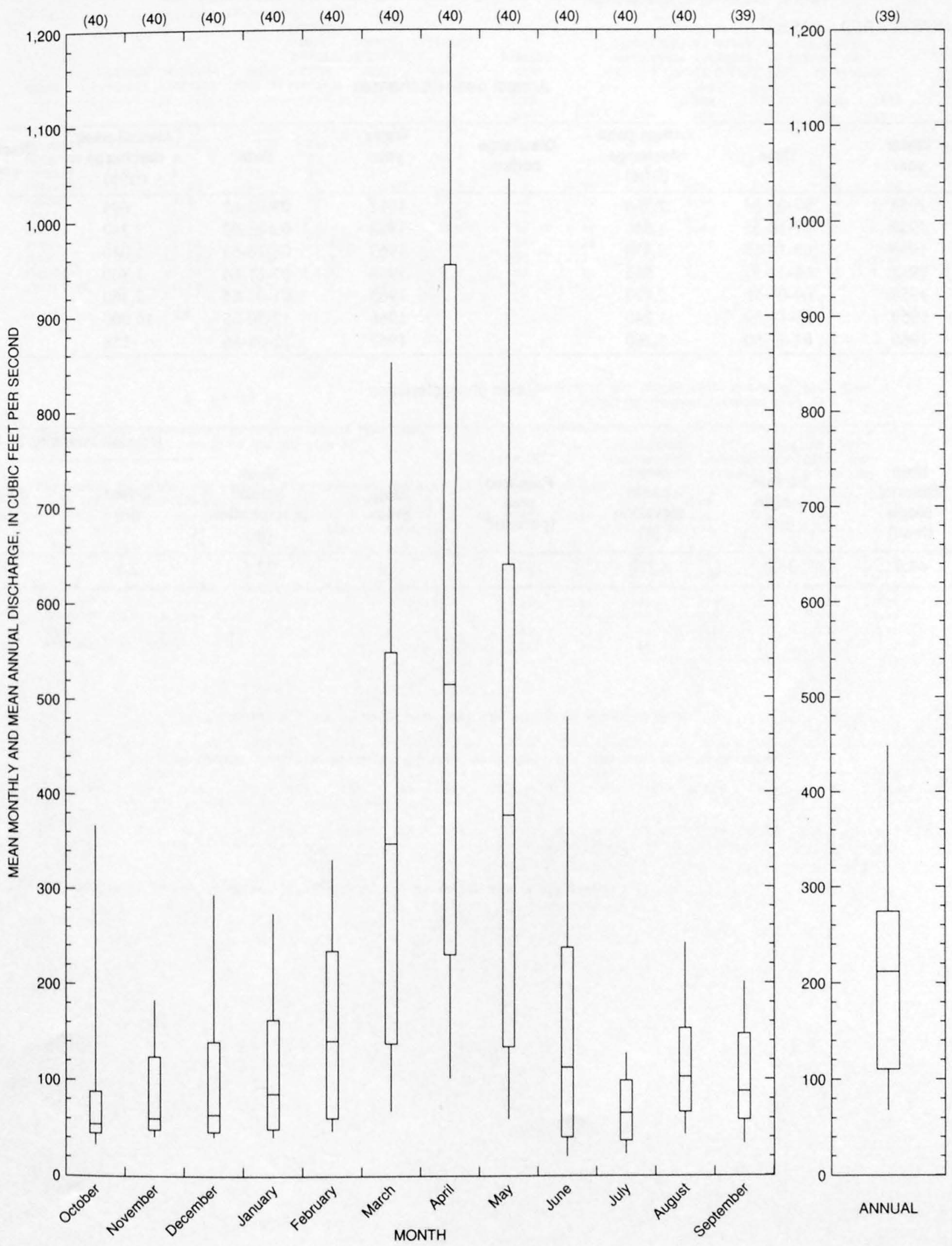


09494300 CARRIZO CREEK ABOVE CORDUROY CREEK, NEAR SHOW LOW, AZ

LOCATION.--Lat 34 00'00", long 110'17'20", in sec.13, T.7 N., R.19 E., Navajo County, Hydrologic Unit 15060104 (unsurveyed), in Fort Apac Indian Reservation, on left bank $0.5 \mathrm{mi}$ upstream from Corduroy Creek and 23 mi southwest of Show Low.

DRAINAGE AREA.--225 $\mathrm{mi}^{2}$.

Annual peak discharges

\begin{tabular}{|c|c|c|c|c|c|c|c|}
\hline $\begin{array}{l}\text { Water } \\
\text { year }\end{array}$ & Date & $\begin{array}{c}\text { Annual peak } \\
\text { discharge } \\
\left(\mathrm{ft}^{3} / \mathrm{s}\right)\end{array}$ & $\begin{array}{l}\text { Discharge } \\
\text { codes }\end{array}$ & $\begin{array}{l}\text { Water } \\
\text { year }\end{array}$ & Date & $\begin{array}{c}\text { Annual peak } \\
\text { discharge } \\
\left(\mathrm{ft}^{3} / \mathrm{s}\right)\end{array}$ & $\begin{array}{c}\text { Discharge } \\
\text { codes }\end{array}$ \\
\hline 1954 & $07-09-54$ & 2,760 & & 1961 & $09-06-61$ & 694 & \\
\hline 1956 & $08-14-56$ & 2,470 & & 1963 & $08-26-63$ & 3,040 & \\
\hline 1957 & $08-31-57$ & 935 & & 1964 & $07-21-64$ & 1,860 & \\
\hline 1960 & $01-11-60$ & 3,260 & & 1967 & $12-08-66$ & 158 & \\
\hline
\end{tabular}

Basin characteristics

\begin{tabular}{ccccccc}
\hline $\begin{array}{c}\text { Main } \\
\text { channel } \\
\text { slope } \\
(\mathrm{ft} / \mathrm{mi})\end{array}$ & $\begin{array}{c}\text { Stream } \\
\text { length } \\
(\mathrm{mi})\end{array}$ & $\begin{array}{c}\text { Mean } \\
\text { basin } \\
\text { elevation } \\
(\mathrm{ft})\end{array}$ & $\begin{array}{c}\text { Forested } \\
\text { area } \\
\text { (percent) }\end{array}$ & $\begin{array}{c}\text { Soil } \\
\text { index }\end{array}$ & $\begin{array}{c}\text { Mean } \\
\text { annual } \\
\text { precipitation } \\
\text { (in) }\end{array}$ & $\begin{array}{c}\text { Rainfall intensity, 24-hour } \\
\text { (in) }\end{array}$ \\
\hline 64.9 & 35.0 & 6,370 & 97.0 & 3.0 & 22.5 & $\begin{array}{c}50 \text {-year } \\
\text { (in) }\end{array}$ \\
\hline
\end{tabular}


09494300 CARRIZO CREEK ABOVE CORDUROY CREEK, NEAR SHOW LOW, AZ--Continued

MEAN MONTHLY AND ANNUAL DISCHARGES $1954-66$

\begin{tabular}{|c|c|c|c|c|c|c|}
\hline MONTH & $\begin{array}{l}\text { MAXIMUM } \\
(\text { FT } 3 / \mathrm{S})\end{array}$ & $\begin{array}{l}\text { MINIMUM } \\
(\text { FT3/S) }\end{array}$ & $\begin{array}{c}\text { MEAN } \\
(\mathrm{FT} 3 / \mathrm{S})\end{array}$ & $\begin{array}{l}\text { STAN- } \\
\text { DARD } \\
\text { DEVIA- } \\
\text { TION } \\
\text { (FT3/S) }\end{array}$ & $\begin{array}{l}\text { COEFFI- } \\
\text { CIENT OF } \\
\text { VARI- } \\
\text { ATION }\end{array}$ & $\begin{array}{c}\text { PERCENT } \\
\text { OF } \\
\text { ANNUAL } \\
\text { RUNOFF }\end{array}$ \\
\hline OCTOBER & 6.8 & 0.12 & 2.8 & 2.2 & 0.77 & 2.0 \\
\hline NOVEMBER & 54 & 0.87 & 7.8 & 14 & 1.8 & 5.5 \\
\hline DECEMBER & 301 & 2.0 & 35 & 85 & 2.4 & 24.6 \\
\hline JANUARY & 135 & 3.5 & 25 & 39 & 1.6 & 17.5 \\
\hline PEBRUARY & 46 & 3.5 & 15 & 14 & 0.94 & 10.3 \\
\hline MARCH & 94 & 3.8 & 29 & 30 & 1.0 & 20.1 \\
\hline APRIL & 44 & 3.2 & 12 & 12 & 1.0 & 8.2 \\
\hline MAY & 8.8 & 1.3 & 4.1 & 2.7 & 0.65 & 2.9 \\
\hline JUNE & 3.5 & 0.01 & 1.2 & 1.3 & 1.1 & 0.8 \\
\hline JULY & 9.7 & 0.00 & 2.0 & 2.9 & 1.4 & 1.4 \\
\hline AUGUST & 31 & 0.00 & 6.4 & 8.3 & 1.3 & 4.5 \\
\hline SEPTEMBER & 10 & 0.00 & 3.1 & 3.4 & 1.1 & 2.2 \\
\hline ANNUAL & 40 & 3.1 & 12 & 13 & 1.1 & 100 \\
\hline
\end{tabular}

MAGNITUDE AND PROBABILITY OF INSTANTANEOUS PEAK FLOW BASED ON PERIOD OF RECORD 1954-67

DISCHARGE, IN FT3/S, FOR INDICATED RECURRENCE INTERVAL IN YEARS, AND EXCEEDANCE PROBABILITY, IN PERCENT

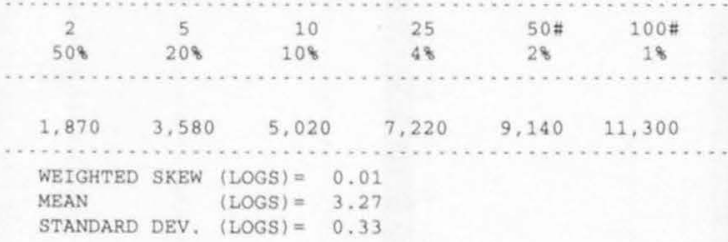

MAGNITUDE AND PROBABILITY OF ANNUAL LOW FLOW BASED ON PERIOD OF RECORD $1955-67$

\begin{tabular}{|c|c|c|c|c|c|c|}
\hline \multirow{4}{*}{$\begin{array}{l}\text { PERIOD } \\
\text { /CON- } \\
\text { SECU. }\end{array}$} & \multicolumn{6}{|c|}{ DISCHARGE, IN FT3/S, FOR INDICATED } \\
\hline & \multirow{2}{*}{\multicolumn{6}{|c|}{$\begin{array}{l}\text { RECURRENCE INTERVAL, IN YEARS, AND } \\
\text { NON-EXCEEDANCE PROBABILITY, IN PERCENT }\end{array}$}} \\
\hline & & & & & & \\
\hline & \multicolumn{6}{|c|}{ 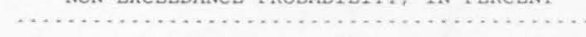 } \\
\hline TIVE & 2 & 5 & $10 \#$ & 20 & $50 \#$ & $100 \#$ \\
\hline DAYS) & 508 & 208 & 108 & 58 & 28 & 18 \\
\hline \multicolumn{7}{|l|}{1} \\
\hline \\
\hline 7 & 0.00 & 0.00 & 0.00 & 0.00 & 0.00 & 0.00 \\
\hline 14 & 0.00 & 0.00 & 0.00 & 0.00 & 0.00 & 0.00 \\
\hline 30 & 0.01 & 0.00 & 0.00 & 0.00 & 0.00 & 0.00 \\
\hline 60 & 0.38 & 0.00 & 0.00 & 0.00 & 0.00 & 0.00 \\
\hline 90 & 0.82 & 0.34 & 0.16 & 0.00 & 0.00 & 0.00 \\
\hline 120 & 1.6 & 0.72 & 0.40 & 0.23 & 0.11 & 0.06 \\
\hline 183 & 3.1 & 1.8 & 1.1 & 0.71 & 0.37 & 0.22 \\
\hline
\end{tabular}

MAGNITUDE AND PROBABILITY OF ANNUAL HIGH FLOW BASED ON PERIOD OF RECORD $1954-66$

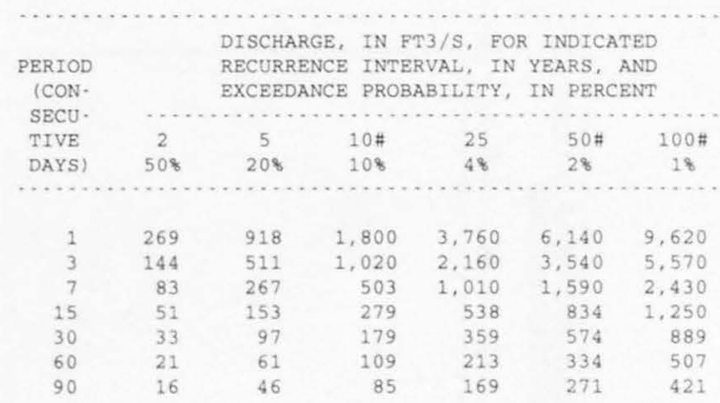

DURATION TABLE OF DAILY MEAN FLOW FOR PERIOD OF RECORD $1954 \cdot 66$

DISCHARGE, IN ET3/S, WHTCH WAS EQUALED OR EXCEEDED FOR INDICATED PERCENT OF TIME

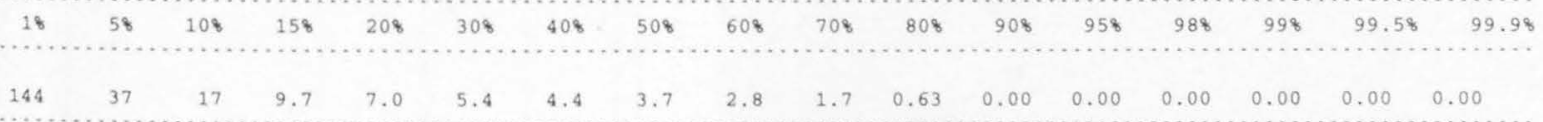

\# Reliability of values in column is uncertain, and potential errors are large. 
09494300 CARRIZO CREEK ABOVE CORDUROY CREEK, NEAR SHOW LOW, AZ--Continued
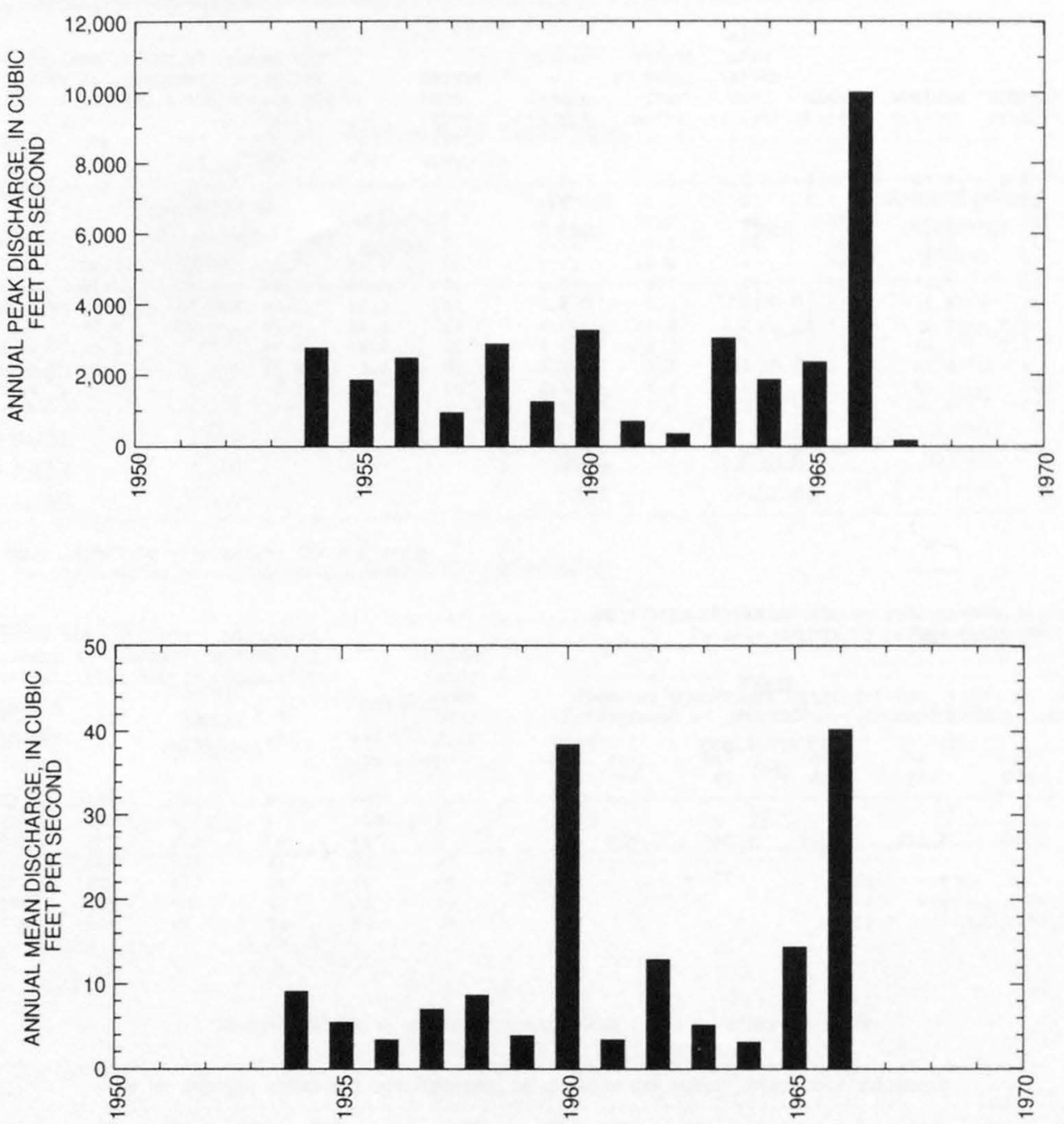
GILA RIVER BASIN

593

09494300 CARRIZO CREEK ABOVE CORDUROY CREEK, NEAR SHOW LOW, AZ--Continued
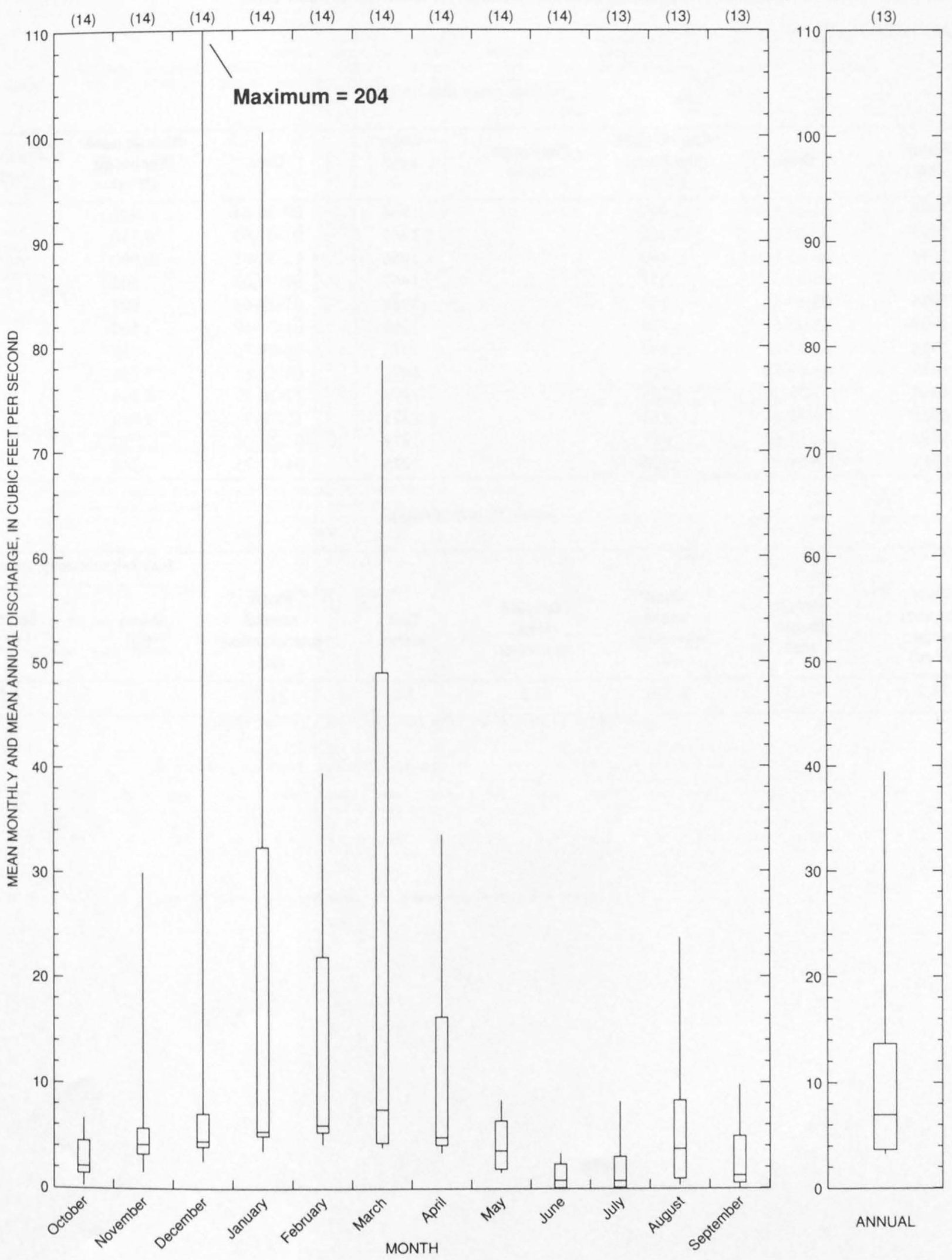

ANNUAL 
09496000 CORDUROY CREEK NEAR MOUTH, NEAR SHOW LOW, AZ

LOCATION.--Lat 34 01'06", long 110 14'30", in sec.8, T.7 N., R.20 E. (unsurveyed), Navajo County, Hydrologic Unit 15060104, in Fort Apact Indian Reservation, on right bank 4 mi upstream from mouth and 20 mi southwest of Show Low.

DRAINAGE AREA.--203 $\mathrm{mi}^{2}$.

Annual peak discharges

\begin{tabular}{|c|c|c|c|c|c|c|c|}
\hline $\begin{array}{l}\text { Water } \\
\text { year }\end{array}$ & Date & $\begin{array}{c}\text { Annual peak } \\
\text { discharge } \\
\left(\mathrm{ft}^{3} / \mathrm{s}\right)\end{array}$ & $\begin{array}{l}\text { Discharge } \\
\text { codes }\end{array}$ & $\begin{array}{l}\text { Water } \\
\text { year }\end{array}$ & Date & $\begin{array}{c}\text { Annual peak } \\
\text { discharge } \\
\left(\mathrm{ft}^{3} / \mathrm{s}\right)\end{array}$ & $\begin{array}{c}\text { Discharge } \\
\text { codes }\end{array}$ \\
\hline 1952 & $01-18-52$ & 10,900 & & 1964 & $07-30-64$ & 402 & \\
\hline 1954 & $03-23-54$ & 2,450 & & 1966 & $12-30-65$ & 10,900 & \\
\hline 1955 & $08-07-55$ & 257 & & 1967 & $07-29-67$ & 505 & \\
\hline 1956 & $08-17-56$ & 149 & & 1968 & $01-28-68$ & 902 & \\
\hline 1958 & $03-22-58$ & 1,140 & & 1970 & $08-09-70$ & 38 & \\
\hline 1959 & $08-19-59$ & 316 & & 1971 & $08-13-71$ & 366 & \\
\hline 1960 & $12-25-59$ & 3,500 & & 1972 & $12-26-71$ & 8,500 & \\
\hline 1961 & $08-22-61$ & 512 & & 1973 & $10-20-72$ & 7,600 & \\
\hline 1962 & $02-13-62$ & 841 & & 1974 & $03-21-74$ & 50 & \\
\hline 1963 & 09-09-63 & 7,150 & & 1975 & $04-12-75$ & 348 & \\
\hline
\end{tabular}

Basin characteristics

\begin{tabular}{cccccccc}
\hline $\begin{array}{c}\text { Main } \\
\text { channel } \\
\text { slope } \\
(\mathrm{ft} / \mathrm{mi})\end{array}$ & $\begin{array}{c}\text { Stream } \\
\text { length } \\
(\mathrm{mi})\end{array}$ & $\begin{array}{c}\text { Mean } \\
\text { basin } \\
\text { elevation } \\
\text { (ft) }\end{array}$ & $\begin{array}{c}\text { Forested } \\
\text { area } \\
\text { (percent) }\end{array}$ & $\begin{array}{c}\text { Soil } \\
\text { index }\end{array}$ & $\begin{array}{c}\text { Mean } \\
\text { annual } \\
\text { precipitation } \\
\text { (in) }\end{array}$ & $\begin{array}{c}\text { Rainfall intensity, 24-hour } \\
\text { (in) }\end{array}$ & $\begin{array}{c}50-y e a r \\
\text { (in) }\end{array}$ \\
\hline 71.2 & 31.5 & 6,370 & 93.0 & 3.0 & 21.7 & 2.1 \\
\hline
\end{tabular}


GILA RIVER BASIN

595

09496000 CORDUROY CREEK NEAR MOUTH, NEAR SHOW LOW, AZ--Continued

MEAN MONTHLY AND ANNUAL DISCHARGES $1952 \cdot 75$

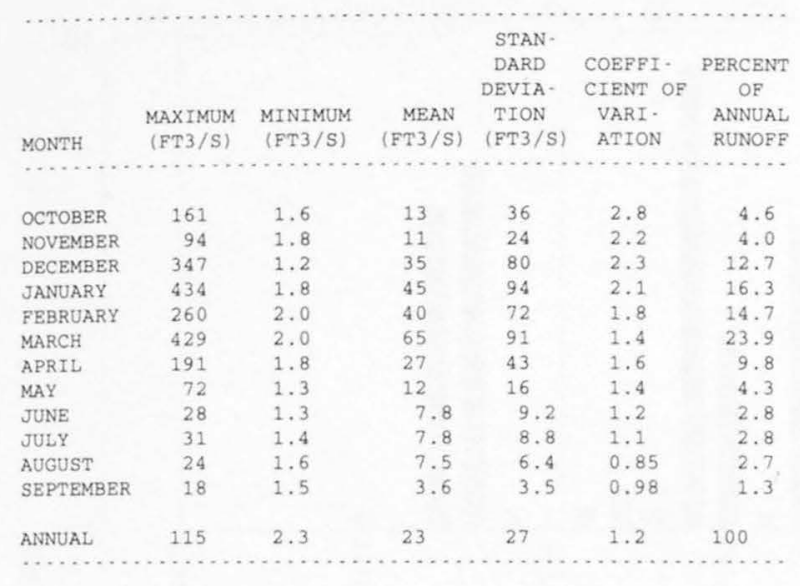

MAGNITUDE AND PROBABILITY OF ANNUAL LOW FLOW BASED ON PERIOD OF RECORD 1953.75

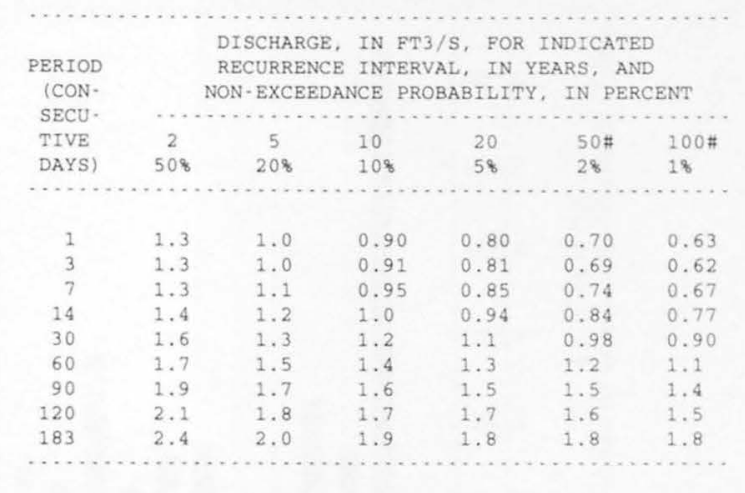

MAGNITUDE AND PROBABILITY OF ANNUAL HIGH FLOW BASED ON PERIOD OF RECORD $1952-75$

MAGNITUDE AND PROBABILITY OF INSTANTANEOUS PEAK FLOW BASED ON PERIOD OF RECORD 1952.75

DISCHARGE, IN FT 3/S, FOR INDICATED RECURRENCE INTERVAL IN YEARS, AND EXCEEDANCE PROBABILITY, IN PERCENT

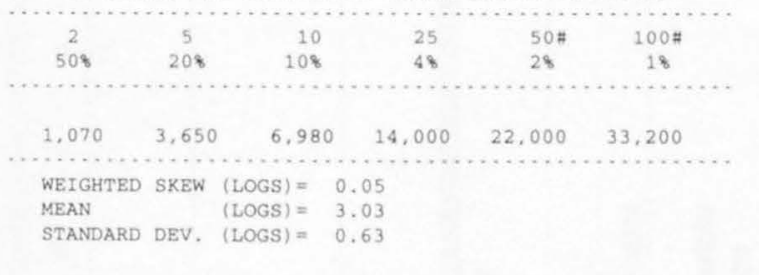

PERIOD (CONSECHDAYS)
DISCHARGE, IN FT 3/S, FOR INDICATED RECURRENCE INTERVAL, IN YEARS, AND EXCEEDANCE PROBABILITY, IN PERCENT

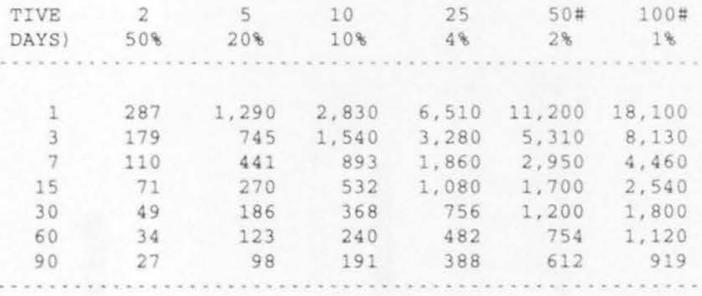

DURATION TABLE OF DAILY MEAN FLOW FOR PERIOD OF RECORD $1952 \cdot 75$

DISCHARGE, IN FT 3/S, WHICH WAS EQUALED OR EXCEEDED FOR INDICATED PERCENT OF TIME

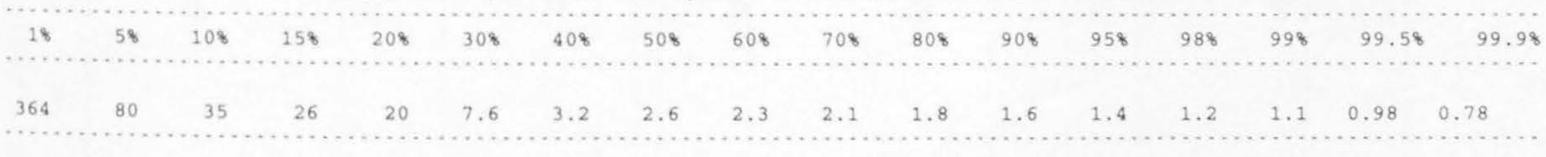

\# Reliability of values in column is uncertain, and potential errors are large. 
09496000 CORDUROY CREEK NEAR MOUTH, NEAR SHOW LOW, AZ--Continued
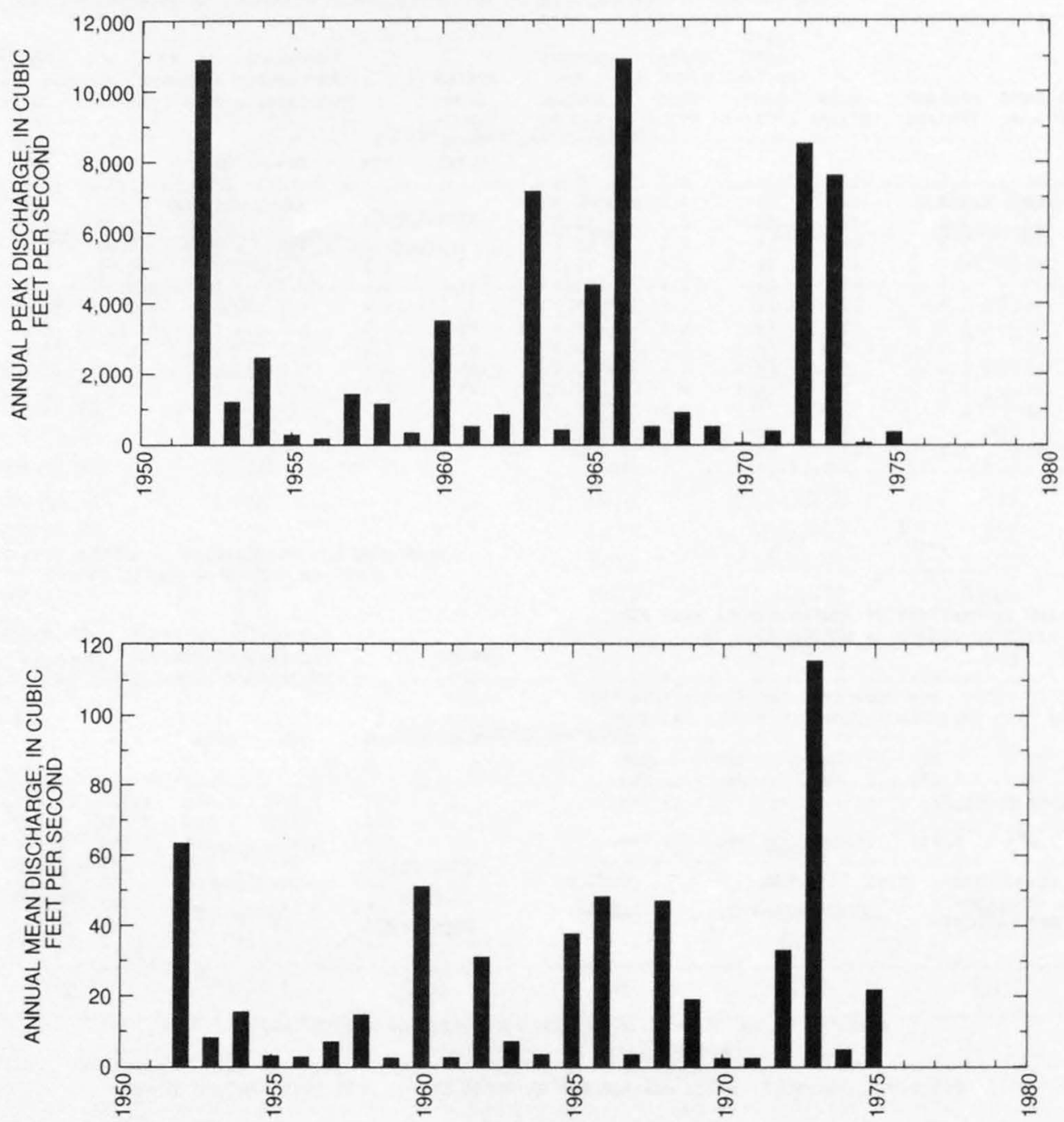
GILA RIVER BASIN

597

09496000 CORDUROY CREEK NEAR MOUTH, NEAR SHOW LOW, AZ--Continued
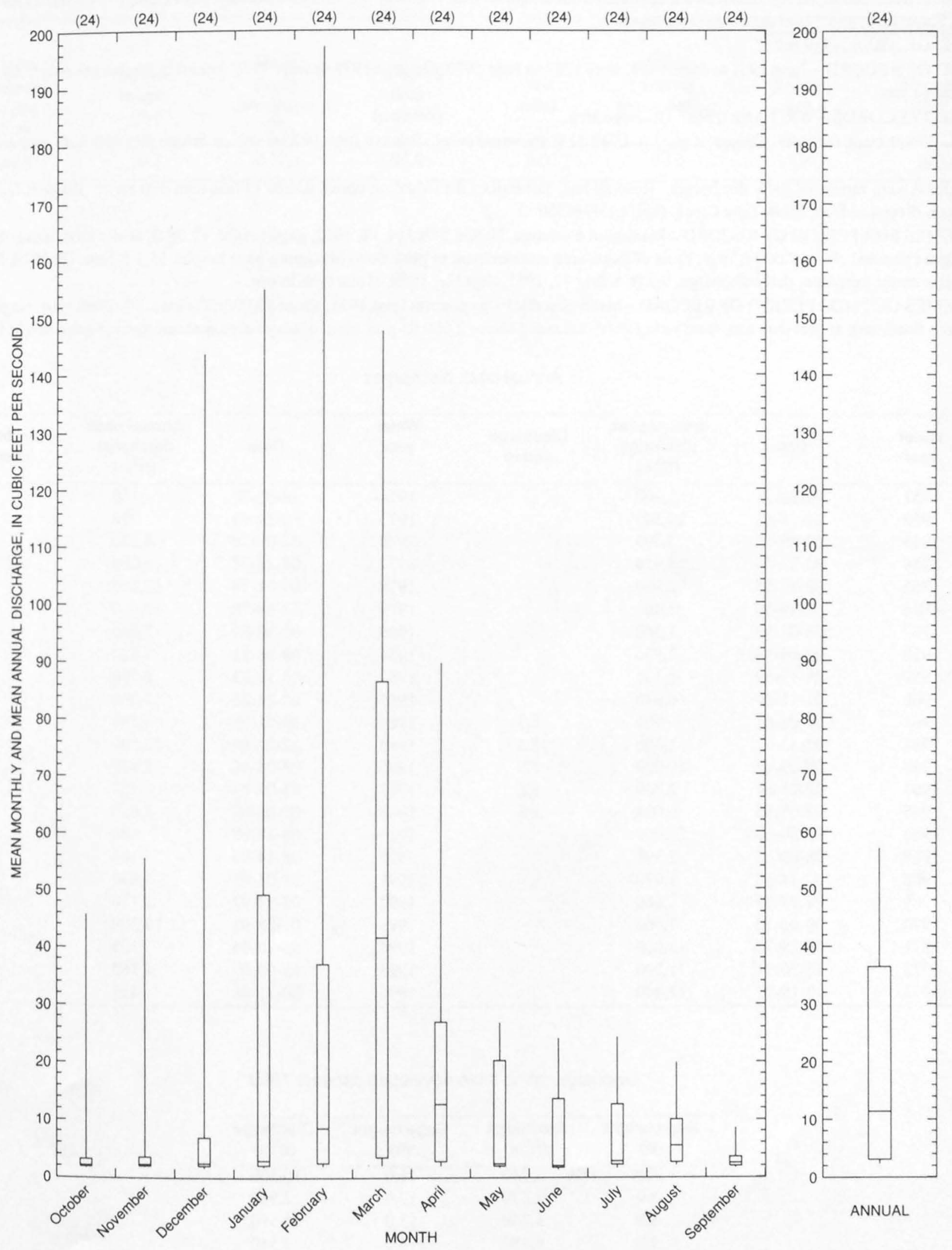
LOCATION.--Lat 33'59'09", long 110 16'49", in sec.24, T.7 N., R.19 E. (unsurveyed), Gila County, Hydrologic Unit 15060104, in Fort Apach Indian Reservation, on right bank $500 \mathrm{ft}$ upstream from bridge on U.S. Highway 60, 1 mi downstream from Corduroy Creek, 23 mi southwes of Show Low, and 24 mi upstream from mouth.

DRAINAGE AREA.--439 $\mathrm{mi}^{2}$.

PERIOD OF RECORD.--June 1951 to June 1961, June 1967 to June 1976, October 1975 to June 1976 (monthly discharges only), April 1977 t current year.

REVISED RECORDS.--WRD Ariz. 1968: Drainage area.

GAGE.--Water-stage recorder. Datum of gage is $4,749.52 \mathrm{ft}$ above sea level. Prior to June 1976 at site on bridge pier $400 \mathrm{ft}$ downstream at sam datum.

REMARKS.--No estimated daily discharges. Records fair. Diversions for irrigation above station of less than 300 acres. Records include tran basin diversion from Show Low Creek. (See sta 09495000.)

EXTREMES FOR PERIOD OF RECORD.--Maximum discharge, 20,500 $\mathrm{ft}^{3} / \mathrm{s} \mathrm{Jan.} \mathrm{18,} \mathrm{1952,} \mathrm{gage} \mathrm{height,} 12.08 \mathrm{ft}$, at site then in use, from ratin curve extended above $2,000 \mathrm{ft}^{3} / \mathrm{s}$ on basis of slope-area measurement of peak flow; maximum gage height, 15.1 ft Dec. 18, 1978, from high water mark; minimum daily discharge, $0.2 \mathrm{ft}^{3} / \mathrm{s}$ July 12, 1951, Sept. 21, 1959, at site then in use.

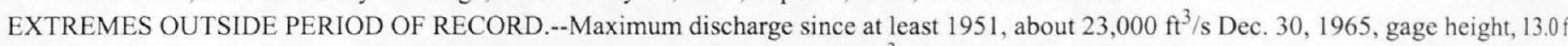
from floodmark at previous site, from rating curve extended above $2,000 \mathrm{ft}^{3} / \mathrm{s}$ on basis of slope-area measurement at gage height $12.08 \mathrm{ft}$.

Annual peak discharges

\begin{tabular}{|c|c|c|c|c|c|c|c|}
\hline $\begin{array}{l}\text { Water } \\
\text { year }\end{array}$ & Date & $\begin{array}{c}\text { Annual peak } \\
\text { discharge } \\
\left(\mathrm{ft}^{3} / \mathrm{s}\right)\end{array}$ & $\begin{array}{l}\text { Discharge } \\
\text { codes }\end{array}$ & $\begin{array}{l}\text { Water } \\
\text { year }\end{array}$ & Date & $\begin{array}{c}\text { Annual peak } \\
\text { discharge } \\
\left(\mathrm{ft}^{3} / \mathrm{s}\right)\end{array}$ & $\begin{array}{c}\text { Discharge } \\
\text { codes }\end{array}$ \\
\hline 1951 & $08-28-51$ & 3,260 & & 1974 & $08-03-74$ & 160 & \\
\hline 1952 & $01-18-52$ & 20,500 & & 1975 & $10-29-74$ & 726 & \\
\hline 1953 & $07-29-53$ & 3,200 & & 1976 & $02-09-76$ & 4,220 & \\
\hline 1954 & $03-23-54$ & 3,910 & & 1977 & $08-11-77$ & 5,060 & \\
\hline 1955 & $08-18-55$ & 2,060 & & 1978 & $03-01-78$ & 12,700 & \\
\hline 1956 & $08-14-56$ & 2,400 & & 1979 & $12-18-78$ & 19,400 & \\
\hline 1957 & $08-02-57$ & 1,360 & & 1980 & $01-30-80$ & 2,880 & \\
\hline 1958 & $09-04-58$ & 2,920 & & 1981 & $08-08-81$ & 1,420 & \\
\hline 1959 & $08-19-59$ & 1,560 & & 1982 & $08-11-82$ & 2,250 & \\
\hline 1960 & $01-11-60$ & 6,980 & & 1983 & $03-24-83$ & 2,060 & \\
\hline 1961 & $09-06-61$ & 900 & ES & 1984 & $10-01-83$ & 3,580 & \\
\hline 1962 & $02-13-62$ & 1,200 & ES & 1985 & $12-27-84$ & 12,800 & \\
\hline 1963 & $09-09-63$ & 10,000 & ES & 1986 & $08-08-86$ & 2,830 & \\
\hline 1964 & $07-21-64$ & 2,000 & ES & 1987 & $03-07-87$ & 763 & \\
\hline 1965 & $01-07-65$ & 6,000 & ES & 1988 & $02-03-88$ & 3,650 & \\
\hline 1966 & $12-30-65$ & 23,000 & & 1989 & $08-18-89$ & 600 & \\
\hline 1967 & $08-09-67$ & 2,590 & & 1990 & $08-14-90$ & 184 & \\
\hline 1968 & $02-14-68$ & 1,070 & & 1991 & $03-01-91$ & 4,490 & \\
\hline 1969 & $01-27-69$ & 1,060 & & 1992 & $02-13-92$ & 2,170 & \\
\hline 1970 & $09-06-70$ & 1,960 & & 1993 & $01-08-93$ & 19,300 & \\
\hline 1971 & $09-29-71$ & 2,000 & & 1994 & $03-20-94$ & 305 & \\
\hline 1972 & $12-26-71$ & 11,200 & & 1995 & $03-06-95$ & 4,160 & \\
\hline 1973 & $10-19-72$ & 12,400 & & 1996 & $09-11-96$ & 452 & \\
\hline
\end{tabular}

Discharge rating table developed January 1993

\begin{tabular}{cccc}
\hline $\begin{array}{c}\text { Gage height } \\
(\mathbf{f t})\end{array}$ & $\begin{array}{c}\text { Discharge } \\
\left(\mathbf{f t}^{\mathbf{3}} / \mathbf{s}\right)\end{array}$ & $\begin{array}{c}\text { Gage height } \\
(\mathbf{f t})\end{array}$ & $\begin{array}{c}\text { Discharge } \\
\left(\mathbf{f t}^{\mathbf{3}} / \mathbf{s}\right)\end{array}$ \\
\hline 6.0 & 2,340 & 11.0 & 10,500 \\
7.0 & 3,470 & 12.0 & 12,910 \\
8.0 & 4,850 & 13.0 & 15,590 \\
9.0 & 6,480 & 14.0 & 18,540 \\
10.0 & 8,360 & 14.4 & 19,800 \\
\hline
\end{tabular}


09496500 CARRIZO CREEK NEAR SHOW LOW, AZ--Continued

Basin characteristics

\begin{tabular}{|c|c|c|c|c|c|c|c|}
\hline \multirow[b]{2}{*}{$\begin{array}{l}\text { Main } \\
\text { channel } \\
\text { slope } \\
\text { (ft/mi) }\end{array}$} & \multirow[b]{2}{*}{$\begin{array}{l}\text { Stream } \\
\text { length } \\
\text { (mi) }\end{array}$} & \multirow[b]{2}{*}{$\begin{array}{c}\text { Mean } \\
\text { basin } \\
\text { elevation } \\
\text { (ft) }\end{array}$} & \multirow[b]{2}{*}{$\begin{array}{l}\text { Forested } \\
\text { area } \\
\text { (percent) }\end{array}$} & \multirow[b]{2}{*}{$\begin{array}{c}\text { Soil } \\
\text { index }\end{array}$} & \multirow[b]{2}{*}{$\begin{array}{c}\text { Mean } \\
\text { annual } \\
\text { precipitation } \\
\text { (in) }\end{array}$} & \multicolumn{2}{|c|}{ Rainfall intensity, 24-hour } \\
\hline & & & & & & $\begin{array}{l}\text { 2-year } \\
\text { (in) }\end{array}$ & $\begin{array}{l}\text { 50-year } \\
\text { (in) }\end{array}$ \\
\hline 61.8 & 36.7 & 6,320 & 95.0 & 3.0 & 22.0 & 2.2 & 4.6 \\
\hline
\end{tabular}


09496500 CARRIZO CREEK NEAR SHOW LOW, AZ--Continued

MEAN MONTHLY AND ANNUAL DISCHARGES $1952 \cdot 60,1968 \cdot 75,1978.96$

\begin{tabular}{|c|c|c|c|c|c|c|}
\hline MONTH & $\begin{array}{l}\text { MAXIMUM } \\
\text { (FT3/S) }\end{array}$ & $\begin{array}{l}\text { MINIMUM } \\
\text { (FT3/S) }\end{array}$ & $\begin{array}{c}\text { MEAN } \\
(\mathrm{FT} 3 / \mathrm{S})\end{array}$ & $\begin{array}{l}\text { STAN- } \\
\text { DARD } \\
\text { DEVIA- } \\
\text { TION } \\
\text { (FT3/S) }\end{array}$ & $\begin{array}{l}\text { COEFFI- } \\
\text { CIENT OF } \\
\text { VARI- } \\
\text { ATION }\end{array}$ & $\begin{array}{c}\text { PERCENT } \\
\text { OF } \\
\text { ANNUAL } \\
\text { RUNOFF }\end{array}$ \\
\hline OCTOBER & 397 & 1.6 & 30 & 75 & 2.5 & 4.7 \\
\hline NOVEMBER & 147 & 2.5 & 23 & 33 & 1.5 & 3.5 \\
\hline DECEMBER & 762 & 3.9 & 78 & 151 & 1.9 & 12.0 \\
\hline JANUARY & 1,030 & 5.4 & 104 & 211 & 2.0 & 16.0 \\
\hline FEBRUARY & 965 & 6.3 & 133 & 214 & 1.6 & 20.4 \\
\hline MARCH & 698 & 6.1 & 157 & 176 & 1.1 & 24.2 \\
\hline APRIL & 350 & 5.3 & 51 & 70 & 1.4 & 7.9 \\
\hline MAY & 154 & 2.4 & 21 & 26 & 1.3 & 3.2 \\
\hline JUNE & 41 & 0.87 & 13 & 11 & 0.88 & 1.9 \\
\hline JULY & 41 & 1.3 & 13 & 11 & 0.86 & 2.0 \\
\hline AUGUST & 42 & 3.2 & 17 & 10 & 0.61 & 2.6 \\
\hline SEPTEMBER & 30 & 0.91 & 11 & 8.0 & 0.74 & 1.7 \\
\hline ANNUAL & 201 & 5.8 & 54 & 54 & 1.0 & 100 \\
\hline
\end{tabular}

MAGNITUDE AND PROBABILITY OF ANNUAL LOW FLOW BASED ON PERIOD OF RECORD $1953.61,1969 \cdot 75,1979.96$

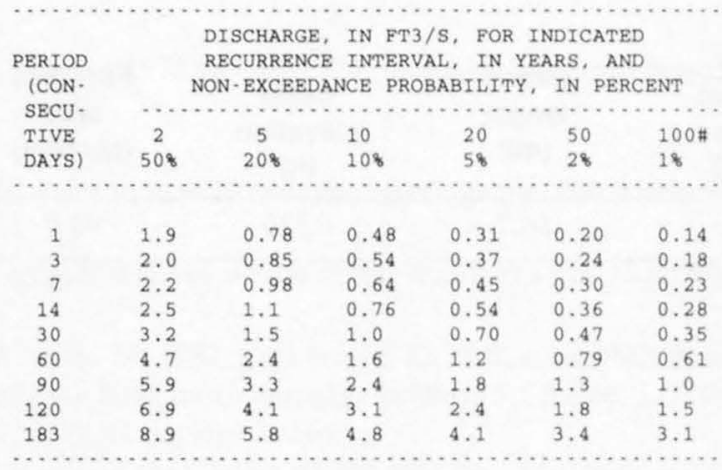

MAGNITUDE AND PROBABILITY OF ANNUAL HIGH FLOW BASED ON PERIOD OF RECORD $1952 \cdot 60,1968 \cdot 75,1978.96$

MAGNITUDE AND PROBABILITY OF INSTANTANEOUS PEAK FLOW BASED ON PERIOD OF RECORD 1951.96

DISCHARGE, IN FT3/S, FOR INDICATED RECURRENCE INTERVAI IN YEARS, AND EXCEEDANCE PROBABILITY, IN PERCENT

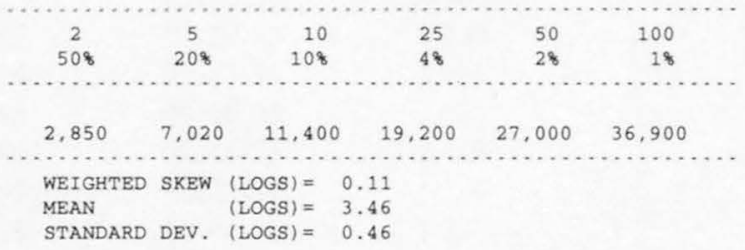

DURATION TABLE OF DAILY MEAN FLOW FOR PERIOD OF RECORD $1952 \cdot 60,1968 \cdot 75,1978.96$

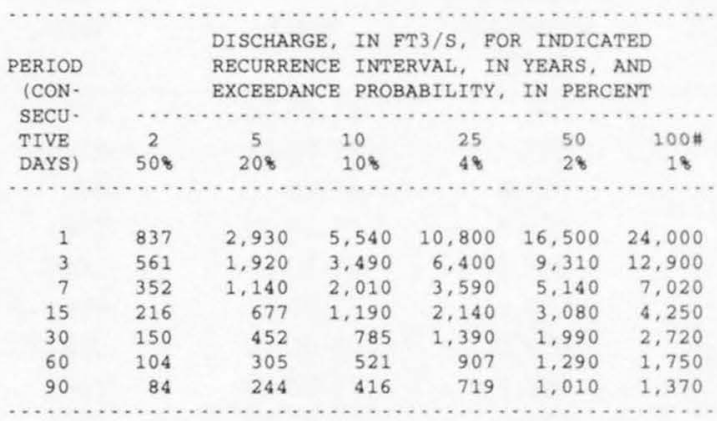

DISCHARGE, IN FT3/S, WHICH WAS EQUALED OR EXCEEDED FOR INDICATED PERCENT OF TIME.

\begin{tabular}{|c|c|c|c|c|c|c|c|c|c|c|c|c|c|c|c|c|}
\hline 18 & 58 & 108 & 158 & 208 & 308 & 408 & 508 & 608 & 708 & $80 \%$ & 908 & 958 & 988 & 998 & 99.58 & 99.98 \\
\hline 738 & 204 & 83 & 48 & 35 & 24 & 17 & 12 & 8.7 & 6.6 & 5.0 & 2.8 & 1.7 & 1.0 & 0.79 & 0.61 & 0.42 \\
\hline
\end{tabular}

\# Reliability of values in column is uncertain, and potential errors are large. 
ANNUAL MEAN DISCHARGE, IN CUBIC REET PER SECOND

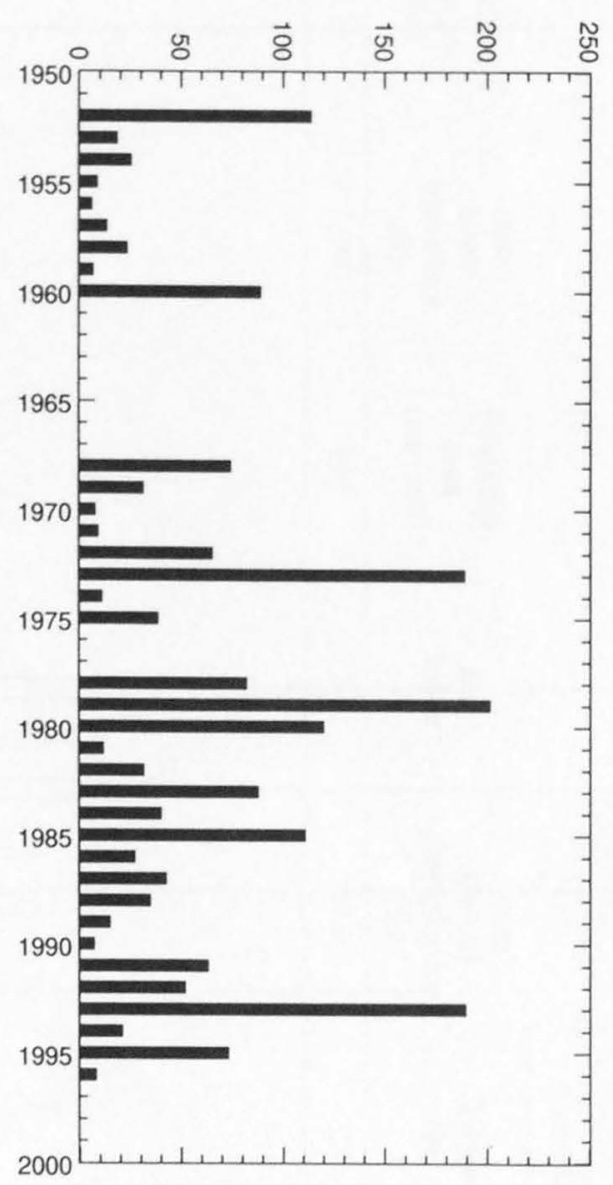

ANNUAL PEAK DISCHARGE, IN CUBIC FEET PER SECOND

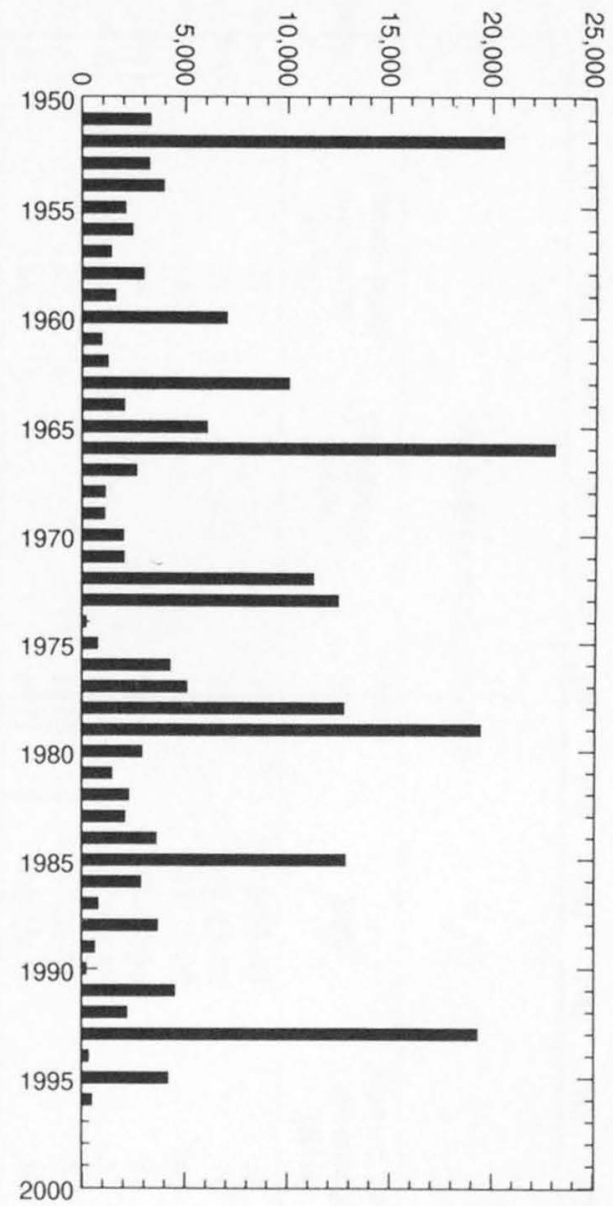


09496500 CARRIZO CREEK NEAR SHOW LOW, AZ--Continued

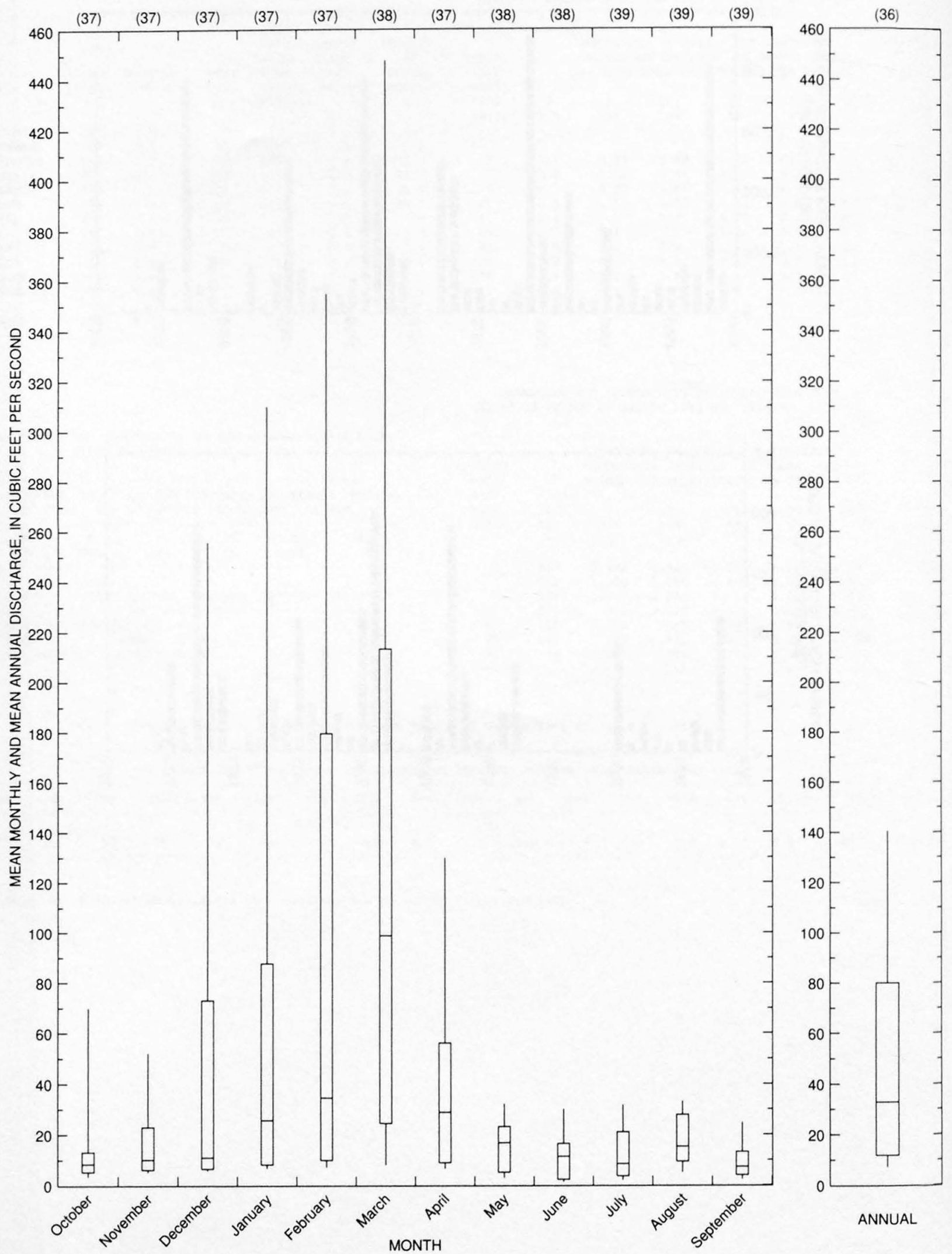


09496600 CIBECUE NO. 1, TRIBUTARY TO CARRIZO CREEK, NEAR SHOW LOW, AZ

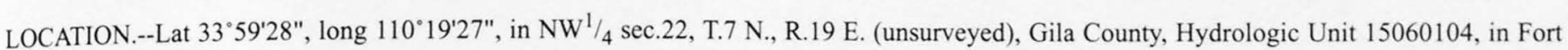
Apache Indian Reservation, on an unnamed tributary to Carrizo Creek, on Cibecue Ridge, 3.0 mi upstream from mouth of main stem, and 25 mi southwest of Show Low.

DRAINAGE AREA.--0.10 $\mathrm{mi}^{2}$.

Annual peak discharges

\begin{tabular}{|c|c|c|c|c|c|c|c|}
\hline $\begin{array}{l}\text { Water } \\
\text { year }\end{array}$ & Date & $\begin{array}{c}\text { Annual peak } \\
\text { discharge } \\
\left(\mathrm{ft}^{3} / \mathrm{s}\right)\end{array}$ & $\begin{array}{l}\text { Discharge } \\
\text { codes }\end{array}$ & $\begin{array}{l}\text { Water } \\
\text { year }\end{array}$ & Date & $\begin{array}{c}\text { Annual peak } \\
\text { discharge } \\
\left(\mathrm{ft}^{3} / \mathrm{s}\right)\end{array}$ & $\begin{array}{c}\text { Discharge } \\
\text { codes }\end{array}$ \\
\hline 1958 & $09-04-58$ & 24 & & 1965 & $09-03-65$ & 38 & \\
\hline 1959 & $08-17-59$ & 39 & & 1966 & $07-23-66$ & 76 & \\
\hline 1960 & $10-29-59$ & 8.3 & & 1967 & $07-27-67$ & 165 & \\
\hline 1961 & $08-18-61$ & 16 & & 1968 & 08-09-68 & 27 & \\
\hline 1962 & $07-26-62$ & 75 & ES & 1969 & $08-02-69$ & 61 & \\
\hline 1963 & $08-26-63$ & 127 & & 1970 & $07-23-70$ & 16 & \\
\hline 1964 & $07-31-64$ & 134 & & 1971 & $08-15-71$ & 40 & \\
\hline
\end{tabular}

\section{Basin characteristics}

\begin{tabular}{cccccccc}
\hline $\begin{array}{c}\text { Main } \\
\text { channel } \\
\text { slope } \\
(\mathrm{ft} / \mathrm{mi})\end{array}$ & $\begin{array}{c}\text { Stream } \\
\text { length } \\
\text { (mi) }\end{array}$ & $\begin{array}{c}\text { Mean } \\
\text { basin } \\
\text { elevation } \\
(\mathrm{ft})\end{array}$ & $\begin{array}{c}\text { Forested } \\
\text { area } \\
\text { (percent) }\end{array}$ & $\begin{array}{c}\text { Soil } \\
\text { index }\end{array}$ & $\begin{array}{c}\text { Mean } \\
\text { annual } \\
\text { precipitation } \\
\text { (in) }\end{array}$ & $\begin{array}{c}\text { R-year } \\
\text { (in) }\end{array}$ & $\begin{array}{c}50 \text {-year } \\
\text { (in) }\end{array}$ \\
\hline 364 & 0.30 & 5,390 & 100 & 3.0 & 18.0 & 1.9 \\
\hline
\end{tabular}


09496600 CIBECUE NO. 1, TRIBUTARY TO CARRIZO CREEK, NEAR SHOW LOW, AZ--Continued

MEAN MONTHLY AND ANNUAL DISCHARGES $1959 \cdot 71$

\begin{tabular}{|c|c|c|c|c|c|c|}
\hline MONTH & $\begin{array}{l}\text { MAXIMUM } \\
(\text { FT } 3 / S)\end{array}$ & $\begin{array}{l}\text { MINIMUM } \\
\text { (FT3/S) }\end{array}$ & $\begin{array}{c}\text { MEAN } \\
(\text { FT3/S) }\end{array}$ & $\begin{array}{l}\text { STAN - } \\
\text { DARD } \\
\text { DEVIA - } \\
\text { TION } \\
(\mathrm{FT} 3 / \mathrm{S})\end{array}$ & $\begin{array}{l}\text { COEFFI- } \\
\text { CIENT OF } \\
\text { VARI- } \\
\text { ATION }\end{array}$ & $\begin{array}{c}\text { PERCENT } \\
\text { OF } \\
\text { ANNUAL } \\
\text { RUNOFF }\end{array}$ \\
\hline OCTOBER & 0.05 & 0.00 & 0.01 & 0.02 & 1.6 & 9.5 \\
\hline NOVEMBER & 0.01 & 0.00 & 0.00 & 0.01 & 1,1 & 3.6 \\
\hline DECEMBER & 0.07 & 0.00 & 0.01 & 0.02 & 2.4 & 6.5 \\
\hline JANUARY & 0.00 & 0.00 & 0.00 & 0.00 & & 0.0 \\
\hline FEBRUARY & 0.00 & 0.00 & 0.00 & 0.00 & & 0.0 \\
\hline MARCH & 0.00 & 0.00 & 0.00 & 0.00 & & 0.0 \\
\hline APRIL & 0.00 & 0.00 & 0.00 & 0.00 & & 0.0 \\
\hline MAY & 0.00 & 0.00 & 0.00 & 0.00 & & 0.0 \\
\hline JUNE & 0.00 & 0.00 & 0.00 & 0.00 & & 0.0 \\
\hline JULY & 0.27 & 0.00 & 0.04 & 0.08 & 1.8 & 34.5 \\
\hline AUGUST & 0.13 & 0.00 & 0.04 & 0.04 & 1.0 & 32.1 \\
\hline SEPTEMBER & 0.06 & 0.00 & 0.02 & 0.02 & 1.0 & 13.7 \\
\hline ANNUAL & 0.03 & 0.00 & 0.01 & 0.01 & 1.1 & 100 \\
\hline
\end{tabular}

MAGNITUDE AND PROBABILITY OF INSTANTANEOUS PEAK FLOW BASED ON PERIOD OF RECORD 1958-71

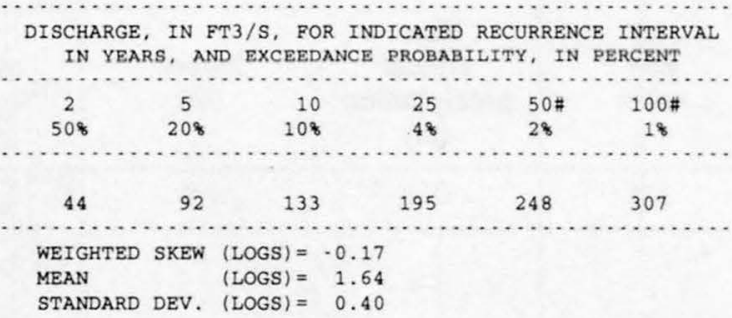

MAGNITUDE AND PROBABILITY OF ANNUAL LOW FLOW BASED ON PERIOD OF RECORD 1960.71

\begin{tabular}{|c|c|c|c|c|c|c|}
\hline \multirow{4}{*}{$\begin{array}{l}\text { PERIOD } \\
\text { (CON- } \\
\text { SECU- } \\
\text { TIVE } \\
\text { DAYS) }\end{array}$} & \multirow{2}{*}{\multicolumn{6}{|c|}{$\begin{array}{l}\text { DISCHARGE, IN FT3/S, FOR INDICATED } \\
\text { RECURRENCE INTERVAL, IN YEARS, AND } \\
\text { NON-EXCEEDANCE PROBABILITY, IN PERCENT }\end{array}$}} \\
\hline & & & & & & \\
\hline & 2 & 5 & 10 & 20 & $50 \#$ & $100 \#$ \\
\hline & 508 & 208 & 108 & 58 & 28 & 18 \\
\hline 1 & 1.9 & 1.1 & 0.61 & 0.45 & 0.36 & 0.23 \\
\hline 3 & 0.75 & 0.41 & 0.22 & 0.16 & 0.12 & 0.07 \\
\hline 7 & 0.41 & 0.22 & 0.12 & 0.09 & 0.07 & 0.04 \\
\hline
\end{tabular}

MAGNITUDE AND PROBABILITY OF ANNUAL HIGH FLOW BASED ON PERIOD OF RECORD $1959 \cdot 71$

\begin{tabular}{|c|c|c|c|c|c|c|}
\hline \multirow{3}{*}{$\begin{array}{l}\text { PERIOD } \\
\text { (CON- } \\
\text { SECU- }\end{array}$} & & \multicolumn{5}{|c|}{ DISCHARGE, IN FT3/S, FOR INDICATED } \\
\hline & & \multicolumn{2}{|c|}{ EXCEEDANCE PR } & BILITY & \multicolumn{2}{|c|}{ YEARS, AND } \\
\hline & . & ...... & ...... & …... & ....... & \\
\hline TIVE & 2 & 5 & 10 & 25 & $50 \#$ & $100=$ \\
\hline DAYS) & 508 & 208 & 108 & 48 & 28 & 18 \\
\hline 1 & & & & & & \\
\hline 3 & & & & & & \\
\hline 7 & & & & & & \\
\hline 15 & 0.12 & 0.24 & 0.35 & 0.54 & 0.73 & 0.97 \\
\hline 30 & 0.07 & 0.13 & 0.20 & 0.32 & 0.44 & 0.60 \\
\hline 60 & 0.04 & 0.07 & 0.11 & 0.17 & 0.24 & 0.33 \\
\hline 90 & 0.03 & 0.05 & 0.08 & 0.12 & 0.17 & 0.23 \\
\hline
\end{tabular}

DURATION TABLE OF DAILY MEAN FLOW FOR PERIOD OF RECORD 1959.71

DISCHARGE, IN PT3/S, WHICH WAS EQUALED OR EXCEEDED FOR INDICATED PERCENT OF TIME

\begin{tabular}{|c|c|c|c|c|c|c|c|c|c|c|c|c|c|c|c|c|}
\hline 18 & 58 & 108 & 158 & 208 & 308 & 408 & 508 & 608 & 708 & 808 & 908 & 958 & 988 & 998 & 99.58 & 99.98 \\
\hline & 0.00 & 0.00 & 0.00 & 0.00 & 0.00 & 0.00 & 0.00 & 0.00 & 0.00 & 0.00 & 0.00 & 0.00 & 0.00 & 0.00 & 0.00 & 0.00 \\
\hline
\end{tabular}

\# Reliability of values in column is uncertain, and potential errors are large. 
09496600 CIBECUE NO. 1, TRIBUTARY TO CARRIZO CREEK, NEAR SHOW LOW, AZ--Continued
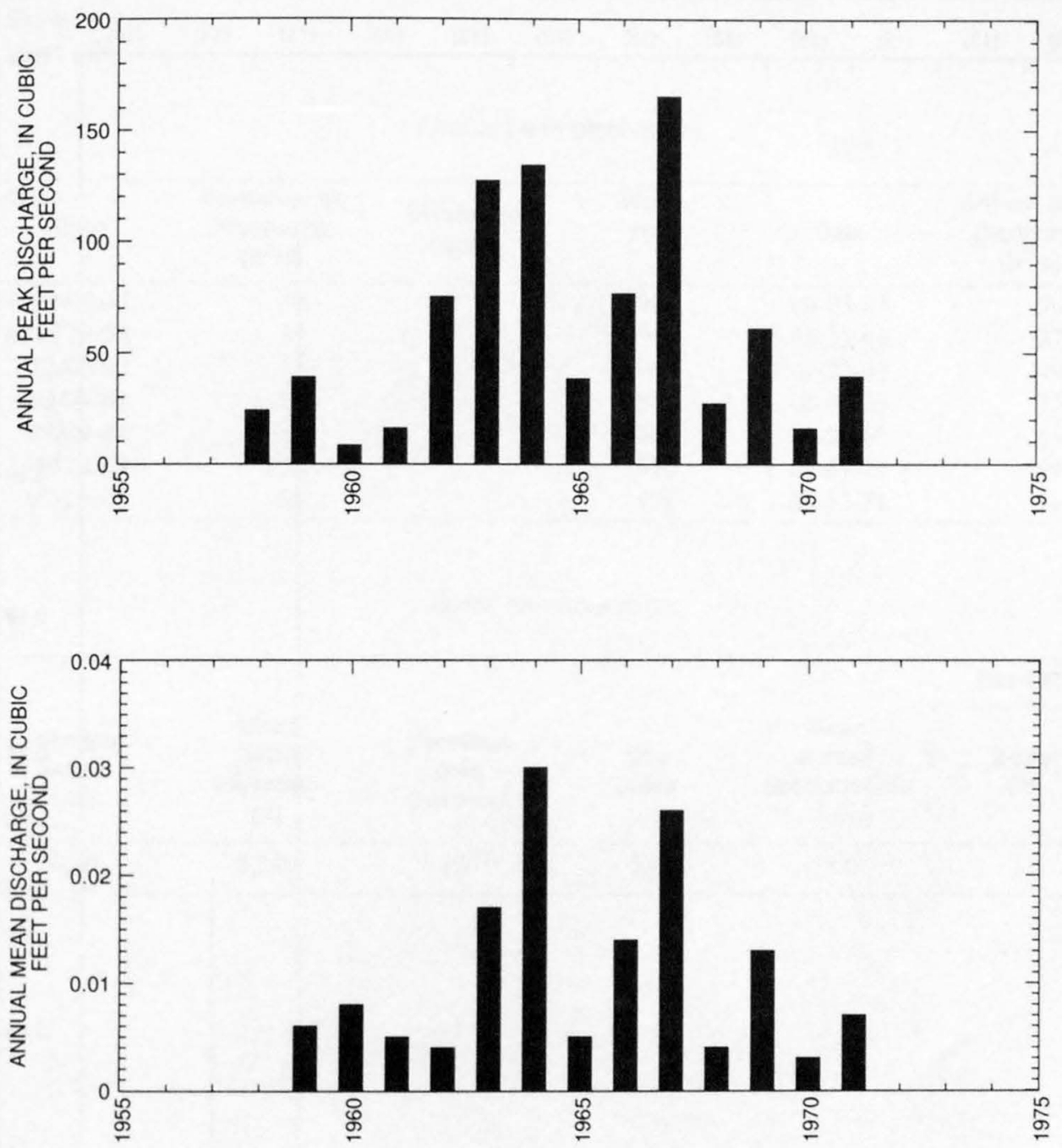
09496600 CIBECUE NO. 1, TRIBUTARY TO CARRIZO CREEK, NEAR SHOW LOW, AZ--Continued
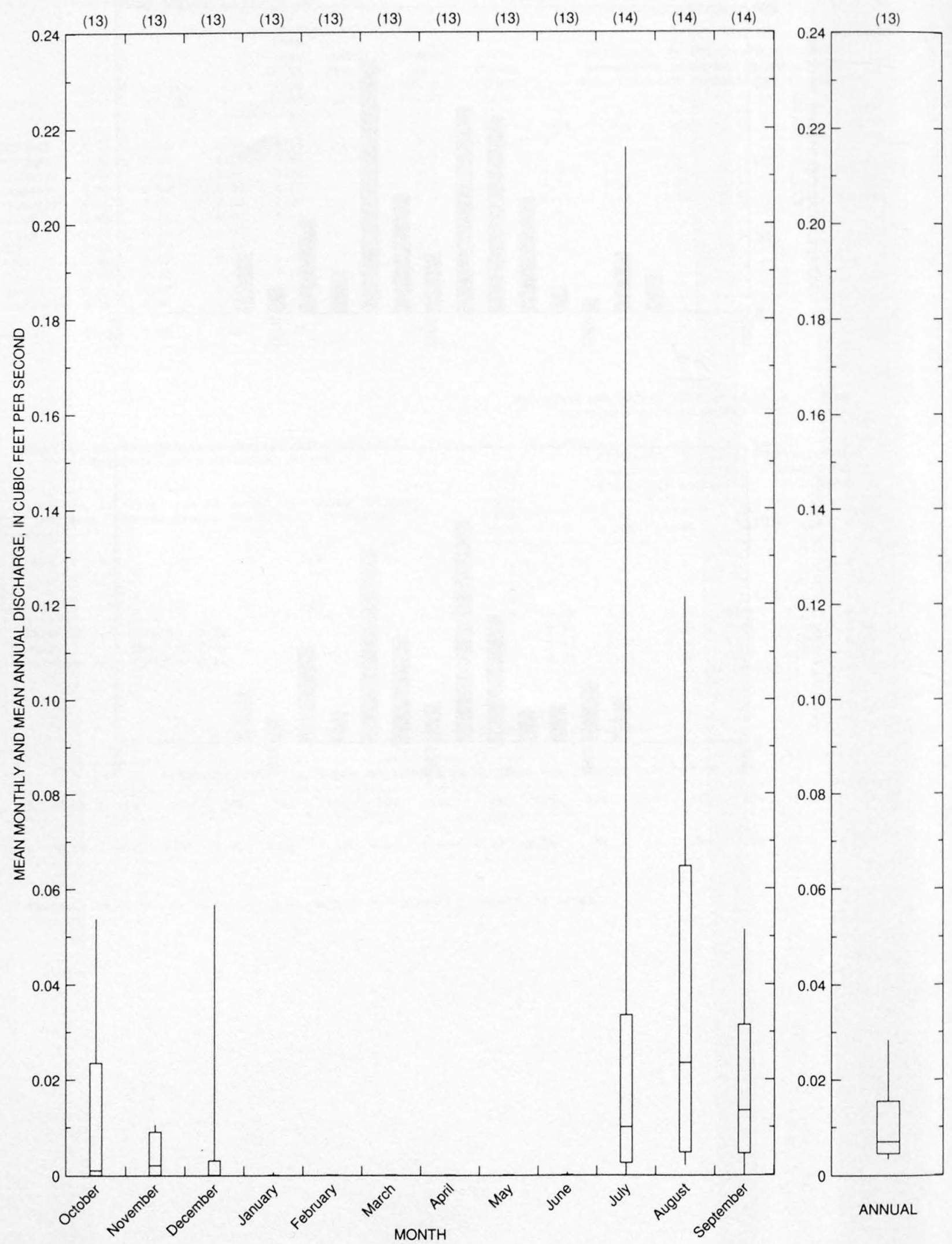
09496700 CIBECUE NO. 2, TRIBUTARY TO CARRIZO CREEK, NEAR SHOW LOW, AZ

LOCATION.--Lat 33'59'17", long 110 18'38", in NE $1 / 4$ sec.22, T.7 N., R.19 E. (unsurveyed), Gila County, Hydrologic Unit 15060104, in Fort Apache Indian Reservation, on an unnamed tributary to Carrizo Creek, on Cibecue Ridge, 2.0 mi upstream from mouth of main stem, and 25 mi southwest of Show Low.

DRAINAGE AREA.--0.07 $\mathrm{mi}^{2}$.

Annual peak discharges

\begin{tabular}{ccccccc}
\hline $\begin{array}{c}\text { Water } \\
\text { year }\end{array}$ & Date & $\begin{array}{c}\text { Annual peak } \\
\text { discharge } \\
\left(\mathrm{ft}^{\mathbf{3}} / \mathbf{s}\right)\end{array}$ & $\begin{array}{c}\text { Discharge } \\
\text { codes }\end{array}$ & $\begin{array}{c}\text { Water } \\
\text { year }\end{array}$ & $\begin{array}{c}\text { Annual peak } \\
\text { Dascharge } \\
\left(\mathrm{ft}^{\mathbf{3}} / \mathbf{s}\right)\end{array}$ & $\begin{array}{c}\text { Discharge } \\
\text { codes }\end{array}$ \\
\hline 1958 & $09-04-58$ & 30 & & 1965 & $09-03-65$ & 96 \\
1959 & $08-19-59$ & 34 & & 1966 & $09-13-66$ & 27 \\
1960 & $12-25-59$ & 11 & & 1967 & $07-27-67$ & 49 \\
1961 & $09-06-61$ & 25 & & 1968 & $08-09-68$ & 22 \\
1962 & $07-29-62$ & 75 & & 1969 & $07-28-69$ & 45 \\
1963 & $08-20-63$ & 120 & & 1970 & $08-09-70$ & 39 \\
1964 & $07-26-64$ & 66 & & 1971 & $08-12-71$ & 51 \\
\hline
\end{tabular}

\section{Basin characteristics}

\begin{tabular}{cccccccc}
\hline $\begin{array}{c}\text { Main } \\
\text { channel } \\
\text { slope } \\
(\mathrm{ft} / \mathrm{mi})\end{array}$ & $\begin{array}{c}\text { Stream } \\
\text { length } \\
(\mathrm{mi})\end{array}$ & $\begin{array}{c}\text { Mean } \\
\text { basin } \\
\text { elevation } \\
(\mathrm{ft})\end{array}$ & $\begin{array}{c}\text { Forested } \\
\text { area } \\
\text { (percent) }\end{array}$ & $\begin{array}{c}\text { Soil } \\
\text { index }\end{array}$ & $\begin{array}{c}\text { Mean } \\
\text { annual } \\
\text { precipitation } \\
\text { (in) }\end{array}$ & $\begin{array}{c}\text { Rainfall intensity, 24-hour } \\
\text { (in) }\end{array}$ & $\begin{array}{c}\text { 50-year } \\
\text { (in) }\end{array}$ \\
\hline 200 & 0.40 & 5,240 & 100 & 3.0 & 18.0 & 2.0 \\
\hline
\end{tabular}


09496700 CIBECUE NO. 2, TRIBUTARY TO CARRIZO CREEK, NEAR SHOW LOW, AZ--Continued

MEAN MONTHLY AND ANNUAL DISCHARGES $1959-71$

\begin{tabular}{|c|c|c|c|c|c|c|}
\hline MONTH & $\begin{array}{l}\text { MAXIMUM } \\
(\text { FT3/S) }\end{array}$ & $\begin{array}{l}\text { MINIMUM } \\
(\text { FT } 3 / \mathrm{S})\end{array}$ & $\begin{array}{c}\text { MEAN } \\
(\text { FT } 3 / S)\end{array}$ & $\begin{array}{l}\text { STAN- } \\
\text { DARD } \\
\text { DEVIA- } \\
\text { TION } \\
(\mathrm{FT} 3 / \mathrm{S})\end{array}$ & $\begin{array}{l}\text { COEFFI- } \\
\text { CIENT OF } \\
\text { VARI- } \\
\text { ATION }\end{array}$ & $\begin{array}{c}\text { PERCENT } \\
\text { OF } \\
\text { ANNUAL } \\
\text { RUNOFF }\end{array}$ \\
\hline OCTOBER & 0.05 & 0.00 & 0.01 & 0.01 & 1.6 & 9.0 \\
\hline NOVEMBER & 0.01 & 0.00 & 0.00 & 0.00 & 1.6 & 3.0 \\
\hline DECEMBER & 0.09 & 0.00 & 0.01 & 0.03 & 2.2 & 11.9 \\
\hline JANUARY & 0.03 & 0.00 & 0.00 & 0.01 & 2.8 & 3.0 \\
\hline FEBRUARY & 0.00 & 0.00 & 0.00 & 0.00 & & 0.0 \\
\hline MARCH & 0.01 & 0.00 & 0.00 & 0.00 & 3.6 & 0.7 \\
\hline APRIL & 0.00 & 0.00 & 0.00 & 0.00 & & 0.0 \\
\hline MAY & 0.00 & 0.00 & 0.00 & 0.00 & & 0.0 \\
\hline JUNE & 0.00 & 0.00 & 0.00 & 0.00 & & 0.0 \\
\hline JULY & 0.13 & 0.00 & 0.02 & 0.04 & 1.8 & 18.7 \\
\hline AUGUST & 0.21 & 0.00 & 0.04 & 0.06 & 1.5 & 36.6 \\
\hline SEPTEMBER & 0.06 & 0.00 & 0.02 & 0.02 & 1.1 & 17.2 \\
\hline ANNUAL & 0.03 & 0.00 & 0.01 & 0.01 & 0.93 & 100 \\
\hline
\end{tabular}

MAGNITUDE AND PROBABILITY OF ANNUAL LOW FLOW BASED ON PERIOD OF RECORD $1960-71$

\begin{tabular}{|c|c|c|c|c|c|c|}
\hline \multirow{2}{*}{$\begin{array}{l}\text { PERIOD } \\
\text { (CON- } \\
\text { SECU. }\end{array}$} & \multicolumn{6}{|c|}{$\begin{array}{l}\text { RECURRENCE INTERVAL, IN YEARS, AND } \\
\text { NON-EXCEEDANCE PROBABILITY, IN PERCENT }\end{array}$} \\
\hline & $\cdots$ & $\cdots \cdots$ & .... & $\cdots \cdots$ & ........... & ..... \\
\hline TIVE & 2 & 5 & 10 & 20 & $50 \#$ & $100 \#$ \\
\hline DAYS) & 508 & 208 & 108 & 58 & 28 & 18 \\
\hline 1 & 1.3 & 0.77 & 0.51 & 0.42 & 0.36 & 0.28 \\
\hline 3 & 0.52 & 0.32 & 0.20 & 0.16 & 0.13 & 0.09 \\
\hline 7 & 0.29 & 0.16 & 0.09 & 0.07 & 0.06 & 0.04 \\
\hline 14 & & & & & & \\
\hline 30 & & & & & & \\
\hline 60 & & & & & & \\
\hline 90 & & & & & & \\
\hline 120 & & & & & & \\
\hline 183 & & & & & & \\
\hline
\end{tabular}

MAGNITUDE AND PROBABILITY OF ANNUAL HIGH FLOW BASED ON PERIOD OF RECORD $1959 \cdot 71$

MAGNITUDE AND PROBABILITY OF INSTANTANEOUS PEAK FLOW BASED ON PERIOD OF RECORD 1958-71

DISCHARGE, IN FT3/S, FOR INDICATED RECURRENCE INTERVAL IN YEARS, AND EXCEEDANCE PROBABILITY, IN PERCENT

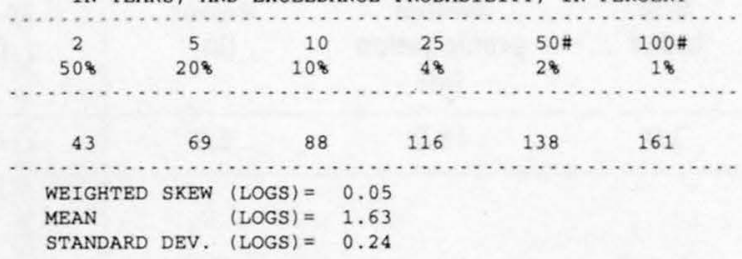

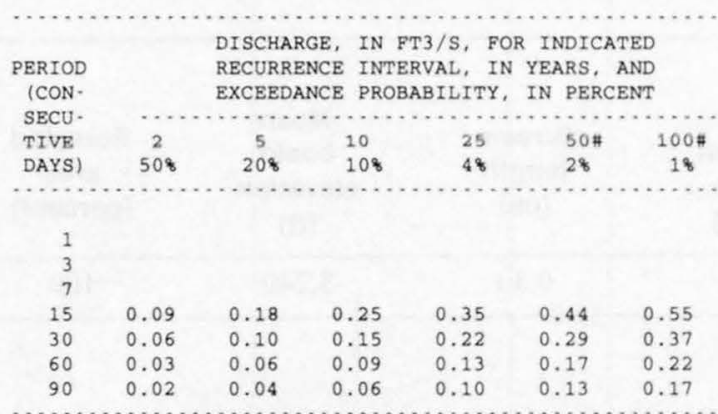

DURATION TABLE OF DAILY MEAN FLOW FOR PERIOD OF RECORD 1959-71

DISCHARGE, IN FT3/S, WHICH WAS EQUALED OR EXCEEDED FOR INDICATED PERCENT OF TIME

\begin{tabular}{|c|c|c|c|c|c|c|c|c|c|c|c|c|c|c|c|c|}
\hline 18 & 58 & 108 & 158 & 208 & 308 & 408 & 508 & 608 & 708 & 808 & 908 & 958 & 988 & 998 & 99.58 & 99.98 \\
\hline 0.29 & 0.00 & 0.00 & 0.00 & 0.00 & 0.00 & 0.00 & 0.00 & 0.00 & 0.00 & 0.00 & 0.00 & 0.00 & 0.00 & 0.00 & 0.00 & 0.00 \\
\hline
\end{tabular}

\# Reliability of values in column is uncertain, and potential errors are large. 
09496700 CIBECUE NO. 2, TRIBUTARY TO CARRIZO CREEK, NEAR SHOW LOW, AZ--Continued
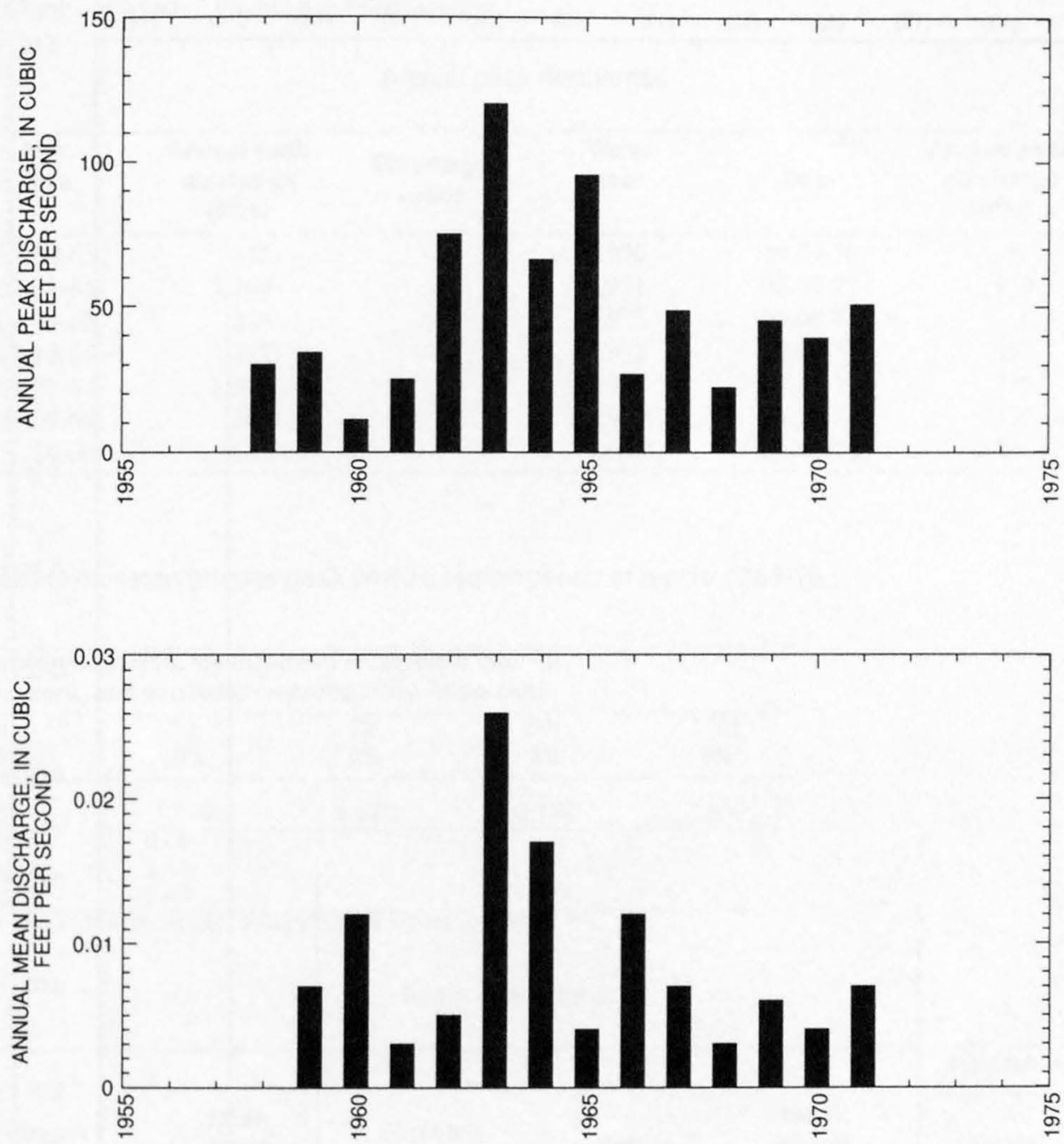
09496700 CIBECUE NO. 2, TRIBUTARY TO CARRIZO CREEK, NEAR SHOW LOW, AZ--Continued

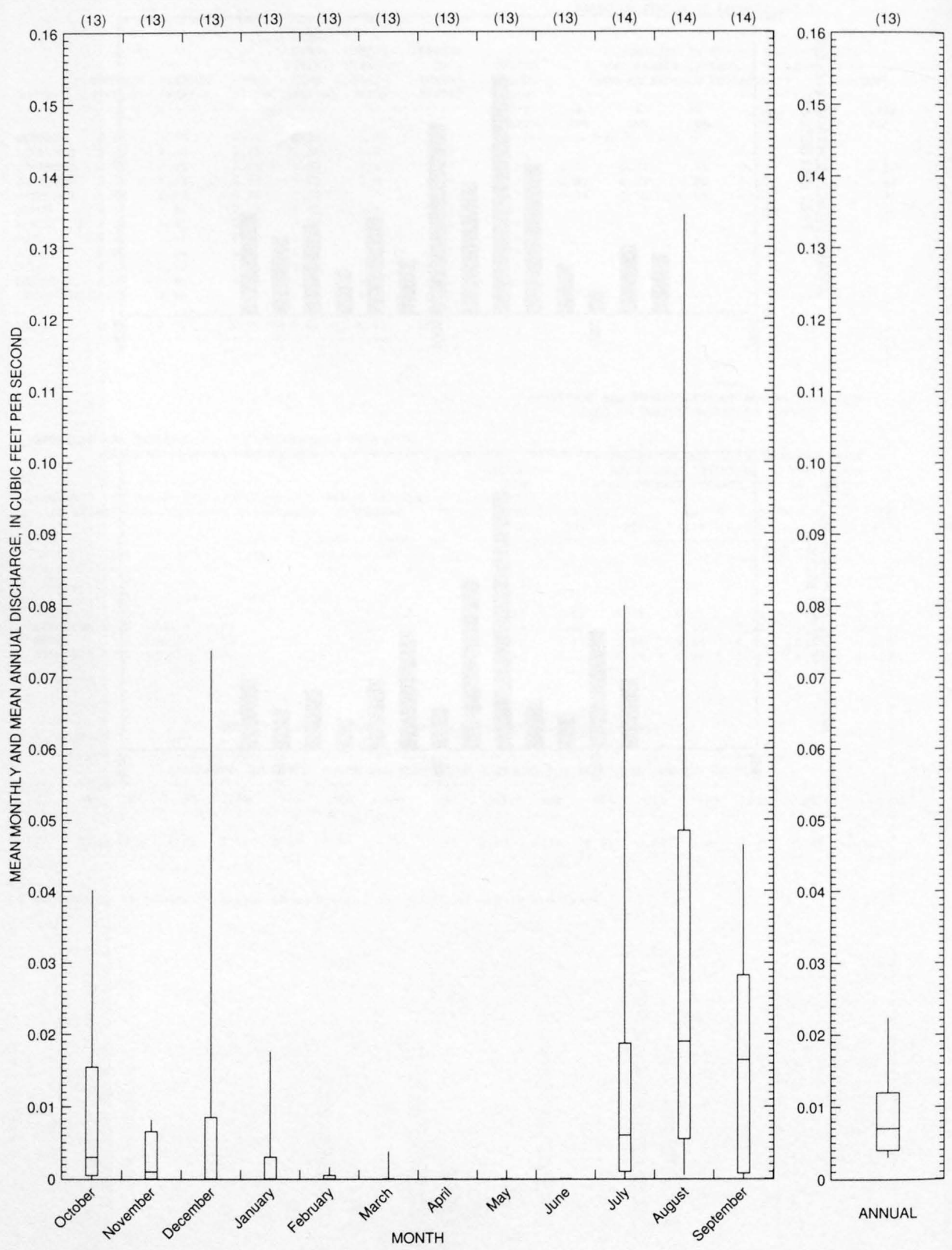


09496800 CARRIZO CREEK TRIBUTARY NEAR SHOW LOW, AZ

LOCATION.--Lat 33'57'16", long 110'19'53", Gila County, Hydrologic Unit 15060104, at U.S. Highway 60, 28 mi southwest of Show Low. DRAINAGE AREA.--4.63 $\mathrm{mi}^{2}$, of which $2.08 \mathrm{mi}^{2}$ is noncontributing.

Annual peak discharges

\begin{tabular}{|c|c|c|c|c|c|c|c|}
\hline $\begin{array}{l}\text { Water } \\
\text { year }\end{array}$ & Date & $\begin{array}{c}\text { Annual peak } \\
\text { discharge } \\
\left(\mathrm{ft}^{3} / \mathbf{s}\right)\end{array}$ & $\begin{array}{l}\text { Discharge } \\
\text { codes }\end{array}$ & $\begin{array}{l}\text { Water } \\
\text { year }\end{array}$ & Date & $\begin{array}{c}\text { Annual peak } \\
\text { discharge } \\
\left(\mathrm{ft}^{3} / \mathrm{s}\right)\end{array}$ & $\begin{array}{c}\text { Discharge } \\
\text { codes }\end{array}$ \\
\hline 1963 & $09-09-63$ & 655 & & 1970 & $08-04-70$ & 290 & \\
\hline 1964 & $07-31-64$ & 1,260 & & 1971 & $08-00-71$ & 930 & \\
\hline 1965 & $09-03-65$ & 286 & & 1972 & $00-00-72$ & 0 & \\
\hline 1966 & $07-24-66$ & 112 & & 1973 & $10-19-72$ & 150 & \\
\hline 1967 & $07-27-67$ & 1,090 & & 1974 & $07-19-74$ & 750 & \\
\hline 1968 & $08-09-68$ & 200 & & 1975 & $10-29-74$ & 130 & \\
\hline 1969 & $11-14-68$ & 150 & & 1976 & $00-00-76$ & 100 & \\
\hline
\end{tabular}

Magnitude and probability of instantaneous peak flow based on period of record 1963-76

\begin{tabular}{|c|c|c|c|c|c|}
\hline \multicolumn{6}{|c|}{$\begin{array}{l}\text { Discharge, in } \mathrm{ft}^{3} / \mathrm{s} \text {, for indicated recurrence interval } \\
\text { in years, and exceedance probablility, in percent }\end{array}$} \\
\hline 2 & 5 & 10 & 25 & $50 t$ & $100 t$ \\
\hline $50 \%$ & $20 \%$ & $10 \%$ & $4 \%$ & $2 \%$ & $1 \%$ \\
\hline 287 & 660 & 1,020 & 1,620 & 2,180 & 2,850 \\
\hline Weighted skew & $(\log s)=$ & 0.00 & & & \\
\hline Mean & $(\log s)=$ & 2.46 & & & \\
\hline Standard dev. & $(\operatorname{logs})=$ & 0.43 & & & \\
\hline
\end{tabular}

f Reliability of values in column is uncertain, and potential errors are large.

Basin characteristics

\begin{tabular}{|c|c|c|c|c|c|c|c|}
\hline \multirow[b]{2}{*}{$\begin{array}{l}\text { Main } \\
\text { channel } \\
\text { slope } \\
\text { (ft/mi) }\end{array}$} & \multirow[b]{2}{*}{$\begin{array}{l}\text { Stream } \\
\text { length } \\
(\mathrm{mi})\end{array}$} & \multirow[b]{2}{*}{$\begin{array}{c}\text { Mean } \\
\text { basin } \\
\text { elevation } \\
(\mathrm{ft})\end{array}$} & \multirow[b]{2}{*}{$\begin{array}{c}\text { Forested } \\
\text { area } \\
\text { (percent) }\end{array}$} & \multirow[b]{2}{*}{$\begin{array}{c}\text { Soil } \\
\text { index }\end{array}$} & \multirow[b]{2}{*}{$\begin{array}{c}\text { Mean } \\
\text { annual } \\
\text { precipitation } \\
\text { (in) }\end{array}$} & \multicolumn{2}{|c|}{ Rainfall intensity, 24-hou } \\
\hline & & & & & & $\begin{array}{l}\text { 2-year } \\
\text { (in) }\end{array}$ & $\begin{array}{l}\text { 50-year } \\
\text { (in) }\end{array}$ \\
\hline 306 & 1.7 & 5,810 & 90.0 & 3.0 & 20.0 & 1.9 & 4.2 \\
\hline
\end{tabular}

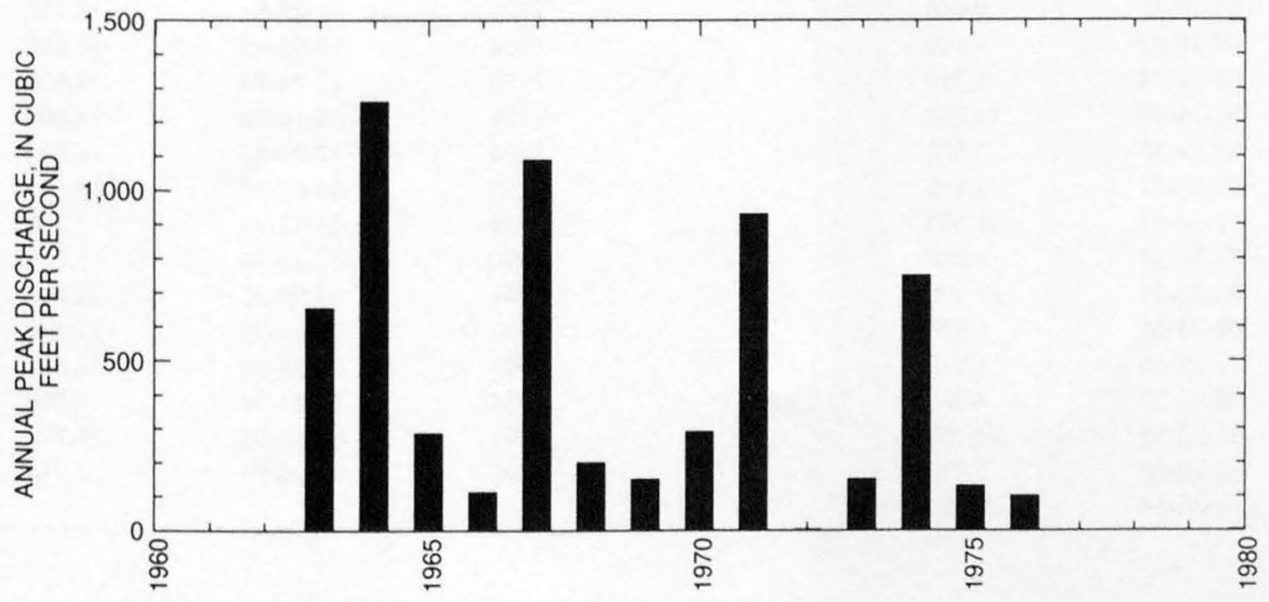


09497500 SALT RIVER NEAR CHRYSOTILE, AZ

LOCATION.--Lat 33'47'53", long 110'29'57", in sec.25, T.5 N., R.17 E. (unsurveyed), Gila County, Hydrologic Unit 15060103, in San Carlos Indian Reservation, on left bank 1,200 ft upstream from bridge on U.S. Highway 60, 5.7 mi northeast of Chrysotile, 8 mi upstream from Cibecue Creek, and 33 mi downstream from confluence of Black and White Rivers.

DRAINAGE AREA.--2,849 $\mathrm{mi}^{2}$.

PERIOD OF RECORD.--September 1924 to current year (monthly discharge only July to December 1954).

REVISED RECORDS.--WSP 859: 1926-27, 1929-30, 1934, 1936. WSP 899: 1927, 1932, 1937, 1938(M). WSP 1313: 1925-26(M), 1929-30(M), 1935-36(M), 1944(M). WSP 1343: Drainage area.

GAGE.--Water-stage recorder. Datum of gage is 3,354.57 ft above sea level.

REMARKS.--No estimated daily discharages. Records fair. Several diversions for irrigation above station of about 3,100 acres, one diversion into the basin (see record of Forestdale Creek diversion from Show Low Creek, near Show Low), and one diversion out of the basin (see record of Willow Creek diversion from Black River, nẻar Morenci).

EXTREMES FOR PERIOD OF RECORD.--Maximum discharge, 76,600 $\mathrm{ft}^{3} / \mathrm{s}$ Jan. 8, 1993, gage height, $18.33 \mathrm{ft}$, from rating curve extended above $52,000 \mathrm{ft}^{3} / \mathrm{s}$; minimum, $49 \mathrm{ft}^{3} / \mathrm{s}$ July $6,7,1955$.

EXTREMES OUTSIDE PERIOD OF RECORD.--Flood peak of $74,000 \mathrm{ft}^{3} / \mathrm{s}$ occurred prior to 1924 and is believed to be the peak of the flood of Jan. 19, 1916, gage height, $18 \mathrm{ft}$, from floodmarks, from rating curve extended above $52,000 \mathrm{ft}^{3} / \mathrm{s}$.

Annual peak discharges

\begin{tabular}{|c|c|c|c|c|c|c|c|}
\hline $\begin{array}{l}\text { Water } \\
\text { year }\end{array}$ & Date & $\begin{array}{c}\text { Annual peak } \\
\text { discharge } \\
\left(\mathrm{ft}^{3} / \mathrm{s}\right)\end{array}$ & $\begin{array}{l}\text { Discharge } \\
\text { codes }\end{array}$ & $\begin{array}{l}\text { Water } \\
\text { year }\end{array}$ & Date & $\begin{array}{c}\text { Annual peak } \\
\text { discharge } \\
\left(\mathrm{ft}^{3} / \mathrm{s}\right)\end{array}$ & $\begin{array}{l}\text { Discharge } \\
\text { codes }\end{array}$ \\
\hline 1916 & $16-01-19$ & ${ }^{1} 74,000$ & HP & 1961 & $08-30-61$ & 2,130 & \\
\hline 1925 & $25-03-08$ & 6,930 & & 1962 & $01-25-62$ & 5,630 & \\
\hline 1926 & $26-04-06$ & 13,600 & & 1963 & $02-11-63$ & 6,220 & \\
\hline 1927 & $27-02-17$ & 19,900 & & 1964 & $07-26-64$ & 2,780 & \\
\hline 1928 & $28-07-21$ & 1,670 & & 1965 & $01-08-65$ & 15,800 & \\
\hline 1929 & $29-09-23$ & 11,500 & & 1966 & $12-30-65$ & 41,100 & \\
\hline 1930 & $30-08-11$ & 11,700 & & 1967 & $08-12-67$ & 5,060 & \\
\hline 1931 & $31-02-15$ & 7,400 & & 1968 & $01-28-68$ & 8,730 & \\
\hline 1932 & $32-02-10$ & 40,000 & & 1969 & $10-04-68$ & 4,940 & \\
\hline 1933 & $33-02-28$ & 2,880 & & 1970 & $09-06-70$ & 5,000 & \\
\hline 1934 & $34-08-20$ & 3,850 & & 1971 & $08-13-71$ & 20,400 & \\
\hline 1935 & $35-04-09$ & 15,700 & & 1972 & $12-26-71$ & 23,300 & \\
\hline 1936 & $36-02-17$ & 13,200 & & 1973 & $10-20-72$ & 42,100 & \\
\hline 1937 & $37-02-07$ & 52,900 & & 1974 & $08-06-74$ & 1,680 & \\
\hline 1938 & $38-03-04$ & 19,000 & & 1975 & $10-29-74$ & 7,080 & \\
\hline 1939 & $39-04-05$ & 8,530 & & 1976 & $02-10-76$ & 6,070 & \\
\hline 1940 & $40-08-15$ & 6,300 & & 1977 & $07-23-77$ & 3,760 & \\
\hline 1941 & $41-03-14$ & 52,200 & & 1978 & $03-02-78$ & 46,700 & \\
\hline 1942 & $42-01-13$ & 5,380 & & 1979 & $12-18-78$ & 70,400 & \\
\hline 1943 & $43-03-05$ & 12,800 & & 1980 & $02-15-80$ & 58,300 & \\
\hline 1944 & $43-10-19$ & 2,380 & & 1981 & $04-14-81$ & 2,060 & \\
\hline 1945 & $03-27-45$ & 4,450 & & 1982 & $02-11-82$ & 13,200 & \\
\hline 1946 & $09-19-46$ & 9,600 & & 1983 & $03-25-83$ & 15,700 & \\
\hline 1947 & $09-18-47$ & 8,160 & & 1984 & $10-02-83$ & 56,600 & \\
\hline 1948 & $04-12-48$ & 5,730 & & 1985 & $12-28-84$ & 34,600 & \\
\hline 1949 & $01-14-49$ & 14,200 & & 1986 & $02-16-86$ & 14,000 & \\
\hline 1950 & $07-21-50$ & 2,500 & & 1987 & $12-08-86$ & 8,370 & \\
\hline 1951 & $08-29-51$ & 5,150 & & 1988 & $09-02-88$ & 10,600 & \\
\hline 1952 & $01-14-52$ & 51,500 & & 1989 & $03-12-89$ & 1,740 & \\
\hline 1953 & $07-30-53$ & 3,680 & & 1990 & $07-22-90$ & 8,610 & \\
\hline 1954 & $03-23-54$ & 28,700 & & 1991 & $03-02-91$ & 38,800 & \\
\hline 1955 & $08-23-55$ & 8,820 & & 1992 & $02-14-92$ & 21,300 & \\
\hline 1956 & $01-29-56$ & 1,640 & & 1993 & $01-08-93$ & ${ }^{2} 76,600$ & \\
\hline 1957 & $08-02-57$ & 3,760 & & 1994 & $03-21-94$ & 7,760 & \\
\hline 1958 & $03-22-58$ & 19,700 & & 1995 & 03-06-95 & 25,700 & \\
\hline 1959 & $08-20-59$ & 7,290 & & 1996 & $09-14-96$ & 2,940 & \\
\hline 1960 & $12-26-59$ & 26,200 & & & & & \\
\hline
\end{tabular}

\footnotetext{
'Highest since 1906.

${ }^{2}$ Highest since 1916.
} 
09497500 SALT RIVER NEAR CHRYSOTILE, AZ--Continued

Discharge rating table developed September 1990

\begin{tabular}{cccc}
\hline $\begin{array}{c}\text { Gage height } \\
(\mathbf{f t})\end{array}$ & $\begin{array}{c}\text { Discharge } \\
\left(\mathbf{f t}^{\mathbf{3}} / \mathbf{s}\right)\end{array}$ & $\begin{array}{c}\text { Gage height } \\
(\mathbf{f t})\end{array}$ & $\begin{array}{c}\text { Discharge } \\
\left(\mathrm{ft}^{3} / \mathbf{s}\right)\end{array}$ \\
\hline 4.0 & 2,020 & 17.0 & 64,270 \\
5.0 & 3,500 & 19.0 & 83,250 \\
7.0 & 7,700 & 21.0 & 105,000 \\
9.0 & 14,060 & 23.0 & 129,500 \\
11.0 & 22,990 & 25.0 & 156,800 \\
13.0 & 34,220 & 27.0 & 187,100 \\
15.0 & 47,960 & 27.8 & 200,000 \\
\hline
\end{tabular}

Basin characteristics

\begin{tabular}{cccccccc}
\hline $\begin{array}{c}\text { Main } \\
\text { channel } \\
\text { slope } \\
(\mathrm{ft} / \mathrm{mi})\end{array}$ & $\begin{array}{c}\text { Stream } \\
\text { length } \\
\text { (mi) }\end{array}$ & $\begin{array}{c}\text { Mean } \\
\text { basin } \\
\text { elevation } \\
(\mathrm{ft})\end{array}$ & $\begin{array}{c}\text { Forested } \\
\text { area } \\
\text { (percent) }\end{array}$ & $\begin{array}{c}\text { Soil } \\
\text { index }\end{array}$ & $\begin{array}{c}\text { Mean } \\
\text { annual } \\
\text { precipitation } \\
\text { (in) }\end{array}$ & $\begin{array}{c}\text { Rainfall intensity, 24-hour } \\
\text { (in) }\end{array}$ & $\begin{array}{c}\text { 50-year } \\
\text { (in) }\end{array}$ \\
\hline 31.9 & 155 & 6,730 & 81.0 & 3.0 & 22.8 & 2.2 \\
\hline
\end{tabular}




\section{SALT RIVER NEAR CHRYSOTILE, AZ--Continued}

MEAN MONTHLY AND ANNUAL DISCHARGES 1925.96

\begin{tabular}{|c|c|c|c|c|c|c|}
\hline MONTH & $\begin{array}{l}\text { MAXIMUM } \\
\text { (FT3/S) }\end{array}$ & $\begin{array}{l}\text { MINIMUM } \\
(\text { FT3/S) }\end{array}$ & $\begin{array}{c}\text { MEAN } \\
(\mathrm{FT} 3 / \mathrm{S})\end{array}$ & $\begin{array}{l}\text { STAN- } \\
\text { DARD } \\
\text { DEVIA- } \\
\text { TION } \\
\text { (FT3/S) }\end{array}$ & $\begin{array}{l}\text { COEFFI- } \\
\text { CIENT OF } \\
\text { VARI- } \\
\text { ATION }\end{array}$ & $\begin{array}{c}\text { PERCENT } \\
\text { OF } \\
\text { ANNUAL } \\
\text { RUNOFF }\end{array}$ \\
\hline OCTOBER & 3,780 & 79 & 342 & 558 & 1.6 & 4.2 \\
\hline NOVEMBER & 1.300 & 113 & 276 & 222 & 0.80 & 3.4 \\
\hline DECEMBER & 3,980 & 113 & 506 & 757 & 1.5 & 6.2 \\
\hline JANUARY & 7,940 & 130 & 659 & 1,110 & 1.7 & 8.1 \\
\hline FEBRUARY & 6,180 & 145 & 917 & 1,110 & 1.2 & 11 \\
\hline MARCH & 6,030 & 175 & 1,500 & 1,260 & 0.84 & 18 \\
\hline APRIL & 4,850 & 170 & 1,740 & 1,300 & 0.75 & 21 \\
\hline MAY & 5,070 & 106 & 923 & 909 & 0.98 & 11 \\
\hline JUNE & 1,180 & 74 & 317 & 253 & 0.80 & 3.9 \\
\hline JULY & 547 & 91 & 223 & 100 & 0.45 & 2.7 \\
\hline AUGUST & 1,250 & 135 & 403 & 240 & 0.60 & 5.0 \\
\hline SEPTEMBER & 1,180 & 68 & 344 & 246 & 0.71 & 4.2 \\
\hline ANNUAL & 2,090 & 184 & 678 & 456 & 0.67 & 100 \\
\hline
\end{tabular}

MAGNITUDE AND PROBABILITY OF INSTANTANEOUS PEAK FLOW BASED ON PERIOD OF RECORD 1916, 1925.96

DISCHARGE, IN FT3/S, FOR INDICATED RECURRENCE INTERVAL IN YEARS, AND EXCEEDANCE PROBABILITY, IN PERCENT

\begin{tabular}{|c|c|c|c|c|c|}
\hline 2 & 5 & 10 & 25 & 50 & 100 \\
\hline 508 & 208 & 108 & 48 & 28 & 18 \\
\hline 10,200 & 24,700 & 39,600 & 65,600 & 91,200 & 122,800 \\
\hline
\end{tabular}

WEIGHTED SKEW $($ LOGS $)=0.07$

MEAN (LOGS) $=4.01$

STANDARD DEV. $($ LOGS $)=0.45$
MAGNITUDE AND PROBABILITY OF ANNUAL LOW FLOW BASED ON PERIOD OF RECORD 1926.96

\begin{tabular}{|c|c|c|c|c|c|c|}
\hline \multirow{3}{*}{$\begin{array}{l}\text { PERIOD } \\
\text { (CON. } \\
\text { SECU. }\end{array}$} & \multirow{2}{*}{\multicolumn{3}{|c|}{$\begin{array}{l}\text { DISCHARGE, I } \\
\text { RECURRENCE II }\end{array}$}} & \multicolumn{3}{|c|}{ IN FT3/S, FOR INDICATED } \\
\hline & & & \multicolumn{4}{|c|}{ NON-EXCEEDANCE PROBABILITY, IN PERCENT } \\
\hline & & ........ & …. & ...... & & \\
\hline TIVE & 2 & 5 & $10 \#$ & $20 \#$ & $50 \#$ & $100 \#$ \\
\hline DAYS) & 508 & 208 & 108 & 58 & 28 & 18 \\
\hline 1 & 103 & 78 & 68 & 60 & 53 & 49 \\
\hline 3 & 105 & 80 & 69 & 61 & 54 & 49 \\
\hline 7 & 109 & 83 & 72 & 64 & 56 & 52 \\
\hline 14 & 117 & 89 & 77 & 69 & 60 & 55 \\
\hline 30 & 130 & 99 & 86 & 77 & 69 & 63 \\
\hline 60 & 148 & 115 & 101 & 92 & 83 & 78 \\
\hline 90 & 167 & 132 & 118 & 109 & 101 & 96 \\
\hline 120 & 182 & 144 & 131 & 122 & 113 & 109 \\
\hline 183 & 218 & 166 & 149 & 139 & 130 & 125 \\
\hline
\end{tabular}

MAGNITUDE AND PROBABILITY OF ANNUAL HIGH FLOW BASED ON PERIOD OF RECORD 1925.96

\begin{tabular}{|c|c|c|c|c|c|c|}
\hline $\begin{array}{l}\text { PERIOD } \\
\text { (CON- }\end{array}$ & & \multicolumn{5}{|c|}{$\begin{array}{l}\text { DISCHARGE, IN FT3/S, FOR INDICATED } \\
\text { RECURRENCE INTERVAL, IN YEARS, AND }\end{array}$} \\
\hline SECU. & & & & & & \\
\hline TIVE & 2 & 5 & $10 \#$ & $25 \#$ & $50 \#$ & $100 \#$ \\
\hline DAYS) & 508 & 208 & 108 & 48 & 28 & 18 \\
\hline 1 & 5,860 & 14,900 & 25,500 & 42,000 & 60,000 & 82,000 \\
\hline 3 & 4,400 & 10,280 & 16,200 & 26,700 & 37,000 & 49,700 \\
\hline 7 & 3,140 & 6,610 & 9,830 & 15,100 & 20,000 & 25,800 \\
\hline 15 & 2.380 & 4,690 & 6,680 & 9,730 & 12,400 & 15,400 \\
\hline 30 & 1,880 & 3,550 & 4,900 & 6.850 & 8,480 & 10,200 \\
\hline 60 & 1,450 & 2,760 & 3,840 & 5,440 & 6,790 & 8,270 \\
\hline 90 & 1,220 & 2,350 & 3,290 & 4.700 & 5,900 & 7,230 \\
\hline
\end{tabular}

DURATION TABLE OF DAILY MEAN FLOW FOR PERIOD OF RECORD 1925.96

DISCHARGE, IN FT3/S, WHICH WAS EQUALED OR EXCEEDED FOR INDICATED PERCENT OF TIME

\begin{tabular}{|c|c|c|c|c|c|c|c|c|c|c|c|c|c|c|c|c|}
\hline 18 & 58 & 108 & 158 & 208 & 308 & 408 & 508 & 608 & 708 & 808 & 908 & 958 & 988 & 998 & 99.58 & 99.98 \\
\hline 30 & 2,650 & 1,610 & 1,080 & 800 & 495 & 355 & 266 & 217 & 184 & 160 & 130 & 109 & 0.00 & 0.00 & 0.00 & 0.00 \\
\hline
\end{tabular}

\# Reliability of values in column is uncertain, and potential errors are large. 
ANNUAL MEAN DISCHARGE, IN CUBIC

FEET PER SECOND

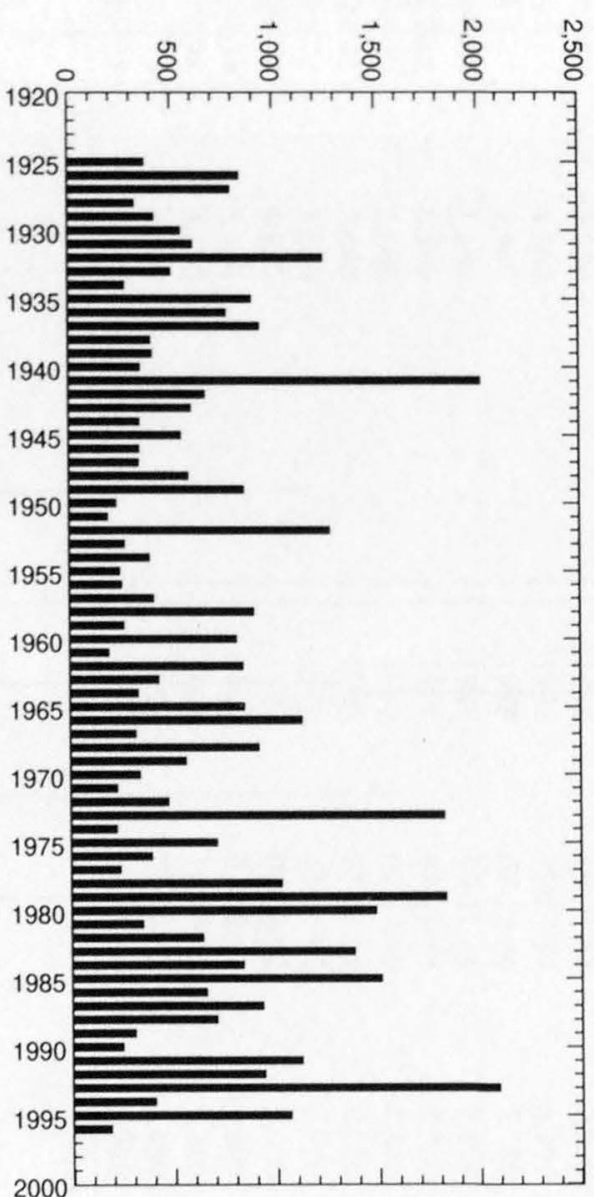

ANNUAL PEAK DISCHARGE, IN CUBIC

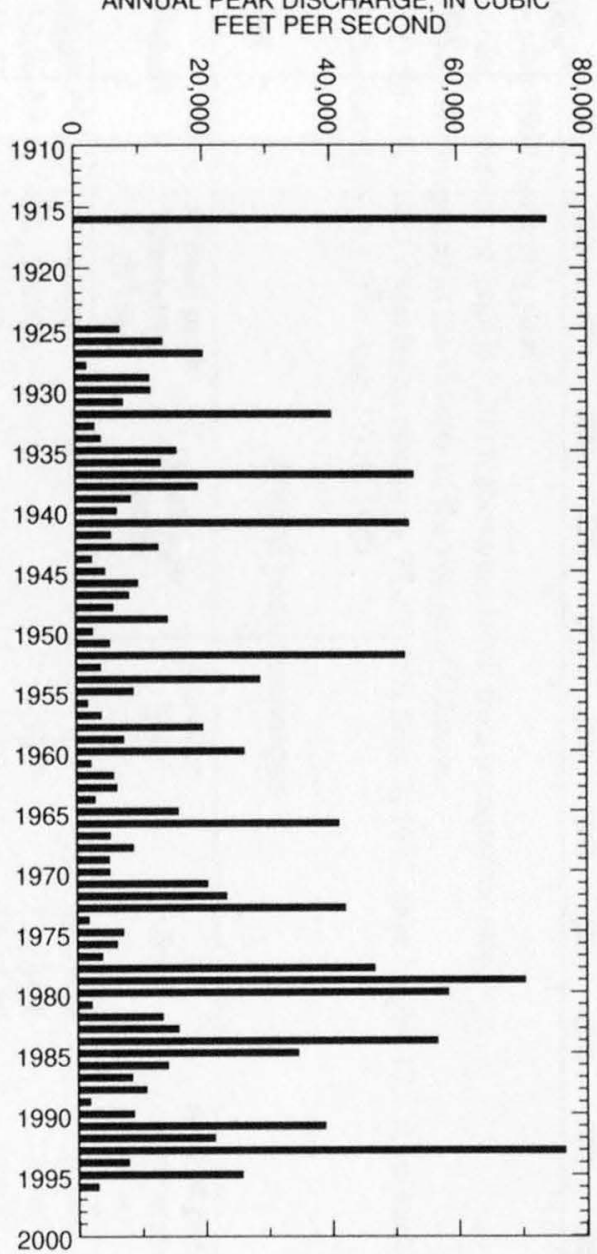


09497500 SALT RIVER NEAR CHRYSOTILE, AZ--Continued

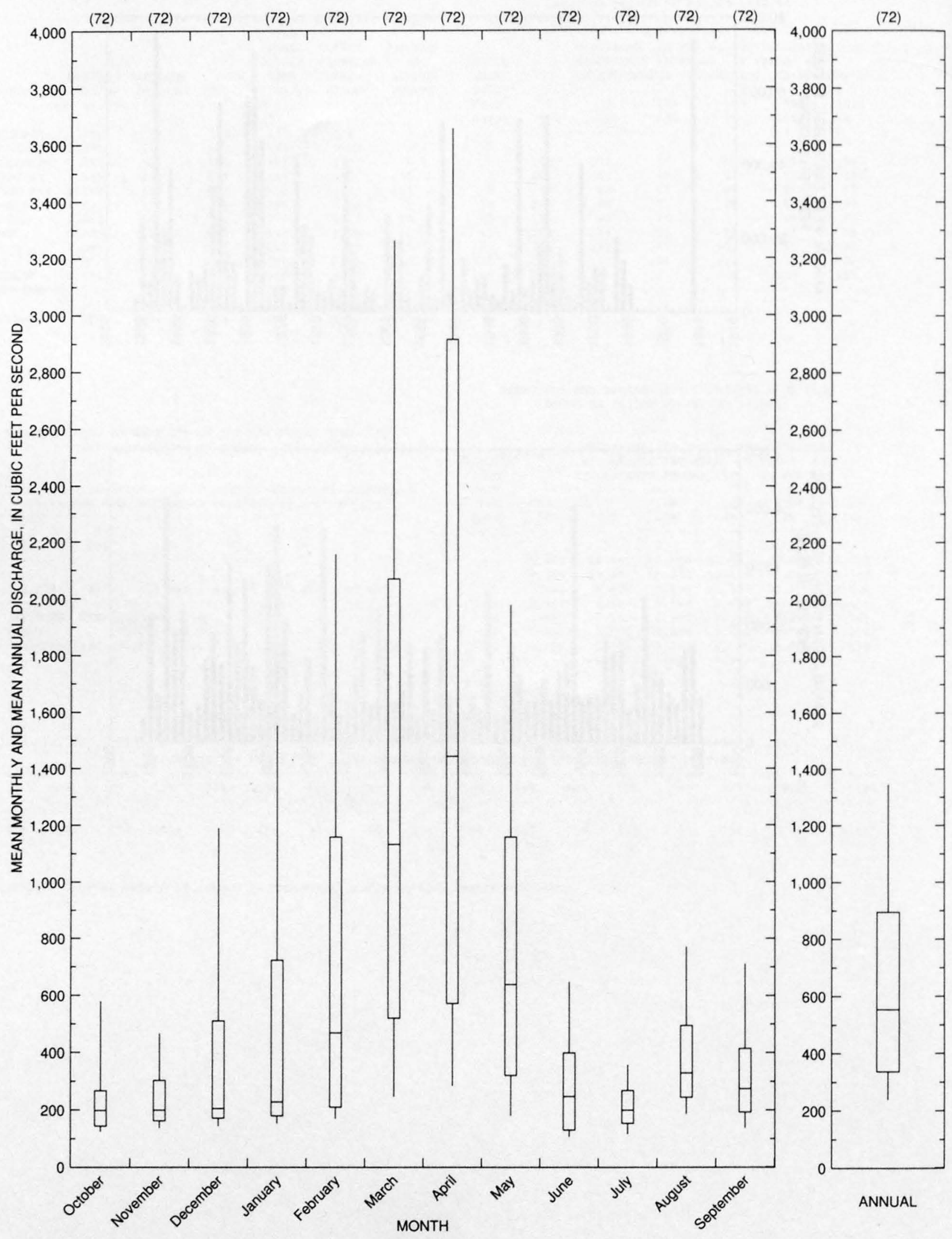




\section{CIBECUE CREEK NEAR CHRYSOTILE, AZ}

LOCATION.--Lat 33.50'35", long 110'33'25", in E $1 / 2$ sec.8, T.5 N., R.17 E. (unsurveyed), Gila County, Hydrologic Unit 15060103, in Fort Apache Indian Reservation, on right bank $0.5 \mathrm{mi}$ upstream from mouth and $7 \mathrm{mi}$ north of Chrysotile.

DRAINAGE AREA.--295 $\mathrm{mi}^{2}$.

PERIOD OF RECORD.--May 1959 to current year.

GAGE.--Water-stage recorder. Elevation of gage is 3,200 ft above sea level, from topographic map.

REMARKS.--Small diversions for irrigation in the vicinity of the village of Cibecue.

EXTREMES FOR PERIOD OF RECORD.--Maximum discharge, 22,200 $\mathrm{ft}^{3} / \mathrm{s}$ Sept. 2, 1977, gage height, $17.3 \mathrm{ft}$, on basis of slope-area measurement of peak flow; minimum daily, $4.1 \mathrm{ft}^{3} / \mathrm{s}$ Aug. 17-19, 1968.

Annual peak discharges

\begin{tabular}{|c|c|c|c|c|c|c|c|}
\hline $\begin{array}{l}\text { Water } \\
\text { year }\end{array}$ & Date & $\begin{array}{c}\text { Annual peak } \\
\text { discharge } \\
\left(\mathrm{ft}^{3} / \mathrm{s}\right)\end{array}$ & $\begin{array}{c}\text { Discharge } \\
\text { codes }\end{array}$ & $\begin{array}{l}\text { Water } \\
\text { year }\end{array}$ & Date & $\begin{array}{c}\text { Annual peak } \\
\text { discharge } \\
\left(\mathrm{ft}^{3} / \mathrm{s}\right)\end{array}$ & $\begin{array}{l}\text { Discharge } \\
\text { codes }\end{array}$ \\
\hline 1959 & $07-29-59$ & 4,600 & & 1978 & $03-01-78$ & 6,540 & \\
\hline 1960 & $12-26-59$ & 7,080 & & 1979 & $12-18-78$ & 7,740 & \\
\hline 1961 & $07-21-61$ & 1,440 & & 1980 & $02-15-80$ & 10,600 & \\
\hline 1962 & $09-24-62$ & 519 & & 1981 & $08-09-81$ & 1,800 & \\
\hline 1963 & $08-31-63$ & 8,180 & & 1982 & $02-11-82$ & 1,530 & \\
\hline 1964 & $07-29-64$ & 7,600 & & 1983 & $10-30-82$ & 5,150 & \\
\hline 1965 & $01-07-65$ & 2,950 & & 1984 & $10-01-83$ & 9,780 & \\
\hline 1966 & $12-30-65$ & 8,800 & & 1985 & $12-27-84$ & 6,190 & \\
\hline 1967 & $07-27-67$ & 2,960 & & 1986 & $07-15-86$ & 2,780 & \\
\hline 1968 & $03-10-68$ & 1,200 & & 1987 & $10-11-86$ & 2,680 & \\
\hline 1969 & $08-12-69$ & 6,580 & & 1988 & $08-31-88$ & 2,300 & \\
\hline 1970 & $09-06-70$ & 3,640 & & 1989 & $07-17-89$ & 1,340 & \\
\hline 1971 & $09-01-71$ & 5,440 & & 1990 & $07-16-90$ & 8,010 & \\
\hline 1972 & $10-17-71$ & 1,650 & & 1991 & $03-01-91$ & 6,750 & \\
\hline 1973 & $10-19-72$ & 6,880 & & 1992 & $08-24-92$ & 3,000 & \\
\hline 1974 & $07-19-74$ & 3,180 & & 1993 & 01-08-93 & 10,600 & \\
\hline 1975 & $10-29-74$ & 3,180 & & 1994 & $10-07-93$ & 1,360 & \\
\hline 1976 & $09-27-76$ & 3,500 & & 1995 & $03-06-95$ & 3,990 & \\
\hline 1977 & $09-02-77$ & 22,200 & & 1996 & $09-14-96$ & 5,760 & \\
\hline
\end{tabular}

Discharge rating table developed September 1992

\begin{tabular}{cccc}
\hline $\begin{array}{c}\text { Gage height } \\
(\mathbf{f t})\end{array}$ & $\begin{array}{c}\text { Discharge } \\
\left(\mathbf{f t}^{\mathbf{3}} / \mathbf{s}\right)\end{array}$ & $\begin{array}{c}\text { Gage height } \\
(\mathbf{f t})\end{array}$ & $\begin{array}{c}\text { Discharge } \\
\left(\mathbf{f t}^{\mathbf{3}} / \mathbf{s}\right)\end{array}$ \\
\hline 5.0 & 1,740 & 12.0 & 12,450 \\
6.0 & 2,880 & 13.0 & 14,300 \\
7.0 & 4,200 & 14.0 & 16,110 \\
8.0 & 5,700 & 15.0 & 18,000 \\
9.0 & 7,300 & 16.0 & 19,800 \\
10.0 & 8,950 & 17.0 & 21,640 \\
11.0 & 10,700 & 17.3 & 22,200 \\
\hline
\end{tabular}


09497800 CIBECUE CREEK NEAR CHRYSOTILE, AZ--Continued

Basin characteristics

\begin{tabular}{|c|c|c|c|c|c|c|c|}
\hline \multirow[b]{2}{*}{$\begin{array}{l}\text { Main } \\
\text { channel } \\
\text { slope } \\
(\mathrm{ft} / \mathrm{mi})\end{array}$} & \multirow[b]{2}{*}{$\begin{array}{c}\text { Stream } \\
\text { length } \\
(\mathrm{mi})\end{array}$} & \multirow[b]{2}{*}{$\begin{array}{c}\text { Mean } \\
\text { basin } \\
\text { elevation } \\
\text { (ft) }\end{array}$} & \multirow[b]{2}{*}{$\begin{array}{l}\text { Forested } \\
\text { area } \\
\text { (percent) }\end{array}$} & \multirow[b]{2}{*}{$\begin{array}{c}\text { Soil } \\
\text { index }\end{array}$} & \multirow[b]{2}{*}{$\begin{array}{c}\text { Mean } \\
\text { annual } \\
\text { precipitation } \\
\text { (in) }\end{array}$} & \multicolumn{2}{|c|}{ Rainfall intensity, 24-hour } \\
\hline & & & & & & $\begin{array}{l}\text { 2-year } \\
\text { (in) }\end{array}$ & $\begin{array}{l}\text { 50-year } \\
\text { (in) }\end{array}$ \\
\hline 79.6 & 38.5 & 5,700 & 78.0 & 3.0 & 20.7 & 2.2 & 4.2 \\
\hline
\end{tabular}


09497800 CIBECUE CREEK NEAR CHRYSOTILE, AZ--Continued

MEAN MONTHLY AND ANNUAL DISCHARGES 1960.96

\begin{tabular}{|c|c|c|c|c|c|c|}
\hline MONTH & $\begin{array}{l}\text { MAXIMUM } \\
\text { (FT3/S) }\end{array}$ & $\begin{array}{l}\text { MINIMUM } \\
\text { (FT3/S) }\end{array}$ & $\begin{array}{c}\text { MEAN } \\
\text { (ET3/S) }\end{array}$ & $\begin{array}{l}\text { STAN- } \\
\text { DARD } \\
\text { DEVIA- } \\
\text { TION } \\
\text { (FT3/S) }\end{array}$ & $\begin{array}{l}\text { COEFFI- } \\
\text { CIENT OF } \\
\text { VARI- } \\
\text { ATION }\end{array}$ & $\begin{array}{c}\text { PERCENT } \\
\text { OF } \\
\text { ANNUAL } \\
\text { RUNOFF }\end{array}$ \\
\hline OCTOBER & 277 & 11 & 36 & 48 & 1.3 & 6.1 \\
\hline NOVEMBER & 186 & 9.1 & 30 & 29 & 0.99 & 5.0 \\
\hline DECEMBER & 368 & 11 & 58 & 77 & 1.3 & 9.9 \\
\hline JANUARY & 209 & 11 & 69 & 142 & 2.1 & 12 \\
\hline FEBRUARY & 550 & 11 & 91 & 141 & 1.6 & 15 \\
\hline MARCH & 477 & 12 & 112 & 118 & 1.1 & 19 \\
\hline APRIL & 274 & 11 & 59 & 57 & 0.97 & 10 \\
\hline MAY & 131 & 5.6 & 26 & 23 & 0.89 & 4.4 \\
\hline JUNE & 40 & 5.0 & 15 & 8.3 & 0.55 & 2.6 \\
\hline JULY & 70 & 6.6 & 25 & 13 & 0.53 & 4.3 \\
\hline AUGUST & 106 & 13 & 37 & 22 & 0.58 & 6.3 \\
\hline SEPTEMBER & 93 & 13 & 33 & 20 & 0.61 & 5.6 \\
\hline ANNUAL & 182 & 16 & 49 & 36 & 0.74 & 100 \\
\hline
\end{tabular}

MAGNITUDE AND PROBABILITY OF INSTANTANEOUS PEAK FLOW BASED ON PERIOD OF RECORD 1959.96

DISCHARGE, IN FT3/S, FOR INDICATED RECURRENCE INTERVAL IN YEARS, AND EXCEEDANCE PROBABILITY, IN PERCENT

$\begin{array}{cccccc}2 & 5 & 10 & 25 & 50 & 100 \\ 508 & 208 & 108 & 48 & 28 & 18 \\ 4,200 & 7,620 & 10,300 & 14,200 & 17,300 & 20,800\end{array}$

WEIGHTED SKEW (LOGS) $=-0.12$

MEAN (LOGS) $=3.62$

STANDARD DEV. (LOGS) $=0.31$
MAGNITUDE AND PROBABILITY OF ANNUAL LOW FLOW BASED ON PERIOD OF RECORD 1961.96

\begin{tabular}{|c|c|c|c|c|c|c|}
\hline PERIOD & \multirow{2}{*}{\multicolumn{6}{|c|}{$\begin{array}{l}\text { DISCHARGE, IN FT3/S, FOR INDICATED } \\
\text { RECURRENCE INTERVAL, IN YEARS, AND } \\
\text { NON-EXCEEDANCE PROBABILITY, IN PERCENT }\end{array}$}} \\
\hline SECU. & & & & & & \\
\hline TIVE & 2 & 5 & $10 \#$ & $20 \#$ & $50 \#$ & $100 \#$ \\
\hline DAYS) & 508 & 208 & 108 & 58 & 28 & 18 \\
\hline 1 & 9.0 & 6.1 & 5.0 & 4.3 & 3.6 & 3.1 \\
\hline 3 & 9.1 & 6.2 & 5.1 & 4.3 & 3.6 & 3.2 \\
\hline 7 & 9.5 & 6.6 & 5.4 & 4.7 & 3.9 & 3.5 \\
\hline 14 & 10 & 7.0 & 5.8 & 5.0 & 4.2 & 3.8 \\
\hline 30 & 11 & 7.6 & 6.3 & 5.4 & 4.5 & 4.0 \\
\hline 60 & 13 & 8.9 & 7.2 & 6.1 & 4.9 & 4.3 \\
\hline 90 & 15 & 10 & 8.6 & 7.3 & 6.0 & 5.3 \\
\hline 120 & 18 & 13 & 11 & 9.5 & 8.0 & 7.1 \\
\hline 183 & 21 & 16 & 14 & 13 & 11 & 11 \\
\hline
\end{tabular}

MAGNITUDE AND PROBABILITY OF ANNUAL HIGH FLOW BASED ON PERIOD OF RECORD 1960.96

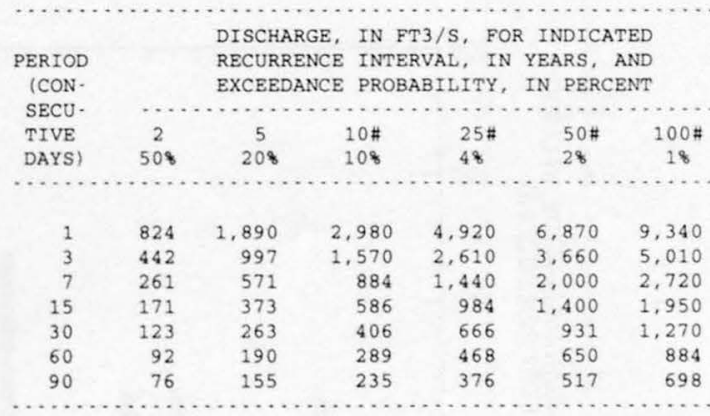

DURATION TABLE OF DAILY MEAN FLOW FOR PERIOD OF RECORD 1960-96

DISCHARGE, IN FT3/S, WHICH WAS EQUALED OR EXCEEDED FOR INDICATED PERCENT OF TIME

\begin{tabular}{|c|c|c|c|c|c|c|c|c|c|c|c|c|c|c|c|c|}
\hline 18 & 58 & 108 & 158 & 208 & 308 & 408 & 508 & 608 & 708 & 808 & 908 & 958 & 988 & 998 & 99.58 & 99.99 \\
\hline 409 & 163 & 92 & 61 & 47 & 32 & 25 & 21 & 18 & 16 & 13 & 10 & 8.0 & 0.00 & 0.00 & 0.00 & 0.00 \\
\hline
\end{tabular}

\# Reliability of values in column is uncertain, and potential errors are large. 
ANNUAL MEAN DISCHARGE, IN CUBIC

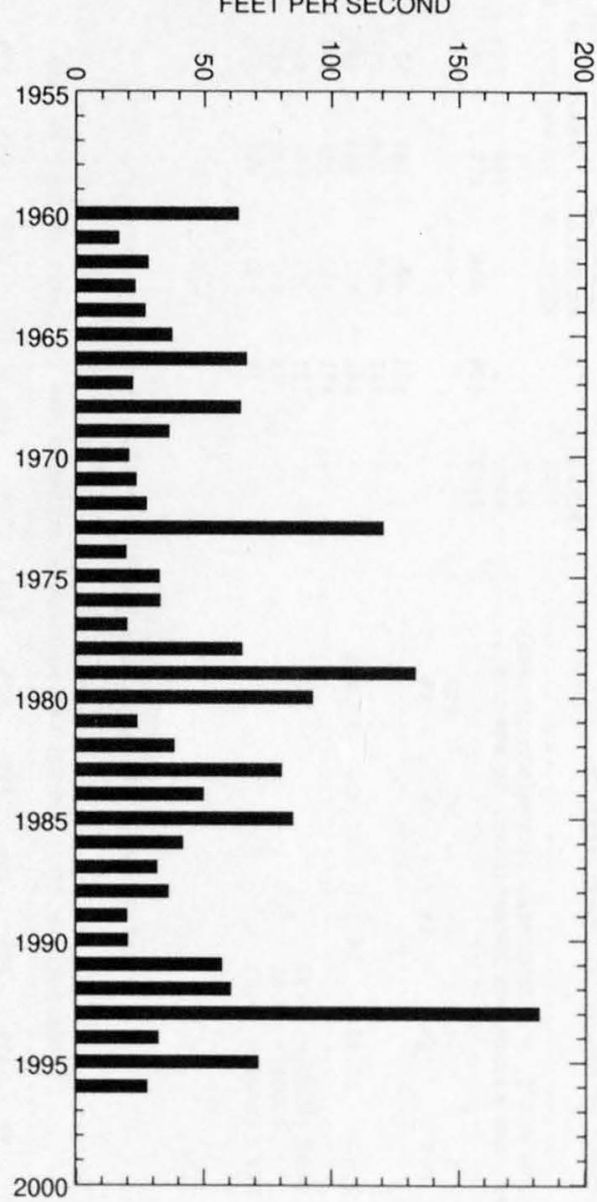

ANNUAL PEAK DISCHARGE, IN CUBIC

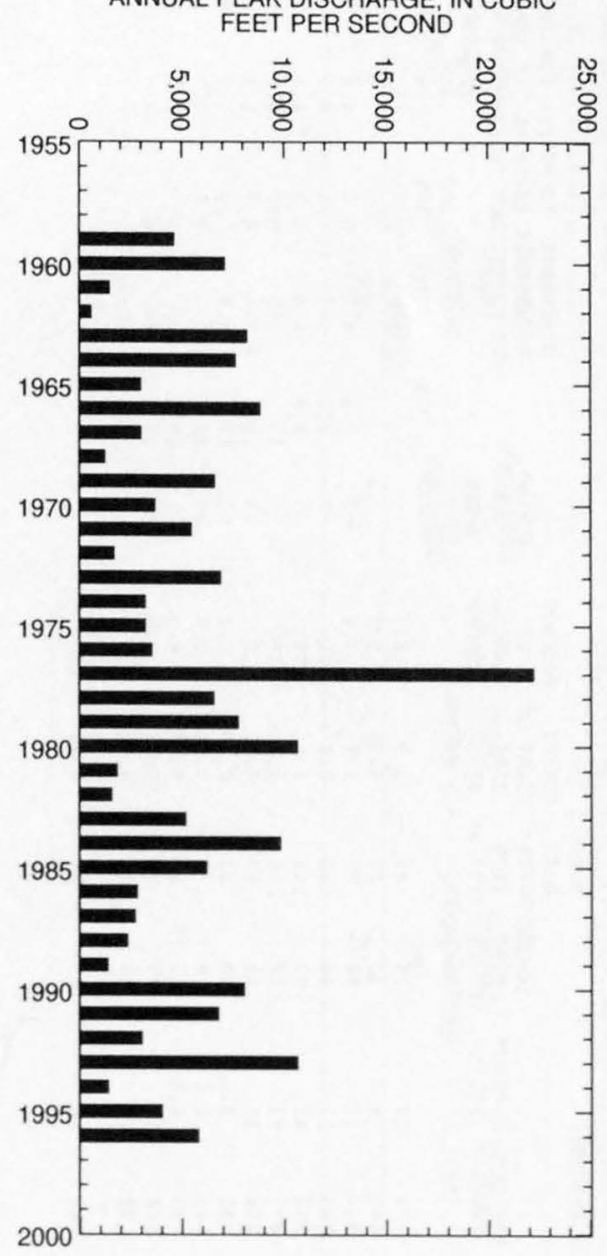


09497800 CIBECUE CREEK NEAR CHRYSOTILE, AZ--Continued
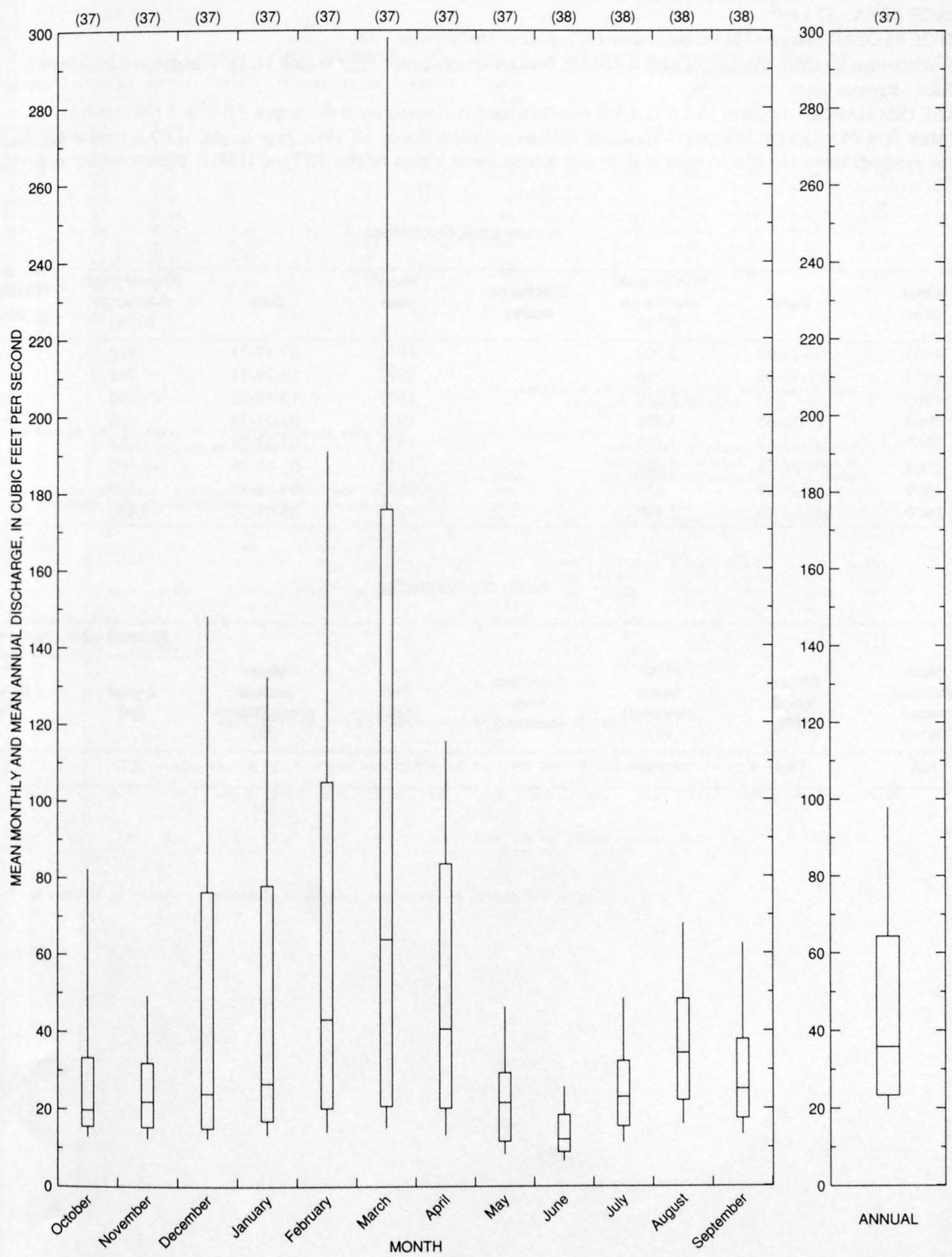


\section{CHERRY CREEK NEAR YOUNG, AZ}

LOCATION.--Lat 34 04'58", long 110 55'25", in SE $1 / 4 \mathrm{NE}^{1 / 4} \mathrm{sec} .32$, T.9 N., R.14 E., Gila County, Hydrologic Unit 15060103 , on left bank 0.3m downstream from Deadman Canyon and $2 \mathrm{mi}$ southeast of Young.

DRAINAGE AREA.--62.1 $\mathrm{mi}^{2}$.

PERIOD OF RECORD.--August 1963 to September 1977 (discontinued).

GAGE.--Water-stage recorder. Altitude of gage is 4,950 ft, from topographic map. Prior to June 11, 1973, at datum $2.07 \mathrm{ft}$ higher.

REMARKS.--Records poor.

AVERAGE DISCHARGE.--14 years, $10.2 \mathrm{ft}^{3} / \mathrm{s}, 7,390$ acre- $\mathrm{ft} / \mathrm{yr}$; median of yearly mean discharges, $7.0 \mathrm{ft}^{3} / \mathrm{s}, 5,100 \mathrm{acre}-\mathrm{ft} / \mathrm{yr}$.

EXTREMES FOR PERIOD OF RECORD.--Maximum discharge, 7,290 ft $3 / \mathrm{s}$ Oct. 19, 1972 (gage height, $11.00 \mathrm{ft}$ present datum), from rating curve extended above $110 \mathrm{ft}^{3} / \mathrm{s}$ on basis of slope-area measurements at gage heights 7.97 and $11.00 \mathrm{ft}$, present datum; no flow July 18,20 1972.

Annual peak discharges

\begin{tabular}{|c|c|c|c|c|c|c|c|}
\hline $\begin{array}{l}\text { Water } \\
\text { year }\end{array}$ & Date & $\begin{array}{c}\text { Annual peak } \\
\text { discharge } \\
\left(\mathrm{ft}^{3} / \mathrm{s}\right)\end{array}$ & $\begin{array}{l}\text { Discharge } \\
\text { codes }\end{array}$ & $\begin{array}{c}\text { Water } \\
\text { year }\end{array}$ & Date & $\begin{array}{c}\text { Annual peak } \\
\text { discharge } \\
\left(\mathrm{ft}^{3} / \mathrm{s}\right)\end{array}$ & $\begin{array}{c}\text { Discharge } \\
\text { codes }\end{array}$ \\
\hline 1963 & $08-22-63$ & 2,700 & & 1971 & $07-17-71$ & 920 & \\
\hline 1965 & $08-17-65$ & 3,280 & & 1973 & $10-19-72$ & 7,290 & \\
\hline 1966 & $12-22-65$ & 3,400 & & 1974 & $08-02-74$ & 950 & \\
\hline 1969 & $01-26-69$ & 616 & & 1977 & $09-02-77$ & 530 & \\
\hline 1970 & $09-05-70$ & 3,100 & & 1978 & $03-01-78$ & 2,480 & \\
\hline
\end{tabular}

Basin characteristics

\begin{tabular}{|c|c|c|c|c|c|c|c|}
\hline \multirow[b]{2}{*}{$\begin{array}{l}\text { Main } \\
\text { channel } \\
\text { slope } \\
\text { (ft/mi) }\end{array}$} & \multirow[b]{2}{*}{$\begin{array}{l}\text { Stream } \\
\text { length } \\
\text { (mi) }\end{array}$} & \multirow[b]{2}{*}{$\begin{array}{c}\text { Mean } \\
\text { basin } \\
\text { elevation } \\
\text { (ft) }\end{array}$} & \multirow[b]{2}{*}{$\begin{array}{l}\text { Forested } \\
\text { area } \\
\text { (percent) }\end{array}$} & \multirow[b]{2}{*}{$\begin{array}{c}\text { Soil } \\
\text { index }\end{array}$} & \multirow[b]{2}{*}{$\begin{array}{c}\text { Mean } \\
\text { annual } \\
\text { precipitation } \\
\text { (in) }\end{array}$} & \multicolumn{2}{|c|}{ Rainfall intensity, 24-hour } \\
\hline & & & & & & $\begin{array}{c}\text { 2-year } \\
\text { (in) }\end{array}$ & $\begin{array}{l}\text { 50-year } \\
\text { (in) }\end{array}$ \\
\hline 106 & 18.6 & 6,030 & 75.0 & 3.0 & 24.8 & 2.7 & 5.5 \\
\hline
\end{tabular}


09497900 CHERRY CREEK NEAR YOUNG, AZ--Continued

MEAN MONTHLY AND ANNUAL DISCHARGES 1964-77

\begin{tabular}{|c|c|c|c|c|c|c|}
\hline MONTH & $\begin{array}{l}\text { MAXIMUM } \\
\text { (FT3/S) }\end{array}$ & $\begin{array}{l}\text { MINIMUM } \\
\text { (FT3/S) }\end{array}$ & $\begin{array}{c}\text { MEAN } \\
(\text { FT3 } / \mathrm{S})\end{array}$ & $\begin{array}{l}\text { STAN- } \\
\text { DARD } \\
\text { DEVIA- } \\
\text { TION } \\
\text { (FT3/S) }\end{array}$ & $\begin{array}{l}\text { COEFFI- } \\
\text { CIENT OF } \\
\text { VARI- } \\
\text { ATION }\end{array}$ & $\begin{array}{c}\text { PERCENT } \\
\text { OF } \\
\text { ANNUAL } \\
\text { RUNOFF }\end{array}$ \\
\hline OCTOBER & 108 & 0.33 & 12 & 31 & 2.4 & 10.2 \\
\hline NOVEMBER & 39 & 0.58 & 4.9 & 10 & 2.1 & 4.0 \\
\hline DECEMBER & 170 & 0.78 & 19 & 45 & 2.4 & 15.4 \\
\hline JANUARY & 81 & 1.2 & 16 & 24 & 1.5 & 13.2 \\
\hline FEBRUARY & 94 & 1.3 & 22 & 32 & 1.4 & 18.1 \\
\hline MARCH & 123 & 1.8 & 22 & 32 & 1.5 & 18.3 \\
\hline APRIL & 63 & 0.86 & 12 & 19 & 1.5 & 10.0 \\
\hline MAY & 15 & 0.58 & 3.0 & 3.6 & 1.2 & 2.5 \\
\hline JUNE & 2.0 & 0.41 & 0.82 & 0.42 & 0.51 & 0.7 \\
\hline JULY & 9.4 & 0.39 & 2.1 & 2.6 & 1.3 & 1.7 \\
\hline AUGUST & 9.5 & 0.46 & 2.7 & 2.7 & 1.0 & 2.2 \\
\hline SEPTEMBER & 46 & 0.12 & 4.6 & 12 & 2.6 & 3.8 \\
\hline ANNUAL & 40 & 1.8 & 10 & 10 & 1.0 & 100 \\
\hline
\end{tabular}

MAGNITUDE AND PROBABILITY OF INSTANTANEOUS PEAK FLOW BASED ON PERIOD OF RECORD 1963.78

DISCHARGE, IN FT $3 / S$, FOR INDICATED RECURRENCE INTERVAL IN YEARS, AND EXCEEDANCE PROBABILITY, IN PERCENT

$\begin{array}{cccccc}2 & 5 & 10 & 25 & 50 & 100 \\ 508 & 208 & 108 & 48 & 28 & 18 \\ 1,390 & 3,120 & 4,660 & 7,080 & 9,200 & 11,600\end{array}$

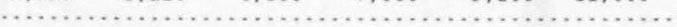

WEIGHTED SKEW (LOGS) $=\cdot 0.19$

MEAN $\quad($ LOGS $)=3.13$

STANDARD DEV. $($ LOGS $)=0.43$
MAGNITUDE AND PROBABILITY OF ANNUAL LOW FLOW BASED ON PERIOD OF RECORD 1965-77

\begin{tabular}{|c|c|c|c|c|c|c|}
\hline $\begin{array}{l}\text { PERIOD } \\
\text { (CON- }\end{array}$ & \multicolumn{6}{|c|}{$\begin{array}{l}\text { DISCHARGE, IN FT3/S, FOR INDICATED } \\
\text { RECURRENCE INTERVAL, IN YEARS, AND } \\
\text { NON-EXCEEDANCE PROBABILITY, IN PERCENT }\end{array}$} \\
\hline SECU- & & $\ldots \ldots$ & $\ldots \ldots$ & ...... & ........ & $\cdots$ \\
\hline TIVE & 2 & 5 & 10 & 20 & $50 \#$ & $100 \#$ \\
\hline DAYS) & 508 & 208 & 108 & 58 & 28 & 18 \\
\hline 1 & 0.17 & 0.08 & 0.04 & 0.00 & 0.00 & 0.00 \\
\hline 3 & 0.17 & 0.09 & 0.06 & 0.00 & 0.00 & 0.00 \\
\hline 7 & 0.19 & 0.11 & 0.08 & 0.06 & 0.05 & 0.04 \\
\hline 14 & 0.25 & 0.17 & 0.14 & 0.12 & 0.09 & 0.08 \\
\hline 30 & 0.39 & 0.28 & 0.22 & 0.16 & 0.11 & 0.08 \\
\hline 60 & 0.53 & 0.35 & 0.28 & 0.22 & 0.18 & 0.15 \\
\hline 90 & 0.66 & 0.47 & 0.40 & 0.35 & 0.31 & 0.29 \\
\hline 120 & 0.88 & 0.58 & 0.49 & 0.42 & 0.37 & 0.34 \\
\hline 183 & 1.2 & 0.79 & 0.66 & 0.59 & 0.52 & 0.49 \\
\hline
\end{tabular}

MAGNITUDE AND PROBABILITY OF ANNUAL HIGH FLOW BASED ON PERIOD OF RECORD 1964.77

\begin{tabular}{|c|c|c|c|c|c|c|}
\hline $\begin{array}{l}\text { PERIOD } \\
(C O N \text { - }\end{array}$ & \multicolumn{6}{|c|}{$\begin{array}{l}\text { DISCHARGE, IN FT3/S, FOR INDICATED } \\
\text { RECURRENCE INTERVAL, IN YEARS, AND }\end{array}$} \\
\hline SECU- & $\cdots$ & 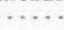 & sere & & ….... & \\
\hline TIVE & 2 & 5 & 10 & $25 \#$ & $50 \#$ & $100 \#$ \\
\hline DAYS) & 508 & 208 & 108 & 48 & 28 & 18 \\
\hline 1 & 261 & 736 & 1,260 & 2,220 & 3,210 & 4,450 \\
\hline 3 & 164 & 421 & 673 & 1,090 & 1.470 & 1,920 \\
\hline 7 & 94 & 229 & 351 & 540 & 703 & 883 \\
\hline 15 & 58 & 139 & 214 & 331 & 433 & 547 \\
\hline 30 & 36 & 90 & 143 & 230 & 310 & 405 \\
\hline 60 & 24 & 62 & 99 & 160 & 217 & 282 \\
\hline 90 & 19 & 48 & 76 & 121 & 161 & 207 \\
\hline
\end{tabular}

DURATION TABLE OF DAILY MEAN FLOW FOR PERIOD OF RECORD 1964-77

DISCHARGE, IN FT3/S, WHICH WAS EQUALED OR EXCEEDED FOR INDICATED PERCENT OF TIME

\begin{tabular}{|c|c|c|c|c|c|c|c|c|c|c|c|c|c|c|c|c|}
\hline 18 & 58 & 108 & 158 & 208 & 308 & 408 & 508 & 608 & 708 & 808 & 908 & 958 & 988 & 998 & 99.58 & 99.98 \\
\hline 162 & 48 & 16 & 8.8 & 4.8 & 2.6 & 1.9 & 1.5 & 1.2 & 0.79 & 0.56 & 0.36 & 0.26 & 0.18 & 0.12 & 0.09 & 0.02 \\
\hline
\end{tabular}

\# Reliability of values in column is uncertain, and potential errors are large. 
09497900 CHERRY CREEK NEAR YOUNG, AZ--Continued
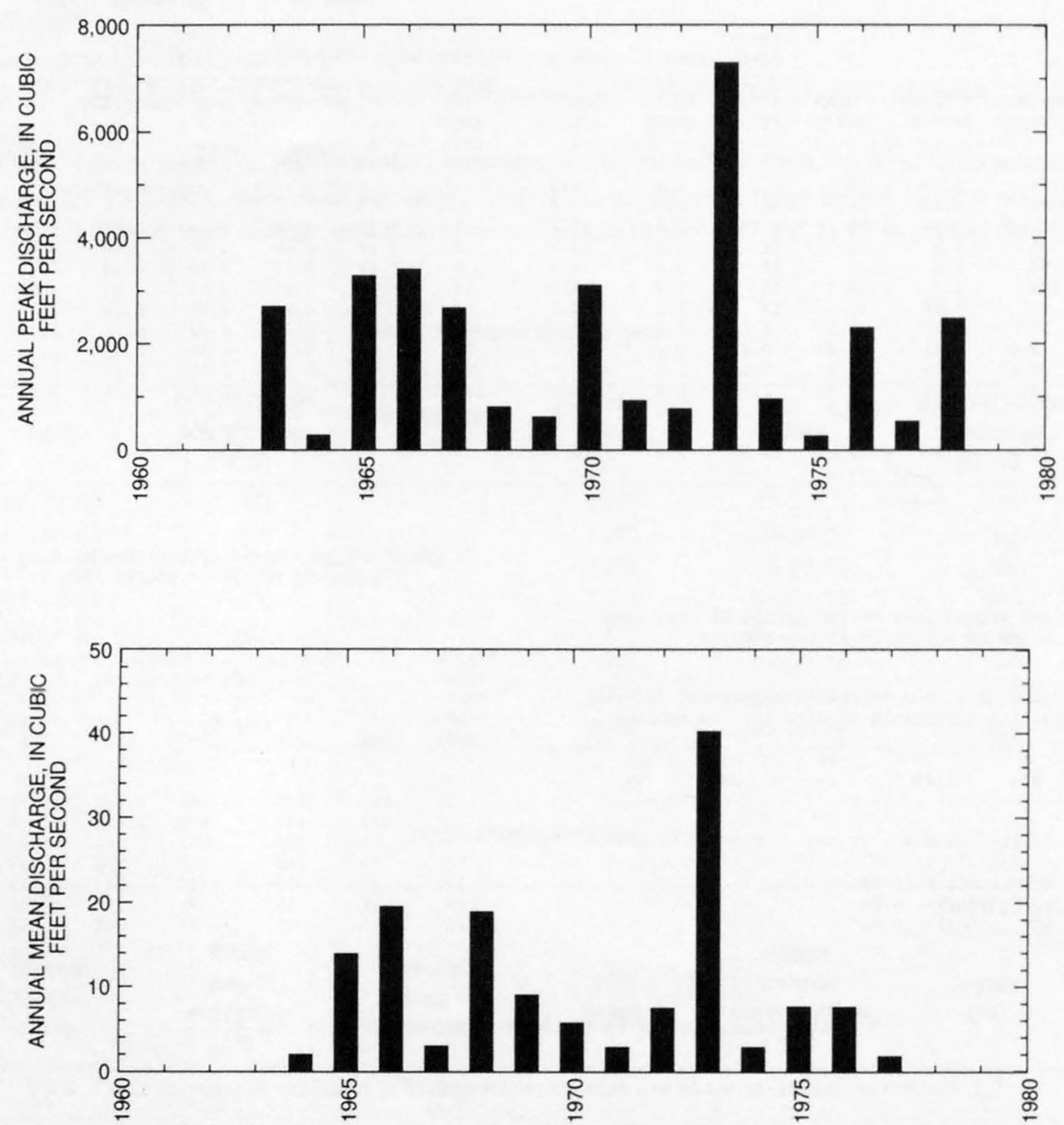
09497900 CHERRY CREEK NEAR YOUNG, AZ--Continued
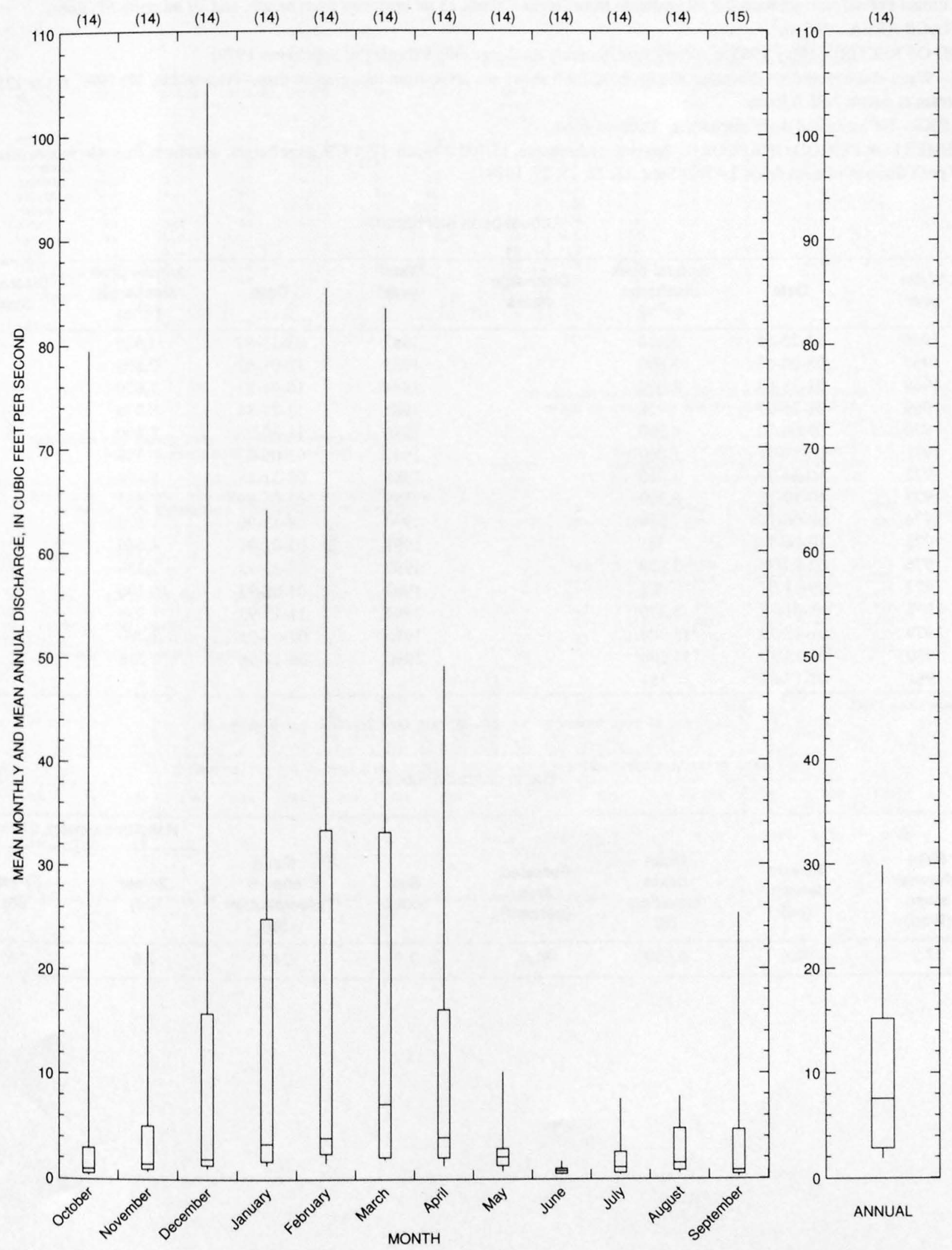
LOCATION.--Lat 33 49'40", long 110 51'20", in SW1/4 sec.30, T.6 N., R.15 E. (unsurveyed), Gila County, Hydrologic Unit 15060103, in Tonto

National Forest, on right bank 0.2 mi upstream from Devils Chasm, $13 \mathrm{mi}$ upstream from mouth, and 30 mi north of Globe.

DRAINAGE AREA.--200 $\mathrm{mi}^{2}$.

PERIOD OF RECORD.--May 1965 to current year (monthly discharge only February to September 1979).

GAGE.--Water-stage recorder. Elevation of gage is 3,200 ft above sea level, from topographic map. Prior to Jan. 17, 1979 , at site $125 \mathrm{ft}$ downstream at datum $2.95 \mathrm{ft}$ lower.

REMARKS.--No estimated daily discharges. Records good.

EXTREMES FOR PERIOD OF RECORD.--Maximum discharge, 15,700 $\mathrm{ft}^{3} / \mathrm{s} \mathrm{Jan.} \mathrm{17,} \mathrm{1979,} \mathrm{gage} \mathrm{height,} \mathrm{unknown,} \mathrm{from} \mathrm{slope-area} \mathrm{measurement}$ of peak flow; minimum daily, $2.4 \mathrm{ft}^{3} / \mathrm{s}$ Sept. 17, 22, 25, 29, 1978.

Annual peak discharges

\begin{tabular}{|c|c|c|c|c|c|c|c|}
\hline $\begin{array}{l}\text { Water } \\
\text { year }\end{array}$ & Date & $\begin{array}{c}\text { Annual peak } \\
\text { discharge } \\
\left(\mathrm{ft}^{3} / \mathrm{s}\right)\end{array}$ & $\begin{array}{c}\text { Discharge } \\
\text { codes }\end{array}$ & $\begin{array}{l}\text { Water } \\
\text { year }\end{array}$ & Date & $\begin{array}{c}\text { Annual peak } \\
\text { discharge } \\
\left(\mathrm{ft}^{3} / \mathrm{s}\right)\end{array}$ & $\begin{array}{l}\text { Discharge } \\
\text { codes }\end{array}$ \\
\hline 1966 & $12-22-65$ & 6,620 & & 1982 & $03-13-82$ & 1,920 & \\
\hline 1967 & $08-06-67$ & 3,600 & & 1983 & $12-01-82$ & 2,800 & \\
\hline 1968 & $01-28-68$ & 1,490 & & 1984 & $10-01-83$ & 1,620 & \\
\hline 1969 & $01-26-69$ & 928 & & 1985 & $12-27-84$ & 8,970 & \\
\hline 1970 & $09-06-70$ & 4,300 & & 1986 & $11-30-85$ & 1,690 & \\
\hline 1971 & $08-19-71$ & 1,260 & & 1987 & $03-05-87$ & 358 & \\
\hline 1972 & $10-24-71$ & 1,010 & & 1988 & $08-31-88$ & 5,170 & \\
\hline 1973 & $10-19-72$ & 8,300 & & 1989 & $02-05-89$ & 563 & \\
\hline 1974 & $08-06-74$ & 596 & & 1990 & $08-13-90$ & 125 & \\
\hline 1975 & $10-24-74$ & 715 & & 1991 & 03-01-91 & 4,640 & \\
\hline 1976 & $02-09-76$ & 3,820 & & 1992 & $08-23-92$ & 2,250 & \\
\hline 1977 & $09-11-77$ & 408 & & 1993 & 01-08-93 & 10,100 & \\
\hline 1978 & $03-01-78$ & 5,370 & & 1994 & $11-15-93$ & 936 & \\
\hline 1979 & $01-17-79$ & ${ }^{\mathrm{l}} 15,700$ & & 1995 & $03-06-95$ & 2,780 & \\
\hline 1980 & $02-15-80$ & 13,500 & & 1996 & $09-12-96$ & 226 & \\
\hline 1981 & $07-17-81$ & 151 & & & & & \\
\hline
\end{tabular}

${ }^{1}$ Highest since 1960.

Basin characteristics

\begin{tabular}{cccccccc}
\hline $\begin{array}{c}\text { Main } \\
\text { channel } \\
\text { slope } \\
(\mathrm{ft} / \mathrm{mi})\end{array}$ & $\begin{array}{c}\text { Stream } \\
\text { length } \\
\text { (mi) }\end{array}$ & $\begin{array}{c}\text { Mean } \\
\text { basin } \\
\text { elevation } \\
(\mathrm{ft})\end{array}$ & $\begin{array}{c}\text { Forested } \\
\text { area } \\
\text { (percent) }\end{array}$ & $\begin{array}{c}\text { Soil } \\
\text { index }\end{array}$ & $\begin{array}{c}\text { Mean } \\
\text { annual } \\
\text { precipitation } \\
\text { (in) }\end{array}$ & $\begin{array}{c}\text { Rainfall intensity, 24-hour } \\
\text { (in) }\end{array}$ & $\begin{array}{c}50 \text {-year } \\
\text { (in) }\end{array}$ \\
\hline 87.9 & 36.4 & 5,600 & 81.0 & 2.9 & 24.0 & 2.6 \\
\hline
\end{tabular}


09497980 CHERRY CREEK NEAR GLOBE, AZ--Continued

MEAN MONTHLY AND ANNUAL DISCHARGES $1966 \cdot 78,1980 \cdot 96$

\begin{tabular}{|c|c|c|c|c|c|c|}
\hline MONTH & $\begin{array}{l}\text { MAXIMUM } \\
\text { (FT3/S) }\end{array}$ & $\begin{array}{l}\text { MINIMUM } \\
\text { (FT3/S) }\end{array}$ & $\begin{array}{c}\text { MEAN } \\
(\text { FT3 } / S)\end{array}$ & $\begin{array}{l}\text { STAN- } \\
\text { DARD } \\
\text { DEVIA- } \\
\text { TION } \\
\text { (FT3/S) }\end{array}$ & $\begin{array}{l}\text { COEFFI- } \\
\text { CIENT OF } \\
\text { VARI- } \\
\text { ATION }\end{array}$ & $\begin{array}{c}\text { PERCENT } \\
\text { OF } \\
\text { ANNUAL } \\
\text { RUNOFF }\end{array}$ \\
\hline OCTOBER & 296 & 4.7 & 24 & 55 & 2.3 & 4.9 \\
\hline NOVEMBER & 101 & 4.5 & 21 & 28 & 1.3 & 4.3 \\
\hline DECEMBER & 537 & 4.8 & 68 & 124 & 1.8 & 14 \\
\hline JANUARY & 652 & 6.7 & 81 & 146 & 1.8 & 17 \\
\hline FEBRUARY & 586 & 6.0 & 97 & 128 & 1.3 & 20 \\
\hline MARCH & 423 & 6.1 & 98 & 120 & 1.2 & 20 \\
\hline APRIL & 195 & 5.3 & 30 & 38 & 1.3 & 6.2 \\
\hline MAY & 66 & 4.9 & 13 & 11 & 0.90 & 2.6 \\
\hline JUNE & 18 & 4.4 & 7.7 & 3.1 & 0.41 & 1.6 \\
\hline JULY & 23 & 5.6 & 9.5 & 4.1 & 0.43 & 2.0 \\
\hline AUGUST & 85 & 5.6 & 18 & 19 & 1.1 & 3.7 \\
\hline SEPTEMBER & 151 & 3.6 & 16 & 26 & 1.7 & 3.2 \\
\hline ANNUAL & 130 & 6.7 & 38 & 32 & 0.84 & 100 \\
\hline
\end{tabular}

MAGNITUDE AND PROBABILITY OF INSTANTANEOUS PEAK FLOW BASED ON PERIOD OF RECORD 1966.96

DISCHARGE, IN FT3/S, FOR INDICATED RECURRENCE INTERVAL IN YEARS, AND EXCEEDANCE PROBABILITY, IN PERCENT

\begin{tabular}{|c|c|c|c|c|c|}
\hline 2 & 5 & 10 & 25 & 50 & 100 \\
\hline 508 & 208 & 108 & 48 & 28 & 18 \\
\hline 2,190 & 5,450 & 8,700 & 14,200 & 19,300 & 25,400 \\
\hline WEIGHTED & SKEW & OGS) $=$ & & & \\
\hline MEAN & & OGS) = & & & \\
\hline STANDARD & DEV. & OGS $)=$ & & & \\
\hline
\end{tabular}

DURATION TABLE OF DAILY MEAN FLOW FOR PERIOD OF RECORD $1966 \cdot 78,1980.96$
MAGNITUDE AND PROBABILITY OF ANNUAL LOW FLOW BASED ON PERIOD OF RECORD $1967-78,1980-96$

\begin{tabular}{|c|c|c|c|c|c|c|}
\hline \multirow{2}{*}{$\begin{array}{l}\text { PERIOD } \\
\text { (CON- } \\
\text { SECU. }\end{array}$} & \multicolumn{6}{|c|}{$\begin{array}{l}\text { DISCHARGE, IN FT3/S, FOR INDICATED } \\
\text { RECURRENCE INTERVAL, IN YEARS, AND }\end{array}$} \\
\hline & $\cdots$ & $\ldots \ldots$ & $\ldots \ldots$ & & $\ldots \ldots$ & $\cdots$ \\
\hline TIVE & 2 & 5 & $10 \#$ & $20 \#$ & $50 \#$ & $100 \#$ \\
\hline DAYS) & 508 & 208 & 108 & 58 & 28 & 18 \\
\hline 1 & 5.2 & 4.2 & 3.8 & 3.5 & 3.2 & 3.0 \\
\hline 3 & 5.3 & 4.3 & 3.9 & 3.6 & 3.4 & 3.2 \\
\hline 7 & 5.4 & 4.4 & 4.0 & 3.7 & 3.4 & 3.3 \\
\hline 14 & 5.6 & 4.6 & 4.1 & 3.8 & 3.5 & 3.4 \\
\hline 30 & 5.9 & 4.9 & 4.4 & 4.1 & 3.8 & 3.6 \\
\hline 60 & 6.5 & 5.3 & 4.8 & 4.4 & 4.1 & 3.9 \\
\hline 90 & 7.0 & 5.7 & 5.1 & 4.7 & 4.4 & 4.1 \\
\hline 120 & 7.5 & 6.2 & 5.7 & 5.3 & 4.9 & 4.7 \\
\hline 183 & 9.2 & 7.0 & 6.2 & 5.7 & 5.2 & 4.9 \\
\hline
\end{tabular}

MAGNITUDE AND PROBABILITY OF ANNUAL HIGH FLOW BASED ON PERIOD OF RECORD $1966.78,1980.96$

\begin{tabular}{|c|c|c|c|c|c|c|}
\hline \multirow{3}{*}{$\begin{array}{l}\text { PERIOD } \\
\text { (CON- } \\
\text { SECU. }\end{array}$} & & \multicolumn{5}{|c|}{ DISCHARGE, IN FT3/S, FOR INDICATED } \\
\hline & & EXCEED & CE PRO & ILITY, & IN PER & ENT \\
\hline & & $\ldots \ldots$ & . & & $\ldots$ & $\ldots$ \\
\hline TIVE & 2 & 5 & $10 \#$ & $25 \#$ & $50 \#$ & $100 \#$ \\
\hline DAYS) & 508 & 208 & 108 & 48 & 28 & 18 \\
\hline 1 & 743 & 2,170 & 3,620 & 6,020 & 8,220 & 10,700 \\
\hline 3 & 472 & 1,250 & 1,990 & 3,130 & 4,110 & 5,190 \\
\hline 7 & 293 & 738 & 1,140 & 1,760 & 2,280 & 2.850 \\
\hline 15 & 186 & 467 & 731 & 1,150 & 1,510 & 1.920 \\
\hline 30 & 123 & 309 & 485 & 767 & 1,020 & 1,310 \\
\hline 60 & 85 & 212 & 334 & 532 & 712 & 919 \\
\hline 90 & 67 & 163 & 254 & 402 & 536 & 692 \\
\hline
\end{tabular}

DISCHARGE, IN FT $3 / \mathrm{S}$, WHICH WAS EQUALED OR EXCEEDED FOR INDICATED PERCENT OF TIME

\begin{tabular}{|c|c|c|c|c|c|c|c|c|c|c|c|c|c|c|c|c|}
\hline 18 & 58 & 108 & 158 & 208 & 308 & 408 & 508 & 608 & 708 & 808 & 908 & 958 & 988 & 998 & 99.58 & 99.98 \\
\hline 46 & 138 & 63 & 37 & 25 & 14 & 11 & 9.0 & 7.2 & 6.8 & 5.8 & 5.3 & 4.7 & 0.00 & 0.001 & 0.00 & 0.00 \\
\hline
\end{tabular}

\# Reliability of values in column is uncertain, and potential errors are large. 
ANNUAL MEAN DISCHARGE, IN CUBIC FEET PER SECOND

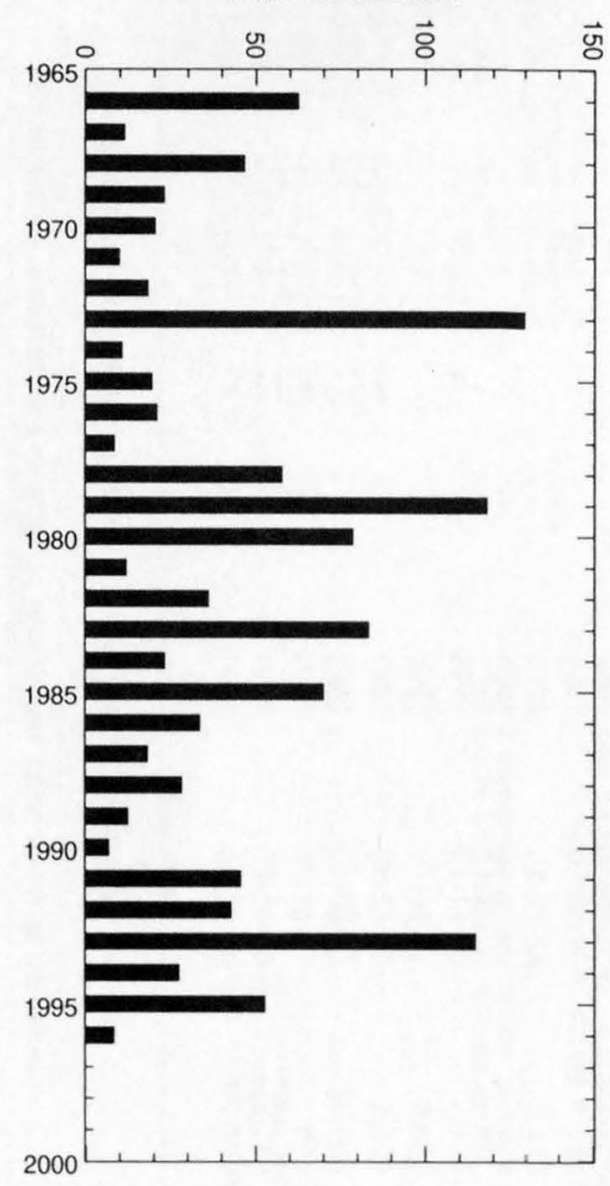

ANNUAL PEAK DISCHARGE, IN CUBIC FEET PER SECOND

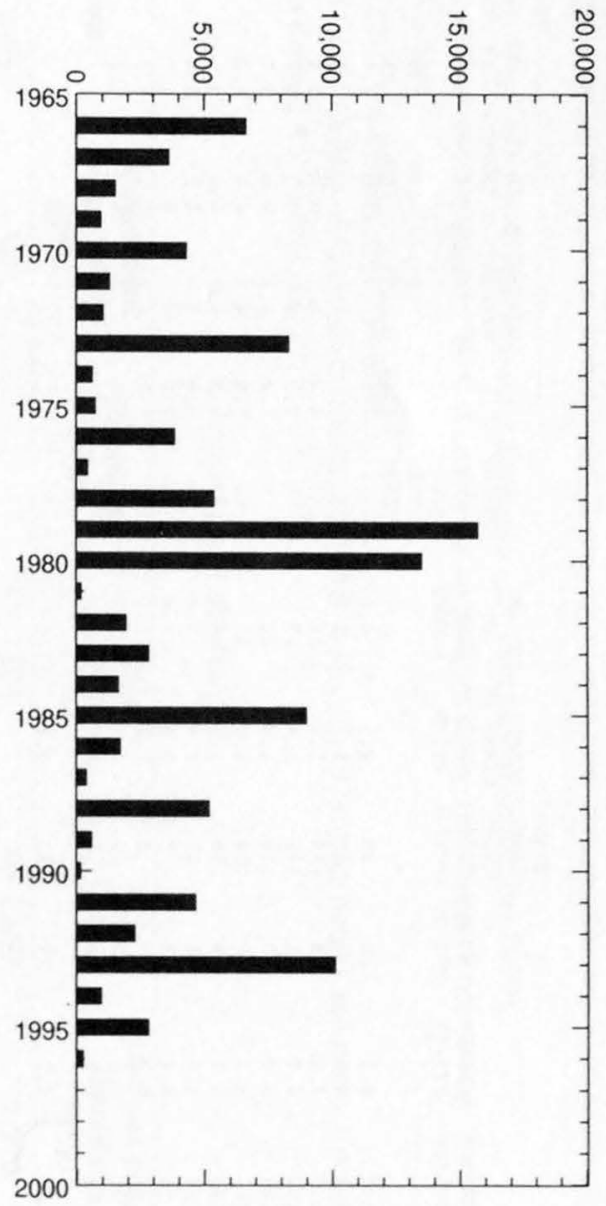


09497980 CHERRY CREEK NEAR GLOBE, AZ--Continued

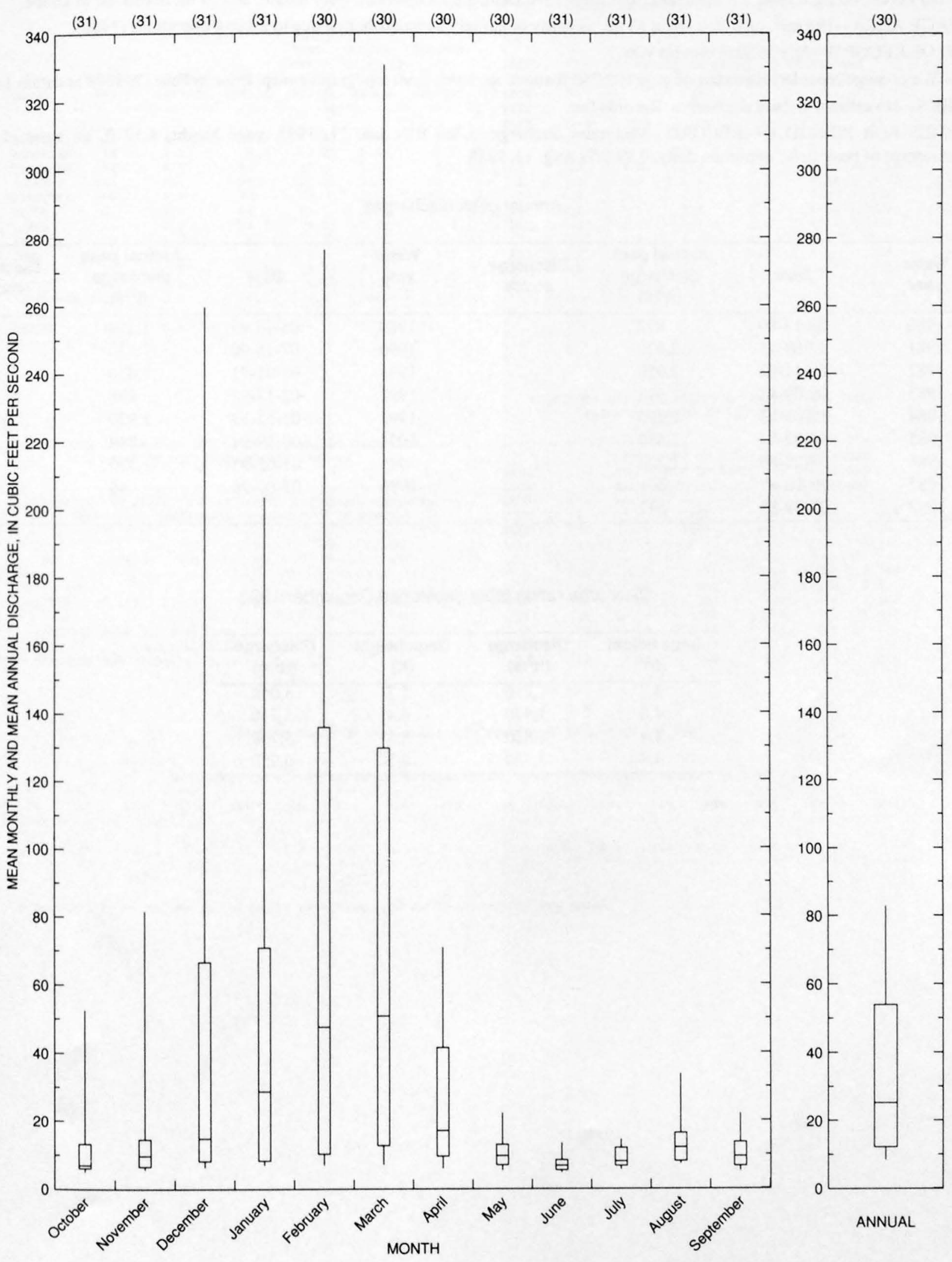




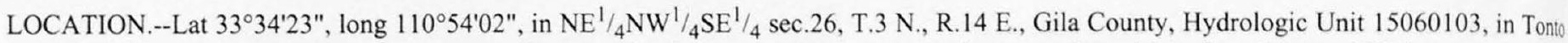
National Forest, on right bank $7 \mathrm{ft}$ upstream from Inspiration Dam, 3.8 mi upstream from mouth, and 14 mi northwest of Globe.

DRAINAGE AREA.--195 $\mathrm{mi}^{2}$, of which about $33 \mathrm{mi}^{2}$ is partly or entirely noncontributing due to mining operations (1988).

PERIOD OF RECORD.--July 1980 to current year.

GAGE.--Water-stage recorder. Elevation of gage is $2,740 \mathrm{ft}$ above sea level, from topographic map. Prior to Feb. 12,1991 at datum $1.0 \mathrm{ft}$ higher. REMARKS.--No estimated daily discharges. Records fair.

EXTREMES FOR PERIOD OF RECORD.--Maximum discharge, 5,700 $\mathrm{ft}^{3} / \mathrm{s} \mathrm{Jan.} \mathrm{11,} \mathrm{1993,} \mathrm{gage} \mathrm{height,} 8.50 \mathrm{ft}$, on basis of slope-area measurement of peak flow; minimum daily, $0.99 \mathrm{ft}^{3} / \mathrm{s}$ Aug. 13, 1988.

Annual peak discharges

\begin{tabular}{|c|c|c|c|c|c|c|c|}
\hline $\begin{array}{l}\text { Water } \\
\text { year }\end{array}$ & Date & $\begin{array}{c}\text { Annual peak } \\
\text { discharge } \\
\left(\mathrm{ft}^{3} / \mathrm{s}\right)\end{array}$ & $\begin{array}{c}\text { Discharge } \\
\text { codes }\end{array}$ & $\begin{array}{l}\text { Water } \\
\text { year }\end{array}$ & Date & $\begin{array}{c}\text { Annual peak } \\
\text { discharge } \\
\left(\mathrm{ft}^{3} / \mathrm{s}\right)\end{array}$ & $\begin{array}{c}\text { Discharge } \\
\text { codes }\end{array}$ \\
\hline 1980 & $08-13-80$ & 872 & & 1989 & $08-18-89$ & 1,250 & \\
\hline 1981 & $07-09-81$ & 2,920 & & 1990 & $07-15-90$ & 1,930 & \\
\hline 1982 & $09-11-82$ & 2,050 & & 1991 & 03-01-91 & 1,430 & \\
\hline 1983 & $09-09-83$ & 673 & & 1992 & $02-13-92$ & 406 & \\
\hline 1984 & $10-01-83$ & 2,290 & & 1993 & $01-12-93$ & 5,950 & \\
\hline 1985 & $10-02-84$ & 1,530 & & 1994 & $08-19-94$ & 849 & \\
\hline 1986 & $08-25-86$ & 1,220 & & 1995 & $01-05-95$ & 1,320 & \\
\hline 1987 & $08-10-87$ & 861 & & 1996 & $07-03-96$ & 46 & \\
\hline 1988 & $07-09-88$ & 547 & & & & & \\
\hline
\end{tabular}

Discharge rating table developed December 1992

\begin{tabular}{cccc}
\hline $\begin{array}{c}\text { Gage height } \\
(\mathbf{f t})\end{array}$ & $\begin{array}{c}\text { Discharge } \\
\left(\mathbf{f t}^{\mathbf{3}} / \mathbf{s}\right)\end{array}$ & $\begin{array}{c}\text { Gage height } \\
(\mathbf{f t})\end{array}$ & $\begin{array}{c}\text { Discharge } \\
\left(\mathbf{f t}^{\mathbf{3}} / \mathbf{s}\right)\end{array}$ \\
\hline 3.7 & 1,210 & 5.7 & 3,050 \\
4.0 & 1,470 & 6.4 & 3,710 \\
4.4 & 1,850 & 8.5 & 5,700 \\
4.9 & 2,320 & 8.8 & 6,050 \\
\hline
\end{tabular}


09498400 PINAL CREEK AT INSPIRATION DAM, NEAR GLOBE, AZ--Continued

MEAN MONTHLY AND ANNUAL DISCHARGES 1980.96

\begin{tabular}{|c|c|c|c|c|c|c|}
\hline MONTH & $\begin{array}{l}\text { MAXIMUM } \\
(\text { FT3/S) }\end{array}$ & $\begin{array}{l}\text { MINIMUM } \\
\text { (FT3/S) }\end{array}$ & $\begin{array}{c}\text { MEAN } \\
(\mathrm{FT} 3 / \mathrm{S})\end{array}$ & $\begin{array}{l}\text { STAN- } \\
\text { DARD } \\
\text { DEVIA- } \\
\text { TION } \\
(\text { FT3/S) }\end{array}$ & $\begin{array}{l}\text { COEFFI- } \\
\text { CIENT OF } \\
\text { VARI- } \\
\text { ATION }\end{array}$ & $\begin{array}{c}\text { PERCENT } \\
\text { OF } \\
\text { ANNUAL } \\
\text { RUNOFF }\end{array}$ \\
\hline OCTOBER & 39 & 4.3 & 11 & 9.0 & 0.82 & 6.0 \\
\hline NOVEMBER & 13 & 5.2 & 8.2 & 2.2 & 0.26 & 4.5 \\
\hline DECEMBER & 58 & 6.0 & 13 & 13 & 1.0 & 6.9 \\
\hline JANUARY & 440 & 6.7 & 39 & 107 & 2.7 & 22 \\
\hline FEBRUARY & 406 & 5.9 & 37 & 99 & 2.7 & 20 \\
\hline MARCH & 67 & 6.1 & 19 & 17 & 0.90 & 10 \\
\hline APRIL & 30 & 7.0 & 11 & 5.8 & 0.52 & 6.2 \\
\hline MAY & 20 & 5.7 & 9.2 & 3.4 & 0.37 & 5.1 \\
\hline JUNE & 16 & 4.5 & 7.3 & 2.8 & 0.38 & 4.0 \\
\hline JULY & 17 & 5.3 & 8.9 & 3.7 & 0.42 & 4.9 \\
\hline AUGUST & 28 & 5.1 & 9.7 & 5.4 & 0.56 & 5.3 \\
\hline SEPTEMBER & 16 & 2.8 & 8.2 & 3.8 & 0.46 & 4.5 \\
\hline ANNUAL & 84 & 6.9 & 15 & 19 & 1.3 & 100 \\
\hline
\end{tabular}

MAGNITUDE AND PROBABILITY OF INSTANTANEOUS PEAK FLOW BASED ON PERIOD OF RECORD $1980-96$

DISCHARGE, IN FT3/S, FOR INDICATED RECURRENCE INTERVAL IN YEARS, AND EXCEEDANCE PROBABILITY, IN PERCENT

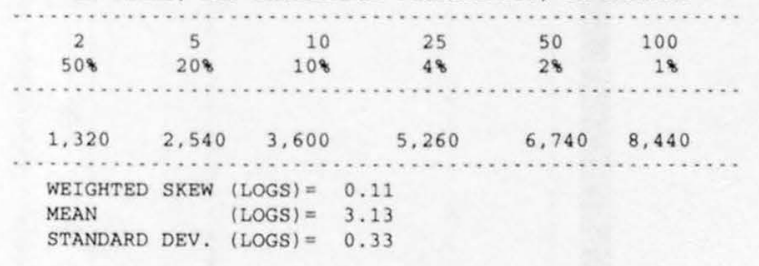

MAGNITUDE AND PROBABILITY OF ANNUAL LOW FLOW BASED ON PERIOD OF RECORD 1980-96

\begin{tabular}{|c|c|c|c|c|c|c|}
\hline \multirow{3}{*}{$\begin{array}{l}\text { PERIOD } \\
\text { /CON - } \\
\text { SECU - }\end{array}$} & \multicolumn{6}{|c|}{ DISCHARGE, IN FT3/S, FOR INDICATED } \\
\hline & \multicolumn{3}{|c|}{ NON-BXCEEDANCE PROBABILITY, IN PERCENT } & & & \\
\hline & & ….... & … & 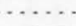 & ..... & ..... \\
\hline TIVE & 2 & 5 & $10 \#$ & $20 \#$ & $50 \#$ & $100 \#$ \\
\hline DAYS) & 508 & 208 & 108 & 58 & 28 & 18 \\
\hline 1 & 3.5 & 2.0 & 1.4 & 1.0 & 0.71 & 0.54 \\
\hline 3 & 3.7 & 2.2 & 1.6 & 1.2 & 0.88 & 0.70 \\
\hline 7 & 4.0 & 2.5 & 2.0 & 1.6 & 1.3 & 1.1 \\
\hline 14 & 4.4 & 3.1 & 2.6 & 2.2 & 1.9 & 1.7 \\
\hline 30 & 5.4 & 4.1 & 3.5 & 3.0 & 2.6 & 2.3 \\
\hline 60 & 6.0 & 4.9 & 4.5 & 4.1 & 3.8 & 3.6 \\
\hline 90 & 6.5 & 5.3 & 4.8 & 4.5 & 4.1 & 3.9 \\
\hline 120 & 7.0 & 5.8 & 5.2 & 4.8 & 4.4 & 4.2 \\
\hline 183 & 7.7 & 6.3 & 5.7 & 5.3 & 4.9 & 4.6 \\
\hline
\end{tabular}

MAGNITUDE AND PROBABILITY OF ANNUAL HIGH FLOW BASED ON PERIOD OF RECORD 1980.96

\begin{tabular}{|c|c|c|c|c|c|c|}
\hline \multirow{4}{*}{$\begin{array}{l}\text { PERIOD } \\
\text { (CON- } \\
\text { SECU. }\end{array}$} & \multirow{3}{*}{\multicolumn{2}{|c|}{$\begin{array}{l}\text { DISCHARGE, } \\
\text { RECURRENCE } \\
\text { EXCEEDANCE }\end{array}$}} & \multicolumn{4}{|c|}{ IN FT3/S, FOR INDICATED } \\
\hline & & & \multirow{2}{*}{\multicolumn{2}{|c|}{$\begin{array}{l}\text { INTERVAL, IN } \\
\text { PROBABILITY, }\end{array}$}} & \multirow{2}{*}{\multicolumn{2}{|c|}{$\begin{array}{l}\text { YEARS, AND } \\
\text { IN PERCENT }\end{array}$}} \\
\hline & & & & & & \\
\hline & $\cdots$ & $\cdots \cdots \cdots$ & . & $\cdots \cdots \cdots$ & $\cdots \cdots$ & ........ \\
\hline TIVE & 2 & 5 & $10 \#$ & $25 \#$ & $50 \#$ & $100 \#$ \\
\hline DAYS) & 508 & 208 & 108 & 48 & 28 & 18 \\
\hline 1 & 184 & 556 & 974 & 1,740 & 2,520 & 3,500 \\
\hline 3 & 94 & 278 & 499 & 945 & 1,440 & 2,110 \\
\hline 7 & 50 & 146 & 278 & 594 & 1,010 & 1,660 \\
\hline 15 & 28 & 79 & 162 & 404 & 792 & 1.530 \\
\hline 30 & 20 & 48 & 91 & 215 & 409 & 775 \\
\hline 60 & 15 & 34 & 69 & 178 & 371 & 779 \\
\hline 90 & 13 & 28 & 54 & 133 & 265 & 536 \\
\hline
\end{tabular}

DURATION TABLE OF DAILY MEAN FLOW FOR PERIOD OF RECORD 1980.96

DISCHARGE, IN FT3/S, WHICH WAS EQUALED OR EXCEEDED FOR INDICATED PERCENT OF TIME

\begin{tabular}{|c|c|c|c|c|c|c|c|c|c|c|c|c|c|c|c|c|}
\hline 18 & 58 & 108 & 158 & 208 & 308 & 408 & 508 & 608 & 708 & 808 & 908 & 958 & 988 & 998 & 99.58 & 99.98 \\
\hline 130 & 24 & 13 & 12 & 11 & 9.8 & 8.9 & 8.1 & 6.9 & 6.5 & 5.7 & 5.2 & 4.4 & 0.00 & 0.00 & 0.00 & 0.00 \\
\hline
\end{tabular}

\# Reliability of values in column is uncertain, and potential errors are large. 
09498400 PINAL CREEK AT INSPIRATION DAM, NEAR GLOBE, AZ--Continued
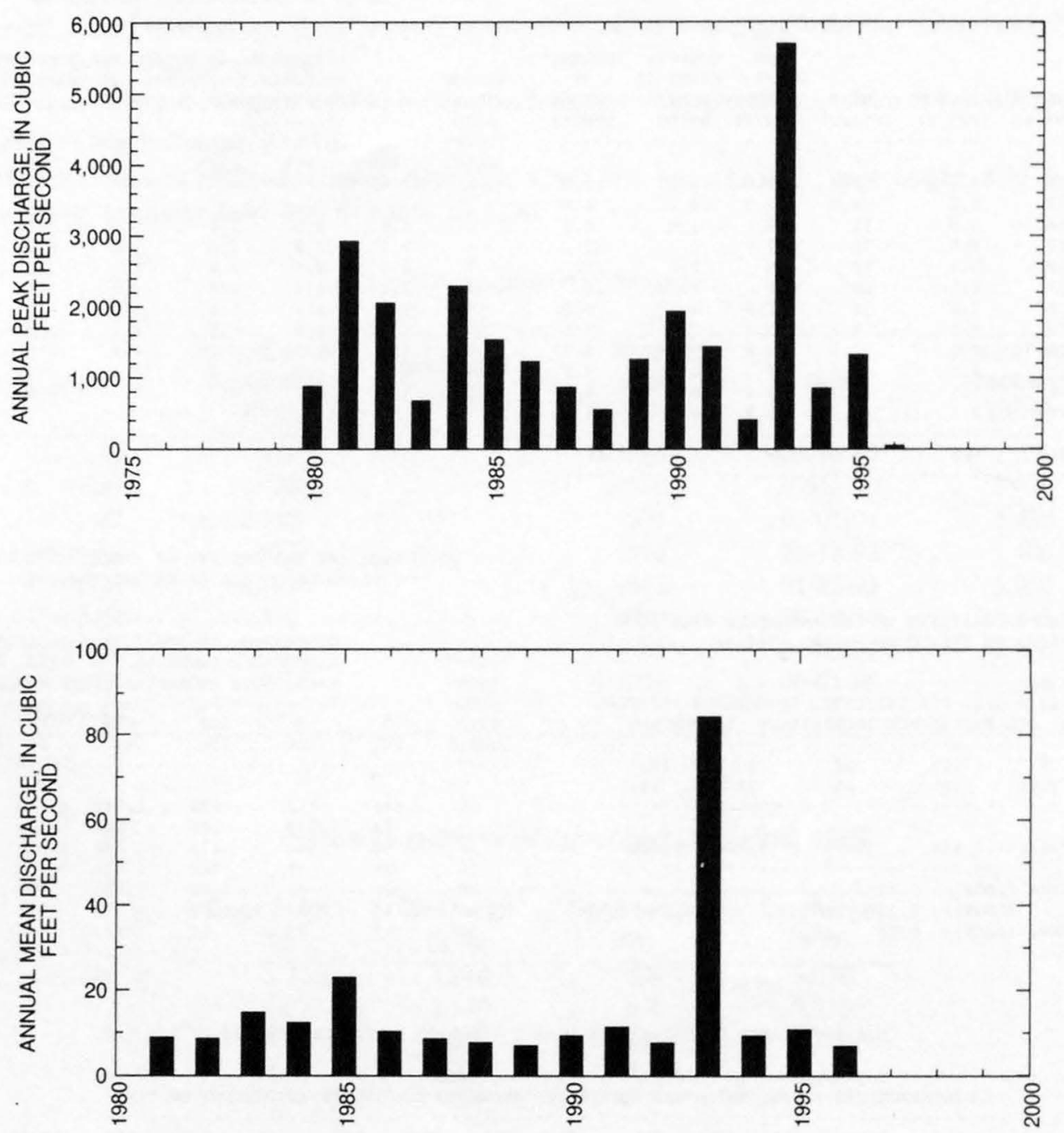
09498400 PINAL CREEK AT INSPIRATION DAM, NEAR GLOBE, AZ--Continued
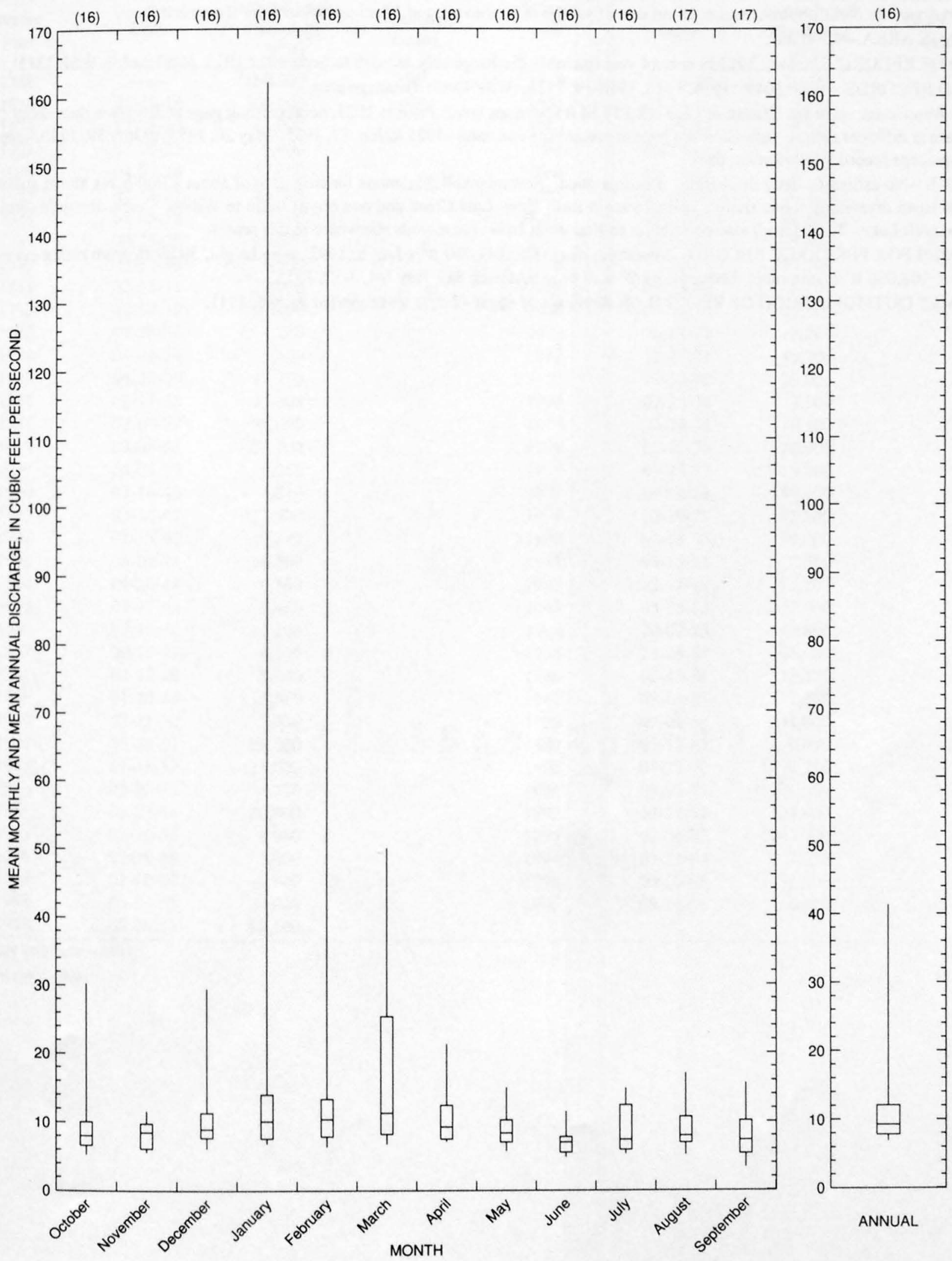


\section{SALT RIVER NEAR ROOSEVELT, AZ}

LOCATION.--Lat 33.37'10", long 110 $55^{\prime} 15^{\prime \prime}$, in $\mathrm{SE}^{1} /{ }_{4} \mathrm{NE}^{1} / 4$ sec.9, T.3 N., R.14 E. (unsurveyed), Gila County, Hydrologic Unit 15060103 , in Tonto National Forest on left bank $100 \mathrm{ft}$ downstream from bridge on State Highway 288, 0.3 mi downstream from Pinal Creek, 1 mi upstream from diversion dam for power canal, 14 mi east of village of Roosevelt, and 17 mi upstream from Roosevelt Dam.

DRAINAGE AREA.--4,306 $\mathrm{mi}^{2}$.

PERIOD OF RECORD.--January 1913 to current year (monthly discharge only January to September 1913, published in WSP 1313).

REVISED RECORDS.--WSP 1049: 1914, 1916, 1918-19, 1926. WSP 1343: Drainage area.

GAGE.--Water-stage recorder. Datum of gage is 2,177.14 ft above sea level. Prior to 1925 , nonrecording gage at diversion dam about 1 mi down stream at different datum. Nonrecording gage at present site and datum 1925 to Jan. 17, 1935. May 20, 1955, to July 30, 1959, supplementary water-stage recorder at diversion dam.

REMARKS.--No estimated daily discharges. Records good. Several small diversions for irrigation of about 4,000 acres above station and two trans basin diversions above station, one into basin from Show Low Creek and one out of basin to Willow Creek. Records show inflow to Roosevelt Lake. Tonto Creek also contributes to Roosevelt Lake; see records elsewhere in this report.

EXTREMES FOR PERIOD OF RECORD.--Maximum discharge, 143,000 ft $3 / \mathrm{s} \mathrm{Jan.} \mathrm{8,} \mathrm{1993,} \mathrm{gage} \mathrm{height,} 30.09 \mathrm{ft}$, from rating curve extended above $108,000 \mathrm{ft}^{3} / \mathrm{s}$; minimum discharge, $59 \mathrm{ft}^{3} / \mathrm{s}$ all or part of each day, July $1-4,7-12,1955$.

EXTREMES OUTSIDE PERIOD OF RECORD.--A discharge of about $42 \mathrm{ft}^{3} / \mathrm{s}$ was reported Aug. $5,1911$. 
GILA RIVER BASIN

09498500 SALT RIVER NEAR ROOSEVELT, AZ--Continued

Annual peak discharges

\begin{tabular}{|c|c|c|c|c|c|c|c|}
\hline $\begin{array}{l}\text { Water } \\
\text { year }\end{array}$ & Date & $\begin{array}{c}\text { Annual peak } \\
\text { discharge } \\
\left(\mathrm{ft}^{3} / \mathrm{s}\right)\end{array}$ & $\begin{array}{l}\text { Discharge } \\
\text { codes }\end{array}$ & $\begin{array}{l}\text { Water } \\
\text { year }\end{array}$ & Date & $\begin{array}{l}\text { Annual peak } \\
\text { discharge } \\
\left(\mathrm{ft}^{3} / \mathrm{s}\right)\end{array}$ & $\begin{array}{l}\text { Discharge } \\
\text { codes }\end{array}$ \\
\hline 1388 & ------ & ${ }^{1} 160,000$ & $\mathrm{PF}$ & 1960 & $12-26-59$ & 78,200 & \\
\hline 1916 & $01-19-16$ & 100,000 & HP & 1961 & $07-28-61$ & 2,590 & \\
\hline 1924 & $12-28-23$ & 43,000 & & 1962 & $01-25-62$ & 8,540 & \\
\hline 1925 & $03-09-25$ & 9,000 & & 1963 & $08-31-63$ & 31,300 & \\
\hline 1926 & $04-07-26$ & 21,000 & & 1964 & $09-15-64$ & 3,620 & \\
\hline 1927 & $02-18-27$ & 40,000 & & 1965 & $01-08-65$ & 20,400 & \\
\hline 1928 & $02-05-28$ & 2,600 & & 1966 & $12-23-65$ & 68,800 & \\
\hline 1929 & $09-23-29$ & 15,000 & & 1967 & $08-06-67$ & 5,600 & \\
\hline 1930 & $03-17-30$ & 8,300 & & 1968 & $12-20-67$ & 17,200 & \\
\hline 1931 & $02-15-31$ & 22,000 & & 1969 & $01-26-69$ & 6,100 & \\
\hline 1932 & $02-10-32$ & 57,000 & & 1970 & $09-06-70$ & 17,300 & \\
\hline 1933 & $02-28-33$ & 4,200 & & 1971 & $08-13-71$ & 12,800 & \\
\hline 1934 & $08-04-34$ & 5,500 & & 1972 & $12-27-71$ & 30,200 & \\
\hline 1935 & $04-09-35$ & 15,200 & & 1973 & $10-20-72$ & 70,000 & \\
\hline 1936 & $02-17-36$ & 13,800 & & 1974 & $07-20-74$ & 1,500 & \\
\hline 1937 & $02-07-37$ & 88,000 & & 1975 & $10-29-74$ & 10,100 & \\
\hline 1938 & $03-04-38$ & 24,100 & & 1976 & $02-10-76$ & 16,000 & \\
\hline 1939 & $04-05-39$ & 9,050 & & 1977 & $09-03-77$ & 10,200 & \\
\hline 1940 & $07-16-40$ & 4,610 & & 1978 & $03-02-78$ & 89,400 & \\
\hline 1941 & $03-14-41$ & 117,000 & & 1979 & $12-19-78$ & 95,800 & \\
\hline 1942 & $01-13-42$ & 5,140 & & 1980 & $02-15-80$ & 99,000 & \\
\hline 1943 & $03-05-43$ & 16,500 & & 1981 & $04-15-81$ & 2,550 & \\
\hline 1944 & $09-26-44$ & 4,560 & & 1982 & $02-12-82$ & 15,200 & \\
\hline 1945 & $03-27-45$ & 5,450 & & 1983 & $03-25-83$ & 17,600 & \\
\hline 1946 & $09-19-46$ & 15,100 & & 1984 & $10-02-83$ & 59,800 & \\
\hline 1947 & $09-19-47$ & 6,170 & & 1985 & $12-28-84$ & 46,600 & \\
\hline 1948 & $04-13-48$ & 5,960 & & 1986 & $02-16-86$ & 13,300 & \\
\hline 1949 & $01-14-49$ & 15,500 & & 1987 & $03-05-87$ & 7,560 & \\
\hline 1950 & $07-21-50$ & 5,930 & & 1988 & $09-01-88$ & 11,000 & \\
\hline 1951 & $08-28-51$ & 27,600 & & 1989 & $03-13-89$ & 2,040 & \\
\hline 1952 & $01-18-52$ & 111,000 & & 1990 & $07-22-90$ & 6,710 & \\
\hline 1953 & $03-09-53$ & 4,320 & & 1991 & $03-01-91$ & 71,300 & \\
\hline 1954 & $03-23-54$ & 40,800 & & 1992 & $02-14-92$ & 24,600 & \\
\hline 1955 & $08-24-55$ & 8,640 & & 1993 & 01-08-93 & ${ }^{2} 143,000$ & \\
\hline 1956 & $01-29-56$ & 1,460 & & 1994 & $03-21-94$ & 7,930 & \\
\hline 1957 & $01-10-57$ & 6,720 & & 1995 & 03-06-95 & 38,000 & \\
\hline 1958 & $03-23-58$ & 24,000 & & 1996 & $09-14-96$ & 6,610 & \\
\hline 1959 & $08-20-59$ & 12,100 & & & & & \\
\hline
\end{tabular}

${ }^{\mathrm{T}}$ Partridge and Baker (1987).

${ }^{2}$ Highest since 1906. 
09498500 SALT RIVER NEAR ROOSEVELT, AZ--Continued

Discharge rating table developed March 1995

\begin{tabular}{cccc}
\hline $\begin{array}{c}\text { Gage height } \\
(\mathbf{f t})\end{array}$ & $\begin{array}{c}\text { Discharge } \\
\left(\mathbf{f t}^{\mathbf{3}} \mathbf{s} \mathbf{s}\right)\end{array}$ & $\begin{array}{c}\text { Gage height } \\
(\mathbf{f t})\end{array}$ & $\begin{array}{c}\text { Discharge } \\
\left(\mathbf{f t}^{3} / \mathbf{s}\right)\end{array}$ \\
\hline 8.0 & 709 & 20.0 & 42,580 \\
10.0 & 3,040 & 22.0 & 56,410 \\
12.0 & 7,150 & 24.0 & 72,290 \\
14.0 & 13,100 & 26.0 & 90,250 \\
16.0 & 20,960 & 28.0 & 110,300 \\
18.0 & 30,780 & 30.0 & 132,500 \\
\hline
\end{tabular}

Basin characteristics

\begin{tabular}{cccccccc}
\hline $\begin{array}{c}\text { Main } \\
\text { channel } \\
\text { slope } \\
(\mathbf{f t} / \mathrm{mi})\end{array}$ & $\begin{array}{c}\text { Stream } \\
\text { length } \\
\text { (mi) }\end{array}$ & $\begin{array}{c}\text { Mean } \\
\text { basin } \\
\text { elevation } \\
\text { (ft) }\end{array}$ & $\begin{array}{c}\text { Forested } \\
\text { area } \\
\text { (percent) }\end{array}$ & $\begin{array}{c}\text { Soil } \\
\text { index }\end{array}$ & $\begin{array}{c}\text { Mean } \\
\text { annual } \\
\text { precipitation } \\
\text { (in) }\end{array}$ & $\begin{array}{c}\text { Rainfall intensity, 24-hour } \\
\text { (in) }\end{array}$ & $\begin{array}{c}50 \text {-year } \\
\text { (in) }\end{array}$ \\
\hline 23.3 & 206 & 6,190 & 71.0 & 2.8 & 22.0 & 2.3 \\
\hline
\end{tabular}


09498500 SALT RIVER NEAR ROOSEVELT, AZ--Continued

MEAN MONTHLY AND ANNUAL DISCHARGES 1914.96

\begin{tabular}{|c|c|c|c|c|c|c|}
\hline MONTH & $\begin{array}{l}\text { MAXIMUM } \\
(\text { FT3/S) }\end{array}$ & $\begin{array}{l}\text { MINIMUM } \\
\text { (FT3/S) }\end{array}$ & $\begin{array}{c}\text { MEAN } \\
(\text { FT } 3 / S)\end{array}$ & $\begin{array}{l}\text { STAN- } \\
\text { DARD } \\
\text { DEVIA- } \\
\text { TION } \\
\text { (FT3/S) }\end{array}$ & $\begin{array}{l}\text { COEFFI- } \\
\text { CIENT OF } \\
\text { VARI- } \\
\text { ATION }\end{array}$ & $\begin{array}{c}\text { PERCENT } \\
\text { OF } \\
\text { ANNUAL } \\
\text { RUNOFF }\end{array}$ \\
\hline OCTOBER & 4,830 & 86 & 443 & 750 & 1.7 & 4.0 \\
\hline NOVEMBER & 2,150 & 122 & 384 & 360 & 0.94 & 3.5 \\
\hline DECEMBER & 6,330 & 127 & 789 & 1,220 & 1.6 & 7.1 \\
\hline JANUARY & 16,000 & 161 & 1.130 & 2,380 & 2.1 & 10 \\
\hline FEBRUARY & 9,070 & 168 & 1,440 & 1,860 & 1.3 & 13 \\
\hline MARCH & 10,400 & 220 & 2.050 & 1,980 & 0.96 & 19 \\
\hline APRIL & 6,280 & 198 & 2.040 & 1,560 & 0.77 & 18 \\
\hline MAY & 5,930 & 127 & 1,050 & 1,020 & 0.97 & 9.5 \\
\hline JUNE & 1,370 & 79 & 372 & 296 & 0.80 & 3.4 \\
\hline JULY & 3,280 & 78 & 335 & 375 & 1.1 & 3.0 \\
\hline AUGUST & 3,610 & 151 & 589 & 476 & 0.81 & 5.3 \\
\hline SEPTEMBER & 1.850 & 78 & 459 & 377 & 0.73 & 4.1 \\
\hline ANNUAL & 130 & 6.7 & 921 & 686 & 0.75 & 100 \\
\hline
\end{tabular}

MAGNITUDE AND PROBABILITY OF INSTANTANEOUS PEAK FLOW BASED ON PERIOD OF RECORD $1388,1924.96$

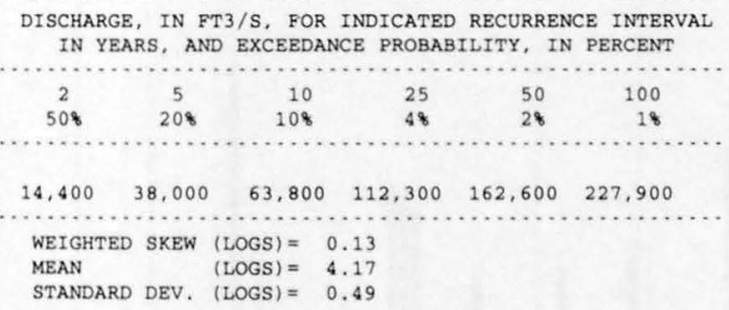

DURATION TABLE OF DAILY MEAN FLOW FOR PERIOD OF RECORD 1914-96
MAGNITUDE AND PROBABILITY OF ANNUAL LOW FLOW BASED ON PERIOD OF RECORD 1915.96

\begin{tabular}{|c|c|c|c|c|c|c|}
\hline PERIOD & \multirow{2}{*}{\multicolumn{6}{|c|}{$\begin{array}{r}\text { DISCHARGE, IN FT3/S, FOR INDICATED } \\
\text { RECURRENCE INTERVAL, IN YEARS, AND } \\
\text { NON-EXCEEDANCE PROBABILITY, IN PERCENT }\end{array}$}} \\
\hline $\begin{array}{l}1 \mathrm{CON}- \\
\mathrm{SECU} \text { - }\end{array}$ & & & & & & \\
\hline TIVE & 2 & 5 & $10 \#$ & $20 \#$ & $50 \#$ & $100=$ \\
\hline DAYS) & 508 & 208 & 108 & 58 & 28 & 18 \\
\hline 1 & 128 & 94 & 80 & 69 & 59 & 53 \\
\hline 3 & 131 & 96 & 81 & 71 & 60 & 54 \\
\hline 7 & 136 & 99 & 84 & 73 & 62 & 56 \\
\hline 14 & 145 & 105 & 89 & 77 & 66 & 59 \\
\hline 30 & 161 & 117 & 99 & 86 & 73 & 66 \\
\hline 60 & 189 & 139 & 118 & 104 & 90 & 82 \\
\hline 90 & 215 & 163 & 142 & 128 & 114 & 106 \\
\hline 120 & 237 & 179 & 159 & 146 & 134 & 127 \\
\hline 183 & 289 & 211 & 185 & 169 & 154 & 147 \\
\hline
\end{tabular}

MAGNITUDE AND PROBABILITY OF ANNUAL HIGH FLOW BASED ON PERIOD OF RECORD 1914.96

PERIOD
(CON

DISCHARGE, IN FT3/S, WHICH WAS EQUALED OR EXCEEDED FOR INDICATED PERCENT OF TIME

\begin{tabular}{|c|c|c|c|c|c|c|c|c|c|c|c|c|c|c|c|c|}
\hline 18 & 58 & 108 & 158 & 208 & 308 & 408 & 508 & 608 & 708 & 808 & 908 & 958 & 988 & 998 & 99.58 & 99.98 \\
\hline 100 & 3,510 & 2,120 & 1,420 & 1,040 & 646 & 453 & 341 & 275 & 234 & 198 & 159 & 130 & 0.00 & 0.00 & 0.00 & 0.00 \\
\hline
\end{tabular}

\# Reliability of values in column is uncertain, and potential errors are large. 
ANNUAL MEAN DISCHARGE, IN CUBIC FEET PER SECOND

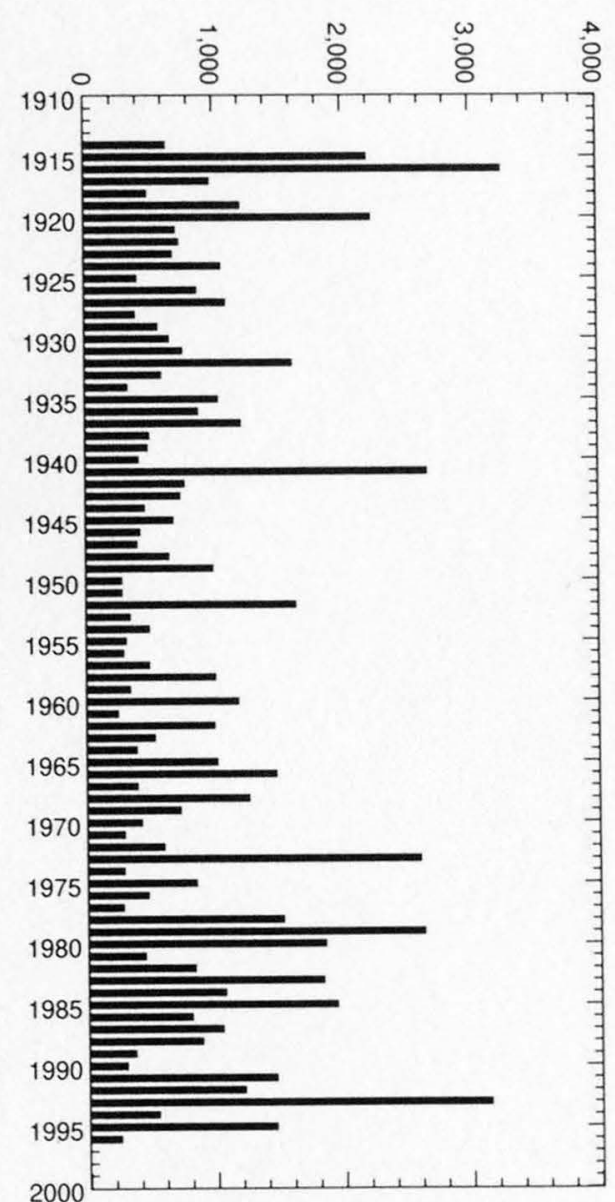

ANNUAL PEAK DISCHARGE IN CUBIC

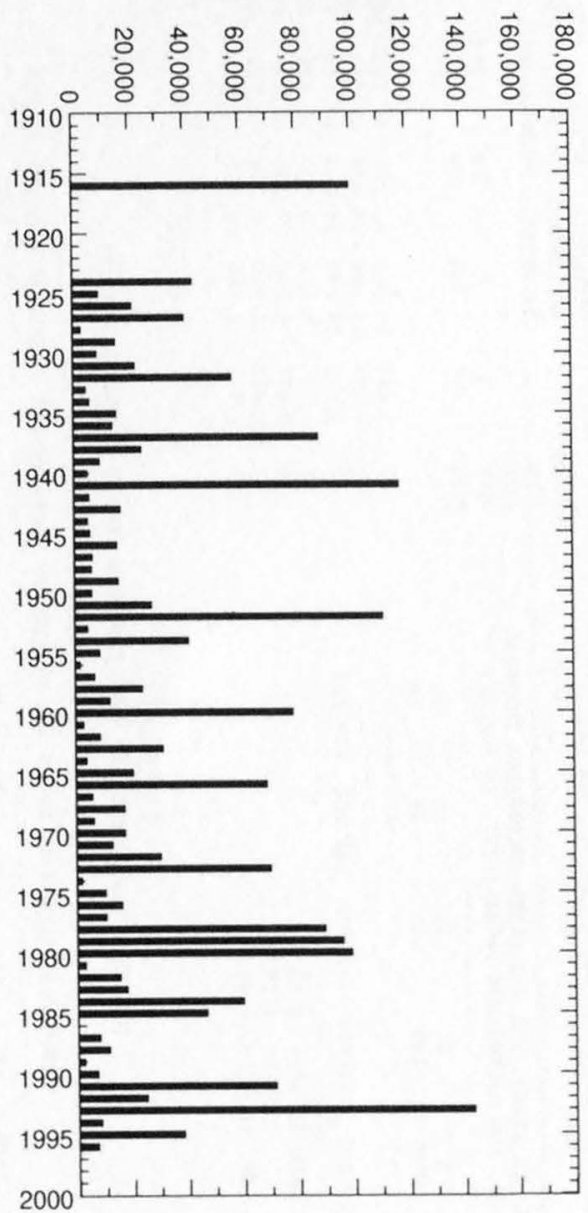

ANNUAL PEAK DISCHARGE IN CUBIC

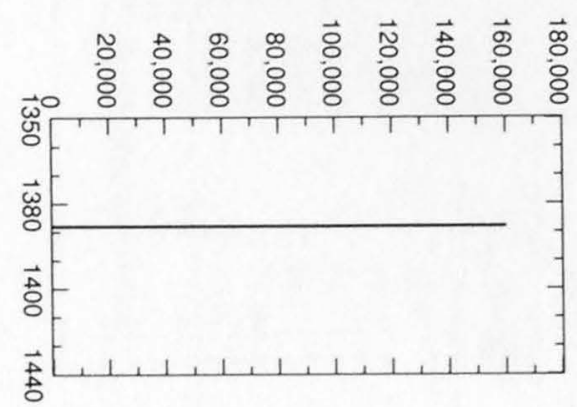


09498500 SALT RIVER NEAR ROOSEVELT, AZ--Continued

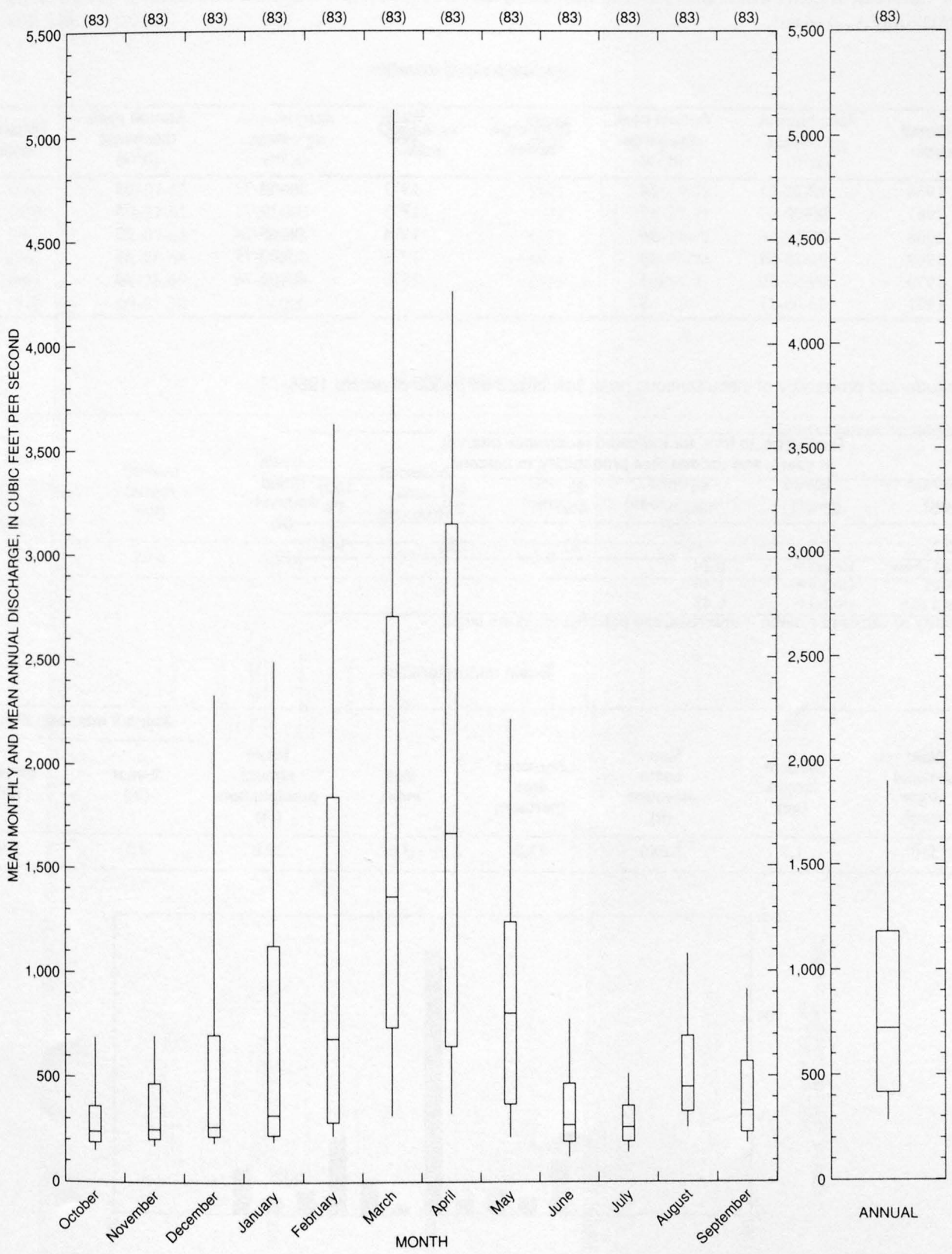


09498600 CHRISTOPHER CREEK TRIBUTARY NEAR KOHL'S RANCH, AZ

LOCATION.--Lat $34^{\circ} 19^{\prime} 20^{\prime \prime}$, long $111^{\circ} 04^{\prime} 00^{\prime \prime}$, in NE $\frac{1}{4} \mathrm{SE}^{1} / 4$ sec.22, T.11 N., R.12 E., Gila County, Hydrologic Unit 15060105, at State Highway $160,1.8 \mathrm{mi}$ east of Kohl's Ranch, and $15.5 \mathrm{mi}$ northeast of Payson.

DRAINAGE AREA.--0.66 $\mathrm{mi}^{2}$.

Annual peak discharges

\begin{tabular}{|c|c|c|c|c|c|c|c|}
\hline $\begin{array}{l}\text { Water } \\
\text { year }\end{array}$ & Date & $\begin{array}{c}\text { Annual peak } \\
\text { discharge } \\
\left(\mathrm{ft}^{3} / \mathrm{s}\right)\end{array}$ & $\begin{array}{c}\text { Discharge } \\
\text { codes }\end{array}$ & $\begin{array}{l}\text { Water } \\
\text { year }\end{array}$ & Date & $\begin{array}{c}\text { Annual peak } \\
\text { discharge } \\
\left(\mathrm{ft}^{3} / \mathrm{s}\right)\end{array}$ & $\begin{array}{c}\text { Discharge } \\
\text { codes }\end{array}$ \\
\hline 1966 & $12-22-65$ & 54 & & 1972 & $08-06-72$ & 35 & \\
\hline 1967 & $00-00-67$ & 17 & & 1973 & $10-19-72$ & 75 & \\
\hline 1968 & $08-10-68$ & 26 & & 1974 & $08-05-74$ & 22 & \\
\hline 1969 & $01-26-69$ & 14 & & 1975 & $07-00-75$ & 16 & \\
\hline 1970 & $09-05-70$ & 265 & & 1976 & $02-09-76$ & 44 & \\
\hline 1971 & $08-00-71$ & 8 & & & & & \\
\hline
\end{tabular}

Magnitude and probability of instantaneous peak flow based on period of record 1966-76

\begin{tabular}{|c|c|c|c|c|c|}
\hline \multicolumn{6}{|c|}{$\begin{array}{l}\text { Discharge, in } \mathrm{ft}^{3} / \mathrm{s} \text {, for indicated recurrence interval } \\
\text { in years, and exceedance probablility, in percent }\end{array}$} \\
\hline 2 & 5 & 10 & 25 & $50 t$ & $100+$ \\
\hline $50 \%$ & $20 \%$ & $10 \%$ & $4 \%$ & $2 \%$ & $1 \%$ \\
\hline 30.2 & 69.5 & 110 & 182 & 256 & 348 \\
\hline Weighted skew & $(\log s)=$ & 0.24 & & & 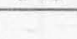 \\
\hline Mean & $(\log s)=$ & 1.50 & & & \\
\hline Standard dev. & $(\log s)=$ & 0.42 & & & \\
\hline
\end{tabular}

Basin characteristics

\begin{tabular}{cccccccc}
\hline $\begin{array}{c}\text { Main } \\
\text { channel } \\
\text { slope } \\
(\mathrm{ft} / \mathrm{mi})\end{array}$ & $\begin{array}{c}\text { Stream } \\
\text { length } \\
(\mathrm{mi})\end{array}$ & $\begin{array}{c}\text { Mean } \\
\text { basin } \\
\text { elevation } \\
(\mathrm{ft})\end{array}$ & $\begin{array}{c}\text { Forested } \\
\text { area } \\
\text { (percent) }\end{array}$ & $\begin{array}{c}\text { Soil } \\
\text { index }\end{array}$ & $\begin{array}{c}\text { Mean } \\
\text { annual } \\
\text { precipitation } \\
\text { (in) }\end{array}$ & $\begin{array}{c}\text { Rainfall intensity, 24-hour } \\
\text { 2-year } \\
\text { (in) }\end{array}$ & $\begin{array}{c}50 \text {-year } \\
\text { (in) }\end{array}$ \\
\hline 510 & 1.7 & 6,080 & 73.0 & 3.0 & 29.0 & 3.5 \\
\hline
\end{tabular}

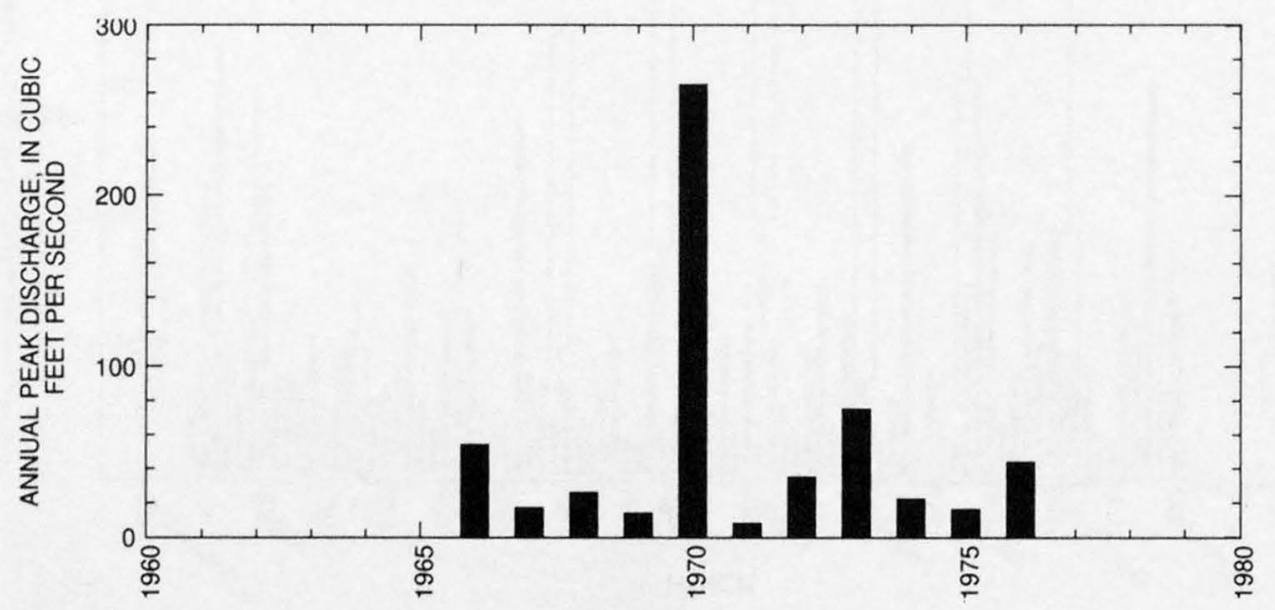


09498800 TONTO CREEK NEAR GISELA, AZ

LOCATION.--Lat $34^{\circ} 07^{\prime} 44^{\prime \prime}$, long $111^{\circ} 15^{\prime} 17^{\prime \prime}$, in NE $\frac{1}{4} \sec ^{2}$ 18, T.9 N., R.11 E., Gila County, Hydrologic Unit 15060105, in Tonto National Forest, on left bank 0.2 mi upstream from Houston Creek, and 1.5 mi northeast of Gisela.

DRAINAGE AREA.--430 $\mathrm{mi}^{2}$.

Annual peak discharges

\begin{tabular}{|c|c|c|c|c|c|c|c|}
\hline $\begin{array}{l}\text { Water } \\
\text { year }\end{array}$ & Date & $\begin{array}{c}\text { Annual peak } \\
\text { discharge } \\
\left(\mathrm{ft}^{3} / \mathbf{s}\right)\end{array}$ & $\begin{array}{l}\text { Discharge } \\
\text { codes }\end{array}$ & $\begin{array}{c}\text { Water } \\
\text { year }\end{array}$ & Date & $\begin{array}{c}\text { Annual peak } \\
\text { discharge } \\
\left(\mathrm{ft}^{3} / \mathbf{s}\right)\end{array}$ & $\begin{array}{c}\text { Discharge } \\
\text { codes }\end{array}$ \\
\hline 1965 & $01-07-65$ & 12,600 & & 1971 & $08-19-71$ & 3,300 & \\
\hline 1966 & $12-22-65$ & 30,000 & & 1972 & $10-17-71$ & 2,750 & \\
\hline 1967 & $12-07-66$ & 8,280 & & 1973 & $10-19-72$ & 26,500 & \\
\hline 1968 & $01-28-68$ & 14,800 & & 1974 & $08-06-74$ & 2,400 & \\
\hline 1969 & $01-26-69$ & 10,100 & & 1975 & $10-29-74$ & 1,860 & \\
\hline 1970 & $09-05-70$ & 38,000 & & & & & \\
\hline
\end{tabular}

Basin characteristics

\begin{tabular}{ccccccccc}
\hline $\begin{array}{c}\text { Main } \\
\text { channel } \\
\text { slope } \\
(\mathrm{ft} / \mathrm{mi})\end{array}$ & $\begin{array}{c}\text { Stream } \\
\text { length }\end{array}$ & $\begin{array}{c}\text { Mean } \\
\text { (mi) }\end{array}$ & $\begin{array}{c}\text { basin } \\
\text { elevation } \\
\text { (ft) }\end{array}$ & $\begin{array}{c}\text { Forested } \\
\text { area } \\
\text { (percent) }\end{array}$ & $\begin{array}{c}\text { Soil } \\
\text { index }\end{array}$ & $\begin{array}{c}\text { Mean } \\
\text { annual } \\
\text { precipitation } \\
\text { (in) }\end{array}$ & $\begin{array}{c}\text { 2-year } \\
\text { (in) }\end{array}$ & $\begin{array}{c}\text { 50-year } \\
\text { (in) }\end{array}$ \\
\hline 126 & 29.6 & 5,810 & 79.0 & 3.0 & 24.7 & 2.8 & 5.5 \\
\hline
\end{tabular}




\section{TONTO CREEK NEAR GISELA, AZ--Continued}

MEAN MONTHLY AND ANNUAL DISCHARGES 1966.75

\begin{tabular}{|c|c|c|c|c|c|c|}
\hline MONTH & $\begin{array}{l}\text { MAXIMUM } \\
(\text { FT3/S) }\end{array}$ & $\begin{array}{l}\text { MINIMUM } \\
\text { (FT3/S) }\end{array}$ & $\begin{array}{c}\text { MEAN } \\
(\mathrm{FT} 3 / \mathrm{S})\end{array}$ & $\begin{array}{l}\text { STAN- } \\
\text { DARD } \\
\text { DEVIA- } \\
\text { TION } \\
\text { (FT3/S) }\end{array}$ & $\begin{array}{l}\text { COEFFI- } \\
\text { CIENT OF } \\
\text { VARI- } \\
\text { ATION }\end{array}$ & $\begin{array}{c}\text { PERCENT } \\
\text { OF } \\
\text { ANNUAL } \\
\text { RUNOFF }\end{array}$ \\
\hline OCTOBER & 761 & 7.8 & 118 & 238 & 2.0 & 8.2 \\
\hline NOVEMBER & 326 & 11 & 67 & 97 & 1.5 & 4.6 \\
\hline DECEMBER & 1.970 & 16 & 344 & 603 & 1.8 & 23.9 \\
\hline JANUARY & 602 & 15 & 182 & 227 & 1.2 & 12.7 \\
\hline FEBRUARY & 753 & 15 & 168 & 248 & 1.5 & 11.7 \\
\hline MARCH & 1,160 & 15 & 242 & 343 & 1.4 & 16.8 \\
\hline APRIL & 568 & 11 & 132 & 186 & 1.4 & 9.2 \\
\hline MAY & 235 & 6.8 & 46 & 68 & 1.5 & 3.2 \\
\hline JUNE & 39 & 3.3 & 14 & 9.6 & 0.68 & 1.0 \\
\hline JULY & 49 & 8.3 & 22 & 13 & 0.59 & 1.5 \\
\hline AUGUST & 103 & 6.7 & 40 & 30 & 0.77 & 2.7 \\
\hline SEPTEMBER & 481 & 8.1 & 64 & 147 & 2.3 & 4.5 \\
\hline ANNUAL & 377 & 24 & 120 & 112 & 0.93 & 100 \\
\hline
\end{tabular}

MAGNITUDE AND PROBABILITY OF ANNUAL LOW FLOW BASED ON PERIOD OF RECORD 1966-75

\begin{tabular}{|c|c|c|c|c|c|c|}
\hline $\begin{array}{l}\text { PERIOD } \\
(\mathrm{CON} \text {. }\end{array}$ & \multicolumn{6}{|c|}{$\begin{array}{l}\text { DISCHARGE, IN FT3/S, FOR INDICATED } \\
\text { RECURRENCE INTERVAL, IN YEARS, AND } \\
\text { NON-EXCEEDANCE PROBABILITY, IN PERCENT }\end{array}$} \\
\hline SECU. & $\cdots$ & $\ldots \ldots$ & $\ldots$. & $\ldots \ldots$ & $\ldots$. & $\ldots$ \\
\hline TIVE & 2 & 5 & 10 & 20 & $50 \#$ & $100 \#$ \\
\hline DAYS) & 508 & 208 & 108 & 58 & 28 & 18 \\
\hline 1 & 5.7 & 3.4 & 2.5 & 1.9 & 1.3 & 1.0 \\
\hline 3 & 5.8 & 3.6 & 2.6 & 2.0 & 1.4 & 1.1 \\
\hline 7 & 6.1 & 3.8 & 2.8 & 2.1 & 1.5 & 1.1 \\
\hline 14 & 6.8 & 4.2 & 3.0 & 2.3 & 1.5 & 1.2 \\
\hline 30 & 8.0 & 5.1 & 3.6 & 2.6 & 1.7 & 1.2 \\
\hline 60 & 9.6 & 6.6 & 5.0 & 3.9 & 2.7 & 2.1 \\
\hline 90 & 13 & 9.2 & 7.4 & 6.1 & 4.6 & 3.8 \\
\hline 120 & 15 & 11 & 9.2 & 7.7 & 6.2 & 5.3 \\
\hline 183 & 20 & 15 & 12 & 10 & 8.4 & 7.1 \\
\hline
\end{tabular}

MAGNITUDE AND PROBABILITY OF ANNUAL HIGH FLOW BASED ON PERIOD OF RECORD 1966.75

MAGNITUDE AND PROBABILITY OF INSTANTANEOUS PEAK FLOW BASED ON PERIOD OF RECORD 1965.75

DISCHARGE, IN FT3/S, FOR INDICATED RECURRENCE INTERVAL IN YEARS, AND EXCEEDANCE PROBABILITY, IN PERCEN

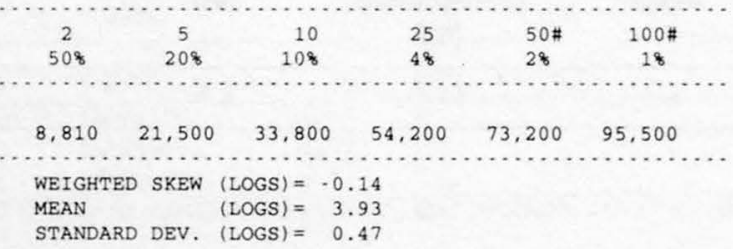

DURATION TABLE OF DAILY MEAN FLOW FOR PERIOD OF RECORD 1966.75

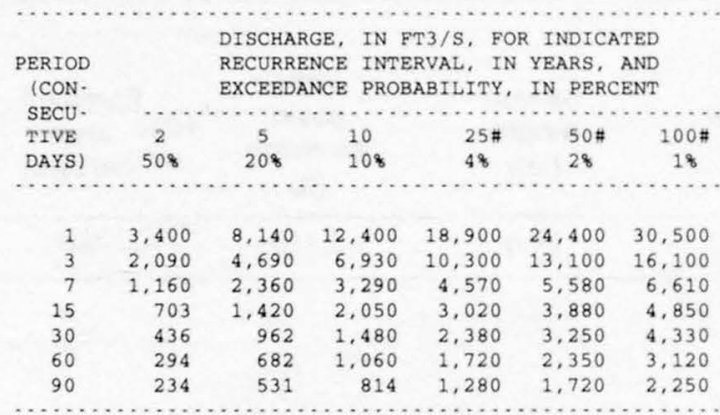

\begin{tabular}{|c|c|c|c|c|c|c|c|c|c|c|c|c|c|c|c|c|}
\hline 18 & 58 & 108 & 158 & 208 & 308 & 408 & 508 & 608 & 708 & $80 \%$ & 908 & 958 & 988 & 998 & 99.58 & 99.98 \\
\hline & 454 & 214 & 130 & 85 & 41 & 27 & 21 & 17 & 14 & 11 & 8.4 & 6.2 & 4.3 & 3.7 & 2.2 & 1.7 \\
\hline
\end{tabular}

\# Reliability of values in column is uncertain, and potential errors are large. 


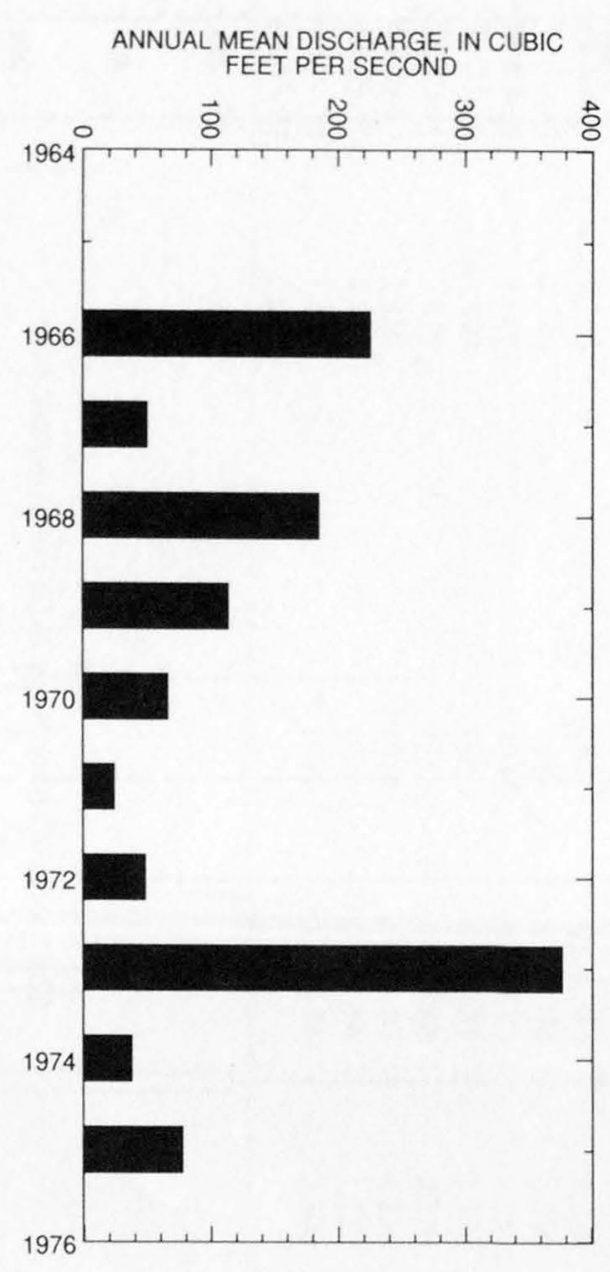

ANNUAL PEAK DISCHARGE, IN CUBIC

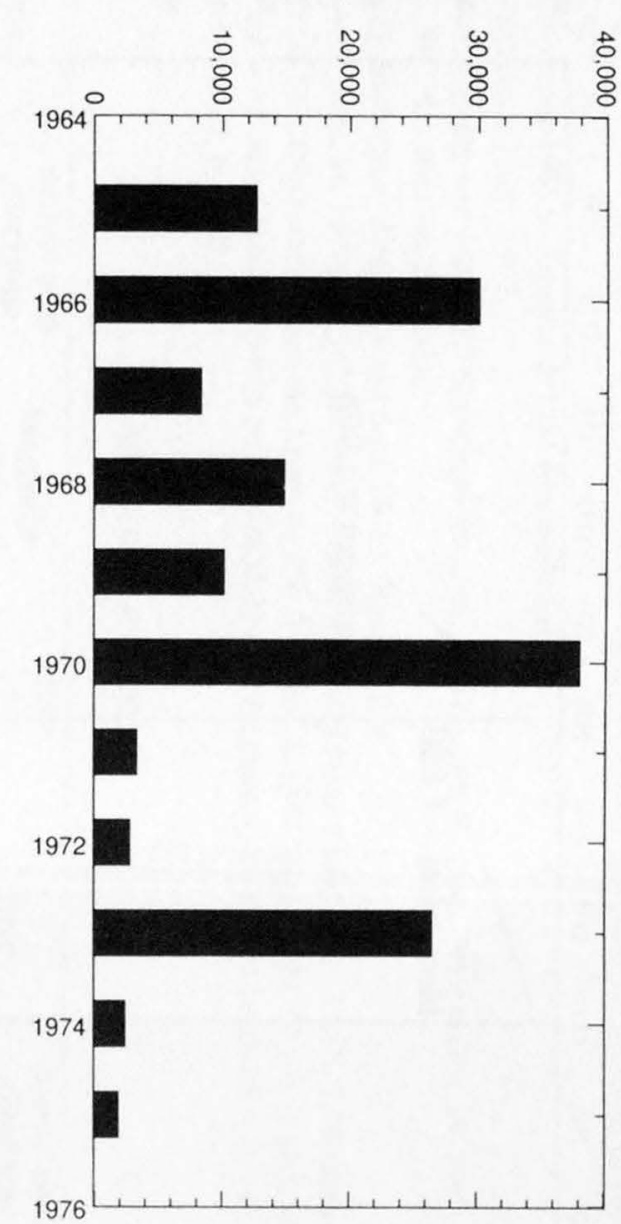


09498800 TONTO CREEK NEAR GISELA, AZ--Continued
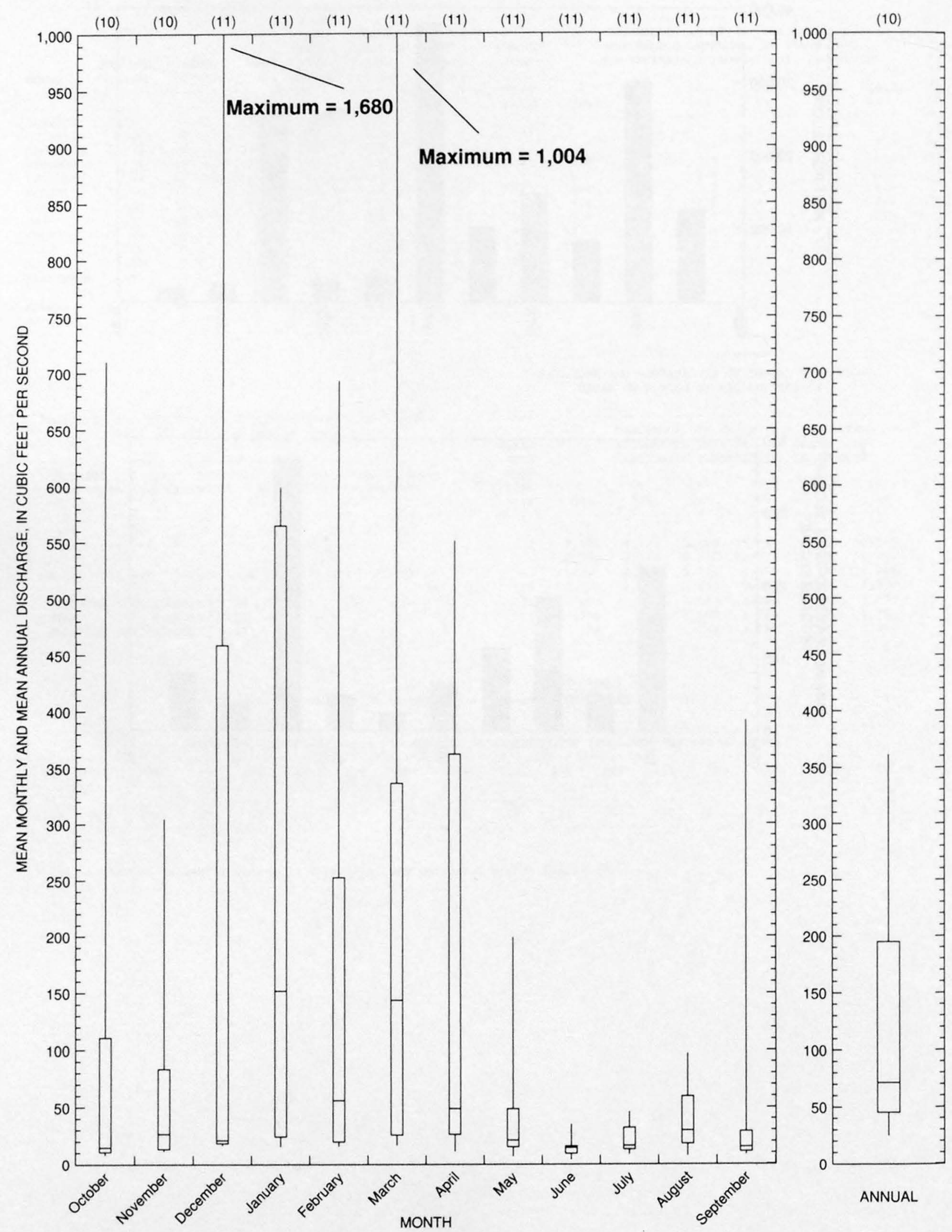


\section{RYE CREEK NEAR GISELA, AZ}

LOCATION.--Lat 34 01'57", long $111^{\circ} 17^{\prime} 26^{\prime \prime}$, in SW $1 / 4$ sec.13, T.8 N., R.10 E., Gila County, Hydrologic Unit 15060105 , in Tonto National Forest, on right bank, $0.5 \mathrm{mi}$ upstream from mouth, $0.8 \mathrm{mi}$ downstream from bridge on county road, and $4.8 \mathrm{mi}$ south of Gisela.

DRAINAGE AREA.--122 $\mathrm{mi}^{2}$.

PERIOD OF RECORD.--December 1965 to September 1985 (discontinued).

REVISED RECORDS.--WRD Ariz. 1969: 1967.

GAGE.--Water-stage recorder. Elevation of gage is 2,730 ft above sea level, from topographic map. Prior to Dec. 19, 1967, at datum 1.00 $\mathrm{ft}$ higher;

Dec. 19,1967 to Sept. 30,1983 at datum 2.0 higher.

REMARKS.--Estimated daily discharges: Aug. 3-23 and Sept. 18-30. Records fair.

AVERAGE DISCHARGE.--19 years, $26.7 \mathrm{ft}^{3} / \mathrm{s}, 19,340$ acre-ft/yr; median of yearly mean discharges, $13 \mathrm{ft}^{3} / \mathrm{s}, 9,400 \mathrm{acre}-\mathrm{ft}^{2} / \mathrm{rr}$.

EXTREMES FOR PERIOD OF RECORD.--Maximum discharge, $44,400 \mathrm{ft}^{3} / \mathrm{s} \mathrm{Sept.} \mathrm{5,} \mathrm{1970,} \mathrm{gage} \mathrm{height,} 14.1 \mathrm{ft}$, in gage well, $18.7 \mathrm{ft}$, from profile past gage, from rating curve extended above $850 \mathrm{ft}^{3} / \mathrm{s}$ on basis of slope-area measurements at gage heights 9.0 and $14.1 \mathrm{ft}$, datum then in use; minimum daily, $0.20 \mathrm{ft}^{3} / \mathrm{s}$ July $5,6,1970$.

Annual peak discharges

\begin{tabular}{ccccccc}
\hline $\begin{array}{l}\text { Water } \\
\text { year }\end{array}$ & Date & $\begin{array}{c}\text { Annual peak } \\
\text { discharge } \\
\left(\mathrm{ft}^{3} / \mathbf{s}\right)\end{array}$ & $\begin{array}{c}\text { Discharge } \\
\text { codes }\end{array}$ & $\begin{array}{c}\text { Water } \\
\text { year }\end{array}$ & $\begin{array}{c}\text { Annual peak } \\
\text { discharge } \\
\left(\mathrm{ft}^{3} / \mathbf{s}\right)\end{array}$ & $\begin{array}{c}\text { Discharge } \\
\text { codes }\end{array}$ \\
\hline 1963 & $08-22-63$ & 10,000 & ES,HP & 1976 & $02-09-76$ & 2,700 \\
1966 & $12-22-65$ & 18,130 & & 1977 & $08-15-77$ & 3,020 \\
1967 & $08-09-67$ & 5,290 & & 1978 & $03-02-78$ & 8,220 \\
1968 & $12-19-67$ & 2,520 & & 1979 & $01-17-79$ & 5,230 \\
1969 & $07-25-69$ & 2,080 & & 1980 & $02-19-80$ & 4,550 \\
1970 & $09-05-70$ & 244,400 & & 1981 & $08-01-81$ & 1,530 \\
1971 & $08-19-71$ & 810 & & 1982 & $08-11-82$ & 5,220 \\
1972 & $09-02-72$ & 1,350 & & 1983 & $09-30-83$ & 4,280 \\
1973 & $10-07-72$ & 4,250 & & 1984 & $09-10-84$ & 1,710 \\
1974 & $07-07-74$ & 1,450 & & 1985 & $12-27-84$ & 2,300 \\
1975 & $07-08-75$ & 1,020 & & & & \\
\hline
\end{tabular}

THighest since 1963.

${ }^{2}$ Highest since 1952.

Discharge rating table developed October 1983

\begin{tabular}{crcc}
\hline $\begin{array}{c}\text { Gage height } \\
(\mathbf{f t})\end{array}$ & $\begin{array}{c}\text { Discharge } \\
\left(\mathrm{ft}^{3} / \mathbf{s}\right)\end{array}$ & $\begin{array}{c}\text { Gage height } \\
(\mathbf{f t})\end{array}$ & $\begin{array}{c}\text { Discharge } \\
\left(\mathrm{ft}^{3} / \mathbf{s}\right)\end{array}$ \\
\hline 4.0 & 565 & 11.0 & 13,970 \\
5.0 & 1,170 & 12.0 & 18,230 \\
6.0 & 2,110 & 13.0 & 23,250 \\
7.0 & 3,440 & 14.0 & 29,110 \\
8.0 & 5,230 & 15.0 & 35,870 \\
9.0 & 7,540 & 16.0 & 43,570 \\
10.0 & 10,430 & 16.1 & 44,400 \\
\hline
\end{tabular}

Basin characteristics

\begin{tabular}{cccccccc}
$\begin{array}{c}\text { Main } \\
\text { channel } \\
\text { slope } \\
\text { (ft/mi) }\end{array}$ & $\begin{array}{c}\text { Stream } \\
\text { length } \\
(\mathrm{mi})\end{array}$ & $\begin{array}{c}\text { Mean } \\
\text { basin } \\
\text { elevation } \\
(\mathrm{ft})\end{array}$ & $\begin{array}{c}\text { Forested } \\
\text { area } \\
\text { (percent) }\end{array}$ & $\begin{array}{c}\text { Soil } \\
\text { index }\end{array}$ & $\begin{array}{c}\text { Mean } \\
\text { annual } \\
\text { precipitation } \\
\text { (in) }\end{array}$ & $\begin{array}{c}\text { Rainfall intensity, 24-hour } \\
\text { (in) }\end{array}$ & $\begin{array}{c}50 \text {-year } \\
\text { (in) }\end{array}$ \\
\hline 91.4 & 15.6 & 4,390 & 21.0 & 3.0 & 24.2 & 2.8 \\
\hline
\end{tabular}


09498870 RYE CREEK NEAR GISELA, AZ--Continued

MEAN MONTHLY AND ANNUAL DISCHARGES 1967.85

\begin{tabular}{|c|c|c|c|c|c|c|}
\hline MONTH & $\begin{array}{l}\text { MAXIMUM } \\
\text { (FT3/S) }\end{array}$ & $\begin{array}{l}\text { MINIMUM } \\
\text { (FT3/S) }\end{array}$ & $\begin{array}{c}\text { MEAN } \\
(\mathrm{FT} 3 / \mathrm{S})\end{array}$ & $\begin{array}{l}\text { STAN- } \\
\text { DARD } \\
\text { DEVIA- } \\
\text { TION } \\
\text { (FT3/S) }\end{array}$ & $\begin{array}{l}\text { COEFFI- } \\
\text { CIENT OF } \\
\text { VARI- } \\
\text { ATION }\end{array}$ & $\begin{array}{c}\text { PERCENT } \\
\text { OF } \\
\text { ANNUAL } \\
\text { RUNOFF }\end{array}$ \\
\hline OCTOBER & 89 & 0.74 & 10 & 21 & 2.0 & 3.2 \\
\hline NOVEMBER & 71 & 0.69 & 11 & 21 & 1.9 & 3.4 \\
\hline DECEMBER & 171 & 0.71 & 36 & 55 & 1.5 & 11.3 \\
\hline JANUARY & 222 & 1.0 & 41 & 61 & 1.5 & 12.7 \\
\hline FEBRUARY & 495 & 1.0 & 80 & 121 & 1.5 & 24.7 \\
\hline MARCH & 608 & 1.2 & 90 & 148 & 1.7 & 27.9 \\
\hline APRIL & 94 & 1.3 & 22 & 27 & 1.2 & 6.9 \\
\hline MAY & 18 & 0.99 & 6.3 & 5.3 & 0.84 & 2.0 \\
\hline JUNE & 6.5 & 0.81 & 3.2 & 1.8 & 0.56 & 1.0 \\
\hline JULY & 12 & 0.63 & 4.5 & 2.7 & 0.60 & 1.4 \\
\hline AUGUST & 22 & 1.0 & 7.1 & 5.6 & 0.78 & 2.2 \\
\hline SEPTEMBER & 97 & 1.1 & 11 & 22 & 2.1 & 3.3 \\
\hline ANNUAL & 77 & 1.6 & 27 & 27 & 1.0 & 100 \\
\hline
\end{tabular}

MAGNITUDE AND PROBABILITY OF INSTANTANEOUS PEAK FLOW BASED ON PERIOD OF RECORD 1963, 1966-85

DISCHARGE, IN FT3/S, FOR INDICATED RECURRENCE INTERVAL IN YEARS, AND EXCEEDANCE PROBABILITY, IN PERCENT

\begin{tabular}{|c|c|c|c|c|c|}
\hline 2 & 5 & 10 & 25 & $50 \#$ & $100 \#$ \\
\hline 508 & 208 & 108 & 48 & 28 & 18 \\
\hline 2,850 & 6,100 & 9,700 & 16,800 & 24,600 & 35,400 \\
\hline WEIGHTED & SKEW & $($ LOGS $)=$ & 0.79 & & \\
\hline MEAN & & $($ LOGS $)=$ & 3.50 & & \\
\hline STANDARD & DEV. & $($ LOGS $)=$ & 0.36 & & \\
\hline
\end{tabular}

MAGNITUDE AND PROBABILITY OF ANNUAL LOW FLOW BASED ON PERIOD OF RECORD 1967-85

\begin{tabular}{|c|c|c|c|c|c|c|}
\hline \multirow{5}{*}{$\begin{array}{l}\text { PERIOD } \\
\text { /CON- } \\
\text { SECU- } \\
\text { TIVE }\end{array}$} & \multirow{2}{*}{\multicolumn{2}{|c|}{$\begin{array}{l}\text { DISCHARGE } \\
\text { RECURREN }\end{array}$}} & \multicolumn{4}{|c|}{ IN FT3/S, FOR INDICATED } \\
\hline & & & INTE & L, IN & ARS, & \\
\hline & \multicolumn{6}{|c|}{ NON-EXCEEDANCE PROBABILITY, IN PERCENT } \\
\hline & & & & & & \\
\hline & 2 & 5 & 10 & 20 & $50 \#$ & $100 \#$ \\
\hline DAYS) & 508 & 208 & 108 & 58 & 28 & 18 \\
\hline 1 & 0.96 & 0.53 & 0.37 & 0.27 & 0.18 & 0.14 \\
\hline 3 & 0.98 & 0.56 & 0.41 & 0.31 & 0.22 & 0.18 \\
\hline 7 & 1.0 & 0.62 & 0.47 & 0.38 & 0.29 & 0.24 \\
\hline 14 & 1.1 & 0.72 & 0.57 & 0.48 & 0.38 & 0.33 \\
\hline 30 & 1.2 & 0.82 & 0.66 & 0.55 & 0.45 & 0.40 \\
\hline 60 & 1.5 & 1.0 & 0.82 & 0.70 & 0.58 & 0.51 \\
\hline 90 & 1.7 & 1.2 & 0.97 & 0.81 & 0.66 & 0.57 \\
\hline 120 & 2.1 & 1.3 & 1.1 & 0.89 & 0.72 & 0.63 \\
\hline 183 & 2.8 & 1.8 & 1.5 & 1.3 & 1.1 & 1.0 \\
\hline
\end{tabular}

MAGNITUDE AND PROBABILITY OF ANNUAL HIGH FLOW BASED ON PERIOD OF RECORD $1967 \cdot 85$

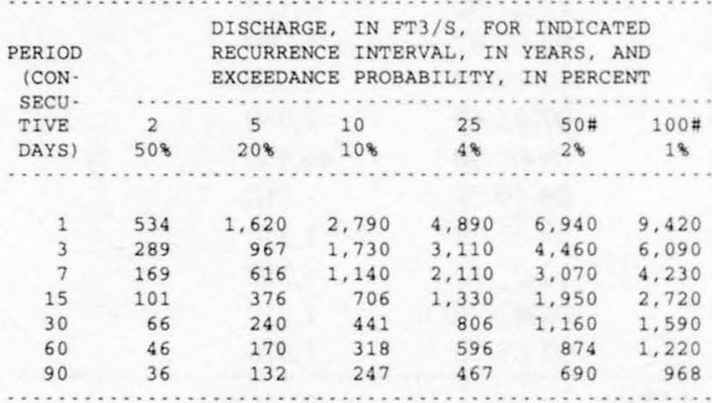

DURATION TABLE OF DAILY MEAN FLOW FOR PERIOD OF RECORD 1967.85

DISCHARGE, IN FT3/S, WHICH WAS EQUALED OR EXCEEDED FOR INDICATED PERCENT OF TIME

\begin{tabular}{|c|c|c|c|c|c|c|c|c|c|c|c|c|c|c|c|c|}
\hline 18 & 58 & 108 & 158 & 208 & 308 & 408 & 508 & 608 & 708 & 808 & 908 & 958 & 988 & 998 & 99.58 & 99.98 \\
\hline 422 & 111 & 49 & 22 & 11 & 5.0 & 3.6 & 2.7 & 2.1 & 1.8 & 1.5 & 1.1 & 0.82 & 0.60 & 0.54 & 0.51 & 0.36 \\
\hline
\end{tabular}

\# Reliability of values in column is uncertain, and potential errors are large. 
09498870 RYE CREEK NEAR GISELA, AZ--Continued
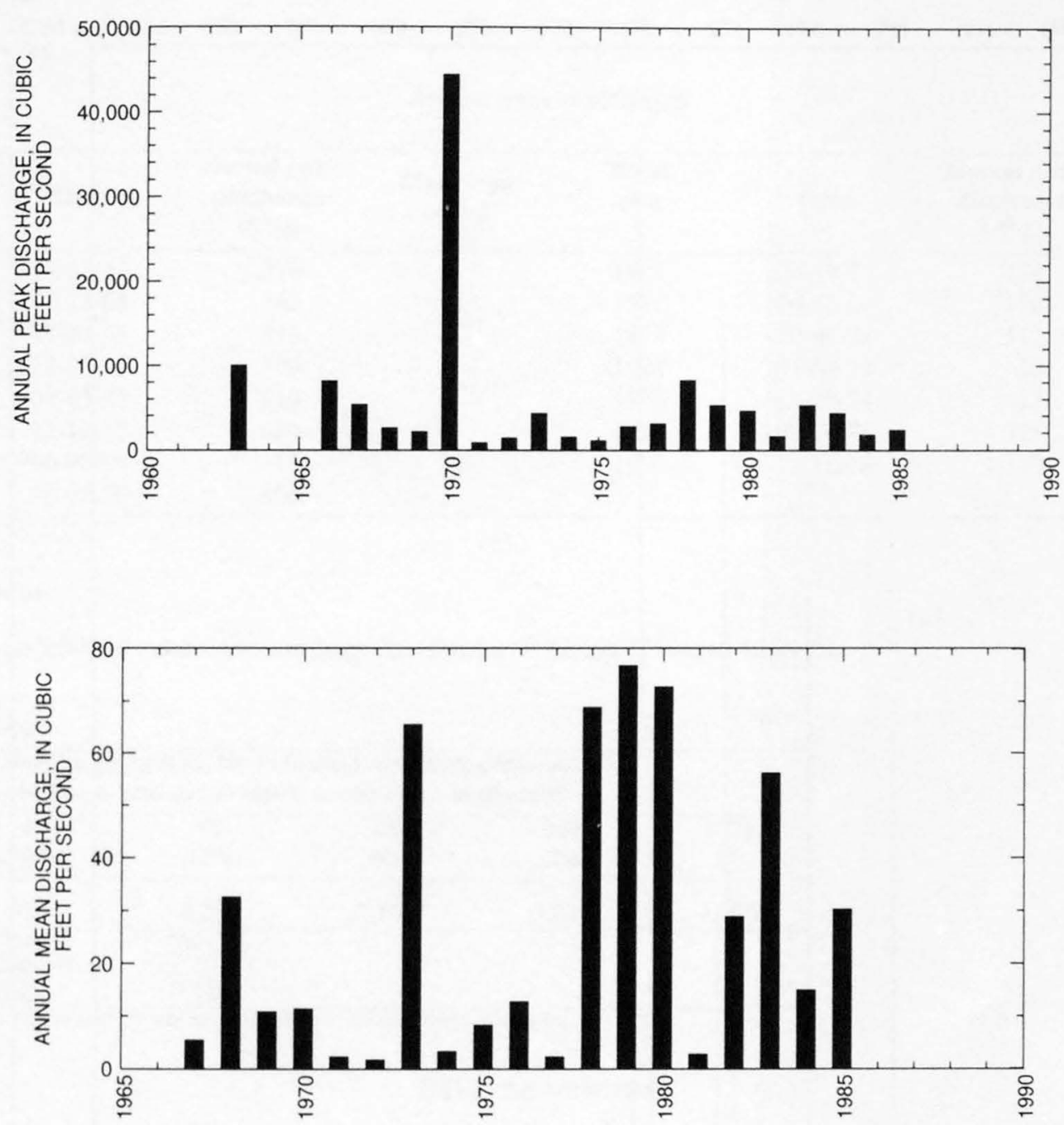
648

GILA RIVER BASIN

09498870 RYE CREEK NEAR GISELA, AZ--Continued

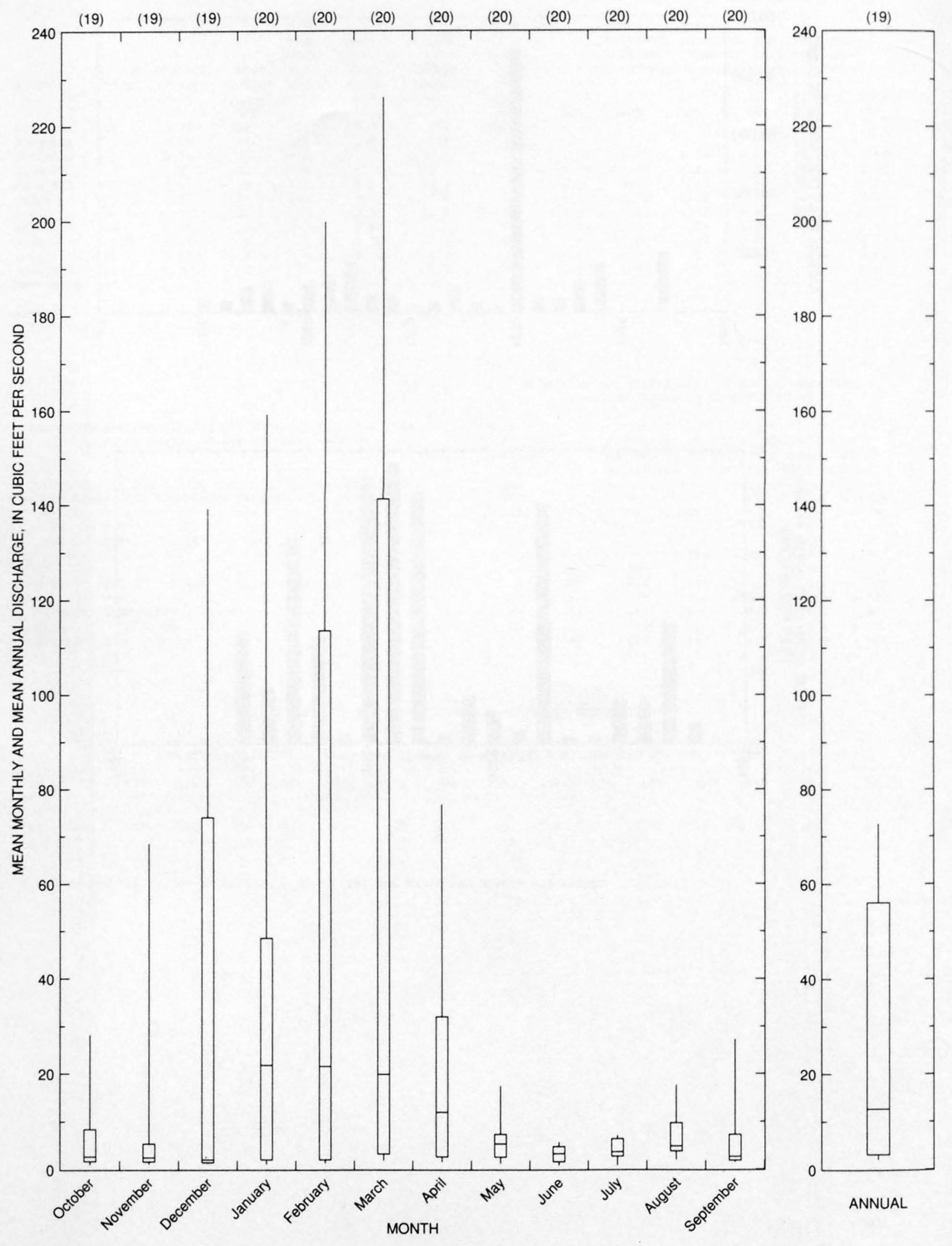


09498900 GOLD CREEK NEAR PAYSON, AZ

LOCATION.--Lat 34 $00^{\prime} 10^{\prime \prime}$, long $111^{\circ} 21^{\prime} 30^{\prime \prime}$, in SW $1 / 4$ sec.29, T.8 N., R.10 E., Gila County, Hydrologic Unit 15060105, at State Highway 87,16 mi south of Payson.

DRAINAGE AREA.--6.44 $\mathrm{mi}^{2}$.

Annual peak discharges

\begin{tabular}{|c|c|c|c|c|c|c|c|}
\hline $\begin{array}{l}\text { Water } \\
\text { year }\end{array}$ & Date & $\begin{array}{c}\text { Annual peak } \\
\text { discharge } \\
\left(\mathrm{ft}^{3} / \mathrm{s}\right)\end{array}$ & $\begin{array}{l}\text { Discharge } \\
\text { codes }\end{array}$ & $\begin{array}{l}\text { Water } \\
\text { year }\end{array}$ & Date & $\begin{array}{c}\text { Annual peak } \\
\text { discharge } \\
\left(\mathrm{ft}^{3} / \mathrm{s}\right)\end{array}$ & $\begin{array}{c}\text { Discharge } \\
\text { codes }\end{array}$ \\
\hline 1963 & $08-22-63$ & 1,370 & & 1971 & $08-19-71$ & 125 & \\
\hline 1964 & $07-15-64$ & 163 & & 1972 & $06-22-72$ & 160 & \\
\hline 1965 & $01-07-65$ & 215 & & 1973 & $10-06-72$ & 515 & \\
\hline 1966 & $12-22-65$ & 500 & & 1974 & $01-08-74$ & 50 & ES \\
\hline 1967 & $08-05-67$ & 510 & & 1975 & $10-29-74$ & 12 & \\
\hline 1968 & $12-19-67$ & 620 & & 1976 & $02-09-76$ & 375 & \\
\hline 1969 & $00-00-69$ & 15 & LT & 1979 & $12-18-78$ & ${ }^{\mathrm{l}} 1,120$ & \\
\hline 1970 & $09-05-70$ & 2,800 & & & & & \\
\hline
\end{tabular}

${ }^{1}$ Highest since 1970.

Magnitude and probability of instantaneous peak flow based on period of record 1963-76, 1979

\begin{tabular}{|c|c|c|c|c|c|}
\hline \multicolumn{6}{|c|}{$\begin{array}{l}\text { Discharge, in } \mathrm{ft}^{3} / \mathrm{s} \text {, for indicated recurrence interval } \\
\text { in years, and exceedance probablility, in percent }\end{array}$} \\
\hline 2 & 5 & 10 & 25 & $50 t$ & $100 \dagger$ \\
\hline $50 \%$ & $20 \%$ & $10 \%$ & $4 \%$ & $2 \%$ & $1 \%$ \\
\hline 314 & 795 & 1,290 & 2,160 & 3,020 & 4,070 \\
\hline Weighted skew & $(\log s)=$ & -0.01 & & & \\
\hline Mean & $(\log s)=$ & 2.50 & & & \\
\hline Standard dev. & $(\log s)=$ & 0.48 & & & \\
\hline
\end{tabular}

†Reliability of values in column is uncertain, and potential errors are large.

Basin characteristics

\begin{tabular}{ccccccccc}
\hline $\begin{array}{c}\text { Main } \\
\text { channel } \\
\text { slope } \\
(\mathrm{ft} / \mathrm{mi})\end{array}$ & $\begin{array}{c}\text { Stream } \\
\text { length } \\
(\mathrm{mi})\end{array}$ & $\begin{array}{c}\text { Mean } \\
\text { basin } \\
\text { elevation } \\
\text { (ft) }\end{array}$ & $\begin{array}{c}\text { Forested } \\
\text { area } \\
\text { (percent) }\end{array}$ & $\begin{array}{c}\text { Soil } \\
\text { index }\end{array}$ & $\begin{array}{c}\text { Mean } \\
\text { annual } \\
\text { precipitation } \\
\text { (in) }\end{array}$ & $\begin{array}{c}\text { 2-year } \\
\text { (in) }\end{array}$ & $\begin{array}{c}\text { 50-year } \\
\text { (in) }\end{array}$ \\
\hline 295 & 4.4 & 4,590 & 6.5 & 3.0 & 21.0 & 2.9 & 5.5 \\
\hline
\end{tabular}


09498900 GOLD CREEK NEAR PAYSON, AZ--Continued

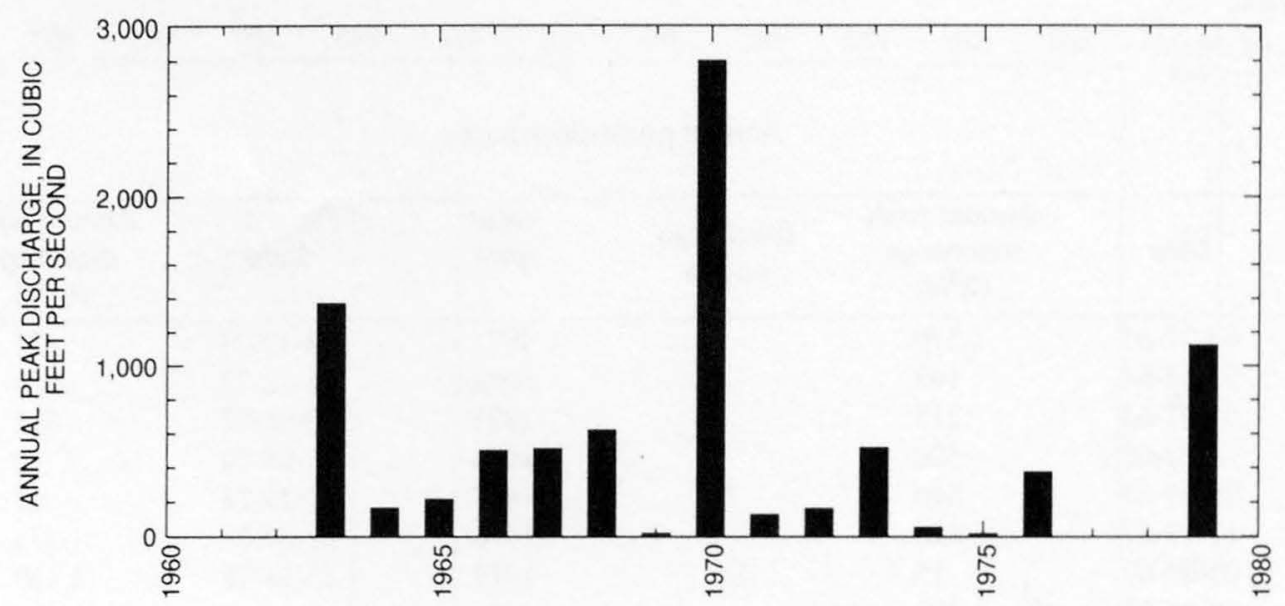


09499000 TONTO CREEK ABOVE GUN CREEK, NEAR ROOSEVELT, AZ

LOCATION.--Lat 33.58'48", long 111 '18'10", in SW $1 / 4 \mathrm{NE}^{1 / 4}$ sec.2, T.7 N., R.10 E., Gila County, Hydrologic Unit 15060105, in Tonto National Forest, on left bank $600 \mathrm{ft}$ upstream from Gun Creek, $25 \mathrm{mi}$ upstream from Roosevelt Dam, and 24 mi northwest of Roosevelt.

DRAINAGE AREA.--675 $\mathrm{mi}^{2}$.

PERIOD OF RECORD.--December 1940 to current year.

REVISED RECORDS.--WSP 1283: Drainage area. WDR AZ-80-1: 1978(M), WDR AZ-88-1: 1979(P).

GAGE.--Water-stage recorder. Datum of gage is 2,523.14 $\mathrm{ft}$ above sea level.

REMARKS.--No estimated daily discharges. Records good. Small diversions above station for irrigation.

EXTREMES FOR PERIOD OF RECORD.--Maximum discharge, 72,500 $\mathrm{ft}^{3} / \mathrm{s} \mathrm{Jan} .8,1993$, gage height, $17.95 \mathrm{ft}$; maximum gage height, $18.2 \mathrm{ft}$

Sept. 5, 1970; no flow at times.

Annual peak discharges

\begin{tabular}{|c|c|c|c|c|c|c|c|}
\hline $\begin{array}{l}\text { Water } \\
\text { year }\end{array}$ & Date & $\begin{array}{c}\text { Annual peak } \\
\text { discharge } \\
\left(\mathrm{ft}^{3} / \mathrm{s}\right)\end{array}$ & $\begin{array}{c}\text { Discharge } \\
\text { codes }\end{array}$ & $\begin{array}{l}\text { Water } \\
\text { year }\end{array}$ & Date & $\begin{array}{c}\text { Annual peak } \\
\text { discharge } \\
\left(\mathrm{ft}^{3} / \mathrm{s}\right)\end{array}$ & $\begin{array}{c}\text { Discharge } \\
\text { codes }\end{array}$ \\
\hline 1941 & $03-14-41$ & 32,000 & & 1969 & $01-26-69$ & 10,600 & \\
\hline 1942 & $41-12-11$ & 1,250 & & 1970 & 09-05-70 & 53,000 & \\
\hline 1943 & $03-05-43$ & 15,800 & & 1971 & $09-09-71$ & 5,280 & \\
\hline 1944 & $02-24-44$ & 2,990 & & 1972 & $12-26-71$ & 2,600 & \\
\hline 1945 & $08-11-45$ & 5320 & & 1973 & $10-19-72$ & 39,800 & \\
\hline 1946 & $09-18-46$ & 10,200 & & 1974 & $08-06-74$ & 3,800 & \\
\hline 1947 & $12-28-46$ & 7,130 & & 1975 & $10-29-74$ & 2,020 & \\
\hline 1948 & $07-26-48$ & 3,240 & & 1976 & $02-09-76$ & 34,900 & \\
\hline 1949 & $01-13-49$ & 9,890 & & 1977 & $08-15-77$ & 2,820 & \\
\hline 1950 & $07-16-50$ & 5,500 & & 1978 & $03-02-78$ & 57,200 & \\
\hline 1951 & $08-28-51$ & 31,100 & & 1979 & $12-18-78$ & 38,700 & \\
\hline 1952 & $01-18-52$ & 45,400 & & 1980 & $02-15-80$ & 61,400 & \\
\hline 1953 & $07-30-53$ & 2,620 & & 1981 & $08-08-81$ & 4,420 & \\
\hline 1954 & $03-23-54$ & 8,100 & & 1982 & $02-11-82$ & 18,000 & \\
\hline 1955 & $08-06-55$ & 15,200 & & 1983 & $11-30-82$ & 32,800 & \\
\hline 1956 & $07-18-56$ & 2,330 & & 1984 & $10-01-83$ & 24,400 & \\
\hline 1957 & $01-09-57$ & 15,000 & & 1985 & $12-27-84$ & 43,300 & \\
\hline 1958 & $03-22-58$ & 10,600 & & 1986 & $11-30-85$ & 10,900 & \\
\hline 1959 & $08-19-59$ & 11,100 & & 1987 & $08-03-87$ & 4,460 & \\
\hline 1960 & $12-26-59$ & 25,200 & & 1988 & $02-03-88$ & 23,900 & \\
\hline 1961 & $09-08-61$ & 12,900 & & 1989 & $02-05-89$ & 6,950 & \\
\hline 1962 & $09-06-62$ & 3,000 & & 1990 & $08-15-90$ & 1,730 & \\
\hline 1963 & $08-22-63$ & 19,700 & & 1991 & 03-03-91 & 43,500 & \\
\hline 1964 & $07-30-64$ & 12,000 & & 1992 & $08-23-92$ & 22,500 & \\
\hline 1965 & $01-07-65$ & 12,900 & & 1993 & $01-08-93$ & ${ }^{\mathrm{I}} 72,500$ & \\
\hline 1966 & $12-22-65$ & 44,700 & & 1994 & $11-15-93$ & 3,150 & \\
\hline 1967 & $12-07-66$ & 7,550 & & 1995 & $02-15-95$ & 43,300 & \\
\hline 1968 & $12-19-67$ & 19,700 & & 1996 & $09-12-96$ & 713 & \\
\hline
\end{tabular}

'Highest since 1708, O’Conner and others (1986). 
09499000 TONTO CREEK ABOVE GUN CREEK, NEAR ROOSEVELT, AZ--Continued

Discharge rating table developed February 1994

\begin{tabular}{cccc}
\hline $\begin{array}{c}\text { Gage height } \\
(\mathrm{ft})\end{array}$ & $\begin{array}{c}\text { Discharge } \\
\left(\mathbf{f t}^{3} \mathbf{s} \mathbf{s}\right)\end{array}$ & $\begin{array}{c}\text { Gage height } \\
(\mathbf{f t})\end{array}$ & $\begin{array}{c}\text { Discharge } \\
\left(\mathbf{f t}^{3} \mathbf{s}\right)\end{array}$ \\
\hline 5.0 & 1,620 & 12.0 & 25,080 \\
6.0 & 3,120 & 13.0 & 31,140 \\
7.0 & 5,180 & 14.0 & 37,960 \\
8.0 & 7,850 & 15.0 & 45,540 \\
9.0 & 11,140 & 16.0 & 53,900 \\
10.0 & 15,100 & 17.0 & 63,050 \\
11.0 & 19,730 & 17.9 & 71,980 \\
\hline
\end{tabular}

Basin characteristics

\begin{tabular}{cccccccc}
\hline $\begin{array}{c}\text { Main } \\
\text { channel } \\
\text { slope } \\
(\mathrm{ft} / \mathrm{mi})\end{array}$ & $\begin{array}{c}\text { Stream } \\
\text { length } \\
(\mathrm{mi})\end{array}$ & $\begin{array}{c}\text { Mean } \\
\text { basin } \\
\text { elevation } \\
(\mathrm{ft})\end{array}$ & $\begin{array}{c}\text { Forested } \\
\text { area } \\
\text { (percent) }\end{array}$ & $\begin{array}{c}\text { Soil } \\
\text { index }\end{array}$ & $\begin{array}{c}\text { Mean } \\
\text { annual } \\
\text { precipitation } \\
\text { (in) }\end{array}$ & $\begin{array}{c}\text { Rainfall intensity, 24-hour } \\
\text { (in) }\end{array}$ & $\begin{array}{c}\text { 50-year } \\
\text { (in) }\end{array}$ \\
\hline 88.4 & 48.3 & 5,020 & 65.0 & 3.0 & 23.9 & 2.8 \\
\hline
\end{tabular}


09499000 TONTO CREEK ABOVE GUN CREEK, NEAR ROOSEVELT, AZ--Continued

MEAN MONTHLY AND ANNUAL DISCHARGES 1942.96

\begin{tabular}{|c|c|c|c|c|c|c|}
\hline MONTH & $\begin{array}{l}\text { MAXIMUM } \\
\text { (FT3/S) }\end{array}$ & $\begin{array}{l}\text { MINIMUM } \\
\text { (FT3/S) }\end{array}$ & $\begin{array}{c}\text { MEAN } \\
(\text { FT3/S) }\end{array}$ & $\begin{array}{l}\text { STAN- } \\
\text { DARD } \\
\text { DEVIA- } \\
\text { TION } \\
\text { (FT3/S) }\end{array}$ & $\begin{array}{l}\text { COEFFI- } \\
\text { CIENT OF } \\
\text { VARI- } \\
\text { ATION }\end{array}$ & $\begin{array}{l}\text { PERCENT } \\
\text { OF } \\
\text { ANNUAL } \\
\text { RUNOFF }\end{array}$ \\
\hline OCTOBER & 1.050 & 1.5 & 61 & 161 & 2.6 & 3.1 \\
\hline NOVEMBER & 438 & 6.5 & 72 & 112 & 1.5 & 3.7 \\
\hline DECEMBER & 2,330 & 9.9 & 249 & 480 & 1.9 & 12.7 \\
\hline JANUARY & 4,270 & 16 & 356 & 665 & 1.9 & 18.2 \\
\hline FEBRUARY & 4,190 & 13 & 368 & 663 & 1.8 & 18.8 \\
\hline MARCH & 4.160 & 12 & 480 & 708 & 1.5 & 24.5 \\
\hline APRIL & 709 & 9.0 & 150 & 172 & 1.2 & 7.7 \\
\hline MAY & 285 & 3.3 & 41 & 47 & 1.1 & 2.1 \\
\hline JUNE & 95 & 0.00 & 14 & 16 & 1.1 & 0.7 \\
\hline JULY & 207 & 0.35 & 22 & 29 & 1.3 & 1.1 \\
\hline AUGUST & 1,090 & 4.5 & 98 & 191 & 1.9 & 5.0 \\
\hline SEPTEMBER & 626 & 0.78 & 45 & 97 & 2.2 & 2.3 \\
\hline ANNUAL & 652 & 15 & 163 & 161 & 0.99 & 100 \\
\hline
\end{tabular}

MAGNITUDE AND PROBABILITY OF ANNUAL LOW FLOW BASED ON PERIOD OF RECORD 1942-96

\begin{tabular}{|c|c|c|c|c|c|c|}
\hline $\begin{array}{l}\text { PERIOD } \\
\text { (CON- }\end{array}$ & \multirow{2}{*}{\multicolumn{6}{|c|}{$\begin{array}{r}\text { DISCHARGE, IN FT3/S, FOR INDICATED } \\
\text { RECURRENCE INTERVAL, IN YEARS, AND } \\
\text { NON-EXCEEDANCE PROBABILITY, IN PERCENT } \\
\cdots \cdots \ldots \ldots \ldots \ldots \ldots \ldots \ldots \ldots \ldots \ldots \ldots \ldots \ldots \ldots \ldots \ldots \ldots\end{array}$}} \\
\hline SECU. & & & & & & \\
\hline TIVE & 2 & 5 & 10 & 20 & 50 & $100 \#$ \\
\hline DAYS) & 508 & 208 & 108 & 58 & 28 & 18 \\
\hline 1 & 1.3 & 0.00 & 0.00 & 0.00 & 0.00 & 0.00 \\
\hline 3 & 1.3 & 0.00 & 0.00 & 0.00 & 0.00 & 0.00 \\
\hline 7 & 1.8 & 0.00 & 0.00 & 0.00 & 0.00 & 0.00 \\
\hline 14 & 2.6 & 0.07 & 0.00 & 0.00 & 0.00 & 0.00 \\
\hline 30 & 4.2 & 0.91 & 0.26 & 0.00 & 0.00 & 0.00 \\
\hline 60 & 7.4 & 3.2 & 1.9 & 1.2 & 0.65 & 0.43 \\
\hline 90 & 11 & 6.5 & 4.9 & 3.8 & 2.8 & 2.3 \\
\hline 120 & 14 & 9.3 & 7.4 & 6.1 & 5.0 & 4.3 \\
\hline 183 & 21 & 13 & 11 & 9.8 & 8.8 & 8.3 \\
\hline
\end{tabular}

MAGNITUDE AND PROBABILITY OF ANNUAL HIGH FLOW BASED ON PERIOD OF RECORD 1942.96

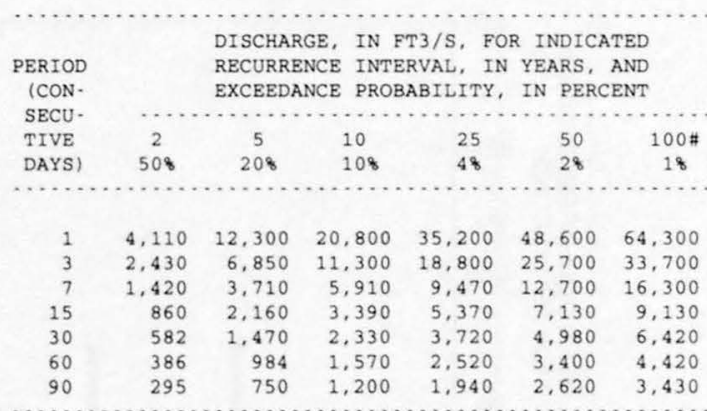

DURATION TABLE OF DAILY MEAN FLOW FOR PERIOD OF RECORD 1942-96

DISCHARGE, IN FT3/S, WHICH WAS EQUALED OR EXCEEDED FOR INDICATED PERCENT OF TIME

\begin{tabular}{|c|c|c|c|c|c|c|c|c|c|c|c|c|c|c|c|c|}
\hline 18 & 58 & 108 & 158 & 208 & 308 & 408 & 508 & 608 & 708 & 808 & 908 & 958 & 988 & 998 & 99.58 & 99.98 \\
\hline 630 & 586 & 261 & 151 & 98 & 49 & 32 & 24 & 18 & 14 & 9.3 & 4.8 & 2.1 & 0.00 & 0.00 & 0.00 & 0.00 \\
\hline
\end{tabular}

\# Reliability of values in column is uncertain, and potential errors are large. 
ANNUAL MEAN DISCHARGE, IN CUBIC

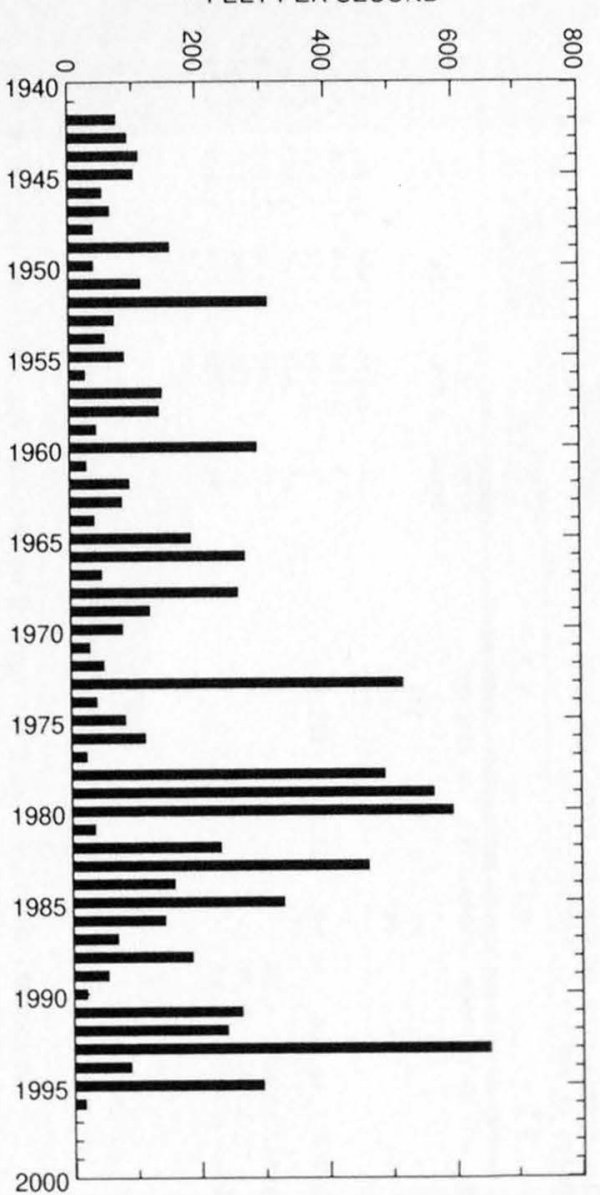

ANNUAL PEAK DISCHARGE, IN CUBIC

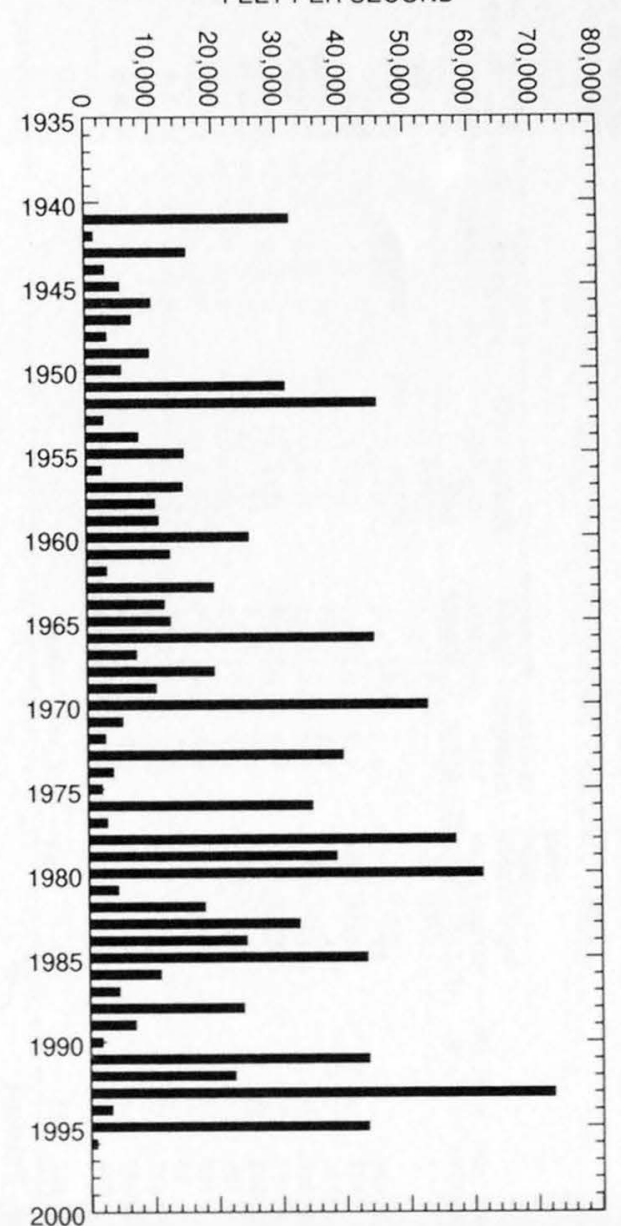


GILA RIVER BASIN

655

09499000 TONTO CREEK ABOVE GUN CREEK, NEAR ROOSEVELT, AZ--Continued

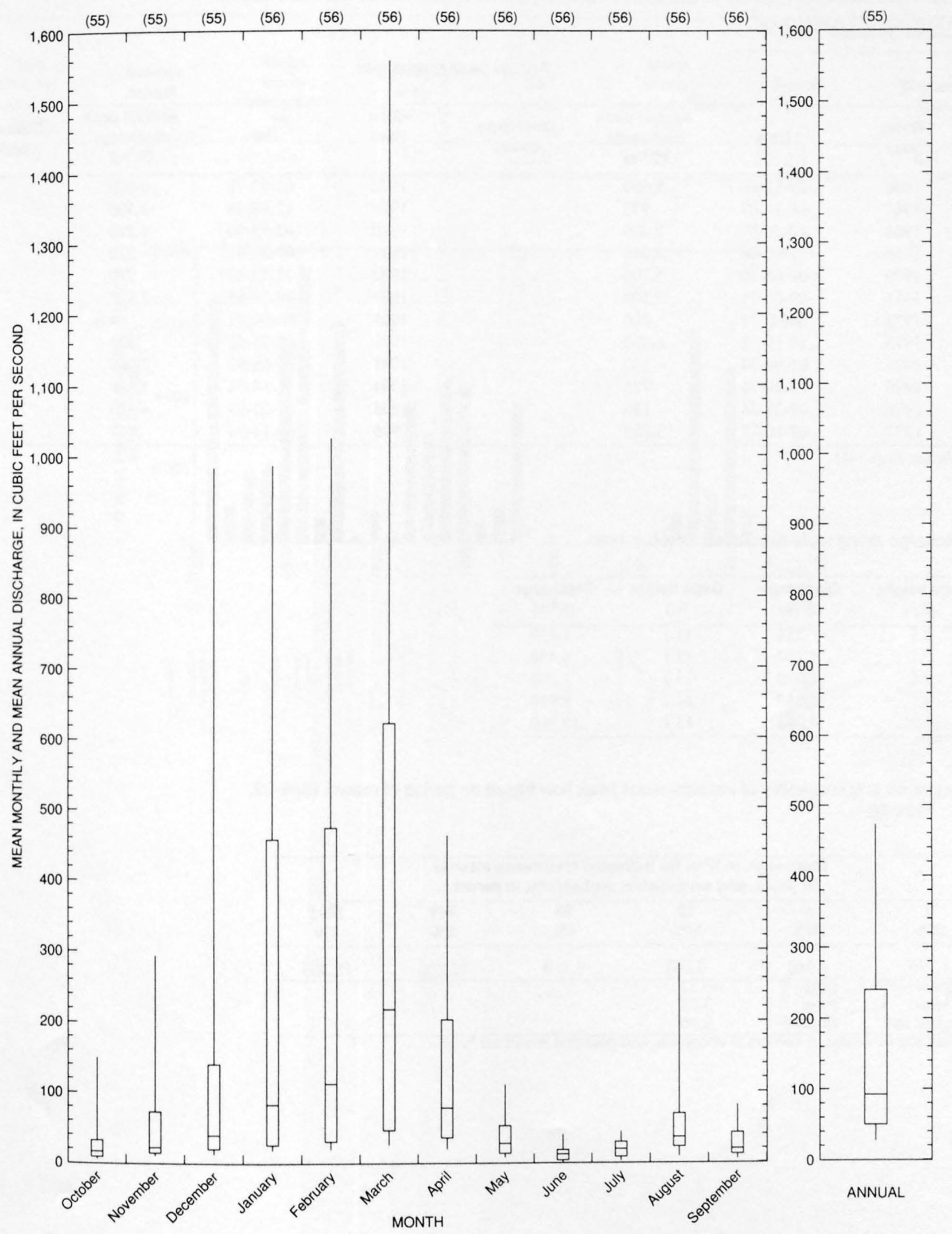




\section{TORTILLA CREEK AT TORTILLA FLAT, AZ}

LOCATION.--Lat 33'31'38", long 111'23'13", in NW¹/4 sec.13, T.2 N., R.9 E. (unsurveyed), Maricopa County, Hydrologic Unit 15060106, 600 upstream from State Highway 88 and Tortilla Flat Store, and 3.7 mi southeast of Mormon Flat Dam.

DRAINAGE AREA.--24.3 $\mathrm{mi}^{2}$.

Annual peak discharges

\begin{tabular}{|c|c|c|c|c|c|c|c|}
\hline $\begin{array}{l}\text { Water } \\
\text { year }\end{array}$ & Date & $\begin{array}{l}\text { Annual peak } \\
\text { discharge } \\
\left(\mathrm{ft}^{3} / \mathrm{s}\right)\end{array}$ & $\begin{array}{c}\text { Discharge } \\
\text { codes }\end{array}$ & $\begin{array}{l}\text { Water } \\
\text { year }\end{array}$ & Date & $\begin{array}{c}\text { Annual peak } \\
\text { discharge } \\
\left(\mathrm{ft}^{3} / \mathrm{s}\right)\end{array}$ & $\begin{array}{c}\text { Discharge } \\
\text { codes }\end{array}$ \\
\hline 1966 & $09-13-66$ & 6,660 & & 1978 & $03-02-78$ & 3,000 & \\
\hline 1967 & $08-18-67$ & 975 & & 1979 & $12-18-78$ & 4,400 & \\
\hline 1968 & $12-19-67$ & 2,000 & & 1980 & $02-15-80$ & 4,250 & \\
\hline 1969 & $11-00-68$ & 2,000 & LT & 1981 & $07-28-81$ & 520 & \\
\hline 1970 & $09-05-70$ & 5,700 & & 1982 & $03-13-82$ & 910 & \\
\hline 1971 & 09-01-71 & 17,500 & & 1983 & $09-30-83$ & 3,800 & \\
\hline 1972 & $08-00-72$ & 600 & & 1991 & $00-00-91$ & 4.0 & \\
\hline 1973 & $10-19-72$ & 6,000 & & 1992 & $03-26-92$ & 823 & \\
\hline 1974 & $01-08-74$ & 150 & & 1993 & $01-08-93$ & 5,900 & \\
\hline 1975 & $10-29-74$ & 775 & & 1994 & $11-14-93$ & 1,320 & \\
\hline 1976 & $09-25-76$ & 160 & & 1995 & $01-05-95$ & 4,840 & \\
\hline 1977 & $08-16-77$ & 3,800 & & 1996 & $09-14-96$ & 847 & \\
\hline
\end{tabular}

${ }^{1}$ Highest since 1941.

Discharge rating table developed October 1991

\begin{tabular}{cccc}
\hline $\begin{array}{c}\text { Gage height } \\
(\mathbf{f t})\end{array}$ & $\begin{array}{c}\text { Discharge } \\
\left(\mathbf{f t}^{\mathbf{3}} \mathbf{/}\right)\end{array}$ & $\begin{array}{c}\text { Gage height } \\
(\mathbf{f t})\end{array}$ & $\begin{array}{c}\text { Discharge } \\
\left(\mathbf{f t}^{3} / \mathbf{s}\right)\end{array}$ \\
\hline 6.1 & 584 & 11.1 & 5,230 \\
7.1 & 1,180 & 12.1 & 6,450 \\
8.1 & 2,050 & 13.1 & 7,700 \\
9.1 & 3,050 & 14.1 & 8,950 \\
10.1 & 4,080 & 15.1 & 10,300 \\
\hline
\end{tabular}

Magnitude and probability of instantaneous peak flow based on period of record 1966-83, 1991-96

\begin{tabular}{|c|c|c|c|c|c|}
\hline \multicolumn{6}{|c|}{$\begin{array}{l}\text { Discharge, in } \mathrm{ft}^{3} / \mathrm{s} \text {, for indicated recurrence interval } \\
\text { in years, and exceedance probablility, in percent }\end{array}$} \\
\hline 2 & 5 & 10 & 25 & $50 t$ & $100 t$ \\
\hline $50 \%$ & $20 \%$ & $10 \%$ & $4 \%$ & $2 \%$ & $1 \%$ \\
\hline 1,740 & 4,080 & 6,180 & 9,410 & 12,200 & 15,300 \\
\hline Weighted skew & $(\log s)=$ & -0.32 & & & \\
\hline Mean & $(\log s)=$ & 3.22 & & & \\
\hline Standard dev. & $(\log s)=$ & 0.46 & & & \\
\hline
\end{tabular}


09501300 TORTILLA CREEK AT TORTILLA FLAT, AZ--Continued

Basin characteristics

\begin{tabular}{ccccccc}
\hline $\begin{array}{c}\text { Main } \\
\text { channel } \\
\text { slope } \\
(\mathrm{ft} / \mathrm{mi})\end{array}$ & $\begin{array}{c}\text { Stream } \\
\text { length } \\
(\mathrm{mi})\end{array}$ & $\begin{array}{c}\text { Mean } \\
\text { basin } \\
\text { elevation } \\
(\mathrm{ft})\end{array}$ & $\begin{array}{c}\text { Forested } \\
\text { area } \\
\text { (percent) }\end{array}$ & $\begin{array}{c}\text { Soil } \\
\text { index }\end{array}$ & $\begin{array}{c}\text { Mean } \\
\text { annual } \\
\text { precipitation } \\
\text { (in) }\end{array}$ & $\begin{array}{c}\text { Rainfall intensity, 24-hour } \\
\text { (in) }\end{array}$ \\
\hline 179 & 13.1 & 2,690 & 1.0 & 1.0 & 15.0 & $\begin{array}{c}50 \text {-year } \\
\text { (in) }\end{array}$ \\
\hline
\end{tabular}

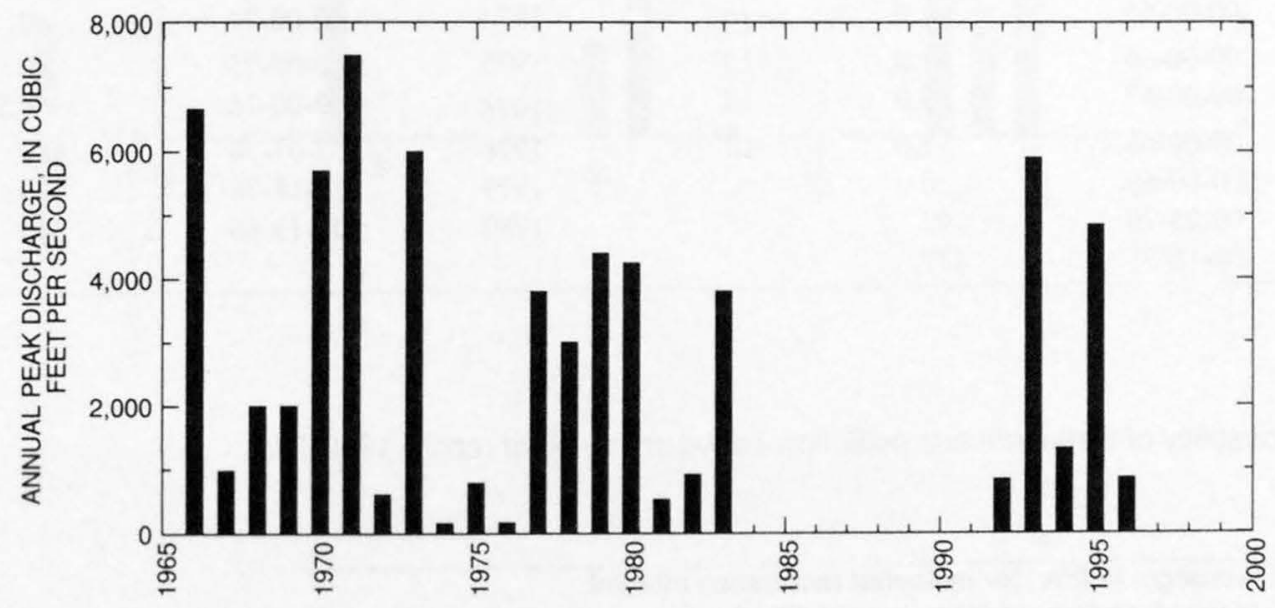


LOCATION.--Lat $35^{\circ} 17^{\prime} 15^{\prime \prime}$, long $112^{\circ} 43^{\prime} 55^{\prime \prime}$, in SE $1 / 4$ sec.17, T.22 N., R.4 W., Yavapai County, Hydrologic Unit 15060201, at U.S. Highway 66,

$9 \mathrm{mi}$ east of Seligman, and $15 \mathrm{mi}$ west of Ashfork.

DRAINAGE AREA.--6.01 $\mathrm{mi}^{2}$.

Annual peak discharges

\begin{tabular}{|c|c|c|c|c|c|c|c|}
\hline $\begin{array}{l}\text { Water } \\
\text { year }\end{array}$ & Date & $\begin{array}{c}\text { Annual peak } \\
\text { discharge } \\
\left(\mathrm{ft}^{3} / \mathrm{s}\right)\end{array}$ & $\begin{array}{c}\text { Discharge } \\
\text { codes }\end{array}$ & $\begin{array}{l}\text { Water } \\
\text { year }\end{array}$ & Date & $\begin{array}{c}\text { Annual peak } \\
\text { discharge } \\
\left(\mathrm{ft}^{3} / \mathrm{s}\right)\end{array}$ & $\begin{array}{l}\text { Discharge } \\
\text { codes }\end{array}$ \\
\hline 1963 & $00-00-63$ & 1.0 & LT & 1972 & $00-00-72$ & 2.0 & ES \\
\hline 1964 & $08-01-64$ & 168 & & 1973 & $00-00-73$ & 10 & \\
\hline 1965 & $00-00-65$ & 1.0 & LT & 1974 & $00-00-74$ & 0 & \\
\hline 1966 & $00-00-66$ & 1.0 & LT & 1975 & $00-00-75$ & 0 & \\
\hline 1967 & $09-00-67$ & 3.0 & LT & 1976 & $00-00-76$ & 6.5 & \\
\hline 1968 & $00-00-68$ & 1.0 & LT & 1978 & $03-01-78$ & ${ }^{1} 480$ & \\
\hline 1969 & $00-00-69$ & 0 & & 1979 & $12-18-78$ & 125 & \\
\hline 1970 & $09-05-70$ & 92 & & 1980 & $02-19-80$ & 240 & \\
\hline 1971 & $08-18-71$ & 477 & & & & & \\
\hline
\end{tabular}

${ }^{T}$ Highest since 1964.

Magnitude and probability of instantaneous peak flow based on period of record 1963-76, $1978-80$

\begin{tabular}{|c|c|c|c|c|c|}
\hline \multicolumn{6}{|c|}{$\begin{array}{l}\text { Discharge, in } \mathrm{ft}^{3} / \mathrm{s} \text {, for indicated recurrence interval } \\
\text { in years, and exceedance probablility, in percent }\end{array}$} \\
\hline 2 & 5 & 10 & 25 & $50 t$ & $100 t$ \\
\hline $50 \%$ & $20 \%$ & $10 \%$ & $4 \%$ & $2 \%$ & $1 \%$ \\
\hline 7.2 & 86.2 & 310 & 1,200 & 2,870 & 6,250 \\
\hline Weighted skew & $(\log s)=$ & -0.05 & & & \\
\hline Mean & $(\log s)=$ & 0.85 & & & \\
\hline Standard dev. & $(\log s)=$ & 1.29 & & & \\
\hline
\end{tabular}

千 Reliability of values in column is uncertain, and potential errors are large.

Basin characteristics

\begin{tabular}{ccccccc}
\hline $\begin{array}{c}\text { Main } \\
\text { channel } \\
\text { slope } \\
(\mathrm{ft} / \mathrm{mi})\end{array}$ & $\begin{array}{c}\text { Stream } \\
\text { length } \\
\text { (mi) }\end{array}$ & $\begin{array}{c}\text { Mean } \\
\text { basin } \\
\text { elevation } \\
\text { (ft) }\end{array}$ & $\begin{array}{c}\text { Forested } \\
\text { area } \\
\text { (percent) }\end{array}$ & $\begin{array}{c}\text { Soil } \\
\text { index }\end{array}$ & $\begin{array}{c}\text { Mean } \\
\text { annual } \\
\text { precipitation } \\
\text { (in) }\end{array}$ & $\begin{array}{c}\text { Rainfall intensity, 24-hour } \\
\text { (in) }\end{array}$ \\
\hline 111 & 4.8 & 5,970 & 27.0 & 3.0 & 15.5 & $\begin{array}{c}50 \text {-year } \\
\text { (in) }\end{array}$ \\
\hline
\end{tabular}


09502700 CROOKTON WASH SELIGMAN, AZ--Continued

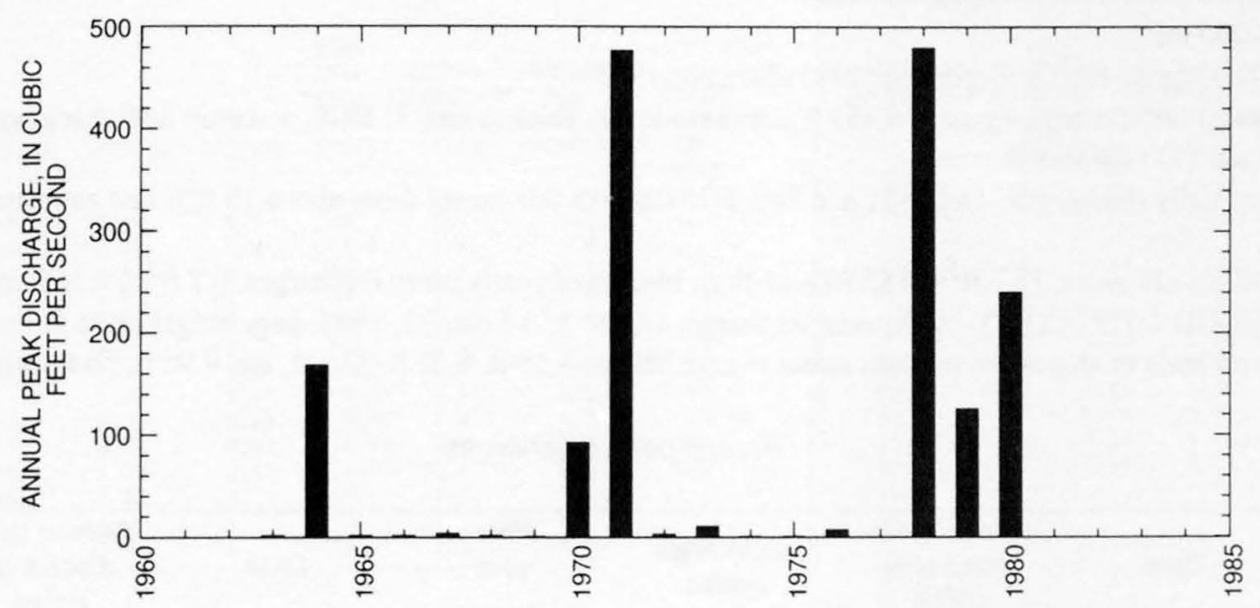




\section{WILLIAMSON VALLEY WASH NEAR PAULDEN, AZ}

LOCATION.--Lat 34 $52^{\prime} 00^{\prime \prime}$, long 112 $36^{\prime} 45^{\prime \prime}$, in $\mathrm{SE}^{1} / \mathrm{SE}^{1} / 4 \mathrm{sec} .7$, T.17 N., R.3 W., Yavapai County, Hydrologic Unit 15060201 , on left bank 3.6 mi north of Simmons and $8.5 \mathrm{mi}$ west of Paulden.

DRAINAGE AREA.--255 $\mathrm{mi}^{2}$.

PERIOD OF RECORD.--March 1965 to September 1985 (discontinued).

GAGE.--Water-stage recorder. Datum of gage is 4,455 ft above sea level. Prior to Oct. 1, 1970, at datum $1.00 \mathrm{ft}$ higher. Datum of $4,447 \mathrm{ft}$ pub. lished in WRD Ariz. 1971-76 was in error.

REMARKS.--Estimated daily discharges: Oct. 9-31 and Feb. 1-7. Records fair except those above $15 \mathrm{ft}^{3} / \mathrm{s}$ and estimated daily dicharges, which are poor.

AVERAGE DISCHARGE.--20 years, $15.7 \mathrm{ft}^{3} / \mathrm{s}, 11,370$ acre- $\mathrm{ft} / \mathrm{yr}$; median of yearly mean discharges, $6.2 \mathrm{ft}^{3} / \mathrm{s}, 4,500 \mathrm{acre}-\mathrm{ft} / \mathrm{yr}$.

EXTREMES FOR PERIOD OF RECORD.--Maximum discharge, 14,800 $\mathrm{ft}^{3} / \mathrm{s}$ Sept. 23, 1983, gage height, $9.96 \mathrm{ft}$ from rating curve extended above $2,200 \mathrm{ft}^{3} / \mathrm{s}$ on basis of slope-area measurements at gage heights $6.38 \mathrm{ft}, 8.22 \mathrm{ft}, 8.93 \mathrm{ft}$, and $9.96 \mathrm{ft}$; no flow at times in most years.

Annual peak discharges

\begin{tabular}{|c|c|c|c|c|c|c|c|}
\hline $\begin{array}{l}\text { Water } \\
\text { year }\end{array}$ & Date & $\begin{array}{c}\text { Annual peak } \\
\text { discharge } \\
\left(\mathrm{ft}^{3} / \mathrm{s}\right)\end{array}$ & $\begin{array}{c}\text { Discharge } \\
\text { codes }\end{array}$ & $\begin{array}{l}\text { Water } \\
\text { year }\end{array}$ & Date & $\begin{array}{c}\text { Annual peak } \\
\text { discharge } \\
\left(\mathrm{ft}^{3} / \mathrm{s}\right)\end{array}$ & $\begin{array}{c}\text { Discharge } \\
\text { codes }\end{array}$ \\
\hline 1965 & $04-10-65$ & 820 & & 1976 & $02-09-76$ & 3,910 & \\
\hline 1966 & $12-30-65$ & 3,630 & & 1977 & $09-12-77$ & 1,300 & \\
\hline 1967 & $12-07-66$ & 1,710 & & 1978 & $03-01-78$ & 7,490 & \\
\hline 1968 & $01-28-68$ & 2,120 & & 1979 & $12-18-78$ & 4,890 & \\
\hline 1969 & $02-26-69$ & 400 & & 1980 & $02-20-80$ & 10,100 & \\
\hline 1970 & $08-19-70$ & 445 & & 1981 & $08-08-81$ & 137 & \\
\hline 1971 & $08-23-71$ & 465 & & 1982 & $02-11-82$ & 572 & \\
\hline 1972 & $08-12-72$ & 254 & & 1983 & $09-23-83$ & 14,800 & \\
\hline 1973 & $10-19-72$ & 1,940 & & 1984 & $10-05-83$ & 1,280 & \\
\hline 1974 & $09-27-74$ & 570 & & 1985 & $12-27-84$ & 2,780 & \\
\hline 1975 & $11-02-74$ & 112 & & & & & \\
\hline
\end{tabular}

${ }^{1}$ Highest since 1964.

Discharge rating table developed October 1982

\begin{tabular}{crcc}
\hline $\begin{array}{c}\text { Gage height } \\
(\mathrm{ft})\end{array}$ & $\begin{array}{c}\text { Discharge } \\
\left(\mathrm{ft}^{3} / \mathbf{s}\right)\end{array}$ & $\begin{array}{c}\text { Gage height } \\
(\mathbf{f t})\end{array}$ & $\begin{array}{c}\text { Discharge } \\
\left(\mathrm{ft}^{3} / \mathbf{s}\right)\end{array}$ \\
\hline 4.0 & 640 & 7.5 & 5,690 \\
5.0 & 1,670 & 8.0 & 7,100 \\
5.5 & 2,280 & 8.5 & 8,760 \\
6.0 & 2,950 & 9.0 & 10,620 \\
6.5 & 3,700 & 9.5 & 12,690 \\
7.0 & 4,590 & 9.9 & 14,510 \\
\hline
\end{tabular}

Basin characteristics

\begin{tabular}{cccccccc}
\hline $\begin{array}{c}\text { Main } \\
\text { channel } \\
\text { slope } \\
\text { (ft/mi) }\end{array}$ & $\begin{array}{c}\text { Stream } \\
\text { length } \\
\text { (mi) }\end{array}$ & $\begin{array}{c}\text { Mean } \\
\text { basin } \\
\text { elevation } \\
\text { (ft) }\end{array}$ & $\begin{array}{c}\text { Forested } \\
\text { area } \\
\text { (percent) }\end{array}$ & $\begin{array}{c}\text { Soil } \\
\text { index }\end{array}$ & $\begin{array}{c}\text { Mean } \\
\text { annual } \\
\text { precipitation } \\
\text { (in) }\end{array}$ & $\begin{array}{c}\text { Rainfall intensity, 24-hour } \\
\text { (in) }\end{array}$ & $\begin{array}{c}50-y e a r \\
\text { (in) }\end{array}$ \\
\hline 48.6 & 19.2 & 5,120 & 42.0 & 2.0 & 17.3 & 2.1 \\
\hline
\end{tabular}


09502800 WILLIAMSON VALLEY WASH NEAR PAULDEN, AZ--Continued

MEAN MONTHLY AND ANNUAL DISCHARGES 1966-85

\begin{tabular}{|c|c|c|c|c|c|c|}
\hline MONTH & $\begin{array}{l}\text { MAXIMUM } \\
\text { (FT3/S) }\end{array}$ & $\begin{array}{l}\text { MINIMUM } \\
\text { (FT3/S) }\end{array}$ & $\begin{array}{c}\text { MEAN } \\
\text { (FT3/S) }\end{array}$ & $\begin{array}{l}\text { STAN- } \\
\text { DARD } \\
\text { DEVIA- } \\
\text { TION } \\
\text { (FT3/S) }\end{array}$ & $\begin{array}{l}\text { COEFFI- } \\
\text { CIENT OF } \\
\text { VARI- } \\
\text { ATION }\end{array}$ & $\begin{array}{c}\text { PERCENT } \\
\text { OF } \\
\text { ANNUAL } \\
\text { RUNOFF }\end{array}$ \\
\hline OCTOBER & 77 & 0.00 & 6.7 & 18 & 2.7 & 3.5 \\
\hline NOVEMBER & 28 & 0.49 & 5.0 & 6.9 & 1.4 & 2.6 \\
\hline DECEMBER & 185 & 0.93 & 26 & 49 & 1.9 & 13.8 \\
\hline JANUARY & 175 & 1.8 & 21 & 41 & 2.0 & 10.9 \\
\hline FEBRUARY & 513 & 1.6 & 58 & 116 & 2.0 & 30.4 \\
\hline MARCH & 381 & 1.3 & 48 & 94 & 2.0 & 25.2 \\
\hline APRIL & 40 & 0.99 & 5.6 & 9.7 & 1.7 & 3.0 \\
\hline MAY & 3.7 & 0.00 & 1.5 & 0.99 & 0.66 & 0.8 \\
\hline JUNE & 2.1 & 0.00 & 0.66 & 0.68 & 1.0 & 0.3 \\
\hline JULY & 3.7 & 0.02 & 0.88 & 0.92 & 1.0 & 0.5 \\
\hline AUGUST & 15 & 0.32 & 3.4 & 4.7 & 1.4 & 1.8 \\
\hline SEPTEMBER & 233 & 0.03 & 14 & 52 & 3.8 & 7.2 \\
\hline ANNUAL & 63 & 1.6 & 16 & 18 & 1.1 & 100 \\
\hline
\end{tabular}

MAGNITUDE AND PROBABILITY OF INSTANTANEOUS PEAK FLOW BASED ON PERIOD OF RECORD $1965 \cdot 85$

DISCHARGE, IN FT3/S, FOR INDICATED RECURRENCE INTERVAL IN YEARS, AND EXCEEDANCE PROBABILITY, IN PERCENT

\begin{tabular}{|c|c|c|c|c|c|}
\hline 2 & 5 & 10 & 25 & $50 \#$ & $100 \#$ \\
\hline 508 & 208 & 108 & 48 & 28 & 18 \\
\hline 1,310 & 4,080 & 7,360 & 13,700 & 20,400 & 29,200 \\
\hline
\end{tabular}

WEIGHTED SKEW (LOGS) $=-0.05$

MEAN $\quad($ LOGS $)=3.11$

STANDARD DEV. $($ LOGS $)=0.59$
MAGNITUDE AND PROBABILITY OF ANNUAL LOW FLOW BASED ON PERIOD OF RECORD $1966-85$

\begin{tabular}{|c|c|c|c|c|c|c|}
\hline \multirow{5}{*}{$\begin{array}{l}\text { PERIOD } \\
\text { (CON- } \\
\text { SECU- } \\
\text { TIVE } \\
\text { DAYS) }\end{array}$} & \multicolumn{6}{|c|}{$\begin{array}{l}\text { DISCHARGE， IN FT3/S, FOR INDICATED } \\
\text { RECURRENCE INTERYAL, IN YEARS, AND }\end{array}$} \\
\hline & \multirow{2}{*}{\multicolumn{6}{|c|}{$\begin{array}{l}\text { RECURRENCE INTERVAL, IN YEARS, AND } \\
\text { NON-EXCEEDANCE PROBABILITY, IN PERCENT }\end{array}$}} \\
\hline & & & & & & \\
\hline & 2 & 5 & 10 & 20 & $50 \#$ & $100 \#$ \\
\hline & 508 & 208 & 108 & 58 & 28. & 18 \\
\hline 1 & 0.00 & 0.00 & 0.00 & 0.00 & 0.00 & 0.00 \\
\hline 3 & 0.00 & 0.00 & 0.00 & 0.00 & 0.00 & 0.00 \\
\hline 7 & 0.00 & 0.00 & 0.00 & 0.00 & 0.00 & 0.00 \\
\hline 14 & 0.00 & 0.00 & 0.00 & 0.00 & 0.00 & 0.00 \\
\hline 30 & 0.15 & 0.00 & 0.00 & 0.00 & 0.00 & 0.00 \\
\hline 60 & 0.33 & 0.04 & 0.00 & 0.00 & 0.00 & 0.00 \\
\hline 90 & 0.56 & 0.20 & 0.09 & 0.00 & 0.00 & 0.00 \\
\hline 120 & 0.73 & 0.34 & 0.22 & 0.14 & 0.08 & 0.06 \\
\hline 183 & 1.3 & 0.52 & 0.31 & 0.20 & 0.12 & 0.08 \\
\hline
\end{tabular}

MAGNITUDE AND PROBABILITY OF ANNUAL HIGH FLOW BASED ON PERIOD OF RECORD $1966 \cdot 85$

\begin{tabular}{|c|c|c|c|c|c|c|}
\hline & & \multicolumn{5}{|c|}{ DISCHARGE, IN FT $3 / S$, FOR INDICATED } \\
\hline $\begin{array}{l}\text { PERIOD } \\
\text { (CON- }\end{array}$ & & \multicolumn{3}{|c|}{$\begin{array}{l}\text { RECURRENCE INTERVAL, IN } \\
\text { EXCEEDANCE PROBABILITY, }\end{array}$} & \multicolumn{2}{|c|}{ IN PERCENT } \\
\hline SECU. & & $\ldots . .$. & & & ........ & \\
\hline TIVE & 2 & 5 & 10 & 25 & $50 \#$ & $100 \#$ \\
\hline DAYS) & 508 & 208 & 108 & 48 & 28 & 18 \\
\hline 1 & 487 & 1,950 & 3,690 & 6.870 & 9,950 & 13,600 \\
\hline 3 & 251 & 1,020 & 1,950 & 3,680 & 5,360 & 7,37 \\
\hline 7 & 130 & 538 & 1.070 & 2,160 & 3,320 & 4,810 \\
\hline 15 & 73 & 293 & 580 & 1,170 & 1,800 & 2,630 \\
\hline 30 & 44 & 171 & 341 & 696 & 1,090 & 1,640 \\
\hline 60 & 28 & 102 & 200 & 409 & 650 & 986 \\
\hline 90 & 20 & 71 & 137 & 279 & 446 & 681 \\
\hline
\end{tabular}

DURATION TABLE OF DAILY MEAN FLOW FOR PERIOD OF RECORD 1966-85

DISCHARGE, IN FT3/S, WHICH WAS EQUALED OR EXCEEDED FOR INDICATED PERCENT OF TIME

\begin{tabular}{|c|c|c|c|c|c|c|c|c|c|c|c|c|c|c|c|c|}
\hline 18 & 58 & 108 & 158 & 208 & 308 & 408 & 508 & 608 & 708 & 808 & 908 & 958 & 988 & 998 & 99.58 & 99.98 \\
\hline 257 & 34 & 7.7 & 4.9 & 4.0 & 3.0 & 2.3 & 1.9 & 1.5 & 1.0 & 0.54 & 0.13 & 0.00 & 0.00 & 0.00 & 0.00 & 0.00 \\
\hline
\end{tabular}

\# Reliability of values in column is uncertain, and potential errors are large. 
09502800 WILLIAMSON VALLEY WASH NEAR PAULDEN, AZ--Continued
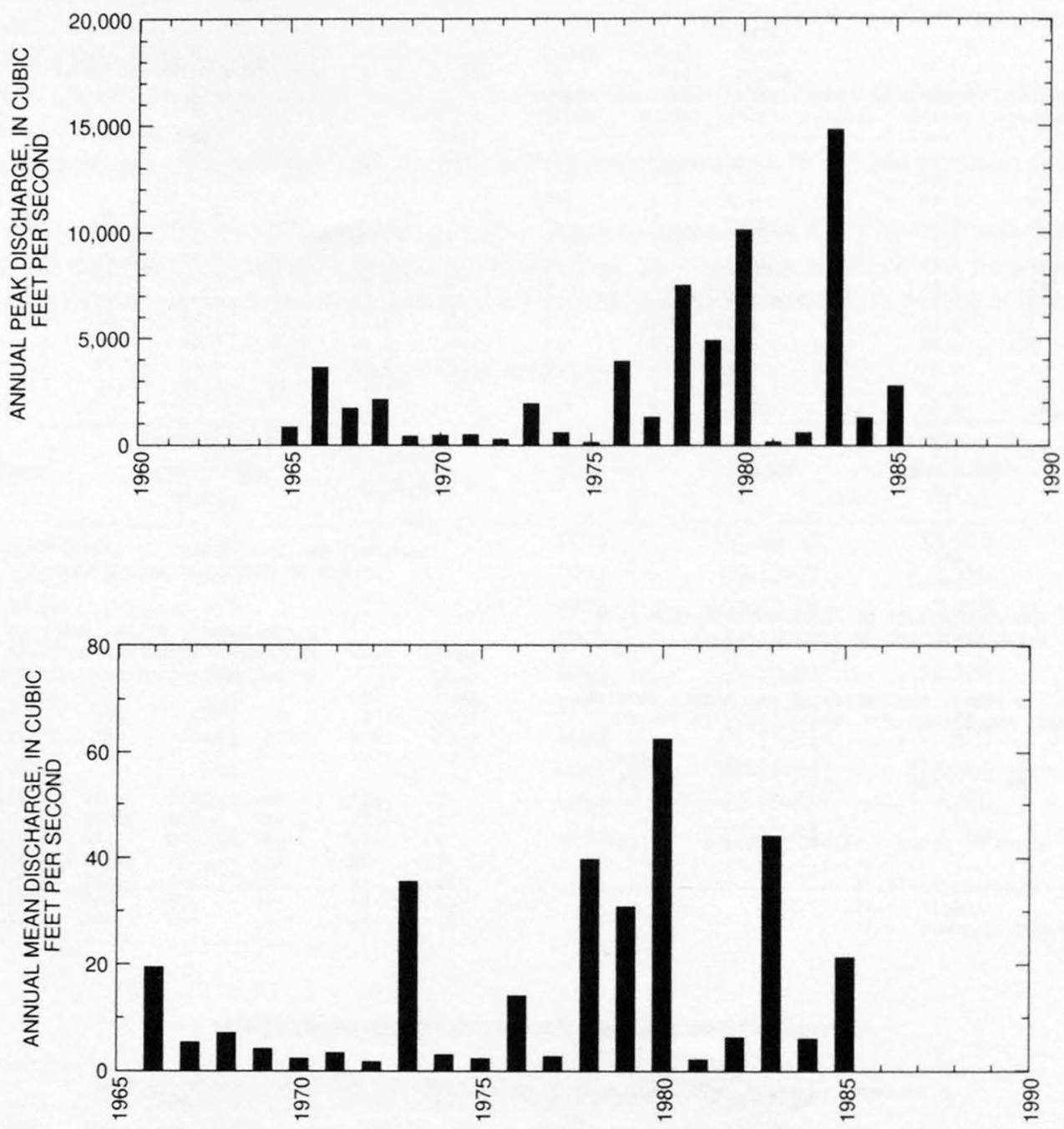
GILA RIVER BASIN

663

09502800 WILLIAMSON VALLEY WASH NEAR PAULDEN, AZ--Continued
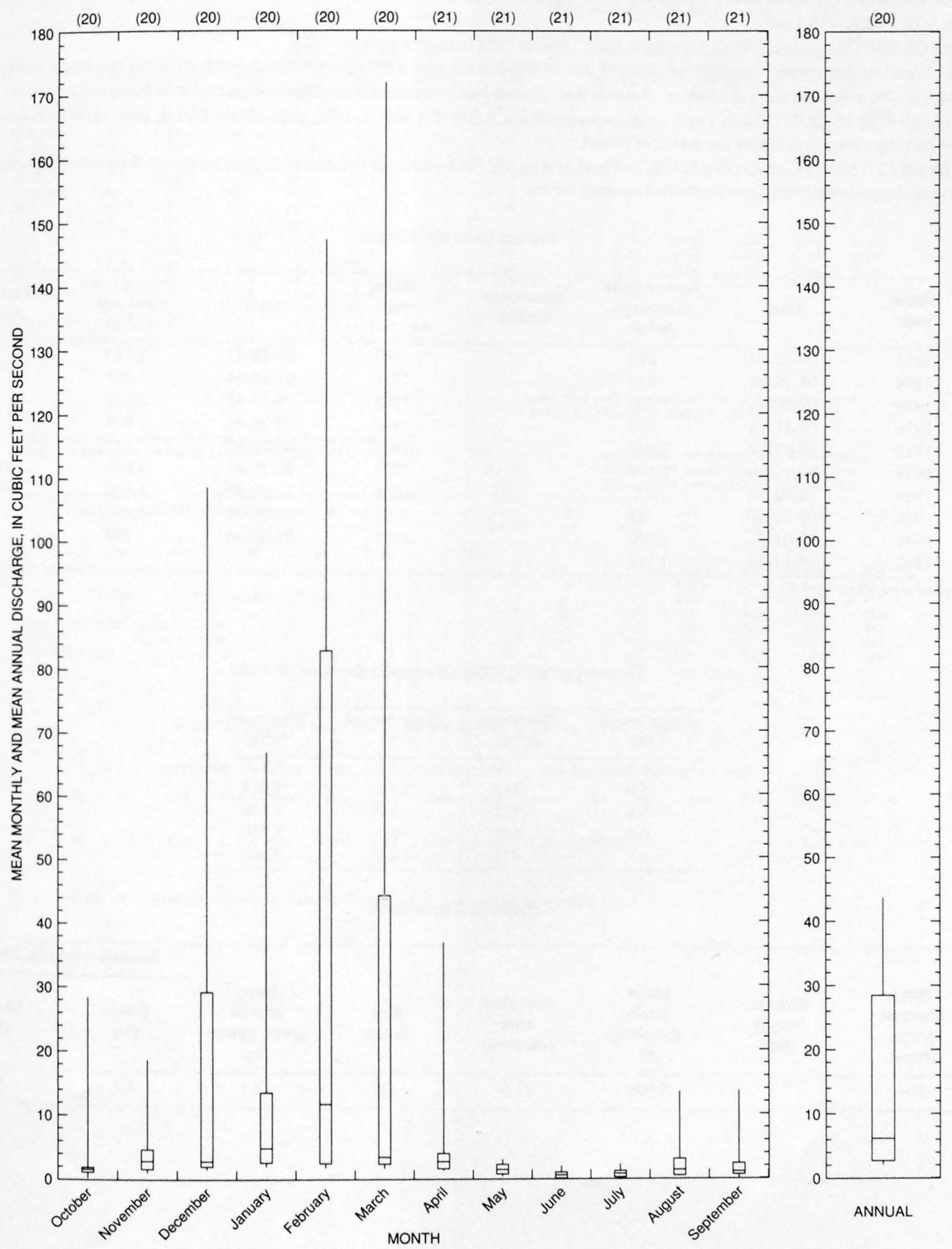

ANNUAL 
09503000 GRANITE CREEK NEAR PRESCOTT, AZ

LOCATION.--Lat $34^{\circ} 34^{\prime}$, long $112^{\circ} 27^{\prime}$, in SW $1 / 4$ sec.26, T.14 N., R.2 W., (unsurveyed), Yavapai County, Hydrologic Unit 15060202 , at bridge 0 n

U.S. Highway 89, 2 mi north of Prescott and 4.5 mi upstream from Willow Creek.

DRAINAGE AREA.--36.3 $\mathrm{mi}^{2}$.

PERIOD OF RECORD.--July 1932 to September 1947, October 1994 to current year.

GAGE.--Water-stage recorder. Elevation of gage is 5,204.29 ft above sea level from surveyed bench-mark elevation and levels survey.

REMARKS.--No estimated daily discharges. Records fair. Flow is partly regulated by Goldwater Reservoirs on Bannon Creek.

EXTREMES FOR PERIOD OF RECORD.--Maximum discharge, 3,200 ft $3 / \mathrm{s} \mathrm{Mar.} \mathrm{6,} \mathrm{1995,} \mathrm{gage-height} 8.90 \mathrm{ft}$, from slope-conveyance survey.

No flow for many days during the period of record.

EXTREMES OUTSIDE PERIOD OF RECORD.--Flood of Aug. 19, 1963, discharge of 6,660 $\mathrm{ft}^{3} / \mathrm{s}$, gage height $9.4 \mathrm{ft}$ (original gage height of 12.4

$\mathrm{ft}$ with datum correction), from contracted opening survey.

Annual peak discharges

\begin{tabular}{|c|c|c|c|c|c|c|c|}
\hline $\begin{array}{l}\text { Water } \\
\text { year }\end{array}$ & Date & $\begin{array}{c}\text { Annual peak } \\
\text { discharge } \\
\left(\mathrm{ft}^{3} / \mathrm{s}\right)\end{array}$ & $\begin{array}{l}\text { Discharge } \\
\text { codes }\end{array}$ & $\begin{array}{l}\text { Water } \\
\text { year }\end{array}$ & Date & $\begin{array}{c}\text { Annual peak } \\
\text { discharge } \\
\left(\mathrm{ft}^{3} / \mathrm{s}\right)\end{array}$ & $\begin{array}{c}\text { Discharge } \\
\text { codes }\end{array}$ \\
\hline 1933 & $09-07-33$ & 230 & & 1943 & $08-28-43$ & 1,780 & \\
\hline 1934 & $08-30-34$ & 450 & & 1944 & $03-14-44$ & 297 & \\
\hline 1935 & $08-30-35$ & 600 & & 1945 & $08-10-45$ & 2,200 & \\
\hline 1936 & $09-11-36$ & 500 & & 1946 & $07-20-46$ & 899 & \\
\hline 1937 & $02-07-37$ & 2,900 & & 1947 & $07-21-47$ & 251 & \\
\hline 1938 & $03-03-38$ & 2,400 & & 1963 & $08-19-63$ & ${ }^{1} 6,660$ & HP \\
\hline 1939 & 08-04-39 & 638 & & 1966 & $12-00-65$ & 1,500 & HP \\
\hline 1940 & $09-29-40$ & 83 & & 1995 & $03-06-95$ & 3,200 & \\
\hline 1941 & $03-01-41$ & 1,530 & & 1996 & $07-09-96$ & 795 & \\
\hline 1942 & $08-17-42$ & 1,110 & & & & & \\
\hline
\end{tabular}

${ }^{1}$ Highest since 1932.

Discharge rating table developed September 1994

\begin{tabular}{cccc}
\hline $\begin{array}{c}\text { Gage height } \\
(\mathbf{f t})\end{array}$ & $\begin{array}{c}\text { Discharge } \\
\left(\mathrm{ft}^{3} / \mathbf{s}\right)\end{array}$ & $\begin{array}{c}\text { Gage height } \\
(\mathbf{f t})\end{array}$ & $\begin{array}{c}\text { Discharge } \\
\left(\mathrm{ft}^{3} / \mathbf{s}\right)\end{array}$ \\
\hline 4.5 & 140 & 7.0 & 1,270 \\
5.0 & 262 & 7.5 & 1,680 \\
5.5 & 431 & 8.0 & 2,160 \\
6.0 & 652 & 8.5 & 2,700 \\
6.5 & 931 & 8.9 & 3,200 \\
\hline
\end{tabular}

Basin characteristics

\begin{tabular}{cccccccc}
\hline $\begin{array}{c}\text { Main } \\
\text { channel } \\
\text { slope } \\
(\mathrm{ft} / \mathrm{mi})\end{array}$ & $\begin{array}{c}\text { Stream } \\
\text { length } \\
(\mathrm{mi})\end{array}$ & $\begin{array}{c}\text { Mean } \\
\text { basin } \\
\text { elevation } \\
(\mathrm{ft})\end{array}$ & $\begin{array}{c}\text { Forested } \\
\text { area } \\
\text { (percent) }\end{array}$ & $\begin{array}{c}\text { Soil } \\
\text { index }\end{array}$ & $\begin{array}{c}\text { Mean } \\
\text { annual } \\
\text { precipitation } \\
\text { (in) }\end{array}$ & $\begin{array}{c}\text { Rainfall intensity, 24-hour } \\
\text { 2-year } \\
\text { (in) }\end{array}$ & $\begin{array}{c}50 \text {-year } \\
\text { (in) }\end{array}$ \\
\hline 104 & 7.3 & 5,900 & 73.0 & 1.0 & 22.1 & 2.2 \\
\hline
\end{tabular}


09503000 GRANITE CREEK NEAR PRESCOTT, AZ--Continued

MEAN MONTHLY AND ANNUAL DISCHARGES 1933-47, 1995-96

\begin{tabular}{|c|c|c|c|c|c|c|}
\hline MONTH & $\begin{array}{l}\text { MAXIMUM } \\
(\text { FT } 3 / S \text { ) }\end{array}$ & $\begin{array}{l}\text { MINIMUM } \\
\text { (FT3/S) }\end{array}$ & $\begin{array}{c}\text { MEAN } \\
(\mathrm{FT} 3 / \mathrm{S})\end{array}$ & $\begin{array}{l}\text { STAN- } \\
\text { DARD } \\
\text { DEVIA- } \\
\text { TION } \\
(\mathrm{FT} 3 / \mathrm{S})\end{array}$ & $\begin{array}{l}\text { COEFFI- } \\
\text { CIENT OF } \\
\text { VARI- } \\
\text { ATION }\end{array}$ & $\begin{array}{c}\text { PERCENT } \\
\text { OF } \\
\text { ANNUAL } \\
\text { RUNOFF }\end{array}$ \\
\hline OCTOBER & 7.3 & 0.00 & 0.73 & 1.8 & 2.4 & 1.0 \\
\hline NOVEMBER & 2.8 & 0.00 & 0.46 & 0.86 & 1.8 & 0.6 \\
\hline DECEMBER & 33 & 0.00 & 3.0 & 7.9 & 2.7 & 4.1 \\
\hline JANUARY & 35 & 0.00 & 5.1 & 11 & 2.1 & 7.0 \\
\hline FEBRUARY & 159 & 0.00 & 22 & 42 & 1.9 & 30.5 \\
\hline MARCH & 79 & 0.00 & 27 & 32 & 1.2 & 36.9 \\
\hline APRIL & 67 & 0.00 & 7.8 & 16 & 2.1 & 10.7 \\
\hline MAY & 7.0 & 0.00 & 0.95 & 1.8 & 1.8 & 1.3 \\
\hline JUNE & 1.2 & 0.00 & 0.19 & 0.32 & 1.6 & 0.3 \\
\hline JULY & 7.8 & 0.00 & 1.2 & 1.8 & 1.6 & 1.6 \\
\hline AUGUST & 8.3 & 0.00 & 2.5 & 2.6 & 1.0 & 3.5 \\
\hline SEPTEMBER & 12 & 0.00 & 1.8 & 3.2 & 1.7 & 2.5 \\
\hline ANNUAL & 24 & 0.37 & 6.0 & 7.2 & 1.2 & 100 \\
\hline
\end{tabular}

MAGNITUDE AND PROBABILITY OF INSTANTANEOUS PEAK FLOW BASED ON PERIOD OF RECORD 1933-47, 1963,1966, 1995-96

DISCHARGE, IN FT3/S, FOR INDICATED RECURRENCE INTERVAL IN YEARS, AND EXCEEDANCE PROBABILITY, IN PERCENT

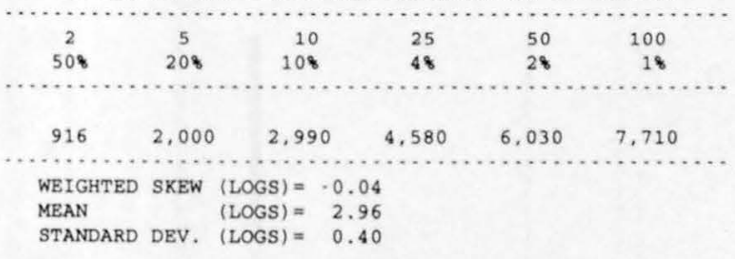

MAGNITUDE AND PROBABILITY OF ANNUAL LOW FLOW BASED ON PERIOD OF RECORD $1934-47,1996$

\begin{tabular}{|c|c|c|c|c|c|c|}
\hline \multirow{3}{*}{$\begin{array}{l}\text { PERIOD } \\
\text { ICON- } \\
\text { SECU- }\end{array}$} & \multicolumn{6}{|c|}{ DISCHARGE, IN FT3/S, FOR INDICATED } \\
\hline & \multicolumn{6}{|c|}{$\begin{array}{l}\text { RECURRENCE INTERVAL, IN YEARS, AND } \\
\text { NON-EXCEEDANCE PROBABILITY, IN PERCENT }\end{array}$} \\
\hline & $\cdots$ & $\ldots \ldots$ & 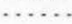 & 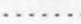 & $\ldots \ldots \ldots$ & $\ldots \ldots$ \\
\hline TIVE & 2 & 5 & $10 \#$ & $20 \#$ & $50 \#$ & $100 \#$ \\
\hline DAYS) & 508 & 208 & 108 & 58 & 28 & 18 \\
\hline 1 & & & & & & \\
\hline 3 & & & & & & \\
\hline 7 & & & & & & \\
\hline 14 & & & & & & \\
\hline 30 & & & & & & \\
\hline 60 & 0.00 & 0.00 & 0.00 & 0.00 & 0.00 & 0.00 \\
\hline 90 & 0.02 & 0.00 & 0.00 & 0.00 & 0.00 & 0.00 \\
\hline 120 & 0.09 & 0.00 & 0.00 & 0.00 & 0.00 & 0.00 \\
\hline 183 & 0.58 & 0.18 & 0.06 & 0.00 & 0.00 & 0.00 \\
\hline
\end{tabular}

MAGNITUDE AND PROBABILITY OF ANNUAL HIGH FLOW BASED ON PERIOD OF RECORD 1933-47, 1995-96

\begin{tabular}{|c|c|c|c|c|c|c|}
\hline \multirow{2}{*}{$\begin{array}{l}\text { PERIOD } \\
\text { (CON- } \\
\text { SECU- }\end{array}$} & \multicolumn{2}{|r|}{$\begin{array}{l}\text { DISCHARGE, } \\
\text { RECURRENCE } \\
\text { EXCEEDANCE }\end{array}$} & $\begin{array}{l}\text { IN } \\
\text { INT } \\
\text { PRO }\end{array}$ & $\begin{array}{l}3 / \mathrm{S}, \text { FOI } \\
\text { VAL, IN } \\
\text { BILITY, }\end{array}$ & $\begin{array}{l}\text { INDICA } \\
\text { YEARS, } \\
\text { IN PERC }\end{array}$ & $\begin{array}{l}\text { ED } \\
\text { ND } \\
\text { NT }\end{array}$ \\
\hline & & $\ldots \ldots \ldots \ldots$ & & & $\ldots \ldots$ & \\
\hline TIVE & 2 & 5 & $10 \#$ & $25 \#$ & $50 \#$ & $100 \#$ \\
\hline DAYS) & 508 & 208 & 108 & 48 & 28 & 18 \\
\hline 1 & 117 & 375 & 748 & 1,660 & 2,880 & 4,850 \\
\hline 3 & 69 & 221 & 426 & 898 & 1,490 & 2,380 \\
\hline 7 & 45 & 130 & 235 & 459 & 721 & 1,090 \\
\hline 15 & 29 & 84 & 155 & 309 & 492 & 759 \\
\hline 30 & 19 & 58 & 109 & 222 & 359 & 563 \\
\hline 60 & 12 & 37 & 69 & 141 & 229 & 362 \\
\hline 90 & 9.1 & 27 & 51 & 104 & 167 & 262 \\
\hline
\end{tabular}

DURATION TABLE OF DAILY MEAN FLOW FOR PERIOD OF RECORD $1933.47,1995.96$

DISCHARGE, IN FT3/S, WHICH WAS EQUALED OR EXCEEDED FOR INDICATED PERCENT OF TIME

\begin{tabular}{|c|c|c|c|c|c|c|c|c|c|c|c|c|c|c|c|c|}
\hline 18 & 58 & 108 & 158 & 208 & 308 & 408 & 508 & 608 & 708 & 808 & 908 & 958 & 988 & 998 & 99.58 & 99.98 \\
\hline 99 & 26 & 10 & 4.8 & 2.6 & 0.80 & 0.31 & 0.12 & 0.00 & 0.00 & 0.00 & 0.00 & 0.00 & 0.00 & 0.00 & 0.00 & 0.00 \\
\hline
\end{tabular}

\# Reliability of values in column is uncertain, and potential errors are large. 
ANNUAL MEAN DISCHARGE, IN CUBIC

FEET PER SECOND

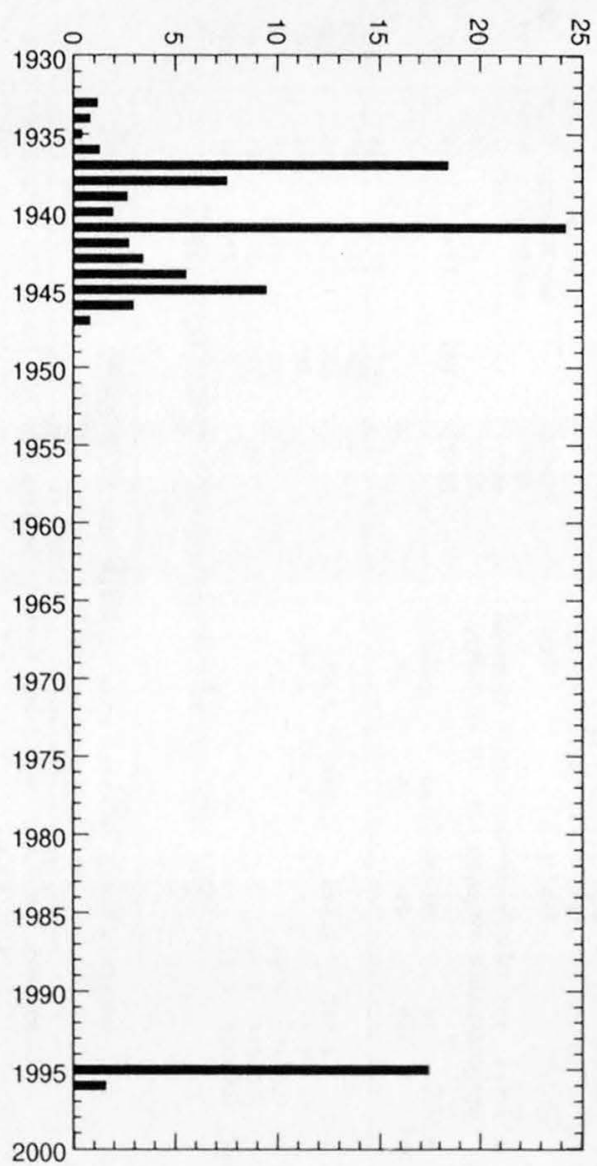

ANNUAL PEAK DISCHARGE, IN CUBIC FEET PER SECOND

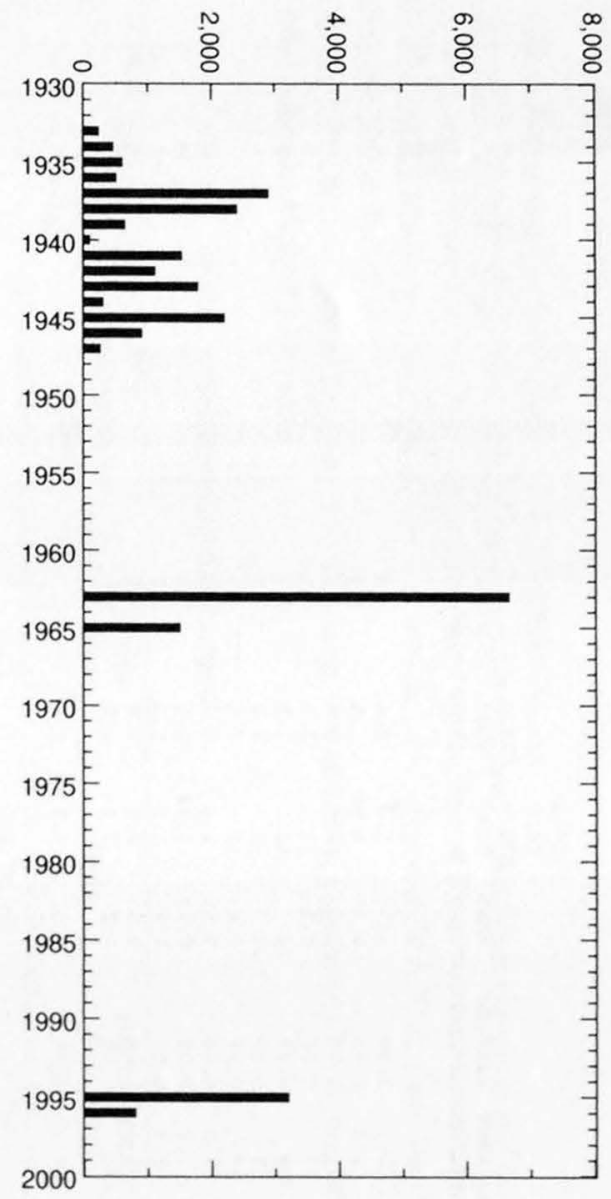


09503000 GRANITE CREEK NEAR PRESCOTT, AZ--Continued
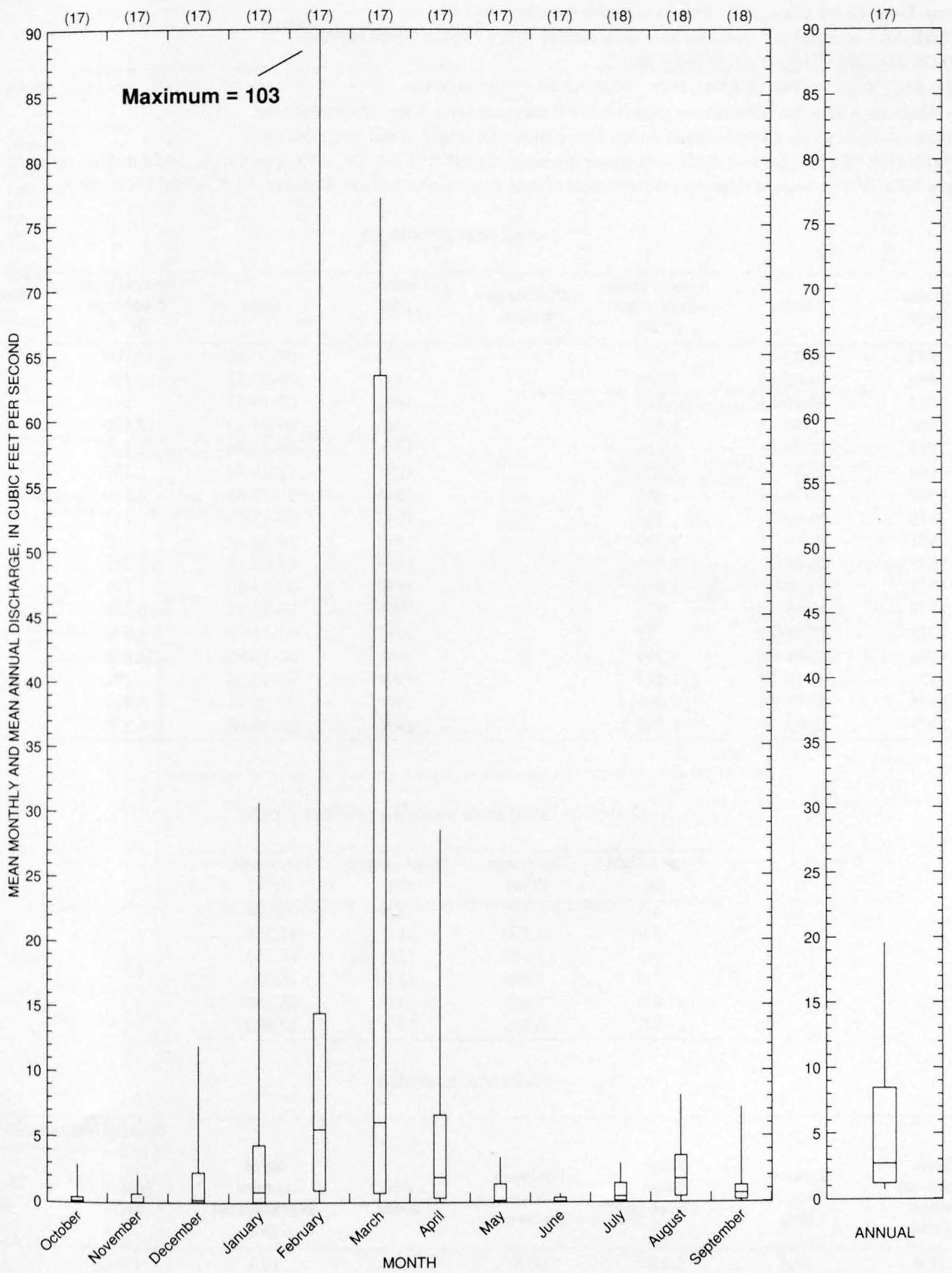


\section{VERDE RIVER NEAR PAULDEN, AZ}

LOCATION.--Lat 34'53'40", long 112'20'32", in SW $1 / 4 \mathrm{SE}^{1} / 4$ sec.39, T.18 N., R.1 W., Yavapai County, Hydrologic Unit 15060202, in Presce National Forest, on right bank 0.3 mi upstream from Verde Valley Ranch, 7 mi east of Paulden, 8 mi upstream from Hell Canyon, 8 mi dowz stream from Granite Creek, and 10 mi downstream from Sullivan Lake.

DRAINAGE AREA.--2,507 $\mathrm{mi}^{2}$ (includes $357 \mathrm{mi}^{2}$ in Aubrey Valley Playa, a closed basin).

PERIOD OF RECORD.--July 1963 to current year.

REVISED RECORDS.--WDR AZ-83-1: 1981. WDR AZ-89-1: Drainage area.

GAGE.--Water-stage recorder. Elevation of gage is $4,117 \mathrm{ft}$ above sea level, from topographic map.

REMARKS.--Records good. Diversions and storage above station for irrigation and municipal use.

EXTREMES FOR PERIOD OF RECORD.--Maximum discharge, 23,200 $\mathrm{ft}^{3} / \mathrm{s} \mathrm{Feb.} \mathrm{20,} \mathrm{1993,} \mathrm{gage} \mathrm{height,} 14.25 \mathrm{ft}$, from rating curve extendet above $7,600 \mathrm{ft}^{3} / \mathrm{s}$ on basis of slope-area measurement of peak flow; minimum daily discharge, $15 \mathrm{ft}^{3} / \mathrm{s} \mathrm{May} 13-23,1964$.

Annual peak discharges

\begin{tabular}{ccccccc}
\hline $\begin{array}{c}\text { Water } \\
\text { year }\end{array}$ & Date & $\begin{array}{c}\text { Annual peak } \\
\text { discharge } \\
\left(\mathbf{f t}^{3} / \mathbf{s}\right)\end{array}$ & $\begin{array}{c}\text { Discharge } \\
\text { codes }\end{array}$ & $\begin{array}{c}\text { Water } \\
\text { year }\end{array}$ & Date & $\begin{array}{c}\text { Annual peak } \\
\text { discharge } \\
\left(\mathrm{ft}^{3} / \mathbf{s}\right)\end{array}$ \\
\hline 1963 & $08-25-63$ & 710 & & 1980 & $02-20-80$ & 15,700 \\
1964 & $08-05-64$ & 1,270 & & 1981 & $08-09-81$ & 195 \\
1965 & $04-10-65$ & 685 & & 1982 & $03-16-82$ & 541 \\
1966 & $12-30-65$ & 6,130 & 1983 & $09-24-83$ & 15,600 \\
1967 & $12-07-66$ & 1,250 & 1984 & $09-01-84$ & 3,650 \\
1968 & $01-28-68$ & 1,800 & 1985 & $12-28-84$ & 2,390 \\
1969 & $07-26-69$ & 465 & 1986 & $11-30-85$ & 1,460 \\
1970 & $08-19-70$ & 705 & 1987 & $08-12-87$ & 217 \\
1971 & $08-13-71$ & 2,270 & 1988 & $04-26-88$ & 342 \\
1972 & $08-08-72$ & 1,620 & 1989 & $07-08-89$ & 263 \\
1973 & $10-20-72$ & 3,040 & 1990 & $07-15-90$ & 123 \\
1974 & $09-05-74$ & 270 & 1991 & $03-02-91$ & 6,320 \\
1975 & $07-09-75$ & 73 & 1992 & $02-14-92$ & 1,590 \\
1976 & $02-09-76$ & 4,340 & 1993 & $02-20-93$ & 23,200 \\
1977 & $09-12-77$ & 1,290 & 1994 & $09-02-94$ & 192 \\
1978 & $03-01-78$ & 8,080 & 1995 & $02-15-95$ & 3,960 \\
1979 & $12-19-78$ & 5,700 & 1996 & $07-15-96$ & 1,030 \\
\hline
\end{tabular}

Discharge rating table developed February 1995

\begin{tabular}{crcc}
\hline $\begin{array}{c}\text { Gage height } \\
(\mathbf{f t})\end{array}$ & $\begin{array}{c}\text { Discharge } \\
\left(\mathrm{ft}^{\mathbf{3}} \mathbf{/} \mathbf{s}\right)\end{array}$ & $\begin{array}{c}\text { Gage height } \\
(\mathbf{f t})\end{array}$ & $\begin{array}{c}\text { Discharge } \\
\left(\mathbf{f t}^{\mathbf{3}} \mathbf{s} \mathbf{s}\right)\end{array}$ \\
\hline 4.0 & 717 & 10.0 & 9,120 \\
5.0 & 1,370 & 11.0 & 11,750 \\
6.0 & 2,280 & 12.0 & 14,780 \\
7.0 & 3,490 & 13.0 & 18,250 \\
8.0 & 5,020 & 14.0 & 22,150 \\
9.0 & 6,890 & 14.2 & 22,990 \\
\hline
\end{tabular}

Basin characteristics

\begin{tabular}{cccccccc}
\hline $\begin{array}{c}\text { Main } \\
\text { channel } \\
\text { slope } \\
(\mathrm{ft} / \mathrm{mi})\end{array}$ & $\begin{array}{c}\text { Stream } \\
\text { length } \\
\text { (mi) }\end{array}$ & $\begin{array}{c}\text { Mean } \\
\text { basin } \\
\text { elevation } \\
\text { (ft) }\end{array}$ & $\begin{array}{c}\text { Forested } \\
\text { area } \\
\text { (percent) }\end{array}$ & $\begin{array}{c}\text { Soil } \\
\text { index }\end{array}$ & $\begin{array}{c}\text { Mean } \\
\text { annual } \\
\text { precipitation } \\
\text { (in) }\end{array}$ & $\begin{array}{c}\text { Rainfall intensity, 24-hour } \\
\text { (in) }\end{array}$ & $\begin{array}{c}50 \text {-year } \\
\text { (in) }\end{array}$ \\
\hline 23.8 & 78.4 & 5,410 & 60.0 & 2.8 & 16.3 & 1.9 \\
\hline
\end{tabular}


09503700 VERDE RIVER NEAR PAULDEN, AZ--Continued

MEAN MONTHLY AND ANNUAL DISCHARGES 1964-96

\begin{tabular}{|c|c|c|c|c|c|c|}
\hline MONTH & $\begin{array}{l}\text { MAXIMUM } \\
(\text { FT3/S) }\end{array}$ & $\begin{array}{l}\text { MINIMUM } \\
\text { (FT3/S) }\end{array}$ & $\begin{array}{c}\text { MEAN } \\
(\mathrm{FT} 3 / \mathrm{S})\end{array}$ & $\begin{array}{l}\text { STAN- } \\
\text { DARD } \\
\text { DEVIA- } \\
\text { TION } \\
(\mathrm{FT} 3 / \mathrm{S})\end{array}$ & $\begin{array}{l}\text { COEFFI- } \\
\text { CIENT OF } \\
\text { VARI- } \\
\text { ATION }\end{array}$ & $\begin{array}{c}\text { PERCENT } \\
\text { OF } \\
\text { ANNUAL } \\
\text { RUNOFF }\end{array}$ \\
\hline OCTOBER & 200 & 19 & 32 & 33 & 1.0 & 5.8 \\
\hline NOVEMBER & 44 & 20 & 27 & 5.2 & 0.19 & 4.9 \\
\hline DECEMBER & 295 & 22 & 44 & 57 & 1.3 & 7.9 \\
\hline JANUARY & 861 & 22 & 63 & 149 & 2.4 & 11.4 \\
\hline FEBRUARY & 1,440 & 20 & 127 & 325 & 2.6 & 23.1 \\
\hline MARCH & 669 & 19 & 80 & 128 & 1.6 & 14.5 \\
\hline APRIL & 155 & 21 & 33 & 25 & 0.76 & 6.0 \\
\hline MAY & 31 & 16 & 25 & 2.8 & 0.11 & 4.6 \\
\hline JUNE & 28 & 20 & 24 & 2.1 & 0.08 & 4.4 \\
\hline JULY & 36 & 21 & 26 & 3.6 & 0.14 & 4.8 \\
\hline AUGUST & 81 & 23 & 31 & 12 & 0.39 & 5.6 \\
\hline SEPTEMBER & 440 & 20 & 39 & 72 & 1.9 & 7.0 \\
\hline ANNUAL & 215 & 24 & 45 & 40 & 0.87 & 100 \\
\hline
\end{tabular}

MAGNITUDE AND PROBABILITY OF INSTANTANEOUS PEAK FLOW BASED ON PERIOD OF RECORD 1963-96

DISCHARGE, IN FT3/S, FOR INDICATED RECURRENCE INTERVAL IN YEARS, AND EXCEEDANCE PROBABILITY, IN PERCENT

\begin{tabular}{|c|c|c|c|c|c|}
\hline 2 & 5 & 10 & 25 & 50 & 100 \\
\hline 508 & 208 & 108 & 48 & 28 & 18 \\
\hline 1,380 & 4,550 & 8,540 & 16,700 & 25,900 & 38,400 \\
\hline
\end{tabular}

WEIGHTED SKEW (LOGS) $=0.03$

MEAN (LOGS) $=3.14$

STANDARD DEV. $($ LOGS $)=0.61$
MAGNITUDE AND PROBABILITY OF ANNUAL LOW FLOW BASED ON PERIOD OF RECORD 1965.96

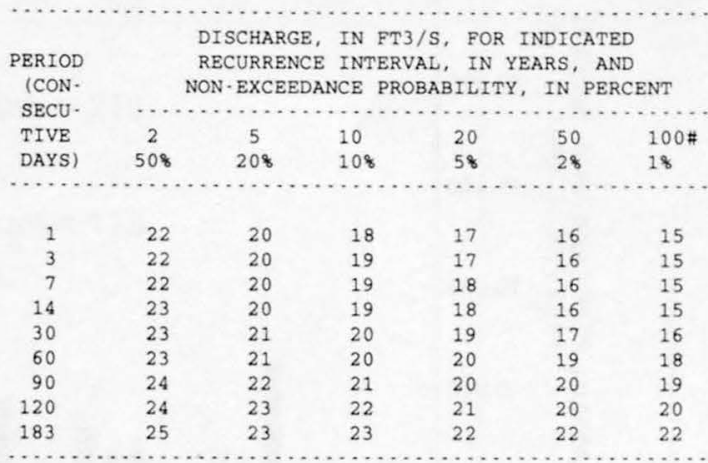

MAGNITUDE AND PROBABILTTY OF ANNUAL HIGH FLOW BASED ON PERTOD OF RECORD 1964.96

\begin{tabular}{|c|c|c|c|c|c|c|}
\hline \multirow{3}{*}{$\begin{array}{l}\text { PERIOD } \\
\text { ICON- } \\
\text { SECU- }\end{array}$} & \multicolumn{6}{|c|}{ DISCHARGE, IN FT3/S, FOR INDICATED } \\
\hline & & EXCEEDA & CE PRO & BILITY, & IN PERC & ENT \\
\hline & & .......... & $\cdots \ldots$ & $\cdots \cdots$ & $\cdots \cdots$ & \\
\hline TIVE & 2 & 5 & 10 & 25 & 50 & $100 \#$ \\
\hline DAYS) & 508 & 208 & 108 & 48 & 28 & 18 \\
\hline 1 & 513 & 2,040 & 4,340 & 9,930 & 17,200 & 28,300 \\
\hline 3 & 318 & 1,200 & 2,500 & 5,660 & 9,750 & 16,100 \\
\hline 7 & 184 & 634 & 1,300 & 2,930 & 5,110 & 8,610 \\
\hline 15 & 115 & 365 & 738 & 1,700 & 3,040 & 5,300 \\
\hline 30 & 81 & 231 & 447 & 990 & 1,740 & 3,000 \\
\hline 60 & 57 & 145 & 270 & 580 & 1,010 & 1,740 \\
\hline 90 & 48 & 110 & 193 & 394 & 663 & 1,110 \\
\hline
\end{tabular}

DURATION TABLE OF DAILY MEAN FLOW FOR PERIOD OF RECORD 1964.96

DISCHARGE, IN FT3/S, WHICH WAS EQUALED OR EXCEEDED FOR INDICATED PERCENT OF TIME

\begin{tabular}{|c|c|c|c|c|c|c|c|c|c|c|c|c|c|c|c|}
\hline 5 \% & 108 & 158 & 208 & 308 & 408 & 508 & 608 & 708 & 808 & 908 & 958 & 988 & 998 & 99.58 & 99.98 \\
\hline 46 & 32 & 31 & 30 & 28 & 27 & 26 & 25 & 24 & 23 & 22 & 21 & 19 & 18 & 18 & 16 \\
\hline
\end{tabular}

\# Reliability of values in column is uncertain, and potential errors are large. 
ANNUAL MEAN DISCHARGE, IN CUBIC

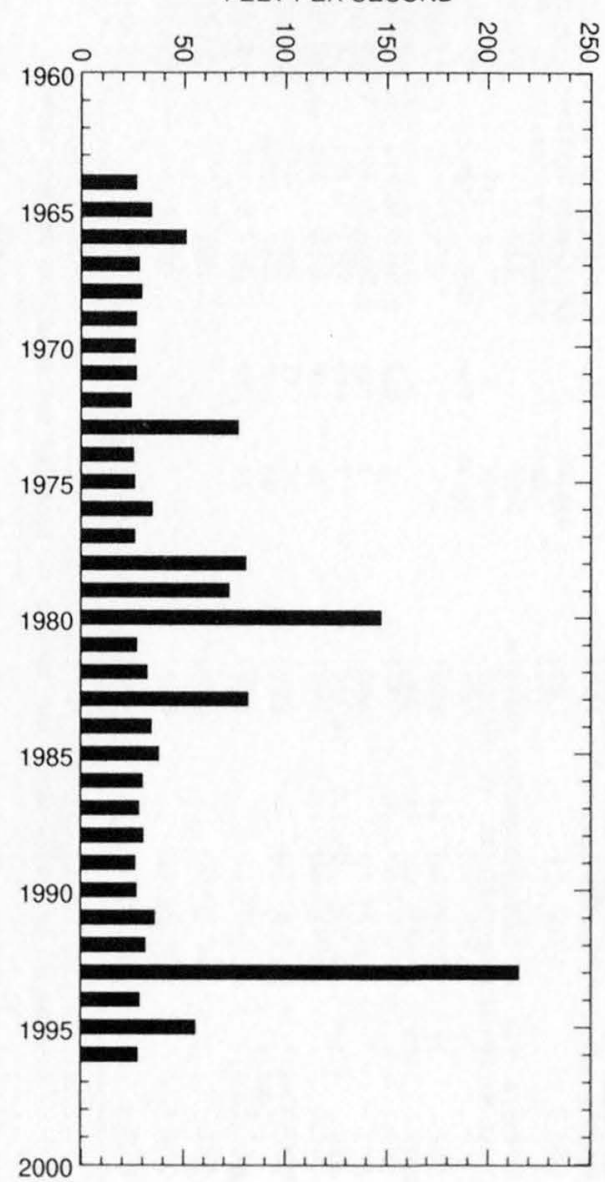

ANNUAL PEAK DISCHARGE IN CUBIC FEET PER SECOND

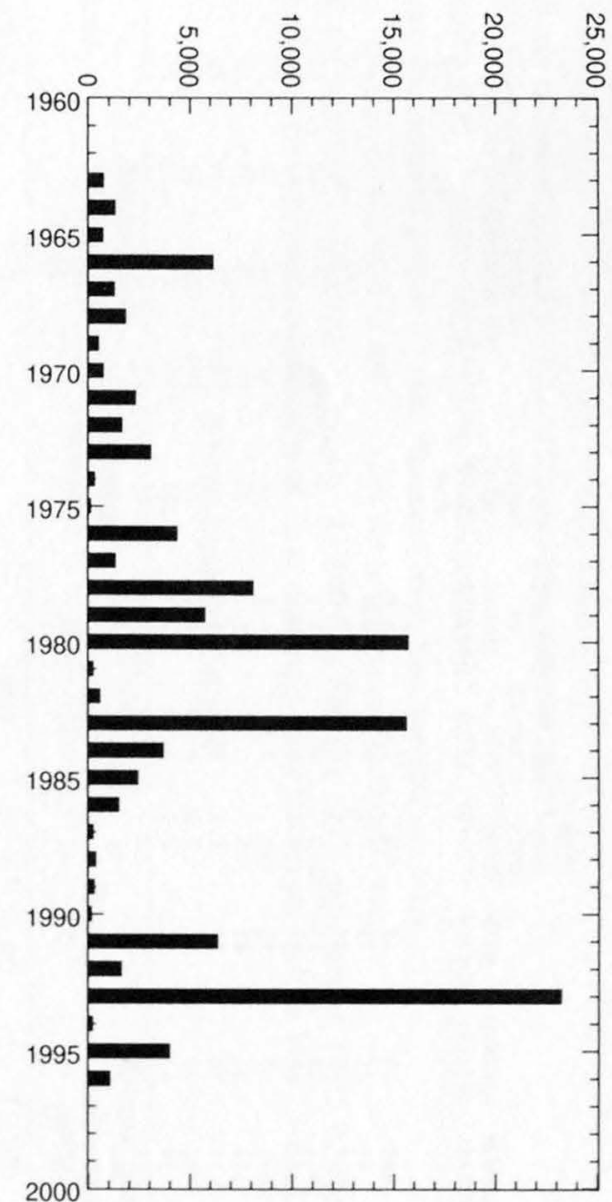


GILA RIVER BASIN

671

09503700 VERDE RIVER NEAR PAULDEN, AZ--Continued
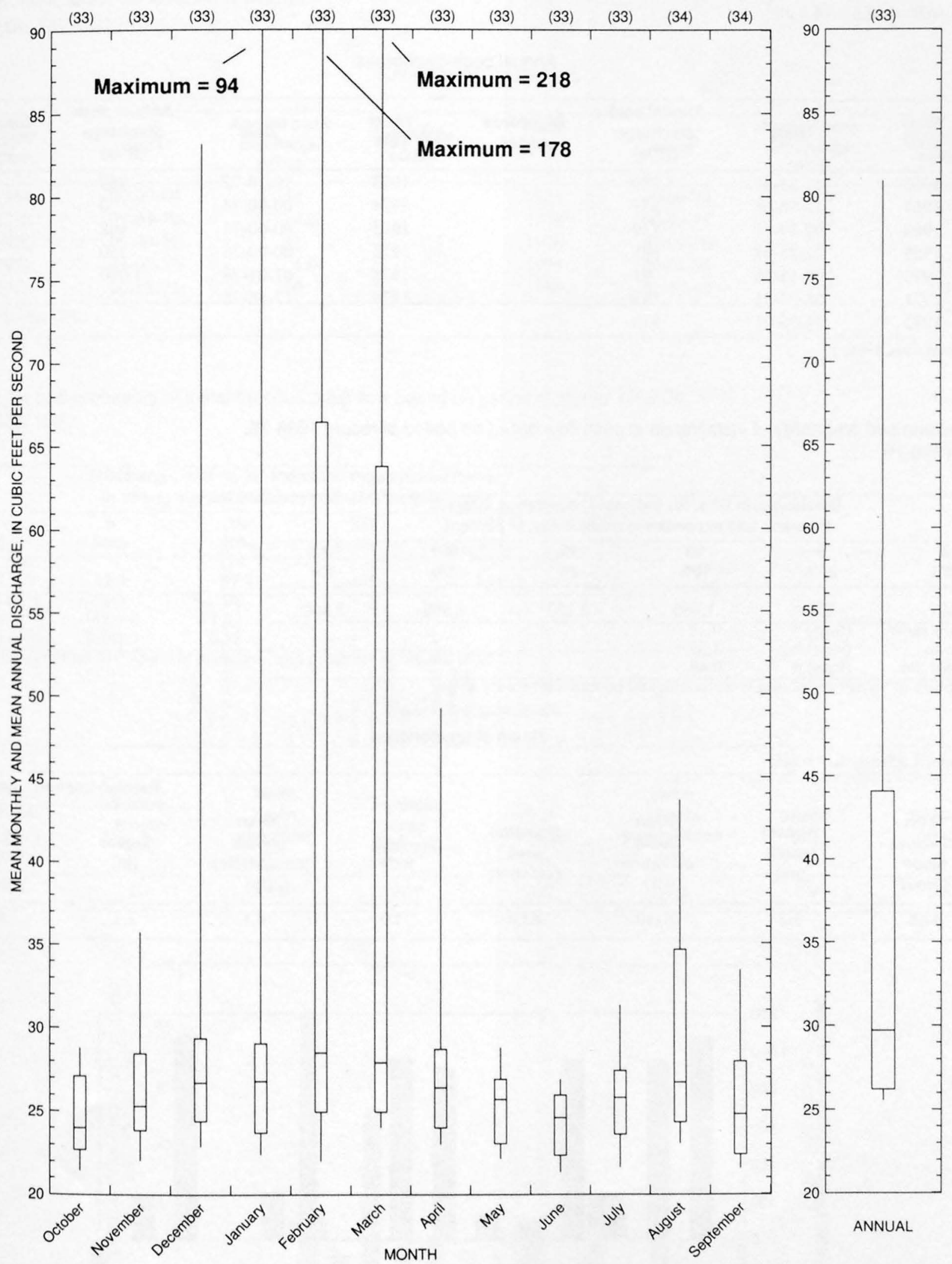
09503720 HELL CANYON NEAR WILLIAMS, AZ

LOCATION.--Lat $35^{\circ} 09^{\prime} 37^{\prime \prime}$, long $112^{\circ} 12^{\prime} 35^{\prime \prime}$, in NW1/4 NW $1 / 4$ sec.32, T.21 N., R.2 E., Coconino County, Hydrologic Unit 15060202 , in Kaibas National Forest, on right bank 6 mi south of Williams.

DRAINAGE AREA.--14.9 $\mathrm{mi}^{2}$.

Annual peak discharges

\begin{tabular}{|c|c|c|c|c|c|c|c|}
\hline $\begin{array}{l}\text { Water } \\
\text { year }\end{array}$ & Date & $\begin{array}{c}\text { Annual peak } \\
\text { discharge } \\
\left(\mathrm{ft}^{3} / \mathrm{s}\right)\end{array}$ & $\begin{array}{l}\text { Discharge } \\
\text { codes }\end{array}$ & $\begin{array}{l}\text { Water } \\
\text { year }\end{array}$ & Date & $\begin{array}{c}\text { Annual peak } \\
\text { discharge } \\
\left(\mathrm{ft}^{3} / \mathrm{s}\right)\end{array}$ & $\begin{array}{c}\text { Discharge } \\
\text { codes }\end{array}$ \\
\hline 1966 & $11-25-65$ & 1,080 & & 1973 & $10-19-72$ & 960 & \\
\hline 1967 & $12-06-66$ & 955 & & 1974 & $00-00-74$ & 0 & \\
\hline 1968 & $02-24-68$ & 139 & & 1975 & $00-00-75$ & 108 & \\
\hline 1969 & $01-25-69$ & 955 & & 1976 & $00-00-76$ & 250 & \\
\hline 1970 & $03-15-70$ & 91 & & 1978 & $03-01-78$ & ${ }^{1} 1,080$ & \\
\hline 1971 & $08-04-71$ & 133 & & 1979 & $12-18-78$ & 220 & \\
\hline 1972 & $12-26-71$ & 910 & & & & & \\
\hline
\end{tabular}

${ }^{1}$ Highest since 1966.

Magnitude and probability of instantaneous peak flow based on period of record 1966-76, 1978-79

\begin{tabular}{|c|c|c|c|c|c|}
\hline \multicolumn{6}{|c|}{$\begin{array}{l}\text { Discharge, in } \mathrm{ft}^{3} / \mathrm{s} \text {, for indicated recurrence interval } \\
\text { in years, and exceedance probablility, in percent }\end{array}$} \\
\hline 2 & 5 & 10 & 25 & $50 \dagger$ & $100 \dagger$ \\
\hline $50 \%$ & $20 \%$ & $10 \%$ & $4 \%$ & $2 \%$ & $1 \%$ \\
\hline 346 & 862 & 1,360 & 2,190 & 2,960 & 3,860 \\
\hline Weighted skew & $(\log s)=$ & -0.18 & & & \\
\hline Mean & $(\log s)=$ & 2.53 & & & \\
\hline Standard dev. & $(\log s)=$ & 0.48 & & & \\
\hline
\end{tabular}

Basin characteristics

\begin{tabular}{ccccccc}
\hline $\begin{array}{c}\text { Main } \\
\text { channel } \\
\text { slope } \\
(\mathrm{ft} / \mathrm{mi})\end{array}$ & $\begin{array}{c}\text { Stream } \\
\text { length } \\
\text { (mi) }\end{array}$ & $\begin{array}{c}\text { Mean } \\
\text { basin } \\
\text { elevation } \\
(\mathrm{ft})\end{array}$ & $\begin{array}{c}\text { Forested } \\
\text { area } \\
\text { (percent) }\end{array}$ & $\begin{array}{c}\text { Soil } \\
\text { index }\end{array}$ & $\begin{array}{c}\text { Mean } \\
\text { annual } \\
\text { precipitation } \\
\text { (in) }\end{array}$ & $\begin{array}{c}\text { Rainfall intensity, 24-hour } \\
\text { (in) }\end{array}$ \\
\hline 47.8 & 5.3 & 7,110 & 88.0 & 3.0 & 24.1 & $\begin{array}{c}50 \text {-year } \\
\text { (in) }\end{array}$ \\
\hline
\end{tabular}

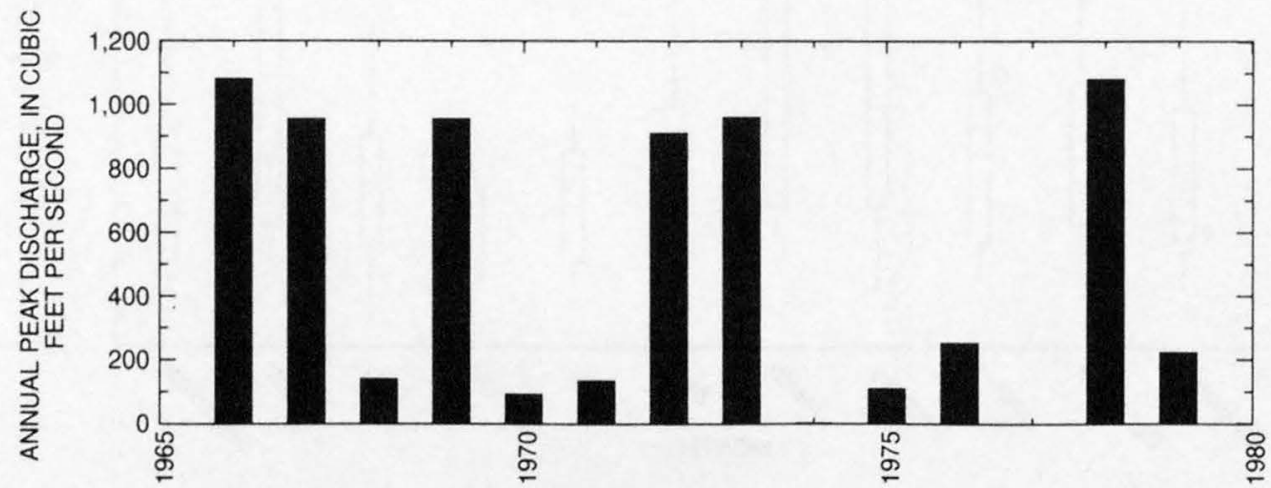


09503740 HELL CANYON TRIBUTARY NEAR ASHFORK, AZ

LOCATION.--Lat $35^{\circ} 05^{\prime} 02^{\prime \prime}$, long $112^{\circ} 24^{\prime} 28^{\prime \prime}$, in SWl/4 sec.30, T.20 N., R.1 W., Yavapai County, Hydrologic Unit $15060202,0.5$ mi upstream from mouth, and $11 \mathrm{mi}$ southeast of Ashfork.

DRAINAGE AREA.--0.75 $\mathrm{mi}^{2}$.

Annual peak discharges

\begin{tabular}{|c|c|c|c|c|c|c|c|}
\hline $\begin{array}{l}\text { Water } \\
\text { year }\end{array}$ & Date & $\begin{array}{c}\text { Annual peak } \\
\text { discharge } \\
\left(\mathrm{ft}^{3} / \mathrm{s}\right)\end{array}$ & $\begin{array}{l}\text { Discharge } \\
\text { codes }\end{array}$ & $\begin{array}{l}\text { Water } \\
\text { year }\end{array}$ & Date & $\begin{array}{c}\text { Annual peak } \\
\text { discharge } \\
\left(\mathrm{ft}^{3} / \mathrm{s}\right)\end{array}$ & $\begin{array}{c}\text { Discharge } \\
\text { codes }\end{array}$ \\
\hline 1969 & $00-00-69$ & 84 & & 1974 & $00-00-74$ & 0 & \\
\hline 1970 & $09-04-70$ & 4.0 & & 1975 & $00-00-75$ & 0 & \\
\hline 1971 & $00-00-71$ & 52 & & 1976 & $02-09-76$ & 10 & \\
\hline 1972 & $04-00-72$ & 4.0 & & 1978 & 03-01-78 & ${ }^{1} 24$ & HP \\
\hline 1973 & $10-19-72$ & 10 & & 1980 & $02-19-80$ & 20 & HP \\
\hline
\end{tabular}

${ }^{1}$ Highest since 1971.

Magnitude and probability of instantaneous peak flow based on period of record 1969-76, 1978,1980

\begin{tabular}{|c|c|c|c|c|c|}
\hline \multicolumn{6}{|c|}{$\begin{array}{l}\text { Discharge, in } \mathrm{ft}^{3} / \mathrm{s} \text {, for indicated recurrence interval } \\
\text { in years, and exceedance probablility, in percent }\end{array}$} \\
\hline 2 & 5 & 10 & $25 \dagger$ & $50 t$ & $100 t$ \\
\hline $50 \%$ & $20 \%$ & $10 \%$ & $4 \%$ & $2 \%$ & $1 \%$ \\
\hline 10.5 & 32.3 & 57.4 & 105 & 156 & 220 \\
\hline Weighted skew & $(\log s)=$ & -0.08 & & & \\
\hline Mean & $(\log s)=$ & 1.02 & & & \\
\hline Standard dev. & $(\log s)=$ & 0.58 & & & \\
\hline
\end{tabular}

fReliability of values in column is uncertain, and potential errors are large.

Basin characteristics

\begin{tabular}{cccccccc}
\hline $\begin{array}{c}\text { Main } \\
\text { channel } \\
\text { slope } \\
(\mathrm{ft} / \mathrm{mi})\end{array}$ & $\begin{array}{c}\text { Stream } \\
\text { length } \\
\text { (mi) }\end{array}$ & $\begin{array}{c}\text { Mean } \\
\text { basin } \\
\text { elevation } \\
\text { (ft) }\end{array}$ & $\begin{array}{c}\text { Forested } \\
\text { area } \\
\text { (percent) }\end{array}$ & $\begin{array}{c}\text { Soil } \\
\text { index }\end{array}$ & $\begin{array}{c}\text { Mean } \\
\text { annual } \\
\text { precipitation } \\
\text { (in) }\end{array}$ & $\begin{array}{c}\text { R-year } \\
\text { (in) }\end{array}$ & $\begin{array}{c}\text { 50-year } \\
\text { (in) }\end{array}$ \\
\hline 78.4 & 1.7 & 5,180 & 91.0 & 3.0 & 17.2 & 1.7 \\
\hline
\end{tabular}

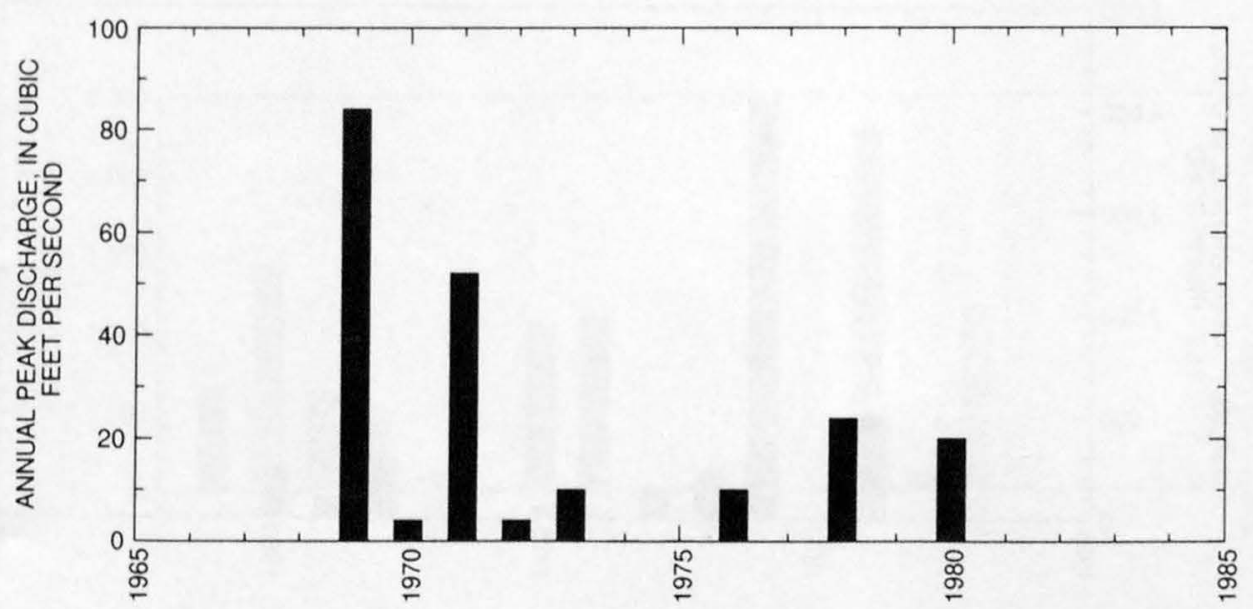




\section{LIMESTONE CANYON NEAR PAULDEN, AZ}

LOCATION.--Lat $34^{\circ} 58^{\prime} 48^{\prime \prime}$, long $112^{\circ} 24^{\prime} 05^{\prime \prime}$, in $\mathrm{S}^{1} / 2 \mathrm{sec} .31$, T.19 N., R.1 W., Yavapai County, Hydrologic Unit 15060202, 1.3 mi upstream fron mouth, $1.5 \mathrm{mi}$ west of drake, and $7.5 \mathrm{mi}$ northeast of Paulden.

DRAINAGE AREA.--14.5 $\mathrm{mi}^{2}$.

Annual peak discharges

\begin{tabular}{cccccccc}
\hline $\begin{array}{l}\text { Water } \\
\text { year }\end{array}$ & Date & $\begin{array}{c}\text { Annual peak } \\
\text { discharge } \\
\left(\mathrm{ft}^{3} / \mathbf{s}\right)\end{array}$ & $\begin{array}{c}\text { Discharge } \\
\text { codes }\end{array}$ & $\begin{array}{c}\text { Water } \\
\text { year }\end{array}$ & Date & $\begin{array}{c}\text { Annual peak } \\
\text { discharge } \\
\left(\mathrm{ft}^{3} / \mathbf{s}\right)\end{array}$ & $\begin{array}{c}\text { Discharge } \\
\text { codes }\end{array}$ \\
\hline 1969 & $00-00-69$ & 1,100 & & 1975 & $11-02-74$ & 125 & \\
1970 & $09-04-70$ & 10 & ES & 1976 & $00-00-76$ & 1.0 & ES \\
1971 & $08-12-71$ & 4,100 & & 1978 & $03-01-78$ & 1580 & \\
1972 & $08-12-72$ & 470 & & 1979 & $12-18-78$ & 200 & \\
1973 & $10-07-72$ & 275 & & 1980 & $02-14-80$ & 500 & \\
\hline
\end{tabular}

${ }^{1}$ Highest since 1971.

Magnitude and probability of instantaneous peak flow based on period of record 1969-76, $1978-80$

\begin{tabular}{|c|c|c|c|c|c|}
\hline \multicolumn{6}{|c|}{$\begin{array}{l}\text { Discharge, in } \mathrm{ft}^{3} / \mathrm{s} \text {, for indicated recurrence interval } \\
\text { in years, and exceedance probablility, in percent }\end{array}$} \\
\hline 2 & 5 & 10 & $25 t$ & $50 t$ & $100 t$ \\
\hline $50 \%$ & $20 \%$ & $10 \%$ & $4 \%$ & $2 \%$ & $1 \%$ \\
\hline 239 & 1,020 & 2,080 & 4,270 & 6,670 & 9,820 \\
\hline Weighted skew & $(\log s)=$ & -0.30 & & & \\
\hline Mean & $(\log s)=$ & 2.34 & & & \\
\hline Standard dev. & $(\log s)=$ & 0.78 & & & \\
\hline
\end{tabular}

f Reliability of values in column is uncertain, and potential errors are large.

Basin characteristics

\begin{tabular}{ccccccc}
\hline $\begin{array}{c}\text { Main } \\
\text { channel } \\
\text { slope } \\
(\mathrm{ft} / \mathrm{mi})\end{array}$ & $\begin{array}{c}\text { Stream } \\
\text { length } \\
\text { (mi) }\end{array}$ & $\begin{array}{c}\text { Mean } \\
\text { basin } \\
\text { elevation } \\
\text { (ft) }\end{array}$ & $\begin{array}{c}\text { Forested } \\
\text { area } \\
\text { (percent) }\end{array}$ & $\begin{array}{c}\text { Soil } \\
\text { index }\end{array}$ & $\begin{array}{c}\text { Mean } \\
\text { annual } \\
\text { precipitation } \\
\text { (in) }\end{array}$ & $\begin{array}{c}\text { Rainfall intensity, 24-hour } \\
\text { (in) }\end{array}$ \\
\hline 87.3 & 8.4 & 5,310 & 100 & 3.0 & 15.5 & $\begin{array}{c}50 \text {-year } \\
\text { (in) }\end{array}$ \\
\hline
\end{tabular}

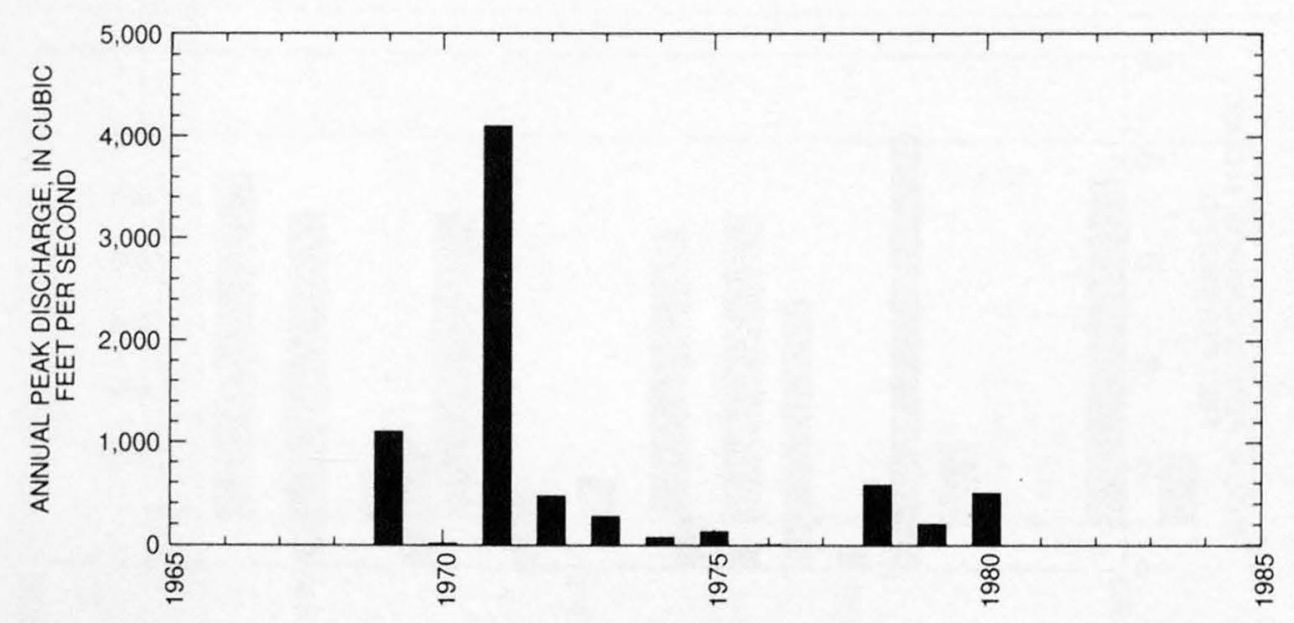




\section{VOLUNTEER WASH NEAR BELLEMONT, AZ}

LOCATION.--Lat 35 $09^{\prime} 02^{\prime \prime}$, long $111^{\circ} 53^{\prime} 54^{\prime \prime}$, in $\mathrm{SE}^{1} / \mathrm{SE}^{1} / 4 \mathrm{sec} .31$, T.21 N., R.5 E., Coconino County, Hydrologic Unit 15060202 , in Kaibab

National Forest, in Navajo Army Depot military reservation, on right bank 7 mi southwest of Bellemont, and 14 mi west of Flagstaff.

DRAINAGE AREA.--131 $\mathrm{mi}^{2}$, of which $6.93 \mathrm{mi}^{2}$ is noncontributing.

Annual peak discharges

\begin{tabular}{|c|c|c|c|c|c|c|c|}
\hline $\begin{array}{l}\text { Water } \\
\text { year }\end{array}$ & Date & $\begin{array}{c}\text { Annual peak } \\
\text { discharge } \\
\left(\mathrm{ft}^{3} / \mathrm{s}\right)\end{array}$ & $\begin{array}{l}\text { Discharge } \\
\text { codes }\end{array}$ & $\begin{array}{l}\text { Water } \\
\text { year }\end{array}$ & Date & $\begin{array}{c}\text { Annual peak } \\
\text { discharge } \\
\left(\mathrm{ft}^{3} / \mathrm{s}\right)\end{array}$ & $\begin{array}{c}\text { Discharge } \\
\text { codes }\end{array}$ \\
\hline 1966 & $03-12-66$ & 660 & & 1973 & $10-19-72$ & 1,100 & \\
\hline 1967 & $12-07-66$ & 1,430 & & 1974 & $00-00-74$ & 0 & \\
\hline 1968 & $02-24-68$ & 632 & & 1975 & $00-00-75$ & 49 & \\
\hline 1969 & $01-26-69$ & 447 & & 1976 & $00-00-76$ & 180 & \\
\hline 1970 & 04-04-70 & 96 & & 1978 & $03-01-78$ & ${ }^{1} 2,300$ & \\
\hline 1971 & $00-00-71$ & 0 & & 1979 & $12-18-78$ & 100 & \\
\hline 1972 & $12-26-71$ & 1,050 & & 1980 & $02-19-80$ & 1,160 & \\
\hline
\end{tabular}

${ }^{1}$ Highest since 1965.

Magnitude and probability of instantaneous peak flow based on period of record 1966-80

\begin{tabular}{|c|c|c|c|c|c|}
\hline \multicolumn{6}{|c|}{$\begin{array}{l}\text { Discharge, in } \mathrm{ft}^{3} / \mathrm{s} \text {, for indicated recurrence interval } \\
\text { in years, and exceedance probablility, in percent }\end{array}$} \\
\hline 2 & 5 & 10 & 25 & $50 \dagger$ & $100 t$ \\
\hline $50 \%$ & $20 \%$ & $10 \%$ & $4 \%$ & $2 \%$ & $1 \%$ \\
\hline 357 & 1,160 & 2,040 & 3,610 & 5,110 & 6,890 \\
\hline Weighted skew & $(\log s)=$ & -0.37 & & & \\
\hline Mean & $(\log s)=$ & 2.51 & & & \\
\hline Standard dev. & $(\log s)=$ & 0.65 & & & \\
\hline
\end{tabular}

Basin characteristics

\begin{tabular}{|c|c|c|c|c|c|c|c|}
\hline \multirow[b]{2}{*}{$\begin{array}{c}\text { Main } \\
\text { channel } \\
\text { slope } \\
(\mathrm{ft} / \mathrm{mi})\end{array}$} & \multirow[b]{2}{*}{$\begin{array}{c}\text { Stream } \\
\text { length } \\
\text { (mi) }\end{array}$} & \multirow[b]{2}{*}{$\begin{array}{c}\text { Mean } \\
\text { basin } \\
\text { elevation } \\
(\mathrm{ft})\end{array}$} & \multirow[b]{2}{*}{$\begin{array}{c}\text { Forested } \\
\text { area } \\
\text { (percent) }\end{array}$} & \multirow[b]{2}{*}{$\begin{array}{c}\text { Soil } \\
\text { index }\end{array}$} & \multirow[b]{2}{*}{$\begin{array}{c}\text { Mean } \\
\text { annual } \\
\text { precipitation } \\
\text { (in) }\end{array}$} & \multicolumn{2}{|c|}{ Rainfall intensity, 24-hou } \\
\hline & & & & & & $\begin{array}{c}\text { 2-year } \\
\text { (in) }\end{array}$ & $\begin{array}{c}\text { 50-year } \\
\text { (in) }\end{array}$ \\
\hline 56.6 & 20.0 & 7,620 & 76.0 & 3.0 & 25.7 & 2.3 & 4.2 \\
\hline
\end{tabular}

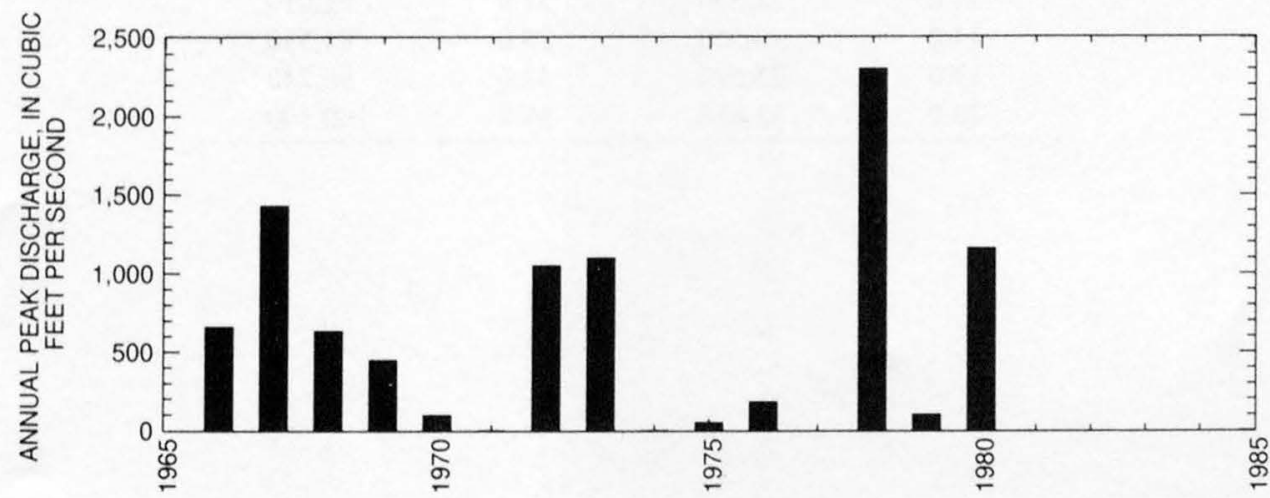


09504000 VERDE RIVER NEAR CLARKDALE, AZ

LOCATION.--Lat 34 '51'08", long $112^{\circ} 03^{\prime} 55^{\prime \prime}$, in SE $\frac{1}{4} \mathrm{NW}^{1} / 4_{4} \mathrm{SE}^{1} / 4$ sec. 17 , T.17 N., R.3 E., Yavapai County, Hydrologic Unit 15060202 , in Pres cott National Forest, on left bank 1.7 mi downstream from Sycamore Creek and 5.6 mi north of Clarkdale.

DRAINAGE AREA.--3,503 mi ${ }^{2}$, of which $364 \mathrm{mi}^{2}$ is noncontributing including $357 \mathrm{mi}^{2}$ in Aubrey Valley Playa, a closed basin.

PERIOD OF RECORD.--June 1915 to October 1916, May 1917 to July 1921, April 1965 to current year.

REVISED RECORDS.--WSP 1213: 1917, 1920. WDR AZ-89-1: Drainage area.

GAGE.--Water-stage recorder. Elevation of gage is 3,500 ft above sea level, from topographic map. June 1915 to June 1921 , at site 2.5 mi down. stream at different datum.

EXTREMES FOR PERIOD OF RECORD.--Maximum discharge, 53,200 $\mathrm{ft}^{3} / \mathrm{s}$ Feb. 20, 1993, gage height, $26.39 \mathrm{ft}$, from rating curve extended above $20,000 \mathrm{ft}^{3} / \mathrm{s}$ on basis of slope-area measurement at $53,200 \mathrm{ft}^{3} / \mathrm{s}$; minimum daily, $55 \mathrm{ft}^{3} / \mathrm{s}$ Aug. 31 , Sept. 1,1920 .

Annual peak discharges

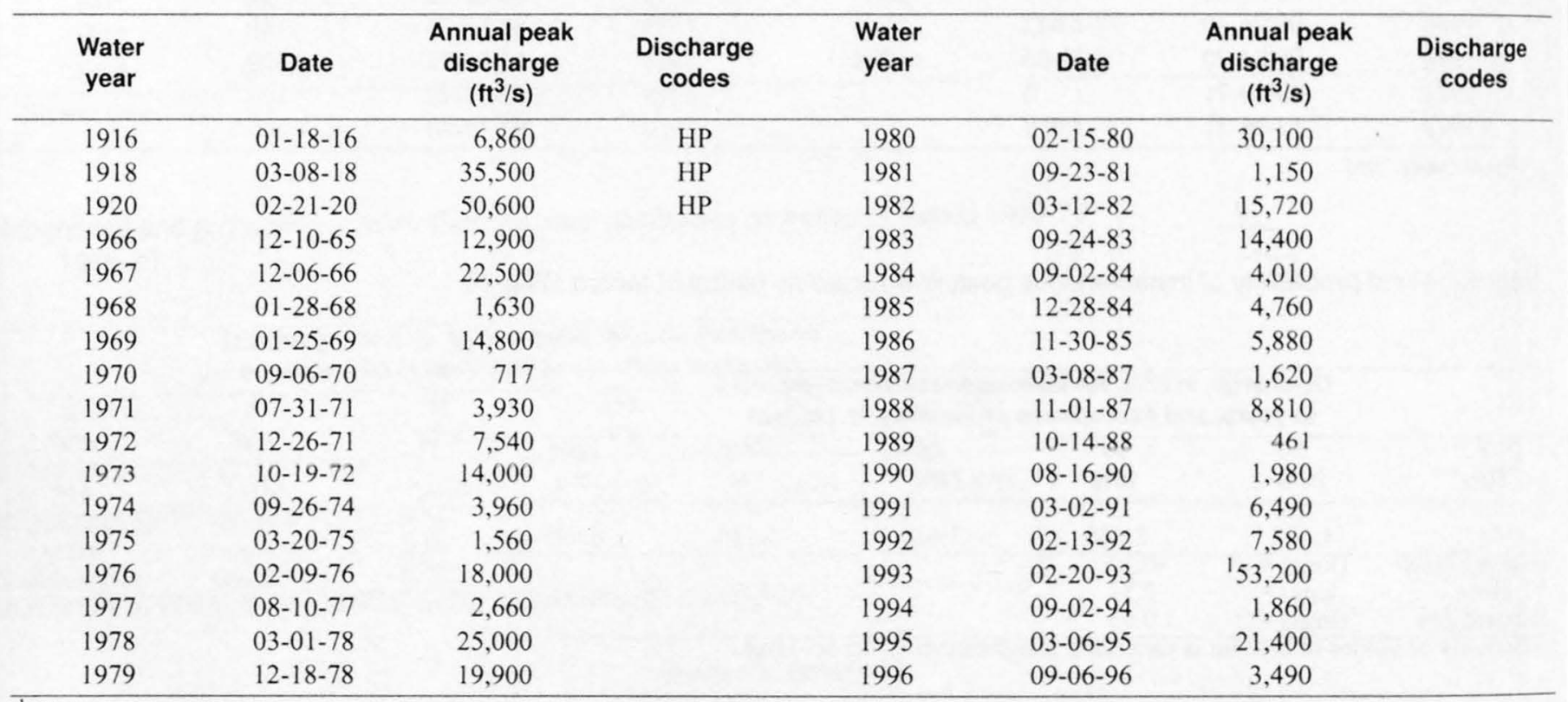

THighest since 1906.

Discharge rating table developed February 1993

\begin{tabular}{cccc}
\hline $\begin{array}{c}\text { Gage height } \\
(\mathbf{f t})\end{array}$ & $\begin{array}{c}\text { Discharge } \\
\left(\mathbf{f t}^{3} / \mathbf{s}\right)\end{array}$ & $\begin{array}{c}\text { Gage height } \\
(\mathbf{f t})\end{array}$ & $\begin{array}{c}\text { Discharge } \\
\left(\mathbf{f t}^{\mathbf{3}} \mathbf{s}\right)\end{array}$ \\
\hline 3.0 & 1,140 & 23.0 & 42,420 \\
5.0 & 3,070 & 26.0 & 51,920 \\
8.0 & 7,120 & 29.0 & 62,130 \\
11.0 & 12,330 & 32.0 & 73,010 \\
14.0 & 18,550 & 35.0 & 84,550 \\
17.0 & 25,690 & 38.0 & 96,740 \\
20.0 & 33,660 & 38.8 & 100,100 \\
\hline
\end{tabular}


GILA RIVER BASIN

09504000 VERDE RIVER NEAR CLARKDALE, AZ--Continued

Basin characteristics

\begin{tabular}{cccccccc}
\hline $\begin{array}{c}\text { Main } \\
\text { channel } \\
\text { slope } \\
(\mathrm{ft} / \mathrm{mi})\end{array}$ & $\begin{array}{c}\text { Stream } \\
\text { length } \\
\text { (mi) }\end{array}$ & $\begin{array}{c}\text { Mean } \\
\text { basin } \\
\text { elevation } \\
\text { (ft) }\end{array}$ & $\begin{array}{c}\text { Forested } \\
\text { area } \\
\text { (percent) }\end{array}$ & $\begin{array}{c}\text { Soil } \\
\text { index }\end{array}$ & $\begin{array}{c}\text { Mean } \\
\text { annual } \\
\text { precipitation } \\
\text { (in) }\end{array}$ & $\begin{array}{c}\text { Rainfall intensity, 24-hour } \\
\text { (in) }\end{array}$ & $\begin{array}{c}\text { 50-year } \\
\text { (in) }\end{array}$ \\
\hline 22.9 & 115 & 5,490 & 73.0 & 2.9 & 19.1 & 2.0 \\
\hline
\end{tabular}


09504000 VERDE RIVER NEAR CLARKDALE, AZ--Continued

MEAN MONTHLY AND ANNUAL DISCHARGES $1916,1918 \cdot 20,1966.96$

\begin{tabular}{|c|c|c|c|c|c|c|}
\hline MONTH & $\begin{array}{l}\text { MAXIMUM } \\
\text { (FT3/S) }\end{array}$ & $\begin{array}{l}\text { MINIMUM } \\
\text { (FT3/S) }\end{array}$ & $\begin{array}{c}\text { MEAN } \\
\text { (ET3/S) }\end{array}$ & $\begin{array}{l}\text { STAN- } \\
\text { DARD } \\
\text { DEVIA- } \\
\text { TION } \\
(\mathrm{FT} 3 / \mathrm{S})\end{array}$ & $\begin{array}{l}\text { COEFFI- } \\
\text { CIENT OF } \\
\text { VARI- } \\
\text { ATION }\end{array}$ & $\begin{array}{c}\text { PERCENT } \\
\text { OF } \\
\text { ANNUAL } \\
\text { RUNOFF }\end{array}$ \\
\hline OCTOBER & 1,080 & 68 & 119 & 173 & 1.5 & 5.0 \\
\hline NOVEMBER & 736 & 70 & 131 & 130 & 0.99 & 5.5 \\
\hline DECEMBER & 1.030 & 75 & 206 & 258 & 1.3 & 8.7 \\
\hline JANUARY & 2,800 & 73 & 222 & 467 & 2.1 & 9.4 \\
\hline FEBRUARY & 3,490 & 74 & 510 & 860 & 1.7 & 21.5 \\
\hline MARCH & 2,760 & 73 & 516 & 561 & 1.1 & 21.7 \\
\hline APRIL & 1,520 & 69 & 189 & 256 & 1.4 & 8.0 \\
\hline MAY & 355 & 69 & 90 & 47 & 0.52 & 3.8 \\
\hline JUNE & 91 & 62 & 77 & 6.8 & 0.09 & 3.2 \\
\hline JULY & 670 & 64 & 105 & 99 & 0.94 & 4.4 \\
\hline AUGUST & 201 & 74 & 103 & 29 & 0.28 & 4.3 \\
\hline SEPTEMBER & 670 & 66 & 106 & 100 & 0.94 & 4.5 \\
\hline ANNUAL & 645 & 82 & 197 & 131 & 0.67 & 100 \\
\hline
\end{tabular}

MAGNITUDE AND PROBABILITY OF INSTANTANEOUS PEAK FLOW BASED ON PERIOD OF RECORD 1916, 1918, 1920, 1966.96 DISCHARGE, IN FT3/S, FOR INDICATED RECURRENCE INTERVAL IN YEARS, AND EXCEEDANCE PROBABILITY, IN PERCENT

\begin{tabular}{|c|c|c|c|c|c|}
\hline 2 & 5 & 10 & 25 & 50 & 100 \\
\hline 508 & 208 & 108 & 48 & 28 & 18 \\
\hline 6,340 & 16.100 & 25,500 & 40,700 & 54,400 & 70,200 \\
\hline
\end{tabular}

WEIGHTED SKEW (LOGS) $=-0.27$

MEAN (LOGS) $=3.78$

STANDARD DEV. $($ LOGS $)=0.50$
MAGNITUDE AND PROBABILITY OF ANNUAL LOW FLOW BASED ON PERIOD OF RECORD 1919-21, 1967-96

\begin{tabular}{|c|c|c|c|c|c|c|}
\hline \multirow{4}{*}{$\begin{array}{l}\text { PERIOD } \\
\text { (CON- } \\
\text { SECU. }\end{array}$} & \multicolumn{6}{|c|}{ DISCHARGE, IN $\mathrm{FT} 3 / \mathrm{S}$, FOR INDICATED } \\
\hline & \multirow{2}{*}{\multicolumn{3}{|c|}{$\begin{array}{l}\text { RECURRENCE IN } \\
\text { NON-EXCEEDANCE }\end{array}$}} & \multicolumn{3}{|c|}{ IN YEARS, AND } \\
\hline & & & & BILI? & IN & ENT \\
\hline & $\cdots$ & … & $\cdots \cdots$ & … & $\cdots$ & .... \\
\hline TIVE & 2 & 5 & 10 & 20 & 50 & $100 \#$ \\
\hline DAYS) & 508 & 208 & 108 & 58 & 28 & 18 \\
\hline 1 & 71 & 65 & 62 & 59 & 56 & 54 \\
\hline 3 & 71 & 66 & 63 & 60 & 57 & 55 \\
\hline 7 & 72 & 66 & 64 & 61 & 58 & 57 \\
\hline 14 & 73 & 68 & 65 & 62 & 60 & 58 \\
\hline 30 & 74 & 69 & 66 & 64 & 61 & 59 \\
\hline 60 & 77 & 72 & 69 & 67 & 64 & 62 \\
\hline 90 & 78 & 73 & 71 & 69 & 66 & 65 \\
\hline 120 & & & & & & \\
\hline 183 & & & & & & \\
\hline
\end{tabular}

MAGNITUDE AND PROBABILITY OF ANNUAL HIGH FLOW BASED ON PERIOD OF RECORD $1916,1918-20,1966-96$

\begin{tabular}{|c|c|c|c|c|c|c|}
\hline \multirow{3}{*}{$\begin{array}{l}\text { PERIOD } \\
\text { (CON- } \\
\text { SECU- } \\
\text { TIVE } \\
\text { DAYS) }\end{array}$} & & $\begin{array}{l}\text { DISCHAF } \\
\text { RECURR } \\
\text { EXCEED }\end{array}$ & $\begin{array}{ll}G E, & \text { IN } \\
\text { NCE } & \text { INT } \\
\text { NCE } & \text { PRO } \\
\ldots . .\end{array}$ & $\begin{array}{l}\text { T3/S, FC } \\
\text { RVAL, IN } \\
\text { ABILITY, } \\
\ldots . . . .\end{array}$ & $\begin{array}{l}\text { R INDIC } \\
\text { YEARS, } \\
\text { IN PER }\end{array}$ & $\begin{array}{l}\text { TED } \\
\text { AND } \\
\text { ENT }\end{array}$ \\
\hline & 2 & 5 & 10 & 25 & 50 & $100 \#$ \\
\hline & 508 & 208 & 108 & 48 & 28 & 18 \\
\hline 1 & 3,140 & 9,930 & 17,500 & 31,200 & 44,700 & 61,300 \\
\hline 3 & 1,940 & 5,850 & 10,100 & 17,600 & 25,000 & 33,900 \\
\hline 7 & 1,220 & 3,580 & 6,080 & 10.500 & 14,700 & 19,800 \\
\hline 15 & 799 & 2,140 & 3,520 & 5,860 & 8,070 & 10.700 \\
\hline 30 & 555 & 1,390 & 2,220 & 3,610 & 4,920 & 6,490 \\
\hline 60 & 389 & 923 & 1,460 & 2,390 & 3.290 & 4,400 \\
\hline 90 & 313 & 693 & 1,060 & 1,690 & 2,300 & 3,030 \\
\hline
\end{tabular}

DURATION TABLE OF DAILY MEAN FLOW FOR PERIOD OF RECORD 1916, $1918 \cdot 20,1966.96$

DISCHARGE, IN FT3/S, WHICH WAS EQUALED OR EXCEEDED FOR INDICATED PERCENT OF TIME

${ }_{18} 8 \begin{gathered}58 \\ \ldots\end{gathered}$

$\begin{array}{lllllllllllllllll}2,120 & 596 & 226 & 127 & 100 & 92 & 89 & 86 & 83 & 80 & 75 & 70 & 68 & 66 & 64 & 59 & 56\end{array}$

\# Reliability of values in column is uncertain, and potential errors are large. 


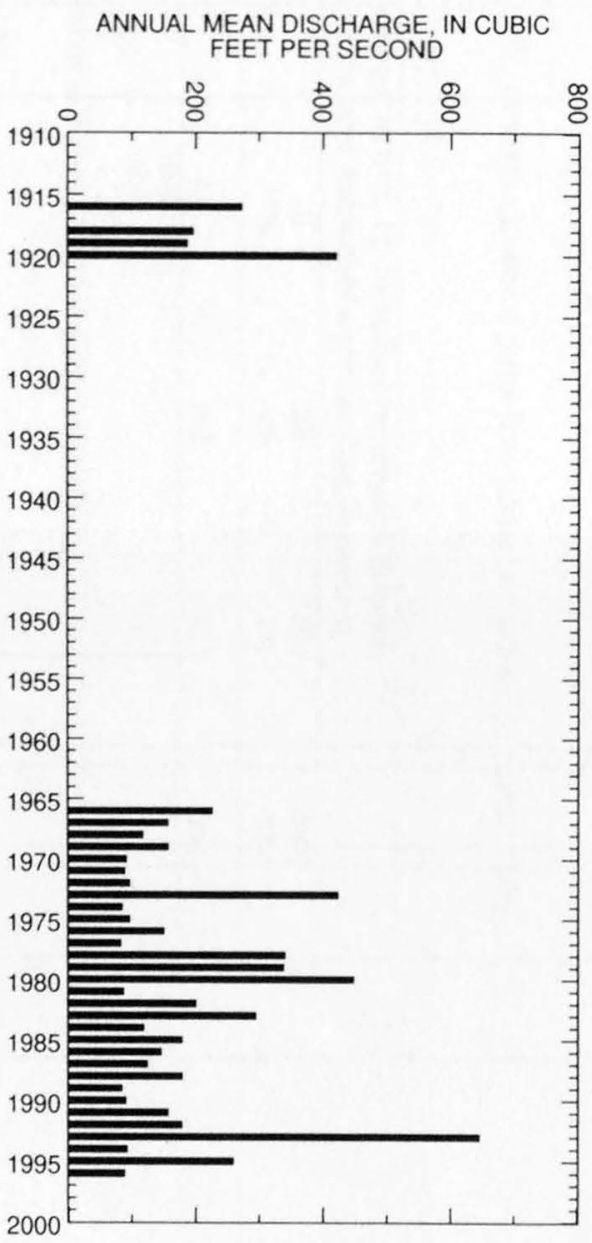

ANNUAL PEAK DISCHARGE, IN CUBIC

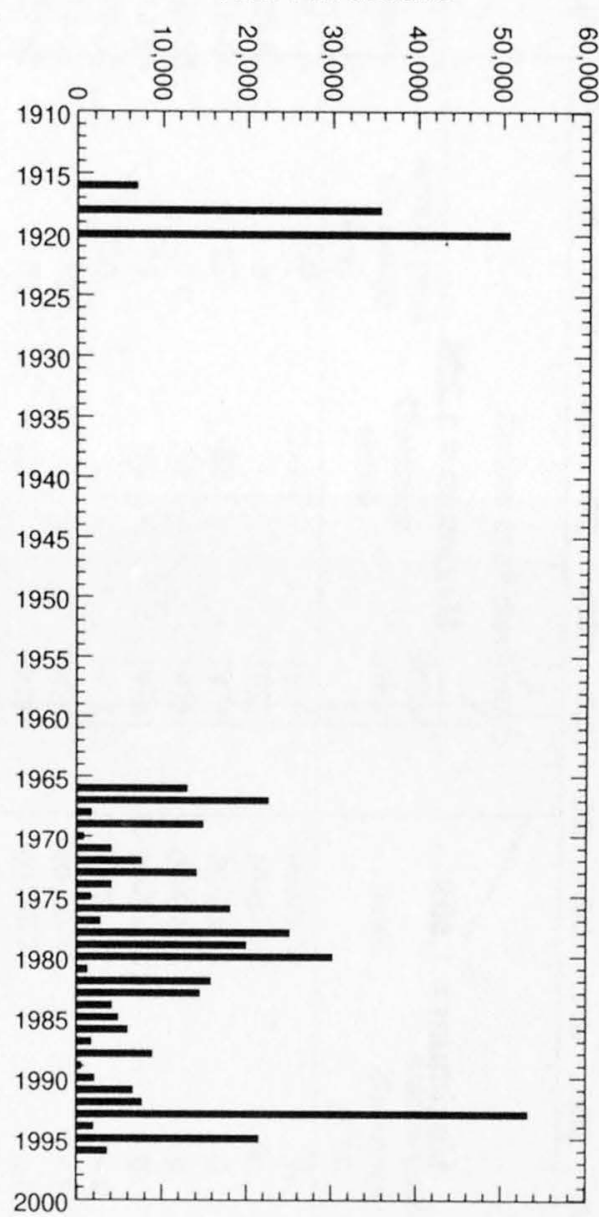


09504000 VERDE RIVER NEAR CLARKDALE, AZ--Continued
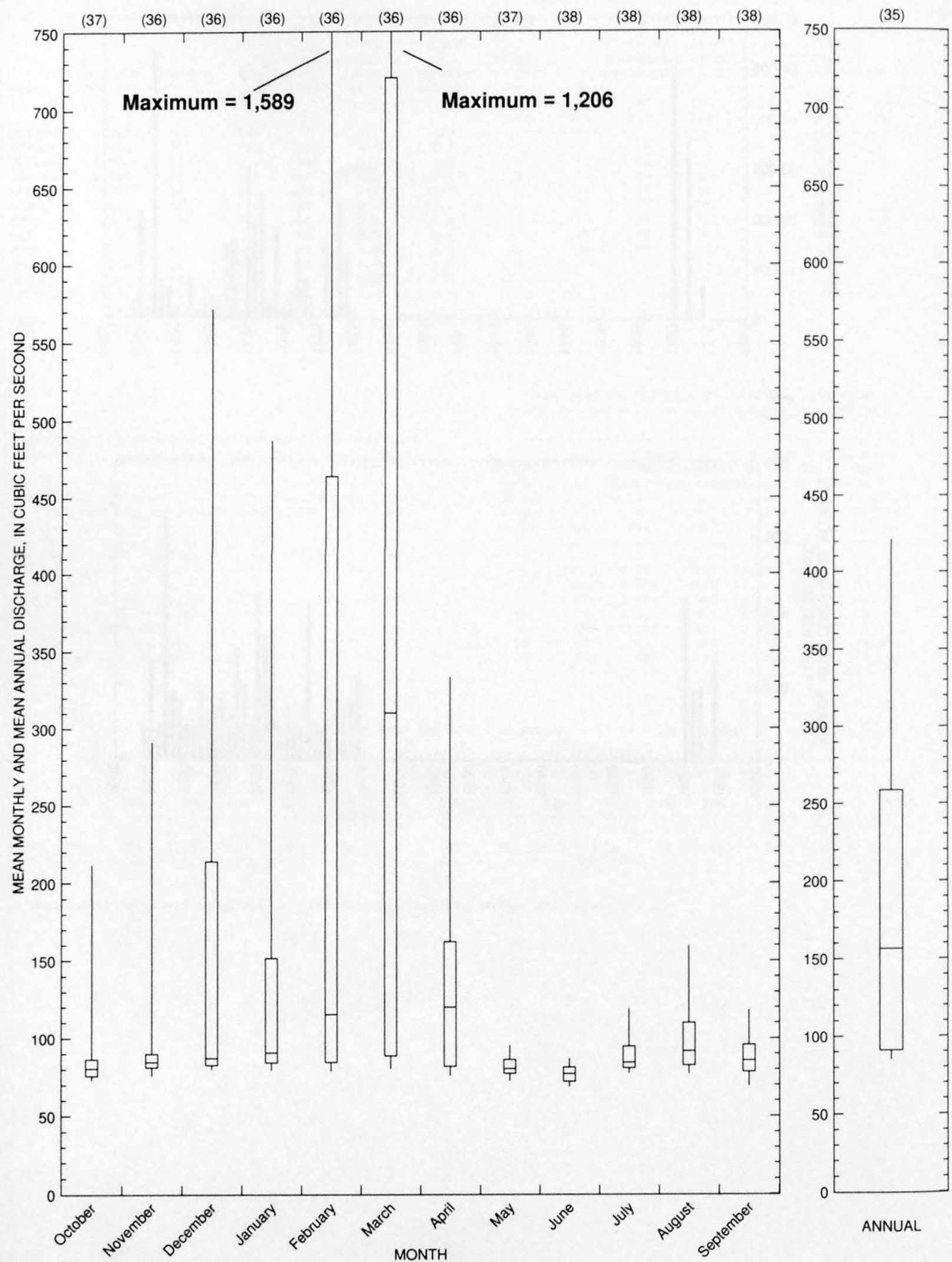
09504100 HULL CANYON NEAR JEROME, AZ

LOCATION.--Lat $34^{\circ} 44^{\prime} 20^{\prime \prime}$, long $112^{\circ} 08^{\prime} 35^{\prime \prime}$, in NW1/4 sec.28, T.16 N., R.2 E., Yavapai County, Hydrologic Unit 15060202, at U.S. Highway Alt. 89, 2 mi west of Jerome.

DRAINAGE AREA.--0.85 $\mathrm{mi}^{2}$.

Annual peak discharges

\begin{tabular}{|c|c|c|c|c|c|c|c|}
\hline $\begin{array}{l}\text { Water } \\
\text { year }\end{array}$ & Date & $\begin{array}{c}\text { Annual peak } \\
\text { discharge } \\
\left(\mathrm{ft}^{3} / \mathrm{s}\right)\end{array}$ & $\begin{array}{l}\text { Discharge } \\
\text { codes }\end{array}$ & $\begin{array}{l}\text { Water } \\
\text { year }\end{array}$ & Date & $\begin{array}{c}\text { Annual peak } \\
\text { discharge } \\
\left(\mathrm{ft}^{3} / \mathrm{s}\right)\end{array}$ & $\begin{array}{c}\text { Discharge } \\
\text { codes }\end{array}$ \\
\hline$\ldots$ & -...-...- & 1500 & ES,HP & 1972 & $00-00-72$ & 0 & \\
\hline 1963 & $00-00-63$ & 0 & & 1973 & $10-07-72$ & 90 & \\
\hline 1964 & $08-10-64$ & 25 & ES & 1974 & $00-00-74$ & 0 & \\
\hline 1965 & $04-19-65$ & 7.0 & ES & 1975 & $00-00-75$ & 0 & \\
\hline 1966 & $09-14-66$ & 25 & ES & 1976 & $00-00-76$ & 0 & \\
\hline 1967 & $00-00-67$ & 0 & & 1977 & $00-00-77$ & 9.0 & LT \\
\hline 1968 & $02-14-68$ & 1.0 & & 1978 & $00-00-78$ & 9.0 & LT \\
\hline 1969 & $07-27-69$ & 0.5 & ES & 1979 & $00-00-79$ & 9.0 & LT \\
\hline 1970 & $09-05-70$ & 100 & ES & 1980 & $02-00-80$ & 10 & \\
\hline 1971 & $00-00-71$ & 0 & & & & & \\
\hline
\end{tabular}

${ }^{1}$ Highest since 1935, year of occurrence unknown.

Magnitude and probability of instantaneous peak flow based on period of record 1935 , 1963-80

\begin{tabular}{|c|c|c|c|c|c|}
\hline \multicolumn{6}{|c|}{$\begin{array}{l}\text { Discharge, in } \mathrm{ft}^{3} / \mathrm{s} \text {, for indicated recurrence interval } \\
\text { in years, and exceedance probablility, in percent }\end{array}$} \\
\hline 2 & 5 & 10 & 25 & $50 t$ & $100 \dagger$ \\
\hline $50 \%$ & $20 \%$ & $10 \%$ & $4 \%$ & $2 \%$ & $1 \%$ \\
\hline 2.6 & 23.5 & 66.3 & 183 & 335 & 561 \\
\hline Weighted skew & $(\log s)=$ & -0.49 & & & \\
\hline Mean & $(\log s)=$ & 0.31 & & & \\
\hline Standard dev. & $(\log s)=$ & 1.24 & & & \\
\hline
\end{tabular}

\section{Basin characteristics}

\begin{tabular}{|c|c|c|c|c|c|c|c|}
\hline \multirow[b]{2}{*}{$\begin{array}{l}\text { Main } \\
\text { channel } \\
\text { slope } \\
\text { (ft/mi) }\end{array}$} & \multirow[b]{2}{*}{$\begin{array}{l}\text { Stream } \\
\text { length } \\
\text { (mi) }\end{array}$} & \multirow[b]{2}{*}{$\begin{array}{c}\text { Mean } \\
\text { basin } \\
\text { elevation } \\
(f t)\end{array}$} & \multirow[b]{2}{*}{$\begin{array}{l}\text { Forested } \\
\text { area } \\
\text { (percent) }\end{array}$} & \multirow[b]{2}{*}{$\begin{array}{c}\text { Soil } \\
\text { index }\end{array}$} & \multirow[b]{2}{*}{$\begin{array}{c}\text { Mean } \\
\text { annual } \\
\text { precipitation } \\
\text { (in) }\end{array}$} & \multicolumn{2}{|c|}{ Rainfall intensity, 24-hour } \\
\hline & & & & & & $\begin{array}{l}\text { 2-year } \\
\text { (in) }\end{array}$ & $\begin{array}{l}\text { 50-year } \\
\text { (in) }\end{array}$ \\
\hline 1,270 & 1.6 & 7,050 & 97.0 & 3.0 & 22.0 & 2.3 & 4.2 \\
\hline
\end{tabular}


09504100 HULL CANYON NEAR JEROME, AZ--Continued

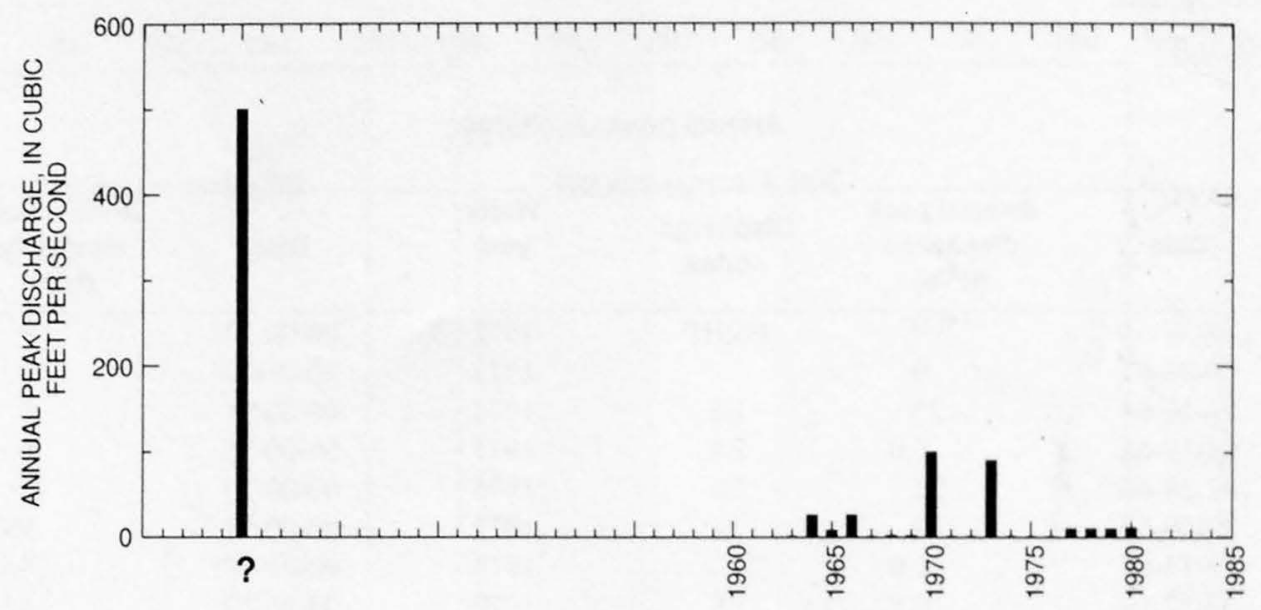


09504400 MUNDS CANYON TRIBUTARY NEAR SEDONA, AZ

LOCATION.--Lat 34.55'20", long $111^{\circ} 38^{\prime} 40^{\prime \prime}$, in SW $\frac{1}{4} \mathrm{sec}^{\prime 22}$, T.18 N., R.7 E., Coconino County, Hydrologic Unit 15060202, at State Highway

79,7 mi northeast of Sedona.

DRAINAGE AREA.--1.15 $\mathrm{mi}^{2}$.

Annual peak discharges

\begin{tabular}{|c|c|c|c|c|c|c|c|}
\hline $\begin{array}{l}\text { Water } \\
\text { year }\end{array}$ & Date & $\begin{array}{c}\text { Annual peak } \\
\text { discharge } \\
\left(\mathrm{ft}^{3} / \mathrm{s}\right)\end{array}$ & $\begin{array}{l}\text { Discharge } \\
\text { codes }\end{array}$ & $\begin{array}{l}\text { Water } \\
\text { year }\end{array}$ & Date & $\begin{array}{c}\text { Annual peak } \\
\text { discharge } \\
\left(\mathrm{ft}^{3} / \mathrm{s}\right)\end{array}$ & $\begin{array}{l}\text { Discharge } \\
\text { codes }\end{array}$ \\
\hline 1964 & $07-00-64$ & 1.0 & ES & 1972 & $06-05-72$ & 44 & \\
\hline 1965 & $09-03-65$ & 222 & & 1973 & $10-07-72$ & 90 & \\
\hline 1966 & $.11-25-65$ & 222 & & 1974 & $04-02-74$ & 20 & \\
\hline 1967 & $12-06-66$ & 192 & & 1975 & $04-00-75$ & 19 & \\
\hline 1968 & $02-00-68$ & 14 & & 1976 & $00-00-76$ & 114 & \\
\hline 1969 & $01-25-69$ & 181 & & 1977 & $00-00-77$ & 18 & \\
\hline 19.70 & $09-05-70$ & 705 & & 1979 & $12-18-78$ & ${ }^{1} 275$ & \\
\hline 1971 & $00-00-71$ & 1.0 & LT & 1980 & $02-00-80$ & 180 & \\
\hline
\end{tabular}

IHighest since 1970.

Magnitude and probability of instantaneous peak flow based on period of record 1964-77, $1979-80$

\begin{tabular}{cccccc}
\hline \multicolumn{5}{c}{$\begin{array}{l}\text { Discharge, in } \mathrm{ft}^{3} \text { /s, for indicated recurrence interval } \\
\text { in years, and exceedance probablility, in percent }\end{array}$} \\
\hline 2 & 5 & 10 & 25 & $50 \dagger$ & $100 \dagger$ \\
$50 \%$ & $20 \%$ & $10 \%$ & $4 \%$ & $2 \%$ & $1 \%$ \\
\hline 73.9 & 232 & 409 & 729 & 1,040 & 1,430 \\
Weighted skew & $($ logs $=$ & -0.26 & & \\
Mean & (logs) $=$ & 1.84 & & \\
Standard dev. & (logs) $=$ & 0.61 & & \\
F Reliability of values in column is uncertain, and potential errors are large.
\end{tabular}

Basin characteristics

\begin{tabular}{|c|c|c|c|c|c|c|c|}
\hline \multirow[b]{2}{*}{$\begin{array}{l}\text { Main } \\
\text { channel } \\
\text { slope } \\
(\mathrm{ft} / \mathrm{mi})\end{array}$} & \multirow[b]{2}{*}{$\begin{array}{l}\text { Stream } \\
\text { length } \\
\text { (mi) }\end{array}$} & \multirow[b]{2}{*}{$\begin{array}{c}\text { Mean } \\
\text { basin } \\
\text { elevation } \\
(\mathrm{ft})\end{array}$} & \multirow[b]{2}{*}{$\begin{array}{l}\text { Forested } \\
\text { area } \\
\text { (percent) }\end{array}$} & \multirow[b]{2}{*}{$\begin{array}{c}\text { Soil } \\
\text { index }\end{array}$} & \multirow[b]{2}{*}{$\begin{array}{c}\text { Mean } \\
\text { annual } \\
\text { precipitation } \\
\text { (in) }\end{array}$} & \multicolumn{2}{|c|}{ Rainfall intensity, 24-hour } \\
\hline & & & & & & $\begin{array}{l}\text { 2-year } \\
\text { (in) }\end{array}$ & $\begin{array}{l}\text { 50-year } \\
\text { (in) }\end{array}$ \\
\hline 269 & 2.2 & 6,880 & 98.0 & 3.0 & 26.0 & 2.8 & 5.5 \\
\hline
\end{tabular}

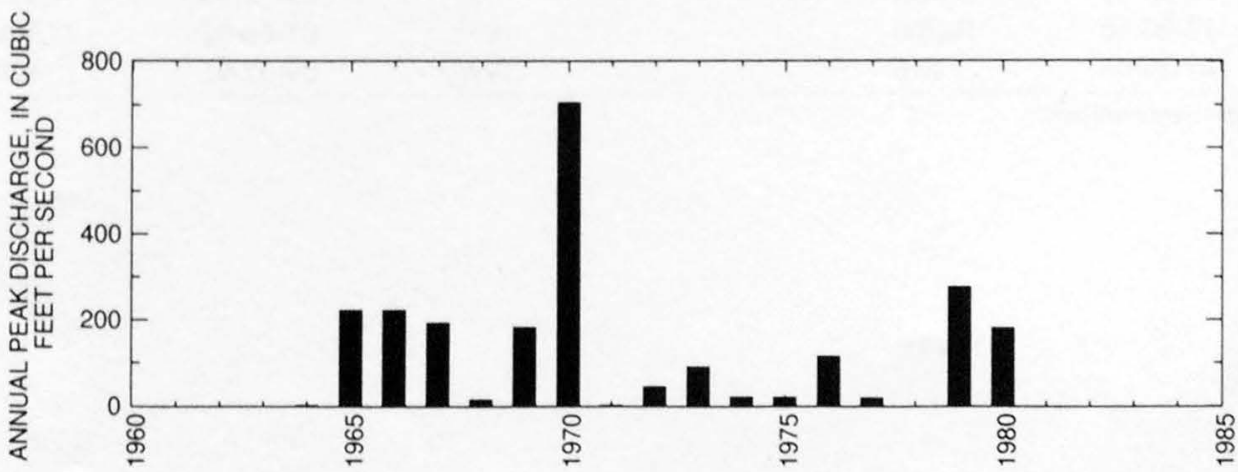


LOCATION.--Lat 34 45'52", long $111^{\circ} 533^{\prime 2} 5^{\prime \prime}$, in NW $1 / 4 \mathrm{SW}^{1} / 4 \mathrm{sec} .23$, T.16 N., R.4 E., Yavapai County, Hydrologic Unit 15060202 , on right bank $250 \mathrm{ft}$ downstream from county highway bridge, $0.2 \mathrm{mi}$ upstream from Page Springs, 4 mi northeast of Cornville, and 15 mi upstream from mouth.

DRAINAGE AREA.---355 $\mathrm{mi}^{2}$.

PERIOD OF RECORD.--July 1940 to September 1945, April 1948 to current year.

REVISED RECORDS.--WSP 1149: 1948(M). WRD Ariz. 1974: 1973. WDR AZ-89-1: Drainage area.

GAGE.--Water-stage recorder and crest-stage gage. Elevation of gage is 3,470 ft above sea level, from topographic map. Prior to March 10, 1981, at site $250 \mathrm{ft}$ upstream at same datum.

REMARKS.--Records fair. Numerous diversions above and below station for irrigation.

EXTREMES FOR PERIOD OF RECORD.--Maximum discharge, 26,400 $\mathrm{ft}^{3} / \mathrm{s} \mathrm{Feb.} \mathrm{19,} \mathrm{1980,} \mathrm{gage} \mathrm{height,} 16.30 \mathrm{ft}$; maximum gage height, 19.15 $\mathrm{ft}$, Feb. 20, 1993; minimum discharge, $6 \mathrm{ft}^{3} / \mathrm{s}$ July 27, 1940.

EXTREMES OUTSIDE PERIOD OF RECORD.--Maximum stage since at least 1885, $23 \mathrm{ft}$ in March 1938, from floodmarks (upstream side of bridge).

Annual peak discharges

\begin{tabular}{|c|c|c|c|c|c|c|c|}
\hline $\begin{array}{l}\text { Water } \\
\text { year }\end{array}$ & Date & $\begin{array}{c}\text { Annual peak } \\
\text { discharge } \\
\left(\mathrm{ft}^{3} / \mathrm{s}\right)\end{array}$ & $\begin{array}{l}\text { Discharge } \\
\text { codes }\end{array}$ & $\begin{array}{l}\text { Water } \\
\text { year }\end{array}$ & Date & $\begin{array}{c}\text { Annual peak } \\
\text { discharge } \\
\left(\mathrm{ft}^{3} / \mathrm{s}\right)\end{array}$ & $\begin{array}{c}\text { Discharge } \\
\text { codes }\end{array}$ \\
\hline 1938 & $03-03-38$ & $\left({ }^{1}\right)$ & HP & 1969 & $01-25-69$ & 15,800 & \\
\hline 1941 & $03-14-41$ & 5,280 & & 1970 & $09-05-70$ & $\mathrm{I}_{24,700}$ & \\
\hline 1942 & $10-13-41$ & 2,580 & & 1971 & $08-27-71$ & 4,050 & \\
\hline 1943 & $03-10-43$ & 3,640 & & 1972 & $12-26-71$ & 4,020 & \\
\hline 1944 & $04-06-44$ & 2,180 & & 1973 & $10-19-72$ & 8,790 & \\
\hline 1945 & $07-30-45$ & 6,020 & & 1974 & $07-07-74$ & 3,220 & \\
\hline 1946 & $00-00-46$ & 1,200 & & 1975 & $07-14-75$ & 4,820 & \\
\hline 1948 & $07-26-48$ & 605 & & 1976 & $02-09-76$ & 12,500 & \\
\hline 1949 & $09-09-49$ & 2,260 & & 1977 & $07-18-77$ & 415 & \\
\hline 1950 & $10-19-49$ & 6,400 & & 1978 & $03-01-78$ & 17,400 & \\
\hline 1951 & $08-29-51$ & 3,440 & & 1979 & $12-19-78$ & 25,100 & \\
\hline 1952 & $12-30-51$ & 17,200 & & 1980 & $02-19-80$ & ${ }^{2} 26,400$ & \\
\hline 1953 & $07-14-53$ & 858 & & 1981 & $08-12-81$ & 830 & \\
\hline 1954 & $03-23-54$ & 7,850 & & 1982 & $03-12-82$ & 13,000 & \\
\hline 1955 & $08-23-55$ & 6,400 & & 1983 & $11-30-82$ & 14,100 & \\
\hline 1956 & $08-17-56$ & 675 & & 1984 & $12-27-83$ & 5,730 & \\
\hline 1957 & $01-10-57$ & 5,150 & & 1985 & $12-27-84$ & 3,070 & \\
\hline 1958 & $11-03-57$ & 9,620 & & 1986 & $11-30-85$ & 6,480 & \\
\hline 1959 & $08-05-59$ & 3,750 & & 1987 & $10-11-86$ & 3,300 & \\
\hline 1960 & $12-25-59$ & 4,340 & & 1988 & $11-01-87$ & 7,640 & \\
\hline 1961 & $07-31-61$ & 4,340 & & 1989 & $07-09-89$ & 1,470 & \\
\hline 1962 & $02-12-62$ & 7,280 & & 1990 & $03-19-90$ & 554 & \\
\hline 1963 & $08-17-63$ & 990 & & 1991 & 03-01-91 & 6,970 & \\
\hline 1964 & $08-14-64$ & 10,300 & & 1992 & $02-13-92$ & 8,800 & \\
\hline 1965 & $04-04-65$ & 3,090 & & 1993 & $02-20-93$ & 26,000 & \\
\hline 1966 & $11-25-65$ & 17,600 & & 1994 & $09-02-94$ & 1,200 & \\
\hline 1967 & $12-06-66$ & 19,200 & & 1995 & $03-06-95$ & 23,000 & \\
\hline 1968 & $02-26-68$ & 816 & & 1996 & $09-02-96$ & 810 & \\
\hline
\end{tabular}

\footnotetext{
${ }^{1}$ Highest since 1885 ; discharge unknown.
}

${ }^{2}$ Highest since 1938. 
09504500 OAK CREEK NEAR CORNVILLE, AZ--Continued

Discharge rating table developed October 1993

\begin{tabular}{cccc}
\hline $\begin{array}{c}\text { Gage height } \\
(\mathbf{f t})\end{array}$ & $\begin{array}{c}\text { Discharge } \\
\left(\mathbf{f t}^{\mathbf{3}} \mathbf{/ s}\right)\end{array}$ & $\begin{array}{c}\text { Gage height } \\
(\mathbf{f t})\end{array}$ & $\begin{array}{c}\text { Discharge } \\
\left(\mathbf{f t}^{\mathbf{3}} \mathbf{s}\right)\end{array}$ \\
\hline 7.0 & 2,330 & 14.0 & 12,980 \\
8.0 & 3,310 & 15.0 & 15,150 \\
9.0 & 4,480 & 16.0 & 17,480 \\
10.0 & 5,860 & 17.0 & 19,990 \\
11.0 & 7,440 & 18.0 & 22,680 \\
12.0 & 9,140 & 19.0 & 25,540 \\
13.0 & 10,990 & 19.6 & 27,340 \\
\hline
\end{tabular}

Basin characteristics

\begin{tabular}{cccccccc}
\hline $\begin{array}{c}\text { Main } \\
\text { channel } \\
\text { slope } \\
(\mathrm{ft} / \mathrm{mi})\end{array}$ & $\begin{array}{c}\text { Stream } \\
\text { length } \\
(\mathrm{mi})\end{array}$ & $\begin{array}{c}\text { Mean } \\
\text { basin } \\
\text { elevation } \\
(\mathrm{ft})\end{array}$ & $\begin{array}{c}\text { Forested } \\
\text { area } \\
\text { (percent) }\end{array}$ & $\begin{array}{c}\text { Soil } \\
\text { index }\end{array}$ & $\begin{array}{c}\text { Mean } \\
\text { annual } \\
\text { precipitation } \\
\text { (in) }\end{array}$ & $\begin{array}{c}\text { Rainfall intensity, 24-hour } \\
\text { (in) }\end{array}$ & $\begin{array}{c}\text { 50-year } \\
\text { (in) }\end{array}$ \\
\hline 85.0 & 40.8 & 6,200 & 66.0 & 2.7 & 22.6 & 2.4 \\
\hline
\end{tabular}


09504500 OAK CREEK NEAR CORNVILLE, AZ--Continued

MEAN MONTHLY AND ANNUAL DISCHARGES 1941.96

\begin{tabular}{|c|c|c|c|c|c|c|}
\hline MONTH & $\begin{array}{l}\text { MAXIMUM } \\
\text { (FT3/S) }\end{array}$ & $\begin{array}{l}\text { MINIMUM } \\
\text { (FT3/S) }\end{array}$ & $\begin{array}{c}\text { MEAN } \\
(\mathrm{FT} 3 / \mathrm{S})\end{array}$ & $\begin{array}{l}\text { STAN- } \\
\text { DARD } \\
\text { DEVIA- } \\
\text { TION } \\
(\mathrm{FT} 3 / \mathrm{S})\end{array}$ & $\begin{array}{l}\text { COEFFI- } \\
\text { CIENT OF } \\
\text { VARI- } \\
\text { ATION }\end{array}$ & $\begin{array}{c}\text { PERCENT } \\
\text { OF } \\
\text { ANNUAL } \\
\text { RUNOFF }\end{array}$ \\
\hline OCTOBER & 571 & 20 & 48 & 78 & 1.6 & 4.4 \\
\hline NOVEMBER & 450 & 23 & 64 & 81 & 1.3 & 6.0 \\
\hline DECEMBER & 881 & 30 & 116 & 175 & 1.5 & 11 \\
\hline JANUARY & 1,300 & 32 & 103 & 190 & 1.8 & 9.5 \\
\hline FEBRUARY & 1,390 & 32 & 190 & 270 & 1.4 & 17 \\
\hline MARCH & 1,320 & 29 & 250 & 241 & 0.96 & 23 \\
\hline APRIL & 1,100 & 25 & 167 & 204 & 1.2 & 15 \\
\hline MAY & 216 & 17 & 33 & 27 & 0.84 & 3.0 \\
\hline JUNE & 58 & 14 & 20 & 6.7 & 0.33 & 1.9 \\
\hline JULY & 41 & 14 & 24 & 7.4 & 0.31 & 2.2 \\
\hline AUGUST & 91 & 13 & 34 & 16 & 0.47 & 3.1 \\
\hline SEPTEMBER & 373 & 15 & 39 & 49 & 1.3 & 3.6 \\
\hline ANNUAL & 256 & 26 & 90 & 58 & 0.64 & 100 \\
\hline
\end{tabular}

MAGNITUDE AND PROBABILITY OF INSTANTANEOUS PEAK FLOW BASED ON PERIOD OF RECORD 1938, 1941.96

DISCHARGE, IN FT3/S, FOR INDICATED RECURRENCE INTERVAL IN YEARS, AND EXCEEDANCE PROBABILITY, IN PERCBNT

\begin{tabular}{|c|c|c|c|c|c|}
\hline 2 & 5 & 10 & 25 & 50 & 100 \\
\hline 508 & 208 & 108 & 48 & 28 & 18 \\
\hline 4,810 & 12,000 & 18,700 & 29,500 & 39,200 & 50,100 \\
\hline WEIGHTED & SKEW & $($ LOGS $)=\cdot$ & 28 & & \\
\hline MEAN & & $($ LOGS $)=$ & 66 & & \\
\hline STANDARD & DEV. & $($ LOGS $)=$ & 49 & & \\
\hline
\end{tabular}

MAGNITUDE AND PROBABILITY OF ANNUAL LOW FLOW BASED ON PERIOD OF RECORD 1941.96

\begin{tabular}{|c|c|c|c|c|c|c|}
\hline \multirow{2}{*}{$\begin{array}{l}\text { PERIOD } \\
\text { (CON- } \\
\text { SECU. }\end{array}$} & \multicolumn{6}{|c|}{$\begin{array}{l}\text { DISCHARGE, IN FT3/S, FOR INDICATED } \\
\text { RECURRENCE INTERVAL, IN YEARS, AND } \\
\text { NON-EXCEEDANCE PROBABILITY, IN PERCENT }\end{array}$} \\
\hline & $\cdots$ & $\cdots \cdots$ & $\ldots \ldots$ & $\ldots \ldots$ & $\ldots \ldots$ & $\ldots \ldots$ \\
\hline TIVE & 2 & 5 & 10 & 20 & 50 & $100=$ \\
\hline DAYS) & 508 & 208 & 108 & 58 & 28 & 18 \\
\hline 1 & 14 & 12 & 11 & 9.9 & 8.9 & 8.1 \\
\hline 3 & 15 & 13 & 11 & 10 & 9.3 & 8.6 \\
\hline 7 & 15 & 13 & 12 & 12 & 11 & 10 \\
\hline 14 & 16 & 14 & 13 & 12 & 12 & 11 \\
\hline 30 & 17 & 15 & 14 & 13 & 13 & 12 \\
\hline 60 & 19 & 17 & 16 & 15 & 14 & 13 \\
\hline 90 & 21 & 18 & 17 & 16 & 15 & 14 \\
\hline 120 & 24 & 20 & 19 & 18 & 17 & 16 \\
\hline 183 & 27 & 23 & 22 & 20 & 19 & 18 \\
\hline
\end{tabular}

MAGNITUDE AND PROBABILITY OF ANNUAL HIGH FLOW BASED ON PERIOD OF RECORD 1941.96

\begin{tabular}{|c|c|c|c|c|c|c|}
\hline \multirow{2}{*}{$\begin{array}{l}\text { PERIOD } \\
\text { ICON- } \\
\text { SECU. }\end{array}$} & & \multicolumn{5}{|c|}{ DISCHARGE, IN FT $3 / S$, FOR INDICATED } \\
\hline & & & & & & \\
\hline TIVE & 2 & 5 & 10 & 25 & 50 & $100 \#$ \\
\hline DAYS) & 508 & 208 & 108 & 48 & 28 & 18 \\
\hline 1 & 1,660 & 4,920 & 8,430 & 14,700 & 20,700 & 28,100 \\
\hline 3 & 1,080 & 2,940 & 4,720 & 7,590 & 10,100 & 12,900 \\
\hline 7 & 691 & 1,720 & 2,640 & 4,010 & 5.140 & 6,360 \\
\hline 15 & 449 & 1,040 & 1.530 & 2,240 & 2.820 & 3,420 \\
\hline 30 & 310 & 700 & 1,030 & 1,500 & 1.900 & 2,310 \\
\hline 60 & 213 & 470 & 694 & 1.030 & 1.330 & 1,650 \\
\hline 90 & 170 & 357 & 518 & 762 & 972 & 1,200 \\
\hline
\end{tabular}

DURATION TABLE OF DAILY MEAN FLOW FOR PERIOD OF RECORD 1941.96

DISCHARGE, IN FT3/S, WHICH WAS EQUALED OR EXCEEDED FOR INDICATED PERCBNT OF TIME

\begin{tabular}{|c|c|c|c|c|c|c|c|c|c|c|c|c|c|c|c|c|}
\hline 18 & 58 & 108 & 158 & 208 & 308 & 408 & 508 & 608 & 708 & 808 & 908 & 958 & 988 & 998 & 99.58 & 99.98 \\
\hline & 332 & 145 & 80 & 59 & 41 & 36 & 32 & 29 & 25 & 22 & 18 & 17 & 0.00 & 0.00 & 0.00 & 0.00 \\
\hline
\end{tabular}

\# Reliability of values in column is uncertain, and potential errors are large. 
ANNUAL MEAN DISCHARGE, IN CUBIC

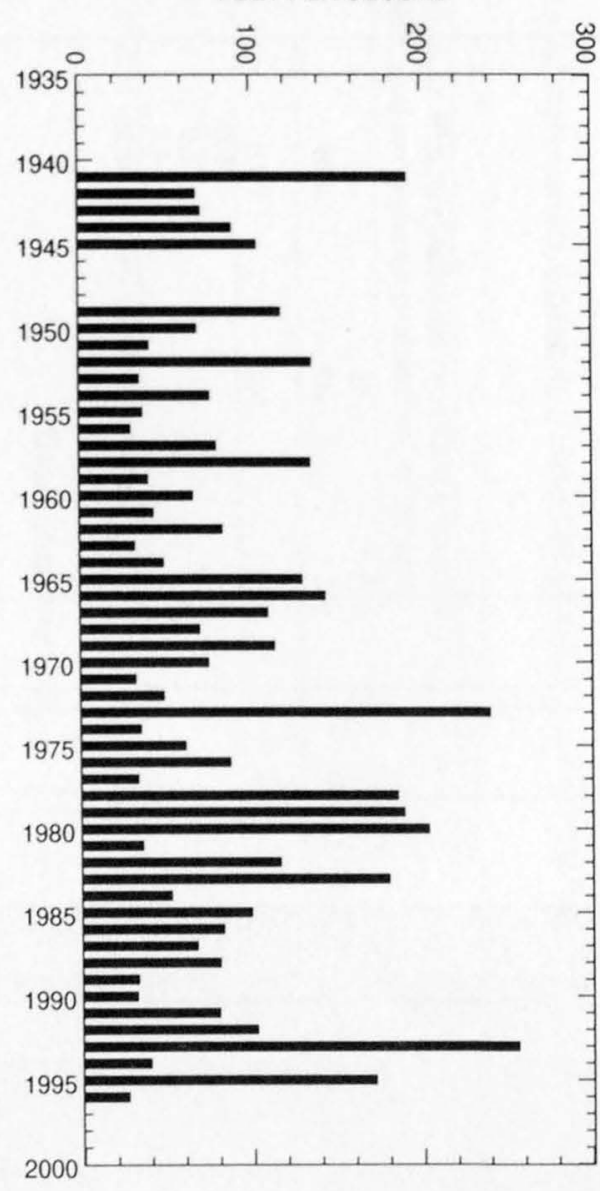

ANNUAL PEAK DISCHARGE, IN CUBIC

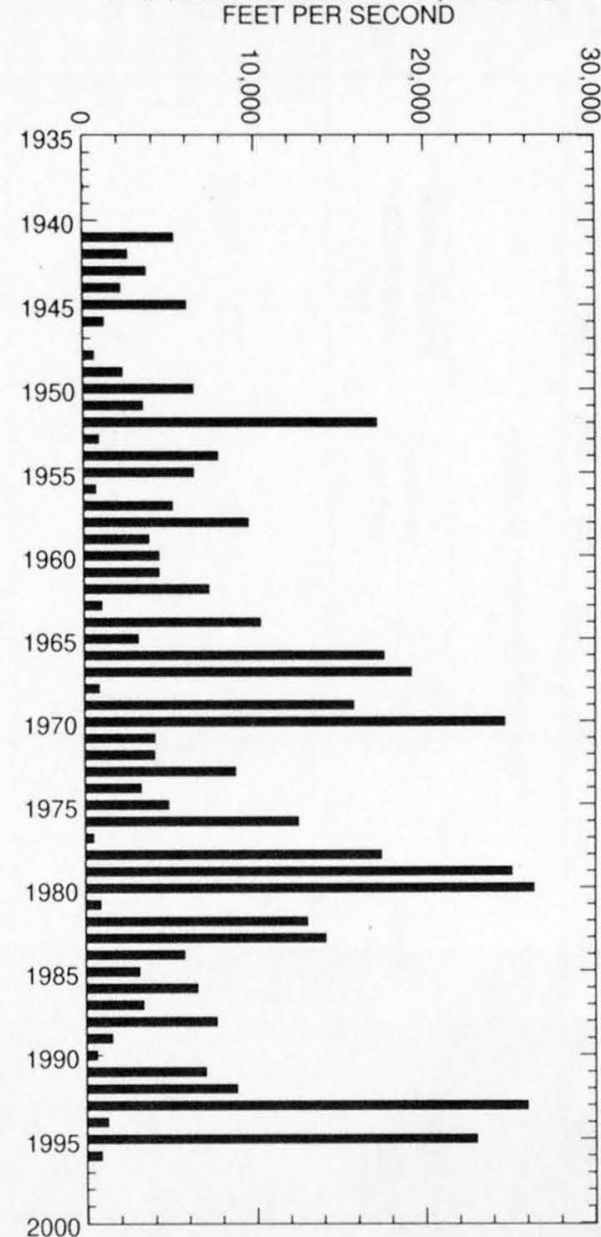


09504500 OAK CREEK NEAR CORNVILLE, AZ--Continued
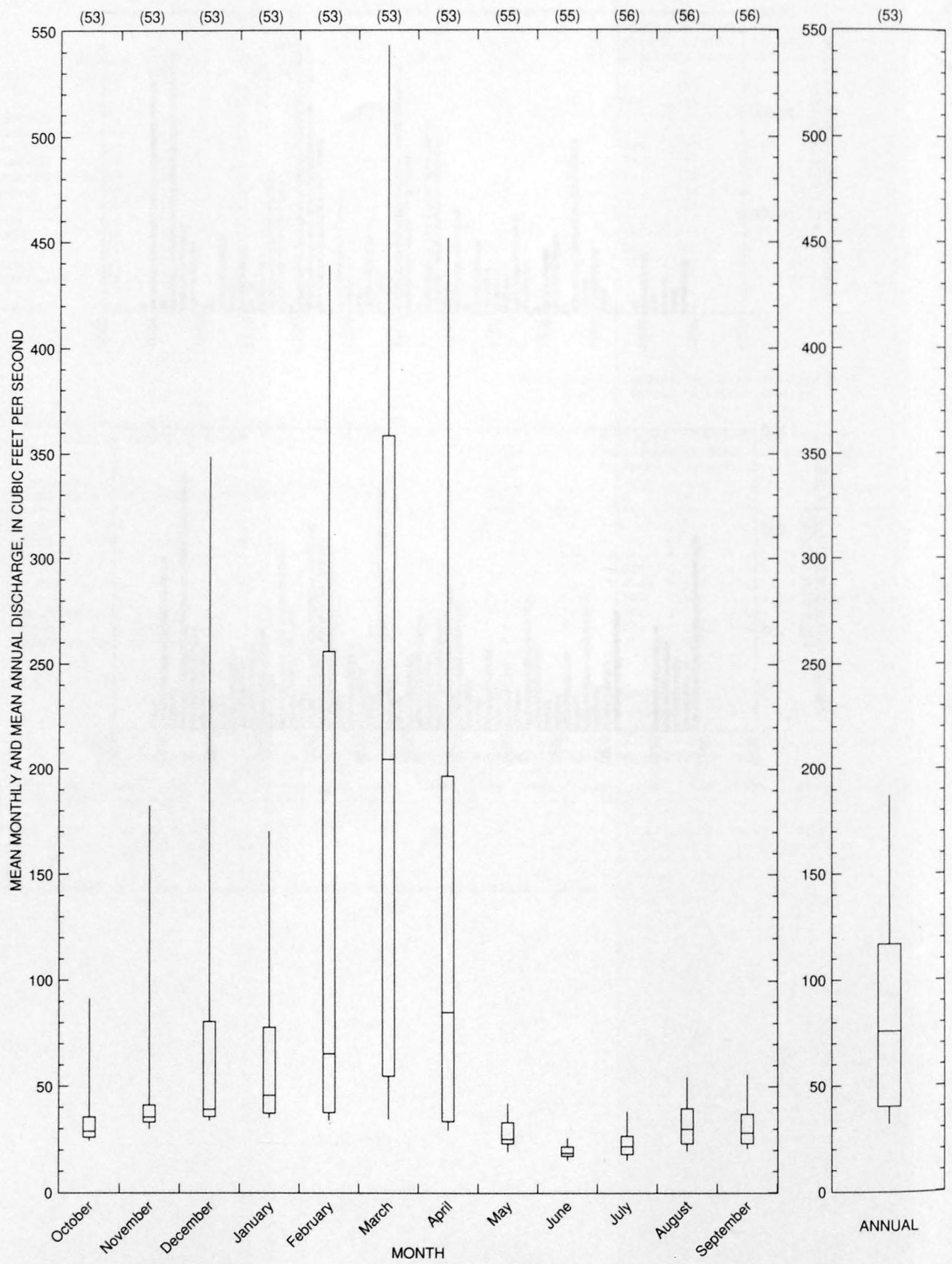
09504800 OAK CREEK TRIBUTARY NEAR CORNVILLE, AZ

LOCATION.--Lat 34 $42^{\prime} 45^{\prime \prime}$, long 111 $52^{\prime} 50^{\prime \prime}$, in NW'/4 sec.12, T.15 N., R.4 E., Yavapai County, Hydrologic Unit 15060202, at county road, 2.5 mi east of Cornville.

DRAINAGE AREA.--0.048 $\mathrm{mi}^{2}$.

Annual peak discharges

\begin{tabular}{|c|c|c|c|c|c|c|c|}
\hline $\begin{array}{l}\text { Water } \\
\text { year }\end{array}$ & Date & $\begin{array}{c}\text { Annual peak } \\
\text { discharge } \\
\left(\mathrm{ft}^{3} / \mathbf{s}\right)\end{array}$ & $\begin{array}{l}\text { Discharge } \\
\text { codes }\end{array}$ & $\begin{array}{l}\text { Water } \\
\text { year }\end{array}$ & Date & $\begin{array}{l}\text { Annual peak } \\
\text { discharge } \\
\left(\mathrm{ft}^{3} / \mathrm{s}\right)\end{array}$ & $\begin{array}{c}\text { Discharge } \\
\text { codes }\end{array}$ \\
\hline 1963 & $09-09-63$ & 19 & & 1971 & $09-29-71$ & 1.0 & LT \\
\hline 1964 & $07-26-64$ & 47 & & 1972 & $00-00-72$ & 0.6 & LT \\
\hline 1965 & $08-00-65$ & 3.0 & ES & 1973 & $10-07-72$ & 5.0 & \\
\hline 1966 & $00-00-66$ & 0 & & 1974 & $08-00-74$ & 1.0 & LT \\
\hline 1967 & $08-00-67$ & 18 & & 1975 & $11-02-74$ & 1.0 & ES \\
\hline 1968 & $00-00-68$ & 1.0 & LT & 1976 & $00-00-76$ & 3.7 & \\
\hline 1969 & $08-08-69$ & 53 & & 1980 & $02-14-80$ & ${ }^{1} 24$ & HP \\
\hline 1970 & $09-05-70$ & 47 & & & & & \\
\hline
\end{tabular}

${ }^{1}$ Highest since 1970.

Magnitude and probability of instantaneous peak flow based on period of record 1963-76, 1980

\begin{tabular}{|c|c|c|c|c|c|}
\hline \multicolumn{6}{|c|}{$\begin{array}{l}\text { Discharge, in } \mathrm{ft}^{3} / \mathrm{s} \text {, for indicated recurrence interval } \\
\text { in years, and exceedance probablility, in percent }\end{array}$} \\
\hline 2 & 5 & 10 & 25 & $50 \dagger$ & $100 t$ \\
\hline $50 \%$ & $20 \%$ & $10 \%$ & $4 \%$ & $2 \%$ & $1 \%$ \\
\hline 4.1 & 25.9 & 61.7 & 144 & 240 & 369 \\
\hline Weighted skew & $(\log s)=$ & -0.48 & & & \\
\hline Mean & $(\log s)=$ & 0.52 & & & \\
\hline Standard dev. & $(\log s)=$ & 1.04 & & & \\
\hline
\end{tabular}

Basin characteristics

\begin{tabular}{cccccccc}
\hline $\begin{array}{c}\text { Main } \\
\text { channel } \\
\text { slope } \\
(\mathrm{ft} / \mathrm{mi})\end{array}$ & $\begin{array}{c}\text { Stream } \\
\text { length } \\
(\mathrm{mi})\end{array}$ & $\begin{array}{c}\text { Mean } \\
\text { basin } \\
\text { elevation } \\
(\mathrm{ft})\end{array}$ & $\begin{array}{c}\text { Forested } \\
\text { area } \\
\text { (percent) }\end{array}$ & $\begin{array}{c}\text { Soil } \\
\text { index }\end{array}$ & $\begin{array}{c}\text { Mean } \\
\text { annual } \\
\text { precipitation } \\
\text { (in) }\end{array}$ & $\begin{array}{c}\text { Rainfall intensity, 24-hour } \\
\text { (in) }\end{array}$ & $\begin{array}{c}50-y e a r \\
\text { (in) }\end{array}$ \\
\hline 65.0 & 0.41 & 3,570 & 0.0 & 1.0 & 12.4 & 1.7 \\
\hline
\end{tabular}


09504800 OAK CREEK TRIBUTARY NEAR CORNVILLE, AZ--Continued

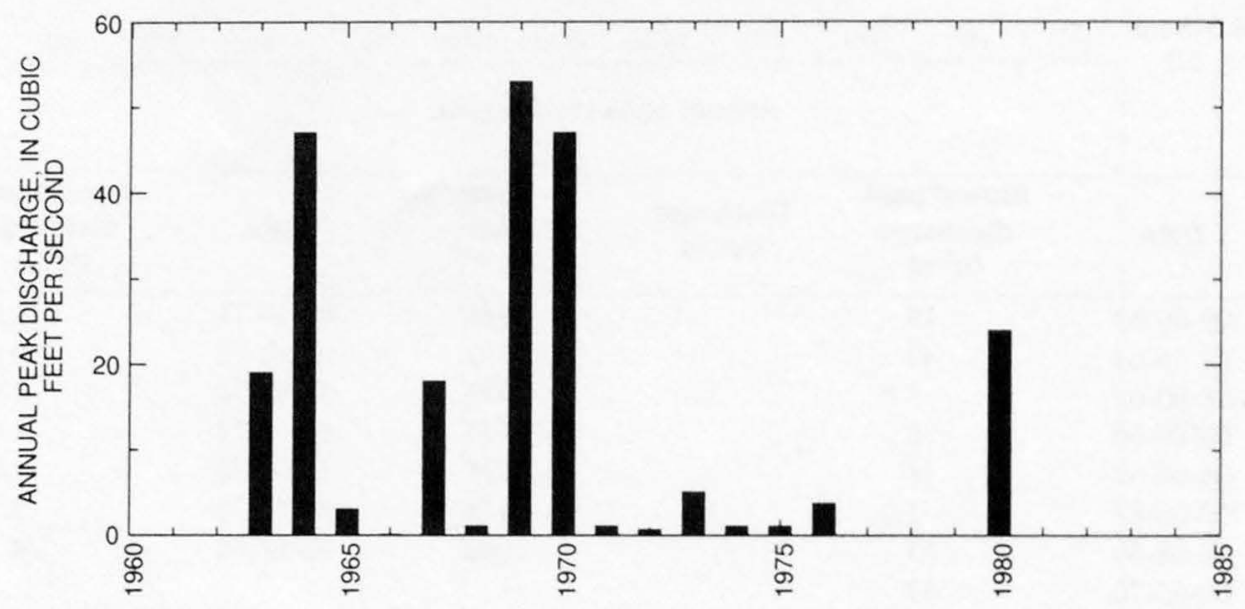




\section{WET BEAVER CREEK NEAR RIMROCK, AZ}

LOCATION.--Lat 34 40'29", long 111 40'17", in NW1/4 SW $1 / 4$ sec.24, T.15 N., R.6 E., Yavapai County, Hydrologic Unit 15060202 , in Coconino National Forest, on right bank 4.5 mi northeast of Rimrock and 5.7 mi upstream from Red Tank Draw.

DRAINAGE AREA.--111 $\mathrm{mi}^{2}$.

PERIOD OF RECORD.--October 1961 to September 1982 (continuous-record), October 1982 to September 1991 (annual maximums only), October 1991 to current year.

REVISED RECORDS.--WRD Ariz. 1969: Drainage area. WRD AZ-93-1, 1993.

GAGE.--Water-stage recorder and concrete control. Datum of gage is $4,020 \mathrm{ft}$ above sea level.

REMARKS.--No estimated daily discharges. Records good. No known diversion or regulation above station.

EXTREMES FOR PERIOD OF RECORD.--Maximum discharge, 16,000 $\mathrm{ft}^{3} / \mathrm{s} \mathrm{Jan.} \mathrm{8,} \mathrm{1993,} \mathrm{gage} \mathrm{height,} 17.21 \mathrm{ft}$ from rating curve extended above

$5,400 \mathrm{ft}^{3} / \mathrm{s}$ on basis of slope-area measurement of peak flow; minimum, $5.4 \mathrm{ft}^{3} / \mathrm{s}$ Aug. 14, 1962, July 1, 2, 5, 8, 9, 12, 21, 1967, June 2-5, 1012, 28, July 5, 6, 1993.

Annual peak discharges

\begin{tabular}{|c|c|c|c|c|c|c|c|}
\hline $\begin{array}{l}\text { Water } \\
\text { year }\end{array}$ & Date & $\begin{array}{c}\text { Annual peak } \\
\text { discharge } \\
\left(\mathrm{ft}^{3} / \mathrm{s}\right)\end{array}$ & $\begin{array}{l}\text { Discharge } \\
\text { codes }\end{array}$ & $\begin{array}{l}\text { Water } \\
\text { year }\end{array}$ & Date & $\begin{array}{c}\text { Annual peak } \\
\text { discharge } \\
\left(\mathrm{ft}^{3} / \mathrm{s}\right)\end{array}$ & $\begin{array}{c}\text { Discharge } \\
\text { codes }\end{array}$ \\
\hline 1962 & $02-12-62$ & 1,870 & & 1980 & $02-19-80$ & 10,900 & \\
\hline 1963 & $08-27-63$ & 748 & & 1981 & $04-05-81$ & 368 & \\
\hline 1964 & $08-06-64$ & 2,030 & & 1982 & $03-12-82$ & 6,880 & \\
\hline 1965 & $01-06-65$ & 6,100 & & 1983 & $11-30-82$ & 5,480 & \\
\hline 1966 & $11-25-65$ & 6,150 & & 1984 & $12-27-83$ & 2,740 & \\
\hline 1967 & $07-31-67$ & 4,340 & & 1985 & $12-27-84$ & 3,960 & \\
\hline 1968 & $03-10-68$ & 982 & & 1986 & $02-18-86$ & 710 & \\
\hline 1969 & $01-25-69$ & 3,500 & & 1987 & $03-18-87$ & 1,180 & \\
\hline 1970 & $09-05-70$ & 7,670 & & 1988 & $02-03-88$ & 3,900 & \\
\hline 1971 & $09-01-71$ & 2,890 & & 1989 & $03-29-89$ & 285 & \\
\hline 1972 & $07-16-72$ & 4,020 & & 1990 & $09-05-90$ & 922 & \\
\hline 1973 & $10-19-72$ & 5,490 & & 1991 & 03-01-91 & 3,030 & \\
\hline 1974 & $03-18-74$ & 119 & & 1992 & $08-23-92$ & 5,280 & \\
\hline 1975 & $04-13-75$ & 1,060 & & 1993 & 01-08-93 & 16,000 & \\
\hline 1976 & $02-09-76$ & 6,880 & & 1994 & $03-20-94$ & 405 & \\
\hline 1977 & $04-07-77$ & 155 & & 1995 & $02-14-95$ & 7,330 & \\
\hline 1978 & $03-01-78$ & 4,360 & & 1996 & $08-02-96$ & 1,050 & \\
\hline 1979 & $12-18-78$ & 7,560 & & & & & \\
\hline
\end{tabular}

Discharge rating table developed January 1993

\begin{tabular}{cccc}
\hline $\begin{array}{c}\text { Gage height } \\
(\mathbf{f t})\end{array}$ & $\begin{array}{c}\text { Discharge } \\
\left(\mathbf{f t}^{3} / \mathbf{s}\right)\end{array}$ & $\begin{array}{c}\text { Gage height } \\
(\mathbf{f t})\end{array}$ & $\begin{array}{c}\text { Discharge } \\
\left(\mathbf{f t}^{3} / \mathbf{s}\right)\end{array}$ \\
\hline 7.0 & 1,060 & 13.0 & 8,110 \\
8.0 & 1,760 & 14.0 & 9,770 \\
9.0 & 2,640 & 15.0 & 11,510 \\
10.0 & 3,720 & 16.0 & 13,440 \\
11.0 & 5,010 & 17.0 & 15,540 \\
12.0 & 6,480 & 17.2 & 16,000 \\
\hline
\end{tabular}


09505200 WET BEAVER CREEK NEAR RIMROCK, AZ--Continued

Basin characteristics

\begin{tabular}{|c|c|c|c|c|c|c|c|}
\hline \multirow[b]{2}{*}{$\begin{array}{l}\text { Main } \\
\text { channel } \\
\text { slope } \\
\text { (ft/mi) }\end{array}$} & \multirow[b]{2}{*}{$\begin{array}{l}\text { Stream } \\
\text { length } \\
\text { (mi) }\end{array}$} & \multirow[b]{2}{*}{$\begin{array}{c}\text { Mean } \\
\text { basin } \\
\text { elevation } \\
(\mathrm{ft})\end{array}$} & \multirow[b]{2}{*}{$\begin{array}{l}\text { Forested } \\
\text { area } \\
\text { (percent) }\end{array}$} & \multirow[b]{2}{*}{$\begin{array}{c}\text { Soil } \\
\text { index }\end{array}$} & \multirow[b]{2}{*}{$\begin{array}{c}\text { Mean } \\
\text { annual } \\
\text { precipitation } \\
\text { (in) }\end{array}$} & \multicolumn{2}{|c|}{ Rainfall intensity, 24-hour } \\
\hline & & & & & & $\begin{array}{l}\text { 2-year } \\
\text { (in) }\end{array}$ & $\begin{array}{l}\text { 50-year } \\
\text { (in) }\end{array}$ \\
\hline 131 & 30.1 & 6,410 & 35.0 & 3.0 & 24.8 & 2.5 & 5.1 \\
\hline
\end{tabular}


09505200 WET BEAVER CREEK NEAR RIMROCK, AZ--Continued

MEAN MONTHLY AND ANNUAL DISCHARGES 1962.82, 1991.96

\begin{tabular}{|c|c|c|c|c|c|c|}
\hline MONTH & $\begin{array}{l}\text { MAXIMUM } \\
\text { (FT3/S) }\end{array}$ & $\begin{array}{l}\text { MINIMUM } \\
(\text { FT3/S) }\end{array}$ & $\begin{array}{c}\text { MEAN } \\
(\mathrm{FT} 3 / \mathrm{S})\end{array}$ & $\begin{array}{l}\text { STAN- } \\
\text { DARD } \\
\text { DEVIA- } \\
\text { TION } \\
\text { (FT3/S) }\end{array}$ & $\begin{array}{l}\text { COEFFI- } \\
\text { CIENT OF } \\
\text { VARI- } \\
\text { ATION }\end{array}$ & $\begin{array}{c}\text { PERCENT } \\
\text { OF } \\
\text { ANNUAL } \\
\text { RUNOFF }\end{array}$ \\
\hline OCTOBER & 231 & 5.7 & 16 & 43 & 2.7 & 3.7 \\
\hline NOVEMBER & 128 & 6.2 & 15 & 26 & 1.7 & 3.6 \\
\hline DECEMBER & 253 & 6.0 & 37 & 68 & 1.8 & 8.4 \\
\hline JANUARY & 601 & 6.0 & 52 & 117 & 2.3 & 11.9 \\
\hline FEBRUARY & 438 & 7.1 & 81 & 106 & 1.3 & 18.6 \\
\hline MARCH & 500 & 7.3 & 112 & 109 & 0.97 & 25.8 \\
\hline APRIL & 433 & 6.8 & 72 & 95 & 1.3 & 16.6 \\
\hline MAY & 109 & 5.8 & 12 & 20 & 1.7 & 2.7 \\
\hline JUNE & 9.9 & 4.5 & 6.9 & 0.98 & 0.14 & 1.6 \\
\hline JULY & 21 & 5.3 & 8.3 & 4.0 & 0.49 & 1.9 \\
\hline AUGUST & 75 & 6.1 & 11 & 14 & 1.2 & 2.6 \\
\hline SEPTEMBER & 82 & 5.8 & 11 & 15 & 1.3 & 2.6 \\
\hline ANNUAL & 103 & 7.7 & 36 & 26 & 0.73 & 100 \\
\hline
\end{tabular}

MAGNITUDE AND PROBABILITY OF INSTANTANEOUS PEAK FLOW BASED ON PERIOD OF RECORD 1962.96

DISCHARGE, IN FT3/S, FOR INDICATED RECURRENCE INTERVAL IN YEARS, AND EXCEEDANCE PROBABILITY, IN PERCENT

$\begin{array}{cccccc}2 & 5 & 10 & 25 & 50 & 100 \\ 508 & 208 & 108 & 48 & 28 & 18 \\ 3,520 & 6,130 & 8,140 & 11,000 & 13,300 & 15,800\end{array} \ldots$

WEIGHTED SKEW (LOGS) $=-0.09$

MEAN (LOGS) $=3.54$

STANDARD DEV. (LOGS) $=0.29$
MAGNITUDE AND PROBABILITY OF ANNUAL LOW FLOW BASED ON PERIOD OF RECORD $1963-82,1992.96$

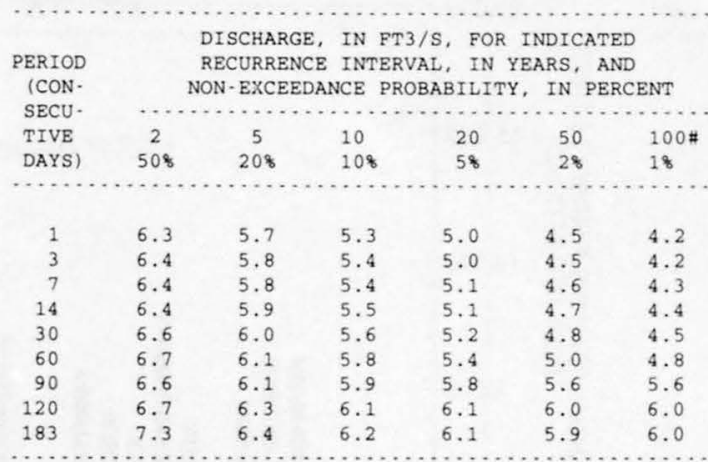

MAGNITUDE AND PROBABILITY OF ANNUAL HIGH FLOW BASED ON PERIOD OF RÉCORD $1962.82,1991.96$

\begin{tabular}{|c|c|c|c|c|c|c|}
\hline $\begin{array}{l}\text { PERIOD } \\
\text { (CON. }\end{array}$ & & $\begin{array}{l}\text { DISCHA } \\
\text { RECURRI } \\
\text { EXCEED }\end{array}$ & $\begin{array}{ll}\text { E, } & \text { IN } \\
\text { CE } & \text { INTE } \\
\text { CE } & \text { PROR }\end{array}$ & \multicolumn{2}{|c|}{ DISCHARGE, IN FT3/S, FOR INDICATED } & $\begin{array}{l}\text { TED } \\
\text { AND } \\
\text { ENT }\end{array}$ \\
\hline SECU - & $\cdots$ & $\ldots \ldots$ & $\cdots \cdots \cdots$ & $\cdots \ldots$ & $\ldots \ldots$ & $\cdots \cdots$ \\
\hline TIVE & 2 & 5 & 10 & 25 & 50 & $100 \#$ \\
\hline DAYS) & 508 & 208 & 108 & 48 & 28 & 18 \\
\hline 1 & 941 & 2.520 & 4,000 & 6,300 & 8,270 & 10,400 \\
\hline 3 & 585 & 1,430 & 2,150 & 3,180 & 4,000 & 4,860 \\
\hline 7 & 356 & 816 & 1,180 & 1,680 & 2,050 & 2,420 \\
\hline 15 & 227 & 509 & 726 & 1,010 & 1,220 & 1,420 \\
\hline 30 & 149 & 340 & 492 & 698 & 855 & 1,010 \\
\hline 60 & 98 & 229 & 336 & 486 & 603 & 724 \\
\hline 90 & 76 & 175 & 259 & 378 & 474 & 574 \\
\hline
\end{tabular}

DURATION TABLE OF DAILY MEAN FLOW FOR PERIOD OF RECORD 1962.82, 1991.96

DISCHARGE, IN FT3/S, WHICH WAS EQUALED OR EXCEEDED FOR INDICATED PERCENT OF TIME

\begin{tabular}{|c|c|c|c|c|c|c|c|c|c|c|c|c|c|c|c|c|}
\hline 18 & 58 & 108 & 158 & 208 & 308 & 408 & 508 & 608 & 708 & 808 & 908 & 958 & 988 & 998 & 99.58 & 99.98 \\
\hline & 156 & 67 & 26 & 12 & 8.3 & 7.8 & 7.5 & 7.2 & 6.9 & 6.6 & 6.2 & 5.7 & 5.4 & 5.3 & 5.2 & 4.4 \\
\hline
\end{tabular}

\# Reliability of values in column is uncertain, and potential errors are large. 
ANNUAL MEAN DISCHARGE IN CUBIC FEET PER SECOND

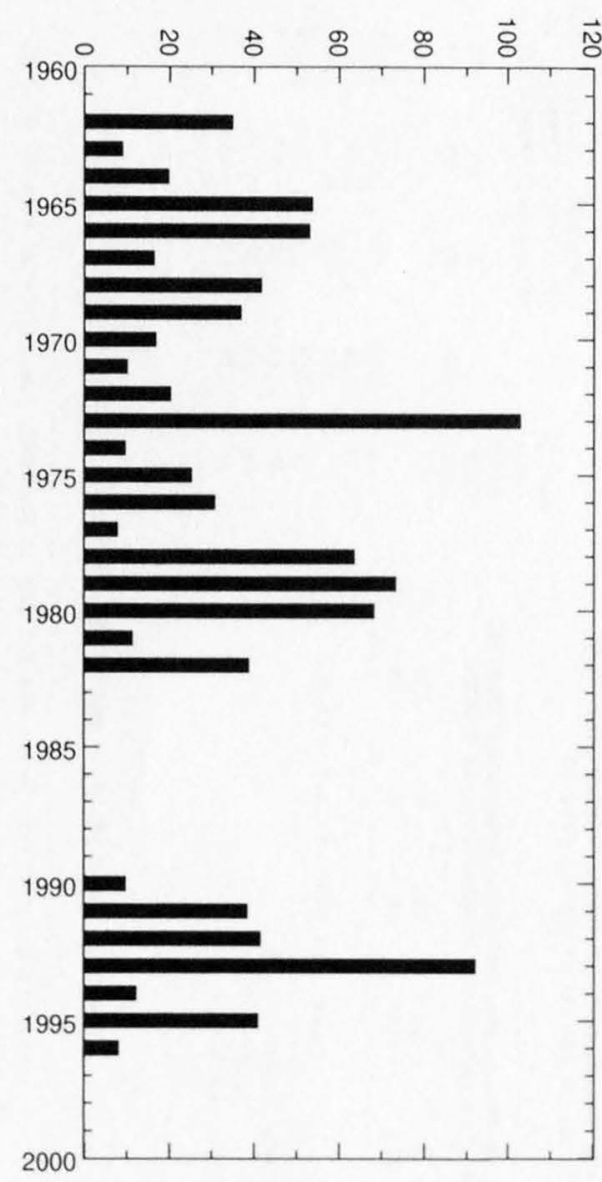

ANNUAL PEAK DISCHARGE, IN CUBIC

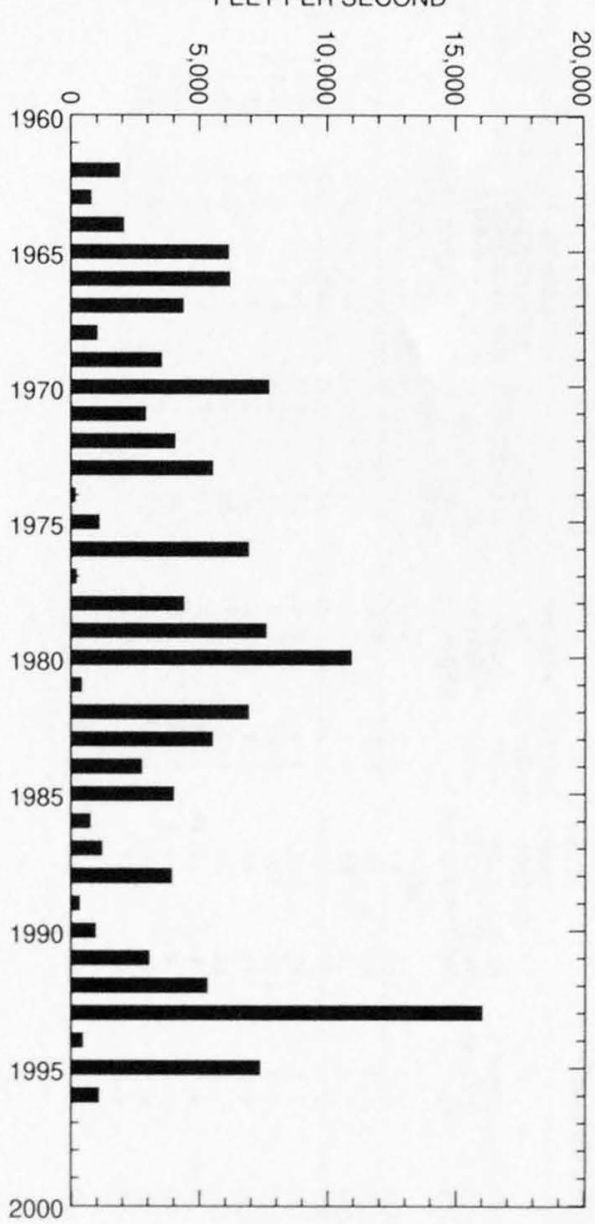


GILA RIVER BASIN

695

09505200 WET BEAVER CREEK NEAR RIMROCK, AZ--Continued
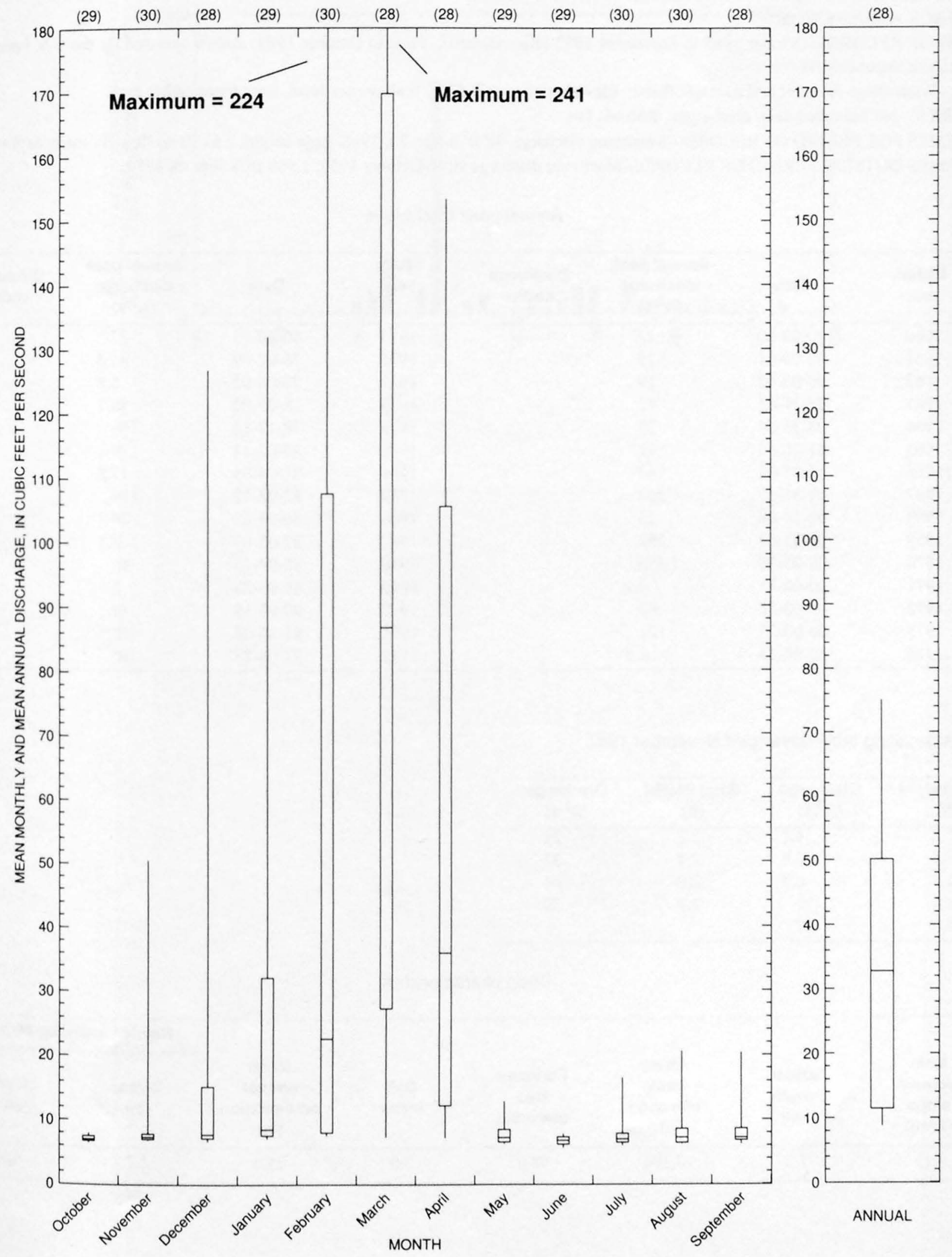

ANNUAL 


\section{ROCKY GULCH NEAR RIMROCK, AZ}

LOCATION.--Lat 34 44'49, long 111 '29'38", in SE $1 /{ }_{4} \mathrm{NW}^{1} / 4$ sec.27, T.16 N., R.8 E., Coconino County, Hydrologic Unit 15060202 , in Coconino

National Forest, on right bank, on headwaters of Rocky Gulch, and 7 mi northwest of Rimrock.

DRAINAGE AREA.--1.40 $\mathrm{mi}^{2}$.

PERIOD OF RECORD.--October 1985 to September 1992 (discontinued). Prior to October 1985, station operated by the U.S. Forest Service (records unpublished).

GAGE.--Water-stage recorder and concrete flume. Elevation of gage is 6,750 ft above sea level, from topographic map.

REMARKS.--No estimated daily discharges. Records fair.

EXTREMES FOR PERIOD OF RECORD.--Maximum discharge, $48 \mathrm{ft}^{3} / \mathrm{s}$ Apr. 25, 1988, gage height, $2.67 \mathrm{ft}$; no flow for many days each year.

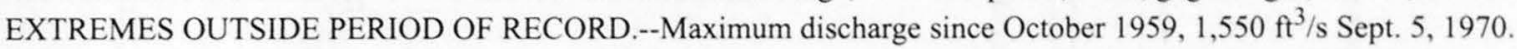

Annual peak discharges

\begin{tabular}{|c|c|c|c|c|c|c|c|}
\hline $\begin{array}{l}\text { Water } \\
\text { year }\end{array}$ & Date & $\begin{array}{c}\text { Annual peak } \\
\text { discharge } \\
\left(\mathrm{ft}^{3} / \mathrm{s}\right)\end{array}$ & $\begin{array}{l}\text { Discharge } \\
\text { codes }\end{array}$ & $\begin{array}{l}\text { Water } \\
\text { year }\end{array}$ & Date & $\begin{array}{c}\text { Annual peak } \\
\text { discharge } \\
\left(\mathrm{ft}^{3} / \mathrm{s}\right)\end{array}$ & $\begin{array}{c}\text { Discharge } \\
\text { codes }\end{array}$ \\
\hline 1960 & $00-00-60$ & 22 & & 1975 & $00-00-75$ & 27 & \\
\hline 1961 & $00-00-61$ & 19 & & 1976 & $76-02-09$ & 45.5 & \\
\hline 1962 & $00-00-62$ & 19 & & 1977 & $77-04-05$ & 5.8 & \\
\hline 1963 & $08-11-63$ & 12 & & 1978 & $78-03-02$ & 98.2 & \\
\hline 1964 & $03-31-64$ & 28 & & 1979 & $78-12-18$ & 166 & \\
\hline 1965 & $01-06-65$ & 82 & & 1980 & $80-02-14$ & 179 & \\
\hline 1966 & $11-25-65$ & 147 & & 1981 & 81-04-05 & 15.8 & \\
\hline 1967 & $07-31-67$ & 363 & & 1982 & $82-03-12$ & 154 & \\
\hline 1968 & $02-24-68$ & 13 & & 1986 & $86-04-02$ & 24 & \\
\hline 1969 & $01-25-69$ & 162 & & 1987 & 87-03-09 & 7.9 & \\
\hline 1970 & $09-05-70$ & 1,550 & & 1988 & $88-04-25$ & 48 & \\
\hline 1971 & $00-00-71$ & 8.1 & & 1989 & $89-03-28$ & 6.5 & \\
\hline 1972 & $00-00-72$ & 69 & & 1990 & $90-03-19$ & 14 & \\
\hline 1973 & $00-00-73$ & 121 & & 1991 & $91-04-06$ & 30 & \\
\hline 1974 & $00-00-74$ & 8.1 & & 1992 & $92-08-23$ & 50 & \\
\hline
\end{tabular}

Discharge rating table developed November 1987

\begin{tabular}{cccc}
\hline $\begin{array}{c}\text { Gage height } \\
(\mathrm{ft})\end{array}$ & $\begin{array}{c}\text { Discharge } \\
\left(\mathrm{ft}^{3} \mathbf{s}\right)\end{array}$ & $\begin{array}{c}\text { Gage height } \\
(\mathrm{ft})\end{array}$ & $\begin{array}{c}\text { Discharge } \\
\left(\mathrm{ft}^{3} / \mathbf{s}\right)\end{array}$ \\
\hline 1.2 & 1.1 & 2.2 & 24 \\
1.4 & 3.4 & 2.4 & 33 \\
1.6 & 6.7 & 2.6 & 44 \\
1.8 & 11 & 2.7 & 50 \\
2.0 & 17 & & \\
\hline
\end{tabular}

Basin characteristics

\begin{tabular}{cccccccr}
\hline $\begin{array}{c}\text { Main } \\
\text { channel } \\
\text { slope } \\
(\mathrm{ft} / \mathrm{mi})\end{array}$ & $\begin{array}{c}\text { Stream } \\
\text { length } \\
(\mathrm{mi})\end{array}$ & $\begin{array}{c}\text { Mean } \\
\text { basin } \\
\text { elevation } \\
(\mathrm{ft})\end{array}$ & $\begin{array}{c}\text { Forested } \\
\text { area } \\
\text { (percent) }\end{array}$ & $\begin{array}{c}\text { Soil } \\
\text { index }\end{array}$ & $\begin{array}{c}\text { Mean } \\
\text { annual } \\
\text { precipitation } \\
\text { (in) }\end{array}$ & $\begin{array}{c}\text { Rainfall intensity, 24-hour } \\
\text { 2-year } \\
\text { (in) }\end{array}$ & $\begin{array}{c}50 \text {-year } \\
\text { (in) }\end{array}$ \\
\hline 421 & 1.5 & 7,190 & 98.0 & 3.0 & 25.0 & 2.7 \\
\hline
\end{tabular}


09505220 ROCKY GULCH NEAR RIMROCK, AZ--Continued

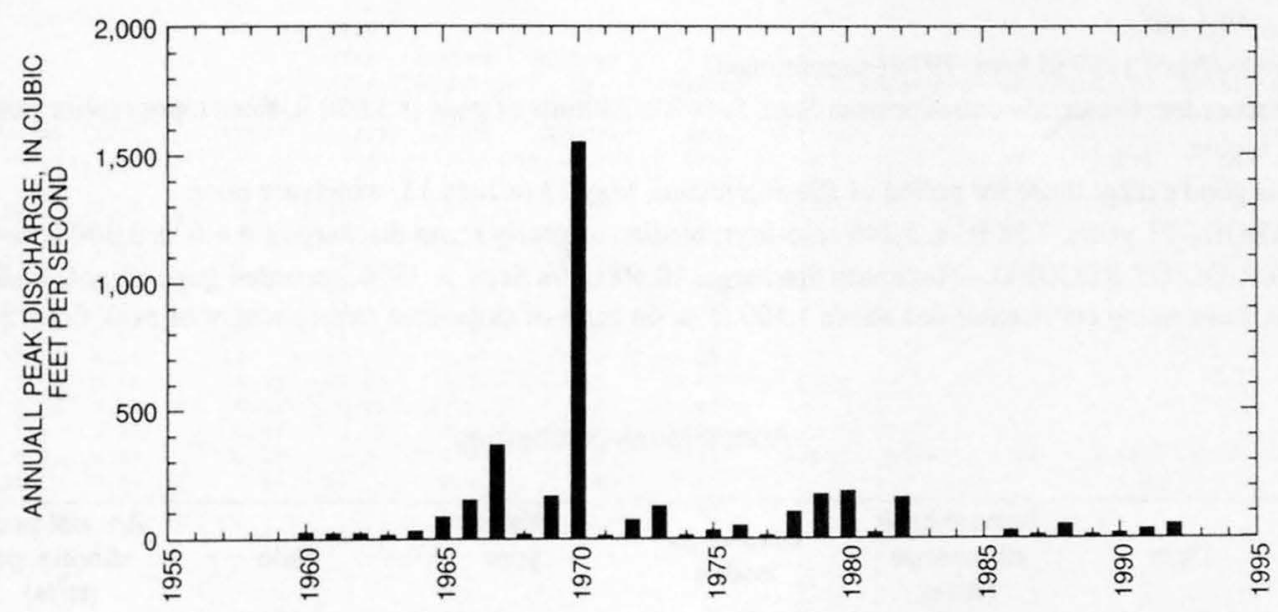




\section{RED TANK DRAW NEAR RIMROCK, AZ}

LOCATION.--Lat 34.41'43", long 111 42'49", in SE $\frac{1}{4} \mathrm{NE}^{1} / 4$ sec.16, T.15 N., R.6 E., Yavapai County, Hydrologic Unit 15060202, in Coconino National Forest, on left bank 2.5 mi downstream from confluence of Rarick and Mullican Canyons, and 3.5 mi northeast of Rimrock.

DRAINAGE AREA.--49.4 $\mathrm{mi}^{2}$.

PERIOD OF RECORD.--April 1957 to Sept. 1978 (discontinued).

GAGE.--Water-stage recorder. Concrete control prior to Sept. 5, 1970. Altitude of gage is 3,920 ft, from topographic map. Prior to Sept. 5, 1970, at datum $0.29 \mathrm{ft}$ higher.

REMARKS.--Records good except those for period of missing record, May 13 to June 13, which are poor.

AVERAGE DISCHARGE.--21 years, $7.24 \mathrm{ft}^{3} / \mathrm{s}, 5,240$ acre- $\mathrm{ft} / \mathrm{yr}$; median of yearly mean discharges, $4.6 \mathrm{ft}^{3} / \mathrm{s} 3,300 \mathrm{acre}-\mathrm{ft} / \mathrm{yr}$.

EXTREMES FOR PERIOD OF RECORD.--Maximum discharge, 10,500 ft $3 / \mathrm{s} \mathrm{Sept.} \mathrm{5,} \mathrm{1970,} \mathrm{recorded} \mathrm{gage} \mathrm{height,} 12.69 \mathrm{ft}$, about $13.3 \mathrm{ft}$, from profile past gage, from rating curve extended above $1,100 \mathrm{ft}^{3} / \mathrm{s}$, on basis of slope-area measurement of peak flow; no flow for many daysin each year.

Annual peak discharges

\begin{tabular}{|c|c|c|c|c|c|c|c|}
\hline $\begin{array}{l}\text { Water } \\
\text { year }\end{array}$ & Date & $\begin{array}{c}\text { Annual peak } \\
\text { discharge } \\
\left(\mathrm{ft}^{3} / \mathrm{s}\right)\end{array}$ & $\begin{array}{l}\text { Discharge } \\
\text { codes }\end{array}$ & $\begin{array}{l}\text { Water } \\
\text { year }\end{array}$ & Date & $\begin{array}{c}\text { Annual peak } \\
\text { discharge } \\
\left(\mathrm{ft}^{3} / \mathrm{s}\right)\end{array}$ & $\begin{array}{c}\text { Discharge } \\
\text { codes }\end{array}$ \\
\hline 1958 & $03-22-58$ & 1,280 & & 1969 & $01-25-69$ & 1,650 & \\
\hline 1959 & $02-17-59$ & 113 & & 1970 & $09-05-70$ & 10,500 & \\
\hline 1960 & $12-25-59$ & 1,230 & & 1971 & $10-03-70$ & 12 & \\
\hline 1961 & $03-31-61$ & 457 & & 1972 & $12-26-71$ & 745 & \\
\hline 1962 & $02-08-62$ & 620 & & 1973 & $10-19-72$ & 2,720 & \\
\hline 1963 & $03-22-63$ & 12 & & 1974 & $01-21-74$ & 34 & \\
\hline 1964 & $08-02-64$ & 1,970 & & 1975 & $04-13-75$ & 407 & \\
\hline 1965 & $04-04-65$ & 1,440 & & 1976 & $02-09-76$ & 1,800 & \\
\hline 1966 & $11-25-65$ & 2,010 & & 1977 & $04-06-77$ & 16 & \\
\hline 1967 & $12-07-66$ & 425 & & 1978 & $02-23-78$ & 441 & \\
\hline 1968 & $02-14-68$ & 327 & & & & & \\
\hline
\end{tabular}

\section{Basin characteristics}

\begin{tabular}{ccccccc}
\hline $\begin{array}{c}\text { Main } \\
\text { channel } \\
\text { slope } \\
(\mathrm{ft} / \mathrm{mi})\end{array}$ & $\begin{array}{c}\text { Stream } \\
\text { length } \\
\text { (mi) }\end{array}$ & $\begin{array}{c}\text { Mean } \\
\text { basin } \\
\text { elevation } \\
\text { (ft) }\end{array}$ & $\begin{array}{c}\text { Forested } \\
\text { area } \\
\text { (percent) }\end{array}$ & $\begin{array}{c}\text { Soil } \\
\text { index }\end{array}$ & $\begin{array}{c}\text { Mean } \\
\text { annual } \\
\text { precipitation } \\
\text { (in) }\end{array}$ & $\begin{array}{c}\text { Rainfall intensity, 24-hour } \\
\text { (in) }\end{array}$ \\
\hline 183 & 19.3 & 5,910 & 23.0 & 3.0 & 21.6 & $\begin{array}{c}50 \text {-year } \\
\text { (in) }\end{array}$ \\
\hline
\end{tabular}


09505250 RED TANK DRAW NEAR RIMROCK, AZ--Continued

MEAN MONTHLY AND ANNUAL DISCHARGES 1958-78

\begin{tabular}{|c|c|c|c|c|c|c|}
\hline MONTH & $\begin{array}{l}\text { MAXIMUM } \\
\text { (FT3/S) }\end{array}$ & $\begin{array}{l}\text { MINIMUM } \\
\text { (FT3/S) }\end{array}$ & $\begin{array}{c}\text { MEAN } \\
(\text { FT3/S) }\end{array}$ & $\begin{array}{l}\text { STAN- } \\
\text { DARD } \\
\text { DEVIA- } \\
\text { TION } \\
\text { (FT3/S) }\end{array}$ & $\begin{array}{l}\text { COEFFI- } \\
\text { CIENT OF } \\
\text { VARI- } \\
\text { ATION }\end{array}$ & $\begin{array}{l}\text { PERCENT } \\
\text { OF } \\
\text { ANNUAL } \\
\text { RUNOFF }\end{array}$ \\
\hline OCTOBER & 80 & 0.00 & 4.2 & 17 & 4.1 & 4.9 \\
\hline NOVEMBER & 41 & 0.00 & 4.4 & 12 & 2.6 & 5.1 \\
\hline DECEMBER & 104 & 0.00 & 10 & 25 & 2.4 & 11.8 \\
\hline JANUARY & 46 & 0.00 & 7.0 & 13 & 1.9 & 8.0 \\
\hline FEBRUARY & 79 & 0.00 & 16 & 23 & 1.4 & 18.5 \\
\hline MARCH & 161 & 0.02 & 27 & 42 & 1.6 & 31.0 \\
\hline APRIL & 110 & 0.00 & 14 & 30 & 2.2 & 16.0 \\
\hline MAY & 3.7 & 0.00 & 0.25 & 0.82 & 3.3 & 0.3 \\
\hline JUNE & 0.05 & 0.00 & 0.01 & 0.01 & 2.7 & 0.0 \\
\hline JULY & 1.5 & 0.00 & 0.07 & 0.32 & 4.6 & 0.1 \\
\hline AUGUST & 8.8 & 0.00 & 0.50 & 1.9 & 3.9 & 0.6 \\
\hline SEPTEMBER & 62 & 0.00 & 3.4 & 14 & 4.0 & 3.8 \\
\hline ANNUAL & 37 & 0.04 & 7.2 & 9.1 & 1.3 & 100 \\
\hline
\end{tabular}

MAGNITUDE AND PROBABILITY OF ANNUAL LOW FLOW BASED ON PERIOD OF RECORD 1959-78

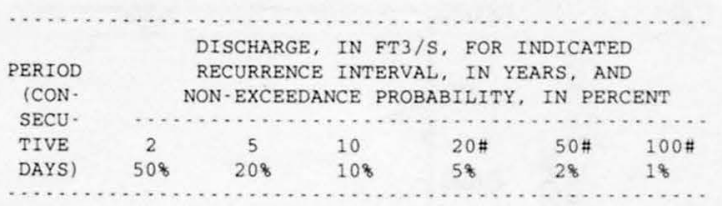

MAGNITUDE AND PROBABILITY OF ANNUAL HIGH FLOW BASED ON PERIOD OF RECORD 1958-78

\begin{tabular}{|c|c|c|c|c|c|c|}
\hline PERIOD & \multicolumn{6}{|c|}{ DISCHARGE, IN FT $3 / S$, FOR INDICATED } \\
\hline$(\mathrm{CON}$. & & \multicolumn{3}{|c|}{ EXCEEDANCE PROBABILITY, } & \multicolumn{2}{|c|}{ IN PERCENT } \\
\hline SECU. & $\cdots$ & ........ & ....... & 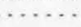 & ....... & ....... \\
\hline TIVE & 2 & 5 & 10 & $25 \#$ & $50 \#$ & $100 \#$ \\
\hline DAYS ) & 508 & 208 & 108 & 48 & 28 & 18 \\
\hline 1 & 208 & 755 & 1,310 & 2,170 & 2,880 & 3,620 \\
\hline 3 & 129 & 424 & 691 & 1.060 & 1,330 & 1.590 \\
\hline 7 & 75 & 247 & 400 & 606 & 755 & 894 \\
\hline 15 & 45 & 151 & 247 & 378 & 474 & 564 \\
\hline 30 & 26 & 96 & 164 & 267 & 349 & 430 \\
\hline 60 & 16 & 61 & 109 & 184 & 248 & 314 \\
\hline 90 & 12 & 46 & 82 & 140 & 189 & 240 \\
\hline
\end{tabular}

DURATION TABLE OF DAILY MEAN FLOW FOR PERIOD OF RECORD 1958.78 DISCHARGE, IN FT3/S, WHICH WAS EQUALED OR EXCEEDED FOR INDICATED PERCENT OF TIME

\begin{tabular}{|c|c|c|c|c|c|c|c|c|c|c|c|c|c|c|c|c|}
\hline 18 & 58 & 108 & 158 & 208 & 308 & 408 & 508 & 608 & 708 & 808 & 908 & 958 & 988 & 998 & 99.58 & 99.98 \\
\hline 174 & 30 & 4.7 & 0.84 & 0.25 & 0.07 & 0.01 & 0.00 & 0.00 & 0.00 & 0.00 & 0.00 & 0.00 & 0.00 & 0.00 & 0.00 & 0.00 \\
\hline
\end{tabular}


09505250 RED TANK DRAW NEAR RIMROCK, AZ--Continued
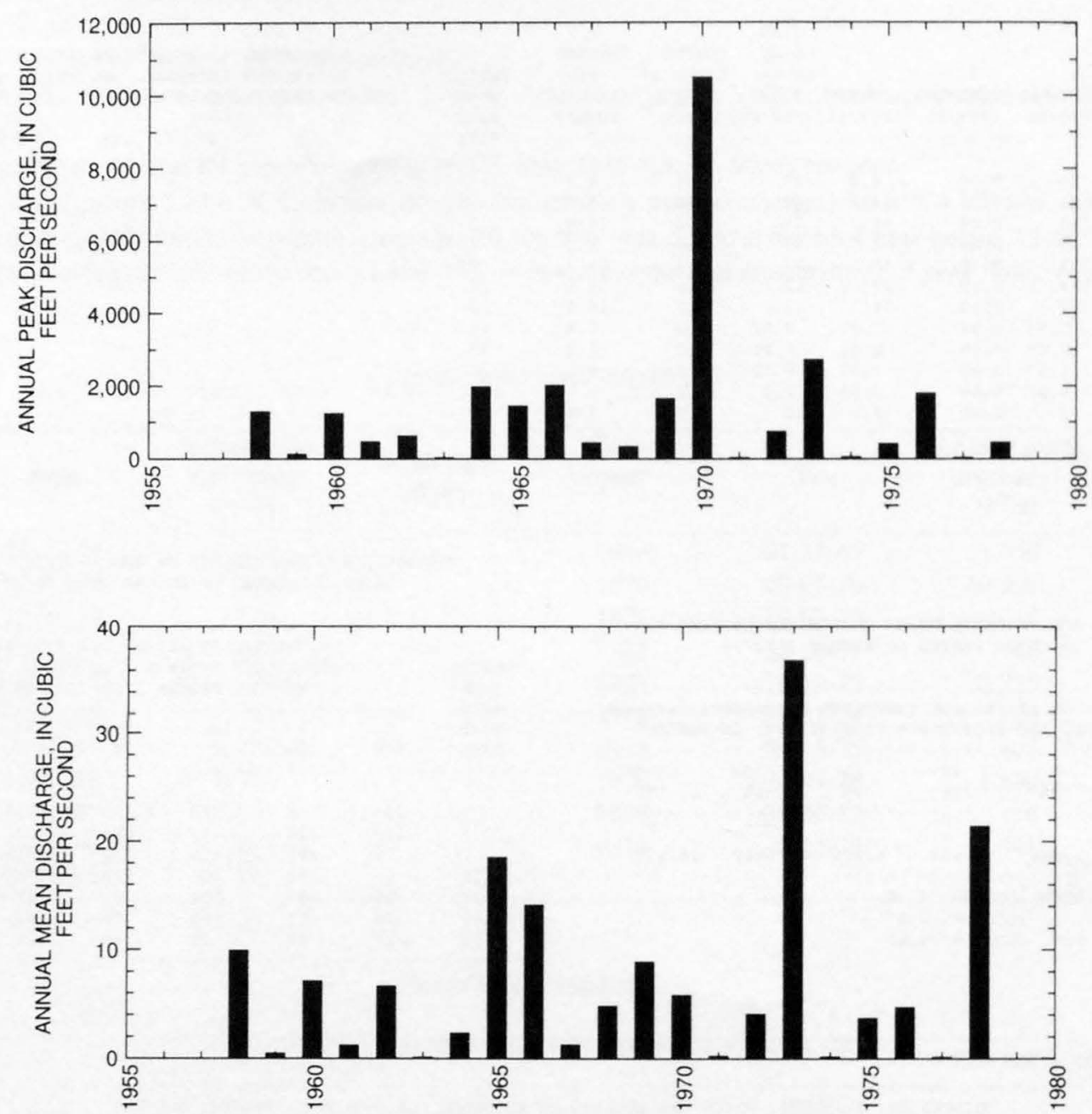
09505250 RED TANK DRAW NEAR RIMROCK, AZ--Continued
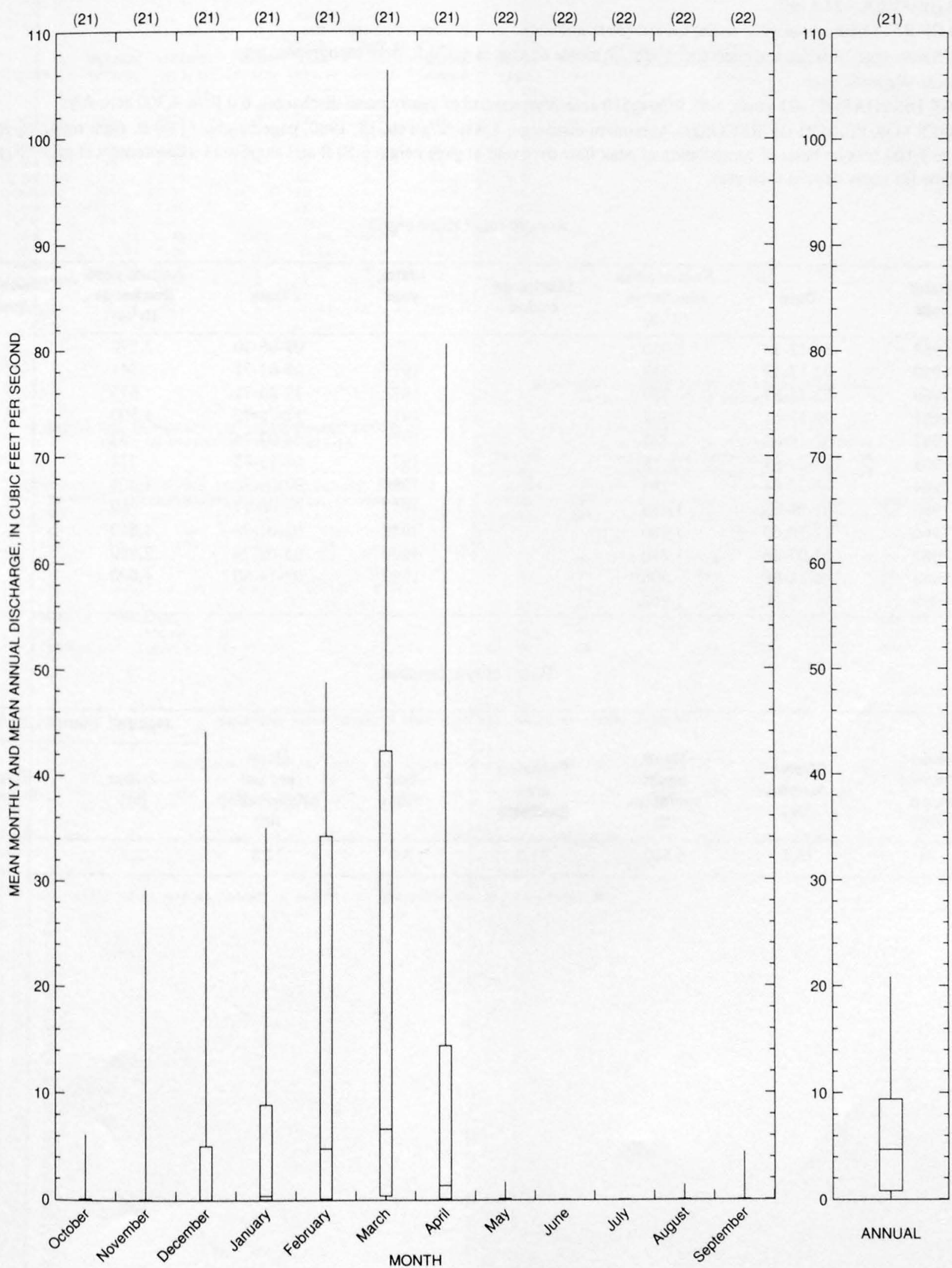
LOCATION.--Lat 34 46'01", long 111 40'23", in NW $1 / 4 \mathrm{SW}^{1} / 4 \mathrm{sec} .24$, T.16 N., R.6 E., Yavapai County, Hydrologic Unit 15060202 , in Coconino National Forest, on left bank 2.6 mi upstream from mouth, 7 mi northeast of Beaver Creek Ranger Station, and 9 mi northeast of Rimrock. DRAINAGE AREA.--24.6 $\mathrm{mi}^{2}$.

PERIOD OF RECORD.--June 1957 to Sept. 1980 (discontinued).

GAGE.--Water-stage recorder and concrete control. Altitude of gage is $4,870 \mathrm{ft}$, from topographic map.

REMARKS.--Records poor.

AVERAGE DISCHARGE.--23 years, $8.98 \mathrm{ft}^{3} / \mathrm{s}, 6,510$ acre- $\mathrm{ft} / \mathrm{yr}$; median of yearly mean discharges, $6.0 \mathrm{ft}^{3} / \mathrm{s}, 4,300 \mathrm{acre}-\mathrm{ft} / \mathrm{yr}$.

EXTREMES FOR PERIOD OF RECORD.--Maximum discharge, 4,000 $\mathrm{ft}^{3} / \mathrm{s}$ Feb. 14, 1980, gage height, $11.90 \mathrm{ft}$, from rating curve extended above $1,100 \mathrm{ft}^{3} / \mathrm{s}$ on basis of computation of peak flow over weir at gage height $8.50 \mathrm{ft}$ and slope-area measurement at gage height $11.50 \mathrm{ft}$ no flow for many days in each year.

Annual peak discharges

\begin{tabular}{|c|c|c|c|c|c|c|c|}
\hline $\begin{array}{l}\text { Water } \\
\text { year }\end{array}$ & Date & $\begin{array}{c}\text { Annual peak } \\
\text { discharge } \\
\left(\mathrm{ft}^{3} / \mathrm{s}\right)\end{array}$ & $\begin{array}{l}\text { Discharge } \\
\text { codes }\end{array}$ & $\begin{array}{l}\text { Water } \\
\text { year }\end{array}$ & Date & $\begin{array}{c}\text { Annual peak } \\
\text { discharge } \\
\left(\mathrm{ft}^{3} / \mathrm{s}\right)\end{array}$ & $\begin{array}{c}\text { Discharge } \\
\text { codes }\end{array}$ \\
\hline 1958 & $09-12-58$ & 1,050 & & 1970 & $09-05-70$ & 3,590 & \\
\hline 1959 & $02-17-59$ & 119 & & 1971 & $09-01-71$ & 741 & \\
\hline 1960 & $12-25-59$ & 590 & & 1972 & $12-26-71$ & 675 & \\
\hline 1961 & $09-17-61$ & 384 & & 1973 & $12-28-72$ & 1,500 & \\
\hline 1962 & $02-12-62$ & 775 & & 1974 & $04-03-74$ & 52 & \\
\hline 1963 & $03-23-63$ & 18 & & 1975 & $04-13-75$ & 311 & \\
\hline 1964 & $03-30-64$ & 288 & & 1976 & $02-09-76$ & 1,200 & \\
\hline 1965 & $01-06-65$ & 1,430 & & 1977 & $04-06-77$ & 69 & \\
\hline 1966 & $12-30-65$ & 1,880 & & 1978 & $03-01-78$ & 1,610 & \\
\hline 1967 & $12-07-66$ & 1,240 & & 1979 & $03-08-79$ & 2,850 & \\
\hline 1968 & $01-28-68$ & 306 & & 1980 & $02-14-80$ & 4,000 & \\
\hline 1969 & $01-25-69$ & 2,160 & & & & & \\
\hline
\end{tabular}

\section{Basin characteristics}

\begin{tabular}{|c|c|c|c|c|c|c|c|}
\hline \multirow[b]{2}{*}{$\begin{array}{l}\text { Main } \\
\text { channel } \\
\text { slope } \\
\text { (ft/mi) }\end{array}$} & \multirow[b]{2}{*}{$\begin{array}{l}\text { Stream } \\
\text { length } \\
\text { (mi) }\end{array}$} & \multirow[b]{2}{*}{$\begin{array}{c}\text { Mean } \\
\text { basin } \\
\text { elevation } \\
\text { (ft) }\end{array}$} & \multirow[b]{2}{*}{$\begin{array}{l}\text { Forested } \\
\text { area } \\
\text { (percent) }\end{array}$} & \multirow[b]{2}{*}{$\begin{array}{l}\text { Soil } \\
\text { index }\end{array}$} & \multirow[b]{2}{*}{$\begin{array}{c}\text { Mean } \\
\text { annual } \\
\text { precipitation } \\
\text { (in) }\end{array}$} & \multicolumn{2}{|c|}{ Rainfall intensity, 24-hour } \\
\hline & & & & & & $\begin{array}{l}\text { 2-year } \\
\text { (in) }\end{array}$ & $\begin{array}{l}\text { 50-year } \\
\text { (in) }\end{array}$ \\
\hline 174 & 15.5 & 6,560 & 33.0 & 3.0 & 22.8 & 2.5 & 5.1 \\
\hline
\end{tabular}


09505300 RATTLESNAKE CANYON NEAR RIMROCK, AZ--Continued

MEAN MONTHLY AND ANNUAL DISCHARGES $1958 \cdot 80$

\begin{tabular}{|c|c|c|c|c|c|c|}
\hline MONTH & $\begin{array}{l}\text { MAXIMUM } \\
(\text { FT } 3 / \mathrm{S})\end{array}$ & $\begin{array}{l}\text { MINIMUM } \\
\text { (FT3/S) }\end{array}$ & $\begin{array}{c}\text { MEAN } \\
\text { (FT3/S) }\end{array}$ & $\begin{array}{l}\text { STAN- } \\
\text { DARD } \\
\text { DEVIA- } \\
\text { TION } \\
(\mathrm{FT} 3 / \mathrm{S})\end{array}$ & $\begin{array}{l}\text { COEFFI- } \\
\text { CIENT OF } \\
\text { VARI- } \\
\text { ATION }\end{array}$ & $\begin{array}{c}\text { PERCENT } \\
\text { OF } \\
\text { ANNUAL } \\
\text { RUNOFF }\end{array}$ \\
\hline OCTOBER & 60 & 0.00 & 2.8 & 12 & 4.5 & 2.5 \\
\hline NOVEMBER & 42 & 0.00 & 4.2 & 10 & 2.5 & 3.9 \\
\hline DECEMBER & 86 & 0.00 & 12 & 25 & 2.1 & 10.8 \\
\hline JANUARY & 78 & 0.00 & 12 & 23 & 1.9 & 10.7 \\
\hline FEBRUARY & 165 & 0.00 & 21 & 36 & 1.7 & 19.6 \\
\hline MARCH & 135 & 0.00 & 31 & 32 & 1.0 & 28.6 \\
\hline APRIL & 116 & 0.00 & 22 & 33 & 1.5 & 20.2 \\
\hline MAY & 37 & 0.00 & 2.1 & 7.7 & 3.7 & 1.9 \\
\hline JUNE & 0.01 & 0.00 & 0.00 & 0.00 & 3.3 & 0.0 \\
\hline JULY & 0.28 & 0.00 & 0.02 & 0.06 & 3.3 & 0.0 \\
\hline AUGUST & 0.88 & 0.00 & 0.10 & 0.24 & 2.3 & 0.1 \\
\hline SEPTEMBER & 29 & 0.00 & 1.7 & 6.1 & 3.6 & 1.6 \\
\hline ANNUAL & 30 & 0.14 & 9.0 & 9.3 & 1.0 & 100 \\
\hline
\end{tabular}

MAGNITUDE AND PROBABILITY OF ANNUAL HIGH FLOW BASED ON PERIOD OF RECORD 1958-80

MAGNITUDE AND PROBABILITY OF INSTANTANEOUS PEAK FLOW BASED ON PERIOD OF RECORD 1958-80

DISCHARGE, IN FT3/S, FOR INDICATED RECURRENCE INTERVAL IN YEARS, AND EXCEEDANCE PROBABILITY, IN PERCENT

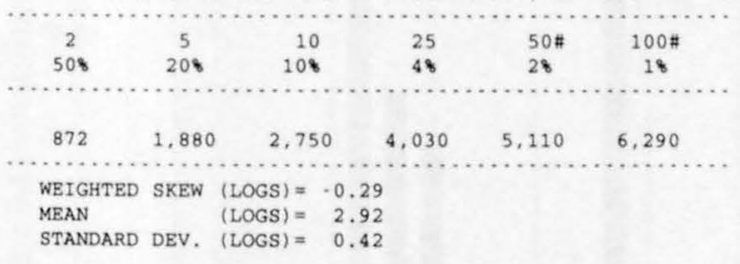

MAGNITUDE AND PROBABILITY OF ANNUAL LOW FLOW BASED ON PERIOD OF RECORD $1959-80$

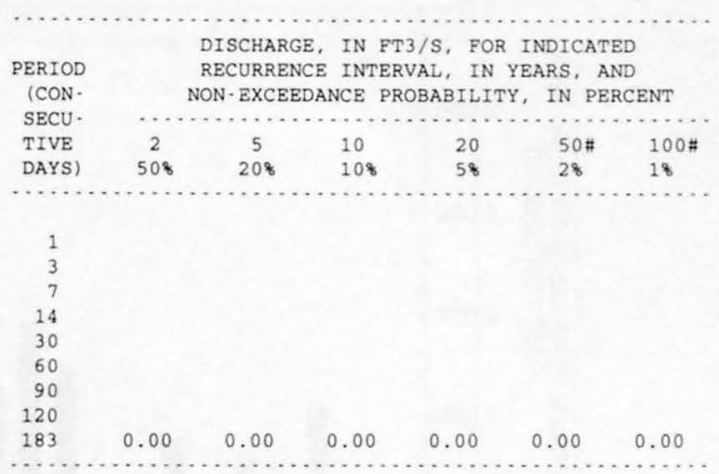

DURATION TABLE OF DAILY MEAN FLOW FOR PERIOD OF RECORD 1958-80 DISCHARGE, IN FT3/S, WHICH WAS EQUALED OR EXCEEDED FOR INDICATED PERCENT OF TIME

\begin{tabular}{|c|c|c|c|c|c|c|c|c|c|c|c|c|c|c|c|c|}
\hline 18 & 58 & 108 & 158 & 208 & 308 & 408 & 508 & 608 & 708 & 808 & 908 & 958 & 988 & 998 & 99.58 & 99.98 \\
\hline 55 & 51 & 21 & 6.7 & 0.91 & 0.02 & 0.00 & 0.00 & 0.00 & 0.00 & 0.00 & 0.00 & 0.00 & 0.00 & 0.00 & 0.00 & 0.00 \\
\hline
\end{tabular}

\# Reliability of values in column is uncertain, and potential errors are large. 
09505300 RATTLESNAKE CANYON NEAR RIMROCK, AZ--Continued
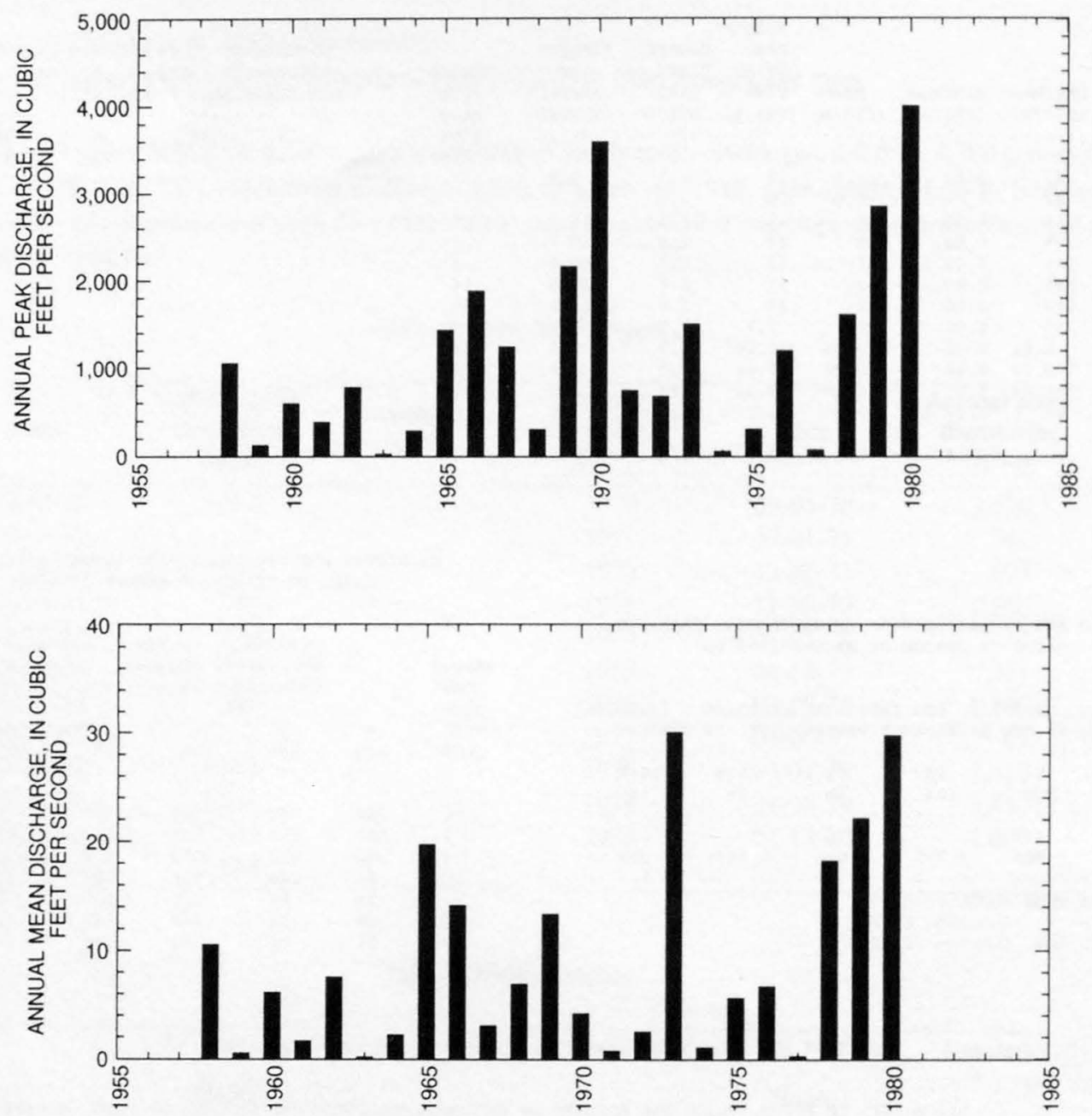
09505300 RATTLESNAKE CANYON NEAR RIMROCK, AZ--Continued
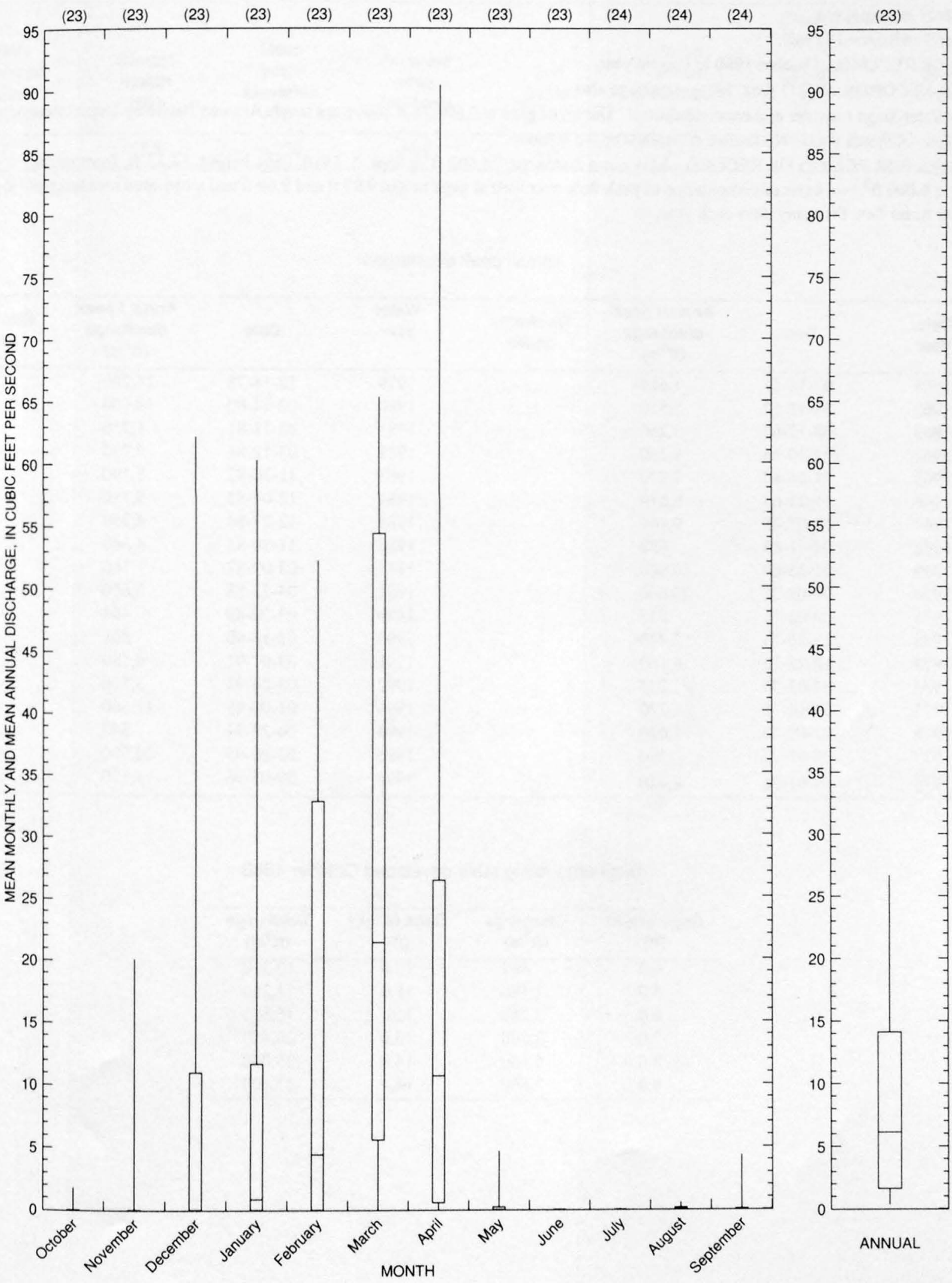


\section{DRY BEAVER CREEK NEAR RIMROCK, AZ}

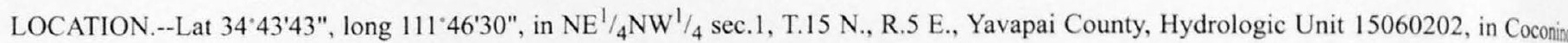
National Forest, on left upstream abutment of abandoned highway bridge, 1,000 ft upstream from present State Highway 179 , and $5.5 \mathrm{~m}$ north of Rimrock.

DRAINAGE AREA.--142 $\mathrm{mi}^{2}$.

PERIOD OF RECORD.--October 1960 to current year.

REVISED RECORDS.--WRD Ariz. 1969: Drainage area.

GAGE.--Water-stage recorder and concrete control. Datum of gage is 3,694.38 ft above sea level (Arizona Highway Department bench mark REMARKS.--Records good. No known diversions above station.

EXTREMES FOR PERIOD OF RECORD.--Maximum discharge, 26,600 $\mathrm{ft}^{3} / \mathrm{s}$ Sept. 5, 1970, gage height, $14.35 \mathrm{ft}$, from rating curve extende above $6,000 \mathrm{ft}^{3} / \mathrm{s}$ on basis of computation of peak flow over weir at gage height $9.07 \mathrm{ft}$ and $9.69 \mathrm{ft}$ and slope-area measurement at gage heigt $14.35 \mathrm{ft}$; no flow for many days each year.

Annual peak discharges

\begin{tabular}{ccccccc}
\hline $\begin{array}{c}\text { Water } \\
\text { year }\end{array}$ & Date & $\begin{array}{c}\text { Annual peak } \\
\text { discharge } \\
\left(\mathrm{ft}^{3} / \mathbf{s}\right)\end{array}$ & $\begin{array}{c}\text { Discharge } \\
\text { codes }\end{array}$ & $\begin{array}{c}\text { Water } \\
\text { year }\end{array}$ & Date & $\begin{array}{c}\text { Annual peak } \\
\text { discharge } \\
\left(\mathbf{f t}^{3} / \mathbf{s}\right)\end{array}$ \\
\hline 1961 & $07-14-61$ & 1,610 & & 1979 & $12-18-78$ & 24,200 \\
1962 & $02-12-62$ & 2,510 & & 1980 & $02-14-80$ & 18,600 \\
1963 & $08-17-63$ & 3,260 & 1981 & $08-11-81$ & 1,250 \\
1964 & $03-30-64$ & 1,160 & 1982 & $03-12-82$ & 7,790 \\
1965 & $01-06-65$ & 7,970 & 1983 & $11-30-82$ & 8,190 \\
1966 & $11-23-65$ & 9,670 & 1984 & $12-04-83$ & 5,780 \\
1967 & $12-07-66$ & 9,460 & 1985 & $12-27-84$ & 4,250 \\
1968 & $02-11-68$ & 652 & 1986 & $11-30-85$ & 4,340 \\
1969 & $01-25-69$ & 10,600 & 1987 & $03-09-87$ & 1,160 \\
1970 & $09-05-70$ & 26,600 & 1988 & $04-25-88$ & 2,650 \\
1971 & $09-01-71$ & 537 & 1989 & $03-29-89$ & 404 \\
1972 & $12-26-71$ & 2,740 & 1990 & $03-18-90$ & 801 \\
1973 & $12-28-72$ & 6,160 & 1991 & $03-01-91$ & 6,180 \\
1974 & $04-03-74$ & 253 & 1992 & $03-04-92$ & 3,770 \\
1975 & $04-14-75$ & 1,220 & 1993 & $01-08-93$ & 11,600 \\
1976 & $02-09-76$ & 7,020 & 1994 & $04-29-94$ & 842 \\
1977 & $04-07-77$ & 304 & 1995 & $03-06-95$ & 20,600 \\
1978 & $03-01-78$ & 8,410 & 1996 & $09-05-96$ & 1,170 \\
\hline
\end{tabular}

Discharge rating table developed October 1988

\begin{tabular}{crcc}
\hline $\begin{array}{c}\text { Gage height } \\
\text { (ft) }\end{array}$ & $\begin{array}{c}\text { Discharge } \\
\left(\mathbf{f t}^{3} / \mathbf{s}\right)\end{array}$ & $\begin{array}{c}\text { Gage height } \\
(\mathbf{f t})\end{array}$ & $\begin{array}{c}\text { Discharge } \\
\left(\mathrm{ft}^{\mathbf{3}} \mathbf{/ s}\right)\end{array}$ \\
\hline 4.0 & 409 & 10.0 & 10,350 \\
5.0 & 1,180 & 11.0 & 13,240 \\
6.0 & 2,280 & 12.0 & 16,580 \\
7.0 & 3,800 & 13.0 & 20,450 \\
8.0 & 5,680 & 14.0 & 25,060 \\
9.0 & 7,860 & 14.4 & 27,100 \\
\hline
\end{tabular}


GILA RIVER BASIN

09505350 DRY BEAVER CREEK NEAR RIMROCK, AZ--Continued

Basin characteristics

\begin{tabular}{cccccccc}
\hline $\begin{array}{c}\text { Main } \\
\text { channel } \\
\begin{array}{c}\text { slope } \\
(\mathrm{ft} / \mathrm{mi})\end{array}\end{array}$ & $\begin{array}{c}\text { Stream } \\
\text { length } \\
(\mathrm{mi})\end{array}$ & $\begin{array}{c}\text { Mean } \\
\text { basin } \\
\text { elevation } \\
(\mathrm{ft})\end{array}$ & $\begin{array}{c}\text { Forested } \\
\text { area } \\
\text { (percent) }\end{array}$ & $\begin{array}{c}\text { Soil } \\
\text { index }\end{array}$ & $\begin{array}{c}\text { Mean } \\
\text { annual } \\
\text { precipitation } \\
\text { (in) }\end{array}$ & $\begin{array}{c}\text { Rainfall intensity, 24-hour } \\
\text { (in) }\end{array}$ & $\begin{array}{c}50 \text {-year } \\
\text { (in) }\end{array}$ \\
\hline 137 & 32.5 & 6,220 & 56.0 & 2.8 & 23.1 & 2.5 & 5.1 \\
\hline
\end{tabular}




\section{DRY BEAVER CREEK NEAR RIMROCK, AZ--Continued}

MEAN MONTHLY AND ANNUAL DISCHARGES 1961.96

\begin{tabular}{|c|c|c|c|c|c|c|}
\hline MONTH & $\begin{array}{l}\text { MAXIMUM } \\
\text { (FT3/S) }\end{array}$ & $\begin{array}{l}\text { MINIMUM } \\
\text { (FT3/S) }\end{array}$ & $\begin{array}{c}\text { MEAN } \\
(\mathrm{FT} 3 / \mathrm{S})\end{array}$ & $\begin{array}{l}\text { STAN- } \\
\text { DARD } \\
\text { DEVIA- } \\
\text { TION } \\
(\mathrm{FT} 3 / \mathrm{S})\end{array}$ & $\begin{array}{l}\text { COEFFI- } \\
\text { CIENT OF } \\
\text { VARI- } \\
\text { ATION }\end{array}$ & $\begin{array}{c}\text { PERCENT } \\
\text { OF } \\
\text { ANNUAL } \\
\text { RUNOFF }\end{array}$ \\
\hline OCTOBER & 246 & 0.00 & 9.4 & 41 & 4.4 & 1.7 \\
\hline NOVEMBER & 251 & 0.00 & 19 & 51 & 2.7 & 3.4 \\
\hline DECEMBER & 602 & 0.00 & 66 & 134 & 2.0 & 11.6 \\
\hline JANUARY & 814 & 0.00 & 63 & 154 & 2.4 & 11.1 \\
\hline FEBRUARY & 850 & 0.00 & 120 & 184 & 1.5 & 21.1 \\
\hline MARCH & 678 & 0.00 & 162 & 159 & 0.98 & 28.6 \\
\hline APRIL & 598 & 0.00 & 106 & 135 & 1.3 & 18.7 \\
\hline MAY & 208 & 0.00 & 8.6 & 35 & 4.1 & 1.5 \\
\hline JUNE & 0.17 & 0.00 & 0.01 & 0.03 & 4.9 & 0.0 \\
\hline JULY & 2.5 & 0.00 & 0.19 & 0.46 & 2.5 & 0.0 \\
\hline AUGUST & 35 & 0.00 & 2.0 & 6.1 & 3.1 & 0.3 \\
\hline SEPTEMBER & 224 & 0.00 & 12 & 46 & 3.9 & 2.1 \\
\hline ANNUAL & 144 & 0.35 & 47 & 43 & 0.92 & 100 \\
\hline
\end{tabular}

MAGNITUDE AND PROBABILITY OF INSTANTANEOUS PEAK FLOW BASED ON PERIOD OF RECORD 1961.96

DISCHARGE, IN FT3/S, FOR INDICATED RECURRENCE INTERVAL

IN YEARS, AND EXCEEDANCE PROBABTUTTY, TN PERCENT

\begin{tabular}{|c|c|c|c|c|c|}
\hline 2 & 5 & 10 & 25 & 50 & 100 \\
\hline 508 & 208 & 108 & 48 & 28 & 18 \\
\hline 3,380 & 9,590 & 16,100 & 27,400 & 38,400 & 51,400 \\
\hline WEIGHTED & SKEW & $($ LOGS $)=-c$ & & & \\
\hline MEAN & & $($ LOGS $)=3$ & & & \\
\hline STANDARD & DEV. & $($ LOGS $)=$ & & & \\
\hline
\end{tabular}

MAGNITUDE AND PROBABILITY OF ANNUAL LOW FLOW BASED ON PERIOD OF RECORD 1962.96

DISCHARGE, IN FT $3 / S$, FOR INDICATED
PERIOD
RECURRENCE INTERVAL, IN YEARS, AND
SECU.
NON-EXCEEDANCE PROBABILITY, IN PERCENT

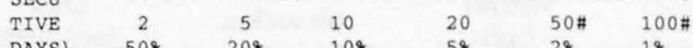

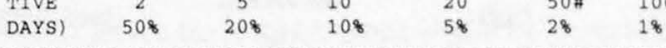

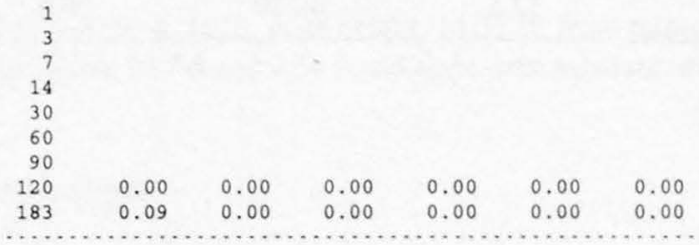

MAGNITUDE AND PROBABILITY OF ANNUAL HIGH FLOW BASED ON PERIOD OF RECORD 1961-96

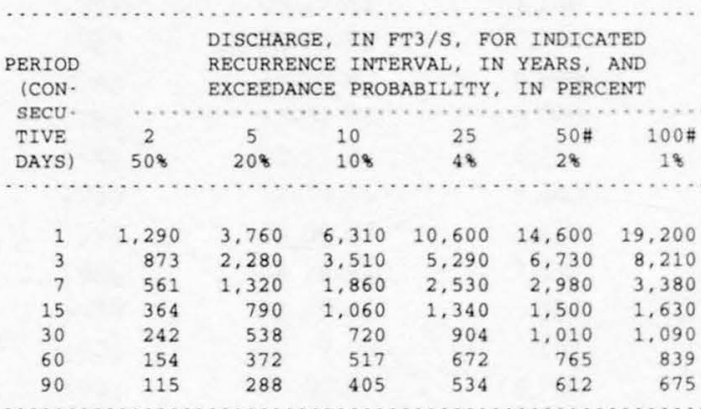

DURATION TABLE OF DAILY MEAN FLOW FOR PERIOD OF RECORD 1961.96

DISCHARGE, IN FT3/S, WHICH WAS EQUALED OR EXCEEDED FOR INDICATED PERCENT OF TIME

$\begin{array}{lllllllllllllllll}18 & 58 & 108 & 158 & 208 & 308 & 408 & 508 & 608 & 708 & 808 & 908 & 958 & 988 & 998 & 99.58 & 99.98 \\ 750 & 244 & 102 & 35 & 8.3 & 0.02 & 0.00 & 0.00 & 0.00 & 0.00 & 0.00 & 0.00 & 0.00 & 0.00 & 0.00 & 0.00 & 0.00\end{array}$

\# Reliability of values in column is uncertain, and potential errors are large. 
09505350 DRY BEAVER CREEK NEAR RIMROCK, AZ--Continued
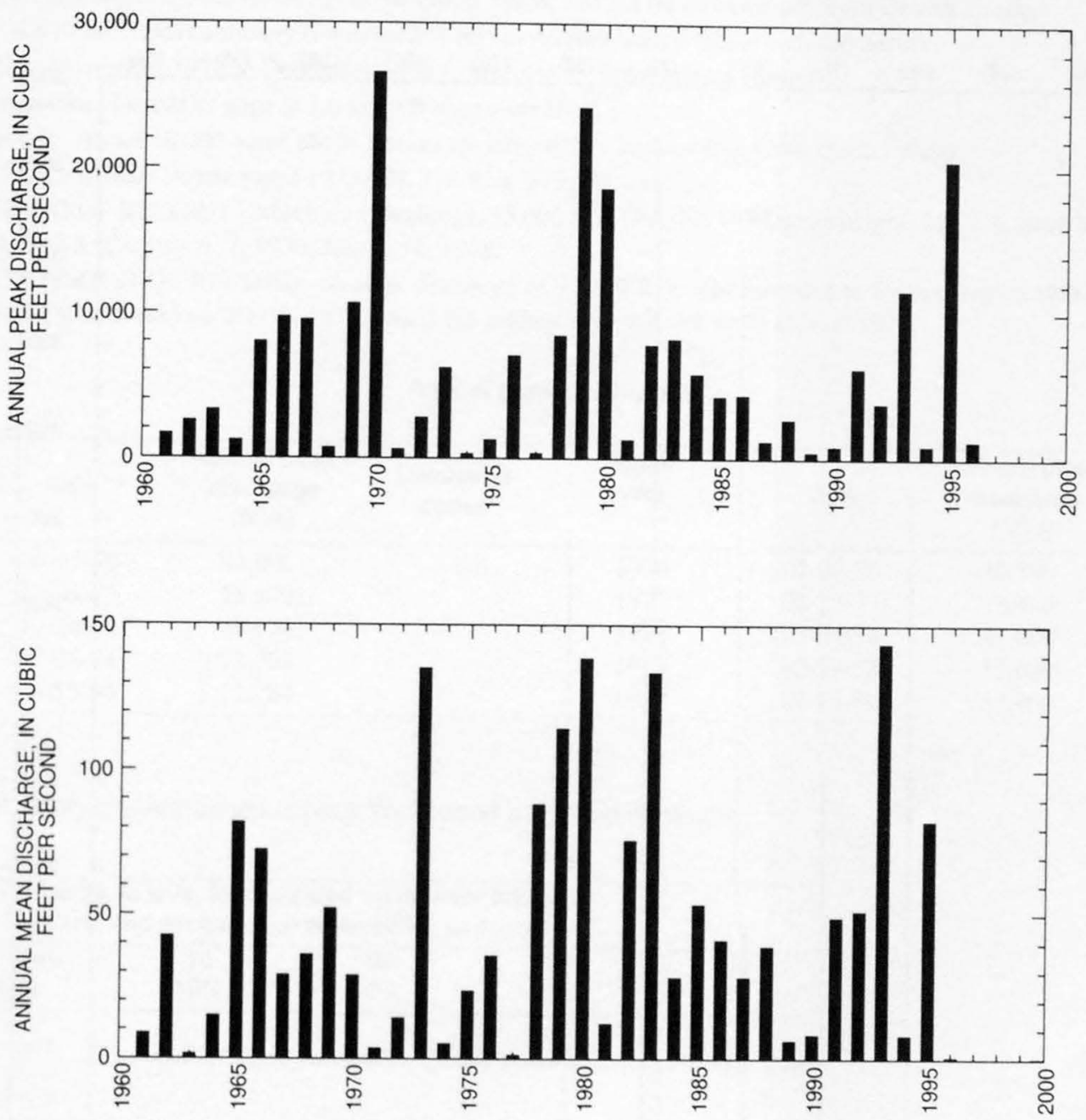
09505350 DRY BEAVER CREEK NEAR RIMROCK, AZ--Continued

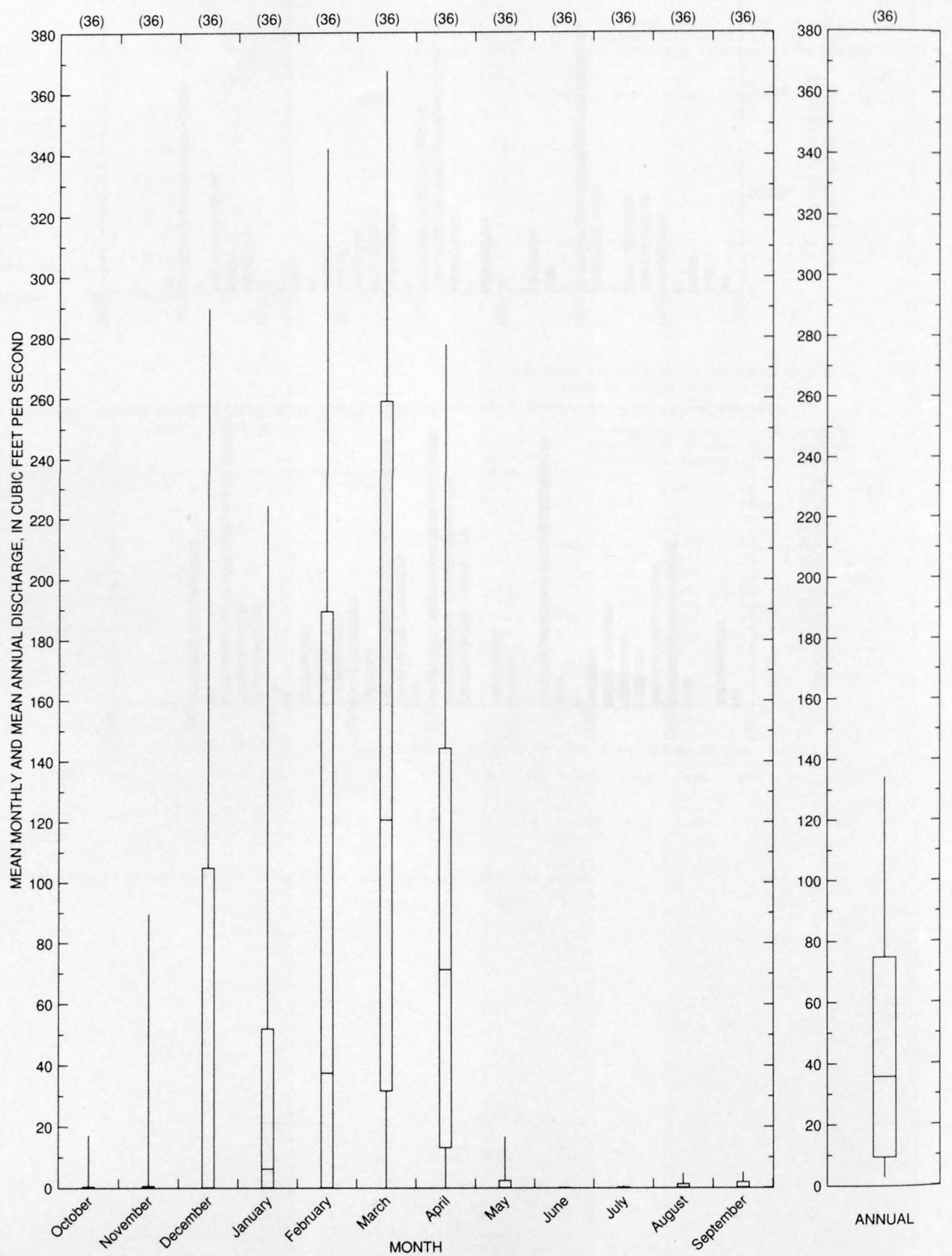


09505550 VERDE RIVER BELOW CAMP VERDE, AZ

LOCATION.--Lat 34'33'02", long 111 '51'02", in SW1/4 NW $1 / 4$ sec.5, T.13 N., R.5 E., Yavapai County, Hydrologic Unit 15060203, on downstream side of bridge on county highway, $0.5 \mathrm{mi}$ southeast of Camp Verde, and 2.2 mi downstream from Beaver Creek.

DRAINAGE AREA.--4,670 $\mathrm{mi}^{2}$, approximately (includes $373 \mathrm{mi}^{2}$ in Aubrey Valley Playa, a closed basin).

PERIOD OF RECORD.--November 1971 to December 1978 (converted to partial-record station).

GAGE.--Water-stage recorder. Datum of gage is 3,045.10 $\mathrm{ft}$ above sea level.

REMARKS.--Records fair. About 10,000 acres above station are irrigated by surface water and ground water.

AVERAGE DISCHARGE.--6 years (water years 1973-78), $378 \mathrm{ft}^{3} / \mathrm{s}, 273,900$ acre- $\mathrm{ft} / \mathrm{yr}$.

EXTREMES FOR PERIOD OF RECORD.--Maximum discharge, 55,000 ft $3 / \mathrm{s} \mathrm{Dec.} \mathrm{19,} \mathrm{1978,} \mathrm{gage} \mathrm{height,} 21.27 \mathrm{ft}$, from high-water mark in gage well; minimum daily, $13 \mathrm{ft}^{3} / \mathrm{s}$ July 6, 7, 1976, July 9, 10, 1978.

EXTREMES OUTSIDE PERIOD OF RECORD.--A peak discharge of $97,000 \mathrm{ft}^{3} / \mathrm{s}$ was recorded at former gaging station at site $8.5 \mathrm{mi}$ downstream (below West Clear Creek) on Mar. 3, 1938, and is the highest near this site since at least 1924

Annual peak discharges

\begin{tabular}{|c|c|c|c|c|c|c|c|}
\hline $\begin{array}{l}\text { Water } \\
\text { year }\end{array}$ & Date & $\begin{array}{c}\text { Annual peak } \\
\text { discharge } \\
\left(\mathrm{ft}^{3} / \mathrm{s}\right)\end{array}$ & $\begin{array}{c}\text { Discharge } \\
\text { codes }\end{array}$ & $\begin{array}{l}\text { Water } \\
\text { year }\end{array}$ & Date & $\begin{array}{c}\text { Annual peak } \\
\text { discharge } \\
\left(\mathrm{ft}^{3} / \mathrm{s}\right)\end{array}$ & $\begin{array}{c}\text { Discharge } \\
\text { codes }\end{array}$ \\
\hline 1970 & $09-05-70$ & 43,000 & HP & 1976 & $02-09-76$ & 30,100 & \\
\hline 1972 & $12-26-71$ & 15,800 & & 1977 & $08-23-77$ & 3,490 & \\
\hline 1973 & $10-20-72$ & 40,600 & & 1978 & $03-01-78$ & 41,000 & \\
\hline 1974 & $07-08-74$ & 2,200 & & 1979 & $12-19-78$ & 55,000 & \\
\hline 1975 & $04-15-75$ & 3,280 & & 1980 & $02-15-80$ & 50,900 & \\
\hline
\end{tabular}

Magnitude and probability of instantaneous peak flow based on period of record

\begin{tabular}{|c|c|c|c|c|c|}
\hline \multicolumn{6}{|c|}{$\begin{array}{l}\text { Discharge, in } \mathrm{ft}^{3} / \mathrm{s} \text {, for indicated recurrence interval } \\
\text { in years, and exceedance probablility, in percent }\end{array}$} \\
\hline 2 & 5 & 10 & 25 & 50 & 100 \\
\hline $50 \%$ & $20 \%$ & $10 \%$ & $4 \%$ & $2 \%$ & $1 \%$ \\
\hline ... & --. & -.. & $\ldots$ & $\ldots$ & $\cdots$ \\
\hline $\begin{array}{l}\text { Weighted skew } \\
\text { Mean } \\
\text { Standard dev. }\end{array}$ & $\begin{array}{l}(\log s)= \\
(\log s)= \\
(\log s)=\end{array}$ & 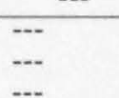 & & & \\
\hline
\end{tabular}

Basin characteristics

\begin{tabular}{|c|c|c|c|c|c|c|c|}
\hline \multirow[b]{2}{*}{$\begin{array}{l}\text { Main } \\
\text { channel } \\
\text { slope } \\
(\mathrm{ft} / \mathrm{mi})\end{array}$} & \multirow[b]{2}{*}{$\begin{array}{l}\text { Stream } \\
\text { length } \\
\text { (mi) }\end{array}$} & \multirow[b]{2}{*}{$\begin{array}{c}\text { Mean } \\
\text { basin } \\
\text { elevation } \\
(f t)\end{array}$} & \multirow[b]{2}{*}{$\begin{array}{c}\text { Forested } \\
\text { area } \\
\text { (percent) }\end{array}$} & \multirow[b]{2}{*}{$\begin{array}{c}\text { Soil } \\
\text { index }\end{array}$} & \multirow[b]{2}{*}{$\begin{array}{c}\text { Mean } \\
\text { annual } \\
\text { precipitation } \\
\text { (in) }\end{array}$} & \multicolumn{2}{|c|}{ Rainfall intensity, 24-hour } \\
\hline & & & & & & $\begin{array}{l}\text { 2-year } \\
\text { (in) }\end{array}$ & $\begin{array}{l}\text { 50-year } \\
\text { (in) }\end{array}$ \\
\hline 17.9 & 153 & 5,544 & 70.0 & 2.5 & 17.6 & 2.1 & 4.1 \\
\hline
\end{tabular}


09505550 VERDE RIVER BELOW CAMP VERDE, AZ--Continued

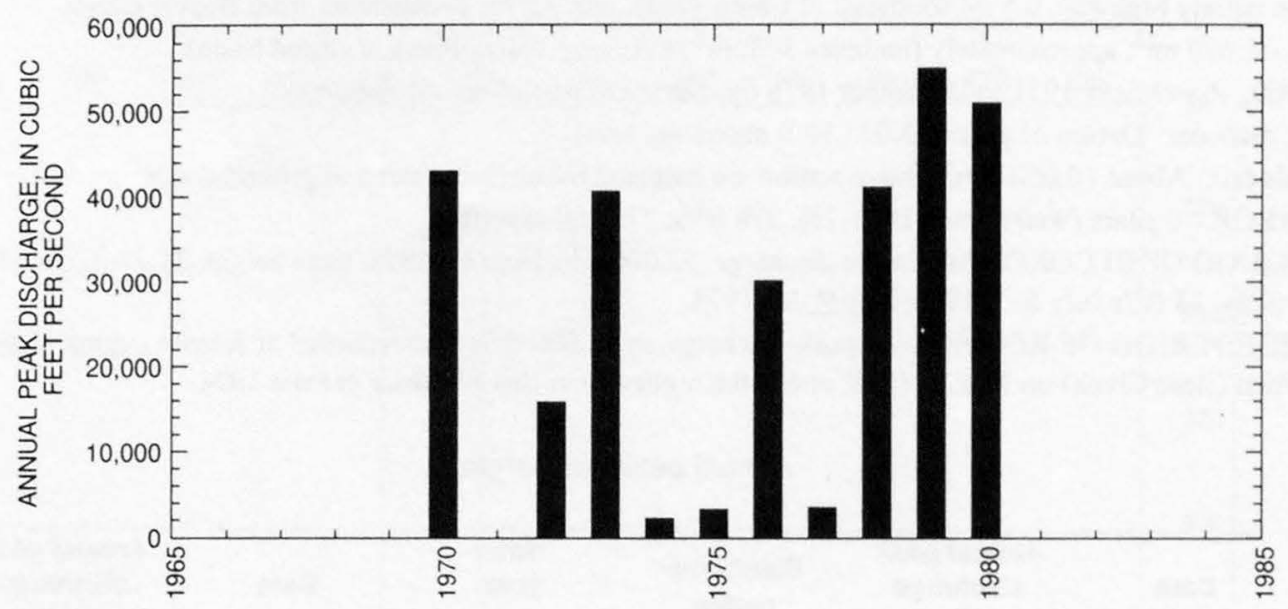


09505600 DIRTY NECK CANYON NEAR CLINTS WELL, AZ

LOCATION.--Lat 34 30'45", long $111^{\circ} 21^{\prime} 30^{\prime \prime}$, in N ${ }^{1} / 2$ sec.23, T.13 N., R.9 E., Coconino County, Hydrologic Unit 15060203 , at State Highway 87 ,

4 mi southwest of Clints Well, and $18 \mathrm{mi}$ south of Payson.

DRAINAGE AREA.--3.42 $\mathrm{mi}^{2}$.

Annual peak discharges

\begin{tabular}{cccccccc}
\hline $\begin{array}{c}\text { Water } \\
\text { year }\end{array}$ & Date & $\begin{array}{c}\text { Annual peak } \\
\text { discharge } \\
\left(\mathrm{ft}^{\mathbf{3}} \mathbf{s} \mathbf{s}\right)\end{array}$ & $\begin{array}{c}\text { Discharge } \\
\text { codes }\end{array}$ & $\begin{array}{c}\text { Water } \\
\text { year }\end{array}$ & Date & $\begin{array}{c}\text { Annual peak } \\
\text { discharge } \\
\left(\mathrm{ft}^{3} / \mathbf{s}\right)\end{array}$ & $\begin{array}{c}\text { Discharge } \\
\text { codes }\end{array}$ \\
\hline 1965 & $01-06-65$ & 85 & & 1971 & $00-00-71$ & 1.0 & LT \\
1966 & $12-30-65$ & 115 & & 1972 & $12-26-71$ & 84 & \\
1967 & $12-06-66$ & 45 & & 1973 & $10-19-72$ & 210 & \\
1968 & $00-00-68$ & 18 & & 1974 & $00-00-74$ & 0 & \\
1969 & $01-25-69$ & 68 & & 1975 & $00-00-75$ & 20 & HP \\
1970 & $09-05-70$ & 210 & & 1979 & $12-18-78$ & 1200 & H 200 \\
\hline
\end{tabular}

Magnitude and probability of instantaneous peak flow based on period of record 1965-75,

1979

\begin{tabular}{|c|c|c|c|c|c|}
\hline \multicolumn{6}{|c|}{$\begin{array}{l}\text { Discharge, in } \mathrm{ft}^{3} / \mathrm{s} \text {, for indicated recurrence interval } \\
\text { in years, and exceedance probablility, in percent }\end{array}$} \\
\hline 2 & 5 & 10 & $25 \dagger$ & $50 \dagger$ & $100 \dagger$ \\
\hline $50 \%$ & $20 \%$ & $10 \%$ & $4 \%$ & $2 \%$ & $1 \%$ \\
\hline 62.7 & 151 & 231 & 353 & 458 & 573 \\
\hline Weighted skew & $(\log s)=$ & -0.37 & & & \\
\hline Mean & $(\log s)=$ & 1.77 & & & \\
\hline Standard dev. & $(\log s)=$ & 0.48 & & & \\
\hline
\end{tabular}

f Reliability of values in column is uncertain, and potential errors are large.

Basin characteristics

\begin{tabular}{cccccccc}
\hline $\begin{array}{c}\text { Main } \\
\text { channel } \\
\text { slope } \\
(\mathrm{ft} / \mathrm{mi})\end{array}$ & $\begin{array}{c}\text { Stream } \\
\text { length } \\
(\mathrm{mi})\end{array}$ & $\begin{array}{c}\text { Mean } \\
\text { basin } \\
\text { elevation } \\
(\mathrm{ft})\end{array}$ & $\begin{array}{c}\text { Forested } \\
\text { area } \\
\text { (percent) }\end{array}$ & $\begin{array}{c}\text { Soil } \\
\text { index }\end{array}$ & $\begin{array}{c}\text { Mean } \\
\text { annual } \\
\text { precipitation } \\
\text { (in) }\end{array}$ & $\begin{array}{c}\text { Rainfall intensity, 24-hour } \\
\text { 2-year } \\
\text { (in) }\end{array}$ & $\begin{array}{c}50 \text {-year } \\
\text { (in) }\end{array}$ \\
\hline 190 & 3.4 & 7,140 & 99.0 & 3.0 & 26.0 & 2.7 \\
\hline
\end{tabular}

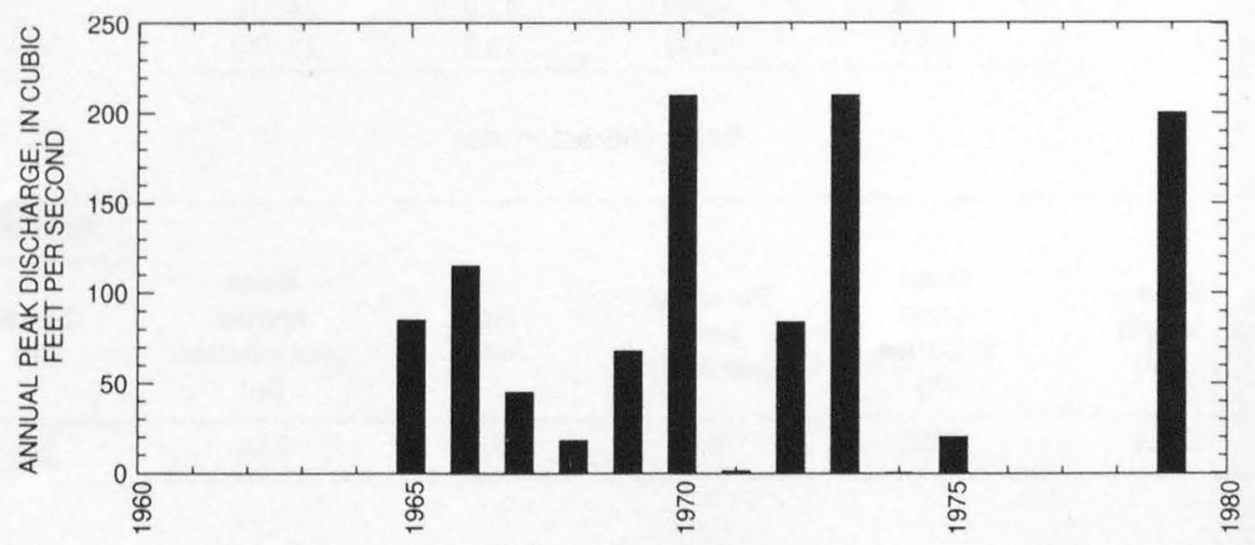


09505800 WEST CLEAR CREEK NEAR CAMP VERDE, AZ

LOCATION.--Lat 34'32'19", long 111 41'36", in NW $1 / 4 \mathrm{NW}^{1} / 4$ sec.11, T.13 N., R.6 E., Yavapai County, Hydrologic Unit 15060203 , in Coconino National Forest, on left bank at Bull Pen Ranch, 9 mi east of Camp Verde, and 11 mi upstream from mouth.

DRAINAGE AREA.--241 $\mathrm{mi}^{2}$.

PERIOD OF RECORD,--December 1964 to current year.

GAGE.--Water-stage recorder. Elevation of gage is 3,630 ft above sea level, from topographic map.

REMARKS.--No estimated daily discharges. Records fair.

EXTREMES FOR PERIOD OF RECORD.--Maximum discharge, 24,800 $\mathrm{ft}^{3} / \mathrm{s}$ Jan. 8, 1993, gage height, $13.22 \mathrm{ft}$, from floodmarks and rating curve extended above $2,700 \mathrm{ft}^{3} / \mathrm{s}$ on basis of slope-area measurements at gage heights $8.3 \mathrm{ft}, 10.15 \mathrm{ft}$, and $13.22 \mathrm{ft}$; minimum daily, $11 \mathrm{ft}^{3} / \mathrm{s}$ Aug. 1, 22, 1986.

Annual peak discharges

\begin{tabular}{rccccrr}
\hline $\begin{array}{l}\text { Water } \\
\text { year }\end{array}$ & Date & $\begin{array}{c}\text { Annual peak } \\
\text { discharge } \\
\left(\mathrm{ft}^{3} / \mathbf{s}\right)\end{array}$ & $\begin{array}{c}\text { Discharge } \\
\text { codes }\end{array}$ & $\begin{array}{c}\text { Water } \\
\text { year }\end{array}$ & Date & $\begin{array}{c}\text { Annual peak } \\
\text { discharge } \\
\left(\mathrm{ft}^{3} / \mathbf{s}\right)\end{array}$ \\
\hline 1965 & $01-06-65$ & 6,510 & & 1981 & $08-12-81$ & 801 \\
1966 & $12-30-65$ & 6,330 & & 1982 & $03-12-82$ & 9,890 \\
1967 & $12-07-66$ & 2,670 & 1983 & $11-30-82$ & 6,700 \\
1968 & $02-26-68$ & 1,300 & 1984 & $12-27-83$ & 3,630 \\
1969 & $01-26-69$ & 3,870 & 1985 & $12-27-84$ & 5,140 \\
1970 & $09-05-70$ & 1,050 & 1986 & $03-12-86$ & 885 \\
1971 & $09-02-71$ & 1,550 & 1987 & $03-18-87$ & 2,110 \\
1972 & $12-26-71$ & 6,660 & 1988 & $08-29-88$ & 6,540 \\
1973 & $10-19-72$ & 11,300 & 1989 & $03-08-89$ & 446 \\
1974 & $01-21-74$ & 308 & 1990 & $09-05-90$ & 249 \\
1975 & $04-15-75$ & 2,730 & 1991 & $03-01-91$ & 6,070 \\
1976 & $02-09-76$ & 8,130 & 1992 & $08-23-92$ & 6,290 \\
1977 & $07-26-77$ & 101 & 1993 & $01-08-93$ & 24,800 \\
1978 & $03-01-78$ & 13,800 & 1994 & $11-24-93$ & 806 \\
1979 & $12-18-78$ & 22,400 & 1995 & $02-15-95$ & 10,900 \\
1980 & $02-19-80$ & 15,100 & 1996 & $09-03-96$ & 750 \\
\hline
\end{tabular}

Discharge rating table developed February 1996

\begin{tabular}{cccc}
\hline $\begin{array}{c}\text { Gage height } \\
(\mathbf{f t})\end{array}$ & $\begin{array}{c}\text { Discharge } \\
\left(\mathbf{f t}^{\mathbf{3}} \mathbf{s}\right)\end{array}$ & $\begin{array}{c}\text { Gage height } \\
(\mathbf{f t})\end{array}$ & $\begin{array}{c}\text { Discharge } \\
\left(\mathbf{f t}^{\mathbf{3}} / \mathbf{s}\right)\end{array}$ \\
\hline 3.0 & 1,250 & 9.0 & 10,990 \\
4.0 & 2,180 & 10.0 & 13,800 \\
5.0 & 3,340 & 11.0 & 16,900 \\
6.0 & 4,820 & 12.0 & 20,410 \\
7.0 & 6,580 & 13.0 & 24,010 \\
8.0 & 8,650 & 13.2 & 24,730 \\
\hline
\end{tabular}

Basin characteristics

\begin{tabular}{cccccccc}
\hline $\begin{array}{c}\text { Main } \\
\text { channel } \\
\text { slope } \\
(\mathrm{ft} / \mathrm{mi})\end{array}$ & $\begin{array}{c}\text { Stream } \\
\text { length } \\
(\mathrm{mi})\end{array}$ & $\begin{array}{c}\text { Mean } \\
\text { basin } \\
\text { elevation } \\
(\mathrm{ft})\end{array}$ & $\begin{array}{c}\text { Forested } \\
\text { area } \\
\text { (percent) }\end{array}$ & $\begin{array}{c}\text { Soil } \\
\text { index }\end{array}$ & $\begin{array}{c}\text { Mean } \\
\text { annual } \\
\text { precipitation } \\
\text { (in) }\end{array}$ & $\begin{array}{c}\text { Rainfall intensity, 24-hour } \\
\text { (in) }\end{array}$ & $\begin{array}{c}50 \text {-year } \\
\text { (in) }\end{array}$ \\
\hline 112 & 34.4 & 6,680 & 78.0 & 3.0 & 23.4 & 2.6 \\
\hline
\end{tabular}


09505800 WEST CLEAR CREEK NEAR CAMP VERDE, AZ--Continued

MEAN MONTHLY AND ANNUAL DISCHARGES 1966-96

\begin{tabular}{|c|c|c|c|c|c|c|}
\hline MONTH & $\begin{array}{l}\text { MAXIMUM } \\
\text { (FT3/S) }\end{array}$ & $\begin{array}{l}\text { MINIMUM } \\
\text { (FT3/S) }\end{array}$ & $\begin{array}{c}\text { MEAN } \\
(\text { FT3 } / S)\end{array}$ & $\begin{array}{l}\text { STAN- } \\
\text { DARD } \\
\text { DEVIA- } \\
\text { TION } \\
\text { (FT3/S) }\end{array}$ & $\begin{array}{l}\text { COEFFI- } \\
\text { CIENT OF } \\
\text { VARI- } \\
\text { ATION }\end{array}$ & $\begin{array}{c}\text { PERCENT } \\
\text { OF } \\
\text { ANNUAL } \\
\text { RUNOFF }\end{array}$ \\
\hline OCTOBER & 458 & 14 & 34 & 79 & 2.3 & 4.2 \\
\hline NOVEMBER & 110 & 15 & 29 & 25 & 0.85 & 3.6 \\
\hline DECEMBER & 758 & 16 & 92 & 155 & 1.7 & 11.3 \\
\hline JANUARY & 1,140 & 16 & 84 & 203 & 2.4 & 10.3 \\
\hline FEBRUARY & 956 & 15 & 155 & 208 & 1.3 & 19.0 \\
\hline MARCH & 886 & 15 & 210 & 208 & 0.99 & 25.8 \\
\hline APRIL & 923 & 15 & 106 & 170 & 1.6 & 13.1 \\
\hline MAY & 157 & 15 & 26 & 25 & 0.97 & 3.2 \\
\hline JUNE & 25 & 13 & 16 & 2.6 & 0.16 & 2.0 \\
\hline JULY & 33 & 14 & 18 & 3.9 & 0.22 & 2.2 \\
\hline AUGUST & 102 & 14 & 22 & 17 & 0.75 & 2.7 \\
\hline SEPTEMBER & 113 & 14 & 22 & 18 & 0.80 & 2.7 \\
\hline ANNUAL & 199 & 16 & 67 & 51 & 0.76 & 100 \\
\hline
\end{tabular}

MAGNITUDE AND PROBABILITY OF INSTANTANEOUS PEAK FLOW BASED ON PERIOD OF RECORD 1965.96

\begin{tabular}{|c|c|c|c|c|c|}
\hline 2 & 5 & 10 & 25 & 50 & 100 \\
\hline 508 & 208 & 108 & 48 & 28 & 18 \\
\hline 4,510 & 9,690 & 14,200 & 20,800 & 26,500 & 32,700 \\
\hline
\end{tabular}

$\begin{array}{ll}\text { WEIGHTED SKEW } & \text { (LOGS) }=-0.25 \\ \text { MEAN } & \text { (LOGS) }=3.64\end{array}$

$\begin{array}{ll}\text { MEAN } & (\text { LOGS })=3.64 \\ \text { STANDARD DEV. } \quad(\text { LOGS })=0.41\end{array}$
MAGNITUDE AND PROBABILITY OF ANNUAL LOW FLOW BASED ON PERIOD OF RECORD 1966.96

\begin{tabular}{|c|c|c|c|c|c|c|}
\hline PERIOD & \multicolumn{6}{|c|}{$\begin{array}{l}\text { DISCHARGE, IN FT3/S, FOR INDICATED } \\
\text { RECURRENCE INTERVAL, IN YEARS, AND }\end{array}$} \\
\hline $\begin{array}{ll}(\mathrm{CON}- \\
\mathrm{SECH} \text { - }\end{array}$ & \multicolumn{6}{|c|}{ NON-EXCEEDANCE PROBABILITY, IN PERCENT } \\
\hline TIVE & 2 & 5 & 10 & 20 & 50 & $100 \#$ \\
\hline DAYS) & 508 & 208 & 108 & 58 & 28 & 18 \\
\hline 1 & 13 & 12 & 12 & 11 & 11 & 11 \\
\hline 3 & 13 & 12 & 12 & 11 & 11 & 11 \\
\hline 7 & 14 & 13 & 12 & 12 & 11 & 11 \\
\hline 14 & 14 & 13 & 12 & 12 & 11 & 11 \\
\hline 30 & 15 & 13 & 13 & 13 & 12 & 12 \\
\hline 60 & 15 & 14 & 14 & 13 & 13 & 13 \\
\hline 90 & 16 & 15 & 14 & 14 & 14 & 13 \\
\hline 120 & 16 & 15 & 15 & 15 & 15 & 14 \\
\hline 183 & 17 & 16 & 15 & 15 & 15 & 15 \\
\hline
\end{tabular}

MAGNITUDE AND PROBABILITY OF ANNUAL HIGH FLOW BASED ON PERIOD OF RECORD 1966.96

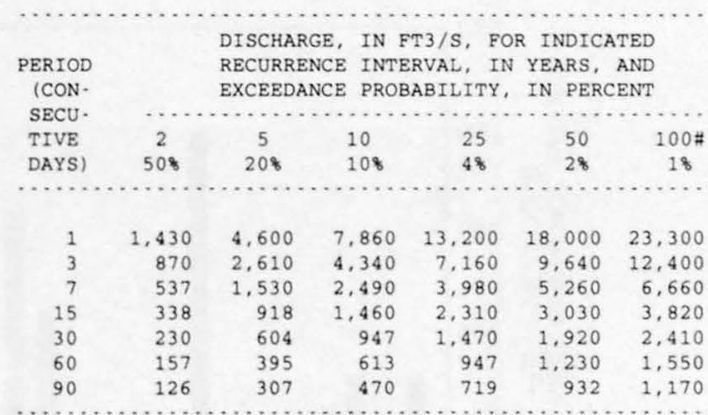

DURATION TABLE OF DAILY MEAN FLOW FOR PERIOD OF RECORD 1966.96

DISCHARGE, IN FT3/S, WHICH WAS EQUALED OR EXCEEDED FOR INDICATED PERCENT OF TIME

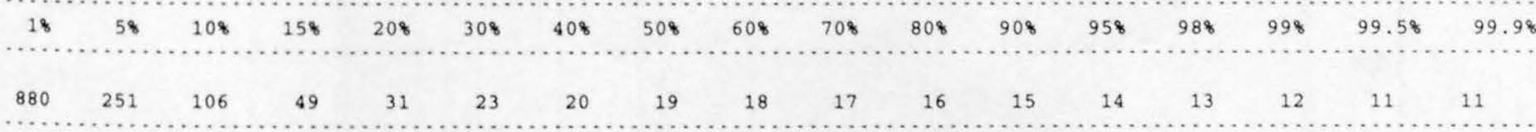

\# Reliability of values in column is uncertain, and potential errors are large. 


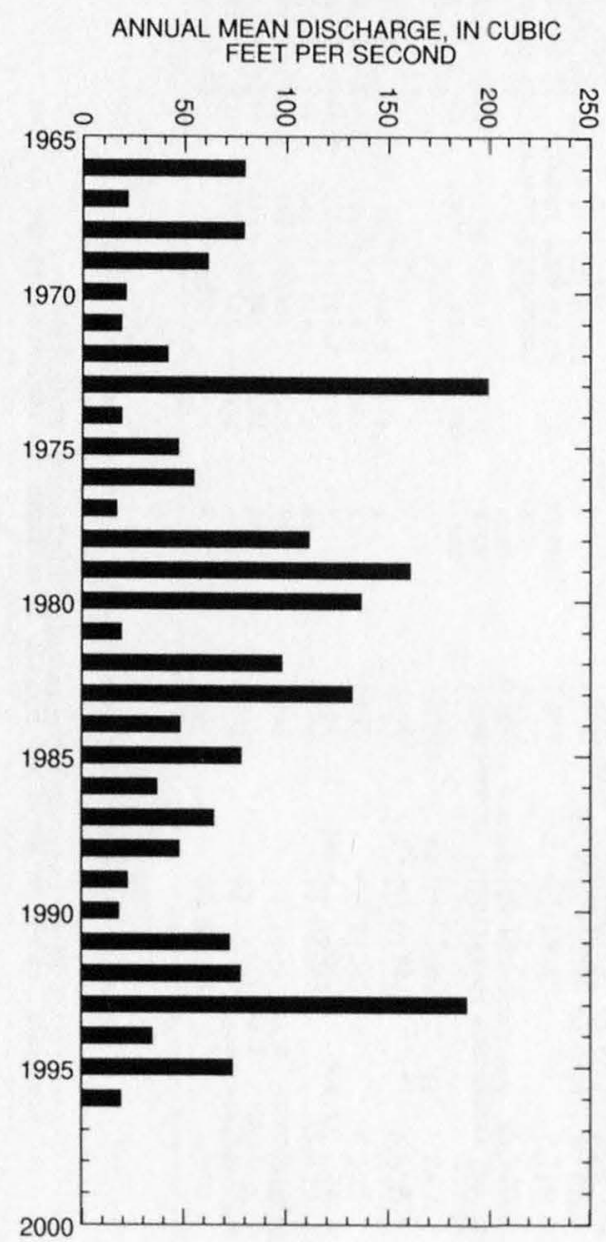

ANNUAL PEAK DISCHARGE, IN CUBIC

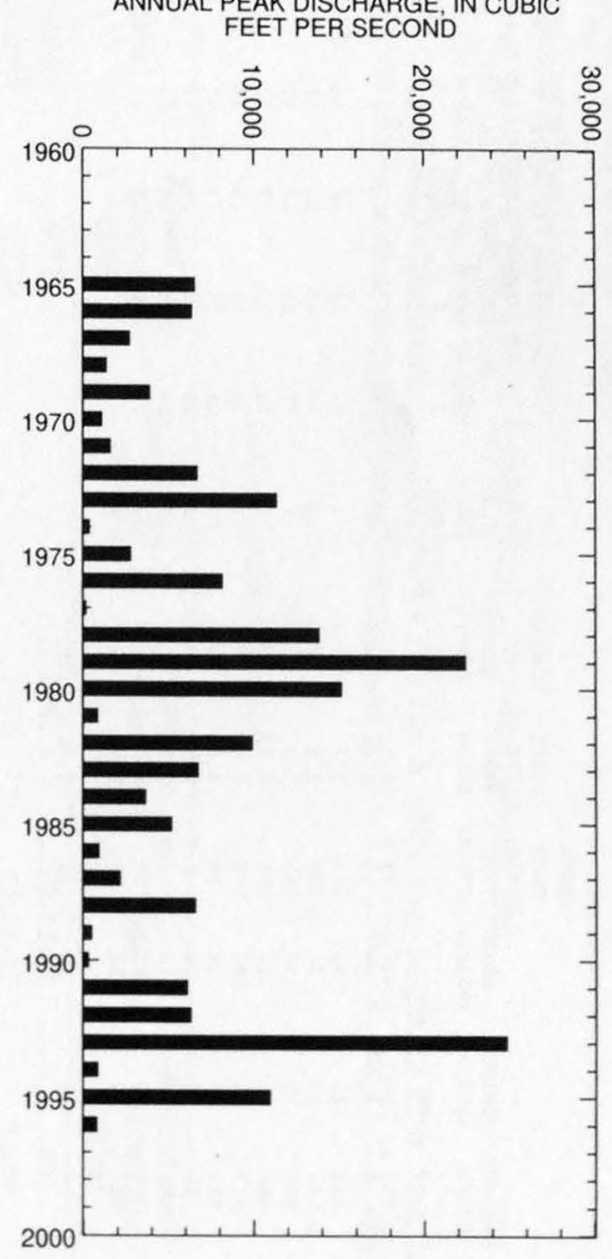


09505800 WEST CLEAR CREEK NEAR CAMP VERDE, AZ--Continued

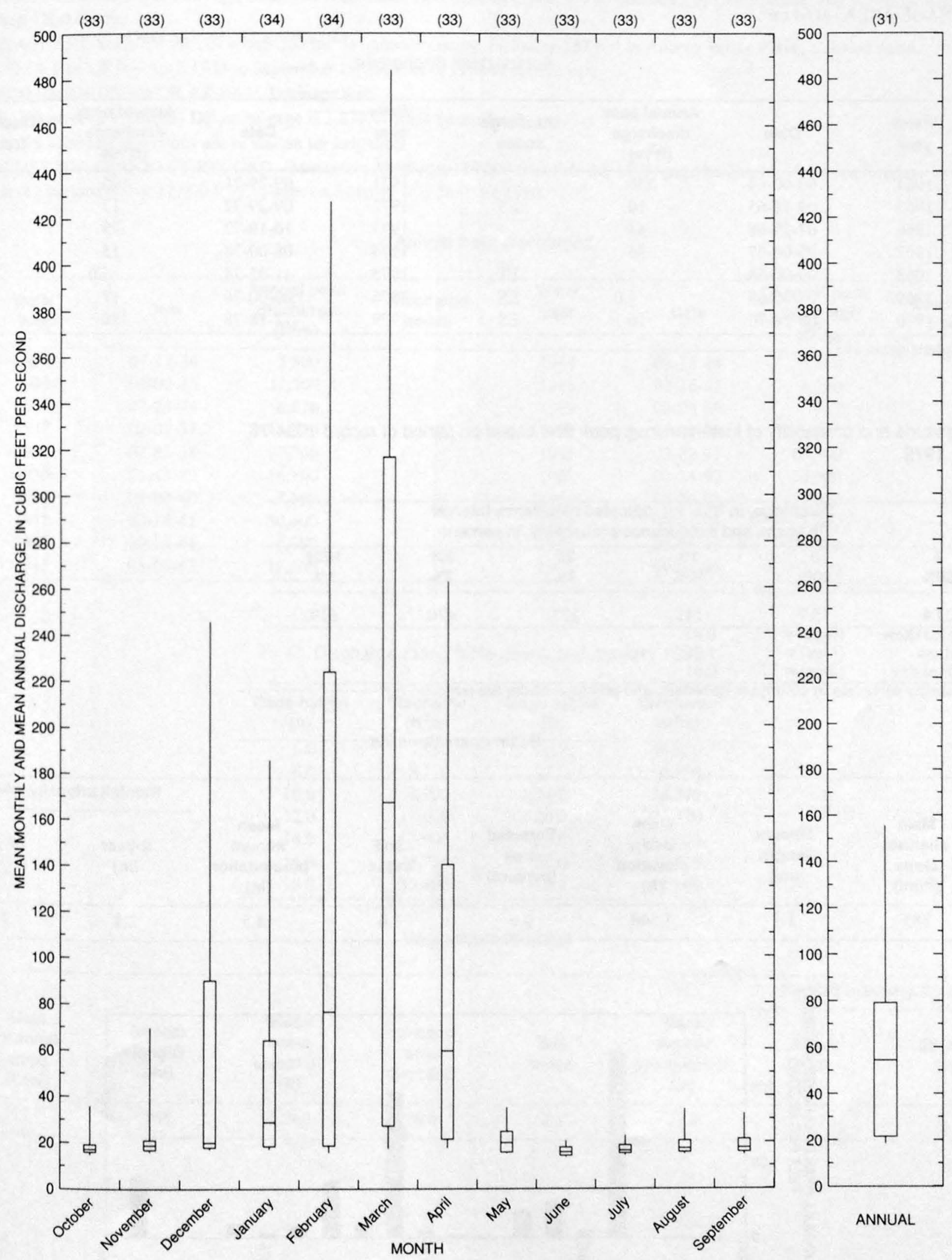


09505900 COTTONWOOD WASH NEAR CAMP VERDE, AZ

LOCATION.--Lat 34 $30^{\prime} 20^{\prime \prime}$, long $111^{\circ} 45^{\prime} 10^{\prime \prime}$, in NE $\frac{1}{4}$ sec.19, T.13 N., R.6 E., Yavapai County, Hydrologic Unit 15060203, at Camp Verde.Pite road, 7 mi southeast of Camp Verde.

DRAINAGE AREA.--0.64 $\mathrm{mi}^{2}$.

Annual peak discharges

\begin{tabular}{|c|c|c|c|c|c|c|c|}
\hline $\begin{array}{l}\text { Water } \\
\text { year }\end{array}$ & Date & $\begin{array}{c}\text { Annual peak } \\
\text { discharge } \\
\left(\mathrm{ft}^{3} / \mathrm{s}\right)\end{array}$ & $\begin{array}{l}\text { Discharge } \\
\text { codes }\end{array}$ & $\begin{array}{l}\text { Water } \\
\text { year }\end{array}$ & Date & $\begin{array}{c}\text { Annual peak } \\
\text { discharge } \\
\left(\mathrm{ft}^{3} / \mathrm{s}\right)\end{array}$ & $\begin{array}{c}\text { Discharge } \\
\text { codes }\end{array}$ \\
\hline 1964 & $00-00-64$ & 250 & & 1971 & $09-29-71$ & 195 & \\
\hline 1965 & $09-18-65$ & 10 & ES & 1972 & $09-29-72$ & 17 & \\
\hline 1966 & $07-29-66$ & 48 & & 1973 & $10-19-72$ & 75 & \\
\hline 1967 & $09-00-67$ & 36 & & 1974 & $08-00-74$ & 15 & \\
\hline 1970 & $08-14-70$ & 10 & ES & 1979 & $12-18-78$ & ${ }^{\prime} 80$ & HP \\
\hline
\end{tabular}

${ }^{1}$ Highest since 1972.

Magnitude and probability of instantaneous peak flow based on period of record 1964-76, 1979

\begin{tabular}{|c|c|c|c|c|c|}
\hline \multicolumn{6}{|c|}{$\begin{array}{l}\text { Discharge, in } \mathrm{ft}^{3} / \mathrm{s} \text {, for indicated recurrence interval } \\
\text { in years, and exceedance probablility, in percent }\end{array}$} \\
\hline 2 & 5 & 10 & 25 & $50 t$ & $100 t$ \\
\hline $50 \%$ & $20 \%$ & $10 \%$ & $4 \%$ & $2 \%$ & $1 \%$ \\
\hline 23.4 & 75.9 & 141 & 273 & 420 & 619 \\
\hline Weighted skew & $(\log s)=$ & 0.03 & & & \\
\hline Mean & $(\log s)=$ & 1.37 & & & \\
\hline Standard dev. & $(\log s)=$ & 0.61 & & & \\
\hline
\end{tabular}

$\mp$ Reliability of values in column is uncertain, and potential errors are large.

Basin characteristics

\begin{tabular}{cccccccc}
\hline $\begin{array}{c}\text { Main } \\
\text { channel } \\
\text { slope } \\
(\mathrm{ft} / \mathrm{mi})\end{array}$ & $\begin{array}{c}\text { Stream } \\
\text { length } \\
(\mathrm{mi})\end{array}$ & $\begin{array}{c}\text { Mean } \\
\text { basin } \\
\text { elevation } \\
(\mathrm{ft})\end{array}$ & $\begin{array}{c}\text { Forested } \\
\text { area } \\
\text { (percent) }\end{array}$ & $\begin{array}{c}\text { Soil } \\
\text { index }\end{array}$ & $\begin{array}{c}\text { Mean } \\
\text { annual } \\
\text { precipitation } \\
\text { (in) }\end{array}$ & $\begin{array}{c}\text { Rainfall intensity, 24-hour } \\
\text { (in) }\end{array}$ & $\begin{array}{c}50 \text {-year } \\
\text { (in) }\end{array}$ \\
\hline 385 & 1.1 & 3,540 & 0.0 & 1.0 & 14.5 & 2.1 \\
\hline
\end{tabular}

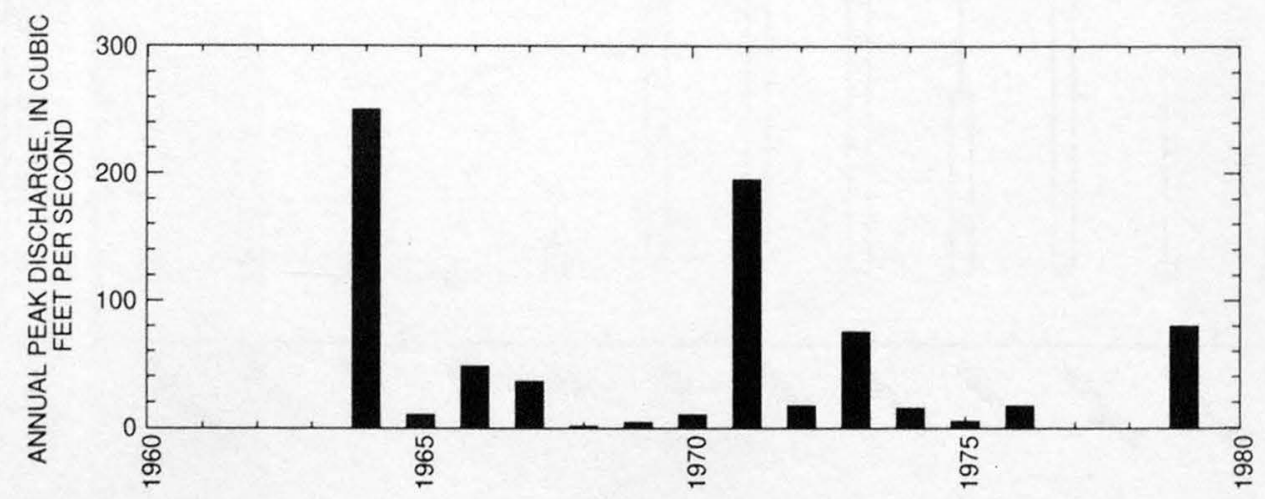


09506000 VERDE RIVER NEAR CAMP VERDE, AZ

LOCATION.--Lat 34'26'54", long 111.47'21", in NW1/4 sec. 11, T.12 N., R.5 E. (unsurveyed), Yavapai County, Hydrologic Unit 15060303, in Prescott National Forest, on right bank $600 \mathrm{ft}$ upstream from Chasm Creek, $9.0 \mathrm{mi}$ southeast of Camp Verde, and $9.7 \mathrm{mi}$ downstream from West Clear Creek.

DRAINAGE AREA.--5,009 $\mathrm{mi}^{2}$, of which $365 \mathrm{mi}^{2}$ is noncontributing, including $357 \mathrm{mi}^{2}$ in Aubrey Valley Playa, a closed basin.

PERIOD OF RECORD.--April 1934 to September 1945, October 1988 to current year.

REVISED RECORDS.--WDR AZ-89-1: Drainage area.

GAGE.--Water-stage recorder. Datum of gage is $2,874.11 \mathrm{ft}$ above sea level.

REMARKS.--Several diversions above station for irrigation.

EXTREMES FOR PERIOD OF RECORD.--Maximum discharge, 119,000 $\mathrm{ft}^{3} / \mathrm{s}$ Feb. 20, 1993, gage-height, $28.36 \mathrm{ft}$ from floodmarks from rating curve extended above $17,000 \mathrm{ft}^{3} / \mathrm{s}$; minimum daily, $40 \mathrm{ft}^{3} / \mathrm{s}$ June $30,1990$.

Annual peak discharges

\begin{tabular}{|c|c|c|c|c|c|c|c|}
\hline $\begin{array}{l}\text { Water } \\
\text { year }\end{array}$ & Date & $\begin{array}{c}\text { Annual peak } \\
\text { discharge } \\
\left(\mathrm{ft}^{3} / \mathrm{s}\right)\end{array}$ & $\begin{array}{l}\text { Discharge } \\
\text { codes }\end{array}$ & $\begin{array}{l}\text { Water } \\
\text { year }\end{array}$ & Date & $\begin{array}{c}\text { Annual peak } \\
\text { discharge } \\
\left(\mathrm{ft}^{3} / \mathrm{s}\right)\end{array}$ & $\begin{array}{l}\text { Discharge } \\
\text { codes }\end{array}$ \\
\hline 1934 & $07-17-34$ & 5,500 & & 1944 & $03-14-44$ & 5,160 & \\
\hline 1935 & $04-09-35$ & 11,500 & & 1945 & $03-16-45$ & 8,380 & \\
\hline 1936 & $02-24-36$ & 6,820 & & 1989 & 03-08-89 & 777 & \\
\hline 1937 & $02-07-37$ & 41,700 & & 1990 & $07-22-90$ & 5,060 & \\
\hline 1938 & $03-03-38$ & 97,000 & & 1991 & $03-02-91$ & 14,300 & \\
\hline 1939 & $09-13-39$ & 16,100 & & 1992 & $02-14-92$ & 14,000 & \\
\hline 1940 & $08-04-40$ & 7,560 & & 1993 & $02-20-93$ & 119,000 & \\
\hline 1941 & $03-14-41$ & 30,000 & & 1994 & $09-03-94$ & 2,860 & \\
\hline 1942 & $10-13-41$ & 6,080 & & 1995 & $03-06-95$ & 75,500 & \\
\hline 1943 & $03-05-43$ & 11,600 & & 1996 & $09-06-96$ & 2,110 & \\
\hline
\end{tabular}

Discharge rating table developed January 1993

\begin{tabular}{cccc}
\hline $\begin{array}{c}\text { Gage height } \\
(\mathbf{f t})\end{array}$ & $\begin{array}{c}\text { Discharge } \\
\left(\mathbf{f t}^{\mathbf{3}} \mathbf{s}\right)\end{array}$ & $\begin{array}{c}\text { Gage height } \\
(\mathbf{f t})\end{array}$ & $\begin{array}{c}\text { Discharge } \\
\left(\mathbf{f t}^{3} / \mathbf{s}\right)\end{array}$ \\
\hline 6.0 & 1,380 & 20.0 & 46,970 \\
8.0 & 3,720 & 22.0 & 60,580 \\
10.0 & 6,900 & 24.0 & 76,340 \\
12.0 & 11,620 & 26.0 & 94,370 \\
14.0 & 17,900 & 28.0 & 114,800 \\
16.0 & 25,750 & 30.0 & 137,500 \\
18.0 & 35,400 & 30.3 & 141,100 \\
\hline
\end{tabular}

Basin characteristics

\begin{tabular}{|c|c|c|c|c|c|c|c|}
\hline \multirow[b]{2}{*}{$\begin{array}{l}\text { Main } \\
\text { channel } \\
\text { slope } \\
(\mathrm{ft} / \mathrm{mi})\end{array}$} & \multirow[b]{2}{*}{$\begin{array}{l}\text { Stream } \\
\text { length } \\
\text { (mi) }\end{array}$} & \multirow[b]{2}{*}{$\begin{array}{c}\text { Mean } \\
\text { basin } \\
\text { elevation } \\
(f t)\end{array}$} & \multirow[b]{2}{*}{$\begin{array}{c}\text { Forested } \\
\text { area } \\
\text { (percent) }\end{array}$} & \multirow[b]{2}{*}{$\begin{array}{c}\text { Soil } \\
\text { index }\end{array}$} & \multirow[b]{2}{*}{$\begin{array}{c}\text { Mean } \\
\text { annual } \\
\text { precipitation } \\
\text { (in) }\end{array}$} & \multicolumn{2}{|c|}{ Rainfall intensity, 24-hour } \\
\hline & & & & & & $\begin{array}{l}\text { 2-year } \\
\text { (in) }\end{array}$ & $\begin{array}{c}\text { 50-year } \\
\text { (in) }\end{array}$ \\
\hline 17.9 & 168 & 5,560 & 70.0 & 2.5 & 17.6 & 2.1 & 4.1 \\
\hline
\end{tabular}


09506000 VERDE RIVER NEAR CAMP VERDE, AZ--Continued

MEAN MONTHLY AND ANNUAL DISCHARGES $1935-45,1989.96$

\begin{tabular}{|c|c|c|c|c|c|c|}
\hline MONTH & $\begin{array}{l}\text { MAXIMUM } \\
(\text { FT3/S) }\end{array}$ & $\begin{array}{l}\text { MINIMUM } \\
\text { (FT3/S) }\end{array}$ & $\begin{array}{c}\text { MEAN } \\
\left(F^{2} 3 / S\right)\end{array}$ & $\begin{array}{l}\text { STAN- } \\
\text { DARD } \\
\text { DEVIA- } \\
\text { TION } \\
\text { (FT3/S) }\end{array}$ & $\begin{array}{l}\text { COBFFI- } \\
\text { CIENT OF } \\
\text { VARI- } \\
\text { ATION }\end{array}$ & $\begin{array}{c}\text { PERCENT } \\
\text { OF } \\
\text { ANNUAL } \\
\text { RUNOFF }\end{array}$ \\
\hline OCTOBER & 551 & 106 & 193 & 114 & 0.59 & 3.4 \\
\hline NOVEMBER & 339 & 169 & 208 & 45 & 0.21 & 3.7 \\
\hline DECEMBER & 1,350 & 199 & 315 & 281 & 0.89 & 5.6 \\
\hline JANUARY & 7,160 & 198 & 683 & 1,580 & 2.3 & 12.1 \\
\hline FEBRUARY & 6,160 & 180 & 1,280 & 1,700 & 1.3 & 22.6 \\
\hline MARCH & 4,030 & 171 & 1,500 & 1,090 & 0.73 & 26.5 \\
\hline APRIL & 3,050 & 118 & 679 & 736 & 1.1 & 12.0 \\
\hline MAY & 337 & 85 & 136 & 62 & 0.45 & 2.4 \\
\hline JUNE & 123 & 61 & 86 & 17 & 0.20 & 1.5 \\
\hline JULY & 209 & 65 & 111 & 44 & 0.39 & 2.0 \\
\hline AUGUST & 616 & 103 & 214 & 129 & 0.60 & 3.8 \\
\hline SEPTEMBER & 1,150 & 83 & 243 & 233 & 0.96 & 4.3 \\
\hline ANNUAL & 1,400 & 159 & 465 & 325 & 0.70 & 100 \\
\hline
\end{tabular}

MAGNITUDE AND PROBABILITY OF INSTANTANEOUS PEAK FLOW BASED ON PERIOD OF RECORD 1934-45, 1989-96

DISCHARGE, IN FT3/S, FOR INDICATED RECURRENCE INTERVAI IN YEARS, AND EXCEEDANCE PROBABILITY, IN PERCENT

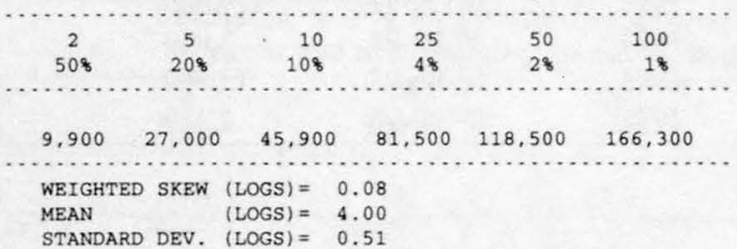

DURATION TABLE OF DAILY MEAN FLOW FOR PERIOD OF RECORD $1935-45,1989.96$
MAGNITUDB AND PROBABILITY OF ANNUAL, LOW FLOW BASED ON PERIOD OF RECORD $1935-45,1990.96$

\begin{tabular}{|c|c|c|c|c|c|c|}
\hline \multirow{4}{*}{$\begin{array}{l}\text { PERIOD } \\
\text { /CON- } \\
\text { SECU- }\end{array}$} & \multirow{2}{*}{\multicolumn{3}{|c|}{$\begin{array}{l}\text { DISCHARGE, I } \\
\text { RECURRENCE II }\end{array}$}} & \multicolumn{3}{|c|}{ - FOR INDICATED } \\
\hline & & & & \multicolumn{3}{|c|}{ IN YEARS, AND } \\
\hline & \multicolumn{6}{|c|}{ NON-EXCEEDANCE PROBABILITY, IN PERCENT } \\
\hline & & 5 & 10 & 20 & & \\
\hline TIVE & 2 & 5 & 10 & 20 & $50 \#$ & $100 \#$ \\
\hline DAYS) & 508 & 208 & 108 & 58 & 28 & 18 \\
\hline 1 & 56 & 47 & 43 & 41 & 38 & 36 \\
\hline 3 & 59 & 49 & 45 & 42 & 39 & 37 \\
\hline 7 & 62 & 53 & 49 & 46 & 43 & 41 \\
\hline 14 & 66 & 57 & 53 & 51 & 48 & 46 \\
\hline 30 & 72 & 63 & 59 & 56 & 54 & 52 \\
\hline 60 & 82 & 73 & 69 & 66 & 64 & 62 \\
\hline 90 & 93 & 82 & 77 & 74 & 72 & 70 \\
\hline 120 & 111 & 97 & 90 & 86 & 81 & 78 \\
\hline 183 & 148 & 118 & 106 & 98 & 90 & 85 \\
\hline
\end{tabular}

MAGNITUDE AND PROBABILITY OF ANNUAL HIGH FLOW BASED ON PERIOD OF RECORD $1935.45,1989 \cdot 96$

\begin{tabular}{|c|c|c|c|c|c|c|}
\hline \multirow{4}{*}{$\begin{array}{l}\text { PERIOD } \\
\text { ICON- } \\
\text { SECU. }\end{array}$} & \multicolumn{6}{|c|}{ DISCHARGE, IN FT3/S, FOR INDICATED } \\
\hline & & \multicolumn{3}{|c|}{ RECURRENCE INTERVAL, IN } & \multicolumn{2}{|c|}{ YEARS, AND } \\
\hline & & EXCEED & NCE PROI & ABILITY, & IN PERC & ENT \\
\hline & & 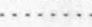 & & 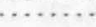 & $\ldots \ldots \ldots$ & $\ldots \ldots \ldots$ \\
\hline TIVE & 2 & 5 & 10 & 25 & $50 \#$ & $100 \#$ \\
\hline DAYS) & 508 & 208 & 108 & 48 & 28 & 18 \\
\hline 1 & 6,200 & 18,900 & 34,000 & 63,800 & 95,900 & 139,000 \\
\hline 3 & 4,230 & 11,900 & 20,600 & 36,800 & 53,700 & 75,500 \\
\hline 7 & 2,900 & 7,300 & 11.700 & 19,000 & 25,900 & 34,100 \\
\hline 15 & 2,020 & 4,850 & 7,670 & 12,500 & 17,100 & 22,800 \\
\hline 30 & 1,560 & 3,510 & 5,300 & 8,150 & 10,700 & 13,700 \\
\hline 60 & .14 & 2,5 & 3,910 & 6,100 & 8,120 & 10.500 \\
\hline 90 & 873 & 1,890 & 2,860 & 4,470 & 5.990 & 7,810 \\
\hline
\end{tabular}

TSCHARGE, IN FT3/S, WHICH WAS EQUALED OR EXCEEDED FOR INDICATED PERCENT OF TIME

\begin{tabular}{|c|c|c|c|c|c|c|c|c|c|c|c|c|c|c|c|c|}
\hline 18 & 58 & 108 & 158 & 208 & 308 & 408 & 508 & 608 & 708 & 808 & 908 & 958 & 988 & 998 & 99.58 & 99.98 \\
\hline 140 & 1,610 & 794 & 444 & 309 & 234 & 211 & 188 & 157 & 127 & 103 & 82 & 70 & 60 & 54 & 51 & 43 \\
\hline
\end{tabular}

\# Reliability of values in column is uncertain, and potential errors are large. 
ANNUAL MEAN DISCHARGE, IN CUBIC FEET PER SECOND

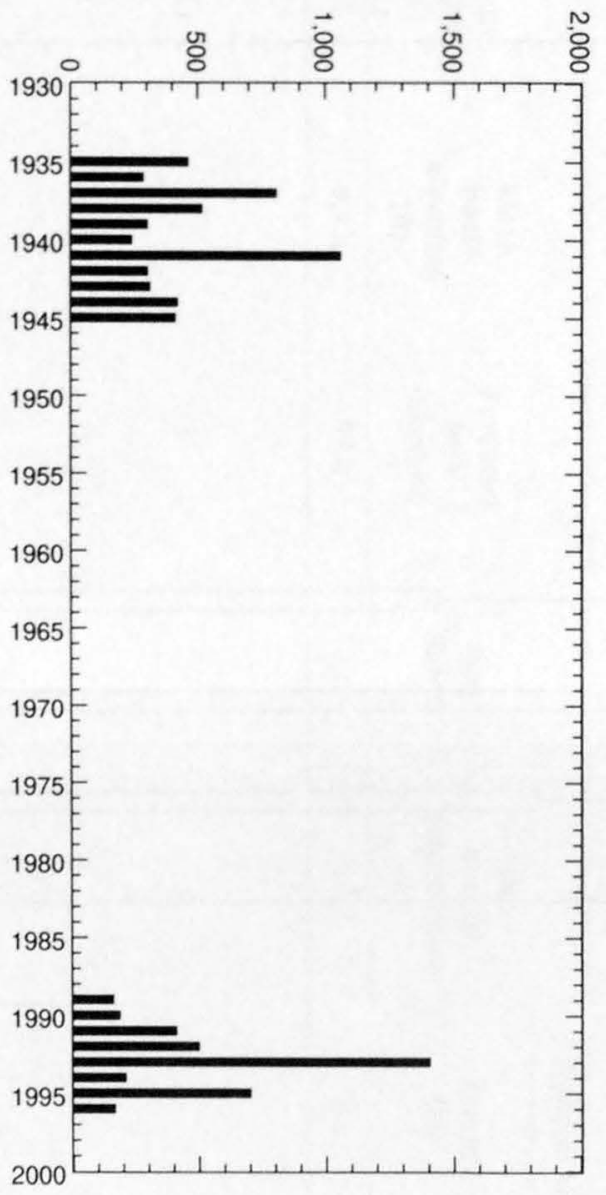

ANNUAL PEAK DISCHARGE, IN CUBIC
FEET PER SECOND

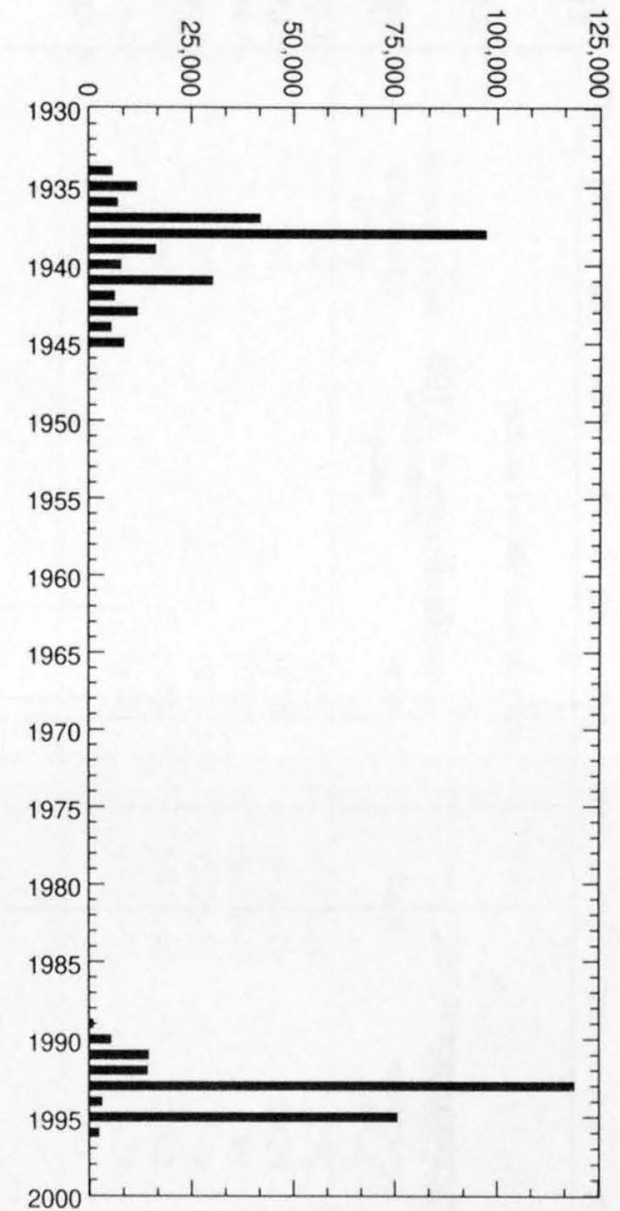


09506000 VERDE RIVER NEAR CAMP VERDE, AZ--Continued

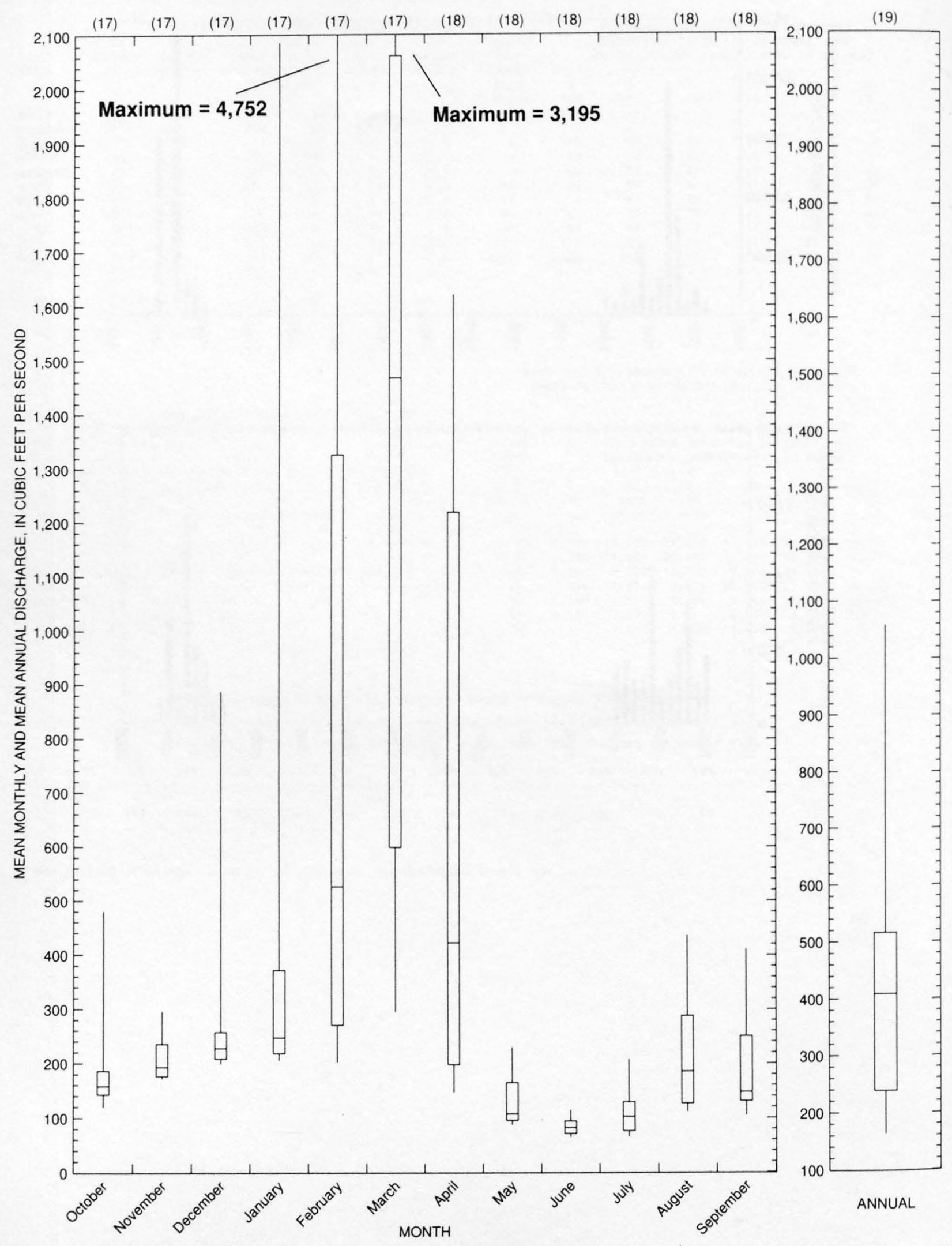


09507600 EAST VERDE RIVER NEAR PINE, AZ

LOCATION.--Lat $34^{\circ} 23^{\prime} 30^{\prime \prime}$, long $111^{\circ} 16^{\prime} 05^{\prime \prime}$, in SE $1 / 4 \mathrm{SW}^{1} / 4$ sec.26, T.12 N., R.10 E., Gila County, Hydrologic Unit 15060203 , at right bank 0.8 mi upstream from Dude Creek, 2.7 mi south of Washington Park, and 10 mi east of Pine.

DRAINAGE AREA.--6.34 $\mathrm{mi}^{2}$.

Annual peak discharges

\begin{tabular}{|c|c|c|c|c|c|c|c|}
\hline $\begin{array}{l}\text { Water } \\
\text { year }\end{array}$ & Date & $\begin{array}{c}\text { Annual peak } \\
\text { discharge } \\
\left(\mathrm{ft}^{3} / \mathrm{s}\right)\end{array}$ & $\begin{array}{l}\text { Discharge } \\
\text { codes }\end{array}$ & $\begin{array}{c}\text { Water } \\
\text { year }\end{array}$ & Date & $\begin{array}{c}\text { Annual peak } \\
\text { discharge } \\
\left(\mathrm{ft}^{3} / \mathrm{s}\right)\end{array}$ & $\begin{array}{c}\text { Discharge } \\
\text { codes }\end{array}$ \\
\hline 1962 & $02-13-62$ & 38 & & 1969 & $01-25-69$ & 298 & \\
\hline 1964 & $08-04-64$ & 143 & & 1971 & $08-28-71$ & 99 & \\
\hline 1965 & $01-06-65$ & 127 & & 1972 & $12-26-71$ & 60 & ES \\
\hline 1966 & $12-30-65$ & 960 & & 1973 & $10-19-72$ & 2,700 & \\
\hline 1968 & $08-02-68$ & 330 & & & & & \\
\hline
\end{tabular}

Basin characteristics

\begin{tabular}{cccccccc}
\hline $\begin{array}{c}\text { Main } \\
\text { channel } \\
\text { slope } \\
(\mathrm{ft} / \mathrm{mi})\end{array}$ & $\begin{array}{c}\text { Stream } \\
\text { length } \\
(\mathrm{mi})\end{array}$ & $\begin{array}{c}\text { Mean } \\
\text { basin } \\
\text { elevation } \\
(\mathrm{ft})\end{array}$ & $\begin{array}{c}\text { Forested } \\
\text { area } \\
\text { (percent) }\end{array}$ & $\begin{array}{c}\text { Soil } \\
\text { index }\end{array}$ & $\begin{array}{c}\text { Mean } \\
\text { annual } \\
\text { precipitation } \\
\text { (in) }\end{array}$ & $\begin{array}{c}\text { Rainfall intensity, 24-hour } \\
\text { (in) }\end{array}$ & $\begin{array}{c}50 \text {-year } \\
\text { (in) }\end{array}$ \\
\hline 303 & 4.4 & 6,430 & 99.0 & 3.0 & 30.0 & 2.6 \\
\hline
\end{tabular}




\section{EAST VERDE RIVER NEAR PINE, AZ--Continued}

MEAN MONTHLY AND ANNUAL DISCHARGES $1962 \cdot 71$

\begin{tabular}{|c|c|c|c|c|c|c|}
\hline MONTH & $\begin{array}{l}\text { MAXIMUM } \\
\text { (FT3/S) }\end{array}$ & $\begin{array}{l}\text { MINIMUM } \\
\text { (FT3/S) }\end{array}$ & $\begin{array}{c}\text { MEAN } \\
(\text { FT3 } / \mathrm{S})\end{array}$ & $\begin{array}{l}\text { STAN - } \\
\text { DARD } \\
\text { DEVIA- } \\
\text { TION } \\
(\mathrm{FT} 3 / \mathrm{S})\end{array}$ & $\begin{array}{l}\text { COEFFI- } \\
\text { CIENT OF } \\
\text { VARI- } \\
\text { ATION }\end{array}$ & $\begin{array}{c}\text { PERCENT } \\
\text { OF } \\
\text { ANNUAL } \\
\text { RUNOFF }\end{array}$ \\
\hline OCTOBER & 29 & 0.49 & 8.8 & 13 & 1.4 & 6.1 \\
\hline NOVEMBER & 29 & 0.69 & 9.2 & 12 & 1.3 & 6.4 \\
\hline DECEMBER & 28 & 0.59 & 8.8 & 12 & 1.4 & 6.1 \\
\hline JANUARY & 28 & 0.60 & 10 & 11 & 1.1 & 7.0 \\
\hline FEBRUARY & 33 & 0.62 & 12 & 12 & 0.98 & 8.7 \\
\hline MARCH & 36 & 1.3 & 15 & 13 & 0.85 & 10.3 \\
\hline APRIL & 38 & 0.84 & 18 & 13 & 0.76 & 12.3 \\
\hline MAY & 33 & 0.29 & 16 & 14 & 0.86 & 11.3 \\
\hline JUNE & 29 & 0.10 & 12 & 13 & 1.1 & 8.1 \\
\hline JULY & 29 & 0.11 & 11 & 13 & 1.2 & 7.6 \\
\hline AUGUST & 30 & 0.04 & 11 & 12 & 1.1 & 7.6 \\
\hline SEPTEMBER & 28 & 0.24 & 12 & 12 & 0.99 & 8.3 \\
\hline ANNUAL & 26 & 0.73 & 12 & 9.3 & 0.78 & 100 \\
\hline
\end{tabular}

MAGNITUDE AND PROBABILITY OF INSTANTANEOUS PEAK FLOW BASED ON PERIOD OF RECORD $1962 \cdot 74$

DISCHARGE, IN FT3/S, FOR INDICATED RECURRENCE INTERVAL IN YEARS, AND EXCEEDANCE PROBABILITY, IN EERCENT

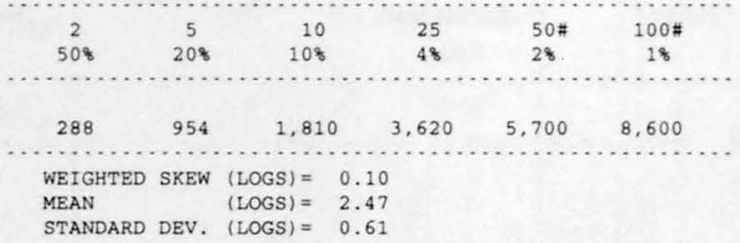

MAGNITUDE AND PROBABILITY OF ANNUAL LOW FLOW BASED ON PERIOD OF RECORD 1963.71

\begin{tabular}{|c|c|c|c|c|c|c|}
\hline $\begin{array}{l}\text { PERIOD } \\
\text { (CON- }\end{array}$ & \multirow{2}{*}{\multicolumn{6}{|c|}{$\begin{array}{l}\text { DISCHARGE, IN FT3/S, FOR INDICATED } \\
\text { RECURRENCE INTERVAL, IN YEARS, AND } \\
\text { NON-EXCBEDANCE PROBABILITY, IN PERCENT }\end{array}$}} \\
\hline SECU. & & & & & & \\
\hline TIVE & 2 & 5 & 10 & $20 \#$ & $50 \#$ & $100 \#$ \\
\hline DAYS) & 508 & 208 & 108 & 58 & 28 & 18 \\
\hline 1 & 0.55 & 0.00 & 0.00 & 0.00 & 0.00 & 0.00 \\
\hline 3 & 0.56 & 0.00 & 0.00 & 0.00 & 0.00 & 0.00 \\
\hline 7 & 0.48 & 0.00 & 0.00 & 0.00 & 0.00 & 0.00 \\
\hline 14 & 0.42 & 0.08 & 0.00 & 0.00 & 0.00 & 0.00 \\
\hline 30 & 0.55 & 0.12 & 0.05 & 0.03 & 0.02 & 0.01 \\
\hline 60 & 0.84 & 0.17 & 0.08 & 0.04 & 0.02 & 0.01 \\
\hline 90 & 1.2 & 0.25 & 0.12 & 0.07 & 0.04 & 0.02 \\
\hline 120 & 1.6 & 0.37 & 0.18 & 0.11 & 0.06 & 0.04 \\
\hline 183 & 3.1 & 0.71 & 0.33 & 0.17 & 0.08 & 0.05 \\
\hline
\end{tabular}

MAGNITUDE AND PROBABILITY OF ANNUAL HIGH FLOW BASED ON PERIOD OF RECORD 1962.71

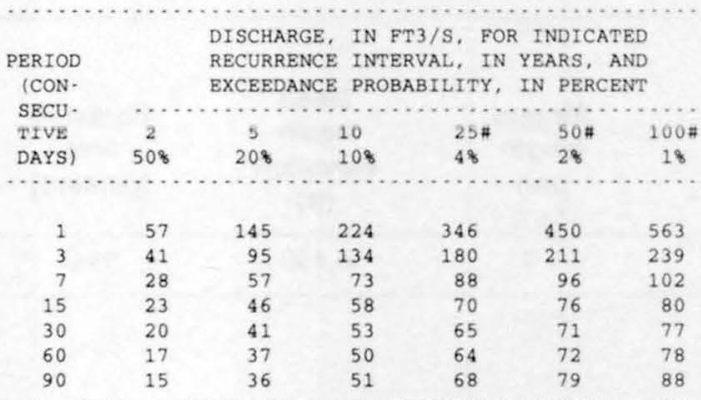

DURATION TABLE OF DAILY MEAN FLOW FOR PERIOD OF RECORD 1962-71

ISCHARGE, IN FT3/S, WHICH WAS EQUALED OR EXCEEDED FOR INDICATED PERCENT OF TIME

\begin{tabular}{|c|c|c|c|c|c|c|c|c|c|c|c|c|c|c|c|c|}
\hline 18 & 58 & 108 & 158 & 208 & 308 & 408 & 508 & 608 & 708 & 808 & 908 & 958 & 988 & 998 & 99.58 & 99.98 \\
\hline 42 & 35 & 32 & 29 & 28 & 25 & 9.5 & 2.8 & 1.2 & 0.78 & 0.64 & 0.47 & 0.22 & 0.11 & 0.10 & 0.00 & 0.00 \\
\hline
\end{tabular}

\# Reliability of values in column is uncertain, and potential errors are large. 
09507600 EAST VERDE RIVER NEAR PINE, AZ--Continued
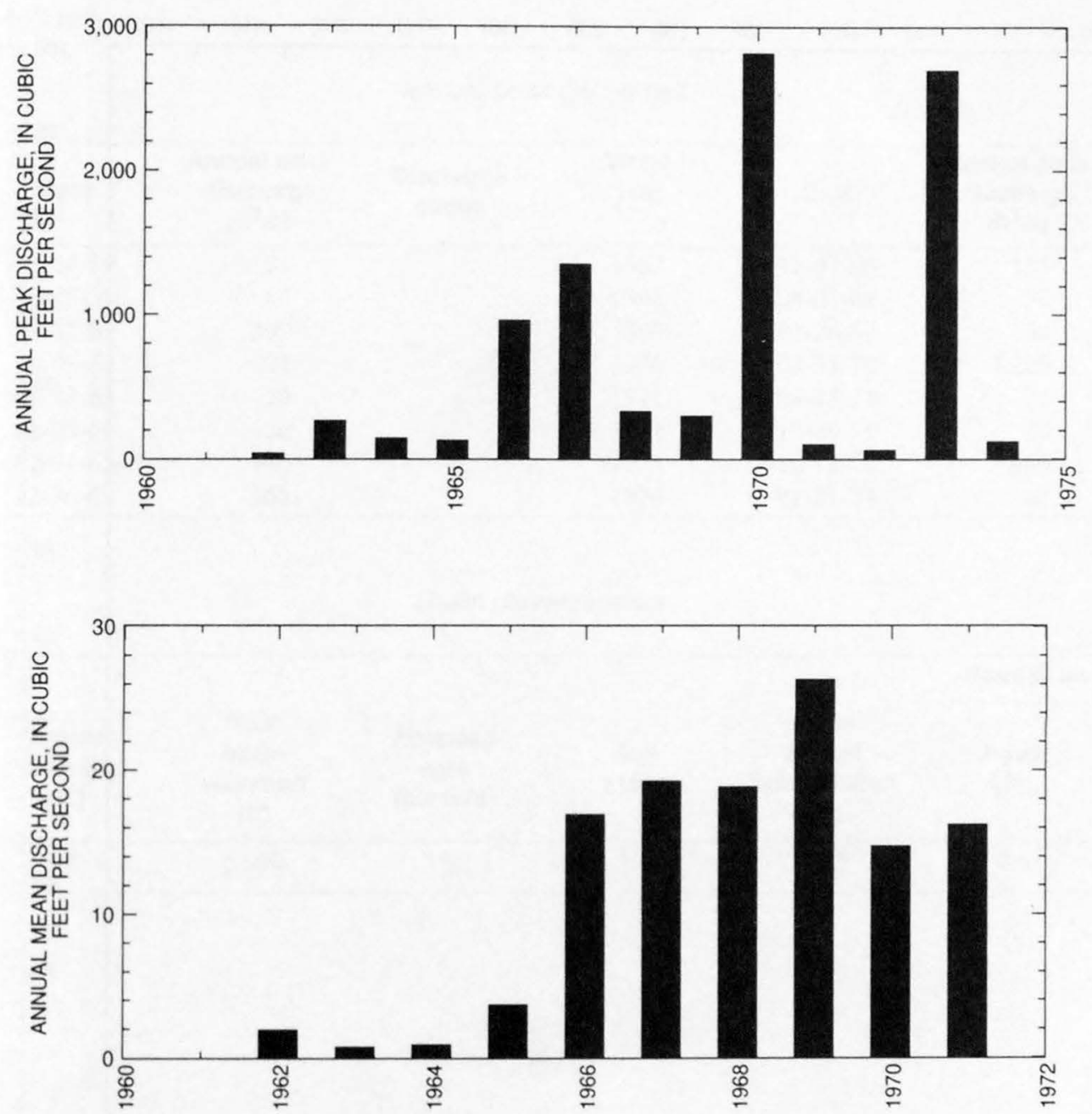
09507600 EAST VERDE RIVER NEAR PINE, AZ--Continued
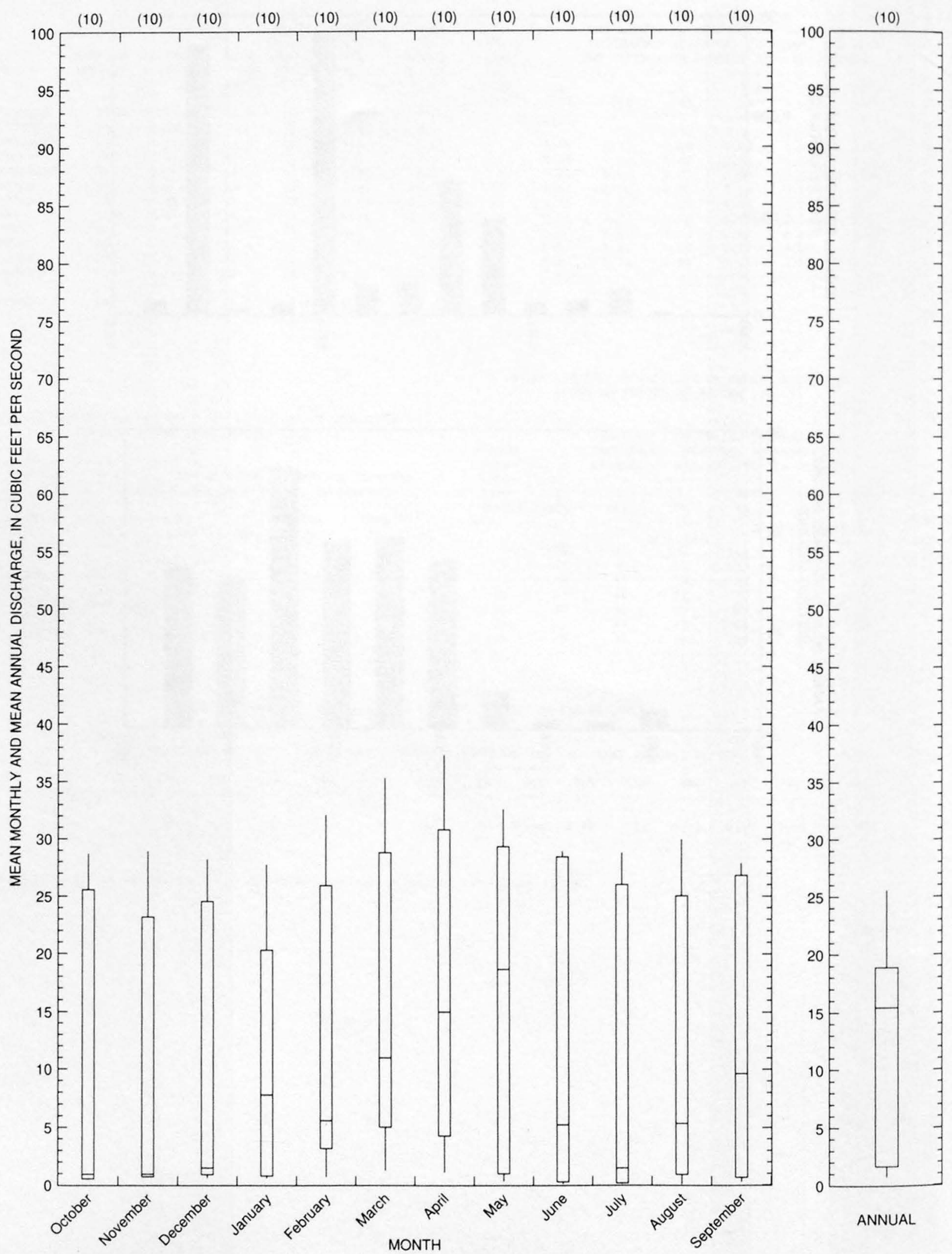
09507700 WEBBER CREEK ABOVE WEST FORK WEBBER CREEK, NEAR PINE, AZ

LOCATION.--Lat $34^{\circ} 24^{\prime} 40^{\prime \prime}$, long $111^{\circ} 22^{\prime} 20^{\prime \prime}$, in SW $\mathrm{SW}^{1} / \mathrm{sec} .23$, T.12 N., R.9 E. (unsurveyed), Gila County, Hydrologic Unit 15060203 , in Tonto National Forest, on left bank $0.2 \mathrm{mi}$ upstream from West Fork, and 4.9 mi northeast of Pine.

DRAINAGE AREA.--4.79 $\mathrm{mi}^{2}$.

Annual peak discharges

\begin{tabular}{|c|c|c|c|c|c|c|c|}
\hline $\begin{array}{l}\text { Water } \\
\text { year }\end{array}$ & Date & $\begin{array}{c}\text { Annual peak } \\
\text { discharge } \\
\left(\mathrm{ft}^{3} / \mathrm{s}\right)\end{array}$ & $\begin{array}{l}\text { Discharge } \\
\text { codes }\end{array}$ & $\begin{array}{c}\text { Water } \\
\text { year }\end{array}$ & Date & $\begin{array}{c}\text { Annual peak } \\
\text { discharge } \\
\left(\mathrm{ft}^{3} / \mathrm{s}\right)\end{array}$ & $\begin{array}{c}\text { Discharge } \\
\text { codes }\end{array}$ \\
\hline 1959 & $08-24-59$ & 21 & & 1967 & $12-07-66$ & 153 & \\
\hline 1960 & $12-25-59$ & 84 & & 1968 & $04-01-68$ & 32 & \\
\hline 1961 & $09-13-61$ & 399 & & 1969 & $01-26-69$ & 134 & \\
\hline 1962 & $04-08-62$ & 32 & & 1970 & $09-05-70$ & 1,220 & \\
\hline 1963 & $08-22-63$ & 19 & & 1971 & $08-25-71$ & 26 & \\
\hline 1964 & $08-08-64$ & 126 & & 1972 & $12-26-71$ & 50 & \\
\hline 1965 & 01-07-65 & 148 & & 1973 & $10-19-72$ & 686 & \\
\hline 1966 & $12-30-65$ & 366 & & 1974 & $01-21-74$ & 12 & \\
\hline
\end{tabular}

Basin characteristics

\begin{tabular}{|c|c|c|c|c|c|c|c|}
\hline \multirow[b]{2}{*}{$\begin{array}{l}\text { Main } \\
\text { channel } \\
\text { slope } \\
(\mathrm{ft} / \mathrm{mi})\end{array}$} & \multirow[b]{2}{*}{$\begin{array}{c}\text { Stream } \\
\text { length } \\
\text { (mi) }\end{array}$} & \multirow[b]{2}{*}{$\begin{array}{c}\text { Mean } \\
\text { basin } \\
\text { elevation } \\
\text { (ft) }\end{array}$} & \multirow[b]{2}{*}{$\begin{array}{l}\text { Forested } \\
\text { area } \\
\text { (percent) }\end{array}$} & \multirow[b]{2}{*}{$\begin{array}{c}\text { Soil } \\
\text { index }\end{array}$} & \multirow[b]{2}{*}{$\begin{array}{c}\text { Mean } \\
\text { annual } \\
\text { precipitation } \\
\text { (in) }\end{array}$} & \multicolumn{2}{|c|}{ Rainfall intensity, 24-hou } \\
\hline & & & & & & $\begin{array}{l}\text { 2-year } \\
\text { (in) }\end{array}$ & $\begin{array}{l}\text { 50-year } \\
\text { (in) }\end{array}$ \\
\hline 634 & 3.8 & 6,980 & 100 & 3.0 & 27.5 & 2.8 & 5.2 \\
\hline
\end{tabular}


09507700 WEBBER CREEK ABOVE WEST FORK WEBBER CREEK, NEAR PINE, AZ--Continued

MEAN MONTHLY AND ANNUAL DISCHARGES $1960-74$

\begin{tabular}{|c|c|c|c|c|c|c|}
\hline MONTH & $\begin{array}{l}\text { MAXIMUM } \\
\text { (FT3/S) }\end{array}$ & $\begin{array}{l}\text { MINIMUM } \\
\text { (FT3/S) }\end{array}$ & $\begin{array}{c}\text { MEAN } \\
(\text { FT3/S) }\end{array}$ & $\begin{array}{l}\text { STAN- } \\
\text { DARD } \\
\text { DEVIA- } \\
\text { TION } \\
(\mathrm{FT} 3 / \mathrm{S})\end{array}$ & $\begin{array}{l}\text { COEFFI- } \\
\text { CIENT OF } \\
\text { VARI- } \\
\text { ATION }\end{array}$ & $\begin{array}{c}\text { PERCENT } \\
\text { OF } \\
\text { ANNUAL } \\
\text { RUNOFF }\end{array}$ \\
\hline & & & & & & \\
\hline OCTOBER & 26 & 0.25 & 2.2 & 6.7 & 3.0 & 7.4 \\
\hline NOVEMBER & 4.6 & 0.31 & 1.1 & 1.3 & 1.2 & 3.7 \\
\hline DECEMBER & 16 & 0.32 & 2.8 & 4.1 & 1.5 & 9.2 \\
\hline JANUARY & 11 & 0.51 & 3.0 & 3.4 & 1.1 & 10.0 \\
\hline FEBRUARY & 8.4 & 0.48 & 2.6 & 2.5 & 0.99 & 8.5 \\
\hline MARCH & 15 & 0.52 & 5.4 & 5.1 & 0.95 & 17.9 \\
\hline APRIL & 29 & 0.50 & 7.7 & 9.2 & 1.2 & 25.6 \\
\hline MAY & 21 & 0.43 & 2.7 & 5.2 & 2.0 & 8.9 \\
\hline JUNE & 1.3 & 0.18 & 0.53 & 0.35 & 0.65 & 1.8 \\
\hline JULY & 0.53 & 0.16 & 0.33 & 0.11 & 0.33 & 1.1 \\
\hline AUGUST & 1.2 & 0.31 & 0.62 & 0.34 & 0.56 & 2.1 \\
\hline SEPTEMBER & 8.8 & 0.24 & 1.1 & 2.2 & 1.9 & 3.8 \\
\hline NNUAL & 9.1 & 0.61 & 2.5 & 2.3 & 0.92 & 100 \\
\hline
\end{tabular}

MAGNITUDE AND PROBABILITY OF ANNUAL LOW FLOW BASED ON PERIOD OF RECORD 1961-74

\begin{tabular}{|c|c|c|c|c|c|c|}
\hline $\begin{array}{l}\text { PERIOD } \\
\text { (CON }-\end{array}$ & \multirow{2}{*}{\multicolumn{6}{|c|}{ 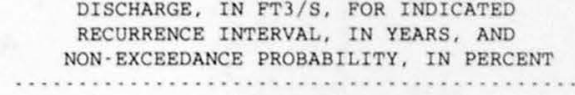 }} \\
\hline SECU - & & & & & & \\
\hline TIVE & 2 & 5 & 10 & 20 & $50 \#$ & $100 \#$ \\
\hline DAYS) & 508 & 208 & 108 & 58 & 28 & 18 \\
\hline 1 & 0.19 & 0.15 & 0.13 & 0.12 & 0.10 & 0.09 \\
\hline 3 & 0.20 & 0.16 & 0.13 & 0.12 & 0.10 & 0.09 \\
\hline 7 & 0.21 & 0.16 & 0.14 & 0.12 & 0.10 & 0.09 \\
\hline 14 & 0.22 & 0.18 & 0.15 & 0.14 & 0.12 & 0.11 \\
\hline 30 & 0.25 & 0.21 & 0.19 & 0.17 & 0.16 & 0.15 \\
\hline 60 & 0.30 & 0.25 & 0.23 & 0.21 & 0.20 & 0.19 \\
\hline 90 & 0.34 & 0.29 & 0.26 & 0.24 & 0.22 & 0.21 \\
\hline 120 & 0.39 & 0.32 & 0.29 & 0.26 & 0.24 & 0.22 \\
\hline 183 & 0.50 & 0.39 & 0.35 & 0.31 & 0.28 & 0.25 \\
\hline
\end{tabular}

MAGNITUDE AND PROBABILITY OF ANNUAL HIGH FLOW BASED ON PERIOD OF RECORD $1960 \cdot 74$

MAGNITUDE AND PROBABILITY OF INSTANTANEOUS PEAK FLOW BASED ON PERIOD OF RECORD 1959.74

DISCHARGE, IN FT3/S, FOR INDICATED RECURRENCE INTERVAL IN YEARS, AND EXCEBDANCE PROBABILITY, IN PERCENT

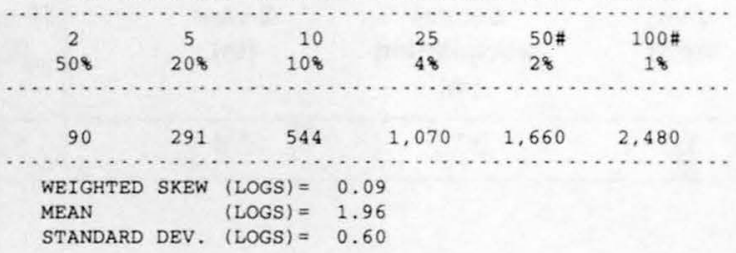

DURATION TABLE OF DAILY MEAN FLOW FOR PERIOD OF RECORD 1960.74

\begin{tabular}{|c|c|c|c|c|c|c|}
\hline \multirow{4}{*}{$\begin{array}{l}\text { PERIOD } \\
\text { (CON- } \\
\text { SECU- }\end{array}$} & \multicolumn{6}{|c|}{ DISCHARGE, IN FT3/S, FOR INDICATED } \\
\hline & \multirow{2}{*}{\multicolumn{3}{|c|}{$\begin{array}{l}\text { RECURRENCE IN } \\
\text { EXCEEDANCE PR }\end{array}$}} & AL, I & \multicolumn{2}{|c|}{ YEARS, AND } \\
\hline & & & & ILITY & IN PERC & \\
\hline & & & & & & \\
\hline TIVE & 2 & 5 & 10 & 25 & $50 \#$ & $100 *$ \\
\hline DAYS) & 508 & 208 & 108 & 48 & 28 & 18 \\
\hline 1 & 39 & 117 & 200 & 343 & 479 & 640 \\
\hline 3 & 27 & 70 & 110 & 171 & 224 & 282 \\
\hline 7 & 18 & 43 & 61 & 85 & 103 & 120 \\
\hline 15 & 12 & 27 & 38 & 51 & 61 & 71 \\
\hline 30 & 8.2 & 19 & 28 & 42 & 53 & 66 \\
\hline 60 & 5.1 & 12 & 19 & 30 & 40 & 52 \\
\hline 90 & 4.1 & 9.8 & 15 & 25 & 33 & 44 \\
\hline
\end{tabular}

DISCHARGE, IN FT3/S, WHICH WAS EQUALED OR EXCEEDED FOR INDICATED PERCENT OF TIME

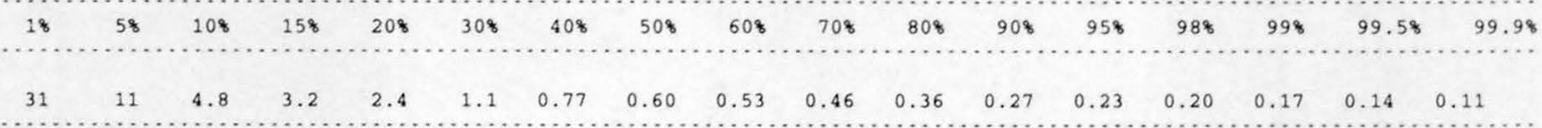

\# Reliability of values in column is uncertain, and potential errors are large. 
09507700 WEBBER CREEK ABOVE WEST FORK WEBBER CREEK, NEAR PINE, AZ--Continued
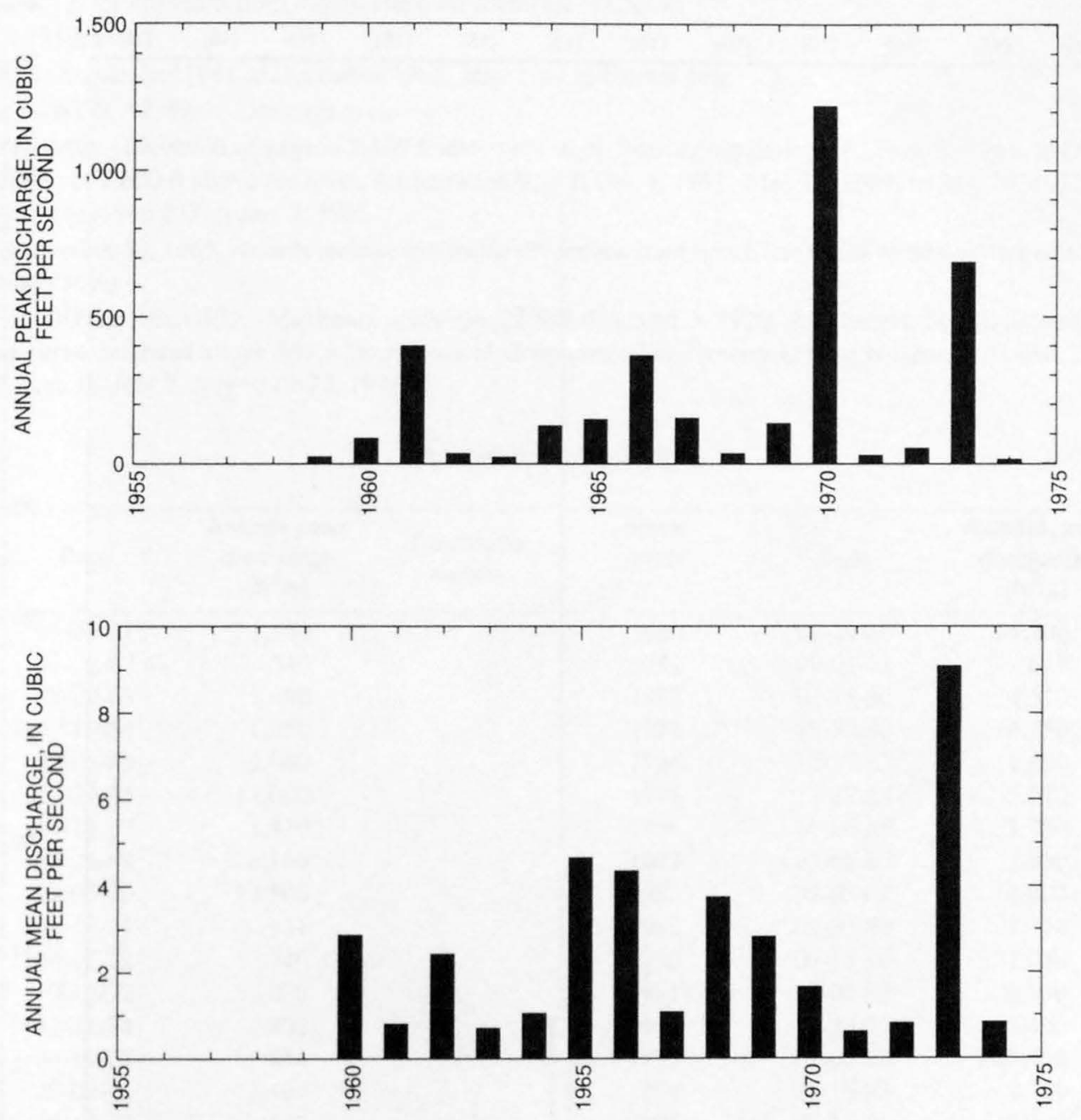
09507700 WEBBER CREEK ABOVE WEST FORK WEBBER CREEK, NEAR PINE, AZ--Continued
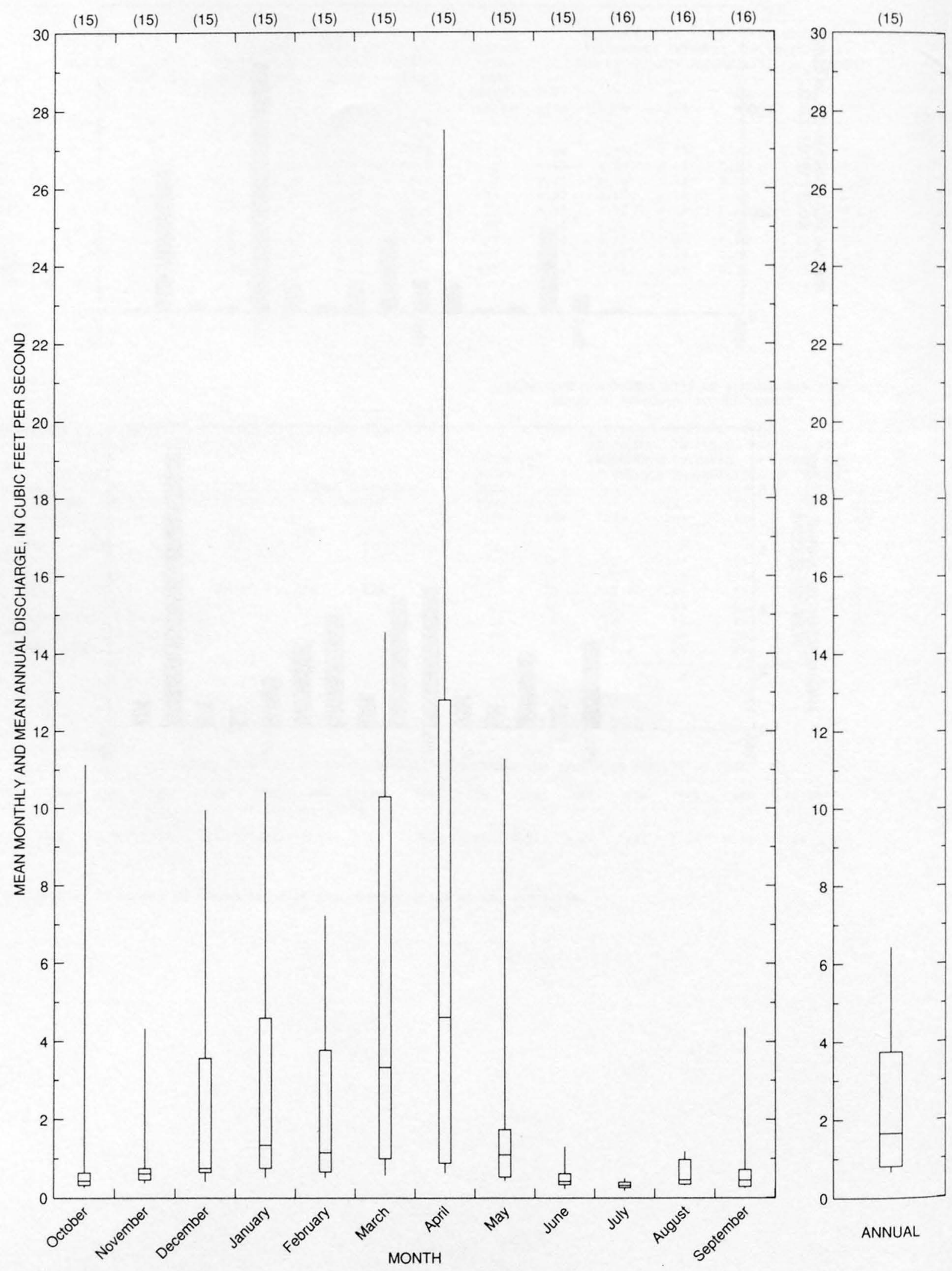


\section{EAST VERDE RIVER NEAR CHILDS, AZ}

LOCATION.--Lat 34'16'35", long 111'38'17", in sec.21, T.11 N., R.7 E. (unsurveyed), Gila County Hydrologic Unit 15060203, in Tonto National Forest, on left bank 1.6 mi upstream from mouth and 6 mi southeast of Childs.

DRAINAGE AREA.--331 $\mathrm{mi}^{2}$.

PERIOD OF RECORD.--September 1961 to December 1965, May 1967 to current year.

REVISED RECORDS.--WDR AZ-89-1: Drainage area.

GAGE.--Water-stage recorder. Elevation of gage is 2,500 ft above sea level, from topographic map. Sept. 1, 1961, to Dec. 15, 1965 , at site $1 \mathrm{mi}$ upstream at elevation of 2,600 ft above sea level, datum raised $0.38 \mathrm{ft}$ Oct. 4, 1963. May 25, 1967, to July 20, 1972, at present site at datum $3.29 \mathrm{ft}$ higher, datum lowered $2.00 \mathrm{ft}$ Jan. 7, 1993.

REMARKS.--Since September 30, 1965, records include transbasin diversions from East Clear Creek to headwaters of East Verde River. (See sta 09507580 and 09398300 .)

EXTREMES FOR PERIOD OF RECORD.--Maximum discharge, $23,500 \mathrm{ft}^{3} / \mathrm{s} \mathrm{Sept} .5,1970$, gage height, $22.5 \mathrm{ft}$, present datum, from profile past gage, from rating curve extended above $960 \mathrm{ft}^{3} / \mathrm{s}$ on basis of slope-area measurements at gage heights 12.11 and $22.5 \mathrm{ft}$, present datum; no flow June 11-13, June 18-July 7, July 9, 19-27, 1996.

Annual peak discharges

\begin{tabular}{|c|c|c|c|c|c|c|c|}
\hline $\begin{array}{l}\text { Water } \\
\text { year }\end{array}$ & Date & $\begin{array}{c}\text { Annual peak } \\
\text { discharge } \\
\left(\mathrm{ft}^{3} / \mathrm{s}\right)\end{array}$ & $\begin{array}{l}\text { Discharge } \\
\text { codes }\end{array}$ & $\begin{array}{l}\text { Water } \\
\text { year }\end{array}$ & Date & $\begin{array}{c}\text { Annual peak } \\
\text { discharge } \\
\left(\mathrm{ft}^{3} / \mathrm{s}\right)\end{array}$ & $\begin{array}{c}\text { Discharge } \\
\text { codes }\end{array}$ \\
\hline 1961 & 09-09-61 & 1,340 & & 1980 & $02-20-80$ & 14,100 & \\
\hline 1962 & $03-22-62$ & 540 & & 1981 & $09-01-81$ & 617 & \\
\hline 1963 & $08-22-63$ & 11,400 & & 1982 & $02-11-82$ & 4,510 & \\
\hline 1964 & $09-13-64$ & 1,280 & & 1983 & $11-30-82$ & 6,250 & \\
\hline 1965 & $01-06-65$ & 5,980 & & 1984 & $12-27-83$ & 1,690 & \\
\hline 1966 & $12-22-65$ & 17,000 & & 1985 & $12-27-84$ & 5,570 & \\
\hline 1968 & $12-19-67$ & 1,410 & & 1986 & $11-26-85$ & 1,760 & \\
\hline 1969 & $01-26-69$ & 6,100 & & 1987 & $03-05-87$ & 1,030 & \\
\hline 1970 & $09-05-70$ & 23,500 & & 1988 & $02-03-88$ & 4,000 & \\
\hline 1971 & $08-11-71$ & 931 & & 1989 & $02-05-89$ & 1,750 & \\
\hline 1972 & $08-11-72$ & 740 & & 1990 & $07-17-90$ & 1,020 & \\
\hline 1973 & $10-19-72$ & 10,000 & & 1991 & $03-01-91$ & 8,790 & \\
\hline 1974 & $01-21-74$ & 802 & & 1992 & $08-23-92$ & 11,000 & \\
\hline 1975 & $04-11-75$ & 814 & & 1993 & $01-08-93$ & 20,100 & \\
\hline 1976 & $02-09-76$ & 11,400 & & 1994 & $11-15-93$ & 2,110 & \\
\hline 1977 & $08-17-77$ & 502 & & 1995 & $02-15-95$ & 11,900 & \\
\hline 1978 & $03-01-78$ & 15,000 & & 1996 & $09-14-96$ & 519 & \\
\hline 1979 & $01-17-79$ & 11,600 & & & & & \\
\hline
\end{tabular}

Discharge rating table developed January 1993

\begin{tabular}{crcc}
\hline $\begin{array}{c}\text { Gage height } \\
(\mathbf{f t})\end{array}$ & $\begin{array}{c}\text { Discharge } \\
\left(\mathbf{f t}^{\mathbf{3}} \mathbf{s}\right)\end{array}$ & $\begin{array}{c}\text { Gage height } \\
(\mathbf{f t})\end{array}$ & $\begin{array}{c}\text { Discharge } \\
\left(\mathbf{f t}^{\mathbf{3}} / \mathbf{s}\right)\end{array}$ \\
\hline 3.0 & 891 & 13.0 & 11,960 \\
4.0 & 1,890 & 15.0 & 14,340 \\
5.0 & 2,930 & 17.0 & 16,740 \\
7.0 & 5,090 & 19.0 & 19,170 \\
9.0 & 7,330 & 21.0 & 21,620 \\
11.0 & 9,630 & 23.0 & 24,100 \\
\hline
\end{tabular}


09507980 EAST VERDE RIVER NEAR CHILDS, AZ--Continued

Basin characteristics

\begin{tabular}{cccccccc}
\hline $\begin{array}{c}\text { Main } \\
\text { channel } \\
\text { slope } \\
(\mathrm{ft} / \mathrm{mi})\end{array}$ & $\begin{array}{c}\text { Stream } \\
\text { length } \\
\text { (mi) }\end{array}$ & $\begin{array}{c}\text { Mean } \\
\text { basin } \\
\text { elevation } \\
\text { (ft) }\end{array}$ & $\begin{array}{c}\text { Forested } \\
\text { area } \\
\text { (percent) }\end{array}$ & $\begin{array}{c}\text { Soil } \\
\text { index }\end{array}$ & $\begin{array}{c}\text { Mean } \\
\text { annual } \\
\text { precipitation } \\
\text { (in) }\end{array}$ & $\begin{array}{c}\text { Rainfall intensity, 24-hour } \\
\text { 2-year } \\
\text { (in) }\end{array}$ & $\begin{array}{c}50 \text {-year } \\
\text { (in) }\end{array}$ \\
\hline 91.6 & 32.0 & 5,140 & 50.0 & 2.8 & 24.7 & 2.7 \\
\hline
\end{tabular}


09507980 EAST VERDE RIVER NEAR CHILDS, AZ--Continued

MEAN MONTHLY AND ANNUAL DISCHARGES $1962-65,1968-96$

\begin{tabular}{|c|c|c|c|c|c|c|}
\hline MONTH & $\begin{array}{l}\text { MAXIMUM } \\
(\text { FT3/S) }\end{array}$ & $\begin{array}{l}\text { MINIMUM } \\
\text { (FT3/S) }\end{array}$ & $\begin{array}{c}\text { MEAN } \\
\text { (FT3/S) }\end{array}$ & $\begin{array}{l}\text { STAN- } \\
\text { DARD } \\
\text { DEVIA- } \\
\text { TION } \\
\text { (FT3/S) }\end{array}$ & $\begin{array}{l}\text { COEFFI- } \\
\text { CIENT OF } \\
\text { VARI- } \\
\text { ATION }\end{array}$ & $\begin{array}{c}\text { PERCENT } \\
\text { OF } \\
\text { ANNUAL } \\
\text { RUNOFF }\end{array}$ \\
\hline OCTOBER & 308 & 0.73 & 30 & 52 & 1.8 & 3.5 \\
\hline NOVBMBER & 157 & 0.83 & 34 & 38 & 1.1 & 4.0 \\
\hline DECEMBER & 443 & 1.4 & 64 & 102 & 1.6 & 7.5 \\
\hline JANUARY & 1,820 & 2.3 & 142 & 321 & 2.3 & 16.6 \\
\hline FEBRUARY & 1,150 & 3.7 & 176 & 259 & 1.5 & 20.6 \\
\hline MARCH & 968 & 6.3 & 184 & 220 & 1.2 & 21.6 \\
\hline APRIL & 371 & 4.3 & 85 & 92 & 1.1 & 9.9 \\
\hline MAY & 115 & 1.8 & 31 & 22 & 0.73 & 3.6 \\
\hline JUNE & 49 & 0.43 & 19 & 14 & 0.74 & 2.2 \\
\hline JULY & 51 & 0.35 & 20 & 15 & 0.75 & 2.4 \\
\hline AUGUST & 203 & 1.2 & 37 & 44 & 1.2 & 4.3 \\
\hline SEPTEMBER & 282 & 0.73 & 32 & 47 & 1.5 & 3.7 \\
\hline ANNUAL & 290 & 11 & 71 & 64 & 0.91 & 100 \\
\hline
\end{tabular}

MAGNITUDE AND PROBABILITY OF ANNUAL LOW FLOW BASED ON PERIOD OF RECORD 1963.65, 1969.96

\begin{tabular}{|c|c|c|c|c|c|c|}
\hline \multirow{4}{*}{$\begin{array}{l}\text { PERIOD } \\
\text { (CON- } \\
\text { SECU- } \\
\text { TIVE }\end{array}$} & \multirow{3}{*}{\multicolumn{2}{|c|}{$\begin{array}{l}\text { DISCHARGE, } \\
\text { RECURRENCE } \\
\text { NON-EXCEEDAN }\end{array}$}} & \multicolumn{4}{|c|}{ IN ET3/S, FOR INDICATED } \\
\hline & & & \multicolumn{4}{|c|}{$\begin{array}{l}\text { INTERVAL, IN YEARS, AND } \\
\text { NCE PROBABILITY, IN PERCENT }\end{array}$} \\
\hline & & & & . & . . . . & …. \\
\hline & 2 & 5 & 10 & 20 & 50 & $100 \#$ \\
\hline DAYS) & 508 & 208 & 108 & 58 & 28 & 18 \\
\hline 1 & 3.6 & 0.96 & 0.42 & 0.20 & 0.08 & 0.04 \\
\hline 3 & 3.8 & 1.0 & 0.46 & 0.22 & 0.09 & 0.05 \\
\hline 7 & 4.3 & 1.2 & 0.51 & 0.24 & 0.09 & 0.05 \\
\hline 14 & 5.2 & 1.5 & 0.65 & 0.31 & 0.12 & 0.06 \\
\hline 30 & 6.8 & 1.9 & 0.88 & 0.42 & 0.17 & 0.09 \\
\hline 60 & 8.9 & 2.8 & 1.3 & 0.68 & 0.30 & 0.16 \\
\hline 90 & 12 & 4.3 & 2.3 & 1.3 & 0.61 & 0.36 \\
\hline 120 & 15 & 6.0 & 3.2 & 1.8 & 0.87 & 0.51 \\
\hline 183 & 21 & 9.3 & 5.3 & 3.1 & 1.6 & 0.93 \\
\hline
\end{tabular}

MAGNITUDE AND PROBABILITY OF INSTANTANEOUS PEAK FLOW BASED ON PBRIOD OF RECORD 1961.66, 1968.96

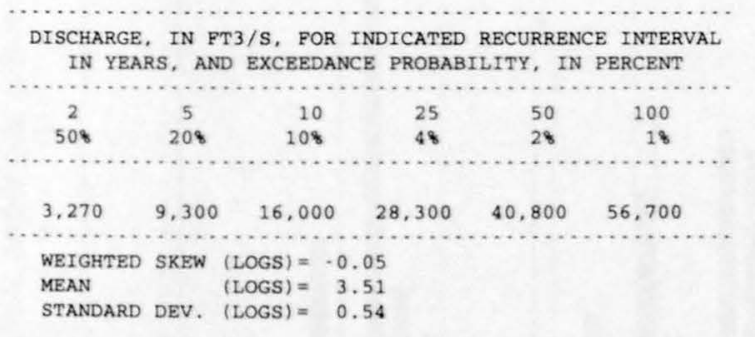

MAGNITUDE AND PROBABILITY OF ANNUAL HIGH FLOW BASED ON PERIOD OF RECORD $1962.65,1968.96$

\begin{tabular}{|c|c|c|c|c|c|c|}
\hline \multirow{2}{*}{$\begin{array}{l}\text { PERIOD } \\
\text { (CON- } \\
\text { SECU. }\end{array}$} & \multicolumn{6}{|c|}{ DISCHARGE, IN FT3/S, FOR INDICATED } \\
\hline & & $\ldots \ldots \ldots$ & $\ldots \ldots$ & & $\ldots \ldots \ldots$ & \\
\hline TIVE & 2 & 5 & 10 & 25 & 50 & $100 \#$ \\
\hline DAYS) & 508 & 208 & 108 & 48 & 28 & 18 \\
\hline 1 & 1,350 & 4,080 & 7.050 & 12,400 & 17,500 & 23.800 \\
\hline 3 & 790 & 2,310 & 3.910 & 6,710 & 9,380 & 12.600 \\
\hline 7 & 463 & 1,320 & 2,210 & 3.740 & 5,170 & 6.870 \\
\hline 15 & 283 & 786 & 1.320 & 2,280 & 3.230 & 4,390 \\
\hline 30 & 197 & 528 & 875 & 1,490 & 2,090 & 2,830 \\
\hline 60 & 138 & 358 & 595 & 1,030 & 1,470 & 2.030 \\
\hline 90 & 112 & 278 & 453 & 768 & 1,090 & 1,480 \\
\hline
\end{tabular}

DURATION TABLE OF DAILY MEAN FLOW FOR PERIOD OF RECORD 1962.65, 1968.96 DISCHARGE, IN FT3/S, WHICH WAS EQUALED OR EXCEEDED FOR INDICATED PERCENT OF TIME

\begin{tabular}{|c|c|c|c|c|c|c|c|c|c|c|c|c|c|c|c|c|}
\hline 18 & 58 & 108 & 158 & 208 & 308 & 408 & 508 & 608 & 708 & 808 & 908 & 958 & 988 & 998 & 99.58 & 99.98 \\
\hline 932 & 244 & 115 & 66 & 50 & 38 & 30 & 25 & 19 & 13 & 7.0 & 2.5 & 1.2 & 0.62 & 0.41 & 0.19 & 0.00 \\
\hline
\end{tabular}

\# Reliability of values in column is uncertain, and potential errors are large. 
ANNUAL MEAN DISCHARGE, IN CUBIC

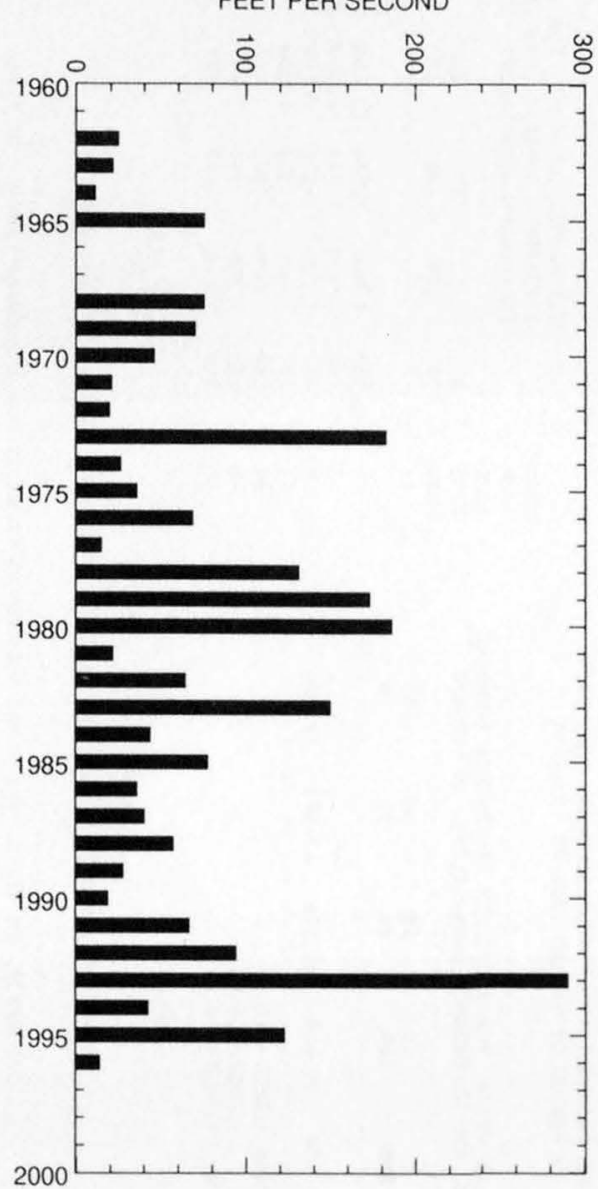

ANNUAL PEAK DISCHARGE, IN CUBIC

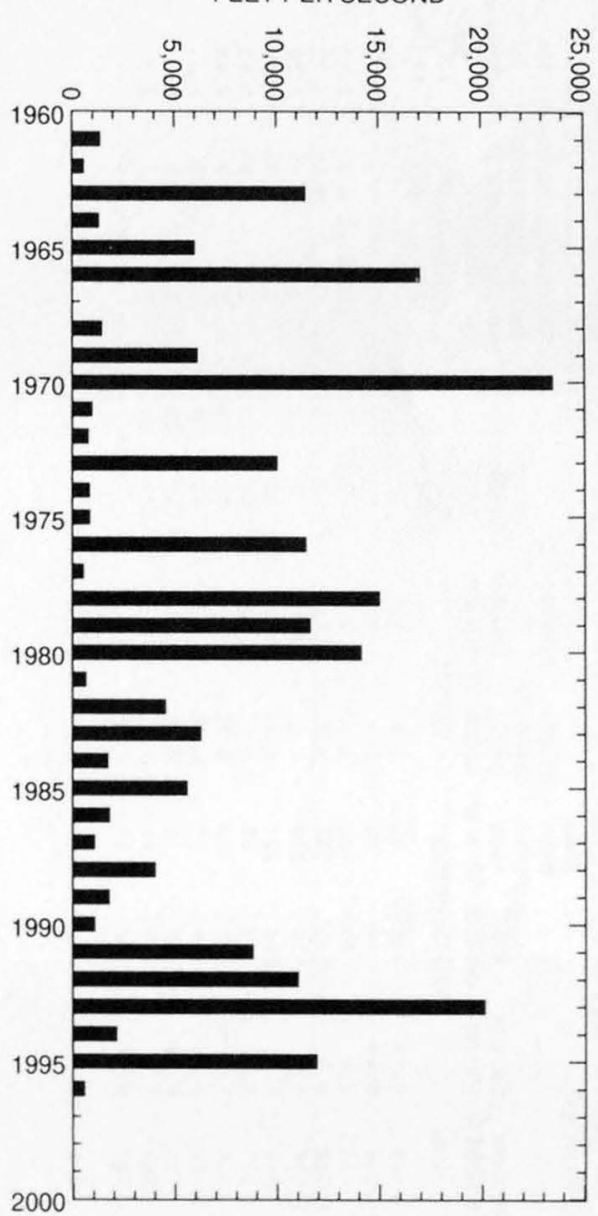


09507980 EAST VERDE RIVER NEAR CHILDS, AZ--Continued
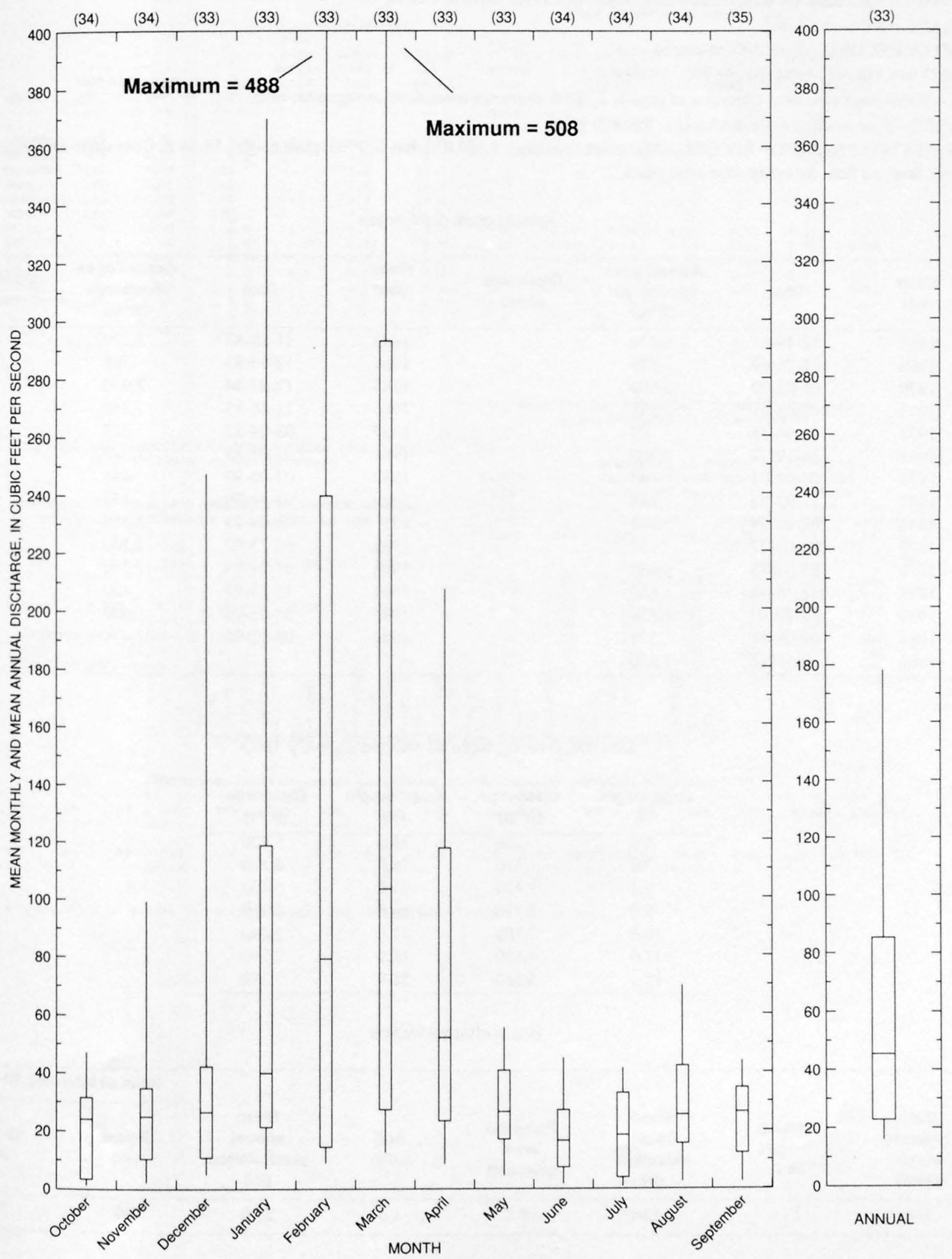


\section{WET BOTTOM CREEK NEAR CHILDS, AZ}

LOCATION.--Lat 34 09'39", long 111 41'32", in sec.36, T.9 N., R.6 E. (unsurveyed), Gila County, Hydrologic Unit 15060203, in Tonto National Forest, on right bank 1.4 mi upstream from mouth and 13 mi south of Childs.

DRAINAGE AREA.--36.4 $\mathrm{mi}^{2}$.

PERIOD OF RECORD.--June 1967 to current year.

REVISED RECORDS.--WRD Ariz. 1970: 1968(M).

GAGE.--Water-stage recorder. Elevation of gage is 2,320 ft above sea level, from topographic map.

REMARKS.--No estimated daily discharges. Records good.

EXTREMES FOR PERIOD OF RECORD.--Maximum discharge, $7,380 \mathrm{ft}^{3} / \mathrm{s} \mathrm{Jan.} 8,1993$, gage height, $18.36 \mathrm{ft}$, from slope-area measurement of peak flow; no flow for many days most years.

Annual peak discharges

\begin{tabular}{|c|c|c|c|c|c|c|c|}
\hline $\begin{array}{l}\text { Water } \\
\text { year }\end{array}$ & Date & $\begin{array}{c}\text { Annual peak } \\
\text { discharge } \\
\left(\mathrm{ft}^{3} / \mathrm{s}\right)\end{array}$ & $\begin{array}{l}\text { Discharge } \\
\text { codes }\end{array}$ & $\begin{array}{l}\text { Water } \\
\text { year }\end{array}$ & Date & $\begin{array}{c}\text { Annual peak } \\
\text { discharge } \\
\left(\mathrm{ft}^{3} / \mathrm{s}\right)\end{array}$ & $\begin{array}{c}\text { Discharge } \\
\text { codes }\end{array}$ \\
\hline 1968 & $12-19-67$ & 5,220 & & 1983 & $11-30-82$ & 3,220 & \\
\hline 1969 & $01-26-69$ & 535 & & 1984 & $12-04-83$ & 768 & \\
\hline 1970 & $09-05-70$ & 5,600 & & 1985 & $12-27-84$ & 2,090 & \\
\hline 1971 & $08-03-71$ & 158 & & 1986 & $11-26-85$ & 1,260 & \\
\hline 1972 & $12-26-71$ & 89 & & 1987 & $03-04-87$ & 522 & \\
\hline 1973 & $10-19-72$ & 3,700 & & 1988 & $02-03-88$ & 1,840 & \\
\hline 1974 & 01-09-74 & 744 & & 1989 & $02-05-89$ & 851 & \\
\hline 1975 & $11-02-74$ & 684 & & 1990 & $03-30-90$ & 157 & \\
\hline 1976 & $02-09-76$ & 5,940 & & 1991 & 03-01-91 & 3,550 & \\
\hline 1977 & $01-03-77$ & 52 & & 1992 & $08-23-92$ & 2,200 & \\
\hline 1978 & $03-02-78$ & 6,660 & & 1993 & $01-08-93$ & 7,380 & \\
\hline 1979 & $12-18-78$ & 6,680 & & 1994 & $11-15-93$ & 420 & \\
\hline 1980 & $02-19-80$ & 6,830 & & 1995 & $02-15-95$ & 6,000 & \\
\hline 1981 & $03-08-81$ & 122 & & 1996 & $08-02-96$ & 2,470 & \\
\hline 1982 & $02-11-82$ & 1,650 & & & & & \\
\hline
\end{tabular}

Discharge rating table developed January 1993

\begin{tabular}{crcc}
\hline $\begin{array}{c}\text { Gage height } \\
\text { (ft) }\end{array}$ & $\begin{array}{c}\text { Discharge } \\
\left(\mathbf{f t}^{\mathbf{3}} \mathbf{/ s}\right)\end{array}$ & $\begin{array}{c}\text { Gage height } \\
(\mathbf{f t})\end{array}$ & $\begin{array}{c}\text { Discharge } \\
\left(\mathbf{f t}^{\mathbf{3}} \mathbf{s}\right)\end{array}$ \\
\hline 6.0 & 286 & 13.0 & 5,720 \\
7.0 & 730 & 14.0 & 6,110 \\
8.0 & 1,470 & 15.0 & 6,460 \\
9.0 & 2,570 & 16.0 & 6,760 \\
10.0 & 3,880 & 17.0 & 7,040 \\
11.0 & 4,680 & 18.0 & 7,290 \\
12.0 & 5,260 & 18.4 & 7,390 \\
\hline
\end{tabular}

Basin characteristics

\begin{tabular}{cccccccc}
\hline $\begin{array}{c}\text { Main } \\
\text { channel } \\
\text { slope } \\
(\mathrm{ft} / \mathrm{mi})\end{array}$ & $\begin{array}{c}\text { Stream } \\
\text { length } \\
\text { (mi) }\end{array}$ & $\begin{array}{c}\text { Mean } \\
\text { basin } \\
\text { elevation } \\
\text { (ft) }\end{array}$ & $\begin{array}{c}\text { Forested } \\
\text { area } \\
\text { (percent) }\end{array}$ & $\begin{array}{c}\text { Soil } \\
\text { index }\end{array}$ & $\begin{array}{c}\text { Mean } \\
\text { annual } \\
\text { precipitation } \\
\text { (in) }\end{array}$ & $\begin{array}{c}\text { R-year } \\
\text { (in) }\end{array}$ & $\begin{array}{c}\text { 50-year } \\
\text { (in) }\end{array}$ \\
\hline 210 & 17.6 & 4,810 & 68.0 & 1.0 & 25.0 & 2.7 \\
\hline
\end{tabular}


09508300 WET BOTTOM CREEK NEAR CHILDS, AZ--Continued

MEAN MONTHLY AND ANNUAL DISCHARGES 1968.96

\begin{tabular}{|c|c|c|c|c|c|c|}
\hline MONTH & $\begin{array}{l}\text { MAXIMUM } \\
\text { (FT3/S) }\end{array}$ & $\begin{array}{l}\text { MINIMUM } \\
\text { (FT3/S) }\end{array}$ & $\begin{array}{c}\text { MEAN } \\
\text { (FT3/S) }\end{array}$ & $\begin{array}{l}\text { STAN- } \\
\text { DARD } \\
\text { DEVIA- } \\
\text { TION } \\
\text { (FT3/S) }\end{array}$ & $\begin{array}{l}\text { COEFFI- } \\
\text { CIENT OF } \\
\text { VARI- } \\
\text { ATION }\end{array}$ & $\begin{array}{c}\text { PERCEN? } \\
\text { OF } \\
\text { ANNUAI } \\
\text { RUNOFF }\end{array}$ \\
\hline OCTOBER & 103 & 0.01 & 5.4 & 19 & 3.6 & 2.8 \\
\hline NOVEMBER & 52 & 0.17 & 8.3 & 13 & 1.6 & 4.3 \\
\hline DECEMBER & 111 & 0.28 & 22 & 35 & 1.6 & 11.6 \\
\hline JANUARY & 373 & 0.26 & 43 & 73 & 1.7 & 22.4 \\
\hline FEBRUARY & 345 & 0.56 & 46 & 70 & 1.5 & 23.8 \\
\hline MARCH & 321 & 0.29 & 49 & 71 & 1.5 & 25.3 \\
\hline APRIL & 38 & 0.09 & 8.8 & 11 & 1.2 & 4.6 \\
\hline MAY & 2.1 & 0.00 & 0.63 & 0.59 & 0.94 & 0.3 \\
\hline JUNE & 0.55 & 0.00 & 0.09 & 0.14 & 1.5 & 0.0 \\
\hline JULY & 12 & 0.00 & 1.0 & 2.6 & 2.6 & 0.5 \\
\hline AUGUST & 48 & 0.00 & 4.8 & 11 & 2.2 & 2.5 \\
\hline SEPTEMBER & 27 & 0.00 & 3.3 & 6.1 & 1.8 & 1.7 \\
\hline NNUAL & 47 & 0.45 & 16 & 14 & 0.87 & 100 \\
\hline
\end{tabular}

MAGNITUDE AND PROBABILITY OF INSTANTANEOUS PEAK FLOW BASED ON PERIOD OF RECORD 1968.96

DISCHARGE, IN FT3/S, FOR INDICATED RECURRENCE INTERVAL IN YEARS, AND EXCEEDANCE PROBABILITY, IN PERCENT

$\begin{array}{cccccc}2 & 5 & 10 & 25 & 50 & 100 \\ 508 & 208 & 108 & 48 & 28 & 18\end{array}$

$1,740 \quad 4,340 \quad 6,760 \quad 10,600 \quad 13,900 \quad 17,600$

WEIGHTED SKEW $($ LOGS $)=-0.33$

MEAN (LOGS) $=3.21$

STANDARD DEV. $($ LOGS $)=0.50$
MAGNITUDE AND PROBABILITY OF ANNUAL LOW FLOW BASED ON PERIOD OF RECORD 1969.96

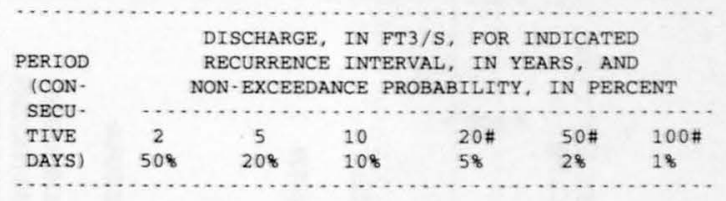

$\begin{array}{rrrrrrr}1 & & & & & & \\ 3 & & & & & & \\ 7 & & & & & & \\ 14 & & & & & & \\ 30 & 0.00 & 0.00 & 0.00 & 0.00 & 0.00 & 0.00 \\ 60 & 0.02 & 0.00 & 0.00 & 0.00 & 0.00 & 0.00 \\ 90 & 0.06 & 0.01 & 0.00 & 0.00 & 0.00 & 0.00 \\ 120 & 0.15 & 0.05 & 0.00 & 0.00 & 0.00 & 0.00 \\ 183 & 0.56 & 0.13 & 0.06 & 0.03 & 0.01 & 0.01\end{array}$

MAGNITUDE AND PROBABILITY OF ANNUAL HIGH FLOW BASED ON PERIOD OF RECORD 1968.96

\begin{tabular}{|c|c|c|c|c|c|c|}
\hline \multirow{2}{*}{$\begin{array}{l}\text { PERIOD } \\
\text { /CON- } \\
\text { SECU- }\end{array}$} & & \multicolumn{5}{|c|}{$\begin{array}{l}\text { DISCHARGE, IN FT3/S, FOR INDICATED } \\
\text { RECURRENCE INTERVAL, IN YEARS, AND }\end{array}$} \\
\hline & & & & & & \\
\hline $\begin{array}{l}\text { TIVE } \\
\text { DAYS) }\end{array}$ & $\begin{array}{c}2 \\
508\end{array}$ & $\begin{array}{c}5 \\
208\end{array}$ & $\begin{array}{l}10 \\
108\end{array}$ & $\begin{array}{l}25 \# \\
48\end{array}$ & $\begin{array}{l}50 \# \\
28\end{array}$ & $\begin{array}{c}100 \# \\
18\end{array}$ \\
\hline 1 & 555 & 1,650 & 2,630 & 4,030 & 5.120 & 6,200 \\
\hline 3 & 327 & 880 & 1.310 & 1.850 & 2.220 & 2,560 \\
\hline 7 & 194 & 498 & 719 & 979 & 1.150 & 1,290 \\
\hline 15 & 109 & 281 & 414 & 583 & 701 & 811 \\
\hline 30 & 67 & 173 & 261 & 379 & 467 & 553 \\
\hline 60 & 45 & 118 & 178 & 260 & 321 & 381 \\
\hline 90 & 33 & 88 & 134 & 198 & 247 & 296 \\
\hline
\end{tabular}

DURATION TABLE OF DAILY MEAN FLOW FOR PERIOD OF RECORD 1968.96

DISCHARGE, IN FT3/S, WHICH WAS EQUALED OR EXCEEDED FOR INDICATED PERCENT OF TIME

\begin{tabular}{|c|c|c|c|c|c|c|c|c|c|c|c|c|c|c|c|c|}
\hline 18 & 58 & 108 & 158 & 208 & 308 & 408 & 508 & 608 & 708 & 808 & 908 & 958 & 988 & 998 & 99.58 & 99.98 \\
\hline 77 & 61 & 24 & 12 & 6.6 & 2.1 & 0.90 & 0.56 & 0.36 & 0.19 & 0.05 & 0.00 & 0.00 & 0.00 & 0.00 & 0.00 & 0.00 \\
\hline
\end{tabular}

\# Reliability of values in column is uncertain, and potential errors are large. 
ANNUAL. MEAN DISCHARGE, IN CUBIC FEET PER SECOND

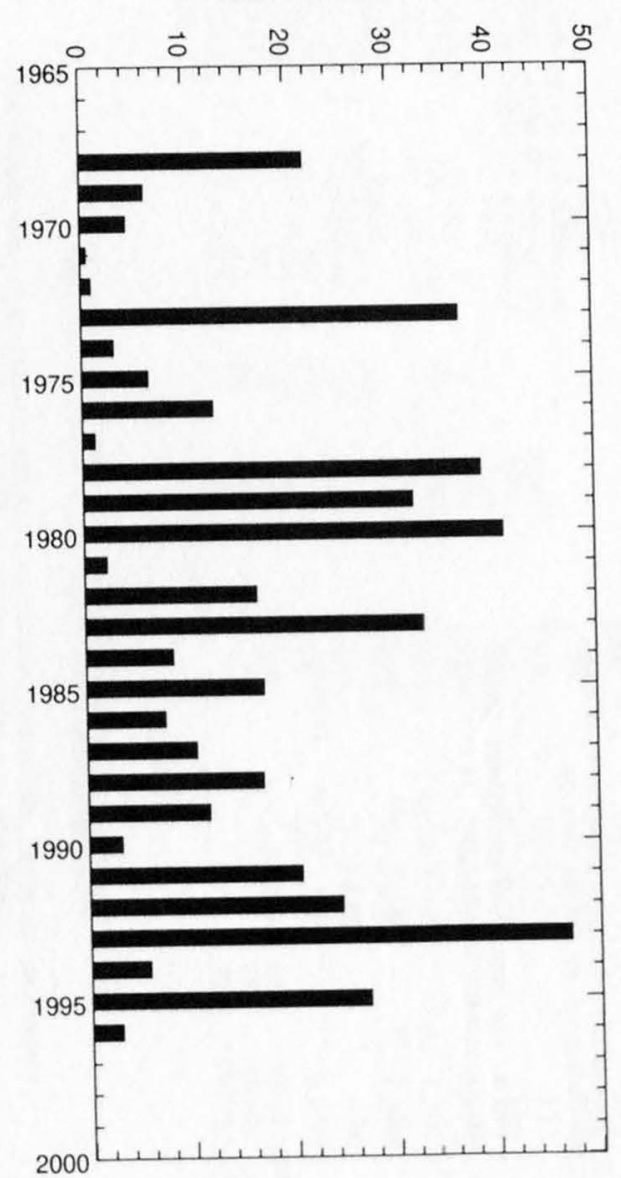

ANNUAL PEAK DISCHARGE, IN CUBIC FEET PER SECOND

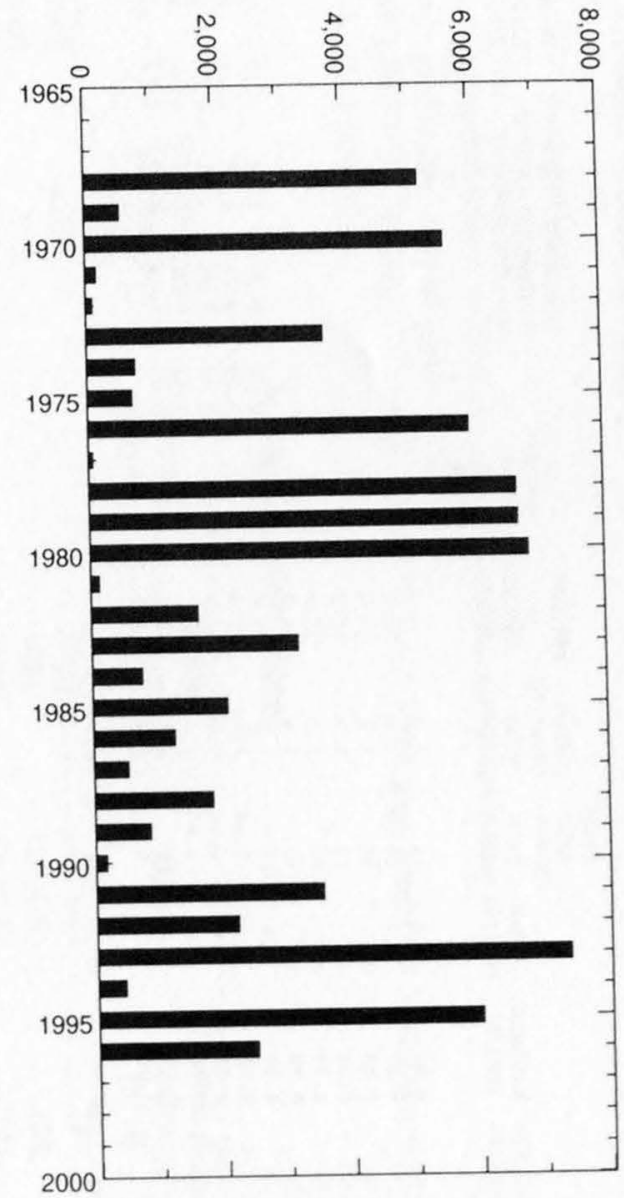


GILA RIVER BASIN

739

09508300 WET BOTTOM CREEK NEAR CHILDS, AZ--Continued
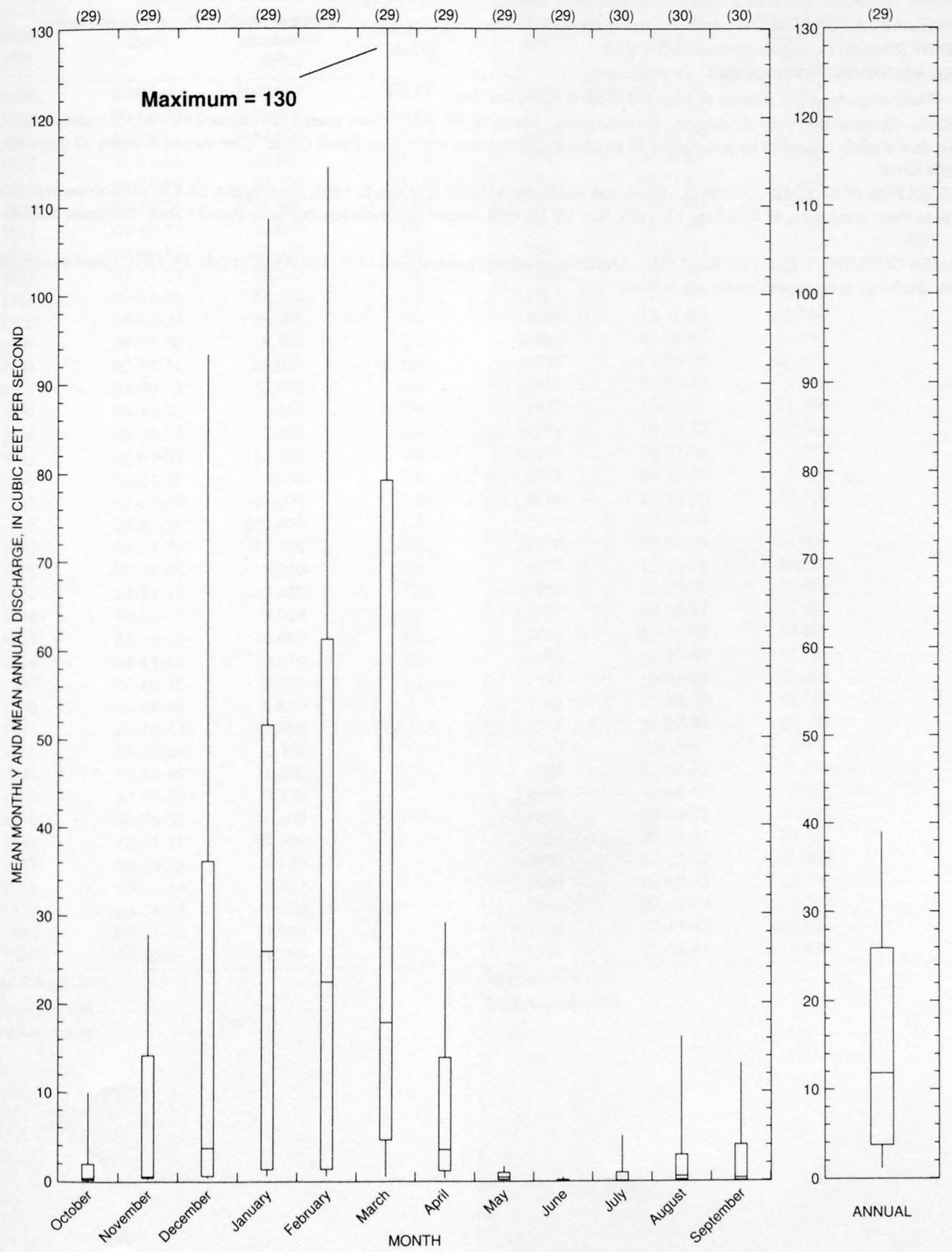

ANNUAL 


\section{VERDE RIVER BELOW TANGLE CREEK, ABOVE HORSESHOE DAM, AZ}

LOCATION.--Lat 34'04'23", long 111 42'56", in sec.35, T.9 N., R.6 E. (unsurveyed), Yavapai County, Hydrologic Unit 15060203, in Tonto National Forest, on right bank 1.3 mi downstream from Tangle Creek and 9 mi upstream from Horseshoe Dam.

DRAINAGE AREA.--5,858 $\mathrm{mi}^{2}$, of which $365 \mathrm{mi}^{2}$ is noncontributing, including $357 \mathrm{mi}^{2}$ in Aubrey Valley Playa, a closed basin.

PERIOD OF RECORD.--August 1945 to current year.

REVISED RECORDS.--WDR AZ-89-1: Drainage area.

GAGE.--Water-stage recorder. Datum of gage is 2,029.0 $\mathrm{ft}$ above sea level.

REMARKS.--No estimated daily discharges. Records good. About 12,500 acres above station are irrigated by surface water and ground water. Low flow slightly regulated by powerplant $32 \mathrm{mi}$ above station, using water from Fossil Creek. This station is above all major reservoirs on Verde River.

EXTREMES FOR PERIOD OF RECORD.--Maximum discharge, 145,000 $\mathrm{ft}^{3} / \mathrm{s}$ Jan. 8, 1993, gage height, $23.4 \mathrm{ft}$, from slope-area measurement of peak flow; minimum, $48 \mathrm{ft}^{3} / \mathrm{s}$ June 17,1956 , July 18, 19, 1958, caused by power regulation on Fossil Creek; minimum daily, $61 \mathrm{ft}^{3} / \mathrm{s}$ July $18,1958$.

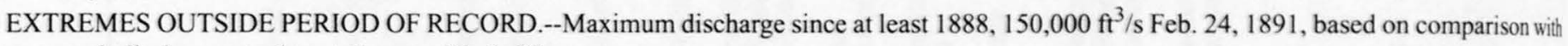
peak discharge at other stations on Verde River. 
GILA RIVER BASIN

09508500 VERDE RIVER BELOW TANGLE CREEK, ABOVE HORSESHOE DAM, AZ

Annual peak discharges

\begin{tabular}{|c|c|c|c|c|c|c|c|}
\hline $\begin{array}{l}\text { Water } \\
\text { year }\end{array}$ & Date & $\begin{array}{c}\text { Annual peak } \\
\text { discharge } \\
\left(\mathrm{ft}^{3} / \mathbf{s}\right)\end{array}$ & $\begin{array}{l}\text { Discharge } \\
\text { codes }\end{array}$ & $\begin{array}{l}\text { Water } \\
\text { year }\end{array}$ & Date & $\begin{array}{c}\text { Annual peak } \\
\text { discharge } \\
\left(\mathrm{ft}^{3} / \mathrm{s}\right)\end{array}$ & $\begin{array}{l}\text { Discharge } \\
\text { codes }\end{array}$ \\
\hline 1000 & $00-00-00$ & ${ }^{\mathrm{I}} 180,000$ & ES,PF & 1958 & $03-23-58$ & 21,100 & \\
\hline 1760 & $00-00-00$ & ${ }^{1} 130,000$ & ES,PF & 1959 & $08-17-59$ & 6,060 & \\
\hline 1891 & $02-2491$ & ${ }^{2} 150,000$ & ES,HP & 1960 & $12-26-59$ & 23,400 & \\
\hline 1906 & $11-27-05$ & ${ }^{3} 96,000$ & ES,HP & 1961 & $08-23-61$ & 2,800 & \\
\hline 1916 & $01-20-16$ & 68,900 & ES,HP & 1962 & $02-13-62$ & 13,300 & \\
\hline 1920 & $02-22-20$ & ${ }^{4} 95,000$ & ES,HP & 1963 & $08-22-63$ & 18,900 & \\
\hline 1925 & $09-17-25$ & 20,000 & ES & 1964 & $08-27-64$ & 6,910 & \\
\hline 1926 & $04-06-26$ & 32,000 & ES & 1965 & $01-07-65$ & 25,700 & \\
\hline 1927 & $02-17-27$ & 70,000 & ES & 1966 & $12-22-65$ & 39,300 & \\
\hline 1928 & $02-05-28$ & 14,000 & ES & 1967 & $12-07-66$ & 53,000 & \\
\hline 1929 & 04-05-29 & 26,000 & ES & 1968 & $12-19-67$ & 32,600 & \\
\hline 1930 & $08-09-30$ & 8,100 & ES & 1969 & $01-26-69$ & 45,800 & \\
\hline 1931 & $02-14-31$ & 34,000 & ES & 1970 & 09-06-70 & 61,900 & \\
\hline 1932 & $02-09-32$ & 53,000 & ES & 1971 & $08-03-71$ & 3,030 & \\
\hline 1933 & $03-13-33$ & 1,660 & ES & 1972 & $12-27-71$ & 21,100 & \\
\hline 1934 & $08-25-34$ & 3,300 & ES & 1973 & $10-20-72$ & 63,400 & \\
\hline 1935 & $02-07-35$ & 14,300 & ES & 1974 & $08-02-74$ & 1500 & \\
\hline 1936 & $02-24-36$ & 12,000 & ES & 1975 & $04-15-75$ & 5,420 & \\
\hline 1937 & $02-07-37$ & 63,000 & ES & 1976 & $02-10-76$ & 39,900 & \\
\hline 1938 & $03-04-38$ & 100,000 & ES & 1977 & $08-24-77$ & 1,620 & \\
\hline 1939 & $09-14-39$ & 17,700 & ES & 1978 & $03-01-78$ & 91,400 & \\
\hline 1940 & $02-27-40$ & 5,020 & ES & 1979 & $12-19-78$ & 94,000 & \\
\hline 1941 & $03-14-41$ & 43,800 & ES & 1980 & $02-15-80$ & 94,800 & \\
\hline 1942 & $10-14-41$ & 3,510 & ES & 1981 & $04-06-81$ & 2,030 & \\
\hline 1943 & $08-14-43$ & 16,600 & ES & 1982 & $03-12-82$ & 42,100 & \\
\hline 1944 & $03-14-44$ & 7,530 & ES & 1983 & $12-23-82$ & 22,400 & \\
\hline 1945 & $03-16-45$ & 9,710 & ES & 1984 & $10-01-83$ & 27,200 & \\
\hline 1946 & $04-08-46$ & 8,660 & & 1985 & $12-28-84$ & 19,300 & \\
\hline 1947 & 09-19-47 & 11,500 & & 1986 & $11-30-85$ & 10,300 & \\
\hline 1948 & $03-25-48$ & 2,560 & & 1987 & $03-10-87$ & 5,000 & \\
\hline 1949 & $01-13-49$ & 11,000 & & 1988 & $02-03-88$ & 19,800 & \\
\hline 1950 & $10-19-49$ & 9,330 & & 1989 & $02-05-89$ & 2,670 & \\
\hline 1951 & $08-30-51$ & 16,400 & & 1990 & 09-03-90 & 2,790 & \\
\hline 1952 & $12-31-51$ & 81,600 & & 1991 & $03-02-91$ & 34,300 & \\
\hline 1953 & $08-29-53$ & 6,390 & & 1992 & $08-23-92$ & 27,200 & \\
\hline 1954 & $03-23-54$ & 19,700 & & 1993 & $01-08-93$ & 145,000 & \\
\hline 1955 & $08-23-55$ & 11,600 & & 1994 & $02-08-94$ & 4,770 & \\
\hline 1956 & $07-31-56$ & 12,800 & & 1995 & $02-15-95$ & ${ }^{5} 108,000$ & \\
\hline 1957 & $01-10-57$ & 14,500 & & 1996 & $08-03-96$ & 2,450 & \\
\hline
\end{tabular}

'Ely and Baker (1985).

${ }^{2}$ Highest since 1888.

${ }^{4}$ Highest since 1906.

${ }^{5}$ Highest since 1920

${ }^{3}$ Highest since 1891. 
09508500 VERDE RIVER BELOW TANGLE CREEK, ABOVE HORSESHOE DAM, AZ--Continued

Discharge rating table developed February 1995

\begin{tabular}{cccc}
\hline $\begin{array}{c}\text { Gage height } \\
(\mathbf{f t})\end{array}$ & $\begin{array}{c}\text { Discharge } \\
\left(\mathbf{f t}^{\mathbf{3}} \mathbf{s}\right)\end{array}$ & $\begin{array}{c}\text { Gage height } \\
(\mathbf{f t})\end{array}$ & $\begin{array}{c}\text { Discharge } \\
\left(\mathbf{f t}^{\mathbf{3}} \mathbf{s}\right)\end{array}$ \\
\hline 8.0 & 2,000 & 17.0 & 40,750 \\
9.0 & 3,260 & 19.0 & 63,390 \\
11.0 & 7,230 & 21.0 & 94,340 \\
13.0 & 14,040 & 23.0 & 135,400 \\
15.0 & 24,780 & 23.5 & 147,500 \\
\hline
\end{tabular}

Basin characteristics

\begin{tabular}{cccccccc}
\hline $\begin{array}{c}\text { Main } \\
\text { channel } \\
\text { slope } \\
(\mathrm{ft} / \mathrm{mi})\end{array}$ & $\begin{array}{c}\text { Stream } \\
\text { length } \\
(\mathrm{mi})\end{array}$ & $\begin{array}{c}\text { Mean } \\
\text { basin } \\
\text { elevation } \\
(\mathrm{ft})\end{array}$ & $\begin{array}{c}\text { Forested } \\
\text { area } \\
\text { (percent) }\end{array}$ & $\begin{array}{c}\text { Soil } \\
\text { index }\end{array}$ & $\begin{array}{c}\text { Mean } \\
\text { annual } \\
\text { precipitation } \\
\text { (in) }\end{array}$ & $\begin{array}{c}\text { Rainfall intensity, 24-hour } \\
\text { (in) }\end{array}$ & $\begin{array}{c}50 \text {-year } \\
\text { (in) }\end{array}$ \\
\hline 16.2 & 209 & 5,470 & 67.0 & 2.4 & 18.4 & 2.1 \\
\hline
\end{tabular}


09508500 VERDE RIVER BELOW TANGLE CREEK, ABOVE HORSESHOE DAM, AZ--Continued

MEAN MONTHLY AND ANNUAL DISCHARGES 1946.96

\begin{tabular}{|c|c|c|c|c|c|c|}
\hline MONTH & $\begin{array}{l}\text { MAXIMUM } \\
(\text { FT } 3 / \mathrm{S})\end{array}$ & $\begin{array}{l}\text { MINIMUM } \\
\text { (FT3/S) }\end{array}$ & $\begin{array}{c}\text { MEAN } \\
(\mathrm{FT} 3 / \mathrm{S})\end{array}$ & $\begin{array}{l}\text { STAN - } \\
\text { DARD } \\
\text { DEVIA- } \\
\text { TION } \\
\text { (FT3/S) }\end{array}$ & $\begin{array}{l}\text { COEFFI- } \\
\text { CIENT OF } \\
\text { VARI- } \\
\text { ATION }\end{array}$ & $\begin{array}{c}\text { PERCENT } \\
\text { OF } \\
\text { ANNUAL } \\
\text { RUNOFF }\end{array}$ \\
\hline OCTOBER & 4,190 & 155 & 333 & 579 & 1.7 & 4.7 \\
\hline NOVEMBER & 1,380 & 192 & 370 & 297 & 0.80 & 5.2 \\
\hline DECEMBER & 4,640 & 227 & 754 & 1,010 & 1.3 & 10.6 \\
\hline JANUARY & 12,400 & 224 & 879 & 1,760 & 2.0 & 12.3 \\
\hline FEBRUARY & 11,000 & 220 & 1,250 & 2,000 & 1.6 & 17.5 \\
\hline MARCH & 10,400 & 194 & 1.560 & 1,810 & 1.2 & 21.8 \\
\hline APRIL & 5,640 & 155 & 849 & 1,030 & 1.2 & 11.9 \\
\hline MAY & 1,320 & 113 & 218 & 173 & 0.80 & 3.1 \\
\hline JUNE & 316 & 83 & 135 & 42 & 0.31 & 1.9 \\
\hline JULY & 430 & 76 & 179 & 71 & 0.40 & 2.5 \\
\hline AUGUST & 1,180 & 127 & 334 & 232 & 0.70 & 4.7 \\
\hline SEPTEMBER & 1,460 & 99 & 273 & 211 & 0.77 & 3.8 \\
\hline ANNUAL & 2.230 & 189 & 591 & 437 & 0.74 & 100 \\
\hline
\end{tabular}

MAGNITUDE AND PROBABILITY OF INSTANTANEOUS PEAK FLOW BASED ON PERIOD OF RECORD 1000, 1925.96

DISCHARGE, IN FT3/S, FOR INDICATED RECURRENCE INTERVAL IN YEARS, AND EXCEEDANCE PROBABILITY, IN PERCENT

$\begin{array}{ccccccc}2 & 5 & 10 & 25 & 50 & 100 \\ 508 & 208 & 108 & 48 & 28 & 18 \\ 16,000 & 41,600 & 67,300 & 110,800 & 151,700 & 200,300\end{array} \ldots$

MAGNITUDE AND PROBABILITY OF ANNUAL LOW PLOW BASED ON PERIOD OF RECORD 1947.96

\begin{tabular}{|c|c|c|c|c|c|c|}
\hline \multirow{3}{*}{$\begin{array}{l}\text { PERIOD } \\
\text { /CON - } \\
\text { SECU- }\end{array}$} & \multicolumn{3}{|c|}{$\begin{array}{l}\text { DISCHARGE, IN } \\
\text { RECURRENCE INI }\end{array}$} & \multicolumn{3}{|c|}{ 3, FOR INDICATED } \\
\hline & \multicolumn{6}{|c|}{ NON-EXCEEDANCE PROBABILITY, IN PERCENT } \\
\hline & $\cdots$ & $\cdots \cdots$ & & & & \\
\hline TIVE & 2 & 5 & 10 & 20 & 50 & 100 \\
\hline DAYS) & 508 & $20 \%$ & 108 & 58 & $2 \%$ & 18 \\
\hline 1 & 91 & 76 & 70 & 65 & 61 & 58 \\
\hline 3 & 94 & 79 & 72 & 68 & 63 & 60 \\
\hline 7 & 98 & 82 & 75 & 70 & 65 & 62 \\
\hline 14 & 103 & 86 & 79 & 73 & 68 & 65 \\
\hline 30 & 111 & 93 & 85 & 79 & 72 & 68 \\
\hline 60 & 128 & 107 & 98 & 91 & 83 & 79 \\
\hline 90 & 146 & 123 & 112 & 104 & 96 & 90 \\
\hline 120 & 173 & 147 & 133 & 122 & 109 & 101 \\
\hline 183 & 198 & 171 & 163 & 158 & 154 & 152 \\
\hline
\end{tabular}

MAGNITUDE AND PROBABILITY OF ANNUAL HIGH FLOW BASED ON PERIOD OF RECORD 1946-96

\begin{tabular}{|c|c|c|c|c|c|c|}
\hline \multirow{3}{*}{$\begin{array}{l}\text { PERIOD } \\
\text { (CON- } \\
\text { SECU- }\end{array}$} & \multicolumn{6}{|c|}{ DISCHARGE, IN FT3/S, FOR INDICATED } \\
\hline & & \multicolumn{3}{|c|}{ EXCEEDANCE PROBABILITY, } & \multicolumn{2}{|c|}{ IN PERCENT } \\
\hline & & & & & & \\
\hline TIVE & 2 & 5 & 10 & 25 & 50 & 100 \\
\hline DAYS) & 508 & 208 & 108 & 48 & 28 & 18 \\
\hline 1 & 8,220 & 25,200 & 44.600 & 81,600 & 120,000 & 169.000 \\
\hline 3 & 5,660 & 16,000 & 27.000 & 46,900 & 66,400 & 90,600 \\
\hline 7 & 3,640 & 9,510 & 15,500 & 25,600 & 35,300 & 47,000 \\
\hline 15 & 2,430 & 5,890 & 9,320 & 15,100 & 20,700 & 27,300 \\
\hline 30 & 1,710 & 3,950 & 6,130 & 9.830 & 13,400 & 17,600 \\
\hline 60 & 1,190 & 2,690 & 4,200 & 6,840 & 9,460 & 12,700 \\
\hline 90 & 976 & 2,130 & 3,260 & 5,230 & 7,150 & 9,540 \\
\hline
\end{tabular}

DURATION TABLE OF DAILY MEAN FLOW FOR PERIOD OF RECORD 1946.96 DISCHARGE, IN FT3/S, WHICH WAS EQUALED OR EXCEEDED FOR INDICATED PERCENT OF TIME

\begin{tabular}{|c|c|c|c|c|c|c|c|c|c|c|c|c|c|c|c|c|}
\hline 18 & 58 & 108 & 158 & 208 & 308 & 408 & 508 & 608 & 708 & 808 & 908 & 958 & 988 & 998 & 99.58 & 99.98 \\
\hline 330 & 2,130 & 942 & 561 & 412 & 310 & 267 & 240 & 212 & 182 & 153 & 123 & 104 & 89 & 81 & 76 & 64 \\
\hline
\end{tabular}




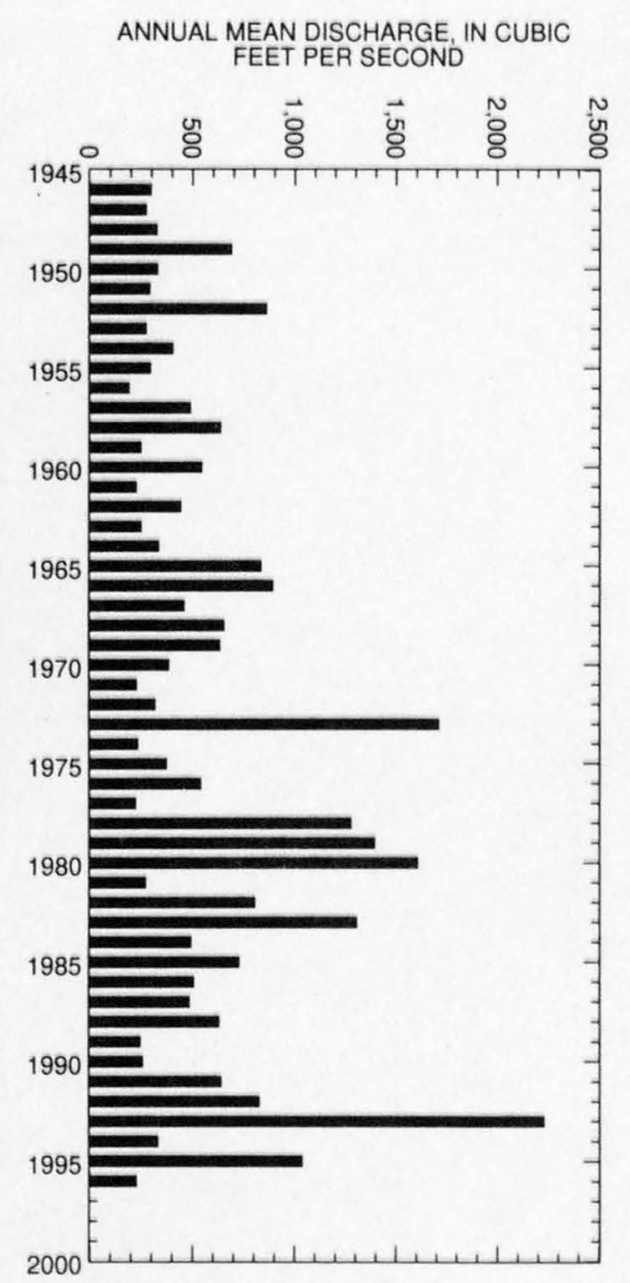

ANNUAL PEAK DISCHARGE, IN CUBIC

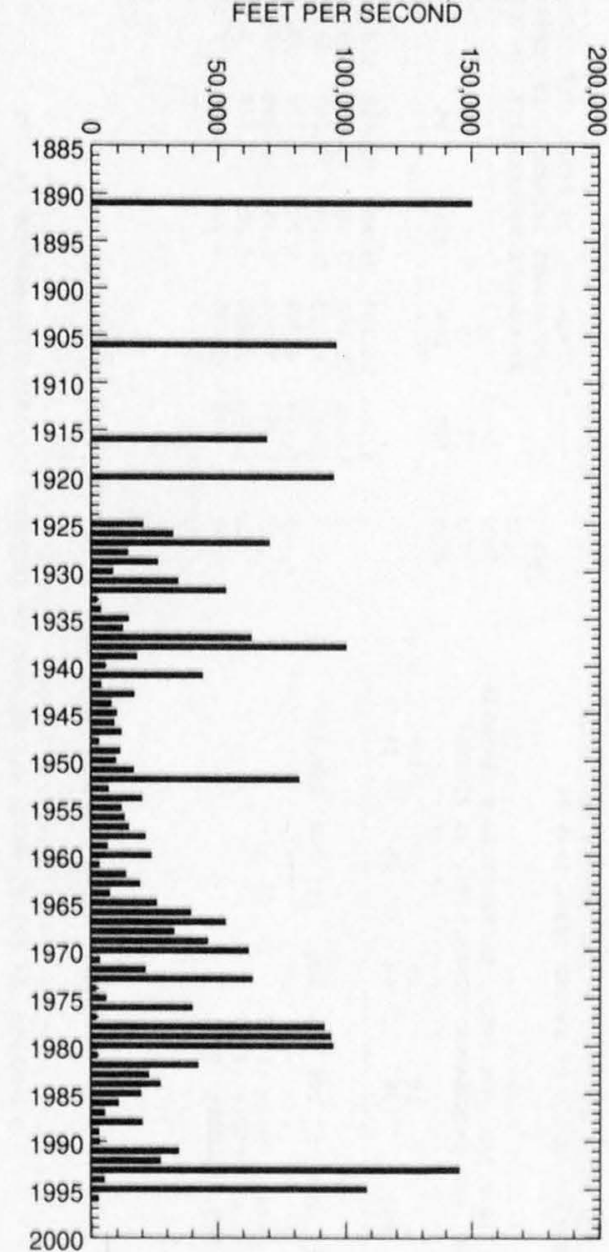

ANNUAL PEAK DISCHARGE, IN CUBIC

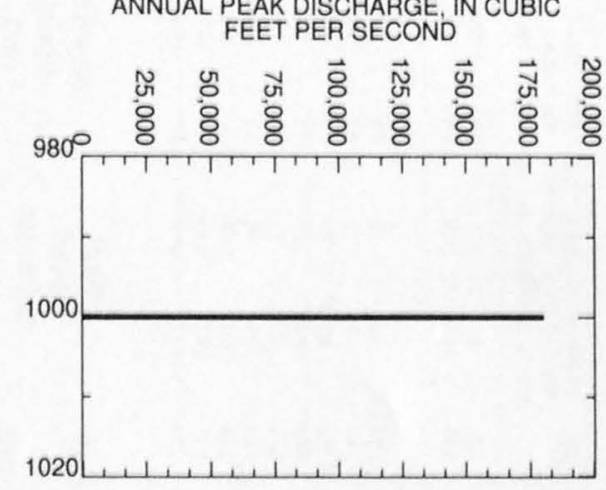

ANNUAL PEAK DISCHARGE, IN CUBIC FEET PER SECOND

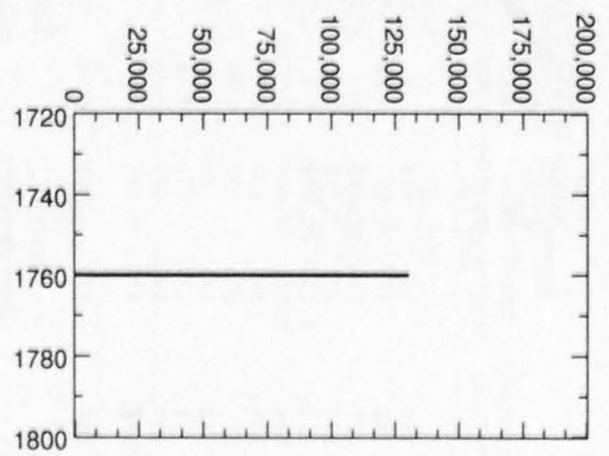


09508500 VERDE RIVER BELOW TANGLE CREEK, ABOVE HORSESHOE DAM, AZ--Continued

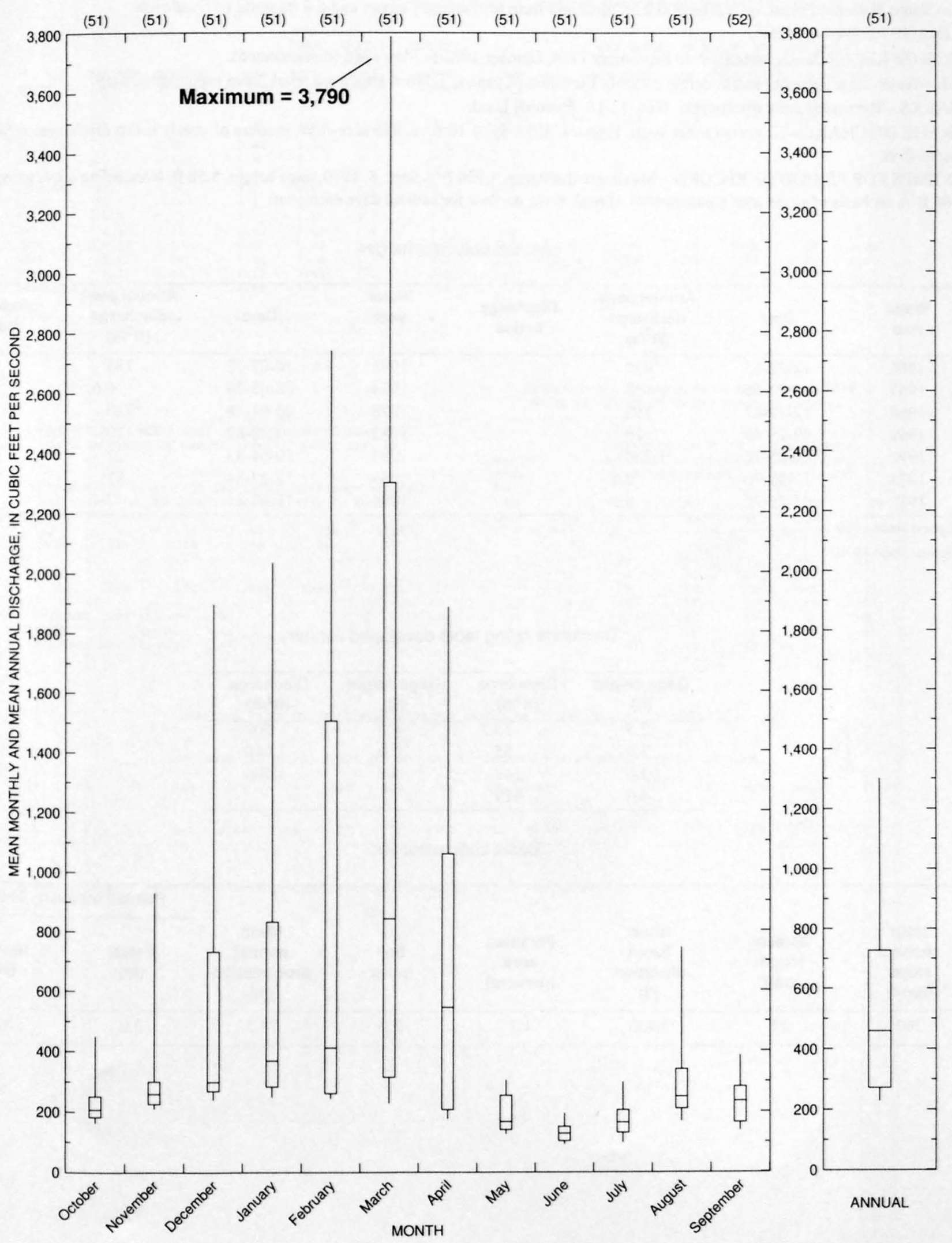




\section{WEST FORK SYCAMORE CREEK ABOVE MCFARLAND CANYON,}

NEAR SUNFLOWER, AZ

LOCATION.--Lat 33.57'38", long $111^{\prime 2} 29^{\prime} 12^{\prime \prime}$, in SE $1 /{ }_{4} \mathrm{SW}^{1} / 4$ sec.12, T.7 N., R.8 E. (unsurveyed), Maricopa County, Hydrologic Unit 15060203 , in Tonto National Forest, on left bank 0.2 mi upstream from McFarland Canyon and 6.8 mi north of Sunflower.

DRAINAGE AREA.--4.58 $\mathrm{mi}^{2}$.

PERIOD OF RECORD.--October 1965 to September 1974, October 1982 to May 1986 (discontinued).

GAGE.--Water-stage recorder and concrete control. Elevation of gage is 4,380 ft above sea level, from topographic map.

REMARKS.--Estimated daily discharges: Nov. 12-18. Records good.

AVERAGE DISCHARE.--13 years (water years 1966-74, 1983-86) $1.18 \mathrm{ft}^{3} / \mathrm{s}, 855$ acre- $\mathrm{ft} / \mathrm{yr}$; median of yearly mean discharges, $0.54 \mathrm{ft}^{3} / \mathrm{s}, 390$ acre-ft/yr.

EXTREMES FOR PERIOD OF RECORD.--Maximum discharge, 1,700 ft ${ }^{3} / \mathrm{s}$ Sept. 5,1970 , gage height, $5.50 \mathrm{ft}$, from rating curve extended above $68 \mathrm{ft}^{3} / \mathrm{s}$ on basis of slope-area measurement of peak flow; no flow for several days each year.

Annual peak discharges

\begin{tabular}{|c|c|c|c|c|c|c|c|}
\hline $\begin{array}{l}\text { Water } \\
\text { year }\end{array}$ & Date & $\begin{array}{c}\text { Annual peak } \\
\text { discharge } \\
\left(\mathrm{ft}^{3} / \mathrm{s}\right)\end{array}$ & $\begin{array}{c}\text { Discharge } \\
\text { codes }\end{array}$ & $\begin{array}{l}\text { Water } \\
\text { year }\end{array}$ & Date & $\begin{array}{c}\text { Annual peak } \\
\text { discharge } \\
\left(\mathrm{ft}^{3} / \mathrm{s}\right)\end{array}$ & $\begin{array}{c}\text { Discharge } \\
\text { codes }\end{array}$ \\
\hline 1966 & $12-22-65$ & ${ }^{\mathrm{I}} 430$ & & 1973 & $10-07-72$ & 185 & \\
\hline 1967 & $12-07-66$ & 13 & & 1974 & $08-05-74$ & 6.6 & \\
\hline 1968 & $12-19-67$ & 152 & & 1978 & $03-01-78$ & 2720 & \\
\hline 1969 & $02-25-69$ & 10 & & 1983 & $11-30-82$ & 178 & \\
\hline 1970 & $09-05-70$ & ${ }^{\mathrm{I}} 1,700$ & & 1984 & $10-01-83$ & 21 & \\
\hline 1971 & $11-30-70$ & 0.3 & & 1985 & $12-27-84$ & 97 & \\
\hline 1972 & $06-22-72$ & 0.3 & & 1986 & $11-30-85$ & 12 & \\
\hline
\end{tabular}

${ }^{1}$ Highest since 1959.

${ }^{2}$ Highest since 1970.

Discharge rating table developed January ---

\begin{tabular}{cccc}
\hline $\begin{array}{c}\text { Gage height } \\
(\mathrm{ft})\end{array}$ & $\begin{array}{c}\text { Discharge } \\
\left(\mathrm{ft}^{3} / \mathbf{s}\right)\end{array}$ & $\begin{array}{c}\text { Gage height } \\
(\mathrm{ft})\end{array}$ & $\begin{array}{c}\text { Discharge } \\
\left(\mathrm{ft}^{3} / \mathbf{s}\right)\end{array}$ \\
\hline 2.5 & 13.7 & 4.5 & 600 \\
3.0 & 55 & 5.0 & 1,040 \\
3.5 & 148 & 5.5 & 1,700 \\
4.0 & 319 & & \\
\hline
\end{tabular}

Basin characteristics

\begin{tabular}{cccccccc}
\hline $\begin{array}{c}\text { Main } \\
\text { channel } \\
\text { slope } \\
(\mathrm{ft} / \mathrm{mi})\end{array}$ & $\begin{array}{c}\text { Stream } \\
\text { length } \\
\text { (mi) }\end{array}$ & $\begin{array}{c}\text { Mean } \\
\text { basin } \\
\text { elevation } \\
\text { (ft) }\end{array}$ & $\begin{array}{c}\text { Forested } \\
\text { area } \\
\text { (percent) }\end{array}$ & $\begin{array}{c}\text { Soil } \\
\text { index }\end{array}$ & $\begin{array}{c}\text { Mean } \\
\text { annual } \\
\text { precipitation } \\
\text { (in) }\end{array}$ & $\begin{array}{c}\text { R-year } \\
\text { (in) }\end{array}$ & $\begin{array}{c}50 \text {-year } \\
\text { (in) }\end{array}$ \\
\hline 260 & 3.8 & 5,430 & 1.3 & 3.0 & 24.5 & 3.0 \\
\hline
\end{tabular}


09510070 WEST FORK SYCAMORE CREEK ABOVE MCFARLAND CANYON, NEAR SUNFLOWER, AZ--Continued

MEAN MONTHLY AND ANNUAL DISCHARGES $1966 \cdot 74,1983 \cdot 85$

\begin{tabular}{|c|c|c|c|c|c|c|}
\hline MONTH & $\begin{array}{l}\text { MAXIMUM } \\
(\text { FT } 3 / S)\end{array}$ & $\begin{array}{l}\text { MINIMUM } \\
\text { (FT3/S) }\end{array}$ & $\begin{array}{c}\text { MEAN } \\
(\mathrm{FT} 3 / \mathrm{S})\end{array}$ & $\begin{array}{l}\text { STAN- } \\
\text { DARD } \\
\text { DEVIA- } \\
\text { TION } \\
(\mathrm{FT} 3 / \mathrm{S})\end{array}$ & $\begin{array}{l}\text { COEFFI- } \\
\text { CIENT OF } \\
\text { VARI- } \\
\text { ATION }\end{array}$ & $\begin{array}{c}\text { PERCENT } \\
\text { OF } \\
\text { ANNUAL } \\
\text { RUNOFF }\end{array}$ \\
\hline OCTOBER & 4.3 & 0.00 & 0.53 & 1.2 & 2.4 & 3.7 \\
\hline NOVEMBER & 2.7 & 0.00 & 0.44 & 0.84 & 1.9 & 3.1 \\
\hline DECEMBER & 17 & 0.00 & 3.4 & 4.9 & 1.5 & 23.7 \\
\hline JANUARY & 7.1 & 0.01 & 1.9 & 2.1 & 1.1 & 13.4 \\
\hline FEBRUARY & 13 & 0.04 & 3.2 & 4.4 & 1.4 & 22.4 \\
\hline MARCH & 12 & 0.04 & 2.8 & 4.3 & 1.5 & 19.6 \\
\hline APRIL & 6.6 & 0.03 & 1.1 & 1.9 & 1.7 & 7.6 \\
\hline MAY & 1.3 & 0.01 & 0.32 & 0.44 & 1.4 & 2.3 \\
\hline JUNE & 0.39 & 0.00 & 0.07 & 0.12 & 1.5 & 0.5 \\
\hline JULY & 0.12 & 0.00 & 0.02 & 0.04 & 1.9 & 0.1 \\
\hline AUGUST & 0.10 & 0.00 & 0.02 & 0.03 & 1.5 & 0.1 \\
\hline SEPTEMBER & 5.5 & 0.00 & 0.48 & 1.6 & 3.3 & 3.4 \\
\hline ANNUAL & 3.6 & 0.02 & 1.2 & 1.3 & 1.1 & 100 \\
\hline
\end{tabular}

MAGNITUDE AND PROBABILITY OF ANNUAL LOW FLOW BASED ON PERIOD OF RECORD 1967-74, 1984-86

\begin{tabular}{|c|c|c|c|c|c|}
\hline \multirow{4}{*}{$\begin{array}{l}\text { PERIOD } \\
\text { /CON- } \\
\text { SECU. }\end{array}$} & \multicolumn{5}{|c|}{ DISCHARGE, IN FT $3 / \mathrm{S}$, FOR INDICATED } \\
\hline & & RECURRENCE INTE & , IN & RS, & \\
\hline & & NON-EXCEEDANCE P & BILI? & IN PI & ENT \\
\hline & & 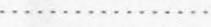 & … & & \\
\hline TIVE & 2 & $10 \#$ & 20 & $50 \#$ & $100 \#$ \\
\hline DAYS) & 508 & 108 & 58 & 28 & 18 \\
\hline
\end{tabular}

\begin{tabular}{|c|c|c|c|c|c|c|}
\hline \\
\hline \multicolumn{7}{|l|}{3} \\
\hline \multicolumn{7}{|l|}{7} \\
\hline \multicolumn{7}{|l|}{14} \\
\hline \multicolumn{7}{|l|}{30} \\
\hline 60 & & & & & & \\
\hline 90 & 0.00 & 0.00 & 0.00 & 0.00 & 0.00 & 0.00 \\
\hline 120 & 0.01 & 0.00 & 0.00 & 0.00 & 0.00 & 0.00 \\
\hline 183 & 0.02 & 0.00 & 0.00 & 0.00 & 0.00 & 0.00 \\
\hline
\end{tabular}

MAGNITUDE AND PROBABILITY OF ANNUAL HIGH FLOW BASED ON PERIOD OF RECORD 1966-74, 1983-85

MAGNITUDE AND PROBABILITY OF INSTANTANEOUS PEAK FLOW BASED ON PERIOD OF RECORD 1966-74, 1978, 1983.86

DISCHARGE, IN FT3/S, FOR INDICATED RECURRENCE INTERVAL IN YEARS, AND EXCEEDANCE PROBABILITY, IN PERCENT

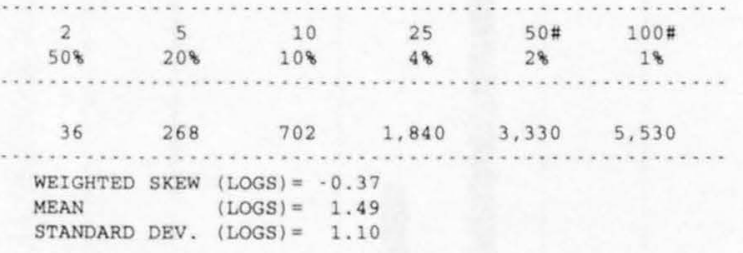

\begin{tabular}{|c|c|c|c|c|c|c|}
\hline \multirow{3}{*}{$\begin{array}{l}\text { PERIOD } \\
\text { (CON- }\end{array}$} & \multicolumn{6}{|c|}{ DISCHARGE, IN FT $3 / S$, FOR INDICATED } \\
\hline & & RECURRENCE & \multicolumn{4}{|c|}{ INTERVAL, IN YEARS, AND } \\
\hline & & EXCEEDANCE & E PRC & & IN PERC & \\
\hline SECU. & & t & & 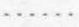 & . $\ldots \ldots$ & $\ldots \ldots$ \\
\hline TIVE & 2 & 5 & 10\# & $25 \#$ & $50 \#$ & $100 \#$ \\
\hline DAYS) & 508 & 208 & $10 \%$ & 48 & 28 & 18 \\
\hline 1 & 17 & 85 & 160 & 270 & 352 & 429 \\
\hline 3 & 11 & 51 & 91 & 145 & 183 & 217 \\
\hline 7 & 7.2 & 29 & 49 & 76 & 94 & 111 \\
\hline 15 & 4.6 & 18 & 32 & 51 & 66 & 80 \\
\hline 30 & 3.3 & 12 & 21 & 35 & 45 & 55 \\
\hline 60 & 2.3 & 8.8 & 16 & 28 & 38 & 49 \\
\hline 90 & 1.8 & 6.7 & 12 & 20 & 27 & 35 \\
\hline
\end{tabular}

DURATION TABLE OF DAILY MEAN FLOW FOR PERIOD OF RECORD $1966 \cdot 74,1983 \cdot 85$

DISCHARGE, IN FT3/S, WHICH WAS EQUALED OR EXCEEDED FOR INDICATED PERCENT OF TIME

\begin{tabular}{|c|c|c|c|c|c|c|c|c|c|c|c|c|c|c|c|c|}
\hline 18 & 58 & 108 & 158 & 208 & 308 & 408 & 508 & 608 & 708 & 808 & 908 & 958 & 988 & 998 & 99.58 & 99.98 \\
\hline 21 & 5.3 & 2.4 & 1.5 & 0.93 & 0.25 & 0.12 & 0.07 & 0.03 & 0.02 & 0.00 & 0.00 & 0.00 & 0.00 & 0.00 & 0.00 & 0.00 \\
\hline
\end{tabular}

\# Reliability of values in column is uncertain, and potential errors are large. 
09510070 WEST FORK SYCAMORE CREEK ABOVE MCFARLAND CANYON, NEAR SUNFLOWER, AZ--Continued
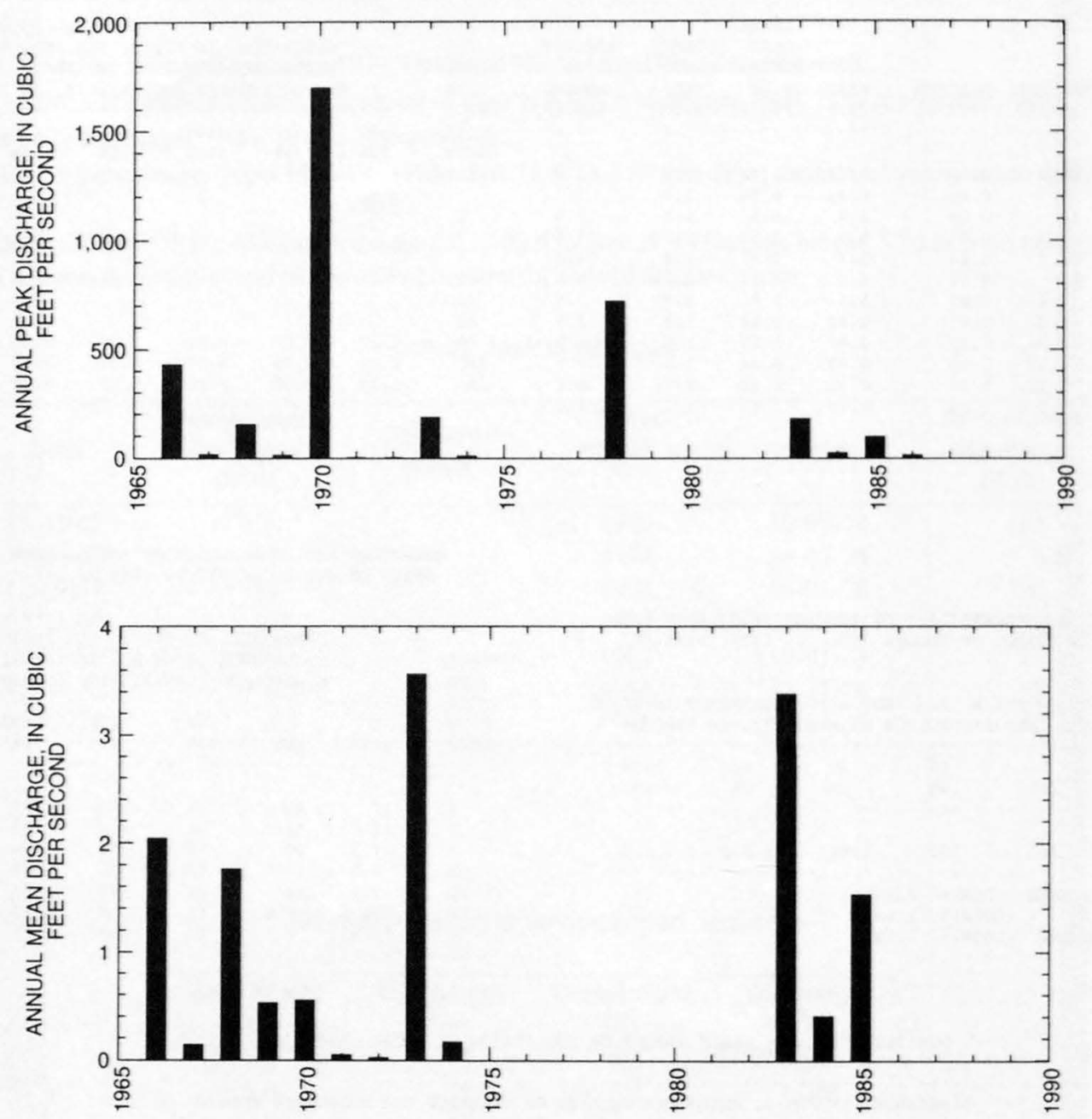
09510070 WEST FORK SYCAMORE CREEK ABOVE MCFARLAND CANYON, NEAR SUNFLOWER, AZ--Continued
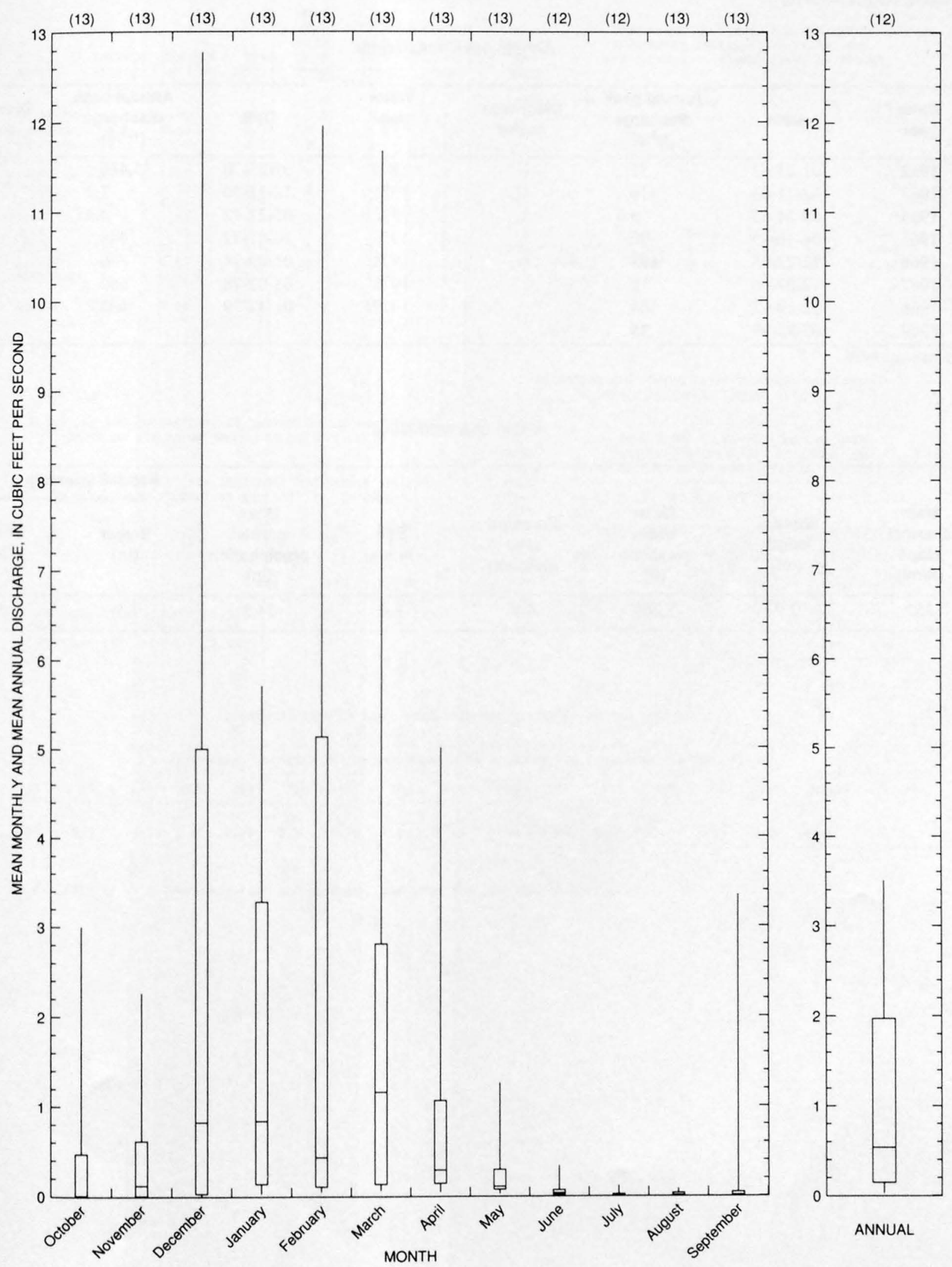


\section{WEST FORK SYCAMORE CREEK NEAR SUNFLOWER, AZ}

LOCATION.--Lat 33 $56^{\prime} 45^{\prime \prime}$, long $111^{\circ} 29^{\prime} 05^{\prime \prime}$, in SE $1 / 4$ sec.13, T.7 N., R.8 E. (unsurveyed), Maricopa County, Hydrologic Unit 15060203 , in Tonto

National Forest, on right bank $1.2 \mathrm{mi}$ upstream from confluence with East Fork, and 5.7 mi north of Sunflower.

DRAINAGE AREA.--9.8 $\mathrm{mi}^{2}$.

Annual peak discharges

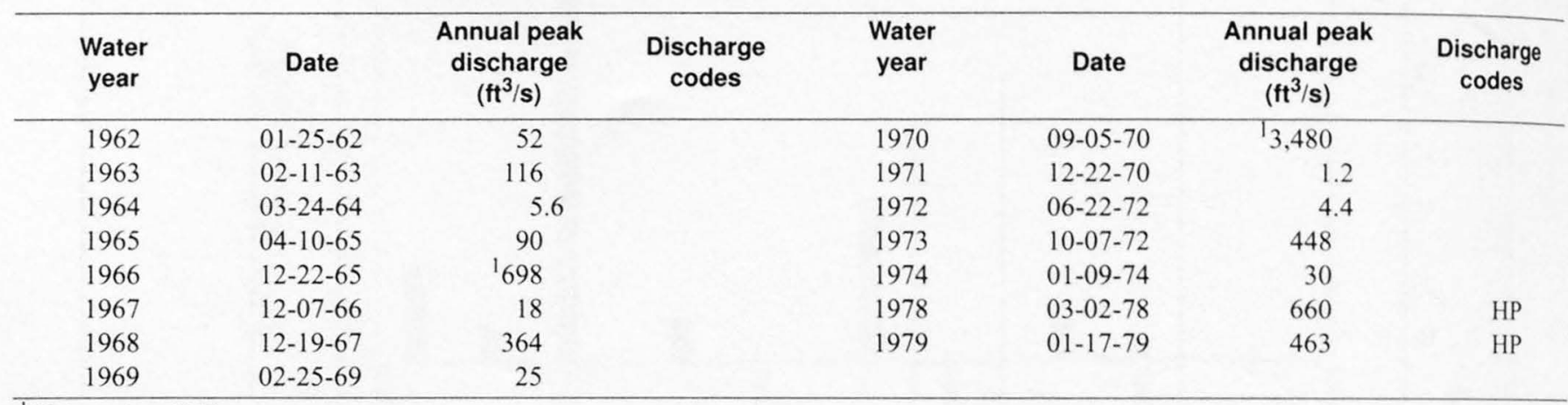

${ }^{1}$ Highest since 1959.

Basin characteristics

\begin{tabular}{|c|c|c|c|c|c|c|c|}
\hline \multirow[b]{2}{*}{$\begin{array}{c}\text { Main } \\
\text { channel } \\
\text { slope } \\
(\mathrm{ft} / \mathrm{mi})\end{array}$} & \multirow[b]{2}{*}{$\begin{array}{c}\text { Stream } \\
\text { length } \\
\text { (mi) }\end{array}$} & \multirow[b]{2}{*}{$\begin{array}{c}\text { Mean } \\
\text { basin } \\
\text { elevation } \\
\text { ( } \mathrm{ft})\end{array}$} & \multirow[b]{2}{*}{$\begin{array}{l}\text { Forested } \\
\text { area } \\
\text { (percent) }\end{array}$} & \multirow[b]{2}{*}{$\begin{array}{c}\text { Soil } \\
\text { index }\end{array}$} & \multirow[b]{2}{*}{$\begin{array}{c}\text { Mean } \\
\text { annual } \\
\text { precipitation } \\
\text { (in) }\end{array}$} & \multicolumn{2}{|c|}{ Rainfall intensity, 24-hou } \\
\hline & & & & & & $\begin{array}{c}\text { 2-year } \\
\text { (in) }\end{array}$ & $\begin{array}{l}\text { 50-year } \\
\text { (in) }\end{array}$ \\
\hline 353 & 3.9 & 5,260 & 0.8 & 3.0 & 24.5 & 2.9 & 5.5 \\
\hline
\end{tabular}


GILA RIVER BASIN

09510080 WEST FORK SYCAMORE CREEK NEAR SUNFLOWER, AZ--Continued

MEAN MONTHLY AND ANNUAL DISCHARGES 1962.74

\begin{tabular}{|c|c|c|c|c|c|c|}
\hline MONTH & $\begin{array}{l}\text { MAXIMUM } \\
\text { (FT3/S) }\end{array}$ & $\begin{array}{l}\text { MINIMUM } \\
\text { (FT3/S) }\end{array}$ & $\begin{array}{c}\text { MEAN } \\
(\text { FT3/S) }\end{array}$ & $\begin{array}{l}\text { STAN- } \\
\text { DARD } \\
\text { DEVIA- } \\
\text { TION } \\
\text { (FT3/S) }\end{array}$ & $\begin{array}{l}\text { COEFFI- } \\
\text { CIENT OF } \\
\text { VARI- } \\
\text { ATION }\end{array}$ & $\begin{array}{c}\text { PERCENT } \\
\text { OF } \\
\text { ANNUAL } \\
\text { RUNOFF }\end{array}$ \\
\hline OCTOBER & 14 & 0.00 & 1.1 & 3.8 & 3.5 & 4.4 \\
\hline NOVEMBER & 8.0 & 0.00 & 0.70 & 2.2 & 3.2 & 2.8 \\
\hline DECEMBER & 33 & 0.01 & 4.7 & 9.7 & 2.1 & 19.1 \\
\hline JANUARY & 21 & 0.03 & 3.4 & 5.8 & 1.7 & 13.7 \\
\hline FEBRUARY & 21 & 0.05 & 4.6 & 7.0 & 1.5 & 18.5 \\
\hline MARCH & 32 & 0.08 & 5.4 & 8.8 & 1.6 & 22.0 \\
\hline APRIL & 16 & 0.04 & 2.8 & 5.5 & 2.0 & 11.4 \\
\hline MAY & 2.1 & 0.02 & 0.36 & 0.55 & 1.5 & 1.5 \\
\hline JUNE & 0.55 & 0.01 & 0.07 & 0.15 & 2.0 & 0.3 \\
\hline JULY & 0.15 & 0.00 & 0.02 & 0.04 & 2.3 & 0.1 \\
\hline AUGUST & 5.5 & 0.00 & 0.44 & 1.5 & 3.4 & 1.8 \\
\hline SEPTEMBER & 11 & 0.00 & 1.1 & 3.1 & 2.9 & 4.3 \\
\hline INUAL & 8.9 & 0.04 & 2.0 & 2.6 & 1.3 & 100 \\
\hline
\end{tabular}

MAGNITUDE AND PROBABILITY OF ANNUAL HIGH FLOW BASED ON PERIOD OF RECORD $1962 \cdot 74$

MAGNITUDE AND PROBABILITY OF INSTANTANEOUS PEAK FLOW BASED ON PERIOD OF RECORD $1962.74,1978.79$

DISCHARGE, IN FTT/S, FOR INDICATED RECURRENCE INTERVAL IN YEARS, AND EXCEEDANCE PROBABILITY, IN PERCENT

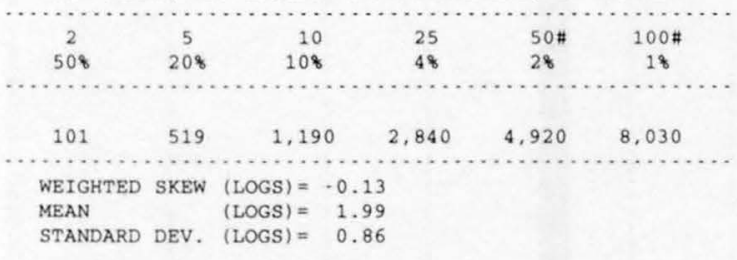

MAGNITUDE AND PROBABILITY OF ANNUAL LOW FLOW BASED ON PERIOD OF RECORD $1963 \cdot 74$

\begin{tabular}{|c|c|c|c|c|c|c|}
\hline \multirow{4}{*}{$\begin{array}{l}\text { PERIOD } \\
\text { /CON- } \\
\text { SECU. }\end{array}$} & \multicolumn{6}{|c|}{ DISCHARGE, IN FT3/S, FOR INDICATED } \\
\hline & \multicolumn{6}{|c|}{ RECURRENCE INTERVAL, IN YEARS, AND } \\
\hline & \multicolumn{6}{|c|}{ NON-EXCEEDANCE PROBABILITY, IN PERCENT } \\
\hline & & & & & & $\cdots \cdots$ \\
\hline TIVE & 2 & 5 & $10 \#$ & 20 & $50 \#$ & $100 \#$ \\
\hline DAYS) & 508 & 208 & 108 & 58 & 28 & 18 \\
\hline 1 & & & & & & \\
\hline 3 & & & & & & \\
\hline 7 & & & & & & \\
\hline 14 & & & & & & \\
\hline 30 & & & & & & \\
\hline 60 & & & & & & \\
\hline 90 & 0.00 & 0.00 & 0.00 & 0.00 & 0.00 & 0.00 \\
\hline 120 & 0.00 & 0.00 & 0.00 & 0.00 & 0.00 & 0.00 \\
\hline 183 & 0.02 & 0.00 & 0.00 & 0.00 & 0.00 & 0.00 \\
\hline
\end{tabular}

DURATION TABLE OF DAILY MEAN FLOW FOR PERIOD OF RECORD 1962.74

DISCHARGE, IN FT $3 / \mathrm{S}$, WHICH WAS EQUALED OR EXCEEDED FOR INDICATED PERCENT OF TIME

$\begin{array}{rrrrrrrrrrrrrrrrrr}18 & 58 & 108 & 158 & 208 & 308 & 408 & 508 & 608 & 708 & 808 & 908 & 958 & 988 & 998 & 99.58 & 99.98 \\ 39 & 9.3 & 3.1 & 1.7 & 0.79 & 0.31 & 0.15 & 0.07 & 0.03 & 0.02 & 0.00 & 0.00 & 0.00 & 0.00 & 0.00 & 0.00 & 0.00\end{array}$

\# Reliability of values in column is uncertain, and potential errors are large. 
09510080 WEST FORK SYCAMORE CREEK NEAR SUNFLOWER, AZ--Continued
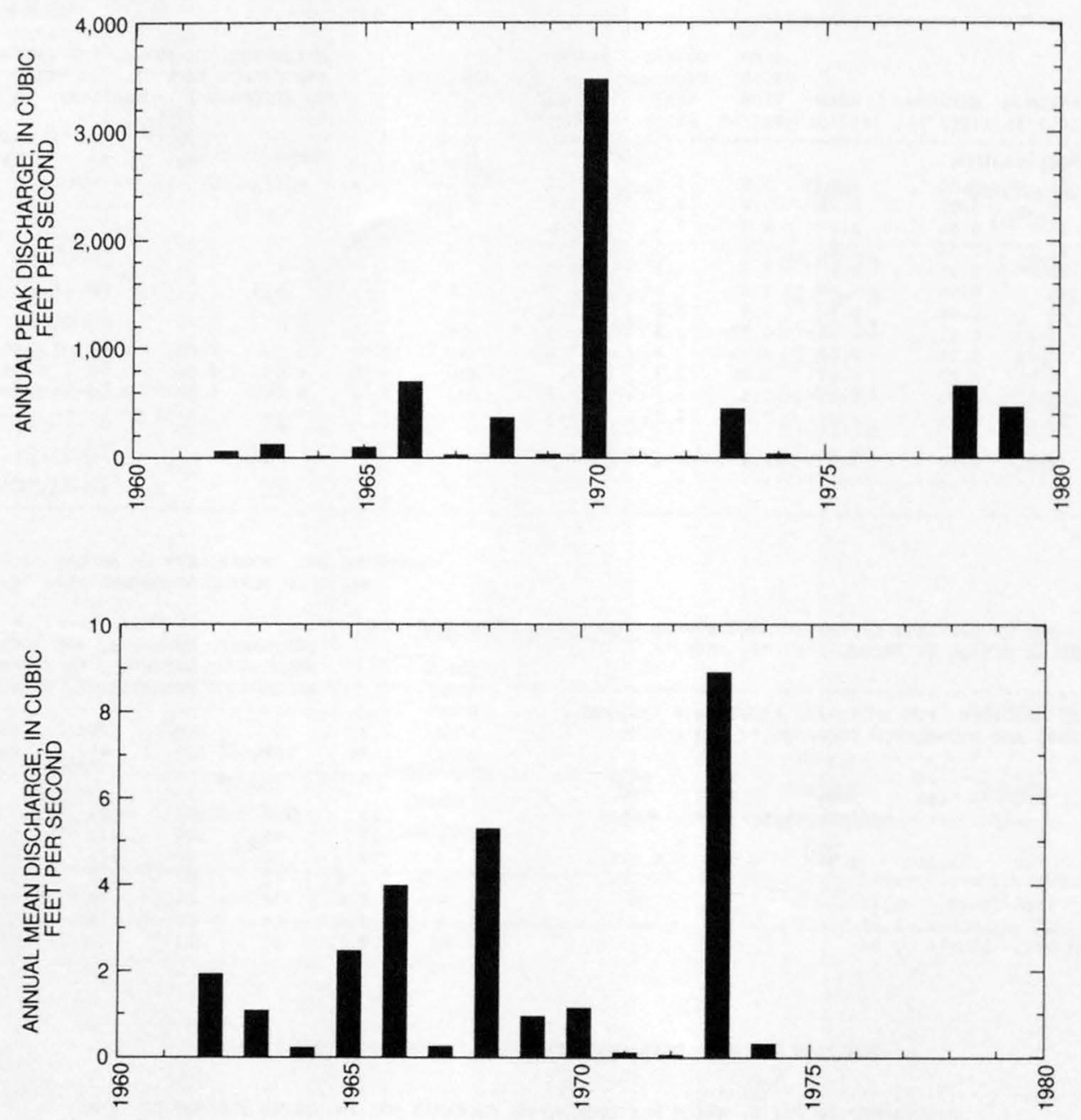
09510080 WEST FORK SYCAMORE CREEK NEAR SUNFLOWER, AZ--Continued
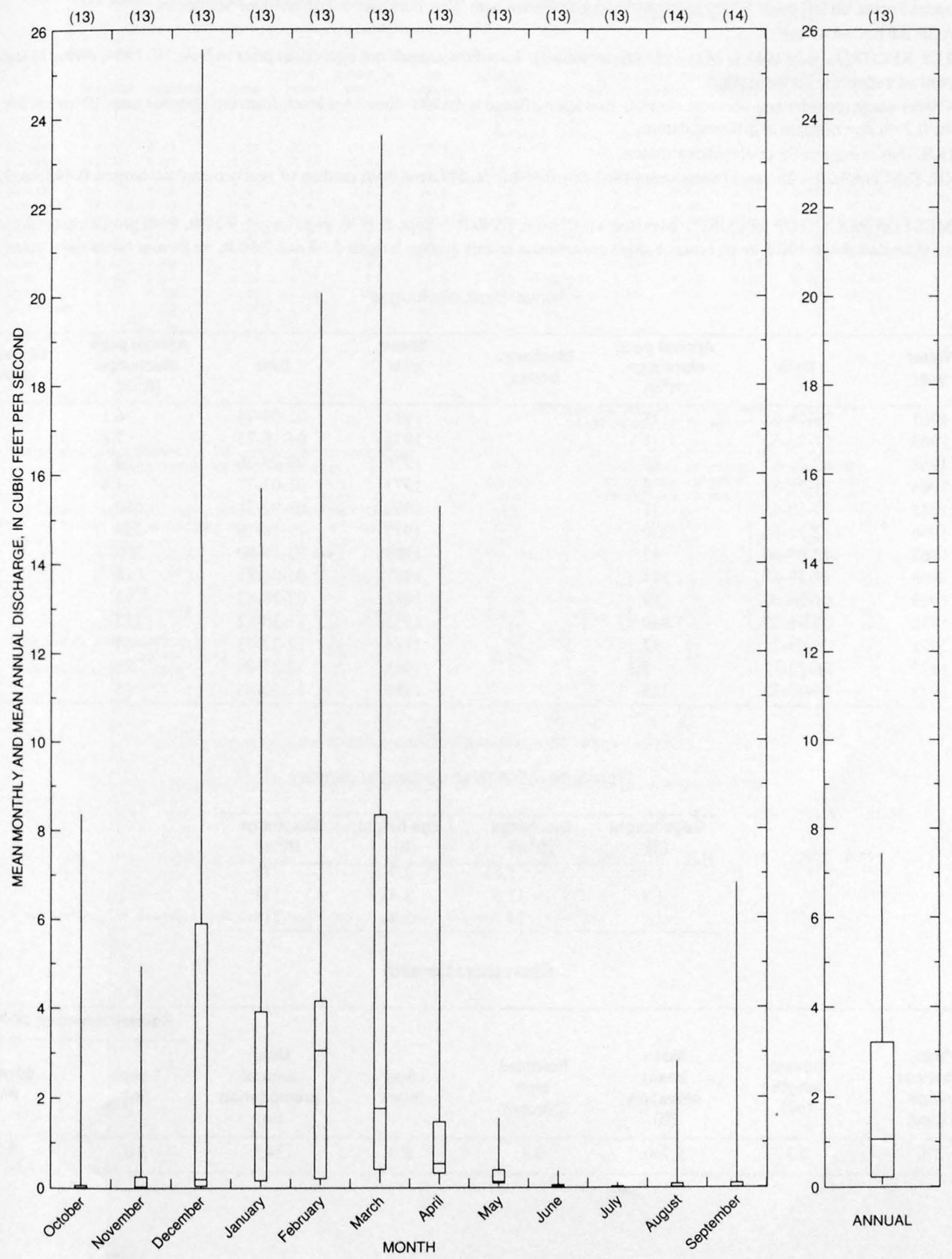


\section{EAST FORK SYCAMORE CREEK NEAR SUNFLOWER, AZ}

LOCATION.--Lat 33.56'58", long 111 27'39", in NE $\frac{1}{4} \mathrm{SE}^{1} / 4$ sec. 18, T.7 N., R.9 E., Maricopa County, Hydrologic Unit 15060203 , in Tonto National Forest, on left bank $1.7 \mathrm{mi}$ upstream from confluence with West Fork and 6.0 mi north of Sunflower.

DRAINAGE AREA.--4.49 $\mathrm{mi}^{2}$.

PERIOD OF RECORD.--July 1961 to May 1986 (discontinued). Low-flow records not equivalent prior to Nov. 10, 1964, owing to undetermined amount of underflow between sites.

GAGE,--Water-stage recorder and concrete control. Elevation of gage is 4,140 ft above sea level, from topographic map. Prior to Nov. 10,1964 . at site $0.2 \mathrm{mi}$ downstream at different datum.

REMARKS.--No storage or diversion above station.

AVERAGE DISCHARGE.--25 years (water years 1962-86), $0.962 \mathrm{ft}^{3} / \mathrm{s}, 697$ acre- $\mathrm{ft} / \mathrm{yr}$; median of yearly mean discharges, $0.44 \mathrm{ft}^{3} / \mathrm{s}, 320$ acre-ft yr.

EXTREMES FOR PERIOD OF RECORD.--Maximum discharge, 1,940 ft $3 / \mathrm{s} \mathrm{Sept.} \mathrm{5,} \mathrm{1970,} \mathrm{gage} \mathrm{height,} 9.50 \mathrm{ft}$, from profile past gage, from rating curve extended above $130 \mathrm{ft}^{3} / \mathrm{s}$ on basis of slope-area measurements at gage heights 5.07 and $9.50 \mathrm{ft}$; no flow at times most years.

Annual peak discharges

\begin{tabular}{|c|c|c|c|c|c|c|c|}
\hline $\begin{array}{l}\text { Water } \\
\text { year }\end{array}$ & Date & $\begin{array}{c}\text { Annual peak } \\
\text { discharge } \\
\left(\mathrm{ft}^{3} / \mathrm{s}\right)\end{array}$ & $\begin{array}{l}\text { Discharge } \\
\text { codes }\end{array}$ & $\begin{array}{c}\text { Water } \\
\text { year }\end{array}$ & Date & $\begin{array}{c}\text { Annual peak } \\
\text { discharge } \\
\left(\mathrm{ft}^{3} / \mathrm{s}\right)\end{array}$ & $\begin{array}{c}\text { Discharge } \\
\text { codes }\end{array}$ \\
\hline 1961 & $09-08-61$ & 15 & & 1974 & 01-09-74 & 6.1 & \\
\hline 1962 & $01-25-62$ & 11 & & 1975 & $04-10-75$ & 7.6 & \\
\hline 1963 & $09-01-63$ & 30 & & 1976 & $02-09-76$ & 150 & \\
\hline 1964 & $11-21-63$ & 0.8 & & 1977 & $01-03-77$ & 4.8 & \\
\hline 1965 & $04-10-65$ & 31 & & 1978 & $03-02-78$ & 550 & \\
\hline 1966 & $12-22-65$ & 330 & & 1979 & $12-18-78$ & 298 & \\
\hline 1967 & $12-07-66$ & 12 & & 1980 & $02-19-80$ & 300 & \\
\hline 1968 & $12-19-67$ & 244 & & 1981 & $05-01-81$ & 16 & \\
\hline 1969 & $01-26-69$ & 19 & & 1982 & $07-28-82$ & 93 & \\
\hline 1970 & $09-05-70$ & 1,940 & & 1983 & $11-30-82$ & 157 & $\mathrm{C}$ \\
\hline 1971 & $08-03-71$ & 32 & & 1984 & $12-27-83$ & 64 & $\mathrm{C}$ \\
\hline 1972 & $06-22-72$ & 3.8 & & 1985 & $12-27-84$ & 78 & $\mathrm{C}$ \\
\hline 1973 & $10-07-72$ & 125 & & 1986 & $11-30-85$ & 15 & $\mathrm{C}$ \\
\hline
\end{tabular}

Discharge rating table developed January --.

\begin{tabular}{cccc}
\hline $\begin{array}{c}\text { Gage height } \\
(\mathbf{f t})\end{array}$ & $\begin{array}{c}\text { Discharge } \\
\left(\mathrm{ft}^{3} / \mathbf{s}\right)\end{array}$ & $\begin{array}{c}\text { Gage height } \\
(\mathbf{f t})\end{array}$ & $\begin{array}{c}\text { Discharge } \\
\left(\mathrm{ft}^{\mathbf{3}} / \mathbf{s}\right)\end{array}$ \\
\hline 1.4 & 1.85 & 2.9 & 73 \\
1.9 & 11.5 & 3.4 & 131 \\
2.4 & 34 & 3.9 & 216 \\
\hline
\end{tabular}

Basin characteristics

\begin{tabular}{cccccccc}
\hline $\begin{array}{c}\text { Main } \\
\text { channel } \\
\text { slope } \\
(\mathrm{ft} / \mathrm{mi})\end{array}$ & $\begin{array}{c}\text { Stream } \\
\text { length } \\
\text { (mi) }\end{array}$ & $\begin{array}{c}\text { Mean } \\
\text { basin } \\
\text { elevation } \\
(\mathrm{ft})\end{array}$ & $\begin{array}{c}\text { Forested } \\
\text { area } \\
\text { (percent) }\end{array}$ & $\begin{array}{c}\text { Soil } \\
\text { index }\end{array}$ & $\begin{array}{c}\text { Mean } \\
\text { annual } \\
\text { precipitation } \\
\text { (in) }\end{array}$ & $\begin{array}{c}\text { Rainfall intensity, 24-hour } \\
\text { (in) }\end{array}$ & $\begin{array}{c}50 \text {-year } \\
\text { (in) }\end{array}$ \\
\hline 370 & 4.3 & 5,760 & 0.4 & 3.0 & 24.5 & 3.0 \\
\hline
\end{tabular}


09510100 EAST FORK SYCAMORE CREEK NEAR SUNFLOWER, AZ--Continued

MEAN MONTHLY AND ANNUAL DISCHARGES $1962 \cdot 85$

\begin{tabular}{|c|c|c|c|c|c|c|}
\hline MONTH & $\begin{array}{l}\text { MAXIMUM } \\
\text { (FT3/S) }\end{array}$ & $\begin{array}{l}\text { MINIMUM } \\
\text { (FT3/S) }\end{array}$ & $\begin{array}{c}\text { MEAN } \\
(\mathrm{FT} 3 / \mathrm{S})\end{array}$ & $\begin{array}{l}\text { STAN- } \\
\text { DARD } \\
\text { DEVIA- } \\
\text { TION } \\
\text { (FT3/S) }\end{array}$ & $\begin{array}{l}\text { COEFFI- } \\
\text { CIENT OF } \\
\text { VARI- } \\
\text { ATION }\end{array}$ & $\begin{array}{c}\text { PERCENT } \\
\text { OF } \\
\text { ANNUAL } \\
\text { RUNOFF }\end{array}$ \\
\hline OCTOBER & 3.7 & 0.00 & 0.21 & 0.78 & 3.7 & 1.8 \\
\hline NOVEMBER & 1.7 & 0.00 & 0.19 & 0.46 & 2.4 & 1.7 \\
\hline DECEMBER & 16 & 0.00 & 1.8 & 3.7 & 2.1 & 15.5 \\
\hline JANUARY & 11 & 0.00 & 1.6 & 2.7 & 1.7 & 14.1 \\
\hline FEBRUARY & 24 & 0.00 & 3.0 & 5.4 & 1.8 & 25.6 \\
\hline MARCH & 20 & 0.01 & 3.0 & 5.1 & 1.7 & 26.0 \\
\hline APRIL & 5.5 & 0.00 & 1.1 & 1.5 & 1.4 & 9.1 \\
\hline MAY & 1.3 & 0.00 & 0.29 & 0.40 & 1.4 & 2.5 \\
\hline JUNE & 0.40 & 0.00 & 0.07 & 0.13 & 1.8 & 0.6 \\
\hline JULY & 0.30 & 0.00 & 0.04 & 0.07 & 1.9 & 0.3 \\
\hline AUGUST & 0.47 & 0.00 & 0.06 & 0.11 & 1.9 & 0.5 \\
\hline SEPTEMBER & 5.3 & 0.00 & 0.26 & 1.1 & 4.1 & 2.3 \\
\hline ANNUAL & 3.3 & 0.01 & 0.96 & 1.2 & 1.2 & 100 \\
\hline
\end{tabular}

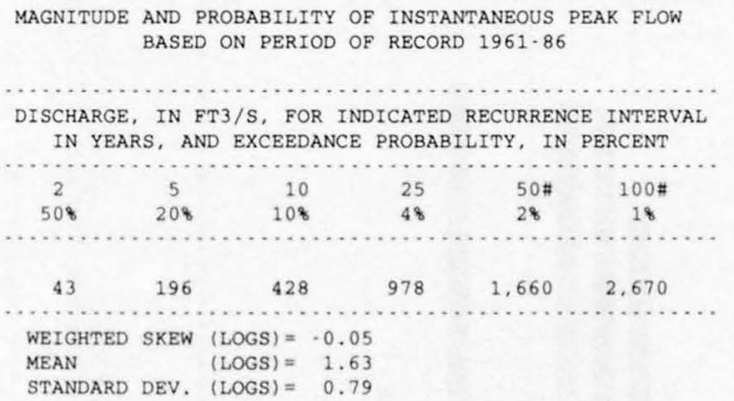

MAGNITUDE AND PROBABILITY OF ANNUAL LOW FLOW BASED ON PERIOD OF RECORD 1963.86

\begin{tabular}{|c|c|c|c|c|c|c|}
\hline \multirow{3}{*}{$\begin{array}{l}\text { PERIOD } \\
\text { (CON- } \\
\text { SECU- }\end{array}$} & \multirow{2}{*}{\multicolumn{6}{|c|}{$\begin{array}{l}\text { DISCHARGE, IN FT3/S, FOR INDICATED } \\
\text { RECURRENCE INTERVAL, IN YEARS, AND }\end{array}$}} \\
\hline & & & & & & \\
\hline & \multicolumn{6}{|c|}{ NON-EXCEEDANCE PROBABILITY, IN PERCENT } \\
\hline & 2 & 5 & $10 \#$ & 20 & $50 \#$ & $100 \#$ \\
\hline DAYS) & 508 & 208 & 108 & 58 & 28 & 18 \\
\hline 1 & 0.00 & 0.00 & 0.00 & 0.00 & 0.00 & 0.00 \\
\hline 3 & 0.00 & 0.00 & 0.00 & 0.00 & 0.00 & 0.00 \\
\hline 7 & 0.00 & 0.00 & 0.00 & 0.00 & 0.00 & 0.00 \\
\hline 14 & 0.00 & 0.00 & 0.00 & 0.00 & 0.00 & 0.00 \\
\hline 30 & 0.00 & 0.00 & 0.00 & 0.00 & 0.00 & 0.00 \\
\hline 60 & 0.00 & 0.00 & 0.00 & 0.00 & 0.00 & 0.00 \\
\hline 90 & 0.00 & 0.00 & 0.00 & 0.00 & 0.00 & 0.00 \\
\hline 120 & 0.00 & 0.00 & 0.00 & 0.00 & 0.00 & 0.00 \\
\hline 183 & 0.01 & 0.00 & 0.00 & 0.00 & 0.00 & 0.00 \\
\hline
\end{tabular}

MAGNITUDE AND PROBABILITY OF ANNUAL HIGH FLOW BASED ON PERIOD OF RECORD $1962 \cdot 85$

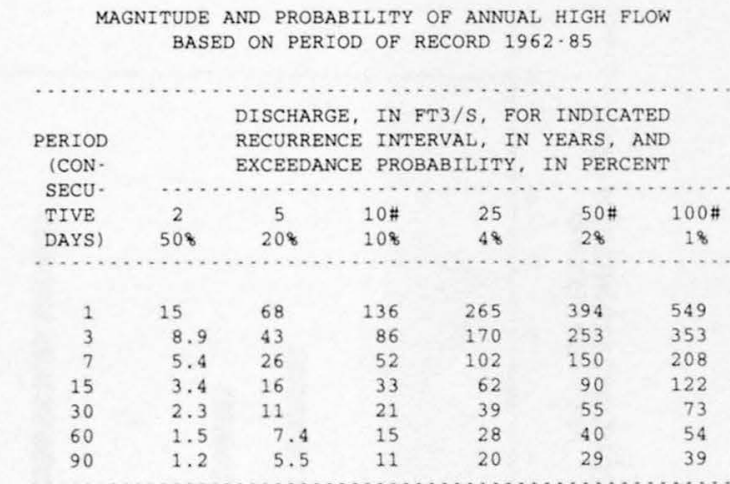

DURATION TABLE OF DAILY MEAN FLOW FOR PERIOD OF RECORD 1962.85

DISCHARGE, IN FT3/S, WHICH WAS EQUALED OR EXCEEDED FOR INDICATED PERCENT OF TIME

\begin{tabular}{|c|c|c|c|c|c|c|c|c|c|c|c|c|c|c|c|c|}
\hline 18 & 58 & 108 & 158 & 208 & 308 & 408 & 508 & 608 & 708 & 808 & 908 & 958 & 988 & 998 & 99.58 & 99.98 \\
\hline 17 & 3.8 & 1.6 & 0.79 & 0.45 & 0.14 & 0.06 & 0.03 & 0.02 & 0.01 & 0.00 & 0.00 & 0.00 & 0.00 & 0.00 & 0.00 & 0.00 \\
\hline
\end{tabular}

\# Reliability of values in column is uncertain, and potential errors are large. 
09510100 EAST FORK SYCAMORE CREEK NEAR SUNFLOWER, AZ--Continued
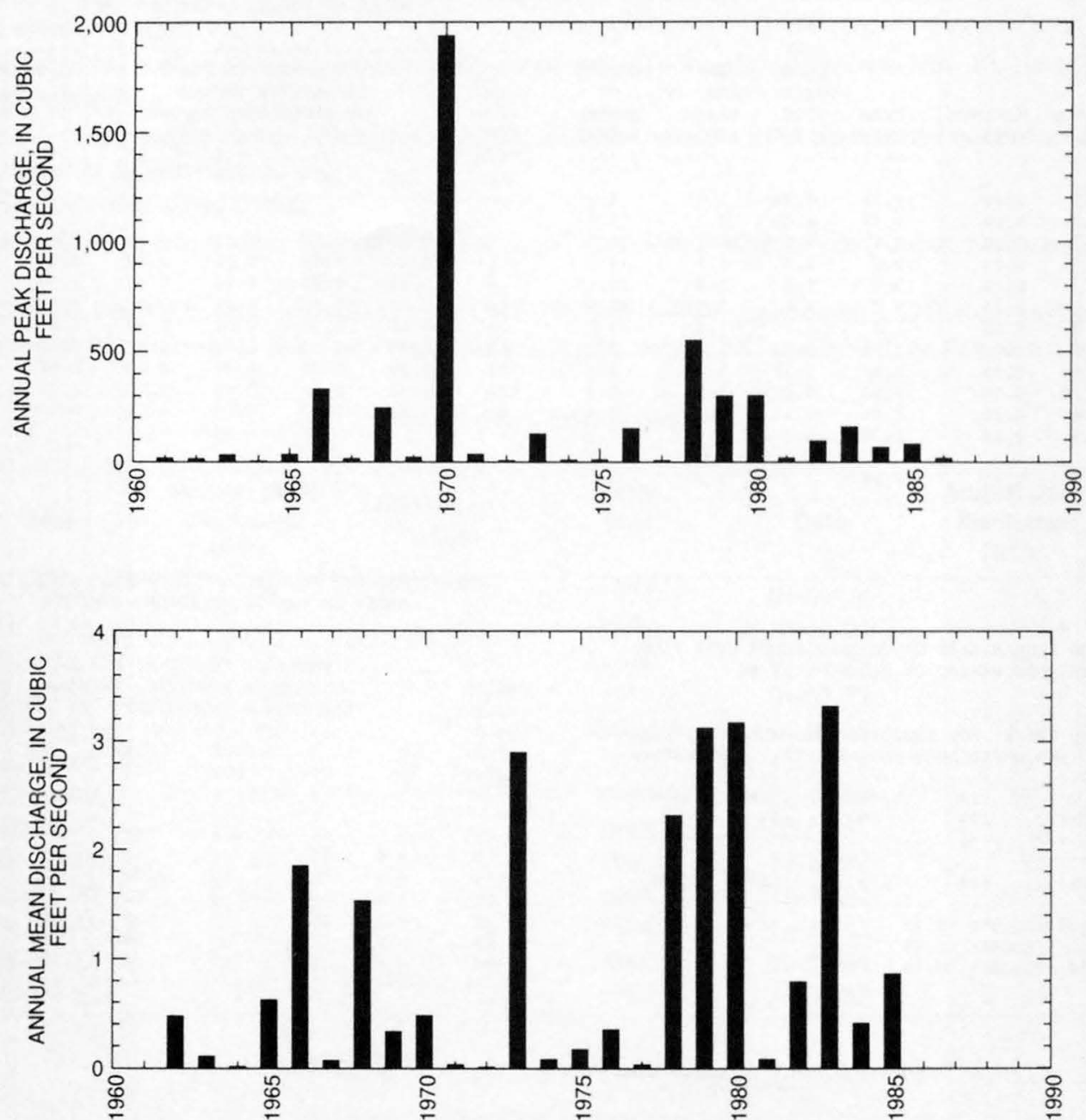
GILA RIVER BASIN

757

09510100 EAST FORK SYCAMORE CREEK NEAR SUNFLOWER, AZ--Continued
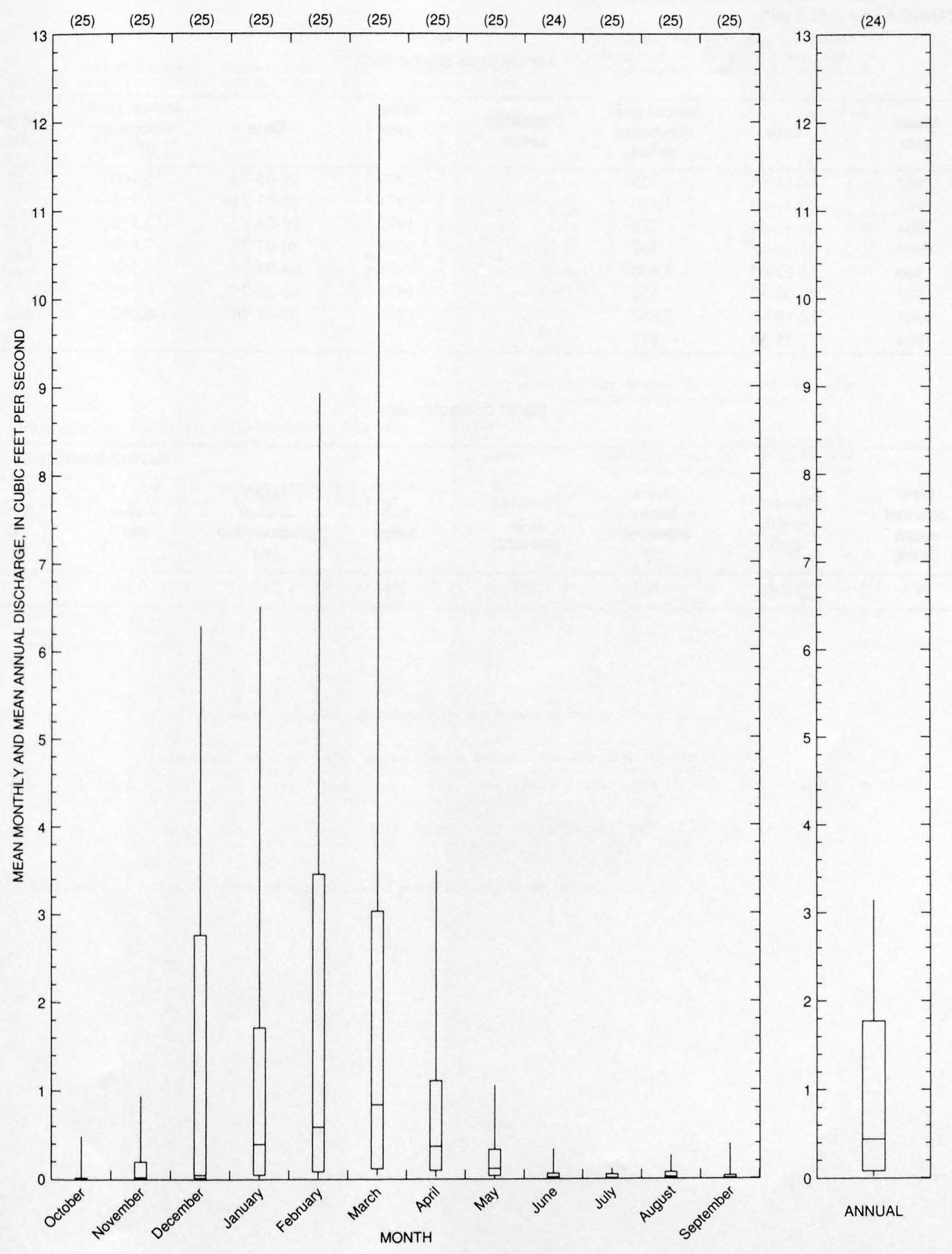


\section{SYCAMORE CREEK NEAR SUNFLOWER, AZ}

LOCATION.--Lat 33 51'05", long $111^{\circ} 27^{\prime} 09^{\prime \prime}$, in NE $1 / 4$ sec.20, T.6 N., R.9 E., Maricopa County, Hydrologic Unit 15060203 , in Tonto National

Forest, on right bank $1.1 \mathrm{mi}$ upstream from Boulder Creek, 1.2 mi north of Crabtree Butte, and 1.2 mi southeast of Sunflower.

DRAINAGE AREA.--52.3 $\mathrm{mi}^{2}$.

Annual peak discharges

\begin{tabular}{cccccrr}
\hline $\begin{array}{c}\text { Water } \\
\text { year }\end{array}$ & Date & $\begin{array}{c}\text { Annual peak } \\
\text { discharge } \\
\left(\mathbf{f t}^{3} / \mathbf{s}\right)\end{array}$ & $\begin{array}{c}\text { Discharge } \\
\text { codes }\end{array}$ & $\begin{array}{c}\text { Water } \\
\text { year }\end{array}$ & $\begin{array}{c}\text { Annual peak } \\
\text { discharge } \\
\left(\mathbf{f t}^{3} / \mathbf{s}\right)\end{array}$ & $\begin{array}{c}\text { Discharge } \\
\text { codes }\end{array}$ \\
\hline 1962 & $12-16-61$ & 325 & & 1970 & $09-05-70$ & 16,100 \\
1963 & $02-11-63$ & 1,120 & & 1971 & $08-03-71$ & 395 \\
1964 & $08-12-64$ & 286 & & 1972 & $08-04-72$ & 2,350 \\
1965 & $04-10-65$ & 762 & & 1973 & $10-07-72$ & 3,810 \\
1966 & $12-22-65$ & 4,800 & & 1974 & $08-05-74$ & 355 \\
1967 & $09-06-67$ & 550 & & 1975 & $03-15-75$ & 69 \\
1968 & $12-19-67$ & 7,650 & & 1976 & $02-09-76$ & 6,000 \\
1969 & $01-25-69$ & 142 & & & & \\
\hline
\end{tabular}

Basin characteristics

\begin{tabular}{|c|c|c|c|c|c|c|c|}
\hline \multirow[b]{2}{*}{$\begin{array}{c}\text { Main } \\
\text { channel } \\
\text { slope } \\
(\mathrm{ft} / \mathrm{mi})\end{array}$} & \multirow[b]{2}{*}{$\begin{array}{c}\text { Stream } \\
\text { length } \\
\text { (mi) }\end{array}$} & \multirow[b]{2}{*}{$\begin{array}{c}\text { Mean } \\
\text { basin } \\
\text { elevation } \\
\text { (ft) }\end{array}$} & \multirow[b]{2}{*}{$\begin{array}{l}\text { Forested } \\
\text { area } \\
\text { (percent) }\end{array}$} & \multirow[b]{2}{*}{$\begin{array}{c}\text { Soil } \\
\text { index }\end{array}$} & \multirow[b]{2}{*}{$\begin{array}{c}\text { Mean } \\
\text { annual } \\
\text { precipitation } \\
\text { (in) }\end{array}$} & \multicolumn{2}{|c|}{ Rainfall intensity, 24-hou } \\
\hline & & & & & & $\begin{array}{l}\text { 2-year } \\
\text { (in) }\end{array}$ & $\begin{array}{l}\text { 50-year } \\
\text { (in) }\end{array}$ \\
\hline 58.6 & 12.4 & 4,260 & 0.6 & 3.0 & 23.5 & 3.0 & 5.5 \\
\hline
\end{tabular}


09510150 SYCAMORE CREEK NEAR SUNFLOWER, AZ--Continued

MEAN MONTHLY AND ANNUAL DISCHARGES 1962.76

\begin{tabular}{|c|c|c|c|c|c|c|}
\hline MONTH & $\begin{array}{l}\text { MAXIMUM } \\
(\text { FT } 3 / \mathrm{S})\end{array}$ & $\begin{array}{l}\text { MINIMUM } \\
\text { (FT3/S) }\end{array}$ & $\begin{array}{c}\text { MEAN } \\
(\text { FT3/S) }\end{array}$ & $\begin{array}{l}\text { STAN- } \\
\text { DARD } \\
\text { DEVIA- } \\
\text { TION } \\
(\text { FT3/S) }\end{array}$ & $\begin{array}{l}\text { COEFFI- } \\
\text { CIENT OF } \\
\text { VARI- } \\
\text { ATION }\end{array}$ & $\begin{array}{c}\text { PERCENT } \\
\text { OF } \\
\text { ANNUAL } \\
\text { RUNOFF }\end{array}$ \\
\hline OCTOBER & 62 & 0.00 & 4.4 & 16 & 3.6 & 4.7 \\
\hline NOVEMBER & 22 & 0.10 & 2.7 & 6.1 & 2.3 & 2.9 \\
\hline DECEMBER & 175 & 0.25 & 24 & 52 & 2.2 & 26.0 \\
\hline JANUARY & 39 & 0.32 & 8.5 & 11 & 1.3 & 9.2 \\
\hline FEBRUARY & 86 & 0.38 & 19 & 27 & 1.5 & 20.1 \\
\hline MARCH & 124 & 0.29 & 16 & 32 & 2.0 & 17.5 \\
\hline APRIL & 55 & 0.28 & 9.6 & 17 & 1.7 & 10.4 \\
\hline MAY & 14 & 0.21 & 2.1 & 3.3 & 1.6 & 2.3 \\
\hline JUNE & 3.7 & 0.13 & 0.89 & 1.1 & 1.3 & 1.0 \\
\hline JULY & 3.5 & 0.01 & 0.57 & 0.91 & 1.6 & 0.6 \\
\hline AUGUST & 11 & 0.04 & 1.7 & 3.0 & 1.8 & 1.8 \\
\hline SEPTEMBER & 42 & 0.00 & 3.4 & 11 & 3.1 & 3.7 \\
\hline ANNUAL & 34 & 0.52 & 7.7 & 9.5 & 1.2 & 100 \\
\hline
\end{tabular}

MAGNITUDE AND PROBABILITY OF INSTANTANEOUS PEAK FLOW BASED ON PERIOD OF RECORD 1962.76

DISCHARGE, IN FT3/S, FOR INDICATED RECURRENCE INTERVAL IN YEARS, AND EXCEEDANCE PROBABILITY, IN PERCENT

\begin{tabular}{|c|c|c|c|c|c|}
\hline 2 & 5 & 10 & 25 & $50 \#$ & $100 \#$ \\
\hline 508 & 208 & 108 & 48 & 28 & 18 \\
\hline 1,050 & 4,050 & 8,160 & 17,200 & 27,800 & 42,700 \\
\hline
\end{tabular}

WEIGHTED SKEW (LOGS) $=-0.02$

MEAN (LOGS) $=3.02$

STANDARD DEV. (LOGS) $=0.70$
MAGNITUDE AND PROBABILITY OF ANNUAL LOW FLOW BASED ON PERIOD OF RECORD 1963.76

\begin{tabular}{|c|c|c|c|c|c|c|}
\hline $\begin{array}{l}\text { PERIOD } \\
\text { (CON- }\end{array}$ & \multirow{2}{*}{\multicolumn{6}{|c|}{ 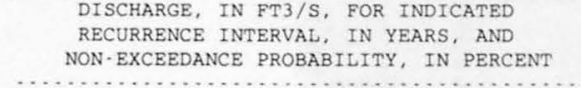 }} \\
\hline SECU. & & & & & & \\
\hline TIVE & 2 & 5 & 10 & 20 & $50 \#$ & $100 \#$ \\
\hline DAYSI & 508 & 208 & 108 & 58 & 28 & 18 \\
\hline 1 & 0.04 & 0.00 & 0.00 & 0.00 & 0.00 & 0.00 \\
\hline 3 & 0.05 & 0.00 & 0.00 & 0.00 & 0.00 & 0.00 \\
\hline 7 & 0.05 & 0.00 & 0.00 & 0.00 & 0.00 & 0.00 \\
\hline 14 & 0.07 & 0.00 & 0.00 & 0.00 & 0.00 & 0.00 \\
\hline 30 & 0.08 & 0.00 & 0.00 & 0.00 & 0.00 & 0.00 \\
\hline 60 & 0.12 & 0.06 & 0.04 & 0.02 & 0.02 & 0.01 \\
\hline 90 & 0.19 & 0.10 & 0.07 & 0.06 & 0.05 & 0.04 \\
\hline 120 & 0.26 & 0.14 & 0.11 & 0.08 & 0.07 & 0.06 \\
\hline 183 & 0.40 & 0.26 & 0.22 & 0.19 & 0.17 & 0.16 \\
\hline
\end{tabular}

MAGNITUDE AND PROBABILTTY OF ANNUAL HIGH FLOW BASED ON PERIOD OF RECORD 1962.76

PERIOD
(CON

DURATION TABLE OF DAILY MEAN FLOW FOR PERIOD OF RECORD 1962.76

DISCHARGE, IN FT3/S, WHICH WAS EQUALED OR EXCEEDED FOR INDICATED PERCENT OF TIME

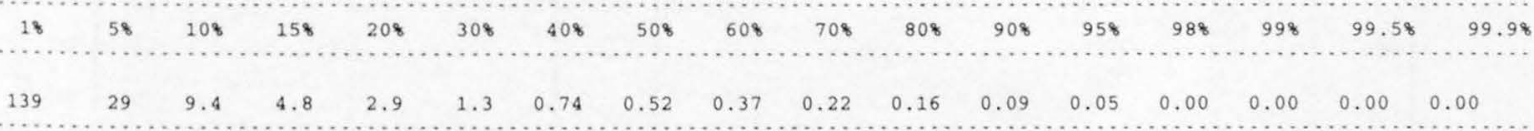

\# Reliability of values in column is uncertain, and potential errors are large. 
09510150 SYCAMORE CREEK NEAR SUNFLOWER, AZ--Continued
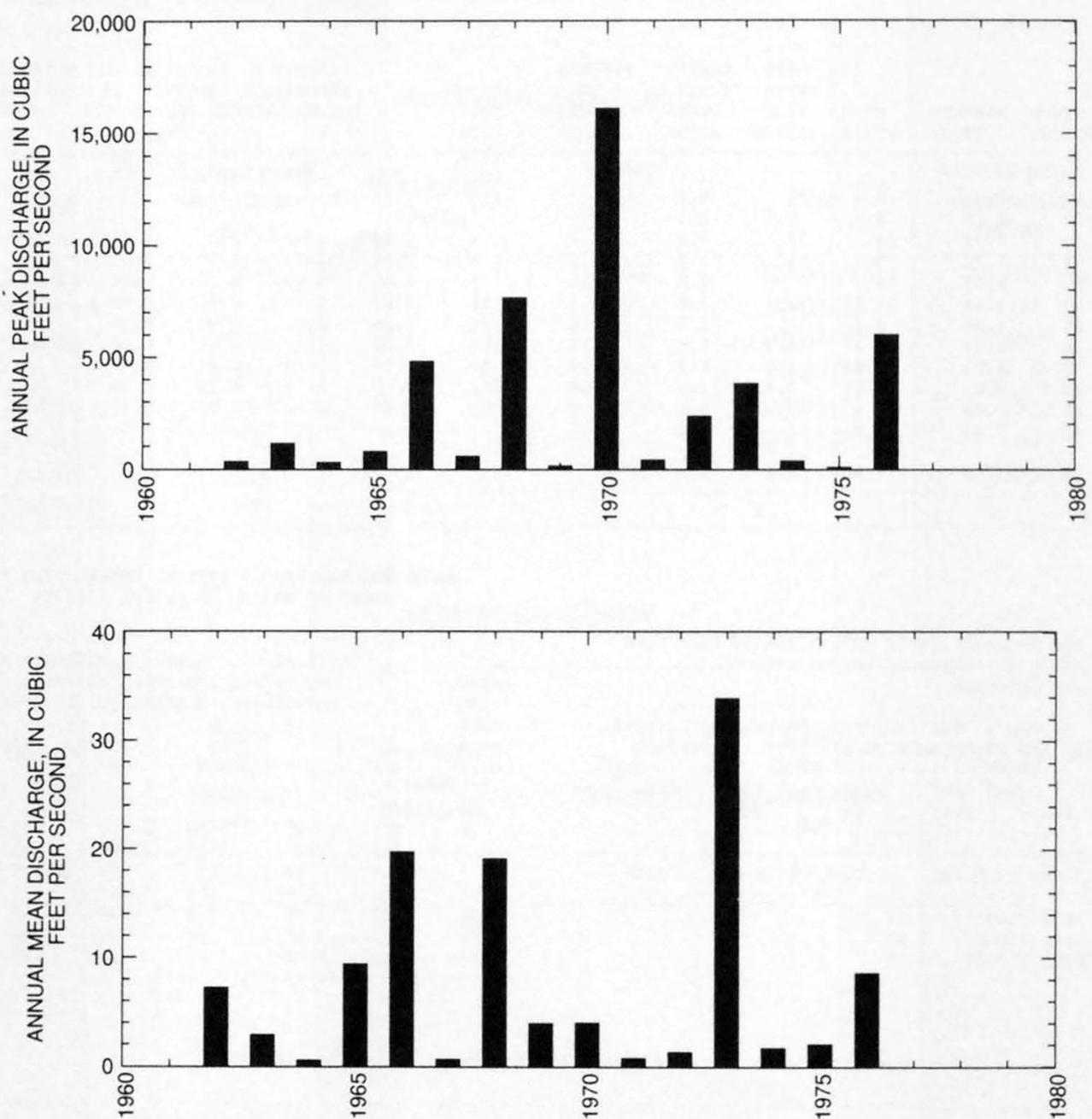
09510150 SYCAMORE CREEK NEAR SUNFLOWER, AZ--Continued
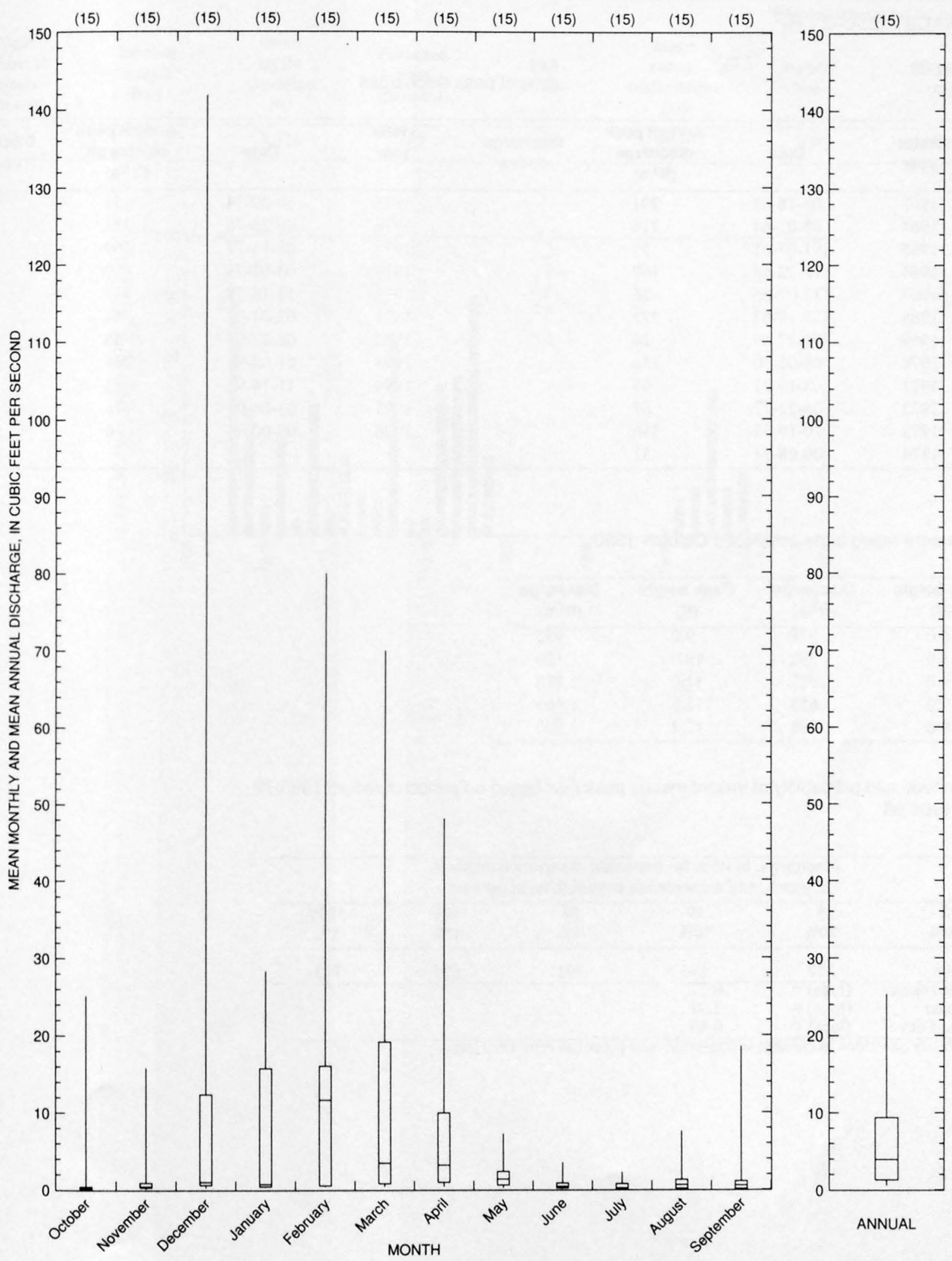


\section{CAMP CREEK NEAR SUNFLOWER, AZ}

LOCATION.--Lat 3345'35", long $11^{\circ} 29^{\prime} 44^{\prime \prime}$, in SW $1 / 4$ sec.24, T.5 N., R.8 E., Maricopa County, Hydrologic Unit 15060203 , on right bank upstream side of culvert of State Highway $87,0.5 \mathrm{mi}$ upstream from mouth, and $7 \mathrm{mi}$ south of Sunflower.

DRAINAGE AREA.--2.6 $\mathrm{mi}^{2}$.

Annual peak discharges

\begin{tabular}{|c|c|c|c|c|c|c|c|}
\hline $\begin{array}{l}\text { Water } \\
\text { year }\end{array}$ & Date & $\begin{array}{c}\text { Annual peak } \\
\text { discharge } \\
\left(\mathrm{ft}^{3} / \mathrm{s}\right)\end{array}$ & $\begin{array}{l}\text { Discharge } \\
\text { codes }\end{array}$ & $\begin{array}{l}\text { Water } \\
\text { year }\end{array}$ & Date & $\begin{array}{c}\text { Annual peak } \\
\text { discharge } \\
\left(\mathrm{ft}^{3} / \mathrm{s}\right)\end{array}$ & $\begin{array}{c}\text { Discharge } \\
\text { codes }\end{array}$ \\
\hline 1963 & $08-16-63$ & 391 & & 1975 & $10-29-74$ & 31 & \\
\hline 1964 & $08-02-64$ & 216 & & 1976 & $09-26-76$ & 252 & \\
\hline 1965 & $01-07-65$ & 51 & & 1977 & $08-16-77$ & 269 & \\
\hline 1966 & $12-22-65$ & 167 & & 1978 & $03-02-78$ & 402 & \\
\hline 1967 & $12-07-66$ & 26 & & 1979 & $12-18-78$ & 134 & \\
\hline 1968 & $12-18-67$ & 221 & & 1991 & $03-01-91$ & 64 & \\
\hline 1969 & $01-27-69$ & 26 & & 1992 & $08-23-92$ & 83 & \\
\hline 1970 & $09-05-70$ & 136 & & 1993 & 01-08-93 & 246 & \\
\hline 1971 & $08-19-71$ & 68 & & 1994 & $11-14-93$ & 83 & \\
\hline 1972 & $06-22-72$ & 67 & & 1995 & $03-06-95$ & 116 & \\
\hline 1973 & $10-19-72$ & 336 & & 1996 & $00-00-96$ & 0 & \\
\hline 1974 & $08-08-74$ & 31 & & & & & \\
\hline
\end{tabular}

Discharge rating table developed October 1990

\begin{tabular}{cccc}
\hline $\begin{array}{c}\text { Gage height } \\
(\mathbf{f t})\end{array}$ & $\begin{array}{c}\text { Discharge } \\
\left(\mathrm{ft}^{\mathbf{3}} \mathbf{s}\right)\end{array}$ & $\begin{array}{c}\text { Gage height } \\
(\mathbf{f t})\end{array}$ & $\begin{array}{c}\text { Discharge } \\
\left(\mathrm{ft}^{\mathbf{3}} / \mathbf{s}\right)\end{array}$ \\
\hline 4.0 & 278 & 9.0 & 952 \\
5.0 & 392 & 10.0 & 1,120 \\
6.0 & 517 & 11.0 & 1,280 \\
7.0 & 653 & 12.0 & 1,460 \\
8.0 & 798 & 12.2 & 1,500 \\
\hline
\end{tabular}

Magnitude and probability of instantaneous peak flow based on period of record 1963-79, 1991-96

\begin{tabular}{|c|c|c|c|c|c|}
\hline \multicolumn{6}{|c|}{$\begin{array}{l}\text { Discharge, in } \mathrm{ft}^{3} / \mathrm{s} \text {, for indicated recurrence interval } \\
\text { in years, and exceedance probablility, in percent }\end{array}$} \\
\hline 2 & 5 & 10 & 25 & $50 t$ & $100 t$ \\
\hline $50 \%$ & $20 \%$ & $10 \%$ & $4 \%$ & $2 \%$ & $1 \%$ \\
\hline 108 & 230 & 334 & 491 & 625 & 773 \\
\hline Weighted skew & $(\log s)=$ & -0.22 & & & \\
\hline Mean & $(\log s)=$ & 2.02 & & & \\
\hline Standard dev. & $(\log s)=$ & 0.40 & & & \\
\hline
\end{tabular}

f Reliability of values in column is uncertain, and potential errors are large. 
09510170 CAMP CREEK NEAR SUNFLOWER, AZ--Continued

Basin characteristics

\begin{tabular}{|c|c|c|c|c|c|c|c|}
\hline \multirow[b]{2}{*}{$\begin{array}{l}\text { Main } \\
\text { channel } \\
\text { slope } \\
(\mathrm{ft} / \mathrm{mi})\end{array}$} & \multirow[b]{2}{*}{$\begin{array}{l}\text { Stream } \\
\text { length } \\
\text { (mi) }\end{array}$} & \multirow[b]{2}{*}{$\begin{array}{c}\text { Mean } \\
\text { basin } \\
\text { elevation } \\
\text { (ft) }\end{array}$} & \multirow[b]{2}{*}{$\begin{array}{c}\text { Forested } \\
\text { area } \\
\text { (percent) }\end{array}$} & \multirow[b]{2}{*}{$\begin{array}{c}\text { Soil } \\
\text { index }\end{array}$} & \multirow[b]{2}{*}{$\begin{array}{c}\text { Mean } \\
\text { annual } \\
\text { precipitation } \\
\text { (in) }\end{array}$} & \multicolumn{2}{|c|}{ Rainfall intensity, 24-hour } \\
\hline & & & & & & $\begin{array}{c}\text { 2-year } \\
\text { (in) }\end{array}$ & $\begin{array}{l}\text { 50-year } \\
\text { (in) }\end{array}$ \\
\hline 498 & 4.7 & 3,520 & 0.0 & 3.0 & 20.0 & 2.5 & 5.0 \\
\hline
\end{tabular}

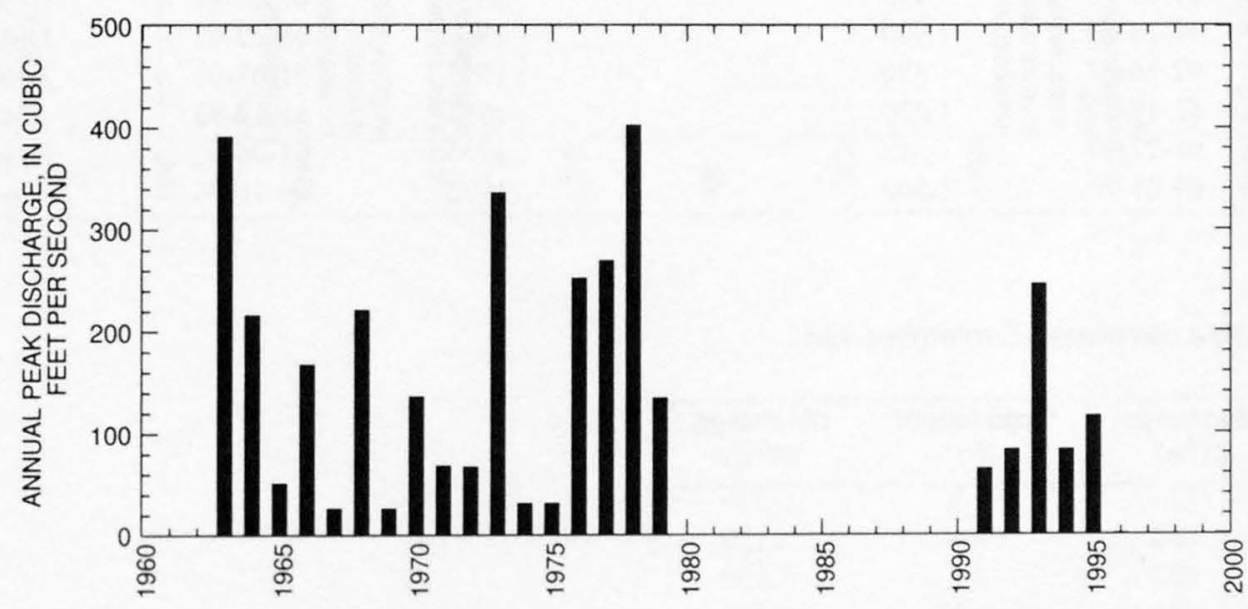




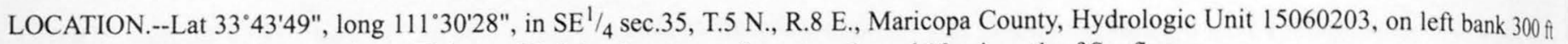
upstream from culvert on State Highway 87, 0.3 mi upstream from mouth, and $10 \mathrm{mi}$ south of Sunflower.

DRAINAGE AREA.--15.2 $\mathrm{mi}^{2}$.

Annual peak discharges

\begin{tabular}{|c|c|c|c|c|c|c|c|}
\hline $\begin{array}{l}\text { Water } \\
\text { year }\end{array}$ & Date & $\begin{array}{c}\text { Annual peak } \\
\text { discharge } \\
\left(\mathrm{ft}^{3} / \mathrm{s}\right)\end{array}$ & $\begin{array}{l}\text { Discharge } \\
\text { codes }\end{array}$ & $\begin{array}{l}\text { Water } \\
\text { year }\end{array}$ & Date & $\begin{array}{c}\text { Annual peak } \\
\text { discharge } \\
\left(\mathrm{ft}^{3} / \mathrm{s}\right)\end{array}$ & $\begin{array}{c}\text { Discharge } \\
\text { codes }\end{array}$ \\
\hline 1963 & $08-16-63$ & 262 & & 1971 & $08-19-71$ & 924 & \\
\hline 1964 & $08-01-64$ & 916 & & 1972 & $06-22-72$ & 151 & \\
\hline 1965 & $01-07-65$ & 175 & & 1991 & $03-01-91$ & 1,140 & \\
\hline 1966 & $12-22-65$ & 1,900 & & 1992 & $08-23-92$ & 1,140 & \\
\hline 1967 & $07-16-67$ & 570 & & 1993 & $01-08-93$ & 2,550 & \\
\hline 1968 & $12-19-67$ & 1,230 & & 1994 & $11-14-93$ & 242 & \\
\hline 1969 & $01-27-69$ & 43 & & 1995 & $03-06-95$ & 1,240 & \\
\hline 1970 & $09-05-70$ & 1,540 & & 1996 & $09-11-96$ & 65 & \\
\hline
\end{tabular}

Discharge rating table developed September 1991

\begin{tabular}{cccc}
\hline $\begin{array}{c}\text { Gage height } \\
(\mathbf{f t})\end{array}$ & $\begin{array}{c}\text { Discharge } \\
\left(\mathbf{f t}^{\mathbf{3}} / \mathbf{s}\right)\end{array}$ & $\begin{array}{c}\text { Gage height } \\
(\mathbf{f t})\end{array}$ & $\begin{array}{c}\text { Discharge } \\
\left(\mathbf{f t}^{\mathbf{3}} / \mathbf{s}\right)\end{array}$ \\
\hline 4.0 & 126 & 6.5 & 1,570 \\
4.5 & 258 & 7.0 & 2,150 \\
5.0 & 458 & 7.5 & 2,850 \\
5.5 & 735 & 8.0 & 3,700 \\
6.0 & 1,100 & 8.5 & 4,700 \\
\hline
\end{tabular}

Magnitude and probability of instantaneous peak flow based on period of record 1963-72, 1991-96

\begin{tabular}{|c|c|c|c|c|c|}
\hline \multicolumn{6}{|c|}{$\begin{array}{l}\text { Discharge, in } \mathrm{ft}^{3} / \mathrm{s} \text {, for indicated recurrence interval } \\
\text { in years, and exceedance probablility, in percent }\end{array}$} \\
\hline 2 & 5 & 10 & 25 & $50 t$ & $100 t$ \\
\hline $50 \%$ & $20 \%$ & $10 \%$ & $4 \%$ & $2 \%$ & $1 \%$ \\
\hline 573 & 1,520 & 2,410 & 3,810 & 5,030 & 6,390 \\
\hline Weighted skew & $(\log s)=$ & -0.42 & & & \\
\hline Mean & $(\log s)=$ & 2.72 & & & \\
\hline Standard dev. & $(\log s)=$ & 0.54 & & & \\
\hline
\end{tabular}

\section{Basin characteristics}

\begin{tabular}{cccccccc}
\hline $\begin{array}{c}\text { Main } \\
\text { channel } \\
\text { slope } \\
\text { (ft/mi) }\end{array}$ & $\begin{array}{c}\text { Stream } \\
\text { length } \\
\text { (mi) }\end{array}$ & $\begin{array}{c}\text { Mean } \\
\text { basin } \\
\text { elevation } \\
\text { (ft) }\end{array}$ & $\begin{array}{c}\text { Forested } \\
\text { area } \\
\text { (percent) }\end{array}$ & $\begin{array}{c}\text { Soil } \\
\text { index }\end{array}$ & $\begin{array}{c}\text { Mean } \\
\text { annual } \\
\text { precipitation } \\
\text { (in) }\end{array}$ & $\begin{array}{c}\text { 2-year } \\
\text { (in) }\end{array}$ & $\begin{array}{c}50 \text {-year } \\
\text { (in) }\end{array}$ \\
\hline 412 & 7.3 & 3,680 & 0.2 & 3.0 & 16.0 & 2.3 \\
\hline
\end{tabular}


09510180 ROCK CREEK NEAR SUNFLOWER, AZ--Continued

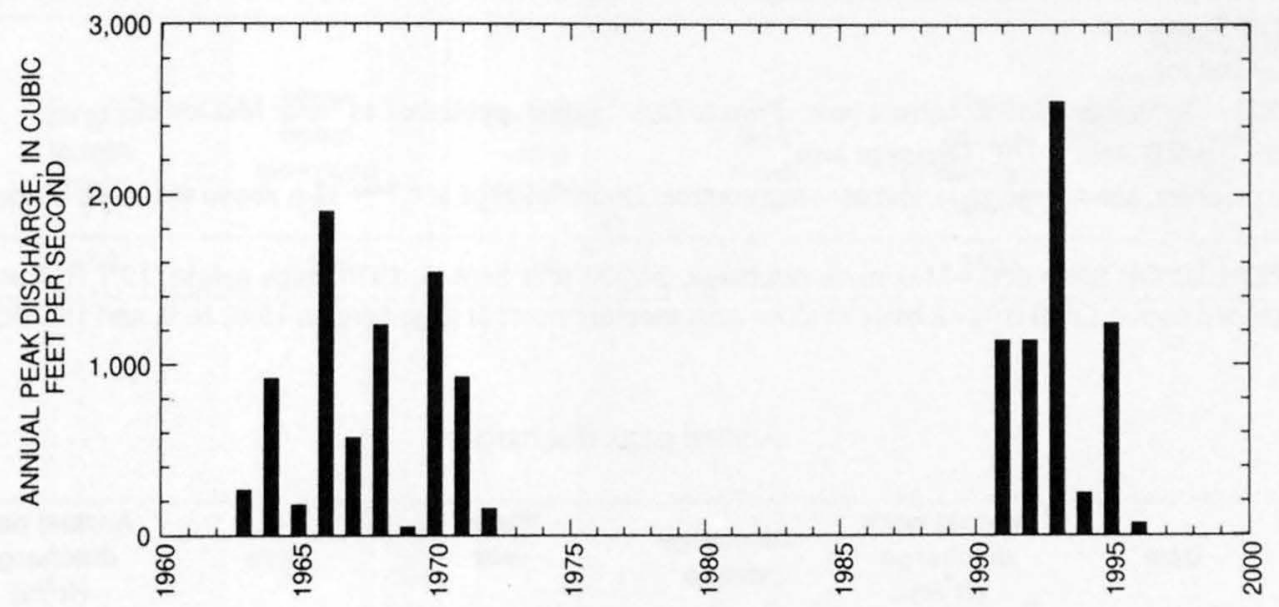


LOCATION.--Lat 33'41'39", long 111'32'28", in sec.16, T.4 N., R.8 E. (unsurveyed), Maricopa County, Hydrologic Unit 15060203, in Tonto National Forest, on right bank 0.7 mi southwest of Sugarloaf Mountain, 9 mi northeast of Fort McDowell, 10 mi upstream from mouth, and 25 mi northeast of Scottsdale.

DRAINAGE AREA.--164 $\mathrm{mi}^{2}$.

PERIOD OF RECORD.--December 1960 to current year. Prior to Oct. 1, 1963, published as "near McDowell."

REVISED RECORDS.--WRD Ariz. 1970: Drainage area.

GAGE.--Water-stage recorder, crest-stage gage, and concrete control. Datum of gage is 1,759.33 ft above sea level. Prior to Oct. 1, 1970, at datum $0.16 \mathrm{ft}$ lower.

EXTREMES FOR PERIOD OF RECORD.--Maximum discharge, 24,200 $\mathrm{ft}^{3} / \mathrm{s}$ Sept. 5, 1970, gage height, $19.7 \mathrm{ft}$, from profile past gage, from rating curve extended above $3,600 \mathrm{ft}^{3} / \mathrm{s}$ on basis of slope-area measurements at gage heights $15.0,16.0$, and 19.7 ft; no flow at times in mos years.

Annual peak discharges

\begin{tabular}{|c|c|c|c|c|c|c|c|}
\hline $\begin{array}{l}\text { Water } \\
\text { year }\end{array}$ & Date & $\begin{array}{c}\text { Annual peak } \\
\text { discharge } \\
\left(\mathrm{ft}^{3} / \mathrm{s}\right)\end{array}$ & $\begin{array}{c}\text { Discharge } \\
\text { codes }\end{array}$ & $\begin{array}{l}\text { Water } \\
\text { year }\end{array}$ & Date & $\begin{array}{c}\text { Annual peak } \\
\text { discharge } \\
\left(\mathrm{ft}^{3} / \mathrm{s}\right)\end{array}$ & $\begin{array}{c}\text { Discharge } \\
\text { codes }\end{array}$ \\
\hline 1960 & $12-25-59$ & 15,800 & & 1979 & $12-18-78$ & 9,520 & \\
\hline 1961 & $07-03-61$ & 248 & & 1980 & $02-15-80$ & 10,400 & \\
\hline 1962 & $12-16-61$ & 674 & & 1981 & $09-23-81$ & 170 & \\
\hline 1963 & $08-16-63$ & 2,860 & & 1982 & $03-14-82$ & 1,290 & \\
\hline 1964 & $08-01-64$ & 1,060 & & 1983 & $11-30-82$ & 7,440 & \\
\hline 1965 & $01-07-65$ & 1,170 & & 1984 & $09-02-84$ & 2,600 & \\
\hline 1966 & $12-14-65$ & 668 & & 1985 & $12-27-84$ & 2,960 & \\
\hline 1967 & $07-16-67$ & 1,060 & & 1986 & $03-17-86$ & 3,000 & \\
\hline 1968 & $12-19-67$ & 9,880 & & 1987 & $10-11-86$ & 319 & \\
\hline 1969 & $01-27-69$ & 216 & & 1988 & $01-18-88$ & 1,410 & \\
\hline 1970 & $09-05-70$ & ${ }^{1} 24,200$ & & 1989 & $02-05-89$ & 893 & \\
\hline 1971 & $08-19-71$ & 876 & & 1990 & $08-14-90$ & 1,740 & \\
\hline 1972 & $06-22-72$ & 1,810 & & 1991 & $03-01-91$ & 9,030 & \\
\hline 1973 & $10-19-72$ & 8,540 & & 1992 & $08-22-92$ & 3,990 & \\
\hline 1974 & $08-05-74$ & 1,030 & & 1993 & $01-08-93$ & 14,100 & \\
\hline 1975 & $04-11-75$ & 188 & & 1994 & $07-18-94$ & 3,410 & \\
\hline 1976 & $02-09-76$ & 5,470 & & 1995 & $01-05-95$ & 6,630 & \\
\hline 1977 & $08-16-77$ & 6,150 & & 1996 & $03-15-96$ & 22 & \\
\hline 1978 & $03-02-78$ & 17,900 & & & & & \\
\hline
\end{tabular}

${ }^{1}$ Highest since 1959.

Discharge rating table developed September 1991

\begin{tabular}{cccc}
\hline $\begin{array}{c}\text { Gage height } \\
(\mathrm{ft})\end{array}$ & $\begin{array}{c}\text { Discharge } \\
\left(\mathrm{ft}^{3} / \mathbf{s}\right)\end{array}$ & $\begin{array}{c}\text { Gage height } \\
(\mathbf{f t})\end{array}$ & $\begin{array}{c}\text { Discharge } \\
\left(\mathrm{ft}^{3} / \mathbf{s}\right)\end{array}$ \\
\hline 5.0 & 2,030 & 14.0 & 14,040 \\
6.0 & 2,890 & 15.0 & 15,920 \\
7.0 & 3,880 & 16.0 & 17,900 \\
8.0 & 4,990 & 17.0 & 19,940 \\
9.0 & 6,220 & 18.0 & 22,080 \\
10.0 & 7,570 & 19.0 & 24,300 \\
11.0 & 9,020 & 20.0 & 26,610 \\
12.0 & 10,590 & 21.0 & 29,010 \\
13.0 & 12,260 & 21.4 & 30,000 \\
\hline
\end{tabular}


GILA RIVER BASIN

09510200 SYCAMORE CREEK NEAR FORT MCDOWELL, AZ--Continued

Basin characteristics

\begin{tabular}{cccccccc}
\hline $\begin{array}{c}\text { Main } \\
\text { channel } \\
\text { slope } \\
(\mathrm{ft} / \mathrm{mi})\end{array}$ & $\begin{array}{c}\text { Stream } \\
\text { length } \\
(\mathrm{mi})\end{array}$ & $\begin{array}{c}\text { Mean } \\
\text { basin } \\
\text { elevation } \\
(\mathrm{ft})\end{array}$ & $\begin{array}{c}\text { Forested } \\
\text { area } \\
\text { (percent) }\end{array}$ & $\begin{array}{c}\text { Soil } \\
\text { index }\end{array}$ & $\begin{array}{c}\text { Mean } \\
\text { annual } \\
\text { precipitation } \\
\text { (in) }\end{array}$ & $\begin{array}{c}\text { Rainfall intensity, 24-hour } \\
\text { (in) }\end{array}$ & $\begin{array}{c}50 \text {-year } \\
\text { (in) }\end{array}$ \\
\hline 116 & 30.0 & 3,820 & 0.6 & 2.3 & 21.2 & 2.7 \\
\hline
\end{tabular}


09510200 SYCAMORE CREEK NEAR FORT MCDOWELL, AZ--Continued

MEAN MONTHLY AND ANNUAL DISCHARGES 1961.96

\begin{tabular}{|c|c|c|c|c|c|c|}
\hline MONTH & $\begin{array}{l}\text { MAXIMUM } \\
\text { (FT3/S) }\end{array}$ & $\begin{array}{l}\text { MINIMUM } \\
\text { (FT3/S) }\end{array}$ & $\begin{array}{c}\text { MEAN } \\
\text { (FT3/S) }\end{array}$ & $\begin{array}{l}\text { STAN- } \\
\text { DARD } \\
\text { DEVIA- } \\
\text { TION } \\
\text { (ET3/S) }\end{array}$ & $\begin{array}{l}\text { COEFFI- } \\
\text { CIENT OF } \\
\text { VARI- } \\
\text { ATION }\end{array}$ & $\begin{array}{c}\text { PERCENT } \\
\text { OF } \\
\text { ANNUAL } \\
\text { RUNOFF }\end{array}$ \\
\hline OCTOBER & 194 & 0.00 & 6.5 & 32 & 5.0 & 1.8 \\
\hline NOVEMBER & 72 & 0.00 & 7.3 & 16 & 2.2 & 2.0 \\
\hline DECEMBER & 426 & 0.00 & 48 & 105 & 2.2 & 13.3 \\
\hline JANUARY & 1,070 & 0.00 & 70 & 186 & 2.7 & 19.2 \\
\hline FEBRUARY & 852 & 0.00 & 85 & 162 & 1.9 & 23.5 \\
\hline MARCH & 881 & 0.19 & 97 & 171 & 1.8 & 26.8 \\
\hline APRIL & 120 & 0.07 & 26 & 36 & 1.4 & 7.3 \\
\hline MAY & 52 & 0.00 & 7.7 & 13 & 1.7 & 2.1 \\
\hline JUNE & 21 & 0.00 & 2.6 & 4.9 & 1.9 & 0.7 \\
\hline JULY & 15 & 0.00 & 2.1 & 3.2 & 1.5 & 0.6 \\
\hline AUGUST & 52 & 0.00 & 4.8 & 9.9 & 2.1 & 1.3 \\
\hline SEPTEMBER & 93 & 0.00 & 4.6 & 16 & 3.4 & 1.3 \\
\hline ANNUAL & 155 & 0.23 & 30 & 40 & 1.3 & 100 \\
\hline
\end{tabular}

MAGNITUDE AND PROBABILITY OF INSTANTANEOUS PEAK FLOW BASED ON PERIOD OF RECORD 1960.96

DISCHARGE, IN FT3/S, FOR INDICATED RECURRENCE INTERVAL IN YEARS, AND EXCEEDANCE PROBABILITY, IN PERCENT

\begin{tabular}{|c|c|c|c|c|c|}
\hline 2 & 5 & 10 & 25 & 50 & 100 \\
\hline 508 & 208 & 108 & $4 \%$ & 28 & 18 \\
\hline 2,330 & 7,320 & 12,900 & 23,200 & 33,600 & 46,300 \\
\hline
\end{tabular}

WEIGHTED SKEW (LOGS) $=-0.23$

MEAN $\quad($ LOGS $)=3.34$

STANDARD DEV. $($ LOGS $)=0.61$
MAGNITUDE AND PROBABILITY OF ANNUAL LOW FLOW BASED ON PERIOD OF RECORD 1962.96

\begin{tabular}{|c|c|c|c|c|c|c|}
\hline $\begin{array}{l}\text { PERIOD } \\
\text { (CON. }\end{array}$ & \multicolumn{6}{|c|}{$\begin{array}{l}\text { DISCHARGE, IN FT3/S, FOR INDICATED } \\
\text { RECURRENCE INTERVAL, IN YEARS, AND } \\
\text { NON-EXCEEDANCE PROBABILITY, IN PERCENT }\end{array}$} \\
\hline SECU. & & $\ldots \ldots$ & $\ldots \ldots$ & $\ldots \ldots$ & & \\
\hline TIVE & 2 & 5 & 10 & 20 & $50 \#$ & $100 \#$ \\
\hline DAYS) & 508 & 208 & 108 & 58 & 28 & 18 \\
\hline 1 & 0.00 & 0.00 & 0.00 & 0.00 & 0.00 & 0.00 \\
\hline 3 & 0.01 & 0.00 & 0.00 & 0.00 & 0.00 & 0.00 \\
\hline 7 & 0.00 & 0.00 & 0.00 & 0.00 & 0.00 & 0.00 \\
\hline 14 & 0.01 & 0.00 & 0.00 & 0.00 & 0.00 & 0.00 \\
\hline 30 & 0.03 & 0.00 & 0.00 & 0.00 & 0.00 & 0.00 \\
\hline 60 & 0.08 & 0.00 & 0.00 & 0.00 & 0.00 & 0.00 \\
\hline 90 & 0.18 & 0.02 & 0.00 & 0.00 & 0.00 & 0.00 \\
\hline 120 & 0.34 & 0.07 & 0.02 & 0.00 & 0.00 & 0.00 \\
\hline 183 & 1.0 & 0.27 & 0.11 & 0.00 & 0.00 & 0.00 \\
\hline
\end{tabular}

MAGNITUDE AND PROBABILITY OF ANNUAL HIGH FLOW BASED ON PERIOD OF RECORD 1961.96

\begin{tabular}{|c|c|c|c|c|c|c|}
\hline & \multicolumn{6}{|c|}{ DISCHARGE, IN FT3/S, FOR INDICATED } \\
\hline $\begin{array}{l}\text { PERIOD } \\
\text { (CON - }\end{array}$ & & $\begin{array}{l}\text { RECURRE } \\
\text { EXCEEDF }\end{array}$ & $\begin{array}{ll}C E & \text { INTE } \\
C E & \text { PROE }\end{array}$ & $\begin{array}{l}\text { VAL, IN } \\
\text { BILITY, }\end{array}$ & $\begin{array}{l}\text { YEARS, } \\
\text { IN PERC }\end{array}$ & $\begin{array}{l}\text { AND } \\
\text { ENT }\end{array}$ \\
\hline SECU - & $\cdots$ & $\ldots \ldots$ & - & - & $\ldots \ldots$ & $\ldots \ldots \ldots$ \\
\hline TIVE & 2 & 5 & 10 & 25 & $50 \#$ & $100 \#$ \\
\hline DAYS) & 508 & 208 & 108 & 48 & 28 & 18 \\
\hline 1 & 631 & 2,350 & 4,380 & 8.170 & 11,900 & 16,500 \\
\hline 3 & 354 & 1,320 & 2,470 & 4,650 & 6,840 & 9,540 \\
\hline 7 & 199 & 766 & 1,460 & 2,770 & 4,100 & 5,740 \\
\hline 15 & 117 & 464 & 892 & 1,700 & 2,520 & 3,530 \\
\hline 30 & 76 & 305 & 579 & 1,080 & 1,560 & 2,130 \\
\hline 60 & 49 & 207 & 400 & 757 & 1,100 & 1,510 \\
\hline 90 & 36 & 153 & 301 & 584 & 869 & 1,220 \\
\hline
\end{tabular}

DURATION TABLE OF DAILY MEAN FLOW FOR PERIOD OF RECORD 1961.96

DISCHARGE, IN FT3/S, WHICH WAS EQUALED OR EXCEEDED FOR INDICATED PERCENT OF TIME

\begin{tabular}{|c|c|c|c|c|c|c|c|c|c|c|c|c|c|c|c|c|}
\hline 18 & 58 & 108 & $15 \%$ & 208 & 308 & 408 & 508 & 608 & 708 & 808 & 908 & 958 & 988 & 998 & 99.58 & 99.98 \\
\hline 515 & 112 & 47 & 25 & 12 & 3.7 & 1.6 & 0.76 & 0.33 & 0.11 & 0.05 & 0.00 & 0.00 & 0.00 & 0.00 & 0.00 & 0.00 \\
\hline
\end{tabular}

\# Reliability of values in column is uncertain, and potential errors are large. 
09510200 SYCAMORE CREEK NEAR FORT MCDOWELL, AZ--Continued
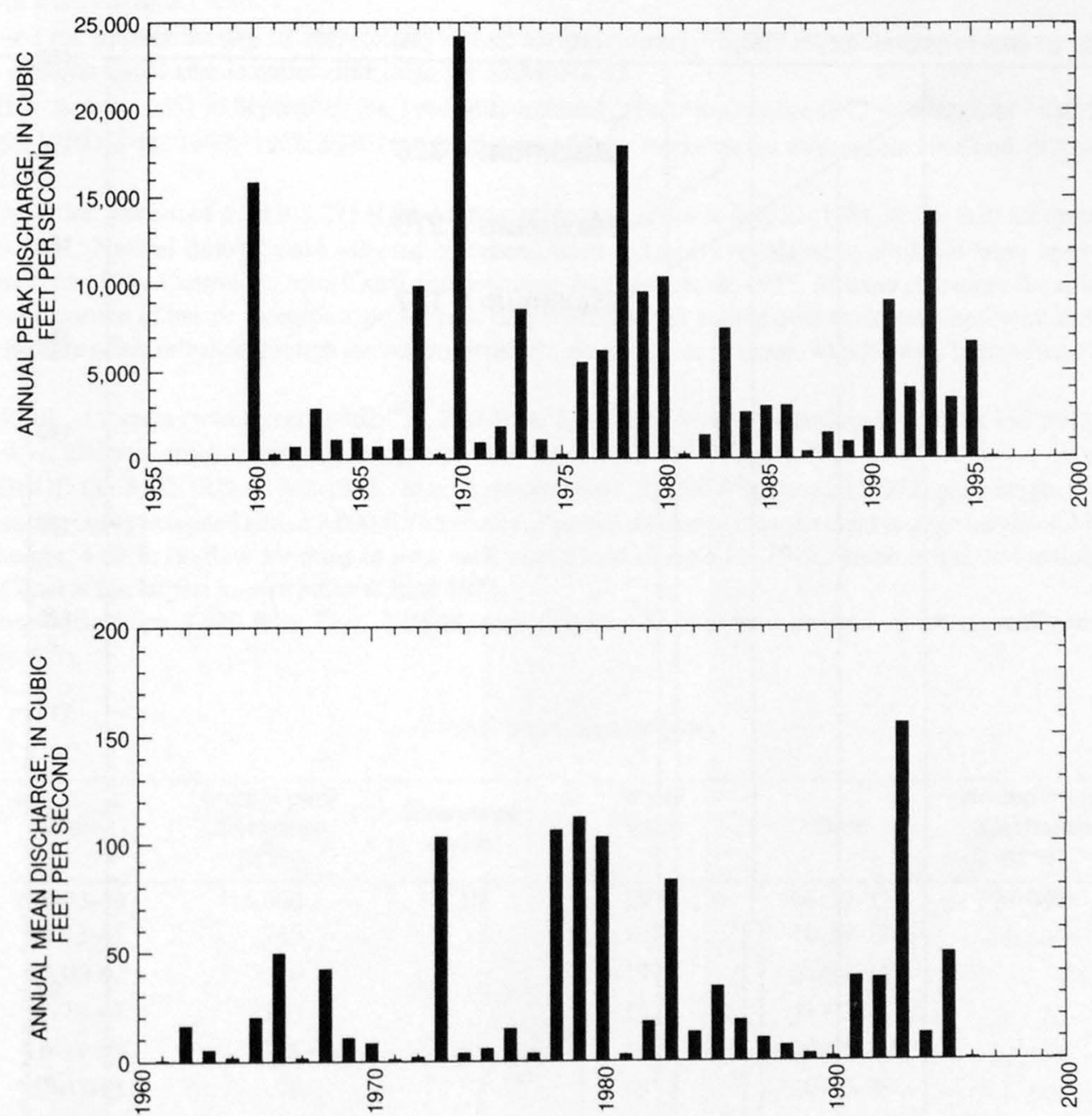
770

GILA RIVER BASIN

09510200 SYCAMORE CREEK NEAR FORT MCDOWELL, AZ--Continued
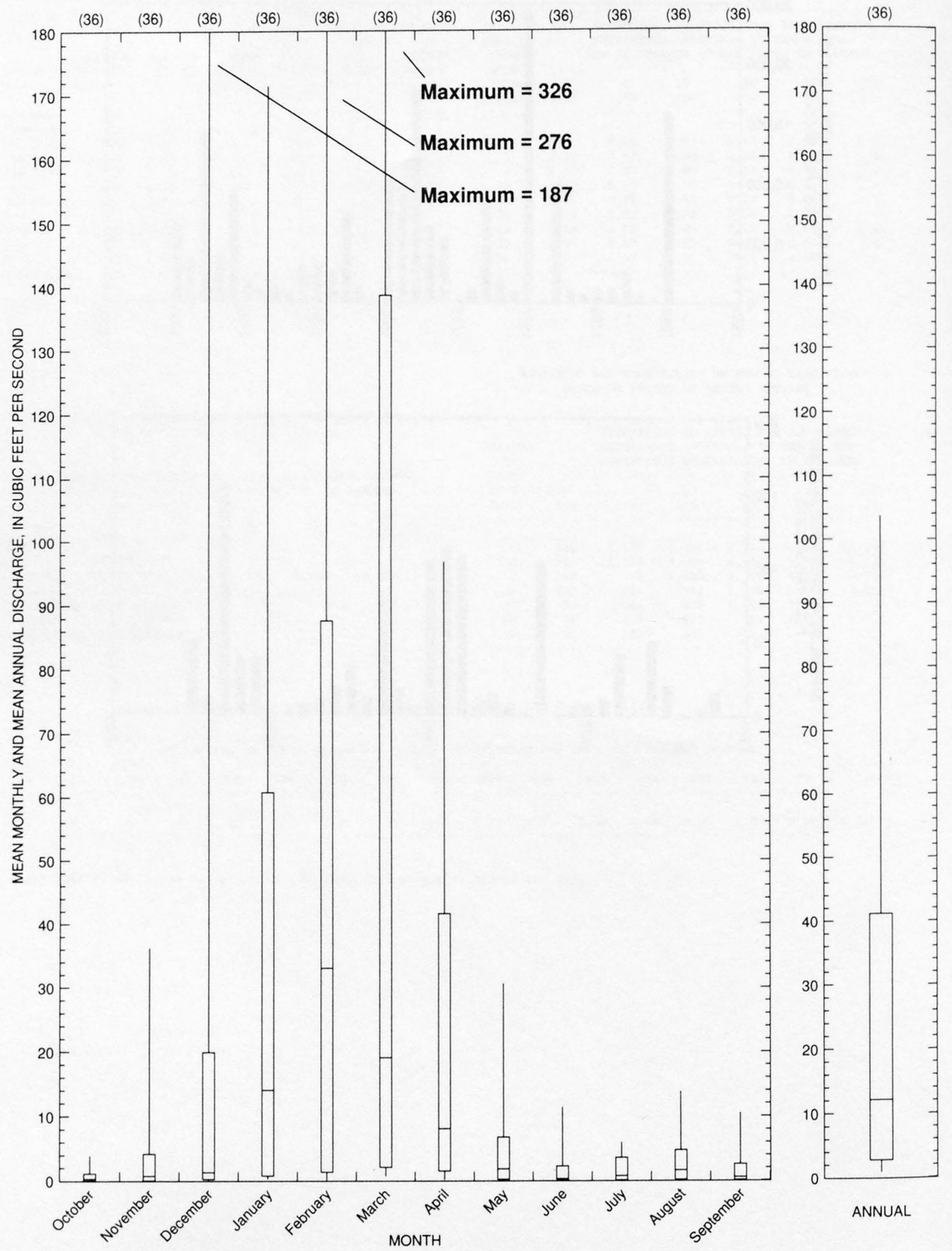


\section{INDIAN BEND WASH AT SCOTTSDALE, AZ}

LOCATION.--Lat 33 $32^{\prime} 08^{\prime \prime}$, long $111^{\circ} 54^{\prime} 50^{\prime \prime}$, in NW $\frac{1}{4} \mathrm{NE}^{1} / 4$ sec.11, T.2 N., R.4 E., Maricopa County, Hydrologic Unit 15060106, on right bank $100 \mathrm{ft}$ downstream from Arizona Canal.

DRAINAGE AREA.--62 $\mathrm{mi}^{2}$ approximately, since October 1975. Prior to October 1975, $139 \mathrm{mi}^{2}$; reduction caused by cutoff of upper portion of basin by Central Arizona Canal and detention dike (also see REMARKS).

PERIOD OF RECORD.--January 1961 to September 30, 1984 (discontinued). Prior to October 1972 published as "near Scottsdale."

REVISED RECORDS.--WRD Ariz., 1972: 1968, 1970 (revised figures of daily discharge for water years 1968 and 1970 were inadvertently omitted from WSP 2126).

GAGE.--Water-stage recorder. Datum of gage is $1,275 \mathrm{ft}$ from topographic map. Prior to July 31, 1981, at site $0.25 \mathrm{mi}$ upstream at different datum.

REMARKS.--Records poor. Natural flow of wash affected by urbanization and partly regulated by artificial lakes upstream. Upper portion of basin (about $77 \mathrm{mi}$ ) cut off by Central Arizona Canal and detention dike in October 1975. Release of excess flood waters may occasionally pass canal into lower portion of basin. Records at present site may include water spilled over emergency spillway along north side of Arizona Canal but do not include water released through the control structure on south side of canal, which enters Indian Bend Wash downstream from the gage.

AVERAGE DISCHARGE.--15 years (water years 1962-75), $2.67 \mathrm{ft} \mathrm{ft}^{3} / \mathrm{s}, 1,930 \mathrm{acre}-\mathrm{ft} / \mathrm{yr}$, representing flow from $139 \mathrm{mi}^{2}$; median of yearly mean discharges, $0.30 \mathrm{ft}^{3} / \mathrm{s}, 220$ acre-ft/yr. 9 years (water years 1976-84), $2.54 \mathrm{ft}^{3} / \mathrm{s}, 1,840$ acre-ft/yr.

EXTREMES FOR PERIOD OF RECORD.--1962-1975: Maximum discharge, 21,000 ft $3 / \mathrm{s}$ June 22, 1972, gage height, $4.90 \mathrm{ft}$, at site and datum then in use, from rating curve extended above $7,000 \mathrm{ft}^{3} / \mathrm{s}$ on basis of partial discharge measurement at gage height, $4.2 \mathrm{ft}$ and slope-conveyence method at gage height, $4.90 \mathrm{ft}$; no flow for most of time each year. Flood of June 22, 1972, which originated mainly downstream from the Central Arizona Canal is the largest known since at least 1922.

1976-84: Maximum discharge, 5,000 ft $3 / \mathrm{s}$, Sept. 2, 1984, gage height, $4.35 \mathrm{ft}$ at new site and datum currently in use, from rating curve extended above $96 \mathrm{ft}^{3} / \mathrm{s}$.

Annual peak discharges

\begin{tabular}{|c|c|c|c|c|c|c|c|}
\hline $\begin{array}{l}\text { Water } \\
\text { year }\end{array}$ & Date & $\begin{array}{c}\text { Annual peak } \\
\text { discharge } \\
\left(\mathrm{ft}^{3} / \mathrm{s}\right)\end{array}$ & $\begin{array}{l}\text { Discharge } \\
\text { codes }\end{array}$ & $\begin{array}{l}\text { Water } \\
\text { year }\end{array}$ & Date & $\begin{array}{c}\text { Annual peak } \\
\text { discharge } \\
\left(\mathrm{ft}^{3} / \mathbf{s}\right)\end{array}$ & $\begin{array}{c}\text { Discharge } \\
\text { codes }\end{array}$ \\
\hline 1943 & $08-03-43$ & 15,000 & HP & 1972 & $06-22-72$ & ' 21,000 & C \\
\hline 1961 & $09-13-61$ & 745 & & 1973 & $10-19-72$ & 9,600 & C \\
\hline 1962 & $00-00-62$ & 0 & & 1974 & $07-31-74$ & 9.4 & C \\
\hline 1963 & $07-19-63$ & 350 & & 1975 & $11-03-74$ & 20 & ES,C \\
\hline 1964 & $10-19-63$ & 328 & & 1976 & $09-25-76$ & 3,500 & C \\
\hline 1965 & $02-07-65$ & 76 & & 1977 & $10-23-76$ & 140 & C \\
\hline 1966 & $09-13-66$ & 596 & & 1978 & $03-02-78$ & 3,180 & C \\
\hline 1967 & $07-17-67$ & 248 & & 1979 & $01-17-79$ & 252 & C \\
\hline 1968 & $12-19-67$ & 5,620 & & 1980 & $02-15-80$ & 125 & C \\
\hline 1969 & $00-00-69$ & 0 & & 1981 & $07-30-81$ & 450 & C \\
\hline 1970 & $09-05-70$ & 2,150 & $\mathrm{C}$ & 1983 & $00-00-82$ & 150 & C \\
\hline 1971 & $08-10-71$ & 85 & $\mathrm{C}$ & 1984 & $08-16-83$ & 3,830 & $\mathrm{C}$ \\
\hline
\end{tabular}

'Highest since 1922.

Discharge rating table developed October 1982

\begin{tabular}{cccc}
\hline $\begin{array}{c}\text { Gage height } \\
(\mathbf{f t})\end{array}$ & $\begin{array}{c}\text { Discharge } \\
\left(\mathrm{ft}^{3} / \mathbf{s}\right)\end{array}$ & $\begin{array}{c}\text { Gage height } \\
(\mathrm{ft})\end{array}$ & $\begin{array}{c}\text { Discharge } \\
\left(\mathrm{ft}^{3} / \mathbf{s}\right)\end{array}$ \\
\hline 1.8 & 110 & 3.0 & 1,310 \\
2.0 & 205 & 3.2 & 1,670 \\
2.2 & 336 & 3.4 & 2,100 \\
2.4 & 509 & 3.6 & 2,590 \\
2.6 & 726 & 3.8 & 3,130 \\
2.8 & 990 & 4.0 & 3,740 \\
\hline
\end{tabular}


09512100 INDIAN BEND WASH AT SCOTTSDALE, AZ--Continued

Basin characteristics

\begin{tabular}{cccccccc}
\hline $\begin{array}{c}\text { Main } \\
\text { channel } \\
\text { slope } \\
(\mathrm{ft} / \mathrm{mi})\end{array}$ & $\begin{array}{c}\text { Stream } \\
\text { length } \\
(\mathrm{mi})\end{array}$ & $\begin{array}{c}\text { Mean } \\
\text { basin } \\
\text { elevation } \\
\text { (ft) }\end{array}$ & $\begin{array}{c}\text { Forested } \\
\text { area } \\
\text { (percent) }\end{array}$ & $\begin{array}{c}\text { Soil } \\
\text { index }\end{array}$ & $\begin{array}{c}\text { Mean } \\
\text { annual } \\
\text { precipitation } \\
\text { (in) }\end{array}$ & $\begin{array}{c}\text { Rainfall intensity, 24-hour } \\
\text { (in) }\end{array}$ & $\begin{array}{c}50 \text {-year } \\
\text { (in) }\end{array}$ \\
\hline 60.0 & 23.3 & 1,780 & 0.0 & 2.4 & 10.9 & 1.7 \\
\hline
\end{tabular}


09512100 INDIAN BEND WASH AT SCOTTSDALE, AZ--Continued

MEAN MONTHLY AND ANNUAL DISCHARGES 1961.84

\begin{tabular}{|c|c|c|c|c|c|c|}
\hline MONTH & $\begin{array}{l}\text { MAXIMUM } \\
(\mathrm{FT} 3 / \mathrm{S})\end{array}$ & $\begin{array}{l}\text { MINIMUM } \\
\text { (FT3/S) }\end{array}$ & $\begin{array}{c}\text { MEAN } \\
(\mathrm{PT} 3 / \mathrm{S})\end{array}$ & $\begin{array}{l}\text { STAN- } \\
\text { DARD } \\
\text { DEVIA- } \\
\text { TION } \\
\text { (FT3/S) }\end{array}$ & $\begin{array}{l}\text { COEFFI- } \\
\text { CIENT OF } \\
\text { VARI- } \\
\text { ATION }\end{array}$ & $\begin{array}{c}\text { PERCENT } \\
\text { OF } \\
\text { ANNUAL } \\
\text { RUNOFF }\end{array}$ \\
\hline OCTOBER & 192 & 0.00 & 8.2 & 39 & 4.8 & 27.1 \\
\hline NOVEMBER & 7.0 & 0.00 & 0.69 & 1.8 & 2.7 & 2.3 \\
\hline DECEMBER & 49 & 0.00 & 2.7 & 9.9 & 3.7 & 8.7 \\
\hline JANUARY & 4.3 & 0.00 & 0.38 & 1.0 & 2.7 & 1.3 \\
\hline FEBRUARY & 9.2 & 0.00 & 0.92 & 2.4 & 2.6 & 3.0 \\
\hline MARCH & 45 & 0.00 & 2.9 & 9.7 & 3.3 & 9.6 \\
\hline APRIL & 0.26 & 0.00 & 0.01 & 0.05 & 4.9 & 0.0 \\
\hline MAY & 1.4 & 0.00 & 0.10 & 0.35 & 3.4 & 0.3 \\
\hline JUNE & 150 & 0.00 & 6.3 & 31 & 4.9 & 20.6 \\
\hline JULY & 6.1 & 0.00 & 0.87 & 1.8 & 2.1 & 2.9 \\
\hline AUGUST & 24 & 0.00 & 1.3 & 4.9 & 3.9 & 4.1 \\
\hline SEPTEMBER & 71 & 0.00 & 6.1 & 16 & 2.6 & 20.0 \\
\hline ANNUAL & 17 & 0.00 & 2.5 & 4.2 & 1.7 & 100 \\
\hline
\end{tabular}

MAGNITUDE AND PROBABILITY OF INSTANTANEOUS PEAK FLOW BASED ON PERIOD OF RECORD 1943, 1961-70

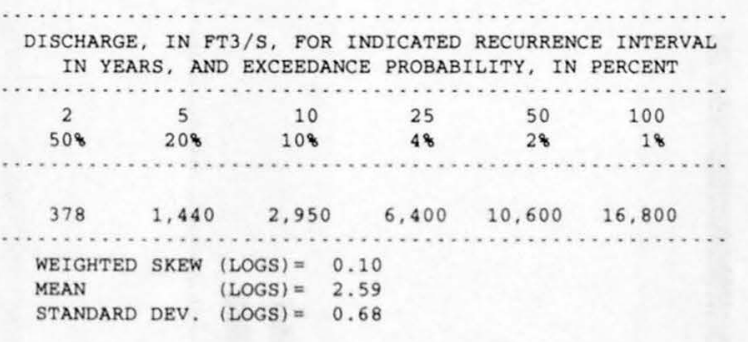

MAGNITUDE AND PROBABILITY OF ANNUAL LOW FLOW BASED ON PERIOD OF RECORD $1962 \cdot 84$

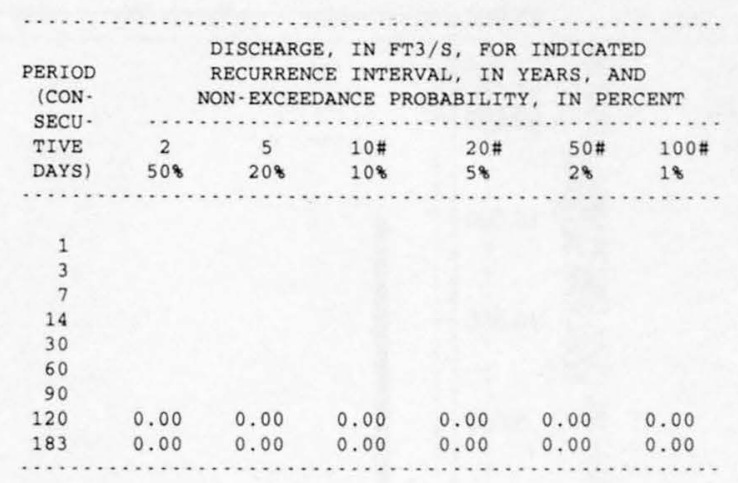

MAGNITUDE AND PROBABILITY OF ANNUAL HIGH FLOW BASED ON PERIOD OF RECORD 1961-84

\begin{tabular}{|c|c|c|c|c|c|c|}
\hline \multirow{3}{*}{$\begin{array}{l}\text { PERIOD } \\
\text { (CON- }\end{array}$} & & \multicolumn{5}{|c|}{ DISCHARGE, IN FT3/S, FOR INDICATED } \\
\hline & & RECURR & E INTE & NAL，IN & YEARS, & AND \\
\hline & & EXCEED & E PROB & BILITY, & IN PERC & ENT \\
\hline SECU - & & ...... & & & $-2,0-0$ & - \\
\hline TIVE & 2 & 5 & $10 \#$ & $25 \#$ & $50 \#$ & $100 \#$ \\
\hline DAYS) & 508 & 208 & 108 & 48 & 28 & 18 \\
\hline 1 & 100 & 618 & 1,490 & 3,560 & 6,170 & 10,100 \\
\hline 3 & 44 & 262 & 610 & 1,390 & 2,320 & 3,650 \\
\hline 7 & 21 & 121 & 275 & 605 & 982 & 1,500 \\
\hline 15 & 11 & 65 & 150 & 335 & 549 & 843 \\
\hline 30 & 5.5 & 34 & 78 & 176 & 290 & 448 \\
\hline 60 & 3.1 & 18 & 42 & 95 & 156 & 243 \\
\hline 90 & 2.1 & 13 & 29 & 64 & 106 & 164 \\
\hline
\end{tabular}

DURATION TABLE OF DAILY MEAN FLOW FOR PERIOD OF RECORD 1961.84 DISCHARGE, IN FT3/S, WHICH WAS EQUALED OR EXCEEDED FOR INDICATED PERCENT OF TIME

\begin{tabular}{|c|c|c|c|c|c|c|c|c|c|c|c|c|c|c|c|c|}
\hline 18 & 58 & 108 & 158 & 208 & 308 & 408 & 508 & 608 & 708 & 808 & 908 & 958 & 988 & 998 & 99.58 & 99.98 \\
\hline 20 & 0.00 & 0.00 & 0.00 & 0.00 & 0.00 & 0.00 & 0.00 & 0.00 & 0.00 & 0.00 & 0.00 & 0.00 & 0.00 & 0.00 & 0.00 & 0.00 \\
\hline
\end{tabular}

\# Reliability of values in column is uncertain, and potential errors are large. 


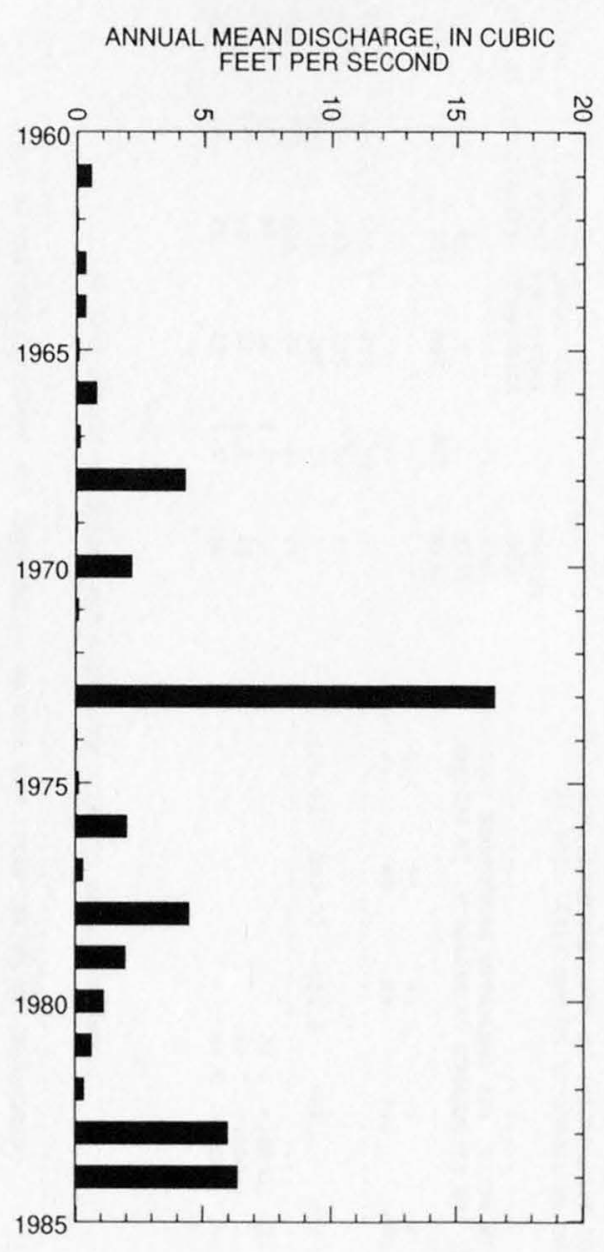

ANNUAL PEAK DISCHARGE, IN CUBIC FEET PER SECOND

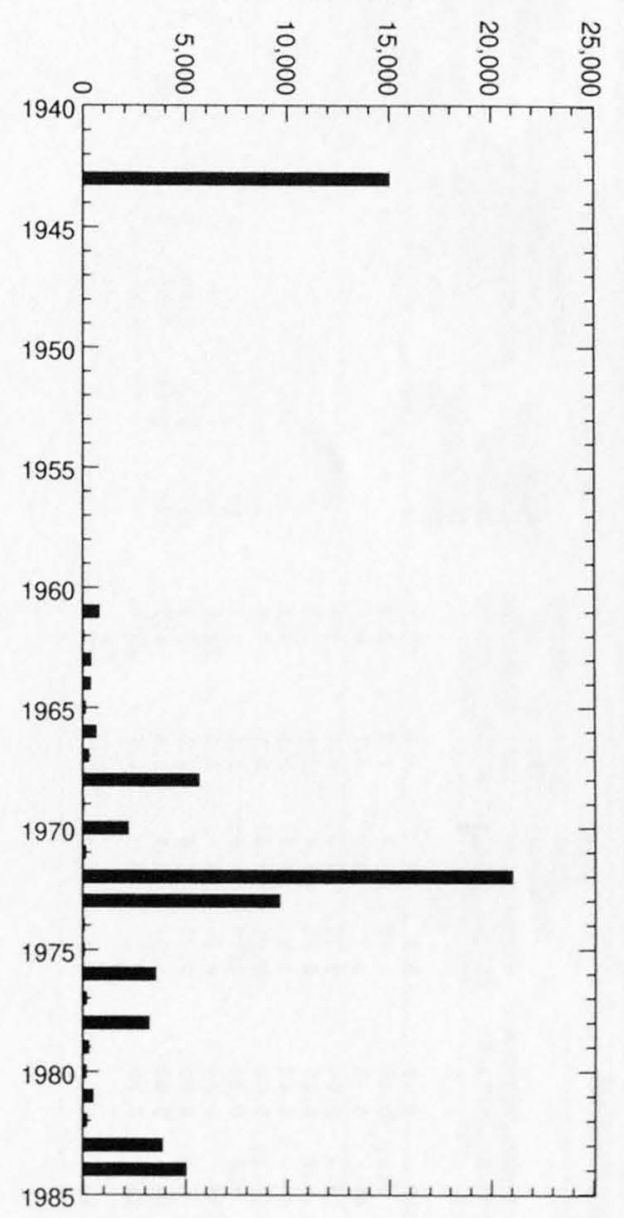


GILA RIVER BASIN

775

09512100 INDIAN BEND WASH AT SCOTTSDALE, AZ--Continued
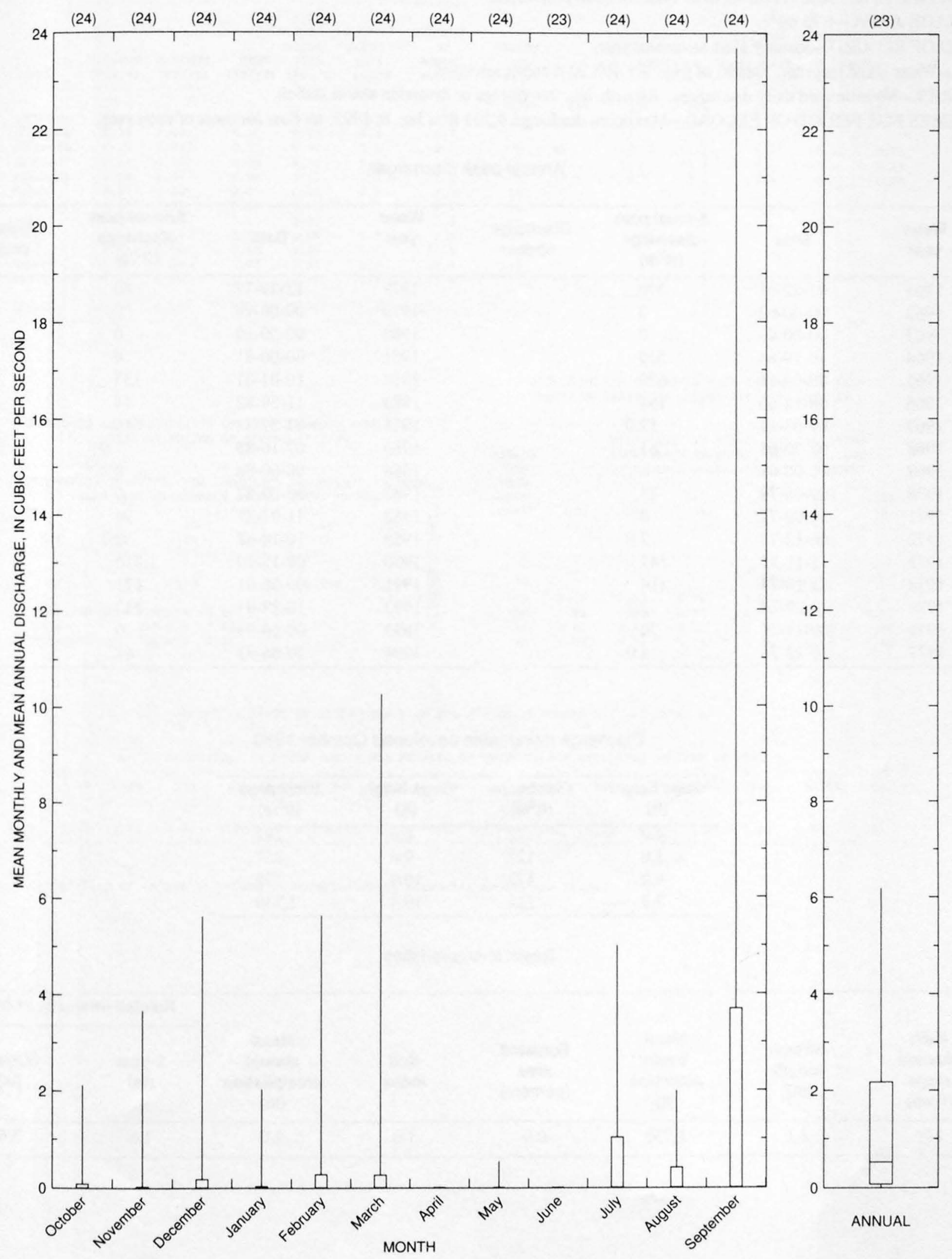


\section{SALT RIVER TRIBUTARY IN SOUTH MOUNTAIN PARK, AT PHOENIX, AZ}

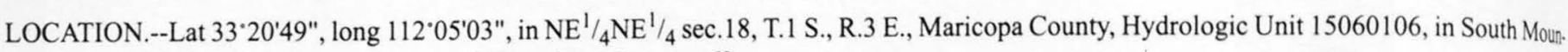
tain Park, on left bank 7.4 mi south of Phoenix main post office.

DRAINAGE AREA.--1.75 $\mathrm{mi}^{2}$.

PERIOD OF RECORD.--January 1961 to current year.

GAGE.--Water-stage recorder. Datum of gage is $1,405.20 \mathrm{ft}$ above sea level.

REMARKS.--No estimated daily discharges. Records fair. No storage or diversion above station.

EXTREMES FOR PERIOD OF RECORD.--Maximum discharge, 9,200 ft³ Jan. 8, 1993; no flow for most of each year.

Annual peak discharges

\begin{tabular}{ccccccc}
\hline $\begin{array}{c}\text { Water } \\
\text { year }\end{array}$ & Date & $\begin{array}{c}\text { Annual peak } \\
\text { discharge } \\
\left(\mathbf{f t}^{3} / \mathbf{s}\right)\end{array}$ & $\begin{array}{c}\text { Discharge } \\
\text { codes }\end{array}$ & $\begin{array}{c}\text { Water } \\
\text { year }\end{array}$ & $\begin{array}{c}\text { Annual peak } \\
\text { Discharge } \\
\left.\mathbf{f t}^{3} / \mathbf{s}\right)\end{array}$ & $\begin{array}{c}\text { Discharge } \\
\text { codes }\end{array}$ \\
\hline 1961 & $07-22-61$ & 370 & & 1978 & $12-29-77$ & 86 \\
1962 & $00-00-62$ & 0 & 1979 & $00-00-79$ & 0 \\
1963 & $00-00-63$ & 0 & 1980 & $00-00-80$ & 0 \\
1964 & $10-19-63$ & 530 & 1981 & $00-00-81$ & 0 \\
1965 & $09-04-65$ & 670 & 1982 & $10-01-81$ & 133 \\
1966 & $08-18-66$ & 194 & 1983 & $11-30-82$ & 44 \\
1967 & $09-03-67$ & 12.0 & 1984 & $07-27-84$ & 644 \\
1968 & $07-30-68$ & 81 & 1985 & $07-16-85$ & 7.0 \\
1969 & $00-00-69$ & 0 & 1986 & $00-00-86$ & 0 \\
1970 & $09-05-70$ & 77 & 1987 & $00-00-87$ & 0 \\
1971 & $00-00-71$ & 0 & 1988 & $11-01-87$ & \\
1972 & $08-12-72$ & 2.0 & 1989 & $10-14-88$ & 94 \\
1973 & $11-11-72$ & 147 & 1990 & $08-15-90$ & 9.2 \\
1974 & $03-20-74$ & 114 & 1991 & $09-05-91$ & 1,210 \\
1975 & $10-29-74$ & 3.5 & 1992 & $10-27-91$ & 171 \\
1976 & $09-23-76$ & 70 & 1993 & $00-00-93$ & 232 \\
1977 & $10-23-76$ & 5.0 & 1994 & $10-06-93$ & 0 \\
\hline
\end{tabular}

Discharge rating table developed October 1989

\begin{tabular}{crcr}
\hline $\begin{array}{c}\text { Gage height } \\
(\mathrm{ft})\end{array}$ & $\begin{array}{c}\text { Discharge } \\
\left(\mathrm{ft}^{3} / \mathbf{s}\right)\end{array}$ & $\begin{array}{c}\text { Gage height } \\
(\mathbf{f t})\end{array}$ & $\begin{array}{c}\text { Discharge } \\
\left(\mathrm{ft}^{\mathbf{3}} / \mathbf{s}\right)\end{array}$ \\
\hline 4.0 & 72 & 8.0 & 262 \\
5.0 & 122 & 9.0 & 357 \\
6.0 & 172 & 10.0 & 792 \\
7.0 & 222 & 10.4 & 1,340 \\
\hline
\end{tabular}

Basin characteristics

\begin{tabular}{|c|c|c|c|c|c|c|c|}
\hline \multirow[b]{2}{*}{$\begin{array}{c}\text { Main } \\
\text { channel } \\
\text { slope } \\
(\mathrm{ft} / \mathrm{mi})\end{array}$} & \multirow[b]{2}{*}{$\begin{array}{l}\text { Stream } \\
\text { length } \\
\text { (mi) }\end{array}$} & \multirow[b]{2}{*}{$\begin{array}{c}\text { Mean } \\
\text { basin } \\
\text { elevation } \\
(f t)\end{array}$} & \multirow[b]{2}{*}{$\begin{array}{l}\text { Forested } \\
\text { area } \\
\text { (percent) }\end{array}$} & \multirow[b]{2}{*}{$\begin{array}{c}\text { Soil } \\
\text { index }\end{array}$} & \multirow[b]{2}{*}{$\begin{array}{c}\text { Mean } \\
\text { annual } \\
\text { precipitation } \\
\text { (in) }\end{array}$} & \multicolumn{2}{|c|}{ Rainfall intensity, 24-hour } \\
\hline & & & & & & $\begin{array}{l}\text { 2-year } \\
\text { (in) }\end{array}$ & $\begin{array}{l}\text { 50-year } \\
\text { (in) }\end{array}$ \\
\hline 244 & 2.1 & 1,730 & 0.0 & 1.0 & 9.0 & 1.6 & 3.6 \\
\hline
\end{tabular}


09512200 SALT RIVER TRIBUTARY IN SOUTH MOUNTAIN PARK, AT PHOENIX, AZ--Continued

MEAN MONTHLY AND ANNUAL DISCHARGES 1961.92, 1994.96

\begin{tabular}{|c|c|c|c|c|c|c|}
\hline MONTH & $\begin{array}{l}\text { MAXIMUM } \\
(\text { FT3/S) }\end{array}$ & $\begin{array}{l}\text { MINIMUM } \\
(\text { FT3/S) }\end{array}$ & $\begin{array}{c}\text { MEAN } \\
(\text { FT3/S) }\end{array}$ & $\begin{array}{l}\text { STAN- } \\
\text { DARD } \\
\text { DEVIA- } \\
\text { TION } \\
\text { (FT3/S) }\end{array}$ & $\begin{array}{l}\text { COEFFI- } \\
\text { CIENT OF } \\
\text { VARI- } \\
\text { ATION }\end{array}$ & $\begin{array}{c}\text { PERCENT } \\
\text { OF } \\
\text { ANNUAL } \\
\text { RUNOFF }\end{array}$ \\
\hline OCTOBER & 0.26 & 0.00 & 0.02 & 0.06 & 3.0 & 13.1 \\
\hline NOVEMBER & 0.22 & 0.00 & 0.01 & 0.05 & 3.4 & 8.7 \\
\hline DECEMBER & 0.12 & 0.00 & 0.01 & 0.02 & 4.0 & 3.3 \\
\hline JANUARY & 0.14 & 0.00 & 0.00 & 0.02 & 5.5 & 2.8 \\
\hline FEBRUARY & 0.08 & 0.00 & 0.00 & 0.01 & 5.9 & 1.5 \\
\hline MARCH & 0.07 & 0.00 & 0.00 & 0.01 & 4.6 & 1.7 \\
\hline APRIL & 0.00 & 0.00 & 0.00 & 0.00 & & 0.0 \\
\hline MAY & 0.00 & 0.00 & 0.00 & 0.00 & & 0.0 \\
\hline JUNE & 0.01 & 0.00 & 0.00 & 0.00 & 5.9 & 0.2 \\
\hline JULY & 0.84 & 0.00 & 0.03 & 0.15 & 4.4 & 21.5 \\
\hline AUGUST & 1.2 & 0.00 & 0.05 & 0.21 & 4.2 & 32.2 \\
\hline SEPTEMBER & 0.27 & 0.00 & 0.02 & 0.06 & 2.8 & 15.0 \\
\hline ANNUAL & 0.10 & 0.00 & 0.01 & 0.02 & 1.8 & 100 \\
\hline
\end{tabular}

MAGNITUDE AND PROBABILITY OF INSTANTANEOUS PEAK FLOW BASED ON PERIOD OF RECORD 1961.94

DISCHARGE, IN FT3/S, FOR INDICATED RECURRENCE INTERVAL IN YEARS, AND EXCEEDANCE PROBABILITY, IN PERCENT

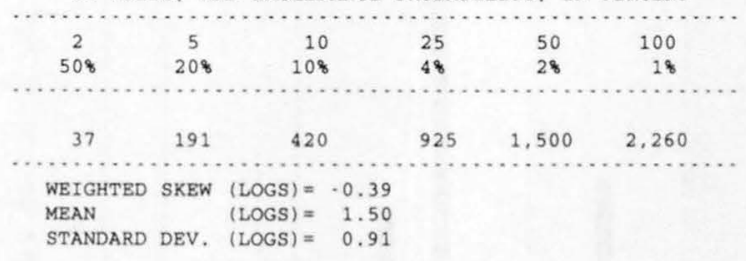

MAGNITUDE AND PROBABILITY OF ANNUAL LOW FLOW BASED ON PERIOD OF RECORD 1962.92, 1995.96

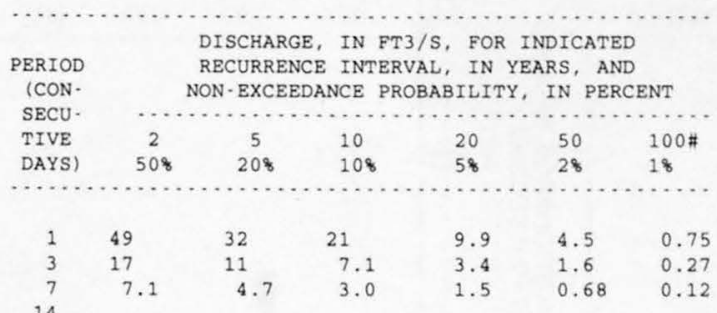

MAGNITUDE AND PROBABILITY OF ANNUAL HIGH FLOW BASED ON PERIOD OF RECORD 1961.92, 1994.96

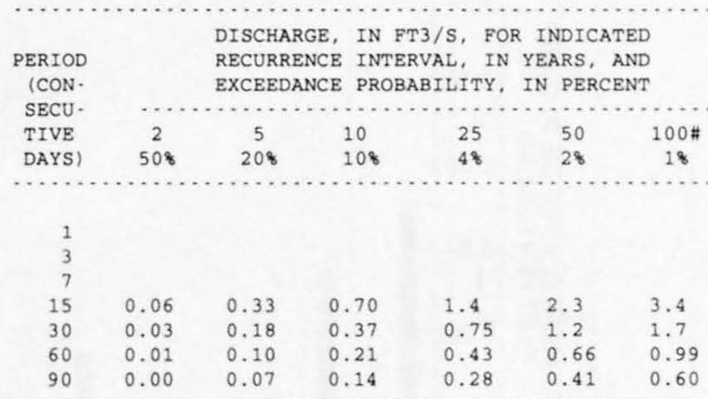

DURATION TABLE OF DAILY MEAN FLOW FOR PERIOD OF RECORD 1961.92, 1994.96

DISCHARGE, IN FT3/S, WHICH WAS EQUALED OR EXCEEDED FOR INDICATED PERCENT OF TIME

\begin{tabular}{|c|c|c|c|c|c|c|c|c|c|c|c|c|c|c|c|c|}
\hline 18 & 58 & 108 & 158 & 208 & 308 & 408 & 508 & 608 & 708 & 808 & 908 & 958 & 988 & 998 & 99.58 & 99.98 \\
\hline 00 & 0.00 & 0.00 & 0.00 & 0.00 & 0.00 & 0.00 & 0.00 & 0.00 & 0.00 & 0.00 & 0.00 & 0.00 & 0.00 & 0.00 & 0.00 & 0.00 \\
\hline
\end{tabular}

\# Reliability of values in column is uncertain, and potential errors are large. 
ANNUAL MEAN DISCHARGE, IN CUBIC FEET PER SECOND

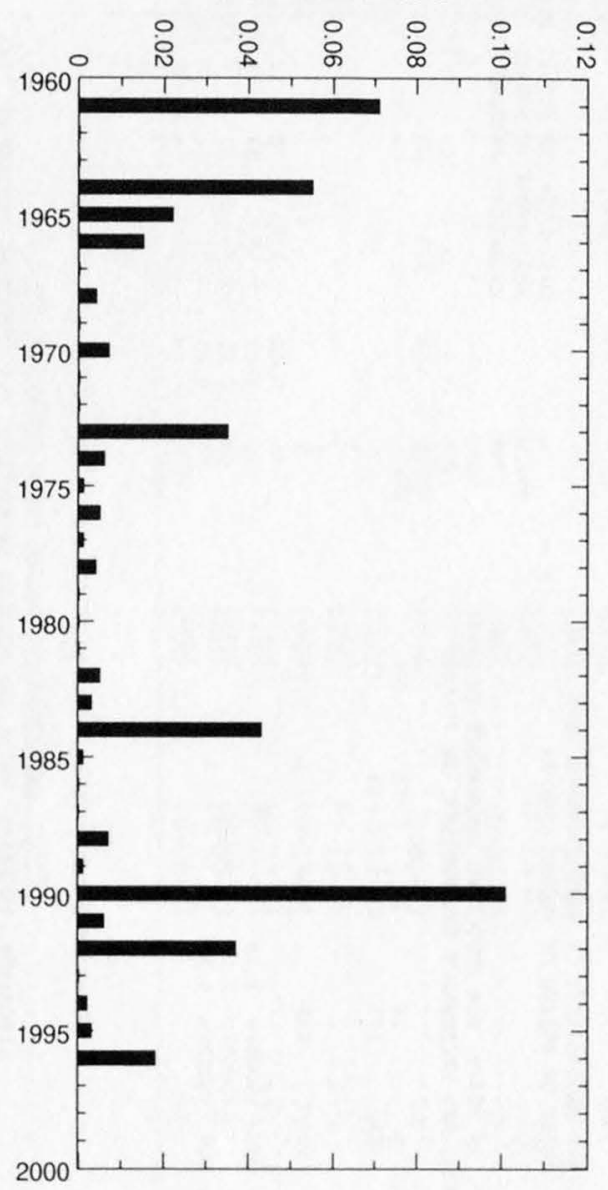

ANNUAL PEAK DISCHARGE, IN CUBIC EET PER SECOND

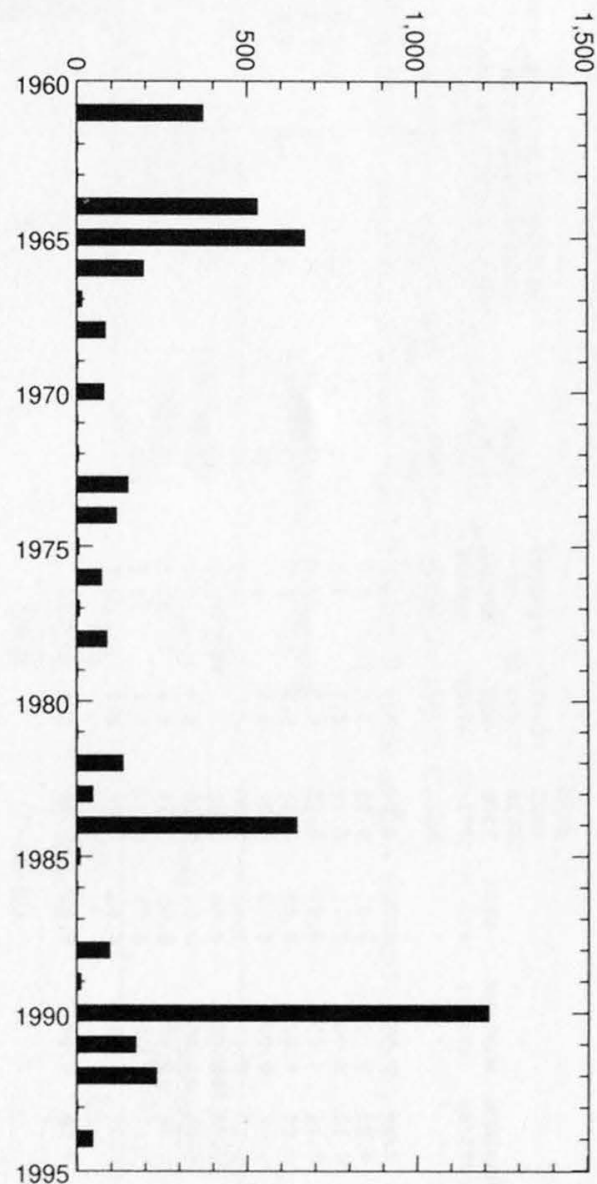


GILA RIVER BASIN

779

09512200 SALT RIVER TRIBUTARY IN SOUTH MOUNTAIN PARK, AT PHOENIX, AZ--Continued

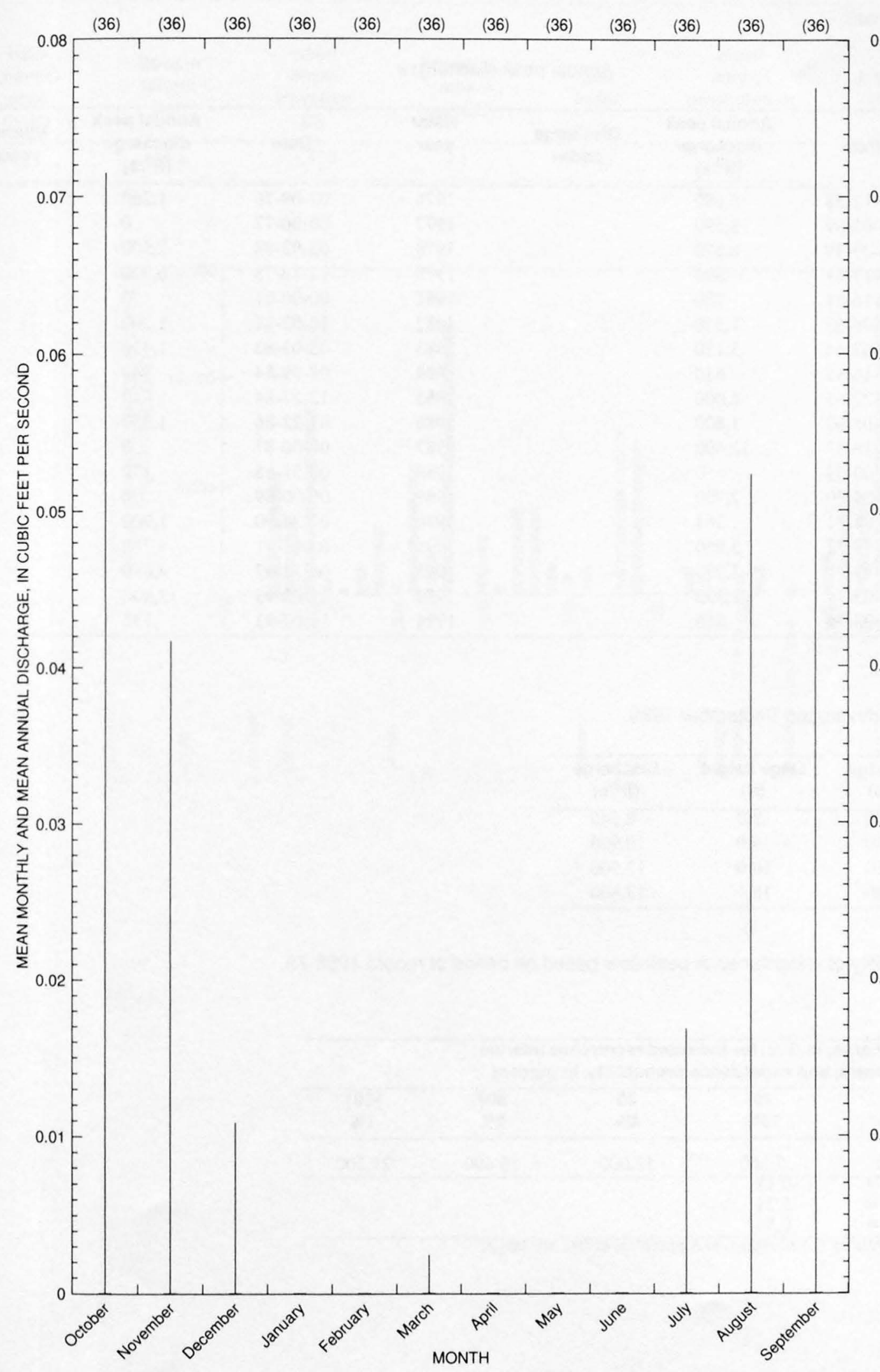

(36)

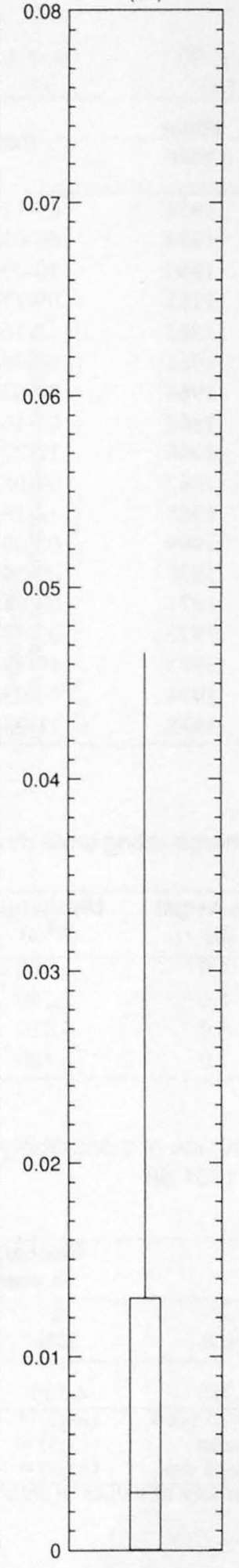

ANNUAL 


\section{CAVE CREEK NEAR CAVE CREEK, AZ}

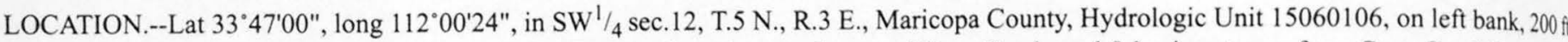
upstream from Prescott-to-Mesa transmission line, 5 mi southwest of town of Cave Creek, and 5.0 mi upstream from Cave Creek Dam.

DRAINAGE AREA.--121 $\mathrm{mi}^{2}$.

Annual peak discharges

\begin{tabular}{|c|c|c|c|c|c|c|c|}
\hline $\begin{array}{l}\text { Water } \\
\text { year }\end{array}$ & Date & $\begin{array}{c}\text { Annual peak } \\
\text { discharge } \\
\left(\mathrm{ft}^{3} / \mathrm{s}\right)\end{array}$ & $\begin{array}{l}\text { Discharge } \\
\text { codes }\end{array}$ & $\begin{array}{l}\text { Water } \\
\text { year }\end{array}$ & Date & $\begin{array}{c}\text { Annual peak } \\
\text { discharge } \\
\left(\mathrm{ft}^{3} / \mathrm{s}\right)\end{array}$ & $\begin{array}{c}\text { Discharge } \\
\text { codes }\end{array}$ \\
\hline 1958 & $09-12-58$ & 5,680 & & 1976 & $02-09-76$ & 1,260 & \\
\hline 1959 & $08-05-59$ & 3,590 & & 1977 & $00-00-77$ & 0 & \\
\hline 1960 & $10-29-59$ & 8,570 & & 1978 & $03-02-78$ & 7,500 & \\
\hline 1961 & $09-17-61$ & 696 & & 1979 & $12-18-78$ & 6,900 & \\
\hline 1962 & $12-16-61$ & 280 & & 1981 & $00-00-81$ & 0 & \\
\hline 1963 & $08-06-63$ & 1,510 & & 1982 & $10-02-81$ & 1,200 & \\
\hline 1964 & $08-02-64$ & 3,120 & & 1983 & $03-03-83$ & 1,420 & \\
\hline 1965 & $07-16-65$ & 610 & & 1984 & $08-09-84$ & 148 & \\
\hline 1966 & $12-22-65$ & 6,000 & & 1985 & $12-27-84$ & 910 & \\
\hline 1967 & $09-06-67$ & 1,800 & & 1986 & $07-22-86$ & 1,350 & \\
\hline 1968 & $12-19-67$ & 12,400 & & 1987 & $00-00-87$ & 0 & \\
\hline 1969 & $00-00-69$ & 0 & & 1988 & $08-21-88$ & 170 & \\
\hline 1970 & $09-05-70$ & 2,700 & & 1989 & $00-00-89$ & 0 & \\
\hline 1971 & 08-04-71 & 364 & & 1990 & $07-24-90$ & 1,900 & \\
\hline 1972 & $07-17-72$ & 3,950 & & 1991 & 03-01-91 & 4,780 & \\
\hline 1973 & $10-19-72$ & 3,950 & & 1992 & $08-22-92$ & 4,680 & \\
\hline 1974 & $08-05-74$ & 1,390 & & 1993 & $01-08-93$ & 13,800 & \\
\hline 1975 & $11-02-74$ & 856 & & 1994 & $10-07-93$ & 132 & \\
\hline
\end{tabular}

Discharge rating table developed September 1989

\begin{tabular}{cccc}
\hline $\begin{array}{c}\text { Gage height } \\
(\mathbf{f t})\end{array}$ & $\begin{array}{c}\text { Discharge } \\
\left(\mathrm{ft}^{\mathbf{3}} \mathbf{s}\right)\end{array}$ & $\begin{array}{c}\text { Gage height } \\
(\mathbf{f t})\end{array}$ & $\begin{array}{c}\text { Discharge } \\
\left(\mathrm{ft}^{\mathbf{3}} / \mathbf{s}\right)\end{array}$ \\
\hline 4.0 & 1,180 & 8.0 & 8,540 \\
5.0 & 2,360 & 9.0 & 10,900 \\
6.0 & 4,010 & 10.0 & 13,500 \\
7.0 & 6,180 & 10.1 & 13,800 \\
\hline
\end{tabular}

Magnitude and probability of instantaneous peak flow based on period of record 1958-79, $1981-86$

\begin{tabular}{|c|c|c|c|c|c|}
\hline \multicolumn{6}{|c|}{$\begin{array}{l}\text { Discharge, in } \mathrm{ft}^{3} / \mathrm{s} \text {, for indicated recurrence interval } \\
\text { in years, and exceedance probablility, in percent }\end{array}$} \\
\hline 2 & 5 & 10 & 25 & $50 \dagger$ & $100 t$ \\
\hline $50 \%$ & $20 \%$ & $10 \%$ & $4 \%$ & $2 \%$ & $1 \%$ \\
\hline 1,780 & 4,580 & 7,360 & 12,000 & 16,400 & 21,500 \\
\hline Weighted skew & $(\log s)=$ & -0.18 & & & \\
\hline Mean & $(\log s)=$ & 3.24 & & & \\
\hline Standard dev. & $(\log s)=$ & 0.50 & & & \\
\hline
\end{tabular}

$\mp$ Reliability of values in column is uncertain, and potential errors are large. 
GILA RIVER BASIN

09512300 CAVE CREEK NEAR CAVE CREEK, AZ--Continued

Basin characteristics

\begin{tabular}{ccccccccc}
\hline $\begin{array}{c}\text { Main } \\
\text { channel } \\
\text { slope } \\
(\mathrm{ft} / \mathrm{mi})\end{array}$ & $\begin{array}{c}\text { Stream } \\
\text { length } \\
(\mathrm{mi})\end{array}$ & $\begin{array}{c}\text { Mean } \\
\text { basin } \\
\text { elevation } \\
\text { (ft) }\end{array}$ & $\begin{array}{c}\text { Forested } \\
\text { area }\end{array}$ & $\begin{array}{c}\text { Soil } \\
\text { (percent) }\end{array}$ & index & $\begin{array}{c}\text { Mean } \\
\text { annual } \\
\text { precipitation } \\
\text { (in) }\end{array}$ & $\begin{array}{c}\text { 2-year } \\
\text { (in) }\end{array}$ & $\begin{array}{c}\text { 50-year } \\
\text { (in) }\end{array}$ \\
\hline 123 & 18.4 & 3,470 & 0.1 & 1.17 & 15.7 & 2.3 & 4.4 \\
\hline
\end{tabular}

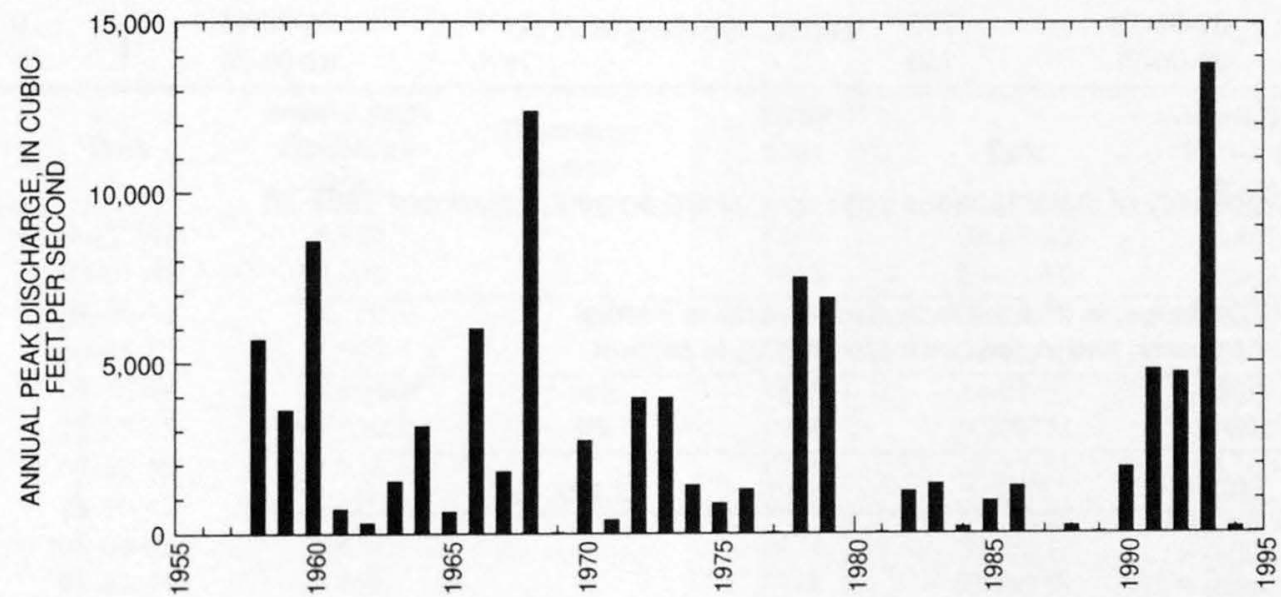




\section{LYNX CREEK TRIBUTARY NEAR PRESCOTT, AZ}

LOCATION.--Lat 34³2'51", long 112 23'58", in SE $1 / 4$ sec.31, T.14 N., R.1 W., Yavapai County, Hydrologic Unit 15060202, on Walker Road,400 $\mathrm{ft}$ south of State Highway 69, and 4 mi east of Prescott.

DRAINAGE AREA.--0.95 $\mathrm{mi}^{2}$.

Annual peak discharges

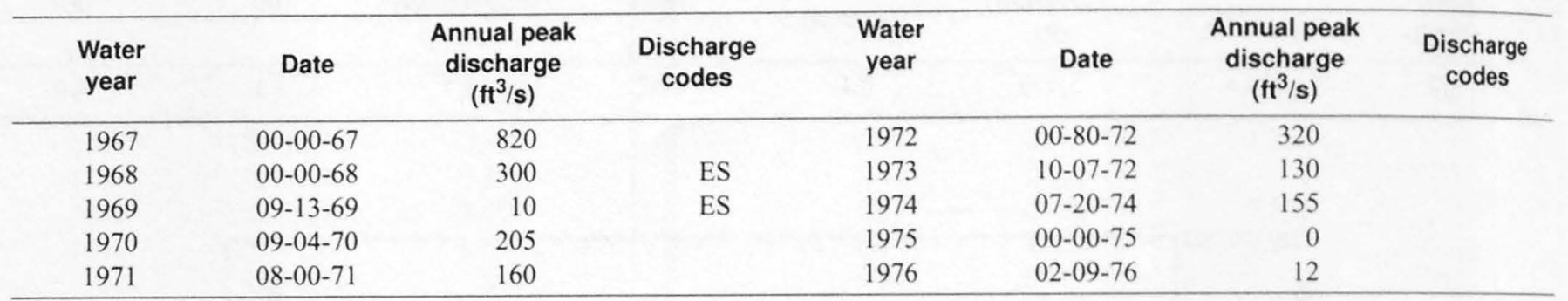

Magnitude and probability of instantaneous peak flow based on period of record 1967-76

\begin{tabular}{|c|c|c|c|c|c|}
\hline \multicolumn{6}{|c|}{$\begin{array}{l}\text { Discharge, in } \mathrm{ft}^{3} / \mathrm{s} \text {, for indicated recurrence interval } \\
\text { in years, and exceedance probablility, in percent }\end{array}$} \\
\hline 2 & 5 & 10 & $25+$ & $50 t$ & $100 t$ \\
\hline $50 \%$ & $20 \%$ & $10 \%$ & $4 \%$ & $2 \%$ & $1 \%$ \\
\hline 106 & 403 & 761 & 1,430 & 2,100 & 2,910 \\
\hline Weighted skew & $(\log s)=$ & -0.41 & & & \\
\hline Mean & $(\log s)=$ & 1.97 & & & \\
\hline Standard dev. & $(\log s)=$ & 0.74 & & & \\
\hline
\end{tabular}

Basin characteristics

\begin{tabular}{cccccccc}
\hline $\begin{array}{c}\text { Main } \\
\text { channel } \\
\text { slope } \\
(\mathrm{ft} / \mathrm{mi})\end{array}$ & $\begin{array}{c}\text { Stream } \\
\text { length } \\
\text { (mi) }\end{array}$ & $\begin{array}{c}\text { Mean } \\
\text { basin } \\
\text { elevation } \\
\text { (ft) }\end{array}$ & $\begin{array}{c}\text { Forested } \\
\text { area } \\
\text { (percent) }\end{array}$ & $\begin{array}{c}\text { Soil } \\
\text { index }\end{array}$ & $\begin{array}{c}\text { Mean } \\
\text { annual } \\
\text { precipitation } \\
\text { (in) }\end{array}$ & $\begin{array}{c}\text { Rainfall intensity, 24-hour } \\
\text { (in) }\end{array}$ & $\begin{array}{c}50 \text {-year } \\
\text { (in) }\end{array}$ \\
\hline 101 & 2.2 & 5,900 & 26.0 & 1.0 & 16.0 & 2.1 \\
\hline
\end{tabular}

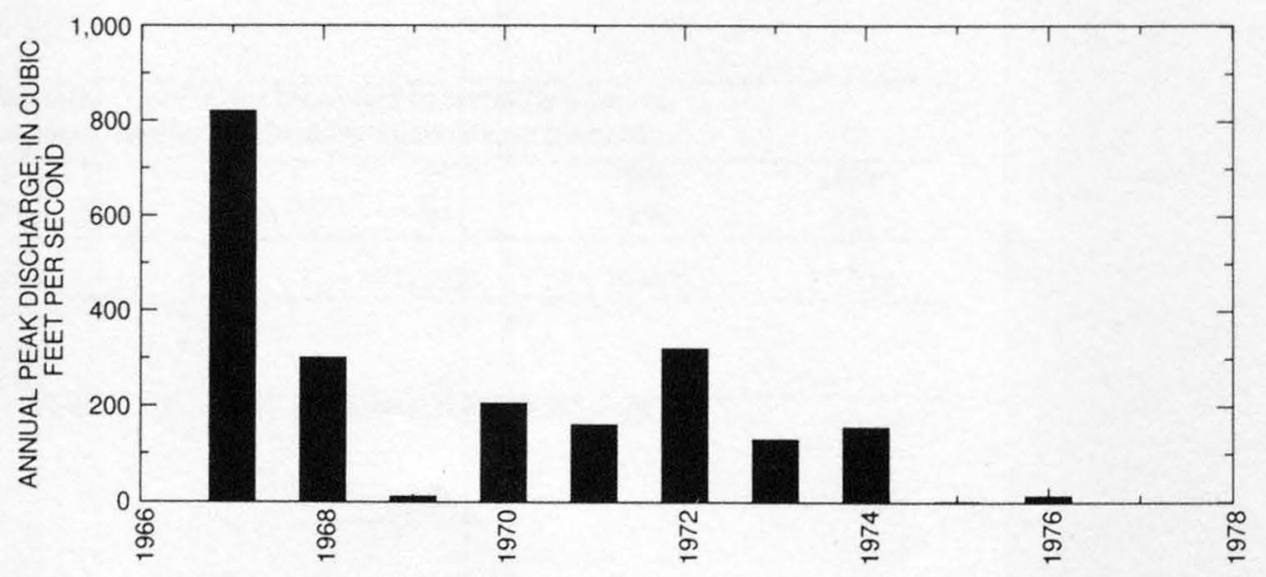


09512500 AGUA FRIA RIVER NEAR MAYER, AZ

LOCATION.--Lat 34'18'55", long 112 03'48", in NW'1/4 SE $1 / 4$ sec.20, T.11 N., R.3 E., Yavapai County, Hydrologic Unit 15070102 , on left bank at Sycamore damsite, $700 \mathrm{ft}$ downstream from Big Bug Creek and $12 \mathrm{mi}$ southeast of Mayer.

DRAINAGE AREA.--585 $\mathrm{mi}^{2}$.

PERIOD OF RECORD.--January 1940 to current year.

REVISED RECORDS.--WDR AZ-88-1: 1987. WDR AZ-89-1: Drainage area.

GAGE.--Water-stage recorder. Datum of gage is 3,434 $\mathrm{ft}$ above sea level.

REMARKS.--Diversions above station for mining and irrigation of about 600 acres.

EXTREMES FOR PERIOD OF RECORD.--Maximum discharge, 33,100 $\mathrm{ft}^{3} / \mathrm{s} \mathrm{Feb.} \mathrm{19,} \mathrm{1980,} \mathrm{from} \mathrm{rating} \mathrm{curve} \mathrm{extended} \mathrm{above} 3,400 \mathrm{ft}^{3} / \mathrm{s}$ on basis of slope-area measurement of peak flow, gage height, $15.76 \mathrm{ft}$, from mean of surge, inside high-water mark $16.03 \mathrm{ft}$, floodmark 18.97 $\mathrm{ft}$; negligible flow at times during the summer months in most previous years when entire flow was diverted to Perry Canal above station and flow past gage was seepage only.

Annual peak discharges

\begin{tabular}{|c|c|c|c|c|c|c|c|}
\hline $\begin{array}{l}\text { Water } \\
\text { year }\end{array}$ & Date & $\begin{array}{c}\text { Annual peak } \\
\text { discharge } \\
\left(\mathrm{ft}^{3} / \mathrm{s}\right)\end{array}$ & $\begin{array}{l}\text { Discharge } \\
\text { codes }\end{array}$ & $\begin{array}{l}\text { Water } \\
\text { year }\end{array}$ & Date & $\begin{array}{l}\text { Annual peak } \\
\text { discharge } \\
\left(\mathrm{ft}^{3} / \mathrm{s}\right)\end{array}$ & $\begin{array}{c}\text { Discharge } \\
\text { codes }\end{array}$ \\
\hline 1940 & $06-26-40$ & 5,920 & & 1969 & $08-07-69$ & 2,490 & \\
\hline 1941 & $03-01-41$ & 13,000 & & 1970 & $09-05-70$ & 19,800 & \\
\hline 1942 & $08-06-42$ & 6,280 & & 1971 & $08-25-71$ & 7,280 & \\
\hline 1943 & $09-25-43$ & 3,500 & & 1972 & $08-12-72$ & 6,800 & \\
\hline 1944 & $09-16-44$ & 3,810 & & 1973 & $10-07-72$ & 10,700 & \\
\hline 1945 & $07-27-45$ & 2,620 & & 1974 & $07-20-74$ & 740 & \\
\hline 1946 & $07-22-46$ & 4,930 & & 1975 & $07-27-75$ & 2,190 & \\
\hline 1947 & $08-16-47$ & 1,610 & & 1976 & $02-09-76$ & 9,700 & \\
\hline 1948 & $08-04-48$ & 6,830 & & 1977 & $08-23-77$ & 5,480 & \\
\hline 1949 & $01-13-49$ & 2,460 & & 1978 & 03-01-78 & 9,900 & \\
\hline 1950 & $07-17-50$ & 2,170 & & 1979 & $12-18-78$ & 18,300 & \\
\hline 1951 & $08-28-51$ & 8,180 & & 1980 & $02-19-80$ & 33,100 & \\
\hline 1952 & $01-18-52$ & 7,500 & & 1981 & $09-23-81$ & 2,850 & \\
\hline 1953 & $07-08-53$ & 5,510 & & 1982 & $09-10-82$ & 3,040 & \\
\hline 1954 & $09-03-54$ & 4,570 & & 1983 & $09-23-83$ & 9,940 & \\
\hline 1955 & $08-03-55$ & 12,800 & & 1984 & $08-14-84$ & 3,620 & \\
\hline 1956 & $07-25-56$ & 6,880 & & 1985 & $12-27-84$ & 2,880 & \\
\hline 1957 & $08-13-57$ & 2,710 & & 1986 & $11-26-85$ & 3,970 & \\
\hline 1958 & $06-21-58$ & 4,620 & & 1987 & $10-11-86$ & 6,070 & \\
\hline 1959 & 08-04-59 & 9,700 & & 1988 & $08-29-88$ & 25,500 & \\
\hline 1960 & $08-08-60$ & 4,820 & & 1989 & $08-18-89$ & 1,280 & \\
\hline 1961 & $07-22-61$ & 10,200 & & 1990 & $09-15-90$ & 3,600 & \\
\hline 1962 & $09-13-62$ & 2,470 & & 1991 & 03-01-91 & 19,200 & \\
\hline 1963 & $08-19-63$ & 12,800 & & 1992 & $08-23-92$ & 5,410 & \\
\hline 1964 & $07-24-64$ & 9,000 & & 1993 & $01-08-93$ & 27,400 & \\
\hline 1965 & $04-04-65$ & 7,470 & & 1994 & $10-06-93$ & 688 & \\
\hline 1966 & $12-22-65$ & 12,100 & & 1995 & $02-15-95$ & 12,500 & \\
\hline 1967 & $08-19-67$ & 6,960 & & 1996 & $07-25-96$ & 5,370 & \\
\hline 1968 & $12-19-67$ & 3,850 & & & & & \\
\hline
\end{tabular}


09512500 AGUA FRIA RIVER NEAR MAYER, AZ--Continued

Discharge rating table developed July 1990

\begin{tabular}{cccc}
\hline $\begin{array}{c}\text { Gaige height } \\
(\mathbf{f t})\end{array}$ & $\begin{array}{c}\text { Discharge } \\
\left(\mathbf{f t}^{\mathbf{3}} / \mathbf{s}\right)\end{array}$ & $\begin{array}{c}\text { Gage height } \\
(\mathbf{f t})\end{array}$ & $\begin{array}{c}\text { Discharge } \\
\left(\mathbf{f t}^{\mathbf{3}} / \mathbf{s}\right)\end{array}$ \\
\hline 5.0 & 1,100 & 11.0 & 13,480 \\
6.0 & 2,320 & 12.0 & 16,820 \\
7.0 & 3,930 & 13.0 & 20,580 \\
8.0 & 5,800 & 14.0 & 24,740 \\
9.0 & 7,980 & 15.0 & 29,330 \\
10.0 & 10,530 & 15.7 & 32,790 \\
\hline
\end{tabular}

Basin characteristics

\begin{tabular}{|c|c|c|c|c|c|c|c|}
\hline \multirow[b]{2}{*}{$\begin{array}{l}\text { Main } \\
\text { channel } \\
\text { slope } \\
\text { (ft/mi) }\end{array}$} & \multirow[b]{2}{*}{$\begin{array}{l}\text { Stream } \\
\text { length } \\
\text { (mi) }\end{array}$} & \multirow[b]{2}{*}{$\begin{array}{c}\text { Mean } \\
\text { basin } \\
\text { elevation } \\
(f t)\end{array}$} & \multirow[b]{2}{*}{$\begin{array}{l}\text { Forested } \\
\text { area } \\
\text { (percent) }\end{array}$} & \multirow[b]{2}{*}{$\begin{array}{c}\text { Soil } \\
\text { index }\end{array}$} & \multirow[b]{2}{*}{$\begin{array}{c}\text { Mean } \\
\text { annual } \\
\text { precipitation } \\
\text { (in) }\end{array}$} & \multicolumn{2}{|c|}{ Rainfall intensity, 24-hour } \\
\hline & & & & & & $\begin{array}{l}\text { 2-year } \\
\text { (in) }\end{array}$ & $\begin{array}{l}\text { 50-year } \\
\text { (in) }\end{array}$ \\
\hline 56.9 & 37.5 & 5,000 & 3.4 & 1.3 & 16.7 & 2.1 & 4.3 \\
\hline
\end{tabular}


09512500 AGUA FRIA RIVER NEAR MAYER, AZ--Continued

MEAN MONTHLY AND ANNUAL DISCHARGES 1941.96

\begin{tabular}{|c|c|c|c|c|c|c|}
\hline MONTH & $\begin{array}{l}\text { MAXIMUM } \\
\text { (FT3/S) }\end{array}$ & $\begin{array}{l}\text { MINIMUM } \\
(\mathrm{FT} 3 / \mathrm{S})\end{array}$ & $\begin{array}{c}\text { MEAN } \\
(\mathrm{FT} 3 / \mathrm{S})\end{array}$ & $\begin{array}{l}\text { STAN- } \\
\text { DARD } \\
\text { DEVIA- } \\
\text { TION } \\
(\text { FT3/S) }\end{array}$ & $\begin{array}{l}\text { COEFFI- } \\
\text { CIENT OF } \\
\text { VARI- } \\
\text { ATION }\end{array}$ & $\begin{array}{c}\text { PERCENT } \\
\text { OF } \\
\text { ANNUAL } \\
\text { RUNOFF }\end{array}$ \\
\hline OCTOBER & 223 & 0.14 & 9.2 & 31 & 3.3 & 3.2 \\
\hline NOVEMBER & 146 & 0.10 & 9.7 & 24 & 2.4 & 3.3 \\
\hline DECEMBER & 453 & 0.08 & 31 & 82 & 2.6 & 10.6 \\
\hline JANUARY & 718 & 0.07 & 35 & 105 & 3.0 & 12.0 \\
\hline FEBRUARY & 1,180 & 0.02 & 67 & 191 & 2.9 & 22.9 \\
\hline MARCH & 389 & 0.01 & 52 & 91 & 1.7 & 18.0 \\
\hline APRIL & 314 & 0.00 & 21 & 55 & 2.6 & 7.1 \\
\hline MAY & 20 & 0.03 & 3.3 & 5.0 & 1.5 & 1.1 \\
\hline JUNE & 23 & 0.01 & 2.2 & 3.5 & 1.6 & 0.8 \\
\hline JULY & 48 & 0.15 & 11 & 12 & 1.1 & 3.9 \\
\hline AUGUST & 244 & 0.31 & 34 & 49 & 1.4 & 11.7 \\
\hline SEPTEMBER & 187 & 0.20 & 16 & 34 & 2.1 & 5.5 \\
\hline ANNUAL & 143 & 1.5 & 24 & 29 & 1.2 & 100 \\
\hline
\end{tabular}

MAGNITUDE AND PROBABILITY OF INSTANTANEOUS PEAK FLOW BASED ON PERIOD OF RECORD 1940-96

DISCHARGE, IN FT3/S, FOR INDICATED RECURRENCE INTERVAL IN YEARS, AND EXCEEDANCE PROBABILITY, IN PERCENT

$\begin{array}{cccccc}2 & 5 & 10 & 25 & 50 & 100 \\ 508 & 208 & 108 & 48 & 28 & 18 \\ 5,920 & 11,000 & 15,500 & 22,400 & 28,700 & 35,900\end{array}$

$\begin{array}{ll}\text { WEIGHTED SKEW } & (\text { LOGS) }=0.19 \\ \text { MEAN } & (\text { LOGS })=3.78\end{array}$

STANDARD DEV. $($ LOGS $)=0.31$
MAGNITUDE AND PROBABILITY OF ANNUAL LOW FLOW BASED ON PERIOD OF RECORD 1941.96

\begin{tabular}{|c|c|c|c|c|c|c|}
\hline \multirow{4}{*}{$\begin{array}{l}\text { PERIOD } \\
\text { (CON- } \\
\text { SECU- } \\
\text { TIVE }\end{array}$} & \multicolumn{6}{|c|}{ DISCHARGE, IN FT3/S, FOR INDICATED } \\
\hline & \multicolumn{6}{|c|}{$\begin{array}{l}\text { RECURRENCE INTERVAL, IN YEARS, AND } \\
\text { NON-EXCEEDANCE PROBABILITY, IN PERCENT }\end{array}$} \\
\hline & & 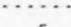 & & & & \\
\hline & 2 & 5 & 10 & 20 & 50 & 100 \\
\hline DAYS) & 508 & 208 & 108 & 58 & 28 & 18 \\
\hline 1 & 0.24 & 0.10 & 0.06 & 0.00 & 0.00 & 0.00 \\
\hline 3 & 0.25 & 0.10 & 0.06 & 0.00 & 0.00 & 0.00 \\
\hline 7 & 0.27 & 0.11 & 0.06 & 0.00 & 0.00 & 0.00 \\
\hline 14 & 0.32 & 0.12 & 0.07 & 0.00 & 0.00 & 0.00 \\
\hline 30 & 0.42 & 0.17 & 0.10 & 0.00 & 0.00 & 0.00 \\
\hline 60 & 0.65 & 0.22 & 0.12 & 0.07 & 0.04 & 0.02 \\
\hline 90 & 1.0 & 0.34 & 0.18 & 0.10 & 0.05 & 0.03 \\
\hline 120 & 2.0 & 0.74 & 0.39 & 0.22 & 0.10 & 0.06 \\
\hline 183 & 4.5 & 1.7 & 0.92 & 0.53 & 0.27 & 0.17 \\
\hline
\end{tabular}

MAGNITUDE AND PROBABILITY OF ANNUAL HIGH FLOW BASED ON PERIOD OF RECORD 1941.96

\begin{tabular}{|c|c|c|c|c|c|c|}
\hline \multirow{3}{*}{$\begin{array}{l}\text { PERIOD } \\
\text { (CON- } \\
\text { SECU - }\end{array}$} & \multicolumn{6}{|c|}{ DISCHARGE, IN FT $3 / S$, FOR INDICATED } \\
\hline & & EXCBED & E PROI & BILITY, & IN PERC & ENT \\
\hline & & & & & ........ & …... \\
\hline TIVE & 2 & 5 & 10 & 25 & 50 & 100 \\
\hline DAYS) & 508 & 208 & 108 & 48 & 28 & 18 \\
\hline 1 & 817 & 2,220 & 3,830 & 6,970 & 10,300 & 14,800 \\
\hline 3 & 399 & 1.090 & 1,880 & 3,460 & 5,170 & 7,490 \\
\hline 7 & 219 & 603 & 1,040 & 1,880 & 2,770 & 3,940 \\
\hline 15 & 130 & 353 & 603 & 1,080 & 1,580 & 2,250 \\
\hline 30 & 84 & 224 & 375 & 652 & 932 & 1.290 \\
\hline 60 & 53 & 144 & 243 & 425 & 611 & 847 \\
\hline 90 & 38 & 103 & 174 & 306 & 443 & 619 \\
\hline
\end{tabular}

DURATION TABLE OF DAILY MEAN FLOW FOR PERIOD OF RECORD 1941-96

DISCHARGE, IN FT3/S, WHICH WAS EQUALED OR EXCEEDED FOR INDICATED PERCENT OF TIME

\begin{tabular}{|c|c|c|c|c|c|c|c|c|c|c|c|c|c|c|c|c|}
\hline 18 & 58 & 108 & 158 & 208 & 308 & 408 & 508 & 608 & 708 & 808 & 908 & 958 & 988 & 998 & 99.58 & 99.98 \\
\hline 08 & 73 & 21 & 11 & 7.4 & 4.5 & 3.1 & 2.2 & 1.5 & 0.92 & 0.56 & 0.32 & 0.15 & 0.10 & 0.09 & 0.00 & 0.00 \\
\hline
\end{tabular}


ANNUAL MEAN DISCHARGE, IN CUBIC

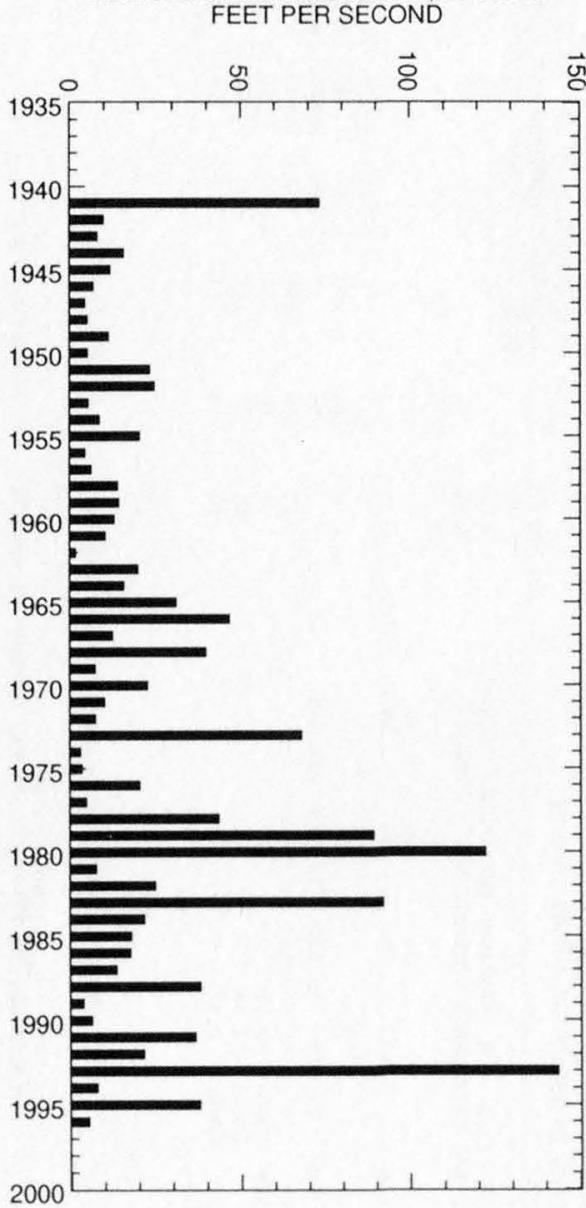

ANNUAL PEAK DISCHARGE, IN CUBIC FEET PER SECOND

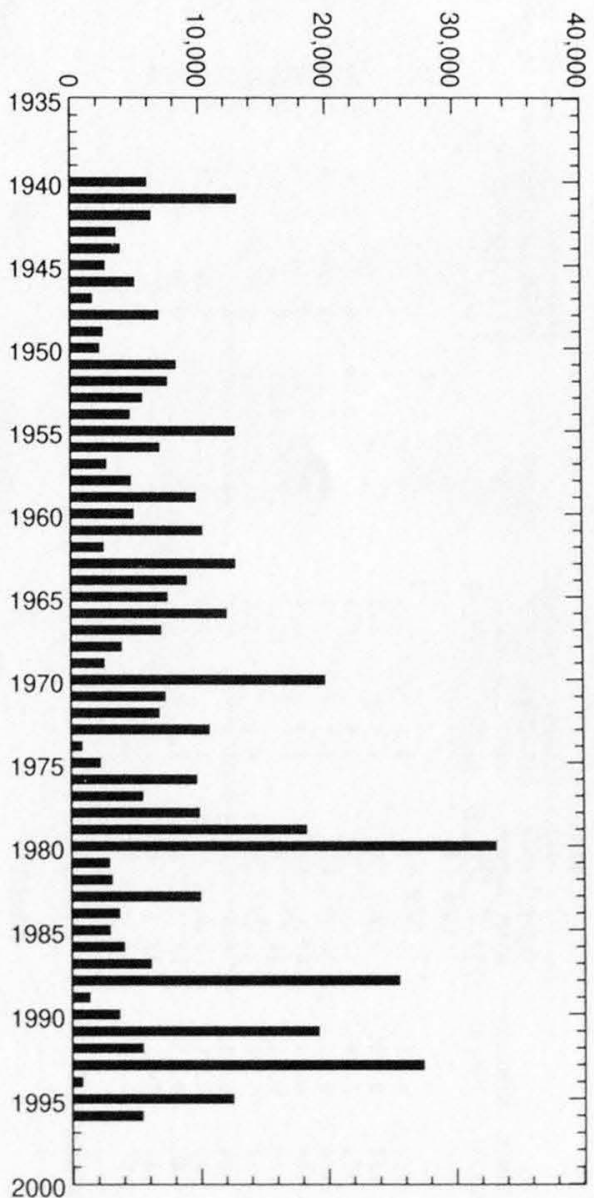


GILA RIVER BASIN

787

09512500 AGUA FRIA RIVER NEAR MAYER, AZ--Continued
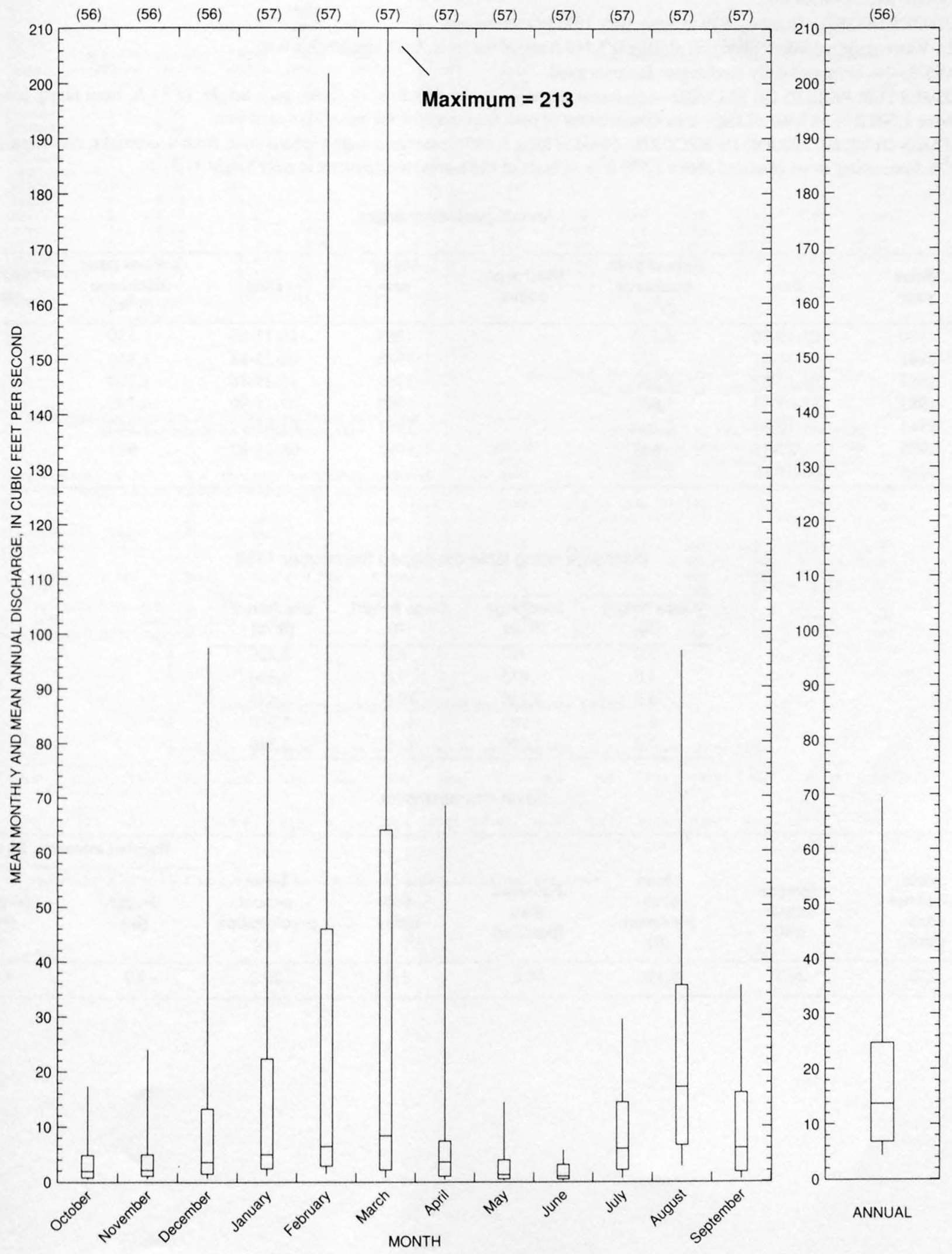

ANNUAL 


\section{TURKEY CREEK NEAR CLEATOR, AZ}

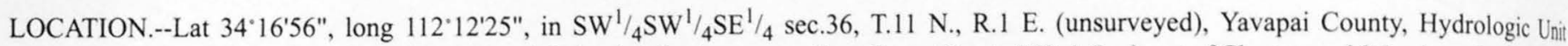
15070102, in Prescott National Forest, on right bank 0.7 mi upstream from Forest Route 259, 1.5 mi east of Cleator, and 9.3 mi south of Mayer. DRAINAGE AREA.--89.4 $\mathrm{mi}^{2}$.

PERIOD OF RECORD.--October 1979 to September 1992 (discontinued).

GAGE.--Water-stage recorder. Elevation of gage is 3,140 ft above sea level, from topographic map.

REMARKS.--No estimated daily discharges. Records good.

EXTREMES FOR PERIOD OF RECORD.--Maximum discharge, 5,230 $\mathrm{ft}^{3} / \mathrm{s}$ Feb. 19, 1980, gage height, $11.51 \mathrm{ft}$, from rating curve extended above $1,500 \mathrm{ft}^{3} / \mathrm{s}$ on basis of slope-area measurement of peak flow; no flow for many days each year.

EXTREMES OUTSIDE PERIOD OF RECORD.--Flood of Sept. 5, 1970, reached a stage of about $16 \mathrm{ft}$, from floodmarks, discharge, about 9,000 $\mathrm{ft}^{3} / \mathrm{s}$, from rating curve extended above $1,500 \mathrm{ft}^{3} / \mathrm{s}$ on basis of slope-area measurement at gage height $11.51 \mathrm{ft}$.

Annual peak discharges

\begin{tabular}{cccccrr}
\hline $\begin{array}{c}\text { Water } \\
\text { year }\end{array}$ & Date & $\begin{array}{c}\text { Annual peak } \\
\text { discharge } \\
\left(\mathrm{ft}^{\mathbf{3}} \mathbf{s}\right)\end{array}$ & $\begin{array}{c}\text { Discharge } \\
\text { codes }\end{array}$ & $\begin{array}{c}\text { Water } \\
\text { year }\end{array}$ & $\begin{array}{c}\text { Date } \\
\text { Annual peak } \\
\text { discharge } \\
\left(\mathrm{ft}^{3} / \mathbf{s}\right)\end{array}$ \\
\hline 1980 & $02-19-80$ & 5,230 & & 1987 & $10-11-86$ & $\begin{array}{c}\text { Discharge } \\
\text { codes }\end{array}$ \\
1981 & $07-31-81$ & 127 & & 1988 & $08-23-88$ & 1,850 \\
1982 & $08-23-82$ & 1,260 & & 1989 & $12-29-88$ & 1,720 \\
1983 & $12-09-83$ & 1,840 & & 1990 & $07-15-90$ & 4,750 \\
1984 & $09-10-84$ & 2,260 & & 1991 & $03-01-91$ & 2,810 \\
1985 & $12-27-85$ & 648 & & 1992 & $08-23-92$ & 603 \\
1986 & $06-01-86$ & 4,110 & & & & \\
\hline
\end{tabular}

Discharge rating table developed September 1988

\begin{tabular}{crcc}
\hline $\begin{array}{c}\text { Gage height } \\
(\mathbf{f t})\end{array}$ & $\begin{array}{c}\text { Discharge } \\
\left(\mathbf{f t}^{\mathbf{3}} \mathbf{s}\right)\end{array}$ & $\begin{array}{c}\text { Gage height } \\
(\mathbf{f t})\end{array}$ & $\begin{array}{c}\text { Discharge } \\
\left(\mathbf{f t}^{\mathbf{3}} \mathbf{s}\right)\end{array}$ \\
\hline 3.0 & 397 & 8.0 & 3,220 \\
4.0 & 835 & 9.0 & 3,830 \\
5.0 & 1,380 & 10.0 & 4,430 \\
6.0 & 1,980 & 11.0 & 4,950 \\
7.0 & 2,620 & 11.5 & 5,230 \\
\hline
\end{tabular}

Basin characteristics

\begin{tabular}{cccccccc}
\hline $\begin{array}{c}\text { Main } \\
\text { channel } \\
\text { slope } \\
(\mathrm{ft} / \mathrm{mi})\end{array}$ & $\begin{array}{c}\text { Stream } \\
\text { length } \\
\text { (mi) }\end{array}$ & $\begin{array}{c}\text { Mean } \\
\text { basin } \\
\text { elevation } \\
(\mathrm{ft})\end{array}$ & $\begin{array}{c}\text { Forested } \\
\text { area } \\
\text { (percent) }\end{array}$ & $\begin{array}{c}\text { Soil } \\
\text { index }\end{array}$ & $\begin{array}{c}\text { Mean } \\
\text { annual } \\
\text { precipitation } \\
\text { (in) }\end{array}$ & $\begin{array}{c}\text { Rainfall intensity, 24-hour } \\
\text { (in) }\end{array}$ & $\begin{array}{c}50 \text {-year } \\
\text { (in) }\end{array}$ \\
\hline 122 & 26.7 & 5,360 & 54.0 & 1.0 & 20.2 & 2.2 \\
\hline
\end{tabular}




\section{TURKEY CREEK NEAR CLEATOR, AZ--Continued}

MEAN MONTHLY AND ANNUAL DISCHARGES 1980-92

\begin{tabular}{|c|c|c|c|c|c|c|}
\hline MONTH & $\begin{array}{l}\text { MAXIMUM } \\
\text { (FT3/S) }\end{array}$ & $\begin{array}{l}\text { MINIMUM } \\
(\text { FT3/S) }\end{array}$ & $\begin{array}{c}\text { MEAN } \\
(\mathrm{FT} 3 / \mathrm{S})\end{array}$ & $\begin{array}{l}\text { STAN- } \\
\text { DARD } \\
\text { DEVIA- } \\
\text { TION } \\
(\text { FT3/S) }\end{array}$ & $\begin{array}{l}\text { COEFFI- } \\
\text { CIENT OF } \\
\text { VARI- } \\
\text { ATION }\end{array}$ & $\begin{array}{c}\text { PBRCENT } \\
\text { OF } \\
\text { ANNUAL } \\
\text { RUNOFF }\end{array}$ \\
\hline OCTOBER & 7.9 & 0.00 & 1.0 & 2.3 & 2.3 & 0.8 \\
\hline NOVEMBER & 15 & 0.00 & 2.0 & 4.1 & 2.1 & 1.4 \\
\hline DECEMBER & 106 & 0.00 & 14 & 29 & 2.2 & 10.0 \\
\hline JANUARY & 52 & 0.00 & 9.1 & 14 & 1.6 & 6.7 \\
\hline FEBRUARY & 420 & 0.00 & 50 & 115 & 2.3 & 37.0 \\
\hline MARCH & 120 & 0.00 & 33 & 38 & 1.2 & 24.5 \\
\hline APRIL & 39 & 0.00 & 9.9 & 12 & 1.2 & 7.3 \\
\hline MAY & 14 & 0.00 & 3.1 & 4.5 & 1.5 & 2.3 \\
\hline JUNE & 3.5 & 0.00 & 0.87 & 1.2 & 1.4 & 0.6 \\
\hline JULY & 35 & 0.00 & 3.7 & 9.8 & 2.6 & 2.7 \\
\hline AUGUST & 47 & 0.00 & 6.8 & 13 & 1.9 & 5.0 \\
\hline SEPTEMBER & 12 & 0.00 & 2.1 & 3.7 & 1.8 & 1.5 \\
\hline ANNUAL & 47 & 0.26 & 11 & 14 & 1.2 & 100 \\
\hline
\end{tabular}

MAGNITUDE AND PROBABILITY OF ANNUAL HIGH FLOW BASED ON PERIOD OF RECORD 1980.92

MAGNITUDE AND PROBABILITY OF INSTANTANEOUS PEAK FLOW BASED ON PERIOD OF RECORD 1980-1992

DISCHARGE, IN FT3/S, FOR INDICATED RECURRENCE INTERVAL IN YEARS, AND EXCEEDANCE PROBABILITY, IN PERCENT

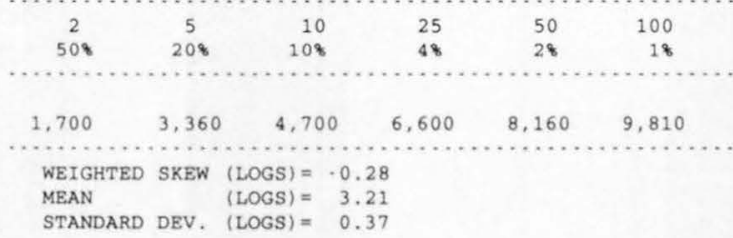

MAGNITUDE AND PROBABILITY OF ANNUAL LOW FLOW BASED ON PERIOD OF RECORD 1981.92

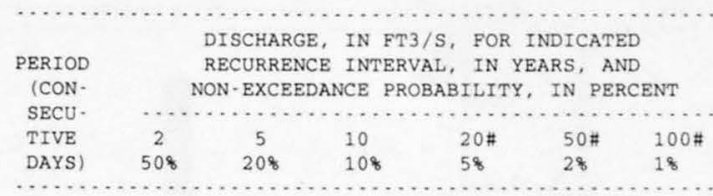

$\begin{array}{llllll}0.00 & 0.00 & 0.00 & 0.00 & 0.00 & 0.00\end{array}$

$\begin{array}{llllll}0.03 & 0.00 & 0.00 & 0.00 & 0.00 & 0.00\end{array}$ $\begin{array}{llllll}0.19 & 0.00 & 0.00 & 0.00 & 0.00 & 0.00\end{array}$

$\begin{array}{llllll}0.57 & 0.00 & 0.00 & 0.00 & 0.00 & 0.00\end{array}$

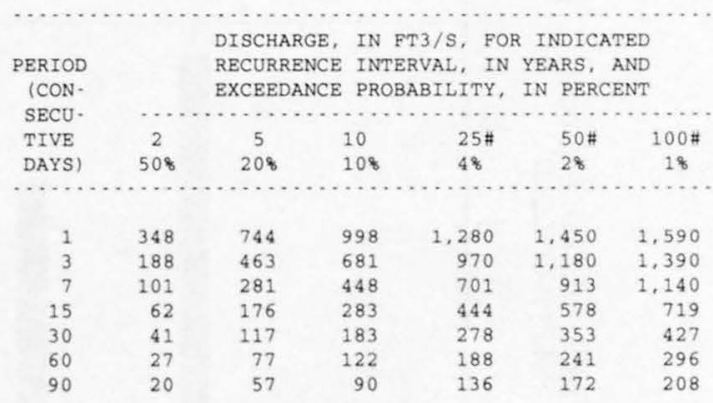

DURATION TABLE OF DAILY MEAN FLOW FOR PERIOD OF RECORD 1980.92

DISCHARGE, IN FT3/S, WHICH WAS EQUALED OR EXCEEDED FOR INDICATED PERCENT OF TIME

\begin{tabular}{|c|c|c|c|c|c|c|c|c|c|c|c|c|c|c|c|c|}
\hline 18 & 58 & 108 & 158 & 208 & 308 & 408 & 508 & 608 & 708 & 808 & $90 \%$ & 958 & 988 & 998 & 99.58 & 99.98 \\
\hline 188 & 45 & 21 & 11 & 6.4 & 2.1 & 0.71 & 0.13 & 0.00 & 0.00 & 0.00 & 0.00 & 0.00 & 0.00 & 0.00 & 0.00 & 0.00 \\
\hline
\end{tabular}

\# Reliability of values in column is uncertain, and potential errors are large. 
09512600 TURKEY CREEK NEAR CLEATOR, AZ--Continued
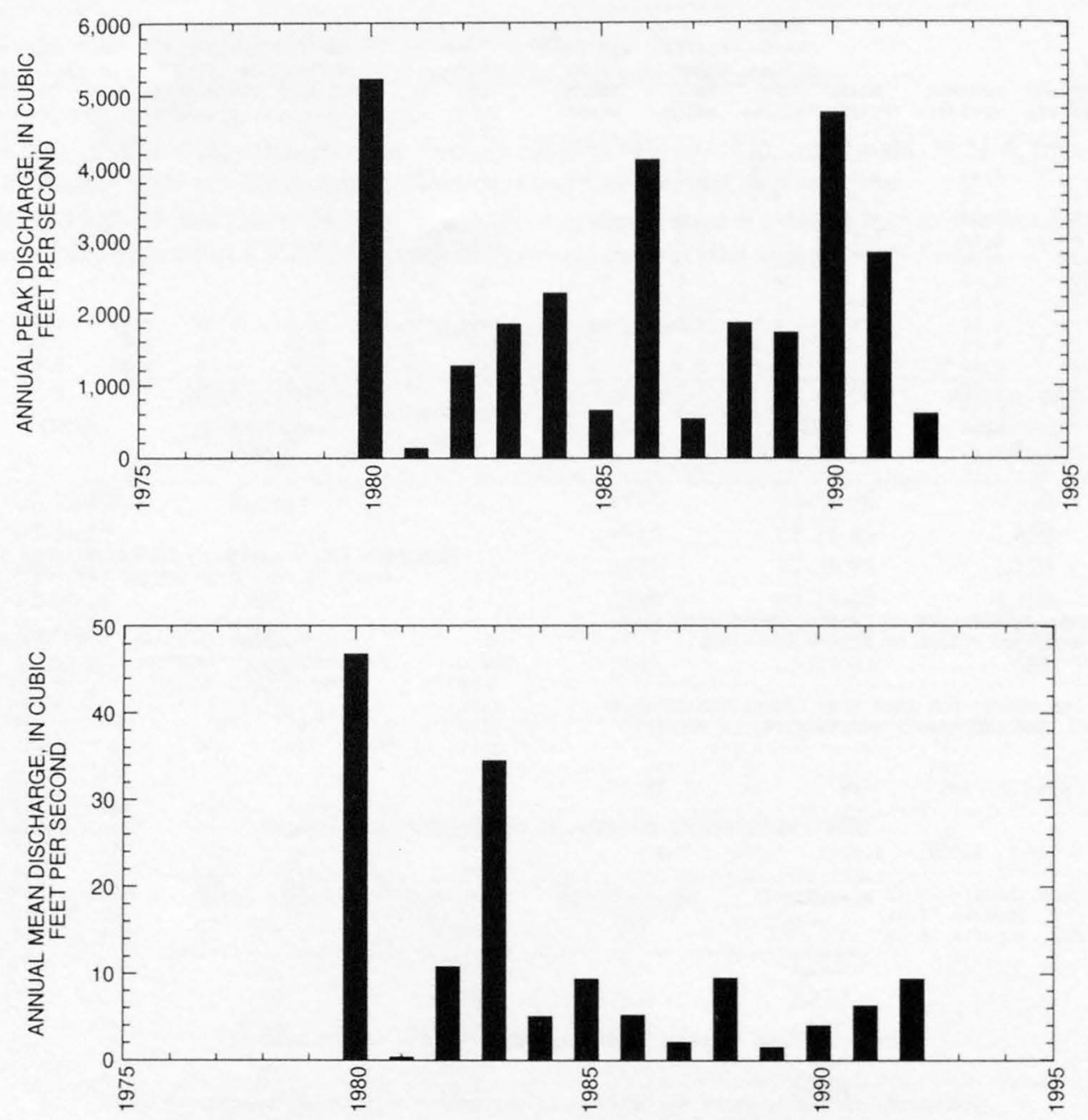
GILA RIVER BASIN

791

09512600 TURKEY CREEK NEAR CLEATOR, AZ--Continued
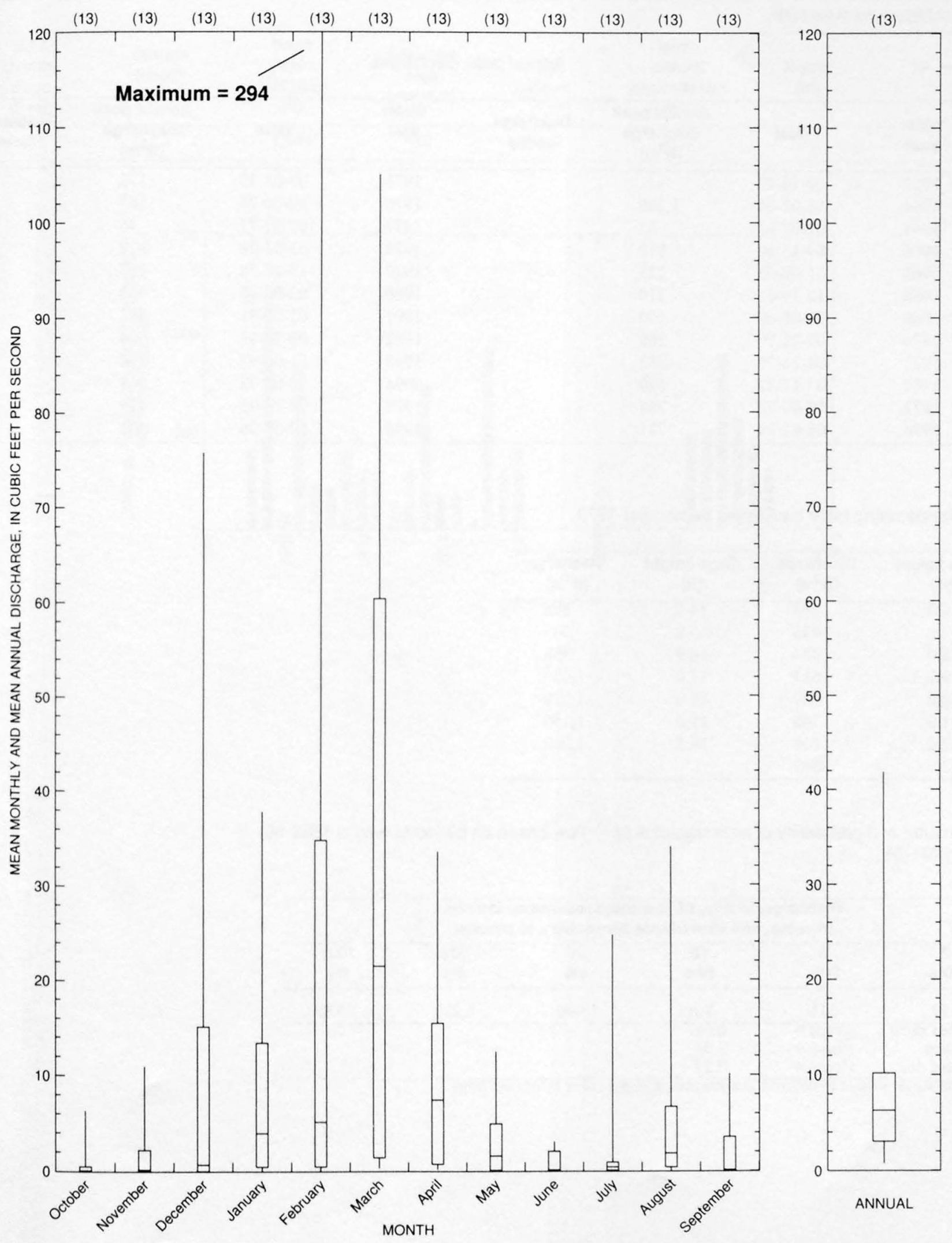
09512700 AGUA FRIA RIVER TRIBUTARY NO. 2 NEAR ROCK SPRINGS, AZ

LOCATION.--Lat $34^{\circ} 02^{\prime} 00^{\prime \prime}$, long $112^{\circ} 08^{\prime} 42^{\prime \prime}$, in SW $\mathrm{SW}_{4}$ sec.15, T.8 N., R.2 E., Maricopa County, Hydrologic Unit 15070102 , at State Highway 69,1 mi south of Rock Springs.

DRAINAGE AREA.--1.07 $\mathrm{mi}^{2}$.

Annual peak discharges

\begin{tabular}{|c|c|c|c|c|c|c|c|}
\hline $\begin{array}{l}\text { Water } \\
\text { year }\end{array}$ & Date & $\begin{array}{c}\text { Annual peak } \\
\text { discharge } \\
\left(\mathrm{ft}^{3} / \mathrm{s}\right)\end{array}$ & $\begin{array}{l}\text { Discharge } \\
\text { codes }\end{array}$ & $\begin{array}{l}\text { Water } \\
\text { year }\end{array}$ & Date & $\begin{array}{c}\text { Annual peak } \\
\text { discharge } \\
\left(\mathrm{ft}^{3} / \mathrm{s}\right)\end{array}$ & $\begin{array}{c}\text { Discharge } \\
\text { codes }\end{array}$ \\
\hline 1963 & $08-16-63$ & 411 & & 1975 & $07-08-75$ & 175 & \\
\hline 1964 & $08-02-64$ & 1,200 & & 1976 & $09-26-76$ & 165 & \\
\hline 1965 & $01-07-65$ & 87 & & 1977 & $09-27-77$ & 46 & \\
\hline 1966 & $09-13-66$ & 812 & & 1978 & $03-02-78$ & 920 & \\
\hline 1967 & $11-08-66$ & 225 & & 1979 & $12-18-78$ & 265 & \\
\hline 1968 & $12-19-67$ & 210 & & 1980 & $02-00-80$ & 405 & \\
\hline 1969 & $09-05-69$ & 400 & & 1991 & $03-01-91$ & 491 & \\
\hline 1970 & $07-21-70$ & 300 & & 1992 & $08-22-92$ & 504 & \\
\hline 1971 & $08-21-71$ & 285 & & 1993 & $01-08-93$ & 898 & \\
\hline 1972 & $07-17-72$ & 140 & & 1994 & $10-06-93$ & 568 & \\
\hline 1973 & $10-07-72$ & 285 & & 1995 & $09-28-95$ & 419 & \\
\hline 1974 & $08-02-74$ & 721 & & 1996 & $09-07-96$ & 305 & \\
\hline
\end{tabular}

Discharge rating table developed September 1973

\begin{tabular}{cccc}
\hline $\begin{array}{c}\text { Gage height } \\
(\mathbf{f t})\end{array}$ & $\begin{array}{c}\text { Discharge } \\
\left(\mathbf{f t}^{\mathbf{3}} \mathbf{s}\right)\end{array}$ & $\begin{array}{c}\text { Gage height } \\
(\mathbf{f t})\end{array}$ & $\begin{array}{c}\text { Discharge } \\
\left(\mathbf{f t}^{\mathbf{3}} / \mathbf{s}\right)\end{array}$ \\
\hline 6.0 & 348 & 14.0 & 898 \\
7.0 & 433 & 15.0 & 945 \\
8.0 & 524 & 16.0 & 988 \\
9.0 & 613 & 17.0 & 1,030 \\
10.0 & 680 & 18.0 & 1,070 \\
11.0 & 740 & 19.0 & 1,150 \\
12.0 & 800 & 19.5 & 1,200 \\
13.0 & 849 & & \\
\hline
\end{tabular}

Magnitude and probability of instantaneous peak flow based on period of record 1963-80, 1991-96

\begin{tabular}{|c|c|c|c|c|c|}
\hline \multicolumn{6}{|c|}{$\begin{array}{l}\text { Discharge, in } \mathrm{ft}^{3} / \mathrm{s} \text {, for indicated recurrence interval } \\
\text { in years, and exceedance probablility, in percent }\end{array}$} \\
\hline 2 & 5 & 10 & 25 & $50 \dagger$ & $100 t$ \\
\hline $50 \%$ & $20 \%$ & $10 \%$ & $4 \%$ & $2 \%$ & $1 \%$ \\
\hline 359 & 611 & 808 & 1,090 & 1,320 & 1,570 \\
\hline Weighted skew & $(\log s)=$ & 0.01 & & & \\
\hline Mean & $(\log s)=$ & 2.56 & & & \\
\hline Standard dev. & $(\log s)=$ & 0.27 & & & \\
\hline
\end{tabular}

$\mp$ Reliability of values in column is uncertain, and potential errors are large. 
09512700 AGUA FRIA RIVER TRIBUTARY NO. 2 NEAR ROCK SPRINGS, AZ--Continued

Basin characteristics

\begin{tabular}{cccccccc}
\hline $\begin{array}{c}\text { Main } \\
\text { channel } \\
\text { slope } \\
(\mathrm{ft} / \mathrm{mi})\end{array}$ & $\begin{array}{c}\text { Stream } \\
\text { length } \\
(\mathrm{mi})\end{array}$ & $\begin{array}{c}\text { Mean } \\
\text { basin } \\
\text { elevation } \\
(\mathrm{ft})\end{array}$ & $\begin{array}{c}\text { Forested } \\
\text { area } \\
\text { (percent) }\end{array}$ & $\begin{array}{c}\text { Soil } \\
\text { index }\end{array}$ & $\begin{array}{c}\text { Mean } \\
\text { annual } \\
\text { precipitation } \\
\text { (in) }\end{array}$ & $\begin{array}{c}\text { Rainfall intensity, 24-hour } \\
\text { (in) }\end{array}$ & $\begin{array}{c}50-y e a r \\
\text { (in) }\end{array}$ \\
\hline 173 & 2.1 & 2,140 & 0.0 & 1.0 & 16.2 & 2.2 & 4.3 \\
\hline
\end{tabular}

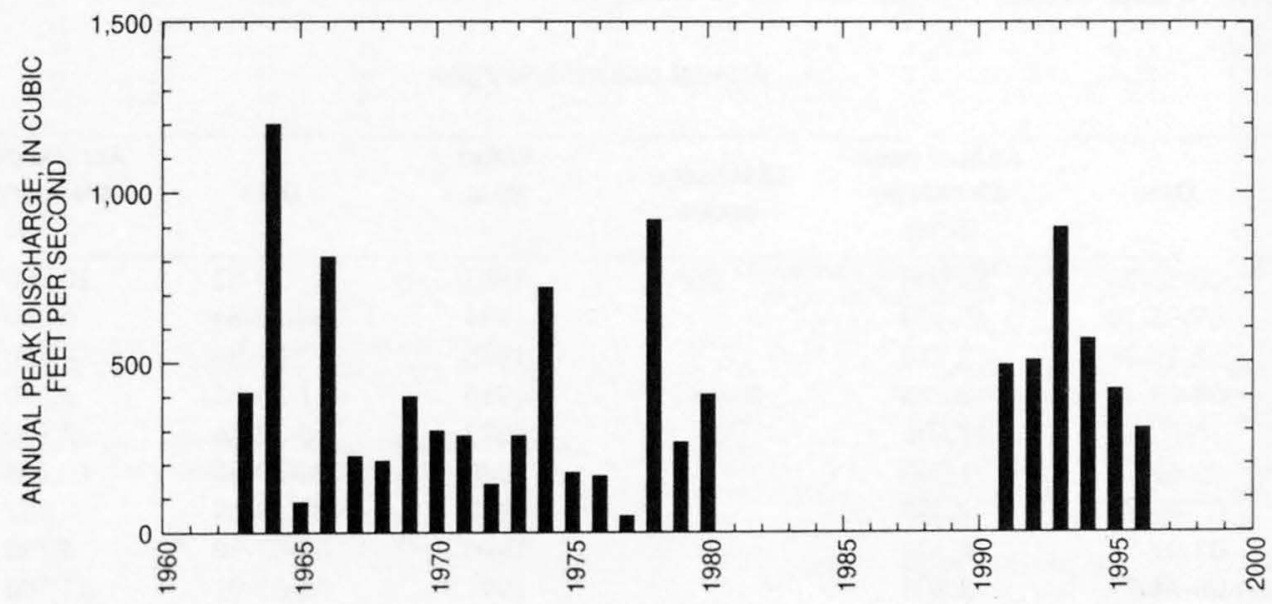


LOCATION.--Lat $34^{\circ} 00^{\prime} 56^{\prime \prime}$, long $112^{\circ} 10^{\prime} 02^{\prime \prime}$, in NW $1 /{ }_{4} \mathrm{NW}^{1} / 4$ sec.28, T.8 N., R.2 E., Yavapai County, Hydrologic Unit 15070102 , on right bank $2.5 \mathrm{mi}$ southwest of Rock Springs and $10 \mathrm{mi}$ upstream from Lake Pleasant.

DRAINAGE AREA.--1,111 $\mathrm{mi}^{2}$.

PERIOD OF RECORD.--January 1970 to current year (monthly discharge only, October 1973 to September 1974). Low-flow records not equivalent prior to Oct. 1, 1974, due to spring flow in streambed between sites in use.

REVISED RECORDS.--WDR AZ-89-1: Drainage area.

GAGE.--Water-stage recorder and crest-stage gage. Elevation of gage is $1,800 \mathrm{ft}$ above sea level, from topographic map. Prior to Oct. 1, 1974, at site $600 \mathrm{ft}$ upstream at datum $10.00 \mathrm{ft}$ higher.

REMARKS.--No estimated daily discharges. Records fair.

EXTREMES FOR PERIOD OF RECORD.--Maximum discharge, 59,500 ft $3 / \mathrm{s} \mathrm{Feb.} \mathrm{19,} \mathrm{1980,} \mathrm{gage} \mathrm{height,} 21.08 \mathrm{ft}$ recorded, $28.15 \mathrm{ft}$ from flood. mark, from rating curve extended above $21,000 \mathrm{ft}^{3} / \mathrm{s}$ on basis of slope-area measurement at gage height $27.2 \mathrm{ft}$; no flow at times each year prior to October 1974; since October 1974, no flow May 27 to July 12, 1977, and for many days in 1990.

Annual peak discharges

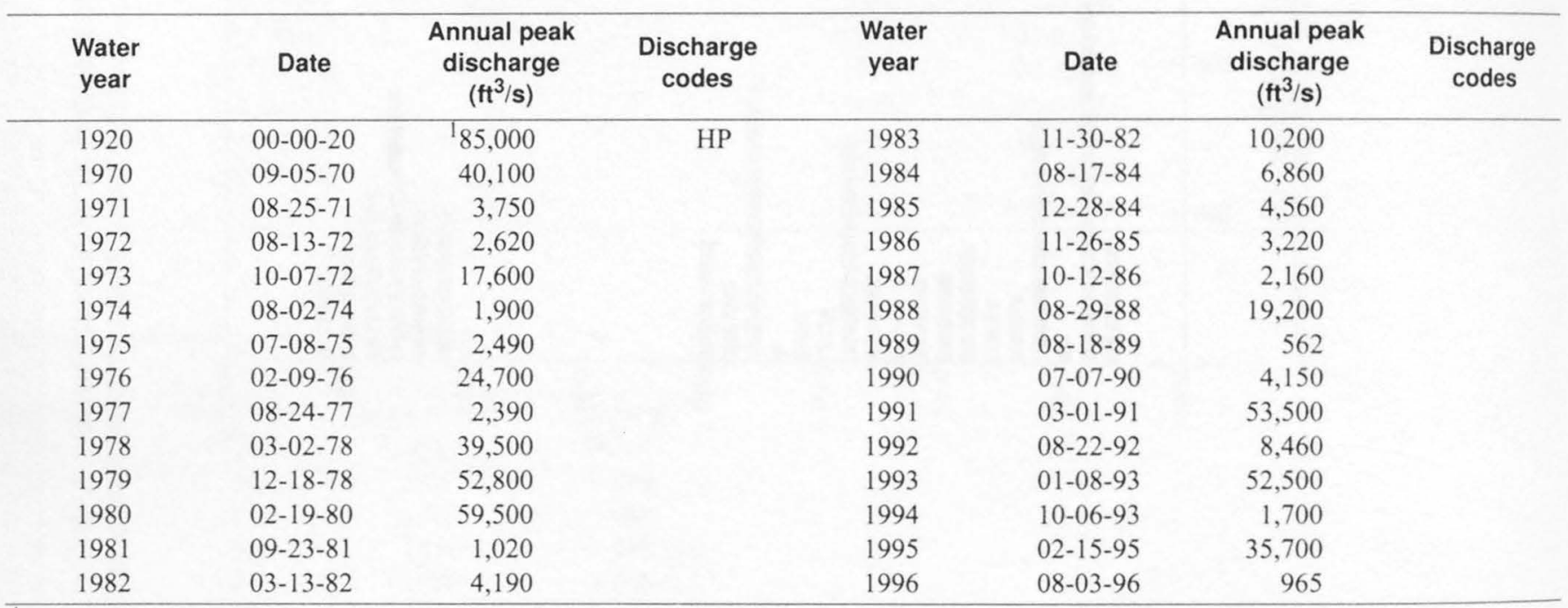

${ }^{1}$ Highest since 1891.

Discharge rating table developed January 1993

\begin{tabular}{cccc}
\hline $\begin{array}{c}\text { Gage height } \\
(\mathrm{ft})\end{array}$ & $\begin{array}{c}\text { Discharge } \\
\left(\mathrm{ft}^{3} / \mathbf{s}\right)\end{array}$ & $\begin{array}{c}\text { Gage height } \\
(\mathrm{ft})\end{array}$ & $\begin{array}{c}\text { Discharge } \\
\left(\mathrm{ft}^{3} / \mathbf{s}\right)\end{array}$ \\
\hline 7.0 & 1,120 & 17.0 & 18,500 \\
8.0 & 1,840 & 19.0 & 25,000 \\
9.0 & 2,770 & 21.0 & 32,500 \\
11.0 & 5,280 & 23.0 & 41,200 \\
13.0 & 8,710 & 25.0 & 51,000 \\
15.0 & 13,100 & 25.3 & 52,600 \\
\hline
\end{tabular}

Basin characteristics

\begin{tabular}{cccccccc}
\hline $\begin{array}{c}\text { Main } \\
\text { channel } \\
\begin{array}{c}\text { slope } \\
(\mathrm{ft} / \mathrm{mi})\end{array}\end{array}$ & $\begin{array}{c}\text { Stream } \\
\text { length } \\
\text { (mi) }\end{array}$ & $\begin{array}{c}\text { Mean } \\
\text { basin } \\
\text { elevation } \\
\text { (ft) }\end{array}$ & $\begin{array}{c}\text { Forested } \\
\text { area } \\
\text { (percent) }\end{array}$ & $\begin{array}{c}\text { Soil } \\
\text { index }\end{array}$ & $\begin{array}{c}\text { Mean } \\
\text { annual } \\
\text { precipitation } \\
\text { (in) }\end{array}$ & $\begin{array}{c}\text { Rainfall intensity, 24-hour } \\
\text { (in) }\end{array}$ & $\begin{array}{c}50 \text {-year } \\
\text { (in) }\end{array}$ \\
\hline 61.2 & 47.4 & 4,770 & 3.6 & 1.0 & 16.6 & 2.3 \\
\hline
\end{tabular}


09512800 AGUA FRIA RIVER NEAR ROCK SPRINGS, AZ--Continued

MEAN MONTHLY AND ANNUAL DISCHARGES 1971-73, 1975-96

\begin{tabular}{|c|c|c|c|c|c|c|}
\hline MONTH & $\begin{array}{l}\text { MAXIMUM } \\
\text { (FT3/S) }\end{array}$ & $\begin{array}{l}\text { MINIMUM } \\
\text { (FT3/S) }\end{array}$ & $\begin{array}{c}\text { MEAN } \\
(\mathrm{FT} 3 / \mathrm{S})\end{array}$ & $\begin{array}{l}\text { STAN- } \\
\text { DARD } \\
\text { DEVIA- } \\
\text { TION } \\
\text { (FT3/S) }\end{array}$ & $\begin{array}{l}\text { COEFFI- } \\
\text { CIENT OF } \\
\text { VARI- } \\
\text { ATION }\end{array}$ & $\begin{array}{l}\text { PERCENT } \\
\text { OF } \\
\text { ANNUAL } \\
\text { RUNOFF }\end{array}$ \\
\hline OCTOBER & 381 & 0.12 & 23 & 76 & 3.3 & 1.9 \\
\hline NOVEMBER & 176 & 0.11 & 24 & 44 & 1.9 & 2.0 \\
\hline DECEMBER & 943 & 0.10 & 89 & 218 & 2.4 & 7.5 \\
\hline JANUARY & 3,300 & 0.22 & 228 & 666 & 2.9 & 19.1 \\
\hline FEBRUARY & 3,320 & 0.66 & 382 & 774 & 2.0 & 32.1 \\
\hline MARCH & 1,970 & 0.19 & 305 & 490 & 1.6 & 25.6 \\
\hline APRIL & 338 & 0.00 & 62 & 79 & 1.3 & 5.2 \\
\hline MAY & 71 & 0.00 & 14 & 19 & 1.4 & 1.2 \\
\hline JUNE & 46 & 0.00 & 5.0 & 9.3 & 1.9 & 0.4 \\
\hline JULY & 36 & 0.00 & 11 & 11 & 1.0 & 0.9 \\
\hline AUGUST & 164 & 0.35 & 33 & 49 & 1.5 & 2.8 \\
\hline SEPTEMBER & 160 & 0.17 & 16 & 32 & 2.0 & 1.3 \\
\hline ANNUAL & 499 & 2.6 & 98 & 126 & 1.3 & 100 \\
\hline
\end{tabular}

MAGNITUDE AND PROBABILITY OF INSTANTANEOUS PEAK FLOW BASED ON PERIOD OF RECORD 1920, 1970-96

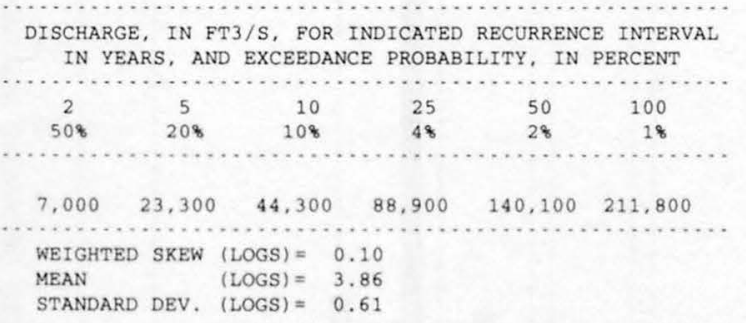

MAGNITUDE AND PROBABILITY OF ANNUAL LOW FLOW BASED ON PERIOD OF RECORD 1971.73, 1976.96

\begin{tabular}{|c|c|c|c|c|c|c|}
\hline $\begin{array}{l}\text { PERIOD } \\
\text { (CON- }\end{array}$ & \multicolumn{6}{|c|}{$\begin{array}{l}\text { DISCHARGE, IN FT3/S, FOR INDICATED } \\
\text { RECURRENCE INTERVAL, IN YEARS, AND } \\
\text { NON-EXCEEDANCE PROBABILITY, IN PERCENT }\end{array}$} \\
\hline SECU - & & & & & & $\ldots$ \\
\hline TIVE & 2 & 5 & 10 & 20 & $50 \#$ & $100 \#$ \\
\hline DAYS) & 508 & 208 & 108 & 58 & 28 & 18 \\
\hline 1 & 0.60 & 0.00 & 0.00 & 0.00 & 0.00 & 0.00 \\
\hline 3 & 0.63 & 0.00 & 0.00 & 0.00 & 0.00 & 0.00 \\
\hline 7 & 0.68 & 0.00 & 0.00 & 0.00 & 0.00 & 0.00 \\
\hline 14 & 0.74 & 0.00 & 0.00 & 0.00 & 0.00 & 0.00 \\
\hline 30 & 0.97 & 0.00 & 0.00 & 0.00 & 0.00 & 0.00 \\
\hline 60 & 1.3 & 0.14 & 0.00 & 0.00 & 0.00 & 0.00 \\
\hline 90 & 1.7 & 0.40 & 0.13 & 0.02 & 0.00 & 0.00 \\
\hline 120 & 3.0 & 0.87 & 0.37 & 0.17 & 0.06 & 0.03 \\
\hline 183 & 5.1 & 1.8 & 0.95 & 0.55 & 0.29 & 0.19 \\
\hline
\end{tabular}

MAGNITUDE AND PROBABILITY OF ANNUAL HIGH FLOW BASED ON PERIOD OF RECORD 1971.73, 1975.96

\begin{tabular}{|c|c|c|c|c|c|c|}
\hline \multirow{4}{*}{$\begin{array}{l}\text { PERIOD } \\
\text { ICON- } \\
\text { SECU- } \\
\text { TIVE }\end{array}$} & & \multicolumn{5}{|c|}{ DISCHARGE, IN FT3/S, FOR INDICATED } \\
\hline & & \multicolumn{3}{|c|}{$\begin{array}{l}\text { RECURRENCE INTERVAL, In } \\
\text { EXCEEDANCE PROBABILITY }\end{array}$} & \multicolumn{2}{|c|}{ YEARS, AND } \\
\hline & & . & , & & ….... & ….... \\
\hline & 2 & 5 & 10 & 25 & $50 \#$ & $100 \#$ \\
\hline DAYS) & 508 & 208 & 108 & 48 & 28 & 18 \\
\hline 1 & 1,860 & 8,200 & 18,100 & 42,900 & 75,400 & 126,000 \\
\hline 3 & 957 & 4,390 & 9.760 & 22,900 & 39,900 & 65,600 \\
\hline 7 & 594 & 2,610 & 5.590 & 12,400 & 20,800 & 32,800 \\
\hline 15 & 347 & 1,540 & 3,300 & 7,410 & 12,400 & 19.700 \\
\hline 30 & 219 & 943 & 2,000 & 4,450 & 7,410 & 11.700 \\
\hline 60 & 145 & 623 & 1,330 & 2,980 & 5,020 & 8,020 \\
\hline 90 & 108 & 456 & 959 & 2,110 & 3,510 & 5.530 \\
\hline
\end{tabular}

DURATION TABLE OF DAILY MEAN FLOW FOR PERIOD OF RECORD 1971.73, 1975.96

DISCHARGE, IN FT3/S, WHICH WAS EQUALED OR EXCEEDED FOR INDICATED PERCENT OF TIME

\begin{tabular}{|c|c|c|c|c|c|c|c|c|c|c|c|c|c|c|c|c|}
\hline 18 & 58 & 108 & 158 & 208 & 308 & 408 & 508 & 608 & 708 & 808 & 908 & 958 & 988 & 998 & 99.58 & 99.98 \\
\hline 1,680 & 309 & 127 & 61 & 34 & 13 & 6.3 & 3.5 & 2.3 & 1.5 & 0.80 & 0.32 & 0.08 & 0.00 & 0.00 & 0.00 & 0.00 \\
\hline
\end{tabular}

\# Reliability of values in column is uncertain, and potential errors are large. 
ANNUAL MEAN DISCHARGE, IN CUBIC FEET PER SECOND

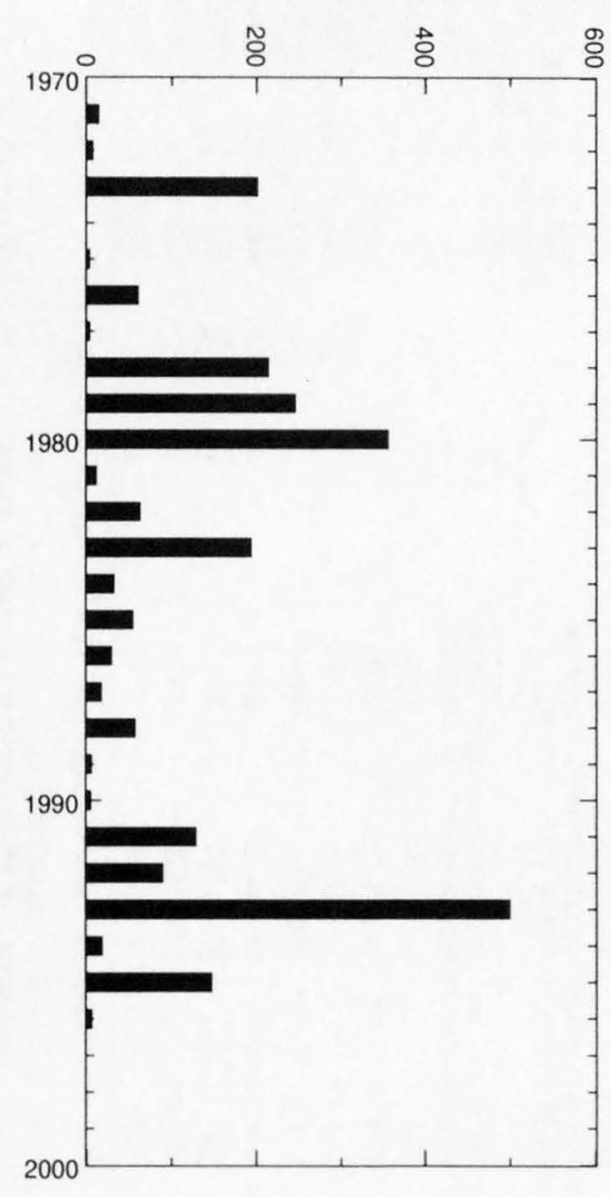

ANNUAL PEAK DISCHARGE, IN CUBIC FEET PER SECOND

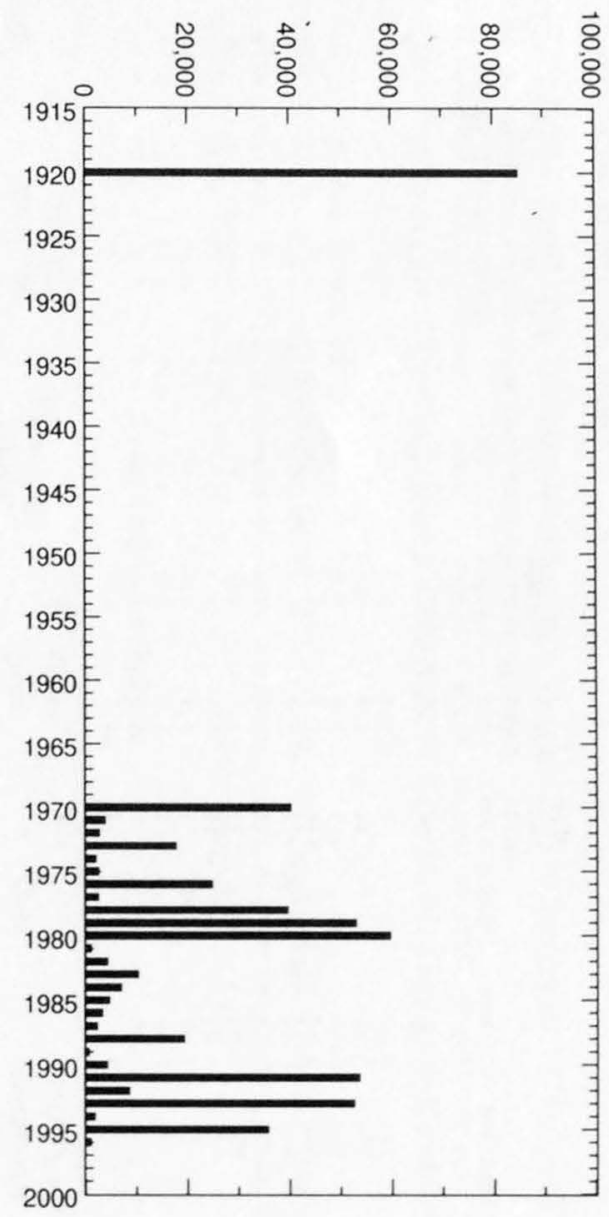


09512800 AGUA FRIA RIVER NEAR ROCK SPRINGS, AZ--Continued
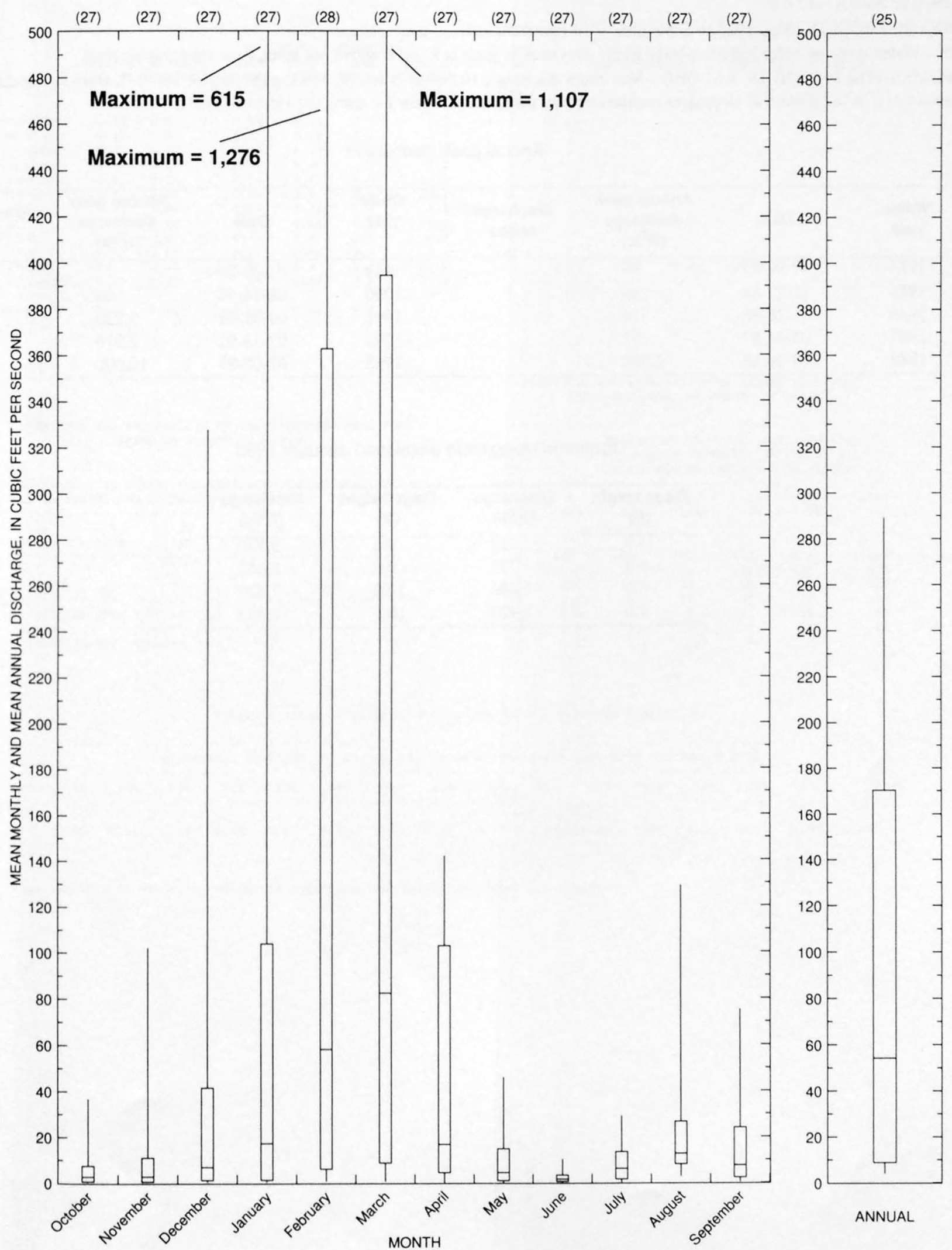


\section{BOULDER CREEK NEAR ROCK SPRINGS, AZ}

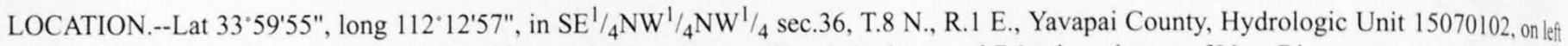
bank 2.2 mi upstream from Agua Fria River, 1.5 mi southwest of Rock Springs, and 7.1 mi northwest of New River.

DRAINAGE AREA.--37.8 $\mathrm{mi}^{2}$.

PERIOD OF RECORD.--May 1983 to September 1993 (discontinued).

GAGE.--Water-stage recorder and crest-stage gage. Elevation of gage is $1,890 \mathrm{ft}$ above sea level, from topographic map.

EXTREMES FOR PERIOD OF RECORD.--Maximum discharge, 10,000 $\mathrm{ft}^{3} / \mathrm{s}$ Jan. 8, 1993, gage height, $10.79 \mathrm{ft}$, from rating curve extended above $31.1 \mathrm{ft}^{3} / \mathrm{s}$ on basis of slope-area measurement of peak flow; no flow for many days in most years.

Annual peak discharges

\begin{tabular}{rrrrrrrr}
\hline $\begin{array}{l}\text { Water } \\
\text { year }\end{array}$ & Date & $\begin{array}{c}\text { Annual peak } \\
\text { discharge } \\
\left(\mathbf{f t}^{3} / \mathbf{s}\right)\end{array}$ & $\begin{array}{c}\text { Discharge } \\
\text { codes }\end{array}$ & $\begin{array}{c}\text { Water } \\
\text { year }\end{array}$ & Date & $\begin{array}{c}\text { Annual peak } \\
\text { discharge } \\
\left(\mathbf{f t}^{3} / \mathbf{s}\right)\end{array}$ & $\begin{array}{c}\text { Discharge } \\
\text { codes }\end{array}$ \\
\hline 1984 & $10-02-83$ & 40 & & 1989 & $02-06-89$ & 21 \\
1985 & $12-27-84$ & 239 & & 1990 & $08-14-90$ & 58 \\
1986 & $11-26-85$ & 376 & & 1991 & $03-01-91$ & 3,230 \\
1987 & $03-04-87$ & 32 & & 1992 & $02-13-92$ & 2,970 \\
1988 & $08-29-88$ & 2,350 & & 1993 & $01-08-93$ & 10,000 \\
\hline
\end{tabular}

Discharge rating table developed January 1993

\begin{tabular}{crcc}
\hline $\begin{array}{c}\text { Gage height } \\
(\mathrm{ft})\end{array}$ & $\begin{array}{c}\text { Discharge } \\
\left(\mathrm{ft}^{3} / \mathbf{s}\right)\end{array}$ & $\begin{array}{c}\text { Gage height } \\
(\mathrm{ft})\end{array}$ & $\begin{array}{c}\text { Discharge } \\
\left(\mathrm{ft}^{3} / \mathbf{s}\right)\end{array}$ \\
\hline 4.0 & 352 & 8.0 & 3,770 \\
5.0 & 772 & 9.0 & 5,550 \\
6.0 & 1,440 & 10.0 & 7,820 \\
7.0 & 2,420 & 10.8 & 10,040 \\
\hline
\end{tabular}


09512830 BOULDER CREEK NEAR ROCK SPRINGS, AZ--Continued

MEAN MONTHLY AND ANNUAL DISCHARGES 1984.92

\begin{tabular}{|c|c|c|c|c|c|c|}
\hline MONTH & $\begin{array}{l}\text { MAXIMUM } \\
\text { (FT3/S) }\end{array}$ & $\begin{array}{l}\text { MINIMUM } \\
\text { (FT3/S) }\end{array}$ & $\begin{array}{c}\text { MEAN } \\
(\mathrm{FT} 3 / \mathrm{S})\end{array}$ & $\begin{array}{l}\text { STAN - } \\
\text { DARD } \\
\text { DEVIA- } \\
\text { TION } \\
\text { (FT3/S) }\end{array}$ & $\begin{array}{l}\text { COEFFI- } \\
\text { CIENT OF } \\
\text { VARI- } \\
\text { ATION }\end{array}$ & $\begin{array}{c}\text { PERCENT } \\
\text { OF } \\
\text { ANNUAL } \\
\text { RUNOFF }\end{array}$ \\
\hline OCTOBER & 0.38 & 0.00 & 0.08 & 0.14 & 1.7 & 1.9 \\
\hline NOVEMBER & 0.37 & 0.00 & 0.11 & 0.14 & 1.2 & 2.7 \\
\hline DECEMBER & 0.70 & 0.00 & 0.23 & 0.23 & 1.0 & 5.4 \\
\hline JANUARY & 2.1 & 0.01 & 0.38 & 0.65 & 1.7 & 9.2 \\
\hline FEBRUARY & 2.8 & 0.01 & 0.64 & 0.97 & 1.5 & 15.3 \\
\hline MARCH & 6.3 & 0.01 & 0.84 & 2.1 & 2.4 & 20.1 \\
\hline APRIL & 0.21 & 0.01 & 0.06 & 0.08 & 1.2 & 1.5 \\
\hline MAY & 0.10 & 0.00 & 0.03 & 0.03 & 0.98 & 0.8 \\
\hline JUNE & 0.02 & 0.00 & 0.00 & 0.01 & 2.1 & 0.1 \\
\hline JULY & 0.11 & 0.00 & 0.01 & 0.04 & 2.7 & 0.3 \\
\hline AUGUST & 13 & 0.00 & 1.7 & 4.3 & 2.5 & 41.5 \\
\hline SEPTEMBER & 0.29 & 0.00 & 0.05 & 0.09 & 2.0 & 1.1 \\
\hline ANNUAL & 1.6 & 0.02 & 0.35 & 0.51 & 1.4 & 100 \\
\hline
\end{tabular}

MAGNITUDE AND PROBABILITY OF INSTANTANEOUS PEAK FLOW BASED ON PERIOD OF RECORD

DISCHARGE, IN FT3/S, FOR INDICATED RECURRENCE INTERVAL IN YEARS, AND EXCEEDANCE PROBABILITY, IN PERCENT

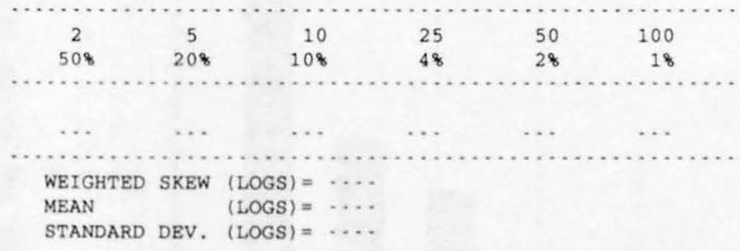

MAGNITUDE AND PROBABILITY OF ANNUAL LOW FLOW BASED ON PERIOD OF RECORD 1985.92

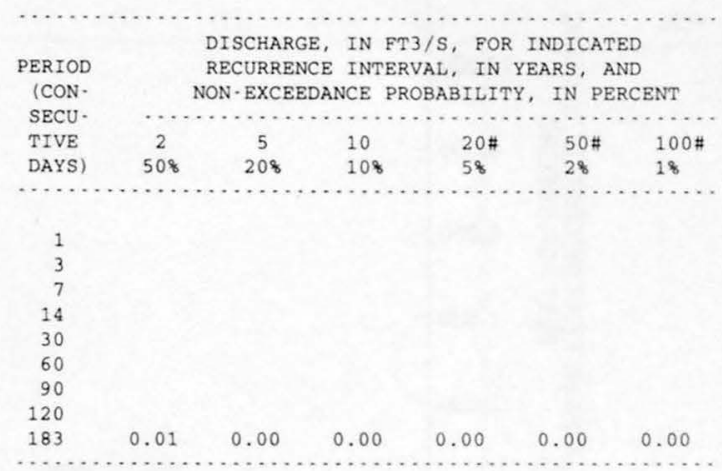

MAGNITUDE AND PROBABILITY OF ANNUAL HIGH FLOW BASED ON PERIOD OF RECORD 1984-92

\begin{tabular}{|c|c|c|c|c|c|c|}
\hline & \multicolumn{6}{|c|}{ DISCHARGE, IN FT3/S, FOR INDICATED } \\
\hline PERIOD & & RECURRE & CE INT & VAL, IN & YEARS, & ND \\
\hline (CON- & & EXCEEDA & CE PRO & BILITY, & IN PERC & NT \\
\hline SECU- & $\cdots \cdots$ & ....... & $\cdots \cdots$ & ......... & $\ldots \ldots$ & $\cdots \ldots$ \\
\hline TIVE & 2 & 5 & 10 & $25 \#$ & $50 \#$ & $100 \#$ \\
\hline DAYS) & 508 & 208 & 108 & 48 & 28 & 18 \\
\hline 1 & 12 & 65 & 161 & 432 & 826 & 1.490 \\
\hline 3 & 5.5 & 30 & 76 & 216 & 436 & 835 \\
\hline 7 & 2.6 & 13 & 33 & 89 & 176 & 327 \\
\hline 15 & 1.4 & 6.5 & 15 & 39 & 74 & 133 \\
\hline 30 & 0.91 & 3.8 & 8.5 & 21 & 39 & 68 \\
\hline 60 & 0.59 & 2.2 & 4.4 & 9.5 & 16 & 26 \\
\hline 90 & 0.47 & 1.6 & 3.0 & 6.1 & 9.7 & 15 \\
\hline
\end{tabular}

DURATION TABLE OF DAILY MEAN FLOW FOR PERIOD OF RECORD 1984.92 DISCHARGE, IN FT3/S, WHICH WAS EQUALED OR EXCEEDED FOR INDICATED PERCENT OF TIME

$\begin{array}{rl}18 & 58 \\ \ldots \ldots\end{array}$

\# Reliability of values in column is uncertain, and potential errors are large. 
09512830 BOULDER CREEK NEAR ROCK SPRINGS, AZ--Continued
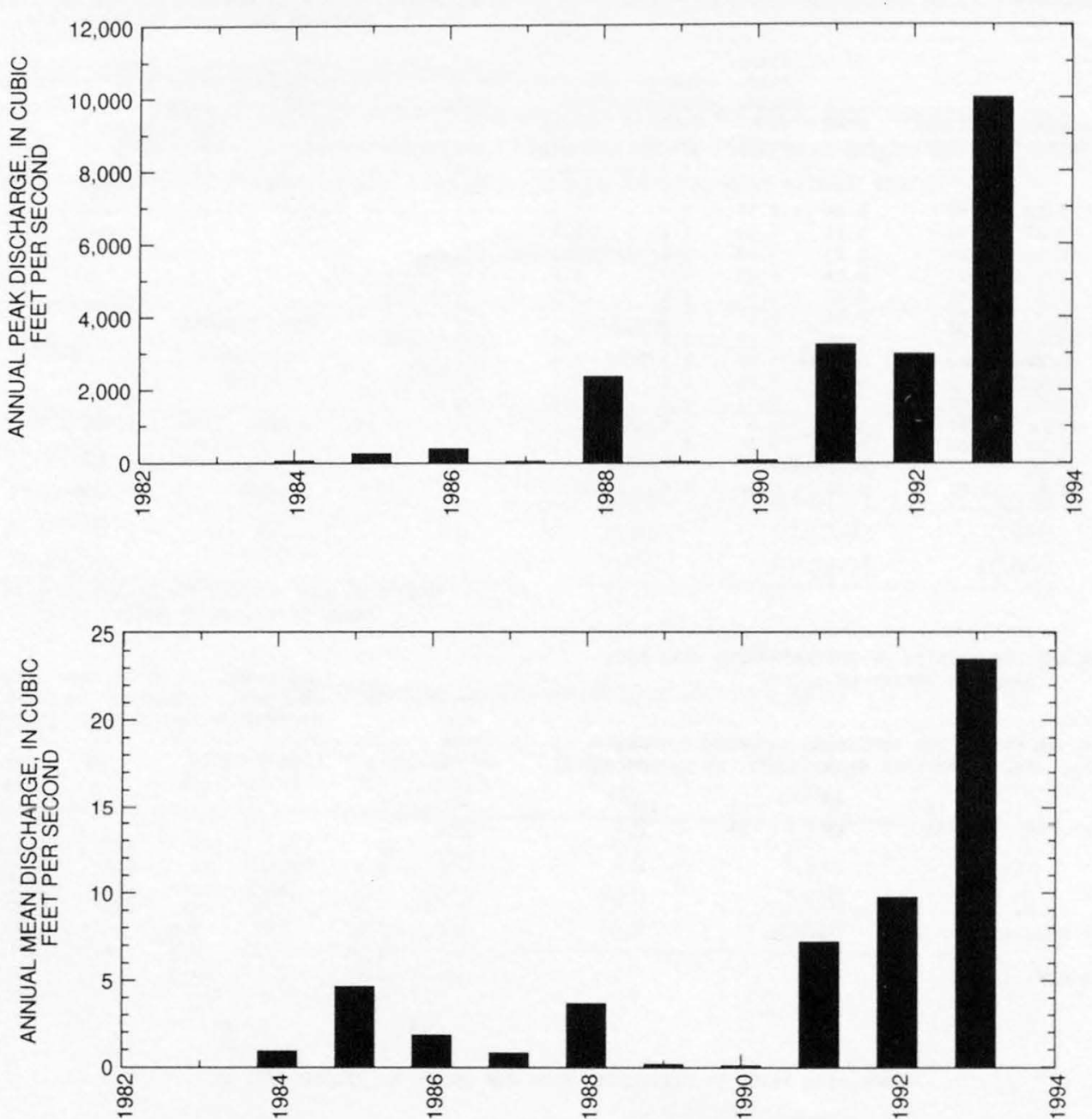
09512830 BOULDER CREEK NEAR ROCK SPRINGS, AZ--Continued
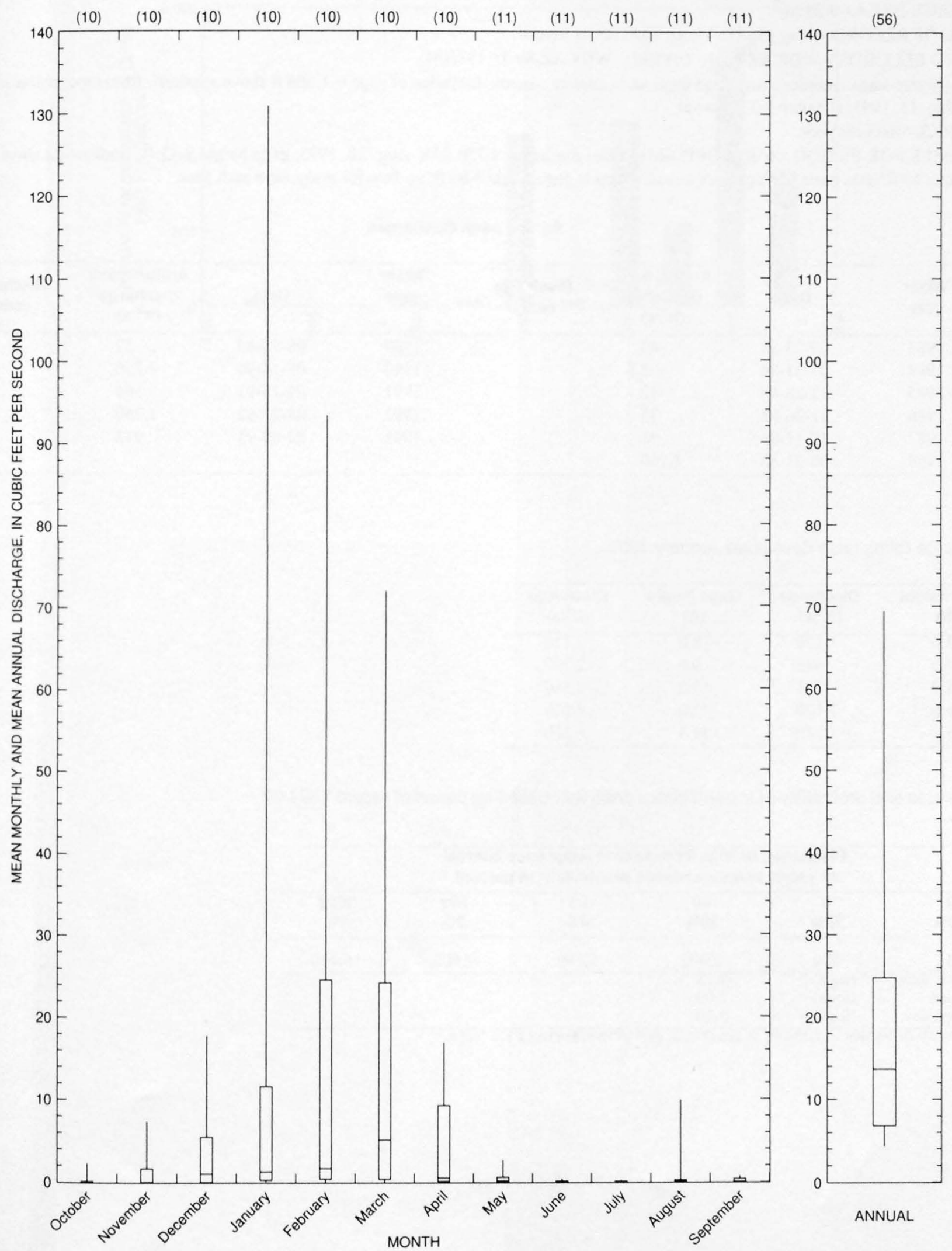
LOCATION.--Lat 33.53'55", long 112.18'39", in SW $1 / 4 \mathrm{SW}^{1} / \mathrm{SE}^{1} / 4 \mathrm{sec} .36$, T.7 N., R.1 W., Yavapai County, Hydrologic Unit 15070102 , on left bank 4 mi northwest of Waddell Dam and 1 mi upstream from the mouth at Lake Pleasant.

DRAINAGE AREA.--9.28 $\mathrm{mi}^{2}$.

PERIOD OF RECORD.--May 1983 to March 1993 (discontinued).

REVISED RECORDS.--WDR AZ-86-1: 1983(M). WDR AZ-89-1: 1985(M).

GAGE.--Water-stage recorder, crest-stage gage, and concrete control. Elevation of gage is 1,660 ft above sea level, from topographic map. Prior to Jan. 11, 1991, at datum $0.33 \mathrm{ft}$ lower.

REMARKS.--Records poor.

EXTREMES FOR PERIOD OF RECORD.--Maximum discharge, 1,750 $\mathrm{ft}^{3} / \mathrm{s}$, Aug. 22, 1992, gage height $7.33 \mathrm{ft}$, from rating curve extended above $40 \mathrm{ft}^{3} / \mathrm{s}$ on basis of slope-area measurement at gage height $5.80 \mathrm{ft}$; no flow for many days each year.

Annual peak discharges

\begin{tabular}{|c|c|c|c|c|c|c|c|}
\hline $\begin{array}{l}\text { Water } \\
\text { year }\end{array}$ & Date & $\begin{array}{c}\text { Annual peak } \\
\text { discharge } \\
\left(\mathrm{ft}^{3} / \mathrm{s}\right)\end{array}$ & $\begin{array}{l}\text { Discharge } \\
\text { codes }\end{array}$ & $\begin{array}{l}\text { Water } \\
\text { year }\end{array}$ & Date & $\begin{array}{l}\text { Annual peak } \\
\text { discharge } \\
\left(\mathrm{ft}^{3} / \mathrm{s}\right)\end{array}$ & $\begin{array}{c}\text { Discharge } \\
\text { codes }\end{array}$ \\
\hline 1983 & $09-23-83$ & 43 & & 1989 & $08-15-89$ & 37 & \\
\hline 1984 & $07-21-84$ & 2.8 & & 1990 & $08-14-90$ & 1,250 & \\
\hline 1985 & $12-28-84$ & 12 & & 1991 & $02-28-91$ & 564 & \\
\hline 1986 & $11-26-85$ & 35 & & 1992 & $08-22-92$ & 1,750 & \\
\hline 1987 & $10-11-86$ & 41 & & 1993 & $02-09-93$ & 912 & \\
\hline 1988 & $08-21-88$ & 1,160 & & & & & \\
\hline
\end{tabular}

Discharge rating table developed January 1991

\begin{tabular}{crcc}
\hline $\begin{array}{c}\text { Gage height } \\
(\mathrm{ft})\end{array}$ & $\begin{array}{c}\text { Discharge } \\
\left(\mathrm{ft}^{\mathbf{3}} / \mathbf{s}\right)\end{array}$ & $\begin{array}{c}\text { Gage height } \\
(\mathrm{ft})\end{array}$ & $\begin{array}{c}\text { Discharge } \\
\left(\mathrm{ft}^{3} / \mathbf{s}\right)\end{array}$ \\
\hline 3.0 & 179 & 8.0 & 2,110 \\
4.0 & 416 & 9.0 & 2,700 \\
5.0 & 731 & 10.0 & 3,360 \\
6.0 & 1,120 & 11.0 & 4,070 \\
7.0 & 1,580 & 11.4 & 4,380 \\
\hline
\end{tabular}

Magnitude and probability of instantaneous peak flow based on period of record 1984-92

\begin{tabular}{|c|c|c|c|c|c|}
\hline \multicolumn{6}{|c|}{$\begin{array}{c}\text { Discharge, in } \mathrm{ft}^{3} / \mathrm{s} \text {, for indicated recurrence interval } \\
\text { in years, and exceedance probablility, in percent }\end{array}$} \\
\hline $\begin{array}{c}2 \\
50 \%\end{array}$ & $\begin{array}{c}5 \\
20 \%\end{array}$ & $\begin{array}{c}10 \\
10 \%\end{array}$ & $\begin{array}{l}25 \\
4 \%\end{array}$ & $\begin{array}{l}50 \dagger \\
2 \%\end{array}$ & $\begin{array}{c}100 \dagger \\
1 \%\end{array}$ \\
\hline 131 & 804 & 2,020 & 5,240 & 9,600 & 16,400 \\
\hline $\begin{array}{l}\text { Weighted skew } \\
\text { Mean } \\
\text { Standard dev. }\end{array}$ & $\begin{array}{l}(\log s)= \\
(\log s)= \\
(\log s)=\end{array}$ & $\begin{array}{r}-0.15 \\
2.09 \\
0.96\end{array}$ & & & \\
\hline
\end{tabular}


09512970 COTTONWOOD CREEK NEAR WADDELL DAM, AZ--Continued

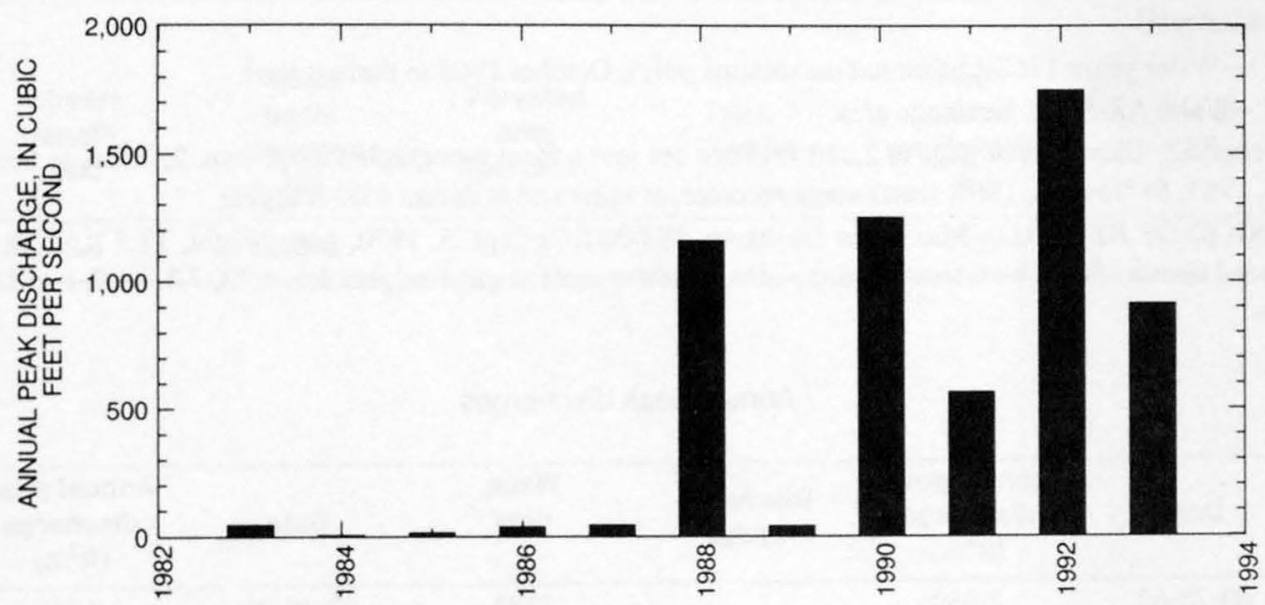




\section{NEW RIVER NEAR ROCK SPRINGS, AZ}

LOCATION.--Lat 33.58'27", long $112^{\circ} 05^{\prime} 54^{\prime \prime}$, in SW $1 / 4 \mathrm{SW}^{1} / 4$ sec.6, T.7 N., R.3 E., Maricopa County, Hydrologic Unit 15070102 , on right bank $180 \mathrm{ft}$ upstream from road crossing and $6 \mathrm{mi}$ southeast of Rock Springs.

DRAINAGE AREA.--68.3 $\mathrm{mi}^{2}$.

PERIOD OF RECORD.--Water years 1962-65 (annual maximums only), October 1965 to current year.

REVISED RECORDS.--WDR AZ-89-1: Drainage area.

GAGE.--Water-stage recorder. Elevation of gage is 2,310 ft above sea level, from topographic map. Jan. 2, 1964, to Sept. 30, 1965, crest-stage gage, and Oct. 28, 1965, to Nov. 16, 1967, water-stage recorder, at same site at datum $1.00 \mathrm{ft}$ higher.

EXTREMES FOR PERIOD OF RECORD.--Maximum discharge, 18,600 $\mathrm{ft}^{3} / \mathrm{s}$ Sept. 5, 1970, gage height, $13.5 \mathrm{ft}$, from profile past gage, from rating curve extended above $380 \mathrm{ft}^{3} / \mathrm{s}$ on basis of slope-area measurements at gage heights $3.6,4.73,7.3,10.7$, and 13.5 ft; no flow for many days in most years.

Annual peak discharges

\begin{tabular}{|c|c|c|c|c|c|c|c|}
\hline $\begin{array}{l}\text { Water } \\
\text { year }\end{array}$ & Date & $\begin{array}{c}\text { Annual peak } \\
\text { discharge } \\
\left(\mathrm{ft}^{3} / \mathrm{s}\right)\end{array}$ & $\begin{array}{c}\text { Discharge } \\
\text { codes }\end{array}$ & $\begin{array}{l}\text { Water } \\
\text { year }\end{array}$ & Date & $\begin{array}{c}\text { Annual peak } \\
\text { discharge } \\
\left(\mathrm{ft}^{3} / \mathrm{s}\right)\end{array}$ & $\begin{array}{c}\text { Discharge } \\
\text { codes }\end{array}$ \\
\hline 1962 & $09-28-62$ & 1,050 & & 1980 & $02-19-80$ & 9,350 & \\
\hline 1963 & $08-16-63$ & 765 & & 1981 & $03-06-81$ & 35 & \\
\hline 1964 & $08-02-64$ & 4,900 & & 1982 & $03-15-82$ & 1,760 & \\
\hline 1965 & $04-04-65$ & 1,510 & & 1983 & $11-30-82$ & 12,500 & \\
\hline 1966 & $12-22-65$ & 4,020 & & 1984 & $12-27-83$ & 692 & \\
\hline 1967 & $09-06-67$ & 245 & & 1985 & $12-27-84$ & 2,310 & \\
\hline 1968 & $12-19-67$ & 10,600 & & 1986 & $11-26-85$ & 3,700 & \\
\hline 1969 & $09-05-69$ & 1,530 & & 1987 & $03-03-87$ & 545 & \\
\hline 1970 & $09-05-70$ & 18,600 & & 1988 & $11-01-87$ & 4,980 & \\
\hline 1971 & $08-03-71$ & 6,320 & & 1989 & $01-05-89$ & 428 & \\
\hline 1972 & $08-12-72$ & 231 & & 1990 & $07-07-90$ & 1,670 & \\
\hline 1973 & $12-28-72$ & 1,550 & & 1991 & 03-01-91 & 9,810 & \\
\hline 1974 & $08-05-74$ & 68 & & 1992 & $02-13-92$ & 5,510 & \\
\hline 1975 & $11-01-74$ & 1,570 & & 1993 & $01-08-93$ & 12,600 & \\
\hline 1976 & $02-09-76$ & 3,230 & & 1994 & $11-15-93$ & 604 & \\
\hline 1977 & $08-16-77$ & 4.0 & & 1995 & $02-15-95$ & 9,710 & \\
\hline 1978 & $03-02-78$ & 13,600 & & 1996 & $07-16-96$ & 77 & \\
\hline 1979 & $03-28-79$ & 6,530 & & & & & \\
\hline
\end{tabular}

Discharge rating table developed January 1993

\begin{tabular}{crcr}
\hline $\begin{array}{c}\text { Gage height } \\
(\mathbf{f t})\end{array}$ & $\begin{array}{c}\text { Discharge } \\
\left(\mathbf{f t}^{\mathbf{3}} / \mathbf{s}\right)\end{array}$ & $\begin{array}{c}\text { Gage height } \\
(\mathbf{f t})\end{array}$ & $\begin{array}{c}\text { Discharge } \\
\left(\mathbf{f t}^{\mathbf{3}} \mathbf{s}\right)\end{array}$ \\
\hline 2.5 & 128 & 7.0 & 3,800 \\
3.0 & 259 & 8.0 & 5,540 \\
4.0 & 697 & 9.0 & 7,670 \\
5.0 & 1,410 & 10.0 & 10,230 \\
6.0 & 2,430 & 11.0 & 13,240 \\
\hline
\end{tabular}


GILA RIVER BASIN

09513780 NEW RIVER NEAR ROCK SPRINGS, AZ--Continued

Basin characteristics

\begin{tabular}{cccccccc}
\hline $\begin{array}{c}\text { Main } \\
\text { channel } \\
\text { slope } \\
(\mathrm{ft} / \mathrm{mi})\end{array}$ & $\begin{array}{c}\text { Stream } \\
\text { length } \\
(\mathrm{mi})\end{array}$ & $\begin{array}{c}\text { Mean } \\
\text { basin } \\
\text { elevation } \\
(\mathrm{ft})\end{array}$ & $\begin{array}{c}\text { Forested } \\
\text { area } \\
\text { (percent) }\end{array}$ & $\begin{array}{c}\text { Soil } \\
\text { index }\end{array}$ & $\begin{array}{c}\text { Mean } \\
\text { annual } \\
\text { precipitation } \\
\text { (in) }\end{array}$ & $\begin{array}{c}\text { Rainfall intensity, 24-hour } \\
\text { (in) }\end{array}$ & $\begin{array}{c}50 \text {-year } \\
\text { (in) }\end{array}$ \\
\hline 140 & 18.6 & 3,970 & 0.2 & 1.0 & 20.0 & 2.4 \\
\hline
\end{tabular}


09513780 NEW RIVER NEAR ROCK SPRINGS, AZ--Continued

MEAN MONTHLY AND ANNUAL DISCHARGES 1966.96

\begin{tabular}{|c|c|c|c|c|c|c|}
\hline MONTH & $\begin{array}{l}\text { MAXIMUM } \\
(\text { FT3/S) }\end{array}$ & $\begin{array}{l}\text { MINIMUM } \\
\text { (FT3/S) }\end{array}$ & $\begin{array}{c}\text { MEAN } \\
(\text { FT3/S) }\end{array}$ & $\begin{array}{l}\text { STAN- } \\
\text { DARD } \\
\text { DEVIA- } \\
\text { TION } \\
\text { (FT3/S) }\end{array}$ & $\begin{array}{l}\text { COEFFI- } \\
\text { CIENT OF } \\
\text { VARI- } \\
\text { ATION }\end{array}$ & $\begin{array}{c}\text { PERCENT } \\
\text { OF } \\
\text { ANNUAL } \\
\text { RUNOFF }\end{array}$ \\
\hline OCTOBER & 32 & 0.00 & 1.3 & 5.7 & 4.3 & 0.8 \\
\hline NOVEMBER & 52 & 0.00 & 5.9 & 12 & 2.1 & 3.3 \\
\hline DECEMBER & 218 & 0.00 & 27 & 57 & 2.1 & 15.3 \\
\hline JANUARY & 573 & 0.00 & 41 & 109 & 2.7 & 23.2 \\
\hline FEBRUARY & 348 & 0.00 & 43 & 76 & 1.8 & 24.7 \\
\hline MARCH & 444 & 0.00 & 45 & 90 & 2.0 & 25.5 \\
\hline APRIL & 30 & 0.00 & 5.0 & 7.8 & 1.6 & 2.8 \\
\hline MAY & 11 & 0.00 & 1.3 & 2.4 & 1.9 & 0.7 \\
\hline JUNE & 2.2 & 0.00 & 0.29 & 0.64 & 2.2 & 0.2 \\
\hline JULY & 8.5 & 0.00 & 0.71 & 1.9 & 2.7 & 0.4 \\
\hline AUGUST & 15 & 0.00 & 1.5 & 3.6 & 2.4 & 0.8 \\
\hline SEPTEMBER & 104 & 0.00 & 4.0 & 19 & 4.6 & 2.3 \\
\hline ANNUAL & 72 & 0.00 & 15 & 19 & 1.3 & 100 \\
\hline
\end{tabular}

MAGNITUDE AND PROBABILITY OF INSTANTANEOUS PEAK FLOW BASED ON PERIOD OF RECORD 1962.96

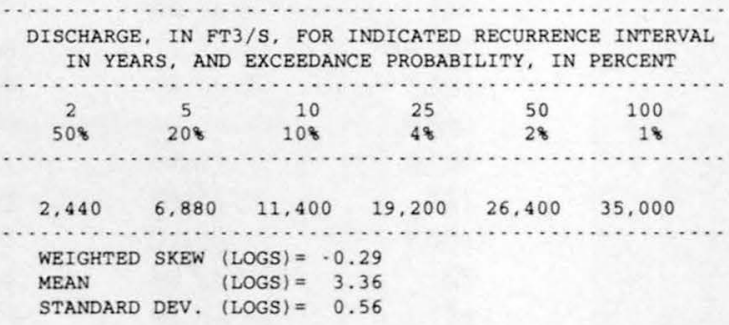

MAGNITUDE AND PROBABILITY OF ANNUAL LOW FLOW BASED ON PERIOD OF RECORD 1967.96

\begin{tabular}{|c|c|c|c|c|c|c|}
\hline \multirow{2}{*}{$\begin{array}{l}\text { PERIOD } \\
\text { (CON- } \\
\text { SECU. }\end{array}$} & \multicolumn{6}{|c|}{$\begin{array}{l}\text { RECURRENCE INTERVAL, IN YEARS, AND } \\
\text { NON-EXCEEDANCE PROBABILITY, IN PERCENT }\end{array}$} \\
\hline & $\cdots$ & $\ldots \ldots$ & $\ldots \ldots$ & ...... & ........ & $\ldots \ldots \ldots$ \\
\hline TIVE & 2 & 5 & 10 & $20 \#$ & $50 \#$ & $100 \#$ \\
\hline DAYS) & 508 & 208 & 108 & 58 & 28 & 18 \\
\hline 1 & & & & & & \\
\hline 3 & & & & & & \\
\hline 7 & & & & & & \\
\hline 14 & & & & & & \\
\hline 30 & & & & & & \\
\hline 60 & & & & & & \\
\hline 90 & 0.00 & 0.00 & 0.00 & 0.00 & 0.00 & 0.00 \\
\hline 120 & 0.00 & 0.00 & 0.00 & 0.00 & 0.00 & 0.00 \\
\hline 183 & 0.10 & 0.00 & 0.00 & 0.00 & 0.00 & 0.00 \\
\hline
\end{tabular}

MAGNITUDE AND PROBABILITY OF ANNUAL HIGH FLOW BASED ON PERIOD OF RECORD 1966.96

\begin{tabular}{|c|c|c|c|c|c|c|}
\hline \multirow{4}{*}{$\begin{array}{l}\text { PERIOD } \\
\text { (CON- } \\
\text { SECU- } \\
\text { TIVE } \\
\text { DAYS) }\end{array}$} & & \multicolumn{4}{|c|}{$\begin{array}{l}\text { DISCHARGE, IN FT3/S, FOR INDICATED } \\
\text { RECURRENCE INTERVAL, IN YEARS, AND }\end{array}$} & $\begin{array}{l}\text { TED } \\
\text { AND } \\
\text { ENT }\end{array}$ \\
\hline & $\cdots$ & ........ & \multicolumn{2}{|c|}{ EXCEEDANCE PROBABILITY, } & $\ldots \ldots \ldots$ & $\ldots \ldots \ldots$ \\
\hline & 2 & 5 & 10 & $25 \#$ & $50 \#$ & $100 \#$ \\
\hline & 508 & 208 & 108 & 48 & 28 & 18 \\
\hline 1 & 480 & 2,190 & 4,000 & 6.650 & 8,670 & 10,600 \\
\hline 3 & 235 & 1,080 & 1.950 & 3,220 & 4.160 & 5,030 \\
\hline 7 & 127 & 585 & 1.050 & 1,690 & 2,140 & 2,560 \\
\hline 15 & 71 & 330 & 587 & 939 & 1.190 & 1,400 \\
\hline 30 & 43 & 199 & 354 & 566 & 715 & 848 \\
\hline 60 & 25 & 116 & 225 & 417 & 594 & 795 \\
\hline 90 & 19 & 85 & 163 & 298 & 421 & 559 \\
\hline
\end{tabular}

DURATION TABLE OF DAILY MEAN FLOW FOR PERIOD OF RECORD 1966.96

DISCHARGE, IN FT3/S, WHICH WAS EQUALED OR EXCEEDED FOR INDICATED PERCENT OF TIME

\begin{tabular}{|c|c|c|c|c|c|c|c|c|c|c|c|c|c|c|c|c|}
\hline 18 & 58 & 108 & 158 & 208 & 308 & 408 & 508 & 608 & 708 & 808 & 908 & 958 & 988 & 998 & 99.58 & 99.98 \\
\hline 265 & 30 & 11 & 5.2 & 2.8 & 0.82 & 0.08 & 0.00 & 0.00 & 0.00 & 0.00 & 0.00 & 0.00 & 0.00 & 0.00 & 0.00 & 0.00 \\
\hline
\end{tabular}

\# Reliability of values in column is uncertain, and potential errors are large. 
09513780 NEW RIVER NEAR ROCK SPRINGS, AZ--Continued
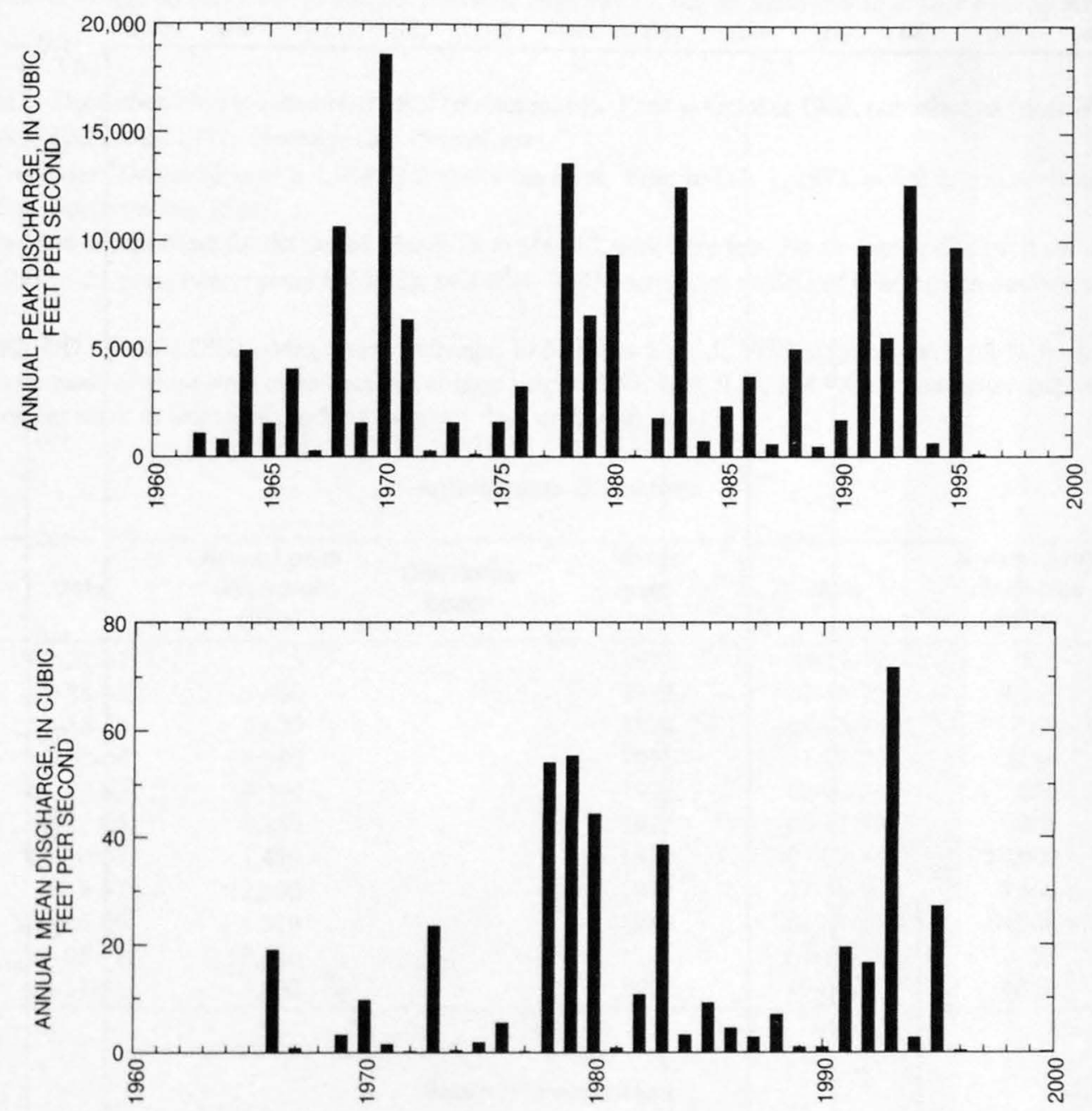
09513780 NEW RIVER NEAR ROCK SPRINGS, AZ--Continued
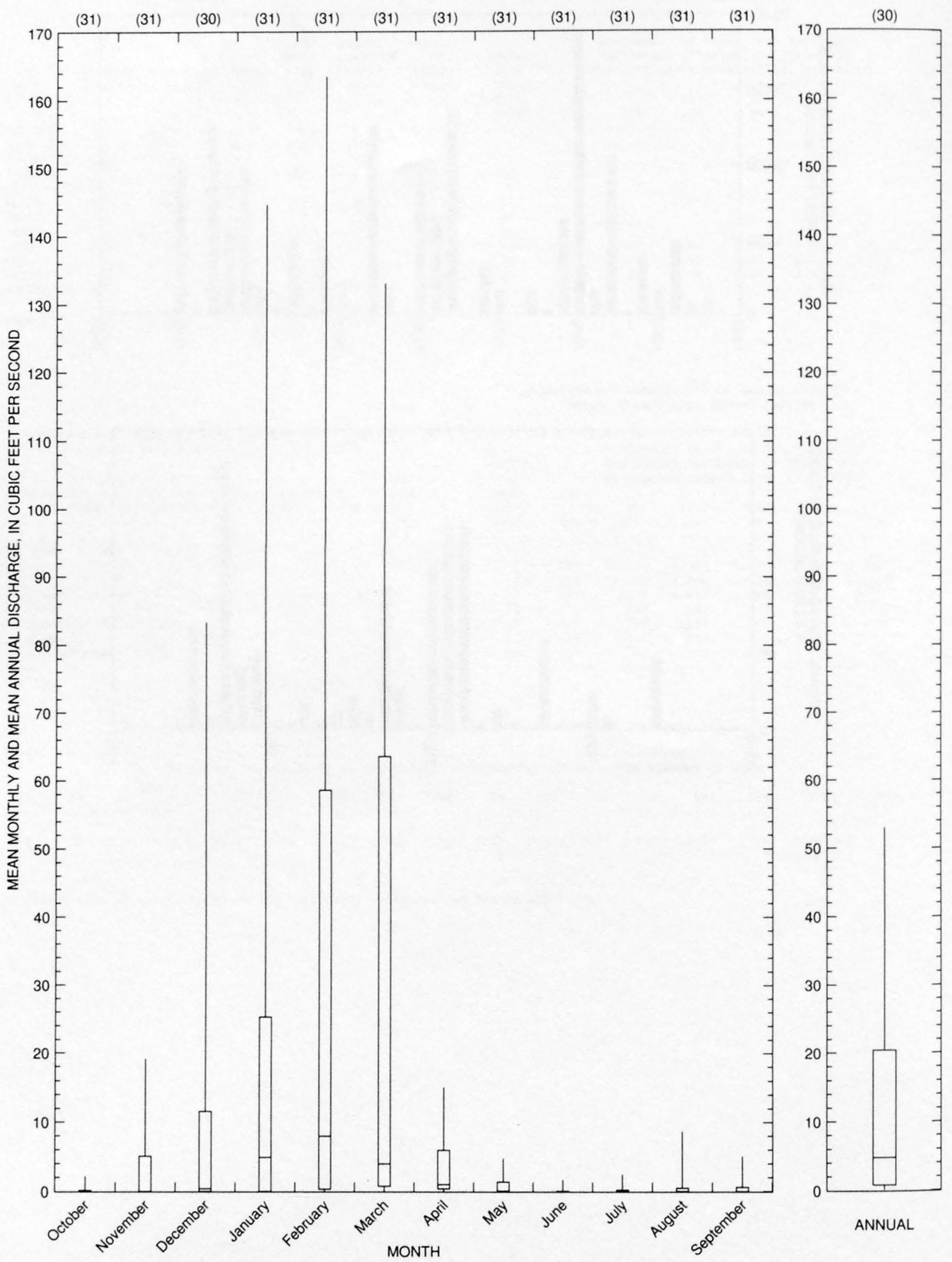
09513800 NEW RIVER AT NEW RIVER, AZ

LOCATION.--Lat 33.54'41", long 112 08'26", in NW $1 / 4^{\prime} \mathrm{NE}^{1} / 4 \mathrm{sec} .34$, T.7 N., R.2 E., Maricopa County, Hydrologic Unit 15070102 , near center of downstream side of bridge on east frontage road of Interstate Highway 17, 0.5 mi southwest of village of New River, and 10 mi south of Rock Springs.

DRAINAGE AREA.--83.3 $\mathrm{mi}^{2}$.

PERIOD OF RECORD.--December 1960 to September 1982 (discontinued). Prior to October 1965, published as "near Black Canyon."

REVISED RECORDS.--WRD Ariz. 1972: Drainage area (former site).

GAGE.--Water-stage recorder. Datum of gage is $1,984.02 \mathrm{ft}$ above sea level. Prior to Feb. 1,1972 , at site $0.3 \mathrm{mi}$ downstream at datum $10.86 \mathrm{ft}$ lower (now used as supplementary gage).

REMARKS.--Records good except those for the period March 28 to May 13, which are fair. No storage or diversion above station.

AVERAGE DISCHARGE.--21 years (water years 1962-82), $14.4 \mathrm{ft}^{3} / \mathrm{s}, 10,430$ acre- $\mathrm{ft} / \mathrm{yr}$; median of yearly mean discharges, $3.1 \mathrm{ft}^{3} / \mathrm{s}, 2,200 \mathrm{acre}$ $\mathrm{ft} / \mathrm{yr}$.

EXTREMES FOR PERIOD OF RECORD.--Maximum discharge, 19,500 $\mathrm{ft}^{3} / \mathrm{s}$ Sept. 5, 1970, gage height, $9.98 \mathrm{ft}$, from rating curve extended above $1,300 \mathrm{ft}^{3} / \mathrm{s}$ on basis of slope-area measurements at gage heights 5.57, 7.33, 9.12, and $9.98 \mathrm{ft}$; maximum gage height, $12.34 \mathrm{ft}$ Mar. 2 , 1978, from high-water mark in gage well; no flow for many days each year.

Annual peak discharges

\begin{tabular}{|c|c|c|c|c|c|c|c|}
\hline $\begin{array}{l}\text { Water } \\
\text { year }\end{array}$ & Date & $\begin{array}{c}\text { Annual peak } \\
\text { discharge } \\
\left(\mathrm{ft}^{3} / \mathrm{s}\right)\end{array}$ & $\begin{array}{c}\text { Discharge } \\
\text { codes }\end{array}$ & $\begin{array}{l}\text { Water } \\
\text { year }\end{array}$ & Date & $\begin{array}{c}\text { Annual peak } \\
\text { discharge } \\
\left(\mathrm{ft}^{3} / \mathrm{s}\right)\end{array}$ & $\begin{array}{l}\text { Discharge } \\
\text { codes }\end{array}$ \\
\hline 1961 & $07-22-61$ & 325 & & 1972 & $08-12-72$ & 525 & \\
\hline 1962 & $09-28-62$ & 1,430 & & 1973 & $02-11-73$ & 4,250 & \\
\hline 1963 & $08-16-63$ & 4,620 & & 1974 & $08-05-74$ & 49 & \\
\hline 1964 & $08-02-64$ & 4,380 & & 1975 & $11-02-74$ & 2,280 & \\
\hline 1965 & $04-04-65$ & 1,990 & & 1976 & $02-09-76$ & 7,050 & \\
\hline 1966 & $12-22-65$ & 4,180 & & 1977 & $08-17-77$ & 805 & \\
\hline 1967 & $08-10-67$ & 1,420 & & 1978 & $03-02-78$ & 18,000 & \\
\hline 1968 & $12-19-67$ & 12,600 & & 1979 & $12-18-78$ & 5,560 & \\
\hline 1969 & $09-05-69$ & 1,310 & & 1980 & $02-19-80$ & 14,900 & \\
\hline 1970 & $09-05-70$ & 19,500 & & 1981 & $09-23-81$ & 20 & \\
\hline 1971 & $08-21-71$ & 5,090 & & 1982 & $10-01-81$ & 2,510 & \\
\hline
\end{tabular}

Basin characteristics

\begin{tabular}{cccccccc}
\hline $\begin{array}{c}\text { Main } \\
\text { channel } \\
\text { slope } \\
(\mathrm{ft} / \mathrm{mi})\end{array}$ & $\begin{array}{c}\text { Stream } \\
\text { length } \\
\text { (mi) }\end{array}$ & $\begin{array}{c}\text { Mean } \\
\text { basin } \\
\text { elevation } \\
\text { (ft) }\end{array}$ & $\begin{array}{c}\text { Forested } \\
\text { area } \\
\text { (percent) }\end{array}$ & $\begin{array}{c}\text { Soil } \\
\text { index }\end{array}$ & $\begin{array}{c}\text { Mean } \\
\text { annual } \\
\text { precipitation } \\
\text { (in) }\end{array}$ & $\begin{array}{c}\text { 2-year } \\
\text { (in) }\end{array}$ & $\begin{array}{c}50 \text {-year } \\
\text { (in) }\end{array}$ \\
\hline 105 & 25.9 & 3,600 & 0.2 & 1.0 & 19.5 & 2.3 & 4.6 \\
\hline
\end{tabular}




\section{NEW RIVER AT NEW RIVER, AZ--Continued}

MEAN MONTHLY AND ANNUAL DISCHARGES 1961.82

\begin{tabular}{|c|c|c|c|c|c|c|}
\hline MONTH & $\begin{array}{l}\text { MAXIMUM } \\
\text { (FT3/S) }\end{array}$ & $\begin{array}{l}\text { MINIMUM } \\
\text { (FT3/S) }\end{array}$ & $\begin{array}{c}\text { MEAN } \\
(\mathrm{FT} 3 / \mathrm{S})\end{array}$ & $\begin{array}{l}\text { STAN- } \\
\text { DARD } \\
\text { DEVIA- } \\
\text { TION } \\
\text { (FT3/S) }\end{array}$ & $\begin{array}{l}\text { COEFFI- } \\
\text { CIENT OF } \\
\text { VARI- } \\
\text { ATION }\end{array}$ & $\begin{array}{c}\text { PERCENT } \\
\text { OF } \\
\text { ANNUAL } \\
\text { RUNOFF }\end{array}$ \\
\hline OCTOBER & 35 & 0.00 & 2.1 & 7.5 & 3.5 & 1.3 \\
\hline NOVEMBER & 44 & 0.00 & 4.4 & 11 & 2.4 & 2.6 \\
\hline DECEMBER & 207 & 0.00 & 28 & 65 & 2.3 & 16.6 \\
\hline JANUARY & 310 & 0.00 & 28 & 73 & 2.6 & 16.8 \\
\hline FEBRUARY & 523 & 0.00 & 46 & 114 & 2.5 & 27.4 \\
\hline MARCH & 443 & 0.00 & 40 & 99 & 2.5 & 24.1 \\
\hline APRIL & 74 & 0.00 & 6.7 & 16 & 2.4 & 4.0 \\
\hline MAY & 20 & 0.00 & 1.6 & 4.9 & 3.1 & 0.9 \\
\hline JUNE & 0.90 & 0.00 & 0.11 & 0.25 & 2.2 & 0.1 \\
\hline JULY & 2.9 & 0.00 & 0.40 & 0.77 & 1.9 & 0.2 \\
\hline AUGUST & 25 & 0.00 & 3.8 & 6.8 & 1.8 & 2.3 \\
\hline SEPTEMBER & 115 & 0.00 & 6.3 & 24 & 3.8 & 3.8 \\
\hline ANNUAL & 74 & 0.01 & 14 & 21 & 1.5 & 100 \\
\hline
\end{tabular}

MAGNITUDE AND PROBABILITY OF ANNUAL LOW FLOW BASED ON PERIOD OF RECORD $1962 \cdot 82$

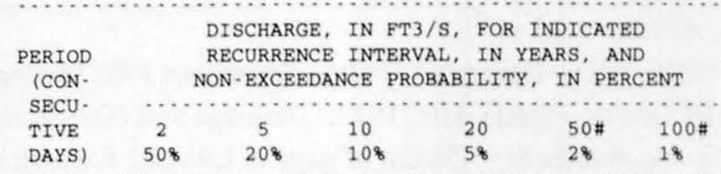

MAGNITUDE AND PROBABILITY OF ANNUAL HIGH FLOW BASED ON PERIOD OF RECORD 1961.82

MAGNITUDE AND PROBABILITY OF INSTANTANEOUS PEAK FLOW BASED ON PERIOD OF RECORD 1961.82

DISCHARGE, IN FT3/S, FOR INDICATED RECURRENCE INTERVAL IN YEARS, AND EXCEEDANCE PROBABILITY, IN PERCENT

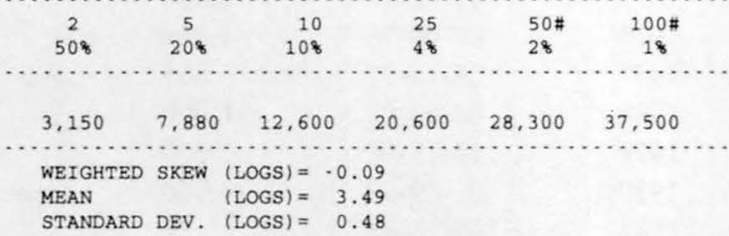

DURATION TABLE OF DAILY MEAN FLOW FOR PERIOD OF RECORD 1961.82

\begin{tabular}{|c|c|c|c|c|c|c|}
\hline \multirow{3}{*}{$\begin{array}{l}\text { PERIOD } \\
\text { ICON- } \\
\text { SECU- }\end{array}$} & \multicolumn{6}{|c|}{ DISCHARGE, IN FT3/S, FOR INDICATED } \\
\hline & & EXCEEDF & CE PROE & BILITY, & IN PERC & ENT \\
\hline & & 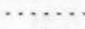 & & & & \\
\hline TIVE & 2 & 5 & 10 & 25 & $50 \#$ & $100 \#$ \\
\hline DAYS) & 508 & 208 & 108 & 48 & 28 & 18 \\
\hline 1 & 410 & 1,980 & 3,800 & 6.790 & 9,310 & 11,900 \\
\hline 3 & 179 & 969 & 1.970 & 3.730 & 5,310 & 7,020 \\
\hline 7 & 93 & 544 & 1.150 & 2,240 & 3.250 & 4,360 \\
\hline 15 & 50 & 304 & 648 & 1,280 & 1,860 & 2,500 \\
\hline 30 & 28 & 174 & 377 & 754 & 1,100 & 1,490 \\
\hline 60 & 16 & 109 & 243 & 499 & 742 & 1,020 \\
\hline 90 & 12 & 79 & 174 & 355 & 524 & 712 \\
\hline
\end{tabular}

DISCHARGE, IN FT3/S, WHICH WAS EQUALED OR EXCEEDED FOR INDICATED PERCENT OF TIME

$\begin{array}{lllllllllllllllllll}18 & 58 & 108 & 158 & 208 & 308 & 408 & 508 & 608 & 708 & 808 & 908 & 958 & 988 & 998 & 99.58 & 99.98\end{array}$

$\begin{array}{lllllllllllllllllllll}298 & 30 & 7.4 & 1.9 & 0.31 & 0.00 & 0.00 & 0.00 & 0.00 & 0.00 & 0.00 & 0.00 & 0.00 & 0.00 & 0.00 & 0.00 & 0.00\end{array}$

\# Reliability of values in column is uncertain, and potential errors are large. 
ANNUAL MEAN DISCHARGE, IN CUBIC

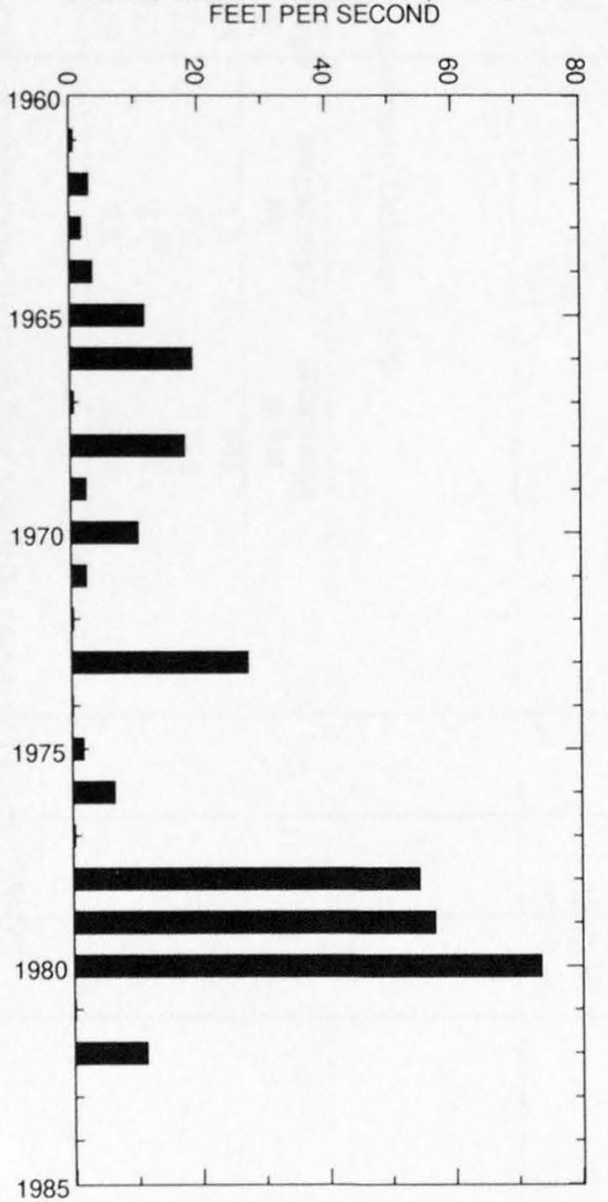

ANNUAL PEAK DISCHARGE, IN CUBIC FEET PER SECOND

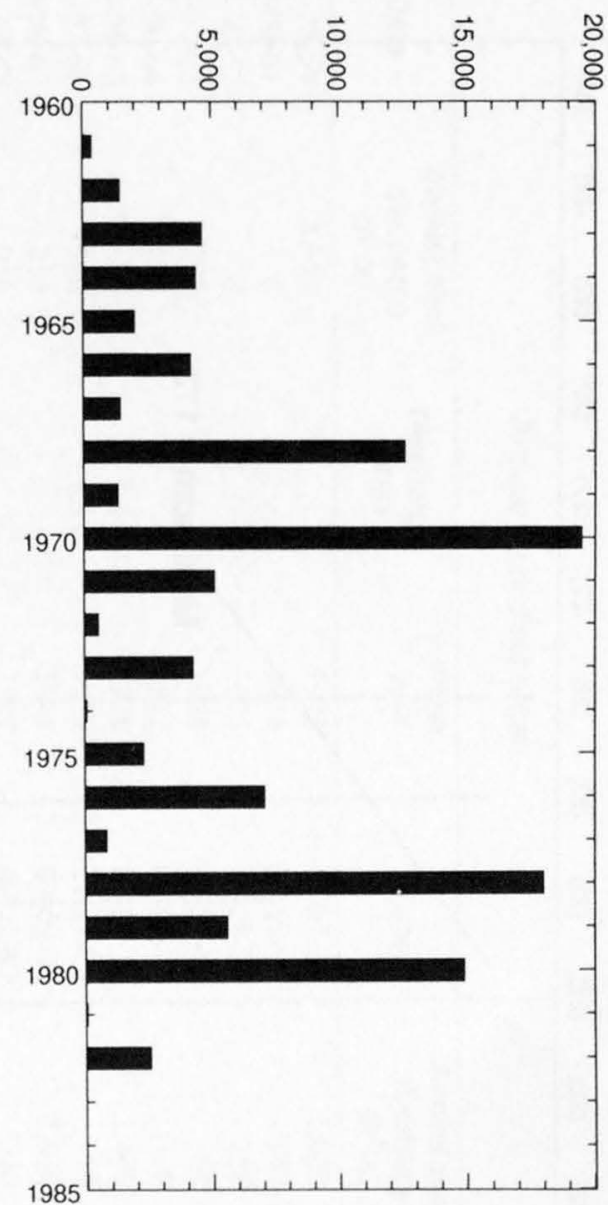


812

GILA RIVER BASIN

09513800 NEW RIVER AT NEW RIVER, AZ--Continued
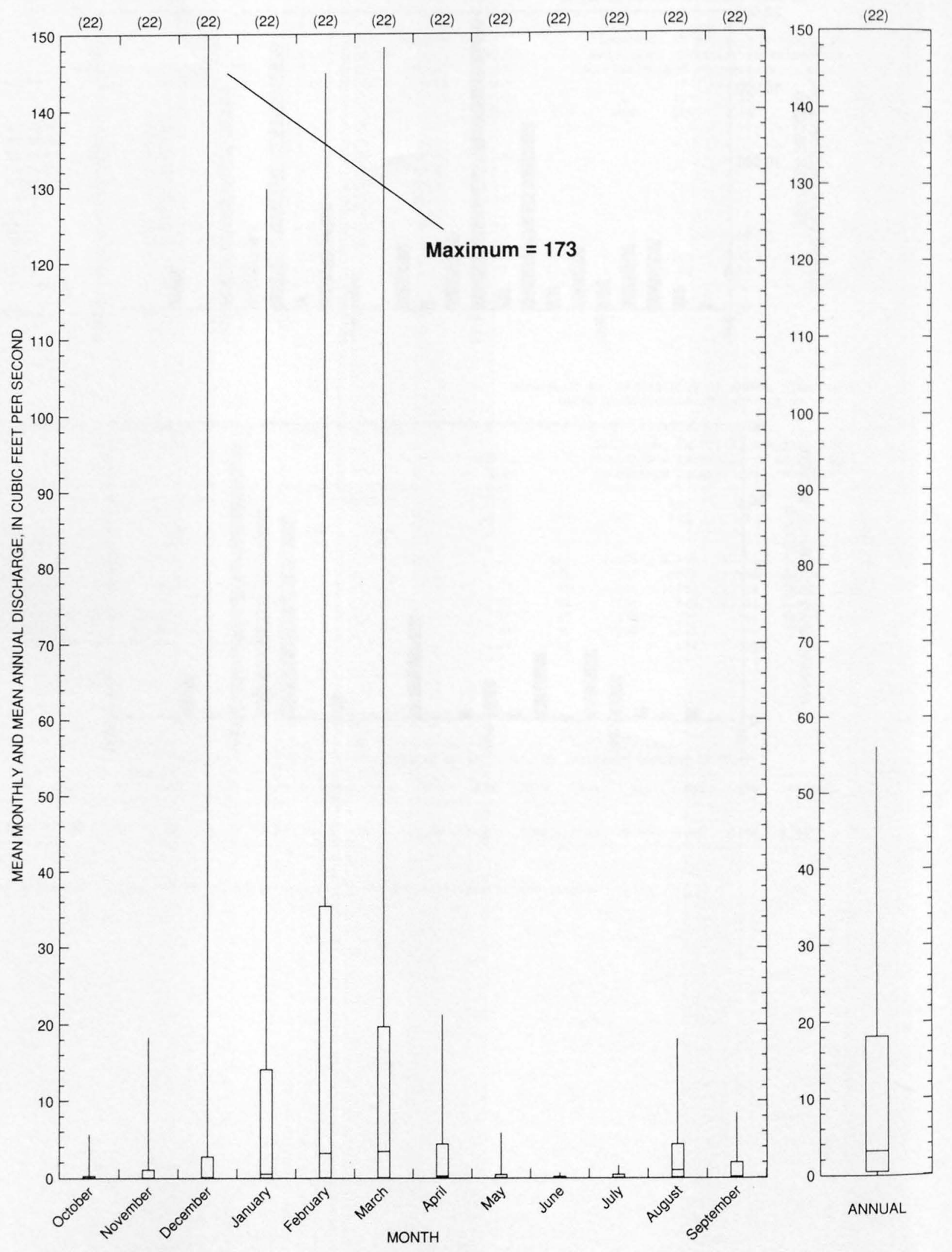
09513820 DEADMAN WASH NEAR NEW RIVER, AZ

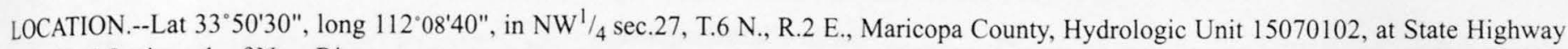
$69,4.5$ mi south of New River.

DRAINAGE AREA.--11.1 $\mathrm{mi}^{2}$.

Annual peak discharges

\begin{tabular}{|c|c|c|c|c|c|c|c|}
\hline $\begin{array}{l}\text { Water } \\
\text { year }\end{array}$ & Date & $\begin{array}{l}\text { Annual peak } \\
\text { discharge } \\
\left(\mathrm{ft}^{3} / \mathrm{s}\right)\end{array}$ & $\begin{array}{l}\text { Discharge } \\
\text { codes }\end{array}$ & $\begin{array}{l}\text { Water } \\
\text { year }\end{array}$ & Date & $\begin{array}{c}\text { Annual peak } \\
\text { discharge } \\
\left(\mathrm{ft}^{3} / \mathrm{s}\right)\end{array}$ & $\begin{array}{c}\text { Discharge } \\
\text { codes }\end{array}$ \\
\hline 1960 & $12-25-59$ & 1,850 & & 1973 & $10-07-72$ & 1,000 & ES \\
\hline 1961 & $00-00-61$ & 0 & & 1974 & $09-19-74$ & 150 & \\
\hline 1962 & $00-00-62$ & 0 & & 1975 & $10-28-74$ & 250 & ES \\
\hline 1963 & $08-00-63$ & 270 & & 1976 & $08-31-76$ & 64 & \\
\hline 1964 & $07-30-64$ & 1,140 & & 1977 & $00-00-77$ & 0 & \\
\hline 1965 & $04-04-65$ & 70 & ES & 1978 & $03-02-78$ & 1,400 & \\
\hline 1966 & $12-22-65$ & 200 & ES & 1979 & $11-11-78$ & 88 & \\
\hline 1967 & $09-00-67$ & 230 & & 1991 & 03-01-91 & 1,030 & \\
\hline 1968 & $12-19-67$ & 950 & & 1992 & $08-22-92$ & 730 & \\
\hline 1969 & $00-00-69$ & 0 & & 1993 & $01-08-93$ & 729 & \\
\hline 1970 & $09-05-70$ & 1,630 & & 1994 & $10-06-93$ & 1,110 & \\
\hline 1971 & $08-21-71$ & 1,300 & & 1995 & $02-15-95$ & 177 & \\
\hline 1972 & $07-17-72$ & 135 & & 1996 & $09-05-96$ & 700 & \\
\hline
\end{tabular}

Discharge rating table developed October 1995

\begin{tabular}{cccc}
\hline $\begin{array}{c}\text { Gage height } \\
(\mathbf{f t})\end{array}$ & $\begin{array}{c}\text { Discharge } \\
\left(\mathbf{f t}^{3} \mathbf{s}\right)\end{array}$ & $\begin{array}{c}\text { Gage height } \\
(\mathbf{f t})\end{array}$ & $\begin{array}{c}\text { Discharge } \\
\left(\mathbf{f t}^{3} \mathbf{s}\right)\end{array}$ \\
\hline 5.0 & 138 & 7.0 & 561 \\
5.5 & 222 & 7.5 & 718 \\
6.0 & 311 & 8.0 & 941 \\
6.5 & 419 & 8.8 & 1,420 \\
\hline
\end{tabular}

Magnitude and probability of instantaneous peak flow based on period of record 1960-79, 1991-96

\begin{tabular}{|c|c|c|c|c|c|}
\hline \multicolumn{6}{|c|}{$\begin{array}{l}\text { Discharge, in } \mathrm{ft}^{3} / \mathrm{s} \text {, for indicated recurrence interval } \\
\text { in years, and exceedance probablility, in percent }\end{array}$} \\
\hline 2 & 5 & 10 & 25 & $50 t$ & $100 t$ \\
\hline $50 \%$ & $20 \%$ & $10 \%$ & $4 \%$ & $2 \%$ & $1 \%$ \\
\hline 348 & 969 & 1,590 & 2,610 & 3,550 & 4,630 \\
\hline Weighted skew & $(\log s)=$ & -0.34 & & & \\
\hline Mean & $(\log s)=$ & 2.51 & & & \\
\hline Standard dev. & $(\log s)=$ & 0.56 & & & \\
\hline
\end{tabular}

fReliability of values in column is uncertain, and potential errors are large. 
09513820 DEADMAN WASH NEAR NEW RIVER, AZ--Continued

Basin characteristics

\begin{tabular}{cccccccc}
\hline $\begin{array}{c}\text { Main } \\
\text { channel } \\
\text { slope } \\
(\mathrm{ft} / \mathrm{mi})\end{array}$ & $\begin{array}{c}\text { Stream } \\
\text { length } \\
(\mathrm{mi})\end{array}$ & $\begin{array}{c}\text { Mean } \\
\text { basin } \\
\text { elevation } \\
(\mathrm{ft})\end{array}$ & $\begin{array}{c}\text { Forested } \\
\text { area } \\
\text { (percent) }\end{array}$ & $\begin{array}{c}\text { Soil } \\
\text { index }\end{array}$ & $\begin{array}{c}\text { Mean } \\
\text { annual } \\
\text { precipitation } \\
\text { (in) }\end{array}$ & $\begin{array}{c}\text { R-year } \\
\text { (in) }\end{array}$ & $\begin{array}{c}\text { 24-hour } \\
\text { (in) }\end{array}$ \\
\hline 124 & 4.2 & 1,980 & 0.0 & 1.0 & 11.0 & 1.8 \\
\hline
\end{tabular}

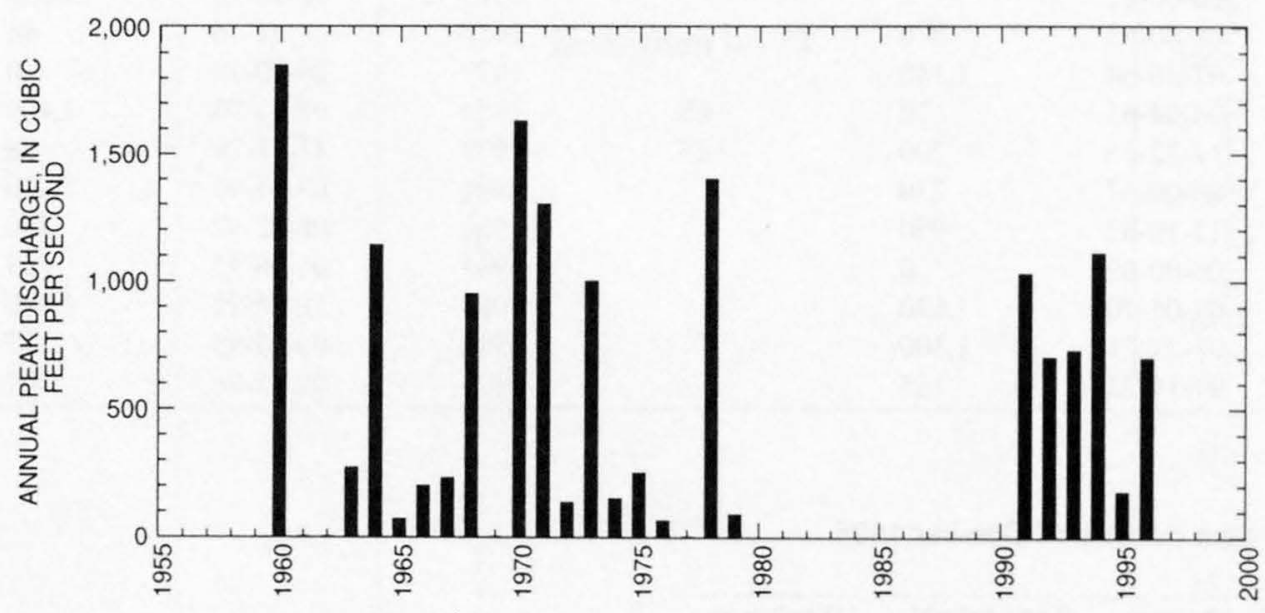




\section{NEW RIVER AT BELL ROAD, NEAR PEORIA, AZ}

LOCATION.--Lat 33'38'18", long 112'14'22", in NE $1 / 4 \mathrm{NE}^{1} / 4$ sec.3, T.3 N., R.1 E., Maricopa County, Hydrologic Unit 15070102, on downstream side of bridge at Bell Road, 1.6 mi upstream from Skunk Creek, 3.1 mi north of Peoria, and 9 mi upstream from mouth.

DRAINAGE AREA.--185 $\mathrm{mi}^{2}$.

PERIOD OF RECORD.--Water years 1963, 1965-67 (annual maximums only), October 1967 to September 1984, June 1990 to September 1993 (discontinued).

REVISED RECORDS.--WDR AZ-89-1: Drainage area.

GAGE.--Water-stage recorder. Datum of gage is 1,190.00 ft, sea level (Arizona State Highway Department bench mark). Oct. 1, 1965, to Sept. 30,1967 , crest-stage gage at present site and at site $50 \mathrm{ft}$ upstream, at datum $5.00 \mathrm{ft}$ higher.

REMARKS.--No estimated daily discharges. Records fair. Flow regulated to some extent since 1990 by flood control dike.

EXTREMES FOR PERIOD OF RECORD.--Maximum discharge, 14,600 ft $3 / \mathrm{s} \mathrm{Dec.} \mathrm{19,1967,} \mathrm{gage} \mathrm{height,} 13.5 \mathrm{ft}$, from rating curve extended above $680 \mathrm{ft}^{3} / \mathrm{s}$ on basis of slope-area measurement of peak flow; no flow for most of time each year.

Annual peak discharges

\begin{tabular}{|c|c|c|c|c|c|c|c|}
\hline $\begin{array}{l}\text { Water } \\
\text { year }\end{array}$ & Date & $\begin{array}{l}\text { Annual peak } \\
\text { discharge } \\
\left(\mathrm{ft}^{3} / \mathrm{s}\right)\end{array}$ & $\begin{array}{l}\text { Discharge } \\
\text { codes }\end{array}$ & $\begin{array}{l}\text { Water } \\
\text { year }\end{array}$ & Date & $\begin{array}{l}\text { Annual peak } \\
\text { discharge } \\
\left(\mathrm{ft}^{3} / \mathrm{s}\right)\end{array}$ & $\begin{array}{c}\text { Discharge } \\
\text { codes }\end{array}$ \\
\hline 1963 & $08-17-63$ & 1,550 & & 1977 & $00-00-77$ & 0 & \\
\hline 1965 & $04-05-65$ & 1,020 & & 1978 & $03-02-78$ & 12,500 & \\
\hline 1966 & $12-22-65$ & 4,060 & & 1979 & $12-19-78$ & 8,410 & \\
\hline 1967 & $06-18-67$ & 100 & & 1980 & $02-20-80$ & 12,100 & \\
\hline 1968 & $12-19-67$ & 14,600 & & 1981 & $09-05-81$ & 21 & \\
\hline 1969 & $00-00-69$ & 0 & & 1982 & $03-15-82$ & 876 & \\
\hline 1970 & $09-05-70$ & 11,900 & & 1983 & $12-01-82$ & 4,240 & \\
\hline 1971 & $08-21-71$ & 4,800 & & 1984 & $00-00-84$ & 0 & \\
\hline 1972 & $07-17-72$ & 1,520 & & 1990 & $09-03-90$ & 2,340 & \\
\hline 1973 & $10-07-72$ & 2,590 & & 1991 & $03-01-91$ & 1,280 & \\
\hline 1974 & $00-00-74$ & 0 & & 1992 & $03-10-92$ & 384 & \\
\hline 1975 & $11-03-74$ & 257 & & 1993 & $01-08-93$ & 2,760 & \\
\hline 1976 & $02-09-76$ & 2,280 & & & & & \\
\hline
\end{tabular}

Discharge rating table developed January 1993

\begin{tabular}{crcc}
\hline $\begin{array}{c}\text { Gage height } \\
(\mathrm{ft})\end{array}$ & $\begin{array}{c}\text { Discharge } \\
\left(\mathrm{ft}^{\mathbf{3}} \mathbf{/} \mathbf{s}\right)\end{array}$ & $\begin{array}{c}\text { Gage height } \\
(\mathbf{f t})\end{array}$ & $\begin{array}{c}\text { Discharge } \\
\left(\mathbf{f t}^{3} \mathbf{s}\right)\end{array}$ \\
\hline 2.8 & 233 & 4.2 & 1,410 \\
3.0 & 331 & 4.4 & 1,680 \\
3.2 & 449 & 4.6 & 1,980 \\
3.4 & 590 & 4.8 & 2,320 \\
3.6 & 756 & 5.0 & 2,680 \\
3.8 & 946 & 5.2 & 3,080 \\
4.0 & 1,160 & 5.4 & 3,510 \\
\hline
\end{tabular}

Basin characteristics

\begin{tabular}{ccccccccc}
\hline $\begin{array}{c}\text { Main } \\
\text { channel } \\
\text { slope } \\
(\mathrm{ft} / \mathrm{mi})\end{array}$ & $\begin{array}{c}\text { Stream } \\
\text { length }\end{array}$ & $\begin{array}{c}\text { Mean } \\
\text { (mi) }\end{array}$ & $\begin{array}{c}\text { basin } \\
\text { elevation } \\
\text { (ft) }\end{array}$ & $\begin{array}{c}\text { Forested } \\
\text { area } \\
\text { (percent) }\end{array}$ & $\begin{array}{c}\text { Soil } \\
\text { index }\end{array}$ & $\begin{array}{c}\text { Mean } \\
\text { annual } \\
\text { precipitation } \\
\text { (in) }\end{array}$ & $\begin{array}{c}\text { 2-year } \\
\text { (in) }\end{array}$ & $\begin{array}{c}50 \text {-year } \\
\text { (in) }\end{array}$ \\
\hline 64.5 & 38.2 & 2,700 & 0.1 & 1.0 & 15.6 & 1.9 & 4.0 \\
\hline
\end{tabular}




\section{NEW RIVER AT BELL ROAD, NEAR PEORIA, AZ--Continued}

MEAN MONTHLY AND ANNUAL DISCHARGES $1968-84,1990 \cdot 93$

\begin{tabular}{|c|c|c|c|c|c|c|}
\hline MONTH & $\begin{array}{l}\text { MAXIMUM } \\
\text { (FT3/S) }\end{array}$ & $\begin{array}{l}\text { MINIMUM } \\
(\text { FT3/S) }\end{array}$ & $\begin{array}{c}\text { MEAN } \\
(\text { FT3 } / \mathrm{S})\end{array}$ & $\begin{array}{l}\text { STAN- } \\
\text { DARD } \\
\text { DEVIA- } \\
\text { TION } \\
\text { (FT3/S) }\end{array}$ & $\begin{array}{l}\text { COBFFI- } \\
\text { CIENT OF } \\
\text { VARI- } \\
\text { ATION }\end{array}$ & $\begin{array}{c}\text { PERCENT } \\
\text { OF } \\
\text { ANNUAL } \\
\text { RUNOFF }\end{array}$ \\
\hline OCTOBER & 33 & 0.00 & 1.9 & 7.4 & 4.0 & 1.1 \\
\hline NOVEMBER & 7.9 & 0.00 & 1.0 & 2.6 & 2.5 & 0.60 \\
\hline DECEMBER & 230 & 0.00 & 25 & 62 & 2.5 & 15 \\
\hline JANUARY & 609 & 0.00 & 42 & 136 & 3.9 & 25 \\
\hline FEBRUARY & 491 & 0.00 & 54 & 116 & 2.2 & 32 \\
\hline MARCH & 446 & 0.00 & 39 & 101 & 2.6 & 23 \\
\hline APRIL & 10 & 0.00 & 0.70 & 2.3 & 3.2 & 0.41 \\
\hline MAY & 0.76 & 0.00 & 0.04 & 0.17 & 4.5 & 0.02 \\
\hline JUNE & 0.00 & 0.00 & 0.00 & 0.00 & $\cdots$ & 0.00 \\
\hline JULY & 2.7 & 0.00 & 0.13 & 0.60 & 4.9 & 0.08 \\
\hline AUGUST & 13 & 0.00 & 1.0 & 3.1 & 3.1 & 0.59 \\
\hline SEPTEMBER & 99 & 0.00 & 6.0 & 22 & 3.7 & 3.5 \\
\hline ANNUAL & 65 & 0.00 & 14 & 19 & 1.4 & 100 \\
\hline
\end{tabular}

MAGNITUDE AND PROBABILITY OF INSTANTANEOUS PEAK FLOW BASED ON PERIOD OF RECORD $1963,1965.84,1990.93$

DISCHARGE, IN FT3/S, FOR INDICATED RECURRENCE INTERVAL IN YEARS, AND EXCEEDANCE PROBABILITY, IN PERCENT

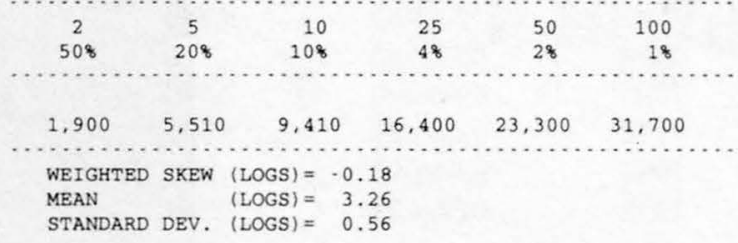

MAGNITUDE AND PROBABILITY OF ANNUAL LOW FLOW BASED ON PERIOD OF RECORD $1969 \cdot 84,1990 \cdot 93$

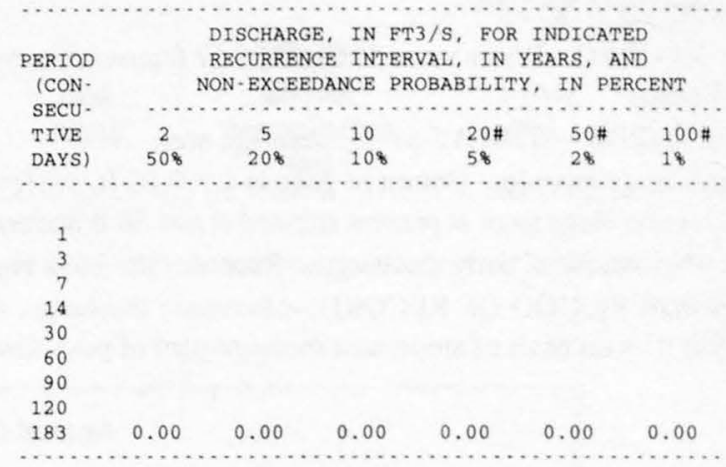

MAGNITUDE AND PROBABILITY OF ANNUAL HIGH FLOW BASED ON PERIOD OF RECORD $1968.84,1990.93$

\begin{tabular}{|c|c|c|c|c|c|c|}
\hline $\begin{array}{l}\text { PERIOD } \\
\text { (CON- }\end{array}$ & & \multicolumn{5}{|c|}{$\begin{array}{l}\text { DISCHARGE, IN FT3/S, FOR INDICATED } \\
\text { RECURRENCE INTERVAL, IN YEARS, AND }\end{array}$} \\
\hline SECU - & & & & & . & $\ldots \ldots$ \\
\hline TIVE & 2 & 5 & 10 & $25 \#$ & $50 \#$ & $100 \#$ \\
\hline DAYS) & 508 & 208 & 108 & 48 & 28 & 18 \\
\hline 1 & 531 & 2,550 & 3,480 & 4,070 & 4,260 & 4,340 \\
\hline 3 & 253 & 1,520 & 2,300 & 2,890 & 3,120 & 3,240 \\
\hline 7 & 134 & 919 & 1,460 & 1,900 & 2,090 & 2,190 \\
\hline 15 & 65 & 491 & 815 & 1,110 & 1,240 & 1,320 \\
\hline 30 & 36 & 275 & 461 & 629 & 706 & 754 \\
\hline 60 & 20 & 163 & 279 & 389 & 440 & 473 \\
\hline 90 & 14 & 112 & 193 & 268 & 304 & 326 \\
\hline
\end{tabular}

DURATION TABLE OF DAILY MEAN FLOW FOR PERIOD OF RECORD 1968-84, 1990-93

DISCHARGE, IN FT3/S, WHICH WAS EQUALED OR EXCEEDED FOR INDICATED PERCENT OF TIME

\begin{tabular}{|c|c|c|c|c|c|c|c|c|c|c|c|c|c|c|c|c|}
\hline 18 & 58 & 108 & 158 & 208 & 308 & 408 & 508 & 608 & 708 & 808 & 908 & 958 & $98 \%$ & 998 & 99.58 & 99.98 \\
\hline 3 & 0.75 & 0.00 & 0.00 & 0.00 & 0.00 & 0.00 & 0.00 & 0.00 & 0.00 & 0.00 & 0.00 & 0.00 & 0.00 & 0.00 & 0.00 & 0.00 \\
\hline
\end{tabular}

\# Reliability of values in column is uncertain, and potential errors are large. 
09513835 NEW RIVER AT BELL ROAD, NEAR PEORIA, AZ--Continued
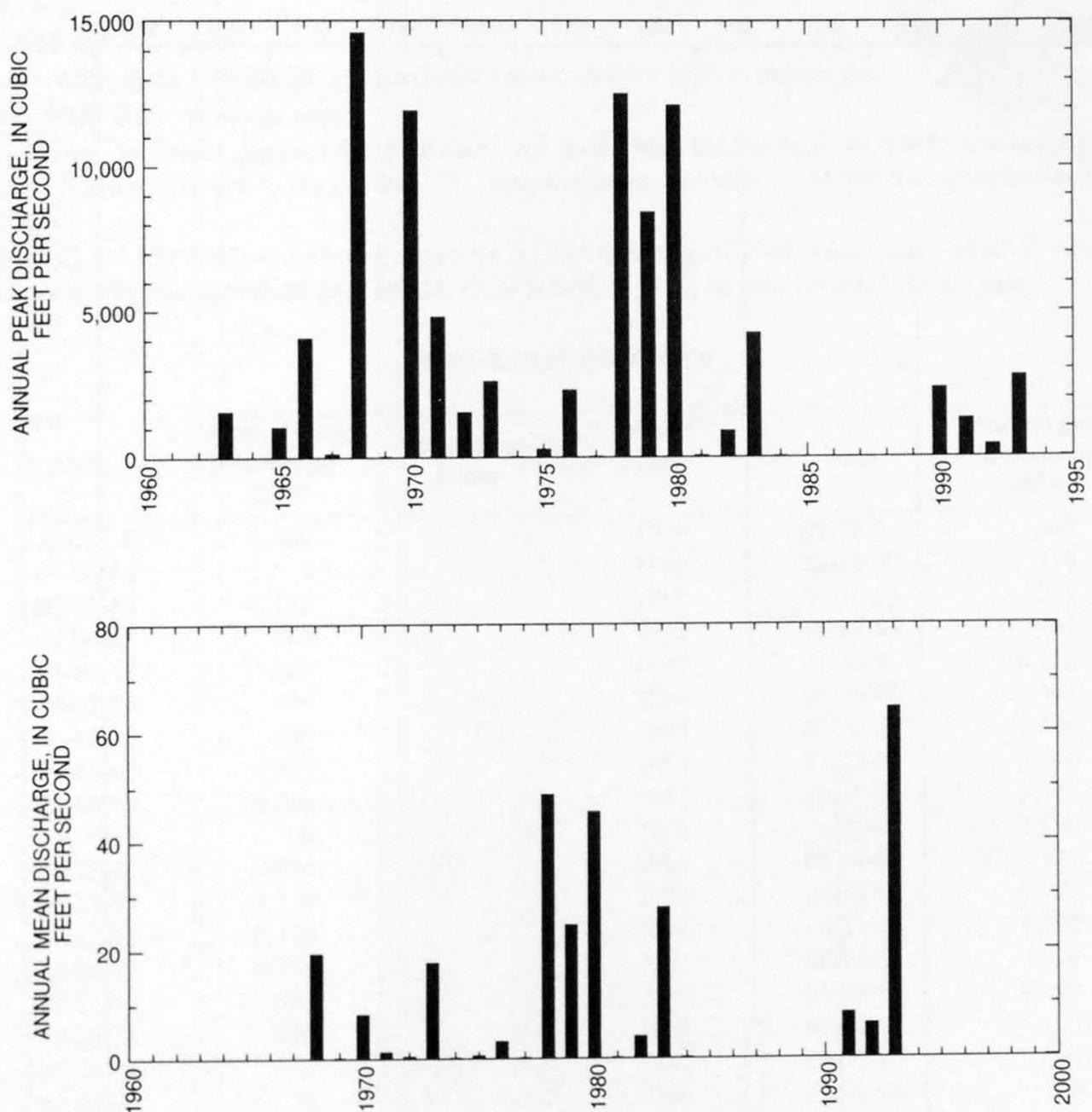
818

GILA RIVER BASIN

09513835 NEW RIVER AT BELL ROAD, NEAR PEORIA, AZ--Continued
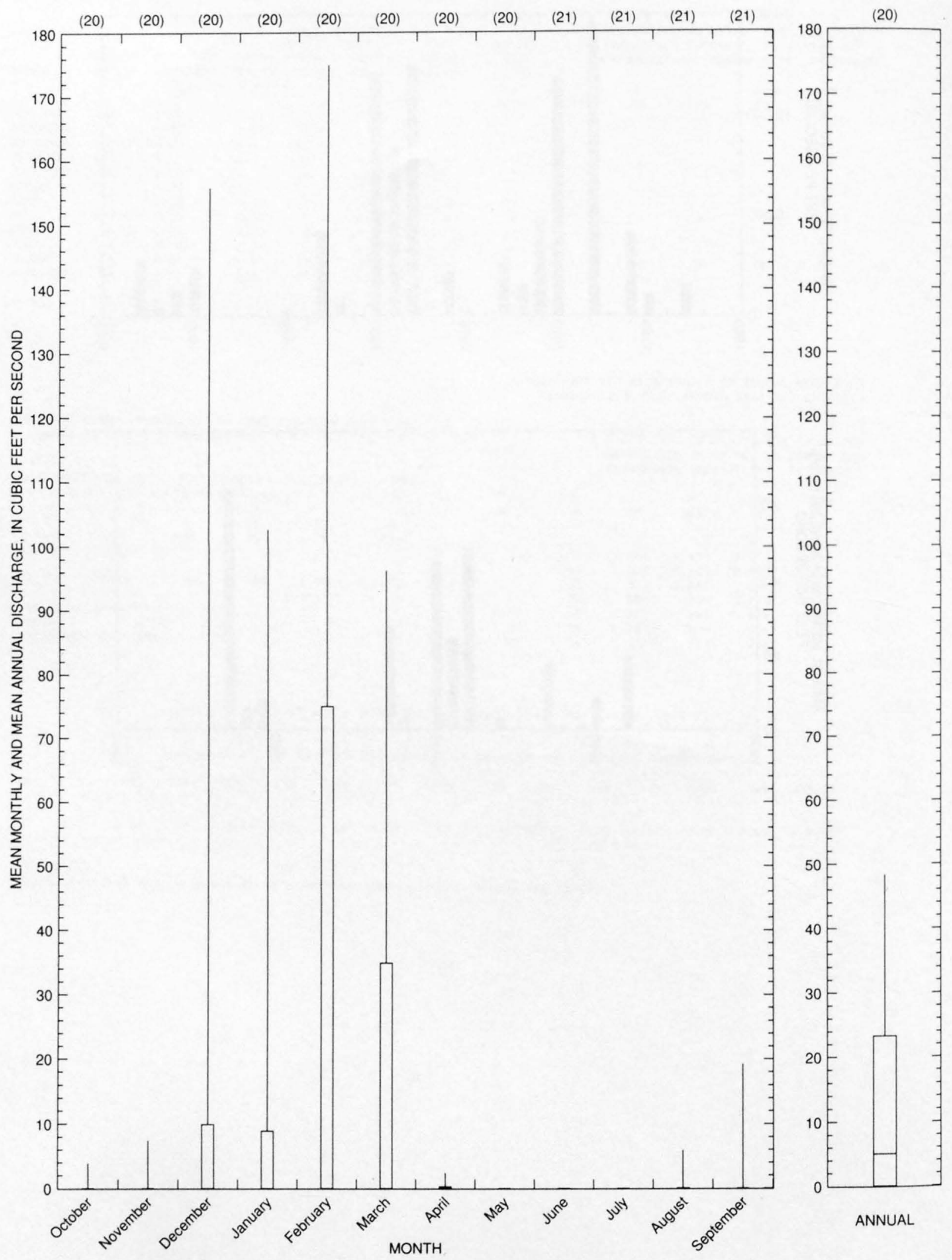


\section{SKUNK CREEK NEAR PHOENIX, AZ}

LOCATION.--Lat 33 43'45", long $112^{\circ} 07^{\prime} 09^{\prime \prime}$, in NW $1 / 4 \mathrm{SE}^{1} / 4 \mathrm{SE}^{1} / 4$ sec.35, T.5 N., R.2 E., Maricopa County, Hydrologic Unit 15070102 , on right bank dike of Skunk Creek flood control channel, $300 \mathrm{ft}$ east of frontage road of Interstate Highway 17,3 mi north of Adobe and 20 mi north of City Hall in Phoenix.

DRAINAGE AREA.--64.9 $\mathrm{mi}^{2}$.

PERIOD OF RECORD.--Water years 1960-67 (annual maximums only), October 1967 to current year.

REVISED RECORDS.--WDR-89-1: Drainage area.

GAGE.--Water-stage recorder. Datum of gage is 1,472.60 ft above sea level. May 1961 to Sept. 30, 1967, crest-stage gage at site $400 \mathrm{ft}$ downstream at datum $6.67 \mathrm{ft}$ lower, and Oct. 1, 1967 to Dec. 29, 1984, water-stage recorder at site $300 \mathrm{ft}$ downstream at datum $12.66 \mathrm{ft}$ lower.

REMARKS.--Records fair.

EXTREMES FOR PERIOD OF RECORD.--Maximum discharge, 11,500 $\mathrm{ft}^{3} / \mathrm{s}$ Aug. 1, 1964, gage height, $10.48 \mathrm{ft}$, present datum, from rating curve extended above $6,200 \mathrm{ft}^{3} / \mathrm{s}$; maximum gage height, $12.24 \mathrm{ft} \mathrm{Sept.} \mathrm{5,} \mathrm{1970;} \mathrm{no} \mathrm{flow} \mathrm{for} \mathrm{most} \mathrm{of} \mathrm{each} \mathrm{year.}$

Annual peak discharges

\begin{tabular}{|c|c|c|c|c|c|c|c|}
\hline $\begin{array}{l}\text { Water } \\
\text { year }\end{array}$ & Date & $\begin{array}{c}\text { Annual peak } \\
\text { discharge } \\
\left(\mathrm{ft}^{3} / \mathrm{s}\right)\end{array}$ & $\begin{array}{l}\text { Discharge } \\
\text { codes }\end{array}$ & $\begin{array}{l}\text { Water } \\
\text { year }\end{array}$ & Date & $\begin{array}{c}\text { Annual peak } \\
\text { discharge } \\
\left(\mathrm{ft}^{3} / \mathrm{s}\right)\end{array}$ & $\begin{array}{l}\text { Discharge } \\
\text { codes }\end{array}$ \\
\hline 1960 & $12-25-59$ & 9,400 & & 1979 & $01-18-79$ & 600 & \\
\hline 1961 & $00-00-61$ & 0 & & 1980 & $02-20-80$ & 1,210 & \\
\hline 1962 & $00-00-62$ & 175 & & 1981 & $07-16-81$ & 311 & \\
\hline 1963 & $00-00-63$ & 480 & & 1982 & $10-02-81$ & 281 & \\
\hline 1964 & $08-01-64$ & 11,500 & & 1983 & $11-30-82$ & 6,170 & \\
\hline 1965 & $02-07-65$ & 400 & ES & 1984 & $09-26-84$ & 565 & \\
\hline 1966 & $08-18-66$ & 700 & ES & 1985 & $01-26-85$ & 1,320 & $\mathrm{C}$ \\
\hline 1967 & $09-02-67$ & 950 & & 1986 & $07-22-86$ & 906 & $\mathrm{C}$ \\
\hline 1968 & $12-19-67$ & 5,900 & & 1987 & $10-10-86$ & 3,440 & $\mathrm{C}$ \\
\hline 1969 & $00-00-69$ & 0 & & 1988 & $11-01-87$ & 2,250 & $\mathrm{C}$ \\
\hline 1970 & $09-05-70$ & 9,650 & & 1989 & 01-04-89 & 111 & $\mathrm{C}$ \\
\hline 1971 & $08-21-71$ & 4,770 & & 1990 & $08-12-90$ & 8,160 & \\
\hline 1972 & $07-17-72$ & 2,380 & & 1991 & $08-11-91$ & 1,250 & \\
\hline 1973 & $10-06-72$ & 4,700 & & 1992 & $08-23-92$ & 7,020 & \\
\hline 1974 & $07-21-74$ & 300 & & 1993 & $01-08-93$ & 4,990 & \\
\hline 1975 & $10-29-74$ & 240 & & 1994 & $00-00-94$ & 0 & \\
\hline 1976 & $07-28-76$ & 13 & & 1995 & $09-28-95$ & 822 & \\
\hline 1977 & $01-03-77$ & 70 & & 1996 & $11-01-95$ & 500 & \\
\hline 1978 & $03-01-78$ & 3,590 & & & & & \\
\hline
\end{tabular}

Discharge rating table developed September 1992

\begin{tabular}{crcc}
\hline $\begin{array}{c}\text { Gage height } \\
(\mathrm{ft})\end{array}$ & $\begin{array}{c}\text { Discharge } \\
\left(\mathrm{ft}^{3} / \mathbf{s}\right)\end{array}$ & $\begin{array}{c}\text { Gage height } \\
(\mathbf{f t})\end{array}$ & $\begin{array}{c}\text { Discharge } \\
\left(\mathrm{ft}^{3} / \mathbf{s}\right)\end{array}$ \\
\hline 2.0 & 331 & 4.5 & 5,100 \\
2.5 & 874 & 5.0 & 6,620 \\
3.0 & 1,640 & 5.5 & 8,320 \\
3.5 & 2,600 & 6.0 & 10,190 \\
4.0 & 3,760 & 6.4 & 11,800 \\
\hline
\end{tabular}


GILA RIVER BASIN

09513860 SKUNK CREEK NEAR PHOENIX, AZ--Continued

Basin characteristics

\begin{tabular}{|c|c|c|c|c|c|c|c|}
\hline \multirow[b]{2}{*}{$\begin{array}{l}\text { Main } \\
\text { channel } \\
\text { slope } \\
(\mathrm{ft} / \mathrm{mi})\end{array}$} & \multirow[b]{2}{*}{$\begin{array}{l}\text { Stream } \\
\text { length } \\
(\mathrm{mi})\end{array}$} & \multirow[b]{2}{*}{$\begin{array}{c}\text { Mean } \\
\text { basin } \\
\text { elevation } \\
(\mathrm{ft})\end{array}$} & \multirow[b]{2}{*}{$\begin{array}{c}\text { Forested } \\
\text { area } \\
\text { (percent) }\end{array}$} & \multirow[b]{2}{*}{$\begin{array}{c}\text { Soil } \\
\text { index }\end{array}$} & \multirow[b]{2}{*}{$\begin{array}{c}\text { Mean } \\
\text { annual } \\
\text { precipitation } \\
\text { (in) }\end{array}$} & \multicolumn{2}{|c|}{ Rainfall intensity, 24-hou } \\
\hline & & & & & & $\begin{array}{l}\text { 2-year } \\
\text { (in) }\end{array}$ & $\begin{array}{l}50 \text {-year } \\
\text { (in) }\end{array}$ \\
\hline 49.2 & 19.7 & 2,180 & 0.0 & 1.0 & 12.2 & 1.9 & 4.2 \\
\hline
\end{tabular}


09513860 SKUNK CREEK NEAR PHOENIX, AZ--Continued

MEAN MONTHLY AND ANNUAL DISCHARGES 1968.96

\begin{tabular}{|c|c|c|c|c|c|c|}
\hline MONTH & $\begin{array}{l}\text { MAXIMUM } \\
(\text { FT } 3 / \mathrm{S})\end{array}$ & $\begin{array}{l}\text { MINIMUM } \\
\text { (FT3/S) }\end{array}$ & $\begin{array}{c}\text { MEAN } \\
(\mathrm{FT} 3 / \mathrm{S})\end{array}$ & $\begin{array}{l}\text { STAN- } \\
\text { DARD } \\
\text { DEVIA- } \\
\text { TION } \\
(\mathrm{FT} 3 / \mathrm{S})\end{array}$ & $\begin{array}{l}\text { COEFFI- } \\
\text { CIENT OF } \\
\text { VARI- } \\
\text { ATION }\end{array}$ & $\begin{array}{c}\text { PERCENT } \\
\text { OF } \\
\text { ANNUAL } \\
\text { RUNOFF }\end{array}$ \\
\hline OCTOBER & 26 & 0.00 & 1.6 & 5.2 & 3.2 & 7.6 \\
\hline NOVEMBER & 41 & 0.00 & 2.0 & 7.8 & 4.0 & 9.4 \\
\hline DECEMBER & 60 & 0.00 & 3.5 & 12 & 3.5 & 16.8 \\
\hline JANUARY & 56 & 0.00 & 3.2 & 11 & 3.3 & 15.1 \\
\hline FEBRUARY & 24 & 0.00 & 1.9 & 5.3 & 2.8 & 8.9 \\
\hline MARCH & 46 & 0.00 & 2.1 & 8.7 & 4.2 & 9.9 \\
\hline APRIL & 0.00 & 0.00 & 0.00 & 0.00 & & 0.0 \\
\hline MAY & 0.03 & 0.00 & 0.00 & 0.01 & 4.2 & 0.0 \\
\hline JUNE & 0.26 & 0.00 & 0.01 & 0.05 & 5.4 & 0.0 \\
\hline JULY & 18 & 0.00 & 1.2 & 3.6 & 3.0 & 5.8 \\
\hline AUGUST & 38 & 0.00 & 3.6 & 9.2 & 2.6 & 17.1 \\
\hline SEPTEMBER & 43 & 0.00 & 2.0 & 7.9 & 4.0 & 9.4 \\
\hline ANNUAL & 8.6 & 0.00 & 1.8 & 2.3 & 1.3 & 100 \\
\hline
\end{tabular}

MAGNITUDE AND PROBABILITY OF INSTANTANEOUS PEAK FLOW BASED ON PERIOD OF RECORD 1960.96

DISCHARGE, IN FT3/S, FOR INDICATED RECURRENCB INTERVAL IN YEARS, AND EXCEEDANCE PROBABILITY, IN PERCENT

\begin{tabular}{|c|c|c|c|c|c|}
\hline 2 & 5 & 10 & 25 & 50 & 100 \\
\hline 508 & 208 & 108 & 48 & 28 & 18 \\
\hline 940 & 3,230 & 6,120 & 12,100 & 18,700 & 27,600 \\
\hline
\end{tabular}

WEIGHTED SKEW (LOGS) $=-0.03$

MEAN (LOGS $)=2.97$

STANDARD DEV. $($ LOGS $)=0.64$

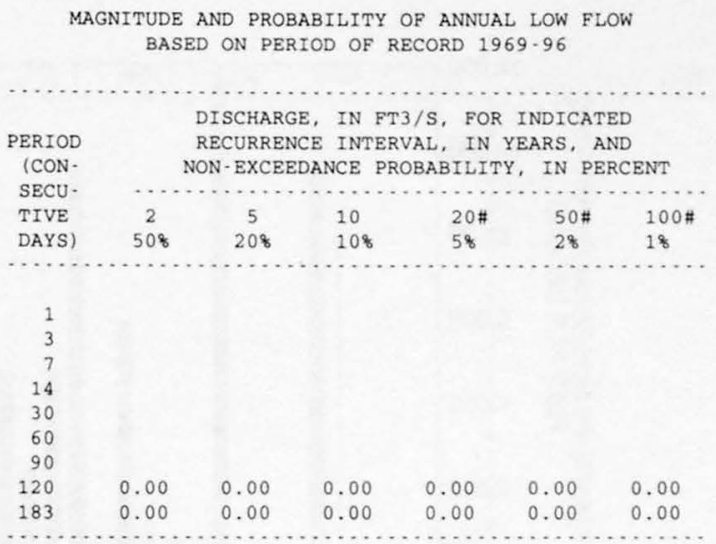

MAGNITUDE AND PROBABILITY OF ANNUAL HIGH FLOW BASED ON PERIOD OF RECORD 1968.96

\begin{tabular}{|c|c|c|c|c|c|c|}
\hline \multirow{4}{*}{$\begin{array}{l}\text { PERIOD } \\
\text { (CON- } \\
\text { SECU- }\end{array}$} & \multicolumn{6}{|c|}{ DISCHARGE, IN FT3/S, FOR INDICATED } \\
\hline & \multirow{2}{*}{\multicolumn{2}{|c|}{$\begin{array}{l}\text { RECURRENCE } \\
\text { EXCEEDANCE }\end{array}$}} & \multicolumn{4}{|c|}{ INTERVAL, IN YEARS, AND } \\
\hline & & & B PRC & BILITY, & IN PERC & NT \\
\hline & $\cdots$ & 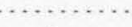 & & & ........ & …. \\
\hline TIVE & 2 & 5 & 10 & $25=$ & $50 \#$ & $100 \#$ \\
\hline DAYS) & 508 & 208 & 108 & 48 & 28 & 18 \\
\hline 1 & 138 & 518 & 880 & 1,390 & 1.790 & 2,170 \\
\hline 3 & 55 & 227 & 404 & 672 & 892 & 1,120 \\
\hline 7 & 25 & 105 & 191 & 323 & 434 & 549 \\
\hline 15 & 12 & 53 & 97 & 168 & 229 & 294 \\
\hline 30 & 6.7 & 29 & 54 & 91 & 122 & 155 \\
\hline 60 & 3.5 & 16 & 30 & 52 & 73 & 95 \\
\hline 90 & 2.4 & 11 & 20 & 36 & 50 & 67 \\
\hline
\end{tabular}

DURATION TABLE OF DAILY MEAN FLOW FOR PERIOD OF RECORD 1968.96

DISCHARGE, IN FT3/S, WHICH WAS EQUALED OR EXCEEDED FOR INDICATED PERCENT OF TIME

\begin{tabular}{|c|c|c|c|c|c|c|c|c|c|c|c|c|c|c|c|c|}
\hline 18 & 58 & 108 & 158 & 208 & 308 & 408 & 508 & 608 & 708 & 808 & 908 & 958 & 988 & 998 & 99.58 & 99.98 \\
\hline 16 & 0.00 & 0.00 & 0.00 & 0.00 & 0.00 & 0.00 & 0.00 & 0.00 & 0.00 & 0.00 & 0.00 & 0.00 & 0.00 & 0.00 & 0.00 & 0.00 \\
\hline
\end{tabular}

\# Reliability of values in column is uncertain, and potential errors are large. 
09513860 SKUNK CREEK NEAR PHOENIX, AZ--Continued
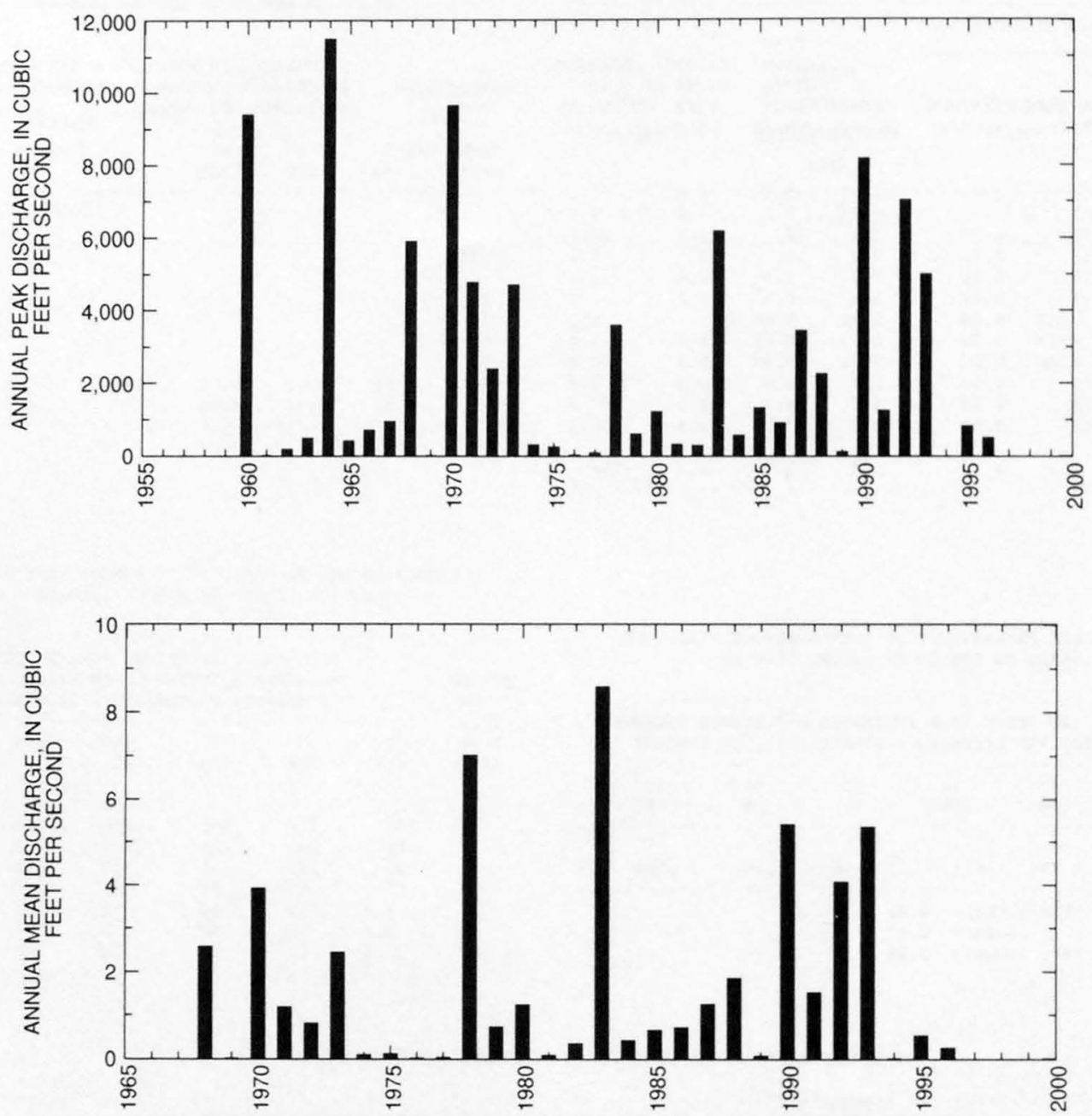
GILA RIVER BASIN

823

09513860 SKUNK CREEK NEAR PHOENIX, AZ--Continued
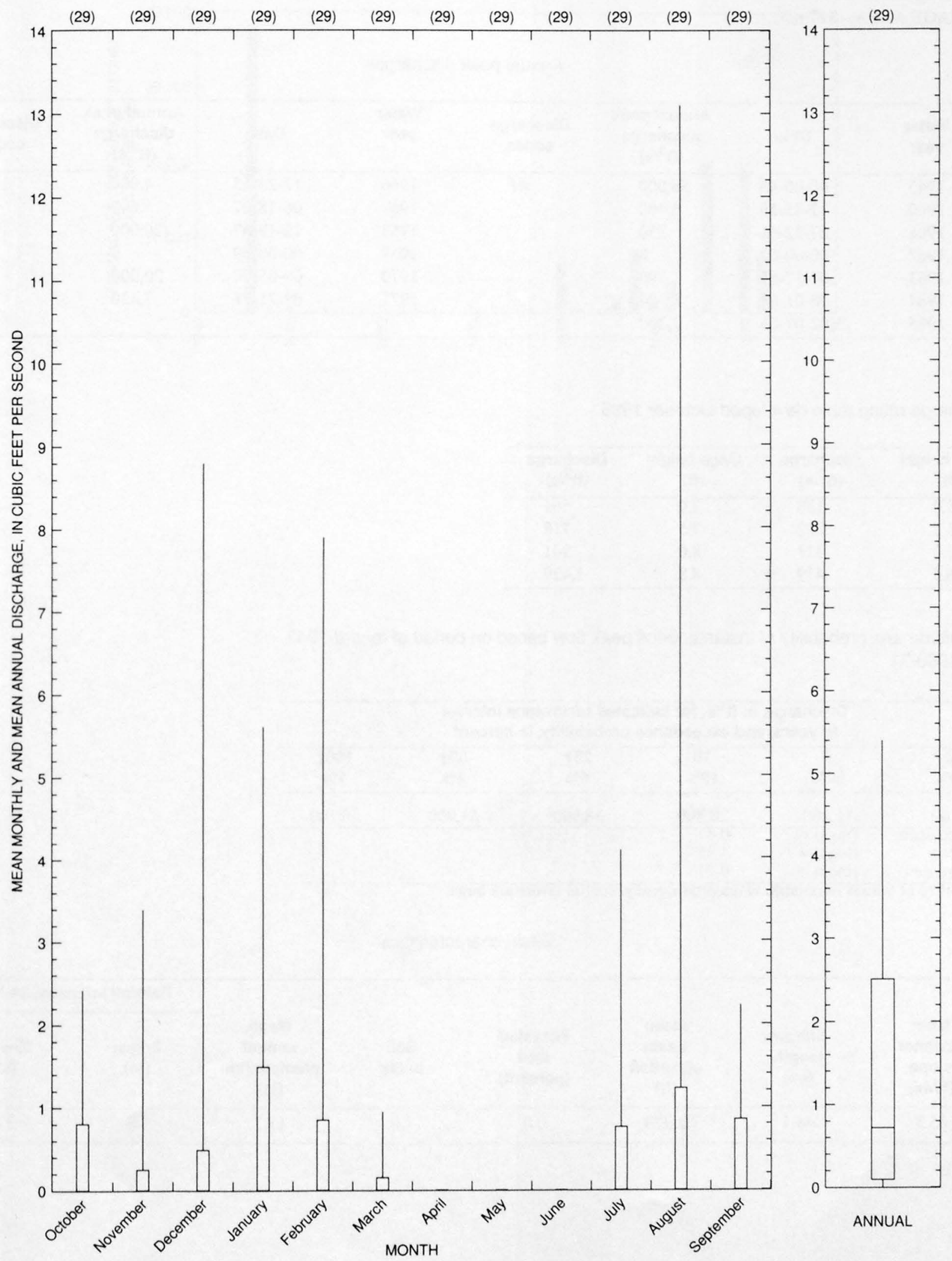


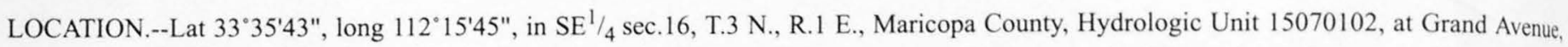
$1.75 \mathrm{mi}$ northwest of Peoria.

DRAINAGE AREA.--317 $\mathrm{mi}^{2}$.

Annual peak discharges

\begin{tabular}{|c|c|c|c|c|c|c|c|}
\hline $\begin{array}{l}\text { Water } \\
\text { year }\end{array}$ & Date & $\begin{array}{c}\text { Annual peak } \\
\text { discharge } \\
\left(\mathrm{ft}^{3} / \mathrm{s}\right)\end{array}$ & $\begin{array}{c}\text { Discharge } \\
\text { codes }\end{array}$ & $\begin{array}{l}\text { Water } \\
\text { year }\end{array}$ & Date & $\begin{array}{c}\text { Annual peak } \\
\text { discharge } \\
\left(\mathrm{ft}^{3} / \mathrm{s}\right)\end{array}$ & $\begin{array}{c}\text { Discharge } \\
\text { codes }\end{array}$ \\
\hline 1943 & $00-00-43$ & 38,000 & \multirow[t]{7}{*}{ HP } & 1966 & $12-23-65$ & 4,000 & \multirow{7}{*}{ ES } \\
\hline 1960 & $12-25-59$ & 6,400 & & 1967 & $06-18-67$ & 600 & \\
\hline 1961 & $07-22-61$ & 250 & & 1968 & $12-19-67$ & 20,000 & \\
\hline 1962 & $00-00-62$ & 0 & & 1969 & $00-00-69$ & 0 & \\
\hline 1963 & $08-17-63$ & 1,390 & & 1970 & $09-05-70$ & 20,000 & \\
\hline 1964 & $08-01-64$ & 8,300 & & 1971 & $08-21-71$ & 7,430 & \\
\hline 1965 & $02-07-65$ & 2,300 & & & & & \\
\hline
\end{tabular}

Discharge rating table developed October 1995

\begin{tabular}{cccc}
\hline $\begin{array}{c}\text { Gage height } \\
(\mathbf{f t})\end{array}$ & $\begin{array}{c}\text { Discharge } \\
\left(\mathbf{f t}^{\mathbf{3}} \mathbf{s}\right)\end{array}$ & $\begin{array}{c}\text { Gage height } \\
(\mathbf{f t})\end{array}$ & $\begin{array}{c}\text { Discharge } \\
\left(\mathbf{f t}^{3} / \mathbf{s}\right)\end{array}$ \\
\hline 5.0 & 138 & 7.0 & 561 \\
5.5 & 222 & 7.5 & 718 \\
6.0 & 311 & 8.0 & 941 \\
6.5 & 419 & 8.8 & 1,420 \\
\hline
\end{tabular}

Magnitude and probability of instantaneous peak flow based on period of record 1943 , 1960-71

\begin{tabular}{|c|c|c|c|c|c|}
\hline \multicolumn{6}{|c|}{$\begin{array}{l}\text { Discharge, in } \mathrm{ft}^{3} / \mathrm{s} \text {, for indicated recurrence interval } \\
\text { in years, and exceedance probablility, in percent }\end{array}$} \\
\hline 2 & 5 & 10 & $25 \dagger$ & $50 \dagger$ & $100 t$ \\
\hline $50 \%$ & $20 \%$ & $10 \%$ & $4 \%$ & $2 \%$ & $1 \%$ \\
\hline 3,160 & 11,300 & 20,800 & 38,000 & 54,900 & 75,100 \\
\hline Weighted skew & $(\log s)=$ & -0.41 & & & \\
\hline Mean & $(\log s)=$ & 3.45 & & & \\
\hline Standard dev. & $(\log s)=$ & 0.71 & & & \\
\hline
\end{tabular}

Basin characteristics

\begin{tabular}{ccccccc}
\hline $\begin{array}{c}\text { Main } \\
\text { channel } \\
\text { slope } \\
\text { (ft/mi) }\end{array}$ & $\begin{array}{c}\text { Stream } \\
\text { length } \\
\text { (mi) }\end{array}$ & $\begin{array}{c}\text { Mean } \\
\text { basin } \\
\text { elevation } \\
\text { (ft) }\end{array}$ & $\begin{array}{c}\text { Forested } \\
\text { area } \\
\text { (percent) }\end{array}$ & $\begin{array}{c}\text { Soil } \\
\text { index }\end{array}$ & $\begin{array}{c}\text { Mean } \\
\text { annual } \\
\text { precipitation } \\
\text { (in) }\end{array}$ & $\begin{array}{c}\text { Rainfall intensity, 24-hour } \\
\text { (in) }\end{array}$ \\
\hline 65.3 & 44.1 & 2,320 & 0.0 & 1.0 & 13.3 & $\begin{array}{c}50-y e a r \\
\text { (in) }\end{array}$ \\
\hline
\end{tabular}


09513890 NEW RIVER AT PEORIA, AZ--Continued

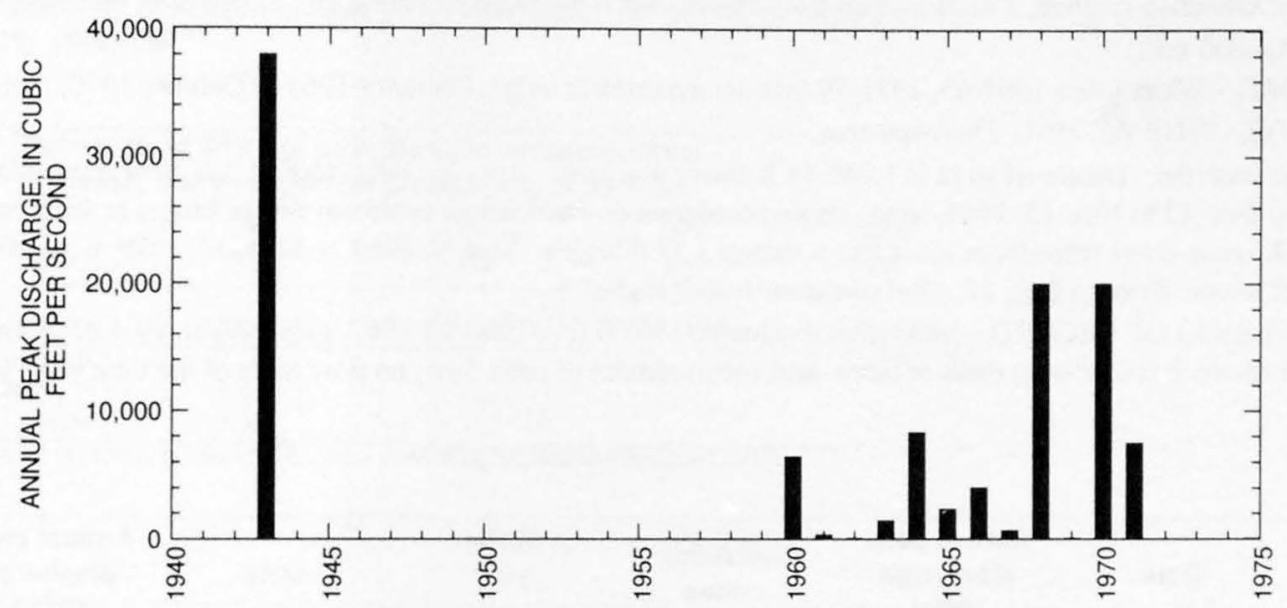




\section{NEW RIVER NEAR GLENDALE, AZ}

LOCATION.--Lat 33 $32^{\prime} 12^{\prime \prime}$, long $112^{\circ} 16^{\prime} 52^{\prime \prime}$, in NE $1 / 4^{N} W^{1} / 4$ sec.8, T.2 N., R.1 E., Maricopa County, Hydrologic Unit 15070102, on downstream side of bridge at Glendale Avenue, 2 mi upstream from mouth, 4 mi southwest of Peoria and 1.5 mi west of Glendale.

DRAINAGE AREA.--600 $\mathrm{mi}^{2}$.

PERIOD OF RECORD.--Water years 1961-63, 1971-79 (annual maximums only); February 1964 to October 1970, April 1990 to current year. REVISED RECORDS.--WDR AZ-89-1: Drainage area.

GAGE.--Water-stage recorder. Datum of gage is 1,046.44 ft above sea level. Apr. 26, 1961, to Feb. 12, 1964, crest-stage gage at same site and different datum. Feb. 13 to Nov. 12, 1964, water-stage recorder on downstream or upstream side of bridge at different datums. Nov. 13, 1964, to Sept. 8, 1969, water-stage recorder at same site at datum 3.47 ft higher. Sept. 9, 1969, to Sept. 30, 1979, water-stage recorder at same site at datum $0.24 \mathrm{ft}$ lower. Prior to Dec. 31,1991 at datum $1.00 \mathrm{ft}$ higher.

EXTREMES FOR PERIOD OF RECORD.--Maximum discharge, 19,800 $\mathrm{ft}^{3} / \mathrm{s} \mathrm{Dec.} \mathrm{19,} \mathrm{1967,} \mathrm{gage} \mathrm{height,} 10.4 \mathrm{ft}$, datum then in use, from rating curve extended above $2,100 \mathrm{ft}^{3} / \mathrm{s}$ on basis of slope-area measurement of peak flow; no flow most of the time each year.

Annual peak discharges

\begin{tabular}{|c|c|c|c|c|c|c|c|}
\hline $\begin{array}{l}\text { Water } \\
\text { year }\end{array}$ & Date & $\begin{array}{c}\text { Annual peak } \\
\text { discharge } \\
\left(\mathrm{ft}^{3} / \mathrm{s}\right)\end{array}$ & $\begin{array}{l}\text { Discharge } \\
\text { codes }\end{array}$ & $\begin{array}{l}\text { Water } \\
\text { year }\end{array}$ & Date & $\begin{array}{c}\text { Annual peak } \\
\text { discharge } \\
\left(\mathrm{ft}^{3} / \mathrm{s}\right)\end{array}$ & $\begin{array}{l}\text { Discharge } \\
\text { codes }\end{array}$ \\
\hline 1943 & $08-00-43$ & 38,000 & ES,HP & 1973 & $10-07-72$ & 8,650 & \\
\hline 1955 & $07-00-55$ & 12,000 & ES,HP & 1974 & $08-05-74$ & 775 & \\
\hline 1960 & $12-25-59$ & 5,500 & ES & 1975 & $10-29-74$ & 490 & \\
\hline 1961 & $00-00-61$ & 0 & & 1976 & $09-25-76$ & 1,550 & \\
\hline 1962 & $00-00-62$ & 0 & & 1977 & $10-23-76$ & 168 & \\
\hline 1963 & $08-30-63$ & 690 & & 1978 & $03-02-78$ & 12,300 & \\
\hline 1964 & $08-01-64$ & 7,000 & & 1979 & $12-19-78$ & 3,620 & \\
\hline 1965 & $01-08-65$ & 1,100 & & 1990 & 09-03-90 & 10,000 & \\
\hline 1966 & $12-23-65$ & 3,000 & & 1991 & $03-02-91$ & 1,400 & \\
\hline 1967 & $00-00-67$ & 0 & & 1992 & $07-24-92$ & 2,110 & \\
\hline 1968 & $12-19-67$ & 19,800 & & 1993 & $01-11-93$ & 5,450 & \\
\hline 1969 & $00-00-69$ & 0 & & 1994 & $10-06-93$ & 1,770 & \\
\hline 1970 & $09-05-70$ & 19,200 & & 1995 & $09-28-95$ & 1,690 & \\
\hline 1971 & $08-21-71$ & 7,000 & ES & 1996 & $11-02-95$ & 2,540 & \\
\hline 1972 & $07-17-72$ & 6,300 & & & & & \\
\hline
\end{tabular}

Discharge rating table developed November 1993

\begin{tabular}{crcc}
\hline $\begin{array}{c}\text { Gage height } \\
(\mathbf{f t})\end{array}$ & $\begin{array}{c}\text { Discharge } \\
\left(\mathrm{ft}^{\mathbf{3}} / \mathbf{s}\right)\end{array}$ & $\begin{array}{c}\text { Gage height } \\
(\mathbf{f t})\end{array}$ & $\begin{array}{c}\text { Discharge } \\
\left(\mathrm{ft}^{\mathbf{3}} / \mathbf{s}\right)\end{array}$ \\
\hline 1.1 & 50 & 1.6 & 2,020 \\
1.2 & 209 & 1.7 & 2,780 \\
1.3 & 483 & 1.8 & 3,660 \\
1.4 & 874 & 1.9 & 4,670 \\
1.5 & 1,390 & 2.0 & 5,800 \\
\hline
\end{tabular}


09513910 NEW RIVER NEAR GLENDALE, AZ--Continued

Magnitude and probability of instantaneous peak flow based on period of record 1943 ,

$1955,1960-79,1990-96$

\begin{tabular}{|c|c|c|c|c|c|}
\hline \multicolumn{6}{|c|}{$\begin{array}{l}\text { Discharge, in } \mathrm{ft}^{3} / \mathrm{s} \text {, for indicated recurrence interval } \\
\text { in years, and exceedance probablility, in percent }\end{array}$} \\
\hline 2 & 5 & 10 & 25 & $50 \dagger$ & $100 \dagger$ \\
\hline $50 \%$ & $20 \%$ & $10 \%$ & $4 \%$ & $2 \%$ & $1 \%$ \\
\hline 2,890 & 8,410 & 14,400 & 25,400 & 36,300 & 49,800 \\
\hline Weighted skew & $(\log s)=$ & -0.14 & & & \\
\hline Mean & $(\log s)=$ & 3.45 & & & \\
\hline Standard dev. & $(\log s)=$ & 0.56 & & & \\
\hline
\end{tabular}

fReliability of values in column is uncertain, and potential errors are large.

Basin characteristics

\begin{tabular}{ccccccc}
\hline $\begin{array}{c}\text { Main } \\
\text { channel } \\
\text { slope } \\
(\mathrm{ft} / \mathrm{mi})\end{array}$ & $\begin{array}{c}\text { Stream } \\
\text { length } \\
(\mathrm{mi})\end{array}$ & $\begin{array}{c}\text { Mean } \\
\text { basin } \\
\text { elevation } \\
(\mathrm{ft})\end{array}$ & $\begin{array}{c}\text { Forested } \\
\text { area } \\
\text { (percent) }\end{array}$ & $\begin{array}{c}\text { Soil } \\
\text { index }\end{array}$ & $\begin{array}{c}\text { Mean } \\
\text { annual } \\
\text { precipitation } \\
\text { (in) }\end{array}$ & $\begin{array}{c}\text { Rainfall intensity, 24-hour } \\
\text { (in) }\end{array}$ \\
\hline 66.9 & 49.6 & 2,130 & 0.0 & 1.5 & 13.8 & $\begin{array}{c}50 \text {-year } \\
\text { (in) }\end{array}$ \\
\hline
\end{tabular}

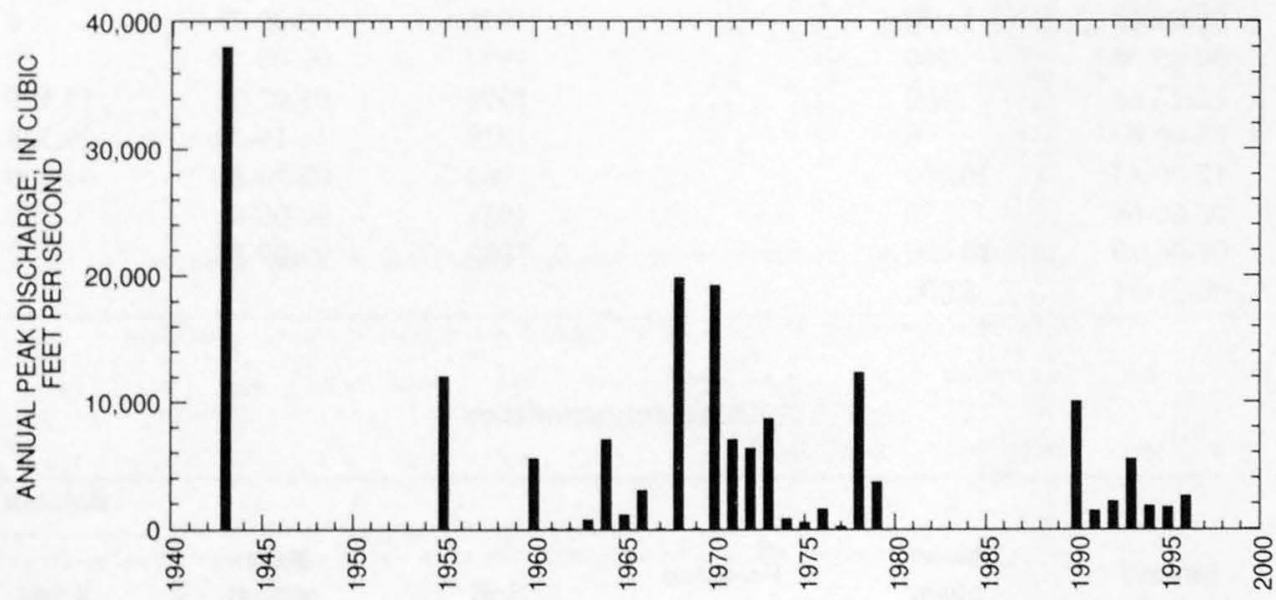




\section{AGUA FRIA RIVER AT AVONDALE, AZ}

LOCATION.--Lat 33 $26^{\prime} 06^{\prime \prime}$, long 112 19'59", in NW $1 / 4$ sec.14, T.1 N., R.1 W., Maricopa County, Hydrologic Unit 15070102, on downstream side of bridge on U.S. Highway $80,0.5$ mi east of Avondale, and 3 mi upstream from mouth.

DRAINAGE AREA.--2,013 $\mathrm{mi}^{2}$, of which 1,459 $\mathrm{mi}^{2}$ above Lake Pleasant is noncontributing except during periods of spill from Waddell Dam. Flood water from drainage area of $247 \mathrm{mi}^{2}$ above McMicken Dam may be diverted into Agua Fria River basin above station.

PERIOD OF RECORD.--Water years 1960-67, 1973 (annual maximums only), October 1967 to September 1972, October 1973 to current year.

GAGE.--Water-stage recorder since Jan. 7, 1974, with supplementary crest-stage gage. Datum of gage is $952.92 \mathrm{ft}$ above sea level (Arizona Highway Department bench mark). Apr. 26, 1961, to Sept. 30, 1967, crest-stage gage; Oct. 1, 1967, to July 21, 1972, water-stage recorder (with supplementary crest-stage gage from Mar. 10 to July 22, 1972) at present site at datum $2.92 \mathrm{ft}$ lower. July 22, 1972, to Jan. 6, 1974, creststage gage only, at railroad bridge upstream at datum $2.92 \mathrm{ft}$ lower.

REMARKS.--No flow during the year. Flow partly regulated by Lake Pleasant, 35 mi upstream. (See elsewhere in this report.) Records at times may include waste water from the Arizona Canal of the Salt River Project. Excess flood water released from McMicken Dam on Trilby Wash may enter Agua Fria River basin above station; this amount generally is negligible.

AVERAGE DISCHARGE.--14 years (water years 1968-72, 1974-82), $31.7 \mathrm{ft}^{3} / \mathrm{s}, 22,970$ acre- $\mathrm{ft} / \mathrm{yr}$; median of yearly mean discharges, $0.7 \mathrm{ft}^{3} / \mathrm{s}, 510$ acre-ft/yr.

EXTREMES FOR PERIOD OF RECORD.--Maximum discharge, 44,200 $\mathrm{ft}^{3} / \mathrm{s} \mathrm{Feb.} \mathrm{20,} \mathrm{1980,} \mathrm{gage} \mathrm{height,} 6.77 \mathrm{ft}$; maximum gage height, 12.70 $\mathrm{ft}$ Dec. 20, 1967, datum then in use; no flow for most of time each year.

Annual peak discharges

\begin{tabular}{|c|c|c|c|c|c|c|c|}
\hline $\begin{array}{l}\text { Water } \\
\text { year }\end{array}$ & Date & $\begin{array}{c}\text { Annual peak } \\
\text { discharge } \\
\left(\mathrm{ft}^{3} / \mathrm{s}\right)\end{array}$ & $\begin{array}{l}\text { Discharge } \\
\text { codes }\end{array}$ & $\begin{array}{l}\text { Water } \\
\text { year }\end{array}$ & Date & $\begin{array}{c}\text { Annual peak } \\
\text { discharge } \\
\left(\mathrm{ft}^{3} / \mathrm{s}\right)\end{array}$ & $\begin{array}{c}\text { Discharge } \\
\text { codes }\end{array}$ \\
\hline 1960 & $12-25-59$ & 4,700 & & 1972 & $07-17-72$ & 5,180 & \\
\hline 1961 & $00-00-61$ & 0 & & 1973 & $10-07-72$ & 5,000 & \\
\hline 1962 & $00-00-62$ & 0 & & 1974 & $00-00-74$ & 0 & \\
\hline 1963 & $08-00-63$ & 63 & & 1975 & $00-00-75$ & 0 & \\
\hline 1964 & $08-01-64$ & 3,000 & & 1976 & $00-00-76$ & 0 & \\
\hline 1965 & $04-05-65$ & 460 & & 1977 & $00-00-77$ & 0 & \\
\hline 1966 & $12-23-65$ & 800 & & 1978 & $03-02-78$ & 13,100 & \\
\hline 1967 & $00-00-67$ & 0 & & 1979 & $12-19-78$ & 29,300 & UR \\
\hline 1968 & $12-20-67$ & 20,000 & & 1980 & $02-20-80$ & 44,200 & UR \\
\hline 1969 & $00-00-69$ & 0 & & 1981 & $00-00-81$ & 0 & $\mathrm{C}, \mathrm{UR}$ \\
\hline 1970 & $08-06-70$ & 20,600 & & 1982 & $00-00-82$ & 0 & C,UR \\
\hline 1971 & $08-21-71$ & 8,200 & & & & & \\
\hline
\end{tabular}

Basin characteristics

\begin{tabular}{cccccccc}
\hline $\begin{array}{c}\text { Main } \\
\text { channel } \\
\text { slope } \\
(\mathrm{ft} / \mathrm{mi})\end{array}$ & $\begin{array}{c}\text { Stream } \\
\text { length } \\
(\mathrm{mi})\end{array}$ & $\begin{array}{c}\text { Mean } \\
\text { basin } \\
\text { elevation } \\
(\mathrm{ft})\end{array}$ & $\begin{array}{c}\text { Forested } \\
\text { area } \\
\text { (percent) }\end{array}$ & $\begin{array}{c}\text { Soil } \\
\text { index }\end{array}$ & $\begin{array}{c}\text { Mean } \\
\text { annual } \\
\text { precipitation } \\
\text { (in) }\end{array}$ & $\begin{array}{c}\text { R-year } \\
\text { (in) }\end{array}$ & $\begin{array}{c}50 \text {-year } \\
\text { (in) }\end{array}$ \\
\hline 38.7 & 96.4 & 3,010 & 0.0 & 1.0 & 16.3 & 1.7 \\
\hline
\end{tabular}


09513970 AGUA FRIA RIVER AT AVONDALE, AZ--Continued

MEAN MONTHLY AND ANNUAL DISCHARGES $1968-72,1974 \cdot 82$

\begin{tabular}{|c|c|c|c|c|c|c|}
\hline MONTH & $\begin{array}{l}\text { MAXIMUM } \\
\text { (FT3/S) }\end{array}$ & $\begin{array}{l}\text { MINIMUM } \\
\text { (FT3/S) }\end{array}$ & $\begin{array}{l}\text { MEAN } \\
(\text { FT3/S) }\end{array}$ & $\begin{array}{l}\text { STAN- } \\
\text { DARD } \\
\text { DEVIA- } \\
\text { TION } \\
(\text { FT } 3 / \mathrm{S})\end{array}$ & $\begin{array}{l}\text { COEFFI- } \\
\text { CIENT OF } \\
\text { VARI- } \\
\text { ATION }\end{array}$ & $\begin{array}{c}\text { PERCENT } \\
\text { OF } \\
\text { ANNUAL } \\
\text { RUNOFF }\end{array}$ \\
\hline OCTOBER & 0.00 & 0.00 & 0.00 & 0.00 & & 0.0 \\
\hline NOVEMBER & 0.00 & 0.00 & 0.00 & 0.00 & & 0.0 \\
\hline DECEMBER & 520 & 0.00 & 54 & 148 & 2.8 & 13.9 \\
\hline JANUARY & 518 & 0.00 & 42 & 138 & 3.3 & 10.9 \\
\hline FEBRUARY 2 & 2,900 & 0.00 & 212 & 773 & 3.6 & 54.6 \\
\hline MARCH & 810 & 0.00 & 68 & 217 & 3.2 & 17.4 \\
\hline APRIL & 0.00 & 0.00 & 0.00 & 0.00 & & 0.0 \\
\hline MAY & 0.00 & 0.00 & 0.00 & 0.00 & & 0.0 \\
\hline JUNE & 1.1 & 0.00 & 0.08 & 0.30 & 3.7 & 0.0 \\
\hline JULY & 15 & 0.00 & 1.0 & 3.9 & 3.7 & 0.3 \\
\hline AUGUST & 24 & 0.00 & 1.7 & 6.4 & 3.7 & 0.4 \\
\hline SEPTEMBER & 133 & 0.00 & 9.5 & 36 & 3.7 & 2.4 \\
\hline ANNUAL & 232 & 0.00 & 32 & 66 & 2.1 & 100 \\
\hline
\end{tabular}

MAGNITUDE AND PROBABILITY OF INSTANTANEOUS PEAK FLOW BASED ON PERIOD OF RECORD

DISCHARGE, IN FT3/S, FOR INDICATED RECURRENCE INTERVAL IN YEARS, AND EXCEEDANCE PROBABILITY, IN PERCENT

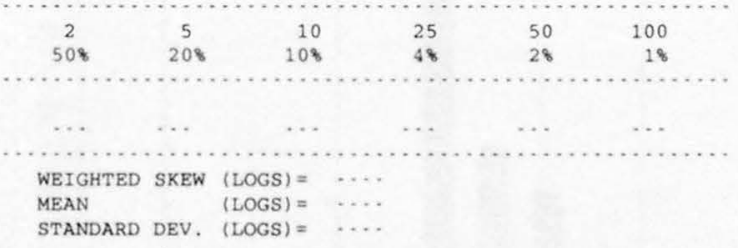

MAGNITUDE AND PROBABILITY OF ANNUAL LOW FLOW BASED ON PERIOD OF RECORD $1969 \cdot 72,1975 \cdot 82$

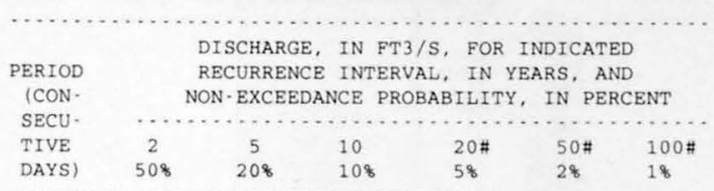

MAGNITUDE AND PROBABILITY OF ANNUAL HIGH FLOW BASED ON PERIOD OF RECORD 1968-72, 1974-82

\begin{tabular}{|c|c|c|c|c|c|c|}
\hline & \multicolumn{6}{|c|}{$\begin{array}{l}\text { DISCHARGE, IN FT3/S, FOR INDICATED } \\
\text { RECURRENCE INTERVAL, IN YEARS, AND }\end{array}$} \\
\hline $\begin{array}{l}\text { PERIOD } \\
(\mathrm{CON} \text { - }\end{array}$ & & $\begin{array}{l}\text { RECURR } \\
\text { EXCEED }\end{array}$ & $\begin{array}{l}\text { NCE INTE } \\
\text { NCE PROE }\end{array}$ & $\begin{array}{l}\text { RVAL, IN } \\
\text { ABILITY }\end{array}$ & YEARS, & \\
\hline SECU. & & ENC..... & .......... & .......... & 1........ & $\ldots$ \\
\hline TIVE & 2 & 5 & 10 & $25 \#$ & $50 \#$ & $100 \#$ \\
\hline DAYS) & 508 & 208 & 108 & 48 & 28 & 18 \\
\hline 1 & 0.00 & 5,630 & 12,100 & 23,400 & 32,900 & 44,100 \\
\hline 3 & 0.00 & 2,820 & 6,890 & 15,400 & 23,500 & 34,000 \\
\hline 7 & 0.00 & 1,460 & 3,970 & 10,100 & 16,400 & 25,500 \\
\hline 15 & 0.00 & 698 & 2,030 & 5,600 & 9,700 & 16,000 \\
\hline 30 & 0.00 & 367 & 1,070 & 2,970 & 5,130 & 8,500 \\
\hline 60 & 0.00 & 206 & 620 & 1,730 & 3,000 & 4,930 \\
\hline 90 & 0.00 & 138 & 412 & 1,150 & 1,990 & 3,260 \\
\hline
\end{tabular}

DURATION TABLE OF DAILY MEAN FLOW FOR PERIOD OF RECORD 1968-72, 1974-82

DISCHARGE, IN FT3/S, WHICH WAS EQUALED OR EXCEEDED FOR INDICATED PERCENT OF TIME

\begin{tabular}{|c|c|c|c|c|c|c|c|c|c|c|c|c|c|c|c|c|}
\hline 18 & 58 & 108 & 158 & 208 & 308 & 408 & 508 & 608 & 708 & 808 & 908 & 958 & 988 & 998 & 99.58 & 99.98 \\
\hline 98 & 4.6 & 4.4 & 4.1 & 3.9 & 3.4 & 2.9 & 2.4 & 1.9 & 1.5 & 0.97 & 0.49 & 0.24 & 0.10 & 0.05 & 0.02 & 0.00 \\
\hline
\end{tabular}


09513970 AGUA FRIA RIVER AT AVONDALE, AZ--Continued
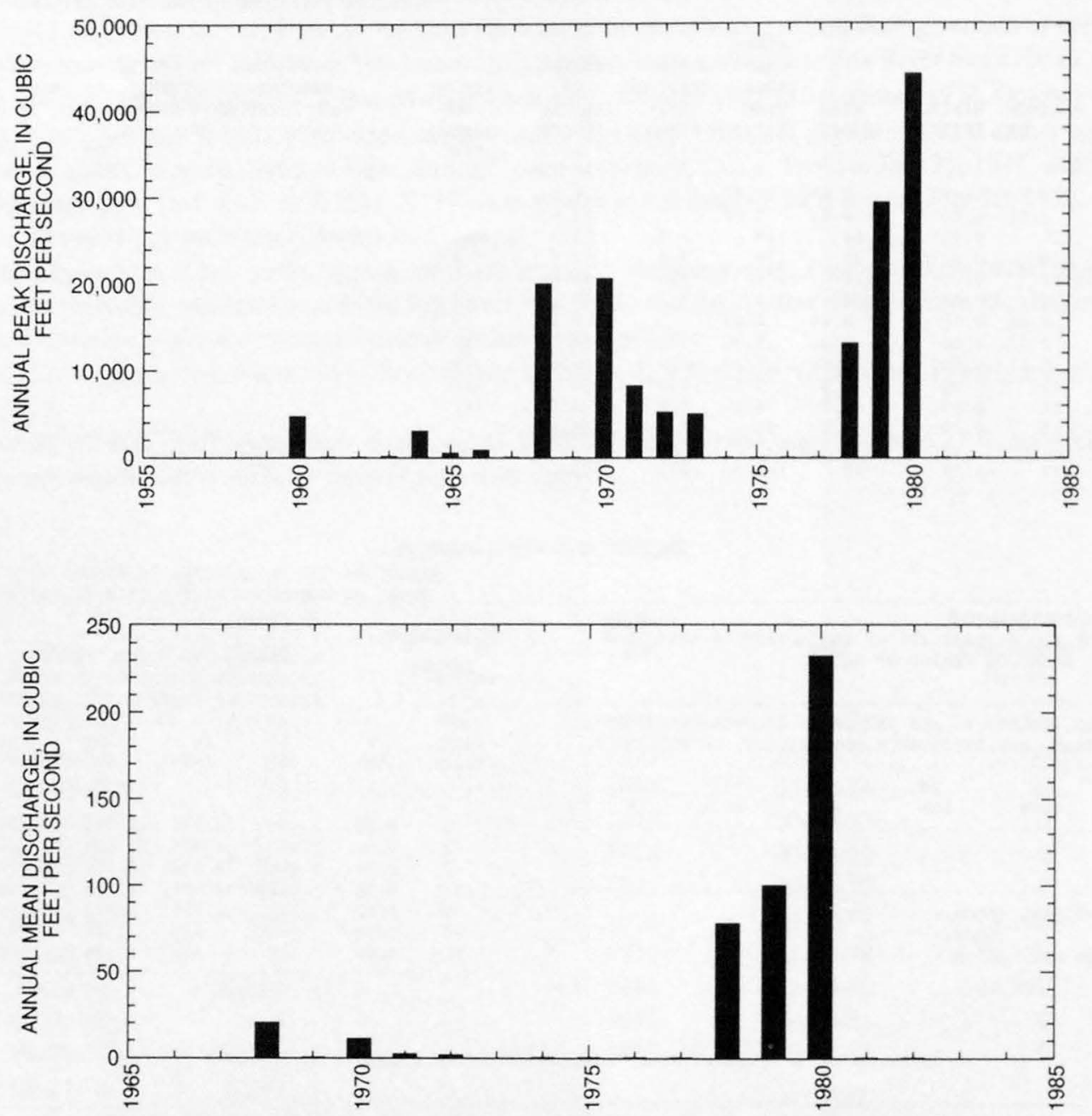
09513970 AGUA FRIA RIVER AT AVONDALE, AZ--Continued
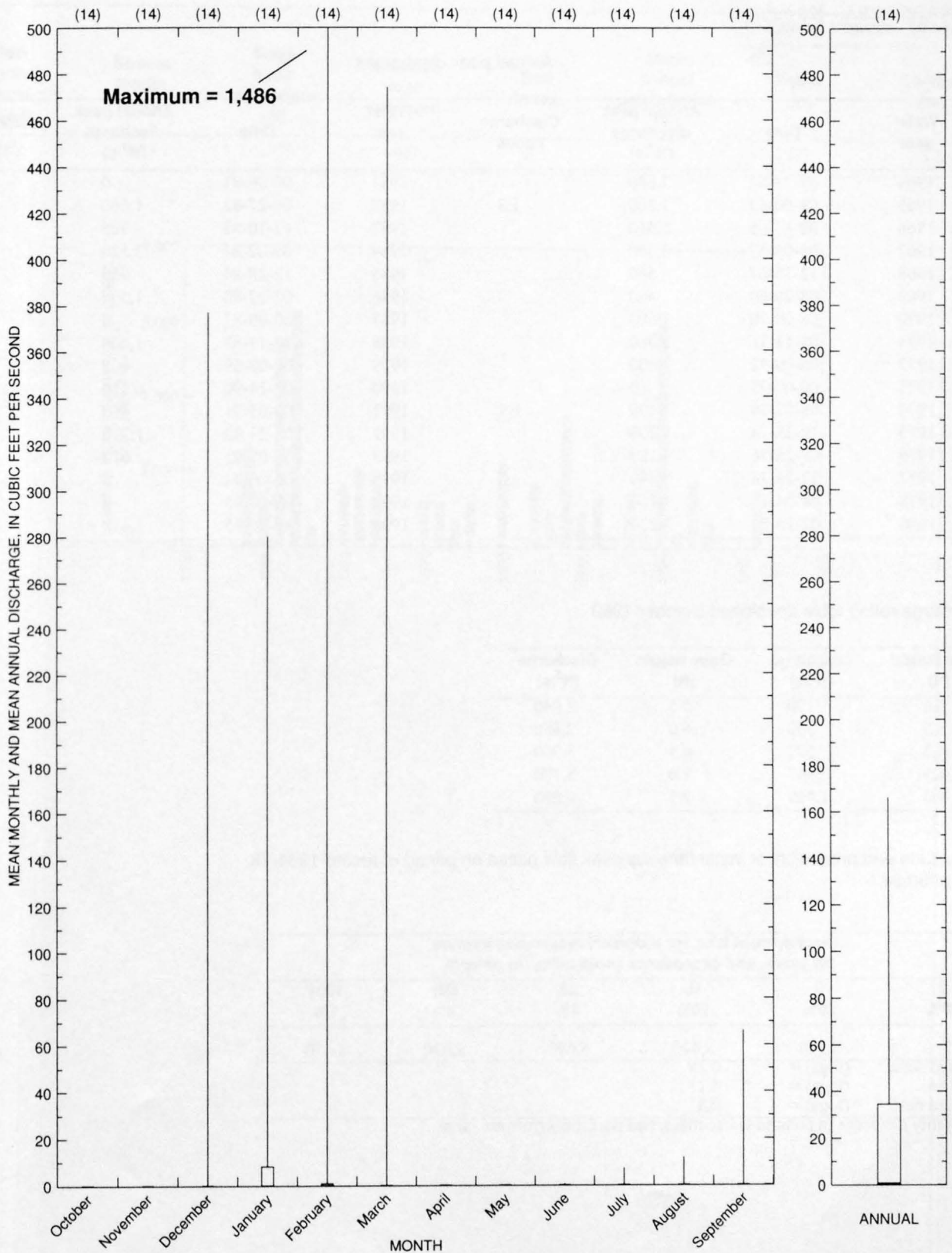


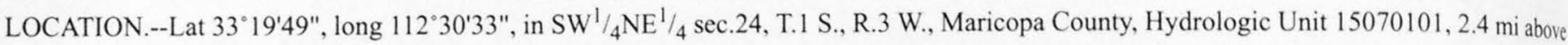
mouth, 5.2 mi southeast of Buckeye.

DRAINAGE AREA.--420 $\mathrm{mi}^{2}$.

Annual peak discharges

\begin{tabular}{|c|c|c|c|c|c|c|c|}
\hline $\begin{array}{l}\text { Water } \\
\text { year }\end{array}$ & Date & $\begin{array}{c}\text { Annual peak } \\
\text { discharge } \\
\left(\mathrm{ft}^{3} / \mathrm{s}\right)\end{array}$ & $\begin{array}{l}\text { Discharge } \\
\text { codes }\end{array}$ & $\begin{array}{l}\text { Water } \\
\text { year }\end{array}$ & Date & $\begin{array}{c}\text { Annual peak } \\
\text { discharge } \\
\left(\mathrm{ft}^{3} / \mathrm{s}\right)\end{array}$ & $\begin{array}{c}\text { Discharge } \\
\text { codes }\end{array}$ \\
\hline 1964 & $09-13-64$ & 2,680 & \multirow{10}{*}{ ES } & 1981 & $00-00-81$ & 0 & \\
\hline 1965 & $08-00-65$ & 1,200 & & 1982 & $09-27-82$ & 1,660 & \\
\hline 1966 & $09-13-66$ & 5,560 & & 1983 & $12-10-82$ & 985 & \\
\hline 1967 & $09-03-67$ & 6,300 & & 1984 & $09-02-84$ & 3,520 & \\
\hline 1968 & $12-15-67$ & 560 & & 1985 & $12-28-84$ & 950 & \\
\hline 1969 & $08-29-69$ & 400 & & 1986 & $07-22-86$ & 1,500 & \\
\hline 1970 & $08-09-70$ & 1,600 & & 1987 & $00-00-87$ & 0 & \\
\hline 1971 & $08-11-71$ & 2,080 & & 1988 & $12-17-87$ & 1,430 & \\
\hline 1972 & $08-00-72$ & 2,000 & & 1989 & $00-00-89$ & 402 & \\
\hline 1973 & $00-00-73$ & 0 & & 1990 & $08-14-90$ & 6,310 & \\
\hline 1974 & $09-03-74$ & 100 & \multirow[t]{6}{*}{ ES } & 1991 & $09-05-91$ & 413 & \\
\hline 1975 & $10-28-74$ & 1,200 & & 1992 & $09-23-92$ & 1,770 & \\
\hline 1976 & $09-26-76$ & 1,180 & & 1993 & $01-08-93$ & 670 & \\
\hline 1977 & $10-23-76$ & 740 & & 1994 & $00-00-94$ & 0 & \\
\hline 1978 & $08-04-78$ & 1,150 & & 1995 & $00-00-95$ & 0 & \\
\hline 1980 & $02-15-80$ & 2,220 & & 1996 & $11-06-95$ & 1,470 & \\
\hline
\end{tabular}

Discharge rating table developed October 1989

\begin{tabular}{crcc}
\hline $\begin{array}{c}\text { Gage height } \\
(\mathbf{f t})\end{array}$ & $\begin{array}{c}\text { Discharge } \\
\left(\mathbf{f t}^{3} / \mathbf{s}\right)\end{array}$ & $\begin{array}{c}\text { Gage height } \\
(\mathbf{f t})\end{array}$ & $\begin{array}{c}\text { Discharge } \\
\left(\mathrm{ft}^{3} / \mathbf{s}\right)\end{array}$ \\
\hline 3.0 & 120 & 5.5 & 2,040 \\
3.5 & 300 & 6.0 & 2,880 \\
4.0 & 572 & 6.5 & 3,900 \\
4.5 & 943 & 7.0 & 5,100 \\
5.0 & 1,420 & 7.5 & 6,600 \\
\hline
\end{tabular}

Magnitude and probability of instantaneous peak flow based on period of record 1964-78, 1980-96

\begin{tabular}{|c|c|c|c|c|c|}
\hline \multicolumn{6}{|c|}{$\begin{array}{l}\text { Discharge, in } \mathrm{ft}^{3} / \mathrm{s} \text {, for indicated recurrence interval } \\
\text { in years, and exceedance probablility, in percent }\end{array}$} \\
\hline 2 & 5 & 10 & 25 & $50 t$ & $100 t$ \\
\hline $50 \%$ & $20 \%$ & $10 \%$ & $4 \%$ & $2 \%$ & $1 \%$ \\
\hline 1,250 & 2,390 & 3,420 & 5,090 & 6,630 & 8,470 \\
\hline Weighted skew & $(\log s)=$ & 0.28 & & & \\
\hline Mean & $(\log s)=$ & 3.11 & & & \\
\hline Standard dev. & $(\log s)=$ & 0.32 & & & \\
\hline
\end{tabular}

$\mp$ Reliability of values in column is uncertain, and potential errors are large. 
09514200 WATERMAN WASH NEAR BUCKEYE, AZ--Continued

Basin characteristics

\begin{tabular}{cccccccc}
\hline $\begin{array}{c}\text { Main } \\
\text { channel } \\
\text { slope } \\
(\mathrm{ft} / \mathrm{mi})\end{array}$ & $\begin{array}{c}\text { Stream } \\
\text { length } \\
(\mathrm{mi})\end{array}$ & $\begin{array}{c}\text { Mean } \\
\text { basin } \\
\text { elevation } \\
(\mathrm{ft})\end{array}$ & $\begin{array}{c}\text { Forested } \\
\text { area } \\
\text { (percent) }\end{array}$ & $\begin{array}{c}\text { Soil } \\
\text { index }\end{array}$ & $\begin{array}{c}\text { Mean } \\
\text { annual } \\
\text { precipitation } \\
\text { (in) }\end{array}$ & $\begin{array}{c}\text { Rainfall intensity, 24-hour } \\
\text { (in) }\end{array}$ & $\begin{array}{c}50 \text {-year } \\
\text { (in) }\end{array}$ \\
\hline 21.2 & 37.7 & 1,570 & 0.0 & 2.5 & 9.2 & 1.7 \\
\hline
\end{tabular}

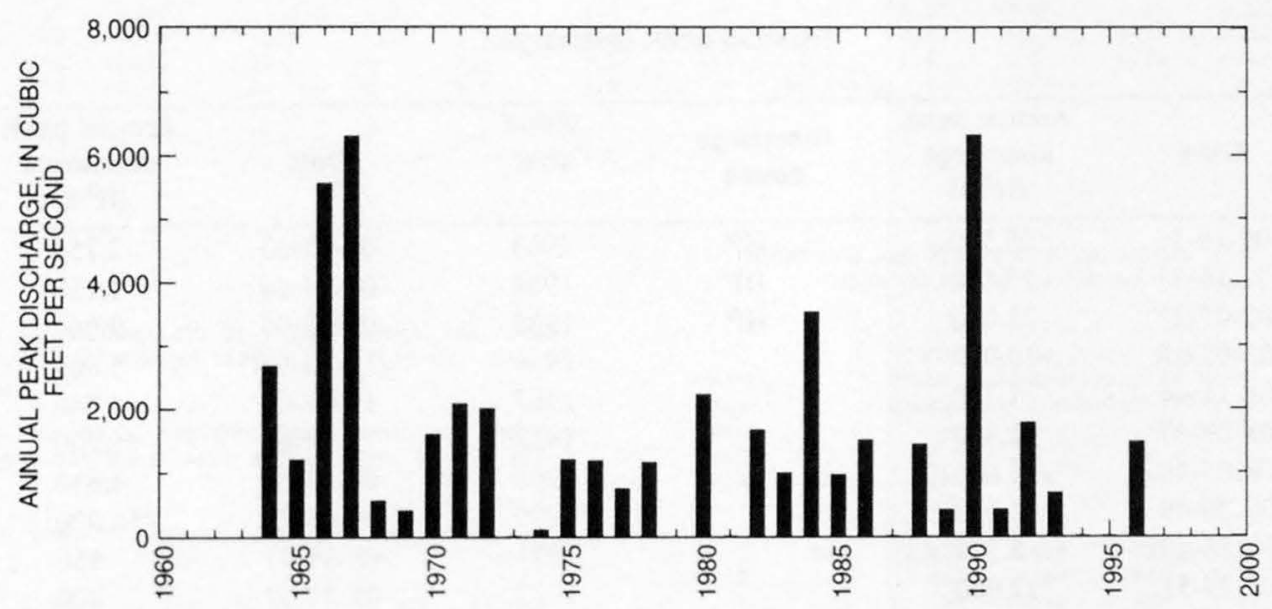




\section{HASSAYAMPA RIVER AT BOX DAMSITE, NEAR WICKENBURG, AZ}

LOCATION.--Lat 34 $02^{\prime} 42^{\prime \prime}$, long $112^{\circ} 42^{\prime} 33^{\prime \prime}$, in SW $1 /{ }_{4} \mathrm{SE}^{1} / 4$ sec.7, T.8 N., R.4 W., Yavapai County, Hydrologic Unit 15070103 , on right bank at Box damsite, 5.5 mi northeast of Wickenburg.

DRAINAGE AREA.--417 $\mathrm{mi}^{2}$.

PERIOD OF RECORD.--January to June 1938, May 1946 to September 1982 (discontinued).

GAGE.--Water-stage recorder. Datum of gage is 2,236.12 ft above sea level. January to June 1938 at site 1 mi downstream at datum $23.76 \mathrm{ft}$ lower. May 1, 1946, to Nov. 17, 1949, at present site at datum $2.16 \mathrm{ft}$ higher.

REMARKS.--Records poor. Small diversions for irrigation and mining above station.

AVERAGE DISCHARGE.--36 years (water years 1947-82), $24.4 \mathrm{ft}^{3} / \mathrm{s}, 17,680$ acre- $\mathrm{ft} / \mathrm{yr}$; median of yearly mean discharges, $8.5 \mathrm{ft}^{3} / \mathrm{s}, 6,200$ acre$\mathrm{ft} / \mathrm{yr}$.

EXTREMES FOR PERIOD OF RECORD.--Maximum discharge, $58,000 \mathrm{ft}^{3} / \mathrm{s}$ Sept. 5,1970 , gage height, $34.6 \mathrm{ft}$, from profile past gage, by slopearea measurement of peak flow; no flow for many days in 1964 and 1965.

Annual peak discharges

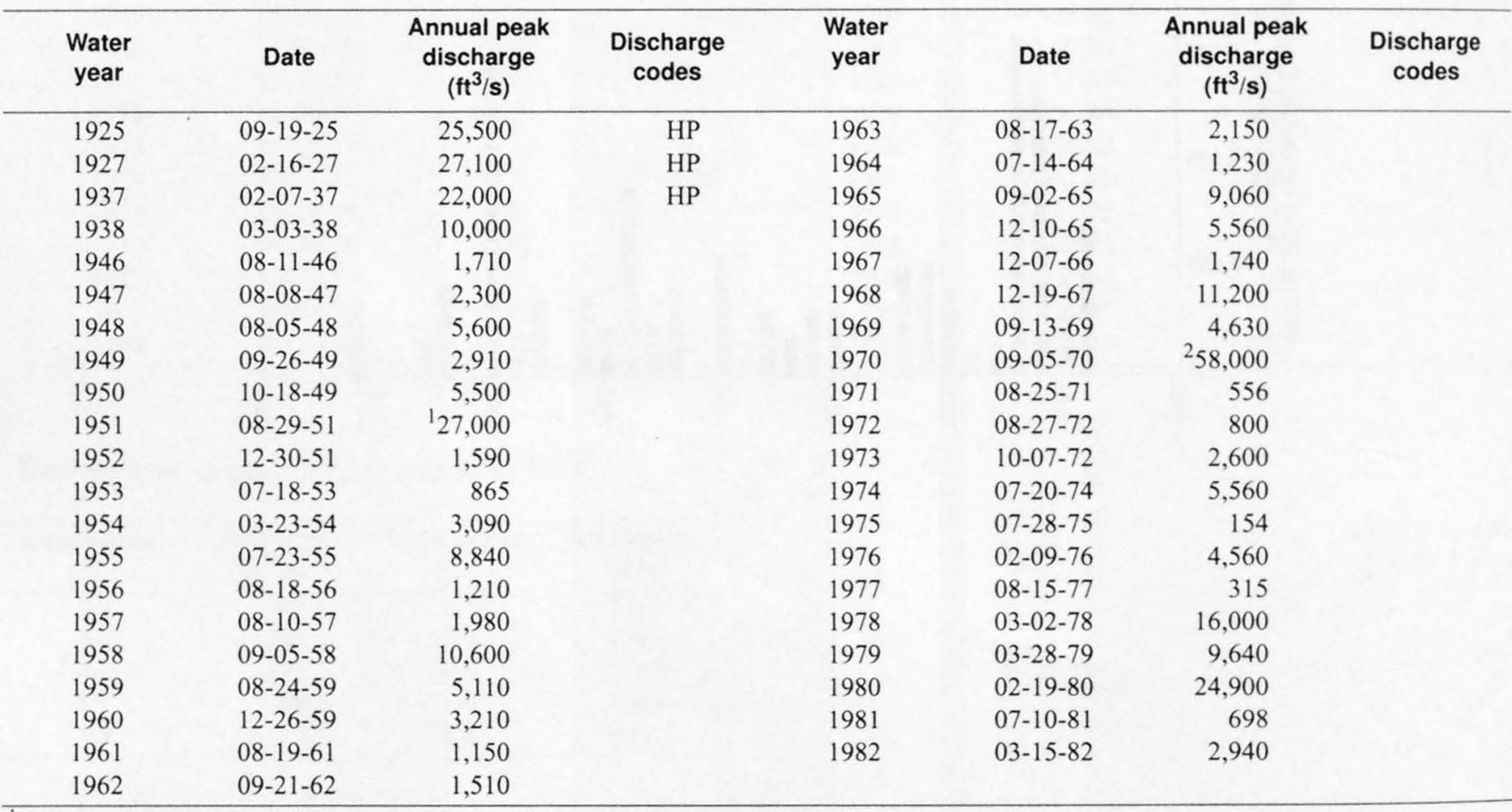

${ }^{1}$ Highest since 1927.

${ }^{2}$ Highest since 1890.

Basin characteristics

\begin{tabular}{cccccccc}
\hline $\begin{array}{c}\text { Main } \\
\text { channel } \\
\text { slope } \\
\text { (ft/mi) }\end{array}$ & $\begin{array}{c}\text { Stream } \\
\text { length } \\
\text { (mi) }\end{array}$ & $\begin{array}{c}\text { Mean } \\
\text { basin } \\
\text { elevation } \\
\text { (ft) }\end{array}$ & $\begin{array}{c}\text { Forested } \\
\text { area } \\
\text { (percent) }\end{array}$ & $\begin{array}{c}\text { Soil } \\
\text { index }\end{array}$ & $\begin{array}{c}\text { Mean } \\
\text { annual } \\
\text { precipitation } \\
\text { (in) }\end{array}$ & $\begin{array}{c}\text { Rainfall intensity, 24-hour } \\
\text { (in) }\end{array}$ & $\begin{array}{c}\text { 50-year } \\
\text { (in) }\end{array}$ \\
\hline 71.0 & 45.0 & 4,750 & 9.6 & 1.0 & 19.3 & 2.4 \\
\hline
\end{tabular}


GILA RIVER BASIN

09515500 HASSAYAMPA RIVER AT BOX DAMSITE, NEAR WICKENBURG, AZ--Continued

MEAN MONTHLY AND ANNUAL DISCHARGES 1947.82

\begin{tabular}{|c|c|c|c|c|c|c|}
\hline MONTH & $\begin{array}{l}\text { MAXIMUM } \\
\text { (FT3/S) }\end{array}$ & $\begin{array}{l}\text { MINIMUM } \\
\text { (FT3/S) }\end{array}$ & $\begin{array}{c}\text { MEAN } \\
(\mathrm{FT} 3 / \mathrm{S})\end{array}$ & $\begin{array}{l}\text { STAN- } \\
\text { DARD } \\
\text { DEVIA- } \\
\text { TION } \\
\text { (FT3/S) }\end{array}$ & $\begin{array}{l}\text { COEFFI- } \\
\text { CIENT OF } \\
\text { VARI- } \\
\text { ATION }\end{array}$ & $\begin{array}{c}\text { PERCENT } \\
\text { OF } \\
\text { ANNUAL } \\
\text { RUNOFF }\end{array}$ \\
\hline OCTOBER & 50 & 0.25 & 4.0 & 9.4 & 2.3 & 1.4 \\
\hline NOVEMBER & 104 & 0.21 & 7.2 & 20 & 2.7 & 2.4 \\
\hline DECEMBER & 298 & 0.22 & 20 & 56 & 2.7 & 7.0 \\
\hline JANUARY & 365 & 0.41 & 28 & 74 & 2.6 & 9.5 \\
\hline FEBRUARY & 1,280 & 0.26 & 63 & 214 & 3.4 & 21.5 \\
\hline MARCH & 1,170 & 0.44 & 84 & 211 & 2.5 & 28.5 \\
\hline APRIL & 247 & 0.34 & 34 & 68 & 2.0 & 11.5 \\
\hline MAY & 77 & 0.03 & 8.1 & 16 & 2.0 & 2.7 \\
\hline JUNE & 27 & 0.00 & 3.5 & 5.8 & 1.7 & 1.2 \\
\hline JULY & 42 & 0.19 & 5.9 & 8.1 & 1.4 & 2.0 \\
\hline AUGUST & 372 & 0.33 & 22 & 62 & 2.8 & 7.5 \\
\hline SEPTEMBER & 178 & 0.71 & 14 & 34 & 2.4 & 4.9 \\
\hline ANNUAL & 169 & 1.2 & 24 & 39 & 1.6 & 100 \\
\hline
\end{tabular}

MAGNITUDE AND PROBABILITY OF INSTANTANEOUS PEAK FLOW BASED ON PERIOD OF RECORD $1925,1927,1937-38,1946 \cdot 82$

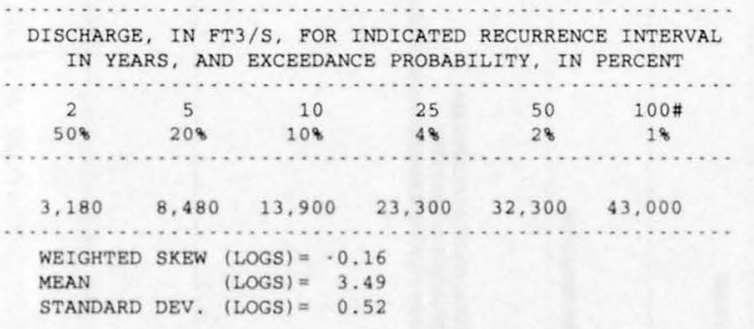

DURATION TABLE OF DAILY MEAN FLOW FOR PERIOD OF RECORD 1947.82
MAGNITUDE AND PROBABILITY OF ANNUAL LOW FLOW BASED ON PERIOD OF RECORD $1948 \cdot 82$

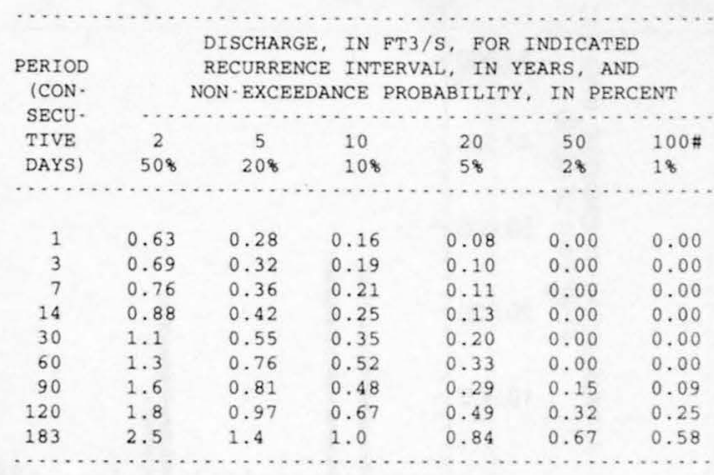

MAGNITUDE AND PROBABILITY OF ANNUAL HIGH PLOW BASED ON PERIOD OF RECORD 1947.82

\begin{tabular}{|c|c|c|c|c|c|c|}
\hline \multirow{4}{*}{$\begin{array}{l}\text { PERIOD } \\
\text { (CON- } \\
\text { SECU. }\end{array}$} & \multicolumn{6}{|c|}{ DISCHARGE, IN FT $3 / \mathrm{S}$, FOR INDICATED } \\
\hline & & RECURRE & CE INTI & VAL, IN & YEARS, & AND \\
\hline & & EXCEEDA & CE PROI & BILITY, & IN PERC & ENT \\
\hline & & 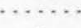 & & & .......... & …..... \\
\hline TIVE & 2 & 5 & 10 & 25 & 50 & $100 \#$ \\
\hline DAYS) & $50 \%$ & 208 & 108 & 48 & 28 & 18 \\
\hline 1 & 598 & 2,010 & 3,680 & 6,880 & 10,200 & 14,400 \\
\hline 3 & 271 & 975 & 1,900 & 3,850 & 6,070 & 9,130 \\
\hline 7 & 142 & 540 & 1,090 & 2,330 & 3,810 & 5,940 \\
\hline 15 & 83 & 335 & 704 & 1,580 & 2,680 & 4,320 \\
\hline 30 & 52 & 208 & 441 & 1,010 & 1,740 & 2,860 \\
\hline 60 & 32 & 124 & 262 & 602 & 1,050 & 1,740 \\
\hline 90 & 24 & 93 & 199 & 462 & 808 & 1.350 \\
\hline
\end{tabular}

ISCHARGE, IN FT3/S, WHICH WAS EQUALED OR EXCEEDED FOR INDICATED PERCENT OF TIME

\begin{tabular}{|c|c|c|c|c|c|c|c|c|c|c|c|c|c|c|c|c|}
\hline 18 & 58 & 108 & 158 & 208 & 308 & 408 & 508 & 608 & 708 & 808 & 908 & 958 & 988 & 998 & 99.58 & 99.98 \\
\hline 04 & 83 & 27 & 12 & 5.6 & 3.1 & 2.3 & 1.9 & 1.7 & 1.5 & 1.2 & 0.67 & 0.41 & 0.24 & 0.12 & 0.00 & 0.00 \\
\hline
\end{tabular}

\# Reliability of values in column is uncertain, and potential errors are large. 
09515500 HASSAYAMPA RIVER AT BOX DAMSITE, NEAR WICKENBURG, AZ--Continued
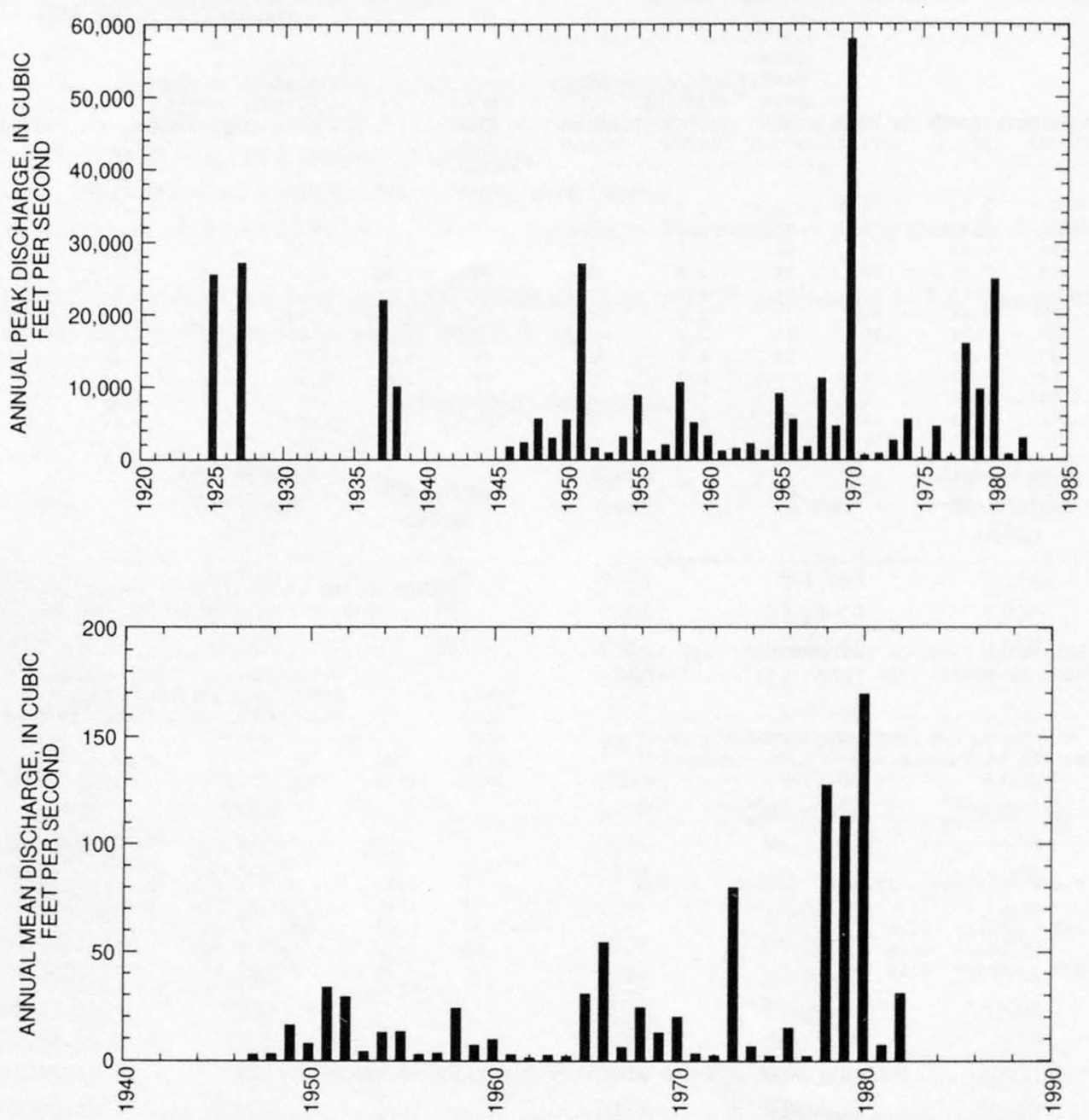
09515500 HASSAYAMPA RIVER AT BOX DAMSITE, NEAR WICKENBURG, AZ--Continued
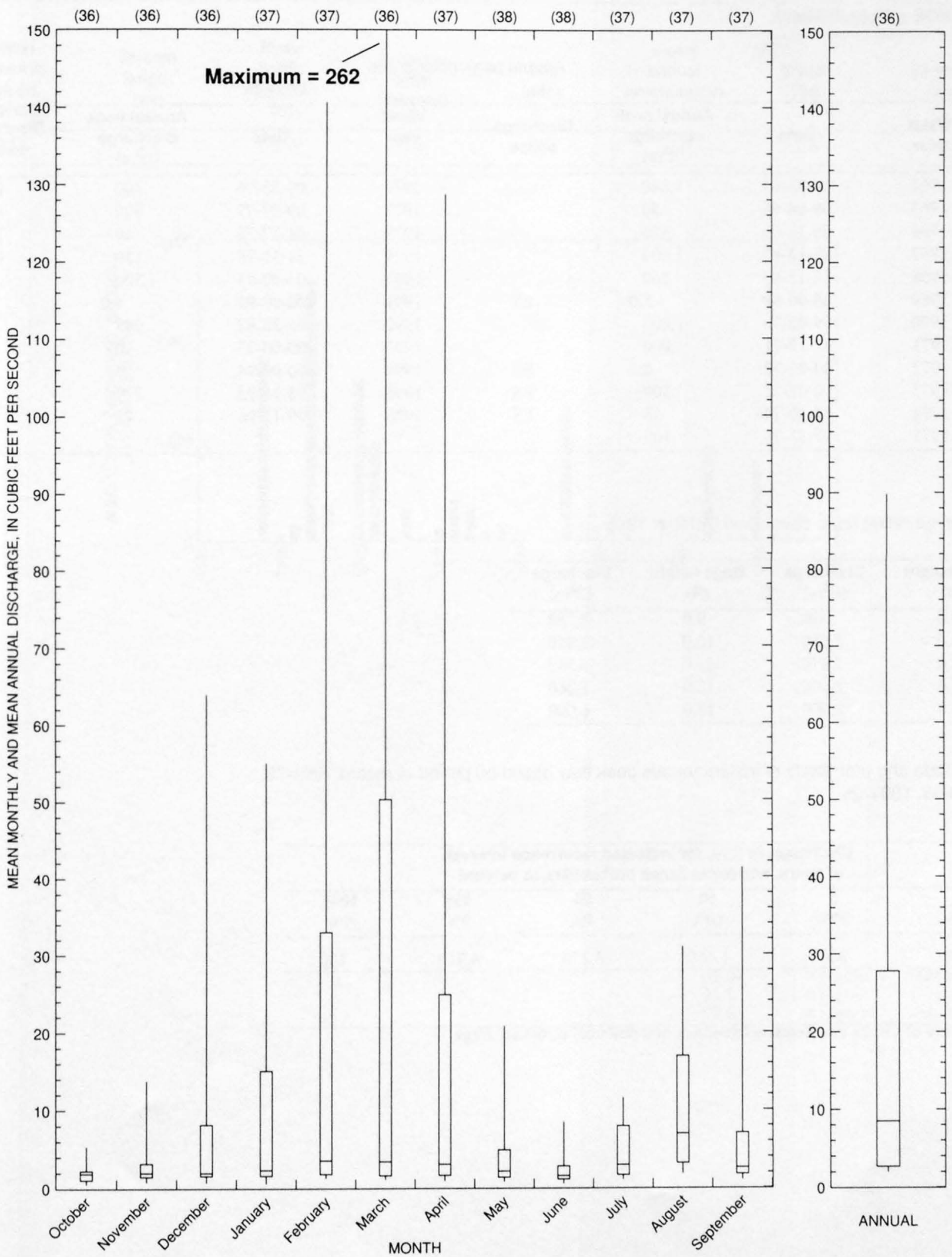
09515800 HARTMAN WASH NEAR WICKENBURG, AZ

LOCATION.--Lat $33^{\circ} 57^{\prime} 46^{\prime \prime}$, long $112^{\circ} 49^{\prime} 40^{\prime \prime}$, in SE $1 / 4$ sec.12, T.7 N., R.6 W., Maricopa County, Hydrologic Unit 15070103, at U.S. Highway 60,

$5.7 \mathrm{mi}$ west of Wickenburg.

DRAINAGE AREA.--5.57 $\mathrm{mi}^{2}$.

Annual peak discharges

\begin{tabular}{|c|c|c|c|c|c|c|c|}
\hline $\begin{array}{l}\text { Water } \\
\text { year }\end{array}$ & Date & $\begin{array}{c}\text { Annual peak } \\
\text { discharge } \\
\left(\mathrm{ft}^{3} / \mathrm{s}\right)\end{array}$ & $\begin{array}{l}\text { Discharge } \\
\text { codes }\end{array}$ & $\begin{array}{l}\text { Water } \\
\text { year }\end{array}$ & Date & $\begin{array}{c}\text { Annual peak } \\
\text { discharge } \\
\left(\mathrm{ft}^{3} / \mathrm{s}\right)\end{array}$ & $\begin{array}{c}\text { Discharge } \\
\text { codes }\end{array}$ \\
\hline 1964 & $08-23-64$ & 1,840 & & 1976 & $09-25-76$ & 400 & ES \\
\hline 1965 & $09-04-65$ & 50 & & 1977 & $09-27-77$ & 320 & ES \\
\hline 1966 & $09-13-66$ & 150 & & 1978 & $01-17-78$ & 60 & ES \\
\hline 1967 & $08-14-67$ & 2,600 & & 1979 & $11-11-78$ & 150 & ES \\
\hline 1968 & $12-19-67$ & 360 & & 1983 & $03-00-83$ & 1,300 & \\
\hline 1969 & $08-00-69$ & 5.0 & ES & 1991 & $00-00-91$ & 4.0 & \\
\hline 1970 & $09-05-70$ & 1,600 & & 1992 & $05-28-92$ & 943 & \\
\hline 1971 & $08-19-71$ & 1,000 & & 1993 & $00-00-93$ & 0 & \\
\hline 1972 & $09-03-72$ & 0.5 & ES & 1994 & $00-00-94$ & 0 & \\
\hline 1973 & $10-07-72$ & 300 & ES & 1995 & $08-14-95$ & 779 & \\
\hline 1974 & $07-30-74$ & 35 & ES & 1996 & $09-11-96$ & 45 & \\
\hline 1975 & $07-13-75$ & 100 & & & & & \\
\hline
\end{tabular}

Discharge rating table developed October 1995

\begin{tabular}{crcc}
\hline $\begin{array}{c}\text { Gage height } \\
(\mathbf{f t})\end{array}$ & $\begin{array}{c}\text { Discharge } \\
\left(\mathrm{ft}^{\mathbf{3}} / \mathbf{s}\right)\end{array}$ & $\begin{array}{c}\text { Gage height } \\
(\mathbf{f t})\end{array}$ & $\begin{array}{c}\text { Discharge } \\
\left(\mathrm{ft}^{\mathbf{3}} / \mathbf{s}\right)\end{array}$ \\
\hline 4.0 & 599 & 9.0 & 3,190 \\
5.0 & 1,050 & 10.0 & 3,850 \\
6.0 & 1,510 & 11.0 & 4,540 \\
7.0 & 2,040 & 12.0 & 5,260 \\
8.0 & 2,600 & 13.0 & 6,000 \\
\hline
\end{tabular}

Magnitude and probability of instantaneous peak flow based on period of record 1964-79, 1983, 1991-96

\begin{tabular}{|c|c|c|c|c|c|}
\hline \multicolumn{6}{|c|}{$\begin{array}{l}\text { Discharge, in } \mathrm{ft}^{3} / \mathrm{s} \text {, for indicated recurrence interval } \\
\text { in years, and exceedance probablility, in percent }\end{array}$} \\
\hline $\begin{array}{c}2 \\
50 \%\end{array}$ & $\begin{array}{c}5 \\
20 \%\end{array}$ & $\begin{array}{c}10 \\
10 \%\end{array}$ & $\begin{array}{l}25 \\
4 \%\end{array}$ & $\begin{array}{l}50 t \\
2 \%\end{array}$ & $\begin{array}{c}100 t \\
1 \%\end{array}$ \\
\hline 239 & 869 & 1,660 & 3,230 & 4,910 & 7,100 \\
\hline $\begin{array}{l}\text { Weighted skew } \\
\text { Mean } \\
\text { Standard dev. }\end{array}$ & $\begin{array}{l}(\log s)= \\
(\log s)= \\
(\log s)=\end{array}$ & $\begin{array}{r}-0.20 \\
2.36 \\
0.69\end{array}$ & & & \\
\hline
\end{tabular}


09515800 HARTMAN WASH NEAR WICKENBURG, AZ--Continued

Basin characteristics

\begin{tabular}{cccccccc}
\hline $\begin{array}{c}\text { Main } \\
\text { channel } \\
\text { slope } \\
(\mathrm{ft} / \mathrm{mi})\end{array}$ & $\begin{array}{c}\text { Stream } \\
\text { length } \\
(\mathrm{mi})\end{array}$ & $\begin{array}{c}\text { Mean } \\
\text { basin } \\
\text { elevation } \\
\text { (ft) }\end{array}$ & $\begin{array}{c}\text { Forested } \\
\text { area } \\
\text { (percent) }\end{array}$ & $\begin{array}{c}\text { Soil } \\
\text { index }\end{array}$ & $\begin{array}{c}\text { Mean } \\
\text { annual } \\
\text { precipitation } \\
\text { (in) }\end{array}$ & $\begin{array}{c}\text { Rainfall intensity, 24-hour } \\
\text { (in) }\end{array}$ & $\begin{array}{c}\text { 50-year } \\
\text { (in) }\end{array}$ \\
\hline 71.6 & 5.4 & 2,690 & 0.0 & 3.0 & 11.0 & 1.6 \\
\hline
\end{tabular}

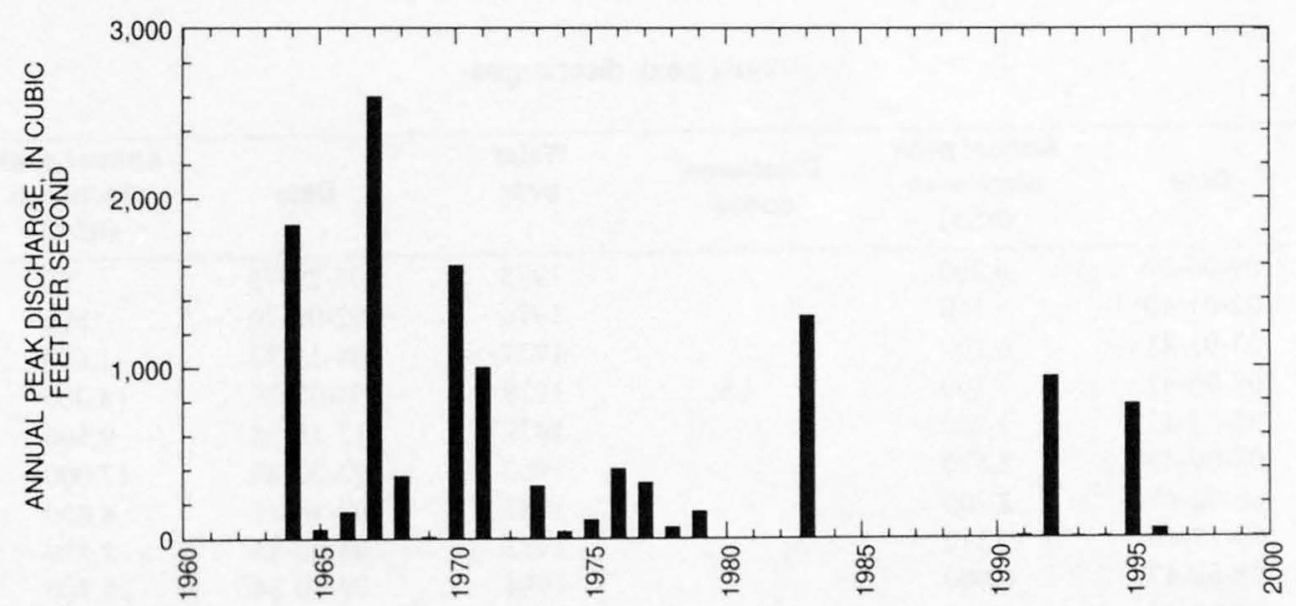




\section{HASSAYAMPA RIVER NEAR MORRISTOWN, AZ}

LOCATION.--Lat 33.53'06", long 112.39'41", in SW $1 /$ SE$^{1} / 4$ sec.3, T.6 N., R.4 W., Maricopa County, Hydrologic Unit 15070103, on left bank $600 \mathrm{ft}$ downstream from mouth of San Domingo Wash, 3.0 mi northwest of Morristown, and 7 mi southeast of Wickenburg.

DRAINAGE AREA.--796 $\mathrm{mi}^{2}$.

PERIOD OF RECORD.--October 1938 to June 1947 (continuous-record), water years 1954, 1956, 1964-81 (annual maximums only), October 1981 to September 1991 (discharge above $500 \mathrm{ft} 3 / \mathrm{s}$ only), October 1991 to current year (continuous-record).

REVISED RECORDS.--WDR AZ-89-1: Drainage area.

GAGE.--Water-stage recorder. Datum of gage is $1,831.16 \mathrm{ft}$ above sea level. Crest-stage gage at same site and datum water years 1954,1956 , and 1964-81.

REMARKS.--Records poor.

EXTREMES FOR PERIOD OF RECORD.--Maximum discharge, 47,500 ft $3 / \mathrm{s}$ Sept. 5, 1970, gage height, $19.0 \mathrm{ft}$, from high-water profile past gage and on basis of slope-area measurement of peak flow; no flow for most of each year.

Annual peak discharges

\begin{tabular}{|c|c|c|c|c|c|c|c|}
\hline $\begin{array}{l}\text { Water } \\
\text { year }\end{array}$ & Date & $\begin{array}{c}\text { Annual peak } \\
\text { discharge } \\
\left(\mathrm{ft}^{3} / \mathrm{s}\right)\end{array}$ & $\begin{array}{l}\text { Discharge } \\
\text { codes }\end{array}$ & $\begin{array}{l}\text { Water } \\
\text { year }\end{array}$ & Date & $\begin{array}{c}\text { Annual peak } \\
\text { discharge } \\
\left(\mathrm{ft}^{3} / \mathrm{s}\right)\end{array}$ & $\begin{array}{l}\text { Discharge } \\
\text { codes }\end{array}$ \\
\hline 1939 & 09-06-39 & 6,200 & & 1975 & $07-29-75$ & 50 & LT \\
\hline 1940 & $02-01-40$ & 160 & & 1976 & $02-09-76$ & 800 & \\
\hline 1941 & $03-02-41$ & 6,100 & & 1977 & $08-15-77$ & 1,600 & ES \\
\hline 1942 & $08-05-42$ & 100 & ES & 1978 & $03-02-78$ & 18,000 & \\
\hline 1943 & $08-03-43$ & 7,700 & & 1979 & $12-18-78$ & 9,600 & \\
\hline 1944 & $08-09-44$ & 3,520 & & 1980 & $02-20-80$ & 17,000 & \\
\hline 1945 & $08-02-45$ & 2,200 & & 1981 & $07-10-81$ & 4,800 & \\
\hline 1946 & $09-17-46$ & 2,310 & & 1983 & $03-03-83$ & 2,520 & \\
\hline 1947 & $08-08-47$ & 6,000 & & 1984 & $09-10-84$ & 26,700 & \\
\hline 1954 & $00-00-54$ & 0 & & 1985 & $12-28-84$ & 848 & \\
\hline 1956 & $00-00-56$ & 0 & & 1986 & $11-26-85$ & 2,740 & \\
\hline 1964 & $07-12-64$ & 4,000 & ES & 1987 & $11-18-86$ & 714 & \\
\hline 1965 & $09-02-65$ & 9,280 & & 1988 & $08-27-88$ & 6,820 & \\
\hline 1966 & $09-13-66$ & 3,210 & & 1989 & 01-04-89 & 1,210 & \\
\hline 1967 & $09-00-67$ & 1,150 & & 1990 & $09-05-90$ & 6,280 & \\
\hline 1968 & $12-19-67$ & 4,800 & & 1991 & $03-02-91$ & 13,700 & \\
\hline 1969 & $09-13-69$ & 650 & & 1992 & $02-13-92$ & 3,380 & \\
\hline 1970 & $09-05-70$ & ' 47,500 & & 1993 & 01-08-93 & 26,300 & \\
\hline 1971 & $08-18-71$ & 2,000 & & 1994 & $08-19-94$ & 25 & \\
\hline 1972 & $08-27-72$ & 700 & & 1995 & $02-15-95$ & 20,000 & \\
\hline 1973 & $10-07-72$ & 2,000 & & 1996 & $07-26-96$ & 1,970 & \\
\hline 1974 & $07-20-74$ & 650 & ES & & & & \\
\hline
\end{tabular}

${ }^{\top}$ Highest since 1916 .

Discharge rating table developed February 1995

\begin{tabular}{cccc}
\hline $\begin{array}{c}\text { Gage height } \\
(\mathbf{f t})\end{array}$ & $\begin{array}{c}\text { Discharge } \\
\left(\mathbf{f t}^{\mathbf{3}} \mathbf{s}\right)\end{array}$ & $\begin{array}{c}\text { Gage height } \\
(\mathbf{f t})\end{array}$ & $\begin{array}{c}\text { Discharge } \\
\left(\mathbf{f t}^{\mathbf{3}} / \mathbf{s}\right)\end{array}$ \\
\hline 9.0 & 1,180 & 15.0 & 20,960 \\
10.0 & 2,730 & 16.0 & 26,750 \\
11.0 & 4,980 & 17.0 & 33,290 \\
12.0 & 7,900 & 18.0 & 40,560 \\
13.0 & 11,540 & 19.0 & 48,580 \\
14.0 & 15,890 & 19.4 & 52,000 \\
\hline
\end{tabular}


09516500 HASSAYAMPA RIVER NEAR MORRISTOWN, AZ--Continued

Basin characteristics

\begin{tabular}{|c|c|c|c|c|c|c|c|}
\hline $\begin{array}{c}\text { Main } \\
\text { channel } \\
\text { slope } \\
(\mathrm{ft} / \mathrm{mi})\end{array}$ & $\begin{array}{c}\text { Stream } \\
\text { length } \\
\text { (mi) }\end{array}$ & $\begin{array}{c}\text { Mean } \\
\text { basin } \\
\text { elevation } \\
\text { (ft) }\end{array}$ & $\begin{array}{l}\text { Forested } \\
\text { area } \\
\text { (percent) }\end{array}$ & $\begin{array}{c}\text { Soil } \\
\text { index }\end{array}$ & $\begin{array}{c}\text { Mean } \\
\text { annual } \\
\text { precipitation } \\
\text { (in) }\end{array}$ & \multicolumn{2}{|c|}{ Rainfall intensity, 24-hour } \\
\hline 84.9 & 58.4 & 3,190 & 5.2 & 1.72 & 16.9 & 2.2 & 4.3 \\
\hline
\end{tabular}


09516500 HASSAYAMPA RIVER NEAR MORRISTOWN, AZ--Continued

MEAN MONTHLY AND ANNUAL DISCHARGES 1939-46, 1992.96

\begin{tabular}{|c|c|c|c|c|c|c|}
\hline MONTH & $\begin{array}{l}\text { MAXIMUM } \\
(\text { ET3/S) }\end{array}$ & $\begin{array}{l}\text { MINIMUM } \\
(\text { FT } 3 / \mathrm{S})\end{array}$ & $\begin{array}{c}\text { MEAN } \\
\text { (FT3/S) }\end{array}$ & $\begin{array}{l}\text { STAN - } \\
\text { DARD } \\
\text { DEVIA- } \\
\text { TION } \\
\text { (FT3/S) }\end{array}$ & $\begin{array}{l}\text { COEFFI- } \\
\text { CIENT OF } \\
\text { VARI- } \\
\text { ATION }\end{array}$ & $\begin{array}{c}\text { PERCENT } \\
\text { OF } \\
\text { ANNUAL } \\
\text { RUNOFF }\end{array}$ \\
\hline OCTOBER & 13 & 0.00 & 2.0 & 4.2 & 2.1 & 0.4 \\
\hline NOVEMBER & 1.9 & 0.00 & 0.70 & 0.59 & 0.84 & 0.2 \\
\hline DECEMBER & 28 & 0.00 & 5.3 & 9.5 & 1.8 & 1.2 \\
\hline JANUARY & 1,080 & 0.00 & 91 & 299 & 3.3 & 20.4 \\
\hline FEBRUARY & 1,290 & 0.00 & 163 & 370 & 2.3 & 36.7 \\
\hline MARCH & 445 & 0.00 & 107 & 160 & 1.5 & 24.1 \\
\hline APRIL & 424 & 0.00 & 52 & 115 & 2.2 & 11.7 \\
\hline MAY & 44 & 0.00 & 6.1 & 12 & 2.0 & 1.4 \\
\hline JUNE & 1.6 & 0.00 & 0.33 & 0.63 & 1.9 & 0.1 \\
\hline JULY & 10 & 0.00 & 1.5 & 3.2 & 2.1 & 0.3 \\
\hline AUGUST & 39 & 0.00 & 8.5 & 11 & 1.3 & 1.9 \\
\hline SEPTEMBER & 71 & 0.00 & 7.4 & 19 & 2.6 & 1.7 \\
\hline ANNUAL & 220 & 0.22 & 36 & 64 & 1.8 & 100 \\
\hline
\end{tabular}

MAGNITUDE AND PROBABILITY OF INSTANTANEOUS PEAK FLOW BASED ON PERIOD OF RECORD $1939 \cdot 47,1964 \cdot 81,1983.96$

DISCHARGE, IN FT3/S, FOR INDICATED RECURRENCE INTERVAL IN YEARS, AND EXCEEDANCE PROBABILITY, IN PERCENT

\begin{tabular}{|c|c|c|c|c|c|}
\hline 2 & 5 & 10 & 25 & 50 & 100 \\
\hline 508 & 208 & 108 & 48 & 28 & 18 \\
\hline 3,030 & 8,540 & 14,300 & 24,400 & 34,000 & 45,600 \\
\hline WEIGHTED & SKEW & DGS) $=$ & & & \\
\hline MEAN & & DGS) $=$ & & & \\
\hline STANDARD & DEV. & DGS) $=$ & & & \\
\hline
\end{tabular}

MAGNITUDE AND PROBABILITY OF ANNUAL LOW FLOW BASED ON PERIOD OF RECORD $1940.47,1993.96$

\begin{tabular}{|c|c|c|c|c|c|c|}
\hline \multirow{3}{*}{$\begin{array}{l}\text { PERIOD } \\
\text { ICON- } \\
\text { SECU- } \\
\text { TIVE }\end{array}$} & \multicolumn{6}{|c|}{$\begin{array}{l}\text { DISCHARGE, IN FT3/S, FOR INDICATED } \\
\text { RECURRENCE INTERVAL, IN YEARS, AND } \\
\text { NON-EXCEEDANCE PROBABILITY, IN PERCENT }\end{array}$} \\
\hline & $\cdots$ & $\cdots \cdots$ & $\ldots \ldots$ & . . . . . & ....... & $\cdots \cdots$ \\
\hline & 2 & 5 & $10 \#$ & $20 \#$ & $50 \#$ & $100 \#$ \\
\hline DAYS) & 508 & 208 & 108 & 58 & 28 & 18 \\
\hline \multicolumn{7}{|l|}{1} \\
\hline \multicolumn{7}{|l|}{3} \\
\hline \multicolumn{7}{|l|}{7} \\
\hline \multicolumn{7}{|l|}{14} \\
\hline \multicolumn{7}{|l|}{30} \\
\hline 60 & 0.00 & 0.00 & 0.00 & 0.00 & 0.00 & 0.00 \\
\hline 90 & 0.00 & 0.00 & 0.00 & 0.00 & 0.00 & 0.00 \\
\hline 120 & 0.19 & 0.00 & 0.00 & 0.00 & 0.00 & 0.00 \\
\hline 183 & 0.62 & 0.13 & 0.05 & 0.02 & 0.01 & 0.00 \\
\hline
\end{tabular}

MAGNITUDE AND PROBABILITY OF ANNUAL HIGH FLOW BASED ON PERIOD OF RECORD 1939-46, 1992.96

\begin{tabular}{|c|c|c|c|c|c|c|}
\hline \multirow{3}{*}{$\begin{array}{l}\text { PERIOD } \\
\text { ICON- } \\
\text { SECU- }\end{array}$} & & \multicolumn{5}{|c|}{ DISCHARGE, IN FT $3 / S$, FOR INDICATED } \\
\hline & & \multicolumn{3}{|c|}{ EXCEEDANCE PROBABILITY, } & \multicolumn{2}{|c|}{ IN PERCENT } \\
\hline & & & 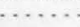 & 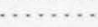 & $\ldots \ldots$ & $\cdots$ \\
\hline TIVE & 2 & 5 & $10 \#$ & $25 \#$ & $50 \#$ & $100 \#$ \\
\hline DAYS) & $50 \%$ & 208 & 108 & 48 & 28 & 18 \\
\hline 1 & 380 & 2,290 & 5,520 & 13,600 & 23,700 & 38,600 \\
\hline 3 & 211 & 1,220 & 2,850 & 6,700 & 11,300 & 17,800 \\
\hline 7 & 116 & 688 & 1,650 & 4,060 & 7,120 & 11,600 \\
\hline 15 & 72 & 434 & 1,090 & 2,840 & 5,220 & 8,980 \\
\hline 30 & 50 & 309 & 784 & 2,090 & 3,890 & 6,780 \\
\hline 60 & 35 & 220 & 577 & 1,620 & 3,170 & 5,790 \\
\hline 90 & 26 & 155 & 401 & 1,120 & 2,180 & 3.990 \\
\hline
\end{tabular}

DURATION TABLE OF DAILY MEAN FLOW FOR PERIOD OF RECORD 1939-46, 1992-96

DISCHARGE, IN FT3/S, WHICH WAS EQUALED OR EXCEEDED FOR INDICATED PERCENT OF TIME

\begin{tabular}{|c|c|c|c|c|c|c|c|c|c|c|c|c|c|c|c|c|}
\hline 18 & 58 & 108 & 158 & 208 & 308 & 408 & 508 & 608 & 708 & 808 & 908 & 958 & 988 & 998 & 99.58 & 99.98 \\
\hline 815 & 135 & 29 & 8.0 & 3.0 & 1.6 & 1.1 & 0.55 & 0.00 & 0.00 & 0.00 & 0.00 & 0.00 & 0.00 & 0.00 & 0.00 & 0.00 \\
\hline
\end{tabular}

\# Reliability of values in column is uncertain, and potential errors are large. 
ANNUAL MEAN DISCHARGE, IN CUBIC FEET PER SECOND

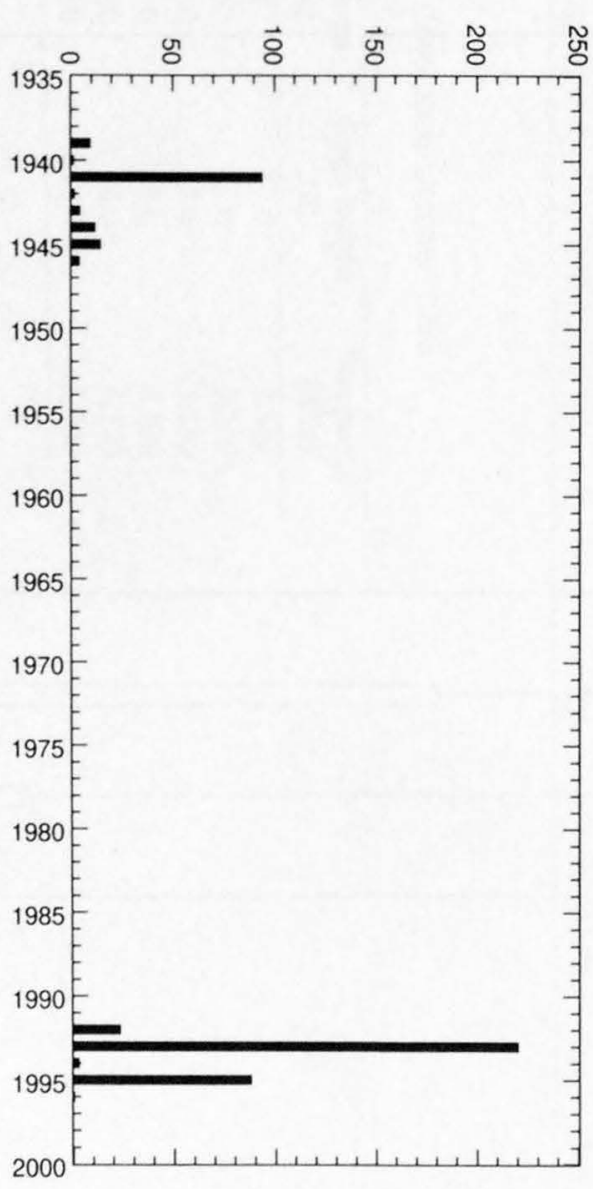

ANNUAL PEAK DISCHARGE, IN CUBIC FEET PER SECOND

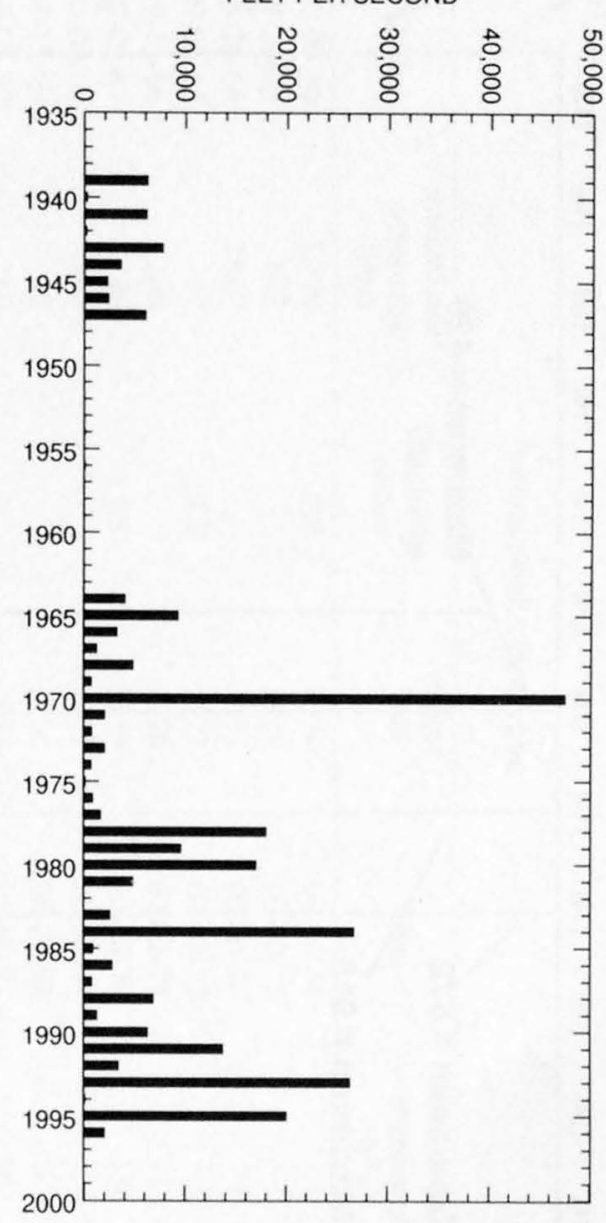


09516500 HASSAYAMPA RIVER NEAR MORRISTOWN, AZ--Continued

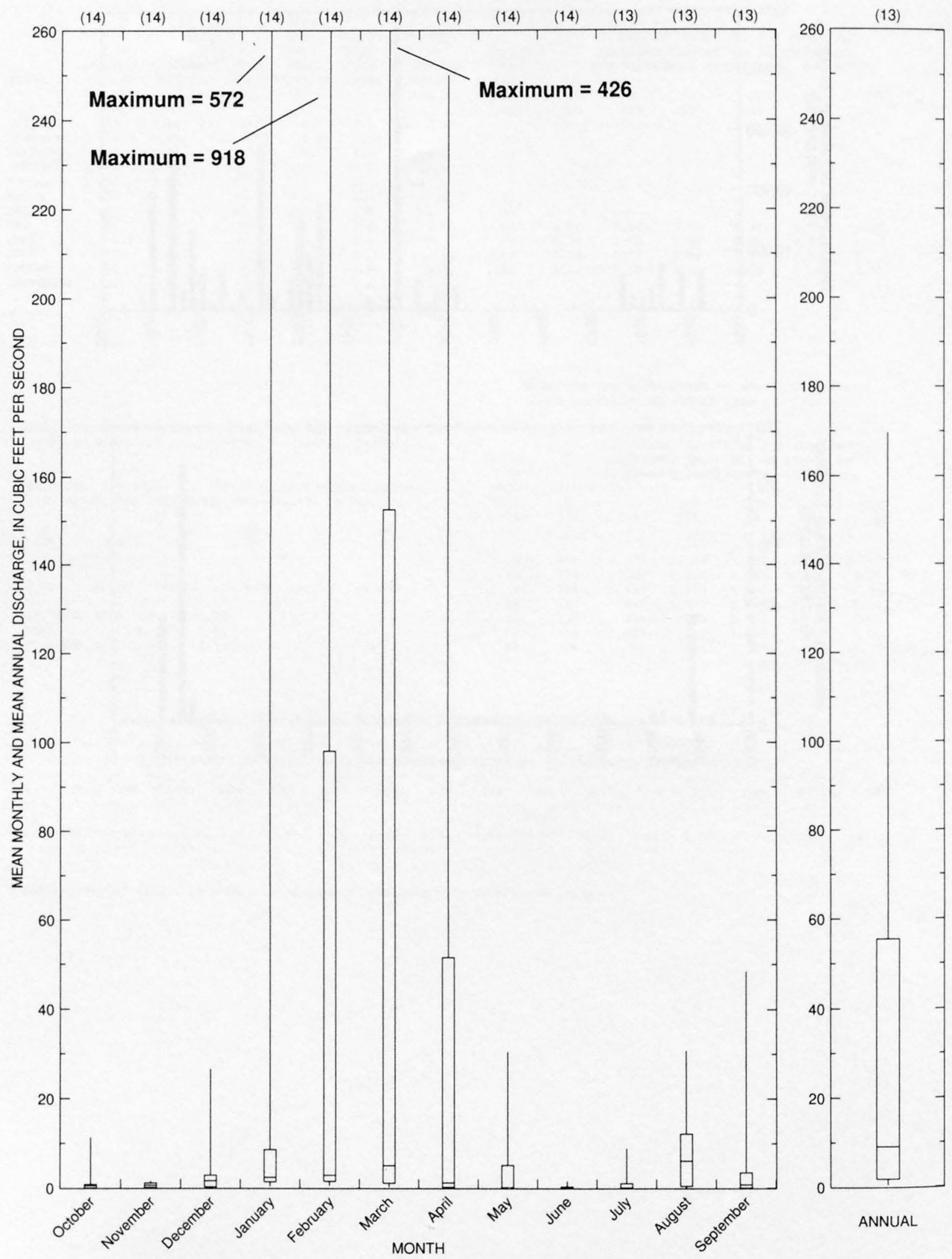


09516600 OX WASH NEAR MORRISTOWN, AZ

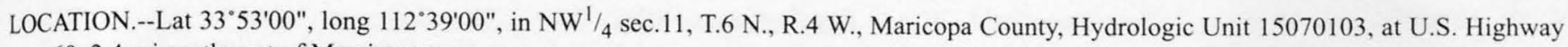
$60,2.4 \mathrm{mi}$ northwest of Morristown.

DRAINAGE AREA.--6.31 $\mathrm{mi}^{2}$.

Annual peak discharges

\begin{tabular}{|c|c|c|c|c|c|c|c|}
\hline $\begin{array}{l}\text { Water } \\
\text { year }\end{array}$ & Date & $\begin{array}{c}\text { Annual peak } \\
\text { discharge } \\
\left(\mathrm{ft}^{3} / \mathrm{s}\right)\end{array}$ & $\begin{array}{l}\text { Discharge } \\
\text { codes }\end{array}$ & $\begin{array}{l}\text { Water } \\
\text { year }\end{array}$ & Date & $\begin{array}{c}\text { Annual peak } \\
\text { discharge } \\
\left(\mathrm{ft}^{3} / \mathrm{s}\right)\end{array}$ & $\begin{array}{l}\text { Discharge } \\
\text { codes }\end{array}$ \\
\hline 1960 & $00-00-60$ & 1,770 & HP & 1974 & $00-00-74$ & 0 & \\
\hline 1963 & $08-26-63$ & 628 & & 1975 & $00-00-75$ & 0 & \\
\hline 1964 & $08-26-64$ & 2,900 & & 1976 & $00-00-76$ & 0 & \\
\hline 1965 & $09-02-65$ & 50 & ES & 1977 & $00-00-77$ & 0 & \\
\hline 1966 & $09-13-66$ & 1,300 & & 1978 & $02-22-78$ & 335 & \\
\hline 1967 & $09-00-67$ & 80 & ES & 1979 & $12-18-78$ & 150 & ES \\
\hline 1968 & $00-00-68$ & 550 & & 1991 & $00-00-91$ & 4.0 & \\
\hline 1969 & $01-15-69$ & 30 & & 1992 & $08-23-92$ & 783 & \\
\hline 1970 & $09-05-70$ & 160 & & 1993 & $01-08-93$ & 661 & \\
\hline 1971 & $08-20-71$ & 250 & & 1994 & $08-27-94$ & 302 & \\
\hline 1972 & $09-00-72$ & 450 & & 1995 & $08-14-95$ & 388 & \\
\hline 1973 & $10-00-72$ & 220 & & 1996 & $00-00-96$ & 369 & \\
\hline
\end{tabular}

Discharge rating table developed October 1996

\begin{tabular}{crcc}
\hline $\begin{array}{c}\text { Gage height } \\
(\mathbf{f t})\end{array}$ & $\begin{array}{c}\text { Discharge } \\
\left(\mathbf{f t}^{\mathbf{3}} / \mathbf{s}\right)\end{array}$ & $\begin{array}{c}\text { Gage height } \\
(\mathbf{f t})\end{array}$ & $\begin{array}{c}\text { Discharge } \\
\left(\mathbf{f t}^{\mathbf{3}} / \mathbf{s}\right)\end{array}$ \\
\hline 4.0 & 687 & 10.0 & 2,810 \\
5.0 & 963 & 11.0 & 3,260 \\
6.0 & 1,270 & 12.0 & 3,730 \\
7.0 & 1,610 & 13.0 & 4,220 \\
8.0 & 1,980 & 14.0 & 4,740 \\
9.0 & 2,380 & 14.5 & 5,000 \\
\hline
\end{tabular}

Magnitude and probability of instantaneous peak flow based on period of record 1960 , 1963-79, 1991-96

\begin{tabular}{|c|c|c|c|c|c|}
\hline \multicolumn{6}{|c|}{$\begin{array}{l}\text { Discharge, in } \mathrm{ft}^{3} / \mathrm{s} \text {, for indicated recurrence interval } \\
\text { in years, and exceedance probablility, in percent }\end{array}$} \\
\hline 2 & 5 & 10 & 25 & $50 \dagger$ & $100 t$ \\
\hline $50 \%$ & $20 \%$ & $10 \%$ & $4 \%$ & $2 \%$ & $1 \%$ \\
\hline 209 & 647 & 1,120 & 1,960 & 2,760 & 3,720 \\
\hline Weighted skew & $(\log s)=$ & -0.32 & & & \\
\hline Mean & $(\log s)=$ & 2.29 & & & \\
\hline Standard dev. & $(\log s)=$ & 0.61 & & & \\
\hline
\end{tabular}

fReliability of values in column is uncertain, and potential errors are large. 
09516600 OX WASH NEAR MORRISTOWN, AZ--Continued

Basin characteristics

Rainfall intensity, 24-hour

\begin{tabular}{|c|c|c|c|c|c|c|c|}
\hline \multirow[b]{2}{*}{$\begin{array}{c}\text { Main } \\
\text { channel } \\
\text { slope } \\
(\mathrm{ft} / \mathrm{mi})\end{array}$} & \multirow[b]{2}{*}{$\begin{array}{l}\text { Stream } \\
\text { length } \\
\text { (mi) }\end{array}$} & \multirow[b]{2}{*}{$\begin{array}{c}\text { Mean } \\
\text { basin } \\
\text { elevation } \\
(f t)\end{array}$} & \multirow[b]{2}{*}{$\begin{array}{l}\text { Forested } \\
\text { area } \\
\text { (percent) }\end{array}$} & \multirow[b]{2}{*}{$\begin{array}{c}\text { Soil } \\
\text { index }\end{array}$} & \multirow[b]{2}{*}{$\begin{array}{c}\text { Mean } \\
\text { annual } \\
\text { precipitation } \\
\text { (in) }\end{array}$} & \multirow[b]{2}{*}{$\begin{array}{l}\text { 2-year } \\
\text { (in) }\end{array}$} & \multirow[b]{2}{*}{$\begin{array}{l}\text { 50-year } \\
\text { (in) }\end{array}$} \\
\hline & & & & & & & \\
\hline 101 & 6.6 & 2,290 & 0.0 & 3.0 & 12.2 & 1.8 & 3.8 \\
\hline
\end{tabular}

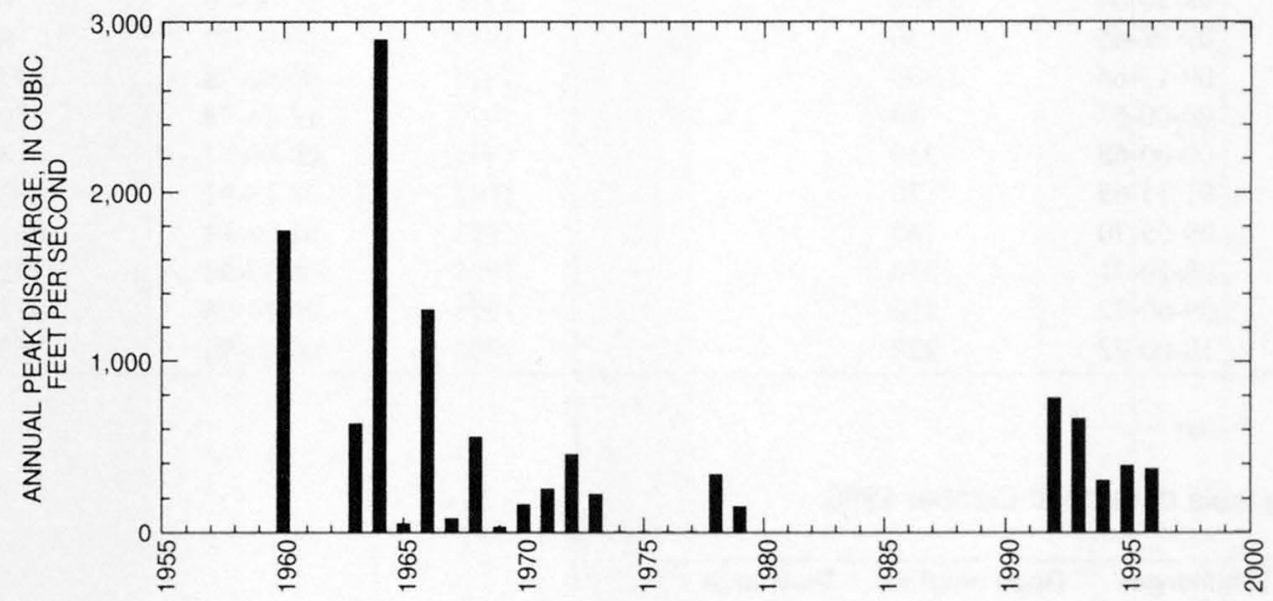




\section{JACK RABBIT WASH NEAR TONOPAH, AZ}

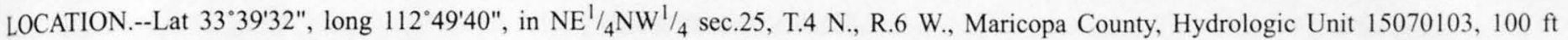
upstream from the Wickenburg-Hassayampa Road, 4.5 mi upstream from Star Wash, and 14 mi northeast of Tonopah. DRAINAGE AREA.--137 $\mathrm{mi}^{2}$.

Annual peak discharges

\begin{tabular}{|c|c|c|c|c|c|c|c|}
\hline $\begin{array}{l}\text { Water } \\
\text { year }\end{array}$ & Date & $\begin{array}{c}\text { Annual peak } \\
\text { discharge } \\
\left(\mathrm{ft}^{3} / \mathrm{s}\right)\end{array}$ & $\begin{array}{c}\text { Discharge } \\
\text { codes }\end{array}$ & $\begin{array}{l}\text { Water } \\
\text { year }\end{array}$ & Date & $\begin{array}{c}\text { Annual peak } \\
\text { discharge } \\
\left(\mathrm{ft}^{3} / \mathrm{s}\right)\end{array}$ & $\begin{array}{c}\text { Discharge } \\
\text { codes }\end{array}$ \\
\hline 1964 & $08-26-64$ & 2,070 & & 1976 & $09-25-76$ & 2,200 & \\
\hline 1965 & $04-04-65$ & 200 & ES & 1977 & $08-16-77$ & 80 & \\
\hline 1966 & $09-13-66$ & 200 & ES & 1978 & $10-06-77$ & 650 & \\
\hline 1967 & $09-03-67$ & 6,040 & & 1979 & $01-18-79$ & 1,510 & ES \\
\hline 1968 & $12-19-67$ & 105 & & 1983 & $09-00-83$ & 13,000 & \\
\hline 1969 & $09-13-69$ & 200 & ES & 1991 & $08-26-91$ & 2,250 & \\
\hline 1970 & $09-05-70$ & 5,000 & ES & 1992 & $08-23-92$ & 2,960 & \\
\hline 1971 & $08-17-71$ & 2,500 & ES & 1993 & $02-08-93$ & 2,670 & \\
\hline 1972 & $09-18-72$ & 100 & ES & 1994 & $10-06-93$ & 191 & \\
\hline 1973 & $10-07-72$ & 6,840 & & 1995 & $12-23-94$ & 612 & \\
\hline 1974 & $07-07-74$ & 75 & & 1996 & $09-10-96$ & 3,000 & \\
\hline 1975 & $03-11-75$ & 6 & ES & & & & \\
\hline
\end{tabular}

Discharge rating table developed October 1990

\begin{tabular}{crcc}
\hline $\begin{array}{c}\text { Gage height } \\
(\mathbf{f t})\end{array}$ & $\begin{array}{c}\text { Discharge } \\
\left(\mathbf{f t}^{3} / \mathbf{s}\right)\end{array}$ & $\begin{array}{c}\text { Gage height } \\
(\mathbf{f t})\end{array}$ & $\begin{array}{c}\text { Discharge } \\
\left(\mathrm{ft}^{\mathbf{3}} / \mathbf{s}\right)\end{array}$ \\
\hline 8.0 & 191 & 10.5 & 7,680 \\
8.5 & 677 & 11.0 & 11,100 \\
9.0 & 1,580 & 11.5 & 15,320 \\
9.5 & 3,000 & 12.0 & 20,410 \\
10.0 & 5,010 & 12.2 & 22,700 \\
\hline
\end{tabular}

Magnitude and probability of instantaneous peak flow based on period of record 1964-79, 1983, 1991-96

\begin{tabular}{|c|c|c|c|c|c|}
\hline \multicolumn{6}{|c|}{$\begin{array}{c}\text { Discharge, in } \mathrm{ft}^{3} / \mathrm{s} \text {, for indicated recurrence interval } \\
\text { in years, and exceedance probablility, in percent }\end{array}$} \\
\hline 2 & 5 & 10 & 25 & $50 \dagger$ & $100 t$ \\
\hline $50 \%$ & $20 \%$ & $10 \%$ & $4 \%$ & $2 \%$ & $1 \%$ \\
\hline 847 & 3,400 & 6,860 & 14,200 & 22,600 & 33,900 \\
\hline Weighted skew & $(\log s)=$ & -0.16 & & & \\
\hline Mean & $($ logs $)=$ & 2.91 & & & \\
\hline Standard dev. & $(\log s)=$ & 0.73 & & & \\
\hline
\end{tabular}

†Reliability of values in column is uncertain, and potential errors are large. 
09516800 JACK RABBIT WASH NEAR TONOPAH, AZ--Continued

Basin characteristics

\begin{tabular}{cccccccc}
\hline $\begin{array}{c}\text { Main } \\
\text { channel } \\
\text { slope } \\
(\mathrm{ft} / \mathrm{mi})\end{array}$ & $\begin{array}{c}\text { Stream } \\
\text { length } \\
(\mathrm{mi})\end{array}$ & $\begin{array}{c}\text { Mean } \\
\text { basin } \\
\text { elevation } \\
(\mathrm{ft})\end{array}$ & $\begin{array}{c}\text { Forested } \\
\text { area } \\
\text { (percent) }\end{array}$ & $\begin{array}{c}\text { Soil } \\
\text { index }\end{array}$ & $\begin{array}{c}\text { Mean } \\
\text { annual } \\
\text { precipitation } \\
\text { (in) }\end{array}$ & $\begin{array}{c}\text { Rainfall intensity, 24-hour } \\
\text { (in) }\end{array}$ & $\begin{array}{c}50 \text {-year } \\
\text { (in) }\end{array}$ \\
\hline 34.4 & 22.5 & 2,260 & 0.0 & 2.5 & 9.2 & 1.5 \\
\hline
\end{tabular}

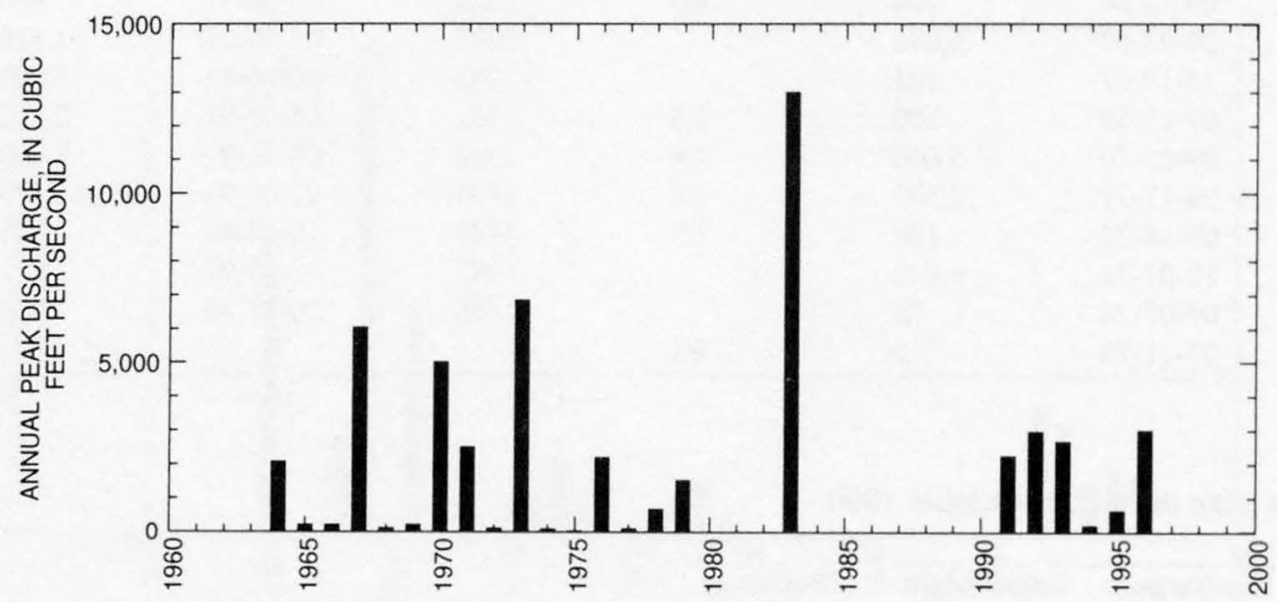


09517000 HASSAYAMPA RIVER NEAR ARLINGTON, AZ

LOCATION.--Lat 33'20'50", long 112 43'30", in NW1/4 sec.13, T.1 S., R.5 W., Maricopa County, Hydrologic Unit 15070103 , at former U.S. Highway $80,1.8 \mathrm{mi}$ upstream from mouth and $2.8 \mathrm{mi}$ northeast of Arlington.

DRAINAGE AREA.--1,471 $\mathrm{mi}^{2}$.

PERIOD OF RECORD.--Water years 1961-77 (annual maximums only), October 1977 to September 1990 (discharge above 500 ft3/s only), October 1990 to current year.

REVISED RECORDS.--WDR AZ-81-1: 1969(M). WDR AZ-89-1: Drainage area.

GAGE.--Water-stage recorder. Datum of gage is $824.75 \mathrm{ft}$ above sea level. Prior to Nov. 11, 1993 at datum $2.07 \mathrm{ft}$ higher.

REMARKS.--Records include irrigation return flow past station. Small diversions above station for irrigation and livestock.

EXTREMES FOR PERIOD OF RECORD.--Maximum discharge, $39,000 \mathrm{ft}^{3} / \mathrm{s} \mathrm{Sept}$. 5, 1970, gage height, $8.40 \mathrm{ft}$, result of slope-area measurement of peak flow; no natural flow for most of time each year.

Annual peak discharges

\begin{tabular}{|c|c|c|c|c|c|c|c|}
\hline $\begin{array}{l}\text { Water } \\
\text { year }\end{array}$ & Date & $\begin{array}{c}\text { Annual peak } \\
\text { discharge } \\
\left(\mathrm{ft}^{3} / \mathrm{s}\right)\end{array}$ & $\begin{array}{c}\text { Discharge } \\
\text { codes }\end{array}$ & $\begin{array}{l}\text { Water } \\
\text { year }\end{array}$ & Date & $\begin{array}{c}\text { Annual peak } \\
\text { discharge } \\
\left(\mathrm{ft}^{3} / \mathrm{s}\right)\end{array}$ & $\begin{array}{l}\text { Discharge } \\
\text { codes }\end{array}$ \\
\hline 1961 & $09-11-61$ & 50 & ES & 1978 & $03-02-78$ & 20,000 & \\
\hline 1962 & $09-06-62$ & 470 & & 1979 & $11-11-78$ & 3,300 & \\
\hline 1963 & $08-00-63$ & 1,930 & & 1980 & $02-20-80$ & 11,200 & \\
\hline 1964 & $08-14-64$ & 6,500 & & 1983 & $09-30-83$ & 3,300 & \\
\hline 1965 & $02-07-65$ & 3,000 & & 1984 & $09-02-84$ & 2,850 & \\
\hline 1966 & $12-10-65$ & 1,600 & & 1985 & $12-28-84$ & 372 & \\
\hline 1967 & $09-05-67$ & 5,270 & & 1986 & $11-26-85$ & 2,610 & \\
\hline 1968 & $12-20-67$ & 4,000 & & 1987 & $10-10-86$ & 404 & \\
\hline 1969 & $09-15-69$ & 500 & ES & 1988 & $11-01-87$ & 2,800 & \\
\hline 1970 & $09-05-70$ & ${ }^{\prime} 39,000$ & & 1989 & $08-11-89$ & 1,510 & \\
\hline 1971 & $08-11-71$ & 1,230 & & 1990 & $08-14-90$ & 2,120 & \\
\hline 1972 & $08-12-72$ & 225 & & 1991 & $03-02-91$ & 7,010 & \\
\hline 1973 & $10-07-72$ & 12,300 & & 1992 & $08-22-92$ & 6,110 & \\
\hline 1974 & $09-00-74$ & 250 & & 1993 & $01-08-93$ & 11,400 & \\
\hline 1975 & $00-00-75$ & 0 & & 1994 & $02-02-94$ & 129 & \\
\hline 1976 & $09-26-76$ & 13,000 & & 1995 & $02-15-95$ & 3,900 & \\
\hline 1977 & $10-24-76$ & 4,300 & & 1996 & $07-15-96$ & 1,730 & \\
\hline
\end{tabular}

${ }^{1}$ Highest since 1916 .

Discharge rating table developed December 1996

\begin{tabular}{crcc}
\hline $\begin{array}{c}\text { Gage height } \\
(\mathbf{f t})\end{array}$ & $\begin{array}{c}\text { Discharge } \\
\left(\mathbf{f t}^{3} / \mathbf{s}\right)\end{array}$ & $\begin{array}{c}\text { Gage height } \\
(\mathbf{f t})\end{array}$ & $\begin{array}{c}\text { Discharge } \\
\left(\mathrm{ft}^{\mathbf{3}} / \mathbf{s}\right)\end{array}$ \\
\hline 6.0 & 189 & 10.0 & 3,270 \\
7.0 & 580 & 11.0 & 4,720 \\
8.0 & 1,210 & 12.0 & 6,450 \\
9.0 & 2,110 & 12.8 & 8,060 \\
\hline
\end{tabular}


Magnitude and probability of instantaneous peak flow based on period of record 1961-80, 1983-96

\begin{tabular}{|c|c|c|c|c|c|}
\hline \multicolumn{6}{|c|}{$\begin{array}{l}\text { Discharge, in } \mathrm{ft}^{3} / \mathrm{s} \text {, for indicated recurrence interval } \\
\text { in years, and exceedance probablility, in percent }\end{array}$} \\
\hline 2 & 5 & 10 & 25 & $50 \dagger$ & $100 \dagger$ \\
\hline $50 \%$ & $20 \%$ & $10 \%$ & $4 \%$ & $2 \%$ & $1 \%$ \\
\hline 3,010 & 7,340 & 12,000 & 20,500 & 29,200 & 40,500 \\
\hline Weighted skew & $(\log s)=$ & 0.22 & & & \\
\hline Mean & $(\log s)=$ & 3.50 & & & \\
\hline Standard dev. & $(\log s)=$ & 0.45 & & & \\
\hline
\end{tabular}

f Reliability of values in column is uncertain, and potential errors are large.

\section{Basin characteristics}

\begin{tabular}{cccccccc}
\hline $\begin{array}{c}\text { Main } \\
\text { channel } \\
\text { slope } \\
(\mathrm{ft} / \mathrm{mi})\end{array}$ & $\begin{array}{c}\text { Stream } \\
\text { length } \\
(\mathrm{mi})\end{array}$ & $\begin{array}{c}\text { Mean } \\
\text { basin } \\
\text { elevation } \\
(\mathrm{ft})\end{array}$ & $\begin{array}{c}\text { Forested } \\
\text { area } \\
\text { (percent) }\end{array}$ & $\begin{array}{c}\text { Soil } \\
\text { index }\end{array}$ & $\begin{array}{c}\text { Mean } \\
\text { annual } \\
\text { precipitation } \\
\text { (in) }\end{array}$ & $\begin{array}{c}\text { Rainfall intensity, 24-hour } \\
\text { 2-year } \\
\text { (in) }\end{array}$ & $\begin{array}{c}50 \text {-year } \\
\text { (in) }\end{array}$ \\
\hline 39.9 & 93.6 & 3,010 & 2.7 & 2.0 & 15.9 & 1.9 \\
\hline
\end{tabular}

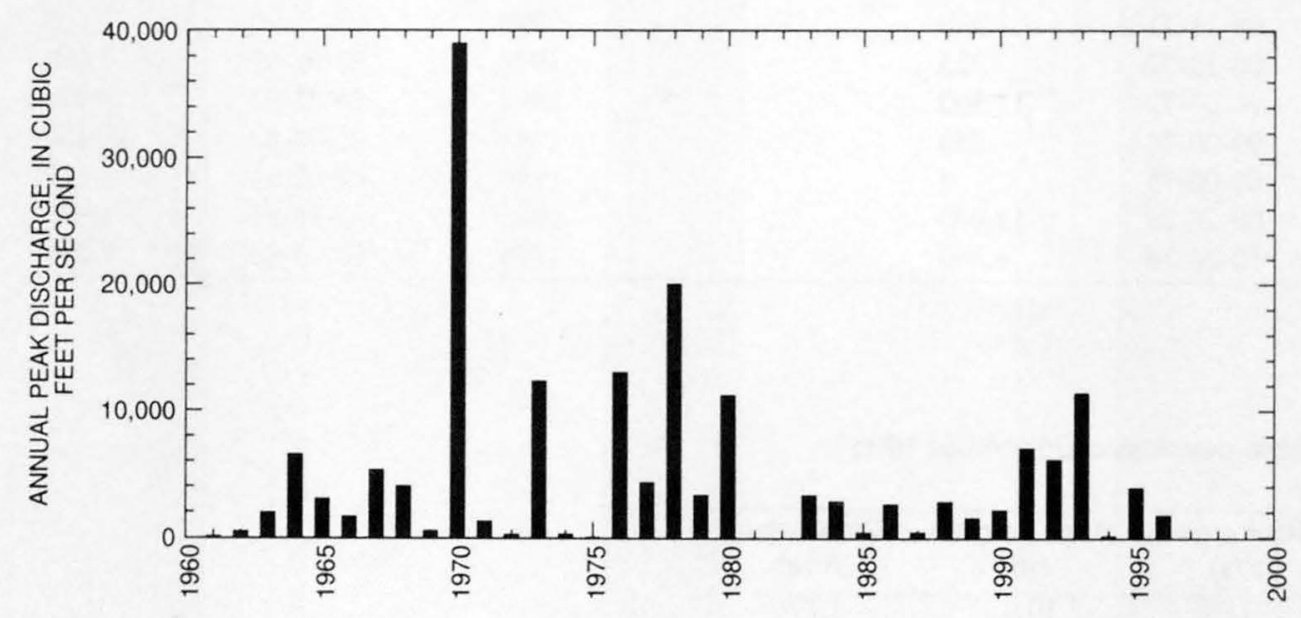


09517200 CENTENNIAL WASH TRIBUTARY NEAR WENDEN, AZ

LOCATION.--Lat $33^{\circ} 50^{\prime} 40^{\prime \prime}$, long $113^{\circ} 27^{\prime} 00^{\prime \prime}$, in SW $\frac{1}{4} \mathrm{SW}^{1} / 4$ sec.24, T.6 N., R. 12 W., Yuma County, Hydrologic Unit 15070104 , at U.S. Highway 60,5 mi northeast of Wenden.

DRAINAGE AREA.--2.79 $\mathrm{mi}^{2}$.

Annual peak discharges

\begin{tabular}{rcrrrrrr}
\hline $\begin{array}{c}\text { Water } \\
\text { year }\end{array}$ & Date & $\begin{array}{c}\text { Annual peak } \\
\text { discharge } \\
\left(\mathbf{f t}^{\mathbf{3}} \mathbf{/ s}\right)\end{array}$ & $\begin{array}{c}\text { Discharge } \\
\text { codes }\end{array}$ & $\begin{array}{c}\text { Water } \\
\text { year }\end{array}$ & Date & $\begin{array}{c}\text { Annual peak } \\
\text { discharge } \\
\left(\mathbf{f t}^{\mathbf{3}} / \mathbf{s}\right)\end{array}$ & $\begin{array}{c}\text { Discharge } \\
\text { codes }\end{array}$ \\
\hline 1963 & $08-16-63$ & 394 & & 1975 & $07-29-75$ & 275 \\
1964 & $08-02-64$ & 30 & ES & 1976 & $09-25-76$ & 220 \\
1965 & $02-07-65$ & 25 & ES & 1977 & $00-00-77$ & 0 \\
1966 & $12-10-65$ & 30 & ES & 1978 & $02-13-78$ & 260 \\
1967 & $09-02-67$ & 90 & & 1979 & $05-21-79$ & 90 \\
1968 & $00-00-68$ & 320 & & 1983 & $09-00-83$ & 435 \\
1969 & $09-16-69$ & 190 & & 1991 & $08-12-91$ & 169 \\
1970 & $09-05-70$ & 720 & & 1992 & $08-23-92$ & 63 \\
1971 & $08-20-71$ & 170 & & 1993 & $02-08-93$ & 15 \\
1972 & $10-00-71$ & 90 & & 1994 & $10-06-93$ & 20 \\
1973 & $02-11-73$ & 75 & & 1995 & $00-00-95$ & 0 \\
1974 & $07-00-74$ & 330 & ES & 1996 & $09-10-96$ & 450 \\
\hline
\end{tabular}

Discharge rating table developed October 1972

\begin{tabular}{cccc}
\hline $\begin{array}{c}\text { Gage height } \\
(\mathrm{ft})\end{array}$ & $\begin{array}{c}\text { Discharge } \\
\left(\mathrm{ft}^{3} / \mathbf{s}\right)\end{array}$ & $\begin{array}{c}\text { Gage height } \\
(\mathbf{f t})\end{array}$ & $\begin{array}{c}\text { Discharge } \\
\left(\mathrm{ft}^{3} / \mathbf{s}\right)\end{array}$ \\
\hline 2.8 & 163 & 4.2 & 464 \\
3.0 & 201 & 4.4 & 513 \\
3.2 & 241 & 4.6 & 563 \\
3.4 & 282 & 4.8 & 614 \\
3.6 & 325 & 5.0 & 667 \\
3.8 & 370 & 5.2 & 720 \\
4.0 & 416 & 5.5 & 800 \\
\hline
\end{tabular}

Magnitude and probability of instantaneous peak flow based on period of record 1963-79, 1983, 1991-96

\begin{tabular}{|c|c|c|c|c|c|}
\hline \multicolumn{6}{|c|}{$\begin{array}{l}\text { Discharge, in } \mathrm{ft}^{3} / \mathrm{s} \text {, for indicated recurrence interval } \\
\text { in years, and exceedance probablility, in percent }\end{array}$} \\
\hline 2 & 5 & 10 & 25 & $50 \dagger$ & $100 t$ \\
\hline $50 \%$ & $20 \%$ & $10 \%$ & $4 \%$ & $2 \%$ & $1 \%$ \\
\hline 135 & 303 & 451 & 676 & 868 & 1,080 \\
\hline Weighted skew & $(\log s)=$ & -0.29 & & & \\
\hline Mean & $(\log s)=$ & 2.11 & & & \\
\hline Standard dev. & $(\log s)=$ & 0.44 & & & \\
\hline
\end{tabular}

fReliability of values in column is uncertain, and potential errors are large. 
09517200 CENTENNIAL WASH TRIBUTARY NEAR WENDEN, AZ--Continued

Basin characteristics

\begin{tabular}{cccccccc}
\hline $\begin{array}{c}\text { Main } \\
\text { channel } \\
\text { slope } \\
\text { (ft/mi) }\end{array}$ & $\begin{array}{c}\text { Stream } \\
\text { length } \\
\text { (mi) }\end{array}$ & $\begin{array}{c}\text { Mean } \\
\text { basin } \\
\text { elevation } \\
\text { (ft) }\end{array}$ & $\begin{array}{c}\text { Forested } \\
\text { area } \\
\text { (percent) }\end{array}$ & $\begin{array}{c}\text { Soil } \\
\text { index }\end{array}$ & $\begin{array}{c}\text { Mean } \\
\text { annual } \\
\text { precipitation } \\
\text { (in) }\end{array}$ & $\begin{array}{c}\text { R-year } \\
\text { (in) }\end{array}$ & $\begin{array}{c}50 \text {-year } \\
\text { (in) }\end{array}$ \\
\hline 193 & 5.4 & 2,480 & 0.0 & 1.0 & 8.0 & 1.6
\end{tabular}

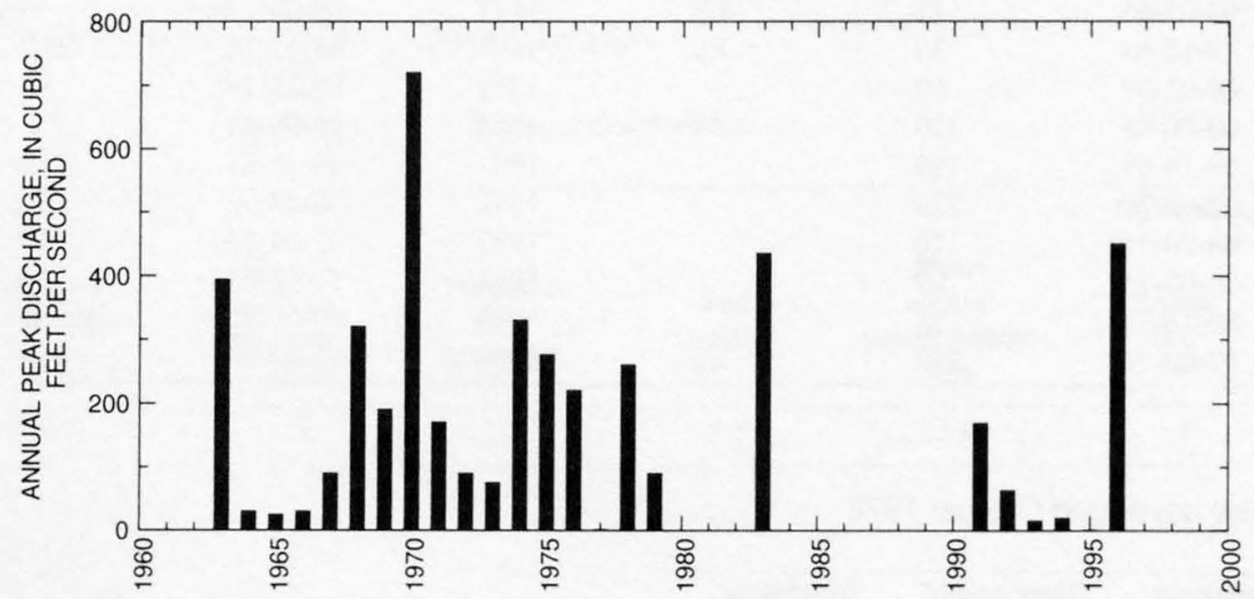


09517280 TIGER WASH NEAR AGUILA, AZ

LOCATION.--Lat 33 44'30", long $113^{\circ} 16^{\prime} 43^{\prime \prime}$, in SW $\frac{1}{4} \mathrm{SW}^{1} / 4$ sec.26, T.5 N., R. 10 W., Maricopa County, Hydrologic Unit 15070104,17 mi south of Aguila.

DRAINAGE AREA.--85.2 $\mathrm{mi}^{2}$.

Annual peak discharges

\begin{tabular}{|c|c|c|c|c|c|c|c|}
\hline $\begin{array}{l}\text { Water } \\
\text { year }\end{array}$ & Date & $\begin{array}{c}\text { Annual peak } \\
\text { discharge } \\
\left(\mathrm{ft}^{3} / \mathrm{s}\right)\end{array}$ & $\begin{array}{l}\text { Discharge } \\
\text { codes }\end{array}$ & $\begin{array}{l}\text { Water } \\
\text { year }\end{array}$ & Date & $\begin{array}{c}\text { Annual peak } \\
\text { discharge } \\
\left(\mathrm{ft}^{3} / \mathrm{s}\right)\end{array}$ & $\begin{array}{c}\text { Discharge } \\
\text { codes }\end{array}$ \\
\hline 1963 & $08-16-63$ & 910 & ES & 1975 & $07-30-75$ & 100 & ES \\
\hline 1964 & $10-19-63$ & 400 & ES & 1976 & $09-25-76$ & 3,000 & \\
\hline 1965 & $08-18-65$ & 1,680 & & 1977 & $08-16-77$ & 870 & \\
\hline 1966 & $09-13-66$ & 1,450 & & 1978 & $03-01-78$ & 1,400 & \\
\hline 1967 & $08-14-67$ & 620 & & 1979 & $12-18-78$ & 60 & ES \\
\hline 1968 & $12-19-67$ & 440 & & 1983 & $09-00-83$ & 3,170 & \\
\hline 1969 & $09-14-69$ & 441 & & 1991 & 09-04-91 & 1 & \\
\hline 1970 & $08-20-70$ & 4,550 & & 1992 & $03-03-92$ & 906 & \\
\hline 1971 & $08-20-71$ & 2,000 & & 1993 & $01-08-93$ & 1,040 & \\
\hline 1972 & $08-00-72$ & 2,770 & & 1994 & $07-18-94$ & 3,640 & \\
\hline 1973 & $10-06-72$ & 1,750 & & 1995 & $02-15-95$ & 185 & \\
\hline 1974 & $08-03-74$ & 45 & ES & 1996 & $00-00-96$ & 0 & \\
\hline
\end{tabular}

Discharge rating table developed October 1990

\begin{tabular}{cccc}
\hline $\begin{array}{c}\text { Gage height } \\
(\mathbf{f t})\end{array}$ & $\begin{array}{c}\text { Discharge } \\
\left(\mathrm{ft}^{3} / \mathbf{s}\right)\end{array}$ & $\begin{array}{c}\text { Gage height } \\
(\mathbf{f t})\end{array}$ & $\begin{array}{c}\text { Discharge } \\
\left(\mathrm{ft}^{3} / \mathbf{s}\right)\end{array}$ \\
\hline 6.0 & 133 & 8.5 & 2,070 \\
6.5 & 345 & 9.0 & 2,700 \\
7.0 & 650 & 9.5 & 3,410 \\
7.5 & 1,040 & 10.0 & 4,210 \\
8.0 & 1,520 & 10.2 & 4,550 \\
\hline
\end{tabular}

Magnitude and probability of instantaneous peak flow based on period of record 1963-79, $1983,1991-96$

\begin{tabular}{|c|c|c|c|c|c|}
\hline \multicolumn{6}{|c|}{$\begin{array}{l}\text { Discharge, in } \mathrm{ft}^{3} / \mathrm{s} \text {, for indicated recurrence interval } \\
\text { in years, and exceedance probablility, in percent }\end{array}$} \\
\hline 2 & 5 & 10 & 25 & $50 \dagger$ & $100 \dagger$ \\
\hline $50 \%$ & $20 \%$ & $10 \%$ & $4 \%$ & $2 \%$ & $1 \%$ \\
\hline 961 & 2,140 & 3,160 & 4,670 & 5,950 & 7,340 \\
\hline Weighted skew & $(\log s)=$ & -0.33 & & & \\
\hline Mean & $(\log s)=$ & 2.96 & & & \\
\hline Standard dev. & $(\log s)=$ & 0.43 & & & \\
\hline
\end{tabular}

fReliability of values in column is uncertain, and potential errors are large. 
09517280 TIGER WASH NEAR AGUILA, AZ--Continued

Basin characteristics

\begin{tabular}{cccccccc}
\hline $\begin{array}{c}\text { Main } \\
\text { channel } \\
\begin{array}{c}\text { slope } \\
(\mathrm{ft} / \mathrm{mi})\end{array}\end{array}$ & $\begin{array}{c}\text { Stream } \\
\text { length } \\
(\mathrm{mi})\end{array}$ & $\begin{array}{c}\text { Mean } \\
\text { basin } \\
\text { elevation } \\
(\mathrm{ft})\end{array}$ & $\begin{array}{c}\text { Forested } \\
\text { area } \\
\text { (percent) }\end{array}$ & $\begin{array}{c}\text { Soil } \\
\text { index }\end{array}$ & $\begin{array}{c}\text { Mean } \\
\text { annual } \\
\text { precipitation } \\
\text { (in) }\end{array}$ & $\begin{array}{c}\text { R-year } \\
\text { (in) }\end{array}$ & $\begin{array}{c}\text { 50-year } \\
\text { (in) }\end{array}$ \\
\hline 35.2 & 15.0 & 2,590 & 0.0 & 1.0 & 9.6 & 1.5 \\
\hline
\end{tabular}

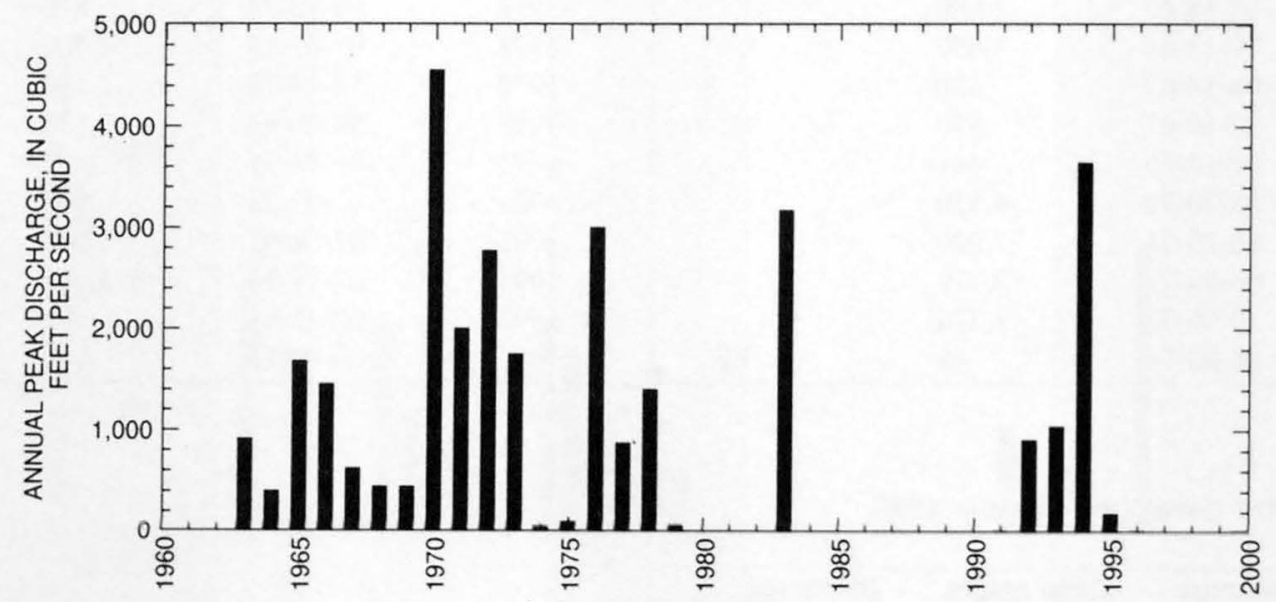


09517400 WINTERS WASH NEAR TONOPAH, AZ

LOCATION.--Lat 33⒉'22", long $112^{\circ} 55^{\prime} 05^{\prime \prime}$, in SW $1 / 4 \mathrm{NW}^{1} / 4 \mathrm{sec} .30$, T.2 N., R.6 W., Maricopa County, Hydrologic Unit $15070104,0.3$ mi downstream from Airline Road, and 1 mi east of Tonopah.

DRAINAGE AREA.---47.8 $\mathrm{mi}^{2}$.

Annual peak discharges

\begin{tabular}{|c|c|c|c|c|c|c|c|}
\hline $\begin{array}{l}\text { Water } \\
\text { year }\end{array}$ & Date & $\begin{array}{c}\text { Annual peak } \\
\text { discharge } \\
\left(\mathrm{ft}^{3} / \mathrm{s}\right)\end{array}$ & $\begin{array}{l}\text { Discharge } \\
\text { codes }\end{array}$ & $\begin{array}{l}\text { Water } \\
\text { year }\end{array}$ & Date & $\begin{array}{c}\text { Annual peak } \\
\text { discharge } \\
\left(\mathrm{ft}^{3} / \mathrm{s}\right)\end{array}$ & $\begin{array}{c}\text { Discharge } \\
\text { codes }\end{array}$ \\
\hline 1962 & $09-05-62$ & 776 & \multirow{9}{*}{ ES } & 1971 & $08-20-71$ & 1,000 & \multirow{9}{*}{ ES } \\
\hline 1963 & $09-03-63$ & 100 & & 1972 & $08-12-72$ & 795 & \\
\hline 1964 & $08-00-64$ & 850 & & 1973 & $10-06-72$ & 2,100 & \\
\hline 1965 & $02-07-65$ & 810 & & 1974 & $03-20-74$ & 900 & \\
\hline 1966 & $12-10-65$ & 390 & & 1975 & $10-28-74$ & 560 & \\
\hline 1967 & $09-03-67$ & 900 & & 1976 & $09-25-76$ & 3,640 & \\
\hline 1968 & $12-19-67$ & 1,350 & & 1977 & $08-16-77$ & 60 & \\
\hline 1969 & $11-15-68$ & 960 & & 1978 & $03-02-78$ & 1,800 & \\
\hline 1970 & $09-05-70$ & 480 & & 1979 & $12-18-78$ & 2,100 & \\
\hline
\end{tabular}

Magnitude and probability of instantaneous peak flow based on period of record 1962-79

\begin{tabular}{|c|c|c|c|c|c|}
\hline \multicolumn{6}{|c|}{$\begin{array}{l}\text { Discharge, in } \mathrm{ft}^{3} / \mathrm{s} \text {, for indicated recurrence interval } \\
\text { in years, and exceedance probablility, in percent }\end{array}$} \\
\hline 2 & 5 & 10 & 25 & $50 \dagger$ & $100 \dagger$ \\
\hline $50 \%$ & $20 \%$ & $10 \%$ & $4 \%$ & $2 \%$ & $1 \%$ \\
\hline 857 & 1,540 & 2,120 & 2,980 & 3,720 & 4,560 \\
\hline Weighted skew & $(\log s)=$ & 0.11 & & & \\
\hline Mean & $(\log s)=$ & 2.94 & & & \\
\hline Standard dev. & $(\log s)=$ & 0.30 & & & \\
\hline
\end{tabular}

FReliability of values in column is uncertain, and potential errors are large.

Basin characteristics

\begin{tabular}{cccccccc}
\hline $\begin{array}{c}\text { Main } \\
\text { channel } \\
\text { slope } \\
(\mathrm{ft} / \mathrm{mi})\end{array}$ & $\begin{array}{c}\text { Stream } \\
\text { length } \\
(\mathrm{mi})\end{array}$ & $\begin{array}{c}\text { Mean } \\
\text { basin } \\
\text { elevation } \\
(\mathrm{ft})\end{array}$ & $\begin{array}{c}\text { Forested } \\
\text { area } \\
\text { (percent) }\end{array}$ & $\begin{array}{c}\text { Soil } \\
\text { index }\end{array}$ & $\begin{array}{c}\text { Mean } \\
\text { annual } \\
\text { precipitation } \\
\text { (in) }\end{array}$ & $\begin{array}{c}\text { Rainfall intensity, 24-hour } \\
\text { (in) }\end{array}$ & $\begin{array}{c}50 \text {-year } \\
\text { (in) }\end{array}$ \\
\hline 83.7 & 18.0 & 1,630 & 0.0 & 1.0 & 9.1 & 1.5 \\
\hline
\end{tabular}

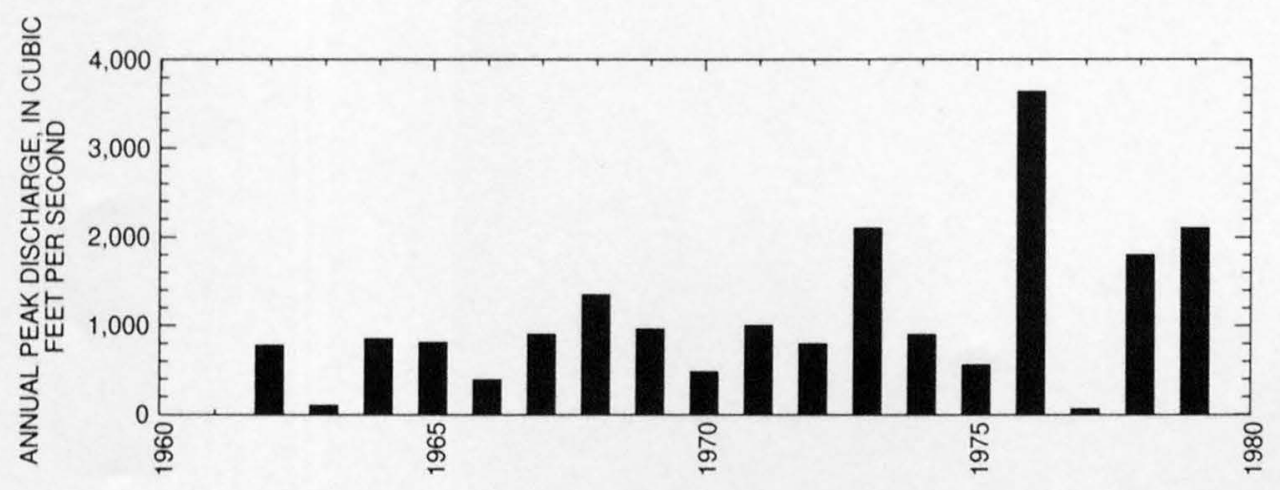




\section{CENTENNIAL WASH NEAR ARLINGTON, AZ}

LOCATION.--Lat 33 ${ }^{\circ} 6^{\prime} 12^{\prime \prime}$, long $112^{\circ} 47^{\prime} 50^{\prime \prime}$, in sec.7, T.2 S., R.5 W., Maricopa County, Hydrologic Unit 15070104, on upstream side of ford on former U.S. Highway 80, 3.0 mi upstream from Gillespie Dam and $4.4 \mathrm{mi}$ southwest of Arlington.

DRAINAGE AREA.--1,810 $\mathrm{mi}^{2}$, approximately.

PERIOD OF RECORD.--January 1961 to September 1979 (discontinued).

GAGE.--Water-stage recorder. Datum of gage is $773.22 \mathrm{ft}$ above sea level.

REMARKS.--Flow regulated by several small retention dams in upper end of basin. Records do not include irrigation return flow past station. AVERAGE DISCHARGE.-- 18 years, $3.73 \mathrm{ft}^{3} / \mathrm{s}, 2,700$ acre- $\mathrm{ft} / \mathrm{yr}$; median of yearly mean discharges, $1.9 \mathrm{ft}^{3} / \mathrm{s}, 1,400 \mathrm{acre}-\mathrm{ft} / \mathrm{yr}$.

EXTREMES FOR PERIOD OF RECORD.--Maximum dsicharge, 14,500 $\mathrm{ft}^{3} / \mathrm{s}$ July 23, 1961, gage height, $4.70 \mathrm{ft}$, from rating curve extended above $5,500 \mathrm{ft}^{3} / \mathrm{s}$; maximum gage height, $4.71 \mathrm{ft}$ Sept. 5, 1970; no flow for most of time each year.

Annual peak discharges

\begin{tabular}{|c|c|c|c|c|c|c|c|}
\hline $\begin{array}{l}\text { Water } \\
\text { year }\end{array}$ & Date & $\begin{array}{c}\text { Annual peak } \\
\text { discharge } \\
\left(\mathrm{ft}^{3} / \mathrm{s}\right)\end{array}$ & $\begin{array}{c}\text { Discharge } \\
\text { codes }\end{array}$ & $\begin{array}{l}\text { Water } \\
\text { year }\end{array}$ & Date & $\begin{array}{c}\text { Annual peak } \\
\text { discharge } \\
\left(\mathrm{ft}^{3} / \mathrm{s}\right)\end{array}$ & $\begin{array}{l}\text { Discharge } \\
\text { codes }\end{array}$ \\
\hline 1961 & $07-23-61$ & 14,500 & & 1971 & $08-20-71$ & 2,040 & \\
\hline 1962 & $09-06-62$ & 1,100 & & 1972 & $00-00-72$ & 0 & \\
\hline 1963 & $00-00-63$ & 0 & & 1973 & $10-07-72$ & 9,340 & \\
\hline 1964 & $07-31-64$ & 2,890 & & 1974 & $08-04-74$ & 105 & \\
\hline 1965 & $02-07-65$ & 1,040 & & 1975 & $10-28-74$ & 755 & \\
\hline 1966 & $09-13-66$ & 5,500 & & 1976 & $09-26-76$ & 7,800 & \\
\hline 1967 & $09-05-67$ & 1,040 & & 1977 & $00-00-77$ & 0 & \\
\hline 1968 & $12-19-67$ & 5,330 & & 1978 & $03-02-78$ & 10,900 & \\
\hline 1969 & $08-29-69$ & 990 & & 1979 & $01-17-79$ & 818 & \\
\hline 1970 & $09-05-70$ & 11,900 & & & & & \\
\hline
\end{tabular}


09517500 CENTENNIAL WASH NEAR ARLINGTON, AZ--Continued

MEAN MONTHLY AND ANNUAL DISCHARGES 1961.79

\begin{tabular}{|c|c|c|c|c|c|c|}
\hline MONTH & $\begin{array}{l}\text { MAXIMUM } \\
\text { (FT3/S) }\end{array}$ & $\begin{array}{l}\text { MINIMUM } \\
\text { (FT3/S) }\end{array}$ & $\begin{array}{c}\text { MEAN } \\
\text { (FT3/S) }\end{array}$ & $\begin{array}{l}\text { STAN- } \\
\text { DARD } \\
\text { DEVIA- } \\
\text { TION } \\
(\mathrm{FT} 3 / \mathrm{S})\end{array}$ & $\begin{array}{l}\text { COEFFI- } \\
\text { CIENT OF } \\
\text { VARI- } \\
\text { ATION }\end{array}$ & $\begin{array}{c}\text { PERCENT } \\
\text { OF } \\
\text { ANNUAL } \\
\text { RUNOFF }\end{array}$ \\
\hline OCTOBER & 121 & 0.00 & 6.6 & 28 & 4.2 & 13.5 \\
\hline NOVEMBER & 0.00 & 0.00 & 0.00 & 0.00 & $\cdots$ & 0.00 \\
\hline DECEMBER & 55 & 0.00 & 3.0 & 13 & 4.2 & 6.2 \\
\hline JANUARY & 6.6 & 0.00 & 0.69 & 2.1 & 3.0 & 1.4 \\
\hline FEBRUARY & 19 & 0.00 & 1.3 & 4.5 & 3.3 & 2.8 \\
\hline MARCH & 115 & 0.00 & 6.1 & 26 & 4.4 & 12.4 \\
\hline APRIL & 0.00 & 0.00 & 0.00 & 0.00 & $\cdots$ & 0.00 \\
\hline MAY & 0.00 & 0.00 & 0.00 & 0.00 & ... & 0.00 \\
\hline JUNE & 0.00 & 0.00 & 0.00 & 0.00 & $\cdots$ & 0.00 \\
\hline JULY & 122 & 0.00 & 8.5 & 28 & 3.3 & 17.5 \\
\hline AUGUST & 55 & 0.00 & 5.3 & 13 & 2.4 & 10.9 \\
\hline SEPTEMBER & 146 & 0.00 & 17 & 40 & 2.3 & 35.4 \\
\hline ANNUAL & 12.7 & 0.00 & 4.1 & 4.7 & 1.1 & 100 \\
\hline
\end{tabular}

MAGNITUDE AND PROBABILITY OF ANNUAL LOW FLOW BASED ON PERIOD OF RECORD 1962.79

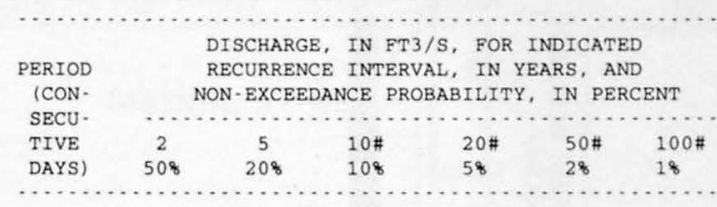

\begin{tabular}{|c|c|c|c|c|c|c|}
\hline \multicolumn{7}{|l|}{1} \\
\hline 3 & & & & & & \\
\hline 14 & & & & & & \\
\hline 30 & & & & & & \\
\hline 60 & 0.00 & 0.00 & 0.00 & 0.00 & 0.00 & 0.00 \\
\hline 90 & 0.00 & 0.00 & 0.00 & 0.00 & 0.00 & 0.00 \\
\hline 120 & 0.00 & 0.00 & 0.00 & 0.00 & 0.00 & 0.00 \\
\hline 183 & 0.00 & 0.00 & 0.00 & 0.00 & 0.00 & 0.00 \\
\hline
\end{tabular}

MAGNITUDE AND PROBABILITY OF ANNUAL HIGH FLOW BASED ON PERIOD OF RECORD 1962.79

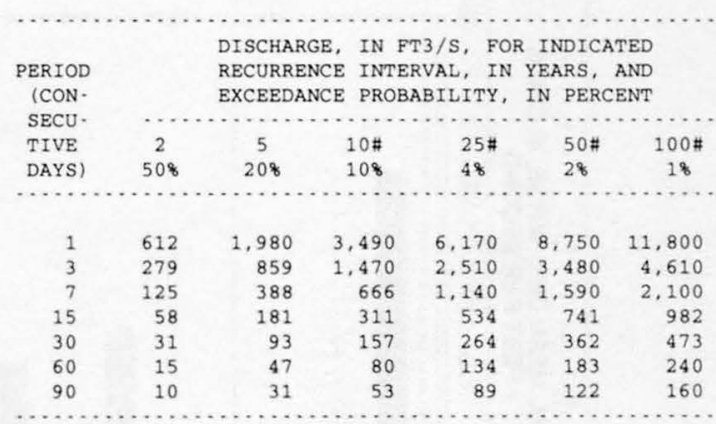

DURATION TABLE OF DAILY MEAN FLOW FOR PERIOD OF RECORD $1962 \cdot 79$ DISCHARGE, IN FT3/S, WHICH WAS EQUALED OR EXCEEDED FOR INDICATED PERCENT OF TIME

\begin{tabular}{|c|c|c|c|c|c|c|c|c|c|c|c|c|c|c|c|c|}
\hline 18 & 58 & 108 & 158 & 208 & 308 & 408 & 508 & 608 & 708 & 808 & 908 & 958 & 988 & 998 & 99.58 & 99.98 \\
\hline .0 & 0.00 & 0.00 & 0.00 & 0.00 & 0.00 & 0.00 & 0.00 & 0.00 & 0.00 & 0.00 & 0.00 & 0.00 & 0.00 & 0.00 & 0.00 & 0.00 \\
\hline
\end{tabular}


09517500 CENTENNIAL WASH NEAR ARLINGTON, AZ--Continued
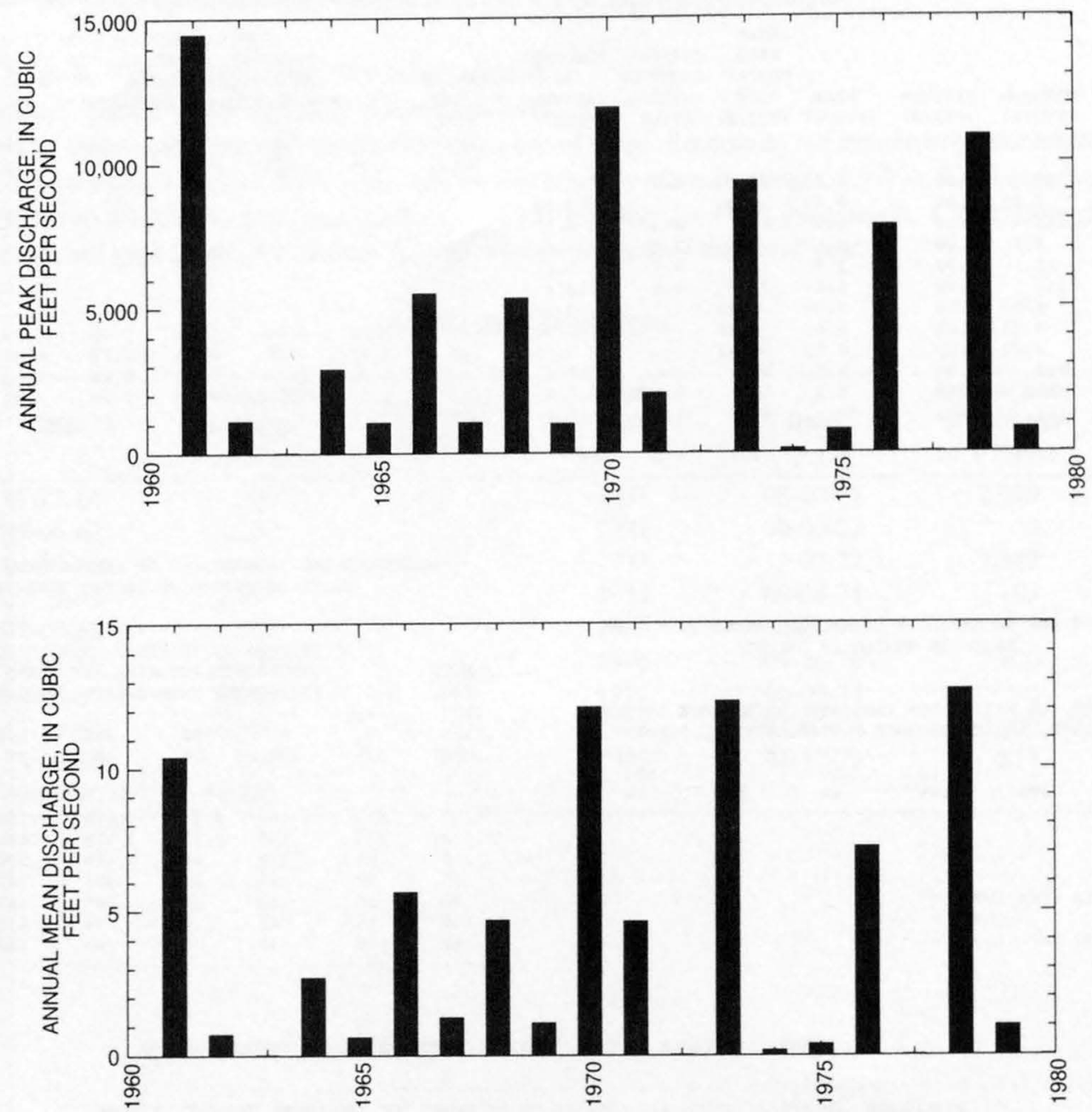
09517500 CENTENNIAL WASH NEAR ARLINGTON, AZ--Continued
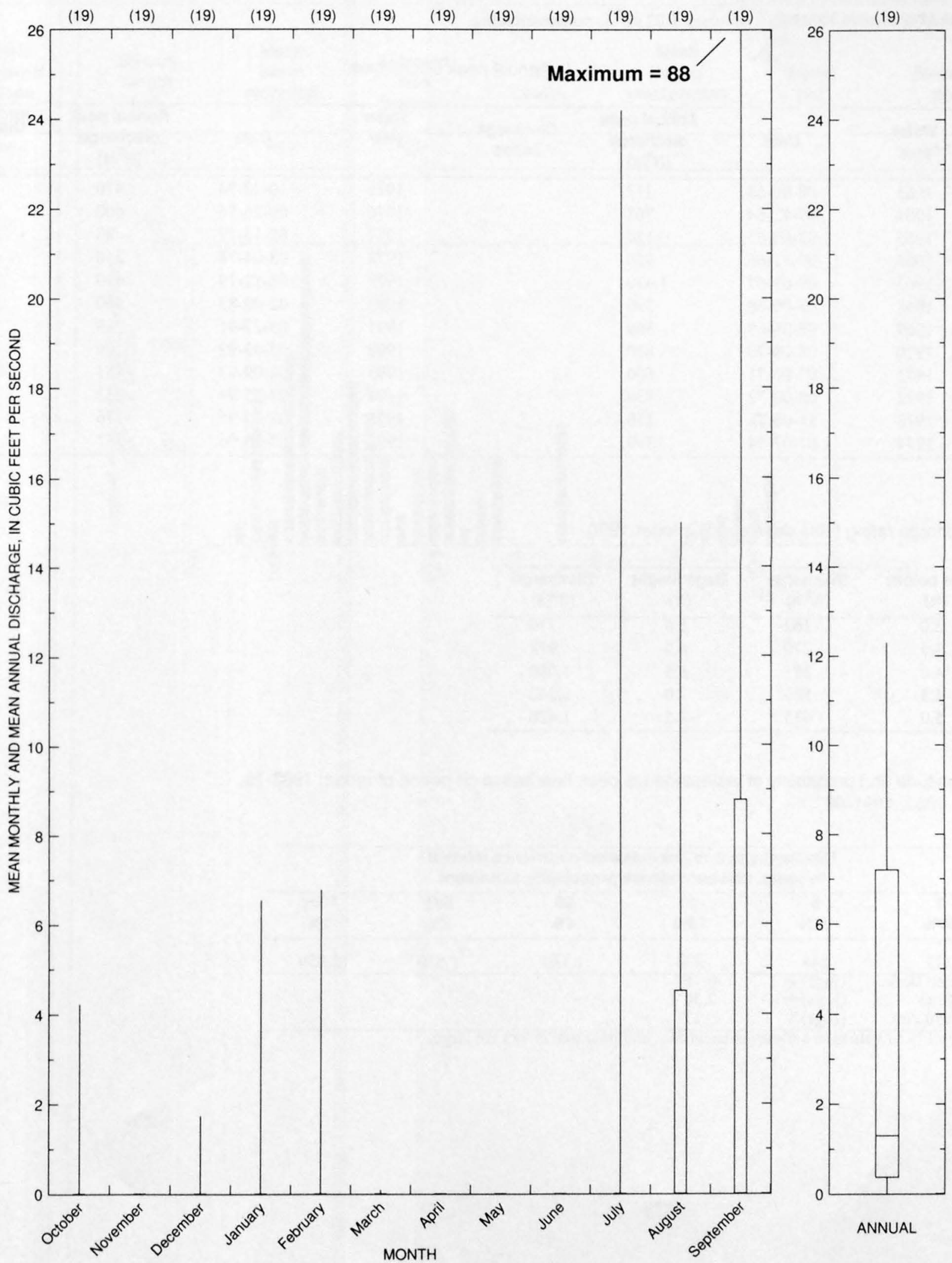
09519600 RAINBOW WASH TRIBUTARY NEAR BUCKEYE, AZ

LOCATION.--Lat 33 14'35", long 112 $38^{\prime} 15^{\prime \prime}$, in NE $1 / 4$ sec.23, T.2 S., R.4 W., Maricopa County, Hydrologic Unit 15070101 , at U.S. Highway 80 9.5 mi southwest of Buckeye.

DRAINAGE AREA.--3.45 $\mathrm{mi}^{2}$, of which $1.02 \mathrm{mi}^{2}$ is noncontributing.

Annual peak discharges

\begin{tabular}{|c|c|c|c|c|c|c|c|}
\hline $\begin{array}{l}\text { Water } \\
\text { year }\end{array}$ & Date & $\begin{array}{c}\text { Annual peak } \\
\text { discharge } \\
\left(\mathrm{ft}^{3} / \mathrm{s}\right)\end{array}$ & $\begin{array}{l}\text { Discharge } \\
\text { codes }\end{array}$ & $\begin{array}{l}\text { Water } \\
\text { year }\end{array}$ & Date & $\begin{array}{c}\text { Annual peak } \\
\text { discharge } \\
\left(\mathrm{ft}^{3} / \mathrm{s}\right)\end{array}$ & $\begin{array}{c}\text { Discharge } \\
\text { codes }\end{array}$ \\
\hline 1963 & $08-00-63$ & 112 & & 1975 & $10-28-74$ & 470 & \\
\hline 1964 & $09-13-64$ & 763 & & 1976 & $09-26-76$ & 660 & \\
\hline 1965 & $02-07-65$ & 120 & & 1977 & $08-16-77$ & 80 & ES \\
\hline 1966 & $09-13-66$ & 950 & & 1978 & $08-04-78$ & 240 & ES \\
\hline 1967 & $09-03-67$ & 1,430 & & 1979 & $08-12-79$ & 430 & ES \\
\hline 1968 & $08-00-68$ & 390 & & 1983 & $02-00-83$ & 600 & \\
\hline 1969 & $08-29-69$ & 560 & & 1991 & $03-27-91$ & 69 & \\
\hline 1970 & $08-08-70$ & 810 & & 1992 & $03-03-92$ & 106 & \\
\hline 1971 & $07-00-71$ & 660 & & 1993 & $02-09-93$ & 151 & \\
\hline 1972 & $08-00-72$ & 430 & & 1994 & $03-25-94$ & 233 & \\
\hline 1973 & $11-00-72$ & 150 & & 1995 & $08-20-95$ & 176 & \\
\hline 1974 & $07-07-74$ & 390 & & 1996 & $07-15-96$ & 351 & \\
\hline
\end{tabular}

Discharge rating table developed October 1990

\begin{tabular}{crcr}
\hline $\begin{array}{c}\text { Gage height } \\
(\mathbf{f t})\end{array}$ & $\begin{array}{c}\text { Discharge } \\
\left(\mathbf{f t}^{3} / \mathbf{s}\right)\end{array}$ & $\begin{array}{c}\text { Gage height } \\
(\mathbf{f t})\end{array}$ & $\begin{array}{c}\text { Discharge } \\
\left(\mathbf{f t}^{3} / \mathbf{s}\right)\end{array}$ \\
\hline 3.0 & 162 & 5.5 & 770 \\
3.5 & 270 & 6.0 & 918 \\
4.0 & 381 & 6.5 & 1,080 \\
4.5 & 505 & 7.0 & 1,240 \\
5.0 & 633 & 7.5 & 1,420 \\
\hline
\end{tabular}

Magnitude and probability of instantaneous peak flow based on period of record 1963-79, 1983, 1991-96

\begin{tabular}{cccccc}
\hline \multicolumn{7}{c}{$\begin{array}{l}\text { Discharge, in } \mathrm{ft}^{3} / \mathbf{s} \text {, for indicated recurrence interval } \\
\text { in years, and exceedance probablility, in percent }\end{array}$} \\
\hline $\mathbf{2}$ & $\mathbf{5}$ & $\mathbf{1 0}$ & $\mathbf{2 5}$ & $\mathbf{5 0 \dagger}$ & $\mathbf{1 0 0 \dagger}$ \\
$\mathbf{5 0 \%}$ & $\mathbf{2 0 \%}$ & $\mathbf{1 0 \%}$ & $\mathbf{4 \%}$ & $\mathbf{2 \%}$ & $\mathbf{1 \%}$ \\
\hline 323 & 648 & 920 & 1,330 & 1,670 & 2,050 \\
Weighted skew & $(\operatorname{logs})=$ & -0.16 & & & \\
Mean & $(\operatorname{logs})=$ & 2.50 & & & \\
Standard dev. & $(\operatorname{logs})=$ & 0.37 & & & \\
\hline
\end{tabular}

$\mp$ Reliability of values in column is uncertain, and potential errors are large. 
09519600 RAINBOW WASH TRIBUTARY NEAR BUCKEYE, AZ--Continued

Basin characteristics

Rainfall intensity, 24-hour

\begin{tabular}{cccccccc}
$\begin{array}{c}\text { Main } \\
\text { channel } \\
\text { slope } \\
(\mathrm{ft} / \mathrm{mi})\end{array}$ & $\begin{array}{c}\text { Stream } \\
\text { length } \\
\text { (mi) }\end{array}$ & $\begin{array}{c}\text { Mean } \\
\text { basin } \\
\text { elevation } \\
\text { (ft) }\end{array}$ & $\begin{array}{c}\text { Forested } \\
\text { area } \\
\text { (percent) }\end{array}$ & $\begin{array}{c}\text { Soil } \\
\text { index }\end{array}$ & $\begin{array}{c}\text { Mean } \\
\text { annual } \\
\text { precipitation } \\
\text { (in) }\end{array}$ & $\begin{array}{c}\text { 2-year } \\
\text { (in) }\end{array}$ & $\begin{array}{c}50 \text {-year } \\
\text { (in) }\end{array}$ \\
\hline 34.4 & 3.1 & 950 & 0.0 & 2.0 & 7.6 & 1.4 \\
\hline
\end{tabular}

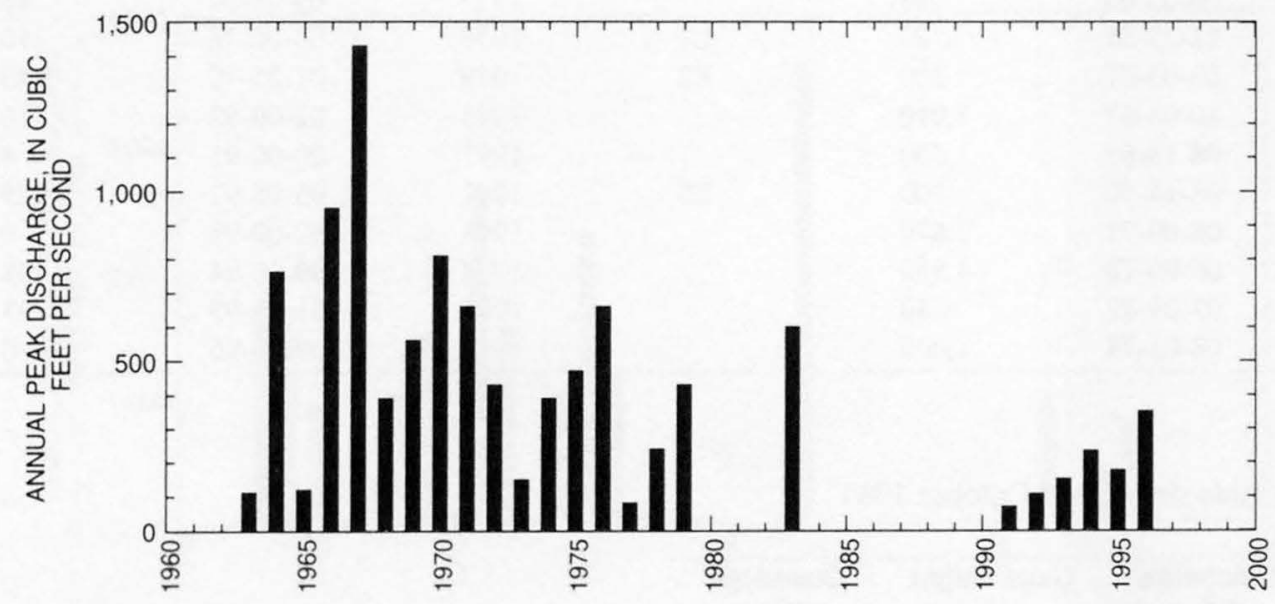


09519750 BENDER WASH NEAR GILA BEND, AZ

LOCATION.--Lat 32 54'25", long 112 33'05", in NW¹/4 sec.15, T.6 S., R.3 W., Maricopa County, Hydrologic Unit 15070101 , along side of Interstate Highway $8,10 \mathrm{mi}$ southeast of Gila Bend. Prior to Oct. 1, 1966, at site 0.65 mi downstream.

DRAINAGE AREA.--68.6 $\mathrm{mi}^{2}$.

Annual peak discharges

\begin{tabular}{|c|c|c|c|c|c|c|c|}
\hline $\begin{array}{l}\text { Water } \\
\text { year }\end{array}$ & Date & $\begin{array}{c}\text { Annual peak } \\
\text { discharge } \\
\left(\mathrm{ft}^{3} / \mathrm{s}\right)\end{array}$ & $\begin{array}{l}\text { Discharge } \\
\text { codes }\end{array}$ & $\begin{array}{c}\text { Water } \\
\text { year }\end{array}$ & Date & $\begin{array}{c}\text { Annual peak } \\
\text { discharge } \\
\left(\mathrm{ft}^{3} / \mathrm{s}\right)\end{array}$ & $\begin{array}{c}\text { Discharge } \\
\text { codes }\end{array}$ \\
\hline 1963 & $08-00-63$ & 1,740 & & 1975 & $08-00-75$ & 2,290 & \\
\hline 1964 & $00-00-64$ & 1,450 & & 1976 & $09-25-76$ & 1,220 & \\
\hline 1965 & $00-00-65$ & 943 & & 1977 & $09-11-77$ & 45 & ES \\
\hline 1966 & $12-22-65$ & 20 & LT & 1978 & $00-00-78$ & 110 & ES \\
\hline 1967 & $09-03-67$ & 300 & ES & 1979 & $01-25-79$ & 40 & ES \\
\hline 1968 & $10-03-67$ & 1,010 & & 1983 & $02-00-83$ & 3,610 & \\
\hline 1969 & $08-18-69$ & 1,500 & & 1991 & $00-00-91$ & 4.0 & \\
\hline 1970 & $08-14-70$ & 100 & ES & 1992 & $05-05-92$ & 975 & \\
\hline 1971 & $08-00-71$ & 2,670 & & 1993 & $00-00-93$ & 0 & \\
\hline 1972 & $06-00-72$ & 1,550 & & 1994 & $09-10-94$ & 408 & \\
\hline 1973 & $07-29-73$ & 40 & & 1995 & $01-05-95$ & 793 & \\
\hline 1974 & $08-03-74$ & 1,000 & & 1996 & $00-00-96$ & 0 & \\
\hline
\end{tabular}

Discharge rating table developed October 1991

\begin{tabular}{crcc}
\hline $\begin{array}{c}\text { Gage height } \\
(\mathbf{f t})\end{array}$ & $\begin{array}{c}\text { Discharge } \\
\left(\mathbf{f t}^{\mathbf{3}} \mathbf{s}\right)\end{array}$ & $\begin{array}{c}\text { Gage height } \\
(\mathbf{f t})\end{array}$ & $\begin{array}{c}\text { Discharge } \\
\left(\mathbf{f t}^{\mathbf{3}} / \mathbf{s}\right)\end{array}$ \\
\hline 4.0 & 207 & 7.0 & 2,420 \\
4.5 & 595 & 7.5 & 2,770 \\
5.0 & 993 & 8.0 & 3,110 \\
5.5 & 1,370 & 8.5 & 3,450 \\
6.0 & 1,730 & 9.0 & 3,790 \\
6.5 & 2,080 & 9.6 & 4,190 \\
\hline
\end{tabular}

Magnitude and probability of instantaneous peak flow based on period of record 1963-79, 1983, 1991-96

Discharge, in $\mathrm{ft}^{3} / \mathrm{s}$, for indicated recurrence interval in years, and exceedance probablility, in percent

\begin{tabular}{|c|c|c|c|c|c|}
\hline $\begin{array}{c}2 \\
50 \%\end{array}$ & $\begin{array}{c}5 \\
20 \%\end{array}$ & $\begin{array}{c}10 \\
10 \%\end{array}$ & $\begin{array}{l}25 \\
4 \%\end{array}$ & $\begin{array}{l}50 \dagger \\
2 \%\end{array}$ & $\begin{array}{c}100 t \\
1 \%\end{array}$ \\
\hline 867 & 1,580 & 2,160 & 2,990 & 3,690 & 4,450 \\
\hline $\begin{array}{l}\text { Weighted skew } \\
\text { Mean } \\
\text { Stand }\end{array}$ & $\begin{array}{l}(\log s)= \\
(\log s)= \\
(\operatorname{logs})=\end{array}$ & $\begin{array}{r}-0.07 \\
2.93 \\
0.31\end{array}$ & & & \\
\hline
\end{tabular}

$\mp$ Reliability of values in column is uncertain, and potential errors are large. 
09519750 BENDER WASH NEAR GILA BEND, AZ--Continued

Basin characteristics

\begin{tabular}{cccccccc}
\hline $\begin{array}{c}\text { Main } \\
\text { channel } \\
\text { slope } \\
(\mathrm{ft} / \mathrm{mi})\end{array}$ & $\begin{array}{c}\text { Stream } \\
\text { length } \\
(\mathrm{mi})\end{array}$ & $\begin{array}{c}\text { Mean } \\
\text { basin } \\
\text { elevation } \\
(\mathrm{ft})\end{array}$ & $\begin{array}{c}\text { Forested } \\
\text { area } \\
\text { (percent) }\end{array}$ & $\begin{array}{c}\text { Soil } \\
\text { index }\end{array}$ & $\begin{array}{c}\text { Mean } \\
\text { annual } \\
\text { precipitation } \\
\text { (in) }\end{array}$ & $\begin{array}{c}\text { Rainfall intensity, 24-hour } \\
\text { (in) }\end{array}$ & $\begin{array}{c}50 \text {-year } \\
\text { (in) }\end{array}$ \\
\hline 73.9 & 18.2 & 1,900 & 0.0 & 2.0 & 8.5 & 1.5 \\
\hline
\end{tabular}

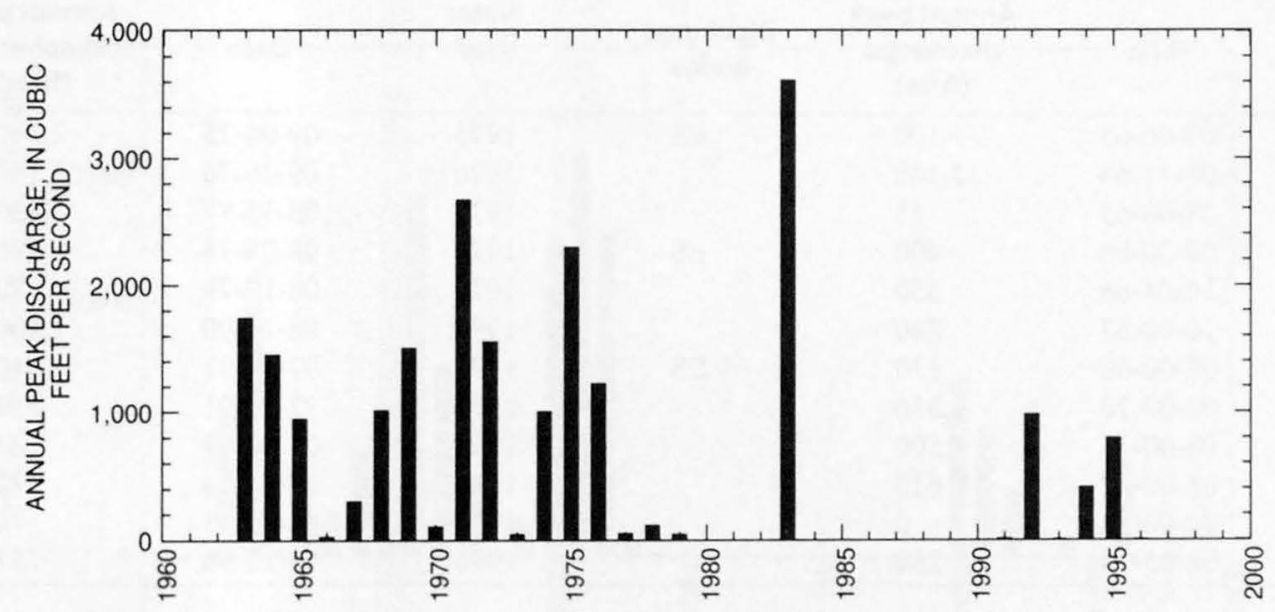




\section{SAUCEDA WASH NEAR GILA BEND, AZ}

LOCATION.--Lat 32 $52^{\prime} 14^{\prime \prime}$, long $112^{\circ} 45^{\prime} 30^{\prime \prime}$, in SE $1 /{ }_{4} \mathrm{SW}^{1} / 4 \mathrm{sec}^{2} 27$, T.6 S., R.5 W., Maricopa County, Hydrologic Unit 15070101 , on right bank wing wall of culvert on State Highway $85,5.3 \mathrm{mi}$ south of Gila Bend.

DRAINAGE AREA.--126 $\mathrm{mi}^{2}$, of which $20 \mathrm{mi}^{2}$ also contributes to an adjoining basin.

PERIOD OF RECORD.--Water years 1964-79 (annual maximums only), October 1989 to current year.

GAGE.--Water-stage recorder. Elevation of gage is $848 \mathrm{ft}$ above sea level, from topographic map. Prior to October 1979 , crest-stage gage at same site and datum.

REMARKS.--No estimated daily discharges. Records fair.

EXTREMES FOR PERIOD OF RECORD.--Maximum discharge, 3,150 $\mathrm{ft}^{3} / \mathrm{s}$, Sept. 26, 1976, gage height, $6.3 \mathrm{ft}$; no flow most of each year.

Annual peak discharges

\begin{tabular}{|c|c|c|c|c|c|c|c|}
\hline $\begin{array}{l}\text { Water } \\
\text { year }\end{array}$ & Date & $\begin{array}{c}\text { Annual peak } \\
\text { discharge } \\
\left(\mathrm{ft}^{3} / \mathrm{s}\right)\end{array}$ & $\begin{array}{l}\text { Discharge } \\
\text { codes }\end{array}$ & $\begin{array}{c}\text { Water } \\
\text { year }\end{array}$ & Date & $\begin{array}{c}\text { Annual peak } \\
\text { discharge } \\
\left(\mathrm{ft}^{3} / \mathrm{s}\right)\end{array}$ & $\begin{array}{c}\text { Discharge } \\
\text { codes }\end{array}$ \\
\hline 1963 & $09-00-63$ & 1,100 & ES & 1975 & $09-08-75$ & 2,500 & \\
\hline 1964 & $08-12-64$ & 2,160 & & 1976 & $09-26-76$ & 3,150 & \\
\hline 1965 & $08-00-65$ & 55 & & 1977 & $08-15-77$ & 2,550 & \\
\hline 1966 & $08-00-66$ & 400 & ES & 1978 & $08-08-78$ & 180 & \\
\hline 1967 & $10-04-66$ & 350 & & 1979 & $08-12-79$ & 20 & ES \\
\hline 1968 & $10-02-67$ & 840 & & 1990 & $08-14-90$ & 1,400 & \\
\hline 1969 & $08-00-69$ & 130 & ES & 1991 & $07-07-91$ & 1,010 & \\
\hline 1970 & $08-00-70$ & 2,850 & & 1992 & $12-20-91$ & 530 & \\
\hline 1971 & $08-00-71$ & 1,500 & & 1993 & $02-09-93$ & 27 & \\
\hline 1972 & $08-00-72$ & 630 & & 1994 & $09-11-94$ & 172 & \\
\hline 1973 & $00-00-73$ & 0 & & 1995 & $00-00-95$ & 0 & \\
\hline 1974 & $08-03-74$ & 250 & & 1996 & $08-15-96$ & 757 & \\
\hline
\end{tabular}

Discharge rating table developed March 1990

\begin{tabular}{crcc}
\hline $\begin{array}{c}\text { Gage height } \\
(\mathbf{f t})\end{array}$ & $\begin{array}{c}\text { Discharge } \\
\left(\mathrm{ft}^{\mathbf{3}} / \mathbf{s}\right)\end{array}$ & $\begin{array}{c}\text { Gage height } \\
(\mathbf{f t})\end{array}$ & $\begin{array}{c}\text { Discharge } \\
\left(\mathrm{ft}^{\mathbf{3}} / \mathbf{s}\right)\end{array}$ \\
\hline 2.0 & 184 & 7.0 & 3,940 \\
3.0 & 671 & 8.0 & 5,030 \\
4.0 & 1,320 & 9.0 & 6,200 \\
5.0 & 2,100 & 10.0 & 7,440 \\
6.0 & 2,960 & 10.2 & 7,700 \\
\hline
\end{tabular}

Magnitude and probability of instantaneous peak flow based on period of record 1963-79, 1990-96

\begin{tabular}{cccccc}
\hline \multicolumn{5}{c}{$\begin{array}{l}\text { Discharge, in } \mathrm{ft}^{3} / \mathbf{s} \text {, for indicated recurrence interval } \\
\text { in years, and exceedance probablility, in percent }\end{array}$} \\
\hline $\mathbf{2}$ & $\mathbf{5}$ & $\mathbf{1 0}$ & $\mathbf{2 5}$ & $\mathbf{5 0 \dagger}$ & $\mathbf{1 0 0 \dagger}$ \\
$\mathbf{5 0 \%}$ & $\mathbf{2 0 \%}$ & $\mathbf{1 0 \%}$ & $\mathbf{4} \%$ & $\mathbf{2 \%}$ & $\mathbf{1 \%}$ \\
\hline 591 & 1,580 & 2,520 & 4,030 & 5,360 & 6,850 \\
Weighted skew & $\begin{array}{l}(\operatorname{logs})= \\
\text { Mean }\end{array}$ & $\begin{array}{c}(\operatorname{logs})= \\
(\operatorname{logs})=\end{array}$ & 2.39 & & \\
Standard dev. & 0.54 & & \\
F Reliability of values in column is uncertain, and potential errors are large.
\end{tabular}

$\mp$ Reliability of values in column is uncertain, and potential errors are large. 
09519760 SAUCEDA WASH NEAR GILA BEND, AZ--Continued

Basin characteristics

\begin{tabular}{cccccccc}
\hline $\begin{array}{c}\text { Main } \\
\text { channel } \\
\text { slope } \\
(\mathrm{ft} / \mathrm{mi})\end{array}$ & $\begin{array}{c}\text { Stream } \\
\text { length } \\
(\mathrm{mi})\end{array}$ & $\begin{array}{c}\text { Mean } \\
\text { basin } \\
\text { elevation } \\
(\mathrm{ft})\end{array}$ & $\begin{array}{c}\text { Forested } \\
\text { area } \\
\text { (percent) }\end{array}$ & $\begin{array}{c}\text { Soil } \\
\text { index }\end{array}$ & $\begin{array}{c}\text { Mean } \\
\text { annual } \\
\text { precipitation } \\
\text { (in) }\end{array}$ & $\begin{array}{c}\text { Rainfall intensity, 24-hour } \\
\text { (in) }\end{array}$ & $\begin{array}{c}50 \text {-year } \\
\text { (in) }\end{array}$ \\
\hline 46.7 & 32.5 & 1,980 & 0.0 & 2.0 & 8.2 & 1.5 \\
\hline
\end{tabular}

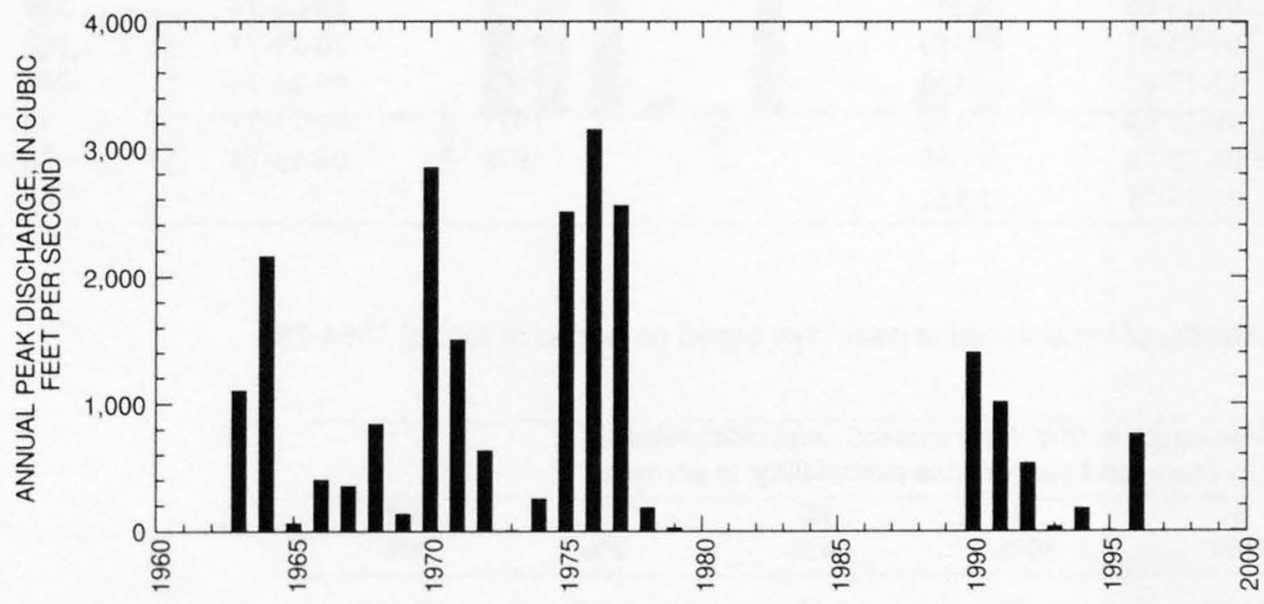


09519780 WINDMILL WASH NEAR GILA BEND, AZ

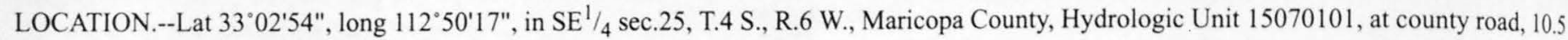
mi mi northwest of Gila Bend.

DRAINAGE AREA.--12.9 $\mathrm{mi}^{2}$.

Annual peak discharges

\begin{tabular}{|c|c|c|c|c|c|c|c|}
\hline $\begin{array}{l}\text { Water } \\
\text { year }\end{array}$ & Date & $\begin{array}{c}\text { Annual peak } \\
\text { discharge } \\
\left(\mathrm{ft}^{3} / \mathrm{s}\right)\end{array}$ & $\begin{array}{l}\text { Discharge } \\
\text { codes }\end{array}$ & $\begin{array}{l}\text { Water } \\
\text { year }\end{array}$ & Date & $\begin{array}{c}\text { Annual peak } \\
\text { discharge } \\
\left(\mathrm{ft}^{3} / \mathrm{s}\right)\end{array}$ & $\begin{array}{c}\text { Discharge } \\
\text { codes }\end{array}$ \\
\hline 1964 & $00-00-64$ & 0 & & 1972 & $06-06-72$ & 5.0 & ES \\
\hline 1965 & $02-09-65$ & 10 & LT & 1973 & $10-00-72$ & 60 & ES \\
\hline 1966 & $09-13-66$ & 967 & & 1974 & $08-03-74$ & 340 & ES \\
\hline 1967 & $09-03-67$ & 2,510 & & 1975 & $10-29-74$ & 1,900 & \\
\hline 1968 & $12-19-67$ & 4,430 & & 1976 & $09-26-76$ & 200 & ES \\
\hline 1969 & $09-15-69$ & 150 & & 1977 & $09-27-77$ & 10 & ES \\
\hline 1970 & $08-12-70$ & 65 & & 1978 & $04-13-78$ & 20 & ES \\
\hline 1971 & $08-15-71$ & 1,850 & & & & . & \\
\hline
\end{tabular}

Magnitude and probability of instantaneous peak flow based on period of record 1964-78

\begin{tabular}{|c|c|c|c|c|c|}
\hline \multicolumn{6}{|c|}{$\begin{array}{l}\text { Discharge, in } \mathrm{ft}^{3} / \mathrm{s} \text {, for indicated recurrence interval } \\
\text { in years, and exceedance probablility, in percent }\end{array}$} \\
\hline 2 & 5 & 10 & 25 & $50 t$ & $100 t$ \\
\hline $50 \%$ & $20 \%$ & $10 \%$ & $4 \%$ & $2 \%$ & $1 \%$ \\
\hline 155 & 1,160 & 3,120 & 8,550 & 16,000 & 27,600 \\
\hline Weighted skew & $(\log s)=$ & -0.28 & & & \\
\hline Mean & $(\log s)=$ & 2.14 & & & \\
\hline Standard dev. & $(\log s)=$ & 1.09 & & & \\
\hline
\end{tabular}

Basin characteristics

\begin{tabular}{cccccccc}
\hline $\begin{array}{c}\text { Main } \\
\text { channel } \\
\text { slope } \\
(\mathrm{ft} / \mathrm{mi})\end{array}$ & $\begin{array}{c}\text { Stream } \\
\text { length } \\
\text { (mi) }\end{array}$ & $\begin{array}{c}\text { Mean } \\
\text { basin } \\
\text { elevation } \\
\text { (ft) }\end{array}$ & $\begin{array}{c}\text { Forested } \\
\text { area } \\
\text { (percent) }\end{array}$ & $\begin{array}{c}\text { Soil } \\
\text { index }\end{array}$ & $\begin{array}{c}\text { Mean } \\
\text { annual } \\
\text { precipitation } \\
\text { (in) }\end{array}$ & $\begin{array}{c}\text { R-year } \\
\text { (in) }\end{array}$ & $\begin{array}{c}\text { 24-hour } \\
\text { (in) }\end{array}$ \\
\hline 64.4 & 9.1 & 1,050 & 0.0 & 1.0 & 6.1 & 1.3 \\
\hline
\end{tabular}


09519780 WINDMILL WASH NEAR GILA BEND, AZ--Continued

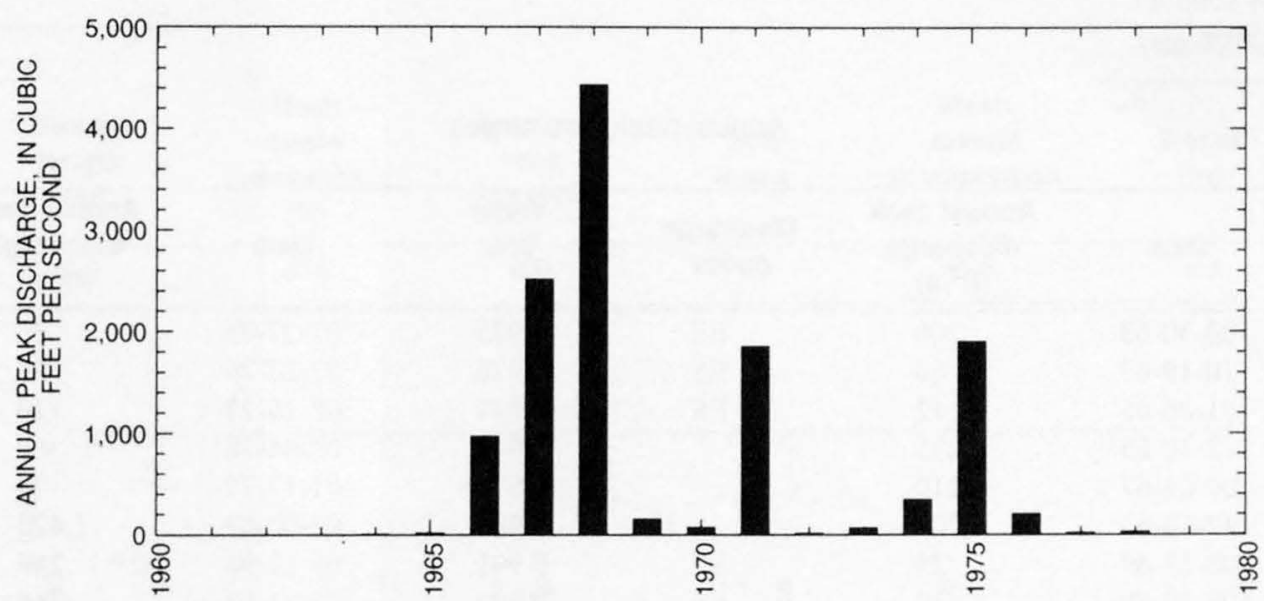




\section{MILITARY WASH NEAR SENTINEL, AZ}

LOCATION.--Lat $32^{\circ} 50^{\prime} 43^{\prime \prime}$, long $113^{\circ} 16^{\prime} 44^{\prime \prime}$, in SW $1 / 4$ sec.3, T.7 S., R.10 W., Maricopa County, Hydrologic Unit 15070201 , at U.S. Highway $80,4.1$ mi west of Sentinel.

DRAINAGE AREA.--8.70 $\mathrm{mi}^{2}$.

Annual peak discharges

\begin{tabular}{|c|c|c|c|c|c|c|c|}
\hline $\begin{array}{l}\text { Water } \\
\text { year }\end{array}$ & Date & $\begin{array}{c}\text { Annual peak } \\
\text { discharge } \\
\left(\mathrm{ft}^{3} / \mathrm{s}\right)\end{array}$ & $\begin{array}{l}\text { Discharge } \\
\text { codes }\end{array}$ & $\begin{array}{l}\text { Water } \\
\text { year }\end{array}$ & Date & $\begin{array}{c}\text { Annual peak } \\
\text { discharge } \\
\left(\mathrm{ft}^{3} / \mathrm{s}\right)\end{array}$ & $\begin{array}{c}\text { Discharge } \\
\text { codes }\end{array}$ \\
\hline 1963 & $08-30-63$ & 200 & ES & 1975 & $07-27-75$ & 30 & \\
\hline 1964 & $10-19-63$ & 60 & ES & 1976 & $07-27-76$ & 550 & ES \\
\hline 1965 & $01-06-65$ & 12 & ES & 1977 & $08-15-77$ & 120 & ES \\
\hline 1966 & $12-10-65$ & 235 & & 1978 & $08-04-78$ & 560 & \\
\hline 1967 & $09-03-67$ & 210 & & 1979 & $01-17-79$ & 20 & ES \\
\hline 1968 & $12-19-67$ & 1,200 & & 1983 & $02-00-83$ & 1,420 & \\
\hline 1969 & $08-13-69$ & 25 & ES & 1991 & $08-12-91$ & 249 & \\
\hline 1970 & $08-20-70$ & 30 & ES & 1992 & $08-11-92$ & 266 & \\
\hline 1971 & $08-20-71$ & 900 & & 1993 & $04-19-93$ & 122 & \\
\hline 1972 & $06-00-72$ & 30 & & 1994 & $09-20-94$ & 19 & \\
\hline 1973 & $11-00-72$ & 100 & & 1995 & $08-14-95$ & 468 & \\
\hline 1974 & $08-02-74$ & 1,530 & & 1996 & $08-15-96$ & 1,000 & \\
\hline
\end{tabular}

Discharge rating table developed October 1982

\begin{tabular}{cccc}
\hline $\begin{array}{c}\text { Gage height } \\
(\mathbf{f t})\end{array}$ & $\begin{array}{c}\text { Discharge } \\
\left(\mathrm{ft}^{3} / \mathbf{s}\right)\end{array}$ & $\begin{array}{c}\text { Gage height } \\
(\mathbf{f t})\end{array}$ & $\begin{array}{c}\text { Discharge } \\
\left(\mathrm{ft}^{3} / \mathbf{s}\right)\end{array}$ \\
\hline 1.8 & 110 & 3.0 & 1,310 \\
2.0 & 205 & 3.2 & 1,670 \\
2.2 & 336 & 3.4 & 2,100 \\
2.4 & 509 & 3.6 & 2,590 \\
2.6 & 726 & 3.8 & 3,130 \\
2.8 & 990 & 4.0 & 3,740 \\
\hline
\end{tabular}

Magnitude and probability of instantaneous peak flow based on period of record 1963-79, $1983,1991-96$

\begin{tabular}{|c|c|c|c|c|c|}
\hline \multicolumn{6}{|c|}{$\begin{array}{l}\text { Discharge, in } \mathrm{ft}^{3} / \mathrm{s} \text {, for indicated recurrence interval } \\
\text { in years, and exceedance probablility, in percent }\end{array}$} \\
\hline 2 & 5 & 10 & 25 & $50 t$ & $100 t$ \\
\hline $50 \%$ & $20 \%$ & $10 \%$ & $4 \%$ & $2 \%$ & $1 \%$ \\
\hline 161 & 577 & 1,120 & 2,240 & 3,500 & 5,220 \\
\hline Weighted skew & $(\log s)=$ & -0.06 & & & \\
\hline Mean & $(\log s)=$ & 2.20 & & & \\
\hline Standard dev. & $(\log s)=$ & 0.67 & & & \\
\hline
\end{tabular}

千 Reliability of values in column is uncertain, and potential errors are large. 
09520100 MILITARY WASH NEAR SENTINEL, AZ--Continued

Basin characteristics

\begin{tabular}{cccccccc}
\hline $\begin{array}{c}\text { Main } \\
\text { channel } \\
\text { slope } \\
(\mathrm{ft} / \mathrm{mi})\end{array}$ & $\begin{array}{c}\text { Stream } \\
\text { length } \\
(\mathrm{mi})\end{array}$ & $\begin{array}{c}\text { Mean } \\
\text { basin } \\
\text { elevation } \\
\text { (ft) }\end{array}$ & $\begin{array}{c}\text { Forested } \\
\text { area } \\
\text { (percent) }\end{array}$ & $\begin{array}{c}\text { Soil } \\
\text { index }\end{array}$ & $\begin{array}{c}\text { Mean } \\
\text { annual } \\
\text { precipitation } \\
\text { (in) }\end{array}$ & $\begin{array}{c}\text { Rainfall intensity, 24-hour } \\
\text { (in) }\end{array}$ & $\begin{array}{c}50 \text {-year } \\
\text { (in) }\end{array}$ \\
\hline 56.0 & 5.0 & 674 & 0.0 & 1.0 & 5.0 & 1.3 \\
\hline
\end{tabular}

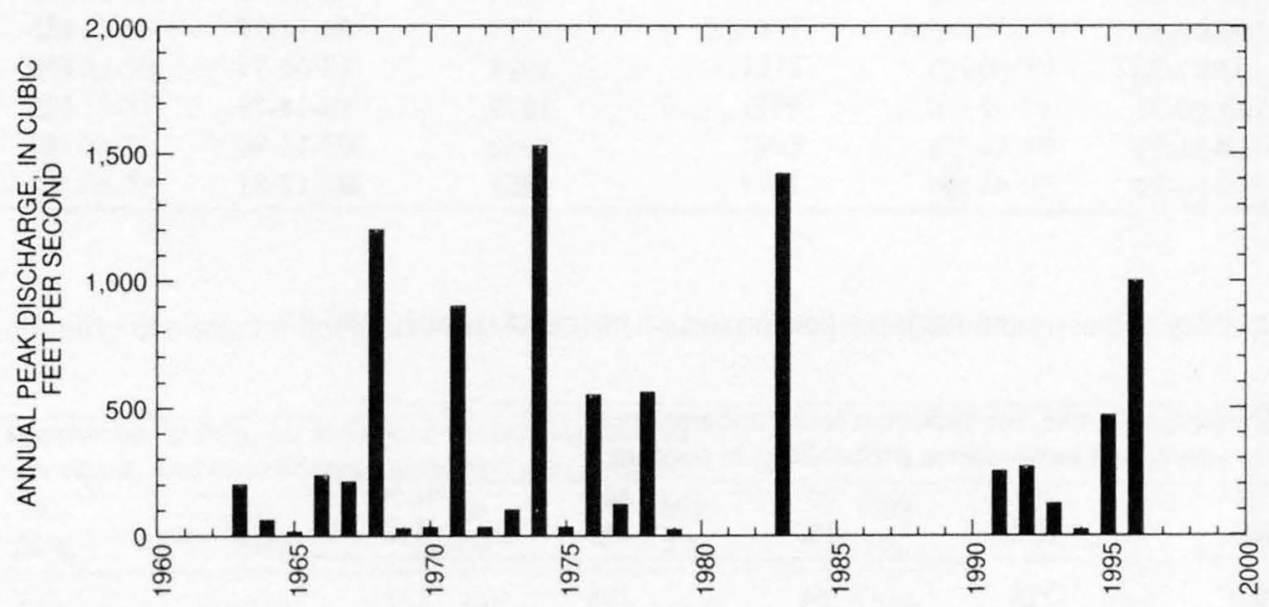




\section{HOT SHOT ARROYO NEAR AJO, AZ}

LOCATION.--Lat $32^{\circ} 20^{\prime} 49^{\prime \prime}$, long $112^{\circ} 48^{\prime} 31^{\prime \prime}$, in SW $\frac{1}{4}$ sec.29, T.12 S., R.5 W., Pima County, Hydrologic Unit 15070202, at State Highway 85, 3 mi southeast of Ajo.

DRAINAGE AREA.--0.44 $\mathrm{mi}^{2}$.

Annual peak discharges

\begin{tabular}{|c|c|c|c|c|c|c|c|}
\hline $\begin{array}{l}\text { Water } \\
\text { year }\end{array}$ & Date & $\begin{array}{l}\text { Annual peak } \\
\text { discharge } \\
\left(\mathrm{ft}^{3} / \mathrm{s}\right)\end{array}$ & $\begin{array}{l}\text { Discharge } \\
\text { codes }\end{array}$ & $\begin{array}{l}\text { Water } \\
\text { year }\end{array}$ & Date & $\begin{array}{c}\text { Annual peak } \\
\text { discharge } \\
\left(\mathrm{ft}^{3} / \mathrm{s}\right)\end{array}$ & $\begin{array}{c}\text { Discharge } \\
\text { codes }\end{array}$ \\
\hline 1966 & $09-13-66$ & 215 & & 1974 & $09-23-74$ & 215 & \\
\hline 1967 & $07-15-67$ & 206 & & 1975 & $10-29-74$ & 110 & \\
\hline 1968 & $10-03-67$ & 175 & & 1976 & $09-05-76$ & 240 & \\
\hline 1969 & $00-00-69$ & 5.0 & ES & 1977 & $09-11-77$ & 155 & \\
\hline 1970 & $07-27-70$ & 170 & & 1978 & $10-06-77$ & 150 & \\
\hline 1971 & $00-00-71$ & 116 & & 1979 & $09-14-79$ & 125 & \\
\hline 1972 & $08-31-72$ & 92 & & 1980 & $08-13-80$ & 40 & \\
\hline 1973 & $10-06-72$ & 60 & & 1981 & $01-12-81$ & 72 & \\
\hline
\end{tabular}

Magnitude and probability of instantaneous peak flow based on period of record 1966-81

\begin{tabular}{|c|c|c|c|c|c|}
\hline \multicolumn{6}{|c|}{$\begin{array}{l}\text { Discharge, in } \mathrm{ft}^{3} / \mathrm{s} \text {, for indicated recurrence interval } \\
\text { in years, and exceedance probablility, in percent }\end{array}$} \\
\hline 2 & 5 & 10 & 25 & $50 \dagger$ & $100 t$ \\
\hline $50 \%$ & $20 \%$ & $10 \%$ & $4 \%$ & $2 \%$ & $1 \%$ \\
\hline 136 & 191 & 226 & 268 & 298 & 327 \\
\hline Weighted skew & $(\log s)=$ & -0.28 & & & \\
\hline Mean & $(\log s)=$ & 2.13 & & & \\
\hline Standard dev. & $(\log s)=$ & 0.18 & & & \\
\hline
\end{tabular}

f Reliability of values in column is uncertain, and potential errors are large.

Basin characteristics

\begin{tabular}{ccccccccc}
\hline $\begin{array}{c}\text { Main } \\
\text { channel } \\
\text { slope } \\
(\mathrm{ft} / \mathrm{mi})\end{array}$ & $\begin{array}{c}\text { Stream } \\
\text { length }\end{array}$ & $\begin{array}{c}\text { Mean } \\
\text { (mi) }\end{array}$ & $\begin{array}{c}\text { basin } \\
\text { elevation } \\
\text { (ft) }\end{array}$ & $\begin{array}{c}\text { Forested } \\
\text { area } \\
\text { (percent) }\end{array}$ & $\begin{array}{c}\text { Soil } \\
\text { index }\end{array}$ & $\begin{array}{c}\text { Mean } \\
\text { annual } \\
\text { precipitation } \\
\text { (in) }\end{array}$ & $\begin{array}{c}\text { 2-year } \\
\text { (in) }\end{array}$ & $\begin{array}{c}\text { 50-year } \\
\text { (in) }\end{array}$ \\
\hline 83.3 & 1.6 & 1,760 & 0.0 & 3.0 & 8.1 & 1.7 & 4.0 \\
\hline
\end{tabular}

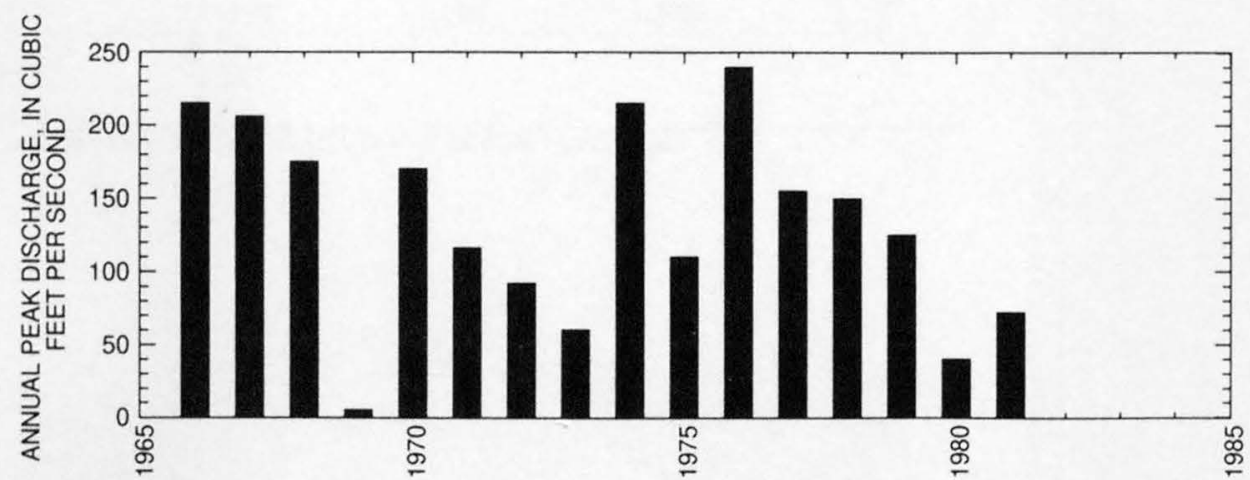


09520130 DARBY ARROYO NEAR AJO, AZ

LOCATION.--Lat 32²1'19", long $112^{\circ} 49^{\prime} 31^{\prime \prime}$, in NW $1 / 4$ sec.30, T.12 S., R.5 W., Pima County, Hydrologic Unit 15070202 , at State Highway 85,

2 mi southeast of Ajo.

DRAINAGE AREA.--4.72 $\mathrm{mi}^{2}$.

Annual peak discharges

\begin{tabular}{|c|c|c|c|c|c|c|c|}
\hline $\begin{array}{l}\text { Water } \\
\text { year }\end{array}$ & Date & $\begin{array}{c}\text { Annual peak } \\
\text { discharge } \\
\left(\mathrm{ft}^{3} / \mathrm{s}\right)\end{array}$ & $\begin{array}{c}\text { Discharge } \\
\text { codes }\end{array}$ & $\begin{array}{l}\text { Water } \\
\text { year }\end{array}$ & Date & $\begin{array}{c}\text { Annual peak } \\
\text { discharge } \\
\left(\mathrm{ft}^{3} / \mathrm{s}\right)\end{array}$ & $\begin{array}{c}\text { Discharge } \\
\text { codes }\end{array}$ \\
\hline 1966 & $09-13-66$ & 1,320 & & 1974 & $09-26-74$ & 470 & \\
\hline 1967 & $09-06-67$ & 1,670 & & 1975 & $10-29-74$ & 270 & \\
\hline 1968 & $07-04-68$ & 480 & & 1976 & $08-11-76$ & 840 & \\
\hline 1969 & $08-11-69$ & 480 & & 1977 & $07-04-77$ & 780 & \\
\hline 1970 & $08-02-70$ & 960 & & 1978 & $10-06-77$ & 1,070 & \\
\hline 1971 & $08-19-71$ & 500 & & 1979 & $11-11-78$ & 320 & \\
\hline 1972 & $10-06-71$ & 450 & & 1980 & $08-13-80$ & 150 & \\
\hline 1973 & $10-06-72$ & 105 & & 1981 & $08-23-81$ & 400 & \\
\hline
\end{tabular}

Magnitude and probability of instantaneous peak flow based on period of record 1966-81

\begin{tabular}{|c|c|c|c|c|c|}
\hline \multicolumn{6}{|c|}{$\begin{array}{l}\text { Discharge, in } \mathrm{ft}^{3} / \mathrm{s} \text {, for indicated recurrence interval } \\
\text { in years, and exceedance probablility, in percent }\end{array}$} \\
\hline 2 & 5 & 10 & 25 & $50 \dagger$ & $100 t$ \\
\hline $50 \%$ & $20 \%$ & $10 \%$ & $4 \%$ & $2 \%$ & $1 \%$ \\
\hline 550 & 922 & 1,210 & 1,640 & 1,990 & 2,380 \\
\hline Weighted skew & $(\log s)=$ & 0.11 & & & \\
\hline Mean & $(\log s)=$ & 2.75 & & & \\
\hline Standard dev. & $(\log s)=$ & 0.26 & & & \\
\hline
\end{tabular}

Basin characteristics

\begin{tabular}{cccccccc}
\hline $\begin{array}{c}\text { Main } \\
\text { channel } \\
\text { slope } \\
(\mathrm{ft} / \mathrm{mi})\end{array}$ & $\begin{array}{c}\text { Stream } \\
\text { length } \\
(\mathrm{mi})\end{array}$ & $\begin{array}{c}\text { Mean } \\
\text { basin } \\
\text { elevation } \\
\text { (ft) }\end{array}$ & $\begin{array}{c}\text { Forested } \\
\text { area } \\
\text { (percent) }\end{array}$ & $\begin{array}{c}\text { Soil } \\
\text { index }\end{array}$ & $\begin{array}{c}\text { Mean } \\
\text { annual } \\
\text { precipitation } \\
\text { (in) }\end{array}$ & $\begin{array}{c}\text { Rainfall intensity, 24-hour } \\
\text { (in) }\end{array}$ & $\begin{array}{c}50 \text {-year } \\
\text { (in) }\end{array}$ \\
\hline 71.1 & 6.0 & 1,920 & 0.0 & 3.0 & 8.1 & 1.6 \\
\hline
\end{tabular}

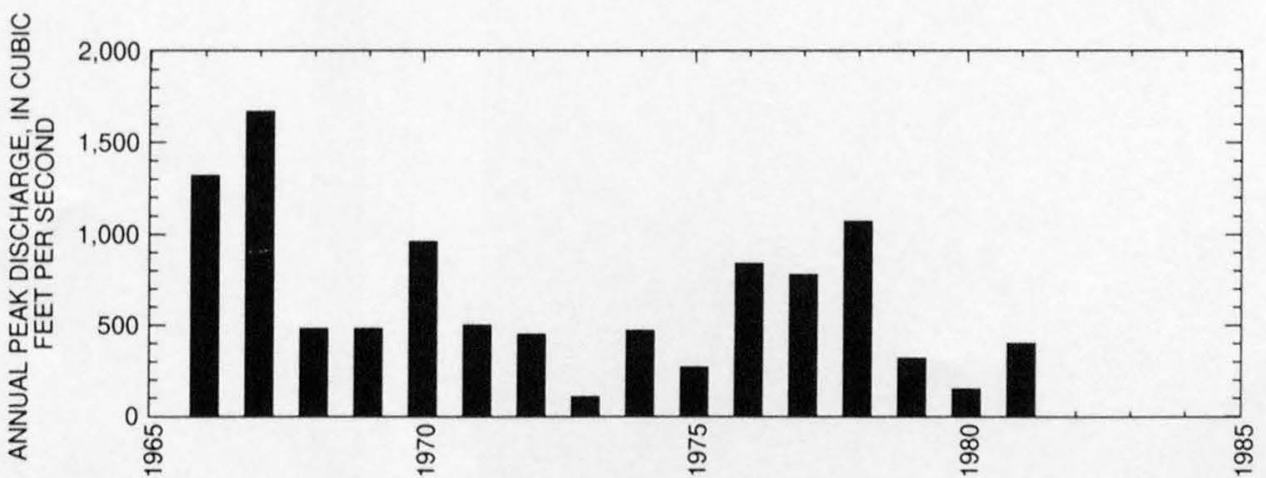


LOCATION.--Lat 32 22'48", long $112^{\circ} 51^{\prime} 40^{\prime \prime}$, in NW $1 / 4 \mathrm{SW}^{1} / 4$ sec.14, T.12 S., R.6 W., Pima County, Hydrologic Unit 15070202 , at 2nd Avenue next to railroad tracks in Ajo.

DRAINAGE AREA.--2.18 $\mathrm{mi}^{2}$.

Annual peak discharges

\begin{tabular}{|c|c|c|c|c|c|c|c|}
\hline $\begin{array}{l}\text { Water } \\
\text { year }\end{array}$ & Date & $\begin{array}{c}\text { Annual peak } \\
\text { discharge } \\
\left(\mathrm{ft}^{3} / \mathrm{s}\right)\end{array}$ & $\begin{array}{l}\text { Discharge } \\
\text { codes }\end{array}$ & $\begin{array}{c}\text { Water } \\
\text { year }\end{array}$ & Date & $\begin{array}{c}\text { Annual peak } \\
\text { discharge } \\
\left(\mathrm{ft}^{3} / \mathrm{s}\right)\end{array}$ & $\begin{array}{c}\text { Discharge } \\
\text { codes }\end{array}$ \\
\hline 1967 & $07-22-67$ & 132 & & 1975 & $10-29-74$ & 320 & \\
\hline 1968 & $07-04-68$ & 553 & & 1976 & $09-05-76$ & 340 & \\
\hline 1969 & $08-11-69$ & 306 & & 1977 & $07-04-77$ & 50 & \\
\hline 1970 & $08-02-70$ & ${ }^{1} 1,800$ & & 1978 & $08-11-78$ & 380 & \\
\hline 1971 & $08-19-71$ & 10 & LT & 1979 & $08-13-79$ & 270 & \\
\hline 1972 & $08-00-72$ & 345 & & 1980 & $08-13-80$ & 70 & \\
\hline 1973 & $08-00-73$ & 240 & & 1981 & $08-23-81$ & 200 & \\
\hline 1974 & $08-02-74$ & 254 & & & & & \\
\hline
\end{tabular}

${ }^{1}$ Highest since 1960.

Magnitude and probability of instantaneous peak flow based on period of record 1967-81

\begin{tabular}{|c|c|c|c|c|c|}
\hline \multicolumn{6}{|c|}{$\begin{array}{l}\text { Discharge, in } \mathrm{ft}^{3} / \mathrm{s} \text {, for indicated recurrence interval } \\
\text { in years, and exceedance probablility, in percent }\end{array}$} \\
\hline 2 & 5 & 10 & 25 & $50 t$ & $100 t$ \\
\hline $50 \%$ & $20 \%$ & $10 \%$ & $4 \%$ & $2 \%$ & $1 \%$ \\
\hline 231 & 474 & 686 & 1,010 & 1,300 & 1,620 \\
\hline Weighted skew & $(\log s)=$ & -0.07 & & & \\
\hline Mean & $(\log s)=$ & 2.36 & & & \\
\hline Standard dev. & $(\log s)=$ & 0.37 & & & \\
\hline
\end{tabular}

$\mp$ Reliability of values in column is uncertain, and potential errors are large.

\section{Basin characteristics}

\begin{tabular}{cccccccc}
\hline $\begin{array}{c}\text { Main } \\
\text { channel } \\
\text { slope } \\
(\mathrm{ft} / \mathrm{mi})\end{array}$ & $\begin{array}{c}\text { Stream } \\
\text { length } \\
(\mathrm{mi})\end{array}$ & $\begin{array}{c}\text { Mean } \\
\text { basin } \\
\text { elevation } \\
(\mathrm{ft})\end{array}$ & $\begin{array}{c}\text { Forested } \\
\text { area } \\
\text { (percent) }\end{array}$ & $\begin{array}{c}\text { Soil } \\
\text { index }\end{array}$ & $\begin{array}{c}\text { Mean } \\
\text { annual } \\
\text { precipitation } \\
\text { (in) }\end{array}$ & $\begin{array}{c}\text { Rainfall intensity, 24-hour } \\
\text { (in) }\end{array}$ & $\begin{array}{c}50 \text {-year } \\
\text { (in) }\end{array}$ \\
\hline 171 & 2.8 & 2,100 & 0.0 & 2.0 & 8.1 & 1.7 \\
\hline
\end{tabular}


09520160 GIBSON ARROYO AT AJO, AZ--Continued

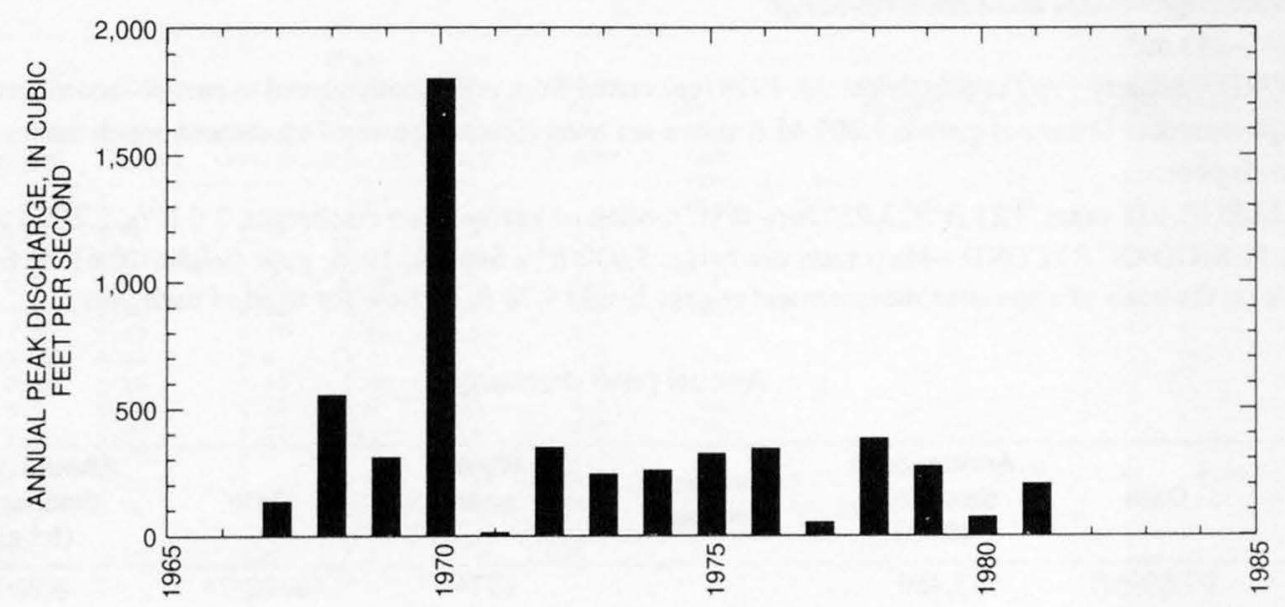




\section{RIO CORNEZ NEAR AJO, AZ}

LOCATION.--Lat 32'29'58", long 112 52'50", in SE $1 / 4 \mathrm{NE}^{1 / 4}$ sec.4, T.11 S., R.6 W., Pima County, Hydrologic Unit 15070202 , on downstream side of bridge on State Highway 85 , and 8 mi north of Ajo.

DRAINAGE AREA.--243 $\mathrm{mi}^{2}$.

PERIOD OF RECORD.--January 1967 to September 30, 1978 (converted from continuous-record to partial-record station).

GAGE.--Water-stage recorder. Datum of gage is $1,309.45 \mathrm{ft}$ above sea level (State Highway Department bench mark).

REMARKS.--Records poor.

AVERAGE DISCHARGE.--11 years, $4.21 \mathrm{ft}^{3} / \mathrm{s}, 3,050$ acre-ft/yr; median of yearly mean discharges, $3.0 \mathrm{ft}^{3} / \mathrm{s}, 2,200 \mathrm{acre}-\mathrm{ft} / \mathrm{yr}$.

EXTREMES FOR PERIOD OF RECORD.--Maximum discharge, $8,030 \mathrm{ft}^{3} / \mathrm{s}$ Sept. 4, 1976, gage height, $10.41 \mathrm{ft}$, from rating curve extended above $520 \mathrm{ft}^{3} / \mathrm{s}$ on the basis of slope-area measurement at gage height $9.70 \mathrm{ft}$; no flow for most of each year.

\section{Annual peak discharges}

\begin{tabular}{|c|c|c|c|c|c|c|c|}
\hline $\begin{array}{l}\text { Water } \\
\text { year }\end{array}$ & Date & $\begin{array}{c}\text { Annual peak } \\
\text { discharge } \\
\left(\mathrm{ft}^{3} / \mathrm{s}\right)\end{array}$ & $\begin{array}{c}\text { Discharge } \\
\text { codes }\end{array}$ & $\begin{array}{l}\text { Water } \\
\text { year }\end{array}$ & Date & $\begin{array}{c}\text { Annual peak } \\
\text { discharge } \\
\left(\mathrm{ft}^{3} / \mathrm{s}\right)\end{array}$ & $\begin{array}{l}\text { Discharge } \\
\text { codes }\end{array}$ \\
\hline 1967 & $07-09-67$ & 3,460 & & 1974 & $08-02-74$ & 6,000 & \\
\hline 1969 & $08-29-69$ & 1,610 & & 1976 & $09-04-76$ & 8,030 & \\
\hline 1970 & $08-16-70$ & 2,300 & & 1977 & $09-10-77$ & 1,390 & \\
\hline 1973 & $08-19-73$ & 2,620 & & 1980 & $00-00-80$ & 0 & \\
\hline
\end{tabular}

Discharge rating table developed January ----

\begin{tabular}{crcc}
\hline $\begin{array}{c}\text { Gage height } \\
(\mathbf{f t})\end{array}$ & $\begin{array}{c}\text { Discharge } \\
\left(\mathbf{f t}^{\mathbf{3}} \mathbf{s}\right)\end{array}$ & $\begin{array}{c}\text { Gage height } \\
(\mathbf{f t})\end{array}$ & $\begin{array}{c}\text { Discharge } \\
\left(\mathbf{f t}^{3} / \mathbf{s}\right)\end{array}$ \\
\hline 6.0 & 214 & 8.5 & 3,160 \\
6.5 & 525 & 9.0 & 4,250 \\
7.0 & 998 & 9.5 & 5,460 \\
7.5 & 1,610 & 10.0 & 6,810 \\
8.0 & 2,300 & 10.5 & 8,300 \\
\hline
\end{tabular}

Basin characteristics

\begin{tabular}{cccccccr}
\hline $\begin{array}{c}\text { Main } \\
\text { channel } \\
\text { slope } \\
(\mathrm{ft} / \mathrm{mi})\end{array}$ & $\begin{array}{c}\text { Stream } \\
\text { length } \\
\text { (mi) }\end{array}$ & $\begin{array}{c}\text { Mean } \\
\text { basin } \\
\text { elevation } \\
\text { (ft) }\end{array}$ & $\begin{array}{c}\text { Forested } \\
\text { area } \\
\text { (percent) }\end{array}$ & $\begin{array}{c}\text { Soil } \\
\text { index }\end{array}$ & $\begin{array}{c}\text { Mean } \\
\text { annual } \\
\text { precipitation } \\
\text { (in) }\end{array}$ & $\begin{array}{c}\text { Rainfall intensity, 24-hour } \\
\text { (in) }\end{array}$ & $\begin{array}{c}50 \text {-year } \\
\text { (in) }\end{array}$ \\
\hline 26.8 & 33.6 & 1,950 & 0.0 & 2.0 & 8.4 & 1.7 \\
\hline
\end{tabular}


09520170 RIO CORNEZ NEAR AJO, AZ--Continued

MEAN MONTHLY AND ANNUAL DISCHARGES 1968.78

\begin{tabular}{|c|c|c|c|c|c|c|}
\hline MONTH & $\begin{array}{l}\text { MAXIMUM } \\
(\text { FT3/S) }\end{array}$ & $\begin{array}{l}\text { MINIMUM } \\
(\mathrm{FT} 3 / \mathrm{S})\end{array}$ & $\begin{array}{c}\text { MEAN } \\
(\mathrm{FT} 3 / \mathrm{S})\end{array}$ & $\begin{array}{l}\text { STAN- } \\
\text { DARD } \\
\text { DEVIA- } \\
\text { TION } \\
(\text { FT3/S) }\end{array}$ & $\begin{array}{l}\text { COEPFI- } \\
\text { CIENT OF } \\
\text { VARI- } \\
\text { ATION }\end{array}$ & $\begin{array}{c}\text { PERCENT } \\
\text { OF } \\
\text { ANNUAL } \\
\text { RUNOFF }\end{array}$ \\
\hline OCTOBER & 41 & 0.00 & 4.6 & 12 & 2.7 & 9.1 \\
\hline NOVEMBER & 3.8 & 0.00 & 0.64 & 1.2 & 1.9 & 1.3 \\
\hline DECEMBER & 2.7 & 0.00 & 0.24 & 0.80 & 3.3 & 0.5 \\
\hline JANUARY & 9.0 & 0.00 & 0.82 & 2.7 & 3.3 & 1.6 \\
\hline FEBRUARY & 3.0 & 0.00 & 0.27 & 0.90 & 3.3 & 0.5 \\
\hline MARCH & 39 & 0.00 & 3.6 & 12 & 3.3 & 7.2 \\
\hline APRIL & 0.00 & 0.00 & 0.00 & 0.00 & & 0.0 \\
\hline MAY & 8.0 & 0.00 & 0.73 & 2.4 & 3.3 & 1.5 \\
\hline JUNE & 1.2 & 0.00 & 0.11 & 0.35 & 3.3 & 0.2 \\
\hline JULY & 35 & 0.00 & 6.5 & 11 & 1.7 & 13.0 \\
\hline AUGUST & 56 & 0.00 & 18 & 18 & 0.98 & 36.5 \\
\hline SEPTEMBER & 89 & 0.00 & 14 & 27 & 1.8 & 28.6 \\
\hline ANNUAL & 12 & 0.96 & 4.2 & 3.3 & 0.79 & 100 \\
\hline
\end{tabular}

MAGNITUDE AND PROBABILITY OF ANNUAL LOW FLOW BASED ON PERIOD OF RECORD 1968.78

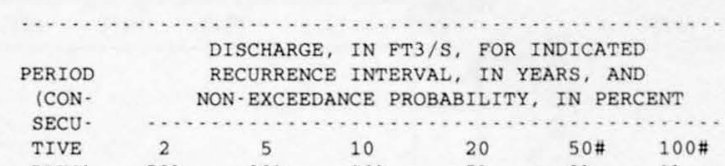

$\begin{array}{lccllll}\text { TIVE } & 2 & 5 & 10 & 20 & 50 \# & 100 \# \\ \text { DAYS) } & 508 & 208 & 108 & 58 & 28 & 18\end{array}$

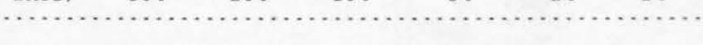

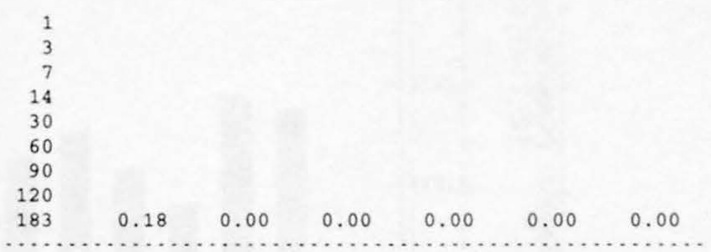

MAGNITUDE AND PROBABILITY OF ANNUAL HIGH FLOW BASED ON PERIOD OF RECORD 1968.78

\begin{tabular}{|c|c|c|c|c|c|c|}
\hline \multirow{4}{*}{$\begin{array}{l}\text { PERIOD } \\
\text { (CON- } \\
\text { SECU - }\end{array}$} & \multicolumn{6}{|c|}{ DISCHARGE, IN FT3/S, FOR INDICATED } \\
\hline & & RECURRENCE & $E$ INT & VAL, IN & YEARS, & ND \\
\hline & & EXCEEDANCE & E PRC & BILITY, & IN PERC & NT \\
\hline & & …… & & & $\ldots \ldots$ & (2-2. \\
\hline TIVE & 2 & 5 & 10 & $25 \#$ & $50 \#$ & $100 \#$ \\
\hline DAYS) & 508 & 208 & 108 & 48 & 28 & 18 \\
\hline 1 & 432 & 761 & 972 & 1.220 & 1,380 & 1,530 \\
\hline 3 & 228 & 396 & 487 & 577 & 628 & 668 \\
\hline 7 & 96 & 170 & 223 & 291 & 343 & 395 \\
\hline 15 & 53 & 87 & 108 & 132 & 148 & 162 \\
\hline 30 & 30 & 53 & 71 & 97 & 119 & 142 \\
\hline 60 & 16 & 29 & 38 & 52 & 64 & 77 \\
\hline 90 & 11 & 21 & 30 & 45 & 60 & 77 \\
\hline
\end{tabular}

DURATION TABLE OF DAILY MEAN FLOW FOR PERIOD OF RECORD 1968-78

DISCHARGE, IN FT3/S, WHICH WAS EQUALED OR EXCEEDED FOR INDICATED PERCENT OF TIME

\begin{tabular}{|c|c|c|c|c|c|c|c|c|c|c|c|c|c|c|c|c|}
\hline 18 & 58 & 108 & 158 & 208 & 308 & 408 & 508 & 608 & 708 & 808 & 908 & 958 & 988 & 998 & 99.58 & 99.98 \\
\hline & 0.00 & 0.00 & 0.00 & 0.00 & 0.00 & 0.00 & 0.00 & 0.00 & 0.00 & 0.00 & 0.00 & 0.00 & 0.00 & 0.00 & 0.00 & 0.00 \\
\hline
\end{tabular}

\# Reliability of values in column is uncertain, and potential errors are large. 
09520170 RIO CORNEZ NEAR AJO, AZ--Continued
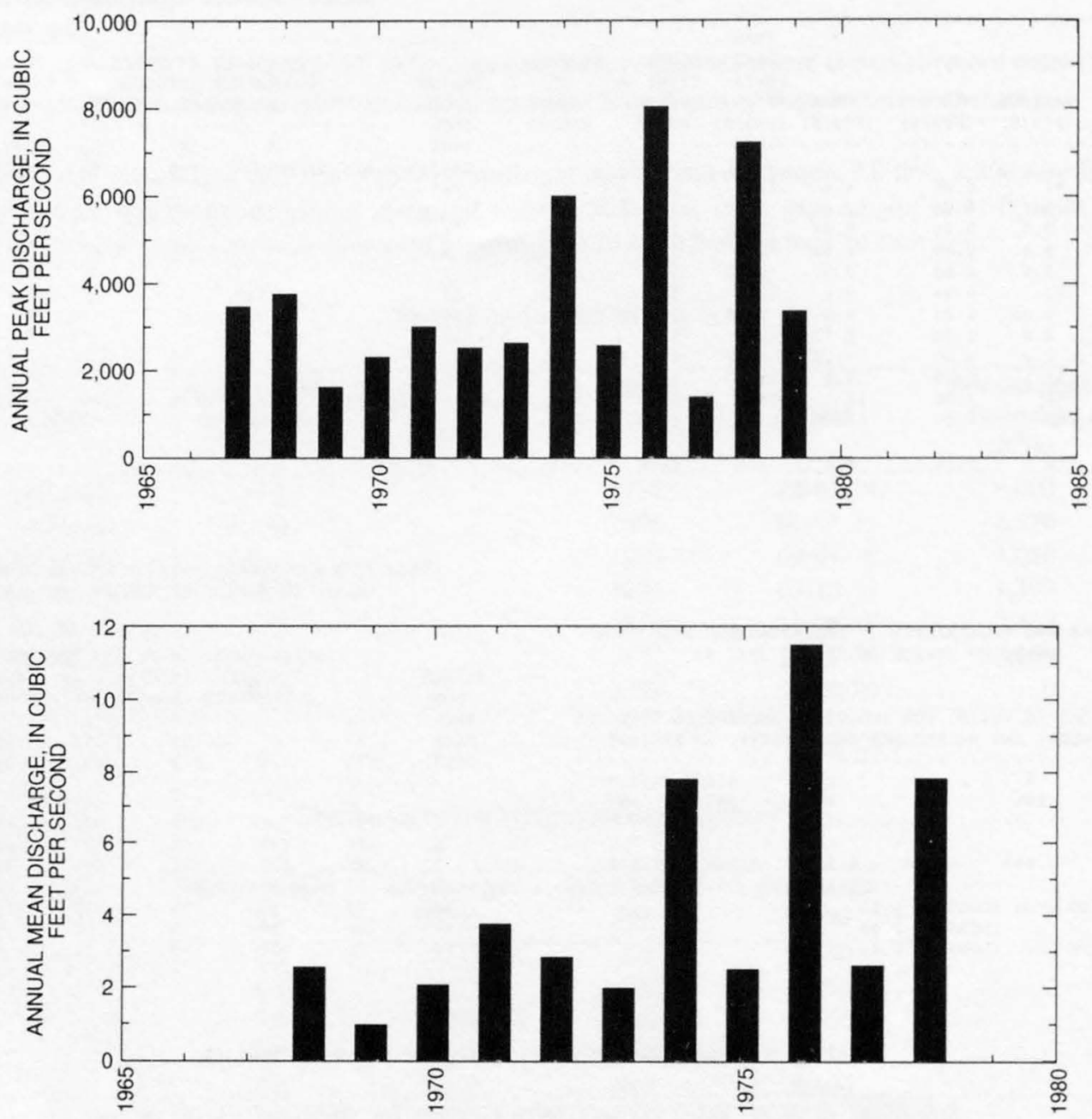
09520170 RIO CORNEZ NEAR AJO, AZ--Continued
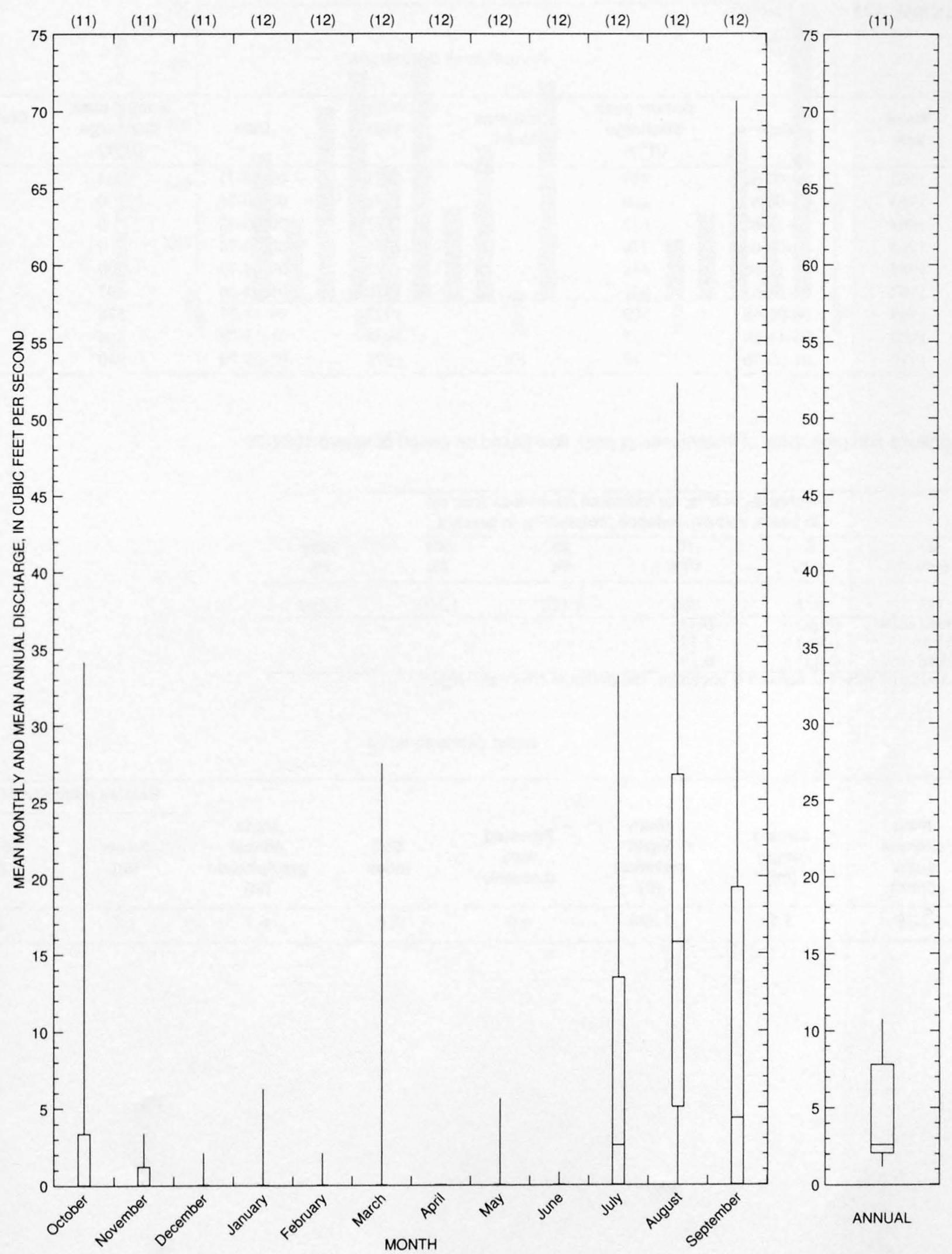
09520200 BLACK GAP WASH NEAR AJO, AZ

LOCATION.--Lat $32^{\circ} 42^{\prime} 23^{\prime \prime}$, long $112^{\circ} 50^{\prime} 43^{\prime \prime}$, in NW'1/4 NE $\frac{1}{4}$ sec.26, T.8 S., R.6 W., Maricopa County, Hydrologic Unit 15070202 , at State High. way $85,5.7 \mathrm{mi}$ north of Midway, and $23 \mathrm{mi}$ north of Ajo.

DRAINAGE AREA.--12.1 $\mathrm{mi}^{2}$.

Annual peak discharges

\begin{tabular}{|c|c|c|c|c|c|c|c|}
\hline $\begin{array}{l}\text { Water } \\
\text { year }\end{array}$ & Date & $\begin{array}{c}\text { Annual peak } \\
\text { discharge } \\
\left(\mathrm{ft}^{3} / \mathrm{s}\right)\end{array}$ & $\begin{array}{l}\text { Discharge } \\
\text { codes }\end{array}$ & $\begin{array}{l}\text { Water } \\
\text { year }\end{array}$ & Date & $\begin{array}{c}\text { Annual peak } \\
\text { discharge } \\
\left(\mathrm{ft}^{3} / \mathrm{s}\right)\end{array}$ & $\begin{array}{c}\text { Discharge } \\
\text { codes }\end{array}$ \\
\hline 1962 & $00-00-62$ & 499 & & 1971 & $09-30-71$ & 735 & \\
\hline 1964 & $10-19-63$ & 652 & & 1973 & $00-00-73$ & 0 & \\
\hline 1965 & $08-00-65$ & 770 & & 1974 & $00-00-74$ & 0 & \\
\hline 1966 & $09-13-66$ & 445 & & 1975 & $09-08-75$ & 200 & \\
\hline 1968 & $08-00-68$ & 309 & & 1977 & $09-11-77$ & 578 & \\
\hline 1969 & $08-14-69$ & 525 & & 1978 & $01-15-78$ & 364 & \\
\hline 1970 & $08-20-70$ & 10 & ES & 1979 & $07-20-79$ & 940 & \\
\hline
\end{tabular}

Magnitude and probability of instantaneous peak flow based on period of record 1962-79

\begin{tabular}{|c|c|c|c|c|c|}
\hline \multicolumn{6}{|c|}{$\begin{array}{l}\text { Discharge, in } \mathrm{ft}^{3} / \mathrm{s} \text {, for indicated recurrence interval } \\
\text { in years, and exceedance probablility, in percent }\end{array}$} \\
\hline 2 & 5 & 10 & 25 & $50 \dagger$ & $100 t$ \\
\hline $50 \%$ & $20 \%$ & $10 \%$ & $4 \%$ & $2 \%$ & $1 \%$ \\
\hline 392 & 672 & 868 & 1,120 & 1,300 & 1,490 \\
\hline Weighted skew & $(\log s)=$ & -0.43 & & & \\
\hline Mean & $(\log s)=$ & 2.57 & & & \\
\hline Standard dev. & $(\log s)=$ & 0.30 & & & \\
\hline
\end{tabular}

f Reliability of values in column is uncertain, and potential errors are large.

\section{Basin characteristics}

\begin{tabular}{ccccccrr}
\hline $\begin{array}{c}\text { Main } \\
\text { channel } \\
\text { slope } \\
(\mathrm{ft} / \mathrm{mi})\end{array}$ & $\begin{array}{c}\text { Stream } \\
\text { length } \\
\text { (mi) }\end{array}$ & $\begin{array}{c}\text { Mean } \\
\text { basin } \\
\text { elevation } \\
\text { (ft) }\end{array}$ & $\begin{array}{c}\text { Forested } \\
\text { area } \\
\text { (percent) }\end{array}$ & $\begin{array}{c}\text { Soil } \\
\text { index }\end{array}$ & $\begin{array}{c}\text { Mean } \\
\text { annual } \\
\text { precipitation } \\
\text { (in) }\end{array}$ & $\begin{array}{c}\text { Rainfall intensity, 24-hour } \\
\text { 2-year } \\
\text { (in) }\end{array}$ & $\begin{array}{c}50 \text {-year } \\
\text { (in) }\end{array}$ \\
\hline 21.8 & 5.5 & 1,280 & 0.0 & 2.0 & 6.7 & 1.5 \\
\hline
\end{tabular}


09520200 BLACK GAP WASH NEAR AJO, AZ--Continued

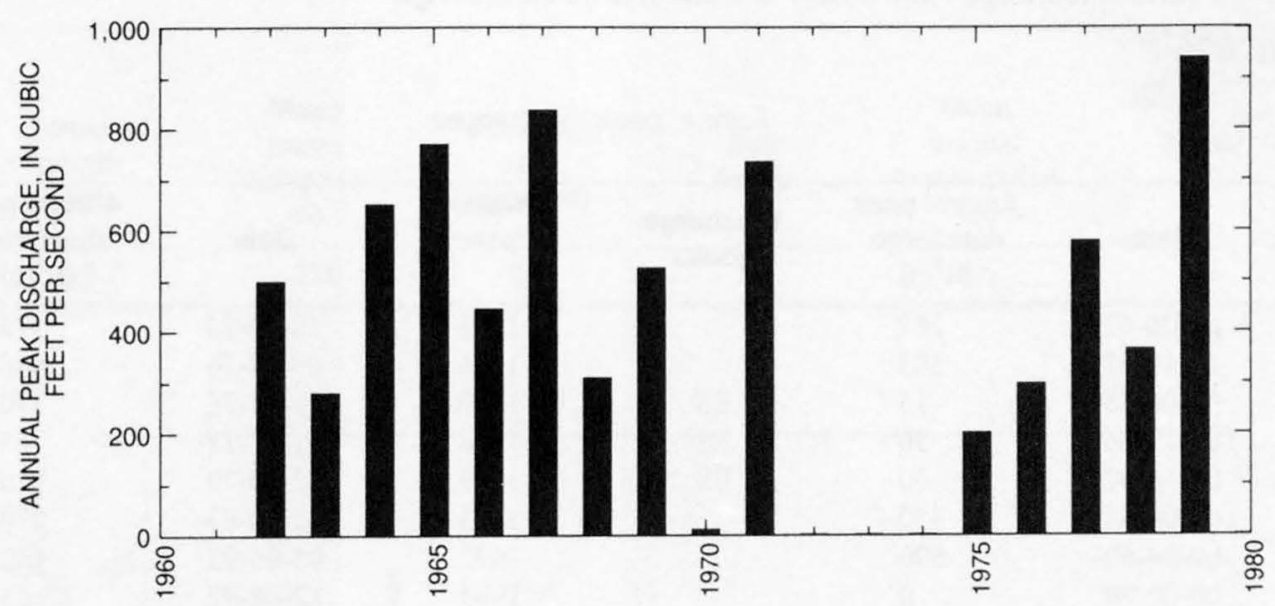




\section{CRATER RANGE WASH NEAR AJO, AZ}

LOCATION.--Lat 32 33'44", long $112^{\circ} 52^{\prime} 37^{\prime \prime}$, in NW1/4NW1/4 sec.15, T.10 S., R.6 W., Maricopa County, Hydrologic Unit 15070202 , at State

Highway $85,4.1$ mi north of Maricopa-Pima County line, and $13.5 \mathrm{mi}$ north of Ajo.

DRAINAGE AREA.--1.49 $\mathrm{mi}^{2}$.

Annual peak discharges

\begin{tabular}{|c|c|c|c|c|c|c|c|}
\hline $\begin{array}{l}\text { Water } \\
\text { year }\end{array}$ & Date & $\begin{array}{c}\text { Annual peak } \\
\text { discharge } \\
\left(\mathrm{ft}^{3} / \mathrm{s}\right)\end{array}$ & $\begin{array}{l}\text { Discharge } \\
\text { codes }\end{array}$ & $\begin{array}{l}\text { Water } \\
\text { year }\end{array}$ & Date & $\begin{array}{c}\text { Annual peak } \\
\text { discharge } \\
\left(\mathrm{ft}^{3} / \mathrm{s}\right)\end{array}$ & $\begin{array}{c}\text { Discharge } \\
\text { codes }\end{array}$ \\
\hline 1963 & $08-06-63$ & 267 & & 1975 & $10-00-74$ & 175 & \\
\hline 1965 & $02-06-65$ & 15 & ES & 1977 & $11-15-76$ & 370 & \\
\hline 1966 & $09-13-66$ & 50 & ES & 1978 & $01-15-78$ & 65 & ES \\
\hline 1967 & $07-15-67$ & 50 & ES & 1979 & $02-20-79$ & 20 & ES \\
\hline 1969 & $09-04-69$ & 590 & & 1992 & $05-05-92$ & 132 & ES \\
\hline 1970 & $00-00-70$ & 0 & & 1993 & $12-08-92$ & 25 & \\
\hline 1971 & $09-00-71$ & 300 & & 1994 & $09-11-94$ & 5.0 & \\
\hline 1972 & $08-12-72$ & 20 & & 1995 & $00-00-95$ & 0 & \\
\hline 1973 & $02-22-73$ & 260 & & 1996 & $07-25-96$ & 133 & \\
\hline 1974 & $08-04-74$ & 12 & ES & & & & \\
\hline
\end{tabular}

Discharge rating table developed October 1990

\begin{tabular}{crcr}
\hline $\begin{array}{c}\text { Gage height } \\
(\mathrm{ft})\end{array}$ & $\begin{array}{c}\text { Discharge } \\
\left(\mathrm{ft}^{3} / \mathbf{s}\right)\end{array}$ & $\begin{array}{c}\text { Gage height } \\
(\mathbf{f t})\end{array}$ & $\begin{array}{c}\text { Discharge } \\
\left(\mathrm{ft}^{3} / \mathbf{s}\right)\end{array}$ \\
\hline 1.6 & 60 & 4.0 & 609 \\
2.0 & 174 & 4.5 & 737 \\
2.5 & 271 & 5.0 & 872 \\
3.0 & 375 & 5.5 & 1,010 \\
3.5 & 488 & 5.8 & 1,100 \\
\hline
\end{tabular}

Magnitude and probability of instantaneous peak flow based on period of record 1963-79,

1983, 1991-96

\begin{tabular}{|c|c|c|c|c|c|}
\hline \multicolumn{6}{|c|}{$\begin{array}{l}\text { Discharge, in } \mathrm{ft}^{3} / \mathrm{s} \text {, for indicated recurrence interval } \\
\text { in years, and exceedance probablility, in percent }\end{array}$} \\
\hline 2 & 5 & 10 & 25 & $50 t$ & $100 t$ \\
\hline $50 \%$ & $20 \%$ & $10 \%$ & $4 \%$ & $2 \%$ & $1 \%$ \\
\hline 70 & 270 & 522 & 1,020 & 1,550 & 2,230 \\
\hline Weighted skew & $(\log s)=$ & -0.28 & & & \\
\hline Mean & $(\log s)=$ & 1.81 & & & \\
\hline Standard dev. & $(\log s)=$ & 0.73 & & & \\
\hline
\end{tabular}

$\mp$ Reliability of values in column is uncertain, and potential errors are large. 
GILA RIVER BASIN

09520230 CRATER RANGE WASH NEAR AJO, AZ

Basin characteristics

\begin{tabular}{ccccccccc}
\hline $\begin{array}{c}\text { Main } \\
\text { channel } \\
\text { slope } \\
(\mathbf{f t} / \mathrm{mi})\end{array}$ & $\begin{array}{c}\text { Stream } \\
\text { length } \\
(\mathbf{m i})\end{array}$ & $\begin{array}{c}\text { Mean } \\
\text { basin } \\
\text { elevation } \\
\text { (ft) }\end{array}$ & $\begin{array}{c}\text { Forested } \\
\text { area } \\
\text { (percent) }\end{array}$ & $\begin{array}{c}\text { Soil } \\
\text { index }\end{array}$ & $\begin{array}{c}\text { Mean } \\
\text { annual } \\
\text { precipitation } \\
\text { (in) }\end{array}$ & $\begin{array}{c}\text { 2-year } \\
\text { (in) }\end{array}$ & $\begin{array}{c}\text { 50-year } \\
\text { (in) }\end{array}$ \\
\hline 69.3 & 2.5 & 1,280 & 0.0 & 1.0 & 6.6 & 1.5 & 3.9 \\
\hline
\end{tabular}

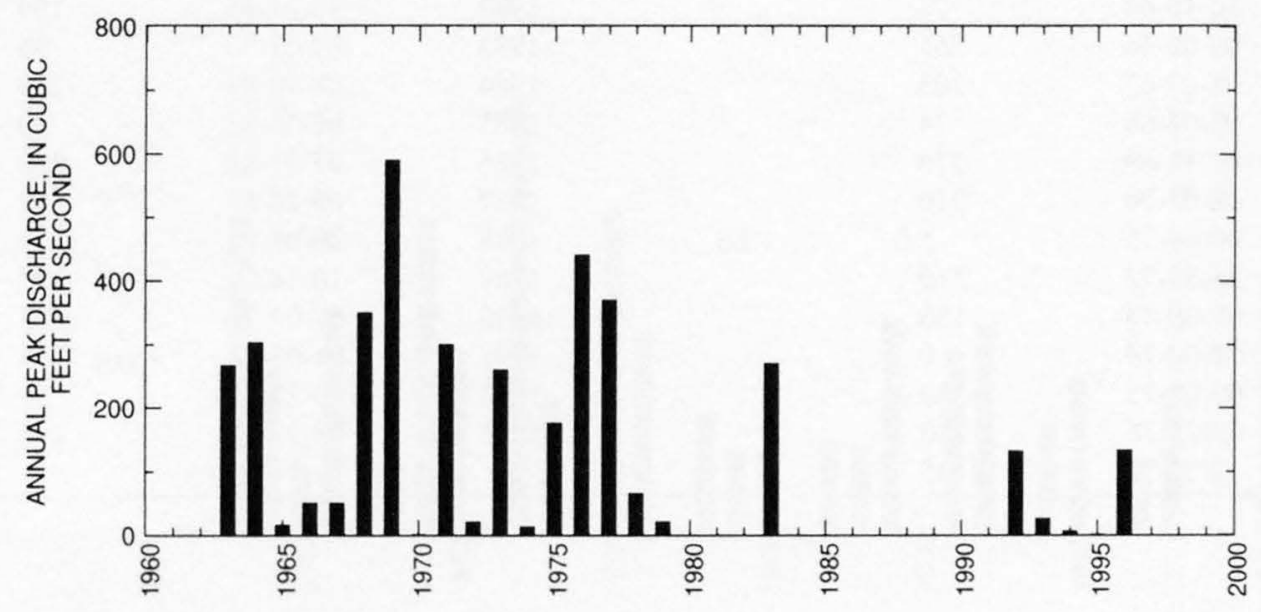


09520300 ALAMO WASH TRIBUTARY NEAR AJO, AZ

LOCATION.--Lat $32^{\circ} 06^{\prime} 00^{\prime \prime}$, long $112^{\circ} 46^{\prime} 15^{\prime \prime}$, in SW $1 / 4$ sec.22, T.15 S., R.5 W. (unsurveyed), Pima County, Hydrologic Unit 15070203, at State

Highway $85,20 \mathrm{mi}$ southeast of Ajo.

DRAINAGE AREA.--0.90 $\mathrm{mi}^{2}$.

Annual peak discharges

\begin{tabular}{|c|c|c|c|c|c|c|c|}
\hline $\begin{array}{l}\text { Water } \\
\text { year }\end{array}$ & Date & $\begin{array}{c}\text { Annual peak } \\
\text { discharge } \\
\left(\mathrm{ft}^{3} / \mathrm{s}\right)\end{array}$ & $\begin{array}{l}\text { Discharge } \\
\text { codes }\end{array}$ & $\begin{array}{l}\text { Water } \\
\text { year }\end{array}$ & Date & $\begin{array}{c}\text { Annual peak } \\
\text { discharge } \\
\left(\mathrm{ft}^{3} / \mathrm{s}\right)\end{array}$ & $\begin{array}{l}\text { Discharge } \\
\text { codes }\end{array}$ \\
\hline 1963 & $08-15-63$ & 375 & & 1979 & $00-00-79$ & 92 & \\
\hline 1964 & $09-09-64$ & 187 & & 1980 & $00-00-80$ & 92 & \\
\hline 1965 & $10-16-64$ & 74 & & 1982 & $10-01-81$ & 104 & \\
\hline 1966 & $00-00-66$ & 261 & & 1983 & $07-21-83$ & 98 & \\
\hline 1967 & $08-00-67$ & 103 & & 1984 & $08-00-84$ & 250 & \\
\hline 1968 & $08-02-68$ & 74 & & 1985 & $00-00-85$ & 0 & \\
\hline 1969 & $08-14-69$ & 370 & & 1986 & $07-21-86$ & 210 & \\
\hline 1970 & $08-02-70$ & 210 & & 1987 & $09-23-87$ & 240 & \\
\hline 1971 & $00-00-71$ & 5.0 & ES & 1988 & $00-00-88$ & 0 & \\
\hline 1972 & $08-31-72$ & 510 & & 1989 & $10-14-88$ & 125 & \\
\hline 1973 & $08-00-73$ & 150 & & 1990 & $07-07-90$ & 173 & \\
\hline 1974 & $00-00-74$ & 0 & & 1991 & $01-05-91$ & 5 & \\
\hline 1975 & $09-08-75$ & 380 & & 1992 & $10-27-91$ & 81 & \\
\hline 1976 & $09-25-76$ & 230 & & 1993 & $08-27-93$ & 154 & \\
\hline 1978 & $10-06-77$ & ${ }^{\mathrm{I}} 135$ & & & & & \\
\hline
\end{tabular}

THighest since 1976.

Discharge rating table developed October 1989

\begin{tabular}{cccc}
\hline $\begin{array}{c}\text { Gage height } \\
(\mathrm{ft})\end{array}$ & $\begin{array}{c}\text { Discharge } \\
\left(\mathrm{ft}^{3} / \mathbf{s}\right)\end{array}$ & $\begin{array}{c}\text { Gage height } \\
(\mathbf{f t})\end{array}$ & $\begin{array}{c}\text { Discharge } \\
\left(\mathrm{ft}^{3} / \mathbf{s}\right)\end{array}$ \\
\hline 2.0 & 107 & 4.5 & 485 \\
2.5 & 166 & 5.0 & 585 \\
3.0 & 233 & 5.5 & 693 \\
3.5 & 308 & 6.0 & 808 \\
4.0 & 392 & 6.5 & 1,020 \\
\hline
\end{tabular}

Magnitude and probability of instantaneous peak flow based on period of record 1963-76, $1978-80,1982-93$

\begin{tabular}{|c|c|c|c|c|c|}
\hline \multicolumn{6}{|c|}{$\begin{array}{l}\text { Discharge, in } \mathrm{ft}^{3} / \mathrm{s} \text {, for indicated recurrence interval } \\
\text { in years, and exceedance probablility, in percent }\end{array}$} \\
\hline 2 & 5 & 10 & 25 & $50 \dagger$ & $100 \dagger$ \\
\hline $50 \%$ & $20 \%$ & $10 \%$ & $4 \%$ & $2 \%$ & $1 \%$ \\
\hline 141 & 240 & 326 & 445 & 546 & 656 \\
\hline Weighted skew & $(\log s)=$ & 0.06 & & & \\
\hline Mean & $(\log s)=$ & 2.15 & & & \\
\hline Standard dev. & $(\log s)=$ & 0.28 & & & \\
\hline
\end{tabular}


GILA RIVER BASIN

09520300 ALAMO WASH TRIBUTARY NEAR AJO, AZ--Continued

Basin characteristics

\begin{tabular}{cccccccr}
\hline $\begin{array}{c}\text { Main } \\
\text { channel } \\
\text { slope } \\
(\mathrm{ft} / \mathrm{mi})\end{array}$ & $\begin{array}{c}\text { Stream } \\
\text { length } \\
\text { (mi) }\end{array}$ & $\begin{array}{c}\text { Mean } \\
\text { basin } \\
\text { elevation } \\
\text { (ft) }\end{array}$ & $\begin{array}{c}\text { Forested } \\
\text { area } \\
\text { (percent) }\end{array}$ & $\begin{array}{c}\text { Soil } \\
\text { index }\end{array}$ & $\begin{array}{c}\text { Mean } \\
\text { annual } \\
\text { precipitation } \\
\text { (in) }\end{array}$ & $\begin{array}{c}\text { Rainfall intensity, 24-hour } \\
\text { (in) }\end{array}$ & $\begin{array}{c}50 \text {-year } \\
\text { (in) }\end{array}$ \\
\hline 64.0 & 2.5 & 2,040 & 0.0 & 2.0 & 9.7 & 1.7 \\
\hline
\end{tabular}

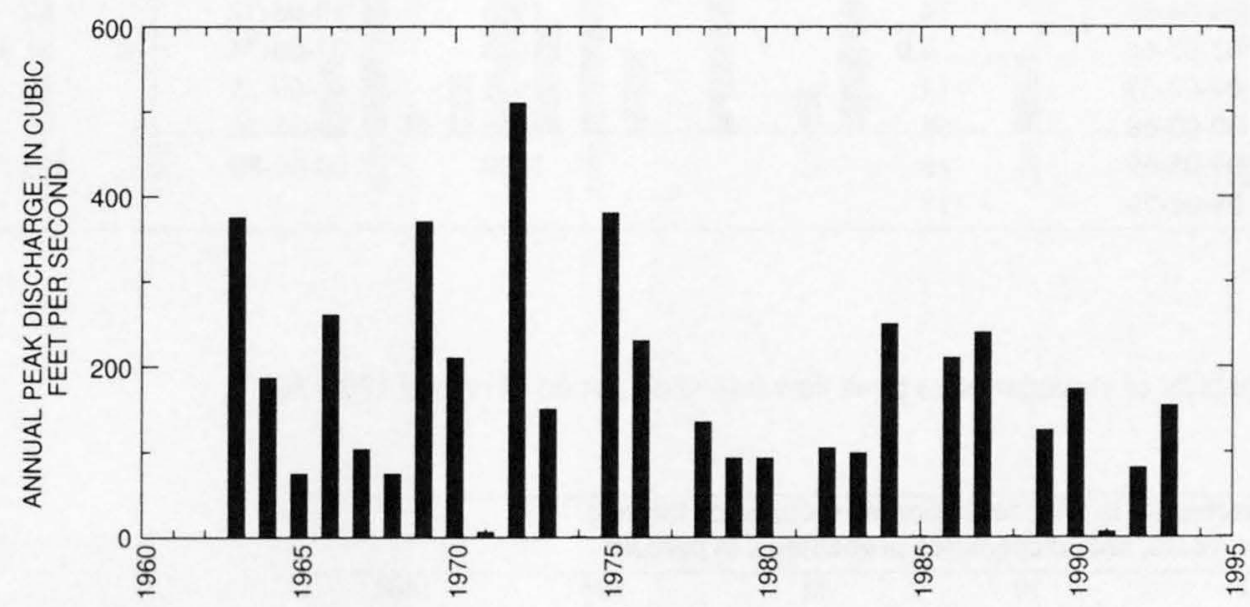


09520350 MOHAWK PASS WASH AT MOHAWK, AZ

LOCATION.--Lat 32 43'44", long $113^{\circ} 44^{\prime} 30^{\prime \prime}$, in SE $\frac{1}{4} \mathrm{SW}^{1} / 4$ sec.17, T.8 S., R.14 S., Yuma County, Hydrologic Unit 15070203 , at Southern Pacific Railroad crossing, 0.6 mi east of Mohawk.

DRAINAGE AREA.--0.09 $\mathrm{mi}^{2}$.

Annual peak discharges

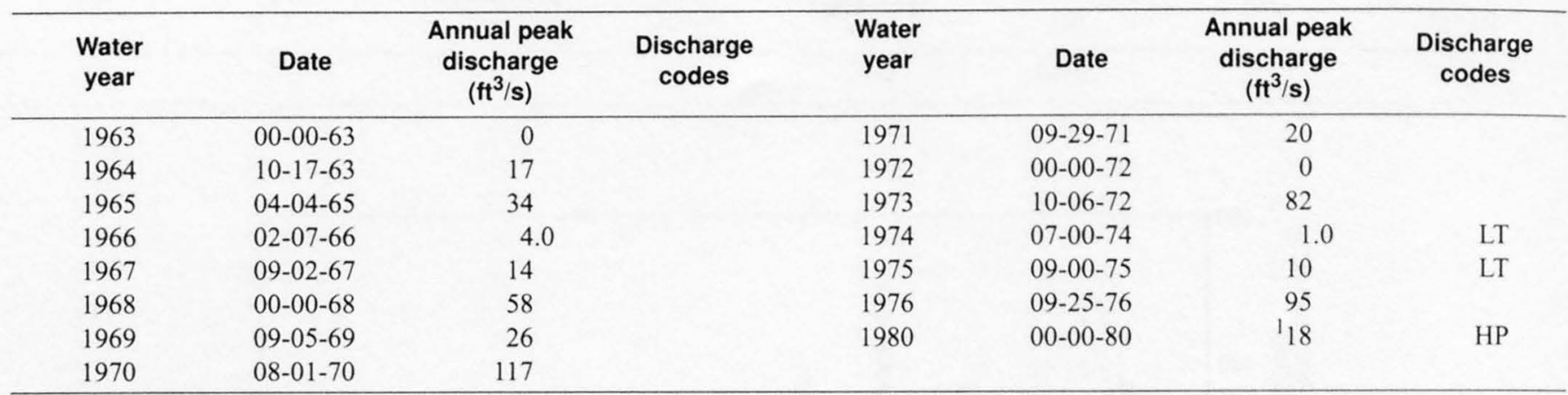

${ }^{1}$ Highest since 1976.

Magnitude and probability of instantaneous peak flow based on period of record 1963-76, 1980

\begin{tabular}{|c|c|c|c|c|c|}
\hline \multicolumn{6}{|c|}{$\begin{array}{l}\text { Discharge, in } \mathrm{ft}^{3} / \mathrm{s} \text {, for indicated recurrence interval } \\
\text { in years, and exceedance probablility, in percent }\end{array}$} \\
\hline 2 & 5 & 10 & 25 & $50 t$ & $100 t$ \\
\hline $50 \%$ & $20 \%$ & $10 \%$ & $4 \%$ & $2 \%$ & $1 \%$ \\
\hline 20.2 & 52.4 & 85.7 & 145 & 202 & 273 \\
\hline Weighted skew & $(\log s)=$ & -0.04 & & & \\
\hline Mean & $(\log s)=$ & 1.30 & & & \\
\hline Standard dev. & $(\log s)=$ & 0.50 & & & \\
\hline
\end{tabular}

Basin characteristics

\begin{tabular}{cccccccc}
\hline $\begin{array}{c}\text { Main } \\
\text { channel } \\
\text { slope } \\
(\mathrm{ft} / \mathrm{mi})\end{array}$ & $\begin{array}{c}\text { Stream } \\
\text { length } \\
(\mathrm{mi})\end{array}$ & $\begin{array}{c}\text { Mean } \\
\text { basin } \\
\text { elevation } \\
(\mathrm{ft})\end{array}$ & $\begin{array}{c}\text { Forested } \\
\text { area } \\
\text { (percent) }\end{array}$ & $\begin{array}{c}\text { Soil } \\
\text { index }\end{array}$ & $\begin{array}{c}\text { Mean } \\
\text { annual } \\
\text { precipitation } \\
\text { (in) }\end{array}$ & $\begin{array}{c}\text { Rainfall intensity, 24-hour } \\
\text { (in) }\end{array}$ & $\begin{array}{c}\text { 2-year } \\
\text { (in) }\end{array}$ \\
\hline 707 & 0.49 & 601 & 0.0 & 1.0 & 4.9 & 1.4 \\
\hline
\end{tabular}


09520350 MOHAWK PASS WASH AT MOHAWK, AZ--Continued

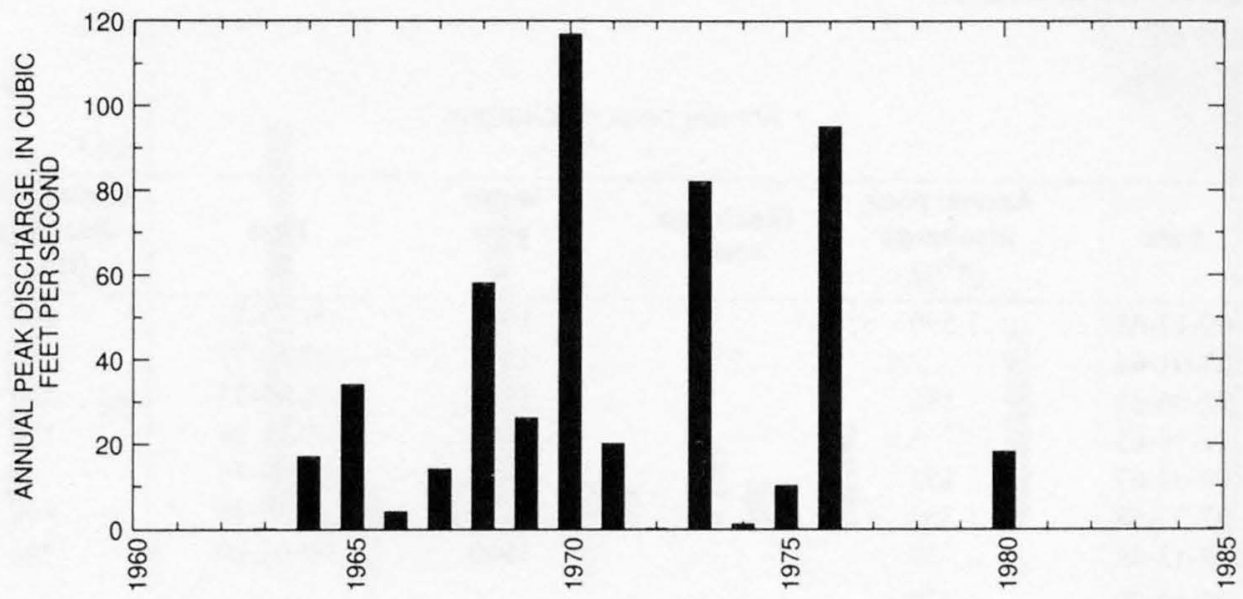


09520400 LIGURTA WASH AT LIGURTA, AZ

LOCATION.--Lat $32^{\circ} 40^{\prime} 33^{\prime \prime}$, long $114^{\circ} 17^{\prime} 38^{\prime \prime}$, in NW $1 /{ }_{4} \mathrm{NW}^{1} / 4$ sec.2, T.9 S., R.20 W., Yuma County, Hydrologic Unit 15070201 , at U.S. Highway 80 at Ligurta, and $9.0 \mathrm{mi}$ west of Wellton.

DRAINAGE AREA.--1.99 $\mathrm{mi}^{2}$.

Annual peak discharges

\begin{tabular}{|c|c|c|c|c|c|c|c|}
\hline $\begin{array}{l}\text { Water } \\
\text { year }\end{array}$ & Date & $\begin{array}{c}\text { Annual peak } \\
\text { discharge } \\
\left(\mathrm{ft}^{3} / \mathrm{s}\right)\end{array}$ & $\begin{array}{l}\text { Discharge } \\
\text { codes }\end{array}$ & $\begin{array}{l}\text { Water } \\
\text { year }\end{array}$ & Date & $\begin{array}{c}\text { Annual peak } \\
\text { discharge } \\
\left(\mathrm{ft}^{3} / \mathrm{s}\right)\end{array}$ & $\begin{array}{c}\text { Discharge } \\
\text { codes }\end{array}$ \\
\hline 1963 & $09-17-63$ & 1,590 & \multirow{8}{*}{ ES } & 1971 & $09-29-71$ & 156 & \multirow{8}{*}{ HP } \\
\hline 1964 & $08-01-64$ & 2.0 & & 1972 & $00-00-72$ & 0 & \\
\hline 1965 & $02-06-65$ & 195 & & 1973 & $08-05-73$ & 330 & \\
\hline 1966 & $11-16-65$ & 8.0 & & 1974 & $09-03-74$ & 190 & \\
\hline 1967 & $09-02-67$ & 194 & & 1975 & $00-00-75$ & 0 & \\
\hline 1968 & $07-27-68$ & 1,330 & & 1976 & $09-25-76$ & 430 & \\
\hline 1969 & $08-13-69$ & 58 & & 1980 & $00-00-80$ & ${ }^{1} 780$ & \\
\hline 1970 & $02-10-70$ & 175 & & & & & \\
\hline
\end{tabular}

${ }^{1}$ Highest since 1976.

Magnitude and probability of instantaneous peak flow based on period of record 1963-76, 1980

\begin{tabular}{|c|c|c|c|c|c|}
\hline \multicolumn{6}{|c|}{$\begin{array}{l}\text { Discharge, in } \mathrm{ft}^{3} / \mathrm{s} \text {, for indicated recurrence interval } \\
\text { in years, and exceedance probablility, in percent }\end{array}$} \\
\hline 2 & 5 & 10 & 25 & $50 \dagger$ & $100 \dagger$ \\
\hline $50 \%$ & $20 \%$ & $10 \%$ & $4 \%$ & $2 \%$ & $1 \%$ \\
\hline 182 & 548 & 980 & 1,820 & 2,730 & 3,930 \\
\hline Weighted skew & $(\log s)=$ & 0.03 & & & \\
\hline Mean & $(\log s)=$ & 2.26 & & & \\
\hline Standard dev. & $(\log s)=$ & 0.57 & & & \\
\hline
\end{tabular}

\section{Basin characteristics}

\begin{tabular}{cccccccc}
\hline $\begin{array}{c}\text { Main } \\
\text { channel } \\
\begin{array}{c}\text { slope } \\
(\mathrm{ft} / \mathrm{mi})\end{array}\end{array}$ & $\begin{array}{c}\text { Stream } \\
\text { length } \\
(\mathrm{mi})\end{array}$ & $\begin{array}{c}\text { Mean } \\
\text { basin } \\
\text { elevation } \\
\text { (ft) }\end{array}$ & $\begin{array}{c}\text { Forested } \\
\text { area } \\
\text { (percent) }\end{array}$ & $\begin{array}{c}\text { Soil } \\
\text { index }\end{array}$ & $\begin{array}{c}\text { Mean } \\
\text { annual } \\
\text { precipitation } \\
\text { (in) }\end{array}$ & $\begin{array}{c}\text { R-year } \\
\text { (in) }\end{array}$ & $\begin{array}{c}50 \text {-year } \\
\text { (in) }\end{array}$ \\
\hline 127 & 2.0 & 395 & 0.0 & 2.0 & 4.0 & 1.4 \\
\hline
\end{tabular}


09520400 LIGURTA WASH AT LIGURTA, AZ--Continued

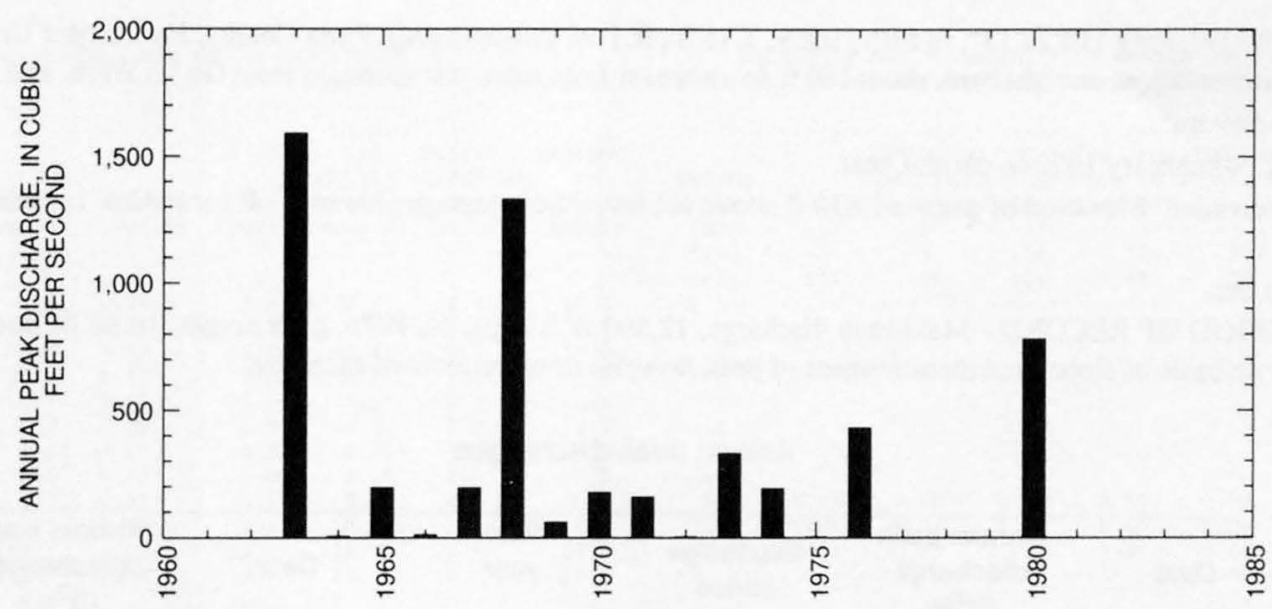




\section{SAN SIMON WASH BASIN}

\section{SAN SIMON WASH NEAR PISINIMO, AZ}

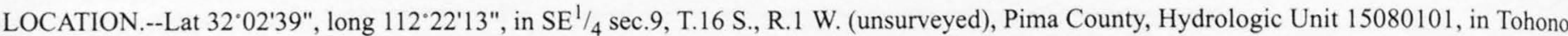
O'Odham Indian Reservation, on right bank about $100 \mathrm{ft}$ downstream from road, just upstream from Gu Vo Wash, and $3.2 \mathrm{mi}$ west of Pisinimo. DRAINAGE AREA.--569 $\mathrm{mi}^{2}$.

PERIOD OF RECORD.--February 1972 to current year.

GAGE.--Water-stage recorder. Elevation of gage is $1,830 \mathrm{ft}$ above sea level, from topographic map. Prior to Oct. 1, 1980 , at site $120 \mathrm{ft}$ upstream at same datum.

REMARKS.--Records fair.

EXTREMES FOR PERIOD OF RECORD.--Maximum discharge, 12,500 $\mathrm{ft}^{3} / \mathrm{s}$ Sept. 24, 1976, gage height, $10.82 \mathrm{ft}$, from rating curve extended above $1,700 \mathrm{ft}^{3} / \mathrm{s}$ on basis of slope-area measurement of peak flow; no flow for most of each year.

Annual peak discharges

\begin{tabular}{|c|c|c|c|c|c|c|c|}
\hline $\begin{array}{l}\text { Water } \\
\text { year }\end{array}$ & Date & $\begin{array}{c}\text { Annual peak } \\
\text { discharge } \\
\left(\mathrm{ft}^{3} / \mathrm{s}\right)\end{array}$ & $\begin{array}{l}\text { Discharge } \\
\text { codes }\end{array}$ & $\begin{array}{c}\text { Water } \\
\text { year }\end{array}$ & Date & $\begin{array}{c}\text { Annual peak } \\
\text { discharge } \\
\left(\mathrm{ft}^{3} / \mathrm{s}\right)\end{array}$ & $\begin{array}{l}\text { Discharge } \\
\text { codes }\end{array}$ \\
\hline 1972 & $08-09-72$ & 1,080 & & 1985 & $07-19-85$ & 790 & \\
\hline 1973 & $10-19-72$ & 1,930 & & 1986 & $07-21-86$ & 943 & \\
\hline 1974 & $07-29-74$ & 1,370 & & 1987 & $08-11-87$ & 641 & \\
\hline 1975 & $09-07-75$ & 1,950 & & 1988 & $08-29-88$ & 1,740 & \\
\hline 1976 & $09-24-76$ & 12,500 & & 1989 & $10-15-88$ & 1,420 & \\
\hline 1977 & $08-08-77$ & 720 & & 1990 & $07-14-90$ & 837 & \\
\hline 1978 & $09-07-78$ & 473 & & 1991 & $08-27-91$ & 648 & \\
\hline 1979 & $11-11-78$ & 512 & & 1992 & $07-11-92$ & 1,100 & \\
\hline 1980 & $08-13-80$ & 101 & & 1993 & $01-08-93$ & 1,050 & \\
\hline 1981 & $07-12-81$ & 2,020 & & 1994 & $09-10-94$ & 431 & \\
\hline 1982 & $08-25-82$ & 1,780 & & 1995 & $08-24-95$ & 1,240 & \\
\hline 1983 & $09-29-83$ & 688 & & 1996 & $09-24-96$ & 418 & \\
\hline 1984 & $08-17-84$ & 8,600 & & & & & \\
\hline
\end{tabular}

Discharge rating table developed October 1985

\begin{tabular}{cccc}
\hline $\begin{array}{c}\text { Gage height } \\
(\mathrm{ft})\end{array}$ & $\begin{array}{c}\text { Discharge } \\
\left(\mathbf{f t}^{3} / \mathbf{s}\right)\end{array}$ & $\begin{array}{c}\text { Gage height } \\
(\mathbf{f t})\end{array}$ & $\begin{array}{c}\text { Discharge } \\
\left(\mathrm{ft}^{\mathbf{3}} / \mathbf{s}\right)\end{array}$ \\
\hline 4.5 & 187 & 7.5 & 1,200 \\
5.0 & 247 & 8.0 & 1,740 \\
5.5 & 337 & 8.5 & 2,580 \\
6.0 & 458 & 9.0 & 4,010 \\
6.5 & 622 & 9.5 & 6,700 \\
7.0 & 854 & 10.0 & 12,500 \\
\hline
\end{tabular}

Basin characteristics

\begin{tabular}{cccccccc}
\hline $\begin{array}{c}\text { Main } \\
\text { channel } \\
\text { slope } \\
(\mathrm{ft} / \mathrm{mi})\end{array}$ & $\begin{array}{c}\text { Stream } \\
\text { length } \\
(\mathrm{mi})\end{array}$ & $\begin{array}{c}\text { Mean } \\
\text { basin } \\
\text { elevation } \\
(\mathrm{ft})\end{array}$ & $\begin{array}{c}\text { Forested } \\
\text { area } \\
\text { (percent) }\end{array}$ & $\begin{array}{c}\text { Soil } \\
\text { index }\end{array}$ & $\begin{array}{c}\text { Mean } \\
\text { annual } \\
\text { precipitation } \\
\text { (in) }\end{array}$ & $\begin{array}{c}\text { Rainfall intensity, 24-hour } \\
\text { (in) }\end{array}$ & $\begin{array}{c}50 \text {-year } \\
\text { (in) }\end{array}$ \\
\hline 17.4 & 39.4 & 2,250 & 0.0 & 0.0 & 10.0 & 1.8 \\
\hline
\end{tabular}


SAN SIMON WASH BASIN

09535100 SAN SIMON WASH NEAR PISINIMO, AZ--Continued

MEAN MONTHLY AND ANNUAL DISCHARGES 1973-96

\begin{tabular}{|c|c|c|c|c|c|c|}
\hline MONTH & $\begin{array}{l}\text { MAXIMUM } \\
\text { (FT3/S) }\end{array}$ & $\begin{array}{l}\text { MINIMUM } \\
\text { (FT3/S) }\end{array}$ & $\begin{array}{c}\text { MEAN } \\
(\mathrm{FT} 3 / \mathrm{S})\end{array}$ & $\begin{array}{l}\text { STAN- } \\
\text { DARD } \\
\text { DEVIA- } \\
\text { TION } \\
\text { (FT3/S) }\end{array}$ & $\begin{array}{l}\text { COEFFI- } \\
\text { CIENT OF } \\
\text { VARI- } \\
\text { ATION }\end{array}$ & $\begin{array}{c}\text { PERCENT } \\
\text { OF } \\
\text { ANNUAL } \\
\text { RUNOFF }\end{array}$ \\
\hline OCTOBER & 44 & 0.00 & 5.2 & 12 & 2.4 & 12.7 \\
\hline NOVEMBER & 14 & 0.00 & 1.4 & 3.5 & 2.5 & 3.3 \\
\hline DECEMBER & 9.0 & 0.00 & 1.2 & 2.7 & 2.2 & 3.0 \\
\hline JANUARY & 39 & 0.00 & 2.7 & 8.0 & 3.0 & 6.5 \\
\hline FEBRUARY & 7.6 & 0.00 & 1.1 & 2.3 & 2.2 & 2.6 \\
\hline MARCH & 8.5 & 0.00 & 1.0 & 2.3 & 2.3 & 2.5 \\
\hline APRIL & 0.20 & 0.00 & 0.02 & 0.05 & 2.8 & 0.0 \\
\hline MAY & 2.0 & 0.00 & 0.09 & 0.40 & 4.6 & 0.2 \\
\hline JUNE & 0.02 & 0.00 & 0.00 & 0.00 & 4.9 & 0.0 \\
\hline JULY & 40 & 0.00 & 8.1 & 10 & 1.3 & 19.6 \\
\hline AUGUST & 93 & 0.01 & 12 & 22 & 1.9 & 28.0 \\
\hline SEPTEMBER & 140 & 0.00 & 8.9 & 28 & 3.2 & 21.6 \\
\hline ANNUAL & 15 & 0.13 & 3.5 & 3.6 & 1.0 & 100 \\
\hline
\end{tabular}

MAGNITUDE AND PROBABILITY OF INSTANTANEOUS PEAK FLOW BASED ON PERIOD OF RECORD 1972.96

DISCHARGE, IN FT3/S, FOR INDICATED RECURRENCE INTERVAL IN YEARS, AND EXCEEDANCE PROBABILITY, IN PERCENT

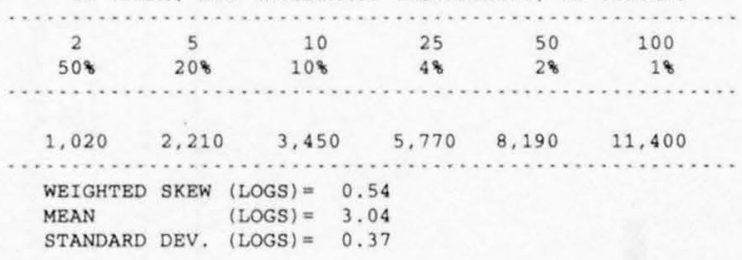

MAGNITUDE AND PROBABILITY OF ANNUAL LOW FLOW BASED ON PERIOD OF RECORD 1973.96

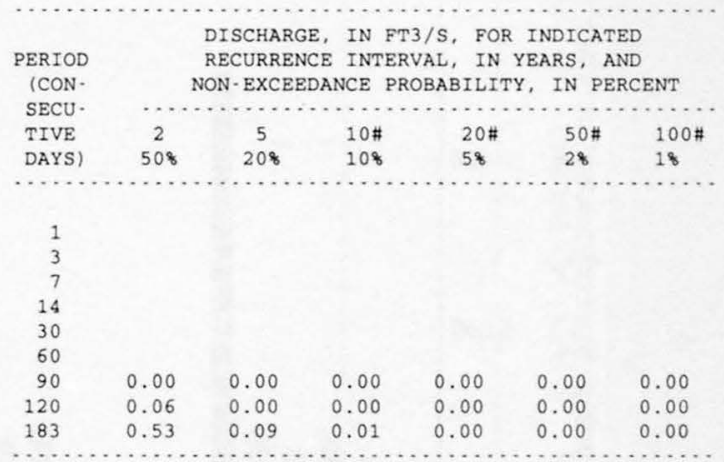

MAGNITUDE AND PROBABILITY OF ANNUAL HIGH FLOW BASED ON PERIOD OF RECORD 1973.96

\begin{tabular}{|c|c|c|c|c|c|c|}
\hline $\begin{array}{l}\text { PERIOD } \\
\text { (CON. }\end{array}$ & \multicolumn{6}{|c|}{ DISCHARGE, IN FT3/S, FOR INDICATED } \\
\hline SECU - & & ....... & , ...... & . . . . . & $\ldots \ldots \ldots$ & \\
\hline TIVE & 2 & 5 & $10 \#$ & $25 \#$ & $50 \#$ & $100 \#$ \\
\hline DAYS) & 508 & 208 & 108 & 48 & 28 & 18 \\
\hline 1 & 306 & 754 & 1,210 & 2,020 & 2,820 & 3,800 \\
\hline 3 & 132 & 334 & 537 & 887 & 1,220 & 1.630 \\
\hline 7 & 62 & 156 & 248 & 399 & 539 & 702 \\
\hline 15 & 32 & 78 & 122 & 192 & 254 & 324 \\
\hline 30 & 18 & 42 & 64 & 95 & 122 & 150 \\
\hline 60 & 11 & 25 & 37 & 54 & 68 & 82 \\
\hline 90 & 7.7 & 18 & 26 & 37 & 46 & 54 \\
\hline
\end{tabular}

DURATION TABLE OF DAILY MEAN FLOW FOR PERIOD OF RECORD 1973.96

DISCHARGE, IN FT3/S, WHICH WAS EQUALED OR EXCEEDED FOR INDICATED PERCENT OF TIME

\begin{tabular}{|c|c|c|c|c|c|c|c|c|c|c|c|c|c|c|c|c|}
\hline 18 & 58 & 108 & 158 & 208 & 308 & 408 & 508 & 608 & 708 & 808 & 908 & 958 & 988 & 998 & 99.58 & 99.98 \\
\hline 76 & 1.2 & 0.00 & 0.00 & 0.00 & 0.00 & 0.00 & 0.00 & 0.00 & 0.00 & 0.00 & 0.00 & 0.00 & 0.00 & 0.00 & 0.00 & 0.00 \\
\hline
\end{tabular}

\# Reliability of values in column is uncertain, and potential errors are large. 
SAN SIMON WASH BASIN

09535100 SAN SIMON WASH NEAR PISINIMO, AZ--Continued
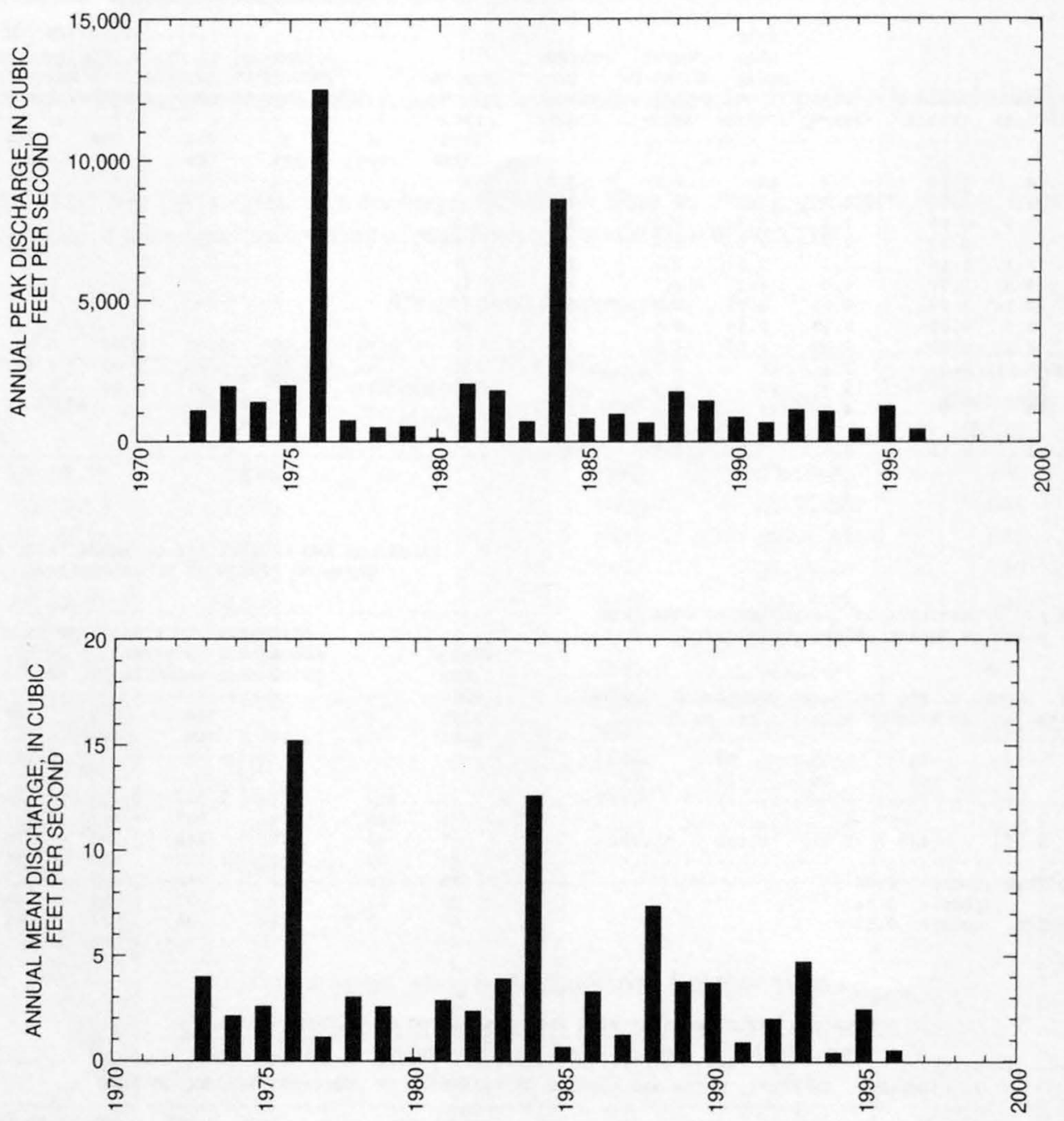
SAN SIMON WASH BASIN

09535100 SAN SIMON WASH NEAR PISINIMO, AZ--Continued
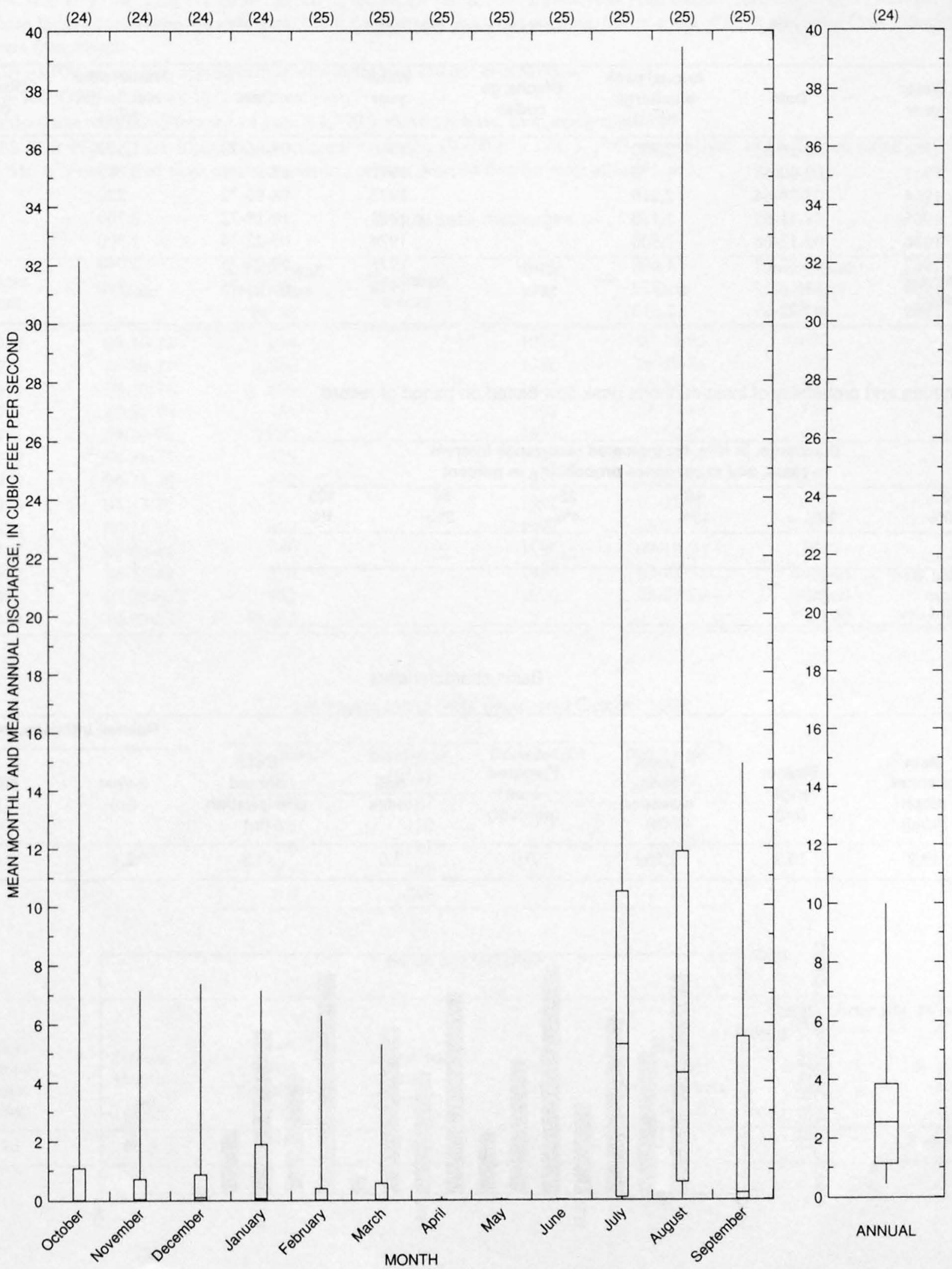
09535200 LITTLE TUCSON WASH AT SELLS, AZ

LOCATION.--Lat $31^{\circ} 54^{\prime} 55^{\prime \prime}$, long $111^{\circ} 52^{\prime} 42^{\prime \prime}$, in SE $1 / 4$ sec.25, T.17 S., R.4 E., Pima County, Hydrologic Unit 15080101, at Sells. DRAINAGE AREA.--26.8 $\mathrm{mi}^{2}$ contributing drainage area not determined.

Annual peak discharges

\begin{tabular}{|c|c|c|c|c|c|c|c|}
\hline $\begin{array}{l}\text { Water } \\
\text { year }\end{array}$ & Date & $\begin{array}{c}\text { Annual peak } \\
\text { discharge } \\
\left(\mathrm{ft}^{3} / \mathrm{s}\right)\end{array}$ & $\begin{array}{l}\text { Discharge } \\
\text { codes }\end{array}$ & $\begin{array}{l}\text { Water } \\
\text { year }\end{array}$ & Date & $\begin{array}{c}\text { Annual peak } \\
\text { discharge } \\
\left(\mathrm{ft}^{3} / \mathrm{s}\right)\end{array}$ & $\begin{array}{l}\text { Discharge } \\
\text { codes }\end{array}$ \\
\hline 1962 & $09-26-62$ & 2,740 & & 1970 & $09-06-70$ & 1,520 & \\
\hline 1963 & $00-00-63$ & 1,770 & & 1971 & $08-19-71$ & 2,080 & \\
\hline 1964 & $07-29-64$ & 2,210 & & 1972 & $08-00-72$ & 220 & \\
\hline 1965 & $08-11-65$ & 1,130 & & 1973 & $10-18-72$ & 2,760 & \\
\hline 1966 & $09-13-66$ & 2,800 & & 1974 & $09-22-74$ & 1,500 & \\
\hline 1967 & $08-07-67$ & 1,690 & & 1975 & $09-05-75$ & 2,040 & \\
\hline 1968 & $12-19-67$ & 770 & & 1976 & $00-00-76$ & 790 & \\
\hline 1969 & $07-22-69$ & 2,510 & & & & & \\
\hline
\end{tabular}

Magnitude and probability of instantaneous peak flow based on period of record

\begin{tabular}{|c|c|c|c|c|c|}
\hline \multicolumn{6}{|c|}{$\begin{array}{l}\text { Discharge, in } \mathrm{ft}^{3} / \mathrm{s} \text {, for indicated recurrence interval } \\
\text { in years, and exceedance probablility, in percent }\end{array}$} \\
\hline 2 & 5 & 10 & 25 & 50 & 100 \\
\hline $50 \%$ & $20 \%$ & $10 \%$ & $4 \%$ & $2 \%$ & $1 \%$ \\
\hline -.- & -.. & $\ldots$ & --. & -- & -.- \\
\hline Weighted skew & $(\log s)=$ & $\cdots$ & & & \\
\hline Mean & $(\log s)=$ & --. & & & \\
\hline Standard dev. & $(\log s)=$ & -- & & & \\
\hline
\end{tabular}

Basin characteristics

\begin{tabular}{cccccccc}
\hline $\begin{array}{c}\text { Main } \\
\text { channel } \\
\text { slope } \\
(\mathrm{ft} / \mathrm{mi})\end{array}$ & $\begin{array}{c}\text { Stream } \\
\text { length } \\
\text { (mi) }\end{array}$ & $\begin{array}{c}\text { Mean } \\
\text { basin } \\
\text { elevation } \\
(\mathrm{ft})\end{array}$ & $\begin{array}{c}\text { Forested } \\
\text { area } \\
\text { (percent) }\end{array}$ & $\begin{array}{c}\text { Soil } \\
\text { index }\end{array}$ & $\begin{array}{c}\text { Mean } \\
\text { annual } \\
\text { precipitation } \\
\text { (in) }\end{array}$ & $\begin{array}{c}\text { Rainfall intensity, 24-hour } \\
\text { (in) }\end{array}$ & $\begin{array}{c}50 \text {-year } \\
\text { (in) }\end{array}$ \\
\hline 49.9 & 10.2 & 2,560 & 0.0 & 3.0 & 11.8 & 2.1 \\
\hline
\end{tabular}

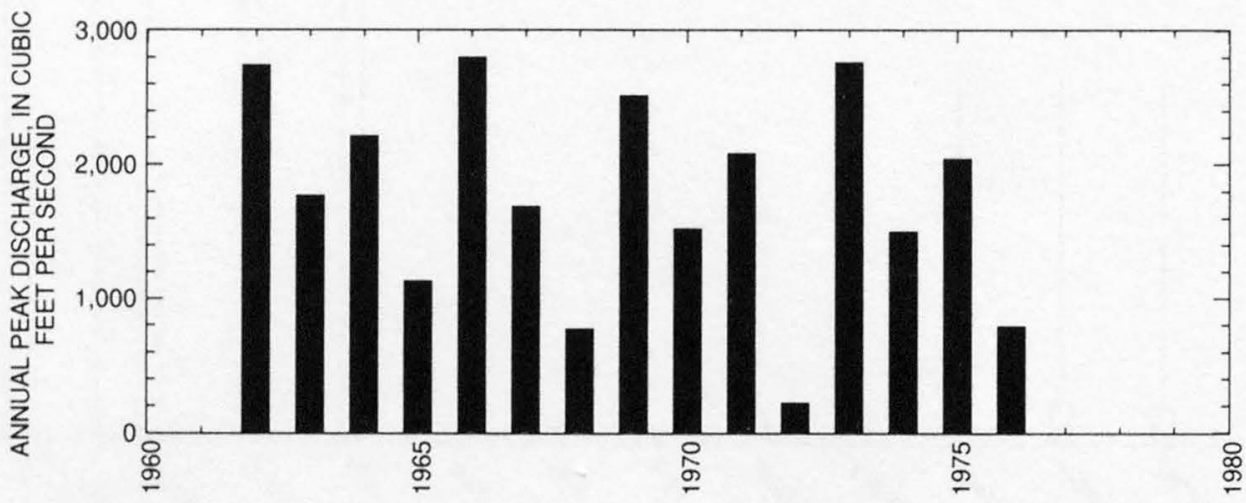




\section{SAN SIMON WASH BASIN}

\section{VAMORI WASH AT KOM VO, AZ}

LOCATION.--Lat $31^{\circ} 57^{\prime} 04^{\prime \prime}$, long 112 $20^{\prime} 50^{\prime \prime}$, in NW $1 / 4$ sec.14, T.17 S., R.1 W (unsurveyed), Pima County, Hydrologic Unit 15080101 , in Tohono O'Odham Indian Reservation, on right bank $200 \mathrm{ft}$ downstream from road crossing, $0.6 \mathrm{mi}$ south of Kom Vo (Santa Cruz Village) and $5 \mathrm{mi}$ upstream from mouth.

DRAINAGE AREA.--1,250 $\mathrm{mi}^{2}$, approximately, of which about $250 \mathrm{mi}^{2}$ is in Mexico.

PERIOD OF RECORD,--February 1972 to current year.

GAGE.--Water-stage recorder. Elevation of gage is $1,770 \mathrm{ft}$ above sea level, from topographic map.

EXTREMES FOR PERIOD OF RECORD.--Maximum discharge, 10,400 $\mathrm{ft}^{3} / \mathrm{s}$ Oct. 3, 1983, gage height, $10.54 \mathrm{ft}$, from rating curve extended above $550 \mathrm{ft}^{3} / \mathrm{s}$ on basis of slope-area measurement of peak flow; no flow for most of each year.

Annual peak discharges

\begin{tabular}{|c|c|c|c|c|c|c|c|}
\hline $\begin{array}{l}\text { Water } \\
\text { year }\end{array}$ & Date & $\begin{array}{c}\text { Annual peak } \\
\text { discharge } \\
\left(\mathrm{ft}^{3} / \mathrm{s}\right)\end{array}$ & $\begin{array}{l}\text { Discharge } \\
\text { codes }\end{array}$ & $\begin{array}{l}\text { Water } \\
\text { year }\end{array}$ & Date & $\begin{array}{c}\text { Annual peak } \\
\text { discharge } \\
\left(\mathrm{ft}^{3} / \mathrm{s}\right)\end{array}$ & $\begin{array}{c}\text { Discharge } \\
\text { codes }\end{array}$ \\
\hline 1972 & $08-10-72$ & 214 & & 1985 & $07-19-85$ & 630 & \\
\hline 1973 & $10-20-72$ & 1,880 & & 1986 & $08-29-86$ & 960 & \\
\hline 1974 & $09-22-74$ & 689 & & 1987 & $07-31-87$ & 762 & \\
\hline 1975 & $09-00-75$ & 751 & & 1988 & $08-29-88$ & 768 & \\
\hline 1976 & $09-26-76$ & 1,650 & & 1989 & $07-28-89$ & 453 & \\
\hline 1977 & $08-14-77$ & 325 & & 1990 & $07-11-90$ & 1,150 & \\
\hline 1978 & $08-13-78$ & 808 & & 1991 & $10-01-90$ & 1,210 & \\
\hline 1979 & $01-17-79$ & 575 & & 1992 & $07-11-92$ & 484 & \\
\hline 1980 & $08-15-80$ & 469 & & 1993 & $08-28-93$ & 1,400 & \\
\hline 1981 & $09-05-81$ & 769 & & 1994 & $09-10-94$ & 1,790 & \\
\hline 1982 & $08-26-82$ & 576 & & 1995 & 09-07-95 & 1,250 & \\
\hline 1983 & $07-23-83$ & 982 & & 1996 & $08-27-96$ & 465 & \\
\hline 1984 & $10-03-83$ & 10,400 & & & & & \\
\hline
\end{tabular}

Discharge rating table developed October 1992

\begin{tabular}{crcr}
\hline $\begin{array}{c}\text { Gage height } \\
(\mathbf{f t})\end{array}$ & $\begin{array}{c}\text { Discharge } \\
\left(\mathbf{f t}^{\mathbf{3}} \mathbf{3}\right)\end{array}$ & $\begin{array}{c}\text { Gage height } \\
(\mathbf{f t})\end{array}$ & $\begin{array}{c}\text { Discharge } \\
\left(\mathbf{f t}^{\mathbf{3}} / \mathbf{s}\right)\end{array}$ \\
\hline 6.0 & 87 & 8.5 & 340 \\
6.5 & 120 & 9.0 & 700 \\
7.0 & 157 & 9.5 & 1,820 \\
7.5 & 197 & 10.0 & 4,360 \\
8.0 & 240 & 10.5 & 9,780 \\
\hline
\end{tabular}

\section{Basin characteristics}

\begin{tabular}{cccccccc}
\hline $\begin{array}{c}\text { Main } \\
\text { channel } \\
\text { slope } \\
(\mathrm{ft} / \mathrm{mi})\end{array}$ & $\begin{array}{c}\text { Stream } \\
\text { length } \\
(\mathrm{mi})\end{array}$ & $\begin{array}{c}\text { Mean } \\
\text { basin } \\
\text { elevation } \\
(\mathrm{ft})\end{array}$ & $\begin{array}{c}\text { Forested } \\
\text { area } \\
\text { (percent) }\end{array}$ & $\begin{array}{c}\text { Soil } \\
\text { index }\end{array}$ & $\begin{array}{c}\text { Mean } \\
\text { annual } \\
\text { precipitation } \\
\text { (in) }\end{array}$ & $\begin{array}{c}\text { Rainfall intensity, 24-hour } \\
\text { (in) }\end{array}$ & $\begin{array}{c}50 \text {-year } \\
\text { (in) }\end{array}$ \\
\hline 14.1 & 64.4 & 2,699 & 1.6 & 0.0 & 12.5 & 2.2 \\
\hline
\end{tabular}




\section{SAN SIMON WASH BASIN}

09535300 VAMORI WASH AT KOM VO, AZ--Continued

MEAN MONTHLY AND ANNUAL DISCHARGES 1973.96

\begin{tabular}{|c|c|c|c|c|c|c|}
\hline MONTH & $\begin{array}{l}\text { MAXIMUN } \\
\text { (FT3/S) }\end{array}$ & $\begin{array}{l}\text { MINIMUM } \\
(\text { FT3/S) }\end{array}$ & $\begin{array}{c}\text { MEAN } \\
(F T 3 / S)\end{array}$ & $\begin{array}{l}\text { STAN- } \\
\text { DARD } \\
\text { DEVIA. } \\
\text { TION } \\
\text { (FT3/S) }\end{array}$ & $\begin{array}{l}\text { COEFFI- } \\
\text { CIENT OF } \\
\text { VARI- } \\
\text { ATION }\end{array}$ & $\begin{array}{c}\text { PERCENT } \\
\text { OF } \\
\text { ANNUAL } \\
\text { RUNOFF }\end{array}$ \\
\hline OCTOBER & 463 & 0.00 & 29 & 95 & 3.3 & 24.5 \\
\hline NOVEMBER & 37 & 0.00 & 3.3 & 9.0 & 2.8 & 2.8 \\
\hline DECEMBER & 26 & 0.00 & 5.4 & 8.4 & 1.5 & 4.6 \\
\hline JANUARY & 61 & 0,00 & 8.9 & 17 & 1.9 & 7.6 \\
\hline FEBRUARY & 33 & 0.00 & 4.5 & 9.6 & 2.1 & 3.9 \\
\hline MARCH & 28 & 0.00 & 2.4 & 6.1 & 2.5 & 2.1 \\
\hline APRIL & 10 & 0.00 & 0.45 & 2.1 & 4,7 & 0.4 \\
\hline MAY & 0.49 & 0.00 & 0.04 & 0.13 & 2.9 & 0.0 \\
\hline JUNE & 0.07 & 0.00 & 0.00 & 0.01 & 4.9 & 0.0 \\
\hline JULY & 113 & 0.00 & 17 & 24 & 1.4 & 14.5 \\
\hline AUGUST & 106 & 0.73 & 31 & 25 & 0.82 & 26.2 \\
\hline SEPTEMBER & 103 & 0.00 & 16 & 22 & 1.4 & 13.4 \\
\hline L & 52 & 0.97 & 9.8 & 10 & 1.0 & 100 \\
\hline
\end{tabular}

MAGNITUDE AND PROBABILITY OF INSTANTANBOUS PEAK FLOW BASED ON PERIOD OF RECORD 1972.96

DISCHARGE, IN FT3/S, FOR INDICATED RECURRENCE INTERVAL IN YEARS, AND EXCEEDANCE PROBABILITY, IN PERCENT

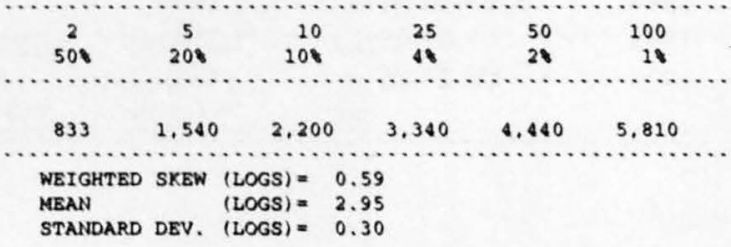

MAGNITUDE AND PROBABILITY OF ANNUAL LOW FLOW BASED ON PERIOD OF RECORD 1973.96

\begin{tabular}{|c|c|c|c|c|c|c|}
\hline \multirow{4}{*}{$\begin{array}{l}\text { PERIOD } \\
\text { (CON- } \\
\text { SECU. } \\
\text { TIVE } \\
\text { DAYS) }\end{array}$} & \multicolumn{6}{|c|}{$\begin{array}{l}\text { DISCHARGE, IN FT3/S, FOR INDICATED } \\
\text { RECURRENCE INTERVAL, IN YEARS, AND } \\
\text { NON-EXCEEDANCE PROBABILITY, IN PERCENT }\end{array}$} \\
\hline & $\cdots$ & $\cdots \cdots$ & $\cdots \cdots$ & & & \\
\hline & 2 & 5 & $10 \#$ & $20 \#$ & $50 \#$ & $100 \#$ \\
\hline & 508 & 208 & 108 & 58 & 28 & 18 \\
\hline $\begin{array}{r}1 \\
3 \\
7 \\
14 \\
30 \\
60\end{array}$ & & & & & & \\
\hline 90 & 0.00 & 0.00 & 0.00 & 0.00 & 0.00 & 0.00 \\
\hline 120 & 0.21 & 0.00 & 0.00 & 0.00 & 0.00 & 0.00 \\
\hline 183 & 2.0 & 0.20 & 0.00 & 0.00 & 0.00 & 0.00 \\
\hline
\end{tabular}

MAGNITUDE AND PROBABILITY OF ANNUAL HIGH FLOW BASED ON PERIOD OF RECORD 1973.96

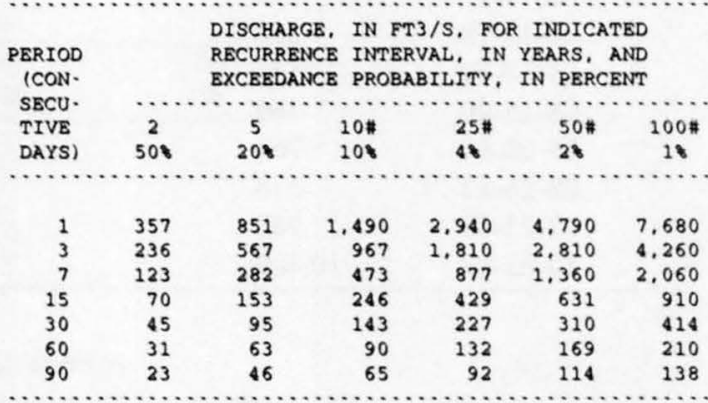

DURATION TABLE OF DAILY MEAN FLOW FOR PERIOD OF RECORD 1973.96 DISCHARGE, IN FT3/S, WHICH WAS EQUALED OR EXCEEDED FOR INDICATED PERCENT OF TIME

\begin{tabular}{|c|c|c|c|c|c|c|c|c|c|c|c|c|c|c|c|c|}
\hline 14 & 58 & 108 & 158 & 208 & 308 & 408 & 508 & 608 & 708 & 808 & 908 & 958 & 988 & 998 & 99.58 & 99.98 \\
\hline & 33 & 5.0 & 0.17 & 0.00 & 0.00 & 0,00 & 0,00 & 0.00 & 0.00 & 0.00 & 0.00 & 0.00 & 0.00 & 0.00 & 0.00 & 0.00 \\
\hline
\end{tabular}

\# Reliability of values in column is uncertain, and potential errors are large. 
SAN SIMON WASH BASIN

09535300 VAMORI WASH AT KOM VO, AZ--Continued
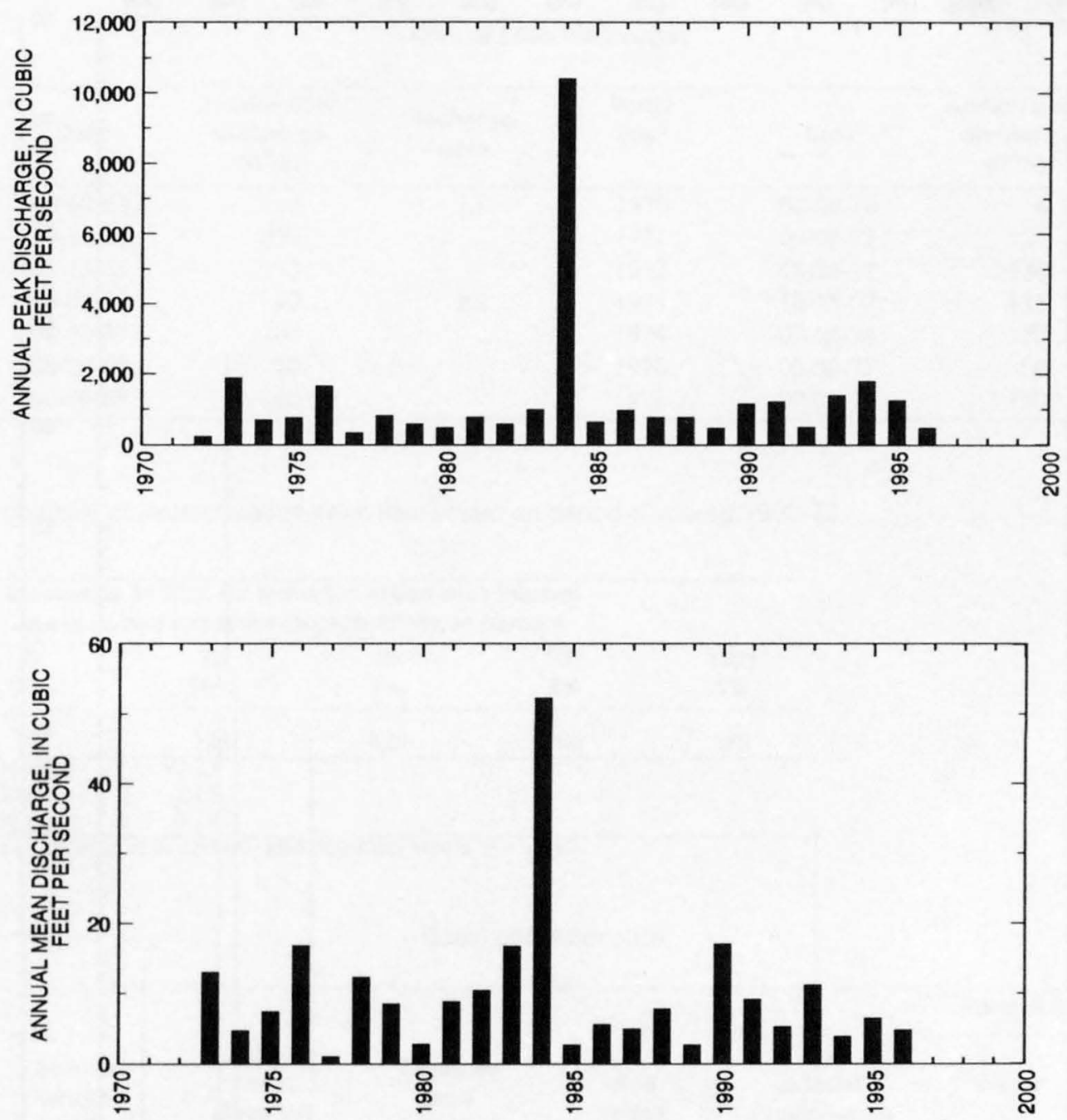
896

RIO SONOYTA BASIN

SAN SIMON WASH BASIN

09535300 VAMORI WASH AT KOM VO, AZ--Continued
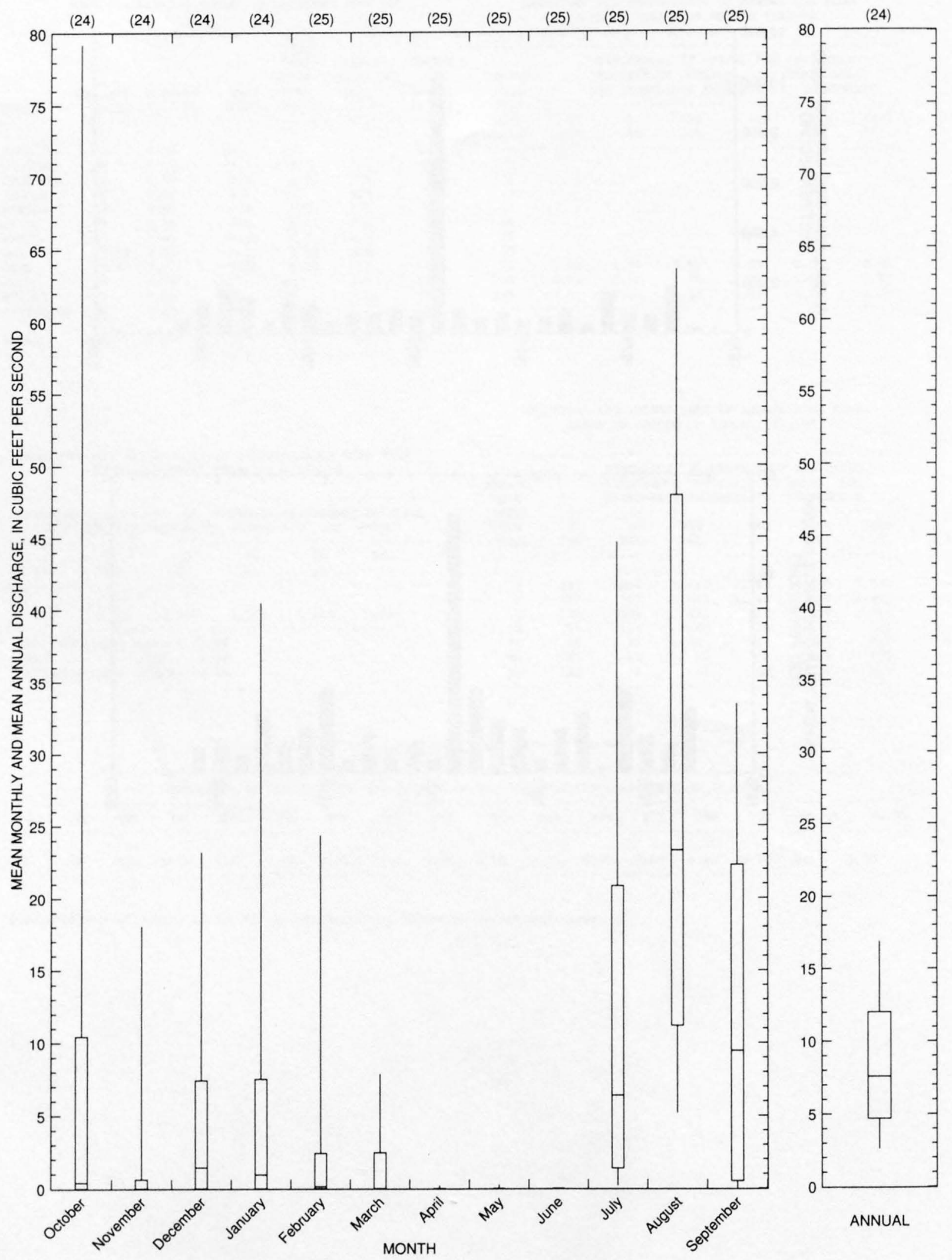
09536100 PITCHFORK CANYON TRIBUTARY NEAR FORT GRANT, AZ

LOCATION.--Lat 32 35'20", long 109 54'40", in SE $1 / 4$ sec.5, T.10 S., R.24 E., Graham County, Hydrologic Unit 15050201 , at State Highway 266 , 3 mi southeast of Fort Grant.

DRAINAGE AREA.--0.81 $\mathrm{mi}^{2}$

Annual peak discharges

\begin{tabular}{|c|c|c|c|c|c|c|c|}
\hline $\begin{array}{l}\text { Water } \\
\text { year }\end{array}$ & Date & $\begin{array}{c}\text { Annual peak } \\
\text { discharge } \\
\left(\mathrm{ft}^{3} / \mathrm{s}\right)\end{array}$ & $\begin{array}{l}\text { Discharge } \\
\text { codes }\end{array}$ & $\begin{array}{l}\text { Water } \\
\text { year }\end{array}$ & Date & $\begin{array}{c}\text { Annual peak } \\
\text { discharge } \\
\left(\mathrm{ft}^{3} / \mathrm{s}\right)\end{array}$ & $\begin{array}{c}\text { Discharge } \\
\text { codes }\end{array}$ \\
\hline 1963 & $00-00-63$ & 10 & LT & 1970 & $00-00-70$ & 0 & \\
\hline 1964 & $07-24-64$ & 354 & & 1971 & $09-08-71$ & 320 & \\
\hline 1965 & $08-15-65$ & 375 & & 1972 & $08-26-72$ & 130 & \\
\hline 1966 & $08-07-66$ & 40 & ES & 1973 & $10-15-72$ & 135 & \\
\hline 1967 & $08-00-67$ & 200 & & 1974 & $07-16-74$ & 120 & \\
\hline 1968 & $08-20-68$ & 60 & & 1975 & $00-00-75$ & 0 & \\
\hline 1969 & $00-00-69$ & 180 & & 1976 & $00-00-76$ & 190 & \\
\hline
\end{tabular}

Magnitude and probability of instantaneous peak flow based on period of record 1963-76

\begin{tabular}{|c|c|c|c|c|c|}
\hline \multicolumn{6}{|c|}{$\begin{array}{l}\text { Discharge, in } \mathrm{ft}^{3} / \mathrm{s} \text {, for indicated recurrence interval } \\
\text { in years, and exceedance probablility, in percent }\end{array}$} \\
\hline 2 & 5 & 10 & 25 & $50 t$ & $100 t$ \\
\hline $50 \%$ & $20 \%$ & $10 \%$ & $4 \%$ & $2 \%$ & $1 \%$ \\
\hline 144 & 247 & 323 & 425 & 506 & 589 \\
\hline Weighted skew & $(\log s)=$ & -0.23 & & & \\
\hline Mean & $(\log s)=$ & 2.15 & & & \\
\hline Standard dev. & $(\log s)=$ & 0.29 & & & \\
\hline
\end{tabular}

fReliability of values in column is uncertain, and potential errors are large.

Basin characteristics

\begin{tabular}{cccccccc}
\hline $\begin{array}{c}\text { Main } \\
\text { channel } \\
\text { slope } \\
\text { (ft/mi) }\end{array}$ & $\begin{array}{c}\text { Stream } \\
\text { length } \\
(\mathrm{mi})\end{array}$ & $\begin{array}{c}\text { Mean } \\
\text { basin } \\
\text { elevation } \\
(\mathrm{ft})\end{array}$ & $\begin{array}{c}\text { Forested } \\
\text { area } \\
\text { (percent) }\end{array}$ & $\begin{array}{c}\text { Soil } \\
\text { index }\end{array}$ & $\begin{array}{c}\text { Mean } \\
\text { annual } \\
\text { precipitation } \\
\text { (in) }\end{array}$ & $\begin{array}{c}\text { Rainfall intensity, 24-hour } \\
\text { (in) }\end{array}$ & $\begin{array}{c}50 \text {-year } \\
\text { (in) }\end{array}$ \\
\hline 459 & 1.8 & 5,210 & 0.0 & 3.0 & 15.0 & 2.1 \\
\hline
\end{tabular}

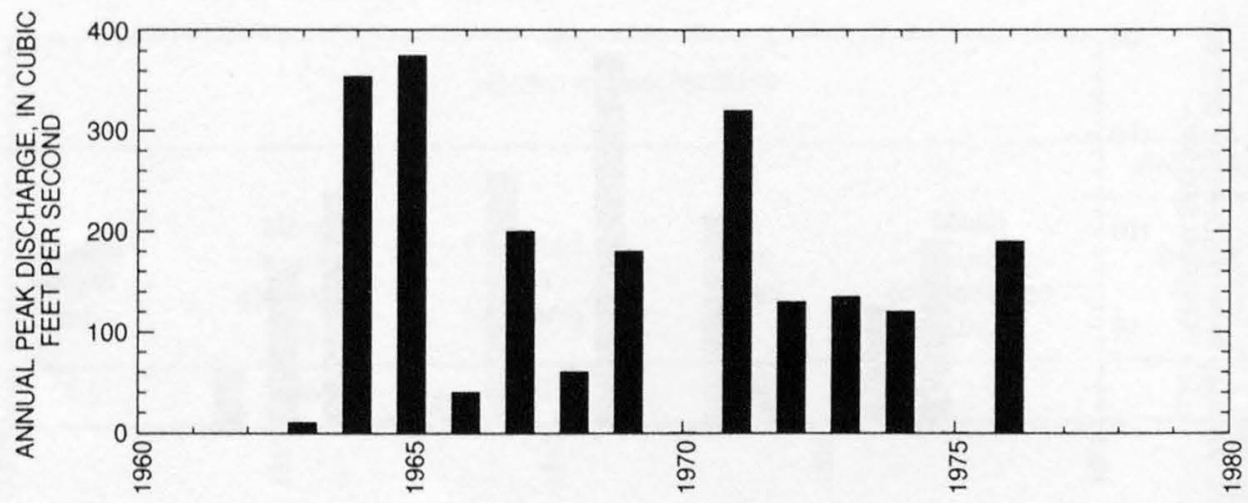




\section{SURPRISE CANYON NEAR DOS CABEZAS, AZ}

LOCATION.--Lat $32^{\circ} 00^{\prime} 40^{\prime \prime}$, long $109^{\circ} 21^{\prime} 12^{\prime \prime}$, in SW1/4 sec.25, T.16 S., R.29 E., Cochise County, Hydrologic Unit 15050201 , at main road through

Chiricahua National Monument, 0.4 mi north of ranger station, and $19 \mathrm{mi}$ southeast of Dos Cabezas.

DRAINAGE AREA.--0.65 $\mathrm{mi}^{2}$.

Annual peak discharges

\begin{tabular}{|c|c|c|c|c|c|c|c|}
\hline $\begin{array}{l}\text { Water } \\
\text { year }\end{array}$ & Date & $\begin{array}{c}\text { Annual peak } \\
\text { discharge } \\
\left(\mathrm{ft}^{3} / \mathrm{s}\right)\end{array}$ & $\begin{array}{c}\text { Discharge } \\
\text { codes }\end{array}$ & $\begin{array}{l}\text { Water } \\
\text { year }\end{array}$ & Date & $\begin{array}{c}\text { Annual peak } \\
\text { discharge } \\
\left(\mathrm{ft}^{3} / \mathrm{s}\right)\end{array}$ & $\begin{array}{c}\text { Discharge } \\
\text { codes }\end{array}$ \\
\hline 1963 & $08-00-63$ & 94 & & 1970 & $00-00-70$ & 0 & \\
\hline 1964 & $08-14-64$ & 61 & & 1971 & $08-19-71$ & 130 & ES \\
\hline 1965 & $00-00-65$ & 0 & & 1972 & $10-25-71$ & 17 & \\
\hline 1966 & $08-18-66$ & 32 & & 1973 & $02-21-73$ & 4.0 & \\
\hline 1967 & $07-26-67$ & 108 & & 1974 & $07-07-74$ & 120 & \\
\hline 1968 & $00-00-68$ & 0 & & 1975 & $07-19-75$ & 80 & \\
\hline 1969 & $07-15-69$ & 191 & & 1976 & $00-00-76$ & 30 & ES \\
\hline
\end{tabular}

Magnitude and probability of instantaneous peak flow based on period of record 1963-76

\begin{tabular}{|c|c|c|c|c|c|}
\hline \multicolumn{6}{|c|}{$\begin{array}{l}\text { Discharge, in } \mathrm{ft}^{3} / \mathrm{s} \text {, for indicated recurrence interval } \\
\text { in years, and exceedance probablility, in percent }\end{array}$} \\
\hline 2 & 5 & 10 & 25 & $50+$ & $100+$ \\
\hline $50 \%$ & $20 \%$ & $10 \%$ & $4 \%$ & $2 \%$ & $1 \%$ \\
\hline 44.9 & 113 & 172 & 261 & 335 & 413 \\
\hline Weighted skew & $(\log s)=$ & -0.51 & & & \\
\hline Mean & $(\log s)=$ & 1.61 & & & \\
\hline Standard dev. & $(\log s)=$ & 0.52 & & & \\
\hline
\end{tabular}

$\mp$ Reliability of values in column is uncertain, and potential errors are large.

Basin characteristics

\begin{tabular}{cccccccc}
\hline $\begin{array}{c}\text { Main } \\
\text { channel } \\
\text { slope } \\
(\mathrm{ft} / \mathrm{mi})\end{array}$ & $\begin{array}{c}\text { Stream } \\
\text { length } \\
\text { (mi) }\end{array}$ & $\begin{array}{c}\text { Mean } \\
\text { basin } \\
\text { elevation } \\
\text { (ft) }\end{array}$ & $\begin{array}{c}\text { Forested } \\
\text { area } \\
\text { (percent) }\end{array}$ & $\begin{array}{c}\text { Soil } \\
\text { index }\end{array}$ & $\begin{array}{c}\text { Mean } \\
\text { annual } \\
\text { precipitation } \\
\text { (in) }\end{array}$ & $\begin{array}{c}\text { Rainfall intensity, 24-hour } \\
\text { (in) }\end{array}$ & $\begin{array}{c}50-y e a r \\
\text { (in) }\end{array}$ \\
\hline 1,040 & 1.7 & 6,280 & 100 & 1.0 & 18.0 & 2.2 \\
\hline
\end{tabular}

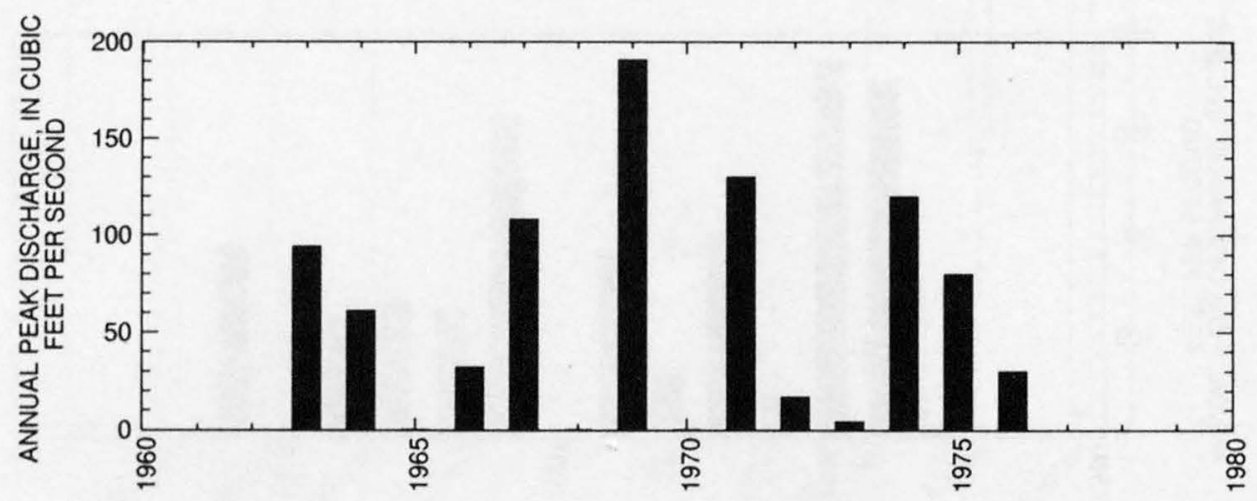




\section{WHITEWATER DRAW BASIN}

\section{LESLIE CREEK NEAR McNEAL, AZ}

LOCATION.--Lat 31.35'24", long 109.30'30", in SE $1 / 4 \mathrm{NE}^{1} / 4$ sec.20, T.21 S., R.28 E., Cochise County, Hydrologic Unit 15080301, on right bank $10 \mathrm{mi}$ east of McNeal.

DRAINAGE AREA.--79.1 $\mathrm{mi}^{2}$.

PERIOD OF RECORD.--October 1969 to September 1977, July 1982 to current year.

GAGE.--Water-stage recorder and concrete control with shallow sharp-crested V-notch weir. Elevation of gage is $4,620 \mathrm{ft}$ above sea level, from topographic map.

REMARKS.--No estimated daily discharges. Records good.

EXTREMES FOR PERIOD OF RECORD.--Maximum discharge, 5,200 ft $3 / \mathrm{s} \mathrm{Sept.} \mathrm{1,} \mathrm{1994,} \mathrm{gage} \mathrm{height,} 9.00 \mathrm{ft}$, from rating curve extended above $12 \mathrm{ft}^{3} / \mathrm{s}$ on basis of slope-area measurements of peak flow at gage height $7.33 \mathrm{ft}$ and $8.54 \mathrm{ft}$; no flow for many days in 1976,1977 , and 1990 . EXTREMES OUTSIDE PERIOD OF RECORD.--Maximum discharge, October 1977 to July $1982,468 \mathrm{ft}^{3} / \mathrm{s}$, date unknown, gage height, $4.76 \mathrm{ft}$ in gage well.

Annual peak discharges

\begin{tabular}{ccccccc}
\hline $\begin{array}{c}\text { Water } \\
\text { year }\end{array}$ & Date & $\begin{array}{c}\text { Annual peak } \\
\text { discharge } \\
\left(\mathbf{f t}^{\mathbf{3}} / \mathbf{s}\right)\end{array}$ & $\begin{array}{c}\text { Discharge } \\
\text { codes }\end{array}$ & $\begin{array}{c}\text { Water } \\
\text { year }\end{array}$ & $\begin{array}{c}\text { Annual peak } \\
\text { Date } \\
\text { discharge } \\
\left.\mathbf{f t}^{\mathbf{3}} / \mathbf{s}\right)\end{array}$ \\
\hline 1970 & $08-10-70$ & 345 & & 1986 & $08-18-86$ & 658 \\
1971 & $08-12-71$ & 1,760 & & 1987 & $08-05-87$ & 307 \\
1972 & $07-15-72$ & 314 & 1988 & $07-20-88$ & 250 \\
1973 & $10-20-72$ & 255 & 1989 & $08-06-89$ & 71 \\
1974 & $07-20-74$ & 162 & 1990 & $07-21-90$ & 1,090 \\
1975 & $07-23-75$ & 132 & 1991 & $08-27-91$ & 300 \\
1976 & $10-21-75$ & 1.0 & 1992 & $07-23-92$ & 750 \\
1977 & $08-08-77$ & 563 & 1993 & $01-19-93$ & 2,410 \\
1982 & $08-15-82$ & 30 & 1994 & $09-01-94$ & 5,200 \\
1983 & $02-04-83$ & 322 & 1995 & $01-05-95$ & 2,660 \\
1984 & $07-21-84$ & 4,600 & 1996 & $07-10-96$ & 4,920 \\
1985 & $10-03-83$ & 709 & & & & \\
\hline
\end{tabular}

Discharge rating table developed October 1991

\begin{tabular}{cccc}
\hline $\begin{array}{c}\text { Gage height } \\
\text { (ft) }\end{array}$ & $\begin{array}{c}\text { Discharge } \\
\left(\mathbf{f t}^{\mathbf{3}} / \mathbf{s}\right)\end{array}$ & $\begin{array}{c}\text { Gage height } \\
(\mathbf{f t})\end{array}$ & $\begin{array}{c}\text { Discharge } \\
\left(\mathbf{f t}^{\mathbf{3}} / \mathbf{s}\right)\end{array}$ \\
\hline 4.5 & 340 & 7.0 & 2,780 \\
5.0 & 700 & 7.5 & 3,400 \\
5.5 & 1,140 & 8.0 & 4,000 \\
6.0 & 1,660 & 8.5 & 4,600 \\
6.5 & 2,220 & 9.0 & 5,200 \\
\hline
\end{tabular}

Basin characteristics

\begin{tabular}{cccccccc}
\hline $\begin{array}{c}\text { Main } \\
\text { channel } \\
\text { slope } \\
(\mathrm{ft} / \mathrm{mi})\end{array}$ & $\begin{array}{c}\text { Stream } \\
\text { length } \\
(\mathrm{mi})\end{array}$ & $\begin{array}{c}\text { Mean } \\
\text { basin } \\
\text { elevation } \\
(\mathrm{ft})\end{array}$ & $\begin{array}{c}\text { Forested } \\
\text { area } \\
\text { (percent) }\end{array}$ & $\begin{array}{c}\text { Soll } \\
\text { index }\end{array}$ & $\begin{array}{c}\text { Mean } \\
\text { annual } \\
\text { precipitation } \\
\text { (in) }\end{array}$ & $\begin{array}{c}\text { Rainfall intensity, 24-hour } \\
\text { (in) }\end{array}$ & $\begin{array}{c}\text { 50-year } \\
\text { (in) }\end{array}$ \\
\hline 62.1 & 14.5 & 5,360 & 30.0 & 2.0 & 18.0 & 2.0 \\
\hline
\end{tabular}


WHITEWATER DRAW BASIN

09537200 LESLIE CREEK NEAR McNEAL, AZ--Continued

MEAN MONTHLY AND ANNUAL DISCHARGES 1970.77, 1983.96

\begin{tabular}{|c|c|c|c|c|c|c|}
\hline MONTH & $\begin{array}{l}\text { MAXIMUM } \\
\text { (FT3/S) }\end{array}$ & $\begin{array}{l}\text { MINIMUM } \\
\text { (FT3/S) }\end{array}$ & $\begin{array}{c}\text { MEAN } \\
(\text { FT3/S) }\end{array}$ & $\begin{array}{l}\text { STAN- } \\
\text { DARD } \\
\text { DEVIA- } \\
\text { TION } \\
\text { (FT3/S) }\end{array}$ & $\begin{array}{l}\text { COEFFI- } \\
\text { CIENT OF } \\
\text { VARI- } \\
\text { ATION }\end{array}$ & $\begin{array}{c}\text { PERCENT } \\
\text { OF } \\
\text { ANNUAL } \\
\text { RUNOFF }\end{array}$ \\
\hline OCTOBER & 31 & 0.00 & 2.5 & 6.5 & 2.6 & 14.5 \\
\hline NOVEMBER & 1.8 & 0.00 & 0.73 & 0.57 & 0.78 & 4.3 \\
\hline DECEMBER & 4.0 & 0.02 & 0.83 & 0.88 & 1.1 & 4.9 \\
\hline JANUARY & 14 & 0.02 & 1.8 & 3.7 & 2.1 & 10.5 \\
\hline FEBRUARY & 3.2 & 0.02 & 0.75 & 0.77 & 1.0 & 4.4 \\
\hline MARCH & 1.6 & 0.02 & 0.54 & 0.42 & 0.78 & 3.2 \\
\hline APRIL & 1.7 & 0.00 & 0.52 & 0.45 & 0.87 & 3.0 \\
\hline MAY & 1.4 & 0.00 & 0.44 & 0.42 & 0.96 & 2.6 \\
\hline JUNE & 1.8 & 0.00 & 0.42 & 0.46 & 1.1 & 2.4 \\
\hline JULY & 26 & 0.00 & 3.3 & 7.2 & 2.2 & 19.2 \\
\hline AUGUST & 17 & 0.00 & 3.2 & 4.0 & 1.3 & 18.9 \\
\hline SEPTEMBER & 26 & 0.00 & 2.1 & 5.5 & 2.7 & 12.2 \\
\hline ANNUAL & 6.6 & 0.07 & 1.4 & 1.5 & 1.0 & 100 \\
\hline
\end{tabular}

MAGNITUDE AND PROBABILITY OF INSTANTANEOUS PEAK FLOW BASED ON PERIOD OF RECORD $1970.77,1982.96$

DISCHARGE, IN FT3/S, FOR INDICATED RECURRENCE INTERVAL IN YEARS, AND EXCEEDANCE PROBABILITY, IN PERCENT

\begin{tabular}{|c|c|c|c|c|c|}
\hline 2 & 5 & 10 & 25 & 50 & 100 \\
\hline 508 & 208 & 108 & 48 & 28 & 18 \\
\hline 612 & 1,910 & 3,430 & 6,380 & 9,490 & 13,500 \\
\hline
\end{tabular}

WEIGHTED SKEW (LOGS $)=0.06$

MEAN (LOGS) $=2.78$

STANDARD DEV. $($ LOGS $)=0.59$
MAGNITUDE AND PROBABILITY OF ANNUAL LOW FLOW BASED ON PERIOD OF RECORD $1971.77,1984.96$

\begin{tabular}{|c|c|c|c|c|c|c|}
\hline \multirow{4}{*}{$\begin{array}{l}\text { PERIOD } \\
\text { (CON- } \\
\text { SECU- } \\
\text { TIVE } \\
\text { DAYS) }\end{array}$} & \multicolumn{6}{|c|}{$\begin{array}{l}\text { DISCHARGE, IN FT3/S, FOR INDICATED } \\
\text { RECURRENCE INTERVAL, IN YEARS, AND } \\
\text { NON-EXCEEDANCE PROBABILITY, IN PERCENT }\end{array}$} \\
\hline & & & & & & \\
\hline & 2 & 5 & 10 & 20 & $50 \#$ & $100 \#$ \\
\hline & 508 & 208 & 108 & 58 & 28 & 18 \\
\hline 1 & 0.13 & 0.02 & 0.00 & 0.00 & 0.00 & 0.00 \\
\hline 3 & 0.13 & 0.02 & 0.00 & 0.00 & 0.00 & 0.00 \\
\hline 7 & 0.14 & 0.03 & 0.00 & 0.00 & 0.00 & 0.00 \\
\hline 14 & 0.15 & 0.03 & 0.00 & 0.00 & 0.00 & 0.00 \\
\hline 30 & 0.17 & 0.04 & 0.00 & 0.00 & 0.00 & 0.00 \\
\hline 60 & 0.21 & 0.07 & 0.00 & 0.00 & 0.00 & 0.00 \\
\hline 90 & 0.25 & 0.08 & 0.04 & 0.00 & 0.00 & 0.00 \\
\hline 120 & 0.35 & 0.16 & 0.10 & 0.00 & 0.00 & 0.00 \\
\hline 183 & 0.53 & 0.22 & 0.13 & 0.00 & 0.00 & 0.00 \\
\hline
\end{tabular}

MAGNITUDE AND PROBABILITY OF ANNUAL HIGH FLOW BASED ON PERIOD OF RECORD 1970-77, 1983.96

\begin{tabular}{|c|c|c|c|c|c|c|}
\hline \multirow{3}{*}{$\begin{array}{l}\text { PERIOD } \\
\text { (CON- } \\
\text { SECU. }\end{array}$} & \multicolumn{6}{|c|}{ DISCHARGE, IN FT3/S, FOR INDICATED } \\
\hline & & \multicolumn{3}{|c|}{ EXCEEDANCE PROBABILITY } & \multicolumn{2}{|c|}{ IN PERCENT } \\
\hline & & & & & & \\
\hline TIVE & 2 & 5 & 10 & 25 & $50 \%$ & $100=$ \\
\hline DAYS) & 508 & 208 & 108 & 48 & 28 & 18 \\
\hline 1 & 82 & 279 & 440 & 636 & 764 & 871 \\
\hline 3 & 32 & 109 & 183 & 288 & 369 & 448 \\
\hline 7 & 14 & 48 & 84 & 142 & 194 & 250 \\
\hline 15 & 7.5 & 25 & 43 & 75 & 105 & 140 \\
\hline 30 & 4.4 & 14 & 24 & 41 & 58 & 78 \\
\hline 60 & 2.7 & 7.6 & 13 & 22 & 30 & 40 \\
\hline 90 & 2.0 & 5.4 & 8.9 & 15 & 20 & 26 \\
\hline
\end{tabular}

DURATION TABLE OF DAILY MEAN FLOW FOR PERIOD OF RECORD 1970-77, 1983.96

DISCHARGE, IN FT3/S, WHICH WAS EQUALED OR EXCEEDED FOR INDICATED PERCENT OF TIME

\begin{tabular}{|c|c|c|c|c|c|c|c|c|c|c|c|c|c|c|c|c|}
\hline 18 & 58 & 108 & $15 \%$ & 208 & 308 & 408 & 508 & 608 & 708 & 808 & 908 & 958 & $98 \%$ & 998 & 99.58 & 99.98 \\
\hline 10 & 1.9 & 1.5 & 1.3 & 1.1 & 0.74 & 0.57 & 0.44 & 0.31 & 0.22 & 0.16 & 0.07 & 0.02 & 0.00 & 0.00 & 0.00 & 0.00 \\
\hline
\end{tabular}

\# Reliability of values in column is uncertain, and potential errors are large. 
WHITEWATER DRAW BASIN

09537200 LESLIE CREEK NEAR McNEAL, AZ--Continued
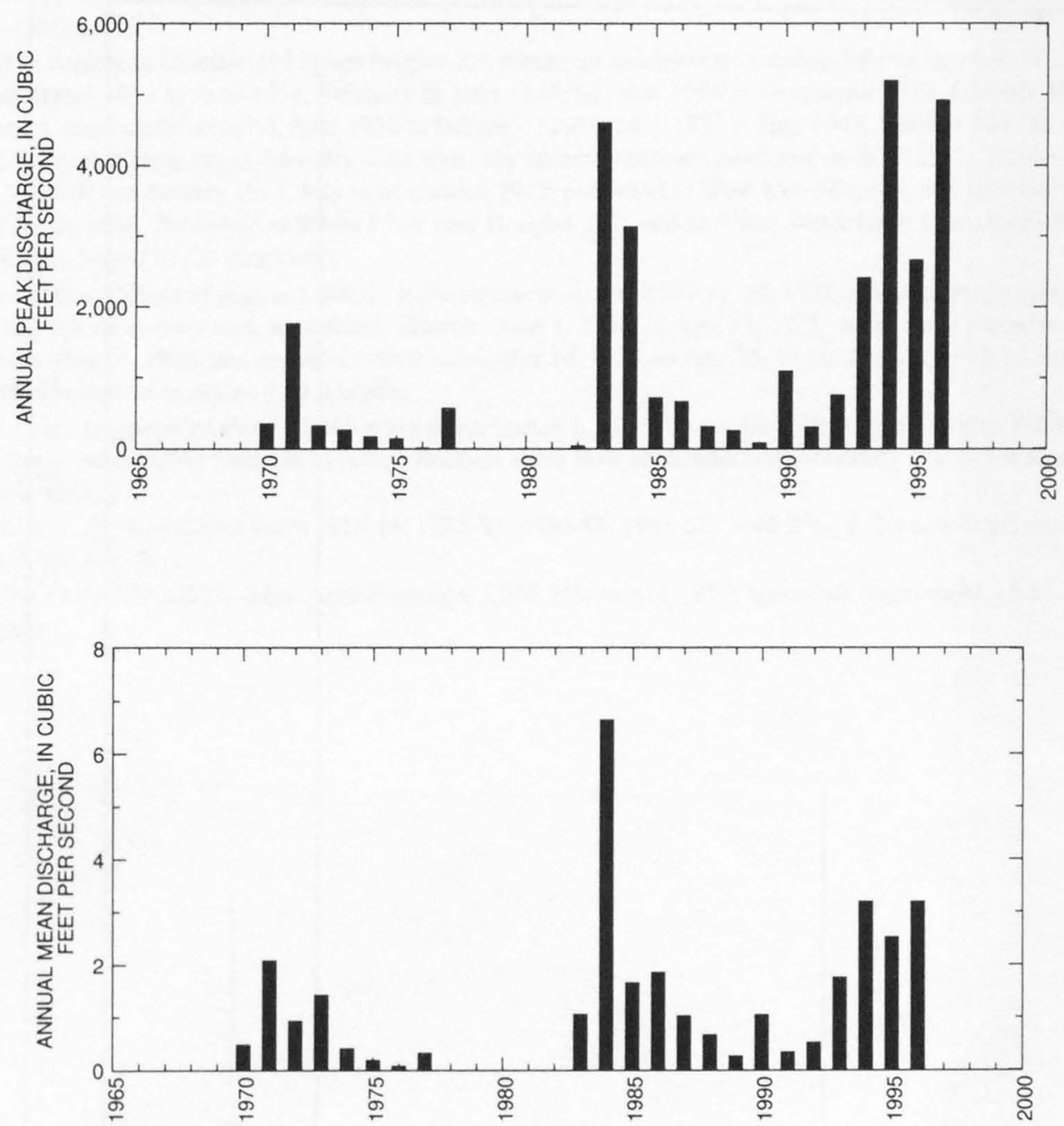
WHITEWATER DRAW BASIN

09537200 LESLIE CREEK NEAR McNEAL, AZ--Continued
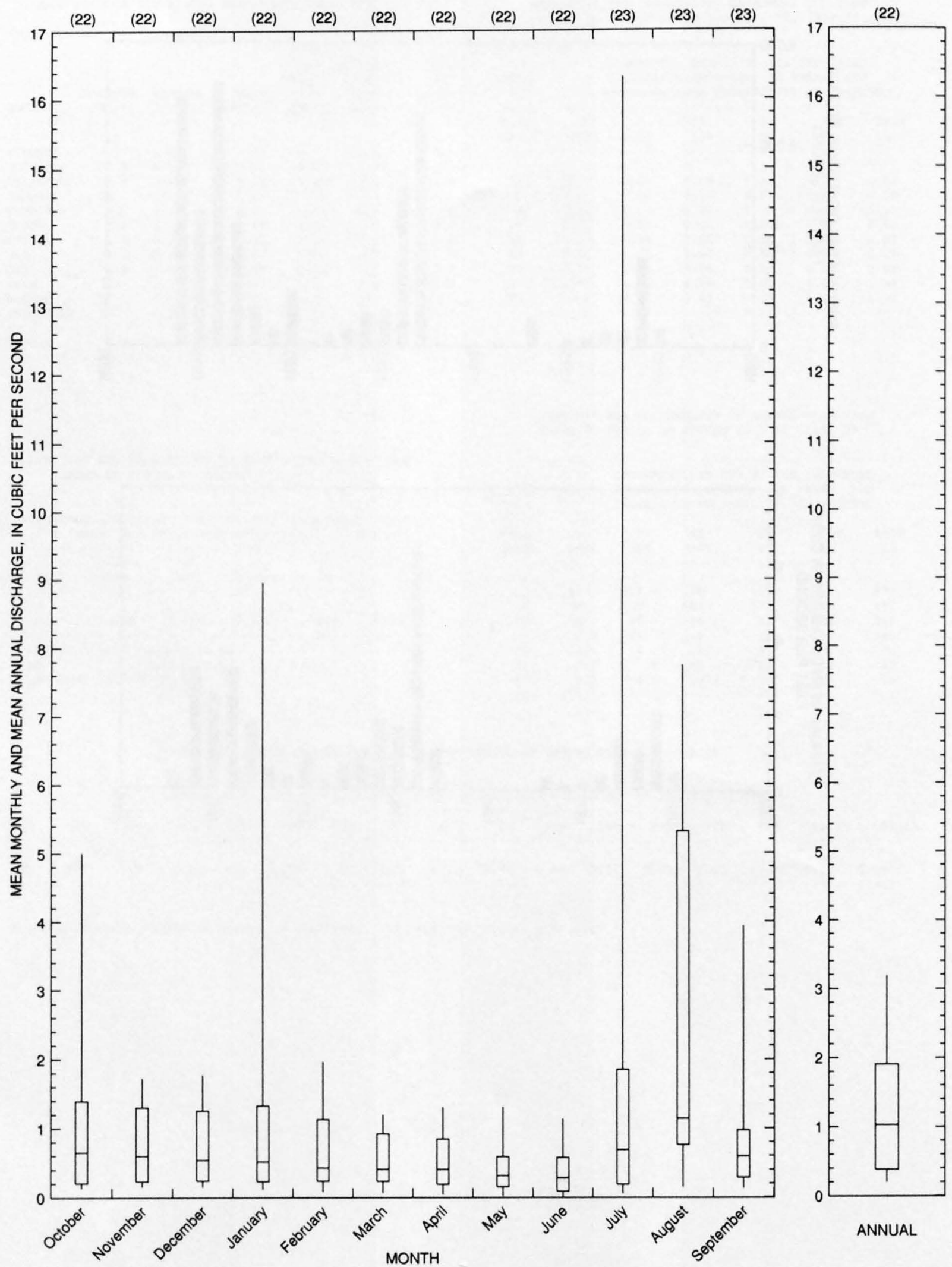


\section{WHITEWATER DRAW BASIN}

09537500 WHITEWATER DRAW NEAR DOUGLAS, AZ

LOCATION.--Lat 31'21'08", long 109.35'04", in SW1/4 $\mathrm{SE}^{1 / 4} \mathrm{sec} .10$, T.24 S., R.27 E., Cochise County, Hydrologic Unit 15080301 , on downstream side of pier of bridge on U.S. Highway $80,1.5 \mathrm{mi}$ upstream from international boundary and 2 mi west of Douglas.

DRAINAGE AREA.--1,023 $\mathrm{mi}^{2}$.

PERIOD OF RECORD.--August to October 191 (gage heights and discharge measurements only), July to October 1912, January to June 1913, October 1913, December 1913 to June 1914, February to June 1915, October 1915 to September 1919, October 1911 to April 1922 (gage heights and discharge measurements only), June 1930 to December 1933, May 1935 to July 1947, October 1947 to current year. (July 1954 to March 1955 monthly discharge only.) Monthly discharge only for some periods, published in WSP 1313. Records for July to September, November 1913, July 1914 to January 1915, July to September 1915, published in WSP $359,389,409$, and 1049 have been found to be unreliable and should not be used. Published as White River near Douglas 1911 and as White Water River near Douglas 1912 . Discontinued.

REVISED RECORDS.--See PERIOD OF RECORD.

GAGE.--Water-stage recorder. Datum of gage is 3,909.14 ft above sea level. Prior to Apr. 30, 1922, nonrecording gages at various sites between $0.3 \mathrm{mi}$ upstream and $0.8 \mathrm{mi}$ downstream, at different datums. June 1, 1930, to Apr. 25, 1972, water-stage recorder at present site, at datum $2.17 \mathrm{ft}$ lower prior to May 14, 1938, and at datum $2.20 \mathrm{ft}$ lower May 14, 1938, to Apr. 25, 1972. Apr. 26, 1972, to Apr. 10, 1974, water- stage recorder at site $200 \mathrm{ft}$ upstream at datum $4.40 \mathrm{ft}$ higher.

REMARKS.--Records poor. Irrigation of about 40,000 acres above station in 1978, by pumping from ground water. Whitewater Draw discharges into Gulf of California through Rio Yaqui in Mexico. Records show flow at international boundary except for smelter waste water, which enters stream below station.

AVERAGE DISCHARGE.--53 years (water years 1916-19, 1931-33, 1936-46, 1948-82), $9.46 \mathrm{ft}^{3} / \mathrm{s}, 6,850$ acre-ft/yr; median of yearly mean discharges, $8.2 \mathrm{ft}^{3} / \mathrm{s}, 5,900$ acre- $\mathrm{ft} / \mathrm{yr}$.

EXTREMES FOR PERIOD OF RECORD.--Maximum discharge, 5,060 $\mathrm{ft}^{3} / \mathrm{s}$ Aug. 7, 1955; maximum gage height, $16.55 \mathrm{ft}$ July 29,1966 ; no flow at times in most years. 
WHITEWATER DRAW BASIN

09537500 WHITEWATER DRAW NEAR DOUGLAS, AZ--Continued

Annual peak discharges

\begin{tabular}{|c|c|c|c|c|c|c|c|}
\hline $\begin{array}{l}\text { Water } \\
\text { year }\end{array}$ & Date & $\begin{array}{l}\text { Annual peak } \\
\text { discharge } \\
\left(\mathrm{ft}^{3} / \mathrm{s}\right)\end{array}$ & $\begin{array}{l}\text { Discharge } \\
\text { codes }\end{array}$ & $\begin{array}{l}\text { Water } \\
\text { year }\end{array}$ & Date & $\begin{array}{c}\text { Annual peak } \\
\text { discharge } \\
\left(\mathrm{ft}^{3} / \mathrm{s}\right)\end{array}$ & $\begin{array}{l}\text { Discharge } \\
\text { codes }\end{array}$ \\
\hline 1916 & $07-11-16$ & 1,600 & & 1961 & $07-29-61$ & 1,380 & \\
\hline 1917 & $08-09-17$ & 720 & & 1962 & $07-28-62$ & 687 & \\
\hline 1918 & $07-15-18$ & 1,050 & & 1963 & $08-01-63$ & 1,260 & \\
\hline 1919 & $07-27-19$ & 4,050 & & 1964 & $07-31-64$ & 1,370 & \\
\hline 1920 & $11-23-19$ & 3,400 & & 1965 & $09-04-65$ & 1,500 & \\
\hline 1930 & $09-07-30$ & 1,700 & & 1966 & $07-29-66$ & 3,760 & \\
\hline 1931 & $08-10-31$ & 3,450 & & 1967 & $08-05-67$ & 2,930 & \\
\hline 1932 & $07-31-32$ & 1,800 & & 1968 & $09-01-68$ & 1,280 & \\
\hline 1933 & $09-20-33$ & 1,730 & & 1969 & $08-25-69$ & 1,130 & \\
\hline 1934 & $08-00-34$ & 3,100 & & 1970 & $08-17-70$ & 2,260 & \\
\hline 1935 & $09-01-35$ & 2,900 & & 1971 & $08-11-71$ & 1,700 & \\
\hline 1936 & $09-11-36$ & 2,000 & ES & 1972 & $08-13-72$ & 2,540 & \\
\hline 1937 & $08-19-37$ & 2,770 & & 1973 & $07-11-73$ & 800 & \\
\hline 1938 & $08-07-38$ & 1,990 & & 1974 & $08-02-74$ & 936 & \\
\hline 1939 & $08-05-39$ & 2,690 & & 1975 & $07-23-75$ & 1,020 & \\
\hline 1940 & $06-24-40$ & 2,750 & & 1976 & $07-24-76$ & 654 & \\
\hline 1941 & $09-29-41$ & 2,750 & & 1977 & $08-19-77$ & 625 & \\
\hline 1942 & $09-13-42$ & 2,300 & & 1978 & $10-09-77$ & 3,020 & \\
\hline 1943 & $06-30-43$ & 2,750 & & 1979 & $07-20-79$ & 1,100 & \\
\hline 1944 & $08-16-44$ & 2,190 & & 1980 & $08-13-80$ & 467 & \\
\hline 1945 & $07-31-45$ & 3,100 & & 1981 & $07-19-81$ & 753 & \\
\hline 1946 & $10-09-45$ & 1,440 & & 1982 & $08-30-82$ & 542 & \\
\hline 1947 & $07-08-47$ & 1,580 & & 1983 & $07-24-83$ & 170 & \\
\hline 1948 & $07-22-48$ & 3,170 & & 1984 & $10-01-83$ & 891 & \\
\hline 1949 & $07-18-49$ & 1,790 & & 1985 & $09-29-85$ & 1,420 & \\
\hline 1950 & $07-19-50$ & 3,400 & & 1986 & $08-18-86$ & 1,540 & \\
\hline 1951 & $08-20-51$ & 1,230 & & 1987 & $08-05-87$ & 381 & \\
\hline 1952 & $06-02-52$ & 1,670 & & 1988 & $08-26-88$ & 172 & \\
\hline 1953 & $07-07-53$ & 2,950 & & 1989 & $10-16-88$ & 95 & \\
\hline 1954 & $08-09-54$ & 3,680 & & 1990 & $07-15-90$ & 126 & \\
\hline 1955 & $08-07-55$ & 5,060 & & 1991 & $11-26-90$ & 52 & \\
\hline 1956 & $08-27-56$ & 513 & & 1992 & $08-25-92$ & 58 & \\
\hline 1957 & $07-24-57$ & 2,720 & & 1993 & $09-02-93$ & 66 & \\
\hline 1958 & $09-23-58$ & 1,280 & & 1994 & $08-10-94$ & 149 & \\
\hline 1959 & $07-27-59$ & 2,760 & & 1995 & $11-13-94$ & 242 & \\
\hline 1960 & $07-31-60$ & 676 & & 1996 & $08-04-96$ & 188 & \\
\hline
\end{tabular}

Basin characteristics

\begin{tabular}{cccccccc}
\hline $\begin{array}{c}\text { Main } \\
\text { channel } \\
\text { slope } \\
(\mathrm{ft} / \mathrm{mi})\end{array}$ & $\begin{array}{c}\text { Stream } \\
\text { length } \\
(\mathrm{mi})\end{array}$ & $\begin{array}{c}\text { Mean } \\
\text { basin } \\
\text { elevation } \\
(\mathrm{ft})\end{array}$ & $\begin{array}{c}\text { Forested } \\
\text { area } \\
\text { (percent) }\end{array}$ & $\begin{array}{c}\text { Soil } \\
\text { index }\end{array}$ & $\begin{array}{c}\text { Mean } \\
\text { annual } \\
\text { precipitation } \\
\text { (in) }\end{array}$ & $\begin{array}{c}\text { R-year } \\
\text { (in) }\end{array}$ & $\begin{array}{c}50 \text {-year } \\
\text { (in) }\end{array}$ \\
\hline 20.5 & 61.7 & 4,740 & 11.0 & 2.3 & 14.8 & 1.8 \\
\hline
\end{tabular}


WHITEWATER DRAW BASIN

09537500 WHITEWATER DRAW NEAR DOUGLAS, AZ--Continued

MEAN MONTHLY AND ANNUAL DISCHARGES $1919,1931 \cdot 33,1936 \cdot 46,1949 \cdot 82$

MAGNITUDE AND PROBABILITY OF ANNUAL LOW FLOW BASED ON PERIOD OF RECORD $1932 \cdot 33,1937 \cdot 47,1949 \cdot 82$

\begin{tabular}{|c|c|c|c|c|c|c|}
\hline MONTH & $\begin{array}{l}\text { MAXIMUM } \\
\text { (FT3/S) }\end{array}$ & $\begin{array}{l}\text { MINIMUM } \\
\text { (FT3/S) }\end{array}$ & $\begin{array}{c}\text { MEAN } \\
\text { (FT3/S) }\end{array}$ & $\begin{array}{l}\text { STAN- } \\
\text { DARD } \\
\text { DEVIA- } \\
\text { TION } \\
(\text { FT3/S) }\end{array}$ & $\begin{array}{l}\text { COEFFI- } \\
\text { CIENT OF } \\
\text { VARI- } \\
\text { ATION }\end{array}$ & $\begin{array}{c}\text { PERCENT } \\
\text { OF } \\
\text { ANNUAL } \\
\text { RUNOFF }\end{array}$ \\
\hline OCTOBER & 99 & 0.00 & 4.9 & 15 & 3.1 & 4.4 \\
\hline NOVEMBER & 9.9 & 0.00 & 0.85 & 1.7 & 2.0 & 0.8 \\
\hline DECEMBER & 38 & 0.00 & 2.0 & 6.0 & 2.9 & 1.8 \\
\hline JANUARY & 7.3 & 0.00 & 0.66 & 1.2 & 1.8 & 0.6 \\
\hline FEBRUARY & 9.1 & 0.00 & 0.55 & 1.3 & 2.4 & 0.5 \\
\hline MARCH & 4.8 & 0.00 & 0.49 & 0.79 & 1.6 & 0.4 \\
\hline APRIL & 2.9 & 0.00 & 0.38 & 0.55 & 1.5 & 0.3 \\
\hline MAY & 2.2 & 0.00 & 0.27 & 0.44 & 1.6 & 0.2 \\
\hline JUNE & 27 & 0.00 & 2.1 & 5.9 & 2.8 & 1.9 \\
\hline JULY & 342 & 0.00 & 38 & 55 & 1.5 & 34.2 \\
\hline AUGUST & 235 & 0.00 & 48 & 58 & 1.2 & 43.3 \\
\hline SEPTEMBER & 53 & 0.00 & 13 & 14 & 1.1 & 11.3 \\
\hline ANNUAL & 33 & 0.32 & 9.3 & 6.9 & 0.75 & 100 \\
\hline
\end{tabular}

\begin{tabular}{|c|c|c|c|c|c|c|}
\hline \multirow{4}{*}{$\begin{array}{l}\text { PERIOD } \\
\text { ICON- } \\
\text { SECU- } \\
\text { TIVE }\end{array}$} & \multicolumn{6}{|c|}{ DISCHARGE, IN FT3/S, FOR INDICATED } \\
\hline & \multicolumn{6}{|c|}{$\begin{array}{l}\text { RECURRENCE INTERVAL, IN YEARS, AND } \\
\text { NON-EXCEEDANCE PROBABILITY, IN PERCENT }\end{array}$} \\
\hline & & & ... & ... & & …… \\
\hline & 2 & 5 & 10 & 20 & $50 \#$ & $100 \#$ \\
\hline DAYS) & 508 & 208 & 108 & 58 & 28 & 18 \\
\hline 1 & 0.00 & 0.00 & 0.00 & 0.00 & 0.00 & 0.00 \\
\hline 3 & 0.00 & 0.00 & 0.00 & 0.00 & 0.00 & 0.00 \\
\hline 7 & 0.00 & 0.00 & 0.00 & 0.00 & 0.00 & 0.00 \\
\hline 14 & 0.00 & 0.00 & 0.00 & 0.00 & 0.00 & 0.00 \\
\hline 30 & 0.00 & 0.00 & 0.00 & 0.00 & 0.00 & 0.00 \\
\hline 60 & 0.02 & 0.00 & 0.00 & 0.00 & 0.00 & 0.00 \\
\hline 90 & 0.06 & 0.00 & 0.00 & 0.00 & 0.00 & 0.00 \\
\hline 120 & 0.25 & 0.00 & 0.00 & 0.00 & 0.00 & 0.00 \\
\hline 183 & 0.52 & 0.10 & 0.00 & 0.00 & 0.00 & 0.00 \\
\hline
\end{tabular}

MAGNITUDE AND PROBABILITY OF INSTANTANEOUS PEAK FLOW BASED ON PERIOD OF RECORD $1916 \cdot 20,1930.96$

DISCHARGE, IN FT3/S, FOR INDICATED RECURRENCE INTERVAL IN YEARS, AND EXCEEDANCE PROBABILITY, IN PERCENT

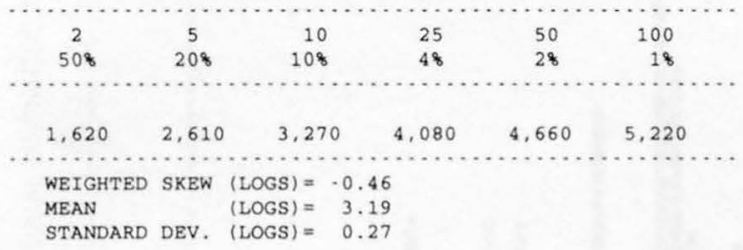

MAGNITUDE AND PROBABILITY OF ANNUAL HIGH FLOW BASED ON PERIOD OF RECORD 1919, 1931-33, 1936-46, 1949-82

\begin{tabular}{|c|c|c|c|c|c|c|}
\hline PERIOD & & \multicolumn{5}{|c|}{ DISCHARGE, IN FT3/S, FOR INDICATED } \\
\hline (CON- & & \multirow{2}{*}{\multicolumn{3}{|c|}{ EXCEEDANCE PROBABILITY, }} & IN PER & \\
\hline SECU - & $\cdots$ & & & & $\ldots \ldots$ & $\ldots \ldots$ \\
\hline TIVE & 2 & 5 & 10 & 25 & $50 \#$ & $100 \#$ \\
\hline DAYS) & 508 & 208 & 108 & 48 & 28 & 18 \\
\hline 1 & 623 & 1,020 & 1,290 & 1,600 & 1,820 & 2,030 \\
\hline 3 & 327 & 574 & 740 & 945 & 1,090 & 1,230 \\
\hline 7 & 176 & 328 & 437 & 579 & 684 & 788 \\
\hline 15 & 102 & 198 & 269 & 362 & 433 & 503 \\
\hline 30 & 63 & 129 & 181 & 253 & 310 & 370 \\
\hline 60 & 39 & 76 & 101 & 133 & 156 & 177 \\
\hline 90 & 29 & 54 & 70 & 89 & 101 & 112 \\
\hline
\end{tabular}

DURATION TABLE OF DAILY MEAN FLOW FOR PERIOD OF RECORD $1519,1931 \cdot 33,1936 \cdot 46,1949 \cdot 82$

DISCHARGE, IN FT3/S, WHICH WAS EQUALED OR EXCEEDED FOR INDICATED PERCENT OF TIME

\begin{tabular}{|c|c|c|c|c|c|c|c|c|c|c|c|c|c|c|c|c|}
\hline 18 & 58 & 108 & 158 & 208 & 308 & 408 & 508 & 608 & 708 & 808 & $90 \%$ & 958 & 988 & 998 & 99.58 & 99.98 \\
\hline 248 & 22 & 3.4 & 1.4 & 1.0 & 0.66 & 0.44 & 0.25 & 0.11 & 0.01 & 0.00 & 0.00 & 0.00 & 0.00 & 0.00 & 0.00 & 0.00 \\
\hline
\end{tabular}

\# Reliability of values in column is uncertain, and potential errors are large. 
WHITEWATER DRAW BASIN

09537500 WHITEWATER DRAW NEAR DOUGLAS, AZ--Continued
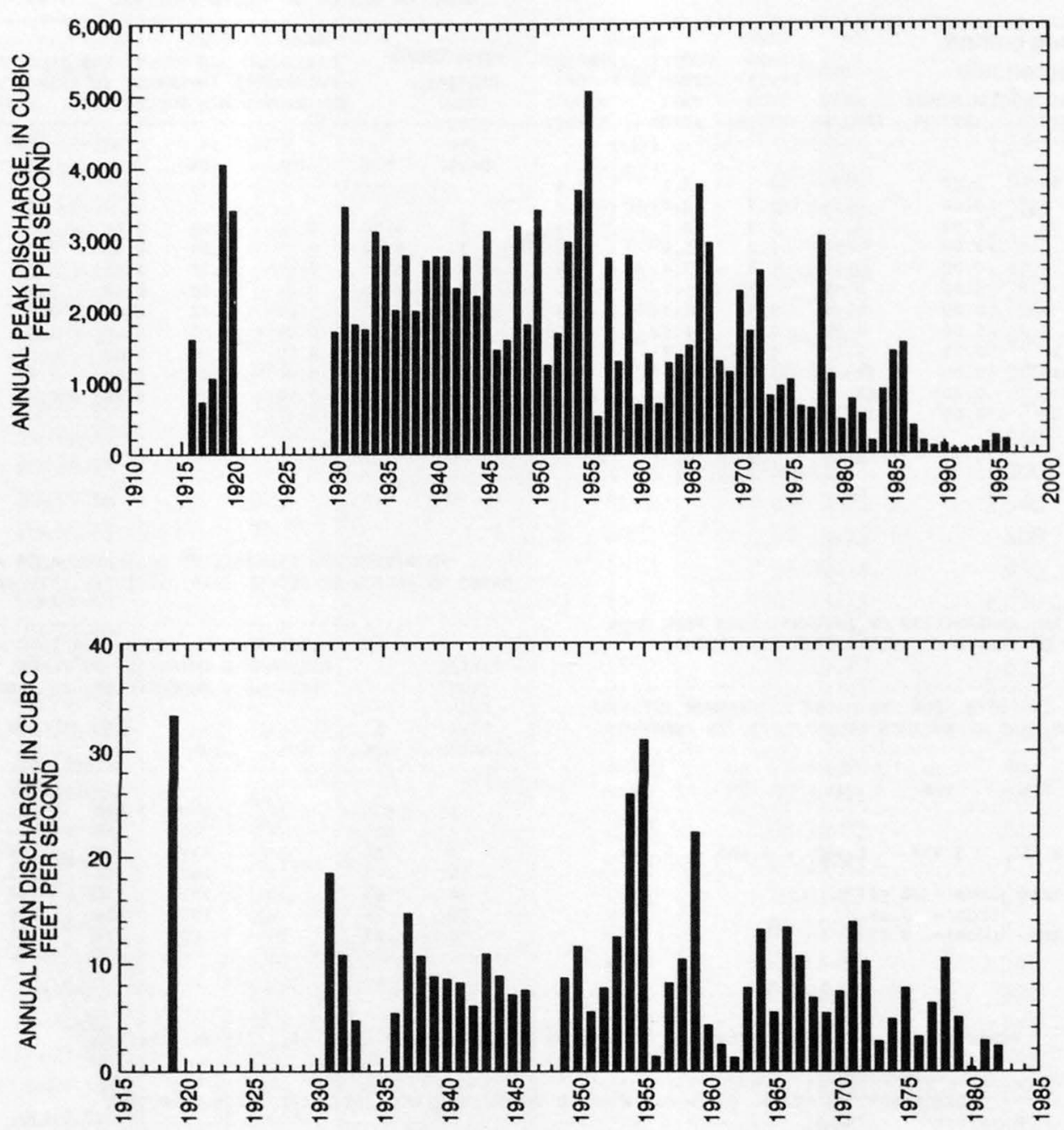
SULPHUR SPRING VALLEY

907

WHITEWATER DRAW BASIN

09537500 WHITEWATER DRAW NEAR DOUGLAS, AZ--Continued

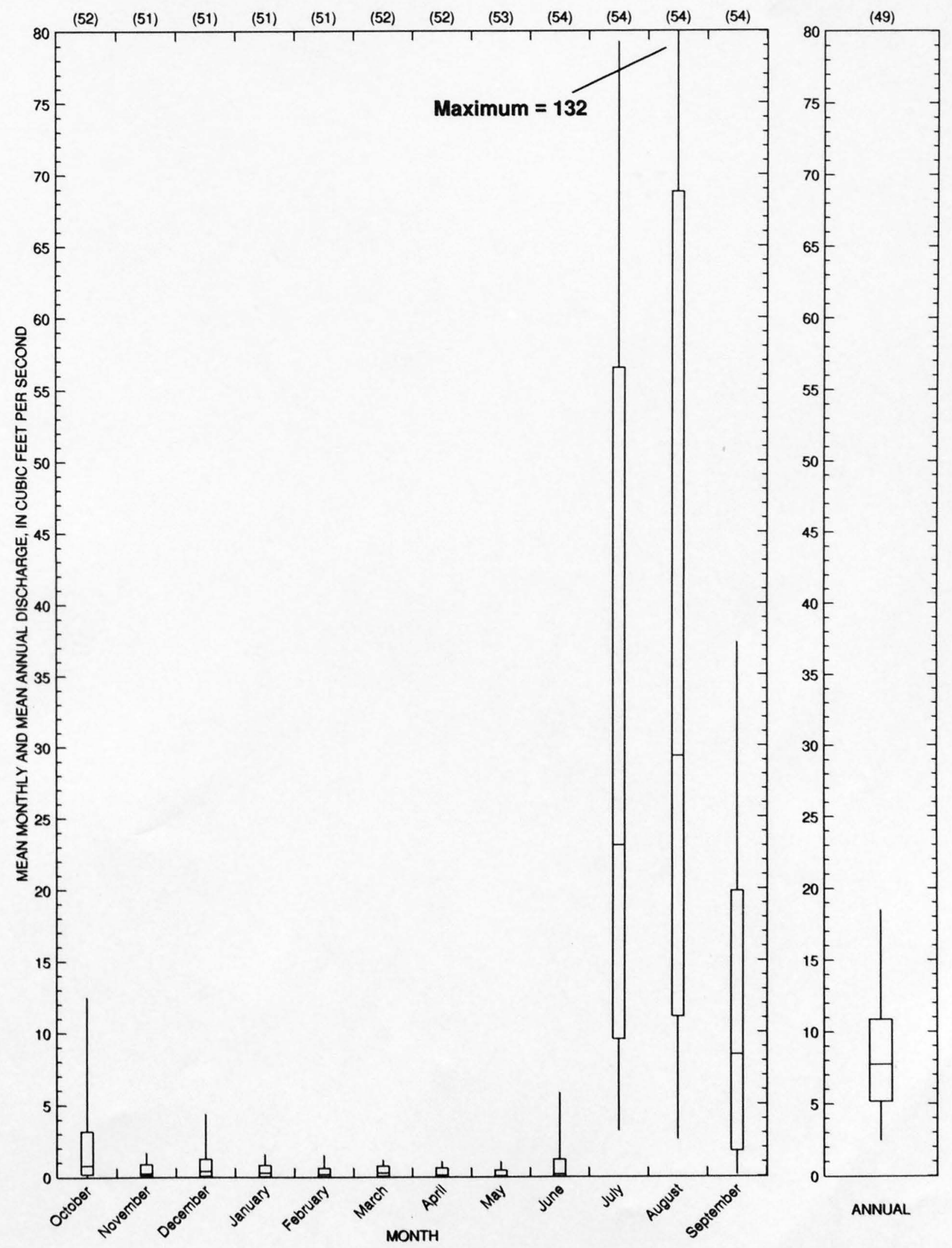

4 0.S. GOVEPANENT PRINTING OFFICE: 1999-783-729 